\title{
Enhancing automated reaction discovery with boxed molecular dynamics in energy space
}

\author{
Rafael A. Jara-Toro, ${ }^{[a]}$ Gustavo A. Pino, ${ }^{[a]}$ David R. Glowacki, ${ }^{[b]}$ Robin J. Shannon, ${ }^{*[b]}$ and Emilio \\ Martínez-Núñez ${ }^{\star[c]}$
}

\begin{abstract}
The rare event acceleration method BXDE is interfaced in the present work with the automated reaction discovery method AutoMeKin. To test the efficiency of the combined AutoMeKin-BXDE procedure, the ozonolysis of $\alpha$-pinene is studied in comparison with standard AutoMeKin. AutoMeKin-BXDE locates intermediates and transition states that are more densely connected with each other and approximately $50 \mathrm{kcal} / \mathrm{mol}$ more stable than those found with standard AutoMeKin. Other than the different density of edges between the nodes, both networks are scale-free and display small-world properties, mimicking the network of organic chemistry. Finally, while AutoMeKin-BXDE finds more transition states than those previously reported for $\mathrm{O}_{3}+\alpha$-pinene, the standard procedure fails to locate some of the previously published reaction pathways using the same simulation time of $2.5 \mathrm{~ns}$. In summary, the mixed procedure is very promising and clearly outperforms the standard simulation algorithms implemented in AutoMeKin. BXDE will be available in the next release of AutoMekin.
\end{abstract}

\section{Introduction}

The study of mechanisms and kinetics of chemical reactions has greatly benefited from the surge of automated methods developed in the last years. ${ }^{1-63}$ Heuristics rules, artificial forces and/or molecular dynamics (MD) are commonly employed in these methods in the quest for new reactions. While knowledgebased methods are usually faster than MD-based methods, this superior efficiency comes at a cost, since they are not always able to find all reaction mechanisms found with methods based on MD simulations. ${ }^{60}$

Automated MD-based methods for reaction discovery involve costly computations, as they require many gradient and Hessian calculations evaluated at an ab initio or semiempirical level of theory. ${ }^{30,45}$ The efficiency of these methods can be enhanced by the use of different accelerated MD strategies like the ones

[a] R. A. Jara, G. A. Pino

INIFIQC (CONICET-UNC) Dpto. De Fisicoquímica-Facultad de Ciencias Químicas-Centro Láser de Ciencias Moleculares Universidad de Córdoba

Ciudad Universitaria, X50000HUA Córdoba, Argentina

[b] R. A. Shanon, D. R. Glowacki

Centre for Computational Chemistry, School of Chemistry, University of Bristol, Cantock's Close, Bristol BS8 1TS, UK

[c] E Martínez-Núñez

Departmento de Química Física, Facultade de Química, Universidade de Santiago de Compostela, 15782, Santiago de Compostela, SPAIN

E-mail: emilio.nunez@usc.es

Supporting information for this article is given via a link at the end of the document. employed in the ab initio nanoreactor, i.e., adding external forces and using high temperatures. ${ }^{30}$

The automated reaction discovery method recently developed by one of the authors is called AutoMeKin ${ }^{64}$ (formerly known as tsscds). This software/method employs a very simple approach to accelerate the MD simulations: the use of high amounts of vibrational energy, which boosts bond breakage/formation in a very short time scale (typically $0.5 \mathrm{ps}$ ). ${ }^{45}$ After the MD step, AutoMeKin employs some graph theoretic tools to identify candidate transition state (TS) structures from the MD geometries. The automated method has been so far successfully employed to study combustion chemistry, ${ }^{65,66}$ photodissociation processes, ${ }^{67-}$ 69 organometallic catalysis, ${ }^{43}$ and the simulation of mass spectrometry experiments. ${ }^{70,71}$ However, a drawback is the large number of trajectories required to safely obtain the important TSs and reaction pathways, which is typically of the order of tens of thousands, rendering the whole procedure inefficient.

The use of powerful rare event acceleration methods, other than adding high amounts of vibrational energy to the system, is therefore highly desirable. For this reason, we present in this paper a new combined methodology where the high-energy MD simulation algorithm of AutoMeKin is substituted by a rare event acceleration technique recently developed by two of the authors. ${ }^{72}$ The accelerated method is called Boxed Molecular Dynamics in Energy space (BXDE) and is based on their previous BXD method. ${ }^{73-76}$ BXDE scans through potential energy "boxes", accelerating the observation of reactive events by many orders of magnitude. ${ }^{72}$

To test the combined AutoMekin-BXDE methodology we have chosen the ozonolysis of $\alpha$-pinene. ${ }^{77-91}$ A comprehensive computational study of this reaction was carried by Zhang and Zhang $^{79}$ (hereinafter ZhZh) using accurate levels of theory. The reaction discovery methods employed in the present work will be benchmarked against the computational study of ZhZh. Additionally, and to facilitate the comparisons, the nomenclature employed by ZhZh is closely followed here. Scheme 1 shows that the reaction follows the "Criegee mechanism" of alkene ozonolysis. ${ }^{92,} 93$ The acronyms of the structures are those previously employed by ZhZh. ${ }^{79}$

The first step of the ozonolysis reaction is the highly exothermic cycloaddition of ozone to the double bond of $\alpha$-pinene to form a primary ozonide (POZ), which rapidly isomerizes yielding two possible Criegee intermediates (Cls): $\mathrm{Cl} 1$ and $\mathrm{Cl} 2$. These intermediates can subsequently undergo $\mathrm{H}$-shifts and ring closure reactions leading to hydroperoxides $\mathrm{HP} 1 \mathrm{a}, \mathrm{HP} 1 \mathrm{~b}, \mathrm{HP} 2$ and dioxiranes DIO1 and DIO2 as shown in scheme 1.

The new methodology proposed in this work will be explained in the Methods section, followed by a discussion of the main results obtained with standard AutoMeKin and AutoMeKin-BXDE. A special focus will be placed on comparing the properties of the 
obtained reaction networks. Also, the relative efficiency/efficacy of both methods will be assessed by comparing their predicted $\alpha$-pinene ozonolysis mechanisms with those previously found by ZhZh. ${ }^{79}$

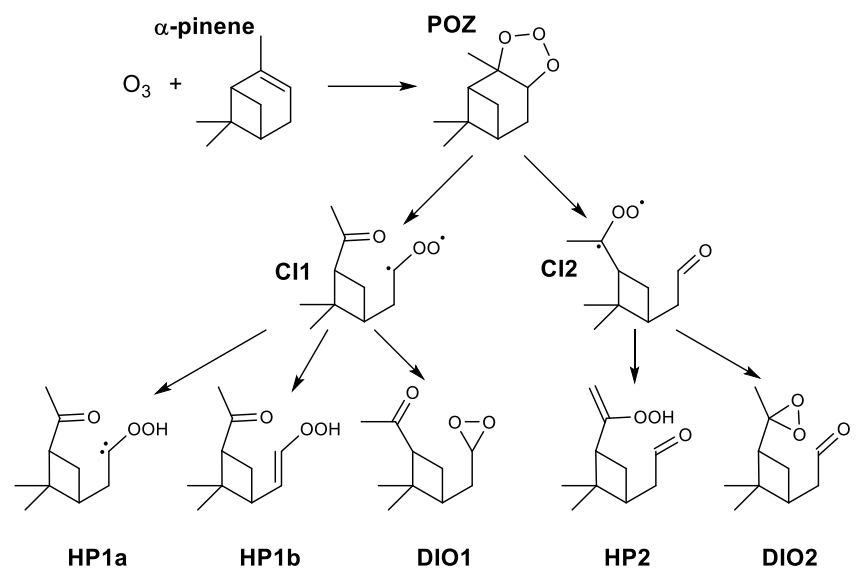

Scheme 1. Summary of the reaction mechanism of the $\alpha$-pinene ozonolysis.

\section{Methods}

A detailed description of AutoMeKin has been given elsewhere, and here we only summarize its main features. The method is based on short-time high-energy molecular dynamics (MD) simulations and a subsequent post-processing analysis. Several graph theoretic tools, like the Adjacency and Laplacian matrices or the SPRINT coordinates, ${ }^{45}$ are employed at various stages to find transition states (TS), screen their structures and construct the reaction network.

Specifically, the time dependence of the Adjacency matrix (constructed from the Cartesian coordinates of the system) is monitored to identify bond breakage/formation processes using our own recognition algorithm. ${ }^{45}$ This is followed by a stepwise optimization consisting of:

(a) A partial optimization where the atoms involved in the reaction are frozen, which facilitates a relaxation of the normal modes perpendicular to the reaction coordinate.

(b) A TS optimization of the resulting geometry in the previous step.

While the trajectories are integrated with MOPAC2016 ${ }^{94}$ using any of the implemented semiempirical methods, the stationary points can be re-optimized using an ab initio or a Density Functional Theory (DFT) level of theory.

One of the shortcomings of AutoMeKin is the fact that chemical reactions are triggered by use of huge amounts of vibrational energy placed in each normal mode of the molecule. While this approach was successfully employed to tackle different problems, its efficiency is compromised because of its bias towards the entropically favored reaction pathways, and consequently tens of thousands of trajectories are typically employed to obtain the relevant TSs.
To alleviate the above drawback, we propose in this paper to replace the current standard MD simulation step of AutoMeKin by the rare-event acceleration method named Boxed Molecular Dynamics in Energy space (BXDE). ${ }^{72}$

BXD introduces several reflective barriers in the phase space of a MD trajectory along a particular collective variable, which is typically a function of the atomic positions. Those boundaries are employed to push the dynamics along the collective variable into regions of phase space which would be rarely sampled in an unbiased trajectory. In standard BXD however, the specification of an appropriate collective variable requires the user to already know the particular reaction or process that they are interested in and as such traditional BXD is poorly suited to the field of mechanism generation, where one wish to accelerate not one particular reaction, but rather reactivity in general.

To remedy this, a generalization of BXD has been recently put forward by two of us, ${ }^{72}$ who showed that the BXD bias can also be introduced along the potential energy $(E)$ of the system, which is referred to as BXDE. By scanning through potential energy "boxes", the energetic "windows" at which different chemical reaction channels switch on or off can be identified. In a previous work, we showed that this procedure accelerated the observation of reactive events by many orders of magnitude, allowing the sampling of reactions at much lower temperature than would typically be required in a MD simulation.

To gauge the performance of the combined methodology, we have chosen the ozonolysis of $\alpha$-pinene as a test system. The aim of this work is to compare the results of AutoMeKin using the standard MD step (hereinafter AMK-stdMD) with those obtained interfacing AutoMeKin with BXDE (hereinafter AMK-BXDE).

Both methods use MOPAC2016 ${ }^{94}$ to run the trajectory simulations, and in this case the chosen semiempirical level of theory was PM7. The trajectories are integrated until one of the following criteria is met: i) fragmentation of the system, or ii) the simulation time reaches a maximum value $t_{\max }$. In AMK-stdMD, 5000 trajectories were integrated with $t_{\max }=0.5 \mathrm{ps}$, while in AMK-BXDE the total number of trajectories was only 500 and $t_{\max }=5 \mathrm{ps}$, which makes in both cases a total simulation time of $2.5 \mathrm{~ns}$.

In the standard MD simulations an NVE ensemble of trajectories is set up using microcanonical normal mode sampling, ${ }^{95}$ with vibrational energies automatically selected by AutoMeKin. ${ }^{47}$ In BXDE, we use an NVT ensemble, and a Langevin thermostat with a friction coefficient of $0.5 \mathrm{ps}^{-1}$ and temperature of $1000 \mathrm{~K}$. We also specify two additional parameters in the BXDE case: The "Adaptive Steps" parameter determines how many MD steps are performed in a new box before the BXDE algorithm adaptively places a new box based upon the sampled energies. The "window" parameter determines the number of consecutive MD steps before considering a reaction to have occurred. This "Window" ensures the reaction criteria is triggered by an actual chemical transformation rather than a transient fluctuation in bond lengths. In the current work both the "Adaptive Limit" and the "Window" were set to 100 .

In both, the stdMD and BXDE simulations, the starting structure is POZ. Once the simulations have ended, the standard algorithms of AutoMeKin are applied to obtain the optimized structures (TSs and minima), and the reaction networks. 
The (reaction) networks are analyzed using NetworkX Python package. ${ }^{96}$ In Graph Theory, vertices (or nodes) are connected with edges, which constitute a graph or network. Here, a node is either a minimum energy structure, or the resulting fragments of a given pathway, while an edge represents a pathway connecting two nodes (via a TS). The number of edges connected to a given node $s$ is called the degree $k$ of $s$.

The networks are constructed in the same way in both cases. Specifically, a family of conformers form a single node of the network, whose label is that of the most stable isomer. Additionally, the networks are weighted, with the edges having weights that represent the number of TSs (or pathways) connecting two nodes. Self-loops are avoided by removing transition states connecting permutation-inversion isomers of the same minimum. Edges connecting nodes that represent fragments are also discarded.

Representative properties of both networks are studied in this work, namely, the degree distribution, the average clustering coefficient, the transitivity, the average shortest path length, and the assortativity. ${ }^{96}$ Similar properties have been studied before for the network of organic chemistry ${ }^{97}$ and for networks of small clusters bound by Morse potentials in two and three dimensions. ${ }^{98}$ The probability of observing a given degree $k$ in our networks was fit to a power law distribution

$$
P(k)=C k^{-\gamma}
$$

Where $C$ and $\gamma$ are parameters. These fits were performed with powerlaw Python package. ${ }^{99}$ Networks that exhibit power law distributions have many nodes with very few connections, while a few nodes have a very large number of connections, and they are termed scale-free. These networks are hierarchical and have a great degree of clustering.

The clustering coefficient provides an idea of the degree to which the neighbors of a node are also neighbors of each other, and an average clustering coefficient can be calculated for the network. ${ }^{100}$ The transitivity, which is pretty much related to the clustering coefficient, is proportional to the ratio of the number of triangles over the number of triads in the network. For any three nodes $r, s$ and $t$ in the network, a triangle is formed when the three possible pairs of nodes $(r-s, r-t$ and $s-t)$ are connected, while in a triad only two pairs are connected.

The shortest path connects a pair of nodes through the least number of edges; the shortest path is calculated here using Dijkstra's algorithm. ${ }^{101}$ Since our networks are connected by construction, a path always exists between every pair of nodes. Networks with average shortest path lengths that scale with the logarithm of the number of nodes or smaller are said to exhibit "small-world" effects.

The assortativity is a measure of the tendency of nodes to have connections with nodes of similar degree. It can be measured through a coefficient ${ }^{102}$ that varies from -1 to 1 , with values close to 1 indicating that nodes have a preference to connect with nodes of similar degree, which is called assortative mixing, and -1 means the opposite (and is called disassortative mixing).

For comparison purposes, the corresponding properties of random networks with the same number of nodes and edges were either calculated with analytical formulae and/or averaged over the values of $10^{3}$ randomly generated networks.

\section{Results and Discussion}

The results obtained with both procedures (AMK-stdMD and AMK-BXDE) are compared here in terms of the obtained structures, properties of the reaction networks, and the underlying reaction mechanisms. To make a meaningful comparison, the involved simulations were carried out for a maximum simulation time of $2.5 \mathrm{~ns}$ in both cases, as stated above.

Structures. The total number of structures obtained with AMK-stdMD is 1088, which breaks down into 470 minima and 618 TSs. By contrast, AMK-BXDE locates 446 structures (224 minima and 222 TSs). The complete AMK-stdMD and AMK-BXDE databases are included in the Supporting Information (SI). The databases gather electronic energies, zero-point vibrational energies (ZPE), Cartesian coordinates, and vibrational frequencies of each structure, as well as the detailed reaction networks, with all the pathways connecting intermediates and fragments.

Owing to the flexibility of the system, the above numbers account for many conformational isomers, and the AMK-stdMD minima and TSs can be lumped together in 265 and 486 families of conformers, respectively; in the case of AMK-BXDE we found 70 and 159 families of minima and TSs, respectively.

Mechanistic computational studies of chemical reactions aim to obtain the (kinetically) relevant structures and paths, rather than locating all possible TSs of a molecular system, primarily because many of them have energies that are way too high to be accessible at the experimental or simulation conditions. In this regard it is interesting to compare the energies of the structures collected in both databases.

Figure 1 shows the energy distributions of the AMK-stdMD and AMK-BXDE structures. As expected, the TS structures (Figure 1b) have average energies $>50 \mathrm{kcal} / \mathrm{mol}$ greater than those of the minima, but the distributions have tails that reach very low energies (close to $-150 \mathrm{kcal} / \mathrm{mol}$ with AMK-BXDE). Of significance, the AMK-BXDE energy distribution for the minima (blue histogram in Figure 1a) falls, almost entirely, to the left of the starting structure, which shows the efficiency of the method finding very stable minima. Additionally, the AMK-BXDE distributions are shifted, on average, $\sim 50-60 \mathrm{kcal} / \mathrm{mol}$ to lower energies, with respect to the AMK-stdMD ones.

Thus, the new AMK-BXDE methodology shows much greater propensity to locate low-energy structures than the standard procedure currently implemented in AutoMeKin. Although AMK-BXDE clearly outperforms AMK-stdMD regarding the relative stability of the structures, it is necessary to study the pathways connecting the different intermediates and product fragments in each of the networks for a comprehensive assessment of both procedures. This is done in the following sections, first by studying the different properties/statistics of the networks, and second by comparing the reaction mechanisms. 


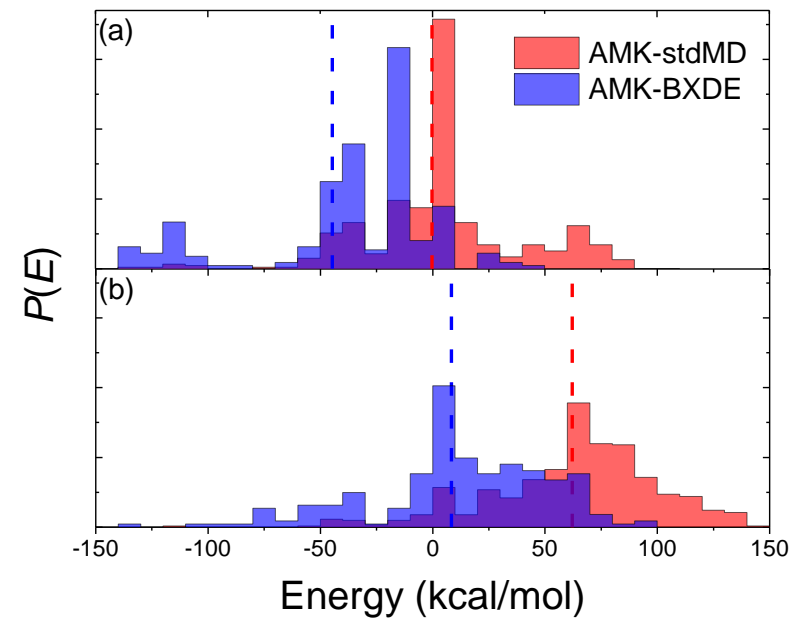

Figure 1. Distributions of energies of (a) minima and (b) TSs, obtained with AMK-stdMD and AMK-BXDE methods. Average values are shown as dashed vertical lines. The energies are relative to the starting structure (POZ) and include the zero-point energy (ZPE) correction.

Properties of the networks. As indicated above, families of conformers are represented in the networks as single nodes, with edges representing paths connecting the nodes. Self-loops and edges between nodes that represent fragments are also avoided in our networks.

With these construction principles, the initial networks contain the following number of nodes and edges in the AMK-stdMD network: 278 and 293, respectively, which compare with the AMK-BXDE numbers: 86 and 95 , respectively.

Table 1 gathers some relevant properties of the AMK-stdMD and AMK-BXDE initial networks. However, to compute some of the properties studied here, the networks need to be connected. In a connected network, no isolated clusters of nodes can exist. To avoid detached nodes, the initial networks were trimmed, a process where only $38 \%$ of the nodes in the AMK-stdMD network survived, compared to a $60 \%$ survival in the AMK-BXDE case. Additionally, the density of edges in the AMK-BXDE network, computed as the fraction of edges with respect to their maximum possible number, is more than three times the AMK-stdMD density.

The above numbers indicate that the AMK-stdMD network is significantly more sparsely connected than the AMK-BXDE one. Therefore, AMK-BXDE not only provides more stable structures than AMK-stdMD, but also creates more densely connected reaction networks. This is very important when dealing with reaction mechanisms and kinetics studies, as groups of nodes isolated from the bulk of the network do not partake in the kinetics. The diagrams of Figure 2 show (reduced) AMK-stdMD and AMK-BXDE networks in the form of graphs. To make the graphs readable, only paths (edges) whose TSs are below the entrance channel $\left(\mathrm{O}_{3}+\alpha\right.$-pinene, i.e., energies below $\left.60 \mathrm{kcal} / \mathrm{mol}\right)$ are included. The nodes are labelled in increasing order of their relative energies, except for those previously found by $\mathrm{ZhZh},{ }^{79}$ where we kept the same acronyms. In general, however, the node labels of both networks do not refer to the same intermediate because of the different number of structures obtained. The geometries of each node (intermediate) are collected in the SI.

Table 1. Properties of the networks obtained with the AMK-stdMD and AMK-BXDE methods

\begin{tabular}{|c|c|c|}
\hline & AMK-stdMD & AMK-BXDE \\
\hline \multicolumn{3}{|c|}{ Initial (unconnected) networks } \\
\hline Percent of connected nodes ${ }^{[a]}$ & 38 & 60 \\
\hline Density of edges ${ }^{[b]}$ & 0.0076 & 0.026 \\
\hline \multicolumn{3}{|c|}{ Connected networks } \\
\hline$\gamma$ & 1.97 & 1.63 \\
\hline Average clustering coefficient ${ }^{[c]}$ & $0.15(6.91)$ & $0.12(3.26)$ \\
\hline Transitivity ${ }^{[c]}$ & $0.06(2.30)$ & $0.12(2.46)$ \\
\hline Average shortest path length ${ }^{[c]}$ & $3.12(0.57)$ & $3.00(0.53)$ \\
\hline Assortativity & -0.33 & -0.44 \\
\hline
\end{tabular}

[a] Percentage of nodes that are connected to the bulk of the network. [b] Fraction of edges with respect to the maximum number of edges between the nodes of the network. [c] In parentheses, the ratio of the value of the property over the corresponding value of a random (Erdős-Rényi) network with the same number of nodes and edges.

The red nodes in Figure 2 refer to the primary ozonide POZ, and the red edges represent the cycloaddition of ozone to the double bond of $\alpha$-pinene. The widths of the edges (weights, as defined above) are a measure of the number of TSs (or paths) connecting a pair of nodes.

A simple inspection of Figure 2 indicates that, besides $\mathrm{POZ}$ nodes $\mathrm{Cl} 1$ and $\mathrm{Cl} 2$ are two important hubs (nodes with high degrees) in both graphs. These structures are Criegee intermediates (Cls) that are formed from POZ (vide infra).

The resulting product fragments obtained in the reduced AMK-stdMD network are: $\mathrm{H}_{2}, \mathrm{O}_{2}, \mathrm{CO}$, ozone, water, methane, and larger fragments (see Figure 2). In the reduced AMD-BXDE network, besides the above products, the following molecules are also obtained: hydrogen peroxide, acetaldehyde, vinyl alcohol, acetic acid, and different $\mathrm{C}_{4} \mathrm{H}_{6} \mathrm{O}_{2}$ and $\mathrm{C}_{6} \mathrm{H}_{10} \mathrm{O}$ isomers. Other products like $\mathrm{OH}$ radicals have been previously suggested, ${ }^{79}$ but they are not included in Figure 2 because AutoMeKin's algorithms aim to locate saddle points and, therefore, the method is blind to barrierless processes. Although the search of dissociations occurring without a barrier could be easily implemented in an automated manner, AutoMeKin is an integrated protocol with a kinetics module, and the automated calculation of rate coefficients for barrierless reactions is not straightforward. 

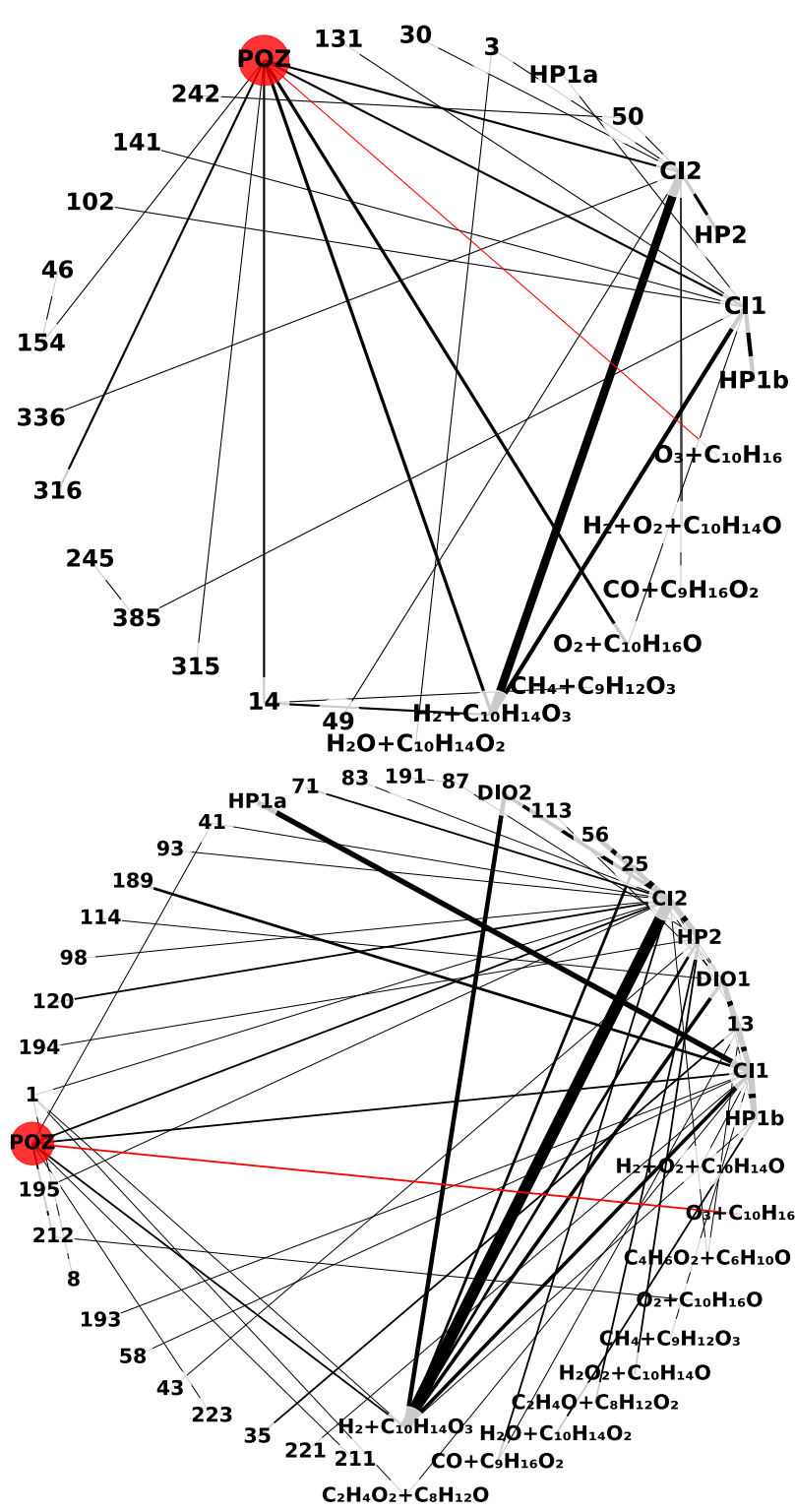

Figure 2. Reduced reaction networks obtained with AMK-stdMD (top) and AMK-BXDE (bottom). They include only paths (edges) whose TSs are below the addition of ozone to the double bond of $\alpha$-pinene (shown in red). Nodes represent different structures (labelled in order of increasing energies, with POZ shown in red; for the structures found in the ZhZh study ${ }^{79}$ we keep the same nomenclature; see also Table 2) and the width of the edges is a measure of the number of paths between a pair of nodes.

An important property of any network is the probability distribution of the degrees in the network, or degree distribution. The degree distributions obtained in this work for the AMK-stdMD and AMKBXDE networks are displayed in Figure 3. To assess whether our networks present scale-free behavior, they were fit to a power law (see eq 1 above) treating the datasets as discrete (integer) distributions. Since power laws are undefined for $k=0$, the minimum values for $k\left(k_{\min }\right)$ were optimized here, obtaining $k_{\text {min }}=2$ and 1 for the AMK-stdMD and AMK-BXDE distributions, respectively. Finally, degree distributions are typically very noisy in their tails, which led us to adopt a logarithmic, rather than linear, binning procedure.

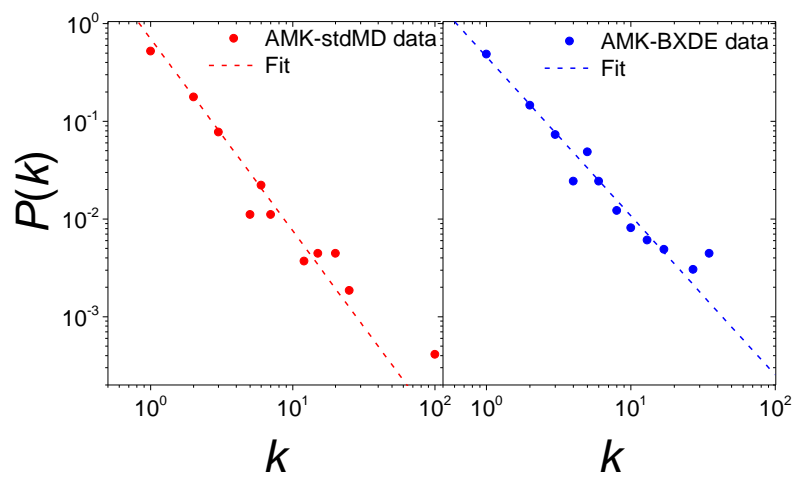

Figure 3. Degree distributions of the AMK-stdMD and AMK-BXDE networks Dashed lines represent fits to power law distributions.

Although apparently a power-law model fits well both distributions (see Figure 3) with $\gamma$ values of 1.97 (standard error of 0.15 ) and 1.63 (standard error of 0.10 ) for AMK-stdMD and AMK-BXDE, respectively, the goodness of the fits should be checked by assessing other candidate distributions. The Python package employed in this analysis offers the possibility to judge whether other distributions, namely, exponential, lognormal, truncated power-law, stretched exponential and lognormal positive, provide better fits than a power law. ${ }^{99}$ The comparisons are made through the calculation of two values: $R$, which is the loglikelihood ratio between any two candidate distributions (with its sign indicating the likelihood of each one), and $p$ that provides the significance of the comparison (if $p>0.05$, the sign of $R$ cannot be trusted if its value is close to 0 ).

The degree distributions are undoubtedly better fit with a power law than with an exponential. However, the comparisons with the other distributions indicate that either $R$ is close to 0 , and/or the values of $p$ are too high for the comparison to be statistically significant. Therefore, it is not possible to firmly conclude that the degree distributions follow a power law. By contrast, the degree distribution for the network of organic chemistry, ${ }^{97}$ made up of millions of nodes, undoubtedly follows a power law. We attribute the uncertainty in our result to the relative scarcity of nodes in our networks. At any rate, other properties of the network studied in this work point to a scale-free behavior, as will be discussed below. The clustering coefficient provides the amount of interlinking between neighbors of a given node. A signature of scale-free networks is their enhanced clustering compared with a similar (same number of nodes and edges) random network. The clustering coefficients obtained in our work for the AMK-stdMD and AMK-BXDE networks are similar to each other and greatly exceed the corresponding values for a random network, which reveals scale-free behavior.

Another interesting feature of networks is whether they present small-world behavior, where every pair of nodes are connected, on average, through a relatively small number of edges. This 
property can be assessed by comparing the values of the transitivity and the average shortest path length with those of random networks. Examples of networks exhibiting small-world behavior are the network of organic chemistry, ${ }^{97}$ and networks of small clusters bound by Morse potentials. ${ }^{98}$

As seen in Table 1, the networks transitivities are more than double the transitivities of equivalent random networks. Regarding the average shortest path lengths, their values are $\sim 3$ for our networks, meaning that, on average, two different nodes are connected by three elementary steps. These numbers are much shorter than those for the corresponding random networks (see Table 1), pointing to small-world behavior. Another signature of small-world networks is the logarithmic dependence of the average shortest path length with the number of nodes. However, such a test cannot be carried out here due to the limited number of edges and nodes in our networks.

Finally, the values obtained for the assortativity are negative and similar in both networks indicating disassortativity mixing, i.e., nodes of different degree tend to be connected. By way of example, the networks of Figure 2 show links between highlyconnected nodes (like $\mathrm{Cl} 1$ and $\mathrm{Cl} 2$ ) with other nodes that are only connected to these hubs. Substantial disassortative mixing has been also observed in biological and technological networks, ${ }^{103}$ while the network of organic chemistry shows modest disassortative mixing. ${ }^{97}$ However, it should be borne in mind that disassortativity can arise, to some extent, from the absence of self-loops in the networks, as pointed out by Park and Newman. ${ }^{104}$

Reaction mechanism. The relative efficiency of AMK-BXDE vs AMK-stdMD can be assessed by comparing the corresponding $\alpha$-pinene ozonolysis reaction mechanisms. Additionally, it is also useful to compare our results with those obtained by ZhZh in their computational study. ${ }^{79}$ These comparisons are summarized in Table 2, which highlights the most important differences among our two methods and the previous study. A complete list of all reaction channels is gathered in the SI.

The initial step in the ozonolysis is the cycloaddition of ozone to the double bond of $\alpha$-pinene to form POZ. ${ }^{79}$ Both AMK-stdMD and AMK-BXDE locate the same TS found by ZhZh for this step. ${ }^{79}$ This structure corresponds to TSs 228, 197 and I in the AMK-stdMD and AMK-BXDE databases and in ZhZh, respectively.

Interestingly, AMK-BXDE finds an alternative saddle point for the cycloaddition reaction, TS 196, which is $0.7 \mathrm{kcal} / \mathrm{mol}$ more stable than TS 197 at the PM7 level of theory. To confirm the nature of this novel TS, its structure was reoptimized at the MP2/6-31(d) level of theory and is shown in Figure 4 in comparison with TS 197. Of significance, TS 196 is $1.5 \mathrm{kcal} / \mathrm{mol}$ more stable than TS 197 at the $\operatorname{CCSD}(\mathrm{T}) / 6-311+\mathrm{G}(\mathrm{d}, \mathrm{p}) / / \mathrm{MP} 2 / 6-31 \mathrm{G}(\mathrm{d})$ level of theory, which corresponds to a factor of $\sim 10$ in terms of rate coefficients. Thus, the new TS for the cycloaddition should be taken into account in future computational kinetics studies of this system. The optimized geometry, electronic energies, and vibrational frequencies of this new TS is in the SI.
Table 2. Main differences between the reaction mechanisms predicted by AMK-stdMD and AMK-BXDE

\begin{tabular}{|c|c|c|c|}
\hline & ZhZh ${ }^{79}$ & AMK-stdMD $[\mathrm{a}]$ & AMK-BXDE[a] \\
\hline Channel & \multicolumn{3}{|c|}{ Labels of the involved TSs } \\
\hline \multirow[t]{2}{*}{$\mathrm{O}_{3}+\alpha$-pinene } & I & 228 & 197 \\
\hline & & & \\
\hline $\mathrm{POZ} \rightleftharpoons \mathrm{Cl} 1$ & $\| 11$ & 89,95 & 133,136 \\
\hline $\mathrm{POZ} \rightleftharpoons \mathrm{Cl} 2$ & 112 & 88,93 & 132,134, stepwise $^{[b]}$ \\
\hline $\mathrm{Cl} 1 \rightleftharpoons \mathrm{HP} 1 \mathrm{a}$ & III1a & 63 & {$[90,92,94,97,99,101]$} \\
\hline $\mathrm{Cl} 1 \rightleftharpoons \mathrm{HP} 1 \mathrm{~b}$ & III1b & {$[26,27,30,34]$} & {$[48,51,52,53,54,55,69]$} \\
\hline $\mathrm{Cl} 1 \rightleftharpoons \mathrm{DIO} 1$ & III1C & Not found & {$[71,72,73,74,75,77,78]$} \\
\hline $\mathrm{Cl} 1 \rightleftharpoons 13^{[c]}$ & Not found & Not found & 105 \\
\hline $\mathrm{Cl} 2 \rightleftharpoons \mathrm{HP} 2$ & $\mathrm{III} 2 \mathrm{a}$ & {$[32,35,36]$} & {$[57,58,60,61,62,67]$} \\
\hline $\mathrm{Cl} 2 \rightleftharpoons \mathrm{DIO} 2$ & III2b & Not found & {$[76,79,80,81]$} \\
\hline $\mathrm{Cl} 2 \rightleftharpoons 56^{[\mathrm{c}]}$ & Not found & Not found & {$[63,64,66,68,70]$} \\
\hline $\mathrm{HP} 2 \rightleftharpoons 25^{[c]}$ & IV & Not found & 85 \\
\hline $\mathrm{DIO} 1 \rightleftharpoons 13^{[\mathrm{c}]}$ & Not found & Not found & 50 \\
\hline $\mathrm{DIO} 1 \rightleftharpoons 25^{[\mathrm{c}]}$ & Not found & Not found & 59 \\
\hline $\mathrm{DIO} 1 \rightleftharpoons \mathrm{Cl} 2$ & Not found & Not found & 83 \\
\hline $\mathrm{DIO} 1 \rightleftharpoons \mathrm{HP} 1 \mathrm{~b}$ & Not found & Not found & 109 \\
\hline $\mathrm{DIO} 1 \rightleftharpoons 114^{[\mathrm{c}]}$ & Not found & Not found & 112 \\
\hline $\mathrm{HP} 1 \mathrm{~b} \rightarrow \mathrm{H}_{2} \mathrm{O}$ & Not found & Not found & 43 \\
\hline $\mathrm{HP} 1 \mathrm{~b} \rightarrow \mathrm{H}_{2} \mathrm{O}$ & Not found & Not found & 56 \\
\hline $\mathrm{HP} 1 \mathrm{~b} \rightarrow \mathrm{H}_{2}$ & Not found & Not found & 93 \\
\hline $\mathrm{DIO} 2 \rightarrow \mathrm{H}_{2}$ & Not found & Not found & 96 \\
\hline $\mathrm{HP} 2 \rightarrow \mathrm{H}_{2}$ & Not found & Not found & 98 \\
\hline $\mathrm{DIO} 1 \rightarrow \mathrm{H}_{2}$ & Not found & Not found & 102 \\
\hline $\mathrm{HP} 2 \rightarrow \mathrm{H}_{2} \mathrm{O}_{2}$ & Not found & Not found & 106 \\
\hline
\end{tabular}

[a] Families of TS conformers are in brackets. Each database has different labels because the number of obtained structures differs, and they are labelled in increasing order of their energies. The table collects only the lowest energy channels. For a full description of all channels see the SI. [b] As discussed in the main text, we found here a stepwise $\mathrm{POZ} \rightarrow \mathrm{Cl} 2$ mechanism via hydroperoxide 41. [c] These labels correspond to the AMK-BXDE database.

The next step in the ozonolysis reaction mechanism is the cleavage of $\mathrm{POZ}$ to form the Criegee intermediates $\mathrm{Cl} 1$ and $\mathrm{Cl} 2$. Zhang and Zhang reported a concerted mechanism where the $\mathrm{O}-\mathrm{O}$ and $\mathrm{C}-\mathrm{C}$ bonds break simultaneously, ${ }^{79}$ a process that involves one concerted TS for the formation of each Criegee intermediate, labelled as TS II1 and TS II2, for the formation of $\mathrm{Cl} 1$ and $\mathrm{Cl} 2$, respectively. ${ }^{79}$ In the present work, two concerted TSs were located for the formation of each Criegee intermediate. These TSs are labelled 133 and 136 (for Cl1) and 132 and 134 (for $\mathrm{Cl} 2$ ) in the AMK-BXDE database and connect $\mathrm{POZ}$ with different conformers of the Cls; the AMK-stdMD calculations provide the same results as seen in Table 2.

Additionally, AMK-BXDE finds an alternative stepwise mechanism for the $\mathrm{POZ} \rightarrow \mathrm{Cl} 2$ reaction through hydroperoxide 41 (see Figure 2). A similar stepwise mechanism was previously reported for ethylene ozonolysis, ${ }^{105}$ but not for $\alpha$-pinene. No further attempts have been made to reoptimize the PM7 structures at higher levels of theory as this is out of the scope of the present work. However, future (computational) studies on the 
ozonolysis of $\alpha$-pinene should take into account the new transition states provided in the present work.

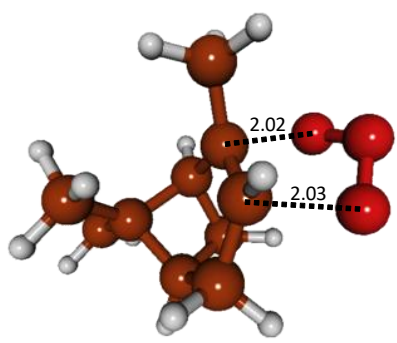

TS 196

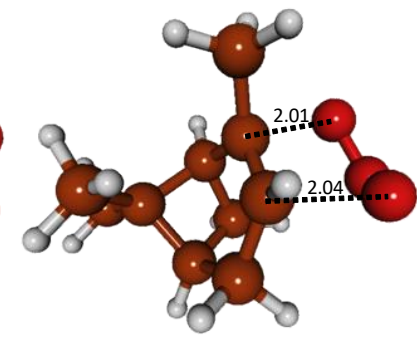

TS 197
Figure 4. TS structures found in this study for the initial addition of ozone to the double bond of $\alpha$-pinene using AMK-BXDE methodology. The structures are optimized at the MP2/6-31G(d) level of theory and correspond to TS 196 and 197 in the AMK-BXDE database collected in the SI.

The networks obtained in this study (see Figure 2) also predict isomerizations of POZ leading to, for instance, intermediates 315 (in the AMK-stdMD network) or 211 (in the AMK-BXDE network) as well as fragmentations of POZ. However, these alternative isomerization/fragmentation mechanisms from $\mathrm{POZ}$ involve highenergy TSs (at the PM7 level of theory) and are probably not very competitive in the decomposition kinetics.

According to the computational study of $\mathrm{ZhZh},{ }^{79}$ the Criegee intermediates $\mathrm{Cl} 1$ and $\mathrm{Cl} 2$ can evolve to the hydroperoxides HP1a, HP1b and HP2, or to the dioxiranes DIO1 and DIO2. While AMK-BXDE predicts the same pathways, AMK-stdMD fails to locate the dioxirane intermediates as indicated in Table 2. According to AMK-BXDE, the pathway leading to HP1b presents the lowest energy barrier, in accordance with previous work. ${ }^{79}$ For these pathways, AutoMeKin finds families of TS conformers, whose labels are inside brackets in Table 2, with the number of isomers being greater with AMK-BXDE. Previous computational work has shown the importance of including all these families of conformers for an accurate kinetics analysis. ${ }^{65}$

Starting from $\mathrm{Cl} 1$, AMK-BXDE predicts an isomerization reaction leading to ketone 13, a channel that is absent in both the AMK-stdMD network and in the previous computational study. ${ }^{79}$ In agreement with $\mathrm{ZhZh},{ }^{79}$ AMK-BXDE finds paths for $\mathrm{Cl} 2$ evolving to HP2 and DIO2. While the DIO2 formation channel is missing in the AMK-stdMD database, four TS conformers were located with AMK-BXDE. Moreover, AMK-BXDE finds five TS conformers connecting $\mathrm{Cl} 2$ to hydroperoxide 56 , a process that can be competitive in the $298 \mathrm{~K}$ kinetics as seen below; this hydroperoxide has not been reported before.

The AMK-BXDE calculations predict that HP2 can isomerize to ketone 25 through TS 85 (using the AMK-BXDE labels), which corresponds to TS IV in the ZhZh computational study. ${ }^{79}$ This transition state is overlooked in the AMK-stdMD calculations.

What follows is a very brief description of some other examples of pathways only found in our AMK-BXDE calculations. These pathways involve DIO1 isomerizations as well as fragmentations from the hydroperoxides HP1b and HP2 and from both dioxiranes, and correspond to the last two entries of Table 2. AMK-BXDE predicts that dioxirane DIO1 can isomerize to ketone 13 , ketone 25, $\mathrm{Cl} 2, \mathrm{HP} 1 \mathrm{~b}$, and intermediate 114. In the same fashion, fragmentations of HP1b, HP2, DIO1 and DIO2 leading to $\mathrm{H}_{2}, \mathrm{H}_{2} \mathrm{O}$ and $\mathrm{H}_{2} \mathrm{O}_{2}$ (and to their corresponding co-products) are possible according to our AMK-BXDE method.

As indicated above, Table 2 shows only some representative examples of the lowest energy channels; a full account of all channels is gathered in the SI. However, from the above results, quite clearly AMK-BXDE is superior to AMK-stdMD in predicting reaction mechanisms.

A final comparison between the two methods can be carried out by running preliminary kinetics simulations on the predicted networks. A detailed kinetics study is out of the scope of this work. Firstly, because the level of electronic structure theory is not accurate enough to provide quantitative results. And secondly, because collisional stabilization, bimolecular reactions of stabilized intermediates with other atmospheric molecules, and barrierless reactions (like $\mathrm{OH}$ radical formation) are all neglected in AutoMeKin. However, and only for the sake of comparison, we ran two separate kinetic simulations using the networks obtained in our study. The Kinetic Monte Carlo ${ }^{106}$ simulations start from POZ using $10^{3}$ molecules and state-to-state rate coefficients calculated with transition state theory at a temperature of $298 \mathrm{~K}$. The kinetics results indicate that, in both networks, nodes POZ, $\mathrm{Cl} 1, \mathrm{Cl} 2, \mathrm{HP} 1 \mathrm{~b}$ and HP2 are populated at some point of the simulations. However, while the AMK-stdMD simulation ends in an equilibrium mixture between $\mathrm{HP} 1 \mathrm{~b}$ and HP2 with no fragmentations, the AMK-BXDE kinetics results provide the following yields: $42 \%$ of $\mathrm{H}_{2} \mathrm{O}$ formation and $58 \%$ of hydroperoxide 56 . The different outcomes of both kinetics simulations stem from the differences between the underlying reaction mechanisms highlighted in Table 2 and discussed in detail above.

The way the AMK-stdMD calculation was performed was the usual one, i.e., a large number (5000) of very short-time ( $0.5 \mathrm{ps})$ trajectories was employed, a strategy that proved to be very efficient in past studies. However, and to make a more realistic comparison between both methods, we decided to perform an additional AMK-stdMD calculation using 500 trajectories and a $t_{\text {max }}$ of $5 \mathrm{ps}$, as in AMK-BXDE. With the new AMK-stdMD settings, the energies of the obtained structures are again much higher than those obtained with AMK-BXDE, and similar to those obtained in the $0.5-p s$ MD simulations. However, only $31 \%$ of the nodes in the new AMK-stdMD network are connected, which can be compared with a $38 \%$ obtained before (see Table 1). Most importantly, not only dioxiranes DIO1 and DIO2, and intermediates 13 and 25 are missing in this new calculation, but also hydroperoxide HP1 and the cycloaddition transition states of Figure 4.

In summary, only the new procedure, AMK-BXDE, is capable of finding the most important reaction pathways using a moderate accumulated simulation time. 


\section{Conclusions}

The reaction discovery method AutoMeKin was interfaced with the rare event acceleration algorithm BXDE. The new combined computational protocol was put to the test in the present work through a detailed analysis of the obtained reaction mechanisms for the $\alpha$-pinene ozonolysis.

AutoMeKin-BXDE brings a real improvement over standard AutoMeKin. The main differences between the results obtained with both methodologies are summarized in the following points:

1) The total number of structures obtained with AMK-stdMD is more than double those found with AMK-BXDE.

2) However, the average energies of the structures obtained with AMK-BXDE are $\sim 50-60 \mathrm{kcal} / \mathrm{mol}$ lower than the AMK-stdMD ones.

3) The reaction networks have similar properties: both display small-world and scale-free behavior, although the AMK-BXDE network is significantly more densely connected than the AMK-stdMD one.

4) AMK-BXDE predicts the previously reported reaction mechanism for the $\alpha$-pinene ozonolysis in its entirety.

5) By contrast, AMK-stdMD fails to find some of the reaction pathways and intermediates found in previous computational work.

6) Furthermore, AMK-BXDE predicts some new TSs and mechanisms not previously reported, which should be taken into account in future kinetics simulations.

In summary, owing to its superior performance, the new accelerated MD procedure BXDE will become standard in the next release of AutoMeKin. ${ }^{64}$

\section{Acknowledgements}

This research was funded by "Consellería de Cultura, Educación e Ordenación Universitaria, Xunta de Galicia", grant ED431C 2017/17. RAJT and GAP akcnowledge the founding of CONICET, FONCyT, SeCyT-UNC and MinCyT-Córdoba. RJS is supported by EPSRC Programme grant EP/P021123/1.

Keywords: MD simulations - rare-event acceleration • BXDE • AutoMeKin $\bullet$ Reaction discovery $\cdot$ ozonolysis of $\alpha$-pinene

[1] H. B. Schlegel Wiley Interdiscip. Rev. Comput. Mol. Sci. 2011, 1, 790-809

[2] H. L. Davis, D. J. Wales, R. S. Berry J. Chem. Phys. 1990, 92, 4308-4319.

[3] J. Q. Sun, K. Ruedenberg J. Chem. Phys. 1993, 98, 9707-9714.

[4] C. J. Tsai, K. D. Jordan J. Phys. Chem. 1993, 97, 11227-11237.

[5] Y. Abashkin, N. Russo J. Chem. Phys. 1994, 100, 4477-4483.

[6] K. Bondensgard, F. Jensen J. Chem. Phys. 1996, 104, 8025-8031.

[7] J. P. K. Doye, D. J. Wales Z. Phys. D. 1997, 40, 194-197.

[8] W. Quapp, M. Hirsch, O. Imig, D. Heidrich J. Comput. Chem. 1998, 19, 10871100.

[9] M. Černohorský, S. Kettou, J. Koča J. Chem. Inf. Comput. Sci. 1999, 39, 705-712.

[10] K. M. Westerberg, C. A. Floudas J. Chem. Phys. 1999, 110, 9259-9295.

[11] D. J. Wales, J. P. Doye, M. A. Miller, P. N. Mortenson, T. R. Walsh Adv. Chem. Phys. 2000, 115, 1-111.
[12] K. K. Irikura, R. D. Johnson J. Phys. Chem. A. 2000, 104, 2191-2194.

[13] E. M. Müller, A. d. Meijere, H. Grubmüller J. Chem. Phys. 2002, 116, $897-$ 905.

[14] M. Dallos, H. Lischka, E. Ventura Do Monte, M. Hirsch, W. Quapp J. Comput. Chem. 2002, 23, 576-583.

[15] J. Baker, K. Wolinski J. Comput. Chem. 2011, 32, 43-53.

[16] P. M. Zimmerman J. Comput. Chem. 2013, 34, 1385-1392.

[17] P. M. Zimmerman J. Chem. Phys. 2013, 138, 184102.

[18] P. Zimmerman J. Chem. Theory Comput. 2013, 9, 3043-3050.

[19] P. M. Zimmerman J. Comput. Chem. 2015, 36, 601-611.

[20] P. M. Zimmerman Mol. Simul. 2015, 41, 43-54.

[21] M. Jafari, P. M. Zimmerman J. Comput. Chem. 2017, 38, 645-658.

[22] A. L. Dewyer, P. M. Zimmerman Org. \& Biomol. Chem. 2017, 15, 501-504.

[23] D. Rappoport, C. J. Galvin, D. Y. Zubarev, A. Aspuru-Guzik J. Chem. Theory Comput. 2014, 10, 897-907.

[24] B. Schaefer, S. Mohr, M. Amsler, S. Goedecker J. Chem. Phys. 2014, 140, 214102.

[25] D. J. Wales J. Chem. Phys. 2015, 142, 130901.

[26] S. Habershon J. Chem. Phys. 2015, 143, 094106.

[27] S. Habershon J. Chem. Theory Comput. 2016, 12, 1786-1798.

[28] X.-J. Zhang, Z.-P. Liu Phys. Chem. Chem. Phys. 2015, 17, 2757-2769.

[29] L.-P. Wang, R. T. McGibbon, V. S. Pande, T. J. Martinez J. Chem. Theory Comput. 2016, 12, 638-649.

[30] L.-P. Wang, A. Titov, R. McGibbon, F. Liu, V. S. Pande, T. J. Martínez Nat. Chem. 2014, 6, 1044-1048.

[31] M. Yang, J. Zou, G. Wang, S. Li J. Phys. Chem. A. 2017, 121, 1351-1361.

[32] L. D. Jacobson, A. D. Bochevarov, M. A. Watson, T. F. Hughes, D. Rinaldo, S. Ehrlich, T. B. Steinbrecher, S. Vaitheeswaran, D. M. Philipp, M. D. Halls, R. A. Friesner J. Chem. Theory Comput. 2017, 13, 5780-5797.

[33] K. Ohno, S. Maeda Chem. Phys. Lett. 2004, 384, 277-282.

[34] S. Maeda, K. Ohno J. Phys. Chem. A. 2005, 109, 5742-5753.

[35] K. Ohno, S. Maeda J. Phys. Chem. A. 2006, 110, 8933-8941.

[36] K. Ohno, S. Maeda Phys. Scr. 2008, 78, 058122.

[37] S. Maeda, K. Morokuma J. Chem. Phys. 2010, 132, 241102.

[38] S. Maeda, K. Morokuma J. Chem. Theory Comput. 2011, 7, 2335-2345.

[39] S. Maeda, K. Ohno, K. Morokuma Phys. Chem. Chem. Phys. 2013, 15, 3683-3701.

[40] S. Maeda, T. Taketsugu, K. Morokuma J. Comput. Chem. 2014, 35, 166173.

[41] S. Maeda, Y. Harabuchi, M. Takagi, T. Taketsugu, K. Morokuma Chem. Rec. 2016, 16, 2232-2248

[42] S. Maeda, Y. Harabuchi, M. Takagi, K. Saita, K. Suzuki, T. Ichino, Y. Sumiya, K. Sugiyama, Y. Ono J. Comput. Chem. 2017, 39, 233-250.

[43] J. A. Varela, S. A. Vazquez, E. Martinez-Nunez Chem. Sci. 2017, 8, 38433851.

[44] E. Martínez-Núñez Phys. Chem. Chem. Phys. 2015, 17, 14912-14921.

[45] E. Martínez-Núñez J. Comput. Chem. 2015, 36, 222-234.

[46] A. Rodríguez, R. Rodríguez-Fernández, S. A. Vázquez, G. L. Barnes, J. J. P. Stewart, E. Martínez-Núñez J. Comput. Chem. 2018, 39, 1922-1930.

[47] S. A. Vazquez, X. L. Otero, E. Martinez-Nunez Molecules. 2018, 23, 3156

[48] L. J. Broadbelt, S. M. Stark, M. T. Klein Ind. Eng. Chem. Res. 1994, 33, 790-799.

[49] D. M. Matheu, A. M. Dean, J. M. Grenda, W. H. Green J. Phys. Chem. A 2003, 107, 8552-8565

[50] C. W. Gao, J. W. Allen, W. H. Green, R. H. West Comput. Phys. Commun. 2016, 203, 212-225

[51] P. L. Bhoorasingh, R. H. West Phys. Chem. Chem. Phys. 2015, 17, 3217332182.

[52] P. L. Bhoorasingh, B. L. Slakman, F. Seyedzadeh Khanshan, J. Y. Cain, R. H. West J. Phys. Chem. A. 2017, 121, 6896-6904.

[53] Y. V. Suleimanov, W. H. Green J. Chem. Theory Comput. 2015, 11, 42484259.

[54] M. Bergeler, G. N. Simm, J. Proppe, M. Reiher J. Chem. Theory Comput. 2015, 11, 5712-5722. 
[55] J. Proppe, T. Husch, G. N. Simm, M. Reiher Faraday Discuss. 2016, 195, 497-520.

[56] G. N. Simm, M. Reiher J. Chem. Theor. Comput. 2017, 13, 6108-6119.

[57] G. N. Simm, M. Reiher J. Chem. Theor. Comput. 2018, 14, 5238-5248.

[58] G. N. Simm, A. C. Vaucher, M. Reiher J. Phys. Chem. A. 2019, 123, 385399

[59] A. L. Dewyer, A. J. Argüelles, P. M. Zimmerman WIREs Comput Mol Sci. 2018, 8:e1354, doi: 1310.1002/wcms.1354.

[60] Y. Kim, J. W. Kim, Z. Kim, W. Y. Kim Chem. Sci. 2018, 9, 825-835.

[61] J. W. Kim, Y. Kim, K. Y. Baek, K. Lee, W. Y. Kim J. Phys. Chem. A. 2019 123, 4796-4805

[62] M. Kawano, S. Koido, T. Nakatomi, Y. Watabe, T. Takayanagi Comput. Theor. Chem. 2019, 1155, 31-37.

[63] C. A. Grambow, A. Jamal, Y.-P. Li, W. H. Green, J. Zádor, Y. V. Suleimanov J. Am. Chem. Soc. 2018, 140, 1035-1048.

[64] https://rxnkin.usc.es/index.php/AutoMeKin.

[65] D. Ferro-Costas, E. Martínez-Núñez, J. Rodríguez-Otero, E. CabaleiroLago, C. M. Estévez, B. Fernández, A. Fernández-Ramos, S. A. Vázquez J. Phys. Chem. A. 2018, 122, 4790-4800.

[66] Y. Fenard, A. Gil, G. Vanhove, H.-H. Carstensen, K. M. Van Geem, P. R. Westmoreland, O. Herbinet, F. Battin-Leclerc Combust. Flame. 2018 , 191, 252-269.

[67] M. J. Wilhelm, E. Martínez-Núñez, J. González-Vázquez, S. A. Vázquez, J. M. Smith, H.-L. Dai ApJ. 2017, 849, 15.

[68] R. Perez-Soto, S. A. Vazquez, E. Martinez-Nunez Phys. Chem. Chem. Phys. 2016, 18, 5019-5026.

[69] S. A. Vazquez, E. Martinez-Nunez Phys. Chem. Chem. Phys. 2015, 17, 6948-6955.

[70] E. Rossich Molina, J.-Y. Salpin, R. Spezia, E. Martinez-Nunez Phys. Chem Chem. Phys. 2016, 18, 14980-14990.

[71] V. Macaluso, D. Scuderi, M. E. Crestoni, S. Fornarini, D. Corinti, E. Dalloz, E. Martinez-Nunez, W. L. Hase, R. Spezia J. Phys. Chem. A. 2019, 123 3685-3696.

[72] R. J. Shannon, S. Amabilino, M. O'Connor, D. V. Shalishilin, D. R. Glowacki J. Chem. Theor. Comput. 2018, 14, 4541-4552.

[73] D. R. Glowacki, E. Paci, D. V. Shalashilin J. Chem. Theory Comput. 2011, 7, 1244-1252.

[74] J. Booth, S. Vazquez, E. Martínez-Núñez, A. Marks, J. Rodgers, D. R. Glowacki, D. V. Shalashilin Phil. Trans. R. Soc. A. 2014, 372, 20130384

[75] M. O'Connor, E. Paci, S. Mclntosh-Smith, D. R. Glowacki Faraday Discuss. 2016, 195, 395.

[76] E. Martínez-Núñez, D. V. Shalashilin J. Chem. Theor. Comput. 2006, 2, 912-919.

[77] J. J. Orlando, G. S. Tyndall, T. J. Wallington Chem. Rev. 2003, 103, $4657-$ 4690.

[78] P. Di Carlo, W. H. Brune, M. Martinez, H. Harder, R. Lesher, X. Ren, T. Thornberry, M. A. Carroll, V. Young, P. B. Shepson, D. Riemer, E. Apel, C. Campbell Science. 2004, 304, 722-725.

[79] D. Zhang, R. Zhang J. Chem. Phys. 2005, 122, 114308.

[80] S. M. Japar, C. H. Wu, H. Niki Environ. Lett. 1974, 7, 245-249.

[81] R. Atkinson, D. Hasegawa, S. M. Aschmann Int. J. Chem. Kinet. 1990, 22 871-887.

[82] M. Witter, T. Berndt, O. Böge, F. Stratmann, J. Heintzenberg Int. J. Chem. Kinet. 2002, 34, 394-403.

[83] F. Nolting, W. Behnke, C. Zetzsch J. Atmos. Chem. 1988, 6, 47-59.

[84] D. Grosjean, E. L. Williams, E. Grosjean, J. M. Andino, J. H. Seinfeld Environ. Sci. Technol. 1993, 27, 2754-2758.

[85] V. G. Khamaganov, R. A. Hites J. Phys. Chem. A. 2001, 105, 815-822.

[86] R. Atkinson, S. M. Aschmann, J. Arey, B. Shorees J. Geophys. Res. 1992 , 97, 6065-6073.

[87] R. Saleh, N. M. Donahue, A. L. Robinson Environ. Sci. Technol. 2013, 47, 5588-5594.

[88] R. K. Pathak, A. A. Presto, T. E. Lane, C. O. Stanier, N. M. Donahue, S. N. Pandis Atmos. Chem. Phys. 2007, 7, 3811-3821.
[89] F. Holloway, H. J. Anderson, W. Rodin Ind. Eng. Chem. 1955, 47, 2111 2113

[90] C. Kidd, V. Perraud, L. M. Wingen, B. J. Finlayson-Pitts PNAS. 2014, 111 7552-7557.

[91] A. L. Putman, J. H. Offenberg, R. Fisseha, S. Kundu, T. A. Rahn, L. R. Mazzoleni Atmos. Environ. 2012, 46, 164-172.

[92] R. Criegee Angew. Chem. Int. Ed. 1975, 14, 745-752.

[93] S. Hatakeyama, K. Izumi, T. Fukuyama, H. Akimoto J. Geophys. Res. 1989 94, 13013-13024.

[94] J. J. P. Stewart, MOPAC2016, Stewart Computational Chemistry, Colorado Springs, CO, USA, HTTP://OpenMOPAC.net (2016).

[95] W. L. Hase, D. G. Buckowski Chem. Phys. Lett. 1980, 74, 284-287.

[96] A. A. Hagberg, D. A. Schult, P. J. Swart, Proceedings of the 7th Python in Science Conference (SciPy2008), G. Varoquaux, T. Vaught, and J. Millman (Eds), (Pasadena, CA USA), pp. 2011-2015, Aug 2008.

[97] P.-M. Jacob, A. Lapkin React. Chem. Eng. 2018, 3, 102-118.

[98] J. W. R. Morgan, D. Mehta, D. J. Wales Phys. Chem. Chem. Phys. 2017 19, 25498-25508.

[99] A. Klaus, S. Yu, D. Plenz PloS ONE. 2011, 6, e19779.

[100] J. Saramäki, M. Kivelä, J.-P. Onnela, K. Kaski, J. Kertész Phys. Rev. E. 2007, 75, 027105

[101] E. W. Dijkstra Numer. Math. 1959, 1, 83-89.

[102] M. E. J. Newman Phys. Rev. E. 2003, 67, 026126.

[103] L. D. F. Costa, F. A. Rodrigues, G. Travieso, P. R. Villas Boas Adv. Phys. 2007, 56, 167-242.

[104] J. Park, M. E. J. Newman Phys. Rev. E. 2003, 68, 026112.

[105] J. M. Anglada, R. Crehuet, J. M. Bofill Chem.: Eur. J. 1999, 5, 1809-1822.

[106] D. T. Gillespie J. Comput. Phys. 1976, 22, 403-434 


\section{Supporting Information for}

\section{Enhancing automated reaction discovery with boxed molecular dynamics in energy space}

Rafael A. Jara-Toro, ${ }^{[a]}$ Gustavo A. Pino, ${ }^{[a]}$ David R. Glowacki, ${ }^{[b]}$ Robin J. Shannon, ${ }^{[b]}$ and Emilio Martínez-Núñez*[c]

[a] INIFIQC (CONICET-UNC) Dpto. De Fisicoquímica-Facultad de Ciencias Químicas-Centro Láser de Ciencias Moleculares, Universidad de Córdoba, Ciudad Universitaria, X50000HUA Córdoba, Argentina

[b] Centre for Computational Chemistry, School of Chemistry, University of Bristol, Cantock's Close, Bristol BS8 1TS, UK

[c] Departmento de Química Física, Facultade de Química, Universidade de Santiago de Compostela, 15782, Santiago de Compostela, SPAIN, E-mail: emilio.nunez@usc.es 


\section{Contents}

CORRESPONDENCE OF THE ACRONYMS EMPLOYED BY ZhZh WITH THE LABELS OF OUR DATABASES

AMK-stdMD NETWORK

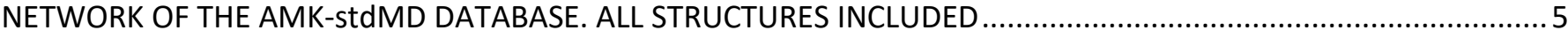

NETWORK OF THE AMK-stdMD DATABASE. FAMILIES OF CONFORMERS FORM A NODE ...................................14

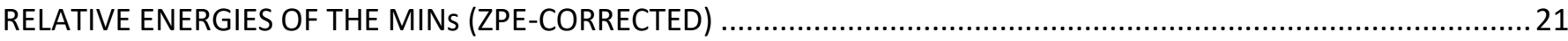

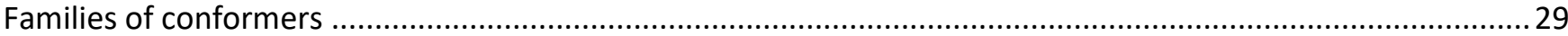

ABSOLUTE ENERGY, ZPE, GIBBS ENERGY CORRECTION, GEOMETRY AND FREQUENCIES OF THE MINs...................30

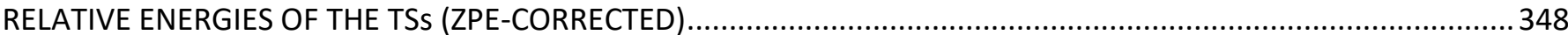

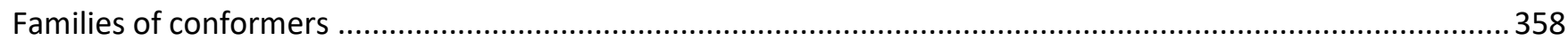

ABSOLUTE ENERGY, ZPE, GIBBS ENERGY CORRECTION, GEOMETRY AND FREQUENCIES OF THE TSS ......................359

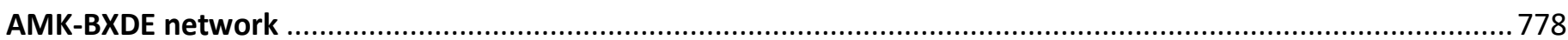

NETWORK OF THE AMK-BXDE DATABASE. ALL STRUCTURES INCLUDED …….................................................

NETWORK OF THE AMK-BXDE DATABASE. FAMILIES OF CONFORMERS FORM A NODE ....................................781

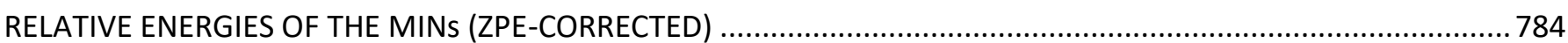

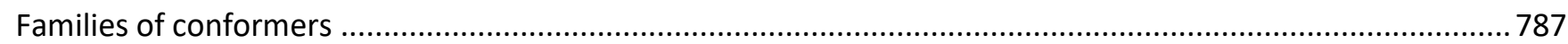

ABSOLUTE ENERGY, ZPE, GIBBS ENERGY CORRECTION, GEOMETRY AND FREQUENCIES OF THE MINs..................788

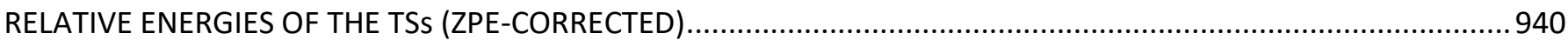

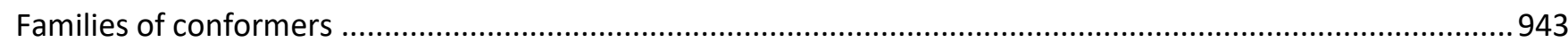

ABSOLUTE ENERGY, ZPE, GIBBS ENERGY CORRECTION, GEOMETRY AND FREQUENCIES OF THE TSs ...................944

TSS FOR THE CYCLOADDITION REACTION OPTIMIZED AT THE MP2/6-31G(D) LEVEL OF THEORY ......................... 1095 
CORRESPONDENCE OF THE ACRONYMS EMPLOYED BY ZhZh WITH THE LABELS OF OUR DATABASES

\begin{tabular}{|c|c|c|}
\hline ZhZh & AMK-stdMD & AMK-BXDE \\
\hline POZ & 232 & 203 \\
\hline Cl1 & 82 & 117 \\
\hline Cl2 & 97 & 131 \\
\hline HP1a & 25 & 42 \\
\hline HP1b & 20 & 44 \\
\hline HP2 & 37 & 65 \\
\hline DIO1 & - & 82 \\
\hline DIO2 & - & 99 \\
\hline
\end{tabular}




\section{AMK-stdMD NETWORK}

NETWORK OF THE AMK-stdMD DATABASE. ALL STRUCTURES INCLUDED

TS \# DE $(\mathrm{kcal} / \mathrm{mol})$

------Path info-------

$-121.580$

$-67.045$

$-58.910$

$-57.317$

$-57.221$

$-56.537$

$-55.435$

$-54.854$

$-53.212$

$-49.425$

$-48.818$

$-45.882$

$-42.054$

$-41.732$

$-41.290$

$-35.209$

$-34.760$

$-27.865$

$-27.373$

$-27.090$

$-26.010$

$-25.875$

$-25.077$

$-16.721$

$-16.310$

$-15.715$

$-13.846$

$-12.853$

$-12.499$

$-12.316$

$-11.842$

$-11.654$

$-10.679$

$-10.112$

$-9.986$

$-9.115$

$-8.988$

$-5.970$

$-4.745$

$-2.668$

$-1.573$

$-1.328$

$-1.164$

$-0.873$

$-0.674$

$-0.465$

$-0.211$

0.088

$\mathrm{C} 2 \mathrm{H} 4 \mathrm{O}+\mathrm{C} 8 \mathrm{H} 12 \mathrm{O} 2<-->$

$\mathrm{C} 2 \mathrm{H} 4 \mathrm{O}+\mathrm{C} 8 \mathrm{H} 12 \mathrm{O} 2$

MIN $2<-->\quad \mathrm{H} 2+\mathrm{ClOH} 4403$

MIN $20<-->$ MIN 20

MIN $20<-->\quad$ MIN 20

MIN $4<-->\quad M I N \quad 8$

MIN $20<-->$ MIN 20

MIN $20<-->\quad$ MIN 20

MIN $29<-->\quad M I N \quad 29$

MIN $\quad 5<-->\quad \mathrm{H} 2 \mathrm{O}+\mathrm{C} 10 \mathrm{H} 14 \mathrm{O} 2$

MIN $37<-->$ MIN 37

MIN $37<-->\quad$ MIN 37

MIN $50<-->$ MIN 53

MIN $50<-->$ MIN 54

MIN $51<-->\quad$ MIN 55

$\mathrm{CH} 2 \mathrm{O}+\mathrm{C} 9 \mathrm{H} 14 \mathrm{O} 2<-->\quad \mathrm{CH} 2 \mathrm{O}+\mathrm{C} 9 \mathrm{H} 14 \mathrm{O} 2$

MIN $6<-->\quad \mathrm{CH} 2 \mathrm{O}+\mathrm{CH} 4+\mathrm{C} 8 \mathrm{H} 10 \mathrm{O} 2$

MIN $\quad 7<-->$ MIN 36

MIN $82<-->\quad$ MIN 82

MIN $82<-->\quad M I N \quad 82$

MIN $84<-->\quad M I N \quad 84$

MIN $84<-->\quad M I N \quad 84$

MIN $85<-->\quad$ MIN 85

MIN $97<-->\quad$ MIN 97

MIN $146<-->$ MIN 146

MIN $129<-->$ MIN 129

MIN $21<-->\quad$ MIN 82

MIN $22<-->\quad$ MIN 87

$\mathrm{H} 2+\mathrm{O} 2+\mathrm{C} 10 \mathrm{H} 14 \mathrm{O}<-->\quad \mathrm{H} 2+\mathrm{O} 2+\mathrm{C} 10 \mathrm{H} 14 \mathrm{O}$

MIN $148<-->\quad 02+\mathrm{C} 10 \mathrm{H} 16 \mathrm{O}$

MIN $31<-->\quad$ MIN 92

MIN $154<-->$ MIN 154

MIN $37<-->\quad$ MIN 97

MIN $\quad 35<-->\quad$ H2+C10H1403

MIN $27<-->$ MIN 90

MIN $41<-->$ MIN 104

MIN $44<-->\quad$ MIN 111

MIN $32<-->\quad$ MIN 181

MIN $45<-->$ MIN 137

MIN $16<-->\quad \mathrm{H} 2+\mathrm{C} 10 \mathrm{H} 1403$

MIN $13<-->\quad \mathrm{H} 2+\mathrm{ClOH} 1403$

MIN $14<-->\quad \mathrm{H} 2+\mathrm{C} 10 \mathrm{H} 1403$

MIN $47<-->\quad \mathrm{H} 2+\mathrm{ClOH} 1403$

MIN $81<-->\quad 02+\mathrm{ClOH} 160$

MIN $219<-->$ MIN 219

MIN $28<-->\quad \mathrm{H} 2+\mathrm{ClOH} 1403$

MIN $\quad 50<-->\quad$ MIN 156

MIN $204<-->\quad 02+\mathrm{ClOH} 16 \mathrm{O}$

MIN $10<-->\quad \mathrm{H} 2+\mathrm{C} 10 \mathrm{H} 1403$

0.516

0.705

1.138

1.188

1.198

1.266

1.298

1.392

1.462

1.726

MIN $226<-->$

MIN $233<-->$

MIN 226

MIN $233<-->$ MIN 233

MIN $233<-->$ MIN 233

MIN $232<-->$ MIN 232

MIN $233<-->$ MIN 233

MIN $\quad 11<-->\quad \mathrm{H} 2+\mathrm{C} 10 \mathrm{H} 1403$

MIN $233<-->\quad$ MIN 233

MIN $233<-->$ MIN 233

MIN $233<-->$ MIN 233

2.250

MIN $228<-->$ MIN 228 


\begin{tabular}{|c|c|c|c|c|c|}
\hline 60 & 2.351 & \multicolumn{2}{|c|}{$\mathrm{O} 2+\mathrm{C} 10 \mathrm{H} 16 \mathrm{O}$} & $<-->$ & $\mathrm{CO}+\mathrm{C} 9 \mathrm{H} 16 \mathrm{O} 2$ \\
\hline 61 & 2.440 & MIN & 233 & $<-->$ & MIN 233 \\
\hline 62 & 2.971 & MIN & 232 & $<-->$ & MIN \\
\hline 63 & 3.355 & MIN & 25 & $<-->$ & MIN \\
\hline 64 & 4.070 & MIN & 280 & $<-->$ & MIN \\
\hline 65 & 4.074 & MIN & 275 & $<-->$ & MIN 275 \\
\hline 66 & 4.287 & \multicolumn{2}{|c|}{$\mathrm{H} 2 \mathrm{O} 2+\mathrm{C} 10 \mathrm{H} 14 \mathrm{O}$} & $<-->$ & $\mathrm{H} 2 \mathrm{O} 2+\mathrm{C} 10 \mathrm{H} 14 \mathrm{O}$ \\
\hline 67 & 4.293 & MIN & 261 & $<-->$ & MIN 261 \\
\hline 68 & 9.224 & MIN & 40 & $<-->$ & MIN \\
\hline 69 & 9.802 & MIN & 43 & $<-->$ & MIN 61 \\
\hline 70 & 10.792 & MIN & 33 & $<-->$ & $\mathrm{H} 2+\mathrm{C} 10 \mathrm{H} 1403$ \\
\hline 71 & 11.441 & $\mathrm{MIN}$ & 3 & $<-->$ & MIN 101 \\
\hline 72 & 11.823 & MIN & 30 & $<-->$ & MIN \\
\hline 73 & 11.902 & MIN & 65 & $<-->$ & MIN \\
\hline 74 & 12.712 & MIN & 83 & $<-->$ & MIN 131 \\
\hline 75 & 12.812 & MIN & 64 & $<-->$ & $\mathrm{C} 4 \mathrm{H} 8 \mathrm{O}+\mathrm{C} 6 \mathrm{H} 8 \mathrm{O} 2$ \\
\hline 76 & 13.303 & MIN & 66 & $<-->$ & $\mathrm{H} 2 \mathrm{O}+\mathrm{C} 10 \mathrm{H} 14 \mathrm{O} 2$ \\
\hline 77 & 15.405 & MIN & 143 & $<-->$ & $\mathrm{O} 2+\mathrm{C} 10 \mathrm{H} 160$ \\
\hline 78 & 16.691 & MIN & 38 & $<-->$ & $\mathrm{H} 2+\mathrm{C} 10 \mathrm{H} 1403$ \\
\hline 79 & 17.766 & MIN & 57 & $<-->$ & $\mathrm{H} 2+\mathrm{C} 10 \mathrm{H} 1403$ \\
\hline 80 & 19.688 & MIN & 78 & $<-->$ & $\mathrm{H} 2 \mathrm{O}+\mathrm{C} 10 \mathrm{H} 14 \mathrm{O} 2$ \\
\hline 81 & 20.390 & MIN & 23 & $<-->$ & $\mathrm{CH} 4+\mathrm{C} 9 \mathrm{H} 12 \mathrm{O} 3$ \\
\hline 82 & 20.503 & MIN & 88 & $<-->$ & MIN 88 \\
\hline 83 & 20.816 & MIN & 134 & $<-->$ & MIN 224 \\
\hline 84 & 20.877 & $\mathrm{H} 2+\mathrm{H} 2+\mathrm{C} 10 \mathrm{H} 1$ & 1203 & $<-->$ & $\mathrm{H} 2+\mathrm{H} 2+\mathrm{C} 10 \mathrm{H} 12 \mathrm{O} 3$ \\
\hline 85 & 21.022 & MIN & 50 & $<-->$ & MIN $\quad 50$ \\
\hline 86 & 21.262 & MIN & 58 & $<-->$ & MIN 74 \\
\hline 87 & 21.550 & MIN & 267 & $<-->$ & $\mathrm{C} 4 \mathrm{H} 6 \mathrm{O}+\mathrm{C} 6 \mathrm{H} 10 \mathrm{O} 2$ \\
\hline 88 & 21.820 & MIN & 112 & $<-->$ & MIN 232 \\
\hline 89 & 21.966 & MIN & 118 & $<-->$ & MIN 232 \\
\hline 90 & 22.144 & MIN & 270 & $<-->$ & $\mathrm{C} 4 \mathrm{H} 6 \mathrm{O} 2+\mathrm{C} 6 \mathrm{H} 10 \mathrm{O}$ \\
\hline 91 & 22.654 & MIN & 271 & $<-->$ & $\mathrm{C} 4 \mathrm{H} 6 \mathrm{O} 2+\mathrm{C} 6 \mathrm{H} 10 \mathrm{O}$ \\
\hline 92 & 22.775 & MIN & 274 & $<-->$ & $\mathrm{C} 4 \mathrm{H} 6 \mathrm{O}+\mathrm{C} 6 \mathrm{H} 10 \mathrm{O} 2$ \\
\hline 93 & 23.135 & MIN & 115 & $<-->$ & MIN 233 \\
\hline 94 & 23.536 & MIN & 47 & $<-->$ & $\mathrm{H} 2+\mathrm{C} 10 \mathrm{H} 1403$ \\
\hline 95 & 23.985 & MIN & 93 & $<-->$ & MIN 233 \\
\hline 96 & 24.146 & MIN & 157 & $<-->$ & $\mathrm{O} 2+\mathrm{C} 10 \mathrm{H} 16 \mathrm{O}$ \\
\hline 97 & 24.215 & MIN & 295 & $<-->$ & $\mathrm{C} 4 \mathrm{H} 6 \mathrm{O} 2+\mathrm{C} 6 \mathrm{H} 10 \mathrm{O}$ \\
\hline 98 & 24.381 & MIN & 52 & $<-->$ & $\mathrm{H} 2+\mathrm{C} 3 \mathrm{H} 4+\mathrm{C} 7 \mathrm{H} 1003$ \\
\hline 99 & 24.829 & MIN & 60 & $<-->$ & $\mathrm{H} 2+\mathrm{C} 10 \mathrm{H} 1403$ \\
\hline 100 & 24.881 & MIN & 94 & $<-->$ & $\mathrm{C} 4 \mathrm{H} 6 \mathrm{O}+\mathrm{C} 6 \mathrm{H} 10 \mathrm{O} 2$ \\
\hline 101 & 24.918 & MIN & 62 & $<-->$ & $\mathrm{H} 2+\mathrm{C} 10 \mathrm{H} 1403$ \\
\hline 102 & 25.198 & MIN & 269 & $<-->$ & $\mathrm{C} 4 \mathrm{H} 6 \mathrm{O}+\mathrm{C} 6 \mathrm{H} 10 \mathrm{O} 2$ \\
\hline 103 & 25.222 & MIN & 108 & $<-->$ & $\mathrm{H} 2+\mathrm{C} 10 \mathrm{H} 1403$ \\
\hline 104 & 26.445 & MIN & 124 & $<-->$ & $\mathrm{H} 2+\mathrm{C} 10 \mathrm{H} 1403$ \\
\hline 105 & 26.531 & MIN & 48 & $<-->$ & MIN 48 \\
\hline 106 & 26.679 & MIN & 68 & $<-->$ & $\mathrm{H} 2+\mathrm{C} 10 \mathrm{H} 1403$ \\
\hline 107 & 26.834 & MIN & 63 & $<-->$ & $\mathrm{H} 2+\mathrm{C} 10 \mathrm{H} 1403$ \\
\hline 108 & 26.926 & MIN & 50 & $<-->$ & MIN 242 \\
\hline 109 & 27.177 & MIN & 297 & $<-->$ & $\mathrm{C} 4 \mathrm{H} 6 \mathrm{O}+\mathrm{C} 6 \mathrm{H} 10 \mathrm{O} 2$ \\
\hline 110 & 27.422 & MIN & 201 & $<-->$ & $\mathrm{H} 2+\mathrm{C} 10 \mathrm{H} 1403$ \\
\hline 111 & 27.858 & MIN & 113 & $<-->$ & $\mathrm{H} 2+\mathrm{C} 10 \mathrm{H} 1403$ \\
\hline 112 & 29.401 & MIN & 115 & $<-->$ & $\mathrm{H} 2+\mathrm{C} 10 \mathrm{H} 1403$ \\
\hline 113 & 29.922 & MIN & 82 & $<-->$ & MIN 141 \\
\hline 114 & 30.697 & MIN & 239 & $<-->$ & $\mathrm{C} 3 \mathrm{H} 6 \mathrm{O} 2+\mathrm{C} 7 \mathrm{H} 10 \mathrm{O}$ \\
\hline 115 & 30.785 & MIN & 75 & $<-->$ & MIN 351 \\
\hline 116 & 31.112 & MIN & 99 & $<-->$ & $\mathrm{O} 2+\mathrm{C} 10 \mathrm{H} 16 \mathrm{O}$ \\
\hline 117 & 32.172 & MIN & 34 & $<-->$ & $\mathrm{O} 2+\mathrm{C} 10 \mathrm{H} 16 \mathrm{O}$ \\
\hline 118 & 34.248 & $\mathrm{H} 2+\mathrm{O} 2+\mathrm{C} 10 \mathrm{H}$ & $\mathrm{H} 14 \mathrm{O}$ & $<-->$ & $\mathrm{O} 2+\mathrm{C} 10 \mathrm{H} 160$ \\
\hline 119 & 34.454 & MIN & 80 & $<-->$ & $\mathrm{H} 2+\mathrm{C} 10 \mathrm{H} 1403$ \\
\hline 120 & 34.547 & MIN & 71 & $<-->$ & MIN 379 \\
\hline 121 & 34.678 & $\mathrm{H} 2+\mathrm{C} 10 \mathrm{H} 1$ & 1403 & $<-->$ & $\mathrm{H} 2+\mathrm{C} 10 \mathrm{H} 14 \mathrm{O} 3$ \\
\hline 122 & 35.383 & $\mathrm{C} 2 \mathrm{H} 4 \mathrm{O}+\mathrm{C} 8 \mathrm{H} 1$ & 1202 & $<-->$ & $\mathrm{C} 2 \mathrm{H} 4 \mathrm{O}+\mathrm{C} 8 \mathrm{H} 12 \mathrm{O} 2$ \\
\hline 123 & 35.651 & MIN & 125 & $<-->$ & $\mathrm{H} 2+\mathrm{C} 10 \mathrm{H} 14 \mathrm{O} 3$ \\
\hline 124 & 35.816 & MIN & 82 & $<-->$ & $\mathrm{H} 2+\mathrm{C} 10 \mathrm{H} 1403$ \\
\hline
\end{tabular}




\begin{tabular}{|c|c|c|c|c|c|}
\hline 125 & 35.986 & MIN & 115 & $<-->$ & MIN \\
\hline 126 & 36.949 & MIN & 191 & $<-->$ & MIN \\
\hline 127 & 37.118 & $\mathrm{MIN}$ & 155 & $<-->$ & MIN \\
\hline 128 & 37.422 & MIN & 1 & $<-->$ & MIN \\
\hline 129 & 37.965 & MIN & 42 & $<-->$ & MIN 79 \\
\hline 130 & 38.008 & $\mathrm{C} 4 \mathrm{H} 6 \mathrm{O}+\mathrm{C} 6 \mathrm{H} 1$ & 1002 & $<-->$ & $\mathrm{C} 4 \mathrm{H} 6 \mathrm{O}+\mathrm{C} 6 \mathrm{H} 10 \mathrm{O} 2$ \\
\hline 131 & 38.496 & MIN & 82 & $<-->$ & MIN 102 \\
\hline 132 & 38.577 & MIN & 111 & $<-->$ & $\mathrm{CO}+\mathrm{C} 9 \mathrm{H} 1602$ \\
\hline 133 & 39.010 & MIN & 83 & $<-->$ & $\mathrm{H} 2+\mathrm{C} 10 \mathrm{H} 14 \mathrm{O} 3$ \\
\hline 134 & 39.147 & MIN & 109 & $<-->$ & $\mathrm{H} 2+\mathrm{O} 2+\mathrm{C} 10 \mathrm{H} 14 \mathrm{O}$ \\
\hline 135 & 39.318 & MIN & 212 & $<-->$ & MIN 260 \\
\hline 136 & 39.364 & MIN & 82 & $<-->$ & $\mathrm{H} 2+\mathrm{C} 10 \mathrm{H} 1403$ \\
\hline 137 & 39.405 & MIN & 203 & $<-->$ & $\mathrm{O} 2+\mathrm{C} 10 \mathrm{H} 160$ \\
\hline 138 & 39.525 & MIN & 84 & $<-->$ & $\mathrm{H} 2+\mathrm{C} 10 \mathrm{H} 1403$ \\
\hline 139 & 39.764 & MIN & 389 & $<-->$ & MIN 389 \\
\hline 140 & 40.288 & MIN & 82 & $<-->$ & MIN \\
\hline 141 & 41.149 & MIN & 46 & $<-->$ & MIN 165 \\
\hline 142 & 41.506 & MIN & 122 & $<-->$ & $\mathrm{H} 2+\mathrm{C} 10 \mathrm{H} 1403$ \\
\hline 143 & 41.830 & MIN & 70 & $<-->$ & $\mathrm{H} 2+\mathrm{C} 10 \mathrm{H} 1403$ \\
\hline 144 & 41.886 & MIN & 98 & $<-->$ & $\mathrm{H} 2+\mathrm{C} 10 \mathrm{H} 1403$ \\
\hline 145 & 42.219 & MIN & 208 & $<-->$ & MIN 305 \\
\hline 146 & 42.384 & MIN & 220 & $<-->$ & MIN \\
\hline 147 & 42.537 & MIN & 103 & $<-->$ & MIN \\
\hline 148 & 42.739 & MIN & 207 & $<-->$ & MIN \\
\hline 149 & 43.207 & MIN & 219 & $<-->$ & MIN 310 \\
\hline 150 & 43.477 & $\mathrm{H} 2+\mathrm{C} 10 \mathrm{H} 1$ & 1403 & $<-->$ & $\mathrm{H} 2+\mathrm{C} 10 \mathrm{H} 1403$ \\
\hline 151 & 43.568 & $\mathrm{H} 2+\mathrm{C} 10 \mathrm{H} 1$ & 1403 & $<-->$ & $\mathrm{H} 2+\mathrm{C} 10 \mathrm{H} 14 \mathrm{O} 3$ \\
\hline 152 & 43.728 & MIN & 78 & $<-->$ & MIN 393 \\
\hline 153 & 44.011 & MIN & 160 & $<-->$ & MIN 286 \\
\hline 154 & 44.123 & MIN & 104 & $<-->$ & $\mathrm{H} 2+\mathrm{C} 10 \mathrm{H} 14 \mathrm{O} 3$ \\
\hline 155 & 44.217 & MIN & 9 & $<-->$ & MIN 158 \\
\hline 156 & 44.742 & MIN & 233 & $<-->$ & MIN 316 \\
\hline 157 & 44.976 & MIN & 291 & $<-->$ & $\mathrm{H} 2+\mathrm{C} 10 \mathrm{H} 14 \mathrm{O} 3$ \\
\hline 158 & 44.980 & MIN & 390 & $<-->$ & $\mathrm{O} 2+\mathrm{C} 10 \mathrm{H} 160$ \\
\hline 159 & 45.372 & MIN & 245 & $<-->$ & MIN 385 \\
\hline 160 & 45.540 & MIN & 104 & $<-->$ & MIN 110 \\
\hline 161 & 45.652 & $\mathrm{H} 2+\mathrm{C} 10 \mathrm{H} 1$ & 1403 & $<-->$ & $\mathrm{H} 2+\mathrm{C} 10 \mathrm{H} 1403$ \\
\hline 162 & 45.796 & MIN & 130 & $<-->$ & $\mathrm{H} 2+\mathrm{C} 10 \mathrm{H} 1403$ \\
\hline 163 & 45.837 & MIN & 372 & $<-->$ & $\mathrm{CH} 4+\mathrm{C} 9 \mathrm{H} 12 \mathrm{O} 3$ \\
\hline 164 & 45.915 & MIN & 107 & $<-->$ & $\mathrm{H} 2+\mathrm{C} 10 \mathrm{H} 1403$ \\
\hline 165 & 45.944 & $\mathrm{H} 2+\mathrm{H} 2+\mathrm{C} 10 \mathrm{H} 1$ & 1203 & $<-->$ & $\mathrm{H} 2+\mathrm{C} 10 \mathrm{H} 1403$ \\
\hline 166 & 46.035 & MIN & 82 & $<-->$ & MIN 387 \\
\hline 167 & 46.190 & MIN & 150 & $<-->$ & $\mathrm{H} 2+\mathrm{C} 10 \mathrm{H} 1403$ \\
\hline 168 & 46.323 & MIN & 18 & $<-->$ & $\mathrm{CH} 2+\mathrm{C} 9 \mathrm{H} 1403$ \\
\hline 169 & 46.781 & MIN & 232 & $<-->$ & MIN 315 \\
\hline 170 & 46.824 & MIN & 47 & $<-->$ & $\mathrm{C} 3 \mathrm{H} 6+\mathrm{C} 7 \mathrm{H} 10 \mathrm{O} 3$ \\
\hline 171 & 47.715 & MIN & 264 & $<-->$ & MIN 337 \\
\hline 172 & 47.803 & MIN & 232 & $<-->$ & MIN 324 \\
\hline 173 & 47.989 & $\mathrm{O} 2+\mathrm{C} 10 \mathrm{H}$ & 160 & $<-->$ & $\mathrm{O} 2+\mathrm{C} 10 \mathrm{H} 16 \mathrm{O}$ \\
\hline 174 & 48.154 & MIN & 95 & $<-->$ & MIN 384 \\
\hline 175 & 48.284 & MIN & 151 & $<-->$ & $\mathrm{H} 2+\mathrm{C} 10 \mathrm{H} 14 \mathrm{O} 3$ \\
\hline 176 & 48.442 & MIN & 185 & $<-->$ & MIN 293 \\
\hline 177 & 48.567 & MIN & 257 & $<-->$ & $\mathrm{H} 2+\mathrm{C} 10 \mathrm{H} 1403$ \\
\hline 178 & 48.780 & MIN & 377 & $<-->$ & $\mathrm{O} 2+\mathrm{C} 10 \mathrm{H} 16 \mathrm{O}$ \\
\hline 179 & 49.262 & MIN & 56 & $<-->$ & $\mathrm{C} 4 \mathrm{H} 6+\mathrm{C} 6 \mathrm{H} 10 \mathrm{O} 3$ \\
\hline 180 & 49.278 & MIN & 39 & $<-->$ & MIN 234 \\
\hline 181 & 49.614 & MIN & 299 & $<-->$ & MIN 320 \\
\hline 182 & 50.498 & MIN & 318 & $<-->$ & $\mathrm{O} 2+\mathrm{C} 10 \mathrm{H} 160$ \\
\hline 183 & 51.498 & MIN & 162 & $<-->$ & $\mathrm{H} 2+\mathrm{C} 10 \mathrm{H} 1403$ \\
\hline 184 & 51.600 & MIN & 106 & $<-->$ & $\mathrm{H} 2+\mathrm{C} 10 \mathrm{H} 1403$ \\
\hline 185 & 51.904 & MIN & 140 & $<-->$ & $\mathrm{H} 2+\mathrm{C} 10 \mathrm{H} 1403$ \\
\hline 186 & 52.357 & MIN & 198 & $<-->$ & $\mathrm{H} 2+\mathrm{C} 10 \mathrm{H} 14 \mathrm{O} 3$ \\
\hline 187 & 52.529 & $\mathrm{H} 2+\mathrm{C} 10 \mathrm{H} 1$ & 1403 & $<-->$ & $\mathrm{C} 4 \mathrm{H} 6 \mathrm{O} 2+\mathrm{C} 6 \mathrm{H} 10 \mathrm{O}$ \\
\hline 188 & 52.659 & $\mathrm{O} 2+\mathrm{C} 10 \mathrm{H}$ & 160 & $<-->$ & $\mathrm{O} 2+\mathrm{C} 10 \mathrm{H} 16 \mathrm{O}$ \\
\hline 189 & 52.714 & MIN & 24 & $<-->$ & MIN 26 \\
\hline
\end{tabular}




\begin{tabular}{|c|c|c|c|c|}
\hline 190 & 52.973 & $\mathrm{H} 2+\mathrm{O} 2+\mathrm{C} 10 \mathrm{H} 14 \mathrm{O}$ & $<-->$ & $\mathrm{O} 2+\mathrm{C} 10 \mathrm{H} 16 \mathrm{O}$ \\
\hline 191 & 52.988 & $\mathrm{O} 2+\mathrm{C} 10 \mathrm{H} 16 \mathrm{O}$ & $<-->$ & $\mathrm{O} 2+\mathrm{C} 10 \mathrm{H} 16 \mathrm{O}$ \\
\hline 192 & 53.070 & MIN 12 & $<-->$ & MIN 223 \\
\hline 193 & 53.136 & $\mathrm{O} 2+\mathrm{C} 10 \mathrm{H} 16 \mathrm{O}$ & $<-->$ & $\mathrm{O} 2+\mathrm{C} 10 \mathrm{H} 16 \mathrm{O}$ \\
\hline 194 & 53.246 & MIN 115 & $<-->$ & MIN 115 \\
\hline 195 & 53.264 & MIN & $<-->$ & $\mathrm{O} 2+\mathrm{C} 10 \mathrm{H} 16 \mathrm{O}$ \\
\hline 196 & 53.277 & MIN & $<-->$ & $\mathrm{C} 3 \mathrm{H} 4 \mathrm{O} 3+\mathrm{C} 7 \mathrm{H} 12$ \\
\hline 97 & 53.326 & MIN & $<-->$ & MIN 233 \\
\hline 98 & 53.395 & MIN 192 & $<-->$ & $\mathrm{H} 2+\mathrm{C} 10 \mathrm{H} 1403$ \\
\hline 99 & 53.403 & $\mathrm{O} 2+\mathrm{C} 10 \mathrm{H} 16 \mathrm{O}$ & $<-->$ & $\mathrm{O} 2+\mathrm{C} 10 \mathrm{H} 160$ \\
\hline 0 & 54.055 & MIN 171 & $<-->$ & $\mathrm{H} 2+\mathrm{C} 10 \mathrm{H} 1403$ \\
\hline 01 & 54.661 & MIN & $<-->$ & MIN 340 \\
\hline 02 & 54.736 & MIN & $<-->$ & $\mathrm{H} 2+\mathrm{C} 10 \mathrm{H} 1403$ \\
\hline 3 & 55.012 & MIN & $<-->$ & $\mathrm{O} 2+\mathrm{C} 10 \mathrm{H} 160$ \\
\hline 04 & 55.071 & MIN & $<-->$ & $\mathrm{H} 2+\mathrm{C} 10 \mathrm{H} 1403$ \\
\hline 05 & 55.095 & MIN & $<-->$ & $\mathrm{H} 2+\mathrm{C} 10 \mathrm{H} 1403$ \\
\hline 06 & 55.277 & MIN & $<-->$ & $\mathrm{O} 2+\mathrm{C} 10 \mathrm{H} 160$ \\
\hline 07 & 55.510 & MIN & $<-->$ & $\mathrm{O} 2+\mathrm{C} 10 \mathrm{H} 16 \mathrm{O}$ \\
\hline 08 & 55.703 & MIN 200 & $<-->$ & MIN 306 \\
\hline 09 & 55.776 & $\mathrm{O} 2+\mathrm{C} 10 \mathrm{H} 16 \mathrm{O}$ & $<-->$ & $\mathrm{O} 2+\mathrm{C} 10 \mathrm{H} 16 \mathrm{O}$ \\
\hline 10 & 55.780 & MIN 233 & $<-->$ & $\mathrm{O} 2+\mathrm{C} 10 \mathrm{H} 160$ \\
\hline 1 & 55.797 & MIN & $<-->$ & $\mathrm{H} 2+\mathrm{C} 10 \mathrm{H} 1403$ \\
\hline 12 & 55.809 & MIN 411 & $<-->$ & $\mathrm{C} 3 \mathrm{H} 4 \mathrm{O} 3+\mathrm{C} 7 \mathrm{H} 12$ \\
\hline 13 & 55.854 & $\mathrm{O} 2+\mathrm{C} 10 \mathrm{H} 16 \mathrm{O}$ & $<-->$ & $\mathrm{O} 2+\mathrm{C} 10 \mathrm{H} 16 \mathrm{O}$ \\
\hline 14 & 55.858 & MIN 15 & $<-->$ & MIN 232 \\
\hline 15 & 55.858 & $\mathrm{O} 2+\mathrm{C} 10 \mathrm{H} 16 \mathrm{O}$ & $<-->$ & $\mathrm{O} 2+\mathrm{C} 10 \mathrm{H} 16 \mathrm{O}$ \\
\hline 16 & 56.003 & MIN 415 & $<-->$ & MIN 415 \\
\hline 217 & 56.230 & MIN & $<-->$ & $\mathrm{H} 2+\mathrm{C} 10 \mathrm{H} 1403$ \\
\hline 218 & 56.418 & MIN & $<-->$ & $\mathrm{H} 2+\mathrm{C} 10 \mathrm{H} 1403$ \\
\hline 19 & 56.439 & MIN & $<-->$ & $\mathrm{H} 2+\mathrm{C} 10 \mathrm{H} 1403$ \\
\hline 220 & 56.532 & MIN & $<-->$ & $\mathrm{H} 2+\mathrm{C} 10 \mathrm{H} 14 \mathrm{O} 3$ \\
\hline 221 & 56.609 & MIN & $<-->$ & $\mathrm{C} 3 \mathrm{H} 6+\mathrm{C} 7 \mathrm{H} 1003$ \\
\hline 222 & 57.339 & MIN & $<-->$ & $\mathrm{C} 4 \mathrm{H} 6 \mathrm{O} 2+\mathrm{C} 6 \mathrm{H} 10 \mathrm{O}$ \\
\hline 223 & 57.691 & MIN & $<-->$ & $\mathrm{H} 2+\mathrm{C} 10 \mathrm{H} 14 \mathrm{O} 3$ \\
\hline 224 & 57.974 & MIN & $<-->$ & $\mathrm{H} 2+\mathrm{C} 10 \mathrm{H} 1403$ \\
\hline 225 & 58.281 & MIN & $<-->$ & $\mathrm{H} 2+\mathrm{C} 10 \mathrm{H} 1403$ \\
\hline 226 & 59.091 & MIN & $<-->$ & MIN 115 \\
\hline 227 & 59.111 & MIN & $<-->$ & MIN 233 \\
\hline 228 & 59.180 & MIN & $<-->$ & $\mathrm{O} 3+\mathrm{C} 10 \mathrm{H} 16$ \\
\hline 229 & 59.340 & MIN & $<-->$ & MIN 392 \\
\hline 230 & 59.376 & MIN & $<-->$ & MIN 133 \\
\hline 231 & 59.782 & MIN & $<-->$ & $\mathrm{H} 2+\mathrm{C} 10 \mathrm{H} 1403$ \\
\hline 232 & 59.916 & MIN & $<-->$ & $\mathrm{H} 2+\mathrm{C} 10 \mathrm{H} 1403$ \\
\hline 233 & 60.121 & MIN & $<-->$ & MIN 117 \\
\hline 234 & 60.233 & MIN & $<-->$ & $\mathrm{H} 2+\mathrm{C} 10 \mathrm{H} 1403$ \\
\hline 235 & 60.307 & MIN & $<-->$ & MIN 186 \\
\hline 236 & 60.455 & MIN & $<-->$ & $\mathrm{C} 4 \mathrm{H} 6 \mathrm{O} 2+\mathrm{C} 6 \mathrm{H} 10 \mathrm{O}$ \\
\hline 237 & 60.460 & MIN & $<-->$ & $\mathrm{H} 2+\mathrm{C} 10 \mathrm{H} 14 \mathrm{O} 3$ \\
\hline 238 & 60.619 & MIN & $<-->$ & MIN 247 \\
\hline 239 & 60.645 & MIN & $<-->$ & $\mathrm{H} 2+\mathrm{C} 10 \mathrm{H} 1403$ \\
\hline 240 & 60.822 & MIN & $<-->$ & MIN 226 \\
\hline 241 & 61.224 & MIN & $<-->$ & $\mathrm{H} 2+\mathrm{C} 10 \mathrm{H} 1403$ \\
\hline 242 & 61.238 & MIN & $<-->$ & MIN 166 \\
\hline 243 & 61.746 & MIN & $<-->$ & $\mathrm{C} 2 \mathrm{H} 4 \mathrm{O}+\mathrm{C} 8 \mathrm{H} 12 \mathrm{O} 2$ \\
\hline 244 & 62.209 & MIN 233 & $<-->$ & $\mathrm{H} 2+\mathrm{C} 10 \mathrm{H} 14 \mathrm{O} 3$ \\
\hline 245 & 62.280 & $\mathrm{O} 2+\mathrm{C} 10 \mathrm{H} 16 \mathrm{O}$ & $<-->$ & $\mathrm{O} 2+\mathrm{C} 10 \mathrm{H} 160$ \\
\hline 246 & 62.345 & MIN 250 & $<-->$ & MIN 395 \\
\hline 247 & 62.836 & MIN & $<-->$ & $\mathrm{H} 2+\mathrm{C} 10 \mathrm{H} 1403$ \\
\hline 248 & 62.841 & MIN & $<-->$ & MIN 176 \\
\hline 249 & 62.896 & MIN & $<-->$ & $\mathrm{H} 2+\mathrm{C} 10 \mathrm{H} 1403$ \\
\hline 250 & 63.018 & MIN & $<-->$ & $\mathrm{H} 2+\mathrm{C} 10 \mathrm{H} 1403$ \\
\hline 251 & 63.192 & MIN & $<-->$ & MIN 142 \\
\hline 252 & 63.266 & MIN 197 & $<-->$ & MIN 282 \\
\hline 253 & 63.598 & $\mathrm{O} 2+\mathrm{C} 10 \mathrm{H} 16 \mathrm{O}$ & $<-->$ & $\mathrm{O} 2+\mathrm{C} 3 \mathrm{H} 6+\mathrm{C} 7 \mathrm{H} 10 \mathrm{O}$ \\
\hline 254 & 63.627 & MIN 217 & $<-->$ & $\mathrm{H} 2+\mathrm{C} 10 \mathrm{H} 14 \mathrm{O} 3$ \\
\hline
\end{tabular}




\begin{tabular}{|c|c|c|c|c|}
\hline 255 & 64.109 & MIN 218 & $<-->$ & MIN 218 \\
\hline 256 & 64.291 & $\mathrm{O} 2+\mathrm{CH} 2 \mathrm{O}+\mathrm{C} 9 \mathrm{H} 14$ & $<-->$ & $\mathrm{O} 2+\mathrm{C} 10 \mathrm{H} 160$ \\
\hline 257 & 64.352 & MIN 232 & $<-->$ & $\mathrm{H} 2+\mathrm{C} 10 \mathrm{H} 14 \mathrm{O} 3$ \\
\hline 258 & 64.353 & MIN & $<-->$ & $\mathrm{H} 2+\mathrm{C} 3 \mathrm{H} 4+\mathrm{C} 7 \mathrm{H} 1003$ \\
\hline 259 & 64.383 & $\mathrm{O} 2+\mathrm{C} 10 \mathrm{H} 16 \mathrm{O}$ & $<-->$ & $\mathrm{O} 2+\mathrm{C} 10 \mathrm{H} 16 \mathrm{O}$ \\
\hline 260 & 64.413 & MIN 410 & $<-->$ & MIN 412 \\
\hline 261 & 64.973 & MIN & $<-->$ & $\mathrm{H} 2+\mathrm{C} 10 \mathrm{H} 1403$ \\
\hline 262 & 65.046 & MIN & $<-->$ & $\mathrm{H} 2+\mathrm{C} 10 \mathrm{H} 1403$ \\
\hline 263 & 65.060 & MIN 248 & $<-->$ & $\mathrm{H} 2+\mathrm{C} 10 \mathrm{H} 1403$ \\
\hline 64 & 65.089 & $\mathrm{H} 2+\mathrm{C} 10 \mathrm{H} 1403$ & $<-->$ & $\mathrm{H} 2+\mathrm{C} 10 \mathrm{H} 1403$ \\
\hline 265 & 65.490 & MIN 233 & $<-->$ & $\mathrm{H} 2+\mathrm{C} 10 \mathrm{H} 1403$ \\
\hline 266 & 65.594 & MIN & $<-->$ & $\mathrm{H} 2+\mathrm{C} 10 \mathrm{H} 1403$ \\
\hline 267 & 65.694 & MIN & $<-->$ & $\mathrm{H} 2+\mathrm{C} 10 \mathrm{H} 1403$ \\
\hline 268 & 65.731 & MIN & $<-->$ & MIN 233 \\
\hline 269 & 65.768 & MIN & $<-->$ & MIN 232 \\
\hline 270 & 65.799 & MIN & $<-->$ & $\mathrm{O} 2+\mathrm{C} 10 \mathrm{H} 16 \mathrm{O}$ \\
\hline 71 & 65.845 & MIN & $<-->$ & MIN 400 \\
\hline 272 & 65.896 & MIN 147 & $<-->$ & $\mathrm{CH} 4+\mathrm{C} 9 \mathrm{H} 12 \mathrm{O} 3$ \\
\hline 273 & 65.953 & $\mathrm{O} 2+\mathrm{C} 10 \mathrm{H} 16 \mathrm{O}$ & $<-->$ & $\mathrm{O} 2+\mathrm{C} 10 \mathrm{H} 160$ \\
\hline 274 & 65.958 & $\mathrm{H} 2+\mathrm{C} 10 \mathrm{H} 1403$ & $<-->$ & $\mathrm{H} 2+\mathrm{C} 10 \mathrm{H} 1403$ \\
\hline 275 & 66.062 & MIN 233 & $<-->$ & $\mathrm{H} 2+\mathrm{C} 10 \mathrm{H} 1403$ \\
\hline 276 & 66.144 & MIN & $<-->$ & $\mathrm{H} 2+\mathrm{C} 10 \mathrm{H} 1403$ \\
\hline 277 & 66.150 & MIN & $<-->$ & MIN 394 \\
\hline 278 & 66.252 & MIN 233 & $<-->$ & $\mathrm{H} 2+\mathrm{C} 10 \mathrm{H} 1403$ \\
\hline 279 & 66.255 & $\mathrm{O} 2+\mathrm{C} 10 \mathrm{H} 16 \mathrm{O}$ & $<-->$ & $\mathrm{H} 2+\mathrm{O} 2+\mathrm{C} 10 \mathrm{H} 14 \mathrm{O}$ \\
\hline 280 & 66.317 & MIN 258 & $<-->$ & $\mathrm{H} 2+\mathrm{C} 10 \mathrm{H} 1403$ \\
\hline 281 & 66.339 & $\mathrm{O} 2+\mathrm{C} 10 \mathrm{H} 16 \mathrm{O}$ & $<-->$ & $\mathrm{O} 2+\mathrm{C} 10 \mathrm{H} 160$ \\
\hline 282 & 66.343 & MIN 232 & $<-->$ & $\mathrm{H} 2+\mathrm{C} 10 \mathrm{H} 1403$ \\
\hline 283 & 66.425 & $\mathrm{O} 2+\mathrm{C} 10 \mathrm{H} 16 \mathrm{O}$ & $<-->$ & $\mathrm{O} 2+\mathrm{C} 10 \mathrm{H} 160$ \\
\hline 284 & 66.449 & $\mathrm{O} 2+\mathrm{C} 10 \mathrm{H} 16 \mathrm{O}$ & $<-->$ & $\mathrm{O} 2+\mathrm{C} 10 \mathrm{H} 16 \mathrm{O}$ \\
\hline 285 & 66.613 & MIN 80 & $<-->$ & MIN 232 \\
\hline 286 & 66.655 & MIN & $<-->$ & $\mathrm{H} 2+\mathrm{C} 10 \mathrm{H} 1403$ \\
\hline 287 & 66.744 & MIN & $<-->$ & MIN 233 \\
\hline 288 & 66.758 & $\mathrm{H} 2+\mathrm{C} 10 \mathrm{H} 1403$ & $<-->$ & $\mathrm{H} 2+\mathrm{C} 10 \mathrm{H} 1403$ \\
\hline 289 & 66.782 & $\mathrm{O} 2+\mathrm{C} 10 \mathrm{H} 160$ & $<-->$ & $\mathrm{O} 2+\mathrm{C} 10 \mathrm{H} 160$ \\
\hline 290 & 66.819 & MIN 232 & $<-->$ & $\mathrm{H} 2+\mathrm{C} 10 \mathrm{H} 1403$ \\
\hline 291 & 66.856 & MIN & $<-->$ & $\mathrm{CH} 4+\mathrm{C} 9 \mathrm{H} 12 \mathrm{O} 3$ \\
\hline 292 & 66.947 & MIN & $<-->$ & MIN 406 \\
\hline 293 & 67.030 & MIN & $<-->$ & $\mathrm{H} 2+\mathrm{C} 10 \mathrm{H} 1403$ \\
\hline 294 & 67.031 & MIN & $<-->$ & MIN 408 \\
\hline 295 & 67.056 & MIN & $<-->$ & $\mathrm{H} 2+\mathrm{C} 10 \mathrm{H} 1403$ \\
\hline 296 & 67.078 & MIN & $<-->$ & $\mathrm{H} 2+\mathrm{C} 10 \mathrm{H} 1403$ \\
\hline 297 & 67.084 & MIN & $<-->$ & $\mathrm{H} 2+\mathrm{C} 10 \mathrm{H} 1403$ \\
\hline 298 & 67.154 & MIN & $<-->$ & $\mathrm{H} 2+\mathrm{C} 10 \mathrm{H} 1403$ \\
\hline 299 & 67.163 & MIN & $<-->$ & MIN 208 \\
\hline 300 & 67.164 & MIN 233 & $<-->$ & MIN 233 \\
\hline 301 & 67.259 & $\mathrm{O} 2+\mathrm{C} 10 \mathrm{H} 16 \mathrm{O}$ & $<-->$ & $\mathrm{O} 2+\mathrm{C} 10 \mathrm{H} 160$ \\
\hline 302 & 67.321 & MIN 290 & $<-->$ & $\mathrm{H} 2+\mathrm{C} 10 \mathrm{H} 1403$ \\
\hline 303 & 67.408 & MIN 268 & $<-->$ & $\mathrm{H} 2+\mathrm{C} 10 \mathrm{H} 1403$ \\
\hline 304 & 67.409 & $\mathrm{O} 2+\mathrm{C} 10 \mathrm{H} 16 \mathrm{O}$ & $<-->$ & $\mathrm{O} 2+\mathrm{C} 10 \mathrm{H} 160$ \\
\hline 305 & 67.581 & MIN 233 & $<-->$ & $\mathrm{H} 2+\mathrm{C} 10 \mathrm{H} 1403$ \\
\hline 306 & 67.796 & $\mathrm{O} 2+\mathrm{C} 10 \mathrm{H} 16 \mathrm{O}$ & $<-->$ & $\mathrm{O} 2+\mathrm{C} 10 \mathrm{H} 160$ \\
\hline 307 & 67.829 & MIN 233 & $<-->$ & MIN 370 \\
\hline 308 & 67.959 & MIN & $<-->$ & MIN 183 \\
\hline 309 & 67.989 & MIN & $<-->$ & $\mathrm{H} 2+\mathrm{C} 10 \mathrm{H} 1403$ \\
\hline 310 & 68.022 & MIN & $<-->$ & MIN 265 \\
\hline 311 & 68.053 & MIN & $<-->$ & $\mathrm{H} 2+\mathrm{O} 2+\mathrm{C} 10 \mathrm{H} 14 \mathrm{O}$ \\
\hline 312 & 68.099 & MIN 233 & $<-->$ & $\mathrm{H} 2+\mathrm{C} 10 \mathrm{H} 1403$ \\
\hline 313 & 68.104 & $\mathrm{O} 2+\mathrm{C} 10 \mathrm{H} 16 \mathrm{O}$ & $<-->$ & $\mathrm{O} 2+\mathrm{C} 10 \mathrm{H} 160$ \\
\hline 314 & 68.118 & $\mathrm{O} 2+\mathrm{C} 10 \mathrm{H} 16 \mathrm{O}$ & $<-->$ & $\mathrm{O} 2+\mathrm{C} 2 \mathrm{H} 2 \mathrm{O}+\mathrm{C} 8 \mathrm{H} 14$ \\
\hline 315 & 68.127 & $\mathrm{O} 2+\mathrm{C} 10 \mathrm{H} 16 \mathrm{O}$ & $<-->$ & $\mathrm{O} 2+\mathrm{C} 10 \mathrm{H} 16 \mathrm{O}$ \\
\hline 316 & 68.195 & MIN 219 & $<-->$ & $\mathrm{H} 2+\mathrm{C} 10 \mathrm{H} 1403$ \\
\hline 31 & 68.276 & MIN & $<-->$ & $\mathrm{H} 2+\mathrm{C} 10 \mathrm{H} 1403$ \\
\hline 3 & 68.316 & MIN & $<-->$ & MIN 226 \\
\hline & 68.318 & MIN & $<-->$ & $\mathrm{H} 2+\mathrm{C} 10 \mathrm{H} 1403$ \\
\hline
\end{tabular}




\begin{tabular}{|c|c|c|c|c|c|}
\hline 320 & 68.350 & MIN & 233 & $<-->$ & $\mathrm{H} 2+\mathrm{C} 10 \mathrm{H} 1403$ \\
\hline 321 & 68.411 & MIN & 233 & $<-->$ & $\mathrm{H} 2+\mathrm{C} 10 \mathrm{H} 1403$ \\
\hline 322 & 68.599 & MIN & 253 & $<-->$ & MIN 253 \\
\hline 323 & 68.626 & $\mathrm{H} 2+\mathrm{C} 10 \mathrm{H} 1$ & 1403 & $<-->$ & $\mathrm{H} 2+\mathrm{C} 10 \mathrm{H} 1403$ \\
\hline 324 & 68.656 & MIN & 219 & $<-->$ & $\mathrm{H} 2+\mathrm{C} 10 \mathrm{H} 1403$ \\
\hline 325 & 68.683 & $\mathrm{O} 2+\mathrm{C} 10 \mathrm{H}$ & $\mathrm{H} 160$ & $<-->$ & $\mathrm{O} 2+\mathrm{C} 10 \mathrm{H} 160$ \\
\hline 326 & 68.691 & MIN & 89 & $<-->$ & $\mathrm{O} 2+\mathrm{C} 10 \mathrm{H} 160$ \\
\hline 327 & 68.757 & MIN & 277 & $<-->$ & $\mathrm{H} 2+\mathrm{C} 10 \mathrm{H} 1403$ \\
\hline 328 & 68.787 & MIN & 273 & $<-->$ & $\mathrm{H} 2+\mathrm{C} 10 \mathrm{H} 1403$ \\
\hline 329 & 68.820 & MIN & 92 & $<-->$ & $\mathrm{C} 3 \mathrm{H} 6+\mathrm{C} 7 \mathrm{H} 1003$ \\
\hline 330 & 68.990 & MIN & 256 & $<-->$ & MIN 256 \\
\hline 331 & 69.026 & MIN & 233 & $<-->$ & MIN 233 \\
\hline 332 & 69.030 & MIN & 233 & $<-->$ & $\mathrm{H} 2+\mathrm{C} 10 \mathrm{H} 1403$ \\
\hline 333 & 69.045 & MIN & 233 & $<-->$ & $\mathrm{H} 2+\mathrm{C} 10 \mathrm{H} 1403$ \\
\hline 334 & 69.064 & MIN & 233 & $<-->$ & $\mathrm{H} 2+\mathrm{C} 10 \mathrm{H} 1403$ \\
\hline 335 & 69.072 & MIN & 232 & $<-->$ & $\mathrm{H} 2+\mathrm{C} 10 \mathrm{H} 1403$ \\
\hline 336 & 69.222 & MIN & 381 & $<-->$ & $\mathrm{H} 2+\mathrm{C} 10 \mathrm{H} 1403$ \\
\hline 337 & 69.239 & MIN & 233 & $<-->$ & MIN 233 \\
\hline 338 & 69.251 & MIN & 232 & $<-->$ & $\mathrm{H} 2+\mathrm{C} 10 \mathrm{H} 1403$ \\
\hline 339 & 69.308 & MIN & 233 & $<-->$ & $\mathrm{H} 2+\mathrm{C} 10 \mathrm{H} 1403$ \\
\hline 340 & 69.361 & MIN & 232 & $<-->$ & $\mathrm{H} 2+\mathrm{C} 10 \mathrm{H} 1403$ \\
\hline 341 & 69.463 & MIN & 276 & $<-->$ & $\mathrm{CO}+\mathrm{C} 3 \mathrm{H} 6+\mathrm{C} 6 \mathrm{H} 10 \mathrm{O} 2$ \\
\hline 342 & 69.504 & MIN & 232 & $<-->$ & MIN 232 \\
\hline 343 & 70.022 & MIN & 149 & $<-->$ & MIN 396 \\
\hline 344 & 70.073 & MIN & 233 & $<-->$ & $\mathrm{H} 2+\mathrm{C} 10 \mathrm{H} 1403$ \\
\hline 345 & 70.109 & MIN & 233 & $<-->$ & $\mathrm{H} 2+\mathrm{C} 10 \mathrm{H} 1403$ \\
\hline 346 & 70.184 & MIN & 226 & $<-->$ & $\mathrm{H} 2+\mathrm{C} 10 \mathrm{H} 1403$ \\
\hline 347 & 70.269 & MIN & 232 & $<-->$ & $\mathrm{H} 2+\mathrm{C} 10 \mathrm{H} 1403$ \\
\hline 348 & 70.371 & MIN & 233 & $<-->$ & $\mathrm{H} 2+\mathrm{C} 10 \mathrm{H} 1403$ \\
\hline 349 & 70.387 & $\mathrm{O} 2+\mathrm{C} 10 \mathrm{H}$ & 1160 & $<-->$ & $\mathrm{O} 2+\mathrm{C} 10 \mathrm{H} 160$ \\
\hline 350 & 70.393 & MIN & 226 & $<-->$ & $\mathrm{H} 2+\mathrm{C} 10 \mathrm{H} 1403$ \\
\hline 351 & 70.424 & MIN & 233 & $<-->$ & $\mathrm{H} 2+\mathrm{C} 10 \mathrm{H} 1403$ \\
\hline 352 & 70.536 & MIN & 233 & $<-->$ & MIN 233 \\
\hline 353 & 70.750 & MIN & 233 & $<-->$ & MIN 329 \\
\hline 354 & 70.853 & MIN & 169 & $<-->$ & $\mathrm{CH} 4+\mathrm{C} 9 \mathrm{H}_{1} 2 \mathrm{O} 3$ \\
\hline 355 & 70.935 & MIN & 233 & $<-->$ & MIN 233 \\
\hline 356 & 71.197 & MIN & 168 & $<-->$ & $\operatorname{MIN}$ \\
\hline 357 & 71.251 & MIN & 232 & $<-->$ & MIN 232 \\
\hline 358 & 71.369 & MIN & 233 & $<-->$ & $\mathrm{H} 2+\mathrm{C} 10 \mathrm{H} 1403$ \\
\hline 359 & 71.396 & MIN & 226 & $<-->$ & MIN 226 \\
\hline 360 & 71.405 & MIN & 281 & $<-->$ & $\operatorname{MIN}$ \\
\hline 361 & 71.529 & MIN & 386 & $<-->$ & MIN \\
\hline 362 & 71.691 & MIN & 219 & $<-->$ & MIN 409 \\
\hline 363 & 71.911 & MIN & 232 & $<-->$ & $\mathrm{H} 2+\mathrm{C} 10 \mathrm{H} 1403$ \\
\hline 364 & 71.978 & MIN & 301 & $<-->$ & $\mathrm{H} 2+\mathrm{C} 10 \mathrm{H} 1403$ \\
\hline 365 & 72.113 & MIN & 397 & $<-->$ & $\mathrm{H} 2+\mathrm{C} 10 \mathrm{H} 1403$ \\
\hline 366 & 72.182 & MIN & 235 & $<-->$ & $\mathrm{H} 2+\mathrm{O} 2+\mathrm{C} 10 \mathrm{H} 14 \mathrm{O}$ \\
\hline 367 & 72.187 & MIN & 225 & $<-->$ & $\mathrm{O} 2+\mathrm{C} 10 \mathrm{H} 160$ \\
\hline 368 & 72.202 & MIN & 110 & $<-->$ & $\mathrm{C} 2 \mathrm{H} 4 \mathrm{O} 2+\mathrm{C} 8 \mathrm{H} 12 \mathrm{O}$ \\
\hline 369 & 72.224 & MIN & 330 & $<-->$ & $\mathrm{H} 2+\mathrm{C} 10 \mathrm{H} 1403$ \\
\hline 370 & 72.301 & MIN & 72 & $<-->$ & MIN 367 \\
\hline 371 & 72.636 & $\mathrm{O} 2+\mathrm{C} 10 \mathrm{H}$ & 1160 & $<-->$ & $\mathrm{C} 4 \mathrm{H} 6 \mathrm{O} 3+\mathrm{C} 6 \mathrm{H} 1 \mathrm{O}$ \\
\hline 372 & 72.648 & MIN & 398 & $<-->$ & $\mathrm{H} 2+\mathrm{C} 10 \mathrm{H} 1403$ \\
\hline 373 & 72.992 & $\mathrm{O} 2+\mathrm{C} 10 \mathrm{H}$ & 160 & $<-->$ & $\mathrm{O} 2+\mathrm{C} 10 \mathrm{H} 16 \mathrm{O}$ \\
\hline 374 & 73.054 & $\mathrm{C} 2 \mathrm{H} 4 \mathrm{O} 2+\mathrm{C} 8 \mathrm{H}$ & $\mathrm{H} 120$ & $<-->$ & $\mathrm{C} 2 \mathrm{H} 4 \mathrm{O} 2+\mathrm{C} 8 \mathrm{H} 12 \mathrm{O}$ \\
\hline 375 & 73.131 & MIN & 233 & $<-->$ & MIN 403 \\
\hline 376 & 73.251 & MIN & 175 & $<-->$ & MIN \\
\hline 377 & 73.341 & MIN & 445 & $<-->$ & MIN 445 \\
\hline 378 & 73.446 & MIN & 326 & $<-->$ & $\mathrm{H} 2+\mathrm{C} 10 \mathrm{H} 1403$ \\
\hline 379 & 73.650 & MIN & 312 & $<-->$ & $\mathrm{H} 2+\mathrm{C} 10 \mathrm{H} 1403$ \\
\hline 380 & 73.747 & $\mathrm{O} 2+\mathrm{C} 1 \mathrm{OH}$ & 1160 & $<-->$ & $\mathrm{O} 2+\mathrm{C} 10 \mathrm{H} 160$ \\
\hline 381 & 73.799 & MIN & 331 & $<-->$ & $\mathrm{H} 2+\mathrm{C} 10 \mathrm{H} 1403$ \\
\hline 382 & 73.884 & MIN & 354 & $<-->$ & $\mathrm{O} 2+\mathrm{C} 10 \mathrm{H} 160$ \\
\hline 383 & 73.896 & MIN & 170 & $<-->$ & $\mathrm{CH} 4+\mathrm{C} 9 \mathrm{H} 12 \mathrm{O} 3$ \\
\hline 384 & 74.066 & MIN & 114 & $<-->$ & MIN 442 \\
\hline
\end{tabular}




\begin{tabular}{|c|c|c|c|c|c|}
\hline 385 & 74.446 & $\mathrm{MIN}$ & 233 & $<-->$ & $\mathrm{MIN}$ \\
\hline 386 & 74.675 & MIN & 177 & $<-->$ & $\mathrm{CH} 4+\mathrm{C} 9 \mathrm{H} 12 \mathrm{O} 3$ \\
\hline 387 & 75.046 & MIN & 233 & $<-->$ & $\mathrm{H} 2+\mathrm{C} 10 \mathrm{H} 1403$ \\
\hline 388 & 75.091 & MIN & 233 & $<-->$ & MIN 414 \\
\hline 389 & 75.108 & $\operatorname{MIN}$ & 233 & $<-->$ & MIN \\
\hline 390 & 75.374 & MIN & 175 & $<-->$ & MIN 423 \\
\hline 91 & 75.432 & $\mathrm{C} 2 \mathrm{H} 2+\mathrm{C} 8 \mathrm{H} 1$ & 1403 & $<-->$ & $\mathrm{C} 2 \mathrm{H} 2+\mathrm{C} 3 \mathrm{H} 4 \mathrm{O} 3+\mathrm{C} 5 \mathrm{H} 1$ \\
\hline 92 & 75.838 & MIN & 254 & $<-->$ & $\mathrm{H} 2+\mathrm{C} 10 \mathrm{H} 1403$ \\
\hline 93 & 76.257 & MIN & 219 & $<-->$ & MIN 417 \\
\hline 94 & 76.827 & MIN & 173 & $<-->$ & MIN \\
\hline 95 & 77.158 & MIN & 67 & $<-->$ & MIN \\
\hline 96 & 77.195 & MIN & 316 & $<-->$ & $\mathrm{H} 2+\mathrm{C} 10 \mathrm{H} 1403$ \\
\hline 97 & 77.392 & MIN & 316 & $<-->$ & $\mathrm{H} 2+\mathrm{C} 10 \mathrm{H} 1403$ \\
\hline 398 & 77.403 & MIN & 317 & $<-->$ & $\mathrm{H} 2+\mathrm{C} 10 \mathrm{H} 1403$ \\
\hline 399 & 77.776 & MIN & 311 & $<-->$ & MIN 457 \\
\hline 00 & 77.986 & MIN & 205 & $<-->$ & $\mathrm{H} 2+\mathrm{C} 10 \mathrm{H} 1403$ \\
\hline 1 & 78.042 & MIN & 237 & $<-->$ & $\mathrm{H} 2+\mathrm{C} 10 \mathrm{H} 1403$ \\
\hline 02 & 78.048 & MIN & 171 & $<-->$ & $\mathrm{H} 2+\mathrm{C} 10 \mathrm{H} 1403$ \\
\hline 403 & 78.190 & MIN & 91 & $<-->$ & $\mathrm{CH} 2+\mathrm{C} 9 \mathrm{H} 1403$ \\
\hline 404 & 78.332 & MIN & 209 & $<-->$ & $\operatorname{MIN} \quad 323$ \\
\hline 105 & 78.451 & MIN & 332 & $<-->$ & MIN 419 \\
\hline 406 & 78.702 & MIN & 232 & $<-->$ & $\mathrm{H} 2+\mathrm{C} 10 \mathrm{H} 1403$ \\
\hline 407 & 78.746 & MIN & 233 & $<-->$ & $\mathrm{H} 2+\mathrm{C} 10 \mathrm{H} 1403$ \\
\hline 408 & 78.949 & MIN & 233 & $<-->$ & MIN 233 \\
\hline 409 & 79.051 & MIN & 232 & $<-->$ & $\mathrm{H} 2+\mathrm{C} 10 \mathrm{H} 1403$ \\
\hline 10 & 79.155 & MIN & 373 & $<-->$ & $\mathrm{O} 3+\mathrm{C} 10 \mathrm{H} 16$ \\
\hline 11 & 79.207 & $\mathrm{C} 2 \mathrm{H} 2+\mathrm{C} 8 \mathrm{H} 1$ & 1403 & $<-->$ & $\mathrm{C} 2 \mathrm{H} 2+\mathrm{C} 8 \mathrm{H} 14 \mathrm{O} 3$ \\
\hline 412 & 79.209 & MIN & 222 & $<-->$ & MIN 429 \\
\hline 413 & 79.209 & MIN & 226 & $<-->$ & $\mathrm{H} 2+\mathrm{C} 10 \mathrm{H} 1403$ \\
\hline 414 & 79.311 & MIN & 206 & $<-->$ & MIN 454 \\
\hline 415 & 79.459 & MIN & 247 & $<-->$ & MIN 302 \\
\hline 416 & 79.700 & $\mathrm{O} 2+\mathrm{C} 5 \mathrm{H} 6 \mathrm{O}+\mathrm{C} 5$ & $5 \mathrm{H} 10$ & $<-->$ & $\mathrm{O} 2+\mathrm{C} 10 \mathrm{H} 16 \mathrm{O}$ \\
\hline 417 & 80.004 & MIN & 233 & $<-->$ & MIN 233 \\
\hline 418 & 80.014 & $\mathrm{H} 2+\mathrm{C} 10 \mathrm{H} 1$ & 1403 & $<-->$ & $\mathrm{H} 2+\mathrm{C} 10 \mathrm{H} 1403$ \\
\hline 419 & 80.024 & MIN & 233 & $<-->$ & $\mathrm{H} 2+\mathrm{C} 10 \mathrm{H} 1403$ \\
\hline 420 & 80.030 & MIN & 214 & $<-->$ & MIN $\quad 464$ \\
\hline 421 & 80.061 & MIN & 219 & $<-->$ & $\mathrm{CH} 4+\mathrm{C} 9 \mathrm{H}_{1} 2 \mathrm{O} 3$ \\
\hline 422 & 80.149 & MIN & 335 & $<-->$ & $\mathrm{H} 2+\mathrm{C} 10 \mathrm{H} 1403$ \\
\hline 423 & 80.152 & MIN & 233 & $<-->$ & $\mathrm{H} 2+\mathrm{C} 10 \mathrm{H} 1403$ \\
\hline 424 & 80.169 & MIN & 247 & $<-->$ & MIN 294 \\
\hline 425 & 80.253 & MIN & 285 & $<-->$ & MIN 436 \\
\hline 426 & 80.346 & MIN & 240 & $<-->$ & $\mathrm{H} 2+\mathrm{C} 10 \mathrm{H} 1403$ \\
\hline 427 & 80.352 & $\mathrm{O} 2+\mathrm{C} 10 \mathrm{H}$ & $\mathrm{H} 160$ & $<-->$ & $\mathrm{H} 2+\mathrm{O} 2+\mathrm{C} 10 \mathrm{H} 14 \mathrm{O}$ \\
\hline 428 & 80.367 & MIN & 321 & $<-->$ & $\mathrm{H} 2+\mathrm{C} 10 \mathrm{H} 1403$ \\
\hline 429 & 80.387 & MIN & 226 & $<-->$ & $\mathrm{CH} 4+\mathrm{C} 9 \mathrm{H} 12 \mathrm{O} 3$ \\
\hline 430 & 80.398 & MIN & 232 & $<-->$ & $\mathrm{H} 2+\mathrm{C} 10 \mathrm{H} 1403$ \\
\hline 431 & 80.448 & MIN & 226 & $<-->$ & MIN 431 \\
\hline 432 & 80.465 & MIN & 285 & $<-->$ & MIN 435 \\
\hline 433 & 80.499 & MIN & 322 & $<-->$ & $\mathrm{H} 2+\mathrm{C} 10 \mathrm{H} 1403$ \\
\hline 434 & 80.659 & MIN & 226 & $<-->$ & $\mathrm{CH} 4+\mathrm{C} 9 \mathrm{H} 12 \mathrm{O} 3$ \\
\hline 435 & 80.753 & MIN & 300 & $<-->$ & $\mathrm{C} 3 \mathrm{H} 4 \mathrm{O} 3+\mathrm{C} 7 \mathrm{H} 12$ \\
\hline 436 & 80.861 & MIN & 233 & $<-->$ & $\mathrm{H} 2+\mathrm{C} 10 \mathrm{H} 1403$ \\
\hline 437 & 81.360 & MIN & 172 & $<-->$ & MIN 247 \\
\hline 438 & 81.662 & MIN & 233 & $<-->$ & MIN 428 \\
\hline 439 & 81.694 & MIN & 233 & $<-->$ & $\mathrm{H} 2+\mathrm{C} 10 \mathrm{H} 1403$ \\
\hline 440 & 81.727 & MIN & 232 & $<-->$ & $\mathrm{H} 2+\mathrm{C} 10 \mathrm{H} 1403$ \\
\hline 441 & 81.827 & MIN & 355 & $<-->$ & MIN 466 \\
\hline 442 & 81.897 & $\mathrm{O} 2+\mathrm{C} 10 \mathrm{H}$ & $\mathrm{H} 16 \mathrm{O}$ & $<-->$ & $\mathrm{O} 2+\mathrm{C} 10 \mathrm{H} 16 \mathrm{O}$ \\
\hline 443 & 82.013 & MIN & 232 & $<-->$ & $\mathrm{H} 2+\mathrm{O} 2+\mathrm{C} 10 \mathrm{H} 14 \mathrm{O}$ \\
\hline 444 & 82.566 & $\mathrm{H} 2+\mathrm{O} 2+\mathrm{C} 10 \mathrm{~F}$ & $\mathrm{H} 14 \mathrm{O}$ & $<-->$ & $\mathrm{O} 2+\mathrm{C} 10 \mathrm{H} 16 \mathrm{O}$ \\
\hline 445 & 82.666 & $\mathrm{H} 2+\mathrm{O} 2+\mathrm{C} 10 \mathrm{H}$ & $\mathrm{H} 14 \mathrm{O}$ & $<-->$ & $\mathrm{O} 2+\mathrm{C} 10 \mathrm{H} 16 \mathrm{O}$ \\
\hline 446 & 82.795 & MIN & 356 & $<-->$ & $\mathrm{H} 2+\mathrm{C} 10 \mathrm{H} 1403$ \\
\hline 447 & 82.859 & $\mathrm{CH} 4+\mathrm{C} 9 \mathrm{H} 1$ & 1203 & $<-->$ & $\mathrm{CH} 4+\mathrm{C} 9 \mathrm{H}_{1} 2 \mathrm{O}_{3}$ \\
\hline 448 & 83.040 & MIN & 233 & $<-->$ & MIN 252 \\
\hline 440 & 83.270 & MIN & 233 & $<-->$ & $\mathrm{H} 2+\mathrm{C} 10 \mathrm{H} 1403$ \\
\hline
\end{tabular}




\begin{tabular}{|c|c|c|c|c|c|}
\hline 450 & 83.300 & MIN & 231 & $<-->$ & MIN \\
\hline 451 & 83.307 & MIN & 147 & $<-->$ & MIN \\
\hline 452 & 83.365 & MIN & 232 & $<-->$ & $\mathrm{MIN}$ \\
\hline 453 & 83.427 & MIN & 232 & $<-->$ & MIN 418 \\
\hline 454 & 83.470 & MIN & 232 & $<-->$ & $\mathrm{H} 2+\mathrm{C} 10 \mathrm{H} 1403$ \\
\hline 455 & 83.504 & MIN & 233 & $<-->$ & MIN 430 \\
\hline 456 & 83.772 & MIN & 174 & $<-->$ & $\mathrm{CH} 4+\mathrm{C} 9 \mathrm{H} 12 \mathrm{O} 3$ \\
\hline 457 & 84.089 & $\mathrm{O} 2+\mathrm{C} 10 \mathrm{H}$ & 1160 & $<-->$ & $\mathrm{O} 2+\mathrm{C} 10 \mathrm{H} 16 \mathrm{O}$ \\
\hline 458 & 84.123 & MIN & 198 & $<-->$ & MIN 461 \\
\hline 459 & 84.320 & MIN & 104 & $<-->$ & $\mathrm{CH} 2+\mathrm{C} 9 \mathrm{H} 1403$ \\
\hline 460 & 84.540 & MIN & 359 & $<-->$ & $\mathrm{H} 2+\mathrm{C} 10 \mathrm{H} 1403$ \\
\hline 461 & 84.703 & $\mathrm{MIN}$ & 82 & $<-->$ & MIN 123 \\
\hline 462 & 84.774 & MIN & 342 & $<-->$ & $\mathrm{H} 2+\mathrm{C} 10 \mathrm{H} 1403$ \\
\hline 463 & 84.813 & MIN & 255 & $<-->$ & MIN $\quad 440$ \\
\hline 464 & 84.897 & MIN & 371 & $<-->$ & MIN 374 \\
\hline 465 & 85.127 & $\mathrm{H} 2+\mathrm{C} 10 \mathrm{H} 1$ & 1403 & $<-->$ & $\mathrm{H} 2+\mathrm{C} 10 \mathrm{H} 1403$ \\
\hline 466 & 85.189 & $\mathrm{CH} 4+\mathrm{C} 9 \mathrm{H} 1$ & 1203 & $<-->$ & $\mathrm{CH} 4+\mathrm{C} 9 \mathrm{H} 12 \mathrm{O} 3$ \\
\hline 467 & 85.266 & MIN & 303 & $<-->$ & $\mathrm{H} 2+\mathrm{C} 10 \mathrm{H} 1403$ \\
\hline 468 & 85.369 & MIN & 196 & $<-->$ & MIN 400 \\
\hline 469 & 85.669 & $\mathrm{O} 2+\mathrm{C} 10 \mathrm{H}$ & 1160 & $<-->$ & $\mathrm{O} 2+\mathrm{C} 10 \mathrm{H} 160$ \\
\hline 470 & 85.831 & MIN & 86 & $<-->$ & $\mathrm{CH} 2+\mathrm{C} 9 \mathrm{H} 1403$ \\
\hline 471 & 85.940 & MIN & 367 & $<-->$ & $\mathrm{H} 2+\mathrm{C} 10 \mathrm{H} 1403$ \\
\hline 472 & 86.122 & MIN & 213 & $<-->$ & MIN 418 \\
\hline 473 & 86.380 & MIN & 232 & $<-->$ & MIN \\
\hline 474 & 86.816 & MIN & 233 & $<-->$ & MIN \\
\hline 475 & 87.512 & MIN & 249 & $<-->$ & MIN 424 \\
\hline 476 & 87.598 & $\mathrm{CH} 4+\mathrm{C} 9 \mathrm{H} 1$ & 1203 & $<-->$ & $\mathrm{CH} 4+\mathrm{C} 9 \mathrm{H} 12 \mathrm{O} 3$ \\
\hline 477 & 88.073 & MIN & 208 & $<-->$ & MIN 468 \\
\hline 478 & 88.094 & MIN & 427 & $<-->$ & $\mathrm{H} 2+\mathrm{C} 10 \mathrm{H} 1403$ \\
\hline 479 & 88.103 & MIN & 210 & $<-->$ & MIN 233 \\
\hline 480 & 88.838 & MIN & 287 & $<-->$ & MIN 432 \\
\hline 481 & 88.926 & MIN & 167 & $<-->$ & $\mathrm{C} 3 \mathrm{H} 6+\mathrm{C} 7 \mathrm{H} 1003$ \\
\hline 482 & 88.942 & MIN & 327 & $<-->$ & $\mathrm{CH} 4+\mathrm{C} 9 \mathrm{H} 12 \mathrm{O} 3$ \\
\hline 483 & 89.030 & MIN & 96 & $<-->$ & $\mathrm{CH} 2 \mathrm{O}+\mathrm{C} 9 \mathrm{H} 14 \mathrm{O} 2$ \\
\hline 484 & 89.197 & MIN & 105 & $<-->$ & $\mathrm{CH} 2+\mathrm{C} 9 \mathrm{H} 1403$ \\
\hline 485 & 89.307 & MIN & 360 & $<-->$ & $\mathrm{H} 2+\mathrm{C} 10 \mathrm{H} 1403$ \\
\hline 486 & 89.404 & MIN & 376 & $<-->$ & $\mathrm{H} 2+\mathrm{C} 10 \mathrm{H} 1403$ \\
\hline 487 & 89.749 & MIN & 313 & $<-->$ & MIN 459 \\
\hline 488 & 89.769 & MIN & 358 & $<-->$ & MIN 358 \\
\hline 489 & 89.907 & MIN & 233 & $<-->$ & $\mathrm{H} 2+\mathrm{C} 10 \mathrm{H} 1403$ \\
\hline 490 & 90.066 & $\operatorname{MIN}$ & 202 & $<-->$ & MIN 463 \\
\hline 491 & 90.101 & $\mathrm{C} 2 \mathrm{H} 2+\mathrm{C} 8 \mathrm{H} 1$ & 1403 & $<-->$ & $\mathrm{C} 2 \mathrm{H} 2+\mathrm{C} 3 \mathrm{H} 6 \mathrm{O}+\mathrm{C} 5 \mathrm{H} 8 \mathrm{O} 2$ \\
\hline 492 & 90.502 & MIN & 233 & $<-->$ & MIN 448 \\
\hline 493 & 91.266 & MIN & 232 & $<-->$ & MIN \\
\hline 494 & 91.682 & MIN & 233 & $<-->$ & MIN $\quad 460$ \\
\hline 495 & 91.732 & MIN & 182 & $<-->$ & $\mathrm{C} 3 \mathrm{H} 6+\mathrm{C} 7 \mathrm{H} 1003$ \\
\hline 496 & 92.095 & MIN & 233 & $<-->$ & $\mathrm{H} 2+\mathrm{C} 10 \mathrm{H} 1403$ \\
\hline 497 & 92.266 & MIN & 380 & $<-->$ & $\mathrm{H} 2+\mathrm{C} 10 \mathrm{H} 1403$ \\
\hline 498 & 92.391 & MIN & 233 & $<-->$ & $\operatorname{MIN} \quad 456$ \\
\hline 499 & 92.492 & MIN & 357 & $<-->$ & MIN 439 \\
\hline 500 & 92.585 & MIN & 233 & $<-->$ & $\mathrm{C} 3 \mathrm{H} 6+\mathrm{C} 7 \mathrm{H} 1003$ \\
\hline 501 & 92.587 & MIN & 233 & $<-->$ & $\mathrm{H} 2+\mathrm{C} 10 \mathrm{H} 1403$ \\
\hline 502 & 93.015 & MIN & 226 & $<-->$ & $\mathrm{CH} 4+\mathrm{C} 9 \mathrm{H} 12 \mathrm{O} 3$ \\
\hline 503 & 93.116 & MIN & 165 & $<-->$ & $\mathrm{CH} 2+\mathrm{C} 9 \mathrm{H} 1403$ \\
\hline 504 & 93.347 & MIN & 233 & $<-->$ & $\mathrm{C} 3 \mathrm{H} 6+\mathrm{C} 7 \mathrm{H} 1003$ \\
\hline 505 & 93.684 & $\mathrm{MIN}$ & 232 & $<-->$ & $\mathrm{C} 3 \mathrm{H} 6+\mathrm{C} 7 \mathrm{H} 1003$ \\
\hline 506 & 93.945 & MIN & 233 & $<-->$ & $\mathrm{CH}_{4}+\mathrm{C} 9 \mathrm{H} 12 \mathrm{O} 3$ \\
\hline 507 & 94.075 & MIN & 246 & $<-->$ & MIN 349 \\
\hline 508 & 94.231 & MIN & 159 & $<-->$ & MIN 469 \\
\hline 509 & 94.935 & MIN & 325 & $<-->$ & $\mathrm{H} 2+\mathrm{C} 10 \mathrm{H} 1403$ \\
\hline 510 & 95.271 & $\mathrm{MIN}$ & 233 & $<-->$ & $\mathrm{H} 2+\mathrm{C} 10 \mathrm{H} 1403$ \\
\hline 511 & 95.528 & MIN & 375 & $<-->$ & MIN 443 \\
\hline 512 & 95.650 & MIN & 233 & $<-->$ & MIN 465 \\
\hline 513 & 95.666 & MIN & 346 & $<-->$ & $\mathrm{H} 2+\mathrm{C} 10 \mathrm{H} 1403$ \\
\hline 514 & 95.676 & MIN & 333 & $<-->$ & MIN 449 \\
\hline
\end{tabular}




\begin{tabular}{|c|c|c|c|c|c|}
\hline 515 & 95.938 & MIN & 328 & $<-->$ & MIN \\
\hline 516 & 95.949 & MIN & 230 & $<-->$ & MIN 470 \\
\hline 517 & 95.968 & MIN & 193 & $<-->$ & $\mathrm{H} 2+\mathrm{C} 10 \mathrm{H} 1403$ \\
\hline 18 & 96.212 & $\mathrm{O} 2+\mathrm{C} 10 \mathrm{H}$ & $\mathrm{H} 16 \mathrm{O}$ & $<-->$ & $\mathrm{O} 2+\mathrm{CH} 4+\mathrm{C} 9 \mathrm{H} 12 \mathrm{O}$ \\
\hline 19 & 96.238 & MIN & 361 & $<-->$ & MIN 447 \\
\hline 20 & 96.298 & MIN & 233 & $<-->$ & $\mathrm{H} 2+\mathrm{C} 10 \mathrm{H} 1403$ \\
\hline 21 & 96.324 & MIN & 233 & $<-->$ & $\mathrm{C} 3 \mathrm{H} 4 \mathrm{O} 3+\mathrm{C} 7 \mathrm{H} 12$ \\
\hline 22 & 96.430 & MIN & 232 & $<-->$ & $\mathrm{H} 2+\mathrm{C} 10 \mathrm{H} 1403$ \\
\hline 23 & 96.802 & MIN & 416 & $<-->$ & $\mathrm{C} 2 \mathrm{H} 6+\mathrm{C} 8 \mathrm{H} 10 \mathrm{O} 3$ \\
\hline 24 & 96.825 & MIN & 232 & $<-->$ & $\mathrm{H} 2+\mathrm{C} 10 \mathrm{H} 1403$ \\
\hline 25 & 97.943 & MIN & 341 & $<-->$ & MIN 441 \\
\hline 26 & 98.271 & $\mathrm{H} 2 \mathrm{O} 2+\mathrm{C} 10 \mathrm{H}$ & $\mathrm{H} 14 \mathrm{O}$ & $<-->$ & $\mathrm{H} 2 \mathrm{O} 2+\mathrm{C} 10 \mathrm{H} 14 \mathrm{O}$ \\
\hline 27 & 98.732 & MIN & 388 & $<-->$ & $\mathrm{C} 2 \mathrm{H} 6+\mathrm{C} 8 \mathrm{H} 10 \mathrm{O} 3$ \\
\hline 528 & 98.754 & MIN & 319 & $<-->$ & $\mathrm{C} 3 \mathrm{H} 6+\mathrm{C} 7 \mathrm{H} 1003$ \\
\hline 529 & 98.891 & MIN & 399 & $<-->$ & failed 0 \\
\hline 30 & 99.160 & MIN & 347 & $<-->$ & $\mathrm{H} 2+\mathrm{C} 10 \mathrm{H} 1403$ \\
\hline 31 & 99.367 & $\mathrm{H} 2+\mathrm{O} 2+\mathrm{C} 10 \mathrm{H}$ & $\mathrm{H} 14 \mathrm{O}$ & $<-->$ & $\mathrm{O} 2+\mathrm{C} 10 \mathrm{H} 160$ \\
\hline 32 & 99.657 & $\operatorname{MIN}$ & 233 & $<-->$ & $\mathrm{CH} 4+\mathrm{C} 9 \mathrm{H} 12 \mathrm{O} 3$ \\
\hline 33 & 99.736 & MIN & 238 & $<-->$ & $\mathrm{C} 3 \mathrm{H} 6+\mathrm{C} 7 \mathrm{H} 1003$ \\
\hline 34 & 100.579 & $\mathrm{H} 2+\mathrm{C} 10 \mathrm{H} 1$ & 1403 & $<-->$ & $\mathrm{CH} 4+\mathrm{C} 9 \mathrm{H} 12 \mathrm{O} 3$ \\
\hline 35 & 100.762 & MIN & 244 & $<-->$ & MIN 308 \\
\hline 36 & 100.857 & MIN & 382 & $<-->$ & MIN \\
\hline 37 & 101.662 & MIN & 353 & $<-->$ & $\operatorname{MIN} \quad 364$ \\
\hline 538 & 101.755 & MIN & 208 & $<-->$ & $\mathrm{CH} 2+\mathrm{C} 9 \mathrm{H} 1403$ \\
\hline 39 & 101.843 & MIN & 233 & $<-->$ & $\mathrm{CH} 4+\mathrm{C} 9 \mathrm{H} 12 \mathrm{O} 3$ \\
\hline 40 & 102.172 & MIN & 208 & $<-->$ & $\mathrm{CH} 4+\mathrm{C} 9 \mathrm{H} 12 \mathrm{O} 3$ \\
\hline 541 & 102.335 & MIN & 163 & $<-->$ & $\mathrm{CH} 4+\mathrm{C} 9 \mathrm{H} 12 \mathrm{O} 3$ \\
\hline 42 & 102.839 & MIN & 414 & $<-->$ & $\mathrm{H} 2+\mathrm{C} 10 \mathrm{H} 1403$ \\
\hline 43 & 103.066 & MIN & 233 & $<-->$ & $\mathrm{CH} 2+\mathrm{C} 9 \mathrm{H} 1403$ \\
\hline 544 & 103.295 & MIN & 217 & $<-->$ & $\mathrm{CH} 2+\mathrm{C} 9 \mathrm{H} 1403$ \\
\hline 545 & 103.887 & $\mathrm{O} 2+\mathrm{C} 10 \mathrm{H}$ & $\mathrm{H} 16 \mathrm{O}$ & $<-->$ & $\mathrm{O} 2+\mathrm{C} 10 \mathrm{H} 16 \mathrm{O}$ \\
\hline 46 & 104.166 & MIN & 233 & $<-->$ & $\mathrm{CH} 2+\mathrm{C} 9 \mathrm{H} 14 \mathrm{O} 3$ \\
\hline 547 & 104.174 & MIN & 119 & $<-->$ & MIN 433 \\
\hline 548 & 104.275 & MIN & 233 & $<-->$ & $\mathrm{H} 2+\mathrm{C} 10 \mathrm{H} 1403$ \\
\hline 49 & 104.380 & $\mathrm{O} 2+\mathrm{C} 1 \mathrm{OH}$ & $\mathrm{H} 16 \mathrm{O}$ & $<-->$ & $\mathrm{O} 2+\mathrm{CH} 2+\mathrm{C} 9 \mathrm{H} 14 \mathrm{O}$ \\
\hline 550 & 104.575 & MIN & 228 & $<-->$ & $\mathrm{CH} 2+\mathrm{C} 9 \mathrm{H} 1403$ \\
\hline 551 & 104.891 & MIN & 233 & $<-->$ & $\operatorname{MIN} \quad 367$ \\
\hline 552 & 105.548 & MIN & 199 & $<-->$ & $\operatorname{MIN} \quad 455$ \\
\hline 553 & 106.425 & MIN & 435 & $<-->$ & $\mathrm{CH} 4+\mathrm{C} 9 \mathrm{H} 12 \mathrm{O} 3$ \\
\hline 554 & 106.618 & MIN & 219 & $<-->$ & $\mathrm{CH} 4+\mathrm{C} 9 \mathrm{H} 12 \mathrm{O} 3$ \\
\hline 555 & 106.673 & MIN & 233 & $<-->$ & $\mathrm{CH} 2+\mathrm{C} 9 \mathrm{H} 1403$ \\
\hline 556 & 106.695 & MIN & 233 & $<-->$ & $\mathrm{CH} 2+\mathrm{C} 9 \mathrm{H} 1403$ \\
\hline 557 & 106.864 & MIN & 227 & $<-->$ & $\operatorname{MIN} 233$ \\
\hline 558 & 107.707 & MIN & 232 & $<-->$ & $\mathrm{CH} 2+\mathrm{C} 9 \mathrm{H} 1403$ \\
\hline 559 & 107.903 & MIN & 233 & $<-->$ & $\mathrm{CH} 2+\mathrm{C} 9 \mathrm{H} 1403$ \\
\hline 560 & 108.386 & MIN & 164 & $<-->$ & MIN 233 \\
\hline 561 & 109.277 & MIN & 251 & $<-->$ & MIN 428 \\
\hline 62 & 109.949 & $\mathrm{H} 2+\mathrm{C} 10 \mathrm{H} 1$ & 1403 & $<-->$ & $\mathrm{C} 3 \mathrm{H} 6+\mathrm{C} 7 \mathrm{H} 1003$ \\
\hline 563 & 110.209 & MIN & 233 & $<-->$ & MIN 402 \\
\hline 564 & 110.245 & MIN & 266 & $<-->$ & $\mathrm{CH} 2+\mathrm{C} 9 \mathrm{H} 1403$ \\
\hline 565 & 110.303 & MIN & 413 & $<-->$ & $\mathrm{H} 2+\mathrm{C} 10 \mathrm{H} 1403$ \\
\hline 566 & 110.422 & MIN & 215 & $<-->$ & $\mathrm{CH} 2+\mathrm{C} 9 \mathrm{H} 14 \mathrm{O} 3$ \\
\hline 567 & 111.279 & MIN & 378 & $<-->$ & $\mathrm{O} 3+\mathrm{C} 10 \mathrm{H} 16$ \\
\hline 568 & 111.320 & MIN & 233 & $<-->$ & $\mathrm{CH} 2+\mathrm{C} 9 \mathrm{H} 1403$ \\
\hline 569 & 111.577 & $\mathrm{H} 2+\mathrm{C} 10 \mathrm{H} 1$ & 1403 & $<-->$ & $\mathrm{CH} 4+\mathrm{C} 9 \mathrm{H} 12 \mathrm{O} 3$ \\
\hline 570 & 112.021 & MIN & 126 & $<-->$ & MIN 348 \\
\hline 571 & 112.521 & MIN & 298 & $<-->$ & MIN \\
\hline 572 & 112.792 & MIN & 139 & $<-->$ & $\operatorname{MIN} 233$ \\
\hline 573 & 113.249 & MIN & 233 & $<-->$ & $\mathrm{CH} 4+\mathrm{C} 9 \mathrm{H} 12 \mathrm{O} 3$ \\
\hline 574 & 113.291 & MIN & 233 & $<-->$ & $\mathrm{CH} 2+\mathrm{C} 9 \mathrm{H} 1403$ \\
\hline 575 & 113.513 & MIN & 233 & $<-->$ & MIN 437 \\
\hline 576 & 113.924 & MIN & 233 & $<-->$ & $\mathrm{CH} 4+\mathrm{C} 9 \mathrm{H} 12 \mathrm{O} 3$ \\
\hline 577 & 114.392 & MIN & 188 & $<-->$ & $\operatorname{MIN} 233$ \\
\hline 578 & 114.867 & MIN & 179 & $<-->$ & MIN \\
\hline & 114.891 & MIN & 422 & $<-->$ & MIN \\
\hline
\end{tabular}




\begin{tabular}{|c|c|c|c|c|c|}
\hline 580 & 116.815 & MIN & 233 & $<-->$ & $\mathrm{CH} 4+\mathrm{C} 9 \mathrm{H} 12 \mathrm{O} 3$ \\
\hline 581 & 117.792 & $\mathrm{H} 2+\mathrm{C} 10 \mathrm{H} 1$ & 1403 & $<-->$ & $\mathrm{C} 2 \mathrm{H} 2+\mathrm{C} 8 \mathrm{H} 14 \mathrm{O} 3$ \\
\hline 582 & 118.043 & MIN & 391 & $<-->$ & $\mathrm{H} 2+\mathrm{C} 10 \mathrm{H} 1403$ \\
\hline 583 & 118.300 & MIN & 233 & $<-->$ & $\mathrm{H} 2+\mathrm{C} 10 \mathrm{H} 1403$ \\
\hline 584 & 118.783 & MIN & 413 & $<-->$ & $\mathrm{H} 2+\mathrm{C} 10 \mathrm{H} 1403$ \\
\hline 585 & 118.831 & MIN & 233 & $<-->$ & $\mathrm{CH} 4+\mathrm{C} 9 \mathrm{H}_{1} 2 \mathrm{O} 3$ \\
\hline 586 & 119.179 & MIN & 413 & $<-->$ & $\mathrm{H} 2+\mathrm{C} 10 \mathrm{H} 1403$ \\
\hline 587 & 119.376 & $\mathrm{CH} 4+\mathrm{C} 9 \mathrm{H}]$ & 1203 & $<-->$ & $\mathrm{H} 2+\mathrm{C} 10 \mathrm{H} 1403$ \\
\hline 588 & 119.497 & MIN & 420 & $<-->$ & $\mathrm{O} 2+\mathrm{CH} 2 \mathrm{O}+\mathrm{C} 9 \mathrm{H} 14$ \\
\hline 589 & 119.831 & MIN & 434 & $<-->$ & $\mathrm{H} 2+\mathrm{C} 10 \mathrm{H} 1403$ \\
\hline 590 & 120.169 & MIN & 161 & $<-->$ & MIN 233 \\
\hline 591 & 121.089 & MIN & 423 & $<-->$ & $\mathrm{H} 2+\mathrm{C} 10 \mathrm{H} 1403$ \\
\hline 592 & 121.268 & MIN & 453 & $<-->$ & MIN 458 \\
\hline 593 & 121.712 & MIN & 138 & $<-->$ & $\mathrm{CH} 2+\mathrm{C} 9 \mathrm{H} 1403$ \\
\hline 594 & 122.463 & MIN & 289 & $<-->$ & $\mathrm{H} 2+\mathrm{C} 10 \mathrm{H} 1403$ \\
\hline 595 & 122.854 & MIN & 369 & $<-->$ & $\mathrm{CH} 2+\mathrm{C} 9 \mathrm{H} 1403$ \\
\hline 596 & 123.203 & MIN & 363 & $<-->$ & $\mathrm{H} 2+\mathrm{C} 10 \mathrm{H} 1403$ \\
\hline 597 & 123.801 & MIN & 365 & $<-->$ & $\mathrm{CH} 2 \mathrm{O}+\mathrm{C} 9 \mathrm{H} 14 \mathrm{O} 2$ \\
\hline 598 & 124.167 & MIN & 216 & $<-->$ & MIN 284 \\
\hline 599 & 125.766 & MIN & 233 & $<-->$ & MIN 338 \\
\hline 600 & 126.100 & $\mathrm{O} 2+\mathrm{C} 10 \mathrm{H}$ & 160 & $<-->$ & $\mathrm{H} 2 \mathrm{O} 2+\mathrm{C} 3 \mathrm{H} 4+\mathrm{C} 7 \mathrm{H} 10 \mathrm{O}$ \\
\hline 601 & 126.553 & $\mathrm{H} 2+\mathrm{C} 10 \mathrm{H} 1$ & 1403 & $<-->$ & $\mathrm{H} 2+\mathrm{H} 2+\mathrm{C} 10 \mathrm{H} 12 \mathrm{O} 3$ \\
\hline 602 & 127.299 & MIN & 121 & $<-->$ & MIN 236 \\
\hline 603 & 127.454 & MIN & 195 & $<-->$ & MIN 350 \\
\hline 604 & 127.519 & MIN & 366 & $<-->$ & $\mathrm{O} 3+\mathrm{C} 10 \mathrm{H} 16$ \\
\hline 605 & 131.407 & MIN & 405 & $<-->$ & $\mathrm{CH} 4+\mathrm{C} 9 \mathrm{H} 12 \mathrm{O} 3$ \\
\hline 606 & 132.663 & MIN & 233 & $<-->$ & $\mathrm{CH} 4+\mathrm{C} 9 \mathrm{H} 12 \mathrm{O} 3$ \\
\hline 607 & 132.839 & MIN & 438 & $<-->$ & $\mathrm{H} 2+\mathrm{C} 10 \mathrm{H} 1403$ \\
\hline 608 & 133.211 & $\mathrm{CH} 4+\mathrm{C} 9 \mathrm{H}_{1}$ & 1203 & $<-->$ & $\mathrm{C} 4 \mathrm{H} 6 \mathrm{O} 2+\mathrm{C} 6 \mathrm{H} 10 \mathrm{O}$ \\
\hline 609 & 133.259 & MIN & 444 & $<-->$ & $\mathrm{H} 2+\mathrm{C} 10 \mathrm{H} 14 \mathrm{O} 3$ \\
\hline 610 & 134.445 & $\mathrm{H} 2+\mathrm{C} 10 \mathrm{H} 1$ & 1403 & $<-->$ & $\mathrm{H} 2+\mathrm{C} 10 \mathrm{H} 14 \mathrm{O} 3$ \\
\hline 611 & 135.388 & MIN & 413 & $<-->$ & MIN 413 \\
\hline 612 & 135.404 & MIN & 446 & $<-->$ & $\mathrm{H} 2+\mathrm{C} 10 \mathrm{H} 1403$ \\
\hline 613 & 135.413 & MIN & 401 & $<-->$ & $\mathrm{CH} 4+\mathrm{C} 9 \mathrm{H} 12 \mathrm{O} 3$ \\
\hline 614 & 135.695 & $\mathrm{H} 4 \mathrm{O} 2+\mathrm{C} 3 \mathrm{H} 4+\mathrm{C} 7$ & $7 \mathrm{H} 8 \mathrm{O}$ & $<-->$ & $\mathrm{O} 2+\mathrm{C} 3 \mathrm{H} 6+\mathrm{C} 7 \mathrm{H} 10 \mathrm{O}$ \\
\hline 615 & 136.374 & MIN & 352 & $<-->$ & MIN 448 \\
\hline 616 & 138.863 & MIN & 452 & $<-->$ & $\mathrm{H} 2+\mathrm{C} 10 \mathrm{H} 1403$ \\
\hline 617 & 139.787 & MIN & 467 & $<-->$ & $\mathrm{H} 2+\mathrm{C} 10 \mathrm{H} 14 \mathrm{O} 3$ \\
\hline 618 & 140.584 & MIN & 233 & $<-->$ & MIN 380 \\
\hline
\end{tabular}

NETWORK OF THE AMK-stdMD DATABASE. FAMILIES OF CONFORMERS FORM A NODE

\begin{tabular}{|c|c|c|c|c|c|c|}
\hline TS \# & $\mathrm{DE}(\mathrm{kcal} / \mathrm{mol})$ & \multicolumn{5}{|c|}{----- Path info------- } \\
\hline 2 & -67.045 & MIN & 2 & $<-->$ & $\mathrm{H} 2+\mathrm{C}$ & $\mathrm{OH} 1403$ \\
\hline 5 & -57.221 & MIN & 4 & $<-->$ & MIN & 8 \\
\hline 9 & -53.212 & MIN & 3 & $<-->$ & $\mathrm{H} 2 \mathrm{O}$ & $10 \mathrm{H} 1402$ \\
\hline 16 & -35.209 & MIN & 6 & $<-->$ & $\mathrm{CH} 2 \mathrm{C}$ & $-\mathrm{CH} 4+\mathrm{C} 8 \mathrm{H} 10 \mathrm{O} 2$ \\
\hline 17 & -34.760 & MIN & 7 & $<-->$ & MIN & 36 \\
\hline 26 & -15.715 & MIN & 20 & $<-->$ & MIN & 82 \\
\hline 27 & -13.846 & MIN & 20 & $<-->$ & MIN & 82 \\
\hline 29 & -12.499 & MIN & 135 & $<-->$ & $\mathrm{O} 2+\mathrm{C}$ & $0 \mathrm{H} 16 \mathrm{O}$ \\
\hline 30 & $-12 \cdot 316$ & MIN & 20 & $<-->$ & MIN & 82 \\
\hline 32 & -11.654 & MIN & 37 & $<-->$ & MIN & 97 \\
\hline 33 & -10.679 & MIN & 35 & $<-->$ & $\mathrm{H} 2+\mathrm{C}$ & $\mathrm{OH} 14 \mathrm{O} 3$ \\
\hline 34 & -10.112 & MIN & 20 & $<-->$ & MIN & 82 \\
\hline 35 & -9.986 & MIN & 37 & $<-->$ & MIN & 97 \\
\hline 36 & -9.115 & MIN & 37 & $<-->$ & MIN & 97 \\
\hline 37 & -8.988 & MIN & 32 & $<-->$ & MIN & 181 \\
\hline 38 & -5.970 & MIN & 45 & $<-->$ & MIN & 136 \\
\hline 39 & -4.745 & MIN & 14 & $<-->$ & $\mathrm{H} 2+\mathrm{C}$ & $\mathrm{OH} 14 \mathrm{O} 3$ \\
\hline 40 & -2.668 & MIN & 13 & $<-->$ & $\mathrm{H} 2+\mathrm{C}$ & $\mathrm{OH} 14 \mathrm{O} 3$ \\
\hline 41 & -1.573 & MIN & 14 & $<-->$ & $\mathrm{H} 2+\mathrm{C}$ & OH1 403 \\
\hline 42 & -1.328 & MIN & 47 & $<-->$ & $\mathrm{H} 2+\mathrm{C}$ & $\mathrm{OH} 1403$ \\
\hline 43 & -1.164 & MIN & 81 & $<-->$ & $\mathrm{O} 2+\mathrm{C}$ & $0 \mathrm{H} 160$ \\
\hline 45 & -0.674 & MIN & 28 & $<-->$ & $\mathrm{H} 2+\mathrm{C}$ & $\mathrm{OH} 14 \mathrm{O} 3$ \\
\hline
\end{tabular}




\begin{tabular}{|c|c|c|c|c|c|}
\hline 46 & -0.465 & MIN & 50 & $<-->$ & MIN 97 \\
\hline 47 & -0.211 & MIN & 204 & $<-->$ & $\mathrm{O} 2+\mathrm{C} 10 \mathrm{H} 160$ \\
\hline 48 & 0.088 & MIN & 10 & $<-->$ & $\mathrm{H} 2+\mathrm{C} 10 \mathrm{H} 1403$ \\
\hline 55 & 1.298 & MIN & 11 & $<-->$ & $\mathrm{H} 2+\mathrm{C} 10 \mathrm{H} 1403$ \\
\hline 63 & 3.355 & MIN & 25 & $<-->$ & MIN 82 \\
\hline 68 & 9.224 & MIN & 40 & $<-->$ & $\operatorname{MIN}$ \\
\hline 69 & 9.802 & MIN & 43 & $<-->$ & MIN \\
\hline 70 & 10.792 & MIN & 33 & $<-->$ & $\mathrm{H} 2+\mathrm{C} 10 \mathrm{H} 1403$ \\
\hline 71 & 11.441 & MIN & 3 & $<-->$ & MIN 97 \\
\hline 72 & 11.823 & MIN & 30 & $<-->$ & MIN \\
\hline 73 & 11.902 & MIN & 65 & $<-->$ & MIN \\
\hline 74 & 12.712 & MIN & 82 & $<-->$ & MIN 131 \\
\hline 75 & 12.812 & MIN & 64 & $<-->$ & $\mathrm{C} 4 \mathrm{H} 8 \mathrm{O}+\mathrm{C} 6 \mathrm{H} 8 \mathrm{O} 2$ \\
\hline 76 & 13.303 & MIN & 66 & $<-->$ & $\mathrm{H} 2 \mathrm{O}+\mathrm{C} 10 \mathrm{H} 14 \mathrm{O} 2$ \\
\hline 77 & 15.405 & MIN & 143 & $<-->$ & $\mathrm{O} 2+\mathrm{C} 10 \mathrm{H} 160$ \\
\hline 78 & 16.691 & MIN & 38 & $<-->$ & $\mathrm{H} 2+\mathrm{C} 10 \mathrm{H} 1403$ \\
\hline 79 & 17.766 & MIN & 57 & $<-->$ & $\mathrm{H} 2+\mathrm{C} 10 \mathrm{H} 1403$ \\
\hline 80 & 19.688 & MIN & 78 & $<-->$ & $\mathrm{H} 2 \mathrm{O}+\mathrm{C} 10 \mathrm{H} 14 \mathrm{O} 2$ \\
\hline 81 & 20.390 & MIN & 14 & $<-->$ & $\mathrm{CH} 4+\mathrm{C} 9 \mathrm{H} 12 \mathrm{O} 3$ \\
\hline 83 & 20.816 & MIN & 134 & $<-->$ & MIN 224 \\
\hline 86 & 21.262 & MIN & 58 & $<-->$ & MIN 74 \\
\hline 87 & 21.550 & MIN & 248 & $<-->$ & $\mathrm{C} 4 \mathrm{H} 6 \mathrm{O}+\mathrm{C} 6 \mathrm{H} 10 \mathrm{O} 2$ \\
\hline 88 & 21.820 & MIN & 97 & $<-->$ & MIN 232 \\
\hline 89 & 21.966 & MIN & 82 & $<-->$ & MIN 232 \\
\hline 90 & 22.144 & MIN & 249 & $<-->$ & $\mathrm{C} 4 \mathrm{H} 6 \mathrm{O} 2+\mathrm{C} 6 \mathrm{H} 10 \mathrm{O}$ \\
\hline 91 & 22.654 & MIN & 248 & $<-->$ & $\mathrm{C} 4 \mathrm{H} 6 \mathrm{O} 2+\mathrm{C} 6 \mathrm{H} 10 \mathrm{O}$ \\
\hline 92 & 22.775 & MIN & 248 & $<-->$ & $\mathrm{C} 4 \mathrm{H} 6 \mathrm{O}+\mathrm{C} 6 \mathrm{H} 10 \mathrm{O} 2$ \\
\hline 93 & 23.135 & MIN & 97 & $<-->$ & MIN 232 \\
\hline 94 & 23.536 & MIN & 47 & $<-->$ & $\mathrm{H} 2+\mathrm{C} 10 \mathrm{H} 1403$ \\
\hline 95 & 23.985 & MIN & 82 & $<-->$ & MIN 232 \\
\hline 96 & 24.146 & MIN & 157 & $<-->$ & $\mathrm{O} 2+\mathrm{C} 10 \mathrm{H} 160$ \\
\hline 97 & 24.215 & MIN & 249 & $<-->$ & $\mathrm{C} 4 \mathrm{H} 6 \mathrm{O} 2+\mathrm{C} 6 \mathrm{H} 10 \mathrm{O}$ \\
\hline 98 & 24.381 & MIN & 52 & $<-->$ & $\mathrm{H} 2+\mathrm{C} 3 \mathrm{H} 4+\mathrm{C} 7 \mathrm{H} 1003$ \\
\hline 99 & 24.829 & MIN & 57 & $<-->$ & $\mathrm{H} 2+\mathrm{C} 10 \mathrm{H} 1403$ \\
\hline 100 & 24.881 & MIN & 94 & $<-->$ & $\mathrm{C} 4 \mathrm{H} 6 \mathrm{O}+\mathrm{C} 6 \mathrm{H} 10 \mathrm{O} 2$ \\
\hline 101 & 24.918 & MIN & 62 & $<-->$ & $\mathrm{H} 2+\mathrm{C} 10 \mathrm{H} 1403$ \\
\hline 102 & 25.198 & MIN & 249 & $<-->$ & $\mathrm{C} 4 \mathrm{H} 6 \mathrm{O}+\mathrm{C} 6 \mathrm{H} 10 \mathrm{O} 2$ \\
\hline 103 & 25.222 & MIN & 97 & $<-->$ & $\mathrm{H} 2+\mathrm{C} 10 \mathrm{H} 1403$ \\
\hline 104 & 26.445 & MIN & 124 & $<-->$ & $\mathrm{H} 2+\mathrm{C} 10 \mathrm{H} 1403$ \\
\hline 106 & 26.679 & MIN & 68 & $<-->$ & $\mathrm{H} 2+\mathrm{C} 10 \mathrm{H} 1403$ \\
\hline 107 & 26.834 & MIN & 62 & $<-->$ & $\mathrm{H} 2+\mathrm{C} 10 \mathrm{H} 1403$ \\
\hline 108 & 26.926 & MIN & 50 & $<-->$ & MIN 242 \\
\hline 109 & 27.177 & MIN & 249 & $<-->$ & $\mathrm{C} 4 \mathrm{H} 6 \mathrm{O}+\mathrm{C} 6 \mathrm{H} 10 \mathrm{O} 2$ \\
\hline 110 & 27.422 & MIN & 201 & $<-->$ & $\mathrm{H} 2+\mathrm{C} 10 \mathrm{H} 1403$ \\
\hline 111 & 27.858 & MIN & 97 & $<-->$ & $\mathrm{H} 2+\mathrm{C} 10 \mathrm{H} 1403$ \\
\hline 112 & 29.401 & MIN & 97 & $<-->$ & $\mathrm{H} 2+\mathrm{C} 10 \mathrm{H} 1403$ \\
\hline 113 & 29.922 & MIN & 82 & $<-->$ & MIN 141 \\
\hline 114 & 30.697 & MIN & 239 & $<-->$ & $\mathrm{C} 3 \mathrm{H} 6 \mathrm{O} 2+\mathrm{C} 7 \mathrm{H} 10 \mathrm{O}$ \\
\hline 115 & 30.785 & MIN & 75 & $<-->$ & MIN 351 \\
\hline 116 & 31.112 & MIN & 82 & $<-->$ & $\mathrm{O} 2+\mathrm{C} 10 \mathrm{H} 16 \mathrm{O}$ \\
\hline 117 & 32.172 & MIN & 33 & $<-->$ & $\mathrm{O} 2+\mathrm{C} 10 \mathrm{H} 160$ \\
\hline 119 & 34.454 & MIN & 80 & $<-->$ & $\mathrm{H} 2+\mathrm{C} 10 \mathrm{H} 1403$ \\
\hline 120 & 34.547 & MIN & 71 & $<-->$ & MIN 379 \\
\hline 123 & 35.651 & MIN & 125 & $<-->$ & $\mathrm{H} 2+\mathrm{C} 10 \mathrm{H} 1403$ \\
\hline 124 & 35.816 & MIN & 82 & $<-->$ & $\mathrm{H} 2+\mathrm{C} 10 \mathrm{H} 1403$ \\
\hline 126 & 36.949 & MIN & 191 & $<-->$ & MIN 243 \\
\hline 127 & 37.118 & MIN & 144 & $<-->$ & MIN \\
\hline 128 & 37.422 & MIN & 1 & $<-->$ & MIN \\
\hline 129 & 37.965 & MIN & 42 & $<-->$ & MIN \\
\hline 131 & 38.496 & MIN & 82 & $<-->$ & MIN 102 \\
\hline 132 & 38.577 & MIN & 97 & $<-->$ & $\mathrm{CO}+\mathrm{C} 9 \mathrm{H} 16 \mathrm{O} 2$ \\
\hline 133 & 39.010 & MIN & 82 & $<-->$ & $\mathrm{H} 2+\mathrm{C} 10 \mathrm{H} 14 \mathrm{O} 3$ \\
\hline 134 & 39.147 & MIN & 97 & $<-->$ & $\mathrm{H} 2+\mathrm{O} 2+\mathrm{C} 10 \mathrm{H} 14 \mathrm{O}$ \\
\hline 135 & 39.318 & MIN & 191 & $<-->$ & MIN 243 \\
\hline 136 & 39.364 & MIN & 82 & $<-->$ & $\mathrm{H} 2+\mathrm{C} 10 \mathrm{H} 1403$ \\
\hline
\end{tabular}




\begin{tabular}{|c|c|c|c|c|c|}
\hline 137 & 39.405 & MIN & 203 & $<-->$ & $\mathrm{O} 2+\mathrm{C} 10 \mathrm{H} 160$ \\
\hline 138 & 39.525 & MIN & 82 & $<-->$ & $\mathrm{H} 2+\mathrm{C} 10 \mathrm{H} 1403$ \\
\hline 141 & 41.149 & MIN & 46 & $<-->$ & MIN 154 \\
\hline 142 & 41.506 & MIN & 97 & $<-->$ & $\mathrm{H} 2+\mathrm{C} 10 \mathrm{H} 1403$ \\
\hline 143 & 41.830 & MIN & 70 & $<-->$ & $\mathrm{H} 2+\mathrm{C} 10 \mathrm{H} 1403$ \\
\hline 144 & 41.886 & MIN & 97 & $<-->$ & $\mathrm{H} 2+\mathrm{C} 10 \mathrm{H} 1403$ \\
\hline 145 & 42.219 & MIN & 208 & $<-->$ & MIN 305 \\
\hline 146 & 42.384 & MIN & 220 & $<-->$ & MIN \\
\hline 147 & 42.537 & MIN & 97 & $<-->$ & MIN \\
\hline 148 & 42.739 & MIN & 207 & $<-->$ & MIN \\
\hline 149 & 43.207 & MIN & 219 & $<-->$ & MIN \\
\hline 152 & 43.728 & MIN & 78 & $<-->$ & MIN \\
\hline 153 & 44.011 & MIN & 160 & $<-->$ & MIN 249 \\
\hline 154 & 44.123 & MIN & 97 & $<-->$ & $\mathrm{H} 2+\mathrm{C} 10 \mathrm{H} 1403$ \\
\hline 155 & 44.217 & MIN & 9 & $<-->$ & MIN 158 \\
\hline 156 & 44.742 & MIN & 232 & $<-->$ & MIN 316 \\
\hline 157 & 44.976 & MIN & 291 & $<-->$ & $\mathrm{H} 2+\mathrm{C} 10 \mathrm{H} 1403$ \\
\hline 158 & 44.980 & MIN & 390 & $<-->$ & $\mathrm{O} 2+\mathrm{C} 10 \mathrm{H} 16 \mathrm{O}$ \\
\hline 159 & 45.372 & MIN & 245 & $<-->$ & MIN 385 \\
\hline 162 & 45.796 & MIN & 130 & $<-->$ & $\mathrm{H} 2+\mathrm{C} 10 \mathrm{H} 1403$ \\
\hline 163 & 45.837 & MIN & 372 & $<-->$ & $\mathrm{CH} 4+\mathrm{C} 9 \mathrm{H} 12 \mathrm{O} 3$ \\
\hline 164 & 45.915 & MIN & 97 & $<-->$ & $\mathrm{H} 2+\mathrm{C} 10 \mathrm{H} 1403$ \\
\hline 166 & 46.035 & MIN & 82 & $<-->$ & MIN 385 \\
\hline 167 & 46.190 & MIN & 150 & $<-->$ & $\mathrm{H} 2+\mathrm{C} 10 \mathrm{H} 1403$ \\
\hline 168 & 46.323 & MIN & 18 & $<-->$ & $\mathrm{CH} 2+\mathrm{C} 9 \mathrm{H} 1403$ \\
\hline 169 & 46.781 & MIN & 232 & $<-->$ & MIN 315 \\
\hline 170 & 46.824 & MIN & 47 & $<-->$ & $\mathrm{C} 3 \mathrm{H} 6+\mathrm{C} 7 \mathrm{H} 1003$ \\
\hline 171 & 47.715 & MIN & 249 & $<-->$ & MIN 337 \\
\hline 172 & 47.803 & MIN & 232 & $<-->$ & MIN \\
\hline 174 & 48.154 & MIN & 95 & $<-->$ & MIN 384 \\
\hline 175 & 48.284 & MIN & 151 & $<-->$ & $\mathrm{H} 2+\mathrm{C} 10 \mathrm{H} 1403$ \\
\hline 176 & 48.442 & MIN & 171 & $<-->$ & MIN 249 \\
\hline 177 & 48.567 & MIN & 249 & $<-->$ & $\mathrm{H} 2+\mathrm{C} 10 \mathrm{H} 1403$ \\
\hline 178 & 48.780 & MIN & 377 & $<-->$ & $\mathrm{O} 2+\mathrm{C} 10 \mathrm{H} 160$ \\
\hline 179 & 49.262 & MIN & 56 & $<-->$ & $\mathrm{C} 4 \mathrm{H} 6+\mathrm{C} 6 \mathrm{H} 1003$ \\
\hline 180 & 49.278 & MIN & 38 & $<-->$ & MIN 234 \\
\hline 181 & 49.614 & MIN & 249 & $<-->$ & MIN 320 \\
\hline 182 & 50.498 & MIN & 318 & $<-->$ & $\mathrm{O} 2+\mathrm{C} 10 \mathrm{H} 160$ \\
\hline 183 & 51.498 & MIN & 162 & $<-->$ & $\mathrm{H} 2+\mathrm{C} 10 \mathrm{H} 1403$ \\
\hline 184 & 51.600 & MIN & 97 & $<-->$ & $\mathrm{H} 2+\mathrm{C} 10 \mathrm{H} 1403$ \\
\hline 185 & 51.904 & MIN & 140 & $<-->$ & $\mathrm{H} 2+\mathrm{C} 10 \mathrm{H} 14 \mathrm{O} 3$ \\
\hline 186 & 52.357 & MIN & 171 & $<-->$ & $\mathrm{H} 2+\mathrm{C} 10 \mathrm{H} 1403$ \\
\hline 189 & 52.714 & MIN & 24 & $<-->$ & MIN 26 \\
\hline 192 & 53.070 & MIN & 12 & $<-->$ & MIN 219 \\
\hline 195 & 53.264 & MIN & 318 & $<-->$ & $\mathrm{O} 2+\mathrm{C} 10 \mathrm{H} 16 \mathrm{O}$ \\
\hline 196 & 53.277 & MIN & 189 & $<-->$ & $\mathrm{C} 3 \mathrm{H} 4 \mathrm{O} 3+\mathrm{C} 7 \mathrm{H} 12$ \\
\hline 197 & 53.326 & MIN & 154 & $<-->$ & MIN 232 \\
\hline 198 & 53.395 & MIN & 171 & $<-->$ & $\mathrm{H} 2+\mathrm{C} 10 \mathrm{H} 1403$ \\
\hline 200 & 54.055 & MIN & 171 & $<-->$ & $\mathrm{H} 2+\mathrm{C} 10 \mathrm{H} 1403$ \\
\hline 201 & 54.661 & MIN & 211 & $<-->$ & MIN 340 \\
\hline 202 & 54.736 & MIN & 203 & $<-->$ & $\mathrm{H} 2+\mathrm{C} 10 \mathrm{H} 1403$ \\
\hline 203 & 55.012 & MIN & 383 & $<-->$ & $\mathrm{O} 2+\mathrm{C} 10 \mathrm{H} 160$ \\
\hline 204 & 55.071 & MIN & 171 & $<-->$ & $\mathrm{H} 2+\mathrm{C} 10 \mathrm{H} 1403$ \\
\hline 205 & 55.095 & MIN & 171 & $<-->$ & $\mathrm{H} 2+\mathrm{C} 10 \mathrm{H} 1403$ \\
\hline 206 & 55.277 & MIN & 232 & $<-->$ & $\mathrm{O} 2+\mathrm{C} 10 \mathrm{H} 160$ \\
\hline 207 & 55.510 & MIN & 232 & $<-->$ & $\mathrm{O} 2+\mathrm{C} 10 \mathrm{H} 16 \mathrm{O}$ \\
\hline 208 & 55.703 & MIN & 200 & $<-->$ & MIN 306 \\
\hline 210 & 55.780 & MIN & 232 & $<-->$ & $\mathrm{O} 2+\mathrm{C} 10 \mathrm{H} 160$ \\
\hline 211 & 55.797 & MIN & 232 & $<-->$ & $\mathrm{H} 2+\mathrm{C} 10 \mathrm{H} 1403$ \\
\hline 212 & 55.809 & MIN & 411 & $<-->$ & $\mathrm{C} 3 \mathrm{H} 4 \mathrm{O} 3+\mathrm{C} 7 \mathrm{H} 12$ \\
\hline 214 & 55.858 & MIN & 14 & $<-->$ & MIN 232 \\
\hline 217 & 56.230 & MIN & 248 & $<-->$ & $\mathrm{H} 2+\mathrm{C} 10 \mathrm{H} 1403$ \\
\hline 218 & 56.418 & MIN & 232 & $<-->$ & $\mathrm{H} 2+\mathrm{C} 10 \mathrm{H} 1403$ \\
\hline 219 & 56.439 & MIN & 191 & $<-->$ & $\mathrm{H} 2+\mathrm{C} 10 \mathrm{H} 1403$ \\
\hline 220 & 56.532 & MIN & 178 & $<-->$ & $\mathrm{H} 2+\mathrm{C} 10 \mathrm{H} 1403$ \\
\hline
\end{tabular}




\begin{tabular}{|c|c|c|c|c|c|}
\hline 221 & 56.609 & MIN & 58 & $<-->$ & $\mathrm{C} 3 \mathrm{H} 6+\mathrm{C} 7 \mathrm{H} 1003$ \\
\hline 222 & 57.339 & MIN & 328 & $<-->$ & $\mathrm{C} 4 \mathrm{H} 6 \mathrm{O} 2+\mathrm{C} 6 \mathrm{H} 10 \mathrm{O}$ \\
\hline 223 & 57.691 & MIN & 249 & $<-->$ & $\mathrm{H} 2+\mathrm{C} 10 \mathrm{H} 1403$ \\
\hline 224 & 57.974 & MIN & 120 & $<-->$ & $\mathrm{H} 2+\mathrm{C} 10 \mathrm{H} 1403$ \\
\hline 225 & 58.281 & MIN & 171 & $<-->$ & $\mathrm{H} 2+\mathrm{C} 10 \mathrm{H} 1403$ \\
\hline 226 & 59.091 & MIN & 49 & $<-->$ & MIN 97 \\
\hline 27 & 59.111 & MIN & 14 & $<-->$ & MIN 232 \\
\hline 28 & 59.180 & MIN & 232 & $<-->$ & $\mathrm{O} 3+\mathrm{C} 10 \mathrm{H} 16$ \\
\hline 229 & 59.340 & MIN & 132 & $<-->$ & MIN 392 \\
\hline 231 & 59.782 & MIN & 171 & $<-->$ & $\mathrm{H} 2+\mathrm{C} 10 \mathrm{H} 1403$ \\
\hline 232 & 59.916 & MIN & 232 & $<-->$ & $\mathrm{H} 2+\mathrm{C} 10 \mathrm{H} 1403$ \\
\hline 234 & 60.233 & MIN & 248 & $<-->$ & $\mathrm{H} 2+\mathrm{C} 10 \mathrm{H} 1403$ \\
\hline 236 & 60.455 & MIN & 328 & $<-->$ & $\mathrm{C} 4 \mathrm{H} 6 \mathrm{O} 2+\mathrm{C} 6 \mathrm{H} 10 \mathrm{O}$ \\
\hline 237 & 60.460 & MIN & 309 & $<-->$ & $\mathrm{H} 2+\mathrm{C} 10 \mathrm{H} 1403$ \\
\hline 238 & 60.619 & MIN & 171 & $<-->$ & MIN 224 \\
\hline 239 & 60.645 & MIN & 232 & $<-->$ & $\mathrm{H} 2+\mathrm{C} 10 \mathrm{H} 1403$ \\
\hline 240 & 60.822 & MIN & 19 & $<-->$ & MIN 226 \\
\hline 241 & 61.224 & MIN & 309 & $<-->$ & $\mathrm{H} 2+\mathrm{C} 10 \mathrm{H} 14 \mathrm{O} 3$ \\
\hline 243 & 61.746 & MIN & 127 & $<-->$ & $\mathrm{C} 2 \mathrm{H} 4 \mathrm{O}+\mathrm{C} 8 \mathrm{H} 12 \mathrm{O} 2$ \\
\hline 244 & 62.209 & MIN & 232 & $<-->$ & $\mathrm{H} 2+\mathrm{C} 10 \mathrm{H} 1403$ \\
\hline 246 & 62.345 & MIN & 250 & $<-->$ & MIN 395 \\
\hline 247 & 62.836 & MIN & 208 & $<-->$ & $\mathrm{H} 2+\mathrm{C} 10 \mathrm{H} 1403$ \\
\hline 249 & 62.896 & MIN & 232 & $<-->$ & $\mathrm{H} 2+\mathrm{C} 10 \mathrm{H} 1403$ \\
\hline 250 & 63.018 & MIN & 232 & $<-->$ & $\mathrm{H} 2+\mathrm{C} 10 \mathrm{H} 1403$ \\
\hline 251 & 63.192 & MIN & 77 & $<-->$ & MIN 142 \\
\hline 252 & 63.266 & MIN & 171 & $<-->$ & MIN 249 \\
\hline 254 & 63.627 & MIN & 208 & $<-->$ & $\mathrm{H} 2+\mathrm{C} 10 \mathrm{H} 1403$ \\
\hline 257 & 64.352 & MIN & 232 & $<-->$ & $\mathrm{H} 2+\mathrm{C} 10 \mathrm{H} 1403$ \\
\hline 258 & 64.353 & MIN & 194 & $<-->$ & $\mathrm{H} 2+\mathrm{C} 3 \mathrm{H} 4+\mathrm{C} 7 \mathrm{H} 10 \mathrm{C}$ \\
\hline 260 & 64.413 & MIN & 410 & $<-->$ & MIN 404 \\
\hline 261 & 64.973 & MIN & 232 & $<-->$ & $\mathrm{H} 2+\mathrm{C} 10 \mathrm{H} 1403$ \\
\hline 262 & 65.046 & MIN & 232 & $<-->$ & $\mathrm{H} 2+\mathrm{C} 10 \mathrm{H} 14 \mathrm{O} 3$ \\
\hline 263 & 65.060 & MIN & 248 & $<-->$ & $\mathrm{H} 2+\mathrm{C} 10 \mathrm{H} 1403$ \\
\hline 265 & 65.490 & MIN & 232 & $<-->$ & $\mathrm{H} 2+\mathrm{C} 10 \mathrm{H} 1403$ \\
\hline 266 & 65.594 & MIN & 249 & $<-->$ & $\mathrm{H} 2+\mathrm{C} 10 \mathrm{H} 1403$ \\
\hline 267 & 65.694 & MIN & 232 & $<-->$ & $\mathrm{H} 2+\mathrm{C} 10 \mathrm{H} 1403$ \\
\hline 268 & 65.731 & MIN & 62 & $<-->$ & MIN 232 \\
\hline 270 & 65.799 & MIN & 232 & $<-->$ & $\mathrm{O} 2+\mathrm{C} 10 \mathrm{H} 16 \mathrm{O}$ \\
\hline 271 & 65.845 & MIN & 138 & $<-->$ & MIN 400 \\
\hline 272 & 65.896 & MIN & 147 & $<-->$ & $\mathrm{CH} 4+\mathrm{C} 9 \mathrm{H} 12 \mathrm{O} 3$ \\
\hline 275 & 66.062 & MIN & 232 & $<-->$ & $\mathrm{H} 2+\mathrm{C} 10 \mathrm{H} 1403$ \\
\hline 276 & 66.144 & MIN & 232 & $<-->$ & $\mathrm{H} 2+\mathrm{C} 10 \mathrm{H} 1403$ \\
\hline 277 & 66.150 & MIN & 135 & $<-->$ & MIN 394 \\
\hline 278 & 66.252 & MIN & 232 & $<-->$ & $\mathrm{H} 2+\mathrm{C} 10 \mathrm{H} 1403$ \\
\hline 280 & 66.317 & MIN & 248 & $<-->$ & $\mathrm{H} 2+\mathrm{C} 10 \mathrm{H} 1403$ \\
\hline 282 & 66.343 & MIN & 232 & $<-->$ & $\mathrm{H} 2+\mathrm{C} 10 \mathrm{H} 1403$ \\
\hline 285 & 66.613 & MIN & 80 & $<-->$ & MIN 232 \\
\hline 286 & 66.655 & MIN & 232 & $<-->$ & $\mathrm{H} 2+\mathrm{C} 10 \mathrm{H} 1403$ \\
\hline 290 & 66.819 & MIN & 232 & $<-->$ & $\mathrm{H} 2+\mathrm{C} 10 \mathrm{H} 1403$ \\
\hline 291 & 66.856 & MIN & 147 & $<-->$ & $\mathrm{CH} 4+\mathrm{C} 9 \mathrm{H} 12 \mathrm{O} 3$ \\
\hline 292 & 66.947 & MIN & 136 & $<-->$ & MIN 406 \\
\hline 293 & 67.030 & MIN & 229 & $<-->$ & $\mathrm{H} 2+\mathrm{C} 10 \mathrm{H} 1403$ \\
\hline 294 & 67.031 & MIN & 145 & $<-->$ & MIN 408 \\
\hline 295 & 67.056 & MIN & 249 & $<-->$ & $\mathrm{H} 2+\mathrm{C} 10 \mathrm{H} 1403$ \\
\hline 296 & 67.078 & MIN & 249 & $<-->$ & $\mathrm{H} 2+\mathrm{C} 10 \mathrm{H} 1403$ \\
\hline 297 & 67.084 & MIN & 221 & $<-->$ & $\mathrm{H} 2+\mathrm{C} 10 \mathrm{H} 1403$ \\
\hline 298 & 67.154 & MIN & 232 & $<-->$ & $\mathrm{H} 2+\mathrm{C} 10 \mathrm{H} 1403$ \\
\hline 302 & 67.321 & MIN & 254 & $<-->$ & $\mathrm{H} 2+\mathrm{C} 10 \mathrm{H} 1403$ \\
\hline 303 & 67.408 & MIN & 248 & $<-->$ & $\mathrm{H} 2+\mathrm{C} 10 \mathrm{H} 1403$ \\
\hline 305 & 67.581 & MIN & 232 & $<-->$ & $\mathrm{H} 2+\mathrm{C} 10 \mathrm{H} 1403$ \\
\hline 307 & 67.829 & MIN & 232 & $<-->$ & MIN 370 \\
\hline 308 & 67.959 & MIN & 144 & $<-->$ & MIN 183 \\
\hline 309 & 67.989 & MIN & 232 & $<-->$ & $\mathrm{H} 2+\mathrm{C} 10 \mathrm{H} 1403$ \\
\hline 311 & 68.053 & MIN & 97 & $<-->$ & $\mathrm{H} 2+\mathrm{O} 2+\mathrm{C} 10 \mathrm{H} 14 \mathrm{O}$ \\
\hline 312 & 68.099 & MIN & 232 & $<-->$ & $\mathrm{H} 2+\mathrm{C} 10 \mathrm{H} 1403$ \\
\hline
\end{tabular}




\begin{tabular}{|c|c|c|c|c|c|}
\hline 316 & 68.195 & MIN & 219 & $<-->$ & $\mathrm{H} 2+\mathrm{C} 10 \mathrm{H} 1403$ \\
\hline 317 & 68.276 & MIN & 248 & $<-->$ & $\mathrm{H} 2+\mathrm{C} 10 \mathrm{H} 1403$ \\
\hline 319 & 68.318 & MIN & 249 & $<-->$ & $\mathrm{H} 2+\mathrm{C} 10 \mathrm{H} 1403$ \\
\hline 320 & 68.350 & MIN & 232 & $<-->$ & $\mathrm{H} 2+\mathrm{C} 10 \mathrm{H} 1403$ \\
\hline 321 & 68.411 & MIN & 232 & $<-->$ & $\mathrm{H} 2+\mathrm{C} 10 \mathrm{H} 1403$ \\
\hline 324 & 68.656 & MIN & 219 & $<-->$ & $\mathrm{H} 2+\mathrm{C} 10 \mathrm{H} 1403$ \\
\hline 326 & 68.691 & MIN & 82 & $<-->$ & $\mathrm{O} 2+\mathrm{C} 10 \mathrm{H} 160$ \\
\hline 327 & 68.757 & MIN & 249 & $<-->$ & $\mathrm{H} 2+\mathrm{C} 10 \mathrm{H} 1403$ \\
\hline 328 & 68.787 & MIN & 248 & $<-->$ & $\mathrm{H} 2+\mathrm{C} 10 \mathrm{H} 1403$ \\
\hline 329 & 68.820 & MIN & 82 & $<-->$ & $\mathrm{C} 3 \mathrm{H} 6+\mathrm{C} 7 \mathrm{H} 1003$ \\
\hline 332 & 69.030 & MIN & 232 & $<-->$ & $\mathrm{H} 2+\mathrm{C} 10 \mathrm{H} 1403$ \\
\hline 333 & 69.045 & MIN & 232 & $<-->$ & $\mathrm{H} 2+\mathrm{C} 10 \mathrm{H} 1403$ \\
\hline 334 & 69.064 & MIN & 232 & $<-->$ & $\mathrm{H} 2+\mathrm{C} 10 \mathrm{H} 1403$ \\
\hline 335 & 69.072 & MIN & 232 & $<-->$ & $\mathrm{H} 2+\mathrm{C} 10 \mathrm{H} 1403$ \\
\hline 336 & 69.222 & MIN & 381 & $<-->$ & $\mathrm{H} 2+\mathrm{C} 10 \mathrm{H} 1403$ \\
\hline 338 & 69.251 & MIN & 232 & $<-->$ & $\mathrm{H} 2+\mathrm{C} 10 \mathrm{H} 1403$ \\
\hline 339 & 69.308 & MIN & 232 & $<-->$ & $\mathrm{H} 2+\mathrm{C} 10 \mathrm{H} 1403$ \\
\hline 340 & 69.361 & MIN & 232 & $<-->$ & $\mathrm{H} 2+\mathrm{C} 10 \mathrm{H} 1403$ \\
\hline 341 & 69.463 & MIN & 248 & $<-->$ & $\mathrm{CO}+\mathrm{C} 3 \mathrm{H} 6+\mathrm{C} 6 \mathrm{H} 10$ \\
\hline 343 & 70.022 & MIN & 149 & $<-->$ & MIN 396 \\
\hline 344 & 70.073 & MIN & 232 & $<-->$ & $\mathrm{H} 2+\mathrm{C} 10 \mathrm{H} 1403$ \\
\hline 345 & 70.109 & MIN & 232 & $<-->$ & $\mathrm{H} 2+\mathrm{C} 10 \mathrm{H} 1403$ \\
\hline 346 & 70.184 & MIN & 226 & $<-->$ & $\mathrm{H} 2+\mathrm{C} 10 \mathrm{H} 1403$ \\
\hline 347 & 70.269 & MIN & 232 & $<-->$ & $\mathrm{H} 2+\mathrm{C} 10 \mathrm{H} 1403$ \\
\hline 348 & 70.371 & MIN & 232 & $<-->$ & $\mathrm{H} 2+\mathrm{C} 10 \mathrm{H} 1403$ \\
\hline 350 & 70.393 & MIN & 226 & $<-->$ & $\mathrm{H} 2+\mathrm{C} 10 \mathrm{H} 1403$ \\
\hline 351 & 70.424 & MIN & 232 & $<-->$ & $\mathrm{H} 2+\mathrm{C} 10 \mathrm{H} 1403$ \\
\hline 353 & 70.750 & MIN & 232 & $<-->$ & MIN 329 \\
\hline 354 & 70.853 & MIN & 169 & $<-->$ & $\mathrm{CH} 4+\mathrm{C} 9 \mathrm{H} 12 \mathrm{O} 3$ \\
\hline 356 & 71.197 & MIN & 168 & $<-->$ & MIN 421 \\
\hline 358 & 71.369 & MIN & 232 & $<-->$ & $\mathrm{H} 2+\mathrm{C} 10 \mathrm{H} 1403$ \\
\hline 361 & 71.529 & MIN & 386 & $<-->$ & MIN 404 \\
\hline 362 & 71.691 & MIN & 219 & $<-->$ & MIN 409 \\
\hline 363 & 71.911 & MIN & 232 & $<-->$ & $\mathrm{H} 2+\mathrm{C} 10 \mathrm{H} 1403$ \\
\hline 364 & 71.978 & MIN & 301 & $<-->$ & $\mathrm{H} 2+\mathrm{C} 10 \mathrm{H} 1403$ \\
\hline 365 & 72.113 & MIN & 397 & $<-->$ & $\mathrm{H} 2+\mathrm{C} 10 \mathrm{H} 1403$ \\
\hline 366 & 72.182 & MIN & 232 & $<-->$ & $\mathrm{H} 2+\mathrm{O} 2+\mathrm{C} 10 \mathrm{H} 14 \mathrm{O}$ \\
\hline 367 & 72.187 & MIN & 225 & $<-->$ & $\mathrm{O} 2+\mathrm{C} 10 \mathrm{H} 160$ \\
\hline 368 & 72.202 & MIN & 97 & $<-->$ & $\mathrm{C} 2 \mathrm{H} 4 \mathrm{O} 2+\mathrm{C} 8 \mathrm{H} 12 \mathrm{O}$ \\
\hline 369 & 72.224 & MIN & 329 & $<-->$ & $\mathrm{H} 2+\mathrm{C} 10 \mathrm{H} 1403$ \\
\hline 370 & 72.301 & MIN & 72 & $<-->$ & MIN 367 \\
\hline 372 & 72.648 & MIN & 398 & $<-->$ & $\mathrm{H} 2+\mathrm{C} 10 \mathrm{H} 1403$ \\
\hline 375 & 73.131 & MIN & 232 & $<-->$ & MIN 400 \\
\hline 376 & 73.251 & MIN & 168 & $<-->$ & $\operatorname{MIN} \quad 421$ \\
\hline 378 & 73.446 & MIN & 315 & $<-->$ & $\mathrm{H} 2+\mathrm{C} 10 \mathrm{H} 1403$ \\
\hline 379 & 73.650 & MIN & 309 & $<-->$ & $\mathrm{H} 2+\mathrm{C} 10 \mathrm{H} 1403$ \\
\hline 381 & 73.799 & MIN & 331 & $<-->$ & $\mathrm{H} 2+\mathrm{C} 10 \mathrm{H} 1403$ \\
\hline 382 & 73.884 & MIN & 354 & $<-->$ & $\mathrm{O} 2+\mathrm{C} 10 \mathrm{H} 160$ \\
\hline 383 & 73.896 & MIN & 170 & $<-->$ & $\mathrm{CH} 4+\mathrm{C} 9 \mathrm{H} 12 \mathrm{O} 3$ \\
\hline 384 & 74.066 & MIN & 97 & $<-->$ & MIN 442 \\
\hline 385 & 74.446 & MIN & 232 & $<-->$ & MIN 398 \\
\hline 386 & 74.675 & MIN & 170 & $<-->$ & $\mathrm{CH} 4+\mathrm{C} 9 \mathrm{H} 12 \mathrm{O} 3$ \\
\hline 387 & 75.046 & MIN & 232 & $<-->$ & $\mathrm{H} 2+\mathrm{C} 10 \mathrm{H} 1403$ \\
\hline 388 & 75.091 & MIN & 232 & $<-->$ & MIN 409 \\
\hline 389 & 75.108 & MIN & 232 & $<-->$ & MIN \\
\hline 390 & 75.374 & MIN & 168 & $<-->$ & MIN 421 \\
\hline 392 & 75.838 & MIN & 254 & $<-->$ & $\mathrm{H} 2+\mathrm{C} 10 \mathrm{H} 1403$ \\
\hline 393 & 76.257 & MIN & 219 & $<-->$ & MIN 417 \\
\hline 394 & 76.827 & MIN & 171 & $<-->$ & MIN \\
\hline 395 & 77.158 & MIN & 67 & $<-->$ & MIN 362 \\
\hline 396 & 77.195 & MIN & 316 & $<-->$ & $\mathrm{H} 2+\mathrm{C} 10 \mathrm{H} 1403$ \\
\hline 397 & 77.392 & MIN & 316 & $<-->$ & $\mathrm{H} 2+\mathrm{C} 10 \mathrm{H} 1403$ \\
\hline 398 & 77.403 & MIN & 316 & $<-->$ & $\mathrm{H} 2+\mathrm{C} 10 \mathrm{H} 1403$ \\
\hline 399 & 77.776 & MIN & 249 & $<-->$ & MIN 457 \\
\hline 0 & 77.986 & MIN & 171 & $<-->$ & $\mathrm{H} 2+\mathrm{C} 10 \mathrm{H} 1403$ \\
\hline
\end{tabular}




\begin{tabular}{|c|c|c|c|c|c|}
\hline 401 & 78.042 & MIN & 237 & $<-->$ & $\mathrm{H} 2+\mathrm{C} 10 \mathrm{H} 1403$ \\
\hline 402 & 78.048 & MIN & 171 & $<-->$ & $\mathrm{H} 2+\mathrm{C} 10 \mathrm{H} 1403$ \\
\hline 403 & 78.190 & MIN & 82 & $<-->$ & $\mathrm{CH} 2+\mathrm{C} 9 \mathrm{H} 1403$ \\
\hline 404 & 78.332 & MIN & 183 & $<-->$ & MIN 323 \\
\hline 405 & 78.451 & MIN & 327 & $<-->$ & MIN 419 \\
\hline 406 & 78.702 & MIN & 232 & $<-->$ & $\mathrm{H} 2+\mathrm{C} 10 \mathrm{H} 1403$ \\
\hline 407 & 78.746 & MIN & 232 & $<-->$ & $\mathrm{H} 2+\mathrm{C} 10 \mathrm{H} 1403$ \\
\hline 409 & 79.051 & MIN & 232 & $<-->$ & $\mathrm{H} 2+\mathrm{C} 10 \mathrm{H} 1403$ \\
\hline 410 & 79.155 & MIN & 373 & $<-->$ & $\mathrm{O} 3+\mathrm{C} 10 \mathrm{H} 16$ \\
\hline 412 & 79.209 & MIN & 222 & $<-->$ & MIN 421 \\
\hline 413 & 79.209 & MIN & 226 & $<-->$ & $\mathrm{H} 2+\mathrm{C} 10 \mathrm{H} 1403$ \\
\hline 414 & 79.311 & MIN & 206 & $<-->$ & MIN 454 \\
\hline 415 & 79.459 & MIN & 224 & $<-->$ & MIN 249 \\
\hline 419 & 80.024 & MIN & 232 & $<-->$ & $\mathrm{H} 2+\mathrm{C} 10 \mathrm{H} 1403$ \\
\hline 420 & 80.030 & MIN & 214 & $<-->$ & $\operatorname{MIN} \quad 464$ \\
\hline 421 & 80.061 & MIN & 219 & $<-->$ & $\mathrm{CH} 4+\mathrm{C} 9 \mathrm{H} 12 \mathrm{O} 3$ \\
\hline 422 & 80.149 & MIN & 335 & $<-->$ & $\mathrm{H} 2+\mathrm{C} 10 \mathrm{H} 1403$ \\
\hline 423 & 80.152 & MIN & 232 & $<-->$ & $\mathrm{H} 2+\mathrm{C} 10 \mathrm{H} 1403$ \\
\hline 424 & 80.169 & MIN & 224 & $<-->$ & MIN 249 \\
\hline 425 & 80.253 & MIN & 249 & $<-->$ & MIN 435 \\
\hline 426 & 80.346 & MIN & 226 & $<-->$ & $\mathrm{H} 2+\mathrm{C} 10 \mathrm{H} 1403$ \\
\hline 428 & 80.367 & MIN & 316 & $<-->$ & $\mathrm{H} 2+\mathrm{C} 10 \mathrm{H} 1403$ \\
\hline 429 & 80.387 & MIN & 226 & $<-->$ & $\mathrm{CH} 4+\mathrm{C} 9 \mathrm{H} 12 \mathrm{O} 3$ \\
\hline 430 & 80.398 & MIN & 232 & $<-->$ & $\mathrm{H} 2+\mathrm{C} 10 \mathrm{H} 1403$ \\
\hline 431 & 80.448 & MIN & 226 & $<-->$ & MIN 431 \\
\hline 432 & 80.465 & MIN & 249 & $<-->$ & MIN 435 \\
\hline 433 & 80.499 & MIN & 316 & $<-->$ & $\mathrm{H} 2+\mathrm{C} 10 \mathrm{H} 1403$ \\
\hline 434 & 80.659 & MIN & 226 & $<-->$ & $\mathrm{CH} 4+\mathrm{C} 9 \mathrm{H} 12 \mathrm{O} 3$ \\
\hline 435 & 80.753 & MIN & 248 & $<-->$ & $\mathrm{C} 3 \mathrm{H} 4 \mathrm{O} 3+\mathrm{C} 7 \mathrm{H} 12$ \\
\hline 436 & 80.861 & MIN & 232 & $<-->$ & $\mathrm{H} 2+\mathrm{C} 10 \mathrm{H} 1403$ \\
\hline 437 & 81.360 & MIN & 170 & $<-->$ & MIN 224 \\
\hline 438 & 81.662 & MIN & 232 & $<-->$ & MIN 421 \\
\hline 439 & 81.694 & MIN & 232 & $<-->$ & $\mathrm{H} 2+\mathrm{C} 10 \mathrm{H} 1403$ \\
\hline 440 & 81.727 & MIN & 232 & $<-->$ & $\mathrm{H} 2+\mathrm{C} 10 \mathrm{H} 1403$ \\
\hline 441 & 81.827 & MIN & 355 & $<-->$ & MIN 466 \\
\hline 443 & 82.013 & MIN & 232 & $<-->$ & $\mathrm{H} 2+\mathrm{O} 2+\mathrm{C} 10 \mathrm{H} 14 \mathrm{O}$ \\
\hline 446 & 82.795 & MIN & 355 & $<-->$ & $\mathrm{H} 2+\mathrm{C} 10 \mathrm{H} 1403$ \\
\hline 448 & 83.040 & MIN & 232 & $<-->$ & MIN 249 \\
\hline 449 & 83.270 & MIN & 232 & $<-->$ & $\mathrm{H} 2+\mathrm{C} 10 \mathrm{H} 1403$ \\
\hline 450 & 83.300 & MIN & 226 & $<-->$ & MIN 249 \\
\hline 451 & 83.307 & MIN & 147 & $<-->$ & MIN \\
\hline 452 & 83.365 & MIN & 232 & $<-->$ & $\operatorname{MIN}$ \\
\hline 453 & 83.427 & MIN & 232 & $<-->$ & MIN 418 \\
\hline 454 & 83.470 & MIN & 232 & $<-->$ & $\mathrm{H} 2+\mathrm{C} 10 \mathrm{H} 1403$ \\
\hline 455 & 83.504 & MIN & 232 & $<-->$ & MIN 430 \\
\hline 456 & 83.772 & MIN & 174 & $<-->$ & $\mathrm{CH} 4+\mathrm{C} 9 \mathrm{H} 12 \mathrm{O} 3$ \\
\hline 458 & 84.123 & MIN & 171 & $<-->$ & MIN 458 \\
\hline 459 & 84.320 & MIN & 97 & $<-->$ & $\mathrm{CH} 2+\mathrm{C} 9 \mathrm{H} 1403$ \\
\hline 460 & 84.540 & MIN & 353 & $<-->$ & $\mathrm{H} 2+\mathrm{C} 10 \mathrm{H} 1403$ \\
\hline 461 & 84.703 & MIN & 82 & $<-->$ & MIN 123 \\
\hline 462 & 84.774 & MIN & 342 & $<-->$ & $\mathrm{H} 2+\mathrm{C} 10 \mathrm{H} 1403$ \\
\hline 463 & 84.813 & MIN & 249 & $<-->$ & MIN 440 \\
\hline 467 & 85.266 & MIN & 249 & $<-->$ & $\mathrm{H} 2+\mathrm{C} 10 \mathrm{H} 1403$ \\
\hline 468 & 85.369 & MIN & 196 & $<-->$ & MIN 400 \\
\hline 470 & 85.831 & MIN & 82 & $<-->$ & $\mathrm{CH} 2+\mathrm{C} 9 \mathrm{H} 1403$ \\
\hline 471 & 85.940 & MIN & 367 & $<-->$ & $\mathrm{H} 2+\mathrm{C} 10 \mathrm{H} 1403$ \\
\hline 472 & 86.122 & MIN & 213 & $<-->$ & MIN 418 \\
\hline 474 & 86.816 & MIN & 232 & $<-->$ & MIN \\
\hline 475 & 87.512 & MIN & 249 & $<-->$ & MIN \\
\hline 477 & 88.073 & MIN & 208 & $<-->$ & MIN 468 \\
\hline 478 & 88.094 & MIN & 424 & $<-->$ & $\mathrm{H} 2+\mathrm{C} 10 \mathrm{H} 1403$ \\
\hline 479 & 88.103 & MIN & 210 & $<-->$ & MIN 232 \\
\hline 480 & 88.838 & MIN & 249 & $<-->$ & MIN 424 \\
\hline 481 & 88.926 & MIN & 167 & $<-->$ & $\mathrm{C} 3 \mathrm{H} 6+\mathrm{C} 7 \mathrm{H} 10 \mathrm{O} 3$ \\
\hline 482 & 88.942 & MIN & 327 & $<-->$ & $\mathrm{CH}_{4}+\mathrm{C} 9 \mathrm{H} 12 \mathrm{O} 3$ \\
\hline
\end{tabular}




\begin{tabular}{|c|c|c|c|c|c|}
\hline 483 & 89.030 & MIN & 82 & $<-->$ & $\mathrm{CH} 2 \mathrm{O}+\mathrm{C} 9 \mathrm{H} 14 \mathrm{O} 2$ \\
\hline 484 & 89.197 & MIN & 82 & $<-->$ & $\mathrm{CH} 2+\mathrm{C} 9 \mathrm{H} 1403$ \\
\hline 485 & 89.307 & MIN & 353 & $<-->$ & $\mathrm{H} 2+\mathrm{C} 10 \mathrm{H} 1403$ \\
\hline 486 & 89.404 & MIN & 376 & $<-->$ & $\mathrm{H} 2+\mathrm{C} 10 \mathrm{H} 1403$ \\
\hline 487 & 89.749 & MIN & 313 & $<-->$ & MIN 459 \\
\hline 489 & 89.907 & MIN & 232 & $<-->$ & $\mathrm{H} 2+\mathrm{C} 10 \mathrm{H} 1403$ \\
\hline 490 & 90.066 & MIN & 171 & $<-->$ & MIN 458 \\
\hline 492 & 90.502 & MIN & 232 & $<-->$ & MIN \\
\hline 493 & 91.266 & MIN & 232 & $<-->$ & MIN \\
\hline 494 & 91.682 & MIN & 232 & $<-->$ & MIN $\quad 460$ \\
\hline 495 & 91.732 & MIN & 182 & $<-->$ & $\mathrm{C} 3 \mathrm{H} 6+\mathrm{C} 7 \mathrm{H} 1003$ \\
\hline 496 & 92.095 & MIN & 232 & $<-->$ & $\mathrm{H} 2+\mathrm{C} 10 \mathrm{H} 1403$ \\
\hline 497 & 92.266 & MIN & 380 & $<-->$ & $\mathrm{H} 2+\mathrm{C} 10 \mathrm{H} 1403$ \\
\hline 498 & 92.391 & MIN & 232 & $<-->$ & MIN 456 \\
\hline 499 & 92.492 & MIN & 355 & $<-->$ & MIN 439 \\
\hline 500 & 92.585 & MIN & 232 & $<-->$ & $\mathrm{C} 3 \mathrm{H} 6+\mathrm{C} 7 \mathrm{H} 1003$ \\
\hline 501 & 92.587 & MIN & 232 & $<-->$ & $\mathrm{H} 2+\mathrm{C} 10 \mathrm{H} 1403$ \\
\hline 502 & 93.015 & MIN & 226 & $<-->$ & $\mathrm{CH} 4+\mathrm{C} 9 \mathrm{H} 12 \mathrm{O} 3$ \\
\hline 503 & 93.116 & MIN & 154 & $<-->$ & $\mathrm{CH} 2+\mathrm{C} 9 \mathrm{H} 1403$ \\
\hline 504 & 93.347 & MIN & 232 & $<-->$ & $\mathrm{C} 3 \mathrm{H} 6+\mathrm{C} 7 \mathrm{H} 1003$ \\
\hline 505 & 93.684 & MIN & 232 & $<-->$ & $\mathrm{C} 3 \mathrm{H} 6+\mathrm{C} 7 \mathrm{H} 1003$ \\
\hline 506 & 93.945 & MIN & 232 & $<-->$ & $\mathrm{CH} 4+\mathrm{C} 9 \mathrm{H} 12 \mathrm{O} 3$ \\
\hline 507 & 94.075 & MIN & 226 & $<-->$ & MIN 349 \\
\hline 508 & 94.231 & MIN & 159 & $<-->$ & MIN 469 \\
\hline 509 & 94.935 & MIN & 309 & $<-->$ & $\mathrm{H} 2+\mathrm{C} 10 \mathrm{H} 1403$ \\
\hline 510 & 95.271 & MIN & 232 & $<-->$ & $\mathrm{H} 2+\mathrm{C} 10 \mathrm{H} 1403$ \\
\hline 511 & 95.528 & MIN & 375 & $<-->$ & MIN 439 \\
\hline 512 & 95.650 & MIN & 232 & $<-->$ & $\operatorname{MIN} \quad 460$ \\
\hline 513 & 95.666 & MIN & 337 & $<-->$ & $\mathrm{H} 2+\mathrm{C} 10 \mathrm{H} 1403$ \\
\hline 514 & 95.676 & MIN & 328 & $<-->$ & MIN 439 \\
\hline 515 & 95.938 & MIN & 328 & $<-->$ & MIN \\
\hline 516 & 95.949 & MIN & 230 & $<-->$ & MIN 470 \\
\hline 517 & 95.968 & MIN & 171 & $<-->$ & $\mathrm{H} 2+\mathrm{C} 10 \mathrm{H} 1403$ \\
\hline 519 & 96.238 & MIN & 355 & $<-->$ & MIN 439 \\
\hline 520 & 96.298 & MIN & 232 & $<-->$ & $\mathrm{H} 2+\mathrm{C} 10 \mathrm{H} 1403$ \\
\hline 521 & 96.324 & MIN & 232 & $<-->$ & $\mathrm{C} 3 \mathrm{H} 4 \mathrm{O} 3+\mathrm{C} 7 \mathrm{H} 12$ \\
\hline 522 & 96.430 & MIN & 232 & $<-->$ & $\mathrm{H} 2+\mathrm{C} 10 \mathrm{H} 1403$ \\
\hline 523 & 96.802 & MIN & 416 & $<-->$ & $\mathrm{C} 2 \mathrm{H} 6+\mathrm{C} 8 \mathrm{H} 1003$ \\
\hline 524 & 96.825 & MIN & 232 & $<-->$ & $\mathrm{H} 2+\mathrm{C} 10 \mathrm{H} 1403$ \\
\hline 525 & 97.943 & MIN & 341 & $<-->$ & MIN 441 \\
\hline 527 & 98.732 & MIN & 385 & $<-->$ & $\mathrm{C} 2 \mathrm{H} 6+\mathrm{C} 8 \mathrm{H} 1003$ \\
\hline 528 & 98.754 & MIN & 316 & $<-->$ & $\mathrm{C} 3 \mathrm{H} 6+\mathrm{C} 7 \mathrm{H} 1003$ \\
\hline 530 & 99.160 & MIN & 309 & $<-->$ & $\mathrm{H} 2+\mathrm{C} 10 \mathrm{H} 1403$ \\
\hline 532 & 99.657 & MIN & 232 & $<-->$ & $\mathrm{CH} 4+\mathrm{C} 9 \mathrm{H} 12 \mathrm{O} 3$ \\
\hline 533 & 99.736 & MIN & 226 & $<-->$ & $\mathrm{C} 3 \mathrm{H} 6+\mathrm{C} 7 \mathrm{H} 1003$ \\
\hline 535 & 100.762 & MIN & 219 & $<-->$ & MIN 248 \\
\hline 536 & 100.857 & MIN & 382 & $<-->$ & MIN \\
\hline 537 & 101.662 & MIN & 353 & $<-->$ & MIN 364 \\
\hline 538 & 101.755 & MIN & 208 & $<-->$ & $\mathrm{CH} 2+\mathrm{C} 9 \mathrm{H} 1403$ \\
\hline 539 & 101.843 & MIN & 232 & $<-->$ & $\mathrm{CH} 4+\mathrm{C} 9 \mathrm{H} 12 \mathrm{O} 3$ \\
\hline 540 & 102.172 & MIN & 208 & $<-->$ & $\mathrm{CH} 4+\mathrm{C} 9 \mathrm{H} 12 \mathrm{O} 3$ \\
\hline 541 & 102.335 & MIN & 163 & $<-->$ & $\mathrm{CH} 4+\mathrm{C} 9 \mathrm{H} 12 \mathrm{O} 3$ \\
\hline 542 & 102.839 & MIN & 409 & $<-->$ & $\mathrm{H} 2+\mathrm{C} 10 \mathrm{H} 1403$ \\
\hline 543 & 103.066 & MIN & 232 & $<-->$ & $\mathrm{CH} 2+\mathrm{C} 9 \mathrm{H} 1403$ \\
\hline 544 & 103.295 & MIN & 208 & $<-->$ & $\mathrm{CH} 2+\mathrm{C} 9 \mathrm{H} 1403$ \\
\hline 546 & 104.166 & MIN & 232 & $<-->$ & $\mathrm{CH} 2+\mathrm{C} 9 \mathrm{H} 1403$ \\
\hline 547 & 104.174 & MIN & 119 & $<-->$ & MIN 421 \\
\hline 548 & 104.275 & MIN & 232 & $<-->$ & $\mathrm{H} 2+\mathrm{C} 10 \mathrm{H} 1403$ \\
\hline 550 & 104.575 & MIN & 226 & $<-->$ & $\mathrm{CH} 2+\mathrm{C} 9 \mathrm{H} 1403$ \\
\hline 551 & 104.891 & MIN & 232 & $<-->$ & MIN 367 \\
\hline 552 & 105.548 & MIN & 171 & $<-->$ & MIN 455 \\
\hline 553 & 106.425 & MIN & 435 & $<-->$ & $\mathrm{CH} 4+\mathrm{C} 9 \mathrm{H} 12 \mathrm{O} 3$ \\
\hline 554 & 106.618 & MIN & 219 & $<-->$ & $\mathrm{CH} 4+\mathrm{C} 9 \mathrm{H} 12 \mathrm{O} 3$ \\
\hline 555 & 106.673 & MIN & 232 & $<-->$ & $\mathrm{CH} 2+\mathrm{C} 9 \mathrm{H} 1403$ \\
\hline 556 & 106.695 & MIN & 232 & $<-->$ & $\mathrm{CH} 2+\mathrm{C} 9 \mathrm{H} 1403$ \\
\hline
\end{tabular}




\begin{tabular}{|c|c|c|c|c|c|}
\hline 557 & 106.864 & MIN & 227 & $<-->$ & MIN \\
\hline 558 & 107.707 & MIN & 232 & $<-->$ & $\mathrm{CH} 2+\mathrm{C} 9 \mathrm{H} 1403$ \\
\hline 559 & 107.903 & MIN & 232 & $<-->$ & $\mathrm{CH} 2+\mathrm{C} 9 \mathrm{H} 1403$ \\
\hline 560 & 108.386 & MIN & 164 & $<-->$ & MIN 232 \\
\hline 561 & 109.277 & MIN & 224 & $<-->$ & MIN \\
\hline 563 & 110.209 & MIN & 232 & $<-->$ & MIN 402 \\
\hline 564 & 110.245 & MIN & 248 & $<-->$ & $\mathrm{CH} 2+\mathrm{C} 9 \mathrm{H} 1403$ \\
\hline 565 & 110.303 & MIN & 409 & $<-->$ & $\mathrm{H} 2+\mathrm{C} 10 \mathrm{H} 1403$ \\
\hline 66 & 110.422 & MIN & 215 & $<-->$ & $\mathrm{CH} 2+\mathrm{C} 9 \mathrm{H} 1403$ \\
\hline 567 & 111.279 & MIN & 378 & $<-->$ & $\mathrm{O} 3+\mathrm{C} 10 \mathrm{H} 16$ \\
\hline 568 & 111.320 & MIN & 232 & $<-->$ & $\mathrm{CH} 2+\mathrm{C} 9 \mathrm{H} 1403$ \\
\hline 570 & 112.021 & MIN & 126 & $<-->$ & MIN 348 \\
\hline 571 & 112.521 & MIN & 249 & $<-->$ & MIN \\
\hline 572 & 112.792 & MIN & 139 & $<-->$ & MIN \\
\hline 573 & 113.249 & MIN & 232 & $<-->$ & $\mathrm{CH} 4+\mathrm{C} 9 \mathrm{H} 12 \mathrm{O} 3$ \\
\hline 574 & 113.291 & MIN & 232 & $<-->$ & $\mathrm{CH} 2+\mathrm{C} 9 \mathrm{H} 14 \mathrm{O} 3$ \\
\hline 575 & 113.513 & MIN & 232 & $<-->$ & MIN 437 \\
\hline 576 & 113.924 & MIN & 232 & $<-->$ & $\mathrm{CH} 4+\mathrm{C} 9 \mathrm{H} 12 \mathrm{O} 3$ \\
\hline 577 & 114.392 & MIN & 170 & $<-->$ & MIN 232 \\
\hline 578 & 114.867 & MIN & 179 & $<-->$ & MIN \\
\hline 579 & 114.891 & MIN & 422 & $<-->$ & MIN 439 \\
\hline 580 & 116.815 & MIN & 232 & $<-->$ & $\mathrm{CH} 4+\mathrm{C} 9 \mathrm{H} 12 \mathrm{O} 3$ \\
\hline 582 & 118.043 & MIN & 389 & $<-->$ & $\mathrm{H} 2+\mathrm{C} 10 \mathrm{H} 1403$ \\
\hline 583 & 118.300 & MIN & 232 & $<-->$ & $\mathrm{H} 2+\mathrm{C} 10 \mathrm{H} 1403$ \\
\hline 584 & 118.783 & MIN & 409 & $<-->$ & $\mathrm{H} 2+\mathrm{C} 10 \mathrm{H} 1403$ \\
\hline 585 & 118.831 & MIN & 232 & $<-->$ & $\mathrm{CH} 4+\mathrm{C} 9 \mathrm{H}_{1} 2 \mathrm{O} 3$ \\
\hline 586 & 119.179 & MIN & 409 & $<-->$ & $\mathrm{H} 2+\mathrm{C} 10 \mathrm{H} 14 \mathrm{O} 3$ \\
\hline 588 & 119.497 & MIN & 420 & $<-->$ & $\mathrm{O} 2+\mathrm{CH} 2 \mathrm{O}+\mathrm{C} 9 \mathrm{H} 14$ \\
\hline 589 & 119.831 & MIN & 430 & $<-->$ & $\mathrm{H} 2+\mathrm{C} 10 \mathrm{H} 14 \mathrm{O} 3$ \\
\hline 590 & 120.169 & MIN & 159 & $<-->$ & MIN 232 \\
\hline 591 & 121.089 & MIN & 421 & $<-->$ & $\mathrm{H} 2+\mathrm{C} 10 \mathrm{H} 1403$ \\
\hline 592 & 121.268 & MIN & 453 & $<-->$ & MIN 458 \\
\hline 593 & 121.712 & MIN & 138 & $<-->$ & $\mathrm{CH} 2+\mathrm{C} 9 \mathrm{H} 1403$ \\
\hline 594 & 122.463 & MIN & 248 & $<-->$ & $\mathrm{H} 2+\mathrm{C} 10 \mathrm{H} 1403$ \\
\hline 595 & 122.854 & MIN & 369 & $<-->$ & $\mathrm{CH} 2+\mathrm{C} 9 \mathrm{H} 1403$ \\
\hline 596 & 123.203 & MIN & 363 & $<-->$ & $\mathrm{H} 2+\mathrm{C} 10 \mathrm{H} 1403$ \\
\hline 597 & 123.801 & MIN & 365 & $<-->$ & $\mathrm{CH} 2 \mathrm{O}+\mathrm{C} 9 \mathrm{H} 14 \mathrm{O} 2$ \\
\hline 598 & 124.167 & MIN & 216 & $<-->$ & MIN 248 \\
\hline 599 & 125.766 & MIN & 232 & $<-->$ & MIN \\
\hline 602 & 127.299 & MIN & 121 & $<-->$ & MIN \\
\hline 603 & 127.454 & MIN & 195 & $<-->$ & MIN 350 \\
\hline 604 & 127.519 & MIN & 366 & $<-->$ & $\mathrm{O} 3+\mathrm{C} 10 \mathrm{H} 16$ \\
\hline 605 & 131.407 & MIN & 404 & $<-->$ & $\mathrm{CH} 4+\mathrm{C} 9 \mathrm{H} 12 \mathrm{O} 3$ \\
\hline 606 & 132.663 & MIN & 232 & $<-->$ & $\mathrm{CH} 4+\mathrm{C} 9 \mathrm{H} 12 \mathrm{O} 3$ \\
\hline 607 & 132.839 & MIN & 438 & $<-->$ & $\mathrm{H} 2+\mathrm{C} 10 \mathrm{H} 1403$ \\
\hline 609 & 133.259 & MIN & 439 & $<-->$ & $\mathrm{H} 2+\mathrm{C} 10 \mathrm{H} 1403$ \\
\hline 612 & 135.404 & MIN & 439 & $<-->$ & $\mathrm{H} 2+\mathrm{C} 10 \mathrm{H} 1403$ \\
\hline 613 & 135.413 & MIN & 400 & $<-->$ & $\mathrm{CH} 4+\mathrm{C} 9 \mathrm{H} 12 \mathrm{O} 3$ \\
\hline 615 & 136.374 & MIN & 352 & $<-->$ & MIN 448 \\
\hline 616 & 138.863 & MIN & 452 & $<-->$ & $\mathrm{H} 2+\mathrm{C} 10 \mathrm{H} 1403$ \\
\hline 617 & 139.787 & MIN & 467 & $<-->$ & $\mathrm{H} 2+\mathrm{C} 10 \mathrm{H} 1403$ \\
\hline 618 & 140.584 & MIN & 232 & $<-->$ & MIN 380 \\
\hline
\end{tabular}

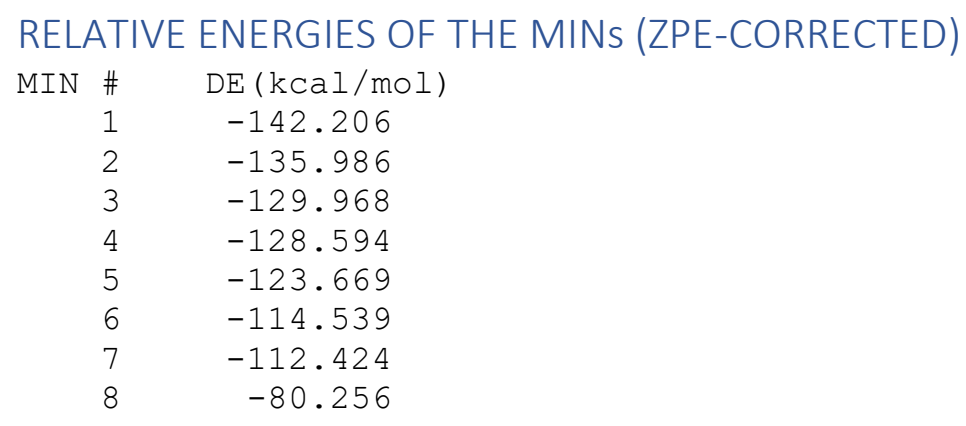


$-72.180$

$-67.368$

$-66.263$

$-61.523$

$-60.688$

$-60.624$

$-60.573$

$-60.064$

$-59.528$

$-59.271$

$-59.199$

$-59.138$

$-59.105$

$-58.870$

$-58.708$

$-57.811$

$-57.104$

$-56.675$

$-55.921$

$-55.782$

$-55.556$

$-55.552$

$-55.442$

$-55.213$

$-53.811$

$-53.549$

$-52.621$

$-52.521$

$-51.213$

$-51.002$

$-50.518$

$-50.049$

$-49.788$

$-49.322$

$-49.155$

$-48.772$

$-48.269$

$-48.221$

$-48.081$

$-47.501$

$-47.073$

$-47.071$

$-46.363$

$-46.015$

$-45.996$

$-45.849$

$-45.710$

$-45.396$

$-44.690$

$-44.365$

$-43.604$

$-43.435$

$-43.258$

$-43.226$

$-42.360$

$-41.769$

$-41.682$

$-41.505$

$-40.750$

$-40.614$

$-40.387$

$-40.247$

$-40.213$

$-39.859$

$-38.974$ 


\begin{tabular}{|c|c|}
\hline 74 & -38.640 \\
\hline 75 & -35.532 \\
\hline 76 & -34.276 \\
\hline 77 & -32.688 \\
\hline 78 & -30.560 \\
\hline 79 & -30.453 \\
\hline 80 & -30.364 \\
\hline 81 & -30.196 \\
\hline 82 & -29.603 \\
\hline 83 & -29.171 \\
\hline 84 & -28.642 \\
\hline 85 & -28.536 \\
\hline 86 & -28.525 \\
\hline 87 & -28.215 \\
\hline 88 & -28.117 \\
\hline 89 & -27.979 \\
\hline 90 & -27.816 \\
\hline 91 & -27.558 \\
\hline 92 & -27.418 \\
\hline 93 & -27.149 \\
\hline 94 & -26.871 \\
\hline 95 & -26.733 \\
\hline 96 & -26.731 \\
\hline 97 & -26.689 \\
\hline 98 & -26.472 \\
\hline 99 & -26.463 \\
\hline 100 & -26.081 \\
\hline 101 & -25.944 \\
\hline 102 & -25.566 \\
\hline 103 & -25.159 \\
\hline 104 & -24.892 \\
\hline 105 & -24.762 \\
\hline 106 & -24.541 \\
\hline 107 & -24.522 \\
\hline 108 & -24.339 \\
\hline 109 & -24.229 \\
\hline 110 & -24.101 \\
\hline 111 & -23.972 \\
\hline 112 & -23.267 \\
\hline 113 & -23.019 \\
\hline 114 & -22.706 \\
\hline 115 & -22.668 \\
\hline 116 & -22.587 \\
\hline 117 & -22.242 \\
\hline 118 & -22.136 \\
\hline 119 & -22.026 \\
\hline 120 & -21.979 \\
\hline 121 & -21.793 \\
\hline 122 & -21.785 \\
\hline 123 & -21.781 \\
\hline 124 & -21.511 \\
\hline 125 & -21.288 \\
\hline 126 & -20.886 \\
\hline 127 & -20.697 \\
\hline 128 & -19.976 \\
\hline 129 & -19.435 \\
\hline 130 & $-19 \cdot 380$ \\
\hline 131 & -19.242 \\
\hline 132 & -18.889 \\
\hline 133 & -18.115 \\
\hline 134 & -17.898 \\
\hline 135 & -17.872 \\
\hline 136 & -17.677 \\
\hline 137 & -17.614 \\
\hline 138 & -17.484 \\
\hline
\end{tabular}




\begin{tabular}{|c|c|}
\hline 139 & -17.478 \\
\hline 140 & -17.472 \\
\hline 141 & -17.363 \\
\hline 142 & -17.267 \\
\hline 143 & -17.195 \\
\hline 144 & -16.779 \\
\hline 145 & -16.773 \\
\hline 146 & -16.748 \\
\hline 147 & -16.694 \\
\hline 148 & -16.658 \\
\hline 149 & -15.616 \\
\hline 150 & -14.641 \\
\hline 151 & -14.494 \\
\hline 152 & -14.060 \\
\hline 153 & -13.637 \\
\hline 154 & -13.336 \\
\hline 155 & -13.176 \\
\hline 156 & -13.066 \\
\hline 157 & -13.009 \\
\hline 158 & -12.426 \\
\hline 159 & -12.120 \\
\hline 160 & -11.787 \\
\hline 161 & -11.720 \\
\hline 162 & -11.585 \\
\hline 163 & -11.163 \\
\hline 164 & -10.910 \\
\hline 165 & -10.641 \\
\hline 166 & -10.638 \\
\hline 167 & -10.489 \\
\hline 168 & -10.355 \\
\hline 169 & -9.663 \\
\hline 170 & -9.578 \\
\hline 171 & -9.293 \\
\hline 172 & -9.037 \\
\hline 173 & -8.937 \\
\hline 174 & -8.927 \\
\hline 175 & -8.920 \\
\hline 176 & -8.822 \\
\hline 177 & -8.739 \\
\hline 178 & -8.648 \\
\hline 179 & -8.631 \\
\hline 180 & -8.544 \\
\hline 181 & -8.441 \\
\hline 182 & -8.009 \\
\hline 183 & -7.931 \\
\hline 184 & -7.829 \\
\hline 185 & -7.685 \\
\hline 186 & -7.675 \\
\hline 187 & -7.573 \\
\hline 188 & -7.166 \\
\hline 189 & -7.041 \\
\hline 190 & -7.015 \\
\hline 191 & -6.989 \\
\hline 192 & -6.960 \\
\hline 193 & -6.868 \\
\hline 194 & -6.770 \\
\hline 195 & -6.635 \\
\hline 196 & -6.574 \\
\hline 197 & -6.435 \\
\hline 198 & -6.195 \\
\hline 199 & -5.997 \\
\hline 200 & -5.737 \\
\hline 201 & -5.561 \\
\hline & -5.334 \\
\hline , & -5.310 \\
\hline
\end{tabular}




\begin{tabular}{|c|c|}
\hline 204 & -5.074 \\
\hline 205 & -5.069 \\
\hline 206 & -4.916 \\
\hline 207 & -3.912 \\
\hline 208 & -3.827 \\
\hline 209 & -3.815 \\
\hline 210 & -3.764 \\
\hline 211 & -3.699 \\
\hline 212 & -3.460 \\
\hline 213 & -3.148 \\
\hline 214 & -2.921 \\
\hline 215 & -2.818 \\
\hline 216 & -2.283 \\
\hline 217 & -2.214 \\
\hline 218 & -1.790 \\
\hline 219 & -1.606 \\
\hline 220 & -1.552 \\
\hline 221 & -1.457 \\
\hline 222 & -1.117 \\
\hline 223 & -1.049 \\
\hline 224 & -1.028 \\
\hline 225 & -0.637 \\
\hline 226 & -0.606 \\
\hline 227 & -0.316 \\
\hline 228 & -0.285 \\
\hline 229 & -0.225 \\
\hline 230 & -0.193 \\
\hline 231 & -0.178 \\
\hline 232 & -0.138 \\
\hline 233 & 0.000 \\
\hline 234 & 0.099 \\
\hline 235 & 0.164 \\
\hline 236 & 0.334 \\
\hline 237 & 0.382 \\
\hline 238 & 0.387 \\
\hline 239 & 0.535 \\
\hline 240 & 0.763 \\
\hline 241 & 0.857 \\
\hline 242 & 0.911 \\
\hline 243 & 0.925 \\
\hline 244 & 1.031 \\
\hline 245 & 1.544 \\
\hline 246 & 1.564 \\
\hline 247 & 1.578 \\
\hline 248 & 1.930 \\
\hline 249 & 1.988 \\
\hline 250 & 2.038 \\
\hline 251 & 2.060 \\
\hline 252 & 2.065 \\
\hline 253 & 2.100 \\
\hline 254 & 2.190 \\
\hline 255 & 2.245 \\
\hline 256 & 2.362 \\
\hline 257 & 2.419 \\
\hline 258 & 2.534 \\
\hline 259 & 2.543 \\
\hline 260 & 2.567 \\
\hline 261 & 2.596 \\
\hline 262 & 2.601 \\
\hline 263 & 2.651 \\
\hline 264 & 2.667 \\
\hline & 2.682 \\
\hline 266 & 2.756 \\
\hline 267 & 2.758 \\
\hline 0 & 2.801 \\
\hline
\end{tabular}




\begin{tabular}{|c|c|}
\hline 269 & 2.818 \\
\hline 270 & 2.840 \\
\hline 271 & 2.930 \\
\hline 272 & 3.023 \\
\hline 273 & 3.060 \\
\hline 274 & 3.063 \\
\hline 275 & 3.166 \\
\hline 276 & 3.227 \\
\hline 277 & 3.248 \\
\hline 278 & 3.306 \\
\hline 279 & 3.313 \\
\hline 280 & 3.363 \\
\hline 281 & 3.390 \\
\hline 282 & 3.440 \\
\hline 283 & 3.452 \\
\hline 284 & 3.512 \\
\hline 285 & 3.579 \\
\hline 286 & 3.609 \\
\hline 287 & 3.635 \\
\hline 288 & 3.639 \\
\hline 289 & 3.652 \\
\hline 290 & 3.658 \\
\hline 291 & 3.717 \\
\hline 292 & 3.731 \\
\hline 293 & 3.732 \\
\hline 294 & 3.850 \\
\hline 295 & 3.956 \\
\hline 296 & 4.040 \\
\hline 297 & 4.061 \\
\hline 298 & 4.104 \\
\hline 299 & 4.210 \\
\hline 300 & 4.253 \\
\hline 301 & 4.554 \\
\hline 302 & 4.717 \\
\hline 303 & 4.799 \\
\hline 304 & 5.190 \\
\hline 305 & 5.468 \\
\hline 306 & 5.599 \\
\hline 307 & 5.762 \\
\hline 308 & 5.894 \\
\hline 309 & 6.038 \\
\hline 310 & 6.642 \\
\hline 311 & 6.941 \\
\hline 312 & 7.076 \\
\hline 313 & 7.166 \\
\hline 314 & 7.249 \\
\hline 315 & 7.567 \\
\hline 316 & 7.847 \\
\hline 317 & 7.860 \\
\hline 318 & 7.875 \\
\hline 319 & 7.897 \\
\hline 320 & 8.060 \\
\hline 321 & 8.093 \\
\hline 322 & 8.257 \\
\hline 323 & 8.264 \\
\hline 324 & 8.364 \\
\hline 325 & 8.461 \\
\hline 326 & 8.479 \\
\hline 327 & 8.653 \\
\hline 328 & 8.674 \\
\hline 329 & 9.148 \\
\hline 330 & 9.156 \\
\hline 331 & 9.436 \\
\hline 332 & 9.490 \\
\hline 333 & 9.64 \\
\hline
\end{tabular}




\begin{tabular}{|c|c|}
\hline 334 & 9.683 \\
\hline 335 & 9.695 \\
\hline 336 & 9.756 \\
\hline 337 & 10.412 \\
\hline 338 & 10.630 \\
\hline 339 & 10.929 \\
\hline 340 & 11.366 \\
\hline 341 & 11.433 \\
\hline 342 & 11.693 \\
\hline 343 & 11.966 \\
\hline 344 & 12.621 \\
\hline 345 & 12.728 \\
\hline 346 & 13.063 \\
\hline 347 & 13.214 \\
\hline 348 & 13.453 \\
\hline 349 & 13.990 \\
\hline 350 & 14.130 \\
\hline 351 & 15.155 \\
\hline 352 & 15.229 \\
\hline 353 & 15.929 \\
\hline 354 & 16.059 \\
\hline 355 & 16.165 \\
\hline 356 & 16.443 \\
\hline 357 & 17.081 \\
\hline 358 & 17.115 \\
\hline 359 & 17.190 \\
\hline 360 & 17.294 \\
\hline 361 & 17.423 \\
\hline 362 & 17.441 \\
\hline 363 & 17.928 \\
\hline 364 & 19.291 \\
\hline 365 & 19.526 \\
\hline 366 & 19.898 \\
\hline 367 & 19.978 \\
\hline 368 & 20.166 \\
\hline 369 & 20.253 \\
\hline 370 & 20.675 \\
\hline 371 & 20.713 \\
\hline 372 & 21.115 \\
\hline 373 & 21.501 \\
\hline 374 & 21.821 \\
\hline 375 & 22.284 \\
\hline 376 & 22.611 \\
\hline 377 & 23.255 \\
\hline 378 & 25.168 \\
\hline 379 & 25.457 \\
\hline 380 & 25.994 \\
\hline 381 & 27.467 \\
\hline 382 & 28.502 \\
\hline 383 & 28.684 \\
\hline 384 & 30.435 \\
\hline 385 & 31.401 \\
\hline 386 & 31.755 \\
\hline 387 & 32.413 \\
\hline 388 & 33.259 \\
\hline 389 & 38.628 \\
\hline 390 & 39.064 \\
\hline 391 & 39.265 \\
\hline 392 & 40.554 \\
\hline 393 & 40.848 \\
\hline 394 & 42.255 \\
\hline 395 & 44.420 \\
\hline 396 & 45.496 \\
\hline 397 & 46.104 \\
\hline & 46.526 \\
\hline
\end{tabular}




\begin{tabular}{|c|c|}
\hline 399 & 47.181 \\
\hline 400 & 47.543 \\
\hline 401 & 48.140 \\
\hline 402 & 48.501 \\
\hline 403 & 48.589 \\
\hline 404 & 49.126 \\
\hline 405 & 49.135 \\
\hline 406 & 49.729 \\
\hline 407 & 49.750 \\
\hline 408 & 50.441 \\
\hline 409 & 50.794 \\
\hline 410 & 51.637 \\
\hline 411 & 51.805 \\
\hline 412 & 52.418 \\
\hline 413 & 52.540 \\
\hline 414 & 52.810 \\
\hline 415 & 52.986 \\
\hline 416 & 54.315 \\
\hline 417 & 56.486 \\
\hline 418 & 57.331 \\
\hline 419 & 57.489 \\
\hline 420 & 61.262 \\
\hline 421 & 61.420 \\
\hline 422 & 61.422 \\
\hline 423 & 61.919 \\
\hline 424 & 62.056 \\
\hline 425 & 62.061 \\
\hline 426 & 62.079 \\
\hline 427 & 62.330 \\
\hline 428 & 62.459 \\
\hline 429 & 62.633 \\
\hline 430 & 63.133 \\
\hline 431 & 63.630 \\
\hline 432 & 63.714 \\
\hline 433 & 64.476 \\
\hline 434 & 64.898 \\
\hline 435 & 65.110 \\
\hline 436 & 65.111 \\
\hline 437 & 65.679 \\
\hline 438 & 67.122 \\
\hline 439 & 67.276 \\
\hline 440 & 67.864 \\
\hline 441 & 67.921 \\
\hline 442 & 67.933 \\
\hline 443 & 68.082 \\
\hline 444 & 68.282 \\
\hline 445 & 69.173 \\
\hline 446 & 69.257 \\
\hline 447 & 69.393 \\
\hline 448 & 69.996 \\
\hline 449 & 70.256 \\
\hline 450 & 70.311 \\
\hline 451 & 71.952 \\
\hline 452 & 72.107 \\
\hline 453 & 72.475 \\
\hline 454 & 72.804 \\
\hline 455 & 73.613 \\
\hline 456 & 73.744 \\
\hline 457 & 75.550 \\
\hline 458 & 75.651 \\
\hline 459 & 76.410 \\
\hline 460 & 76.570 \\
\hline 461 & 77.450 \\
\hline 462 & 77.466 \\
\hline 463 & 78.045 \\
\hline
\end{tabular}




$\begin{array}{ll}464 & 78.120 \\ 465 & 80.398 \\ 466 & 81.550 \\ 467 & 82.461 \\ 468 & 83.013 \\ 469 & 86.402 \\ 470 & 86.995\end{array}$

Families of conformers

[3 5]

$\left[\begin{array}{lllll}14 & 15 & 16 & 17 & 23\end{array}\right]$

$\left[\begin{array}{lllll}20 & 21 & 22 & 27 & 31\end{array}\right]$

$\left[\begin{array}{ll}33 & 34\end{array}\right]$

$\left[\begin{array}{lll}37 & 41 & 44\end{array}\right]$

$\left[\begin{array}{ll}38 & 39\end{array}\right]$

$\left[\begin{array}{lll}50 & 53 & 54\end{array}\right]$

$\left[\begin{array}{ll}51 & 55\end{array}\right]$

$\left[\begin{array}{ll}52 & 61\end{array}\right]$

$\left[\begin{array}{ll}57 & 60\end{array}\right]$

$\left[\begin{array}{ll}58 & 59\end{array}\right]$

$\left[\begin{array}{lll}62 & 63 & 76\end{array}\right]$

$\left[\begin{array}{lllllllllllllllll}82 & 83 & 84 & 85 & 86 & 87 & 88 & 89 & 90 & 91 & 92 & 93 & 96 & 99 & 100 & 105 & 118\end{array}\right]$

$\left[\begin{array}{lllllllllllllllllll}97 & 98 & 101 & 103 & 104 & 106 & 107 & 108 & 109 & 110 & 111 & 112 & 113 & 114 & 115 & 116 & 122 & 129 & 156\end{array}\right]$

$\left[\begin{array}{ll}127 & 128\end{array}\right]$

$\left[\begin{array}{ll}130 & 133\end{array}\right]$

$\left[\begin{array}{ll}135 & 148\end{array}\right]$

$\left[\begin{array}{ll}136 & 137\end{array}\right]$

$[138$ 153

$[144$ 155]

$\left[\begin{array}{ll}147 & 152\end{array}\right]$

$\left[\begin{array}{ll}154 & 165\end{array}\right]$

$[159161]$

$\left[\begin{array}{ll}164 & 166\end{array}\right]$

$\left[\begin{array}{ll}168 & 175\end{array}\right]$

$\left[\begin{array}{lllll}170 & 172 & 177 & 186 & 188\end{array}\right]$

$\left[\begin{array}{llllllllllllll}171 & 173 & 180 & 184 & 185 & 187 & 190 & 192 & 193 & 197 & 198 & 199 & 202 & 205\end{array}\right]$

$\left[\begin{array}{ll}183 & 209\end{array}\right]$

$\left[\begin{array}{ll}191 & 212\end{array}\right]$

$\left[\begin{array}{ll}203 & 358\end{array}\right]$

$\left[\begin{array}{ll}208 & 217\end{array}\right]$

$\left[\begin{array}{lll}219 & 223 & 244\end{array}\right]$

$\left[\begin{array}{lll}224 & 247 & 251\end{array}\right]$

$\left[\begin{array}{llllll}226 & 228 & 231 & 238 & 240 & 246\end{array}\right]$

$\left[\begin{array}{lll}232 & 233 & 235\end{array}\right]$

$\left[\begin{array}{ll}243 & 260\end{array}\right]$

$\left[\begin{array}{llllllllllllllll}248 & 258 & 262 & 266 & 267 & 268 & 271 & 273 & 274 & 276 & 283 & 284 & 289 & 300 & 307 & 308\end{array}\right]$

$\left[\begin{array}{lllllllllllllllllllll}249 & 252 & 253 & 255 & 256 & 257 & 259 & 261 & 263 & 264 & 265 & 269 & 270 & 275 & 277 & 278 & 279 & 281 & 282 & 285 & 286\end{array}\right.$ $287288292293294295297298299302303 \quad 304$ 311]

[254 290]

$\left[\begin{array}{lll}272 & 280 & 296\end{array}\right]$

$\left[\begin{array}{lllll}309 & 312 & 314 & 325 & 347\end{array}\right]$

$\left[\begin{array}{ll}315 & 326\end{array}\right]$

$\left[\begin{array}{llllll}316 & 317 & 319 & 321 & 322 & 324\end{array}\right]$

$\left[\begin{array}{ll}318 & 343\end{array}\right]$

$\left[\begin{array}{ll}327 & 332\end{array}\right]$

$\left[\begin{array}{llll}328 & 333 & 344 & 345\end{array}\right]$

[329 330]

[ [337 346]

$\left[\begin{array}{lll}353 & 359 & 360\end{array}\right]$

$\left[\begin{array}{llll}355 & 356 & 357 & 361\end{array}\right]$

[ [362 368]

$\left[\begin{array}{lll}367 & 371 & 374\end{array}\right]$

$\left[\begin{array}{lll}385 & 387 & 388\end{array}\right]$

[ [389 391]

$\left[\begin{array}{lll}400 & 401 & 403\end{array}\right]$

[ $404 \quad 405$ 412] 


\section{name $=$ MIN1 \\ energy (in $\mathrm{kcal} / \mathrm{mol})=-148.611528$ \\ $\operatorname{ZPE}($ in $\mathrm{kcal} / \mathrm{mol})=144.473$ \\ $\mathrm{G}($ in $\mathrm{kcal} / \mathrm{mol})=118.772$ \\ Geometry \\ C $-2.47350588-1.20096022-0.57495262$ \\ C $-1.11672329-1.431769050 .00466742$ \\ C $-0.57298203 \quad 1.60793154-0.70208497$ \\ C $0.915694081 .65034415-0.82740159$ \\ $\begin{array}{llll}\text { C } & 1.59250391 & 0.33622762 & -0.41141793\end{array}$ \\ C $1.48022146-0.67407948-1.55473844$ \\ C $-0.53795681-0.27048551 \quad 0.82551440$ \\ C $0.99348275-0.15285703 \quad 0.93022410$ \\ $\begin{array}{llll}\text { C } 1.29539119 & 0.88834798 & 2.03022329\end{array}$ \\ C $1.61757319-1.478429491 .37099963$ \\ H $-2.49782175-0.31967732-1.23849204$ \\ $\mathrm{H}-3.21397500-0.999541830 .21442076$ \\ $\mathrm{H}-2.82356005-2.06852160-1.15224996$ \\ $\mathrm{H}-0.98617610 \quad-0.34010964 \quad 1.85331579$ \\ H $1.294383782 .49615039-0.21104037$ \\ H $1.19365637 \quad 1.91488611-1.87188987$ \\ H $2.68168100 \quad 0.53523069-0.24006409$ \\ H $1.84591088-1.66715571-1.26120633$ \\ H $2.07139372-0.35741464-2.42201607$ \\ $\mathrm{H} \quad 0.44805178-0.80840554-1.89605752$ \\ H $2.35854678 \quad 1.14708837 \quad 2.05591747$ \\ H $1.03490190 \quad 0.50359816 \quad 3.02325092$ \\ H $0.72577081 \quad 1.81350082 \quad 1.88773035$ \\ H $1.15601867-1.85882350 \quad 2.28902349$ \\ H $2.69127308-1.370300461 .55773319$ \\ H $1.49963012 \quad-2.271518890 .61754158$ \\ $\begin{array}{lllll}0 & -1.34699748 & 2.21696693 & -1.39230106\end{array}$ \\ $\begin{array}{lllll}0 & -1.13989348 & 0.91407107 & 0.33677764\end{array}$ \\ $0-0.54695314-2.48678391-0.10041377$ \\ Vibrational frequencies (in $\mathrm{cm}-1$ )}

ABSOLUTE ENERGY, ZPE, GIBBS ENERGY CORRECTION, GEOMETRY AND FREQUENCIES OF THE MINS

$\begin{array}{lllllllllllllll}33.9 & 56.2 & 75.0 & 104.5 & 114.9 & 166.4 & 200.3 & 220.4 & 234.8 & 258.2 & 267.2 & 322.5 & 330.6 & 360.7\end{array}$ $387.2411 .2 \quad 449.6 \quad 472.0 \quad 476.6 \quad 501.2 \quad 552.5 \quad 592.1 \quad 627.3 \quad 740.9 \quad 806.3 \quad 879.9 \quad 896.7 \quad 935.5$ $974.7995 .4 \quad 1001.8 \quad 1008.2 \quad 1033.3 \quad 1045.5 \quad 1054.6 \quad 1076.3 \quad 1104.5 \quad 1123.3 \quad 1170.6 \quad 1187.2$

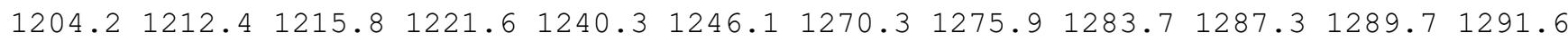
$1298.71302 .91306 .81311 .2 \quad 1325.0 \quad 1346.3 \quad 1366.6 \quad 1381.21401 .01404 .7 \quad 1416.01848 .8$ 1860.32648 .52650 .72654 .22661 .52664 .82671 .52672 .12672 .82676 .62681 .32698 .5 $2734.2 \quad 2774.92778 .6 \quad 2780.4 \quad 2781.3$

name $=$ MIN2

energy (in $\mathrm{kcal} / \mathrm{mol})=-141.833619$

$\operatorname{ZPE}($ in $\mathrm{kcal} / \mathrm{mol})=143.915$

G $($ in $\mathrm{kcal} / \mathrm{mol})=117.448$

Geometry

C $-3.13021255-1.27400685-0.68616711$

C $-1.62702529-1.28970786-0.68246378$ 
C $2.12270226 \quad 0.79366222-0.67850990$

C $1.88235118 \quad 0.92565314 \quad 0.80106513$

C $0.785328110 .02866862 \quad 1.34946503$

C $0.55421247-1.36250213 \quad 0.70310891$

C $-0.97219705-1.10423631 \quad 0.65572378$

C $-0.72621153 \quad 0.391162691 .08256648$

C $-0.98131719 \quad 1.39555687-0.02457539$

C $-1.47166954 \quad 0.788113592 .34641196$

$\mathrm{H}-3.52215005-1.04996616-1.68976636$

$\mathrm{H}-3.54329299-0.53794118 \quad 0.01170767$

$\mathrm{H}-3.52446647-2.26069374-0.40469444$

H $1.26895014 \quad 2.57062287-0.97426071$

$\mathrm{H} \quad 1.67655352 \quad 1.97589714 \quad 1.09058460$

H 2.84253252 $0.66423213 \quad 1.31056502$

$\mathrm{H} \quad 0.95499173-0.07631670 \quad 2.44367916$

H $1.00681395-1.49423731-0.29341018$

$\mathrm{H} \quad 0.86388552-2.21313781 \quad 1.31265534$

$\mathrm{H}-1.51930018-1.674035341 .42817700$

$\mathrm{H}-2.05437937 \quad 1.49251381-0.23696345$

$\mathrm{H}-0.50548707 \quad 1.09129414 \quad-0.97154402$

$\mathrm{H}-0.61885509 \quad 2.39311662 \quad 0.24206937$

$\mathrm{H}-1.11892354 \quad 1.75359498 \quad 2.72891217$

$\mathrm{H}-1.34564837 \quad 0.054474593 .15111776$

$\mathrm{H}-2.54863253 \quad 0.884405422 .16358054$

○ $2.66123343-0.11748889-1.23928996$

O $1.75710168 \quad 1.86499677-1.44382740$

O $-0.99848876-1.46099814-1.69501754$

Vibrational frequencies (in $\mathrm{cm}-1$ )

$\begin{array}{lllllllllllllll}35.5 & 45.2 & 52.2 & 98.0 & 110.1 & 145.8 & 173.5 & 174.5 & 199.4 & 233.3 & 276.4 & 294.8 & 324.7 & 360.3\end{array}$ $380.4 \quad 388.7 \quad 401.9 \quad 445.2 \quad 509.7 \quad 524.6 \quad 568.4 \quad 596.3 \quad 663.7 \quad 697.6 \quad 786.9 \quad 816.8 \quad 905.8 \quad 926.3$ $952.0986 .2998 .2 \quad 1007.6 \quad 1015.6 \quad 1034.4 \quad 1047.2 \quad 1055.5 \quad 1090.8 \quad 1107.8 \quad 1139.2 \quad 1146.51178 .1$

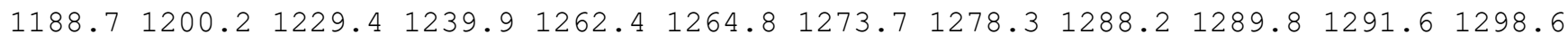

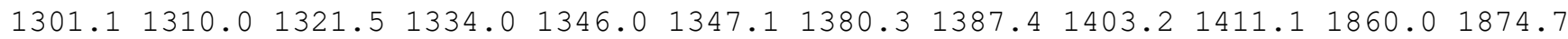
$2650.62652 .92663 .2 \quad 2668.5 \quad 2675.3 \quad 2676.02678 .12679 .92707 .2 \quad 2726.72733 .02756 .5$ $2774.92781 .0 \quad 2783.0 \quad 2786.5$

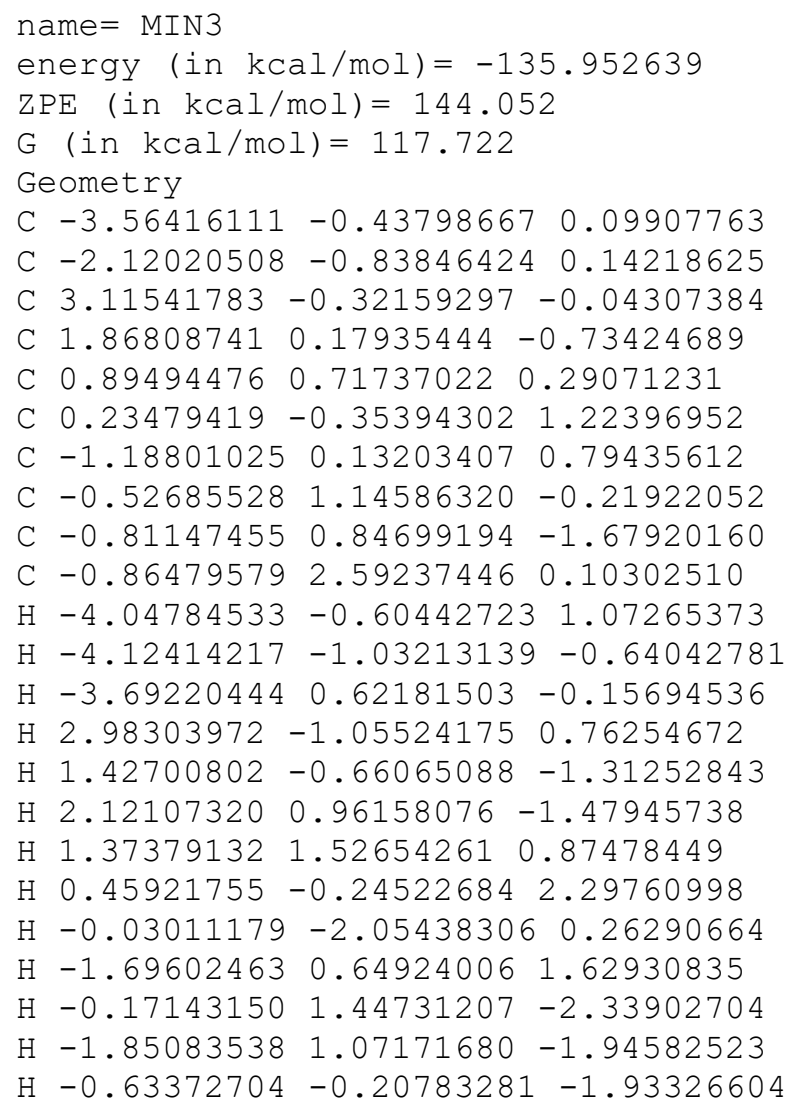


$\mathrm{H}-1.920539972 .81271519-0.09351626$

$\mathrm{H}-0.27021597 \quad 3.28145910-0.51186347$

$\mathrm{H}-0.667760762 .844864591 .15119052$

O $0.59619508-1.65454568 \quad 0.91866719$

$04.208812590 .06524092-0.37228617$

o $-1.74935655-1.88218069-0.34749160$

Vibrational frequencies (in $\mathrm{cm}-1$ )

$\begin{array}{lllllllllllllll}34.2 & 51.5 & 61.8 & 81.5 & 111.3 & 161.8 & 180.2 & 194.1 & 212.4 & 229.3 & 251.5 & 287.3 & 323.7 & 341.0\end{array}$ $382.1 \quad 436.0 \quad 449.7 \quad 476.0 \quad 498.0 \quad 521.4 \quad 558.1 \quad 606.1 \quad 648.5 \quad 752.1828 .6 \quad 871.9886 .1 \quad 900.2$ $917.2998 .1 \quad 1005.5 \quad 1016.0 \quad 1037.4 \quad 1041.1 \quad 1047.7 \quad 1050.3 \quad 1100.41109 .11152 .11165 .4$

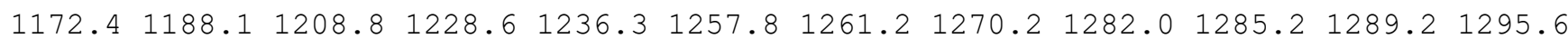

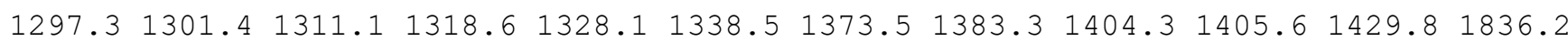
$1837.12646 .82662 .12664 .8 \quad 2665.12670 .7 \quad 2672.8 \quad 2675.82678 .5 \quad 2686.02705 .8 \quad 2716.0$ $2722.62742 .12778 .2 \quad 2778.8 \quad 2782.6$

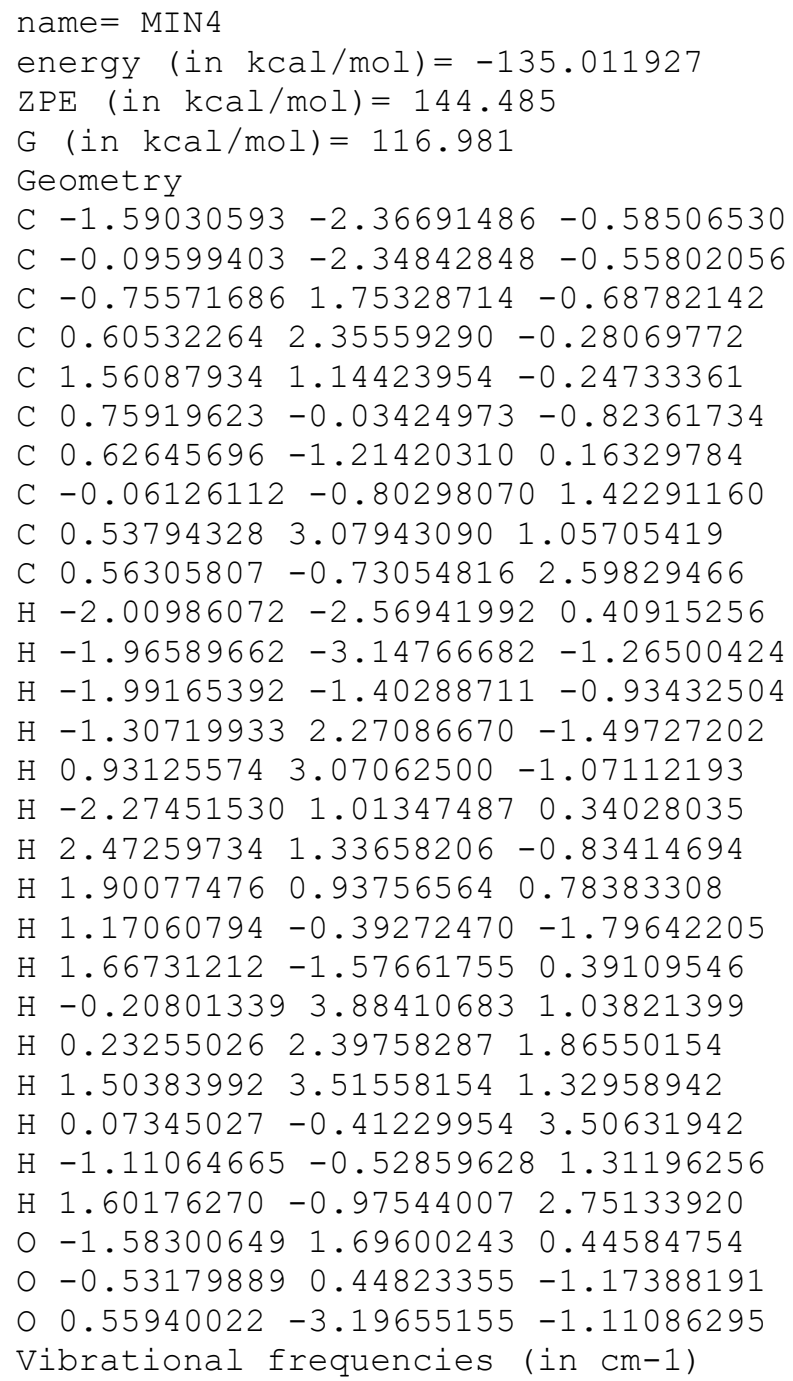
$\begin{array}{lllllllllllllll}375.9 & 403.7 & 449.1 & 475.0 & 522.7 & 553.2 & 607.6 & 664.0 & 679.2 & 742.8 & 866.9 & 899.6 & 934.5 & 964.8\end{array}$ $977.9991 .1 \quad 1012.3 \quad 1025.3 \quad 1042.4 \quad 1053.0 \quad 1085.6 \quad 1093.8 \quad 1106.4 \quad 1125.01138 .21153 .0$

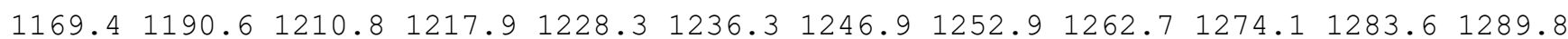
$1296.51303 .91308 .2 \quad 1325.6 \quad 1331.1 \quad 1333.2 \quad 1356.5 \quad 1375.81380 .3 \quad 1392.21403 .11836 .1$ $1854.62658 .32663 .6 \quad 2664.92665 .5 \quad 2670.3 \quad 2677.72682 .42683 .82709 .42714 .82746 .4$ $2754.62776 .2 \quad 2780.6 \quad 2787.92810 .9$

name $=$ MIN5

energy (in $\mathrm{kcal} / \mathrm{mol})=-129.110655$

$\mathrm{ZPE}($ in $\mathrm{kcal} / \mathrm{mol})=143.509$

$\mathrm{G}(\mathrm{in} \mathrm{kcal} / \mathrm{mol})=116.966$

Geometry 
C $-2.46845838-1.53094455 \quad 0.76948908$

C $-0.97042038-1.53271687 \quad 0.75835676$

C $0.14708300 \quad 2.09273475 \quad 0.74744893$

C $0.82811768 \quad 1.94466668-0.59232459$

C $0.65218980 \quad 0.61435601-1.28749069$

C $-0.78538818 \quad 0.01027525-1.32469134$

C $-0.30300106-1.25377369-0.56044136$

C $1.16751828-0.69025644 \quad-0.56504754$

C $1.84312451-0.51099705 \quad 0.77553448$

C $2.07321298-1.51037281-1.48115208$

$\mathrm{H}-2.83944337-0.53305794 \quad 1.06609264$

$\mathrm{H}-2.86467567-2.24980773 \quad 1.49991222$

$\mathrm{H}-2.90680066-1.74656378-0.21049230$

$\mathrm{H}-0.38925513 \quad 1.23348160 \quad 1.17004296$

H $1.91579954 \quad 2.14690713-0.44979996$

$\mathrm{H} 0.485274392 .77048140-1.25436825$

H $1.07960151 \quad 0.69211069-2.30782936$

$\mathrm{H}-1.19063750-0.17711226-2.33083640$

$\mathrm{H}-1.92048994 \quad 1.58101910-0.95504428$

$\mathrm{H}-0.38845178-2.17566274-1.17168927$

H $2.77630125 \quad 0.05566403 \quad 0.68541213$

H $2.08177055-1.48664390 \quad 1.22831329$

$\mathrm{H} \quad 1.21389192-0.005903201 .51827572$

H $3.04281605-1.01683669-1.62102651$

H $1.63907129-1.66718355-2.47316277$

H $2.27442766-2.50006604-1.05111578$

$\begin{array}{lllll}0 & -1.74337765 & 0.69730681 & -0.57062955\end{array}$

0.201389003 .145591251 .33554540

$0-0.34030599-1.806877471 .74934887$

Vibrational frequencies (in $\mathrm{cm}-1$ )

$\begin{array}{llllllllllllllll}26.7 & 32.6 & 42.8 & 133.1 & 152.6 & 181.5 & 202.4 & 208.2 & 226.6 & 236.7 & 240.3 & 275.5 & 301.5 & 324.7\end{array}$ $331.9 \quad 349.2 \quad 386.0 \quad 430.8 \quad 465.3 \quad 504.8 \quad 533.0 \quad 592.7 \quad 655.7 \quad 742.7 \quad 780.6 \quad 825.0 \quad 859.2 \quad 904.4$ $919.3 \quad 990.7 \quad 1009.7 \quad 1020.5 \quad 1029.4 \quad 1045.1 \quad 1050.1 \quad 1065.4 \quad 1077.5 \quad 1095.4 \quad 1146.7 \quad 1173.8$

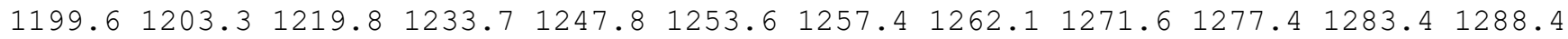

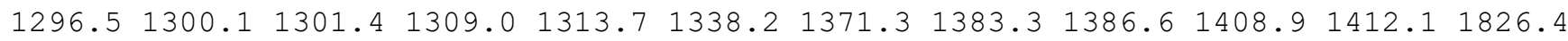
$1849.32639 .5 \quad 2649.8 \quad 2656.92660 .8 \quad 2674.2 \quad 2678.52681 .02681 .62692 .82709 .12713 .0$ $2737.22779 .6 \quad 2780.6 \quad 2782.92803 .5$

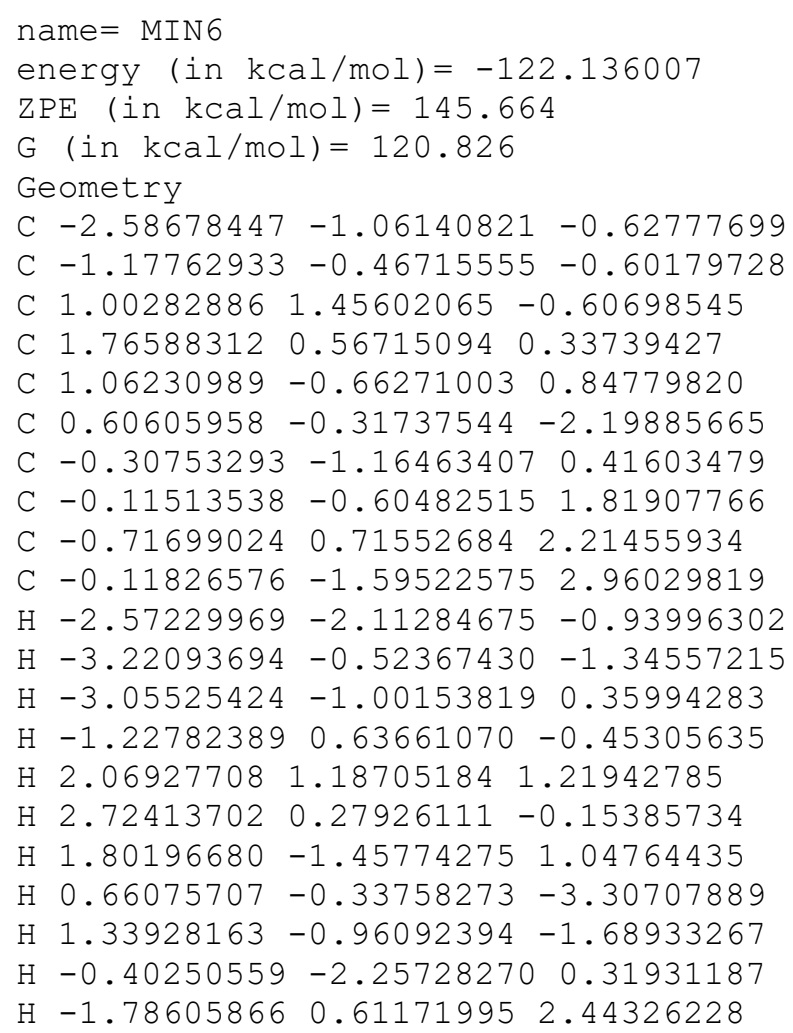


$\mathrm{H}-0.628405141 .487205501 .43692334$

$\mathrm{H}-0.22699124 \quad 1.12262170 \quad 3.11017754$

$\mathrm{H}-1.13940679-1.772371313 .32389067$

$\mathrm{H} \quad 0.47198799-1.22413662 \quad 3.80854144$

$\mathrm{H} \quad 0.29560652-2.57006437 \quad 2.67825053$

$0.805882291 .04752145-1.89520446$

$0 \quad 0.64297358 \quad 2.57325804-0.34452663$

$0-0.70265654-0.70801918-1.92499343$

Vibrational frequencies (in $\mathrm{cm}-1$ )

$\begin{array}{lllllllllllllllll}71.3 & 97.4 & 111.5 & 120.9 & 126.5 & 141.0 & 167.3 & 172.9 & 224.9 & 241.9 & 303.5 & 324.0 & 359.7 & 397.4\end{array}$ $407.1426 .2 \quad 470.1 \quad 502.2 \quad 515.2 \quad 583.9 \quad 609.5 \quad 742.4 \quad 774.3 \quad 801.0 \quad 874.3 \quad 895.4 \quad 927.4 \quad 977.6$ $1006.41009 .3 \quad 1013.0 \quad 1019.3 \quad 1035.1 \quad 1063.3 \quad 1075.2 \quad 1106.91120 .8 \quad 1140.7 \quad 1156.31168 .3$

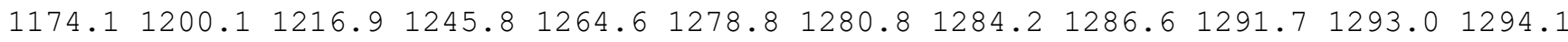
$1295.71299 .31321 .1 \quad 1327.8 \quad 1336.6 \quad 1365.4 \quad 1368.6 \quad 1389.81398 .4 \quad 1401.4 \quad 1416.81516 .4$ $1846.22631 .8 \quad 2643.0 \quad 2660.12664 .2 \quad 2673.12676 .4 \quad 2677.4 \quad 2678.6 \quad 2682.02684 .12724 .7$ $2732.8 \quad 2745.12780 .4 \quad 2781.5 \quad 2782.9$

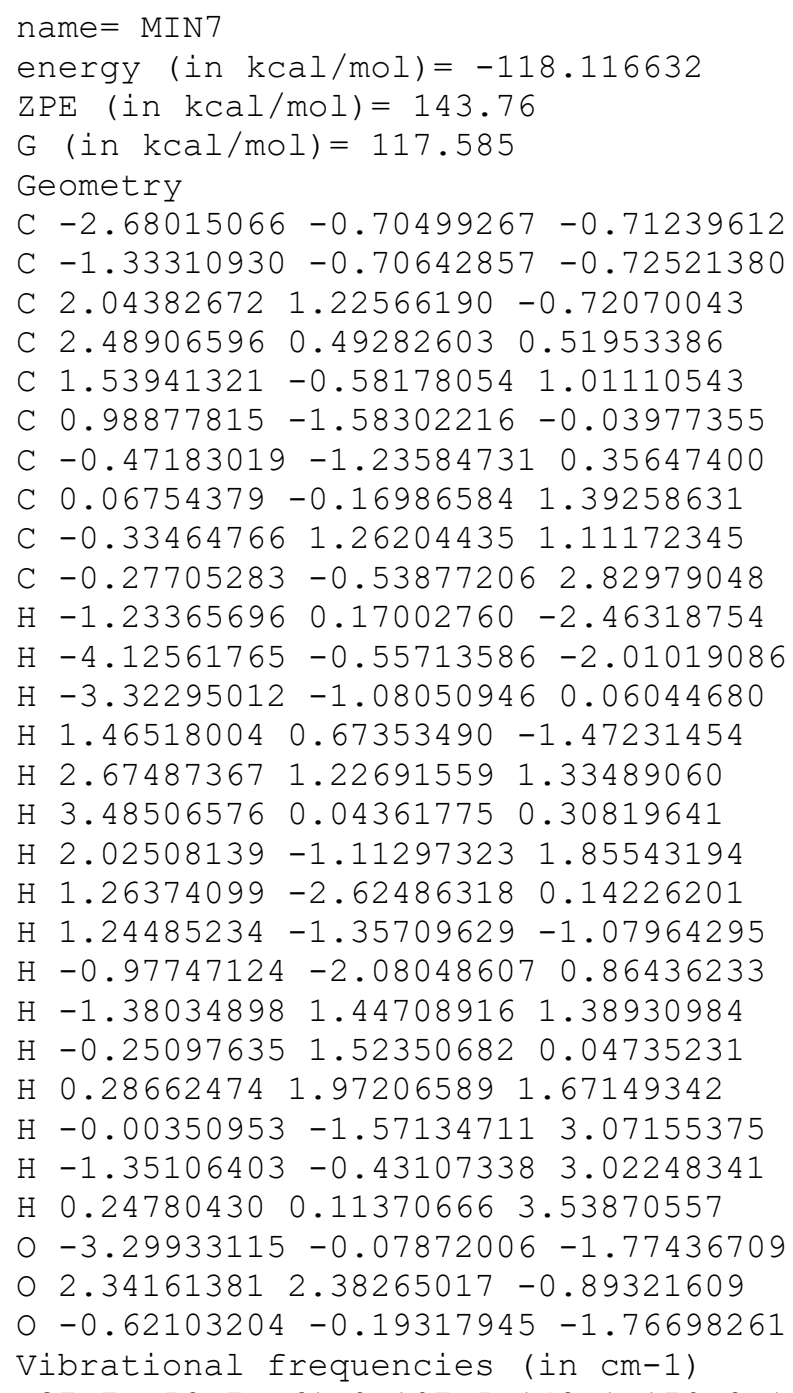


$\mathrm{G}($ in $\mathrm{kcal} / \mathrm{mol})=116.746$

Geometry

C $-1.60748015-2.35957411-0.70787373$

C $-0.11820244-2.35182133-0.62151913$

C $-0.786128161 .65980531-0.55620738$

$\begin{array}{lllll}\text { C } & 0.58292917 & 2.32974021 & -0.29607822\end{array}$

C $1.558593891 .13184192-0.26272825$

C $0.75863280-0.05240337-0.84223570$

C $0.56016501-1.21780694 \quad 0.14859085$

C $-0.31040831-0.82630694 \quad 1.28576684$

C $0.59478165 \quad 3.15564247 \quad 0.97964003$

C $0.31865072-0.367758372 .52067491$

$\mathrm{H}-2.06755771-2.34679837 \quad 0.29317216$

$\mathrm{H}-1.98079755-3.23868260-1.24989630$

$\mathrm{H}-1.96865364-1.45765909-1.22914516$

$\mathrm{H}-1.508658362 .20237658-1.19112461$

$\mathrm{H} 0.82724555 \quad 2.98551175-1.16435801$

$\mathrm{H}-1.90652234 \quad 0.53484857 \quad 0.64628143$

H $2.46173617 \quad 1.33571514 \quad-0.85973023$

H $1.91195997 \quad 0.93119439 \quad 0.76123873$

$\mathrm{H} 1.19249398-0.43408843-1.79609986$

$\mathrm{H} \quad 1.57252279-1.57509418 \quad 0.46717329$

$\mathrm{H}-0.13682806 \quad 3.97265498 \quad 0.93006629$

H $0.32095059 \quad 2.55319178 \quad 1.85720937$

$\mathrm{H} 1.57992093 \quad 3.59585506 \quad 1.16698014$

$\mathrm{H}-0.09291753-0.929507053 .37836512$

$\mathrm{H}-0.00854152 \quad 0.68156101 \quad 2.68994600$

$\mathrm{H} 1.40981452-0.396365612 .59100044$

$0-1.38096938 \quad 1.374315120 .68062915$

$0-0.509420350 .45610905-1.23875427$

$0.56801094-3.19571059-1.14133619$

Vibrational frequencies (in $\mathrm{cm}-1$ )

$\begin{array}{llllllllllllllll}48.8 & 57.3 & 72.2 & 98.6 & 114.1 & 143.8 & 151.9 & 163.8 & 172.6 & 200.0 & 259.7 & 272.4 & 304.2 & 353.7\end{array}$

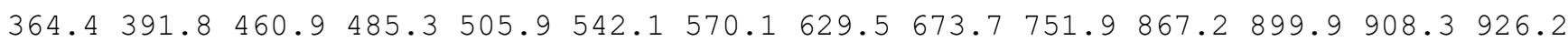
$960.8 \quad 975.3 \quad 988.2 \quad 1014.4 \quad 1037.5 \quad 1051.2 \quad 1079.2 \quad 1084.7 \quad 1112.2 \quad 1125.2 \quad 1134.6 \quad 1146.0 \quad 1174.8$

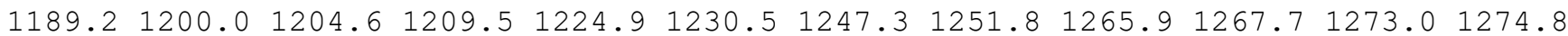

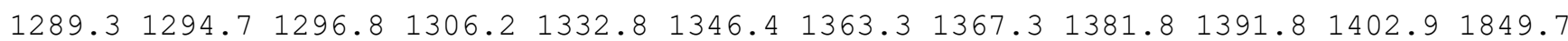
$2636.82656 .62661 .3 \quad 2664.2 \quad 2669.4 \quad 2675.02675 .2 \quad 2678.42680 .5 \quad 2688.02709 .42736 .7$ $2750.6 \quad 2763.3 \quad 2774.92780 .2$

name $=$ MIN9

energy (in $\mathrm{kcal} / \mathrm{mol})=-77.706005$

$\mathrm{ZPE}(\mathrm{in} \mathrm{kcal} / \mathrm{mol})=143.593$

$\mathrm{G}($ in $\mathrm{kcal} / \mathrm{mol})=116.632$

Geometry

C $-1.68509681 \quad 0.39502413-0.54310416$

C $-0.18605510 \quad 0.24333767-0.64253161$

C $0.566965391 .58363346 \quad-0.57482978$

C $1.75351572 \quad 1.47827715 \quad 0.34263660$

C $-0.94056309-2.009749831 .95027877$

C $1.86260234-0.00404044 \quad 0.72229701$

C $0.49671689-0.66839217 \quad 0.41185825$

C $-0.33710151-0.85157147 \quad 1.64516122$

C $-0.46292362 \quad 0.31921918 \quad 2.56882613$

C $-0.88244315-3.24898166 \quad 1.12871201$

$\mathrm{H}-2.00857702 \quad 0.792916190 .42520495$

$\mathrm{H}-2.18412220-0.57431658-0.68712244$

$\mathrm{H}-2.068132821 .07187742-1.32320285$

$\mathrm{H}-0.09444151 \quad 1.09372741-3.03562400$

H 2.67005814 1.85130602 -0.15243927

H $1.618084532 .12614050 \quad 1.22930417$

$\mathrm{H}-1.53051739-2.10142646 \quad 2.86255363$

$\mathrm{H} 2.14078775-0.138707051 .78008669$

H $2.65957908-0.49655158 \quad 0.13605446$ 
$\mathrm{H} \quad 0.68608820 \quad-1.66716377-0.06179447$

$\mathrm{H}-0.42512482 \quad 1.27899685 \quad 2.03731564$

$\mathrm{H} \quad 0.35159938 \quad 0.32425089 \quad 3.30794274$

$\mathrm{H}-1.40621303 \quad 0.304445523 .13185810$

$\mathrm{H}-1.50252595-4.052389171 .54815463$

H $0.14284253-3.64158566 \quad 1.05847043$

$\mathrm{H}-1.23799290-3.07491217 \quad 0.10154793$

$\begin{array}{llll}0 & -0.52664063 & 0.20418104 & -2.94568412\end{array}$

○ $0.26759888 \quad 2.55211758-1.21710609$

O $0.20155207-0.36792335-1.87498079$

Vibrational frequencies (in $\mathrm{cm}-1$ )

$\begin{array}{llllllllllllllll}28.7 & 40.2 & 79.3 & 83.3 & 104.6 & 130.0 & 146.7 & 162.4 & 193.1 & 210.3 & 225.5 & 247.9 & 281.2 & 300.2\end{array}$ $319.2355 .4 \quad 412.2 \quad 475.4 \quad 486.6 \quad 519.7 \quad 535.6 \quad 594.3 \quad 630.9 \quad 718.4 \quad 799.6 \quad 823.0 \quad 857.3 \quad 897.0$

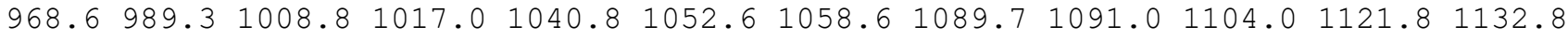

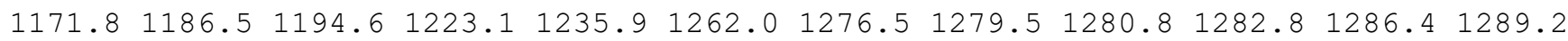

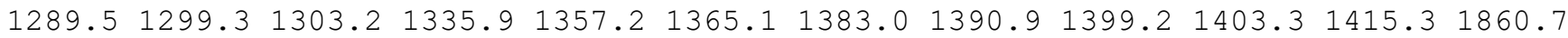
$1877.02652 .7 \quad 2665.5 \quad 2666.7 \quad 2669.5 \quad 2671.7 \quad 2672.62674 .72675 .42686 .92747 .22748 .7$ $2752.52777 .6 \quad 2779.2 \quad 2780.0 \quad 2842.3$

\section{name $=$ MIN10}

energy (in $\mathrm{kcal} / \mathrm{mol})=-73.514793$

$\mathrm{ZPE}($ in $\mathrm{kcal} / \mathrm{mol})=144.214$

$\mathrm{G}($ in $\mathrm{kcal} / \mathrm{mol})=114.579$

Geometry

C $-1.61383193-1.97096314-0.23586216$

C $-0.13367926-1.97655208-0.46527565$

C $1.05048893 \quad 0.25042086-0.48470964$

C $0.31077800 \quad 1.53052146-0.08748857$

C $-1.138075651 .44849114-0.42387636$

C $2.06473679-1.557081690 .73133310$

C $0.68157228 \quad-0.99845743 \quad 0.35695134$

C $-2.11735007 \quad 1.95943939 \quad 0.33547484$

C $-1.86779410 \quad 2.68714974 \quad 1.61442648$

C $-3.55135871 \quad 1.83941269-0.06362673$

$\mathrm{H}-2.14271202-2.54120546-1.01489666$

$\mathrm{H}-2.02867236-0.95274340-0.22701702$

$\mathrm{H}-1.86534057-2.43407584 \quad 0.72816856$

$\mathrm{H} \quad 0.98242796 \quad 0.04204372-1.57370979$

$\mathrm{H} \quad 0.76740508 \quad 2.39683875-0.61998750$

$\mathrm{H} \quad 0.48087043 \quad 1.74004638 \quad 0.98900453$

$\mathrm{H}-1.35847850 \quad 0.95618108-1.37020610$

H $2.26625351-1.485533891 .81099504$

H $2.24325984 \quad-2.58872343 \quad 0.37602439$

$\mathrm{H} \quad 0.11912093 \quad-0.70293878 \quad 1.26906734$

$\mathrm{H}-1.25101307 \quad 3.583340121 .44603503$

$\mathrm{H}-1.33578980 \quad 2.05754309 \quad 2.34211758$

$\mathrm{H}-2.79193389 \quad 3.02519800 \quad 2.10002833$

$\mathrm{H}-4.13804820 \quad 1.32506756 \quad 0.71100788$

$\mathrm{H}-3.69274654 \quad 1.28451501-1.00029410$

$\mathrm{H}-4.00567190 \quad 2.83084344 \quad-0.20875170$

$03.02340350-0.77272017 \quad 0.02635916$

$\begin{array}{lllll}0 & 2.42008791 & 0.51240604 & -0.16331540\end{array}$

O $0.38339655-2.72827174-1.25068625$

Vibrational frequencies (in $\mathrm{cm}-1$ )

$\begin{array}{lllllllllllllll}-8.3 & 11.0 & 25.3 & 71.6 & 77.4 & 108.8 & 110.1 & 117.2 & 179.9 & 188.9 & 231.4 & 254.3 & 267.6 & 302.1\end{array}$

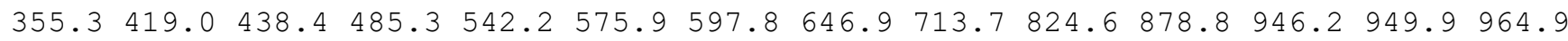
$993.21020 .91025 .1 \quad 1034.7 \quad 1053.9 \quad 1066.0 \quad 1075.8 \quad 1095.0 \quad 1109.7 \quad 1114.21143 .11152 .7$ $1171.21179 .0 \quad 1215.2 \quad 1229.8 \quad 1237.5 \quad 1258.6 \quad 1261.3 \quad 1271.1 \quad 1272.8 \quad 1278.3 \quad 1280.01286 .0$

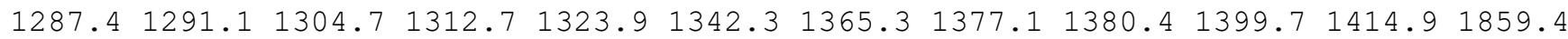
$1869.92645 .12650 .6 \quad 2662.0 \quad 2667.2 \quad 2669.3 \quad 2670.3 \quad 2672.62675 .8 \quad 2676.82712 .5 \quad 2713.5$ $2732.62756 .5 \quad 2777.7 \quad 2778.12780 .2$

name $=$ MIN11 
energy (in $\mathrm{kcal} / \mathrm{mol})=-72.85618$

$\mathrm{ZPE}(\mathrm{in} \mathrm{kcal} / \mathrm{mol})=144.66$

$\mathrm{G}($ in $\mathrm{kcal} / \mathrm{mol})=119.962$

Geometry

C $-3.01400625-0.37325210 \quad 0.51173687$

C $-1.52602990-0.364820640 .53130906$

C $-0.90002401 \quad 1.006976150 .53076106$

C $0.57204075 \quad 0.97793336 \quad 0.95730571$

C $1.30377366-0.126430720 .18321913$

C $1.189551130 .19825148-1.29458903$

C $-0.79282929-1.483449690 .52063191$

C $0.71085618-1.51123106 \quad 0.54617572$

C $1.15084315-1.89447556 \quad 1.97359042$

C $1.22320827-2.58976606-0.42400294$

$\mathrm{H}-3.40217706 \quad 0.27414148 \quad-0.29271984$

$\mathrm{H}-3.43017761 \quad 0.00322554 \quad 1.45773999$

$\mathrm{H}-3.43365011-1.37455464 \quad 0.34917926$

$\mathrm{H}-1.47787786 \quad 1.735463851 .14093235$

$\mathrm{H} \quad 0.65459134 \quad 0.81543266 \quad 2.04710430$

$\mathrm{H} 1.04370768 \quad 1.96344756 \quad 0.76358937$

$\mathrm{H} 2.38883916-0.11313184 \quad 0.45465456$

$\mathrm{H} \quad 0.73282297-0.53941946-1.96355443$

$\mathrm{H} \quad 0.33935004 \quad 2.60518851-1.33598558$

$\mathrm{H}-1.25975203-2.466942620 .52218681$

H $0.79508391-1.16957278 \quad 2.71376940$

H 2.24083071 -1.95205081 2.05474329

H $0.74609699-2.870562332 .26354533$

$\mathrm{H} 1.01593316-3.59662103-0.04163139$

H $2.30618575-2.51721186-0.56915642$

$\mathrm{H} \quad 0.74208991-2.52441851-1.40589746$

$\begin{array}{lllll}0 & -0.56480453 & 2.73335120 & -0.94520870\end{array}$

$0-0.99035530 \quad 1.38240415-0.84884621$

o $1.58868686 \quad 1.25259676-1.73467005$

Vibrational frequencies (in cm-1)

$\begin{array}{lllllllllllllllll}58.7 & 115.9 & 125.1 & 131.6 & 155.2 & 178.6 & 190.1 & 208.6 & 220.1 & 257.7 & 284.3 & 304.7 & 331.0 & 357.3\end{array}$ $376.9391 .5 \quad 427.5 \quad 461.3 \quad 470.4 \quad 512.4 \quad 551.2 \quad 565.3 \quad 609.0 \quad 716.8 \quad 854.2 \quad 925.7 \quad 929.9 \quad 946.5$ $967.2972 .0 \quad 981.7 \quad 986.0 \quad 1021.9 \quad 1029.1 \quad 1046.0 \quad 1056.4 \quad 1073.8 \quad 1085.0 \quad 1149.8 \quad 1154.7 \quad 1180.6$

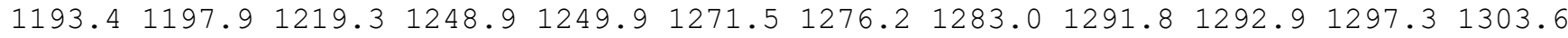
$1305.11313 .5 \quad 1335.91339 .7 \quad 1361.7 \quad 1380.8 \quad 1392.8 \quad 1398.31406 .2 \quad 1414.1 \quad 1813.3 \quad 1871.9$ $2649.72660 .32663 .92670 .0 \quad 2673.92674 .92676 .52679 .62687 .72698 .22740 .82753 .9$ $2777.6 \quad 2779.7 \quad 2781.7 \quad 2801.7$

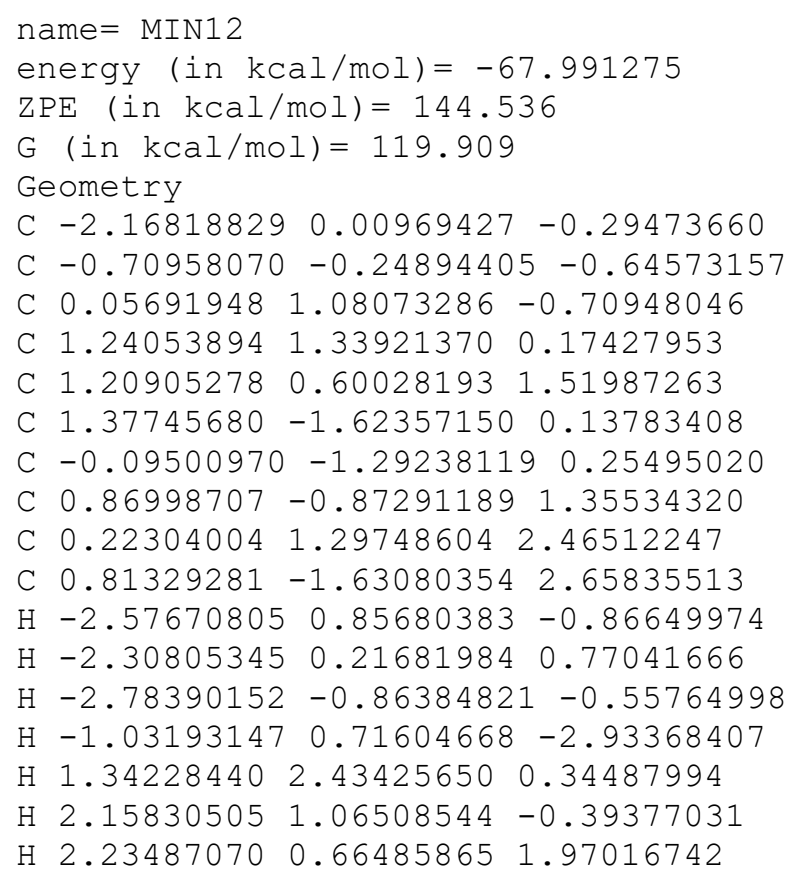


$\mathrm{H} \quad 1.69963201-2.656694390 .23776478$

$\mathrm{H} 1.98893283-1.12742579-0.60826739$

$\mathrm{H}-0.78892611-2.11833398 \quad 0.45675571$

$\mathrm{H}-0.811218601 .18513542 \quad 2.12163312$

$\mathrm{H} \quad 0.43266095 \quad 2.37105537 \quad 2.53914470$

$\mathrm{H} \quad 0.28126308 \quad 0.88977402 \quad 3.48044294$

$\mathrm{H} 1.72028603-1.46829980 \quad 3.25502475$

$\mathrm{H} \quad 0.71997641-2.71369453 \quad 2.50591227$

$\mathrm{H}-0.04660915-1.31971522 \quad 3.26646776$

$0-1.44362783-0.18544128-2.85700972$

O $-0.26793864 \quad 1.89145731-1.54703172$

o $-0.57367790-0.83143131-1.94724105$

Vibrational frequencies (in $\mathrm{cm}-1$ )

$\begin{array}{lllllllllllllll}52.3 & 98.5 & 109.3 & 154.1 & 158.1 & 171.5 & 193.5 & 216.8 & 255.4 & 267.8 & 306.2 & 332.1 & 351.2 & 358.9\end{array}$ $394.0 \quad 404.1 \quad 439.4 \quad 447.8 \quad 494.2 \quad 518.4 \quad 603.8 \quad 627.1 \quad 696.6 \quad 735.5 \quad 780.7 \quad 869.9 \quad 888.0 \quad 928.0$

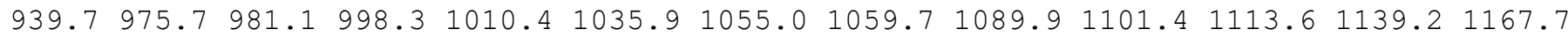
$\begin{array}{lllllllllllll}1170.5 & 1204.8 & 1221.7 & 1228.7 & 1275.3 & 1275.6 & 1281.2 & 1283.6 & 1285.9 & 1290.6 & 1291.7 & 1295.4\end{array}$ $1298.61304 .7 \quad 1309.51358 .8 \quad 1374.3 \quad 1378.8 \quad 1396.7 \quad 1400.11409 .91424 .21497 .7 \quad 1821.4$ $2646.22662 .32671 .62672 .6 \quad 2674.9 \quad 2675.6 \quad 2678.02697 .22710 .5 \quad 2732.92750 .12777 .4$ $2781.02781 .32787 .5 \quad 2804.9$

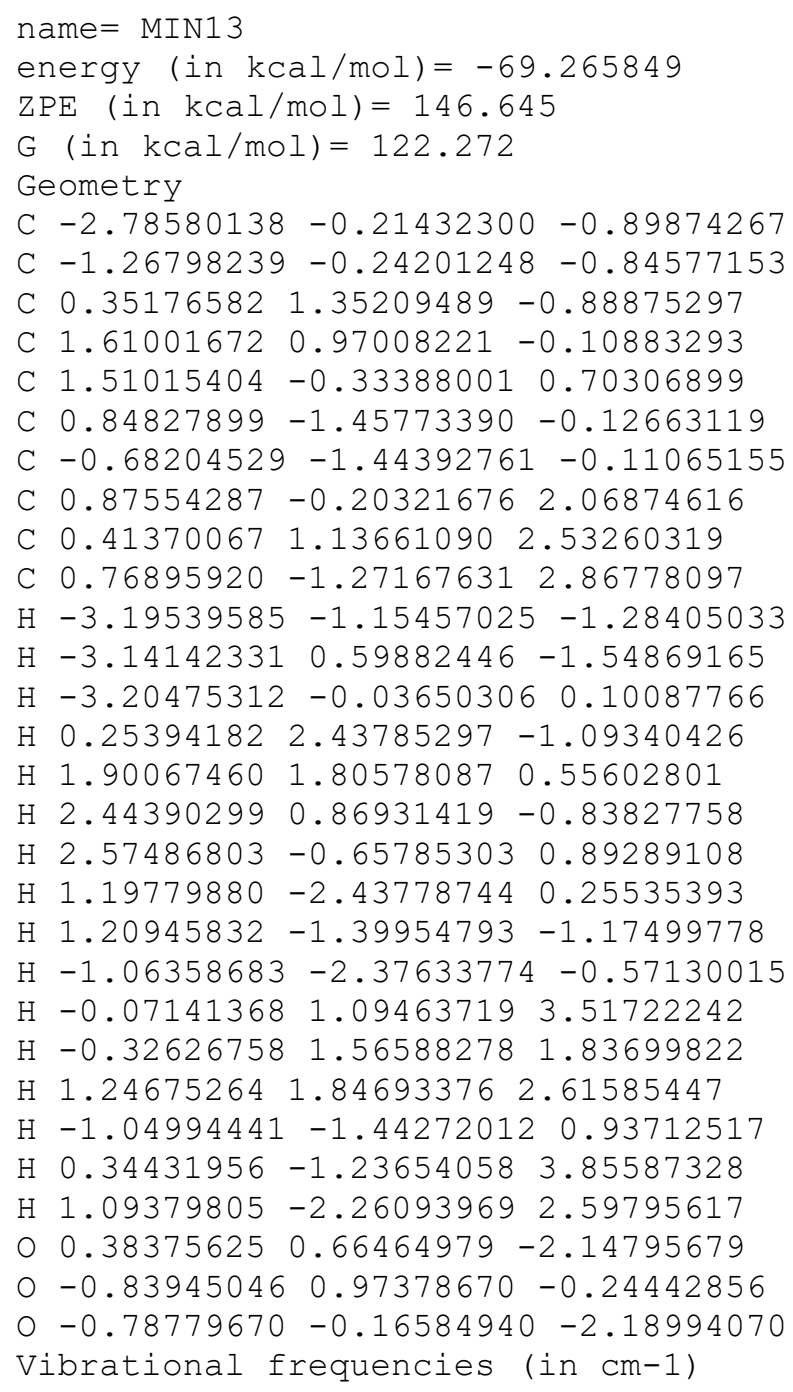


name $=\operatorname{MIN} 14$

energy (in $\mathrm{kcal} / \mathrm{mol})=-67.095323$

$\mathrm{ZPE}(\mathrm{in} \mathrm{kcal} / \mathrm{mol})=144.539$

$\mathrm{G}($ in $\mathrm{kcal} / \mathrm{mol})=119.942$

Geometry

C $-2.02280239-0.30946006-0.71177268$

C $-0.50651568-0.29397551-0.69043298$

C $0.07411337 \quad 1.14371999-0.68361937$

C $1.03365944 \quad 1.55779290 \quad 0.41107534$

C $1.41470644 \quad 0.36878015 \quad 1.29044490$

C $1.67331110-0.857033990 .35940934$

C $0.13265086-1.124366630 .42772123$

C $0.11636922-0.36097420 \quad 1.81207544$

C $-1.06326416 \quad 0.50430563 \quad 2.20110250$

C $0.41852193-1.31901645 \quad 2.96150672$

$\mathrm{H}-2.43548587 \quad 0.58838728-1.19337701$

$\mathrm{H}-2.46654819-0.39868691 \quad 0.28374494$

$\mathrm{H}-2.38277271-1.16874620-1.30247222$

$\mathrm{H}-0.44513666 \quad 0.49654022-3.07509249$

$\mathrm{H} 0.583618932 .374418261 .01188033$

$\mathrm{H} \quad 1.93817562 \quad 2.01085548 \quad-0.04764647$

H $2.17330608 \quad 0.60034950 \quad 2.03803226$

H $2.28527197-1.644055090 .80454986$

$\mathrm{H} 2.08825667-0.64051459-0.62599023$

$\mathrm{H}-0.20089458-2.162052790 .43941229$

$\mathrm{H}-0.82325350 \quad 1.110772493 .08525273$

$\mathrm{H}-1.93734800-0.108498352 .45936922$

$\mathrm{H}-1.37812018 \quad 1.20088051 \quad 1.41731342$

$\mathrm{H}-0.43238486-1.98293868 \quad 3.15519807$

$\mathrm{H} 0.62930182-0.772543153 .88811663$

$\mathrm{H} 1.28546132-1.958434662 .76096482$

$0-0.72137748-0.45520581-3.00118483$

$0-0.197836431 .88892795-1.59286253$

o $0.02177912-0.89439483-1.87921932$

Vibrational frequencies (in $\mathrm{cm}-1$ )

$\begin{array}{lllllllllllllll}34.1 & 91.8 & 112.1 & 147.7 & 170.9 & 190.4 & 211.8 & 243.6 & 262.8 & 290.8 & 305.2 & 331.2 & 375.3 & 381.1\end{array}$

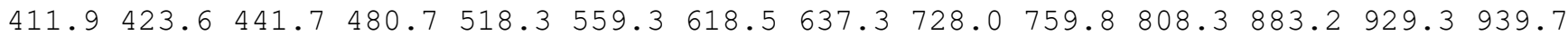

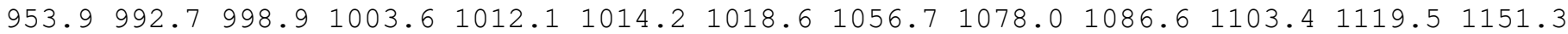

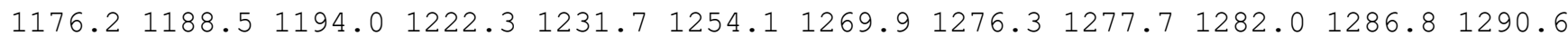

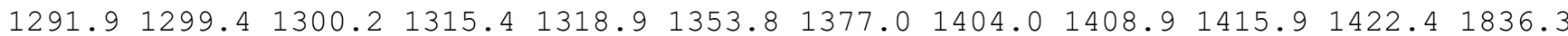
$2654.32660 .22670 .12672 .4 \quad 2674.3 \quad 2675.8 \quad 2679.22688 .02739 .2 \quad 2746.92750 .82766 .3$ $2777.6 \quad 2780.7 \quad 2782.7 \quad 2815.9$

name $=$ MIN15

energy (in $\mathrm{kcal} / \mathrm{mol})=-67.145062$

$\operatorname{ZPE}($ in $\mathrm{kcal} / \mathrm{mol})=144.639$

$\mathrm{G}($ in $\mathrm{kcal} / \mathrm{mol})=120.644$

Geometry

C $-2.11540543-0.05274274-0.54989060$

C $-0.60989928-0.19095407-0.65121565$

C $0.12007133 \quad 1.17692838-0.59869712$

C $1.32277301 \quad 1.342142990 .30645670$

C $1.599548890 .07242045 \quad 1.10714726$

C $1.55143549-1.139482530 .12558648$

C $0.00558090-1.16030394 \quad 0.36437898$

C $0.26368175-0.459780351 .75805754$

C $-0.71306662 \quad 0.56570672 \quad 2.29363749$

C $0.52987361-1.49393148 \quad 2.84841359$

$\mathrm{H}-2.47743350 \quad 0.83776093-1.08609200$

$\mathrm{H}-2.470575110 .00743062 \quad 0.48276133$

$\mathrm{H}-2.60827107-0.91985757-1.01835234$

$\mathrm{H}-0.69418023 \quad 0.70619594 \quad-3.01028795$

$\mathrm{H} \quad 1.16817155 \quad 2.21227858 \quad 0.97625920$ 
$\mathrm{H} 2.204916201 .61315071-0.31215913$

H $2.46388486 \quad 0.14743670 \quad 1.76660748$

$\mathrm{H} \quad 2.07018201-2.032715820 .48002727$

$\mathrm{H} \quad 1.88785349-0.95228945-0.89493665$

$\mathrm{H}-0.48917597-2.13127345 \quad 0.39024528$

$\mathrm{H}-1.63939810 \quad 0.08873356 \quad 2.64051487$

$\mathrm{H}-1.001789631 .331146351 .56596058$

$\mathrm{H}-0.281240221 .097701893 .15251653$

$\mathrm{H} 0.93541388-1.021064363 .75049106$

$\mathrm{H} \quad 1.24686497 \quad-2.261281392 .53648077$

$\mathrm{H}-0.39311075-2.012910643 .13368746$

$0-1.03488127-0.22463582-2.94322171$

$0-0.243979132 .07204502-1.31983420$

o $-0.23044780-0.76092708-1.90975508$

Vibrational frequencies (in $\mathrm{cm}-1$ )

$\begin{array}{lllllllllllllll}73.8 & 126.7 & 130.7 & 158.3 & 170.7 & 185.8 & 207.9 & 232.7 & 269.5 & 292.1 & 299.1 & 323.9 & 369.3 & 380.2\end{array}$ $405.0 \quad 417.0 \quad 438.4 \quad 477.9 \quad 517.8 \quad 564.0 \quad 607.8 \quad 638.2 \quad 727.5 \quad 757.3 \quad 809.3 \quad 884.5 \quad 927.6 \quad 940.2$ $951.2989 .3 \quad 1001.3 \quad 1007.9 \quad 1012.6 \quad 1014.6 \quad 1019.8 \quad 1056.8 \quad 1077.6 \quad 1087.6 \quad 1102.4 \quad 1121.1$ $\begin{array}{lllllllllllll}1153.2 & 1178.9 & 1189.8 & 1193.5 & 1223.6 & 1229.7 & 1256.2 & 1269.1 & 1275.8 & 1280.6 & 1283.2 & 1287.0\end{array}$

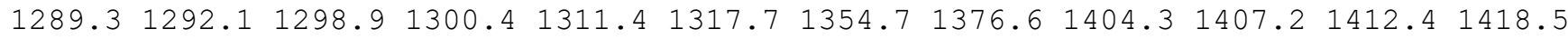
$1839.82655 .42662 .12669 .0 \quad 2673.12675 .0 \quad 2676.0 \quad 2679.22687 .92739 .82746 .92751 .3$ $2766.4 \quad 2778.1 \quad 2780.8 \quad 2782.9 \quad 2833.2$

\section{name $=$ MIN16}

energy (in $\mathrm{kcal} / \mathrm{mol})=-66.711203$

$\mathrm{ZPE}($ in $\mathrm{kcal} / \mathrm{mol})=144.714$

$\mathrm{G}($ in $\mathrm{kcal} / \mathrm{mol})=120.083$

Geometry

C $-2.01746056-0.33063203-0.65414717$

C $-0.50115199-0.26986462-0.69467407$

C $0.02881848 \quad 1.18640945-0.63862932$

C $0.97197755 \quad 1.59880067 \quad 0.47060397$

C $1.40711700 \quad 0.39216676 \quad 1.29865275$

C $1.72052659-0.77697584 \quad 0.31231015$

C $0.19689619-1.122350720 .37342445$

C $0.14686483-0.42214027 \quad 1.78915418$

C $-1.083720620 .35534877 \quad 2.20419146$

C $0.49913091-1.40792227 \quad 2.89968246$

$\mathrm{H}-2.47814037 \quad 0.62302478-0.37323070$

$\mathrm{H}-2.38032294-1.09807053 \quad 0.03951376$

$\mathrm{H}-2.42367911-0.58932228-1.64863398$

H $0.49435575 \quad 2.37287065 \quad 1.10450704$

$\mathrm{H}-1.38361553 \quad 1.12512202 \quad 1.48669855$

H $1.85558072 \quad 2.10507296 \quad 0.02563598$

H $2.15665870 \quad 0.62484878 \quad 2.05480863$

$\mathrm{H} \quad 2.11821604-0.49835774-0.66456712$

$\mathrm{H}-0.46440920 \quad 0.67499655-2.99279683$

$\mathrm{H}-0.08313952-2.17543961 \quad 0.33709737$

$\mathrm{H}-1.94578706-0.31186317 \quad 2.33617571$

$\mathrm{H}-0.91741670 \quad 0.86630242 \quad 3.16216964$

$\mathrm{H}-0.32569715-2.106947213 .08274058$

$\mathrm{H} \quad 0.70432553-0.88567491 \quad 3.84150357$

H $2.37244390-1.55222246 \quad 0.72026192$

H $1.38462338-2.00910465 \quad 2.66554189$

$0-0.69316378-0.29142710-3.02242809$

$0-0.265488161 .95164184-1.52484006$

○ $0.01873587-0.80938256-1.91371273$

Vibrational frequencies (in $\mathrm{cm}-1$ )

$23.9 \quad 77.0 \quad 141.2 \quad 170.1 \quad 188.9 \quad 194.1 \quad 218.5 \quad 240.0 \quad 265.8 \quad 300.2 \quad 317.4 \quad 332.2 \quad 379.6 \quad 388.0$

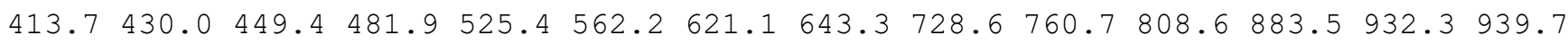

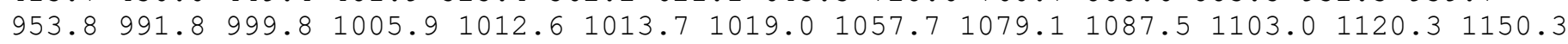
$1176.0 \quad 1189.3 \quad 1194.1 \quad 1223.5 \quad 1233.8 \quad 1248.3 \quad 1267.7 \quad 1276.2 \quad 1278.9 \quad 1281.5 \quad 1284.9 \quad 1288.2$ $1292.91299 .2 \quad 1300.1 \quad 1315.6 \quad 1318.2 \quad 1353.5 \quad 1376.2 \quad 1405.3 \quad 1412.4 \quad 1420.01438 .7 \quad 1833.9$ 
$2649.82654 .12670 .6 \quad 2673.5 \quad 2674.5 \quad 2675.8 \quad 2679.42688 .12739 .3 \quad 2746.02750 .7 \quad 2766.4$ $2774.72781 .3 \quad 2783.12801 .7$

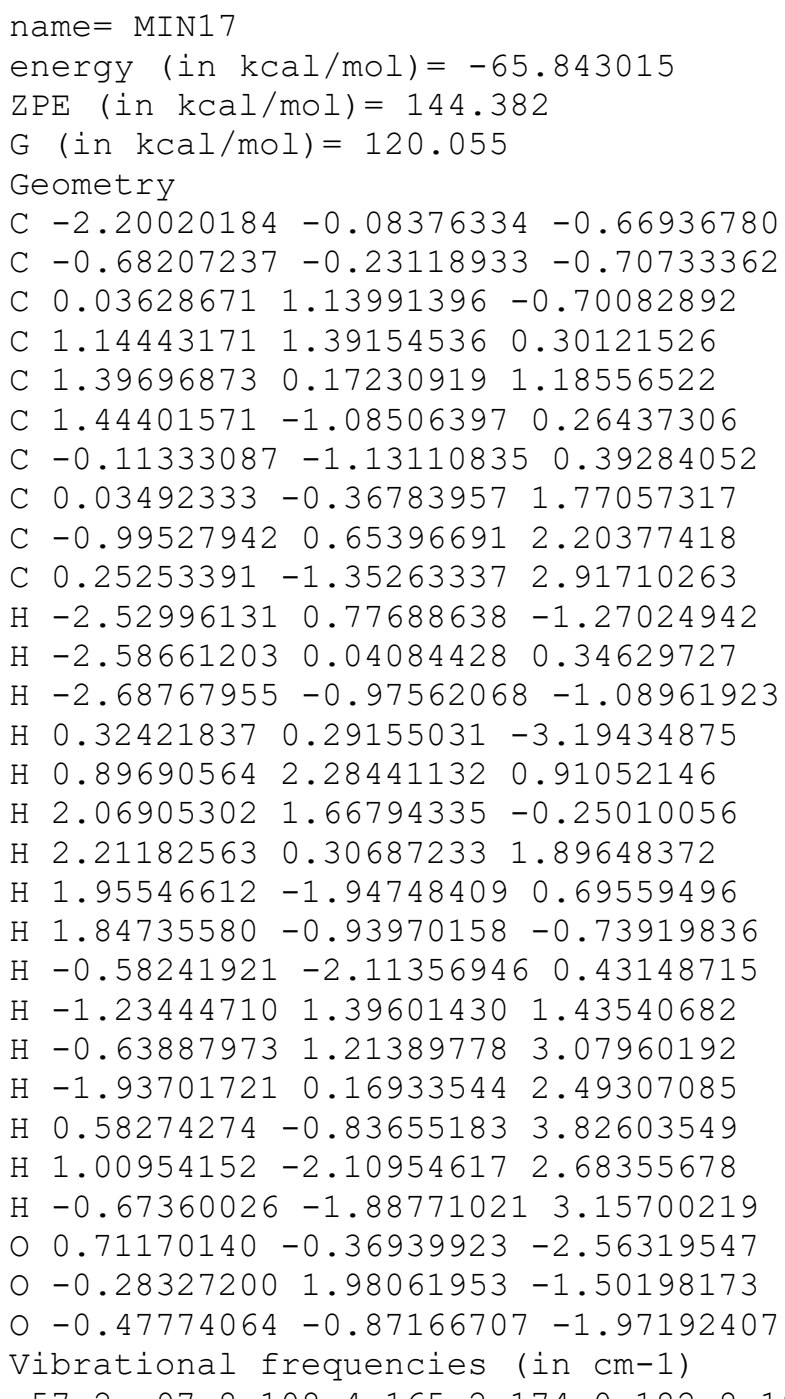

$\begin{array}{lllllllllllllllll}57.2 & 97.8 & 108.4 & 165.2 & 174.0 & 182.8 & 197.3 & 223.1 & 271.7 & 283.8 & 298.0 & 334.3 & 355.8 & 379.9\end{array}$ $404.4 \quad 418.4 \quad 437.7 \quad 491.4 \quad 516.2 \quad 550.9 \quad 589.9 \quad 640.6 \quad 717.3 \quad 759.1 \quad 810.2 \quad 885.9 \quad 928.6 \quad 941.8$ $950.9977 .6 \quad 1000.5 \quad 1004.7 \quad 1013.5 \quad 1018.6 \quad 1030.5 \quad 1052.8 \quad 1078.4 \quad 1087.7 \quad 1100.4 \quad 1121.9$ $1162.91177 .2 \quad 1188.4 \quad 1199.9 \quad 1201.6 \quad 1229.5 \quad 1243.6 \quad 1265.91276 .5 \quad 1279.2 \quad 1283.4 \quad 1287.8$ $1288.41296 .5 \quad 1299.8 \quad 1303.8 \quad 1314.7 \quad 1318.6 \quad 1343.9 \quad 1356.1 \quad 1380.2 \quad 1402.1 \quad 1407.1 \quad 1416.2$ $1843.82655 .02664 .5 \quad 2669.4 \quad 2672.0 \quad 2673.3 \quad 2675.6 \quad 2679.82687 .5 \quad 2738.6 \quad 2748.7 \quad 2752.3$ $2768.22776 .82780 .62782 .7 \quad 2845.0$

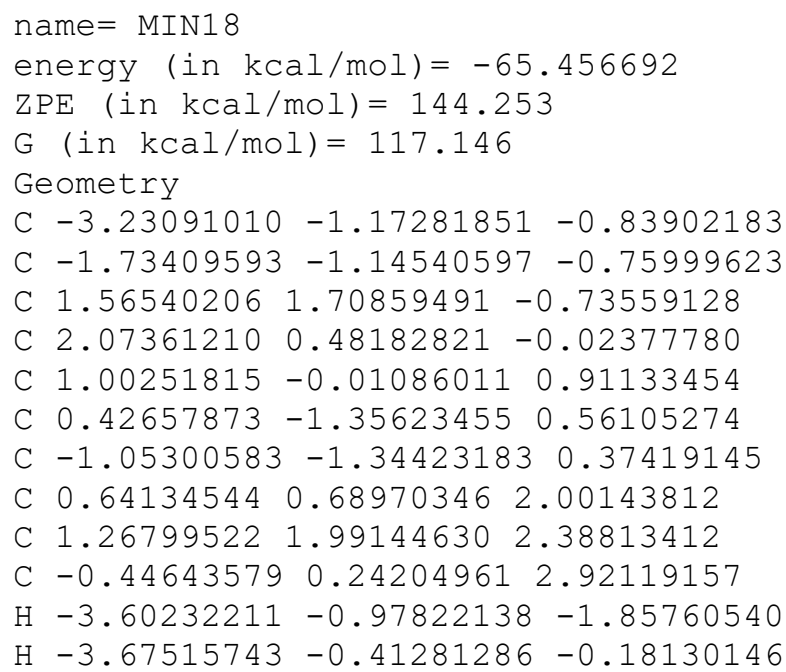


$\mathrm{H}-3.62026904-2.15585689-0.53659976$

$\mathrm{H} \quad 1.83863544 \quad 2.69205067 \quad-0.33528544$

H $3.01200847 \quad 0.687165640 .53048489$

$\mathrm{H} 2.34630947-0.30210087-0.76648529$

$\mathrm{H} \quad 0.31043438 \quad 0.05847169-2.06175888$

H $0.69058895 \quad-2.07468453 \quad 1.37318018$

$\mathrm{H} \quad 0.91792573-1.78324333-0.34256465$

$\mathrm{H}-1.61332151-1.54524907 \quad 1.28702154$

$\mathrm{H} 0.64695986 \quad 2.83701609 \quad 2.05851006$

H 2.27515490 2.12952004 1.97781556

$\mathrm{H} \quad 1.37068330 \quad 2.07938290 \quad 3.47999795$

$\mathrm{H}-1.43127545 \quad 0.385688402 .44746578$

$\mathrm{H}-0.46385197 \quad 0.79537762 \quad 3.86763513$

$\mathrm{H}-0.35810954-0.82456303 \quad 3.17272990$

$00.09536764-0.90950464-2.16456662$

$00.867628231 .63035906-1.72102413$

$\begin{array}{llll}0 & -1.31366624 & -0.88983820 & -2.03227017\end{array}$

Vibrational frequencies (in $\mathrm{cm}-1$ )

$\begin{array}{lllllllllllllll}20.3 & 64.6 & 81.4 & 87.7 & 104.4 & 105.9 & 119.8 & 154.4 & 164.1 & 200.8 & 240.8 & 263.4 & 307.0 & 324.7\end{array}$

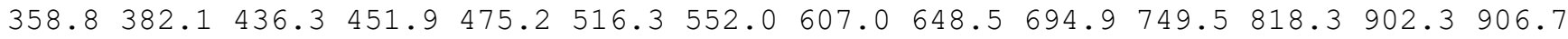

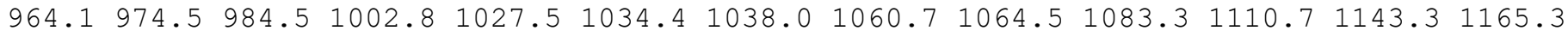

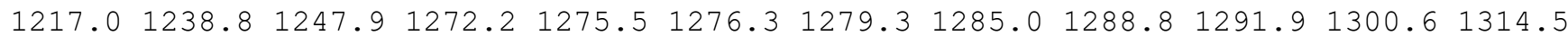

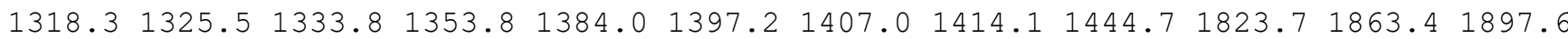
$2642.32645 .8 \quad 2663.1 \quad 2663.6 \quad 2665.8 \quad 2666.92671 .02672 .12674 .92727 .72733 .2 \quad 2747.8$ $2757.92776 .12776 .3 \quad 2777.8$

name $=$ MIN19

energy (in $\mathrm{kcal} / \mathrm{mol})=-64.80604$

$\mathrm{ZPE}($ in $\mathrm{kcal} / \mathrm{mol})=143.674$

$\mathrm{G}($ in $\mathrm{kcal} / \mathrm{mol})=118.117$

Geometry

C $-2.32837554 \quad 0.01376643 \quad-0.86780937$

C $-0.82342527-0.19462742-0.97188590$

C $-0.07962860 \quad 1.16173835 \quad-0.97359857$

C $0.766395791 .31446841 \quad 0.26888953$

C $0.68961052-0.022155790 .99592196$

C $1.24692578-1.197176050 .21823408$

C $-0.24420332-0.95127158 \quad 0.20775191$

C $0.71262204 \quad 0.06363710 \quad 2.50511851$

C $-0.64548944 \quad 0.56070607 \quad 3.02364414$

C $1.05420879-1.273930683 .17226290$

$\mathrm{H}-2.689426690 .69600609-1.65164589$

$\mathrm{H}-2.606608350 .43846299 \quad 0.10429168$

$\mathrm{H}-2.86946257-0.93481076-0.98473731$

$\mathrm{H} 0.46458253 \quad 0.08089805-3.35043623$

$\mathrm{H} \quad 0.39407133 \quad 2.15343364 \quad 0.88758338$

$\mathrm{H} 1.80871106 \quad 1.58184836 \quad 0.00767861$

H $1.50166537 \quad 0.80485142 \quad 2.79834035$

H $1.63646604 \quad-2.06786962 \quad 0.73671459$

$\mathrm{H} 1.83116150-1.02348693-0.68483129$

$\mathrm{H}-0.90872814-1.65699499 \quad 0.69464740$

$\mathrm{H}-1.44838267-0.13896903 \quad 2.76667670$

$\mathrm{H}-0.90962782 \quad 1.53547077 \quad 2.60139752$

$\mathrm{H}-0.637382320 .66673005 \quad 4.11387360$

H $0.34401922 \quad-2.05898664 \quad 2.89155512$

H 2.05945982 -1.61333661 2.89779688

$\mathrm{H} \quad 1.02927780-1.18630760 \quad 4.26436024$

$00.66068241-0.62142901-2.67744202$

o $-0.17200158 \quad 1.96734258-1.85548458$

o $-0.65557062-0.87889778-2.20309219$

Vibrational frequencies (in $\mathrm{cm}-1$ )

$\begin{array}{llllllllllllllll}45.8 & 64.1 & 92.3 & 99.6 & 154.4 & 163.7 & 174.1 & 183.2 & 218.6 & 244.1 & 254.9 & 303.5 & 320.0 & 335.8\end{array}$ $356.3 \quad 410.2 \quad 430.8 \quad 469.2 \quad 506.7 \quad 554.6 \quad 575.1 \quad 584.2 \quad 629.4 \quad 773.3 \quad 786.9 \quad 869.4 \quad 880.8 \quad 956.4$ $960.1976 .0 \quad 981.0 \quad 987.2 \quad 1007.3 \quad 1012.5 \quad 1025.9 \quad 1034.2 \quad 1052.4 \quad 1117.7 \quad 1124.5 \quad 1153.0 \quad 1170.7$ 
$\begin{array}{lllllllllllllll}1174.2 & 1179.1 & 1190.6 & 1213.3 & 1243.6 & 1275.4 & 1278.9 & 1282.9 & 1283.6 & 1292.4 & 1295.4 & 1300.0\end{array}$

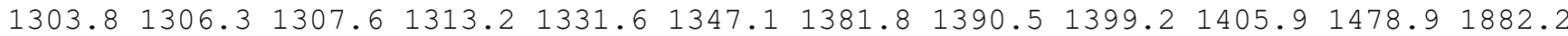
$2660.62668 .42672 .12673 .9 \quad 2675.92677 .6 \quad 2679.42703 .32704 .72745 .02771 .22777 .7$ $2781.4 \quad 2782.2 \quad 2783.2 \quad 2847.1$

\section{name $=$ MIN20}

energy (in $\mathrm{kcal} / \mathrm{mol})=-63.961618$

$\mathrm{ZPE}($ in $\mathrm{kcal} / \mathrm{mol})=142.891$

$\mathrm{G}($ in $\mathrm{kcal} / \mathrm{mol})=114.818$

Geometry

C $-3.32561399-1.83068110-0.10834961$

C $-1.82247377-1.84297838-0.11006542$

C $2.218964181 .66369490-0.10834299$

C $1.47300178 \quad 0.55661723-0.12463536$

C $0.63252291 \quad 0.15276150 \quad 1.02446034$

C $0.37704883-1.37737700 \quad 1.13874263$

C $-1.15264096-1.158189391 .04526465$

C $-0.92054266 \quad 0.38640743 \quad 0.85019520$

C $-1.33144173 \quad 0.93045288-0.50475745$

C -1.525386801 .216157391 .97149030$

$\mathrm{H}-3.72602286-2.04948625-1.10952504$

$\mathrm{H}-3.73253011-0.86636725 \quad 0.21598258$

$\mathrm{H}-3.71659194 \quad-2.60381474 \quad 0.56743001$

H 2.324385392 .381061420 .70117082

$\mathrm{H} 1.45457731-0.10668647-0.99032152$

H $2.47419288 \quad 1.67020302-2.85298060$

$\mathrm{H} \quad 1.00339264 \quad 0.58358080 \quad 1.97269383$

$\mathrm{H} \quad 0.71912588-1.82811465 \quad 2.07168173$

$\mathrm{H} \quad 0.79126434-1.966853890 .30889928$

$\mathrm{H}-1.67833055-1.391839561 .98699646$

$\mathrm{H}-2.420151170 .99495329-0.60636409$

$\mathrm{H}-0.958806190 .30301754 \quad-1.32745769$

$\mathrm{H}-1.21018768 \quad 2.26439262 \quad 1.90066155$

$\mathrm{H}-1.22724050 \quad 0.85206307 \quad 2.96133892$

$\mathrm{H}-2.62088502 \quad 1.20148305 \quad 1.93353599$

○ $3.03857907 \quad 1.19222546-2.19647672$

0 $3.009798282 .12788466-1.11909209$

$0-1.20379323-2.39621401-0.98444241$

$\mathrm{H}-0.92421038 \quad 1.93675209-0.66417987$

Vibrational frequencies (in $\mathrm{cm}-1$ )

$\begin{array}{lllllllllllllll}25.9 & 29.5 & 42.1 & 68.9 & 81.4 & 133.3 & 161.5 & 163.0 & 177.2 & 196.4 & 204.3 & 224.1 & 262.7 & 286.4\end{array}$ $319.7 \quad 328.4 \quad 389.8 \quad 410.2 \quad 448.3 \quad 471.3 \quad 567.6 \quad 598.5 \quad 659.6 \quad 686.6 \quad 820.4 \quad 832.8 \quad 902.1 \quad 923.4$

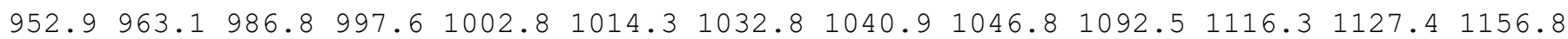

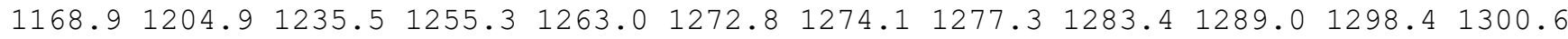

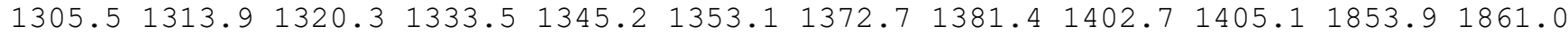
$2667.52669 .7 \quad 2676.7 \quad 2676.8 \quad 2678.2 \quad 2679.5 \quad 2681.22719 .42727 .6 \quad 2729.5 \quad 2755.42760 .9$ 2779.82780 .92782 .92869 .4

name $=$ MIN21

energy (in $\mathrm{kcal} / \mathrm{mol})=-64.089796$

$\mathrm{ZPE}(\mathrm{in} \mathrm{kcal} / \mathrm{mol})=143.052$

$\mathrm{G}($ in $\mathrm{kcal} / \mathrm{mol})=116.068$

Geometry

C $-3.46222544-0.52940208-0.93530088$

C $-1.96536907-0.64713191-0.91483040$

C $1.650700230 .58597006-0.79752055$

C $1.68249826 \quad 0.19285054 \quad 0.47892909$

C $0.66879112-0.696134301 .09128806$

C $0.06423627-1.78433884 \quad 0.15516051$

C $-1.35696291-1.200567790 .34003851$

C $-0.78054414-0.107503391 .31989404$

C $-0.99199118 \quad 1.33131717 \quad 0.89858145$

C $-1.26452897-0.315591332 .74910906$ 
$\mathrm{H}-3.80358171 \quad 0.07963142-1.78589490$

$\mathrm{H}-3.84975849-0.07243554-0.01689091$

$\mathrm{H}-3.92841584-1.51868055-1.03942303$

$\mathrm{H} \quad 0.87916415 \quad 0.36888130-1.54434973$

H 2.48444353 $0.51432254 \quad 1.14228339$

H $4.347555201 .07157481-0.90897520$

H $1.07033187-1.13530427 \quad 2.02574431$

$\mathrm{H} \quad 0.18402212-2.80539565 \quad 0.52362753$

$\mathrm{H} \quad 0.43428731-1.75691358-0.87603541$

$\mathrm{H}-2.04901789-1.896050890 .84241978$

$\mathrm{H}-2.038441111 .637489581 .00259478$

$\mathrm{H}-0.69736581 \quad 1.50940379-0.14510494$

$\mathrm{H}-0.38321458 \quad 2.01151004 \quad 1.51029067$

$\mathrm{H}-1.12324964-1.34806636 \quad 3.08764907$

$\mathrm{H}-2.32964065-0.079070662 .85026660$

$\mathrm{H}-0.71798220 \quad 0.33281662 \quad 3.44602098$

$03.652701871 .68321091-0.56144018$

o $2.577382311 .34629113-1.44036673$

$0-1.30200525-0.32205869-1.86966406$

Vibrational frequencies (in $\mathrm{cm}-1$ )

$\begin{array}{llllllllllllllll}36.0 & 59.6 & 59.8 & 89.9 & 99.7 & 143.6 & 160.6 & 169.7 & 177.9 & 199.5 & 216.5 & 224.9 & 237.4 & 278.9\end{array}$ $329.2 \quad 342.9 \quad 374.5 \quad 398.0 \quad 458.4 \quad 537.9 \quad 574.8 \quad 597.6 \quad 677.7 \quad 701.1 \quad 814.8 \quad 827.4 \quad 908.9 \quad 916.6$

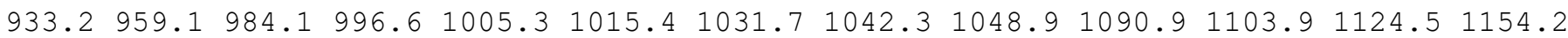
$1168.41203 .7 \quad 1227.8 \quad 1240.6 \quad 1260.5 \quad 1263.4 \quad 1273.7 \quad 1283.0 \quad 1285.0 \quad 1294.6 \quad 1298.2 \quad 1299.0$ $1305.61316 .51324 .51343 .2 \quad 1350.6 \quad 1356.91371 .0 \quad 1381.11404 .6 \quad 1405.6 \quad 1847.3 \quad 1860.8$ $2664.82670 .32675 .5 \quad 2676.0 \quad 2678.12681 .2 \quad 2682.12686 .32720 .22731 .62757 .62762 .0$ $2780.0 \quad 2780.7 \quad 2782.7 \quad 2867.3$

\footnotetext{
name $=$ MIN22

energy (in $\mathrm{kcal} / \mathrm{mol})=-63.739197$

$\mathrm{ZPE}($ in $\mathrm{kcal} / \mathrm{mol})=142.936$

$\mathrm{G}($ in $\mathrm{kcal} / \mathrm{mol})=115.157$

Geometry

C $-3.09738149-1.99123086-0.07403866$

C $-1.59515007-1.94255009-0.06912165$

C $1.88889306 \quad 1.86151528-0.26011301$

C $1.503034220 .59123205-0.11747166$

C $0.76291480 \quad 0.12204968 \quad 1.07569521$

C $0.58651358-1.41874598 \quad 1.18238403$

C $-0.95408164-1.27799271 \quad 1.11494000$

C $-0.80754321 \quad 0.28288446 \quad 0.98012813$

C $-1.30457842 \quad 0.86384171-0.33032522$

C $-1.39835106 \quad 1.033683192 .16237119$

$\mathrm{H}-3.48573601-2.10303052-1.09744188$

$\mathrm{H}-3.54630970-1.09207681 \quad 0.36241267$

$\mathrm{H}-3.45585504 \quad-2.85770118 \quad 0.49868826$

$\mathrm{H} \quad 1.77601528 \quad 2.67323901 \quad 0.45317356$

$\mathrm{H} 1.69764024-0.15382824-0.88957944$

H $1.95456365 \quad 1.60443644 \quad-2.95423294$

$\mathrm{H} \quad 1.16901414 \quad 0.56249357 \quad 2.00459619$

$\mathrm{H} \quad 0.96247348 \quad-1.85534613 \quad 2.10978571$

H $1.01843916-1.98465144 \quad 0.34592876$

$\mathrm{H}-1.45856705-1.580476592 .04789953$

$\mathrm{H}-2.39731985 \quad 0.84943759-0.39655934$

$\mathrm{H}-0.91100551 \quad 0.31027813-1.19551647$

$\mathrm{H}-0.98091081 \quad 1.90583550-0.44715395$

$\mathrm{H}-1.12369193 \quad 2.09540897 \quad 2.13301878$

$\mathrm{H}-1.04901888 \quad 0.63390058 \quad 3.12135231$

H $-2.49315884 \quad 0.98047091 \quad 2.16392530$

o $2.71555146 \quad 1.41066654 \quad-2.35195544$

O $2.504312602 .40451503-1.35103319$

$0-0.95335686-2.42722461-0.96737603$

Vibrational frequencies (in $\mathrm{cm}-1$ )
} 
$\begin{array}{llllllllllllllllll}27.5 & 35.5 & 43.0 & 74.2 & 83.7 & 131.7 & 162.6 & 178.5 & 180.2 & 203.1 & 210.6 & 230.5 & 265.0 & 292.2\end{array}$

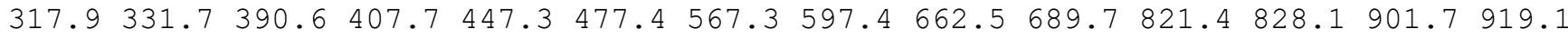
$950.4962 .0 \quad 986.3 \quad 998.3 \quad 1002.3 \quad 1014.2 \quad 1033.91040 .3 \quad 1048.1 \quad 1093.6 \quad 1114.7 \quad 1124.01157 .0$

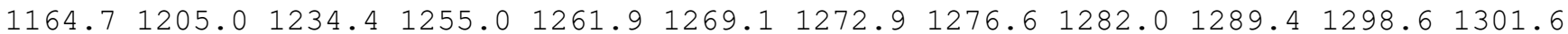
$1306.31308 .0 \quad 1319.0 \quad 1337.4 \quad 1345.4 \quad 1354.0 \quad 1369.3 \quad 1380.91401 .91404 .11853 .2 \quad 1859.3$ $2667.3 \quad 2669.4 \quad 2676.5 \quad 2676.8 \quad 2678.0 \quad 2679.6 \quad 2681.12719 .82728 .2 \quad 2730.3 \quad 2754.3 \quad 2760.5$ $2779.52780 .8 \quad 2782.8 \quad 2867.3$

name $=$ MIN23

energy (in $\mathrm{kcal} / \mathrm{mol})=-64.869036$

$\operatorname{ZPE}($ in $\mathrm{kcal} / \mathrm{mol})=144.228$

$\mathrm{G}($ in $\mathrm{kcal} / \mathrm{mol})=119.67$

Geometry

C $-2.05140777-0.56280150-0.57811403$

C $-0.54580338-0.38050079-0.64097425$

C $-0.164495021 .12002328-0.69682335$

C $0.99795051 \quad 1.608207920 .15132809$

C $1.51458086 \quad 0.51794209 \quad 1.08521452$

C $1.74695054 \quad-0.76819843 \quad 0.23319861$

C $0.23492865-1.093757820 .47377383$

C $0.31106349-0.22298281 \quad 1.78917715$

C $-0.88167769 \quad 0.59982497 \quad 2.22627673$

C $0.77534615-1.07024606 \quad 2.97216393$

$\mathrm{H}-2.54124958-0.01396395-1.39911179$

$\mathrm{H}-2.48099765-0.184414120 .35569296$

$\mathrm{H}-2.32792190-1.61987236-0.66251702$

$\mathrm{H}-1.20606534-1.12182355-3.24083468$

$\mathrm{H} \quad 0.68302140 \quad 2.50575027 \quad 0.72114857$

H $1.80732803 \quad 1.96344122-0.52038253$

H 2.32742018 $0.84352942 \quad 1.73440634$

H 2.43794233 -1.48890440 0.67321515

H 2.05231999 $-0.61011438-0.80320262$

$\mathrm{H}-0.04294304 \quad-2.13908952 \quad 0.59935427$

$\mathrm{H}-1.26304551 \quad 1.278009851 .45574340$

$\mathrm{H}-0.625439861 .227520913 .09091783$

$\mathrm{H}-1.71538516-0.04681743 \quad 2.53051412$

$\mathrm{H} 1.05855924-0.43999702 \quad 3.82323647$

$\mathrm{H} 1.64380648-1.69267110 \quad 2.73009145$

$\mathrm{H}-0.02021895-1.74473732 \quad 3.30980522$

$0-0.58369783-0.40873825-2.95321593$

$0-0.790102291 .88419233-1.38149321$

o $-0.02151676-1.02280406-1.80549518$

Vibrational frequencies (in cm-1)

$\begin{array}{lllllllllllllll}56.8 & 81.8 & 134.9 & 151.9 & 166.2 & 179.7 & 187.3 & 220.0 & 233.1 & 267.7 & 281.9 & 298.1 & 321.3 & 380.9\end{array}$ $398.6 \quad 411.8 \quad 433.8 \quad 478.2 \quad 508.1 \quad 565.6 \quad 596.5 \quad 640.6 \quad 727.3 \quad 757.4 \quad 810.2 \quad 886.1926 .5 \quad 939.7$ $949.0 \quad 988.1 \quad 1002.5 \quad 1009.1 \quad 1014.0 \quad 1018.9 \quad 1026.3 \quad 1055.5 \quad 1075.5 \quad 1087.3 \quad 1096.4 \quad 1121.7$

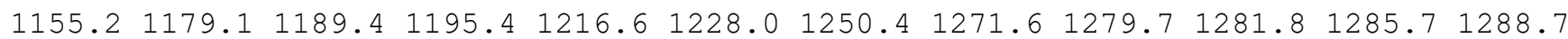

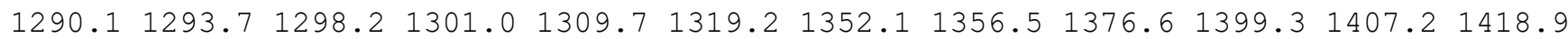
$1852.12657 .72664 .22668 .5 \quad 2673.5 \quad 2675.3 \quad 2678.32679 .62687 .0 \quad 2740.62747 .92751 .5$ $2765.22778 .92781 .2 \quad 2782.92873 .9$

name $=$ MIN24

energy $($ in $\mathrm{kcal} / \mathrm{mol})=-64.484117$

$\mathrm{ZPE}(\mathrm{in} \mathrm{kcal} / \mathrm{mol})=144.74$

$\mathrm{G}($ in $\mathrm{kcal} / \mathrm{mol})=120.187$

Geometry

C $-3.47057837 \quad 0.439206690 .93467329$

C $-1.13848504 \quad 0.55215117 \quad 0.58503232$

C $-0.035106291 .56258276 \quad 0.37203415$

C $1.28058840 \quad 0.90945760 \quad 0.82362111$

C $1.38362831-0.13024523-0.31726961$

C $2.80598647-0.54788087-0.64347160$

C $-0.95305841-0.75733296 \quad 0.37682661$ 
C $0.38195104-1.30624388-0.06129079$

C $0.91526863-2.239623301 .03612766$

C $0.17787965-2.09670087-1.36188839$

$\mathrm{H}-4.22849064 \quad 1.18911260 \quad 1.19518015$

$\mathrm{H}-3.45307472-0.36810760 \quad 1.67195301$

$\mathrm{H}-3.64346443 \quad 0.05147536-0.07494565$

$\mathrm{H}-0.250013292 .57157436 \quad 0.76504819$

H $1.23892647 \quad 0.46395642 \quad 1.82525747$

H $2.12073847 \quad 1.620483020 .81303901$

H $3.30736588-0.98291386 \quad 0.22736627$

H $2.83398458-1.28671227-1.45471224$

H $3.39885340 \quad 0.31433720 \quad-0.98045877$

$\mathrm{H}-1.73284562-1.495842770 .49945299$

$\mathrm{H} 1.08094954-1.703659791 .97622757$

$\mathrm{H} \quad 1.85890603-2.710007790 .74237992$

$\mathrm{H} 0.20163903-3.04488030 \quad 1.24505649$

$\mathrm{H}-0.19088702-1.43994328-2.16229901$

$\mathrm{H}-0.55791342-2.89653111-1.23030108$

H $1.10757859-2.55606135-1.70968235$

O $0.91609720 \quad 0.57592310-1.47664255$

O $0.14128417 \quad 1.71823672-1.03007646$

o $-2.26479250 \quad 1.191709290 .98534161$

Vibrational frequencies (in cm-1)

$\begin{array}{llllllllllllllll}58.7 & 97.7 & 123.0 & 145.3 & 170.6 & 182.7 & 187.8 & 199.1 & 226.0 & 252.2 & 295.8 & 305.6 & 324.6 & 361.9\end{array}$ $379.1400 .0 \quad 464.1 \quad 508.6 \quad 535.1 \quad 573.9 \quad 613.1 \quad 675.0 \quad 754.9 \quad 773.9 \quad 909.3 \quad 916.4 \quad 938.4 \quad 952.0$

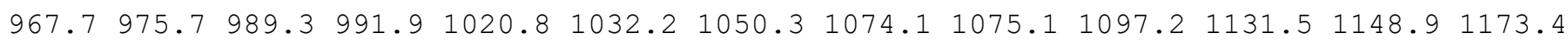

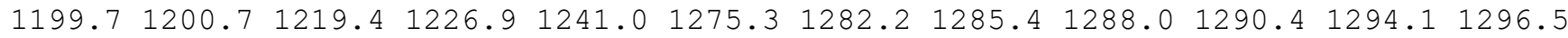
$1299.51305 .6 \quad 1308.7 \quad 1313.91324 .4 \quad 1341.6 \quad 1368.6 \quad 1385.7 \quad 1394.01399 .7 \quad 1410.51864 .7$ $2652.52658 .5 \quad 2670.42671 .7 \quad 2676.7 \quad 2678.8 \quad 2679.12681 .32682 .12707 .72746 .62756 .9$ $2772.6 \quad 2779.7 \quad 2780.6 \quad 2782.2$

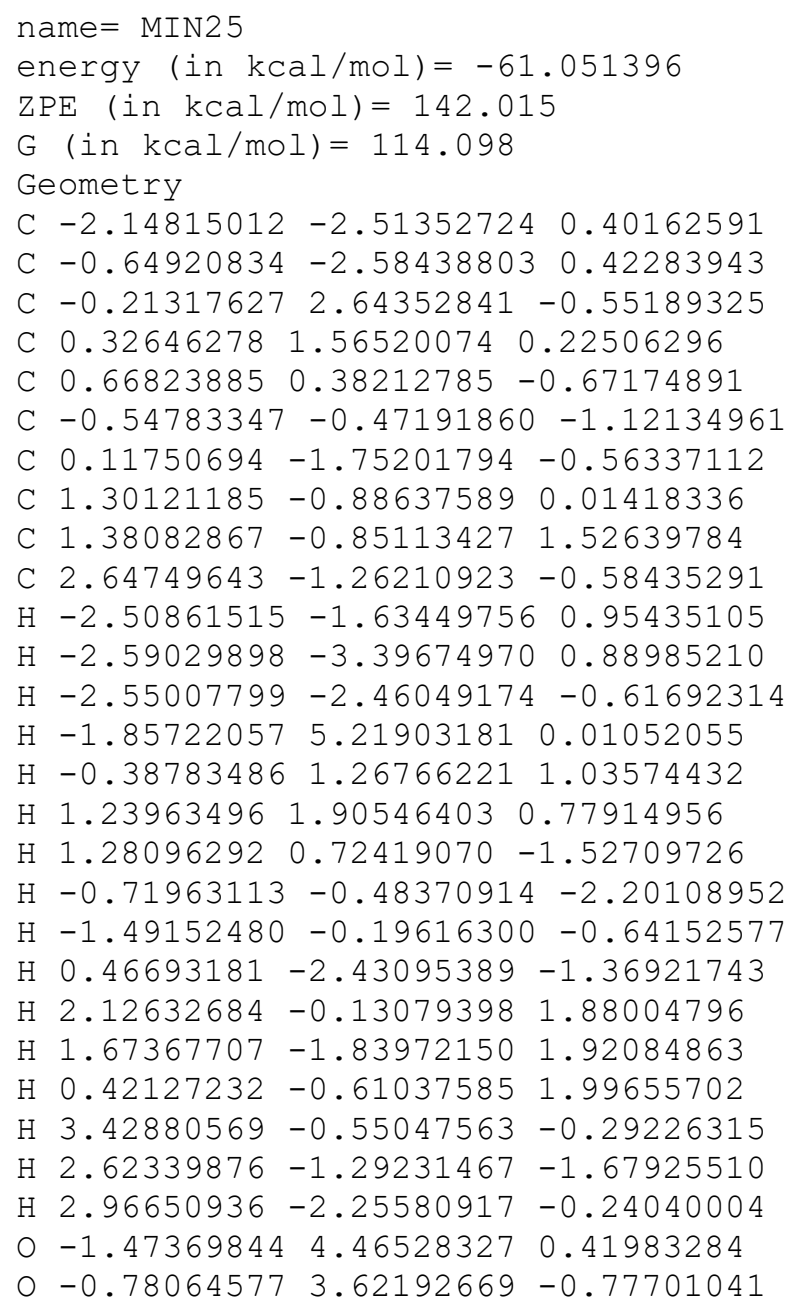


$0-0.07687740-3.328009091 .18196421$

Vibrational frequencies (in $\mathrm{cm}-1$ )

$\begin{array}{llllllllllllllll}21.8 & 36.0 & 41.3 & 73.1 & 79.4 & 141.5 & 164.0 & 190.5 & 195.1 & 211.1 & 231.8 & 247.9 & 263.9 & 282.5\end{array}$ $326.6 \quad 366.4 \quad 412.9 \quad 430.6 \quad 472.6 \quad 493.6 \quad 501.2 \quad 514.9 \quad 592.3 \quad 625.1 \quad 665.5 \quad 808.6 \quad 861.5 \quad 896.4$ $937.7 \quad 938.5 \quad 994.2 \quad 1000.3 \quad 1008.5 \quad 1017.7 \quad 1025.5 \quad 1043.4 \quad 1055.7 \quad 1066.2 \quad 1089.6 \quad 1110.3 \quad 1131.4$ $1155.3 \quad 1172.4 \quad 1198.7 \quad 1220.9 \quad 1249.8 \quad 1261.9 \quad 1263.0 \quad 1266.7 \quad 1275.1 \quad 1285.6 \quad 1290.1 \quad 1292.7$

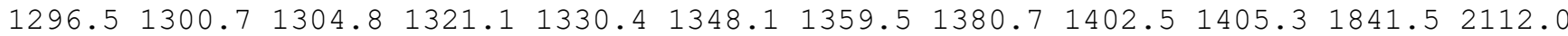
$2627.02658 .22665 .7 \quad 2673.4 \quad 2675.0 \quad 2677.42681 .62683 .22710 .62715 .12725 .52764 .0$ $2778.5 \quad 2779.4 \quad 2782.0 \quad 2841.8$

\author{
name $=$ MIN26 \\ energy (in $\mathrm{kcal} / \mathrm{mol})=-65.152547$ \\ $\mathrm{ZPE}($ in $\mathrm{kcal} / \mathrm{mol})=146.545$ \\ $\mathrm{G}($ in $\mathrm{kcal} / \mathrm{mol})=122.57$ \\ Geometry \\ C $-3.35186218 \quad 0.47616890 \quad 0.54254561$ \\ C $-0.98504235 \quad 0.48092037 \quad 0.50904081$ \\ C $0.17128487 \quad 1.44655781 \quad 0.52850438$ \\ C $1.42622643 \quad 0.69844251 \quad 1.00117918$ \\ C $1.64133632-0.464270890 .01561597$ \\ C $1.73664355 \quad 0.17203296-1.38255250$ \\ C $-0.85551092-0.837925990 .32517700$ \\ C $0.48526547-1.49818326 \quad 0.15805103$ \\ C $0.75181710-2.35742636 \quad 1.41378453$ \\ C $0.42809790-2.43033278-1.06480698$ \\ $\mathrm{H}-4.101546591 .26567357 \quad 0.68380956$ \\ $\mathrm{H}-3.46062407-0.30363781 \quad 1.30169419$ \\ $\mathrm{H}-3.41845105 \quad 0.05932765-0.46792697$ \\ $\mathrm{H}-0.04333901 \quad 2.36706701 \quad 1.11731549$ \\ H $1.30461724 \quad 0.33477592 \quad 2.03553142$ \\ H 2.29909497 1.37467799 1.00915052 \\ $\mathrm{H} 2.60298923-0.97931165 \quad 0.23701782$ \\ $\mathrm{H} \quad 1.78129237-0.56827135-2.20049803$ \\ H $2.61528778 \quad 0.83665054-1.45534950$ \\ $\mathrm{H}-1.70049461-1.513882590 .29507849$ \\ $\mathrm{H} \quad 0.79339241-1.74153218 \quad 2.31749054$ \\ H $1.69530648-2.90409091 \quad 1.33143895$ \\ $\mathrm{H}-0.04475001-3.094005451 .56314072$ \\ $\mathrm{H} \quad 0.05289168-1.89866843-1.94795378$ \\ $\mathrm{H}-0.25211621-3.27103727-0.88841437$ \\ $\mathrm{H} 1.41111636-2.84629455-1.30169085$ \\ $00.55851993 \quad 0.89217523-1.69007289$ \\ $0.40805454 \quad 1.96835400-0.76843959$ \\ O -2.130467401 .180718550 .71853693$ \\ Vibrational frequencies (in $\mathrm{cm}-1$ )
}

$\begin{array}{llllllllllllllll}60.6 & 95.3 & 124.8 & 176.8 & 185.3 & 192.9 & 208.8 & 230.8 & 272.0 & 295.1 & 330.9 & 360.7 & 374.3 & 401.2\end{array}$

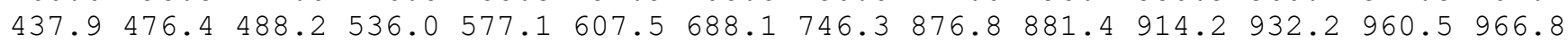
$977.1981 .91010 .7 \quad 1027.9 \quad 1032.2 \quad 1050.2 \quad 1074.2 \quad 1088.7 \quad 1106.6 \quad 1110.8 \quad 1151.11161 .4$ $1173.11187 .41191 .6 \quad 1200.3 \quad 1220.6 \quad 1249.2 \quad 1262.9 \quad 1280.6 \quad 1288.6 \quad 1293.7 \quad 1297.7 \quad 1300.2$

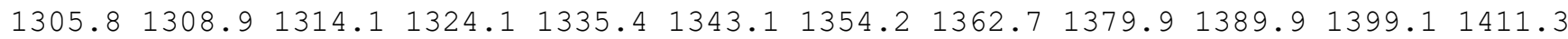
$1873.02648 .6 \quad 2652.5 \quad 2658.0 \quad 2672.92673 .4 \quad 2678.12680 .52683 .2 \quad 2691.02707 .02717 .9$ $2746.02749 .3 \quad 2769.6 \quad 2780.12782 .5$

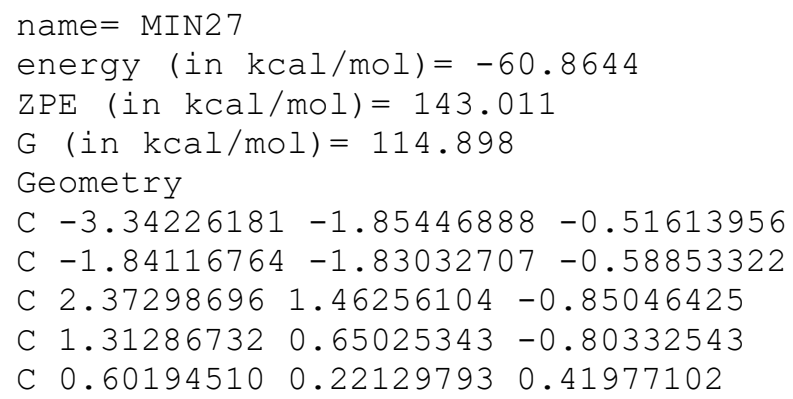


C $0.40939050-1.31795147 \quad 0.54063407$

C $-1.13039006-1.15479153 \quad 0.54743222$

C $-0.96828063 \quad 0.40330725 \quad 0.38676497$

C $-1.53340985 \quad 0.98880674 \quad-0.89366280$

C $-1.48333603 \quad 1.17174341 \quad 1.59439367$

$\mathrm{H}-3.75393199-0.92011774-0.11746975$

$\mathrm{H}-3.68428425-2.67422636 \quad 0.13026422$

$\mathrm{H}-3.78438418-2.02475575-1.50886937$

H $2.85307717 \quad 1.81274087-1.76305824$

$\mathrm{H} 4.077251151 .048617421 .31114370$

$\mathrm{H} 0.906523020 .24249772-1.73485575$

$\mathrm{H} 1.03096726 \quad 0.66759972 \quad 1.33659147$

$\mathrm{H} \quad 0.82576697-1.75360728 \quad 1.45098609$

$\mathrm{H} \quad 0.79384569-1.88974332-0.31369091$

$\mathrm{H}-1.58974448-1.43561916 \quad 1.51075316$

$\mathrm{H}-2.62514987 \quad 1.07324254-0.85573491$

$\mathrm{H}-1.28277096 \quad 0.38423275-1.77561238$

$\mathrm{H}-1.13283275 \quad 1.99617289-1.07085127$

$\mathrm{H}-1.08682063 \quad 0.774036332 .53605760$

$\mathrm{H}-2.57649431 \quad 1.14080537 \quad 1.65679282$

$\mathrm{H}-1.18467561 \quad 2.22719344 \quad 1.54128792$

$\begin{array}{llll}0 & 3.09949357 & 1.19281049 & 1.30637048\end{array}$

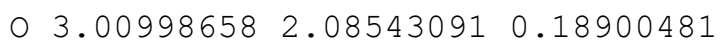

o $-1.25371294-2.34292513-1.50863388$

Vibrational frequencies (in $\mathrm{cm}-1$ )

$\begin{array}{lllllllllllllll}16.8 & 36.4 & 42.0 & 51.8 & 104.1 & 138.8 & 144.3 & 157.1 & 182.5 & 194.3 & 198.2 & 237.7 & 276.6 & 307.3\end{array}$

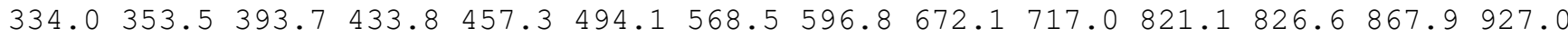
$946.5973 .3 \quad 996.8 \quad 1000.4 \quad 1005.1 \quad 1016.6 \quad 1034.2 \quad 1043.9 \quad 1048.0 \quad 1089.3 \quad 1102.4 \quad 1124.6 \quad 1127.0$ $\begin{array}{lllllllllllllll}1162.9 & 1198.0 & 1218.1 & 1235.9 & 1262.8 & 1265.1 & 1272.7 & 1283.9 & 1287.6 & 1292.3 & 1298.5 & 1300.1\end{array}$ $1302.3 \quad 1308.7 \quad 1326.91338 .8 \quad 1352.0 \quad 1370.0 \quad 1380.7 \quad 1395.2 \quad 1404.3 \quad 1411.3 \quad 1851.91852 .5$ $2667.42670 .4 \quad 2674.4 \quad 2676.4 \quad 2676.8 \quad 2680.92682 .12713 .22721 .3 \quad 2728.7 \quad 2745.5 \quad 2761.7$ $2779.02781 .0 \quad 2782.3 \quad 2865.1$

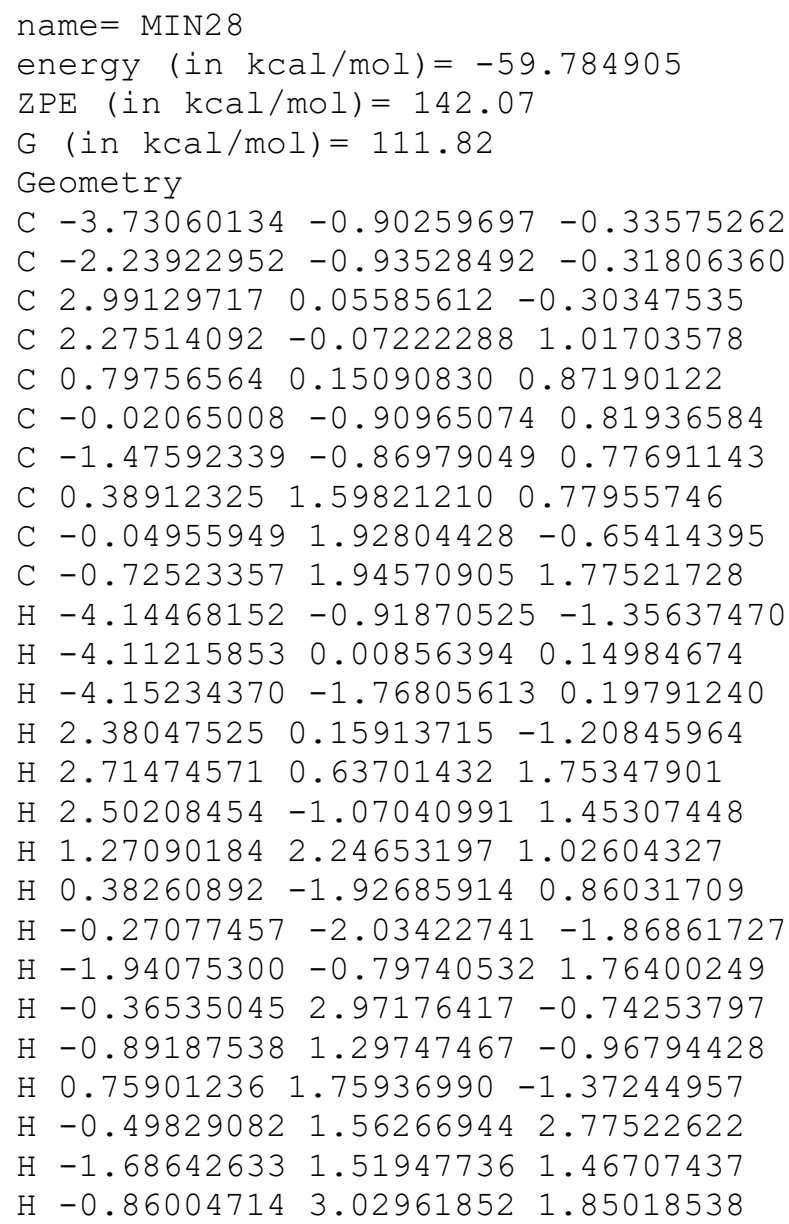


$0-0.41710505-1.06690119-1.72030843$

$04.195664890 .03477151-0.36249279$

o $-1.83664848-1.04487414-1.62464947$

Vibrational frequencies (in $\mathrm{cm}-1$ )

$\begin{array}{lllllllllllllllll}10.7 & 30.2 & 44.6 & 51.2 & 57.1 & 69.2 & 84.4 & 98.8 & 143.3 & 150.9 & 177.1 & 182.0 & 241.1 & 255.7\end{array}$

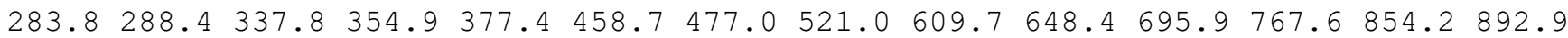
$919.4974 .6 \quad 980.1 \quad 986.5 \quad 1015.5 \quad 1022.0 \quad 1041.4 \quad 1053.8 \quad 1060.0 \quad 1106.8 \quad 1145.2 \quad 1149.9 \quad 1170.5$ $\begin{array}{llllllllllllll}1184.6 & 1226.9 & 1228.8 & 1258.7 & 1271.0 & 1272.5 & 1274.9 & 1281.8 & 1292.6 & 1295.8 & 1300.8 & 1301.7\end{array}$

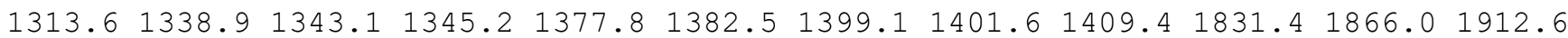
$2648.62651 .02664 .82665 .92668 .3 \quad 2673.92681 .52684 .02700 .02736 .02741 .42751 .1$ 2774.62782 .02783 .62870 .8

name $=$ MIN29

energy (in $\mathrm{kcal} / \mathrm{mol})=-60.420148$

$\mathrm{ZPE}($ in $\mathrm{kcal} / \mathrm{mol})=142.931$

$\mathrm{G}($ in $\mathrm{kcal} / \mathrm{mol})=114.515$

Geometry

C $-2.89876870-0.83390008-0.40068380$

C $-1.41623346-0.88165405-0.38933178$

C $-1.559932242 .35824071-0.37284356$

C $1.46250937-0.15398206-1.51075652$

C $1.44056989-0.66706666-0.27302336$

C $0.80316254-2.00345660 \quad-0.00780204$

C $-0.68691203-1.94876326-0.02367459$

C 2.083468930 .050224460 .88622668

C $1.11207568 \quad 0.19806869 \quad 2.06097238$

C $3.35103108-0.703170191 .31621280$

$\mathrm{H}-3.28544472-0.51716570-1.38293031$

$\mathrm{H}-3.28091964-0.12263570 \quad 0.34811175$

$\mathrm{H}-3.34848091-1.81224161-0.17753458$

$\mathrm{H}-2.21159937 \quad 3.12603397-0.80394280$

$\mathrm{H} \quad 1.89461831 \quad 0.80236646-1.75497831$

H $1.05297828-0.65599958-2.37293525$

H 2.38268968 1.080978490 .55968995

H $1.14710070-2.41628846 \quad 0.96754813$

$\mathrm{H} \quad 1.15278942-2.74076444 \quad-0.76794637$

$\mathrm{H}-1.17898870 \quad-2.86750768 \quad 0.28590230$

$\mathrm{H} \quad 0.80170245 \quad-0.76850213 \quad 2.46767027$

$\mathrm{H} \quad 0.20067162 \quad 0.72805690 \quad 1.74565514$

H $1.55958127 \quad 0.77837668 \quad 2.87405516$

H $3.11779426-1.69501657 \quad 1.71632533$

H $3.88512345-0.15131388 \quad 2.09736337$

H $4.03660760 \quad-0.83643221 \quad 0.47217148$

O $-1.66557102 \quad 1.27103835-1.17282930$

$0-0.88361453 \quad 2.441415330 .61453879$

$\begin{array}{lllll}0 & -0.71981626 & 0.23568681 & -0.79403782\end{array}$

Vibrational frequencies (in $\mathrm{cm}-1$ )

$\begin{array}{lllllllllllllllll}13.7 & 23.2 & 45.0 & 81.4 & 107.8 & 133.8 & 135.2 & 147.0 & 166.6 & 184.0 & 193.5 & 230.3 & 250.4 & 277.6\end{array}$ $299.5 \quad 332.2 \quad 340.8 \quad 428.0 \quad 490.2 \quad 514.7 \quad 539.3 \quad 569.5 \quad 645.0 \quad 696.9 \quad 727.6 \quad 814.8 \quad 856.2 \quad 918.2$ $931.4964 .2 \quad 976.3 \quad 981.3 \quad 985.6 \quad 1010.0 \quad 1014.6 \quad 1040.1 \quad 1062.8 \quad 1082.1 \quad 1101.1 \quad 1148.9 \quad 1158.8$

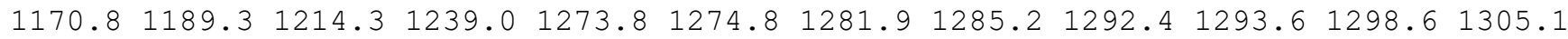
$1305.71308 .31349 .6 \quad 1363.5 \quad 1383.5 \quad 1392.1 \quad 1399.2 \quad 1405.91427 .0 \quad 1834.01842 .6 \quad 1856.2$ $2637.02650 .5 \quad 2665.7 \quad 2667.2 \quad 2671.4 \quad 2674.0 \quad 2679.5 \quad 2681.12703 .92722 .8 \quad 2731.7 \quad 2759.9$ $2777.8 \quad 2780.8 \quad 2782.3 \quad 2789.7$

name $=$ MIN30

energy (in $\mathrm{kcal} / \mathrm{mol})=-63.471758$

$\operatorname{ZPE}($ in $\mathrm{kcal} / \mathrm{mol})=145.987$

$\mathrm{G}($ in $\mathrm{kcal} / \mathrm{mol})=122.23$

Geometry

C $-2.61920642-1.00601271-1.17441444$

C $-1.36182339-0.28744784-0.67118479$

C $0.873151521 .31222769-0.83427989$ 
C $1.912645690 .83460060 \quad 0.14316957$

C $1.44553207 \quad-0.43287849 \quad 0.86333243$

C $0.87960696-1.54715710-0.06762082$

C $-0.57100043-1.14075390 \quad 0.32030668$

C $0.03479903-0.369665551 .56604608$

C $-0.52575134 \quad 0.97506640 \quad 1.96631480$

C $0.01757883-1.28068470 \quad 2.79812078$

$\mathrm{H}-3.13495545-0.41067067-1.94094158$

$\mathrm{H}-3.32617766-1.17125154-0.35391815$

$\mathrm{H}-2.37627922-1.97670597-1.62260460$

$\mathrm{H}-1.64365108 \quad 0.68910425 \quad-0.20520095$

H 2.12695435 $1.63677153 \quad 0.88119454$

H $2.86830940 \quad 0.63225367 \quad-0.38447607$

H $2.24454337-0.78073501 \quad 1.53784116$

H $1.13861547 \quad-2.55648253 \quad 0.27454203$

$\mathrm{H} \quad 1.14793704-1.51376311-1.12367458$

$\mathrm{H}-1.18172634-2.00570226 \quad 0.63094748$

$\mathrm{H}-0.54057591 \quad 1.71808815 \quad 1.16085507$

$\mathrm{H} \quad 0.06182355 \quad 1.418809392 .78163086$

$\mathrm{H}-1.55883251 \quad 0.88108442 \quad 2.32691105$

$\mathrm{H} \quad 0.61570366-0.85705144 \quad 3.61284758$

$\mathrm{H} \quad 0.41840599-2.27756005 \quad 2.58485004$

$\mathrm{H}-1.00284021-1.414884953 .17515748$

$0 \quad 0.64589962 \quad 0.31799146-1.76822083$

$00.28607255 \quad 2.35772867-0.83056493$

o $-0.740049490 .04639667-1.91973388$

Vibrational frequencies (in $\mathrm{cm}-1$ )

$\begin{array}{llllllllllllllllll}75.1 & 123.8 & 154.3 & 158.5 & 186.0 & 196.6 & 209.3 & 228.6 & 249.3 & 279.7 & 301.4 & 345.3 & 374.5 & 397.6\end{array}$

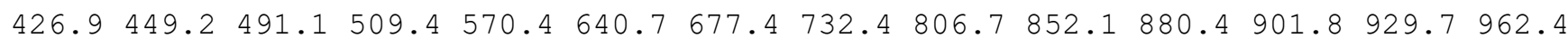
$988.0 \quad 997.1 \quad 1000.1 \quad 1015.9 \quad 1017.3 \quad 1021.2 \quad 1052.2 \quad 1072.2 \quad 1103.3 \quad 1108.4 \quad 1122.3 \quad 1135.4$ $\begin{array}{lllllllllllll}1174.9 & 1193.1 & 1199.4 & 1211.0 & 1237.7 & 1245.3 & 1262.6 & 1276.3 & 1282.5 & 1287.2 & 1289.7 & 1292.4\end{array}$

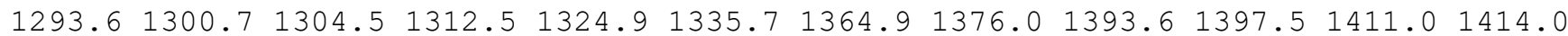
$1872.22649 .12660 .42668 .0 \quad 2671.2 \quad 2673.2 \quad 2674.02676 .72679 .02681 .32719 .92724 .5$ $2738.22760 .92778 .92781 .4 \quad 2785.2$

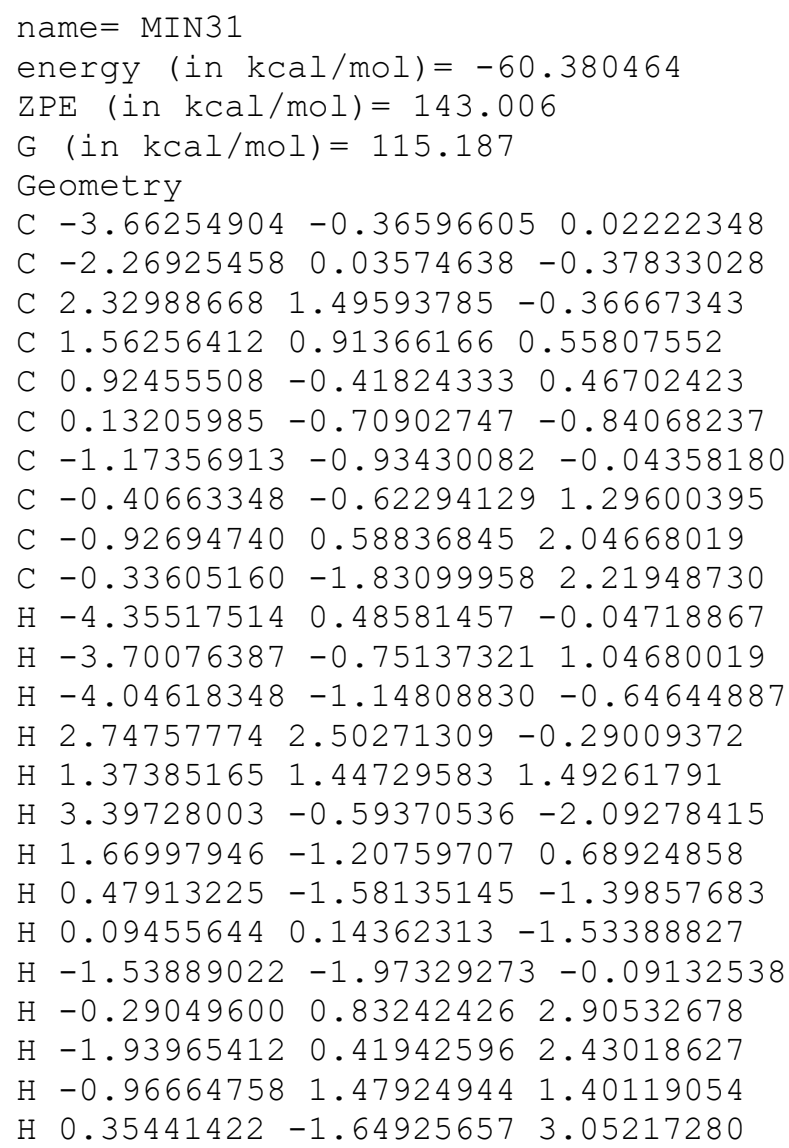


$\mathrm{H} \quad 0.01421021-2.728579841 .69710468$

$\mathrm{H}-1.31644949-2.06365247 \quad 2.65050050$

$02.51621775-0.34871700-1.71876124$

$02.734205631 .06099615-1.59069618$

O $-2.067186481 .07654639-0.95204605$

Vibrational frequencies (in $\mathrm{cm}-1$ )

$\begin{array}{llllllllllllllll}31.2 & 33.8 & 41.6 & 73.4 & 85.0 & 99.9 & 144.5 & 165.3 & 176.2 & 193.8 & 214.2 & 252.5 & 277.0 & 288.0\end{array}$ $334.0 \quad 343.0 \quad 386.0 \quad 404.9 \quad 463.9 \quad 522.2 \quad 574.4 \quad 595.4 \quad 676.3 \quad 732.6 \quad 805.5 \quad 827.3 \quad 873.4 \quad 922.3$ $941.1954 .1994 .6 \quad 994.8 \quad 1002.3 \quad 1015.1 \quad 1033.0 \quad 1045.1 \quad 1048.7 \quad 1091.1 \quad 1113.8 \quad 1121.8 \quad 1130.3$ $1157.91194 .5 \quad 1214.2 \quad 1232.5 \quad 1256.7 \quad 1265.2 \quad 1274.3 \quad 1275.91290 .1 \quad 1292.6 \quad 1301.2 \quad 1302.0$

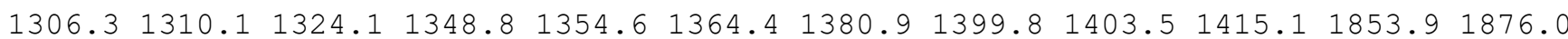
$2665.12671 .62676 .2 \quad 2677.8 \quad 2678.5 \quad 2678.7 \quad 2681.22714 .62718 .02731 .42747 .82758 .9$ $2778.0 \quad 2781.5 \quad 2782.7 \quad 2874.1$

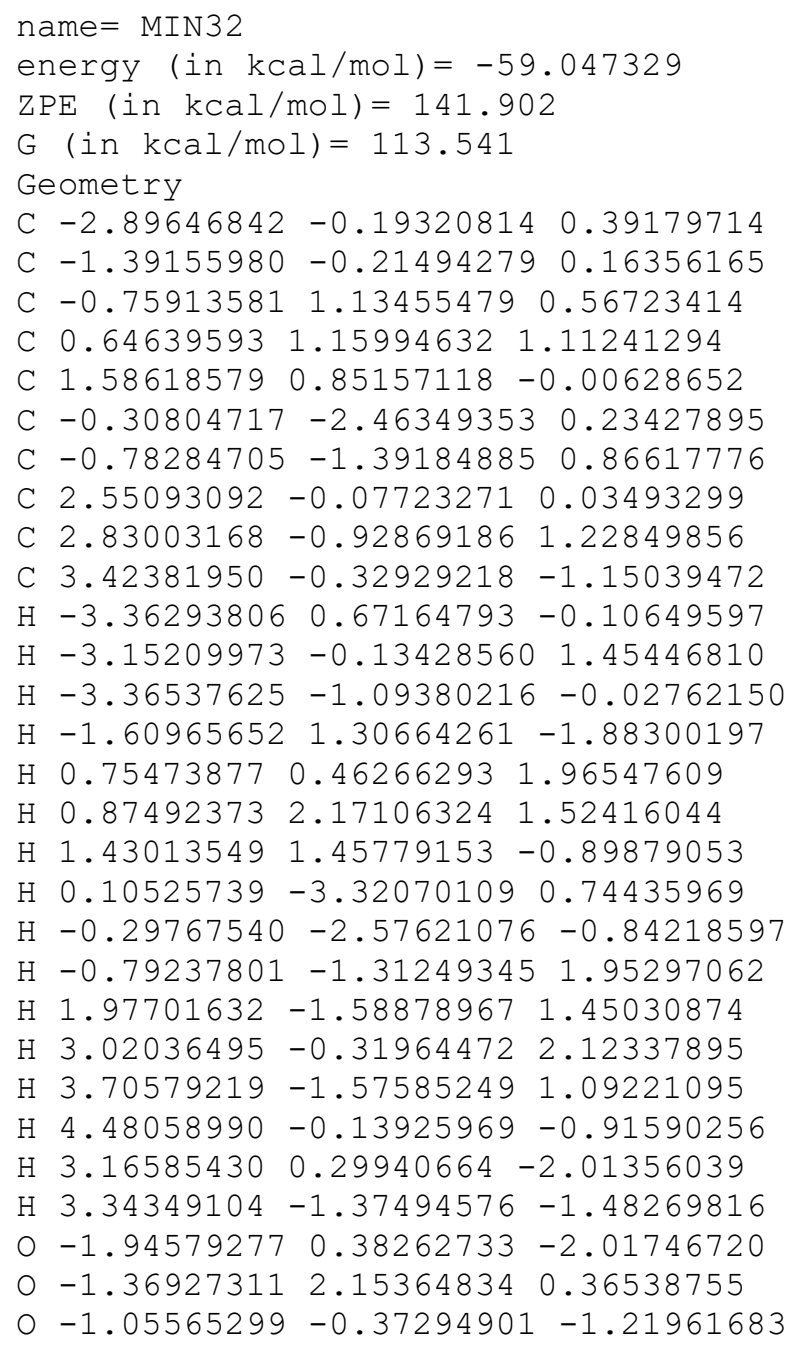


C $-1.01896639-1.54991730-0.63574099$

C $1.372335342 .28780499 \quad-0.58968116$

$\begin{array}{lllll}\text { C } 1.63405556 & 1.88817077 & 0.83875854\end{array}$

$\begin{array}{llll}\text { C } & 1.01531424 & 0.56920521 & 1.25134526\end{array}$

$\begin{array}{llll}\text { C } & 1.23883609 & -0.64190914 & 0.28905254\end{array}$

C $-0.24975376-0.76105671 \quad 0.10329661$

C $-0.55166400 \quad 0.377092291 .06587009$

C $-1.31061475 \quad 1.54671677 \quad 0.46636872$

C $-1.21711537-0.094930012 .35326786$

$\mathrm{H}-2.91600910-0.57480982-0.37459358$

$\mathrm{H}-2.95440701-2.33267419-0.12461831$

$\mathrm{H}-2.85169048-1.66828674-1.76762703$

H $1.189488791 .49445873-1.32543687$

H $1.28603298 \quad 2.69762699 \quad 1.51847267$

H 2.736421651 .849780420 .99049976

$\begin{array}{lllll}\mathrm{H} & 1.32138954 & 0.33378452 & 2.28696054\end{array}$

H $1.71150121-1.50708881 \quad 0.76661499$

$\mathrm{H} \quad 1.80311031-0.42735310-0.62475904$

H $0.94858649-3.45692326-0.91934553$

$\mathrm{H}-1.05322212 \quad 1.71257310 \quad-0.58698271$

$\mathrm{H}-1.103722082 .47699612 \quad 1.01076418$

$\mathrm{H}-2.39476142 \quad 1.387687290 .50289825$

$\mathrm{H}-1.24295620 \quad 0.70485613 \quad 3.10194149$

H $-0.68825723 \quad-0.94825658 \quad 2.79354349$

$\mathrm{H}-2.24878779-0.41749782 \quad 2.17294225$

$00.83555058-2.63506288-1.45699496$

$\begin{array}{lllll}0 & 1.38285561 & 3.44784493 & -0.92281512\end{array}$

$0-0.58666565-2.55378293-1.47202195$

Vibrational frequencies (in $\mathrm{cm}-1$ )

$\begin{array}{llllllllllllllll}38.4 & 46.9 & 48.5 & 63.0 & 117.3 & 138.0 & 145.1 & 163.3 & 181.3 & 185.0 & 210.1 & 224.5 & 246.1 & 271.5\end{array}$

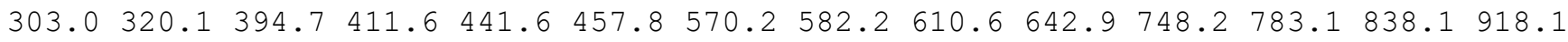

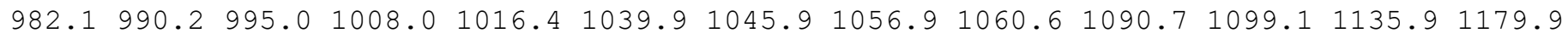

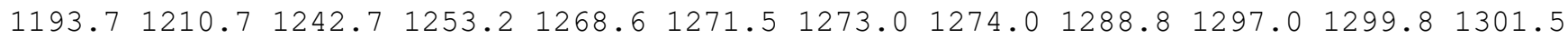

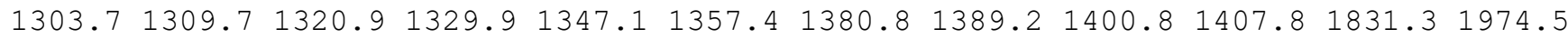

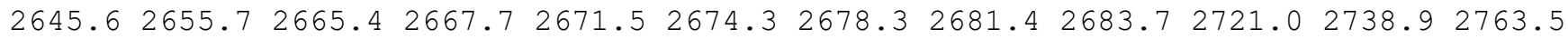
2774.92780 .02783 .02869 .6

name $=$ MIN34

energy (in $\mathrm{kcal} / \mathrm{mol})=-58.027678$

$\mathrm{ZPE}($ in $\mathrm{kcal} / \mathrm{mol})=142.546$

$\mathrm{G}(\mathrm{in} \mathrm{kcal} / \mathrm{mol})=115.134$

Geometry

C $-2.66498567-0.99466672-0.49595641$

C $-1.20940374-1.19374881-0.73993606$

C $1.267137372 .16749039-0.43067640$

C $1.89463514 \quad 1.554306690 .79608142$

C $1.40352244 \quad 0.15528774 \quad 1.11387206$

C $1.29142377-0.81926553-0.10533060$

C $-0.20739355-0.79668798 \quad 0.03354924$

C $-0.15841514-0.03044857 \quad 1.34589065$

C $-1.00138551 \quad 1.22691813 \quad 1.41433750$

C $-0.43584404-0.929074392 .54790091$

$\mathrm{H}-2.92869494 \quad 0.07313042 \quad-0.57464056$

$\mathrm{H}-2.95259156-1.336355590 .50894107$

$\mathrm{H}-3.29337381-1.53532192-1.22078232$

$\mathrm{H} 1.029236341 .50860999-1.27557016$

$\mathrm{H} \quad 1.72543468 \quad 2.22001068 \quad 1.67120420$

$\mathrm{H} \quad 2.99754005 \quad 1.54468095 \quad 0.65292859$

H $1.99351998-0.265854291 .94470597$

H $1.74613511-1.79993020 \quad 0.07661680$

$\mathrm{H} \quad 1.68587998-0.44843822-1.05613044$

H $0.44046840 \quad-2.92133809-2.03384391$

$\mathrm{H}-2.05285837 \quad 0.99153636 \quad 1.61912009$

$\mathrm{H}-0.98137778 \quad 1.79539556 \quad 0.47640324$ 
$\mathrm{H}-0.65532275 \quad 1.896470442 .21157671$

$\mathrm{H}-0.19765756-0.419875663 .48821269$

$\mathrm{H} 0.15482412-1.851847192 .50840811$

$\mathrm{H}-1.48996877-1.225345762 .58646311$

$0.26687650-1.99048021-2.31714056$

O $1.045243103 .35208182-0.49249446$

o $-1.09846698-1.86052885-1.93745345$

Vibrational frequencies (in $\mathrm{cm}-1$ )

$\begin{array}{lllllllllllllllll}38.2 & 48.4 & 60.3 & 74.8 & 98.1 & 119.9 & 143.3 & 161.8 & 171.6 & 180.5 & 200.7 & 222.0 & 248.0 & 269.2\end{array}$ $309.1324 .4 \quad 391.6 \quad 414.5 \quad 446.2 \quad 455.0 \quad 558.7 \quad 574.3 \quad 609.5 \quad 649.6 \quad 750.8 \quad 787.0 \quad 845.5 \quad 913.5$ $983.1989 .3 \quad 995.7 \quad 1009.2 \quad 1014.5 \quad 1033.3 \quad 1040.1 \quad 1052.0 \quad 1053.9 \quad 1089.1 \quad 1096.5 \quad 1127.91175 .8$

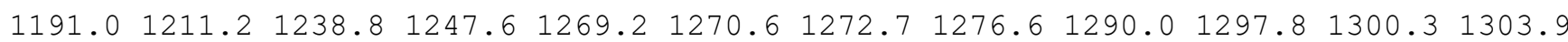

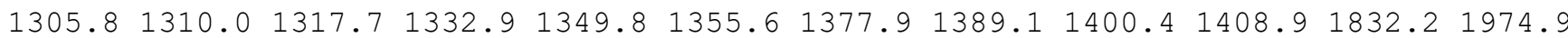
$2643.92658 .2 \quad 2662.1 \quad 2668.8 \quad 2672.7 \quad 2676.6 \quad 2678.82681 .82683 .8 \quad 2724.5 \quad 2739.3 \quad 2762.3$ $2773.5 \quad 2781.4 \quad 2783.2 \quad 2869.2$

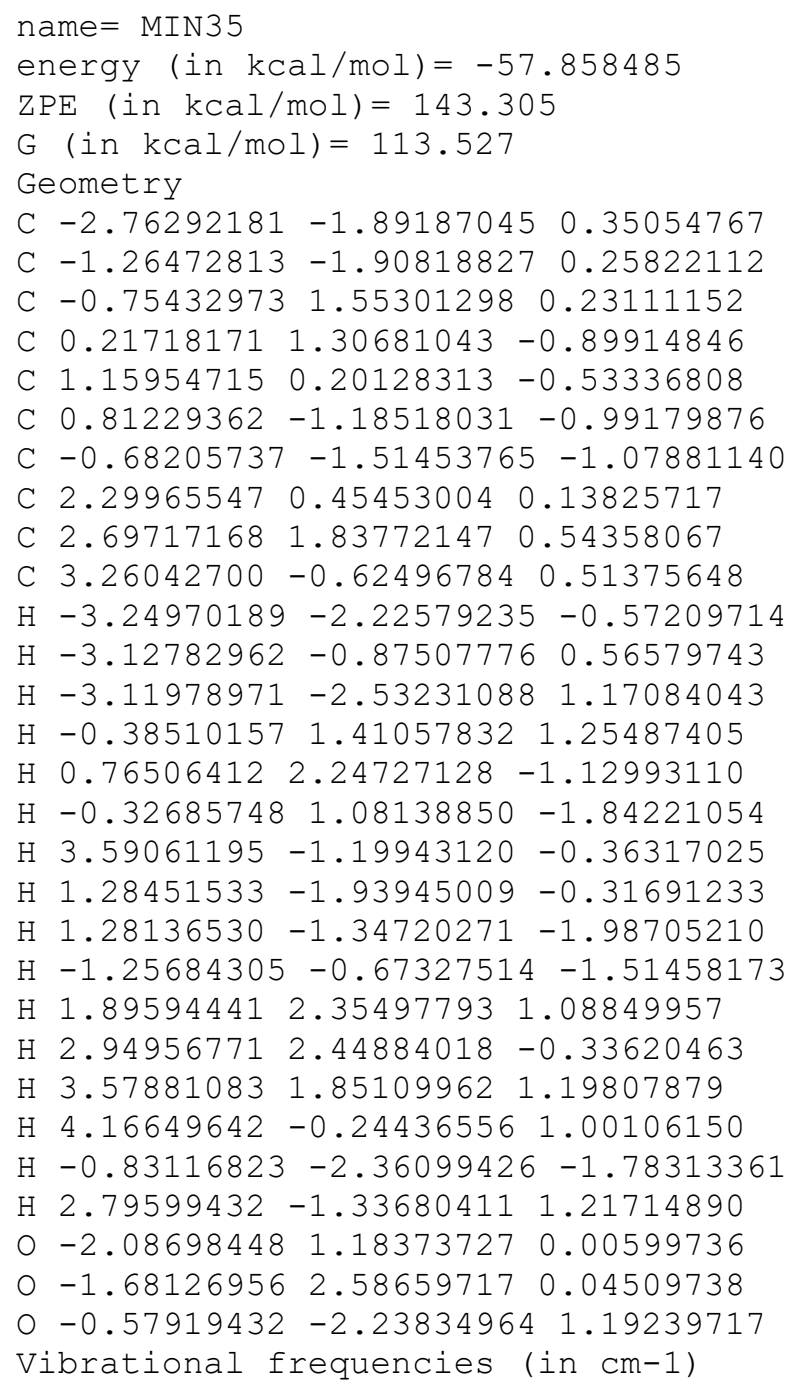




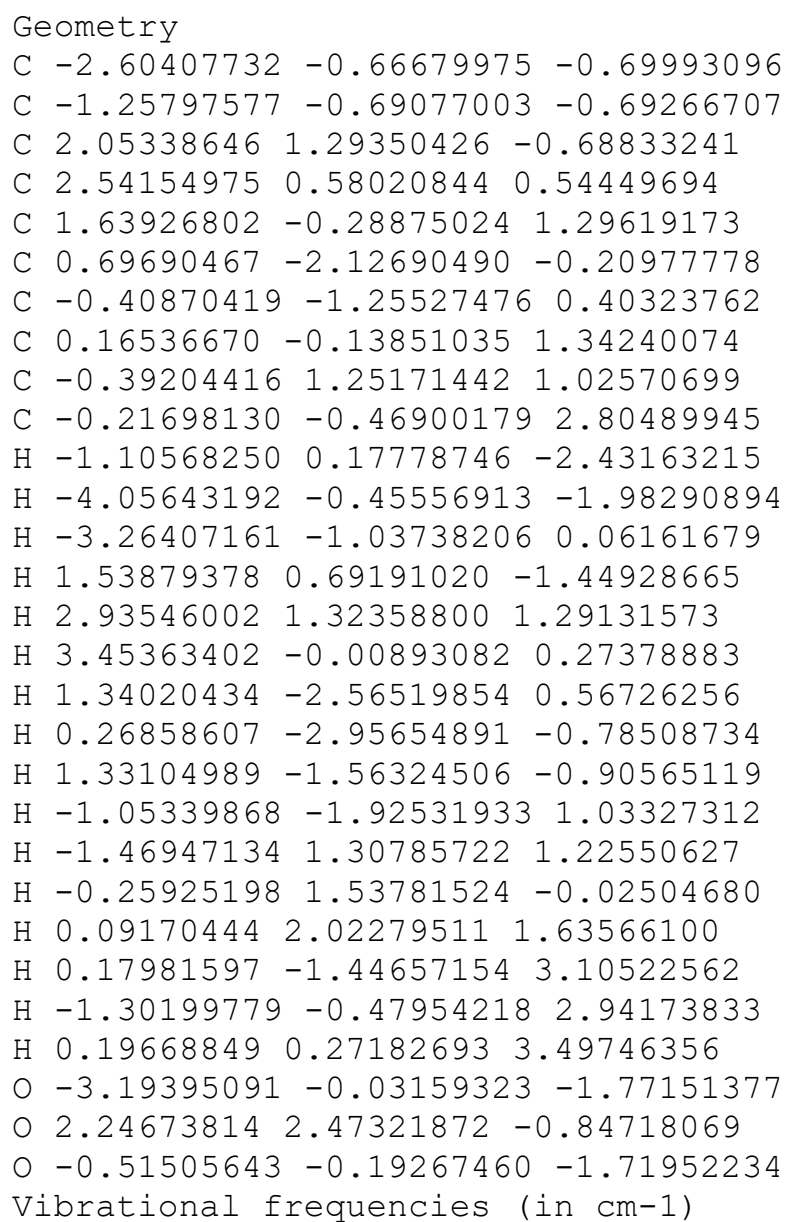

$\begin{array}{lllllllllllllllll}27.9 & 52.1 & 57.3 & 77.1 & 110.9 & 128.3 & 174.9 & 194.8 & 201.7 & 205.5 & 241.9 & 245.3 & 255.3 & 280.6\end{array}$ $303.0 \quad 309.8 \quad 330.2 \quad 356.6 \quad 397.2 \quad 430.2 \quad 449.6 \quad 463.9 \quad 526.1 \quad 586.6 \quad 611.8 \quad 672.4 \quad 780.2 \quad 819.4$ $840.7 \quad 872.8 \quad 956.6 \quad 969.2 \quad 977.2 \quad 993.0 \quad 1028.3 \quad 1067.8 \quad 1075.6 \quad 1092.3 \quad 1123.7 \quad 1136.0 \quad 1154.9$ $1177.8 \quad 1188.2 \quad 1208.1 \quad 1220.0 \quad 1223.6 \quad 1258.1 \quad 1267.1 \quad 1275.6 \quad 1290.8 \quad 1298.4 \quad 1300.7 \quad 1303.4$

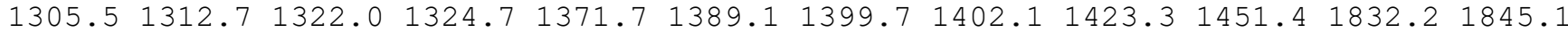
$2627.62649 .22662 .3 \quad 2663.4 \quad 2667.4 \quad 2674.4 \quad 2676.82680 .72687 .62721 .12752 .12762 .9$ 2775.22779 .02781 .12803 .8

\footnotetext{
name $=$ MIN37

energy (in $\mathrm{kcal} / \mathrm{mol})=-56.349392$

$\mathrm{ZPE}($ in $\mathrm{kcal} / \mathrm{mol})=143.204$

$\mathrm{G}(\mathrm{in} \mathrm{kcal} / \mathrm{mol})=115.682$

Geometry

C $-3.10446945-0.93697799-0.20118220$

C $-1.77147210-0.95358795-0.20197062$

C $2.708978421 .44379555-0.18509576$

C $2.065012890 .08629543-0.26165392$

C $1.24408834-0.22404716 \quad 0.97445007$

C $0.47761292-1.57203501 \quad 0.88545638$

C $-0.88483058-0.83420996 \quad 0.98736096$

C $-0.12420931 \quad 0.53963620 \quad 1.14229637$

C $-0.45660140 \quad 1.589396390 .10255672$

C $-0.28497133 \quad 1.11949070 \quad 2.54184387$

$\mathrm{H}-1.77450313-0.35890617-2.85599298$

$\mathrm{H}-3.69809783-0.832457760 .68861000$

$\mathrm{H}-3.70192761-1.04490212-1.09136092$

H $2.87325247 \quad 1.87545092 \quad 0.80963814$

$\mathrm{H} 2.85769928-0.68230528-0.39960039$

H $1.449807690 .00642932-1.18499159$

$\mathrm{H} 1.87051724-0.150599821 .88119916$

$\mathrm{H} \quad 0.67254474 \quad-2.25527018 \quad 1.71684797$

$\mathrm{H} \quad 0.63476621-2.13547634-0.03916076$

$\mathrm{H}-1.44916095-1.105060461 .90056088$
} 
$\mathrm{H} \quad 0.17626710 \quad 2.479172910 .21075311$

$\mathrm{H}-1.50102539 \quad 1.917139920 .19362046$

$\mathrm{H} \quad 0.38313492 \quad 1.97461833 \quad 2.69825928$

$\mathrm{H}-0.06311644 \quad 0.38328333 \quad 3.32280339$

$\mathrm{H}-1.310000331 .47304154 \quad 2.70944049$

o $-1.79116702-1.27256376-2.47571429$

O $3.048607312 .03721093-1.17873710$

O $-0.98131801-1.07671804-1.32009712$

$\mathrm{H}-0.325232491 .22413738-0.92327121$

Vibrational frequencies (in $\mathrm{cm}-1$ )

$\begin{array}{lllllllllllllll}24.1 & 35.2 & 45.4 & 74.5 & 88.0 & 139.5 & 162.6 & 177.0 & 188.2 & 208.6 & 223.4 & 243.3 & 302.4 & 313.1\end{array}$

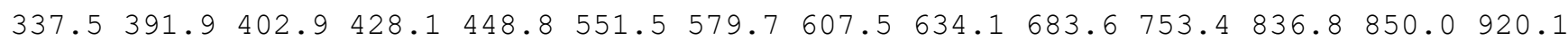
$954.1992 .1 \quad 1005.6 \quad 1010.5 \quad 1016.3 \quad 1022.5 \quad 1035.6 \quad 1046.4 \quad 1049.3 \quad 1088.3 \quad 1116.51123 .2$ $1130.61157 .0 \quad 1178.6 \quad 1206.5 \quad 1227.8 \quad 1248.2 \quad 1267.3 \quad 1281.5 \quad 1285.6 \quad 1289.2 \quad 1297.6 \quad 1301.0$

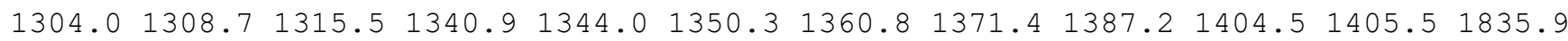
$1877.12646 .42655 .8 \quad 2670.2 \quad 2673.3 \quad 2677.0 \quad 2678.4 \quad 2682.3 \quad 2717.6 \quad 2722.5 \quad 2729.5 \quad 2740.4$ $2763.42779 .8 \quad 2782.2 \quad 2798.2 \quad 2867.4$

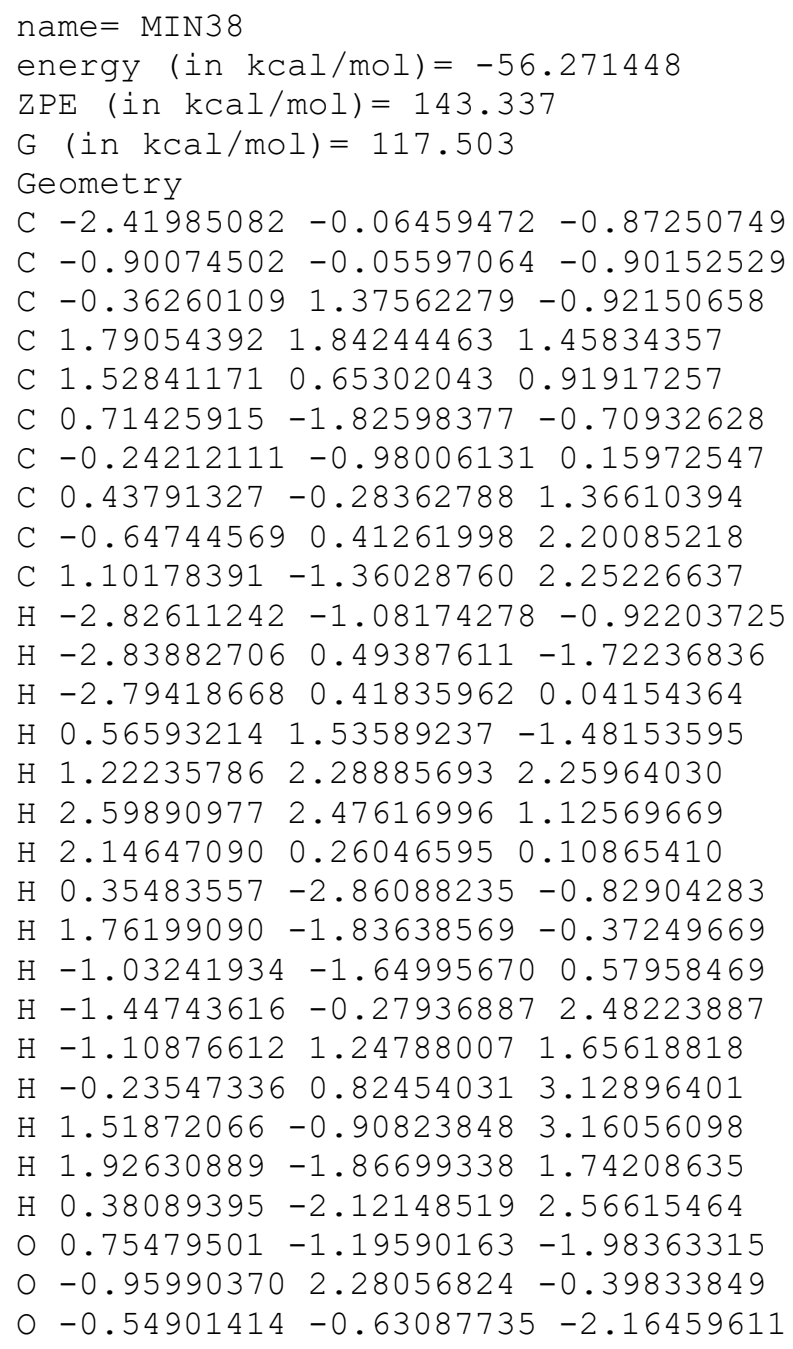


$\mathrm{ZPE}($ in $\mathrm{kcal} / \mathrm{mol})=143.434$

$\mathrm{G}(\mathrm{in} \mathrm{kcal} / \mathrm{mol})=117.817$

Geometry

C $-2.35823385 \quad 0.11828472-0.83243300$

C $-0.84226751 \quad 0.12283573-0.90676473$

C $-0.27036464 \quad 1.52454991 \quad-0.69648919$

C $1.555243551 .61632071 \quad 1.66302493$

C $1.54734723 \quad 0.33910490 \quad 1.28606291$

C $0.93800200-1.48308702-1.02838014$

C $-0.15086345-0.97684967-0.05702910$

C $0.36655186-0.59356915 \quad 1.35091349$

C $-0.79172733-0.02261363 \quad 2.18094304$

C $0.84571753-1.889319832 .04664350$

$\mathrm{H}-2.77639555-0.88384077-0.98197061$

$\mathrm{H}-2.79662100 \quad 0.77359273-1.59926573$

$\mathrm{H}-2.70241104 \quad 0.49642992 \quad 0.14160415$

$\mathrm{H} \quad 0.68552857 \quad 1.73631574-1.18997181$

$\mathrm{H} \quad 0.69462095 \quad 2.15226629 \quad 2.03838192$

H 2.43694902 2.23926409 1.61944826

$\mathrm{H} 2.46101686-0.13194987 \quad 0.91631610$

$\mathrm{H} \quad 0.80372052-2.54492019-1.28656823$

H $1.97152848-1.31246984 \quad-0.68834342$

$\mathrm{H}-0.89757151-1.79759003 \quad 0.09540718$

$\mathrm{H}-0.47257730 \quad 0.18929434 \quad 3.20847241$

$\mathrm{H}-1.63296066-0.72042192 \quad 2.23608008$

$\mathrm{H}-1.17281697 \quad 0.91995851 \quad 1.76471786$

$\mathrm{H} 1.26311636-1.665664963 .03598519$

$\mathrm{H} 1.62170840 \quad-2.40342488 \quad 1.47285871$

$\mathrm{H} \quad 0.02014131-2.59270570 \quad 2.19584062$

$\begin{array}{llllll}0 & 0.82289674 & -0.70854510 & -2.21874520\end{array}$

$0-0.863702932 .35830202-0.06191682$

o $-0.53126860-0.24078411-2.25531875$

Vibrational frequencies (in $\mathrm{cm}-1$ )

$\begin{array}{llllllllllllll}55.6 & 60.3 & 101.8 & 105.3 & 116.6 & 162.8 & 181.8 & 186.7 & 225.5 & 239.1 & 252.5 & 269.1 & 271.8 & 313.0\end{array}$ $335.3 \quad 376.2 \quad 431.6 \quad 438.9 \quad 458.8 \quad 515.5 \quad 545.4 \quad 662.9 \quad 669.5 \quad 707.8 \quad 777.2 \quad 891.2 \quad 929.9 \quad 950.2$ $965.3969 .7 \quad 976.2986 .0991 .7 \quad 1002.0 \quad 1027.3 \quad 1039.8 \quad 1070.6 \quad 1096.4 \quad 1099.2 \quad 1106.01142 .6$

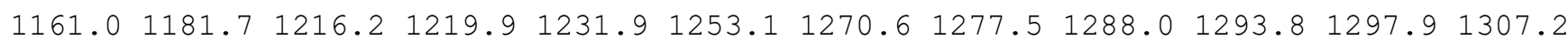

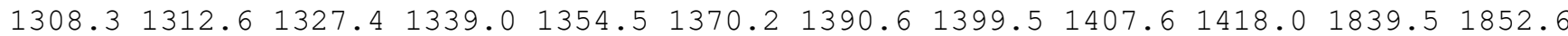
$2645.52653 .8 \quad 2662.92665 .1 \quad 2674.3 \quad 2675.2 \quad 2678.5 \quad 2679.32689 .12704 .4 \quad 2717.5 \quad 2744.4$ $2778.0 \quad 2779.4 \quad 2780.1 \quad 2782.7$

\section{name $=$ MIN40}

energy (in $\mathrm{kcal} / \mathrm{mol})=-54.814349$

$\mathrm{ZPE}($ in $\mathrm{kcal} / \mathrm{mol})=142.833$

$\mathrm{G}(\mathrm{in} \mathrm{kcal} / \mathrm{mol})=114.181$

Geometry

C $-3.80282414 \quad 0.23692695 \quad-0.16779672$

C $-2.33103035 \quad 0.50305848-0.21570479$

C $2.68305368 \quad 0.82489236-0.16820786$

C $2.33816422-0.42035371-0.94222629$

C $0.86168302-0.84734096-0.88018644$

C $-0.05504071 \quad 0.32656187-1.30130643$

C $-1.505352850 .02867257-1.15506097$

C $0.48793175-1.438602190 .45637336$

C $0.99953735-1.06582908 \quad 1.63266508$

C $-0.50311925-2.557673750 .38183338$

$\mathrm{H}-4.295090820 .741170350 .68049008$

$\mathrm{H}-4.01078055-0.83749165-0.06739457$

$\mathrm{H}-4.29837605 \quad 0.59638411-1.08194624$

H $1.86407857 \quad 1.46540050 \quad 0.18731138$

H 2.98352333 -1.25530288 -0.58969695

H 2.64024642 -0.26403363 -2.00056346

$\mathrm{H} \quad 0.72861778-1.65536391-1.65220937$

$\mathrm{H} \quad 0.15642918 \quad 0.56366259-2.36915490$ 
$\mathrm{H} \quad 0.21525573 \quad 1.25567017-0.75987625$

$\mathrm{H}-1.90515035-0.62198138-1.93325709$

$\mathrm{H} \quad 0.72580156-1.52950330 \quad 2.56763521$

$\mathrm{H}-0.43114158 \quad 0.77261376 \quad 1.58319117$

$\mathrm{H} \quad 1.74953574-0.30760005 \quad 1.77268324$

$\mathrm{H}-1.38059586-2.28593195-0.22260480$

$\mathrm{H}-0.87603435-2.860940191 .36879028$

$\mathrm{H}-0.05382610-3.44970223-0.07845245$

O $-0.71498073 \quad 1.57999583 \quad 1.07481126$

0
$0.83220328 \quad 1.12137054 \quad 0.04911168$

o $-2.08863520 \quad 1.303002090 .86799069$

Vibrational frequencies (in $\mathrm{cm}-1$ )

$\begin{array}{lllllllllllllll}16.8 & 32.3 & 52.9 & 60.8 & 69.7 & 90.2 & 117.0 & 122.5 & 155.5 & 194.1 & 208.6 & 275.5 & 290.1 & 308.1\end{array}$ $\begin{array}{lllllllllllllllll}322.1 & 334.8 & 357.7 & 414.1 & 448.7 & 481.8 & 524.7 & 573.0 & 618.9 & 631.9 & 673.9 & 761.4 & 824.9 & 899.6\end{array}$ $934.5988 .2993 .6 \quad 998.6 \quad 1028.7 \quad 1038.8 \quad 1049.8 \quad 1054.0 \quad 1060.01081 .1 \quad 1095.41109 .7 \quad 1169.0$

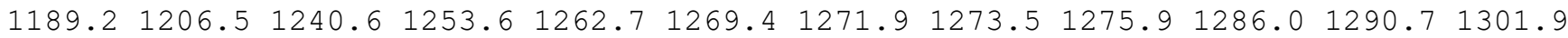
$1306.01338 .21351 .91367 .1 \quad 1380.3 \quad 1386.2 \quad 1400.7 \quad 1403.11411 .51832 .31861 .61896 .2$ $2630.62642 .6 \quad 2652.12662 .0 \quad 2667.0 \quad 2669.6 \quad 2672.5 \quad 2685.62718 .82728 .12737 .5 \quad 2758.6$ $2774.7 \quad 2778.2 \quad 2792.7 \quad 2822.6$

name $=$ MIN41

energy $($ in $\mathrm{kcal} / \mathrm{mol})=-55.278224$

$\operatorname{ZPE}($ in $\mathrm{kcal} / \mathrm{mol})=143.557$

$\mathrm{G}($ in $\mathrm{kcal} / \mathrm{mol})=116.884$

Geometry

C $-2.62156948-1.25548347-0.48978220$

C $-1.30134452-1.10155024-0.59428418$

C $1.57628351 \quad 1.63280981-0.65032729$

C $2.142142691 .06613961 \quad 0.62461574$

C $1.47238679-0.198923531 .12354033$

C $1.20155765-1.326110050 .09069175$

C $-0.30218788-1.35079471 \quad 0.47861626$

C $-0.06177310-0.15248374 \quad 1.47812759$

C $-0.79787710 \quad 1.12613489 \quad 1.13568001$

C $-0.33486978-0.54282652 \quad 2.92355407$

$\mathrm{H}-3.10980744-1.58287963 \quad 0.41048594$

$\mathrm{H}-3.31107681-1.08149176-1.29871798$

$\mathrm{H}-1.70484440 \quad 0.45495736-2.77638359$

H $1.19901095 \quad 0.93271062-1.40639528$

H $2.10743367 \quad 1.84277562 \quad 1.42194806$

H $3.22722563 \quad 0.87634506 \quad 0.46198839$

H $2.05633196-0.58110763 \quad 1.98697703$

H $1.72581561-2.263525190 .30191987$

H $1.42273684-1.07406332-0.94885361$

$\mathrm{H}-0.57216470-2.28556705 \quad 1.01200327$

$\mathrm{H}-0.73806026 \quad 1.38016516 \quad 0.07072262$

$\mathrm{H}-0.400976731 .980373091 .69867331$

$\mathrm{H}-1.86764630 \quad 1.04443947 \quad 1.37300164$

$\mathrm{H}-0.00918924 \quad 0.24491204 \quad 3.61379545$

$\mathrm{H} \quad 0.18264120-1.464940113 .21079059$

$\mathrm{H}-1.40629156-0.705012403 .09584802$

$\begin{array}{lllll}0 & -1.54926830 & -0.52270426 & -2.80338369\end{array}$

O $1.55665502 \quad 2.82287889-0.84916487$

o- $-0.63969763-0.66818316-1.71735628$

Vibrational frequencies (in $\mathrm{cm}-1$ )

$\begin{array}{llllllllllllll}32.3 & 37.0 & 59.9 & 93.2 & 116.5 & 164.4 & 172.9 & 194.2 & 213.1 & 219.5 & 228.0 & 268.9 & 287.4 & 327.7\end{array}$

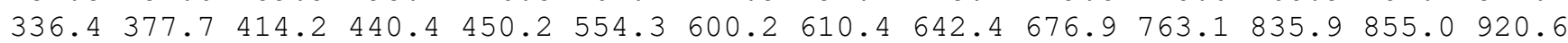
$932.6996 .2998 .5 \quad 1008.2 \quad 1013.2 \quad 1018.4 \quad 1033.7 \quad 1048.9 \quad 1052.3 \quad 1076.4 \quad 1100.1 \quad 1127.6 \quad 1136.1$ $1161.21191 .8 \quad 1209.91238 .7 \quad 1259.6 \quad 1268.4 \quad 1283.8 \quad 1286.1 \quad 1290.1 \quad 1296.8 \quad 1300.5 \quad 1301.6$ $1313.51323 .8 \quad 1335.0 \quad 1347.4 \quad 1357.5 \quad 1366.91381 .0 \quad 1387.6 \quad 1406.1 \quad 1409.4 \quad 1832.6 \quad 1874.8$ $2644.12655 .8 \quad 2668.92674 .3 \quad 2676.7 \quad 2677.5 \quad 2678.8 \quad 2710.32716 .92729 .32737 .62762 .9$ $2780.3 \quad 2782.4 \quad 2798.4 \quad 2866.5$ 
name $=$ MIN42

energy (in $\mathrm{kcal} / \mathrm{mol})=-54.327653$

$\mathrm{ZPE}($ in $\mathrm{kcal} / \mathrm{mol})=143.073$

$\mathrm{G}($ in $\mathrm{kcal} / \mathrm{mol})=117.296$

Geometry

C $-3.06903665-1.12660690-0.84567536$

C $-1.59371812-0.88668382-0.75942717$

C $0.27419424 \quad 1.92829215 \quad-0.34426597$

C $1.11584895 \quad 0.86193795 \quad 0.24229700$

C $0.51231598-0.508792520 .57911900$

C $1.33134267-1.74558778 \quad 0.29285022$

C $-0.93103774-0.73794371 \quad 0.39590275$

C $0.97176084 \quad 0.43925609 \quad 1.69631450$

$\begin{array}{llll}\text { C } 2.23645746 & 0.13139927 & 2.46034776\end{array}$

C $-0.056787351 .11575424 \quad 2.56835602$

$\mathrm{H}-3.62952365-0.30717339-0.37226665$

$\mathrm{H}-3.34750020-2.06395609-0.34278608$

$\mathrm{H}-3.42381463-1.19977668-1.88676661$

$\mathrm{H}-0.15600878 \quad 2.69145467 \quad 0.31586897$

H $2.12416626 \quad 0.86321031-0.19879373$

H $0.28415128 \quad 0.35770964 \quad-2.14420949$

H $2.39209412-1.52907284 \quad 0.12945913$

H $1.25410818-2.45995421 \quad 1.12244426$

$\mathrm{H} \quad 0.96935200 \quad-2.25453948 \quad-0.61473084$

$\mathrm{H}-1.47912757-0.84083714 \quad 1.33435906$

H $2.62735794 \quad 1.02850405 \quad 2.95798534$

$\mathrm{H} \quad 3.03769041-0.25348131 \quad 1.81773474$

$\mathrm{H} 2.05323412-0.625293183 .23444356$

H $0.304710962 .08697293 \quad 2.93010461$

$\mathrm{H}-0.28117866 \quad 0.50094222 \quad 3.45131510$

$\mathrm{H}-1.009459791 .286966252 .05246306$

$0.23920582-0.63876828-2.16023724$

$0.07796711 \quad 1.97974849-1.54090740$

o $-1.15846495-0.83933859-2.04867414$

Vibrational frequencies (in $\mathrm{cm}-1$ )

$\begin{array}{lllllllllllllllll}76.7 & 88.7 & 96.9 & 109.8 & 124.8 & 146.9 & 151.0 & 164.7 & 175.8 & 192.3 & 218.2 & 259.7 & 269.5 & 298.1\end{array}$

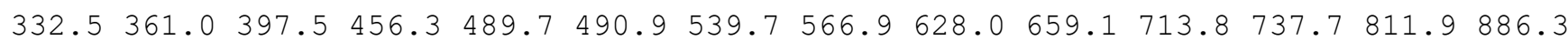
$893.0 \quad 967.0 \quad 985.3 \quad 1002.0 \quad 1011.1 \quad 1012.9 \quad 1035.7 \quad 1043.3 \quad 1048.4 \quad 1063.2 \quad 1094.6 \quad 1105.91148 .6$

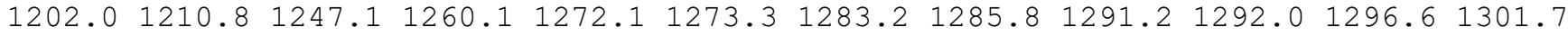
$1307.41327 .4 \quad 1358.1 \quad 1381.7 \quad 1399.0 \quad 1400.5 \quad 1406.9 \quad 1417.4 \quad 1431.1 \quad 1503.2 \quad 1805.4 \quad 1885.2$ $2659.12663 .5 \quad 2666.0 \quad 2669.12671 .0 \quad 2673.6 \quad 2674.72678 .12679 .72739 .12742 .22754 .6$ $2775.4 \quad 2778.6 \quad 2779.5 \quad 2781.9$

name $=$ MIN43

energy (in $\mathrm{kcal} / \mathrm{mol})=-53.872816$

$\mathrm{ZPE}($ in $\mathrm{kcal} / \mathrm{mol})=142.785$

$\mathrm{G}($ in $\mathrm{kcal} / \mathrm{mol})=114.011$

Geometry

C $-3.21581292-1.70669923-0.77642063$

C $-1.72253327-1.58720634-0.79073657$

C $0.96647188 \quad 2.44136914 \quad-0.42369628$

C $0.73822538 \quad 0.98944373-0.39858311$

C $0.79388804 \quad 0.16246756 \quad 0.65127585$

C $0.50026380-1.30543637 \quad 0.41723831$

C $-0.97518603-1.48945163 \quad 0.31289663$

C 1.150993520 .506420632 .07328193

C $1.549177851 .96698054 \quad 2.30308586$

C $-0.022318420 .15648397 \quad 3.00382720$

$\mathrm{H}-3.65991241-1.60571715-1.77917851$

$\mathrm{H}-3.66969750-0.93050180-0.14342519$

$\mathrm{H}-3.52642080-2.68769620-0.38638551$

$\mathrm{H} \quad 0.22719681 \quad 3.08111356 \quad 0.08017636$

$\mathrm{H} \quad 0.51034278 \quad 0.59638764-1.39436796$

$\mathrm{H} \quad 0.15525775-0.90781604-2.89225514$ 
$\mathrm{H} \quad 2.03392821-0.13102265 \quad 2.35518874$

$\mathrm{H} \quad 0.90392797-1.93589344 \quad 1.23857205$

$\mathrm{H} 1.04040139-1.66974589-0.48246131$

$\mathrm{H}-1.47675031-1.51557006 \quad 1.28292659$

$\mathrm{H} \quad 1.92373298 \quad 2.11303977 \quad 3.32402333$

H $0.69885352 \quad 2.64607064 \quad 2.18042072$

$\mathrm{H} 2.35312738 \quad 2.28186069 \quad 1.62515571$

$\mathrm{H}-0.95414069 \quad 0.61929786 \quad 2.66033785$

$\mathrm{H} \quad 0.16455591 \quad 0.50615961 \quad 4.02497838$

$\mathrm{H}-0.18838389-0.92495848 \quad 3.05761111$

○ $0.02020601-1.69742010-2.30975118$

O $1.89233247 \quad 2.93773307 \quad-1.02201472$

o $-1.38739030-1.59265280-2.11666586$

Vibrational frequencies (in $\mathrm{cm}-1$ )

$\begin{array}{lllllllllllllll}26.0 & 36.4 & 40.1 & 59.6 & 76.2 & 85.6 & 98.7 & 150.9 & 164.0 & 176.5 & 194.0 & 246.9 & 276.9 & 289.9\end{array}$ $298.2 \quad 321.0 \quad 337.7 \quad 363.8 \quad 418.7 \quad 447.2 \quad 513.7 \quad 537.4 \quad 580.4 \quad 621.4 \quad 662.2 \quad 816.1 \quad 888.9 \quad 897.2$ $936.3 \quad 973.9 \quad 984.5 \quad 994.1 \quad 997.8 \quad 1014.7 \quad 1036.2 \quad 1058.3 \quad 1068.4 \quad 1123.3 \quad 1143.0 \quad 1163.9 \quad 1170.0$

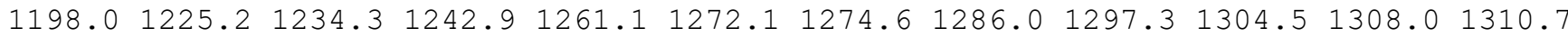
$1324.21333 .61339 .21350 .6 \quad 1369.6 \quad 1382.2 \quad 1398.3 \quad 1408.21413 .2 \quad 1829.6 \quad 1876.91904 .6$ $2649.02650 .42664 .2 \quad 2666.2 \quad 2667.8 \quad 2668.42674 .52677 .12696 .22737 .32745 .92753 .1$ $2775.02777 .32781 .5 \quad 2860.6$

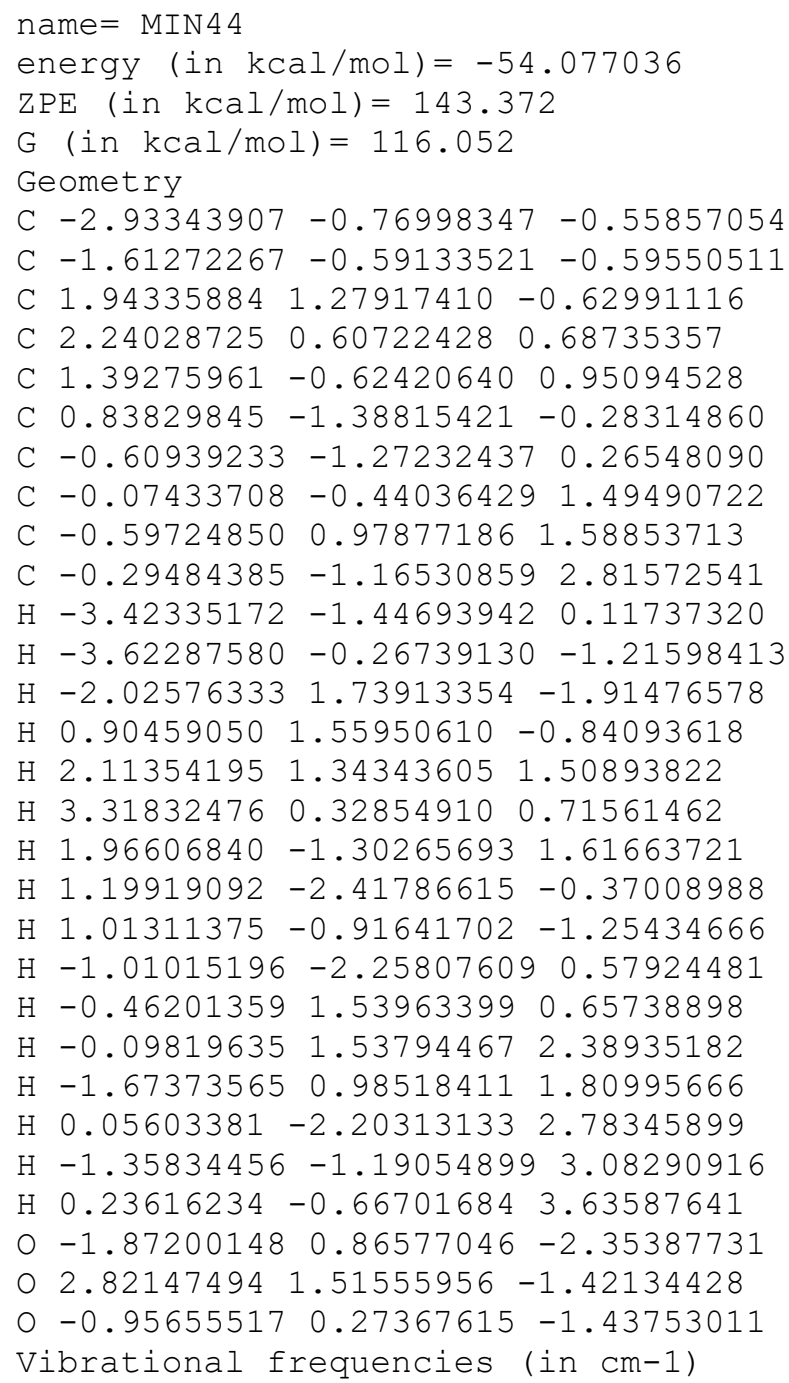


name $=$ MIN4 5

energy $($ in $\mathrm{kcal} / \mathrm{mol})=-52.936195$

$\mathrm{ZPE}(\mathrm{in} \mathrm{kcal} / \mathrm{mol})=142.734$

$\mathrm{G}($ in $\mathrm{kcal} / \mathrm{mol})=115.61$

Geometry

C $-2.89488191-0.80503566-1.37534344$

C $-1.39979400-0.92517922-1.31885347$

C $1.22750750 \quad 0.92876206-1.10657579$

C $0.70088899 \quad 0.79230547 \quad 0.11497908$

C $0.23188826-0.47653110 \quad 0.70082757$

C $0.61788162-1.78753227 \quad 0.05130522$

C $-0.82582636-1.34495994-0.01491648$

$\begin{array}{lllll}\text { C } 0.12038832 & -0.36345111 & 2.22628087\end{array}$

C $-1.17593495 \quad 0.37505656 \quad 2.58853929$

C $0.17195269-1.707954952 .95819876$

$\mathrm{H}-3.22590435-0.39264561-2.34065844$

$\mathrm{H}-3.27628676-0.14695964-0.58393751$

$\mathrm{H}-3.37556697-1.78529377-1.25713250$

$\mathrm{H} \quad 1.32252578 \quad 0.17259152 \quad-1.89170108$

$\mathrm{H} \quad 0.61517111 \quad 1.66691508 \quad 0.76467333$

H $2.65066553 \quad 3.20257567 \quad-0.45803551$

H $0.99227961 \quad 0.24575680 \quad 2.58341863$

$\mathrm{H} \quad 0.88871701-2.64967988 \quad 0.65220059$

H $1.21137783-1.77913875-0.86314325$

$\mathrm{H}-1.55049488-1.85739620 \quad 0.62895058$

$\mathrm{H}-2.06049770-0.21658241 \quad 2.33093310$

$\mathrm{H}-1.25631296 \quad 1.32977810 \quad 2.05484234$

$\mathrm{H}-1.22251210 \quad 0.591629013 .66078962$

$\mathrm{H} \quad 0.05989531-1.56528428 \quad 4.03963315$

$\mathrm{H}-0.62633942-2.38400300 \quad 2.63649863$

$\mathrm{H} \quad 1.13079612-2.214046492 .79807711$

$01.691089693 .13792633-0.68844620$

○ $1.745266232 .06825255-1.63545475$

o - $0.71415068-0.71345855-2.29048198$

Vibrational frequencies (in $\mathrm{cm}-1$ )

$\begin{array}{lllllllllllllll}45.5 & 57.6 & 64.2 & 96.6 & 109.1 & 113.5 & 132.2 & 163.4 & 172.0 & 180.3 & 216.2 & 226.0 & 257.1 & 265.0\end{array}$ $297.0 \quad 340.9 \quad 355.3 \quad 396.1 \quad 466.4 \quad 494.4 \quad 555.4 \quad 575.9 \quad 613.6 \quad 646.5 \quad 795.5 \quad 822.0 \quad 850.7 \quad 923.0$

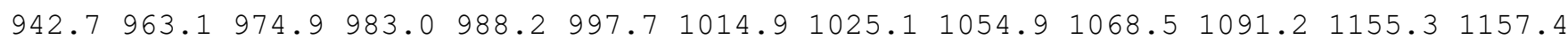

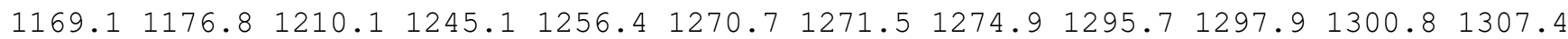
$1308.31317 .5 \quad 1344.8 \quad 1367.3 \quad 1380.5 \quad 1386.0 \quad 1387.5 \quad 1402.1 \quad 1404.8 \quad 1493.4 \quad 1843.21855 .1$ $2670.02671 .22673 .8 \quad 2674.6 \quad 2676.3 \quad 2679.3 \quad 2690.3 \quad 2700.82704 .2 \quad 2746.4 \quad 2750.3 \quad 2779.7$ $2780.5 \quad 2781.0 \quad 2782.5 \quad 2867.7$

name $=$ MIN46

energy $($ in $\mathrm{kcal} / \mathrm{mol})=-56.881876$

$\mathrm{ZPE}(\mathrm{in} \mathrm{kcal} / \mathrm{mol})=146.728$

$\mathrm{G}($ in $\mathrm{kcal} / \mathrm{mol})=123.454$

Geometry

C $-2.43953031 \quad 0.17065823 \quad 0.93708789$

C $-1.20912906 \quad 0.30435669 \quad 0.07122324$

C $-0.34886661 \quad 1.58349996 \quad 0.28975848$

C 1.167377451 .452116290 .33608044

C $1.648787290 .03600206-0.00572521$

C $1.27123142-0.31356678-1.46553547$

C $-0.44306484-1.01837828-0.08887481$

C $0.83562036-1.02491375 \quad 0.80058098$

C $0.60829949-0.68799012 \quad 2.26754874$

C $1.51174318-2.397706690 .71794313$

$\mathrm{H}-2.97817639 \quad 1.12504324 \quad 1.02173585$

$\mathrm{H}-2.19689828-0.17382231 \quad 1.94805170$

$\mathrm{H}-3.15018871-0.54650700 \quad 0.49990027$

$\mathrm{H}-0.73500254 \quad 2.27062427 \quad 1.05978390$ 
H $1.54714014 \quad 1.74375881 \quad 1.33404832$

$\mathrm{H} \quad 1.612380612 .18184894 \quad-0.37388281$

$\mathrm{H} 2.73400116-0.05729584 \quad 0.17357017$

$\mathrm{H} 2.00926502-0.95137464-1.97144277$

H $1.03635912 \quad 0.56829834 \quad-2.08364131$

$\mathrm{H}-1.08679669-1.907869150 .01232988$

$\mathrm{H} 0.03683093-1.47912667 \quad 2.76899533$

$\mathrm{H} \quad 0.05959656 \quad 0.24684819 \quad 2.41723090$

$\mathrm{H} 1.56440603-0.58996560 \quad 2.79687180$

$\mathrm{H} \quad 0.92158795-3.16564796 \quad 1.22985775$

H $2.50575770-2.382162411 .17595008$

$\mathrm{H} \quad 1.63014190-2.72821350-0.32288593$

$\begin{array}{llll}0 & -1.58963397 & 0.84448210 & -1.22823008\end{array}$

O $-0.786784532 .05383136-1.01416099$

O $0.07272692-1.10003085-1.40891594$

Vibrational frequencies (in cm-1)

$104.3125 .5 \quad 132.9 \quad 164.1 \quad 183.0 \quad 205.9 \quad 225.4 \quad 251.6 \quad 282.2 \quad 321.3 \quad 333.5 \quad 346.3 \quad 402.0420 .6$ $460.7485 .8 \quad 511.3 \quad 583.4 \quad 687.7 \quad 704.1 \quad 720.7 \quad 875.1 \quad 877.3 \quad 910.0 \quad 928.1960 .1969 .1977 .0$ $997.91007 .0 \quad 1010.3 \quad 1019.8 \quad 1032.4 \quad 1046.1 \quad 1052.0 \quad 1096.91117 .5 \quad 1125.7 \quad 1137.9 \quad 1158.0$

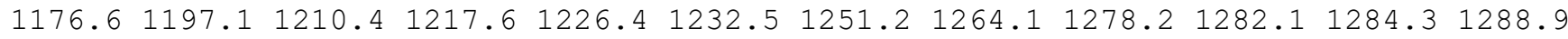
$1292.21296 .6 \quad 1305.6 \quad 1307.7 \quad 1313.4 \quad 1323.4 \quad 1336.4 \quad 1344.91353 .3 \quad 1378.5 \quad 1397.5 \quad 1407.9$ $1415.72654 .12659 .8 \quad 2667.92670 .2 \quad 2675.2 \quad 2676.42678 .62681 .5 \quad 2700.02709 .12722 .0$ $2726.7 \quad 2739.6 \quad 2779.9 \quad 2780.92783 .1$

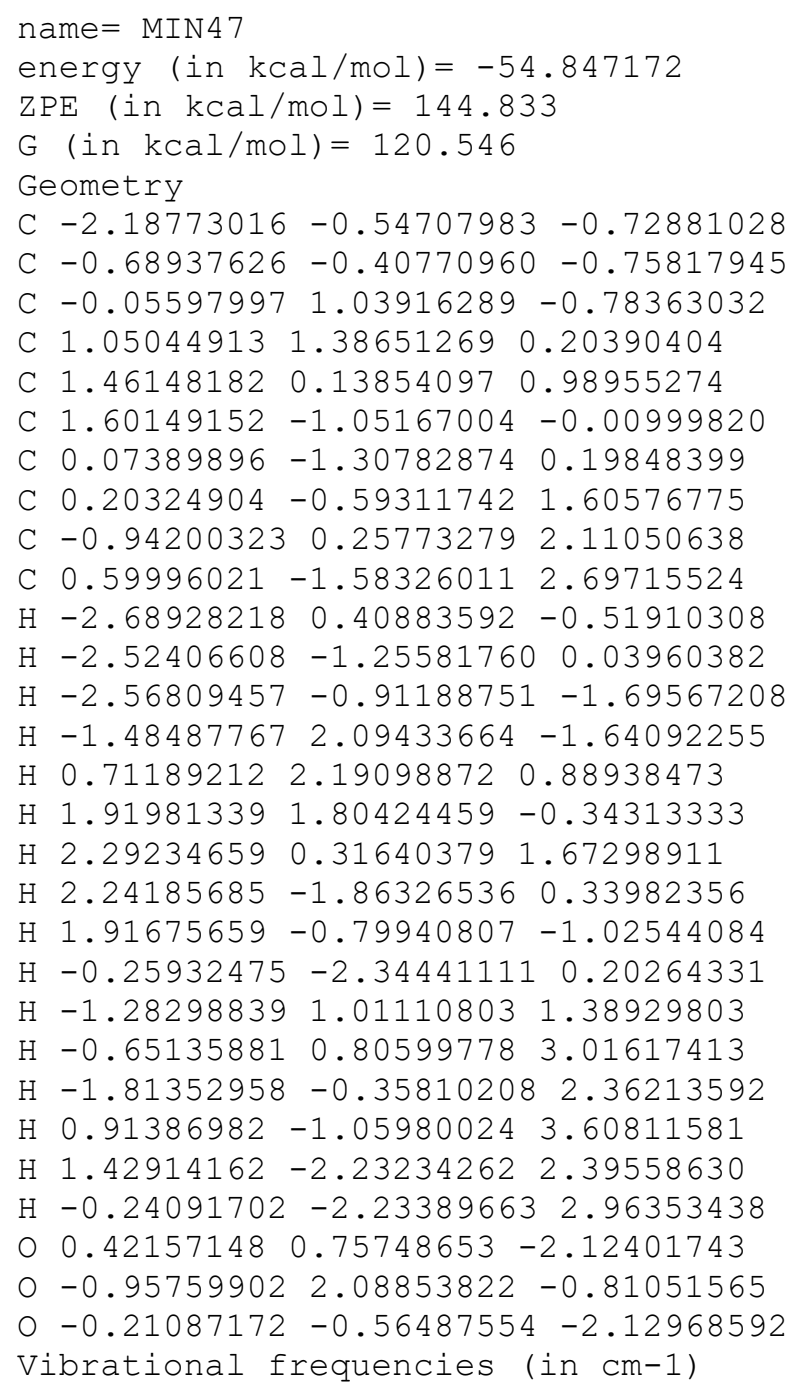


$1420.52659 .8 \quad 2662.0 \quad 2666.4 \quad 2668.92676 .2 \quad 2676.92681 .32684 .92739 .3 \quad 2747.5 \quad 2752.3$ $2764.6 \quad 2774.5 \quad 2781.4 \quad 2783.5 \quad 2795.3$

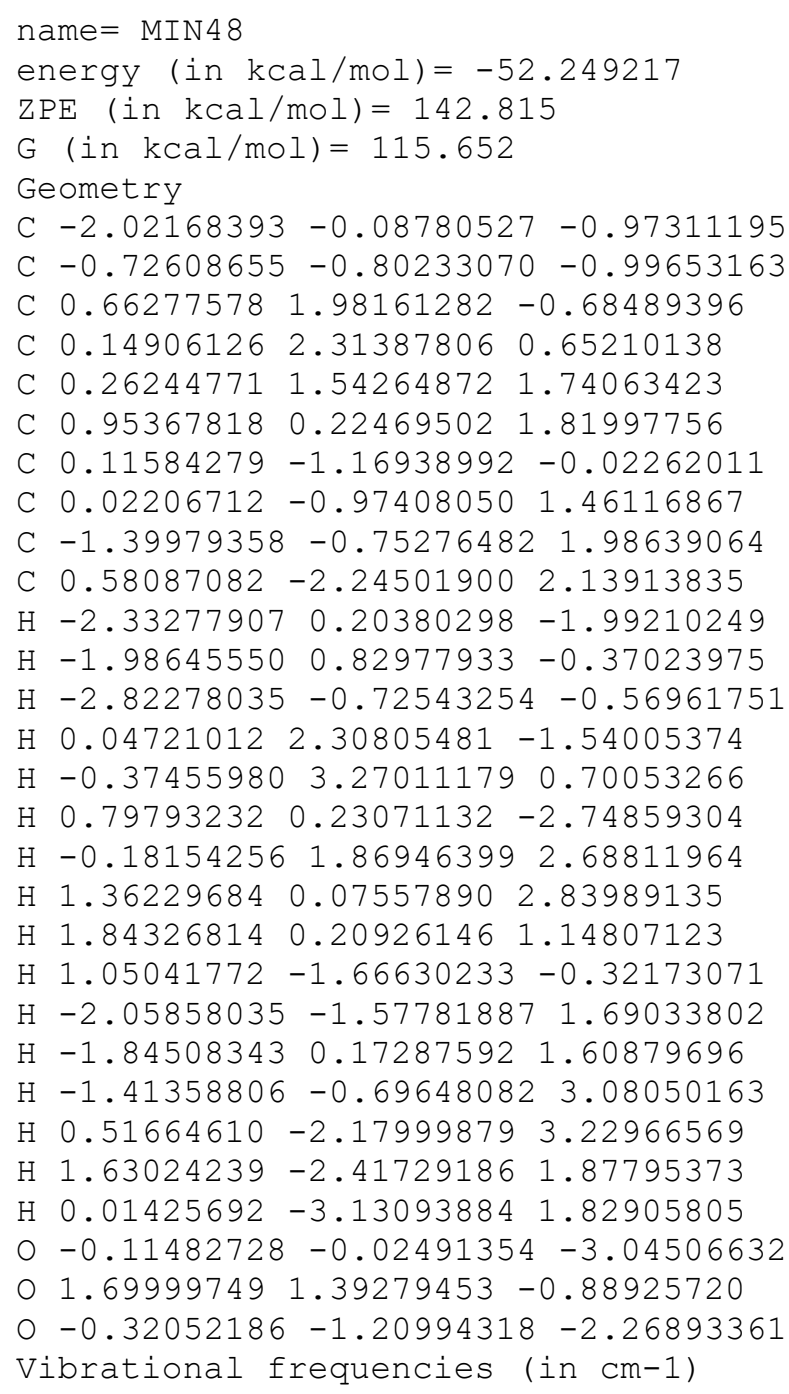


$\mathrm{H}-2.691413230 .15237269-1.74349090$

$\mathrm{H}-0.07219191 \quad 1.97941791-1.72098121$

$\mathrm{H}-0.89973241 \quad 2.16188259 \quad 0.92593533$

$\mathrm{H} \quad 0.67958316 \quad 2.86462944 \quad 0.55962463$

H $1.45222783 \quad 1.17339721 \quad 2.16345647$

H $2.40863170 \quad-0.51949735 \quad 0.27410022$

$\mathrm{H} 1.25438294-0.56312903-2.36492095$

$\mathrm{H} 0.10920843-1.91714288-0.01686590$

$\mathrm{H}-1.98209032 \quad 0.77078437 \quad 1.72239147$

$\mathrm{H}-1.48920190 \quad 0.10688992 \quad 3.26940151$

$\mathrm{H}-2.18167542-0.958751812 .03857651$

$\mathrm{H} \quad 0.65587825-0.97561515 \quad 3.61360747$

$\mathrm{H} 1.55064304 \quad-1.67921708 \quad 2.26632411$

$\mathrm{H}-0.04058572-2.31257523 \quad 2.68615290$

$00.60506125-1.31337123-2.36097514$

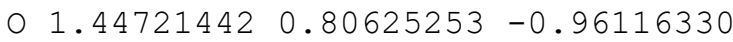

$0-0.61889160-0.62797811-2.17481318$

Vibrational frequencies (in cm-1)

$\begin{array}{lllllllllllllll}137.7 & 145.3 & 172.2 & 183.8 & 200.4 & 214.7 & 228.4 & 256.3 & 302.2 & 324.1 & 367.7 & 385.1 & 400.0 & 424.4\end{array}$ $445.6 \quad 502.7 \quad 517.4 \quad 566.5 \quad 577.7 \quad 627.2 \quad 730.7 \quad 764.9 \quad 783.3 \quad 836.5 \quad 882.3 \quad 914.2 \quad 938.8 \quad 963.4$ $972.0 \quad 993.4 \quad 1002.8 \quad 1012.6 \quad 1015.9 \quad 1022.0 \quad 1035.9 \quad 1040.5 \quad 1050.5 \quad 1074.3 \quad 1096.51113 .8$ $1128.51148 .51161 .1 \quad 1169.5 \quad 1178.2 \quad 1193.4 \quad 1209.2 \quad 1228.7 \quad 1256.3 \quad 1276.4 \quad 1280.11281 .9$

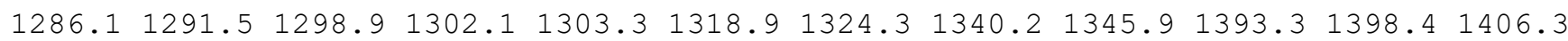
$1413.52658 .12664 .6 \quad 2673.4 \quad 2675.0 \quad 2675.92678 .0 \quad 2679.4 \quad 2732.6 \quad 2745.3 \quad 2748.3 \quad 2753.7$ $2760.72774 .12778 .3 \quad 2782.2 \quad 2815.8$

name $=$ MIN50

energy $($ in $\mathrm{kcal} / \mathrm{mol})=-53.705066$

$\operatorname{ZPE}($ in $\mathrm{kcal} / \mathrm{mol})=144.701$

$\mathrm{G}($ in $\mathrm{kcal} / \mathrm{mol})=120.478$

Geometry

C $-2.34843786-0.23197113-0.72874659$

C $-0.81527632-0.30926624-0.79810520$

C $0.71507525 \quad 1.64544151 \quad-0.79276955$

C $1.542739851 .44046280 \quad 0.23952882$

C $1.444829430 .29321766 \quad 1.17158695$

C $1.36041355-1.025842810 .34362968$

C $-0.18736313-0.93512204 \quad 0.43185905$

C $-0.03114793 \quad 0.03056582 \quad 1.66941663$

C $-0.93298771 \quad 1.23772354 \quad 1.81553925$

C $-0.04258437-0.760846532 .97635685$

$\mathrm{H}-2.69683918-0.11864280 \quad 0.30310009$

$\mathrm{H}-2.80934575-1.13845894-1.14518827$

$\mathrm{H}-2.72250804 \quad 0.62304282-1.30973423$

H $0.78345102 \quad 2.48082831-1.49484248$

$\begin{array}{lllll}\mathrm{H} & 2.36862397 & 2.12417851 & 0.41862195\end{array}$

$\mathrm{H} \quad 0.61241638-0.23990308-3.02104191$

H $2.19683571 \quad 0.30995474 \quad 1.96823945$

H $1.77837488-1.90100427 \quad 0.84703510$

$\mathrm{H} \quad 1.80031687-0.98113114-0.65419620$

$\mathrm{H}-0.68658569-1.88849851 \quad 0.66277261$

$\mathrm{H}-0.44186767 \quad 2.00824148 \quad 2.42565318$

$\mathrm{H}-1.88052110 \quad 0.98387053 \quad 2.30425602$

$\mathrm{H} \quad 0.26334902-0.12935122 \quad 3.81882864$

H $0.63826352-1.618818852 .94840842$

$\mathrm{H}-1.04444701-1.14571240 \quad 3.19779338$

○ $0.68283627-1.04201987-2.44244676$

$\begin{array}{lllll}0 & -0.40839170 & 0.99770148 & -1.17888590\end{array}$

$0-0.64448261-1.12178975-1.96152535$

$\mathrm{H}-1.18174293 \quad 1.71387629 \quad 0.85964847$

Vibrational frequencies (in cm-1)

$\begin{array}{lllllllllllllll}51.7 & 116.6 & 127.6 & 160.0 & 170.0 & 194.5 & 210.6 & 240.2 & 246.7 & 283.9 & 310.4 & 328.3 & 348.6 & 362.5\end{array}$ $380.1 \quad 408.6 \quad 439.3 \quad 467.0 \quad 487.3 \quad 561.7 \quad 607.9 \quad 703.7 \quad 774.0 \quad 796.3 \quad 809.3 \quad 858.7 \quad 903.1 \quad 926.3$ $941.1956 .2960 .2 \quad 1006.0 \quad 1010.2 \quad 1019.5 \quad 1023.8 \quad 1035.0 \quad 1062.0 \quad 1092.0 \quad 1097.4 \quad 1119.1 \quad 1138.9$ 


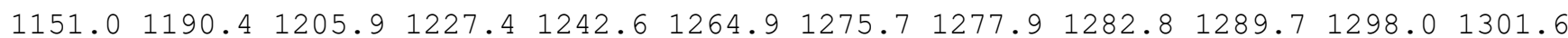

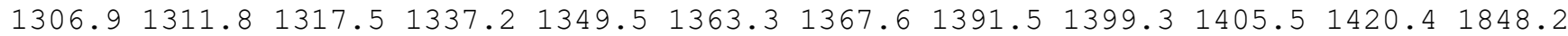
$2666.12668 .02671 .92676 .2 \quad 2677.6 \quad 2680.5 \quad 2687.32719 .52730 .92744 .52765 .92767 .0$ $2774.8 \quad 2781.3 \quad 2783.3 \quad 2861.7$

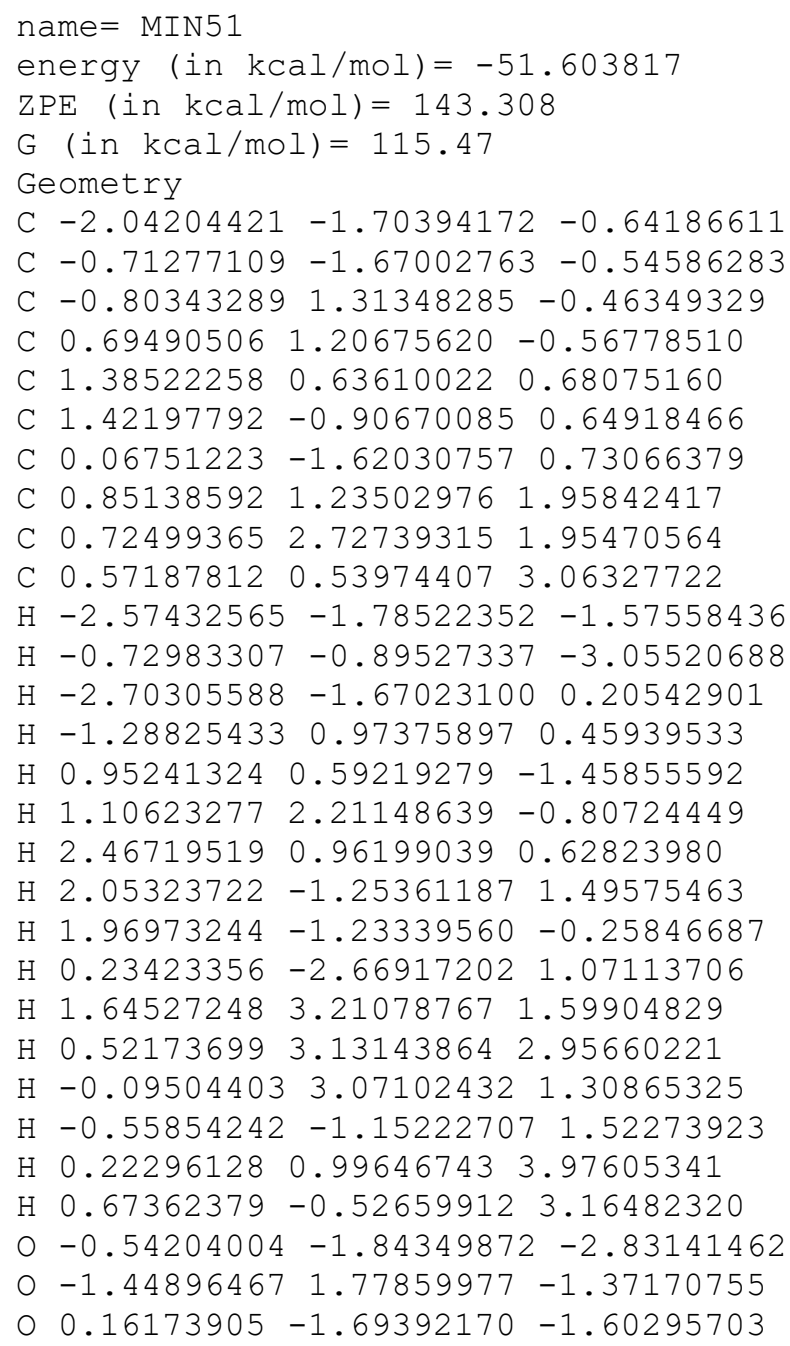


$\mathrm{H}-1.69442834 \quad-2.24392231 \quad 0.80838872$

$\mathrm{H}-1.72229201-3.15556776-0.71074353$

$\mathrm{H}-1.68088948-1.38177288-0.74243801$

$\mathrm{H}-2.86845695 \quad 1.20700954-0.91981357$

$\mathrm{H}-0.75643843 \quad 1.90945625-1.75896118$

H $2.20074498-3.38451105-1.72790341$

$\mathrm{H} 1.33475904 \quad 0.84816021-0.94878952$

$\mathrm{H}-0.42261564-0.170069361 .35065711$

H $1.25327663 \quad 0.23311445 \quad 1.69950717$

H $2.10844078-1.49066014 \quad 0.23406231$

$\mathrm{H} \quad 0.50450399 \quad 4.48895083-0.56862308$

$\mathrm{H} \quad 1.28356645 \quad 3.27459092-1.58791802$

$\mathrm{H} 2.01224253 \quad 3.72312975-0.04373776$

$\mathrm{H}-0.62199205 \quad 2.06128438 \quad 2.11515423$

$\mathrm{H}-0.75586828 \quad 3.73623897 \quad 1.58700010$

$\mathrm{H} \quad 0.80242989 \quad 3.10975213 \quad 2.15885907$

O $2.00027588-3.58183742-0.77997130$

$\begin{array}{lllll}0 & -2.30176988 & 1.03291604 & 0.98265703\end{array}$

o $0.58059522-3.45828723-0.79933709$

Vibrational frequencies (in $\mathrm{cm}-1$ )

$\begin{array}{llllllllllllllll}11.9 & 34.5 & 63.9 & 87.9 & 98.4 & 122.8 & 127.4 & 140.6 & 156.3 & 180.4 & 198.1 & 202.6 & 231.7 & 252.0\end{array}$ $291.0 \quad 345.8 \quad 371.5 \quad 401.7 \quad 449.0 \quad 483.1 \quad 493.8 \quad 504.3 \quad 618.3 \quad 688.4 \quad 732.6 \quad 833.7 \quad 885.8 \quad 910.6$ $935.3 \quad 953.8 \quad 981.5 \quad 1014.3 \quad 1014.7 \quad 1019.7 \quad 1034.3 \quad 1042.4 \quad 1053.8 \quad 1059.5 \quad 1084.4 \quad 1148.5 \quad 1179.9$

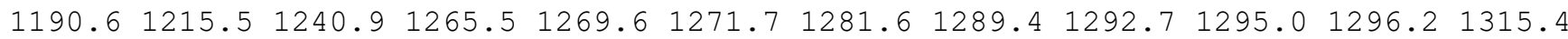
$1316.41349 .31351 .0 \quad 1367.2 \quad 1378.2 \quad 1385.5 \quad 1393.7 \quad 1400.11411 .91498 .3 \quad 1836.01894 .8$ $2647.42651 .5 \quad 2658.92660 .7 \quad 2668.8 \quad 2674.4 \quad 2676.62680 .22728 .02742 .02751 .12760 .4$ 2773.72778 .92782 .12870 .6

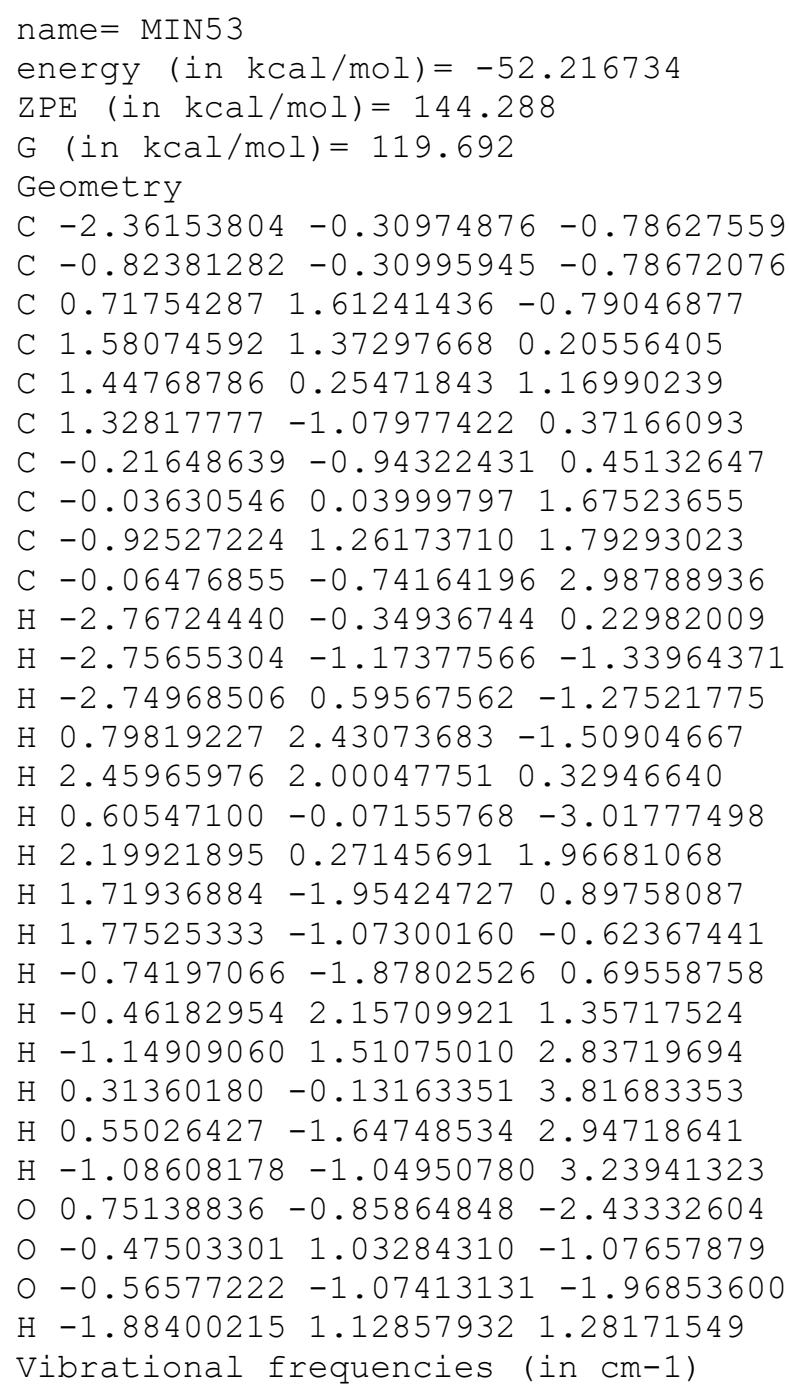


$\begin{array}{llllllllllllllllll}-127.8 & 46.9 & 86.2 & 117.8 & 163.7 & 190.7 & 207.6 & 226.2 & 252.1 & 273.3 & 295.6 & 324.2 & 347.0 & 366.2\end{array}$

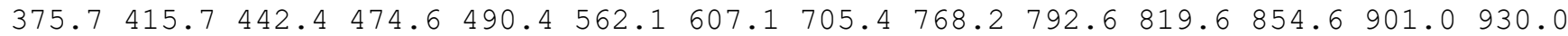

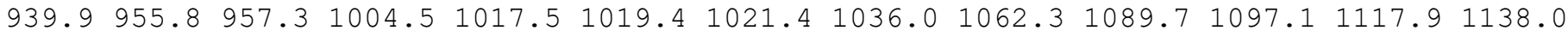

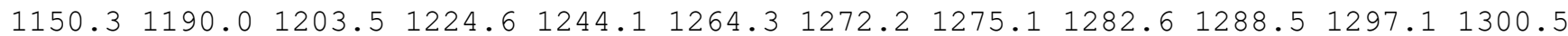

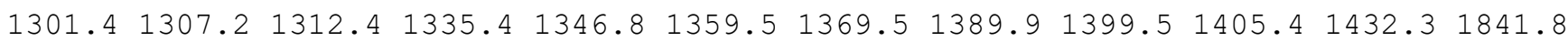
$2663.32665 .2 \quad 2668.5 \quad 2674.0 \quad 2677.5 \quad 2679.4 \quad 2687.4 \quad 2720.82731 .92744 .0 \quad 2765.3 \quad 2767.8$ $2771.92781 .12783 .2 \quad 2862.5$

name $=$ MIN54

energy (in $\mathrm{kcal} / \mathrm{mol})=-52.051435$

$\mathrm{ZPE}(\mathrm{in} \mathrm{kcal} / \mathrm{mol})=144.27$

$\mathrm{G}($ in $\mathrm{kcal} / \mathrm{mol})=119.243$

Geometry

C $-2.11771529-1.15749603-0.49678323$

C $1.47956925-1.19637245-0.48331878$

C $2.07382840 \quad 0.00449779-0.49251463$

C $1.38440280 \quad 1.27668764-0.17429893$

C $0.639367831 .10981576 \quad 1.18754952$

C $-0.63931955 \quad 0.72469244 \quad 0.39439694$

C $-0.020229821 .42274834-0.87948946$

C $-0.17887154 \quad 0.79717551 \quad-2.24809539$

C $-0.452410602 .88732016-0.95430667$

$\mathrm{H}-2.29224196-0.47983242-1.33835981$

$\mathrm{H}-2.99747810-1.12693640 \quad 0.15783687$

$\mathrm{H}-2.03657584-2.17987874-0.89499958$

$\mathrm{H} \quad 1.98109137-2.14785964-0.68186875$

H $3.13613183 \quad 0.08404863-0.70434323$

$\mathrm{H}-0.26380120-1.13279374 \quad 3.14548087$

H $2.02973292 \quad 2.15775848 \quad-0.26172398$

$\mathrm{H} \quad 0.54018776 \quad 2.03433070 \quad 1.76039207$

H $1.06085780 \quad 0.34926618 \quad 1.84623473$

$\mathrm{H}-1.56306214 \quad 1.19737658 \quad 0.75852413$

H $0.55400754 \quad 1.22053302 \quad-2.94848295$

$\mathrm{H}-1.17619282 \quad 0.97676238 \quad-2.66515729$

$\mathrm{H} \quad 0.13273958 \quad 3.42922990-1.70690616$

$\mathrm{H}-0.31866523 \quad 3.41008958-0.00126785$

$\mathrm{H}-1.50880872 \quad 2.97522803-1.23145034$

o $0.08514803-1.485694252 .29011118$

$00.17842597-1.53913203-0.35501577$

O $-1.10957424-1.39425603 \quad 1.52533819$

$\mathrm{H}-0.01546663-0.28749333-2.25192416$

C $-0.83711037-0.77604929 \quad 0.26836484$

Vibrational frequencies (in $\mathrm{cm}-1$ )

$\begin{array}{lllllllllllllll}32.8 & 82.2 & 118.2 & 135.1 & 153.4 & 171.2 & 180.9 & 206.8 & 228.1 & 249.7 & 288.5 & 322.6 & 344.7 & 359.6\end{array}$

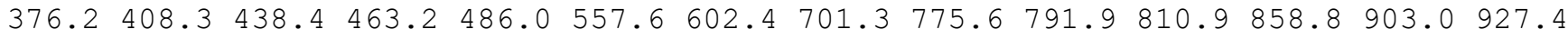
$939.0 \quad 950.2955 .6 \quad 1003.7 \quad 1009.2 \quad 1017.0 \quad 1024.8 \quad 1057.6 \quad 1062.9 \quad 1091.9 \quad 1097.3 \quad 1119.0 \quad 1133.5$ $1147.91189 .0 \quad 1204.4 \quad 1227.2 \quad 1239.8 \quad 1259.0 \quad 1275.3 \quad 1279.0 \quad 1280.9 \quad 1289.4 \quad 1297.91301 .2$

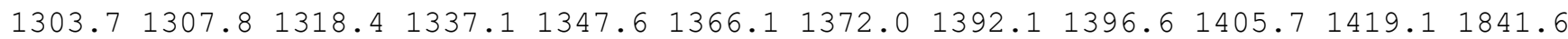
$2667.32669 .12673 .2 \quad 2676.62677 .7 \quad 2680.7 \quad 2689.72718 .32732 .3 \quad 2744.62766 .2 \quad 2768.6$ $2776.22781 .2 \quad 2783.2 \quad 2878.3$

name $=$ MIN55

energy (in $\mathrm{kcal} / \mathrm{mol})=-51.077176$

$\mathrm{ZPE}($ in $\mathrm{kcal} / \mathrm{mol})=143.434$

$\mathrm{G}($ in $\mathrm{kcal} / \mathrm{mol})=115.963$

Geometry

C $-2.02868408-1.65405962-0.65906575$

C $-0.70124109-1.70153293-0.54914184$

C $-0.83141200 \quad 1.34892672-0.45875788$

C $0.65369140 \quad 1.15611999-0.61504271$

$\begin{array}{llll}\text { C } & 1.36062381 & 0.61694926 & 0.63910777\end{array}$

C $1.42267049-0.925825290 .63898732$

C $0.07848736-1.65935281 \quad 0.72798278$ 
C $0.82653377 \quad 1.22950453 \quad 1.91068463$

C $0.70363744 \quad 2.72150348 \quad 1.89113026$

C $0.54436479 \quad 0.54627440 \quad 3.02260080$

$\mathrm{H}-2.55369788-1.68304369-1.59982539$

$\mathrm{H}-0.58360347-2.71360221-3.06572133$

$\mathrm{H}-2.69493697-1.56402928 \quad 0.18039882$

$\mathrm{H}-1.34433641 \quad 0.79634401 \quad 0.33685243$

$\mathrm{H} \quad 0.82474842 \quad 0.47234298-1.47920115$

H $1.114860342 .11468267-0.93372428$

H $2.43699327 \quad 0.958049920 .57263726$

H 2.05618674 -1.246852311 .49356361$

$\mathrm{H} \quad 1.97609443-1.25743252-0.26317833$

$\mathrm{H} \quad 0.25842049-2.70521528 \quad 1.06562398$

$\mathrm{H} 1.64686669 \quad 3.19902576 \quad 1.59179007$

$\mathrm{H} 0.43478466 \quad 3.13270877 \quad 2.87404446$

$\mathrm{H}-0.069650813 .06622327 \quad 1.18867763$

$\mathrm{H}-0.54971055-1.199161201 .52375146$

$\mathrm{H} \quad 0.19389123 \quad 1.01293721 \quad 3.92960753$

$\mathrm{H} \quad 0.64465330-0.51836551 \quad 3.13672831$

$\begin{array}{lllll}0 & -0.51255290 & -1.75424760 & -2.83373837\end{array}$

O $-1.438413862 .09436352-1.18847105$

o $0.17956916-1.84604128-1.59201417$

Vibrational frequencies (in $\mathrm{cm}-1$ )

$\begin{array}{llllllllllllll}32.6 & 49.0 & 60.7 & 64.3 & 78.4 & 96.9 & 127.5 & 152.2 & 178.2 & 195.9 & 232.1 & 261.8 & 304.5 & 314.0\end{array}$ $355.9 \quad 386.0 \quad 408.3 \quad 437.5 \quad 496.4 \quad 523.4 \quad 529.2 \quad 582.4 \quad 606.2 \quad 615.9 \quad 703.9 \quad 802.4 \quad 842.4 \quad 890.1$ $927.3972 .2995 .1 \quad 1006.2 \quad 1018.3 \quad 1038.8 \quad 1042.6 \quad 1050.0 \quad 1057.4 \quad 1074.7 \quad 1080.8 \quad 1104.91149 .0$

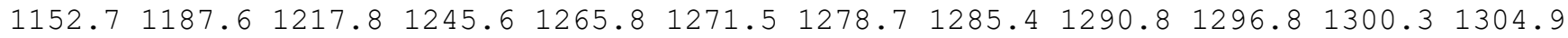

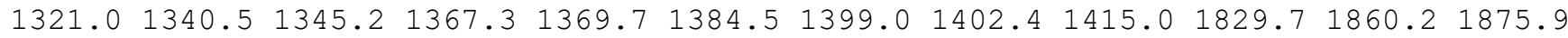
$2641.22644 .7 \quad 2653.1 \quad 2654.9 \quad 2662.7 \quad 2668.4 \quad 2673.8 \quad 2720.12724 .82728 .8 \quad 2733.4 \quad 2735.5$ $2777.92793 .22797 .3 \quad 2870.3$

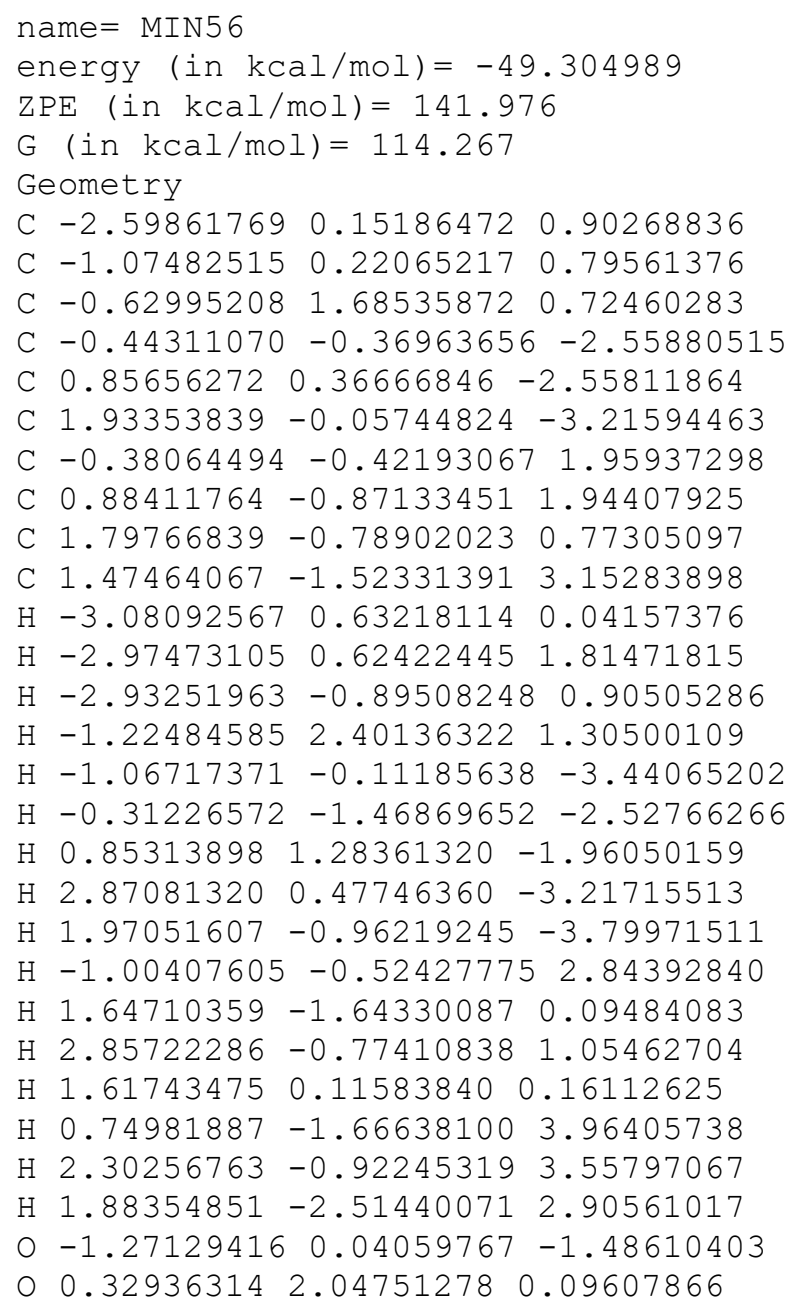


$0-0.63293013-0.52936627-0.32009343$

Vibrational frequencies (in $\mathrm{cm}-1$ )

$\begin{array}{llllllllllllllll}37.1 & 55.2 & 70.2 & 82.2 & 96.0 & 98.2 & 124.7 & 138.5 & 144.9 & 159.6 & 181.7 & 212.9 & 262.2 & 285.2\end{array}$ $306.4 \quad 312.0 \quad 344.6 \quad 361.0 \quad 422.2 \quad 461.8 \quad 494.0 \quad 540.2 \quad 584.7 \quad 600.2 \quad 645.7 \quad 872.1 \quad 885.5 \quad 923.4$ $940.7962 .4 \quad 965.4983 .6 \quad 989.0 \quad 1012.5 \quad 1018.8 \quad 1025.6 \quad 1056.6 \quad 1094.9 \quad 1100.6 \quad 1118.4 \quad 1132.0$

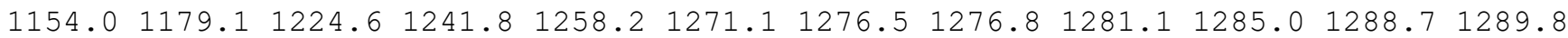
$1295.71315 .91331 .4 \quad 1334.3 \quad 1380.0 \quad 1389.4 \quad 1399.0 \quad 1401.7 \quad 1425.6 \quad 1843.2 \quad 1854.91856 .5$ $2641.52642 .7 \quad 2649.2 \quad 2668.7 \quad 2671.12673 .8 \quad 2676.12680 .72708 .02716 .22744 .22756 .8$ $2774.8 \quad 2779.8 \quad 2781.6 \quad 2790.8$

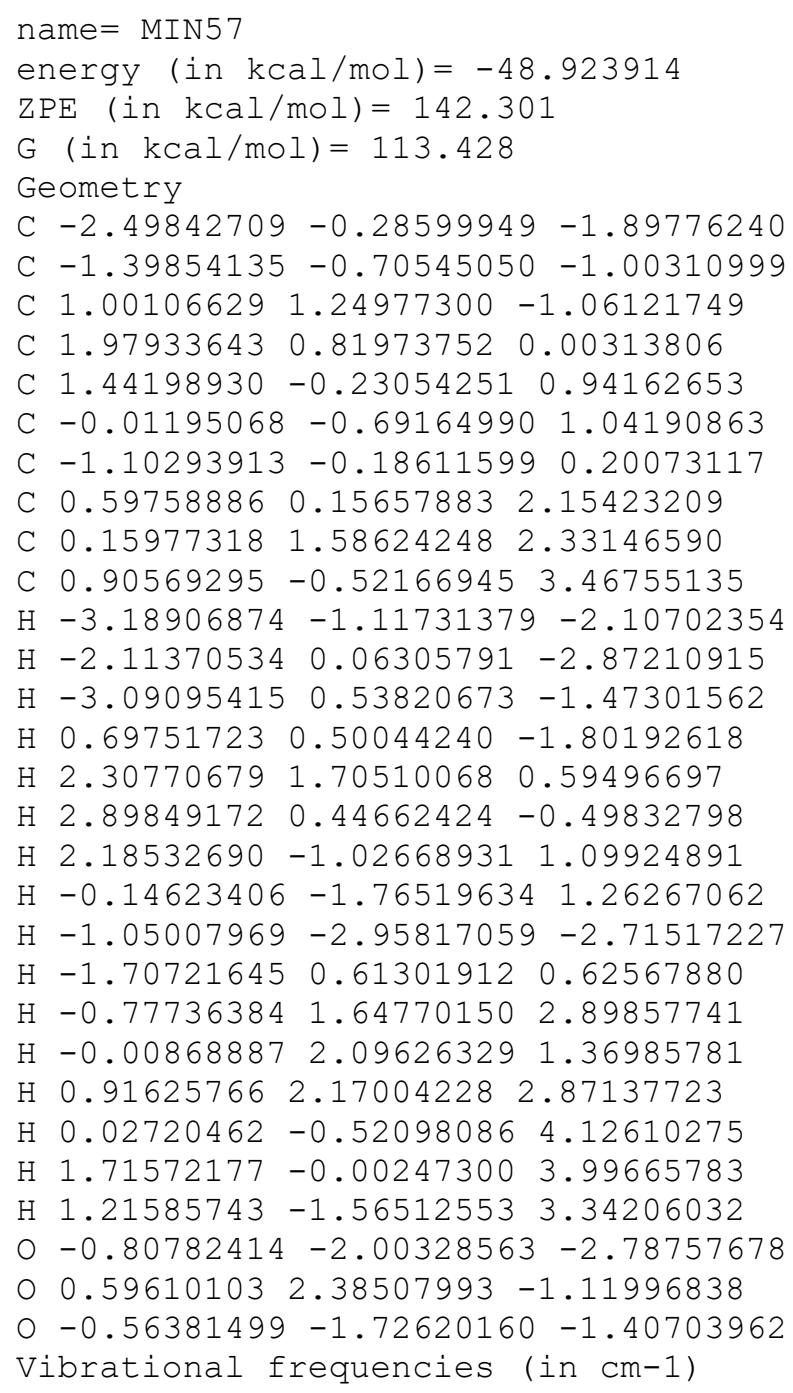


C $1.07411731-1.51778926-0.31980184$

C $-0.34858123-1.10641310-0.00960018$

C $1.12269613-0.568520562 .16874389$

$\begin{array}{llll}C & -0.01407270 & 0.06717991 & 2.98349667\end{array}$

C $1.30031648-2.02984250 \quad 2.59777322$

$\mathrm{H}-2.90278466 \quad 0.69514279-1.58010044$

$\mathrm{H}-2.82573050 \quad 0.28750815 \quad 0.14391145$

$\mathrm{H}-2.97668714-0.99536422-1.07461046$

$\mathrm{H}-1.32289240 \quad 2.61187107-1.45679305$

$\mathrm{H} \quad 0.92649084 \quad 1.76740044 \quad 0.95206380$

H $1.989503961 .15077871-0.32072206$

H $2.07630060-0.02407582 \quad 2.39592441$

$\mathrm{H} 1.42663033-2.49397734-0.00268293$

$\mathrm{H} 1.49913083-1.27128510-1.29423485$

$\mathrm{H}-1.00411716-1.78999730 \quad 0.52011330$

$\mathrm{H}-0.96467587-0.44859240 \quad 2.80900569$

$\mathrm{H}-0.16418049 \quad 1.11982693 \quad 2.71775303$

H $0.19563587 \quad 0.02531011 \quad 4.05704051$

$\mathrm{H} \quad 1.45711207-2.10380423 \quad 3.67995480$

H $2.16868017-2.48847947 \quad 2.11227542$

H $0.41917151 \quad-2.633276132 .35495366$

$\begin{array}{lllll}0 & 0.26550867 & 0.94668442 & -2.23638657\end{array}$

$\begin{array}{llllll}0 & -0.72681704 & 2.39968242 & -0.70211543\end{array}$

o $-0.56850884-0.25898382-2.32118307$

Vibrational frequencies (in $\mathrm{cm}-1$ )

$\begin{array}{lllllllllllllll}55.3 & 89.6 & 103.8 & 123.3 & 161.0 & 203.6 & 215.5 & 222.7 & 234.1 & 284.2 & 293.4 & 319.4 & 321.0 & 372.4\end{array}$

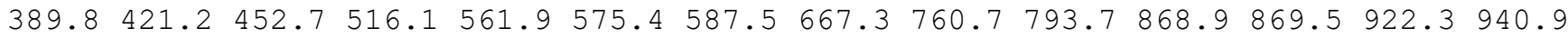

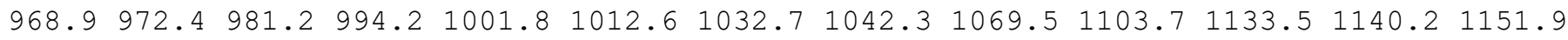

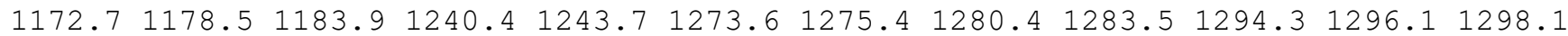

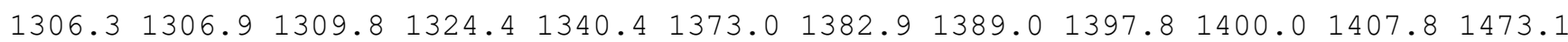
$2663.72671 .22672 .42674 .2 \quad 2675.3 \quad 2677.12680 .12703 .72704 .12744 .12770 .52779 .6$ $2780.22781 .3 \quad 2783.12796 .3$

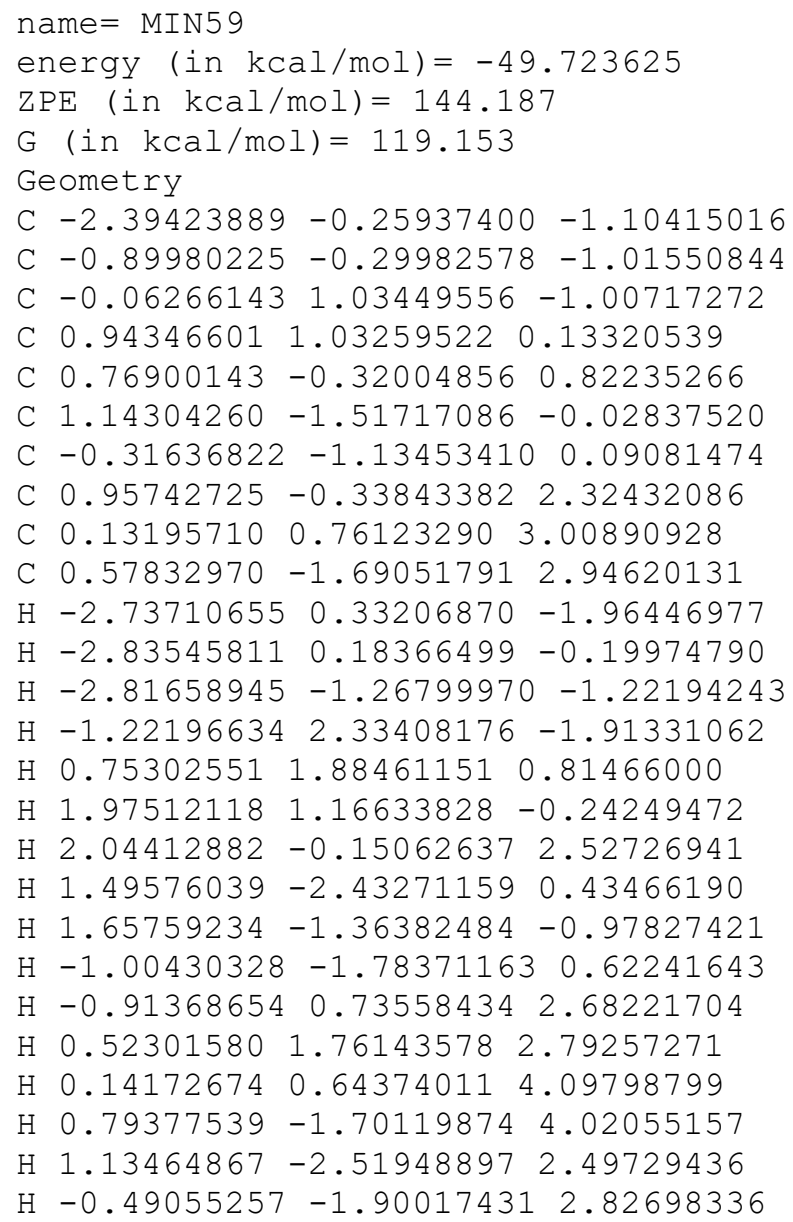


$0.48319952 \quad 0.65082632-2.29507367$

$0-0.71103070 \quad 2.24220601-1.07613311$

o $-0.30952033-0.58505201-2.31748661$

Vibrational frequencies (in $\mathrm{cm}-1$ )

$\begin{array}{llllllllllllllll}25.6 & 86.1 & 105.6 & 119.7 & 164.9 & 198.1 & 212.8 & 220.0 & 248.0 & 285.6 & 296.8 & 321.7 & 337.7 & 380.5\end{array}$

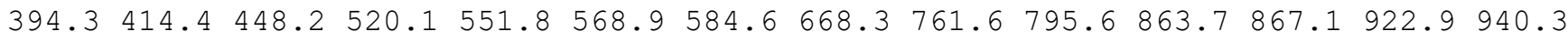
$966.7971 .8 \quad 980.9 \quad 993.3 \quad 1000.2 \quad 1012.6 \quad 1034.8 \quad 1044.7 \quad 1067.7 \quad 1103.4 \quad 1133.8 \quad 1138.0 \quad 1148.4$

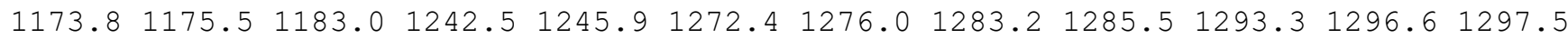

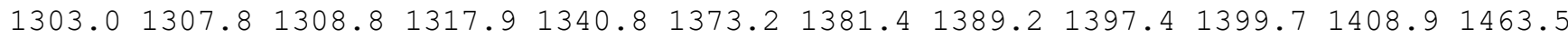
$2660.32670 .02672 .02673 .5 \quad 2674.4 \quad 2676.3 \quad 2678.52703 .22703 .5 \quad 2742.2 \quad 2769.32779 .2$ 2780.12780 .62782 .22796 .4

\section{name $=$ MIN60}

energy $($ in $\mathrm{kcal} / \mathrm{mol})=-47.667016$

$\mathrm{ZPE}($ in $\mathrm{kcal} / \mathrm{mol})=142.299$

$\mathrm{G}($ in $\mathrm{kcal} / \mathrm{mol})=113.97$

Geometry

C $-3.15945480-1.20768395-0.45108642$

C $-1.66603204-1.20641779-0.44301660$

C $-0.274101402 .00066666-0.46489585$

C $0.91011643 \quad 1.23981780 \quad-0.99555340$

C $1.43059264 \quad 0.13504783-0.11491837$

C $0.56784316-0.888071150 .61679080$

C $-0.89927779-0.87392674 \quad 0.60166973$

C $1.382796160 .13551407 \quad 1.40950005$

C $0.71350341 \quad 1.251892292 .16532135$

C $2.61758063-0.371513312 .12106212$

$\mathrm{H}-3.56226105-0.24042883-0.11489829$

$\mathrm{H}-3.55688844-1.98700713 \quad 0.21666847$

$\mathrm{H}-3.57804200-1.39802312-1.45232221$

$\mathrm{H}-1.168248201 .42988293-0.18646223$

$\mathrm{H} \quad 1.73969721 \quad 1.95247417-1.20964716$

$\mathrm{H} \quad 0.62372027 \quad 0.80429900 \quad-1.98365071$

H 2.35311809 $-0.27623628-0.56093321$

$\mathrm{H} \quad 0.95014811-1.92310268 \quad 0.61595055$

$\mathrm{H} \quad 0.20315677-2.58858298-2.15241983$

$\mathrm{H}-1.36234922-0.60731371 \quad 1.55352439$

$\mathrm{H} 1.16830247 \quad 2.22225492 \quad 1.91438009$

$\mathrm{H} \quad 0.80594195 \quad 1.11893431 \quad 3.25101227$

$\mathrm{H}-0.35987232 \quad 1.33030362 \quad 1.95541339$

H $3.35495237 \quad 0.43327726 \quad 2.24132940$

H $3.11106727-1.187439521 .58100638$

$\mathrm{H} \quad 2.37033992-0.75014908 \quad 3.12087595$

○ $0.13506449-1.66698606-1.79918194$

o $-0.264288693 .20529098-0.38390069$

o $-1.28674265-1.57863227-1.70550618$

Vibrational frequencies (in $\mathrm{cm}-1$ )

$\begin{array}{lllllllllllllll}42.3 & 48.0 & 61.9 & 67.3 & 75.5 & 89.3 & 99.6 & 118.3 & 124.8 & 135.8 & 180.1 & 208.6 & 231.0 & 265.6\end{array}$ $310.9 \quad 324.2 \quad 331.9 \quad 404.2 \quad 459.3 \quad 484.4 \quad 496.4 \quad 535.5 \quad 594.7 \quad 661.7 \quad 763.0 \quad 801.8 \quad 865.5 \quad 892.3$ $949.2971 .6 \quad 980.4 \quad 1008.3 \quad 1014.1 \quad 1023.7 \quad 1030.3 \quad 1048.7 \quad 1059.6 \quad 1065.11091 .5 \quad 1113.21156 .8$

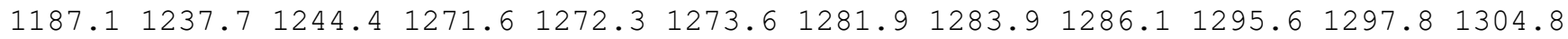
$1306.31341 .7 \quad 1351.91367 .3 \quad 1379.2 \quad 1398.4 \quad 1402.01408 .41420 .4 \quad 1524.4 \quad 1831.91895 .8$ $2644.62658 .62664 .2 \quad 2666.1 \quad 2667.4 \quad 2671.6 \quad 2677.4 \quad 2678.02727 .4 \quad 2732.4 \quad 2739.92753 .9$ $2774.7 \quad 2778.3 \quad 2781.7 \quad 2873.0$

name $=$ MIN61

energy (in $\mathrm{kcal} / \mathrm{mol})=-47.932862$

$\operatorname{ZPE}($ in $\mathrm{kcal} / \mathrm{mol})=142.742$

$\mathrm{G}($ in $\mathrm{kcal} / \mathrm{mol})=114.97$

Geometry

C $-2.99248400-1.05066490-0.98323905$

C $-1.50016595-1.14297165-0.89084992$

C $-0.031781581 .59596883-0.40084067$ 
C $1.15896743 \quad 1.21626730 \quad 0.39417176$

C $1.43504632-0.21108497 \quad 0.84376695$

C $0.62501061-1.43024178 \quad 0.46290795$

C $-0.83734238-1.24525518 \quad 0.26747960$

C $1.04966457 \quad 0.84202386 \quad 1.87102603$

$\begin{array}{lllll}\text { C } 2.14670567 & 1.34321541 & 2.78431166\end{array}$

C -0.286354390 .837540122 .56385686$

$\mathrm{H}-3.33995858-0.84051921-2.00770928$

$\mathrm{H}-3.38424576-0.25018612-0.34073038$

$\mathrm{H}-3.46187201-1.99571462-0.67095591$

$\mathrm{H}-0.95456549 \quad 1.85616189 \quad 0.13454677$

$\mathrm{H} 2.03705891 \quad 1.80202396 \quad 0.07503038$

$\mathrm{H} \quad 0.54682707-0.13833538-2.30890082$

H $2.50730853-0.487731520 .84101116$

$\mathrm{H} \quad 0.77920747 \quad-2.19933597 \quad 1.25892970$

$\mathrm{H} 1.08285119-1.88624101-0.44792174$

$\mathrm{H}-1.40807449-1.266700131 .19700915$

H $2.20490730 \quad 0.73537591 \quad 3.69676203$

$\mathrm{H} \quad 1.962869892 .38124790 \quad 3.09070977$

H $3.13668074 \quad 1.31580671 \quad 2.31435654$

$\mathrm{H}-1.123530320 .63378063 \quad 1.88534340$

$\mathrm{H}-0.489204691 .80331105 \quad 3.04564140$

$\mathrm{H}-0.316305560 .065572013 .34542900$

$0.35985215-1.11727600-2.28262359$

$0 \quad 0.01268541 \quad 1.68673105-1.60686195$

O $-1.05490732-1.10791050-2.18027518$

Vibrational frequencies (in cm-1)

$\begin{array}{llllllllllllllllll}-68.6 & 24.1 & 49.0 & 64.6 & 81.3 & 110.8 & 126.2 & 134.1 & 145.0 & 195.0 & 208.1 & 241.7 & 284.7 & 293.6\end{array}$ $319.0 \quad 365.2 \quad 408.8 \quad 414.4 \quad 428.1 \quad 471.3 \quad 521.9 \quad 541.1620 .7 \quad 638.3 \quad 749.1 \quad 803.7 \quad 878.8 \quad 911.6$

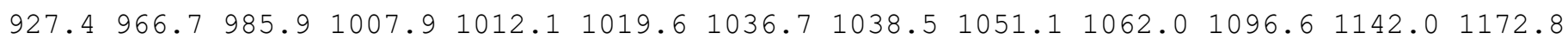
$1213.3 \quad 1227.7 \quad 1241.4 \quad 1253.2 \quad 1273.1 \quad 1274.2 \quad 1283.1 \quad 1291.6 \quad 1294.7 \quad 1295.6 \quad 1300.01300 .6$ $1309.3 \quad 1352.3 \quad 1356.5 \quad 1379.3 \quad 1381.8 \quad 1398.4 \quad 1401.2 \quad 1409.4 \quad 1423.91521 .6 \quad 1820.7 \quad 1893.6$ $2640.82652 .2 \quad 2663.8 \quad 2666.6 \quad 2669.8 \quad 2673.5 \quad 2676.12677 .92720 .02727 .62739 .2 \quad 2753.3$ $2775.22779 .2 \quad 2782.02806 .3$

\footnotetext{
name $=$ MIN62

energy (in $\mathrm{kcal} / \mathrm{mol})=-47.821248$

$\mathrm{ZPE}(\mathrm{in} \mathrm{kcal} / \mathrm{mol})=142.662$

$\mathrm{G}($ in $\mathrm{kcal} / \mathrm{mol})=115.632$

Geometry

C $-2.76416346-1.071068981 .43528005$

C $-1.27412350-1.076298241 .43123499$

C $-0.54919746 \quad 0.26879356 \quad 1.42314657$

C $-0.11781972 \quad 0.631928350 .00071246$

C $1.05935018-0.20772584-0.44599555$

C $2.442695820 .33903432-0.09364066$

C $2.849909130 .46410376-1.36773786$

C $1.53183619-0.04035331-1.97609579$

C $0.79078650 \quad 0.99427746 \quad-2.79581357$

C $1.64741248-1.36442653-2.70391143$

$\mathrm{H}-3.16437034-0.62270477 \quad 0.51305616$

$\mathrm{H}-3.16378962-0.47183797 \quad 2.26923423$

$\mathrm{H}-3.17572996-2.087127851 .51940967$

$\mathrm{H} \quad 0.32601711 \quad 0.23884449 \quad 2.12327849$

$\mathrm{H} \quad 0.13545460 \quad 1.71098757-0.03908536$

$\mathrm{H}-0.96914491 \quad 0.50214492-0.69930035$

$\mathrm{H} \quad 0.92647849-1.27207672-0.17508671$

H 2.85409557 $0.51306951 \quad 0.86445355$

$\mathrm{H}-2.19023489 \quad 2.57830194 \quad 1.00731364$

H $3.72484132 \quad 0.77466984-1.87119696$

$\mathrm{H}-0.23851471 \quad 0.67862722-3.00473589$

H $1.285385121 .15727040-3.76215393$

H $0.74814693 \quad 1.96564229-2.29005973$

H 2.14782855 -1.24775347 -3.67241266
} 
$\mathrm{H} \quad 0.65948490-1.80335587-2.89089725$

$\mathrm{H} 2.22553677-2.09786878-2.12701916$

O -2.392932411 .620873541 .14216102$

$0-1.317690191 .289188302 .02124764$

O $-0.62770525-2.094267581 .42359055$

Vibrational frequencies (in $\mathrm{cm}-1$ )

$\begin{array}{llllllllllllllll}34.2 & 39.2 & 61.1 & 95.1 & 130.4 & 141.2 & 165.4 & 186.0 & 190.7 & 204.9 & 208.3 & 222.6 & 269.7 & 294.4\end{array}$ $310.2 \quad 345.7 \quad 359.1 \quad 383.8 \quad 458.5 \quad 489.1 \quad 547.0 \quad 601.8 \quad 690.7 \quad 698.4 \quad 811.4 \quad 835.5 \quad 877.1 \quad 916.4$ $929.2 \quad 968.3 \quad 982.9 \quad 998.4 \quad 1008.4 \quad 1018.5 \quad 1039.3 \quad 1049.2 \quad 1067.7 \quad 1072.8 \quad 1099.2 \quad 1122.3 \quad 1146.1$ $1166.91182 .91218 .6 \quad 1230.1 \quad 1246.1 \quad 1249.7 \quad 1265.5 \quad 1271.8 \quad 1277.4 \quad 1280.5 \quad 1287.3 \quad 1293.5$

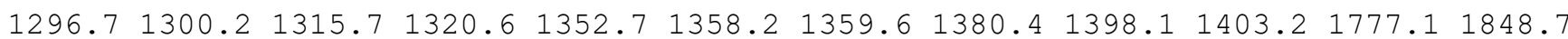
$2656.22660 .62672 .0 \quad 2674.1 \quad 2674.9 \quad 2675.8 \quad 2679.5 \quad 2680.72705 .2 \quad 2742.72777 .5 \quad 2781.3$ $2782.92799 .3 \quad 2812.8 \quad 2867.1$

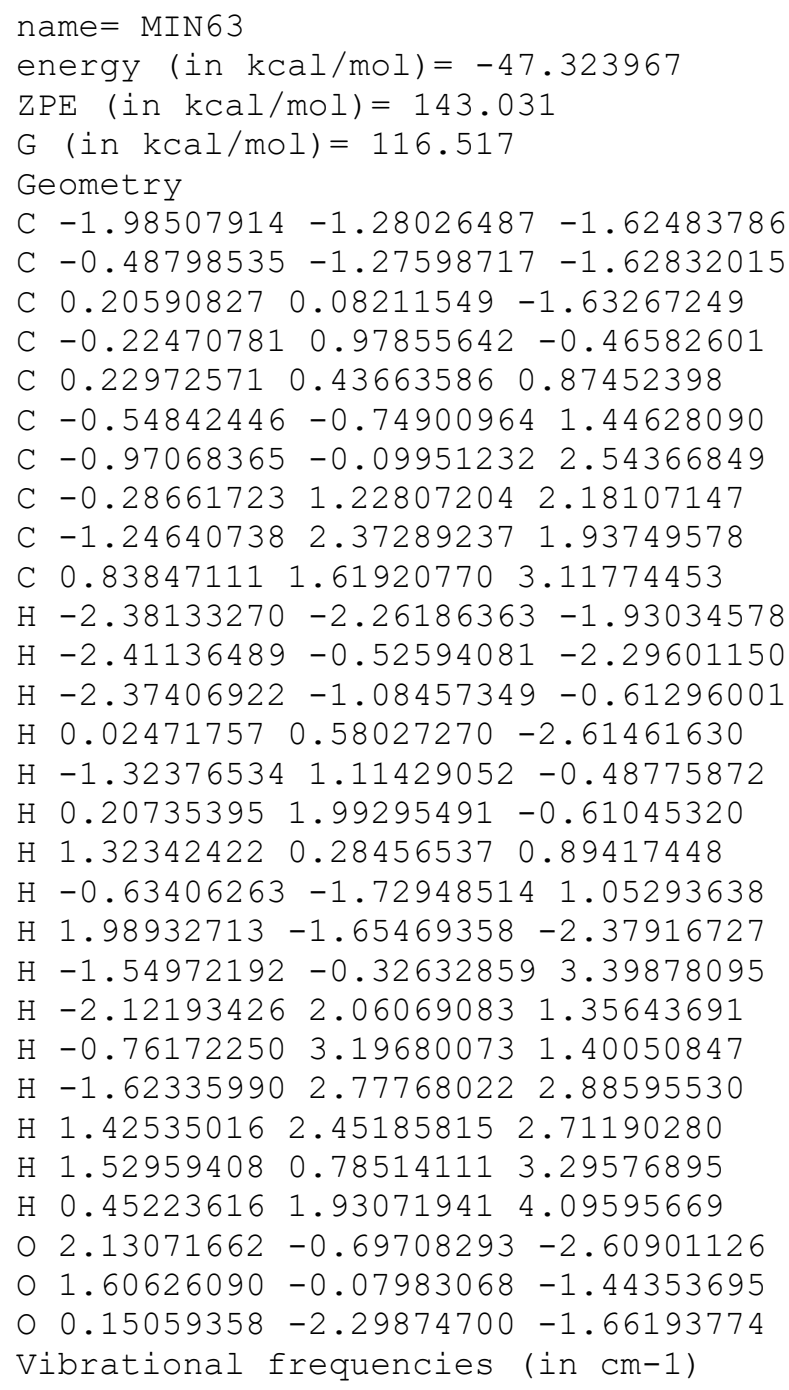


C $-1.57476346-1.11086321-0.79706644$

C $-0.55075661 \quad 1.02802792-0.85381828$

C $0.54291235 \quad 0.91717882 \quad 0.15657482$

C $0.32095671 \quad 0.40192658 \quad 1.36953022$

C $0.38216944-2.53160483-0.19527292$

C $-1.07003984-2.22323047-0.24636597$

C $1.38217008 \quad 0.19838200 \quad 2.35978270$

C $1.05929418 \quad 0.68233969 \quad 3.73468272$

C $2.53203025-0.409105732 .05149053$

$\mathrm{H}-3.22615347-0.07881588-1.71032600$

$\mathrm{H}-3.34647084-0.228237110 .05636737$

$\mathrm{H}-3.65485480-1.62743947-0.98060708$

$\mathrm{H}-1.515654491 .43438236-0.47429810$

H $1.52545753 \quad 1.25198884-0.17809954$

$\mathrm{H} \quad 0.77377156 \quad 0.85853753-3.08808930$

H $0.98986538-1.77337512-0.71423678$

$\mathrm{H} \quad 0.74280079-2.57482580 \quad 0.84386361$

$\mathrm{H} \quad 0.60399402 \quad-3.50066975-0.66337155$

$\mathrm{H}-0.67294350 \quad 0.07977847 \quad 1.68851803$

$\mathrm{H} \quad 1.90444917 \quad 0.57584888 \quad 4.42735329$

$\mathrm{H} \quad 0.21524716 \quad 0.12485175 \quad 4.16541730$

$\mathrm{H} \quad 0.77918765 \quad 1.74598684 \quad 3.72674192$

$\mathrm{H}-1.72282173-2.974891850 .19135645$

H $2.76509780 \quad-0.76923209 \quad 1.06130247$

H $3.32023382-0.59669827 \quad 2.76082864$

$\begin{array}{llll}0 & 1.01072184 & 1.58883492 & -2.45981513\end{array}$

$0-0.268680551 .89541103-1.92365243$

o $-0.72853628-0.25829238-1.46635040$

Vibrational frequencies (in cm-1)

$\begin{array}{lllllllllllllll}22.3 & 37.2 & 60.8 & 85.9 & 96.3 & 96.9 & 105.0 & 105.8 & 114.8 & 116.2 & 192.3 & 211.2 & 224.5 & 266.5\end{array}$ $289.8 \quad 321.1 \quad 367.1 \quad 398.4 \quad 415.1 \quad 426.5 \quad 512.7 \quad 552.3 \quad 569.3 \quad 592.4 \quad 627.5 \quad 699.1 \quad 863.9 \quad 923.1$ $955.9971 .2975 .5 \quad 987.9 \quad 1017.3 \quad 1028.0 \quad 1046.5 \quad 1050.3 \quad 1054.6 \quad 1072.8 \quad 1080.6 \quad 1090.4 \quad 1099.5$ $1120.61171 .5 \quad 1213.5 \quad 1233.0 \quad 1268.1 \quad 1274.6 \quad 1276.7 \quad 1279.5 \quad 1280.0 \quad 1280.6 \quad 1285.1 \quad 1286.5$ $\begin{array}{lllllllllllll}1312.1 & 1342.7 & 1362.8 & 1368.7 & 1375.4 & 1388.0 & 1392.7 & 1400.4 & 1407.0 & 1848.6 & 1861.3 & 1864.1\end{array}$ $2655.32665 .12670 .3 \quad 2671.1 \quad 2671.3 \quad 2671.6 \quad 2675.52722 .12733 .5 \quad 2755.32759 .12777 .1$ $2779.12779 .5 \quad 2789.5 \quad 2857.6$

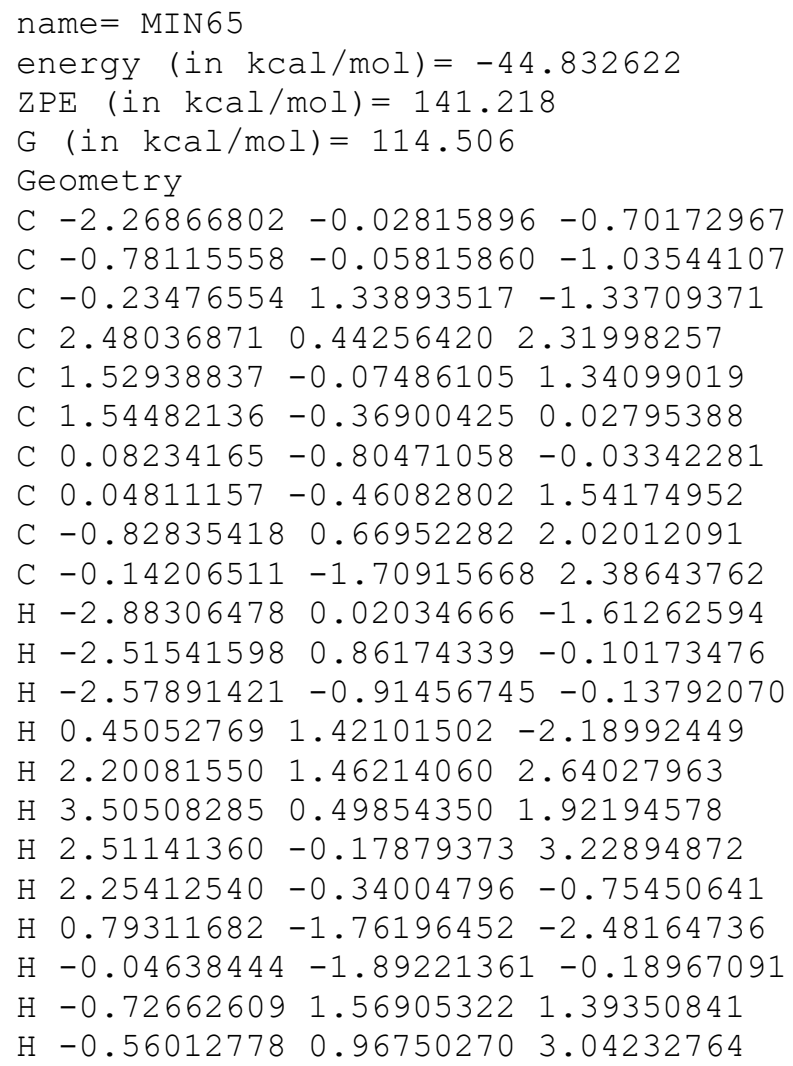


$\mathrm{H}-1.88811413 \quad 0.391755392 .02840165$

$\mathrm{H} 0.02426670-1.49589644 \quad 3.44990838$

$\mathrm{H} \quad 0.55655274 \quad-2.50607047 \quad 2.10494430$

$\mathrm{H}-1.15838603-2.10662943 \quad 2.28548610$

O $0.52990184-0.85556004-2.77615956$

$\begin{array}{lllll}0 & -0.55030439 & 2.30172434 & -0.68718689\end{array}$

o $-0.79304016-0.79434208-2.26780235$

Vibrational frequencies (in $\mathrm{cm}-1$ )

$\begin{array}{lllllllllllllllll}51.9 & 73.1 & 80.0 & 95.2 & 106.8 & 135.5 & 155.6 & 159.6 & 183.0 & 203.8 & 227.1 & 232.4 & 244.5 & 247.8\end{array}$ $264.7 \quad 288.3 \quad 304.8 \quad 360.7 \quad 384.9 \quad 463.5 \quad 491.3 \quad 518.2 \quad 573.0 \quad 619.0 \quad 666.0 \quad 784.2 \quad 801.6 \quad 892.1$ $908.6953 .6 \quad 974.7 \quad 992.3 \quad 997.5 \quad 1005.5 \quad 1016.3 \quad 1027.4 \quad 1035.5 \quad 1058.7 \quad 1076.01111 .1 \quad 1161.1$

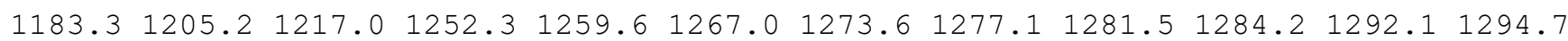

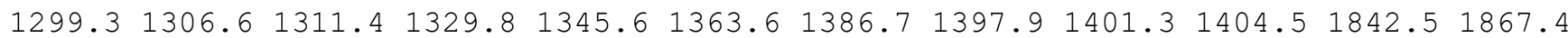
$2649.92657 .42662 .3 \quad 2663.92673 .92674 .5 \quad 2676.22677 .82681 .22714 .12774 .02776 .5$ $2780.32782 .8 \quad 2807.2 \quad 2860.5$

\section{name $=$ MIN66}

energy (in $\mathrm{kcal} / \mathrm{mol})=-47.230135$

$\mathrm{ZPE}($ in $\mathrm{kcal} / \mathrm{mol})=143.792$

$\mathrm{G}($ in $\mathrm{kcal} / \mathrm{mol})=116.951$

Geometry

C $-2.16669323-1.84389755-0.75546432$

C $-0.72293733-1.57072816-0.94068237$

C $0.068475581 .34899803-0.84426650$

C 1.282714621 .486880110 .03136453

C $1.23409525 \quad 0.57739343 \quad 1.27283387$

C $1.01822699-0.863051130 .87645288$

C $0.13621325-1.22265949-0.01306677$

C $0.14733104 \quad 1.00295944 \quad 2.29508826$

C $0.39505584 \quad 2.42839913 \quad 2.80099989$

C $0.14719574 \quad 0.03684147 \quad 3.48733107$

$\mathrm{H}-2.77081485-1.28789670-1.49431211$

$\mathrm{H}-2.52597484-1.57548053 \quad 0.24577601$

$\mathrm{H}-2.38665919-2.91182686-0.91700775$

$\mathrm{H}-0.91823964 \quad 1.32086092-0.36553345$

$\mathrm{H} \quad 1.40343148 \quad 2.54845598 \quad 0.32933342$

H 2.19572095 $1.24927238-0.56002646$

H $2.23000465 \quad 0.66240828 \quad 1.78144877$

$\mathrm{H} \quad 1.64564757-1.59022946 \quad 1.39555614$

$\mathrm{H}-0.58210175-0.02466899-2.96755216$

$\mathrm{H}-0.85188340 \quad 0.95898288 \quad 1.80118352$

H $0.27927651 \quad 3.17328863 \quad 2.00770719$

$\mathrm{H} 1.40070888 \quad 2.53886247 \quad 3.22145260$

$\mathrm{H}-0.31554683 \quad 2.69266443 \quad 3.59348308$

$\mathrm{H} \quad 1.12669519-0.003860313 .97535424$

$\mathrm{H}-0.11415886-0.98274813 \quad 3.18058403$

$\mathrm{H}-0.58253244 \quad 0.34255583 \quad 4.24509024$

$\begin{array}{lllll}0 & -1.00867470 & -0.90979294 & -3.09703790\end{array}$

○ $0.172180421 .29209939-2.04939871$

o $-0.21270933-1.71338578-2.22432141$

Vibrational frequencies (in $\mathrm{cm}-1$ )

$\begin{array}{llllllllllllllll}41.5 & 46.7 & 82.1 & 88.0 & 100.6 & 125.4 & 143.3 & 156.1 & 168.9 & 195.5 & 245.1 & 261.5 & 271.5 & 335.8\end{array}$ $361.5 \quad 392.8 \quad 398.0 \quad 432.4 \quad 450.8 \quad 475.3 \quad 526.3 \quad 588.2 \quad 618.7 \quad 628.1 \quad 729.0 \quad 835.6 \quad 843.6 \quad 907.0$ $926.4976 .6 \quad 985.7 \quad 987.3 \quad 1001.1 \quad 1013.4 \quad 1028.7 \quad 1057.6 \quad 1062.91097 .1 \quad 1149.61168 .1 \quad 1180.9$ $1191.21207 .5 \quad 1260.5 \quad 1264.4 \quad 1274.3 \quad 1277.4 \quad 1279.4 \quad 1288.6 \quad 1288.9 \quad 1293.6 \quad 1296.7 \quad 1300.9$

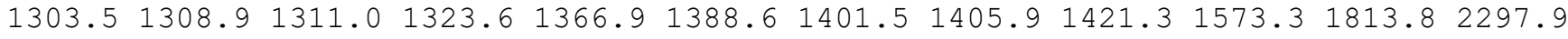
$2645.62657 .6 \quad 2658.3 \quad 2672.2 \quad 2674.1 \quad 2676.2 \quad 2677.3 \quad 2678.22687 .92711 .92736 .12741 .1$ $2776.32780 .92782 .8 \quad 2803.5$

name $=$ MIN67

energy $($ in $\mathrm{kcal} / \mathrm{mol})=-45.511626$

$\mathrm{ZPE}($ in $\mathrm{kcal} / \mathrm{mol})=142.829$

$\mathrm{G}($ in $\mathrm{kcal} / \mathrm{mol})=114.671$ 


\begin{tabular}{|c|c|c|}
\hline \multicolumn{3}{|c|}{ eome } \\
\hline & -1.89972968 & $-0.85695348-0.59637814$ \\
\hline & & -1.01171034 \\
\hline & 5410 & $0.37701392-0.69$ \\
\hline & & 0.748413550 .374 \\
\hline & & 1.649553361 .443 \\
\hline & 378 & $-2.94556746 \quad 0.15$ \\
\hline & 97 & -1.912041260 .3 \\
\hline & 791747 & 1.485545681 .9 \\
\hline & -0.33243311 & $2.68726514 \quad 1.12$ \\
\hline & -0.96411037 & $1.65861770 \quad 3.43$ \\
\hline & -2.2 & $-0.13970088-1$. \\
\hline & -2.22221568 & $-0.50837650 \quad 0.3$ \\
\hline & -2.40147804 & $-1.81588862-0$ \\
\hline & & $265-3.0$ \\
\hline & 2.14 & $8149-0.11$ \\
\hline & 1.7 & .7600 .8 \\
\hline & 1.4 & 92.17 \\
\hline & 1.31 & $6790 \quad 0.91$ \\
\hline & $1.2 \xi$ & $5176-0 . \varepsilon$ \\
\hline & -0.1 & 0307951.3 \\
\hline & -0.71389967 & 2.773718040 .10 \\
\hline & -1.33295887 & $0.71582948 \quad 1.51$ \\
\hline & -0.27366536 & 3.671711861 .57 \\
\hline & -0.76306546 & 0.731529223 .98 \\
\hline & -2.01258807 & 1.932011023 .61 \\
\hline & -0.34527853 & 2.445467513 .8849056 \\
\hline & & $-1.29405813-2.41$ \\
\hline & & $163867-1.594$ \\
\hline & $\begin{array}{l}-0.20276904 \\
\text { brational f }\end{array}$ & $\begin{array}{l}-1.59740530-1.9753851 \\
\text { requencies (in cm-1) }\end{array}$ \\
\hline
\end{tabular}

Vibrational frequencies (in $\mathrm{cm}-1$ )

$\begin{array}{llllllllllllllllll}9.3 & 26.0 & 60.3 & 83.2 & 102.6 & 131.5 & 137.7 & 148.1 & 178.6 & 197.4 & 230.2 & 263.4 & 277.2 & 323.1\end{array}$ $335.2 \quad 361.4 \quad 399.3 \quad 429.7 \quad 440.2 \quad 471.7 \quad 528.1 \quad 603.1 \quad 634.4 \quad 728.0 \quad 804.1 \quad 829.3 \quad 839.0 \quad 903.2$ $928.9952 .1968 .8 \quad 982.8 \quad 987.6 \quad 1018.7 \quad 1029.8 \quad 1058.2 \quad 1063.4 \quad 1082.9 \quad 1099.7 \quad 1101.4 \quad 1112.0$

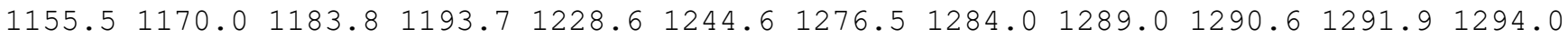
$1323.31332 .31338 .21348 .4 \quad 1376.8 \quad 1379.6 \quad 1387.9 \quad 1395.4 \quad 1396.6 \quad 1500.2 \quad 1836.01854 .1$ $2648.62661 .7 \quad 2666.1 \quad 2674.92677 .0 \quad 2705.8 \quad 2705.92729 .12736 .6 \quad 2753.8 \quad 2754.3 \quad 2773.3$ $2778.72781 .8 \quad 2786.3 \quad 2844.4$

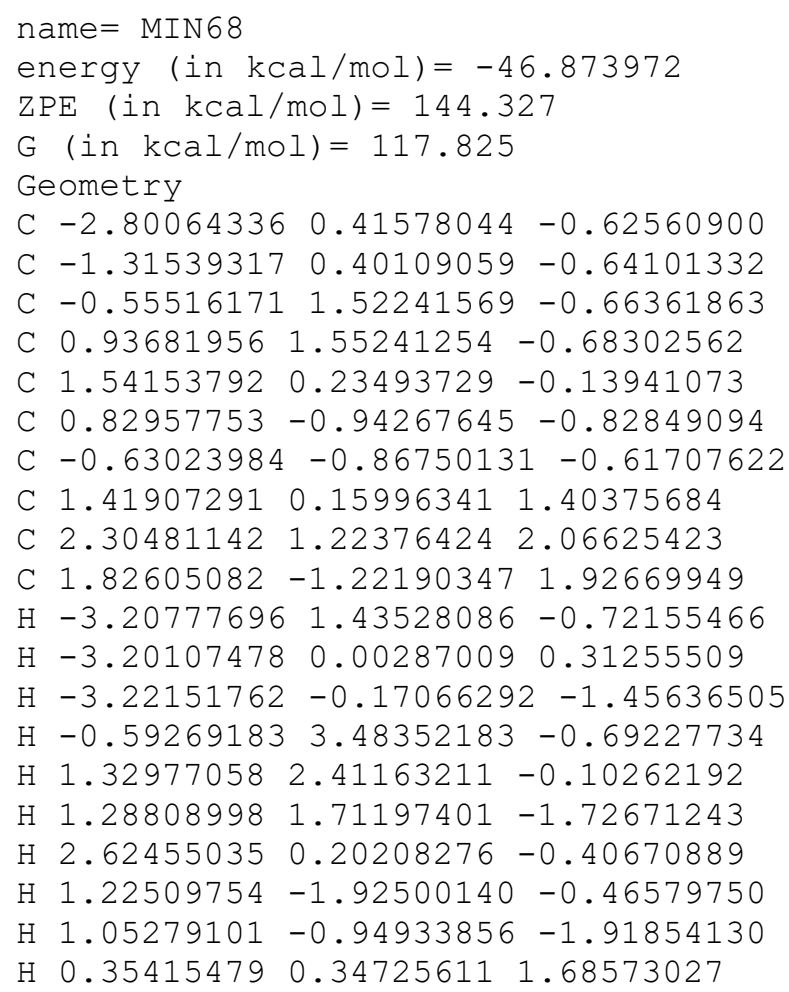


H 2.24641291 $1.15236375 \quad 3.15940322$

H $2.00302491 \quad 2.23968824 \quad 1.79829685$

$\mathrm{H} \quad 3.35724730 \quad 1.09879517 \quad 1.79020034$

$\mathrm{H} \quad 1.85717929-1.23860613 \quad 3.02167560$

$\mathrm{H} 1.10925308-1.999123321 .62212069$

H $2.81436803-1.520848751 .56402128$

$0-0.78010802-3.03874908-0.32031272$

$\begin{array}{llll}0 & -1.20580568 & 2.71073172 & -0.69027086\end{array}$

o $-1.37344554-1.91586152-0.37597820$

Vibrational frequencies (in $\mathrm{cm}-1$ )

$\begin{array}{llllllllllllllll}27.2 & 47.1 & 75.3 & 79.8 & 97.7 & 144.9 & 189.1 & 193.6 & 206.4 & 274.6 & 276.1 & 296.7 & 325.8 & 338.1\end{array}$ $\begin{array}{lllllllllllllll}371.0 & 385.0 & 412.9 & 436.9 & 452.2 & 482.6 & 553.6 & 600.8 & 635.4 & 747.1 & 813.2 & 863.1 & 918.7 & 967.8\end{array}$

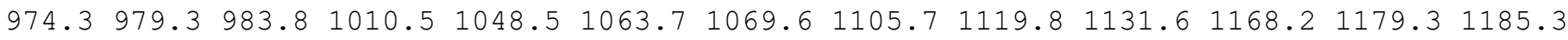

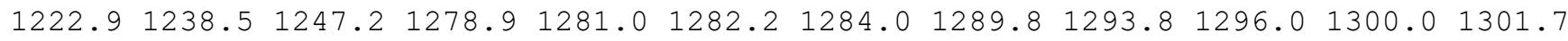
$1304.61310 .6 \quad 1319.5 \quad 1332.8 \quad 1358.7 \quad 1389.3 \quad 1396.6 \quad 1400.8 \quad 1406.1 \quad 1500.4 \quad 1579.5 \quad 1830.5$ $2628.22649 .32658 .0 \quad 2665.8 \quad 2667.9 \quad 2674.3 \quad 2675.02680 .02693 .5 \quad 2711.82723 .32730 .1$ $2775.32778 .12781 .7 \quad 2788.4$

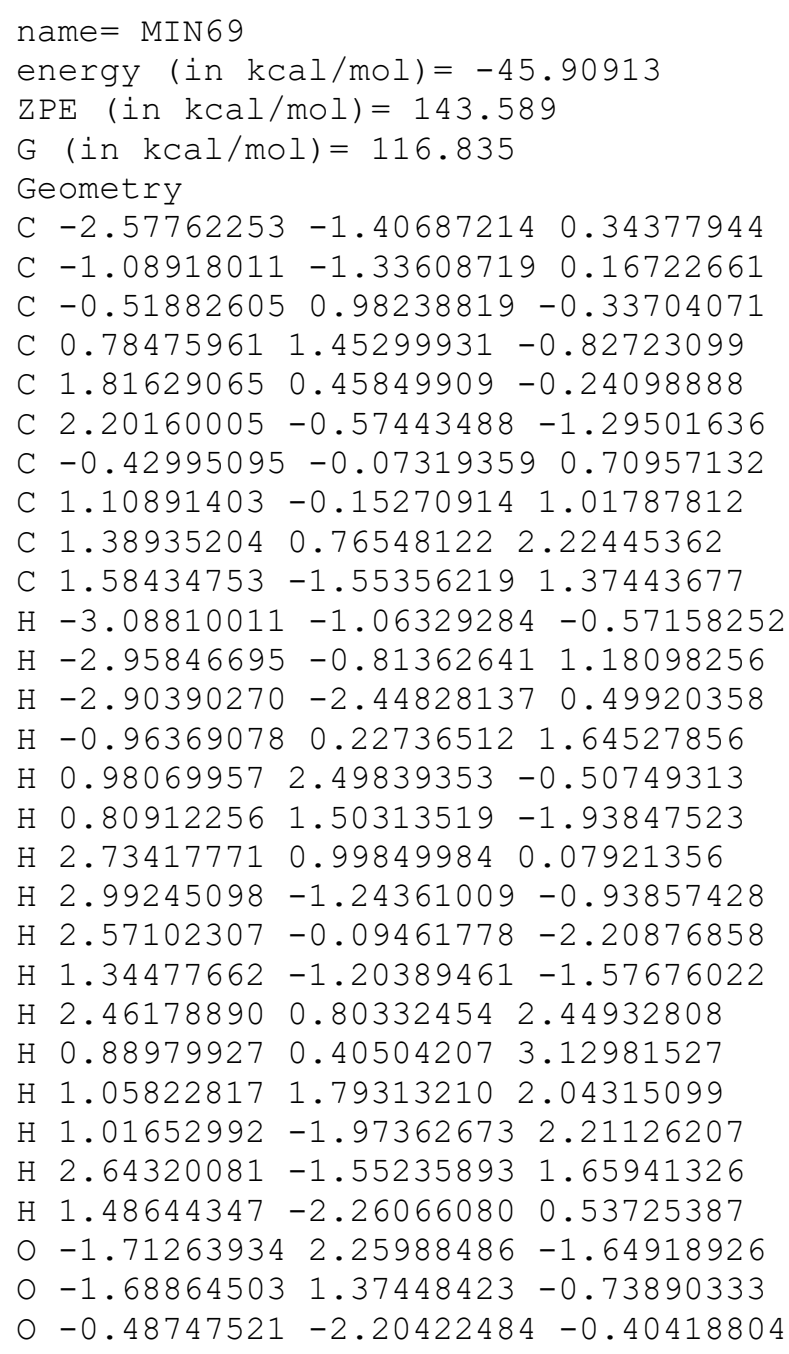


$\mathrm{ZPE}($ in $\mathrm{kcal} / \mathrm{mol})=145.29$

$\mathrm{G}($ in $\mathrm{kcal} / \mathrm{mol})=121.706$

Geometry

C $-2.52796472-0.22215489-0.73810483$

C $-1.02205175-0.23841337-0.75277642$

C $-0.270902151 .13141375 \quad-0.77625914$

C $0.93576736 \quad 1.27959003 \quad 0.18128712$

C $1.23464706-0.033190590 .92091092$

C $1.16336530-1.21461400-0.09009124$

C $-0.37625310-1.248209920 .18290108$

C $-0.08229773-0.58495082 \quad 1.58945558$

C $-1.064996590 .41566864 \quad 2.16224067$

C $0.21738960-1.63855314 \quad 2.65145144$

$\mathrm{H}-2.932627320 .56338302-1.39136504$

$\mathrm{H}-2.93125051-0.06719414 \quad 0.26943753$

$\mathrm{H}-2.93055707-1.17661021-1.10867780$

$\mathrm{H}-0.902859512 .02890428-0.79831305$

$\mathrm{H} \quad 0.77856276 \quad 2.11712949 \quad 0.89996640$

$\mathrm{H} 2.248871031 .10708550-1.28969917$

$\mathrm{H} 2.121623690 .03187372 \quad 1.55202902$

$\mathrm{H} 1.69350540-2.11469587 \quad 0.22890500$

H $1.46947890-1.00314782-1.11667469$

$\mathrm{H}-0.86117123-2.22280402 \quad 0.19439730$

$\mathrm{H}-1.37817257 \quad 1.18836469 \quad 1.45469304$

$\mathrm{H}-0.62899685 \quad 0.93464524 \quad 3.02719038$

$\mathrm{H}-1.97784678-0.084637092 .51141975$

$\mathrm{H} \quad 0.66657349-1.183143823 .54236003$

$\mathrm{H} \quad 0.91394932-2.406540022 .29664353$

$\mathrm{H}-0.69672018-2.15401084 \quad 2.96779250$

$\begin{array}{lllll}0 & 0.16267848 & 0.82739090 & -2.12764757\end{array}$

$\begin{array}{lllll}0 & 2.06647389 & 1.70727664 & -0.53209717\end{array}$

o $-0.56387509-0.44773300-2.12653159$

Vibrational frequencies (in $\mathrm{cm}-1$ )

$100.9108 .0 \quad 144.5 \quad 153.2 \quad 181.9 \quad 203.6 \quad 215.8 \quad 253.2 \quad 268.8 \quad 316.4 \quad 326.8 \quad 345.1 \quad 350.8 \quad 383.3$ $429.7 \quad 477.6 \quad 488.3 \quad 505.4 \quad 541.7 \quad 647.9 \quad 714.3 \quad 751.6 \quad 832.2 \quad 850.6 \quad 900.1 \quad 909.8 \quad 939.8 \quad 964.7$ $981.9997 .5 \quad 1004.8 \quad 1012.3 \quad 1013.4 \quad 1017.1 \quad 1054.1 \quad 1071.4 \quad 1083.0 \quad 1095.51121 .81136 .3$

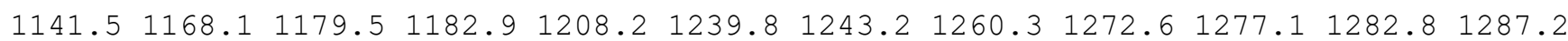

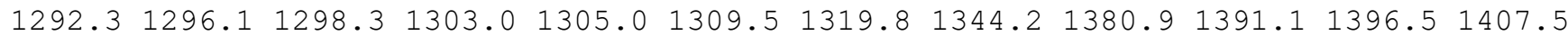
$1418.12670 .0 \quad 2672.6 \quad 2673.4 \quad 2675.5 \quad 2677.12679 .4 \quad 2684.52685 .7 \quad 2705.4 \quad 2748.2 \quad 2753.0$ $2764.2 \quad 2780.7 \quad 2781.8 \quad 2783.4 \quad 2796.3$

\section{name $=$ MIN71}

energy (in $\mathrm{kcal} / \mathrm{mol})=-45.492918$

$\mathrm{ZPE}($ in $\mathrm{kcal} / \mathrm{mol})=143.347$

$\mathrm{G}(\mathrm{in} \mathrm{kcal} / \mathrm{mol})=115.912$

Geometry

$\begin{array}{lllll}\text { C }-2.05315172 & -0.28223771 & 0.02744931\end{array}$

C $-0.76419826-0.08158786 \quad 0.31364091$

C -0.079918901 .230675090 .03184427$

C $1.36453406 \quad 1.14598104-0.47787055$

C $1.75709396 \quad 0.16817681-1.53180752$

C $1.04658050-0.72726363-2.22554450$

C $0.06104947-1.102458710 .96231961$

C $0.23416723-1.21142510 \quad 2.28524729$

C $-0.38764687-0.28168526 \quad 3.27299101$

C $1.07791752-2.296565462 .87051055$

$\mathrm{H}-2.67947699 \quad 0.45744122-0.44859645$

$\mathrm{H}-2.57216249-1.19951712 \quad 0.24899453$

$\mathrm{H}-0.87053262 \quad 0.81497533-2.18625936$

$\mathrm{H}-0.08972705 \quad 1.83390210 \quad 0.98581257$

H 2.02083981 $0.96626386 \quad 0.40721107$

$\mathrm{H} 1.627749302 .16762229-0.85503286$

$\mathrm{H} \quad 2.83043714 \quad 0.23333050-1.75672824$

$\mathrm{H}-0.69490100-1.54765595-2.59388180$ 
Н $1.44965641-1.39250116-2.97915257$

H $0.53409719-1.79091894 \quad 0.25985799$

$\mathrm{H}-0.72725351-0.80876253 \quad 4.17448353$

$\mathrm{H}-1.26400654 \quad 0.23884635 \quad 2.85831506$

$\mathrm{H} \quad 0.32952573 \quad 0.48710181 \quad 3.59350045$

$\mathrm{H} \quad 0.45485925-3.02861454 \quad 3.40538576$

$\mathrm{H} 1.80666293-1.89836037 \quad 3.58921814$

$\mathrm{H} \quad 1.64194201-2.851168452 .10919747$

$\begin{array}{lllll}0 & -0.75194151 & 1.79428014 & -2.14858132\end{array}$

$0-0.890406862 .07697788-0.76585660$

$0-0.30091520-0.86056505-2.00844907$

Vibrational frequencies (in $\mathrm{cm}-1$ )

$\begin{array}{llllllllllllllll}33.0 & 37.8 & 57.8 & 86.1 & 100.5 & 110.2 & 118.7 & 132.7 & 161.3 & 198.7 & 207.8 & 277.0 & 296.3 & 327.2\end{array}$ $366.0 \quad 376.8 \quad 404.3 \quad 429.4 \quad 492.0 \quad 508.3 \quad 528.1 \quad 578.0 \quad 623.5 \quad 646.9 \quad 702.2 \quad 776.4 \quad 812.9 \quad 859.8$ $913.8 \quad 918.8 \quad 947.4 \quad 976.0 \quad 982.4 \quad 998.4 \quad 1006.4 \quad 1016.5 \quad 1027.2 \quad 1076.7 \quad 1090.2 \quad 1117.3 \quad 1121.2$

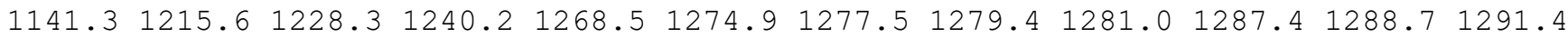

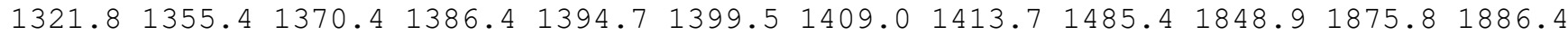
$2643.82655 .7 \quad 2668.4 \quad 2670.92675 .3 \quad 2676.8 \quad 2718.3 \quad 2721.82726 .12740 .3 \quad 2751.8 \quad 2777.8$ $2780.82787 .5 \quad 2798.2 \quad 2815.3$

name $=$ MIN72

energy (in $\mathrm{kcal} / \mathrm{mol})=-43.544337$

$\operatorname{ZPE}($ in $\mathrm{kcal} / \mathrm{mol})=141.753$

$\mathrm{G}($ in $\mathrm{kcal} / \mathrm{mol})=115.673$

Geometry

C $-1.89377475 \quad 0.14704297-0.64429380$

C $-0.373297610 .07410798-0.70788920$

C $0.232325371 .48942073-0.59621066$

C $1.59703517 \quad 1.71285392 \quad-0.03597944$

C $1.50976847-1.11337462 \quad 1.80998377$

C $1.63504059-1.151778880 .47259895$

C $0.14015714-1.03800947 \quad 0.19076432$

C $-0.02106347-0.96621610 \quad 1.79383003$

C $-0.54128276 \quad 0.31313542 \quad 2.40764018$

C $-0.74558441-2.178882962 .35033754$

$\mathrm{H}-2.28594411 \quad 0.97481403-1.25681170$

H $-2.25668771 \quad 0.29925403 \quad 0.37699621$

$\mathrm{H}-2.34495492-0.77454787-1.03575667$

$\mathrm{H}-0.48246628 \quad 1.10785020-3.04558637$

$\mathrm{H} 1.688872101 .374545691 .00589540$

$\mathrm{H} \quad 1.86727884 \quad 2.78068087 \quad-0.06020157$

H $2.15307548-1.173186712 .64628538$

$\mathrm{H} \quad 2.42515297-1.25884549-0.22447827$

$\mathrm{H} 2.356183151 .16891629-0.61885357$

$\mathrm{H}-0.27323439-1.99714288-0.19315724$

$\mathrm{H}-0.13516663 \quad 1.21259721 \quad 1.93273564$

$\mathrm{H}-0.27683067 \quad 0.36746382 \quad 3.47295522$

$\mathrm{H}-1.63538768 \quad 0.38005512 \quad 2.34576929$

$\mathrm{H}-0.68907872-2.20731521 \quad 3.44563597$

$\mathrm{H}-0.31501579-3.11879187 \quad 1.98336865$

$\mathrm{H}-1.80677520-2.17272005 \quad 2.07420694$

$\begin{array}{lllll}0 & -0.76229792 & 0.15556593 & -3.01326595\end{array}$

$0-0.38672574 \quad 2.40763336-1.07980041$

O $0.07700865-0.36627910-2.00358223$

Vibrational frequencies (in $\mathrm{cm}-1$ )

$\begin{array}{lllllllllllllll}-30.2 & 90.9 & 109.9 & 119.1 & 131.6 & 151.3 & 158.2 & 179.9 & 188.1 & 213.0 & 245.6 & 270.6 & 284.4 & 299.3\end{array}$ $312.4 \quad 335.7 \quad 355.6 \quad 371.2 \quad 417.0 \quad 436.1 \quad 481.2 \quad 536.9608 .3 \quad 686.5 \quad 713.0 \quad 744.7 \quad 825.7 \quad 883.6$ $917.3961 .6 \quad 972.8 \quad 992.7 \quad 997.4 \quad 1009.1 \quad 1019.3 \quad 1020.6 \quad 1045.2 \quad 1052.1 \quad 1072.8 \quad 1106.6 \quad 1124.8$ $1137.11178 .6 \quad 1201.8 \quad 1226.4 \quad 1238.1 \quad 1259.0 \quad 1264.6 \quad 1272.4 \quad 1277.5 \quad 1284.4 \quad 1290.2 \quad 1292.2$ $1294.51298 .0 \quad 1300.5 \quad 1316.3 \quad 1364.8 \quad 1377.2 \quad 1398.8 \quad 1402.3 \quad 1403.91408 .91775 .2 \quad 1829.9$ $2657.32660 .02666 .5 \quad 2670.7 \quad 2672.8 \quad 2676.1 \quad 2676.62678 .92702 .72774 .12777 .22781 .6$ $2783.02794 .12809 .0 \quad 2842.3$ 
name $=$ MIN73

energy (in $\mathrm{kcal} / \mathrm{mol})=-45.270224$

$\mathrm{ZPE}($ in $\mathrm{kcal} / \mathrm{mol})=144.363$

$\mathrm{G}($ in $\mathrm{kcal} / \mathrm{mol})=120.376$

Geometry

C $-2.49319569-0.34408609-0.99776395$

C $-0.99210836-0.31485981-0.94982627$

C $-0.25299073 \quad 1.05783950-1.07841243$

C $2.32230548 \quad 0.79516212 \quad 1.47442813$

C $1.14367998 \quad 0.27662158 \quad 0.70906288$

C $1.21336632-1.04837077-0.10195020$

C $-0.33153390-1.15654168 \quad 0.13028822$

C $-0.14649537-0.28816962 \quad 1.44271756$

C $-1.205795380 .71621702 \quad 1.83556036$

C $0.18171406-1.168578292 .64071318$

$\mathrm{H}-2.88783318 \quad 0.32041083-1.77989531$

$\mathrm{H}-2.94439061-0.04688623-0.04314214$

$\mathrm{H}-2.85863443-1.35557053-1.23072700$

$\mathrm{H}-0.80248161 \quad 1.99623901-1.21897086$

H $2.04772945 \quad 1.67102425 \quad 2.07953319$

H $3.12393967 \quad 1.11522100 \quad 0.79134421$

H $2.73377867 \quad 0.03322775 \quad 2.14659759$

H $1.79688301-1.84215957 \quad 0.36532542$

$\mathrm{H} \quad 1.55397415-0.96117317-1.13765584$

$\mathrm{H}-0.75306333-2.15160072 \quad 0.25457407$

$\mathrm{H}-1.398516151 .46974504 \quad 1.06427471$

$\mathrm{H}-0.89735427 \quad 1.27472335 \quad 2.73043992$

$\mathrm{H}-2.15686414 \quad 0.22245710 \quad 2.06917683$

$\mathrm{H} \quad 0.55993948-0.57163573 \quad 3.47937453$

$\mathrm{H} \quad 0.93947340-1.926889292 .41367621$

$\mathrm{H}-0.71140985-1.700838792 .99078016$

$\begin{array}{lllll}0 & 0.29637517 & 0.59657251 & -2.34094250\end{array}$

O $0.73999823 \quad 1.34305710-0.14900690$

o $-0.45872045-0.65936984-2.26572746$

Vibrational frequencies (in cm-1)

$\begin{array}{lllllllllllllllll}89.7 & 103.5 & 139.8 & 142.9 & 163.6 & 168.9 & 198.2 & 207.1 & 250.6 & 291.8 & 292.8 & 319.7 & 344.8 & 393.6\end{array}$

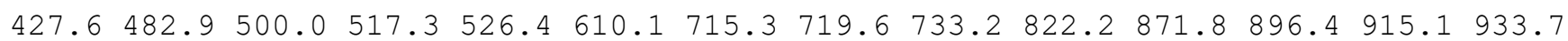
$960.3980 .1988 .3 \quad 1004.0 \quad 1011.8 \quad 1015.6 \quad 1039.1 \quad 1047.5 \quad 1068.4 \quad 1078.7 \quad 1122.31134 .1 \quad 1146.5$ $\begin{array}{llllllllllllllll}1169.9 & 1191.0 & 1206.4 & 1229.6 & 1249.0 & 1260.4 & 1273.3 & 1277.0 & 1279.8 & 1281.9 & 1282.4 & 1289.0\end{array}$

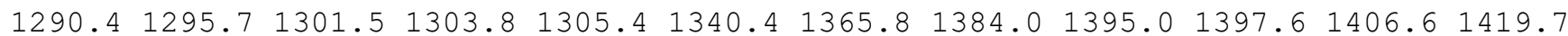
$2669.32670 .8 \quad 2673.1 \quad 2673.9 \quad 2674.8 \quad 2677.1 \quad 2679.72680 .22682 .8 \quad 2685.92753 .7 \quad 2765.0$ $2779.92780 .92781 .8 \quad 2783.6$

\footnotetext{
name $=$ MIN74

energy (in $\mathrm{kcal} / \mathrm{mol})=-44.044905$

$\mathrm{ZPE}(\mathrm{in} \mathrm{kcal} / \mathrm{mol})=143.472$

$\mathrm{G}(\mathrm{in} \mathrm{kcal} / \mathrm{mol})=116.308$

Geometry

C $-2.48417151-0.36921078-1.03497411$

C $-1.00080528-0.08709455-0.97668685$

C $-0.69007622 \quad 1.40216650-1.18076083$

C $1.70652526 \quad 0.872829150 .34311790$

C $1.01752317-0.400058900 .76536486$

C $1.02814114-1.54963300-0.21553123$

C $-0.28298673-0.85130963 \quad 0.08281836$

C $1.12946039-0.62642776 \quad 2.27367732$

C $-0.01279356 \quad 0.10414594 \quad 2.99361027$

C $1.13328160-2.10612201 \quad 2.67274083$

$\mathrm{H}-2.961257120 .11664156-1.89935373$

$\mathrm{H}-2.99547654-0.01027986-0.13020010$

$\mathrm{H}-2.68492251-1.44608282-1.12734130$

H $1.21249091 \quad 1.76018047 \quad 0.76543038$

H $2.75066891 \quad 0.88943064 \quad 0.68595352$

H $1.74501405 \quad 0.99682653-0.74753391$
} 
H $2.10071530-0.18442668 \quad 2.61828964$

$\mathrm{H} 1.17660566-2.572718320 .11397655$

$\mathrm{H} 1.49819437-1.41003600-1.18900381$

$\mathrm{H}-0.98500050-1.389597510 .74102261$

$\mathrm{H}-0.98505168-0.35034273 \quad 2.77697167$

$\mathrm{H}-0.06955352 \quad 1.15602558 \quad 2.68937795$

H $0.12525897 \quad 0.08116825 \quad 4.07964166$

H $1.21211251-2.21621515 \quad 3.76066183$

H $1.98555542 \quad-2.63566096 \quad 2.23169240$

H $0.21798589-2.61886194 \quad 2.36092726$

$\begin{array}{llll}0 & -0.14681527 & 1.14888059 & -2.44723748\end{array}$

$\begin{array}{lllll}0 & -0.81425330 & 2.46131866 & -0.66528226\end{array}$

o $-0.39491864-0.29618399-2.30042969$

Vibrational frequencies (in $\mathrm{cm}-1$ )

$\begin{array}{lllllllllllllllll}-4.7 & 47.0 & 88.3 & 138.8 & 143.5 & 150.0 & 152.9 & 189.8 & 205.0 & 234.5 & 249.3 & 265.0 & 280.6 & 327.5\end{array}$

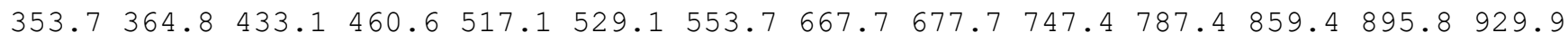
$948.8 \quad 969.8 \quad 978.0 \quad 986.8 \quad 989.8 \quad 1012.3 \quad 1016.5 \quad 1057.8 \quad 1078.7 \quad 1094.6 \quad 1097.4 \quad 1149.6 \quad 1159.1$ $\begin{array}{llllllllllllllll}1174.3 & 1182.9 & 1192.5 & 1215.5 & 1269.8 & 1273.5 & 1275.5 & 1280.6 & 1287.5 & 1296.9 & 1298.2 & 1305.7\end{array}$

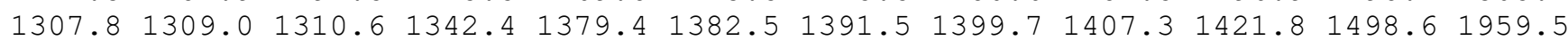
$2664.62665 .52666 .82669 .5 \quad 2670.72675 .62676 .72679 .22701 .82704 .02736 .32775 .9$ $2776.7 \quad 2780.7 \quad 2781.7 \quad 2782.8$

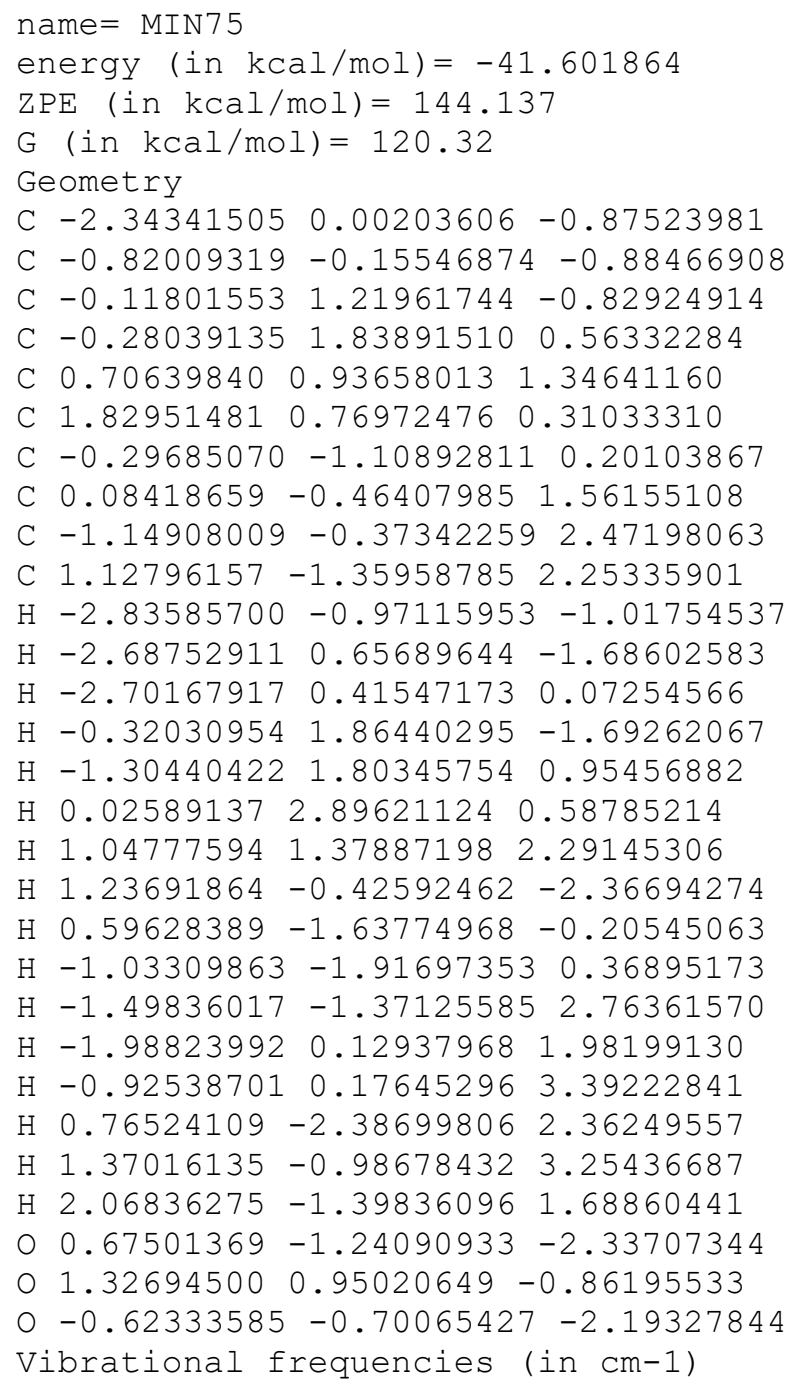


name $=$ MIN76

energy $($ in $\mathrm{kcal} / \mathrm{mol})=-39.084212$

$\mathrm{ZPE}(\mathrm{in} \mathrm{kcal} / \mathrm{mol})=142.875$

$\mathrm{G}($ in $\mathrm{kcal} / \mathrm{mol})=116.215$

Geometry

C $-2.22975022-0.10057206-1.48618316$

C $-0.74252889-0.04657451-1.70257343$

C $-0.05398718 \quad 1.23864725 \quad-1.26096395$

C $0.04710537 \quad 1.40616840 \quad 0.25867916$

C $0.65351463 \quad 0.26305427 \quad 1.05511345$

C $0.77659094-1.130980230 .44060875$

C $0.02788712-1.74738209 \quad 1.36918144$

C $-0.27375629-0.471599292 .16477660$

C $-1.74118951-0.102541832 .19515575$

C $0.33643917-0.450192153 .55273165$

$\mathrm{H}-2.75225142-0.10119625-2.45514487$

$\mathrm{H}-2.62403714 \quad 0.72672550-0.89069731$

$\mathrm{H}-2.50885391-1.04313263-0.98866618$

$\mathrm{H}-0.61782827 \quad 2.11102624 \quad-1.69612824$

$\mathrm{H}-0.96087171 \quad 1.64639362 \quad 0.65586343$

$\mathrm{H} \quad 0.64387870 \quad 2.32670399 \quad 0.45129648$

H $1.60674195 \quad 0.58758748 \quad 1.51131009$

$\mathrm{H} 1.31683144-1.42526960-0.42765510$

H $2.601922620 .85289132-0.85104903$

$\mathrm{H}-0.28778947-2.73548127 \quad 1.56842379$

$\mathrm{H}-2.19603347-0.15542882 \quad 1.19877954$

$\mathrm{H}-1.89865649 \quad 0.90707986 \quad 2.59119409$

$\mathrm{H}-2.30305579-0.795519412 .83540332$

$\mathrm{H} \quad 0.31275358 \quad 0.55561591 \quad 3.98642338$

$\mathrm{H} 1.38059546-0.78618558 \quad 3.54532421$

$\mathrm{H}-0.20819258-1.11700446 \quad 4.23329300$

○ $2.09857827 \quad 0.41764507-1.58329810$

○ $1.180897631 .44330235-1.91149053$

o - $0.15861264-0.94405551-2.24558007$

Vibrational frequencies (in $\mathrm{cm}-1$ )

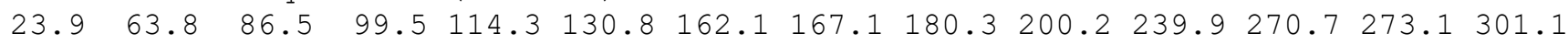
$325.4 \quad 353.4 \quad 368.5 \quad 386.0 \quad 453.8 \quad 502.4 \quad 567.6 \quad 599.1 \quad 695.9 \quad 733.1 \quad 751.8 \quad 865.9 \quad 894.4 \quad 905.5$ $947.0951 .9979 .7 \quad 996.2 \quad 998.8 \quad 1011.2 \quad 1018.3 \quad 1047.5 \quad 1054.7 \quad 1082.6 \quad 1094.01114 .1 \quad 1141.3$

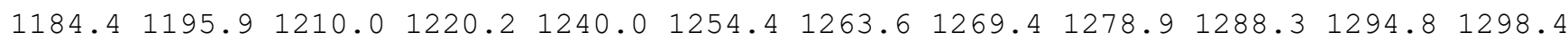
$1302.31312 .8 \quad 1316.0 \quad 1322.0 \quad 1343.4 \quad 1360.8 \quad 1380.7 \quad 1396.01396 .2 \quad 1405.01780 .7 \quad 1863.6$ $2647.0 \quad 2659.8 \quad 2662.3 \quad 2673.4 \quad 2677.1 \quad 2678.5 \quad 2679.4 \quad 2682.3 \quad 2717.3 \quad 2732.4 \quad 2771.12778 .8$ $2782.0 \quad 2783.5 \quad 2809.5 \quad 2877.3$

\section{name $=$ MIN77}

energy (in $\mathrm{kcal} / \mathrm{mol})=-40.064361$

$\mathrm{ZPE}($ in $\mathrm{kcal} / \mathrm{mol})=145.444$

$\mathrm{G}($ in $\mathrm{kcal} / \mathrm{mol})=119.73$

Geometry

C $-0.28367386-0.77513506-0.99176501$

C $1.07404555-1.31060285-0.62830385$

C $-1.95273200 \quad 0.93998482-0.70227422$

C $-0.54118339 \quad 0.62677232 \quad-0.40307897$

C $-0.12232304 \quad 0.78028777 \quad 1.08631878$

C $2.78158078-0.221665520 .76789670$

C $1.53888516-1.11259582 \quad 0.80087182$

C $0.38568825-0.560604931 .66354837$

C $-1.23202335 \quad 1.36309690 \quad 1.96521226$

C $0.84057436-0.425096933 .12004204$

$\mathrm{H}-0.38231415-0.72788069-2.10611334$

$\mathrm{H}-0.45603338-1.296983521 .63420305$

$\mathrm{H}-1.05386888-1.49879123-0.65905029$

$\mathrm{H}-2.74313336 \quad 0.96844922 \quad 0.03044400$ 
$\mathrm{H} \quad 0.09445131 \quad 1.35007046-1.00216478$

H $2.57160593 \quad 0.76695053 \quad 0.34481639$

$\mathrm{H} 0.732829221 .504942321 .11154063$

$\mathrm{H} \quad 3.56822930-0.67849027 \quad 0.14814102$

$\mathrm{H} \quad 3.19679149-0.07452865 \quad 1.77057934$

H $1.83513229-2.11528845 \quad 1.20658003$

$\mathrm{H}-0.850035221 .610557892 .96470780$

$\mathrm{H}-2.05881344 \quad 0.66005815 \quad 2.10829748$

$\mathrm{H}-1.62894088 \quad 2.29702174 \quad 1.55147766$

$\mathrm{H} \quad 1.52084675 \quad 0.42185658 \quad 3.25934331$

$\mathrm{H} 1.36531455-1.327400523 .45672062$

$\mathrm{H}-0.01063520-0.28127934 \quad 3.79452183$

O $-1.564145131 .16452373-2.85226970$

O $-2.38384428 \quad 1.18603208-1.90056703$

o $1.75051976-1.90432653-1.42862141$

Vibrational frequencies (in cm-1)

$\begin{array}{lllllllllllllllll}-32.1 & 51.1 & 71.7 & 122.2 & 157.9 & 166.6 & 187.1 & 236.5 & 247.2 & 255.3 & 267.0 & 291.6 & 329.0 & 353.7\end{array}$

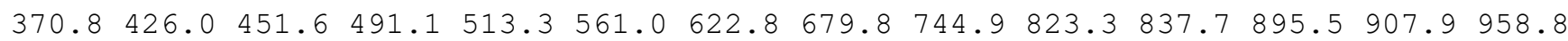
$997.7 \quad 1035.91056 .1 \quad 1060.0 \quad 1063.0 \quad 1069.9 \quad 1080.6 \quad 1129.4 \quad 1142.11161 .3 \quad 1166.8 \quad 1182.1$ $1203.8 \quad 1216.7 \quad 1244.3 \quad 1245.2 \quad 1250.6 \quad 1265.0 \quad 1270.5 \quad 1285.4 \quad 1293.5 \quad 1296.4 \quad 1298.7 \quad 1301.0$ $1303.01306 .01312 .1 \quad 1316.0 \quad 1316.5 \quad 1325.1 \quad 1331.6 \quad 1372.91398 .8 \quad 1400.61407 .11580 .7$ $1850.42630 .5 \quad 2651.7 \quad 2665.0 \quad 2665.8 \quad 2671.8 \quad 2673.92674 .32675 .12687 .72697 .22705 .6$ $2718.2 \quad 2727.0 \quad 2777.8 \quad 2778.9 \quad 2780.8$

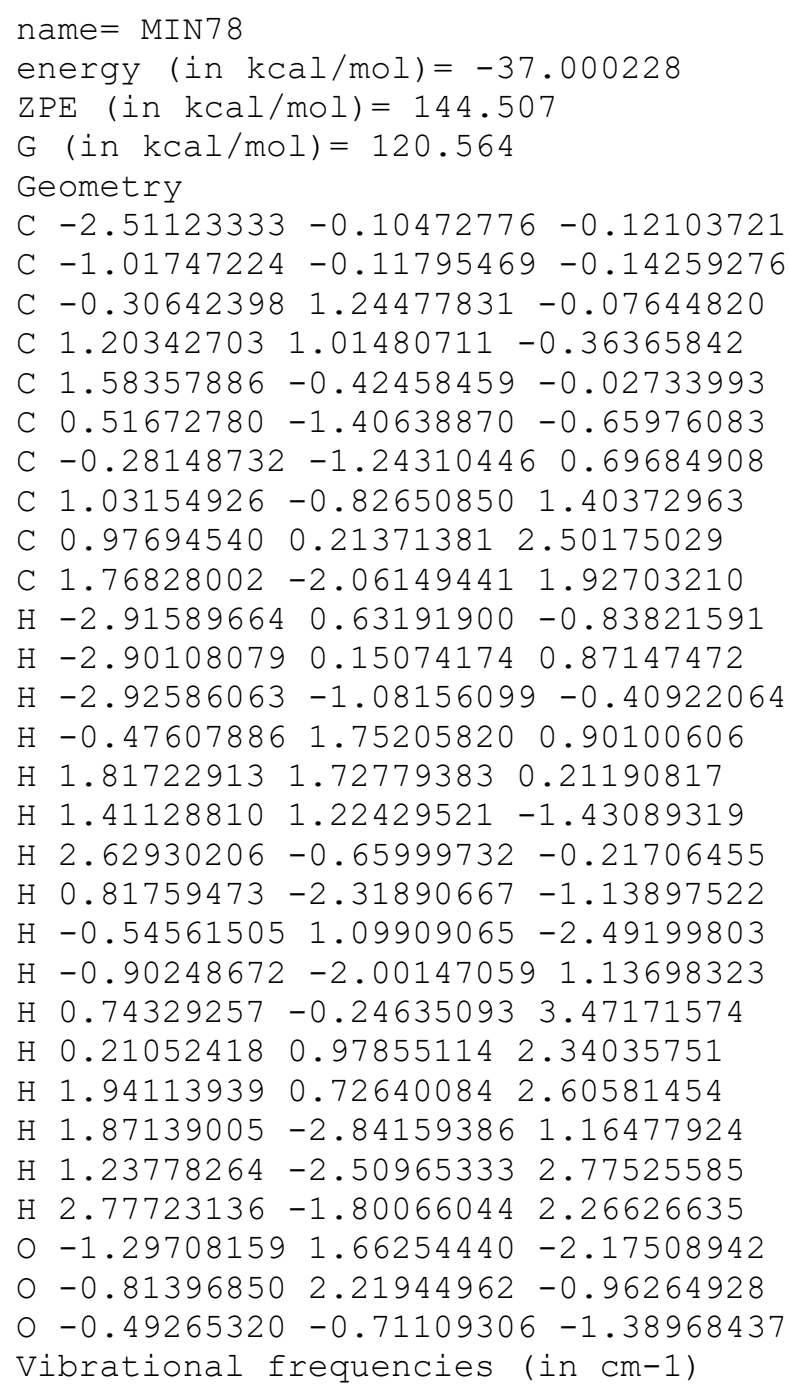


$2649.52658 .5 \quad 2665.3 \quad 2674.1 \quad 2677.0 \quad 2678.5 \quad 2679.82681 .5 \quad 2745.42746 .32751 .02770 .1$ $2778.6 \quad 2778.92782 .92825 .1$

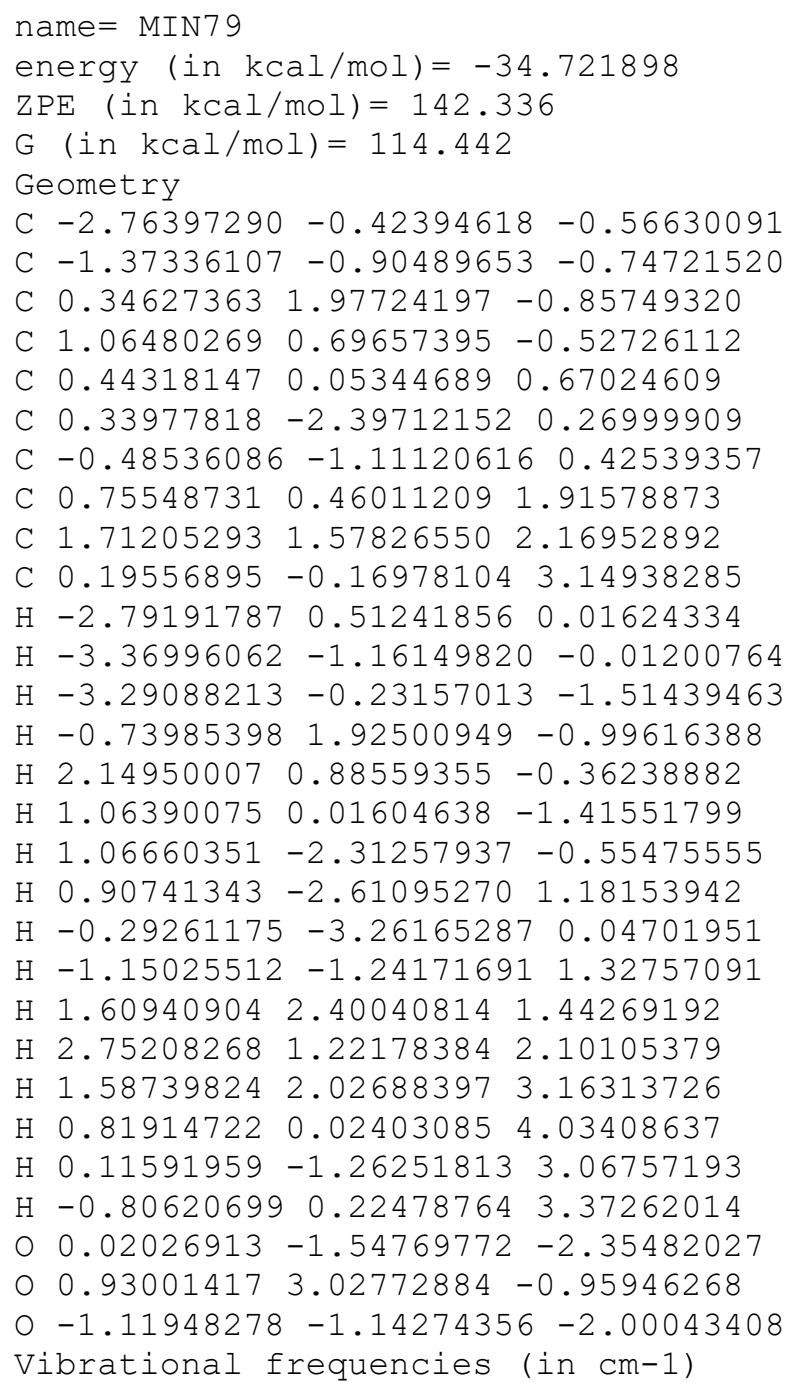

$\begin{array}{lllllllllllllllll}35.1 & 57.9 & 64.2 & 70.0 & 90.7 & 102.0 & 109.4 & 128.9 & 148.7 & 157.2 & 199.0 & 204.9 & 256.0 & 276.3\end{array}$ $280.7 \quad 333.2 \quad 340.3 \quad 407.2 \quad 427.3 \quad 469.6 \quad 494.3 \quad 527.5 \quad 603.7 \quad 674.3 \quad 699.5 \quad 812.3 \quad 887.3 \quad 914.5$ $976.7991 .81011 .3 \quad 1028.8 \quad 1037.91043 .4 \quad 1064.7 \quad 1084.7 \quad 1097.41110 .11116 .61141 .1$ $1154.51201 .7 \quad 1252.3 \quad 1262.7 \quad 1265.2 \quad 1267.3 \quad 1275.2 \quad 1282.0 \quad 1284.8 \quad 1287.0 \quad 1288.6 \quad 1295.0$ $1298.61301 .8 \quad 1326.3 \quad 1337.3 \quad 1342.0 \quad 1375.6 \quad 1388.1 \quad 1398.21407 .2 \quad 1410.61609 .01835 .3$ $1849.72613 .0 \quad 2652.1 \quad 2654.4 \quad 2656.2 \quad 2656.8 \quad 2660.6 \quad 2666.92670 .92671 .7 \quad 2680.5 \quad 2682.3$ $2725.42770 .6 \quad 2775.2 \quad 2777.8 \quad 2778.7$

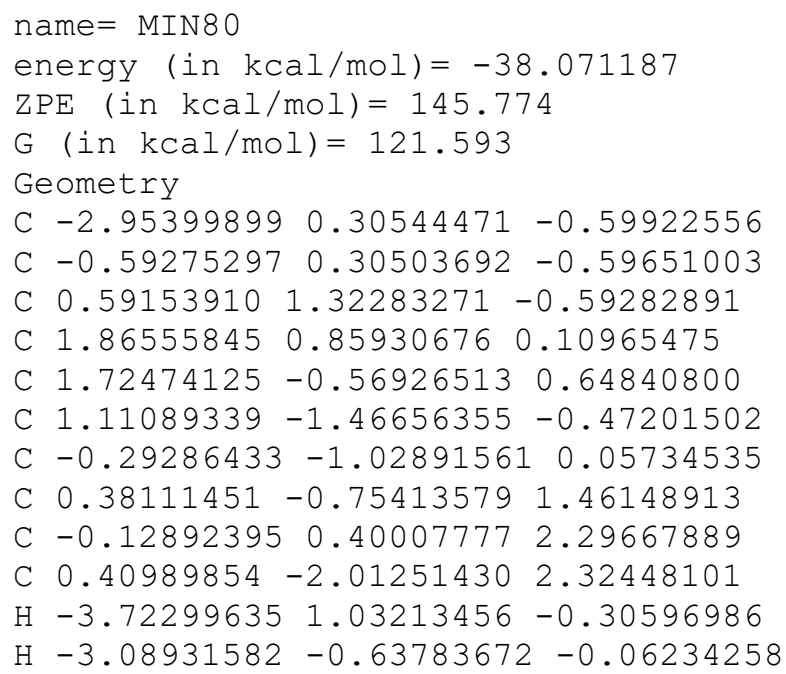


$\mathrm{H}-2.96733916 \quad 0.15213764-1.68347771$

$\mathrm{H} \quad 0.31139752 \quad 2.36362028 \quad-0.37058951$

H $2.122721531 .54888790 \quad 0.93603299$

H $2.71455932 \quad 0.90674553-0.60370044$

H 2.62936086 -0.933553431 .13606272$

$\mathrm{H} \quad 1.31628961-2.53261136-0.36433998$

$\mathrm{H} 1.34901781-1.18366313-1.50084138$

$\mathrm{H}-1.09937717-1.75595632 \quad 0.02755466$

$\mathrm{H}-1.12463848 \quad 0.17932643 \quad 2.70434939$

$\mathrm{H}-0.23742357 \quad 1.33707236 \quad 1.73987289$

H $0.54183132 \quad 0.60240618 \quad 3.14064722$

$\mathrm{H} \quad 1.06131159-1.875455093 .19595231$

$\mathrm{H} \quad 0.77745503 \quad-2.88861478 \quad 1.77980262$

$\mathrm{H}-0.59171466-2.25775514 \quad 2.69641346$

$\begin{array}{lllll}0 & -0.49352508 & 0.24947459 & -2.04711524\end{array}$

$0 \quad 0.66650868 \quad 1.15239150 \quad-2.03734313$

$\begin{array}{lllll}0 & -1.75388877 & 0.94060341 & -0.18868359\end{array}$

Vibrational frequencies (in cm-1)

$\begin{array}{lllllllllllllll}46.4 & 99.2 & 126.9 & 137.9 & 165.8 & 187.0 & 192.4 & 217.4 & 254.2 & 317.0 & 335.6 & 347.9 & 391.8 & 432.4\end{array}$ $\begin{array}{llllllllllllllllll}447.9 & 456.4 & 494.7 & 557.0 & 626.1 & 692.7 & 725.6 & 799.6 & 868.8 & 892.7 & 914.4 & 932.4 & 975.7 & 982.9\end{array}$ $998.61008 .2 \quad 1013.8 \quad 1019.6 \quad 1045.1 \quad 1059.4 \quad 1076.6 \quad 1080.1 \quad 1089.8 \quad 1094.01108 .2 \quad 1142.4$

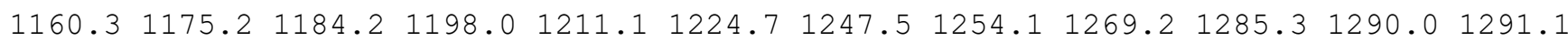

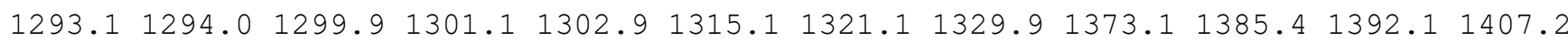

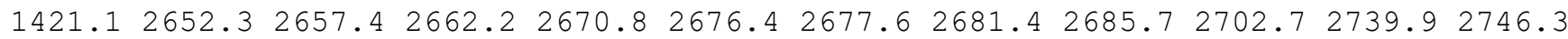
$\begin{array}{llllll}2747.9 & 2756.7 & 2764.3 & 2781.9 & 2785.2\end{array}$

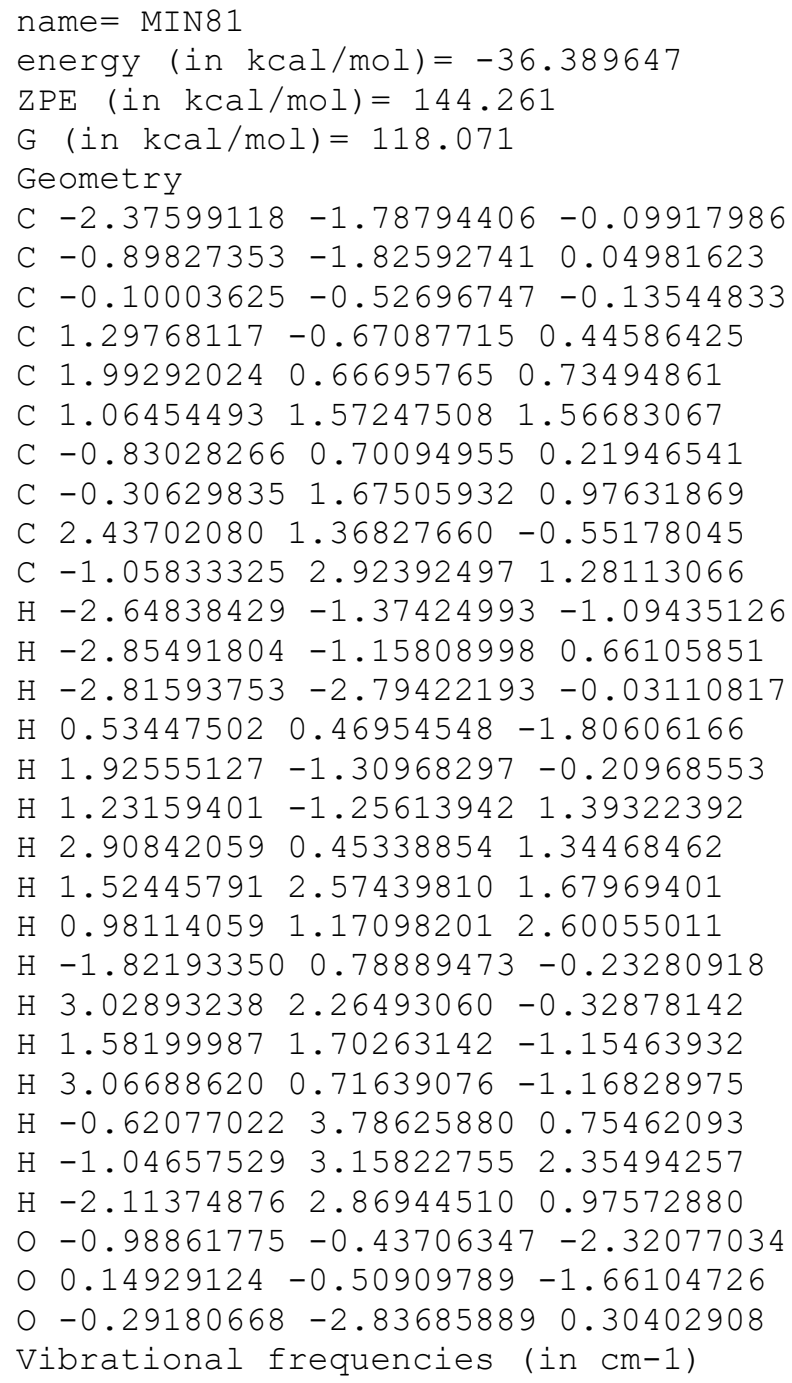


$\begin{array}{llllllllllllllll}1223.0 & 1239.1 & 1248.5 & 1250.9 & 1265.8 & 1268.1 & 1272.6 & 1278.9 & 1283.6 & 1288.5 & 1291.5 & 1294.8\end{array}$

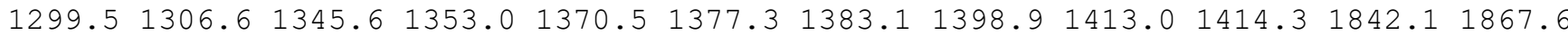
$2632.32641 .62643 .6 \quad 2658.5 \quad 2667.2 \quad 2671.12673 .42674 .02695 .92713 .5 \quad 2732.5 \quad 2738.5$ $2741.0 \quad 2769.92775 .0 \quad 2778.4$

\section{name $=$ MIN82}

energy (in $\mathrm{kcal} / \mathrm{mol})=-35.352588$

$\mathrm{ZPE}($ in $\mathrm{kcal} / \mathrm{mol})=143.817$

$\mathrm{G}($ in $\mathrm{kcal} / \mathrm{mol})=117.546$

Geometry

C $-3.22761995-1.12259466-0.84216210$

C $-1.72872373-1.08476311-0.88433977$

C $1.46821987 \quad 0.89212274 \quad-0.86991204$

C $1.75188195 \quad 0.51639418 \quad 0.51919483$

C $0.86313791-0.55268424 \quad 1.12565859$

C $0.49701623-1.75245996 \quad 0.21104882$

C $-1.01375031-1.45652540 \quad 0.38116479$

C $-0.66843819-0.235511851 .31777145$

C $-1.148965491 .11932345 \quad 0.83847369$

C $-1.12412695-0.47025526 \quad 2.75214575$

$\mathrm{H}-3.66615202-0.60190726-1.70731330$

$\mathrm{H}-3.62441072-0.65529961 \quad 0.06780784$

$\mathrm{H}-3.59424939-2.15818037-0.87143674$

H $0.71560984 \quad 0.41599933-1.49102797$

H $1.73624771 \quad 1.43985204 \quad 1.15728997$

$\mathrm{H} \quad 2.82744772 \quad 0.199146890 .58485734$

$\mathrm{H} 1.31725963-0.887001252 .08028509$

$\mathrm{H} \quad 0.81132775-2.72536355 \quad 0.59766712$

$\mathrm{H} \quad 0.85895464 \quad-1.67827064 \quad-0.82102976$

$\mathrm{H}-1.55328050 \quad-2.258075190 .91321529$

$\mathrm{H}-2.23009464 \quad 1.23796457 \quad 0.97964649$

$\mathrm{H}-0.942163571 .29311381-0.22472481$

$\mathrm{H}-0.65984575 \quad 1.93069110 \quad 1.39531286$

$\mathrm{H}-2.21615537-0.43955582 \quad 2.83821404$

$\mathrm{H}-0.72258278 \quad 0.30012494 \quad 3.42342762$

$\mathrm{H}-0.79356426-1.441008813 .13900092$

$03.024975572 .43117135-0.82216866$

O $2.120120121 .83284562-1.47705444$

o $-1.13848334-0.77363923-1.89111896$

Vibrational frequencies (in $\mathrm{cm}-1$ )

$\begin{array}{lllllllllllllll}33.5 & 55.6 & 71.7 & 104.2 & 114.6 & 147.5 & 171.7 & 190.2 & 202.4 & 220.8 & 232.3 & 265.3 & 308.5 & 337.3\end{array}$

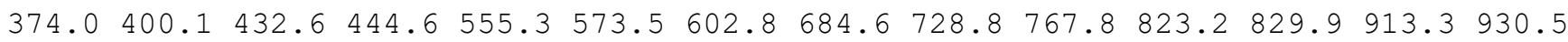
$986.9996 .6 \quad 1008.2 \quad 1017.1 \quad 1033.3 \quad 1042.7 \quad 1049.1 \quad 1053.1 \quad 1087.11105 .01127 .31152 .3$ $\begin{array}{llllllllllllll}1163.5 & 1194.5 & 1203.1 & 1210.5 & 1237.7 & 1242.6 & 1257.6 & 1261.4 & 1270.9 & 1278.5 & 1286.4 & 1287.5\end{array}$ $1295.51299 .0 \quad 1303.6 \quad 1307.7 \quad 1329.6 \quad 1341.7 \quad 1358.7 \quad 1379.2 \quad 1387.6 \quad 1406.2 \quad 1408.91591 .3$ $1846.82618 .12667 .8 \quad 2668.4 \quad 2672.3 \quad 2674.0 \quad 2675.5 \quad 2676.42679 .12679 .3 \quad 2712.4 \quad 2719.6$ $2729.8 \quad 2761.1 \quad 2779.7 \quad 2779.9 \quad 2782.7$

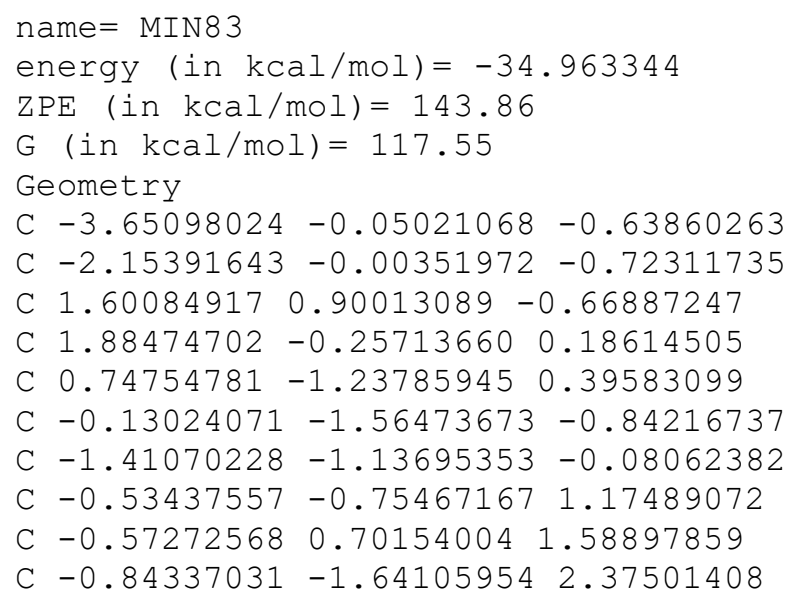


$\mathrm{H}-4.10269481 \quad 0.89985904 \quad-0.96277311$

$\mathrm{H}-4.00203608-0.25717126 \quad 0.37962604$

$\mathrm{H}-4.05232859-0.83633732-1.29471484$

$\mathrm{H} \quad 0.64363050 \quad 1.08402512-1.14949396$

H 2.26713659 $0.10692943 \quad 1.17628221$

$\mathrm{H} \quad 2.77773671-0.79398626-0.23399383$

$\mathrm{H} 1.15880071-2.15928383 \quad 0.85640247$

$\mathrm{H}-0.11540845-2.61526974-1.14326646$

$\mathrm{H} \quad 0.08574203-0.96447626-1.73307048$

$\mathrm{H}-2.08441333-1.98521274 \quad 0.12966108$

$\mathrm{H}-1.53078766 \quad 0.96303976 \quad 2.05435420$

$\mathrm{H}-0.43466906 \quad 1.38802087 \quad 0.74307013$

$\mathrm{H} \quad 0.21708966 \quad 0.92774200 \quad 2.31728209$

$\mathrm{H}-0.10448812-1.492998963 .17311691$

$\mathrm{H}-0.83612792 \quad-2.706341352 .11893584$

$\mathrm{H}-1.82864092-1.413698202 .79815015$

$03.637054141 .66060896-0.39860365$

o $2.49204694 \quad 1.80536953-0.92293133$

o - $1.58916904 \quad 0.89265163-1.30383203$

Vibrational frequencies (in $\mathrm{cm}-1$ )

$\begin{array}{lllllllllllllllll}37.3 & 47.0 & 62.8 & 111.7 & 121.4 & 149.6 & 175.2 & 187.8 & 200.9 & 221.8 & 234.9 & 269.7 & 303.3 & 337.3\end{array}$ $372.1389 .6 \quad 436.6 \quad 447.8 \quad 557.1 \quad 590.0 \quad 599.7 \quad 680.0 \quad 727.1 \quad 771.1 \quad 821.0 \quad 828.9 \quad 916.0 \quad 929.5$ $987.9996 .8 \quad 1008.0 \quad 1018.0 \quad 1034.5 \quad 1041.6 \quad 1049.7 \quad 1052.8 \quad 1085.6 \quad 1103.4 \quad 1132.7 \quad 1155.2$

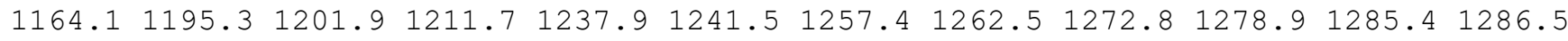

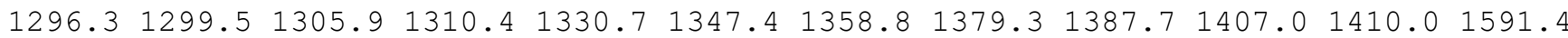
$1847.52618 .8 \quad 2665.12667 .02671 .2 \quad 2673.8 \quad 2674.12677 .02679 .12680 .52711 .92719 .3$ $2728.92761 .5 \quad 2779.5 \quad 2780.12782 .8$

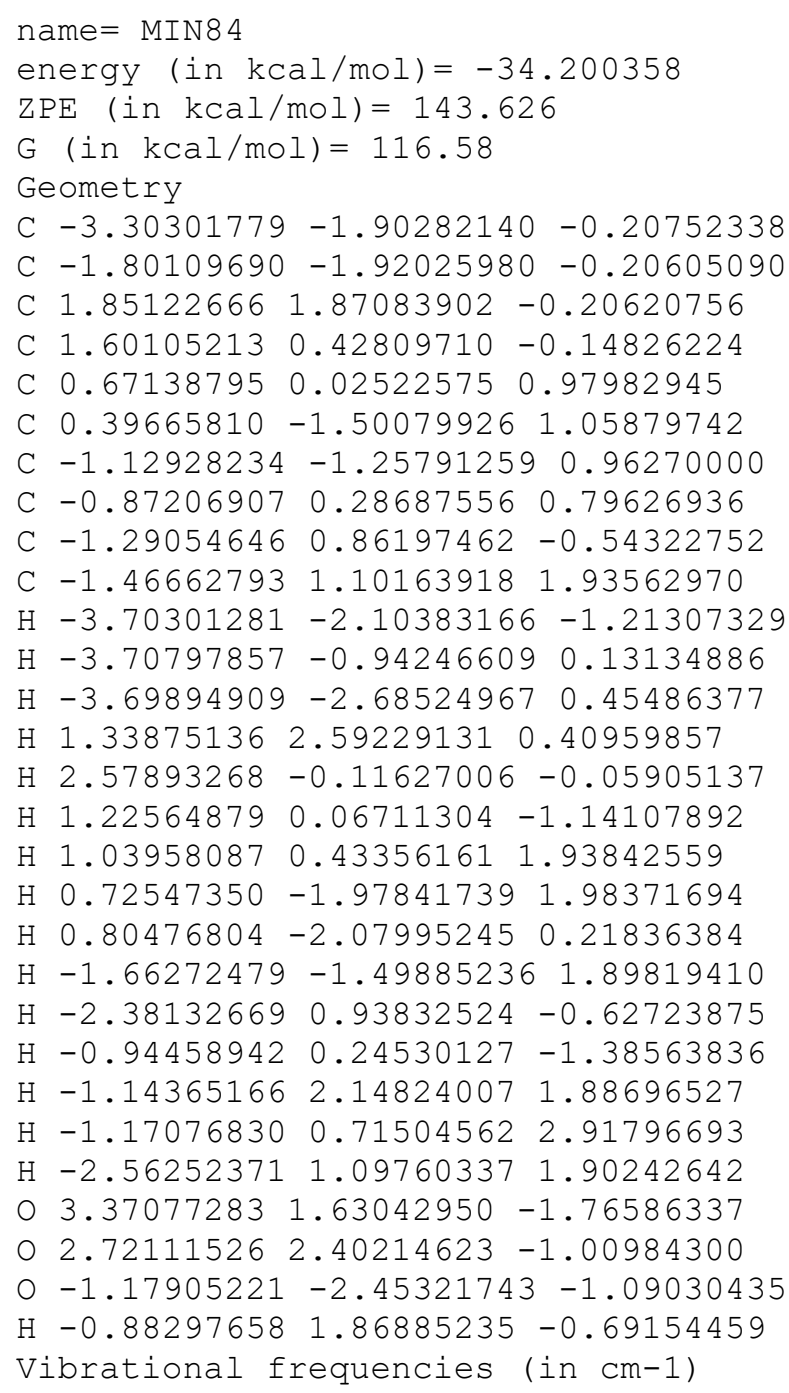


$\begin{array}{lllllllllllllllll}29.2 & 37.8 & 52.7 & 75.9 & 109.4 & 128.3 & 162.4 & 183.0 & 191.9 & 225.5 & 244.2 & 272.8 & 305.8 & 332.4\end{array}$

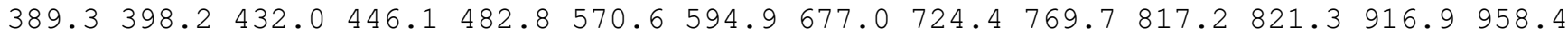
$987.6998 .5 \quad 1005.6 \quad 1016.1 \quad 1035.4 \quad 1042.1 \quad 1048.3 \quad 1056.8 \quad 1094.11117 .01130 .21149 .2$ $1179.6 \quad 1193.2 \quad 1201.7 \quad 1213.5 \quad 1228.2 \quad 1240.9 \quad 1259.0 \quad 1262.3 \quad 1269.1 \quad 1272.2 \quad 1284.4 \quad 1288.6$

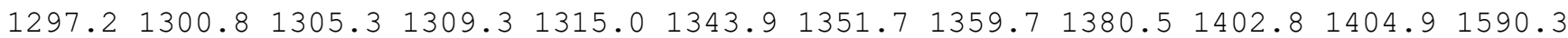
$1855.22613 .62664 .52668 .62675 .6 \quad 2675.6 \quad 2677.3 \quad 2678.12680 .12704 .32722 .62729 .2$ $2735.22759 .92779 .12780 .6 \quad 2783.0$

name $=$ MIN85

energy (in $\mathrm{kcal} / \mathrm{mol})=-34.300871$

$\operatorname{ZPE}($ in $\mathrm{kcal} / \mathrm{mol})=143.832$

$\mathrm{G}($ in $\mathrm{kcal} / \mathrm{mol})=116.861$

Geometry

C $-3.55228545-1.44621451-0.49636610$

C $-2.05074802-1.47589551-0.49277887$

C $2.75697658-0.04525756-0.51457932$

C $1.39553596 \quad 0.43566740 \quad-0.78754563$

C $0.54675103 \quad 0.31057792 \quad 0.47554132$

C $0.12290230-1.14228518 \quad 0.81855200$

C $-1.37180599-0.77447989 \quad 0.64790324$

C $-0.96611058 \quad 0.71798874 \quad 0.33803363$

C $-1.402640721 .24350213-1.01557197$

C $-1.40210728 \quad 1.671998391 .44104896$

$\mathrm{H}-3.95580208-2.15194796 \quad 0.24268384$

$\mathrm{H}-3.95406592-1.74126203-1.47702818$

$\mathrm{H}-3.94458927-0.45083956-0.25570535$

$\mathrm{H} 3.14768764-0.96819207-0.91606399$

$\mathrm{H} \quad 0.92364113-0.11104235-1.63186624$

H $1.432414191 .51334085-1.08867018$

$\mathrm{H} \quad 1.04511546 \quad 0.83686598 \quad 1.31430337$

$\mathrm{H} \quad 0.39506301-1.46547558 \quad 1.82612514$

$\mathrm{H} \quad 0.47928757-1.900386950 .11131013$

$\mathrm{H}-1.95507975-0.87305807 \quad 1.57787994$

$\mathrm{H}-0.91511268 \quad 2.20099623-1.24212689$

$\mathrm{H}-2.484902931 .41615236-1.04994792$

$\mathrm{H}-0.942197952 .66093780 \quad 1.30846069$

$\mathrm{H}-1.106811661 .31561497 \quad 2.43529372$

$\mathrm{H}-2.48832040 \quad 1.81230885 \quad 1.44980361$

$\begin{array}{llll}0 & 3.26232444 & 1.67025515 & 0.76169149\end{array}$

$\begin{array}{llll}0 & 3.60861696 & 0.57972447 & 0.23565694\end{array}$

O $-1.43530124-2.04364577-1.36148062$

$\mathrm{H}-1.15742884 \quad 0.55232614 \quad-1.83271714$

Vibrational frequencies (in $\mathrm{cm}-1$ )

$\begin{array}{llllllllllllllll}30.7 & 40.0 & 47.5 & 67.8 & 111.9 & 150.5 & 157.2 & 183.7 & 196.6 & 218.1 & 244.8 & 286.4 & 307.6 & 327.1\end{array}$ $362.7 \quad 392.8 \quad 429.4 \quad 467.5 \quad 509.2 \quad 569.3 \quad 599.1 \quad 678.4 \quad 721.5 \quad 809.7 \quad 826.1 \quad 892.2 \quad 917.2 \quad 936.2$ $952.7996 .3 \quad 1006.6 \quad 1016.7 \quad 1035.3 \quad 1041.7 \quad 1047.5 \quad 1067.3 \quad 1088.1 \quad 1107.7 \quad 1125.91140 .1$

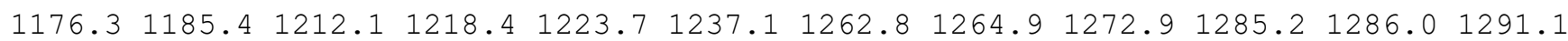
$1295.8 \quad 1299.6 \quad 1306.5 \quad 1309.4 \quad 1317.7 \quad 1348.4 \quad 1352.8 \quad 1358.91380 .3 \quad 1402.7 \quad 1405.5 \quad 1602.8$ $1851.0 \quad 2645.7 \quad 2667.6 \quad 2670.4 \quad 2672.2 \quad 2675.12676 .02680 .12683 .12703 .4 \quad 2721.62728 .4$ $2730.92761 .8 \quad 2778.7 \quad 2780.6 \quad 2782.1$

name $=$ MIN86

energy (in $\mathrm{kcal} / \mathrm{mol})=-34.481441$

$\mathrm{ZPE}(\mathrm{in} \mathrm{kcal} / \mathrm{mol})=144.024$

$\mathrm{G}($ in $\mathrm{kcal} / \mathrm{mol})=117.091$

Geometry

C $-3.41590284 \quad 0.37481614 \quad 0.33558513$

C $-1.99611286 \quad 0.55433989-0.11228145$

C $1.47270673 \quad 1.29636050 \quad 0.01109711$

C $1.916703490 .08047507-0.68695923$

C $0.79209361-0.79670038-1.21878121$

C $-0.36049619-0.07018846-1.96250778$

C $-1.38826840-0.56177869-0.91074241$ 
C $-0.24664745-1.37544651-0.18342071$

C $-0.01409723-1.05809156 \quad 1.27637801$

C $-0.43117652-2.87877238-0.36396772$

$\mathrm{H}-3.722920621 .17860015 \quad 1.02331687$

$\mathrm{H}-3.56699003-0.58153086 \quad 0.85105998$

$\mathrm{H}-4.10297103 \quad 0.40522370-0.52153878$

H $0.75881902 \quad 1.99708377-0.41128240$

H $2.56143592-0.52895486 \quad 0.00322693$

H $2.59137035 \quad 0.36478899-1.52903487$

$\mathrm{H} 1.23858154-1.60432516-1.83102463$

$\mathrm{H}-0.54656576-0.44488768-2.97266457$

$\mathrm{H}-0.26042000 \quad 1.01754717-2.02739744$

$\mathrm{H}-2.16552074-1.21951220-1.33216352$

$\mathrm{H}-0.73678391-1.562592361 .92728040$

$\mathrm{H}-0.07429890 \quad 0.01434795 \quad 1.50057923$

H $0.99124969-1.374874551 .59817112$

$\mathrm{H} \quad 0.45581772-3.42495828-0.01676271$

$\mathrm{H}-0.59552672-3.15504166-1.41105800$

$\mathrm{H}-1.28684318-3.25030332 \quad 0.21126734$

○ $2.67218814 \quad 0.88452180 \quad 1.80774132$

○ 1.864824921 .642187311 .19408180

$0-1.392369791 .570600280 .13882047$

Vibrational frequencies (in $\mathrm{cm}-1$ )

$\begin{array}{lllllllllllllll}13.7 & 38.7 & 44.9 & 115.1 & 117.1 & 165.4 & 174.6 & 203.5 & 213.1 & 229.6 & 255.8 & 279.8 & 299.6 & 333.7\end{array}$ $368.2 \quad 394.5 \quad 433.6 \quad 454.3 \quad 568.6 \quad 591.9 \quad 599.1 \quad 687.7 \quad 712.8 \quad 785.9 \quad 824.4 \quad 858.2 \quad 909.2 \quad 933.3$

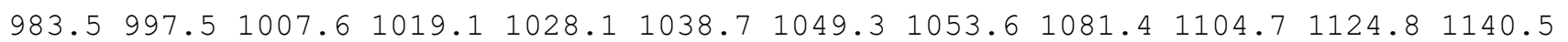

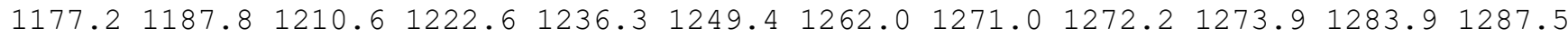

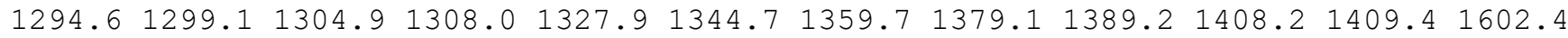
$1844.82627 .8 \quad 2660.0 \quad 2667.92673 .4 \quad 2674.4 \quad 2676.0 \quad 2679.4 \quad 2683.0 \quad 2690.92713 .3 \quad 2721.2$ $2731.2 \quad 2762.9 \quad 2779.7 \quad 2780.4 \quad 2782.8$

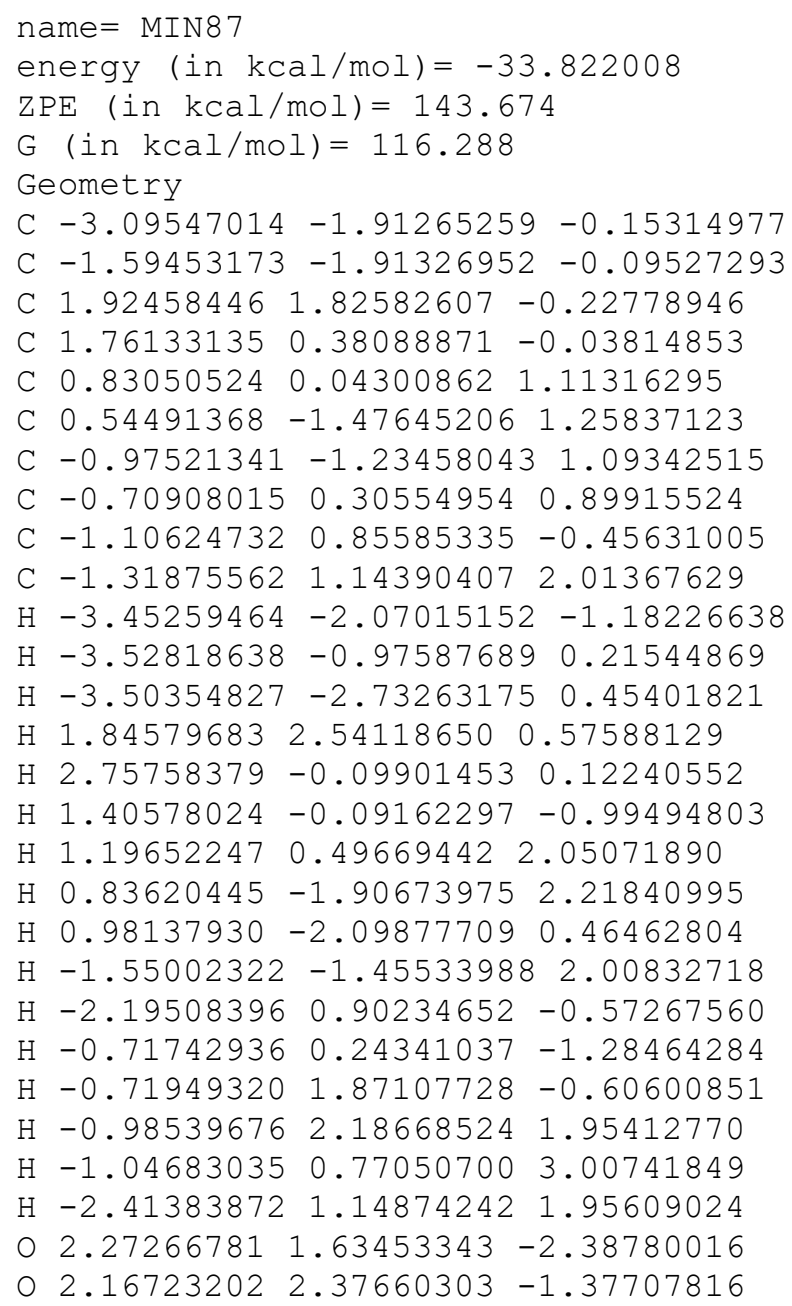


$0-0.93396232-2.44546854-0.95158185$

Vibrational frequencies (in $\mathrm{cm}-1$ )

$\begin{array}{llllllllllllllll}21.3 & 27.5 & 51.4 & 70.7 & 101.0 & 132.1 & 160.9 & 187.0 & 204.5 & 232.0 & 256.4 & 274.9 & 303.2 & 327.0\end{array}$ $380.8 \quad 392.9 \quad 439.6 \quad 446.1 \quad 491.7 \quad 570.8 \quad 595.1 \quad 680.6 \quad 730.6 \quad 760.3 \quad 822.7 \quad 826.4 \quad 914.5 \quad 961.4$ $986.1997 .5 \quad 1005.6 \quad 1015.8 \quad 1036.0 \quad 1041.8 \quad 1048.8 \quad 1057.9 \quad 1094.4 \quad 1114.5 \quad 1129.2 \quad 1146.1$ $\begin{array}{lllllllllllllll}1174.9 & 1193.3 & 1207.4 & 1220.7 & 1233.8 & 1245.9 & 1261.3 & 1262.2 & 1269.0 & 1272.6 & 1285.2 & 1288.4\end{array}$ $1296.91300 .41303 .8 \quad 1308.2 \quad 1313.3 \quad 1344.6 \quad 1354.3 \quad 1358.01380 .3 \quad 1401.8 \quad 1404.51599 .9$ $1855.02613 .92660 .3 \quad 2668.8 \quad 2675.8 \quad 2676.3 \quad 2678.3 \quad 2678.72680 .3 \quad 2707.12722 .82730 .1$ $\begin{array}{llllll}2734.0 & 2759.9 & 2778.6 & 2780.8 & 2783.2\end{array}$

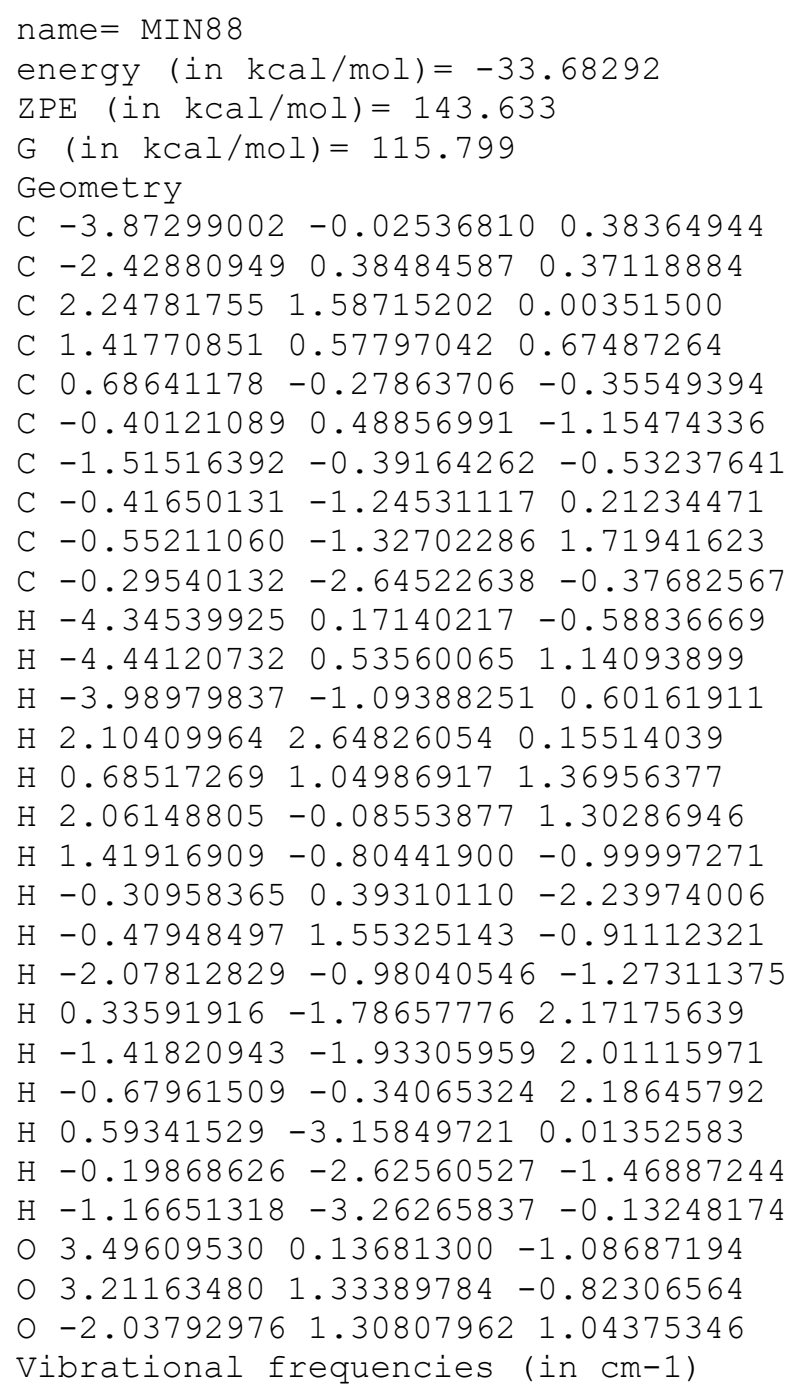


C $1.00329320-1.13601244 \quad 0.39967209$

C $-0.28458519-1.051162691 .25572734$

C $0.04031314 \quad 0.45594511 \quad 1.58647366$

C $-1.03187940 \quad 1.46449492 \quad 1.22778234$

C $0.44783982 \quad 0.63094351 \quad 3.04503126$

$\mathrm{H}-3.70465309-1.20957327 \quad 0.72819398$

$\mathrm{H}-2.89946468-2.54102904 \quad 1.56201898$

$\mathrm{H}-2.80934161-0.901288292 .23237003$

$\mathrm{H}-0.64373994 \quad 0.61854170-1.52141506$

$\mathrm{H} 1.111129552 .30159008-0.34372789$

H 2.37419426 1.25801059 -1.01885719

H $2.23279998 \quad 0.53510296 \quad 1.20121774$

H $1.76717438-1.80607668 \quad 0.80435377$

$\mathrm{H} \quad 0.83985406-1.42292138-0.64590042$

$\mathrm{H}-0.25990625-1.701525912 .14587822$

$\mathrm{H}-1.41507729 \quad 1.33464848 \quad 0.20892067$

$\mathrm{H}-0.64762258 \quad 2.49066141 \quad 1.30076134$

$\mathrm{H}-1.89095822 \quad 1.39570377 \quad 1.90680621$

$\mathrm{H} \quad 0.84071743 \quad 1.63876176 \quad 3.22846808$

$\mathrm{H} \quad 1.22731139-0.07999754 \quad 3.34420511$

$\mathrm{H}-0.402347410 .48448296 \quad 3.72076840$

$\begin{array}{lllll}0 & 1.99527405 & 0.66063789 & -3.20285398\end{array}$

○ $0.78226425 \quad 0.51975151-2.88835380$

o $-1.60293946-1.22203768-0.71579356$

Vibrational frequencies (in cm-1)

$\begin{array}{lllllllllllllll}-6.0 & 49.3 & 71.6 & 107.2 & 111.5 & 161.3 & 176.0 & 193.3 & 195.3 & 225.5 & 235.1 & 267.0 & 320.5 & 332.6\end{array}$ $369.2 \quad 400.8 \quad 435.6 \quad 447.4 \quad 565.2 \quad 579.2 \quad 602.9691 .1 \quad 700.3 \quad 815.7 \quad 828.1 \quad 840.3 \quad 907.3 \quad 928.3$ $982.9993 .6 \quad 1007.3 \quad 1016.3 \quad 1036.7 \quad 1046.2 \quad 1051.01054 .4 \quad 1087.31106 .11124 .1 \quad 1132.2$

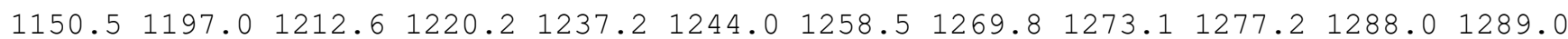
$1296.01298 .3 \quad 1303.8 \quad 1307.8 \quad 1328.2 \quad 1344.7 \quad 1359.6 \quad 1379.8 \quad 1387.2 \quad 1404.2 \quad 1408.31603 .4$ $1846.72627 .02666 .62669 .0 \quad 2673.12675 .3 \quad 2676.2 \quad 2677.62679 .12694 .32714 .62722 .4$ $2729.7 \quad 2760.0 \quad 2779.6 \quad 2780.0 \quad 2782.7$

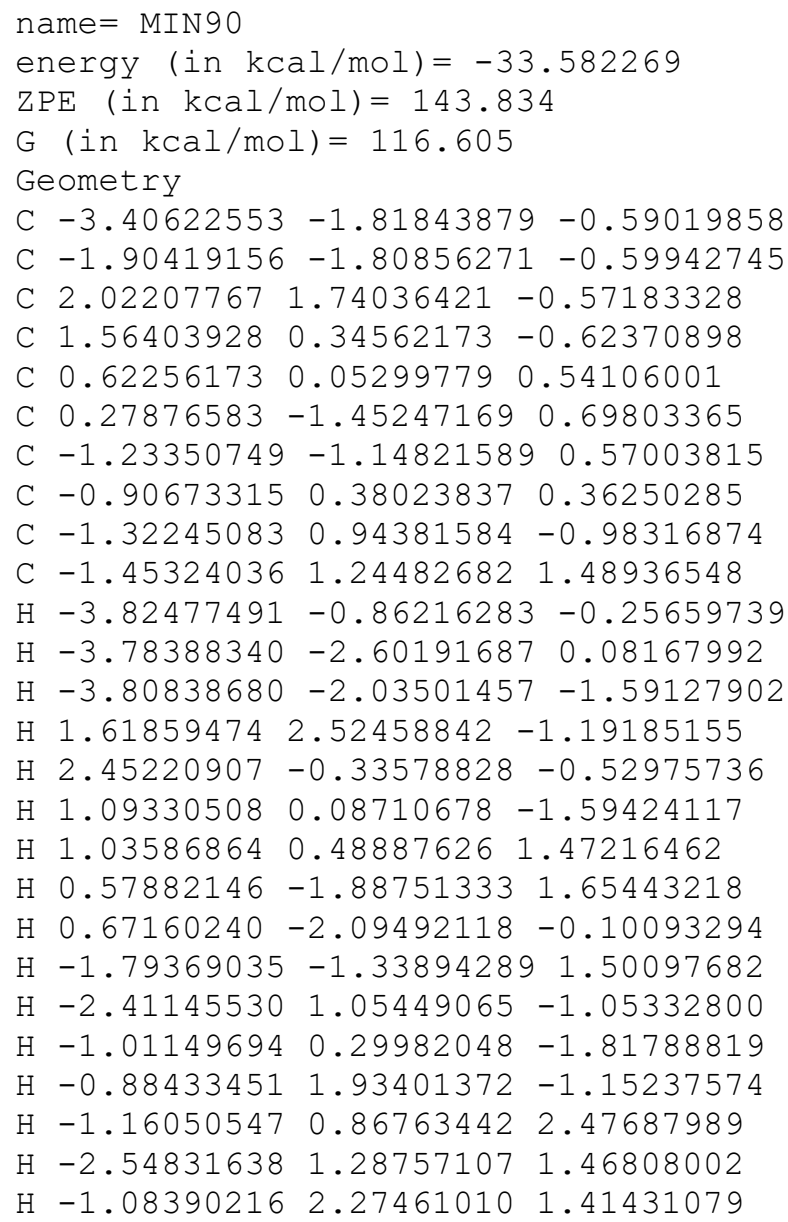


03.487802621 .329236371 .01160347

$\begin{array}{llll}0 & 2.95040202 & 2.15729323 & 0.23193912\end{array}$

O $-1.28241307-2.31776019-1.49877392$

Vibrational frequencies (in $\mathrm{cm}-1$ )

$\begin{array}{lllllllllllllll}28.3 & 33.0 & 43.5 & 56.3 & 112.2 & 144.1 & 155.6 & 185.1 & 195.1 & 235.0 & 259.9 & 289.2 & 309.5 & 325.2\end{array}$

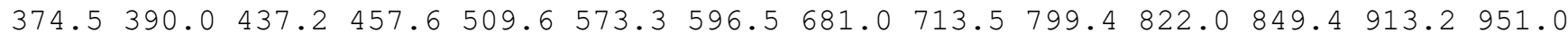
$990.4 \quad 998.9 \quad 1005.7 \quad 1016.1 \quad 1035.4 \quad 1045.6 \quad 1047.8 \quad 1057.7 \quad 1093.2 \quad 1109.5 \quad 1124.5 \quad 1137.9$ $\begin{array}{llllllllllllll}1164.5 & 1192.9 & 1208.2 & 1221.2 & 1228.6 & 1239.6 & 1262.7 & 1266.7 & 1272.9 & 1285.1 & 1288.3 & 1290.6\end{array}$ $1299.31300 .21306 .1 \quad 1315.6 \quad 1316.7 \quad 1345.0 \quad 1350.5 \quad 1359.51380 .4 \quad 1402.01405 .01597 .0$ $1852.62638 .52665 .52669 .12675 .42676 .62677 .2 \quad 2679.62680 .92707 .22725 .42729 .3$ $2731.32760 .12779 .4 \quad 2780.92782 .8$

\section{name $=$ MIN91}

energy (in $\mathrm{kcal} / \mathrm{mol})=-33.476317$

$\mathrm{ZPE}(\mathrm{in} \mathrm{kcal} / \mathrm{mol})=143.986$

$\mathrm{G}(\mathrm{in} \mathrm{kcal} / \mathrm{mol})=117.386$

Geometry

C $-2.60267098-2.05081496-0.68692942$

C $-1.23731133-1.42794601-0.68978424$

C $0.80638548 \quad 1.42231844-1.00547800$

C $1.036531951 .59164703 \quad 0.43847521$

C $0.72478453 \quad 0.33674257 \quad 1.24891855$

C $0.98512372-1.01267795 \quad 0.52461372$

C $-0.51411798-1.38659077 \quad 0.62414913$

C $-0.78390111-0.06974840 \quad 1.44825173$

C $-1.83259995 \quad 0.86687111 \quad 0.88217528$

C $-1.09940916-0.37728528 \quad 2.90714393$

$\mathrm{H}-3.23027860-1.63446683-1.48950203$

$\mathrm{H}-3.12911563-1.90798298 \quad 0.26348353$

$\mathrm{H}-2.53281144-3.13273280-0.87244370$

$\mathrm{H}-0.178236091 .32436986-1.44482204$

$\mathrm{H} \quad 0.45566406 \quad 2.45153823 \quad 0.83623381$

H 2.11630536 $1.86004308 \quad 0.60134089$

H $1.25403106 \quad 0.40314510 \quad 2.21851235$

H $1.65455210-1.687823521 .06495781$

$\mathrm{H} \quad 1.37157389-0.92896808-0.49887654$

$\mathrm{H}-0.69813178-2.30594078 \quad 1.20367358$

$\mathrm{H}-2.84468974 \quad 0.46636742 \quad 1.01969133$

$\mathrm{H}-1.70350504 \quad 1.05073470-0.19105662$

$\mathrm{H}-1.802030361 .84259677 \quad 1.38413890$

$\mathrm{H}-2.08157512-0.85138673 \quad 3.01451647$

$\mathrm{H}-1.11079622 \quad 0.53849694 \quad 3.51093333$

$\mathrm{H}-0.36107099-1.052884773 .35538607$

$\begin{array}{llll}0 & 2.94727219 & 1.39157649 & -1.50581982\end{array}$

O $1.74845870 \quad 1.32563025-1.88779249$

o - $0.76031074-0.98065773-1.70511477$

Vibrational frequencies (in $\mathrm{cm}-1$ )

$\begin{array}{llllllllllllllll}-17.4 & 53.2 & 69.5 & 93.1 & 108.4 & 160.9 & 179.3 & 192.2 & 197.2 & 227.4 & 241.1 & 278.5 & 324.8 & 332.8\end{array}$ $369.3 \quad 402.6 \quad 435.9 \quad 445.6 \quad 564.6 \quad 581.7 \quad 603.7 \quad 691.6 \quad 698.1 \quad 813.2 \quad 828.9 \quad 849.7 \quad 905.9 \quad 925.4$ $983.1 \quad 994.1 \quad 1006.7 \quad 1016.3 \quad 1036.6 \quad 1043.8 \quad 1051.4 \quad 1059.3 \quad 1086.1 \quad 1105.6 \quad 1123.6 \quad 1126.5$

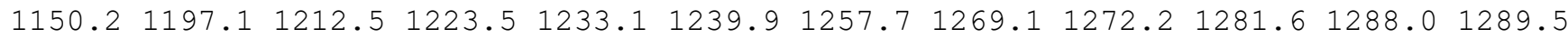
$1297.61298 .8 \quad 1305.0 \quad 1310.7 \quad 1327.2 \quad 1341.9 \quad 1359.7 \quad 1380.1 \quad 1386.7 \quad 1404.4 \quad 1407.91603 .7$ $1846.92632 .92666 .2 \quad 2667.5 \quad 2674.0 \quad 2675.6 \quad 2676.02676 .72679 .4 \quad 2703.82716 .22725 .5$ $2730.5 \quad 2760.3 \quad 2779.5 \quad 2780.2 \quad 2782.8$

name $=$ MIN92

energy (in $\mathrm{kcal} / \mathrm{mol})=-33.515469$

$\mathrm{ZPE}(\mathrm{in} \mathrm{kcal} / \mathrm{mol})=144.165$

$\mathrm{G}($ in $\mathrm{kcal} / \mathrm{mol})=118.068$

Geometry

C $-3.31849223 \quad 0.09094962 \quad-0.45839814$

C $-1.81954913 \quad 0.11178398-0.51885690$

C $1.390696161 .31203885-0.46732313$ 
C $1.90919713 \quad 0.357980190 .52759595$

C $1.01900673-0.86840537 \quad 0.72165362$

C $0.26461122-1.36585086-0.54155462$

C $-1.10236448-1.04459150 \quad 0.11401641$

C $-0.36092264-0.67871479 \quad 1.45723879$

C $-0.66148319 \quad 0.68558598 \quad 2.04155212$

C $-0.57705461-1.752632852 .51848579$

$\mathrm{H}-3.74668334 \quad 1.04462345 \quad-0.80344633$

$\mathrm{H}-3.68224063-0.08934346 \quad 0.56055010$

$\mathrm{H}-3.72763680-0.69904949-1.10362443$

$\mathrm{H} \quad 0.68337500 \quad 2.09668513-0.23173897$

H 2.07911542 $0.85271116 \quad 1.50690512$

H $2.91767895 \quad 0.00167037 \quad 0.18153005$

$\mathrm{H} 1.62281833-1.666506721 .19374091$

$\mathrm{H} \quad 0.41763448-2.42688083-0.76003951$

$\mathrm{H} \quad 0.48181115-0.81794789-1.46660491$

$\mathrm{H}-1.76035184 \quad-1.92251849 \quad 0.21202398$

$\mathrm{H} \quad 0.01310546 \quad 0.91496084 \quad 2.87623968$

$\mathrm{H}-1.68602699 \quad 0.73814747 \quad 2.43014433$

$\mathrm{H}-0.55467721 \quad 1.49383838 \quad 1.30787418$

$\mathrm{H} \quad 0.08599883-1.59675786 \quad 3.37825697$

$\mathrm{H}-0.37784042-2.759736622 .13317580$

$\mathrm{H}-1.60606322-1.74486432 \quad 2.89497552$

$02.509737250 .40827892-2.12863807$

o $1.70404210 \quad 1.29409647-1.72261793$

o $-1.233621091 .02502434-1.05041989$

Vibrational frequencies (in $\mathrm{cm}-1$ )

$\begin{array}{llllllllllllllll}32.4 & 43.8 & 80.0 & 113.3 & 119.2 & 160.8 & 174.7 & 198.9 & 200.0 & 227.5 & 247.2 & 288.0 & 316.0 & 333.2\end{array}$

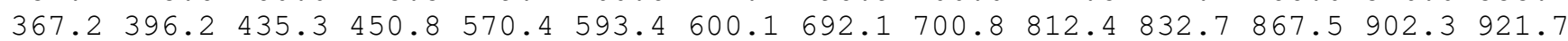

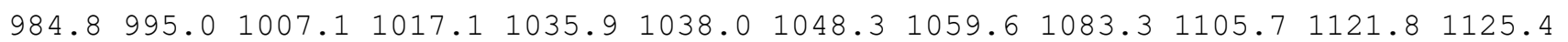
$\begin{array}{lllllllllllll}1151.9 & 1201.7 & 1211.6 & 1222.1 & 1230.2 & 1240.1 & 1261.2 & 1267.7 & 1273.1 & 1282.6 & 1287.2 & 1288.3\end{array}$ $1298.51299 .4 \quad 1305.1 \quad 1314.2 \quad 1329.8 \quad 1342.8 \quad 1359.8 \quad 1379.2 \quad 1388.0 \quad 1405.91408 .91605 .5$ $1845.22638 .22668 .5 \quad 2668.7 \quad 2674.1 \quad 2675.3 \quad 2675.72676 .42679 .8 \quad 2707.92715 .92727 .3$ $2731.82761 .92780 .12780 .6 \quad 2783.0$

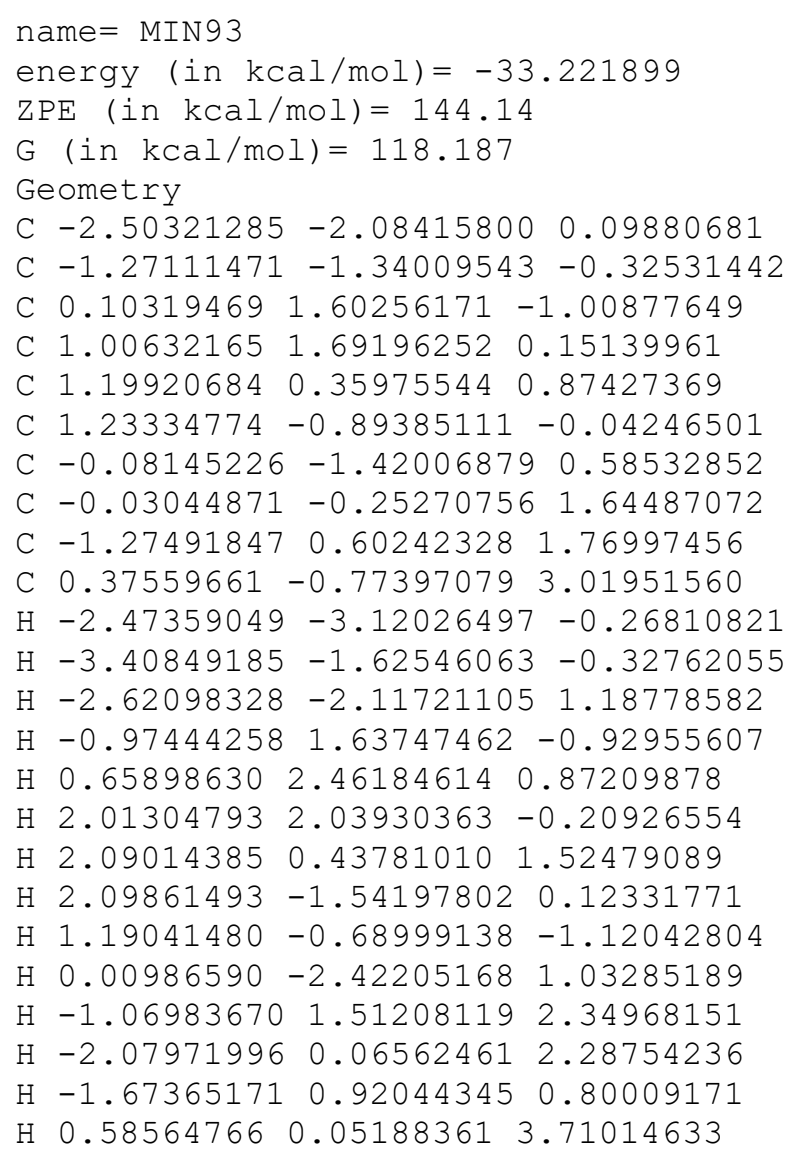


H $1.27706275-1.396913932 .97364566$

$\mathrm{H}-0.41742083-1.38228260 \quad 3.46863964$

o $1.73674870 \quad 1.37301826-2.45926641$

$0.498812971 .44120908-2.23057598$

o $-1.25910801-0.69927135-1.34959900$

Vibrational frequencies (in $\mathrm{cm}-1$ )

$\begin{array}{llllllllllllllll}35.8 & 55.7 & 79.3 & 105.4 & 112.3 & 164.1 & 179.7 & 194.1 & 201.1 & 227.3 & 243.4 & 287.2 & 318.4 & 339.8\end{array}$

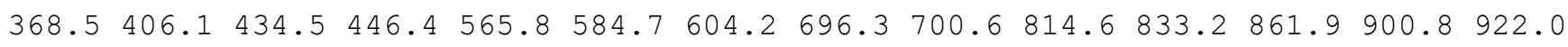
$982.6 \quad 994.1 \quad 1006.7 \quad 1016.2 \quad 1036.6 \quad 1040.0 \quad 1050.4 \quad 1062.1 \quad 1085.0 \quad 1104.6 \quad 1119.4 \quad 1122.5$

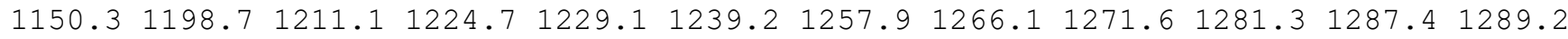

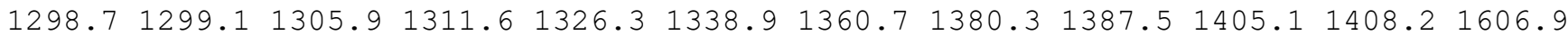
$1843.92637 .5 \quad 2666.8 \quad 2668.7 \quad 2673.5 \quad 2674.12675 .72676 .92679 .62711 .52717 .02727 .6$ $2732.12760 .3 \quad 2779.9 \quad 2780.5 \quad 2782.7$

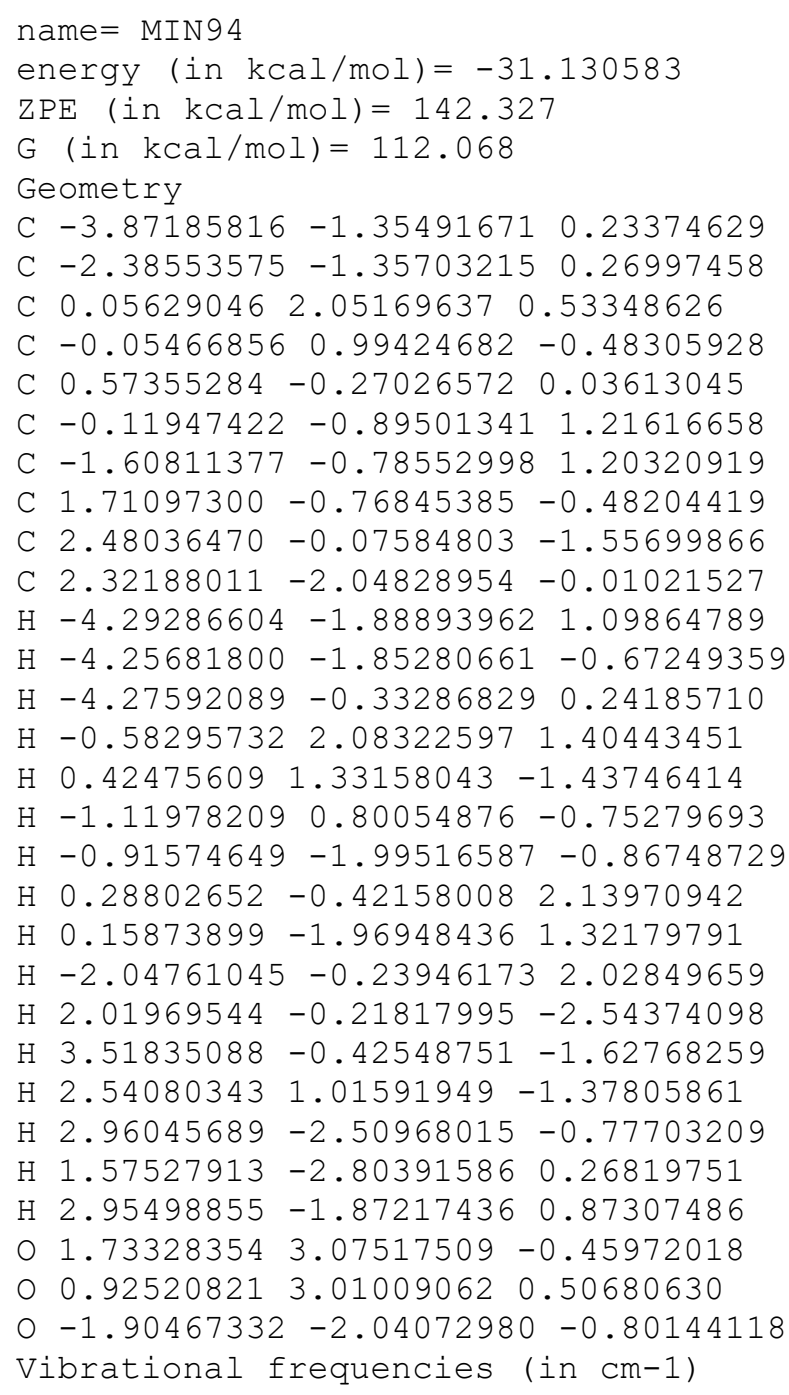


C $-1.20328172-1.87922785-0.12428777$

C $-0.34713630 \quad 1.53396869-0.04554914$

C $1.15079431 \quad 1.25383413 \quad 0.09695841$

C $1.54768097-0.18312740 \quad-0.19332158$

C $0.76101019-0.89691568-1.32505123$

C $0.20865170-1.94669260-0.31298371$

C $1.09317419-1.295094330 .82639911$

C $0.35631479-0.84264882 \quad 2.06789339$

C $2.25020888-2.20935797 \quad 1.21609867$

$\mathrm{H}-3.42316647-1.77099731 \quad 0.13873110$

$\mathrm{H}-1.845965792 .44967181 \quad-2.52602787$

$\mathrm{H}-1.43509709 \quad 3.024149920 .68219806$

$\mathrm{H}-1.02952923 \quad 0.66381553 \quad 0.06472944$

$\mathrm{H} 1.459578601 .533690291 .13058129$

$\mathrm{H} 1.718245591 .94258469-0.56539998$

H $2.64198182-0.21940472-0.35965070$

$\mathrm{H} 1.39286702-1.32752816-2.10551281$

$\mathrm{H}-0.00259805-0.29139499-1.82218570$

$\mathrm{H} \quad 0.52706008 \quad-2.98399834 \quad-0.54864619$

$\mathrm{H} 1.00980154-0.26331656 \quad 2.73226510$

$\mathrm{H}-0.02303199-1.69955858 \quad 2.63882298$

$\mathrm{H}-0.51091825-0.20831467 \quad 1.84332970$

H 2.96105244 $-1.69113037 \quad 1.87061424$

H $2.80789505-2.56661150 \quad 0.34352841$

H $1.89093455-3.092440201 .75808271$

$0-1.878165592 .02267899-1.63700884$

$0-0.67223248 \quad 2.47670287 \quad 0.95184967$

o $-0.481860082 .13682088-1.32054557$

Vibrational frequencies (in $\mathrm{cm}-1$ )

$\begin{array}{lllllllllllllll}41.6 & 61.6 & 63.8 & 86.2 & 145.8 & 149.9 & 164.5 & 178.6 & 190.8 & 216.3 & 240.7 & 255.2 & 267.9 & 304.5\end{array}$

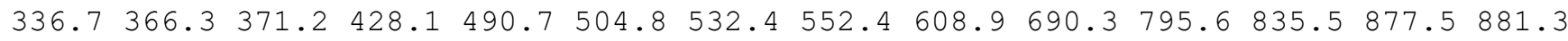
$900.3 \quad 927.7 \quad 952.7 \quad 993.5 \quad 1007.4 \quad 1018.7 \quad 1037.3 \quad 1043.5 \quad 1055.1 \quad 1069.3 \quad 1087.1 \quad 1117.1 \quad 1131.3$ $1164.7 \quad 1183.3 \quad 1199.9 \quad 1215.2 \quad 1222.7 \quad 1245.6 \quad 1249.4 \quad 1258.3 \quad 1283.7 \quad 1285.1 \quad 1291.6 \quad 1296.8$ $1299.51302 .6 \quad 1307.91332 .4 \quad 1344.6 \quad 1354.1 \quad 1366.1 \quad 1388.3 \quad 1395.11406 .1 \quad 1410.2 \quad 2432.8$ $2637.62650 .5 \quad 2667.12675 .8 \quad 2678.12680 .2 \quad 2682.02715 .32720 .52733 .72763 .62781 .0$ $2783.0 \quad 2806.5 \quad 2871.0 \quad 2897.5$

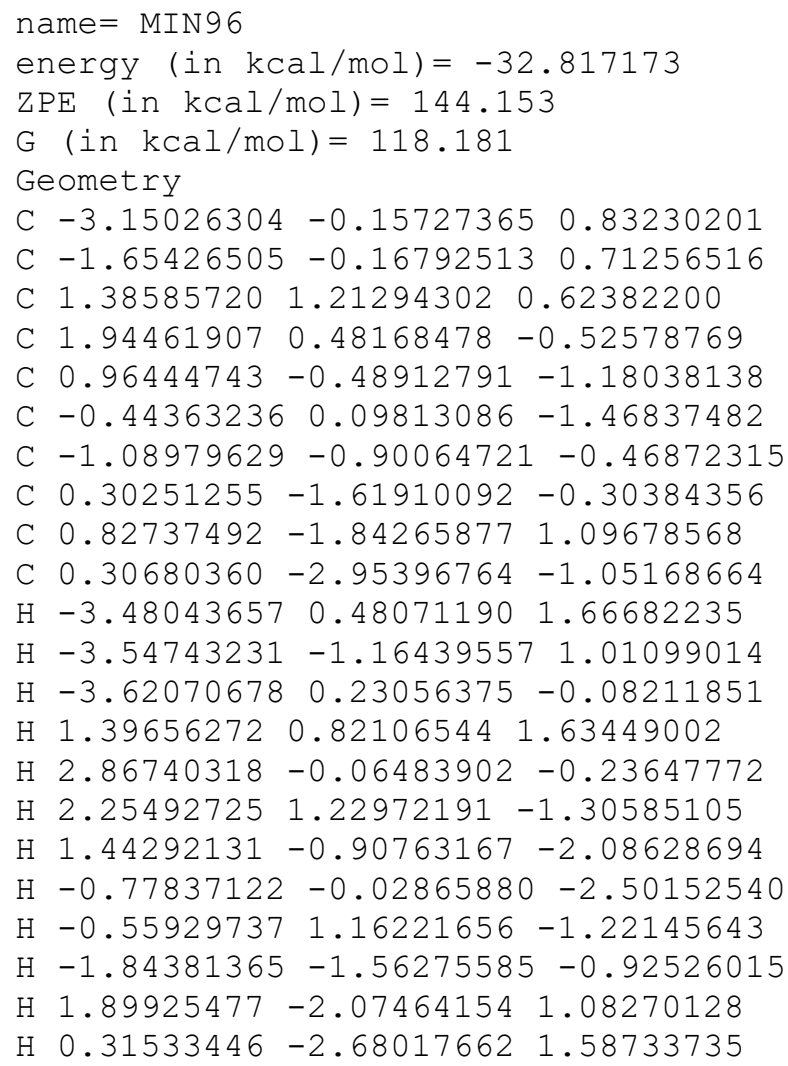


$\mathrm{H} \quad 0.68940547-0.975037751 .75408523$

$\mathrm{H} 1.31773980-3.37621184-1.09545968$

$\mathrm{H}-0.05074641-2.85385229-2.08242413$

$\mathrm{H}-0.33336577-3.69119075-0.55386214$

$0.729151942 .94048552-0.56828666$

$0.81752308 \quad 2.37181977 \quad 0.55979308$

O $-0.96913694 \quad 0.42974476 \quad 1.50868193$

Vibrational frequencies (in cm-1)

$\begin{array}{llllllllllllllll}27.8 & 58.7 & 85.5 & 113.4 & 123.9 & 149.1 & 177.1 & 206.6 & 211.9 & 227.1 & 247.6 & 274.0 & 312.5 & 317.5\end{array}$

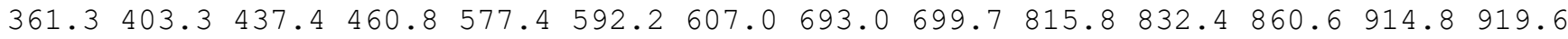
$978.8 \quad 991.0 \quad 1007.6 \quad 1020.4 \quad 1028.4 \quad 1035.1 \quad 1043.1 \quad 1057.2 \quad 1086.01100 .3 \quad 1116.3 \quad 1127.3$

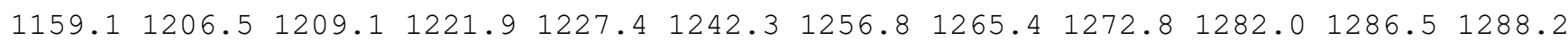
$1298.91299 .6 \quad 1305.4 \quad 1315.5 \quad 1331.3 \quad 1348.3 \quad 1357.1 \quad 1379.3 \quad 1390.2 \quad 1410.4 \quad 1411.3 \quad 1612.3$ $1845.82639 .02660 .2 \quad 2668.02669 .02672 .3 \quad 2675.92677 .22680 .32702 .92714 .62726 .6$ $2729.02757 .02779 .3 \quad 2780.0 \quad 2782.6$

name $=$ MIN97

energy (in $\mathrm{kcal} / \mathrm{mol})=-32.013048$

$\mathrm{ZPE}($ in $\mathrm{kcal} / \mathrm{mol})=143.391$

$\mathrm{G}($ in $\mathrm{kcal} / \mathrm{mol})=116.272$

Geometry

C $-2.85904737-1.51638873-0.13518079$

C $-1.39362189-1.51483387-0.12835984$

C $2.228191652 .37811651-0.11735945$

C $2.087535690 .87901213-0.15882768$

C $1.20284384 \quad 0.36405758 \quad 0.95630204$

C $0.93895521-1.166196550 .92846058$

C $-0.59430075-0.92586762 \quad 0.95875415$

C $-0.34518451 \quad 0.63366136 \quad 0.85129472$

C $-0.822417121 .25334436-0.44653362$

C $-0.894870791 .39751287 \quad 2.04408091$

$\mathrm{H}-3.24485636-1.25133947-1.14313502$

$\mathrm{H}-3.31046106-0.83756945 \quad 0.59766459$

$\mathrm{H}-3.25231413-2.53514303 \quad 0.04912178$

H $1.82759342 \quad 2.905383610 .75695218$

$\mathrm{H} \quad 3.09848797 \quad 0.41769940 \quad-0.09392428$

$\mathrm{H} 1.71629214 \quad 0.56737516-1.15954878$

$\mathrm{H} \quad 1.60067881 \quad 0.69197118 \quad 1.93619066$

H $1.32413092-1.70922711 \quad 1.79652846$

$\mathrm{H} 1.31955751-1.672704340 .03713498$

$\mathrm{H}-1.03685009-1.20131998 \quad 1.93912828$

$\mathrm{H}-1.91922758 \quad 1.27665116-0.49940001$

$\mathrm{H}-0.474810590 .70261230-1.33057449$

$\mathrm{H}-0.47335975 \quad 2.28827469-0.55322737$

$\mathrm{H}-1.99213614 \quad 1.40802713 \quad 2.04151915$

$\mathrm{H}-0.56307024 \quad 2.44339998 \quad 2.03485343$

o $-1.34737216-2.56070594-2.05797670$

$\begin{array}{lllll}0 & 2.76571160 & 2.98397602 & -1.01040371\end{array}$

$0-0.70038512-2.04550716-1.09838225$

$\mathrm{H}-0.57744477 \quad 0.96468834 \quad 2.99966776$

Vibrational frequencies (in $\mathrm{cm}-1$ )

$\begin{array}{lllllllllllllllll}30.2 & 38.0 & 50.5 & 92.6 & 107.0 & 128.8 & 135.0 & 182.0 & 211.4 & 221.2 & 238.4 & 272.0 & 283.5 & 302.1\end{array}$ $317.7 \quad 394.3 \quad 405.0 \quad 437.7 \quad 464.1 \quad 544.3 \quad 556.5 \quad 669.2 \quad 686.0 \quad 753.4 \quad 823.4 \quad 836.7 \quad 919.2 \quad 950.5$ $993.21004 .4 \quad 1016.6 \quad 1026.6 \quad 1035.1 \quad 1045.2 \quad 1045.6 \quad 1071.7 \quad 1110.5 \quad 1119.5 \quad 1128.2 \quad 1131.5$ $1154.31184 .6 \quad 1202.1 \quad 1228.3 \quad 1231.9 \quad 1259.4 \quad 1261.4 \quad 1264.6 \quad 1282.4 \quad 1284.01289 .1 \quad 1295.9$ $1299.31301 .21309 .1 \quad 1315.51340 .0 \quad 1351.6 \quad 1355.4 \quad 1384.11401 .51403 .7 \quad 1446.61594 .3$ $1837.82634 .6 \quad 2646.8 \quad 2653.12667 .7 \quad 2671.5 \quad 2673.12676 .42676 .7 \quad 2680.62712 .3 \quad 2719.7$ $\begin{array}{llllll}2740.2 & 2763.3 & 2768.8 & 2779.4 & 2782.6\end{array}$

name $=$ MIN98

energy (in $\mathrm{kcal} / \mathrm{mol})=-31.826989$

$\mathrm{ZPE}(\mathrm{in} \mathrm{kcal} / \mathrm{mol})=143.422$

$\mathrm{G}($ in $\mathrm{kcal} / \mathrm{mol})=116.227$ 


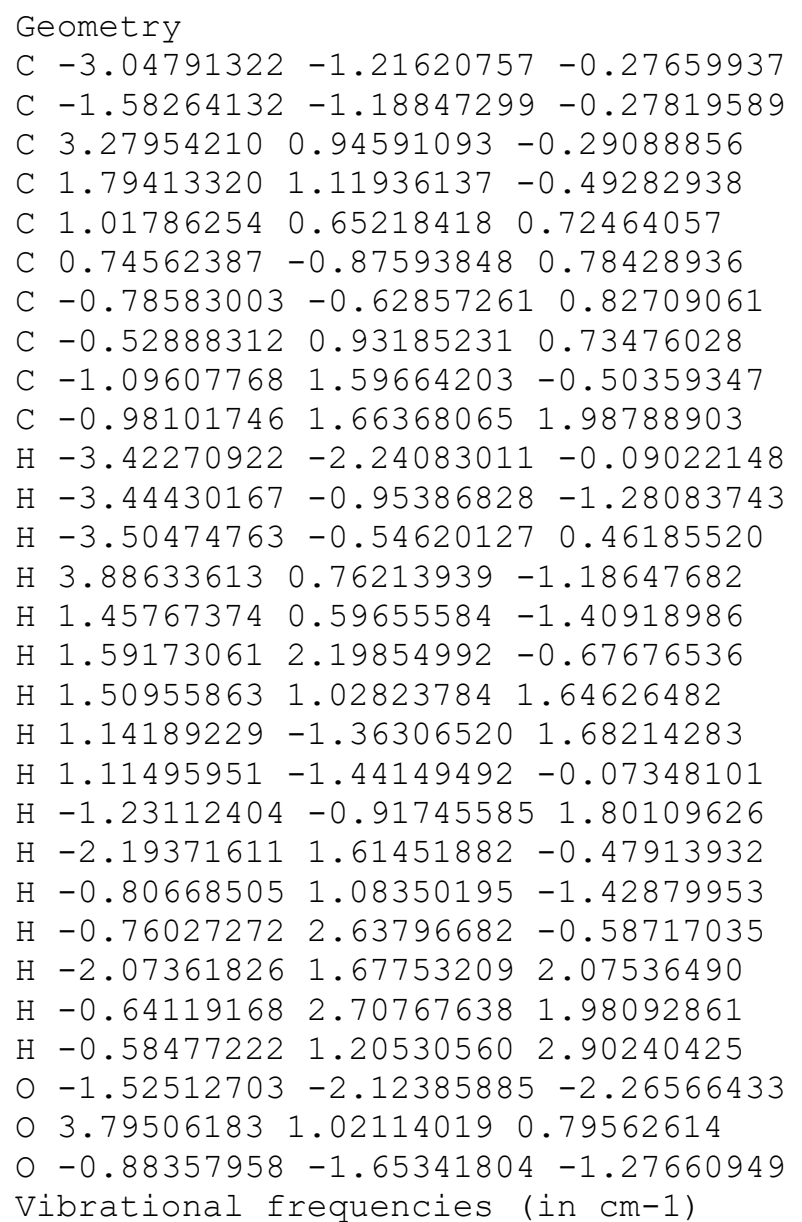

$\begin{array}{llllllllllllllll}28.9 & 38.3 & 53.1 & 84.4 & 102.2 & 121.7 & 160.5 & 176.0 & 188.4 & 222.3 & 229.4 & 253.2 & 276.8 & 295.0\end{array}$ $327.2 \quad 379.3 \quad 428.1 \quad 452.0 \quad 464.2 \quad 542.3 \quad 608.7 \quad 665.6 \quad 684.4 \quad 804.3 \quad 826.7 \quad 871.7 \quad 927.3 \quad 937.2$ $972.2995 .6 \quad 1008.3 \quad 1016.8 \quad 1027.2 \quad 1044.7 \quad 1058.9 \quad 1069.01081 .01112 .5 \quad 1124.11124 .8$

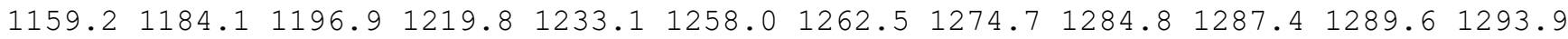
$1297.71305 .01306 .91311 .1 \quad 1340.5 \quad 1352.2 \quad 1360.4 \quad 1384.21402 .91404 .21441 .51595 .1$ $1843.0 \quad 2636.2 \quad 2649.3 \quad 2658.7 \quad 2670.7 \quad 2672.3 \quad 2672.72675 .62678 .5 \quad 2680.02705 .72717 .0$ $2740.02763 .8 \quad 2768.7 \quad 2780.3 \quad 2782.5$

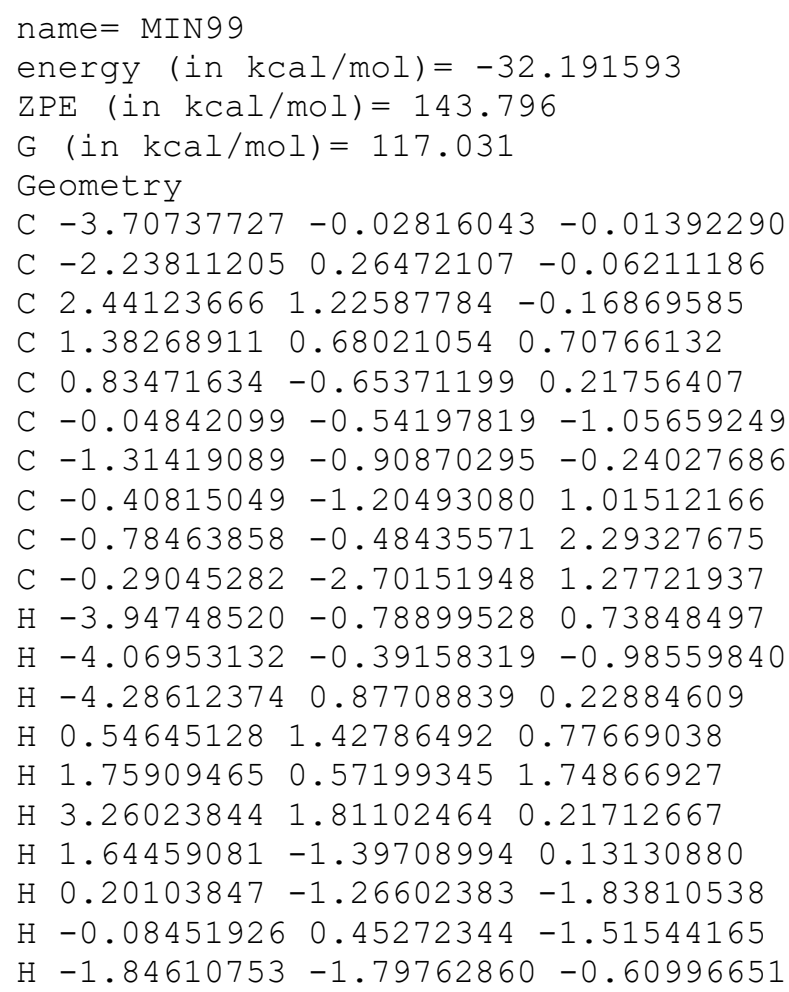


$\mathrm{H}-0.02283569-0.62641184 \quad 3.07016159$

$\mathrm{H}-1.73295012-0.858544062 .69860076$

$\mathrm{H}-0.90442083 \quad 0.59889699 \quad 2.15278602$

$\mathrm{H} \quad 0.47903522-2.912385132 .03036815$

$\mathrm{H}-0.02237196-3.26082294 \quad 0.37324757$

$\mathrm{H}-1.23306737-3.11809177 \quad 1.65112320$

O $3.150917331 .45419076-2.22369442$

O $2.291991930 .98037531-1.43451795$

$0-1.822099001 .394320650 .02980322$

Vibrational frequencies (in $\mathrm{cm}-1$ )

$\begin{array}{llllllllllllllll}35.9 & 38.1 & 67.3 & 81.6 & 118.8 & 124.2 & 163.6 & 186.2 & 207.5 & 208.4 & 250.7 & 263.3 & 286.7 & 320.6\end{array}$ $354.6 \quad 359.5 \quad 397.0 \quad 467.6 \quad 488.6 \quad 573.9 \quad 598.0 \quad 659.4 \quad 687.7 \quad 835.7 \quad 860.1 \quad 874.2 \quad 909.2 \quad 933.4$ $978.0 \quad 997.3 \quad 1005.8 \quad 1017.7 \quad 1035.0 \quad 1037.9 \quad 1048.5 \quad 1078.2 \quad 1096.7 \quad 1109.3 \quad 1124.3 \quad 1148.9$

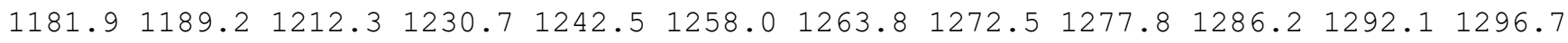
$1298.71306 .21311 .2 \quad 1313.0 \quad 1314.2 \quad 1343.5 \quad 1350.1 \quad 1355.6 \quad 1379.5 \quad 1402.6 \quad 1406.7 \quad 1578.5$

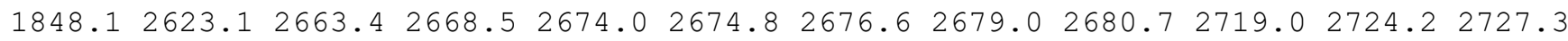
$2734.8 \quad 2757.7 \quad 2779.3 \quad 2780.2 \quad 2782.9$

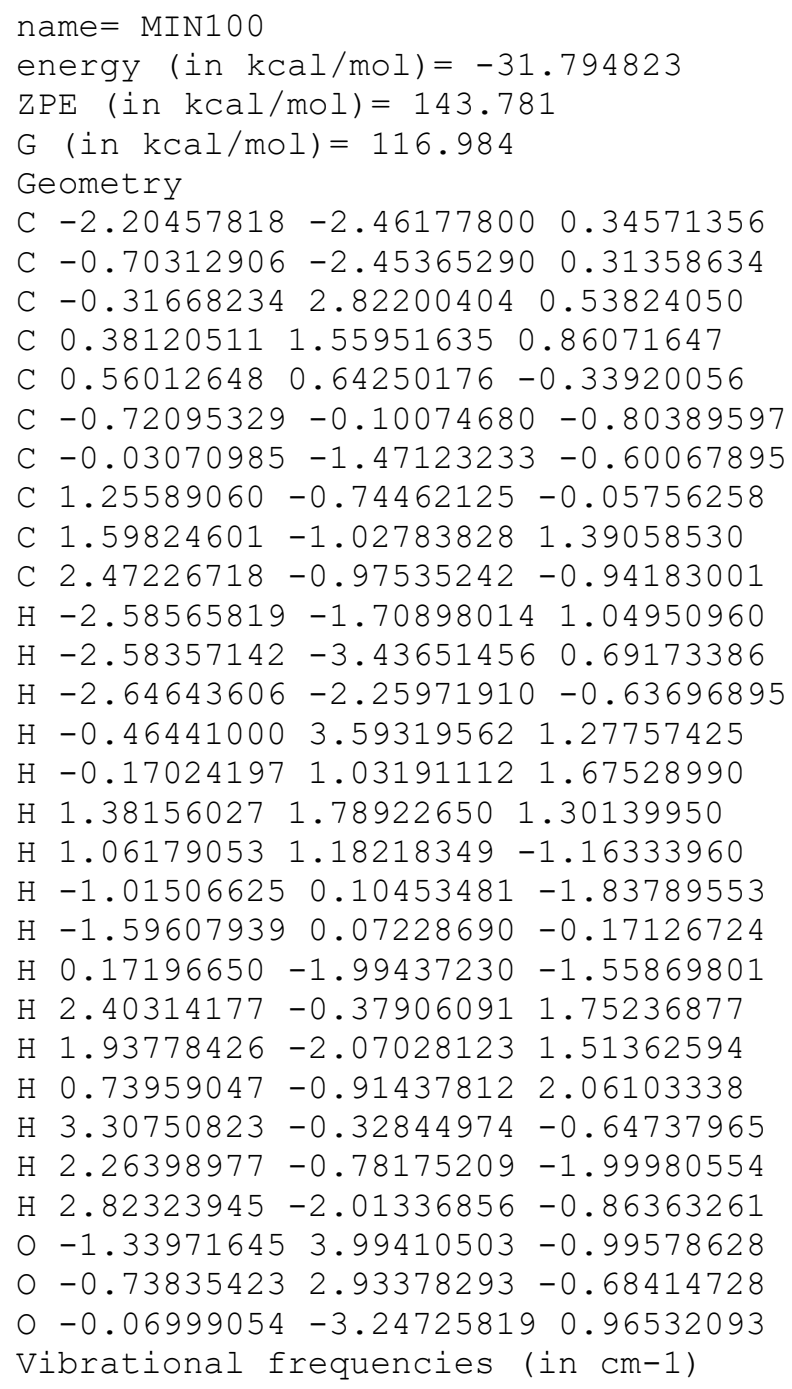

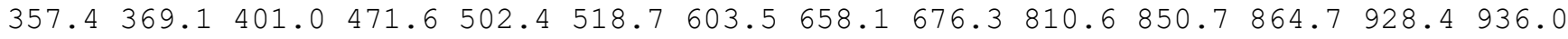
$971.2994 .1 \quad 1007.8 \quad 1018.3 \quad 1026.5 \quad 1043.7 \quad 1050.4 \quad 1063.5 \quad 1082.6 \quad 1112.2 \quad 1132.01153 .5$

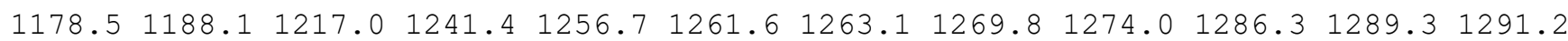
$1301.31301 .4 \quad 1304.5 \quad 1312.3 \quad 1320.5 \quad 1346.3 \quad 1349.2 \quad 1358.3 \quad 1381.0 \quad 1402.2 \quad 1405.7 \quad 1580.1$ 1845.12638 .32660 .32666 .62674 .02675 .72677 .12681 .62682 .32710 .62717 .12722 .8 $2729.6 \quad 2762.3 \quad 2779.3 \quad 2779.8 \quad 2782.1$

name $=$ MIN101

energy $($ in $\mathrm{kcal} / \mathrm{mol})=-31.750528$ 
$\mathrm{ZPE}($ in $\mathrm{kcal} / \mathrm{mol})=143.874$

$\mathrm{G}($ in $\mathrm{kcal} / \mathrm{mol})=117.894$

Geometry

C $-3.44440201-0.79014738 \quad 0.36719422$

$\begin{array}{lllll}\text { C } & -1.96201027 & -0.79921527 & 0.30626879\end{array}$

C $3.15512677-0.14231638 \quad 0.30923662$

C $1.927892320 .23426505-0.47172310$

C $0.90516157 \quad 0.92961771 \quad 0.40371253$

C $0.19812225 \quad 0.00942556 \quad 1.43667224$

C $-1.19033479 \quad 0.33308837 \quad 0.82066978$

C $-0.48395389 \quad 1.25071223-0.26223735$

C $-0.66412004 \quad 0.80693845-1.69845262$

C $-0.87593902 \quad 2.71397484-0.10724383$

$\mathrm{H}-3.80016408-0.74762886 \quad 1.41074174$

$\mathrm{H}-3.90993011-1.67483284-0.09444261$

$\mathrm{H}-3.85434770 \quad 0.09588868 \quad-0.14731596$

$\mathrm{H} 3.02012485-0.43874451 \quad 1.35682097$

$\mathrm{H} 1.49668831-0.70131916-0.91169468$

H $2.19524616 \quad 0.86333894 \quad-1.34479599$

$\mathrm{H} \quad 1.34992709 \quad 1.82869160 \quad 0.86879961$

$\mathrm{H} \quad 0.30541863 \quad 0.32400823 \quad 2.47678906$

$\mathrm{H} \quad 0.48946230-1.04760729 \quad 1.37002480$

$\mathrm{H}-1.80829025 \quad 0.94257817 \quad 1.51231151$

$\mathrm{H}-0.03959156 \quad 1.40069685-2.37856304$

$\mathrm{H}-1.702501690 .91645994 \quad-2.02834431$

$\mathrm{H}-0.37879704-0.24665990-1.84560365$

$\mathrm{H}-1.915380832 .88624979-0.40738155$

$\mathrm{H}-0.24423377 \quad 3.35733254 \quad-0.73397769$

$\mathrm{H}-0.76956856 \quad 3.06606603 \quad 0.92516468$

O $-0.28427434-2.08116437-0.39238307$

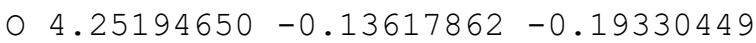

o $-1.52341178-1.89998774-0.23315490$

Vibrational frequencies (in $\mathrm{cm}-1$ )

$\begin{array}{lllllllllllllll}45.4 & 56.4 & 89.2 & 107.1 & 128.3 & 153.0 & 170.1 & 183.6 & 206.6 & 226.6 & 252.8 & 278.7 & 292.5 & 313.9\end{array}$ $330.2381 .6 \quad 411.4 \quad 433.0 \quad 471.6 \quad 546.6 \quad 592.5 \quad 642.3 \quad 697.3 \quad 810.7 \quad 817.8 \quad 876.6 \quad 914.5 \quad 941.5$ $982.8 \quad 992.0 \quad 1004.01014 .6 \quad 1017.8 \quad 1036.5 \quad 1068.2 \quad 1083.2 \quad 1098.8 \quad 1125.11131 .7 \quad 1148.8$

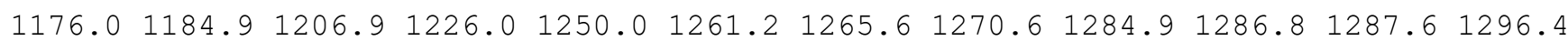

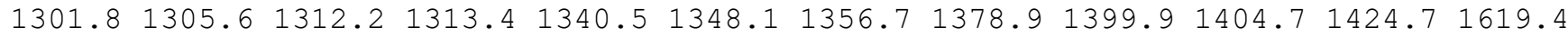
$1835.72632 .7 \quad 2654.92655 .5 \quad 2657.3 \quad 2661.8 \quad 2672.92674 .2 \quad 2677.92681 .02715 .92721 .2$ $2735.12756 .3 \quad 2771.02777 .9 \quad 2782.2$

\section{name $=$ MIN102}

energy (in $\mathrm{kcal} / \mathrm{mol})=-32.12739$

$\mathrm{ZPE}($ in $\mathrm{kcal} / \mathrm{mol})=144.629$

$\mathrm{G}($ in $\mathrm{kcal} / \mathrm{mol})=118.75$

Geometry

C $-2.92099603-1.10401168-1.23368069$

C $-1.57371880-1.04908305-0.60762480$

C $0.35701479 \quad 1.63628347 \quad-0.55195624$

C $1.47954784 \quad 0.78811756-0.14400358$

C $1.14948132-0.68747617 \quad 0.10645939$

C $0.69061735-1.37101704-1.18762016$

C $-1.29075094-0.997438120 .69917835$

C $0.10813140-0.95369281 \quad 1.23386663$

C $0.18543284 \quad 0.11304359 \quad 2.33834101$

C $0.41813076-2.329375811 .86601744$

$\mathrm{H}-3.24667907-2.14900361-1.36132055$

$\mathrm{H}-2.92648772-0.64703926-2.23640118$

$\mathrm{H}-3.67967527-0.59112408-0.62718110$

$\mathrm{H}-0.62836562 \quad 1.26066834-0.79154017$

H $1.96701738 \quad 1.240009320 .76115818$

$\mathrm{H} 2.29164018 \quad 0.87363513-0.91229299$

H 2.11154095 -1.17440795 0.42025410

$\mathrm{H} \quad 0.71756498-2.47203606-1.09502133$ 
H $1.28440243-1.05902875-2.06727256$

$\mathrm{H}-2.07384162-1.00857168 \quad 1.44816336$

$\mathrm{H}-0.44732925-0.158344613 .19138062$

$\mathrm{H}-0.16445100 \quad 1.08833020 \quad 1.98159632$

$\mathrm{H} \quad 1.20627562 \quad 0.23403764 \quad 2.71419477$

$\mathrm{H} 1.40009635-2.33814690 \quad 2.34865005$

$\mathrm{H} 0.39541650 \quad-3.12799511 \quad 1.11876314$

$\mathrm{H}-0.32501606-2.58466267 \quad 2.62965387$

O $1.59715234 \quad 3.43560592-0.39619916$

$\begin{array}{lllll}0 & 0.46777849 & 2.92272698 & -0.66271635\end{array}$

$0-0.62702422-1.01538879-1.59031275$

Vibrational frequencies (in $\mathrm{cm}-1$ )

$\begin{array}{lllllllllllllll}35.2 & 68.3 & 82.1 & 87.2 & 108.8 & 158.7 & 187.0 & 195.5 & 221.5 & 240.5 & 279.8 & 291.5 & 321.1 & 347.8\end{array}$ $402.1 \quad 435.8 \quad 452.9 \quad 471.2 \quad 507.7 \quad 551.0 \quad 601.3 \quad 703.3 \quad 750.3 \quad 776.8 \quad 850.5 \quad 898.7 \quad 924.2 \quad 942.9$

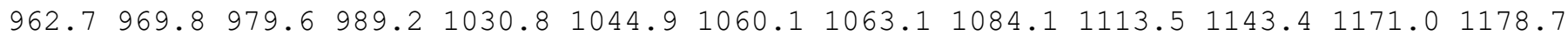

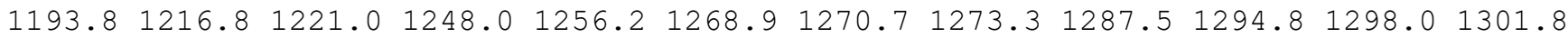
$1310.41313 .1 \quad 1319.4 \quad 1344.4 \quad 1356.4 \quad 1382.0 \quad 1385.6 \quad 1400.7 \quad 1406.2 \quad 1420.4 \quad 1589.8 \quad 1877.8$ $2614.52648 .8 \quad 2664.5 \quad 2673.8 \quad 2674.0 \quad 2677.0 \quad 2678.92681 .22684 .32699 .12715 .12722 .8$ $2770.8 \quad 2778.6 \quad 2780.8 \quad 2782.8$

name $=$ MIN103

energy $($ in $\mathrm{kcal} / \mathrm{mol})=-31.139065$

$\operatorname{ZPE}(\mathrm{in} \mathrm{kcal} / \mathrm{mol})=144.047$

$\mathrm{G}($ in $\mathrm{kcal} / \mathrm{mol})=118.633$

Geometry

C $-2.00549498-1.58561606 \quad 0.20298373$

C $-0.53061729-1.59033202 \quad 0.20161763$

C $-1.28389818 \quad 2.38532618 \quad 0.23552714$

C $-0.169444512 .58150100-0.75731406$

C $0.53623960 \quad 1.30601327-1.19006623$

C $-0.35265395 \quad 0.14681069-1.71443567$

C $0.31534567-0.84451817-0.73184317$

C $1.14841893 \quad 0.34727749-0.10085741$

C $0.81481848 \quad 0.64886913 \quad 1.34649691$

C $2.64651197 \quad 0.14880159-0.26310216$

$\mathrm{H}-2.40912360-1.91941264-0.76865925$

$\mathrm{H}-2.40307927-0.56195934 \quad 0.36262010$

$\mathrm{H}-2.44939590 \quad-2.225903130 .97974925$

$\mathrm{H}-1.19261746 \quad 2.90147264 \quad 1.20140972$

$\mathrm{H} \quad 0.58340770 \quad 3.29092825-0.35603135$

$\mathrm{H}-0.59668146 \quad 3.07080948-1.66354278$

$\mathrm{H} \quad 1.311417191 .58298987-1.93554834$

$\mathrm{H}-0.20694011-0.09493727-2.76997306$

$\mathrm{H}-1.42711144 \quad 0.30066569-1.55857002$

$\mathrm{H} 0.99744053-1.56612745-1.24428082$

$\mathrm{H}-0.26046487 \quad 0.63292845 \quad 1.54917958$

H $1.21550282 \quad 1.61856686 \quad 1.65840148$

H $1.26484875-0.11711806 \quad 2.00421827$

H $3.21068131 \quad 1.01290258 \quad 0.10187756$

H $2.93755157-0.02832890-1.30342187$

$\mathrm{H} \quad 2.97494321-0.73234643 \quad 0.31588715$

o $1.24343734-2.458958621 .17979047$

$0-2.26786628 \quad 1.72836194 \quad-0.00297265$

o $-0.02407505-2.375857021 .11316808$

Vibrational frequencies (in $\mathrm{cm}-1$ )

$\begin{array}{lllllllllllllll}50.5 & 67.3 & 95.0 & 124.6 & 144.5 & 158.5 & 186.8 & 208.6 & 216.1 & 236.0 & 258.1 & 277.2 & 300.5 & 318.2\end{array}$ $332.2381 .2 \quad 429.3 \quad 459.7 \quad 478.4 \quad 549.5 \quad 612.2 \quad 651.4 \quad 684.0 \quad 790.1 \quad 808.2 \quad 897.3 \quad 913.0 \quad 924.4$ $990.7 \quad 998.2 \quad 1009.0 \quad 1017.9 \quad 1019.6 \quad 1031.3 \quad 1057.9 \quad 1066.8 \quad 1081.8 \quad 1089.5 \quad 1118.3 \quad 1136.1$ $1163.31195 .21208 .5 \quad 1227.2 \quad 1249.9 \quad 1261.3 \quad 1263.0 \quad 1276.4 \quad 1284.7 \quad 1288.4 \quad 1296.7 \quad 1298.7$ $1304.11306 .51322 .1 \quad 1326.2 \quad 1338.7 \quad 1356.3 \quad 1381.3 \quad 1384.6 \quad 1405.0 \quad 1408.8 \quad 1442.7 \quad 1580.2$ $1838.52635 .82644 .32653 .02661 .0 \quad 2663.3 \quad 2664.32674 .02680 .72683 .72693 .52711 .2$ $2736.6 \quad 2761.0 \quad 2767.8 \quad 2775.4 \quad 2778.7$ 
name $=$ MIN104

energy (in $\mathrm{kcal} / \mathrm{mol})=-30.386728$

$\mathrm{ZPE}($ in $\mathrm{kcal} / \mathrm{mol})=143.562$

$\mathrm{G}($ in $\mathrm{kcal} / \mathrm{mol})=116.9$

Geometry

C $-2.69791247-1.14183340-0.50745452$

C $-1.24334276-1.05932757-0.65989804$

C $1.58294283 \quad 1.66047064-0.63342017$

C 2.148500431 .061597330 .62936655

C $1.46723276-0.19832961 \quad 1.12275073$

C $1.21326900-1.32685490 \quad 0.08750256$

C $-0.29969807-1.340096960 .43518049$

C $-0.07171967-0.14938710 \quad 1.45422000$

C $-0.79713143 \quad 1.13664137 \quad 1.12011717$

C $-0.36599293-0.559483512 .88929630$

$\mathrm{H}-3.03689901-1.153106910 .53546649$

$\mathrm{H}-3.09664427-2.04401819-1.00917093$

$\mathrm{H}-3.19337215-0.29312683-1.02743283$

$\mathrm{H} \quad 1.180201990 .98299219-1.39522966$

H $2.13626587 \quad 1.83010377 \quad 1.43605057$

$\mathrm{H} 3.23001238 \quad 0.85944408 \quad 0.45501060$

$\mathrm{H} 2.03845590-0.57785823 \quad 1.99665309$

H $1.72768106-2.26600027 \quad 0.31771104$

$\mathrm{H} 1.46710988-1.07617774-0.94444229$

$\mathrm{H}-0.59991390 \quad-2.27254459 \quad 0.95735757$

$\mathrm{H}-0.75356766 \quad 1.39541758 \quad 0.05465049$

$\mathrm{H}-0.37569858 \quad 1.98746192 \quad 1.67358353$

$\mathrm{H}-1.86220107 \quad 1.07829404 \quad 1.38098301$

$\mathrm{H}-0.04756758 \quad 0.21894117 \quad 3.59539418$

$\mathrm{H} 0.14816992-1.483846253 .17557573$

$\mathrm{H}-1.43901154-0.72059616 \quad 3.04969063$

O $-1.45554102-0.46641822-2.76539783$

o $1.59678843 \quad 2.85297897-0.81013497$

o $-0.68625279-0.72930428-1.79290709$

Vibrational frequencies (in cm-1)

$\begin{array}{llllllllllllllll}34.7 & 42.2 & 56.1 & 95.1 & 112.1 & 129.4 & 163.5 & 194.4 & 212.5 & 224.1 & 256.7 & 278.8 & 280.7 & 296.2\end{array}$ $\begin{array}{llllllllllllll}333.5 & 386.4 & 418.0 & 448.5 & 452.6 & 542.0 & 598.4 & 669.9 & 683.8 & 763.7 & 825.1 & 853.7 & 913.0 & 931.9\end{array}$ $995.61002 .2 \quad 1008.91017 .3 \quad 1029.3 \quad 1043.5 \quad 1051.0 \quad 1068.9 \quad 1076.9 \quad 1113.4 \quad 1124.8 \quad 1131.2$ $\begin{array}{lllllllllllllll}1162.0 & 1193.3 & 1207.1 & 1232.2 & 1237.9 & 1257.0 & 1262.2 & 1265.9 & 1282.4 & 1287.8 & 1290.1 & 1293.7\end{array}$ $1297.61300 .61309 .01312 .8 \quad 1338.1 \quad 1354.4 \quad 1383.2 \quad 1384.21405 .2 \quad 1406.3 \quad 1445.81591 .8$

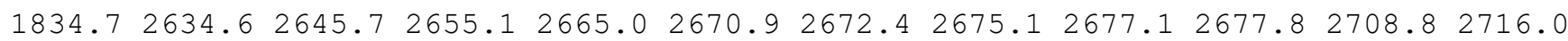
$2736.52762 .7 \quad 2768.4 \quad 2779.2 \quad 2782.2$

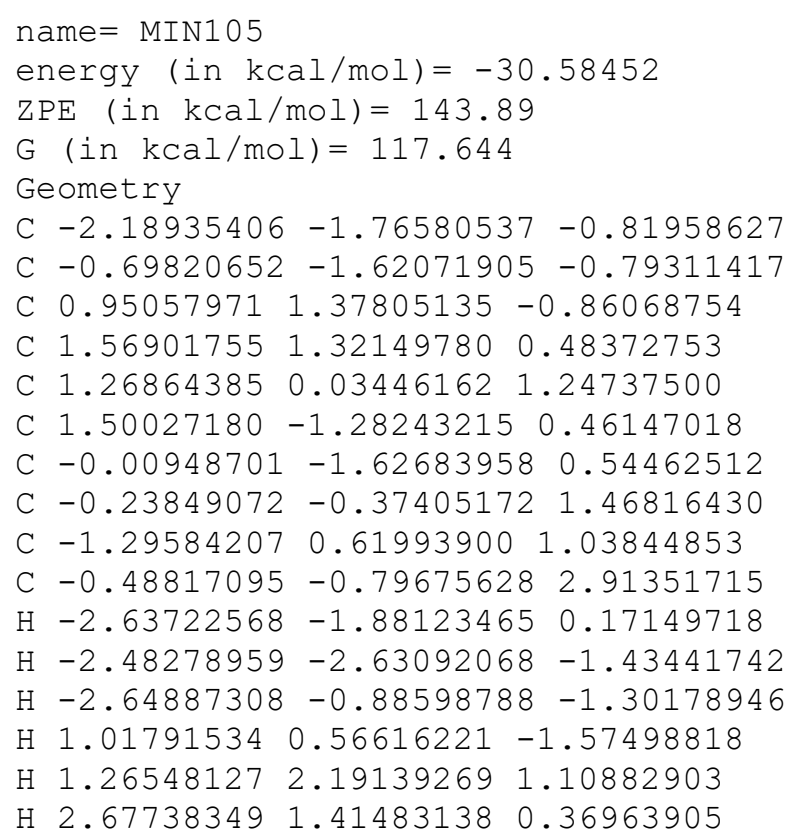


H $1.82386388 \quad 0.05872103 \quad 2.20425563$

$\mathrm{H} \quad 2.14960604 \quad-1.99990880 \quad 0.97019670$

H $1.88517143-1.16453198 \quad-0.55891547$

$\mathrm{H}-0.21843077-2.58304966 \quad 1.05563552$

$\mathrm{H}-2.30574440 \quad 0.26277434 \quad 1.28083652$

$\mathrm{H}-1.28771637 \quad 0.81762594-0.04100586$

$\mathrm{H}-1.167606361 .581466591 .55128321$

$\mathrm{H}-1.46640442-1.279203463 .02555931$

$\mathrm{H}-0.47072007 \quad 0.06651929 \quad 3.58903038$

$\mathrm{H} \quad 0.26532933-1.507489653 .27229006$

$\begin{array}{lllll}0 & -0.26554191 & 2.58973056 & -2.22137142\end{array}$

$\begin{array}{lllll}0 & 0.30827161 & 2.47934057 & -1.10287399\end{array}$

o - $0.06553529-1.53447622-1.81766204$

Vibrational frequencies (in cm-1)

$\begin{array}{llllllllllllllll}33.6 & 52.2 & 63.0 & 98.5 & 112.6 & 168.8 & 183.0 & 194.6 & 207.6 & 215.1 & 271.0 & 292.9 & 300.6 & 319.2\end{array}$ $343.4 \quad 376.6 \quad 421.6 \quad 436.7 \quad 549.2 \quad 561.2 \quad 580.1 \quad 612.1 \quad 695.0 \quad 791.8 \quad 817.3 \quad 902.9 \quad 919.2 \quad 936.4$ $989.51002 .8 \quad 1007.7 \quad 1015.8 \quad 1027.4 \quad 1039.8 \quad 1051.7 \quad 1071.5 \quad 1092.2 \quad 1104.31119 .61146 .3$ $1170.5 \quad 1189.4 \quad 1209.5 \quad 1229.7 \quad 1238.8 \quad 1249.3 \quad 1264.9 \quad 1271.3 \quad 1279.9 \quad 1287.4 \quad 1289.8 \quad 1292.0$

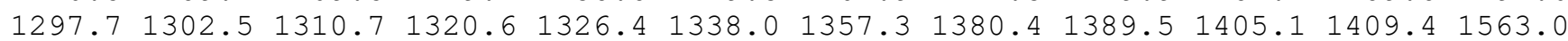
$1843.72645 .52656 .92664 .72673 .8 \quad 2675.8 \quad 2676.2 \quad 2678.42680 .4 \quad 2687.82715 .62724 .5$ $2729.72760 .12778 .8 \quad 2779.5 \quad 2782.6$

name $=$ MIN106

energy (in $\mathrm{kcal} / \mathrm{mol})=-29.883644$

$\mathrm{ZPE}(\mathrm{in} \mathrm{kcal} / \mathrm{mol})=143.41$

$\mathrm{G}($ in $\mathrm{kcal} / \mathrm{mol})=116.458$

Geometry

C $-2.34731976-1.54976669-0.53405961$

C $-0.88364401-1.50027501-0.52245489$

C $1.334208692 .08643400 \quad-0.74787607$

C $1.749198461 .80170526 \quad 0.67194215$

C $1.24653805 \quad 0.49929554 \quad 1.25765216$

C $1.41437705-0.796679370 .41955317$

C $-0.07587509-1.163014850 .66083710$

C $-0.30691523 \quad 0.24458428 \quad 1.34168788$

C $-1.17549598 \quad 1.20767483 \quad 0.55937117$

C $-0.80630830 \quad 0.12327888 \quad 2.77262414$

$\mathrm{H}-2.81200314-1.339490920 .43607965$

$\mathrm{H}-2.70092539-2.54150265-0.88605095$

$\mathrm{H}-2.75766986-0.83853429-1.27968165$

$\mathrm{H} 1.189073251 .23868510-1.42767649$

H $1.429842162 .64752808 \quad 1.32342695$

H 2.86238650 $1.82695836 \quad 0.71298930$

$\mathrm{H} \quad 1.70592695 \quad 0.37678628 \quad 2.26265971$

H $2.13113998-1.51380438 \quad 0.82952053$

$\mathrm{H} \quad 1.68752177-0.62969694-0.62600057$

$\mathrm{H}-0.18130583-1.983979121 .40650296$

$\mathrm{H}-1.008728892 .24566940 \quad 0.87959829$

$\mathrm{H}-2.24366340 \quad 0.99957098 \quad 0.70369829$

$\mathrm{H}-0.99202838 \quad 1.16555712 \quad-0.52132257$

$\mathrm{H}-0.810196301 .09975286 \quad 3.27395832$

$\mathrm{H}-0.18627700-0.54800786 \quad 3.37760338$

$\mathrm{H}-1.83226068-0.263308332 .80855059$

O $-0.82361611-2.18067626-2.60910412$

O $1.188584993 .21986052-1.13345816$

o $-0.18224568-1.82785572-1.57478046$

Vibrational frequencies (in $\mathrm{cm}-1$ )

$\begin{array}{lllllllllllllll}30.1 & 37.3 & 47.5 & 99.0 & 109.6 & 142.3 & 167.0 & 181.5 & 217.7 & 220.9 & 256.1 & 263.5 & 274.6 & 289.6\end{array}$

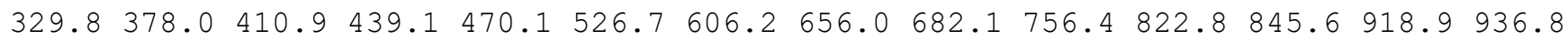
$993.9999 .8 \quad 1007.0 \quad 1015.4 \quad 1027.8 \quad 1038.9 \quad 1051.9 \quad 1069.5 \quad 1074.11112 .8 \quad 1128.4 \quad 1133.5$ $1162.51193 .2 \quad 1204.6 \quad 1228.4 \quad 1239.9 \quad 1258.5 \quad 1262.8 \quad 1265.0 \quad 1285.7 \quad 1286.7 \quad 1290.7 \quad 1294.7$

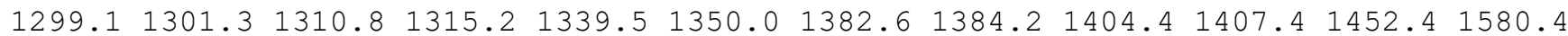

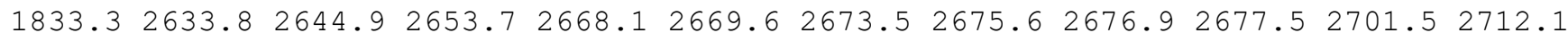
$2736.22761 .6 \quad 2768.5 \quad 2778.9 \quad 2782.2$ 
name $=$ MIN107

energy (in $\mathrm{kcal} / \mathrm{mol})=-30.198524$

$\mathrm{ZPE}(\mathrm{in} \mathrm{kcal} / \mathrm{mol})=143.744$

$\mathrm{G}($ in $\mathrm{kcal} / \mathrm{mol})=117.625$

Geometry

C $-3.19156779-1.05460001-0.47448667$

C $-1.73463731-1.02497211-0.18822036$

C $2.503051310 .00168385-0.31149356$

$\begin{array}{llll}\text { C } 2.15022614 & 0.91323117 & 0.83982687\end{array}$

C $0.71524003 \quad 0.85638325 \quad 1.31784506$

C $0.07691142-0.529430891 .60110410$

C $-1.20469831-0.11517781 \quad 0.82586853$

C $-0.45238018 \quad 1.18562399 \quad 0.31361687$

C $-0.09312938 \quad 1.17057213-1.15618033$

C $-1.19333129 \quad 2.46699616 \quad 0.66411634$

$\mathrm{H}-3.76737785-1.35022608 \quad 0.41893868$

$\mathrm{H}-3.46957661-1.75384291-1.27852160$

$\mathrm{H}-3.55557365-0.05718481-0.77463845$

$\mathrm{H} 2.05088592-0.99759104-0.34895540$

H 2.40488349 1.961514990 .56519383

H $2.83177398 \quad 0.66247844 \quad 1.68386620$

$\mathrm{H} \quad 0.63248335 \quad 1.50981099 \quad 2.21451099$

$\mathrm{H}-0.08649979-0.75624890 \quad 2.65591449$

$\mathrm{H} 0.61615424-1.37853000 \quad 1.15948440$

$\mathrm{H}-2.01206403 \quad 0.16950148 \quad 1.53663972$

$\mathrm{H}-0.964215451 .35812816-1.79283994$

$\mathrm{H} \quad 0.33590856 \quad 0.20696933-1.47497634$

$\mathrm{H} 0.656100331 .93917496-1.39173287$

$\mathrm{H}-0.57119792 \quad 3.34686959 \quad 0.45235777$

$\mathrm{H}-1.47228200 \quad 2.51445098 \quad 1.72223472$

$\mathrm{H}-2.11258697 \quad 2.572325310 .07685679$

$\begin{array}{lllll}0 & 0.15376198 & -2.00277237 & -0.83777142\end{array}$

$\begin{array}{lllll}0 & 3.29444290 & 0.36555398 & -1.14754391\end{array}$

o - $1.09996003-1.88453155-0.93028463$

Vibrational frequencies (in $\mathrm{cm}-1$ )

$\begin{array}{lllllllllllllll}37.0 & 61.8 & 62.6 & 110.0 & 120.8 & 154.7 & 164.1 & 193.0 & 215.1 & 227.8 & 267.0 & 282.5 & 299.8 & 310.3\end{array}$

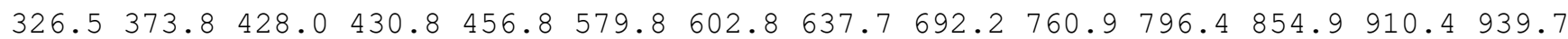
$991.4 \quad 1000.7 \quad 1008.4 \quad 1015.4 \quad 1017.3 \quad 1036.0 \quad 1047.1 \quad 1068.8 \quad 1086.6 \quad 1112.6 \quad 1134.4 \quad 1150.2$ $\begin{array}{lllllllllllll}1170.1 & 1194.1 & 1204.7 & 1239.6 & 1255.2 & 1259.9 & 1265.5 & 1268.9 & 1280.6 & 1281.9 & 1285.8 & 1294.3\end{array}$

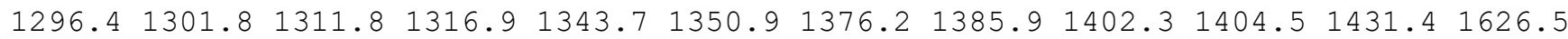
$1825.52640 .62651 .5 \quad 2655.7 \quad 2657.7 \quad 2661.0 \quad 2667.2 \quad 2674.32677 .0 \quad 2679.8 \quad 2706.02714 .1$ $\begin{array}{llllll}2737.0 & 2757.1 & 2770.9 & 2777.4 & 2782.4\end{array}$

name $=$ MIN108

energy (in $\mathrm{kcal} / \mathrm{mol})=-29.63789$

$\mathrm{ZPE}(\mathrm{in} \mathrm{kcal} / \mathrm{mol})=143.366$

$\mathrm{G}($ in $\mathrm{kcal} / \mathrm{mol})=116.122$

Geometry

C $-2.88014283-1.06675890-0.59070730$

C $-1.41468464-1.07150804-0.61420805$

C $2.208649251 .34321885-0.54619419$

C $2.12195528 \quad 1.07200003 \quad 0.93222591$

C $1.15629714-0.006734261 .37291441$

C $0.94363932-1.244365100 .45829326$

C $-0.59584185-1.10611373 \quad 0.60705975$

C $-0.39185243 \quad 0.28082960 \quad 1.34256691$

C $-0.83010997 \quad 1.48580944 \quad 0.53501470$

C $-1.01910599 \quad 0.311881712 .72572909$

$\mathrm{H}-3.28097589-0.32452470-1.31354273$

$\mathrm{H}-3.31128039-0.85820104 \quad 0.39533256$

$\mathrm{H}-3.28311283-2.03582583-0.94522560$

H $1.440339290 .92409624-1.20593212$ 
H $1.87527163 \quad 2.02824833 \quad 1.44883099$

H $3.14619918 \quad 0.82675342 \quad 1.29985990$

H $1.45347501-0.33139075 \quad 2.39500976$

$\mathrm{H} 1.35274746-2.17804516 \quad 0.85626763$

$\mathrm{H} 1.32823818-1.13839038-0.55890193$

$\mathrm{H}-1.00584182-1.87213796 \quad 1.30121125$

$\mathrm{H}-0.473653341 .45596950-0.50160495$

$\mathrm{H}-0.46715437 \quad 2.42068924 \quad 0.98033877$

$\mathrm{H}-1.925568351 .55697016 \quad 0.48697033$

$\mathrm{H}-0.73095766-0.55150695 \quad 3.33623116$

$\mathrm{H}-2.11465182 \quad 0.31713077 \quad 2.66958033$

$\mathrm{H}-0.71892756 \quad 1.21241990 \quad 3.27682201$

o $-1.41714122-1.03770476-2.80827467$

$03.105916392 .01513254-0.99177853$

o $-0.74480366-1.06149540-1.73383900$

Vibrational frequencies (in cm-1)

$\begin{array}{llllllllllllllllll}28.5 & 30.6 & 42.3 & 93.0 & 119.6 & 134.9 & 152.6 & 180.1 & 218.3 & 219.8 & 229.4 & 267.3 & 275.0 & 289.0\end{array}$ $332.1372 .4 \quad 405.0 \quad 450.3 \quad 453.9 \quad 544.3 \quad 611.8 \quad 656.5 \quad 684.6 \quad 757.5 \quad 817.3 \quad 827.3 \quad 913.2 \quad 940.8$ $993.7 \quad 1002.4 \quad 1009.9 \quad 1016.0 \quad 1030.9 \quad 1043.4 \quad 1052.4 \quad 1072.0 \quad 1073.01111 .11127 .7 \quad 1135.0$ $\begin{array}{lllllllllllllll}1162.6 & 1201.9 & 1206.9 & 1230.9 & 1239.1 & 1252.1 & 1261.7 & 1268.6 & 1282.6 & 1286.3 & 1288.9 & 1294.6\end{array}$ $1298.51299 .2 \quad 1304.2 \quad 1319.0 \quad 1345.1 \quad 1351.8 \quad 1383.9 \quad 1385.4 \quad 1403.3 \quad 1407.6 \quad 1451.6 \quad 1593.8$

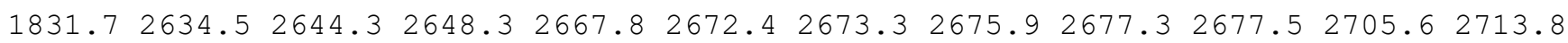
$\begin{array}{llllll}2735.3 & 2762.5 & 2768.9 & 2779.8 & 2782.8\end{array}$

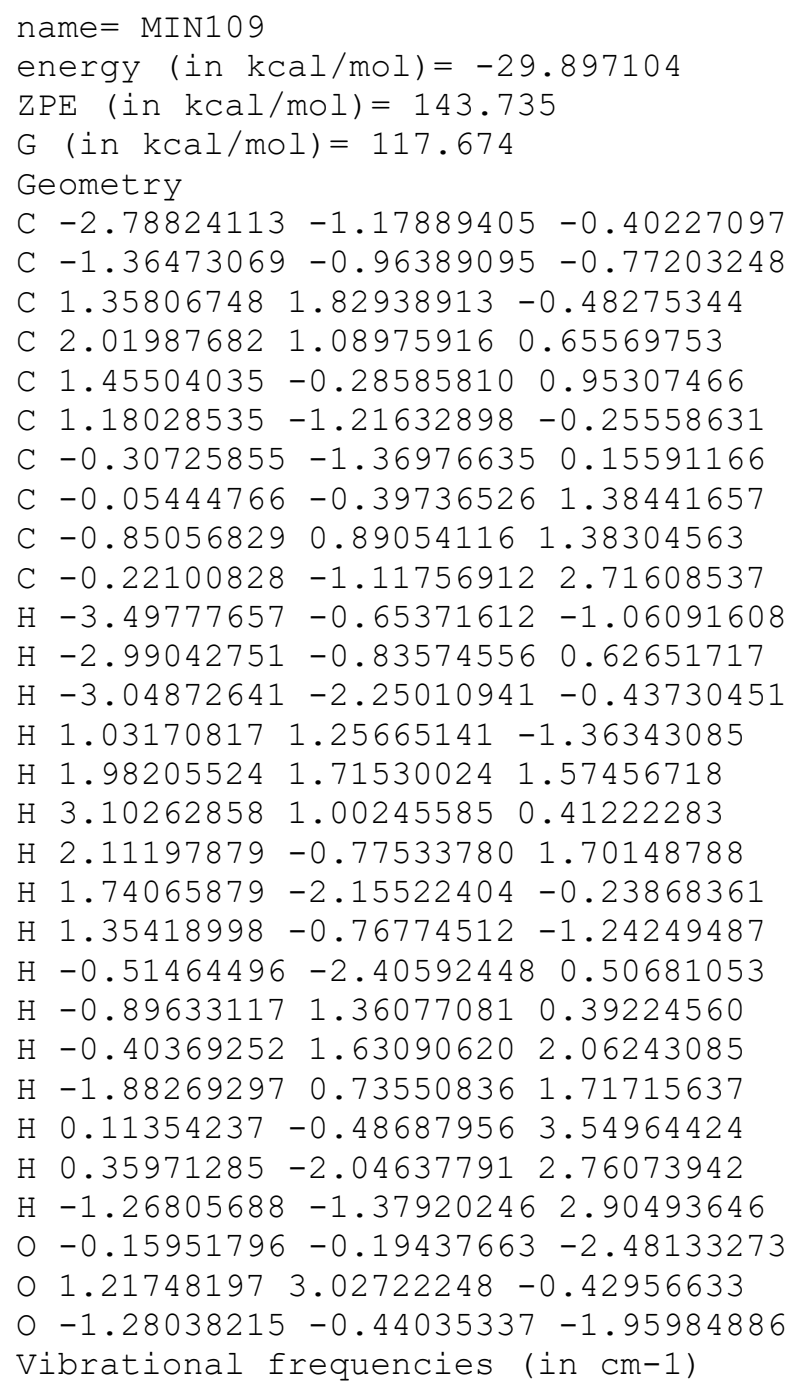


$1827.62623 .0 \quad 2654.0 \quad 2656.7 \quad 2658.5 \quad 2659.92666 .4 \quad 2672.82675 .3 \quad 2678.92705 .6 \quad 2713.3$ $2738.22755 .6 \quad 2770.2 \quad 2779.0 \quad 2782.4$

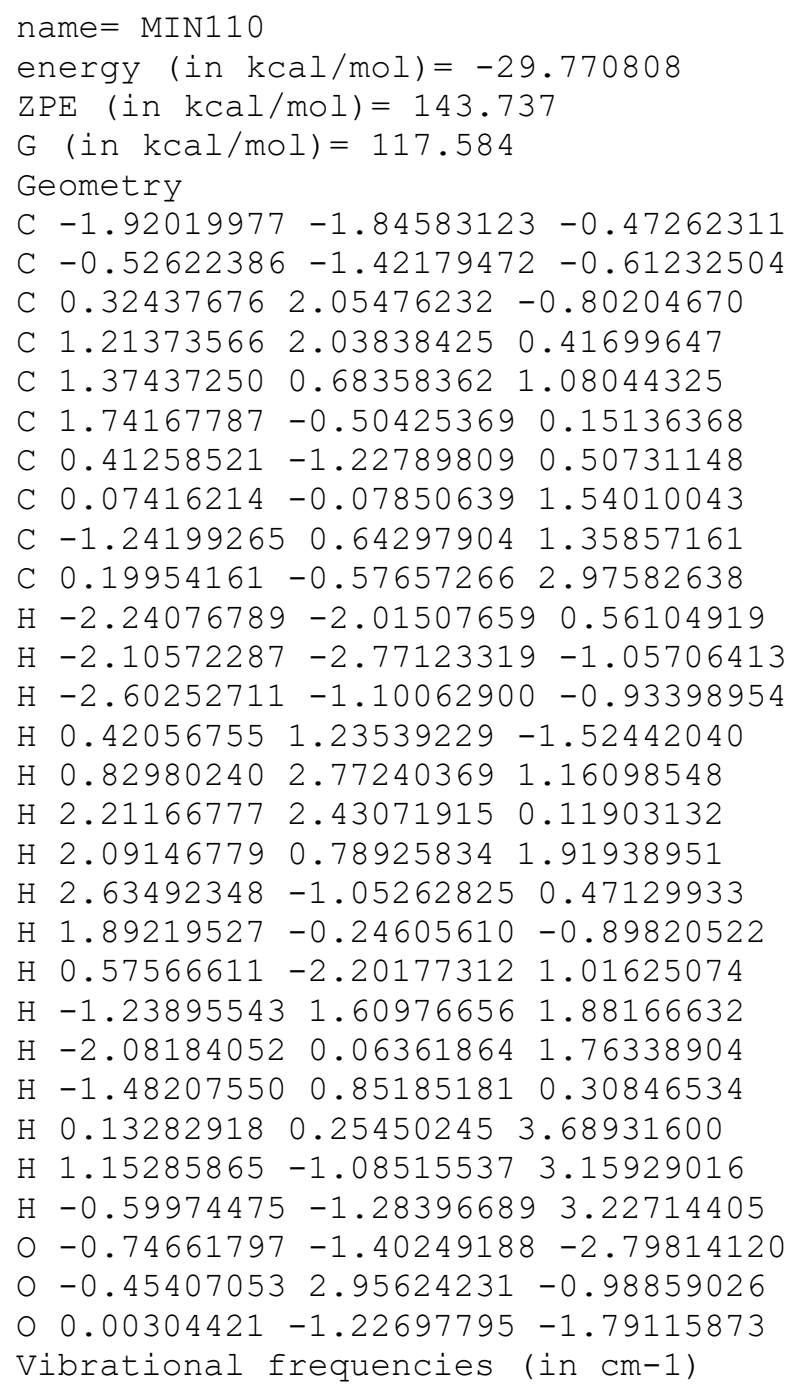


$\mathrm{H}-3.49999877 \quad 0.32286863-0.67832943$

$\mathrm{H} \quad 0.92625101 \quad 1.65641869-0.82040292$

H $2.069927121 .37633798 \quad 1.51984557$

$\mathrm{H} \quad 3.30264175 \quad 0.37367322 \quad 0.75489321$

H $1.96098234-1.27488211 \quad 1.63153193$

$\begin{array}{lllll}\mathrm{H} & 1.22355607 & -2.40766132 & -0.35109897\end{array}$

$\mathrm{H} 1.07266398-0.91525257-1.25457943$

$\mathrm{H}-1.02318992-2.23775757 \quad 0.51020210$

$\mathrm{H}-0.468518081 .564106260 .67743195$

$\mathrm{H}-0.140210291 .520179722 .41269095$

$\mathrm{H}-1.70052405 \quad 0.97853583 \quad 1.79060918$

$\mathrm{H} \quad 0.02431240-2.22766178 \quad 2.73469105$

$\mathrm{H}-1.39659849-1.223535693 .03284461$

$\mathrm{H} \quad 0.18741870 \quad-0.70945156 \quad 3.61933493$

$\begin{array}{lllll}0 & -1.76091803 & 0.97847795 & -2.22695813\end{array}$

O $2.818555721 .43602767-1.44316509$

O $-0.99247448 \quad 0.28042888-1.50085499$

Vibrational frequencies (in $\mathrm{cm}-1$ )

$\begin{array}{llllllllllllll}34.4 & 35.2 & 64.6 & 91.2 & 94.5 & 117.2 & 166.9 & 189.2 & 212.6 & 225.3 & 251.2 & 280.3 & 288.5 & 304.9\end{array}$ $333.4 \quad 385.8 \quad 418.5 \quad 446.2 \quad 470.2 \quad 551.8 \quad 558.6 \quad 673.3 \quad 686.0 \quad 801.4 \quad 827.0 \quad 872.8 \quad 909.2 \quad 949.2$ $992.6 \quad 999.7 \quad 1008.91017 .1 \quad 1029.2 \quad 1046.2 \quad 1051.8 \quad 1068.1 \quad 1076.2 \quad 1108.6 \quad 1124.81129 .2$

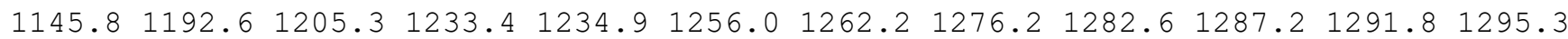
$1296.61298 .7 \quad 1305.6 \quad 1313.8 \quad 1344.0 \quad 1356.0 \quad 1379.6 \quad 1385.3 \quad 1403.5 \quad 1405.7 \quad 1443.4 \quad 1596.5$ $1838.72634 .4 \quad 2639.8 \quad 2658.5 \quad 2666.8 \quad 2671.6 \quad 2672.12674 .22675 .92677 .3 \quad 2711.02718 .7$ $2738.5 \quad 2760.8 \quad 2768.5 \quad 2779.12782 .6$

name $=$ MIN112

energy (in $\mathrm{kcal} / \mathrm{mol})=-28.832496$

$\operatorname{ZPE}(\mathrm{in} \mathrm{kcal} / \mathrm{mol})=143.633$

$\mathrm{G}($ in $\mathrm{kcal} / \mathrm{mol})=116.916$

Geometry

C $-2.23450698-1.68381055-0.36910173$

C $-0.88286821-1.20552875-0.67223800$

C $0.58539701 \quad 2.21054069-0.51290728$

C $1.33600697 \quad 1.917085390 .76521130$

C $1.431779550 .43218677 \quad 1.07371778$

C $1.54022240 \quad-0.51976689-0.14846359$

C $0.24210103-1.25797966 \quad 0.27668962$

C $0.13950491-0.32994900 \quad 1.55285460$

C $-1.126178190 .48831610 \quad 1.69396711$

C $0.39339941-1.11685786 \quad 2.83329879$

$\mathrm{H}-2.97985030-0.87884708-0.53295451$

$\mathrm{H}-2.35834914-2.07055761 \quad 0.64799033$

$\mathrm{H}-2.53514342-2.47619745-1.08568967$

$\mathrm{H}-0.50480660 \quad 2.09196829-0.50533494$

$\mathrm{H} \quad 0.86605809 \quad 2.45452888 \quad 1.61284267$

H 2.36454410 2.33696941 0.68155602

$\mathrm{H} \quad 2.26520010 \quad 0.27952678 \quad 1.78920911$

$\mathrm{H} 2.43295883-1.15447598-0.13777870$

$\mathrm{H} 1.52323535-0.02601523-1.12476084$

H $0.42955602 \quad-2.31753357 \quad 0.55592379$

$\mathrm{H}-0.99785824 \quad 1.29269576 \quad 2.42974380$

$\mathrm{H}-1.96532286-0.12940825 \quad 2.04153383$

$\mathrm{H}-1.44428593 \quad 0.94746910 \quad 0.75074131$

$\mathrm{H} \quad 0.48965298-0.44822340 \quad 3.69755076$

$\mathrm{H} 1.31297250-1.711636962 .78087569$

$\mathrm{H}-0.42843043-1.80997437 \quad 3.04862711$

$\begin{array}{lllll}0 & -1.52087330 & -0.69336090 & -2.70562772\end{array}$

O $1.163675392 .57013277-1.50667708$

o $-0.58645486-0.73250335-1.85394095$

Vibrational frequencies (in $\mathrm{cm}-1$ )

$\begin{array}{lllllllllllllllll}37.7 & 38.6 & 47.3 & 82.4 & 118.0 & 148.3 & 165.8 & 199.9 & 221.6 & 227.0 & 243.0 & 266.7 & 280.3 & 296.4\end{array}$

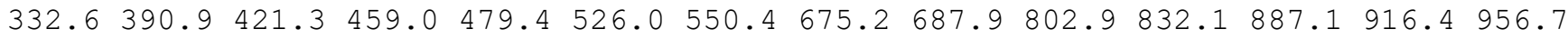
$984.8 \quad 990.0 \quad 1008.8 \quad 1017.2 \quad 1029.5 \quad 1041.1 \quad 1057.9 \quad 1068.8 \quad 1077.2 \quad 1104.9 \quad 1122.7 \quad 1125.1$ 
$\begin{array}{lllllllllllllll}1146.3 & 1191.7 & 1204.4 & 1229.4 & 1230.8 & 1247.2 & 1264.2 & 1280.4 & 1285.5 & 1291.1 & 1293.3 & 1296.3\end{array}$

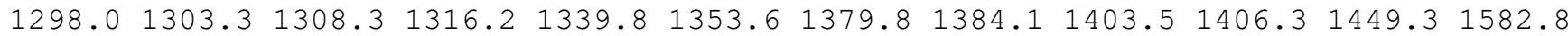
$1840.82634 .62638 .0 \quad 2661.6 \quad 2667.8 \quad 2672.1 \quad 2672.92674 .92676 .3 \quad 2678.2 \quad 2706.3 \quad 2714.5$ $2740.0 \quad 2759.2 \quad 2769.8 \quad 2778.2 \quad 2782.4$

\section{name $=$ MIN113}

energy (in $\mathrm{kcal} / \mathrm{mol})=-28.494703$

$\mathrm{ZPE}($ in $\mathrm{kcal} / \mathrm{mol})=143.543$

$\mathrm{G}($ in $\mathrm{kcal} / \mathrm{mol})=116.449$

Geometry

C $-2.43075008-1.44736491-0.74007964$

C $-0.95096370-1.50692733-0.70070321$

C $1.12423447 \quad 2.21563024 \quad-0.65478589$

C $1.493682891 .87601888 \quad 0.76480914$

C $1.07045847 \quad 0.49736698 \quad 1.23110367$

C $1.29790518 \quad-0.69758961 \quad 0.27249987$

C $-0.16620799-1.17543030 \quad 0.48891646$

C $-0.46701884 \quad 0.15157388 \quad 1.29198853$

C $-1.37641370 \quad 1.15155875 \quad 0.60623582$

C $-0.95515643-0.12194572 \quad 2.70663708$

$\mathrm{H}-2.87670398-2.26498547-1.32906322$

$\mathrm{H}-2.78867291-0.50266853-1.18500129$

$\mathrm{H}-2.86420253-1.50847493 \quad 0.27164197$

H $1.13051357 \quad 1.41020731-1.40013218$

$\mathrm{H} 2.59883617 \quad 1.97960704 \quad 0.86267430$

$\mathrm{H} 1.07710876 \quad 2.64627590 \quad 1.45191417$

$\mathrm{H} 1.54224125 \quad 0.308152462 .21881976$

H $2.05859080-1.417034050 .58713906$

H $1.54019411-0.43081349-0.76325397$

$\mathrm{H}-0.19218179-2.05987398 \quad 1.17121086$

$\mathrm{H}-2.43429479 \quad 0.89016735 \quad 0.72396477$

$\mathrm{H}-1.17553216 \quad 1.23379465 \quad-0.46845175$

$\mathrm{H}-1.25217334 \quad 2.15668520 \quad 1.03471552$

$\mathrm{H}-1.00171074 \quad 0.80402360 \quad 3.29385222$

$\mathrm{H}-0.29859606-0.814181713 .24666569$

$\mathrm{H}-1.95985583-0.559646492 .70793323$

O $0.78211396-2.16935398-1.91011578$

$00.849899793 .34706094-0.97258380$

O $-0.46227709-1.99140398-1.80924862$

Vibrational frequencies (in $\mathrm{cm}-1$ )

$\begin{array}{lllllllllllllll}17.3 & 35.9 & 48.2 & 99.8 & 107.6 & 162.2 & 181.5 & 202.3 & 216.4 & 233.6 & 256.7 & 276.1 & 284.1 & 299.4\end{array}$ $\begin{array}{llllllllllllllll}314.6 & 391.1 & 406.9 & 435.1 & 465.4 & 553.8 & 609.2 & 655.8 & 673.1 & 757.4 & 795.4 & 849.2 & 928.7 & 938.3\end{array}$ $991.61000 .4 \quad 1006.4 \quad 1010.1 \quad 1016.0 \quad 1035.5 \quad 1053.1 \quad 1065.0 \quad 1084.8 \quad 1120.2 \quad 1130.11150 .0$ $1158.81190 .4 \quad 1200.4 \quad 1238.3 \quad 1257.0 \quad 1260.3 \quad 1265.7 \quad 1268.8 \quad 1287.5 \quad 1289.4 \quad 1292.2 \quad 1296.6$ $1301.01304 .21314 .11320 .6 \quad 1332.7 \quad 1341.3 \quad 1375.01383 .31404 .2 \quad 1404.61425 .11595 .0$ $1832.02641 .0 \quad 2653.0 \quad 2654.2 \quad 2661.6 \quad 2668.0 \quad 2668.6 \quad 2673.72676 .0 \quad 2678.72694 .92712 .6$ $2736.7 \quad 2761.4 \quad 2770.7 \quad 2779.3 \quad 2782.4$

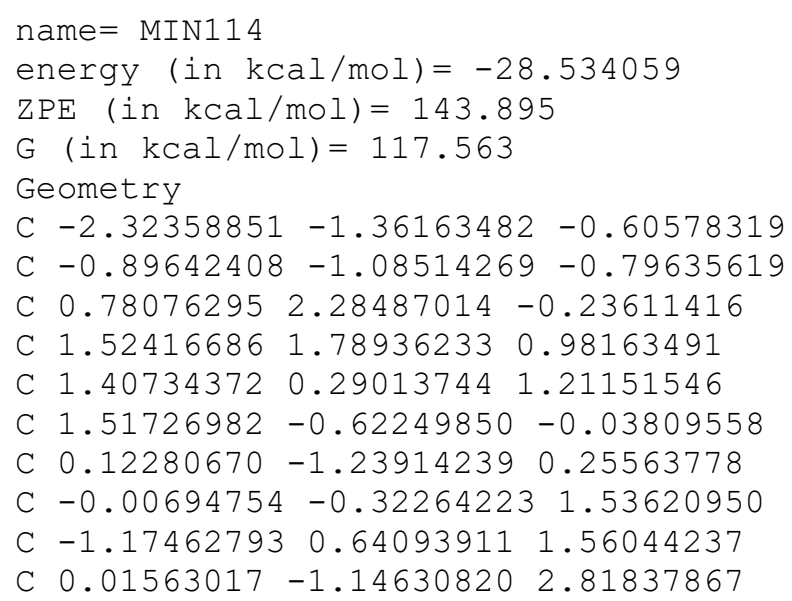


$\mathrm{H}-2.92899318-0.46239285-0.83772323$

$\mathrm{H}-2.58438636-1.706461490 .40030513$

$\mathrm{H}-2.67306507-2.11756172-1.33956891$

$\mathrm{H}-0.17174780 \quad 2.80406679-0.07434929$

$\mathrm{H} 1.18855535 \quad 2.32992468 \quad 1.88837229$

H 2.602147312 .044622010 .85797944

H $2.14497871 \quad 0.00567774 \quad 1.99005307$

H 2.34093095 -1.34169039 0.00390914

$\mathrm{H} 1.61663644-0.08568762-0.98918310$

H $0.18770058-2.314611330 .53282996$

$\mathrm{H}-1.35884894 \quad 1.10488923 \quad 0.58440771$

$\mathrm{H}-1.011163601 .44299399 \quad 2.29105503$

$\mathrm{H}-2.10640518 \quad 0.13463984 \quad 1.84670217$

$\mathrm{H} \quad 0.09454806-0.50208720 \quad 3.70242923$

H $0.86101048-1.843831792 .84953845$

$\mathrm{H}-0.89868872-1.741335412 .92926513$

o $-1.26686352-0.64887735-2.91028920$

O $1.22255490 \quad 2.14333354-1.34821543$

o $-0.43130793-0.73354112-1.96648585$

Vibrational frequencies (in $\mathrm{cm}-1$ )

$\begin{array}{llllllllllllllll}39.2 & 40.1 & 53.0 & 112.3 & 126.4 & 153.1 & 174.5 & 199.6 & 222.8 & 230.2 & 263.8 & 274.7 & 288.4 & 300.8\end{array}$

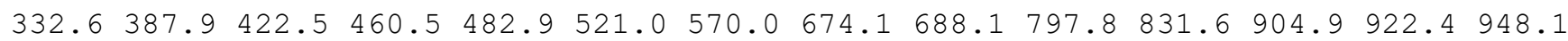
$987.0 \quad 994.8 \quad 1008.3 \quad 1018.0 \quad 1030.7 \quad 1040.1 \quad 1055.8 \quad 1066.3 \quad 1079.01103 .5 \quad 1124.6 \quad 1127.3$

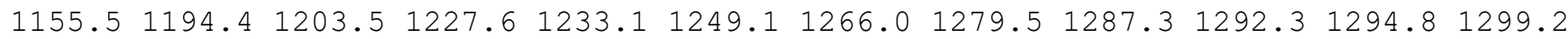
$1299.91306 .6 \quad 1314.5 \quad 1317.2 \quad 1338.2 \quad 1352.6 \quad 1381.7 \quad 1384.8 \quad 1404.6 \quad 1406.8 \quad 1450.6 \quad 1582.3$ $1842.0 \quad 2636.0 \quad 2645.0 \quad 2665.5 \quad 2667.6 \quad 2668.7 \quad 2674.4 \quad 2675.0 \quad 2677.3 \quad 2678.7 \quad 2704.2 \quad 2712.7$ $2739.42757 .2 \quad 2770.12779 .3 \quad 2782.5$

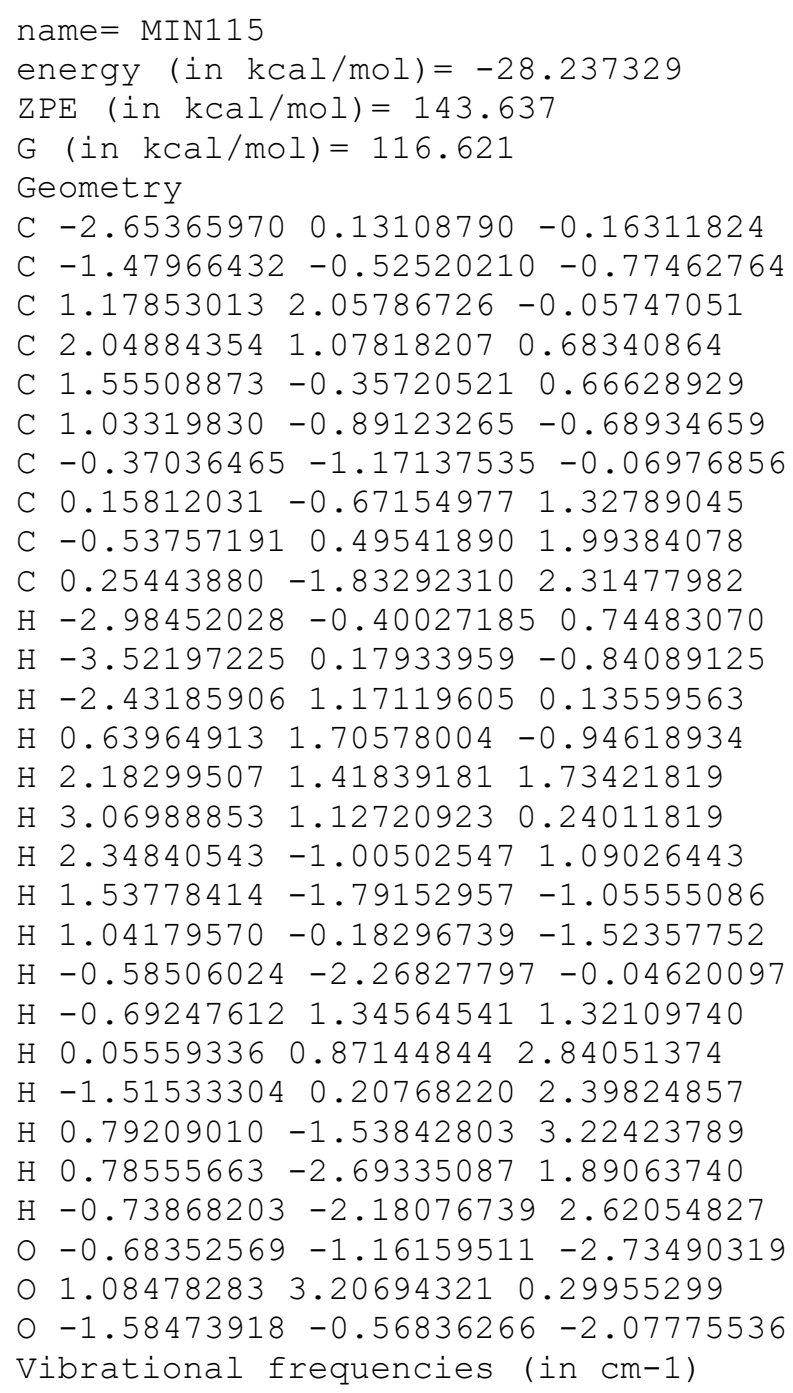


$\begin{array}{lllllllllllllllll}16.9 & 28.6 & 46.6 & 101.4 & 144.2 & 170.9 & 187.5 & 200.1 & 228.9 & 241.0 & 250.1 & 262.9 & 294.8 & 312.0\end{array}$ $327.7 \quad 386.0 \quad 425.2 \quad 438.9 \quad 470.4 \quad 548.2 \quad 600.1 \quad 664.1681 .5 \quad 760.3 \quad 798.5 \quad 851.0 \quad 925.5 \quad 940.6$ $986.21001 .91009 .41011 .0 \quad 1016.6 \quad 1030.9 \quad 1051.6 \quad 1057.7 \quad 1084.91113 .01121 .81141 .1$ $1155.8 \quad 1188.1 \quad 1200.9 \quad 1236.9 \quad 1239.9 \quad 1260.2 \quad 1263.1 \quad 1269.2 \quad 1288.5 \quad 1290.01294 .21298 .0$ $1304.31305 .91316 .91326 .7 \quad 1336.3 \quad 1344.6 \quad 1378.7 \quad 1382.01406 .0 \quad 1407.4 \quad 1437.6 \quad 1570.8$ $\begin{array}{llllllllllllll}1832.7 & 2642.3 & 2648.6 & 2654.1 & 2660.1 & 2668.4 & 2670.7 & 2674.0 & 2675.7 & 2678.9 & 2691.8 & 2715.4\end{array}$ $2737.3 \quad 2762.8 \quad 2768.7 \quad 2779.9 \quad 2782.3$

\section{name $=$ MIN116}

energy (in $\mathrm{kcal} / \mathrm{mol})=-28.448336$

$\mathrm{ZPE}($ in $\mathrm{kcal} / \mathrm{mol})=143.929$

$\mathrm{G}($ in $\mathrm{kcal} / \mathrm{mol})=117.534$

Geometry

C $-2.38985953-1.31540833-0.28142297$

C $-1.01044135-1.02449454-0.68564155$

C $0.72441335 \quad 2.29918140 \quad-0.21379056$

C $1.71319094 \quad 1.73128965 \quad 0.77608934$

C $1.616652290 .22517582 \quad 0.96757600$

C $1.50570119-0.63756980-0.31682594$

C $0.16443139-1.24630843 \quad 0.17458890$

C $0.26640148-0.38846390 \quad 1.49749049$

C $-0.86602162 \quad 0.58259828 \quad 1.75718901$

C $0.48866914-1.27128761 \quad 2.71985522$

$\mathrm{H}-3.02045304-0.40792302-0.37167589$

$\mathrm{H}-2.48743986-1.70728823 \quad 0.73624883$

$\mathrm{H}-2.85476752-2.03723052-0.98437428$

$\mathrm{H}-0.061094892 .95773548 \quad 0.17768656$

$\mathrm{H} \quad 1.61169061 \quad 2.23362120 \quad 1.75826943$

$\mathrm{H} 2.739121651 .97572576 \quad 0.41472056$

$\mathrm{H} 2.46909056-0.094022051 .60170473$

$\mathrm{H} 2.31005273-1.36961404-0.43529117$

$\mathrm{H} \quad 1.46013610-0.06269364-1.25003346$

H $0.24846077-2.33438357 \quad 0.38904650$

$\mathrm{H}-1.20720518 \quad 1.08574569 \quad 0.84438592$

$\mathrm{H}-0.57034138 \quad 1.353278612 .47951225$

$\mathrm{H}-1.74327258 \quad 0.07126842 \quad 2.17624812$

$\mathrm{H} \quad 0.72337705-0.67042303 \quad 3.60662758$

$\mathrm{H} 1.31611227-1.976479412 .57761011$

$\mathrm{H}-0.40406485-1.863540962 .95377885$

$0-1.72022102-0.45675508-2.67686225$

○ $0.801957122 .07348403-1.39480882$

o $-0.74168897-0.59439030-1.89060743$

Vibrational frequencies (in cm-1)

$\begin{array}{lllllllllllllll}32.1 & 39.7 & 52.5 & 116.8 & 128.1 & 154.1 & 177.0 & 201.8 & 220.9 & 228.2 & 264.1 & 276.9 & 292.9 & 301.1\end{array}$ $\begin{array}{llllllllllllllll}332.7 & 387.9 & 424.6 & 457.3 & 475.8 & 525.6 & 584.6 & 674.7 & 689.7 & 798.0 & 832.3 & 905.7 & 922.3 & 932.4\end{array}$ $990.0 \quad 998.4 \quad 1008.1 \quad 1018.1 \quad 1031.6 \quad 1041.6 \quad 1053.9 \quad 1065.6 \quad 1079.5 \quad 1103.2 \quad 1125.2 \quad 1126.9$

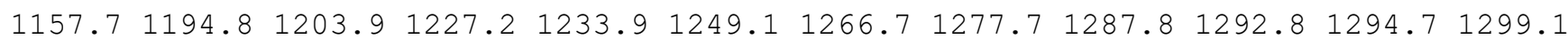
$1301.61306 .41315 .3 \quad 1317.2 \quad 1337.1 \quad 1354.4 \quad 1382.1 \quad 1385.1 \quad 1405.0 \quad 1406.7 \quad 1450.61583 .8$ $1843.02636 .5 \quad 2645.72665 .12666 .92668 .0 \quad 2674.92675 .12677 .7 \quad 2679.02705 .02712 .9$ $2738.92756 .6 \quad 2770.3 \quad 2779.2 \quad 2782.4$

\section{name $=$ MIN117}

energy (in $\mathrm{kcal} / \mathrm{mol})=-26.775304$

$\mathrm{ZPE}($ in $\mathrm{kcal} / \mathrm{mol})=142.601$

$\mathrm{G}($ in $\mathrm{kcal} / \mathrm{mol})=116.81$

Geometry

C $-2.38993897-0.34248371-0.38982929$

C $-0.91103586-0.09025665-0.72158793$

C $-0.69647511 \quad 1.41194204 \quad-0.86748068$

$\begin{array}{llll}\text { C } 2.07382024 & 0.71585591 & 0.42402242\end{array}$

C $1.71391933-0.22750586 \quad 1.54632798$

C $1.53643858-0.65305907 \quad 0.07998999$

C $0.01157066-0.78881379 \quad 0.26722624$ 
C $0.18249568-0.31605575 \quad 1.78662551$

C $-0.46119691 \quad 0.97661809 \quad 2.24197828$

C $-0.16896855-1.444105652 .74858981$

$\mathrm{H}-3.05157577 \quad 0.19756985-1.08028446$

$\mathrm{H}-2.62928016-0.03702422 \quad 0.63352978$

$\mathrm{H}-2.63783759-1.40871758-0.48279452$

$\mathrm{H}-1.09683608 \quad 2.03377176-0.05542964$

H $3.12671310 \quad 0.91709501 \quad 0.23017531$

$\mathrm{H} 1.49887232 \quad 1.61468070 \quad 0.22782194$

H $2.40864386-0.55782338 \quad 2.28908079$

$\mathrm{H} 2.08513860-1.36379391-0.50379058$

H $0.85627565-1.43334076-2.43689157$

$\mathrm{H}-0.26838448-1.86666130 \quad 0.25756975$

$\mathrm{H}-0.06217365 \quad 1.85142592 \quad 1.71473954$

$\mathrm{H}-0.25908433 \quad 1.15347562 \quad 3.30877932$

$\mathrm{H}-1.54912234 \quad 0.96077509 \quad 2.11988180$

$\mathrm{H} 0.14717023-1.20448580 \quad 3.77160962$

H $0.32360581 \quad-2.38539830 \quad 2.47724471$

$\mathrm{H}-1.24864836-1.628223892 .77170265$

O $0.48586161-0.51977441-2.52134272$

$0-0.16362706 \quad 1.91434880-1.81951810$

o $-0.81040814-0.73696028-1.99102295$

Vibrational frequencies (in $\mathrm{cm}-1$ )

$\begin{array}{lllllllllllllll}38.5 & 57.9 & 100.2 & 124.3 & 130.3 & 172.2 & 180.9 & 199.8 & 204.3 & 238.3 & 248.6 & 257.6 & 271.2 & 294.2\end{array}$ $337.6 \quad 357.2 \quad 377.1406 .9 \quad 482.0 \quad 494.1 \quad 574.2 \quad 646.2 \quad 677.0 \quad 786.8 \quad 797.8 \quad 838.8 \quad 893.2 \quad 932.1$

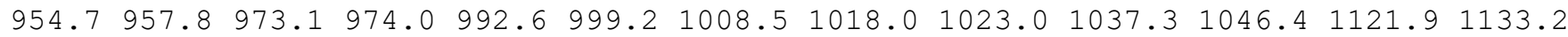
$\begin{array}{lllllllllllllll}1154.7 & 1162.9 & 1187.8 & 1217.6 & 1226.7 & 1245.9 & 1254.4 & 1276.0 & 1283.1 & 1286.8 & 1290.6 & 1296.3\end{array}$ $1297.31303 .31311 .41322 .8 \quad 1364.2 \quad 1376.2 \quad 1379.2 \quad 1392.01405 .91409 .2 \quad 1429.7 \quad 1850.3$ $2639.32664 .92668 .1 \quad 2673.4 \quad 2674.8 \quad 2676.3 \quad 2680.82701 .42708 .2 \quad 2776.5 \quad 2778.52782 .1$ 2784.52786 .12792 .02880 .6
name $=$ MIN118
energy $($ in $\mathrm{kcal} / \mathrm{mol})=-28.187765$
$\operatorname{ZPE}($ in $\mathrm{kcal} / \mathrm{mol})=144.119$
$\mathrm{G}($ in $\mathrm{kcal} / \mathrm{mol})=117.554$
Geometry
C $-2.31412284-1.91471302-0.30120965$
C $-0.87124899-1.61525749-0.58199418$
C $0.45940147 \quad 1.98991720 \quad-0.65733824$
C $1.27210470 \quad 1.76342903 \quad 0.55920275$
C $1.200702370 .36451347 \quad 1.16456900$
C $1.43261117-0.830568390 .20713125$
C $0.05686784-1.43620017 \quad 0.58760243$
C $-0.19907242-0.237154421 .57081841$
C $-1.41384627 \quad 0.62356191 \quad 1.29430661$
C $-0.21057855-0.697958013 .02521326$
$\mathrm{H}-2.62615630-2.82875736-0.83019181$
$\mathrm{H}-2.95312617-1.10673099-0.69197001$
$\mathrm{H}-2.53588968-2.04123978 \quad 0.76198842$
$\mathrm{H}-0.469578962 .53433419-0.63536601$
$\mathrm{H} \quad 0.96968453 \quad 2.50063947 \quad 1.33997254$
H 2.34390347 1.993633820 .34725739
H $1.89917664 \quad 0.33704319 \quad 2.02643458$
H $2.28872969-1.459277880 .46704400$
$\mathrm{H} \quad 1.52930963-0.58012352-0.85592752$
$\mathrm{H} 0.14080725-2.404081431 .11739667$
$\mathrm{H}-2.333615690 .15559100 \quad 1.66816298$
$\mathrm{H}-1.56297086 \quad 0.80664608 \quad 0.22265659$
$\mathrm{H}-1.32808218 \quad 1.59889188 \quad 1.78954621$
$\mathrm{H}-0.21551594 \quad 0.15599137 \quad 3.71266033$
$\mathrm{H} \quad 0.66460982-1.31019407 \quad 3.27216857$
$\mathrm{H}-1.09920722-1.300774893 .24633851$
○ $0.33337142 \quad 1.75898717 \quad-2.82258866$
O $0.96159475 \quad 1.52755821-1.76337093$ 
$0-0.45733796-1.57323583-1.71367846$

Vibrational frequencies (in $\mathrm{cm}-1$ )

$\begin{array}{llllllllllllllll}24.2 & 41.8 & 56.0 & 88.8 & 125.1 & 171.1 & 182.2 & 197.6 & 207.8 & 234.3 & 274.7 & 295.0 & 306.7 & 313.8\end{array}$

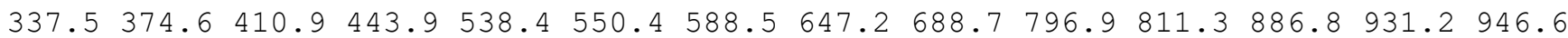
$983.1990 .8 \quad 1008.0 \quad 1017.0 \quad 1032.7 \quad 1044.0 \quad 1054.3 \quad 1082.2 \quad 1090.5 \quad 1102.5 \quad 1128.7 \quad 1154.6$

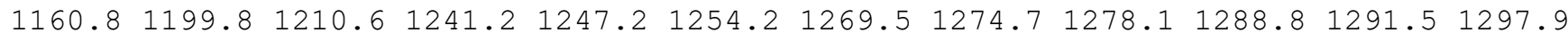

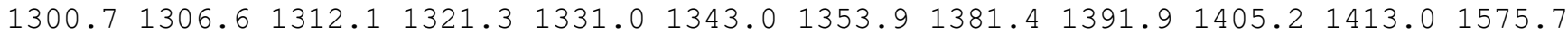
$1848.52647 .22661 .4 \quad 2667.4 \quad 2674.3 \quad 2675.92676 .52678 .92681 .82710 .02720 .32724 .8$ $2731.8 \quad 2761.2 \quad 2778.9 \quad 2780.3 \quad 2782.7$

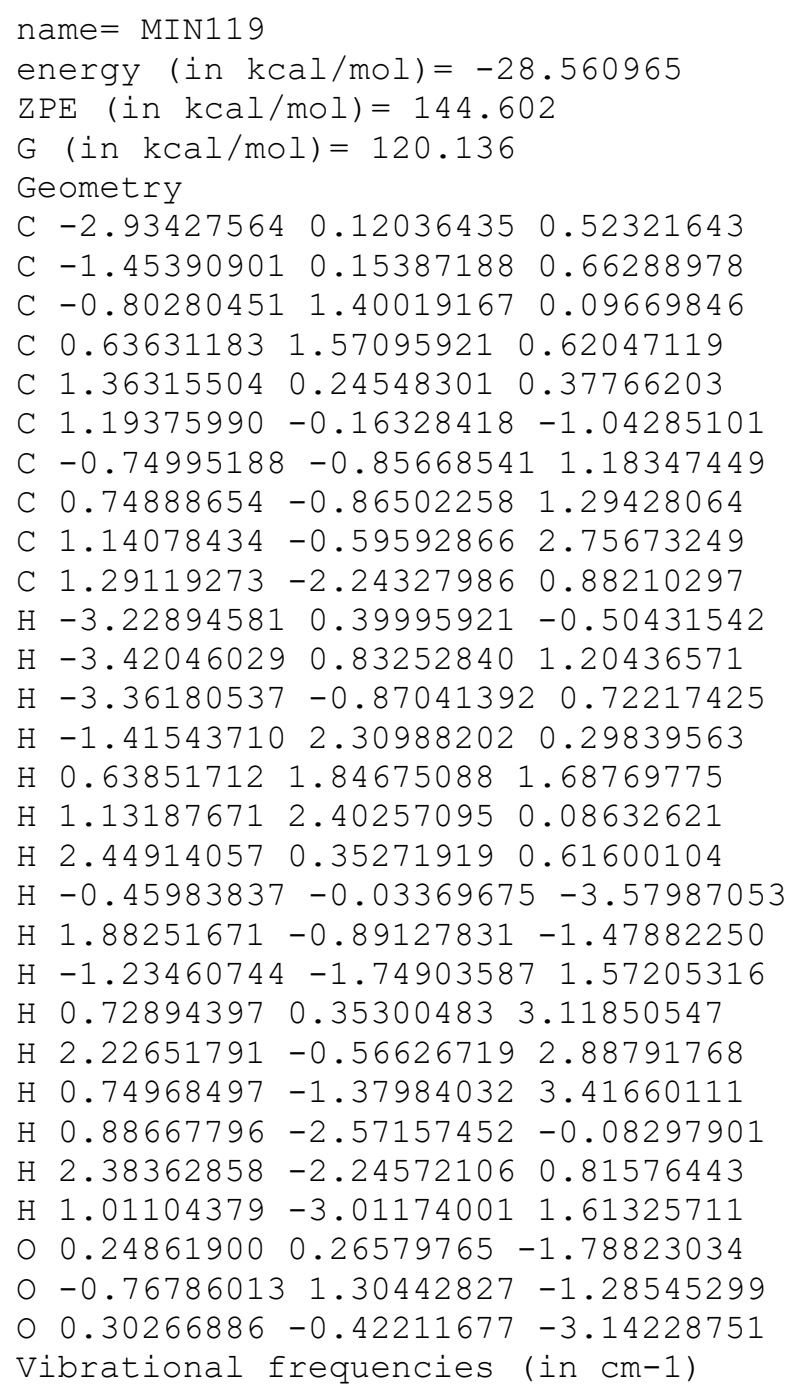


$\begin{array}{llll}\text { C } 1.69618805 & -0.23384007 & -0.60578257\end{array}$

C $0.88675282-1.46012392-0.16737524$

C $1.315438222 .08272413-1.42692444$

C $0.49318692 \quad 3.31618542-1.60768254$

C $2.693587162 .12457906-2.00248537$

$\mathrm{H}-2.95302826-1.548073312 .01700315$

$\mathrm{H} \quad 0.62744894 \quad-1.47923846 \quad 2.01154330$

$\mathrm{H}-2.76505569-1.09147880 \quad 0.24527319$

$\mathrm{H} \quad 0.01944334 \quad 0.87016001 \quad 1.91937457$

$\mathrm{H}-0.99807007 \quad 1.91275042-0.09872181$

$\mathrm{H}-1.18625132 \quad 0.35570992-0.90343697$

H $2.49513600-0.023873630 .13681383$

$\mathrm{H} \quad 0.18835167-1.76944096-0.98657106$

$\mathrm{H} \quad 2.22676141-0.47699115-1.55151971$

$\mathrm{H} \quad 1.55152771-2.33560129-0.03436212$

$\mathrm{H} \quad 0.26830988 \quad 3.78952409-0.63996837$

$\mathrm{H}-0.46453063 \quad 3.09520164-2.10066580$

H $0.99334901 \quad 4.07542139-2.22246057$

$\mathrm{H} 2.96033142 \quad 3.11208615-2.39951375$

$\mathrm{H} \quad 2.79812876 \quad 1.40866197 \quad-2.83174065$

H $3.45388271 \quad 1.87044480-1.24998733$

$\begin{array}{lllll}0 & -1.23774371 & -2.89130261 & 0.17576922\end{array}$

$0-1.79389117 \quad 0.06672786 \quad 1.67589843$

O $-1.11404443-2.122360081 .19015348$

Vibrational frequencies (in $\mathrm{cm}-1$ )

$\begin{array}{lllllllllllllll}27.7 & 70.1 & 88.9 & 110.1 & 118.8 & 146.8 & 181.6 & 222.2 & 260.8 & 325.5 & 342.4 & 361.4 & 425.8 & 441.1\end{array}$ $\begin{array}{llllllllllllllll}453.9 & 503.6 & 505.2 & 572.0 & 582.8 & 622.2 & 708.9 & 800.8 & 839.8 & 885.9 & 912.2 & 939.7 & 981.9 & 985.3\end{array}$

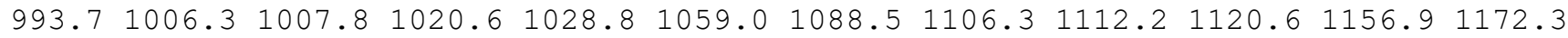

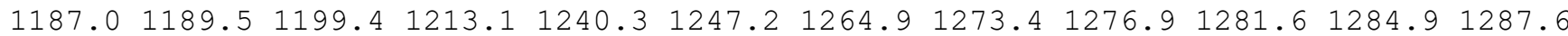

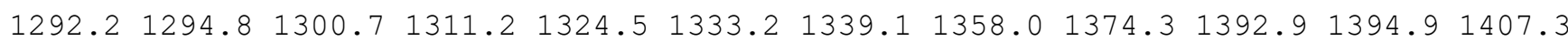
$1865.02628 .02646 .12655 .5 \quad 2657.6 \quad 2667.0 \quad 2668.72670 .12674 .2 \quad 2691.82699 .7 \quad 2703.5$ $\begin{array}{llllll}2732.9 & 2734.2 & 2740.5 & 2776.7 & 2777.8\end{array}$

\section{name $=$ MIN121}

energy (in $\mathrm{kcal} / \mathrm{mol})=-27.889279$

$\mathrm{ZPE}($ in $\mathrm{kcal} / \mathrm{mol})=144.164$

$\mathrm{G}($ in $\mathrm{kcal} / \mathrm{mol})=116.647$

Geometry

C $-3.069373290 .67896741-1.01214059$

C $-1.61670678 \quad 0.32390102-1.11119907$

C $-0.639319541 .43491168-0.82471649$

C $-0.236336961 .49882210 \quad 0.63685066$

$\begin{array}{lllll}\text { C } & 0.98104877 & 0.55144230 & 0.78762260\end{array}$

C $1.37923529 \quad 0.17423804 \quad 0.63922676$

C $-0.59627597-1.35999367 \quad 1.50237115$

C $0.73438874-0.69868300 \quad 1.59453697$

C $1.65488983-2.49355673 \quad 3.07113812$

C $1.74548515-1.21872177 \quad 2.30772754$

$\mathrm{H}-3.28182898 \quad 1.37618390 \quad-0.19498873$

$\mathrm{H}-3.68726925-0.22027078-0.86150093$

$\mathrm{H}-3.41503421 \quad 1.14182199-1.94937554$

$\mathrm{H}-0.96889524 \quad 2.39420747 \quad-1.25706348$

$\mathrm{H}-1.06107524 \quad 1.22271628 \quad 1.31761533$

$\mathrm{H} \quad 0.04027650 \quad 2.52854908 \quad 0.92878666$

$\mathrm{H} 1.80842511 \quad 1.13090263 \quad 1.26990118$

H 2.44067372 $0.29502495 \quad-0.87352034$

H $1.07259703-0.84135930-0.93492206$

$\mathrm{H}-0.64769972-1.99954859 \quad 0.60214149$

H $2.62703445-2.80870762 \quad 3.47073840$

$\mathrm{H} 1.28558266-3.31637158 \quad 2.43925199$

$\mathrm{H} \quad 0.96579573-2.404693393 .92380565$

$\mathrm{H}-1.43072768-0.65236397 \quad 1.43001495$

$\mathrm{H}-0.80822453-2.002909372 .36723379$

H $2.71596767-0.72886655 \quad 2.35647289$ 
$0.57520567 \quad 0.81726900-2.70726295$

○ $0.700395431 .19819380-1.52094393$

o $-1.24853684-0.78758830-1.38380137$

Vibrational frequencies (in $\mathrm{cm}-1$ )

$\begin{array}{llllllllllllllll}13.0 & 32.0 & 78.6 & 85.1 & 89.5 & 103.1 & 135.7 & 155.7 & 187.6 & 196.2 & 262.6 & 272.3 & 302.9 & 353.3\end{array}$

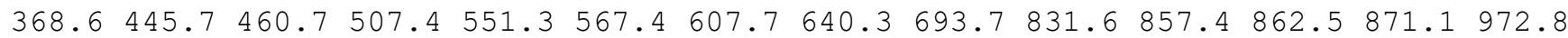
$984.91007 .31020 .5 \quad 1043.0 \quad 1045.0 \quad 1051.0 \quad 1063.0 \quad 1084.11090 .51099 .31104 .61128 .1$

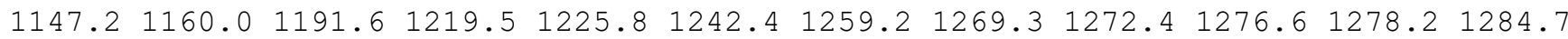

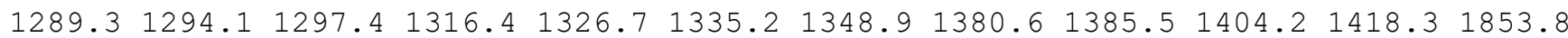
1861.42646 .72653 .62655 .32663 .92667 .02669 .62674 .52677 .62697 .12710 .02731 .5 $2742.4 \quad 2755.9 \quad 2774.4 \quad 2779.0 \quad 2780.1$

name $=\operatorname{MIN} 122$

energy $($ in $\mathrm{kcal} / \mathrm{mol})=-27.753201$

$\mathrm{ZPE}($ in $\mathrm{kcal} / \mathrm{mol})=144.035$

G $($ in $\mathrm{kcal} / \mathrm{mol})=118.001$

Geometry

C $-1.40935368-1.73333017-0.27043250$

C $0.04482241-1.57635737-0.45708616$

C $-1.66045372 \quad 1.79476484-0.81241018$

C $-0.58862695 \quad 2.49694820 \quad-0.02264976$

C $0.65764529 \quad 1.65717229 \quad 0.20924568$

C $1.07783536 \quad 0.67571039-0.91498316$

C $0.93745804-0.53507322 \quad 0.05869862$

C $0.56604929 \quad 0.45083203 \quad 1.22502491$

C $-0.74283262 \quad 0.26219296 \quad 1.95694513$

C $1.69960538 \quad 0.50889673 \quad 2.25020274$

$\mathrm{H}-1.95815684-0.80010286-0.49299899$

$\mathrm{H}-1.65595729-2.00285803 \quad 0.77185014$

$\mathrm{H}-1.84801926-2.51580929-0.91132246$

$\mathrm{H}-1.42624521 \quad 1.50464575-1.84543472$

$\mathrm{H}-0.99429965 \quad 2.82830047 \quad 0.95943767$

$\mathrm{H}-0.31395626 \quad 3.43298455-0.55636107$

H $1.486961362 .33948761 \quad 0.48716286$

H $2.101921690 .82072615-1.27904011$

$\mathrm{H} \quad 0.43377993 \quad 0.63643864 \quad-1.79323234$

H $1.93654431-1.01426904 \quad 0.25236342$

$\mathrm{H}-1.62147728 \quad 0.334846351 .30602968$

$\mathrm{H}-0.87703767 \quad 1.03166582 \quad 2.73013925$

$\mathrm{H}-0.77992090-0.71046003 \quad 2.46259490$

H $1.55783776 \quad 1.33746937 \quad 2.95354308$

H $2.680362290 .64503169 \quad 1.77955663$

$\mathrm{H} \quad 1.74781724 \quad-0.41629990 \quad 2.83756922$

$\begin{array}{lllll}0 & 1.76662511 & -2.51431750 & -1.47004424\end{array}$

O $-2.75171766 \quad 1.56701434-0.35008548$

O $0.52675355-2.52666687-1.21919318$

Vibrational frequencies (in $\mathrm{cm}-1$ )

$\begin{array}{llllllllllllllll}-20.4 & 61.1 & 89.4 & 103.9 & 168.6 & 171.6 & 186.2 & 207.8 & 223.3 & 242.6 & 253.5 & 275.9 & 295.5 & 312.2\end{array}$ $335.4 \quad 385.9 \quad 440.8 \quad 469.3 \quad 507.6 \quad 534.2 \quad 584.0 \quad 654.1 \quad 685.7 \quad 776.5 \quad 801.5 \quad 904.6 \quad 923.2 \quad 938.9$ $985.4 \quad 1004.6 \quad 1009.3 \quad 1018.9 \quad 1021.4 \quad 1029.7 \quad 1039.1 \quad 1053.8 \quad 1079.9 \quad 1117.9 \quad 1122.11135 .4$ $\begin{array}{lllllllllllll}1158.1 & 1182.2 & 1206.6 & 1233.5 & 1239.3 & 1257.8 & 1261.2 & 1279.4 & 1284.0 & 1291.8 & 1293.5 & 1298.4\end{array}$

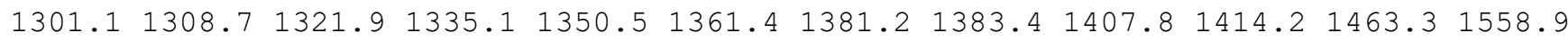
1836.02641 .12646 .32656 .82659 .02660 .52668 .82673 .62676 .32679 .12684 .62712 .6 $2737.4 \quad 2764.12768 .5 \quad 2780.6 \quad 2784.4$

name $=$ MIN123

energy (in $\mathrm{kcal} / \mathrm{mol})=-27.472122$

$\operatorname{ZPE}($ in $\mathrm{kcal} / \mathrm{mol})=143.758$

$\mathrm{G}($ in $\mathrm{kcal} / \mathrm{mol})=117.167$

Geometry

C $-2.03627242-1.28629985-1.06480251$

C $-0.59536002-1.02386166-1.36583516$

C $-0.14715606 \quad 0.39694140-1.27923766$ 
C $0.87418103 \quad 0.99816921-0.41276618$

C $1.32257825 \quad 0.345671390 .88362186$

C $1.62925895-1.17506522 \quad 0.85698649$

C $0.43811617-1.44227503 \quad 1.80894330$

C $0.25608504 \quad 0.09609491 \quad 2.01774789$

C $-1.13925558 \quad 0.64174038 \quad 1.79874451$

C $0.77544925 \quad 0.54381750 \quad 3.38137572$

$\mathrm{H}-2.39825943-2.17549486-1.60910037$

$\mathrm{H}-2.69763027-0.44793813-1.31760469$

$\mathrm{H}-2.18169781-1.506846390 .00661520$

$\mathrm{H}-0.41107126-1.935312591 .33362572$

$\mathrm{H} \quad 0.53418820 \quad 2.04881720-0.17130276$

$\mathrm{H} \quad 1.78294008 \quad 1.17259208-1.05448827$

H $2.18132187 \quad 0.93083581 \quad 1.27278276$

H 2.60262661 -1.42938461 1.28939361

$\mathrm{H} 1.58634827-1.65926033-0.11863708$

$\mathrm{H} 0.68421278 \quad-2.00372263 \quad 2.71101607$

$\mathrm{H}-1.83964865 \quad 0.238786892 .54099390$

$\mathrm{H}-1.537658290 .40687546 \quad 0.80951799$

$\mathrm{H}-1.155602951 .73584245 \quad 1.89608950$

$\mathrm{H} \quad 1.77648862 \quad 0.14650651 \quad 3.58634390$

$\mathrm{H} \quad 0.11868690 \quad 0.19499383 \quad 4.18679533$

$\mathrm{H} \quad 0.83284534 \quad 1.63558838 \quad 3.45080652$

$\begin{array}{llll}0 & -0.41846472 & 2.34515748 & -2.26380272\end{array}$

$0-0.73887274 \quad 1.13604140-2.18715103$

O $0.15902616-1.88956731-1.72481964$

Vibrational frequencies (in $\mathrm{cm}-1$ )

$\begin{array}{llllllllllllllll}-19.4 & 50.6 & 75.6 & 96.2 & 117.5 & 158.6 & 168.8 & 190.1 & 194.6 & 202.7 & 238.8 & 263.1 & 307.2 & 343.2\end{array}$

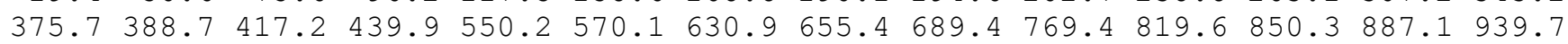
$956.1990 .8 \quad 1000.7 \quad 1008.6 \quad 1024.8 \quad 1036.4 \quad 1045.7 \quad 1059.6 \quad 1086.7 \quad 1089.3 \quad 1100.01144 .0$ $\begin{array}{llllllllllllll}1154.9 & 1195.2 & 1211.9 & 1226.9 & 1244.2 & 1249.6 & 1250.8 & 1257.3 & 1263.9 & 1288.5 & 1299.7 & 1300.7\end{array}$ $1302.01308 .21319 .7 \quad 1333.6 \quad 1361.6 \quad 1370.3 \quad 1374.4 \quad 1393.2 \quad 1406.4 \quad 1410.6 \quad 1415.51521 .1$ $1855.12600 .72648 .5 \quad 2665.3 \quad 2674.92676 .8 \quad 2679.82680 .62686 .5 \quad 2692.72702 .8 \quad 2715.2$ $2769.62771 .92774 .2 \quad 2782.3 \quad 2789.3$

\footnotetext{
name $=$ MIN124

energy (in $\mathrm{kcal} / \mathrm{mol})=-22.658931$

$\mathrm{ZPE}(\mathrm{in} \mathrm{kcal} / \mathrm{mol})=139.215$

$\mathrm{G}($ in $\mathrm{kcal} / \mathrm{mol})=110.471$

Geometry

C $-2.81166826 \quad 0.59449001 \quad 0.08067780$

C $-1.29749095 \quad 0.60249360 \quad 0.05250755$

$\begin{array}{lllll}\text { C } & 0.38969170 & 2.25312731 & 0.02054730\end{array}$

C 1.145251412 .588543321 .06497422

C $1.04475544 \quad-2.14272679 \quad 0.12017077$

C $1.27615683-0.88387627-0.73392411$

C $0.09866772-0.56373748-1.60428227$

C $0.42329588-1.72267082 \quad 1.39582137$

C $0.91160355-2.30132605 \quad 2.64115039$

C $0.05391952-3.11906151-0.53563938$

$\mathrm{H}-3.192460291 .14162596 \quad 0.95365093$

$\mathrm{H}-3.19696970-0.43179783 \quad 0.11683742$

$\mathrm{H}-3.22853818 \quad 1.07537249-0.81651050$

$\mathrm{H}-0.78729463 \quad 0.05335214 \quad 0.87306803$

$\mathrm{H} 0.77653945 \quad 2.59473545 \quad 2.07489766$

H $2.16927051 \quad 2.90573266 \quad 0.96989360$

H $2.02613305-2.665253820 .23765567$

H 2.14515549 -1.04802941 -1.40682791

$\mathrm{H} \quad 1.56989012-0.02566461-0.09718421$

H $0.17094043 \quad 2.04009912-1.91226970$

$\mathrm{H} 0.05916490-2.674254313 .23816096$

$\mathrm{H} 1.34878806-1.489918193 .25722063$

$\mathrm{H} 1.65498622-3.10358740 \quad 2.57577743$

$\mathrm{H} \quad 0.00654879-4.070064590 .00453956$
} 
$\mathrm{H} \quad 0.35784342-3.33799972-1.56858423$

$\mathrm{H}-0.96318627-2.70676618-0.57992399$

$0.88357780 \quad 2.20909453-1.24072990$

$0-0.94586897 \quad 1.98105823 \quad 0.08374088$

$0-0.92067126 \quad 0.07708900-1.23375616$

Vibrational frequencies (in $\mathrm{cm}-1$ )

$\begin{array}{lllllllllllllll}-23.6 & 39.8 & 55.3 & 65.8 & 81.2 & 92.7 & 97.2 & 113.5 & 119.7 & 169.8 & 184.7 & 187.4 & 284.2 & 294.7\end{array}$ $313.1338 .5 \quad 369.7 \quad 379.5 \quad 416.0 \quad 441.5 \quad 485.9 \quad 535.4 \quad 575.5 \quad 592.7 \quad 606.9 \quad 679.8 \quad 848.7 \quad 876.2$ $903.0 \quad 911.7 \quad 949.0 \quad 950.8 \quad 973.2 \quad 990.8 \quad 1020.1 \quad 1025.5 \quad 1056.6 \quad 1076.9 \quad 1101.2 \quad 1107.7 \quad 1153.6$

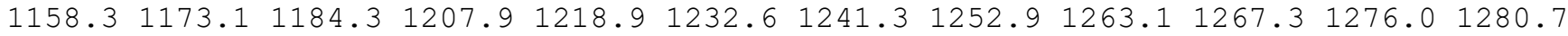

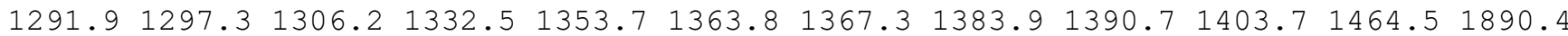
$2603.42648 .42652 .92663 .2 \quad 2668.0 \quad 2673.7 \quad 2674.32678 .52694 .6 \quad 2734.62736 .52760 .7$ $2767.02776 .7 \quad 2780.2 \quad 2801.6$

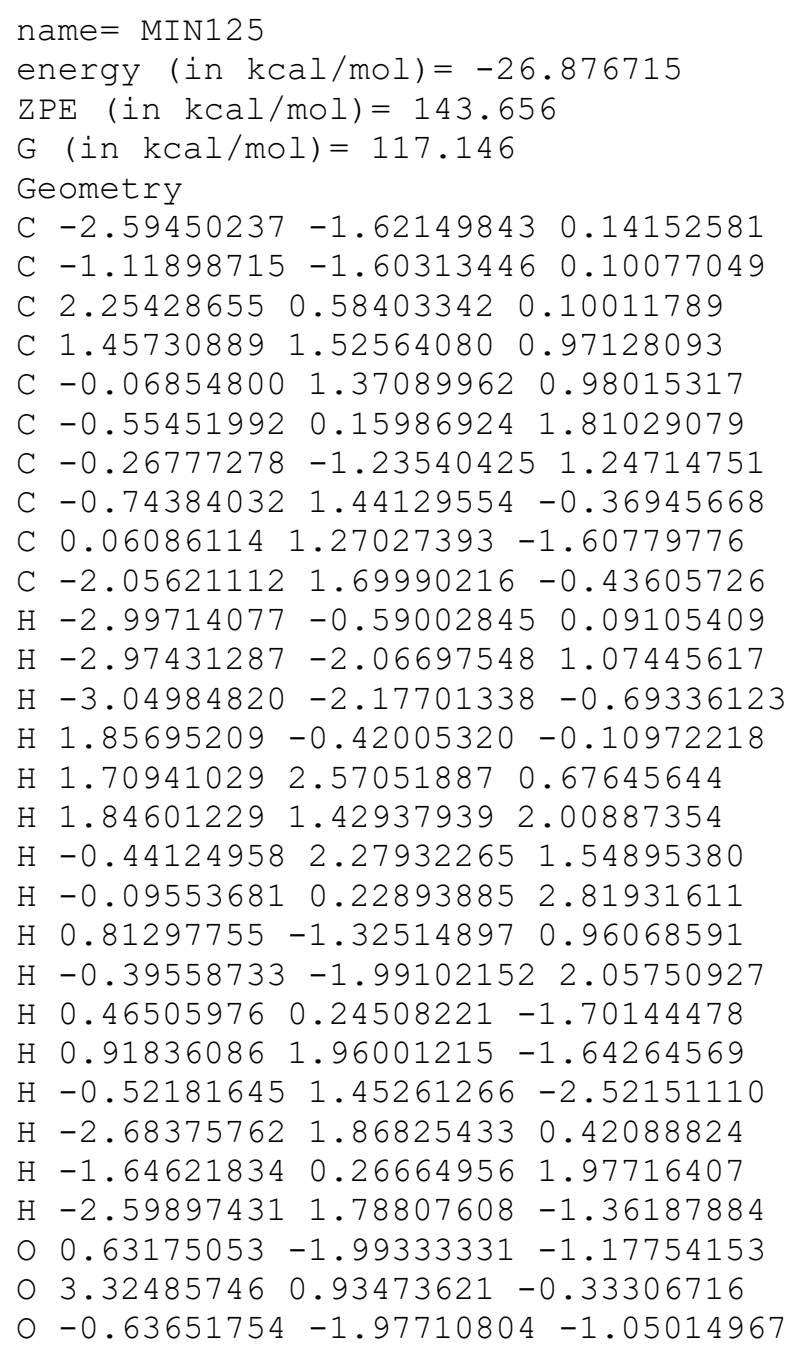


C $-1.569523390 .30032136-0.46863465$

C $1.85053411 \quad 1.25548006-0.93878486$

C 2.325404460 .505025850 .19065671

C $1.73004038 \quad-0.58418627 \quad 0.69865735$

C $0.42367214 \quad-1.15320220 \quad 0.24439048$

C $-0.72005740 \quad-0.26892096 \quad 0.64182432$

C $-1.01496946-0.01905591 \quad 1.93005092$

C $-2.13164960 \quad 0.88005070 \quad 2.35112950$

C $-0.25758046-0.62639528 \quad 3.06628084$

$\mathrm{H}-3.34905780-0.24656376-1.59158030$

$\mathrm{H}-3.45991348-0.60199666 \quad 0.14265076$

$\mathrm{H}-2.48630543-1.63463262-0.91122187$

$\mathrm{H}-1.878639221 .35396299-0.25124697$

$\mathrm{H} 1.70161078 \quad 2.33004824-0.88606862$

H $3.25165285 \quad 0.88910914 \quad 0.63176950$

H 2.19167464 -1.11863061 1.53320536

H $0.26997266-2.17372660 \quad 0.66089587$

$\mathrm{H} \quad 0.46172895-1.32240792-0.86306151$

$\mathrm{H}-0.52401031-0.36034502-2.04537510$

$\mathrm{H}-2.30534384 \quad 1.70505503 \quad 1.64610352$

$\mathrm{H}-1.93862950 \quad 1.34538674 \quad 3.32759276$

$\mathrm{H}-3.07394752 \quad 0.31927260 \quad 2.43811879$

$\mathrm{H} \quad 0.62954620-0.02297625 \quad 3.30953177$

$\mathrm{H} 0.08851376-1.645109222 .84207061$

$\mathrm{H}-0.86427458-0.69565962 \quad 3.97895139$

O $1.66832615-0.43834732-2.34757544$

$\begin{array}{llll}0 & 1.56159303 & 0.79859969 & -2.11539677\end{array}$

o $-0.86493547 \quad 0.48539198-1.67164423$

Vibrational frequencies (in $\mathrm{cm}-1$ )

$\begin{array}{lllllllllllllll}21.6 & 63.4 & 80.3 & 95.7 & 106.1 & 122.9 & 134.2 & 142.5 & 150.4 & 175.1 & 228.7 & 235.8 & 249.6 & 339.1\end{array}$

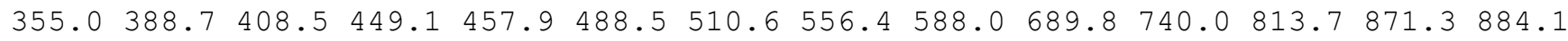
$952.3 \quad 985.4 \quad 992.6 \quad 1004.3 \quad 1018.9 \quad 1028.2 \quad 1031.5 \quad 1044.0 \quad 1066.0 \quad 1090.4 \quad 1109.51139 .1 \quad 1147.6$ $1165.21199 .3 \quad 1229.2 \quad 1240.8 \quad 1273.7 \quad 1279.2 \quad 1281.6 \quad 1286.6 \quad 1290.3 \quad 1291.4 \quad 1293.9 \quad 1303.6$ $1306.21321 .21331 .2 \quad 1339.7 \quad 1371.2 \quad 1392.7 \quad 1398.6 \quad 1404.41406 .1 \quad 1625.1 \quad 1845.51858 .4$ $2607.52660 .72667 .42668 .5 \quad 2671.3 \quad 2672.92680 .62686 .32713 .42716 .12733 .62748 .3$ $2749.8 \quad 2775.4 \quad 2777.5 \quad 2783.8$

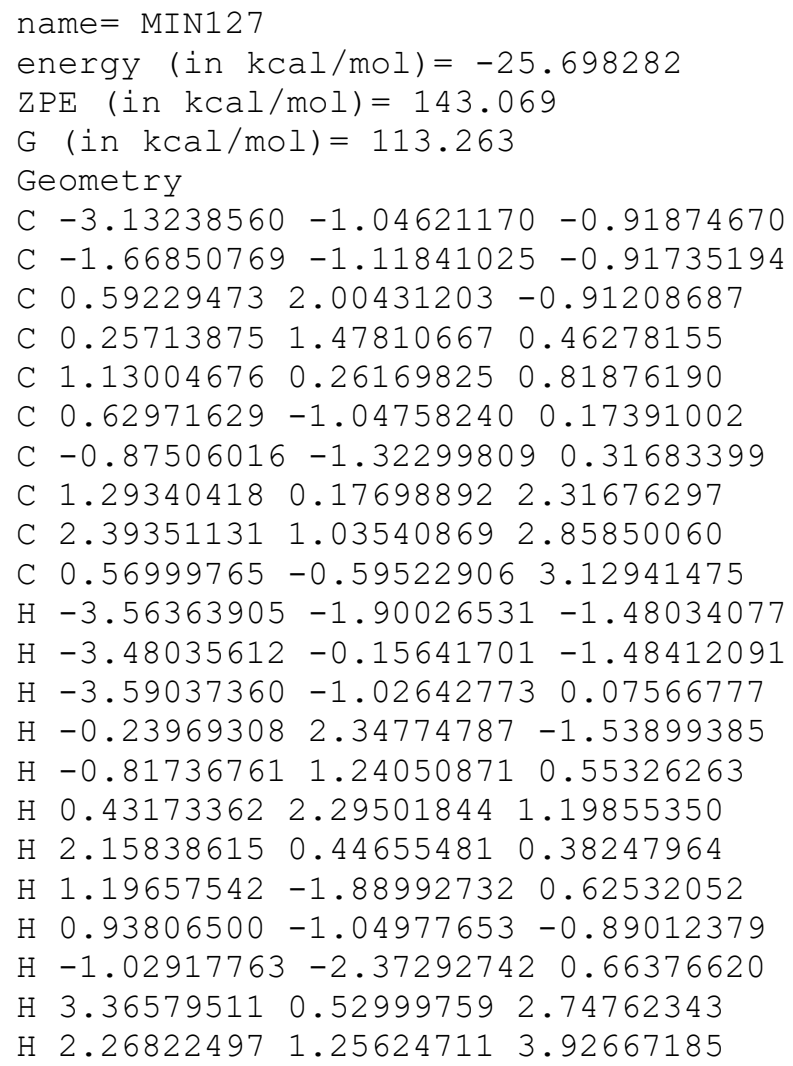


$\mathrm{H} 2.47351196 \quad 1.99864764 \quad 2.33646338$

$\mathrm{H}-1.29924937-0.69909907 \quad 1.13863025$

$\mathrm{H} \quad 0.72029396-0.630584994 .19693565$

$\mathrm{H}-0.21529589-1.257327602 .81565222$

$0-1.64744600-0.88693949-3.09712642$

O $1.729847402 .06277711-1.30596644$

o $-0.98549512-1.04068627-2.02620531$

Vibrational frequencies (in $\mathrm{cm}-1$ )

$\begin{array}{lllllllllllllll}-10.2 & 22.4 & 32.3 & 39.1 & 70.9 & 86.6 & 93.1 & 107.6 & 153.9 & 201.0 & 221.4 & 241.1 & 279.1 & 290.0\end{array}$

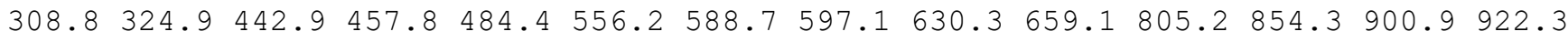
$975.7990 .5 \quad 996.8 \quad 1031.5 \quad 1038.9 \quad 1052.8 \quad 1056.4 \quad 1069.6 \quad 1084.1 \quad 1098.6 \quad 1104.7 \quad 1125.7 \quad 1140.4$

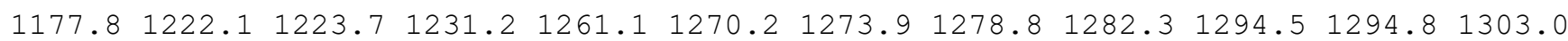

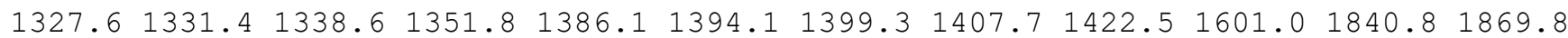
$2631.62634 .02645 .12649 .2 \quad 2655.2 \quad 2662.32666 .12673 .82674 .92720 .2 \quad 2724.32736 .2$ $2739.62770 .3 \quad 2778.02796 .9$

\section{name $=$ MIN128}

energy (in $\mathrm{kcal} / \mathrm{mol})=-25.231376$

$\mathrm{ZPE}($ in $\mathrm{kcal} / \mathrm{mol})=143.323$

$\mathrm{G}(\mathrm{in} \mathrm{kcal} / \mathrm{mol})=115.556$

Geometry

C $-3.80062295 \quad 0.36758878-0.07336340$

C $-2.32509848 \quad 0.54710335-0.13451432$

C $2.57006122 \quad 0.80553577-0.06800978$

C $2.31798439-0.42705522-0.90144078$

C $0.86410830-0.92646424-0.91874931$

C $-0.087693790 .21980464-1.32513959$

C $-1.57375830-0.13425193-1.20354971$

C $0.46678764-1.59724060 \quad 0.37580790$

C $0.88966038-0.988328521 .66570296$

C $-0.24916829-2.72802801 \quad 0.35184101$

$\mathrm{H}-4.26637855 \quad 0.85201805 \quad 0.79990035$

$\mathrm{H}-4.07457445-0.69930858-0.02805175$

$\mathrm{H}-4.28943340 \quad 0.78978274 \quad-0.96758667$

H $1.71121770 \quad 1.33343556 \quad 0.37155321$

$\mathrm{H} \quad 2.98936978-1.24145637-0.55196005$

H 2.65454535 -0.21779689 -1.93976263

$\mathrm{H} \quad 0.80416766-1.70413314-1.73072375$

$\mathrm{H} \quad 0.13826123 \quad 0.50299684-2.37248961$

$\mathrm{H} \quad 0.13568081 \quad 1.13525785 \quad-0.73573780$

$\mathrm{H}-2.07303683 \quad 0.06342030 \quad-2.18150699$

H $0.51050623-1.53773914 \quad 2.53763311$

H $0.51336590 \quad 0.04774940 \quad 1.76195221$

$\mathrm{H} \quad 1.98367951-0.95438631 \quad 1.76598347$

$\mathrm{H}-1.68443217-1.24022562-1.06394687$

$\mathrm{H}-0.56445339-3.24959356 \quad 1.23952714$

$\mathrm{H}-0.56528021-3.22897614-0.54525389$

$\begin{array}{lllll}0 & -0.72558563 & 1.64818256 & 0.97735224\end{array}$

$\begin{array}{lllll}0 & 3.69657881 & 1.20489846 & 0.10132984\end{array}$

$\begin{array}{llll}0 & -1.93626060 & 1.32538964 & 0.83075197\end{array}$

Vibrational frequencies (in cm-1)

$\begin{array}{lllllllllllllllll}-46.4 & 22.6 & 35.3 & 46.0 & 87.8 & 125.9 & 174.6 & 184.0 & 195.3 & 212.1 & 262.1 & 271.6 & 298.1 & 300.3\end{array}$ $320.3 \quad 339.2 \quad 412.1 \quad 444.5 \quad 460.9 \quad 478.1 \quad 570.2 \quad 622.1 \quad 657.4 \quad 693.9 \quad 763.7 \quad 789.4 \quad 890.3 \quad 908.1$ $988.6997 .6998 .1 \quad 1011.4 \quad 1033.8 \quad 1044.5 \quad 1051.9 \quad 1070.4 \quad 1092.0 \quad 1106.8 \quad 1121.5 \quad 1160.5 \quad 1179.3$ $1189.51208 .4 \quad 1231.0 \quad 1256.8 \quad 1259.1 \quad 1265.5 \quad 1270.1 \quad 1274.6 \quad 1279.2 \quad 1285.3 \quad 1289.3 \quad 1296.5$ $1300.71328 .1 \quad 1362.7 \quad 1370.2 \quad 1374.6 \quad 1390.2 \quad 1400.8 \quad 1403.91413 .0 \quad 1627.4 \quad 1827.9 \quad 1841.3$ $2619.02626 .22629 .5 \quad 2640.92653 .3 \quad 2656.6 \quad 2659.02670 .92681 .92719 .32726 .02726 .6$ $2738.4 \quad 2771.0 \quad 2772.7 \quad 2796.1$

name $=$ MIN129

energy (in $\mathrm{kcal} / \mathrm{mol})=-25.277672$

$\mathrm{ZPE}(\mathrm{in} \mathrm{kcal} / \mathrm{mol})=143.91$

$\mathrm{G}($ in $\mathrm{kcal} / \mathrm{mol})=117.444$ 


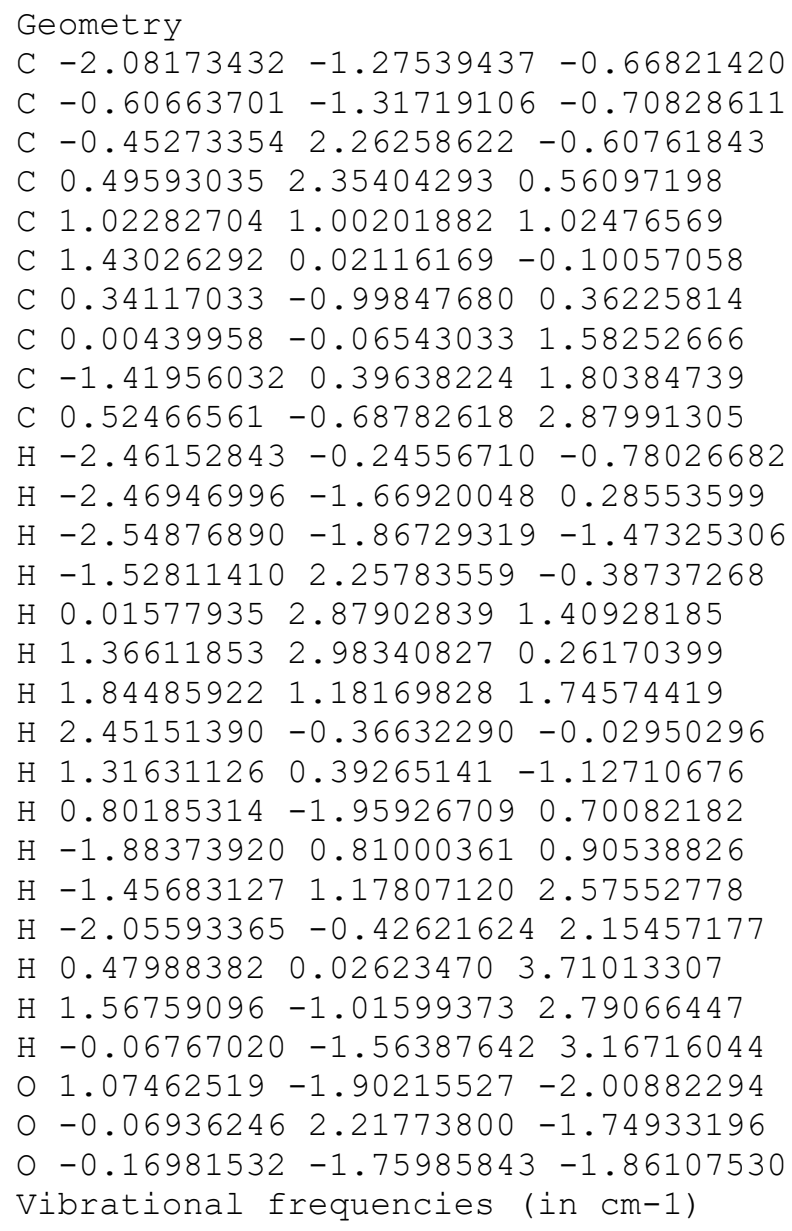

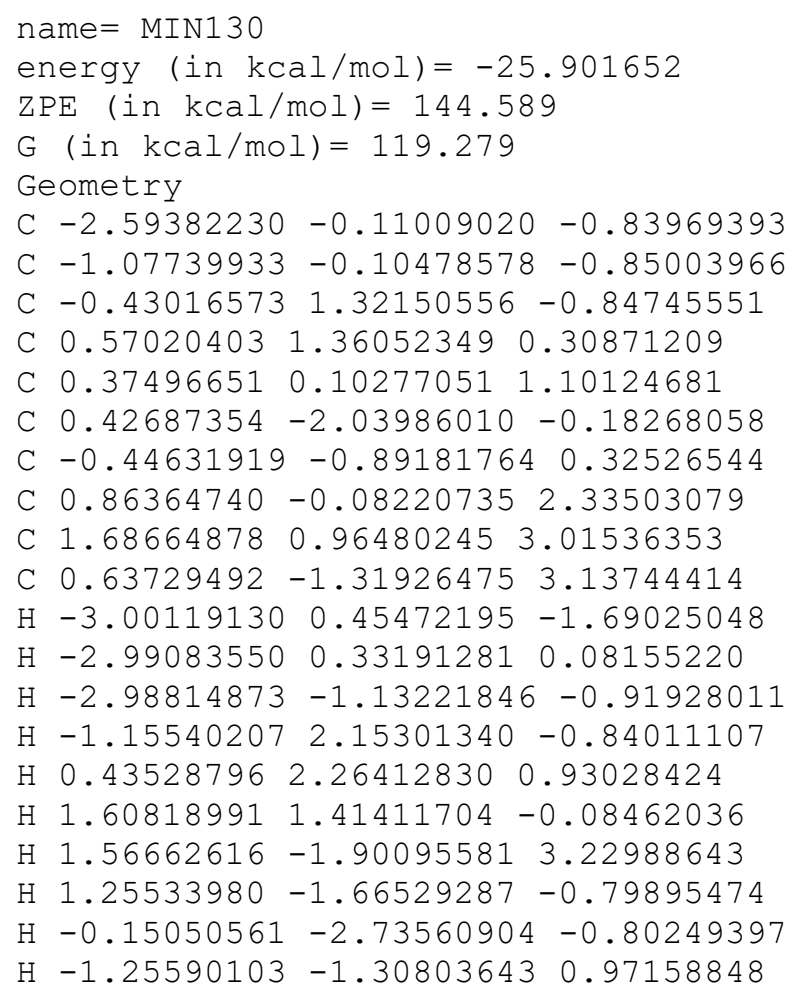


$\mathrm{H} \quad 1.05757397 \quad 1.79921337 \quad 3.35802218$

H $2.451737551 .38267142 \quad 2.34568538$

H $2.21526748 \quad 0.577106293 .89570354$

$\mathrm{H} \quad 0.30058255-1.07682355 \quad 4.15571636$

$\mathrm{H} \quad 0.86712885-2.60346368 \quad 0.64666953$

$\mathrm{H}-0.11855602-1.98576827 \quad 2.70287900$

○ $0.47975758 \quad 0.06591713-2.47329335$

$00.211692591 .41800036-2.11167859$

o $-0.70529144-0.63069333-2.12709033$

Vibrational frequencies (in $\mathrm{cm}-1$ )

$\begin{array}{lllllllllllllllll}46.8 & 71.8 & 98.4 & 118.8 & 125.7 & 169.9 & 183.9 & 202.7 & 210.6 & 228.6 & 258.1 & 285.5 & 327.3 & 351.4\end{array}$ $392.4 \quad 436.1 \quad 470.4 \quad 522.8 \quad 555.9 \quad 575.1 \quad 609.9 \quad 669.3 \quad 717.6 \quad 833.9 \quad 858.5 \quad 873.4 \quad 919.4 \quad 967.4$

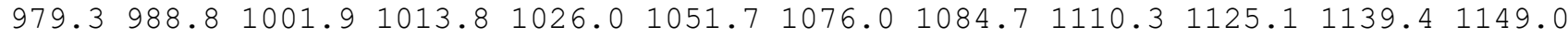

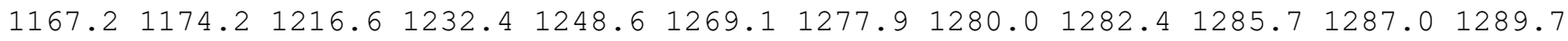

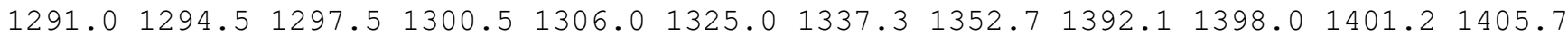
$1892.42660 .3 \quad 2669.02669 .7 \quad 2670.4 \quad 2671.2 \quad 2672.02675 .02677 .4 \quad 2680.22701 .52702 .6$ $2742.12777 .5 \quad 2779.0 \quad 2780.22781 .4$

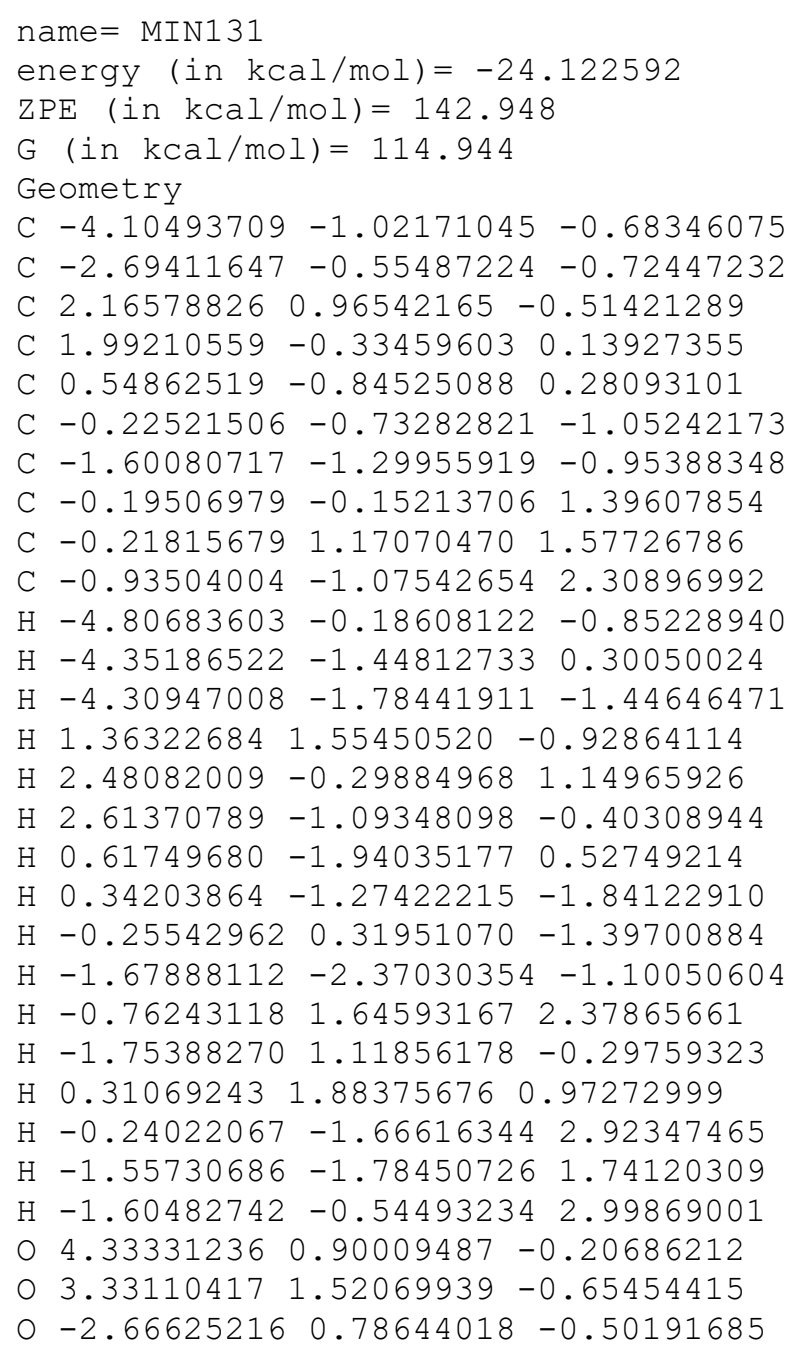


$\mathrm{ZPE}($ in $\mathrm{kcal} / \mathrm{mol})=142.156$

$\mathrm{G}($ in $\mathrm{kcal} / \mathrm{mol})=115.253$

Geometry

C $-2.67010172 \quad 0.30710217 \quad 0.11299791$

$\begin{array}{llll}\text { C }-1.16300154 & 0.28567147 & -0.08120548\end{array}$

C $1.083129162 .86460667-0.01667456$

C $1.460764952 .95816681-1.29012858$

C $0.16590049-2.37974237 \quad 0.90770960$

C $1.07529073-3.048981351 .61319617$

C $-1.749007890 .28423800 \quad 1.31262938$

C $0.50953077-1.47624302-0.24842233$

C $0.88026496-2.31558039-1.48103174$

C $1.59244665-0.46443984 \quad 0.09670183$

$\mathrm{H}-3.22358362 \quad 1.21028507-0.13070638$

$\mathrm{H}-1.67921460-0.60915933 \quad 1.92656134$

$\mathrm{H}-3.24376447-0.57887725-0.14926297$

$\mathrm{H} 1.50148943 \quad 3.39968409 \quad 0.83657504$

H 2.25566420 3.60060451 -1.62622009

$\mathrm{H} 1.001213972 .40631099-2.09425527$

$\mathrm{H}-0.90287831-2.470402691 .10090859$

H $2.13825587 \quad-2.99092783 \quad 1.43638047$

$\mathrm{H} \quad 0.82562081 \quad-3.71428227 \quad 2.42511751$

$\mathrm{H}-1.65521787 \quad 1.17223501 \quad 1.93456092$

H $1.76438294-2.92979092-1.28841192$

$\mathrm{H} \quad 1.08284195-1.67047559-2.34465735$

$\mathrm{H} \quad 0.05388060-2.98101598-1.76245655$

$\mathrm{H} 1.36179647 \quad 0.07524776 \quad 1.02342499$

$\mathrm{H} \quad 1.69625976 \quad 0.28649480 \quad-0.70062462$

$\begin{array}{lllll}\mathrm{H} & 2.56703005 & -0.94582901 & 0.22750935\end{array}$

$0-0.604218821 .43820236-0.60987566$

○ $0.10196335 \quad 2.07926160 \quad 0.49295609$

o $-0.69769531-0.84510520-0.70156621$

Vibrational frequencies (in $\mathrm{cm}-1$ )

$\begin{array}{lllllllllllllll}23.0 & 52.7 & 63.7 & 85.4 & 119.7 & 141.0 & 148.1 & 185.0 & 206.3 & 223.5 & 250.4 & 257.4 & 275.22 & 287.3\end{array}$ $328.8 \quad 354.5 \quad 386.9 \quad 463.5 \quad 477.9 \quad 514.9 \quad 587.5 \quad 606.4 \quad 618.1 \quad 659.6 \quad 800.1 \quad 819.7 \quad 859.4 \quad 885.8$

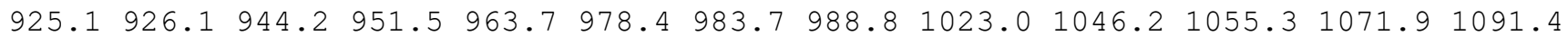
$1097.7 \quad 1109.5 \quad 1129.2 \quad 1193.0 \quad 1200.6 \quad 1209.1 \quad 1246.8 \quad 1276.4 \quad 1283.3 \quad 1290.2 \quad 1292.7 \quad 1294.9$

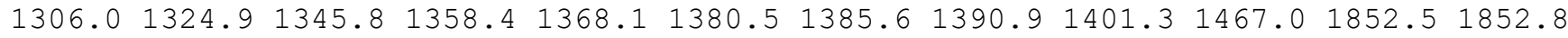
$2665.92675 .92678 .0 \quad 2683.6 \quad 2700.2 \quad 2711.7 \quad 2714.12723 .32735 .5 \quad 2751.7 \quad 2779.4 \quad 2779.5$ $2781.92785 .8 \quad 2788.12796 .4$

\section{name $=$ MIN133}

energy (in $\mathrm{kcal} / \mathrm{mol})=-24.631883$

$\mathrm{ZPE}(\mathrm{in} \mathrm{kcal} / \mathrm{mol})=144.584$

$\mathrm{G}($ in $\mathrm{kcal} / \mathrm{mol})=119.061$

Geometry

C $-2.82955857 \quad 0.09889968-0.48347860$

C $-1.316168110 .06740621-0.57332670$

C $-0.624273221 .46921594-0.59139230$

C $0.51578624 \quad 1.41614406 \quad 0.42383650$

C $0.54832065 \quad 0.03227742 \quad 1.00717037$

C $-0.29055732-2.154593230 .00541859$

C $-0.63930071-0.767526790 .54385159$

C $1.51495282-0.36352701 \quad 1.84818431$

C $2.65760745 \quad 0.54315004 \quad 2.18228761$

C $1.55043566-1.70266744 \quad 2.50293358$

$\mathrm{H}-3.265438170 .64707892 \quad-1.33156704$

$\mathrm{H}-3.17355062 \quad 0.57617880 \quad 0.44026913$

$\mathrm{H}-3.24912549-0.91603976-0.51400641$

$\mathrm{H}-1.30706700 \quad 2.32590247-0.46855117$

$\mathrm{H} \quad 0.38446991 \quad 2.17262412 \quad 1.22057108$

$\mathrm{H} 1.47708117 \quad 1.66337538-0.07479235$

H $2.05198350-2.43802758 \quad 1.85467483$

$\mathrm{H}-1.12675599-2.57996819-0.56441805$ 
$\mathrm{H} \quad 0.57171196-2.10925136-0.67273791$

$\mathrm{H}-1.34868013-0.87774409 \quad 1.40199134$

H $2.31367600 \quad 1.44027695 \quad 2.71607497$

$\mathrm{H} \quad 3.17773590 \quad 0.88047618 \quad 1.27274115$

H $3.41225472 \quad 0.06029785 \quad 2.81521133$

H $2.09706048-1.686391573 .45512229$

$\mathrm{H}-0.05032141-2.85916343 \quad 0.80651010$

$\mathrm{H} \quad 0.54448293-2.08444056 \quad 2.72438339$

$\begin{array}{lllll}0 & 0.12083930 & 0.24771565 & -2.31257183\end{array}$

$0-0.126851551 .59214360-1.91846844$

o $-1.02956083-0.45741553-1.87570109$

Vibrational frequencies (in $\mathrm{cm}-1$ )

$28.165 .6 \quad 104.0 \quad 112.0 \quad 152.5 \quad 165.2 \quad 178.0 \quad 204.8 \quad 216.1233 .0 \quad 270.3 \quad 300.9 \quad 323.7 \quad 354.7$ $380.9 \quad 447.1 \quad 483.1 \quad 515.3 \quad 548.1 \quad 569.3 \quad 626.3 \quad 668.9 \quad 719.0 \quad 830.0 \quad 859.2 \quad 878.6 \quad 920.4 \quad 966.1$ $976.1986 .8 \quad 1002.0 \quad 1006.6 \quad 1026.6 \quad 1054.0 \quad 1071.6 \quad 1083.2 \quad 1110.11121 .01140 .01153 .9$

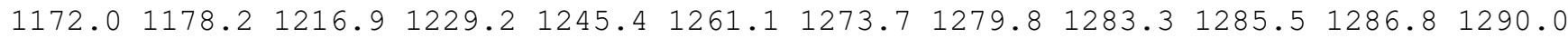
$1292.21294 .21296 .3 \quad 1302.3 \quad 1310.1 \quad 1319.3 \quad 1332.2 \quad 1356.1 \quad 1392.4 \quad 1393.91402 .51409 .0$ $1886.02658 .8 \quad 2666.12668 .92670 .02671 .4 \quad 2671.82675 .12675 .92678 .82699 .3 \quad 2704.7$ $2739.7 \quad 2776.5 \quad 2777.7 \quad 2780.6 \quad 2781.6$

name $=$ MIN134

energy $($ in $\mathrm{kcal} / \mathrm{mol})=-22.575552$

$\operatorname{ZPE}($ in $\mathrm{kcal} / \mathrm{mol})=142.745$

$\mathrm{G}($ in $\mathrm{kcal} / \mathrm{mol})=114.501$

Geometry

C $-3.02354462-1.13285272-0.41262607$

C $-1.56169890-1.12495274-0.48082350$

C $-0.24662838 \quad 2.24450964-0.73345628$

C $0.39384585 \quad 1.76544567 \quad 0.55077250$

C $1.26788735 \quad 0.57030618 \quad 0.29378264$

C $0.75077276-0.86028751 \quad 0.37482088$

C $-0.68411801-1.17526122 \quad 0.71433614$

C $1.72722691-0.34016204 \quad 1.42715530$

C $1.27805403-0.10237470 \quad 2.84395986$

C $3.14312296-0.86279885 \quad 1.36030994$

$\mathrm{H}-3.43329792-2.04568510-0.89133009$

$\mathrm{H}-3.44742974-0.30332614-1.01836444$

$\mathrm{H}-3.43378168-1.065628890 .60059262$

$\mathrm{H}-0.684130931 .48684245-1.39509859$

$\mathrm{H}-0.40248857 \quad 1.56251716 \quad 1.29425894$

H $0.99963094 \quad 2.58299065 \quad 1.00437407$

H $2.00599004 \quad 0.77805694 \quad-0.49749404$

$\mathrm{H}-0.74157727-2.18996330 \quad 1.17875892$

$\mathrm{H} \quad 1.16127869-1.57393039-0.35507415$

$\mathrm{H}-1.07743486-0.49013838 \quad 1.49941838$

$\mathrm{H} \quad 0.26175562 \quad 0.29958188 \quad 2.91779011$

H $1.94047617 \quad 0.61379803 \quad 3.35127263$

H $1.30176649-1.034529593 .42521346$

H $3.48909217-1.02047366 \quad 0.33114338$

H $3.23648029-1.823569891 .88311211$

$\mathrm{H} 3.84657622-0.162990761 .83111922$

o $-1.63189326-1.02634183-2.67044620$

$0-0.282539563 .41666284-1.00730239$

o $-0.92607911-1.07641690-1.62013658$

Vibrational frequencies (in $\mathrm{cm}-1$ )

$\begin{array}{lllllllllllllll}23.8 & 32.6 & 49.2 & 77.9 & 86.4 & 93.2 & 119.7 & 146.8 & 167.3 & 186.0 & 205.3 & 224.4 & 270.8 & 278.7\end{array}$ $293.1333 .5 \quad 410.9 \quad 452.8 \quad 478.8 \quad 484.9 \quad 517.9 \quad 569.6 \quad 679.1 \quad 760.8 \quad 830.7 \quad 862.6 \quad 896.5 \quad 944.2$ $959.9996 .2 \quad 1010.4 \quad 1013.2 \quad 1030.7 \quad 1033.1 \quad 1048.6 \quad 1059.1 \quad 1079.5 \quad 1110.3 \quad 1115.01150 .7$

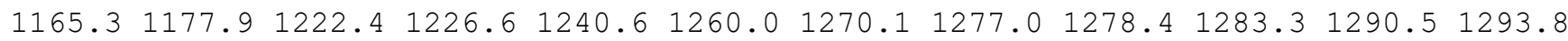
$1295.71300 .6 \quad 1322.4 \quad 1335.8 \quad 1368.5 \quad 1374.8 \quad 1385.1 \quad 1400.01410 .8 \quad 1419.2 \quad 1511.3 \quad 1599.4$ $1839.82631 .72640 .12645 .6 \quad 2658.72670 .3 \quad 2672.42674 .12674 .52676 .82724 .12730 .8$ $2741.2 \quad 2746.8 \quad 2769.8 \quad 2779.7 \quad 2782.1$ 
name $=$ MIN135

energy (in $\mathrm{kcal} / \mathrm{mol})=-21.675876$

$\mathrm{ZPE}($ in $\mathrm{kcal} / \mathrm{mol})=141.871$

$\mathrm{G}($ in $\mathrm{kcal} / \mathrm{mol})=114.057$

Geometry

C $-2.739263321 .10999618-0.30187923$

C $-1.250588501 .03330216-0.23656848$

C $-0.57296432 \quad 2.37160974-0.22260814$

C $-0.38450002 \quad 3.03103496-1.54667899$

C $1.06387756-1.387792221 .62063907$

C $0.78462782 \quad-0.13113460 \quad 0.86079521$

C $-0.60154687-0.15632547 \quad 0.30253727$

C $1.34786826-2.57051598 \quad 1.05911357$

C $1.56106314-3.789736531 .89965188$

$\begin{array}{llll}\text { C } 1.47523213 & -2.80119722 & -0.40793178\end{array}$

$\mathrm{H}-3.091087341 .74428452-1.12842652$

$\mathrm{H}-3.14540078 \quad 1.539229010 .63142531$

$\mathrm{H}-3.213151290 .12601923-0.42460793$

$\mathrm{H} \quad 0.03079136 \quad 4.04390230-1.44152282$

H $0.32339406 \quad 2.44978562 \quad-2.17147697$

$\mathrm{H}-1.318330573 .10522072-2.11658545$

$\mathrm{H} 1.00013429-1.279514992 .70148956$

H $1.52871424 \quad 0.01327771 \quad 0.03295636$

H $0.92745844 \quad 0.76271913 \quad 1.51290242$

$\mathrm{H}-1.21924582-0.996242620 .63658509$

$\mathrm{H} \quad 0.92878466-4.62269846 \quad 1.56249745$

$\mathrm{H} 1.33245601-3.621662062 .96002343$

$\mathrm{H} 2.60456578-4.130520491 .84364101$

$\mathrm{H} \quad 0.73866834-3.53228620-0.76671224$

H 2.47101871 $-3.19192286-0.66243099$

H $1.33606541-1.88568008-1.00527067$

$\begin{array}{lllll}0 & 0.32937095 & 0.21229278 & -1.88334954\end{array}$

$0-0.22630063 \quad 2.87191547 \quad 0.81634156$

o $-0.70226604-0.02512126-1.25294894$

Vibrational frequencies (in cm-1)

$\begin{array}{lllllllllllllll}35.1 & 51.8 & 59.7 & 78.1 & 91.5 & 105.7 & 115.8 & 123.6 & 128.6 & 176.9 & 202.9 & 233.4 & 260.8 & 278.8\end{array}$ $300.6 \quad 348.4 \quad 383.8 \quad 428.8 \quad 467.4 \quad 483.9 \quad 550.0 \quad 556.7 \quad 601.9631 .9648 .2 \quad 712.9 \quad 828.3 \quad 886.0$ $919.7 \quad 960.1 \quad 996.1 \quad 1012.7 \quad 1028.5 \quad 1034.2 \quad 1041.5 \quad 1056.6 \quad 1071.1 \quad 1085.3 \quad 1118.5 \quad 1135.2 \quad 1180.9$

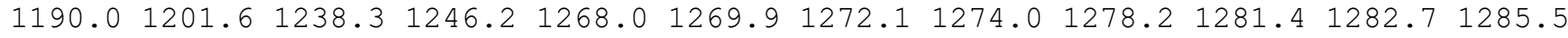
$1289.61298 .91346 .3 \quad 1361.91377 .9 \quad 1378.4 \quad 1400.3 \quad 1401.91414 .3 \quad 1499.8 \quad 1853.51874 .4$ $2616.62638 .2 \quad 2657.92658 .7 \quad 2668.3 \quad 2671.0 \quad 2675.42675 .72676 .02708 .32731 .72757 .7$ $2773.6 \quad 2775.3 \quad 2777.12781 .5$

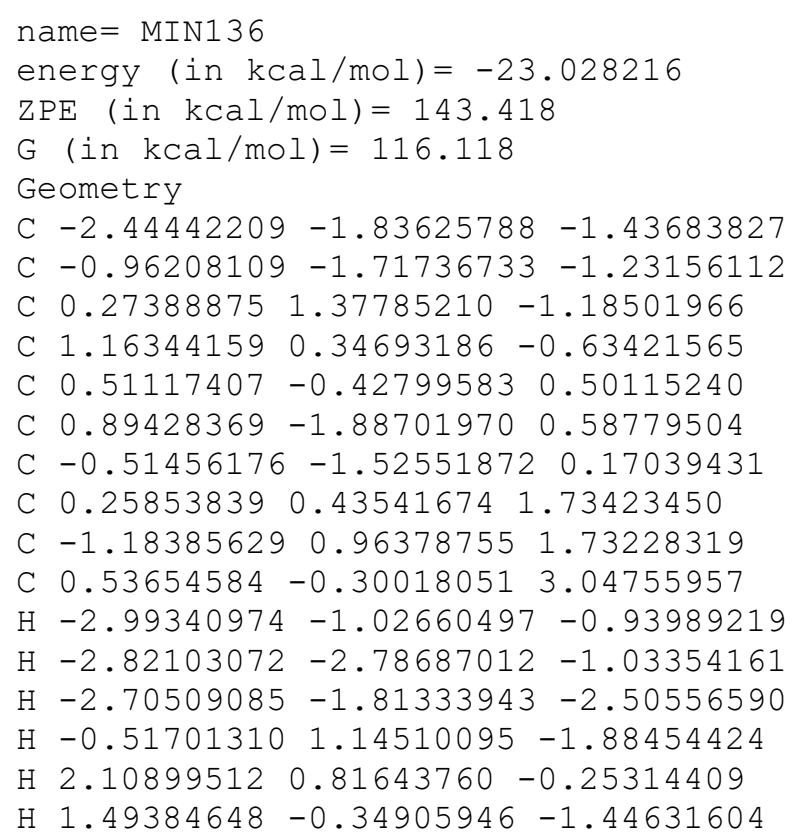


H $0.949890891 .32391135 \quad 1.68434370$

$\mathrm{H} 1.57482884-2.30504605-0.15556312$

$\mathrm{H} 1.05781656-2.370250881 .54692524$

$\mathrm{H}-0.11965114-1.166205713 .18064428$

$\mathrm{H}-1.90960775 \quad 0.17246888 \quad 1.94318258$

$\mathrm{H}-1.44676590 \quad 1.41215070 \quad 0.76935378$

$\mathrm{H}-1.31349428 \quad 1.738363192 .49815058$

$\mathrm{H} \quad 0.37511538 \quad 0.36418671 \quad 3.90550798$

$\mathrm{H}-1.31027824-1.69652444 \quad 0.90705417$

H $1.57496464 \quad-0.64711314 \quad 3.09869135$

$01.24079586 \quad 3.02861505-0.10124127$

$00.35797188 \quad 2.64074457-0.91055933$

o $-0.19414739-1.75795468-2.16286904$

Vibrational frequencies (in cm-1)

$\begin{array}{lllllllllllllll}32.2 & 35.5 & 56.8 & 64.5 & 95.0 & 113.4 & 141.6 & 164.7 & 192.1 & 222.8 & 232.8 & 254.1 & 293.9 & 322.9\end{array}$

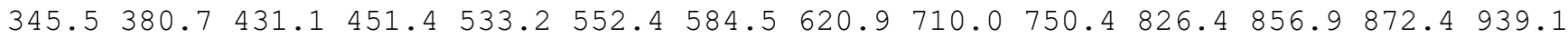
$973.9986 .0 \quad 988.1 \quad 1001.6 \quad 1016.4 \quad 1028.4 \quad 1039.2 \quad 1057.4 \quad 1073.2 \quad 1084.3 \quad 1130.2 \quad 1154.4 \quad 1170.9$ $\begin{array}{lllllllllllll}1176.5 & 1192.1 & 1212.7 & 1227.1 & 1244.3 & 1260.8 & 1270.1 & 1272.5 & 1280.1 & 1297.6 & 1299.3 & 1304.1\end{array}$

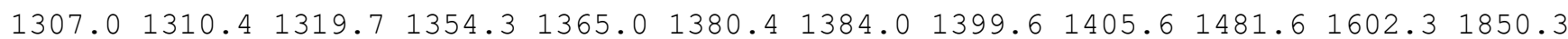
$2623.52658 .62669 .12673 .7 \quad 2674.6 \quad 2677.8 \quad 2680.22690 .42700 .82705 .42720 .52748 .5$ $2777.42780 .3 \quad 2780.6 \quad 2782.3$

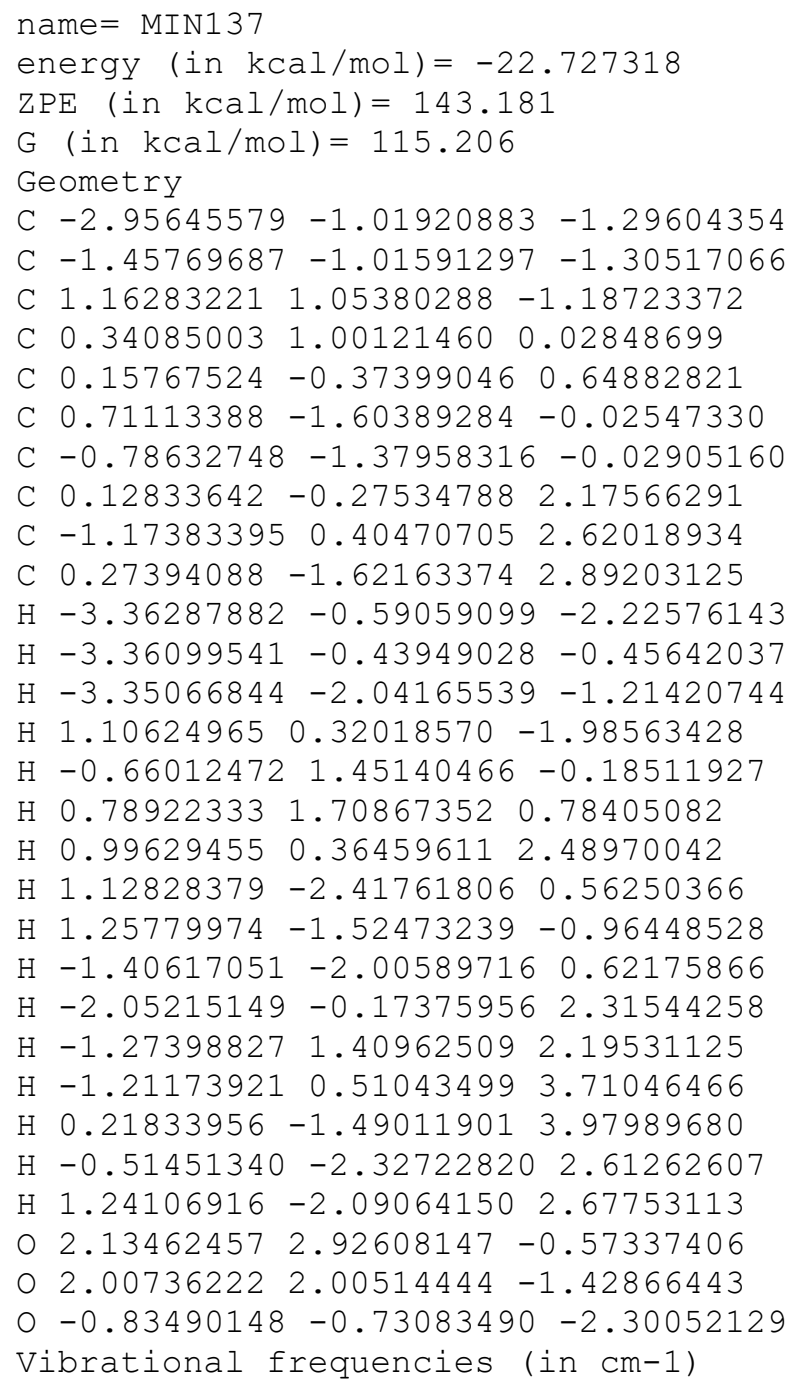


name $=$ MIN138

energy (in $\mathrm{kcal} / \mathrm{mol})=-27.362479$

$\mathrm{ZPE}($ in $\mathrm{kcal} / \mathrm{mol})=147.946$

$\mathrm{G}($ in $\mathrm{kcal} / \mathrm{mol})=124.333$

Geometry

C $-2.26281126-0.23358218-0.66267843$

C $-0.73587306-0.21536304-0.62984934$

C $-0.17656980 \quad 1.24099849-0.75473499$

C $1.107653501 .58350036 \quad 0.01067026$

C $1.47350080 \quad 0.58233483 \quad 1.11356347$

C $1.37673825-0.84047923 \quad 0.53155286$

C $-0.14778594-1.04742661 \quad 0.51834278$

C $-0.60229932-0.50666636 \quad 1.90148148$

$\begin{array}{llll}\text { C } & 0.42863115 & 0.59596778 & 2.25393329\end{array}$

C $-0.61099321-1.625910372 .94213475$

$\mathrm{H}-2.70397620 \quad 0.32873657 \quad 0.16548614$

$\mathrm{H}-2.64810143-1.25990662-0.61480686$

$\mathrm{H}-2.63535776 \quad 0.20102159-1.60204219$

$\mathrm{H}-0.966530661 .99915399-0.58097231$

$\mathrm{H} 1.02927142 \quad 2.60113188 \quad 0.43584084$

H $1.944742601 .63573446-0.72016583$

H $2.48708726 \quad 0.803233331 .50045136$

$\mathrm{H} 1.88106711-1.580904651 .16996040$

$\mathrm{H} \quad 1.82498337-0.92782701-0.47025923$

$\mathrm{H}-0.42341257-2.111155290 .38055762$

$\mathrm{H} \quad 0.91028983 \quad 0.39676597 \quad 3.22335193$

$\mathrm{H}-1.62119894-0.06979241 \quad 1.84020890$

$\mathrm{H}-0.055310291 .57809736 \quad 2.35030913$

H $0.37262395 \quad-2.10222788 \quad 3.02956658$

$\mathrm{H}-1.33299044-2.40846434 \quad 2.68240156$

$\mathrm{H}-0.88272686-1.245547693 .93280879$

$0.56466372 \quad 0.04984173-2.49367433$

$00.085860331 .33685765-2.15877042$

o $-0.34783493-0.84558812-1.86587959$

Vibrational frequencies (in $\mathrm{cm}-1$ )

$\begin{array}{lllllllllllllll}44.3 & 82.7 & 137.5 & 170.3 & 184.8 & 220.2 & 250.2 & 279.0 & 313.3 & 341.8 & 358.0 & 415.5 & 451.4 & 465.9\end{array}$

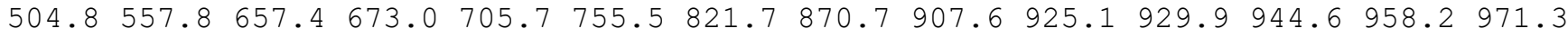
$976.3996 .51011 .0 \quad 1045.0 \quad 1058.2 \quad 1068.5 \quad 1082.91108 .4 \quad 1117.3 \quad 1141.11152 .11160 .7$

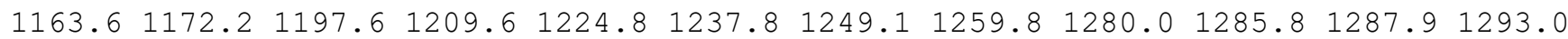

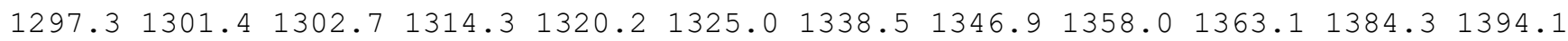
$1401.42655 .2 \quad 2669.8 \quad 2672.7 \quad 2675.92676 .5 \quad 2679.6 \quad 2679.82692 .8 \quad 2709.7 \quad 2720.92722 .9$ $2738.2 \quad 2753.8 \quad 2756.0 \quad 2779.2 \quad 2782.0$

name $=$ MIN139

energy (in $\mathrm{kcal} / \mathrm{mol})=-27.357247$

$\mathrm{ZPE}(\mathrm{in} \mathrm{kcal} / \mathrm{mol})=147.946$

$\mathrm{G}($ in $\mathrm{kcal} / \mathrm{mol})=124.321$

Geometry

C $-2.26124138 \quad 0.03987897-1.18319254$

C $-0.78514958-0.17721219-0.85804886$

C $-0.009499421 .17596971-0.74446231$

C $1.15718705 \quad 1.24806484 \quad 0.24943648$

C $1.18012658 \quad 0.12846443 \quad 1.29858195$

C $0.94713586-1.21879906 \quad 0.59180032$

C $-0.56477635-1.14511796 \quad 0.31183690$

C $-0.02235962 \quad 0.25402963 \quad 2.27495667$

C $-1.15303036-0.585810591 .62655064$

C $0.34168082-0.27631083 \quad 3.66135928$

$\mathrm{H}-2.78618488 \quad 0.58525760 \quad-0.39392845$

$\mathrm{H}-2.77709332-0.91756122-1.33093692$

$\mathrm{H}-2.372859390 .60583252-2.11961278$

$\mathrm{H}-0.698089412 .03646063-0.62851280$ 
H $1.15870074 \quad 2.23360573 \quad 0.75157950$

H $2.106865291 .21480824-0.32931345$

$\mathrm{H} 2.14367055 \quad 0.14911931 \quad 1.84362130$

$\mathrm{H} \quad 1.19830458-2.07062942 \quad 1.24118718$

$\mathrm{H} \quad 1.54357754-1.33250670-0.32660556$

$\mathrm{H}-0.99090148 \quad-2.13590430 \quad 0.06119237$

$\mathrm{H}-0.337117301 .31490757 \quad 2.36375790$

$\mathrm{H}-2.05480834 \quad 0.01911713 \quad 1.45932625$

$\mathrm{H}-1.46373539-1.41261482 \quad 2.28535894$

$\mathrm{H}-0.51789288 \quad-0.24190942 \quad 4.33937034$

H $1.14399040 \quad 0.31336258 \quad 4.11833099$

$\mathrm{H} \quad 0.68388586-1.317056893 .61576915$

$\begin{array}{lllll}0 & 0.87086503 & 0.00675174 & -2.42964968\end{array}$

$0 \quad 0.50864310 \quad 1.32452113-2.07080693$

o $-0.24596711-0.79178870-2.04367608$

Vibrational frequencies (in $\mathrm{cm}-1$ )

$\begin{array}{lllllllllllllllll}50.2 & 69.2 & 137.4 & 176.7 & 181.8 & 222.4 & 252.5 & 288.0 & 322.6 & 331.2 & 345.5 & 412.7 & 454.4 & 471.4\end{array}$

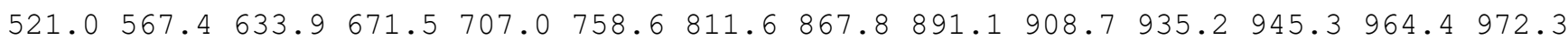
$981.11005 .2 \quad 1023.7 \quad 1045.2 \quad 1053.1 \quad 1068.0 \quad 1089.5 \quad 1110.6 \quad 1122.1 \quad 1140.6 \quad 1148.9 \quad 1161.5$

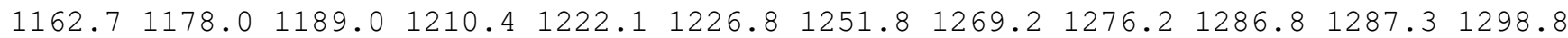

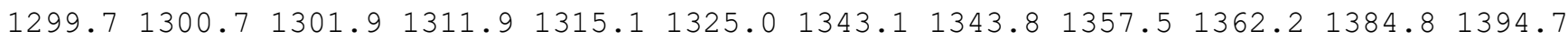
$1402.02654 .22670 .12670 .2 \quad 2675.5 \quad 2677.5 \quad 2680.02680 .22693 .8 \quad 2714.5 \quad 2721.22723 .7$ $2737.12751 .2 \quad 2756.02779 .92782 .3$

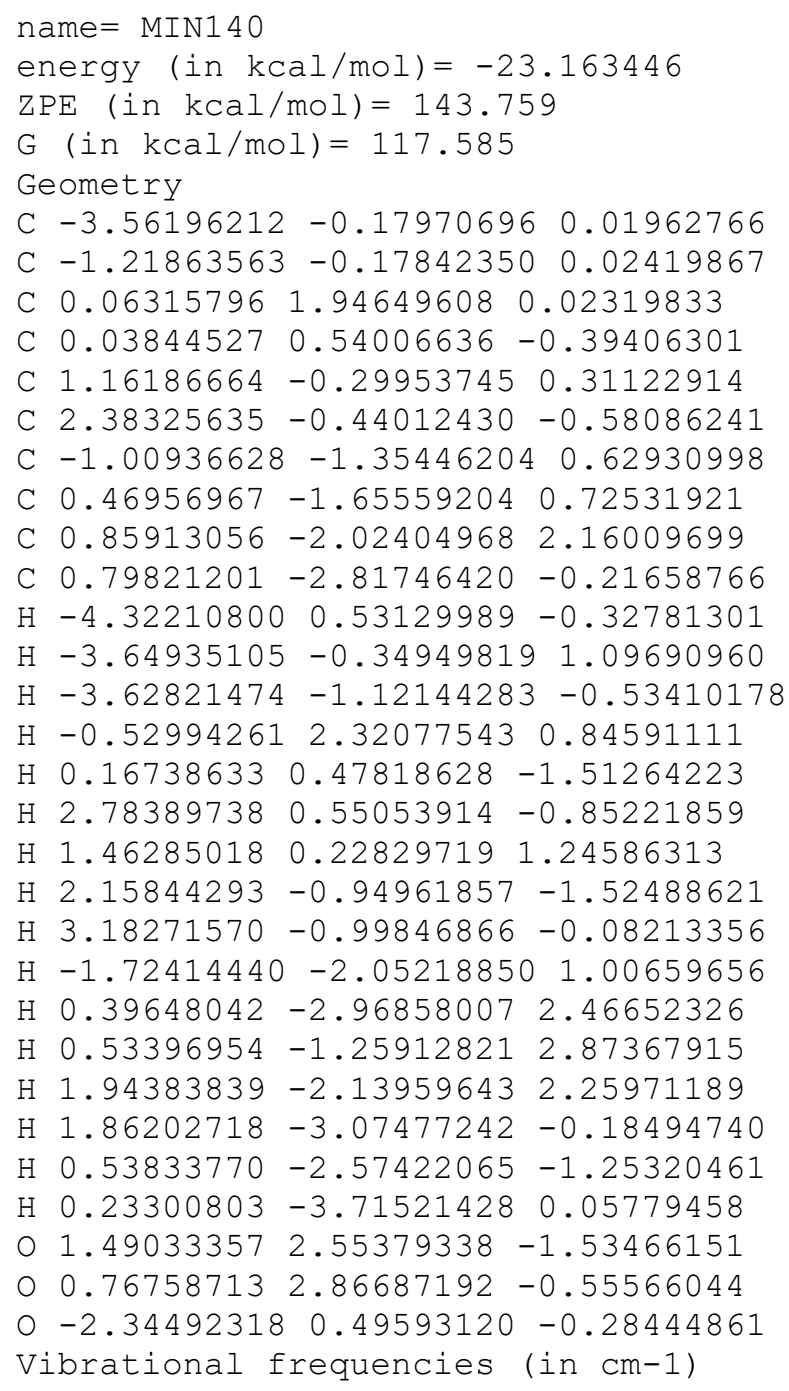


$2653.22658 .92659 .2 \quad 2663.4 \quad 2676.8 \quad 2677.4 \quad 2679.12680 .82682 .6 \quad 2701.02720 .2 \quad 2747.2$ 2778.62781 .62783 .22800 .8

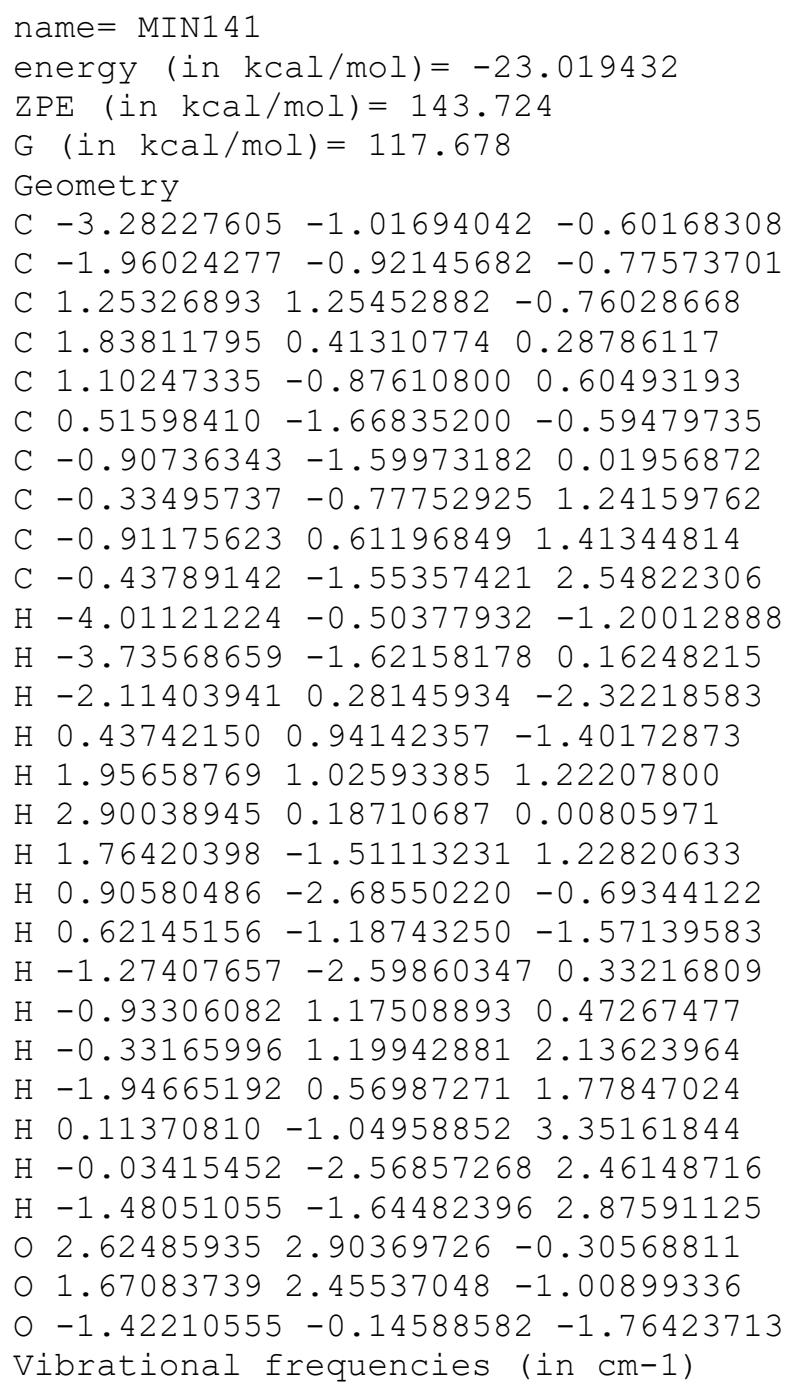


H $-0.05563898-2.86932598-1.81643294$

$\mathrm{H}-2.58339430 \quad 1.22391788 \quad 0.54189456$

$\mathrm{H} 0.15194479 \quad 1.09193532-0.92596753$

H $2.50278063 \quad 0.86406724-0.36877323$

H $1.18105431 \quad 1.99246701 \quad 1.16958381$

H $3.53256067-0.48113536 \quad 0.10393036$

H 2.93312661 $0.63129356 \quad 1.34273933$

$\mathrm{H} \quad 1.68719361-1.525589851 .41446687$

$\mathrm{H}-0.060511312 .02421980 \quad 3.35487189$

$\mathrm{H}-1.56811667 \quad 1.613749992 .54052823$

$\mathrm{H}-0.77479633 \quad 3.15451794 \quad 2.19461092$

$\mathrm{H}-0.34270001-0.849842712 .95281095$

$\mathrm{H}-0.73323383-1.92437060 \quad 1.60570137$

$\mathrm{H}-1.74689756-0.522858491 .93389203$

$0-1.62895579 \quad 0.69538553-2.39013653$

$\begin{array}{lllll}0 & -2.38073377 & 0.92081578 & -1.40637481\end{array}$

O $2.24201874-1.96509886-1.27428955$

Vibrational frequencies (in cm-1)

$\begin{array}{lllllllllllllll}-22.3 & 68.2 & 71.9 & 94.2 & 125.9 & 144.9 & 176.0 & 180.5 & 196.5 & 209.8 & 232.7 & 235.5 & 259.4 & 308.9\end{array}$ $318.4 \quad 350.6 \quad 397.6 \quad 448.3 \quad 475.3 \quad 523.3 \quad 538.7 \quad 572.9 \quad 643.7 \quad 703.2 \quad 732.7 \quad 822.5 \quad 855.9861 .6$ $952.6979 .6 \quad 1009.8 \quad 1016.6 \quad 1043.0 \quad 1049.3 \quad 1051.4 \quad 1076.0 \quad 1096.2 \quad 1100.51105 .41131 .4$ $1144.8 \quad 1220.0 \quad 1222.5 \quad 1238.6 \quad 1239.7 \quad 1253.4 \quad 1256.2 \quad 1265.8 \quad 1268.5 \quad 1284.91287 .8 \quad 1292.7$ $1295.51302 .5 \quad 1307.8 \quad 1318.7 \quad 1367.2 \quad 1376.0 \quad 1383.0 \quad 1399.4 \quad 1402.4 \quad 1406.91502 .11603 .9$ $1836.12628 .12657 .12664 .7 \quad 2670.92672 .5 \quad 2672.92674 .12676 .4 \quad 2688.2 \quad 2690.92727 .2$ $2735.4 \quad 2773.1 \quad 2776.2 \quad 2779.2 \quad 2781.0$

name $=$ MIN143

energy $($ in $\mathrm{kcal} / \mathrm{mol})=-22.967423$

$\mathrm{ZPE}($ in $\mathrm{kcal} / \mathrm{mol})=143.84$

$\mathrm{G}($ in $\mathrm{kcal} / \mathrm{mol})=116.181$

Geometry

C $-3.40141958-0.36241444-0.85425079$

C $-1.93846874-0.35193589-1.16985342$

C $-1.277240170 .96196500-1.17981715$

C $0.02984760 \quad 1.21657273-1.02725931$

C $1.148804590 .26492500-0.82507955$

C $1.08562543-0.520225950 .47553033$

$\begin{array}{llll}\text { C } 1.44955627 & 0.34921783 & 1.63132250\end{array}$

C $0.58087699 \quad 0.81766095 \quad 2.53727354$

C $1.03164112 \quad 1.68660167 \quad 3.66581340$

C -0.882570860 .520094322 .50861155$

$\mathrm{H}-3.76802183-1.38675847-0.67391096$

$\mathrm{H}-3.98763226 \quad 0.03904201-1.69528477$

$\mathrm{H}-3.64405661 \quad 0.23773317 \quad 0.03216529$

$\mathrm{H}-1.97164947 \quad 1.79957740-1.30890171$

$\mathrm{H} \quad 0.36260184 \quad 2.26709427 \quad-1.02611721$

$\mathrm{H} \quad 0.24558787-1.25253336-1.84798374$

H $2.14859113 \quad 0.74271743 \quad-0.94344609$

H $1.81131162-1.377452530 .39484194$

$\mathrm{H} \quad 0.09882567-1.005699120 .60572980$

H $2.511441610 .58446686 \quad 1.69558654$

H $0.75105134 \quad 1.25400175 \quad 4.63709421$

$\mathrm{H} \quad 0.57105325 \quad 2.68274032 \quad 3.60599685$

H 2.11970688 $1.83448175 \quad 3.68311937$

$\mathrm{H}-1.31752447 \quad 0.72696167 \quad 1.52133469$

$\mathrm{H}-1.07128339-0.54023045 \quad 2.73697833$

$\mathrm{H}-1.449435531 .110050093 .23948532$

$02.07079983-1.62191598-1.84869744$

O $1.14739252-0.69663780-2.01388408$

o $-1.36337666-1.39322212-1.42241314$

Vibrational frequencies (in $\mathrm{cm}-1$ )

$\begin{array}{lllllllllllllll}29.7 & 41.3 & 61.5 & 80.5 & 100.6 & 102.7 & 109.7 & 132.7 & 157.4 & 174.9 & 191.0 & 278.2 & 304.3 & 319.4\end{array}$

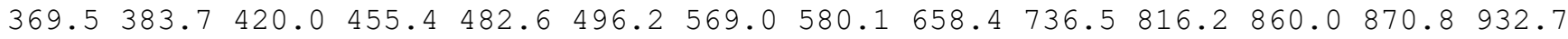
$934.6 \quad 957.9 \quad 987.9 \quad 998.3 \quad 1010.8 \quad 1025.5 \quad 1048.3 \quad 1063.2 \quad 1072.2 \quad 1089.5 \quad 1115.11138 .2 \quad 1184.4$ 
$\begin{array}{lllllllllllll}1191.2 & 1236.7 & 1250.1 & 1261.2 & 1268.5 & 1276.5 & 1279.2 & 1280.3 & 1287.0 & 1288.8 & 1292.2 & 1298.6\end{array}$

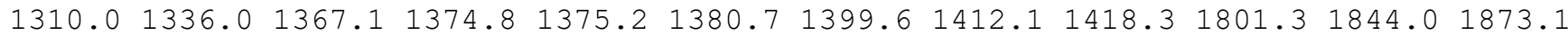
$2571.82621 .32661 .0 \quad 2667.6 \quad 2669.5 \quad 2672.0 \quad 2672.92676 .2 \quad 2686.8 \quad 2714.92724 .8 \quad 2743.4$ 2756.92777 .02778 .02780 .2

\section{name $=\operatorname{MIN} 144$}

energy (in $\mathrm{kcal} / \mathrm{mol})=-25.058452$

$\mathrm{ZPE}($ in $\mathrm{kcal} / \mathrm{mol})=146.347$

$\mathrm{G}($ in $\mathrm{kcal} / \mathrm{mol})=123.463$

Geometry

C $-2.24169127-0.216749640 .34150546$

C $-0.74542590-0.13175275 \quad 0.22796674$

C $-0.18480627 \quad 1.32339393 \quad 0.14101618$

C $1.318289891 .16777980 \quad 0.48588733$

C $1.47452667-0.357229190 .71524861$

C $1.34956413-1.03300951-0.67407849$

C $-0.15198470-0.86320741-1.01823281$

C $0.11035564-0.73770558 \quad 1.39702364$

C $-0.11128035-0.06396487 \quad 2.74949504$

C $-0.07568120-2.240412651 .59798640$

$\mathrm{H}-2.727106150 .22253895 \quad-0.54365544$

$\mathrm{H}-2.61640706 \quad 0.32110828 \quad 1.22097487$

$\mathrm{H}-2.58601803-1.25510930 \quad 0.42351425$

H $-0.72439118 \quad 2.03593553 \quad 0.79245329$

H $1.60071818 \quad 1.74604615 \quad 1.37575799$

$\mathrm{H} \quad 1.95716604 \quad 1.53180120 \quad-0.33360495$

$\mathrm{H} 2.36729413-0.63982008 \quad 1.27568517$

$\mathrm{H} \quad 1.64879328-2.08933590-0.64185091$

$\mathrm{H} \quad 1.99087056-0.55318378-1.42930577$

$\mathrm{H}-0.66945349-1.81264812-1.25187127$

$\mathrm{H}-1.12699250 \quad-0.25253700 \quad 3.11813925$

$\mathrm{H} \quad 0.02131544 \quad 1.02124718 \quad 2.72090417$

$\mathrm{H} \quad 0.59009569-0.452084523 .49765925$

H $0.70417556-2.644377312 .25437049$

$\mathrm{H}-0.03707435-2.81113905 \quad 0.66586897$

$\mathrm{H}-1.04516507-2.45743115 \quad 2.06277871$

$00.26626806 \quad 1.11857387-2.09963667$

$0-0.407599521 .88315565-1.13781371$

o $-0.37388612-0.12362534-2.20218234$

Vibrational frequencies (in $\mathrm{cm}-1$ )

$\begin{array}{llllllllllllll}111.3 & 149.2 & 156.3 & 169.1 & 206.0 & 207.9 & 228.3 & 258.5 & 307.3 & 327.4 & 396.8 & 397.3 & 434.1 & 487.6\end{array}$

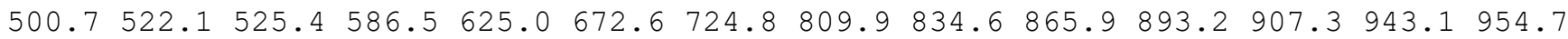

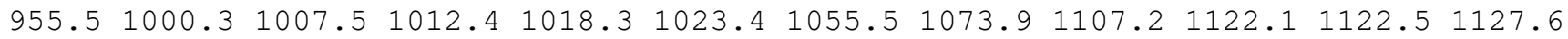

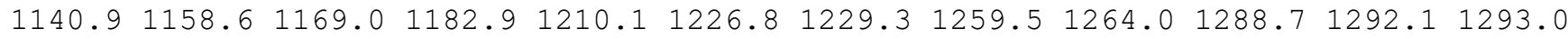

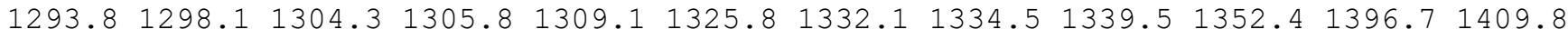
$1418.8 \quad 2671.8 \quad 2672.2 \quad 2672.92675 .6 \quad 2676.0 \quad 2676.6 \quad 2678.22680 .3 \quad 2699.5 \quad 2700.5 \quad 2749.5$ $2753.3 \quad 2754.92780 .4 \quad 2781.6 \quad 2783.3$

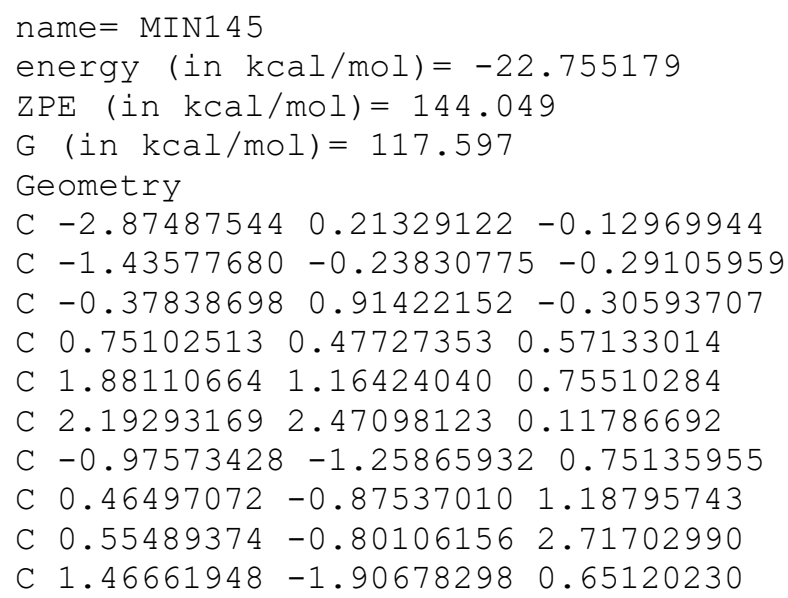


$\mathrm{H}-3.15935078 \quad 0.92099700 \quad-0.92181473$

$\mathrm{H}-3.04324640 \quad 0.69988694 \quad 0.83691188$

$\mathrm{H}-3.56592440-0.63761846-0.20348430$

$\mathrm{H}-0.78203957 \quad 1.91391499-0.06264396$

$\mathrm{H}-0.10952420-0.02803715 \quad 3.11856438$

H $2.36189906 \quad 3.25263350 \quad 0.87249776$

H $2.67188843 \quad 0.77775410 \quad 1.40124828$

H $3.108938812 .39934842-0.48993646$

H $1.401740292 .82792635-0.55536217$

$\mathrm{H}-1.00752050-2.27844671 \quad 0.32747528$

$\mathrm{H}-1.65849007-1.27740225 \quad 1.61662120$

$\mathrm{H} \quad 1.57149267-0.55838595 \quad 3.04613958$

$\mathrm{H} \quad 0.28060960-1.755140503 .17913193$

$\mathrm{H} 1.26648372-2.905786691 .05069254$

H $2.49605014 \quad-1.642357090 .91437148$

H $1.41558323-1.96528521-0.44435911$

o $-0.11119682-0.34184494-2.13445965$

$00.00634666 \quad 0.99529648-1.67547030$

o - $1.35831529-0.78733556-1.61375555$

Vibrational frequencies (in $\mathrm{cm}-1$ )

$\begin{array}{lllllllllllllll}15.4 & 33.0 & 58.8 & 138.4 & 154.4 & 168.0 & 179.5 & 198.6 & 199.6 & 240.2 & 275.9 & 292.8 & 309.4 & 337.4\end{array}$

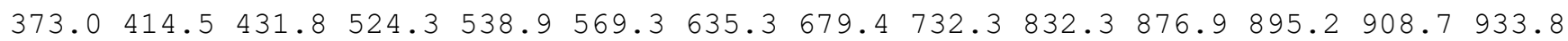
$943.8965 .8 \quad 982.8 \quad 986.1 \quad 992.5 \quad 1010.4 \quad 1029.4 \quad 1066.1 \quad 1079.9 \quad 1083.4 \quad 1096.7 \quad 1139.1 \quad 1171.3$ $1189.21206 .51240 .7 \quad 1251.8 \quad 1254.8 \quad 1271.7 \quad 1276.2 \quad 1277.0 \quad 1284.0 \quad 1285.5 \quad 1287.6 \quad 1293.9$ $1295.21297 .4 \quad 1305.1 \quad 1306.7 \quad 1326.0 \quad 1366.3 \quad 1375.2 \quad 1395.6 \quad 1398.6 \quad 1400.1 \quad 1408.6 \quad 1887.2$ $2666.72667 .12670 .7 \quad 2672.7 \quad 2673.8 \quad 2677.3 \quad 2679.3 \quad 2680.3 \quad 2682.6 \quad 2700.02747 .22748 .2$ $2778.8 \quad 2780.3 \quad 2780.8 \quad 2782.7$

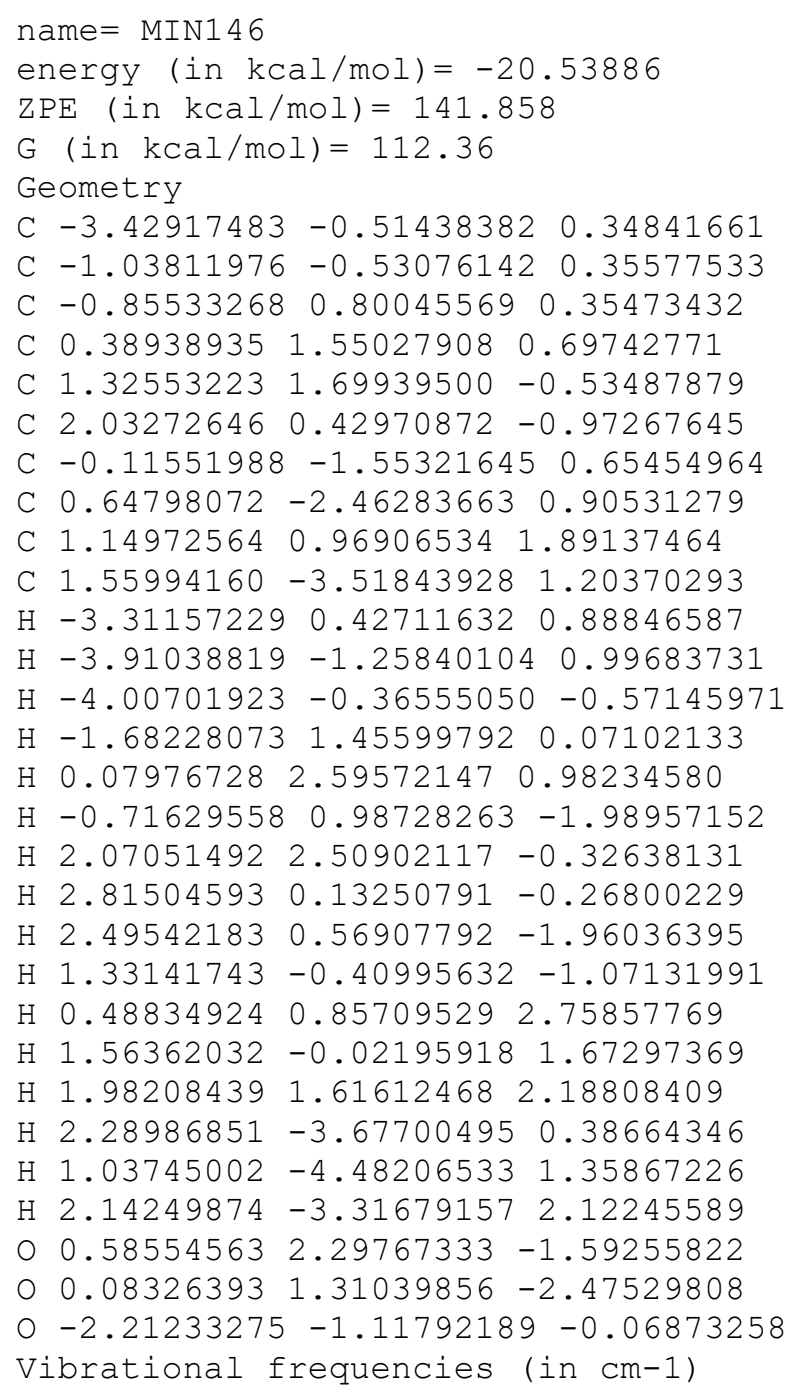


$\begin{array}{llllllllllllllllll}-138.2 & 14.6 & 24.4 & 40.4 & 45.5 & 81.3 & 88.3 & 139.6 & 161.8 & 174.3 & 192.7 & 202.0 & 252.0 & 265.8\end{array}$ $289.8 \quad 315.6 \quad 328.7 \quad 345.7 \quad 381.2 \quad 413.5 \quad 431.5 \quad 499.7 \quad 575.9 \quad 635.4 \quad 662.4 \quad 702.7 \quad 757.3 \quad 851.6$

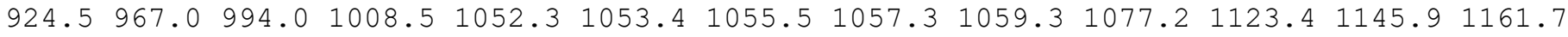
$1189.31206 .3 \quad 1238.6 \quad 1258.0 \quad 1258.8 \quad 1259.2 \quad 1282.5 \quad 1287.2 \quad 1291.4 \quad 1295.91296 .6 \quad 1299.3$ $1308.31311 .8 \quad 1319.8 \quad 1348.3 \quad 1381.5 \quad 1382.91394 .4 \quad 1399.1 \quad 1406.11478 .3 \quad 1867.92564 .2$ $2648.62654 .0 \quad 2657.8 \quad 2659.0 \quad 2660.4 \quad 2667.0 \quad 2676.2 \quad 2680.02684 .42690 .22741 .02744 .2$ $2775.8 \quad 2781.7 \quad 2782.2 \quad 2848.7$

name $=\operatorname{MIN} 147$

energy (in $\mathrm{kcal} / \mathrm{mol})=-21.701381$

$\mathrm{ZPE}($ in $\mathrm{kcal} / \mathrm{mol})=143.075$

$\mathrm{G}($ in $\mathrm{kcal} / \mathrm{mol})=116.267$

Geometry

C $-2.89775860-0.59515660-0.88030382$

C $-1.45524260-0.94455083-0.87516378$

C $0.297017391 .76340910-0.66286113$

C $1.15996450 \quad 0.58759200 \quad-0.31249274$

C $0.54570111-0.31942156 \quad 0.71935197$

C $0.59233584 \quad-1.80685744 \quad 0.47671334$

C $-0.75010690-1.101867290 .37932253$

$\begin{array}{llll}\text { C } & 0.64028601 & 0.15448185 & 2.16850433\end{array}$

C $2.09448022 \quad 0.42427208 \quad 2.56876799$

C $-0.22048397 \quad 1.40144103 \quad 2.39695393$

$\mathrm{H}-3.098300250 .30096079-0.26954584$

$\mathrm{H}-3.50350418-1.41362455-0.45310942$

$\mathrm{H}-3.29908729-0.39607662-1.88650764$

$\mathrm{H}-0.78476880 \quad 1.66404890 \quad-0.50897794$

H $2.16751792 \quad 0.92911798 \quad 0.01307616$

$\mathrm{H} \quad 1.37874836 \quad 0.02312680 \quad-1.26141423$

$\mathrm{H} \quad 0.25073095 \quad-0.65998260 \quad 2.83384977$

H $0.76497243-2.49730260 \quad 1.29701679$

H $1.06204140 \quad-2.19359393-0.43321304$

$\mathrm{H}-1.44017227-1.25520651 \quad 1.22767402$

H 2.49022309 1.329063492 .09203685

H $2.74635973-0.411916112 .29027218$

H 2.18299608 $0.56769829 \quad 3.65165147$

$\mathrm{H}-0.23692998 \quad 1.67719341 \quad 3.45811742$

$\mathrm{H}-1.25628452 \quad 1.237869892 .08411385$

$\mathrm{H} \quad 0.16533243 \quad 2.26957198 \quad 1.84890936$

O $0.20383101-1.41416021-2.27637062$

$\begin{array}{lllll}0 & 0.76179389 & 2.78022877 & -1.11642178\end{array}$

o -1.00403213-1.10534802 -2.08843583

Vibrational frequencies (in $\mathrm{cm}-1$ )

$\begin{array}{lllllllllllllll}37.6 & 48.8 & 62.4 & 97.4 & 104.4 & 109.5 & 154.4 & 176.9 & 191.9 & 200.3 & 236.9 & 268.2 & 298.6 & 305.4\end{array}$

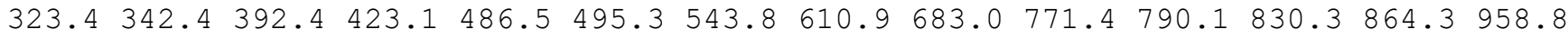
$971.5979 .6 \quad 986.91003 .1 \quad 1013.4 \quad 1015.8 \quad 1044.8 \quad 1064.4 \quad 1086.91109 .6 \quad 1129.2 \quad 1144.4 \quad 1168.7$ $\begin{array}{llllllllllll}1179.9 & 1184.1 & 1194.7 & 1258.2 & 1259.7 & 1264.7 & 1282.6 & 1286.9 & 1295.8 & 1297.6 & 1301.7 & 1306.6\end{array}$ $1309.81312 .2 \quad 1336.5 \quad 1350.91377 .4 \quad 1380.6 \quad 1398.6 \quad 1400.7 \quad 1424.7 \quad 1481.2 \quad 1627.7 \quad 1834.6$ $2600.62650 .82654 .2 \quad 2660.6 \quad 2666.0 \quad 2670.5 \quad 2677.5 \quad 2680.72687 .2 \quad 2699.32719 .92738 .0$ $2770.7 \quad 2772.1 \quad 2779.4 \quad 2781.1$

name $=$ MIN148

energy (in $\mathrm{kcal} / \mathrm{mol})=-20.659224$

$\mathrm{ZPE}(\mathrm{in} \mathrm{kcal} / \mathrm{mol})=142.068$

$\mathrm{G}($ in $\mathrm{kcal} / \mathrm{mol})=114.482$

Geometry

C $-2.57208695-0.10380893-0.57597570$

C $-1.08174742-0.10777121-0.70450116$

C $-0.49410190 \quad 1.27019262-0.63205252$

C $0.47268627 \quad 1.69202039-1.68389483$

C $1.58032365-0.24685588 \quad 0.88249300$

C $1.14053420-1.31918472-0.06047545$

C $-0.32685565-1.30882710-0.35914759$ 
C $1.13090463-0.094969892 .13619274$

C 1.631134791 .012321153 .00455702

C $0.09178358-0.96889708 \quad 2.75401541$

$\mathrm{H}-3.05006759 \quad 0.45809612-1.39311182$

$\mathrm{H}-2.87335207 \quad 0.39712772 \quad 0.36334659$

$\mathrm{H}-3.01344851-1.10730945-0.56084195$

$\mathrm{H} \quad 0.051409191 .57976388-2.69659646$

$\mathrm{H} \quad 0.77798618 \quad 2.74018164 \quad-1.55472777$

$\mathrm{H} \quad 1.38352167 \quad 1.07106125-1.67161793$

H 2.33520432 $0.42505123 \quad 0.47893333$

H $1.44562887-2.31160002 \quad 0.34332147$

$\mathrm{H} \quad 1.70291962-1.23116863-1.02947773$

$\mathrm{H}-0.87544959-2.17498404 \quad 0.02082809$

$\mathrm{H} \quad 0.81007670 \quad 1.69727915 \quad 3.26982109$

H $2.40518921 \quad 1.62054460 \quad 2.51789206$

H $2.05836711 \quad 0.62872760 \quad 3.94092745$

$\mathrm{H} \quad 0.12285337-0.94924217 \quad 3.85098058$

H $0.19332971-2.01724517 \quad 2.44448383$

$\mathrm{H}-0.91393737-0.633376152 .45355359$

o $0.21469445-0.80381884-2.65090872$

$0-0.85322207 \quad 2.01378497 \quad 0.24781175$

O $-0.68325806-1.07966234-1.86508191$

Vibrational frequencies (in $\mathrm{cm}-1$ )

$\begin{array}{lllllllllllllll}22.0 & 54.6 & 62.6 & 76.9 & 105.1 & 112.3 & 120.2 & 139.2 & 168.9 & 191.8 & 211.8 & 234.8 & 275.9 & 286.2\end{array}$ $296.6 \quad 314.3 \quad 396.2 \quad 436.4 \quad 471.2 \quad 480.4 \quad 555.8 \quad 563.3 \quad 620.4 \quad 629.8 \quad 667.8 \quad 700.0 \quad 799.6 \quad 883.0$ $922.8 \quad 977.9 \quad 993.3 \quad 996.0 \quad 1021.0 \quad 1026.0 \quad 1044.1 \quad 1055.6 \quad 1067.4 \quad 1082.8 \quad 1116.0 \quad 1143.4 \quad 1144.8$ $\begin{array}{lllllllllllllll}1195.1 & 1215.1 & 1249.5 & 1253.8 & 1268.6 & 1270.5 & 1273.2 & 1275.2 & 1278.9 & 1282.5 & 1284.7 & 1286.2\end{array}$ $1289.81303 .2 \quad 1346.8 \quad 1365.3 \quad 1378.4 \quad 1382.6 \quad 1398.8 \quad 1400.6 \quad 1418.8 \quad 1507.8 \quad 1841.2 \quad 1870.8$ $2620.82652 .12654 .0 \quad 2666.8 \quad 2669.3 \quad 2671.92672 .92674 .32677 .92717 .5 \quad 2731.02756 .3$ $2773.92775 .92777 .8 \quad 2780.0$

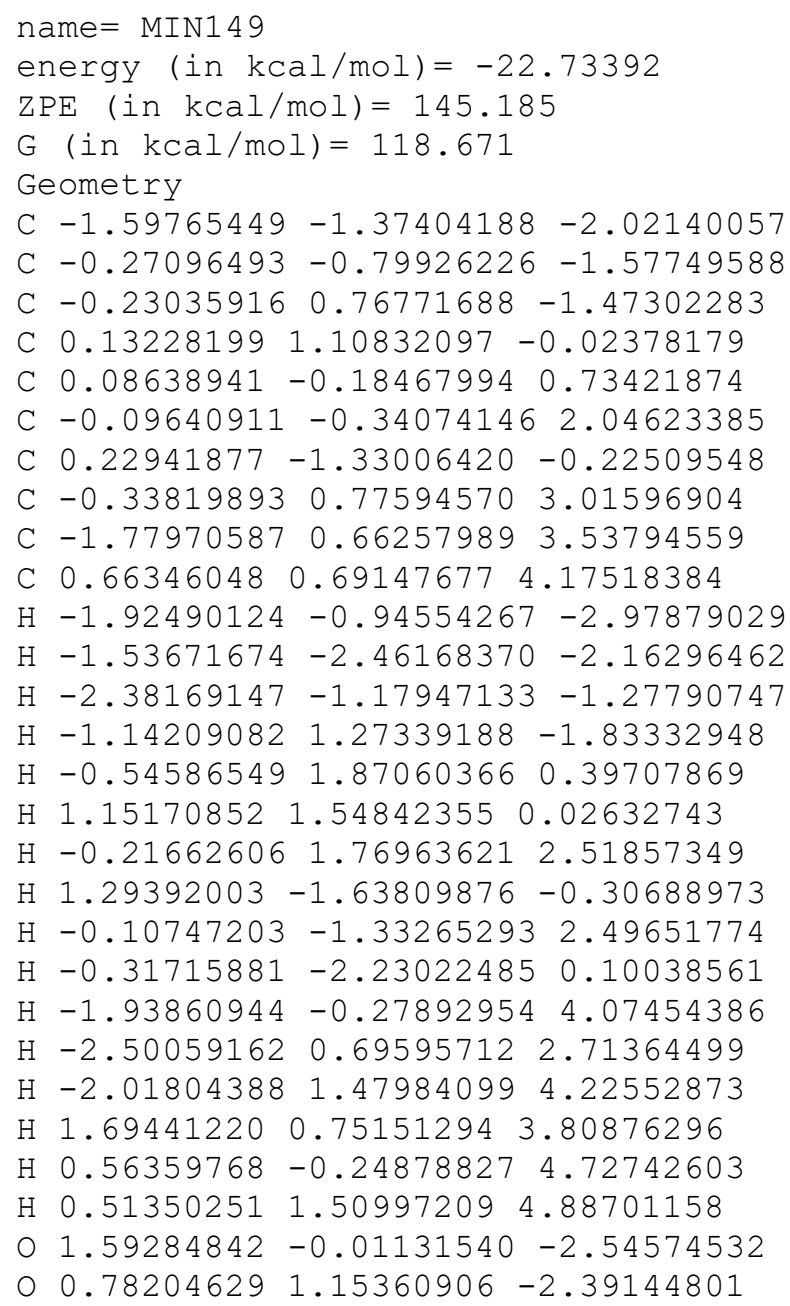


$0.66541666-1.07893574-2.62165300$

Vibrational frequencies (in cm-1)

$\begin{array}{lllllllllllllllll}13.0 & 40.3 & 77.0 & 113.9 & 132.3 & 150.5 & 156.5 & 174.3 & 217.0 & 255.3 & 294.4 & 322.8 & 374.3 & 392.9\end{array}$

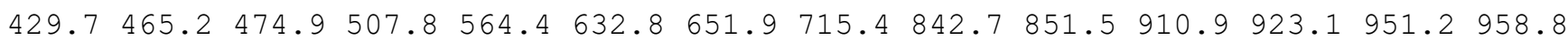

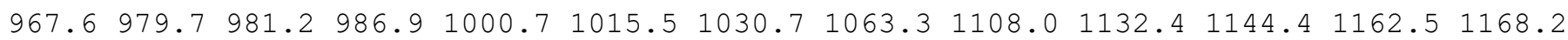
$\begin{array}{llllllllllllllll}1170.9 & 1194.9 & 1248.9 & 1253.7 & 1260.6 & 1275.4 & 1280.0 & 1282.3 & 1286.9 & 1296.0 & 1297.7 & 1298.5\end{array}$

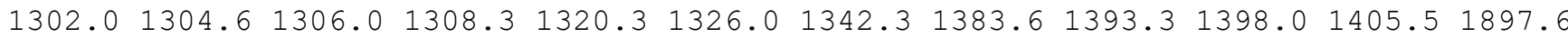
$2660.12664 .12672 .2 \quad 2674.3 \quad 2675.2 \quad 2678.6 \quad 2679.72682 .42701 .8 \quad 2706.02743 .42746 .4$ $2758.92780 .5 \quad 2782.12783 .7$

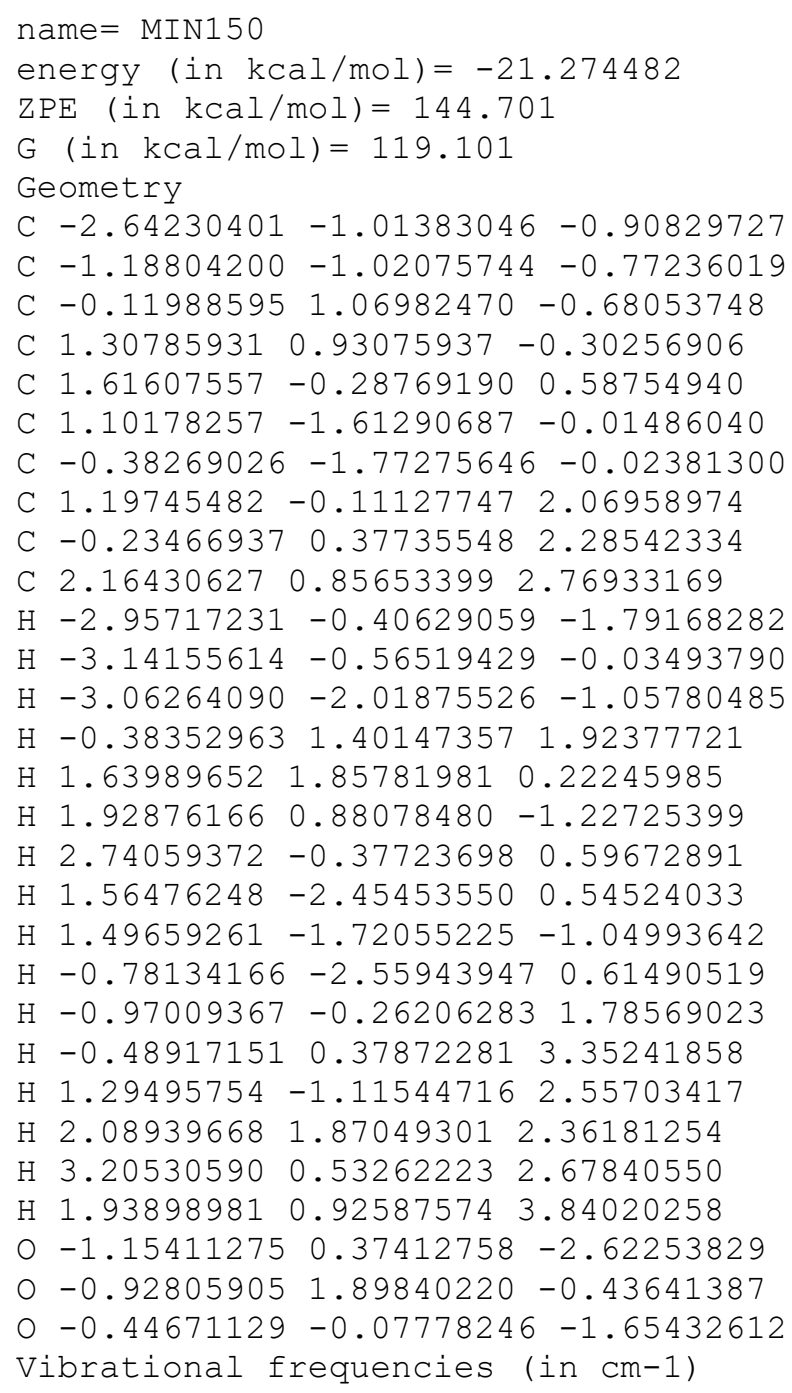


C $1.32831397 \quad 0.78130149 \quad 2.75342462$

C $-0.43306718-1.47644408 \quad 0.31086107$

C $0.13764449-1.02812297 \quad 1.43629508$

$\begin{array}{lllll}\text { C } 0.98874053 & 2.59477855 & 0.42802091\end{array}$

C $0.59699357 \quad-1.97590874 \quad 2.48961796$

$\mathrm{H}-2.90162878 \quad 0.04041800-1.54960901$

$\mathrm{H}-2.89025410 \quad-0.210103690 .21314439$

$\mathrm{H}-2.88711266-1.59563441-0.88768010$

$\mathrm{H}-1.16559924 \quad 1.64608440-0.68094407$

$\mathrm{H} 1.65775378 \quad 0.58722586-0.04547254$

$\mathrm{H} \quad 1.05815169 \quad 0.31867112 \quad 3.70910137$

$\mathrm{H}-0.68322672 \quad 0.87524457 \quad 1.96872868$

H $2.33273259 \quad 0.43606691 \quad 2.48366380$

$\mathrm{H} 1.38743750 \quad 1.86145248 \quad 2.93257940$

$\mathrm{H}-0.54910417-2.53585040 \quad 0.09468993$

$\mathrm{H} 0.16342966 \quad 3.12028953 \quad 0.91903423$

H $1.12869333 \quad 3.04405719-0.56501395$

H $1.90186511 \quad 2.79999251 \quad 0.99830654$

$\mathrm{H} \quad 0.10449871-1.77535753 \quad 3.45186260$

$\mathrm{H} 1.68291251-1.90151947 \quad 2.65104678$

H $0.38666428-3.024228692 .23412794$

O $0.52403477-0.30442063-2.42235326$

$00.17262718 \quad 1.01992899 \quad-2.04364965$

o $-0.59956006-1.07796907-2.04494788$

Vibrational frequencies (in $\mathrm{cm}-1$ )

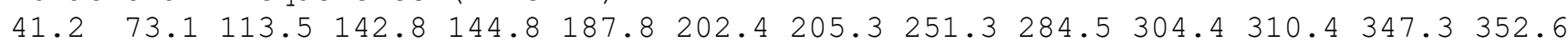
$399.1409 .2 \quad 445.5 \quad 476.9 \quad 533.4 \quad 600.8 \quad 614.9 \quad 674.0 \quad 717.1 \quad 847.8 \quad 874.5 \quad 909.8 \quad 922.8 \quad 930.7$ $968.3 \quad 995.7 \quad 1001.7 \quad 1018.3 \quad 1039.7 \quad 1050.3 \quad 1060.8 \quad 1095.4 \quad 1101.81120 .01148 .7 \quad 1169.8$

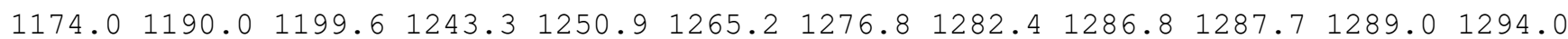

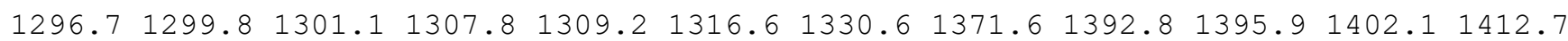
$1860.62668 .72670 .72671 .2 \quad 2671.6 \quad 2672.2 \quad 2673.82675 .12679 .72683 .82693 .4 \quad 2711.1$ $2759.7 \quad 2778.12779 .7 \quad 2780.6 \quad 2781.1$

name $=$ MIN152

energy (in $\mathrm{kcal} / \mathrm{mol})=-19.059401$

$\operatorname{ZPE}(\mathrm{in} \mathrm{kcal} / \mathrm{mol})=143.067$

$\mathrm{G}($ in $\mathrm{kcal} / \mathrm{mol})=116.238$

Geometry

C $-2.14099228-0.98003092-1.13245288$

C $-0.66075203-0.96779080-1.09538523$

C $0.36566008 \quad 1.76200866-1.10230337$

C $0.206648491 .61287923 \quad 0.39308937$

C $0.45366181 \quad 0.23017418 \quad 0.95596283$

C $1.50288401-0.65292221 \quad 0.33166307$

C $0.04437642-1.03142255 \quad 0.17280237$

C $0.21382531 \quad 0.22209132 \quad 2.46904687$

C $-1.29541005 \quad 0.22115930 \quad 2.75398275$

C $0.86876352-0.960244173 .19123493$

$\mathrm{H}-2.55656998-0.90427842-2.15005956$

$\mathrm{H}-2.55257933-0.13027094-0.55751895$

$\mathrm{H}-2.54945037-1.89894565-0.68083078$

$\mathrm{H} \quad 1.21508887 \quad 1.26617526-1.59401857$

$\mathrm{H}-0.81100337 \quad 1.96041466 \quad 0.68349699$

$\mathrm{H} \quad 0.90480788 \quad 2.33647475 \quad 0.87476121$

$\mathrm{H} \quad 0.65705173 \quad 1.16026843 \quad 2.89360118$

H $2.14744903-1.279214640 .94299406$

H $2.07422931-0.31232871-0.53522886$

$\mathrm{H}-0.31278280-1.88031699 \quad 0.78524558$

$\mathrm{H}-1.78882449-0.64427267 \quad 2.29899176$

$\mathrm{H}-1.78383864 \quad 1.12154628 \quad 2.36625252$

$\mathrm{H}-1.49490186 \quad 0.18334707 \quad 3.83073467$

$\mathrm{H} \quad 0.65994706 \quad-0.92389687 \quad 4.26720001$

$\mathrm{H} 1.95889311-0.94939368 \quad 3.07604035$

$\mathrm{H} \quad 0.50004096-1.923922932 .82504381$ 
$01.11576609-0.89653001-2.41622731$

$0-0.416880862 .43563368-1.72632561$

o $-0.13820537-0.93496675-2.28920691$

Vibrational frequencies (in $\mathrm{cm}-1$ )

$\begin{array}{llllllllllllllll}38.5 & 54.0 & 64.0 & 89.4 & 96.9 & 103.8 & 155.9 & 158.5 & 190.1 & 211.3 & 231.7 & 268.0 & 286.4 & 317.3\end{array}$ $\begin{array}{lllllllllllllllll}335.5 & 345.1 & 409.9 & 442.9 & 459.1 & 496.1 & 564.7 & 579.7 & 678.7 & 759.4 & 808.4 & 829.7 & 895.2 & 952.1\end{array}$ $971.5980 .51002 .2 \quad 1010.0 \quad 1013.4 \quad 1025.4 \quad 1042.7 \quad 1047.6 \quad 1090.01103 .1 \quad 1118.91162 .3$

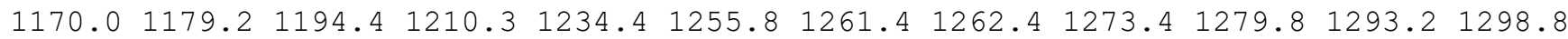

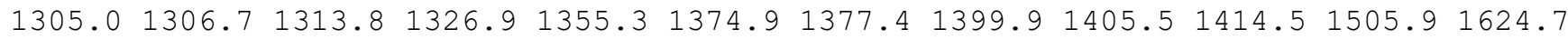
$1829.02624 .42648 .92653 .42661 .12669 .52673 .12675 .42677 .2 \quad 2684.62701 .12729 .3$ $2731.22770 .12772 .8 \quad 2780.2 \quad 2782.1$

name $=$ MIN153

energy $($ in $\mathrm{kcal} / \mathrm{mol})=-23.678471$

$\mathrm{ZPE}(\mathrm{in} \mathrm{kcal} / \mathrm{mol})=148.109$

$\mathrm{G}($ in $\mathrm{kcal} / \mathrm{mol})=124.783$

Geometry

C $-2.16209814-0.31442964-0.66157026$

C $-0.63878752-0.24359713-0.59412542$

C $-0.144593391 .23979278-0.50734520$

C $1.139799501 .52415862 \quad 0.28090499$

C $1.56477824 \quad 0.37507227 \quad 1.20171840$

C $1.53071510-0.93259982 \quad 0.38350825$

C $0.01486637-1.23282733 \quad 0.38162344$

C $-0.31367026-1.063243751 .89061623$

C $-1.77869315-0.954322062 .28247986$

$\begin{array}{lllll}\text { C } 0.52436790 & 0.16256970 & 2.32935693\end{array}$

$\mathrm{H}-2.520648850 .09594392-1.61885926$

$\mathrm{H}-2.64378454 \quad 0.25472820 \quad 0.13885552$

$\mathrm{H}-2.51946738-1.34890278-0.60459515$

$\mathrm{H}-0.96169447 \quad 1.92785922-0.21167352$

H $1.025066652 .45632936 \quad 0.86438922$

$\mathrm{H} 1.95802726 \quad 1.73269417-0.44298560$

H $2.57032088 \quad 0.57394708 \quad 1.61902000$

H $2.10650849-1.73603620 \quad 0.86468179$

$\mathrm{H} \quad 1.94335874-0.81541408-0.63020834$

$\mathrm{H}-0.21138305-2.264411180 .04492250$

$\mathrm{H}-2.37907486-1.73716192 \quad 1.80392935$

$\mathrm{H}-2.21255163 \quad 0.01539318 \quad 2.01736044$

H $0.09991492-1.97155372 \quad 2.40163530$

$\mathrm{H}-1.89956954-1.06958388 \quad 3.36727294$

$\mathrm{H} 1.01313728-0.01930873 \quad 3.29704138$

$\mathrm{H}-0.10603731 \quad 1.05301281 \quad 2.46900824$

$\begin{array}{lllll}0 & 0.62252969 & 0.36173635 & -2.41363298\end{array}$

○ $0.08448003 \quad 1.55685689-1.88465986$

o $-0.22879870-0.66369586-1.91700264$

Vibrational frequencies (in $\mathrm{cm}-1$ )

$\begin{array}{lllllllllllllllll}43.4 & 88.2 & 160.9 & 191.8 & 219.8 & 236.1 & 251.9 & 307.7 & 333.7 & 347.1 & 378.1 & 423.8 & 457.3 & 472.4\end{array}$ $480.2 \quad 549.3 \quad 612.8 \quad 684.8 \quad 703.2 \quad 752.4 \quad 812.3 \quad 872.6 \quad 895.0 \quad 907.1 \quad 929.7 \quad 949.2 \quad 962.4 \quad 965.1$ $981.2999 .6 \quad 1012.2 \quad 1038.8 \quad 1063.9 \quad 1067.5 \quad 1071.3 \quad 1106.4 \quad 1118.5 \quad 1135.2 \quad 1146.11161 .8$

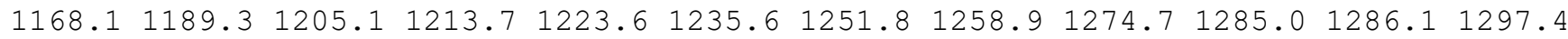

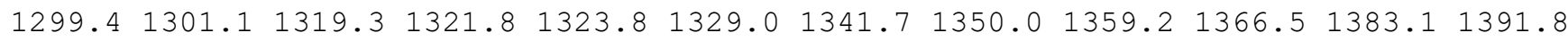
$1404.32655 .22665 .12667 .2 \quad 2673.6 \quad 2675.4 \quad 2677.62678 .42693 .42699 .32718 .42723 .0$ $2738.4 \quad 2754.3 \quad 2756.0 \quad 2776.92780 .4$

name $=$ MIN154

energy $($ in $\mathrm{kcal} / \mathrm{mol})=-21.435869$

$\operatorname{ZPE}($ in $\mathrm{kcal} / \mathrm{mol})=146.167$

$\mathrm{G}($ in $\mathrm{kcal} / \mathrm{mol})=122.406$

Geometry

C $-2.84100812-0.213227790 .65918151$

C $-1.35038918-0.21110222 \quad 0.65961265$

C $-0.73174204 \quad 1.15831950 \quad 0.66843789$ 
$\begin{array}{lllll}\text { C } & 0.79863271 & 1.15987111 & 0.76666097\end{array}$

C $1.42270767-0.036486330 .03817252$

C $1.15990942 \quad 0.05494254-1.47909177$

C $-0.63120721-1.33834109 \quad 0.64657771$

C $0.87123478-1.35774451 \quad 0.64415887$

C $1.35015967-1.507696112 .10286330$

C $1.38672011-2.57045668-0.15067181$

$\mathrm{H}-3.23709241 \quad 0.48317973-0.09796278$

$\mathrm{H}-3.24124831 \quad 0.10254868 \quad 1.63316438$

$\mathrm{H}-3.26360133-1.20190466 \quad 0.43849910$

$\mathrm{H}-1.17719255 \quad 1.78849621 \quad 1.47957382$

H $1.11048848 \quad 1.16295593 \quad 1.82733413$

H $1.18764048 \quad 2.10742277 \quad 0.33673977$

H $2.52623714 \quad-0.01346600 \quad 0.20477817$

$\mathrm{H} \quad 0.52708965-0.77385523-1.84108127$

$\mathrm{H} \quad 2.10198378 \quad 0.06287813 \quad-2.05772123$

$\mathrm{H}-1.10348560-2.31734488 \quad 0.65402050$

H $1.03614075-2.46968222 \quad 2.52250710$

$\mathrm{H} \quad 0.93020050-0.72769577 \quad 2.74674606$

$\mathrm{H} 2.44058117-1.454365582 .17287708$

$\mathrm{H} \quad 0.94656940-2.62372621-1.15178700$

$\mathrm{H} \quad 1.13793436-3.510248320 .35530515$

H $2.47525354-2.53723573-0.26263321$

o $-0.807024321 .21564621-1.64924053$

$0-1.11886547 \quad 1.90702715-0.48038544$

$0.575117311 .28996056-1.86510015$

Vibrational frequencies (in $\mathrm{cm}-1$ )

$\begin{array}{llllllllllllllll}75.8 & 115.1 & 125.9 & 143.4 & 176.1 & 191.7 & 218.6 & 264.1 & 296.7 & 309.1 & 326.9 & 364.2 & 386.3 & 412.1\end{array}$

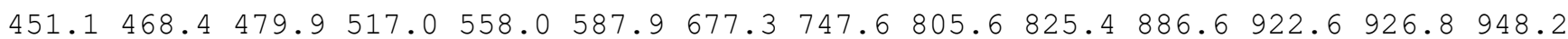
$970.0 \quad 984.5 \quad 986.8 \quad 1010.8 \quad 1027.5 \quad 1038.7 \quad 1060.4 \quad 1071.0 \quad 1084.3 \quad 1108.0 \quad 1136.0 \quad 1158.6 \quad 1181.2$ $\begin{array}{lllllllllllll}1193.4 & 1203.0 & 1231.5 & 1240.2 & 1247.4 & 1276.6 & 1276.8 & 1283.7 & 1286.7 & 1293.2 & 1296.8 & 1301.0\end{array}$ $1302.31306 .51309 .2 \quad 1333.6 \quad 1346.6 \quad 1355.8 \quad 1373.7 \quad 1384.51398 .6 \quad 1406.5 \quad 1414.81875 .2$ $2642.12660 .2 \quad 2666.2 \quad 2672.0 \quad 2674.5 \quad 2676.0 \quad 2678.5 \quad 2680.02681 .72700 .92712 .72739 .1$ $2757.22778 .0 \quad 2780.3 \quad 2781.9$

\footnotetext{
name $=$ MIN155

energy (in $\mathrm{kcal} / \mathrm{mol})=-21.278994$

$\mathrm{ZPE}(\mathrm{in} \mathrm{kcal} / \mathrm{mol})=146.17$

$\mathrm{G}($ in $\mathrm{kcal} / \mathrm{mol})=123.008$

Geometry

C $-2.19349763-0.11031724 \quad 0.08455673$

C $-0.68952107-0.097313710 .13038956$

C -0.042598551 .318313620 .18465559$

C $1.40944475 \quad 1.04237422 \quad 0.66829970$

C $1.43873173-0.49389688 \quad 0.86881717$

C $1.43700213-1.12594782-0.54390768$

C $0.00639517-0.81777174-1.06579621$

C $-0.01682910 \quad-0.79877938 \quad 1.37068722$

C $-0.34927015-0.15260330 \quad 2.71370058$

C $-0.33184332-2.28818427 \quad 1.48854172$

$\mathrm{H}-2.57506108 \quad 0.40204239-0.81003618$

$\mathrm{H}-2.63075856 \quad 0.39077439 \quad 0.95761132$

$\mathrm{H}-2.58923553-1.134209350 .06693128$

$\mathrm{H}-0.59676075 \quad 2.02283166 \quad 0.82948255$

H $1.646344291 .58455141 \quad 1.59191376$

H $2.14202874 \quad 1.37391045-0.08636663$

H $2.23833291-0.848533121 .52192612$

H $1.64055217-2.20450555-0.51432135$

$\mathrm{H} 2.20330535-0.68221719-1.20028372$

$\mathrm{H}-0.54211130-1.70930979-1.41418708$

$\mathrm{H} \quad 0.22335004 \quad-0.62220434 \quad 3.52273514$

$\mathrm{H}-1.41333922-0.26720717 \quad 2.95268307$

$\mathrm{H}-0.126298220 .91776001 \quad 2.74790703$

$\mathrm{H} \quad 0.33143574 \quad-2.77041943 \quad 2.21648914$
} 
$\mathrm{H}-0.22226322-2.831339590 .54511100$

$\mathrm{H}-1.36288072-2.446943081 .82757104$

$0-0.589971121 .17468200-2.04900912$

$0.030575201 .96877330-1.07858837$

$0.154120650 .00833866-2.21896353$

Vibrational frequencies (in $\mathrm{cm}-1$ )

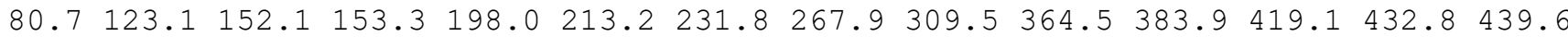
$486.2513 .2 \quad 531.7 \quad 562.7 \quad 594.2 \quad 686.5 \quad 697.2 \quad 823.4 \quad 836.0 \quad 862.5 \quad 896.7 \quad 911.3 \quad 942.9 \quad 956.0$

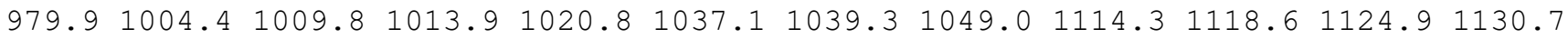

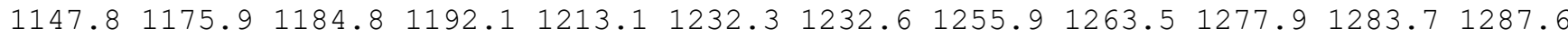
$1292.21293 .0 \quad 1296.7 \quad 1303.8 \quad 1305.3 \quad 1325.8 \quad 1327.6 \quad 1335.3 \quad 1340.7 \quad 1349.4 \quad 1400.91409 .8$ $1419.0 \quad 2670.12672 .2 \quad 2672.7 \quad 2674.3 \quad 2675.0 \quad 2675.82676 .42679 .92695 .32696 .12749 .3$ $2751.52753 .6 \quad 2780.82781 .5 \quad 2783.2$

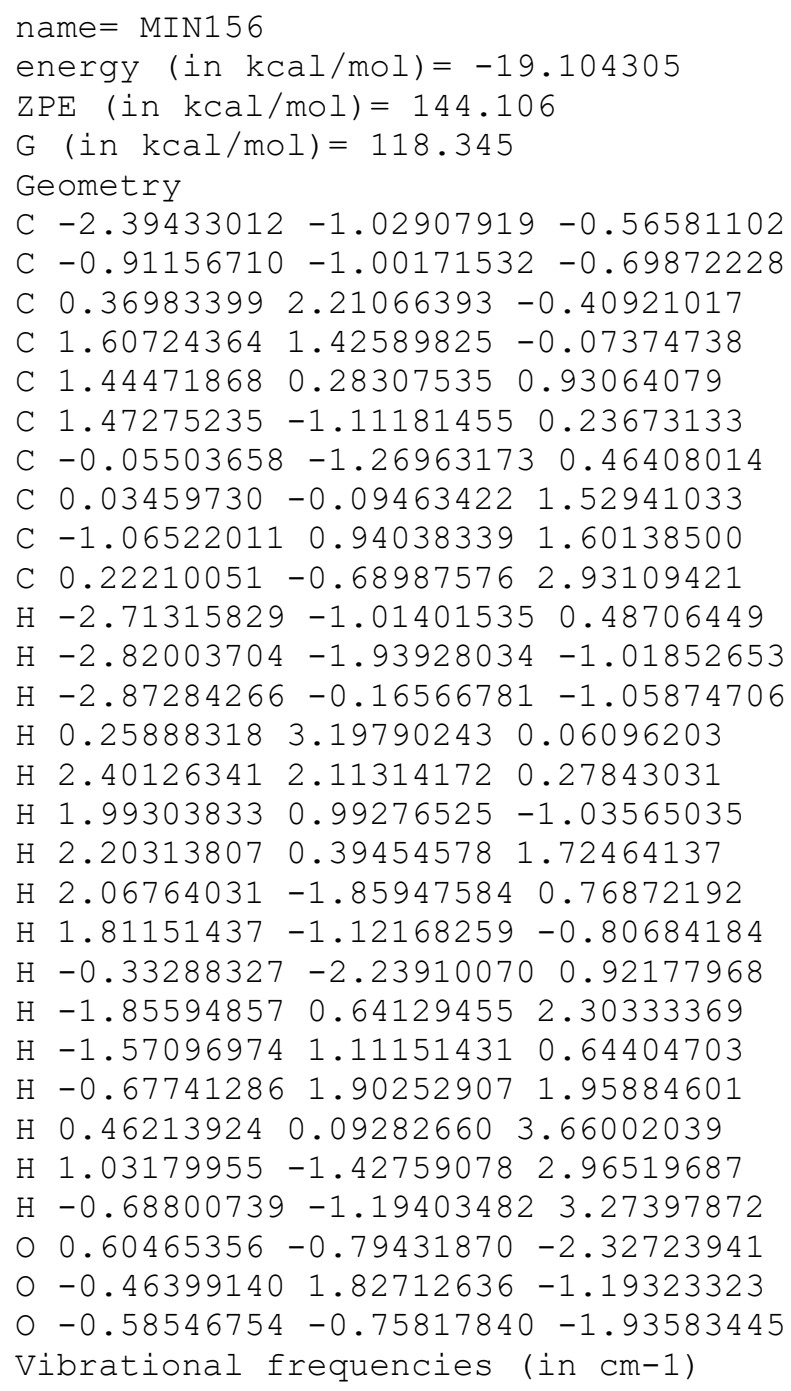


$\begin{array}{llll}\text { C }-1.27472991 & -0.42828671 & 0.59616590\end{array}$

C 0.419100871 .400496250 .70333025

C $1.26003707 \quad 1.31272312-0.51040772$

C $1.18577160-0.03959932-1.20972584$

C $-0.24654691-0.32924178-1.73998308$

C $-0.51604660-1.18365297-0.46981138$

C $1.04578774-1.29219114 \quad-0.26126431$

C $1.62573772-1.23843503 \quad 1.13429541$

C $1.58895937-2.54128275-0.95939988$

$\mathrm{H}-3.32298166-0.01770508 \quad 1.21450463$

$\mathrm{H}-3.08572072-1.63097943 \quad 0.51933214$

$\mathrm{H}-3.11304934-0.19327509-0.52724273$

$\mathrm{H}-0.93223374-0.69521396 \quad 1.61936893$

H 2.31524515 $1.55534270-0.25035395$

$\mathrm{H} \quad 0.928641712 .11001745-1.22661273$

$\mathrm{H} 1.98587817-0.13411632-1.95695511$

$\mathrm{H}-0.26943955-0.90514973-2.66905314$

$\mathrm{H}-0.898608590 .53953112-1.89566050$

$\mathrm{H}-0.99105355-2.15742454-0.66875414$

$\mathrm{H} \quad 1.10993124 \quad-0.56767876 \quad 1.82524259$

H $2.67775320-0.923315951 .10669490$

H $1.59983261-2.22869505 \quad 1.61194579$

H 2.68475480 $-2.53465063-0.98491036$

$\mathrm{H} \quad 1.24066751-2.62480850-1.99529209$

H $1.27609165-3.45481539-0.44061523$

$0-1.54458447 \quad 1.69328733-0.40637806$

$00.69807528 \quad 1.79395370 \quad 1.79501560$

o $-1.08439300 \quad 1.08342518 \quad 0.62800691$

Vibrational frequencies (in cm-1)

$\begin{array}{lllllllllllllll}39.1 & 115.5 & 141.5 & 147.4 & 172.5 & 185.9 & 193.5 & 236.0 & 257.3 & 278.7 & 283.0 & 355.8 & 379.3 & 393.2\end{array}$

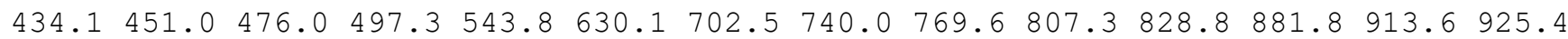

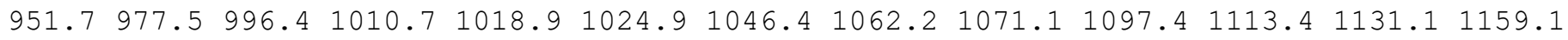
$1166.41189 .2 \quad 1206.2 \quad 1222.2 \quad 1234.4 \quad 1243.4 \quad 1272.7 \quad 1279.5 \quad 1282.6 \quad 1283.0 \quad 1283.9 \quad 1288.6$ $1293.8 \quad 1299.4 \quad 1302.0 \quad 1307.8 \quad 1327.6 \quad 1343.7 \quad 1367.6 \quad 1376.01396 .3 \quad 1407.2 \quad 1418.91896 .3$ $2639.52653 .72656 .32667 .6 \quad 2674.6 \quad 2675.2 \quad 2676.02679 .12681 .32721 .92727 .92733 .6$ $2754.92777 .02781 .3 \quad 2785.4$

\section{name $=$ MIN158}

energy (in $\mathrm{kcal} / \mathrm{mol})=-19.540743$

$\operatorname{ZPE}(\mathrm{in} \mathrm{kcal} / \mathrm{mol})=145.182$

$\mathrm{G}(\mathrm{in} \mathrm{kcal} / \mathrm{mol})=119.359$

Geometry

C $-1.85979234 \quad 0.22689850 \quad-0.42407143$

C $-0.35472504 \quad 0.22623496-0.57088839$

C $0.29984226 \quad 1.64342272-0.55445125$

C $1.58927154 \quad 1.54368033 \quad 0.26525593$

C $-0.87488136-2.081219041 .99324209$

C $1.78385235 \quad 0.06102008 \quad 0.62820137$

C $0.43618319-0.66561094 \quad 0.40798138$

C $-0.31156584-0.90337227 \quad 1.68693547$

C $-0.40716660 \quad 0.24006069 \quad 2.64729814$

C $-0.84478372-3.29392140 \quad 1.13087995$

$\mathrm{H}-2.18418082 \quad 0.64930762 \quad 0.53390207$

$\mathrm{H}-2.25913384-0.79603492-0.47758499$

$\mathrm{H}-2.335910390 .80181323-1.23027629$

$\mathrm{H}-0.380543372 .44763110-0.23124495$

H $2.448450381 .92059945-0.32356039$

$\mathrm{H} \quad 1.53934204 \quad 2.17435087 \quad 1.16739476$

$\mathrm{H}-1.40470453-2.211619372 .93667315$

H $2.14276500 \quad-0.06039506 \quad 1.66234835$

$\mathrm{H} \quad 2.55826003-0.39088329-0.01864285$

$\mathrm{H} \quad 0.64054422-1.64566371-0.09587204$

$\mathrm{H}-0.37597782 \quad 1.21370740 \quad 2.14137999$

$\mathrm{H} \quad 0.42898211 \quad 0.22021520 \quad 3.36193133$ 
$\mathrm{H}-1.33360943 \quad 0.21260550 \quad 3.23607102$

$\mathrm{H}-1.44315902-4.113447121 .55050672$

$\mathrm{H} 0.18003168-3.675988251 .00895418$

$\begin{array}{lllll}\mathrm{H} & -1.24074981 & -3.09185522 & 0.12464674\end{array}$

$\begin{array}{lllll}0 & -0.03131020 & 0.91497902 & -2.67810666\end{array}$

$00.667234061 .90049146-1.91003687$

o $-0.01098184-0.24955852-1.88515810$

Vibrational frequencies (in cm-1)

$\begin{array}{llllllllllllllll}41.9 & 49.9 & 68.2 & 84.2 & 132.9 & 152.7 & 170.9 & 199.2 & 223.1 & 228.7 & 256.3 & 284.0 & 325.6 & 362.0\end{array}$ $418.8 \quad 461.2 \quad 511.5 \quad 529.8 \quad 615.6 \quad 636.4 \quad 675.6 \quad 746.5 \quad 807.6 \quad 844.0 \quad 857.7 \quad 884.1934 .6 \quad 971.7$ $981.9995 .3 \quad 1009.9 \quad 1031.3 \quad 1045.7 \quad 1053.9 \quad 1077.3 \quad 1088.9 \quad 1102.3 \quad 1107.6 \quad 1136.6 \quad 1154.0$

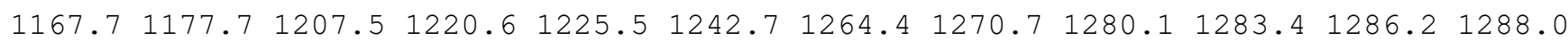

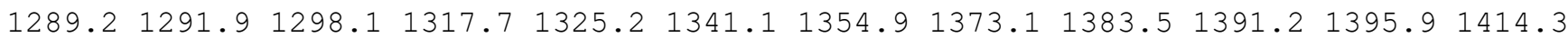
$1861.12655 .32667 .0 \quad 2670.0 \quad 2671.4 \quad 2672.7 \quad 2675.02676 .92677 .72692 .12699 .02746 .3$ $\begin{array}{llllll}2748.3 & 2753.8 & 2778.2 & 2780.2 & 2781.1\end{array}$

\section{name $=$ MIN159}

energy (in $\mathrm{kcal} / \mathrm{mol})=-19.907106$

$\mathrm{ZPE}($ in $\mathrm{kcal} / \mathrm{mol})=145.854$

$\mathrm{G}($ in $\mathrm{kcal} / \mathrm{mol})=121.558$

Geometry

C $-2.55813411-0.22808973 \quad 0.05416027$

C $-1.11585252-0.25125168-0.41255605$

C $-0.478356411 .15646726-0.67354377$

C $0.934054561 .16728192-0.08005209$

C $1.18813425-0.19393690 \quad 0.57303193$

C $2.42181163-0.87616710 \quad 0.02190419$

C $-0.14224755-1.02667693 \quad 0.45848354$

C $1.07444335-0.271690012 .13888819$

C $1.09248077 \quad 1.01730816 \quad 2.93053225$

C $-0.31787191-0.95464861 \quad 1.99510342$

$\mathrm{H}-3.21254801 \quad 0.20832171 \quad-0.71545164$

$\mathrm{H}-2.68463891 \quad 0.35513723 \quad 0.97200406$

$\mathrm{H}-2.92639965-1.24474868 \quad 0.24849509$

$\mathrm{H}-1.105323222 .00724861-0.35991150$

$\mathrm{H} 1.06344642 \quad 1.99679424 \quad 0.63351807$

$\mathrm{H} \quad 1.66923076 \quad 1.36502597 \quad-0.88599501$

$\mathrm{H} 1.82957320-0.968629252 .55088552$

$\mathrm{H} 2.35648560-0.99261150-1.06872003$

$\mathrm{H} 3.32869600-0.30049941 \quad 0.24230434$

$\mathrm{H}-0.00626455-2.05288565 \quad 0.08455054$

H $0.29146370 \quad 1.70331247 \quad 2.63227292$

$\mathrm{H} 2.04816372 \quad 1.543870792 .80945935$

$\mathrm{H} \quad 0.96302203 \quad 0.81979612 \quad 4.00226710$

$\mathrm{H}-0.40134107-1.92496826 \quad 2.48786711$

H $2.55823339-1.879708130 .44208468$

$\mathrm{H}-1.15085656-0.33524976 \quad 2.33561097$

o $-0.24412697-0.10101599-2.50689510$

$\begin{array}{lllll}0 & -0.43637211 & 1.24387644 & -2.09339591\end{array}$

o $-1.16524018-0.84454398-1.72234315$

Vibrational frequencies (in $\mathrm{cm}-1$ )

$\begin{array}{llllllllllllllll}41.3 & 60.1 & 137.8 & 163.6 & 179.1 & 186.9 & 215.6 & 240.9 & 279.9 & 294.7 & 313.5 & 361.3 & 390.0 & 416.3\end{array}$ $449.2508 .6 \quad 551.4 \quad 570.3 \quad 661.0 \quad 671.8 \quad 707.6 \quad 812.7 \quad 840.0 \quad 858.0 \quad 898.0 \quad 909.7 \quad 927.9 \quad 964.4$ $967.4977 .8 \quad 986.5 \quad 1007.2 \quad 1048.9 \quad 1058.7 \quad 1065.2 \quad 1074.4 \quad 1109.6 \quad 1130.0 \quad 1135.7 \quad 1140.8 \quad 1158.0$

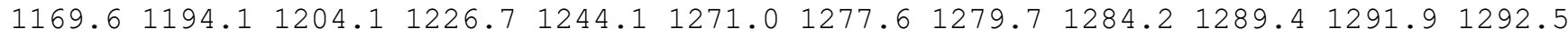
$1295.91298 .0 \quad 1303.0 \quad 1306.5 \quad 1313.5 \quad 1321.9 \quad 1350.3 \quad 1356.3 \quad 1368.7 \quad 1392.5 \quad 1393.7 \quad 1408.5$ $2660.42670 .22674 .0 \quad 2674.2 \quad 2676.8 \quad 2677.4 \quad 2679.5 \quad 2689.92704 .62722 .92735 .02745 .8$ $2767.8 \quad 2779.8 \quad 2780.4 \quad 2781.6$

name $=$ MIN160

energy (in $\mathrm{kcal} / \mathrm{mol})=-18.634956$

$\mathrm{ZPE}(\mathrm{in} \mathrm{kcal} / \mathrm{mol})=144.915$

$\mathrm{G}($ in $\mathrm{kcal} / \mathrm{mol})=119.548$ 
Geometry

C $-2.76478726 \quad 0.15747477-0.39041142$

C $-1.25911919 \quad 0.10823421-0.54548637$

C $-0.53841004 \quad 1.50222809 \quad-0.57162787$

C $0.66276285 \quad 1.39634372 \quad 0.37215474$

C $0.89265179-0.11576090 \quad 0.57548256$

C $-0.52420445-2.21364565 \quad 0.20091037$

C $-0.53307005-0.72862338 \quad 0.52902704$

C $1.62265041-0.49713271 \quad 1.82833489$

C $1.36923684 \quad 0.29811996 \quad 3.06565940$

C $2.46625960-1.534317531 .82802477$

$\mathrm{H}-3.22913124 \quad 0.73094760-1.20609151$

$\mathrm{H}-3.06532461 \quad 0.61323602 \quad 0.55844658$

$\mathrm{H}-3.19804322-0.85216071-0.42885760$

$\mathrm{H}-1.19232396 \quad 2.36843678-0.38286331$

$\mathrm{H} \quad 0.45982753 \quad 1.91519964 \quad 1.32240580$

$\mathrm{H} \quad 1.55429404 \quad 1.88119794-0.06222383$

$\mathrm{H} 1.46326440-0.49585689-0.31292329$

$\mathrm{H}-1.53596045-2.63441988 \quad 0.21596282$

$\mathrm{H}-0.10633664-2.40839952-0.79593052$

$\mathrm{H}-1.03199127-0.57167787 \quad 1.51342455$

$\mathrm{H} \quad 0.30099520 \quad 0.50652926 \quad 3.21022087$

H $1.89722011 \quad 1.26285088 \quad 3.02584509$

$\mathrm{H} \quad 1.72068429-0.21754235 \quad 3.97018188$

H $2.68285784-2.12961127 \quad 0.95594365$

$\mathrm{H} \quad 0.08065851-2.77293033 \quad 0.92660722$

$\mathrm{H} 3.00560943-1.869015672 .69738100$

$\begin{array}{lllll}0 & 0.09345791 & 0.30809227 & -2.35458031\end{array}$

$0-0.12602410 \quad 1.64475391-1.92399164$

o $-1.02741944-0.41081051-1.86003966$

Vibrational frequencies (in $\mathrm{cm}-1$ )

$\begin{array}{lllllllllllllll}38.6 & 60.6 & 77.7 & 133.7 & 137.9 & 160.4 & 161.3 & 211.4 & 220.5 & 256.4 & 282.6 & 310.6 & 340.9 & 381.3\end{array}$ $450.2475 .8 \quad 525.6 \quad 543.0 \quad 558.5 \quad 597.9 \quad 620.4 \quad 675.6 \quad 716.6 \quad 849.9 \quad 871.1 \quad 881.7 \quad 927.0 \quad 964.1$

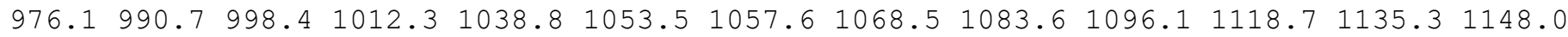
$\begin{array}{lllllllllllll}1170.9 & 1188.3 & 1211.8 & 1221.1 & 1250.8 & 1265.2 & 1277.8 & 1283.3 & 1283.8 & 1286.0 & 1287.7 & 1292.3\end{array}$ $1295.11298 .11313 .4 \quad 1320.6 \quad 1331.3 \quad 1348.4 \quad 1368.51391 .1 \quad 1397.4 \quad 1401.6 \quad 1403.81850 .6$ $2667.72668 .92669 .42670 .0 \quad 2674.8 \quad 2676.4 \quad 2679.5 \quad 2690.3 \quad 2705.3 \quad 2712.42724 .52750 .9$ 2778.12779 .32781 .12792 .9

\footnotetext{
name $=$ MIN161

energy (in $\mathrm{kcal} / \mathrm{mol})=-19.619836$

$\mathrm{ZPE}($ in $\mathrm{kcal} / \mathrm{mol})=145.967$

$\mathrm{G}(\mathrm{in} \mathrm{kcal} / \mathrm{mol})=121.902$

Geometry

C $-2.45103789-0.25128042-1.20708759$

C $-0.97118507-0.13324166-0.88615757$

C $-0.43857421 \quad 1.34240617-0.84212790$

C $0.33585102 \quad 1.54093673 \quad 0.46296344$

C $1.36351250-0.62516884 \quad 1.46328621$

C $0.71363455-1.62921748 \quad 0.46504232$

C $-0.58428661-0.786222190 .43268201$

C $0.10106365 \quad 0.30149499 \quad 1.33799605$

C $-0.63890964 \quad 0.63625051 \quad 2.61235324$

C $1.63807646-1.209939212 .83420051$

$\mathrm{H}-2.69686158 \quad 0.25039482 \quad-2.15322740$

$\mathrm{H}-3.06972673 \quad 0.18894559-0.41701639$

$\mathrm{H}-2.74825653-1.30312568-1.31872775$

$\mathrm{H}-1.21636756 \quad 2.10656577-1.01527817$

H $0.02725628 \quad 2.466642690 .97544889$

H $1.412461481 .67783392 \quad 0.24092730$

H $2.27581561-0.151414551 .06185833$

$\mathrm{H} \quad 0.58393724 \quad-2.64249481 \quad 0.84911034$

$\mathrm{H} 1.23492054-1.70179563-0.49499096$

$\mathrm{H}-1.43128616-1.298933350 .90841319$
} 
$\mathrm{H}-1.572516931 .17306788 \quad 2.40269782$

$\mathrm{H}-0.03182688 \quad 1.26765493 \quad 3.27289537$

$\mathrm{H}-0.90687033-0.263569203 .17939406$

$\mathrm{H} 2.47997153-1.912642112 .79959026$

$\mathrm{H} \quad 0.77480552-1.75956630 \quad 3.22777698$

H $1.88875425-0.42778135 \quad 3.56044541$

$0.863805250 .10316824-2.18656506$

$0.406949151 .43458797-1.98116294$

o $-0.29346451-0.70028503-2.01134507$

Vibrational frequencies (in $\mathrm{cm}-1$ )

$\begin{array}{llllllllllllllll}50.0 & 68.9 & 159.4 & 162.7 & 174.3 & 199.1 & 228.9 & 235.2 & 272.1 & 285.6 & 320.5 & 340.2 & 363.1 & 442.1\end{array}$ $458.4 \quad 504.6 \quad 567.6 \quad 596.9 \quad 621.7 \quad 687.2 \quad 713.7 \quad 819.5 \quad 849.0 \quad 864.0 \quad 895.4 \quad 910.0938 .5 \quad 959.7$

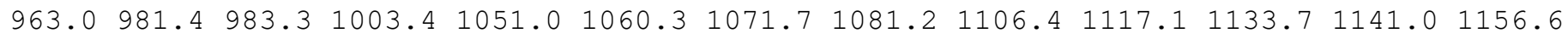
$1168.91187 .2 \quad 1215.7 \quad 1219.0 \quad 1239.1 \quad 1245.6 \quad 1276.4 \quad 1284.0 \quad 1285.8 \quad 1288.7 \quad 1290.9 \quad 1292.0$

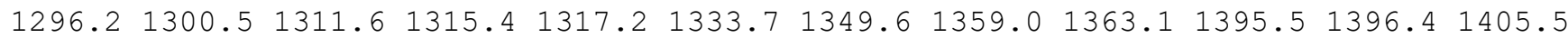
$2662.02671 .32674 .0 \quad 2676.3 \quad 2677.8 \quad 2678.8 \quad 2680.2 \quad 2687.32700 .82725 .72738 .12746 .6$ 2766.62780 .52781 .62782 .6

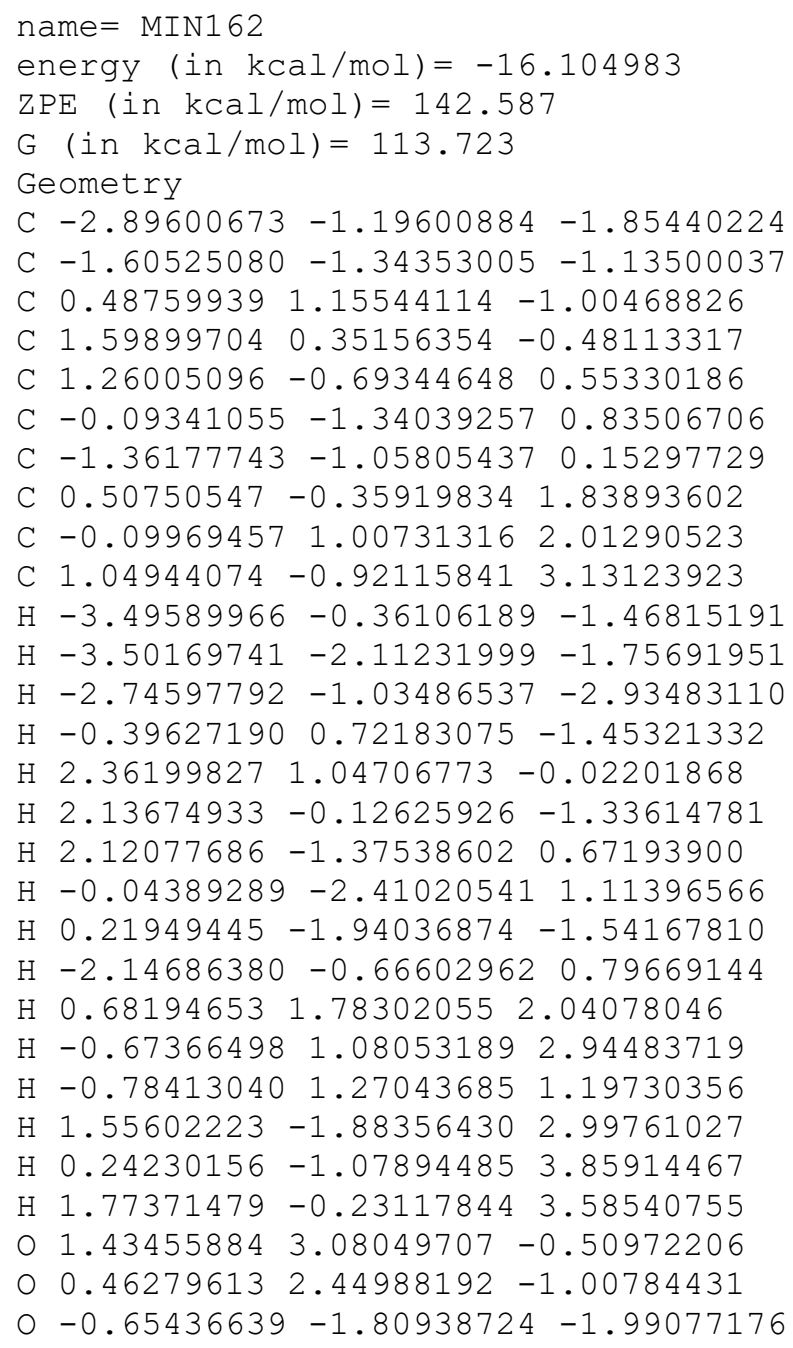


$\mathrm{ZPE}($ in $\mathrm{kcal} / \mathrm{mol})=145.37$

$\mathrm{G}($ in $\mathrm{kcal} / \mathrm{mol})=119.672$

Geometry

C $-1.89449848-0.69878859-1.24221783$

C $-0.39627693-0.61303175-1.03361077$

C $0.179094190 .83882785-1.04225920$

C $1.03398075 \quad 1.00984633 \quad 0.21116131$

C $0.08822912-0.02153581 \quad 2.37696449$

C $0.83583860-0.243514551 .09058540$

C $0.10850003-1.307595820 .23191379$

C $-0.94453532 \quad 0.79669157 \quad 2.61737369$

C $-1.622992461 .71465243 \quad 1.66475551$

C $0.59838529-0.888422913 .49284322$

$\mathrm{H}-2.21004722-0.14690755-2.13713358$

$\mathrm{H}-2.45224436-0.30734495-0.38282898$

$\mathrm{H}-2.20865150-1.74225074-1.38746186$

$\mathrm{H}-0.58567424 \quad 1.62002617-1.18381017$

H $0.79219972 \quad 1.934130750 .75917068$

$\mathrm{H} 2.10147105 \quad 1.10905323-0.07497054$

$\mathrm{H}-1.37647622 \quad 0.83185925 \quad 3.62148173$

$\mathrm{H} \quad 0.80472445-2.12626731-0.03773611$

$\mathrm{H} \quad 1.85641838-0.63286548 \quad 1.34407285$

$\mathrm{H}-0.71127520-1.78543720 \quad 0.79365676$

$\mathrm{H}-1.07688632 \quad 2.66547121 \quad 1.56964278$

$\mathrm{H}-2.639917921 .96573873 \quad 1.99762116$

$\mathrm{H}-1.716765151 .281510570 .66053257$

$\mathrm{H}-0.05938375-0.88280842 \quad 4.37128979$

$\mathrm{H} 1.58828290-0.552181153 .83136499$

$\mathrm{H} \quad 0.69356849-1.936229423 .17588400$

$0.0 .56702567-0.16712808-3.01802225$

$\begin{array}{lllll}0 & 1.05601647 & 0.86855227 & -2.17261308\end{array}$

O $0.27348109-1.23789268-2.14048917$

Vibrational frequencies (in $\mathrm{cm}-1$ )

$\begin{array}{lllllllllllllll}-23.9 & 59.7 & 79.0 & 105.1 & 149.0 & 161.3 & 174.7 & 212.8 & 226.3 & 253.1 & 287.6 & 302.2 & 336.5 & 361.3\end{array}$

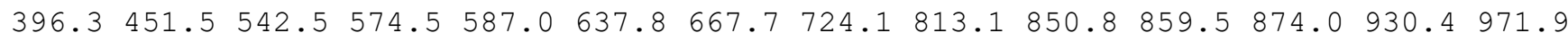
$976.1987 .2 \quad 1001.4 \quad 1009.1 \quad 1051.5 \quad 1069.8 \quad 1083.2 \quad 1083.7 \quad 1089.11109 .6 \quad 1135.3 \quad 1156.9$

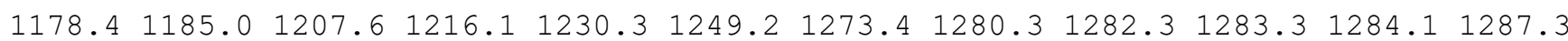

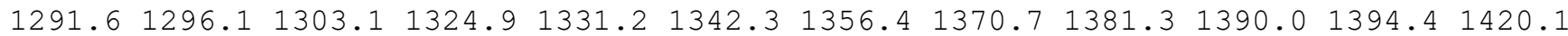
$1881.42656 .12661 .1 \quad 2667.3 \quad 2668.6 \quad 2671.12672 .3 \quad 2673.02674 .7 \quad 2693.2 \quad 2697.3 \quad 2742.5$ $2744.12746 .0 \quad 2776.6 \quad 2779.2 \quad 2781.2$

\section{name $=\operatorname{MIN} 164$}

energy (in $\mathrm{kcal} / \mathrm{mol})=-17.961913$

$\mathrm{ZPE}($ in $\mathrm{kcal} / \mathrm{mol})=145.119$

$\mathrm{G}($ in $\mathrm{kcal} / \mathrm{mol})=119.947$

Geometry

C $-2.21606325-0.31335743-0.33313248$

C $-0.72342384-0.11146285-0.50354497$

C $-0.27527784 \quad 1.36621974 \quad-0.74808956$

C $0.92564706 \quad 1.64055640 \quad 0.15432060$

C $1.37068878 \quad 0.29814831 \quad 0.77252129$

C $2.62766480-0.242162420 .09034039$

C $0.18250136-0.690240690 .60124207$

C $-0.49980521-0.909120221 .92140878$

C $-1.14201572 \quad 0.261643602 .59336162$

C $-0.49188702-2.112320502 .50360182$

$\mathrm{H}-2.74650966-0.13450287-1.28100105$

$\mathrm{H}-2.64237717 \quad 0.35908127 \quad 0.41825215$

$\mathrm{H}-2.44679537-1.34256672-0.02474418$

$\mathrm{H}-1.08299674 \quad 2.11322795 \quad-0.66472497$

$\mathrm{H} \quad 0.67165150 \quad 2.36789582 \quad 0.94297090$

$\mathrm{H} \quad 1.74372723 \quad 2.10427729-0.42867491$

$\mathrm{H} \quad 1.58919234 \quad 0.43871172 \quad 1.85603409$

$\mathrm{H} \quad 3.47030326 \quad 0.44943617 \quad 0.19671946$ 
$\mathrm{H} 2.46309856-0.39881363-0.98423709$

H $0.57110698-1.673218930 .22484773$

$\mathrm{H}-1.04394230 \quad 1.191940912 .02090410$

$\mathrm{H}-0.69750887 \quad 0.44400618 \quad 3.58265939$

$\mathrm{H}-2.21562081 \quad 0.08592271 \quad 2.75618695$

$\mathrm{H}-0.94080520-2.31222671 \quad 3.46224148$

H 2.93507062 $-1.20240930 \quad 0.51972072$

$\mathrm{H}-0.03463412-2.987710892 .07377710$

$\begin{array}{lllll}0 & 0.53659268 & 0.07050370 & -2.38720543\end{array}$

$00.08547106 \quad 1.39176997-2.12406567$

o $-0.41909369-0.76213688-1.74969411$

Vibrational frequencies (in $\mathrm{cm}-1$ )

$\begin{array}{llllllllllllllll}44.9 & 48.9 & 90.9 & 117.5 & 138.1 & 179.5 & 193.8 & 196.8 & 234.1 & 247.5 & 317.2 & 325.7 & 349.9 & 409.1\end{array}$ $421.2 \quad 450.1 \quad 496.8 \quad 568.9 \quad 602.2 \quad 605.9 \quad 636.3 \quad 705.9 \quad 713.9 \quad 852.2 \quad 862.3 \quad 914.0 \quad 935.6 \quad 957.0$ $972.4 \quad 978.9 \quad 984.7 \quad 1009.6 \quad 1031.9 \quad 1041.5 \quad 1044.3 \quad 1060.4 \quad 1091.9 \quad 1102.5 \quad 1113.6 \quad 1140.1 \quad 1147.2$ $\begin{array}{llllllllllllll}1152.9 & 1201.9 & 1212.0 & 1227.5 & 1241.9 & 1267.3 & 1274.3 & 1280.9 & 1283.5 & 1286.6 & 1295.5 & 1296.7\end{array}$

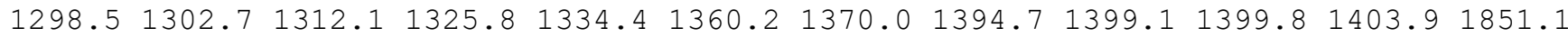
$2665.52668 .62669 .2 \quad 2670.5 \quad 2675.12675 .3 \quad 2678.52685 .22702 .62707 .92724 .72747 .4$ 2778.52780 .12780 .92793 .3

name $=$ MIN165

energy $($ in $\mathrm{kcal} / \mathrm{mol})=-18.726091$

$\operatorname{ZPE}(\mathrm{in} \mathrm{kcal} / \mathrm{mol})=146.152$

$\mathrm{G}($ in $\mathrm{kcal} / \mathrm{mol})=122.307$

Geometry

C $-2.86980591 \quad 0.08362462 \quad 0.21689183$

C $-1.40041277 \quad 0.13233875 \quad 0.46369818$

C $-0.715061261 .34385990-0.11780586$

C $0.79209514 \quad 1.40952701 \quad 0.13770928$

$\begin{array}{lllll}\text { C } 1.42651808 & 0.01246461 & 0.22450037\end{array}$

C $1.38638891-0.65952438-1.15729493$

C $-0.75228611-0.83343616 \quad 1.12162601$

C $0.73119045-0.81163288 \quad 1.34286372$

C $1.00185148 \quad-0.15864368 \quad 2.71571162$

C $1.28296394 \quad-2.24727116 \quad 1.38501572$

$\mathrm{H}-3.09403330 \quad 0.28338807 \quad-0.84537649$

$\mathrm{H}-3.40583581 \quad 0.83363618 \quad 0.81301227$

$\mathrm{H}-3.30422301-0.89725566 \quad 0.44989109$

$\mathrm{H}-1.218411412 .278047290 .23958539$

H $0.99141851 \quad 1.96966450 \quad 1.07115495$

$\mathrm{H} \quad 1.27637634 \quad 1.99737702-0.66843986$

H 2.50246763 $0.12553409 \quad 0.50813208$

$\mathrm{H} \quad 1.51342326-1.75760840-1.10200972$

H $2.17182571-0.24716317-1.81478810$

$\mathrm{H}-1.27203845-1.69176725 \quad 1.53781025$

$\mathrm{H} \quad 0.54364150 \quad 0.83335237 \quad 2.78538592$

$\mathrm{H} \quad 2.07332141-0.05432186 \quad 2.90605600$

H $\quad 0.57529904 \quad-0.76132258 \quad 3.52513878$

H $\quad 0.92729991 \quad-2.78071725 \quad 2.27417131$

H 2.37671170 $-2.25453142 \quad 1.41516081$

$\mathrm{H} \quad 0.95948390-2.83220618 \quad 0.51693302$

$0-0.026372850 .74316071-2.27301785$

$0-1.01731304 \quad 1.38594193-1.50940258$

O $0.12830015-0.56395749-1.81236027$

Vibrational frequencies (in $\mathrm{cm}-1$ )

$\begin{array}{llllllllllllllll}67.1 & 111.7 & 121.1 & 134.6 & 178.6 & 201.9 & 237.1 & 262.8 & 280.3 & 300.8 & 329.2 & 354.7 & 388.5 & 400.9\end{array}$ $447.0 \quad 470.7 \quad 474.6511 .7 \quad 548.2 \quad 589.8 \quad 709.3 \quad 744.3 \quad 845.8 \quad 855.1 \quad 882.7 \quad 919.3 \quad 924.7 \quad 942.5$

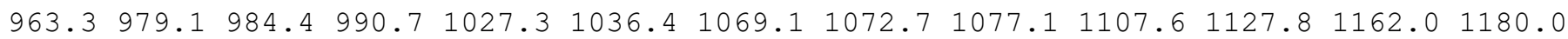
$1190.61205 .6 \quad 1224.6 \quad 1250.0 \quad 1252.9 \quad 1266.9 \quad 1275.2 \quad 1275.8 \quad 1287.9 \quad 1294.7 \quad 1296.91300 .2$ $1306.51307 .1 \quad 1311.0 \quad 1336.4 \quad 1348.0 \quad 1365.2 \quad 1369.7 \quad 1382.91397 .6 \quad 1406.4 \quad 1413.4 \quad 1880.0$ $2643.02659 .92663 .3 \quad 2671.5 \quad 2674.7 \quad 2676.0 \quad 2678.5 \quad 2679.72682 .92695 .72712 .6 \quad 2739.7$ $2759.62777 .4 \quad 2780.0 \quad 2782.0$ 
name $=$ MIN166

energy (in $\mathrm{kcal} / \mathrm{mol})=-17.679916$

$\mathrm{ZPE}($ in $\mathrm{kcal} / \mathrm{mol})=145.109$

$\mathrm{G}($ in $\mathrm{kcal} / \mathrm{mol})=119.633$

Geometry

C $-2.14247294 \quad 0.11118863 \quad-0.57572017$

C $-0.62923601 \quad 0.15394972-0.61606828$

C $-0.02651427 \quad 1.60154789-0.64582910$

C $1.13372151 \quad 1.63589956 \quad 0.34633025$

C $0.97332774 \quad 0.41087221 \quad 1.26534512$

C $2.32184312-0.16278609 \quad 1.69277379$

C $0.15198194 \quad-0.63106960 \quad 0.45882137$

C $-0.66587719-1.50124884 \quad 1.36708551$

C $-1.65388443-0.863010272 .29064962$

C $-0.48501317-2.826004521 .38360040$

$\mathrm{H}-2.56964195 \quad 0.53734397 \quad-1.49685307$

$\mathrm{H}-2.54990006 \quad 0.67226380 \quad 0.27111817$

$\mathrm{H}-2.51057699-0.92235334-0.50440164$

$\mathrm{H}-0.76681780 \quad 2.40700136-0.50917405$

H $1.16029945 \quad 2.57195168 \quad 0.92426104$

$\mathrm{H} 2.09476188 \quad 1.59511294-0.20444705$

$\mathrm{H} \quad 0.40582145 \quad 0.71146928 \quad 2.17652675$

H $2.89627620 \quad 0.56159770 \quad 2.28010914$

$\mathrm{H} 2.93602070-0.45065080 \quad 0.83095416$

$\mathrm{H} \quad 0.87707873-1.28310015-0.10500368$

$\mathrm{H}-2.67555501-1.21157204 \quad 2.07784220$

$\mathrm{H}-1.66366018 \quad 0.23178770 \quad 2.22741845$

$\mathrm{H}-1.44146230-1.12245028 \quad 3.33809649$

$\mathrm{H}-1.02662851-3.492568202 .03399570$

H $2.18884786-1.05977648 \quad 2.31097614$

$\mathrm{H} \quad 0.21520610-3.34732530 \quad 0.75231541$

$\begin{array}{lllll}0 & 0.78441809 & 0.43065687 & -2.37022092\end{array}$

○ $0.429756821 .75223712-1.98422170$

o $-0.29558112-0.36818733-1.91452639$

Vibrational frequencies (in $\mathrm{cm}-1$ )

$\begin{array}{lllllllllllllll}26.0 & 52.7 & 68.2 & 121.0 & 172.6 & 179.5 & 197.7 & 215.6 & 244.7 & 263.0 & 276.3 & 305.8 & 379.7 & 404.5\end{array}$ $446.4 \quad 474.0 \quad 502.3 \quad 527.0 \quad 570.0 \quad 585.9 \quad 614.6 \quad 709.8 \quad 713.6 \quad 826.5 \quad 858.4 \quad 912.6 \quad 949.5 \quad 966.6$

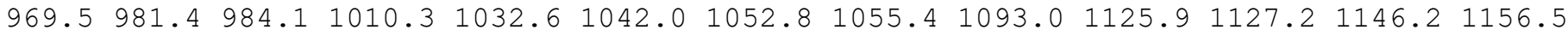
$1164.21185 .41211 .2 \quad 1225.1 \quad 1248.2 \quad 1272.8 \quad 1276.8 \quad 1282.21285 .5 \quad 1288.4 \quad 1296.51298 .6$

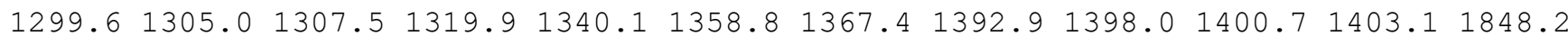
$2663.72666 .8 \quad 2669.0 \quad 2670.7 \quad 2672.0 \quad 2673.5 \quad 2676.12678 .72703 .72709 .52724 .12750 .0$ $2777.32778 .92781 .0 \quad 2792.6$

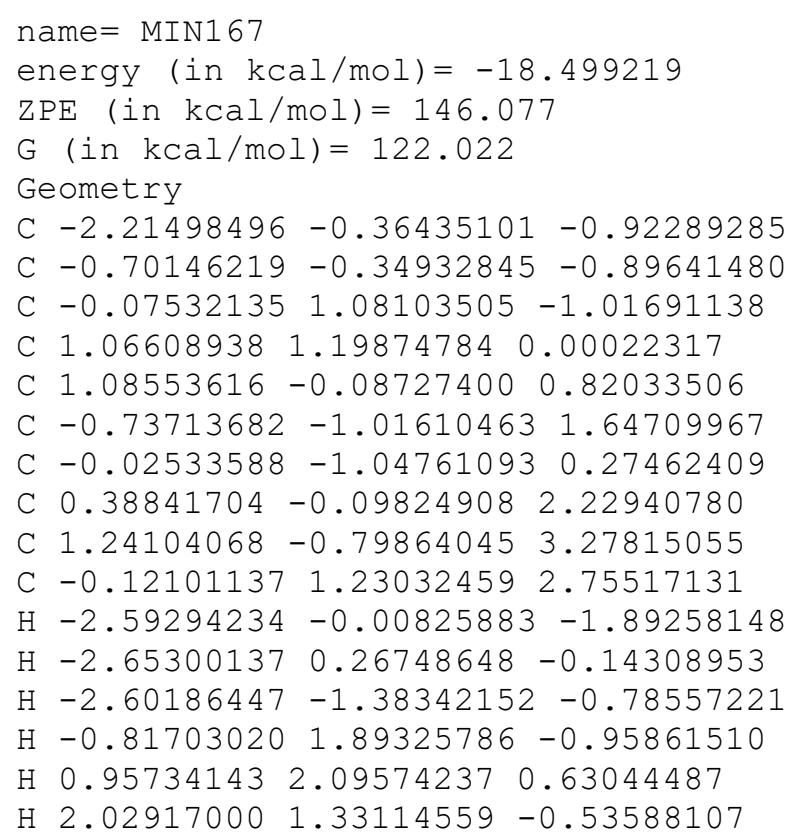


$\mathrm{H} 2.09105816-0.530063730 .84607616$

$\mathrm{H}-1.72914967-0.56105340 \quad 1.65015161$

$\mathrm{H}-0.82231825-1.991792122 .13044960$

$\mathrm{H} \quad 0.33324886-2.04970751-0.01021876$

$\mathrm{H} \quad 1.60189578-1.77278173 \quad 2.92709630$

H $0.67336563-0.97596899 \quad 4.19869998$

H 2.11980228 -0.198681723 .54163230$

$\mathrm{H}-0.77301179 \quad 1.73616911 \quad 2.03379523$

$\mathrm{H} \quad 0.70602111 \quad 1.90999966 \quad 2.99370486$

$\mathrm{H}-0.70462704 \quad 1.09121493 \quad 3.67369856$

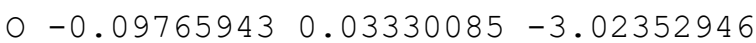

○ $0.515283651 .11298766-2.31792428$

o - $0.20400275-1.00639779-2.07667757$

Vibrational frequencies (in cm-1)

$\begin{array}{llllllllllllllll}47.4 & 56.7 & 166.4 & 186.6 & 197.7 & 204.9 & 228.8 & 240.7 & 281.0 & 300.1 & 330.1 & 354.9 & 387.1 & 411.7\end{array}$ $447.2 \quad 491.3 \quad 571.6 \quad 585.3 \quad 650.2 \quad 700.7 \quad 739.0 \quad 801.0 \quad 840.5 \quad 880.7 \quad 896.5 \quad 923.7 \quad 948.0 \quad 964.8$ $980.4 \quad 991.8 \quad 996.8 \quad 1004.0 \quad 1013.8 \quad 1023.2 \quad 1059.4 \quad 1081.8 \quad 1085.9 \quad 1101.9 \quad 1124.6 \quad 1156.7 \quad 1159.4$

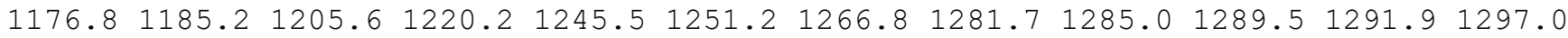

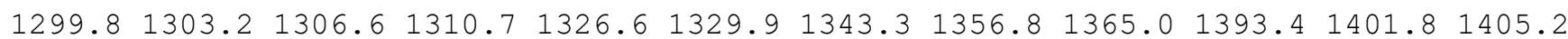
$2659.52670 .62675 .42676 .92677 .5 \quad 2679.02681 .42690 .42698 .4 \quad 2727.12735 .82745 .0$ $2767.6 \quad 2780.5 \quad 2781.2 \quad 2783.3$

\section{name $=$ MIN168}

energy (in $\mathrm{kcal} / \mathrm{mol})=-17.441958$

$\mathrm{ZPE}($ in $\mathrm{kcal} / \mathrm{mol})=145.154$

$\mathrm{G}($ in $\mathrm{kcal} / \mathrm{mol})=121.095$

Geometry

C $-2.89423008-0.03803954-0.21915908$

C $-1.36741064-0.03784461-0.21603024$

C $-0.776757651 .40562285 \quad-0.22287508$

C $0.375862081 .58050928 \quad 0.76139042$

C $1.32264345 \quad 0.41988502 \quad 0.69075344$

C $2.61412415 \quad 0.60230313 \quad 0.40691450$

C $-0.81879775-0.90893694 \quad 0.91177242$

C $0.72604275-0.93372491 \quad 1.00632299$

C $1.11940839-1.289615612 .45528649$

C $1.27184524 \quad-2.01394734 \quad 0.06039383$

$\mathrm{H}-3.30355922 \quad 0.370166630 .70978720$

$\mathrm{H}-3.28843453-1.05496189-0.34303910$

$\mathrm{H}-3.28637196 \quad 0.55463679-1.05748393$

$\mathrm{H}-1.54728844 \quad 2.19367026-0.09847299$

$\mathrm{H}-0.01281838 \quad 1.68494630 \quad 1.79559222$

$\mathrm{H} \quad 0.89863572 \quad 2.53639074 \quad 0.54306462$

H $3.04827084 \quad 1.56502324 \quad 0.19135176$

$\mathrm{H}-1.19017582-1.94518327 \quad 0.78643245$

$\mathrm{H} \quad 3.33811687-0.19382047 \quad 0.35557997$

$\mathrm{H}-1.24419221-0.55632094 \quad 1.87186327$

H 2.20769356 -1.376366992 .55559257$

$\mathrm{H} \quad 0.68241876 \quad-2.24395866 \quad 2.76364172$

$\mathrm{H} \quad 0.78964597 \quad-0.52176775 \quad 3.16233252$

H 2.36110090 -2.101961030 .12912749$

$\mathrm{H} 1.02557335-1.78195442-0.98369706$

H $0.84877420 \quad-2.997315820 .28826342$

$0 \quad 0.05305698 \quad 0.26909055-1.96431654$

$0-0.32936977 \quad 1.57411970-1.56555017$

o $-1.00028007-0.56558773-1.49899012$

Vibrational frequencies (in $\mathrm{cm}-1$ )

$\begin{array}{lllllllllllllll}57.8 & 98.0 & 123.0 & 163.8 & 174.2 & 204.0 & 229.4 & 240.4 & 252.0 & 281.3 & 313.4 & 326.4 & 373.8 & 386.6\end{array}$ $424.9 \quad 448.7 \quad 478.9 \quad 520.2 \quad 561.7 \quad 622.1 \quad 654.9699 .5 \quad 722.1 \quad 800.4 \quad 865.3 \quad 900.6 \quad 914.7 \quad 938.2$

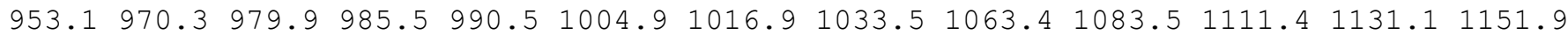

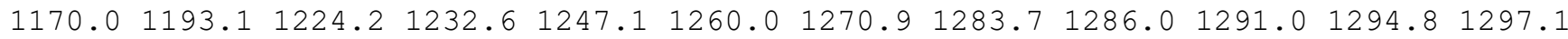
$1303.0 \quad 1309.0 \quad 1313.9 \quad 1323.5 \quad 1342.4 \quad 1361.6 \quad 1382.4 \quad 1395.2 \quad 1398.3 \quad 1410.2 \quad 1422.7 \quad 1856.7$ $2661.22663 .12670 .82674 .12676 .8 \quad 2678.5 \quad 2680.52682 .52689 .12721 .72739 .92741 .2$ $2780.92781 .5 \quad 2782.5 \quad 2791.0$ 
name $=$ MIN169

energy (in $\mathrm{kcal} / \mathrm{mol})=-15.667172$

$\mathrm{ZPE}($ in $\mathrm{kcal} / \mathrm{mol})=144.071$

$\mathrm{G}(\mathrm{in} \mathrm{kcal} / \mathrm{mol})=117.499$

Geometry

C $-2.072812350 .06807762-0.72890661$

C $-0.56329930 \quad 0.05143094-0.66179255$

C $0.08779742 \quad 1.49229883-0.69461204$

C $1.20786331 \quad 1.42276318 \quad 0.29189493$

C $1.28409072 \quad 0.22417028 \quad 0.88787363$

C $2.34151396-0.21284928 \quad 1.82463442$

C $0.17041845-0.72179101 \quad 0.47085591$

C $-0.62473001-1.18295906 \quad 1.70997636$

C $-1.35127727-0.04173656 \quad 2.42806232$

C $-1.58701504-2.32917437 \quad 1.38379288$

$\mathrm{H}-2.414218120 .64671056-1.60156504$

$\mathrm{H}-2.52397126 \quad 0.51394354 \quad 0.16457754$

$\mathrm{H}-2.48077694-0.94516751-0.84971071$

$\mathrm{H}-0.61052876 \quad 2.33354407 \quad-0.56474216$

$\mathrm{H} \quad 1.86627361 \quad 2.26125184 \quad 0.41611234$

H 2.92353570 $0.63252823 \quad 2.22045204$

$\mathrm{H} 0.13616931-1.59616003 \quad 2.42921513$

H $1.94244940 \quad-0.760786732 .69032647$

$\mathrm{H} 3.05467828-0.884403031 .31789050$

$\mathrm{H} 0.62206005-1.636073110 .00332096$

$\mathrm{H}-2.30246419 \quad 0.21283777 \quad 1.94916218$

$\mathrm{H}-0.73930345 \quad 0.86797620 \quad 2.45238073$

$\mathrm{H}-1.58018915-0.313522373 .46435608$

$\mathrm{H}-2.00205611-2.76163662 \quad 2.30167232$

$\mathrm{H}-1.08275268-3.13485722 \quad 0.83851065$

$\mathrm{H}-2.43452307-2.00174941 \quad 0.77391920$

$0.93156483 \quad 0.27681777-2.36641574$

$0 \quad 0.56696204 \quad 1.60432702 \quad-2.02998814$

o - $0.16254082-0.51595469-1.92052989$

Vibrational frequencies (in $\mathrm{cm}-1$ )

$\begin{array}{lllllllllllllll}-24.3 & 30.9 & 45.3 & 123.5 & 125.1 & 131.0 & 164.9 & 187.2 & 213.9 & 254.2 & 277.8 & 280.7 & 316.1 & 353.9\end{array}$ $389.1436 .8 \quad 482.2 \quad 511.2 \quad 533.4 \quad 583.3 \quad 644.6 \quad 708.0 \quad 716.3 \quad 845.3 \quad 855.2 \quad 894.6 \quad 940.6 \quad 951.2$ $965.9971 .3 \quad 981.0 \quad 1003.4 \quad 1015.3 \quad 1019.3 \quad 1039.6 \quad 1060.2 \quad 1083.4 \quad 1103.6 \quad 1136.4 \quad 1156.5 \quad 1170.2$ $\begin{array}{lllllllllllll}1190.1 & 1208.2 & 1237.1 & 1240.1 & 1249.7 & 1265.1 & 1269.4 & 1279.0 & 1283.0 & 1284.9 & 1288.6 & 1294.5\end{array}$ $1295.01299 .21300 .7 \quad 1305.3 \quad 1325.91340 .91356 .1 \quad 1388.91391 .11405 .7 \quad 1407.2 \quad 1849.2$ $2655.22661 .12663 .3 \quad 2665.6 \quad 2670.5 \quad 2672.6 \quad 2675.6 \quad 2677.92685 .0 \quad 2689.4 \quad 2708.2 \quad 2771.2$ $2775.3 \quad 2779.0 \quad 2782.7 \quad 2789.1$

\section{name $=$ MIN170}

energy (in $\mathrm{kcal} / \mathrm{mol})=-17.354485$

$\mathrm{ZPE}(\mathrm{in} \mathrm{kcal} / \mathrm{mol})=145.844$

G $($ in $\mathrm{kcal} / \mathrm{mol})=120.812$

Geometry

C $-1.96912429-0.83932991-1.05223854$

C $-0.44613981-0.77945404-0.98893242$

$\begin{array}{lllll}\text { C } & 0.09897674 & 0.68667397 & -0.93957523\end{array}$

C $1.12458308 \quad 0.937287120 .16234226$

C $0.733096890 .25707793 \quad 1.48546856$

C $0.59132267-1.214883931 .22470655$

C $0.07695788-1.67859896 \quad 0.08392834$

C $-0.58086870 \quad 0.82428064 \quad 2.08083818$

C $-0.43273727 \quad 2.31178233 \quad 2.41679137$

C $-0.967922890 .04619845 \quad 3.34468432$

$\mathrm{H}-2.36020899-0.21973580-1.87033296$

$\mathrm{H}-2.42320715-0.50043432-0.11420269$

$\mathrm{H}-2.31733713-1.86513310-1.23462067$

$\mathrm{H}-0.71600977 \quad 1.43840215-0.93141245$ 
H $1.27160537 \quad 2.02283234 \quad 0.30744997$

H $2.11250076 \quad 0.54953029 \quad-0.16782987$

$\mathrm{H} 1.55346805 \quad 0.43134289 \quad 2.22483978$

$\mathrm{H}-1.39537001 \quad 0.70429430 \quad 1.32667147$

H $0.94747339-1.87344052 \quad 2.01221239$

$\mathrm{H} 0.00337823-2.74069439-0.14154112$

$\mathrm{H}-1.34021178 \quad 2.69902604 \quad 2.89430247$

$\mathrm{H}-0.258317332 .91450200 \quad 1.51909033$

$\mathrm{H} \quad 0.39987649 \quad 2.48895608 \quad 3.10540461$

$\mathrm{H}-0.17026667 \quad 0.06997813 \quad 4.09388566$

$\mathrm{H}-1.18111090-1.00503688 \quad 3.11849695$

$\mathrm{H}-1.86727182 \quad 0.46630393 \quad 3.80801248$

O $1.12378040-0.45047398-2.58238309$

$0 \quad 0.71569561 \quad 0.86021382-2.21587919$

o $0.00275260-1.25746603-2.27152124$

Vibrational frequencies (in cm-1)

$\begin{array}{llllllllllllllllll}38.7 & 61.2 & 89.7 & 134.5 & 143.6 & 159.6 & 181.6 & 208.6 & 272.0 & 283.0 & 326.2 & 351.7 & 374.6 & 415.2\end{array}$ $435.8 \quad 448.1 \quad 476.6 \quad 517.1 \quad 556.1 \quad 645.1 \quad 715.8 \quad 770.7 \quad 821.4 \quad 831.1 \quad 857.4 \quad 890.7 \quad 962.5 \quad 972.9$ $980.9983 .4986 .01003 .2 \quad 1011.0 \quad 1021.7 \quad 1054.1 \quad 1074.4 \quad 1111.6 \quad 1140.4 \quad 1150.7 \quad 1182.4 \quad 1184.5$ $1191.7 \quad 1200.4 \quad 1215.2 \quad 1248.91251 .5 \quad 1273.6 \quad 1278.2 \quad 1283.0 \quad 1286.5 \quad 1289.8 \quad 1293.6 \quad 1296.5$ $1300.81302 .6 \quad 1305.0 \quad 1309.7 \quad 1322.2 \quad 1333.3 \quad 1368.7 \quad 1375.6 \quad 1394.8 \quad 1401.5 \quad 1405.0 \quad 1860.8$ $2657.52670 .5 \quad 2672.4 \quad 2674.4 \quad 2674.7 \quad 2678.2 \quad 2679.12691 .5 \quad 2694.92712 .92741 .72749 .9$ $2763.32778 .62781 .2 \quad 2783.0$

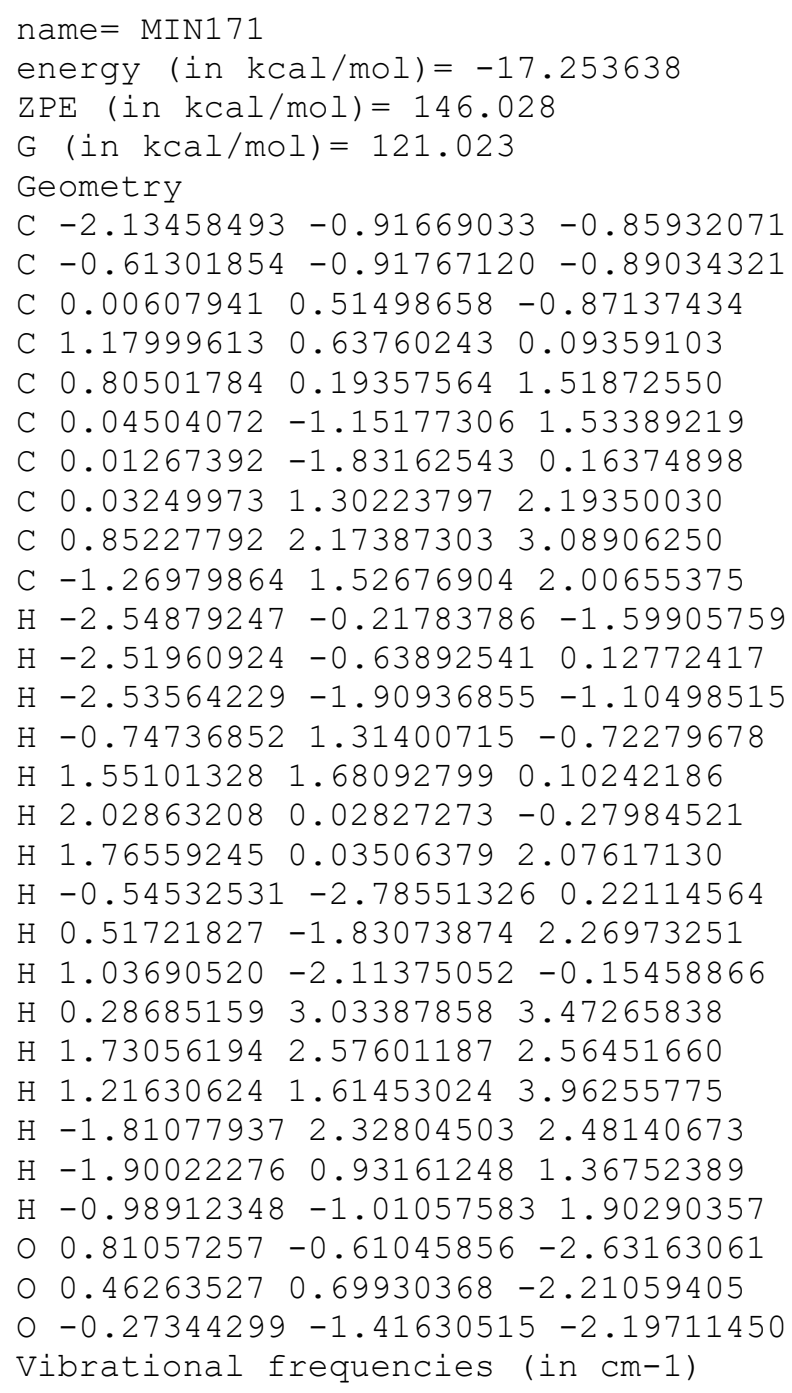

$\begin{array}{lllllllllllllllll}37.7 & 56.0 & 97.3 & 106.4 & 139.2 & 156.2 & 200.7 & 251.7 & 286.8 & 299.7 & 323.1 & 345.7 & 385.2 & 436.1\end{array}$

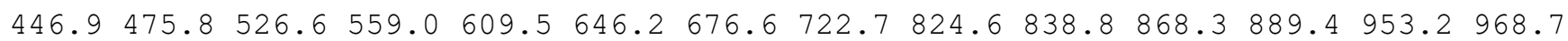

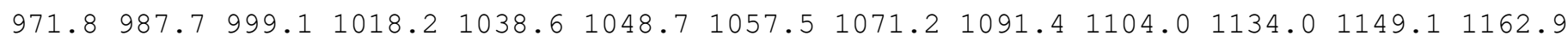
$1189.71198 .2 \quad 1213.2 \quad 1231.3 \quad 1256.8 \quad 1264.9 \quad 1274.2 \quad 1280.7 \quad 1282.9 \quad 1285.9 \quad 1287.5 \quad 1291.5$ $1295.21310 .8 \quad 1325.91335 .8 \quad 1342.9 \quad 1350.7 \quad 1369.8 \quad 1387.1 \quad 1393.8 \quad 1396.1 \quad 1402.3 \quad 1868.0$ 
$2651.02658 .8 \quad 2667.0 \quad 2671.2 \quad 2671.9 \quad 2673.5 \quad 2674.42691 .5 \quad 2694.4 \quad 2720.02740 .32742 .0$ 2745.82778 .62779 .12790 .8

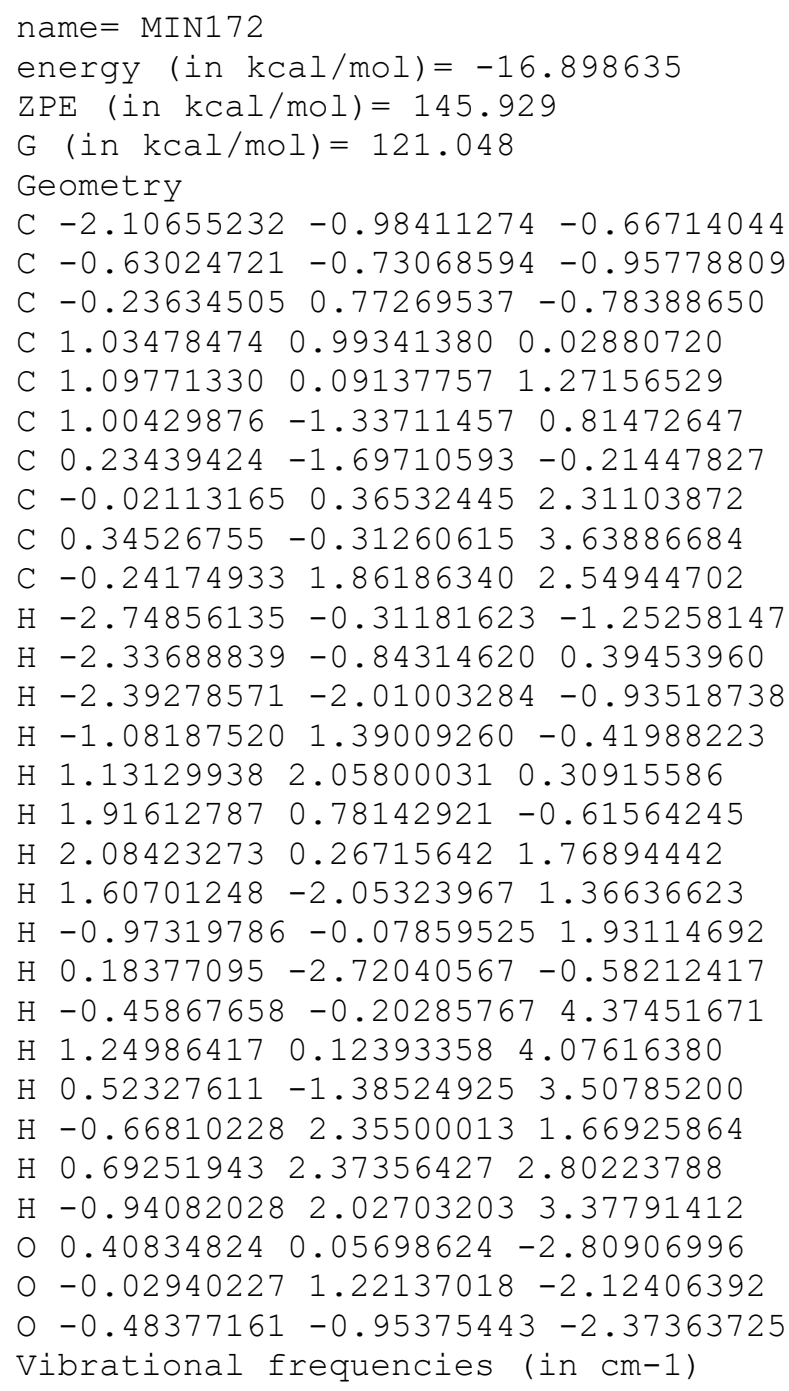


$\mathrm{H}-3.52978606-0.07869247-1.36062167$

$\mathrm{H}-1.848773021 .68475681-0.60542655$

$\mathrm{H}-0.51508485 \quad 1.28756817 \quad 1.49558800$

$\mathrm{H} \quad 0.42244130 \quad 2.23782548 \quad 0.33780970$

H $1.66798618 \quad 0.27382250-0.43512246$

H $0.97829176-1.78172013 \quad 1.49808940$

$\mathrm{H} \quad 0.93875451-1.83260873-0.26275096$

$\mathrm{H}-1.28369843-2.39102749 \quad 0.57331307$

H $3.99635599 \quad 0.28940936 \quad 0.83905427$

H $3.910671181 .48030442 \quad 2.14272052$

H $3.25435557 \quad 1.87141676 \quad 0.54979462$

$\mathrm{H}-1.34482989-0.96093190 \quad 1.61079084$

$\mathrm{H} \quad 0.74965639-0.09907097 \quad 3.27122110$

H $2.34041263 \quad 0.53694783 \quad 3.78929862$

$0-0.08455202-0.32295768-2.19018625$

$0-0.450262161 .01432120-1.87473676$

o -1.19012113-1.10058982 -1.76610752

Vibrational frequencies (in $\mathrm{cm}-1$ )

$\begin{array}{llllllllllllll}29.1 & 43.4 & 94.3 & 99.8 & 143.4 & 145.2 & 195.2 & 255.2 & 259.0 & 273.3 & 292.9 & 341.4 & 400.2 & 414.7\end{array}$ $461.2509 .0 \quad 541.2 \quad 568.3 \quad 594.4 \quad 623.5 \quad 676.5 \quad 714.1 \quad 810.6 \quad 843.6 \quad 876.1 \quad 938.7944 .1963 .7$

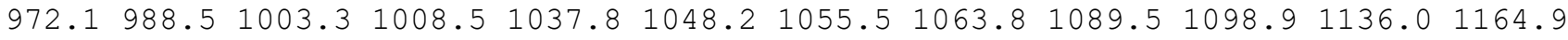
$1170.6 \quad 1189.0 \quad 1195.8 \quad 1208.2 \quad 1239.6 \quad 1258.5 \quad 1269.5 \quad 1275.6 \quad 1280.2 \quad 1282.8 \quad 1284.8 \quad 1285.8$

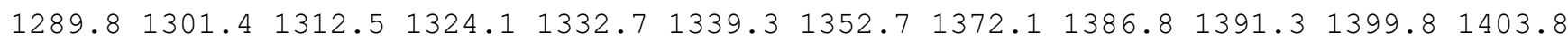

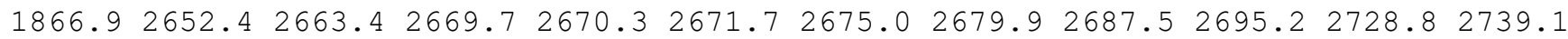
$2742.92745 .3 \quad 2779.12781 .5 \quad 2796.9$

\section{name $=$ MIN174}

energy (in $\mathrm{kcal} / \mathrm{mol})=-15.557236$

$\mathrm{ZPE}(\mathrm{in} \mathrm{kcal} / \mathrm{mol})=144.697$

$\mathrm{G}($ in $\mathrm{kcal} / \mathrm{mol})=118.787$

Geometry

C $-2.08013423-0.01555481-0.69774762$

C $-0.569453820 .04352775-0.64210465$

C $-0.00417996 \quad 1.50946834-0.65640601$

C 1.081047321 .589919990 .41553962

C $1.302405520 .19377153 \quad 0.90989415$

C $2.34964672-0.177838401 .64357942$

C $0.20345858-0.734497360 .44720436$

C $-0.54805970-1.28670014 \quad 1.67995708$

C $-1.30251893-0.213863332 .46895907$

C $-1.46984807-2.454921551 .31776501$

$\mathrm{H}-2.461961650 .57160389-1.54706292$

$\mathrm{H}-2.54502632 \quad 0.37447231 \quad 0.21549561$

$\mathrm{H}-2.43531641-1.04485869-0.84748116$

$\mathrm{H}-0.769748592 .29963825-0.58800475$

$\mathrm{H} \quad 0.78705094 \quad 2.26019078 \quad 1.24492325$

H $2.01122500 \quad 2.02375475-0.00733911$

$\mathrm{H} \quad 0.24777347 \quad-1.69795639 \quad 2.36291652$

H $2.51468377-1.18493486 \quad 1.98733655$

H $3.12830283 \quad 0.49729410 \quad 1.95640241$

$\mathrm{H} \quad 0.67216963-1.61883873-0.06808171$

$\mathrm{H}-2.17282946 \quad 0.16987596 \quad 1.92765457$

$\mathrm{H}-0.65216001 \quad 0.63777464 \quad 2.69971167$

$\mathrm{H}-1.66827769-0.61583006 \quad 3.42092268$

$\mathrm{H}-1.82402942-2.96542306 \quad 2.22101422$

$\mathrm{H}-2.35728385-2.13073848 \quad 0.76649985$

$\mathrm{H}-0.95247341-3.199467090 .70202952$

$\begin{array}{lllll}0 & 0.93862785 & 0.33145162 & -2.31318991\end{array}$

○ $0.54637454 \quad 1.65231356-1.95944509$

o $-0.16196745-0.47062960-1.92652966$

Vibrational frequencies (in $\mathrm{cm}-1$ )

$\begin{array}{lllllllllllllll}17.5 & 42.4 & 69.1 & 131.4 & 139.6 & 163.9 & 183.5 & 211.2 & 235.2 & 272.6 & 292.0 & 317.0 & 354.8 & 390.8\end{array}$ $442.8 \quad 450.2 \quad 504.5 \quad 543.5 \quad 563.0 \quad 601.9 \quad 632.7 \quad 703.7 \quad 714.6 \quad 824.9 \quad 857.2 \quad 914.4 \quad 948.7959 .8$ $964.2975 .3 \quad 979.4 \quad 986.3 \quad 1009.9 \quad 1010.9 \quad 1021.2 \quad 1056.7 \quad 1083.6 \quad 1113.4 \quad 1133.2 \quad 1148.91156 .7$ 


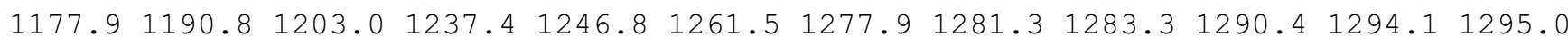
$1300.11300 .51304 .81313 .0 \quad 1326.6 \quad 1343.8 \quad 1360.2 \quad 1387.91388 .8 \quad 1404.91406 .61880 .9$ $2650.32661 .7 \quad 2662.92665 .8 \quad 2672.92674 .2 \quad 2677.8 \quad 2679.82690 .3 \quad 2704.0 \quad 2726.2 \quad 2744.4$ $2769.42779 .3 \quad 2782.8 \quad 2795.1$

\section{name $=$ MIN175}

energy (in $\mathrm{kcal} / \mathrm{mol})=-15.99026$

$\mathrm{ZPE}($ in $\mathrm{kcal} / \mathrm{mol})=145.138$

$\mathrm{G}(\mathrm{in} \mathrm{kcal} / \mathrm{mol})=120.596$

Geometry

C $-2.48896715-0.22042330-0.76145452$

C $-0.96606412-0.23968711-0.77597125$

C $-0.348291931 .19433659-0.78328599$

C $0.52437245 \quad 1.61100628 \quad 0.39757339$

C $1.12600883 \quad 0.46958136 \quad 1.15878051$

C $2.42724555 \quad 0.45783746 \quad 1.45285801$

C $-0.37228652-1.23222677 \quad 0.22314630$

C $0.14693628-0.614804851 .53724755$

C $-1.00082306-0.026570852 .37524265$

C $0.80142438-1.73884594 \quad 2.36049753$

$\mathrm{H}-2.89314915 \quad 0.44339507 \quad-1.53787137$

$\mathrm{H}-2.878139330 .10718309 \quad 0.20852649$

$\mathrm{H}-2.89920857-1.21998731-0.96286134$

$\mathrm{H}-1.13785010 \quad 1.96063224-0.96382027$

$\mathrm{H}-0.06443457 \quad 2.24065564 \quad 1.09933325$

$\mathrm{H} \quad 1.32280611 \quad 2.286341890 .01056405$

H 2.92175052 -0.32104472 2.00758399

$\mathrm{H} \quad 0.47317887-1.75895309-0.27604531$

$\mathrm{H} \quad 3.11128285 \quad 1.23432387 \quad 1.14905921$

$\mathrm{H}-1.10940287-2.02495403 \quad 0.45208603$

$\mathrm{H}-0.61467690 \quad 0.46177249 \quad 3.27716004$

$\mathrm{H}-1.69723903-0.80896000 \quad 2.69575856$

$\mathrm{H}-1.57842307 \quad 0.71701779 \quad 1.81838547$

H $1.20457690-1.354932313 .30413086$

$\mathrm{H} 1.62418049-2.214240331 .81465847$

$\mathrm{H} \quad 0.07710378-2.52208910 \quad 2.60833861$

O $0.61668344-0.11455964-2.36513142$

$00.43159636 \quad 1.24570714-1.98178018$

o $-0.63149118-0.71161087-2.10098092$

Vibrational frequencies (in $\mathrm{cm}-1$ )

$\begin{array}{lllllllllllllll}41.2 & 61.1 & 113.0 & 140.0 & 182.8 & 219.1 & 225.4 & 242.6 & 258.4 & 291.3 & 324.5 & 337.5 & 374.6 & 408.3\end{array}$ $437.5 \quad 447.0 \quad 467.5 \quad 516.5 \quad 553.0 \quad 625.4 \quad 647.4 \quad 695.8 \quad 706.7 \quad 793.0 \quad 850.6 \quad 872.9 \quad 907.8 \quad 940.6$ $948.1960 .6 \quad 983.8 \quad 987.9 \quad 992.6 \quad 1009.5 \quad 1020.0 \quad 1028.5 \quad 1068.9 \quad 1091.0 \quad 1119.1 \quad 1140.9 \quad 1161.4$ $1194.41210 .5 \quad 1220.9 \quad 1232.0 \quad 1243.1 \quad 1273.1 \quad 1278.3 \quad 1285.0 \quad 1288.8 \quad 1293.2 \quad 1298.01304 .0$

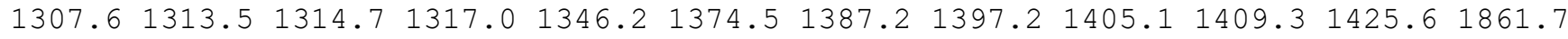
$2650.02653 .5 \quad 2667.92673 .92674 .8 \quad 2675.6 \quad 2676.52678 .02680 .72720 .12732 .12735 .7$ $2778.8 \quad 2780.4 \quad 2781.7 \quad 2791.4$

name $=$ MIN176

energy (in $\mathrm{kcal} / \mathrm{mol})=-17.391805$

$\mathrm{ZPE}(\mathrm{in} \mathrm{kcal} / \mathrm{mol})=146.637$

$\mathrm{G}(\mathrm{in} \mathrm{kcal} / \mathrm{mol})=123.336$

Geometry

C $-2.31929838-0.02195144-0.29315472$

C $-0.80563954-0.03575882-0.28852515$

$\begin{array}{llll}\text { C } & -0.17973541 & 1.38051124 & -0.29187784\end{array}$

C $1.07714901 \quad 1.57182485 \quad 0.58508707$

C $1.310289490 .37886474 \quad 1.51308635$

C $1.34620475-0.914290310 .64718732$

C $-0.20950194-0.93864996 \quad 0.76986174$

C $-0.07535941-0.08822602 \quad 2.08821899$

C $0.39473036 \quad 0.44100474 \quad-2.31384608$

C $0.00993559-0.91294716 \quad 3.35395599$ 
$\mathrm{H}-2.72846182-1.02568493-0.48972750$

$\mathrm{H}-2.73230238 \quad 0.65810281 \quad-1.04939051$

$\mathrm{H}-2.71576330 \quad 0.29084091 \quad 0.68280622$

$\mathrm{H}-0.93450520 \quad 2.16655092-0.06715878$

$\mathrm{H} \quad 0.98484689 \quad 2.50256884 \quad 1.17292665$

$\mathrm{H} \quad 1.95846641 \quad 1.72279542 \quad-0.07076277$

H $2.11827333 \quad 0.52487156 \quad 2.22862726$

H $1.83264368-1.76859474 \quad 1.12444244$

$\mathrm{H} \quad 1.75759931-0.83011137-0.35817467$

$\mathrm{H}-0.67656002-1.922983890 .83740132$

$\mathrm{H}-0.81768772 \quad 0.71398173 \quad 2.19930757$

H $1.43759184 \quad 0.12124473-2.22110259$

$\mathrm{H} \quad 0.05839170 \quad 0.55945457 \quad-3.35033245$

$\mathrm{H}-0.96771637-1.334008213 .61934068$

$\mathrm{H} \quad 0.35076679-0.30523662 \quad 4.20089307$

$\mathrm{H} \quad 0.70763696-1.75402247 \quad 3.25379262$

○ $0.01996899-1.73263808-1.69426926$

○ $0.140540111 .64617258-1.65516028$

O $-0.54495151-0.58902847-1.70690574$

Vibrational frequencies (in cm-1)

$\begin{array}{lllllllllllllll}77.7 & 136.1 & 147.0 & 150.4 & 159.8 & 202.1 & 244.9 & 265.5 & 276.6 & 331.9 & 374.5 & 402.1 & 433.8 & 461.0\end{array}$ $493.8 \quad 542.3 \quad 593.7 \quad 615.5 \quad 645.4 \quad 777.2 \quad 786.9 \quad 836.0 \quad 867.0 \quad 890.0 \quad 913.4 \quad 930.8 \quad 936.1 \quad 967.8$ $987.8 \quad 1004.7 \quad 1009.6 \quad 1034.8 \quad 1050.9 \quad 1061.6 \quad 1065.0 \quad 1074.1 \quad 1088.7 \quad 1100.1 \quad 1118.3 \quad 1136.8$

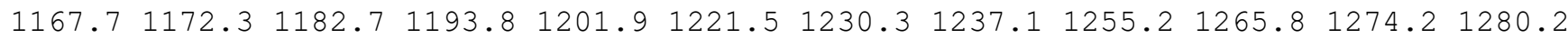

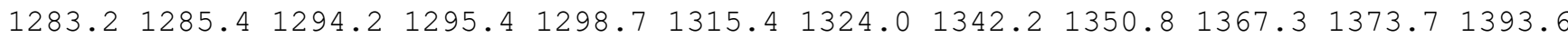
$1403.22645 .02660 .0 \quad 2662.92670 .3 \quad 2673.7 \quad 2679.2 \quad 2686.42689 .72702 .12729 .62743 .2$ $2747.92754 .12770 .02775 .6 \quad 2782.4$

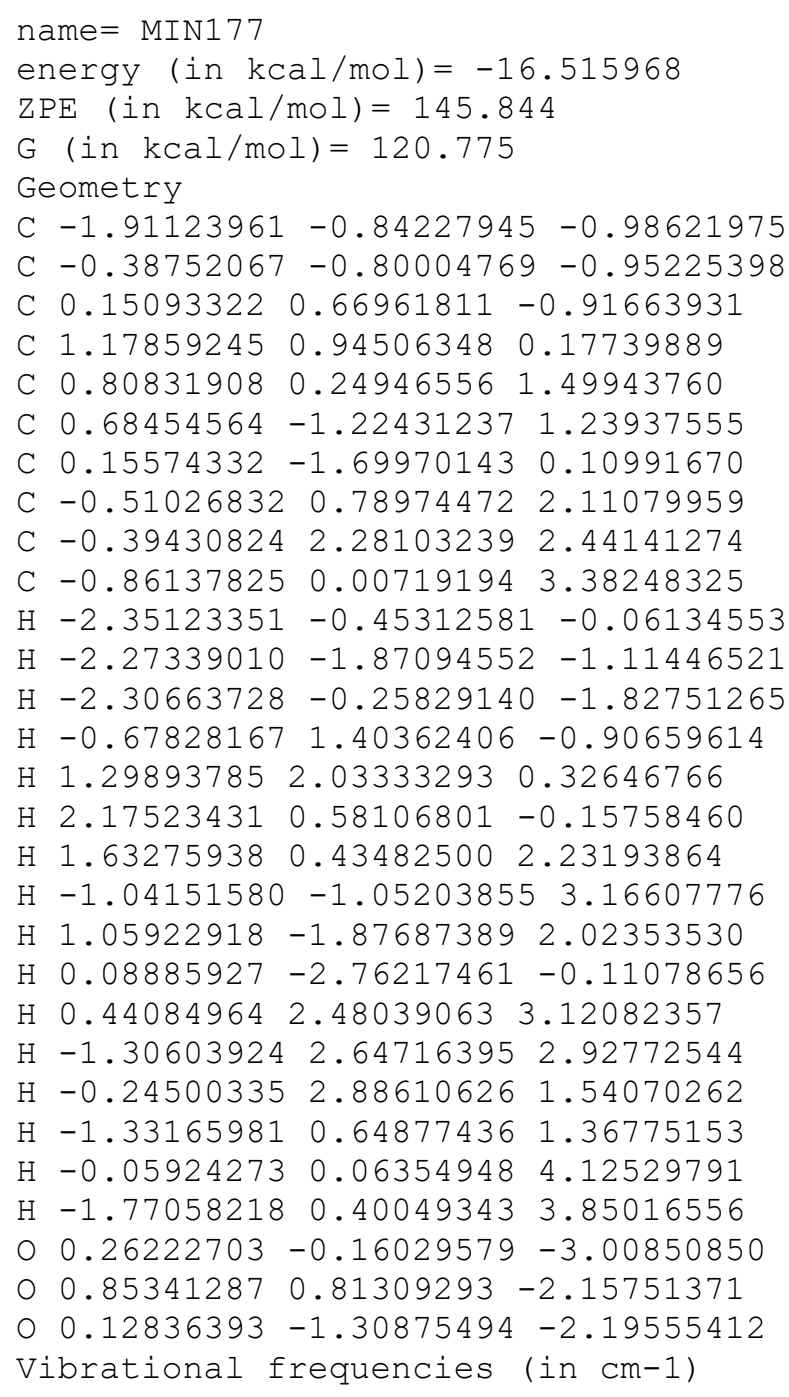


$\begin{array}{lllllllllllllllll}36.9 & 61.4 & 88.0 & 134.8 & 143.0 & 160.8 & 182.6 & 230.2 & 251.9 & 281.5 & 325.9 & 349.0 & 373.6 & 395.2\end{array}$

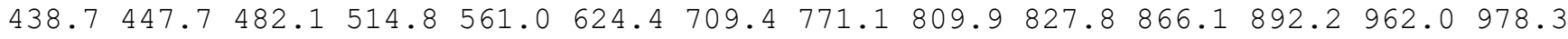
$980.8 \quad 984.6 \quad 988.7 \quad 1010.8 \quad 1012.9 \quad 1018.4 \quad 1061.5 \quad 1091.3 \quad 1119.8 \quad 1148.4 \quad 1154.8 \quad 1163.7 \quad 1180.6$

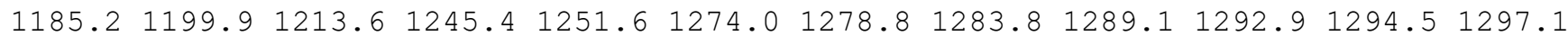
$1301.61303 .6 \quad 1306.6 \quad 1309.91323 .2 \quad 1346.2 \quad 1370.0 \quad 1376.2 \quad 1396.5 \quad 1401.51405 .21860 .4$ $2654.92671 .2 \quad 2672.0 \quad 2674.5 \quad 2674.8 \quad 2678.1 \quad 2678.92686 .92694 .02711 .72739 .6 \quad 2750.9$ $2764.62779 .3 \quad 2781.2 \quad 2783.1$

\section{name $=$ MIN178}

energy (in $\mathrm{kcal} / \mathrm{mol})=-15.477148$

$\operatorname{ZPE}($ in $\mathrm{kcal} / \mathrm{mol})=144.896$

$\mathrm{G}($ in $\mathrm{kcal} / \mathrm{mol})=120.844$

Geometry

C $-2.30683375-0.14035379-0.57671637$

C $-0.77936240 \quad-0.13776454-0.60970828$

C $-0.188689561 .31259574 \quad-0.61655654$

C $0.97920180 \quad 1.52829992 \quad 0.28303143$

C $1.176139190 .79488776 \quad 1.37964685$

C $1.01443165-1.84214410-0.16479416$

C $-0.17125860-1.08910299 \quad 0.44692521$

C $0.24503480-0.32840145 \quad 1.74113839$

C $-0.98850620 \quad 0.26829269 \quad 2.44573001$

C $0.92794785-1.29052814 \quad 2.72762234$

$\mathrm{H}-2.70138008 \quad 0.157607190 .39864704$

$\mathrm{H}-2.70092063-1.14071983-0.80420282$

$\mathrm{H}-2.72048864 \quad 0.53608218-1.33758801$

$\mathrm{H}-0.97355980 \quad 2.08377019-0.44519243$

H $1.650710892 .32620625-0.02662733$

$\mathrm{H} \quad 1.81798618-1.15267526-0.45240747$

H $2.008720110 .96690752 \quad 2.05502472$

$\mathrm{H} \quad 0.71572956-2.38548565-1.07166456$

$\mathrm{H} \quad 1.43020432-2.582214930 .52524900$

$\mathrm{H}-0.94684324-1.84144415 \quad 0.73146340$

$\mathrm{H}-1.73114174-0.50401176 \quad 2.66955685$

$\mathrm{H}-1.470117801 .041167591 .83842445$

$\mathrm{H}-0.70784545 \quad 0.74002201 \quad 3.39455524$

$\mathrm{H} 1.09758873-0.81112975 \quad 3.69793984$

H $1.90353170-1.626101292 .35856240$

H $\quad 0.31427659-2.180322192 .90411671$

$0 \quad 0.565769320 .21147218-2.41036862$

O $0.196557121 .51085300-1.97895516$

$0-0.48301454-0.61537958-1.93541560$

Vibrational frequencies (in cm-1)

$63.2100 .0 \quad 146.2 \quad 173.1 \quad 179.7 \quad 190.5 \quad 207.0 \quad 229.5 \quad 253.6 \quad 277.6 \quad 294.3 \quad 325.8 \quad 363.4 \quad 372.1$

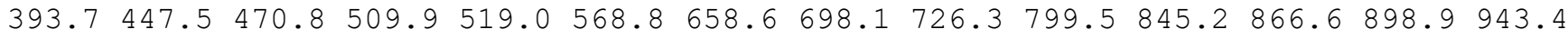

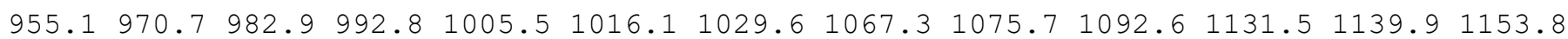

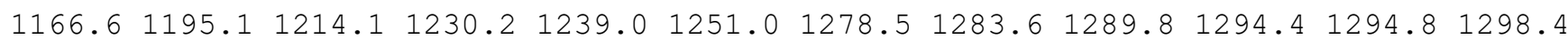
$1302.71307 .21309 .4 \quad 1313.3 \quad 1330.3 \quad 1357.0 \quad 1376.0 \quad 1394.81401 .5 \quad 1406.4 \quad 1416.01862 .3$ $2667.02667 .92672 .2 \quad 2674.4 \quad 2675.4 \quad 2676.7 \quad 2678.12680 .52681 .0 \quad 2699.32751 .0 \quad 2764.5$ 2778.22779 .62780 .62781 .8

\section{name $=$ MIN179}

energy (in $\mathrm{kcal} / \mathrm{mol})=-16.861391$

$\mathrm{ZPE}(\mathrm{in} \mathrm{kcal} / \mathrm{mol})=146.298$

$\mathrm{G}($ in $\mathrm{kcal} / \mathrm{mol})=123.222$

Geometry

C $-2.22862032-0.04938417-0.67017101$

C $-0.71861552-0.15313822-0.70242458$

C $0.11985675 \quad 1.18192731-0.67647978$

C $1.31227394 \quad 0.83753020 \quad 0.25180124$

$\begin{array}{lllll}\text { C } & 0.78185171 & 0.88586213 & 1.70352688\end{array}$

C $1.47697396-0.69353520 \quad 0.04915829$

C $-0.01162479-1.017603320 .37540403$ 
C $-0.13281641-0.38412222 \quad 1.80852055$

C $-1.54562105-0.003009292 .24276477$

C $0.41841970-1.38102186 \quad 2.83749926$

$\mathrm{H}-2.63339249 \quad 0.01389153-1.69539382$

$\mathrm{H}-2.58142367 \quad 0.84168499-0.14154220$

$\mathrm{H}-2.68581567-0.93065260-0.20402989$

$\mathrm{H}-0.42888334 \quad 2.09307487 \quad-0.40254493$

$\mathrm{H} \quad 0.23594118 \quad 1.81180497 \quad 1.92127554$

H 2.20361088 1.441867910 .06258668

$\begin{array}{lllll}\mathrm{H} & 1.60993707 & 0.84355777 & 2.42592709\end{array}$

H $2.19380026-1.14999848 \quad 0.74022946$

$\mathrm{H} \quad 1.78966110-0.98880097-0.95898127$

$\mathrm{H}-0.29276609-2.07221455 \quad 0.33592845$

$\mathrm{H}-1.92342464 \quad 0.87398337 \quad 1.70752372$

$\mathrm{H}-1.57341702 \quad 0.24991945 \quad 3.31034763$

$\mathrm{H}-2.24962147-0.82712736 \quad 2.08618643$

$\mathrm{H} \quad 0.46750528 \quad-0.935424713 .83726741$

H $1.42736107-1.722322012 .58423467$

$\mathrm{H}-0.21865027-2.26998234 \quad 2.90587684$

$0.641791190 .05663520-2.54160160$

O $0.50299851 \quad 1.37107982-2.02436442$

O $-0.45391071-0.68265722-2.01128309$

Vibrational frequencies (in $\mathrm{cm}-1$ )

$\begin{array}{llllllllllllllll}81.1 & 121.3 & 172.2 & 182.6 & 204.4 & 222.3 & 240.6 & 273.9 & 302.8 & 342.4 & 353.9 & 390.5 & 432.3 & 438.8\end{array}$

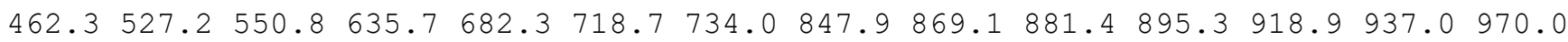
$975.3980 .6 \quad 988.8 \quad 1000.6 \quad 1011.7 \quad 1014.2 \quad 1029.2 \quad 1047.1 \quad 1058.11097 .1 \quad 1108.51123 .1 \quad 1137.1$ $\begin{array}{lllllllllllllll}1145.7 & 1155.3 & 1184.7 & 1209.5 & 1229.3 & 1238.9 & 1253.0 & 1264.9 & 1273.0 & 1279.9 & 1283.7 & 1285.8\end{array}$ $1289.71296 .8 \quad 1300.2 \quad 1305.1 \quad 1308.6 \quad 1326.5 \quad 1342.7 \quad 1354.01390 .4 \quad 1395.01403 .91410 .4$ $2659.72669 .42674 .92675 .1 \quad 2676.0 \quad 2677.7 \quad 2680.12680 .72708 .8 \quad 2745.72747 .92755 .2$ 2760.12775 .92780 .02781 .7
name $=$ MIN180
energy (in $\mathrm{kcal} / \mathrm{mol})=-16.388491$
$\mathrm{ZPE}($ in $\mathrm{kcal} / \mathrm{mol})=145.912$
$\mathrm{G}($ in $\mathrm{kcal} / \mathrm{mol})=119.988$
Geometry
C $-2.99747755-0.49014695-0.60157108$
C $-1.47624784-0.48950098-0.60421688$
C $-0.88665206 \quad 0.95635582 \quad-0.60071670$
C $0.06284075 \quad 1.21990802 \quad 0.55907372$
C $1.17347301 \quad 0.15545792 \quad 0.64248255$
C $0.64046286-1.28775032 \quad 0.49326761$
C $-0.88763251-1.37189848 \quad 0.49674637$
C $1.91614730 \quad 0.32873531 \quad 1.94516602$
C $3.30752695 \quad 0.85440614 \quad 1.81907784$
C $1.36898063 \quad 0.048369693 .13037699$
$\mathrm{H}-3.39637943 \quad 0.05560403-1.46733967$
$\mathrm{H}-3.40627529-0.03341067 \quad 0.30538788$
$\mathrm{H}-3.39278712-1.51218976-0.66833895$
$\mathrm{H}-1.68271295 \quad 1.72513317-0.66609738$
$\mathrm{H}-0.51233824 \quad 1.25206756 \quad 1.50570570$
$\mathrm{H} \quad 0.52395034 \quad 2.22192164 \quad 0.45164323$
H $1.87011957 \quad 0.33808869-0.21778501$
$\mathrm{H} \quad 1.05208004-1.93365297 \quad 1.29150792$
$\mathrm{H} \quad 1.02089745-1.71272747-0.45915239$
$\mathrm{H}-1.20477302-2.42289254 \quad 0.34734781$
$\mathrm{H} \quad 3.30239021 \quad 1.89987910 \quad 1.47731063$
H $3.89516567 \quad 0.27587581 \quad 1.09196039$
H $3.85718412 \quad 0.82732659 \quad 2.76914303$
$\mathrm{H} \quad 0.36799115 \quad-0.32898103 \quad 3.25083493$
$\mathrm{H}-1.29154429-1.067231261 .48038780$
$\mathrm{H} \quad 1.87993601 \quad 0.16999759 \quad 4.06938833$
$\begin{array}{llllll}0 & -0.75133064 & 0.08546028 & -2.66380135\end{array}$
o $-0.112801021 .01806826-1.80422699$ 
$0-1.00657443-1.04260513-1.84316502$

Vibrational frequencies (in $\mathrm{cm}-1$ )

$\begin{array}{lllllllllllllllll}17.4 & 31.4 & 92.8 & 98.1 & 137.8 & 151.8 & 202.0 & 246.7 & 258.0 & 285.8 & 288.6 & 324.2 & 391.5 & 408.6\end{array}$ $464.1510 .0 \quad 547.7 \quad 574.4 \quad 597.0 \quad 626.4 \quad 673.2 \quad 702.3 \quad 805.2 \quad 829.6 \quad 874.8 \quad 929.4 \quad 940.5 \quad 956.9$ $984.5990 .1 \quad 1004.8 \quad 1011.2 \quad 1038.3 \quad 1045.5 \quad 1052.0 \quad 1070.2 \quad 1095.3 \quad 1107.8 \quad 1143.21162 .9$

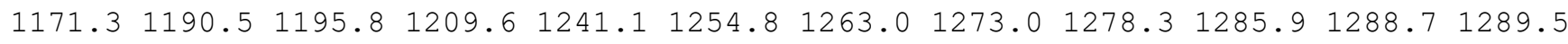

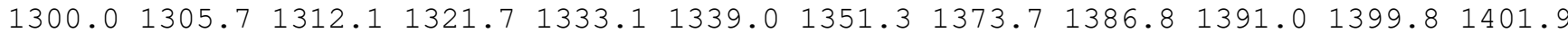
$1865.42652 .72661 .1 \quad 2670.2 \quad 2670.7 \quad 2672.2 \quad 2674.72680 .82683 .2 \quad 2695.72728 .12738 .1$ $2740.8 \quad 2745.3 \quad 2779.1 \quad 2782.5 \quad 2796.2$

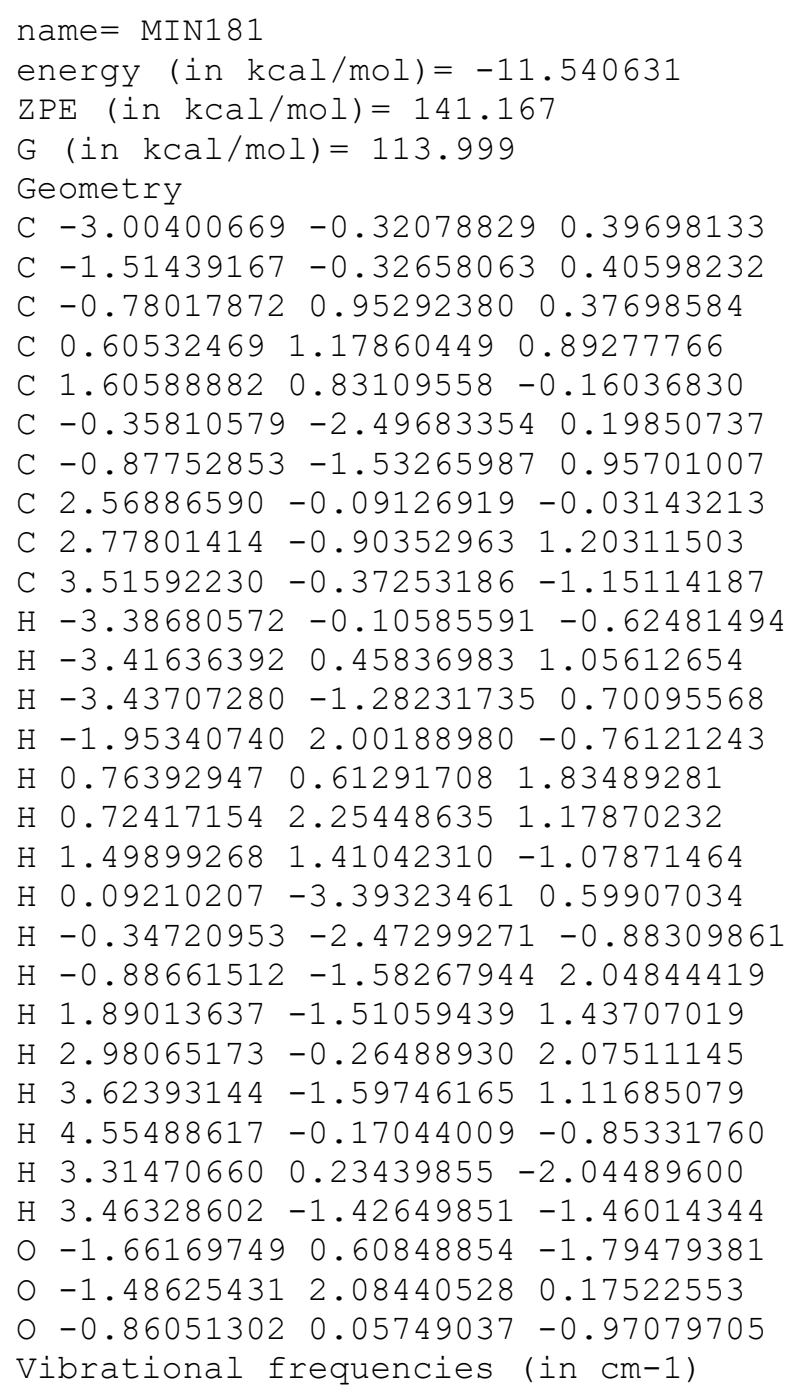


C $1.71493607-0.956101831 .01702503$

C $-0.41656174-0.40796861 \quad 0.99183594$

C $0.38932676-1.72335131 \quad 0.69522272$

C $0.07862860-2.786029591 .74820929$

C $0.29414927-2.32505577-0.69033968$

$\mathrm{H}-2.72005597 \quad 1.30388794 \quad-1.01212549$

$\mathrm{H}-2.78744731 \quad 1.138438650 .75295925$

$\mathrm{H}-2.92966276-0.29040342-0.28553800$

$\mathrm{H}-0.72529716 \quad 2.69313023 \quad 0.22020744$

H $1.20533064 \quad 2.41605987 \quad 1.59031225$

$\mathrm{H} \quad 2.03864869 \quad 1.61193073 \quad 0.26205181$

$\mathrm{H} \quad 0.92582379 \quad 0.34090958 \quad 2.63450791$

$\mathrm{H} \quad 2.35965150-1.43313166 \quad 1.75438593$

$\mathrm{H} 2.31696673-0.738759030 .12986839$

$\mathrm{H}-1.20490207-0.55874700 \quad 1.74258321$

$\mathrm{H}-0.92504735-3.20162260 \quad 1.60720663$

$\mathrm{H} \quad 0.13219361-2.384372792 .76627022$

$\mathrm{H} \quad 0.79110371-3.61730858 \quad 1.69119058$

$\mathrm{H} \quad 0.83870625-1.73359367-1.44054007$

$\mathrm{H}-0.74901965-2.40005581-1.02294678$

$\mathrm{H} \quad 0.72237430-3.33432268-0.72392292$

$\begin{array}{lllll}0 & -0.33724041 & 1.12277954 & -2.20475775\end{array}$

○ $0.381866332 .05115878-1.38195946$

o $-0.47981312-0.03236387-1.40961213$

Vibrational frequencies (in cm-1)

$\begin{array}{llllllllllllll}50.9 & 67.4 & 170.2 & 179.3 & 198.9 & 211.6 & 225.3 & 259.1 & 268.1 & 306.0 & 350.3 & 360.0 & 393.6 & 417.3\end{array}$ $432.6 \quad 501.2 \quad 554.0 \quad 586.0 \quad 657.1 \quad 677.9 \quad 736.9 \quad 843.1 \quad 852.5 \quad 873.3 \quad 882.9 \quad 927.2 \quad 944.6 \quad 964.2$

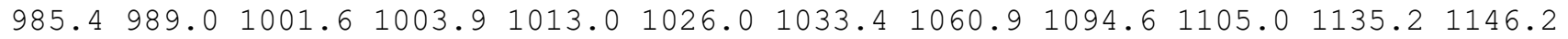

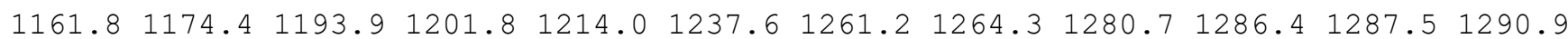

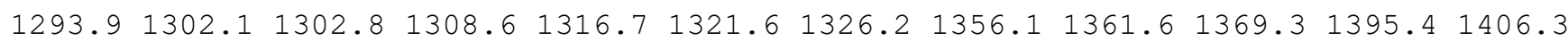
$1409.32662 .92663 .62671 .3 \quad 2675.3 \quad 2678.42679 .72681 .32692 .72697 .12727 .12739 .0$ $2746.7 \quad 2767.2 \quad 2777.5 \quad 2781.4 \quad 2782.9$

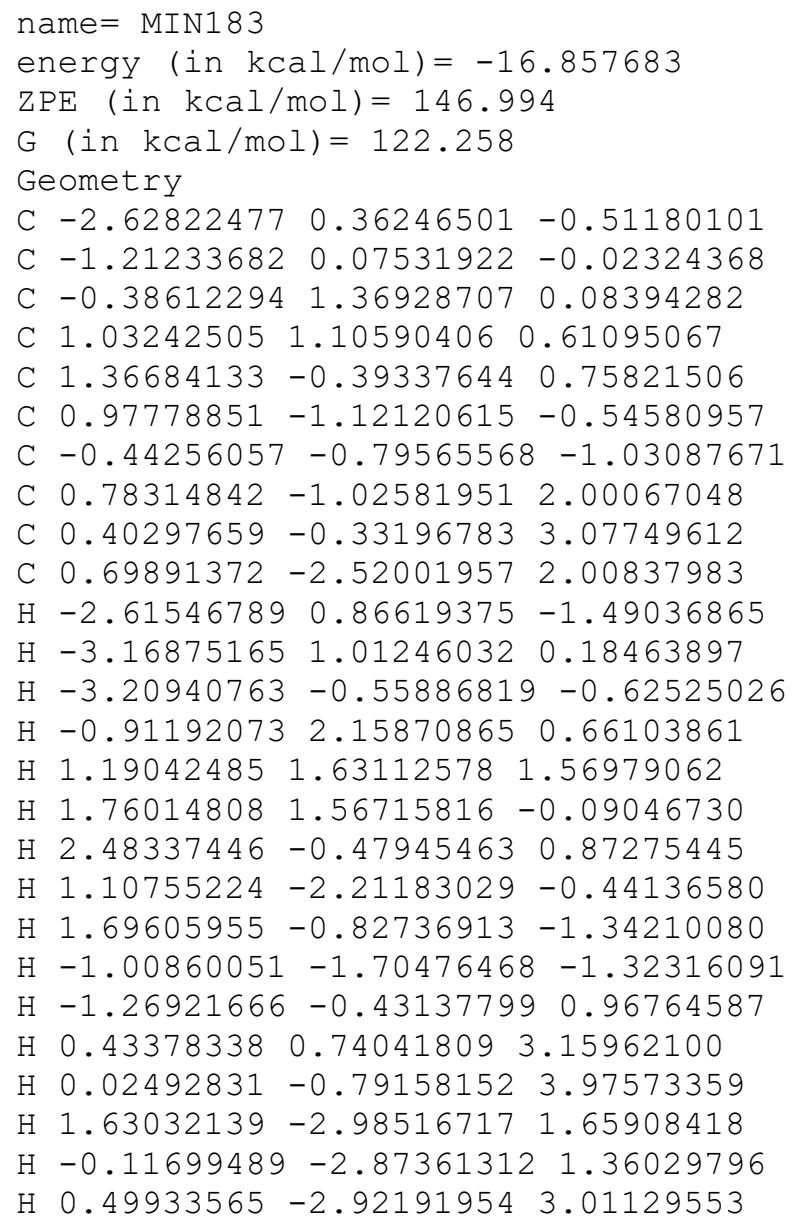


$0.361867021 .08977287-2.07576601$

$0-0.33217544 \quad 1.95537394-1.21760823$

o $-0.39068589-0.07911506-2.26574019$

Vibrational frequencies (in $\mathrm{cm}-1$ )

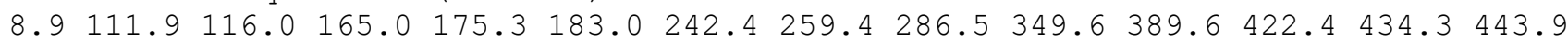
$490.1504 .1 \quad 543.0 \quad 568.4 \quad 602.1 \quad 624.0 \quad 746.5 \quad 804.5 \quad 810.1 \quad 872.0 \quad 880.6 \quad 901.4 \quad 947.3 \quad 957.6$ $975.3991 .4 \quad 1017.9 \quad 1034.2 \quad 1047.5 \quad 1051.7 \quad 1060.3 \quad 1076.1 \quad 1097.8 \quad 1117.6 \quad 1128.21146 .2$

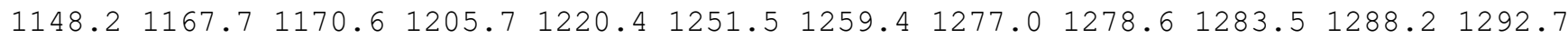

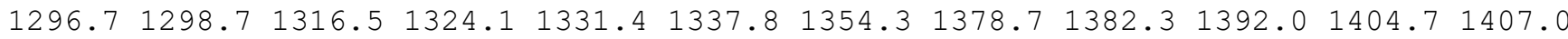
$1852.42652 .02654 .32666 .32668 .42673 .2 \quad 2680.42681 .22695 .42696 .12705 .82720 .5$ $2736.22739 .3 \quad 2777.8 \quad 2780.2 \quad 2792.6$

name $=\operatorname{MIN} 184$

energy (in $\mathrm{kcal} / \mathrm{mol})=-15.690915$

$\mathrm{ZPE}(\mathrm{in} \mathrm{kcal} / \mathrm{mol})=145.929$

$\mathrm{G}($ in $\mathrm{kcal} / \mathrm{mol})=120.144$

Geometry

C $-2.45268175-0.51928168-0.77810457$

C $-0.92284044-0.52023092-0.78208046$

C $-0.36675078 \quad 0.93607402-0.77730370$

C $0.966514521 .19315945-0.07352173$

C $1.33568984 \quad 0.20890168 \quad 1.04509588$

C $1.10719469-1.22703964 \quad 0.53975619$

C $-0.37754461-1.46669528 \quad 0.28237997$

C $0.614321450 .44297495 \quad 2.34827122$

C $1.22516020-0.25706768 \quad 3.52246062$

C $-0.467306151 .21077632 \quad 2.49498650$

$\mathrm{H}-2.85689390-0.132816450 .16239549$

$\mathrm{H}-2.84816197-1.53253401-0.92357041$

$\mathrm{H}-2.84761947 \quad 0.09258614-1.60098866$

$\mathrm{H}-1.14029861 \quad 1.67192589-0.47446681$

$\mathrm{H} \quad 0.98363385 \quad 2.22810067 \quad 0.32156945$

$\mathrm{H} \quad 1.77024860 \quad 1.16454008 \quad-0.84439394$

$\mathrm{H} \quad 2.43453018 \quad 0.33720853 \quad 1.24469047$

H $1.49580402-1.963634921 .26545164$

$\mathrm{H} 1.68168603-1.38864155-0.39528980$

$\mathrm{H}-0.54502514-2.51437827-0.04018658$

H $0.61436513-0.16470787 \quad 4.43034566$

$\mathrm{H} \quad 2.21568510 \quad 0.15634288 \quad 3.75838546$

H $1.35385986-1.33202118 \quad 3.33439147$

$\mathrm{H}-0.96044690 \quad 1.366138513 .44060646$

$\mathrm{H}-0.94896181-1.34337427 \quad 1.22501094$

$\mathrm{H}-0.94852957 \quad 1.74713786 \quad 1.69684628$

$0.29031726-0.04991390-2.66968760$

O $-0.194916891 .18239752 \quad-2.17824498$

o $-0.54950449-1.03667462-2.07223824$

Vibrational frequencies (in $\mathrm{cm}-1$ )

$\begin{array}{lllllllllllllll}-21.7 & 43.9 & 54.6 & 80.1 & 144.9 & 167.2 & 194.0 & 267.1 & 273.5 & 310.1 & 331.8 & 336.0 & 393.0 & 431.5\end{array}$ $449.7 \quad 470.2 \quad 535.2 \quad 553.0 \quad 604.0 \quad 638.2 \quad 697.3 \quad 727.1 \quad 818.1 \quad 862.5 \quad 877.3 \quad 897.0 \quad 948.8 \quad 963.9$ $976.0990 .9992 .3 \quad 1006.2 \quad 1036.5 \quad 1041.6 \quad 1048.7 \quad 1069.9 \quad 1081.8 \quad 1092.91136 .4 \quad 1147.4 \quad 1156.3$

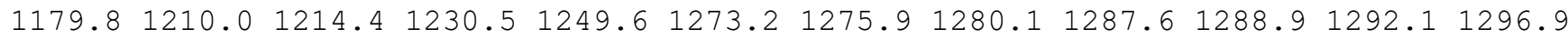
$1307.11310 .6 \quad 1319.2 \quad 1331.3 \quad 1337.4 \quad 1357.4 \quad 1378.5 \quad 1389.8 \quad 1396.4 \quad 1403.9 \quad 1410.4 \quad 1867.4$ $2650.02657 .22670 .12670 .3 \quad 2671.1 \quad 2673.4 \quad 2680.02687 .02689 .42723 .62734 .02739 .4$ $2743.6 \quad 2779.02780 .8 \quad 2794.8$

name $=$ MIN185

energy (in $\mathrm{kcal} / \mathrm{mol})=-15.608247$

$\operatorname{ZPE}($ in $\mathrm{kcal} / \mathrm{mol})=145.99$

$\mathrm{G}($ in $\mathrm{kcal} / \mathrm{mol})=120.368$

Geometry

C $-2.50259436-0.53840098-0.72055822$

C $-0.97338009-0.51164875-0.77200663$

C $-0.444685820 .95348396-0.73014011$ 
C $0.90134083 \quad 1.21565176-0.05470908$

C $1.35915627 \quad 0.18549666 \quad 0.98710331$

C $1.10997398-1.23889222 \quad 0.46194343$

C $-0.37919249-1.48569262 \quad 0.23944969$

C $0.73532392 \quad 0.36483670 \quad 2.34704968$

C $1.60412285-0.057173993 .49060776$

C $-0.48482070 \quad 0.86136996 \quad 2.56269784$

$\mathrm{H}-2.88520566-1.55185768-0.89571499$

$\mathrm{H}-2.93330028 \quad 0.09925385-1.50501905$

$\mathrm{H}-2.88367506-0.19847380 \quad 0.24705648$

$\mathrm{H}-1.22223010 \quad 1.66426020-0.38007663$

$\mathrm{H} \quad 0.89099878 \quad 2.22285803 \quad 0.40753508$

H $1.67609411 \quad 1.26992485-0.85379756$

$\mathrm{H} 2.47030109 \quad 0.31877322 \quad 1.10072589$

$\mathrm{H} 1.51686322-1.98458580 \quad 1.16977797$

H $1.65996679-1.38375126-0.49020389$

$\mathrm{H}-0.54239023-2.52392015-0.11546044$

H 2.31591125 $-0.84531595 \quad 3.21419225$

$\mathrm{H} \quad 1.01731659-0.439933314 .33651699$

H 2.18907899 $0.79604396 \quad 3.86517937$

$\mathrm{H}-0.92208986-1.406476151 .20376463$

$\mathrm{H}-0.91260924 \quad 0.97754667 \quad 3.54434889$

$\mathrm{H}-1.151087201 .19054434 \quad 1.78593789$

$00.17193607 \quad 0.04893360-2.67660788$

$\begin{array}{lllll}0 & -0.31854641 & 1.25360707 & -2.12588370\end{array}$

$0-0.63396342-0.97294626-2.09207057$

Vibrational frequencies (in $\mathrm{cm}-1$ )

$\begin{array}{lllllllllllllll}28.9 & 46.8 & 63.4 & 67.4 & 154.8 & 166.9 & 191.2 & 251.0 & 272.2 & 300.4 & 313.4 & 334.1 & 396.1 & 425.1\end{array}$ $447.7 \quad 470.3 \quad 536.0 \quad 565.7 \quad 614.5 \quad 638.2 \quad 695.3 \quad 724.6 \quad 817.8 \quad 861.7 \quad 875.6 \quad 896.5 \quad 948.0 \quad 964.1$ $974.3 \quad 988.9 \quad 990.3 \quad 1006.4 \quad 1036.3 \quad 1042.7 \quad 1056.2 \quad 1071.7 \quad 1084.2 \quad 1092.7 \quad 1139.3 \quad 1149.01157 .2$

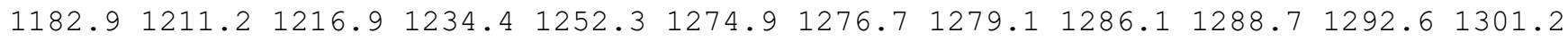
$1306.71312 .4 \quad 1318.8 \quad 1332.3 \quad 1341.5 \quad 1359.6 \quad 1380.5 \quad 1392.4 \quad 1397.5 \quad 1403.0 \quad 1408.91867 .3$ $2649.82656 .0 \quad 2670.4 \quad 2671.0 \quad 2671.2 \quad 2674.5 \quad 2680.02685 .42689 .2 \quad 2726.8 \quad 2733.5 \quad 2738.7$ $2743.12779 .5 \quad 2780.9 \quad 2796.7$

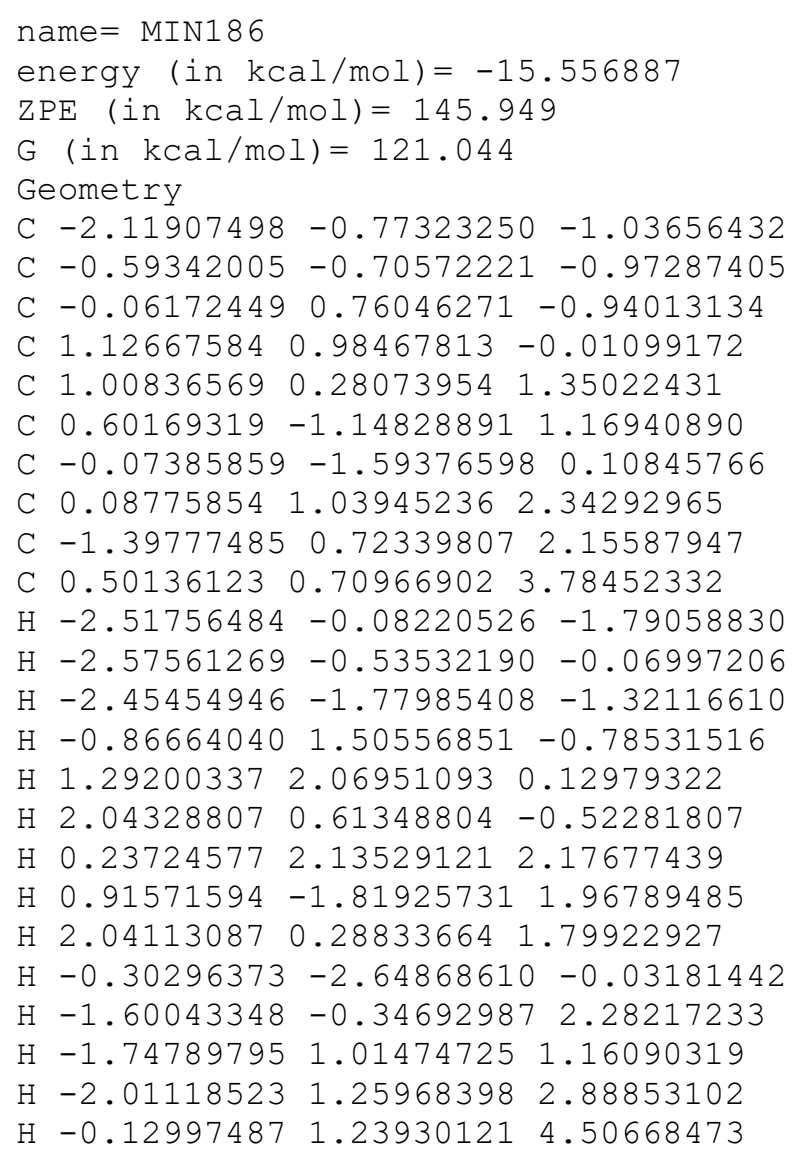


$\mathrm{H} \quad 1.53759827 \quad 1.00086464 \quad 3.98482148$

$\mathrm{H} \quad 0.40754856-0.361159603 .99354609$

$0.84278584-0.29490642-2.69146135$

$0.35802214 \quad 0.97517053-2.29041656$

O $-0.15439109-1.20526777-2.25188214$

Vibrational frequencies (in $\mathrm{cm}-1$ )

$\begin{array}{llllllllllllllll}37.4 & 79.1 & 89.2 & 137.8 & 146.0 & 167.1 & 194.6 & 204.8 & 257.6 & 283.0 & 303.5 & 325.6 & 363.4 & 405.4\end{array}$

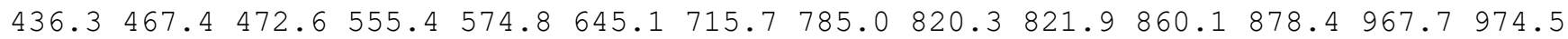

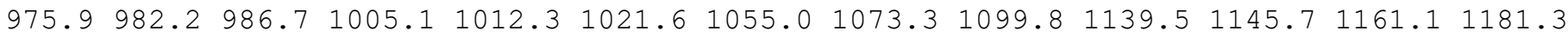
$1189.3 \quad 1198.7 \quad 1234.6 \quad 1246.1 \quad 1254.7 \quad 1260.4 \quad 1278.6 \quad 1281.4 \quad 1284.9 \quad 1290.91296 .7 \quad 1300.5$

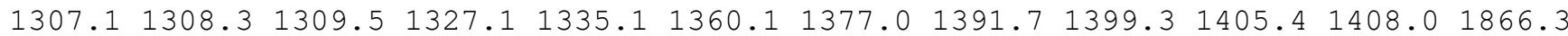
$2653.92670 .42670 .92674 .92676 .0 \quad 2677.4 \quad 2679.3 \quad 2682.02693 .6 \quad 2703.72738 .12747 .3$ $2761.92778 .7 \quad 2781.2 \quad 2782.9$

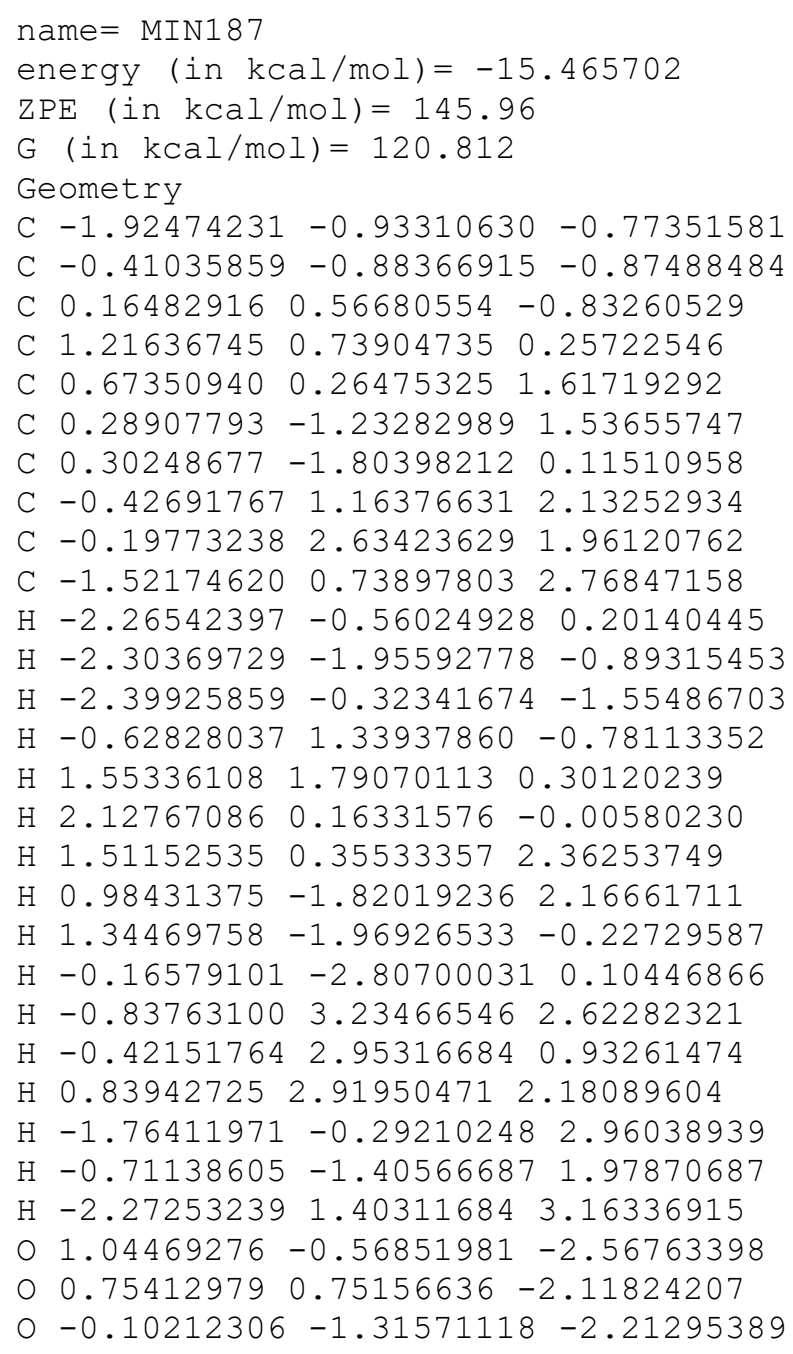


C $-0.77496614 \quad-0.44861769-0.97395455$

C $-0.167209620 .96101252-0.69843676$

C $1.14571907 \quad 0.97439672 \quad 0.08208216$

C $1.26419819-0.08257621 \quad 1.19146241$

C $0.76936370-1.41475598 \quad 0.72460169$

C $-0.11186160-1.57821080-0.26232990$

C $0.61106717 \quad 0.35587256 \quad 2.52931494$

C $-0.86566191 \quad 0.73170361 \quad 2.39628237$

C $0.76043815-0.758428013 .57498666$

$\mathrm{H}-2.78772148 \quad 0.32291135 \quad-1.30356251$

$\mathrm{H}-2.54571105-0.40221484 \quad 0.30301262$

$\mathrm{H}-2.71307966-1.43031975-1.12764445$

$\mathrm{H}-0.91441943 \quad 1.66393005-0.27751697$

$\mathrm{H} 1.31444357 \quad 1.98395112 \quad 0.50341157$

$\mathrm{H} \quad 1.97572067 \quad 0.81658014 \quad-0.64354974$

H $2.36506367-0.19218816 \quad 1.40891449$

H $1.79459311-1.10733642 \quad 3.65296378$

H $1.20458170-2.27041843 \quad 1.24144202$

$\mathrm{H}-0.40759953-2.56450976-0.61644815$

$\mathrm{H}-1.43572674-0.06767464 \quad 1.90918600$

$\mathrm{H}-0.99792111 \quad 1.65015180 \quad 1.81550013$

$\mathrm{H}-1.31892904 \quad 0.90565183 \quad 3.37906971$

$\mathrm{H} \quad 1.16981970 \quad 1.25591261 \quad 2.89017636$

$\mathrm{H} \quad 0.45470930 \quad-0.41071214 \quad 4.56765038$

H $0.13366242-1.623769213 .32889531$

$0.38964359 \quad 0.31228259-2.76935818$

$00.07496348 \quad 1.47044067 \quad-2.01359698$

o $-0.60674435-0.62426413-2.39582027$

Vibrational frequencies (in $\mathrm{cm}-1$ )

$\begin{array}{lllllllllllllll}31.8 & 65.0 & 99.2 & 130.9 & 139.0 & 190.0 & 194.6 & 215.4 & 258.1 & 282.3 & 297.2 & 331.0 & 370.7 & 403.0\end{array}$

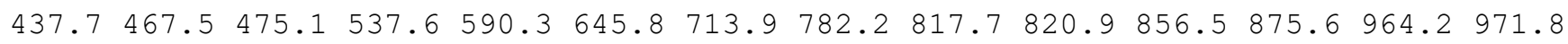
$976.7981 .2 \quad 987.3 \quad 1005.4 \quad 1010.2 \quad 1022.8 \quad 1045.4 \quad 1070.9 \quad 1100.01140 .7 \quad 1149.3 \quad 1168.6 \quad 1184.7$ $1188.91199 .5 \quad 1240.2 \quad 1246.7 \quad 1264.6 \quad 1265.0 \quad 1279.3 \quad 1280.3 \quad 1284.2 \quad 1291.6 \quad 1297.2 \quad 1301.9$ $1305.91309 .5 \quad 1312.1 \quad 1324.0 \quad 1333.1 \quad 1353.6 \quad 1379.7 \quad 1392.2 \quad 1400.3 \quad 1402.1 \quad 1408.1 \quad 1874.3$ $2652.62666 .2 \quad 2670.92671 .8 \quad 2674.4 \quad 2675.6 \quad 2678.5 \quad 2681.72688 .7 \quad 2702.82736 .8 \quad 2745.2$ $2761.02776 .0 \quad 2780.02782 .4$

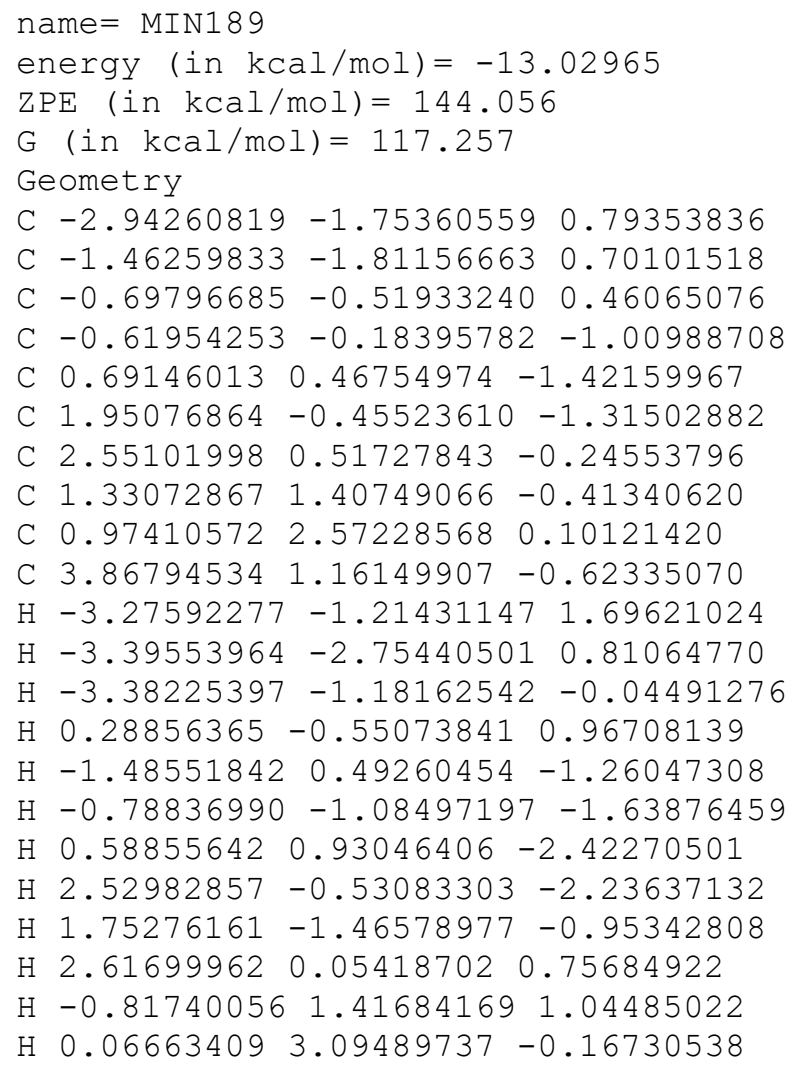


$\mathrm{H} \quad 1.58252981 \quad 3.12228409 \quad 0.80285941$

$\mathrm{H} \quad 3.814284561 .66194381-1.59826926$

$\mathrm{H} \quad 4.67181609 \quad 0.41673878-0.68039865$

$\mathrm{H} \quad 4.16759631 \quad 1.91786920 \quad 0.11284600$

$0-2.528252330 .96145102 \quad 0.54369880$

$0-1.44741071 \quad 0.57579887 \quad 1.20694657$

O $-0.83202598-2.835008730 .79786014$

Vibrational frequencies (in $\mathrm{cm}-1$ )

$\begin{array}{lllllllllllllll}25.2 & 40.2 & 65.8 & 80.5 & 120.7 & 151.7 & 163.4 & 166.5 & 190.7 & 215.2 & 227.6 & 254.5 & 317.6 & 331.8\end{array}$

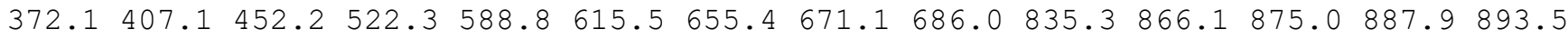
$918.7 \quad 950.1 \quad 956.8 \quad 1005.4 \quad 1042.1 \quad 1049.7 \quad 1054.4 \quad 1068.1 \quad 1078.2 \quad 1090.6 \quad 1122.91141 .8 \quad 1154.3$

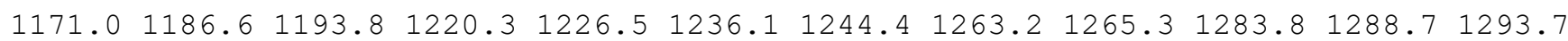
$1295.3 \quad 1296.81313 .0 \quad 1318.2 \quad 1338.5 \quad 1353.0 \quad 1368.1 \quad 1379.4 \quad 1386.1 \quad 1401.11849 .01943 .5$ $2597.62644 .02674 .12674 .7 \quad 2677.6 \quad 2678.2 \quad 2688.92712 .22714 .3 \quad 2719.92722 .5 \quad 2726.2$ $2767.0 \quad 2774.7 \quad 2782.0 \quad 2786.1$

name $=$ MIN190

energy (in $\mathrm{kcal} / \mathrm{mol})=-15.000127$

$\operatorname{ZPE}(\mathrm{in} \mathrm{kcal} / \mathrm{mol})=146.052$

$\mathrm{G}(\mathrm{in} \mathrm{kcal} / \mathrm{mol})=120.456$

Geometry

C $-2.39290247-0.59903880-0.74361984$

C $-0.86202812-0.59375304-0.74020668$

C $-0.317413220 .86526874-0.75003288$

C $1.055437831 .09242819-0.12037763$

C $1.37138177 \quad 0.19160699 \quad 1.08022502$

C $1.15099458-1.27161297 \quad 0.65048510$

C $-0.32216887-1.52526306 \quad 0.34103408$

$\begin{array}{lllll}\text { C } & 0.61963383 & 0.50127731 & 2.35225639\end{array}$

C $-0.42351301 \quad 1.56842180 \quad 2.34087564$

C $0.89782401-0.16471916 \quad 3.47821280$

$\mathrm{H}-2.78431760 \quad 0.01302270 \quad-1.56818719$

$\mathrm{H}-2.80700232-0.218832910 .19404030$

$\mathrm{H}-2.78122789-1.61418256-0.89678136$

$\mathrm{H}-1.06766360 \quad 1.59872345-0.39259236$

$\mathrm{H} \quad 1.17077295 \quad 2.15756361 \quad 0.15612761$

$\mathrm{H} 1.82864651 \quad 0.91583013-0.90348622$

H $2.46452276 \quad 0.32467806 \quad 1.31176214$

$\mathrm{H} \quad 1.49603588-1.96181249 \quad 1.44283551$

$\mathrm{H} \quad 1.76916956-1.49655766-0.24147274$

$\mathrm{H}-0.46330294-2.57711677 \quad 0.01688263$

$\mathrm{H}-1.307281961 .249172821 .77045533$

$\mathrm{H}-0.052770302 .50150282 \quad 1.89486745$

$\mathrm{H}-0.77253160 \quad 1.82094517 \quad 3.35224989$

$\mathrm{H} \quad 0.40180813 \quad 0.01480710 \quad 4.41658095$

$\mathrm{H} \quad 1.64013865-0.94071880 \quad 3.55170220$

$\mathrm{H}-0.92037217-1.41201879 \quad 1.26817792$

O $0.26918086-0.09195957-2.67829066$

o $-0.22948610 \quad 1.12114125 \quad-2.15578321$

o $-0.47599398-1.12124036-2.02013382$

Vibrational frequencies (in $\mathrm{cm}-1$ )

$\begin{array}{llllllllllllllll}-11.5 & 50.8 & 78.9 & 135.7 & 159.8 & 164.7 & 192.1 & 268.8 & 277.4 & 314.0 & 336.7 & 346.3 & 384.5 & 434.4\end{array}$

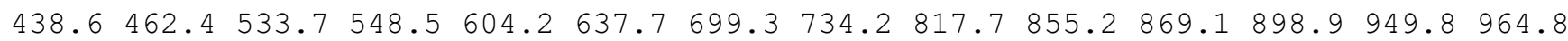

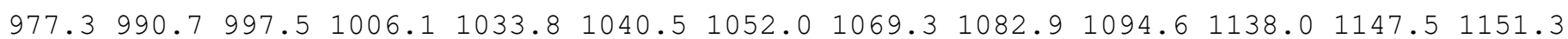
$1177.11209 .51211 .91230 .2 \quad 1247.0 \quad 1273.1 \quad 1280.3 \quad 1286.6 \quad 1288.5 \quad 1289.6 \quad 1292.6 \quad 1299.2$ $1304.51312 .91323 .5 \quad 1334.5 \quad 1346.91363 .7 \quad 1380.2 \quad 1384.91392 .0 \quad 1402.4 \quad 1407.8 \quad 1844.3$ $2650.02656 .42669 .6 \quad 2670.2 \quad 2670.7 \quad 2671.0 \quad 2681.4 \quad 2684.02692 .3 \quad 2726.02735 .5 \quad 2739.0$ $2743.02777 .02781 .2 \quad 2795.2$

name $=$ MIN191

energy (in $\mathrm{kcal} / \mathrm{mol})=-14.992481$

$\mathrm{ZPE}(\mathrm{in} \mathrm{kcal} / \mathrm{mol})=146.071$

$\mathrm{G}($ in $\mathrm{kcal} / \mathrm{mol})=121.298$ 


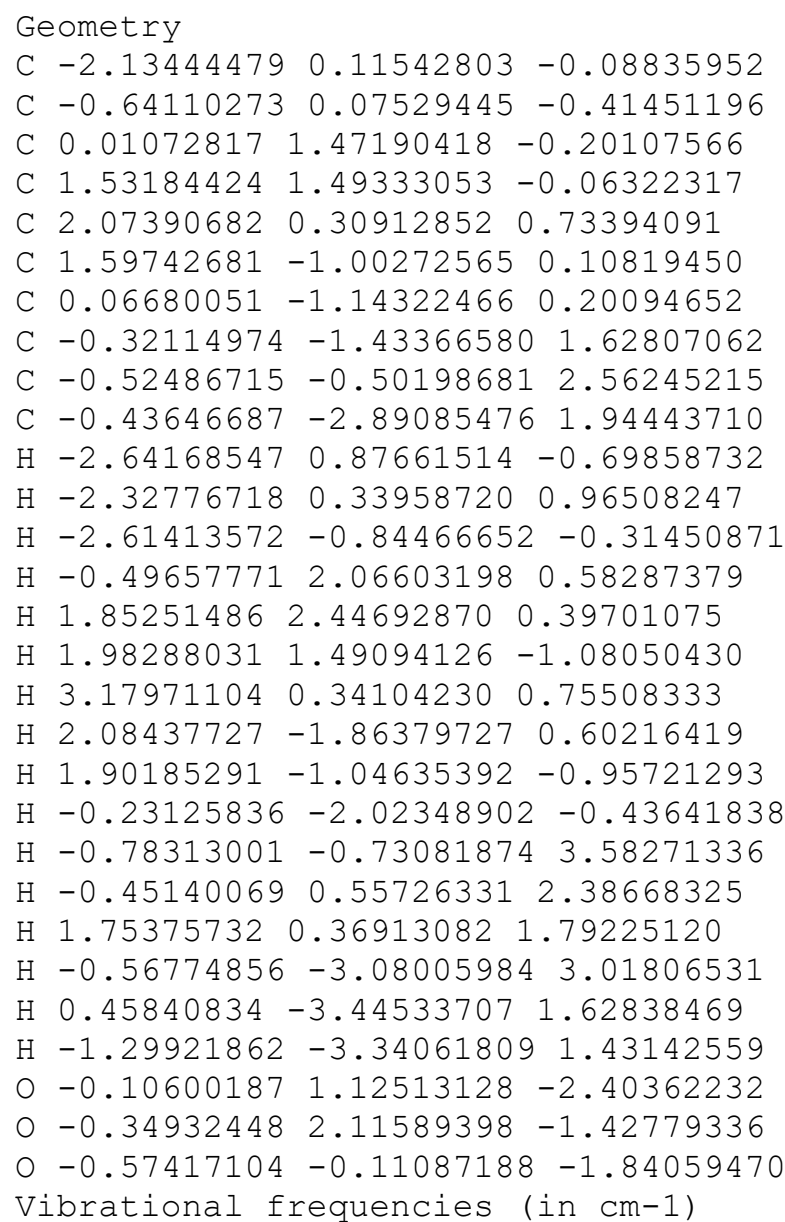

$\begin{array}{lllllllllllllll}56.3 & 62.3 & 88.7 & 96.0 & 143.1 & 191.3 & 208.5 & 215.2 & 271.5 & 298.3 & 309.8 & 334.1 & 351.5 & 424.8\end{array}$

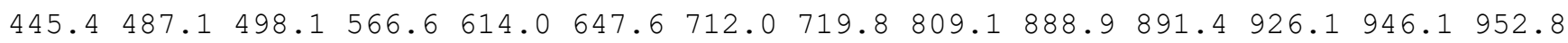
$983.1988 .6 \quad 1001.3 \quad 1018.8 \quad 1038.2 \quad 1041.91051 .2 \quad 1080.0 \quad 1091.11114 .8 \quad 1137.6 \quad 1140.7$

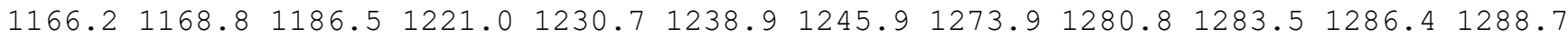

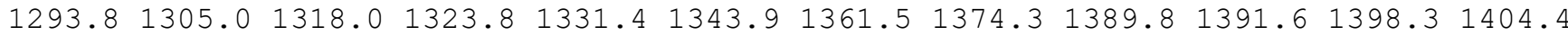
$1866.32651 .62661 .6 \quad 2669.8 \quad 2671.4 \quad 2672.5 \quad 2673.6 \quad 2675.42681 .2 \quad 2698.72724 .72739 .7$ 2741.32746 .12778 .82781 .02794 .0

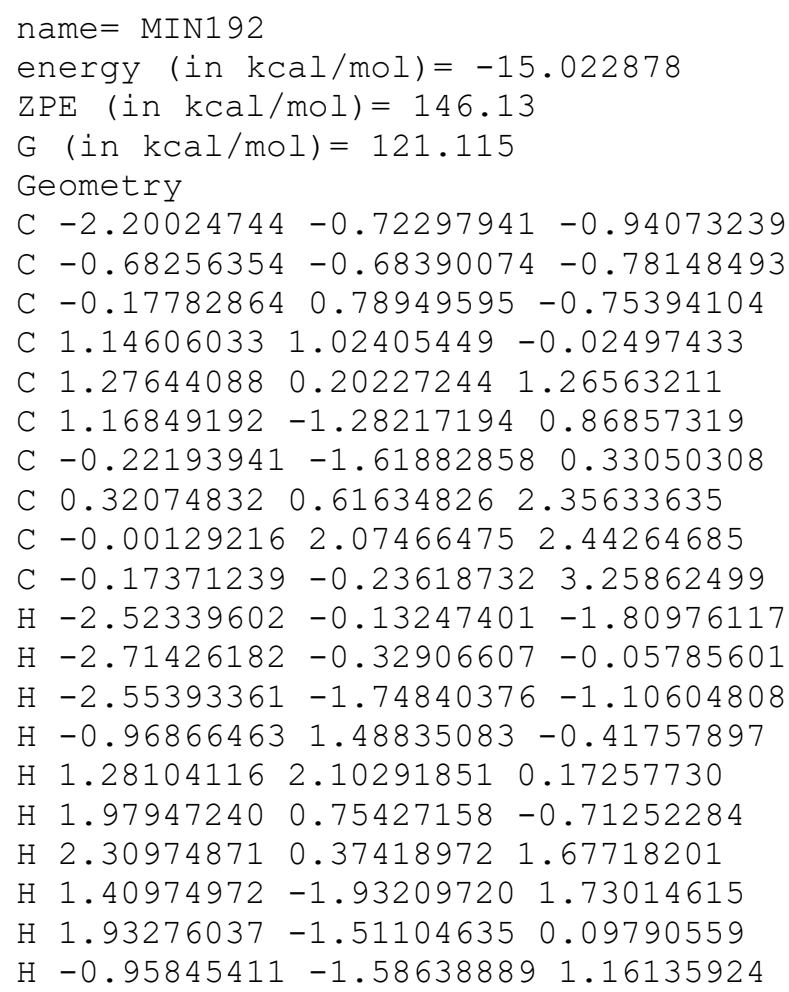


$\mathrm{H}-0.65328078 \quad 2.39089815 \quad 1.61613711$

$\mathrm{H} \quad 0.90584517 \quad 2.69336225 \quad 2.40698993$

$\mathrm{H}-0.525487852 .33143004 \quad 3.37360540$

$\mathrm{H}-0.23680730-2.66412268-0.04363298$

$\mathrm{H} \quad 0.03218406-1.29215994 \quad 3.27800727$

$\mathrm{H}-0.82758140 \quad 0.06177354 \quad 4.06102852$

$0-0.21219574-0.09046621-2.88100495$

$0.08104747 \quad 1.10202808-2.12522083$

o $-0.05990198-1.16066009-1.98643262$

Vibrational frequencies (in $\mathrm{cm}-1$ )

$\begin{array}{llllllllllllllll}31.4 & 47.4 & 83.9 & 133.8 & 149.0 & 172.6 & 224.0 & 243.5 & 294.8 & 312.6 & 336.0 & 368.4 & 378.8 & 423.9\end{array}$

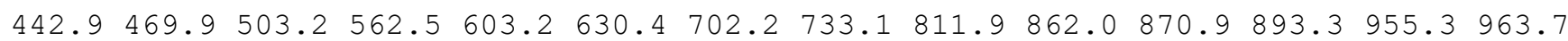

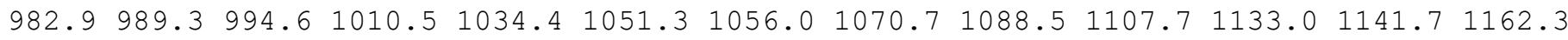

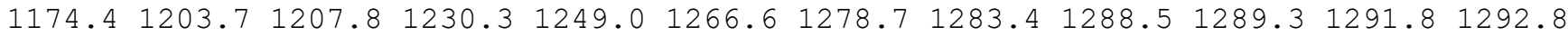

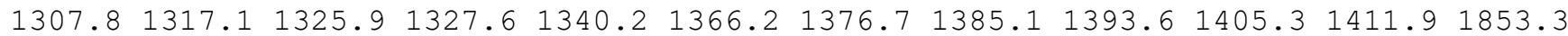
$2650.02653 .12668 .2 \quad 2669.2 \quad 2669.9 \quad 2672.7 \quad 2678.92682 .92688 .12723 .02735 .02739 .2$ $2741.52778 .0 \quad 2780.8 \quad 2793.9$

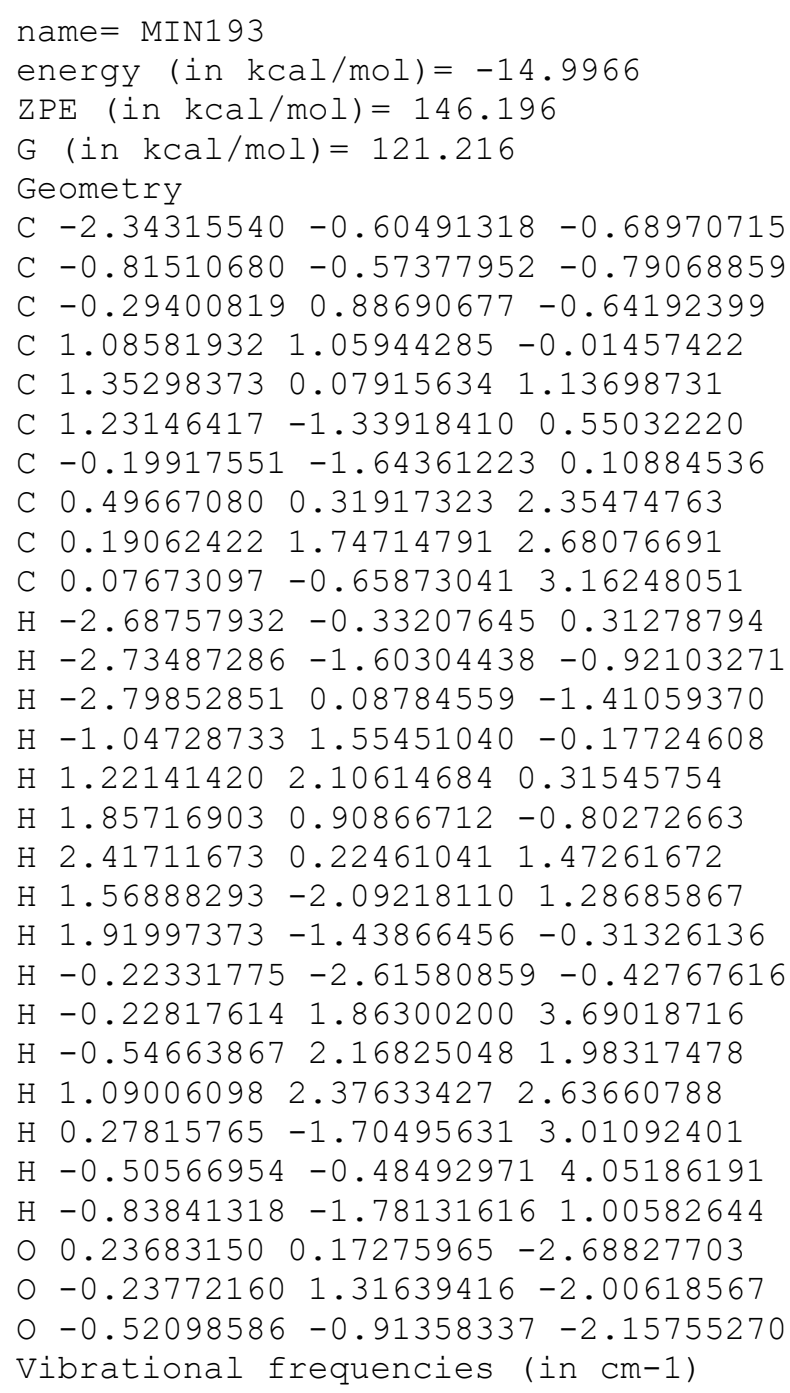


$\mathrm{ZPE}($ in $\mathrm{kcal} / \mathrm{mol})=142.768$

$\mathrm{G}($ in $\mathrm{kcal} / \mathrm{mol})=116.436$

Geometry

C $-2.79650379 \quad 0.00352055 \quad-0.42730954$

C $-1.29113508-0.19982083-0.19848866$

C $1.66079243 \quad 1.27554874-0.34431152$

C $1.78796625-0.17401578-0.54357023$

C $0.88884400-0.74473090-1.61476362$

C $-0.01451691 \quad 0.10371897-2.48351483$

C $-0.62374404-0.79119501-1.43020928$

C $-1.04258979-1.068274931 .01909382$

C $-0.51536837-0.596976492 .14890450$

C $-1.40847426-2.50812201 \quad 0.86827194$

$\mathrm{H}-3.28910273 \quad 0.37606136 \quad 0.47738090$

$\mathrm{H}-3.29708298-0.92252880-0.72241233$

$\mathrm{H}-2.96058559 \quad 0.74095267 \quad-1.22375801$

H $1.423914791 .96375826-1.14618579$

H $1.56984888-0.69327351 \quad 0.43518561$

$\mathrm{H} 2.85445452-0.42919933-0.75053649$

H $1.34620078-1.62425860-2.09067104$

$\mathrm{H}-0.13253828-0.14655384-3.53381978$

$\mathrm{H}-0.06465515 \quad 1.18223776 \quad-2.35441359$

$\mathrm{H}-1.14559927-1.69511894-1.76664156$

$\mathrm{H}-0.33658425-1.205946693 .02244705$

$\mathrm{H}-0.17219093 \quad 0.41814627 \quad 2.30052931$

$\mathrm{H}-1.001707661 .494391890 .81211850$

$\mathrm{H}-0.64806154-3.040024290 .27554304$

$\mathrm{H}-2.37480453-2.64684668 \quad 0.36789424$

$\mathrm{H}-1.47217519-3.023096601 .83747608$

○ $2.06550750 \quad 1.16250276 \quad 1.81905927$

O $1.79572542 \quad 1.87211575 \quad 0.79532138$

O $-0.72181175 \quad 1.09419620-0.03613727$

Vibrational frequencies (in $\mathrm{cm}-1$ )

$\begin{array}{llllllllllllll}28.9 & 41.9 & 87.4 & 108.1 & 124.5 & 136.3 & 150.6 & 190.9 & 217.5 & 235.6 & 246.5 & 276.6 & 303.7 & 314.8\end{array}$

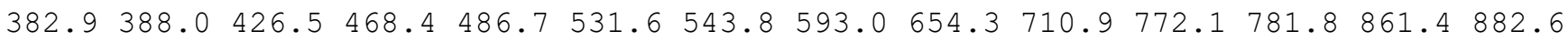
$936.9953 .9963 .9983 .0991 .7 \quad 1011.3 \quad 1030.1 \quad 1047.6 \quad 1056.5 \quad 1059.3 \quad 1065.3 \quad 1097.11133 .9$

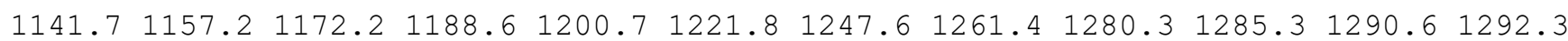

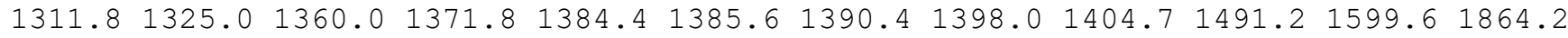
$2611.72668 .32673 .82677 .5 \quad 2680.4 \quad 2700.6 \quad 2703.72708 .42721 .02741 .52753 .72776 .1$ $2781.7 \quad 2783.1 \quad 2784.92797 .6$

\section{name $=$ MIN195}

energy (in $\mathrm{kcal} / \mathrm{mol})=-14.459362$

$\mathrm{ZPE}($ in $\mathrm{kcal} / \mathrm{mol})=145.892$

$\mathrm{G}(\mathrm{in} \mathrm{kcal} / \mathrm{mol})=120.437$

Geometry

C $-2.25908869-0.29552868-0.99767332$

C $-0.75827704-0.20468500-0.78765144$

C $-0.150672331 .21557377-1.04118717$

C $0.79903782 \quad 1.50766631 \quad 0.11721963$

C $0.41464712 \quad 0.548132801 .26192481$

C $0.60514782-1.89754634 \quad 0.53095529$

C $-0.30499983-0.66579373 \quad 0.61539438$

C $-0.498652051 .25358460 \quad 2.21944967$

C $-0.129718951 .59594176 \quad 3.45290842$

C $0.81326542-2.536543421 .90339409$

$\mathrm{H}-2.54008631 \quad 0.04043262 \quad-2.00624673$

$\mathrm{H}-2.80939529 \quad 0.31179199-0.27137265$

$\mathrm{H}-2.60846430-1.33312618-0.90203432$

$\mathrm{H}-0.89496830 \quad 2.00695668-1.23034764$

$\mathrm{H} \quad 1.845141621 .32890069-0.20046390$

$\mathrm{H} \quad 0.75910001 \quad 2.56171903 \quad 0.43574788$

$\mathrm{H} \quad 1.33612358 \quad 0.20514597 \quad 1.78745052$

Н $1.57674390-1.62378282 \quad 0.07681498$ 
$\mathrm{H} \quad 0.16026421-2.64332327-0.15984039$

$\mathrm{H}-1.19735314-0.93616729 \quad 1.22691002$

$\mathrm{H}-1.48908983 \quad 1.48067167 \quad 1.82797356$

$\mathrm{H} \quad 0.84333905 \quad 1.38980321 \quad 3.87053019$

$\mathrm{H}-0.77540948 \quad 2.11378697 \quad 4.14470645$

H $1.27494797-1.840317812 .61199282$

$\mathrm{H} 1.46488823-3.415246531 .83375426$

$\mathrm{H}-0.13613144-2.865605522 .34112310$

$\begin{array}{llll}0 & 0.96162552 & -0.28797149 & -2.26324577\end{array}$

○ $0.55045506 \quad 1.07282111-2.26981270$

O $-0.19105363-0.99967875-1.83554801$

Vibrational frequencies (in $\mathrm{cm}-1$ )

$\begin{array}{llllllllllllllll}27.8 & 60.4 & 79.1 & 104.3 & 162.9 & 171.3 & 174.3 & 217.9 & 243.4 & 246.5 & 299.3 & 318.5 & 357.4 & 367.7\end{array}$

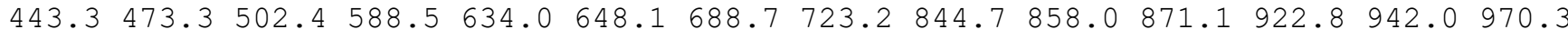

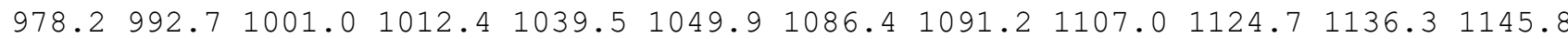
$1158.1 \quad 1178.8 \quad 1201.51221 .4 \quad 1230.3 \quad 1234.1 \quad 1244.4 \quad 1263.3 \quad 1284.6 \quad 1285.6 \quad 1287.7 \quad 1299.0$

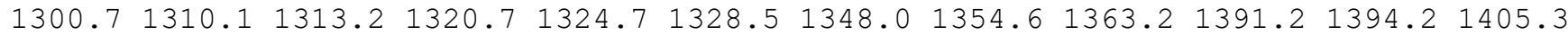
$1848.92655 .12664 .92669 .8 \quad 2677.8 \quad 2678.4 \quad 2682.92696 .42703 .12706 .02716 .12744 .3$ $2748.3 \quad 2759.6 \quad 2780.3 \quad 2782.3 \quad 2789.0$

name $=$ MIN196

energy (in $\mathrm{kcal} / \mathrm{mol})=-13.498461$

$\operatorname{ZPE}($ in $\mathrm{kcal} / \mathrm{mol})=144.992$

$\mathrm{G}($ in $\mathrm{kcal} / \mathrm{mol})=120.233$

Geometry

C $-2.11433270-0.16465279-0.54694468$

C $-0.60158449-0.18220811-0.56940244$

C $0.07108477 \quad 1.23391275-0.49189410$

C $2.05129748 \quad 2.05211380 \quad 0.81414055$

C 1.304680051 .056152170 .34848171

C $1.54396127-0.40622693 \quad 0.56132134$

C $0.15620555-1.088753370 .42650606$

C $-0.45492523-1.29697254 \quad 1.82902652$

C $-0.78931247 \quad 0.01523188 \quad 2.54449907$

C $-1.67751174-2.22181385 \quad 1.80282893$

$\mathrm{H}-2.50729906 \quad 0.46353897 \quad-1.36121523$

H $-2.51410282 \quad 0.22194286 \quad 0.39681232$

$\mathrm{H}-2.52397287-1.17266259-0.70272404$

$\mathrm{H}-0.59435082 \quad 2.05473988-0.18002087$

$\mathrm{H} \quad 2.93767005 \quad 1.90530600 \quad 1.40960052$

H $1.84165421 \quad 3.09335742 \quad 0.62879659$

$\mathrm{H} \quad 0.33451886-1.81908596 \quad 2.43628049$

$\mathrm{H} \quad 2.02697265-0.62650910 \quad 1.52673282$

H 2.23495789 $-0.78909801-0.21875192$

$\mathrm{H} \quad 0.27454986-2.09352616-0.04575242$

$\mathrm{H}-1.55242517 \quad 0.59386970 \quad 2.01564689$

$\mathrm{H} \quad 0.09672994 \quad 0.65560676 \quad 2.63726704$

$\mathrm{H}-1.16508640 \quad-0.17372185 \quad 3.55593174$

$\mathrm{H}-1.88512348-2.61819592 \quad 2.80372382$

$\mathrm{H}-1.52416831-3.07753407 \quad 1.13704157$

$\mathrm{H}-2.58398289-1.704845341 .47479390$

$0.74773606 \quad 0.23762167-2.36731126$

$0.410771761 .51253849-1.84517275$

O $-0.28480900-0.62416467-1.90446100$

Vibrational frequencies (in $\mathrm{cm}-1$ )

$\begin{array}{llllllllllllllll}57.0 & 71.9 & 76.9 & 148.3 & 161.2 & 179.7 & 193.6 & 222.9 & 231.7 & 274.4 & 305.0 & 308.7 & 353.0 & 375.3\end{array}$ $397.4 \quad 463.9 \quad 486.0 \quad 551.2 \quad 578.1 \quad 613.8 \quad 652.5 \quad 712.2 \quad 728.8 \quad 826.8 \quad 863.1 \quad 924.7 \quad 940.2 \quad 958.0$ $964.1976 .0 \quad 978.6 \quad 985.9 \quad 1005.0 \quad 1013.3 \quad 1018.8 \quad 1077.7 \quad 1081.8 \quad 1105.0 \quad 1110.7 \quad 1136.2 \quad 1156.6$ $1161.21185 .7 \quad 1202.3 \quad 1237.3 \quad 1251.3 \quad 1255.2 \quad 1273.0 \quad 1283.01284 .5 \quad 1288.01296 .3 \quad 1300.5$ $1301.51304 .7 \quad 1308.41314 .5 \quad 1323.1 \quad 1339.7 \quad 1365.5 \quad 1385.6 \quad 1388.7 \quad 1408.01408 .61897 .1$ $2662.02664 .22667 .6 \quad 2669.0 \quad 2672.9 \quad 2676.4 \quad 2679.62694 .42698 .62707 .92724 .12745 .9$ $2774.5 \quad 2780.4 \quad 2783.6 \quad 2792.2$ 
name $=$ MIN197

energy (in $\mathrm{kcal} / \mathrm{mol})=-14.502466$

$\mathrm{ZPE}($ in $\mathrm{kcal} / \mathrm{mol})=146.135$

$\mathrm{G}($ in $\mathrm{kcal} / \mathrm{mol})=120.217$

Geometry

C $-2.17866276-0.61480440-0.88141692$

C $-0.64867756-0.58915768-0.85761371$

C $-0.12644817 \quad 0.88147551 \quad-0.78957354$

C 1.041013791 .182012250 .14749370

C $1.12010228 \quad 0.246475351 .36132900$

C $1.20897595-1.18675710 \quad 0.80456435$

C $-0.11262461-1.59705834 \quad 0.15970118$

C $0.00942991 \quad 0.42089363 \quad 2.36792499$

C $-0.68232690 \quad 1.74518182 \quad 2.41101658$

C $-0.32259475-0.54059848 \quad 3.23522880$

$\mathrm{H}-2.59852359-0.25122871 \quad 0.06280613$

$\mathrm{H}-2.55670273-1.63150689-1.04659188$

$\mathrm{H}-2.57479579 \quad 0.00570307-1.69643065$

$\mathrm{H}-0.95996124 \quad 1.60046215-0.63949392$

H $1.00339525 \quad 2.23979524 \quad 0.46837105$

H $1.988242611 .09751576-0.43185820$

$\mathrm{H} 2.076604820 .47329652 \quad 1.90732396$

$\mathrm{H} 1.47961522-1.90037077 \quad 1.60446156$

H 2.02965554 -1.24670494 0.06197753

$\mathrm{H} \quad 0.00697329-2.57882473-0.34620310$

$\mathrm{H}-1.35515467 \quad 1.83582149 \quad 3.27536529$

$\mathrm{H}-1.29826262 \quad 1.90372298 \quad 1.51479163$

$\mathrm{H} \quad 0.03330818 \quad 2.57602868 \quad 2.47725739$

$\mathrm{H}-1.09126273-0.43031268 \quad 3.98120204$

$\mathrm{H} 0.14141084-1.51105143 \quad 3.27262684$

$\mathrm{H}-0.86804260-1.76397151 \quad 0.95584099$

O $0.75263653-0.12331385-2.59005467$

O $0.29092057 \quad 1.13632417 \quad-2.13550503$

o $-0.25389152-1.03006007-2.17226827$

Vibrational frequencies (in cm-1)

$\begin{array}{lllllllllllllllll}-9.6 & 28.2 & 75.1 & 140.7 & 152.9 & 181.9 & 205.5 & 272.2 & 298.5 & 319.4 & 351.7 & 366.2 & 405.0 & 432.5\end{array}$

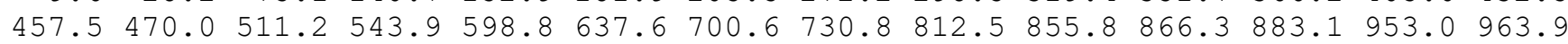
$976.1988 .0 \quad 997.1 \quad 1004.0 \quad 1033.7 \quad 1044.3 \quad 1051.3 \quad 1068.8 \quad 1085.0 \quad 1096.3 \quad 1136.4 \quad 1154.4 \quad 1160.9$ $\begin{array}{lllllllllllll}1182.1 & 1207.4 & 1218.0 & 1232.4 & 1241.8 & 1269.5 & 1279.7 & 1282.9 & 1288.5 & 1289.7 & 1292.4 & 1293.9\end{array}$

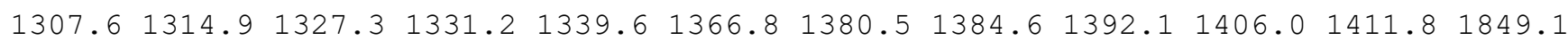
$2649.32651 .3 \quad 2669.0 \quad 2669.6 \quad 2671.8 \quad 2672.4 \quad 2677.8 \quad 2684.5 \quad 2685.7 \quad 2723.92734 .42737 .2$ $2742.8 \quad 2777.92780 .2 \quad 2794.0$

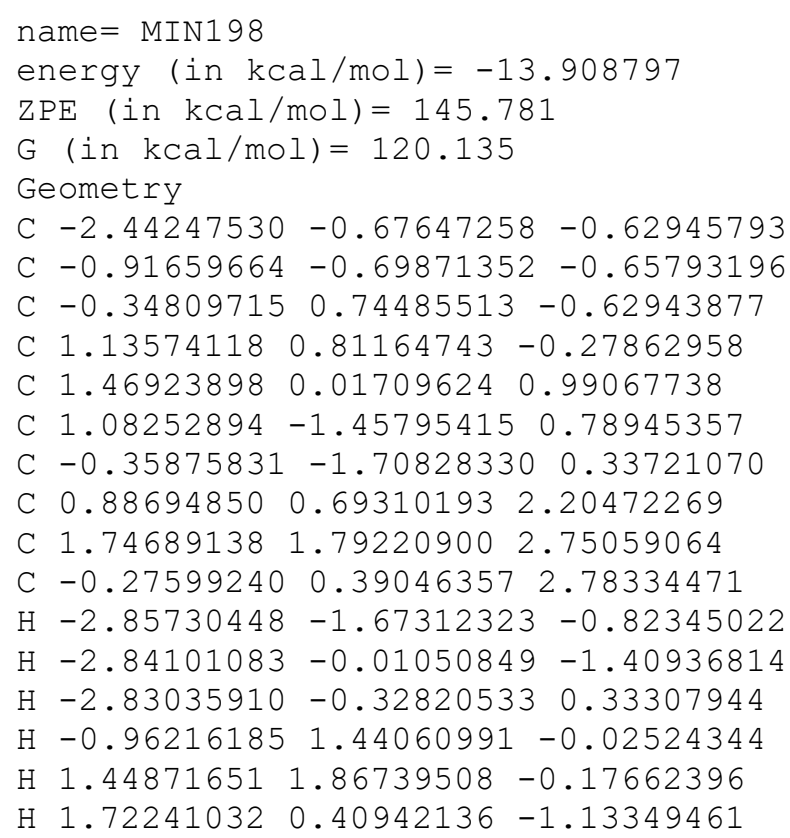


$\mathrm{H} 2.589853650 .02909002 \quad 1.10884635$

$\mathrm{H} 1.26848141-2.014867791 .72919232$

$\mathrm{H} 1.77248908-1.89338326 \quad 0.03577097$

$\mathrm{H}-0.42394481-2.72337122-0.11416572$

H 2.07205861 2.48532999 1.96268387

$\mathrm{H} 2.65047484 \quad 1.38399082 \quad 3.22486956$

H 1.229402062 .392428663 .51042686

$\mathrm{H}-1.02388777-1.74733676 \quad 1.22560823$

$\mathrm{H}-0.65723624 \quad 0.90287730 \quad 3.65093447$

$\mathrm{H}-0.94008151-0.38666038 \quad 2.44983245$

o $-0.69622420-0.00352136-2.78425303$

o $-0.47590298 \quad 1.18159199-1.97829265$

o $-0.46558492-1.11743779-1.95662695$

Vibrational frequencies (in cm-1)

$\begin{array}{llllllllllllllll}-30.8 & 41.5 & 73.7 & 84.4 & 134.3 & 168.1 & 183.4 & 218.2 & 271.2 & 282.6 & 292.7 & 352.8 & 374.3 & 405.8\end{array}$ $427.2 \quad 458.4 \quad 536.9 \quad 560.2 \quad 610.3 \quad 636.0 \quad 702.9 \quad 739.5 \quad 813.7 \quad 841.2 \quad 871.5 \quad 903.9 \quad 959.0 \quad 965.6$ $981.4 \quad 986.6 \quad 990.7 \quad 1006.7 \quad 1035.7 \quad 1051.6 \quad 1059.6 \quad 1072.2 \quad 1091.2 \quad 1107.8 \quad 1135.9 \quad 1155.9 \quad 1165.1$ $1175.81209 .7 \quad 1214.2 \quad 1234.5 \quad 1260.1 \quad 1265.3 \quad 1276.8 \quad 1281.2 \quad 1287.4 \quad 1288.2 \quad 1290.3 \quad 1298.6$

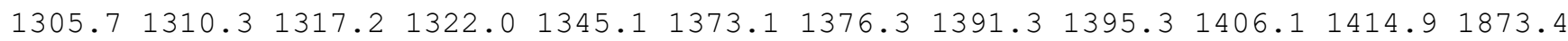
$2640.42658 .32667 .3 \quad 2668.02671 .4 \quad 2673.3 \quad 2679.82682 .42694 .82716 .62730 .22737 .6$ $2741.5 \quad 2778.8 \quad 2780.5 \quad 2791.4$

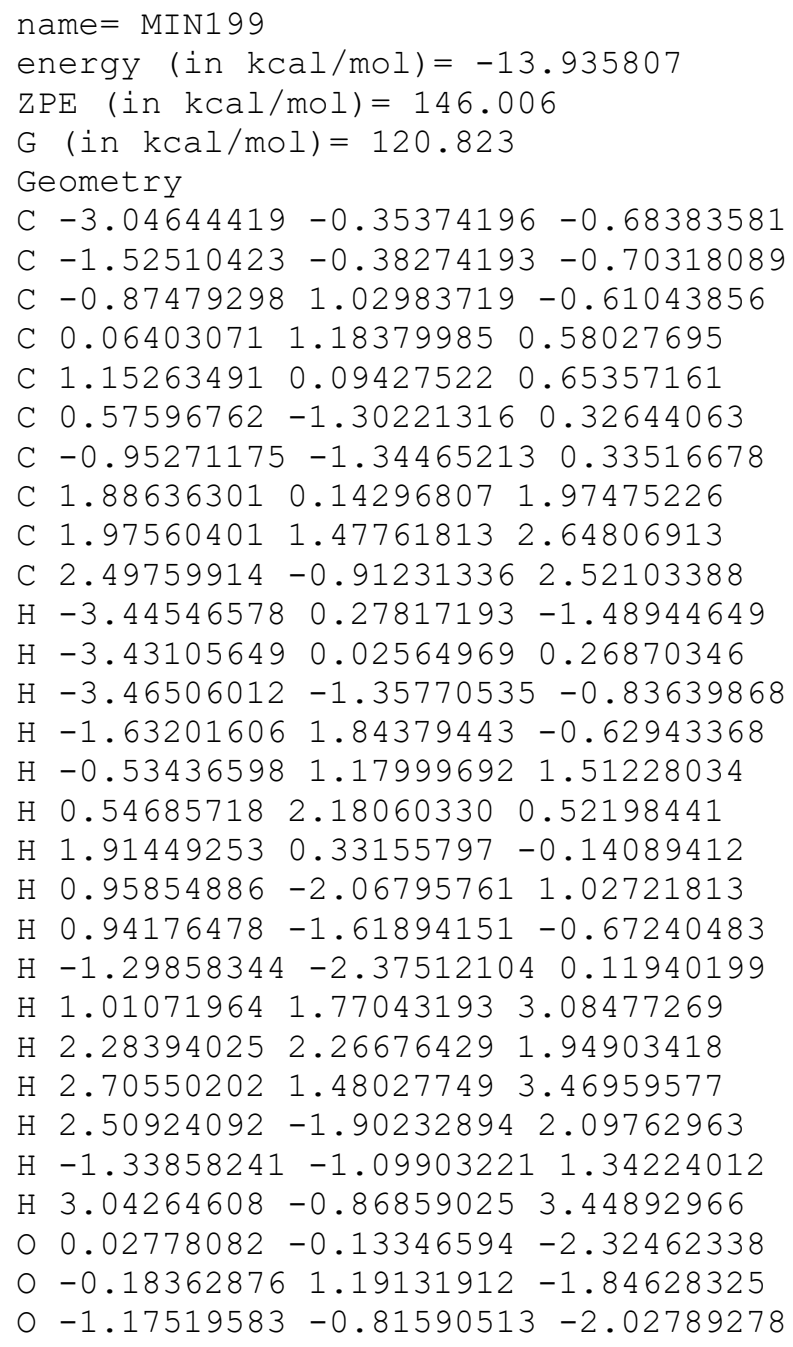




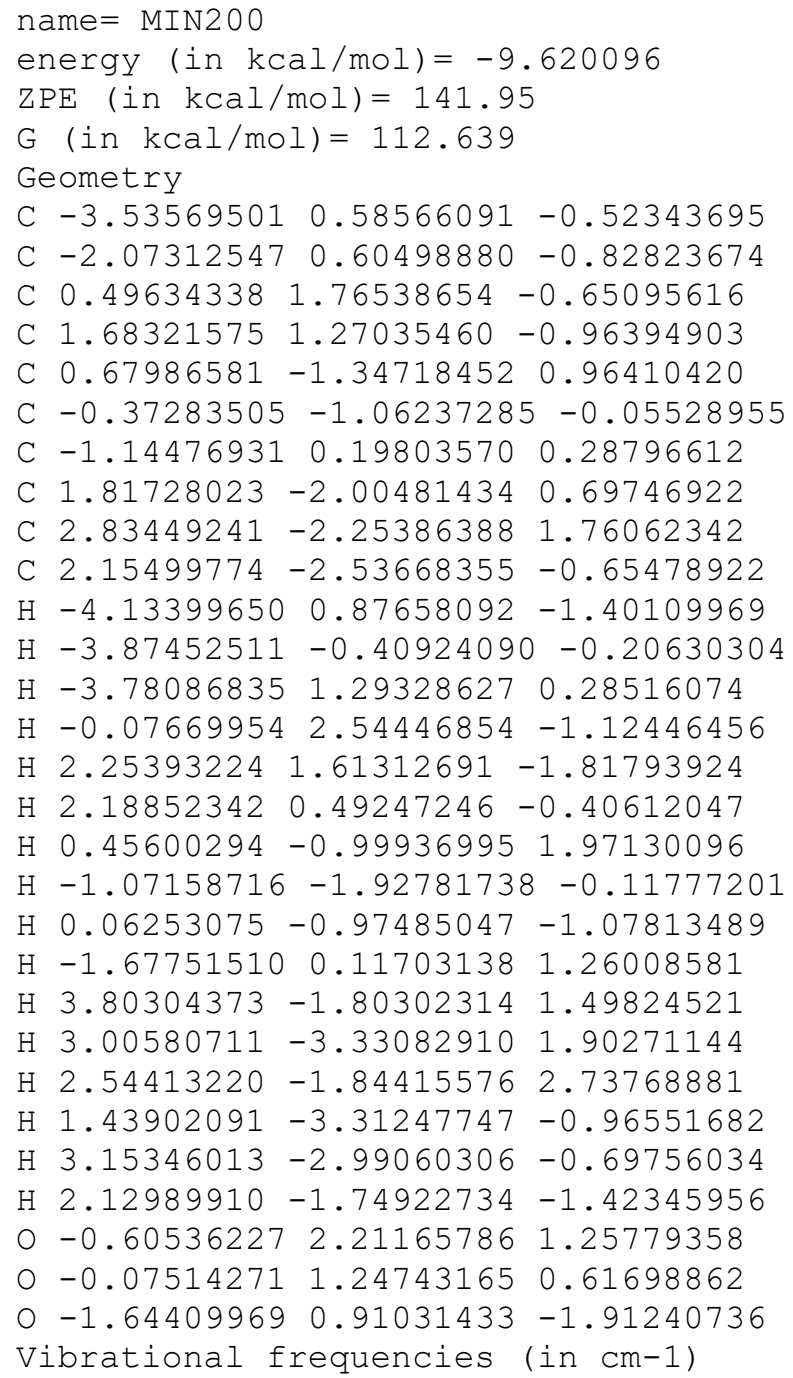


H $1.60162207 \quad 1.73813711 \quad 1.14552014$

$\mathrm{H} 1.565162001 .89602052-0.60573332$

$\mathrm{H} 2.58207670-0.37078827 \quad 0.03158560$

$\mathrm{H} \quad 0.93027366-1.79720873-1.40048150$

$\mathrm{H}-0.47194907 \quad 0.62453618-1.88724784$

$\mathrm{H}-1.15552825-1.83842205 \quad 0.12187290$

$\mathrm{H}-0.03798840 \quad 0.52840950 \quad 2.54635287$

$\mathrm{H} 1.47224879-0.23051174 \quad 3.05604440$

$\mathrm{H}-0.06586311-1.09393028 \quad 3.20981696$

H $2.21189162-2.42300298 \quad 1.91891159$

H $1.49062670-2.91994576 \quad 0.38829502$

$\mathrm{H} \quad 0.57186622-3.082552291 .88213142$

$0 \quad 0.37636042 \quad 0.03969635-2.13233588$

$0-0.72823992 \quad 2.72829903 \quad 0.33809961$

o $-0.12600647-0.67430317-3.14360450$

Vibrational frequencies (in cm-1)

$\begin{array}{llllllllllllllll}-2.5 & 61.2 & 110.1 & 113.8 & 131.5 & 147.8 & 156.0 & 184.1 & 228.9 & 280.8 & 284.9 & 338.5 & 364.7 & 377.8\end{array}$ $402.9411 .1 \quad 448.0 \quad 464.1 \quad 504.5 \quad 544.3 \quad 592.3 \quad 606.7 \quad 734.2 \quad 768.8 \quad 826.2 \quad 848.2 \quad 876.5 \quad 924.9$ $959.5974 .8 \quad 1008.3 \quad 1011.1 \quad 1017.0 \quad 1020.5 \quad 1045.8 \quad 1066.2 \quad 1099.3 \quad 1103.2 \quad 1119.81130 .0$

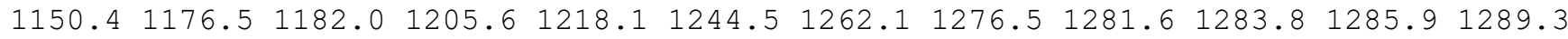

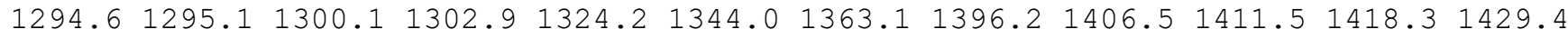
$1840.62654 .3 \quad 2658.0 \quad 2667.8 \quad 2669.4 \quad 2672.6 \quad 2674.42679 .12702 .2 \quad 2724.02740 .3 \quad 2745.2$ $2751.4 \quad 2769.0 \quad 2774.5 \quad 2780.7 \quad 2783.2$

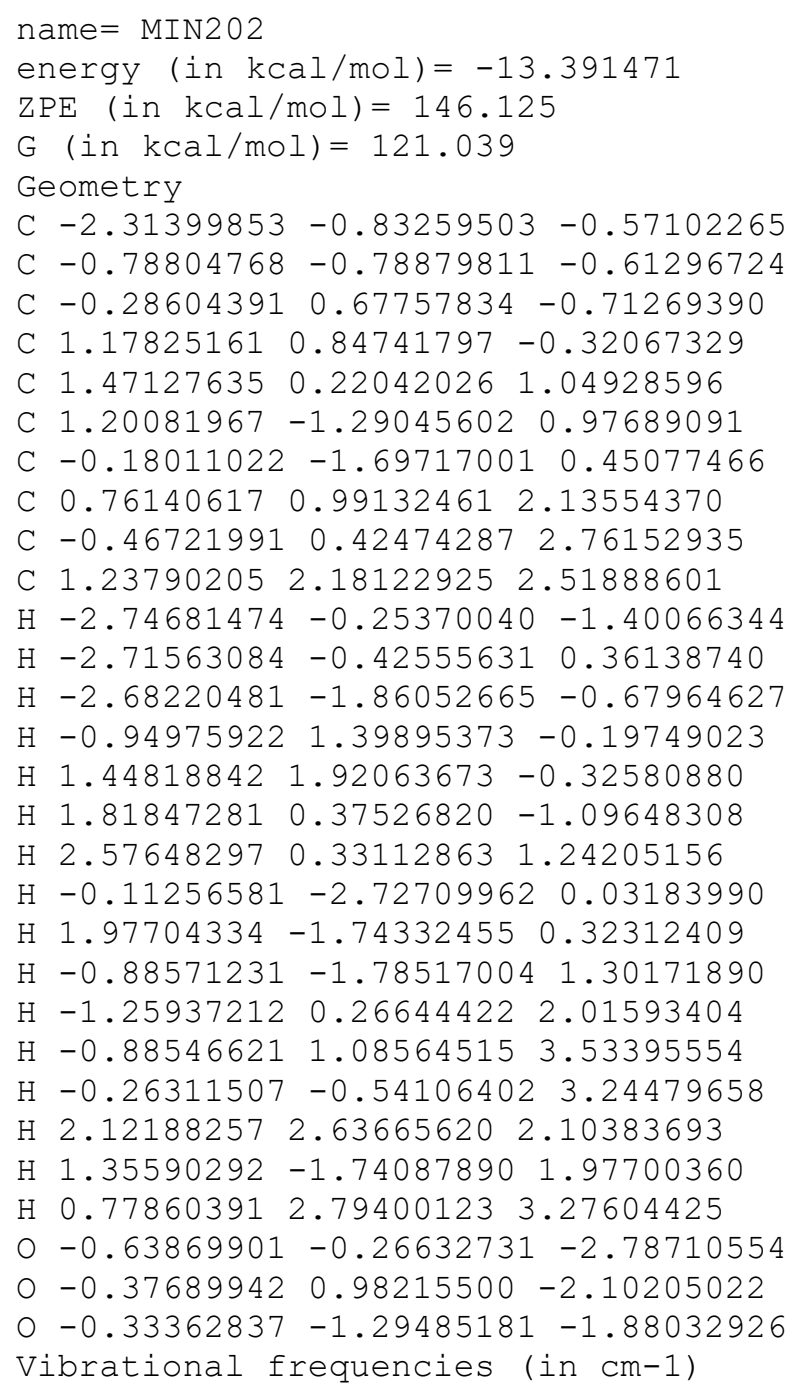


$2642.62659 .0 \quad 2666.5 \quad 2667.3 \quad 2669.1 \quad 2672.1 \quad 2679.4 \quad 2680.3 \quad 2693.3 \quad 2724.02732 .4 \quad 2737.1$ $2741.82777 .02780 .3 \quad 2792.6$

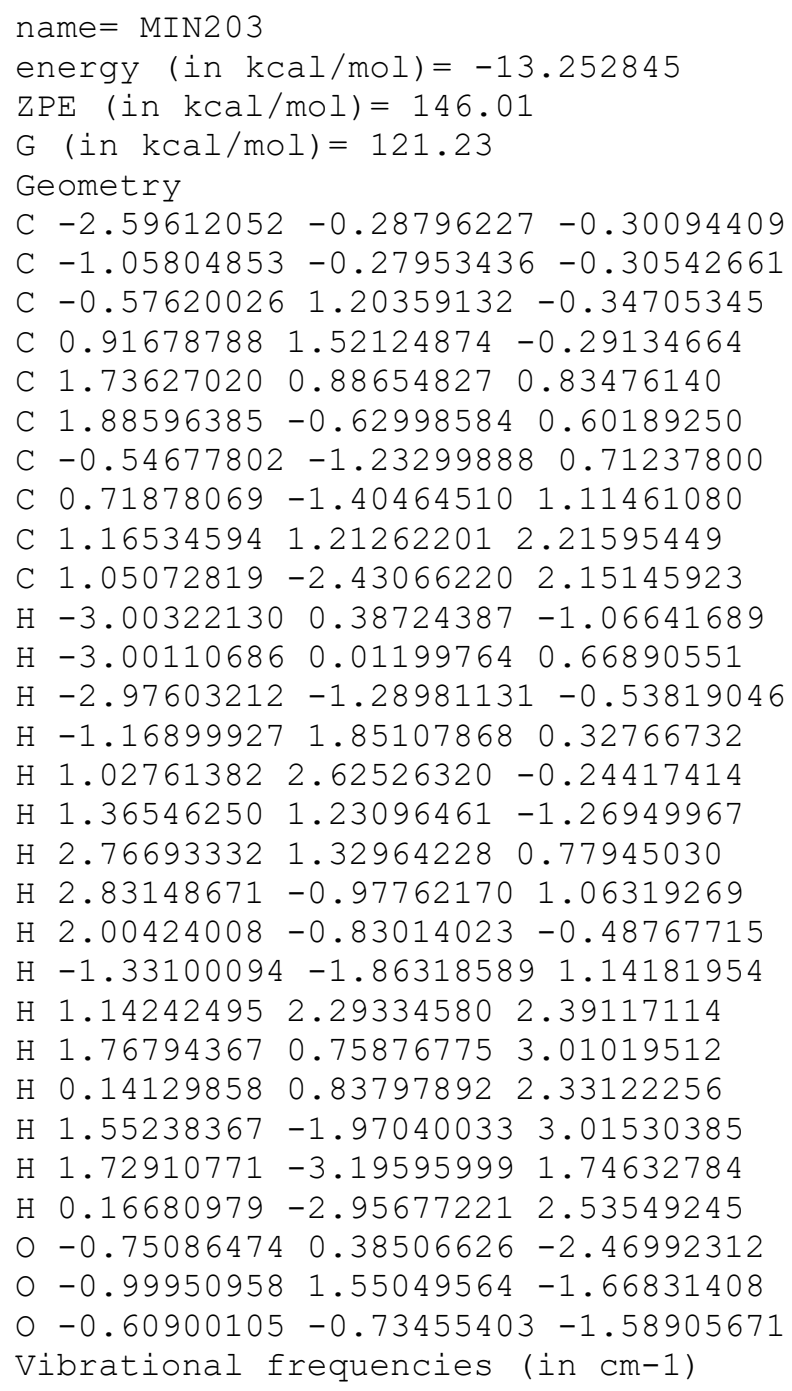


$\mathrm{H}-3.86055692 \quad 1.37449505 \quad 0.22743859$

$\mathrm{H}-0.66981848 \quad 2.28601247 \quad 1.94744543$

$\mathrm{H} \quad 0.38030188-1.67480829-2.19886889$

$\mathrm{H}-0.60177814-2.28928302-0.82800486$

$\mathrm{H} \quad 1.99493219 \quad 1.12668621-0.02079838$

H $0.64787049 \quad 0.45507434 \quad 1.59453833$

H $0.54002946-1.297167131 .76158861$

$\mathrm{H}-1.76350791-1.364933931 .01844753$

H $1.24778507 \quad 2.23033467 \quad-2.11862457$

$\mathrm{H}-0.175250751 .71338930-1.21046303$

H $\quad 0.34652812 \quad 0.79559247 \quad-2.62229362$

H 2.50624955 -0.66078671 -2.49515792

$\mathrm{H} \quad 3.48970549-0.62004291-1.01519606$

H $3.39089774 \quad 0.79443068 \quad-2.05789048$

O $1.57336553-2.01773601-0.40104558$

$\begin{array}{lllll}0 & -1.69545297 & 2.95266459 & 0.36193009\end{array}$

o $1.55242925-2.795469110 .55194663$

Vibrational frequencies (in $\mathrm{cm}-1$ )

$\begin{array}{lllllllllllllll}21.4 & 40.7 & 75.8 & 83.0 & 100.6 & 116.2 & 146.7 & 167.1 & 176.3 & 218.6 & 248.2 & 258.9 & 278.7 & 297.3\end{array}$ $324.0 \quad 334.0 \quad 387.7 \quad 440.9 \quad 469.6 \quad 500.4 \quad 529.1 \quad 604.3 \quad 642.8 \quad 696.0 \quad 705.7 \quad 828.6 \quad 881.0 \quad 926.3$

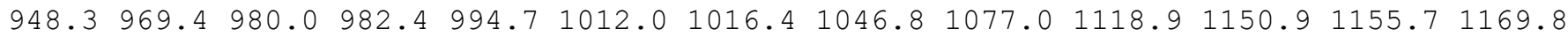

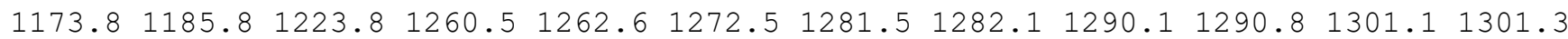

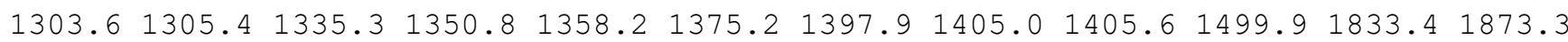
$2601.32649 .12661 .3 \quad 2667.2 \quad 2670.92671 .7 \quad 2672.72676 .92700 .42702 .5 \quad 2715.42744 .0$ $2769.12776 .02779 .7 \quad 2783.6$

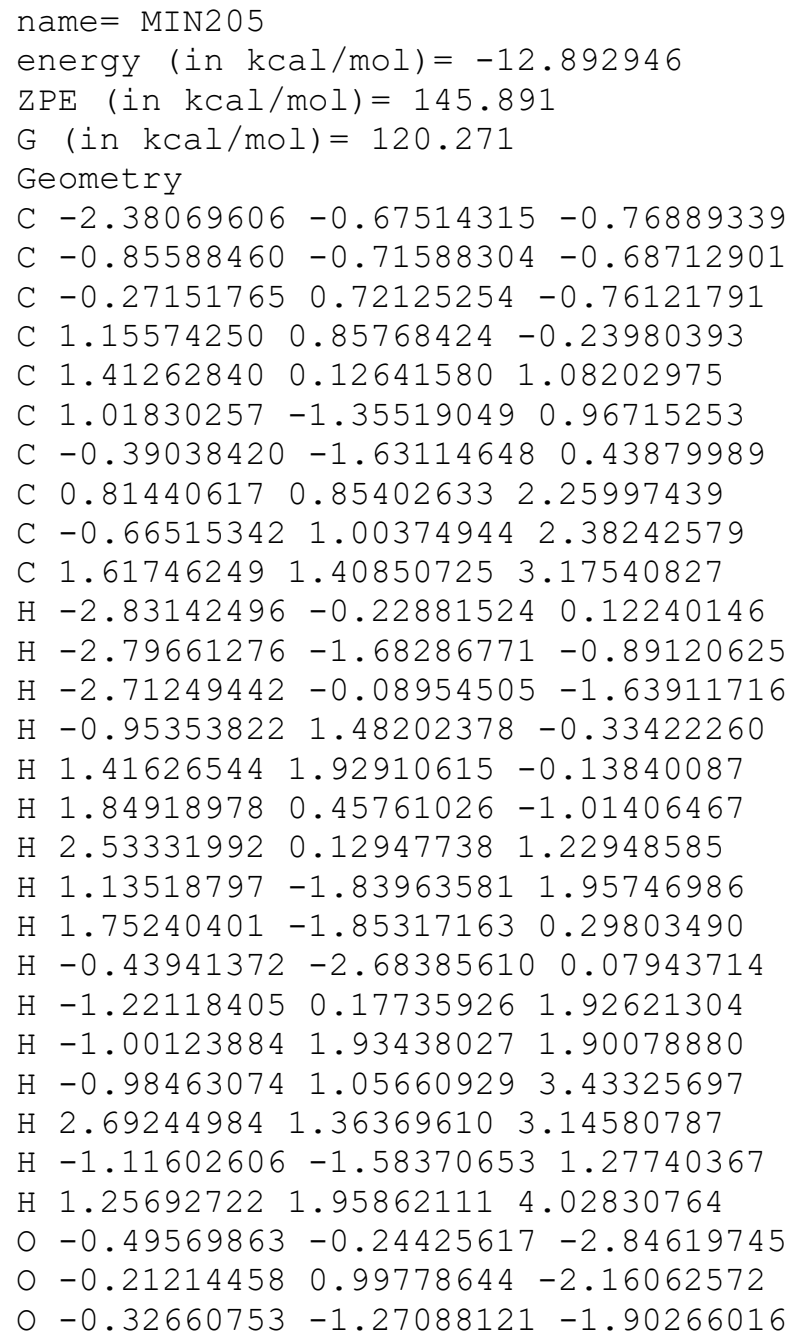


$\begin{array}{lllllllllllllll}1183.3 & 1208.2 & 1218.1 & 1238.9 & 1264.0 & 1269.8 & 1280.9 & 1285.2 & 1286.0 & 1290.8 & 1295.0 & 1303.9\end{array}$ $1309.11311 .21316 .1 \quad 1334.2 \quad 1366.91378 .5 \quad 1383.6 \quad 1391.61404 .7 \quad 1407.01414 .4 \quad 1841.5$ $2641.32655 .02664 .72667 .7 \quad 2668.72671 .42673 .5 \quad 2679.82691 .72724 .92730 .02736 .8$ 2739.22779 .02780 .82794 .3

\section{name $=$ MIN206}

energy (in $\mathrm{kcal} / \mathrm{mol})=-10.61792$

$\mathrm{ZPE}($ in $\mathrm{kcal} / \mathrm{mol})=143.769$

$\mathrm{G}(\mathrm{in} \mathrm{kcal} / \mathrm{mol})=116.065$

Geometry

C $-2.47177395-0.51557002-0.71531967$

C $-1.14583345-0.38972629-0.62053328$

C $-0.51412696 \quad 0.97623060 \quad-0.58805883$

C $0.70847307 \quad 1.04962478 \quad 0.34208209$

C $0.71395238-0.12928735 \quad 1.26438945$

C $0.88343444-1.41456225 \quad 0.51352382$

C $-0.24611341-1.58453815-0.52672641$

C $0.54823571-0.04839968 \quad 2.59347731$

C $0.30297423 \quad 1.24714471 \quad 3.29756567$

C $0.58551887-1.250815893 .48125845$

$\mathrm{H}-0.46727820-0.75812317-3.08336882$

$\mathrm{H}-3.151311090 .31976754 \quad-0.78635561$

$\mathrm{H}-2.98730836-1.46251731-0.72358201$

$\mathrm{H}-1.26577197 \quad 1.75779228-0.30241664$

H $0.72072401 \quad 2.01413480 \quad 0.88249070$

$\mathrm{H} \quad 1.64182009 \quad 1.05937842 \quad-0.26145424$

H $1.87058395-1.419987910 .00649659$

H $\quad 0.89891954 \quad-2.29224763 \quad 1.18581042$

$\mathrm{H} \quad 0.20047453-1.79603536-1.52053974$

$\mathrm{H}-0.83700005-2.48876220-0.27223735$

$\mathrm{H}-0.16721604 \quad 1.10399427 \quad 4.27964353$

$\mathrm{H}-0.36712258 \quad 1.90677767 \quad 2.72865264$

$\mathrm{H} \quad 1.24496062 \quad 1.78949675 \quad 3.46312063$

$\mathrm{H} \quad 0.72548713-0.98882528 \quad 4.53795009$

$\mathrm{H} 1.40476146-1.934253893 .21872594$

$\mathrm{H}-0.35491830-1.81717307 \quad 3.40866190$

$\begin{array}{lllll}0 & 0.72936579 & 0.58709591 & -2.50966500\end{array}$

$\begin{array}{llll}0 & -0.17680638 & 1.44967345 & -1.88773989\end{array}$

O $0.05753871-0.05883823-3.54905034$

Vibrational frequencies (in $\mathrm{cm}-1$ )

$\begin{array}{llllllllllllllll}-52.4 & 43.0 & 47.4 & 52.8 & 56.4 & 67.9 & 101.9 & 124.5 & 171.3 & 224.1 & 286.7 & 304.8 & 361.9 & 370.3\end{array}$ $383.2 \quad 402.3 \quad 442.1 \quad 467.9 \quad 495.5 \quad 527.1 \quad 589.4 \quad 604.9 \quad 683.4 \quad 704.2 \quad 829.6 \quad 838.3 \quad 853.4 \quad 896.8$ $961.4973 .4 \quad 982.2 \quad 988.5 \quad 1007.9 \quad 1026.8 \quad 1040.8 \quad 1077.4 \quad 1083.6 \quad 1108.0 \quad 1109.4 \quad 1126.2 \quad 1157.8$

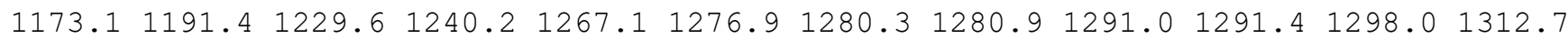
$1324.51329 .91335 .6 \quad 1356.0 \quad 1361.6 \quad 1382.4 \quad 1386.2 \quad 1395.81402 .4 \quad 1409.21851 .11884 .6$ $2644.22652 .92668 .92669 .7 \quad 2671.6 \quad 2672.5 \quad 2674.22676 .92718 .2 \quad 2733.12741 .42742 .6$ $2777.3 \quad 2778.4 \quad 2787.6 \quad 2832.2$

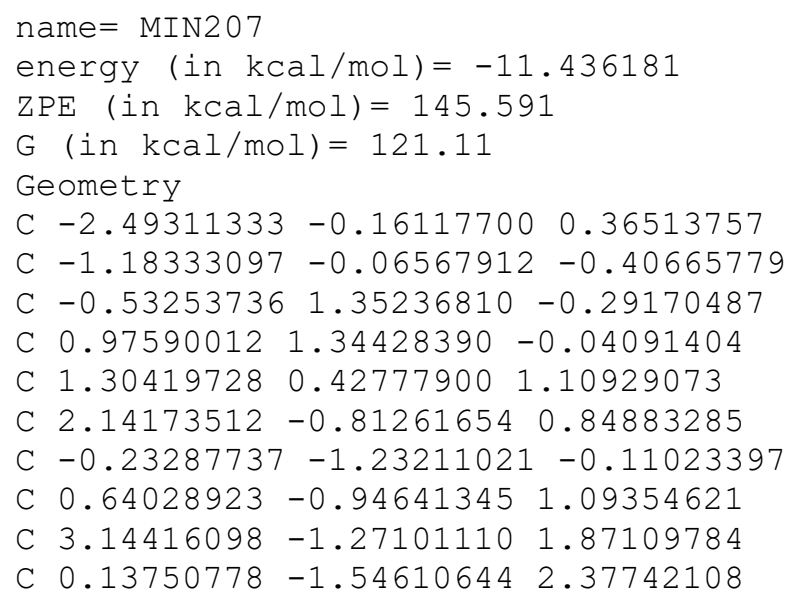


$\mathrm{H}-2.32488211-0.118546951 .44654510$

$\mathrm{H}-3.01857919-1.099155050 .14223970$

$\mathrm{H}-3.17662780 \quad 0.65446444 \quad 0.09078903$

$\mathrm{H}-1.06611458 \quad 2.00229450 \quad 0.43059668$

$\mathrm{H} \quad 1.33321728 \quad 2.37382919 \quad 0.15587832$

$\mathrm{H} 1.50192456 \quad 1.03315691-0.96813022$

$\mathrm{H} 1.423041330 .942435852 .06465657$

$\mathrm{H} \quad 0.38832791-1.42410895-1.01129901$

H 2.49722009 $-0.98199061-0.17589814$

$\mathrm{H}-0.80851102-2.16692299 \quad 0.03264095$

H $4.13612475-0.84561902 \quad 1.66608345$

$\mathrm{H} 0.69895439-1.193525293 .25174646$

$\mathrm{H} \quad 2.87495987 \quad-0.97816680 \quad 2.89272986$

$\mathrm{H}-0.91703506-1.296225252 .55221209$

$\mathrm{H} \quad 0.22176742-2.641218552 .36021556$

$\mathrm{H} \quad 3.24372667-2.364929901 .86102311$

$0-0.79428803 \quad 0.83833793-2.45476593$

$0-0.799876271 .94179351-1.56395528$

$0-1.57009585-0.14719826-1.79200465$

Vibrational frequencies (in $\mathrm{cm}-1$ )

$\begin{array}{lllllllllllllllll}59.0 & 89.8 & 100.5 & 119.4 & 154.6 & 167.4 & 219.0 & 237.7 & 253.9 & 280.1 & 293.0 & 349.0 & 366.3 & 415.8\end{array}$

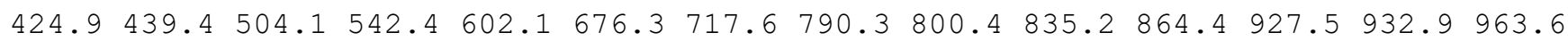
$977.1986 .7 \quad 998.5 \quad 1014.3 \quad 1024.4 \quad 1040.9 \quad 1074.5 \quad 1089.8 \quad 1096.3 \quad 1121.2 \quad 1130.91150 .6 \quad 1164.5$ $\begin{array}{lllllllllllll}1191.7 & 1216.8 & 1219.2 & 1225.4 & 1252.1 & 1268.3 & 1276.4 & 1283.9 & 1284.9 & 1289.4 & 1291.1 & 1292.9\end{array}$

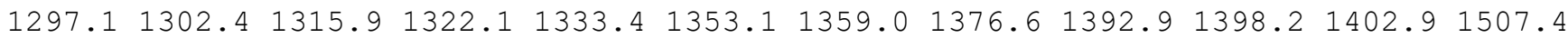
$2657.52659 .22670 .6 \quad 2672.92674 .2 \quad 2675.0 \quad 2676.62677 .02693 .72741 .42743 .62745 .2$ $2764.22779 .02780 .4 \quad 2781.9$

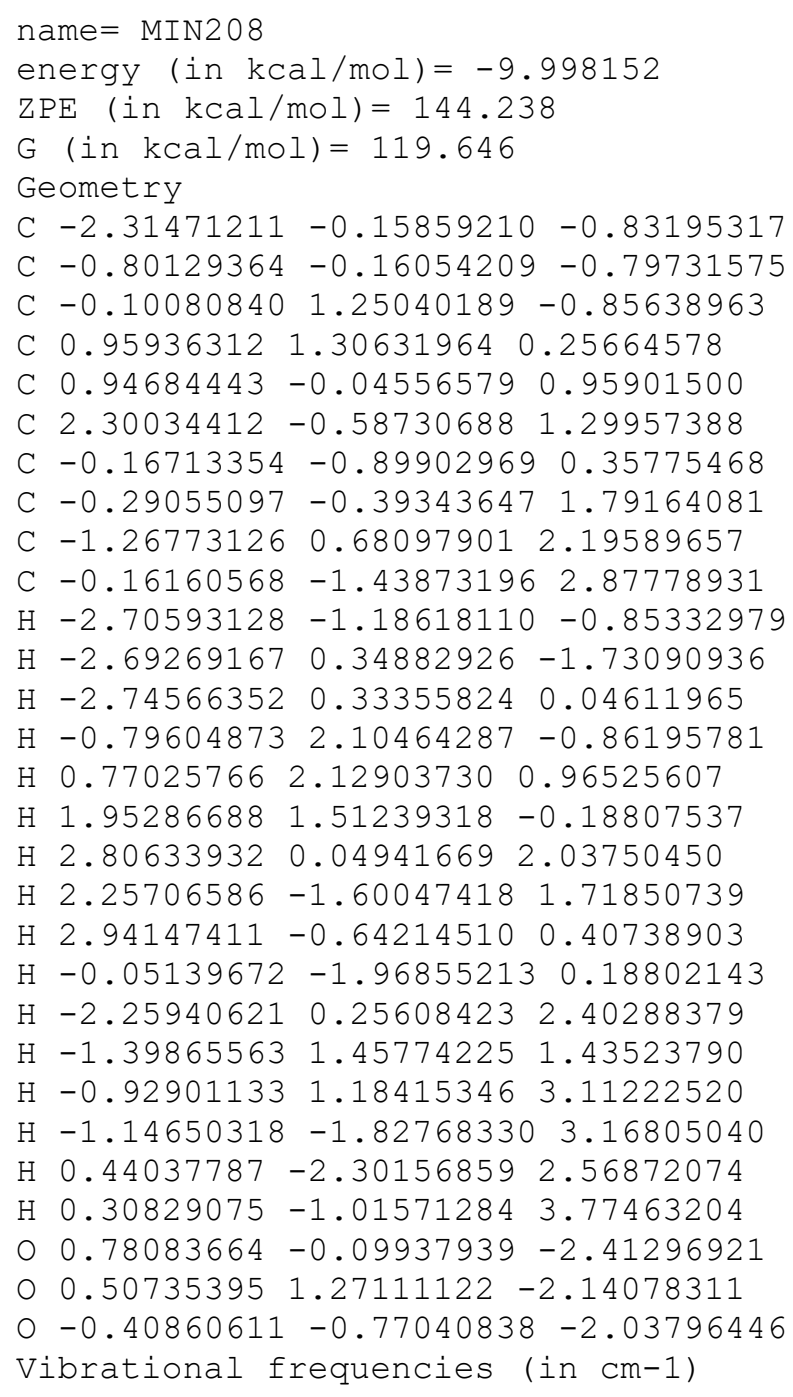


$\begin{array}{lllllllllllllllll}73.1 & 96.1 & 114.2 & 118.4 & 130.2 & 157.6 & 187.0 & 198.0 & 245.0 & 262.3 & 279.4 & 318.8 & 351.6 & 384.1\end{array}$ $416.2 \quad 453.3 \quad 473.1 \quad 495.5 \quad 581.7 \quad 607.2 \quad 673.8 \quad 705.2 \quad 744.9 \quad 768.4 \quad 850.8 \quad 866.0 \quad 940.9 \quad 960.6$ $973.2986 .4996 .81003 .2 \quad 1011.5 \quad 1017.5 \quad 1021.1 \quad 1042.6 \quad 1056.01101 .4 \quad 1130.81135 .8 \quad 1149.9$

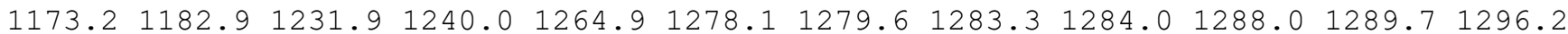
$1297.3 \quad 1301.3 \quad 1305.8 \quad 1311.7 \quad 1323.3 \quad 1332.5 \quad 1340.7 \quad 1392.7 \quad 1396.7 \quad 1403.81406 .3 \quad 1511.7$ $2666.02671 .0 \quad 2671.7 \quad 2672.12673 .7 \quad 2675.6 \quad 2676.8 \quad 2677.22679 .12703 .82747 .6 \quad 2762.3$ $2779.4 \quad 2780.4 \quad 2781.4 \quad 2782.2$

\section{name $=$ MIN209}

energy (in $\mathrm{kcal} / \mathrm{mol})=-12.171287$

$\operatorname{ZPE}($ in $\mathrm{kcal} / \mathrm{mol})=146.424$

$\mathrm{G}($ in $\mathrm{kcal} / \mathrm{mol})=120.492$

Geometry

C $-2.41310554 \quad 0.36516783-1.29108528$

C $-1.16999076 \quad 0.10016744 \quad-0.44728393$

C $-0.21800590 \quad 1.29686115-0.38013787$

C $0.97110661 \quad 1.019211120 .56159322$

C $1.08417084-0.420579951 .11111740$

C $0.72686272-1.496339230 .06634372$

C $-0.31243373-1.05506196-0.97540839$

C $0.32732077-0.54765625 \quad 2.41168259$

C $0.93690056 \quad 0.18751320 \quad 3.56758800$

C $-0.78239892-1.267446442 .59517773$

$\mathrm{H}-2.17245017 \quad 0.63649927-2.32604499$

$\mathrm{H}-3.005761891 .18834069-0.87391895$

$\mathrm{H}-3.06087081-0.51879545-1.33063721$

$\mathrm{H}-0.742553512 .23827484 \quad-0.11247389$

H $0.94683637 \quad 1.73667902 \quad 1.40178492$

$\mathrm{H} \quad 1.90294006 \quad 1.25069988-0.00199178$

H $2.17099433-0.58443700 \quad 1.36369894$

H $0.38944207 \quad-2.41989494 \quad 0.57202596$

H $1.65387769-1.78693894-0.47625166$

$\mathrm{H}-0.92233153-1.90461083-1.34300532$

H $1.19403508 \quad-0.50739198 \quad 4.38073557$

$\mathrm{H} \quad 0.23581724 \quad 0.92402000 \quad 3.98506855$

$\mathrm{H} \quad 1.85612685 \quad 0.72636294 \quad 3.30764660$

$\mathrm{H}-1.27670958-1.84222703 \quad 1.83073338$

$\mathrm{H}-1.51644448-0.13983765 \quad 0.59157642$

$\mathrm{H}-1.28825611-1.33977179 \quad 3.54429851$

$\begin{array}{lllll}0 & 0.04096101 & 0.57805759 & -2.57989159\end{array}$

$0 \quad 0.35953628 \quad 1.59083592-1.65702690$

O $0.48577681-0.64554882-2.10382770$

Vibrational frequencies (in cm-1)

$\begin{array}{llllllllllllllll}-35.3 & 11.6 & 66.9 & 98.6 & 131.6 & 186.9 & 213.6 & 250.7 & 272.7 & 327.5 & 342.1 & 385.6 & 408.4 & 445.0\end{array}$ $494.5 \quad 511.7 \quad 547.5 \quad 553.7 \quad 583.0 \quad 623.3 \quad 752.2 \quad 797.2 \quad 826.9 \quad 836.2 \quad 879.7 \quad 905.7 \quad 936.5 \quad 970.6$ $975.0 \quad 985.1 \quad 1016.7 \quad 1037.4 \quad 1039.2 \quad 1048.0 \quad 1063.1 \quad 1087.4 \quad 1096.7 \quad 1131.01142 .7 \quad 1156.1$

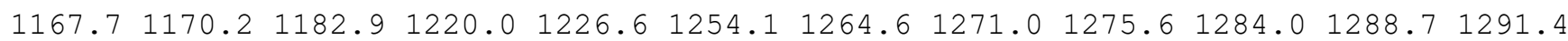
$1298.31304 .7 \quad 1314.1 \quad 1321.0 \quad 1326.6 \quad 1340.5 \quad 1349.2 \quad 1384.8 \quad 1396.3 \quad 1399.01403 .1 \quad 1412.2$ $1862.02650 .02651 .12667 .12669 .6 \quad 2672.6 \quad 2676.72678 .32688 .4 \quad 2689.12695 .92721 .3$ $2732.5 \quad 2733.9 \quad 2778.4 \quad 2781.5 \quad 2792.1$

name $=$ MIN210

energy (in $\mathrm{kcal} / \mathrm{mol})=-11.538787$

$\mathrm{ZPE}(\mathrm{in} \mathrm{kcal} / \mathrm{mol})=145.842$

$\mathrm{G}($ in $\mathrm{kcal} / \mathrm{mol})=120.945$

Geometry

C $-2.18537027 \quad 0.03113606-0.57270201$

C $-0.66308031-0.03908341-0.53299683$

C $-0.00863280 \quad 1.37408561-0.66203563$

C $1.33791691 \quad 1.50120634 \quad 0.02939341$

C 2.034553120 .440098820 .38885630

C $1.47350207-0.94192086 \quad 0.31962773$

C $-0.05301114-0.91972009 \quad 0.57536435$ 
C $-0.32190878-0.465498962 .03269560$

C $-1.80730135-0.313242632 .37562719$

C $0.28200812-1.491614793 .00827440$

$\mathrm{H}-2.59443386 \quad 0.77336442 \quad 0.11856591$

$\mathrm{H}-2.63989341-0.93935769-0.34250607$

$\mathrm{H}-2.52357640 \quad 0.30819757-1.58469766$

$\mathrm{H}-0.69554042 \quad 2.19681536-0.37936527$

$\mathrm{H} \quad 0.18004443 \quad 0.52040436 \quad 2.19229541$

$\mathrm{H} \quad 1.72893002 \quad 2.51394880 \quad 0.01688110$

H $3.03714031 \quad 0.52971429 \quad 0.80172226$

$\mathrm{H} \quad 1.97850724-1.625020121 .02675290$

H $1.67425243-1.37250932-0.68871423$

$\mathrm{H}-0.45809258-1.952074890 .44861424$

$\mathrm{H}-2.22546095 \quad 0.61297323 \quad 1.96859591$

$\mathrm{H}-1.95741557-0.26584489 \quad 3.46157511$

$\mathrm{H}-2.39879651-1.15552124 \quad 2.00425910$

$\mathrm{H} 0.05400239-1.228844714 .04743796$

H $1.37245558-1.54175200 \quad 2.92815480$

$\mathrm{H}-0.11647183-2.495506062 .82866890$

$\begin{array}{lllll}0 & 0.54022829 & 0.20779834 & -2.48036597\end{array}$

O $0.13296866 \quad 1.49852132 \quad-2.08316054$

o $-0.33613800-0.68200021-1.78715320$

Vibrational frequencies (in $\mathrm{cm}-1$ )

$\begin{array}{llllllllllllllll}-22.9 & 60.8 & 113.3 & 148.3 & 168.4 & 193.9 & 202.3 & 245.9 & 253.5 & 310.1 & 325.2 & 337.2 & 352.2 & 390.1\end{array}$ $433.1475 .9 \quad 480.8 \quad 541.8 \quad 557.5 \quad 690.0 \quad 707.4 \quad 739.2 \quad 809.7 \quad 866.8 \quad 896.8 \quad 916.4 \quad 940.1962 .8$ $973.7981 .8 \quad 994.1 \quad 1005.6 \quad 1009.4 \quad 1025.1 \quad 1052.4 \quad 1054.6 \quad 1096.91122 .3 \quad 1142.1 \quad 1156.4 \quad 1166.6$

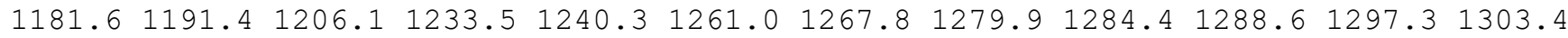
$1307.91309 .21316 .0 \quad 1324.2 \quad 1325.2 \quad 1329.8 \quad 1363.8 \quad 1378.91391 .5 \quad 1401.91409 .41854 .9$ $2650.62663 .42667 .7 \quad 2671.5 \quad 2674.0 \quad 2675.92678 .5 \quad 2693.32696 .42711 .12736 .42749 .4$ $2764.7 \quad 2776.0 \quad 2779.7 \quad 2781.1$

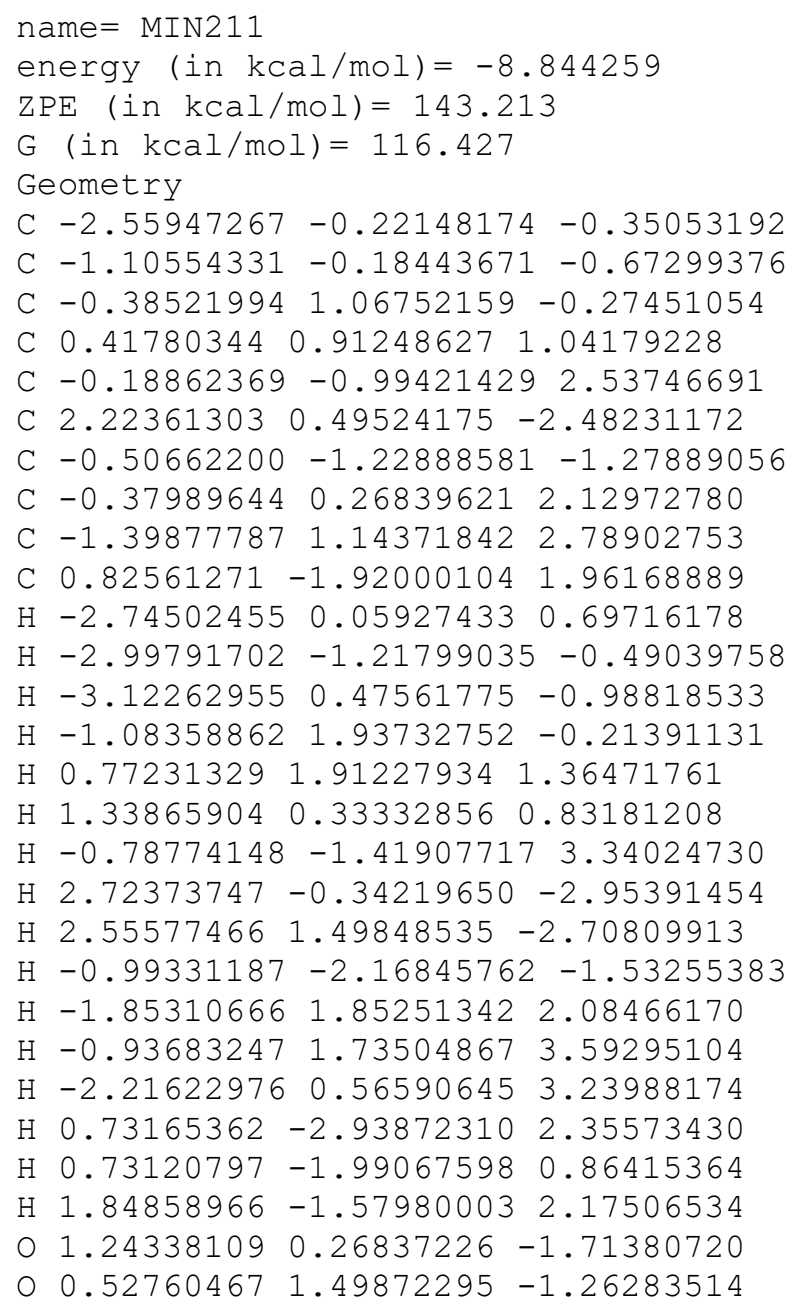


$0.76423716-1.25509329-1.66388129$

Vibrational frequencies (in $\mathrm{cm}-1$ )

$\begin{array}{llllllllllllllll}29.4 & 59.4 & 87.4 & 90.4 & 108.4 & 125.8 & 130.7 & 144.2 & 171.9 & 226.6 & 233.2 & 263.8 & 290.5 & 311.3\end{array}$ $331.9 \quad 340.1 \quad 389.4 \quad 424.6 \quad 484.9 \quad 526.4 \quad 534.3 \quad 602.9 \quad 623.3 \quad 638.4 \quad 707.7 \quad 722.5 \quad 853.2 \quad 868.8$ $912.3 \quad 939.6 \quad 1006.8 \quad 1030.3 \quad 1047.2 \quad 1054.6 \quad 1065.8 \quad 1079.2 \quad 1089.9 \quad 1093.9 \quad 1108.51135 .0$ $\begin{array}{llllllllllllllll}1144.0 & 1166.1 & 1229.7 & 1238.5 & 1243.2 & 1272.1 & 1279.9 & 1282.3 & 1287.2 & 1289.5 & 1291.1 & 1293.5\end{array}$ $1296.41302 .4 \quad 1314.6 \quad 1336.4 \quad 1366.3 \quad 1379.1 \quad 1390.1 \quad 1394.6 \quad 1406.8 \quad 1412.01694 .2 \quad 1815.6$ $1868.52656 .62658 .8 \quad 2660.8 \quad 2669.5 \quad 2672.12674 .72676 .12676 .92685 .12704 .22728 .2$ $2743.6 \quad 2757.92777 .6 \quad 2778.0 \quad 2780.2$

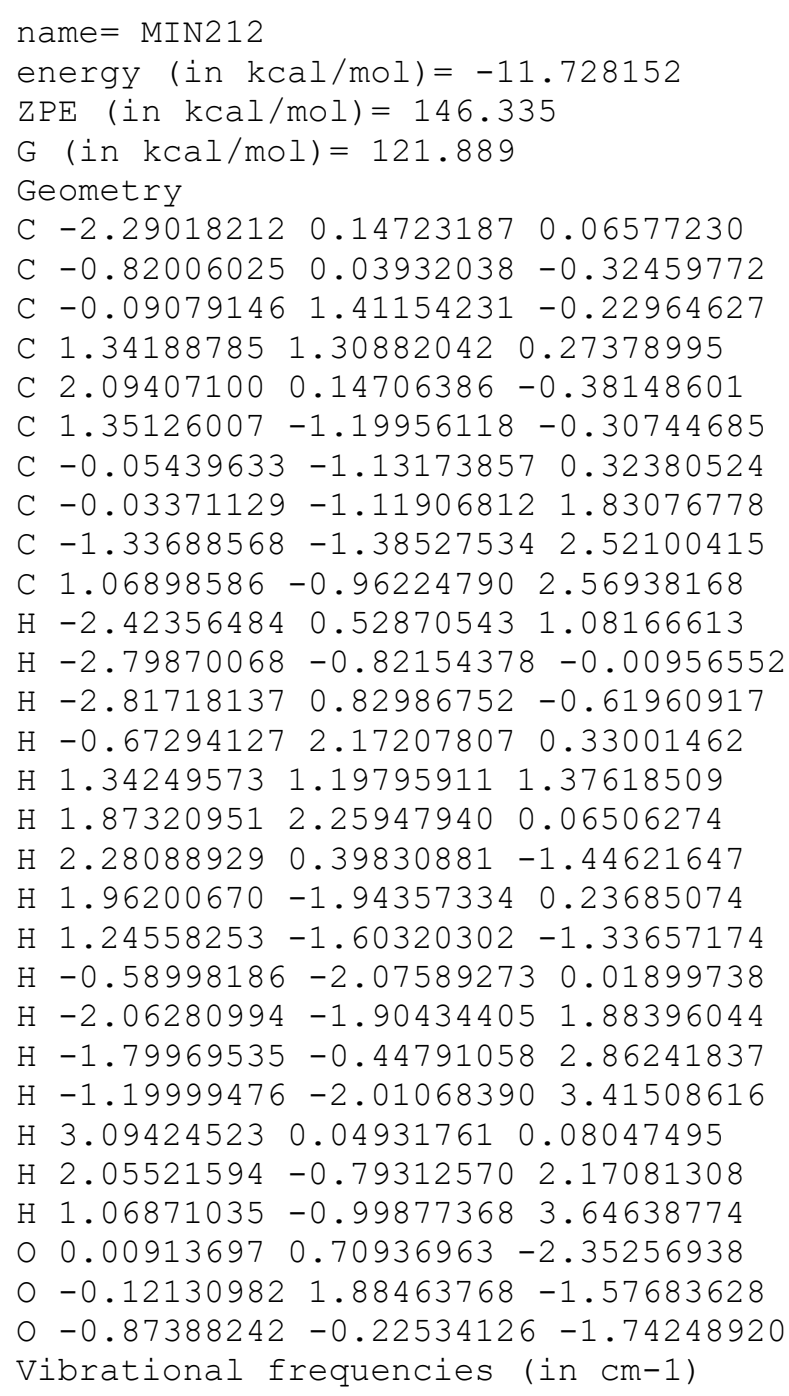


C $1.25340401-0.44150841-0.50171837$

C $-0.15489303-0.82452357-0.13377282$

C $0.03914353-0.45757018 \quad 1.33339403$

C $-0.96532640 \quad 0.53130293 \quad 1.88725159$

C $0.14408725-1.68680653 \quad 2.22640591$

$\mathrm{H}-3.28430182-0.87531173-0.92170641$

$\mathrm{H}-2.70780526-1.54100850 \quad 0.61103545$

$\mathrm{H}-2.85136900-2.59575834-0.79905192$

$\mathrm{H} \quad 0.24975846 \quad 1.95416052-0.64376649$

H $1.53622471 \quad 1.93453964 \quad 2.10403091$

H 2.75635752 $1.84577296 \quad 0.84042253$

H $2.27598470 \quad-0.42210114 \quad 1.48198225$

$\mathrm{H} \quad 1.89983851-1.28445750 \quad-0.77226999$

H $1.33960624 \quad 0.28160272-1.32794354$

$\mathrm{H}-0.66712582-2.30198509-2.45581826$

$\mathrm{H}-1.184543931 .34426658 \quad 1.18379645$

$\mathrm{H}-0.60327084 \quad 0.98727961 \quad 2.81775294$

$\mathrm{H}-1.922706330 .046208602 .11495720$

$\mathrm{H} \quad 0.48021970-1.41592395 \quad 3.23467565$

$\mathrm{H} \quad 0.85372298-2.42272924 \quad 1.82928690$

$\mathrm{H}-0.82241999-2.19385509 \quad 2.32618544$

$0-0.14594971-0.56625356-2.83046838$

$0 \quad 0.79669702 \quad 3.64909625 \quad 0.27707925$

o $-1.09050344-1.33622505-2.33067783$

Vibrational frequencies (in $\mathrm{cm}-1$ )

$\begin{array}{llllllllllllllll}18.3 & 27.9 & 39.4 & 44.9 & 95.0 & 130.7 & 139.1 & 156.0 & 167.8 & 195.7 & 218.8 & 232.8 & 267.7 & 305.4\end{array}$ $\begin{array}{lllllllllllllll}315.6 & 367.1 & 387.7 & 423.9 & 463.7 & 561.0 & 571.9 & 612.9 & 647.0 & 732.3 & 766.0 & 827.5 & 881.2 & 918.0\end{array}$

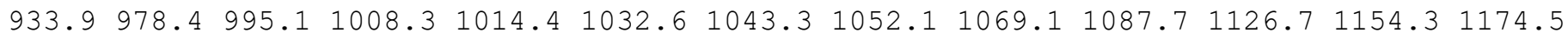

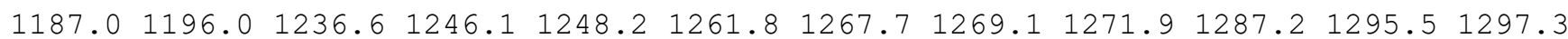

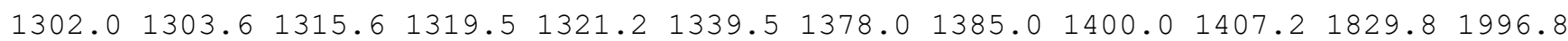
$2639.12651 .4 \quad 2659.12663 .0 \quad 2666.2 \quad 2671.4 \quad 2674.52676 .72679 .2 \quad 2721.32737 .62755 .6$ $2764.12774 .02780 .4 \quad 2782.6$

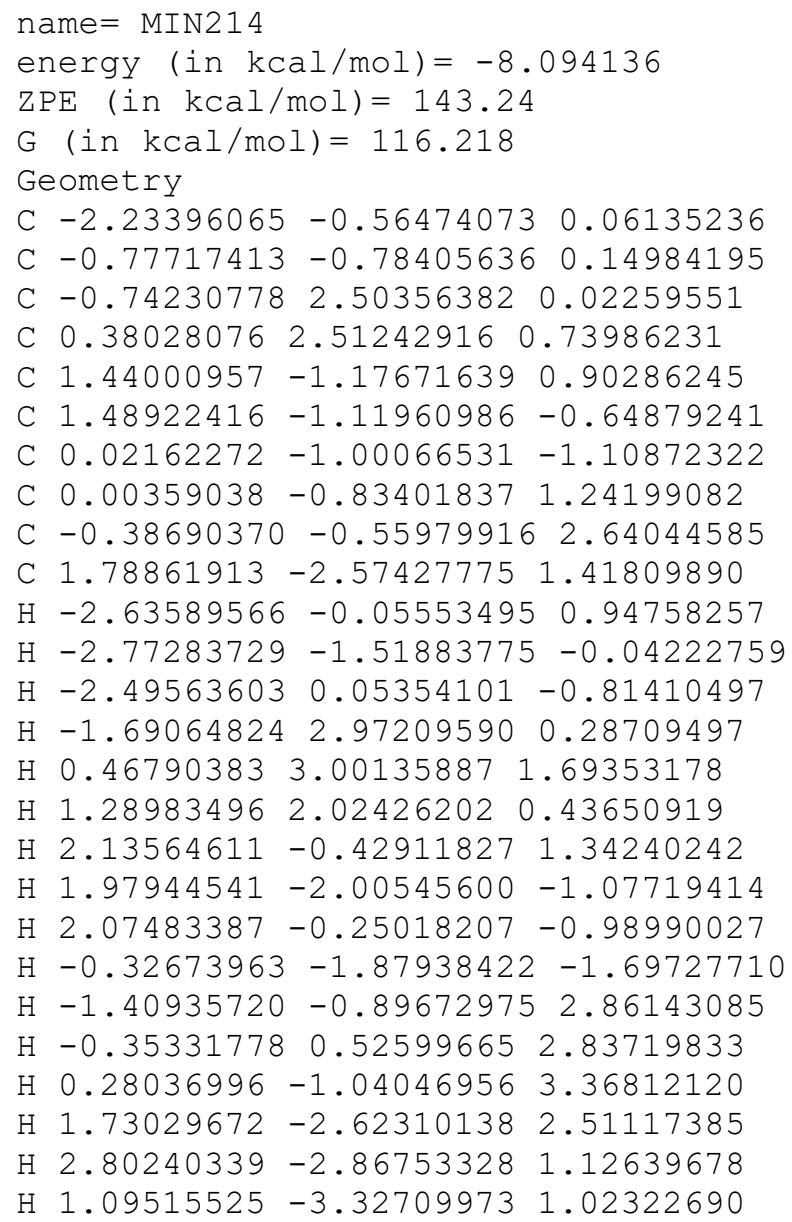


$0.24827487 \quad 1.25604736-1.64329539$

$0-0.932946351 .95048564-1.20168531$

O $-0.16449870 \quad 0.02463756-2.07887774$

Vibrational frequencies (in $\mathrm{cm}-1$ )

$\begin{array}{llllllllllllllll}35.4 & 54.5 & 64.6 & 76.8 & 86.9 & 97.2 & 131.5 & 162.1 & 178.4 & 197.3 & 225.1 & 254.0 & 276.1 & 285.8\end{array}$ $325.2 \quad 418.5 \quad 452.2 \quad 508.5 \quad 529.3 \quad 581.7 \quad 598.9 \quad 614.8 \quad 620.3 \quad 693.4 \quad 813.9 \quad 826.5 \quad 901.5 \quad 925.5$ $948.4 \quad 958.4 \quad 970.0 \quad 1012.1 \quad 1021.3 \quad 1033.1 \quad 1046.4 \quad 1050.7 \quad 1063.3 \quad 1087.0 \quad 1099.8 \quad 1118.6 \quad 1135.6$ $1171.31197 .91201 .1 \quad 1210.2 \quad 1243.0 \quad 1263.2 \quad 1274.4 \quad 1276.7 \quad 1279.4 \quad 1283.0 \quad 1287.01291 .5$

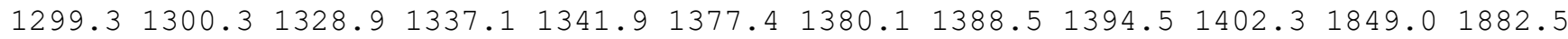
$2663.82665 .12668 .42674 .2 \quad 2675.42677 .12680 .22691 .12715 .22717 .22739 .62751 .7$ $2776.12778 .4 \quad 2782.2 \quad 2800.8$

name $=$ MIN215

energy $($ in $\mathrm{kcal} / \mathrm{mol})=-8.970667$

$\mathrm{ZPE}(\mathrm{in} \mathrm{kcal} / \mathrm{mol})=144.22$

$\mathrm{G}(\mathrm{in} \mathrm{kcal} / \mathrm{mol})=119.646$

Geometry

C $-2.13156725-0.16653692-0.69247491$

C $-0.62156539-0.04294820-0.68952600$

C $-0.04367771 \quad 1.42340227-0.67331039$

C 1.060169921 .492995460 .39692681

C $1.13473454 \quad 0.12659599 \quad 1.04587670$

C $0.38259854 \quad-2.23741401 \quad 0.07650263$

C $0.10382068-0.80483987 \quad 0.41261819$

C $-0.05013603-0.35597859 \quad 1.87262926$

C $-1.12917109 \quad 0.59847975 \quad 2.31683689$

C $0.23291926-1.380059632 .95037933$

$\mathrm{H}-2.56933343 \quad 0.33448315-1.56778279$

$\mathrm{H}-2.585696650 .26172805 \quad 0.20693395$

$\mathrm{H}-2.43543304-1.22232863-0.73846054$

$\mathrm{H}-0.80700845 \quad 2.21328895 \quad-0.58802441$

$\mathrm{H} \quad 0.86196179 \quad 2.28426548 \quad 1.13959174$

$\mathrm{H} 2.026001361 .75493716-0.07961015$

H 2.13387542 $-0.21296822 \quad 1.30310504$

H $1.05696494-2.71849132 \quad 0.79487382$

$\mathrm{H} \quad 0.85820622-2.32843878-0.91409924$

$\mathrm{H}-0.54592775 \quad-2.82430124 \quad 0.04868402$

$\mathrm{H}-1.34833906 \quad 1.38009857 \quad 1.58210487$

$\mathrm{H}-0.83455005 \quad 1.11239958 \quad 3.24310361$

$\mathrm{H}-2.06591028 \quad 0.06330164 \quad 2.52316781$

$\mathrm{H} 0.36209779-0.89986573 \quad 3.92888057$

H $1.14541977-1.957108572 .75908445$

$\mathrm{H}-0.59550113-2.095018123 .03655209$

$\begin{array}{lllll}0 & 0.88169597 & 0.27095827 & -2.35606090\end{array}$

○ $0.500335821 .58837579-1.97577006$

O $-0.22509929-0.52899198-1.98227332$

Vibrational frequencies (in $\mathrm{cm}-1$ )

$\begin{array}{lllllllllllllll}69.3 & 94.5 & 106.3 & 117.4 & 137.9 & 156.4 & 202.5 & 210.2 & 231.5 & 268.2 & 289.4 & 323.4 & 328.1 & 377.6\end{array}$

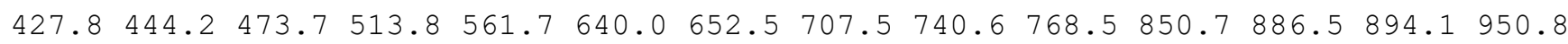
$970.3 \quad 976.0 \quad 1002.4 \quad 1010.1 \quad 1014.0 \quad 1027.4 \quad 1029.0 \quad 1055.6 \quad 1099.5 \quad 1112.3 \quad 1124.8 \quad 1142.3$

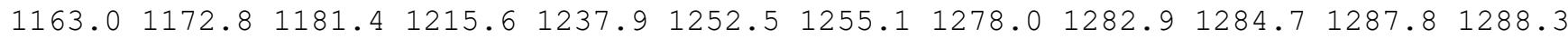
$1291.21298 .21302 .6 \quad 1306.4 \quad 1307.9 \quad 1315.1 \quad 1325.91349 .2 \quad 1392.01400 .91405 .01406 .3$ $1504.62664 .52666 .6 \quad 2670.6 \quad 2670.8 \quad 2673.2 \quad 2674.72676 .02676 .82678 .82702 .82747 .1$ $2768.3 \quad 2777.2 \quad 2779.2 \quad 2780.92781 .8$

name $=$ MIN216

energy $($ in $\mathrm{kcal} / \mathrm{mol})=-10.135479$

$\operatorname{ZPE}($ in $\mathrm{kcal} / \mathrm{mol})=145.92$

$\mathrm{G}($ in $\mathrm{kcal} / \mathrm{mol})=121.169$

Geometry

C $-2.12450361-0.05864905-0.42438841$

C $-0.60442623-0.09118307-0.45348646$

C $0.028709801 .32097894-0.64197619$ 
C $1.418755491 .47594574-0.00613504$

C $2.16836664 \quad 0.18181116 \quad 0.02493729$

C $1.54725989-0.96657085 \quad 0.31374048$

C $0.07035875-0.97392484 \quad 0.61363770$

C $-0.09454752-0.57887987 \quad 2.11168243$

C $-1.20032061 \quad 0.42811842 \quad 2.42693787$

C $-0.32569481-1.85374060 \quad 2.93764877$

$\mathrm{H}-2.521800050 .14149331-1.43269390$

$\mathrm{H}-2.52944468 \quad 0.71528500 \quad 0.23327212$

$\mathrm{H}-2.54027192-1.02236482-0.10551560$

$\mathrm{H}-0.65667136 \quad 2.13668449-0.34186697$

$\mathrm{H} \quad 1.31776309 \quad 1.87292521 \quad 1.02555982$

H $1.98559972 \quad 2.24529251-0.57348380$

H $3.22847315 \quad 0.23615084 \quad-0.20298123$

$\mathrm{H} \quad 0.87199185-0.11938990 \quad 2.44918582$

H $2.05019661-1.925274720 .34514079$

$\mathrm{H}-0.34305614-2.00579940 \quad 0.47291538$

$\mathrm{H}-1.08610440 \quad 1.35246799 \quad 1.85218715$

$\mathrm{H}-1.17390258 \quad 0.70752558 \quad 3.48821685$

$\mathrm{H}-2.19612128 \quad 0.01599902 \quad 2.23288235$

$\mathrm{H} 0.47212263-2.586461722 .77406584$

$\mathrm{H}-1.27775282-2.33197985 \quad 2.68350992$

$\mathrm{H}-0.34868946-1.630585214 .00986518$

$\begin{array}{lllll}0 & -0.35925058 & 0.28709936 & -2.64735850\end{array}$

$0.22880798 \quad 1.45435637-2.05081691$

O $-0.21194746-0.72951290-1.69403223$

Vibrational frequencies (in cm-1)

$\begin{array}{lllllllllllllllll}-34.7 & 68.7 & 101.9 & 119.7 & 176.6 & 187.3 & 231.5 & 247.6 & 262.2 & 281.9 & 302.5 & 319.9 & 356.7 & 377.9\end{array}$

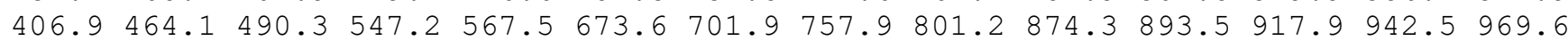
$970.2983 .2993 .3 \quad 1009.1 \quad 1011.4 \quad 1031.4 \quad 1043.2 \quad 1080.3 \quad 1103.6 \quad 1133.7 \quad 1143.3 \quad 1160.51170 .3$ $1177.31199 .8 \quad 1213.0 \quad 1226.0 \quad 1253.0 \quad 1266.1 \quad 1273.9 \quad 1280.6 \quad 1284.2 \quad 1290.2 \quad 1290.91300 .3$ $1303.11307 .0 \quad 1309.6 \quad 1326.2 \quad 1353.8 \quad 1365.3 \quad 1368.3 \quad 1375.7 \quad 1401.8 \quad 1403.01409 .81846 .3$ $2658.92663 .6 \quad 2665.42673 .6 \quad 2676.1 \quad 2678.0 \quad 2679.42685 .32687 .6 \quad 2700.02736 .72755 .5$ $2769.62778 .62780 .8 \quad 2782.2$

\author{
name $=$ MIN217 \\ energy (in $\mathrm{kcal} / \mathrm{mol})=-8.369357$ \\ $\mathrm{ZPE}(\mathrm{in} \mathrm{kcal} / \mathrm{mol})=144.223$ \\ $\mathrm{G}($ in $\mathrm{kcal} / \mathrm{mol})=119.601$ \\ Geometry \\ C $-2.27974250-0.15867428-0.73079290$ \\ C $-0.76761585-0.15756392-0.74318681$ \\ C $-0.09969516 \quad 1.26633510 \quad-0.72977008$ \\ C $1.01858301 \quad 1.26331933 \quad 0.32657576$ \\ C $1.04691525-0.13274276 \quad 0.93923417$ \\ C $2.41823256-0.67970944 \quad 1.18688431$ \\ C $-0.08468085-0.96488258 \quad 0.33492048$ \\ C $-0.15068866-0.54683961 \quad 1.79998467$ \\ C $-1.11907155 \quad 0.488282312 .31261182$ \\ C $0.03770025-1.65466000 \quad 2.81312827$ \\ $\mathrm{H}-2.68562560 \quad 0.39530045 \quad-1.58838891$ \\ $\mathrm{H}-2.69014937 \quad 0.28075517 \quad 0.18432535$ \\ $\mathrm{H}-2.66811025-1.18511303-0.79940375$ \\ $\mathrm{H}-0.82106735 \quad 2.09109210-0.62494550$ \\ $\mathrm{H} 0.86033641 \quad 2.03797554 \quad 1.09445741$ \\ H $1.988621021 .50402733-0.15394076$ \\ $\mathrm{H} 2.40427877-1.735307361 .48605446$ \\ H $2.92875675-0.12007135 \quad 1.98192382$ \\ H $3.03919926-0.61954597 \quad 0.28027142$ \\ $\mathrm{H} \quad 0.03396001-2.02081845 \quad 0.09628938$ \\ $\mathrm{H}-0.73366129 \quad 0.95240793 \quad 3.23131228$ \\ $\mathrm{H}-2.09026325 \quad 0.03629478 \quad 2.55573267$ \\ $\mathrm{H}-1.30772216 \quad 1.29868414 \quad 1.60070244$ \\ $\mathrm{H} \quad 0.66040145-2.47504580 \quad 2.43651573$
}


$\mathrm{H}-0.92684638-2.094029823 .09941319$

H $0.51594011-1.274130293 .72466318$

$0-0.142511350 .42086255-2.82093764$

$0.529425201 .37969300-2.00645302$

O $-0.30681059-0.70876865-1.99095634$

Vibrational frequencies (in $\mathrm{cm}-1$ )

$\begin{array}{lllllllllllllllll}73.5 & 104.8 & 110.7 & 118.7 & 130.1 & 155.3 & 177.0 & 197.9 & 254.0 & 258.8 & 274.6 & 313.0 & 351.7 & 378.3\end{array}$ $397.4 \quad 439.4 \quad 460.1 \quad 499.0 \quad 589.7 \quad 613.7 \quad 654.9 \quad 718.9 \quad 743.5 \quad 763.7 \quad 859.2 \quad 876.1 \quad 946.4 \quad 948.1$ $982.8 \quad 987.2 \quad 997.4 \quad 1002.1 \quad 1010.9 \quad 1019.2 \quad 1020.1 \quad 1051.7 \quad 1067.7 \quad 1110.5 \quad 1130.5 \quad 1143.1 \quad 1149.0$ $1172.0 \quad 1189.4 \quad 1225.3 \quad 1241.3 \quad 1260.0 \quad 1273.0 \quad 1280.5 \quad 1283.0 \quad 1284.8 \quad 1287.6 \quad 1289.6 \quad 1296.5$

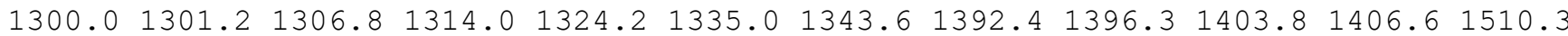
$2663.12670 .62671 .4 \quad 2672.1 \quad 2673.5 \quad 2675.4 \quad 2677.02677 .72679 .02697 .22745 .12761 .8$ $2779.52780 .12781 .7 \quad 2782.6$

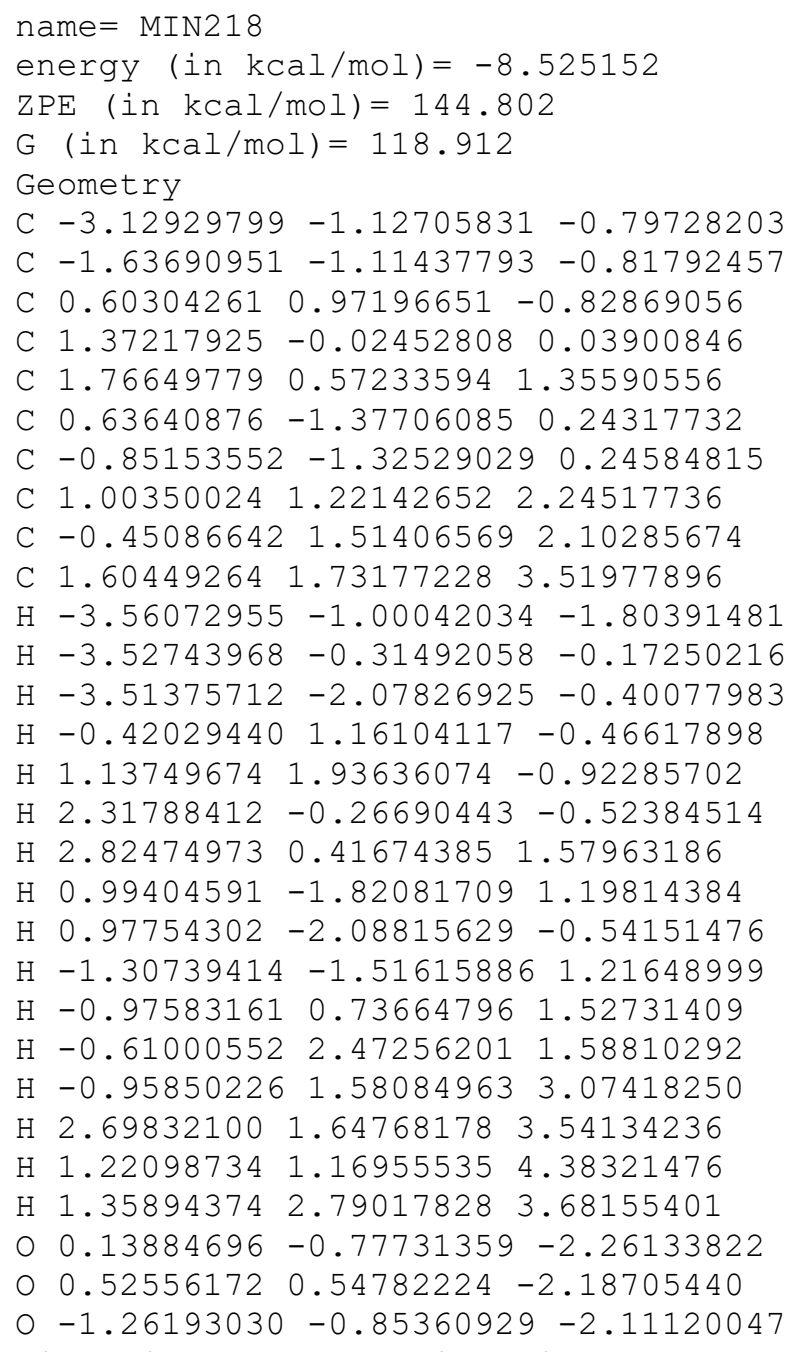


C $-0.78978029-0.32865427-0.71472923$

C $-0.23999608 \quad 1.13652585-0.71808072$

C $0.913644591 .42869035 \quad 0.23625538$

C $0.71084974 \quad 0.80744616 \quad 1.62628064$

C $1.25938376-1.47371478 \quad 0.44753781$

C $-0.23448995-1.255334110 .33612770$

C $0.53059636-0.69896928 \quad 1.52930991$

C $-0.49075343 \quad 1.45946885 \quad 2.32354954$

C $0.35733735-1.37989830 \quad 2.86415715$

$\mathrm{H}-2.72768137 \quad 0.31372044 \quad-1.48562854$

$\mathrm{H}-2.71912685-0.03998780 \quad 0.25894533$

$\mathrm{H}-2.70025628-1.35587512-0.92736758$

$\mathrm{H}-1.05584704 \quad 1.88133822-0.60903198$

H $1.04917434 \quad 2.52408808 \quad 0.32854457$

H $1.86297372 \quad 1.06381094-0.20899049$

$\mathrm{H} \quad 1.62975133 \quad 1.00987977 \quad 2.23399524$

$\mathrm{H} \quad 1.63767672 \quad-2.46917960 \quad 0.65992740$

H $1.92824142 \quad-0.97207806-0.24610347$

$\mathrm{H}-0.87994332-2.126680920 .50142572$

$\mathrm{H}-0.43756358 \quad 2.55214960 \quad 2.27128410$

$\mathrm{H}-0.53537691 \quad 1.18651854 \quad 3.38338566$

$\mathrm{H}-1.43560467 \quad 1.14844636 \quad 1.86427249$

$\mathrm{H}-0.58025261-1.08054064 \quad 3.34970915$

H $1.18140237-1.13069970 \quad 3.54538756$

H $0.33531406-2.47325010 \quad 2.77227808$

$\begin{array}{lllll}0 & 0.62949185 & 0.01422888 & -2.45225436\end{array}$

$0 \quad 0.21193107 \quad 1.31351535-2.06252959$

o $-0.42020247-0.82967701-2.01442622$

Vibrational frequencies (in cm-1)

$\begin{array}{llllllllllllll}49.3 & 108.7 & 118.5 & 139.2 & 159.1 & 168.4 & 227.8 & 250.7 & 271.5 & 302.8 & 327.8 & 350.8 & 378.6 & 417.1\end{array}$ $454.2460 .3 \quad 496.8 \quad 542.5 \quad 636.2 \quad 669.1 \quad 712.9 \quad 761.0 \quad 796.6 \quad 859.4 \quad 871.9 \quad 889.6 \quad 934.6 \quad 965.4$

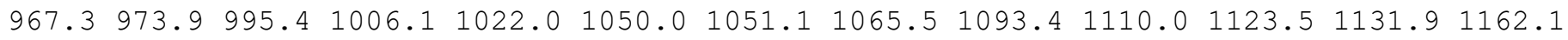

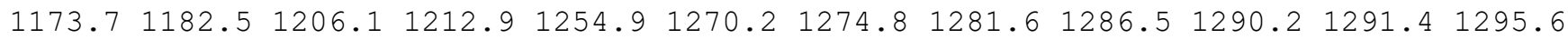
$1300.41303 .1 \quad 1307.6 \quad 1314.1 \quad 1325.3 \quad 1367.9 \quad 1376.01381 .2 \quad 1396.5 \quad 1400.7 \quad 1409.4 \quad 1498.2$ $2653.72669 .82673 .32674 .3 \quad 2676.3 \quad 2678.12679 .32690 .12701 .62708 .72740 .32751 .6$ $2779.7 \quad 2780.8 \quad 2781.7 \quad 2785.3$

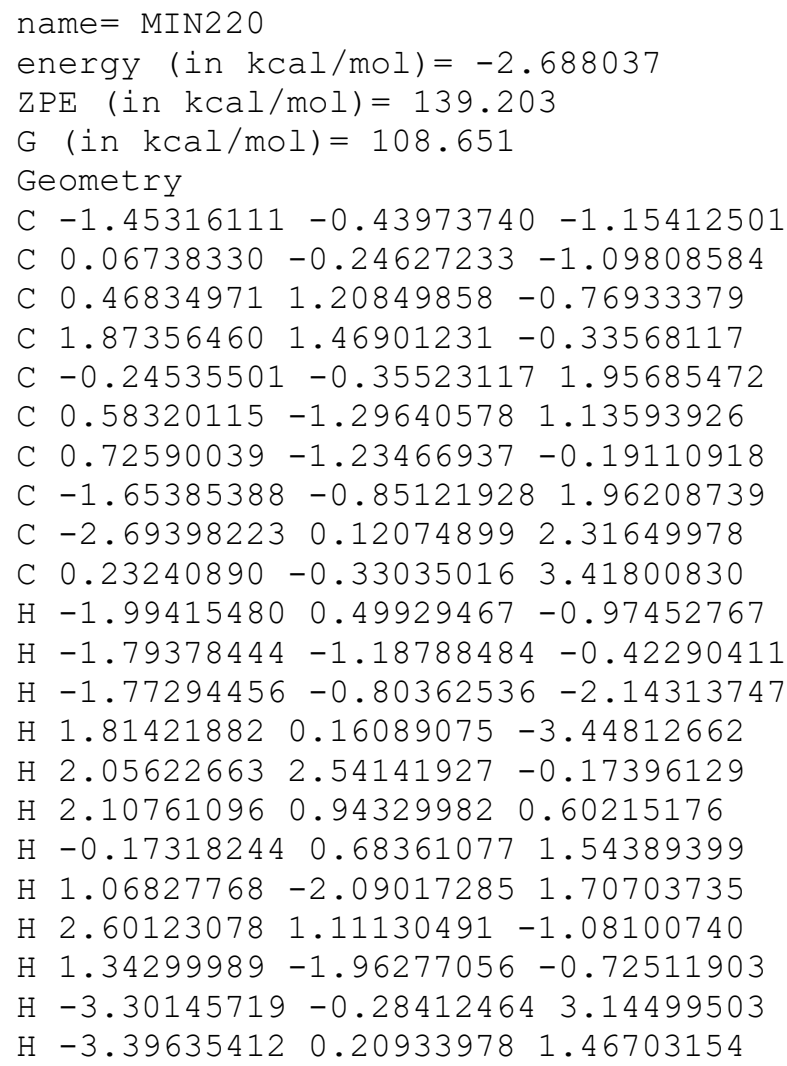


$\mathrm{H}-2.37966647 \quad 1.13327215 \quad 2.59022637$

$\mathrm{H}-0.313046150 .41535158 \quad 4.00743036$

$\mathrm{H} 1.29848274-0.079269013 .47489784$

$\mathrm{H} \quad 0.09842465-1.30580700 \quad 3.90265909$

O $1.81281076-0.37169165-2.61559811$

$0-0.344575332 .09326899-0.85611894$

o $0.39985861-0.50003375-2.46356152$

Vibrational frequencies (in cm-1)

$\begin{array}{llllllllllllllll}-19.2 & 8.1 & 40.7 & 55.3 & 69.3 & 82.5 & 104.5 & 112.2 & 141.0 & 155.5 & 169.8 & 177.3 & 201.1 & 235.9\end{array}$ $260.0 \quad 273.7 \quad 304.5 \quad 352.1 \quad 356.7 \quad 403.4 \quad 437.0 \quad 473.5 \quad 547.0 \quad 568.6 \quad 606.5 \quad 697.2 \quad 765.0 \quad 821.5$ $883.8 \quad 899.1 \quad 960.2 \quad 981.6 \quad 1008.9 \quad 1012.3 \quad 1014.4 \quad 1043.8 \quad 1051.5 \quad 1061.4 \quad 1094.8 \quad 1119.4 \quad 1142.1$

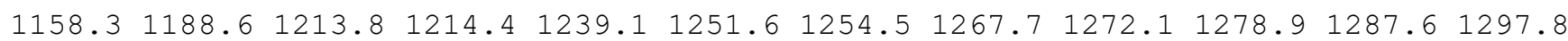

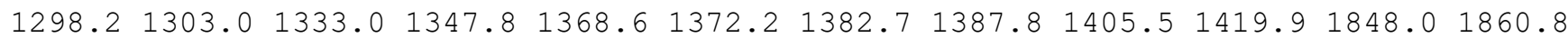
$2647.72654 .12656 .32657 .2 \quad 2667.6 \quad 2670.2 \quad 2671.32674 .22690 .12736 .4 \quad 2752.4 \quad 2762.3$ $2770.0 \quad 2775.7 \quad 2778.12865 .1$

\section{name $=$ MIN221}

energy (in $\mathrm{kcal} / \mathrm{mol})=-9.303538$

$\mathrm{ZPE}($ in $\mathrm{kcal} / \mathrm{mol})=145.914$

$\mathrm{G}($ in $\mathrm{kcal} / \mathrm{mol})=121.358$

Geometry

C $-2.11036490-0.68481717-0.59714569$

C $1.27813101-0.75004136-0.55193442$

C $0.78114987 \quad 0.67100911-0.61112232$

C $0.367721621 .13197265 \quad 0.77237881$

C $-0.63674711 \quad 0.12278138 \quad 1.37671602$

C $-0.01931832-1.297731941 .45284832$

C $0.91203379-1.667017050 .34167361$

C $-2.04846343 \quad 0.115928890 .71039992$

C $-2.52192388 \quad 1.55335141 \quad 0.43241450$

C $-3.03978212-0.51954345 \quad 1.71090954$

$\mathrm{H}-1.70356649-1.69540133-0.47813838$

$\mathrm{H}-1.55033786-0.20531274-1.40547712$

$\mathrm{H}-3.14401549-0.78966320-0.94546903$

$\mathrm{H}-0.01351026 \quad 0.82043406-1.37489366$

$\mathrm{H}-0.05654532 \quad 2.15135886 \quad 0.74860844$

$\mathrm{H} 1.258610031 .19880554 \quad 1.43216301$

$\mathrm{H}-0.80281439 \quad 0.46074545 \quad 2.43459013$

$\mathrm{H}-0.82796006-2.05291280 \quad 1.53676580$

H $0.55164559-1.38015198 \quad 2.40620316$

H $1.30093320-2.678319410 .35167458$

$\mathrm{H}-3.56845185 \quad 1.56365147 \quad 0.10650435$

$\mathrm{H}-1.93886559 \quad 2.02897684 \quad-0.36261974$

$\mathrm{H}-2.45349128 \quad 2.18075288 \quad 1.32633256$

$\mathrm{H}-3.07576567 \quad 0.03769083 \quad 2.65173110$

$\mathrm{H}-2.77262315-1.55466974 \quad 1.94442901$

$\mathrm{H}-4.05598454-0.53384387 \quad 1.30290301$

$\begin{array}{lllll}0 & 2.49278624 & 0.44213301 & -2.00687578\end{array}$

O $1.970077691 .34736361-1.05521774$

o $2.29992652-0.89816430-1.45117931$

Vibrational frequencies (in cm-1)

$\begin{array}{llllllllllllllll}40.0 & 76.6 & 104.5 & 158.4 & 178.5 & 201.7 & 217.2 & 230.1 & 274.5 & 295.3 & 301.8 & 327.6 & 372.7 & 382.2\end{array}$ $409.2446 .7 \quad 485.4 \quad 517.2 \quad 568.7 \quad 663.1 \quad 716.5 \quad 754.9 \quad 779.6 \quad 848.5 \quad 865.2 \quad 879.4 \quad 925.4 \quad 961.6$ $963.2970 .3 \quad 979.1993 .8 \quad 995.6 \quad 1032.1 \quad 1036.9 \quad 1043.8 \quad 1088.7 \quad 1130.0 \quad 1148.9 \quad 1176.3 \quad 1191.7$

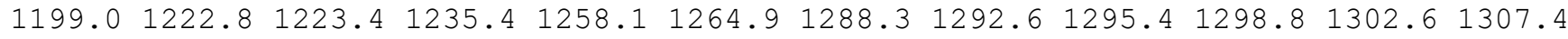
$1309.81310 .7 \quad 1315.7 \quad 1329.0 \quad 1337.8 \quad 1352.8 \quad 1371.4 \quad 1383.1 \quad 1402.7 \quad 1411.2 \quad 1414.6 \quad 1915.1$ $2646.52652 .92665 .42673 .4 \quad 2674.2 \quad 2676.92678 .3 \quad 2679.02679 .92683 .72728 .62743 .2$ $2768.5 \quad 2779.4 \quad 2780.8 \quad 2782.5$

name $=$ MIN222

energy (in $\mathrm{kcal} / \mathrm{mol})=-8.699315$

$\mathrm{ZPE}(\mathrm{in} \mathrm{kcal} / \mathrm{mol})=145.65$

$\mathrm{G}($ in $\mathrm{kcal} / \mathrm{mol})=121.681$ 


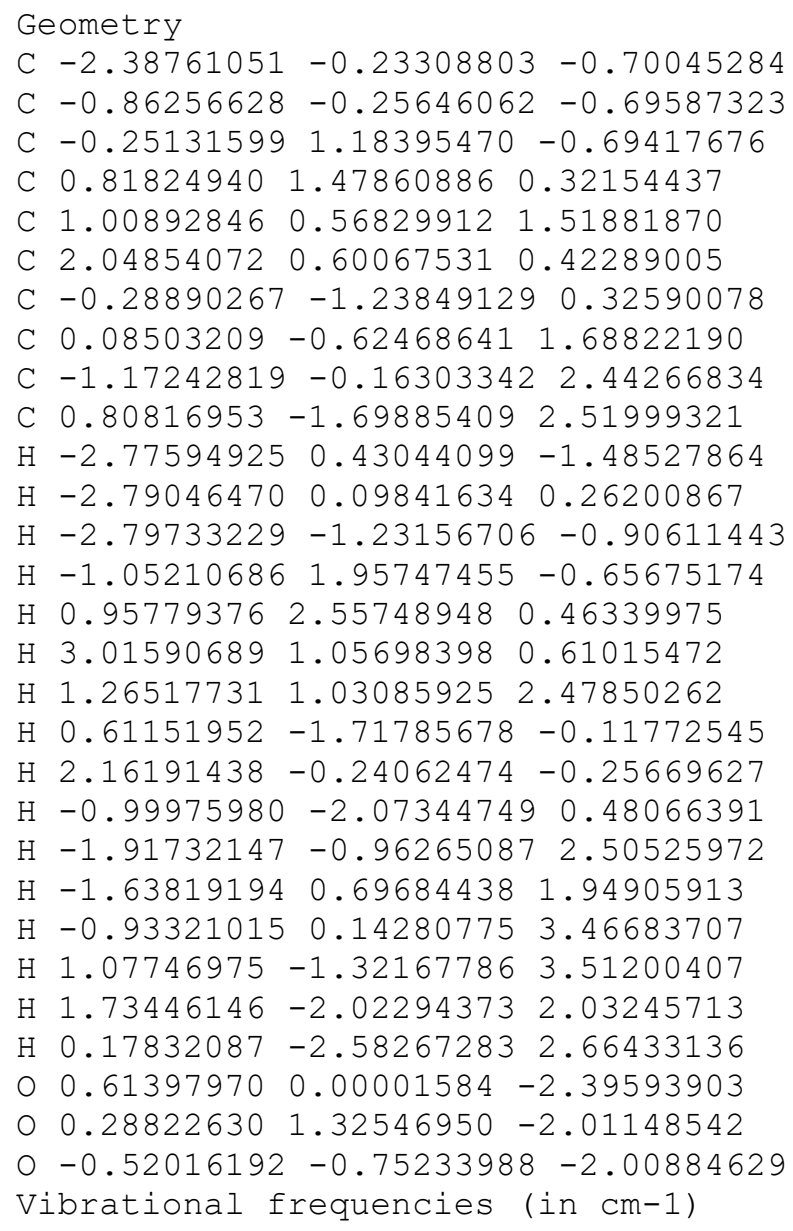

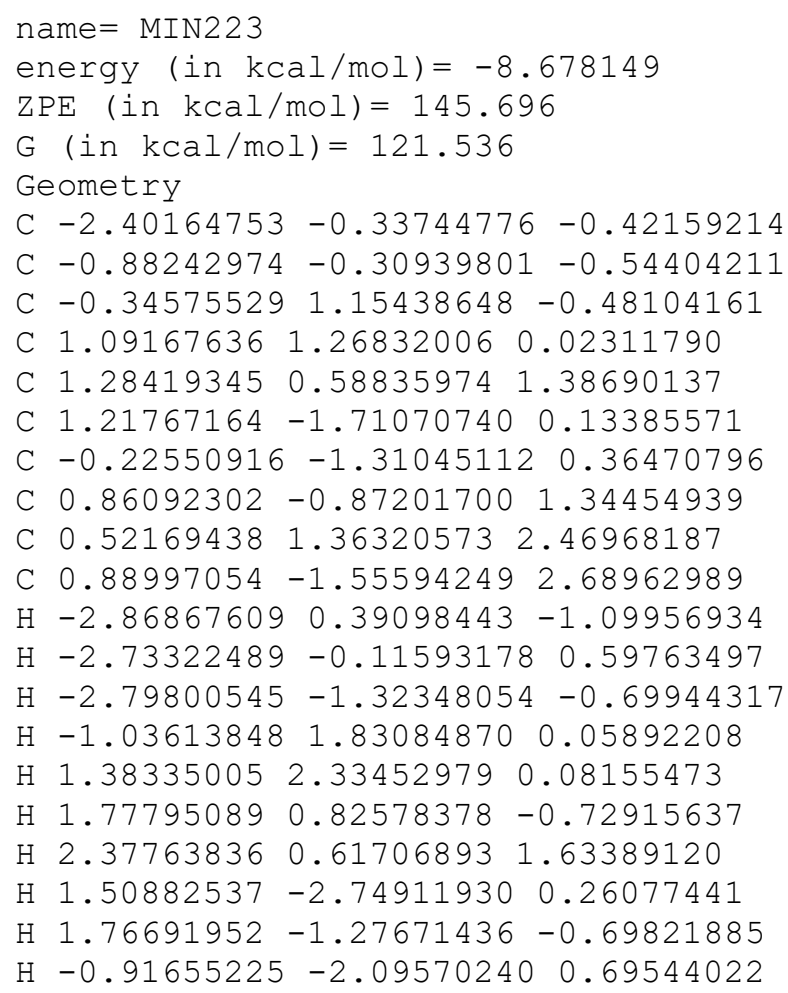


$\mathrm{H}-0.55979570 \quad 1.21463999 \quad 2.38262955$

$\mathrm{H} \quad 0.71717381 \quad 2.43923307 \quad 2.39955758$

$\mathrm{H} 0.81960047 \quad 1.04617043 \quad 3.47500954$

$\mathrm{H} \quad 1.84642869-1.38670764 \quad 3.20130672$

$\mathrm{H} \quad 0.75774276-2.64201151 \quad 2.60735944$

$\mathrm{H} 0.09065650-1.18422023 \quad 3.34413342$

$\begin{array}{llll}0 & -0.79536656 & 0.47833732 & -2.63035889\end{array}$

$0-0.322122391 .58925237-1.84055335$

o $-0.50343010-0.67312235-1.88541648$

Vibrational frequencies (in $\mathrm{cm}-1$ )

$\begin{array}{llllllllllllll}41.5 & 110.6 & 129.5 & 150.9 & 174.2 & 180.9 & 211.7 & 251.2 & 274.9 & 288.8 & 320.3 & 344.5 & 378.5 & 391.9\end{array}$

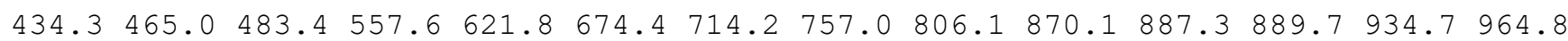
$972.5 \quad 978.4 \quad 998.1 \quad 1000.9 \quad 1025.6 \quad 1054.2 \quad 1057.4 \quad 1077.4 \quad 1097.5 \quad 1110.2 \quad 1125.5 \quad 1127.1 \quad 1154.2$ $1170.51177 .9 \quad 1203.3 \quad 1212.8 \quad 1241.0 \quad 1273.4 \quad 1277.9 \quad 1286.2 \quad 1289.0 \quad 1290.4 \quad 1292.2 \quad 1296.0$

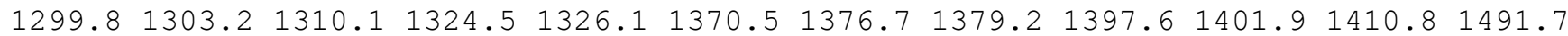
$2656.32668 .5 \quad 2673.92674 .3 \quad 2675.7 \quad 2677.1 \quad 2680.62691 .12699 .5 \quad 2708.2 \quad 2742.5 \quad 2749.4$ $2780.2 \quad 2780.7 \quad 2781.8 \quad 2784.5$

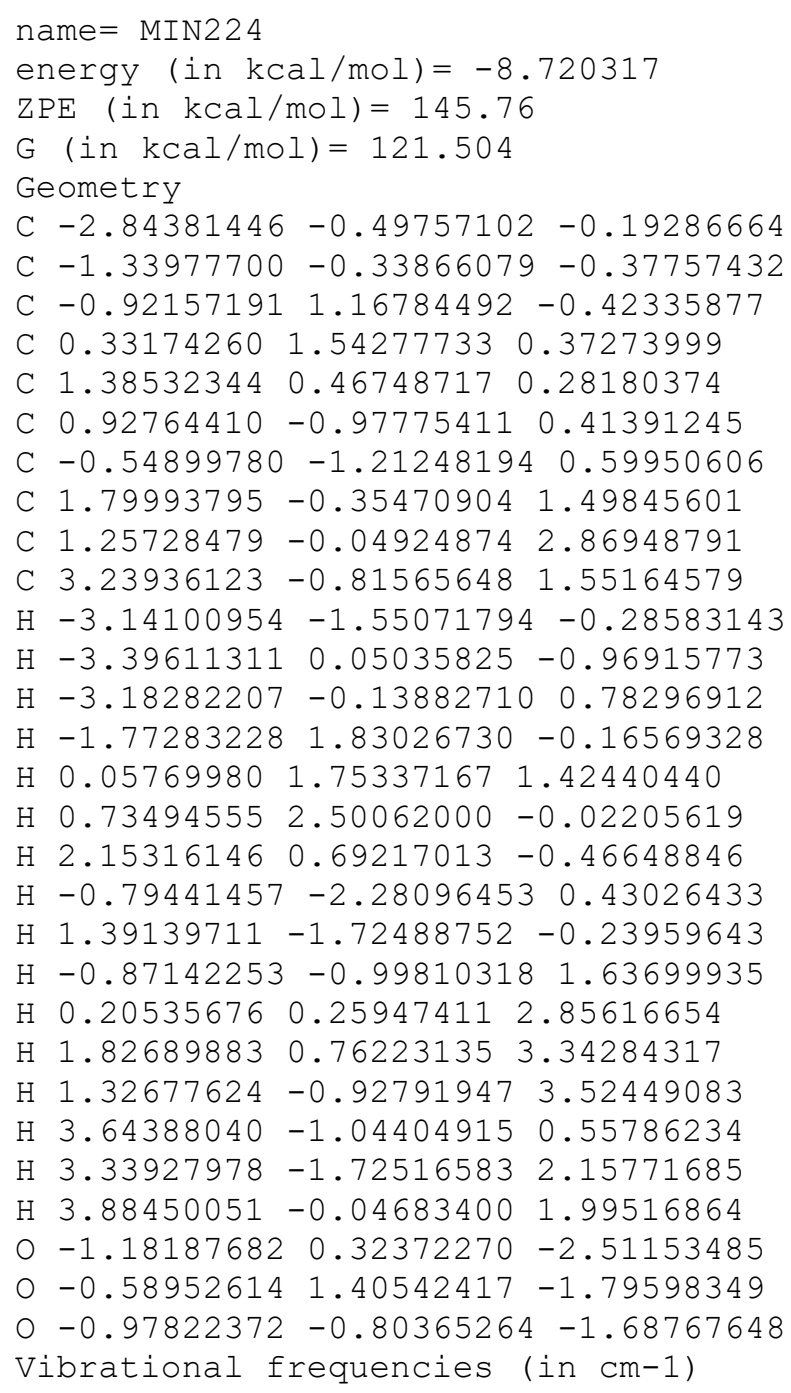


$\mathrm{ZPE}($ in $\mathrm{kcal} / \mathrm{mol})=144.086$

$\mathrm{G}($ in $\mathrm{kcal} / \mathrm{mol})=118.129$

Geometry

C $-2.76770851-0.78563100 \quad 0.13503520$

C $-1.27732233-0.75293482 \quad 0.11584932$

C $-0.69760053 \quad 0.62790880 \quad 0.26241083$

C $0.80203244 \quad 0.70299056 \quad 0.15109189$

C $1.62419741-0.56051895-0.01562307$

C $0.94804107-1.90925232-0.06857638$

C $-0.54323301-1.86185623-0.02235144$

C $1.70188220 \quad 0.23051133 \quad 1.28755164$

C $2.96049268 \quad 1.02660661 \quad 1.54062573$

C $1.10735065-0.319748462 .55603972$

$\mathrm{H}-3.17811791-1.799807210 .05927774$

$\mathrm{H}-3.17629194-0.20407831-0.71227486$

$\mathrm{H}-3.17295241-0.33632778 \quad 1.05223977$

H -1.044852551 .093284951 .22386036$

H $1.12240114 \quad 1.59885904-0.39893861$

$\mathrm{H}-0.82658708-0.46398830-2.32484263$

H 2.49467852 $-0.50960606-0.68049302$

H $1.32225725 \quad-2.53895048 \quad 0.77211244$

H $1.26302277-2.44745112-0.99120740$

$\mathrm{H}-1.01730273-2.84044790-0.10936824$

H $3.72528492 \quad 0.409580362 .02959680$

H $2.75858864 \quad 1.88485422 \quad 2.19501765$

$\mathrm{H} \quad 3.40326657 \quad 1.41996636 \quad 0.61770109$

$\mathrm{H} \quad 0.19838903-0.90892460 \quad 2.37484585$

$\mathrm{H} 0.84084562 \quad 0.48690367 \quad 3.25128583$

$\mathrm{H} \quad 1.81889004 \quad-0.97882074 \quad 3.07095397$

$\begin{array}{lllll}0 & -0.74809497 & 1.37077438 & -1.93985301\end{array}$

$\begin{array}{llll}0 & -1.32059177 & 1.50346879 & -0.68168084\end{array}$

$\begin{array}{llll}0 & -1.36131417 & 0.31768082 & -2.62204630\end{array}$

Vibrational frequencies (in $\mathrm{cm}-1$ )

$\begin{array}{lllllllllllllll}38.4 & 50.0 & 100.1 & 111.1 & 115.5 & 126.6 & 159.1 & 179.2 & 199.8 & 229.7 & 256.6 & 260.5 & 348.6 & 376.6\end{array}$ $405.2427 .0 \quad 456.8 \quad 496.6 \quad 504.6 \quad 510.8 \quad 535.8 \quad 694.2 \quad 761.4 \quad 765.3 \quad 810.0 \quad 829.6 \quad 867.3 \quad 902.2$

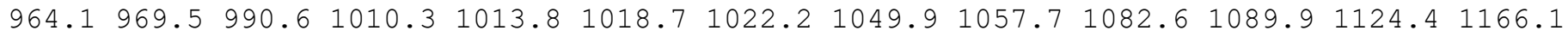

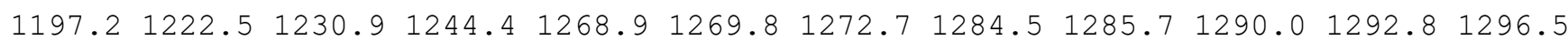

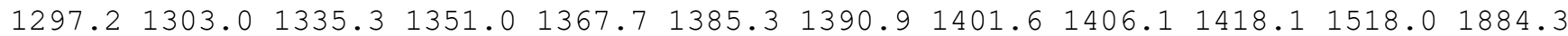
$2651.52656 .62669 .42670 .5 \quad 2675.0 \quad 2676.6 \quad 2677.5 \quad 2679.52732 .6 \quad 2739.6 \quad 2749.92751 .5$ $2776.7 \quad 2780.12782 .2 \quad 2827.4$

\section{name $=$ MIN226}

energy (in kcal/mol) $=-7.852061$

$\mathrm{ZPE}(\mathrm{in} \mathrm{kcal} / \mathrm{mol})=145.313$

$\mathrm{G}(\mathrm{in} \mathrm{kcal} / \mathrm{mol})=120.741$

Geometry

C $-2.45990068-0.21280091-0.88760221$

C $-0.94457534-0.20320758-0.91576998$

C $-0.29556267 \quad 1.23296826 \quad-0.92704858$

C $0.71196342 \quad 1.31711175 \quad 0.22918236$

C $0.65846863-0.01554724 \quad 0.96964690$

C $1.17955661-1.20116222 \quad 0.18497344$

C $-0.31094913-0.94735796 \quad 0.23842061$

C $0.73969937 \quad 0.08248032 \quad 2.47697868$

C $-0.59872472 \quad 0.58874045 \quad 3.03718812$

C $1.09977199-1.249646913 .14537141$

$\mathrm{H}-2.88099817 \quad 0.36927390 \quad-1.71934894$

$\mathrm{H}-2.84475459 \quad 0.20391166 \quad 0.05062108$

$\mathrm{H}-2.85217033-1.23493331-0.98470548$

$\mathrm{H}-1.02994638 \quad 2.05659577 \quad-0.92971637$

$\mathrm{H} \quad 0.47689091 \quad 2.16290967 \quad 0.89679811$

H $1.730416931 .51135234-0.15958466$

$\mathrm{H} \quad 1.54057550 \quad 0.82356510 \quad 2.73382506$

H $1.58623155 \quad-2.06746984 \quad 0.69696239$ 
$\mathrm{H} \quad 1.72393901-1.03560743-0.74331184$

$\mathrm{H}-0.95166811-1.647691550 .76485862$

$\mathrm{H}-1.40990247-0.115589612 .82116495$

$\mathrm{H}-0.87915006 \quad 1.55397851 \quad 2.60363169$

$\mathrm{H}-0.55096869 \quad 0.71510304 \quad 4.12367796$

H $0.37774559-2.03503537 \quad 2.89676908$

H 2.09361345 -1.59520721 2.83951349

H $1.11155765-1.15068360 \quad 4.23662593$

O $0.59979519-0.03849231-2.55448269$

$\begin{array}{lllll}0 & 0.33592817 & 1.31571963 & -2.19558857\end{array}$

$0-0.57449459-0.74124264-2.18894270$

Vibrational frequencies (in $\mathrm{cm}-1$ )

$\begin{array}{lllllllllllllll}46.2 & 77.0 & 94.5 & 147.3 & 150.4 & 186.1 & 207.0 & 211.0 & 262.1 & 281.5 & 316.6 & 335.0 & 380.4 & 417.8\end{array}$

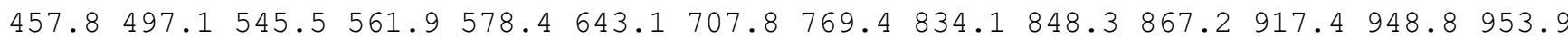
$974.8 \quad 979.3 \quad 983.2 \quad 1002.2 \quad 1005.8 \quad 1011.2 \quad 1030.4 \quad 1042.9 \quad 1079.6 \quad 1124.8 \quad 1134.1 \quad 1146.7 \quad 1167.5$

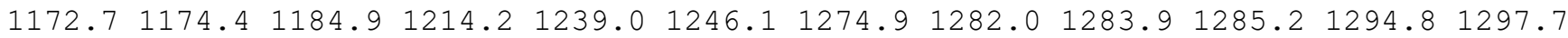

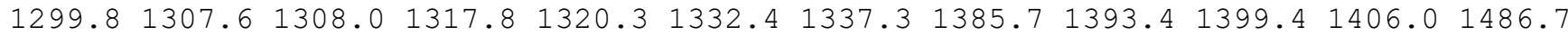
$2665.62670 .5 \quad 2672.1 \quad 2676.7 \quad 2677.0 \quad 2677.7 \quad 2680.72700 .52704 .42707 .52746 .82770 .6$ $2780.32781 .0 \quad 2782.6 \quad 2783.4$

name $=$ MIN227

energy (in $\mathrm{kcal} / \mathrm{mol})=-8.044299$

$\operatorname{ZPE}($ in $\mathrm{kcal} / \mathrm{mol})=145.796$

$\mathrm{G}($ in $\mathrm{kcal} / \mathrm{mol})=121.994$

Geometry

C $-2.276722120 .12154973-0.68932745$

C $-0.76364746-0.08683951-0.65324534$

C $-0.00297131 \quad 1.28008446-0.63279034$

C $1.18147292 \quad 1.33717528 \quad 0.33008101$

C $0.71773197 \quad 0.90534182 \quad 1.69612129$

C $0.79489432-1.99000952-0.03476546$

C $-0.36455777-1.11020813 \quad 0.43888915$

C $-0.08476597-0.393885691 .75325719$

C $-1.23147386-0.474265892 .73034792$

C $1.31700211-0.326750252 .33283033$

$\mathrm{H}-2.56124075 \quad 0.81700449-1.49121657$

$\mathrm{H}-2.65153542 \quad 0.51870005 \quad 0.25915517$

$\mathrm{H}-2.80232834-0.82044812-0.89469263$

$\mathrm{H}-0.68974605 \quad 2.13897696-0.48718299$

$\mathrm{H} \quad 1.61066720 \quad 2.357171890 .35122435$

$\mathrm{H} \quad 2.001249210 .69070355-0.04719363$

H $0.42452222 \quad 1.73470755 \quad 2.34387147$

$\mathrm{H} \quad 0.49578704 \quad-2.59956125 \quad-0.89855700$

$\mathrm{H} \quad 1.65941144-1.39711796-0.35358312$

$\mathrm{H}-1.23908260-1.79728138 \quad 0.59432342$

$\mathrm{H}-1.42877970-1.51371828 \quad 3.02447035$

$\mathrm{H}-2.15558056-0.06782756 \quad 2.29993248$

$\mathrm{H}-1.03267383 \quad 0.08717707 \quad 3.65181412$

H $2.14034962-0.843206691 .85024624$

$\mathrm{H} 1.11946647-2.67595804 \quad 0.75560779$

$\mathrm{H} \quad 1.44693020-0.363968893 .41033019$

$0.69645574 \quad 0.08608349-2.38896575$

$0.459472211 .42118507-1.97481165$

$0-0.45275206-0.62283824-1.95056916$

Vibrational frequencies (in $\mathrm{cm}-1$ )

$\begin{array}{llllllllllllllll}54.9 & 105.6 & 136.7 & 170.1 & 183.8 & 213.9 & 227.5 & 252.6 & 260.5 & 304.4 & 316.8 & 347.4 & 407.5 & 423.9\end{array}$

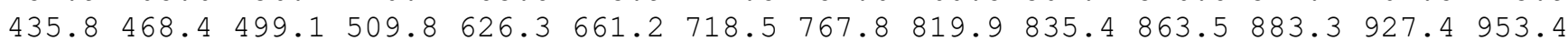
$969.7 \quad 976.2 \quad 990.2 \quad 1009.9 \quad 1041.5 \quad 1060.4 \quad 1077.6 \quad 1082.3 \quad 1093.2 \quad 1103.2 \quad 1145.8 \quad 1149.6 \quad 1159.2$ $1169.01184 .6 \quad 1197.5 \quad 1224.2 \quad 1233.2 \quad 1256.1 \quad 1260.5 \quad 1283.91285 .0 \quad 1292.2 \quad 1292.6 \quad 1296.1$

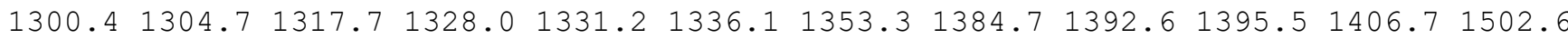
$2658.22664 .52669 .02673 .8 \quad 2675.0 \quad 2676.8 \quad 2677.82691 .52691 .92712 .72745 .12761 .2$ $2778.0 \quad 2779.3 \quad 2780.3 \quad 2788.1$ 
name $=$ MIN228

energy (in $\mathrm{kcal} / \mathrm{mol})=-7.498017$

$\mathrm{ZPE}(\mathrm{in} \mathrm{kcal} / \mathrm{mol})=145.28$

$\mathrm{G}($ in $\mathrm{kcal} / \mathrm{mol})=120.632$

Geometry

C $-2.54715084-0.34886527-1.09141188$

C $-1.03610738-0.28089527-0.99731529$

C $-0.446431751 .17936301-0.98396337$

C $0.42411473 \quad 1.33598079 \quad 0.27085777$

C $0.38298404 \quad 0.00880033 \quad 1.02139898$

C $1.02945274-1.15713080 \quad 0.30628421$

C $-0.47149154-0.97820691 \quad 0.22032577$

C $0.31207365 \quad 0.11302566 \quad 2.52915944$

C $1.47695101 \quad 0.95885418 \quad 3.06380119$

C $0.32646833-1.25661899 \quad 3.22075137$

$\mathrm{H}-2.92050010 \quad 0.20475943-1.96418784$

$\mathrm{H}-3.021495320 .06824988-0.19479693$

$\mathrm{H}-2.89253375-1.38618172-1.19997987$

$\mathrm{H}-1.207037421 .97259801-1.08844589$

$\mathrm{H} \quad 0.05946760 \quad 2.16673671 \quad 0.89867030$

$\mathrm{H} 1.46332406 \quad 1.59739420 \quad-0.00891685$

$\mathrm{H} \quad 1.42849317 \quad 1.99220868 \quad 2.70562425$

H $1.43030972-1.99255580 \quad 0.87200126$

$\mathrm{H} \quad 1.64846948-0.97754467-0.57077428$

$\mathrm{H}-1.12259011-1.70559699 \quad 0.69452952$

$\mathrm{H}-0.65159963 \quad 0.62345280 \quad 2.78994226$

$\mathrm{H} \quad 1.46960940 \quad 0.99355455 \quad 4.15866410$

$\begin{array}{lllll}\mathrm{H} & 2.44292664 & 0.54611934 & 2.75145153\end{array}$

$\mathrm{H} \quad 0.16473047 \quad-1.15193517 \quad 4.29941588$

H $1.28813924 \quad-1.76496864 \quad 3.08528547$

$\mathrm{H}-0.45784966-1.91527894 \quad 2.83456269$

$00.64330684-0.09470020-2.49099946$

o $0.312286601 .25784592-2.18122011$

o $-0.54074294-0.82390763-2.22444112$

Vibrational frequencies (in $\mathrm{cm}-1$ )

$\begin{array}{llllllllllllllll}39.6 & 79.0 & 98.0 & 151.2 & 157.1 & 182.5 & 189.4 & 228.6 & 254.6 & 289.1 & 325.4 & 336.0 & 356.0 & 411.4\end{array}$

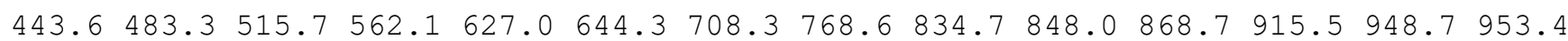
$976.0 \quad 979.9982 .2 \quad 1002.1 \quad 1003.4 \quad 1011.1 \quad 1033.2 \quad 1050.1 \quad 1073.1 \quad 1122.4 \quad 1130.7 \quad 1147.7 \quad 1164.7$

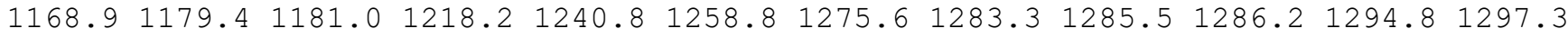
$1298.71304 .7 \quad 1308.41313 .7 \quad 1321.0 \quad 1332.2 \quad 1338.3 \quad 1384.4 \quad 1393.8 \quad 1400.91406 .7 \quad 1483.2$ $2665.82670 .92671 .5 \quad 2676.3 \quad 2676.92677 .2 \quad 2680.02699 .42703 .12708 .32746 .42768 .9$ $2780.6 \quad 2780.92782 .4 \quad 2783.9$

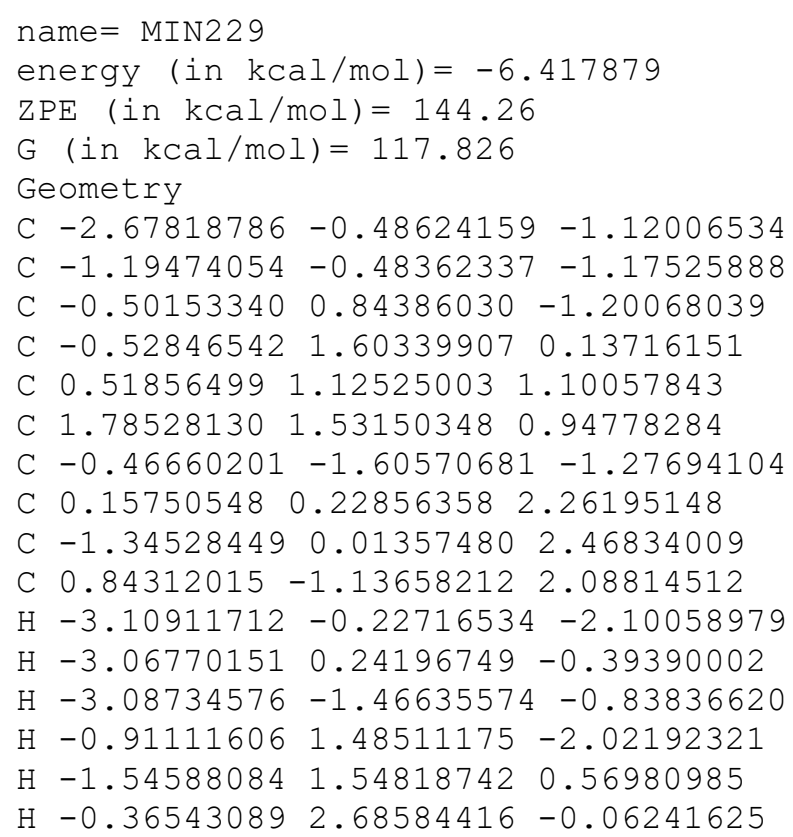


$\mathrm{H} \quad 0.56207973 \quad 0.70918771 \quad 3.19372118$

$\mathrm{H} 2.590190551 .25020434 \quad 1.60608114$

H $2.11416176 \quad 2.16934410 \quad 0.14334229$

$\mathrm{H}-0.82159812-2.62675286-1.33680918$

$\mathrm{H}-1.80140888-0.47484612 \quad 1.59899829$

$\mathrm{H}-1.86776892 \quad 0.95817747 \quad 2.64966114$

$\mathrm{H}-1.53004969-0.63113198 \quad 3.33558180$

$\mathrm{H} 1.93104874 \quad-1.03159438 \quad 2.00711937$

$\mathrm{H} \quad 0.49646769-1.63889128 \quad 1.17729690$

$\mathrm{H} \quad 0.63444642-1.797548192 .93472537$

O $1.41568403-0.35855593-0.91957365$

$\begin{array}{lllll}0 & 0.84197939 & 0.65963267 & -1.65772987\end{array}$

O $0.89309522-1.60944463-1.38618201$

Vibrational frequencies (in cm-1)

$\begin{array}{llllllllllllllll}25.1 & 42.1 & 62.9 & 81.9 & 88.0 & 153.1 & 183.0 & 196.1 & 228.1 & 246.9 & 274.2 & 312.2 & 337.9 & 362.5\end{array}$ $381.6 \quad 429.3 \quad 463.8 \quad 481.6 \quad 499.6 \quad 545.0 \quad 600.1 \quad 639.3 \quad 715.2 \quad 770.6 \quad 795.9 \quad 834.0 \quad 897.1 \quad 909.0$

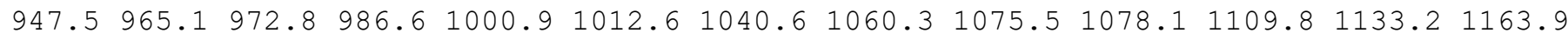

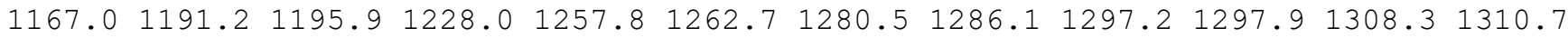

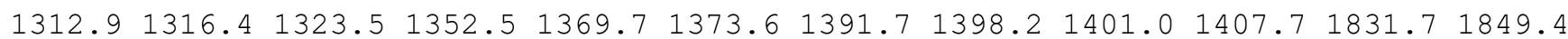
$2653.92665 .52667 .82672 .5 \quad 2674.02677 .12677 .72681 .12697 .52723 .12737 .42739 .6$ $2777.4 \quad 2779.4 \quad 2782.2 \quad 2790.5$

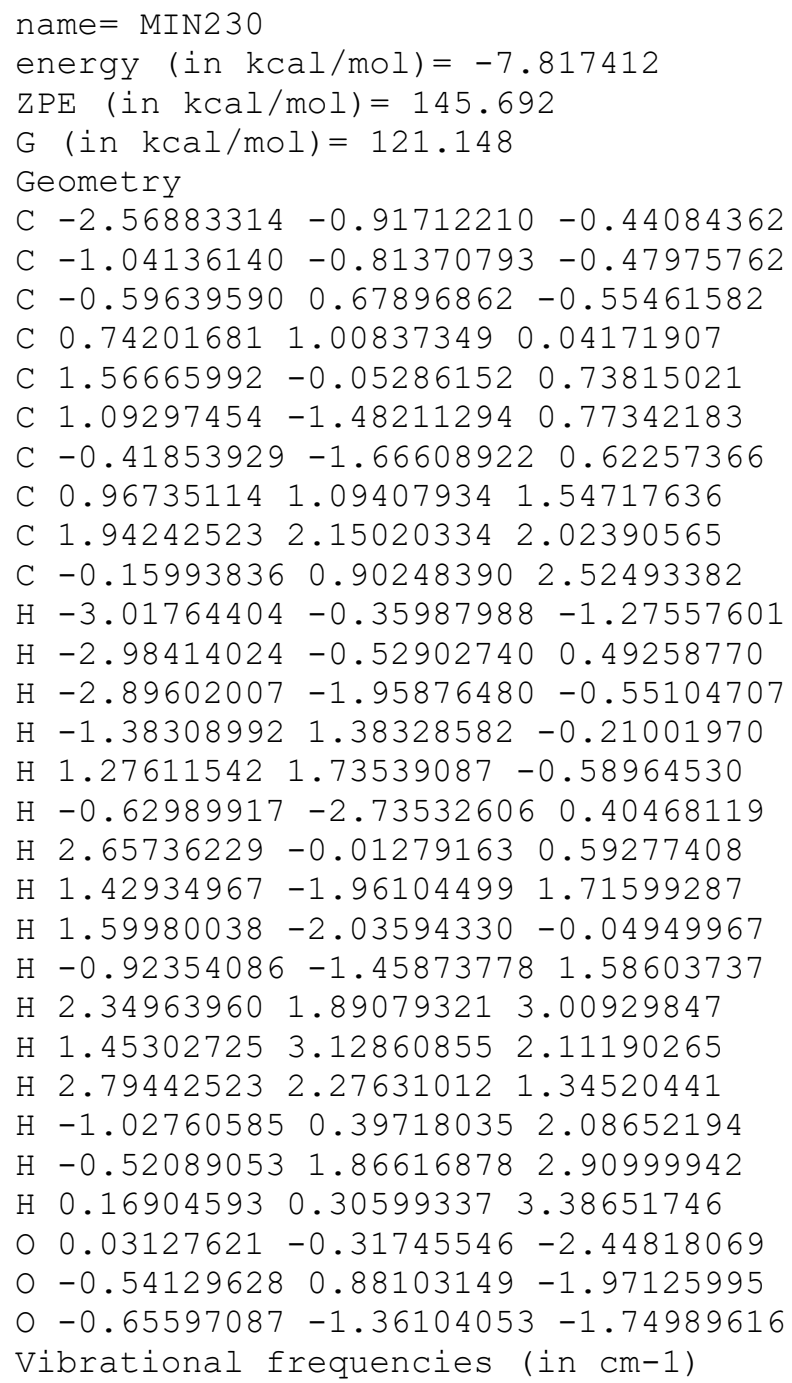


name $=$ MIN231

energy (in $\mathrm{kcal} / \mathrm{mol})=-7.276647$

$\mathrm{ZPE}(\mathrm{in} \mathrm{kcal} / \mathrm{mol})=145.166$

$\mathrm{G}($ in $\mathrm{kcal} / \mathrm{mol})=120.058$

Geometry

C $-2.52258613-0.37401750-0.64881077$

C $-1.01316552-0.33583947-0.78417585$

C $-0.397219401 .10113719-0.97932049$

C $0.79631052 \quad 1.24436699-0.02183482$

C $0.78249093 \quad 0.02273400 \quad 0.89127944$

C $1.18522373-1.27262364 \quad 0.21403581$

C $-0.28299159-0.95356813 \quad 0.38748264$

C $1.05854013 \quad 0.28487480 \quad 2.35561952$

C $0.05347012 \quad 1.29957324 \quad 2.92358846$

C $1.00434838-0.991183153 .20656298$

$\mathrm{H}-3.012693190 .12955698-1.49391553$

$\mathrm{H}-2.85747870 \quad 0.10554436 \quad 0.27759968$

$\mathrm{H}-2.89029295-1.41026007-0.64145549$

$\mathrm{H}-1.13088997 \quad 1.92023482-0.89966170$

$\mathrm{H} \quad 0.74441756 \quad 2.18865396 \quad 0.54338217$

H $1.74237375 \quad 1.29342578 \quad-0.59692350$

$\mathrm{H} 2.09048396 \quad 0.71514538 \quad 2.43484086$

H $1.62374314 \quad-2.08176188 \quad 0.78916566$

$\mathrm{H} 1.62701342-1.24798937-0.78084642$

$\mathrm{H}-0.88453334-1.55720443 \quad 1.05980347$

$\mathrm{H}-0.97783659 \quad 0.97789160 \quad 2.74280385$

$\mathrm{H} 0.17568816 \quad 2.29061929 \quad 2.47458382$

$\mathrm{H} 0.17954506 \quad 1.41830481 \quad 4.00505177$

$\mathrm{H} 1.21600513-0.770004724 .25883616$

$\mathrm{H} 1.74070561-1.73168742 \quad 2.87741540$

H $0.01483733-1.45953886 \quad 3.16492307$

$00.33711416-0.25084051-2.60513958$

o $0.01239964 \quad 1.10811020 \quad-2.33795376$

o $-0.72324624-0.99421712-2.02216722$

Vibrational frequencies (in $\mathrm{cm}-1$ )

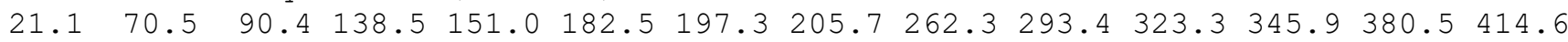
$470.1 \quad 487.1 \quad 546.6 \quad 562.3 \quad 565.2 \quad 642.5 \quad 709.0 \quad 772.4 \quad 831.8 \quad 848.2 \quad 866.9 \quad 913.4 \quad 950.0 \quad 953.5$

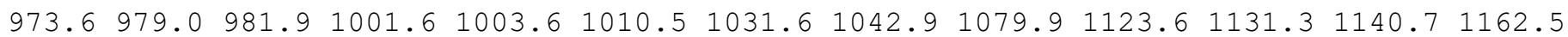

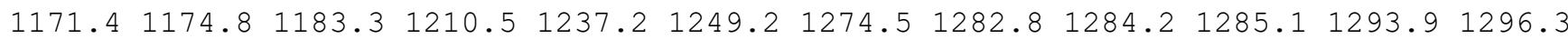
$1298.31303 .7 \quad 1308.1 \quad 1315.6 \quad 1321.4 \quad 1330.3 \quad 1336.3 \quad 1382.21393 .2 \quad 1398.91407 .11480 .0$ $2662.02670 .02671 .6 \quad 2674.4 \quad 2676.8 \quad 2678.8 \quad 2679.4 \quad 2703.22704 .52707 .72745 .22769 .1$ $2780.4 \quad 2780.4 \quad 2782.1 \quad 2782.8$

name $=$ MIN232

energy (in $\mathrm{kcal} / \mathrm{mol})=-8.005261$

$\mathrm{ZPE}(\mathrm{in} \mathrm{kcal} / \mathrm{mol})=145.935$

$\mathrm{G}($ in $\mathrm{kcal} / \mathrm{mol})=122.542$

Geometry

C $-2.30320941-0.20913289-0.36201418$

C $-0.79650103-0.22117653-0.55220249$

C $-0.156274851 .21995437-0.58364896$

C $1.11837782 \quad 1.42210040 \quad 0.26138163$

C $1.51866428 \quad 0.156822101 .02075067$

C $1.46671403-1.044290690 .02784222$

C $-0.05006689-1.163199920 .38896861$

C $0.27688969-0.464302091 .76804051$

C $-0.71959446 \quad 0.48325811 \quad 2.40303657$

C $0.69466757-1.49441405 \quad 2.81576164$

$\mathrm{H}-2.789980550 .46122461-1.08328402$

$\mathrm{H}-2.59964557 \quad 0.09717780 \quad 0.64525733$

$\mathrm{H}-2.72023291-1.21182284-0.53467381$

$\mathrm{H}-0.91692722 \quad 1.99509745-0.37132329$ 
$\mathrm{H} \quad 0.98079308 \quad 2.26325340 \quad 0.96361226$

H $1.95055024 \quad 1.72423396-0.41161447$

H 2.42623448 $0.27543776 \quad 1.61279947$

H $2.06845846-1.906296250 .31802298$

$\mathrm{H} \quad 1.69249543-0.81388497-1.01712591$

$\begin{array}{lllll}\mathrm{H} & -0.47832910 & -2.16361244 & 0.44057084\end{array}$

$\mathrm{H}-1.60223235-0.057842962 .76754978$

$\mathrm{H}-1.07522284 \quad 1.26777179 \quad 1.72963983$

$\mathrm{H}-0.26977607 \quad 0.99351213 \quad 3.26562203$

$\mathrm{H} 1.14875103-1.00851718 \quad 3.68696434$

H $1.42493618-2.214146532 .42999198$

$\mathrm{H}-0.16962473-2.06901889 \quad 3.16894534$

$\begin{array}{llll}0 & -0.52151559 & 0.45203969 & -2.67820892\end{array}$

○ $0.26244555 \quad 1.38354048-1.94317119$

o $-0.52723425-0.71242410-1.87918488$

Vibrational frequencies (in $\mathrm{cm}-1$ )

$82.6116 .6 \quad 148.8 \quad 163.3 \quad 174.8 \quad 203.2 \quad 226.8 \quad 267.5 \quad 279.4 \quad 323.2 \quad 352.5 \quad 377.8 \quad 414.2 \quad 432.4$ $451.4 \quad 490.9 \quad 529.6 \quad 602.8 \quad 632.0 \quad 710.8 \quad 718.0 \quad 793.3 \quad 834.5 \quad 874.5 \quad 891.8 \quad 916.7 \quad 936.7 \quad 952.0$ $981.3995 .6 \quad 1001.7 \quad 1010.21013 .7 \quad 1016.8 \quad 1053.6 \quad 1069.2 \quad 1083.8 \quad 1090.7 \quad 1103.4 \quad 1134.2$ $1158.11170 .0 \quad 1185.1 \quad 1193.1 \quad 1216.3 \quad 1227.3 \quad 1247.7 \quad 1259.01271 .5 \quad 1277.2 \quad 1283.4 \quad 1285.9$ $1289.41294 .0 \quad 1298.8 \quad 1301.5 \quad 1308.5 \quad 1316.0 \quad 1321.1 \quad 1324.4 \quad 1373.8 \quad 1378.9 \quad 1398.2 \quad 1406.5$ $1417.22656 .32670 .0 \quad 2672.92674 .8 \quad 2676.8 \quad 2678.72680 .22683 .5 \quad 2686.62737 .92748 .0$ $2751.5 \quad 2763.9 \quad 2780.8 \quad 2782.2 \quad 2783.9$

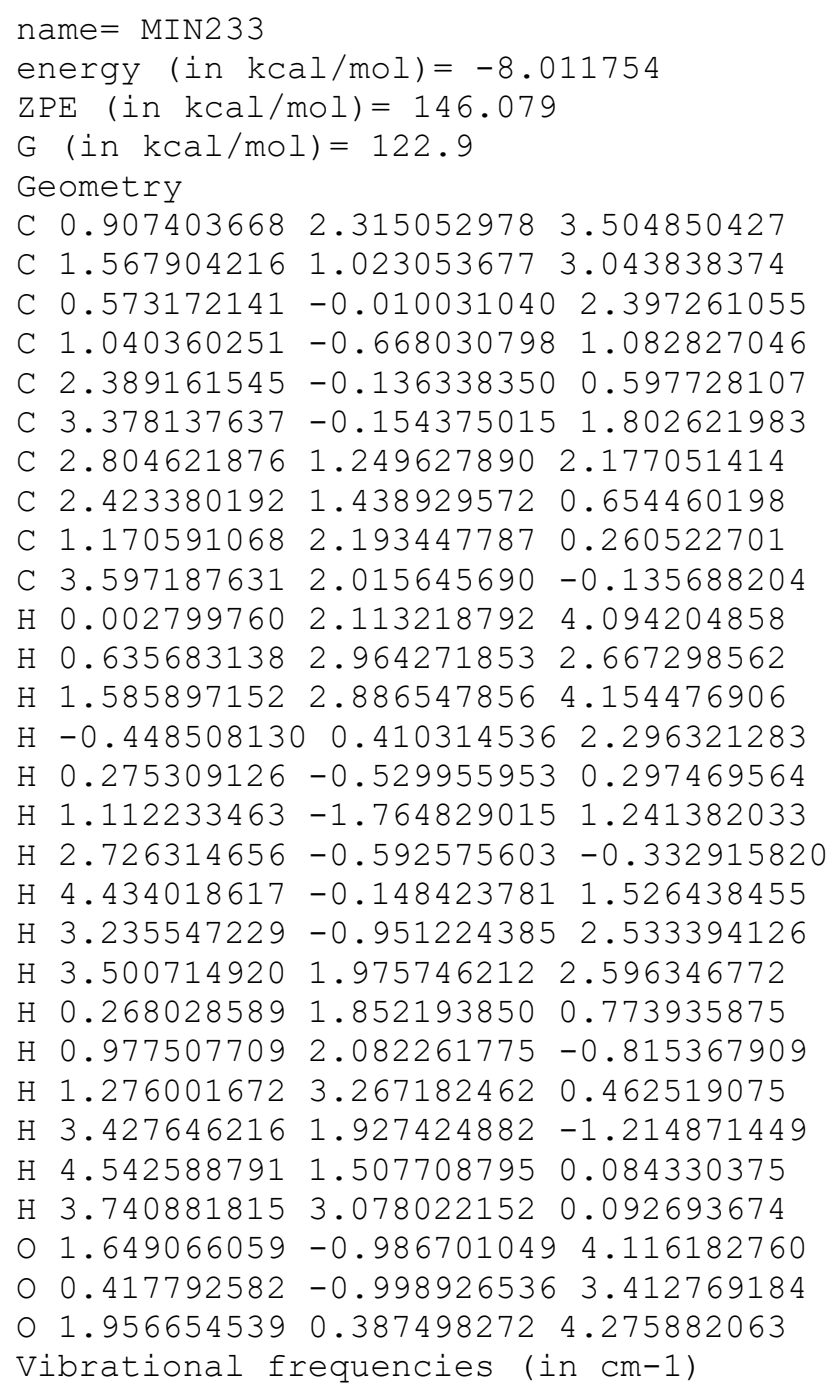


$1417.22659 .0 \quad 2669.8 \quad 2672.92674 .5 \quad 2676.5 \quad 2678.12680 .32686 .02689 .2 \quad 2740.02748 .3$ $2751.82768 .12780 .5 \quad 2782.12784 .0$

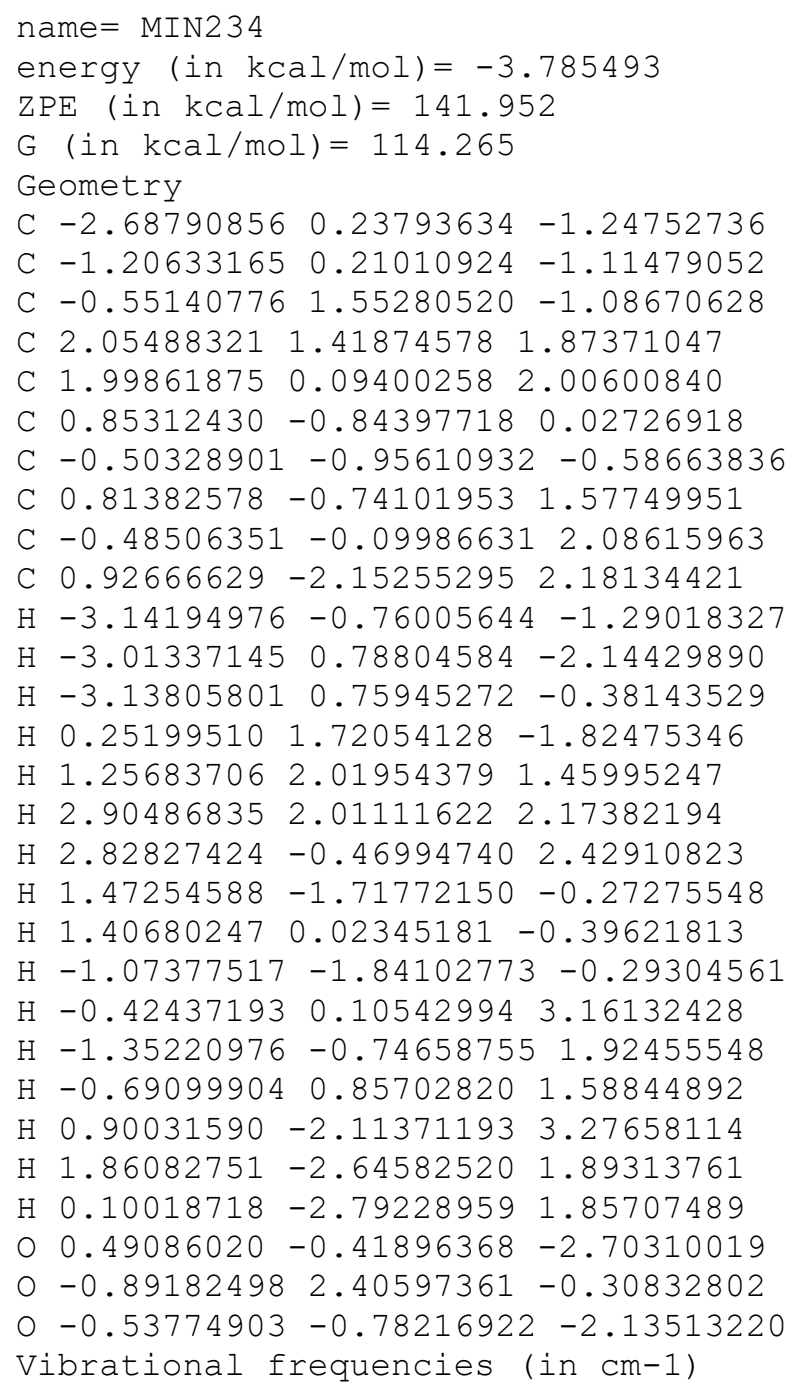


$\mathrm{H}-2.38375141-1.30693976-1.60626662$

$\mathrm{H}-1.14297914 \quad 1.87696841-0.65744596$

$\mathrm{H} 0.323441112 .40721095 \quad 1.05987030$

$\mathrm{H} \quad 1.64969279 \quad 2.03536247-0.04435689$

$\begin{array}{lllll}\mathrm{H} & 1.86656431 & 0.68120883 & 2.09143632\end{array}$

H $2.16223556-1.53059193 \quad 0.83159053$

$\mathrm{H} 1.96869178-0.50911876-0.58788590$

$\mathrm{H}-0.25637447-2.184571190 .34525549$

$\mathrm{H}-1.71659615 \quad 1.03801880 \quad 1.33813137$

$\mathrm{H}-1.196386110 .99054799 \quad 3.01656567$

$\mathrm{H}-2.19704159-0.31210005 \quad 2.36185080$

$\mathrm{H} \quad 0.34413000 \quad-0.81135125 \quad 3.85839448$

$\mathrm{H} 1.12855458-1.92793396 \quad 2.74244910$

$\mathrm{H}-0.59797447-2.08233692 \quad 3.06584000$

$\begin{array}{lllll}0 & 0.82939945 & 0.20081808 & -2.37072627\end{array}$

O $0.310050991 .45539653-1.95732643$

o $-0.17270578-0.73302214-2.01543204$

Vibrational frequencies (in $\mathrm{cm}-1$ )

$\begin{array}{lllllllllllllll}-11.0 & 96.3 & 141.3 & 163.9 & 174.2 & 209.8 & 226.7 & 265.4 & 289.2 & 322.1 & 353.8 & 382.9 & 416.8 & 439.4\end{array}$ $\begin{array}{lllllllllllllllllllll}462.9 & 492.7 & 519.1 & 593.8 & 654.5 & 702.0 & 719.6 & 799.7 & 843.7 & 870.3 & 894.7 & 913.2 & 937.4 & 955.1\end{array}$ $980.7997 .2 \quad 1002.51006 .1 \quad 1014.4 \quad 1016.6 \quad 1044.2 \quad 1059.1 \quad 1087.5 \quad 1089.6 \quad 1099.61141 .7$

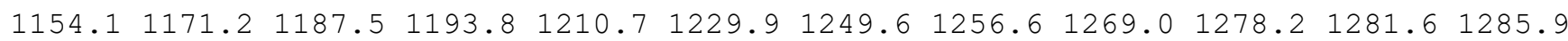
$1289.81294 .0 \quad 1299.0 \quad 1301.0 \quad 1305.3 \quad 1310.3 \quad 1319.4 \quad 1322.2 \quad 1373.6 \quad 1384.2 \quad 1405.6 \quad 1409.3$ $1418.82658 .7 \quad 2662.5 \quad 2673.6 \quad 2674.4 \quad 2675.0 \quad 2678.42680 .42684 .2 \quad 2689.42739 .62748 .0$ $\begin{array}{llllll}2751.5 & 2768.1 & 2777.2 & 2781.8 & 2784.5\end{array}$

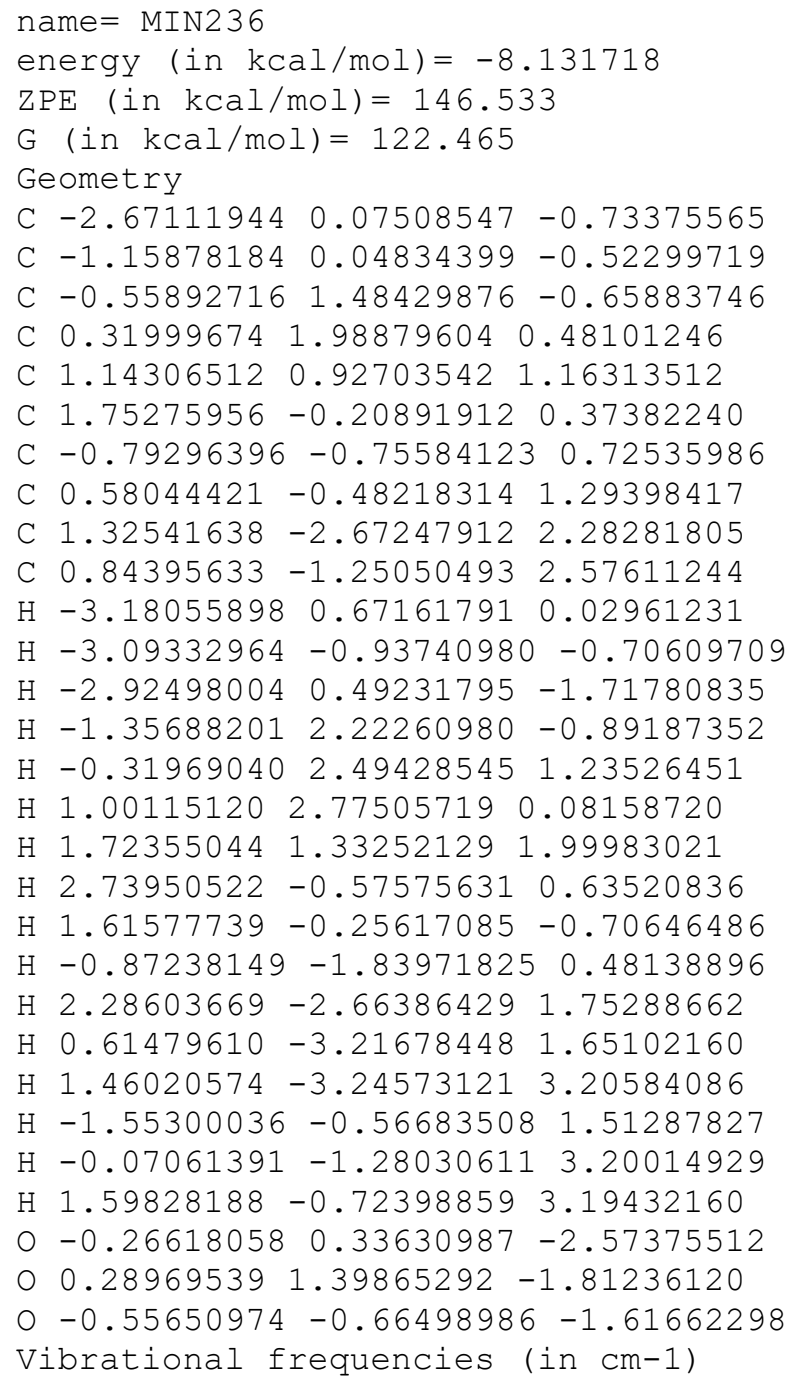




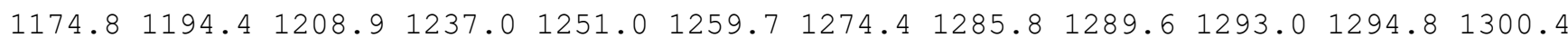

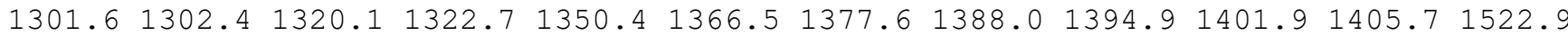
$2655.32657 .32657 .72670 .4 \quad 2675.12680 .0 \quad 2680.32683 .62705 .5 \quad 2733.12734 .62743 .4$ $2753.7 \quad 2781.4 \quad 2782.2 \quad 2783.9$

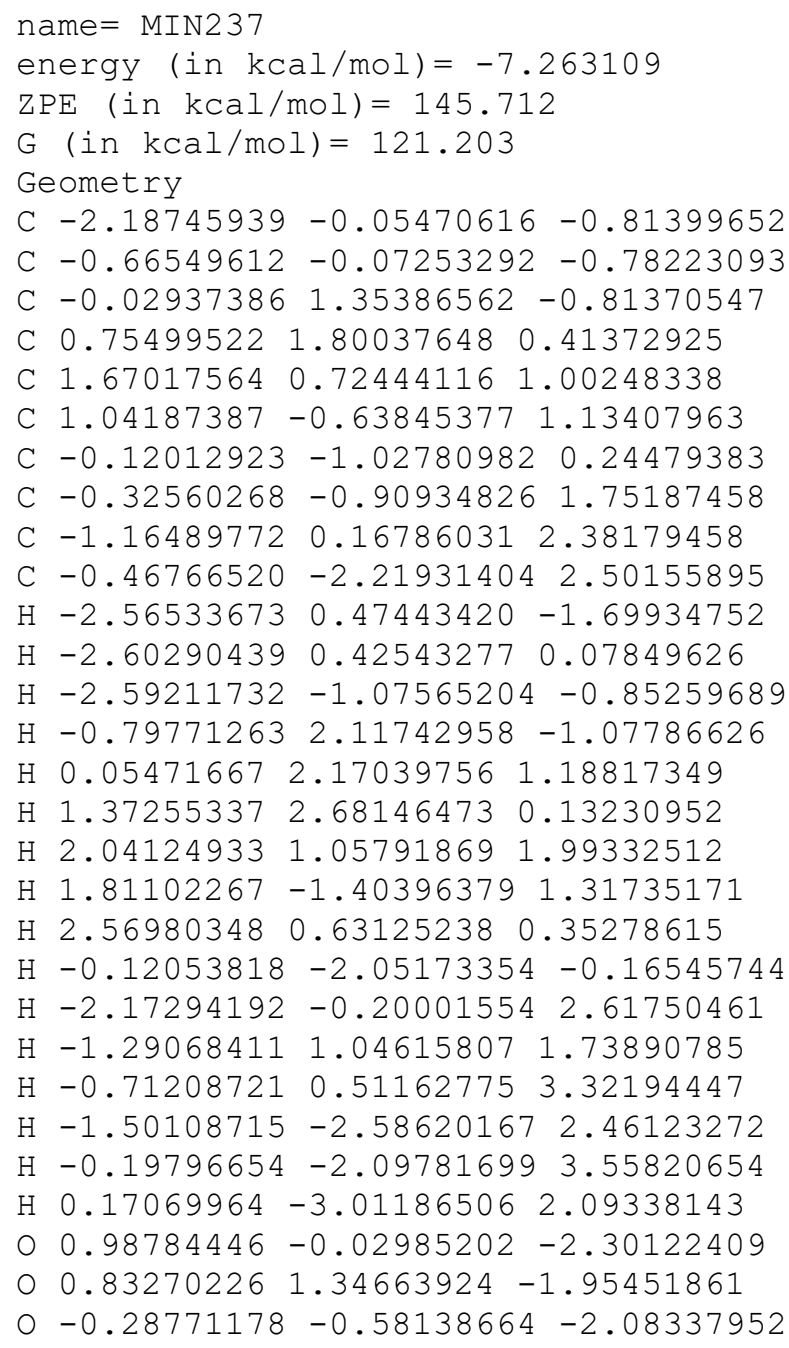
$425.8 \quad 484.5 \quad 523.6 \quad 549.1 \quad 635.0 \quad 682.8 \quad 705.2 \quad 781.1 \quad 824.3 \quad 827.5 \quad 862.7 \quad 890.0 \quad 938.1956 .7$ $974.4 \quad 999.0 \quad 1005.2 \quad 1008.9 \quad 1018.7 \quad 1019.2 \quad 1026.3 \quad 1074.4 \quad 1083.01111 .2 \quad 1151.11174 .7$

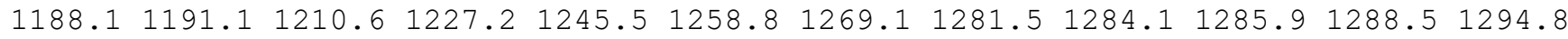
1297.61302 .31308 .51315 .91321 .41336 .01375 .51383 .31394 .81400 .31404 .01417 .3 $1530.02645 .92664 .6 \quad 2669.7 \quad 2670.7 \quad 2674.2 \quad 2674.72675 .5 \quad 2676.2 \quad 2678.8 \quad 2732.8 \quad 2733.9$ $2736.52745 .12778 .3 \quad 2780.42781 .6$

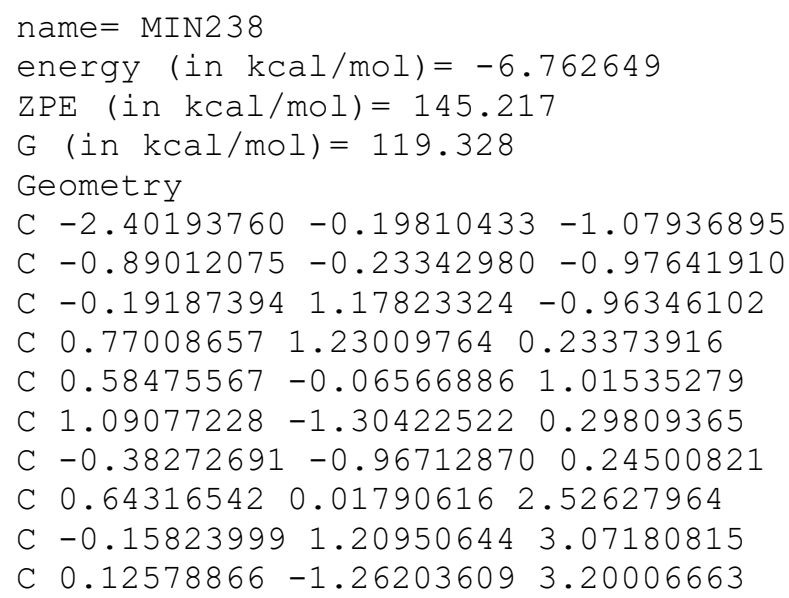


$\mathrm{H}-2.73143861 \quad 0.38463698-1.95097173$

$\mathrm{H}-2.854747390 .24041345 \quad-0.18272524$

$\mathrm{H}-2.81352125-1.21033476-1.20060784$

$\mathrm{H}-0.89535558 \quad 2.02735180 \quad-0.99914161$

$\mathrm{H} \quad 0.57776655 \quad 2.12250670 \quad 0.85112085$

H $1.815889001 .33052572-0.11734155$

H $1.71975725 \quad 0.15416240 \quad 2.80935225$

$\mathrm{H} \quad 1.41958212-2.169068490 .86499030$

$\mathrm{H} \quad 1.70138625-1.19952967-0.59694190$

$\mathrm{H}-1.09782138-1.61595104 \quad 0.74108862$

H $0.32084150 \quad 2.16550727 \quad 2.83559661$

$\mathrm{H}-0.243911631 .15921116 \quad 4.16325683$

$\mathrm{H}-1.17361504 \quad 1.22842132 \quad 2.66220983$

$\mathrm{H} \quad 0.26981003-1.21893347 \quad 4.28534947$

H $\quad 0.64664181-2.15501794 \quad 2.83972659$

$\mathrm{H}-0.94559833-1.40347218 \quad 3.01838092$

$00.76905973-0.13343343-2.51178962$

$\begin{array}{lllll}0 & 0.49602031 & 1.22991323 & -2.20372307\end{array}$

o $-0.43290672-0.81854090-2.20015753$

Vibrational frequencies (in cm-1)

$\begin{array}{lllllllllllllllll}-4.5 & 70.5 & 93.8 & 151.2 & 161.7 & 189.4 & 198.8 & 213.3 & 264.2 & 295.7 & 324.4 & 349.4 & 388.3 & 406.2\end{array}$

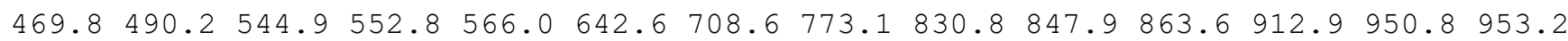

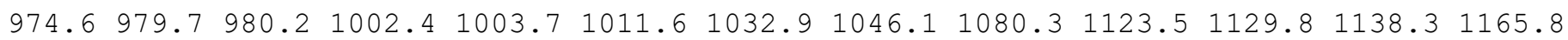

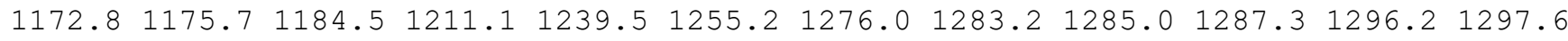

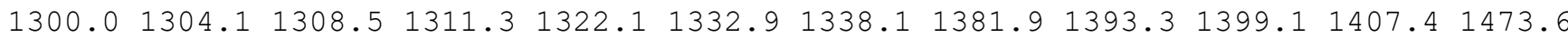
$2661.72670 .12671 .1 \quad 2673.3 \quad 2677.0 \quad 2678.0 \quad 2678.82701 .42703 .2 \quad 2707.92745 .12768 .4$ $2780.12780 .2 \quad 2781.8 \quad 2783.2$

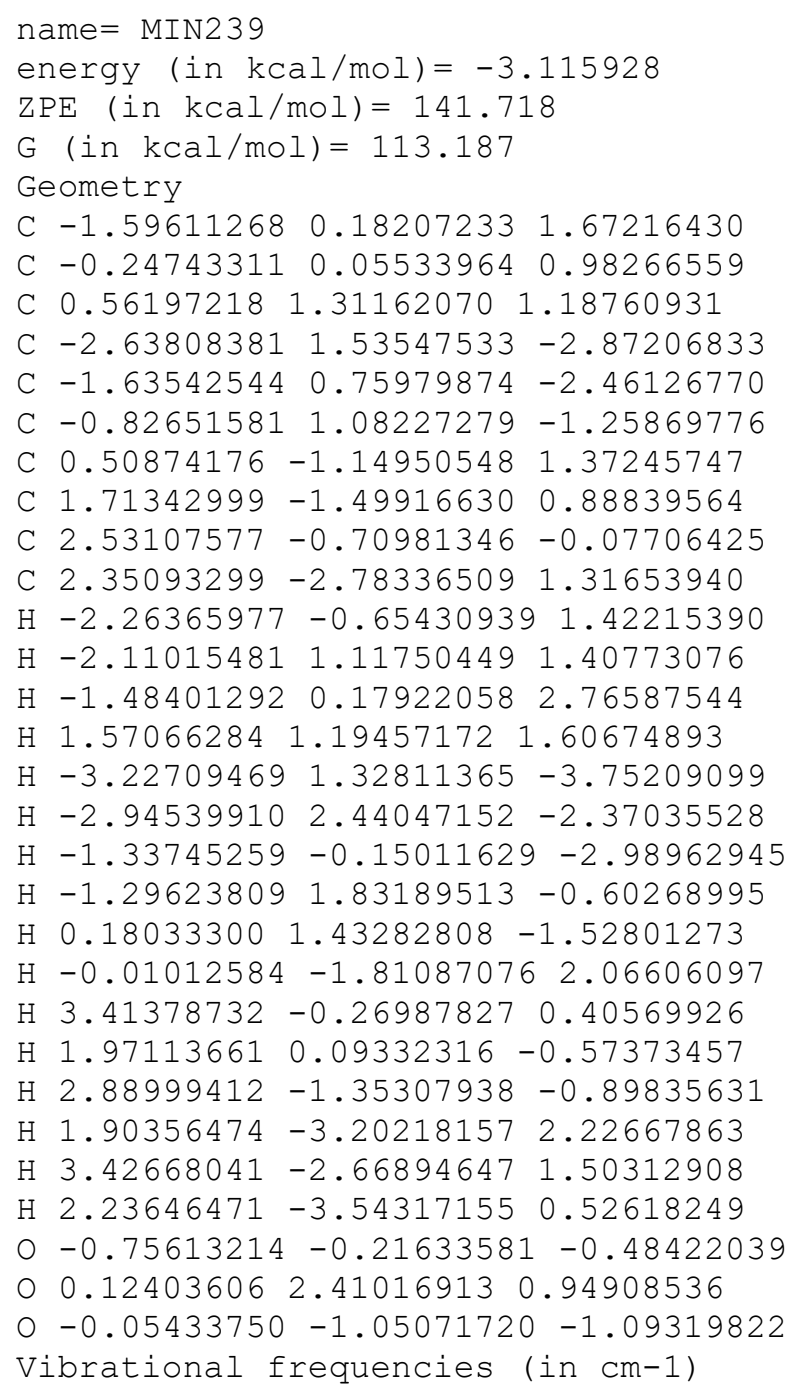


$\begin{array}{lllllllllllllllll}14.8 & 42.2 & 58.2 & 68.5 & 83.4 & 85.2 & 93.4 & 149.8 & 163.1 & 183.0 & 203.0 & 244.0 & 279.1 & 289.7\end{array}$ $303.4 \quad 329.6 \quad 376.7 \quad 393.4 \quad 422.1 \quad 483.0 \quad 491.0 \quad 524.4 \quad 569.5 \quad 601.8 \quad 673.6 \quad 762.0 \quad 894.4 \quad 904.8$ $930.8954 .2963 .6 \quad 976.2993 .0 \quad 1002.2 \quad 1013.8 \quad 1030.8 \quad 1035.3 \quad 1061.5 \quad 1091.31094 .11112 .9$

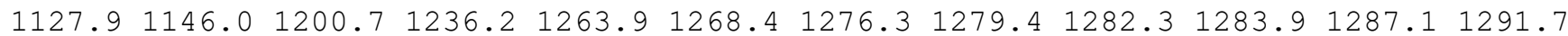

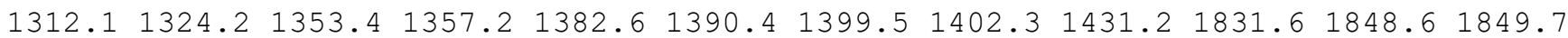
$2645.3 \quad 2649.6 \quad 2661.8 \quad 2662.8 \quad 2666.5 \quad 2667.4 \quad 2674.92677 .92711 .92721 .92746 .62754 .7$ $2773.72777 .6 \quad 2780.4 \quad 2786.6$

name $=$ MIN240

energy (in $\mathrm{kcal} / \mathrm{mol})=-6.588944$

$\mathrm{ZPE}(\mathrm{in} \mathrm{kcal} / \mathrm{mol})=145.419$

$\mathrm{G}($ in $\mathrm{kcal} / \mathrm{mol})=120.895$

Geometry

C $-2.46922085-0.35367579-0.73922554$

C $-0.96039176-0.30626957-0.83554732$

C $-0.37184470 \quad 1.14669410 \quad-0.97969796$

C $0.59259932 \quad 1.40141623 \quad 0.18415984$

C $0.71359930 \quad 0.08876492 \quad 0.95559025$

C $1.26873940-1.07782441 \quad 0.16647336$

C $-0.23010254-0.94352672 \quad 0.32922881$

$\begin{array}{llll}\text { C } & 0.92431234 & 0.25031385 & 2.44578313\end{array}$

C $-0.42111870 \quad 0.48936655 \quad 3.14626061$

C $1.63211893-0.949961493 .08510326$

$\mathrm{H}-2.941480650 .12210290-1.60973762$

$\mathrm{H}-2.83432909 \quad 0.148852590 .16375665$

$\mathrm{H}-2.83044833-1.39133103-0.71203564$

$\mathrm{H}-1.14427188 \quad 1.92185253-1.10223983$

$\mathrm{H} \quad 0.21902486 \quad 2.21402554 \quad 0.83129090$

$\mathrm{H} \quad 1.58223826 \quad 1.73201844 \quad-0.18745414$

H $1.57413185 \quad 1.15057229 \quad 2.60640627$

H $1.78946737-1.88881721 \quad 0.66336114$

H $1.71354535-0.89722958-0.81399004$

$\mathrm{H}-0.78505724 \quad-1.66237640 \quad 0.91990682$

$\mathrm{H}-0.95365618 \quad 1.342859292 .71472108$

$\mathrm{H}-0.28066861 \quad 0.69036971 \quad 4.21356828$

$\mathrm{H}-1.07605431-0.38475576 \quad 3.05891760$

$\mathrm{H} 1.05964493-1.87470149 \quad 2.95566744$

H $1.76846200 \quad-0.79756413 \quad 4.16154771$

H 2.62658189 -1.103543342 .65003273$

O $-0.252531630 .07966856-2.94685616$

O $0.406090091 .07389996-2.17654746$

o $-0.52819927-0.96983120 \quad-2.02996244$

Vibrational frequencies (in $\mathrm{cm}-1$ )

$\begin{array}{llllllllllllll}42.4 & 78.0 & 94.1 & 164.3 & 172.7 & 181.8 & 213.7 & 229.3 & 258.4 & 266.9 & 317.2 & 330.9 & 371.4 & 409.5\end{array}$ $451.6 \quad 489.5 \quad 538.6 \quad 573.9 \quad 593.8 \quad 633.0 \quad 708.0 \quad 765.0 \quad 836.8 \quad 862.8 \quad 879.8 \quad 906.9 \quad 944.7 \quad 965.1$ $976.2980 .8 \quad 983.4 \quad 1001.6 \quad 1012.1 \quad 1013.1 \quad 1029.9 \quad 1044.3 \quad 1109.4 \quad 1116.1 \quad 1148.8 \quad 1150.4 \quad 1172.2$

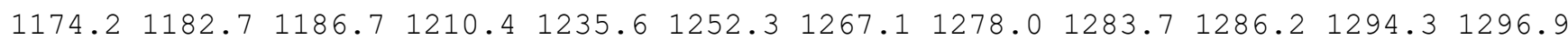

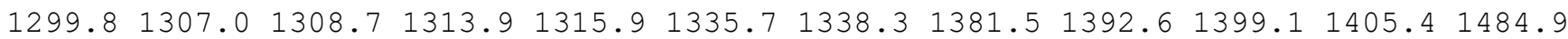
$2665.12671 .32671 .8 \quad 2676.6 \quad 2677.4 \quad 2679.4 \quad 2681.2 \quad 2697.92702 .92704 .52744 .92773 .1$ $2780.12781 .2 \quad 2781.7 \quad 2783.4$

name $=$ MIN241

energy (in $\mathrm{kcal} / \mathrm{mol})=-5.258319$

$\mathrm{ZPE}(\mathrm{in} \mathrm{kcal} / \mathrm{mol})=144.183$

$\mathrm{G}($ in $\mathrm{kcal} / \mathrm{mol})=118.326$

Geometry

C $-2.79079764-0.16891358-0.33011242$

C $-1.39399923-0.01505036 \quad 0.16923858$

C $-0.77616566 \quad 1.32480860 \quad-0.11229619$

C $0.71284897 \quad 1.50605663 \quad 0.21969575$

C $1.32683219 \quad 0.44872874 \quad 1.10246393$

C $1.75383665-0.87559199 \quad 0.49065332$

C $-0.78383450-0.98422145 \quad 0.86047629$ 
C $0.55954294-0.82190206 \quad 1.44365122$

C $3.05025891-1.512309160 .90493406$

C $0.66238729-1.401372212 .83114297$

$\mathrm{H}-3.49287274 \quad 0.41562323 \quad 0.28315835$

$\mathrm{H}-3.13799606-1.20961272-0.31488396$

$\mathrm{H}-2.89851103 \quad 0.19322560-1.36806858$

$\mathrm{H}-1.38368261 \quad 2.11712417 \quad 0.40331816$

$\mathrm{H} \quad 0.85194120 \quad 2.50335867 \quad 0.68760405$

$\mathrm{H} \quad 1.296448381 .56302655-0.72661908$

H $1.965303490 .87492692 \quad 1.88403734$

$\mathrm{H}-0.66024706-0.84121634-2.03480778$

$\mathrm{H} 1.53083185-1.03861124-0.57064141$

$\mathrm{H}-1.26273130-1.942792231 .05510478$

H $3.87653172-1.17340150 \quad 0.26503320$

H $1.58736335-1.108184593 .34124540$

H $3.32720107-1.276552351 .93963148$

$\mathrm{H}-0.17324162-1.06565548 \quad 3.46067674$

$\mathrm{H} \quad 0.63826091-2.49896228 \quad 2.80146187$

$\mathrm{H} 2.99522926-2.60647598 \quad 0.82529305$

$0-0.21806253 \quad 0.96160761-2.34486897$

$0-1.013481581 .69374530-1.47390379$

O $-0.90194544-0.19002474-2.74262182$

Vibrational frequencies (in $\mathrm{cm}-1$ )

$\begin{array}{llllllllllllllll}-28.2 & 66.8 & 88.6 & 123.5 & 131.2 & 144.6 & 154.3 & 185.4 & 221.4 & 244.4 & 267.4 & 282.4 & 309.9 & 384.5\end{array}$ $400.3 \quad 417.7 \quad 444.5 \quad 474.1 \quad 498.1 \quad 523.4 \quad 564.9 \quad 666.0 \quad 702.7 \quad 782.3 \quad 811.5 \quad 824.7 \quad 831.8 \quad 930.0$ $945.6963 .6 \quad 974.1 \quad 1012.9 \quad 1022.8 \quad 1037.4 \quad 1066.4 \quad 1076.1 \quad 1091.4 \quad 1093.0 \quad 1100.91130 .8 \quad 1143.9$

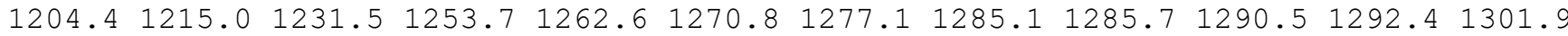

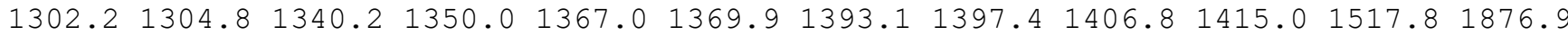
$2645.12654 .2 \quad 2670.2 \quad 2673.5 \quad 2674.6 \quad 2675.7 \quad 2675.8 \quad 2677.4 \quad 2733.92745 .2 \quad 2756.3 \quad 2759.5$ $2775.4 \quad 2780.92782 .0 \quad 2830.4$

name $=$ MIN242

energy (in $\mathrm{kcal} / \mathrm{mol})=-4.010557$

$\mathrm{ZPE}($ in $\mathrm{kcal} / \mathrm{mol})=142.989$

$\mathrm{G}($ in $\mathrm{kcal} / \mathrm{mol})=117.908$

Geometry

C $-2.28616291-0.22149636-0.85328547$

C $-0.75772121-0.15646142-0.68963916$

C $0.70507142 \quad 1.78513034-0.49526259$

C $1.864975861 .18522429-0.17456102$

C $2.17018185-0.20341696-0.15835693$

C $1.26660685-1.218121050 .37591302$

C $-0.21280418-0.83301175 \quad 0.57381239$

C $-0.35960056-0.01668515 \quad 1.88368077$

C $-1.62405081 \quad 0.84125815 \quad 1.96776174$

C $-0.34845484-0.98437709 \quad 3.07893184$

$\mathrm{H}-2.76843198-0.71757924-0.00522520$

$\mathrm{H}-2.57077074-0.76813230-1.76597483$

$\mathrm{H}-2.71092985 \quad 0.78990198-0.94536945$

$\mathrm{H} \quad 0.55516674 \quad 2.86210772 \quad-0.61956444$

$\mathrm{H} 2.74403646 \quad 1.81213246 \quad 0.02693627$

H $1.09547754 \quad 0.09014303 \quad-2.68336960$

H $1.70781951-1.58723477 \quad 1.33218404$

H $1.33322867-2.11284063-0.29445859$

$\mathrm{H}-0.79852901-1.78210909 \quad 0.69396714$

$\mathrm{H}-1.65216950 \quad 1.39336896 \quad 2.91595858$

$\mathrm{H}-2.535585090 .23892588 \quad 1.91746804$

$\mathrm{H}-0.35744852-0.43560948 \quad 4.02756139$

$\mathrm{H} \quad 0.54148219-1.62168855 \quad 3.08155772$

$\mathrm{H}-1.22658772-1.63942107 \quad 3.07316195$

$01.03611542-0.71768672-2.11034697$

$0-0.47719918 \quad 1.23664799-0.81007430$

$0-0.36459057-0.78008480-1.90293529$

$\mathrm{H}-1.66427679 \quad 1.58871447 \quad 1.16663166$ 
$\mathrm{H} \quad 0.52402006 \quad 0.66622351 \quad 1.96628421$

Vibrational frequencies (in $\mathrm{cm}-1$ )

$\begin{array}{llllllllllllllllll}65.1 & 85.2 & 96.3 & 110.0 & 143.5 & 165.4 & 179.0 & 189.4 & 242.8 & 264.5 & 277.0 & 301.5 & 326.5 & 352.0\end{array}$

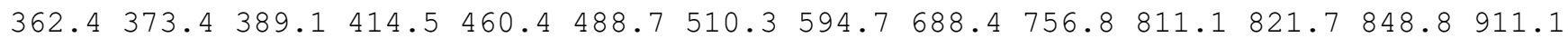

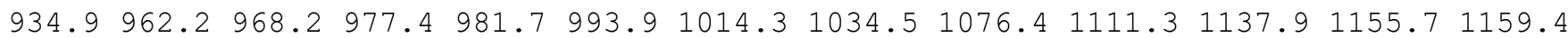

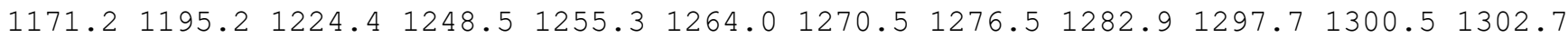

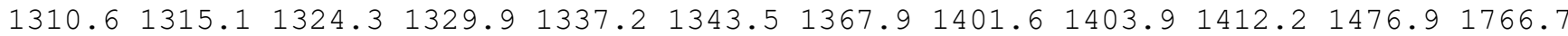
$2634.42654 .5 \quad 2665.12669 .1 \quad 2671.0 \quad 2676.3 \quad 2676.42682 .32703 .5 \quad 2711.4 \quad 2723.32745 .0$ $2768.8 \quad 2779.8 \quad 2781.7 \quad 2862.6$

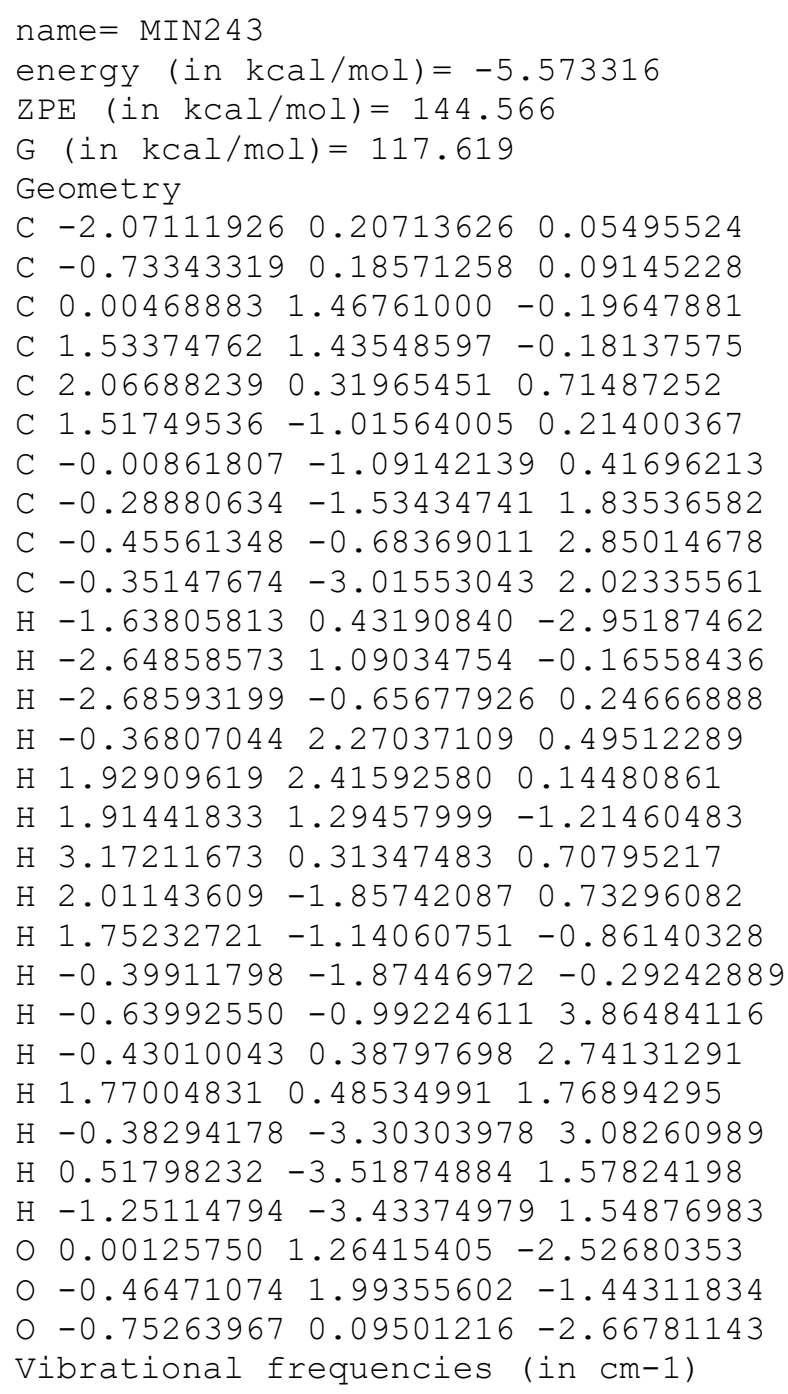


C $2.31318869 \quad 0.47437718 \quad 0.89193637$

C $-0.90902575-0.93177419 \quad 0.55565457$

C $0.07779345-0.48264394 \quad 1.63322921$

C $-0.37203492-0.64802373 \quad 3.06690131$

C $0.33781744 \quad-1.74181748 \quad 0.83660761$

$\mathrm{H}-2.591868120 .31711882 \quad-2.20465348$

$\mathrm{H}-3.13559746 \quad 0.38580077-0.51217054$

$\mathrm{H}-2.90436625-1.17509839-1.32243710$

$\mathrm{H}-1.085867891 .96789776-1.24567965$

$\mathrm{H}-0.69071265 \quad 2.19349915 \quad 1.09897961$

H $0.82886764 \quad 2.62171922 \quad 0.29102207$

$\mathrm{H} \quad 1.04142755 \quad 1.27799089 \quad 2.41590687$

$\mathrm{H} 2.29478136 \quad 0.13072577 \quad-0.14888878$

H $2.94660085 \quad 1.36768724 \quad 0.92023953$

$\mathrm{H}-1.86215649-1.346724210 .90620850$

$\mathrm{H}-0.96892861-1.55596266 \quad 3.21590305$

$\mathrm{H}-0.98862806 \quad 0.20027114 \quad 3.39025274$

$\mathrm{H} \quad 0.48900930-0.71650287 \quad 3.74494865$

H $1.13398112-1.767375150 .09528339$

H $2.80267154-0.303200161 .48898226$

$\mathrm{H} \quad 0.24948782-2.71309704 \quad 1.31326940$

○ $0.90596032-0.28113576-1.88847335$

$00.63466878 \quad 1.11282212-1.78101464$

o $-0.35873315-0.89991551-1.79858765$

Vibrational frequencies (in $\mathrm{cm}-1$ )

$\begin{array}{llllllllllllll}56.0 & 136.1 & 141.6 & 171.3 & 186.8 & 190.7 & 224.6 & 248.1 & 267.0 & 292.9 & 304.3 & 345.6 & 360.5 & 422.5\end{array}$

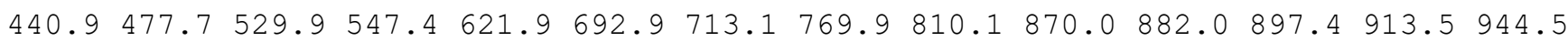

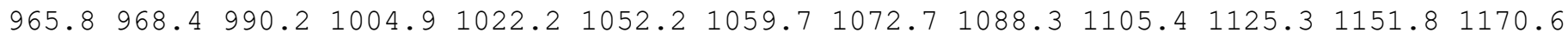

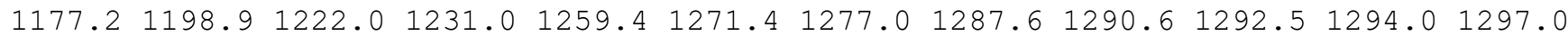
$1301.0 \quad 1303.4 \quad 1313.7 \quad 1316.91344 .9 \quad 1379.6 \quad 1379.91391 .6 \quad 1397.5 \quad 1406.1 \quad 1418.01506 .6$ $2660.52667 .92669 .32671 .6 \quad 2674.92676 .92679 .02680 .82690 .92709 .2 \quad 2736.2 \quad 2748.6$ $2779.62780 .6 \quad 2784.4 \quad 2785.6$

\section{name $=$ MIN245}

energy (in $\mathrm{kcal} / \mathrm{mol})=-0.733482$

$\mathrm{ZPE}($ in $\mathrm{kcal} / \mathrm{mol})=140.345$

G $($ in $\mathrm{kcal} / \mathrm{mol})=112.263$

Geometry

$\begin{array}{lllll}\text { C }-3.17856948 & 0.38119871 & 0.57038877\end{array}$

C $-1.67868822 \quad 0.48836057 \quad 0.67956976$

C 1.336979291 .298219820 .58554456

C $1.28167460 \quad 0.24565339-0.24321897$

C $1.03671100-1.16104728 \quad 0.22428509$

C $2.14082824-2.10732318-0.23348689$

C $-0.98226282-0.20767833-0.33487156$

C $-0.39296350-1.53940456-0.34627385$

C $-1.08534503-2.56170308 \quad 0.55958500$

C $-0.34395235-2.07017675-1.78306895$

$\mathrm{H}-3.51640708-0.49102607 \quad 0.00279920$

$\mathrm{H}-3.58211821 \quad 1.28134726 \quad 0.08083530$

$\mathrm{H}-3.62840872 \quad 0.34043672 \quad 1.57364831$

$\mathrm{H} 1.241727791 .300366761 .67492314$

$\mathrm{H} \quad 1.43703932 \quad 0.35888497-1.31527335$

$\mathrm{H} \quad 0.583362392 .80900222-1.42473704$

$\mathrm{H} \quad 0.97643186-1.202195451 .34060665$

$\mathrm{H} 1.90749542-3.14855200 \quad 0.01462988$

H $3.09151070-1.85173128 \quad 0.25378158$

H $2.31289281-2.05067596-1.31475057$

$\mathrm{H}-2.08470758-2.811901790 .18583728$

$\mathrm{H}-1.19695989-2.19612637 \quad 1.58656650$

$\mathrm{H}-0.51397456-3.496894950 .60485866$

$\mathrm{H} \quad 0.13683133-3.05166067-1.83812836$

$\mathrm{H} \quad 0.20861869-1.38380295-2.43854578$

$\mathrm{H}-1.35226221-2.16744467-2.20124490$ 
$01.54600407 \quad 2.72071491-1.20343366$

O $1.47428154 \quad 2.59451904 \quad 0.21627229$

o -1.154263821 .248095081 .47941437$

Vibrational frequencies (in $\mathrm{cm}-1$ )

$\begin{array}{llllllllllllllll}21.3 & 60.3 & 64.6 & 86.6 & 93.1 & 102.7 & 113.5 & 161.8 & 176.4 & 187.7 & 197.2 & 206.6 & 242.1 & 265.4\end{array}$ $269.2309 .9 \quad 315.3 \quad 330.1 \quad 373.4 \quad 405.0 \quad 422.9 \quad 486.2 \quad 534.3 \quad 563.8 \quad 604.2 \quad 668.7 \quad 789.0 \quad 812.1$

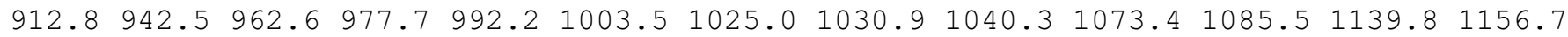
$\begin{array}{llllllllllll}1171.1 & 1181.1 & 1245.1 & 1250.3 & 1262.4 & 1269.5 & 1274.3 & 1288.2 & 1292.8 & 1294.6 & 1298.9 & 1302.5\end{array}$ $1308.91314 .31328 .51336 .41346 .2 \quad 1373.1 \quad 1394.51400 .21404 .61500 .11776 .21826 .2$ $2664.52669 .22673 .0 \quad 2673.92675 .72677 .92679 .72681 .42695 .2 \quad 2701.82758 .02778 .2$ $2779.92780 .6 \quad 2782.2 \quad 2855.6$

name $=$ MIN246

energy $($ in $\mathrm{kcal} / \mathrm{mol})=-5.72029$

$\mathrm{ZPE}(\mathrm{in} \mathrm{kcal} / \mathrm{mol})=145.352$

$\mathrm{G}($ in $\mathrm{kcal} / \mathrm{mol})=120.687$

Geometry

C $-2.48984309-0.31033125-0.87803386$

C $-0.97705529-0.29016923-0.85552297$

C $-0.35344321 \quad 1.14707746 \quad-0.99986807$

C $0.68299717 \quad 1.33943249 \quad 0.11591031$

C $0.63150659 \quad 0.09181561 \quad 0.99522047$

C $1.12429232-1.18333906 \quad 0.34206129$

C $-0.36046409-0.90582441 \quad 0.38032374$

C $0.76441972 \quad 0.34761393 \quad 2.48361810$

C $-0.50734581-0.059586043 .23887559$

C $1.98370381-0.369253263 .07723701$

$\mathrm{H}-2.88200103 \quad 0.15868504-1.79098879$

$\mathrm{H}-2.91809794 \quad 0.21277201-0.01561531$

$\mathrm{H}-2.86916261-1.34184705-0.86480587$

$\mathrm{H}-1.10902040 \quad 1.94746967 \quad-1.04661534$

$\mathrm{H} \quad 0.47376141 \quad 2.25619497 \quad 0.69197673$

$\mathrm{H} \quad 1.69697305 \quad 1.47330655-0.31270670$

$\mathrm{H} \quad 0.91415689 \quad 1.44869857 \quad 2.64128969$

H $1.52722527-1.98798018 \quad 0.94969578$

$\mathrm{H} \quad 1.65400729-1.13274214-0.60957561$

$\mathrm{H}-1.01213489-1.53196055 \quad 0.98057468$

$\mathrm{H}-1.39499838 \quad 0.41448205 \quad 2.80583945$

$\mathrm{H}-0.45220473 \quad 0.23853026 \quad 4.29152248$

$\mathrm{H}-0.66249042-1.143437953 .21519488$

$\mathrm{H} \quad 2.16197597-0.05137838 \quad 4.11025167$

H $2.89035291-0.15148505 \quad 2.50113475$

$\mathrm{H} 1.85087023-1.456141413 .08997358$

$\begin{array}{lllll}0 & -0.27540662 & 0.04056181 & -2.96098848\end{array}$

○ $0.343526371 .10811499-2.24473376$

o $-0.45901916-0.98101633-2.00063630$

Vibrational frequencies (in $\mathrm{cm}-1$ )

$\begin{array}{llllllllllllllll}-37.3 & 64.2 & 94.3 & 171.3 & 184.8 & 194.5 & 212.9 & 231.7 & 257.8 & 265.7 & 293.9 & 319.9 & 359.3 & 413.2\end{array}$ $452.7 \quad 488.6 \quad 528.2 \quad 590.9 \quad 602.0 \quad 629.2 \quad 707.9 \quad 763.9 \quad 838.2 \quad 864.5 \quad 880.7 \quad 909.3 \quad 944.1965 .5$ $976.2978 .4 \quad 983.9 \quad 1000.3 \quad 1014.1 \quad 1016.0 \quad 1030.7 \quad 1048.1 \quad 1106.5 \quad 1117.0 \quad 1145.01156 .3 \quad 1164.0$

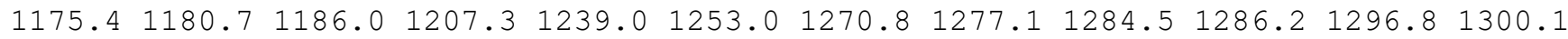
$1303.81306 .6 \quad 1309.8 \quad 1314.7 \quad 1319.1 \quad 1336.91345 .5 \quad 1373.8 \quad 1393.6 \quad 1397.7 \quad 1404.01485 .8$ $2662.22669 .32671 .42676 .5 \quad 2678.4 \quad 2679.7 \quad 2681.52697 .12699 .72706 .72743 .82768 .2$ $2780.22781 .4 \quad 2781.8 \quad 2783.4$

name $=$ MIN247

energy (in $\mathrm{kcal} / \mathrm{mol})=-6.143726$

$\operatorname{ZPE}($ in $\mathrm{kcal} / \mathrm{mol})=145.789$

$\mathrm{G}(\mathrm{in} \mathrm{kcal} / \mathrm{mol})=121.202$

Geometry

C $-2.40684118-0.55590001-0.64495774$

C $-0.87563660-0.53147292-0.72337789$

C $-0.36587349 \quad 0.93704064-0.73028003$ 
C $1.07774434 \quad 1.16515041-0.29347444$

C $1.55229092 \quad 0.29472144 \quad 0.83420259$

C $0.89622541-1.041943151 .09479274$

C $-0.25797566-1.52878494 \quad 0.26081368$

C $0.76489629 \quad 0.08187944 \quad 2.12451353$

C $1.58181961-0.06461532 \quad 3.38975941$

C $-0.54146570 \quad 0.78625098 \quad 2.37528594$

$\mathrm{H}-2.84589579 \quad 0.10694042-1.40436150$

$\mathrm{H}-2.77874420-0.24738035 \quad 0.33535414$

$\mathrm{H}-2.79241580-1.56255120-0.85275210$

$\mathrm{H}-1.06919158 \quad 1.63592660-0.23479499$

H $1.218864592 .23354038-0.02688443$

H $1.73915110 \quad 0.99184368-1.17676632$

$\mathrm{H} 2.64626435 \quad 0.34739124 \quad 0.93705225$

H $1.55575440-1.87641205 \quad 1.37446774$

$\mathrm{H} \quad 0.07843707-2.41516910-0.33019116$

$\mathrm{H}-1.04201112-1.924283270 .94258283$

$\mathrm{H} \quad 1.06054507-0.69176077 \quad 4.12428261$

$\mathrm{H} \quad 1.76311620 \quad 0.91329274 \quad 3.85424933$

H 2.56090554 $-0.52405128 \quad 3.21121905$

$\mathrm{H}-0.44939222 \quad 1.86388874 \quad 2.18568041$

$\mathrm{H}-0.87301226 \quad 0.66862363 \quad 3.41574031$

$\mathrm{H}-1.353928790 .39983000 \quad 1.74944072$

$\begin{array}{llllll}0 & 0.00149834 & 0.09676540 & -2.76513221\end{array}$

$\begin{array}{llllll}0 & -0.48109940 & 1.25624034 & -2.12119908\end{array}$

$0-0.56401636-1.00678732-2.04603141$

Vibrational frequencies (in $\mathrm{cm}-1$ )

$\begin{array}{lllllllllllllll}47.0 & 71.5 & 114.3 & 146.7 & 155.9 & 174.2 & 187.2 & 210.8 & 236.2 & 283.2 & 312.8 & 350.3 & 371.0 & 413.2\end{array}$

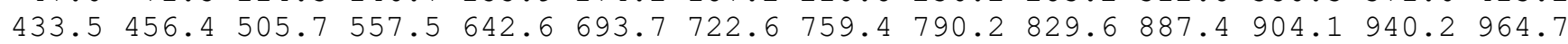
$978.9 \quad 982.3 \quad 1012.0 \quad 1014.3 \quad 1022.1 \quad 1026.4 \quad 1039.3 \quad 1063.6 \quad 1086.7 \quad 1115.5 \quad 1135.7 \quad 1155.4$

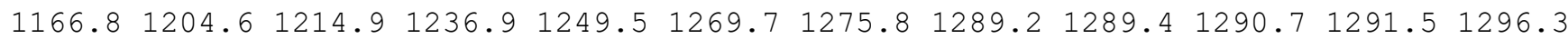
$1297.81299 .3 \quad 1301.21303 .2 \quad 1308.3 \quad 1355.6 \quad 1369.4 \quad 1388.4 \quad 1395.1 \quad 1403.91408 .2 \quad 1419.8$ $1532.82645 .92650 .2 \quad 2668.5 \quad 2670.3 \quad 2675.02676 .62678 .02678 .62694 .02727 .32733 .0$ $2740.4 \quad 2749.0 \quad 2778.4 \quad 2781.0 \quad 2781.9$

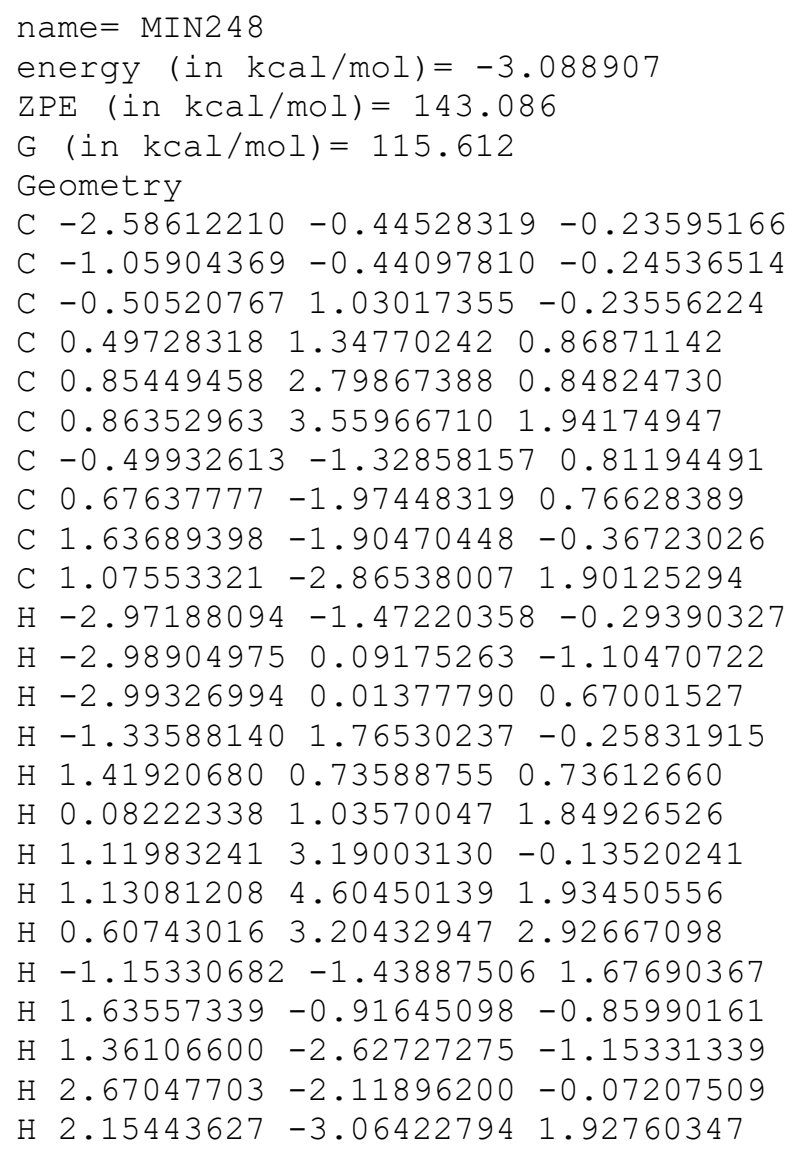


$\mathrm{H} \quad 0.57201617-3.841111231 .81841939$

$\mathrm{H} 0.80220520-2.44274593 \quad 2.87734730$

$0-0.44596104 \quad 0.20351227-2.32154053$

O $0.212072711 .13136031-1.47160625$

O $-0.61602709-0.94857553-1.51631173$

Vibrational frequencies (in $\mathrm{cm}-1$ )

$\begin{array}{lllllllllllllllll}-71.3 & 35.1 & 48.4 & 54.7 & 62.5 & 83.8 & 140.9 & 168.7 & 183.5 & 198.8 & 246.8 & 259.7 & 306.6 & 315.3\end{array}$ $348.5 \quad 374.2 \quad 421.8 \quad 467.8 \quad 493.1 \quad 505.2 \quad 589.8 \quad 613.4 \quad 661.5 \quad 705.8 \quad 868.5 \quad 889.4 \quad 903.8 \quad 908.5$ $949.1 \quad 978.7 \quad 982.0 \quad 985.4 \quad 998.4 \quad 1005.8 \quad 1018.0 \quad 1029.4 \quad 1080.1 \quad 1103.6 \quad 1106.3 \quad 1115.3 \quad 1139.9$

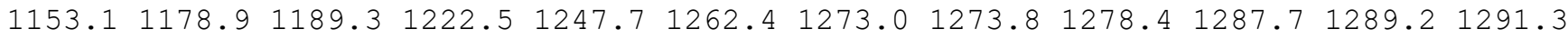

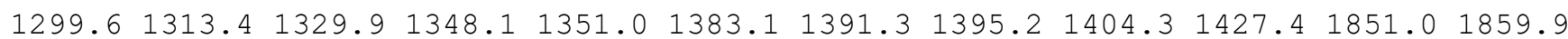
$2651.0 \quad 2652.12667 .8 \quad 2671.5 \quad 2672.6 \quad 2676.1 \quad 2680.02681 .5 \quad 2716.72734 .3 \quad 2753.5 \quad 2756.0$ $2775.22779 .7 \quad 2781.62790 .7$

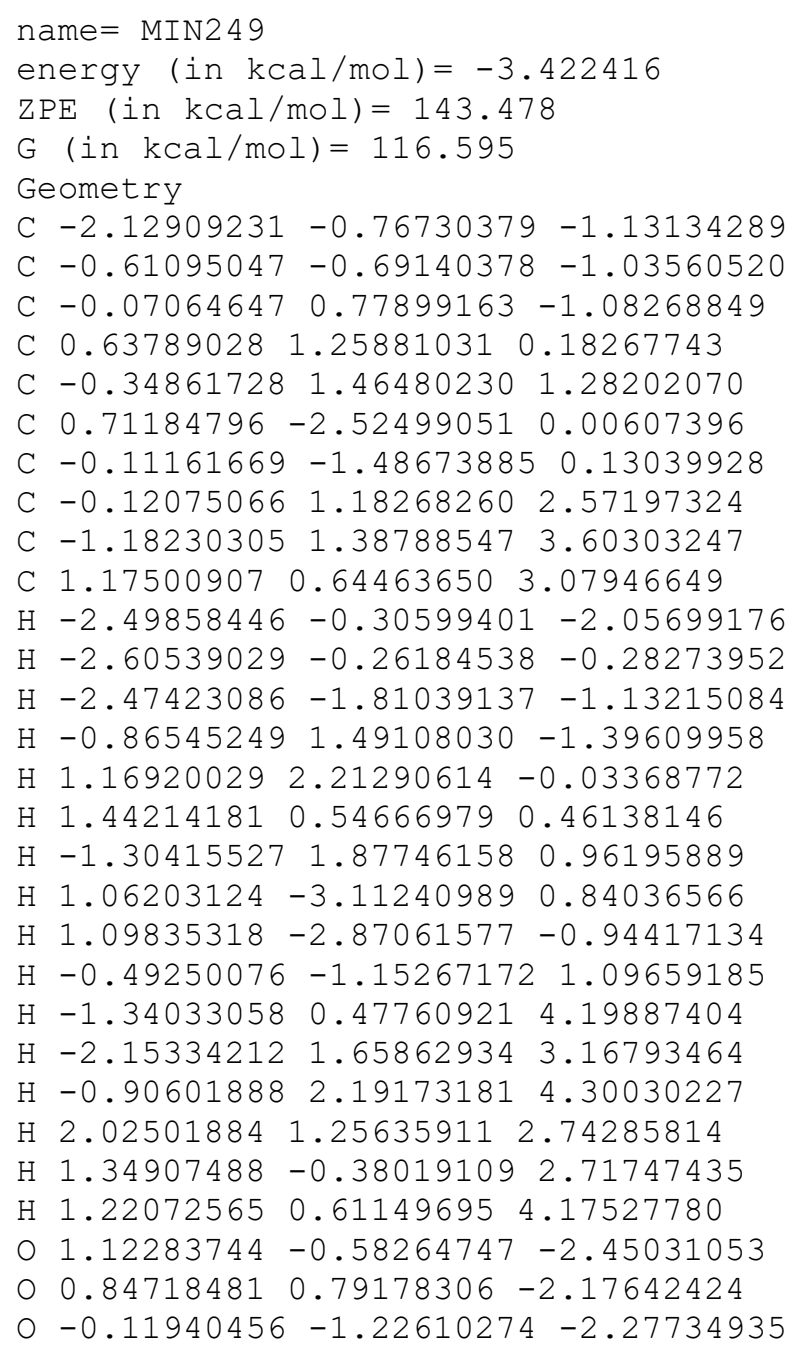


C $-0.85668714 \quad 0.33891232 \quad-0.84631054$

C $-0.45198277 \quad 1.74466042-0.50470536$

C $2.67467815-0.89972071 \quad 1.64937666$

C $1.47935315-1.477378031 .54165822$

C $0.73190895-1.10221522-1.10379824$

C $-0.32712637-0.85662135-0.02275522$

C $0.16074122-0.74472794 \quad 1.44249077$

C $0.26983691 \quad 0.68624239 \quad 1.96847070$

C $-0.85207738-1.48469596 \quad 2.34858072$

$\mathrm{H}-2.962839260 .58053651-0.51248309$

$\mathrm{H}-2.57984289-0.75229798-1.61818551$

$\mathrm{H}-2.46705449 \quad 0.93191249-2.16614543$

$\mathrm{H} \quad 0.55639704 \quad 2.05753873-0.82863949$

H $2.84003422 \quad 0.16626137 \quad 1.68190594$

H $3.59615850-1.45836454 \quad 1.71663710$

H $1.38164961-2.563544331 .51135242$

H $0.76321900-2.07665437-1.58105880$

$\mathrm{H} \quad 1.74410902-0.75899130-0.87814937$

$\mathrm{H}-1.09195101-1.67133943-0.04515039$

$\mathrm{H} \quad 0.62922229 \quad 0.69248031 \quad 3.00710382$

$\mathrm{H}-0.70683283 \quad 1.18951258 \quad 1.97831768$

$\mathrm{H} \quad 0.97334413 \quad 1.29650044 \quad 1.39354915$

$\mathrm{H}-0.52202983-1.47198515 \quad 3.39416737$

$\mathrm{H}-0.97392173-2.53377763 \quad 2.06240719$

$\mathrm{H}-1.83820951-1.00923348 \quad 2.31195383$

$\begin{array}{lllll}0 & 0.75942271 & 0.72644898 & -2.56585282\end{array}$

$0-1.20896793 \quad 2.50605645 \quad 0.04162641$

o $0.01757979-0.14961833-2.07144510$

Vibrational frequencies (in cm-1)

$\begin{array}{lllllllllllllll}-36.9 & 54.0 & 70.8 & 108.8 & 123.0 & 179.2 & 208.7 & 219.5 & 223.0 & 251.2 & 260.5 & 294.5 & 311.9 & 333.4\end{array}$

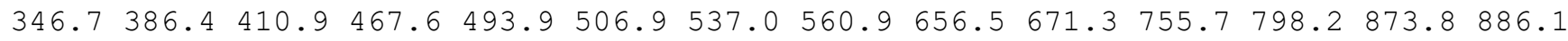
$933.4962 .3971 .6 \quad 982.1 \quad 999.1 \quad 1005.9 \quad 1024.4 \quad 1025.9 \quad 1043.6 \quad 1068.6 \quad 1070.8 \quad 1091.3 \quad 1106.7$

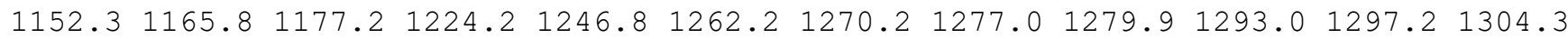

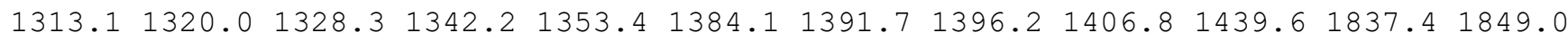
$2619.42653 .6 \quad 2663.7 \quad 2667.12670 .2 \quad 2672.3 \quad 2674.92677 .82692 .2 \quad 2710.2 \quad 2746.02751 .2$ $2774.12775 .8 \quad 2780.3 \quad 2785.6$

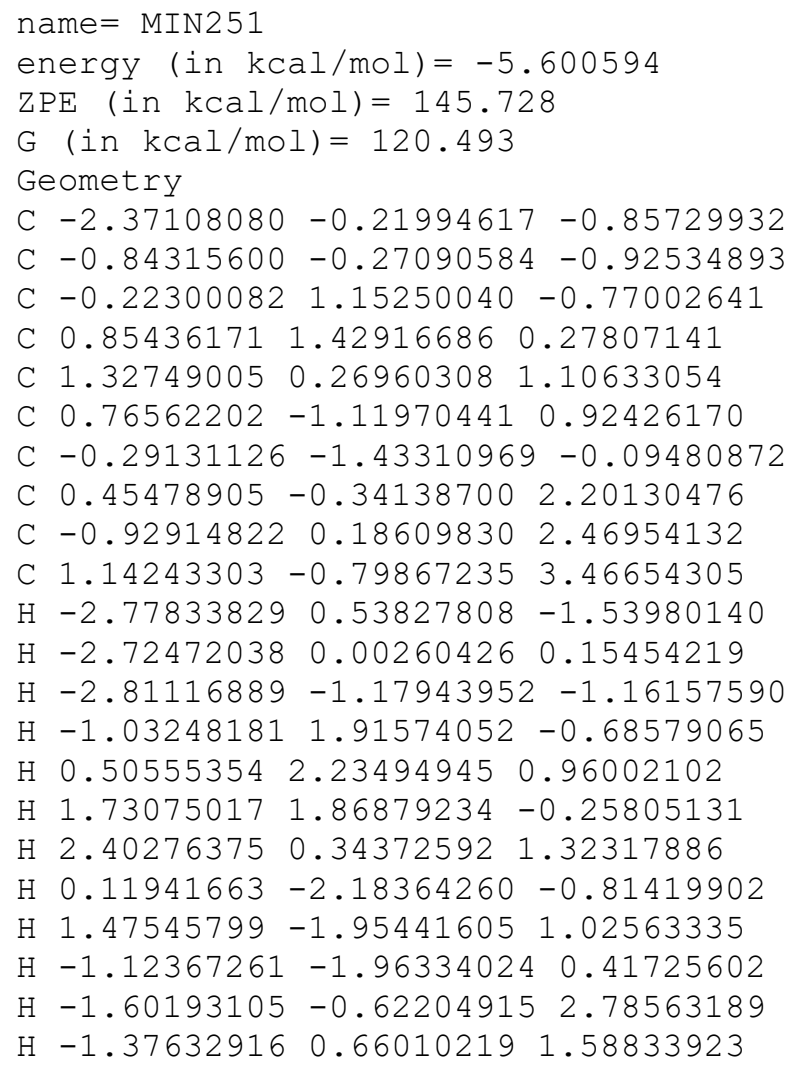


$\mathrm{H}-0.91822126 \quad 0.93920910 \quad 3.26836177$

$\mathrm{H} \quad 1.244029330 .02712953 \quad 4.18207526$

$\mathrm{H} 2.14838389-1.19276297 \quad 3.28150333$

$\mathrm{H} \quad 0.56799244-1.59441959 \quad 3.95871451$

$0 \quad 0.58494201 \quad 0.15224095-2.62651357$

$0.331698981 .42988847-2.06371038$

O $-0.57862001-0.58085875-2.31386994$

Vibrational frequencies (in $\mathrm{cm}-1$ )

$\begin{array}{lllllllllllllll}-44.7 & 23.4 & 107.5 & 129.3 & 164.1 & 172.9 & 196.2 & 207.4 & 250.0 & 277.1 & 322.0 & 347.3 & 402.7 & 420.6\end{array}$

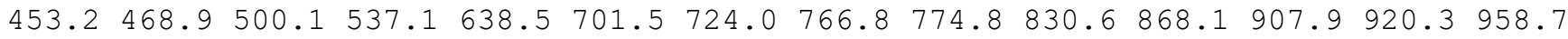
$970.8 \quad 977.7 \quad 1011.3 \quad 1012.2 \quad 1014.9 \quad 1015.8 \quad 1035.2 \quad 1061.7 \quad 1084.9 \quad 1115.3 \quad 1148.11163 .7$

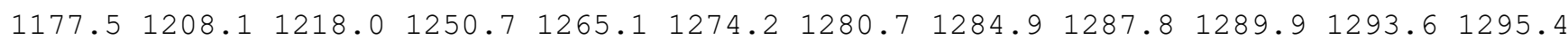
$1297.3 \quad 1298.6 \quad 1302.8 \quad 1307.7 \quad 1325.5 \quad 1362.4 \quad 1375.4 \quad 1389.1 \quad 1399.6 \quad 1403.5 \quad 1408.11419 .2$ $1537.92644 .72645 .02667 .8 \quad 2673.2 \quad 2673.5 \quad 2675.2 \quad 2676.02678 .8 \quad 2679.42727 .12729 .0$ $2740.7 \quad 2750.0 \quad 2777.8 \quad 2781.6 \quad 2783.1$

\section{name $=$ MIN252}

energy $($ in $\mathrm{kcal} / \mathrm{mol})=-3.148811$

$\mathrm{ZPE}(\mathrm{in} \mathrm{kcal} / \mathrm{mol})=143.281$

$\mathrm{G}(\mathrm{in} \mathrm{kcal} / \mathrm{mol})=116.103$

Geometry

C $-2.18275468-0.32460456-1.11781515$

C $-0.65986988-0.29321960-1.11812139$

C $-0.08527357 \quad 1.11648206-0.74387461$

C $0.74591632 \quad 1.17012747 \quad 0.53783711$

C $-0.15017974 \quad 1.05713099 \quad 1.72435636$

C $0.90598479-2.18925112-0.71181166$

C $-0.09491992-1.41332684-0.30148134$

C $-0.02196038 \quad 0.17169055 \quad 2.72122158$

C $-1.01901781 \quad 0.12863801 \quad 3.83609748$

C $1.07460077-0.832193062 .83770734$

$\mathrm{H}-2.56005215-1.31586778-1.40311359$

$\mathrm{H}-2.60014131 \quad 0.39608137-1.83407359$

$\mathrm{H}-2.58387079-0.08920654-0.12456234$

$\mathrm{H}-0.88527552 \quad 1.88908660-0.73791441$

$\mathrm{H} \quad 1.30365179 \quad 2.13280628 \quad 0.57295775$

H $1.53676653 \quad 0.39299794 \quad 0.51702713$

$\mathrm{H}-0.96740326 \quad 1.77914021 \quad 1.72905249$

$\mathrm{H} \quad 1.30600290-3.00378007-0.12822562$

H $1.40076190-2.07328608-1.66708042$

$\mathrm{H}-0.57618315-1.546355490 .66856713$

$\mathrm{H}-1.60835781-0.799148913 .79738826$

$\mathrm{H}-1.731275340 .96310857 \quad 3.80500231$

$\mathrm{H}-0.526811190 .16088505 \quad 4.81753061$

H $1.83109595 \quad-0.49718118 \quad 3.56286827$

H $1.59787944-1.00993709 \quad 1.88675442$

$\mathrm{H} 0.70300048-1.80671126 \quad 3.18298033$

$\begin{array}{lllll}0 & 0.95739286 & 0.26383665 & -2.56927111\end{array}$

O $0.728432021 .48073186-1.85901338$

O $-0.27517314-0.41751782-2.49851683$

Vibrational frequencies (in cm-1)

$\begin{array}{llllllllllllllll}33.4 & 47.0 & 54.6 & 73.2 & 86.6 & 108.1 & 121.2 & 156.5 & 198.8 & 225.4 & 230.6 & 275.7 & 306.0 & 325.3\end{array}$ $352.2 \quad 388.9 \quad 432.7 \quad 456.9 \quad 486.2 \quad 548.1 \quad 559.3 \quad 604.3 \quad 678.8 \quad 724.8 \quad 806.1 \quad 861.4 \quad 891.3 \quad 936.9$ $950.2971 .7990 .3 \quad 995.0 \quad 1003.7 \quad 1011.6 \quad 1020.5 \quad 1063.1 \quad 1071.0 \quad 1094.2 \quad 1094.61115 .91132 .0$ $1158.01184 .5 \quad 1206.2 \quad 1229.3 \quad 1255.3 \quad 1266.8 \quad 1274.91278 .4 \quad 1282.6 \quad 1285.1 \quad 1286.91288 .2$

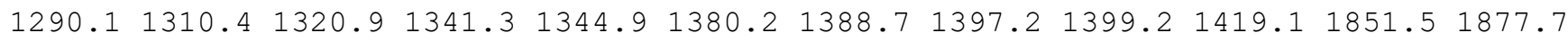
$2649.32661 .7 \quad 2670.0 \quad 2671.1 \quad 2673.9 \quad 2675.4 \quad 2676.12680 .52710 .02734 .02749 .92755 .1$ $2775.82779 .2 \quad 2780.8 \quad 2782.8$

name $=$ MIN253

energy (in $\mathrm{kcal} / \mathrm{mol})=-3.017161$

$\mathrm{ZPE}(\mathrm{in} \mathrm{kcal} / \mathrm{mol})=143.184$

$\mathrm{G}($ in $\mathrm{kcal} / \mathrm{mol})=115.278$ 


\begin{tabular}{|c|c|c|}
\hline \multicolumn{3}{|c|}{ Geometry } \\
\hline & -2.04873573 & $-1.14526748-0.67307112$ \\
\hline & -0.52348836 & $6-1.14794294-0.66$ \\
\hline & & $0.29855634-0.665$ \\
\hline & 1.0 & 0.59687904 \\
\hline & & $0.94305934 \quad 1.711$ \\
\hline & & -3.078739620 .19 \\
\hline & 0.0 & -2.031356470 .4 \\
\hline & 0706815 & 52.118170001 .9 \\
\hline & -0.23650127 & $7 \quad 3.26807238 \quad 1.01$ \\
\hline & -0.91276606 & $62.36413964 \quad 3.25$ \\
\hline & -2.44349159 & $9-0.60179351-1$. \\
\hline & -2.45163712 & $2-0.679630230 .23$ \\
\hline & -2.44539848 & $8-2.16817382-0$. \\
\hline & -0.71667518 & $2853-0.7$ \\
\hline & 1.75277884 & 51160.111 \\
\hline & 1.78045157 & 020.5 \\
\hline & 0.38011172 & 22.454 \\
\hline & 1.15650475 & $874 \quad 0.97$ \\
\hline & 1.130 & $270-0.7$ \\
\hline & -0.333784 & 1791.4 \\
\hline & 0.47764368 & 9480.183 \\
\hline & 0.00 & 31.517 \\
\hline & -1.23445543 & 33.386811150 .56470 \\
\hline & -0.34815130 & 3.069 \\
\hline & -1.05105959 & 91.44911148 \\
\hline & -1.90950117 & $7 \quad 2.79771403 \quad 3.09563741$ \\
\hline & 1.02488196 & $-0.93479361-2.29552032$ \\
\hline & 0.72110882 & $0.40693635-1.93675116$ \\
\hline & & \\
\hline
\end{tabular}

Vibrational frequencies (in $\mathrm{cm}-1$ )

$\begin{array}{lllllllllllllll}28.0 & 33.3 & 48.0 & 66.4 & 74.3 & 82.9 & 101.4 & 151.4 & 201.9 & 211.5 & 235.9 & 282.7 & 318.4 & 325.1\end{array}$

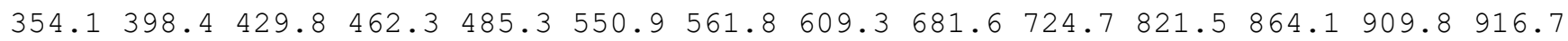
$946.8971 .1987 .6 \quad 995.2 \quad 1003.9 \quad 1013.6 \quad 1020.2 \quad 1059.2 \quad 1066.3 \quad 1084.01095 .11116 .1 \quad 1133.7$

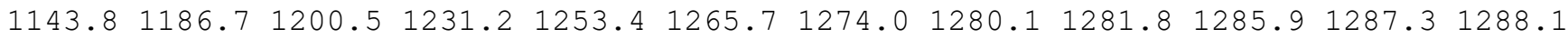
$1290.31312 .31326 .91335 .6 \quad 1342.5 \quad 1378.4 \quad 1386.51395 .11400 .6 \quad 1414.8 \quad 1851.4 \quad 1873.5$ $2651.22667 .8 \quad 2669.8 \quad 2670.7 \quad 2673.6 \quad 2675.8 \quad 2677.32680 .92710 .62739 .22754 .62755 .9$ $2777.7 \quad 2779.5 \quad 2781.0 \quad 2782.8$

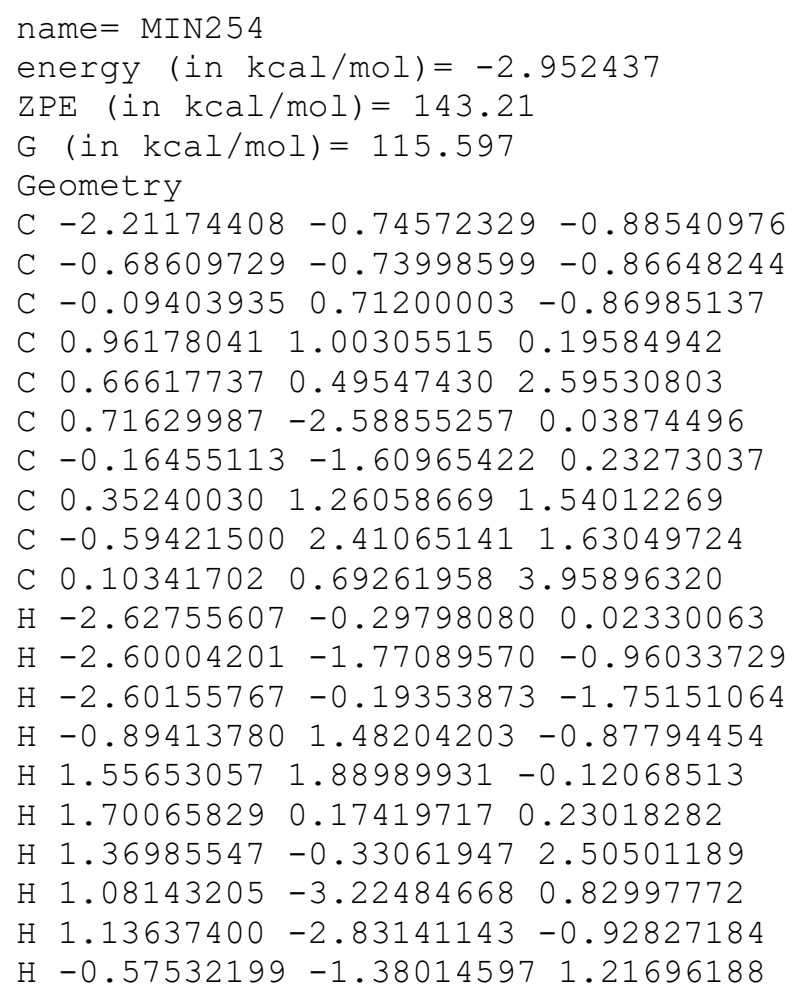


$\mathrm{H}-0.514802893 .095653650 .77658970$

$\mathrm{H}-0.41368588 \quad 3.01113476 \quad 2.53452578$

$\mathrm{H}-1.63622332 \quad 2.062708481 .68072313$

$\mathrm{H} \quad 0.488320691 .61564038 \quad 4.41816238$

$\mathrm{H} \quad 0.35630215-0.134173194 .63512613$

H $-0.99292455 \quad 0.77310525 \quad 3.94128689$

$0.84639473-0.50480964-2.51030707$

$0.50535667 \quad 0.82956933-2.16126702$

o $-0.30062117-1.25222942-2.15503718$

Vibrational frequencies (in $\mathrm{cm}-1$ )

$\begin{array}{lllllllllllllll}27.9 & 36.2 & 45.1 & 69.9 & 87.9 & 89.2 & 140.4 & 153.0 & 204.8 & 224.9 & 231.1 & 255.8 & 285.2 & 314.8\end{array}$ $344.0 \quad 391.7 \quad 416.8 \quad 440.6 \quad 520.4 \quad 560.4 \quad 568.7 \quad 611.3 \quad 680.1 \quad 722.8 \quad 822.2 \quad 863.0 \quad 873.3 \quad 930.1$ $938.6 \quad 969.3 \quad 987.3 \quad 992.8 \quad 1011.1 \quad 1034.6 \quad 1051.6 \quad 1062.8 \quad 1079.8 \quad 1091.4 \quad 1094.6 \quad 1096.2 \quad 1133.3$ $1146.91188 .3 \quad 1198.8 \quad 1230.7 \quad 1249.6 \quad 1264.6 \quad 1272.0 \quad 1275.9 \quad 1285.9 \quad 1287.6 \quad 1290.2 \quad 1291.9$

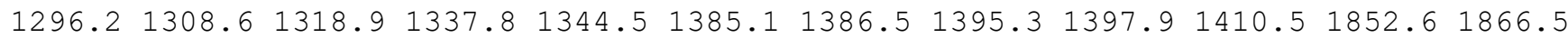
$2650.72668 .0 \quad 2669.2 \quad 2670.2 \quad 2673.8 \quad 2675.12678 .42683 .22710 .72736 .5 \quad 2749.42754 .6$ $2777.8 \quad 2779.4 \quad 2779.9 \quad 2782.8$

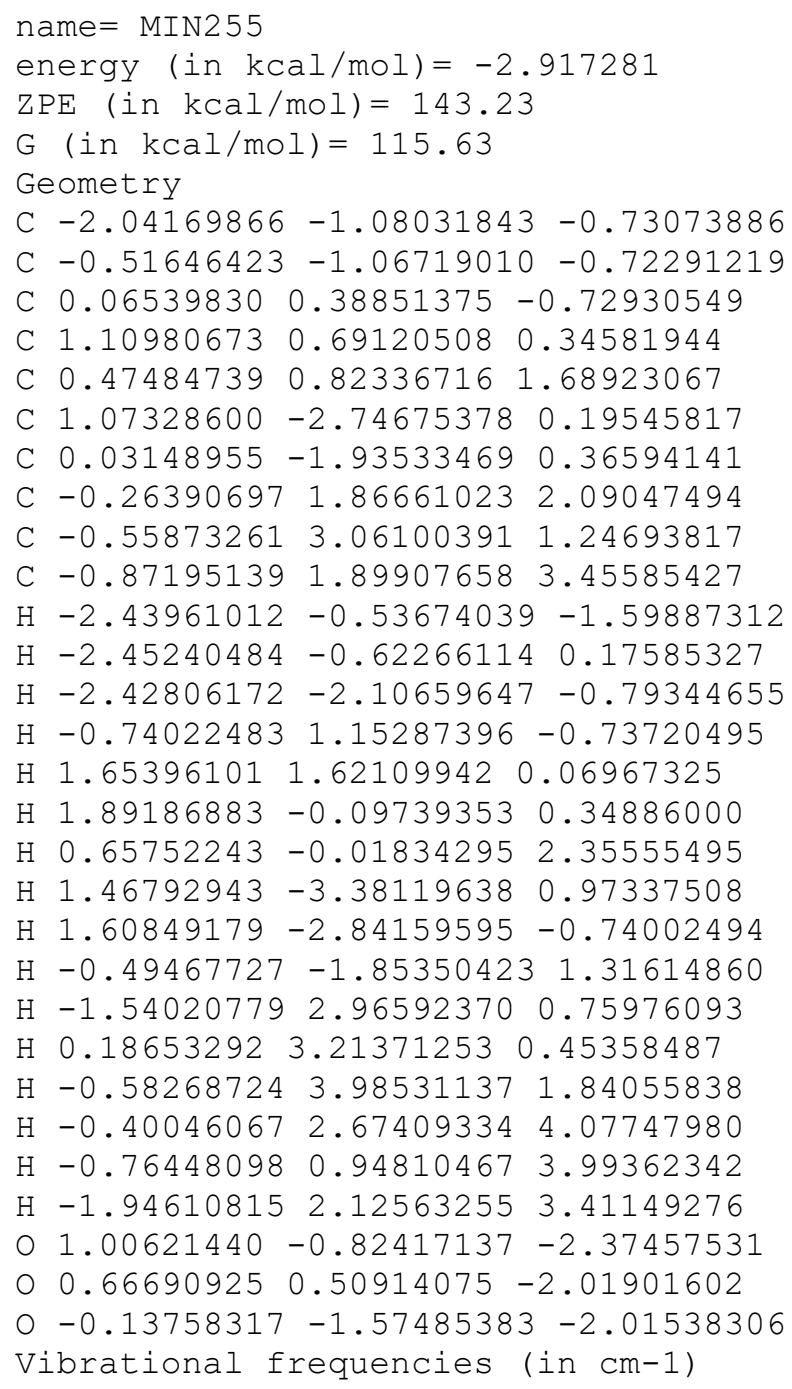


$\mathrm{ZPE}($ in $\mathrm{kcal} / \mathrm{mol})=143.207$

$\mathrm{G}($ in $\mathrm{kcal} / \mathrm{mol})=115.484$

Geometry

C $-1.94090243-1.22791006-0.76352116$

C $-0.41879962-1.22810842-0.76221210$

C $0.18186630 \quad 0.21999968 \quad-0.76142719$

C $1.15576892 \quad 0.546717620 .37166428$

C $0.40379412 \quad 0.91397166 \quad 1.60707109$

C $-0.30037886-2.32128523 \quad 1.48840287$

C $0.18477328-2.13896218 \quad 0.26185176$

C $-0.174020562 .10222930 \quad 1.82758544$

C $-0.13682254 \quad 3.25013778 \quad 0.87587607$

C -0.927218552 .368809973 .09188286$

$\mathrm{H}-2.33863993-0.70866813-1.64681050$

$\begin{array}{lllll}\mathrm{H} & -2.34187705 & -0.73452061 & 0.12950038\end{array}$

$\mathrm{H}-2.33812793-2.25184118-0.78719953$

$\mathrm{H}-0.616791620 .99069145 \quad-0.83434051$

$\mathrm{H} \quad 1.82362657 \quad 1.37516401 \quad 0.04657097$

$\mathrm{H} \quad 1.84574858-0.301000320 .55877269$

$\mathrm{H} \quad 0.34289658 \quad 0.11672230 \quad 2.34686703$

$\mathrm{H}-1.17858372-1.824167331 .87262418$

$\mathrm{H} \quad 0.14884627 \quad-2.98985600 \quad 2.20851765$

$\mathrm{H} \quad 1.07282559-2.66753800 \quad-0.09505688$

$\mathrm{H}-1.12651768 \quad 3.42724205 \quad 0.43061165$

$\mathrm{H} \quad 0.56790695 \quad 3.10011927 \quad 0.04650199$

H $0.16193643 \quad 4.17961914 \quad 1.38172352$

$\mathrm{H}-0.370293423 .060353263 .74082600$

$\mathrm{H}-1.11299298 \quad 1.45849504 \quad 3.67639039$

$\mathrm{H}-1.90470017 \quad 2.82808088 \quad 2.89016611$

$\begin{array}{llll}0 & 1.14231345 & -1.03176702 & -2.37501798\end{array}$

$\begin{array}{lllll}0 & 0.86391927 & 0.31601868 & -2.01301424\end{array}$

o $-0.05273067-1.71765517-2.07028861$

Vibrational frequencies (in $\mathrm{cm}-1$ )

$\begin{array}{llllllllllllll}28.2 & 32.2 & 48.5 & 64.8 & 83.4 & 99.3 & 109.0 & 162.3 & 200.3 & 219.3 & 235.9 & 271.5 & 312.5 & 325.3\end{array}$ $378.0 \quad 403.2 \quad 419.0 \quad 464.4 \quad 480.5 \quad 496.7 \quad 571.0 \quad 630.1 \quad 682.5 \quad 717.6 \quad 816.5 \quad 864.1 \quad 913.4 \quad 914.1$ $940.3971 .8 \quad 984.6 \quad 995.8 \quad 1003.7 \quad 1012.8 \quad 1020.3 \quad 1056.1 \quad 1068.0 \quad 1084.5 \quad 1093.91116 .4 \quad 1141.5$

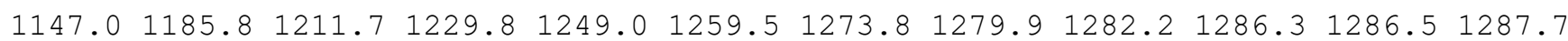

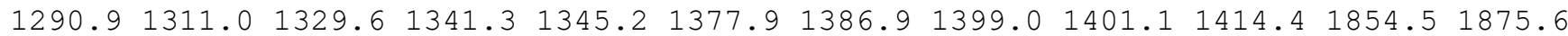
$2652.12668 .22669 .62670 .62673 .52675 .2 \quad 2675.82679 .72710 .12740 .22746 .32752 .5$ $2777.7 \quad 2778.7 \quad 2780.7 \quad 2783.8$

\section{name $=$ MIN257}

energy (in $\mathrm{kcal} / \mathrm{mol})=-2.725888$

$\mathrm{ZPE}(\mathrm{in} \mathrm{kcal} / \mathrm{mol})=143.212$

$\mathrm{G}($ in $\mathrm{kcal} / \mathrm{mol})=115.431$

Geometry

C $-2.22746533-1.16983162-0.56195685$

C $-0.70114751-1.20871158-0.57068471$

C $-0.06743476 \quad 0.22333866 \quad-0.59796930$

C $1.09870395 \quad 0.45617247 \quad 0.36438697$

C 0.636779551 .024665181 .66407961

C $0.51341945-3.190022210 .32000970$

C $-0.19094956-2.08068408 \quad 0.53303083$

C $0.23297148 \quad 2.29130023 \quad 1.83637868$

C $0.14178079 \quad 3.29474599 \quad 0.73583905$

C $-0.172767362 .79957776 \quad 3.18215461$

$\mathrm{H}-2.61457141-0.60307132-1.41996119$

$\mathrm{H}-2.61560529-0.71427673 \quad 0.35479092$

$\mathrm{H}-2.64617953-2.18319558-0.63572549$

$\mathrm{H}-0.83623914 \quad 1.01929724-0.50949038$

H $1.827926651 .14387293-0.12312851$

$\mathrm{H} \quad 1.67592717-0.47913796 \quad 0.51847704$

$\mathrm{H} \quad 0.66602729 \quad 0.32810509 \quad 2.49916972$

$\mathrm{H} \quad 0.87068036-3.83173111 \quad 1.11088084$ 
$\mathrm{H} \quad 0.78683948-3.54345977-0.66635393$

$\mathrm{H}-0.45884520-1.73768972 \quad 1.53214046$

$\mathrm{H}-0.86350639 \quad 3.29400294 \quad 0.28816550$

$\mathrm{H} \quad 0.85700808 \quad 3.09746410-0.07610076$

$\mathrm{H} \quad 0.33528825 \quad 4.31671052 \quad 1.08790377$

H $0.54082085 \quad 3.55464061 \quad 3.54478850$

$\mathrm{H}-0.216446892 .00926685 \quad 3.94249550$

$\mathrm{H}-1.16248282 \quad 3.27575695 \quad 3.15126282$

$00.74705233-0.98137903-2.30219782$

$00.40321875 \quad 0.34601751-1.94150609$

$0-0.35674740-1.75230074-1.85799731$

Vibrational frequencies (in $\mathrm{cm}-1$ )

$\begin{array}{llllllllllllllll}23.7 & 36.2 & 49.3 & 70.0 & 85.0 & 97.3 & 104.5 & 153.6 & 194.0 & 207.6 & 238.6 & 278.9 & 317.2 & 321.2\end{array}$

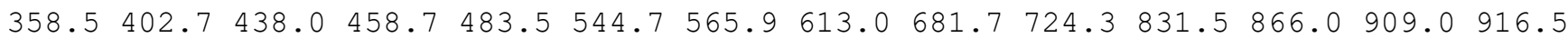
$951.0 \quad 971.9986 .2 \quad 993.1 \quad 1001.8 \quad 1017.0 \quad 1018.5 \quad 1059.8 \quad 1065.3 \quad 1077.9 \quad 1096.2 \quad 1115.2 \quad 1130.0$

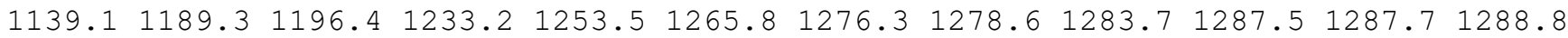

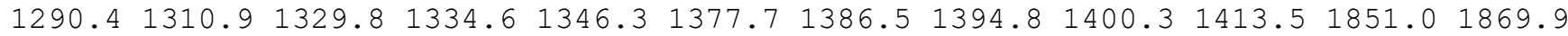
$2647.12664 .92669 .5 \quad 2669.8 \quad 2673.0 \quad 2676.2 \quad 2678.3 \quad 2683.52708 .92736 .02754 .82758 .1$ $2776.92779 .02780 .2 \quad 2781.4$

name $=$ MIN258

energy (in $\mathrm{kcal} / \mathrm{mol})=-2.535789$

$\operatorname{ZPE}($ in $\mathrm{kcal} / \mathrm{mol})=143.137$

$\mathrm{G}($ in $\mathrm{kcal} / \mathrm{mol})=115.55$

Geometry

C $-2.12973039-0.31675110-0.98304191$

C $-0.60780990-0.34039426-0.85759506$

C $0.00985706 \quad 1.10231614 \quad-0.84324441$

C $1.19954548 \quad 1.30885616 \quad 0.09424105$

C $0.80269094 \quad 1.78057300 \quad 1.45282270$

C $1.527545831 .52198440 \quad 2.54197565$

C $-0.09542064-1.25410081 \quad 0.20126504$

C $-0.48620773-1.32659216 \quad 1.47987127$

C $-1.54186325-0.47927418 \quad 2.10595411$

C $0.14468720-2.31326867 \quad 2.41084009$

$\mathrm{H}-2.60221751 \quad 0.30125459-0.21528084$

$\mathrm{H}-2.54448444-1.32971723-0.89880346$

$\mathrm{H}-2.43146173 \quad 0.07476351-1.96580883$

$\mathrm{H}-0.761779091 .88852275-0.72072136$

$\mathrm{H} \quad 1.88241574 \quad 2.06441593-0.36460093$

H $1.81156537 \quad 0.38320715 \quad 0.14781065$

$\mathrm{H}-0.10808740 \quad 2.37624859 \quad 1.49943354$

H $1.26928842 \quad 1.87454988 \quad 3.52703072$

H 2.43607036 $0.94071794 \quad 2.53420122$

$\mathrm{H} \quad 0.68205332-1.92759623-0.17339304$

$\mathrm{H}-1.60397316 \quad 0.51847999 \quad 1.65114284$

$\mathrm{H}-1.36439557-0.32260715 \quad 3.17926452$

$\mathrm{H}-2.52942752-0.95429998 \quad 2.00755195$

$\mathrm{H} \quad 0.76210359-1.79805148 \quad 3.16221542$

$\mathrm{H} \quad 0.79689612-3.03110731 \quad 1.89627056$

$\mathrm{H}-0.61268552-2.89572025 \quad 2.95308369$

$0.88265123-0.03319393-2.55938953$

$0.459164831 .26789504-2.18976369$

$0-0.17620913-0.87176123-2.13501839$

Vibrational frequencies (in $\mathrm{cm}-1$ )

$\begin{array}{llllllllllllllll}22.2 & 37.2 & 47.4 & 74.7 & 99.5 & 102.7 & 128.8 & 149.5 & 178.0 & 206.9 & 247.5 & 277.1 & 303.8 & 334.5\end{array}$ $345.4 \quad 392.1 \quad 419.2 \quad 471.1 \quad 487.4 \quad 494.8 \quad 559.1 \quad 588.9 \quad 698.4 \quad 713.4 \quad 840.6 \quad 869.5 \quad 908.8 \quad 925.2$ $952.4964 .2970 .5 \quad 992.0 \quad 1000.2 \quad 1018.7 \quad 1019.4 \quad 1025.3 \quad 1082.5 \quad 1090.3 \quad 1094.1 \quad 1115.8 \quad 1133.8$

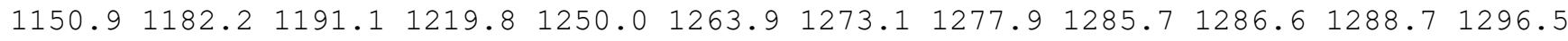

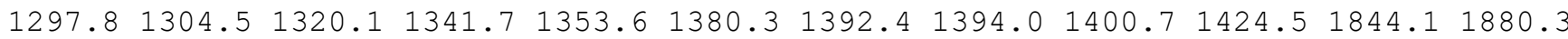
$2645.92668 .62669 .5 \quad 2670.6 \quad 2672.3 \quad 2675.2 \quad 2677.12688 .12717 .2 \quad 2730.2 \quad 2741.22758 .3$ $2777.32779 .8 \quad 2780.12791 .0$ 
name $=$ MIN259

energy (in $\mathrm{kcal} / \mathrm{mol})=-2.592493$

$\mathrm{ZPE}($ in $\mathrm{kcal} / \mathrm{mol})=143.203$

$\mathrm{G}($ in $\mathrm{kcal} / \mathrm{mol})=115.589$

Geometry

C $-2.99053374-1.14334190 \quad-0.06494008$

C $-1.46802331-1.18838456 \quad 0.02914248$

C $-0.82694320 \quad 0.24355821 \quad 0.02679357$

C $0.26563195 \quad 0.44351216 \quad 1.07662388$

C 0.732216391 .861880121 .05954078

C $-0.14599756-3.03273750 \quad 1.05131613$

C $-1.02649780-2.04208530 \quad 1.17671270$

C $1.82297371 \quad 2.29180880 \quad 0.41001446$

C $2.714864621 .39732274 \quad-0.38408114$

C $2.22195687 \quad 3.73182180 \quad 0.43836675$

$\mathrm{H}-3.31369601-0.61814269-0.97503669$

$\mathrm{H}-3.43843789-0.64058263 \quad 0.79767077$

$\mathrm{H}-3.41182640-2.15651627-0.11861298$

$\mathrm{H}-1.58767479 \quad 1.05016377 \quad 0.06230060$

$\mathrm{H} 1.10014846-0.26409593 \quad 0.88429996$

$\mathrm{H}-0.11278899 \quad 0.181352892 .08593235$

$\mathrm{H} \quad 0.10443640 \quad 2.54826284 \quad 1.62201846$

$\mathrm{H} \quad 0.16496247 \quad-3.66177413 \quad 1.87114941$

$\mathrm{H} \quad 0.32799936-3.29431072 \quad 0.11392035$

$\mathrm{H}-1.49898991-1.79943642 \quad 2.12755431$

H $3.25077899 \quad 0.68990220 \quad 0.26440210$

$\mathrm{H} 3.468871541 .94731672-0.96002707$

$\mathrm{H} 2.134286190 .79928277-1.10645659$

$\mathrm{H} \quad 3.25135112 \quad 3.85209818 \quad 0.80395052$

$\mathrm{H} \quad 1.57583695 \quad 4.341609531 .08238364$

H $2.17834636 \quad 4.16940983-0.57008426$

O $0.08782882-0.98048431-1.60961445$

$\begin{array}{lllll}0 & -0.27902906 & 0.34838885 & -1.28734196\end{array}$

o $-1.03922263-1.75305884-1.22095937$

Vibrational frequencies (in $\mathrm{cm}-1$ )

$\begin{array}{llllllllllllll}24.8 & 41.7 & 56.0 & 72.2 & 82.2 & 93.7 & 112.8 & 151.2 & 187.6 & 213.9 & 241.0 & 272.7 & 295.6 & 309.2\end{array}$ $337.7401 .5 \quad 434.4 \quad 474.0 \quad 493.6 \quad 553.1 \quad 565.3 \quad 596.6 \quad 684.2 \quad 714.1 \quad 851.3 \quad 864.4 \quad 908.7 \quad 926.4$

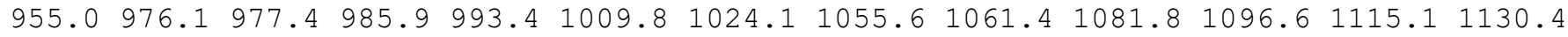

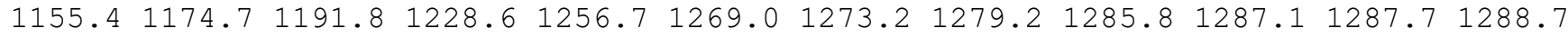

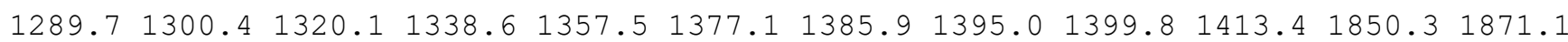
$2658.32663 .4 \quad 2669.4 \quad 2671.5 \quad 2675.0 \quad 2677.7 \quad 2678.92688 .72710 .2 \quad 2740.22756 .32762 .3$ $2777.8 \quad 2779.8 \quad 2780.4 \quad 2782.1$

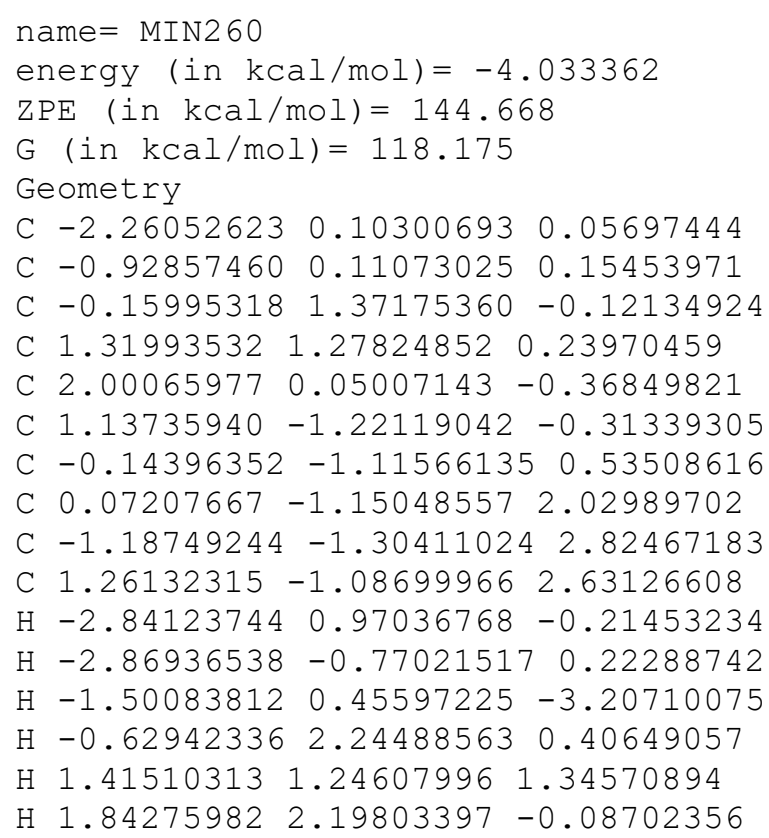


Н $2.26835811 \quad 0.25994881-1.42320258$

H $1.74352434-2.072426390 .04856309$

$\mathrm{H} \quad 0.83128424-1.48493158-1.34894910$

$\mathrm{H}-0.75939372 \quad-2.02674168 \quad 0.28015272$

$\mathrm{H}-1.74963723-2.19692568 \quad 2.51900811$

$\mathrm{H}-1.85132688-0.437700662 .68437160$

$\mathrm{H}-0.99805017-1.39474452 \quad 3.90167299$

$\mathrm{H} 2.96439959-0.12413044 \quad 0.14788131$

H $2.19952846 \quad-0.98540854 \quad 2.11325288$

$\mathrm{H} 1.38852395-1.13432907 \quad 3.70003211$

$0.14383406 \quad 0.88816210-2.38945217$

$0-0.349004681 .79880659-1.46993745$

O $-0.84516609-0.06016371-2.67826126$

Vibrational frequencies (in $\mathrm{cm}-1$ )

$\begin{array}{lllllllllllllll}29.2 & 46.2 & 53.9 & 80.6 & 86.1 & 135.6 & 170.3 & 177.4 & 207.4 & 268.4 & 277.4 & 304.4 & 345.4 & 382.5\end{array}$ $415.2 \quad 420.4 \quad 468.3 \quad 512.8 \quad 546.0 \quad 589.8 \quad 603.5 \quad 631.0 \quad 727.4 \quad 780.4 \quad 797.4 \quad 834.1 \quad 873.3 \quad 893.2$ $919.6962 .1969 .4991 .9998 .0 \quad 1039.1 \quad 1044.4 \quad 1058.1 \quad 1067.3 \quad 1079.3 \quad 1093.01107 .9 \quad 1158.1$ $\begin{array}{llllllllllllllll}1184.9 & 1199.1 & 1202.7 & 1219.7 & 1261.1 & 1269.1 & 1275.0 & 1277.2 & 1288.1 & 1298.1 & 1306.3 & 1318.9\end{array}$

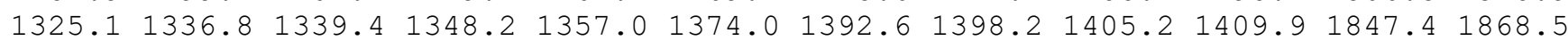
$2644.12661 .12667 .8 \quad 2669.6 \quad 2674.8 \quad 2677.5 \quad 2681.12723 .22723 .4 \quad 2735.92739 .02739 .7$ $2778.72789 .3 \quad 2793.3 \quad 2850.1$

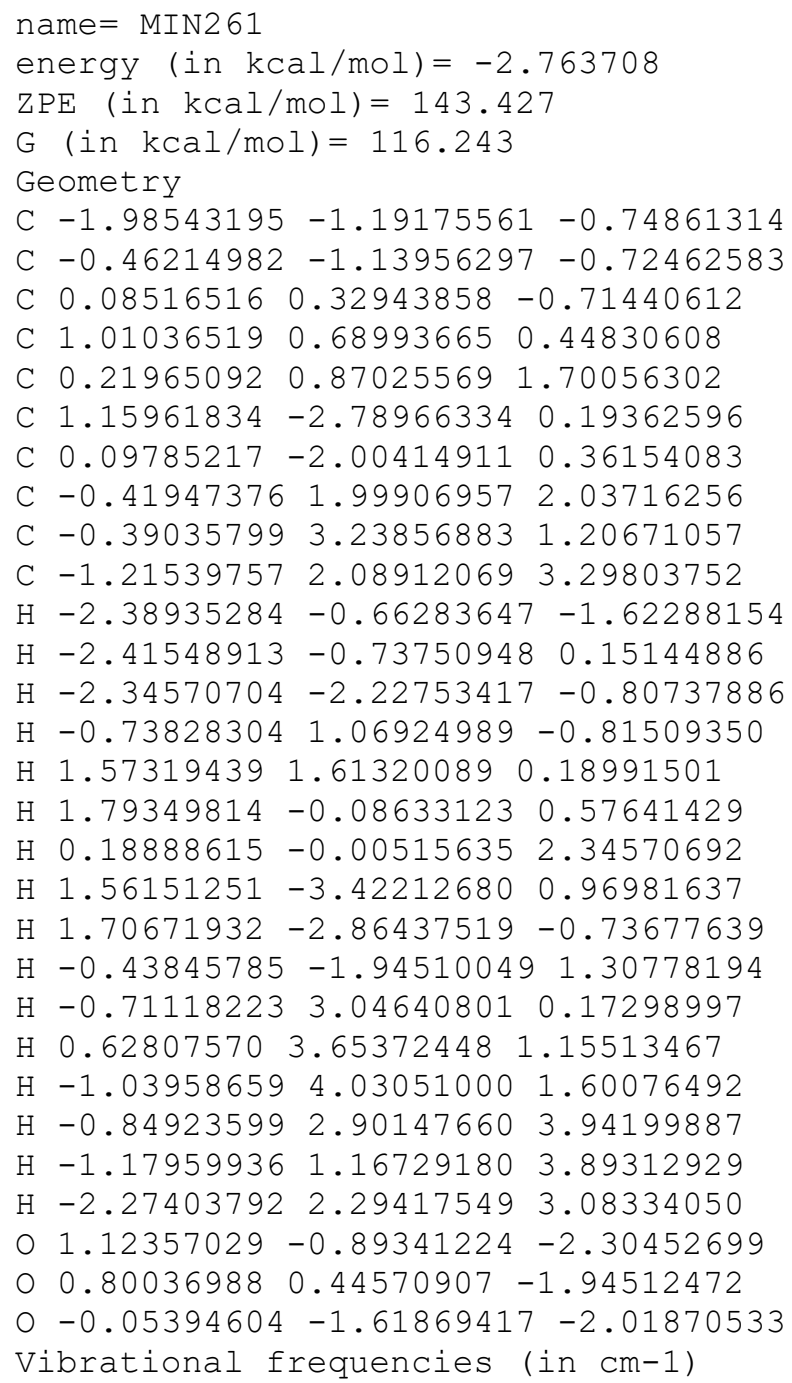


name $=$ MIN262

energy (in $\mathrm{kcal} / \mathrm{mol})=-2.387207$

$\mathrm{ZPE}($ in $\mathrm{kcal} / \mathrm{mol})=143.055$

$\mathrm{G}($ in $\mathrm{kcal} / \mathrm{mol})=115.115$

Geometry

C $-2.04321014-0.65538666-0.91051628$

C $-0.53004544-0.48905727-0.81114306$

C $-0.120382661 .02543733-0.77970610$

C 1.087924321 .381858550 .08906195

C $0.70038550 \quad 2.01463977 \quad 1.38335952$

C $1.411260091 .86204412 \quad 2.50114493$

C $0.09719729-1.37874878 \quad 0.20415625$

C $-0.15353779-1.41116634 \quad 1.51905459$

C $-1.11787714 \quad-0.52989318 \quad 2.23664086$

C $0.55630483-2.388583972 .40202861$

$\mathrm{H}-2.56412628-0.29920767-0.01734951$

$\mathrm{H}-2.30483757-1.71444845-1.04186853$

$\mathrm{H}-2.44404876-0.11561225-1.77906413$

$\mathrm{H}-0.99528374 \quad 1.67211161-0.56853265$

$\mathrm{H} \quad 1.74184811 \quad 2.08964997 \quad-0.47814047$

$\mathrm{H} \quad 1.73007509 \quad 0.48890477 \quad 0.24379644$

$\mathrm{H}-0.19363456 \quad 2.63750640 \quad 1.35383081$

H 2.30298401 $1.25909034 \quad 2.56795526$

H $1.15776413 \quad 2.33333532 \quad 3.43673642$

$\mathrm{H} \quad 0.82851196-2.06749178-0.23303052$

$\mathrm{H}-2.00346443-1.095732202 .56004668$

$\mathrm{H}-1.46615351 \quad 0.31407876 \quad 1.62697349$

$\mathrm{H}-0.66423816-0.09131913 \quad 3.13908144$

$\mathrm{H} 1.09329794-1.87644787 \quad 3.21331326$

H $1.29495968-2.996178301 .86234924$

$\mathrm{H}-0.15412321-3.084949982 .87021934$

$0-0.12501874 \quad 0.19810567-2.90574665$

$0.299115261 .31373458-2.11933232$

$0.04753216-0.91422976-2.06823202$

Vibrational frequencies (in $\mathrm{cm}-1$ )

$\begin{array}{lllllllllllllll}21.7 & 31.4 & 55.7 & 57.5 & 78.5 & 102.6 & 124.8 & 140.6 & 170.8 & 218.3 & 250.4 & 263.9 & 308.1 & 335.8\end{array}$

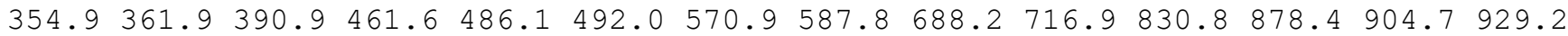
$961.6 \quad 976.0 \quad 977.9 \quad 992.7 \quad 1005.7 \quad 1010.8 \quad 1017.7 \quad 1029.6 \quad 1083.4 \quad 1096.3 \quad 1111.2 \quad 1120.9 \quad 1135.4$ $1145.01175 .1 \quad 1191.3 \quad 1207.5 \quad 1250.6 \quad 1266.7 \quad 1268.6 \quad 1279.5 \quad 1283.5 \quad 1286.5 \quad 1288.3 \quad 1292.8$ $1298.6 \quad 1308.7 \quad 1335.0 \quad 1352.7 \quad 1358.0 \quad 1382.1 \quad 1392.7 \quad 1396.0 \quad 1403.4 \quad 1429.0 \quad 1845.4 \quad 1882.8$ $2641.42665 .3 \quad 2669.92671 .1 \quad 2672.7 \quad 2673.92676 .5 \quad 2678.32716 .72728 .12736 .72756 .5$ $2776.8 \quad 2780.3 \quad 2780.7 \quad 2790.7$

name $=$ MIN263

energy (in $\mathrm{kcal} / \mathrm{mol})=-2.480405$

$\mathrm{ZPE}(\mathrm{in} \mathrm{kcal} / \mathrm{mol})=143.199$

G $($ in $\mathrm{kcal} / \mathrm{mol})=115.182$

Geometry

C $-1.93374739-1.18752317-0.63159661$

C $-0.40830271-1.18818179-0.62737184$

C $0.16350415 \quad 0.27207000 \quad-0.63207381$

C $1.14034392 \quad 0.62892874 \quad 0.48783245$

C $0.39716687 \quad 0.95583641 \quad 1.74005028$

C $0.97229408 \quad-3.066430390 .26359341$

C $0.11991177-2.06376575 \quad 0.46547278$

C $-0.269480452 .09727413 \quad 1.96001146$

C $-0.36124436 \quad 3.22221003 \quad 0.98489876$

C $-1.00991582 \quad 2.32194125 \quad 3.23947413$

$\mathrm{H}-2.34308153-0.69422853 \quad 0.25639956$

$\mathrm{H}-2.32645637-2.21292439-0.65554137$

$\mathrm{H}-2.32755266-0.67634423-1.51960391$

$\mathrm{H}-0.658370401 .01431896-0.70612130$ 
H $1.77391647 \quad 1.48451524 \quad 0.16360322$

$\mathrm{H} 1.86073840 \quad-0.200681220 .65056520$

$\mathrm{H} 0.42848578 \quad 0.17445888 \quad 2.49713126$

$\mathrm{H} \quad 1.33806082-3.70352738 \quad 1.05429937$

$\mathrm{H} \quad 1.37197050-3.33146144 \quad-0.70663943$

$\mathrm{H}-0.26619628-1.81571814 \quad 1.45422542$

$\mathrm{H}-1.34466911 \quad 3.23765677 \quad 0.49251941$

$\mathrm{H}-0.23192193 \quad 4.19677429 \quad 1.47637491$

$\begin{array}{lllll}\mathrm{H} & 0.39711521 & 3.16520351 & 0.19157892\end{array}$

$\mathrm{H}-0.61980028 \quad 3.19773661 \quad 3.77701084$

$\mathrm{H}-0.94395629 \quad 1.46873772 \quad 3.92695704$

$\mathrm{H}-2.07760620 \quad 2.50458333 \quad 3.05223061$

$\begin{array}{llll}0 & 0.28689645 & -0.59982119 & -2.69507883\end{array}$

$0 \quad 0.92931199 \quad 0.33207321-1.84216439$

o $0.05998771-1.73177728-1.87303395$

Vibrational frequencies (in $\mathrm{cm}-1$ )

$\begin{array}{lllllllllllllllll}28.1 & 31.9 & 43.1 & 61.2 & 72.8 & 85.9 & 101.5 & 161.7 & 199.0 & 227.9 & 232.1 & 273.1 & 308.5 & 311.7\end{array}$ $347.6 \quad 407.5 \quad 428.5 \quad 470.8 \quad 485.3 \quad 528.6 \quad 563.6 \quad 605.4 \quad 674.6 \quad 705.0 \quad 800.5 \quad 874.4 \quad 908.8 \quad 916.7$

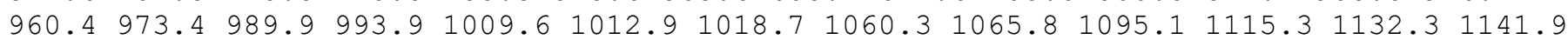
$\begin{array}{lllllllllllll}1147.0 & 1176.9 & 1189.4 & 1224.8 & 1248.6 & 1269.8 & 1273.3 & 1280.1 & 1284.3 & 1285.6 & 1288.7 & 1290.2\end{array}$ $1290.81319 .8 \quad 1338.1 \quad 1341.1 \quad 1345.8 \quad 1378.0 \quad 1388.8 \quad 1398.8 \quad 1401.0 \quad 1415.2 \quad 1850.7 \quad 1873.3$ $2650.32667 .7 \quad 2669.6 \quad 2671.92673 .7 \quad 2675.4 \quad 2677.92679 .3 \quad 2708.8 \quad 2737.92753 .2 \quad 2756.2$ $2777.8 \quad 2780.2 \quad 2780.92782 .3$

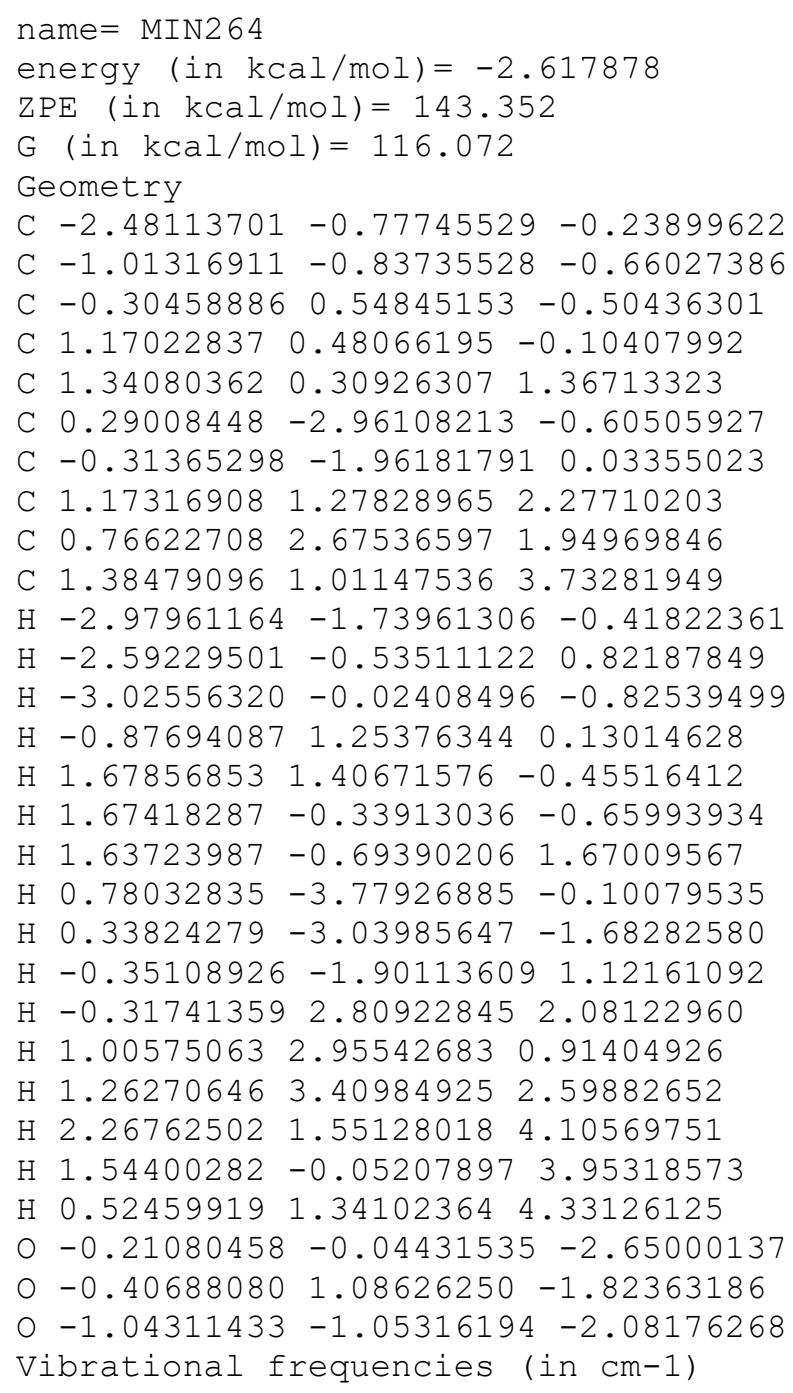


$2651.02667 .6 \quad 2669.92670 .5 \quad 2673.6 \quad 2676.1 \quad 2680.02690 .52711 .5 \quad 2737.3 \quad 2753.7 \quad 2754.8$ $2777.7 \quad 2780.3 \quad 2781.0 \quad 2783.8$

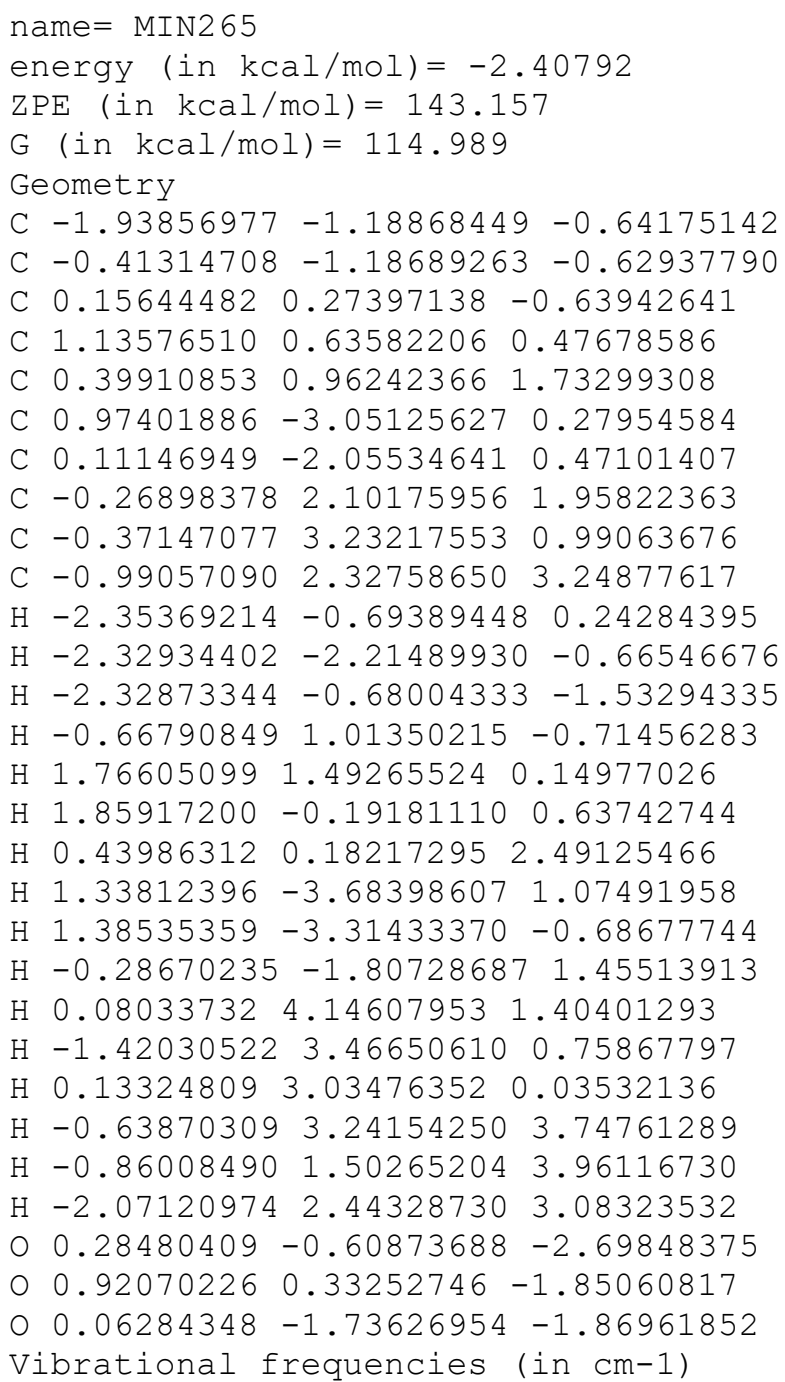

$\begin{array}{llllllllllllllll}-31.1 & 31.1 & 32.9 & 50.3 & 74.4 & 89.5 & 106.3 & 162.6 & 199.9 & 228.4 & 232.0 & 273.1 & 307.9 & 312.6\end{array}$ $347.7406 .3 \quad 428.9 \quad 471.4 \quad 484.2 \quad 529.7 \quad 564.2 \quad 606.5 \quad 675.0 \quad 705.2 \quad 801.4 \quad 874.6910 .1915 .7$ $960.8 \quad 973.7 \quad 990.2 \quad 995.9 \quad 1009.3 \quad 1011.6 \quad 1020.1 \quad 1060.5 \quad 1065.7 \quad 1095.8 \quad 1116.1 \quad 1132.01141 .9$ $1146.81176 .3 \quad 1189.7 \quad 1224.5 \quad 1248.5 \quad 1270.5 \quad 1272.1 \quad 1280.51284 .3 \quad 1284.5 \quad 1288.6 \quad 1291.0$

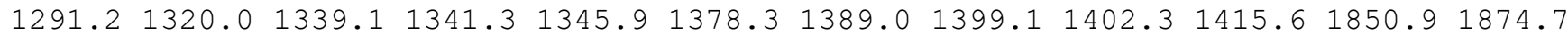
$2650.82668 .32669 .5 \quad 2671.72673 .8 \quad 2675.12676 .92678 .92707 .82738 .52752 .5 \quad 2755.4$ $2778.12779 .5 \quad 2780.8 \quad 2781.8$

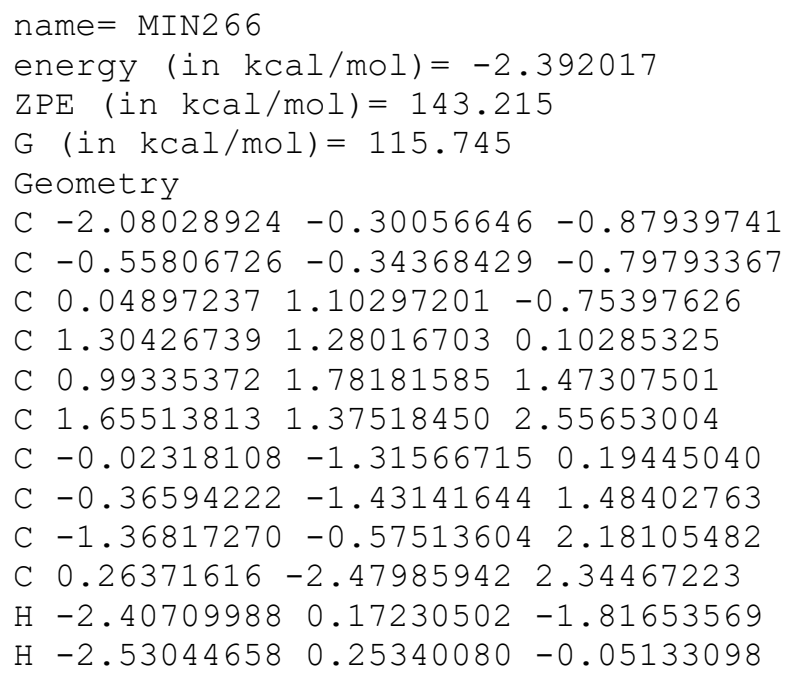


$\mathrm{H}-2.49874176-1.31565623-0.86892959$

$\mathrm{H}-0.72731400 \quad 1.85827745-0.52070452$

$\mathrm{H} 1.985128632 .00707034-0.40409899$

$\mathrm{H} \quad 1.88674167 \quad 0.33510754 \quad 0.12926162$

$\mathrm{H} \quad 0.20240324 \quad 2.52946198 \quad 1.52986685$

H $2.44457416 \quad 0.64032815 \quad 2.53719084$

$\mathrm{H} 1.45779052 \quad 1.74641467 \quad 3.54907822$

$\mathrm{H} \quad 0.72432443-1.99031496-0.23736569$

$\mathrm{H}-1.23643662-0.57789370 \quad 3.27169160$

$\mathrm{H}-2.39092306-0.92832712 \quad 1.98101398$

$\mathrm{H}-1.30644318 \quad 0.47499526 \quad 1.86076974$

$\mathrm{H} \quad 0.86664022 \quad-2.02262994 \quad 3.14316948$

$\mathrm{H} \quad 0.92745954 \quad-3.15293890 \quad 1.78572314$

$\mathrm{H}-0.49833225-3.10763458 \quad 2.82825030$

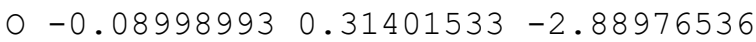

$\begin{array}{llll}0 & 0.48890009 & 1.35155309 & -2.09470258\end{array}$

o $-0.05856740 \quad-0.82350907-2.06933919$

Vibrational frequencies (in $\mathrm{cm}-1$ )

$\begin{array}{lllllllllllllll}23.2 & 37.4 & 52.2 & 74.7 & 98.7 & 105.6 & 139.9 & 146.1 & 190.2 & 216.9 & 247.4 & 269.7 & 299.3 & 341.0\end{array}$ $357.5 \quad 361.2 \quad 396.5 \quad 466.2 \quad 486.9 \quad 489.4 \quad 566.5 \quad 591.6 \quad 692.2 \quad 717.3 \quad 826.3 \quad 878.0 \quad 905.8 \quad 931.8$

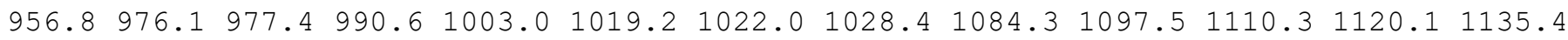
$1146.01176 .3 \quad 1186.4 \quad 1208.7 \quad 1247.6 \quad 1267.8 \quad 1274.3 \quad 1279.2 \quad 1285.7 \quad 1286.4 \quad 1288.8 \quad 1297.7$ $1300.21310 .91333 .2 \quad 1350.3 \quad 1355.5 \quad 1381.5 \quad 1392.4 \quad 1395.91403 .4 \quad 1428.9 \quad 1847.2 \quad 1880.4$ $2644.62667 .72669 .12670 .62671 .8 \quad 2673.7 \quad 2677.42680 .62716 .72730 .22737 .12757 .4$ $2776.92779 .6 \quad 2780.92790 .3$

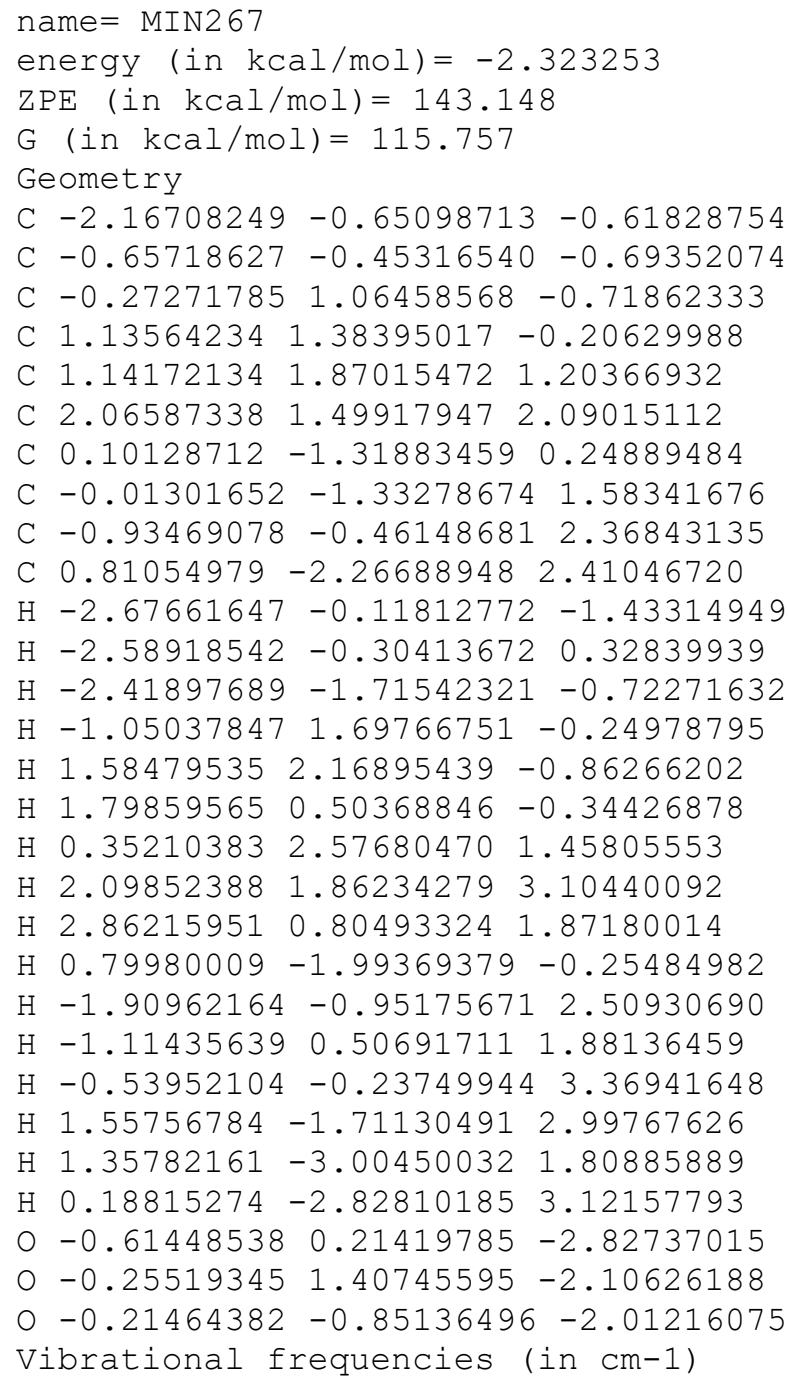


$\begin{array}{lllllllllllllll}1135.7 & 1171.2 & 1188.6 & 1212.3 & 1251.2 & 1266.6 & 1273.2 & 1278.6 & 1284.9 & 1286.5 & 1289.5 & 1291.8\end{array}$

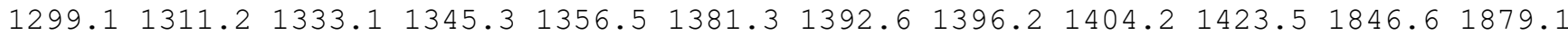
$2644.62667 .92669 .3 \quad 2670.6 \quad 2671.5 \quad 2674.6 \quad 2678.82683 .72716 .92730 .42741 .02757 .9$ $2777.32779 .92781 .6 \quad 2790.4$

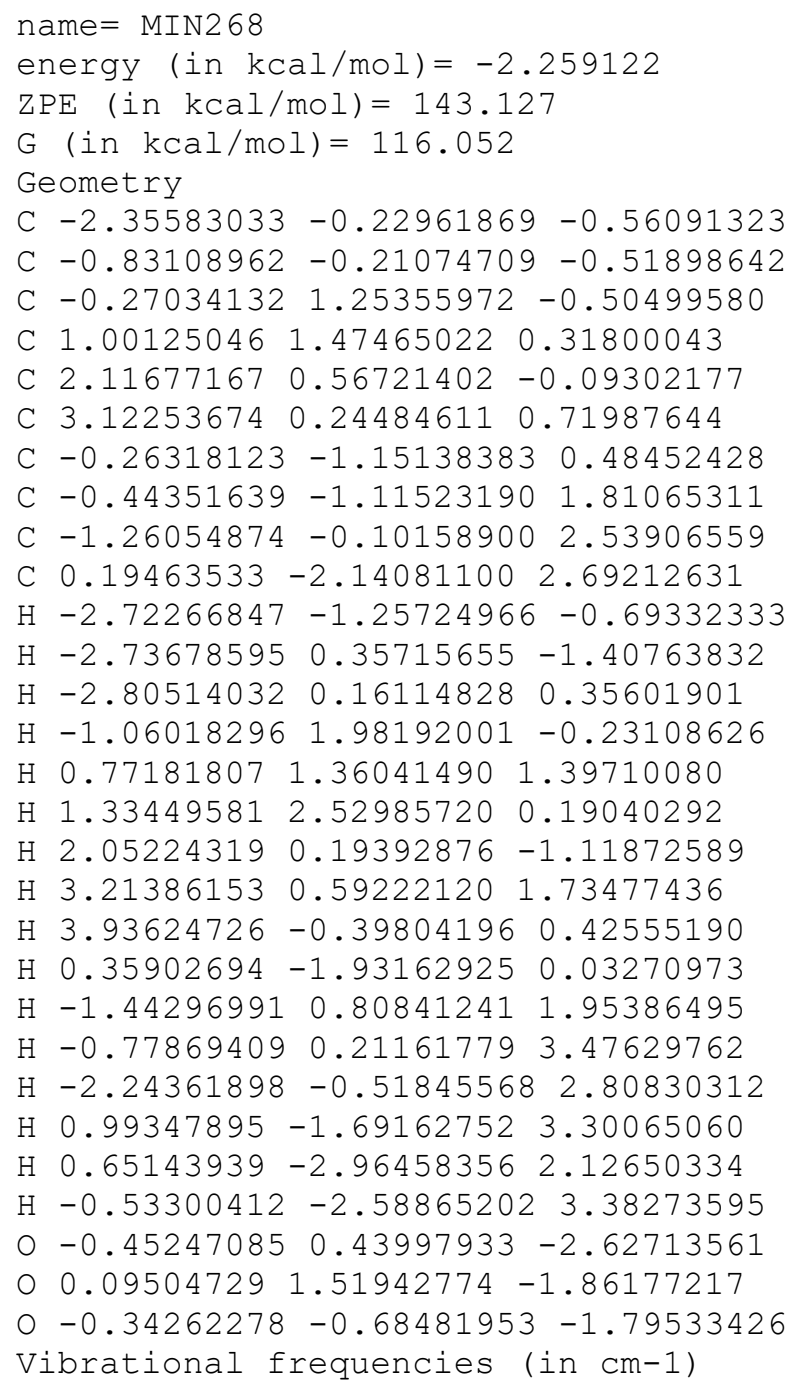


$\mathrm{H}-2.82087205-0.88402689-1.14497383$

$\mathrm{H}-2.62308685-0.95620640 \quad 0.62109466$

$\mathrm{H}-2.55744097-2.43614067-0.35132463$

$\begin{array}{llll}\mathrm{H} & -1.13105375 & 0.98949547 & -0.31244957\end{array}$

$\mathrm{H} \quad 1.428407261 .51498782-0.65031481$

$\mathrm{H} 1.74911833-0.18219456-0.32564614$

$\mathrm{H} 1.28194062 \quad 0.05802268 \quad 1.98872874$

$\mathrm{H} 1.41044182-3.417592371 .10365026$

$\mathrm{H} \quad 1.16428391-3.20754230-0.66966812$

$\mathrm{H}-0.29361217-1.63588554 \quad 1.57567837$

$\mathrm{H} \quad 0.53016070 \quad 4.20601612 \quad 1.62673654$

$\mathrm{H}-0.89217777 \quad 3.31326740 \quad 1.07234108$

$\mathrm{H} \quad 0.60296671 \quad 3.27992843 \quad 0.12708792$

$\mathrm{H} 0.81033540 \quad 1.30978128 \quad 3.95186137$

$\mathrm{H}-0.29953813 \quad 2.67330311 \quad 3.77284648$

$\mathrm{H} \quad 1.44979081 \quad 2.93781528 \quad 3.71173442$

$00.22361825-0.78263417-2.52695239$

$\begin{array}{lllll}0 & -0.32316522 & 0.44191244 & -2.07940230\end{array}$

o $-0.48812133-1.78252558-1.79935013$

Vibrational frequencies (in cm-1)

$\begin{array}{lllllllllllllll}25.4 & 37.3 & 51.4 & 80.6 & 89.7 & 100.4 & 116.7 & 167.8 & 193.3 & 205.7 & 241.6 & 275.5 & 306.1 & 319.9\end{array}$ $360.4 \quad 405.8 \quad 436.8 \quad 452.7 \quad 489.6 \quad 540.7 \quad 569.0 \quad 617.4 \quad 676.5 \quad 726.3 \quad 831.8 \quad 872.7 \quad 910.9 \quad 924.3$ $955.2974 .7 \quad 987.5 \quad 996.3 \quad 1000.9 \quad 1017.5 \quad 1020.5 \quad 1059.1 \quad 1061.1 \quad 1080.1 \quad 1095.91114 .8 \quad 1124.0$ $1139.21180 .3 \quad 1197.9 \quad 1238.4 \quad 1247.5 \quad 1264.9 \quad 1275.2 \quad 1278.4 \quad 1283.0 \quad 1286.6 \quad 1287.0 \quad 1289.2$

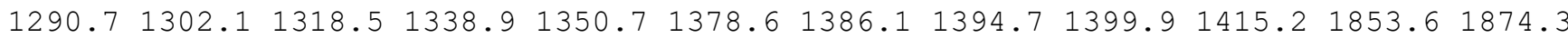
$2649.92666 .72669 .8 \quad 2670.1 \quad 2673.8 \quad 2676.1 \quad 2680.5 \quad 2692.32710 .5 \quad 2736.2 \quad 2753.4 \quad 2754.7$ $2777.62780 .12780 .8 \quad 2782.6$

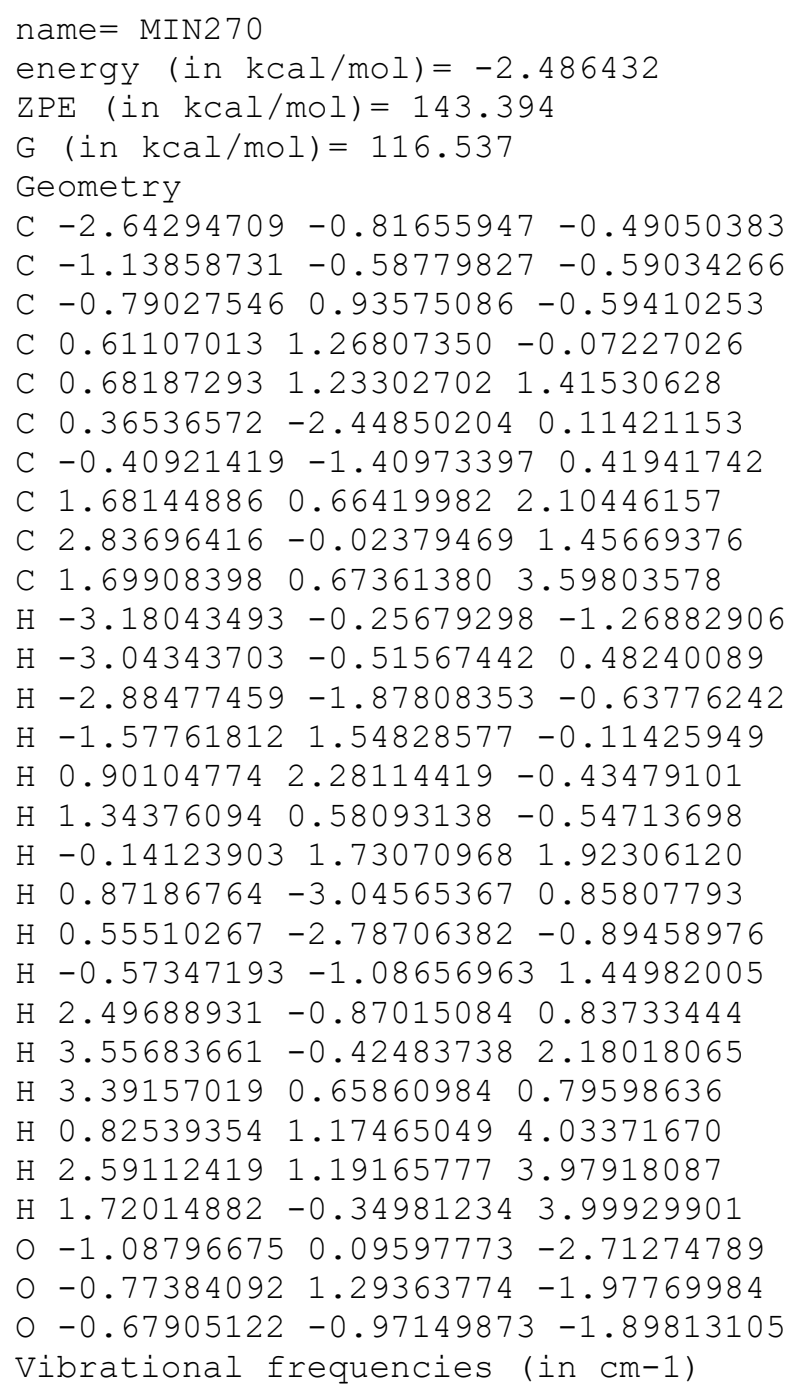


$\begin{array}{lllllllllllllllll}31.0 & 48.7 & 62.6 & 84.7 & 121.9 & 124.5 & 142.9 & 167.4 & 187.9 & 216.9 & 243.6 & 257.3 & 277.6 & 288.2\end{array}$

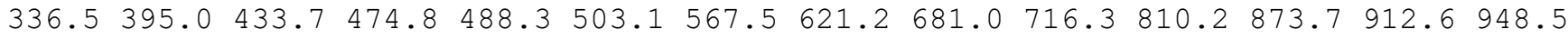

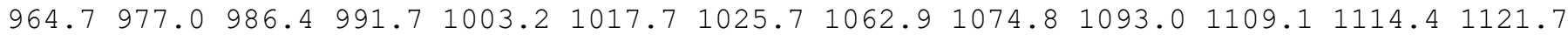
$1150.11168 .8 \quad 1207.2 \quad 1218.2 \quad 1249.2 \quad 1273.2 \quad 1277.2 \quad 1279.91285 .7 \quad 1286.5 \quad 1288.01289 .6$

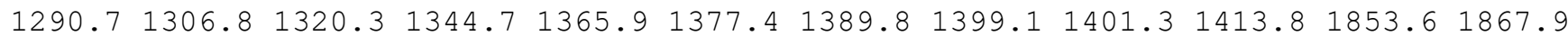
$2650.62660 .12669 .2 \quad 2671.5 \quad 2673.6 \quad 2677.0 \quad 2679.3 \quad 2687.2 \quad 2709.7 \quad 2733.4 \quad 2747.92761 .1$ 2776.22780 .22780 .32783 .2

\section{name $=$ MIN271}

energy (in $\mathrm{kcal} / \mathrm{mol})=-2.311912$

$\mathrm{ZPE}(\mathrm{in} \mathrm{kcal} / \mathrm{mol})=143.309$

$\mathrm{G}(\mathrm{in} \mathrm{kcal} / \mathrm{mol})=115.48$

Geometry

C $-2.14209472-0.80035675-0.65617575$

C $-0.66187713-0.47441110-0.46949764$

C $-0.41440603 \quad 1.07655284-0.51979978$

C $0.05393253 \quad 1.74696475 \quad 0.76708855$

C $-1.123429691 .97587291 \quad 1.66046320$

C $-1.62158764 \quad 3.18464407 \quad 1.91619831$

C $-0.11191683-1.13494223 \quad 0.75014373$

C $1.06857715-1.75988844 \quad 0.86796137$

C $2.05812565-1.93027770-0.23070368$

C $1.48060949-2.356565152 .17725912$

$\mathrm{H}-2.50135604-0.46803973-1.63819646$

$\mathrm{H}-2.76102471-0.32719586 \quad 0.11331233$

$\mathrm{H}-2.31187690-1.88377876-0.60307686$

$\mathrm{H}-1.29873538 \quad 1.59783741-0.94553683$

H $0.57484466 \quad 2.69684228 \quad 0.51897963$

$\mathrm{H} \quad 0.82212162 \quad 1.12825420 \quad 1.28036292$

$\mathrm{H}-1.55547290 \quad 1.07069231 \quad 2.08709363$

$\mathrm{H}-2.47492754 \quad 3.35289269 \quad 2.55465247$

$\mathrm{H}-1.22057376 \quad 4.10098292 \quad 1.51191555$

$\mathrm{H}-0.78453896-1.067051291 .60578051$

$\mathrm{H} 1.68089591-2.62784244-0.99635751$

H $2.24837808-0.97807537-0.75649352$

$\mathrm{H} \quad 3.02608072-2.31369747 \quad 0.11108604$

$\mathrm{H} 1.66114202-3.436933672 .07808493$

$\mathrm{H} 0.73144087-2.225219532 .96773493$

$\mathrm{H} 2.41532305-1.902680092 .53855140$

$0 \quad 0.368843410 .18127736-2.39799403$

○ $0.66567598 \quad 1.19030002-1.45394712$

o $0.04923795-0.96657741-1.61517032$

Vibrational frequencies (in $\mathrm{cm}-1$ )

$\begin{array}{lllllllllllllll}-22.9 & 26.0 & 43.0 & 56.8 & 96.8 & 100.3 & 161.9 & 177.6 & 197.2 & 214.2 & 241.5 & 261.6 & 312.0 & 338.1\end{array}$

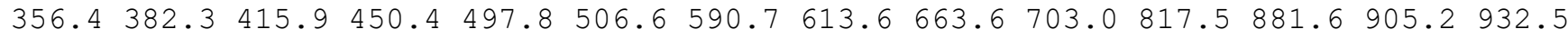

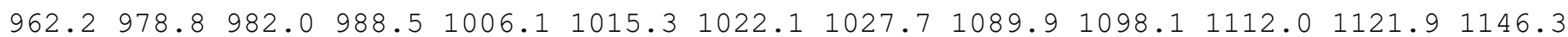

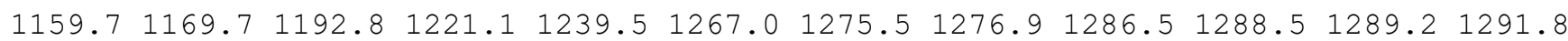

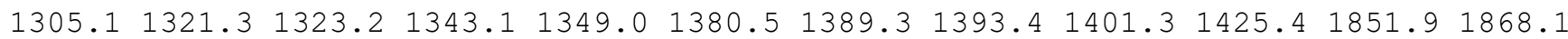
$2651.32654 .72669 .72673 .5 \quad 2673.6 \quad 2676.5 \quad 2678.02679 .62714 .82736 .2 \quad 2751.4 \quad 2756.8$ $2775.5 \quad 2779.6 \quad 2781.5 \quad 2788.5$

\section{name $=$ MIN272}

energy (in $\mathrm{kcal} / \mathrm{mol})=-2.731189$

$\mathrm{ZPE}($ in $\mathrm{kcal} / \mathrm{mol})=143.821$

$\mathrm{G}($ in $\mathrm{kcal} / \mathrm{mol})=119.252$

Geometry

$\begin{array}{llll}\text { C } & -2.34249050 & -0.20670787 & 0.35403207\end{array}$

C $-0.84416058-0.25539086 \quad 0.26935298$

C $-0.151862361 .14051037 \quad 0.09571227$

C $1.34224841 \quad 0.85636404 \quad 0.38454361$

C $1.34899127-0.65266634 \quad 0.77959235$

C $1.03583945-1.37931865-0.52069730$

C $-0.25318195-1.13764447-0.82633438$ 
C $-0.03828232-0.80688758 \quad 1.52784369$

C $-0.17210844 \quad 0.02010382 \quad 2.79948925$

C $-0.37532667-2.25606071 \quad 1.86643234$

$\mathrm{H}-2.772643120 .29402101-0.52483528$

$\mathrm{H}-2.684889890 .33850011 \quad 1.24199620$

$\mathrm{H}-2.77450384-1.214742890 .40163189$

$\mathrm{H}-0.590429161 .90704916 \quad 0.76997269$

$\mathrm{H} 1.741620331 .489400531 .18592730$

$\mathrm{H} \quad 1.96653040 \quad 1.04139267 \quad-0.50547218$

H $2.22617620-0.98317637 \quad 1.33028181$

$\mathrm{H} 1.77409434-1.93590543-1.05399746$

$\mathrm{H} 1.010452522 .16937233-3.17743936$

$\mathrm{H}-0.82991913-1.45907721-1.66508218$

$\mathrm{H} 0.41776623-0.41882828 \quad 3.61402971$

$\mathrm{H}-1.21654635 \quad 0.05583934 \quad 3.13267176$

$\mathrm{H} \quad 0.16908659 \quad 1.05295538 \quad 2.68706290$

$\mathrm{H} \quad 0.32029335-2.66116482 \quad 2.60921192$

$\mathrm{H}-0.32650157-2.91287725 \quad 0.98922386$

$\mathrm{H}-1.38941590 \quad-2.33986512 \quad 2.27287237$

$\begin{array}{lllll}0 & -0.06254301 & 0.95147249 & -2.22781131\end{array}$

O $-0.41442978 \quad 1.75353741-1.14829668$

O $1.203985711 .33613177-2.68324178$

Vibrational frequencies (in $\mathrm{cm}-1$ )

$\begin{array}{llllllllllllllll}49.9 & 88.9 & 123.9 & 140.7 & 160.4 & 172.3 & 199.2 & 210.4 & 226.0 & 263.7 & 302.0 & 329.2 & 352.6 & 392.2\end{array}$ $401.7 \quad 425.4 \quad 475.9 \quad 514.0 \quad 545.4 \quad 568.9 \quad 620.2 \quad 673.2 \quad 706.7 \quad 765.1831 .1 \quad 839.6 \quad 876.2 \quad 894.4$ $948.1966 .9968 .8 \quad 995.4 \quad 1005.6 \quad 1013.7 \quad 1018.1 \quad 1021.3 \quad 1031.8 \quad 1070.0 \quad 1103.7 \quad 1107.3 \quad 1129.5$ $\begin{array}{llllllllllllll}1133.8 & 1160.3 & 1174.9 & 1199.2 & 1223.8 & 1231.9 & 1250.8 & 1270.8 & 1281.3 & 1288.9 & 1292.3 & 1293.7\end{array}$

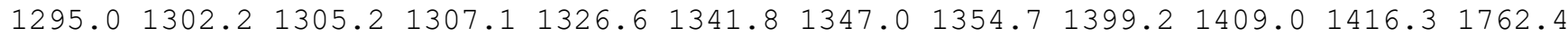
$2669.22670 .8 \quad 2675.12675 .7 \quad 2676.6 \quad 2678.7 \quad 2682.32688 .92752 .12758 .52781 .12781 .6$ $2782.92785 .2 \quad 2797.2 \quad 2850.8$

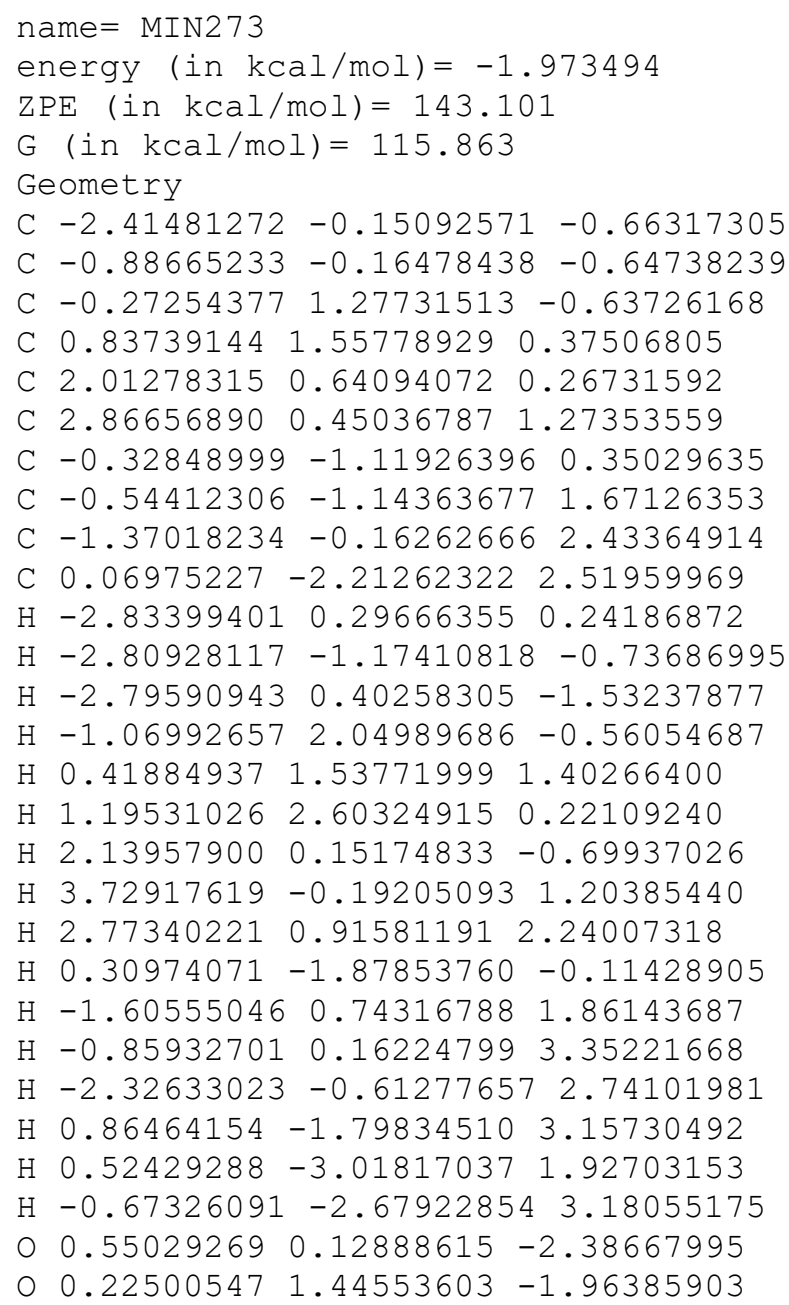


$0-0.55173922-0.64892964-1.97034780$

Vibrational frequencies (in $\mathrm{cm}-1$ )

$\begin{array}{lllllllllllllll}35.0 & 46.6 & 56.9 & 70.0 & 87.2 & 101.5 & 128.2 & 147.2 & 180.1 & 194.0 & 248.2 & 264.4 & 299.2 & 320.0\end{array}$ $348.2 \quad 384.8 \quad 411.1 \quad 471.0 \quad 482.4 \quad 504.8 \quad 564.7 \quad 632.5 \quad 698.7 \quad 719.3 \quad 841.9 \quad 877.1 \quad 898.5 \quad 907.7$

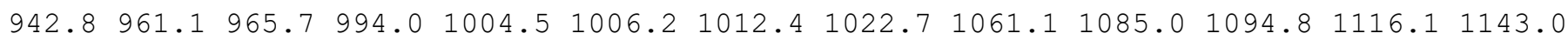
$1153.41176 .6 \quad 1194.5 \quad 1219.3 \quad 1258.9 \quad 1270.2 \quad 1271.6 \quad 1279.3 \quad 1284.6 \quad 1285.3 \quad 1288.5 \quad 1290.1$

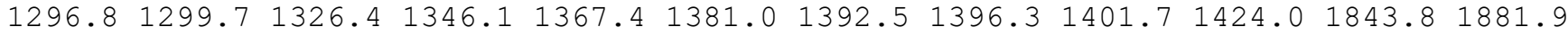
$2654.42667 .62669 .8 \quad 2670.3 \quad 2672.1 \quad 2674.7 \quad 2676.92679 .62718 .5 \quad 2730.72739 .2 \quad 2755.2$ 2776.82780 .02780 .12793 .5

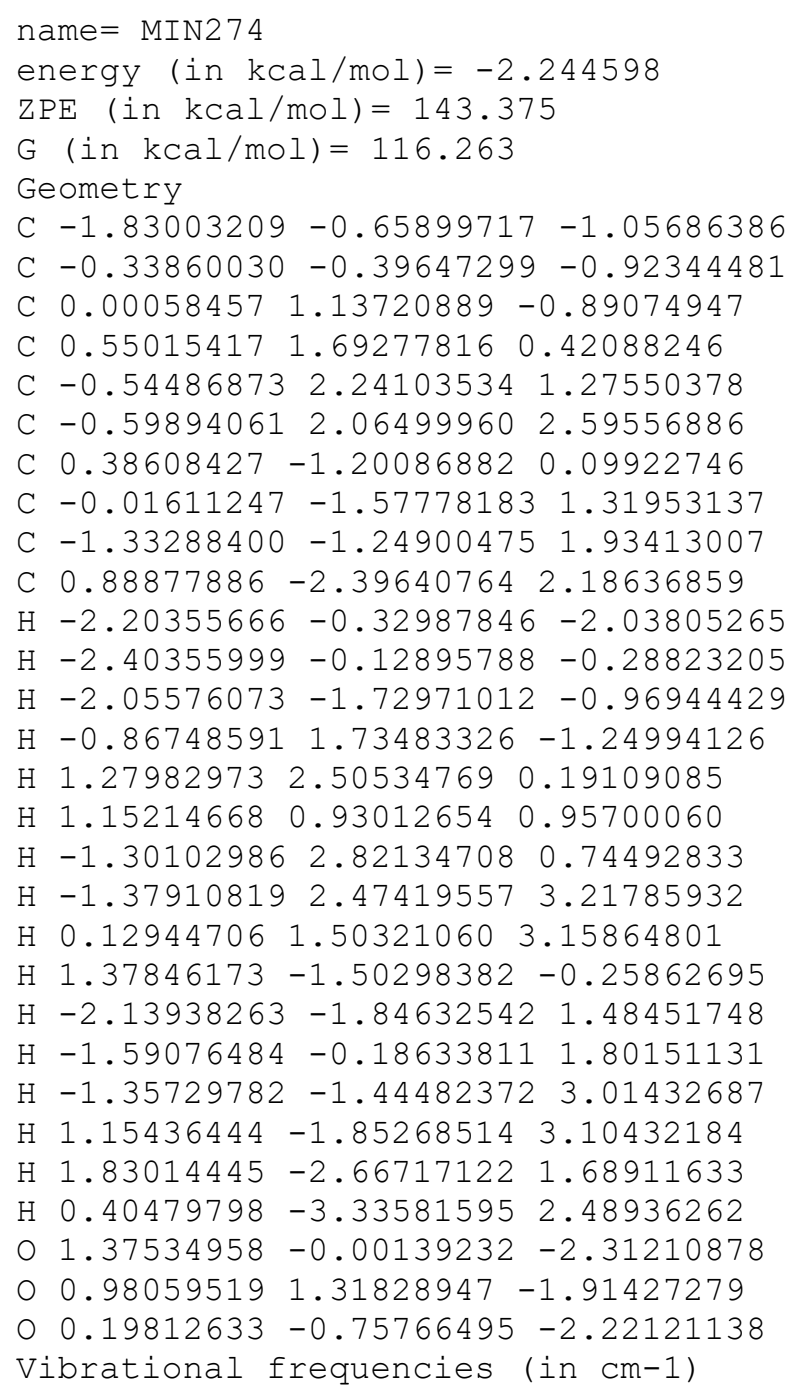


C $-1.08819194 \quad-2.165033192 .29343210$

C $-0.74819470 \quad-2.18186077 \quad 1.00607194$

$\begin{array}{llll}\text { C } 2.01234840 & 2.12738667 & 0.29659852\end{array}$

C $2.48705958 \quad 3.53295596 \quad 0.11953904$

C $2.935522691 .07137036-0.21299188$

$\mathrm{H}-3.26358676-2.26950375-0.06624158$

$\mathrm{H}-3.25940573-0.69968901-0.86854802$

$\mathrm{H}-3.25332540-0.79015795 \quad 0.90611860$

$\mathrm{H}-1.543989850 .94574508-0.05923801$

$\mathrm{H} 1.09270039-0.280277651 .04556996$

$\mathrm{H}-0.18964562 \quad 0.38765580 \quad 2.05911487$

$\mathrm{H} \quad 0.18204778 \quad 2.65602277 \quad 1.22705471$

$\mathrm{H}-0.65498061-2.82913864 \quad 3.02762018$

$\mathrm{H}-1.82172318-1.49677757 \quad 2.71883962$

$\mathrm{H}-0.00300363-2.877595390 .60980193$

H $3.45737118 \quad 3.69024318 \quad 0.61110710$

$\mathrm{H} \quad 1.79020058 \quad 4.27442151 \quad 0.53069681$

H 2.61672768 $3.76976057-0.94731272$

$\mathrm{H} 2.42083003 \quad 0.40673610-0.92787005$

H 3.310459210 .438569720 .60387998

$\mathrm{H} 3.809596621 .48165432-0.73291745$

$0-0.70163604-0.66828389-2.10829428$

$\begin{array}{lllll}0 & -0.02030510 & 0.24699076 & -1.27073498\end{array}$

o $-0.90211522-1.80726498-1.28834907$

Vibrational frequencies (in $\mathrm{cm}-1$ )

$\begin{array}{llllllllllllll}28.0 & 36.7 & 64.2 & 79.7 & 91.7 & 95.3 & 118.8 & 159.5 & 184.4 & 213.7 & 240.2 & 265.7 & 288.3 & 303.2\end{array}$ $346.2 \quad 411.0 \quad 436.7 \quad 465.4 \quad 485.9 \quad 504.4 \quad 561.6 \quad 599.7 \quad 683.6 \quad 703.8 \quad 832.1 \quad 876.4 \quad 898.1 \quad 925.6$ $957.6 \quad 972.4 \quad 978.1 \quad 991.0 \quad 993.8 \quad 1015.9 \quad 1024.3 \quad 1054.8 \quad 1062.4 \quad 1092.7 \quad 1115.2 \quad 1120.1 \quad 1145.1$

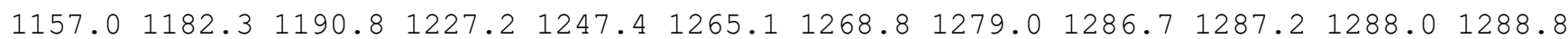

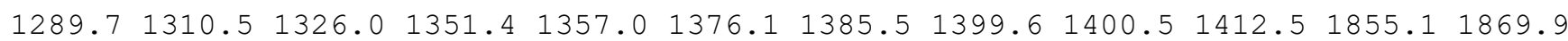
$2656.22660 .62670 .52670 .92674 .6 \quad 2677.3 \quad 2678.72683 .32709 .62738 .32743 .72762 .6$ 2776.82779 .92780 .12784 .1

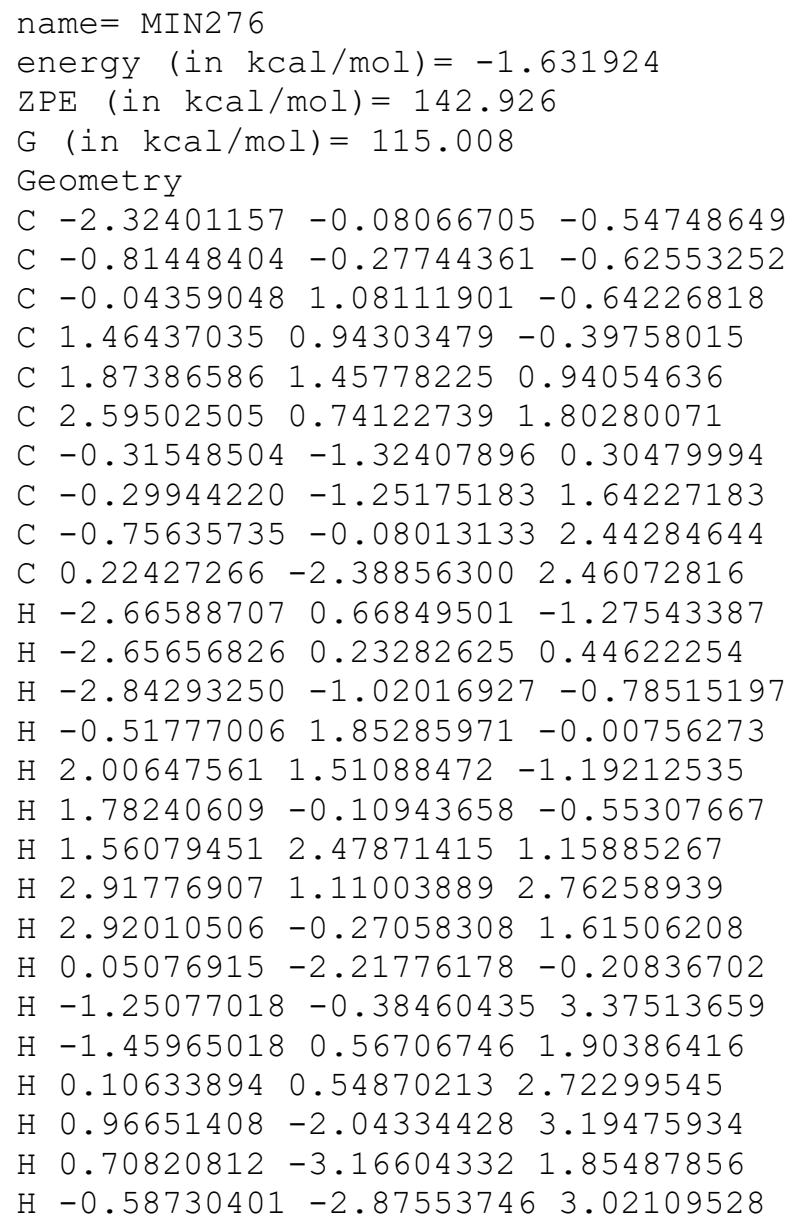


$0-0.63488024 \quad 0.39355813-2.76478793$

$0-0.20827607 \quad 1.53625787-1.98004304$

o $-0.48034668-0.74050095-1.95338916$

Vibrational frequencies (in $\mathrm{cm}-1$ )

$\begin{array}{lllllllllllllll}19.4 & 42.3 & 51.2 & 73.0 & 86.6 & 99.4 & 121.8 & 129.2 & 135.7 & 210.0 & 230.8 & 254.4 & 309.4 & 319.7\end{array}$ $342.7 \quad 366.4 \quad 382.2 \quad 456.0 \quad 482.8 \quad 488.3 \quad 564.0 \quad 612.5 \quad 703.0 \quad 713.9 \quad 858.1 \quad 902.2 \quad 904.8946 .1$ $964.5968 .6981 .0 \quad 990.8 \quad 1001.7 \quad 1014.6 \quad 1015.5 \quad 1030.3 \quad 1083.0 \quad 1093.2 \quad 1100.8 \quad 1114.1 \quad 1117.9$

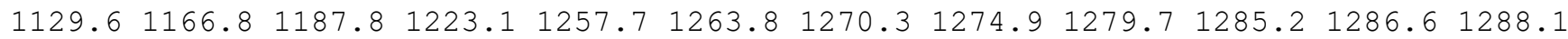

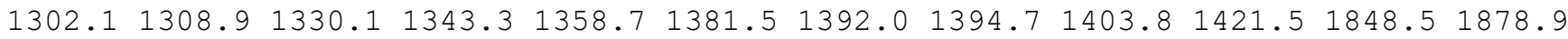
2645.02656 .42667 .72669 .12671 .02673 .12675 .62690 .02716 .62731 .32742 .62758 .2 $2773.5 \quad 2779.0 \quad 2779.92789 .6$

name $=$ MIN277

energy (in $\mathrm{kcal} / \mathrm{mol})=-2.074436$

$\mathrm{ZPE}(\mathrm{in} \mathrm{kcal} / \mathrm{mol})=143.39$

G $($ in $\mathrm{kcal} / \mathrm{mol})=116.078$

Geometry

C $-2.24955449-0.87646169-0.72197187$

C $-0.72174913-0.87638348-0.78102065$

C $-0.14468419 \quad 0.57832080 \quad-0.74639754$

C $1.15702055 \quad 0.76849926 \quad 0.03450674$

C $0.98632815 \quad 0.55517690 \quad 1.49985200$

C $0.73739551-2.74651965-0.02016987$

C $-0.15573561-1.797583550 .25085636$

C $0.37442177 \quad 1.39107449 \quad 2.34866547$

C $-0.22278641 \quad 2.70350197 \quad 1.97036212$

C $0.24959872 \quad 1.04286082 \quad 3.79843157$

$\mathrm{H}-2.67426123-0.26855637-1.53287591$

$\mathrm{H}-2.62023749-0.486960090 .23093753$

$\mathrm{H}-2.64627809-1.89299737-0.84706472$

$\mathrm{H}-0.91379053 \quad 1.32417897-0.46141126$

$\mathrm{H} 1.552629621 .78748816-0.17101236$

$\mathrm{H} \quad 1.92875988 \quad 0.07889572 \quad-0.37322288$

H $1.42420555-0.37555953 \quad 1.86322656$

H $1.13221145-3.420917850 .72329800$

$\mathrm{H} \quad 1.13740520-2.92470242-1.00908911$

$\mathrm{H}-0.54980252-1.63619370 \quad 1.25475682$

$\mathrm{H}-1.207218692 .85179800 \quad 2.43545608$

$\mathrm{H}-0.35470212 \quad 2.82427356 \quad 0.88787655$

$\mathrm{H} \quad 0.42226055 \quad 3.53041272 \quad 2.30553205$

$\mathrm{H} \quad 0.44580747 \quad 1.90837123 \quad 4.44477415$

$\mathrm{H} \quad 0.94515150 \quad 0.25033679 \quad 4.10514511$

$\mathrm{H}-0.76579046 \quad 0.68778794 \quad 4.02722826$

O $0.54031539-0.41409724-2.62437908$

$00.10986308 \quad 0.83966660-2.12845145$

o - $0.40504748-1.34183105-2.10514763$

Vibrational frequencies (in $\mathrm{cm}-1$ )

$\begin{array}{lllllllllllllll}23.7 & 39.0 & 63.3 & 81.0 & 96.1 & 107.7 & 109.6 & 172.2 & 200.3 & 216.5 & 248.0 & 276.5 & 284.4 & 326.2\end{array}$ $359.1400 .2 \quad 436.4 \quad 457.2 \quad 489.0 \quad 548.9 \quad 574.9 \quad 617.8 \quad 675.8 \quad 724.1 \quad 821.7 \quad 867.0 \quad 913.6 \quad 923.7$ $953.2975 .9990 .1 \quad 1000.3 \quad 1002.2 \quad 1016.3 \quad 1022.7 \quad 1055.4 \quad 1059.7 \quad 1083.7 \quad 1094.5 \quad 1113.4 \quad 1127.9$ $1141.41181 .7 \quad 1197.2 \quad 1238.0 \quad 1249.6 \quad 1267.2 \quad 1275.1 \quad 1279.4 \quad 1281.1 \quad 1287.0 \quad 1287.6 \quad 1290.6$ $1291.51305 .6 \quad 1315.0 \quad 1339.91353 .3 \quad 1381.3 \quad 1386.1 \quad 1395.3 \quad 1400.91417 .8 \quad 1853.3 \quad 1878.8$ $2653.62668 .52670 .22671 .32674 .3 \quad 2676.12679 .42686 .52712 .42738 .82750 .12753 .1$ $2778.92780 .12781 .2 \quad 2784.2$

name $=$ MIN278

energy $($ in $\mathrm{kcal} / \mathrm{mol})=-1.881996$

$\operatorname{ZPE}(\mathrm{in} \mathrm{kcal} / \mathrm{mol})=143.255$

$\mathrm{G}($ in $\mathrm{kcal} / \mathrm{mol})=115.645$

Geometry

C $-1.59618503-1.40618808-0.53153057$

C $-0.08114495-1.27408508-0.42415771$

C $0.37940280 \quad 0.21748550 \quad-0.54140468$ 
C $0.88869963 \quad 0.88998022 \quad 0.72917340$

C $-0.27385217 \quad 1.26200610 \quad 1.59009928$

C $1.40521185-2.888097990 .76122195$

C $0.43214092-1.979803590 .79529323$

C $-1.10967751 \quad 2.27956843 \quad 1.33920225$

C $-0.97542310 \quad 3.206684410 .17785170$

C $-2.284109122 .55090247 \quad 2.22294040$

$\mathrm{H}-1.96037571-1.05852636-1.50633892$

$\mathrm{H}-2.10404636-0.82692466 \quad 0.24881549$

$\mathrm{H}-1.90954330-2.45373804-0.42951309$

H $-0.412715140 .82513740-1.03021606$

$\mathrm{H} \quad 1.49287556 \quad 1.78432327 \quad 0.45951157$

H $1.59896921 \quad 0.22786748 \quad 1.26736825$

$\mathrm{H}-0.41531540 \quad 0.62205434 \quad 2.45847227$

$\mathrm{H} 1.76822978-3.40728226 \quad 1.63497047$

$\mathrm{H} 1.91676461-3.18393080-0.14708285$

$\mathrm{H}-0.07013659-1.69405090 \quad 1.71857149$

$\mathrm{H}-1.57897374 \quad 2.86102619-0.67496216$

$\mathrm{H} \quad 0.06244038 \quad 3.29635208-0.17417763$

$\mathrm{H}-1.31625397 \quad 4.222669910 .41998445$

$\mathrm{H}-2.18071835 \quad 3.51915555 \quad 2.73344594$

$\mathrm{H}-2.41825383 \quad 1.78930043 \quad 3.00215304$

$\mathrm{H}-3.217910562 .58740685 \quad 1.64317166$

o $1.05930048-0.84916642-2.36402067$

o $1.49259283 \quad 0.12326721-1.43782517$

o $0.51539730-1.90518096-1.56785494$

Vibrational frequencies (in cm-1)

$\begin{array}{lllllllllllllll}30.1 & 37.0 & 50.9 & 68.9 & 90.0 & 93.6 & 100.7 & 159.4 & 198.0 & 211.2 & 244.4 & 273.2 & 310.6 & 314.5\end{array}$

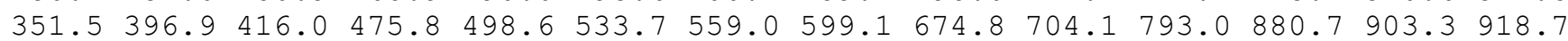

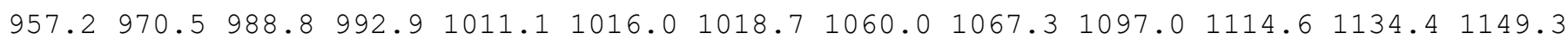

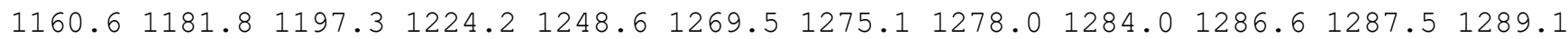
$1290.61321 .0 \quad 1336.1 \quad 1341.6 \quad 1342.3 \quad 1377.3 \quad 1388.4 \quad 1396.91400 .2 \quad 1413.5 \quad 1848.6 \quad 1869.8$ $2651.02665 .5 \quad 2669.6 \quad 2672.1 \quad 2672.7 \quad 2674.9 \quad 2677.02677 .22707 .12738 .82754 .5 \quad 2757.7$ 2776.72778 .62780 .02781 .0

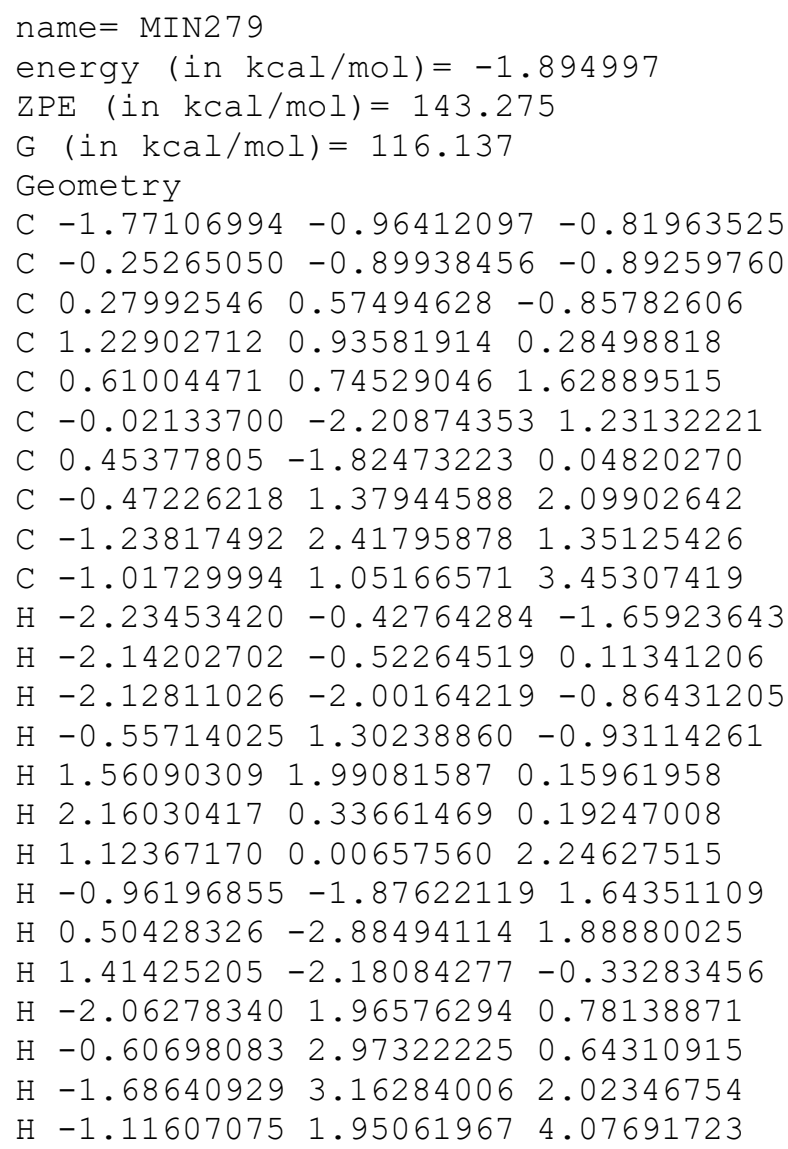


$\mathrm{H}-0.38382990 \quad 0.34700001 \quad 4.00796744$

$\mathrm{H}-2.01484741 \quad 0.59688254 \quad 3.37382662$

$01.25170913-0.59733214-2.53350580$

$\begin{array}{lllll}0 & 0.97205208 & 0.73146378 & -2.09904792\end{array}$

O $0.07000198-1.30641656-2.24046704$

Vibrational frequencies (in $\mathrm{cm}-1$ )

$\begin{array}{lllllllllllllll}29.7 & 34.8 & 69.5 & 83.2 & 87.3 & 112.8 & 127.0 & 173.6 & 207.8 & 219.0 & 236.7 & 258.6 & 279.0 & 321.1\end{array}$

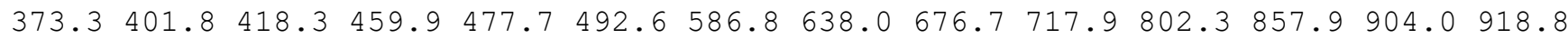

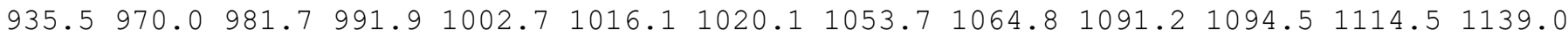

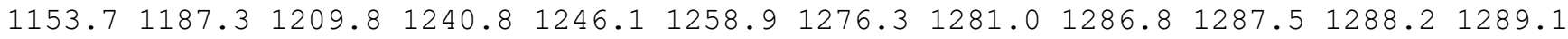

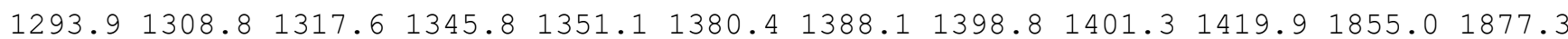
$2652.62668 .92670 .2 \quad 2671.4 \quad 2673.1 \quad 2674.4 \quad 2675.02681 .32711 .6 \quad 2739.32748 .02749 .5$ $2777.32778 .32780 .4 \quad 2785.5$

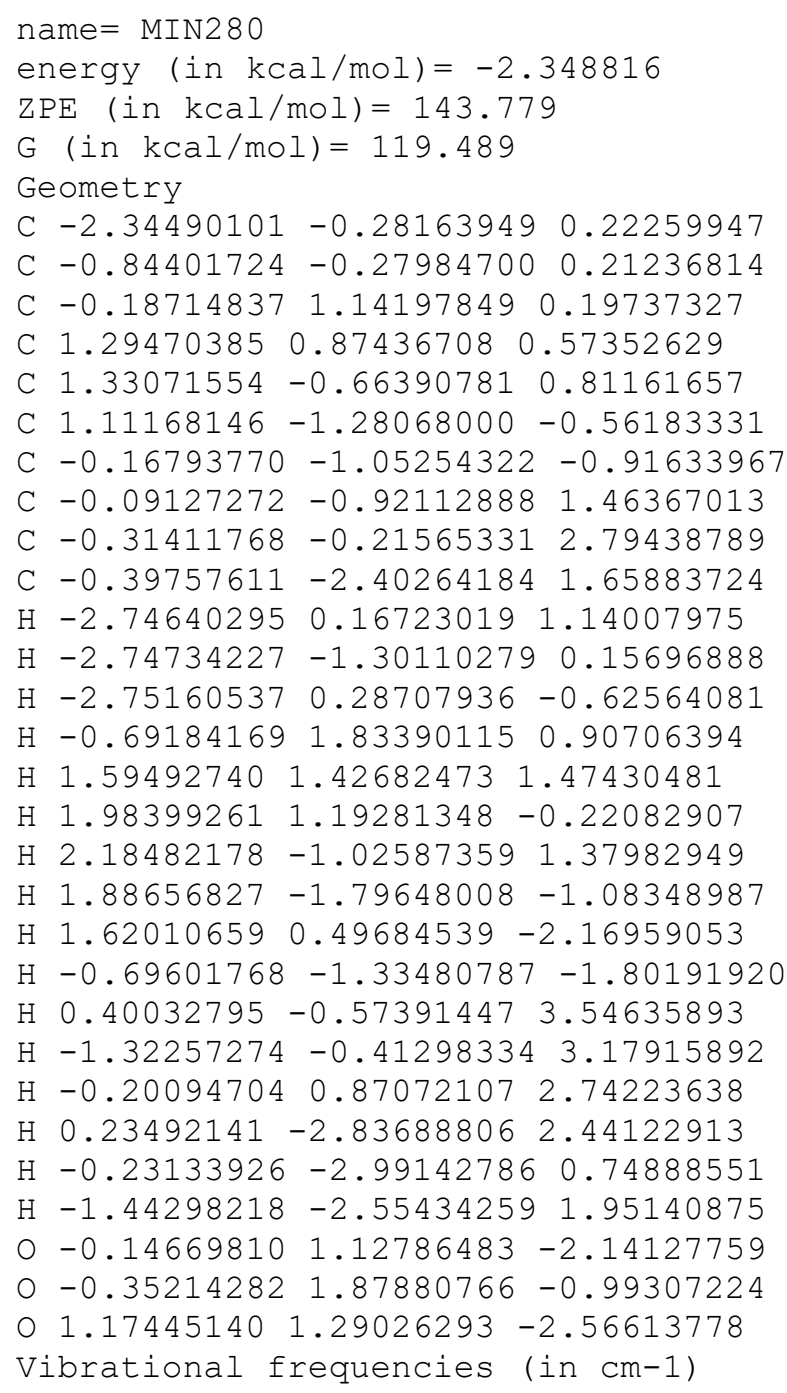


C $-1.46065857-0.32430074-0.62217489$

C $-0.859577731 .12304113-0.62604022$

C $0.079810161 .48152177 \quad 0.52492473$

C $1.24977646 \quad 0.56385610 \quad 0.64447447$

C $-0.42603247-2.41861633 \quad 0.22646983$

C $-0.94994329-1.21793154 \quad 0.46426056$

C $1.93668787 \quad 0.36654423 \quad 1.77736851$

C 1.604554941 .040842073 .06800576

C $3.09856993-0.571044111 .82475059$

$\mathrm{H}-3.37950603-1.34595011-0.71351108$

$\mathrm{H}-3.38492660 \quad 0.24234836-1.48072118$

$\mathrm{H}-3.39274158 \quad 0.11002120 \quad 0.29317788$

$\mathrm{H}-1.66449029 \quad 1.88723044 \quad-0.71866258$

$\mathrm{H}-0.49978192 \quad 1.52204404 \quad 1.47006984$

H $0.45884364 \quad 2.51772292 \quad 0.36132715$

$\mathrm{H} \quad 1.52779620 \quad 0.05829891-0.28106915$

H $-0.07157885-3.07774230 \quad 1.00406455$

$\mathrm{H}-0.30376867-2.83213595-0.76609877$

$\mathrm{H}-1.05852497-0.81897408 \quad 1.47165307$

$\mathrm{H} \quad 0.73913171 \quad 0.56200419 \quad 3.54853058$

$\mathrm{H} \quad 1.35883004 \quad 2.10197949 \quad 2.92586334$

$\mathrm{H} 2.43432843 \quad 1.00156085 \quad 3.78554845$

H $4.04226701-0.021937531 .95351970$

H $3.19603445-1.170503710 .90922458$

H $3.01014208-1.27638277 \quad 2.66244969$

O $0.10292535-0.13388050-2.23492566$

$\begin{array}{lllll}0 & -0.17285313 & 1.21463717 & -1.87372930\end{array}$

o - $-1.08907259-0.82311067-1.91983535$

Vibrational frequencies (in $\mathrm{cm}-1$ )

$\begin{array}{llllllllllllll}26.2 & 41.1 & 52.3 & 64.0 & 71.7 & 106.2 & 126.1 & 145.0 & 201.0 & 214.1 & 229.0 & 245.7 & 295.1 & 316.0\end{array}$ $323.2405 .9 \quad 430.2 \quad 474.5 \quad 497.3 \quad 535.5 \quad 574.7 \quad 610.0 \quad 694.4 \quad 728.6 \quad 819.4 \quad 849.1880 .1944 .6$ $961.3 \quad 966.1978 .7 \quad 983.5 \quad 999.6 \quad 1006.9 \quad 1022.9 \quad 1050.8 \quad 1065.7 \quad 1088.7 \quad 1094.6 \quad 1113.6 \quad 1135.3$ $\begin{array}{lllllllllllll}1153.4 & 1185.7 & 1197.7 & 1233.7 & 1265.4 & 1271.5 & 1278.7 & 1282.0 & 1285.3 & 1288.0 & 1288.3 & 1290.9\end{array}$ $1294.31303 .21313 .3 \quad 1335.7 \quad 1376.2 \quad 1380.2 \quad 1386.91395 .61400 .4 \quad 1419.3 \quad 1849.61872 .0$ $2655.52669 .12671 .5 \quad 2672.6 \quad 2674.2 \quad 2676.12676 .52678 .92710 .82728 .62753 .2 \quad 2757.6$ $2778.62779 .5 \quad 2780.3 \quad 2782.6$

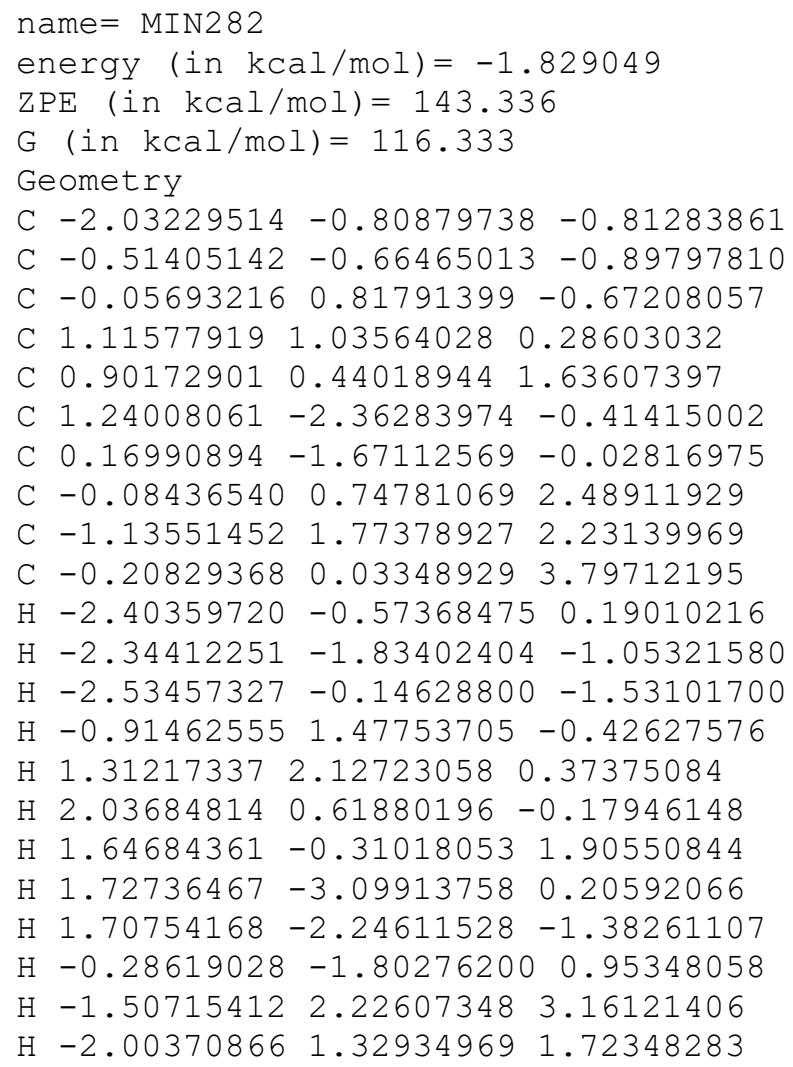


$\mathrm{H}-0.76722134 \quad 2.59785123 \quad 1.60424153$

$\mathrm{H}-0.07017517 \quad 0.72308299 \quad 4.64170789$

$\mathrm{H} \quad 0.53118988 \quad-0.76959648 \quad 3.91597687$

$\mathrm{H}-1.20138777-0.42419390 \quad 3.90630641$

$\begin{array}{lllll}0 & 0.79458800 & 0.05378394 & -2.60195996\end{array}$

○ $0.336227331 .24344225-1.97909107$

o $-0.20005789-0.90262367-2.28292277$

Vibrational frequencies (in $\mathrm{cm}-1$ )

$\begin{array}{llllllllllllllll}31.3 & 41.7 & 63.9 & 88.3 & 92.8 & 109.7 & 128.6 & 163.7 & 203.4 & 227.9 & 240.6 & 259.6 & 274.2 & 329.3\end{array}$

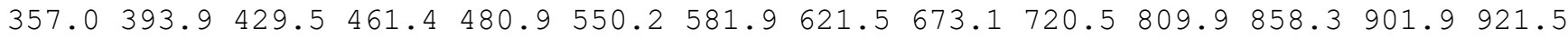
$944.6 \quad 972.4 \quad 988.6 \quad 993.8 \quad 1002.8 \quad 1016.9 \quad 1021.3 \quad 1052.6 \quad 1062.4 \quad 1088.9 \quad 1094.1 \quad 1112.6 \quad 1130.2$

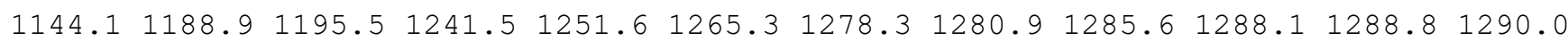

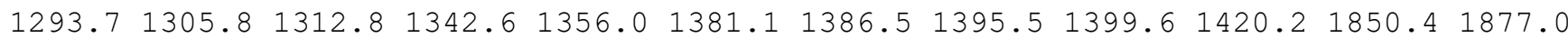
$2651.92669 .0 \quad 2670.7 \quad 2671.2 \quad 2673.7 \quad 2675.0 \quad 2677.72685 .92712 .12737 .12750 .02752 .6$ $2777.4 \quad 2779.5 \quad 2780.4 \quad 2783.2$

name $=$ MIN283

energy (in $\mathrm{kcal} / \mathrm{mol})=-1.581697$

$\mathrm{ZPE}($ in $\mathrm{kcal} / \mathrm{mol})=143.101$

$\mathrm{G}(\mathrm{in} \mathrm{kcal} / \mathrm{mol})=115.469$

Geometry

C $-2.40662083-0.37290698-0.71393751$

C $-0.88330800-0.28480955-0.67569328$

C $-0.365193601 .19631301-0.71707489$

C $0.58413131 \quad 1.63766127 \quad 0.39479004$

C $1.91049592 \quad 0.948873890 .37377477$

C 2.897366861 .284522491 .20522413

C $-0.26296816-1.162330870 .35546801$

C $-0.52217101-1.21414723 \quad 1.66759284$

C $-1.49507940-0.35054375 \quad 2.39679177$

C $0.19138949-2.20200188 \quad 2.53658522$

$\mathrm{H}-2.87123326 \quad 0.14639002 \quad 0.12910432$

$\mathrm{H}-2.73800172-1.41982709-0.67993552$

$\mathrm{H}-2.80393790 \quad 0.05978880 \quad-1.64319558$

$\mathrm{H}-1.217280411 .90789886-0.80280949$

$\mathrm{H} 0.10096206 \quad 1.489115331 .38416020$

$\mathrm{H} 0.743008662 .73670509 \quad 0.30663676$

$\mathrm{H} 2.021211890 .15700021-0.36825163$

$\mathrm{H} \quad 2.82474674 \quad 2.06595576 \quad 1.94309840$

$\mathrm{H} \quad 3.86098281 \quad 0.80186304 \quad 1.20629668$

H $0.47598325-1.84535216-0.07940119$

H $-2.42245502-0.90493127 \quad 2.60846496$

$\mathrm{H}-1.77645801 \quad 0.55173045 \quad 1.83972908$

$\mathrm{H}-1.09288979-0.01784115 \quad 3.36501266$

$\mathrm{H}-0.49843690-2.71368085 \quad 3.22105949$

H $0.95477028-1.70195976 \quad 3.15119574$

$\mathrm{H} \quad 0.70696879-2.98168447 \quad 1.95916951$

○ $0.65004069-0.02798350-2.31800998$

$0.286470371 .30665192-1.98238340$

o $-0.48894000-0.78433607-1.97684355$

Vibrational frequencies (in $\mathrm{cm}-1$ )

$\begin{array}{lllllllllllllll}16.0 & 36.3 & 60.9 & 79.4 & 97.5 & 117.9 & 126.9 & 145.9 & 176.1 & 200.0 & 248.4 & 262.5 & 288.1 & 317.5\end{array}$ $349.1385 .4 \quad 416.3 \quad 479.2 \quad 482.3 \quad 515.2 \quad 563.2 \quad 629.1 \quad 695.7 \quad 716.3 \quad 829.8 \quad 868.8 \quad 898.6 \quad 910.1$ $939.9958 .6 \quad 967.6 \quad 994.0 \quad 1004.4 \quad 1008.1 \quad 1014.7 \quad 1022.3 \quad 1064.4 \quad 1086.7 \quad 1096.91115 .8 \quad 1147.4$

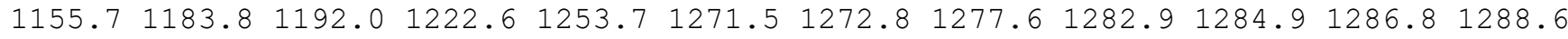

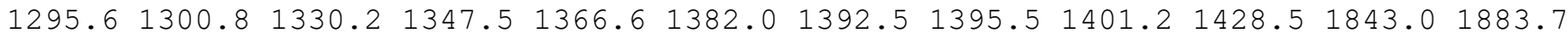
$2653.82667 .3 \quad 2668.9 \quad 2670.2 \quad 2671.7 \quad 2674.3 \quad 2675.42677 .72717 .7 \quad 2730.32736 .5 \quad 2754.3$ $2776.5 \quad 2779.6 \quad 2779.92793 .2$

name $=\operatorname{MIN} 284$

energy (in $\mathrm{kcal} / \mathrm{mol})=-1.483383$

$\mathrm{ZPE}(\mathrm{in} \mathrm{kcal} / \mathrm{mol})=143.063$

$\mathrm{G}($ in $\mathrm{kcal} / \mathrm{mol})=115.722$ 


\begin{tabular}{|c|c|}
\hline & \\
\hline & $0.14757424-0.63289421$ \\
\hline & $-0.08695164-0$. \\
\hline & $-0.000150411 .24726782-0.5$ \\
\hline & $1.34323204 \quad 1.22690670 \quad 0.180$ \\
\hline & $0.08972770-0.28$ \\
\hline & $2.75600383-0.79309678 \quad 0.54$ \\
\hline & $142050-1.001305620 .6$ \\
\hline & $-0.64320976-0.745365591 .9$ \\
\hline & $\begin{array}{llll}-1.16951629 & 0.52665229 & 2.53\end{array}$ \\
\hline & $-0.27822720-1.7$ \\
\hline & 098360.776 \\
\hline & $\begin{array}{lllll}-2.75693997 & 0.61740572 & 0.24\end{array}$ \\
\hline & $-2.83225969-0.80893935-0$. \\
\hline & -0.612473012 .12 \\
\hline & 1.168892001 .190136 \\
\hline & 1.878673052 .183559 \\
\hline & 2.320781310 .033 \\
\hline & $2.63632830-0.77$ \\
\hline & $966899-1.61$ \\
\hline & $-0.08400174-1.96$ \\
\hline & -1.436449131 .271 \\
\hline & 275060.99 \\
\hline & $\begin{array}{llll}-2.07118875 & 0.34266318 & 3.13\end{array}$ \\
\hline & $0.42362321-2.52673812 \quad 2.58$ \\
\hline & $7202999-2.31797668 \quad 3.315$ \\
\hline & $0.19294391-1.330789613 .86283414$ \\
\hline & $-0.284345290 .27536200 \quad-2.61923646$ \\
\hline & $171521.40830842-1.94339893$ \\
\hline & $-0.33272972-0.75097327-1.6685169$ \\
\hline
\end{tabular}

Vibrational frequencies (in $\mathrm{cm}-1$ )

$\begin{array}{lllllllllllllll}35.9 & 45.9 & 57.9 & 69.1 & 81.8 & 105.4 & 127.9 & 139.4 & 147.2 & 202.3 & 240.8 & 255.7 & 298.0 & 326.2\end{array}$ $352.8 \quad 372.6 \quad 400.0 \quad 458.8 \quad 486.1 \quad 489.6 \quad 568.5 \quad 653.3 \quad 703.9 \quad 720.7 \quad 839.2 \quad 886.0 \quad 904.5 \quad 925.6$ $956.1970 .2978 .6 \quad 993.3 \quad 1000.7 \quad 1009.0 \quad 1012.6 \quad 1028.0 \quad 1071.3 \quad 1086.3 \quad 1097.3 \quad 1113.2 \quad 1131.0$ $1136.4 \quad 1168.4 \quad 1170.8 \quad 1213.4 \quad 1247.5 \quad 1265.0 \quad 1271.4 \quad 1278.91285 .0 \quad 1285.5 \quad 1287.3 \quad 1290.7$ $1305.81310 .81331 .01339 .8 \quad 1362.3 \quad 1383.0 \quad 1392.2 \quad 1396.61404 .3 \quad 1422.2 \quad 1850.7 \quad 1882.0$ $2661.32666 .8 \quad 2669.7 \quad 2669.8 \quad 2672.1 \quad 2674.1 \quad 2677.92682 .82717 .8 \quad 2735.8 \quad 2738.6 \quad 2752.9$ $2777.5 \quad 2780.4 \quad 2780.5 \quad 2792.1$

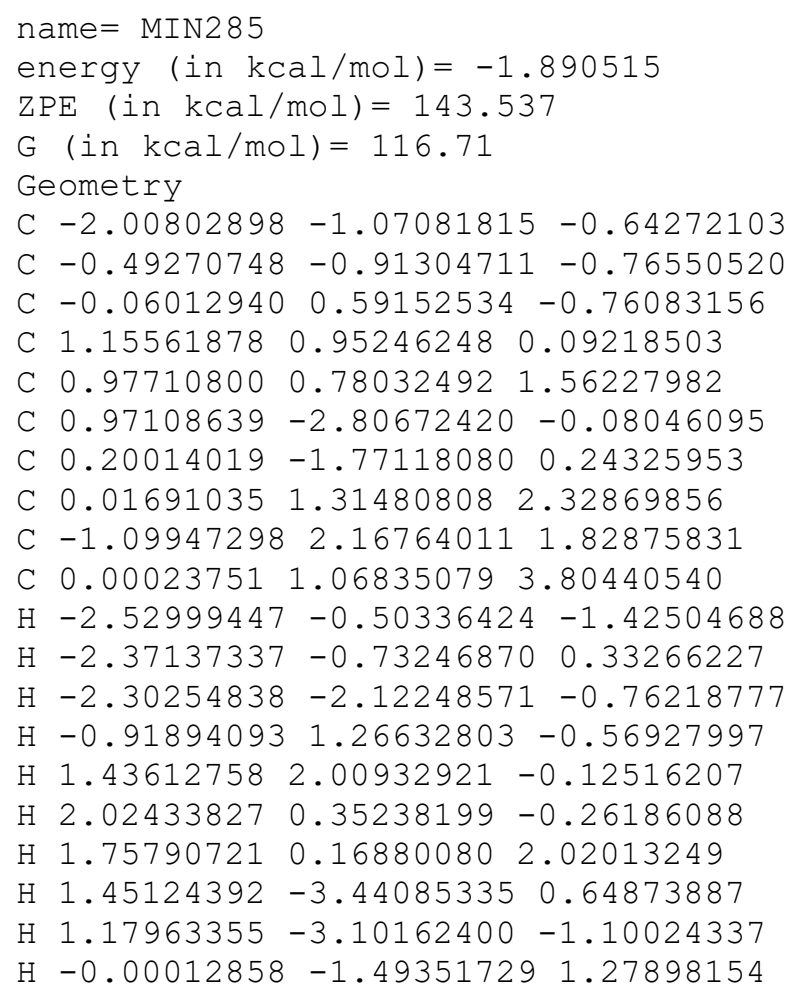


$\mathrm{H}-0.784185662 .821482151 .00333895$

$\mathrm{H}-1.50634376 \quad 2.822599202 .61134549$

$\mathrm{H}-1.935195581 .548614301 .46946139$

$\mathrm{H} \quad 0.79923498 \quad 0.39193109 \quad 4.13494805$

$\mathrm{H}-0.95246223 \quad 0.62496755 \quad 4.12489025$

$\mathrm{H} \quad 0.12806907 \quad 2.00813778 \quad 4.36105540$

$0.75627337-0.40889409-2.59185279$

○ $0.266147320 .83906425-2.13155363$

o $-0.18818915-1.34668670-2.10428014$

Vibrational frequencies (in $\mathrm{cm}-1$ )

$\begin{array}{lllllllllllllllll}29.0 & 40.3 & 57.3 & 89.6 & 122.7 & 128.3 & 162.6 & 168.9 & 205.9 & 224.5 & 237.5 & 259.2 & 293.4 & 323.2\end{array}$ $352.2398 .7 \quad 440.3 \quad 462.1 \quad 481.8 \quad 544.7 \quad 585.6 \quad 624.6 \quad 682.5 \quad 724.6 \quad 810.6 \quad 861.5 \quad 903.2 \quad 919.1$

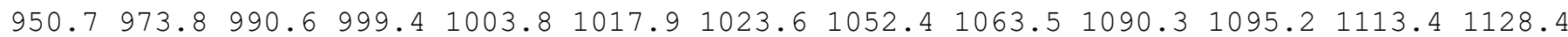
$1143.21188 .2 \quad 1205.7 \quad 1240.8 \quad 1256.4 \quad 1266.8 \quad 1278.6 \quad 1281.8 \quad 1285.3 \quad 1287.3 \quad 1289.0 \quad 1289.4$

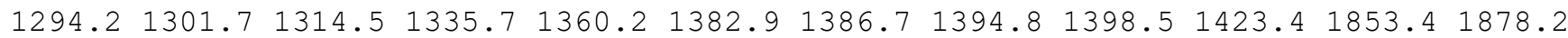
$2649.32667 .6 \quad 2670.02670 .5 \quad 2672.6 \quad 2675.4 \quad 2678.92686 .12710 .52734 .42747 .42749 .9$ $2776.8 \quad 2779.5 \quad 2780.4 \quad 2783.1$

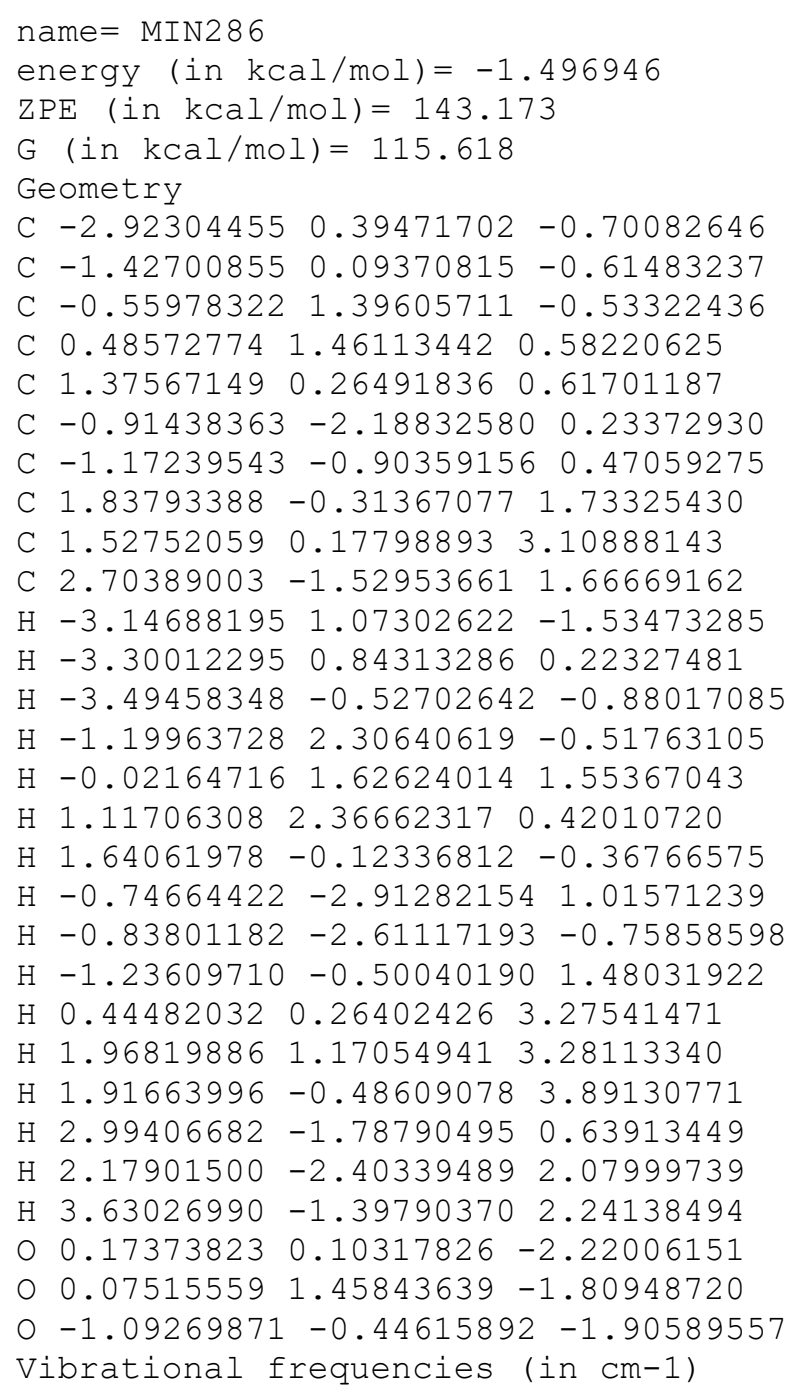


$\mathrm{ZPE}($ in $\mathrm{kcal} / \mathrm{mol})=143.285$

$\mathrm{G}(\mathrm{in} \mathrm{kcal} / \mathrm{mol})=115.947$

Geometry

C $-2.13971421-0.95198074-0.56644608$

C $-0.62509028-0.81695427-0.73219133$

C $-0.18190622 \quad 0.68355475-0.76925730$

C $1.127924851 .03094812-0.06101704$

C $1.15974867 \quad 0.73921962 \quad 1.40027625$

C $0.93615498-2.63250269-0.04242597$

C $0.08480757-1.66042857 \quad 0.27651848$

C $0.30378135 \quad 1.18586855 \quad 2.32907707$

C $-0.880639812 .04750362 \quad 2.04937972$

$\begin{array}{lllll}\text { C } & 0.48556511 & 0.81659041 & 3.76724771\end{array}$

$\mathrm{H}-2.67256093-0.39144658-1.34677071$

$\mathrm{H}-2.47589071-0.591846650 .41023540$

$\mathrm{H}-2.45031694-2.00140085-0.66141922$

$\mathrm{H}-1.00563644 \quad 1.36730696-0.48191880$

$\mathrm{H} 1.345253942 .11067343-0.22995348$

$\mathrm{H} \quad 1.956279890 .49237258-0.57575158$

H 2.00228576 $0.10976856 \quad 1.69486764$

H $1.42942809-3.252677320 .69012827$

H $1.20273764-2.88420948-1.05998605$

$\mathrm{H}-0.16969825-1.42496619 \quad 1.31112940$

$\mathrm{H}-0.68485878 \quad 2.77284140 \quad 1.24724528$

$\mathrm{H}-1.74608992 \quad 1.43826066 \quad 1.74902267$

$\mathrm{H}-1.19143537 \quad 2.62636384 \quad 2.92981426$

$\mathrm{H} \quad 1.31586504 \quad 0.11610436 \quad 3.92716597$

$\mathrm{H}-0.41827291 \quad 0.34515911 \quad 4.17672510$

$\mathrm{H} \quad 0.69701550 \quad 1.70643580 \quad 4.37768149$

$0.48960104-0.31195280-2.65388270$

$0-0.00925948 \quad 0.92117613 \quad-2.16939513$

O $-0.36183781-1.28646859-2.06709295$

Vibrational frequencies (in $\mathrm{cm}-1$ )

$\begin{array}{lllllllllllllll}28.4 & 36.6 & 46.0 & 83.4 & 91.4 & 115.7 & 138.5 & 157.9 & 202.2 & 219.5 & 233.9 & 255.7 & 282.8 & 320.6\end{array}$

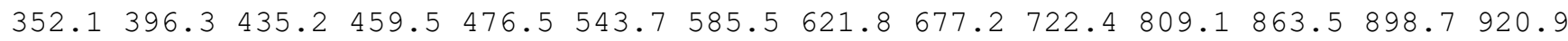
$951.7974 .8 \quad 989.0 \quad 996.8 \quad 1002.4 \quad 1017.1 \quad 1022.4 \quad 1050.3 \quad 1060.4 \quad 1088.6 \quad 1094.11111 .2 \quad 1124.3$

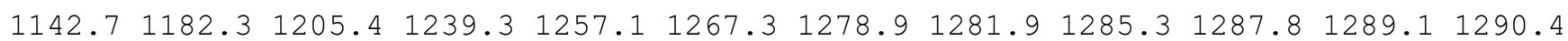

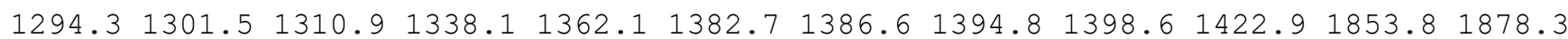
$2649.42668 .2 \quad 2670.2 \quad 2671.0 \quad 2673.1 \quad 2675.3 \quad 2679.72688 .12711 .32734 .22748 .12750 .5$ $2777.12779 .7 \quad 2780.2 \quad 2783.1$

\section{name $=$ MIN28 8}

energy (in $\mathrm{kcal} / \mathrm{mol})=-1.415894$

$\operatorname{ZPE}($ in $\mathrm{kcal} / \mathrm{mol})=143.122$

G $($ in $\mathrm{kcal} / \mathrm{mol})=115.135$

Geometry

C $-3.06249712-0.60209197-0.55670290$

C $-1.53720365-0.55430980-0.54202761$

C $-0.98337534 \quad 0.91076954 \quad-0.61006367$

C $-0.09702572 \quad 1.37363996 \quad 0.54440341$

C $1.16787805 \quad 0.595708670 .68603856$

C $-0.43187737-2.569263390 .40230886$

C $-1.00415374-1.381004110 .58557725$

C $2.08730024 \quad 0.85677985 \quad 1.62515450$

C $1.93933194 \quad 1.96392482 \quad 2.61662056$

C $3.34052226 \quad 0.05279863 \quad 1.73406562$

$\mathrm{H}-3.48855096-0.13920876 \quad 0.33956196$

$\mathrm{H}-3.42168503-1.63971654-0.59724374$

$\mathrm{H}-3.47072305-0.08961986-1.43812008$

$\mathrm{H}-1.811762851 .63807456-0.77164166$

$\mathrm{H}-0.67480427 \quad 1.34899318 \quad 1.49275845$

$\mathrm{H} \quad 0.15609048 \quad 2.44792914 \quad 0.38248707$

$\mathrm{H} \quad 1.30873207-0.20372611-0.04021634$

$\mathrm{H}-0.05953333-3.18106774 \quad 1.20955779$ 
$\mathrm{H}-0.28535631-3.01835186-0.57103180$

$\mathrm{H}-1.13670626-0.94535705 \quad 1.57494517$

H $1.94079302 \quad 2.94350137 \quad 2.11541469$

H 2.74953339 $1.98196720 \quad 3.35647490$

$\mathrm{H} \quad 0.99621301 \quad 1.88394052 \quad 3.17462236$

H $4.23020253 \quad 0.68724780 \quad 1.61046912$

H $3.40289202-0.74002047 \quad 0.97666156$

$\mathrm{H} \quad 3.41667084-0.432405522 .71722913$

$0.04984847-0.40233828-2.13017725$

$\begin{array}{lllll}0 & -0.25920634 & 0.95655307 & -1.83950578\end{array}$

O $-1.13844695-1.09701613-1.81366373$

Vibrational frequencies (in $\mathrm{cm}-1$ )

$\begin{array}{lllllllllllllll}15.9 & 40.1 & 56.2 & 63.8 & 70.1 & 105.6 & 128.1 & 142.9 & 197.5 & 206.3 & 230.8 & 244.4 & 293.4 & 312.3\end{array}$ $323.9 \quad 403.7 \quad 430.7 \quad 478.2 \quad 491.1 \quad 536.3 \quad 578.5 \quad 611.9693 .6 \quad 730.6 \quad 814.3 \quad 840.1 \quad 878.6 \quad 945.7$ $960.9966 .8 \quad 980.0 \quad 985.3 \quad 998.2 \quad 1006.7 \quad 1023.5 \quad 1054.5 \quad 1066.9 \quad 1084.1 \quad 1094.4 \quad 1113.9 \quad 1138.2$

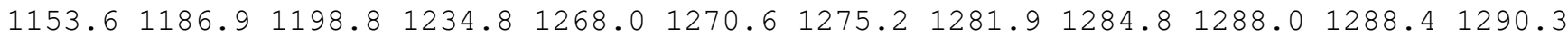
$1291.81303 .3 \quad 1315.5 \quad 1335.8 \quad 1375.7 \quad 1380.1 \quad 1386.7 \quad 1395.7 \quad 1400.6 \quad 1419.2 \quad 1848.91868 .2$ $2651.52669 .02669 .8 \quad 2672.5 \quad 2673.6 \quad 2675.5 \quad 2676.82678 .52711 .12726 .02753 .12757 .1$ $2778.0 \quad 2779.7 \quad 2779.9 \quad 2782.8$

name $=$ MIN289

energy (in $\mathrm{kcal} / \mathrm{mol})=-1.329567$

$\operatorname{ZPE}($ in $\mathrm{kcal} / \mathrm{mol})=143.049$

$\mathrm{G}($ in $\mathrm{kcal} / \mathrm{mol})=115.601$

Geometry

C $-2.30683227-0.11374664-0.64982359$

C $-0.78169252-0.22506607-0.65980290$

C $-0.101073651 .18062980-0.68377683$

C $1.37806967 \quad 1.18679959-0.29796960$

C $1.61799128 \quad 1.14117743 \quad 1.17318769$

C 2.658092240 .499379191 .70664468

C $-0.24589471-1.18036777 \quad 0.34792558$

C $-0.46430401-1.159921331 .66917784$

C $-1.30110379-0.15424572 \quad 2.38738760$

C $0.15056477-2.19410641 \quad 2.55778674$

$\mathrm{H}-2.65656861 \quad 0.45175531 \quad-1.52552879$

$\mathrm{H}-2.69108266 \quad 0.37412392 \quad 0.24776049$

$\mathrm{H}-2.76320157-1.11096823-0.71040088$

$\mathrm{H}-0.688125291 .96258499-0.16898003$

H $1.84871651 \quad 2.10797000 \quad-0.71588022$

H $1.89325665 \quad 0.34757390 \quad-0.81689906$

$\mathrm{H} \quad 0.89742663 \quad 1.68038657 \quad 1.78560655$

$\mathrm{H} \quad 2.86118170 \quad 0.46781470 \quad 2.76423173$

$\mathrm{H} \quad 3.38471089-0.049893521 .12821031$

$\mathrm{H} \quad 0.38215732-1.95836181-0.09611440$

$\mathrm{H}-1.330295190 .81723725 \quad 1.87766684$

$\mathrm{H}-0.93630146 \quad 0.03413525 \quad 3.40692962$

$\mathrm{H}-2.33865050-0.511525892 .47968258$

$\mathrm{H} \quad 0.97494114 \quad-1.75945815 \quad 3.14395523$

$\mathrm{H} \quad 0.56796728-3.042707291 .99985855$

$\mathrm{H}-0.57951629-2.60289240 \quad 3.26934575$

$\begin{array}{lllll}0 & 0.16216111 & 0.28978920 & -2.70979182\end{array}$

$\begin{array}{llll}0 & -0.20476429 & 1.49678804 & -2.07352168\end{array}$

o $-0.46603742-0.76198453-1.96252527$

Vibrational frequencies (in $\mathrm{cm}-1$ )

$\begin{array}{lllllllllllllll}24.2 & 49.2 & 58.9 & 76.5 & 92.7 & 97.2 & 127.0 & 143.3 & 165.6 & 197.8 & 219.1 & 260.5 & 305.9 & 337.3\end{array}$ $345.2 \quad 362.0 \quad 415.2 \quad 457.5 \quad 486.4 \quad 491.7 \quad 553.3 \quad 594.1 \quad 704.9 \quad 723.1 \quad 831.0 \quad 896.4 \quad 905.5 \quad 935.6$ $957.3 \quad 972.0 \quad 976.2 \quad 992.4 \quad 1003.3 \quad 1017.8 \quad 1019.8 \quad 1024.5 \quad 1083.0 \quad 1085.2 \quad 1093.5 \quad 1114.4 \quad 1128.1$

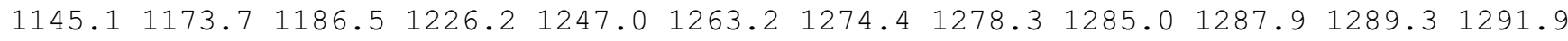
$1296.01301 .91324 .4 \quad 1332.8 \quad 1359.5 \quad 1380.1 \quad 1393.0 \quad 1394.7 \quad 1401.8 \quad 1420.3 \quad 1844.2 \quad 1878.2$ $2648.12667 .2 \quad 2669.7 \quad 2671.3 \quad 2671.7 \quad 2675.8 \quad 2680.72699 .02717 .4 \quad 2730.5 \quad 2742.8 \quad 2760.4$ $2777.02779 .7 \quad 2781.8 \quad 2790.8$ 
name $=$ MIN290

energy (in $\mathrm{kcal} / \mathrm{mol})=-1.480399$

$\mathrm{ZPE}($ in $\mathrm{kcal} / \mathrm{mol})=143.206$

$\mathrm{G}(\mathrm{in} \mathrm{kcal} / \mathrm{mol})=115.25$

Geometry

C $-2.66284891-1.37011733-0.11942070$

C $-1.13730659-1.36491045-0.12003231$

C $-0.54822772 \quad 0.08840326-0.12035394$

C $0.54525207 \quad 0.33721265 \quad 0.91724673$

C $0.87521514 \quad 1.803619950 .96906415$

C $0.32348001-3.17743663 \quad 0.75663215$

C $-0.59489258-2.237160890 .96876487$

C $1.50057640 \quad 2.38684985-0.06167887$

C $1.845704253 .82938253-0.16809409$

C $0.43211752 \quad 2.51239262 \quad 2.20409430$

$\mathrm{H}-3.07110844 \quad-0.920651690 .79132171$

$\mathrm{H}-3.05377584-2.39424285-0.19204471$

$\mathrm{H}-3.06083816-0.81801465-0.98274816$

$\mathrm{H}-1.34188204 \quad 0.86333449-0.07258479$

$\mathrm{H} 1.44940579-0.254989340 .65743119$

$\mathrm{H} \quad 0.22990271-0.03697434 \quad 1.91052972$

$\mathrm{H} \quad 1.79314811 \quad 1.79665213-0.93479329$

$\mathrm{H} \quad 0.70798036-3.82206572 \quad 1.53177270$

$\mathrm{H} \quad 0.75752973-3.37905666-0.21458367$

$\mathrm{H}-1.02663323-2.05580978 \quad 1.95176309$

H $1.45416773 \quad 4.43561633 \quad 0.65760225$

$\mathrm{H} 1.44342408 \quad 4.25857815-1.09872243$

$\mathrm{H} 2.936413523 .97069962-0.19062463$

$\mathrm{H}-0.626128832 .802089712 .12888240$

$\mathrm{H} \quad 0.53915021 \quad 1.88774579 \quad 3.10093383$

H $1.00819374 \quad 3.42861745 \quad 2.39188530$

o $0.34962449-1.09199362-1.80409976$

$0-0.01437408 \quad 0.22838286-1.43735034$

o $-0.76693769-1.87379569-1.41255664$

Vibrational frequencies (in $\mathrm{cm}-1$ )

$\begin{array}{lllllllllllllll}25.0 & 45.7 & 52.1 & 62.0 & 64.4 & 70.5 & 107.5 & 163.7 & 199.5 & 216.7 & 237.7 & 256.7 & 284.9 & 300.1\end{array}$

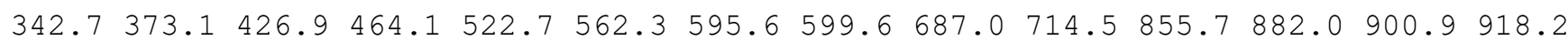
$939.8 \quad 962.5 \quad 976.9986 .6 \quad 1009.0 \quad 1030.4 \quad 1050.3 \quad 1061.7 \quad 1067.5 \quad 1086.0 \quad 1096.3 \quad 1097.8 \quad 1138.8$

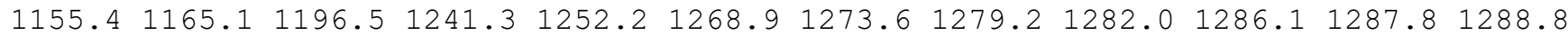

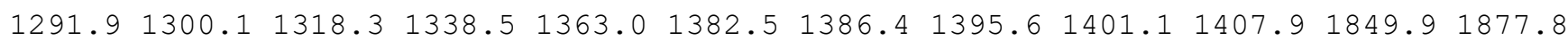
$2658.62669 .22669 .52670 .82673 .02673 .2 \quad 2678.32686 .12709 .72740 .52744 .62756 .6$ $2778.3 \quad 2778.6 \quad 2779.8 \quad 2782.1$

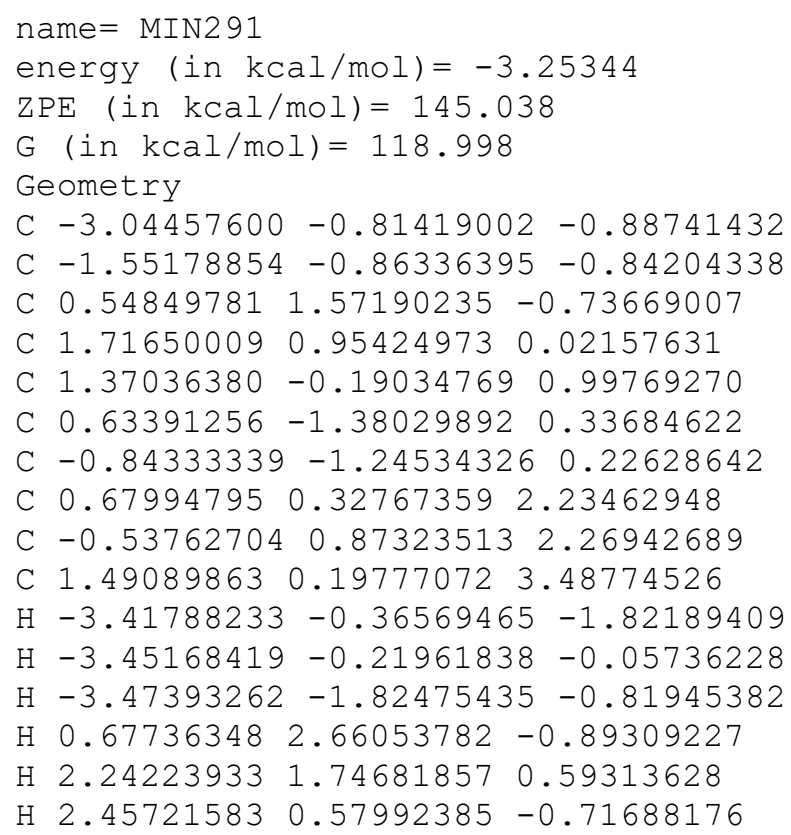


$\mathrm{H} \quad 2.36930980-0.611226691 .31415095$

$\mathrm{H} 0.85232208 \quad-2.29096175 \quad 0.94195481$

$\mathrm{H} 1.09961158-1.58657954-0.64848065$

$\mathrm{H}-1.37342580-1.51333058 \quad 1.14335302$

$\mathrm{H}-0.42343107 \quad 1.39814977-0.24462580$

$\mathrm{H}-1.17652353 \quad 0.98729185 \quad 1.40946657$

$\mathrm{H}-0.998511361 .24303283 \quad 3.16989005$

H 2.44703839 $0.73210182 \quad 3.40331761$

H $1.71571089-0.85554272 \quad 3.70615282$

$\mathrm{H} \quad 0.97425699 \quad 0.60321624 \quad 4.36759800$

O $0.28370183-0.25588278-2.12171538$

$0 \quad 0.48545319 \quad 1.11030893-2.08258971$

o $-1.10652881-0.49816892-2.08689572$

Vibrational frequencies (in $\mathrm{cm}-1$ )

$\begin{array}{llllllllllllll}37.6 & 46.0 & 57.7 & 101.3 & 110.8 & 117.9 & 182.9 & 211.9 & 226.6 & 274.5 & 307.5 & 317.9 & 331.5 & 363.2\end{array}$ $405.1449 .5 \quad 495.8 \quad 528.9 \quad 540.6 \quad 575.3 \quad 627.1 \quad 630.2 \quad 680.7 \quad 809.7 \quad 820.9 \quad 869.3 \quad 900.0 \quad 920.8$

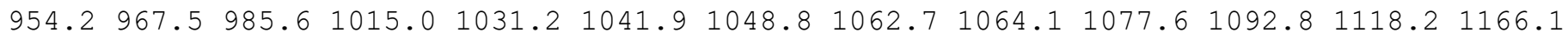
$\begin{array}{llllllllllllllll}1194.0 & 1219.1 & 1229.4 & 1238.5 & 1250.7 & 1274.2 & 1275.9 & 1278.8 & 1289.1 & 1293.6 & 1300.2 & 1310.8\end{array}$

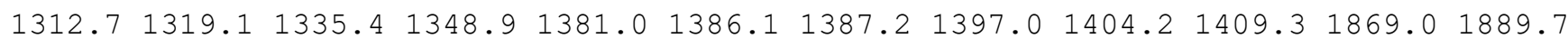
$2637.82649 .62658 .12666 .8 \quad 2669.4 \quad 2669.7 \quad 2673.42675 .42710 .52719 .62731 .42736 .5$ 2753.12775 .92778 .92788 .8

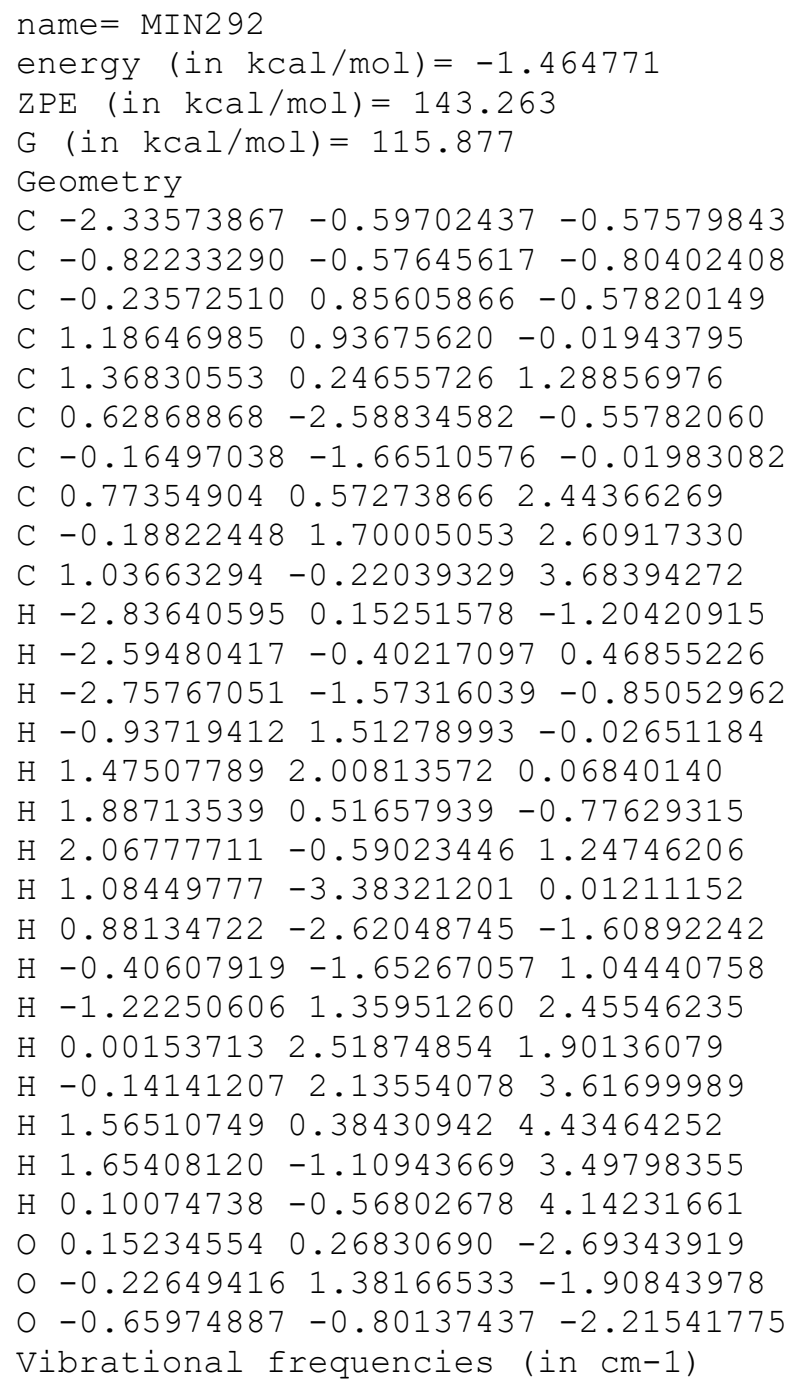


name $=$ MIN293

energy (in $\mathrm{kcal} / \mathrm{mol})=-1.47801$

$\mathrm{ZPE}($ in $\mathrm{kcal} / \mathrm{mol})=143.277$

$\mathrm{G}($ in $\mathrm{kcal} / \mathrm{mol})=115.978$

Geometry

C $-2.19649584-0.94121249-0.41716811$

C $-0.72632670-0.69341518-0.76065220$

C $-0.344451690 .81364750-0.58168166$

C $1.061763381 .10810804 \quad-0.05687265$

C $1.378215810 .49412158 \quad 1.26380820$

C $1.05476496-2.43125128-0.63132778$

C $0.15113903-1.65909931-0.03221377$

$\begin{array}{llll}\text { C } 0.71613956 & 0.68746160 & 2.41226338\end{array}$

C $1.15389428-0.00031595 \quad 3.66606554$

C -0.489113761 .553347732 .55804506$

$\mathrm{H}-2.48370274-1.97446710-0.65469625$

$\mathrm{H}-2.85302180-0.28642705-1.00677037$

$\mathrm{H}-2.40181391-0.77268536 \quad 0.64379941$

$\mathrm{H}-1.12214023 \quad 1.37762910-0.02910220$

$\mathrm{H} \quad 1.19713778 \quad 2.21199108-0.00192089$

H $1.79807047 \quad 0.77048949-0.82167778$

H $2.25389237-0.157409091 .24027038$

$\mathrm{H} 1.67319649-3.13992585-0.10252281$

$\mathrm{H} 1.24250335-2.41512754-1.69634990$

$\mathrm{H}-0.02106658-1.69213080 \quad 1.04467050$

$\mathrm{H} \quad 1.99393020-0.68883992 \quad 3.50424515$

H $0.33572151-0.58713606 \quad 4.10574056$

$\mathrm{H} \quad 1.47913379 \quad 0.72798160 \quad 4.42268986$

$\mathrm{H}-1.40476276 \quad 0.99457729 \quad 2.31435708$

$\mathrm{H}-0.60912368 \quad 1.92847106 \quad 3.58352937$

$\mathrm{H}-0.44890895 \quad 2.43360479 \quad 1.90133287$

$0.03492831 \quad 0.23160964-2.70315247$

$0-0.437404911 .31101419-1.91962608$

$0-0.64265644-0.90541729-2.18151831$

Vibrational frequencies (in $\mathrm{cm}-1$ )

$\begin{array}{llllllllllllll}27.0 & 35.9 & 56.2 & 85.0 & 90.8 & 109.5 & 132.4 & 160.2 & 200.3 & 218.0 & 237.6 & 257.6 & 276.4 & 321.6\end{array}$ $353.7 \quad 395.8 \quad 433.0 \quad 459.0 \quad 477.4 \quad 545.6 \quad 585.2 \quad 619.9 \quad 674.7 \quad 721.5 \quad 810.7 \quad 865.6 \quad 899.2 \quad 922.5$

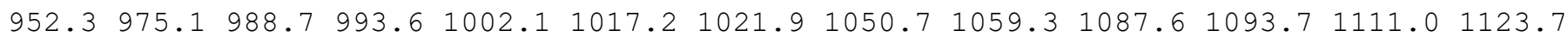
$\begin{array}{lllllllllllll}1142.9 & 1179.8 & 1203.1 & 1239.3 & 1255.3 & 1268.1 & 1279.0 & 1281.9 & 1285.7 & 1288.1 & 1289.0 & 1290.9\end{array}$

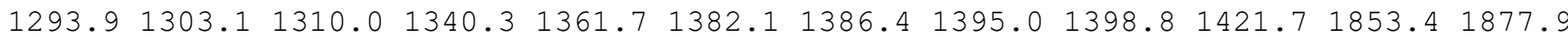
$2650.12668 .8 \quad 2670.2 \quad 2671.3 \quad 2673.5 \quad 2675.3 \quad 2680.12689 .32711 .92734 .82749 .22751 .8$ $2777.52780 .02780 .3 \quad 2783.3$

name $=$ MIN294

energy (in $\mathrm{kcal} / \mathrm{mol})=-1.350074$

$\operatorname{ZPE}($ in $\mathrm{kcal} / \mathrm{mol})=143.267$

G $($ in $\mathrm{kcal} / \mathrm{mol})=115.929$

Geometry

C $-2.24957068-0.87872580-0.39928147$

C $-0.76617140-0.69559212-0.72829256$

C $-0.338496450 .80401392-0.60484604$

C $1.087015891 .07386968-0.12037810$

C $1.41690613 \quad 0.49647611 \quad 1.21333481$

C $0.92876506-2.50793271-0.49419438$

C $0.06417554-1.65869646 \quad 0.05613693$

$\begin{array}{lllll}\text { C } & 0.79089666 & 0.75232961 & 2.36975190\end{array}$

C $1.24050380 \quad 0.09852990 \quad 3.63742837$

C $-0.38415425 \quad 1.65934058 \quad 2.51183795$

$\mathrm{H}-2.87290028-0.22353454-1.02351480$

$\mathrm{H}-2.46523314-0.66424885 \quad 0.65107000$

$\mathrm{H}-2.56964550-1.90924479-0.60441215$

$\mathrm{H}-1.08773595 \quad 1.40872743-0.05654014$ 
$\mathrm{H} \quad 1.25544274 \quad 2.17453281-0.10686921$

$\mathrm{H} \quad 1.79576628 \quad 0.68865409 \quad-0.88891873$

H $2.27052467-0.183786321 .19213768$

$\mathrm{H} \quad 1.51136158-3.213952050 .07722450$

$\mathrm{H} \quad 1.11788781-2.56321859-1.55761460$

$\mathrm{H}-0.10958682-1.62057552 \quad 1.13271147$

$\mathrm{H} \quad 0.41734093-0.44684318 \quad 4.11908444$

$\mathrm{H} \quad 1.60624336 \quad 0.84433263 \quad 4.35763857$

$\mathrm{H} \quad 2.05577625-0.62037710 \quad 3.48100359$

$\mathrm{H}-0.333466512 .514160461 .82303954$

$\mathrm{H}-0.46854738 \quad 2.07388729 \quad 3.52559477$

$\mathrm{H}-1.32148893 \quad 1.120473012 .30920500$

$\begin{array}{lllll}0 & 0.00962021 & 0.14559097 & -2.70727418\end{array}$

o $-0.445328951 .25534594-1.95806981$

$0-0.66619872-0.97121922-2.13677789$

Vibrational frequencies (in cm-1)

$\begin{array}{lllllllllllllllll}26.6 & 34.6 & 56.3 & 84.8 & 91.4 & 108.4 & 132.6 & 159.9 & 199.4 & 215.5 & 237.5 & 257.6 & 275.7 & 320.0\end{array}$

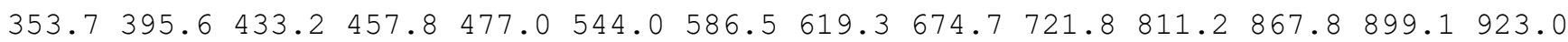
$954.1975 .6 \quad 988.7 \quad 993.2 \quad 1002.1 \quad 1017.2 \quad 1021.9 \quad 1050.4 \quad 1058.9 \quad 1087.3 \quad 1093.7 \quad 1110.61122 .6$

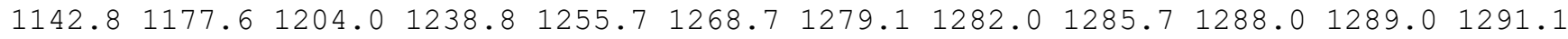

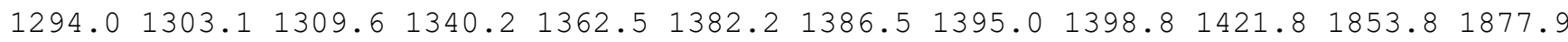
$2649.92668 .92670 .02671 .3 \quad 2673.5 \quad 2675.2 \quad 2680.52690 .22711 .92734 .52749 .12751 .6$ $2777.52780 .02780 .2 \quad 2783.3$

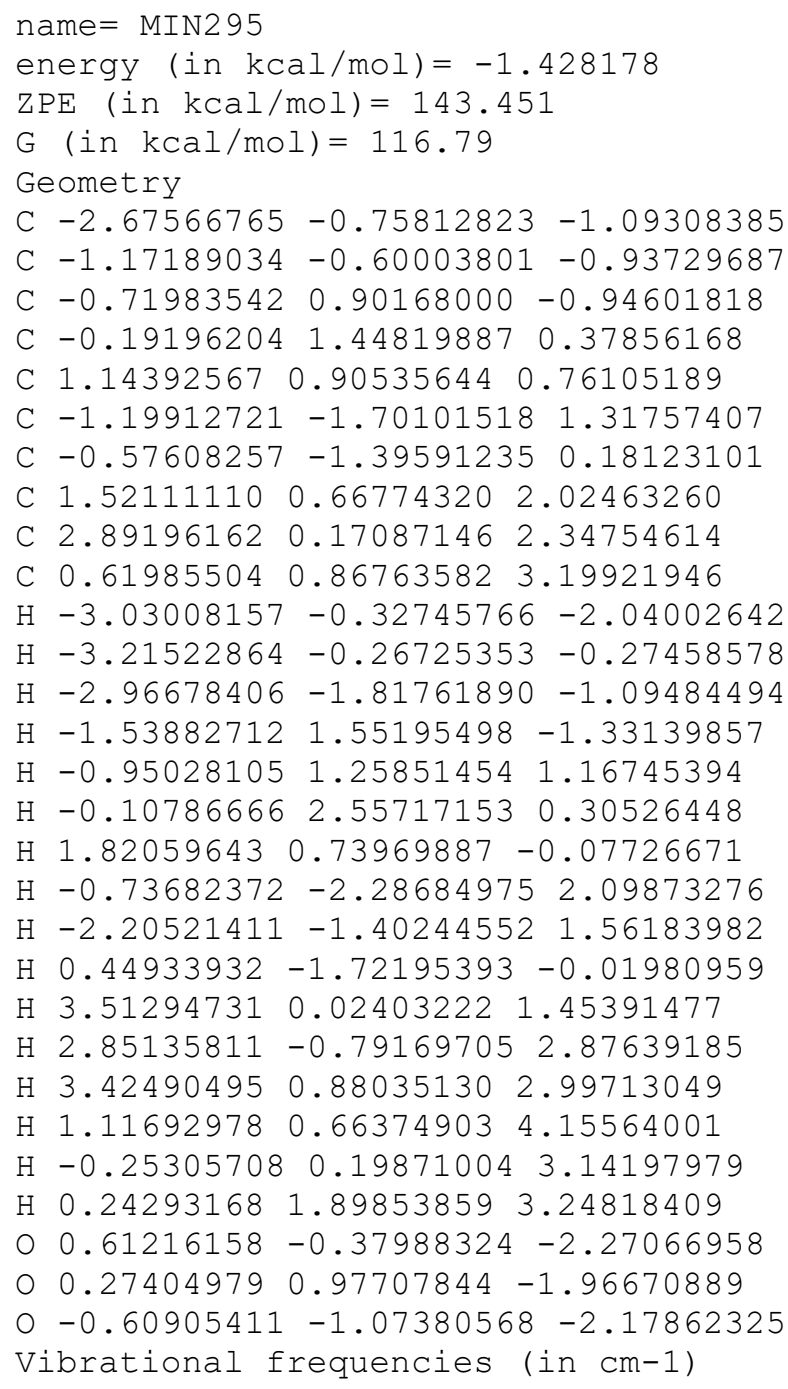


$2654.32665 .7 \quad 2669.4 \quad 2672.3 \quad 2673.6 \quad 2673.9 \quad 2675.42677 .0 \quad 2712.92728 .92744 .92754 .2$ $2777.22779 .3 \quad 2780.0 \quad 2787.6$

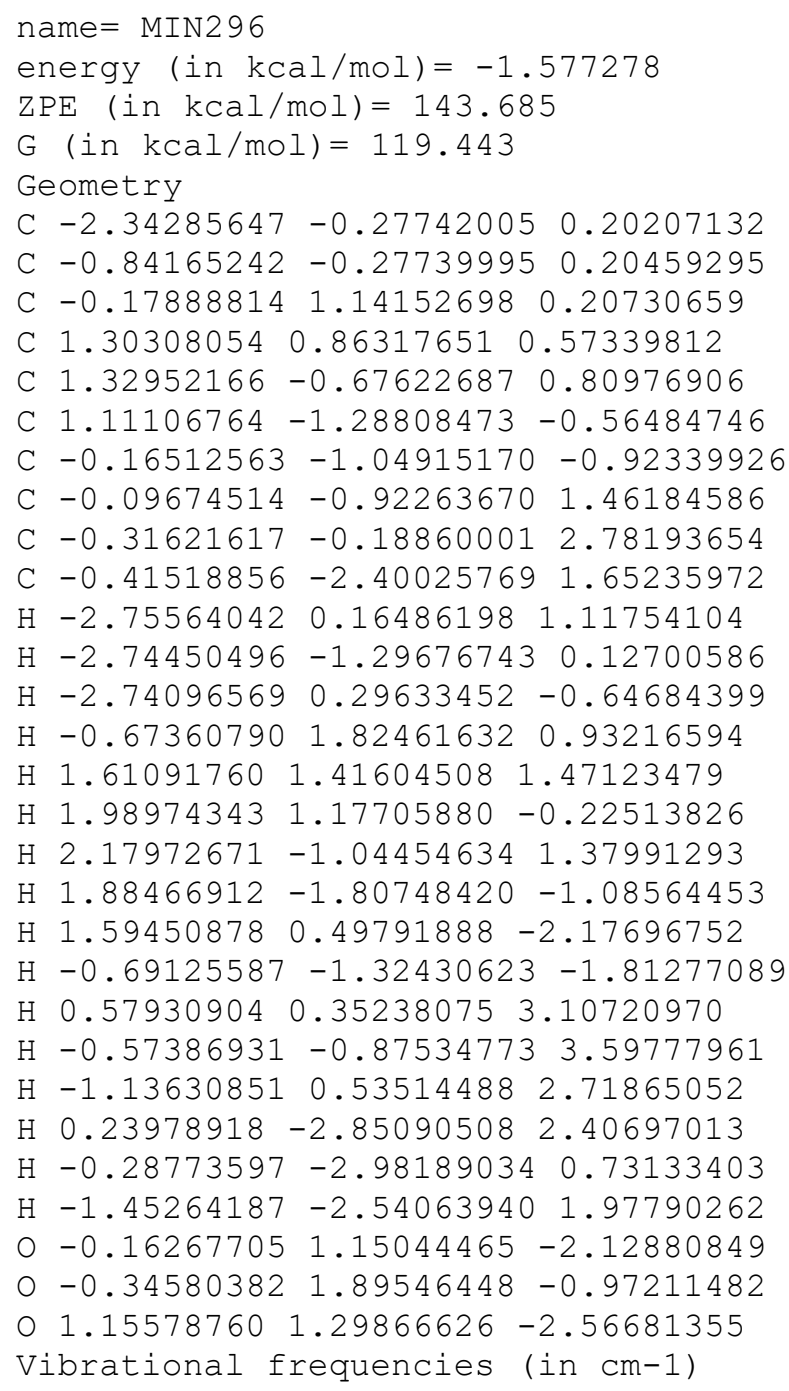


$\mathrm{H}-3.46503489-0.458051221 .14391088$

$\mathrm{H}-2.06786116 \quad 1.53900258 \quad 0.19885195$

$\mathrm{H}-0.05888211 \quad 1.24459136 \quad 1.69374914$

$\mathrm{H} \quad 0.23032901 \quad 2.39975325 \quad 0.38260661$

$\mathrm{H} \quad 1.11192409-0.19959669-0.60268661$

$\mathrm{H} 0.47067219-2.940359451 .62302611$

$\mathrm{H} \quad 0.14375934-2.79839692-0.14434390$

$\mathrm{H}-1.02769089-0.988367692 .08641829$

$\mathrm{H} 3.40651205-0.76110500-0.58497582$

H $4.13264089-0.598046691 .01779444$

H $4.45027933 \quad 0.61960595 \quad-0.23129575$

$\mathrm{H} 3.84771166 \quad 1.80493378 \quad 2.00840204$

H $2.213147791 .57640505 \quad 2.63737168$

$\mathrm{H} 2.52105434 \quad 2.78851558 \quad 1.38251260$

$0-0.60722813-0.14368136-1.99676900$

o $-1.137395231 .07321663-1.51223654$

O $-1.30942898-1.16479849-1.28980709$

Vibrational frequencies (in $\mathrm{cm}-1$ )

$\begin{array}{lllllllllllllll}-13.6 & 41.8 & 43.9 & 56.9 & 75.8 & 102.8 & 126.5 & 150.7 & 189.8 & 211.7 & 227.8 & 241.3 & 278.6 & 308.3\end{array}$ $320.9 \quad 406.8 \quad 424.7 \quad 475.5 \quad 488.2 \quad 513.2 \quad 578.8 \quad 613.5 \quad 685.1 \quad 731.9 \quad 824.5 \quad 857.6 \quad 891.4 \quad 953.6$

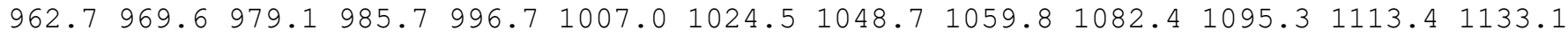

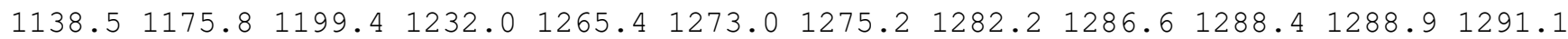
$1291.4 \quad 1303.4 \quad 1308.5 \quad 1339.7 \quad 1373.9 \quad 1383.3 \quad 1386.5 \quad 1395.1 \quad 1400.2 \quad 1429.01853 .2 \quad 1870.5$ $2652.72669 .4 \quad 2670.2 \quad 2672.7 \quad 2674.3 \quad 2677.0 \quad 2681.12682 .92711 .32726 .12752 .2 \quad 2756.6$ $2778.4 \quad 2780.0 \quad 2780.2 \quad 2782.8$

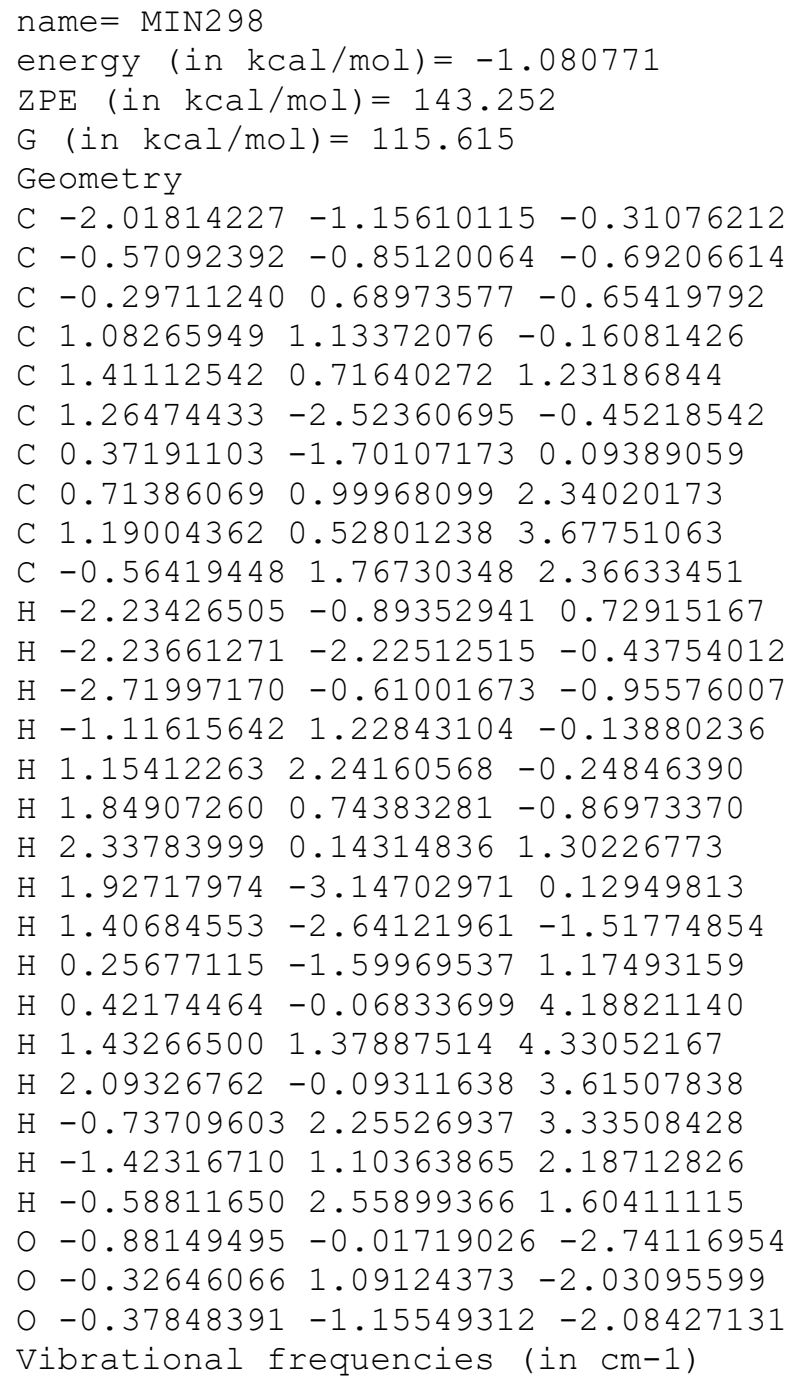


$\begin{array}{llllllllllllllll}1152.2 & 1161.9 & 1196.4 & 1229.4 & 1257.6 & 1274.0 & 1278.9 & 1282.5 & 1285.7 & 1288.2 & 1289.0 & 1292.5\end{array}$

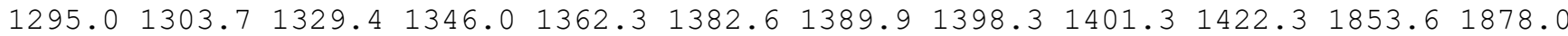
$2649.62668 .22670 .0 \quad 2670.92673 .3 \quad 2675.5 \quad 2679.4 \quad 2682.72709 .5 \quad 2734.82747 .8 \quad 2749.6$ $2777.32780 .0 \quad 2780.3 \quad 2782.7$

\section{name $=$ MIN299}

energy (in $\mathrm{kcal} / \mathrm{mol})=-0.942802$

$\mathrm{ZPE}(\mathrm{in} \mathrm{kcal} / \mathrm{mol})=143.22$

$\mathrm{G}(\mathrm{in} \mathrm{kcal} / \mathrm{mol})=115.668$

Geometry

C $-2.70003784-0.49220313-1.10057405$

C $-1.19047518-0.52845630-0.89750989$

C $-0.517655590 .86989570-1.10791758$

C $0.01498388 \quad 1.595896890 .12477834$

C $0.98104680 \quad 0.80125956 \quad 0.93626390$

C $-0.21694435-2.38085350 \quad 0.44191855$

C $-0.85191003-1.21127250 \quad 0.39034652$

C $1.17777651 \quad 0.99264584 \quad 2.24793726$

C $0.45387806 \quad 2.03620252 \quad 3.03523167$

C $2.14169564 \quad 0.15737028 \quad 3.02385223$

$\mathrm{H}-3.13762790-1.49430147-0.98891349$

$\mathrm{H}-2.96572342-0.13821407-2.10551368$

$\mathrm{H}-3.18434676 \quad 0.16259587-0.36717731$

$\mathrm{H}-1.206857091 .54259767-1.67215036$

$\mathrm{H}-0.84605316 \quad 1.93146447 \quad 0.74030909$

H $0.52441267 \quad 2.53048712 \quad-0.21125912$

$\mathrm{H} \quad 1.53700502 \quad 0.04951742 \quad 0.37559303$

$\mathrm{H} \quad 0.01787834 \quad-2.89123146 \quad 1.36284493$

$\mathrm{H} \quad 0.12347945-2.91629809-0.43492075$

$\mathrm{H}-1.18109231-0.68516994 \quad 1.28543535$

$\mathrm{H} 0.782646112 .08124782 \quad 4.08105203$

$\mathrm{H}-0.62937348 \quad 1.84987470 \quad 3.04823571$

$\mathrm{H} 0.61066199 \quad 3.03726646 \quad 2.60802156$

$\mathrm{H} 1.63525606-0.364498923 .84859605$

H $2.93761717 \quad 0.77398521 \quad 3.46452441$

H $2.62995467 \quad-0.61025147 \quad 2.40872022$

$\begin{array}{lllll}0 & 0.65858181 & -0.79273305 & -2.12722703\end{array}$

$\begin{array}{lllll}0 & 0.54531691 & 0.63060362 & -2.03073244\end{array}$

o $-0.66592716-1.25912470-2.02164826$

Vibrational frequencies (in $\mathrm{cm}-1$ )

$\begin{array}{llllllllllllllll}29.0 & 34.7 & 57.6 & 68.8 & 83.6 & 112.7 & 121.6 & 141.6 & 196.0 & 214.3 & 234.0 & 247.4 & 271.0 & 318.4\end{array}$ $339.1401 .0 \quad 418.2 \quad 480.2 \quad 495.4 \quad 554.4 \quad 572.0 \quad 608.0 \quad 696.9 \quad 725.8 \quad 807.6 \quad 850.7 \quad 882.1 \quad 942.0$ $958.6962 .6 \quad 978.7 \quad 988.5 \quad 998.5 \quad 1006.3 \quad 1023.2 \quad 1054.8 \quad 1068.1 \quad 1094.0 \quad 1096.9 \quad 1113.7 \quad 1137.0$ $1165.11193 .6 \quad 1205.1 \quad 1236.7 \quad 1269.6 \quad 1270.5 \quad 1278.0 \quad 1282.01284 .1 \quad 1288.0 \quad 1288.5 \quad 1289.6$

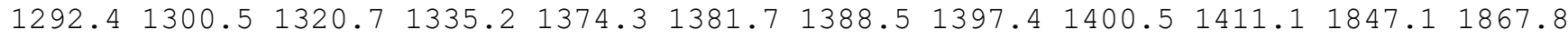
$2653.22667 .4 \quad 2670.0 \quad 2671.3 \quad 2672.8 \quad 2673.4 \quad 2676.72677 .12710 .62726 .92753 .12757 .4$ $2778.32779 .5 \quad 2780.0 \quad 2782.8$

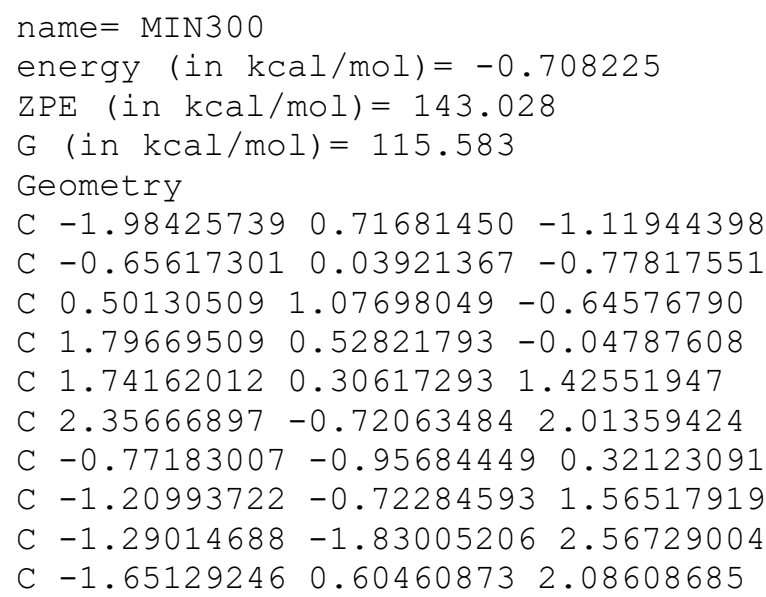


$\mathrm{H}-2.74793143-0.03929599-1.34819917$

$\mathrm{H}-1.879315461 .34478436-2.01557696$

$\mathrm{H}-2.36537088 \quad 1.33807966-0.30717886$

$\mathrm{H} \quad 0.18616825 \quad 2.03710033-0.19920291$

H $2.623222121 .24003281-0.27976241$

$\mathrm{H} 2.07179126-0.40818623-0.58495533$

H $1.17952527 \quad 1.04522923 \quad 1.99353446$

$\mathrm{H} 2.34618021-0.89350520 \quad 3.07678331$

$\mathrm{H} 2.91310528-1.47335600 \quad 1.47662034$

$\mathrm{H}-0.45952402-1.96196958 \quad 0.02307139$

$\mathrm{H}-2.27196334-1.85534328 \quad 3.06010146$

$\mathrm{H}-0.53048256-1.70152807 \quad 3.35293207$

$\mathrm{H}-1.12592209-2.821031732 .12368321$

$\mathrm{H}-1.18568108 \quad 1.44712891 \quad 1.55928736$

$\mathrm{H}-1.41422255 \quad 0.72763997 \quad 3.15231566$

$\mathrm{H}-2.74248622 \quad 0.71521855 \quad 1.98690621$

$\begin{array}{lllll}0 & 0.70905241 & 0.09759246 & -2.64927992\end{array}$

$\begin{array}{llllll}0 & 0.73352131 & 1.36791914 & -2.02432991\end{array}$

o $-0.28237133-0.68895084-1.96430225$

Vibrational frequencies (in $\mathrm{cm}-1$ )

$\begin{array}{lllllllllllllll}25.0 & 56.4 & 61.7 & 79.5 & 85.2 & 95.2 & 123.9 & 139.6 & 155.5 & 192.9 & 212.8 & 252.6 & 307.5 & 332.9\end{array}$

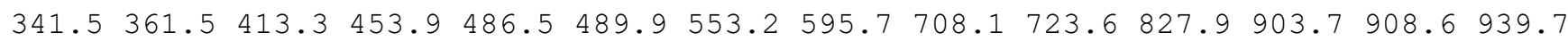
$960.2 \quad 972.4980 .0 \quad 992.6 \quad 1006.6 \quad 1017.0 \quad 1021.2 \quad 1024.8 \quad 1082.8 \quad 1087.5 \quad 1093.5 \quad 1114.4 \quad 1129.5$ $1143.31169 .2 \quad 1186.7 \quad 1225.2 \quad 1249.0 \quad 1263.2 \quad 1273.6 \quad 1279.1 \quad 1285.2 \quad 1288.1 \quad 1288.91291 .2$

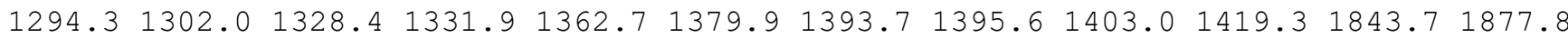
$2647.72667 .12669 .92670 .7 \quad 2671.9 \quad 2675.2 \quad 2681.4 \quad 2699.82717 .5 \quad 2729.92742 .7 \quad 2760.8$ 2777.12780 .02782 .02790 .8

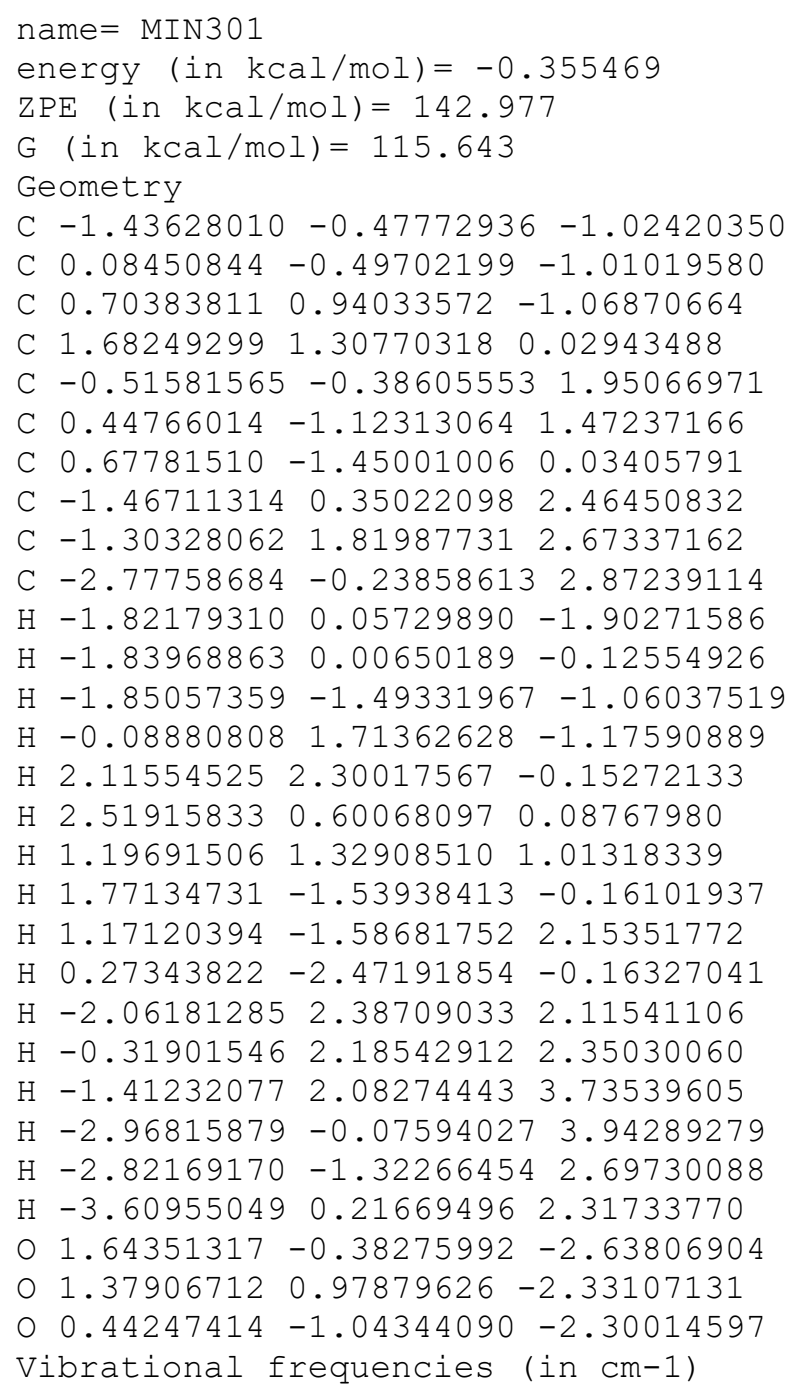


$\begin{array}{llllllllllllllllll}26.4 & 46.5 & 69.4 & 75.7 & 83.6 & 111.4 & 123.7 & 159.7 & 195.9 & 198.3 & 243.1 & 253.9 & 295.9 & 307.1\end{array}$ $317.1351 .9 \quad 415.0 \quad 455.4 \quad 463.2 \quad 515.8 \quad 594.9 \quad 623.5 \quad 675.6 \quad 706.5 \quad 806.9 \quad 830.8 \quad 869.0 \quad 879.1$ $949.9965 .2 \quad 979.4 \quad 983.9 \quad 994.4 \quad 1009.7 \quad 1012.8 \quad 1041.5 \quad 1071.0 \quad 1109.7 \quad 1120.8 \quad 1142.1 \quad 1155.1$

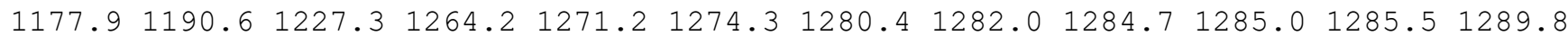
$1292.21293 .3 \quad 1298.3 \quad 1308.2 \quad 1322.4 \quad 1374.8 \quad 1390.91393 .1 \quad 1395.51401 .11598 .12312 .1$ $2645.72666 .8 \quad 2668.8 \quad 2669.7 \quad 2671.9 \quad 2672.4 \quad 2673.2 \quad 2674.12676 .02688 .8 \quad 2728.12735 .7$ $2777.7 \quad 2779.4 \quad 2780.3 \quad 2781.6$

\section{name $=$ MIN302}

energy (in $\mathrm{kcal} / \mathrm{mol})=-0.67728$

$\operatorname{ZPE}($ in $\mathrm{kcal} / \mathrm{mol})=143.462$

$\mathrm{G}($ in $\mathrm{kcal} / \mathrm{mol})=116.563$

Geometry

C $-2.18111398-0.87153099-0.50472941$

C $-0.68381218-0.69071882-0.74605691$

C $-0.276178550 .81231828-0.59869860$

C $1.140320801 .09335941-0.08562167$

C $1.45716438 \quad 0.47834181 \quad 1.23419784$

C $1.01213185-2.49674744-0.45278178$

C $0.10447325-1.66872908 \quad 0.05985819$

$\begin{array}{lllll}\text { C } & 0.81022088 & 0.68775893 & 2.38845593\end{array}$

C $-0.38201607 \quad 1.57010314 \quad 2.54130442$

C $1.25359528 \quad 0.00355602 \quad 3.64240549$

$\mathrm{H}-2.46264956-0.64969425 \quad 0.52888762$

$\mathrm{H}-2.48774949-1.90380390-0.72101143$

$\mathrm{H}-2.76911516-0.22015275-1.16622753$

$\mathrm{H}-1.04152679 \quad 1.38503645-0.03936188$

H $1.29128980 \quad 2.19502366-0.03654394$

H $1.86469407 \quad 0.74264355-0.85661679$

H $2.32176193-0.187616121 .20486956$

H $1.27985464 \quad-2.52766270-1.49986411$

$\mathrm{H} 1.56166253-3.21123934 \quad 0.14174794$

$\mathrm{H}-0.13689644-1.65607588 \quad 1.12466306$

$\mathrm{H}-0.338651872 .44389225 \quad 1.87586920$

$\mathrm{H}-0.48531410 \quad 1.95540085 \quad 3.56468128$

$\mathrm{H}-1.30727771 \quad 1.02122952 \quad 2.31212162$

$\mathrm{H} 2.08086985-0.69905616 \quad 3.47550238$

$\mathrm{H} \quad 0.43276316-0.56633260 \quad 4.09859699$

$\mathrm{H} \quad 1.60046640 \quad 0.73375978 \quad 4.38760863$

$\begin{array}{llllll}0 & -0.80074984 & 0.26103914 & -2.75978702\end{array}$

$\begin{array}{lllll}0 & -0.27752973 & 1.31544794 & -1.94041354\end{array}$

o $-0.39646692-0.93016862-2.13455662$

Vibrational frequencies (in $\mathrm{cm}-1$ )

$\begin{array}{lllllllllllllll}28.3 & 37.5 & 67.6 & 90.8 & 111.8 & 126.7 & 151.7 & 173.5 & 202.1 & 226.0 & 237.9 & 247.8 & 274.1 & 297.9\end{array}$ $349.2 \quad 403.0 \quad 424.4 \quad 465.2 \quad 479.4 \quad 532.4 \quad 586.3 \quad 623.8 \quad 674.2 \quad 712.9 \quad 807.2 \quad 882.1 \quad 901.3 \quad 923.2$ $962.3 \quad 976.2989 .3 \quad 992.7 \quad 1004.5 \quad 1017.8 \quad 1022.9 \quad 1061.1 \quad 1062.8 \quad 1093.8 \quad 1109.5 \quad 1114.2 \quad 1118.6$

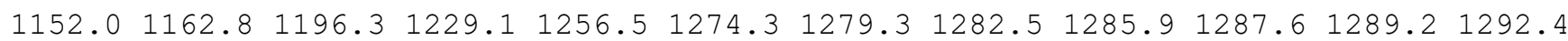

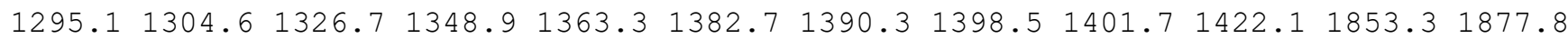
$2649.92668 .92669 .5 \quad 2671.12673 .7 \quad 2675.3 \quad 2679.2 \quad 2683.62709 .92735 .2 \quad 2748.4 \quad 2750.4$ $2777.6 \quad 2780.12780 .4 \quad 2782.9$

name $=$ MIN303

energy $($ in $\mathrm{kcal} / \mathrm{mol})=-0.099634$

$\mathrm{ZPE}(\mathrm{in} \mathrm{kcal} / \mathrm{mol})=142.966$

$\mathrm{G}($ in $\mathrm{kcal} / \mathrm{mol})=113.216$

Geometry

C $-2.93617865-0.56462850-0.63518400$

C $-1.41223136-0.48199790-0.60328976$

C $-0.929108791 .01061942-0.53544859$

C $-0.15511629 \quad 1.43642466 \quad 0.71103402$

C $1.26835839 \quad 0.98824838 \quad 0.70819229$

C $0.24328467-2.12649083 \quad 0.25918222$

C $-0.84282059-1.379624750 .45084809$ 
C $1.913518690 .43825707 \quad 1.74476956$

C $3.36581948 \quad 0.09172133 \quad 1.63540333$

C $1.302548290 .12550679 \quad 3.06968998$

$\mathrm{H}-3.38806048-0.13422967 \quad 0.26397245$

$\mathrm{H}-3.27049077-1.60813919-0.71029140$

$\mathrm{H}-3.34486508-0.04108128-1.50924098$

$\mathrm{H}-1.79020928 \quad 1.69264000 \quad-0.71044745$

$\mathrm{H}-0.70079308 \quad 1.07517458 \quad 1.60803654$

$\mathrm{H}-0.16819494 \quad 2.54858574 \quad 0.78503806$

$\mathrm{H} 1.78778861 \quad 1.16419779-0.23630126$

$\mathrm{H} \quad 0.65668445-2.78168421 \quad 1.00996413$

$\mathrm{H} \quad 0.81006455-2.12979461-0.66398253$

$\mathrm{H}-1.39246149-1.38061250 \quad 1.38980001$

H $3.62319257-0.80388758 \quad 2.21456812$

H $3.98825691 \quad 0.91613085 \quad 2.01298086$

$\mathrm{H} 3.67352510-0.097337650 .59780041$

H $1.26737162-0.96239023 \quad 3.23212449$

$\mathrm{H} \quad 0.27609933 \quad 0.49760665 \quad 3.17409805$

$\mathrm{H} \quad 1.88720960 \quad 0.56081251 \quad 3.89196674$

$\begin{array}{lllll}0 & -0.61928834 & 0.23551311 & -2.60421873\end{array}$

$0-0.049448821 .12199694-1.65863766$

o $-0.89968580-0.94457201-1.86311823$

Vibrational frequencies (in $\mathrm{cm}-1$ )

$\begin{array}{lllllllllllllll}-21.9 & 7.6 & 25.7 & 34.5 & 59.7 & 63.8 & 114.9 & 162.7 & 192.9 & 228.1 & 233.4 & 250.4 & 280.6 & 297.4\end{array}$ $343.6 \quad 403.8 \quad 433.2 \quad 472.9 \quad 508.4 \quad 527.1 \quad 579.5 \quad 605.5 \quad 683.1 \quad 701.9 \quad 776.3 \quad 869.9 \quad 882.5 \quad 947.0$ $960.2967 .4983 .8 \quad 992.2 \quad 1002.7 \quad 1011.4 \quad 1014.4 \quad 1049.8 \quad 1060.7 \quad 1095.9 \quad 1113.4 \quad 1117.91144 .1$ $\begin{array}{llllllllllllll}1153.9 & 1172.0 & 1211.1 & 1236.0 & 1253.6 & 1267.2 & 1277.3 & 1280.7 & 1284.3 & 1288.4 & 1288.8 & 1290.5\end{array}$ $1294.11308 .61332 .91345 .91376 .7 \quad 1383.7 \quad 1388.1 \quad 1399.01400 .11421 .6 \quad 1846.6 \quad 1877.8$ $2649.32669 .32670 .0 \quad 2670.7 \quad 2673.4 \quad 2674.0 \quad 2676.82680 .32709 .6 \quad 2726.02749 .02758 .7$ $2777.6 \quad 2779.8 \quad 2780.7 \quad 2782.2$

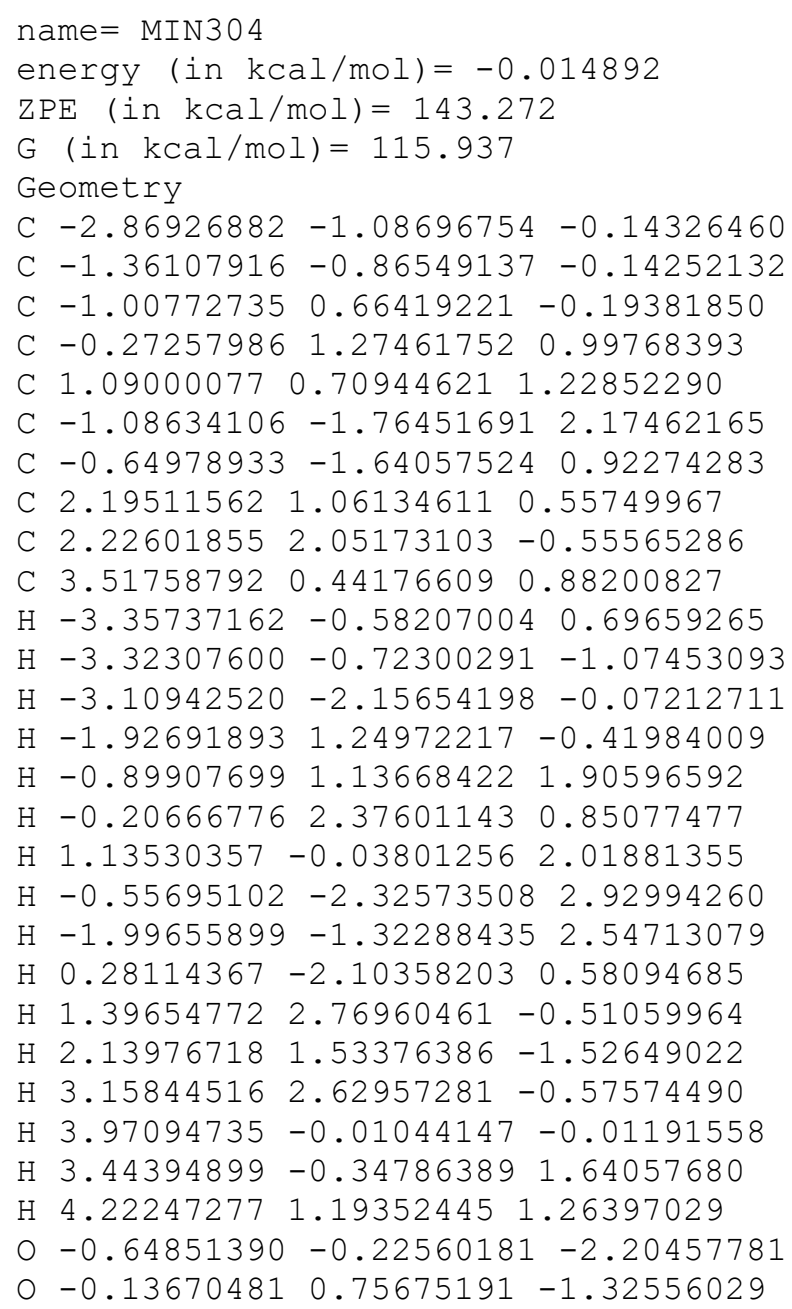


$0-0.83060239-1.37191164-1.38260640$

Vibrational frequencies (in $\mathrm{cm}-1$ )

$\begin{array}{llllllllllllllll}24.0 & 36.2 & 66.5 & 85.2 & 88.1 & 95.0 & 136.6 & 160.0 & 196.9 & 213.9 & 237.3 & 259.6 & 296.9 & 310.3\end{array}$

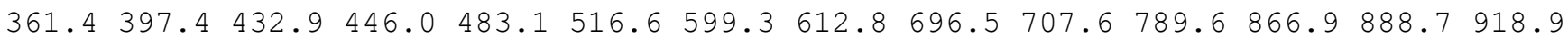

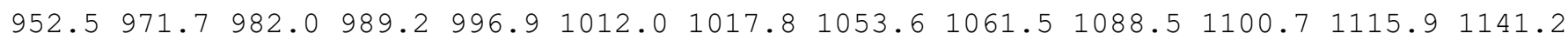

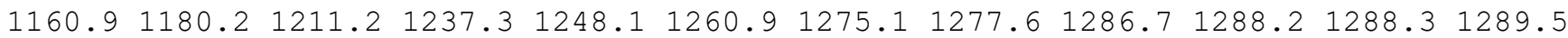

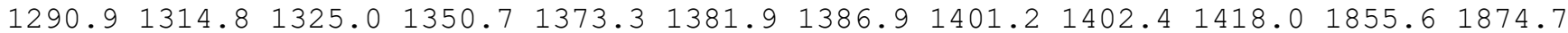
$2654.62661 .8 \quad 2668.7 \quad 2672.4 \quad 2672.6 \quad 2674.6 \quad 2677.92679 .12712 .92733 .2 \quad 2744.2 \quad 2755.1$ $2776.92780 .3 \quad 2780.7 \quad 2787.9$

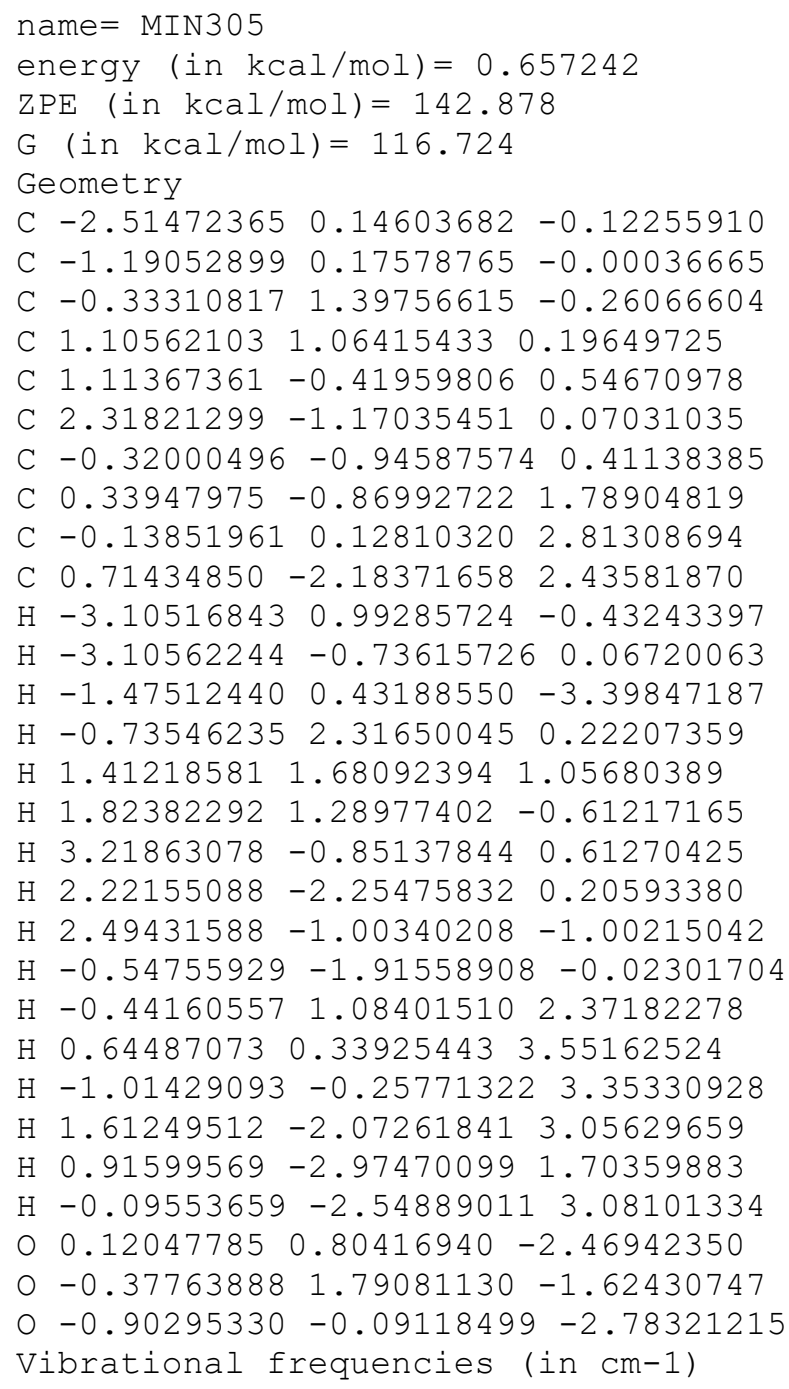


C $-0.35023439-1.05194260-0.03148392$

C $-1.14548235 \quad 0.18695024 \quad 0.34638842$

C $1.84170111-2.010645240 .68916913$

C $2.86557902-2.27990457 \quad 1.74137624$

C $2.16190797-2.53156418-0.67180480$

$\mathrm{H}-2.19896250 \quad 1.01562911-2.58507641$

$\mathrm{H}-4.16808371 \quad 0.94619578-1.26669219$

$\mathrm{H}-3.94952259 \quad 0.57233017 \quad 0.46714174$

$\mathrm{H}-0.170090732 .53743974-1.11774833$

H $2.162282591 .64541199-1.87610723$

H $2.16168586 \quad 0.54690867-0.44834135$

$\mathrm{H} \quad 0.50370202-1.002137121 .98456965$

$\mathrm{H}-1.04069921-1.92327017-0.11178321$

$\mathrm{H} \quad 0.07162045-0.93628352-1.05634383$

$\mathrm{H}-1.62184038 \quad 0.09104357 \quad 1.35176736$

H $3.83616753-1.83651004 \quad 1.47584275$

$\mathrm{H} 3.02623266-3.359858491 .87206290$

H $2.58630124 \quad-1.87646160 \quad 2.72428117$

$\mathrm{H} \quad 1.42734525-3.28678007-0.98977618$

$\mathrm{H} 3.15029710-3.00526272-0.72572648$

H $2.14886080-1.73215974-1.42815305$

$\begin{array}{lllll}0 & -0.61056653 & 2.22743834 & 1.28095595\end{array}$

$0-0.08465245 \quad 1.26576063 \quad 0.63207527$

O $-1.56004917 \quad 0.71694190-1.89431163$

Vibrational frequencies (in $\mathrm{cm}-1$ )

$\begin{array}{lllllllllllllll}39.0 & 43.3 & 52.9 & 56.3 & 93.3 & 95.2 & 117.2 & 134.3 & 177.7 & 200.2 & 232.1 & 267.5 & 274.0 & 312.7\end{array}$

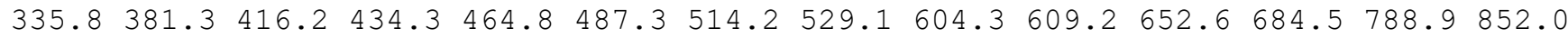
$900.3951 .9970 .9985 .0 \quad 993.2 \quad 1004.6 \quad 1018.5 \quad 1026.4 \quad 1048.3 \quad 1058.2 \quad 1076.4 \quad 1085.5 \quad 1108.9$

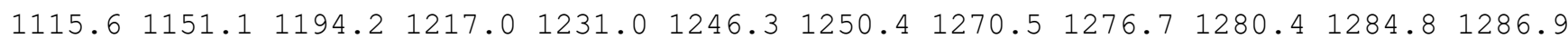

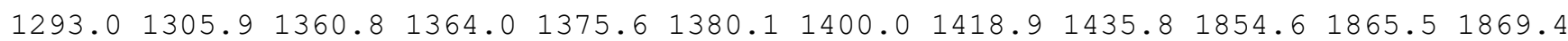
$2639.82664 .02670 .92671 .6 \quad 2674.92677 .7 \quad 2704.02724 .92727 .3 \quad 2752.0 \quad 2754.92777 .1$ $2777.5 \quad 2780.2 \quad 2788.5 \quad 2804.9$

name $=$ MIN307

energy $($ in $\mathrm{kcal} / \mathrm{mol})=0.59544$

$\mathrm{ZPE}($ in $\mathrm{kcal} / \mathrm{mol})=143.234$

$\mathrm{G}($ in $\mathrm{kcal} / \mathrm{mol})=116.182$

Geometry

C $-2.35654707-0.19027964-0.68195297$

C $-0.83249137-0.16856547-0.64382119$

C $-0.24390444 \quad 1.28596862-0.68644843$

C $0.56807888 \quad 1.77839125 \quad 0.51221608$

C $1.68164073 \quad 0.87747924 \quad 0.94444737$

C $2.71443544 \quad 0.55201439 \quad 0.16783243$

C $-0.23504059-1.07694340 \quad 0.37329277$

C $-0.54301106-1.19191731 \quad 1.67076162$

C $-1.56409766-0.375914252 .38859526$

C $0.16091452-2.198911432 .52521118$

$\mathrm{H}-2.79580765 \quad 0.41485726 \quad 0.11645092$

$\mathrm{H}-2.73688597-1.21496364-0.57072469$

$\mathrm{H}-2.73378068 \quad 0.18966365-1.64262469$

$\mathrm{H}-1.054505342 .01829332-0.91350123$

$\mathrm{H}-0.12358838 \quad 1.97116730 \quad 1.35781059$

$\mathrm{H} \quad 0.99738564 \quad 2.77371661 \quad 0.24818967$

$\mathrm{H} \quad 1.59829644 \quad 0.51142044 \quad 1.96750804$

$\mathrm{H} 3.51773183-0.09085588 \quad 0.48924172$

$\mathrm{H} \quad 2.82416439 \quad 0.89066349-0.85198771$

H $0.54350132-1.71628371-0.06087685$

$\mathrm{H}-2.54885393-0.86587888 \quad 2.34907362$

$\mathrm{H}-1.67841451 \quad 0.62844404 \quad 1.96066254$

$\mathrm{H}-1.31431401-0.242951253 .45034588$

$\mathrm{H} \quad 0.82833175-1.70602070 \quad 3.24662582$

$\mathrm{H} \quad 0.77920187-2.89454555 \quad 1.94089639$

$\mathrm{H}-0.55202763-2.80757294 \quad 3.09804497$ 
$0.78206395-0.05167483-2.20487410$

$0 \quad 0.562799221 .31793883-1.86371200$

O $-0.45661602-0.66451597-1.95307171$

Vibrational frequencies (in $\mathrm{cm}-1$ )

$\begin{array}{lllllllllllllll}26.6 & 48.1 & 62.3 & 91.4 & 98.5 & 119.5 & 130.6 & 139.1 & 177.7 & 214.3 & 234.5 & 248.9 & 274.7 & 319.1\end{array}$ $355.5 \quad 404.5 \quad 421.0 \quad 478.1 \quad 489.7 \quad 497.2 \quad 564.4 \quad 663.4 \quad 697.7 \quad 714.2 \quad 825.9 \quad 874.1908 .1910 .4$

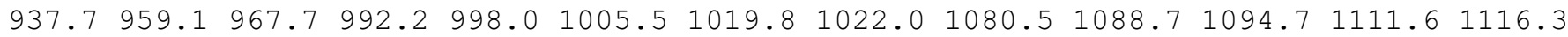
$\begin{array}{lllllllllllll}1161.0 & 1182.3 & 1199.7 & 1234.4 & 1249.5 & 1261.1 & 1274.0 & 1278.4 & 1285.3 & 1286.3 & 1288.5 & 1296.6\end{array}$ $1301.7 \quad 1309.6 \quad 1330.4 \quad 1335.2 \quad 1379.4 \quad 1382.5 \quad 1389.7 \quad 1395.7 \quad 1401.3 \quad 1431.1 \quad 1852.8 \quad 1884.1$ 2655.62667 .82668 .92669 .62671 .02673 .12674 .12676 .42715 .92734 .12735 .02755 .6 $2777.22779 .3 \quad 2779.92786 .8$

name $=$ MIN308

energy $($ in $\mathrm{kcal} / \mathrm{mol})=0.776809$

$\mathrm{ZPE}($ in $\mathrm{kcal} / \mathrm{mol})=143.184$

$\mathrm{G}($ in $\mathrm{kcal} / \mathrm{mol})=115.961$

Geometry

C $-2.37155038-0.30789960-0.58437305$

C $-0.85184795-0.20462672-0.62800515$

C $-0.341873391 .27926011-0.66159866$

C $0.43838041 \quad 1.81009771 \quad 0.54120483$

C $1.62656117 \quad 0.99406877 \quad 0.94180075$

C $2.68120756 \quad 0.789744170 .15340349$

C $-0.14741028-1.107371210 .32338646$

C $-0.37015247-1.28194671 \quad 1.63141517$

C $-1.38899642-0.551979522 .43914742$

C $0.43955015-2.274027562 .40635478$

$\mathrm{H}-2.82168077 \quad 0.06886188-1.51445622$

$\mathrm{H}-2.79702647 \quad 0.25862025 \quad 0.24949234$

$\mathrm{H}-2.69140398-1.35310656-0.47348505$

$\mathrm{H}-1.19345956 \quad 1.96562304 \quad-0.88446626$

$\mathrm{H}-0.25891876 \quad 1.92521388 \quad 1.39634529$

$\mathrm{H} \quad 0.78112708 \quad 2.84342588 \quad 0.29806837$

H $1.57794866 \quad 0.58421113 \quad 1.95027844$

H $2.75598563 \quad 1.17406582-0.85341059$

H $3.53942348 \quad 0.20936413 \quad 0.45050809$

$\mathrm{H} \quad 0.63984524-1.68620470-0.17589915$

$\mathrm{H}-2.32313308-1.13063548 \quad 2.49914316$

$\mathrm{H}-1.63778126 \quad 0.43130765 \quad 2.01992171$

$\mathrm{H}-1.04947960-0.381520613 .47044060$

H $1.09851889-1.769318463 .12719764$

$\mathrm{H} 1.08247433-2.89247844 \quad 1.76461374$

$\mathrm{H}-0.20426206-2.96087814 \quad 2.97258934$

$\begin{array}{lllll}0 & 0.69714616 & 0.01102977 & -2.23751101\end{array}$

○ $0.46395678 \quad 1.36452448-1.83686662$

o $-0.51949723-0.63680678-1.97153725$

Vibrational frequencies (in $\mathrm{cm}-1$ )

$\begin{array}{llllllllllllllll}26.9 & 46.6 & 55.9 & 86.3 & 93.0 & 113.1 & 126.1 & 137.7 & 178.2 & 214.0 & 233.5 & 249.6 & 270.0 & 318.0\end{array}$ $356.5 \quad 401.5 \quad 421.0 \quad 477.5 \quad 491.0 \quad 496.6 \quad 564.6 \quad 662.5 \quad 697.3 \quad 713.2 \quad 821.9 \quad 874.1 \quad 906.9 \quad 911.1$ $937.5958 .8 \quad 968.5 \quad 992.3 \quad 998.4 \quad 1005.6 \quad 1018.0 \quad 1022.2 \quad 1082.1 \quad 1088.5 \quad 1094.7 \quad 1112.6 \quad 1117.8$ $1162.21183 .8 \quad 1201.6 \quad 1236.0 \quad 1249.8 \quad 1260.8 \quad 1272.8 \quad 1278.2 \quad 1284.8 \quad 1286.0 \quad 1288.0 \quad 1296.3$

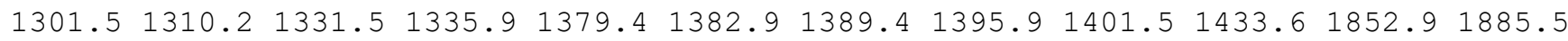
$2655.62666 .92668 .92669 .2 \quad 2670.5 \quad 2673.0 \quad 2674.02675 .52716 .12733 .5 \quad 2734.52755 .8$ 2777.22779 .02779 .92786 .9

name $=$ MIN309

energy $($ in $\mathrm{kcal} / \mathrm{mol})=0.32838$

$\operatorname{ZPE}($ in $\mathrm{kcal} / \mathrm{mol})=143.777$

$\mathrm{G}($ in $\mathrm{kcal} / \mathrm{mol})=116.675$

Geometry

C $-2.09424038-0.64412520-0.83810967$

C $-0.56820731-0.66416133-0.82391134$

C $0.04468377 \quad 0.77940149-0.79894369$ 
C $1.13259465 \quad 1.05163540 \quad 0.23821180$

C $0.62253802 \quad 1.88685418 \quad 1.41969517$

C $0.82162611-2.54345961 \quad 0.03369115$

C $-0.06314084-1.57298868 \quad 0.25143349$

C $-0.23210062 \quad 1.08830966 \quad 2.36064681$

C $-1.55623198 \quad 1.24870977 \quad 2.42483377$

C $0.49755636 \quad 0.13664519 \quad 3.25088395$

$\mathrm{H}-2.47747458-0.07473482-1.69573980$

$\mathrm{H}-2.49674830-0.198036590 .07918164$

$\mathrm{H}-2.50145697-1.66080690-0.92278598$

$\mathrm{H}-0.75060396 \quad 1.55502306-0.77192442$

$\mathrm{H} \quad 1.96087460 \quad 1.59911830 \quad-0.26451307$

H $1.59986091 \quad 0.11419691 \quad 0.59500435$

$\mathrm{H} 1.490354832 .30485343 \quad 1.97333948$

$\mathrm{H} \quad 1.17384621-3.21365147 \quad 0.80227476$

$\mathrm{H} 1.25784425-2.74611979-0.93617697$

$\mathrm{H}-0.49685536-1.38278924 \quad 1.23436832$

$\mathrm{H}-2.10764351 \quad 1.94663035 \quad 1.81852979$

$\mathrm{H} \quad 0.07228321 \quad 2.77432954 \quad 1.04411148$

$\mathrm{H}-2.19228041 \quad 0.70256770 \quad 3.10088837$

$\mathrm{H} \quad 1.01625589 \quad 0.67763843 \quad 4.05629664$

H $1.26070704 \quad-0.43735788 \quad 2.70634085$

$\mathrm{H}-0.17202228-0.58863579 \quad 3.73206959$

O $0.94423263-0.40455318-2.48615595$

$0.60806060 \quad 0.92100193-2.10631448$

o - $0.19629151-1.15938361-2.12329906$

Vibrational frequencies (in cm-1)

$\begin{array}{llllllllllllllll}22.9 & 35.8 & 55.3 & 62.5 & 92.0 & 113.1 & 166.2 & 180.0 & 200.5 & 236.0 & 283.6 & 306.9 & 333.3 & 369.8\end{array}$ $398.8 \quad 418.6 \quad 439.0 \quad 456.8 \quad 551.6 \quad 565.0 \quad 599.8 \quad 628.0 \quad 683.7 \quad 718.5 \quad 835.0 \quad 870.6 \quad 882.2 \quad 929.4$ $951.9971 .9 \quad 989.7 \quad 993.0 \quad 1002.0 \quad 1017.6 \quad 1037.6 \quad 1052.7 \quad 1062.1 \quad 1079.6 \quad 1095.01095 .91117 .5$

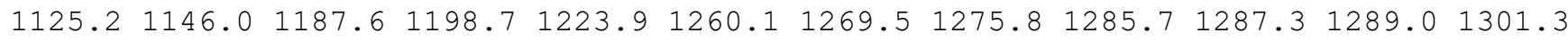

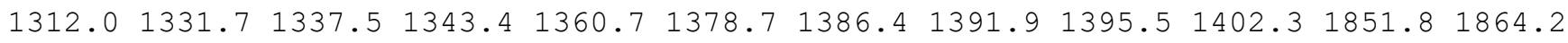
$2647.52659 .92667 .5 \quad 2670.6 \quad 2673.0 \quad 2673.7 \quad 2680.2 \quad 2709.52725 .62735 .62741 .42749 .4$ $2777.32778 .3 \quad 2781.8 \quad 2795.2$

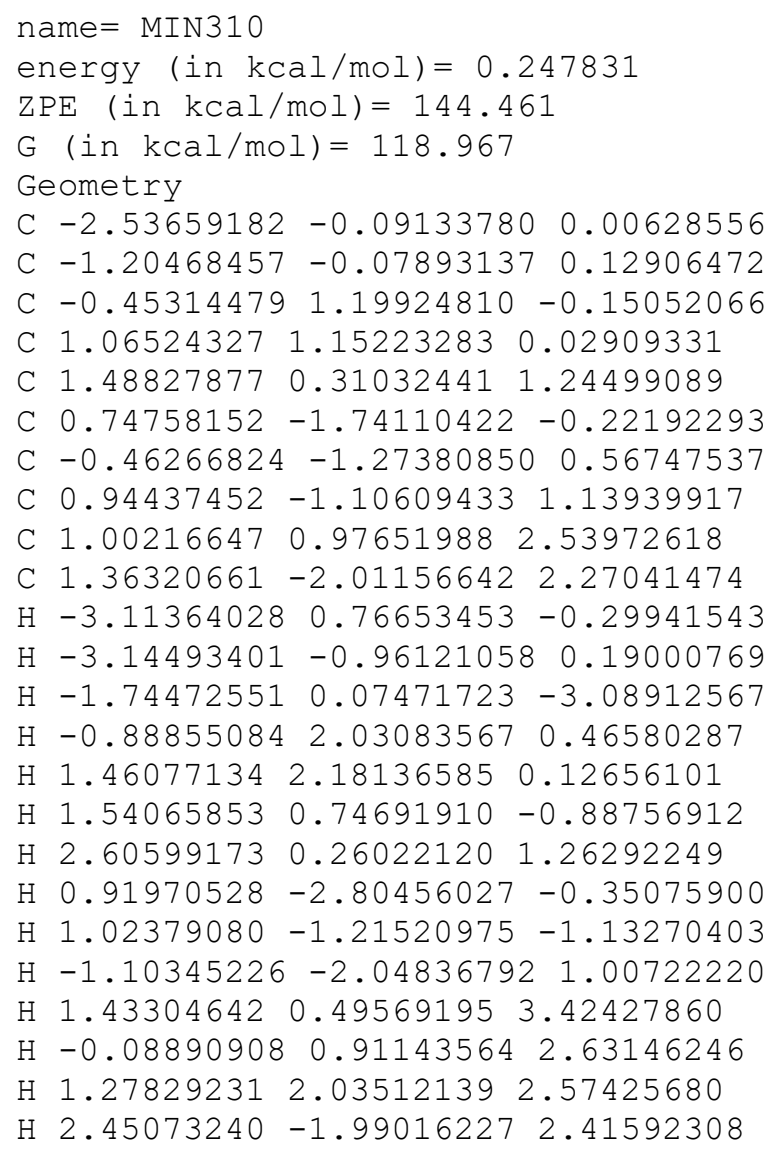


$\mathrm{H} \quad 1.08268045-3.05675287 \quad 2.08892479$

$\mathrm{H} \quad 0.89257827-1.71288570 \quad 3.21623321$

$0-0.18720788 \quad 0.93425723-2.46208822$

$0-0.78862757 \quad 1.67555170-1.45490486$

O $-0.90544312-0.24454440-2.67285559$

Vibrational frequencies (in $\mathrm{cm}-1$ )

$\begin{array}{llllllllllllllll}34.1 & 50.6 & 83.2 & 118.9 & 126.1 & 154.0 & 189.8 & 222.1 & 253.2 & 277.5 & 304.4 & 312.5 & 365.7 & 376.6\end{array}$ $419.4 \quad 423.1 \quad 474.2 \quad 497.0 \quad 530.1 \quad 557.5 \quad 622.8 \quad 670.9 \quad 720.2 \quad 758.5 \quad 805.1 \quad 837.8 \quad 872.5 \quad 892.1$

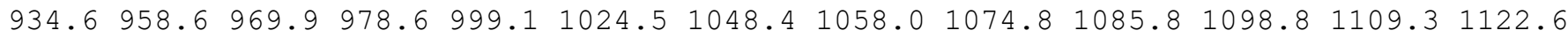

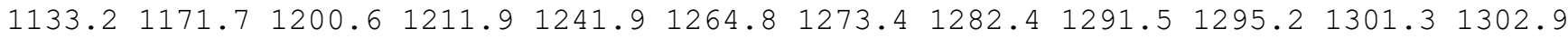

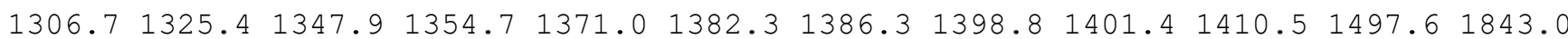
$2651.12672 .5 \quad 2673.7 \quad 2674.7 \quad 2677.0 \quad 2679.92703 .12707 .52724 .02739 .52754 .42780 .8$ $2781.4 \quad 2784.4 \quad 2790.2 \quad 2848.3$

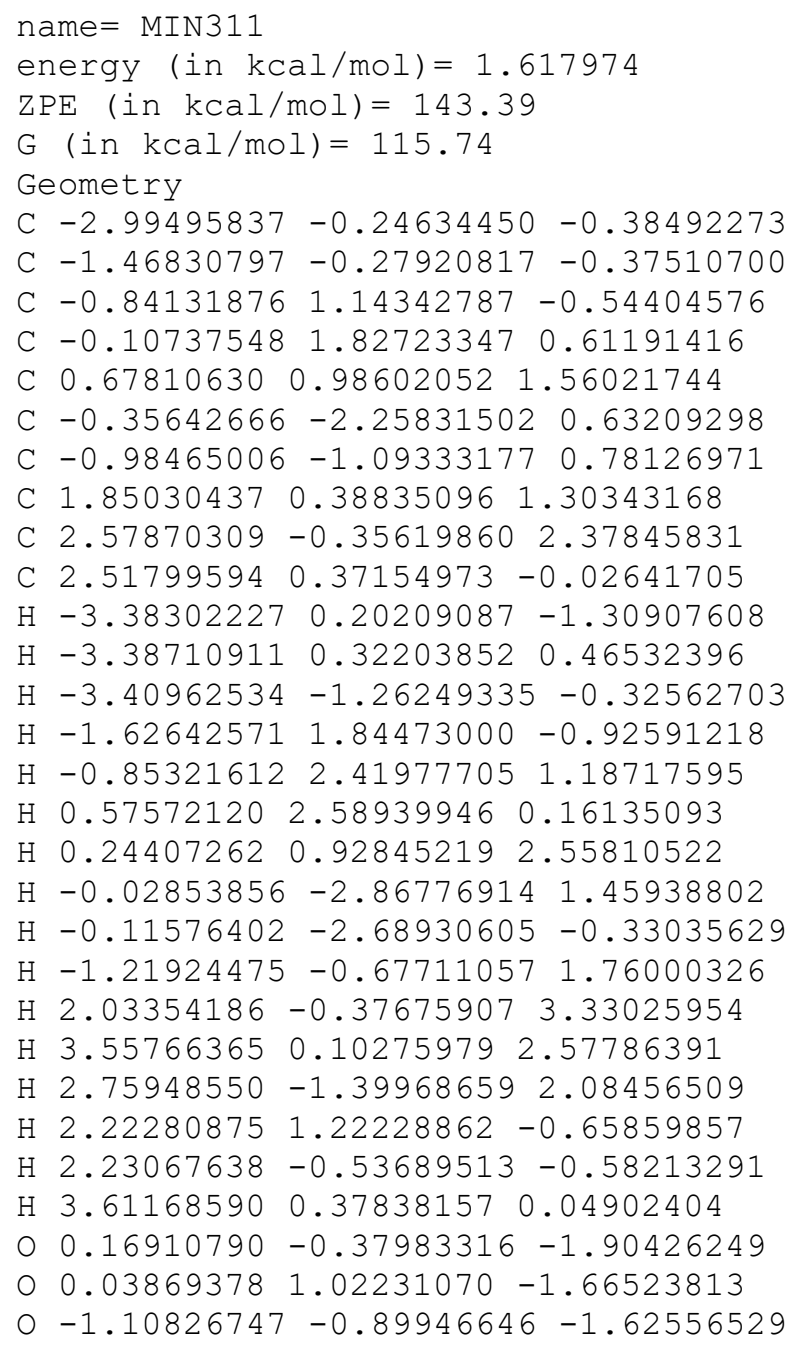


C $-0.10045340-1.30561180-0.60181292$

C $0.31295785 \quad 0.20858578 \quad-0.58314611$

C $1.08599854 \quad 0.71348025 \quad 0.62977675$

C $0.15021928 \quad 1.02080892 \quad 1.80640351$

C $-0.03645421-2.44050056 \quad 1.63325346$

C $0.54120530-2.12669867 \quad 0.47564611$

C $-0.77737765 \quad 2.15981864 \quad 1.49493054$

C -2.043533091 .959719521 .12145220$

C $-0.19683215 \quad 3.52620275 \quad 1.64468921$

$\mathrm{H}-1.88696721-2.53380344-0.74656448$

$\mathrm{H}-2.00159597-1.00199363-1.61286006$

$\mathrm{H}-2.12746544-1.02825960 \quad 0.15722157$

$\mathrm{H}-0.56671837 \quad 0.85371540 \quad-0.79987992$

$\mathrm{H} \quad 1.65222607 \quad 1.62536098 \quad 0.34344598$

H $1.85718197 \quad-0.02011922 \quad 0.93772010$

$\mathrm{H} \quad 0.75552456 \quad 1.25676499 \quad 2.70627958$

$\mathrm{H} 0.45266914 \quad-3.03087530 \quad 2.39455413$

$\mathrm{H}-1.03514804-2.14641669 \quad 1.91694096$

H $1.55152560-2.46314956 \quad 0.22284500$

$\mathrm{H}-0.421242110 .10861525 \quad 2.07715759$

$\mathrm{H}-2.73041377 \quad 2.75584024 \quad 0.88811674$

$\mathrm{H}-2.49503814 \quad 0.98787654 \quad 1.02131696$

$\mathrm{H}-0.01875058 \quad 3.76358735 \quad 2.70377942$

$\mathrm{H}-0.85542582 \quad 4.30987926 \quad 1.24639479$

$\mathrm{H} \quad 0.76606507 \quad 3.61971878 \quad 1.12316905$

$0.0 .73540831-0.64090265-2.60759159$

$01.22635785 \quad 0.31015719-1.68452510$

○ $0.48780311-1.79807742-1.82255127$

Vibrational frequencies (in cm-1)

$\begin{array}{llllllllllllll}31.8 & 46.8 & 59.4 & 92.3 & 99.9 & 118.2 & 153.7 & 181.4 & 214.1 & 252.9 & 267.3 & 296.1 & 339.2 & 385.4\end{array}$ $401.9417 .6 \quad 432.3 \quad 444.6 \quad 499.5 \quad 573.0 \quad 619.2 \quad 637.6 \quad 674.0 \quad 705.6 \quad 775.9 \quad 872.9 \quad 884.8 \quad 946.4$

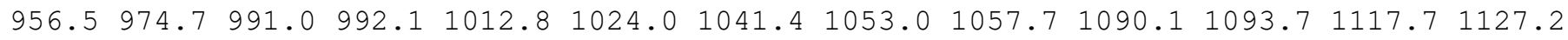

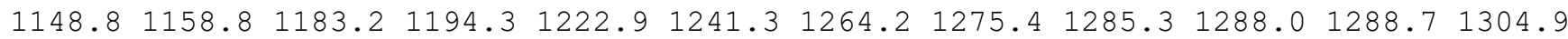

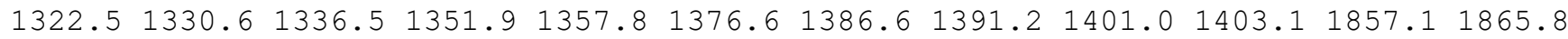
$2650.72661 .0 \quad 2669.3 \quad 2670.5 \quad 2672.8 \quad 2674.3 \quad 2675.32709 .52724 .62738 .92742 .2 \quad 2743.9$ $2779.12779 .3 \quad 2784.8 \quad 2794.7$

\section{name $=$ MIN313}

energy $($ in $\mathrm{kcal} / \mathrm{mol})=1.620396$

$\operatorname{ZPE}(\mathrm{in} \mathrm{kcal} / \mathrm{mol})=143.613$

$\mathrm{G}(\mathrm{in} \mathrm{kcal} / \mathrm{mol})=118.224$

Geometry

C $-2.26585571 \quad 0.09495936-0.93346732$

C $-0.74224897 \quad 0.10628144 \quad-0.92435141$

C $-0.143085551 .53935741-0.92662728$

C $2.12637949-0.10124185 \quad 2.62252087$

C $1.25753838-0.43771016 \quad 1.68528570$

C $1.39492860 \quad-0.833580390 .23872160$

C $-0.16158859-0.85248103 \quad 0.10191805$

C $-0.26585288-0.52810451 \quad 1.65060591$

C $-0.97107114 \quad 0.74090902 \quad 2.08581791$

C $-0.79530056-1.72549198 \quad 2.43133411$

$\mathrm{H}-2.660903650 .70743461-1.75619448$

$\mathrm{H}-2.68005203 \quad 0.47685081 \quad 0.00548808$

$\mathrm{H}-2.65658258-0.92068616-1.07930145$

$\mathrm{H}-0.898650462 .33622095-0.86165029$

$\mathrm{H} \quad 0.63221324 \quad 1.68461260-0.15839839$

H $3.19285786-0.077026552 .46426660$

H $1.84140528 \quad 0.17698672 \quad 3.62429557$

H $1.86756802-1.809268650 .07459857$

H $1.91472559-0.11421536-0.40421674$

$\mathrm{H}-0.54186993-1.86841944-0.11955220$

$\mathrm{H}-2.05054521 \quad 0.68918278 \quad 1.90315309$

$\mathrm{H}-0.582715751 .632583821 .58397913$ 
$\mathrm{H}-0.83296878 \quad 0.90653521 \quad 3.16378571$

$\mathrm{H}-0.68390060-1.574326753 .51178293$

$\mathrm{H}-0.25470818-2.646557152 .18092733$

$\mathrm{H}-1.85778315-1.89675954 \quad 2.22597879$

$\begin{array}{lllll}0 & 0.76282577 & 0.35440499 & -2.59976300\end{array}$

○ $0.443498891 .68764689-2.21834139$

o $-0.37810307-0.39312119-2.23172236$

Vibrational frequencies (in $\mathrm{cm}-1$ )

$\begin{array}{lllllllllllllllll}44.9 & 62.0 & 87.4 & 117.9 & 142.8 & 158.8 & 178.5 & 187.3 & 248.0 & 257.5 & 264.3 & 296.0 & 323.4 & 361.4\end{array}$ $372.1 \quad 390.3 \quad 491.3 \quad 523.4 \quad 540.6 \quad 615.3 \quad 653.2 \quad 665.6 \quad 707.5 \quad 815.1 \quad 824.5 \quad 857.2 \quad 906.8 \quad 934.1$ $950.6957 .7 \quad 975.4989 .9998 .6 \quad 1004.9 \quad 1010.5 \quad 1015.0 \quad 1054.9 \quad 1077.6 \quad 1084.1 \quad 1087.8 \quad 1127.9$

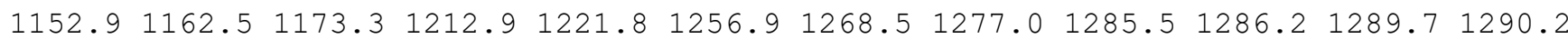
$1295.8 \quad 1301.51303 .7 \quad 1315.2 \quad 1328.5 \quad 1345.4 \quad 1365.1 \quad 1378.2 \quad 1393.91401 .81406 .81951 .6$ $2646.92670 .5 \quad 2670.92672 .7 \quad 2675.7 \quad 2678.0 \quad 2679.02680 .82717 .12717 .8 \quad 2725.7 \quad 2761.2$ $2778.3 \quad 2781.5 \quad 2782.4 \quad 2793.3$

name $=\operatorname{MIN} 314$

energy $($ in $\mathrm{kcal} / \mathrm{mol})=1.633988$

$\mathrm{ZPE}(\mathrm{in} \mathrm{kcal} / \mathrm{mol})=143.682$

G $($ in $\mathrm{kcal} / \mathrm{mol})=116.398$

Geometry

C $-2.55125615-0.57677295-0.99732339$

C $-1.02689853-0.62173701-0.96690488$

C $-0.384466170 .80914561-0.89827566$

C $0.64760876 \quad 1.01302944 \quad 0.20657130$

C $-0.03468347 \quad 1.29735279 \quad 1.54918053$

C $0.31710288-2.55186117-0.14625050$

C $-0.54604934-1.56646195 \quad 0.09100372$

C $0.86366551 \quad 1.08876661 \quad 2.73488143$

C $0.86626505 \quad 1.94805098 \quad 3.75806913$

C $1.71303968-0.13892910 \quad 2.74251197$

$\mathrm{H}-2.915140110 .01255019-1.85055643$

$\mathrm{H}-2.96389425-0.14269932-0.08088175$

$\mathrm{H}-2.97276253-1.58574337-1.10773104$

$\mathrm{H}-1.15543226 \quad 1.60836437-0.89520387$

$\mathrm{H} \quad 1.31522595 \quad 1.85695274 \quad-0.06522410$

$\mathrm{H} \quad 1.31877253 \quad 0.13387819 \quad 0.26413734$

$\mathrm{H}-0.43170080 \quad 2.33488313 \quad 1.54210182$

$\mathrm{H} \quad 0.65049527 \quad-3.24756150 \quad 0.60870269$

$\mathrm{H} 0.75164074-2.74412428-1.11892232$

$\mathrm{H}-0.97544890 \quad-1.39686794 \quad 1.07817633$

$\mathrm{H} 0.26971492 \quad 2.84307218 \quad 3.79406027$

$\mathrm{H}-0.92834272 \quad 0.64815676 \quad 1.67321874$

$\mathrm{H} 1.466293991 .82003031 \quad 4.64287010$

H 2.64896505 $0.03050663 \quad 2.18785534$

$\mathrm{H} 1.20766430-0.99766903 \quad 2.27827310$

H $1.99690119-0.443311463 .75873592$

O $0.54143541-0.37442984-2.57269682$

$\begin{array}{lllll}0 & 0.25013650 & 0.95599391 & -2.16976623\end{array}$

o $-0.64449494-1.08618741-2.27353522$

Vibrational frequencies (in $\mathrm{cm}-1$ )

$\begin{array}{lllllllllllllllll}25.9 & 34.8 & 42.3 & 59.8 & 110.0 & 132.1 & 150.1 & 156.9 & 220.0 & 231.3 & 251.1 & 292.3 & 320.5 & 345.9\end{array}$

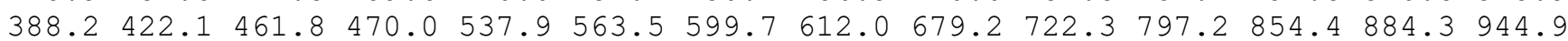
$954.1971 .9984 .7 \quad 993.4 \quad 1003.8 \quad 1023.6 \quad 1039.5 \quad 1058.4 \quad 1064.8 \quad 1080.2 \quad 1095.51097 .4 \quad 1136.0$

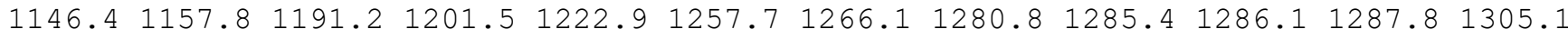

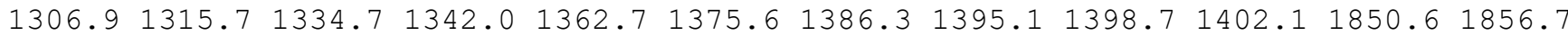
$2643.42664 .12667 .0 \quad 2669.1 \quad 2672.9 \quad 2677.9 \quad 2685.42708 .82726 .02730 .62746 .62751 .1$ $2777.22779 .3 \quad 2781.4 \quad 2795.9$

name $=$ MIN315

energy $($ in $\mathrm{kcal} / \mathrm{mol})=1.924364$

$\mathrm{ZPE}($ in $\mathrm{kcal} / \mathrm{mol})=143.71$

$\mathrm{G}(\mathrm{in} \mathrm{kcal} / \mathrm{mol})=118.769$ 


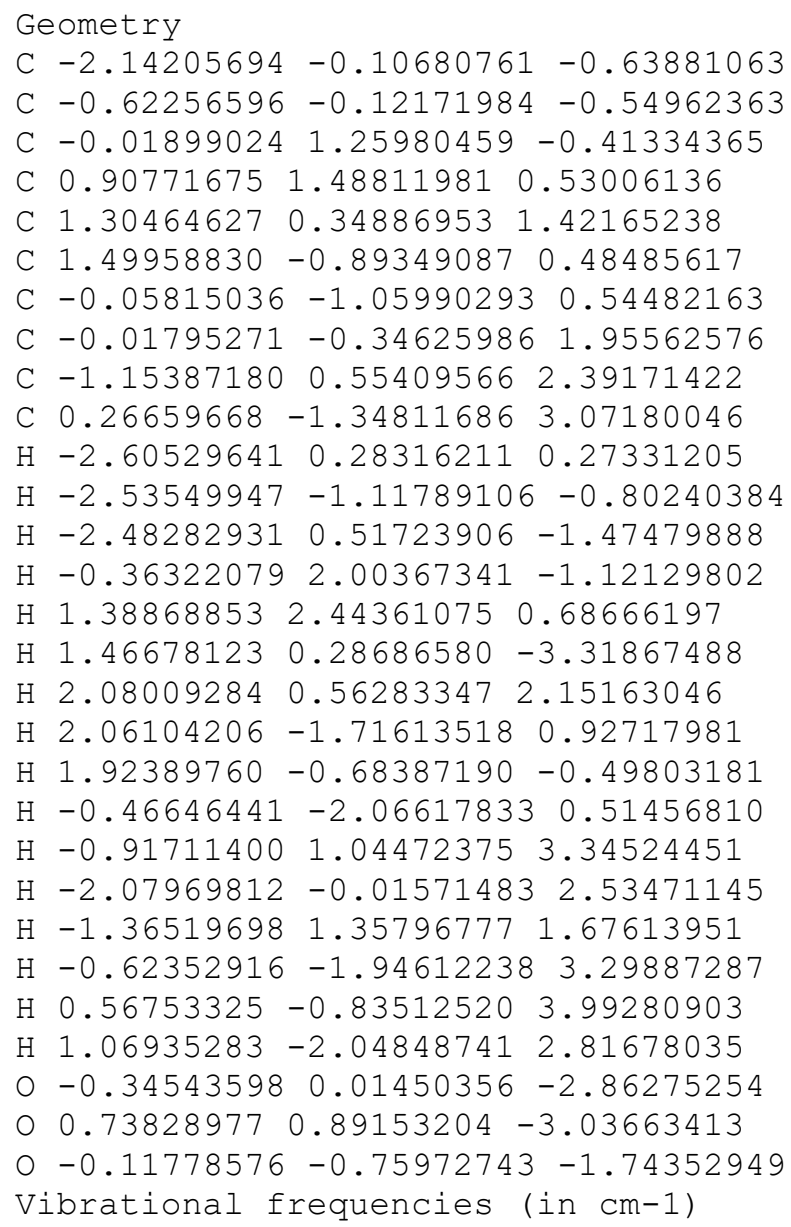


$\mathrm{H} \quad 0.22212156 \quad 0.30690898 \quad 2.56675260$

$\mathrm{H} 1.75332250-0.406620473 .06843233$

$\mathrm{H} 0.23650307-1.300677453 .26465209$

$\mathrm{H} \quad 2.64356949-2.50273779 \quad 1.90443153$

$\mathrm{H} 1.82746556-3.142871550 .47701903$

H $1.06006316-3.275498012 .05967663$

$0-0.494948811 .73431125-1.96052649$

$0-0.81322972 \quad 2.09062489-0.65959901$

O $-1.468962330 .86320778-2.45741423$

Vibrational frequencies (in $\mathrm{cm}-1$ )

$\begin{array}{llllllllllllllll}56.6 & 70.1 & 86.8 & 88.9 & 135.2 & 159.7 & 189.4 & 209.2 & 249.4 & 284.3 & 319.4 & 340.5 & 387.7 & 412.4\end{array}$ $436.2446 .1 \quad 485.9 \quad 513.3 \quad 552.8 \quad 587.9 \quad 626.0 \quad 710.6 \quad 727.4 \quad 803.5 \quad 830.7 \quad 854.7 \quad 890.0 \quad 909.8$ $941.0 \quad 961.3 \quad 967.7 \quad 996.6 \quad 1004.2 \quad 1012.6 \quad 1015.4 \quad 1050.3 \quad 1068.4 \quad 1075.8 \quad 1086.3 \quad 1090.6 \quad 1102.8$

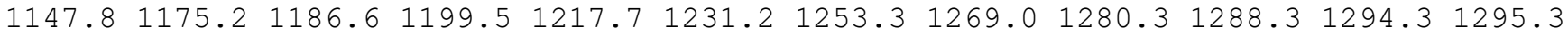

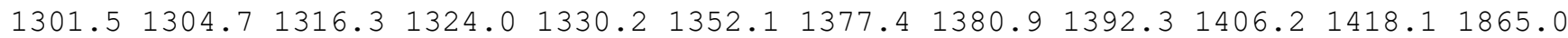
$2654.52671 .22674 .22676 .72678 .82681 .3 \quad 2693.62724 .32741 .62749 .52753 .92769 .7$ 2781.92785 .12790 .12850 .0

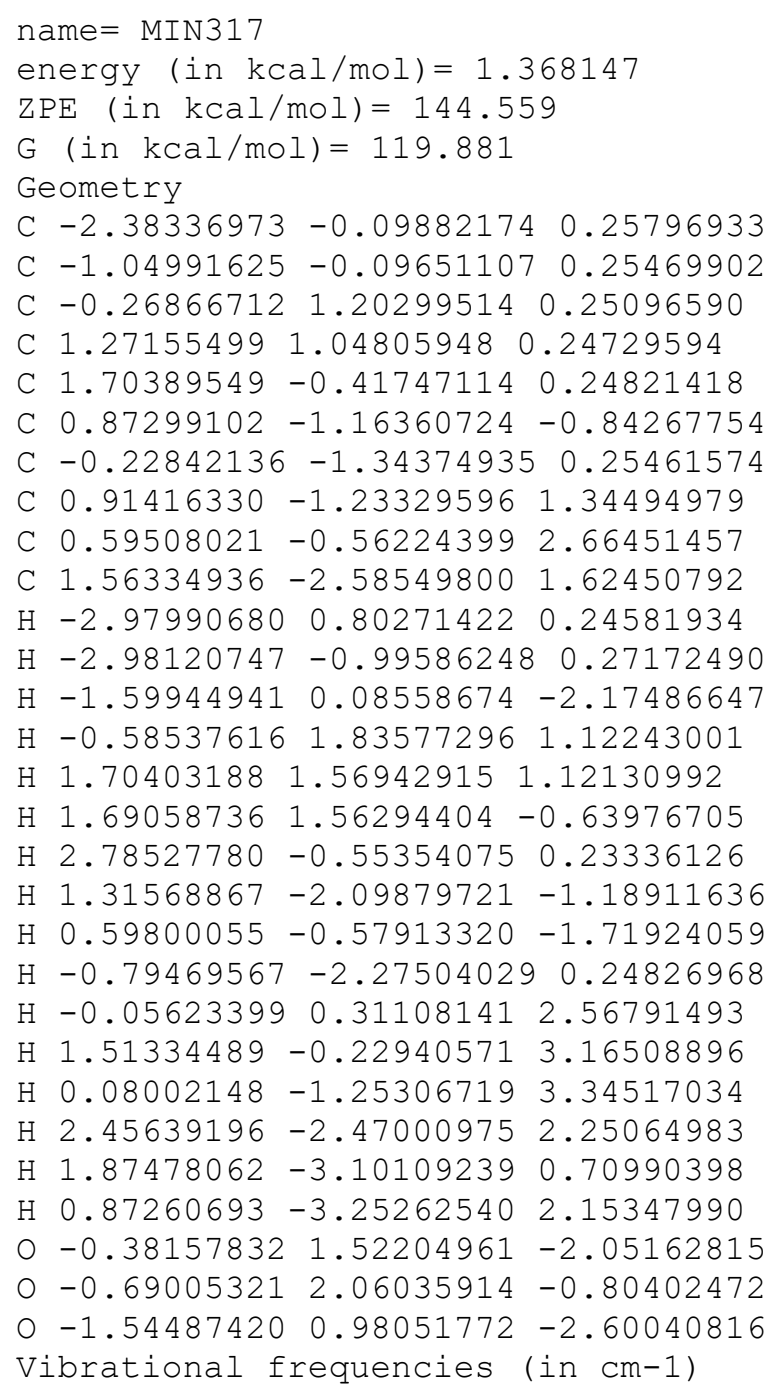


$\mathrm{ZPE}($ in $\mathrm{kcal} / \mathrm{mol})=142.761$

$\mathrm{G}($ in $\mathrm{kcal} / \mathrm{mol})=115.13$

Geometry

C $-2.34262278 \quad 0.10075995-1.48747345$

C $-0.01721805 \quad 0.10300698-0.96722004$

C $0.254177951 .53461493-0.98463151$

C $2.69124051 \quad 0.105361330 .50352870$

C $0.91429719-0.57902368 \quad 2.20849458$

C $1.54502061-0.86011699 \quad 0.81264259$

C $0.48738475-0.89315472-0.24278993$

C $0.25048008 \quad 0.76609174 \quad 2.25703023$

C $-1.06323718 \quad 0.98304999 \quad 2.29753422$

C $-0.02121269-1.728018812 .59885470$

$\mathrm{H}-2.47106569-0.19646603-0.45275641$

$\mathrm{H}-3.07442643-0.42450960-2.10407348$

$\mathrm{H}-2.42742867 \quad 1.18115966-1.60473758$

H $0.920816351 .94567461-0.21384727$

H $3.02066035 \quad 0.00681081-0.53939935$

$\mathrm{H} 2.42220006 \quad 1.15329443 \quad 0.66946576$

$\mathrm{H} 1.75924951-0.56390540 \quad 2.95048067$

H $1.99809175-1.891338110 .86801858$

$\mathrm{H} \quad 3.56177622-0.10244745 \quad 1.13843801$

$\mathrm{H} \quad 0.08540875-1.91266384-0.43298622$

$\mathrm{H} \quad 0.95494125 \quad 1.60050778 \quad 2.27748689$

$\mathrm{H}-1.81122772 \quad 0.20600367 \quad 2.29148671$

$\mathrm{H}-1.49751837 \quad 1.97066245 \quad 2.34921840$

H $\quad 0.51123260 \quad-2.68495375 \quad 2.63779012$

$\mathrm{H}-0.85226247-1.84236185 \quad 1.89354644$

$\mathrm{H}-0.45737698-1.558390673 .59132235$

O $-0.93248203-1.47535446-2.34156983$

$\begin{array}{llll}0 & -0.24775659 & 2.26094899 & -1.81335370\end{array}$

o $-0.99196538-0.24828866-2.04874098$

Vibrational frequencies (in $\mathrm{cm}-1$ )

$\begin{array}{llllllllllllll}22.6 & 51.9 & 66.0 & 71.0 & 83.2 & 115.9 & 132.1 & 154.1 & 180.1 & 206.3 & 214.1 & 238.9 & 247.7 & 254.1\end{array}$ $307.4 \quad 345.8 \quad 393.0 \quad 439.5 \quad 478.1 \quad 510.5 \quad 559.2 \quad 565.0 \quad 623.4 \quad 639.7 \quad 707.5 \quad 816.0 \quad 855.0 \quad 939.4$

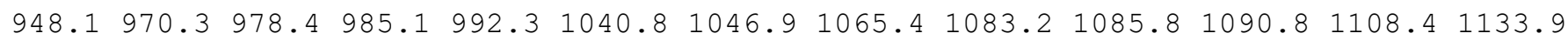
$1147.7 \quad 1167.3 \quad 1200.5 \quad 1219.9 \quad 1253.2 \quad 1264.8 \quad 1280.8 \quad 1287.4 \quad 1292.3 \quad 1296.6 \quad 1300.5 \quad 1302.3$

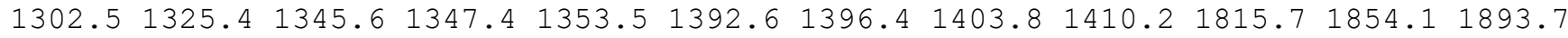
$2645.02660 .3 \quad 2661.8 \quad 2667.12668 .2 \quad 2668.92671 .72675 .32686 .6 \quad 2692.42710 .62747 .5$ $2762.32777 .3 \quad 2780.5 \quad 2786.3$

\section{name $=$ MIN319}

energy $($ in $\mathrm{kcal} / \mathrm{mol})=1.540674$

$\mathrm{ZPE}($ in $\mathrm{kcal} / \mathrm{mol})=144.424$

$\mathrm{G}($ in $\mathrm{kcal} / \mathrm{mol})=119.427$

Geometry

C $-2.413329090 .08460038-0.03966969$

C $-1.09273115-0.006608730 .12094185$

C $-0.220034361 .23308000 \quad 0.14402468$

C $1.29345966 \quad 0.97774785 \quad 0.33097358$

C $1.60801922-0.510993350 .48584060$

C $0.86559785-1.27079718-0.65785951$

C $-0.37400307-1.30376073 \quad 0.29611263$

C $0.62744723-1.195636691 .51659691$

C $0.17662401-0.42892690 \quad 2.74244405$

C $1.13262395-2.56721833 \quad 1.95634159$

$\mathrm{H}-2.93462568 \quad 1.01872792 \quad-0.17454158$

$\mathrm{H}-3.07036531-0.76960962-0.06270336$

$\mathrm{H}-1.57452125 \quad 1.23133641 \quad-3.06523027$

$\mathrm{H}-0.59282715 \quad 1.93779707 \quad 0.93446902$

$\mathrm{H} \quad 1.66777364 \quad 1.53778811 \quad 1.20658154$

$\mathrm{H} 1.84520480 \quad 1.38260653-0.54052647$

H $2.66838644 \quad-0.72108140 \quad 0.62016714$

H $1.27479245-2.25256926-0.89689972$ 
$\mathrm{H} \quad 0.73220952-0.72653669-1.59446112$

$\mathrm{H}-1.00467991-2.192276120 .26450874$

$\mathrm{H} \quad 0.96824090-0.395142113 .50104195$

$\mathrm{H}-0.70021735-0.909865523 .19678458$

$\mathrm{H}-0.10808538 \quad 0.605600732 .53572363$

H $1.99811905-2.47317941 \quad 2.62181461$

$\mathrm{H} 1.44030253-3.191937931 .11067685$

$\mathrm{H} \quad 0.35446322-3.117580242 .49816186$

O $0.056339491 .53379083-2.16595811$

$0-0.47217967 \quad 2.05221625-0.99477207$

O $-0.855131310 .63898669-2.73426118$

Vibrational frequencies (in $\mathrm{cm}-1$ )

$\begin{array}{llllllllllllllll}59.1 & 70.8 & 76.9 & 92.8 & 138.5 & 161.1 & 190.1 & 208.4 & 251.0 & 283.6 & 320.0 & 342.2 & 387.2 & 413.5\end{array}$ $435.5 \quad 445.2 \quad 484.3 \quad 513.4 \quad 554.9 \quad 590.0 \quad 626.7 \quad 711.5 \quad 726.0 \quad 804.1 \quad 830.8 \quad 853.7 \quad 889.2 \quad 909.5$ $940.9961 .6967 .1998 .3 \quad 1004.7 \quad 1011.6 \quad 1016.1 \quad 1050.6 \quad 1068.2 \quad 1075.8 \quad 1087.01090 .21102 .3$

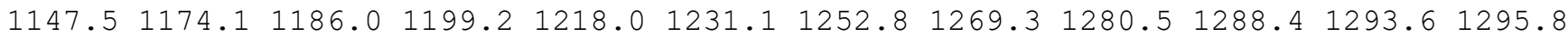

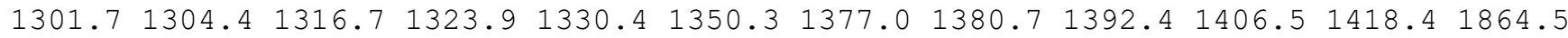
$2655.12671 .6 \quad 2674.7 \quad 2676.6 \quad 2679.02681 .4 \quad 2693.92724 .12741 .72749 .82753 .7 \quad 2770.7$ $2781.92785 .5 \quad 2790.02848 .7$

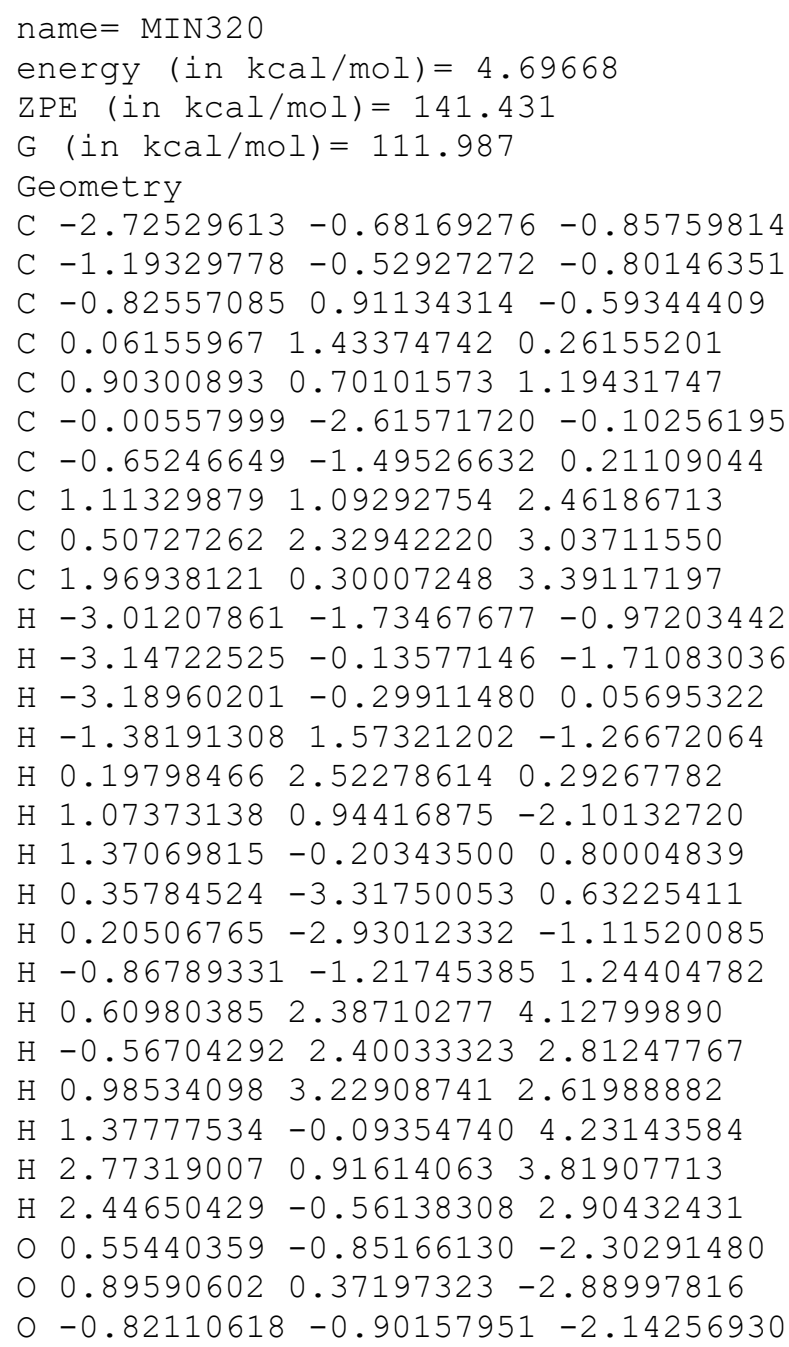


name $=$ MIN321

energy $($ in $\mathrm{kcal} / \mathrm{mol})=1.784692$

$\mathrm{ZPE}($ in $\mathrm{kcal} / \mathrm{mol})=144.376$

$\mathrm{G}($ in $\mathrm{kcal} / \mathrm{mol})=118.84$

Geometry

C $-2.43719603-0.118125890 .13494132$

C $-1.10660043-0.15097281 \quad 0.21164587$

C $-0.29883685 \quad 1.115349850 .42233292$

C $1.23371496 \quad 0.95296792 \quad 0.30843183$

C $1.63919819-0.51116507 \quad 0.13786191$

C $0.77217831-1.11839093-1.00858765$

C $-0.30663010-1.407059290 .08872467$

C $0.85808430-1.427713251 .15962303$

C $0.58235938-0.88426420 \quad 2.54845969$

C $1.49816903-2.80727524 \quad 1.28017863$

$\mathrm{H}-3.01508834 \quad 0.79014823 \quad 0.19895869$

$\mathrm{H}-3.04720830-0.99409003-0.01504122$

$\mathrm{H}-2.00189088 \quad 1.79509362-2.54998509$

$\mathrm{H}-0.56678617 \quad 1.56267239 \quad 1.41640390$

H $1.72629965 \quad 1.38485337 \quad 1.19773079$

$\mathrm{H} \quad 1.60739794 \quad 1.53964924 \quad-0.55434829$

$\mathrm{H} 2.71660389-0.66250600 \quad 0.08150860$

$\mathrm{H} \quad 1.18849392-2.01031575-1.47652660$

$\mathrm{H} 0.46871475-0.43039605-1.80118524$

$\mathrm{H}-0.88916124-2.32313786-0.00679962$

$\mathrm{H}-0.28677180-0.22003094 \quad 2.58622240$

$\mathrm{H} \quad 1.44683884-0.32754139 \quad 2.92934734$

$\mathrm{H} \quad 0.37302911-1.68969987 \quad 3.26432549$

H $2.41294153-2.761763701 .88287259$

H $1.77105388-3.23040424 \quad 0.30737083$

$\mathrm{H} 0.81661250-3.518429631 .76134246$

$0-0.330119552 .05903824-1.72348771$

$\begin{array}{lllll}0 & -0.77465740 & 2.16561048 & -0.41549110\end{array}$

O $-1.19353557 \quad 1.23437365-2.44893058$

Vibrational frequencies (in $\mathrm{cm}-1$ )

$\begin{array}{llllllllllllllll}26.8 & 54.0 & 79.0 & 91.4 & 137.3 & 170.1 & 189.8 & 211.1 & 255.3 & 282.0 & 321.4 & 338.4 & 390.0 & 410.9\end{array}$ $440.2445 .7 \quad 487.6 \quad 509.9 \quad 556.5 \quad 584.7 \quad 626.0 \quad 711.9 \quad 725.6 \quad 800.5 \quad 831.5 \quad 852.8 \quad 891.0 \quad 909.8$ $941.6961 .1967 .5 \quad 995.7 \quad 1010.4 \quad 1013.4 \quad 1019.3 \quad 1050.4 \quad 1070.1 \quad 1076.6 \quad 1086.7 \quad 1092.3 \quad 1105.2$ $1147.41176 .6 \quad 1187.6 \quad 1202.2 \quad 1219.4 \quad 1231.2 \quad 1253.7 \quad 1267.3 \quad 1280.3 \quad 1290.1 \quad 1292.6 \quad 1300.3$

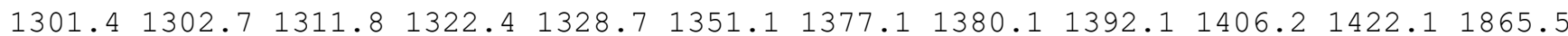
$2654.22669 .5 \quad 2671.7 \quad 2676.7 \quad 2677.8 \quad 2680.5 \quad 2691.92723 .92741 .5 \quad 2749.5 \quad 2753.62767 .5$ $2781.32782 .6 \quad 2789.8 \quad 2849.2$

name $=$ MIN322

energy $($ in $\mathrm{kcal} / \mathrm{mol})=1.870499$

$\mathrm{ZPE}(\mathrm{in} \mathrm{kcal} / \mathrm{mol})=144.454$

$\mathrm{G}($ in $\mathrm{kcal} / \mathrm{mol})=119.192$

Geometry

C $-2.35475522-0.12617704 \quad 0.15978443$

C $-1.02377483-0.17814738 \quad 0.21711771$

C $-0.19154366 \quad 1.06929920 \quad 0.44121925$

C $1.33381775 \quad 0.88689910 \quad 0.24828191$

C $1.71338620-0.58186474 \quad 0.06771573$

C $0.80965746-1.16970453-1.06158010$

C $-0.24747798-1.44520734 \quad 0.05960215$

C $0.94231555-1.492687491 .10308300$

C $0.70130926-0.954470292 .50052986$

C $1.55921445-2.88419763 \quad 1.19833894$

$\mathrm{H}-1.78098550 \quad 0.65385136 \quad-2.10541632$

$\mathrm{H}-2.91861820 \quad 0.79231188 \quad 0.25124263$

$\mathrm{H}-2.98248091-0.99099348 \quad 0.01668851$

$\mathrm{H}-0.40055792 \quad 1.473230561 .46758619$

$\mathrm{H} 1.875906491 .32114090 \quad 1.10756118$

$\mathrm{H} \quad 1.66866060 \quad 1.46881416-0.63324817$ 
$\mathrm{H} \quad 2.78705431-0.75033684-0.01397402$

$\mathrm{H} \quad 1.20291564-2.06925483-1.53731095$

$\mathrm{H} \quad 0.51037644-0.47539556-1.84620928$

$\mathrm{H}-0.84571238-2.35245767-0.02460952$

$\mathrm{H}-0.22045094-0.368311392 .58064509$

H $1.53309863-0.31984903 \quad 2.82887869$

$\mathrm{H} 0.60284271-1.76180127 \quad 3.23802979$

$\mathrm{H} 2.48512646-2.86185747 \quad 1.78597192$

$\mathrm{H} \quad 1.80843039-3.30415867 \quad 0.21812221$

H $0.87297368-3.58723231 \quad 1.68550569$

O $-0.47033775 \quad 1.94164500-1.69369837$

$\begin{array}{lllll}0 & -0.66233283 & 2.16739907 & -0.32911921\end{array}$

o $-1.70425065 \quad 1.63018764 \quad-2.26297197$

Vibrational frequencies (in cm-1)

$\begin{array}{lllllllllllllll}-32.1 & 59.5 & 72.6 & 107.4 & 146.8 & 175.9 & 190.2 & 213.8 & 244.9 & 293.2 & 340.2 & 388.8 & 399.3 & 428.0\end{array}$

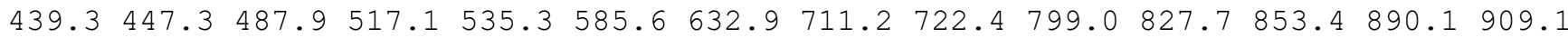
$942.2959 .8 \quad 977.5 \quad 996.5 \quad 1011.0 \quad 1015.7 \quad 1020.0 \quad 1048.9 \quad 1068.01081 .2 \quad 1087.7 \quad 1091.9 \quad 1113.8$

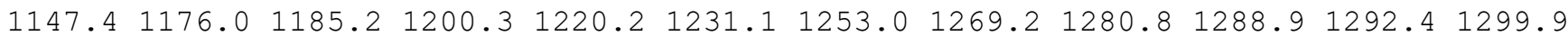

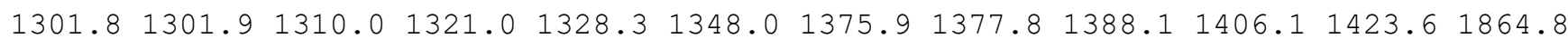
$2653.72667 .32671 .72676 .12676 .6 \quad 2678.92694 .12716 .62742 .2 \quad 2748.32752 .42767 .9$ 2780.62781 .82784 .32829 .8

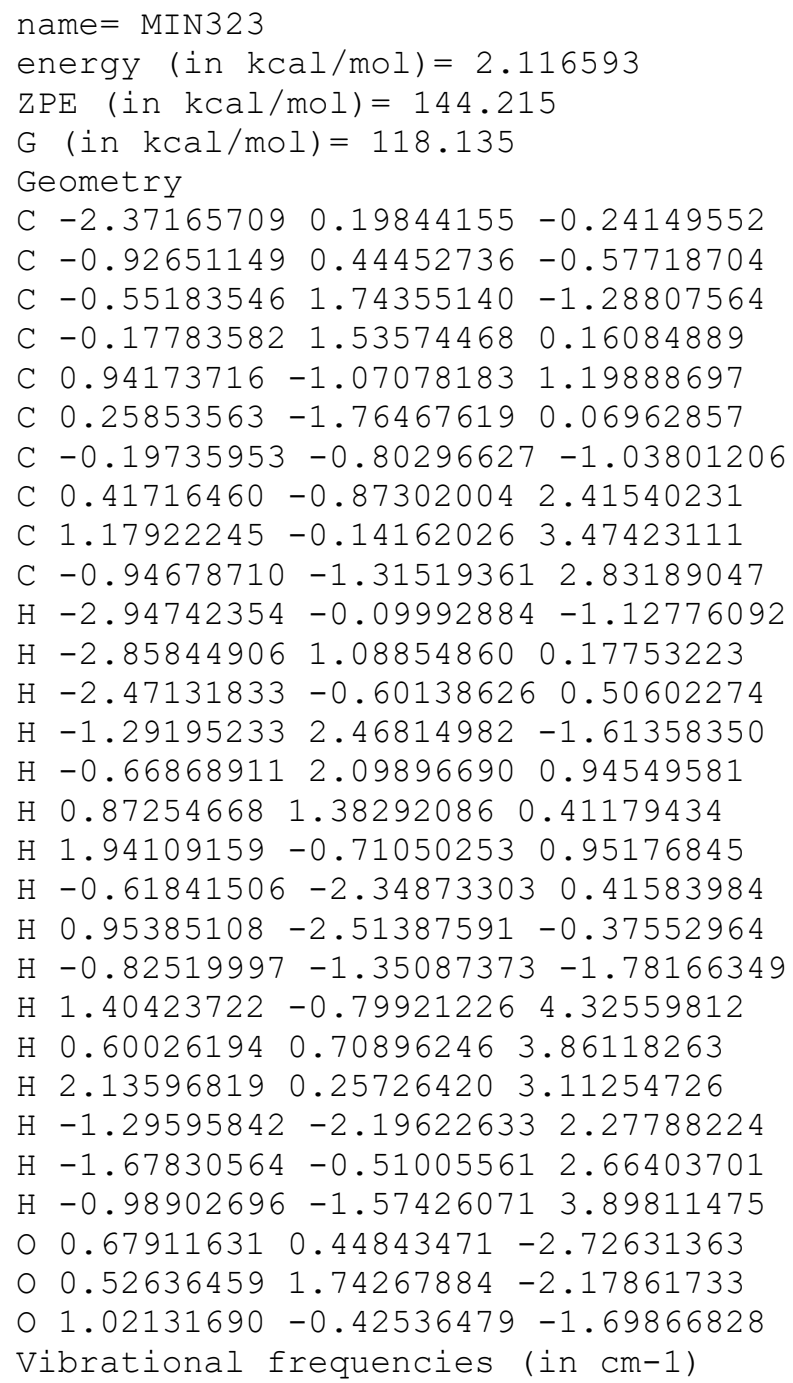




\section{name $=$ MIN324}

energy $($ in $\mathrm{kcal} / \mathrm{mol})=1.833816$

$\mathrm{ZPE}(\mathrm{in} \mathrm{kcal} / \mathrm{mol})=144.597$

$\mathrm{G}($ in $\mathrm{kcal} / \mathrm{mol})=119.816$

Geometry

C $-2.23680319-0.06535185-0.53584637$

C $-0.95401513-0.02140127-0.17292950$

C $-0.12255724 \quad 1.23452087-0.34247993$

C $1.12785778 \quad 1.30206564 \quad 0.57394330$

C $1.49066164-0.08083796 \quad 1.11682417$

C $1.25450258-1.12210512-0.02330111$

C $-0.23396952-1.170658220 .45310516$

C $0.26553960-0.712957191 .88636466$

C -0.639597350 .198202452 .68891366$

C $0.67956477-1.89867788 \quad 2.74978073$

$\mathrm{H}-2.85963686-0.93861076-0.41971381$

$\mathrm{H}-0.91586765-0.72597430-2.52376792$

$\mathrm{H}-2.77075138 \quad 0.76911545-0.96339189$

$\mathrm{H}-0.749086112 .14553455-0.22680349$

$\mathrm{H} \quad 0.95443910 \quad 2.01271483 \quad 1.40079066$

H $1.98111855 \quad 1.71419507-0.00547249$

$\mathrm{H} 2.45006138-0.111082331 .63212190$

$\mathrm{H} \quad 1.79571429-2.06092375 \quad 0.09933844$

$\mathrm{H} \quad 1.42470471-0.76991476-1.04426617$

$\mathrm{H}-0.74586518-2.13077687 \quad 0.40173501$

$\mathrm{H}-1.51018323-0.351630143 .07007760$

$\mathrm{H}-1.03420533 \quad 1.03890554 \quad 2.10868291$

$\mathrm{H}-0.109306520 .61872104 \quad 3.55208307$

$\mathrm{H}-0.19443798-2.476622213 .07233114$

H $1.20696958-1.56439547 \quad 3.65088617$

H $1.34656485-2.58921923 \quad 2.22151581$

$\begin{array}{lllll}0 & -0.47421360 & 1.10470391 & -2.64010187\end{array}$

○ $0.47887477 \quad 1.28851389-1.64636734$

o $-0.33943631-0.19645310-3.13313330$

Vibrational frequencies (in $\mathrm{cm}-1$ )

$\begin{array}{llllllllllllll}33.0 & 80.1 & 118.3 & 138.2 & 159.3 & 170.6 & 182.5 & 201.0 & 238.3 & 332.3 & 340.0 & 363.3 & 390.2 & 409.3\end{array}$

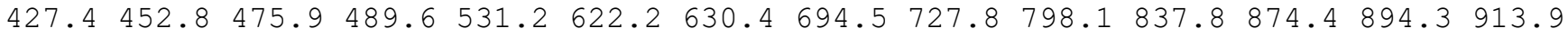
$930.0955 .2984 .3 \quad 994.1 \quad 1005.4 \quad 1009.5 \quad 1015.8 \quad 1039.0 \quad 1064.5 \quad 1076.6 \quad 1081.8 \quad 1087.9 \quad 1111.6$

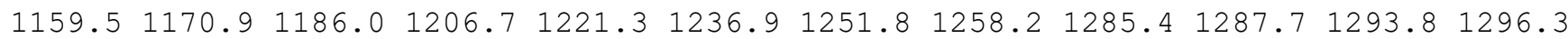
$1302.21304 .41313 .91328 .6 \quad 1334.3 \quad 1361.2 \quad 1371.1 \quad 1386.61392 .11406 .21415 .11864 .1$ $2657.22672 .8 \quad 2676.2 \quad 2677.4 \quad 2681.02681 .42683 .32718 .92737 .4 \quad 2749.12753 .72762 .6$ $2781.7 \quad 2783.4 \quad 2787.4 \quad 2826.6$

\section{name $=$ MIN325}

energy (in $\mathrm{kcal} / \mathrm{mol})=2.703077$

$\mathrm{ZPE}(\mathrm{in} \mathrm{kcal} / \mathrm{mol})=143.825$

$\mathrm{G}($ in $\mathrm{kcal} / \mathrm{mol})=116.991$

Geometry

C $-2.19699509-1.05099472-0.75256139$

C $-0.67450987-1.04927788-0.85129440$

C $-0.09934801 \quad 0.40405194 \quad-0.73853256$

C $1.20667851 \quad 0.57609183 \quad 0.03236983$

C $1.02878261 \quad 0.57196735 \quad 1.55162248$

C $-0.49882073-2.49224387 \quad 1.18765342$

C $-0.00708818-2.060682060 .02744775$

C $0.45912036 \quad 1.81946687 \quad 2.15756432$

C $0.54418573 \quad 1.83691409 \quad 3.65322778$

C $-0.078711312 .83676329 \quad 1.48266361$

$\mathrm{H}-2.63977642-0.42379010-1.54014796$

$\mathrm{H}-2.54455201-0.67571984 \quad 0.21541116$

$\mathrm{H}-2.60105726-2.06288976-0.88816411$

$\mathrm{H}-0.87308337 \quad 1.12809294-0.40458015$ 
$\mathrm{H} \quad 1.68265003 \quad 1.52969180-0.28664317$

$\mathrm{H} 1.92628226-0.21259462-0.26577937$

H $2.018923220 .36660784 \quad 2.01801472$

$\mathrm{H}-1.42663411-2.15039251 \quad 1.62111885$

$\mathrm{H}-0.00673255-3.23402502 \quad 1.79933728$

$\mathrm{H} \quad 0.93071337-2.44552205-0.38112563$

$\mathrm{H}-0.09258771 \quad 2.61033653 \quad 4.10224210$

$\mathrm{H} 1.574462592 .04167119 \quad 3.98047173$

$\mathrm{H} \quad 0.24521117 \quad 0.87784354 \quad 4.09551672$

$\mathrm{H}-0.49182271 \quad 3.71047019 \quad 1.95987252$

$\mathrm{H}-0.14454681 \quad 2.89517914 \quad 0.41029414$

$\mathrm{H} \quad 0.38946686-0.28849581 \quad 1.85065433$

O $0.55165998-0.47612817-2.68628949$

$\begin{array}{llll}0 & 0.13604200 & 0.74611702 & -2.10780661\end{array}$

o $-0.40227985-1.42036789-2.21958636$

Vibrational frequencies (in cm-1)

$\begin{array}{llllllllllllllll}26.1 & 34.3 & 48.1 & 95.3 & 111.9 & 117.5 & 186.7 & 194.4 & 206.6 & 245.0 & 267.0 & 297.5 & 332.6 & 363.5\end{array}$ $402.6 \quad 432.4 \quad 449.6 \quad 461.0 \quad 486.7 \quad 547.4 \quad 609.5 \quad 637.0 \quad 679.8 \quad 718.0 \quad 808.4 \quad 863.7 \quad 899.1 \quad 910.3$ $948.3976 .6987 .3 \quad 989.5 \quad 1003.0 \quad 1029.3 \quad 1038.8 \quad 1051.8 \quad 1060.5 \quad 1071.4 \quad 1091.6 \quad 1094.01127 .4$ $1155.21174 .2 \quad 1195.5 \quad 1199.3 \quad 1247.1 \quad 1252.5 \quad 1256.7 \quad 1276.6 \quad 1284.5 \quad 1288.0 \quad 1289.4 \quad 1290.6$

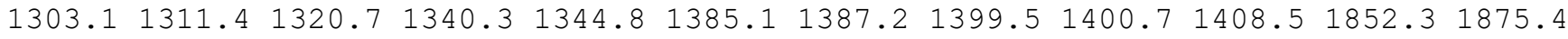
$2649.22654 .7 \quad 2669.5 \quad 2670.92675 .7 \quad 2676.3 \quad 2679.02709 .92721 .32734 .82739 .92748 .0$ 2779.22779 .92784 .92793 .5

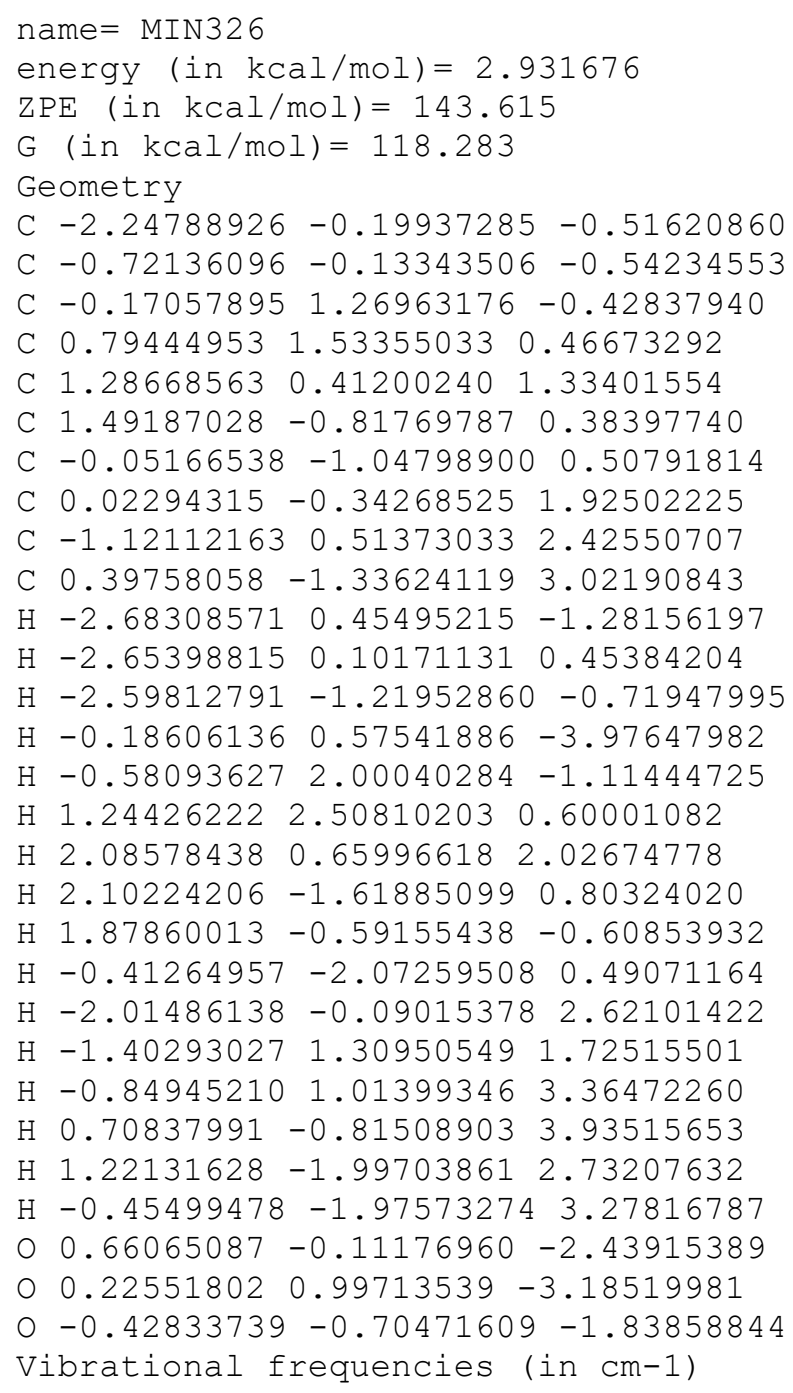


$2665.92675 .2 \quad 2675.8 \quad 2676.5 \quad 2678.0 \quad 2680.8 \quad 2692.0 \quad 2753.4 \quad 2756.0 \quad 2758.5 \quad 2768.7 \quad 2771.1$ $2779.02782 .2 \quad 2783.3 \quad 2852.5$

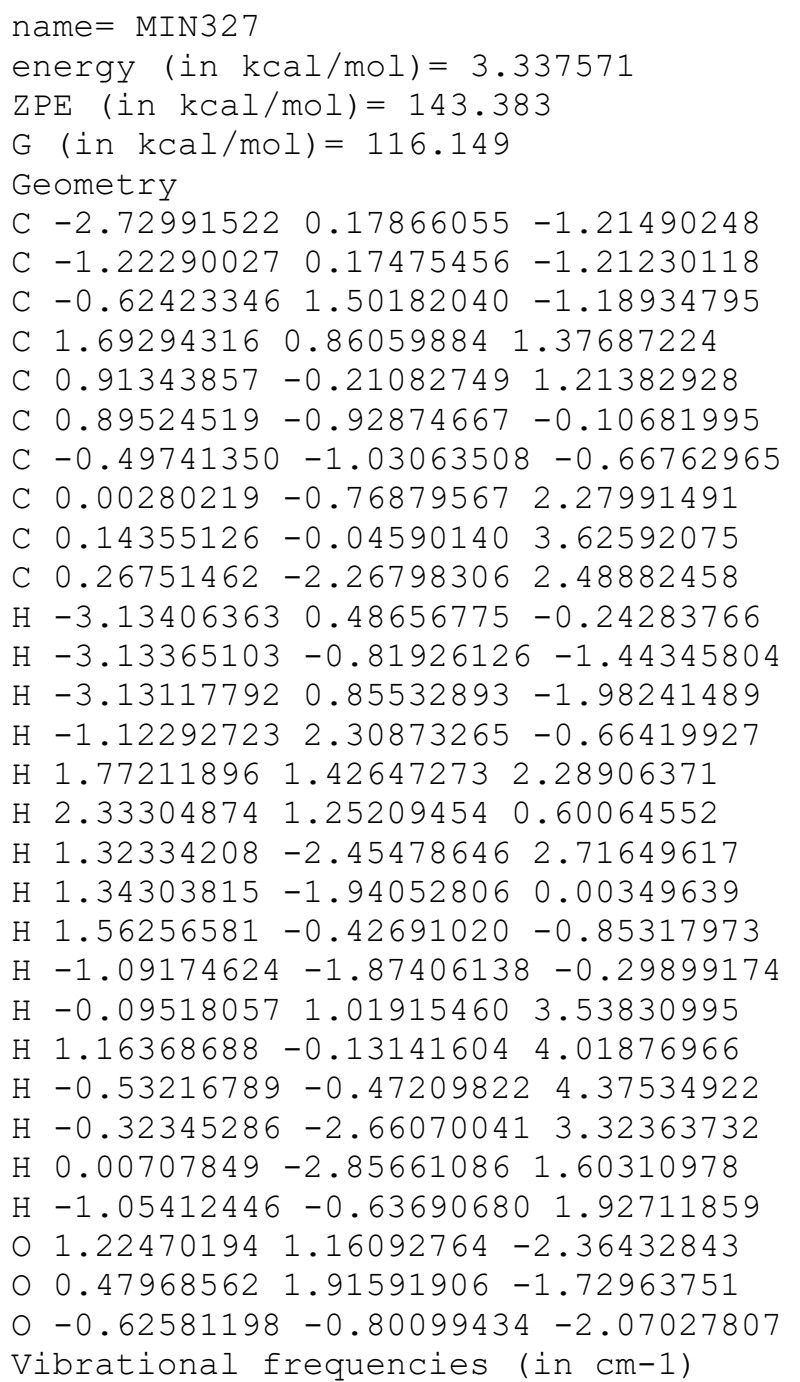

$\begin{array}{lllllllllllllll}25.5 & 43.0 & 47.2 & 78.4 & 89.2 & 125.8 & 147.4 & 179.5 & 183.3 & 223.9 & 234.9 & 293.2 & 312.3 & 345.5\end{array}$ $369.3 \quad 403.4 \quad 421.9 \quad 438.3 \quad 460.5 \quad 506.4 \quad 542.5 \quad 620.9 \quad 657.2 \quad 719.8 \quad 828.2 \quad 846.9 \quad 860.9 \quad 874.5$

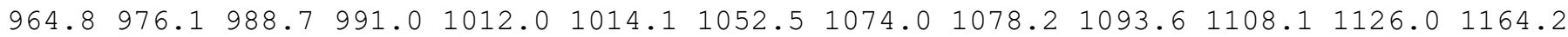
$1173.01194 .2 \quad 1202.91236 .3 \quad 1268.2 \quad 1275.7 \quad 1280.6 \quad 1282.0 \quad 1284.6 \quad 1296.0 \quad 1299.7 \quad 1307.7$

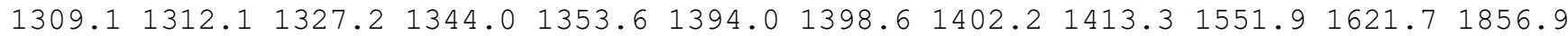
$2628.22667 .72670 .12675 .42676 .4 \quad 2676.8 \quad 2679.82699 .62717 .2 \quad 2718.22720 .82727 .7$ $2779.92780 .5 \quad 2781.92788 .9$

name $=\operatorname{MIN} 328$

energy (in $\mathrm{kcal} / \mathrm{mol})=5.06072$

$\mathrm{ZPE}($ in $\mathrm{kcal} / \mathrm{mol})=141.681$

$\mathrm{G}(\mathrm{in} \mathrm{kcal} / \mathrm{mol})=112.156$

Geometry

C $-2.61730016-1.22666185-0.97484746$

C $-1.13508193-1.13230855-0.94444310$

C $-1.02991728 \quad 2.37806743-0.85382245$

C $-1.14903467 \quad 1.88703320 \quad 0.37588913$

C $1.54724518-0.32812120 \quad 0.72924033$

C $1.16657169-1.54618164-0.05466183$

C $-0.32009226-1.70201508-0.04753282$

C $1.53462824-0.24972275 \quad 2.06671608$

C $1.85877927 \quad 1.03038795 \quad 2.76534105$

C $1.19659831-1.401292062 .95386123$

$\mathrm{H}-2.99481583-1.34550097-2.00262743$

$\mathrm{H}-3.07069090-0.30409248-0.57514714$ 
$\mathrm{H}-2.99794947-2.06894271-0.38342643$

$\mathrm{H}-1.42548077 \quad 3.32030568-1.23104617$

$\mathrm{H}-1.673601892 .38818591 \quad 1.16999868$

$\mathrm{H}-0.72285331 \quad 0.94310268 \quad 0.68753588$

$\mathrm{H} \quad 1.82582827 \quad 0.53132085 \quad 0.12206865$

$\mathrm{H} \quad 1.65346161-2.45299366 \quad 0.36672605$

$\mathrm{H} 1.55144211-1.47619509-1.09670299$

$\mathrm{H}-0.71932712-2.30723996 \quad 0.76309308$

H $1.96317283 \quad 1.87664121 \quad 2.07244244$

H 2.80127294 $0.94954352 \quad 3.32433287$

$\mathrm{H} 1.071050691 .30169778 \quad 3.48239041$

H $1.79837321-2.28798232 \quad 2.70580433$

$\mathrm{H} 0.14000462-1.68641004 \quad 2.84738977$

H $1.36769361-1.182982394 .01521176$

$00.22146645 \quad 0.57945808-1.60178713$

$0-0.37833422 \quad 1.80962113-1.91140712$

o $-0.66723970-0.42668704-2.03894181$

Vibrational frequencies (in $\mathrm{cm}-1$ )

$\begin{array}{lllllllllllllll}-22.5 & 30.4 & 43.4 & 65.2 & 67.3 & 71.0 & 73.9 & 94.0 & 102.4 & 160.5 & 173.5 & 206.4 & 242.9 & 284.2\end{array}$

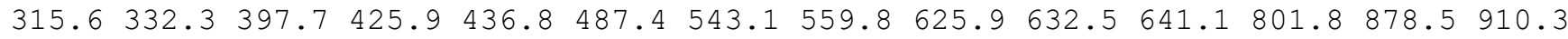

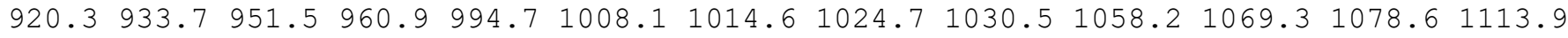
$1161.51170 .3 \quad 1182.0 \quad 1239.8 \quad 1261.2 \quad 1270.0 \quad 1272.4 \quad 1277.4 \quad 1279.8 \quad 1281.8 \quad 1287.1 \quad 1290.0$ $1290.4 \quad 1326.91336 .1 \quad 1369.9 \quad 1372.4 \quad 1379.8 \quad 1394.4 \quad 1399.6 \quad 1417.7 \quad 1857.4 \quad 1858.4 \quad 1871.5$ $2655.22662 .92668 .7 \quad 2672.4 \quad 2674.0 \quad 2676.2 \quad 2677.0 \quad 2705.72732 .6 \quad 2735.3 \quad 2751.82759 .1$ $2777.62778 .32780 .2 \quad 2789.9$

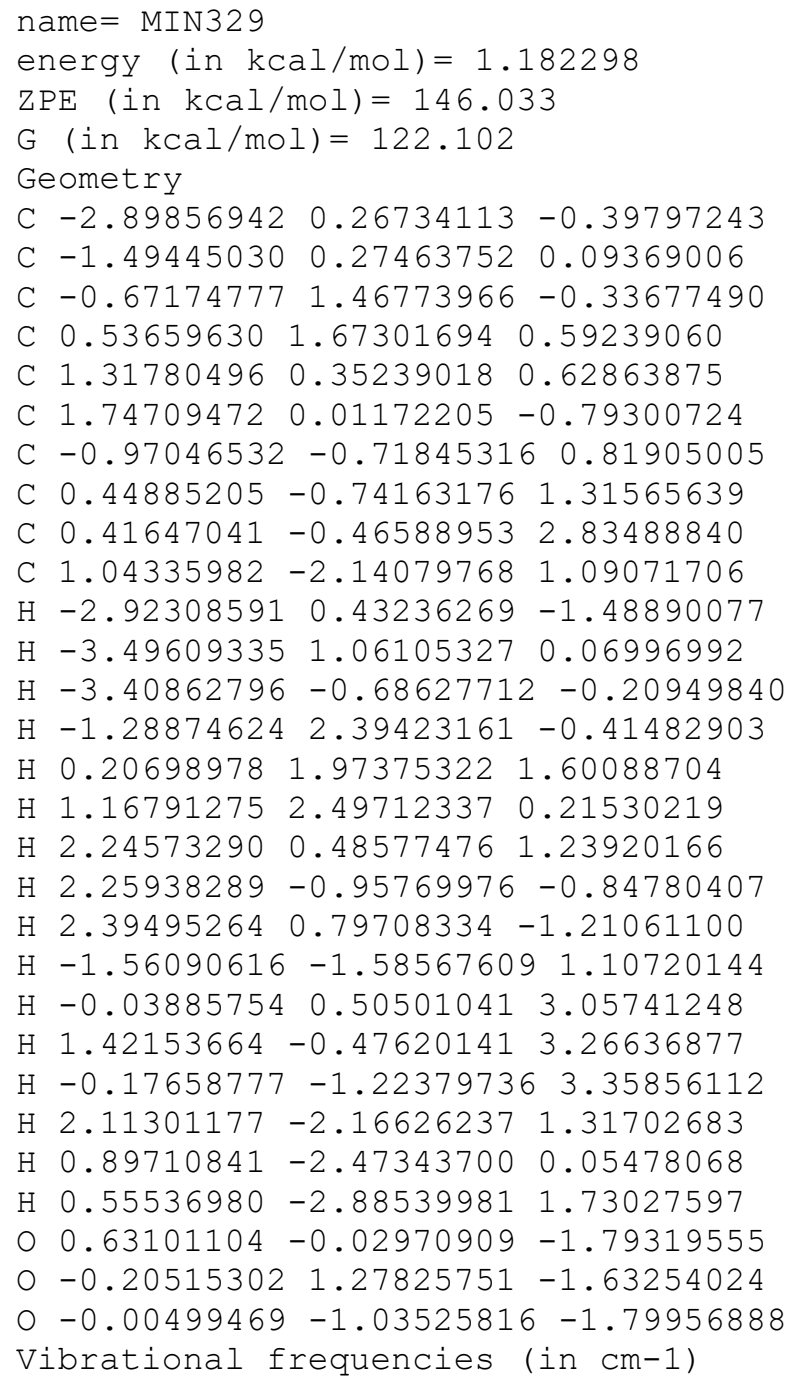




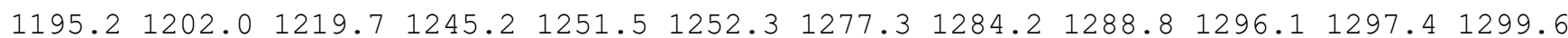

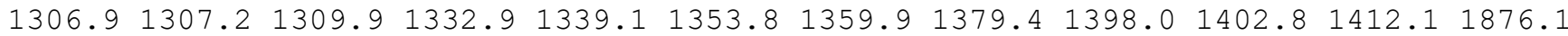
$2660.82666 .92669 .3 \quad 2674.12675 .0 \quad 2677.12679 .2 \quad 2682.12686 .5 \quad 2695.92725 .42750 .1$ $2756.7 \quad 2778.0 \quad 2779.3 \quad 2781.2$

name $=$ MIN330

energy (in $\mathrm{kcal} / \mathrm{mol})=1.13697$

$\mathrm{ZPE}($ in $\mathrm{kcal} / \mathrm{mol})=146.086$

$\mathrm{G}($ in $\mathrm{kcal} / \mathrm{mol})=122.237$

Geometry

C $-2.92326893-0.26953088 \quad 0.42982597$

C $-1.44343141-0.14640535 \quad 0.52440031$

C $-0.91269690 \quad 1.26851692 \quad 0.52384352$

C 0.473545141 .331663251 .18740163

C $1.38389867 \quad 0.34173086 \quad 0.44724866$

C $1.443845690 .75604677-1.01706375$

C $-0.62574617-1.202633890 .57407389$

C $0.87179437-1.110144450 .68061226$

C $1.26492741-1.54577416 \quad 2.10926470$

C $1.51047139-2.09048149-0.31582940$

$\mathrm{H}-3.32789433 \quad 0.42831287 \quad-0.32181318$

$\mathrm{H}-3.40561793-0.040486921 .39005112$

$\mathrm{H}-3.24411825-1.276293390 .13053540$

$\mathrm{H}-1.62605883 \quad 1.98330068 \quad 0.99907572$

$\mathrm{H} \quad 0.40187839 \quad 1.08682197 \quad 2.26034608$

$\mathrm{H} \quad 0.87488514 \quad 2.35871253 \quad 1.13044586$

H $2.42053386 \quad 0.410042200 .86190500$

$\mathrm{H} \quad 2.05224963 \quad 0.06821371-1.61807049$

$\mathrm{H} \quad 1.82992627 \quad 1.78103564-1.12507888$

$\mathrm{H}-1.01031598-2.22035817 \quad 0.55205019$

$\mathrm{H} 0.81930733-0.89145798 \quad 2.86561814$

$\begin{array}{lllll}\mathrm{H} & 2.34995274 & -1.53472544 & 2.24701303\end{array}$

$\mathrm{H} \quad 0.91574025-2.56289442 \quad 2.31940907$

H 2.59561430 -1.96658483 -0.36593635

$\mathrm{H} 1.09711063-1.95920462-1.32480171$

$\mathrm{H} \quad 1.31131696-3.12996688-0.03077212$

O $0.10551375 \quad 0.85534573-1.68459599$

$\begin{array}{lllll}0 & -0.80842054 & 1.73945227 & -0.77949207\end{array}$

$0-0.36974408-0.18079953-2.02472576$

Vibrational frequencies (in $\mathrm{cm}-1$ )

$\begin{array}{lllllllllllllllll}88.1 & 96.7 & 109.9 & 121.6 & 167.8 & 217.0 & 218.6 & 250.3 & 281.0 & 313.0 & 336.8 & 345.7 & 379.8 & 435.9\end{array}$ $451.7 \quad 470.0 \quad 485.2 \quad 523.3 \quad 561.5 \quad 616.7 \quad 660.9 \quad 673.9 \quad 757.7 \quad 846.5 \quad 914.2 \quad 924.6 \quad 935.0 \quad 958.3$ $962.9978 .5981 .5988 .5 \quad 1029.4 \quad 1049.8 \quad 1063.3 \quad 1070.4 \quad 1075.6 \quad 1105.3 \quad 1159.01171 .4 \quad 1179.0$ $1195.01202 .4 \quad 1219.6 \quad 1244.6 \quad 1251.2 \quad 1251.8 \quad 1277.7 \quad 1283.6 \quad 1289.9 \quad 1296.2 \quad 1298.01300 .2$

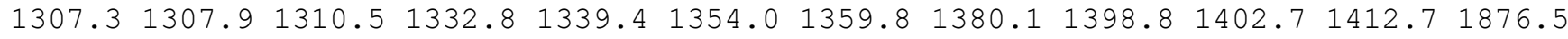
$2661.02668 .0 \quad 2668.6 \quad 2674.8 \quad 2675.4 \quad 2675.8 \quad 2679.5 \quad 2682.42686 .12696 .52726 .02750 .5$ $2756.6 \quad 2777.8 \quad 2779.5 \quad 2781.5$

name $=$ MIN331

energy $($ in $\mathrm{kcal} / \mathrm{mol})=3.256756$

$\mathrm{ZPE}(\mathrm{in} \mathrm{kcal} / \mathrm{mol})=144.246$

$\mathrm{G}($ in $\mathrm{kcal} / \mathrm{mol})=119.437$

Geometry

C $-2.21423940-0.28215804-0.42999005$

C $-0.69600477-0.25026195-0.41293749$

C $-0.074807601 .19168105-0.42775548$

C $1.10145728 \quad 1.20501434 \quad 0.58092884$

C $1.07560070-0.122461791 .30778630$

C $1.44976269-0.20931487-3.06686207$

C $-0.05180523-0.98117384 \quad 0.75461317$

C $-0.12868103-0.45842318 \quad 2.18283400$

C $-1.10052566 \quad 0.60390677 \quad 2.62124332$

C $0.09575450-1.49003598 \quad 3.26795849$ 
$\mathrm{H}-2.646408890 .06321480 \quad 0.51373212$

$\mathrm{H}-2.56736419-1.31001760-0.59868626$

$\mathrm{H}-2.61655780 \quad 0.33167681-1.24466291$

$\mathrm{H}-0.81903675 \quad 1.98364401-0.21604292$

$\mathrm{H} \quad 2.05340318 \quad 1.34556433 \quad 0.03605031$

H $1.02530106 \quad 2.05444304 \quad 1.27860694$

H 2.04652105 $-0.53399724 \quad 1.56430423$

$\mathrm{H} \quad 2.160497120 .53293373-3.38471011$

$\mathrm{H} 1.66460411-1.24360423-3.26989033$

$\mathrm{H} 0.05058309-2.05265127 \quad 0.58865119$

$\mathrm{H}-1.29458294 \quad 1.35615440 \quad 1.84884208$

$\mathrm{H}-0.71570300 \quad 1.14195345 \quad 3.49900886$

$\mathrm{H}-2.06721982 \quad 0.16433593 \quad 2.90032970$

$\mathrm{H} 0.47966374 \quad-1.02192868 \quad 4.18312238$

$\mathrm{H} \quad 0.81627703-2.261644842 .97156699$

$\mathrm{H}-0.84100134-2.002403863 .52141508$

$\begin{array}{lllll}0 & 0.43131565 & 0.12453179 & -2.37980282\end{array}$

O $0.410042461 .48618429-1.71512085$

$0-0.25223345-0.94092931-1.58050857$

Vibrational frequencies (in $\mathrm{cm}-1$ )

$\begin{array}{llllllllllllllllll}40.1 & 73.2 & 126.1 & 140.6 & 153.9 & 167.9 & 184.6 & 220.7 & 261.0 & 273.1 & 276.4 & 333.6 & 346.1 & 383.9\end{array}$ $426.3 \quad 445.2 \quad 476.5 \quad 500.7 \quad 551.7 \quad 597.0 \quad 621.9660 .0 \quad 753.1 \quad 767.7 \quad 790.1 \quad 822.2 \quad 879.4 \quad 920.7$ $967.6 \quad 984.6 \quad 1002.2 \quad 1011.7 \quad 1017.1 \quad 1018.9 \quad 1041.3 \quad 1062.1 \quad 1100.01106 .91115 .2 \quad 1134.3$

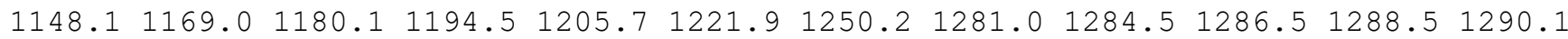

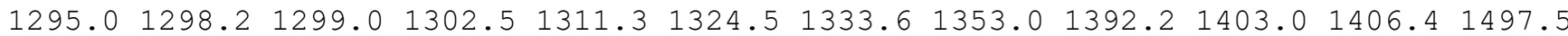
$1668.42669 .5 \quad 2672.92674 .7 \quad 2675.1 \quad 2677.3 \quad 2677.92679 .6 \quad 2682.2 \quad 2690.92728 .22752 .5$ $2762.2 \quad 2771.4 \quad 2780.7 \quad 2782.8 \quad 2783.2$

\footnotetext{
name $=$ MIN332

energy $($ in $\mathrm{kcal} / \mathrm{mol})=4.254668$

$\mathrm{ZPE}($ in $\mathrm{kcal} / \mathrm{mol})=143.303$

$\mathrm{G}($ in $\mathrm{kcal} / \mathrm{mol})=116.14$

Geometry

C $-2.90945318-0.77428278-0.85203466$

C $-1.45208537-0.56593888-0.55451281$

C $-1.02077547 \quad 0.83050127-0.56872076$

C $1.94495774-0.29028170-0.63874765$

C $1.54901954-0.375501750 .63305720$

C $0.49234882-1.325616301 .11155693$

C $-0.69036324-1.60605852 \quad 0.21630411$

C $2.23511380 \quad 0.46490905 \quad 1.68947579$

C $1.42131865 \quad 1.73650343 \quad 1.96654420$

C $2.47445432-0.308718282 .99200135$

$\mathrm{H}-3.20586817-0.24008607-1.77476695$

$\mathrm{H}-3.54662802-0.41408471-0.03626787$

$\mathrm{H}-3.14354823-1.83317097-1.02741935$

$\mathrm{H}-0.46628647 \quad 1.254689190 .26045823$

H $2.72562368 \quad 0.36919293-0.98185730$

H $1.52117124-0.87282544-1.44548536$

H $3.23955407 \quad 0.77561715 \quad 1.29531389$

$\mathrm{H} \quad 0.08648804 \quad-0.98565018 \quad 2.09270089$

H $1.00375362-2.296714231 .32853622$

$\mathrm{H}-1.23566704 \quad-2.51500345 \quad 0.51478637$

$\mathrm{H} \quad 0.41796221 \quad 1.50371482 \quad 2.33674439$

H $1.328416112 .35044895 \quad 1.06382542$

H $1.91122865 \quad 2.35546381 \quad 2.72777314$

$\mathrm{H} 2.93407114-1.284037192 .79616442$

$\mathrm{H} \quad 1.54690084-0.48026425 \quad 3.54781567$

$\mathrm{H} \quad 3.14814800 \quad 0.24403031 \quad 3.65683963$

o $-1.82448191 \quad 1.38652288-2.54965183$

o $-1.208973751 .69263236-1.51601465$

o $-0.55573874-1.48531221-1.19740835$

Vibrational frequencies (in $\mathrm{cm}-1$ )
} 
$\begin{array}{lllllllllllllllll}25.2 & 42.0 & 59.3 & 77.8 & 105.2 & 116.4 & 184.0 & 188.4 & 205.4 & 210.5 & 232.0 & 239.5 & 255.7 & 268.6\end{array}$

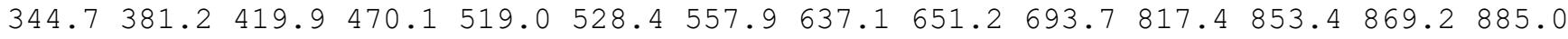
$929.0 \quad 968.2977 .5 \quad 985.0 \quad 1013.3 \quad 1017.0 \quad 1053.4 \quad 1068.8 \quad 1078.2 \quad 1089.5 \quad 1110.01121 .7 \quad 1169.8$

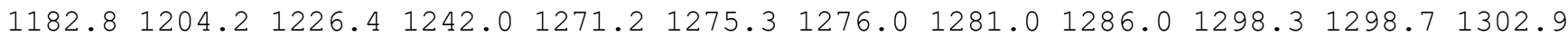
$1307.61312 .3 \quad 1333.3 \quad 1345.1 \quad 1393.9 \quad 1396.1 \quad 1398.1 \quad 1403.31408 .7 \quad 1559.01624 .11857 .3$ $2644.72646 .7 \quad 2668.8 \quad 2673.7 \quad 2677.5 \quad 2678.8 \quad 2679.2 \quad 2700.22704 .22709 .72710 .82725 .6$ 2775.82779 .02780 .92783 .6

name $=$ MIN333

energy $($ in $\mathrm{kcal} / \mathrm{mol})=5.984453$

$\mathrm{ZPE}(\mathrm{in} \mathrm{kcal} / \mathrm{mol})=141.727$

$\mathrm{G}($ in $\mathrm{kcal} / \mathrm{mol})=111.914$

Geometry

C $-2.69486428-1.45148781-0.65987195$

C $-1.20957993-1.37265687-0.66881369$

C $-0.595371821 .81525257-0.64271522$

C $-1.05557514 \quad 3.06681434 \quad-0.62627036$

C $1.56860439-0.33892888 \quad 0.70236775$

C $1.09670986-1.693326720 .27111046$

C $-0.39485510-1.77712592 \quad 0.31290524$

C $1.46851938 \quad 0.14593128 \quad 1.94751784$

C $1.90893567 \quad 1.53682496 \quad 2.26901027$

C $0.91505214-0.634500013 .09230864$

$\mathrm{H}-3.10617146-1.52859237-1.67997867$

$\mathrm{H}-3.13380423-0.54587806-0.21198317$

$\mathrm{H}-3.06270112-2.31809968-0.09481850$

$\mathrm{H}-0.46150087 \quad 1.15053280 \quad 0.20388227$

$\mathrm{H}-1.16438435 \quad 3.67412329-1.51050109$

$\mathrm{H}-1.35653298 \quad 3.57144079 \quad 0.27497093$

H $2.01091461 \quad 0.25751951 \quad-0.09420251$

H $1.54311777-2.47941151 \quad 0.92057589$

H $1.46967460-1.92806010 \quad-0.75138998$

$\mathrm{H}-0.80626933-2.20037189 \quad 1.22728700$

H $1.10760527 \quad 2.10319812 \quad 2.76442598$

H $2.20118408 \quad 2.10819804 \quad 1.37719266$

H 2.77439402 1.53113569 2.94633725

$\mathrm{H} 1.06745102-0.13690324 \quad 4.05800632$

$\mathrm{H} 1.38284330-1.627595423 .16346191$

$\mathrm{H}-0.16675160-0.795029892 .97672188$

$\begin{array}{lllll}0 & 0.27852191 & 0.01138079 & -1.72989756\end{array}$

$0-0.24491598 \quad 1.30965911-1.87557153$

o $-0.78605387-0.88820044-1.89284836$

Vibrational frequencies (in $\mathrm{cm}-1$ )

$\begin{array}{lllllllllllllll}17.9 & 32.0 & 40.3 & 47.7 & 64.1 & 77.7 & 83.3 & 90.0 & 110.0 & 132.1 & 170.7 & 214.7 & 249.2 & 290.5\end{array}$ $321.1342 .6 \quad 399.4 \quad 416.2 \quad 430.6 \quad 487.7 \quad 536.3 \quad 565.6 \quad 578.4 \quad 612.6 \quad 647.9 \quad 817.8 \quad 873.4 \quad 910.4$ $922.4937 .5 \quad 952.3 \quad 962.1 \quad 992.6 \quad 1002.3 \quad 1019.3 \quad 1024.4 \quad 1029.6 \quad 1056.0 \quad 1064.3 \quad 1075.6 \quad 1113.4$

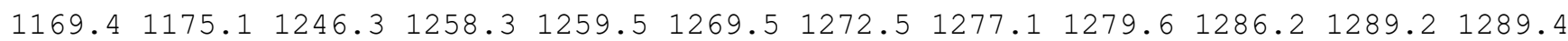

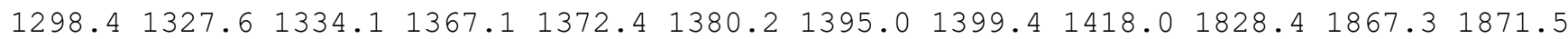
$2653.92662 .92668 .2 \quad 2672.0 \quad 2673.0 \quad 2673.3 \quad 2676.92708 .12734 .2 \quad 2734.92750 .4 \quad 2758.0$ 2776.82778 .22780 .12794 .8

name $=$ MIN334

energy $($ in $\mathrm{kcal} / \mathrm{mol})=3.188734$

$\mathrm{ZPE}(\mathrm{in} \mathrm{kcal} / \mathrm{mol})=144.562$

$\mathrm{G}($ in $\mathrm{kcal} / \mathrm{mol})=118.727$

Geometry

C $-2.54138428-1.50744965-1.61707588$

C $-1.42582758-1.16606228-0.68831104$

C $1.60297984 \quad 1.20472314 \quad-0.94513551$

C $1.46390220-0.14889101-0.36108771$

C $0.92166617-0.43317409 \quad 0.83301396$

C $0.36507076-1.82574961 \quad 1.00019822$

C $-0.78563205-2.06736120 \quad 0.07431732$ 
C $0.73599326 \quad 0.47983393 \quad 2.01434740$

C $2.02731429 \quad 1.23891014 \quad 2.34963494$

C -0.428511621 .450080501 .78760985$

$\mathrm{H}-3.46213684-1.73377276-1.05770441$

$\mathrm{H}-2.31268470-2.37660504-2.24612432$

$\mathrm{H}-2.78067502-0.66733822-2.28679872$

$\mathrm{H} 1.88103199 \quad 1.99737662-0.22553291$

H 2.28819195 1.23975993-1.80966086

$\mathrm{H} 1.65123056-0.95216095-1.07717150$

$\mathrm{H} \quad 0.48100170 \quad-0.14444206 \quad 2.91349511$

H $0.03824167 \quad-2.01760283 \quad 2.04562539$

H $1.16120962 \quad-2.58071458 \quad 0.80794102$

$\mathrm{H}-1.11669799-3.10828631 \quad 0.05783499$

H 2.25434974 2.01024413 1.60632955

H $2.88536519 \quad 0.56007507 \quad 2.39679567$

$\mathrm{H} 1.94173612 \quad 1.740248113 .32020426$

$\mathrm{H}-0.54987826 \quad 2.13439498 \quad 2.63212298$

$\mathrm{H}-1.37341727 \quad 0.90727842 \quad 1.65497287$

$\mathrm{H}-0.28647777 \quad 2.05200278 \quad 0.88117765$

$\begin{array}{llll}0 & -0.48911312 & 0.60804590 & -1.78787948\end{array}$

$00.30579893 \quad 1.64724189-1.41132791$

o $-1.19843356 \quad 0.18357077-0.57437027$

Vibrational frequencies (in $\mathrm{cm}-1$ )

$\begin{array}{lllllllllllllll}42.5 & 55.9 & 57.3 & 101.7 & 148.3 & 156.4 & 193.9 & 200.2 & 230.0 & 236.4 & 243.3 & 267.4 & 308.0 & 337.6\end{array}$

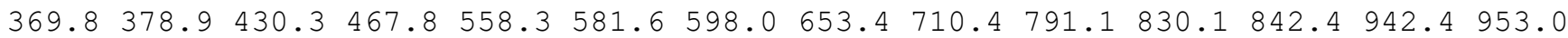
$964.2974 .9979 .6 \quad 986.0 \quad 1010.5 \quad 1018.7 \quad 1034.4 \quad 1049.0 \quad 1072.1 \quad 1108.2 \quad 1143.51147 .11161 .5$

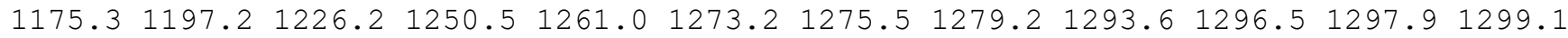

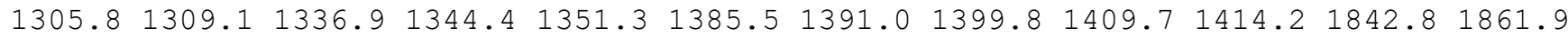
$2642.12651 .12663 .4 \quad 2666.92669 .2 \quad 2675.92679 .52681 .52695 .32714 .62731 .92746 .0$ $2751.4 \quad 2779.12779 .3 \quad 2781.7$

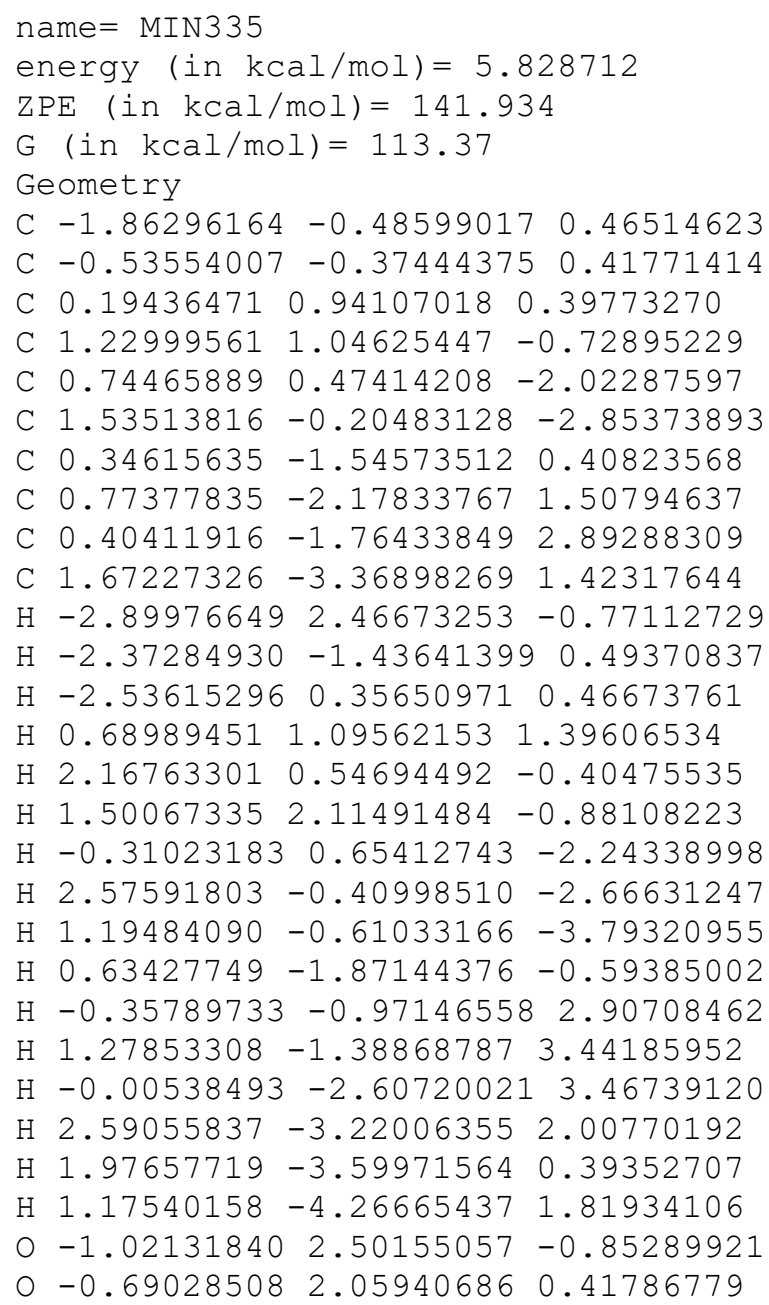


O $-2.22955207 \quad 1.92447800-1.25549979$

Vibrational frequencies (in $\mathrm{cm}-1$ )

$\begin{array}{lllllllllllllll}26.3 & 36.3 & 50.8 & 61.9 & 80.4 & 92.7 & 110.7 & 119.9 & 132.3 & 158.7 & 191.0 & 238.3 & 269.1 & 307.8\end{array}$

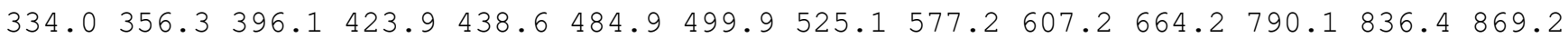

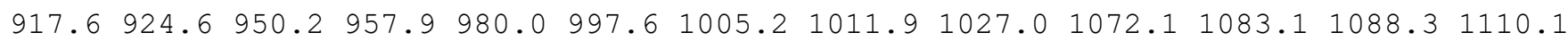

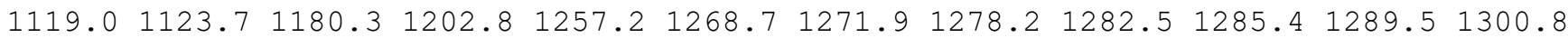

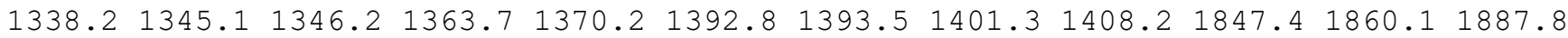
$2655.52663 .0 \quad 2668.8 \quad 2670.6 \quad 2674.4 \quad 2675.9 \quad 2716.4 \quad 2722.72731 .8 \quad 2744.5 \quad 2752.2 \quad 2778.1$ $2780.82788 .3 \quad 2792.0 \quad 2850.0$

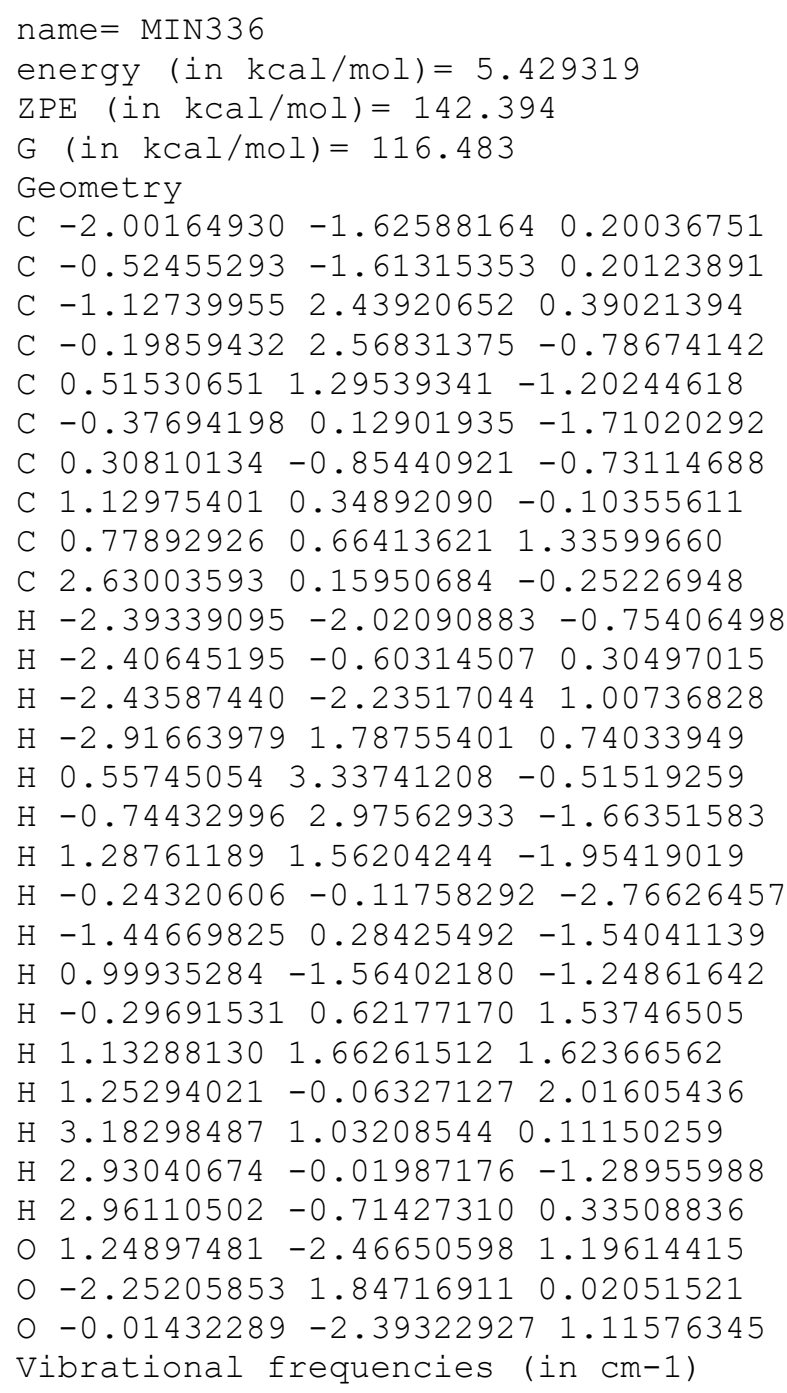


C $0.18333903-2.81022182-0.54153998$

C $-0.37048408-1.973304650 .33527469$

C $1.25074546 \quad 1.28278844 \quad 2.25446473$

$\begin{array}{llll}\text { C } 0.78802811 & 2.66427952 & 1.93318761\end{array}$

C $1.52033876 \quad 1.035146413 .70426994$

$\mathrm{H}-3.06027733-1.70035238 \quad 0.06700162$

$\mathrm{H}-3.07564606 \quad 0.03517243-0.40562477$

$\mathrm{H}-1.69908707-0.48117761-3.47349665$

$\mathrm{H}-0.85067200 \quad 1.34057585 \quad 0.20346652$

$\mathrm{H} 1.589653691 .35425662-0.53049874$

$\mathrm{H} \quad 1.56922350-0.40189654-0.68006310$

$\mathrm{H} \quad 1.75442472-0.67934167 \quad 1.64697555$

$\mathrm{H} \quad 0.74464695-3.68617940 \quad-0.25935257$

$\mathrm{H} \quad 0.11054747-2.67938258-1.61339983$

$\mathrm{H}-0.29055203-2.11790314 \quad 1.41460100$

$\mathrm{H}-0.29597584 \quad 2.76180048 \quad 2.09297213$

$\mathrm{H} \quad 0.99256387 \quad 2.94657620 \quad 0.89109300$

H $1.27546026 \quad 3.41726092 \quad 2.56768946$

H $2.378160961 .62591161 \quad 4.05495742$

$\mathrm{H} 1.74304713-0.01739155 \quad 3.92264018$

$\mathrm{H} \quad 0.65526307 \quad 1.31555757 \quad 4.32189255$

$0-0.099198450 .05776805-2.63427917$

$\begin{array}{lllll}0 & -0.65617557 & 0.91478262 & -1.69494967\end{array}$

o $-1.01798617-0.95314426-2.93336560$

Vibrational frequencies (in $\mathrm{cm}-1$ )

$\begin{array}{llllllllllllll}24.6 & 25.1 & 44.5 & 61.0 & 80.8 & 83.5 & 92.1 & 96.6 & 102.8 & 184.8 & 198.7 & 237.5 & 275.1 & 300.1\end{array}$ $311.2 \quad 319.4 \quad 385.8 \quad 404.0 \quad 461.0 \quad 484.2 \quad 505.5 \quad 550.1 \quad 585.2 \quad 638.6 \quad 651.9 \quad 730.0 \quad 831.7 \quad 847.8$

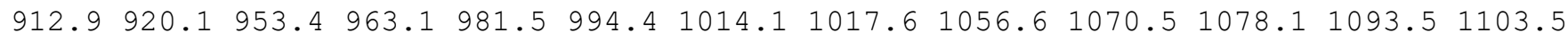

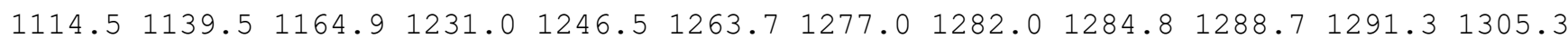

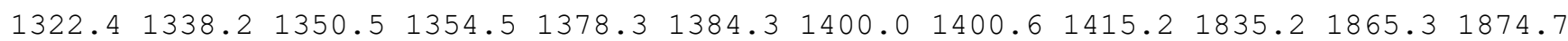
$2653.02668 .4 \quad 2670.8 \quad 2671.3 \quad 2673.7 \quad 2675.92711 .62723 .32739 .12751 .02754 .92777 .5$ $2780.7 \quad 2782.8 \quad 2788.8 \quad 2848.2$

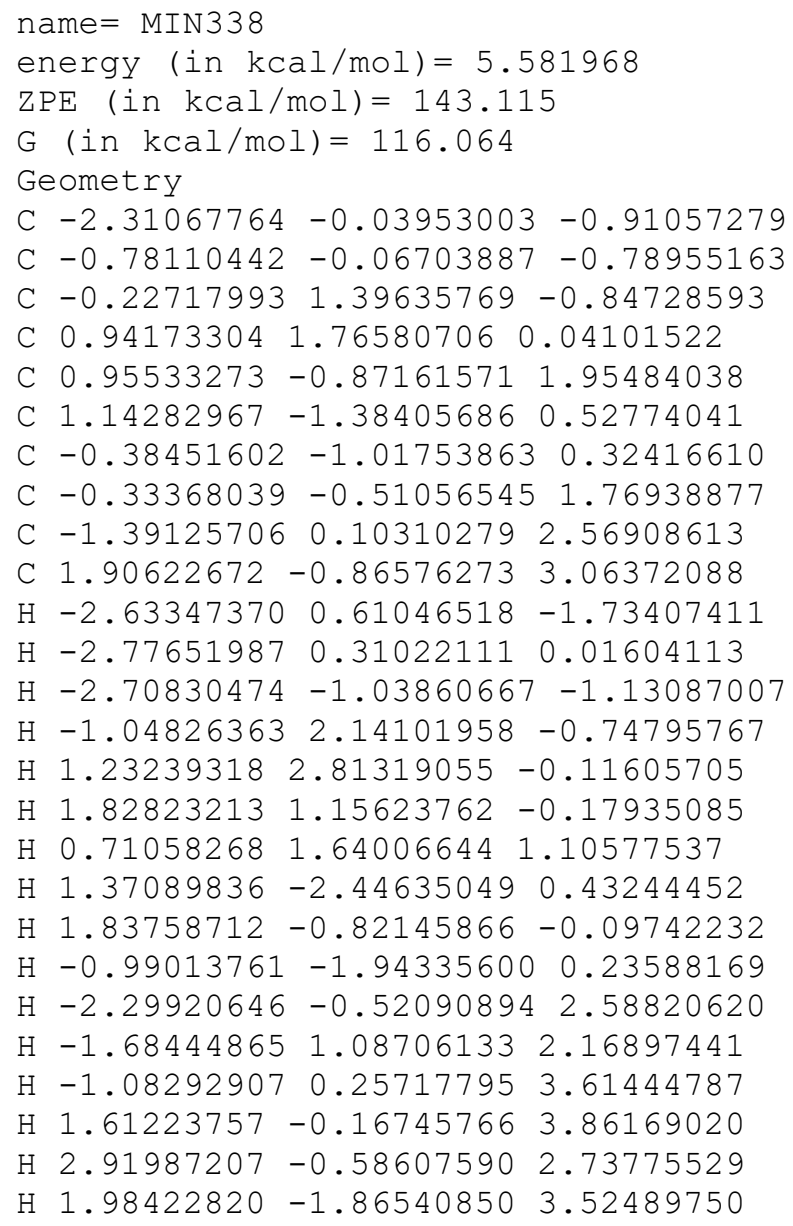


$0.66073640 \quad 0.22944811-2.53488991$

○ $0.168947921 .51499216-2.21921060$

o $-0.32656048-0.65745179-2.02352289$

Vibrational frequencies (in $\mathrm{cm}-1$ )

$\begin{array}{llllllllllllllll}16.7 & 48.1 & 57.5 & 83.6 & 86.7 & 122.9 & 167.5 & 187.4 & 215.8 & 233.6 & 243.1 & 268.0 & 310.8 & 317.6\end{array}$ $323.9 \quad 365.9 \quad 459.5 \quad 476.0 \quad 500.8 \quad 575.2 \quad 641.8 \quad 677.5 \quad 703.4 \quad 795.5 \quad 837.3 \quad 870.6 \quad 913.5 \quad 949.5$ $954.5967 .6 \quad 979.8 \quad 986.1 \quad 1006.0 \quad 1016.1 \quad 1045.7 \quad 1055.5 \quad 1077.8 \quad 1086.1 \quad 1107.01121 .1 \quad 1143.6$

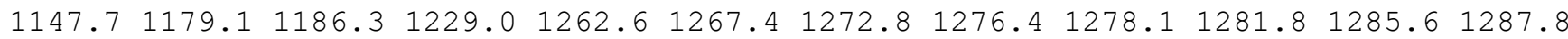

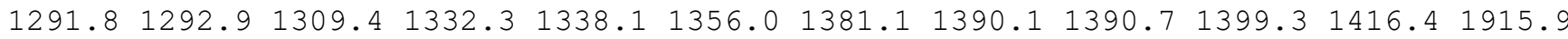
$2662.52665 .82668 .0 \quad 2670.0 \quad 2672.92674 .0 \quad 2676.8 \quad 2679.82687 .8 \quad 2688.5 \quad 2716.3 \quad 2765.5$ $2776.8 \quad 2779.3 \quad 2780.12780 .7$

name $=\operatorname{MIN} 339$

energy $($ in $\mathrm{kcal} / \mathrm{mol})=8.797494$

$\mathrm{ZPE}($ in $\mathrm{kcal} / \mathrm{mol})=140.199$

$\mathrm{G}($ in $\mathrm{kcal} / \mathrm{mol})=112.475$

Geometry

C $-1.42272514-0.42520043-1.07758629$

C $0.08907119-0.44769406-1.07652338$

C $0.802896120 .95120413-1.18114572$

C $1.90577254 \quad 1.37302170 \quad-0.25326721$

C $-0.25942161-0.37763934 \quad 1.93982314$

C $0.53093543-1.408881631 .19328401$

C $0.68115245-1.42650588-0.13437336$

C $-1.68578118-0.81538448 \quad 1.96405995$

$\begin{array}{llll}\text { C }-2.68227118 & 0.19262380 & 2.34672259\end{array}$

C $0.22826494-0.25385702 \quad 3.39093149$

$\mathrm{H}-1.81702552 \quad 0.46123784-0.56307429$

$\mathrm{H}-1.84526497-1.30967966-0.57266179$

$\mathrm{H}-1.82180803-0.42239631-2.10175432$

$\mathrm{H}-0.60409990 \quad 1.96885230 \quad-2.12467093$

H $1.53008205 \quad 2.03428456 \quad 0.54184475$

H $2.39649154 \quad 0.51152891 \quad 0.21940092$

$\mathrm{H}-0.14835765 \quad 0.61959514 \quad 1.43633124$

H $0.97368678-2.18783901 \quad 1.81546751$

$\mathrm{H} \quad 2.67753537 \quad 1.93843640-0.79973432$

$\mathrm{H} \quad 1.26234746-2.21076330-0.62743525$

$\mathrm{H}-3.22622560-0.167308413 .23849165$

$\mathrm{H}-3.450580890 .253424151 .55500566$

$\mathrm{H}-2.33277075 \quad 1.20880319 \quad 2.55342294$

$\mathrm{H}-0.28647884 \quad 0.55459346 \quad 3.92156397$

H $1.30297441-0.03773393 \quad 3.42319609$

H $0.06381951-1.182602613 .95175405$

$\begin{array}{lllll}0 & 1.28603370 & 0.56171544 & -2.49157438\end{array}$

$0-0.08082627 \quad 2.01609823-1.29319600$

O $0.57557244-0.71556239-2.43266408$

Vibrational frequencies (in $\mathrm{cm}-1$ )

$\begin{array}{lllllllllllllll}26.5 & 49.1 & 54.8 & 72.6 & 102.3 & 118.1 & 126.5 & 153.8 & 165.5 & 174.0 & 218.2 & 258.6 & 261.9 & 277.6\end{array}$ $318.7 \quad 342.4 \quad 362.2 \quad 369.9 \quad 415.5 \quad 441.0 \quad 463.1 \quad 513.4 \quad 536.3 \quad 621.6 \quad 725.2 \quad 757.8 \quad 827.5 \quad 885.2$ $900.9934 .0 \quad 950.8 \quad 978.4 \quad 991.8 \quad 1006.7 \quad 1012.1 \quad 1018.3 \quad 1027.5 \quad 1090.6 \quad 1120.2 \quad 1132.5 \quad 1146.3$

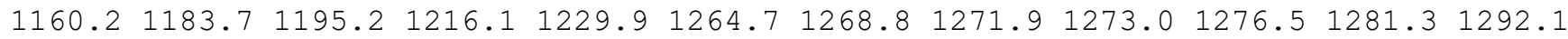
$1298.51299 .5 \quad 1319.91330 .3 \quad 1342.8 \quad 1370.9 \quad 1376.8 \quad 1387.01401 .3 \quad 1407.01414 .8 \quad 1861.4$ $2654.72656 .22665 .3 \quad 2666.02667 .92670 .92671 .82675 .02685 .32738 .02753 .02770 .3$ $2770.7 \quad 2776.6 \quad 2778.8 \quad 2796.8$

name $=$ MIN340

energy $($ in $\mathrm{kcal} / \mathrm{mol})=5.591876$

$\mathrm{ZPE}($ in $\mathrm{kcal} / \mathrm{mol})=143.841$

$\mathrm{G}($ in $\mathrm{kcal} / \mathrm{mol})=118.933$

Geometry

C $-2.60529087 \quad 0.29453063-0.07442201$

C $-1.10146676 \quad 0.08432165-0.08665935$

C $-0.30825898 \quad 1.41885786-0.36835428$ 
C $0.40289637 \quad 1.29423284 \quad 1.00611402$

C $0.39546761-1.233850851 .56473811$

C $2.27684686-0.01922517-2.15661521$

C $-0.78978764-1.06918707-1.04213895$

C $-0.40712186 \quad 0.00444918 \quad 1.33479209$

$\begin{array}{llll}\text { C }-1.34127322 & 0.18556948 & 2.52720948\end{array}$

C $1.60687373-1.25632647 \quad 2.12003166$

$\mathrm{H}-2.89658278 \quad 1.15930626 \quad 0.53375171$

$\mathrm{H}-3.13057467-0.578853190 .33011759$

$\mathrm{H}-2.98850668 \quad 0.47597899-1.08712938$

$\mathrm{H}-0.94278918 \quad 2.30856695-0.50245682$

$\mathrm{H} \quad 0.23537352 \quad 2.12820399 \quad 1.68756136$

$\mathrm{H} 1.48212150 \quad 1.14559545 \quad 0.92508230$

$\mathrm{H}-0.10468108-2.15624498 \quad 1.26366588$

H $2.73149526-0.96677495-2.40511346$

$\mathrm{H} \quad 2.74178370 \quad 0.89188979-2.50605262$

$\mathrm{H}-1.22051553-0.87298372-2.04361647$

$\mathrm{H}-1.90535974 \quad 1.12266497 \quad 2.46792181$

$\mathrm{H}-0.77116035 \quad 0.20888648 \quad 3.46525785$

$\mathrm{H}-2.06287293-0.63547243 \quad 2.59980710$

$\mathrm{H} 2.15538772-2.16772211 \quad 2.29580423$

$\mathrm{H}-1.19345410-2.02744289-0.65965335$

H $2.14323131-0.378593942 .43933146$

o $1.19539676 \quad 0.02992144 \quad-1.49707683$

$0.546126961 .42463922-1.44175760$

o $0.57400189-1.32539402-1.19819571$

Vibrational frequencies (in $\mathrm{cm}-1$ )

$\begin{array}{lllllllllllllllll}39.5 & 79.1 & 84.5 & 136.0 & 178.3 & 191.3 & 219.4 & 227.2 & 255.6 & 266.5 & 293.6 & 324.6 & 330.7 & 337.1\end{array}$ $365.7 \quad 394.0 \quad 458.5 \quad 497.5 \quad 549.4 \quad 588.1 \quad 615.3 \quad 640.7 \quad 654.2 \quad 672.4 \quad 717.1 \quad 760.3 \quad 834.1 \quad 900.9$ $933.2964 .6 \quad 982.6 \quad 989.5 \quad 990.4 \quad 1001.0 \quad 1058.2 \quad 1072.8 \quad 1085.4 \quad 1094.6 \quad 1113.1 \quad 1127.9 \quad 1133.8$

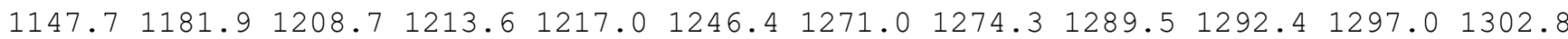
$1305.21315 .41318 .91338 .21341 .3 \quad 1358.2 \quad 1370.1 \quad 1390.51399 .51406 .81695 .01848 .9$ $2642.92664 .12672 .4 \quad 2675.2 \quad 2676.8 \quad 2680.3 \quad 2692.42709 .42710 .5 \quad 2712.3 \quad 2718.02752 .3$ $2770.92780 .02780 .7 \quad 2791.2$

name $=\operatorname{MIN} 341$

energy $($ in $\mathrm{kcal} / \mathrm{mol})=7.252861$

$\mathrm{ZPE}($ in $\mathrm{kcal} / \mathrm{mol})=142.247$

$\mathrm{G}($ in $\mathrm{kcal} / \mathrm{mol})=112.616$

Geometry

C $-2.51686709-1.19379631-0.73965012$

C $-1.03907822-1.16241769-0.68315455$

$\begin{array}{llll}\text { C }-0.19625553 & 2.49548034 & -0.73504237\end{array}$

C $0.90794705 \quad 2.42467955-1.47268514$

C $1.71521263-0.477726210 .69155926$

C $1.24739222-1.81195852 \quad 0.07747207$

C $-0.23996615-1.93593403 \quad 0.06480545$

C $1.36681796-0.40175604 \quad 2.14928304$

C $0.16861390 \quad 0.42197215 \quad 2.48801279$

C $2.09215051-1.02629390 \quad 3.08077864$

$\mathrm{H}-2.88094991-1.26269524-1.77735752$

$\mathrm{H}-2.95454456-0.27871886-0.30705919$

$\mathrm{H}-2.93946057-2.04705074-0.18981065$

$\mathrm{H}-0.40850613 \quad 3.180706790 .08347411$

H $1.75599124 \quad 3.07405967-1.33634958$

$\mathrm{H} 1.05093848 \quad 1.71215527-2.27045686$

H $1.26724926 \quad 0.36807788 \quad 0.12851989$

H $1.70197120-2.653002690 .64054843$

$\mathrm{H} 1.64537033-1.89544501-0.95645996$

$\mathrm{H}-0.65498047-2.71188193 \quad 0.70222326$

$\mathrm{H}-0.20048632 \quad 0.23263825 \quad 3.50373573$

$\mathrm{H}-0.66359771 \quad 0.21804131 \quad 1.79661945$

$\mathrm{H} 0.39896649 \quad 1.49387621 \quad 2.41624255$

$\mathrm{H} \quad 1.86871299-0.99612903 \quad 4.13238169$ 
$\mathrm{H} 2.80973082-0.373536350 .55119511$

$\mathrm{H} 2.96242448-1.618926132 .85797648$

$0-1.21461544 \quad 0.77832231-1.90825118$

$0-1.303376851 .70171863-0.84615621$

$0-0.38458853-0.27106737-1.52192094$

Vibrational frequencies (in $\mathrm{cm}-1$ )

$\begin{array}{llllllllllllllll}7.5 & 32.5 & 32.9 & 61.2 & 73.6 & 86.6 & 95.6 & 115.3 & 125.9 & 159.9 & 182.5 & 218.1 & 244.7 & 344.8\end{array}$ $352.2405 .5 \quad 421.5 \quad 427.3 \quad 465.7 \quad 546.6 \quad 579.6 \quad 610.7 \quad 627.2 \quad 634.2 \quad 661.7 \quad 811.3 \quad 845.4 \quad 891.4$ $908.3930 .4 \quad 945.4 \quad 977.2 \quad 990.8 \quad 1012.1 \quad 1019.2 \quad 1035.2 \quad 1048.7 \quad 1057.2 \quad 1060.9 \quad 1063.4 \quad 1080.6$ $1091.61156 .41170 .1 \quad 1183.6 \quad 1232.6 \quad 1269.7 \quad 1276.4 \quad 1277.5 \quad 1281.2 \quad 1287.91291 .01301 .9$

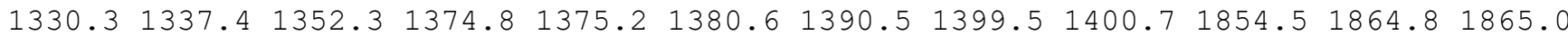
$2654.12663 .0 \quad 2664.2 \quad 2668.4 \quad 2672.3 \quad 2677.7 \quad 2713.6 \quad 2730.22737 .12737 .82741 .02759 .9$ $2778.12778 .32793 .3 \quad 2797.5$

name $=\operatorname{MIN} 342$

energy (in $\mathrm{kcal} / \mathrm{mol})=8.263909$

$\mathrm{ZPE}(\mathrm{in} \mathrm{kcal} / \mathrm{mol})=141.496$

$\mathrm{G}(\mathrm{in} \mathrm{kcal} / \mathrm{mol})=112.626$

Geometry

C $-2.74903585-0.87115902-0.55054523$

C $-1.26387015-0.85721740-0.54691735$

C $-1.00285962 \quad 2.61212194-0.53498898$

C $-1.00930094 \quad 2.17296400 \quad 0.72007582$

$\begin{array}{lllll}\text { C } 1.02954874 & -1.40931474 & 0.29372925\end{array}$

$\begin{array}{lllll}\text { C } 2.31625623 & 0.66070424 & 0.75338364\end{array}$

C $-0.46593499-1.43371152 \quad 0.36029187$

C $1.57956931-0.349880861 .22161339$

C $1.26645480-0.49238280 \quad 2.67425273$

C $1.59502553-2.791709590 .65775450$

$\mathrm{H}-3.14770283 \quad 0.08031613-0.16136417$

$\mathrm{H}-3.16368844-1.68254152 \quad 0.06127088$

$\mathrm{H}-3.14912926-0.98845602-1.57054631$

$\mathrm{H}-1.434574493 .53624436-0.91622218$

$\mathrm{H}-1.46425344 \quad 2.70542668 \quad 1.53624224$

$\mathrm{H}-0.545168331 .24711928 \quad 1.03008162$

$\mathrm{H} 1.35611703-1.18345334-0.75919260$

H $2.56326765 \quad 0.79668672 \quad-0.28681437$

H 2.72879899 $1.43790254 \quad 1.37527949$

$\mathrm{H}-0.88505755-1.97373936 \quad 1.20703564$

H $1.74878298-1.38593182 \quad 3.09705135$

$\mathrm{H} \quad 0.18595691-0.59011624 \quad 2.84791916$

H $1.61186422 \quad 0.36793200 \quad 3.26361076$

H $1.26884998-3.12056611 \quad 1.64942336$

H $2.69107324 \quad-2.778051170 .65460967$

$\mathrm{H} \quad 1.26801339-3.54745160-0.06576495$

$0.15322103 \quad 0.76452280-1.30413695$

O $-0.43698673 \quad 1.99893307-1.61749173$

o $-0.77611644-0.22948497-1.68069282$

Vibrational frequencies (in $\mathrm{cm}-1$ )

$\begin{array}{llllllllllllll}23.6 & 39.9 & 46.5 & 61.4 & 68.3 & 84.3 & 93.7 & 97.6 & 146.4 & 163.3 & 169.5 & 234.1 & 235.5 & 285.0\end{array}$ $310.5 \quad 349.4 \quad 382.9 \quad 436.2 \quad 447.9 \quad 527.7 \quad 546.0 \quad 572.2 \quad 616.5 \quad 622.2 \quad 631.8 \quad 647.8 \quad 798.9 \quad 871.3$ $919.8 \quad 932.7 \quad 951.3 \quad 970.4 \quad 991.8 \quad 1007.6 \quad 1011.4 \quad 1040.7 \quad 1046.2 \quad 1054.6 \quad 1062.5 \quad 1077.5 \quad 1087.2$

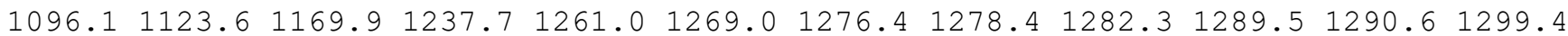

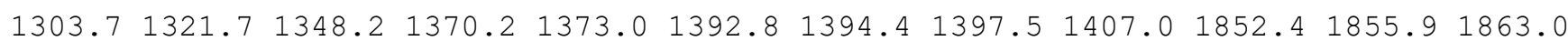
$2662.82669 .22670 .2 \quad 2674.12675 .3 \quad 2678.42689 .12708 .72722 .02734 .12756 .82777 .3$ $2778.3 \quad 2780.92790 .3 \quad 2792.5$

name $=$ MIN343

energy $($ in $\mathrm{kcal} / \mathrm{mol})=7.273833$

$\mathrm{ZPE}($ in $\mathrm{kcal} / \mathrm{mol})=142.759$

$\mathrm{G}($ in $\mathrm{kcal} / \mathrm{mol})=114.78$

Geometry

C $-2.61288169-0.42305751-1.19332332$ 
C $-0.25442101-0.04145591-1.07753722$

C $0.048567681 .36385587-1.37997959$

C $2.35957915 \quad 0.24629724 \quad 0.48913278$

C $0.75068691-0.73321606 \quad 2.17274863$

C $1.35265800-0.87103501 \quad 0.74082376$

C $0.24119863-0.96517763-0.25847504$

C $-0.26481047 \quad 0.37060957 \quad 2.22099266$

C $-0.00056318 \quad 1.58080120 \quad 2.71147145$

C $0.11390381-2.049330372 .63468137$

$\mathrm{H}-2.54331585-1.05268619-0.31400513$

$\mathrm{H}-3.39723327-0.76116735-1.87399425$

$\mathrm{H}-2.81367406 \quad 0.61001757-0.92079979$

$\mathrm{H}-0.82193472 \quad 1.98969791-1.64387195$

H $2.67200774 \quad 0.27967148 \quad-0.56507999$

H $1.94399869 \quad 1.23654247 \quad 0.72143638$

$\mathrm{H} \quad 1.60078390 \quad-0.49789368 \quad 2.86487578$

H $1.91913778-1.84317810 \quad 0.70627999$

H $3.26248367 \quad 0.12119991 \quad 1.09541832$

$\mathrm{H}-0.21875744-1.96741671-0.25519500$

$\mathrm{H}-1.24390555 \quad 0.11365587 \quad 1.82107470$

$\mathrm{H} 0.95703438 \quad 1.87210986 \quad 3.11548515$

$\mathrm{H}-0.72437846 \quad 2.38052452 \quad 2.74464570$

$\mathrm{H} \quad 0.84326240 \quad-2.86693260 \quad 2.64322222$

$\mathrm{H}-0.72202191-2.35248840 \quad 1.99789299$

$\mathrm{H}-0.27597312-1.95364704 \quad 3.65638233$

$\begin{array}{lllll}0 & -1.38544027 & 0.07648279 & -3.05338581\end{array}$

O $1.158458831 .83281749-1.38065713$

o - $1.33327233-0.57666018-1.97231947$

Vibrational frequencies (in $\mathrm{cm}-1$ )

$\begin{array}{lllllllllllllll}20.5 & 38.8 & 46.1 & 74.4 & 89.3 & 103.5 & 114.6 & 174.5 & 185.3 & 203.1 & 240.0 & 249.9 & 267.1 & 302.8\end{array}$ $327.0 \quad 337.0 \quad 376.1 \quad 394.0 \quad 424.3 \quad 513.9 \quad 538.7 \quad 570.0 \quad 609.5 \quad 660.1 \quad 687.8 \quad 844.1 \quad 887.3 \quad 934.4$ $946.3950 .9971 .2987 .9997 .2 \quad 1014.9 \quad 1032.1 \quad 1054.0 \quad 1070.0 \quad 1090.6 \quad 1100.41113 .11122 .0$ $1140.81146 .81199 .6 \quad 1227.2 \quad 1264.8 \quad 1270.1 \quad 1275.2 \quad 1290.51296 .6 \quad 1298.4 \quad 1300.61304 .0$ $1314.71321 .7 \quad 1332.0 \quad 1344.2 \quad 1347.8 \quad 1386.5 \quad 1389.4 \quad 1402.91411 .4 \quad 1841.4 \quad 1848.01900 .6$ $2642.52658 .92660 .12671 .4 \quad 2672.8 \quad 2675.8 \quad 2677.5 \quad 2686.82697 .2 \quad 2711.52714 .42759 .2$ 2765.12779 .12781 .92787 .3

\section{name $=\operatorname{MIN} 344$}

energy $($ in $\mathrm{kcal} / \mathrm{mol})=8.973123$

$\operatorname{ZPE}(\mathrm{in} \mathrm{kcal} / \mathrm{mol})=141.715$

$\mathrm{G}(\mathrm{in} \mathrm{kcal} / \mathrm{mol})=111.682$

Geometry

C $-2.97415764-0.87457530-1.02860038$

C $-1.50660593-1.03382129-0.82078608$

C $0.957166421 .90423578-1.24376622$

C $2.002857061 .41900930-0.57752507$

C $1.06927207-0.85794837 \quad 1.72589855$

C $0.50959159-1.64241553 \quad 0.57978801$

C $-0.94081062-1.43317746 \quad 0.32234450$

C $0.59251654 \quad 0.26028697 \quad 2.28903516$

C $1.33066507 \quad 0.90762202 \quad 3.42135225$

C $-0.65787928 \quad 0.96993047 \quad 1.89263376$

$\mathrm{H}-3.22227943-0.64249821-2.07685528$

$\mathrm{H}-3.37418489-0.05134231-0.41793696$

$\mathrm{H}-3.52234223-1.78915852-0.76163527$

$\mathrm{H} \quad 0.63946013 \quad 2.94563065-1.29548069$

$\mathrm{H} \quad 2.65463636 \quad 2.028567110 .02501165$

H $2.28113835 \quad 0.37926799-0.55323898$

H $2.00255271-1.28820740 \quad 2.09831712$

H $0.66639423-2.73216821 \quad 0.78102727$

$\mathrm{H} 1.12884144-1.44001876-0.32240156$

$\mathrm{H}-1.57296100-1.65642626 \quad 1.18605635$

$\mathrm{H} \quad 1.64961170 \quad 1.92420575 \quad 3.14993152$

H 2.23172394 $0.35633848 \quad 3.71719347$ 
H $0.69311525 \quad 0.98934103 \quad 4.31240843$

$\mathrm{H}-0.72623466 \quad 1.97928575 \quad 2.31700947$

$\mathrm{H}-1.54796144 \quad 0.41929117 \quad 2.22756497$

$\mathrm{H}-0.72862303 \quad 1.07725822 \quad 0.79863028$

O $0.35722816-0.20267057-1.92197576$

$0 \quad 0.09617247 \quad 1.21521123-2.03603881$

o $-0.87932947-0.80026142-2.03391102$

Vibrational frequencies (in cm-1)

$\begin{array}{llllllllllllllll}-18.9 & 14.5 & 33.4 & 45.8 & 67.1 & 70.8 & 94.0 & 106.5 & 154.0 & 159.6 & 176.0 & 220.4 & 253.0 & 265.1\end{array}$ $301.3 \quad 315.8 \quad 375.1 \quad 409.8 \quad 435.8 \quad 472.1 \quad 531.4 \quad 585.2 \quad 604.5 \quad 631.8 \quad 654.0 \quad 819.2 \quad 844.8 \quad 893.3$ $924.2928 .0 \quad 956.8 \quad 977.6 \quad 991.0 \quad 1005.8 \quad 1025.0 \quad 1025.9 \quad 1038.2 \quad 1052.0 \quad 1053.8 \quad 1066.1 \quad 1112.8$

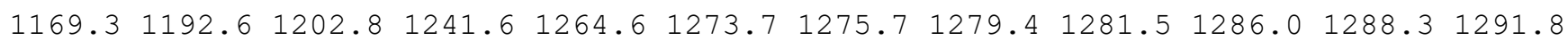

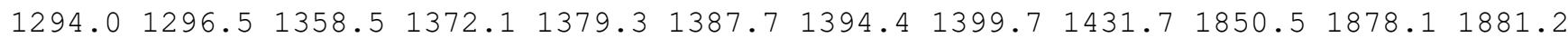
$2644.42664 .1 \quad 2665.7 \quad 2670.3 \quad 2672.2 \quad 2674.6 \quad 2677.0 \quad 2712.5 \quad 2725.5 \quad 2735.3 \quad 2745.4 \quad 2750.9$ $2776.0 \quad 2776.9 \quad 2780.0 \quad 2797.1$

name $=$ MIN345

energy (in $\mathrm{kcal} / \mathrm{mol})=9.028679$

$\mathrm{ZPE}($ in $\mathrm{kcal} / \mathrm{mol})=141.767$

$\mathrm{G}(\mathrm{in} \mathrm{kcal} / \mathrm{mol})=112.517$

Geometry

C $-2.70860546-1.42115077-0.56969462$

C $-1.22687594-1.35231796-0.60806237$

C $0.24938957 \quad 1.67073984-0.90032481$

C $1.345172832 .42482908-0.80258654$

C $1.65506002-0.521715181 .25973777$

C $1.08886598-1.52542940 \quad 0.30559964$

C $-0.38701008-1.71448343 \quad 0.37099528$

C $1.04866388 \quad 0.22731106 \quad 2.18971345$

C $1.85004624 \quad 1.20346441 \quad 2.99819627$

C $-0.400983350 .20217545 \quad 2.54065910$

$\mathrm{H}-3.10790854-1.89517004-1.48097970$

$\mathrm{H}-3.15308386-0.41565381-0.51151635$

$\mathrm{H}-3.08381903-1.995526690 .28733809$

$\mathrm{H}-0.43267461 \quad 1.39563302-0.10744733$

$\mathrm{H} \quad 1.67148621 \quad 2.86708875 \quad 0.12281589$

$\mathrm{H} \quad 1.99679357 \quad 2.64283734-1.63269278$

$\mathrm{H} 2.73622838-0.41057944 \quad 1.11708415$

H $1.59128888-2.507554750 .48070958$

H $1.39939397-1.22866380 \quad-0.72791218$

$\mathrm{H}-0.75375951-2.19434594 \quad 1.27753610$

H $2.92001328 \quad 1.18619190 \quad 2.75549889$

$\mathrm{H} \quad 1.75991550 \quad 0.99578423 \quad 4.07312645$

$\mathrm{H} \quad 1.49983866 \quad 2.23201714 \quad 2.83097755$

$\mathrm{H}-0.66465292-0.735212123 .05150585$

$\mathrm{H}-1.04402074 \quad 0.28706380 \quad 1.65345384$

$\mathrm{H}-0.685991151 .02156132 \quad 3.21397877$

$\begin{array}{lllll}0 & -1.16537592 & 0.35283756 & -2.11692265\end{array}$

$0-0.03599858 \quad 1.17844130 \quad-2.15634324$

o $-0.70023716-0.95521671-1.82560086$

Vibrational frequencies (in $\mathrm{cm}-1$ )

$\begin{array}{lllllllllllllll}-33.1 & 25.2 & 45.1 & 54.8 & 69.0 & 75.2 & 87.1 & 104.2 & 107.9 & 165.0 & 193.4 & 223.4 & 232.5 & 270.0\end{array}$ $318.7 \quad 351.3 \quad 366.3 \quad 414.5 \quad 452.0 \quad 469.7 \quad 537.8 \quad 573.0 \quad 601.8 \quad 614.2 \quad 666.0 \quad 814.4 \quad 825.0 \quad 900.0$ $924.3 \quad 933.5 \quad 949.5 \quad 973.3 \quad 990.4 \quad 999.8 \quad 1021.8 \quad 1027.3 \quad 1034.2 \quad 1050.8 \quad 1055.0 \quad 1069.4 \quad 1111.1$ $\begin{array}{lllllllllllll}1156.6 & 1190.7 & 1253.1 & 1256.9 & 1271.2 & 1272.8 & 1277.5 & 1279.5 & 1282.7 & 1286.7 & 1288.0 & 1291.0\end{array}$

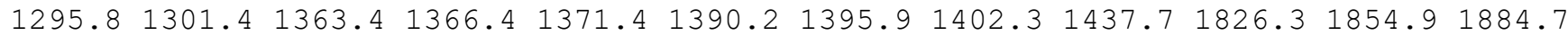
$2641.32665 .52666 .6 \quad 2670.8 \quad 2673.2 \quad 2675.7 \quad 2675.82717 .82722 .02738 .62741 .22753 .1$ $2777.12779 .2 \quad 2780.02794 .6$

name $=$ MIN346

energy $($ in $\mathrm{kcal} / \mathrm{mol})=9.24941$

$\mathrm{ZPE}(\mathrm{in} \mathrm{kcal} / \mathrm{mol})=141.881$

$\mathrm{G}($ in $\mathrm{kcal} / \mathrm{mol})=112.943$ 


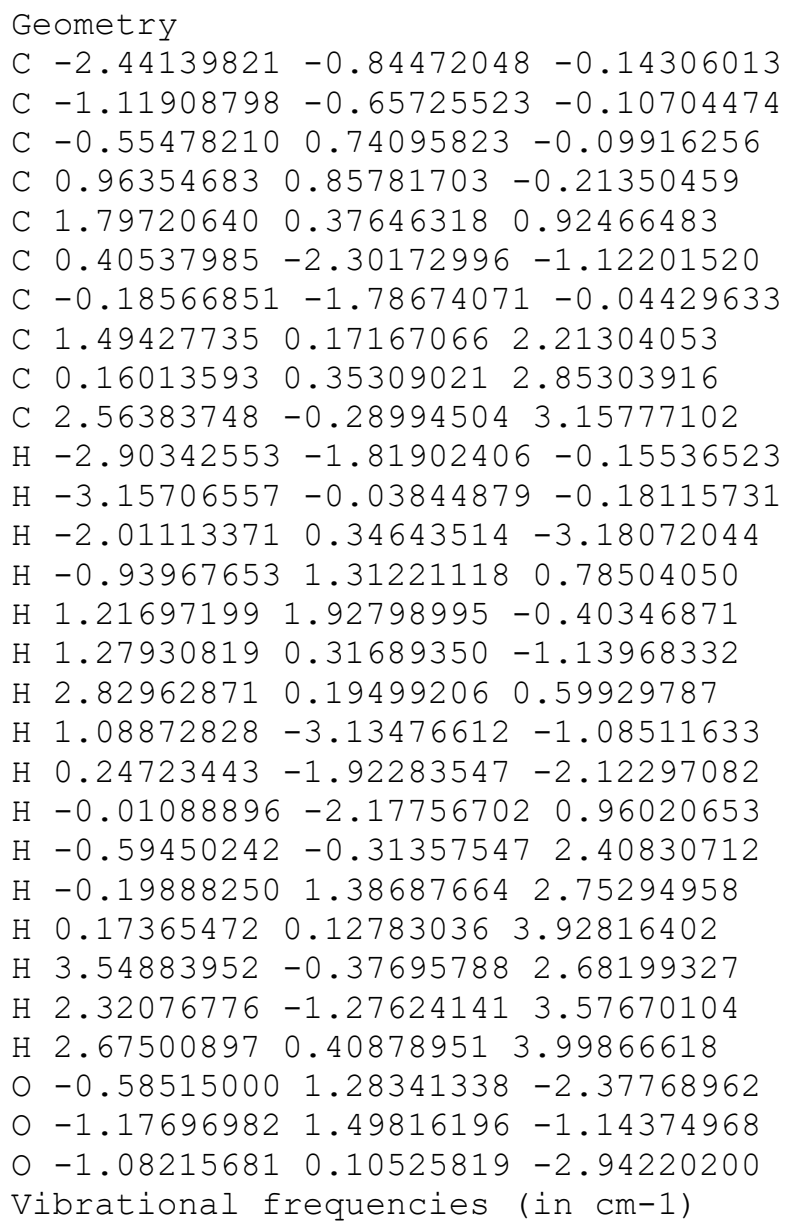

$\begin{array}{llllllllllllllll}12.9 & 34.3 & 52.1 & 58.0 & 77.3 & 88.2 & 101.6 & 126.6 & 160.5 & 190.0 & 216.4 & 234.3 & 238.2 & 280.9\end{array}$ $300.7 \quad 321.2 \quad 386.5 \quad 416.7 \quad 449.3 \quad 471.4 \quad 493.9 \quad 559.6 \quad 632.3 \quad 645.7 \quad 661.7 \quad 722.2 \quad 808.4 \quad 841.5$ $896.9918 .5953 .5961 .2 \quad 975.0 \quad 990.1 \quad 1006.7 \quad 1028.3 \quad 1046.5 \quad 1069.7 \quad 1082.3 \quad 1093.6 \quad 1103.2$ $1113.0 \quad 1134.6 \quad 1198.7 \quad 1236.6 \quad 1260.6 \quad 1270.0 \quad 1278.5 \quad 1284.2 \quad 1289.2 \quad 1291.1 \quad 1294.7 \quad 1298.3$

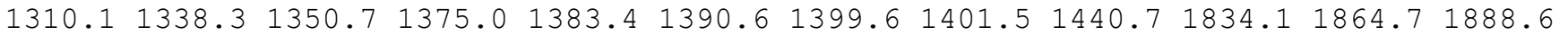
$2640.42663 .8 \quad 2667.92670 .8 \quad 2673.4 \quad 2675.2 \quad 2712.12722 .2 \quad 2723.92737 .5 \quad 2750.5 \quad 2776.4$ $2779.92782 .4 \quad 2788.0 \quad 2847.8$

\footnotetext{
name $=$ MIN347

energy (in $\mathrm{kcal} / \mathrm{mol})=7.384699$

$\mathrm{ZPE}(\mathrm{in} \mathrm{kcal} / \mathrm{mol})=143.897$

$\mathrm{G}(\mathrm{in} \mathrm{kcal} / \mathrm{mol})=117.41$

Geometry

C $-2.36194166-0.79563193-0.52283427$

C $-0.83778495-0.70169600-0.67421046$

C $-0.38259213 \quad 0.79221390 \quad-0.62950602$

C $1.03258231 \quad 1.15747179-0.17918132$

C $1.58297417 \quad 0.596827381 .13203698$

C $0.63564230-2.62534672-0.09569489$

C $-0.18412136-1.645352850 .27946983$

C $0.87971421 \quad 0.92549885 \quad 2.41601307$

C $-0.51211018 \quad 1.457288312 .38640009$

C $1.506028390 .70921935 \quad 3.57871104$

$\mathrm{H}-2.86680071-0.16000757-1.26365682$

$\mathrm{H}-2.69881958-0.50100454 \quad 0.47418382$

$\mathrm{H}-2.70632453-1.82284507-0.70399366$

$\mathrm{H}-1.14641040 \quad 1.43693724-0.14692632$

H $1.09546195 \quad 2.26754940-0.14738523$

H $1.73184017 \quad 0.85298005-0.99411296$

$\mathrm{H} \quad 2.64067415 \quad 0.94817806 \quad 1.21522677$

$\mathrm{H} 1.08323982-3.322354150 .59681288$

$\mathrm{H} \quad 0.92228089-2.80549936-1.12339590$

$\mathrm{H}-0.45990601-1.484644461 .32373788$
} 
$\mathrm{H}-1.20925904 \quad 0.72349290 \quad 1.95785056$

$\mathrm{H}-0.57637127 \quad 2.38211152 \quad 1.79603308$

$\mathrm{H}-0.88771966 \quad 1.697475113 .39167268$

H 2.50813840 $0.32432552 \quad 3.65854171$

$\mathrm{H} 1.68303796-0.50923827 \quad 1.05503031$

H $1.06642980 \quad 0.90145466 \quad 4.54161105$

$0.04420966 \quad 0.00523584-2.67277843$

$0-0.482380241 .13853118-2.01940652$

o $-0.57243290-1.11204121-2.02659150$

Vibrational frequencies (in $\mathrm{cm}-1$ )

$\begin{array}{llllllllllllllll}-42.4 & 28.5 & 49.4 & 93.3 & 112.7 & 160.6 & 195.1 & 201.3 & 205.1 & 237.1 & 256.7 & 275.6 & 319.8 & 346.1\end{array}$ $406.8 \quad 433.4 \quad 458.6 \quad 470.6 \quad 534.0 \quad 563.6 \quad 602.5 \quad 612.4 \quad 677.8 \quad 715.8 \quad 792.6 \quad 870.4 \quad 893.2 \quad 902.7$

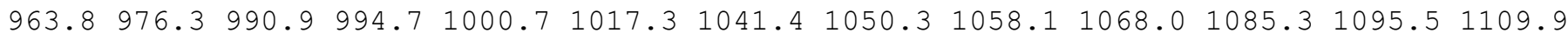

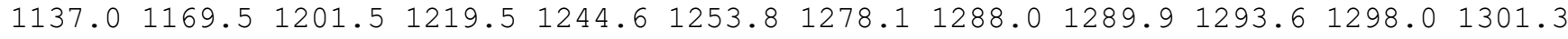
$1304.11317 .8 \quad 1335.8 \quad 1343.4 \quad 1377.8 \quad 1387.1 \quad 1394.6 \quad 1397.4 \quad 1407.6 \quad 1420.2 \quad 1842.01851 .2$ $2636.12648 .5 \quad 2668.6 \quad 2669.02671 .7 \quad 2679.8 \quad 2685.12709 .12725 .72729 .12731 .22748 .3$ $2776.4 \quad 2779.12781 .4 \quad 2796.6$

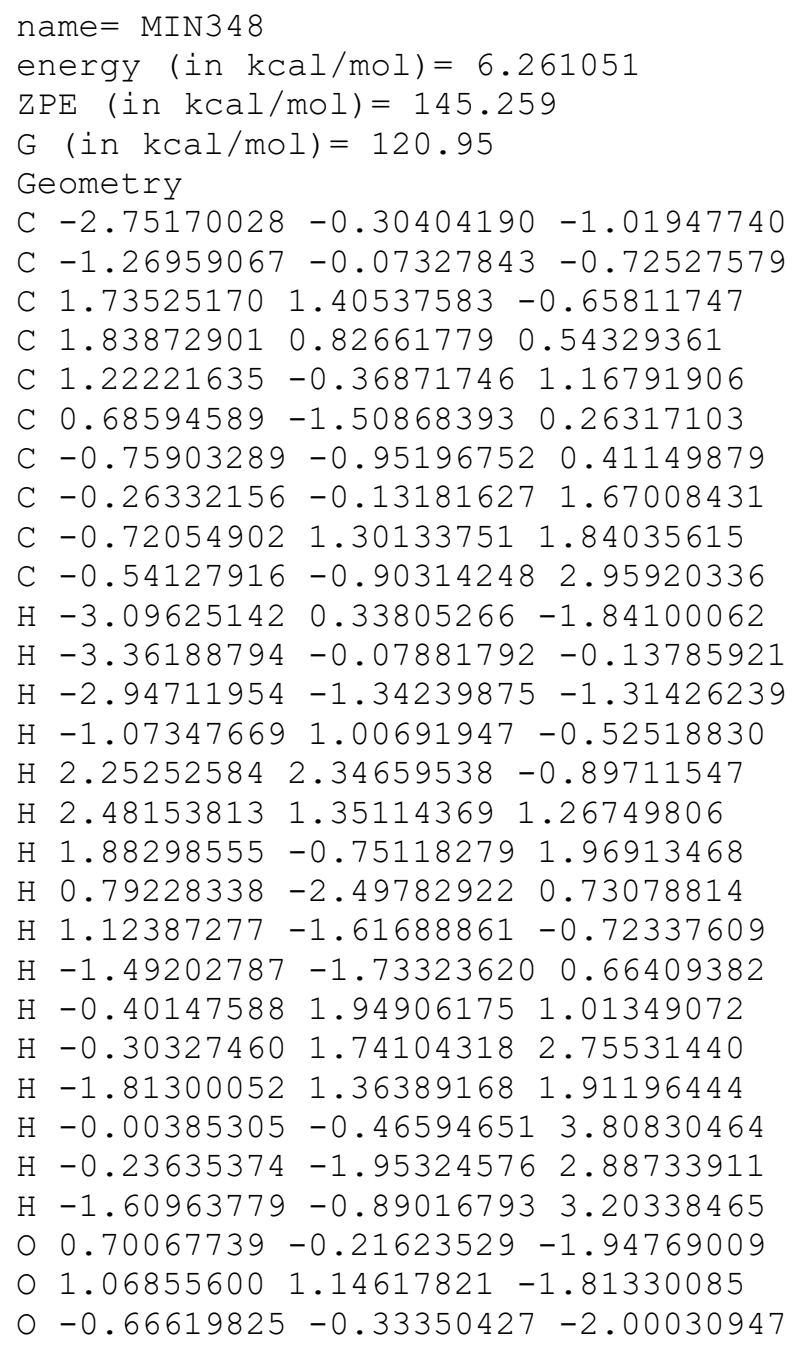


$\mathrm{ZPE}($ in $\mathrm{kcal} / \mathrm{mol})=143.163$

$\mathrm{G}(\mathrm{in} \mathrm{kcal} / \mathrm{mol})=116.686$

Geometry

C $-2.35999472-0.29890642-0.74817398$

C $-0.83125453-0.26848962-0.82388610$

C $-0.353985621 .22077142-0.98291192$

C $0.73296372 \quad 1.76377145 \quad-0.07940694$

C $0.59641397-0.77535258 \quad 1.34749410$

C $1.26331185-1.485481520 .25314918$

C $-0.24250620-1.19689626 \quad 0.21421905$

C $0.66282519-0.21934367 \quad 2.54282861$

C $-0.54257575 \quad 0.34938239 \quad 3.21432451$

C $1.94844511-0.12151850 \quad 3.29308902$

$\mathrm{H}-2.81168036 \quad 0.27191427-1.56994016$

$\mathrm{H}-2.72359394 \quad 0.11155020 \quad 0.19906303$

$\mathrm{H}-2.74109230-1.32416862-0.83940066$

$\mathrm{H}-1.236895091 .89880369-0.97580485$

H $1.02761716 \quad 2.77610704 \quad-0.38923952$

$\mathrm{H} \quad 1.64217974 \quad 1.14812432-0.10902144$

$\mathrm{H} \quad 0.40062640 \quad 1.81714760 \quad 0.96453669$

$\mathrm{H} 1.60561075-2.50957373 \quad 0.38870341$

$\mathrm{H} \quad 1.92233991-0.96109389-0.43836443$

$\mathrm{H}-0.90660528-2.06112581 \quad 0.38966670$

$\mathrm{H}-1.43780934 \quad 0.298815392 .57923828$

$\mathrm{H}-0.393379231 .40302926 \quad 3.48826773$

$\mathrm{H}-0.77161068-0.19802695 \quad 4.14029620$

H $2.14580014 \quad 0.90739775 \quad 3.62464855$

H $2.80976124 \quad-0.43907435 \quad 2.68775892$

$\mathrm{H} \quad 1.93192195-0.75674410 \quad 4.19029130$

$\begin{array}{llll}0 & -0.42543853 & 0.19795573 & -2.99289336\end{array}$

O $0.211145221 .26182156-2.30229061$

o $-0.41577871-0.86790160-2.06671734$

Vibrational frequencies (in $\mathrm{cm}-1$ )

$\begin{array}{lllllllllllllll}35.8 & 39.8 & 85.9 & 93.9 & 100.5 & 120.3 & 166.0 & 184.2 & 225.4 & 233.5 & 245.6 & 270.9 & 294.0 & 328.4\end{array}$ $353.3 \quad 363.0 \quad 427.5 \quad 459.4 \quad 505.0 \quad 553.6 \quad 590.0 \quad 615.4 \quad 705.8 \quad 733.8 \quad 765.5 \quad 876.6 \quad 902.4 \quad 955.0$

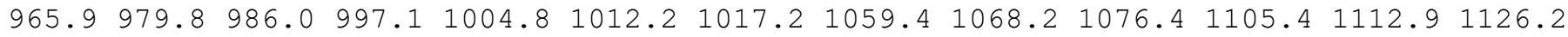

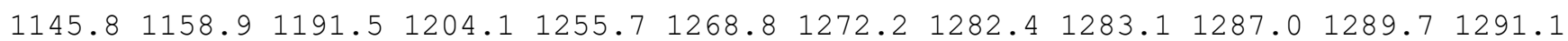

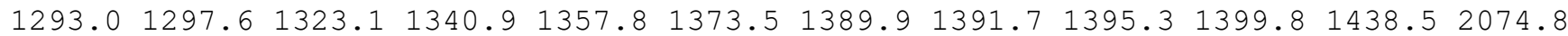
$2659.52669 .5 \quad 2669.7 \quad 2672.92673 .8 \quad 2673.92676 .32680 .12681 .5 \quad 2702.92735 .8 \quad 2778.0$ $2778.6 \quad 2780.12781 .3 \quad 2782.2$

\section{name $=$ MIN350}

energy $($ in $\mathrm{kcal} / \mathrm{mol})=8.35095$

$\mathrm{ZPE}(\mathrm{in} \mathrm{kcal} / \mathrm{mol})=143.846$

$\mathrm{G}(\mathrm{in} \mathrm{kcal} / \mathrm{mol})=117.099$

Geometry

C $-2.27432059-0.39991763-0.88165843$

C $-0.75609405-0.54803121-0.95320311$

C $-0.01444727 \quad 0.73297929-0.43389488$

$\begin{array}{lllll}\text { C } & 1.15228541 & 0.52642104 & 0.52702182\end{array}$

C $0.81121492 \quad 0.50782639 \quad 2.03295383$

C $0.69546550-2.56507853-0.82132019$

C $-0.31178491-1.83863749-0.34007433$

C -0.524298451 .117870512 .34332388$

C $-0.66468344 \quad 2.22279396 \quad 3.07448173$

C $0.83522402-0.91754590 \quad 2.60453482$

$\mathrm{H}-2.61254514 \quad 0.48429071-1.44031617$

$\mathrm{H}-2.62758951-0.30831817 \quad 0.15003303$

$\mathrm{H}-2.77520985-1.26788953-1.33243882$

$\mathrm{H}-0.72753920 \quad 1.50250737 \quad-0.06652033$

H $1.70893197 \quad-0.39312117 \quad 0.25633219$

$\mathrm{H} \quad 1.876507301 .352257890 .34240172$

H $1.61687547 \quad 1.09085964 \quad 2.55435467$

$\mathrm{H} \quad 1.00662370-3.50749024-0.39723985$ 
$\mathrm{H} \quad 1.28068092-2.27533528-1.68444661$

$\mathrm{H}-0.89486259-2.156170150 .52394264$

$\mathrm{H}-1.38250274 \quad 0.57288775 \quad 1.95242437$

$\mathrm{H} 0.16042579 \quad 2.78019377 \quad 3.48973479$

$\mathrm{H}-1.62135325 \quad 2.65560721 \quad 3.32132287$

H $0.70995954 \quad-0.90430271 \quad 3.69340798$

$\mathrm{H} 1.78354424-1.42024288 \quad 2.38694265$

$\mathrm{H} \quad 0.03134345-1.535780162 .19238162$

$0.71895744 \quad 0.21374633-2.49524830$

O $0.49815173 \quad 1.31906901-1.63700637$

$0-0.46169493-0.55700142-2.36338503$

Vibrational frequencies (in $\mathrm{cm}-1$ )

$\begin{array}{llllllllllllllll}-39.0 & 37.7 & 48.4 & 59.9 & 108.8 & 158.8 & 171.6 & 175.1 & 214.3 & 232.3 & 254.4 & 291.6 & 339.0 & 345.4\end{array}$

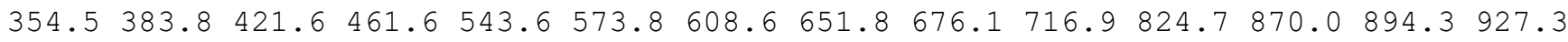
$961.6973 .8 \quad 981.2986 .9998 .9 \quad 1011.0 \quad 1027.3 \quad 1060.4 \quad 1072.9 \quad 1090.1 \quad 1095.5 \quad 1106.7 \quad 1126.3$

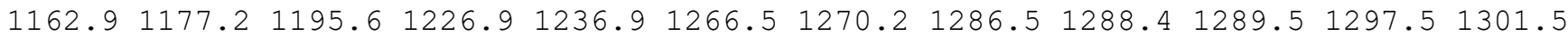

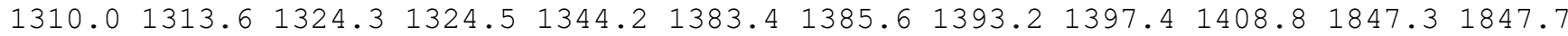
$2644.82668 .32669 .1 \quad 2675.5 \quad 2677.6 \quad 2678.8 \quad 2694.2 \quad 2709.82715 .32732 .92750 .02756 .0$ $2779.0 \quad 2781.12781 .6 \quad 2788.7$

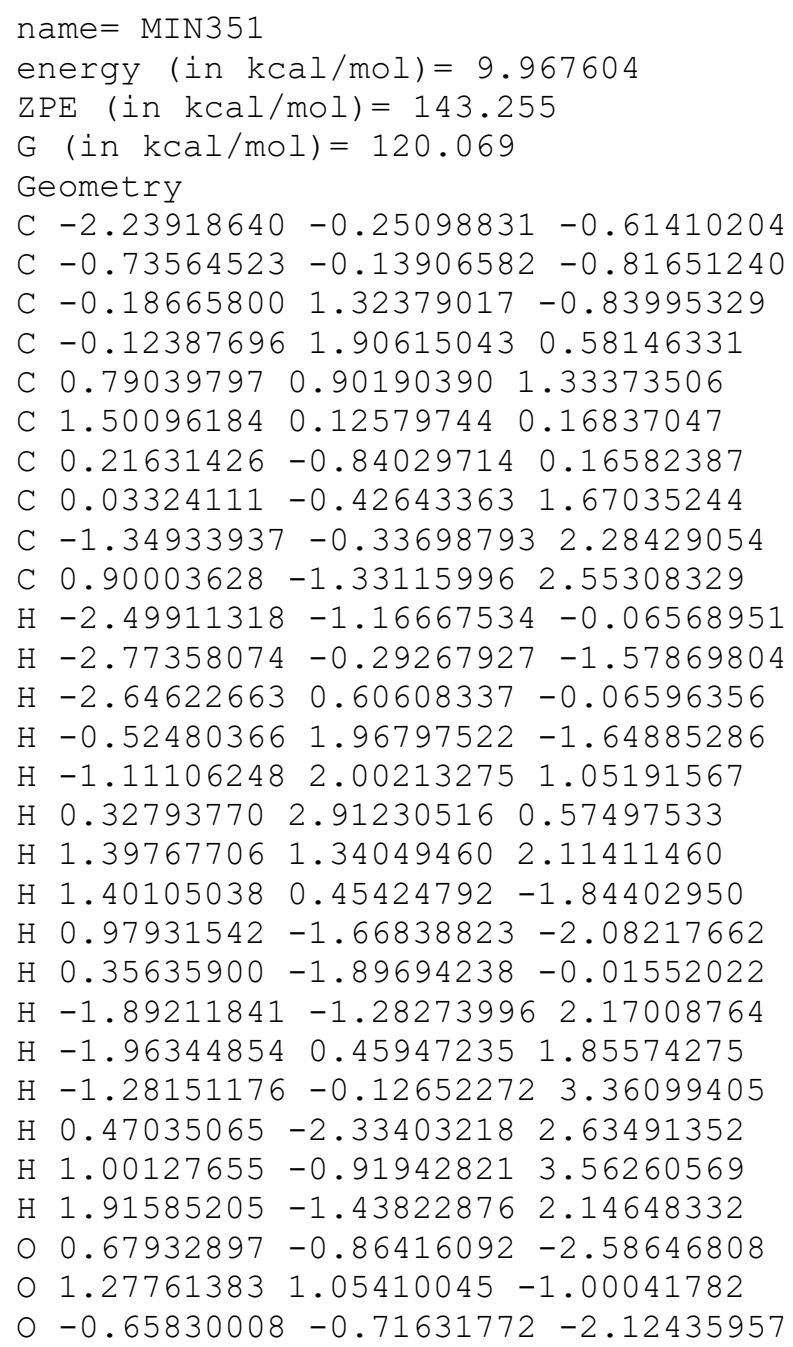


name $=$ MIN352

energy $($ in $\mathrm{kcal} / \mathrm{mol})=9.550387$

$\mathrm{ZPE}($ in $\mathrm{kcal} / \mathrm{mol})=143.746$

$\mathrm{G}($ in $\mathrm{kcal} / \mathrm{mol})=119.721$

Geometry

C $-2.41014106-0.55588915-0.62911153$

C $-0.90105197-0.48673088-0.66693545$

C $-0.16317743 \quad 0.92208979-0.55619978$

C $1.84425223 \quad 1.64245327 \quad 0.94526194$

C $0.87552965 \quad 0.58398373 \quad 0.55626786$

C $1.34229544 \quad-0.85701612 \quad 0.13107076$

C $-0.14483159-1.23793346 \quad 0.46094152$

C $-0.06775276-0.14541778 \quad 1.62605622$

C $-1.31451035 \quad 0.58307973 \quad 2.08594703$

C $0.65311947-0.65453566 \quad 2.86732228$

$\mathrm{H}-2.87011208 \quad 0.34097751 \quad-1.06296662$

$\mathrm{H}-2.79997973-0.692291420 .38554722$

$\mathrm{H}-2.77109200-1.41228949-1.22284427$

$\mathrm{H}-0.786495591 .80131048-0.35352402$

$\mathrm{H} \quad 2.37231124 \quad 2.04157938 \quad 0.06564220$

$\mathrm{H} 2.608241781 .261108291 .63628402$

$\mathrm{H} \quad 1.34744127 \quad 2.48672302 \quad 1.44168968$

H $2.09417469-1.30817401 \quad 0.77876031$

$\mathrm{H} \quad 1.68796186-0.99781608-0.88996190$

$\mathrm{H}-0.42536098-2.25112545 \quad 0.68143097$

$\mathrm{H}-1.852853351 .10586031 \quad 1.29062629$

$\mathrm{H}-1.060113891 .34651553 \quad 2.83462169$

$\mathrm{H}-2.02291930-0.11081178 \quad 2.55675260$

$\mathrm{H} \quad 1.00843505 \quad 0.18101738 \quad 3.48274873$

$\mathrm{H} 1.52580649-1.276176292 .63962281$

$\mathrm{H}-0.01582346-1.26526578 \quad 3.48580796$

O $0.53983138-0.14939965-2.41203869$

$0.367329371 .14393131-1.84086146$

- $-0.57137307-0.92507800-1.98264434$

Vibrational frequencies (in $\mathrm{cm}-1$ )

$93.3104 .5 \quad 126.9131 .2 \quad 154.4 \quad 183.2 \quad 201.2 \quad 207.7 \quad 259.5 \quad 272.2 \quad 291.5 \quad 331.2362 .2 \quad 379.8$

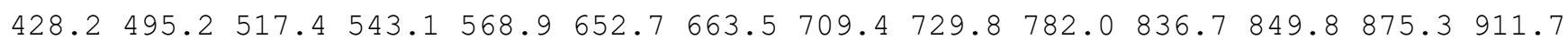
$942.8977 .8 \quad 986.2 \quad 997.2 \quad 1004.0 \quad 1007.5 \quad 1014.7 \quad 1026.0 \quad 1038.5 \quad 1060.8 \quad 1087.8 \quad 1113.7 \quad 1127.7$ $\begin{array}{llllllllllllllllll}1142.9 & 1171.1 & 1197.1 & 1203.2 & 1229.6 & 1233.8 & 1265.0 & 1275.1 & 1279.7 & 1283.3 & 1285.0 & 1285.5\end{array}$

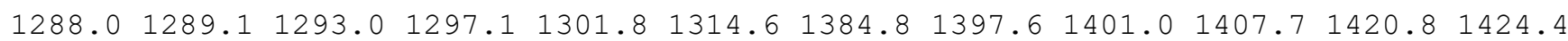
$2663.7 \quad 2667.3 \quad 2670.92673 .6 \quad 2674.0 \quad 2677.1 \quad 2678.4 \quad 2679.4 \quad 2682.4 \quad 2705.5 \quad 2767.02772 .1$ $2778.3 \quad 2780.12781 .12782 .4$

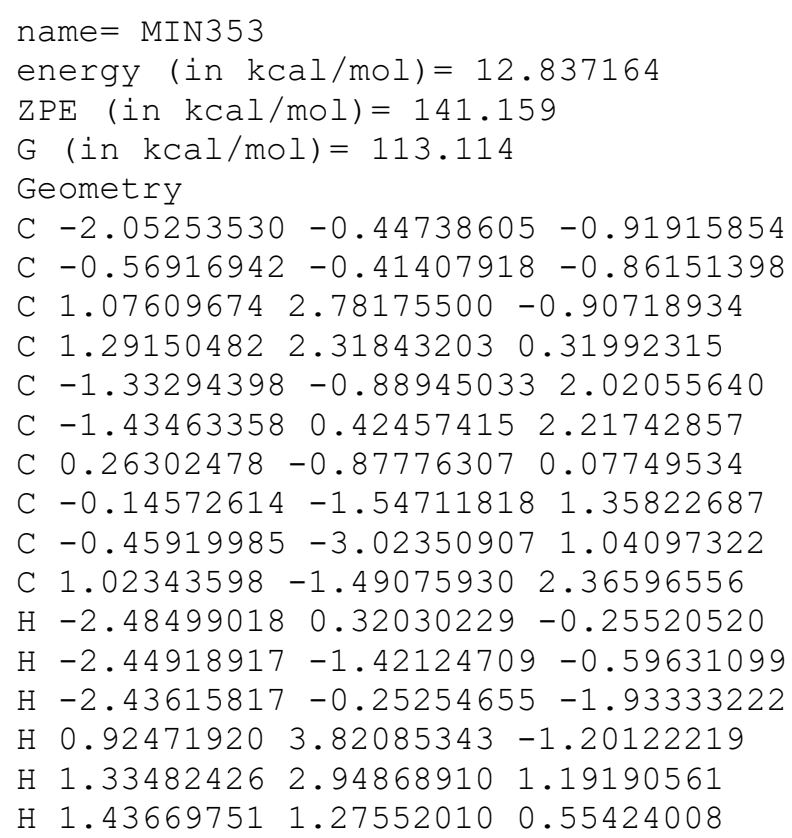


$\mathrm{H}-2.10466289-1.57762870 \quad 2.36227434$

$\mathrm{H}-2.26865359 \quad 0.88946506 \quad 2.71651314$

$\mathrm{H}-0.690519651 .136635161 .89194890$

$\mathrm{H} 1.34152573-0.80897126-0.06522132$

$\mathrm{H}-1.30927256-3.112945910 .35733862$

$\mathrm{H}-0.69300465-3.58788763 \quad 1.94852790$

$\mathrm{H} \quad 0.39732720 \quad-3.50953244 \quad 0.55955729$

$\mathrm{H} 0.74481293-1.95409102 \quad 3.31815427$

$\mathrm{H} 1.31165742-0.454806792 .57589070$

H $1.90476498-2.01626746 \quad 1.98552407$

O $1.16948058 \quad 0.68611923-1.89922299$

O $1.022759112 .07438798-2.07303655$

o $-0.11953310 \quad 0.14171105-2.04601960$

Vibrational frequencies (in $\mathrm{cm}-1$ )

$\begin{array}{llllllllllllllll}29.2 & 41.6 & 57.7 & 68.8 & 82.7 & 103.4 & 130.2 & 149.9 & 166.3 & 173.7 & 196.7 & 202.5 & 248.4 & 293.3\end{array}$ $295.8 \quad 347.2 \quad 364.6 \quad 419.4 \quad 432.4 \quad 477.7 \quad 519.3 \quad 544.4 \quad 590.7 \quad 616.2 \quad 625.8 \quad 673.9 \quad 812.3 \quad 847.7$ $914.2930 .7934 .9963 .5 \quad 974.2 \quad 979.8 \quad 991.4 \quad 1007.2 \quad 1015.4 \quad 1029.4 \quad 1053.9 \quad 1060.2 \quad 1067.5$

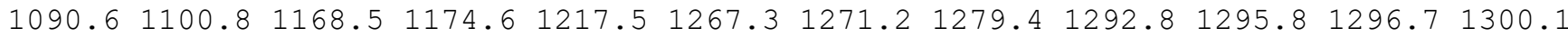

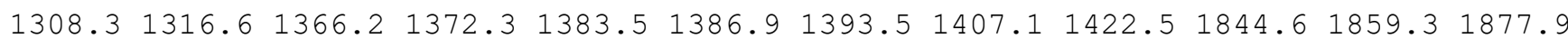
$2659.02668 .22676 .72678 .0 \quad 2680.5 \quad 2682.2 \quad 2708.92714 .02732 .6 \quad 2746.2 \quad 2753.42772 .3$ $2780.8 \quad 2782.0 \quad 2787.2 \quad 2791.9$

\author{
name $=$ MIN354 \\ energy (in $\mathrm{kcal} / \mathrm{mol})=12.172063$ \\ $\mathrm{ZPE}(\mathrm{in} \mathrm{kcal} / \mathrm{mol})=141.954$ \\ $\mathrm{G}($ in $\mathrm{kcal} / \mathrm{mol})=114.103$ \\ Geometry \\ C $-2.93893976-0.78948178 \quad 0.38406128$ \\ C $-1.44171445-0.75995733 \quad 0.44331511$ \\ C $-0.89352531 \quad 0.64166708 \quad 0.53666967$ \\ C 0.568236351 .000780310 .21167680 \\ C $1.53570075-0.10541399-0.07333458$ \\ C $1.59528554-0.72518778-1.25562549$ \\ C $-0.75927640-1.902249620 .51640820$ \\ C $2.48014745-0.408847521 .02065040$ \\ C $3.91955503-0.189411330 .68965078$ \\ C $2.07369560-0.82846608 \quad 2.22141259$ \\ $\mathrm{H}-3.38290396-0.28959682 \quad 1.25641288$ \\ $\mathrm{H}-3.34140747-1.809235890 .35250945$ \\ $\mathrm{H}-3.32694998-0.26340555-0.50481967$ \\ $\mathrm{H}-1.124028651 .00723025 \quad 1.57906959$ \\ H $0.960335651 .62258675 \quad 1.04959490$ \\ $\mathrm{H} \quad 0.57988288 \quad 1.70194363-0.65729372$ \\ $\mathrm{H}-1.36151120-0.25842567-2.06968096$ \\ $\mathrm{H} \quad 0.93588408-0.50877691-2.07933246$ \\ H 2.30427439 $-1.50544610-1.48399930$ \\ $\mathrm{H}-1.23655257-2.87112946 \quad 0.49642907$ \\ H $4.25956284 \quad-0.88947419-0.08764397$ \\ $\mathrm{H} \quad 4.57772238-0.32316147 \quad 1.55815838$ \\ H $4.09045775 \quad 0.82684020 \quad 0.30493653$ \\ $\mathrm{H} \quad 1.03798502-0.980718812 .48323139$ \\ $\mathrm{H} \quad 0.30877237-1.995596810 .60696148$ \\ H 2.74380518 $-1.05514460 \quad 3.03405817$ \\ O $-1.411953431 .54834039-1.55371076$ \\ $0-1.753319461 .51635899-0.20913040$ \\ o $-2.00673154 \quad 0.47955113-2.22688180$ \\ Vibrational frequencies (in $\mathrm{cm}-1$ )
}

$\begin{array}{llllllllllllllll}-50.7 & 19.4 & 39.6 & 67.0 & 97.4 & 107.9 & 123.1 & 147.4 & 170.6 & 188.4 & 250.8 & 277.3 & 290.0 & 329.2\end{array}$ $343.2 \quad 417.0 \quad 434.7 \quad 456.1 \quad 473.4 \quad 501.9 \quad 526.7 \quad 570.6 \quad 608.8 \quad 620.8 \quad 647.5 \quad 678.5 \quad 738.1 \quad 817.9$ $835.1874 .9 \quad 962.3 \quad 972.0 \quad 989.1 \quad 996.3 \quad 1005.3 \quad 1044.6 \quad 1050.6 \quad 1053.9 \quad 1059.2 \quad 1069.4 \quad 1082.7$

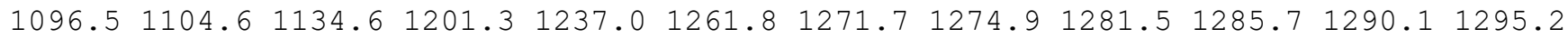

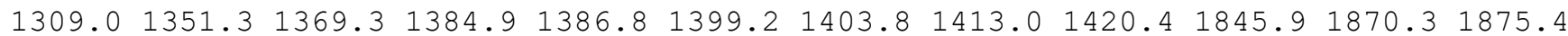
$2631.82653 .72657 .62669 .12675 .2 \quad 2676.12713 .72719 .92721 .72724 .52776 .62778 .2$ $2786.8 \quad 2790.4 \quad 2793.2 \quad 2827.2$ 
name $=$ MIN355

energy $($ in $\mathrm{kcal} / \mathrm{mol})=11.66913$

$\mathrm{ZPE}(\mathrm{in} \mathrm{kcal} / \mathrm{mol})=142.563$

$\mathrm{G}($ in $\mathrm{kcal} / \mathrm{mol})=114.484$

Geometry

C $-2.69995533-1.27646484-0.44506417$

C $-1.22022896-1.26355054-0.43992760$

C $-0.499243992 .42754502-0.43567823$

C $0.598049212 .43419842-1.18686085$

C $1.61625853-0.61828376 \quad 0.67798324$

C $1.07529513-2.000351990 .24674237$

C $-0.41410931-2.07712672 \quad 0.25724548$

C $1.07494640-0.20067713 \quad 2.01436368$

C $1.35403734 \quad 0.97624142 \quad 2.57554610$

C $3.15034309-0.666993220 .71482798$

$\mathrm{H}-3.11369914 \quad-2.15038179 \quad 0.07792318$

$\mathrm{H}-3.10224715-1.28797854-1.47056744$

$\mathrm{H}-3.10800305-0.37882572 \quad 0.04976890$

$\mathrm{H}-0.73454948 \quad 3.07414908 \quad 0.40806164$

$\mathrm{H} 1.41473086 \quad 3.11936096-1.03662909$

$\mathrm{H} 0.767078791 .75432810-2.00718556$

$\mathrm{H} \quad 1.30029777 \quad 0.14274717 \quad-0.08504398$

H $1.50437543-2.78081773 \quad 0.90784854$

$\mathrm{H} 1.45498713-2.23604952-0.77080149$

$\mathrm{H}-0.83540614 \quad-2.87369464 \quad 0.86536479$

$\mathrm{H} \quad 0.41541950 \quad-0.92182473 \quad 2.49615684$

$\mathrm{H} \quad 0.96300497 \quad 1.28619894 \quad 3.52997700$

$\mathrm{H} \quad 1.99705702 \quad 1.71597845 \quad 2.12768054$

H $3.50834008-1.34459111 \quad 1.49762416$

H $3.57075484 \quad 0.32353495 \quad 0.92146803$

H $3.56181969-1.00810174-0.24067362$

$0-1.437928520 .68970593-1.64162864$

$\begin{array}{lllll}0 & -1.57032245 & 1.58796642 & -0.56491836\end{array}$

$0-0.58350629-0.34545750-1.25864450$

Vibrational frequencies (in $\mathrm{cm}-1$ )

$\begin{array}{llllllllllllllll}28.1 & 38.2 & 48.5 & 68.6 & 87.7 & 102.1 & 108.6 & 139.2 & 170.8 & 179.7 & 193.4 & 227.2 & 244.2 & 296.5\end{array}$ $335.2 \quad 357.3 \quad 392.5 \quad 426.6 \quad 490.7 \quad 493.4 \quad 594.0 \quad 622.2 \quad 634.2 \quad 648.7 \quad 662.0 \quad 815.3 \quad 841.3 \quad 902.8$

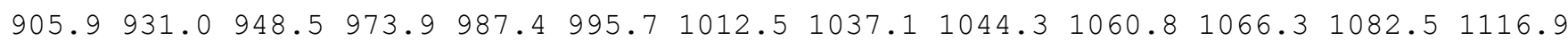
$1147.6 \quad 1169.2 \quad 1176.8 \quad 1222.2 \quad 1240.3 \quad 1269.6 \quad 1271.3 \quad 1277.8 \quad 1280.3 \quad 1290.2 \quad 1295.5 \quad 1302.4$ $1303.61320 .8 \quad 1354.0 \quad 1364.3 \quad 1374.7 \quad 1382.1 \quad 1389.9 \quad 1404.4 \quad 1408.8 \quad 1842.8 \quad 1854.4 \quad 1864.5$ $2657.0 \quad 2662.2 \quad 2671.0 \quad 2672.8 \quad 2679.4 \quad 2689.8 \quad 2714.12719 .72737 .12739 .92754 .7 \quad 2759.5$ $2777.9 \quad 2781.7 \quad 2792.6 \quad 2794.9$

\section{name $=$ MIN356}

energy (in $\mathrm{kcal} / \mathrm{mol})=12.008118$

$\mathrm{ZPE}(\mathrm{in} \mathrm{kcal} / \mathrm{mol})=142.502$

$\mathrm{G}(\mathrm{in} \mathrm{kcal} / \mathrm{mol})=114.102$

Geometry

C $-2.67725451-1.16038399-1.00577706$

C $-1.21360445-1.03355989-0.82324883$

C $0.47618803 \quad 2.23739645-0.81143574$

C $1.38347430 \quad 1.98747510-1.75151450$

C $1.40341834-1.03492360 \quad 1.69793165$

C $1.00670175-1.386596550 .24572906$

C $-0.45481412-1.640211230 .09926921$

C $0.60860411 \quad 0.14828048 \quad 2.17052357$

C $1.164013691 .30451345 \quad 2.53135262$

C $1.18142687-2.21458687 \quad 2.65256945$

$\mathrm{H}-2.91601470-1.73783502-1.91280631$

$\mathrm{H}-3.15461419-0.17291658-1.11842700$

$\mathrm{H}-3.16577170-1.65882409-0.15750661$

$\mathrm{H} \quad 0.60283687 \quad 2.82590680 \quad 0.09689622$ 
H $2.38977390 \quad 2.36962827-1.72105582$

H $1.19037325 \quad 1.38116684-2.62248796$

$\mathrm{H} 2.49618649-0.78785257 \quad 1.69582743$

$\mathrm{H} 1.57830202-2.27708154-0.08890401$

H $1.33816307-0.55677930-0.41935066$

$\mathrm{H}-0.87947533-2.36734278 \quad 0.78761476$

H $2.22557007 \quad 1.49323282 \quad 2.51919940$

$\mathrm{H} \quad 0.59449095 \quad 2.15300024 \quad 2.87637655$

$\mathrm{H}-0.47018579-0.007181552 .19218848$

H $0.12184697 \quad-2.478115712 .73648948$

$\mathrm{H} 1.53742080-1.97269543 \quad 3.66107396$

H $1.71988756-3.105797912 .31377895$

$\begin{array}{lllll}0 & -1.15172256 & 0.99691829 & -1.89492910\end{array}$

$0-0.81263768 \quad 1.78417631-0.77900811$

$0-0.54668076-0.25747800-1.76111914$

Vibrational frequencies (in cm-1)

$\begin{array}{lllllllllllllll}33.0 & 39.0 & 47.1 & 52.3 & 83.6 & 88.8 & 94.3 & 121.5 & 152.3 & 168.2 & 202.3 & 218.7 & 265.6 & 330.7\end{array}$ $\begin{array}{llllllllllllll}337.4 & 346.7 & 376.4 & 425.7 & 445.7 & 538.2 & 588.4 & 625.5 & 643.7 & 658.0 & 681.3 & 803.3 & 846.8 & 904.3\end{array}$

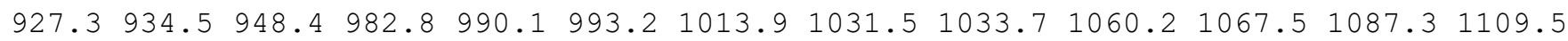

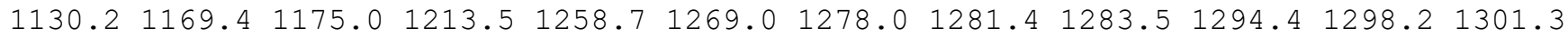

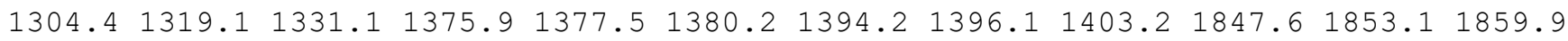
$2648.8 \quad 2663.92673 .1 \quad 2674.6 \quad 2679.8 \quad 2699.2 \quad 2712.02716 .82728 .6 \quad 2735.2 \quad 2756.0 \quad 2756.9$ $2778.5 \quad 2781.7 \quad 2789.2 \quad 2794.0$

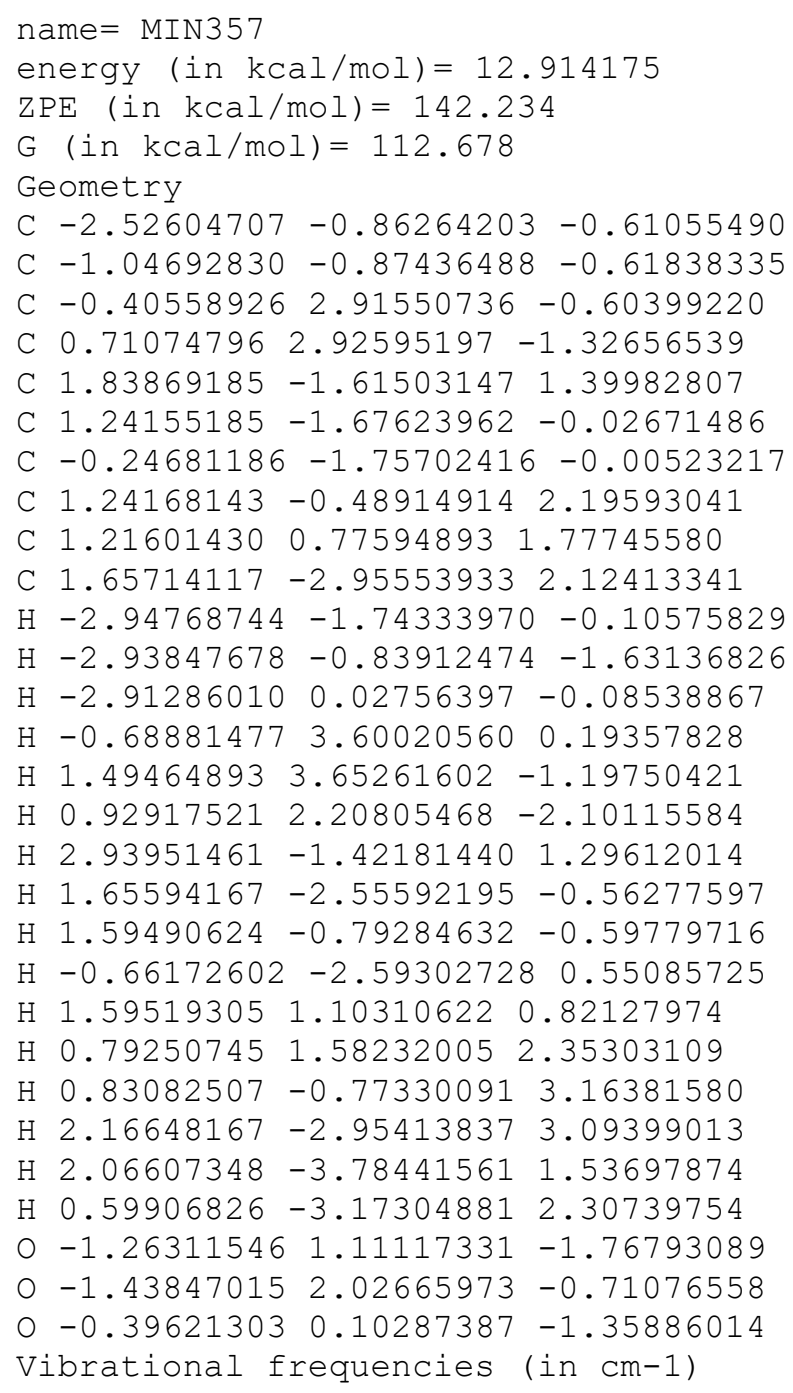


$2657.82662 .5 \quad 2672.2 \quad 2673.92680 .12694 .7 \quad 2713.8 \quad 2715.12734 .5 \quad 2737.5 \quad 2755.5 \quad 2762.6$ $2778.22781 .8 \quad 2788.2 \quad 2794.5$

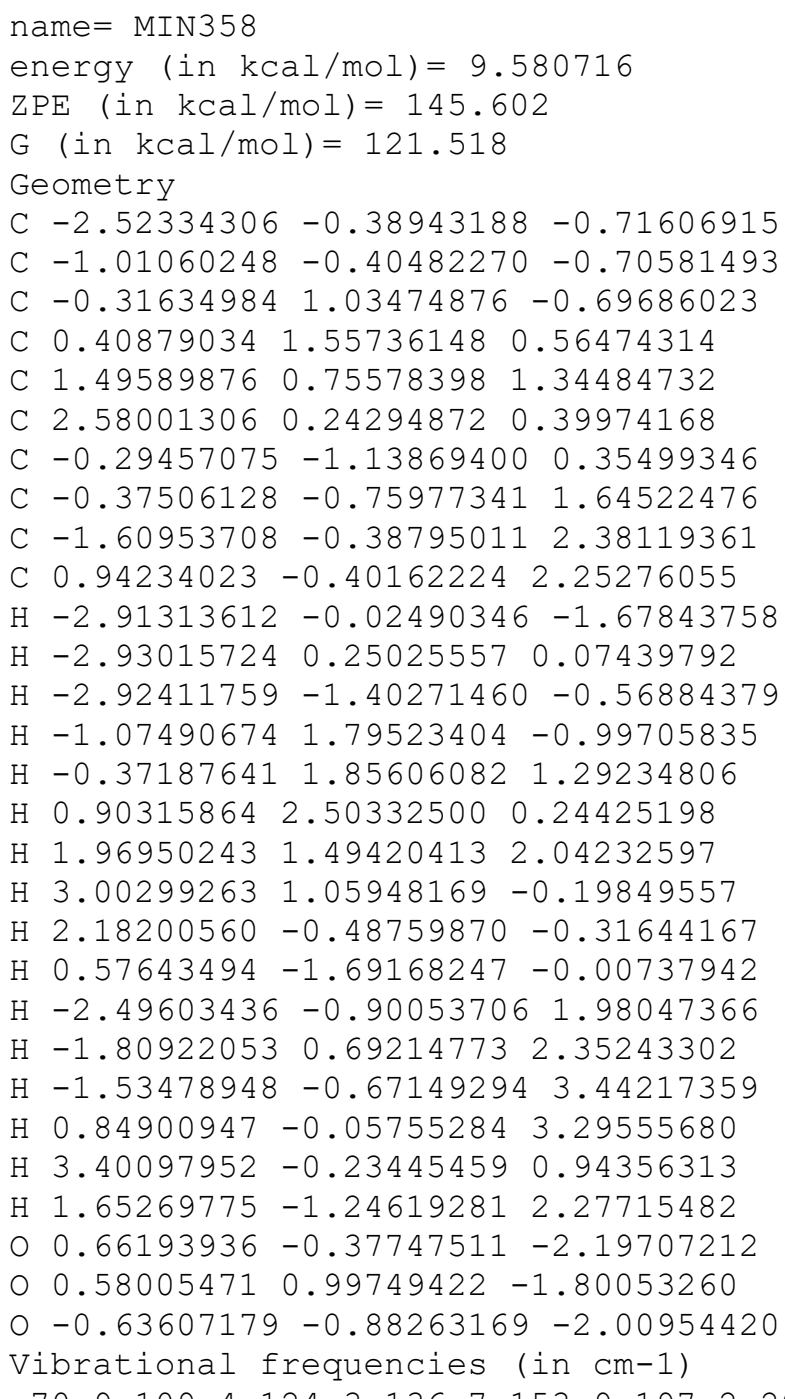

$\begin{array}{lllllllllllllll}70.9 & 109.4 & 124.3 & 136.7 & 153.9 & 197.2 & 202.6 & 234.4 & 240.7 & 281.5 & 305.7 & 343.0 & 350.8 & 400.3\end{array}$ $444.8 \quad 456.8 \quad 480.8 \quad 516.1 \quad 593.2 \quad 654.1 \quad 692.7 \quad 712.7 \quad 775.9 \quad 843.8 \quad 882.6 \quad 899.6 \quad 907.6 \quad 924.0$

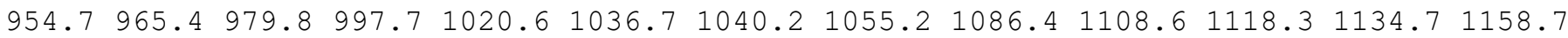
$1192.81206 .91235 .1 \quad 1268.3 \quad 1271.2 \quad 1274.4 \quad 1280.7 \quad 1284.0 \quad 1287.0 \quad 1292.7 \quad 1294.5 \quad 1297.6$

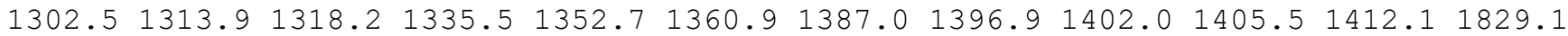
2650.52662 .12663 .52667 .32669 .12670 .12672 .02676 .32680 .82690 .92725 .52738 .9 2751.02777 .22778 .92781 .0

name $=$ MIN359

energy (in $\mathrm{kcal} / \mathrm{mol})=14.25991$

$\mathrm{ZPE}(\mathrm{in} \mathrm{kcal} / \mathrm{mol})=140.997$

$\mathrm{G}(\mathrm{in} \mathrm{kcal} / \mathrm{mol})=112.379$

Geometry

C $-2.64163998 \quad 0.20884624-0.73468916$

C $-1.15745698 \quad 0.13636909-0.72885545$

C $2.06596998 \quad 2.00485878-0.72434993$

C $2.86881020 \quad 0.94669473-0.62836267$

C $0.62149299-1.57080737 \quad 2.10709955$

C $1.43595251-2.42122031 \quad 1.48332379$

C $-0.30365300-0.370044290 .16231607$

C $-0.63950846-1.01016608 \quad 1.47878169$

$\begin{array}{llll}C & -1.24276004 & 0.05725450 & 2.41274407\end{array}$

C $-1.62834383-2.18079458 \quad 1.31843264$

$\mathrm{H}-2.99853183 \quad 1.03551079-0.10075637$

$\mathrm{H}-3.09551997-0.72310954-0.36554787$ 
$\mathrm{H}-3.046070910 .38287152-1.74626061$

$\mathrm{H} \quad 2.18720541 \quad 2.96886104 \quad-0.22976028$

$\mathrm{H} 3.74828354 \quad 0.92939950-0.00736189$

H $2.71907027 \quad 0.01753249-1.15066817$

$\mathrm{H} \quad 0.81603391-1.23725821 \quad 3.12433469$

H $1.27503984 \quad-2.77491806 \quad 0.47637515$

$\mathrm{H} \quad 2.32685091-2.82947731 \quad 1.93243751$

$\mathrm{H} \quad 0.77159738-0.30781303-0.03162805$

$\mathrm{H}-1.48824083-0.363045313 .39288585$

$\mathrm{H}-2.16134887 \quad 0.47943129 \quad 1.99376385$

$\mathrm{H}-0.541700150 .88587421 \quad 2.56391784$

$\mathrm{H}-2.64357033-1.83217840 \quad 1.11009048$

$\mathrm{H}-1.67274970 \quad-2.78438508 \quad 2.23199751$

$\mathrm{H}-1.32157674 \quad-2.84176452 \quad 0.49995273$

$00.60746078 \quad 0.84525608-2.06439099$

$\begin{array}{lllll}0 & 0.95356007 & 2.12157487 & -1.49636165\end{array}$

$\begin{array}{llllll}0 & -0.75989961 & 0.73826234 & -1.91732145\end{array}$

Vibrational frequencies (in cm-1)

$\begin{array}{lllllllllllllll}-53.1 & 20.2 & 50.3 & 57.9 & 67.5 & 85.7 & 126.4 & 132.0 & 156.3 & 175.2 & 192.1 & 216.2 & 236.3 & 280.6\end{array}$ $297.1354 .6 \quad 362.8 \quad 405.4 \quad 415.6 \quad 486.7 \quad 525.9 \quad 547.4 \quad 588.6 \quad 609.3 \quad 622.8 \quad 659.7 \quad 836.2 \quad 861.6$

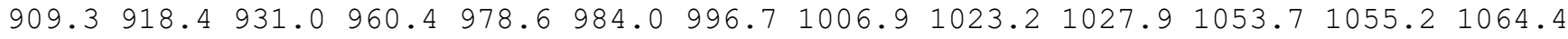
$1089.51104 .0 \quad 1187.6 \quad 1195.9 \quad 1218.8 \quad 1269.3 \quad 1269.8 \quad 1274.5 \quad 1287.3 \quad 1295.8 \quad 1297.5 \quad 1298.5$ $1308.01311 .4 \quad 1364.4 \quad 1377.4 \quad 1385.3 \quad 1386.7 \quad 1400.0 \quad 1408.01427 .4 \quad 1843.6 \quad 1848.11900 .1$ $2656.62662 .6 \quad 2674.2 \quad 2677.92679 .5 \quad 2681.8 \quad 2712.6 \quad 2716.32729 .12735 .82755 .12769 .4$ $2779.4 \quad 2782.2 \quad 2788.5 \quad 2797.8$

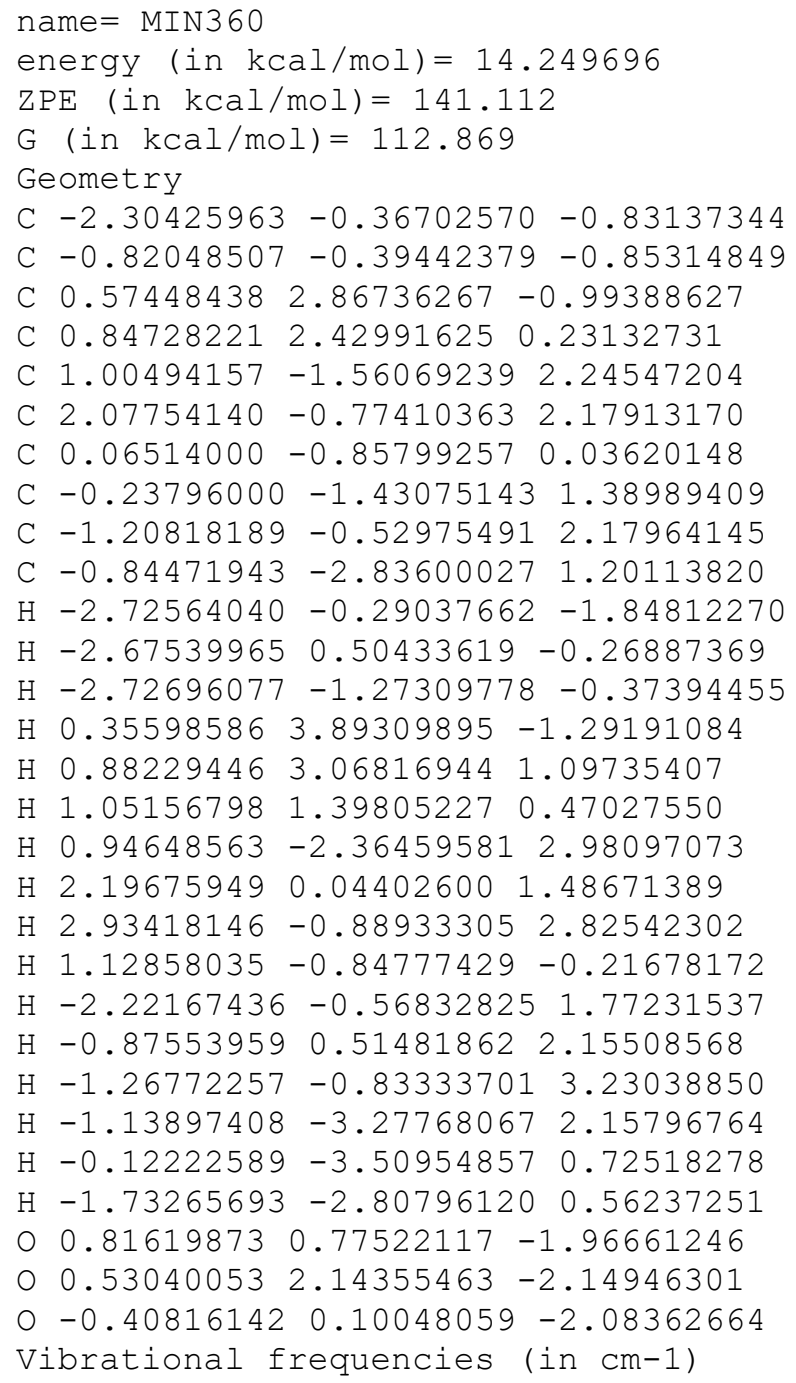




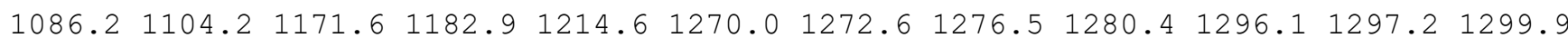
$1309.21310 .4 \quad 1370.7 \quad 1374.1 \quad 1387.4 \quad 1387.9 \quad 1400.0 \quad 1412.1 \quad 1419.7 \quad 1850.0 \quad 1859.6 \quad 1875.2$ $2662.02664 .82675 .02676 .62679 .5 \quad 2680.82707 .02709 .62732 .02734 .82750 .12771 .6$ $2780.5 \quad 2781.6 \quad 2785.5 \quad 2791.7$

\section{name $=$ MIN361}

energy $($ in $\mathrm{kcal} / \mathrm{mol})=13.091644$

$\mathrm{ZPE}($ in $\mathrm{kcal} / \mathrm{mol})=142.399$

$\mathrm{G}($ in $\mathrm{kcal} / \mathrm{mol})=112.733$

Geometry

C $-3.27360212-0.551223650 .42326062$

C $-1.78125093-0.52982041 \quad 0.43821372$

C $0.05978983 \quad 3.02765289 \quad 0.44409272$

C $-0.38585984 \quad 3.47122872 \quad-0.72781831$

C $1.08348369-1.71352860-0.35667547$

C $0.48518221-1.45584087 \quad 1.04892213$

C $-1.00400887-1.45024917 \quad 1.01796126$

C $2.35509197-0.92948669-0.50965709$

C $2.55537115-0.05861705-1.49792103$

C $1.38100643-3.20499218-0.55958989$

$\mathrm{H}-3.68029807-0.44674282 \quad 1.44031348$

$\mathrm{H}-3.65626482-1.49179796 \quad 0.00104298$

$\mathrm{H}-3.69882937 \quad 0.26926451 \quad-0.17715084$

$\mathrm{H} 0.372637693 .61398182 \quad 1.30538178$

$\mathrm{H}-0.49287912 \quad 4.51489370-0.96683288$

$\mathrm{H}-0.683527952 .82348434-1.53739264$

$\mathrm{H} \quad 0.33903380-1.39849767-1.13016052$

$\mathrm{H} \quad 0.86537449-0.49635083 \quad 1.45997705$

H $\quad 0.85538088-2.22351931 \quad 1.75776362$

$\mathrm{H}-1.46941775-2.292669691 .52940205$

H $3.10863872-1.125800590 .25157151$

H $1.831605290 .15633052 \quad-2.26795308$

H $3.46435308 \quad 0.50978913-1.61484330$

$\mathrm{H} 2.12041024 \quad-3.57373893 \quad 0.15867314$

H $1.77538578-3.39216471-1.56491129$

$\mathrm{H} \quad 0.47161775-3.80409972-0.44118795$

$\begin{array}{lllll}0 & -0.03831815 & 0.83495872 & -0.25643579\end{array}$

$\begin{array}{lllll}0 & 0.21558523 & 1.71962665 & 0.80975924\end{array}$

O $-1.40846653 \quad 0.57735101-0.29181114$

Vibrational frequencies (in $\mathrm{cm}-1$ )

$\begin{array}{lllllllllllllll}-13.1 & 34.2 & 40.1 & 47.3 & 55.3 & 66.1 & 80.4 & 103.7 & 155.2 & 164.4 & 173.9 & 247.8 & 287.2 & 295.0\end{array}$ $\begin{array}{llllllllllllllllll}328.1 & 366.1 & 379.7 & 429.4 & 479.1 & 521.7 & 556.4 & 619.2 & 638.7 & 645.4 & 662.0 & 807.3 & 862.1 & 894.1\end{array}$ $931.6938 .4 \quad 960.5 \quad 969.5 \quad 988.1 \quad 1001.9 \quad 1010.1 \quad 1022.1 \quad 1032.2 \quad 1058.5 \quad 1061.2 \quad 1087.5 \quad 1125.0$ $1143.51172 .41201 .5 \quad 1236.0 \quad 1245.3 \quad 1253.1 \quad 1273.4 \quad 1274.51282 .5 \quad 1300.91301 .01302 .1$ $1313.11320 .6 \quad 1350.91360 .4 \quad 1375.4 \quad 1379.8 \quad 1388.5 \quad 1398.01401 .5 \quad 1847.4 \quad 1854.2 \quad 1891.2$ $2654.62665 .0 \quad 2669.0 \quad 2675.1 \quad 2680.6 \quad 2698.7 \quad 2715.72717 .02738 .92741 .12757 .82760 .1$ $2775.4 \quad 2781.92789 .6 \quad 2795.5$

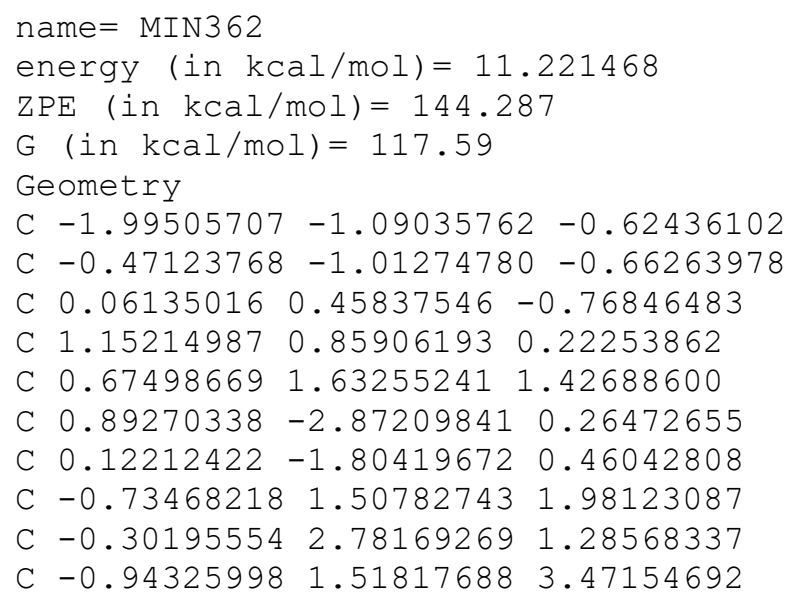


$\mathrm{H}-2.44347015-0.56051128-1.47564778$

$\mathrm{H}-2.40303484 \quad-0.66691863 \quad 0.29914930$

$\mathrm{H}-2.33290987-2.13487793-0.68614176$

$\mathrm{H}-0.77062402 \quad 1.19432440-0.79168155$

H $1.89997381 \quad 1.48271958-0.32380743$

H $\quad 1.72994404 \quad-0.02994284 \quad 0.55313851$

$\mathrm{H} 1.485133391 .75734654 \quad 2.16010669$

$\mathrm{H} \quad 1.31114450-3.46256977 \quad 1.06507412$

$\mathrm{H} 1.16387402-3.24044716-0.71687847$

$\mathrm{H}-0.14167439-1.444559251 .45484528$

$\mathrm{H}-0.70930231 \quad 3.03626048 \quad 0.31283962$

$\mathrm{H}-1.44293087 \quad 0.852549891 .46417140$

$\mathrm{H}-0.15015298 \quad 3.68764329 \quad 1.86403881$

$\mathrm{H}-0.801262390 .51811102 \quad 3.90082519$

$\mathrm{H}-1.96010773 \quad 1.847407393 .72445293$

$\mathrm{H}-0.24979245 \quad 2.19441778 \quad 3.98728539$

O $0.98359858-0.80830082-2.38154452$

$0.59404088 \quad 0.52535901-2.09418185$

$0-0.11348235-1.58259246-1.93533062$

Vibrational frequencies (in $\mathrm{cm}-1$ )

$\begin{array}{llllllllllllllll}26.2 & 42.9 & 51.3 & 65.5 & 116.6 & 142.5 & 154.1 & 185.5 & 219.0 & 234.7 & 274.4 & 305.5 & 326.5 & 344.0\end{array}$

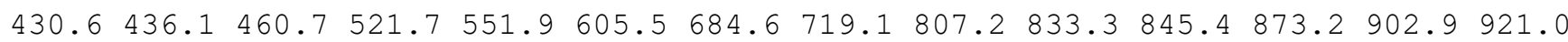
$952.3968 .6986 .2 \quad 986.5 \quad 1009.6 \quad 1022.0 \quad 1048.4 \quad 1056.8 \quad 1065.1 \quad 1095.9 \quad 1096.4 \quad 1100.2 \quad 1129.1$

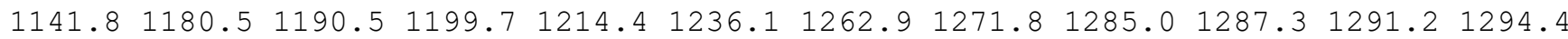
$1301.71320 .6 \quad 1330.2 \quad 1333.3 \quad 1345.3 \quad 1379.8 \quad 1381.6 \quad 1386.91394 .8 \quad 1397.2 \quad 1501.41849 .6$ $2644.02668 .82674 .5 \quad 2675.2 \quad 2676.2 \quad 2677.7 \quad 2708.82712 .62730 .92741 .5 \quad 2753.42757 .9$ $2778.12781 .2 \quad 2782.4 \quad 2789.8$

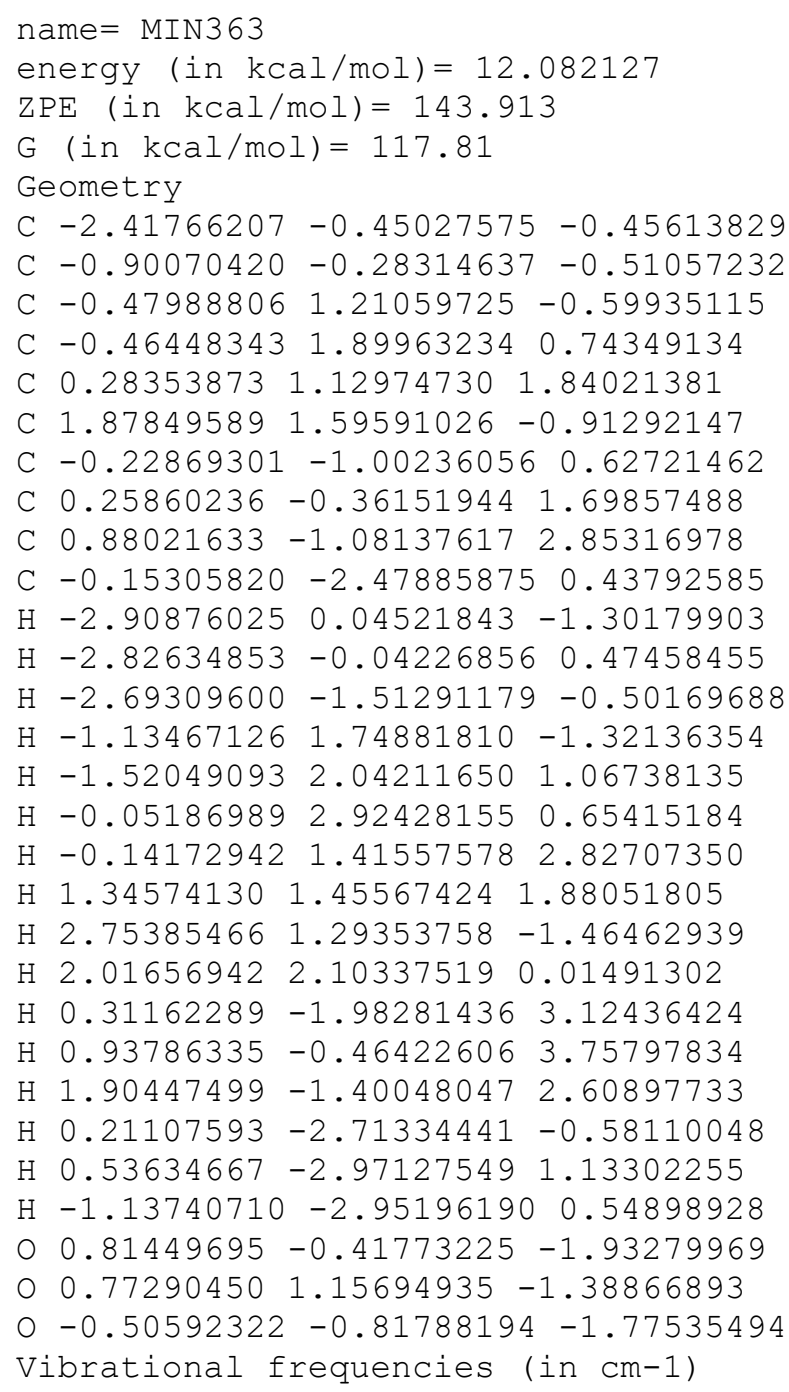


$\begin{array}{lllllllllllllllll}-25.5 & 39.8 & 74.1 & 87.9 & 120.2 & 150.4 & 165.8 & 224.2 & 250.7 & 294.5 & 300.9 & 328.5 & 336.2 & 343.8\end{array}$ $\begin{array}{llllllllllllllllll}374.1 & 410.4 & 429.1 & 468.3 & 503.9 & 549.5 & 565.1 & 608.4 & 684.8 & 711.1 & 761.5 & 821.3 & 888.3 & 916.2\end{array}$

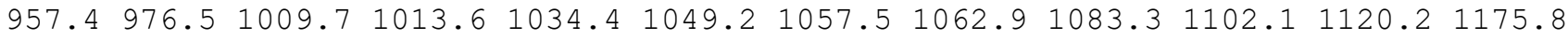

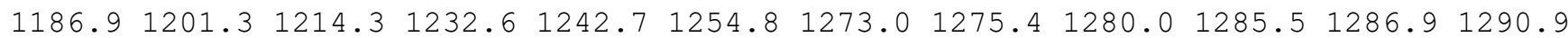

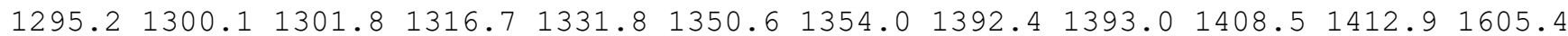

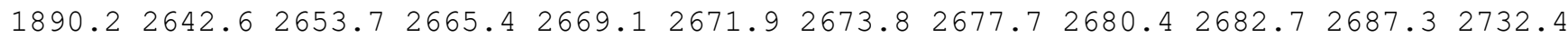
$2733.12736 .8 \quad 2774.8 \quad 2777.8 \quad 2780.7$

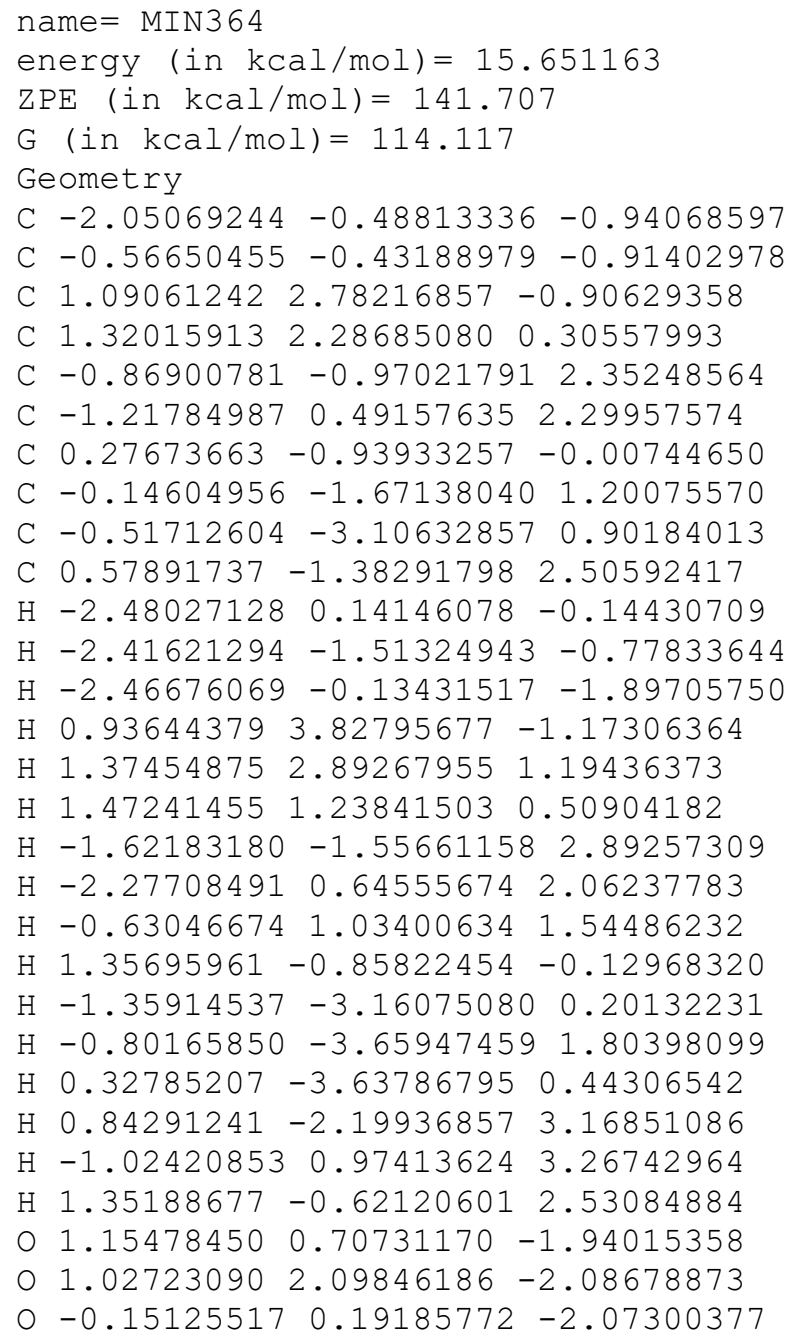

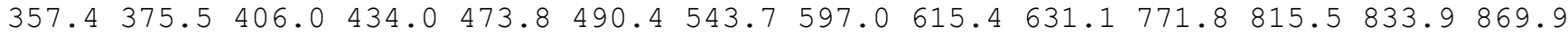
$915.1933 .0 \quad 942.6 \quad 956.4 \quad 980.0 \quad 1004.7 \quad 1010.0 \quad 1017.9 \quad 1030.2 \quad 1053.8 \quad 1061.4 \quad 1077.6 \quad 1091.1$ $1106.01146 .6 \quad 1168.6 \quad 1179.5 \quad 1223.1 \quad 1262.2 \quad 1270.6 \quad 1277.91281 .1 \quad 1290.1 \quad 1291.8 \quad 1295.5$ $1296.61304 .7 \quad 1365.4 \quad 1371.0 \quad 1375.4 \quad 1380.8 \quad 1391.2 \quad 1396.01414 .1 \quad 1503.7 \quad 1858.6 \quad 1880.9$ $2660.32666 .82668 .22675 .62677 .3 \quad 2678.5 \quad 2708.62718 .02732 .4 \quad 2750.42754 .12772 .7$ $2779.5 \quad 2780.92790 .7 \quad 2792.0$

name $=$ MIN365

energy (in $\mathrm{kcal} / \mathrm{mol})=14.559169$

$\mathrm{ZPE}(\mathrm{in} \mathrm{kcal} / \mathrm{mol})=143.034$

$\mathrm{G}($ in $\mathrm{kcal} / \mathrm{mol})=117.873$

Geometry

C $-2.71399600 \quad 0.74280335-0.43640966$

C $-1.18650838 \quad 0.73505431-0.47229539$

C $1.85091476 \quad 1.21093916-0.56840804$

C $1.67646248 \quad 0.57095864 \quad 0.71454939$

C $0.79160669-0.66208134 \quad 0.61037076$

C $1.60251878-1.87717581 \quad 0.22975328$

C $-0.62590819-0.56024004 \quad 0.05099944$ 
C $-0.42516964-0.81977196 \quad 1.53440685$

C $-0.74813253 \quad 0.24920873 \quad 2.54976919$

C $-0.76245263-2.19249773 \quad 2.06738273$

$\mathrm{H}-3.114663751 .67883533-0.84407622$

$\mathrm{H}-3.08371917 \quad 0.62630641 \quad 0.58759525$

$\mathrm{H}-3.12644880-0.07705985-1.03929954$

$\mathrm{H}-0.77165372 \quad 1.621410450 .05494201$

H $1.27348471 \quad 1.31002370 \quad 1.44957373$

H $2.66885593 \quad 0.292984561 .14980430$

H $2.27651785-2.18100283 \quad 1.03990143$

$\mathrm{H} \quad 0.97398724-2.74399900-0.01307241$

$\mathrm{H} 2.20811693-1.68150490-0.66681124$

$\mathrm{H}-0.95992652-1.40257995-0.57554916$

$\mathrm{H}-0.65302255 \quad 1.26752526 \quad 2.15657152$

$\mathrm{H}-0.09254107 \quad 0.16724940 \quad 3.42713851$

$\mathrm{H}-1.78354136 \quad 0.150682392 .90615405$

$\mathrm{H}-0.12781828-2.447495832 .92606931$

$\mathrm{H}-0.63221592-2.98380282 \quad 1.31959928$

$\mathrm{H}-1.80843585-2.23965784 \quad 2.39912391$

$\begin{array}{lllll}0 & 0.27940500 & 0.31781298 & -2.20876489\end{array}$

O $1.46109611 \quad 1.28375026-1.67296213$

$0-0.880896220 .92843385-1.87126403$

Vibrational frequencies (in $\mathrm{cm}-1$ )

$\begin{array}{llllllllllllllll}77.0 & 98.7 & 110.7 & 119.8 & 140.4 & 144.7 & 152.0 & 171.1 & 207.4 & 248.3 & 255.3 & 267.9 & 291.1 & 323.7\end{array}$ $391.3 \quad 415.5 \quad 433.8 \quad 456.4 \quad 485.2 \quad 520.2 \quad 533.3 \quad 569.6 \quad 585.3 \quad 647.1 \quad 775.8 \quad 787.2 \quad 895.8 \quad 931.9$ $960.2986 .2993 .91006 .3 \quad 1009.6 \quad 1017.7 \quad 1032.6 \quad 1055.0 \quad 1069.4 \quad 1101.4 \quad 1117.01135 .4 \quad 1144.5$

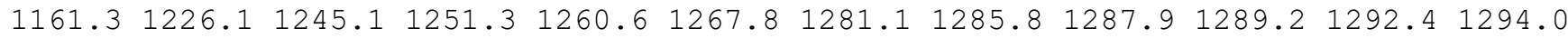
$1296.91297 .91305 .3 \quad 1312.8 \quad 1332.0 \quad 1362.4 \quad 1392.1 \quad 1397.31401 .3 \quad 1407.91513 .01934 .6$ $2634.82665 .8 \quad 2667.12668 .0 \quad 2672.6 \quad 2673.6 \quad 2676.5 \quad 2678.22680 .92684 .42728 .22742 .0$ $2777.92779 .2 \quad 2780.5 \quad 2781.8$
name $=$ MIN366
energy $($ in $\mathrm{kcal} / \mathrm{mol})=12.453321$
$\mathrm{ZPE}($ in $\mathrm{kcal} / \mathrm{mol})=145.512$
$\mathrm{G}($ in $\mathrm{kcal} / \mathrm{mol})=121.353$
Geometry
C $-2.39044633-0.11699085-0.24860594$
C $-0.88165529-0.07445684-0.35090599$
C $-0.297033351 .30188250-0.33617532$
C $1.18123503 \quad 1.44472804 \quad-0.64782592$
C $0.85801546 \quad 0.02153722 \quad 1.46581711$
C $0.55249667-2.03415840-0.06907293$
C $-0.13892959-0.90808451 \quad 0.71732450$
C 0.732208261 .393598850 .79714074
C $0.85333017 \quad 2.619245691 .64639718$
C $0.55271016 \quad 0.04173077 \quad 2.96195975$
$\mathrm{H}-2.87947320 \quad 0.45370941 \quad-1.04947382$
$\mathrm{H}-2.72665163 \quad 0.32331703 \quad 0.70196717$
$\mathrm{H}-2.77760061-1.14618094-0.27824719$
$\mathrm{H}-0.96936819 \quad 2.12079549-0.57536644$
H $1.536293092 .37899498-1.07348643$
$\mathrm{H} \quad 1.73258454 \quad 0.61540355-1.08561926$
$\mathrm{H} 1.90320055-0.346731331 .32659856$
$\mathrm{H} \quad 1.60080801-1.79791074-0.32739117$
$\mathrm{H} \quad 0.52034815-3.01049777 \quad 0.44575606$
$\mathrm{H}-0.86671942-1.340145451 .43613240$
$\mathrm{H} \quad 0.06357589 \quad 2.66439041 \quad 2.40858550$
$\mathrm{H} \quad 0.77718228 \quad 3.54031706 \quad 1.05279082$
H $1.821067312 .64849716 \quad 2.16597364$
$\mathrm{H} \quad 1.27374165 \quad 0.66168177 \quad 3.50807263$
$\mathrm{H} \quad 0.60466518-0.96649543 \quad 3.39015991$
$\mathrm{H}-0.44720306 \quad 0.43957799 \quad 3.16771302$
o $-0.69185068-0.79165983-1.69646435$
O $0.02397344-0.24285085-2.47343883$ 
$0-0.17425316-2.20186738-1.24505288$

Vibrational frequencies (in $\mathrm{cm}-1$ )

$\begin{array}{lllllllllllllllll}37.4 & 109.9 & 129.8 & 170.4 & 182.1 & 193.1 & 225.1 & 228.2 & 250.1 & 292.7 & 318.4 & 333.9 & 382.1 & 415.0\end{array}$ $436.0 \quad 490.6 \quad 530.7 \quad 542.5 \quad 566.8 \quad 631.4 \quad 669.2 \quad 728.5 \quad 781.7 \quad 803.2 \quad 859.7 \quad 863.2904 .1933 .3$ $975.1993 .8 \quad 1001.5 \quad 1010.1 \quad 1050.2 \quad 1063.5 \quad 1091.6 \quad 1104.9 \quad 1110.6 \quad 1112.01126 .91156 .1$

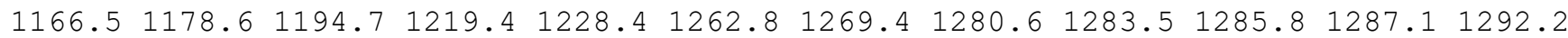
$1302.91306 .8 \quad 1312.4 \quad 1319.1 \quad 1324.6 \quad 1331.1 \quad 1339.1 \quad 1356.4 \quad 1372.0 \quad 1390.2 \quad 1399.1 \quad 1405.0$ $1477.12641 .52664 .5 \quad 2668.3 \quad 2673.12674 .12675 .42676 .52697 .2 \quad 2707.22711 .42716 .2$ $2769.6 \quad 2775.7 \quad 2780.5 \quad 2781.5 \quad 2784.4$

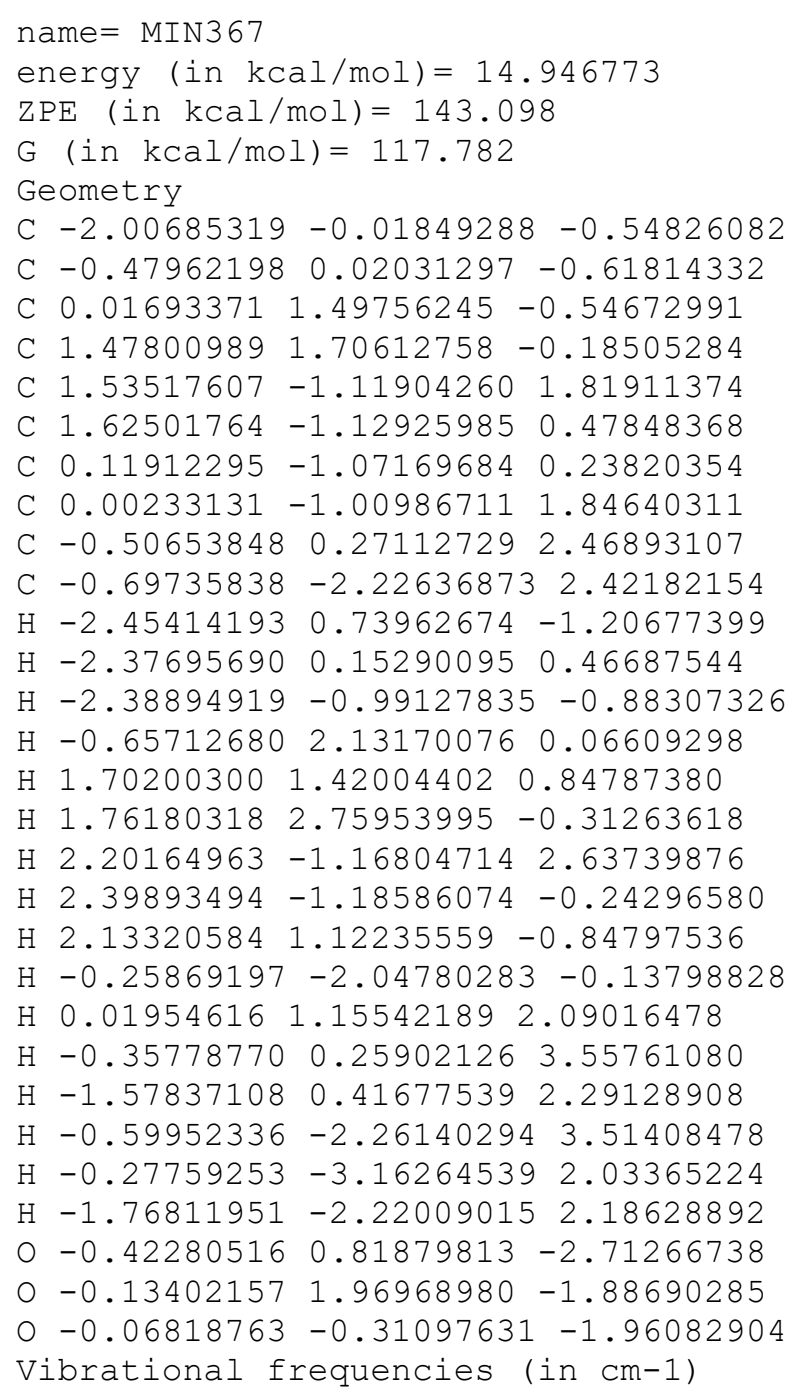


C $1.14146276-2.51192531-0.36542261$

C $0.16530622-1.752516820 .12751549$

C $0.43052364 \quad 0.62562225 \quad 2.66075431$

C $1.69589813 \quad 1.45258867 \quad 2.60380164$

C $-0.91248591 \quad 1.29824173 \quad 2.61671672$

$\mathrm{H}-2.46426859-0.869160350 .51720500$

$\mathrm{H}-2.37542055-2.09519433-0.75732145$

$\mathrm{H}-2.72553925-0.41877238-1.17976125$

$\mathrm{H}-1.14381510 \quad 1.24645506 \quad 0.02575709$

$\mathrm{H} 1.12097425 \quad 2.21161720 \quad 0.11005122$

$\mathrm{H} \quad 1.84028598 \quad 0.78376909-0.62549487$

H $1.93797806-0.39775101 \quad 1.37093648$

$\mathrm{H} \quad 1.69951322-3.21889750 \quad 0.23046746$

$\mathrm{H} 1.46078538-2.48987145-1.39868087$

$\mathrm{H}-0.13300212-1.79237714 \quad 1.17711005$

$\mathrm{H} \quad 0.42750317 \quad-0.24475270 \quad 3.33207704$

H 2.53095089 $1.21360639 \quad 3.25362417$

$\mathrm{H} 1.62635495 \quad 2.52815768 \quad 2.47243203$

$\mathrm{H}-1.22924955 \quad 1.59959748 \quad 3.62516225$

$\mathrm{H}-1.68332903 \quad 0.62332718 \quad 2.22200120$

$\mathrm{H}-0.90850650 \quad 2.20507930 \quad 1.99903310$

$0-0.723025610 .19271602-2.68598036$

$0-0.27486861 \quad 1.25064559-1.83076763$

o $-0.28604141-0.99080695-2.06743408$

Vibrational frequencies (in $\mathrm{cm}-1$ )

$\begin{array}{lllllllllllllll}17.9 & 38.4 & 69.1 & 92.5 & 134.0 & 163.5 & 179.5 & 199.9 & 218.7 & 225.2 & 272.6 & 295.6 & 298.6 & 351.7\end{array}$ $385.4 \quad 440.9 \quad 461.1 \quad 530.8 \quad 556.2 \quad 614.5 \quad 671.9 \quad 711.7 \quad 805.9 \quad 816.8 \quad 834.8 \quad 883.7 \quad 898.9 \quad 939.8$ $963.1976 .6 \quad 989.4 \quad 993.3 \quad 1010.3 \quad 1018.2 \quad 1060.0 \quad 1063.5 \quad 1076.1 \quad 1094.4 \quad 1096.5 \quad 1111.91120 .4$

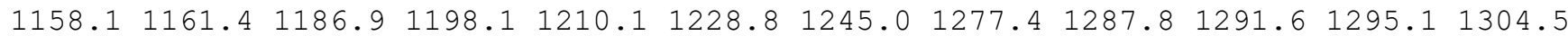

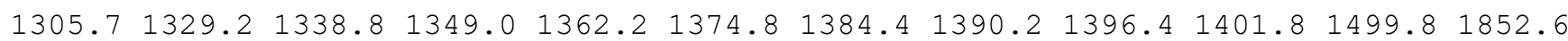
$2648.72669 .7 \quad 2672.6 \quad 2674.5 \quad 2678.4 \quad 2681.8 \quad 2708.7 \quad 2714.22734 .12738 .92749 .4 \quad 2752.7$ $2779.42779 .6 \quad 2781.8 \quad 2790.6$

name $=$ MIN369

energy $($ in $\mathrm{kcal} / \mathrm{mol})=15.104303$

$\operatorname{ZPE}(\mathrm{in} \mathrm{kcal} / \mathrm{mol})=143.216$

$\mathrm{G}($ in $\mathrm{kcal} / \mathrm{mol})=117.798$

Geometry

C $-2.54750334-0.65539263-0.80203327$

C $-1.04979087-0.60427972-0.77776894$

C $-0.21375049 \quad 0.77292412 \quad-0.76018423$

C 2.320217691 .873921421 .12109138

C $0.57800212 \quad 0.24292614 \quad 0.45734596$

C $2.04195494 \quad 0.48104843 \quad 0.54824906$

C $-0.25990252-1.061216960 .45639188$

C $-0.32607193-0.09939498 \quad 1.63622071$

C $-1.53763995 \quad 0.73520315 \quad 1.96303506$

C $0.35032788-0.59658425 \quad 2.89673967$

$\mathrm{H}-2.97510917 \quad 0.17480852-1.38013847$

$\mathrm{H}-2.97730249-0.63196121 \quad 0.20770503$

$\mathrm{H}-2.90210925-1.58719361-1.27337715$

$\mathrm{H}-0.71153919 \quad 1.73657743-0.69990742$

$\mathrm{H} 1.83120315 \quad 2.00882742 \quad 2.09242916$

H $1.944705192 .65804706 \quad 0.45348073$

H $3.39349038 \quad 2.03954147 \quad 1.25907563$

$\mathrm{H} 2.53188936-0.292897531 .17284530$

$\mathrm{H} \quad 2.50783025 \quad 0.38590298 \quad-0.45774971$

$\mathrm{H} \quad 0.04740605 \quad-2.08321519 \quad 0.55053840$

$\mathrm{H}-2.31893328 \quad 0.126943292 .43838735$

$\mathrm{H}-1.98376951 \quad 1.21796308 \quad 1.08689661$

$\mathrm{H}-1.27569892 \quad 1.539875112 .66481223$

$\mathrm{H} \quad 0.77319232 \quad 0.23665197 \quad 3.47241600$

H $1.17052475-1.294699912 .68771857$

$\mathrm{H}-0.36333819-1.123425323 .54307018$ 
$0.66355431-0.64905104-2.24757342$

O $0.50774751 \quad 0.74399538-1.96292404$

- $-0.60514695-1.22522367-1.97347994$

Vibrational frequencies (in $\mathrm{cm}-1$ )

$\begin{array}{lllllllllllllll}54.4 & 61.5 & 93.2 & 128.0 & 135.3 & 144.4 & 156.4 & 194.2 & 198.8 & 231.5 & 259.1 & 286.5 & 304.9 & 350.8\end{array}$ $369.6 \quad 445.9 \quad 450.9 \quad 485.6 \quad 526.3 \quad 624.1 \quad 666.6 \quad 702.3 \quad 738.8 \quad 764.9 \quad 828.2 \quad 838.9 \quad 895.0 \quad 942.3$ $969.0 \quad 974.5 \quad 986.5 \quad 993.0 \quad 1007.0 \quad 1013.1 \quad 1016.5 \quad 1046.4 \quad 1059.1 \quad 1082.3 \quad 1117.4 \quad 1124.4 \quad 1143.8$ $1154.41157 .6 \quad 1196.5 \quad 1209.3 \quad 1266.6 \quad 1275.0 \quad 1278.3 \quad 1279.4 \quad 1282.8 \quad 1291.91295 .9 \quad 1297.8$

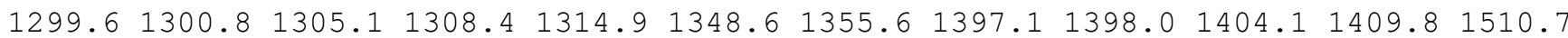
2655.32664 .62670 .92673 .42674 .12676 .02678 .52681 .22685 .22724 .12741 .22778 .3 $2779.92781 .12782 .7 \quad 2785.1$

name $=$ MIN370

energy $($ in $\mathrm{kcal} / \mathrm{mol})=14.984161$

$\mathrm{ZPE}($ in $\mathrm{kcal} / \mathrm{mol})=143.758$

$\mathrm{G}($ in $\mathrm{kcal} / \mathrm{mol})=119.322$

Geometry

C $-2.38498074-0.32919521 \quad 0.26201813$

C $-0.89131178-0.291564690 .24551036$

C -0.240803991 .088463520 .37547775$

C $1.19070138 \quad 0.94380847-0.19337793$

C $1.39573422-0.56154835-0.37136897$

C $-0.02775203-1.14450650-0.66962925$

C $-0.16171755-1.52499872 \quad 0.80581534$

C $1.35300821-1.31164024 \quad 1.03688854$

C $1.75153833-0.552093032 .28361412$

C $2.10696649-2.634503910 .95889699$

$\mathrm{H}-2.82645983 \quad 0.51274847 \quad-0.29925957$

$\mathrm{H}-2.76738202-0.25375175 \quad 1.29035191$

$\mathrm{H}-2.78657088-1.25358595-0.17032982$

$\mathrm{H}-0.26819693 \quad 1.47075727 \quad 1.41932361$

H $1.94693353 \quad 1.38801668 \quad 0.47258390$

H $1.29274997 \quad 1.47270131-1.15697839$

H 2.20794441 -0.84638458 -1.03308319

$\mathrm{H}-0.29407705-1.66178927-1.55620623$

$\mathrm{H}-1.32594068 \quad 0.36413484 \quad-2.22586226$

$\mathrm{H}-0.68974066-2.330218551 .27079715$

$\mathrm{H} 1.69458337-1.198000943 .17024306$

$\mathrm{H} \quad 1.09711188 \quad 0.30318043 \quad 2.48336514$

$\mathrm{H} 2.78041875-0.179494192 .21389538$

H $3.19021892-2.470609430 .93137849$

H $1.83615920-3.20608330 \quad 0.06314072$

$\mathrm{H} \quad 1.88849426-3.269253351 .82538995$

$\begin{array}{lllll}0 & -0.81310380 & 2.07952297 & -1.65751392\end{array}$

O $-0.99927027 \quad 2.09194983-0.28083881$

O $-1.76495200 \quad 1.25334326-2.25682733$

Vibrational frequencies (in $\mathrm{cm}-1$ )

$\begin{array}{llllllllllllllll}69.0 & 82.9 & 127.7 & 142.4 & 148.9 & 164.7 & 175.9 & 221.4 & 237.8 & 264.4 & 306.9 & 352.4 & 367.6 & 415.6\end{array}$ $429.5 \quad 437.6 \quad 493.8 \quad 511.7 \quad 558.2 \quad 600.5 \quad 655.0 \quad 706.1 \quad 762.4 \quad 795.6 \quad 827.3 \quad 880.7 \quad 898.1921 .7$ $936.6966 .5972 .9993 .3 \quad 1007.5 \quad 1012.0 \quad 1024.6 \quad 1043.3 \quad 1051.4 \quad 1070.6 \quad 1097.1 \quad 1103.91106 .3$

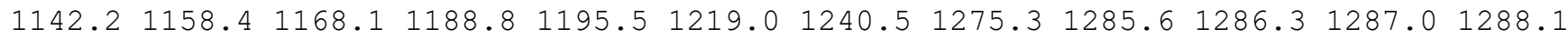
$1295.91298 .41301 .91302 .6 \quad 1323.2 \quad 1334.0 \quad 1346.6 \quad 1356.7 \quad 1396.5 \quad 1399.5 \quad 1407.7 \quad 1459.9$ $2654.92664 .72671 .92677 .2 \quad 2677.8 \quad 2678.2 \quad 2681.72688 .32749 .32756 .32777 .22781 .3$ $2782.8 \quad 2787.7 \quad 2799.7 \quad 2831.7$

name $=$ MIN371

energy $($ in $\mathrm{kcal} / \mathrm{mol})=15.59609$

$\operatorname{ZPE}($ in $\mathrm{kcal} / \mathrm{mol})=143.184$

$\mathrm{G}($ in $\mathrm{kcal} / \mathrm{mol})=117.576$

Geometry

C $-2.03444530 \quad 0.08475451-0.78190158$

C $-0.50771060 \quad 0.11707607-0.75196991$

C $0.02443098 \quad 1.59220611-0.76047692$ 
C $0.90577310 \quad 2.05976175 \quad 0.37929167$

C $1.10600463-0.850859652 .04901647$

C $1.47003952-0.74479824 \quad 0.76005183$

C $0.06026813-0.915065950 .20304479$

C $-0.39090682-1.02691270 \quad 1.74627066$

C $-1.28510609 \quad 0.04924774 \quad 2.31762385$

C $-0.91006582-2.413146792 .08957614$

$\mathrm{H}-2.415341350 .45092914 \quad-1.74714500$

$\mathrm{H}-2.42156750-0.93310538-0.65077778$

$\mathrm{H}-2.46959932 \quad 0.707606920 .00656061$

$\mathrm{H}-0.82256634 \quad 2.30060579-0.91712814$

$\mathrm{H} \quad 1.00691958 \quad 3.15294503 \quad 0.37298729$

$\mathrm{H} 0.514593821 .765864021 .36064866$

$\mathrm{H} \quad 1.58751527-0.84620873 \quad 2.98885167$

H $2.37247847 \quad-0.62058678 \quad 0.21850396$

$\mathrm{H} \quad 1.92342598 \quad 1.65256992 \quad 0.29503888$

$\mathrm{H}-0.05417319-1.90824389-0.29018652$

$\mathrm{H}-2.33877453-0.123589652 .06639696$

$\mathrm{H}-1.01826841 \quad 1.05062373 \quad 1.95860607$

$\mathrm{H}-1.21342058 \quad 0.06978704 \quad 3.41359992$

$\mathrm{H}-1.03064887-2.534254913 .17290342$

$\mathrm{H}-0.22412856-3.201114721 .75379093$

$\mathrm{H}-1.88377625-2.603305421 .62398711$

o $1.06689470 \quad 0.35951953 \quad-2.34811179$

O $0.765645691 .70055444-1.98292228$

o- $0.12731874-0.33440121-2.07764052$

Vibrational frequencies (in $\mathrm{cm}-1$ )

$\begin{array}{lllllllllllllllll}17.8 & 81.6 & 113.6 & 135.8 & 150.0 & 172.0 & 180.6 & 188.6 & 227.7 & 242.0 & 277.4 & 285.4 & 315.9 & 321.8\end{array}$

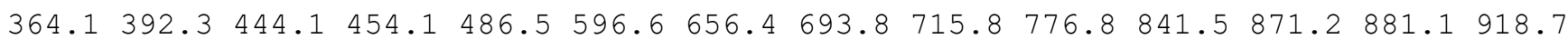

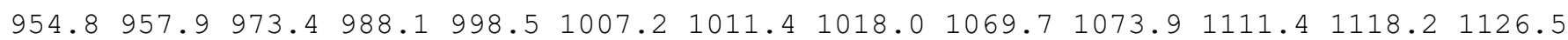

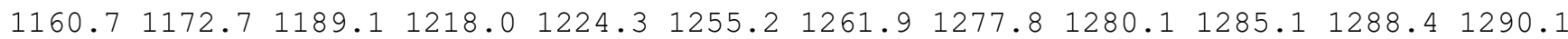

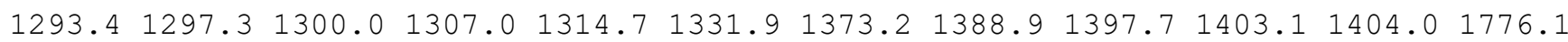
$2652.62660 .52668 .42672 .2 \quad 2674.4 \quad 2674.4 \quad 2675.92680 .42682 .5 \quad 2696.62772 .32778 .6$ $2781.32782 .3 \quad 2792.92810 .5$

\author{
name $=$ MIN372 \\ energy (in $\mathrm{kcal} / \mathrm{mol})=18.176601$ \\ $\mathrm{ZPE}($ in $\mathrm{kcal} / \mathrm{mol})=141.006$ \\ $\mathrm{G}($ in $\mathrm{kcal} / \mathrm{mol})=114.588$ \\ Geometry \\ C $-2.664712320 .18160343-0.32476878$ \\ C $-1.15380293 \quad 0.02741387-0.48187921$ \\ C $-0.566941891 .41424744 \quad-0.73136539$ \\ C $1.92744492 \quad 0.27012594 \quad 0.70172712$ \\ C $1.79507982 \quad 0.39040303 \quad 0.70612372$ \\ C $-0.16404704-2.10420807 \quad 0.06305988$ \\ C $-0.52250733-0.74703426 \quad 0.72051497$ \\ C $0.61220982-0.08217298 \quad 1.38914541$ \\ C 2.450639721 .655403231 .26841223 \\ C $0.53664294 \quad-0.02704393 \quad 2.86997596$ \\ $\mathrm{H}-3.09450151 \quad 0.70239506-1.19344604$ \\ $\mathrm{H}-2.93897053 \quad 0.73669294 \quad 0.57610342$ \\ $\mathrm{H}-3.14879260-0.80422998-0.28500995$ \\ $\mathrm{H}-0.27407071 \quad 1.97887304 \quad 0.16264439$ \\ H $2.58615513 \quad 0.89525243-1.26674217$ \\ H $1.39866890-0.45112871-1.29117291$ \\ $\mathrm{H} 2.47580954-0.55880587 \quad 1.01721084$ \\ $\mathrm{H}-0.57340279-2.97024566 \quad 0.60515557$ \\ $\mathrm{H} \quad 0.91615886 \quad-2.23761500 \quad-0.08897104$ \\ $\mathrm{H}-1.33313214 \quad-0.92432159 \quad 1.47466415$ \\ H 2.48837969 $1.66953774 \quad 2.36167622$ \\ $\mathrm{H} \quad 1.87707514 \quad 2.53934984 \quad 0.95316283$ \\ H $3.47290101 \quad 1.78393967 \quad 0.89388545$ \\ H $1.47301810 \quad 0.28162262 \quad 3.35096396$
}


$\mathrm{H} \quad 0.26163993-0.99720964 \quad 3.30993839$

$\mathrm{H}-0.23087392 \quad 0.69759534 \quad 3.19189868$

$0-0.82648667-2.11877334-1.19248954$

$0-0.498195641 .88589271-1.83568494$

O $-0.87509070-0.75320895-1.63721210$

Vibrational frequencies (in $\mathrm{cm}-1$ )

$\begin{array}{llllllllllllllll}32.3 & 61.1 & 80.9 & 104.6 & 119.6 & 128.9 & 153.1 & 169.0 & 178.4 & 217.3 & 238.4 & 278.8 & 303.9 & 320.8\end{array}$

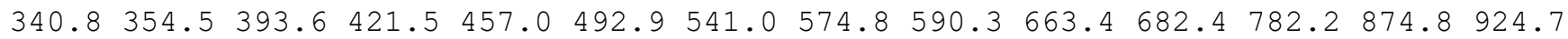

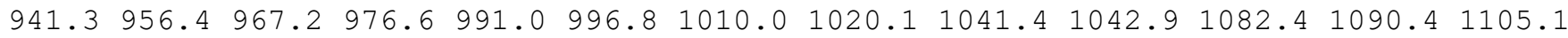

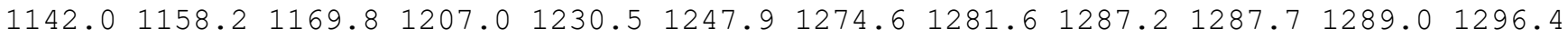
$1306.11311 .2 \quad 1325.5 \quad 1336.7 \quad 1368.0 \quad 1383.3 \quad 1389.0 \quad 1397.3 \quad 1407.5 \quad 1437.4 \quad 1573.91836 .2$ $2441.22643 .6 \quad 2651.5 \quad 2658.5 \quad 2666.0 \quad 2667.1 \quad 2668.3 \quad 2673.92681 .2 \quad 2691.3 \quad 2718.02722 .7$ $2769.7 \quad 2777.5 \quad 2778.8 \quad 2779.5$

\author{
name $=$ MIN373 \\ energy (in $\mathrm{kcal} / \mathrm{mol})=14.40948$ \\ $\mathrm{ZPE}(\mathrm{in} \mathrm{kcal} / \mathrm{mol})=145.159$ \\ $\mathrm{G}(\mathrm{in} \mathrm{kcal} / \mathrm{mol})=120.976$ \\ Geometry \\ C $-2.24380010 \quad 0.31063661 \quad 0.17330453$ \\ C $-0.878917190 .14509442-0.48061668$ \\ C $-0.13203447 \quad 1.54936081-0.56717414$ \\ C $0.93642774 \quad 2.00681379 \quad 0.46422327$ \\ C $0.774739591 .00864216 \quad 1.54900956$ \\ C $1.33630821-0.189858331 .32190493$ \\ C $-0.19720980-1.19292668-0.10603500$ \\ C $0.45396318-1.395054321 .31335272$ \\ C $-0.58078454-1.45758285 \quad 2.43488071$ \\ C $1.25925844 \quad-2.70082290 \quad 1.31362118$ \\ $\mathrm{H}-2.15693309 \quad 0.75768465 \quad 1.17042258$ \\ $\mathrm{H}-2.76345744 \quad-0.649901330 .28259028$ \\ $\mathrm{H}-2.90050986 \quad 0.95716108-0.42750049$ \\ $\mathrm{H}-0.92521154 \quad 2.33206847 \quad-0.62838996$ \\ $\mathrm{H} \quad 0.75896884 \quad 3.04923883 \quad 0.77685620$ \\ H $1.94316366 \quad 1.99930261-0.00152797$ \\ $\mathrm{H}-0.01722920 \quad 1.18389507 \quad 2.26555051$ \\ $\mathrm{H} \quad 0.59291479-1.38038280-0.86586429$ \\ $\mathrm{H} 2.27863816-0.29574273 \quad 0.78758064$ \\ $\mathrm{H}-0.94368732-1.99701110-0.25339815$ \\ $\mathrm{H}-1.23842073-0.58293348 \quad 2.44565011$ \\ $\mathrm{H}-0.08805291-1.50302595 \quad 3.41414288$ \\ $\mathrm{H}-1.21884067-2.34297812 \quad 2.34254840$ \\ $\begin{array}{llll}\mathrm{H} & 1.79558262 & -2.82380261 & 2.26235391\end{array}$ \\ H $2.00671565-2.724441110 .51333744$ \\ $\mathrm{H} \quad 0.61113572-3.57402244 \quad 1.18545602$ \\ $\begin{array}{lllll}0 & 0.02208759 & 0.36391751 & -2.52795913\end{array}$ \\ ○ $0.49756420 \quad 1.54258042-1.84292128$ \\ O $-1.176347490 .01255303-1.89576225$ \\ Vibrational frequencies (in $\mathrm{cm}-1$ )
}

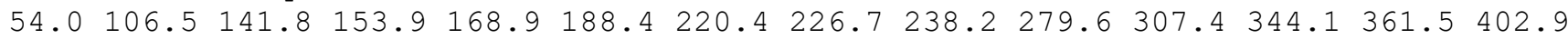
$408.3 \quad 439.4 \quad 473.4 \quad 490.8 \quad 572.0 \quad 603.1 \quad 618.0 \quad 689.2 \quad 767.1 \quad 859.8 \quad 882.0 \quad 905.2 \quad 920.5 \quad 946.5$ $953.5974 .3977 .6 \quad 990.4 \quad 1000.0 \quad 1009.1 \quad 1023.6 \quad 1034.0 \quad 1088.1 \quad 1094.8 \quad 1121.7 \quad 1145.6 \quad 1170.7$ $1181.21186 .91209 .3 \quad 1238.7 \quad 1258.9 \quad 1270.6 \quad 1281.91282 .6 \quad 1286.91290 .4 \quad 1298.7 \quad 1300.9$ $1302.21313 .11315 .6 \quad 1325.6 \quad 1333.5 \quad 1354.6 \quad 1362.6 \quad 1389.11400 .01414 .21424 .8 \quad 1804.7$ $2650.22652 .82666 .92671 .62672 .72673 .2 \quad 2677.02677 .92681 .92729 .42746 .42746 .6$ $2771.32777 .2 \quad 2781.8 \quad 2783.4$

name $=$ MIN374

energy (in $\mathrm{kcal} / \mathrm{mol})=16.554659$

$\mathrm{ZPE}(\mathrm{in} \mathrm{kcal} / \mathrm{mol})=143.334$

$\mathrm{G}($ in $\mathrm{kcal} / \mathrm{mol})=118.178$

Geometry

C $-2.069867860 .15078804-0.75025475$ 
C $-0.54628155 \quad 0.14331611-0.76151211$

C $0.039007951 .59375762-0.81553900$

C $0.66007459 \quad 2.21760060 \quad 0.41679021$

C $1.14688099-0.89051940 \quad 1.97306849$

C $1.46413155-0.76362795 \quad 0.67337299$

C $0.03185757-0.90594915 \quad 0.16763881$

C $-0.36364198-1.03309882 \quad 1.72388432$

$\begin{array}{lllll}\text { C }-1.21030127 & 0.05483161 & 2.34245202\end{array}$

C $-0.90265798-2.41126625 \quad 2.06823042$

$\mathrm{H}-2.471744220 .51733784 \quad-1.70665420$

$\mathrm{H}-2.47923182-0.85575032-0.59600709$

$\mathrm{H}-2.46235161 \quad 0.79321641 \quad 0.04584235$

$\mathrm{H}-0.73921004 \quad 2.27985229-1.23437063$

$\mathrm{H}-0.10281425 \quad 2.59956686 \quad 1.10583384$

H $1.29817786 \quad 3.06847397 \quad 0.12941511$

$\mathrm{H} \quad 1.66283682-0.911318552 .89433144$

$\mathrm{H} 2.34516687-0.62675518 \quad 0.09945996$

$\mathrm{H} 1.306042951 .528604590 .97520974$

$\mathrm{H}-0.11565107-1.89233448-0.33146254$

$\mathrm{H}-2.27210041-0.068236372 .09814005$

$\mathrm{H}-0.90543681 \quad 1.05482403 \quad 2.01543098$

$\mathrm{H}-1.124823390 .03659523 \quad 3.43758258$

$\mathrm{H}-0.99365402-2.54044386 \quad 3.15371004$

$\mathrm{H}-0.24478534-3.21056471 \quad 1.70480778$

$\mathrm{H}-1.89426183-2.57394304 \quad 1.63099162$

$\begin{array}{lllll}0 & 1.09791359 & 0.19095129 & -2.28506177\end{array}$

o $1.041175391 .55239785-1.83978928$

o - $0.20438352-0.29796287-2.10332142$

Vibrational frequencies (in cm-1)

$\begin{array}{llllllllllllll}44.1 & 89.6 & 110.1 & 135.3 & 147.2 & 156.9 & 176.2 & 186.5 & 215.6 & 243.7 & 264.0 & 286.1 & 315.4 & 326.6\end{array}$

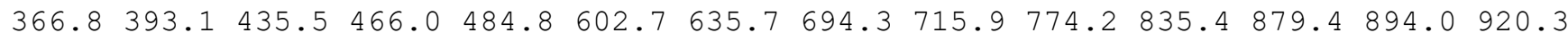

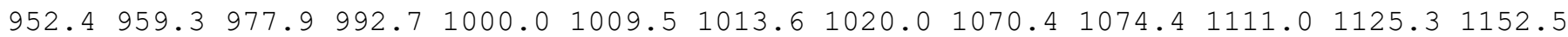

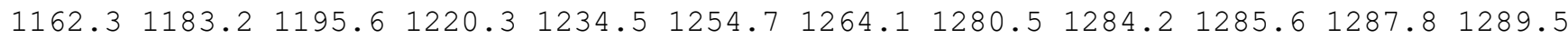
$1290.01294 .0 \quad 1298.6 \quad 1305.3 \quad 1315.3 \quad 1339.9 \quad 1374.7 \quad 1396.6 \quad 1401.4 \quad 1403.8 \quad 1408.31774 .9$ $2654.62665 .12668 .2 \quad 2672.5 \quad 2673.8 \quad 2675.5 \quad 2676.02678 .42680 .72695 .72776 .42778 .5$ $2782.12784 .4 \quad 2792.1 \quad 2810.2$

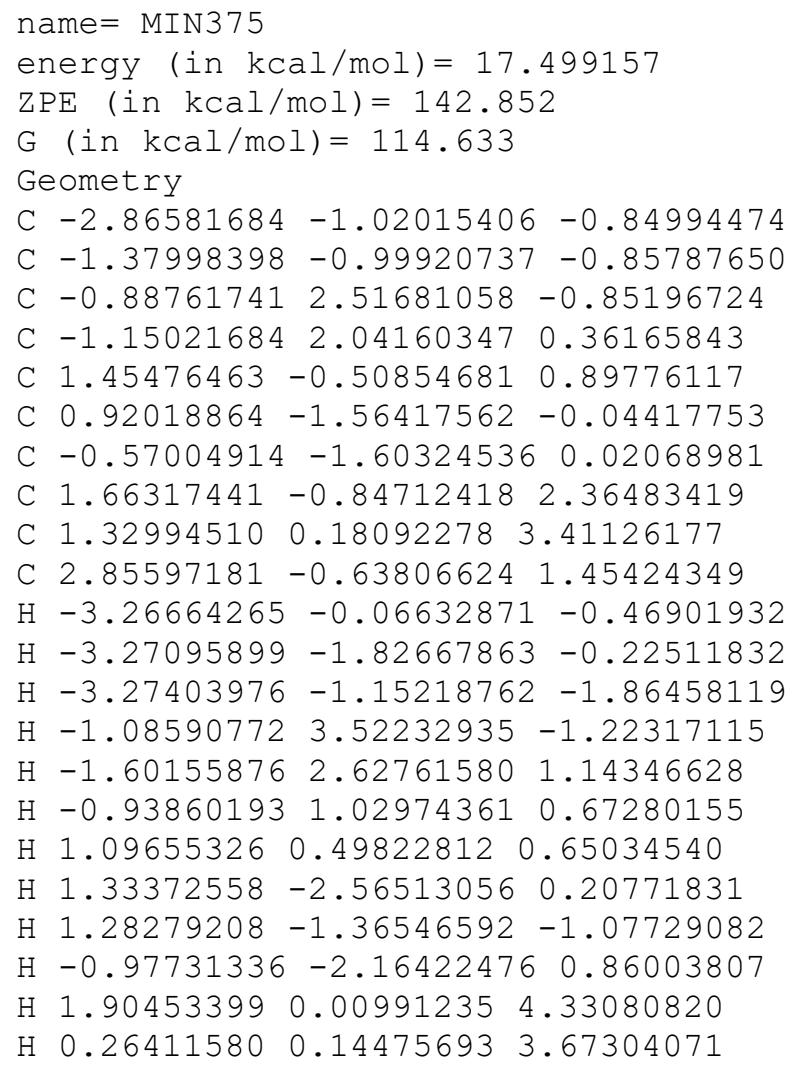


$\mathrm{H} \quad 1.54678111 \quad 1.20342083 \quad 3.07763885$

$\mathrm{H} \quad 3.46923755 \quad 0.25086710 \quad 1.56368984$

$\mathrm{H} 3.47495333-1.483909251 .17545877$

$\mathrm{H} \quad 1.44385756-1.86956144 \quad 2.69128060$

$0.11525283 \quad 0.56080524-1.58347743$

$0-0.318955001 .85376898-1.90145416$

O $-0.91862619-0.32388492-1.97255617$

Vibrational frequencies (in $\mathrm{cm}-1$ )

$\begin{array}{lllllllllllllll}25.1 & 42.2 & 45.7 & 66.3 & 72.2 & 89.5 & 104.7 & 118.9 & 169.1 & 180.8 & 201.8 & 251.6 & 291.7 & 325.0\end{array}$

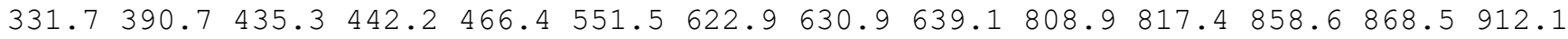
$934.7942 .3 \quad 949.5 \quad 956.6 \quad 1006.2 \quad 1009.7 \quad 1022.0 \quad 1024.2 \quad 1054.6 \quad 1057.4 \quad 1070.7 \quad 1095.2 \quad 1102.1$

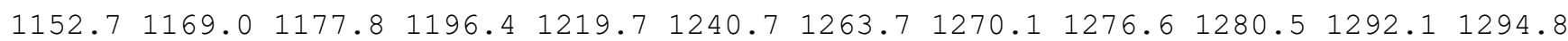

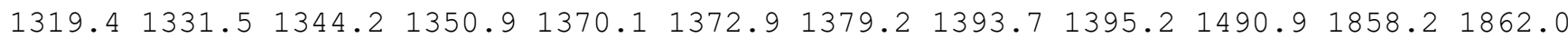
$2652.72663 .12675 .0 \quad 2675.92677 .92707 .0 \quad 2716.8 \quad 2730.4 \quad 2732.6 \quad 2743.5 \quad 2758.5 \quad 2761.9$ $2777.6 \quad 2782.5 \quad 2790.8 \quad 2791.8$

\section{name $=$ MIN376}

energy $($ in $\mathrm{kcal} / \mathrm{mol})=16.441916$

$\mathrm{ZPE}(\mathrm{in} \mathrm{kcal} / \mathrm{mol})=144.236$

$\mathrm{G}($ in $\mathrm{kcal} / \mathrm{mol})=118.845$

Geometry

C $-2.62875154 \quad 0.01395245 \quad-0.55018058$

C $-1.29141400 \quad 0.01326898-0.53888549$

C $-0.574813151 .36070619-0.53620167$

C $0.21086074 \quad 1.70033910 \quad 0.77052566$

C $-0.21580486-1.816766930 .91151271$

C 0.752601130 .377285561 .16857756

C $-0.54112343-1.29765870-0.53921227$

C $-0.09384824-0.53400870 \quad 1.69013906$

C $-1.16222173-0.24177468 \quad 2.68119776$

C $1.03582214 \quad-2.68700983 \quad 0.92098488$

$\mathrm{H}-3.23838286 \quad 0.90155333-0.56024232$

$\mathrm{H}-3.22248064-0.88613312-0.55116131$

H $1.92783839 \quad 0.13719610 \quad-2.92236194$

$\mathrm{H}-1.31462896 \quad 2.18142384 \quad-0.73520071$

$\mathrm{H}-0.46972984 \quad 2.14360179 \quad 1.51917244$

$\mathrm{H} \quad 0.98644083 \quad 2.45875983 \quad 0.56785585$

$\mathrm{H} 1.27717463-3.012978371 .93962103$

$\mathrm{H} \quad 0.40783454-1.18502826-1.10142228$

$\mathrm{H} \quad 1.67292582 \quad 0.08044844 \quad 0.66827209$

$\mathrm{H}-1.11612707-2.07201962-1.07718132$

$\mathrm{H}-0.97259288 \quad 0.68480464 \quad 3.23906551$

$\mathrm{H}-1.23940461-1.05274663 \quad 3.42030227$

$\mathrm{H}-2.14814028-0.14267905 \quad 2.20154090$

H $1.91094664 \quad-2.14650348 \quad 0.53798755$

$\mathrm{H}-1.07901713-2.417259531 .27937363$

H $0.90610570-3.581437110 .30214416$

O $1.574599641 .30611493-1.49029516$

O $0.222950361 .50442472-1.70878581$

O $1.946631290 .03319073-1.93926968$

Vibrational frequencies (in $\mathrm{cm}-1$ )

$\begin{array}{lllllllllllllll}38.5 & 67.0 & 110.8 & 118.6 & 136.1 & 153.3 & 172.0 & 176.3 & 232.4 & 259.1 & 274.2 & 308.1 & 349.6 & 366.6\end{array}$ $388.1410 .4 \quad 468.0 \quad 492.3 \quad 499.5 \quad 541.2611 .2623 .6 \quad 671.5 \quad 706.4 \quad 825.1 \quad 851.8 \quad 868.6 \quad 916.1$ $938.0950 .6966 .1978 .0 \quad 1001.1 \quad 1018.1 \quad 1049.6 \quad 1061.5 \quad 1077.3 \quad 1080.3 \quad 1091.7 \quad 1106.2 \quad 1136.6$

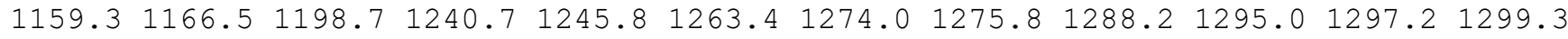

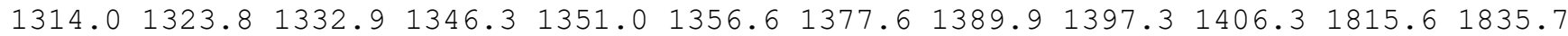
$2655.92659 .2 \quad 2666.6 \quad 2669.9 \quad 2672.6 \quad 2675.5 \quad 2681.12711 .72721 .4 \quad 2741.32745 .82755 .9$ $2776.92781 .12790 .9 \quad 2846.6$

name $=$ MIN377

energy $($ in $\mathrm{kcal} / \mathrm{mol})=17.693042$

$\mathrm{ZPE}(\mathrm{in} \mathrm{kcal} / \mathrm{mol})=143.629$

$\mathrm{G}($ in $\mathrm{kcal} / \mathrm{mol})=118.408$ 


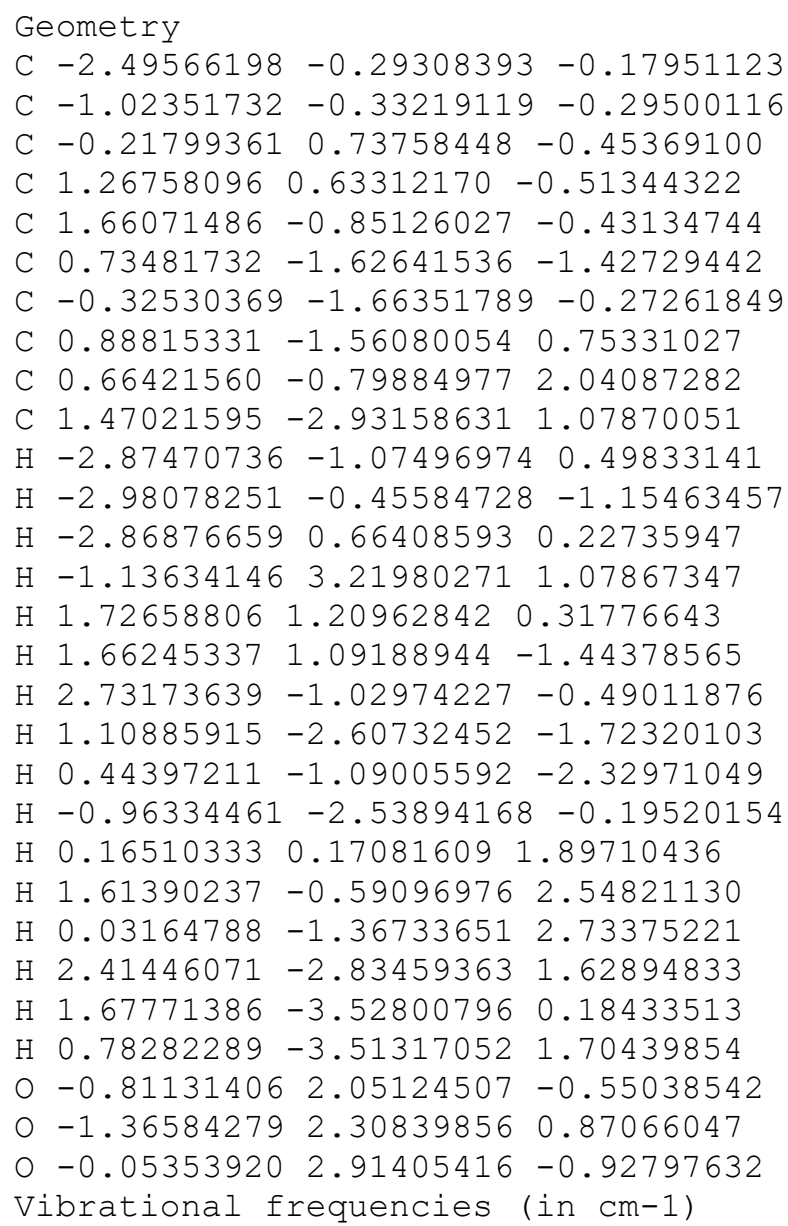

$\begin{array}{llllllllllllllll}48.8 & 71.1 & 80.6 & 120.0 & 135.1 & 164.6 & 167.0 & 202.5 & 237.7 & 268.1 & 294.5 & 311.5 & 341.0 & 382.4\end{array}$ $422.2 \quad 440.5 \quad 461.0 \quad 499.3 \quad 507.6 \quad 576.5 \quad 608.7 \quad 630.6 \quad 710.5 \quad 748.6 \quad 778.3 \quad 843.1 \quad 866.7 \quad 925.7$ $942.8 \quad 968.4 \quad 993.6 \quad 1009.6 \quad 1011.9 \quad 1016.5 \quad 1045.9 \quad 1055.1 \quad 1074.5 \quad 1084.3 \quad 1111.3 \quad 1125.7 \quad 1136.7$ $1168.8 \quad 1173.7 \quad 1176.3 \quad 1214.8 \quad 1234.5 \quad 1257.3 \quad 1263.4 \quad 1268.3 \quad 1273.6 \quad 1277.8 \quad 1286.2 \quad 1288.5$

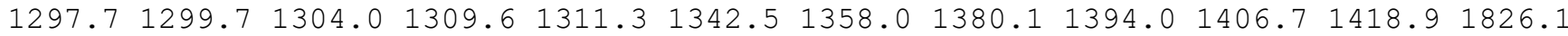
$2651.02655 .22656 .72663 .92675 .8 \quad 2676.72681 .52694 .22738 .92751 .52757 .42768 .2$ $2771.92779 .4 \quad 2782.5 \quad 2812.7$

\footnotetext{
name $=$ MIN378

energy (in $\mathrm{kcal} / \mathrm{mol})=16.471878$

$\mathrm{ZPE}($ in $\mathrm{kcal} / \mathrm{mol})=146.763$

$\mathrm{G}($ in $\mathrm{kcal} / \mathrm{mol})=122.528$

Geometry

C $-2.19302743 \quad 0.32322885-0.85275995$

C $-0.75822583 \quad 0.09728479-1.31022807$

C $0.06368718 \quad 1.39968237 \quad-1.27719814$

C $0.74751702 \quad 1.62875996 \quad 0.04914233$

C $1.41718465 \quad 0.43611023 \quad 0.71535786$

C $2.25912807 \quad 1.54165098 \quad 0.12210258$

C $-0.25783027-1.02427928-0.36160890$

C $1.23294970-0.92300305 \quad 0.06620795$

C $1.51367963-2.029488561 .09621570$

C $2.12864250-1.15940225-1.15619338$

$\mathrm{H}-2.90008666-0.39252028-1.28975806$

$\mathrm{H}-0.74773026-0.28324384-2.35917190$

$\mathrm{H}-2.555773251 .34302154-1.01972693$

$\mathrm{H} \quad 0.81606738 \quad 1.37846660-2.09204624$

$\mathrm{H}-0.58593650 \quad 2.26392814 \quad-1.52079527$

$\mathrm{H} \quad 0.23035632 \quad 2.34988815 \quad 0.68936827$

H $1.36437166 \quad 0.38084106 \quad 1.81103221$

H $2.83780478 \quad 1.37322780 \quad-0.77896676$

H $2.80114648 \quad 2.207125110 .78789395$

$\mathrm{H}-0.45854989-2.02434696-0.80837366$
} 
H $2.53559076-1.95912111 \quad 1.48243404$

$\mathrm{H} 1.38396680-3.02634856 \quad 0.66478355$

$\mathrm{H} \quad 0.82510728-1.95479996 \quad 1.95024579$

$\mathrm{H} 3.18744441-1.07831065-0.88151924$

$\mathrm{H} \quad 1.93873865-0.43525943-1.95375094$

H $1.97597105-2.16011696-1.57443841$

$0-2.23732016-0.00778772 \quad 0.61194355$

$0-1.03115687-0.959141250 .80522005$

O -2.249288130 .933837791 .34275493$

Vibrational frequencies (in $\mathrm{cm}-1$ )

$\begin{array}{lllllllllllllllll}27.9 & 55.9 & 114.2 & 183.5 & 194.9 & 209.7 & 249.9 & 282.0 & 303.3 & 343.7 & 365.5 & 411.1 & 436.3 & 445.4\end{array}$ $473.5480 .2 \quad 541.5 \quad 591.3 \quad 611.5 \quad 720.6 \quad 774.4 \quad 798.4 \quad 856.4 \quad 872.2 \quad 891.1 \quad 906.1936 .8 \quad 962.4$

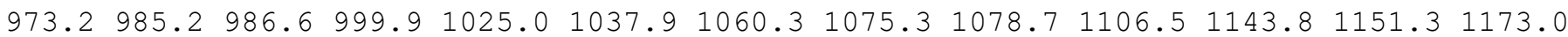

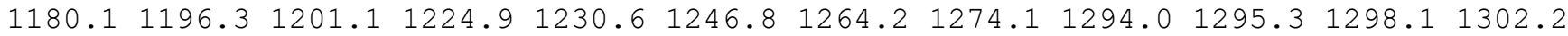

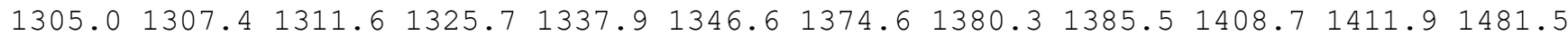
$2655.62664 .5 \quad 2670.1 \quad 2671.3 \quad 2676.8 \quad 2678.0 \quad 2683.5 \quad 2702.52714 .12732 .8 \quad 2738.8 \quad 2744.4$ 2758.22779 .42781 .62789 .8

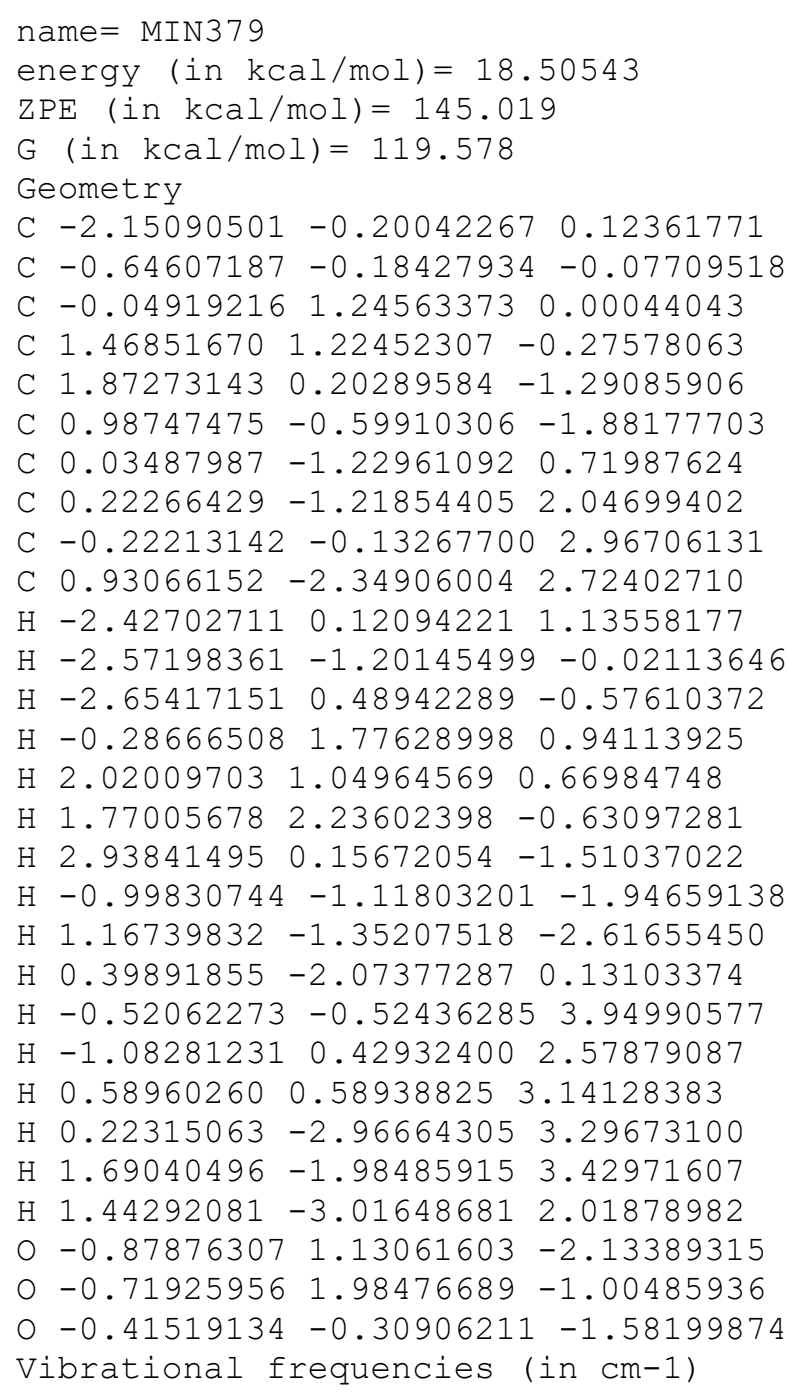


$\mathrm{ZPE}($ in $\mathrm{kcal} / \mathrm{mol})=144.409$

$\mathrm{G}($ in $\mathrm{kcal} / \mathrm{mol})=119.763$

Geometry

C $-2.23567161-0.11010052-0.71106744$

C $-0.70356981-0.06858083-0.69191161$

C $-0.208360961 .41415664-0.81630393$

C $0.94658915 \quad 1.88955004 \quad 0.04197298$

C $1.35426324-0.73547747 \quad 1.93161666$

C $1.38761834-1.18213207 \quad 0.45884032$

C $-0.17277790-1.008070330 .37997006$

C $-0.18048234-0.55960698 \quad 1.86444246$

C $-1.08867278 \quad 0.46295723 \quad 2.43260387$

C $0.44699656-1.63871974 \quad 2.73453428$

$\mathrm{H}-2.64050998 \quad 0.49486042 \quad-1.53327493$

$\mathrm{H}-2.66063568 \quad 0.25148045 \quad 0.23030973$

$\mathrm{H}-2.60271123-1.13275105-0.86901639$

$\mathrm{H}-1.065144712 .12213551-0.74535614$

H $1.108121612 .96802351-0.08928617$

$\mathrm{H} 1.888164451 .39683726-0.23763003$

$\mathrm{H} 0.785417311 .706167831 .10980062$

$\mathrm{H} 1.73592290 \quad-2.20475653 \quad 0.29298257$

H $1.94750921-0.52688263-0.21229967$

$\mathrm{H}-0.67805841-1.988975390 .25150350$

$\mathrm{H}-1.24891217 \quad 1.31276353 \quad 1.75493821$

$\mathrm{H}-0.70328876 \quad 0.87278736 \quad 3.37762698$

$\mathrm{H}-2.07727216 \quad 0.02937359 \quad 2.65117327$

$\mathrm{H} \quad 0.41022088-1.51685810 \quad 3.81341210$

H $2.11232469-0.11455160 \quad 2.35655358$

$\mathrm{H} \quad 0.34610556-2.68844046 \quad 2.47872865$

$\begin{array}{lllll}0 & 0.66832033 & 0.20879612 & -2.48834069\end{array}$

○ $0.17928758 \quad 1.50231533-2.19372656$

o $-0.31962170-0.65647123-1.95516082$

Vibrational frequencies (in $\mathrm{cm}-1$ )

$\begin{array}{lllllllllllllll}36.4 & 86.5 & 123.3 & 143.1 & 155.1 & 169.3 & 181.7 & 240.8 & 248.9 & 274.9 & 320.9 & 322.5 & 337.2 & 378.1\end{array}$

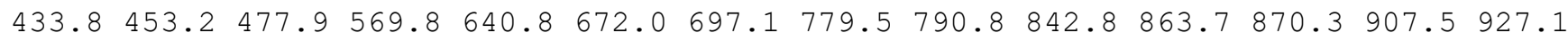
$953.8 \quad 965.3 \quad 985.2 \quad 1002.0 \quad 1010.2 \quad 1014.2 \quad 1035.8 \quad 1058.5 \quad 1069.6 \quad 1090.3 \quad 1107.91114 .01129 .1$

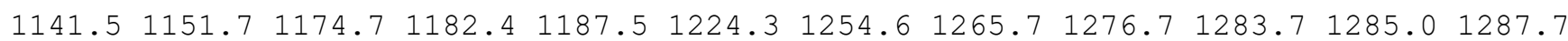

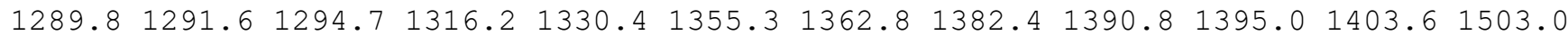
$2659.92669 .0 \quad 2669.8 \quad 2672.6 \quad 2675.1 \quad 2678.2 \quad 2683.0 \quad 2685.12711 .3 \quad 2716.7 \quad 2762.7 \quad 2777.9$ $2779.92782 .4 \quad 2788.0 \quad 2792.5$

\section{name $=\operatorname{MIN} 381$}

energy $($ in $\mathrm{kcal} / \mathrm{mol})=23.520491$

$\mathrm{ZPE}(\mathrm{in} \mathrm{kcal} / \mathrm{mol})=142.014$

$\mathrm{G}(\mathrm{in} \mathrm{kcal} / \mathrm{mol})=114.659$

Geometry

C $-2.46392354-1.94069895 \quad 1.36215454$

C $-1.01122590-1.93122986 \quad 1.63429112$

C $1.15473296 \quad 0.39453527 \quad 1.54636554$

C $1.32981100 \quad 0.89033474 \quad 0.17180769$

C $0.44215471 \quad 0.20312815-0.85806958$

C $-1.064509210 .27052734 \quad-0.65235874$

C $-0.35304655-1.04154389-0.49877754$

C $1.01663789 \quad 0.35345884 \quad-2.26576862$

C $0.34932080-0.55897367-3.30151073$

C $0.883007131 .81547228-2.71529728$

$\mathrm{H}-2.97006844 \quad-2.67777408 \quad 2.00276370$

$\mathrm{H}-2.72808519-2.15752100 \quad 0.32105397$

$\mathrm{H}-2.90180522-0.95863393 \quad 1.60822692$

H $1.71354159-0.44599288 \quad 1.94190134$

$\begin{array}{lllll}\mathrm{H} & 2.39743471 & 0.80547674 & -0.13108153\end{array}$

$\mathrm{H} \quad 1.08709304 \quad 1.98904162 \quad 0.14917872$

$\mathrm{H} 2.10320023 \quad 0.08276379 \quad-2.22890064$

$\mathrm{H}-1.72585193 \quad 0.44596930-1.49421262$ 
$\mathrm{H}-1.44335105 \quad 0.79800953 \quad 0.22776945$

$\mathrm{H}-0.47818156-1.87647059-1.17889291$

$\mathrm{H}-0.73097430-0.38893633-3.36168736$

$\mathrm{H} \quad 0.51752602-1.61767931-3.07819323$

$\mathrm{H} \quad 0.75975762-0.37487999-4.30236572$

$\mathrm{H} \quad 1.255365491 .95392939-3.73578812$

$\mathrm{H} \quad 1.44575280 \quad 2.49433532-2.06595430$

$\mathrm{H}-0.16424124 \quad 2.14156206-2.69703355$

O $-0.40359074 \quad 1.86335335 \quad 2.04905971$

$\begin{array}{lllll}0 & 0.32649338 & 0.89266371 & 2.40622004\end{array}$

o $-0.14145719-1.534866930 .81578158$

Vibrational frequencies (in $\mathrm{cm}-1$ )

$\begin{array}{lllllllllllllllll}28.1 & 44.0 & 52.1 & 75.6 & 88.4 & 108.0 & 146.4 & 169.8 & 196.2 & 215.0 & 226.0 & 241.1 & 279.5 & 301.7\end{array}$ $\begin{array}{llllllllllllll}338.8 & 351.7 & 418.0 & 445.4 & 497.7 & 506.6 & 579.0 & 632.9 & 712.6 & 757.7 & 840.5 & 874.9 & 881.9 & 935.0\end{array}$ $946.9966 .6 \quad 975.7984 .5 \quad 993.9 \quad 1015.9 \quad 1022.4 \quad 1041.1 \quad 1043.0 \quad 1059.6 \quad 1119.1 \quad 1138.2 \quad 1156.5$

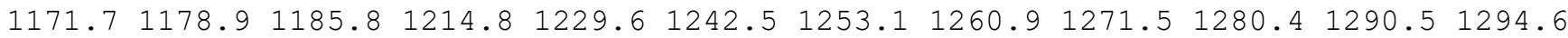
$1301.21306 .31310 .81320 .7 \quad 1357.41361 .91367 .5 \quad 1400.71406 .6 \quad 1465.8 \quad 1506.91606 .1$ $2632.52662 .8 \quad 2669.5 \quad 2673.5 \quad 2675.6 \quad 2677.3 \quad 2679.32691 .22701 .52708 .42719 .82739 .8$ $2774.0 \quad 2777.92780 .2 \quad 2782.4$

name $=$ MIN382

energy (in $\mathrm{kcal} / \mathrm{mol})=22.431672$

$\operatorname{ZPE}($ in $\mathrm{kcal} / \mathrm{mol})=144.138$

$\mathrm{G}($ in $\mathrm{kcal} / \mathrm{mol})=119.388$

Geometry

C $-2.24755895 \quad 0.09364231-0.79293464$

C $-0.71971918 \quad 0.11806460-0.80187931$

C $-0.154771821 .56037511-0.85422805$

C $2.11987577 \quad 0.07959552 \quad 1.04197950$

C $1.28090031-0.833594381 .89974940$

C $1.36247979-1.04607767 \quad 0.37680819$

C $-0.15790921-0.83263550 \quad 0.24015138$

C $-0.24020270-0.52602492 \quad 1.80227808$

C $-0.65135761 \quad 0.86767649 \quad 2.23124126$

C $-1.07581866-1.570487492 .52963103$

$\mathrm{H}-2.66121320 \quad 0.75935745 \quad-1.56219737$

$\mathrm{H}-2.64943330 \quad 0.39617467 \quad 0.17952774$

$\mathrm{H}-2.62954881-0.91257324-1.01144411$

$\mathrm{H}-0.925839332 .34219866-0.78760368$

$\mathrm{H} \quad 0.63684805 \quad 1.74548019-0.11315539$

H $3.20448810-0.004318331 .10166541$

H $1.67304971-1.41471356 \quad 2.70696469$

$\mathrm{H} \quad 1.85193997-1.80705126-0.19950280$

$\mathrm{H} \quad 1.85107490 \quad 1.12048914 \quad 0.89323269$

$\mathrm{H}-0.62869372-1.82634800 \quad 0.04338551$

$\mathrm{H} \quad 0.04105696 \quad 1.63960338 \quad 1.87847648$

$\mathrm{H}-0.66968481 \quad 0.94981674 \quad 3.32671909$

$\mathrm{H}-1.65283227 \quad 1.12423406 \quad 1.86801996$

H $-0.97781996-1.46901495 \quad 3.61737671$

$\mathrm{H}-0.76924297-2.59211538 \quad 2.27493836$

$\mathrm{H}-2.13893730-1.47171932 \quad 2.28258694$

$\begin{array}{lllll}0 & 0.75213242 & 0.35451264 & -2.50724714\end{array}$

○ $0.394653691 .68789698-2.16477850$

$0-0.36261492-0.41259470-2.09871469$

Vibrational frequencies (in $\mathrm{cm}-1$ )

$\begin{array}{lllllllllllllll}44.5 & 61.2 & 128.6 & 146.6 & 151.6 & 166.5 & 190.5 & 235.2 & 242.3 & 259.2 & 285.1 & 342.8 & 355.0 & 376.2\end{array}$

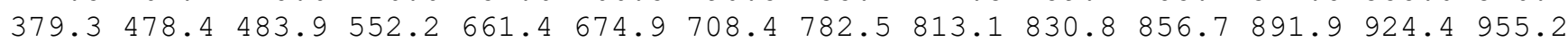
$959.2963 .1975 .0 \quad 992.6 \quad 999.0 \quad 1005.6 \quad 1010.2 \quad 1018.2 \quad 1025.8 \quad 1043.1 \quad 1079.7 \quad 1133.3 \quad 1137.8$ $1152.01153 .5 \quad 1169.9 \quad 1195.2 \quad 1210.2 \quad 1227.3 \quad 1250.2 \quad 1270.4 \quad 1281.3 \quad 1286.5 \quad 1290.91291 .5$

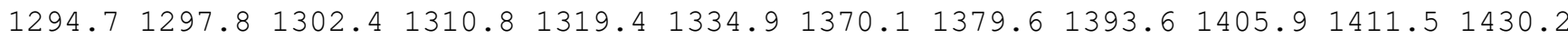
$2629.22669 .5 \quad 2669.92674 .6 \quad 2675.5 \quad 2676.7 \quad 2680.42695 .02696 .62716 .02778 .12778 .5$ $2780.5 \quad 2781.1 \quad 2782.5 \quad 2790.5$ 
name $=$ MIN383

energy (in $\mathrm{kcal} / \mathrm{mol})=21.094795$

$\mathrm{ZPE}($ in $\mathrm{kcal} / \mathrm{mol})=145.656$

$\mathrm{G}($ in $\mathrm{kcal} / \mathrm{mol})=122.383$

Geometry

C $-2.40511296-0.13865238-0.65190769$

C $-0.89012582-0.20008127-0.63757923$

C $-0.144099861 .16993867 \quad-0.62418037$

C 1.024685411 .320434460 .32216015

C $1.25855482 \quad 0.05417043 \quad 1.15643986$

C $1.23398376-1.17490354 \quad 0.19740003$

C $-0.31762799-1.17868386 \quad 0.38470143$

C $-0.09642805-0.46513451 \quad 1.77733684$

C $-1.09358198 \quad 0.563482912 .27087045$

C $0.13819993-1.483649992 .89084270$

$\mathrm{H}-2.78186193 \quad 0.65354660 \quad-1.30696338$

$\mathrm{H}-2.82432322 \quad 0.01115236 \quad 0.34812768$

$\mathrm{H}-2.81057904-1.08593040-1.04089012$

$\mathrm{H}-0.78166818 \quad 2.05568586-0.68599379$

$\mathrm{H} \quad 0.87800159 \quad 2.18845680 \quad 0.99353396$

H $1.952413991 .54515467-0.25689886$

H $2.10214725 \quad 0.13812291 \quad 1.84066089$

H $1.73496671-2.06434457 \quad 0.58271012$

H $1.59762426-1.01238699-0.82007054$

$\mathrm{H}-0.81849364-2.14479916 \quad 0.39737026$

$\mathrm{H}-2.03123170 \quad 0.08632345 \quad 2.58285421$

$\mathrm{H}-1.349515261 .320111121 .52411107$

$\mathrm{H}-0.694297861 .10095942 \quad 3.14114294$

$\mathrm{H} \quad 0.51965105-0.997500393 .79595968$

$\mathrm{H} \quad 0.86312154 \quad-2.25488858 \quad 2.60744547$

$\mathrm{H}-0.79291394 \quad-1.99781384 \quad 3.15569940$

$\begin{array}{lllll}0 & 1.43759522 & 0.90913616 & -2.33536417\end{array}$

o $0.28141591 \quad 0.90084252-2.09411848$

o $-0.43013502-0.51540366-1.94659652$

Vibrational frequencies (in cm-1)

$96.4114 .8 \quad 160.4 \quad 166.0 \quad 183.3 \quad 201.1229 .1 \quad 282.9299 .6 \quad 327.1340 .1390 .7420 .1435 .1$ $444.2505 .3 \quad 529.2 \quad 558.2 \quad 600.8 \quad 658.5 \quad 713.9 \quad 736.4 \quad 789.2 \quad 801.7 \quad 855.8 \quad 898.3 \quad 920.9 \quad 938.6$ $950.5994 .81002 .61006 .2 \quad 1015.2 \quad 1018.2 \quad 1051.61076 .4 \quad 1080.11087 .41109 .21133 .3$

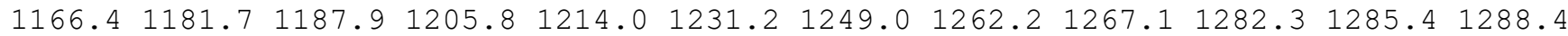

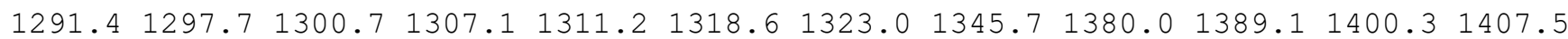
$1417.62640 .3 \quad 2671.3 \quad 2674.0 \quad 2675.5 \quad 2678.0 \quad 2680.82682 .52685 .2 \quad 2716.22730 .02749 .5$ $2754.3 \quad 2765.92781 .5 \quad 2783.2 \quad 2784.5$

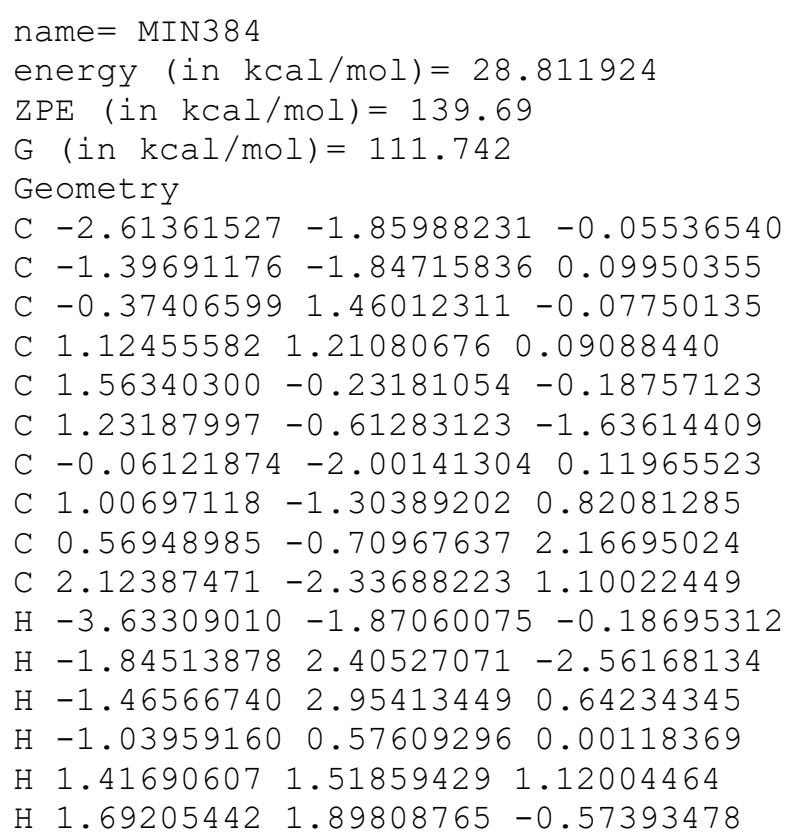


H $2.68017244-0.24948973-0.07658999$

$\mathrm{H} 1.77606107 \quad 0.01622311-2.34871032$

$\mathrm{H} \quad 0.16420256-0.49754366-1.86577198$

H $1.49691030-1.65833693-1.84834959$

H $1.38895728-0.17562127 \quad 2.65926313$

$\mathrm{H} \quad 0.23563335-1.497849692 .85198093$

$\mathrm{H}-0.26641526-0.006409852 .06253556$

$\mathrm{H} 2.97288152-1.87763310 \quad 1.61448527$

H $2.48471804 \quad-2.78880563 \quad 0.16864954$

$\mathrm{H} 1.75543003-3.15474515 \quad 1.72914758$

$0-1.89366732 \quad 2.00706737-1.65977125$

$\begin{array}{lllll}0 & -0.74041890 & 2.36874737 & 0.93763664\end{array}$

O $-0.49597627 \quad 2.08874904-1.34022169$

Vibrational frequencies (in cm-1)

$\begin{array}{lllllllllllllll}30.2 & 39.9 & 65.2 & 70.7 & 85.1 & 98.8 & 141.9 & 173.9 & 197.3 & 218.3 & 224.6 & 232.2 & 247.4 & 255.4\end{array}$ $276.5 \quad 304.5 \quad 324.4 \quad 372.6 \quad 384.5 \quad 404.3 \quad 436.2 \quad 473.8 \quad 488.3 \quad 520.8 \quad 532.3 \quad 639.1737 .6 \quad 750.9$

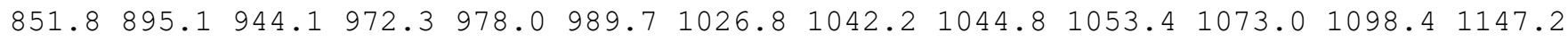

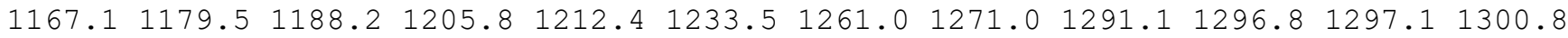

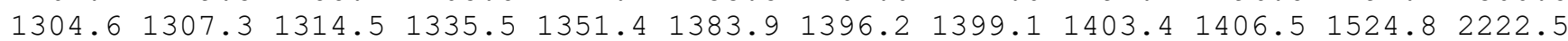
$2644.82654 .42659 .2 \quad 2666.2 \quad 2672.3 \quad 2674.92677 .12681 .32686 .4 \quad 2731.52777 .02779 .1$ $2781.52802 .8 \quad 2859.2 \quad 2869.2$

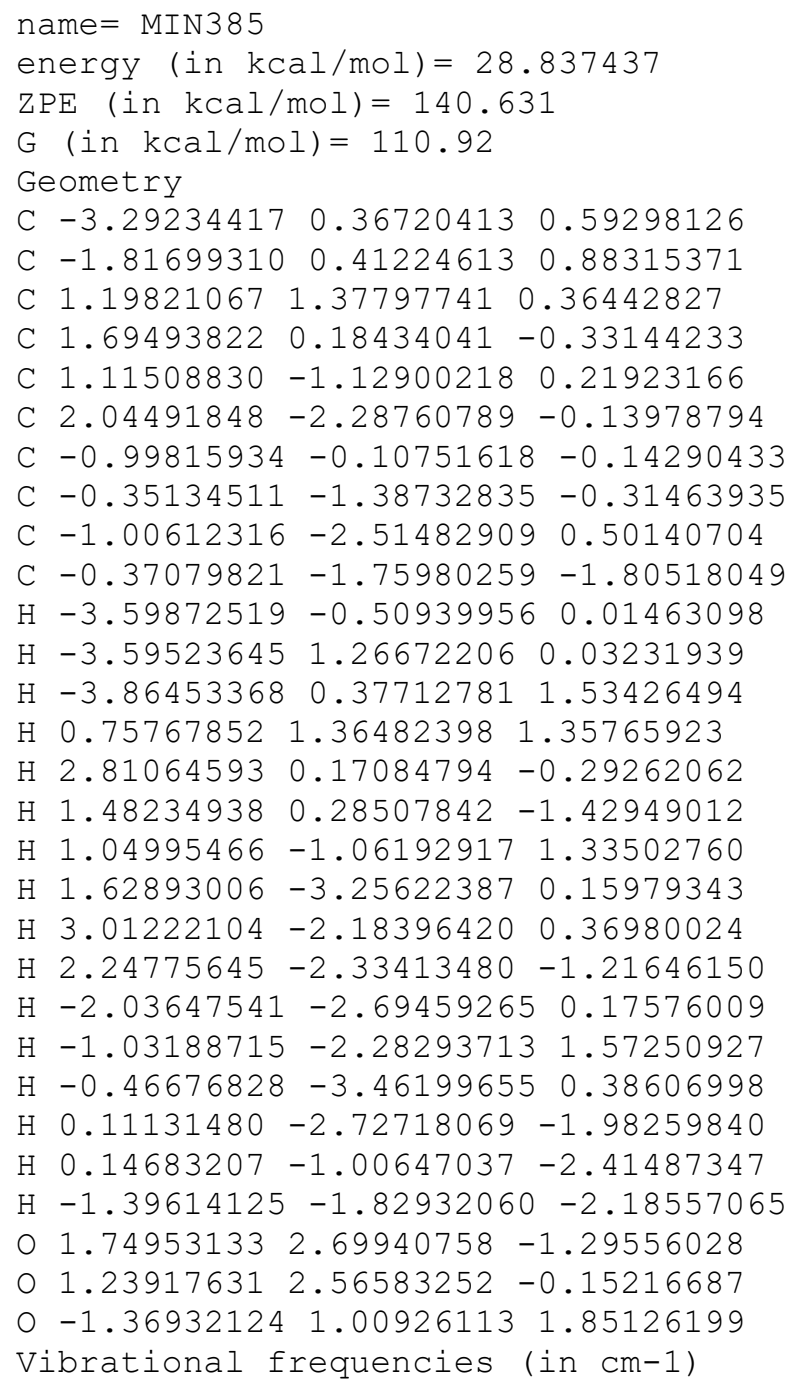


name $=$ MIN386

energy $($ in $\mathrm{kcal} / \mathrm{mol})=27.994346$

$\mathrm{ZPE}($ in $\mathrm{kcal} / \mathrm{mol})=141.828$

$\mathrm{G}($ in $\mathrm{kcal} / \mathrm{mol})=113.513$

Geometry

C $-2.42308155-1.84543200 \quad 0.77451644$

C $-1.28294992-1.67122214-0.19806436$

C $0.052975110 .91842776-1.26056901$

C $1.26986913 \quad 0.87255520-0.44209368$

C $1.13784345 \quad 0.24982555 \quad 0.95578551$

C $1.10357674-1.29405506 \quad 0.85930053$

C $-0.01801542-1.968040610 .32649727$

C $-0.05369897 \quad 0.80500183 \quad 1.76738217$

C $-0.02687437 \quad 2.33752260 \quad 1.79727725$

C $-0.01231544 \quad 0.27655478 \quad 3.20825582$

$\mathrm{H}-2.14504605-1.68402724 \quad 1.81957265$

$\mathrm{H}-2.83490237-2.86376322 \quad 0.68599401$

$\mathrm{H}-3.24351729-1.15606727 \quad 0.52261919$

$\mathrm{H}-0.94942828 \quad 0.79583856-0.88013993$

$\mathrm{H} 1.684382101 .90938551-0.35890958$

H $2.07057688 \quad 0.34155688 \quad-1.02497457$

H $2.07293000 \quad 0.51887214 \quad 1.51639491$

H $1.33549105-1.738097821 .86901168$

$\mathrm{H} 2.00131482-1.646874110 .26488753$

$\mathrm{H}-1.00834666 \quad 0.47431985 \quad 1.29338639$

$\mathrm{H} \quad 0.92295508 \quad 2.71876028 \quad 2.18840243$

$\mathrm{H}-0.82341016 \quad 2.73524565 \quad 2.43728281$

$\mathrm{H}-0.17257120 \quad 2.76849187 \quad 0.80030659$

H $0.93422585 \quad 0.52493786 \quad 3.70165862$

$\mathrm{H}-0.14069714 \quad-0.80815706 \quad 3.25590515$

$\mathrm{H}-0.81225267 \quad 0.72156316 \quad 3.81282782$

○ $1.21990061 \quad 1.27641340-3.07531294$

○ $0.090154461 .10398401-2.54391312$

o - $1.49125313-1.44216767-1.38036096$

Vibrational frequencies (in $\mathrm{cm}-1$ )

$\begin{array}{llllllllllllllll}16.0 & 30.7 & 63.7 & 72.6 & 86.3 & 104.0 & 125.3 & 154.2 & 167.3 & 171.4 & 192.9 & 207.1 & 243.9 & 315.5\end{array}$ $344.6 \quad 370.8 \quad 400.6 \quad 435.1 \quad 452.6 \quad 471.6 \quad 489.4 \quad 563.6 \quad 654.1 \quad 735.4 \quad 800.3 \quad 818.3 \quad 839.0 \quad 887.0$ $945.0 \quad 979.5 \quad 983.7 \quad 1012.2 \quad 1025.5 \quad 1040.4 \quad 1049.2 \quad 1058.4 \quad 1079.8 \quad 1118.1 \quad 1151.7 \quad 1164.8 \quad 1173.0$ $\begin{array}{llllllllllll}1184.6 & 1197.0 & 1210.1 & 1223.9 & 1245.7 & 1258.6 & 1260.9 & 1267.2 & 1270.4 & 1291.2 & 1294.6 & 1297.0\end{array}$ $1300.51303 .8 \quad 1304.51311 .7 \quad 1341.6 \quad 1380.0 \quad 1383.3 \quad 1402.01407 .1 \quad 1593.8 \quad 1608.51783 .4$ $2590.72615 .92661 .8 \quad 2670.3 \quad 2672.8 \quad 2674.6 \quad 2677.12678 .42680 .92693 .32702 .2 \quad 2709.4$ $2726.02777 .5 \quad 2780.8 \quad 2782.7$

name $=$ MIN387

energy (in $\mathrm{kcal} / \mathrm{mol})=29.506381$

$\mathrm{ZPE}(\mathrm{in} \mathrm{kcal} / \mathrm{mol})=140.974$

G $($ in $\mathrm{kcal} / \mathrm{mol})=113.008$

Geometry

C $-2.60873606-1.87010016-0.98315396$

C $-1.14817688-1.50144641-0.94769605$

C $0.868878161 .24353472-0.96972394$

C $1.35439997 \quad 1.181050320 .41234566$

C $1.18609828-0.161922921 .13277685$

C $1.95879026-1.265360010 .40216535$

C $-0.58262583-1.54357510 \quad 0.33448792$

C $-0.31793790-0.58872964 \quad 1.37884224$

C $-1.29084231 \quad 0.59719024 \quad 1.38534348$

C $-0.40923687-1.301771832 .74413199$

$\mathrm{H}-3.17443101-1.10644489-1.53918665$

$\mathrm{H}-3.06495323-1.98885198 \quad 0.00448207$

$\mathrm{H}-2.74262238-2.81703888-1.53146023$

$\mathrm{H} \quad 0.70906470 \quad 0.37469272 \quad-1.60253895$ 
$\mathrm{H} \quad 0.88464478 \quad 2.00955377 \quad 1.00707668$

$\mathrm{H} 2.439194141 .46536002 \quad 0.41934334$

$\mathrm{H} 1.63767708-0.039509522 .15296124$

H $3.03141258-1.04162547 \quad 0.36353010$

$\mathrm{H} \quad 1.63234327-1.40048129-0.64154104$

H $1.84825875 \quad-2.23853961 \quad 0.89970977$

$\mathrm{H}-2.30854070 \quad 0.27331935 \quad 1.63336780$

$\mathrm{H}-1.33882092 \quad 1.103694850 .41384985$

$\mathrm{H}-1.00452807 \quad 1.348398292 .13171996$

$\mathrm{H}-0.17125169-0.62047146 \quad 3.56768985$

H $0.27930925-2.154589502 .79517992$

$\mathrm{H}-1.41646039-1.69453522 \quad 2.92158411$

$0 \quad 0.76567257 \quad 3.43112292-0.90981497$

$\begin{array}{lllll}0 & 0.60479831 & 2.36346001 & -1.56527795\end{array}$

o $-0.50362238-1.33949660-1.97509938$

Vibrational frequencies (in cm-1)

$\begin{array}{llllllllllllllll}11.9 & 51.2 & 60.3 & 89.2 & 100.3 & 105.8 & 141.3 & 177.2 & 187.6 & 191.7 & 218.8 & 229.9 & 245.8 & 258.7\end{array}$ $307.9344 .3 \quad 381.6 \quad 399.2 \quad 417.0 \quad 444.0 \quad 480.3 \quad 559.1 \quad 577.5 \quad 616.6 \quad 728.0 \quad 751.4 \quad 802.7 \quad 834.9$ $957.3968 .7 \quad 982.3 \quad 993.4 \quad 1025.4 \quad 1031.0 \quad 1040.8 \quad 1047.4 \quad 1067.9 \quad 1089.0 \quad 1100.01137 .5 \quad 1161.2$

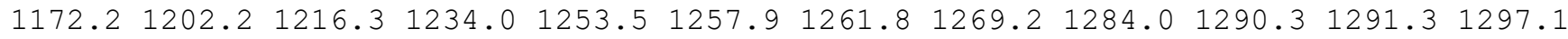
$1303.01304 .1 \quad 1306.4 \quad 1333.5 \quad 1375.3 \quad 1384.3 \quad 1396.91402 .2 \quad 1406.8 \quad 1556.3 \quad 1594.4 \quad 1775.8$ $2614.22644 .42660 .3 \quad 2665.42668 .92670 .42671 .22673 .92676 .62677 .62682 .42713 .8$ $2773.12776 .12778 .6 \quad 2781.3$

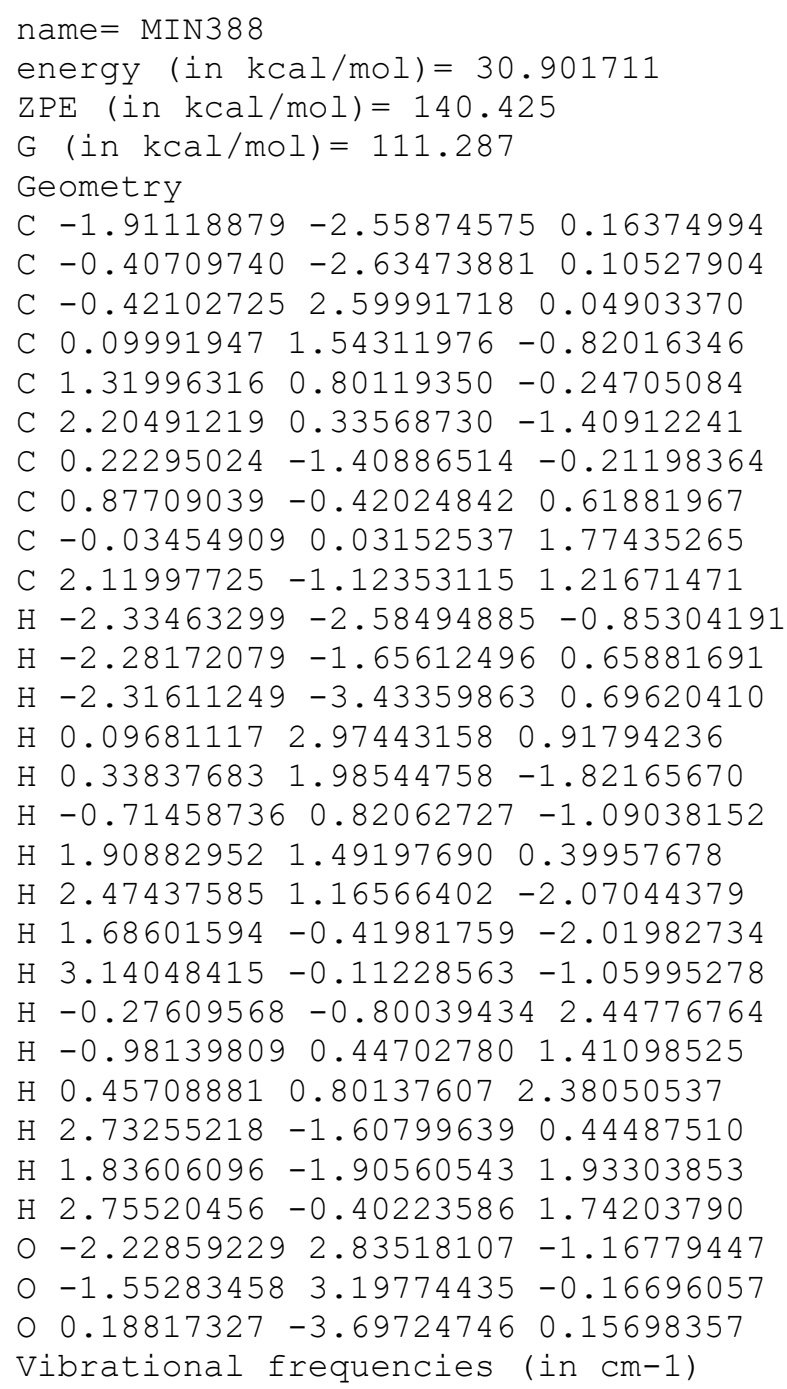


$2609.72660 .82661 .6 \quad 2662.2 \quad 2668.3 \quad 2670.3 \quad 2673.42675 .92676 .42698 .42709 .62732 .7$ 2774.32776 .62777 .62778 .4

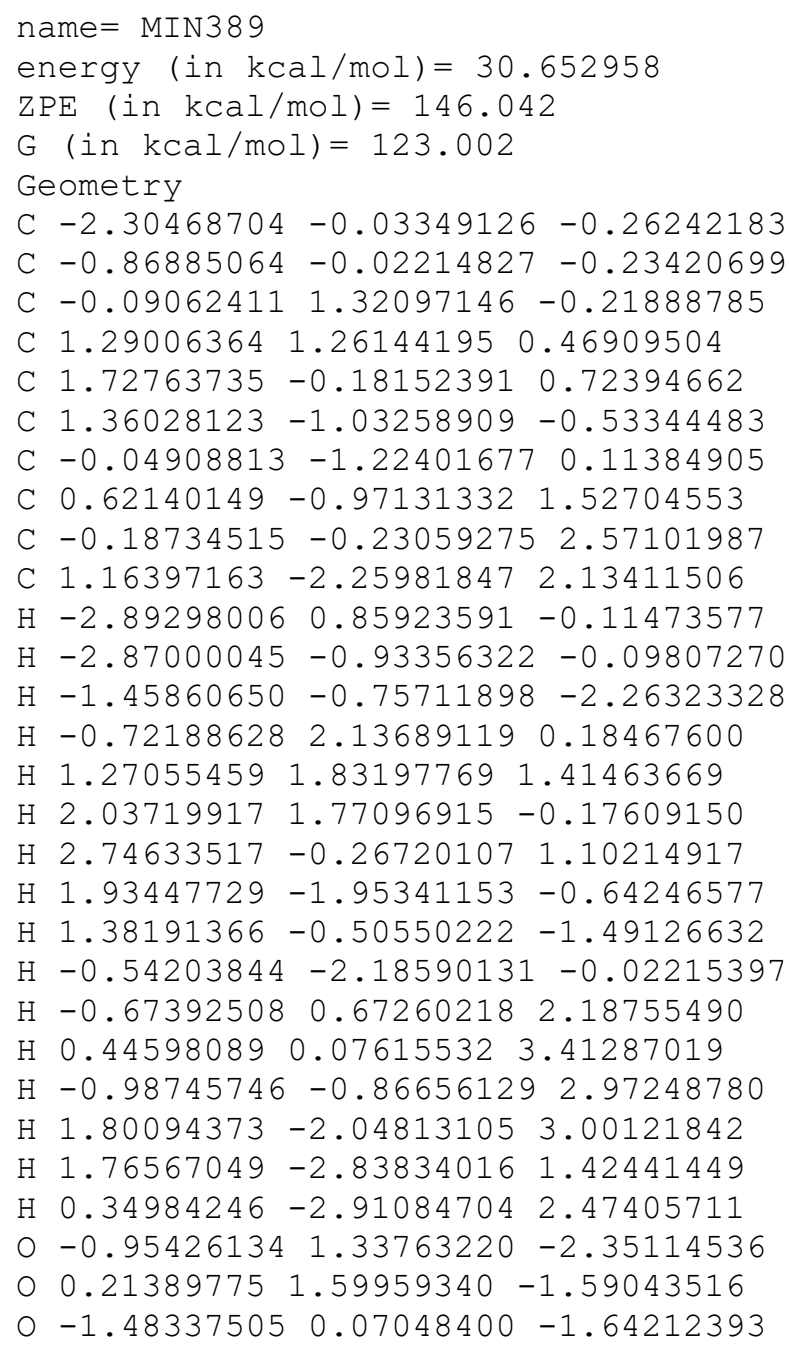


$\mathrm{H}-3.03857471 \quad 0.73261373 \quad 0.10160071$

$\mathrm{H}-0.35125668 \quad 1.57605476 \quad 1.08044924$

$\mathrm{H} 1.326765591 .86568335-1.13980372$

$\mathrm{H} \quad 1.242170990 .11241552-1.09967150$

$\mathrm{H} \quad 1.98428286 \quad 1.85767102 \quad 1.36606174$

$\begin{array}{lllll}\text { H } & 3.77012114 & 0.12125018 & 1.62391404\end{array}$

H $3.18972947-0.726548820 .15140523$

$\mathrm{H}-0.26397075-1.90872119-0.88098819$

$\mathrm{H} \quad 0.95338584-0.611490392 .68179006$

$\mathrm{H} \quad 0.14881491-2.03914095 \quad 3.35769336$

$\mathrm{H}-0.81415195-0.732278772 .65947152$

$\mathrm{H} \quad 0.47009457-4.020107941 .72785627$

H $1.97929355-3.104019811 .57284809$

$\mathrm{H} 1.09995551-3.61554724 \quad 0.12651056$

$0-1.270218051 .63630608-2.16624600$

$\begin{array}{llll}0 & -1.02353194 & 2.08089228 & -0.67769857\end{array}$

$\begin{array}{lllll}0 & -0.59992488 & 0.67998336 & -2.55110883\end{array}$

Vibrational frequencies (in $\mathrm{cm}-1$ )

$\begin{array}{llllllllllllllll}25.6 & 48.7 & 55.4 & 64.8 & 73.8 & 104.1 & 111.4 & 124.5 & 130.6 & 149.2 & 167.1 & 255.5 & 272.4 & 331.7\end{array}$

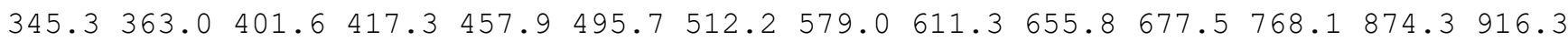
$933.3954 .2979 .0981 .1 \quad 995.6 \quad 1017.0 \quad 1018.1 \quad 1024.7 \quad 1078.6 \quad 1080.9 \quad 1091.01108 .91118 .9$ $1131.61148 .91156 .6 \quad 1223.3 \quad 1259.4 \quad 1268.6 \quad 1273.1 \quad 1278.6 \quad 1283.9 \quad 1287.2 \quad 1288.1 \quad 1309.7$ $1333.01351 .6 \quad 1359.7 \quad 1368.0 \quad 1391.3 \quad 1391.91398 .6 \quad 1406.6 \quad 1420.3 \quad 1840.7 \quad 1849.8 \quad 1880.9$ $2648.22655 .7 \quad 2663.8 \quad 2671.92673 .7 \quad 2676.8 \quad 2717.82721 .92733 .12734 .62749 .2 \quad 2757.8$ $2776.6 \quad 2780.02787 .5 \quad 2790.9$

\section{name $=$ MIN391}

energy (in $\mathrm{kcal} / \mathrm{mol})=31.454411$

$\mathrm{ZPE}(\mathrm{in} \mathrm{kcal} / \mathrm{mol})=145.878$

$\mathrm{G}($ in $\mathrm{kcal} / \mathrm{mol})=122.315$

Geometry

C $-2.37640384-0.08157280-0.03637596$

C $-0.93914279-0.06528768-0.03881307$

C $-0.19754160 \quad 1.29039851-0.03723610$

C $1.341321061 .16514211-0.05992580$

C $1.80689716-0.25780904 \quad 0.24640708$

$\begin{array}{llll}\text { C } 1.09634400 & -1.21409079 & -0.76328500\end{array}$

C $-0.08898789-1.26203873 \quad 0.25628281$

C $0.94678127-0.90975376 \quad 1.40186255$

C $0.47380515-0.02877386 \quad 2.54277964$

C $1.61151655-2.163744451 .95849732$

H $-2.95681572 \quad 0.81786571 \quad 0.10845757$

$\mathrm{H}-2.93863823-0.97554105 \quad 0.16112226$

$\mathrm{H}-1.59988663-0.85782716-2.02492306$

H $-0.52994067 \quad 1.92481521 \quad 0.81544446$

$\mathrm{H} 1.79005134 \quad 1.883198290 .64868865$

$\mathrm{H} \quad 1.70650742 \quad 1.46975322-1.06421931$

H $2.88860489-0.35169783 \quad 0.34416229$

$\mathrm{H} \quad 1.59823871-2.16956636-0.91833533$

$\mathrm{H} \quad 0.87412299-0.78383302-1.74272131$

$\mathrm{H}-0.62653027-2.20260633 \quad 0.36845807$

$\mathrm{H}-0.58573093 \quad 0.23822434 \quad 2.45243792$

$\mathrm{H} \quad 1.05188496 \quad 0.90145769 \quad 2.60203679$

$\mathrm{H} \quad 0.57743147-0.52170542 \quad 3.51813140$

H $2.47706085-1.903885692 .58087748$

$\mathrm{H} 1.97122915-2.83610097 \quad 1.17223910$

$\mathrm{H} \quad 0.91450919-2.738187392 .57995816$

O $-0.92530132 \quad 1.14565503 \quad-2.22854902$

$\begin{array}{lllll}0 & -0.66292884 & 2.03599012 & -1.16035666\end{array}$

o $-1.58507667-0.00802029-1.43586836$

Vibrational frequencies (in $\mathrm{cm}-1$ )

$\begin{array}{lllllllllllllllll}50.5 & 93.1 & 132.6 & 179.5 & 189.7 & 214.0 & 250.6 & 287.9 & 305.4 & 358.7 & 387.1 & 424.5 & 434.4 & 442.6\end{array}$ $\begin{array}{llllllllllllll}458.0 & 510.9 & 569.8 & 581.5 & 623.7 & 707.9 & 722.8 & 752.3 & 794.8 & 815.2 & 851.4 & 888.9 & 914.8 & 935.3\end{array}$ $940.6959 .0 \quad 974.9999 .3 \quad 1016.0 \quad 1019.4 \quad 1021.2 \quad 1045.2 \quad 1058.91085 .7 \quad 1088.81099 .11111 .6$ 


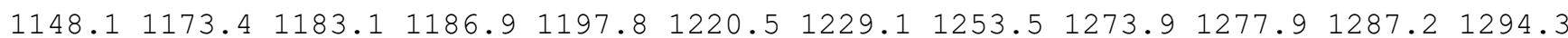
$1297.51299 .3 \quad 1300.6 \quad 1306.6 \quad 1312.4 \quad 1322.4 \quad 1362.8 \quad 1370.5 \quad 1376.6 \quad 1406.1 \quad 1424.91543 .4$ $2656.72666 .92673 .12675 .6 \quad 2678.0 \quad 2682.0 \quad 2687.5 \quad 2721.02740 .12747 .72752 .3 \quad 2763.3$ $2779.32781 .7 \quad 2786.12905 .3$

name $=$ MIN392

energy (in $\mathrm{kcal} / \mathrm{mol})=39.960646$

$\mathrm{ZPE}($ in $\mathrm{kcal} / \mathrm{mol})=138.661$

$\mathrm{G}($ in $\mathrm{kcal} / \mathrm{mol})=109.579$

Geometry

$\begin{array}{lllll}\text { C }-2.72865240 & 0.30277107 & -0.29331341\end{array}$

C $-1.22646815 \quad 0.277983690 .08336126$

C $1.055203582 .86506527-0.05677637$

C $1.39130967 \quad 2.89345740-1.34402282$

C $0.18800907-2.33186858 \quad 0.98177018$

C $1.09194586-3.12334034 \quad 1.55576802$

C $-1.218668650 .15767109 \quad 1.54344648$

C $0.48799855-1.45846061-0.20742851$

C $0.81147756-2.30795078-1.44692834$

C $1.59389301-0.452232160 .09554309$

$\mathrm{H}-3.111872091 .32788291-0.33571299$

$\mathrm{H}-3.33709197-0.28992459 \quad 0.40187972$

$\mathrm{H}-2.85775432-0.14772228-1.29107926$

H $1.50719950 \quad 3.42838740 \quad 0.75993375$

H $2.18770523 \quad 3.50354788-1.73520252$

$\mathrm{H} 0.89316186 \quad 2.31432005-2.10575549$

$\mathrm{H}-0.84313914-2.26664681 \quad 1.33910560$

H $2.11543089-3.20903021 \quad 1.22840674$

H $0.87242933-3.75112348 \quad 2.40465013$

$\mathrm{H}-1.16914792 \quad 1.06314396 \quad 2.12467922$

H $1.70422428-2.91820234-1.28472617$

H $0.97682008-1.67106477-2.32409902$

$\mathrm{H}-0.02400545-2.97848781-1.68521912$

$\mathrm{H} 1.40867993 \quad 0.086668361 .03132200$

H $1.67119754 \quad 0.29503901-0.70774541$

$\mathrm{H} \quad 2.56749881-0.945004160 .18878549$

$\begin{array}{lllll}0 & -0.68242044 & 1.45585528 & -0.51776610\end{array}$

$\begin{array}{lllll}0 & 0.07398983 & 2.12099002 & 0.52015218\end{array}$

$0-0.71110388-0.80703436-0.63743983$

Vibrational frequencies (in $\mathrm{cm}-1$ )

$\begin{array}{llllllllllllllll}-5.4 & 47.8 & 54.1 & 67.3 & 71.6 & 109.4 & 126.1 & 141.1 & 181.3 & 195.7 & 218.2 & 232.6 & 256.0 & 265.9\end{array}$ $274.5 \quad 299.4 \quad 341.6 \quad 347.3 \quad 402.3 \quad 451.2 \quad 487.9 \quad 505.3 \quad 534.6 \quad 606.2 \quad 613.7 \quad 620.3 \quad 658.2 \quad 838.7$

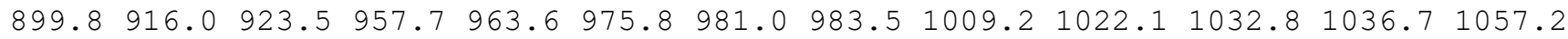
$1073.01076 .8 \quad 1088.1 \quad 1174.6 \quad 1209.1 \quad 1225.2 \quad 1244.6 \quad 1274.2 \quad 1277.0 \quad 1279.3 \quad 1284.2 \quad 1288.2$

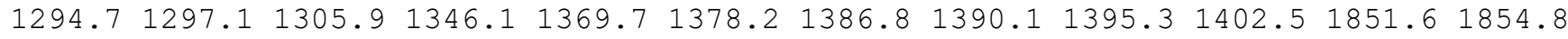
$2656.42667 .3 \quad 2670.7 \quad 2672.5 \quad 2677.1 \quad 2677.9 \quad 2683.82711 .12714 .02734 .42742 .3 \quad 2769.1$ $2778.5 \quad 2781.92790 .12793 .7$

name $=$ MIN393

energy (in $\mathrm{kcal} / \mathrm{mol})=32.994663$

$\mathrm{ZPE}(\mathrm{in} \mathrm{kcal} / \mathrm{mol})=145.921$

$\mathrm{G}($ in $\mathrm{kcal} / \mathrm{mol})=122.815$

Geometry

C $-2.56941180 \quad 0.02756376 \quad 0.19035141$

C $-1.09235454-0.01589954 \quad 0.01388307$

C $-0.36624426 \quad 1.36650784-0.01534571$

C $1.15852832 \quad 1.15532408 \quad-0.20152248$

C $1.52214338-0.32008202-0.07246784$

C $0.46013962-1.22589151-0.77770012$

C $-0.31051616-1.225893050 .58601363$

C $1.00636771-0.86962476 \quad 1.32727596$

C $0.91800595 \quad 0.07519088 \quad 2.50608048$

C $1.76638150 \quad-2.12847280 \quad 1.73497135$ 
$\mathrm{H}-3.03146080 \quad 0.76635607 \quad-0.49046121$

$\mathrm{H}-2.83979147 \quad 0.32590291 \quad 1.21445791$

$\mathrm{H}-3.05239897-0.939859320 .00182155$

$\mathrm{H}-0.63198395 \quad 2.03376777 \quad 0.81791558$

H $1.73287795 \quad 1.76544862 \quad 0.51322500$

H $1.43802481 \quad 1.52506177-1.21461847$

$\mathrm{H} 2.56355590-0.53791726-0.31374476$

$\mathrm{H} \quad 0.76113314-2.08328723-1.33303198$

$\mathrm{H}-1.31907294-0.99412323-1.89640133$

$\mathrm{H}-0.89059972-2.06185201 \quad 0.94572797$

$\mathrm{H} \quad 0.64687178-0.46217802 \quad 3.42442746$

$\mathrm{H} \quad 0.16201388 \quad 0.85546354 \quad 2.37348512$

$\mathrm{H} \quad 1.87949316 \quad 0.57121337 \quad 2.68763468$

H $1.87448120 \quad-2.83529721 \quad 0.90422123$

$\mathrm{H} 1.25407435-2.65871367 \quad 2.54595890$

H $2.77476110-1.880882272 .08662776$

$0-0.670086491 .05636051-2.23042333$

$0-0.88912196 \quad 1.98581705-1.17800265$

$0-0.63242687-0.35793915-1.42408008$

Vibrational frequencies (in $\mathrm{cm}-1$ )

$\begin{array}{lllllllllllllllll}116.1 & 134.4 & 156.8 & 159.8 & 168.6 & 198.6 & 211.6 & 268.3 & 281.5 & 334.7 & 369.9 & 392.6 & 432.4 & 455.8\end{array}$

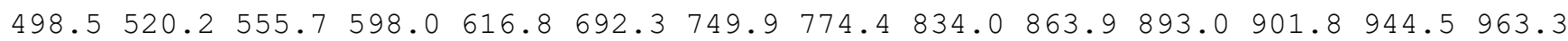
$969.1992 .1 \quad 1001.1 \quad 1013.6 \quad 1015.8 \quad 1019.0 \quad 1033.1 \quad 1056.7 \quad 1073.8 \quad 1096.5 \quad 1113.21123 .6$ $1130.4 \quad 1131.3 \quad 1163.6 \quad 1179.2 \quad 1189.6 \quad 1192.9 \quad 1205.3 \quad 1216.91249 .7 \quad 1260.91273 .8 \quad 1281.3$

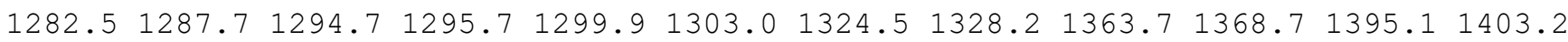
$1413.92655 .0 \quad 2657.6 \quad 2671.92672 .7 \quad 2677.3 \quad 2677.7 \quad 2680.92707 .4 \quad 2740.3 \quad 2746.5 \quad 2763.5$ $2771.12776 .0 \quad 2782.12783 .92878 .5$

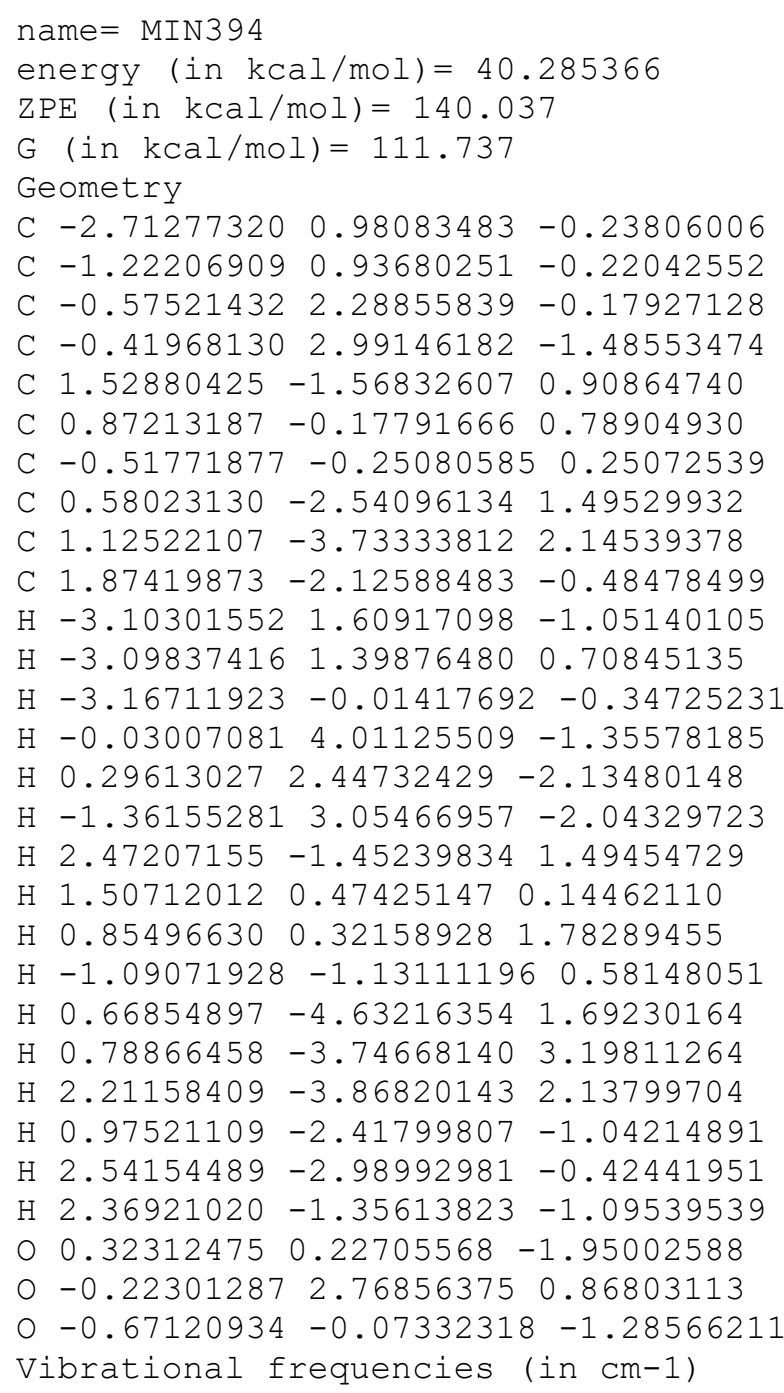


$\begin{array}{lllllllllllllllllll}22.7 & 48.6 & 61.9 & 79.5 & 97.9 & 104.2 & 120.3 & 128.3 & 135.0 & 144.7 & 185.9 & 205.5 & 231.8 & 257.1\end{array}$

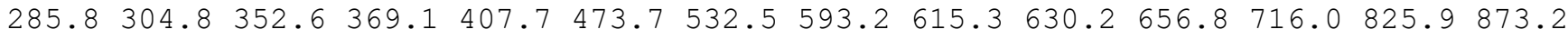

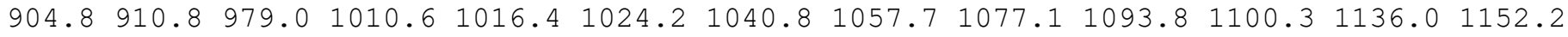

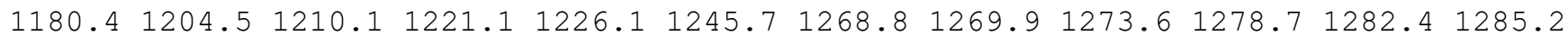
$1289.01294 .21301 .6 \quad 1325.2 \quad 1357.0 \quad 1371.7 \quad 1378.6 \quad 1394.21400 .6 \quad 1409.61503 .1 \quad 1851.8$ $2636.42642 .6 \quad 2656.0 \quad 2660.2 \quad 2661.1 \quad 2669.2 \quad 2671.3 \quad 2677.02677 .82691 .82700 .5 \quad 2728.9$ $2770.12773 .7 \quad 2775.6 \quad 2777.8$

\section{name $=$ MIN395}

energy (in $\mathrm{kcal} / \mathrm{mol})=41.004937$

$\operatorname{ZPE}($ in $\mathrm{kcal} / \mathrm{mol})=141.482$

$\mathrm{G}($ in $\mathrm{kcal} / \mathrm{mol})=115.664$

Geometry

C $-2.16666567 \quad 0.11664987-0.71706615$

C $-0.66458935 \quad 0.07248247-0.85545092$

C $0.07972404 \quad 1.53216349-0.79915796$

C $2.31592031-0.933616330 .79035038$

C $1.37538242-0.93577406 \quad 1.89834576$

C $1.47691119-1.23521586-0.46986477$

C $-0.01180991-1.06318834-0.07290661$

C $-0.02177691-0.83615184 \quad 1.46888308$

C $-0.177381790 .64464793 \quad 1.95605460$

C $-1.01835158-1.72292638 \quad 2.18934511$

$\mathrm{H}-2.52021026-0.05822946 \quad 0.29804165$

$\mathrm{H}-2.65104629-0.59638091-1.39247822$

$\mathrm{H}-2.512133531 .13066503-1.01163232$

H $1.127556581 .44477344-0.44854110$

H $2.83863053 \quad 0.05789406 \quad 0.67570004$

H $3.16751670-1.63831110 \quad 0.91928593$

H $1.68229395-0.98606167 \quad 2.94359787$

$\mathrm{H} \quad 1.67108344-2.24929713-0.85244756$

H $1.73382156-0.55551950-1.31595084$

$\mathrm{H}-0.56277500-2.00286605-0.31014127$

$\mathrm{H}-0.42416158 \quad 0.72613698 \quad 3.01313814$

$\mathrm{H}-1.02799715 \quad 1.11294117 \quad 1.37229856$

$\mathrm{H} \quad 0.622499491 .35139606 \quad 1.68663357$

$\mathrm{H}-0.99005134-1.61440953 \quad 3.27938399$

$\mathrm{H}-0.87477419-2.78467200 \quad 1.95061124$

$\mathrm{H}-2.04840445-1.47060868 \quad 1.87456104$

$0.170913201 .36466517-2.29951497$

$0-0.590256152 .48367478-0.34310835$

$0-0.31389854-0.00639325-2.24410427$

Vibrational frequencies (in $\mathrm{cm}-1$ )

$\begin{array}{llllllllllllllll}-16.3 & 77.3 & 87.8 & 107.2 & 130.0 & 166.6 & 178.7 & 218.6 & 236.9 & 267.3 & 274.8 & 283.1 & 308.5 & 348.4\end{array}$

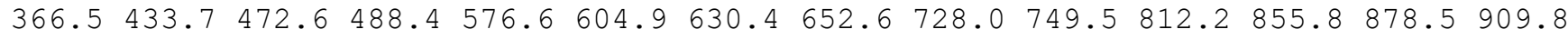
$951.6 \quad 969.5 \quad 975.3 \quad 981.4 \quad 1005.8 \quad 1016.4 \quad 1022.8 \quad 1037.6 \quad 1056.1 \quad 1068.5 \quad 1083.3 \quad 1098.6 \quad 1130.5$

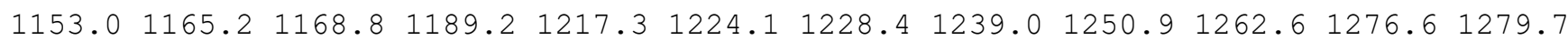

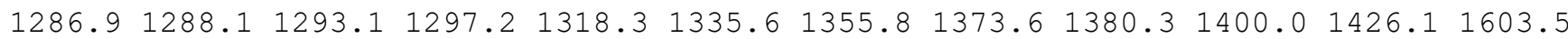
$2486.22575 .82585 .5 \quad 2628.8 \quad 2648.5 \quad 2649.92658 .52671 .62689 .32689 .82697 .62700 .6$ $2734.3 \quad 2747.0 \quad 2774.0 \quad 2775.7$

name $=$ MIN396

energy $($ in $\mathrm{kcal} / \mathrm{mol})=39.611897$

$\mathrm{ZPE}($ in $\mathrm{kcal} / \mathrm{mol})=143.951$

$\mathrm{G}($ in $\mathrm{kcal} / \mathrm{mol})=118.769$

Geometry

C $-1.93424637-0.31497916-1.07799554$

C $-0.42208181-0.44754673-0.92929921$

C $0.29803367 \quad 0.93573303 \quad-0.97573047$

C $1.521767961 .04657549-0.07421458$

C $1.48474662 \quad 0.39948453 \quad 1.23669831$

C $0.39185812-0.561178191 .50188283$

C $-0.01786828-1.340385220 .24400667$ 
C $-0.76806651 \quad 0.24552728 \quad 2.14736182$

C $-1.85504868 \quad-0.691524852 .68116661$

C $-0.226492021 .11418600 \quad 3.29002386$

$\mathrm{H}-2.19466866 \quad 0.33525876-1.92531353$

$\mathrm{H}-2.39516070-1.29138535-1.28539829$

$\mathrm{H}-2.40369786 \quad 0.08888534-0.17572033$

$\mathrm{H}-0.40200696 \quad 1.78343550-0.83616180$

H 1.806090312 .116127910 .05876210

$\mathrm{H} \quad 2.41676949 \quad 0.61854606-0.60365373$

$\mathrm{H}-1.21530661 \quad 0.91170058 \quad 1.37117604$

$\mathrm{H} \quad 0.83207292-1.98414256-0.07228898$

$\mathrm{H} 0.75643510 \quad-1.30160548 \quad 2.26296674$

$\mathrm{H}-0.83174417-2.05116998 \quad 0.48355344$

$\mathrm{H}-1.46153581-1.373184313 .44332245$

$\mathrm{H}-2.29247635-1.303064421 .88437440$

$\mathrm{H}-2.67111142-0.12337388 \quad 3.14310844$

$\mathrm{H} \quad 0.51416094 \quad 1.84314346 \quad 2.93598172$

$\mathrm{H} \quad 0.27186194 \quad 0.50525248 \quad 4.05352246$

$\mathrm{H}-1.02970148 \quad 1.67225964 \quad 3.78134927$

O $1.05833914-0.29838523-2.66931427$

$0 \quad 0.69895007 \quad 1.03048652-2.34328101$

o $0.00018794-1.09477787-2.14607262$

Vibrational frequencies (in $\mathrm{cm}-1$ )

$\begin{array}{lllllllllllllll}46.3 & 57.5 & 95.0 & 105.5 & 155.2 & 162.2 & 179.6 & 208.3 & 228.0 & 276.8 & 292.0 & 345.2 & 356.2 & 376.8\end{array}$ $399.8 \quad 431.6 \quad 461.6 \quad 498.2 \quad 522.4 \quad 634.7 \quad 710.8 \quad 732.6 \quad 776.7 \quad 848.9 \quad 868.5 \quad 908.2 \quad 915.1967 .6$ $974.6 \quad 975.4 \quad 980.5 \quad 990.3 \quad 1009.4 \quad 1014.8 \quad 1064.4 \quad 1095.9 \quad 1110.0 \quad 1137.9 \quad 1141.91158 .2 \quad 1165.1$

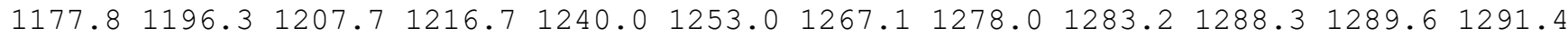

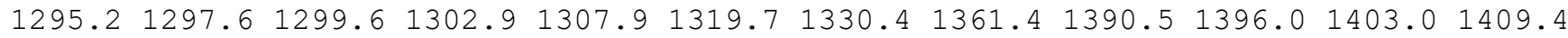
$2629.42653 .5 \quad 2666.3 \quad 2666.8 \quad 2672.7 \quad 2674.4 \quad 2675.8 \quad 2679.22689 .5 \quad 2693.5 \quad 2708.62726 .0$ $2737.4 \quad 2778.0 \quad 2779.4 \quad 2781.7$

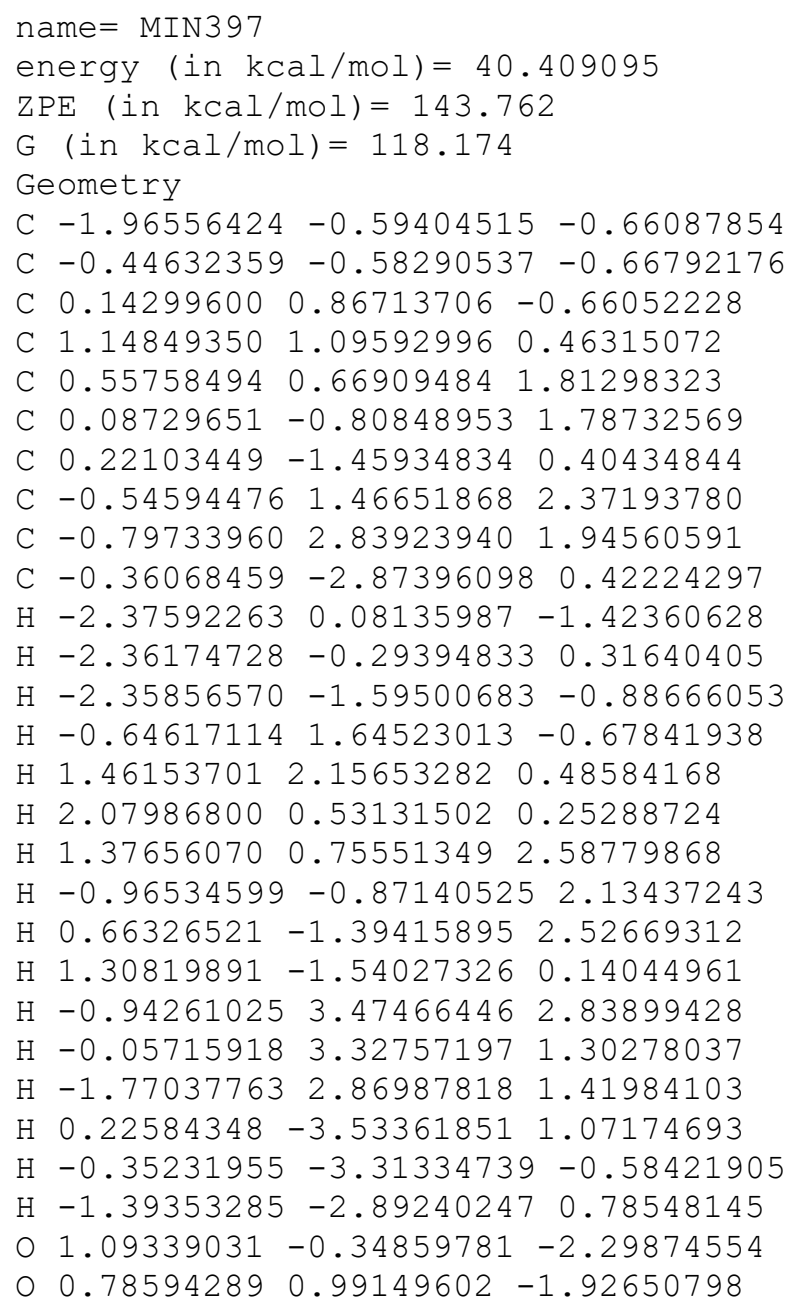


$0-0.07257203-1.07531827-1.96732919$

Vibrational frequencies (in $\mathrm{cm}-1$ )

$\begin{array}{lllllllllllllllll}26.0 & 59.1 & 92.9 & 111.1 & 123.3 & 155.5 & 188.0 & 208.5 & 232.9 & 269.6 & 286.4 & 307.7 & 352.2 & 372.7\end{array}$

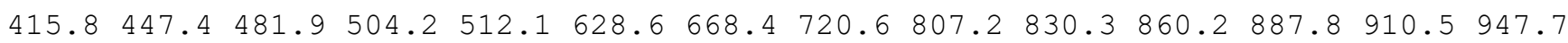
$955.3 \quad 971.3 \quad 985.0 \quad 1005.5 \quad 1031.4 \quad 1050.2 \quad 1066.6 \quad 1080.5 \quad 1121.4 \quad 1135.0 \quad 1138.91161 .1 \quad 1169.4$ $\begin{array}{llllllllllllll}1183.3 & 1196.5 & 1213.7 & 1216.9 & 1222.7 & 1228.4 & 1247.3 & 1264.9 & 1266.0 & 1283.5 & 1286.7 & 1291.9\end{array}$

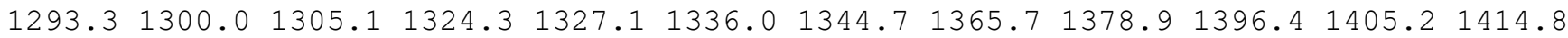
$2649.92654 .0 \quad 2656.2 \quad 2664.7 \quad 2669.12671 .2 \quad 2674.42675 .12675 .92691 .82698 .42740 .0$ $2742.02767 .2 \quad 2778.8 \quad 2780.4$

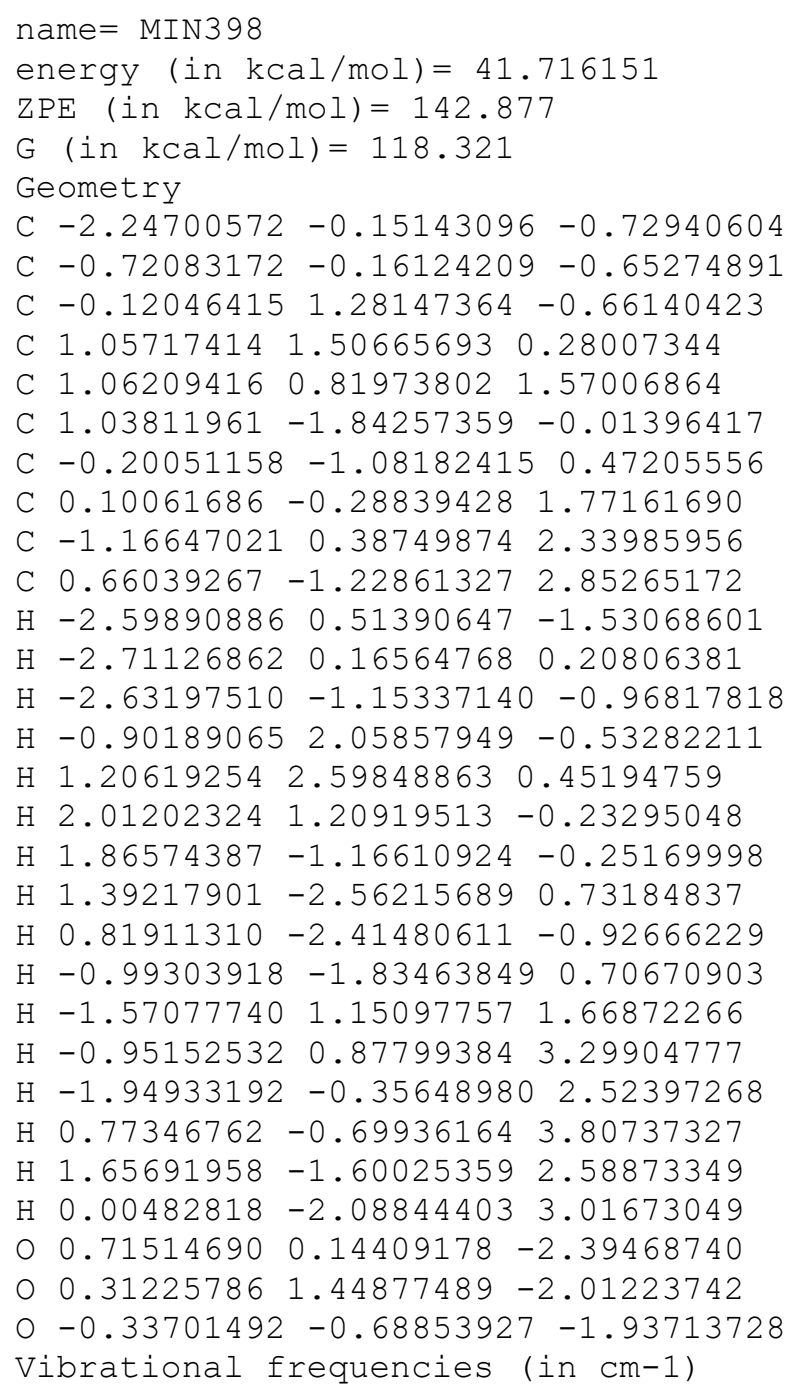


C $1.18920490-0.55065560 \quad 1.11477946$

C $-0.08779971-0.83783400 \quad 0.34641183$

C $-0.13045166-0.419367121 .80943782$

$\begin{array}{llll}\text { C }-0.99800076 & 0.53037777 & 2.51205161\end{array}$

C $0.77327623-2.86868563 \quad 2.35289129$

$\mathrm{H}-2.62223851 \quad 0.44117124-1.60345003$

$\mathrm{H}-2.62261045 \quad 0.25447497 \quad 0.16884576$

$\mathrm{H}-2.47249951-1.15792018-0.88482257$

$\mathrm{H}-1.16945288 \quad 2.20075417 \quad-0.82555244$

$\mathrm{H} \quad 0.69553003 \quad 2.01228258 \quad 1.00755378$

$\mathrm{H} \quad 1.00171830 \quad 3.18845029-0.27735088$

$\mathrm{H} \quad 1.44075489-1.02698695 \quad 3.27483604$

$\mathrm{H} 2.06026530-0.02484386 \quad 0.83478824$

$\mathrm{H} 1.822103221 .63271942-0.31626625$

$\mathrm{H}-0.29249017-1.89435468 \quad 0.09626717$

$\mathrm{H}-1.95972697 \quad 0.05612827 \quad 2.77385723$

$\mathrm{H}-1.23187140 \quad 1.41896891 \quad 1.90667428$

$\mathrm{H}-0.54967764 \quad 0.88142874 \quad 3.45402626$

$\mathrm{H} \quad 0.39645807-3.23703103 \quad 3.31637540$

H $1.74153331-3.354478192 .16553922$

$\mathrm{H} \quad 0.07910124-3.224150211 .58165693$

$0.69031613 \quad 0.39134885-2.55587844$

$0 \quad 0.09544119 \quad 1.64506190-2.28438535$

o $-0.21999606-0.54175946-1.99763563$

Vibrational frequencies (in $\mathrm{cm}-1$ )

$\begin{array}{llllllllllllll}29.1 & 63.4 & 68.5 & 116.9 & 124.2 & 144.5 & 155.9 & 191.5 & 231.3 & 233.7 & 243.5 & 262.5 & 315.6 & 327.5\end{array}$

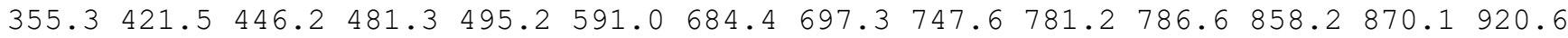
$965.6974 .4 \quad 984.7 \quad 986.4 \quad 1004.8 \quad 1013.3 \quad 1021.4 \quad 1067.1 \quad 1079.2 \quad 1087.1 \quad 1101.6 \quad 1109.5 \quad 1144.8$

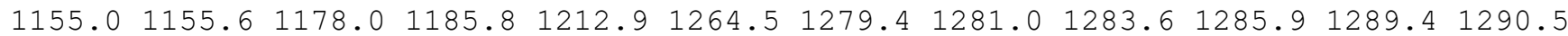

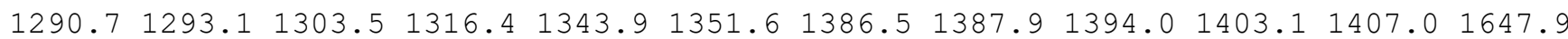
$2661.32666 .42669 .3 \quad 2670.6 \quad 2671.92674 .42675 .62678 .32685 .92718 .12742 .32776 .4$ $2779.02780 .12780 .5 \quad 2801.0$

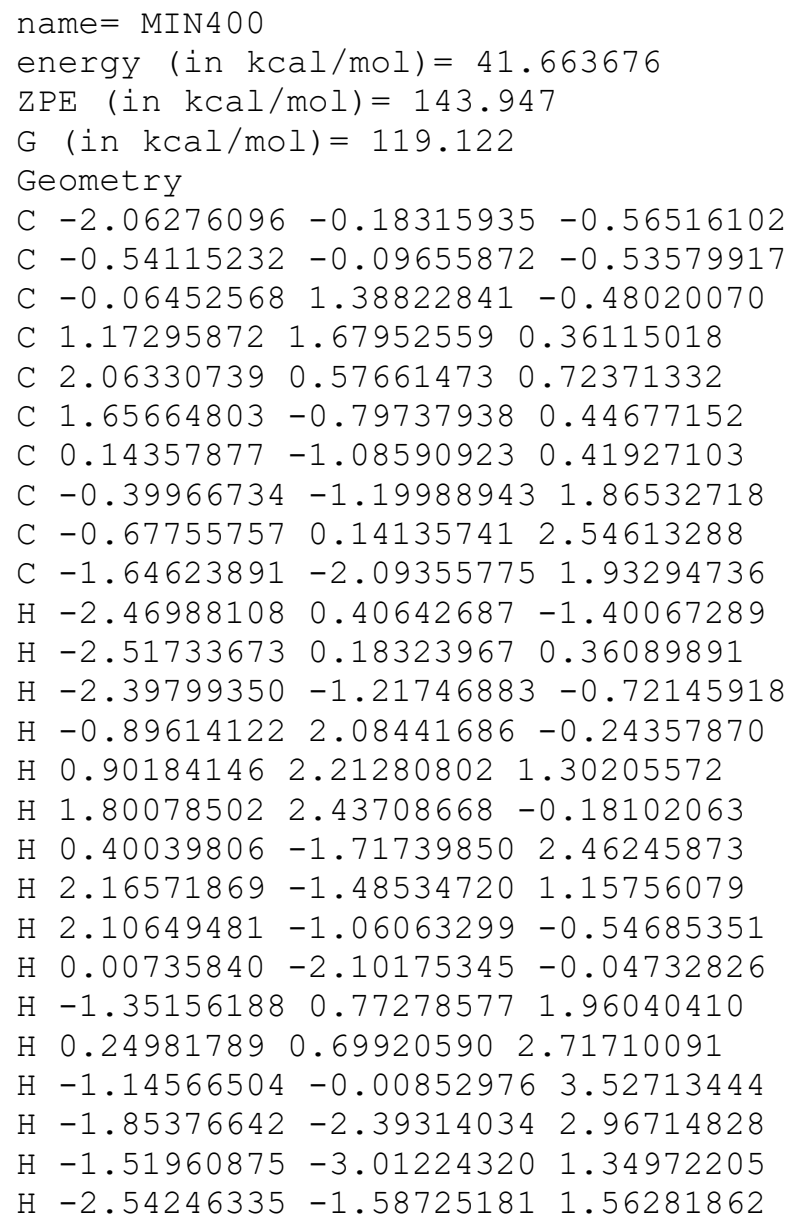


$0.74825348 \quad 0.45799706-2.34596681$

$0.230603501 .67764503-1.85058602$

o $-0.15643209-0.52372588-1.86662642$

Vibrational frequencies (in $\mathrm{cm}-1$ )

$\begin{array}{llllllllllllllll}41.8 & 62.7 & 115.7 & 134.5 & 154.7 & 188.6 & 205.7 & 220.7 & 237.0 & 252.2 & 286.9 & 309.5 & 332.3 & 364.0\end{array}$ $427.3 \quad 451.8 \quad 478.0 \quad 535.1 \quad 555.1 \quad 678.8 \quad 693.9 \quad 711.0 \quad 802.8 \quad 824.5 \quad 842.6 \quad 885.7 \quad 922.6 \quad 939.9$

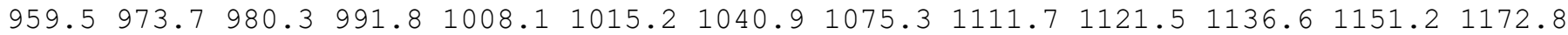
$1176.91184 .2 \quad 1198.5 \quad 1218.1 \quad 1242.7 \quad 1247.7 \quad 1256.4 \quad 1263.0 \quad 1273.6 \quad 1285.2 \quad 1287.4 \quad 1294.4$

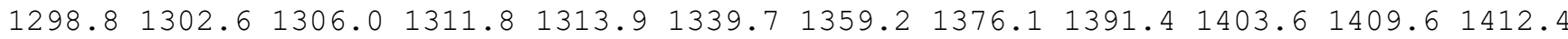
$2633.02638 .42664 .22666 .22671 .2 \quad 2672.62674 .52675 .02678 .32687 .12694 .02725 .1$ $2727.4 \quad 2775.7 \quad 2779.7 \quad 2782.5$

name $=$ MIN401

energy $($ in $\mathrm{kcal} / \mathrm{mol})=42.247209$

$\mathrm{ZPE}($ in $\mathrm{kcal} / \mathrm{mol})=143.96$

$\mathrm{G}($ in $\mathrm{kcal} / \mathrm{mol})=118.836$

Geometry

C $-2.18151702-0.04727334-0.63335187$

C $-0.66012308-0.07438968-0.53957741$

C $-0.02966938 \quad 1.34314459 \quad-0.68285452$

C 1.310149231 .533817690 .02603073

C 2.119800490 .338989990 .30802123

C $1.45511844 \quad-0.96168884 \quad 0.36976526$

C $-0.07024359-0.91984558 \quad 0.60257233$

C $-0.33845122-0.428279872 .04790128$

C $-1.79819809-0.070160852 .33682331$

C $0.08714979-1.524880393 .03995054$

$\mathrm{H}-2.49316140 \quad 0.15340672-1.67257416$

$\mathrm{H}-2.63656769 \quad 0.72605173-0.00856199$

$\mathrm{H}-2.62142583-1.01277197-0.35533867$

$\mathrm{H}-0.74499392 \quad 2.15924549-0.46418865$

H $1.18468094 \quad 2.08055850 \quad 0.98841559$

$\mathrm{H} \quad 1.93740734 \quad 2.22829422 \quad-0.58998679$

H $0.28430924 \quad 0.47952746 \quad 2.23547414$

H $1.95414309-1.599906591 .13076661$

$\mathrm{H} \quad 1.68411389-1.48776014-0.59350453$

$\mathrm{H}-0.48852917-1.951360250 .50307366$

$\mathrm{H}-2.09257746 \quad 0.86423591 \quad 1.84863151$

$\mathrm{H}-1.960668190 .07457732 \quad 3.41241556$

$\mathrm{H}-2.48211231-0.862003412 .01253262$

$\mathrm{H}-0.05072362-1.193942494 .07526129$

H $1.14297723-1.79300496 \quad 2.92824869$

$\mathrm{H}-0.50433351-2.43709978 \quad 2.90502452$

$\begin{array}{lllll}0 & 0.62134031 & 0.13763395 & -2.44068266\end{array}$

$0.18064587 \quad 1.43396467 \quad-2.09459017$

o- $0.27602293-0.74209725-1.76709970$

Vibrational frequencies (in $\mathrm{cm}-1$ )

$\begin{array}{llllllllllllllll}18.9 & 60.5 & 118.0 & 154.0 & 164.1 & 174.4 & 213.3 & 240.6 & 251.6 & 263.7 & 317.9 & 327.7 & 341.6 & 372.7\end{array}$

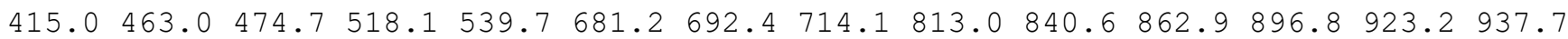
$961.5973 .2980 .0 \quad 990.5 \quad 1008.1 \quad 1014.9 \quad 1034.3 \quad 1066.9 \quad 1098.6 \quad 1120.7 \quad 1131.4 \quad 1146.2 \quad 1157.8$

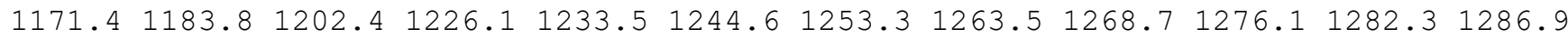

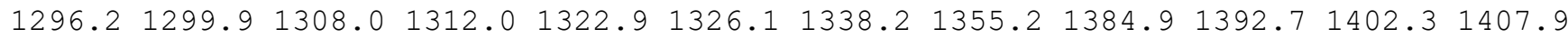
$2633.92640 .82660 .82669 .12670 .72672 .7 \quad 2674.72676 .82691 .52694 .62710 .72726 .2$ $2728.42775 .8 \quad 2779.2 \quad 2780.9$

name $=$ MIN402

energy $($ in $\mathrm{kcal} / \mathrm{mol})=42.50524$

$\operatorname{ZPE}(\mathrm{in} \mathrm{kcal} / \mathrm{mol})=144.063$

$\mathrm{G}($ in $\mathrm{kcal} / \mathrm{mol})=119.14$

Geometry

C $-2.43011591-0.13362252-0.73029723$

C $-0.91155215-0.21357349-0.63086924$

C $-0.21578647 \quad 1.17862885-0.60560271$ 
C $0.79376346 \quad 1.37868032 \quad 0.51777742$

C $1.74227610 \quad 0.19242255 \quad 0.77777303$

C $1.12789832-1.180292470 .44643761$

C $-0.41701749-1.17537154 \quad 0.45636731$

C $-0.80462838-1.05622033 \quad 1.86599491$

C $-1.63799026 \quad 0.01903623 \quad 2.39664252$

$\begin{array}{llll}\text { C } 2.18613138 & 0.22277940 & 2.24770013\end{array}$

$\mathrm{H}-2.73336625 \quad 0.39040432 \quad-1.64971387$

$\mathrm{H}-2.87897554 \quad 0.39261935 \quad 0.11629067$

$\mathrm{H}-2.87876135-1.13444604-0.77804366$

$\mathrm{H}-0.953399492 .01222670-0.62292809$

H $0.24654572 \quad 1.62971325 \quad 1.44777376$

$\mathrm{H} \quad 1.40602370 \quad 2.276011150 .28551049$

H $2.64455638 \quad 0.32406920 \quad 0.12941100$

H $1.49913369-1.94891418 \quad 1.15380946$

$\mathrm{H} \quad 1.47264581-1.51024415-0.55426400$

$\mathrm{H}-0.75739370-2.21263803 \quad 0.15134668$

$\mathrm{H}-1.824925330 .89280353 \quad 1.76483364$

$\mathrm{H}-1.20118141 \quad 0.37687944 \quad 3.34742947$

$\mathrm{H}-2.61696767-0.410808332 .68216135$

H $2.56117536 \quad 1.20895221 \quad 2.53761424$

H $2.98646999-0.50124644 \quad 2.43671863$

H $1.35575228-0.03430352 \quad 2.91866127$

○ $0.66040346-0.06198222-2.26840799$

○ $0.413840231 .28043743-1.88234127$

o $-0.52373562-0.75220365-1.91330435$

Vibrational frequencies (in cm-1)

$\begin{array}{lllllllllllllllll}46.2 & 69.6 & 100.8 & 128.0 & 142.9 & 163.8 & 193.0 & 197.7 & 243.3 & 260.7 & 299.9 & 304.1 & 340.5 & 389.2\end{array}$

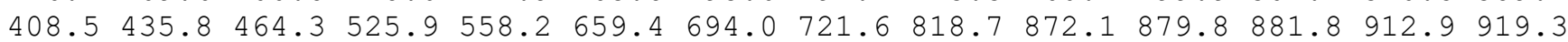
$931.8 \quad 964.0 \quad 986.8 \quad 990.0 \quad 1009.3 \quad 1046.1 \quad 1055.8 \quad 1062.9 \quad 1100.5 \quad 1125.8 \quad 1131.2 \quad 1160.0 \quad 1180.6$ $1188.21205 .0 \quad 1216.8 \quad 1225.2 \quad 1234.5 \quad 1245.8 \quad 1268.8 \quad 1274.1 \quad 1282.7 \quad 1284.3 \quad 1286.2 \quad 1291.5$ $1298.4 \quad 1299.8 \quad 1305.6 \quad 1314.3 \quad 1318.1 \quad 1340.2 \quad 1362.0 \quad 1373.0 \quad 1394.2 \quad 1395.91409 .3 \quad 1414.5$ $2653.52654 .32656 .8 \quad 2664.2 \quad 2665.8 \quad 2667.3 \quad 2669.12675 .52680 .22680 .82703 .12735 .9$ $2737.3 \quad 2766.2 \quad 2779.12779 .7$

\footnotetext{
name $=$ MIN403

energy (in $\mathrm{kcal} / \mathrm{mol})=42.648193$

$\mathrm{ZPE}(\mathrm{in} \mathrm{kcal} / \mathrm{mol})=144.008$

$\mathrm{G}(\mathrm{in} \mathrm{kcal} / \mathrm{mol})=118.683$

Geometry

C $-2.17885929-0.07283761-0.58582962$

C $-0.65508604-0.06618869-0.53306247$

C $-0.048324701 .36622070-0.61834329$

C $1.26022534 \quad 1.55747667 \quad 0.14527379$

C $2.12465048 \quad 0.37849575 \quad 0.31859329$

C $1.50244955-0.94669088 \quad 0.30322146$

C $-0.02209464-0.96186895 \quad 0.54489887$

C $-0.26896738-0.60385962 \quad 2.03530362$

C $-1.57621962 \quad 0.12819707 \quad 2.33823319$

C $-0.22523907-1.898923462 .86380908$

$\mathrm{H}-2.52292743 \quad 0.09329429 \quad-1.62123338$

$\mathrm{H}-2.63421855 \quad 0.70924384 \quad 0.02765976$

$\mathrm{H}-2.59167301-1.03768672-0.26724374$

$\mathrm{H}-0.78473474 \quad 2.16407982-0.39972397$

$\mathrm{H} 1.07589990 \quad 1.97986299 \quad 1.15991891$

$\mathrm{H} 1.858208672 .35012014-0.37021572$

$\mathrm{H} \quad 0.56653005 \quad 0.05655788 \quad 2.38093645$

H $2.02054797-1.607680291 .03157015$

$\mathrm{H} 1.74596095-1.40988787-0.68656796$

$\mathrm{H}-0.41161043-1.995481250 .36543769$

$\mathrm{H}-1.622953691 .099767151 .83682351$

$\mathrm{H}-1.67037760 \quad 0.32125915 \quad 3.41490340$

$\mathrm{H}-2.45176552-0.46092498 \quad 2.04402580$

$\mathrm{H}-0.28127120-1.684081853 .93649263$
} 
$\mathrm{H} \quad 0.70021877-2.45861714 \quad 2.69223223$

$\mathrm{H}-1.06426869-2.560111532 .61918128$

$0.64266702 \quad 0.22385221-2.40698507$

$0.209029891 .51150637-2.01753515$

O $-0.29216853-0.66267241-1.80418810$

Vibrational frequencies (in $\mathrm{cm}-1$ )

$\begin{array}{lllllllllllllllll}15.1 & 61.0 & 113.4 & 137.7 & 154.5 & 172.6 & 220.0 & 246.6 & 251.0 & 272.3 & 306.2 & 324.3 & 338.7 & 365.6\end{array}$ $414.1462 .6 \quad 479.5 \quad 517.1 \quad 545.4 \quad 682.8 \quad 692.3 \quad 713.9 \quad 814.3 \quad 841.3 \quad 859.3 \quad 890.0 \quad 919.7 \quad 938.0$

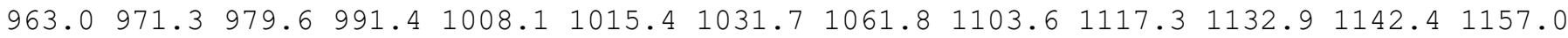

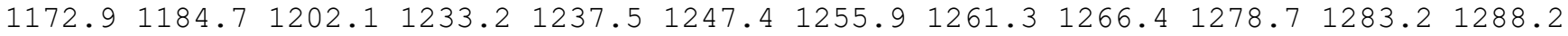
$1298.61301 .61307 .6 \quad 1310.0 \quad 1330.2 \quad 1350.3 \quad 1350.6 \quad 1365.31380 .5 \quad 1396.91404 .61408 .5$ $2639.52643 .92660 .4 \quad 2667.2 \quad 2672.6 \quad 2674.5 \quad 2676.62678 .52687 .12692 .82703 .5 \quad 2729.1$ $2730.5 \quad 2776.7 \quad 2779.7 \quad 2781.6$

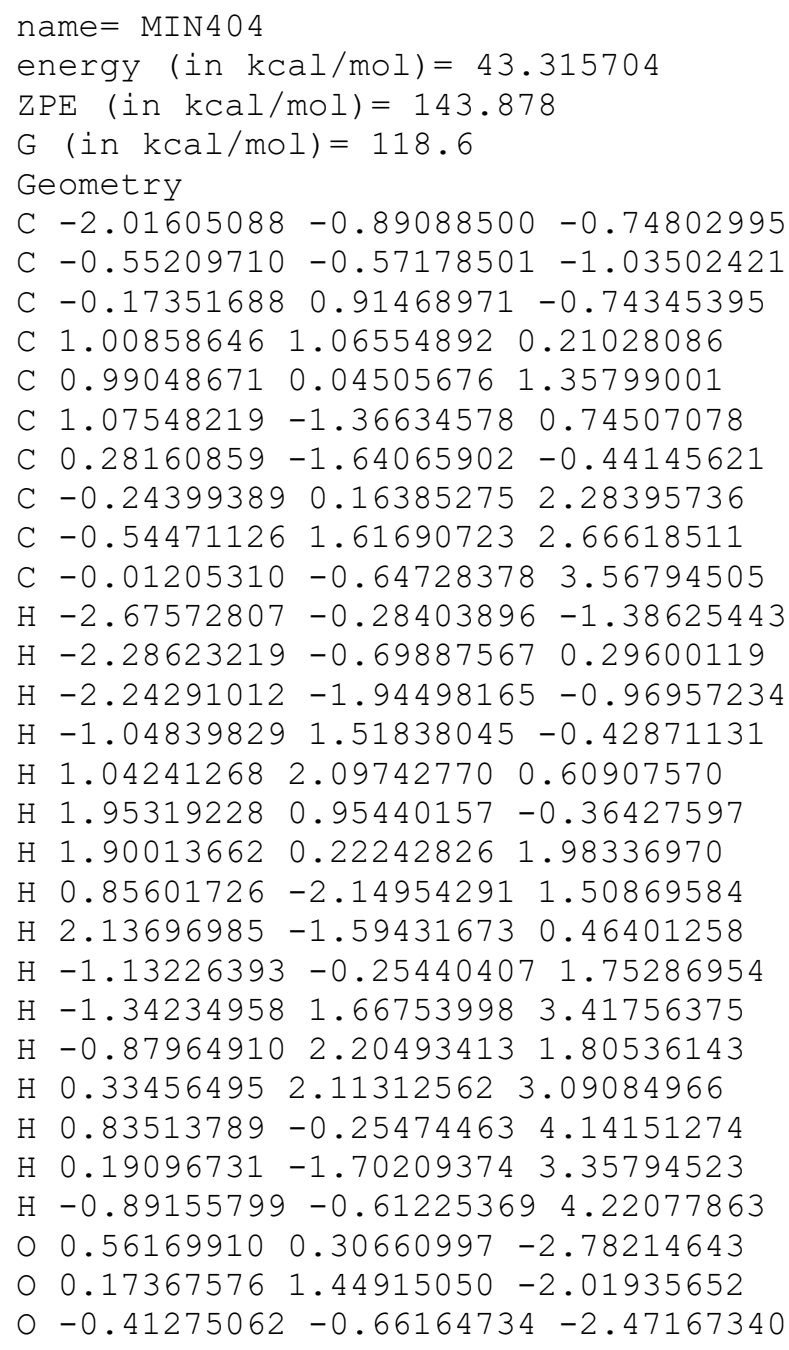


C $-0.35076837-0.83034470-0.95663058$

C $0.26687156 \quad 0.60415648 \quad-0.96529636$

C $1.17536337 \quad 0.86213037 \quad 0.23477277$

C $0.55863442 \quad 0.34786161 \quad 1.54706199$

C $0.42142341-1.18227905 \quad 1.43229142$

C $0.01341437-1.73847746 \quad 0.15247119$

C $-0.78183977 \quad 1.05142640 \quad 1.86881433$

C $-0.55786363 \quad 2.55495198 \quad 2.07950346$

C $-1.43640928 \quad 0.46720681 \quad 3.12762248$

$\mathrm{H}-2.19815371-0.29516647-1.97138098$

$\mathrm{H}-2.36613295-0.41490814-0.20504571$

$\mathrm{H}-2.24055932-1.88160944-1.19665892$

$\mathrm{H}-0.49671997 \quad 1.39691560-1.09470229$

$\mathrm{H} 1.40946507 \quad 1.940717290 .30746015$

$\begin{array}{lllll}\mathrm{H} & 2.15551290 & 0.36947534 & 0.06473165\end{array}$

H $1.26669286 \quad 0.57618252 \quad 2.38113292$

$\mathrm{H}-0.24946397-1.592143412 .22391264$

$\mathrm{H} 1.40257524-1.66423095 \quad 1.68291952$

$\mathrm{H}-1.481244450 .911607121 .01111353$

$\mathrm{H}-1.49492948 \quad 3.06086757 \quad 2.33977939$

$\mathrm{H}-0.17084694 \quad 3.04053674 \quad 1.17740815$

$\mathrm{H} \quad 0.15208820 \quad 2.74534273 \quad 2.89190327$

$\mathrm{H}-0.74257518 \quad 0.45413733 \quad 3.97459940$

$\mathrm{H}-1.79356255-0.55523447 \quad 2.96710988$

$\mathrm{H}-2.307175891 .06183545 \quad 3.42949295$

O $1.34582398-0.71090900-2.43406948$

$\begin{array}{llll}0 & 1.02763531 & 0.65481894 & -2.16858259\end{array}$

O $0.14172417-1.40095535-2.18907064$

Vibrational frequencies (in $\mathrm{cm}-1$ )

$\begin{array}{lllllllllllllll}39.7 & 71.0 & 86.7 & 116.1 & 126.9 & 167.7 & 186.5 & 213.7 & 262.4 & 283.0 & 310.4 & 344.9 & 351.4 & 385.5\end{array}$

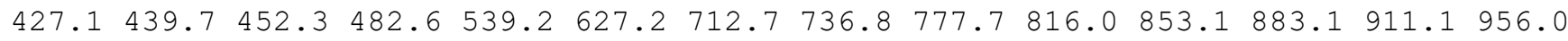

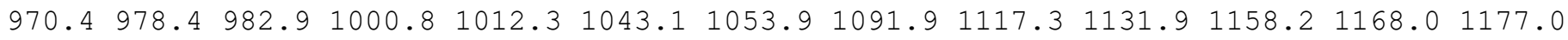

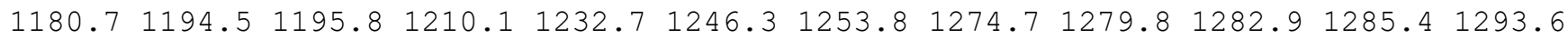

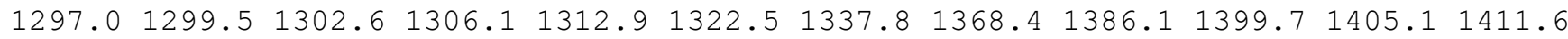
$2627.72656 .62663 .5 \quad 2667.5 \quad 2671.5 \quad 2672.7 \quad 2675.92677 .32691 .4 \quad 2691.52710 .22722 .2$ $2742.42773 .6 \quad 2779.9 \quad 2781.8$

\section{name $=$ MIN406}

energy $($ in $\mathrm{kcal} / \mathrm{mol})=46.653116$

$\operatorname{ZPE}(\mathrm{in} \mathrm{kcal} / \mathrm{mol})=141.143$

$\mathrm{G}(\mathrm{in} \mathrm{kcal} / \mathrm{mol})=114.374$

Geometry

C $-2.61249749-1.69966764-1.01480380$

C $-1.13393536-1.48931988-1.16707429$

C $0.396310331 .23537293-1.24240215$

C $1.26124725 \quad 0.16227823-0.73332317$

C $0.68124534-0.63001064 \quad 0.46468383$

C $1.76485253-1.28354500 \quad 1.24025577$

C $-0.26309327-1.77172133 \quad 0.02637974$

C $0.04965394 \quad 0.32460836 \quad 1.53731700$

C $-1.41772568 \quad 0.65526958 \quad 1.26396733$

C $0.17478077-0.287790012 .94091418$

$\mathrm{H}-3.04557693-1.02205276-0.26794537$

$\mathrm{H}-2.84349810-2.72638318-0.70387403$

$\mathrm{H}-3.13924391-1.51923345-1.96519839$

$\mathrm{H}-0.36411847 \quad 1.07277614-1.99918582$

H $2.23850138 \quad 0.62037986-0.42630942$

H $1.50341064-0.55550301-1.55364878$

$\mathrm{H} \quad 0.63913991 \quad 1.28284600 \quad 1.51525629$

$\mathrm{H} \quad 0.35626387-2.66899785-0.21838866$

$\mathrm{H} \quad 2.69270281-0.73792997 \quad 1.32848524$

$\mathrm{H}-0.23530551-1.304547792 .98238731$

$\mathrm{H}-2.07778222-0.18150098 \quad 1.51692022$

$\begin{array}{llll}\mathrm{H} & -1.59353608 & 0.92130995 & 0.21763958\end{array}$ 
$\mathrm{H}-1.737099921 .512088521 .87379219$

$\mathrm{H}-0.36812395 \quad 0.31389537 \quad 3.68028737$

$\mathrm{H}-0.86530577-2.08640034 \quad 0.90290072$

$\mathrm{H} \quad 1.21514109-0.33535687 \quad 3.29252294$

$01.351093662 .78908964-0.01350664$

$0.492240312 .47633103-0.88761595$

o $-0.67261180-1.15497380-2.23231155$

Vibrational frequencies (in $\mathrm{cm}-1$ )

$\begin{array}{llllllllllllllll}19.0 & 71.3 & 94.8 & 96.0 & 105.3 & 116.3 & 172.7 & 181.0 & 204.7 & 208.3 & 223.9 & 265.5 & 291.3 & 298.4\end{array}$ $332.7 \quad 348.0 \quad 389.0 \quad 429.6 \quad 442.8 \quad 494.0 \quad 521.6 \quad 524.4 \quad 561.0 \quad 616.7 \quad 717.1 \quad 769.0 \quad 841.5 \quad 897.5$ $923.3964 .8 \quad 978.1982 .5 \quad 996.8 \quad 1014.1 \quad 1026.5 \quad 1039.5 \quad 1055.7 \quad 1074.6 \quad 1099.3 \quad 1147.3 \quad 1148.6$

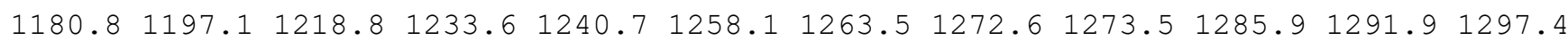

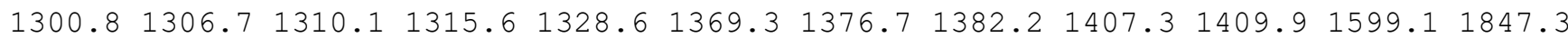
$2627.32644 .72648 .12659 .4 \quad 2661.12667 .8 \quad 2669.72671 .62676 .4 \quad 2682.82695 .72709 .9$ $2732.4 \quad 2773.8 \quad 2778.6 \quad 2780.3$

\section{name $=$ MIN407}

energy $($ in $\mathrm{kcal} / \mathrm{mol})=43.047714$

$\mathrm{ZPE}(\mathrm{in} \mathrm{kcal} / \mathrm{mol})=144.77$

$\mathrm{G}($ in $\mathrm{kcal} / \mathrm{mol})=118.461$

Geometry

C $-3.19868924-0.47834853-0.57734761$

C $-1.67699559-0.53827465-0.56405044$

C $-1.011400650 .87214888-0.52012345$

C $0.01602425 \quad 1.00707867 \quad 0.59805871$

C $1.04584153-0.13031994 \quad 0.55251129$

C $0.38160258-1.530241630 .51416798$

C $-1.14644777-1.46752411 \quad 0.52631686$

C $2.08056893-0.175148921 .59119895$

C $3.49341250 \quad 1.71890327 \quad 1.96800814$

C $2.28829863 \quad 0.94458486 \quad 2.52342041$

$\mathrm{H}-3.59614061-0.069155190 .35725025$

$\mathrm{H}-3.63502485-1.47576282-0.71906349$

$\mathrm{H}-3.56426103 \quad 0.14444098-1.40624457$

$\mathrm{H}-1.75408580 \quad 1.69714453-0.49755317$

$\mathrm{H}-0.49671327 \quad 1.02543579 \quad 1.57774898$

$\mathrm{H} \quad 0.53577553 \quad 1.98163977 \quad 0.50245949$

$\mathrm{H} 1.62680662-0.00963437-0.41509499$

$\mathrm{H} \quad 0.73685904-2.14802471 \quad 1.36365906$

$\mathrm{H} \quad 0.72250445-2.06509881-0.39485397$

$\mathrm{H}-1.55787878-2.48608952 \quad 0.37977910$

H $4.35918613 \quad 1.05702414 \quad 1.82493524$

H $3.79637111 \quad 2.51363155 \quad 2.65897606$

H $3.26426476 \quad 2.18543173 \quad 1.00313363$

$\mathrm{H}-1.51129926-1.13427734 \quad 1.51629190$

$\mathrm{H} \quad 1.43420279 \quad 1.62412055 \quad 2.68620407$

$\mathrm{H} \quad 2.53785105 \quad 0.53187599 \quad 3.52621667$

$0-0.14237009-0.32984455-2.21586837$

$0-0.395211251 .00642886-1.79882297$

O $-1.31517371-1.03950516-1.86069855$

Vibrational frequencies (in $\mathrm{cm}-1$ )

$\begin{array}{lllllllllllllll}16.6 & 32.0 & 63.9 & 111.5 & 147.2 & 158.0 & 160.6 & 200.1 & 230.2 & 262.8 & 279.2 & 314.7 & 378.5 & 416.6\end{array}$ $452.4 \quad 463.5 \quad 505.1 \quad 549.5 \quad 584.1 \quad 678.0 \quad 715.6 \quad 796.0 \quad 842.2 \quad 870.1 \quad 890.7 \quad 924.4 \quad 936.4 \quad 960.8$ $966.4 \quad 988.4 \quad 1003.9 \quad 1007.3 \quad 1037.4 \quad 1056.2 \quad 1082.7 \quad 1091.8 \quad 1102.7 \quad 1133.11156 .4 \quad 1160.6$ $1168.81177 .6 \quad 1190.6 \quad 1195.8 \quad 1216.1 \quad 1238.6 \quad 1258.2 \quad 1262.3 \quad 1277.8 \quad 1281.2 \quad 1284.6 \quad 1288.2$ $1289.51297 .6 \quad 1298.41311 .7 \quad 1323.7 \quad 1331.2 \quad 1337.9 \quad 1348.6 \quad 1366.6 \quad 1378.6 \quad 1390.3 \quad 1400.8$ $1410.02644 .02654 .42659 .2 \quad 2668.5 \quad 2670.5 \quad 2673.5 \quad 2675.32679 .72681 .02685 .52737 .6$ $\begin{array}{llllll}2739.7 & 2741.4 & 2745.8 & 2777.8 & 2781.4\end{array}$

name $=$ MIN408

energy $($ in $\mathrm{kcal} / \mathrm{mol})=45.56934$

$\mathrm{ZPE}($ in $\mathrm{kcal} / \mathrm{mol})=142.939$

$\mathrm{G}($ in $\mathrm{kcal} / \mathrm{mol})=118.08$ 


\begin{tabular}{|c|c|c|}
\hline \multicolumn{3}{|c|}{ Geometry } \\
\hline & -2.86752184 & $-0.25894107-0.21227871$ \\
\hline & -1.33975612 & $-0.20697377-0.2$ \\
\hline & & $1.22964438-0.49$ \\
\hline & 72544 & 1.609609630 .04 \\
\hline & 3656116 & 0.83980801 \\
\hline & 5343960 & 1.074542181 .00 \\
\hline & 15750 & -0.855204301 \\
\hline & 0.7 & -0.670318891 .1 \\
\hline & 1.14333530 & -1.215522302 .5 \\
\hline & 1.52563361 & -1.481222990 .1 \\
\hline & 368804 & $0.18483616-1$ \\
\hline & 8527233 & $0.26855430 \quad 0.64$ \\
\hline & 2426926 & $-1.29720229-0$ \\
\hline & -1.5505130 & $40-0$. \\
\hline & 0.60929788 & 32963.3 \\
\hline & 2.88656705 & 391.09 \\
\hline & 0.78397576 & 622.0 \\
\hline & 3.20482399 & $0.54225106 \quad 1.78$ \\
\hline & 76273 & 3360.03 \\
\hline & -0.97822443 & -1.941320570 . \\
\hline & -1.27390768 & -0.459399211 \\
\hline & 2.21560147 & -1.109062952 .7 \\
\hline & 0.90532026 & -2.282109332 .66 \\
\hline & 1.13566806 & -2.503424540 .0 \\
\hline & 2.59462452 & -1.554892110 .35 \\
\hline & 1.42631049 & $-1.03397163-0.87072718$ \\
\hline & & $-0.02114673-2.23$ \\
\hline & & $1.25721729-1.92854262$ \\
\hline & & $-0.93715012-1.41331632$ \\
\hline
\end{tabular}

Vibrational frequencies (in cm-1)

$\begin{array}{lllllllllllllll}39.5 & 71.9 & 114.3 & 159.4 & 162.2 & 176.7 & 215.8 & 220.0 & 243.8 & 270.5 & 278.9 & 303.7 & 323.9 & 338.9\end{array}$ $374.6 \quad 413.3 \quad 450.5 \quad 476.9 \quad 504.1 \quad 545.0 \quad 628.7 \quad 661.6 \quad 711.8 \quad 792.2 \quad 868.6 \quad 876.0 \quad 900.4 \quad 922.2$ $963.9970 .5 \quad 982.6 \quad 992.1 \quad 1000.6 \quad 1017.2 \quad 1026.9 \quad 1033.8 \quad 1066.3 \quad 1077.2 \quad 1104.91117 .2 \quad 1139.7$ $1149.7 \quad 1179.2 \quad 1200.2 \quad 1208.0 \quad 1235.2 \quad 1249.1 \quad 1263.3 \quad 1281.4 \quad 1285.9 \quad 1287.8 \quad 1289.8 \quad 1296.0$ $1297.91303 .6 \quad 1304.4 \quad 1310.8 \quad 1318.0 \quad 1322.0 \quad 1375.4 \quad 1378.4 \quad 1397.8 \quad 1405.7 \quad 1412.4 \quad 1418.8$ 2658.62660 .52668 .52673 .22674 .42677 .72678 .92679 .22680 .12681 .12683 .02739 .1 $2779.22780 .62781 .2 \quad 2783.4$

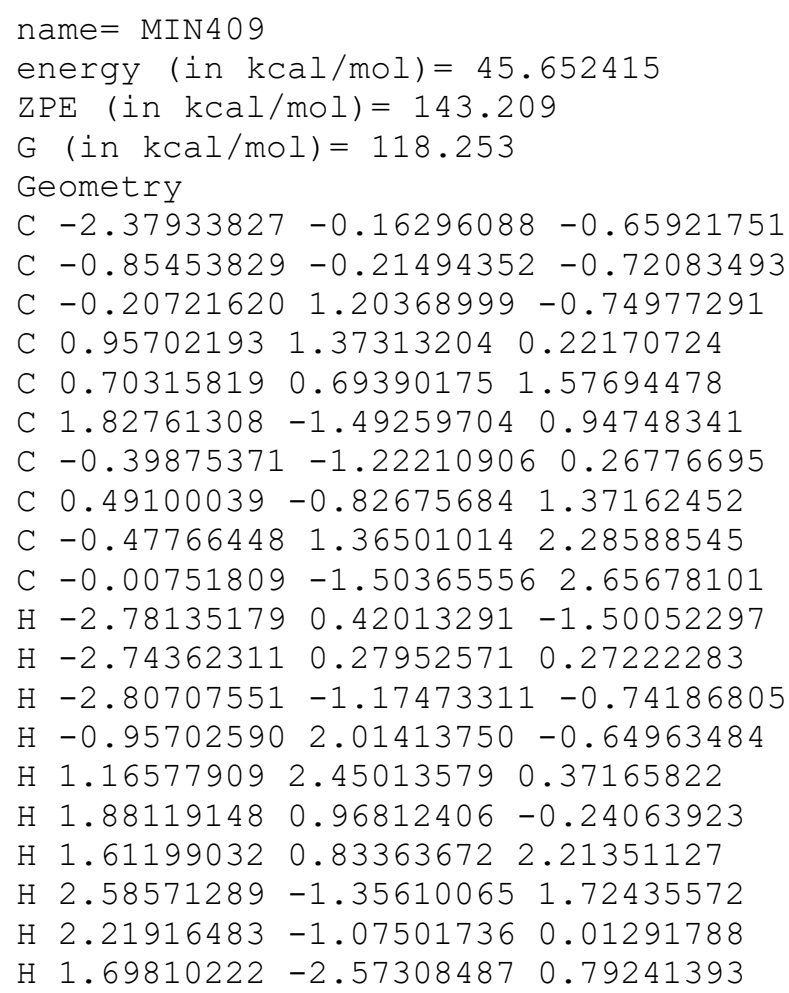


$\mathrm{H}-1.431227691 .13406027 \quad 1.80186116$

$\mathrm{H}-0.36650967 \quad 2.45601735 \quad 2.29214078$

$\mathrm{H}-0.55113187 \quad 1.04687142 \quad 3.33246750$

$\mathrm{H}-0.05284328-2.594340332 .53150505$

$\mathrm{H} \quad 0.65769189-1.29053104 \quad 3.49974908$

$\mathrm{H}-1.01569205-1.173771692 .92756269$

$00.57652782-0.00272520-2.46434404$

○ $0.266901651 .33274042 \quad-2.08944984$

o $-0.53705874-0.75618827-2.02434532$

Vibrational frequencies (in $\mathrm{cm}-1$ )

$\begin{array}{llllllllllllllll}39.8 & 68.3 & 131.8 & 136.1 & 157.9 & 186.8 & 193.7 & 198.8 & 221.3 & 260.7 & 267.0 & 309.8 & 330.0 & 360.5\end{array}$ $399.8 \quad 424.8 \quad 455.8 \quad 472.1 \quad 518.4 \quad 534.8 \quad 636.1 \quad 676.5 \quad 718.5 \quad 774.2 \quad 856.5 \quad 857.2 \quad 902.2 \quad 956.0$ $967.4970 .9 \quad 984.3 \quad 991.3 \quad 1002.1 \quad 1020.6 \quad 1046.6 \quad 1050.0 \quad 1074.2 \quad 1111.9 \quad 1126.8 \quad 1142.0 \quad 1156.0$

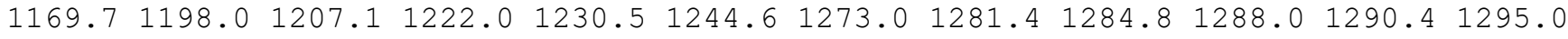

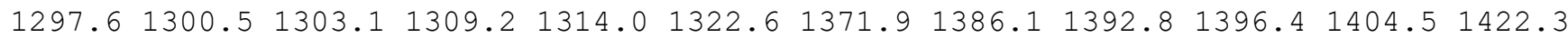
$2654.52663 .5 \quad 2665.0 \quad 2672.7 \quad 2672.8 \quad 2674.0 \quad 2674.6 \quad 2675.72680 .4 \quad 2692.92702 .12741 .0$ $2774.9 \quad 2775.7 \quad 2780.4 \quad 2781.1$

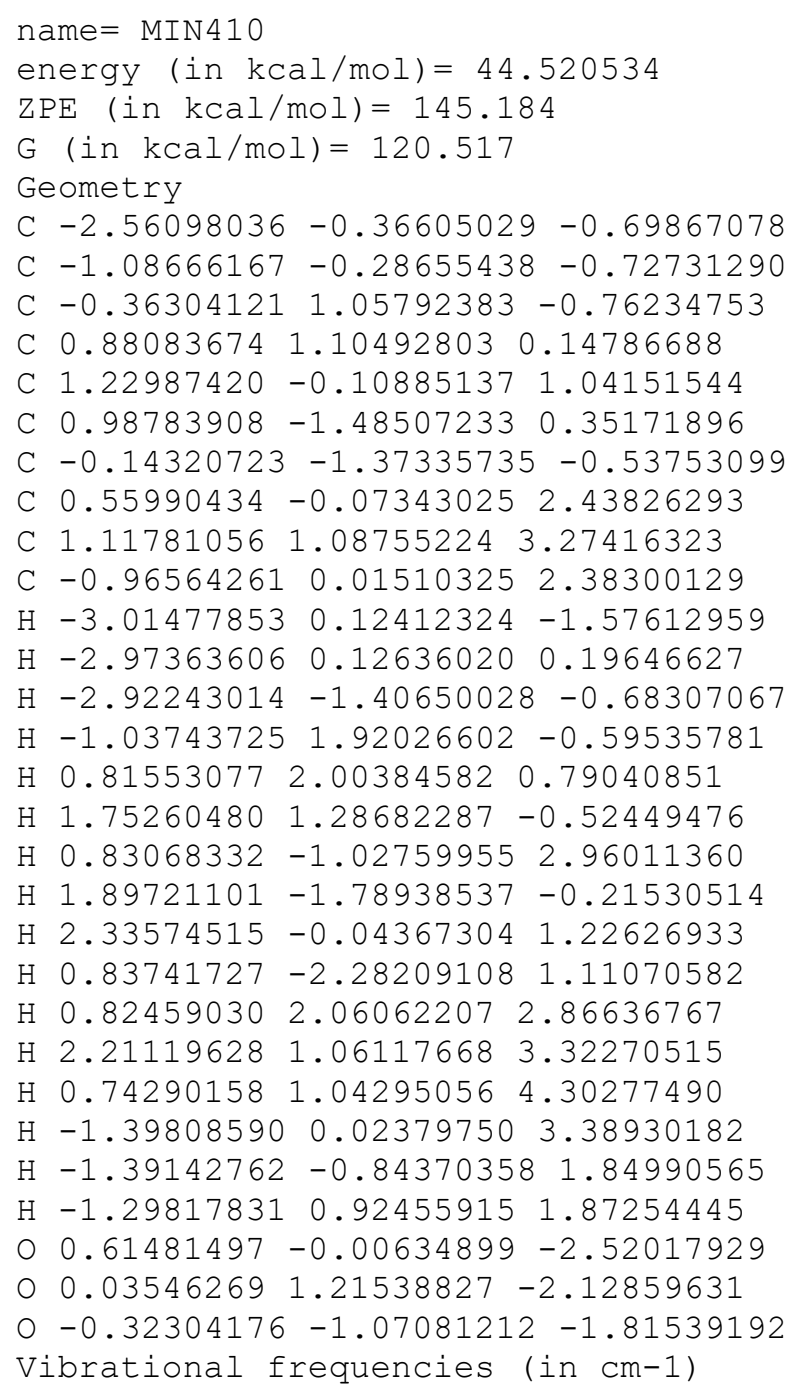


$\mathrm{ZPE}($ in $\mathrm{kcal} / \mathrm{mol})=141.312$

$\mathrm{G}($ in $\mathrm{kcal} / \mathrm{mol})=113.757$

Geometry

C $-3.24801253-1.53282316 \quad 0.63134129$

C $-1.76289072-1.604455620 .68884697$

C $-0.99886682-0.28797599 \quad 0.57396295$

C $0.07202916-0.45688382-0.51028320$

C $1.95243308 \quad 0.40175837-1.66688378$

C $3.13210530-0.03502966-0.89000134$

C $2.36257454 \quad 0.32531692 \quad 0.43812433$

C $1.11440116 \quad 0.68539316-0.48213393$

C $0.52027827 \quad 2.06721440-0.36653934$

C $2.97029628 \quad 1.458892591 .22514839$

$\mathrm{H}-3.65924860-0.89331410 \quad 1.42380047$

$\mathrm{H}-3.71350666-2.52416428 \quad 0.70626057$

$\mathrm{H}-3.57265447-1.07207306-0.32517724$

$\mathrm{H}-0.58746268 \quad 0.00926662 \quad 1.55770701$

$\mathrm{H}-0.46710057-0.44554865-1.49245721$

$\mathrm{H} \quad 0.56045356-1.44604346-0.41481575$

$\mathrm{H} \quad 1.76844380 \quad 0.46968485 \quad-2.70909282$

H $4.05524333 \quad 0.53826224-1.06249835$

H $3.41399362-1.09524271-0.98420149$

H $2.17214834 \quad-0.54894216 \quad 1.08211171$

$\mathrm{H}-0.34708176 \quad 2.18932139-1.04370902$

$\mathrm{H} \quad 1.245237642 .85117541-0.60645703$

H $0.14364459 \quad 2.26218648 \quad 0.64629798$

H $3.20710605 \quad 2.327849120 .59921984$

H $3.90131597 \quad 1.14910441 \quad 1.71789693$

H $2.28768353 \quad 1.80777524 \quad 2.01295028$

$\begin{array}{lllll}0 & -2.26341070 & 0.73334311 & -0.99807388\end{array}$

$\begin{array}{llllll}0 & -1.86154631 & 0.79726386 & 0.22091712\end{array}$

o $-1.15759074-2.638482230 .83152856$

Vibrational frequencies (in $\mathrm{cm}-1$ )

$\begin{array}{llllllllllllllll}11.8 & 38.1 & 57.3 & 77.2 & 111.0 & 140.7 & 162.4 & 172.4 & 197.0 & 227.6 & 243.6 & 265.3 & 278.3 & 291.3\end{array}$ $340.3 \quad 376.4 \quad 414.5 \quad 449.7 \quad 473.6 \quad 509.4 \quad 568.9 \quad 675.1 \quad 714.1 \quad 721.3 \quad 782.6 \quad 870.0 \quad 892.3 \quad 906.9$ $930.6945 .3 \quad 967.7 \quad 982.6 \quad 997.2 \quad 1039.6 \quad 1044.9 \quad 1051.5 \quad 1057.9 \quad 1066.3 \quad 1074.2 \quad 1096.6 \quad 1117.3$

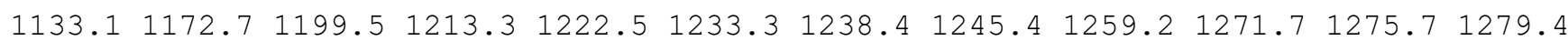

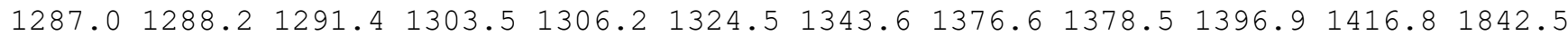
$2626.92636 .3 \quad 2641.7 \quad 2650.4 \quad 2671.4 \quad 2674.2 \quad 2678.0 \quad 2679.52698 .8 \quad 2713.4 \quad 2721.8 \quad 2737.3$ $2749.42773 .5 \quad 2774.5 \quad 2781.6$

\section{name $=$ MIN412}

energy $($ in $\mathrm{kcal} / \mathrm{mol})=46.282667$

$\mathrm{ZPE}(\mathrm{in} \mathrm{kcal} / \mathrm{mol})=144.203$

$\mathrm{G}($ in $\mathrm{kcal} / \mathrm{mol})=119.093$

Geometry

C $-2.31391783-0.61758124-0.70149217$

C $-0.80814687-0.51614954-0.93008302$

C $-0.24886714 \quad 0.90512868 \quad-0.63395366$

C $1.06942917 \quad 0.93371256 \quad 0.13585443$

C $1.27536330-0.16619640 \quad 1.18448382$

C $0.94909896-1.55229688 \quad 0.60348508$

C $-0.14062224-1.70093221-0.34215865$

C $0.55349600 \quad 0.05912604 \quad 2.53608069$

C $1.07436610 \quad 1.32693489 \quad 3.22516809$

C $-0.96979323 \quad 0.11851642 \quad 2.42485927$

$\mathrm{H}-2.85304449 \quad 0.02286040-1.41585288$

$\mathrm{H}-2.60899808-0.31884100 \quad 0.30796798$

$\mathrm{H}-2.66736098-1.64682487-0.86636501$

$\mathrm{H}-1.009261161 .58032950-0.19302370$

$\mathrm{H} \quad 1.18878927 \quad 1.92713175 \quad 0.61386466$

$\mathrm{H} \quad 1.89912081 \quad 0.87344351-0.60458502$

$\mathrm{H} \quad 0.81366078-0.81447507 \quad 3.19020287$

H $1.84912703-1.949899390 .05712351$ 
$\mathrm{H} 2.37378118-0.160343421 .43003954$

H $0.81600818-2.297037191 .42399815$

H $0.78291822 \quad 2.23425047 \quad 2.68530336$

H 2.16640464 $1.32383492 \quad 3.30682667$

H $0.67159102 \quad 1.41321571 \quad 4.24116019$

$\mathrm{H}-1.43293060 \quad 0.19790045 \quad 3.41582555$

$\mathrm{H}-1.36866764-0.78570807 \quad 1.95165830$

$\mathrm{H}-1.29988016 \quad 0.98715631 \quad 1.84613262$

$\begin{array}{lllll}0 & 0.32521297 & 0.31649687 & -2.71450628\end{array}$

$\begin{array}{lllll}0 & -0.02894667 & 1.45020748 & -1.93838007\end{array}$

$0-0.63567433-0.66189365-2.36205022$

Vibrational frequencies (in $\mathrm{cm}-1$ )

$\begin{array}{lllllllllllllll}50.8 & 54.2 & 85.6 & 113.6 & 131.8 & 174.3 & 200.3 & 232.0 & 254.7 & 271.2 & 290.4 & 313.5 & 348.1 & 395.7\end{array}$ $410.3 \quad 438.9 \quad 461.5 \quad 500.0 \quad 563.5 \quad 632.7 \quad 709.8 \quad 762.3 \quad 766.6 \quad 811.3 \quad 842.9 \quad 867.5 \quad 922.7 \quad 959.7$ $969.3980 .7984 .0 \quad 1002.2 \quad 1012.9 \quad 1033.9 \quad 1061.1 \quad 1093.8 \quad 1118.6 \quad 1127.6 \quad 1142.8 \quad 1175.0 \quad 1183.5$

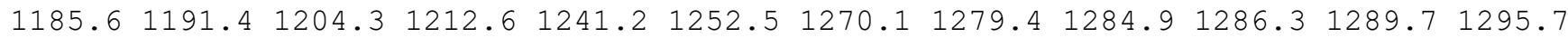

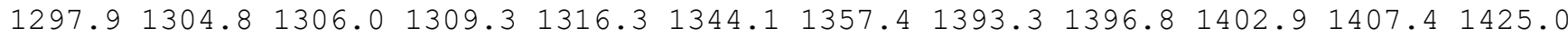
$2626.62648 .82664 .62667 .82671 .6 \quad 2673.82675 .42677 .22680 .12690 .82698 .52720 .0$ $2733.8 \quad 2775.5 \quad 2779.5 \quad 2781.5$

name $=$ MIN413

energy (in $\mathrm{kcal} / \mathrm{mol})=47.345471$

$\operatorname{ZPE}($ in $\mathrm{kcal} / \mathrm{mol})=143.262$

$\mathrm{G}($ in $\mathrm{kcal} / \mathrm{mol})=118.541$

Geometry

C $-2.52289830-0.20974701-0.69703574$

C $-1.00065768-0.16598960-0.72586207$

C $-0.421562651 .27780355-0.69238526$

C $0.22980844 \quad 1.67142588 \quad 0.62845696$

C $1.01725577 \quad 0.54989142 \quad 1.32915099$

C $2.32458417 \quad 0.283986390 .57745142$

C $-0.48974994-1.17571998 \quad 0.23264871$

C $0.15685871-0.731624921 .48184161$

C $-1.00240243-0.48925360 \quad 2.47511354$

C $1.00861858-1.900977452 .00502596$

$\mathrm{H}-2.94413584 \quad 0.28179012 \quad-1.58676784$

$\mathrm{H}-2.93027583 \quad 0.28585763 \quad 0.19010219$

$\mathrm{H}-2.89068061-1.24799033-0.70681450$

$\mathrm{H}-1.188931242 .03040365-0.98678832$

$\mathrm{H}-0.54928654 \quad 2.05922836 \quad 1.31387761$

H $0.91130742 \quad 2.52991818 \quad 0.44412606$

H $1.27831600 \quad 0.90580620 \quad 2.35773381$

H 2.14395869 $-0.18357470-0.39835189$

H $2.86851766 \quad 1.21702483 \quad 0.38570581$

H $2.99478652-0.37074943 \quad 1.14382807$

$\mathrm{H}-1.62129995 \quad 0.36631177 \quad 2.19086584$

$\mathrm{H}-0.60665374-0.29414754 \quad 3.47907403$

$\mathrm{H}-1.65273209-1.370338532 .55062113$

H $1.56381710-1.625320532 .90544542$

$\mathrm{H} 1.72704462-2.24017468 \quad 1.24824739$

$\mathrm{H} \quad 0.37904851 \quad-2.76763334 \quad 2.24227680$

○ $0.65953930-0.03027805-2.21496963$

$00.51796366 \quad 1.32652064-1.76327315$

$0-0.61248133-0.60056000-2.04863027$

Vibrational frequencies (in $\mathrm{cm}-1$ )

$\begin{array}{lllllllllllllll}50.1 & 77.1 & 129.0 & 133.9 & 177.9 & 188.4 & 198.4 & 209.6 & 217.4 & 241.5 & 279.8 & 308.9 & 338.5 & 370.1\end{array}$ $389.8 \quad 403.3 \quad 433.9 \quad 480.3 \quad 507.3 \quad 518.5 \quad 629.3 \quad 691.2 \quad 710.9 \quad 797.9 \quad 842.4 \quad 863.6 \quad 898.0 \quad 920.3$

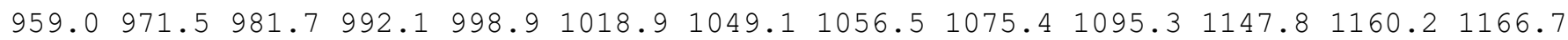

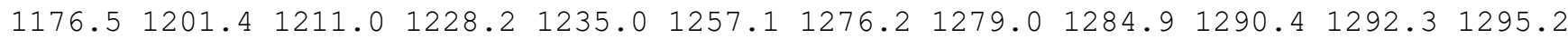

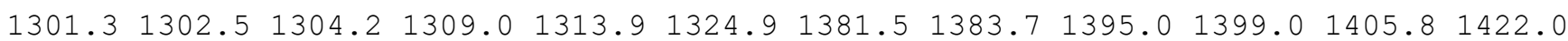
$2657.12661 .8 \quad 2669.6 \quad 2669.8 \quad 2671.2 \quad 2673.7 \quad 2674.72675 .82675 .92683 .02698 .52736 .9$ $2775.12776 .8 \quad 2780.8 \quad 2782.0$ 
name $=\operatorname{MIN} 414$

energy $($ in $\mathrm{kcal} / \mathrm{mol})=47.707104$

$\mathrm{ZPE}($ in $\mathrm{kcal} / \mathrm{mol})=143.17$

$\mathrm{G}($ in $\mathrm{kcal} / \mathrm{mol})=118.495$

Geometry

C $-2.41149352 \quad 0.04778553-0.84277479$

C $-0.89404235-0.09798417-0.73537432$

C $-0.134537851 .26347460 \quad-0.73040712$

C $0.83249464 \quad 1.49410768 \quad 0.43338310$

C $1.16138836 \quad 0.26362624 \quad 1.29870646$

C $2.20342113-0.613796790 .59699886$

C $-0.62365399-1.081169380 .34215418$

C $-0.12647965-0.54693459 \quad 1.62955371$

C $-1.18087763 \quad 0.337968392 .31437661$

C $0.18910663-1.722479482 .56700060$

$\mathrm{H}-2.68417973 \quad 0.56177722-1.77633260$

$\mathrm{H}-2.83756976 \quad 0.60707358-0.00500302$

$\mathrm{H}-2.89787389-0.94011950-0.87565937$

$\mathrm{H}-0.83480416 \quad 2.12161145-0.83498541$

$\mathrm{H} 0.43746883 \quad 2.29814297 \quad 1.08465468$

$\mathrm{H} 1.78022631 \quad 1.90375070 \quad 0.01691380$

$\begin{array}{lllll}\mathrm{H} & 1.59483507 & 0.62195485 & 2.26444842\end{array}$

H $3.11781559-0.05029808 \quad 0.37827130$

$\mathrm{H} 1.83091156-1.00509590-0.35990626$

H 2.49140173 -1.47444889 1.21071741

$\mathrm{H}-1.45621241 \quad 1.20815363 \quad 1.71125618$

$\mathrm{H}-0.80673903 \quad 0.712058293 .27535430$

$\mathrm{H}-2.09561367-0.230155252 .52203308$

$\mathrm{H} \quad 0.69764029-1.391174693 .47648033$

$\mathrm{H} \quad 0.82130826-2.470768712 .07311397$

$\mathrm{H}-0.72988903-2.24325659 \quad 2.86187921$

O $0.75576544-0.10370466-2.28236212$

○ $0.57500738 \quad 1.27309384-1.96742105$

o $-0.48913292-0.69658989-1.98777361$

Vibrational frequencies (in cm-1)

$\begin{array}{lllllllllllllllll}47.7 & 93.7 & 131.1 & 134.6 & 163.7 & 185.5 & 191.7 & 212.4 & 219.3 & 262.5 & 270.4 & 318.0 & 326.7 & 359.3\end{array}$

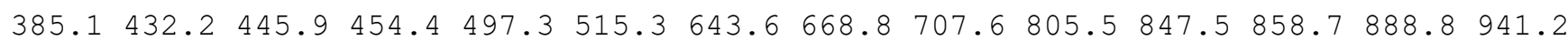
$967.2975 .0 \quad 981.2996 .5 \quad 1003.3 \quad 1021.9 \quad 1045.5 \quad 1052.6 \quad 1077.6 \quad 1093.5 \quad 1133.6 \quad 1146.01168 .5$

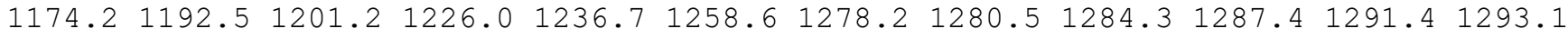

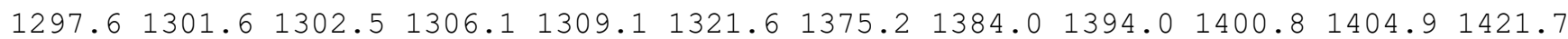
$2650.72661 .62662 .6 \quad 2670.9 \quad 2672.7 \quad 2674.6 \quad 2674.82675 .22680 .72683 .02699 .92734 .2$ 2775.22778 .02778 .92781 .5

name $=$ MIN415

energy $($ in $\mathrm{kcal} / \mathrm{mol})=50.66192$

$\mathrm{ZPE}($ in $\mathrm{kcal} / \mathrm{mol})=140.391$

$\mathrm{G}($ in $\mathrm{kcal} / \mathrm{mol})=112.942$

Geometry

C $-2.445970130 .44861926-0.92481063$

C $-0.95546765 \quad 0.38838526-0.93346983$

C $-0.29635294 \quad 1.72418452-0.96965514$

C $2.03732720 \quad 0.22512188-0.15617205$

C $0.90200646-1.06031637 \quad 1.69760206$

C $1.08218600-0.925328360 .15652612$

C $-0.27787364-0.82089826-0.47905317$

C $-0.33168877-0.30819886 \quad 2.06483373$

C $-0.18376036 \quad 0.93758367 \quad 2.80808436$

C $0.65240940 \quad-2.51633228 \quad 2.11228628$

H $-2.91837580-0.50801356-1.18299173$

$\mathrm{H}-2.80225418 \quad 0.71235424 \quad 0.09027841$

$\mathrm{H}-2.84240862 \quad 1.20974854-1.61143387$

$\mathrm{H} \quad 0.31555730 \quad 1.94455607 \quad-1.86111381$

H $2.12268058 \quad 0.39628690-1.24014882$

$\mathrm{H} \quad 1.73892084 \quad 1.16248373 \quad 0.32546698$ 
$\mathrm{H} 1.83352333-0.70532202 \quad 2.19533731$

$\mathrm{H} 1.55761408-1.87090565-0.22531008$

$\mathrm{H} \quad 3.05211219-0.00257444 \quad 0.19639589$

$\mathrm{H}-0.92396802-1.66673443-0.18530376$

$\mathrm{H}-0.43671156 \quad 1.77514691 \quad 2.11780044$

$\mathrm{H} \quad 0.79403346 \quad 1.16384974 \quad 3.24427850$

$\mathrm{H}-0.942125690 .99821130 \quad 3.60628318$

$\mathrm{H} \quad 0.54178841-2.60107460 \quad 3.20023902$

$\mathrm{H} 1.48124847-3.16569351 \quad 1.81302198$

$\mathrm{H}-0.26916075-2.90923397 \quad 1.66544939$

O $0.56423758-0.28619137-2.67980222$

$\begin{array}{llll}0 & -0.44701169 & 2.53182382 & -0.08772755\end{array}$

o $-0.41354208-0.61705081-2.01144836$

Vibrational frequencies (in $\mathrm{cm}-1$ )

$\begin{array}{lllllllllllllll}30.8 & 46.3 & 68.4 & 81.8 & 106.7 & 110.8 & 141.7 & 152.4 & 163.9 & 200.4 & 220.4 & 243.0 & 245.9 & 277.7\end{array}$ $292.6 \quad 300.5 \quad 346.8 \quad 357.4 \quad 397.8 \quad 445.5 \quad 522.7 \quad 549.1 \quad 599.4 \quad 615.0 \quad 698.5 \quad 722.6 \quad 852.6 \quad 882.2$ $902.3 \quad 919.7974 .1 \quad 996.5 \quad 1001.8 \quad 1021.9 \quad 1047.0 \quad 1050.4 \quad 1074.01106 .7 \quad 1116.4 \quad 1121.2 \quad 1149.3$

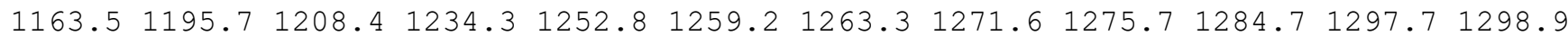

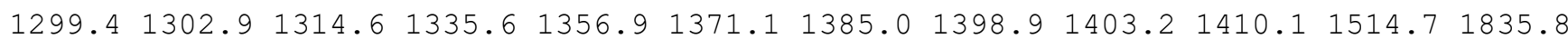
$2628.52630 .72653 .8 \quad 2657.0 \quad 2664.0 \quad 2671.5 \quad 2672.5 \quad 2672.82677 .5 \quad 2687.42699 .22707 .4$ $2765.22774 .4 \quad 2775.12780 .1$

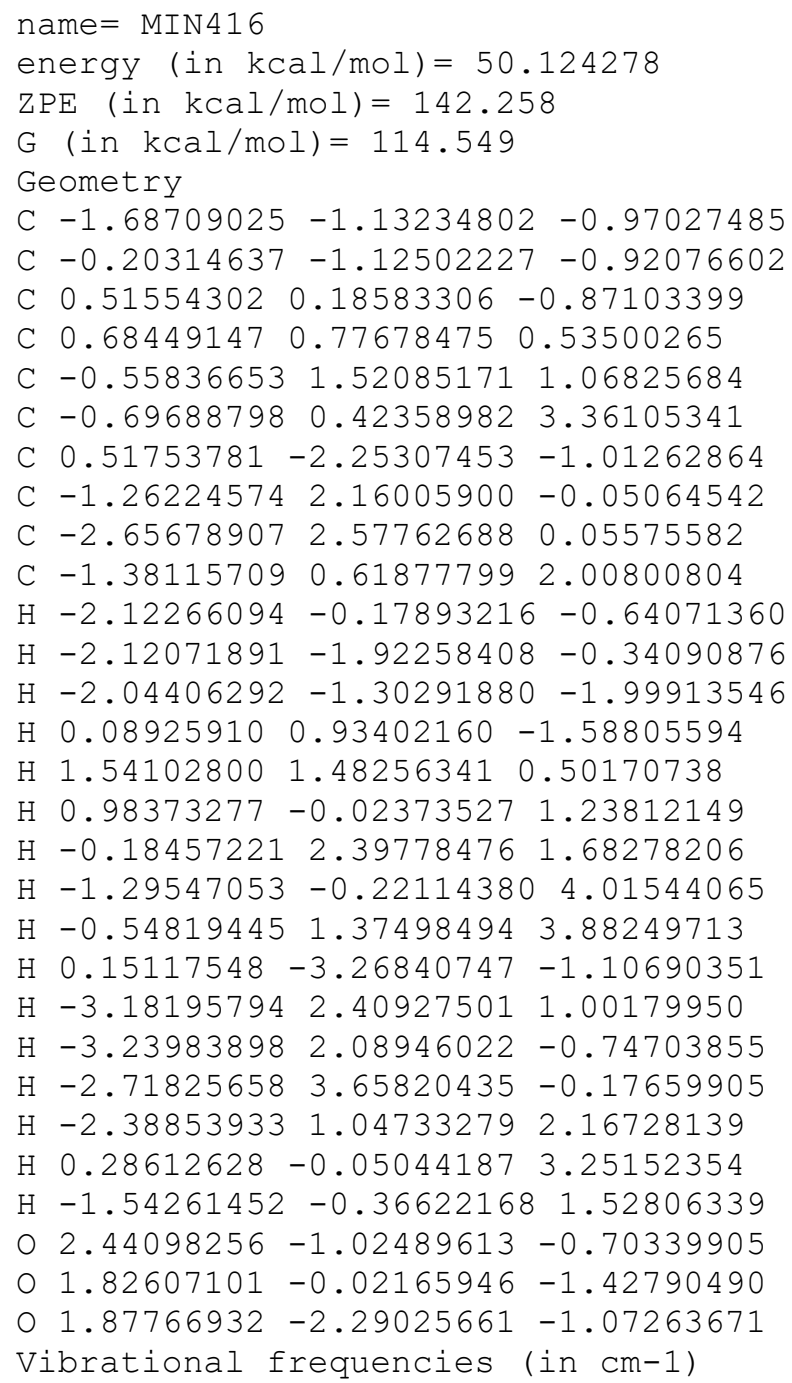


name $=$ MIN417

energy $($ in $\mathrm{kcal} / \mathrm{mol})=51.817607$

$\mathrm{ZPE}(\mathrm{in} \mathrm{kcal} / \mathrm{mol})=142.736$

$\mathrm{G}($ in $\mathrm{kcal} / \mathrm{mol})=117.297$

Geometry

C $-2.11096077-0.70837426-0.82466503$

C $-0.60600581-0.48057044-0.79344226$

C $-0.213781021 .03050221-0.82581764$

C $0.50067930 \quad 1.527090390 .34335642$

C $0.385614610 .90661556 \quad 1.64870242$

C $1.55705112-1.46805028 \quad 0.10488435$

C $0.06388580-1.24396026 \quad 0.38836001$

C $-0.12278751-0.44564682 \quad 1.62018567$

C $-0.25032426 \quad 1.83188954 \quad 2.69783176$

C $-0.86835337-1.04170797 \quad 2.74170255$

$\mathrm{H}-2.57086523-0.21300567-1.69124392$

$\mathrm{H}-2.58727616-0.31803023 \quad 0.08206291$

$\mathrm{H}-2.35646993-1.77463948-0.90468722$

$\mathrm{H}-1.105942731 .67365682-1.04533899$

H $1.14733149 \quad 2.37254490 \quad 0.20911860$

$\mathrm{H} \quad 2.07052091-0.52161917-0.11699286$

$\mathrm{H} \quad 1.50317562 \quad 0.75870181 \quad 1.95348373$

H $1.69264234 \quad-2.11379886 \quad-0.77297222$

H 2.05512229 $-1.94367338 \quad 0.95398362$

$\mathrm{H}-0.42310523-2.24736512 \quad 0.47193369$

$\mathrm{H}-1.32838660 \quad 1.92167988 \quad 2.51168521$

$\mathrm{H} \quad 0.17111108 \quad 2.84290437 \quad 2.62734502$

$\mathrm{H}-0.10632378 \quad 1.47163197 \quad 3.71928111$

$\mathrm{H}-0.79479980 \quad-2.13986089 \quad 2.76432428$

$\mathrm{H}-1.94392355-0.797486532 .67327770$

$\mathrm{H}-0.52752662-0.67917054 \quad 3.72315082$

$00.99433635-0.14799827-2.34243824$

○ $0.55339056 \quad 1.16052454 \quad-2.03827305$

o-0.11529193-0.98378228-2.03658593

Vibrational frequencies (in $\mathrm{cm}-1$ )

$\begin{array}{llllllllllllllll}40.0 & 60.3 & 84.3 & 91.8 & 161.0 & 164.7 & 195.6 & 215.1 & 239.1 & 244.2 & 264.5 & 302.9 & 319.1 & 339.1\end{array}$

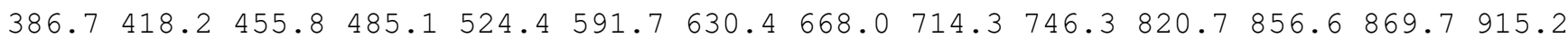
$949.2973 .1975 .2982 .5 \quad 1000.3 \quad 1024.5 \quad 1039.2 \quad 1064.4 \quad 1067.5 \quad 1073.3 \quad 1114.6 \quad 1115.3 \quad 1122.1$

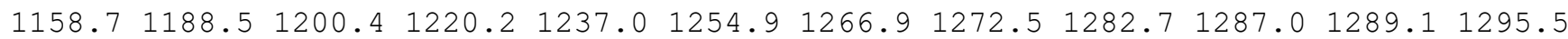
$1298.61302 .3 \quad 1304.1 \quad 1307.8 \quad 1320.7 \quad 1364.8 \quad 1382.3 \quad 1389.1 \quad 1392.8 \quad 1409.11433 .01522 .8$ $2557.32651 .12652 .7 \quad 2659.4 \quad 2666.8 \quad 2671.12673 .3 \quad 2678.32679 .3 \quad 2680.3 \quad 2703.8 \quad 2768.5$ $2775.4 \quad 2779.0 \quad 2780.0 \quad 2782.0$

\section{name $=$ MIN418}

energy (in $\mathrm{kcal} / \mathrm{mol})=52.595999$

$\mathrm{ZPE}(\mathrm{in} \mathrm{kcal} / \mathrm{mol})=142.802$

G $($ in $\mathrm{kcal} / \mathrm{mol})=117.106$

Geometry

C $-2.35084435-0.26723536-0.29233876$

C $-0.88372490-0.13333181-0.24992228$

C $-0.237435491 .19847010-0.55307316$

C 0.700233501 .520039520 .69094046

C $1.288406990 .24465645 \quad 1.34320629$

C $1.48740374-0.74439470 \quad 0.14790742$

C $-0.00725347-0.965370290 .36865846$

C $0.13806950 \quad-0.81681418 \quad 1.88457630$

C $-1.02391630-0.223557152 .64916569$

C $0.73265937-1.98033344 \quad 2.65251693$

$\mathrm{H}-2.67173195-0.43668315-1.33740252$

$\mathrm{H}-2.88129978 \quad 0.62447318 \quad 0.06634513$

$\mathrm{H}-2.70668109-1.12688872 \quad 0.29388190$

$\mathrm{H}-0.985380662 .00018059-0.70557209$ 
$\mathrm{H} \quad 0.12071245 \quad 2.09934377 \quad 1.42511343$

$\mathrm{H} \quad 1.52629876 \quad 2.16663648 \quad 0.34593586$

H $2.10219626 \quad 0.41340252 \quad 2.03376044$

H $2.11558084-1.61300646 \quad 0.35846317$

$\mathrm{H} \quad 1.82847939-0.33461991-0.80165608$

$\mathrm{H} \quad 0.45847775-0.76905024-3.07654656$

$\mathrm{H}-1.42121583 \quad 0.68122327 \quad 2.17353014$

$\mathrm{H}-0.73055393 \quad 0.04623201 \quad 3.67080049$

$\mathrm{H}-1.85460596-0.93789572 \quad 2.71799306$

H $1.11136263-1.66634645 \quad 3.63167596$

H $1.56173649-2.46126034 \quad 2.11954569$

$\mathrm{H}-0.02450608-2.75827744 \quad 2.81887051$

O $-0.04315375 \quad 1.03788289-2.85100129$

$0.65888361 \quad 1.20321952-1.66048934$

$0-0.41037765-0.30362828-2.98517418$

Vibrational frequencies (in $\mathrm{cm}-1$ )

$\begin{array}{llllllllllllllllll}30.5 & 67.0 & 104.1 & 111.5 & 114.5 & 149.7 & 171.7 & 180.7 & 197.8 & 247.3 & 286.9 & 300.0 & 360.7 & 369.2\end{array}$ $391.7 \quad 419.2 \quad 449.0 \quad 477.6 \quad 506.7 \quad 557.2 \quad 595.2 \quad 629.6 \quad 651.8 \quad 763.7 \quad 836.4 \quad 870.8 \quad 892.0 \quad 898.1$ $921.0 \quad 933.3 \quad 970.1987 .0 \quad 990.1 \quad 1001.8 \quad 1010.7 \quad 1017.2 \quad 1041.8 \quad 1051.2 \quad 1087.7 \quad 1097.7 \quad 1113.7$

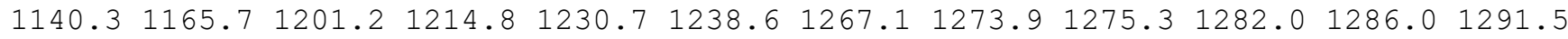
$1294.11297 .81305 .1 \quad 1320.6 \quad 1341.6 \quad 1348.4 \quad 1359.2 \quad 1388.2 \quad 1397.7 \quad 1404.4 \quad 1416.31771 .1$ $2659.82667 .4 \quad 2669.7 \quad 2673.4 \quad 2677.1 \quad 2677.7 \quad 2681.6 \quad 2685.02689 .72740 .72752 .32768 .0$ $2774.7 \quad 2780.2 \quad 2782.0 \quad 2846.3$

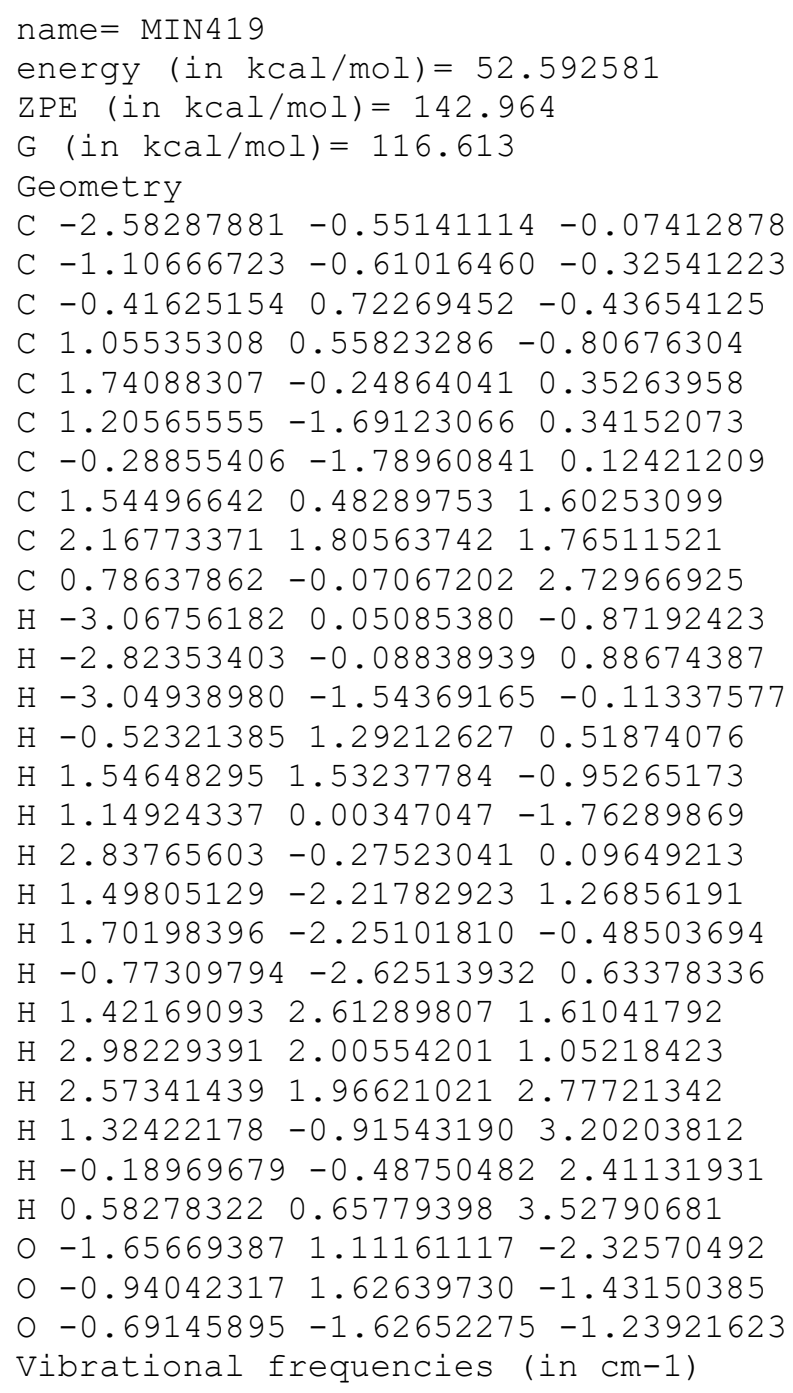


$1555.42628 .2 \quad 2629.2 \quad 2639.6 \quad 2648.0 \quad 2654.6 \quad 2658.42662 .72667 .7 \quad 2668.12686 .2 \quad 2730.4$ $2737.82740 .02760 .12762 .6 \quad 2776.2$

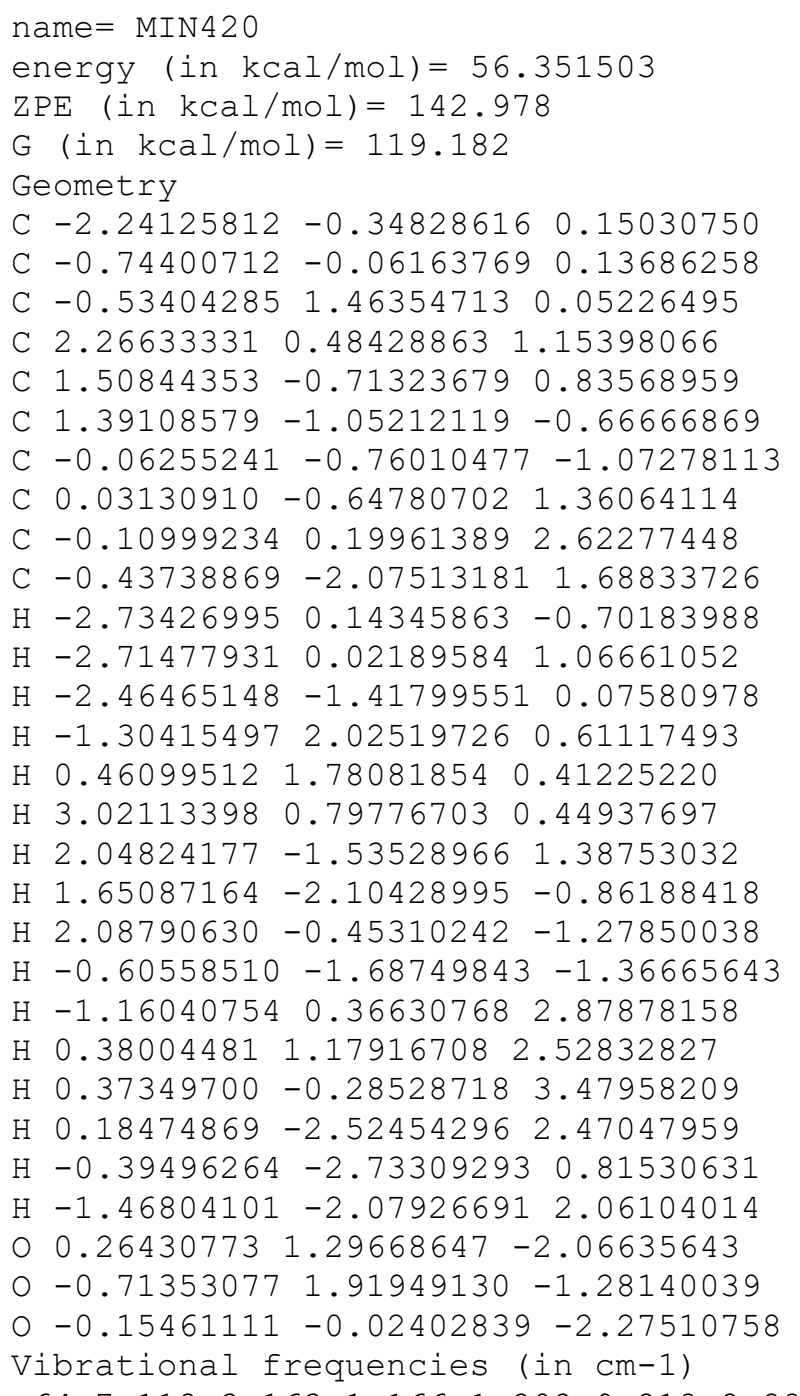

$\begin{array}{lllllllllllllllll}64.7 & 112.3 & 163.1 & 166.1 & 202.9 & 212.2 & 228.9 & 244.4 & 260.3 & 294.7 & 300.0 & 331.4 & 366.2 & 383.8\end{array}$ $421.8 \quad 429.8 \quad 470.0 \quad 506.1 \quad 514.1 \quad 548.9 \quad 592.9 \quad 617.5 \quad 694.2 \quad 797.5 \quad 821.4 \quad 851.6 \quad 891.6 \quad 934.7$ $938.5 \quad 957.0 \quad 983.6 \quad 989.1 \quad 990.8 \quad 999.3 \quad 1027.6 \quad 1063.9 \quad 1078.5 \quad 1091.4 \quad 1104.0 \quad 1107.9 \quad 1142.8$ $1155.3 \quad 1167.5 \quad 1176.5 \quad 1194.8 \quad 1215.9 \quad 1235.4 \quad 1247.1 \quad 1258.3 \quad 1276.4 \quad 1292.1 \quad 1294.3 \quad 1296.8$ $1301.61303 .7 \quad 1306.3 \quad 1315.51316 .4 \quad 1324.6 \quad 1338.6 \quad 1349.2 \quad 1360.51400 .51404 .81414 .3$ $2637.82658 .82661 .62665 .12669 .3 \quad 2672.6 \quad 2675.62676 .62679 .5 \quad 2683.72688 .02712 .9$ $2748.62778 .62779 .7 \quad 2781.8$

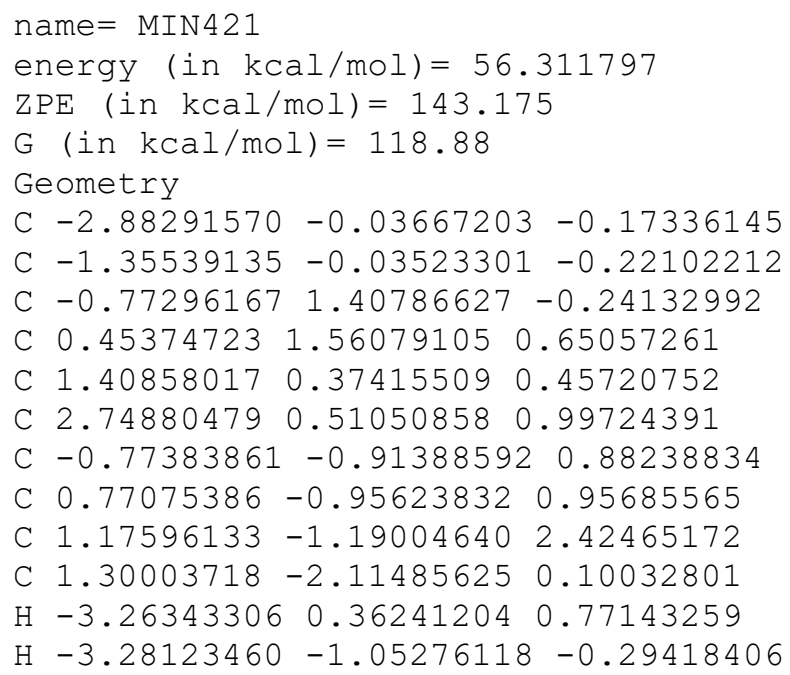


$\mathrm{H}-3.30259527 \quad 0.56262460 \quad-0.99399805$

$\mathrm{H}-1.535287332 .18888573-0.03878054$

$\mathrm{H} 0.14904738 \quad 1.63798742 \quad 1.71059935$

$\mathrm{H} \quad 0.968621092 .51073518 \quad 0.40847646$

$\mathrm{H} \quad 3.05440234 \quad 1.48622174 \quad 1.33986542$

$\mathrm{H}-1.15303237-1.948974220 .75784453$

$\mathrm{H} \quad 1.59277910 \quad 0.26151699-0.66344540$

$\mathrm{H}-1.18299140 \quad-0.56904368 \quad 1.85309713$

H $2.26560579-1.32036258 \quad 2.51093917$

$\mathrm{H} \quad 0.71395744 \quad-2.09650571 \quad 2.82779079$

$\mathrm{H} \quad 0.88809879-0.35269548 \quad 3.06526719$

$\mathrm{H} 2.39786836-2.101982350 .06407986$

$\mathrm{H} \quad 0.92845013-2.05226201-0.92983616$

H $0.99729556-3.08671311 \quad 0.50126973$

$\begin{array}{lllll}0 & -0.08015774 & 0.32264111 & -2.08459594\end{array}$

$0-0.44052831 \quad 1.61006622-1.61258700$

o $-1.03411440-0.56573745-1.51533181$

Vibrational frequencies (in cm-1)

$\begin{array}{lllllllllllllll}42.3 & 91.7 & 136.0 & 156.6 & 201.1 & 227.0 & 235.2 & 243.5 & 251.4 & 263.5 & 290.3 & 309.3 & 352.7 & 364.4\end{array}$ $409.4 \quad 422.5 \quad 440.2 \quad 480.2 \quad 532.7 \quad 562.8 \quad 616.8 \quad 678.5 \quad 717.3 \quad 797.1 \quad 873.8 \quad 894.0 \quad 901.8 \quad 936.7$

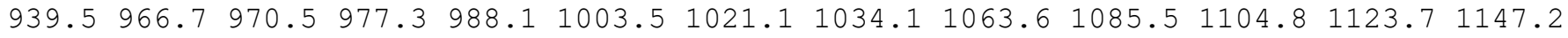
$\begin{array}{llllllllllll}1158.6 & 1182.4 & 1220.5 & 1227.9 & 1231.6 & 1245.6 & 1267.1 & 1274.8 & 1282.8 & 1285.0 & 1288.8 & 1296.0\end{array}$

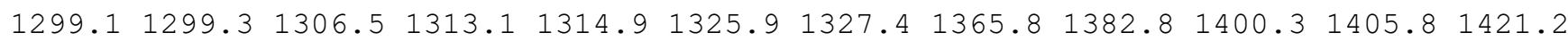
$2626.52658 .62663 .5 \quad 2666.7 \quad 2669.1 \quad 2670.2 \quad 2672.52677 .52679 .92682 .82687 .72737 .9$ $2744.4 \quad 2777.0 \quad 2779.2 \quad 2780.9$

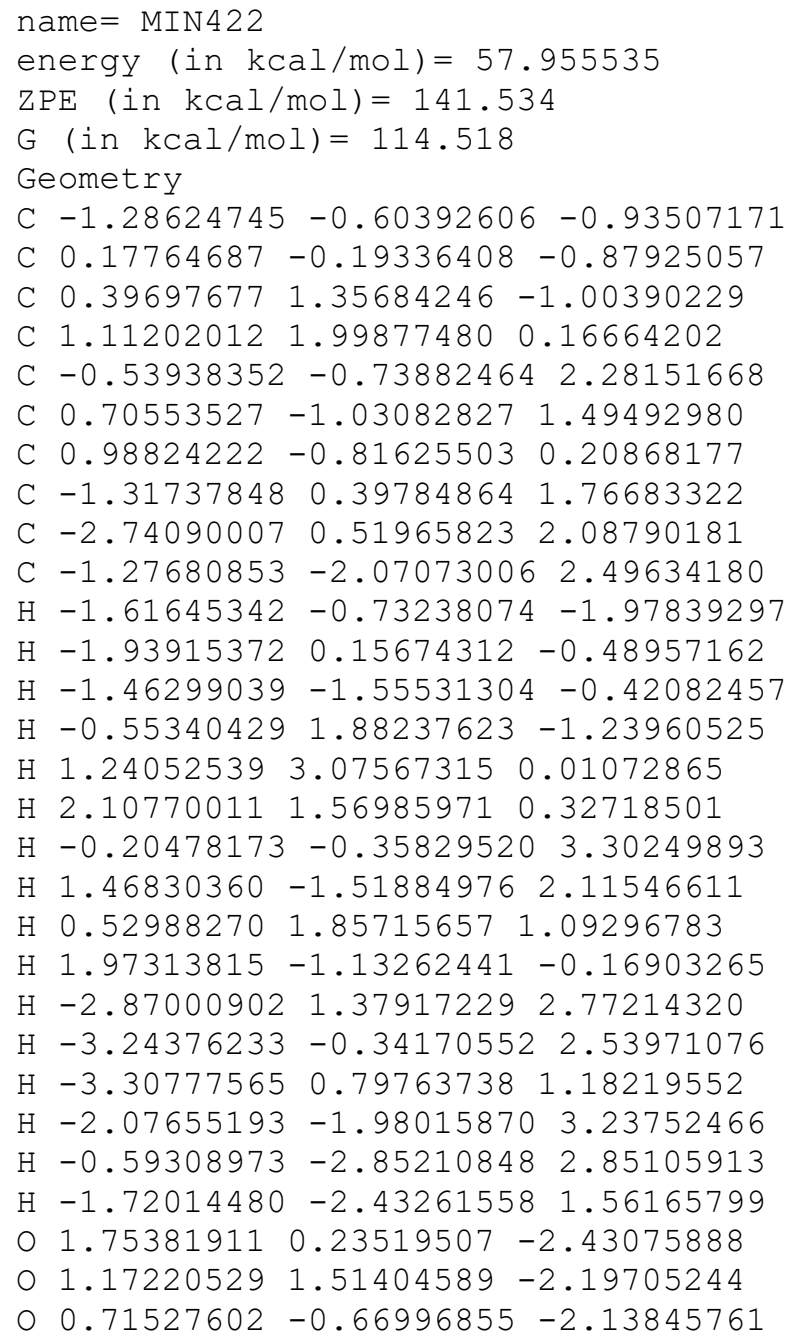


$\begin{array}{llllllllllllllll}1171.1 & 1178.9 & 1202.1 & 1210.8 & 1219.5 & 1237.6 & 1241.2 & 1265.6 & 1271.0 & 1283.4 & 1284.3 & 1291.1\end{array}$

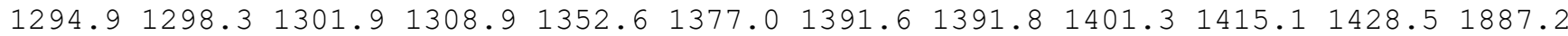
$2648.92654 .92659 .3 \quad 2660.7 \quad 2665.92671 .0 \quad 2672.4 \quad 2674.12678 .82692 .4 \quad 2717.92736 .4$ $2769.4 \quad 2773.4 \quad 2778.7 \quad 2779.1$

\section{name $=$ MIN423}

energy (in $\mathrm{kcal} / \mathrm{mol})=57.014688$

$\mathrm{ZPE}($ in $\mathrm{kcal} / \mathrm{mol})=142.972$

$\mathrm{G}($ in $\mathrm{kcal} / \mathrm{mol})=118.46$

Geometry

C $-2.44914064-0.24896811-0.75931830$

C $-0.92778528-0.22944423-0.74840987$

C $-0.338995631 .21623648-0.74693907$

C $0.37440171 \quad 1.70036698 \quad 0.51056680$

C $1.082324590 .58819112 \quad 1.29579577$

$\begin{array}{llll}\text { C } 2.30140859 & 0.06293381 & 0.68445839\end{array}$

C $-0.31925500-1.20443867 \quad 0.25776846$

C $0.10163904-0.57969162 \quad 1.59917188$

C $-1.11810259-0.085882352 .39040503$

C $0.81590899-1.650212952 .44109400$

$\mathrm{H}-2.85820130 \quad 0.39051265 \quad-1.55380394$

$\mathrm{H}-2.86112260 \quad 0.08931409 \quad 0.19819550$

$\mathrm{H}-2.83136475-1.26284884-0.94388496$

$\mathrm{H}-1.12245958 \quad 1.95295796-1.04595458$

$\mathrm{H}-0.350317892 .22203281 \quad 1.16603842$

$\mathrm{H} 1.11390209 \quad 2.47674368 \quad 0.21882049$

$\mathrm{H} 1.42418258 \quad 1.01095097 \quad 2.28790438$

$\mathrm{H} \quad 0.57761011-1.67760069-0.20708903$

H $2.75550091 \quad 0.64940418-0.09847382$

$\mathrm{H}-1.01657888-2.04419586 \quad 0.43961106$

$\mathrm{H}-0.82025544 \quad 0.352748913 .34834897$

$\mathrm{H}-1.80203144-0.91382946 \quad 2.60988142$

$\mathrm{H}-1.68772105 \quad 0.67162390 \quad 1.84441311$

$\mathrm{H} 1.09878566-1.264738593 .42476967$

H $1.74436919-1.98345123 \quad 1.95178273$

$\mathrm{H} \quad 0.18374451-2.52886666 \quad 2.59540214$

$00.71659582-0.11850894-2.26701010$

o $0.577701061 .24270953-1.84428965$

o $-0.55737376-0.67674263-2.07259141$

Vibrational frequencies (in $\mathrm{cm}-1$ )

$\begin{array}{llllllllllllllll}48.8 & 78.8 & 128.9 & 139.2 & 173.4 & 197.1 & 206.9 & 236.1 & 243.0 & 259.6 & 304.3 & 326.9 & 340.2382 .6\end{array}$

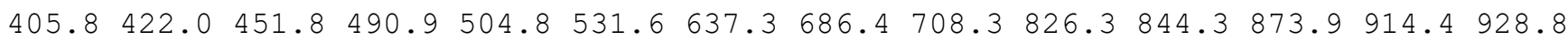

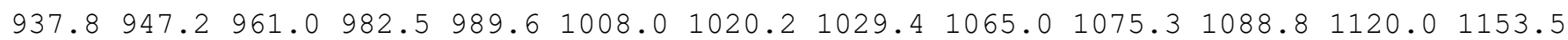
$\begin{array}{llllllllllllll}1162.3 & 1190.3 & 1212.0 & 1219.8 & 1232.2 & 1260.1 & 1273.5 & 1276.9 & 1281.8 & 1286.2 & 1292.9 & 1294.1\end{array}$

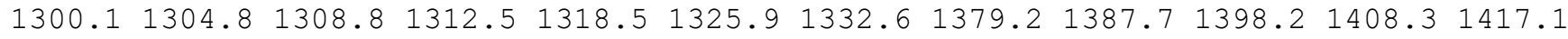
$2647.92655 .42662 .5 \quad 2663.8 \quad 2667.2 \quad 2671.3 \quad 2672.6 \quad 2673.02675 .72679 .02685 .02734 .3$ $2736.0 \quad 2777.9 \quad 2779.92782 .1$

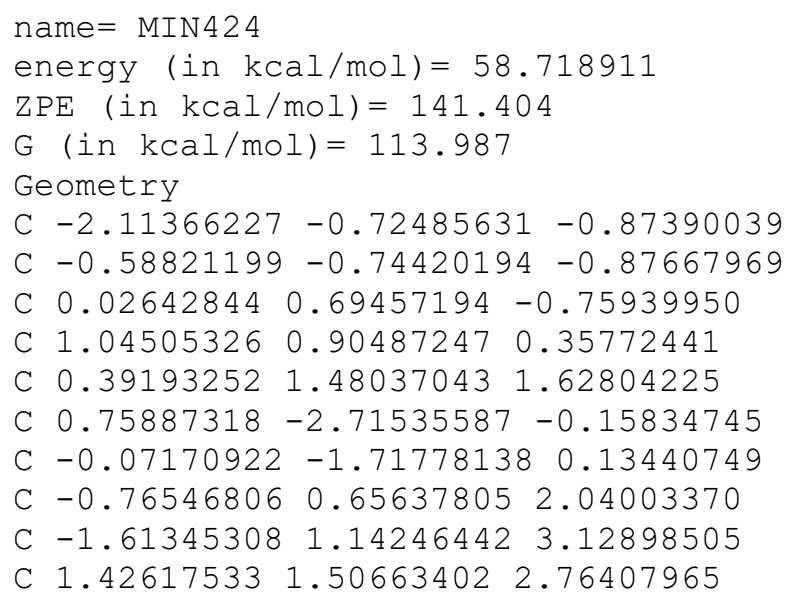


$\mathrm{H}-2.50976239-0.07951103-1.66756559$

$\mathrm{H}-2.49541277-0.368223390 .09184504$

$\mathrm{H}-2.52094582-1.73040198-1.04084227$

$\mathrm{H}-0.766157751 .47211399-0.74231539$

$\mathrm{H} 1.833761021 .59728382-0.00335288$

H $1.57924389-0.03691413 \quad 0.58955163$

$\mathrm{H} 0.064351962 .53035501 \quad 1.41145697$

H $1.11689890-3.422912820 .57169010$

H $1.14130942-2.90287520-1.15145327$

$\mathrm{H}-0.44787419-1.538345251 .14718582$

$\mathrm{H}-1.58249796 \quad 0.40850191 \quad 3.95572424$

$\mathrm{H}-2.66665511 \quad 1.14022535 \quad 2.79345682$

$\mathrm{H}-1.39561325 \quad 2.12897199 \quad 3.54953249$

H 2.32250093 2.06304267 2.46326251

H $1.74900010 \quad 0.49305278 \quad 3.03350751$

$\mathrm{H} \quad 1.02972654 \quad 1.98431499 \quad 3.66587615$

$00.95586967-0.41495804-2.48828559$

$\begin{array}{llllll}0 & 0.66784774 & 0.89700281 & -2.02103001\end{array}$

o $-0.22358784-1.14111679-2.21220523$

Vibrational frequencies (in cm-1)

$\begin{array}{lllllllllllllllll}-70.1 & 28.7 & 37.0 & 62.4 & 97.6 & 122.5 & 145.2 & 156.1 & 170.1 & 196.9 & 234.1 & 261.5 & 273.5 & 298.9\end{array}$ $330.5 \quad 347.9 \quad 385.8 \quad 420.2 \quad 481.9 \quad 524.2 \quad 559.2 \quad 599.0 \quad 682.7 \quad 720.1 \quad 830.2 \quad 857.4 \quad 876.3 \quad 895.8$ $935.6962 .7 \quad 976.8 \quad 989.5 \quad 996.3 \quad 1010.3 \quad 1022.0 \quad 1063.6 \quad 1075.6 \quad 1079.2 \quad 1091.6 \quad 1119.2 \quad 1137.2$ $1156.21173 .3 \quad 1192.0 \quad 1201.1 \quad 1207.3 \quad 1221.8 \quad 1255.2 \quad 1262.1 \quad 1269.0 \quad 1273.5 \quad 1288.8 \quad 1290.6$

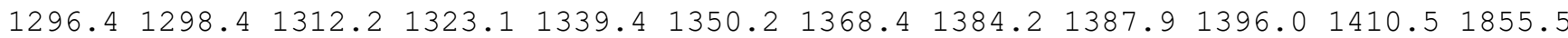
$2652.12654 .22667 .8 \quad 2671.2 \quad 2672.8 \quad 2675.12675 .72683 .92694 .22709 .62735 .62740 .0$ 2769.52778 .52778 .62786 .6

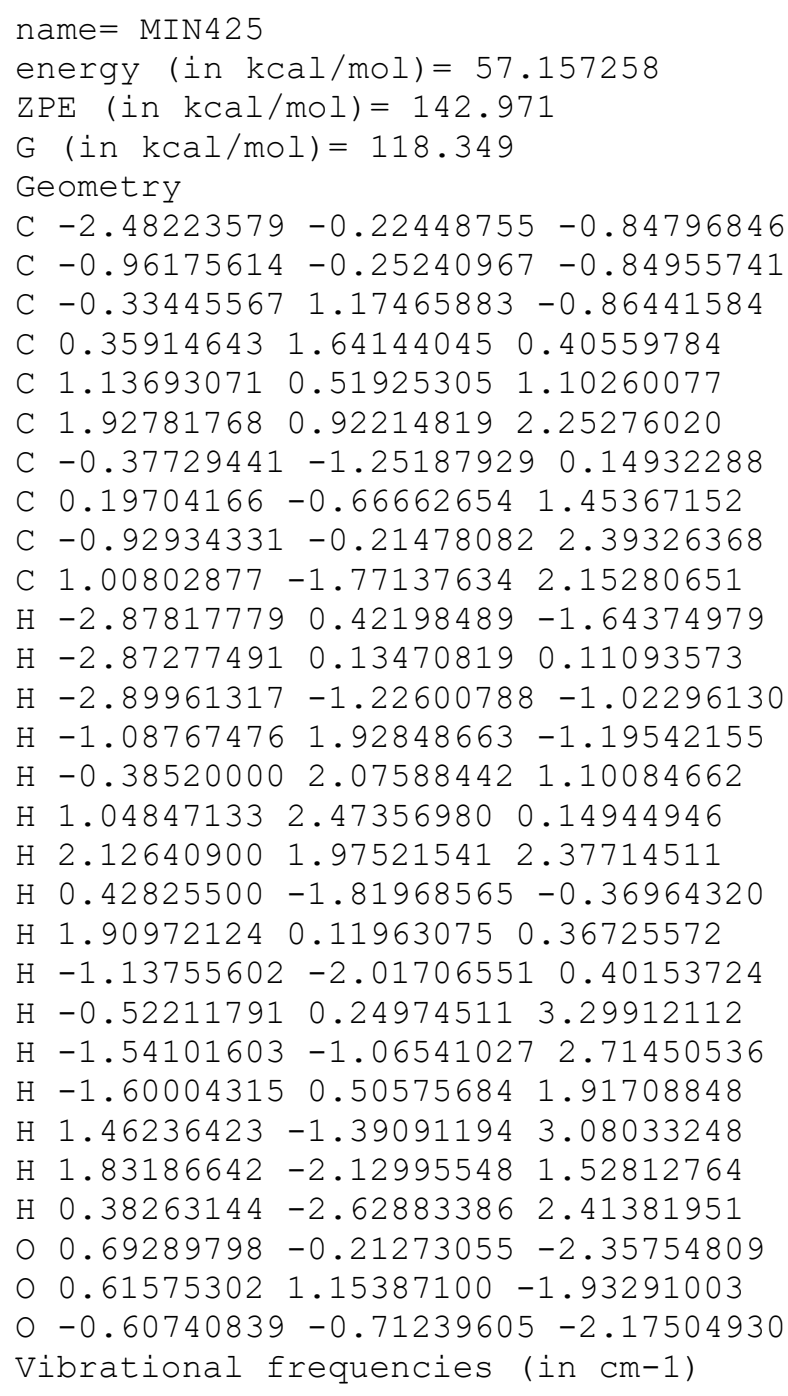


$\begin{array}{lllllllllllllllll}50.1 & 66.6 & 123.8 & 139.1 & 175.4 & 197.6 & 216.4 & 226.4 & 259.7 & 269.8 & 306.2 & 319.9 & 329.1 & 366.9\end{array}$

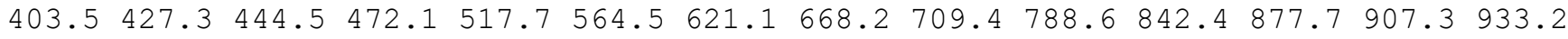

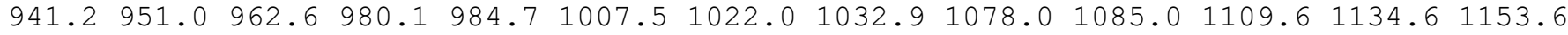
$\begin{array}{llllllllllllll}1166.4 & 1208.0 & 1209.1 & 1227.7 & 1233.7 & 1242.8 & 1271.2 & 1278.6 & 1282.2 & 1286.1 & 1290.3 & 1293.4\end{array}$ $1299.11303 .6 \quad 1308.4 \quad 1314.6 \quad 1318.0 \quad 1324.4 \quad 1330.4 \quad 1378.2 \quad 1394.2 \quad 1398.4 \quad 1407.81423 .8$ $2640.32650 .8 \quad 2661.92665 .6 \quad 2666.7 \quad 2667.5 \quad 2672.2 \quad 2675.42676 .4 \quad 2677.8 \quad 2684.5 \quad 2734.4$ $2738.12778 .8 \quad 2780.02781 .4$

\section{name $=$ MIN426}

energy $($ in $\mathrm{kcal} / \mathrm{mol})=57.2728$

$\operatorname{ZPE}($ in $\mathrm{kcal} / \mathrm{mol})=142.873$

$\mathrm{G}($ in $\mathrm{kcal} / \mathrm{mol})=116.16$

Geometry

C $-2.51272844-0.40143007-0.97488206$

C $-1.01424060-0.39793457-0.86117643$

C $-0.360541350 .96067888-0.83537700$

C $0.71250032 \quad 1.197850110 .24503153$

C $1.09399708 \quad 0.00323586 \quad 1.11784126$

C $1.08988809-1.31527793 \quad 0.29182447$

C $-0.28176490-1.56769908-0.27559865$

C $0.25033366-0.18447802 \quad 2.30448390$

C $-1.06554773 \quad 0.43471078 \quad 2.43880417$

C $0.73467516-1.02967275 \quad 3.40586920$

$\mathrm{H}-2.82519480 \quad 0.10493682 \quad-1.90430954$

$\mathrm{H}-2.998399110 .11382061-0.13986506$

$\mathrm{H}-2.91803243-1.42130397-1.02680983$

$\mathrm{H}-1.112849571 .77639846-0.78434268$

$\mathrm{H} \quad 0.42169105 \quad 2.05801401 \quad 0.87372689$

$\mathrm{H} \quad 1.62563997 \quad 1.56373677-0.30110055$

H $2.16207831 \quad 0.16599629 \quad 1.45335213$

H $1.44275814 \quad-2.16332745 \quad 0.90227229$

H $1.81908873-1.20657730-0.54633626$

$\mathrm{H}-0.81552933-2.43923566 \quad 0.10145642$

$\mathrm{H}-1.674328170 .30299818 \quad 1.51890642$

$\mathrm{H}-0.99235750 \quad 1.535192592 .56591835$

$\mathrm{H}-1.65771301 \quad 0.05569122 \quad 3.28362025$

$\mathrm{H} \quad 0.62776322-0.534512524 .38808456$

$\mathrm{H} 1.78873786-1.331557093 .31271960$

$\mathrm{H} \quad 0.14441254-1.96382292 \quad 3.48291321$

$\begin{array}{lllll}0 & 0.91164680 & 2.17722446 & -2.25049269\end{array}$

O $0.29497421 \quad 1.07123020 \quad-2.13936943$

o $-0.37578421-1.38112685-1.68794666$

Vibrational frequencies (in cm-1)

$\begin{array}{llllllllllllllll}-47.8 & 26.5 & 71.2 & 85.0 & 93.9 & 107.8 & 131.1 & 167.7 & 183.1 & 223.5 & 266.9 & 303.5 & 324.7 & 366.8\end{array}$ $413.9416 .8 \quad 474.6 \quad 507.2 \quad 521.3 \quad 544.6 \quad 573.6 \quad 723.3 \quad 826.4 \quad 846.2 \quad 852.9 \quad 896.6 \quad 922.2 \quad 927.4$

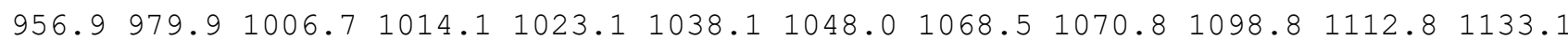

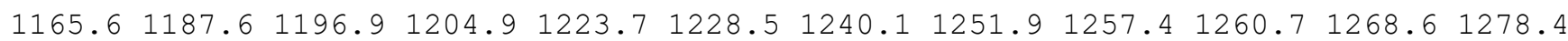

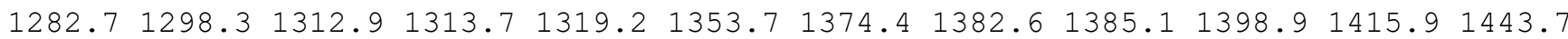
$1559.72604 .02610 .72635 .5 \quad 2650.2 \quad 2653.42657 .12661 .32663 .32678 .12682 .12723 .6$ $2735.02736 .7 \quad 2754.6 \quad 2762.8 \quad 2778.6$

\section{name $=$ MIN427}

energy (in $\mathrm{kcal} / \mathrm{mol})=58.822913$

$\mathrm{ZPE}(\mathrm{in} \mathrm{kcal} / \mathrm{mol})=141.574$

$\mathrm{G}(\mathrm{in} \mathrm{kcal} / \mathrm{mol})=114.143$

Geometry

C $-1.95666140-0.90672085-0.68117301$

C $-0.43002298-0.89492032-0.67981296$

C $0.15417190 \quad 0.56100333-0.66107851$

C $1.15654023 \quad 0.88720877 \quad 0.44325959$

C $0.50684550 \quad 1.56376917 \quad 1.66991370$

C $0.77845890 \quad-2.91395898 \quad 0.12878521$

C $0.09855395-1.79928964 \quad 0.38636508$ 
C $-0.44013487 \quad 0.62523890 \quad 2.28041550$

C $0.013088692 .97274182 \quad 1.32501775$

C $-1.71406420 \quad 1.05447894 \quad 2.84244654$

$\mathrm{H}-2.36083082-0.32955603-1.52333045$

$\mathrm{H}-2.35930764-0.49405321 \quad 0.24949794$

$\mathrm{H}-2.34002936-1.93165855-0.78238793$

$\mathrm{H}-0.65591762 \quad 1.31985673-0.71473107$

$\mathrm{H} \quad 1.93566073 \quad 1.558339420 .02302790$

$\mathrm{H} 1.70431076-0.02277810 \quad 0.76181014$

H $1.32164513 \quad 1.66717932 \quad 2.45298629$

H $1.14131038-3.57668500 \quad 0.89816477$

$\mathrm{H} 1.02286216-3.25048028-0.86868141$

$\mathrm{H}-0.13608742-1.47941177 \quad 1.40944551$

$\mathrm{H}-0.85547423 \quad 2.94839270 \quad 0.65813271$

H $0.79175258 \quad 3.55673887 \quad 0.81971586$

$\mathrm{H}-0.27593249 \quad 3.52781362 \quad 2.22369674$

H $-1.99307337 \quad 2.10919464 \quad 2.75070934$

$\mathrm{H}-1.732774150 .79075992 \quad 3.91700593$

$\mathrm{H}-2.52724146 \quad 0.45105245 \quad 2.39713854$

O $1.11506144 \quad-0.65133477 \quad-2.30272129$

$0 \quad 0.80691100 \quad 0.68528131-1.92745067$

o $-0.05943204-1.36873004-1.98866261$

Vibrational frequencies (in $\mathrm{cm}-1$ )

$\begin{array}{llllllllllllllll}29.8 & 40.6 & 49.3 & 63.7 & 89.4 & 111.5 & 146.3 & 167.6 & 174.6 & 214.0 & 241.6 & 246.3 & 288.1 & 303.9\end{array}$ $330.4 \quad 392.9 \quad 421.0 \quad 457.9 \quad 496.8 \quad 532.8 \quad 553.4 \quad 603.3 \quad 685.9 \quad 720.4 \quad 828.4 \quad 839.5 \quad 869.9 \quad 908.2$ $928.9957 .0 \quad 973.5 \quad 984.9 \quad 992.9 \quad 1009.8 \quad 1035.7 \quad 1052.6 \quad 1057.7 \quad 1067.0 \quad 1092.6 \quad 1102.8 \quad 1123.3$

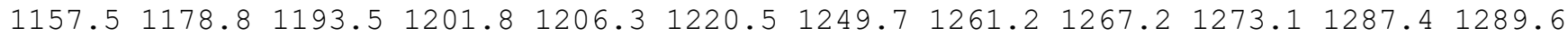

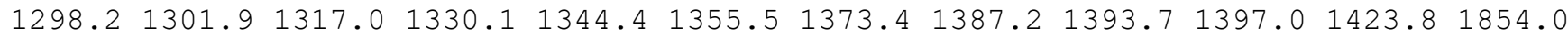
$2650.32652 .12658 .12668 .0 \quad 2670.4 \quad 2674.92677 .62678 .22681 .22697 .32726 .62735 .2$ $2768.22779 .4 \quad 2780.2 \quad 2785.6$

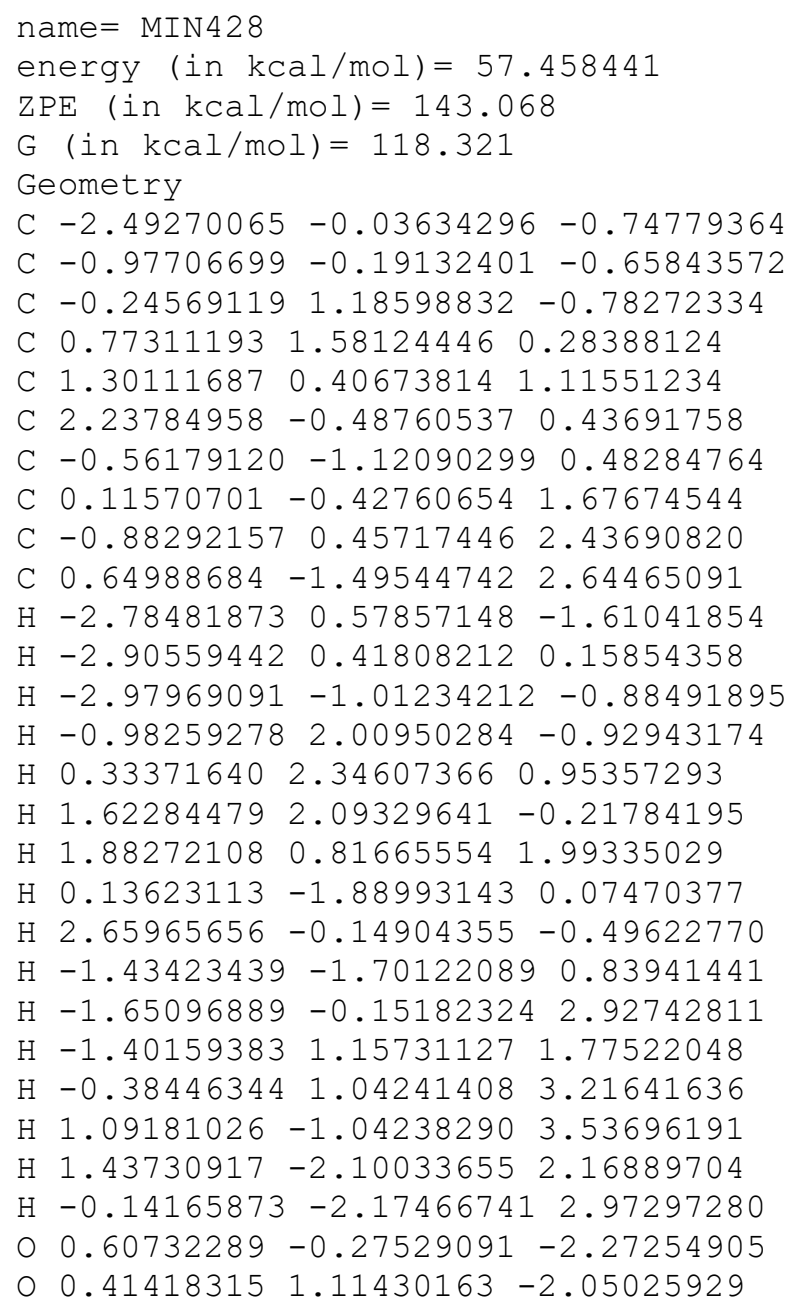


$0-0.62586900-0.84894318-1.89764913$

Vibrational frequencies (in $\mathrm{cm}-1$ )

$\begin{array}{llllllllllllllll}27.0 & 69.4 & 134.7 & 164.9 & 180.8 & 199.7 & 208.7 & 239.6 & 250.3 & 267.3 & 303.5 & 337.8 & 339.2 & 383.1\end{array}$ $401.4 \quad 440.0 \quad 458.5 \quad 488.5 \quad 501.3 \quad 532.7 \quad 652.5 \quad 679.1 \quad 702.9 \quad 831.7 \quad 849.8 \quad 867.2 \quad 915.4 \quad 932.0$

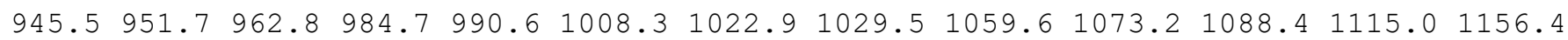

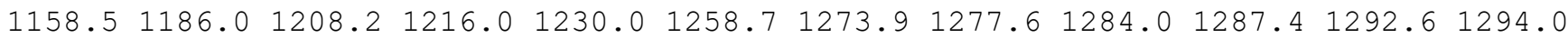
$1299.11303 .3 \quad 1308.2 \quad 1309.9 \quad 1319.5 \quad 1325.3 \quad 1333.3 \quad 1380.8 \quad 1388.5 \quad 1397.5 \quad 1409.01417 .6$ $2644.82653 .4 \quad 2662.6 \quad 2664.4 \quad 2666.7 \quad 2674.7 \quad 2675.72676 .72677 .8 \quad 2679.8 \quad 2684.82732 .1$ $2735.8 \quad 2778.7 \quad 2779.7 \quad 2782.8$

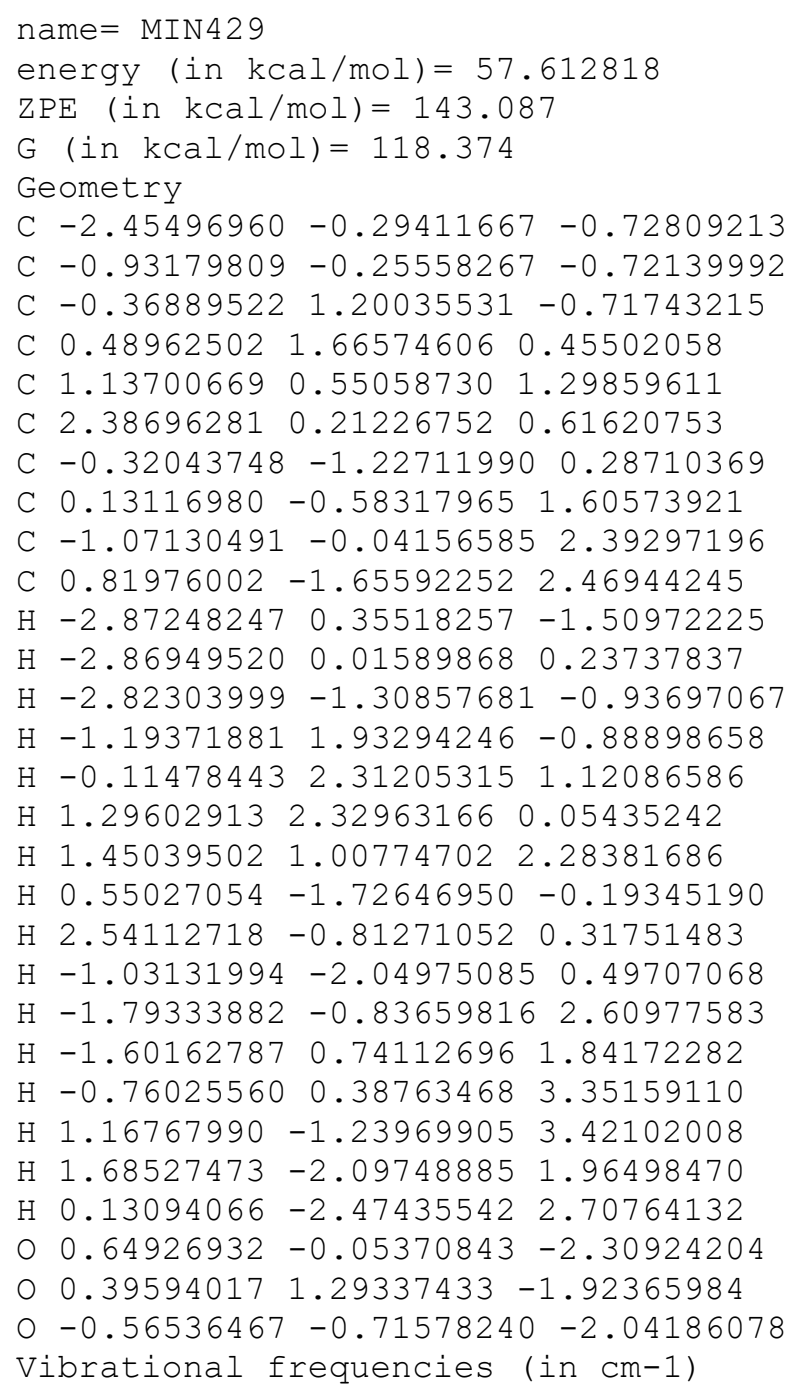


C $1.05136108-1.841018990 .12347988$

C $-0.16360543-1.097364750 .44836860$

C $0.07998742-0.31867343 \quad 1.77974465$

C $-1.21706050 \quad 0.33127462 \quad 2.29037470$

C $0.55806248-1.30776788 \quad 2.85644615$

$\mathrm{H}-2.57322509 \quad 0.52239946-1.53846238$

$\mathrm{H}-2.698491390 .19555873 \quad 0.20102348$

$\mathrm{H}-2.64148224-1.13827741-0.95980984$

$\mathrm{H}-0.772419812 .04042845-0.34008425$

H 1.643768462 .321489820 .11825543

H $2.004542920 .68966053-0.43379514$

$\mathrm{H} \quad 0.96193635 \quad 1.61702674 \quad 2.25502362$

H $2.14478653 \quad 0.37665488 \quad 1.86419596$

H $1.42894237-1.78978465-0.88496153$

$\mathrm{H}-0.93170472-1.89953719 \quad 0.66264833$

$\mathrm{H}-2.03883382-0.39075386 \quad 2.33488105$

$\mathrm{H}-1.53130141 \quad 1.17119187 \quad 1.66328832$

$\mathrm{H}-1.08210998 \quad 0.72503854 \quad 3.30504969$

$\mathrm{H} \quad 0.83454145 \quad-0.793741693 .78061703$

H $1.43259727-1.88151535 \quad 2.51262975$

$\mathrm{H}-0.21831977-2.040164353 .09950235$

$\begin{array}{lllll}0 & 0.60050864 & 0.21255595 & -2.49767977\end{array}$

○ $0.18414796 \quad 1.47666455-2.02331040$

o $-0.33193906-0.71309360-1.93741381$

Vibrational frequencies (in cm-1)

$\begin{array}{llllllllllllll}60.2 & 96.9 & 123.3 & 132.3 & 179.5 & 198.5 & 218.5 & 235.6 & 258.4 & 272.8 & 293.5 & 310.4 & 348.4 & 379.5\end{array}$ $390.5 \quad 417.6 \quad 454.9 \quad 467.6 \quad 525.5 \quad 545.1 \quad 637.8 \quad 672.8 \quad 726.5 \quad 786.7 \quad 852.9 \quad 880.8 \quad 885.7 \quad 917.2$ $943.8 \quad 972.5 \quad 982.5 \quad 986.6 \quad 1000.7 \quad 1024.4 \quad 1031.5 \quad 1050.0 \quad 1071.1 \quad 1079.7 \quad 1106.1 \quad 1133.9 \quad 1153.1$

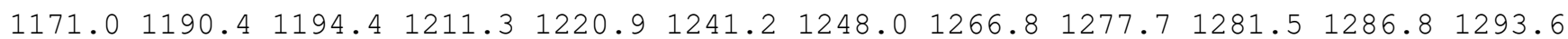

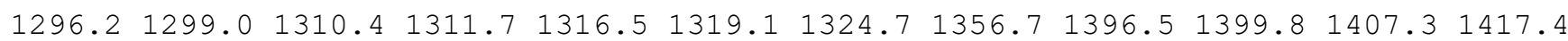
$2644.82658 .72661 .2 \quad 2666.62668 .2 \quad 2672.4 \quad 2672.82676 .42678 .72684 .62699 .52738 .5$ 2739.82778 .22779 .92781 .6

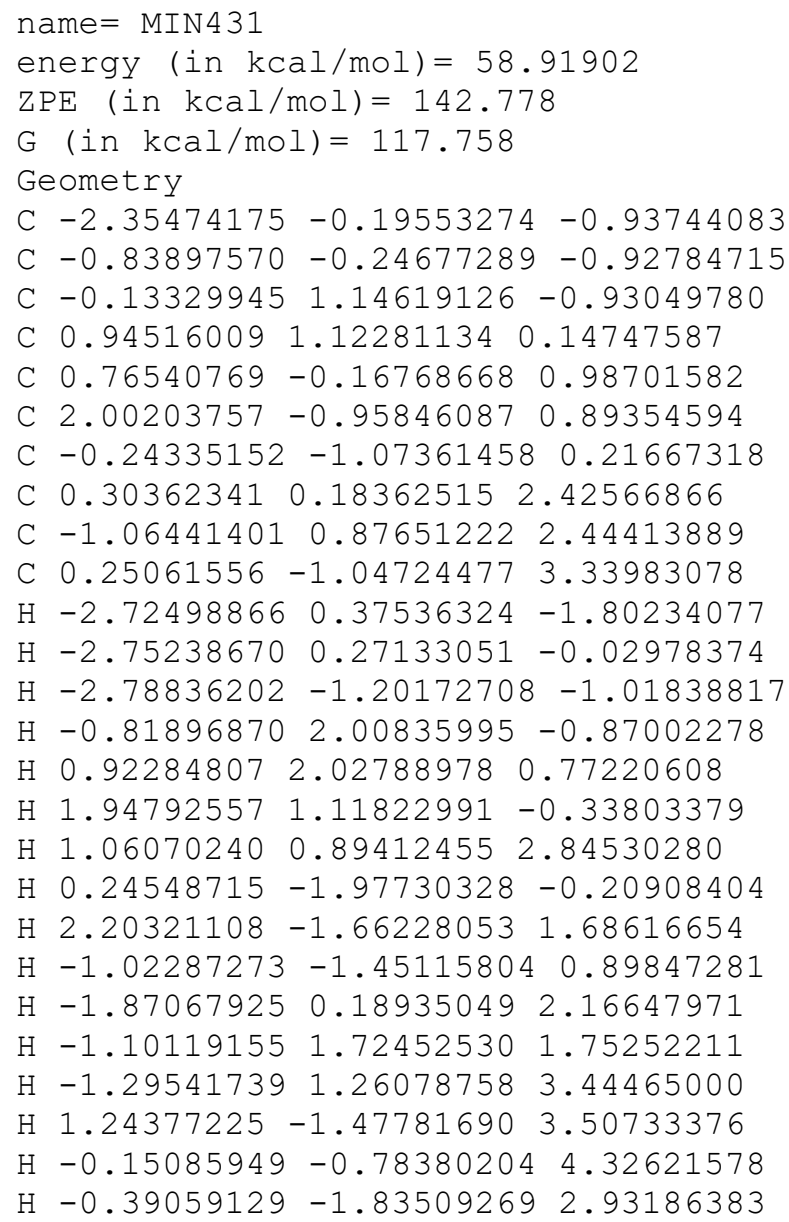


$0.70329934-0.11664903-2.57915339$

$0.432669051 .23415689-2.23482480$

o $-0.47318071-0.81447929-2.19288956$

Vibrational frequencies (in $\mathrm{cm}-1$ )

$\begin{array}{llllllllllllllll}49.3 & 64.8 & 80.4 & 161.9 & 165.9 & 178.9 & 190.4 & 196.8 & 227.2 & 277.1 & 285.7 & 291.8 & 345.2 & 359.2\end{array}$

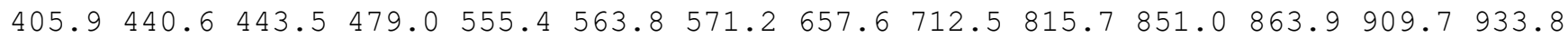

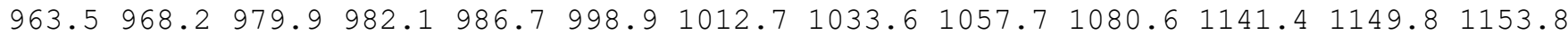
$\begin{array}{lllllllllllll}1176.9 & 1183.6 & 1184.6 & 1208.9 & 1227.7 & 1248.1 & 1276.7 & 1283.8 & 1284.5 & 1288.0 & 1289.7 & 1292.6\end{array}$ $1298.31303 .7 \quad 1305.6 \quad 1307.6 \quad 1319.8 \quad 1323.7 \quad 1333.6 \quad 1365.01382 .91394 .2 \quad 1401.91407 .4$ $2645.72648 .12653 .3 \quad 2668.12669 .12671 .92674 .2 \quad 2676.82677 .4 \quad 2701.32703 .4 \quad 2728.8$ $2739.62779 .4 \quad 2779.92781 .6$

name $=\operatorname{MIN} 432$

energy $($ in $\mathrm{kcal} / \mathrm{mol})=60.385735$

$\operatorname{ZPE}($ in $\mathrm{kcal} / \mathrm{mol})=141.396$

$\mathrm{G}($ in $\mathrm{kcal} / \mathrm{mol})=113.552$

Geometry

C $-2.21287120-0.54928113-0.62097014$

C $-0.69580850-0.69546039-0.71125762$

C $0.03523060 \quad 0.69023776-0.72701858$

C $1.24428077 \quad 0.830188550 .19796749$

C $0.905285831 .34988953 \quad 1.61664585$

C $0.66573619-2.63477897 \quad 0.05550220$

C $-0.19407166-1.653995590 .32313052$

C $-0.47334011 \quad 1.85105551 \quad 1.64047139$

C $-1.36420978 \quad 1.63429114 \quad 2.77342698$

C $1.32275319 \quad 0.33168313 \quad 2.68358055$

$\mathrm{H}-2.600661890 .05416094-1.45385802$

$\mathrm{H}-2.51713300-0.06862847 \quad 0.31454156$

$\mathrm{H}-2.70777196-1.52785424-0.67792280$

$\mathrm{H}-0.67734084 \quad 1.54299075-0.61347941$

$\mathrm{H} \quad 1.95646122 \quad 1.53249943-0.28579932$

$\mathrm{H} 1.79334424 \quad-0.12933436 \quad 0.25918799$

$\mathrm{H} \quad 1.52388216 \quad 2.28619137 \quad 1.78481316$

H $1.01635726-3.33993756 \quad 0.79353252$

$\mathrm{H} 1.08004572-2.81147553-0.92915779$

$\mathrm{H}-0.60652531-1.49535951 \quad 1.31848388$

$\mathrm{H}-2.39601631 \quad 1.47878307 \quad 2.40990389$

$\mathrm{H}-1.12284520 \quad 0.81940845 \quad 3.46433436$

$\mathrm{H}-1.40940340 \quad 2.56836132 \quad 3.36538853$

H $2.37819908 \quad 0.05324451 \quad 2.58081972$

H $0.73767058 \quad-0.59169273 \quad 2.61012855$

$\mathrm{H} \quad 1.19076709 \quad 0.73091810 \quad 3.69439541$

$\begin{array}{lllll}0 & 0.69192001 & -0.52747644 & -2.49587978\end{array}$

$0 \quad 0.47673205 \quad 0.81046779-2.08146027$

o $-0.45738487-1.21589681-2.03167717$

Vibrational frequencies (in $\mathrm{cm}-1$ )

$\begin{array}{llllllllllllllll}-26.6 & 32.6 & 42.0 & 54.0 & 76.8 & 111.6 & 152.5 & 167.3 & 175.4 & 216.1 & 225.8 & 265.1 & 276.2 & 308.9\end{array}$ $341.4 \quad 361.5 \quad 416.1457 .5 \quad 498.9 \quad 508.2 \quad 558.1 \quad 606.0 \quad 677.4 \quad 719.1 \quad 825.2 \quad 840.7 \quad 869.8 \quad 887.4$ $929.1963 .6 \quad 974.1981 .3 \quad 985.3 \quad 1010.1 \quad 1033.0 \quad 1055.0 \quad 1060.5 \quad 1071.6 \quad 1095.2 \quad 1103.7 \quad 1128.2$

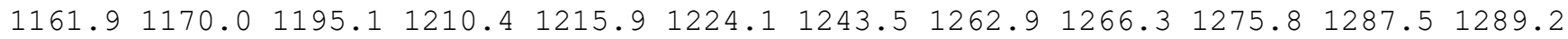

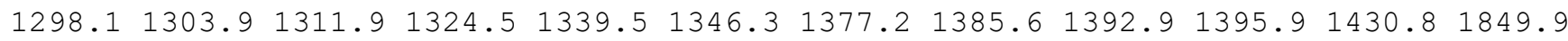
$2640.12650 .7 \quad 2654.92662 .1 \quad 2668.0 \quad 2671.0 \quad 2675.62677 .82680 .72709 .12735 .02753 .0$ $2768.8 \quad 2778.8 \quad 2779.92781 .1$

name $=$ MIN433

energy $($ in $\mathrm{kcal} / \mathrm{mol})=58.812037$

$\operatorname{ZPE}($ in $\mathrm{kcal} / \mathrm{mol})=143.731$

$\mathrm{G}($ in $\mathrm{kcal} / \mathrm{mol})=119.911$

Geometry

C $-2.75976614-0.01123287 \quad 0.05571466$

C $-1.25000542-0.04244004-0.17193741$

C $-0.638253831 .39411216-0.07316600$ 
C $0.67661157 \quad 1.50683592 \quad 0.68723058$

C $1.50599416 \quad 0.23366422 \quad 0.47999386$

C $1.75858421-0.03412656-0.94919637$

C $-0.59969104-1.19552543 \quad 0.59421764$

C $0.80692198-0.95773348 \quad 1.18122573$

C $0.68939833-0.696414692 .69639073$

C $1.66424842 \quad-2.219623190 .98029974$

$\mathrm{H}-3.24833727 \quad 0.71907648-0.60444535$

$\mathrm{H}-3.00673848 \quad 0.24337834 \quad 1.09138355$

$\mathrm{H}-3.21375121-0.98589510-0.16829503$

$\mathrm{H}-1.381728692 .14457862 \quad 0.26488428$

$\mathrm{H} \quad 0.49608661 \quad 1.69054427 \quad 1.76208609$

H $1.23356153 \quad 2.39579903 \quad 0.32541538$

H $2.51579572 \quad 0.363673120 .96182640$

$\mathrm{H}-0.54025942-2.05239531-0.12037205$

H $2.167360990 .79921617-1.50629491$

$\mathrm{H}-1.27456960-1.54811374 \quad 1.39660119$

$\mathrm{H}-0.047693230 .08016002 \quad 2.92104030$

$\mathrm{H} \quad 1.64657964-0.38687621 \quad 3.12632708$

$\mathrm{H} 0.37436656-1.602325953 .22606310$

$\mathrm{H} \quad 1.85650161-2.38684237-0.09166847$

H $2.63519779-2.13059155 \quad 1.47507169$

H $1.17027646-3.111420361 .37474111$

$\begin{array}{lllll}0 & -0.07198082 & 0.48583413 & -2.04267262\end{array}$

$0-0.383540581 .74151075-1.43822163$

o $-1.11031822-0.35259479-1.57928856$

Vibrational frequencies (in cm-1)

$\begin{array}{llllllllllllllllll}-56.8 & 87.7 & 138.3 & 186.3 & 207.0 & 231.6 & 241.6 & 242.8 & 280.5 & 284.0 & 329.0 & 348.6 & 362.4 & 407.8\end{array}$

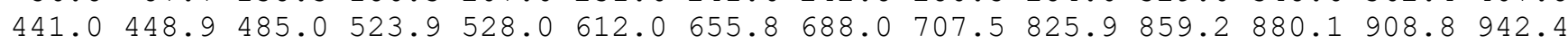

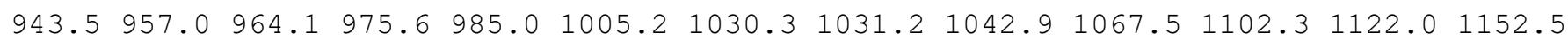
$\begin{array}{llllllllllll}1157.1 & 1172.1 & 1194.3 & 1206.9 & 1223.0 & 1251.8 & 1266.3 & 1279.8 & 1283.7 & 1288.9 & 1290.4 & 1296.7\end{array}$ $1300.0 \quad 1303.1 \quad 1308.1 \quad 1312.91319 .4 \quad 1330.8 \quad 1336.4 \quad 1367.6 \quad 1391.2 \quad 1402.81406 .11436 .4$ $2643.92650 .12659 .7 \quad 2663.4 \quad 2668.8 \quad 2676.12677 .3 \quad 2678.82680 .5 \quad 2685.42688 .42729 .3$ $2740.12778 .4 \quad 2780.0 \quad 2781.8$

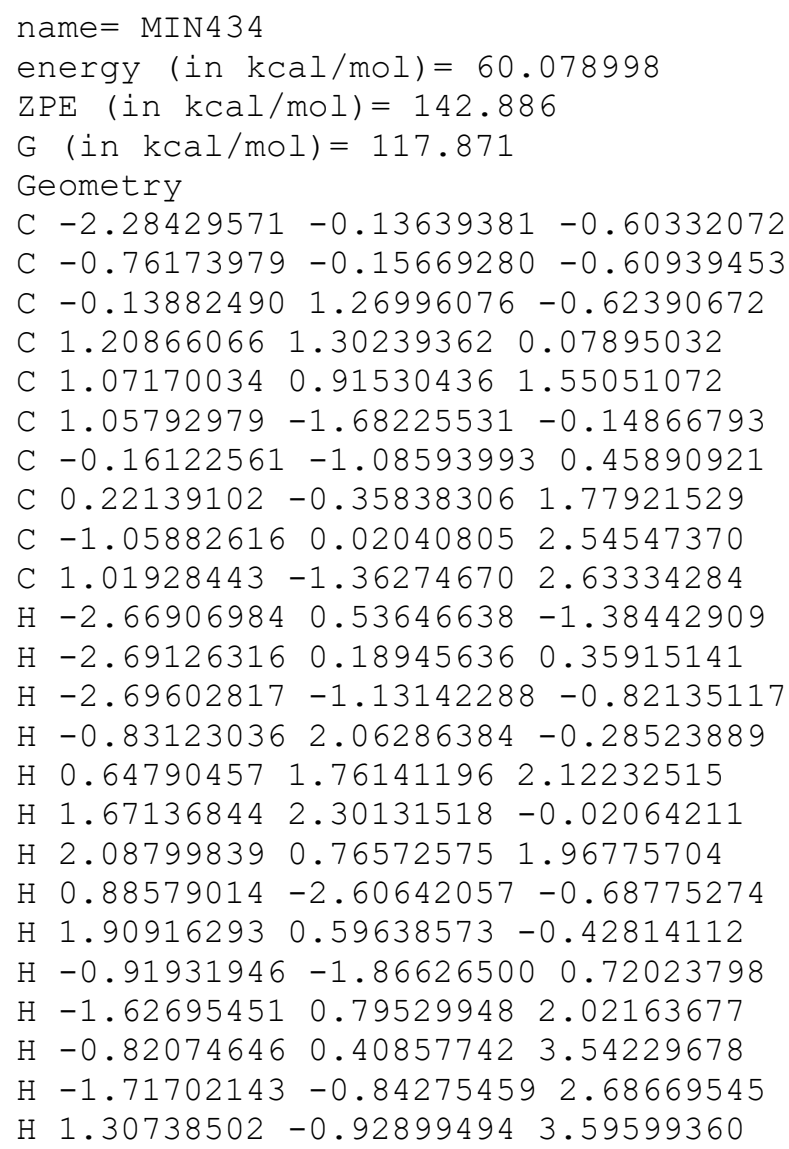


$\mathrm{H} \quad 1.94470257-1.666094372 .12231355$

$\mathrm{H} \quad 0.43930113-2.26585705 \quad 2.84380620$

$0.45450016 \quad 0.26045002-2.51963989$

○ $0.026931081 .50785345-2.02305274$

$0-0.40642184-0.70127824-1.89796928$

Vibrational frequencies (in $\mathrm{cm}-1$ )

$\begin{array}{llllllllllllllll}-19.9 & 79.5 & 135.5 & 147.5 & 173.3 & 208.1 & 229.3 & 239.0 & 245.2 & 262.2 & 273.8 & 289.4 & 307.1 & 354.3\end{array}$ $381.6 \quad 422.4 \quad 446.2 \quad 456.2 \quad 508.8 \quad 535.6 \quad 629.7 \quad 672.4 \quad 725.0 \quad 782.8 \quad 859.1 \quad 884.4 \quad 888.0 \quad 914.5$ $963.5975 .2979 .6 \quad 987.8 \quad 1006.0 \quad 1022.1 \quad 1034.3 \quad 1042.3 \quad 1069.7 \quad 1114.2 \quad 1128.4 \quad 1141.5 \quad 1163.6$ $1165.61178 .6 \quad 1195.91212 .1 \quad 1220.3 \quad 1241.2 \quad 1255.4 \quad 1274.51278 .51281 .5 \quad 1286.01296 .3$

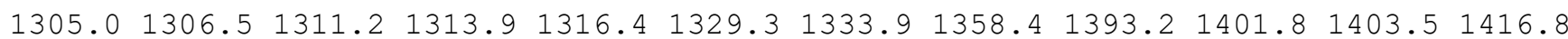
$2642.02648 .72663 .6 \quad 2666.4 \quad 2666.7 \quad 2673.2 \quad 2676.02677 .92680 .12686 .22701 .22737 .4$ $2739.72777 .12778 .0 \quad 2781.2$

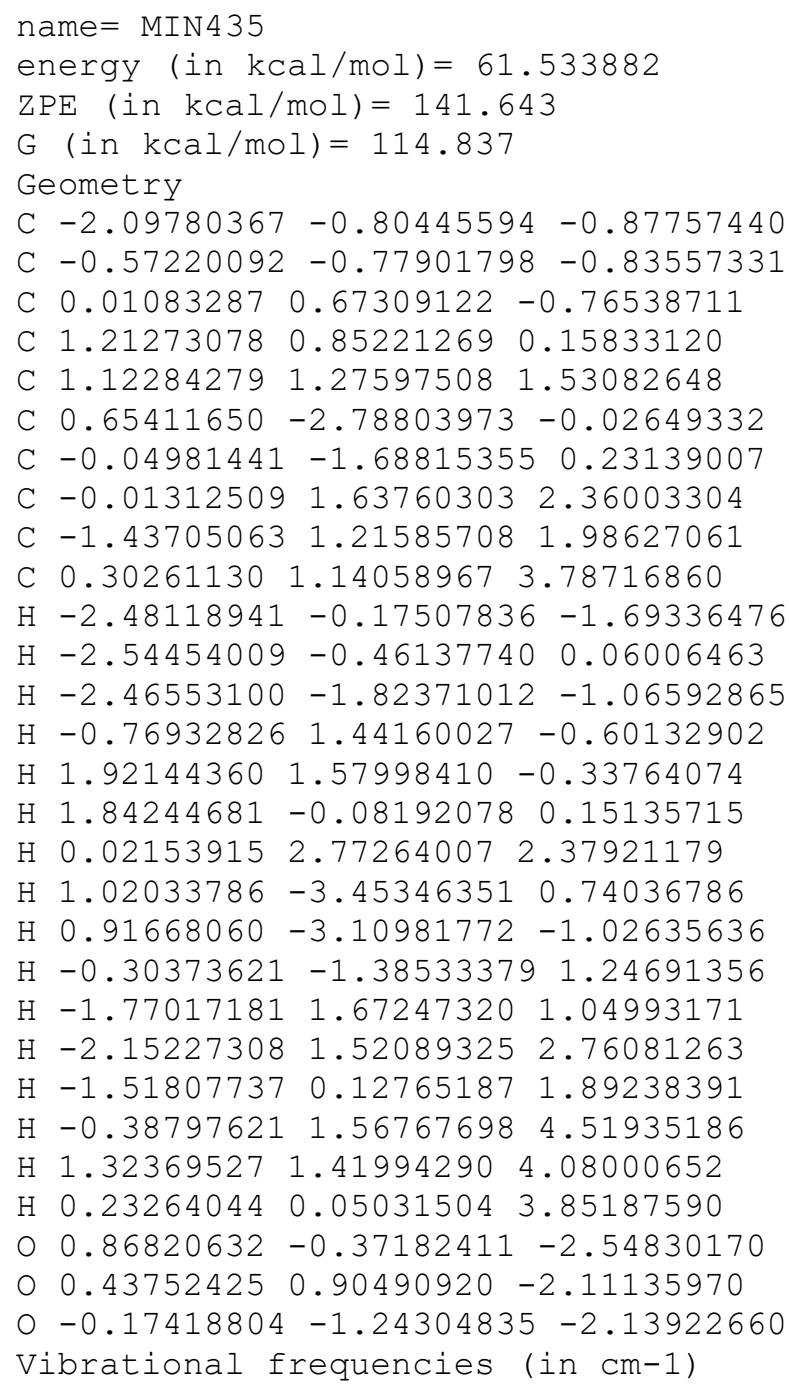


C $-0.67106023-0.82469441-0.78497604$

C $-0.11430247 \quad 0.63900188 \quad-0.76712193$

C $1.17885883 \quad 0.83086802 \quad 0.01991385$

C $1.25394001 \quad 0.96429492 \quad 1.45410559$

C $0.80926114 \quad-2.64686960 \quad 0.04522254$

C $-0.10030988-1.70408544 \quad 0.28191305$

C $0.19823454 \quad 1.00520763 \quad 2.45450216$

C $0.70151568 \quad 1.88797149 \quad 3.61532222$

C $-1.21371720 \quad 1.449102112 .06126351$

$\mathrm{H}-2.62042429-0.53604063 \quad 0.17735745$

$\mathrm{H}-2.55639080-1.89471095-0.95372792$

$\mathrm{H}-2.61597296-0.24629029-1.57713342$

$\mathrm{H}-0.88483097 \quad 1.39409677-0.52132399$

$\mathrm{H} \quad 1.711692121 .73381535-0.39695615$

H $1.89599287 \quad 0.00677471 \quad-0.25851722$

$\mathrm{H} 0.14582152-0.06053622 \quad 2.83962937$

$\mathrm{H} 1.21215456-3.29228474 \quad 0.81157426$

H $1.21767622-2.84972299-0.93698185$

$\mathrm{H}-0.50486394-1.52281395 \quad 1.27661993$

$\mathrm{H} \quad 0.70159680 \quad 2.94614700 \quad 3.33585895$

$\mathrm{H} \quad 1.73219337 \quad 1.62347387 \quad 3.88697528$

$\mathrm{H} \quad 0.07638396 \quad 1.77228762 \quad 4.50468163$

$\mathrm{H}-1.85278142 \quad 1.556771292 .94670745$

$\mathrm{H}-1.70342696 \quad 0.72580386 \quad 1.40319712$

$\mathrm{H}-1.198788992 .42242798 \quad 1.55925096$

$00.63185499-0.38259083-2.60666482$

$\begin{array}{lllll}0 & 0.16339671 & 0.87183684 & -2.15153993\end{array}$

o - $0.30806244-1.31627785-2.08796886$

Vibrational frequencies (in $\mathrm{cm}-1$ )

$\begin{array}{lllllllllllllll}34.5 & 40.0 & 70.6 & 87.8 & 120.7 & 137.6 & 156.1 & 165.0 & 193.1 & 220.1 & 221.9 & 249.0 & 274.3 & 320.6\end{array}$

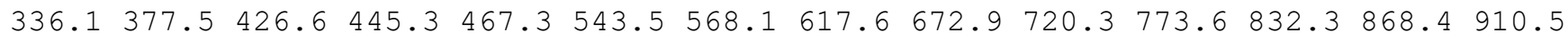
$958.6968 .6975 .5983 .1 \quad 986.9 \quad 1009.8 \quad 1011.7 \quad 1051.4 \quad 1059.0 \quad 1078.6 \quad 1097.9 \quad 1114.6 \quad 1123.6$

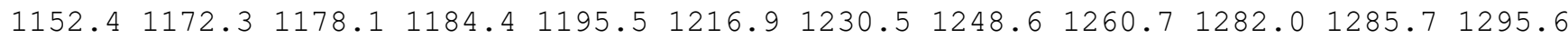
$1299.21300 .8 \quad 1307.4 \quad 1313.5 \quad 1331.2 \quad 1339.9 \quad 1384.8 \quad 1391.0 \quad 1394.8 \quad 1401.01484 .1 \quad 1851.0$ $2610.02655 .12666 .52667 .5 \quad 2674.12676 .12679 .62687 .82689 .2 \quad 2707.92709 .62753 .7$ 2775.22779 .12780 .82781 .8

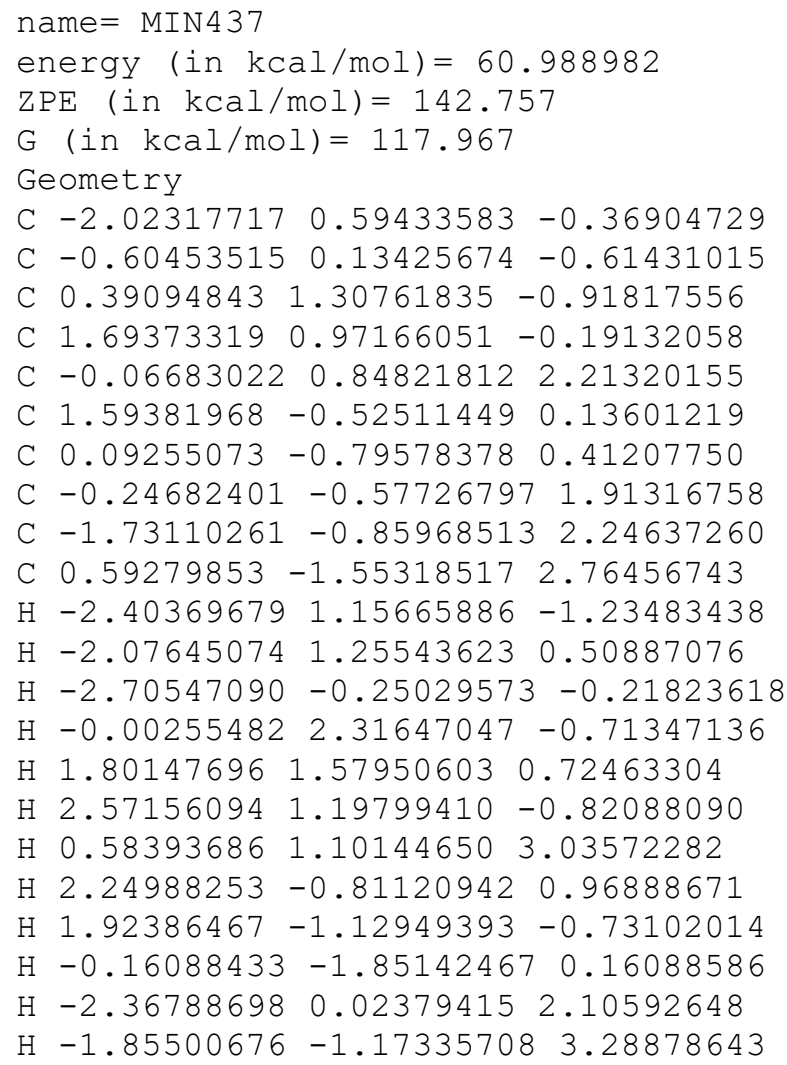


$\mathrm{H}-2.12570594-1.661067531 .61282293$

$\mathrm{H} 0.37332684-1.44092300 \quad 3.83122080$

$\mathrm{H} 1.66845079-1.393338762 .64278479$

$\mathrm{H} \quad 0.38307595-2.594359812 .49448092$

$0.44432129-0.13481095-2.62458308$

$0.553301061 .24895114-2.32934773$

O $-0.67371241-0.57677577-1.86549486$

Vibrational frequencies (in $\mathrm{cm}-1$ )

$\begin{array}{lllllllllllllllll}49.1 & 56.4 & 123.5 & 146.7 & 150.8 & 164.0 & 219.7 & 242.7 & 255.6 & 269.1 & 279.6 & 316.5 & 324.6 & 360.2\end{array}$ $426.2 \quad 431.5 \quad 450.4 \quad 471.0 \quad 544.7 \quad 586.2 \quad 631.1 \quad 703.2 \quad 716.3 \quad 818.5 \quad 853.4 \quad 864.9 \quad 918.9 \quad 932.9$ $947.4965 .1972 .5 \quad 995.6 \quad 999.6 \quad 1014.6 \quad 1025.0 \quad 1030.8 \quad 1043.2 \quad 1073.8 \quad 1082.4 \quad 1116.9 \quad 1132.8$

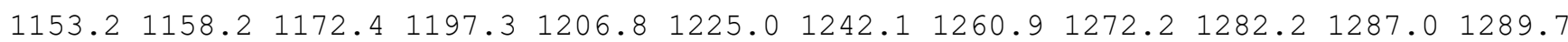

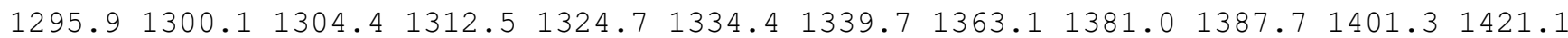
$2655.92660 .32662 .92664 .82668 .2 \quad 2671.92674 .22677 .02678 .02700 .32705 .42744 .8$ $2747.22773 .8 \quad 2776.12778 .6$

\section{name $=$ MIN438}

energy $($ in $\mathrm{kcal} / \mathrm{mol})=63.234254$

$\mathrm{ZPE}($ in $\mathrm{kcal} / \mathrm{mol})=141.955$

$\mathrm{G}($ in $\mathrm{kcal} / \mathrm{mol})=112.361$

Geometry

C $-3.04260255-0.23892030 \quad 0.54142945$

C $-1.53852546-0.27838623 \quad 0.54677497$

C $-0.34311805 \quad 0.55803748 \quad 0.53767230$

C $0.53621723 \quad 0.30235986-0.70728391$

C $1.37028697-0.92486561-0.50402417$

C $0.85587635-2.14336532-1.19904369$

C $-2.14408480-1.44292244 \quad 0.57245636$

C $2.49259413-0.93272244 \quad 0.23587428$

C $3.038829420 .27741875 \quad 0.91920332$

C $3.29749194-2.17623932 \quad 0.44463304$

$\mathrm{H}-2.52270725 \quad 2.20917330 \quad 0.20984321$

$\mathrm{H}-3.60061144 \quad 0.05541538 \quad 1.42480434$

$\mathrm{H}-3.58885580 \quad 0.01471881-0.36500588$

$\mathrm{H} \quad 0.26599133 \quad 0.38858329 \quad 1.47607289$

$\mathrm{H} \quad 1.28055967-2.21957391-2.21082363$

$\mathrm{H}-0.09314744 \quad 0.21599860-1.61723486$

$\mathrm{H} 1.164463051 .19825328-0.89243687$

$\mathrm{H} 1.10233786-3.07268055-0.66978800$

$\mathrm{H}-0.23854979-2.12258721-1.30612762$

$\mathrm{H}-2.15879608-2.48462350 \quad 0.59435755$

H $4.12680601 \quad 0.355639110 .78641457$

$\mathrm{H} \quad 2.84083580 \quad 0.23899716 \quad 1.99986209$

H $2.60818281 \quad 1.21693489 \quad 0.54629458$

H $3.78118119-2.49344402-0.49023923$

H 2.67143243 -3.011953420 .78927959$

$\mathrm{H} \quad 4.08957638-2.046096931 .19203602$

$0-1.678921812 .42473142-0.39993381$

$0-0.651423701 .906631930 .66911688$

O $-1.804789461 .73414735-1.40705448$

Vibrational frequencies (in $\mathrm{cm}-1$ )

$\begin{array}{llllllllllllll}16.5 & 31.5 & 45.6 & 49.0 & 71.2 & 79.5 & 86.9 & 91.8 & 118.8 & 139.0 & 170.5 & 203.6 & 249.0 & 291.4\end{array}$ $306.8 \quad 365.9 \quad 390.1 \quad 441.1 \quad 456.8 \quad 501.3 \quad 524.8 \quad 605.3 \quad 661.3 \quad 696.1 \quad 754.7 \quad 825.1 \quad 867.7 \quad 920.5$ $950.9960 .5986 .01001 .5 \quad 1010.5 \quad 1027.0 \quad 1027.7 \quad 1033.7 \quad 1054.0 \quad 1059.9 \quad 1108.2 \quad 1117.5 \quad 1135.4$

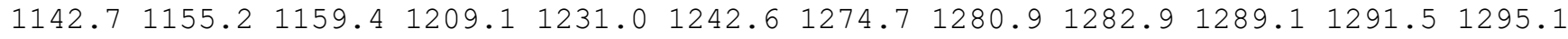
$1299.11315 .8 \quad 1319.8 \quad 1333.3 \quad 1358.8 \quad 1386.3 \quad 1397.7 \quad 1401.51407 .5 \quad 1426.9 \quad 1873.5 \quad 2049.5$ $2640.92656 .02665 .5 \quad 2668.1 \quad 2669.0 \quad 2670.4 \quad 2673.62676 .32703 .4 \quad 2738.82742 .32774 .9$ $2775.5 \quad 2777.3 \quad 2778.6 \quad 2830.2$

name $=$ MIN439

energy $($ in $\mathrm{kcal} / \mathrm{mol})=65.360601$

$\mathrm{ZPE}($ in $\mathrm{kcal} / \mathrm{mol})=139.983$

$\mathrm{G}($ in $\mathrm{kcal} / \mathrm{mol})=110.219$ 


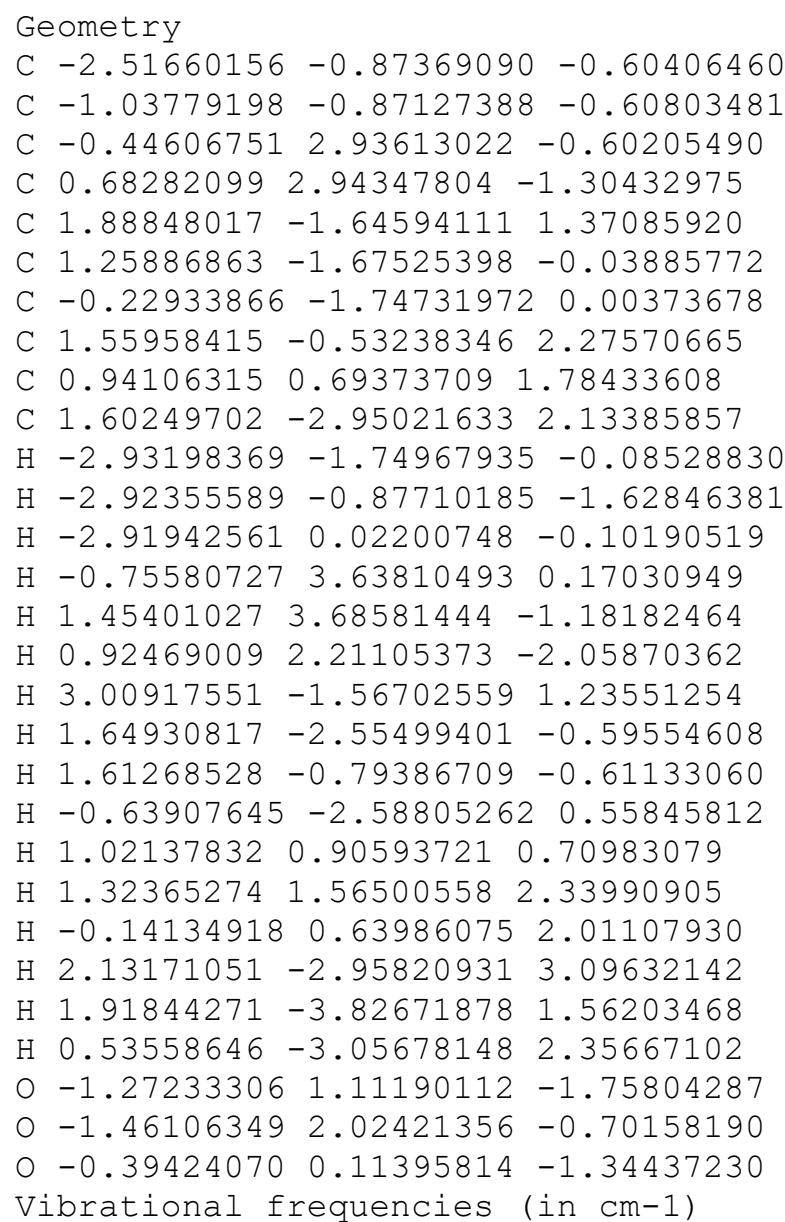

Vibrational frequencies (in $\mathrm{cm}-1$ )

$\begin{array}{lllllllllllllll}17.2 & 32.5 & 46.7 & 49.0 & 62.4 & 78.3 & 84.5 & 113.8 & 132.3 & 144.5 & 155.8 & 173.2 & 221.7 & 236.9\end{array}$ $302.7 \quad 356.7 \quad 366.0 \quad 420.8 \quad 442.6 \quad 470.5 \quad 520.6 \quad 573.1 \quad 616.8 \quad 641.2 \quad 655.6 \quad 809.3 \quad 814.7 \quad 853.0$

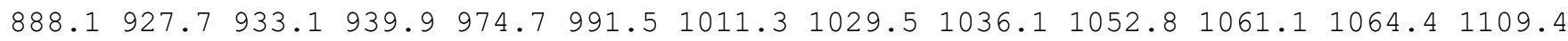
$\begin{array}{llllllllllll}1124.3 & 1168.6 & 1176.7 & 1193.6 & 1219.7 & 1224.9 & 1259.5 & 1268.7 & 1277.6 & 1278.9 & 1280.5 & 1291.7\end{array}$ $1301.01304 .11318 .21358 .3 \quad 1373.7 \quad 1376.91379 .8 \quad 1398.61402 .4 \quad 1410.11855 .41867 .9$ $2653.12655 .92661 .6 \quad 2663.4 \quad 2666.42671 .7 \quad 2679.42685 .62712 .52733 .12735 .92759 .0$ $2767.62777 .4 \quad 2780.7 \quad 2792.5$

\footnotetext{
name $=$ MIN440

energy $($ in $\mathrm{kcal} / \mathrm{mol})=64.792976$

$\mathrm{ZPE}(\mathrm{in} \mathrm{kcal} / \mathrm{mol})=141.138$

$\mathrm{G}(\mathrm{in} \mathrm{kcal} / \mathrm{mol})=113.907$

Geometry

C $-2.06793722-1.04229615-0.76523612$

C $-0.54374625-1.08861335-0.73180581$

C $0.06867967 \quad 0.35011202-0.70547826$

C $1.11589055 \quad 0.65311856 \quad 0.36703327$

C $0.48811520 \quad 0.81344027 \quad 1.71075100$

C $1.32702449-2.54379737 \quad 0.25575302$

C $-0.03721916-2.05854778 \quad 0.33856125$

C $-0.26106582 \quad 1.85753651 \quad 2.09110141$

C $-0.56665936 \quad 3.03522410 \quad 1.22892978$

C -0.865090621 .910349093 .45767823$

$\mathrm{H}-2.43049162-0.40568334-1.58507016$

$\mathrm{H}-2.47773486-0.65409520 \quad 0.17361408$

$\mathrm{H}-2.49680890-2.03849218-0.93426907$

$\mathrm{H}-0.72761847 \quad 1.12817233-0.70323919$

H $1.66255210 \quad 1.580369720 .07700508$

H $1.90378928-0.12682957 \quad 0.38056822$

$\mathrm{H} \quad 0.69360263-0.00151848 \quad 2.40404835$

$\mathrm{H}-0.68353907-2.97760788 \quad 0.35477149$

$\mathrm{H} 1.90559752-2.29532874 \quad-0.61983936$

$\mathrm{H}-0.20653425-1.63092757 \quad 1.35787326$
} 
$\mathrm{H}-1.55107898 \quad 2.93046701 \quad 0.74957048$

$\mathrm{H} 0.17364417 \quad 3.18051452 \quad 0.42941885$

$\mathrm{H}-0.59064882 \quad 3.96921068 \quad 1.80855943$

$\mathrm{H}-0.391846302 .694478364 .06670208$

$\mathrm{H}-0.75691445 \quad 0.96792000 \quad 4.01002267$

$\mathrm{H}-1.939425292 .13736056 \quad 3.41279489$

$\begin{array}{llll}0 & 0.99066654 & -0.84054962 & -2.37735375\end{array}$

$\begin{array}{lllll}0 & 0.66711420 & 0.48643491 & -1.99649258\end{array}$

o $-0.15804694-1.59153156-2.02292729$

Vibrational frequencies (in $\mathrm{cm}-1$ )

$\begin{array}{lllllllllllllll}29.3 & 37.9 & 68.2 & 84.9 & 94.7 & 102.3 & 119.0 & 159.3 & 206.6 & 230.9 & 247.3 & 251.8 & 304.2 & 343.4\end{array}$

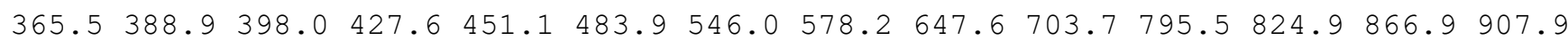
$917.2939 .7 \quad 968.6 \quad 992.3 \quad 1003.3 \quad 1016.2 \quad 1018.0 \quad 1020.4 \quad 1061.2 \quad 1084.2 \quad 1087.2 \quad 1115.2 \quad 1134.9$

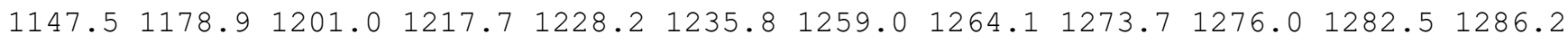

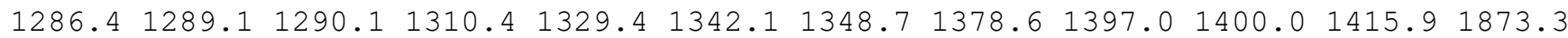
$2624.22647 .32666 .3 \quad 2667.5 \quad 2668.2 \quad 2669.0 \quad 2672.5 \quad 2675.42677 .02678 .72720 .42737 .7$ $2750.12777 .22779 .8 \quad 2780.8$

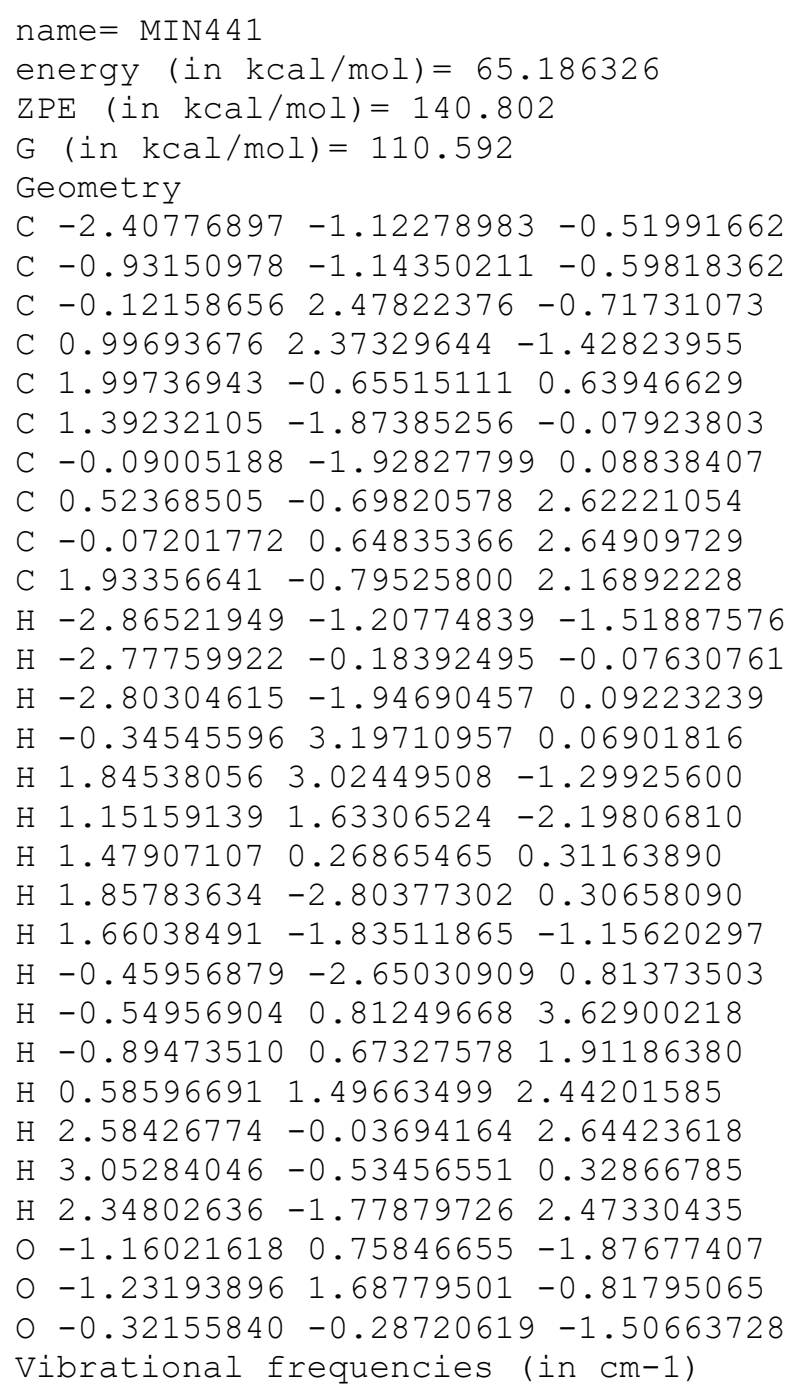
$314.6 \quad 350.4 \quad 413.9 \quad 420.4 \quad 452.9 \quad 474.4 \quad 563.1 \quad 613.7 \quad 634.7 \quad 665.7 \quad 815.2 \quad 846.1874 .0 \quad 882.1$ $902.0913 .7931 .0941 .6 \quad 966.2 \quad 985.6 \quad 1014.3 \quad 1021.1 \quad 1036.8 \quad 1061.8 \quad 1066.0 \quad 1099.3 \quad 1109.3$

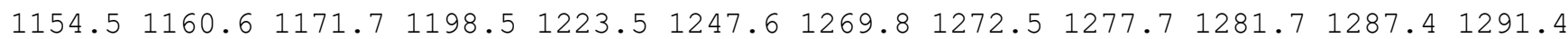
$1305.3 \quad 1313.0 \quad 1333.8 \quad 1341.5 \quad 1354.4 \quad 1368.5 \quad 1375.0 \quad 1377.4 \quad 1384.4 \quad 1398.3 \quad 1856.4 \quad 1866.0$ $2653.12659 .22662 .7 \quad 2663.6 \quad 2667.2 \quad 2671.92674 .92712 .72735 .0 \quad 2736.3 \quad 2740.4 \quad 2743.9$ $2754.92771 .02777 .8 \quad 2792.7$

name $=$ MIN4 42

energy $($ in $\mathrm{kcal} / \mathrm{mol})=64.095643$ 
$\mathrm{ZPE}($ in $\mathrm{kcal} / \mathrm{mol})=141.905$

$\mathrm{G}(\mathrm{in} \mathrm{kcal} / \mathrm{mol})=116.669$

Geometry

C $-2.22363960 \quad 0.18209122 \quad-0.82223584$

C $-0.74404277 \quad 0.17325399-0.79313306$

C $0.07254080 \quad 1.52914514-0.73316056$

C $0.74746849 \quad 1.64849429 \quad 0.75427302$

C $1.16521785 \quad 0.30159090 \quad 1.32248152$

C $1.38667481-0.71859923 \quad 0.14350382$

C $0.04615278-1.01084539-0.44791689$

C $0.18781004 \quad-0.32548943 \quad 2.21583127$

C $-1.077152910 .31196120 \quad 2.54381555$

C $0.44603898-1.65383856 \quad 2.78063764$

$\mathrm{H}-2.64281740 \quad 1.18896867 \quad-0.69770982$

$\mathrm{H}-2.68007679-0.48370643-0.08323263$

$\mathrm{H}-2.57736487-0.17996180-1.81536772$

$\mathrm{H}-0.62683810 \quad 2.38476038-0.83394647$

$\mathrm{H} \quad 0.10313618 \quad 2.23323479 \quad 1.40542917$

$\mathrm{H} \quad 1.64004324 \quad 2.27124031 \quad 0.54912153$

H $2.15657551 \quad 0.41433036 \quad 1.84613684$

$\mathrm{H} 1.94067389-1.61039930 \quad 0.45935201$

H $2.02797302-0.17902365-0.62089592$

$\mathrm{H}-0.40064397-1.97493840-0.25924199$

$\mathrm{H}-1.64885466 \quad 0.53813246 \quad 1.59550522$

$\mathrm{H}-0.94961231 \quad 1.32800297 \quad 2.97939198$

$\mathrm{H}-1.75078988-0.25201672 \quad 3.20317935$

$\mathrm{H} \quad 0.07617545-1.78339146 \quad 3.81296449$

H $1.50656025-1.96304502 \quad 2.76763366$

$\mathrm{H}-0.09040522-2.43252450 \quad 2.18402777$

O $-0.74222650-1.27296840-2.68444578$

O $1.005331761 .48110526-1.61498596$

o $-0.05452780-0.57726128-1.95353789$

Vibrational frequencies (in $\mathrm{cm}-1$ )

$\begin{array}{llllllllllllllll}25.2 & 95.8 & 109.9 & 127.6 & 132.0 & 155.4 & 190.1 & 199.9 & 235.8 & 276.0 & 307.2 & 316.0 & 339.5 & 368.3\end{array}$ $\begin{array}{llllllllllllll}395.0 & 419.6 & 489.7 & 494.1 & 530.1 & 548.1 & 621.0 & 669.5 & 713.1 & 736.0 & 786.8 & 811.5 & 848.3 & 876.2\end{array}$ $890.3 \quad 927.5 \quad 944.7 \quad 984.0 \quad 997.3 \quad 1015.8 \quad 1034.1 \quad 1038.5 \quad 1074.4 \quad 1080.8 \quad 1117.3 \quad 1139.4 \quad 1152.7$ $1159.21188 .4 \quad 1188.8 \quad 1197.6 \quad 1201.8 \quad 1219.8 \quad 1226.2 \quad 1242.5 \quad 1249.5 \quad 1264.3 \quad 1275.1 \quad 1290.0$

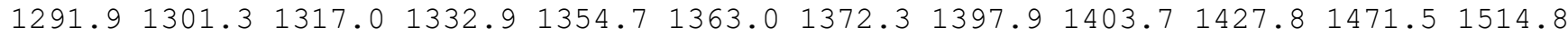
$2544.12576 .5 \quad 2602.8 \quad 2628.7 \quad 2638.6 \quad 2639.3 \quad 2645.32651 .12675 .12681 .5 \quad 2727.72742 .3$ $2750.2 \quad 2755.6 \quad 2767.92770 .5$

\section{name $=$ MIN443}

energy (in $\mathrm{kcal} / \mathrm{mol})=66.222914$

$\mathrm{ZPE}(\mathrm{in} \mathrm{kcal} / \mathrm{mol})=139.926$

G $($ in $\mathrm{kcal} / \mathrm{mol})=110.092$

Geometry

C $-2.82246353-0.98584958-0.80416276$

C $-1.33690873-0.98773251-0.79423168$

C $-0.88307444 \quad 2.50378904 \quad-0.83698347$

C $-1.10436074 \quad 2.04692354 \quad 0.39241576$

C $1.54546411-0.56262008 \quad 1.03461220$

C $0.94356949-1.58822878 \quad 0.05137103$

C $-0.54555099-1.572112420 .11352855$

C $1.14408464 \quad-0.921734692 .41794902$

C $1.05681098 \quad 0.17201393 \quad 3.39382963$

C $3.08063905-0.642402851 .01631472$

$\mathrm{H}-3.21558582-0.01784069-0.45311214$

$\mathrm{H}-3.24607609-1.77130885-0.16467327$

$\mathrm{H}-3.21919628-1.13895601-1.82059693$

$\mathrm{H}-1.12280401 \quad 3.49508217-1.22431891$

$\mathrm{H}-1.562408532 .63452206 \quad 1.16982569$

$\mathrm{H}-0.85333350 \quad 1.04922026 \quad 0.71708544$

H $1.22837868 \quad 0.460685330 .72116662$

H $1.31219523-2.605333250 .31253693$ 
Н $1.31632609-1.39277164-0.97427729$

$\mathrm{H}-0.96795629-2.09452171 \quad 0.97311161$

$\mathrm{H} 1.65053844 \quad-0.09275233 \quad 4.28592671$

$\begin{array}{lllll}\mathrm{H} & 0.01443322 & 0.23924881 & 3.75284026\end{array}$

H 1.362285101 .170974253 .06700069

H $3.53126725 \quad 0.14470687 \quad 1.63055439$

$\mathrm{H} 3.46287131-0.52479385-0.00468866$

$\mathrm{H} 3.43711893-1.611073031 .39086003$

○ $0.15743342 \quad 0.55832200-1.54421144$

O $-0.311931931 .83783571-1.88133420$

O $-0.84530584-0.35930201-1.92586056$

Vibrational frequencies (in $\mathrm{cm}-1$ )

$\begin{array}{llllllllllllllll}16.0 & 30.3 & 40.3 & 54.6 & 70.2 & 72.3 & 101.1 & 106.4 & 108.5 & 151.7 & 172.7 & 184.2 & 241.7 & 274.1\end{array}$ $279.3 \quad 317.7 \quad 330.0 \quad 353.2 \quad 435.3 \quad 464.9 \quad 482.3 \quad 559.5 \quad 624.6 \quad 631.9639 .6 \quad 804.7 \quad 854.6 \quad 890.2$ $899.3934 .7 \quad 939.6951 .4 \quad 980.2 \quad 1005.3 \quad 1010.3 \quad 1017.7 \quad 1029.8 \quad 1055.8 \quad 1071.1 \quad 1097.9 \quad 1113.4$

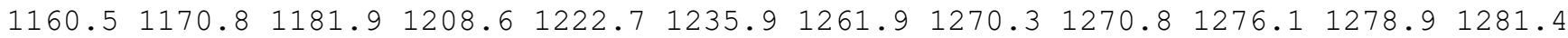

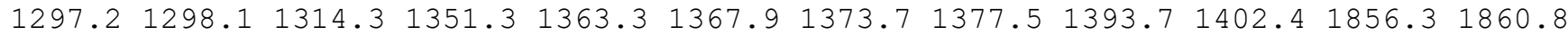
$2655.52659 .82663 .4 \quad 2668.2 \quad 2672.02674 .82675 .62693 .32707 .92731 .4 \quad 2734.92751 .6$ 2770.92777 .22778 .02790 .6

name $=$ MIN4 44

energy $($ in $\mathrm{kcal} / \mathrm{mol})=66.339129$

$\mathrm{ZPE}($ in $\mathrm{kcal} / \mathrm{mol})=140.01$

$\mathrm{G}($ in $\mathrm{kcal} / \mathrm{mol})=109.58$

Geometry

C $-2.50063430-0.96864028-0.57990743$

C $-1.02179477-0.96536418-0.56814024$

C $-0.680463302 .88873312-0.58233557$

C $0.48009703 \quad 2.99973236-1.22213234$

C $1.83975360-1.573199291 .43538965$

C $1.26379426-1.804794730 .01766752$

C $-0.22609596-1.86446574 \quad 0.02560355$

C $1.28692554-0.30759277 \quad 1.98915625$

C $1.79314616 \quad 0.94458169 \quad 1.41421130$

C $1.48433405-2.72256193 \quad 2.38576477$

$\mathrm{H}-2.91905959-1.87730058-0.12257356$

$\mathrm{H}-2.90281564-0.90396122-1.60335715$

$\mathrm{H}-2.90614646-0.11000743-0.01778112$

$\mathrm{H}-1.09153065 \quad 3.55227840 \quad 0.17656848$

$\mathrm{H} \quad 1.17642683 \quad 3.80429129-1.05535931$

H $0.82295479 \quad 2.29843719 \quad-1.96736623$

H $2.95437904-1.52306927 \quad 1.34876607$

$\mathrm{H} \quad 1.66842122-2.76006290-0.38265502$

$\mathrm{H} \quad 1.64299793-1.01892379-0.66618370$

$\mathrm{H}-0.65120090 \quad-2.70923089 \quad 0.56386222$

H $1.960992091 .67863886 \quad 2.21855344$

$\mathrm{H} \quad 0.99242831 \quad 1.37811495 \quad 0.78090558$

H $2.69640879 \quad 0.89465928 \quad 0.79973105$

$\mathrm{H} 0.40624748 \quad-2.74336042 \quad 2.59041578$

$\mathrm{H} \quad 1.98454860-2.59618170 \quad 3.35256180$

$\mathrm{H} \quad 1.77337243-3.69348604 \quad 1.97542450$

$\begin{array}{lllll}0 & -1.25542543 & 0.98945483 & -1.76495429\end{array}$

$0-1.60689273 \quad 1.89684654-0.74606892$

o $-0.365077510 .05410638-1.24466042$

Vibrational frequencies (in $\mathrm{cm}-1$ )

$\begin{array}{llllllllllllllll}-7.0 & 16.5 & 37.6 & 55.8 & 78.2 & 83.0 & 109.1 & 125.3 & 138.5 & 143.8 & 159.8 & 169.6 & 222.6 & 243.1\end{array}$ $279.8 \quad 337.2 \quad 358.1 \quad 407.2 \quad 441.7 \quad 475.2 \quad 512.0 \quad 561.6 \quad 612.4 \quad 635.4 \quad 652.8 \quad 810.2 \quad 840.2 \quad 866.0$ $890.4916 .5 \quad 930.8 \quad 939.9 \quad 971.2 \quad 992.8 \quad 1011.1 \quad 1029.6 \quad 1033.6 \quad 1060.1 \quad 1063.9 \quad 1095.2 \quad 1111.1$

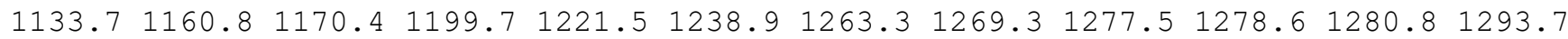
$1301.61304 .41321 .8 \quad 1345.2 \quad 1369.8 \quad 1373.3 \quad 1377.2 \quad 1381.51403 .7 \quad 1404.61854 .11871 .3$ $2645.72656 .8 \quad 2661.7 \quad 2671.1 \quad 2671.6 \quad 2674.5 \quad 2684.5 \quad 2695.12712 .6 \quad 2734.02736 .02759 .0$ $2768.62777 .4 \quad 2782.0 \quad 2792.6$ 
name $=$ MIN445

energy (in $\mathrm{kcal} / \mathrm{mol})=66.021476$

$\mathrm{ZPE}($ in $\mathrm{kcal} / \mathrm{mol})=141.219$

$\mathrm{G}($ in $\mathrm{kcal} / \mathrm{mol})=113.388$

Geometry

C $-2.94942966-0.18833352-0.15656669$

C $-1.45397048-0.17209508-0.13843275$

C $-0.81300104 \quad 1.16836432-0.12161716$

C $0.64352887 \quad 1.15027416 \quad 0.39824876$

C $1.07477637-0.25453034 \quad 0.70016329$

C $1.32629347-1.11554787-0.30574916$

C $-0.82374104-1.33866818-0.18698111$

C $1.15057299-0.609505872 .16303241$

C $1.08393044 \quad-2.120190192 .41023541$

C $2.44248931-0.031880352 .75670531$

$\mathrm{H}-3.33685101 \quad 0.44324854 \quad-0.96935223$

$\mathrm{H}-3.35848001 \quad 0.189952390 .79076202$

$\mathrm{H}-3.35715093-1.19707009-0.30918408$

$\mathrm{H}-1.42509912 \quad 1.91254188 \quad 0.45082778$

$\mathrm{H} \quad 0.73164029 \quad 1.80483237 \quad 1.28662835$

$\mathrm{H} 1.31404179 \quad 1.61046954-0.35844647$

$\mathrm{H} \quad 0.27303654 \quad-0.140083112 .68344382$

$\mathrm{H} \quad 1.64668488-2.13756617-0.16401014$

H $1.32205147-0.82733785-1.35209040$

$\mathrm{H}-1.37467934 \quad 0.50899130 \quad-3.48789927$

$\mathrm{H} 1.98194981-2.63286777 \quad 2.04887488$

$\mathrm{H} \quad 0.21887147 \quad-2.56792283 \quad 1.90122784$

$\mathrm{H} \quad 0.98789992-2.34115894 \quad 3.47840956$

H $2.51909332-0.26225263 \quad 3.82540972$

$\mathrm{H} \quad 2.48737546 \quad 1.05741382 \quad 2.65114721$

H $3.32916149-0.448561792 .26567577$

$0-0.04198703 \quad 1.17217360-2.33305523$

$\begin{array}{lllll}0 & -0.87482318 & 1.78435438 & -1.40982295\end{array}$

o $-0.694795310 .07958376-2.91251059$

Vibrational frequencies (in cm-1)

$\begin{array}{lllllllllllllll}30.6 & 40.6 & 54.5 & 56.8 & 87.1 & 113.6 & 140.5 & 145.9 & 185.7 & 198.3 & 209.9 & 246.6 & 270.2277 .1\end{array}$ $311.1322 .1 \quad 367.2 \quad 397.5 \quad 437.4 \quad 473.6 \quad 500.4 \quad 520.0 \quad 554.7 \quad 679.2 \quad 700.2 \quad 735.2 \quad 836.2 \quad 854.1$

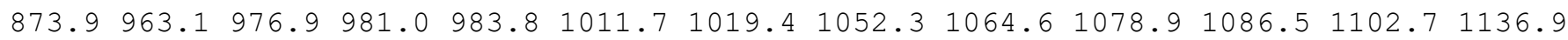

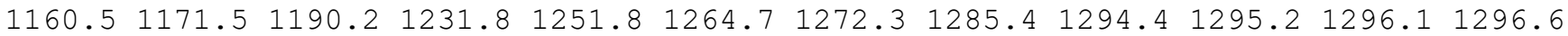
$1302.21306 .91322 .1 \quad 1337.6 \quad 1350.91366 .0 \quad 1384.5 \quad 1399.3 \quad 1401.0 \quad 1406.3 \quad 1804.4 \quad 1838.5$ $2653.12666 .8 \quad 2671.7 \quad 2672.0 \quad 2672.9 \quad 2676.6 \quad 2677.02678 .12699 .92705 .6 \quad 2739.5 \quad 2775.2$ $2776.42778 .92782 .2 \quad 2849.9$

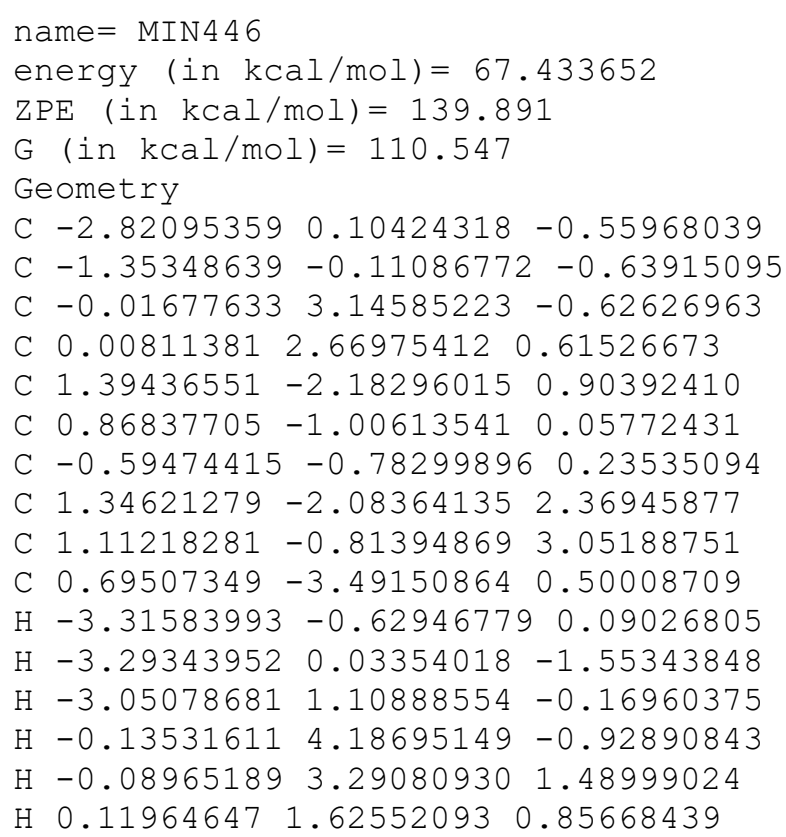


Н $2.49694330-2.30107242 \quad 0.67513603$

H $1.09674985-1.19897005-1.01447497$

$\mathrm{H} 1.43653041-0.08748268 \quad 0.31537643$

$\mathrm{H}-1.03441450-1.21605828 \quad 1.13198622$

$\mathrm{H} 1.93843100 \quad-0.63257220 \quad 3.76334008$

$\mathrm{H} \quad 0.21141317 \quad-0.91791499 \quad 3.68485204$

$\begin{array}{lllll}\mathrm{H} & 0.98689422 & 0.08773921 & 2.44324847\end{array}$

$\mathrm{H} 1.06834074-4.33522652 \quad 1.09473745$

$\mathrm{H} \quad 0.85909667-3.71943870 \quad-0.55779815$

$\mathrm{H}-0.38719216-3.43338222 \quad 0.65988365$

○ $0.352275351 .07524022-1.59990339$

$00.107943622 .44779483-1.79146228$

o $-0.86788048 \quad 0.41361967-1.82430840$

Vibrational frequencies (in $\mathrm{cm}-1$ )

$\begin{array}{llllllllllllllll}30.0 & 35.5 & 52.5 & 61.0 & 64.2 & 70.2 & 96.1 & 103.0 & 129.5 & 144.4 & 150.2 & 163.1 & 231.2 & 259.0\end{array}$ $299.6 \quad 348.3 \quad 355.6 \quad 370.8 \quad 438.3 \quad 447.9 \quad 533.7 \quad 590.6 \quad 619.5 \quad 625.1 \quad 626.1 \quad 803.9 \quad 826.8 \quad 831.7$ $904.5932 .3 \quad 947.2966 .5977 .1 \quad 1001.6 \quad 1011.01023 .3 \quad 1041.9 \quad 1054.9 \quad 1056.7 \quad 1068.2 \quad 1107.3$

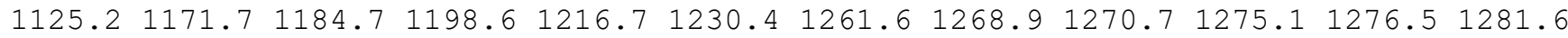

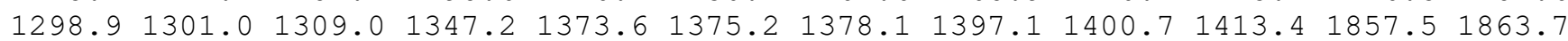
$2648.02656 .8 \quad 2662.6 \quad 2663.4 \quad 2665.4 \quad 2673.7 \quad 2678.82683 .42710 .5 \quad 2724.5 \quad 2733.92757 .9$ $2768.12777 .02780 .4 \quad 2793.4$

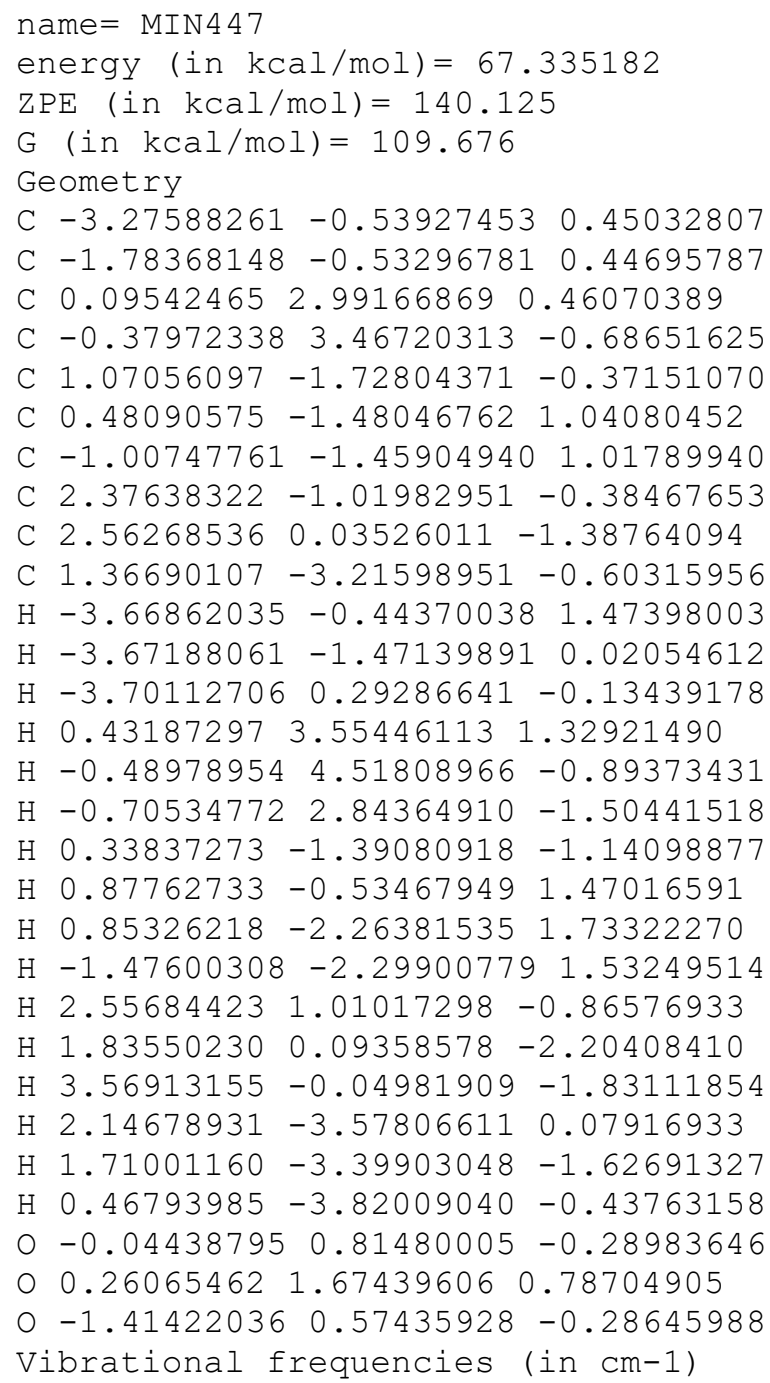


name $=$ MIN 448

energy (in $\mathrm{kcal} / \mathrm{mol})=66.120365$

$\mathrm{ZPE}(\mathrm{in} \mathrm{kcal} / \mathrm{mol})=141.943$

$\mathrm{G}($ in $\mathrm{kcal} / \mathrm{mol})=116.718$

Geometry

C $-2.17899738-0.31412107-0.50334809$

C $-0.65823904-0.18471318-0.62273707$

C $-0.265807571 .29063600-0.99252326$

C $0.89674735 \quad 1.97319664 \quad-0.29595428$

C $1.55269732-0.11281872 \quad 1.81472742$

C $1.58390735-0.88154752 \quad 0.51305807$

C $0.03075236-0.94824606 \quad 0.49928868$

C $0.04320907-0.39103152 \quad 1.97331067$

C $-0.83742724 \quad 0.77596200 \quad 2.34689502$

C $-0.11652329-1.51896087 \quad 2.99107681$

$\mathrm{H}-2.68577578 \quad 0.12490783-1.37440096$

$\mathrm{H}-2.55855787 \quad 0.17134277 \quad 0.40069733$

$\mathrm{H}-2.48649669-1.36794373-0.46622067$

$\mathrm{H}-1.16757720 \quad 1.94540600 \quad-0.97520077$

$\mathrm{H} 0.94954453 \quad 3.03126669-0.59177603$

H $1.86075480 \quad 1.53023298-0.58241175$

H $0.82979292 \quad 1.95280327 \quad 0.79716617$

H $2.07734061-1.85785332 \quad 0.57929955$

$\mathrm{H} 2.05431867-0.38144308-0.33855681$

$\mathrm{H}-0.34153628-1.992827110 .46398220$

$\mathrm{H}-0.946114821 .511183221 .54292542$

$\mathrm{H}-0.42688966 \quad 1.305721393 .21926329$

$\mathrm{H}-1.84984306 \quad 0.44691453 \quad 2.61708183$

$\mathrm{H} \quad 0.09543914 \quad-1.15865455 \quad 4.00656219$

H $0.55879349-2.36008705 \quad 2.79607008$

$\mathrm{H}-1.13969532-1.913035292 .98598781$

$00.57799997-0.10487300-2.53121061$

○ $0.04790373 \quad 1.19932877-2.38828800$

o - $0.34440217-0.91734194-1.83119804$

Vibrational frequencies (in $\mathrm{cm}-1$ )

$\begin{array}{llllllllllllllll}38.0 & 71.9 & 112.1 & 143.1 & 152.3 & 167.6 & 174.9 & 207.4 & 238.3 & 248.1 & 274.6 & 282.6 & 311.1 & 332.3\end{array}$ $351.1 \quad 372.5 \quad 399.4 \quad 468.7 \quad 490.8 \quad 571.8 \quad 642.1 \quad 689.7 \quad 695.3 \quad 778.4 \quad 807.9 \quad 862.2 \quad 868.2 \quad 907.9$ $952.0961 .1979 .7991 .7 \quad 996.0 \quad 1009.8 \quad 1012.4 \quad 1016.6 \quad 1043.4 \quad 1078.0 \quad 1107.9 \quad 1114.4 \quad 1118.3$

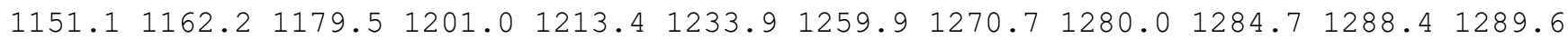
$1295.31297 .5 \quad 1299.3 \quad 1301.6 \quad 1306.8 \quad 1323.91331 .01371 .4 \quad 1390.2 \quad 1397.11404 .91413 .0$ $2656.92663 .5 \quad 2666.8 \quad 2670.6 \quad 2671.5 \quad 2673.7 \quad 2675.02675 .92677 .0 \quad 2682.12710 .92763 .7$ 2775.52778 .32779 .62781 .7

\section{name $=$ MIN449}

energy (in $\mathrm{kcal} / \mathrm{mol})=68.282034$

$\mathrm{ZPE}(\mathrm{in} \mathrm{kcal} / \mathrm{mol})=140.041$

$\mathrm{G}($ in $\mathrm{kcal} / \mathrm{mol})=110.264$

Geometry

C $-2.66525634-1.33712331-0.65068180$

C $-1.17808668-1.29012267-0.61693256$

C $-0.73974672 \quad 1.91115403-0.71536551$

C $-1.10920674 \quad 3.19222256-0.75250464$

C $1.65630841-0.753309791 .49995861$

C $1.08146242-1.687797790 .41251276$

C $-0.40828271-1.69546228 \quad 0.39985068$

C $0.91962976 \quad 0.53265081 \quad 1.52012685$

C $1.65901706 \quad 1.73517908 \quad 1.91019381$

C $1.45516572-1.339620492 .90906433$

$\mathrm{H}-3.04951105-1.37099053-1.68244014$

$\mathrm{H}-3.09724764-0.44011684-0.18006387$

$\mathrm{H}-3.06402311-2.21516621-0.12630790$

$\mathrm{H}-0.77510784 \quad 1.23601545 \quad 0.14088915$ 
$\mathrm{H}-1.04152417 \quad 3.80923669-1.63154508$

$\mathrm{H}-1.51198740 \quad 3.70838745 \quad 0.10005986$

$\mathrm{H} 2.75143114-0.644682651 .30908826$

$\mathrm{H} \quad 1.44823407 \quad-2.723235690 .58962149$

$\mathrm{H} \quad 1.50369300-1.40756329-0.57307373$

$\mathrm{H}-0.86651578-2.06683904 \quad 1.31671594$

H $1.10355985 \quad 2.26298250 \quad 2.70540837$

H 1.659439892 .438454161 .05443830

H 2.69480985 $1.61435832 \quad 2.24136944$

$\mathrm{H} \quad 1.92935991-0.71756924 \quad 3.67598817$

H $1.89322546-2.34171450 \quad 2.97707073$

$\mathrm{H} \quad 0.39004268-1.429375923 .15886740$

$00.30593434 \quad 0.11624814-1.64969160$

$0-0.263041541 .37983076-1.89236311$

o $-0.69404573-0.85087451-1.83768858$

Vibrational frequencies (in cm-1)

$\begin{array}{llllllllllllllll}19.8 & 32.8 & 37.9 & 43.1 & 61.8 & 82.2 & 95.1 & 98.4 & 117.1 & 145.3 & 184.0 & 208.7 & 232.0 & 272.3\end{array}$ $308.0 \quad 334.9 \quad 346.2 \quad 375.2 \quad 406.4 \quad 412.9 \quad 534.8 \quad 579.7 \quad 589.2 \quad 613.2 \quad 645.6 \quad 813.5 \quad 854.1 \quad 881.6$

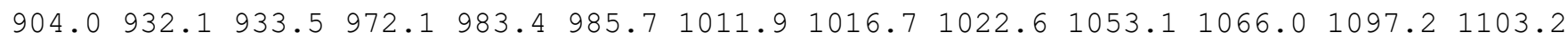
$1150.01190 .5 \quad 1209.2 \quad 1213.3 \quad 1225.4 \quad 1259.2 \quad 1259.9 \quad 1268.3 \quad 1272.8 \quad 1279.1 \quad 1290.5 \quad 1293.5$ $1296.91298 .6 \quad 1314.0 \quad 1349.3 \quad 1368.3 \quad 1372.3 \quad 1374.8 \quad 1394.7 \quad 1395.8 \quad 1404.4 \quad 1827.2 \quad 1865.9$ $2650.32654 .5 \quad 2665.6 \quad 2668.8 \quad 2669.0 \quad 2672.3 \quad 2674.72676 .22696 .3 \quad 2734.42735 .32757 .7$ $2768.3 \quad 2777.8 \quad 2778.5 \quad 2799.4$

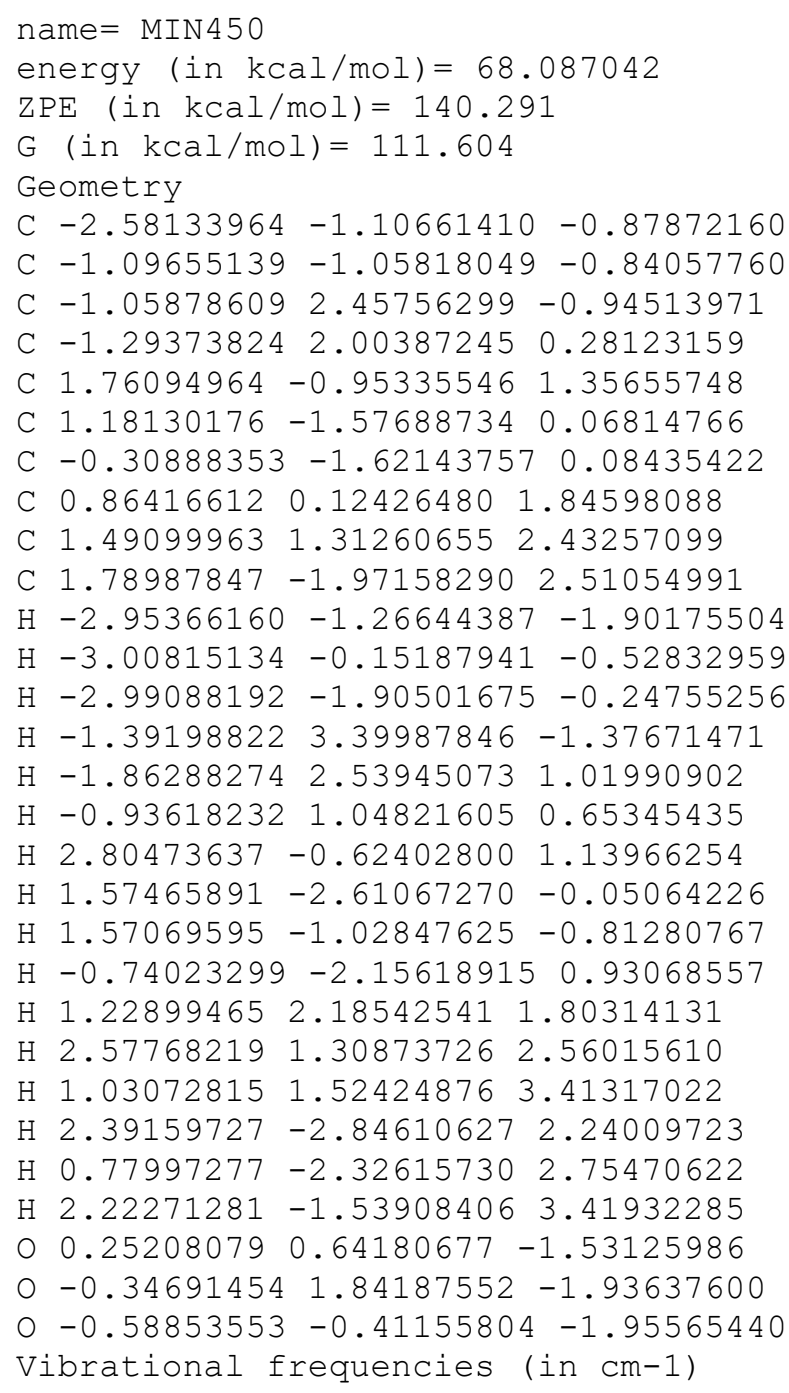
$313.2 \quad 324.7 \quad 338.5 \quad 371.6 \quad 410.9 \quad 436.2 \quad 542.5 \quad 586.2 \quad 630.6 \quad 639.8 \quad 652.1 \quad 799.7 \quad 857.5 \quad 876.0$ $909.7933 .9937 .0 \quad 947.1 \quad 979.2 \quad 996.3 \quad 1005.2 \quad 1018.1 \quad 1024.8 \quad 1063.5 \quad 1084.7 \quad 1096.8 \quad 1105.9$ $1150.0 \quad 1167.8 \quad 1193.0 \quad 1208.7 \quad 1214.7 \quad 1229.3 \quad 1264.3 \quad 1269.0 \quad 1272.9 \quad 1279.2 \quad 1280.7 \quad 1296.3$

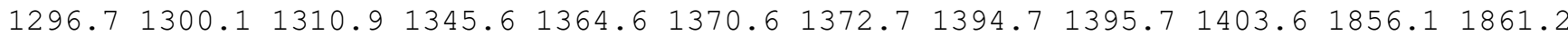


$2652.22653 .3 \quad 2663.6 \quad 2670.02671 .7 \quad 2676.5 \quad 2678.12686 .02697 .92731 .02733 .7 \quad 2757.5$ 2768.62778 .12778 .92785 .2

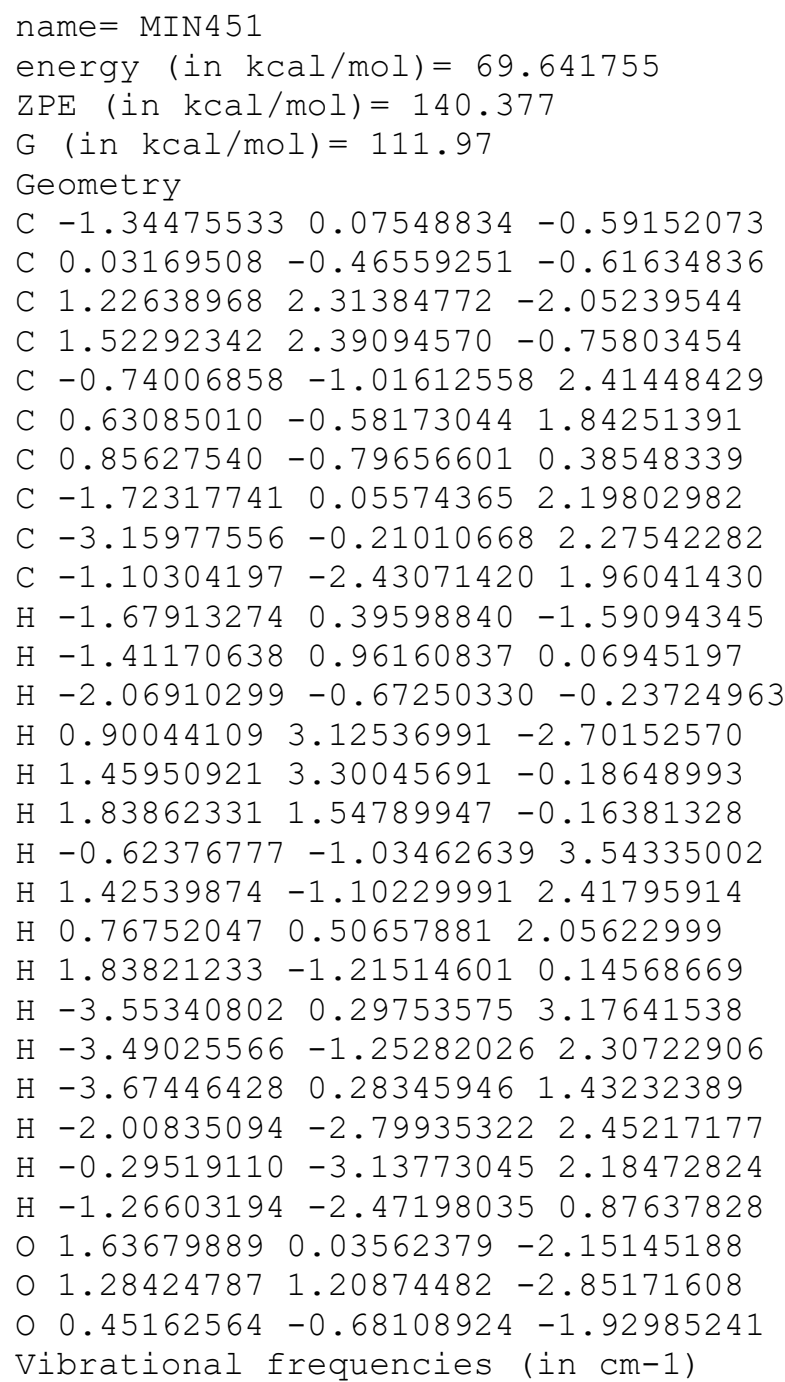

$\begin{array}{llllllllllllllll}18.0 & 37.9 & 48.1 & 79.1 & 86.5 & 106.4 & 136.0 & 145.2 & 172.2 & 177.1 & 183.4 & 218.8 & 250.1 & 261.9\end{array}$ $283.9 \quad 339.2 \quad 353.0 \quad 427.4 \quad 440.4 \quad 465.9 \quad 493.5 \quad 599.6 \quad 622.5 \quad 631.1 \quad 651.6 \quad 783.9 \quad 808.1 \quad 877.8$

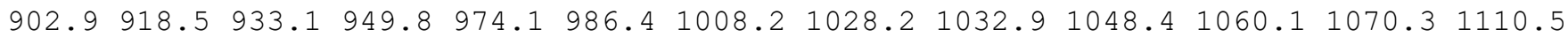
$1128.91173 .2 \quad 1195.6 \quad 1206.7 \quad 1213.5 \quad 1235.2 \quad 1258.6 \quad 1268.7 \quad 1277.1 \quad 1281.1 \quad 1283.4 \quad 1296.1$ $1300.01304 .6 \quad 1308.5 \quad 1353.0 \quad 1373.3 \quad 1374.6 \quad 1382.1 \quad 1394.51400 .7 \quad 1414.3 \quad 1855.11871 .5$ $2641.32648 .8 \quad 2654.8 \quad 2656.8 \quad 2665.3 \quad 2669.12675 .8 \quad 2679.82713 .2 \quad 2727.92736 .6 \quad 2743.5$ $2765.72769 .8 \quad 2780.0 \quad 2794.9$

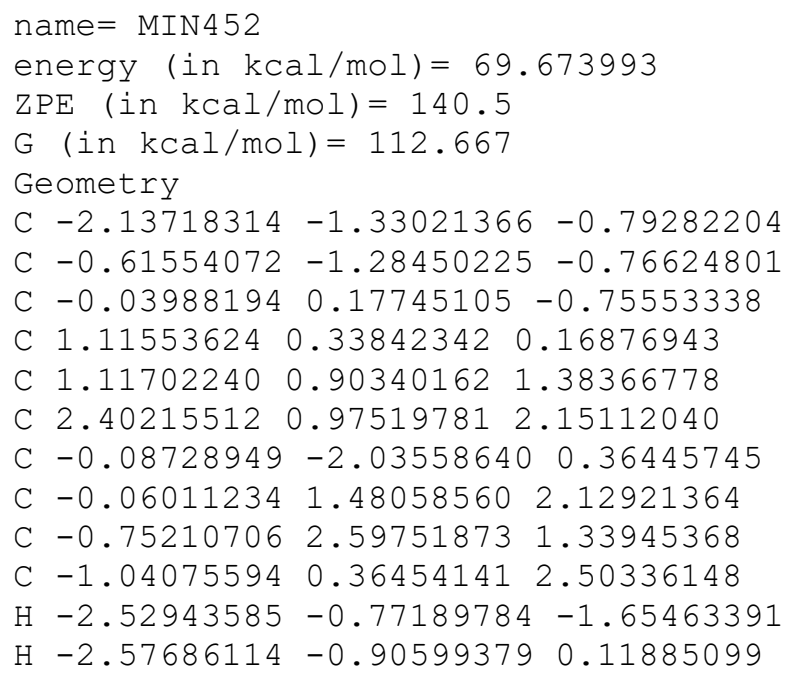


$\mathrm{H}-2.50335069-2.36117478-0.88771034$

$\mathrm{H}-0.844514410 .92893275-0.62094608$

$\mathrm{H} 2.04959483-0.03885468-0.26202524$

$\mathrm{H} 3.25268903 \quad 0.53783100 \quad 1.61114562$

$\mathrm{H} \quad 0.30726384 \quad 1.93890009 \quad 3.08712996$

$\mathrm{H} \quad 2.67017127 \quad 2.01746196 \quad 2.37696752$

H $2.32047467 \quad 0.439291293 .10803828$

$\mathrm{H} \quad 0.61476056-2.82744586 \quad 0.15962823$

$\mathrm{H}-1.31493281 \quad 2.21436299 \quad 0.48095306$

$\mathrm{H}-0.02423502 \quad 3.32375890 \quad 0.95927596$

$\mathrm{H}-1.46436321 \quad 3.13912090 \quad 1.97184529$

$\mathrm{H}-0.55611438-0.39378582 \quad 3.13135868$

$\mathrm{H}-1.41275267-0.16765663 \quad 1.61958918$

$\mathrm{H}-1.90282502 \quad 0.75566691 \quad 3.05043332$

$0-0.08354002-0.68372560-2.86405355$

$0 \quad 0.50113885 \quad 0.35021374-2.07597809$

o - $0.10183825-1.80249226-2.00746057$

Vibrational frequencies (in $\mathrm{cm}-1$ )

$\begin{array}{lllllllllllllll}-14.0 & 51.9 & 67.5 & 78.9 & 110.8 & 118.0 & 132.4 & 136.0 & 150.1 & 173.2 & 226.9 & 233.4 & 254.3 & 288.7\end{array}$ $309.0 \quad 351.9 \quad 388.7 \quad 407.3 \quad 449.2 \quad 498.5 \quad 550.5 \quad 579.8 \quad 598.4 \quad 667.3 \quad 699.7 \quad 843.4 \quad 850.7 \quad 876.3$

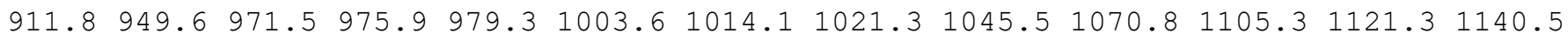

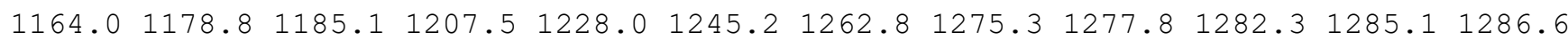
$1289.51294 .8 \quad 1297.3 \quad 1303.3 \quad 1347.7 \quad 1350.3 \quad 1380.4 \quad 1396.4 \quad 1399.2 \quad 1400.7 \quad 1419.6 \quad 1873.3$ $2642.72664 .02668 .5 \quad 2669.7 \quad 2670.3 \quad 2670.4 \quad 2672.2 \quad 2673.62678 .5 \quad 2683.5 \quad 2698.2 \quad 2739.9$ $2775.62777 .22778 .3 \quad 2784.3$

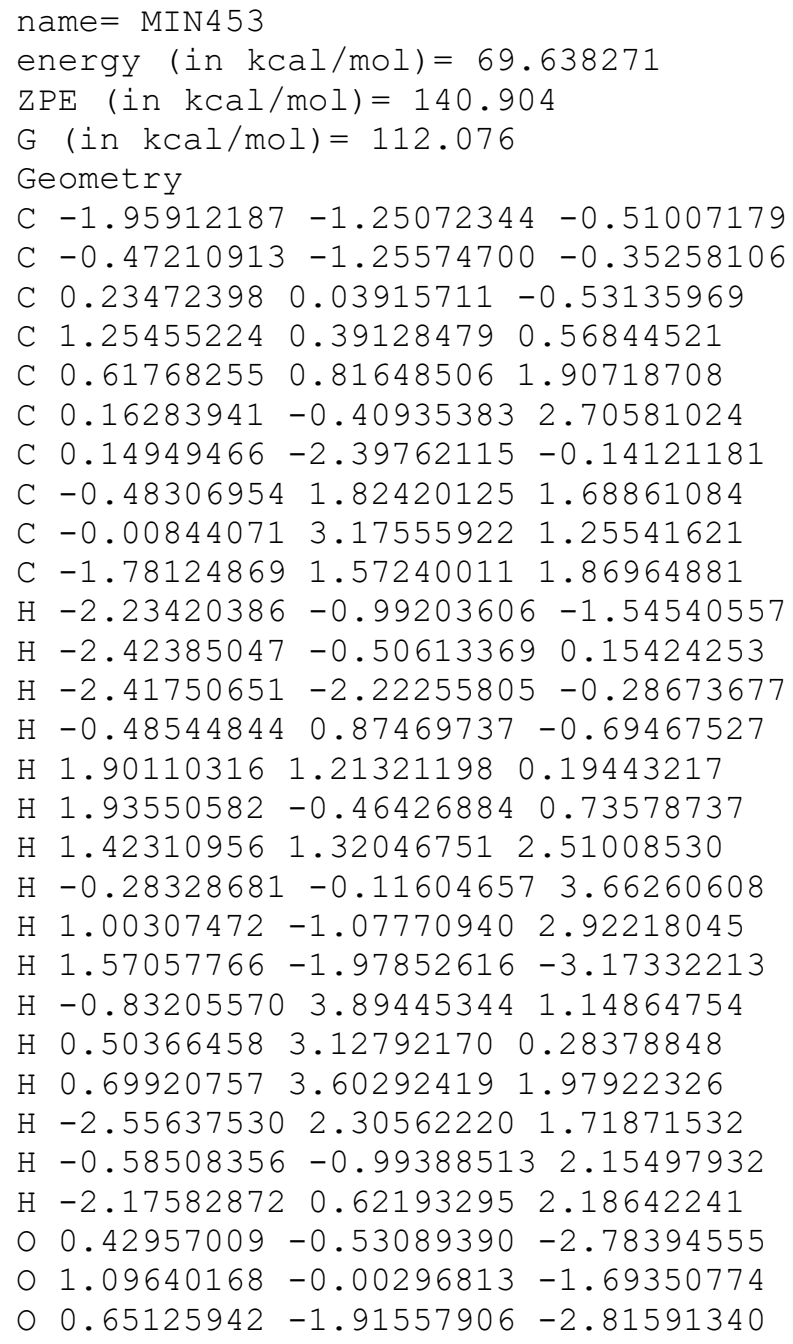


$\begin{array}{lllllllllllllll}1151.1 & 1187.1 & 1224.3 & 1235.5 & 1252.7 & 1271.5 & 1276.2 & 1284.6 & 1290.5 & 1291.2 & 1291.7 & 1298.8\end{array}$

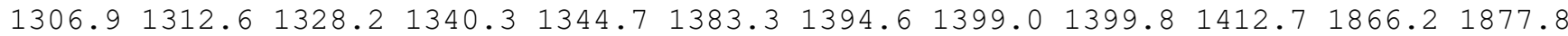
$2655.92662 .82665 .72670 .2 \quad 2670.8 \quad 2673.92677 .82679 .72690 .6 \quad 2722.0 \quad 2738.8 \quad 2772.5$ $2778.62780 .4 \quad 2792.3 \quad 2850.0$

\section{name $=$ MIN454}

energy (in $\mathrm{kcal} / \mathrm{mol})=71.001604$

$\mathrm{ZPE}(\mathrm{in} \mathrm{kcal} / \mathrm{mol})=139.87$

$\mathrm{G}(\mathrm{in} \mathrm{kcal} / \mathrm{mol})=111.644$

Geometry

C $-2.67965265-0.17845844-0.47646351$

C $-1.33825523-0.27058850-0.51639780$

C $-0.49084487 \quad 0.98100321-0.53248583$

C $0.84810967 \quad 0.90172002 \quad 0.22047817$

C $0.93792929-0.22417633 \quad 1.20403212$

C $1.61964113-1.44831564 \quad 0.68361850$

C $-0.71342167-1.50899606-0.76636351$

C $0.50322799-0.122236392 .47193901$

C $-0.15439471 \quad 1.10825265 \quad 3.00521722$

C $0.65379293-1.23100164 \quad 3.46200304$

$\mathrm{H}-0.03671903-1.06268955-2.71004183$

$\mathrm{H}-3.21311034 \quad 0.75900047-0.49681550$

$\mathrm{H}-3.33935848-1.03044434-0.45865226$

$\mathrm{H}-1.094111521 .84412983-0.14138656$

H $1.03617269 \quad 1.87197450 \quad 0.72875317$

$\mathrm{H} \quad 1.68084256 \quad 0.82491864 \quad-0.51365002$

H 2.68986275 $-1.43257168 \quad 0.94432904$

H $1.20413359-2.376775351 .09534738$

$\mathrm{H} \quad 1.57689642-1.52486968-0.41660802$

$\mathrm{H}-0.84616475-2.35798470-0.12839640$

$\mathrm{H}-0.78813744 \quad 0.89721151 \quad 3.87741664$

$\mathrm{H}-0.79821953 \quad 1.59719837 \quad 2.26273891$

$\mathrm{H} 0.60061193 \quad 1.84336511 \quad 3.32478773$

$\mathrm{H} 0.68101436-0.86189908 \quad 4.49675908$

$\mathrm{H} 1.57932280-1.80394167 \quad 3.31256838$

$\mathrm{H}-0.18687514-1.93589648 \quad 3.39062352$

$0 \quad 0.67590787 \quad 0.67009070-2.53620216$

$\begin{array}{llll}0 & -0.29902897 & 1.42010929 & -1.87192218\end{array}$

O $0.05867632-0.28097776-3.33257704$

Vibrational frequencies (in $\mathrm{cm}-1$ )

$\begin{array}{llllllllllllll}-30.4 & 44.4 & 48.6 & 50.6 & 77.5 & 81.7 & 92.9 & 157.1 & 191.5 & 199.1 & 216.2 & 240.9 & 269.5 & 300.4\end{array}$

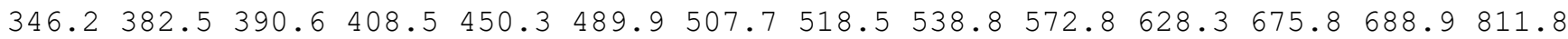

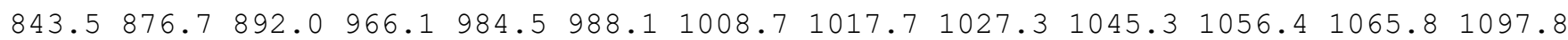
$1109.81138 .8 \quad 1170.8 \quad 1242.8 \quad 1253.9 \quad 1270.6 \quad 1274.7 \quad 1278.4 \quad 1283.4 \quad 1290.2 \quad 1293.7 \quad 1309.8$ $1313.01322 .6 \quad 1336.91355 .6 \quad 1373.2 \quad 1387.9 \quad 1395.91398 .31405 .5 \quad 1446.8 \quad 1770.01872 .8$ $2642.52647 .3 \quad 2667.4 \quad 2668.6 \quad 2669.2 \quad 2670.1 \quad 2672.0 \quad 2674.3 \quad 2694.8 \quad 2716.3 \quad 2730.62733 .8$ $2767.52777 .6 \quad 2779.0 \quad 2782.9$

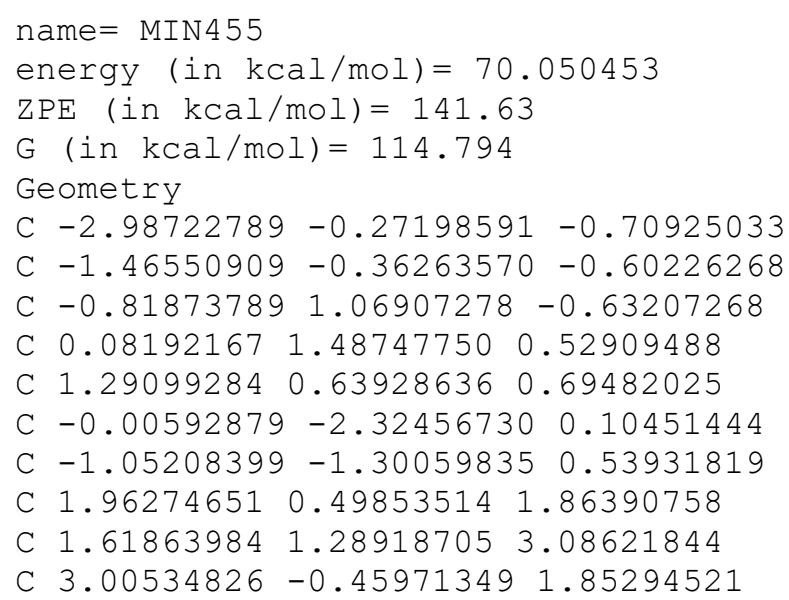


$\mathrm{H}-3.29756214 \quad 0.34377853-1.56420986$

$\mathrm{H}-3.43123954 \quad 0.15017052 \quad 0.19792412$

$\mathrm{H}-3.43008423-1.26516836-0.86875148$

$\mathrm{H}-1.60631076 \quad 1.84414012-0.78767979$

$\mathrm{H}-0.51570518 \quad 1.54210544 \quad 1.46425028$

H $0.42490452 \quad 2.53799057 \quad 0.35016954$

$\mathrm{H} 1.623547130 .10257521-0.20482757$

$\mathrm{H}-0.38720687-2.97204620-0.69565166$

$\mathrm{H} \quad 0.90503310-1.84818488-0.28084243$

$\mathrm{H}-1.94176142-1.84534261 \quad 0.91880228$

$\mathrm{H} 0.62099326 \quad 1.03793076 \quad 3.46965850$

$\mathrm{H} \quad 1.63462178 \quad 2.369044792 .88327536$

$\mathrm{H} 2.32941516 \quad 1.11158824 \quad 3.90393369$

$\mathrm{H} \quad 0.29119611 \quad-2.96853091 \quad 0.93983394$

$\mathrm{H}-0.68496058-0.72966271 \quad 1.41203082$

$\mathrm{H} \quad 3.36177904-0.87389172 \quad 2.77559444$

O $0.16551148-0.26954009-2.15904676$

$0-0.078346821 .10059576-1.85000610$

o $-1.05863051-0.90596604-1.87137948$

Vibrational frequencies (in $\mathrm{cm}-1$ )

$\begin{array}{llllllllllllllll}33.6 & 39.2 & 53.9 & 97.2 & 100.8 & 124.6 & 185.0 & 188.5 & 203.5 & 215.8 & 233.8 & 254.0 & 299.4 & 315.9\end{array}$ $368.6 \quad 387.7 \quad 406.8 \quad 440.6 \quad 480.2 \quad 496.3 \quad 517.5 \quad 585.5 \quad 673.7 \quad 710.1 \quad 809.6 \quad 843.1866 .6 \quad 878.3$ $891.7 \quad 938.2 \quad 969.5 \quad 985.1 \quad 1003.5 \quad 1008.3 \quad 1048.8 \quad 1055.6 \quad 1066.7 \quad 1077.7 \quad 1083.1 \quad 1132.8 \quad 1143.9$

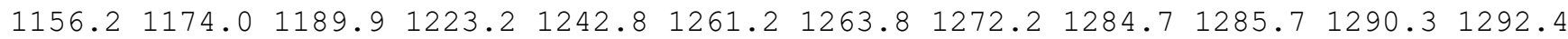

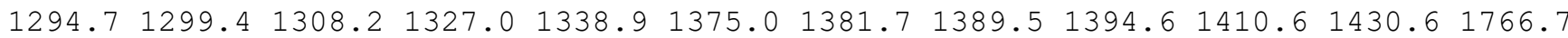
$2641.92647 .3 \quad 2666.0 \quad 2670.2 \quad 2671.5 \quad 2672.1 \quad 2673.82678 .32679 .02686 .92706 .62731 .0$ $2740.22778 .5 \quad 2779.5 \quad 2780.4$

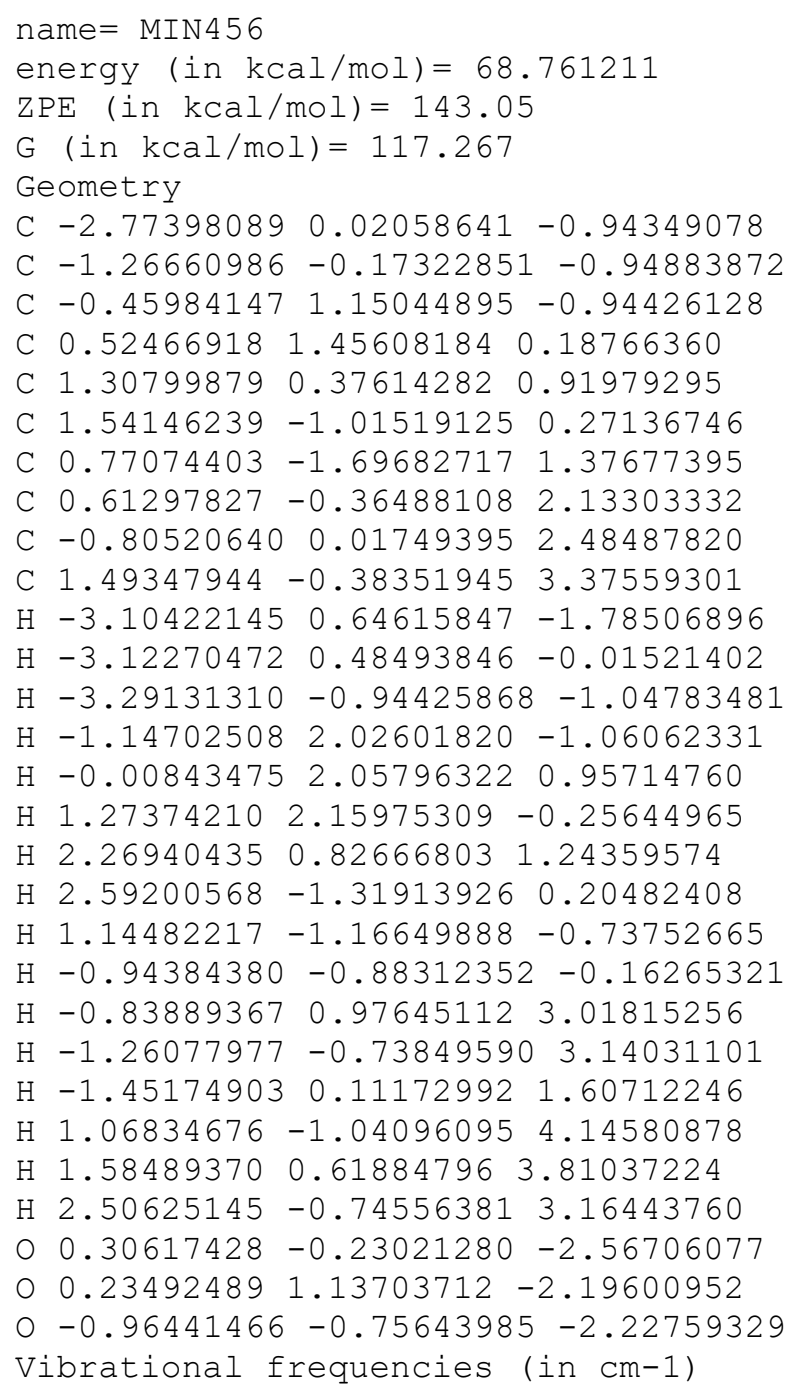


$\begin{array}{lllllllllllllllll}33.8 & 69.8 & 83.9 & 106.6 & 128.2 & 138.4 & 161.3 & 179.8 & 205.9 & 259.2 & 265.6 & 294.0 & 317.4 & 337.2\end{array}$

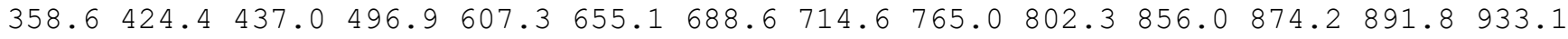

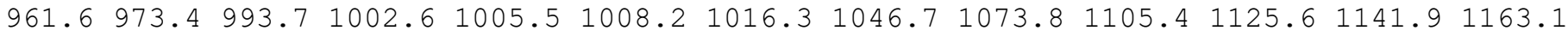

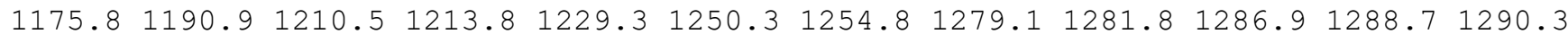

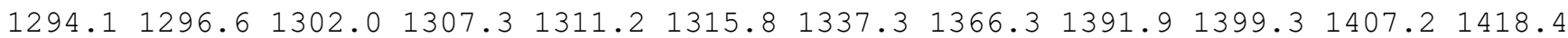
$2640.92662 .6 \quad 2665.3 \quad 2672.4 \quad 2673.3 \quad 2674.92676 .3 \quad 2677.3 \quad 2678.6 \quad 2696.02709 .3 \quad 2723.4$ $2768.62779 .3 \quad 2779.8 \quad 2783.3$

\section{name $=$ MIN457}

energy (in $\mathrm{kcal} / \mathrm{mol})=72.776036$

$\mathrm{ZPE}(\mathrm{in} \mathrm{kcal} / \mathrm{mol})=140.841$

$\mathrm{G}($ in $\mathrm{kcal} / \mathrm{mol})=114.174$

Geometry

C $-2.96401320-0.33361022-0.40161932$

C $-1.43677422-0.35297901-0.43288100$

C $-0.82687458 \quad 1.09717434-0.45482987$

C $-0.30963641 \quad 1.65849657 \quad 0.77611869$

C $0.841583791 .37090607 \quad 1.59458600$

C $-0.51843524-2.49093221 \quad 0.44523917$

C $-0.91588362-1.23686968 \quad 0.65310282$

C $1.89728433 \quad 0.43916359 \quad 1.31948547$

C $2.82599686 \quad 0.09831151 \quad 2.42710945$

C $2.17580208-0.16327058 \quad 0.02427309$

$\mathrm{H}-3.37284530 \quad 0.18186344 \quad-1.28086925$

$\mathrm{H}-3.32879470 \quad 0.17881775 \quad 0.49595063$

$\mathrm{H}-3.37311477-1.35169939-0.40194924$

$\mathrm{H}-1.605202241 .78266671-0.88933861$

$\mathrm{H}-0.901461192 .49014464 \quad 1.14221668$

$\mathrm{H} 1.41471117 \quad 2.38009658 \quad 1.64501457$

$\mathrm{H} \quad 0.50290605 \quad 1.27655282 \quad 2.67106254$

$\mathrm{H}-0.16282007-3.14244352 \quad 1.22593767$

$\mathrm{H}-0.52444432-2.96116410-0.52870699$

$\mathrm{H}-0.90892659-0.76836094 \quad 1.63816167$

H 2.68423574 $0.69760570 \quad 3.33552970$

H $3.88118575 \quad 0.20980358 \quad 2.12702795$

$\mathrm{H} 2.70331675-0.959685392 .72191366$

$\mathrm{H} 2.13584038 \quad 0.58908657 \quad-0.79568765$

$\mathrm{H} 1.37889655-0.89125603-0.25710097$

$\mathrm{H} 3.13328027-0.69234492-0.04362053$

$0.16049715-0.28375546-2.02734843$

$0.155138361 .04607954-1.50902886$

o $-1.10442861-0.84051758-1.73651390$

Vibrational frequencies (in cm-1)

$\begin{array}{lllllllllllllll}-31.7 & 58.0 & 69.3 & 93.7 & 119.6 & 131.4 & 143.2 & 159.6 & 188.1 & 203.3 & 227.4 & 254.0 & 291.1 & 312.5\end{array}$ $321.5 \quad 370.0 \quad 393.2 \quad 420.0 \quad 496.4 \quad 535.5 \quad 562.0 \quad 609.3 \quad 660.4 \quad 700.5 \quad 736.0 \quad 760.4 \quad 815.9 \quad 902.2$

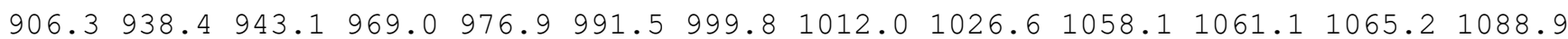

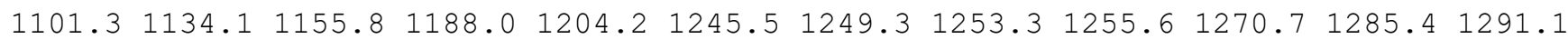
$1293.7 \quad 1327.6 \quad 1352.2 \quad 1373.0 \quad 1379.3 \quad 1385.7 \quad 1392.6 \quad 1396.8 \quad 1417.5 \quad 1459.7 \quad 1616.3 \quad 1849.3$ $2529.72586 .42648 .5 \quad 2650.2 \quad 2657.2 \quad 2659.3 \quad 2667.92673 .52678 .92714 .52750 .72754 .1$ $2762.4 \quad 2771.2 \quad 2779.3 \quad 2786.1$

\section{name $=$ MIN458}

energy $($ in $\mathrm{kcal} / \mathrm{mol})=72.473098$

$\mathrm{ZPE}($ in $\mathrm{kcal} / \mathrm{mol})=141.245$

$\mathrm{G}($ in $\mathrm{kcal} / \mathrm{mol})=114.101$

Geometry

C $-1.80227352-1.38434060-0.74700616$

C $-0.27597511-1.35318064-0.81504093$

C $0.29388510 \quad 0.08962252 \quad-0.61380390$

C $1.28187651 \quad 0.30902367 \quad 0.52784151$

C $0.62445024 \quad 0.72659963 \quad 1.86297308$

C $0.12356460 \quad-0.50239486 \quad 2.62488948$

C $0.24016407-2.34641492 \quad 0.11490089$ 
C $-0.44831251 \quad 1.76595008 \quad 1.64829210$

C $0.06093876 \quad 3.11134657 \quad 1.23749472$

C $-1.754342561 .54114560 \quad 1.80994660$

$\mathrm{H}-2.23563046-0.82080484-1.58567318$

$\mathrm{H}-2.16991155-0.93853279 \quad 0.18778324$

$\mathrm{H}-2.19860859-2.40521419-0.80970367$

$\mathrm{H}-0.53592205 \quad 0.82990083-0.59967087$

H 2.00113303 1.099309290 .21619199

$\mathrm{H} 1.90448730-0.592409720 .69045348$

H $1.42850712 \quad 1.19860605 \quad 2.49197425$

$\mathrm{H}-0.35992984-0.22260530 \quad 3.56571590$

$\mathrm{H} \quad 0.94577598 \quad-1.18729677 \quad 2.86276796$

$\mathrm{H} \quad 0.78444457 \quad-3.18191294 \quad-0.29634271$

$\mathrm{H}-0.74549357 \quad 3.84781010 \quad 1.11928503$

$\mathrm{H} \quad 0.59300456 \quad 3.06321632 \quad 0.27603287$

$\mathrm{H} \quad 0.76110546 \quad 3.52016993 \quad 1.97851712$

$\mathrm{H}-2.510807362 .29458878 \quad 1.66477442$

$\mathrm{H}-0.59695889-1.08512520 \quad 2.03347003$

$\mathrm{H}-2.17551966 \quad 0.59561757 \quad 2.10840173$

○ $0.39825359-0.46016907-2.79361468$

O $1.06415731 \quad 0.30805862-1.80631824$

o $0.15017994-1.69578274-2.15055408$

Vibrational frequencies (in $\mathrm{cm}-1$ )

$\begin{array}{lllllllllllllll}20.9 & 49.5 & 56.8 & 77.7 & 91.7 & 124.8 & 139.8 & 165.9 & 219.0 & 227.4 & 251.7 & 280.3 & 285.2 & 340.9\end{array}$ $394.6 \quad 396.3 \quad 412.8 \quad 448.2 \quad 467.6 \quad 501.0 \quad 533.8 \quad 586.4 \quad 631.5 \quad 680.3 \quad 699.9 \quad 807.1 \quad 850.5 \quad 870.4$ $917.9958 .4 \quad 969.4 \quad 984.4 \quad 996.3 \quad 1010.8 \quad 1020.4 \quad 1038.6 \quad 1046.9 \quad 1076.8 \quad 1086.9 \quad 1102.7 \quad 1127.2$

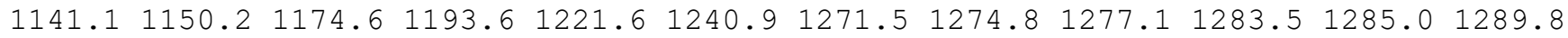
$1290.61294 .8 \quad 1306.0 \quad 1325.3 \quad 1333.2 \quad 1366.4 \quad 1381.4 \quad 1394.51399 .5 \quad 1403.7 \quad 1412.7 \quad 1863.8$ $2648.62660 .92664 .42666 .4 \quad 2668.8 \quad 2670.0 \quad 2671.52673 .62682 .12690 .82721 .02733 .5$ $2775.02778 .5 \quad 2781.5 \quad 2791.5$

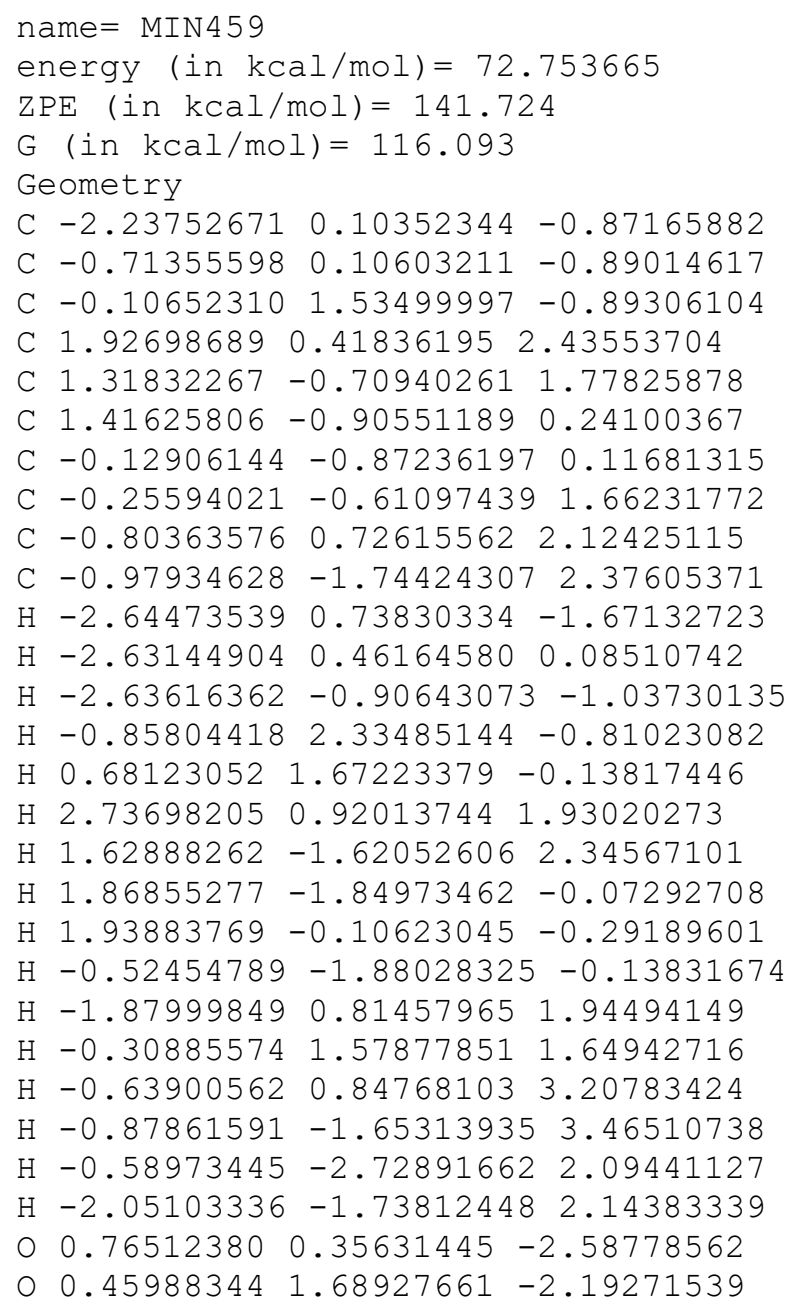


$0-0.37544974-0.38472348-2.20763018$

Vibrational frequencies (in $\mathrm{cm}-1$ )

$\begin{array}{llllllllllllllll}36.7 & 56.5 & 79.6 & 125.2 & 148.1 & 168.5 & 172.4 & 199.2 & 212.4 & 249.3 & 263.5 & 315.6 & 338.0 & 345.0\end{array}$ $363.1377 .4 \quad 408.9 \quad 462.7 \quad 527.3 \quad 539.7 \quad 641.0 \quad 669.8 \quad 707.6 \quad 810.7 \quad 820.0 \quad 856.7 \quad 908.6 \quad 925.9$ $941.6956 .2972 .6 \quad 979.4 \quad 990.1 \quad 1004.8 \quad 1008.4 \quad 1013.6 \quad 1042.0 \quad 1064.4 \quad 1073.1 \quad 1083.9 \quad 1126.3$

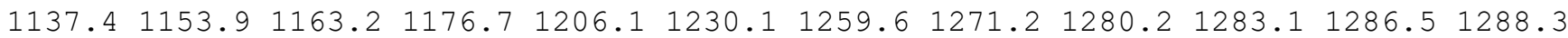
$1294.21296 .61300 .6 \quad 1303.8 \quad 1316.51334 .7 \quad 1350.2 \quad 1366.31378 .51394 .2 \quad 1405.51409 .4$ $2648.42664 .12666 .6 \quad 2669.8 \quad 2673.12676 .2 \quad 2676.52679 .12679 .92698 .02711 .62717 .7$ $2763.82777 .5 \quad 2780.2 \quad 2781.9$

\author{
name $=$ MIN460 \\ energy (in $\mathrm{kcal} / \mathrm{mol})=72.356819$ \\ $\mathrm{ZPE}($ in $\mathrm{kcal} / \mathrm{mol})=142.28$ \\ $\mathrm{G}(\mathrm{in} \mathrm{kcal} / \mathrm{mol})=117.293$ \\ Geometry \\ C $-2.17385156-0.13625495-0.58888984$ \\ C $-0.65631952-0.04140075-0.69707387$ \\ C $-0.16980690 \quad 1.43878358-0.88249538$ \\ C 0.684515431 .982088710 .14503686 \\ C $1.46106831-0.340508991 .91574709$ \\ C $1.59809137-0.72649094 \quad 0.42565548$ \\ C $0.06044170 \quad-0.91905441 \quad 0.31793391$ \\ C $-0.06853259-0.66270650 \quad 1.86932624$ \\ C $-0.95713227 \quad 0.46202396 \quad 2.35526207$ \\ C $-0.41295733-1.95221328 \quad 2.61066106$ \\ $\mathrm{H}-2.66117098 \quad 0.23578988-1.50200668$ \\ $\mathrm{H}-2.55723870 \quad 0.43974104 \quad 0.26080730$ \\ $\mathrm{H}-2.50172810-1.17590002-0.45656900$ \\ $\mathrm{H}-1.05581830 \quad 2.12710899-0.99442067$ \\ $\mathrm{H} \quad 1.67908052 \quad 0.71103103 \quad 2.13070627$ \\ $\mathrm{H} 1.66946236 \quad 2.31975760 \quad-0.12740688$ \\ $\mathrm{H} 2.03832114 \quad-0.95208844 \quad 2.60933780$ \\ H 2.16436193 -1.64858385 0.25355975 \\ $\mathrm{H} \quad 2.03192127 \quad 0.03168805-0.22938742$ \\ $\mathrm{H}-0.20051330-1.971826510 .07397617$ \\ $\mathrm{H}-0.761050351 .40455517 \quad 1.82107312$ \\ $\mathrm{H}-0.77673759 \quad 0.66806410 \quad 3.41773247$ \\ $\mathrm{H}-2.01938588 \quad 0.22246295 \quad 2.24644360$ \\ $\mathrm{H}-0.31388062-1.820516193 .69422693$ \\ H $\quad 0.24605578 \quad-2.77919927 \quad 2.32321696$ \\ $\mathrm{H}-1.44338631-2.26452885 \quad 2.40967281$ \\ $\begin{array}{lllll}0 & 0.74028759 & 0.10720154 & -2.47478170\end{array}$ \\ $0.423008391 .46161463-2.19601533$ \\ $0-0.37561752-0.60805136-1.99980524$ \\ Vibrational frequencies (in $\mathrm{cm}-1$ )
}

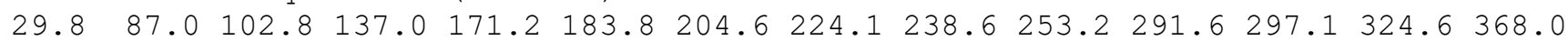
$388.4414 .3 \quad 457.6 \quad 492.9 \quad 548.0 \quad 588.3 \quad 641.2 \quad 681.7 \quad 700.0 \quad 802.7 \quad 853.8 \quad 866.3 \quad 880.5 \quad 910.0$ $915.4947 .4 \quad 959.8 \quad 986.0 \quad 1002.4 \quad 1006.6 \quad 1008.8 \quad 1024.2 \quad 1035.6 \quad 1061.9 \quad 1074.91099 .9 \quad 1103.1$

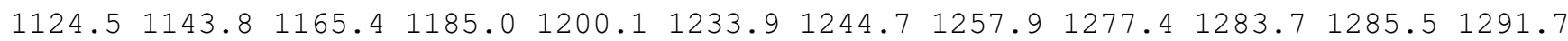
$1295.11302 .7 \quad 1303.8 \quad 1306.3 \quad 1314.1 \quad 1322.2 \quad 1360.4 \quad 1369.5 \quad 1374.91396 .01406 .11410 .5$ $2644.22656 .62668 .32670 .3 \quad 2672.6 \quad 2678.02678 .62682 .12682 .72696 .52709 .32761 .3$ $2766.3 \quad 2776.7 \quad 2779.7 \quad 2782.9$

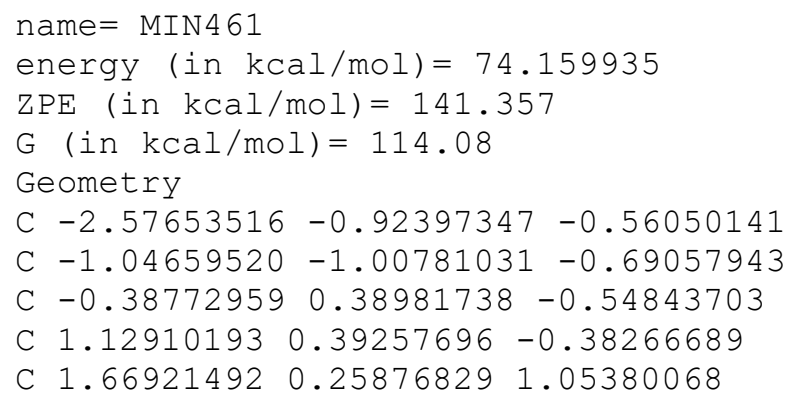


C $1.62517736-1.185849981 .55920683$

C $-0.62907510 \quad-2.05521371 \quad 0.22089825$

C 0.968322121 .205094711 .99798456

$\begin{array}{llll}\text { C } 1.69962932 & 2.48251681 & 2.25808796\end{array}$

C $-0.21031153 \quad 0.94495978 \quad 2.56849378$

$\mathrm{H}-2.97010533-0.17896714-1.26880949$

$\mathrm{H}-2.88384392-0.63548737 \quad 0.45116515$

$\mathrm{H}-3.05618122-1.87880017-0.80725686$

$\mathrm{H}-0.90605460 \quad 1.05214106 \quad 0.17650668$

$\mathrm{H} 1.505017331 .35033966-0.81060765$

$\mathrm{H} \quad 1.57664188-0.39506909-1.02273498$

$\mathrm{H} 2.756664090 .54736209 \quad 1.00476586$

H $2.30553163-1.32445916 \quad 2.40816134$

H $1.92379314 \quad-1.89902078 \quad 0.78470982$

$\mathrm{H}-0.21603725-2.95832783-0.19629426$

H $1.96209696 \quad 2.99661799 \quad 1.32299185$

H 2.63498059 2.29662526 2.80567992

$\mathrm{H} 1.11370007 \quad 3.18919219 \quad 2.86049256$

$\mathrm{H}-0.76349828 \quad 0.02987627 \quad 2.41449662$

$\mathrm{H} \quad 0.62604967-1.46838966 \quad 1.93111833$

$\mathrm{H}-0.71051710 \quad 1.61897743 \quad 3.24209551$

$\begin{array}{lllll}0 & -0.48595308 & -0.11784840 & -2.75240601\end{array}$

$\begin{array}{lllll}0 & -0.70263938 & 0.93969771 & -1.82795962\end{array}$

o $-0.73709361-1.34696375-2.05092955$

Vibrational frequencies (in $\mathrm{cm}-1$ )

$\begin{array}{lllllllllllllll}-17.2 & 41.5 & 59.3 & 93.2 & 105.2 & 118.2 & 129.6 & 154.9 & 194.9 & 249.3 & 251.8 & 275.3 & 294.8 & 319.9\end{array}$ $338.4 \quad 377.1 \quad 406.8 \quad 451.6 \quad 486.7 \quad 548.6 \quad 598.7 \quad 615.4 \quad 655.3 \quad 680.0 \quad 720.5 \quad 834.7 \quad 858.1 \quad 907.5$

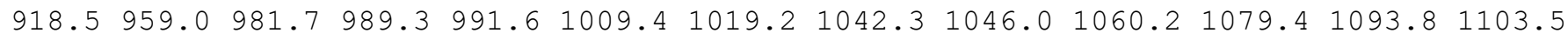

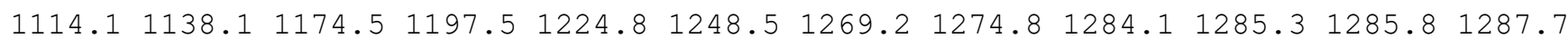

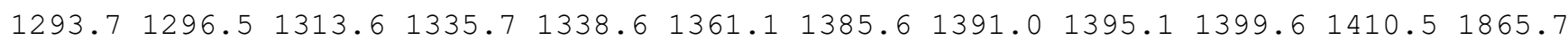
$2633.62649 .02663 .82671 .3 \quad 2674.12674 .5 \quad 2675.42678 .12678 .82686 .12716 .22734 .5$ $2770.22775 .92778 .7 \quad 2788.2$

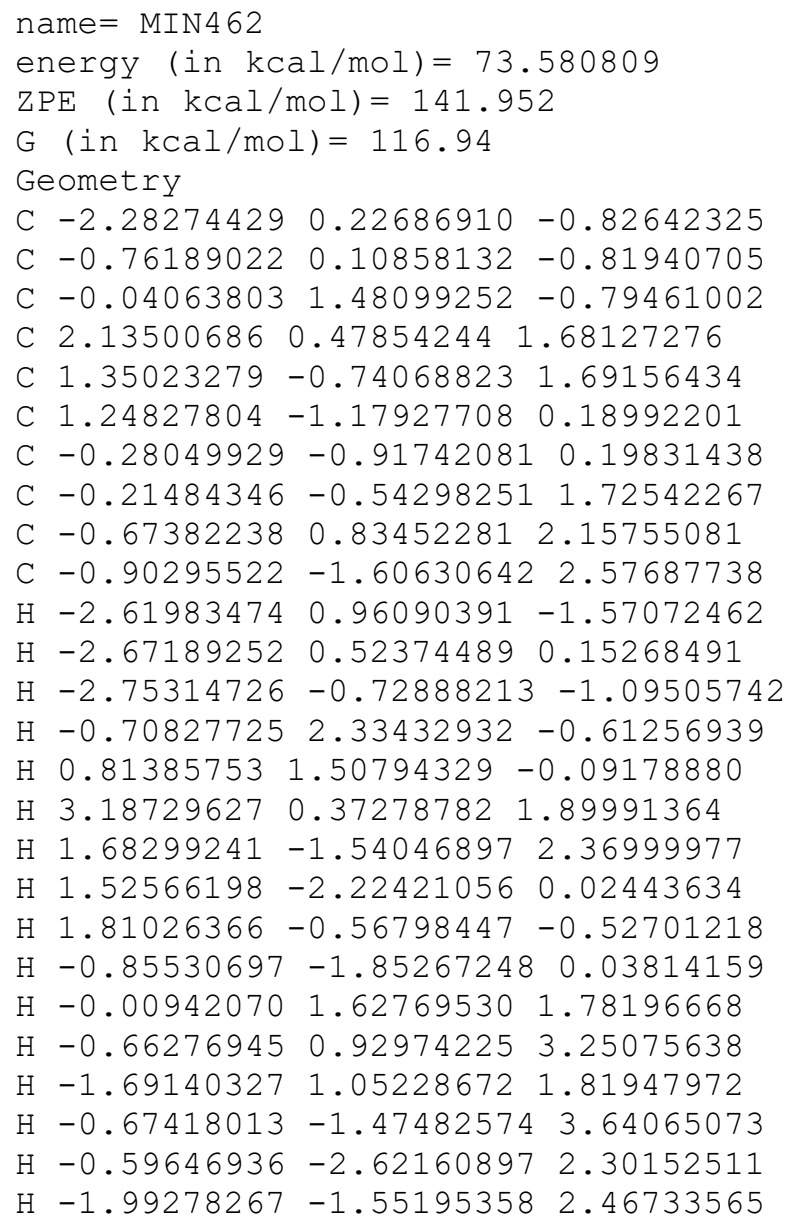


$0.69367587 \quad 0.32663680-2.55762074$

$0 \quad 0.434612251 .65338045-2.12867509$

O $-0.44124451-0.40936887-2.12947040$

Vibrational frequencies (in $\mathrm{cm}-1$ )

$\begin{array}{lllllllllllllllll}56.3 & 76.6 & 98.1 & 127.4 & 161.8 & 173.0 & 191.9 & 207.3 & 243.5 & 251.2 & 266.2 & 299.7 & 327.5 & 338.1\end{array}$

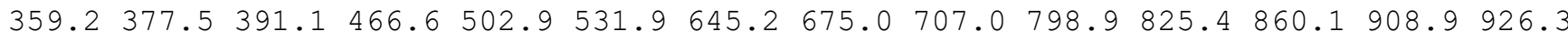
$950.1958 .9966 .2978 .9996 .1 \quad 1005.6 \quad 1007.6 \quad 1015.8 \quad 1039.4 \quad 1069.5 \quad 1081.5 \quad 1111.4 \quad 1122.4$ $\begin{array}{llllllllllll}1151.2 & 1160.4 & 1172.9 & 1197.9 & 1209.7 & 1238.3 & 1255.5 & 1272.8 & 1281.6 & 1283.0 & 1287.8 & 1290.9\end{array}$ $1298.31298 .4 \quad 1304.6 \quad 1306.4 \quad 1319.1 \quad 1333.2 \quad 1348.4 \quad 1362.8 \quad 1374.7 \quad 1394.6 \quad 1408.6 \quad 1410.7$ $2622.42656 .92665 .5 \quad 2669.92672 .7 \quad 2673.5 \quad 2676.52677 .52679 .92712 .72713 .72724 .5$ $2753.6 \quad 2775.8 \quad 2779.8 \quad 2782.2$

name $=\operatorname{MIN} 463$

energy $($ in $\mathrm{kcal} / \mathrm{mol})=74.713347$

$\mathrm{ZPE}($ in $\mathrm{kcal} / \mathrm{mol})=141.399$

$\mathrm{G}($ in $\mathrm{kcal} / \mathrm{mol})=114.33$

Geometry

C $-2.49225120-0.81367827-0.70400917$

C $-0.95989923-0.90124197-0.73789556$

C $-0.301851420 .49438461-0.57976372$

C $1.20382226 \quad 0.48899175-0.32619514$

C $1.66731085 \quad 0.29042199 \quad 1.12950059$

C $1.73529310-1.18781297 \quad 1.52382956$

C $-0.60272085-1.94437372 \quad 0.20279168$

C $0.84577096 \quad 1.11769316 \quad 2.08862674$

C $-0.38114591 \quad 0.51720468 \quad 2.68890339$

$\begin{array}{llll}\text { C } 1.22688000 & 2.35967434 & 2.40473894\end{array}$

$\mathrm{H}-2.84081195-0.06576516-1.43354061$

$\mathrm{H}-2.86358936-0.52456350 \quad 0.28606600$

$\mathrm{H}-2.95951123-1.76669704-0.98124657$

$\mathrm{H}-0.852708691 .16709138 \quad 0.11057216$

$\mathrm{H} 1.596870091 .46887559-0.68566540$

H $1.68801506-0.26279065-0.98196109$

$\begin{array}{lllll}\mathrm{H} & 2.72740914 & 0.67420961 & 1.16811768\end{array}$

$\mathrm{H} \quad 2.09058793-1.81223136 \quad 0.69797578$

H $2.42714947-1.33534137 \quad 2.36227951$

$\mathrm{H}-0.19631105-2.86316375-0.18639331$

$\mathrm{H}-0.85586421-0.232664192 .03472622$

$\mathrm{H}-1.14112776 \quad 1.27179491 \quad 2.92753515$

$\mathrm{H}-0.13660690-0.000948293 .62880528$

H $2.11030824 \quad 2.83649805 \quad 2.01682178$

$\mathrm{H} \quad 0.76777507 \quad-1.58375180 \quad 1.86850275$

$\mathrm{H} \quad 0.68335656 \quad 2.99547729 \quad 3.08237623$

$0-0.53092382-0.03720557-2.81143022$

$0-0.515085751 .05528809-1.87196076$

$0-0.52930262-1.24366024-2.06381803$

Vibrational frequencies (in $\mathrm{cm}-1$ )

$\begin{array}{lllllllllllllll}-43.5 & 25.6 & 58.9 & 70.9 & 101.6 & 126.0 & 131.9 & 175.5 & 196.0 & 245.3 & 247.7 & 276.9 & 310.5 & 313.3\end{array}$

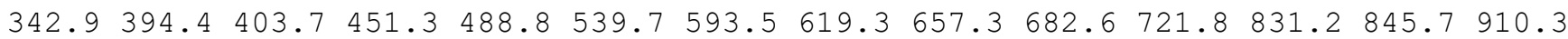
$923.3965 .8 \quad 982.5 \quad 986.1 \quad 992.7 \quad 1003.1 \quad 1020.2 \quad 1038.1 \quad 1055.7 \quad 1064.9 \quad 1086.91089 .7 \quad 1097.7$ $1108.71140 .3 \quad 1177.3 \quad 1196.5 \quad 1226.5 \quad 1239.5 \quad 1266.2 \quad 1277.8 \quad 1282.0 \quad 1283.3 \quad 1286.5 \quad 1291.9$

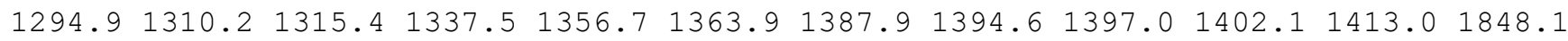
$2636.32647 .8 \quad 2652.6 \quad 2661.4 \quad 2673.7 \quad 2674.4 \quad 2674.92678 .52681 .4 \quad 2683.82726 .92734 .0$ $2771.22774 .8 \quad 2775.4 \quad 2795.8$

name $=$ MIN464

energy (in $\mathrm{kcal} / \mathrm{mol})=75.243767$

$\operatorname{ZPE}($ in $\mathrm{kcal} / \mathrm{mol})=140.943$

$\mathrm{G}($ in $\mathrm{kcal} / \mathrm{mol})=114.22$

Geometry

C $-1.97634712-1.08198544 \quad 0.10148282$

C $-0.51718131-1.03535784 \quad 0.09608194$

C $-0.81262758 \quad 2.50132540 \quad 0.10734980$ 
C $0.37837472 \quad 2.63652720 \quad 0.68996194$

C $1.72467035-0.89022268 \quad 0.77111534$

C $1.74650619-0.77734868-0.69778671$

C $0.27528618-0.93545040-1.15019633$

C $0.36944775-1.14509510 \quad 1.25979120$

C $-0.02627318-0.30354760 \quad 2.47302430$

C $0.36274399-2.668406251 .65547954$

$\mathrm{H}-2.40935301-0.86441566 \quad 1.08773686$

$\mathrm{H}-2.35936223-2.06725490-0.21429704$

$\mathrm{H}-2.39556328-0.34226769-0.60757346$

$\mathrm{H}-1.76417925 \quad 2.89637027 \quad 0.46341649$

$\mathrm{H} \quad 0.53158633 \quad 3.17113385 \quad 1.60970592$

$\mathrm{H} 1.28728405 \quad 2.20930020 \quad 0.30142824$

$\mathrm{H} \quad 2.58150347-0.79101014 \quad 1.38809502$

H 2.40194243 -1.53078377 -1.17418234

H $2.15828163 \quad 0.20580391-1.01660519$

$\mathrm{H} 0.12040566-1.80691649-1.83450245$

$\mathrm{H}-1.01126774-0.59033512 \quad 2.85612552$

$\mathrm{H}-0.07247919 \quad 0.76079712 \quad 2.19750651$

$\mathrm{H} \quad 0.70243693-0.40525423 \quad 3.28430511$

H $1.04234727-2.837816712 .49639534$

$\mathrm{H} \quad 0.68250347 \quad-3.29720473 \quad 0.81726860$

$\mathrm{H}-0.64877163-2.967544521 .94870806$

○ $0.12863466 \quad 1.34963037-1.64548956$

$0-1.084975021 .87463602-1.06230984$

$\begin{array}{lllll}0 & -0.15006054 & 0.08566439 & -2.05837011\end{array}$

Vibrational frequencies (in cm-1)

$\begin{array}{llllllllllllllll}40.9 & 46.7 & 83.5 & 90.4 & 95.3 & 127.4 & 148.8 & 161.6 & 175.3 & 204.7 & 215.5 & 244.0 & 273.4 & 291.7\end{array}$

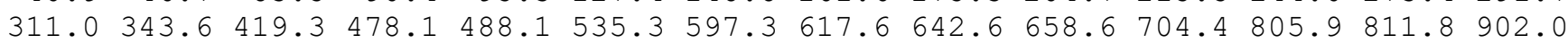
$911.6928 .0 \quad 943.5 \quad 975.5 \quad 993.4 \quad 1003.5 \quad 1014.9 \quad 1023.4 \quad 1025.1 \quad 1041.1 \quad 1049.4 \quad 1074.6 \quad 1083.9$ $1115.41148 .8 \quad 1177.3 \quad 1195.3 \quad 1207.2 \quad 1243.0 \quad 1261.6 \quad 1265.1 \quad 1270.3 \quad 1274.5 \quad 1291.6 \quad 1294.3$ $1295.21296 .41305 .7 \quad 1323.7 \quad 1350.6 \quad 1376.1 \quad 1380.7 \quad 1387.5 \quad 1388.8 \quad 1418.2 \quad 1485.5 \quad 1846.3$ $2639.62648 .8 \quad 2666.42667 .7 \quad 2669.3 \quad 2672.4 \quad 2674.32680 .12715 .02728 .02738 .92766 .5$ 2772.12778 .52791 .62800 .6

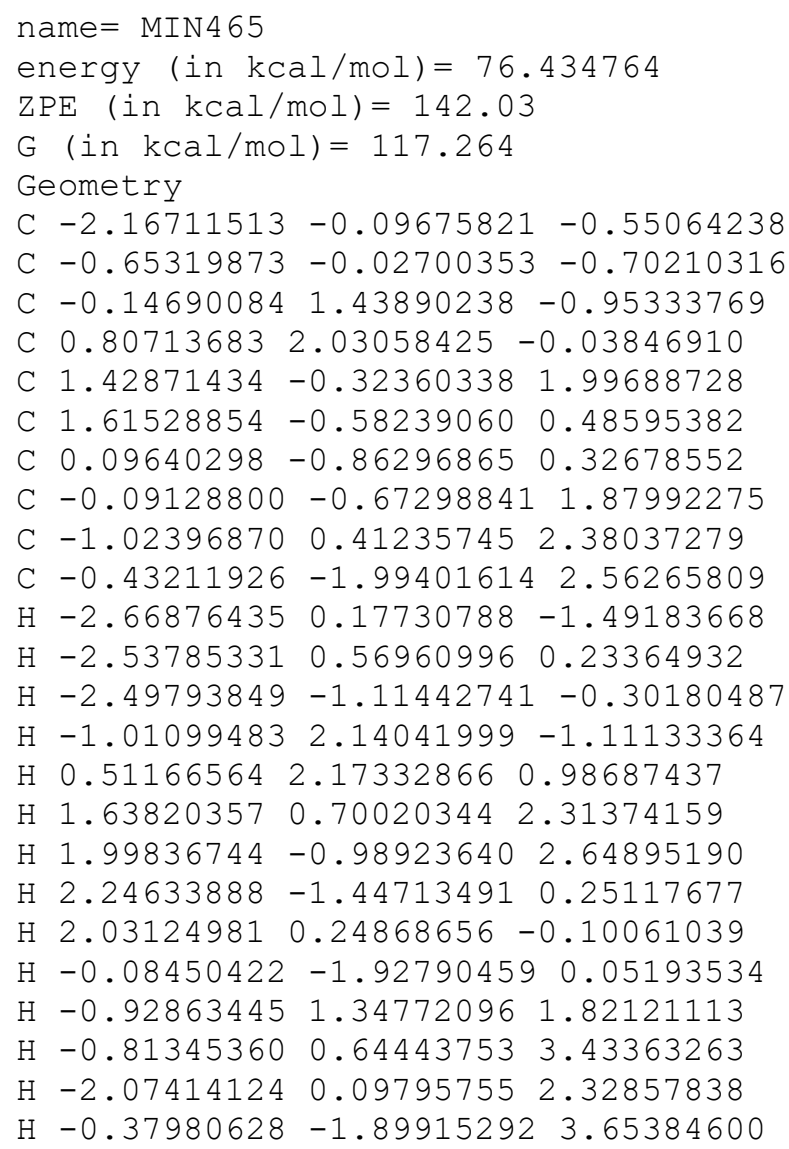


$\mathrm{H} \quad 0.26092458-2.793863362 .27499918$

$\mathrm{H}-1.44407121-2.328634132 .30795662$

$0.73867047 \quad 0.01930171-2.47458307$

O $0.433433881 .38267339-2.27471361$

O $-0.40375974-0.65409515-1.97901027$

Vibrational frequencies (in $\mathrm{cm}-1$ )

$\begin{array}{lllllllllllllllll}42.6 & 90.0 & 111.4 & 137.1 & 163.5 & 189.3 & 196.7 & 215.4 & 238.8 & 256.4 & 292.4 & 303.1 & 336.1 & 368.3\end{array}$ $391.4 \quad 415.3 \quad 475.2 \quad 504.3 \quad 509.2 \quad 571.4 \quad 647.0 \quad 682.3 \quad 696.7 \quad 793.8 \quad 822.0 \quad 857.1 \quad 877.0 \quad 890.7$ $904.1951 .7 \quad 965.3 \quad 980.6 \quad 996.7 \quad 1004.7 \quad 1006.9 \quad 1022.2 \quad 1036.8 \quad 1045.1 \quad 1064.2 \quad 1093.2 \quad 1101.7$ $1123.0 \quad 1143.7 \quad 1157.5 \quad 1181.8 \quad 1195.4 \quad 1235.5 \quad 1244.0 \quad 1258.91279 .1 \quad 1282.9 \quad 1284.7 \quad 1290.9$ $1296.91300 .3 \quad 1304.2 \quad 1307.3 \quad 1315.4 \quad 1330.0 \quad 1360.1 \quad 1374.51389 .5 \quad 1396.2 \quad 1406.21408 .3$ $2641.82647 .7 \quad 2654.8 \quad 2665.8 \quad 2671.7 \quad 2674.0 \quad 2674.82677 .82679 .5 \quad 2693.6 \quad 2700.92748 .3$ $2768.32777 .4 \quad 2779.2 \quad 2781.9$

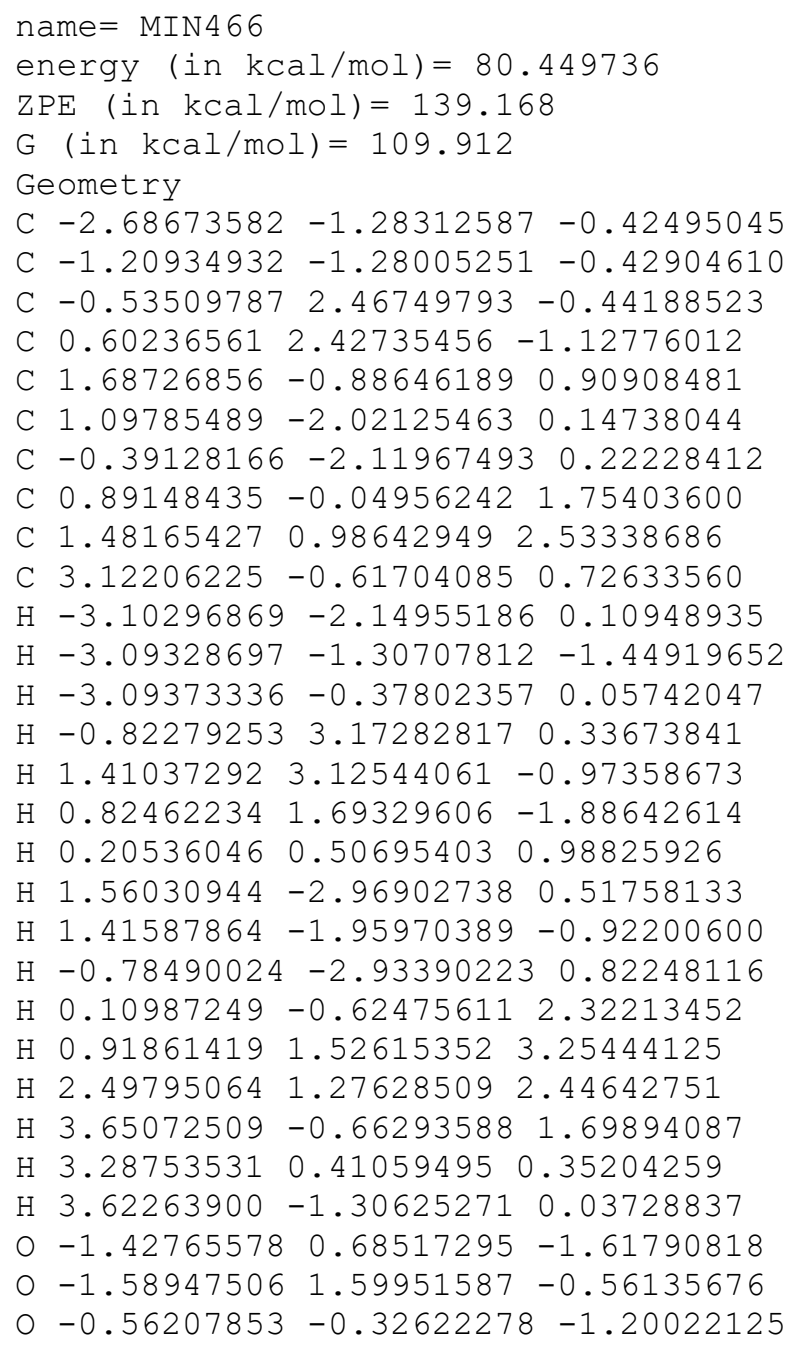


C $-0.76812884-0.96537825-0.94816660$

C $0.17184268 \quad 1.80795721-0.94874449$

C $0.97387206 \quad 1.47538854 \quad 0.31123505$

C $0.09740857 \quad 0.89928672 \quad 1.45744341$

C $0.48696050 \quad 1.53024788 \quad 2.70782997$

C $0.13795216-1.08402075 \quad 0.03428714$

C $0.00707129-0.67509068 \quad 1.46263199$

C $-1.30742170-1.11707587 \quad 2.11668399$

C $1.17644832-1.275894372 .26487873$

$\mathrm{H}-2.65724184-0.04323595-0.48489329$

$\mathrm{H}-2.64982806-1.81914363-0.38511647$

$\mathrm{H}-2.65890393-1.01318857-1.96361689$

$\mathrm{H}-0.88820421 \quad 1.51276346-0.86831601$

H $0.20968138 \quad 2.89169527-1.18599265$

H $1.79955765 \quad 0.78183981 \quad 0.06674047$

$\mathrm{H}-0.95685581 \quad 1.28923102 \quad 1.29983857$

$\mathrm{H} \quad 0.35261431 \quad 0.97623534 \quad 3.62234897$

$\mathrm{H} \quad 1.48008051 \quad 2.39441010 \quad 0.68105748$

$\mathrm{H} 1.17333102-1.30977129-0.25040123$

$\mathrm{H}-2.18148012-0.65664541 \quad 1.64829123$

$\mathrm{H}-1.33302567-0.86276581 \quad 3.18162394$

$\mathrm{H}-1.42906853-2.204861312 .03653431$

$\mathrm{H} 1.08390788-1.08198204 \quad 3.33784568$

H $2.14022809-0.87274809 \quad 1.93592454$

H $1.21332061-2.36509952 \quad 2.13714152$

$0.0 .92666945-0.07311838-2.14798163$

$0 \quad 0.684343371 .28177953-2.17201097$

o - $0.30188751-0.79712097-2.24408279$

Vibrational frequencies (in cm-1)

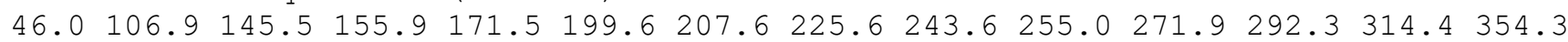
$388.7 \quad 441.0 \quad 452.2 \quad 468.6 \quad 483.7 \quad 488.6 \quad 527.7 \quad 567.1 \quad 619.8 \quad 642.1 \quad 764.2 \quad 775.2 \quad 861.9 \quad 893.7$ $944.4 \quad 949.7 \quad 971.6 \quad 973.0 \quad 985.9 \quad 995.9 \quad 1016.8 \quad 1026.3 \quad 1033.3 \quad 1056.4 \quad 1069.8 \quad 1099.6 \quad 1111.5$ $1143.31160 .21185 .4 \quad 1202.3 \quad 1221.3 \quad 1253.4 \quad 1272.1 \quad 1276.6 \quad 1292.1 \quad 1296.3 \quad 1301.11306 .4$

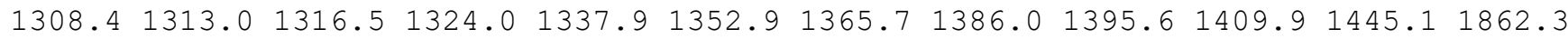
$2633.92644 .32653 .2 \quad 2663.2 \quad 2666.02667 .8 \quad 2672.82675 .62677 .92678 .92710 .02729 .8$ $2736.92773 .8 \quad 2779.9 \quad 2782.3$

\section{name $=$ MIN468}

energy $($ in $\mathrm{kcal} / \mathrm{mol})=79.998573$

$\operatorname{ZPE}(\mathrm{in} \mathrm{kcal} / \mathrm{mol})=141.082$

$\mathrm{G}(\mathrm{in} \mathrm{kcal} / \mathrm{mol})=114.863$

Geometry

C $-2.14565954 \quad 0.10144796-0.70099907$

C $-0.62541244 \quad 0.14793326-0.76123553$

C $-0.071920361 .60111601-0.95093493$

C $0.64431350 \quad 2.16560871 \quad 0.16316678$

C $0.93520384-0.24629171 \quad 1.41926433$

C $2.25716856-0.92412154 \quad 1.65035101$

C $0.05700464 \quad-0.71361515 \quad 0.27291243$

C $-0.39601606-0.92344067 \quad 1.71719436$

C $-1.46605756-0.057473812 .33340629$

C $-0.44297773-2.34122705 \quad 2.23015995$

H $-2.589279110 .36085036-1.67395042$

$\mathrm{H}-2.54534291 \quad 0.79499949 \quad 0.04728006$

$\mathrm{H}-2.50137688-0.90591228-0.44392267$

$\mathrm{H}-0.913559532 .30410534-1.21635182$

$\mathrm{H} \quad 0.98134670 \quad 0.82348712 \quad 1.69243065$

H $1.665215112 .47298894 \quad 0.01385018$

H $2.57140873-0.826942582 .69841856$

H $2.23190839-1.99601694 \quad 1.41846984$

$\mathrm{H} 3.04225885-0.47841480 \quad 1.02395541$

$\mathrm{H} \quad 0.41298542-1.63572417-0.23255017$

$\mathrm{H}-1.41370667 \quad 0.98256480 \quad 1.97982838$

$\mathrm{H}-1.35617243-0.02488358 \quad 3.42573258$ 
$\mathrm{H}-2.47063830-0.43908820 \quad 2.11672178$

$\mathrm{H}-0.24614148 \quad-2.37592580 \quad 3.30960087$

$\mathrm{H} \quad 0.29520627-2.992407551 .74643849$

$\mathrm{H}-1.42970785-2.79034818 \quad 2.05753013$

$\begin{array}{lllll}0 & 0.89425454 & 0.16127230 & -2.42753103\end{array}$

$\begin{array}{lllll}0 & 0.70052150 & 1.54671877 & -2.16616326\end{array}$

o $-0.32638949-0.42942828-2.05616683$

Vibrational frequencies (in $\mathrm{cm}-1$ )

$\begin{array}{lllllllllllllll}-27.4 & 44.9 & 96.9 & 112.8 & 132.5 & 157.0 & 183.9 & 188.1 & 203.6 & 233.1 & 249.4 & 259.9 & 269.8 & 298.4\end{array}$

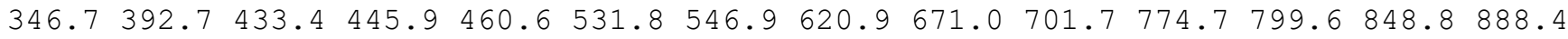
$922.4938 .6 \quad 964.9 \quad 1001.5 \quad 1007.1 \quad 1008.1 \quad 1018.8 \quad 1025.0 \quad 1055.4 \quad 1057.0 \quad 1080.2 \quad 1107.3 \quad 1126.7$

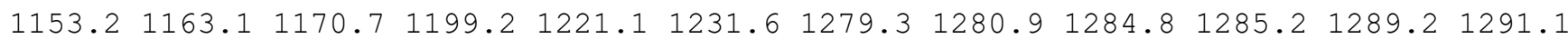

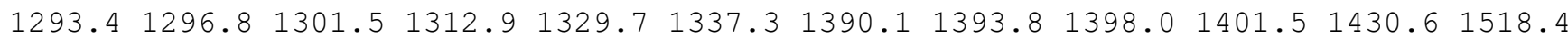
$2642.42664 .92667 .2 \quad 2667.8 \quad 2672.2 \quad 2674.12675 .52676 .12679 .82686 .32687 .42717 .2$ 2777.22778 .22780 .32781 .6

\section{name $=$ MIN469}

energy (in $\mathrm{kcal} / \mathrm{mol})=82.362882$

$\mathrm{ZPE}($ in $\mathrm{kcal} / \mathrm{mol})=142.106$

$\mathrm{G}($ in $\mathrm{kcal} / \mathrm{mol})=116.371$

Geometry

C $-2.86258814-0.33346523-0.82985017$

C $-1.33542444-0.44084177-0.65958481$

C $-0.681764410 .95666313-0.51509827$

C $0.648091701 .07789668 \quad 0.23554070$

C $1.16085439-0.154634240 .94149666$

C $1.88446892-1.115428620 .02878251$

C $-1.14269025-1.39058274 \quad 0.42491764$

C $1.74841393-0.01003528 \quad 2.35037008$

C $1.81496468 \quad 1.31130533 \quad 3.06530013$

C $0.41887381-0.701028532 .15639772$

$\mathrm{H}-3.10683963 \quad 0.35868608-1.64710869$

$\mathrm{H}-3.34468462 \quad 0.02084114 \quad 0.08921747$

$\mathrm{H}-3.30584632-1.30253030-1.09130647$

$\mathrm{H}-1.41182151 \quad 1.71401445 \quad-0.15325645$

$\mathrm{H} \quad 0.56518095 \quad 1.91991341 \quad 0.95518053$

$\mathrm{H} 1.424161421 .41609852-0.49308563$

H $2.61795090-0.640636612 .57850504$

$\mathrm{H} \quad 1.33139825-1.28883728-0.90646520$

$\mathrm{H} 2.86852076-0.71679516-0.25507256$

$\mathrm{H}-0.70931843-2.34660202 \quad 0.18377947$

$\mathrm{H} 0.86687469 \quad 1.86245193 \quad 3.02958040$

$\mathrm{H} 2.59417474 \quad 1.95616777 \quad 2.63755453$

$\mathrm{H} 2.054051621 .16716542 \quad 4.12780754$

$\mathrm{H} \quad 0.34882376-1.771081532 .33527459$

H $2.05298749-2.09307975 \quad 0.49478656$

$\mathrm{H}-0.49377187-0.21403600 \quad 2.49778114$

$0-0.03917360 \quad 0.11579628-2.49402908$

$0-0.486418051 .30943638-1.88732624$

$0-0.82947006-0.93114270-1.91874553$

Vibrational frequencies (in $\mathrm{cm}-1$ )

$\begin{array}{lllllllllllllllll}23.7 & 78.9 & 92.3 & 132.9 & 149.1 & 164.7 & 170.3 & 182.1 & 195.0 & 233.9 & 244.7 & 281.8 & 317.5 & 360.3\end{array}$

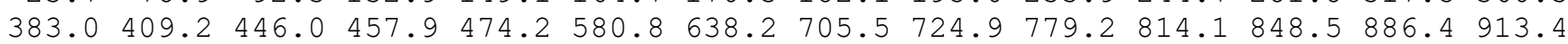
$927.0951 .1955 .0 \quad 981.4 \quad 982.6 \quad 1005.0 \quad 1018.5 \quad 1023.9 \quad 1027.4 \quad 1064.0 \quad 1092.1 \quad 1095.8 \quad 1098.8$ $1131.31162 .3 \quad 1169.91190 .2 \quad 1196.5 \quad 1244.1 \quad 1250.2 \quad 1280.6 \quad 1285.7 \quad 1287.0 \quad 1289.5 \quad 1291.5$ $1292.31296 .41300 .91315 .0 \quad 1356.5 \quad 1360.6 \quad 1365.4 \quad 1393.7 \quad 1396.3 \quad 1401.5 \quad 1406.91490 .2$ $2641.52664 .72668 .12671 .5 \quad 2674.7 \quad 2675.4 \quad 2676.62678 .32685 .22706 .12728 .12750 .5$ $\begin{array}{lllll}2776.7 & 2779.1 & 2779.6 & 2783.3\end{array}$

name $=$ MIN470

energy $($ in $\mathrm{kcal} / \mathrm{mol})=83.376235$

$\mathrm{ZPE}($ in $\mathrm{kcal} / \mathrm{mol})=141.686$

$\mathrm{G}($ in $\mathrm{kcal} / \mathrm{mol})=116.197$ 


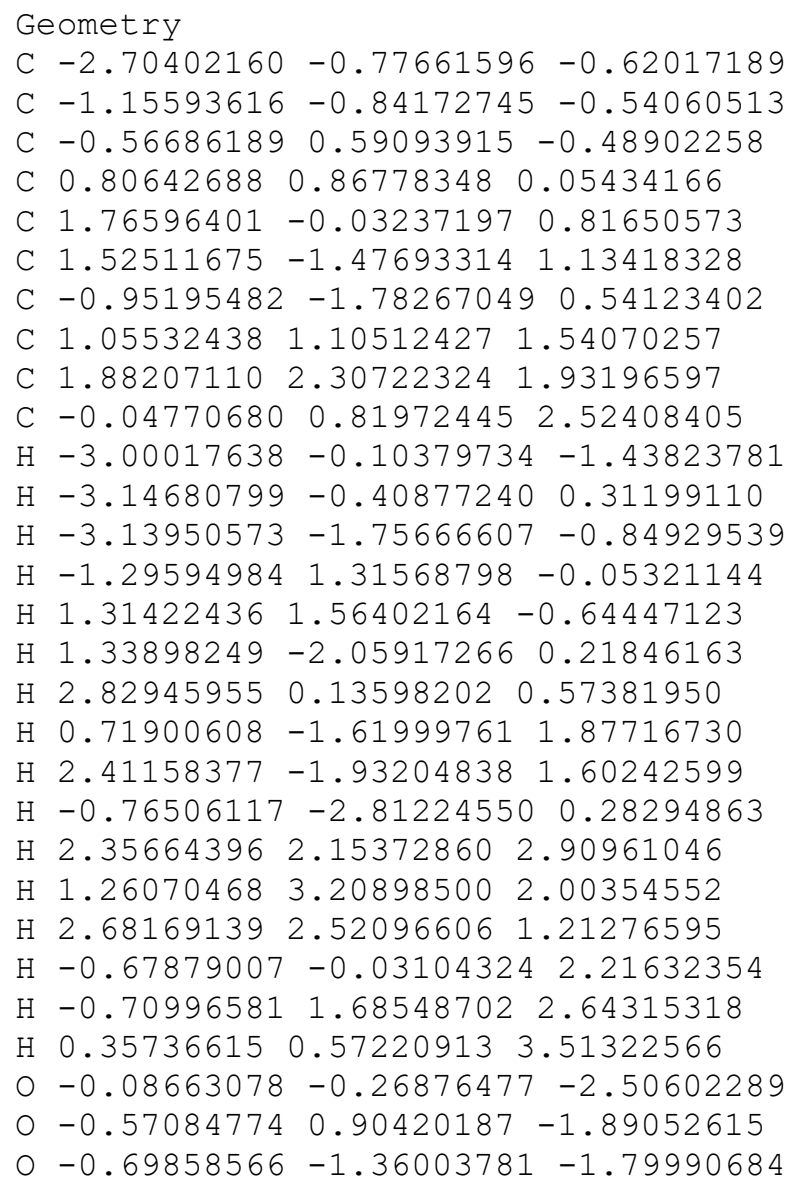

\section{RELATIVE ENERGIES OF THE TSS (ZPE-CORRECTED)}

$\begin{array}{rc}\text { TS } & \text { DE (kcal/mol) } \\ 1 & -121.580 \\ 2 & -67.045 \\ 3 & -58.910 \\ 4 & -57.317 \\ 5 & -57.221 \\ 6 & -56.537 \\ 7 & -55.435 \\ 8 & -54.854 \\ 9 & -53.212 \\ 10 & -49.425 \\ 11 & -48.818 \\ 12 & -45.882 \\ 13 & -42.054 \\ 14 & -41.732 \\ 15 & -41.290 \\ 16 & -35.209 \\ 17 & -34.760 \\ 18 & -27.865 \\ 19 & -27.373 \\ 20 & -27.090 \\ 21 & -26.010 \\ 22 & -25.875 \\ 23 & -25.077 \\ & \end{array}$




\begin{tabular}{|c|c|}
\hline 24 & -16.721 \\
\hline 25 & -16.310 \\
\hline 26 & -15.715 \\
\hline 27 & -13.846 \\
\hline 28 & -12.853 \\
\hline 29 & -12.499 \\
\hline 30 & -12.316 \\
\hline 31 & -11.842 \\
\hline 32 & -11.654 \\
\hline 33 & -10.679 \\
\hline 34 & -10.112 \\
\hline 35 & -9.986 \\
\hline 36 & -9.115 \\
\hline 37 & -8.988 \\
\hline 38 & -5.970 \\
\hline 39 & -4.745 \\
\hline 40 & -2.668 \\
\hline 41 & -1.573 \\
\hline 42 & $-1 \cdot 328$ \\
\hline 43 & -1.164 \\
\hline 44 & -0.873 \\
\hline 45 & -0.674 \\
\hline 46 & -0.465 \\
\hline 47 & -0.211 \\
\hline 48 & 0.088 \\
\hline 49 & 0.516 \\
\hline 50 & 0.705 \\
\hline 51 & 1.138 \\
\hline 52 & 1.188 \\
\hline 53 & 1.198 \\
\hline 54 & 1.266 \\
\hline 55 & 1.298 \\
\hline 56 & 1.392 \\
\hline 57 & 1.462 \\
\hline 58 & 1.726 \\
\hline 59 & 2.250 \\
\hline 60 & 2.351 \\
\hline 61 & 2.440 \\
\hline 62 & 2.971 \\
\hline 63 & 3.35 \\
\hline 64 & 4.07 \\
\hline 65 & 4.07 \\
\hline 66 & 4.28 \\
\hline 67 & 4.29 \\
\hline 68 & 9.22 \\
\hline 69 & 9.80 \\
\hline 70 & 10.79 \\
\hline 71 & 11.44 \\
\hline 72 & 11.82 \\
\hline 73 & 11.90 \\
\hline 74 & 12.71 \\
\hline 75 & 12.81 \\
\hline 76 & 13.30 \\
\hline 77 & 15.40 \\
\hline 78 & 16.69 \\
\hline 79 & 17.76 \\
\hline 80 & 19.68 \\
\hline 81 & 20.39 \\
\hline 82 & 20.50 \\
\hline 83 & 20.81 \\
\hline 84 & 20.87 \\
\hline 85 & 21.02 \\
\hline 86 & 21.26 \\
\hline 87 & 21.55 \\
\hline 88 & 21.8 \\
\hline
\end{tabular}




\begin{tabular}{|c|c|}
\hline 89 & 21.966 \\
\hline 90 & 22.144 \\
\hline 91 & 22.654 \\
\hline 92 & 22.775 \\
\hline 93 & 23.135 \\
\hline 94 & 23.536 \\
\hline 95 & 23.985 \\
\hline 96 & 24.146 \\
\hline 97 & 24.215 \\
\hline 98 & 24.381 \\
\hline 99 & 24.829 \\
\hline 100 & 24.881 \\
\hline 101 & 24.918 \\
\hline 102 & 25.198 \\
\hline 103 & 25.222 \\
\hline 104 & 26.445 \\
\hline 105 & 26.531 \\
\hline 106 & 26.679 \\
\hline 107 & 26.834 \\
\hline 108 & 26.926 \\
\hline 109 & 27.177 \\
\hline 110 & 27.422 \\
\hline 111 & 27.858 \\
\hline 112 & 29.401 \\
\hline 113 & 29.922 \\
\hline 114 & 30.697 \\
\hline 115 & 30.785 \\
\hline 116 & 31.112 \\
\hline 117 & 32.172 \\
\hline 118 & 34.248 \\
\hline 119 & 34.454 \\
\hline 120 & 34.547 \\
\hline 121 & 34.678 \\
\hline 122 & 35.383 \\
\hline 123 & 35.651 \\
\hline 124 & 35.816 \\
\hline 125 & 35.986 \\
\hline 126 & 36.949 \\
\hline 127 & 37.118 \\
\hline 128 & 37.422 \\
\hline 129 & 37.965 \\
\hline 130 & 38.008 \\
\hline 131 & 38.496 \\
\hline 132 & 38.577 \\
\hline 133 & 39.010 \\
\hline 134 & 39.147 \\
\hline 135 & 39.318 \\
\hline 136 & 39.364 \\
\hline 137 & 39.405 \\
\hline 138 & 39.525 \\
\hline 139 & 39.764 \\
\hline 140 & 40.288 \\
\hline 141 & 41.149 \\
\hline 142 & 41.506 \\
\hline 143 & 41.830 \\
\hline 144 & 41.886 \\
\hline 145 & 42.219 \\
\hline 146 & 42.384 \\
\hline 147 & 42.537 \\
\hline 148 & 42.739 \\
\hline 149 & 43.207 \\
\hline 150 & 43.477 \\
\hline 151 & 43.568 \\
\hline 152 & 43.728 \\
\hline 153 & 44.011 \\
\hline
\end{tabular}




\begin{tabular}{|c|c|}
\hline 154 & 44.123 \\
\hline 155 & 44.217 \\
\hline 156 & 44.742 \\
\hline 157 & 44.976 \\
\hline 158 & 44.980 \\
\hline 159 & 45.372 \\
\hline 160 & 45.540 \\
\hline 161 & 45.652 \\
\hline 162 & 45.796 \\
\hline 163 & 45.837 \\
\hline 164 & 45.915 \\
\hline 165 & 45.944 \\
\hline 166 & 46.035 \\
\hline 167 & 46.190 \\
\hline 168 & 46.323 \\
\hline 169 & 46.781 \\
\hline 170 & 46.824 \\
\hline 171 & 47.715 \\
\hline 172 & 47.803 \\
\hline 173 & 47.989 \\
\hline 174 & 48.154 \\
\hline 175 & 48.284 \\
\hline 176 & 48.442 \\
\hline 177 & 48.567 \\
\hline 178 & 48.780 \\
\hline 179 & 49.262 \\
\hline 180 & 49.278 \\
\hline 181 & 49.614 \\
\hline 182 & 50.498 \\
\hline 183 & 51.498 \\
\hline 184 & 51.600 \\
\hline 185 & 51.904 \\
\hline 186 & 52.357 \\
\hline 187 & 52.529 \\
\hline 188 & 52.659 \\
\hline 189 & 52.714 \\
\hline 190 & 52.973 \\
\hline 191 & 52.988 \\
\hline 192 & 53.070 \\
\hline 193 & 53.136 \\
\hline 194 & 53.246 \\
\hline 195 & 53.264 \\
\hline 196 & 53.277 \\
\hline 197 & 53.326 \\
\hline 198 & 53.395 \\
\hline 199 & 53.403 \\
\hline 200 & 54.055 \\
\hline 201 & 54.661 \\
\hline 202 & 54.736 \\
\hline 203 & 55.012 \\
\hline 204 & 55.071 \\
\hline 205 & 55.095 \\
\hline 206 & 55.277 \\
\hline 207 & 55.510 \\
\hline 208 & 55.703 \\
\hline 209 & 55.776 \\
\hline 210 & 55.780 \\
\hline 211 & 55.797 \\
\hline 212 & 55.809 \\
\hline 213 & 55.854 \\
\hline 214 & 55.858 \\
\hline 215 & 55.858 \\
\hline 216 & 56.003 \\
\hline 217 & 56.230 \\
\hline 218 & 56.418 \\
\hline
\end{tabular}




\begin{tabular}{|c|c|}
\hline 219 & 56.439 \\
\hline 220 & 56.532 \\
\hline 221 & 56.609 \\
\hline 222 & 57.339 \\
\hline 223 & 57.691 \\
\hline 224 & 57.974 \\
\hline 225 & 58.281 \\
\hline 226 & 59.091 \\
\hline 227 & 59.111 \\
\hline 228 & 59.180 \\
\hline 229 & 59.340 \\
\hline 230 & 59.376 \\
\hline 231 & 59.782 \\
\hline 232 & 59.916 \\
\hline 233 & 60.121 \\
\hline 234 & 60.233 \\
\hline 235 & 60.307 \\
\hline 236 & 60.455 \\
\hline 237 & 60.460 \\
\hline 238 & 60.619 \\
\hline 239 & 60.645 \\
\hline 240 & 60.822 \\
\hline 241 & 61.224 \\
\hline 242 & 61.238 \\
\hline 243 & 61.746 \\
\hline 244 & 62.209 \\
\hline 245 & 62.280 \\
\hline 246 & 62.345 \\
\hline 247 & 62.836 \\
\hline 248 & 62.841 \\
\hline 249 & 62.896 \\
\hline 250 & 63.018 \\
\hline 251 & 63.192 \\
\hline 252 & 63.266 \\
\hline 253 & 63.598 \\
\hline 254 & 63.627 \\
\hline 255 & 64.109 \\
\hline 256 & 64.291 \\
\hline 257 & 64.352 \\
\hline 258 & 64.353 \\
\hline 259 & 64.383 \\
\hline 260 & 64.413 \\
\hline 261 & 64.973 \\
\hline 262 & 65.046 \\
\hline 263 & 65.060 \\
\hline 264 & 65.089 \\
\hline 265 & 65.490 \\
\hline 266 & 65.594 \\
\hline 267 & 65.694 \\
\hline 268 & 65.731 \\
\hline 269 & 65.768 \\
\hline 270 & 65.799 \\
\hline 271 & 65.845 \\
\hline 272 & 65.896 \\
\hline 273 & 65.953 \\
\hline 274 & 65.958 \\
\hline 275 & 66.062 \\
\hline 276 & 66.144 \\
\hline 277 & 66.150 \\
\hline 278 & 66.252 \\
\hline 279 & 66.255 \\
\hline 280 & 66.317 \\
\hline 281 & 66.339 \\
\hline 282 & 66.343 \\
\hline 283 & 66.425 \\
\hline
\end{tabular}




\begin{tabular}{|c|c|}
\hline 349 & 70.387 \\
\hline 350 & 70.393 \\
\hline 351 & 70.424 \\
\hline 352 & 70.536 \\
\hline 353 & 70.750 \\
\hline 354 & 70.853 \\
\hline 355 & 70.935 \\
\hline 356 & 71.197 \\
\hline 357 & 71.251 \\
\hline 358 & 71.369 \\
\hline 359 & 71.396 \\
\hline 360 & 71.405 \\
\hline 361 & 71.529 \\
\hline 362 & 71.691 \\
\hline 363 & 71.911 \\
\hline 364 & 71.978 \\
\hline 365 & 72.113 \\
\hline 366 & 72.182 \\
\hline 367 & 72.187 \\
\hline 368 & 72.202 \\
\hline 369 & 72.224 \\
\hline 370 & 72.301 \\
\hline 371 & 72.636 \\
\hline 372 & 72.648 \\
\hline 373 & 72.992 \\
\hline 374 & 73.054 \\
\hline 375 & 73.131 \\
\hline 376 & 73.251 \\
\hline 377 & 73.341 \\
\hline 378 & 73.446 \\
\hline 379 & 73.650 \\
\hline 380 & 73.747 \\
\hline 381 & 73.799 \\
\hline 382 & 73.884 \\
\hline 383 & 73.896 \\
\hline 384 & 74.066 \\
\hline 385 & 74.446 \\
\hline 386 & 74.675 \\
\hline 387 & 75.046 \\
\hline 388 & 75.091 \\
\hline 389 & 75.108 \\
\hline 390 & 75.374 \\
\hline 391 & 75.432 \\
\hline 392 & 75.838 \\
\hline 393 & 76.257 \\
\hline 394 & 76.827 \\
\hline 395 & 77.158 \\
\hline 396 & 77.195 \\
\hline 397 & 77.392 \\
\hline 398 & 77.403 \\
\hline 399 & 77.776 \\
\hline 400 & 77.986 \\
\hline 401 & 78.042 \\
\hline 402 & 78.048 \\
\hline 403 & 78.190 \\
\hline 404 & 78.332 \\
\hline 405 & 78.451 \\
\hline 406 & 78.702 \\
\hline 407 & 78.746 \\
\hline 408 & 78.949 \\
\hline 409 & 79.051 \\
\hline 410 & 79.155 \\
\hline 411 & 79.207 \\
\hline 412 & 79.209 \\
\hline & 79.209 \\
\hline
\end{tabular}




\begin{tabular}{|c|c|}
\hline 414 & 79.311 \\
\hline 415 & 79.459 \\
\hline 416 & 79.700 \\
\hline 417 & 80.004 \\
\hline 418 & 80.014 \\
\hline 419 & 80.024 \\
\hline 420 & 80.030 \\
\hline 421 & 80.061 \\
\hline 422 & 80.149 \\
\hline 423 & 80.152 \\
\hline 424 & 80.169 \\
\hline 425 & 80.253 \\
\hline 426 & 80.346 \\
\hline 427 & 80.352 \\
\hline 428 & 80.367 \\
\hline 429 & 80.387 \\
\hline 430 & 80.398 \\
\hline 431 & 80.448 \\
\hline 432 & 80.465 \\
\hline 433 & 80.499 \\
\hline 434 & 80.659 \\
\hline 435 & 80.753 \\
\hline 436 & 80.861 \\
\hline 437 & 81.360 \\
\hline 438 & 81.662 \\
\hline 439 & 81.694 \\
\hline 440 & 81.727 \\
\hline 441 & 81.827 \\
\hline 442 & 81.897 \\
\hline 443 & 82.013 \\
\hline 444 & 82.566 \\
\hline 445 & 82.666 \\
\hline 446 & 82.795 \\
\hline 447 & 82.859 \\
\hline 448 & 83.040 \\
\hline 449 & 83.270 \\
\hline 450 & 83.300 \\
\hline 451 & 83.307 \\
\hline 452 & 83.365 \\
\hline 453 & 83.427 \\
\hline 454 & 83.470 \\
\hline 455 & 83.504 \\
\hline 456 & 83.772 \\
\hline 457 & 84.089 \\
\hline 458 & 84.123 \\
\hline 459 & 84.320 \\
\hline 460 & 84.540 \\
\hline 461 & 84.703 \\
\hline 462 & 84.774 \\
\hline 463 & 84.813 \\
\hline 464 & 84.897 \\
\hline 465 & 85.127 \\
\hline 466 & 85.189 \\
\hline 467 & 85.266 \\
\hline 468 & 85.369 \\
\hline 469 & 85.669 \\
\hline 470 & 85.831 \\
\hline 471 & 85.940 \\
\hline 472 & 86.122 \\
\hline 473 & 86.380 \\
\hline 474 & 86.816 \\
\hline 475 & 87.512 \\
\hline 476 & 87.598 \\
\hline 477 & 88.073 \\
\hline 478 & 88.094 \\
\hline
\end{tabular}




\begin{tabular}{|c|c|}
\hline 479 & 88.103 \\
\hline 480 & 88.838 \\
\hline 481 & 88.926 \\
\hline 482 & 88.942 \\
\hline 483 & 89.030 \\
\hline 484 & 89.197 \\
\hline 485 & 89.307 \\
\hline 486 & 89.404 \\
\hline 487 & 89.749 \\
\hline 488 & 89.769 \\
\hline 489 & 89.907 \\
\hline 490 & 90.066 \\
\hline 491 & 90.101 \\
\hline 492 & 90.502 \\
\hline 493 & 91.266 \\
\hline 494 & 91.682 \\
\hline 495 & 91.732 \\
\hline 496 & 92.095 \\
\hline 497 & 92.266 \\
\hline 498 & 92.391 \\
\hline 499 & 92.492 \\
\hline 500 & 92.585 \\
\hline 501 & 92.587 \\
\hline 502 & 93.015 \\
\hline 503 & 93.116 \\
\hline 504 & 93.347 \\
\hline 505 & 93.684 \\
\hline 506 & 93.945 \\
\hline 507 & 94.075 \\
\hline 508 & 94.231 \\
\hline 509 & 94.935 \\
\hline 510 & 95.271 \\
\hline 511 & 95.528 \\
\hline 512 & 95.650 \\
\hline 513 & 95.666 \\
\hline 514 & 95.676 \\
\hline 515 & 95.938 \\
\hline 516 & 95.949 \\
\hline 517 & 95.968 \\
\hline 518 & 96.212 \\
\hline 519 & 96.238 \\
\hline 520 & 96.298 \\
\hline 521 & 96.324 \\
\hline 522 & 96.430 \\
\hline 523 & 96.802 \\
\hline 524 & 96.825 \\
\hline 525 & 97.943 \\
\hline 526 & 98.271 \\
\hline 527 & 98.732 \\
\hline 528 & 98.754 \\
\hline 529 & 98.891 \\
\hline 530 & 99.160 \\
\hline 531 & 99.367 \\
\hline 532 & 99.657 \\
\hline 533 & 99.736 \\
\hline 534 & 100.579 \\
\hline 535 & 100.762 \\
\hline 536 & 100.857 \\
\hline 537 & 101.662 \\
\hline 538 & 101.755 \\
\hline 539 & 101.843 \\
\hline 540 & 102.172 \\
\hline 541 & 102.335 \\
\hline 542 & 102.839 \\
\hline 543 & 103.066 \\
\hline
\end{tabular}




\begin{tabular}{|c|c|}
\hline 544 & 103.295 \\
\hline 545 & 103.887 \\
\hline 546 & 104.166 \\
\hline 547 & 104.174 \\
\hline 548 & 104.275 \\
\hline 549 & 104.380 \\
\hline 550 & 104.575 \\
\hline 551 & 104.891 \\
\hline 552 & 105.548 \\
\hline 553 & 106.425 \\
\hline 554 & 106.618 \\
\hline 555 & 106.673 \\
\hline 556 & 106.695 \\
\hline 557 & 106.864 \\
\hline 558 & 107.707 \\
\hline 559 & 107.903 \\
\hline 560 & 108.386 \\
\hline 561 & 109.277 \\
\hline 562 & 109.949 \\
\hline 563 & 110.209 \\
\hline 564 & 110.245 \\
\hline 565 & 110.303 \\
\hline 566 & 110.422 \\
\hline 567 & 111.279 \\
\hline 568 & 111.320 \\
\hline 569 & 111.577 \\
\hline 570 & 112.021 \\
\hline 571 & 112.521 \\
\hline 572 & 112.792 \\
\hline 573 & 113.249 \\
\hline 574 & 113.291 \\
\hline 575 & 113.513 \\
\hline 576 & 113.924 \\
\hline 577 & 114.392 \\
\hline 578 & 114.867 \\
\hline 579 & 114.891 \\
\hline 580 & 116.815 \\
\hline 581 & 117.792 \\
\hline 582 & 118.043 \\
\hline 583 & 118.300 \\
\hline 584 & 118.783 \\
\hline 585 & 118.831 \\
\hline 586 & 119.179 \\
\hline 587 & 119.376 \\
\hline 588 & 119.497 \\
\hline 589 & 119.831 \\
\hline 590 & 120.169 \\
\hline 591 & 121.089 \\
\hline 592 & 121.268 \\
\hline 593 & 121.712 \\
\hline 594 & 122.463 \\
\hline 595 & 122.854 \\
\hline 596 & 123.203 \\
\hline 597 & 123.801 \\
\hline 598 & 124.167 \\
\hline 599 & 125.766 \\
\hline 600 & 126.100 \\
\hline 601 & 126.553 \\
\hline 602 & 127.299 \\
\hline 603 & 127.454 \\
\hline 604 & 127.519 \\
\hline 605 & 131.407 \\
\hline 606 & 132.663 \\
\hline 607 & 132.839 \\
\hline 8 & 133.211 \\
\hline
\end{tabular}




$\begin{array}{ll}609 & 133.259 \\ 610 & 134.445 \\ 611 & 135.388 \\ 612 & 135.404 \\ 613 & 135.413 \\ 614 & 135.695 \\ 615 & 136.374 \\ 616 & 138.863 \\ 617 & 139.787 \\ 618 & 140.584\end{array}$

Families of conformers

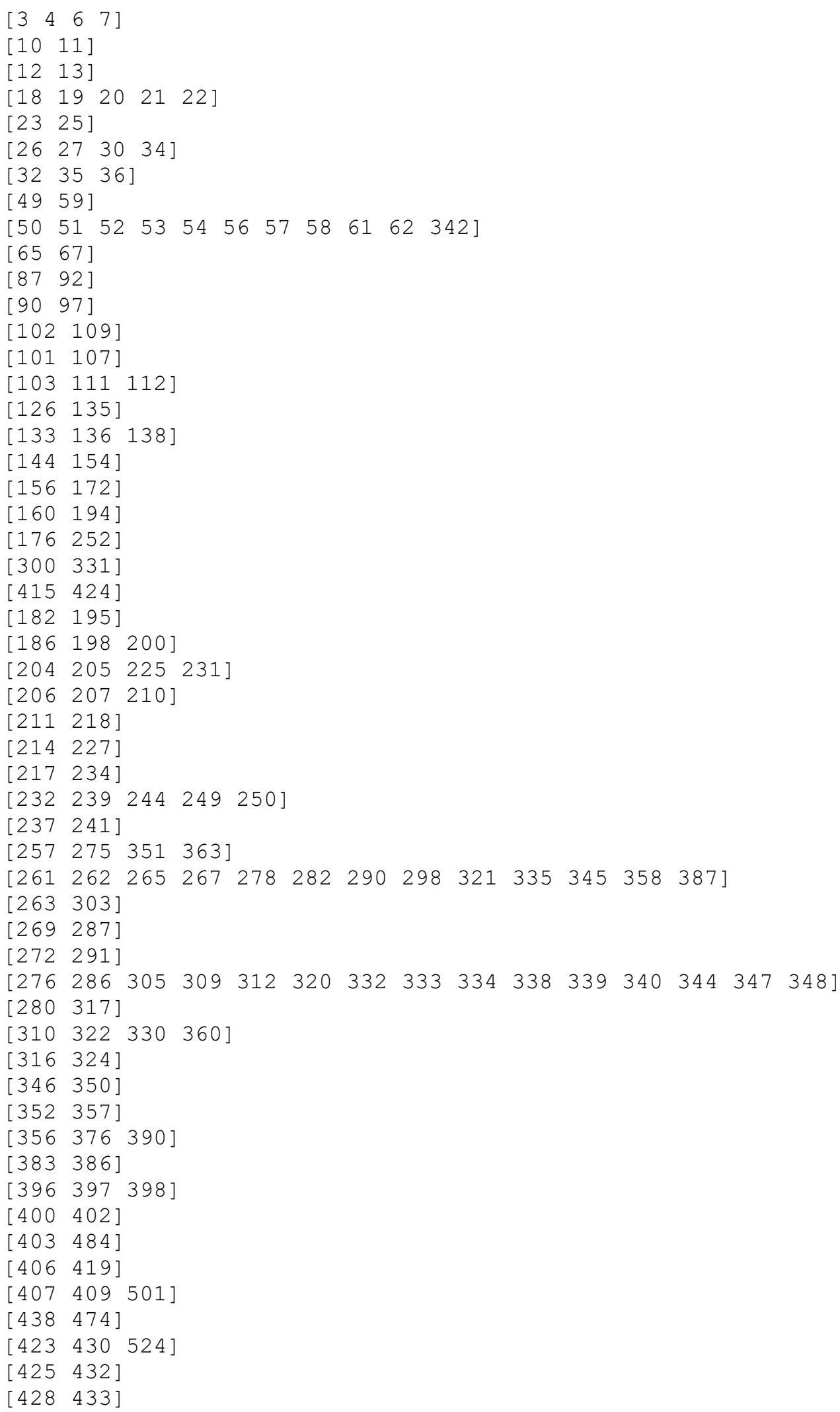




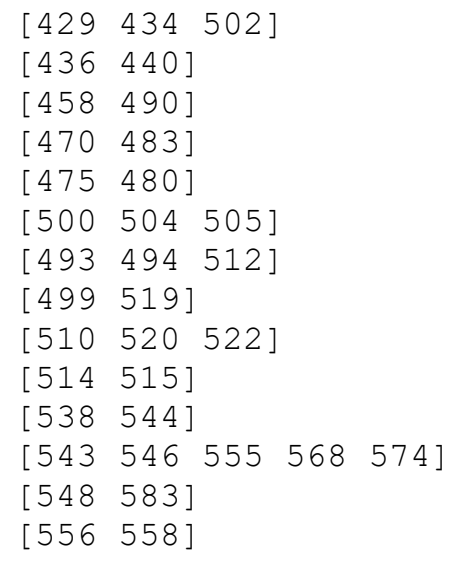

ABSOLUTE ENERGY, ZPE, GIBBS ENERGY CORRECTION, GEOMETRY AND FREQUENCIES OF THE TSS name $=$ TS1

energy (in $\mathrm{kcal} / \mathrm{mol})=-123.870772$

$\mathrm{ZPE}($ in $\mathrm{kcal} / \mathrm{mol})=140.358$

$\mathrm{G}($ in $\mathrm{kcal} / \mathrm{mol})=110.267$

Geometry

C $-1.87481251-4.26569563-1.16309141$

C $-1.75678482-2.93858953-0.47504879$

C $-2.145473861 .46806588-0.66414744$

C $-1.854114682 .69819523-0.23293488$

C $0.44362543-1.66747873 \quad 2.38274288$

C $-0.18258867-1.88556137 \quad 1.08770219$

C $-1.02840099-2.89474305 \quad 0.78949228$

C $0.56730042-0.485685363 .01090447$

C $1.19759972-0.41017818 \quad 4.36284112$

C $0.08062958 \quad 0.81324224 \quad 2.47306970$

$\mathrm{H}-0.89141452-4.74222393-1.27977955$

$\mathrm{H}-2.31771257-4.16160866-2.16507363$

$\mathrm{H}-2.51134662-4.95420698-0.59162106$

$\mathrm{H}-2.525738641 .18324609-1.63876648$

$\mathrm{H}-1.97337403 \quad 3.57791041-0.83703480$

$\mathrm{H}-1.49020660 \quad 2.90714935 \quad 0.75693869$

$\mathrm{H} \quad 0.80677698 \quad-2.58558721 \quad 2.85208876$

$\mathrm{H}-0.09768059-0.127676490 .20944876$

$\mathrm{H}-2.15451752-0.46960327-0.22204202$

$\mathrm{H}-1.22414233-3.69825980 \quad 1.49074317$

$\mathrm{H} \quad 0.56177501 \quad 0.13960132 \quad 5.07312010$

$\mathrm{H} 2.16315269 \quad 0.11707092 \quad 4.31579464$

H $1.39227291-1.39633615 \quad 4.80522598$

$\mathrm{H} \quad 0.37323431 \quad 0.98201979 \quad 1.42401330$

$\mathrm{H} 0.452671891 .68002493 \quad 3.03574756$

$\mathrm{H}-1.02542894 \quad 0.86340867 \quad 2.49488134$

○ $0.19670776-1.073955570 .06629155$

$0-1.95103445 \quad 0.436813410 .19708117$

o $-2.29689654-1.95629485-0.95395972$

Vibrational frequencies (in $\mathrm{cm}-1$ )

$\begin{array}{llllllllllllllll}-294.8 & 6.3 & 43.8 & 54.8 & 63.7 & 81.0 & 82.2 & 91.4 & 101.7 & 110.0 & 123.4 & 128.7 & 167.8 & 219.2\end{array}$ $237.7 \quad 281.5 \quad 361.2 \quad 373.4 \quad 399.2 \quad 440.6 \quad 480.4 \quad 517.1 \quad 556.5 \quad 591.4 \quad 627.8 \quad 696.1 \quad 793.0 \quad 875.4$ $884.5921 .3 \quad 948.8949 .6 \quad 983.3 \quad 985.1 \quad 1000.0 \quad 1025.7 \quad 1042.5 \quad 1049.6 \quad 1059.5 \quad 1066.8 \quad 1108.2$

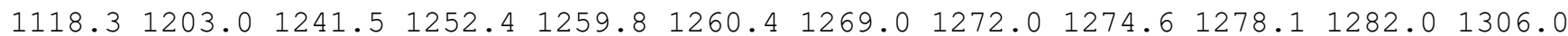

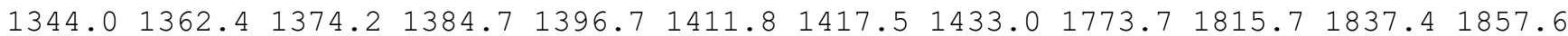
$2481.42630 .6 \quad 2650.62663 .02666 .3 \quad 2671.6 \quad 2671.62672 .32720 .42745 .12749 .12767 .9$ $2771.22778 .2 \quad 2780.92805 .1$

name $=$ TS2

energy (in $\mathrm{kcal} / \mathrm{mol})=-69.045429$

$\mathrm{ZPE}(\mathrm{in} \mathrm{kcal} / \mathrm{mol})=140.068$

$\mathrm{G}($ in $\mathrm{kcal} / \mathrm{mol})=113.664$ 


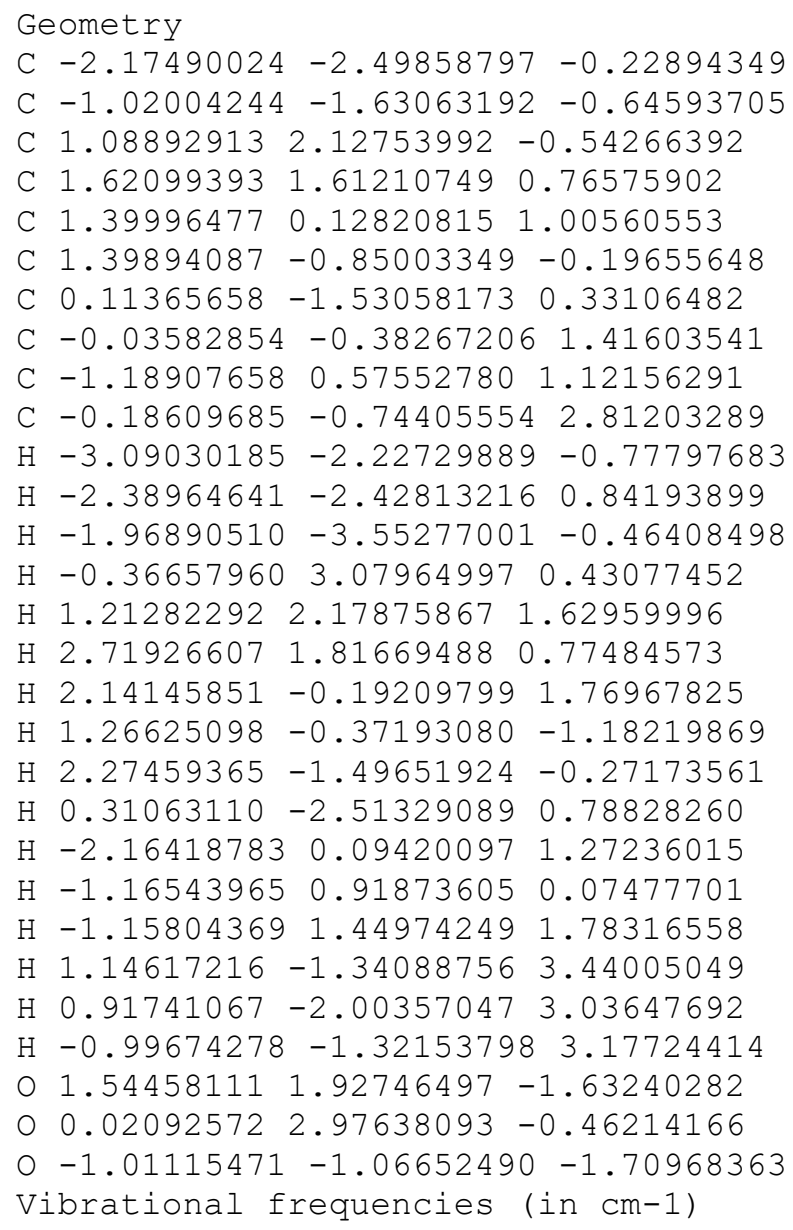
$350.5 \quad 373.0 \quad 387.9 \quad 401.8 \quad 436.9 \quad 503.5 \quad 514.1 \quad 524.5 \quad 567.8 \quad 593.1 \quad 630.6 \quad 668.9 \quad 698.4 \quad 756.1$

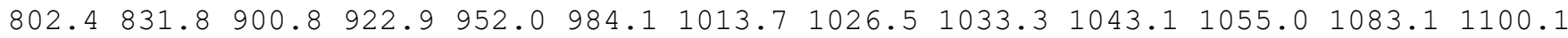

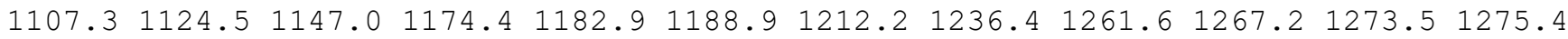

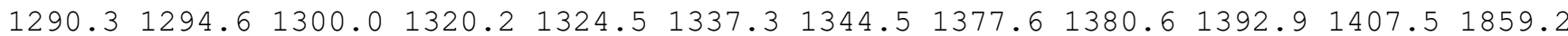
$1874.72649 .12651 .4 \quad 2661.2 \quad 2665.7 \quad 2673.4 \quad 2678.62705 .52725 .52729 .82731 .42756 .6$ $2772.02780 .4 \quad 2784.3 \quad 4770.6$

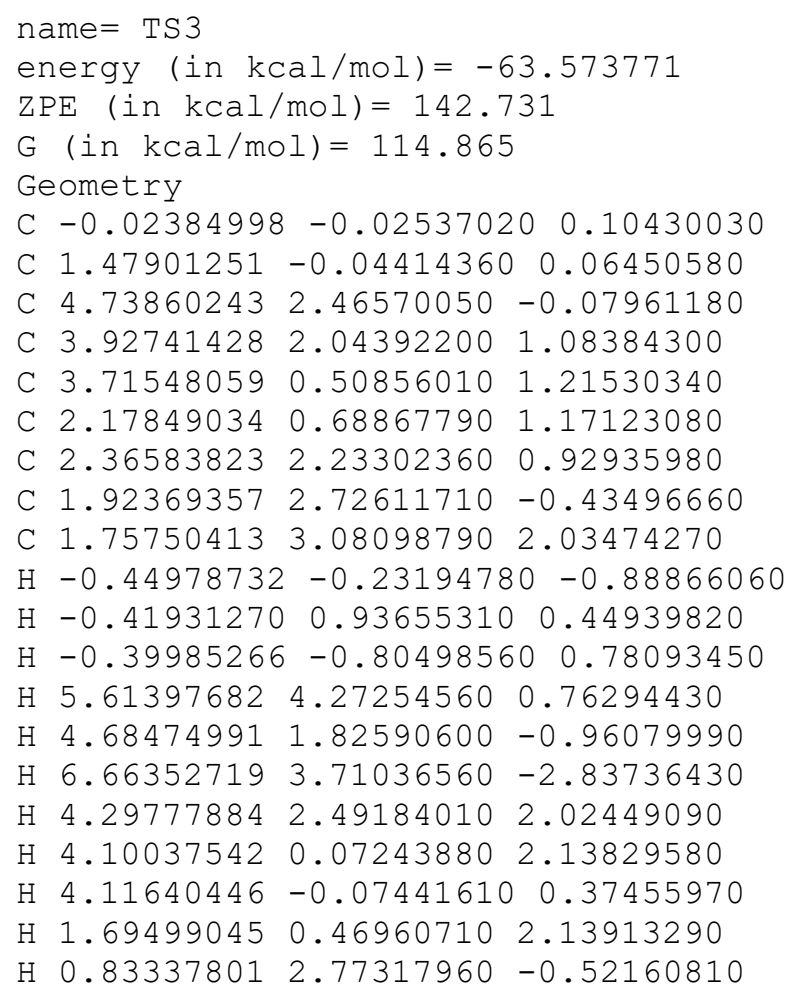


$\mathrm{H} 2.29404265 \quad 2.07953730-1.24414290$

$\mathrm{H} 2.316632153 .73156050-0.63572830$

$\mathrm{H} 2.04814307 \quad 4.13357650 \quad 1.92846740$

$\mathrm{H} 2.07746260 \quad 2.75355780 \quad 3.03041820$

$\mathrm{H} \quad 0.66236468 \quad 3.04136730 \quad 2.01278490$

$06.12663897 \quad 3.17715200-2.20257800$

$06.258036614 .05637090-1.07163690$

O $2.07345884-0.63709290-0.80045770$

C $5.48738184 \quad 3.57116217-0.05829289$

Vibrational frequencies (in cm-1)

$\begin{array}{llllllllllllllll}-108.0 & 31.5 & 32.7 & 43.8 & 63.6 & 83.5 & 133.5 & 162.1 & 176.6 & 187.2 & 204.1 & 220.0 & 262.2 & 285.8\end{array}$

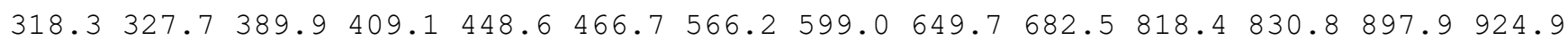
$953.1963 .3 \quad 996.1 \quad 998.7 \quad 1003.5 \quad 1014.5 \quad 1032.5 \quad 1041.2 \quad 1047.6 \quad 1091.7 \quad 1116.8 \quad 1128.6 \quad 1158.4$ $1183.41204 .2 \quad 1235.5 \quad 1252.3 \quad 1262.8 \quad 1267.8 \quad 1273.5 \quad 1278.5 \quad 1283.4 \quad 1289.0 \quad 1297.8 \quad 1300.1$

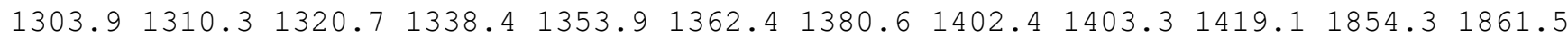
$2665.82670 .12676 .8 \quad 2676.92678 .3 \quad 2680.2 \quad 2681.4 \quad 2719.3 \quad 2726.3 \quad 2729.02756 .92761 .4$ $2779.8 \quad 2781.1 \quad 2782.9 \quad 2874.5$

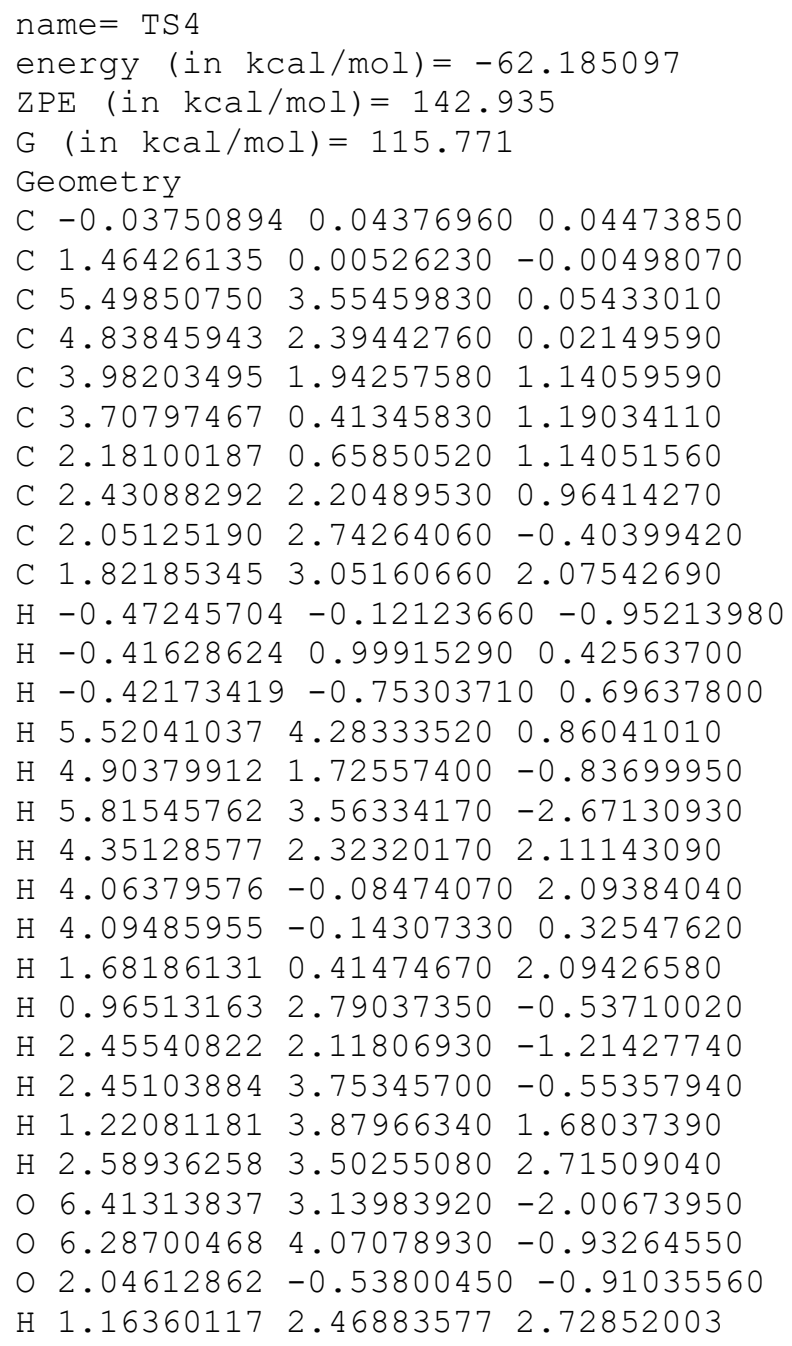


$\operatorname{ZPE}($ in $\mathrm{kcal} / \mathrm{mol})=141.216$

$\mathrm{G}($ in $\mathrm{kcal} / \mathrm{mol})=115.49$

Geometry

C $-0.70160405-1.01895626-2.36132353$

C $0.13027567-1.75185221-1.36341873$

C $0.478383421 .98786683 \quad 0.13289323$

C $1.074345201 .84357104 \quad 1.55050538$

C 1.425405120 .342407891 .64440608

C $1.25102245-0.20711588 \quad 0.21432250$

C $0.13634129-1.264692640 .09053999$

C $-1.19042564 \quad-0.757824350 .43552391$

$\begin{array}{llll}\text { C } 0.12925131 & 2.31543097 & 2.64341041\end{array}$

C $-1.83040330-0.81489136 \quad 1.64103642$

$\mathrm{H}-1.74239278-0.92514619-2.00814759$

$\mathrm{H}-0.70752723-1.52260267-3.33672737$

$\mathrm{H}-0.31883262 \quad 0.00282658-2.50426415$

H $0.727359462 .90260221-0.43476471$

H $2.01333375 \quad 2.44657208 \quad 1.59668582$

$\mathrm{H}-1.288999251 .40693495-0.57710765$

H $2.45848048 \quad 0.19817885 \quad 2.00206539$

$\mathrm{H} \quad 0.78607258 \quad-0.18337734 \quad 2.37114604$

H 2.20366480 $-0.59967388-0.21217866$

H $0.42713325 \quad-2.14164765 \quad 0.73281637$

$\mathrm{H}-0.15721340 \quad 3.36575145 \quad 2.49265829$

$\mathrm{H}-0.80586583 \quad 1.74267868 \quad 2.66648795$

H $0.59171250 \quad 2.23665544 \quad 3.63470982$

$\mathrm{H}-2.89768186-0.61989118 \quad 1.72963813$

$\mathrm{H}-1.408637350 .467570851 .14505894$

$\mathrm{H}-1.44047738-1.30226092 \quad 2.52680353$

$\begin{array}{llll}0 & -0.92968494 & 1.89715943 & 0.21257614\end{array}$

$\begin{array}{lllll}0 & 0.96383196 & 0.91042820 & -0.62447891\end{array}$

O $0.81086425-2.70308293-1.65861401$

Vibrational frequencies (in cm-1)

$\begin{array}{lllllllllllllll}-1438.2 & 41.7 & 54.8 & 66.0 & 98.5 & 135.1 & 141.1 & 153.1 & 162.3 & 194.5 & 247.7 & 260.9 & 304.6 & 328.6\end{array}$ $369.4 \quad 375.5 \quad 446.8 \quad 477.8 \quad 506.2 \quad 537.6 \quad 569.8 \quad 577.5 \quad 672.9 \quad 676.5 \quad 753.9 \quad 857.3 \quad 898.9 \quad 931.8$ $956.1967 .4 \quad 976.8 \quad 1010.5 \quad 1020.4 \quad 1043.4 \quad 1049.0 \quad 1078.3 \quad 1091.8 \quad 1113.7 \quad 1121.01134 .3 \quad 1147.0$

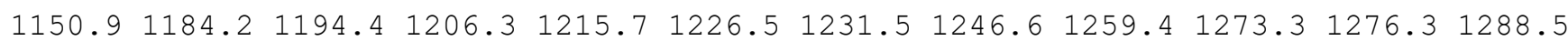

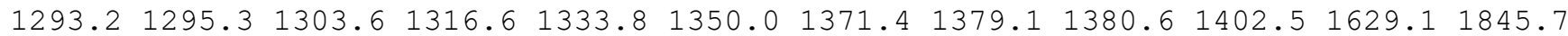
$2009.02659 .62660 .2 \quad 2665.5 \quad 2674.42676 .02677 .3 \quad 2678.92681 .02687 .12706 .52741 .5$ $2750.0 \quad 2755.4 \quad 2775.4 \quad 2779.4$

name $=$ TS 6

energy $($ in $\mathrm{kcal} / \mathrm{mol})=-61.381038$

$\mathrm{ZPE}($ in $\mathrm{kcal} / \mathrm{mol})=142.911$

$\mathrm{G}(\mathrm{in} \mathrm{kcal} / \mathrm{mol})=115.391$

Geometry

C $0.05569850-0.09758831-0.04588758$

C $1.55906163-0.06690486-0.01518239$

C $5.51701374 \quad 3.55979533-0.02937119$

C $4.81814498 \quad 2.42202722 \quad-0.02234203$

C $3.935468462 .04504984 \quad 1.10410105$

C $3.70994373 \quad 0.51549004 \quad 1.26200977$

C $2.18042005 \quad 0.695012991 .11836791$

C $2.377919512 .24275051 \quad 0.88855975$

C $1.95107420 \quad 2.79075622-0.46428127$

C $1.75229046 \quad 3.05708374 \quad 2.01495613$

$\mathrm{H}-0.31699212-0.33763499-1.05246455$

$\mathrm{H}-0.38715678 \quad 0.85654969 \quad 0.26204054$

$\mathrm{H}-0.32590064-0.87655303 \quad 0.62873939$

H $5.54870078 \quad 4.32387904 \quad 0.74337798$

H $4.874916611 .71142128-0.84811125$

H $5.92464833 \quad 3.43548568 \quad-2.75442828$

H $4.26902534 \quad 2.51421317 \quad 2.04920751$

$\mathrm{H} \quad 4.03531613 \quad 0.10347194 \quad 2.21890777$ 
H $4.16154895-0.091792790 .46501120$

H $1.63624920 \quad 0.48135938 \quad 2.05612688$

H $1.33473745 \quad 3.69269432 \quad-0.36852744$

$\mathrm{H} \quad 1.37012472 \quad 2.07582837-1.05589319$

$\mathrm{H} \quad 2.06153904 \quad 4.10807477 \quad 1.95993588$

$\mathrm{H} 2.04234084 \quad 2.68598047 \quad 3.00462559$

$\mathrm{H} 0.65768647 \quad 3.03175491 \quad 1.96443067$

$\begin{array}{lllll}0 & 6.46693413 & 3.01437357 & -2.04263511\end{array}$

○ $6.34135857 \quad 4.00392753-1.02165587$

$02.21155039-0.64723148-0.84597617$

H $2.810933353 .07068896-1.08713496$

Vibrational frequencies (in $\mathrm{cm}-1$ )

$\begin{array}{llllllllllllllll}-205.9 & 24.1 & 32.1 & 48.4 & 71.6 & 98.2 & 134.2 & 157.7 & 170.3 & 200.8 & 208.4 & 217.2 & 266.3 & 293.2\end{array}$ $333.7 \quad 344.7 \quad 400.6 \quad 423.4 \quad 458.9 \quad 482.5 \quad 570.1 \quad 599.1 \quad 660.7 \quad 688.9 \quad 811.7 \quad 826.6 \quad 903.2 \quad 924.2$ $954.0 \quad 964.6 \quad 987.5 \quad 1002.5 \quad 1018.4 \quad 1025.9 \quad 1035.5 \quad 1044.8 \quad 1055.7 \quad 1093.1 \quad 1121.01131 .7 \quad 1158.3$

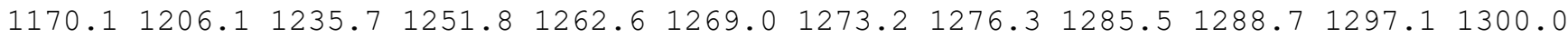

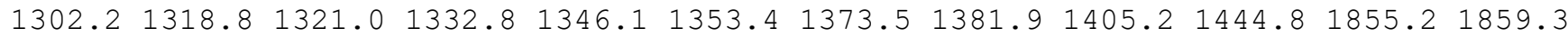
$2664.02669 .62671 .0 \quad 2676.2 \quad 2677.3 \quad 2678.92679 .12716 .62725 .32726 .6 \quad 2754.42760 .7$ $2780.4 \quad 2781.5 \quad 2782.5 \quad 2869.6$

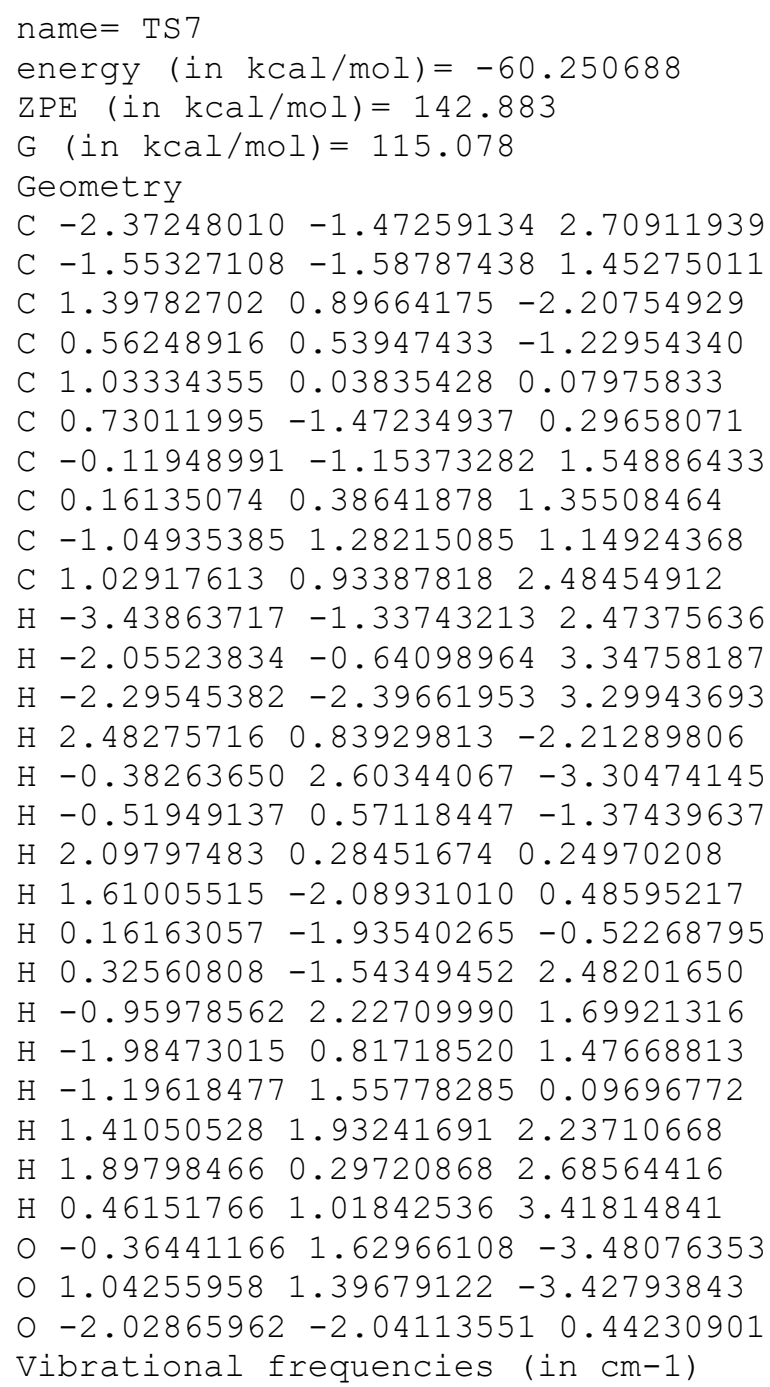




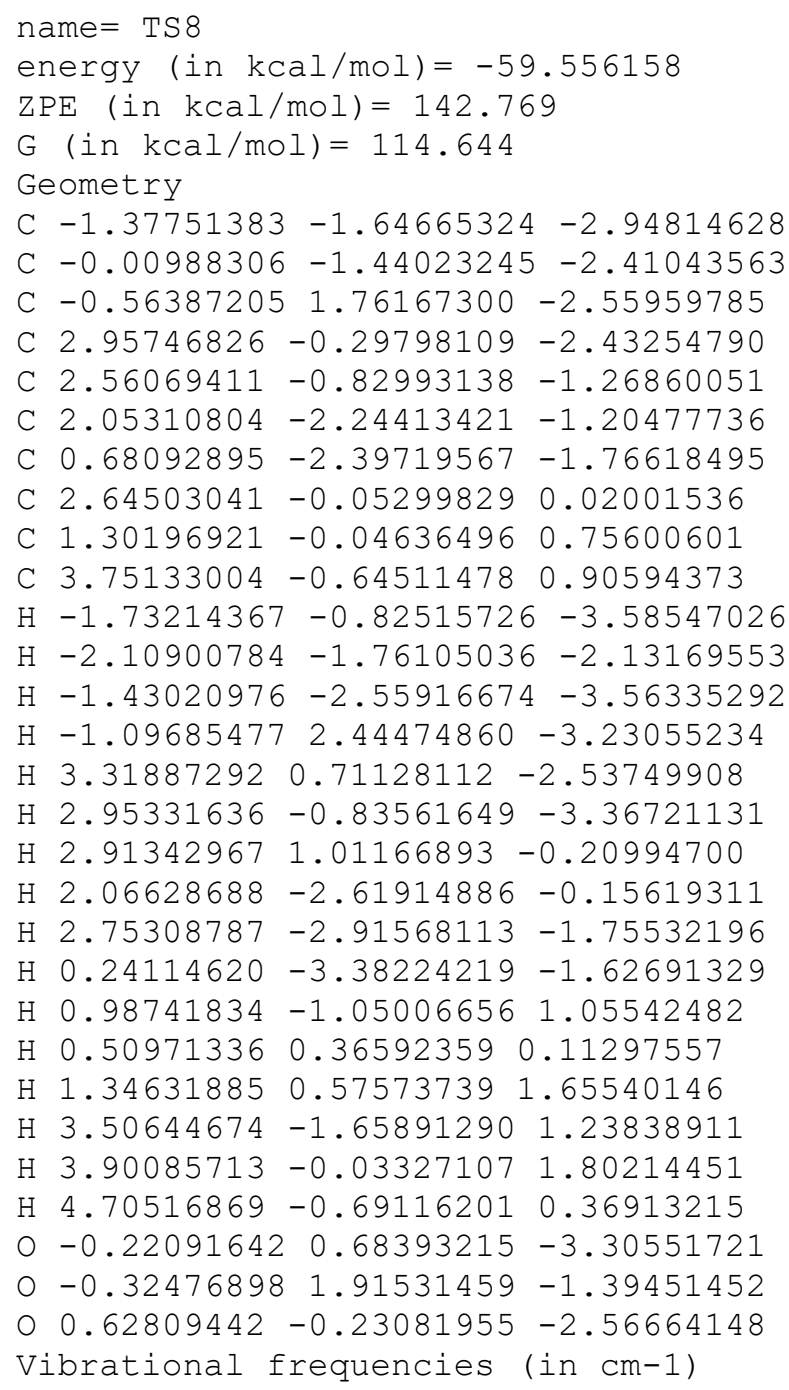


$\mathrm{H} \quad 0.89546772 \quad 1.26787828 \quad 0.85315344$

$\mathrm{H} 2.67979098-1.02963490-0.62701951$

$\mathrm{H} 1.62584493-0.66705639-1.94620889$

$\mathrm{H} \quad 0.73186516-1.927637931 .90019224$

$\mathrm{H}-2.52434572 \quad 0.51980644 \quad 0.48264973$

$\mathrm{H}-2.65171029-1.105377651 .18980442$

$\mathrm{H}-2.09172889-0.92711877-0.44572640$

$\mathrm{H}-1.17759605 \quad 1.11168271 \quad 2.60267558$

$\mathrm{H} \quad 0.31890436 \quad 0.24465143 \quad 2.96753115$

$\mathrm{H}-1.23969728-0.564344523 .16072044$

O $1.75125204-1.28724953-1.18868863$

$\begin{array}{lllll}0 & -0.97568703 & 0.58726725 & -3.16937213\end{array}$

o $-1.39253594-3.097060390 .19597123$

Vibrational frequencies (in cm-1)

$\begin{array}{lllllllllllllll}-1103.1 & 3.3 & 31.4 & 35.6 & 124.6 & 128.6 & 158.1 & 163.5 & 197.0 & 203.0 & 214.6 & 231.2243 .7 & 291.4\end{array}$ $316.1320 .6 \quad 337.9 \quad 382.7 \quad 414.8 \quad 448.2 \quad 498.6 \quad 533.4 \quad 556.1 \quad 625.6 \quad 638.4 \quad 733.1 \quad 769.8 \quad 817.4$ $848.0910 .2964 .2979 .5 \quad 993.4 \quad 1014.1 \quad 1019.9 \quad 1039.3 \quad 1048.51061 .6 \quad 1087.2 \quad 1103.91113 .0$ $1161.4 \quad 1198.2 \quad 1210.0 \quad 1220.4 \quad 1228.8 \quad 1242.8 \quad 1253.2 \quad 1264.51266 .3 \quad 1276.6 \quad 1280.6 \quad 1284.8$

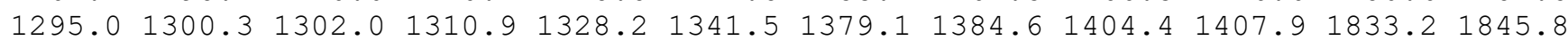
$2500.82639 .82645 .92660 .6 \quad 2667.3 \quad 2675.8 \quad 2678.2 \quad 2680.72681 .72706 .62709 .92735 .8$ $2772.32780 .12781 .2 \quad 2782.4$

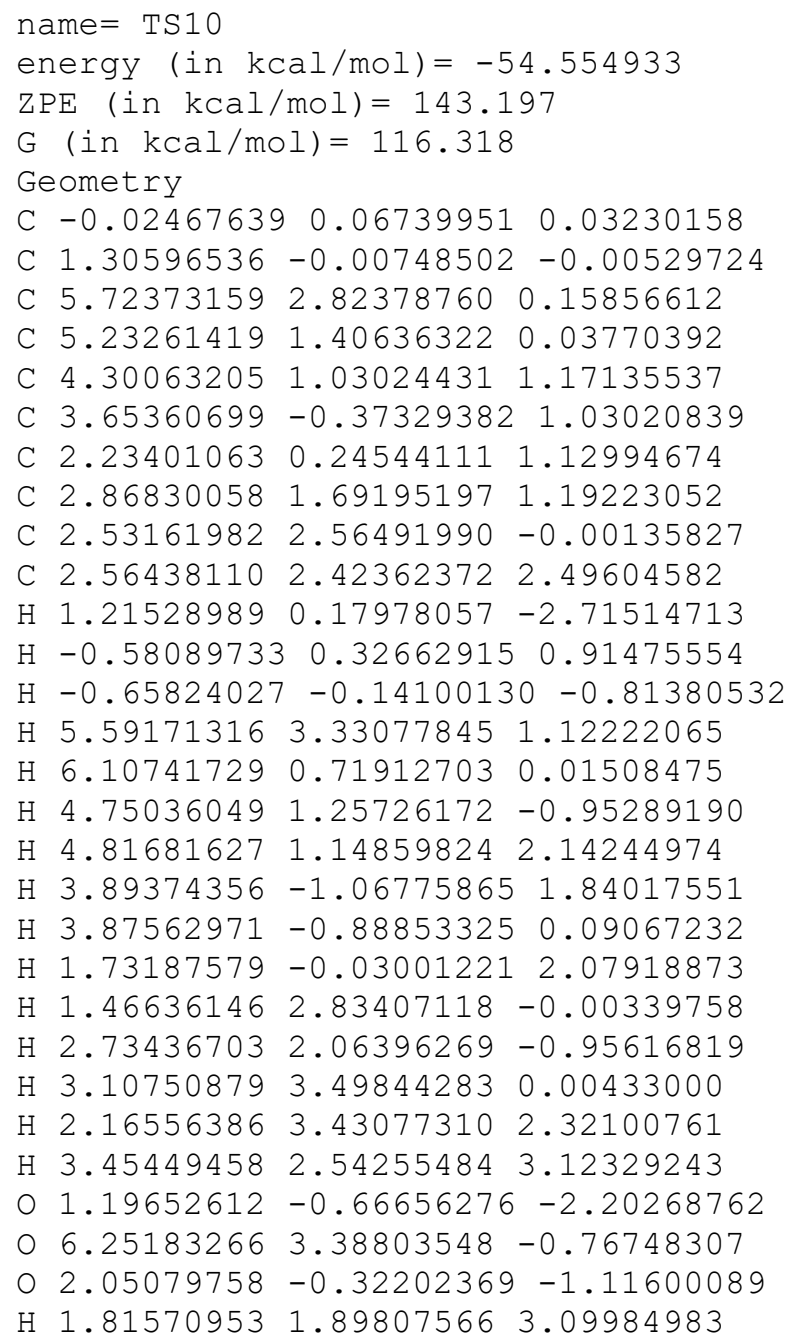




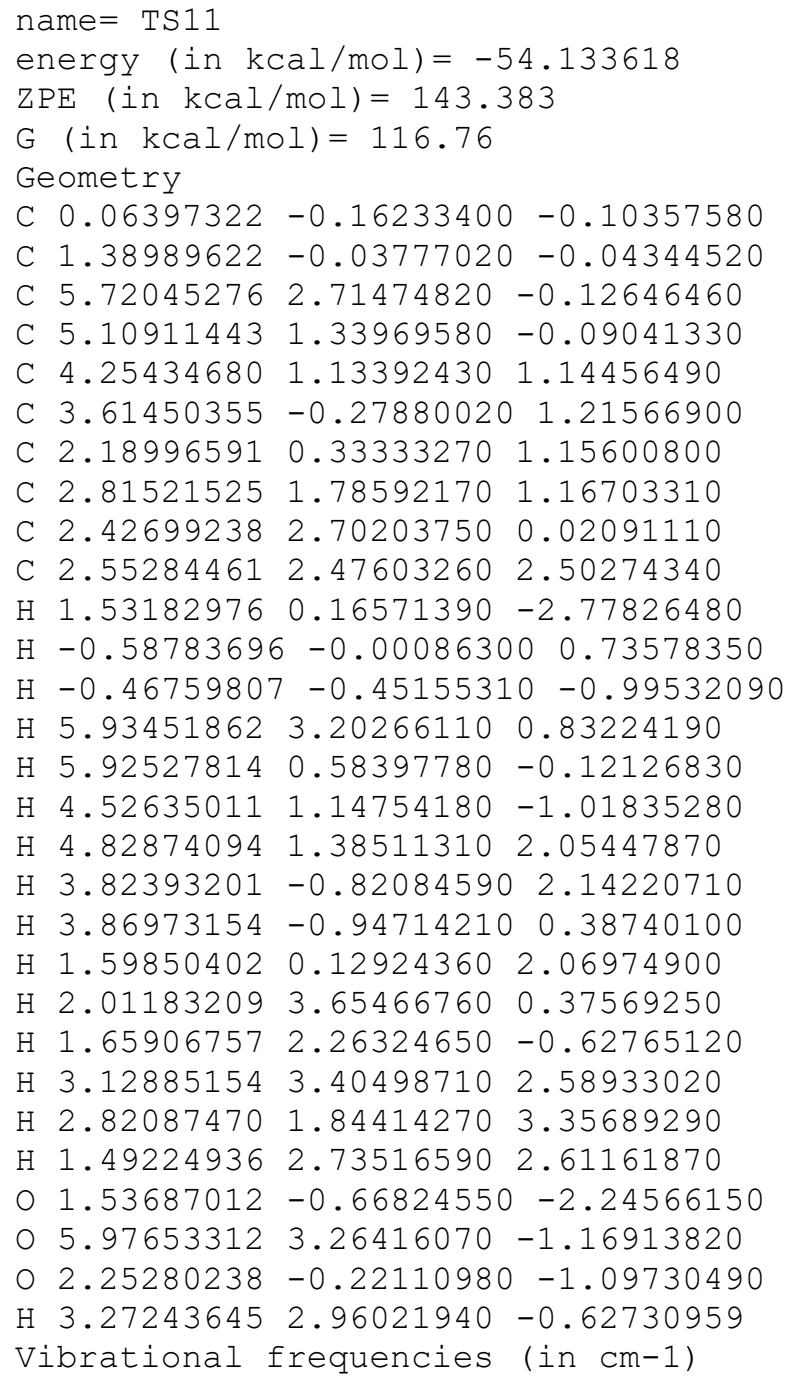

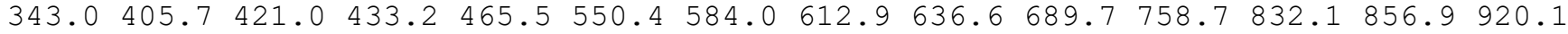
$956.71003 .91010 .8 \quad 1015.1 \quad 1024.8 \quad 1031.4 \quad 1041.8 \quad 1048.81054 .11090 .91116 .71128 .6$

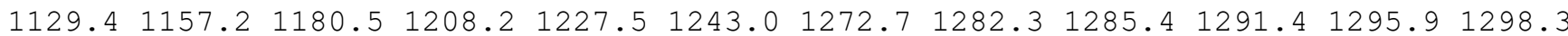

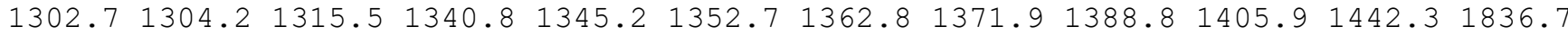
$1877.22646 .5 \quad 2656.5 \quad 2665.6 \quad 2666.7 \quad 2675.8 \quad 2677.32681 .42715 .62720 .5 \quad 2728.72740 .4$ $2763.12780 .6 \quad 2782.4 \quad 2797.8 \quad 2867.9$

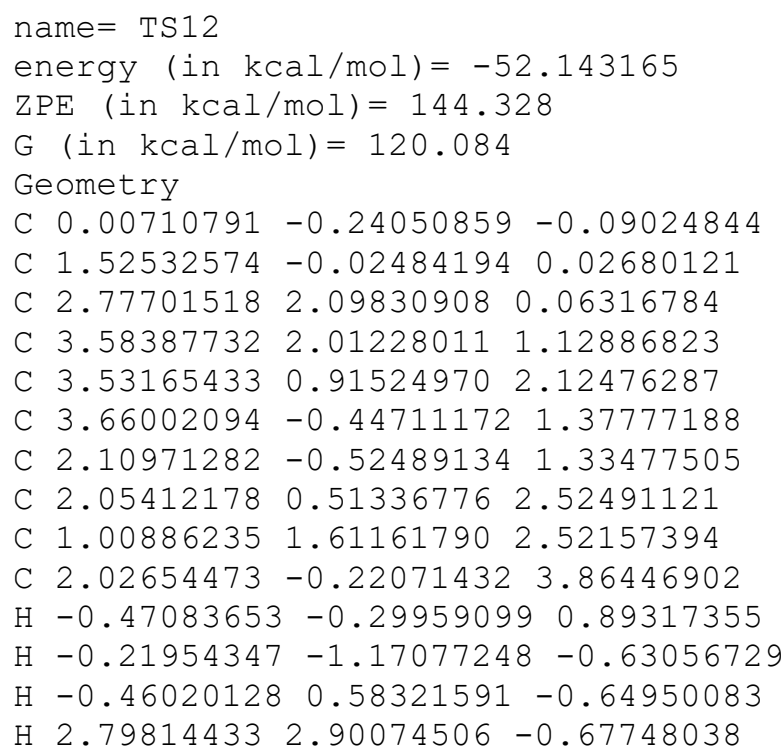


$\mathrm{H} 4.353055662 .76236975 \quad 1.29344720$

H $3.080794610 .34584895-2.09535902$

H $4.20722246 \quad 1.06204087 \quad 2.97442270$

$\mathrm{H} \quad 4.12304039-1.241312921 .96870622$

$\mathrm{H} \quad 4.18125951-0.409536390 .41988391$

H $1.69875919-1.51535461 \quad 1.57950451$

H $1.43594631 \quad 2.58150385 \quad 2.23211399$

$\mathrm{H} \quad 0.554446931 .756849403 .50859086$

H 2.24156143 $0.46559839 \quad 4.69215781$

$\mathrm{H} 2.76700526-1.02692918 \quad 3.91305903$

$\mathrm{H} 1.04291449-0.667801494 .04819823$

$03.29012539-0.39428041-1.47030435$

o $1.70832498 \quad 1.34377362-0.29428409$

O $1.98327011-0.78283514-1.09685496$

H $0.19205657 \quad 1.41417350 \quad 1.82014662$

Vibrational frequencies (in cm-1)

$\begin{array}{llllllllllllllllll}-144.5 & 47.7 & 98.9 & 117.6 & 163.2 & 192.4 & 208.7 & 223.7 & 252.3 & 274.0 & 296.5 & 324.8 & 347.4 & 365.9\end{array}$

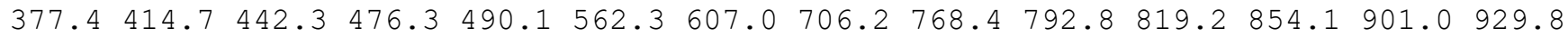

$940.0955 .7957 .91003 .4 \quad 1019.0 \quad 1020.6 \quad 1022.5 \quad 1035.6 \quad 1062.6 \quad 1090.3 \quad 1097.1 \quad 1117.91137 .6$

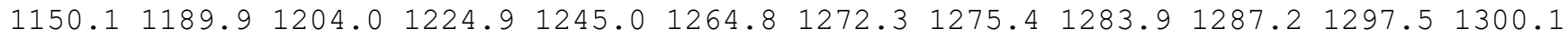

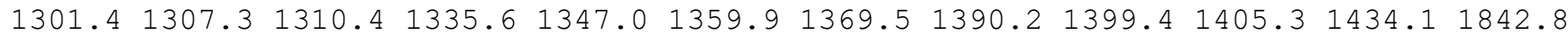
$2663.22666 .12668 .92674 .0 \quad 2677.6 \quad 2679.3 \quad 2687.5 \quad 2720.52731 .72744 .42765 .62767 .9$ $2772.0 \quad 2781.2 \quad 2784.02862 .8$

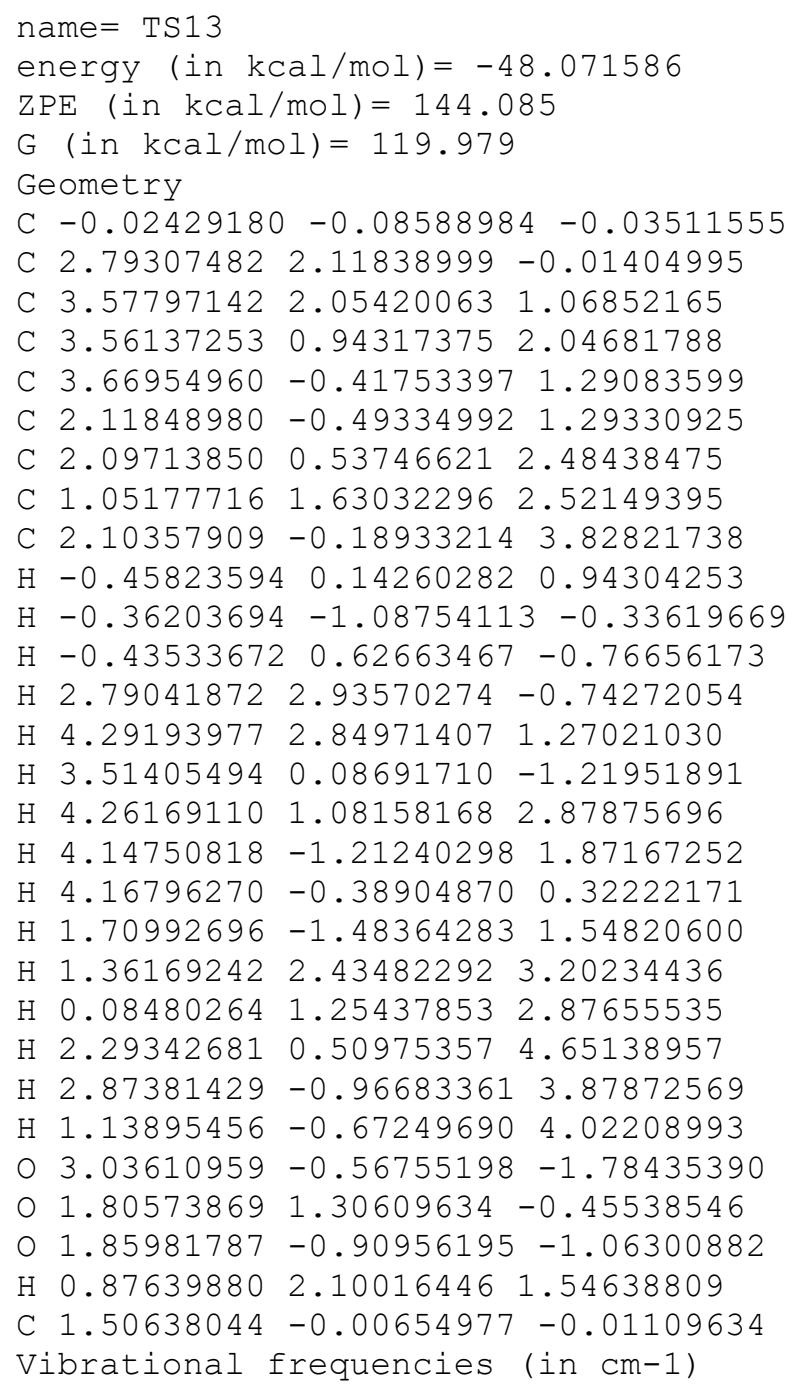


$2664.52669 .12673 .1 \quad 2675.4 \quad 2676.8 \quad 2679.7 \quad 2680.0 \quad 2714.92729 .5 \quad 2742.92760 .12764 .0$ $2775.02781 .22783 .2 \quad 2830.7$

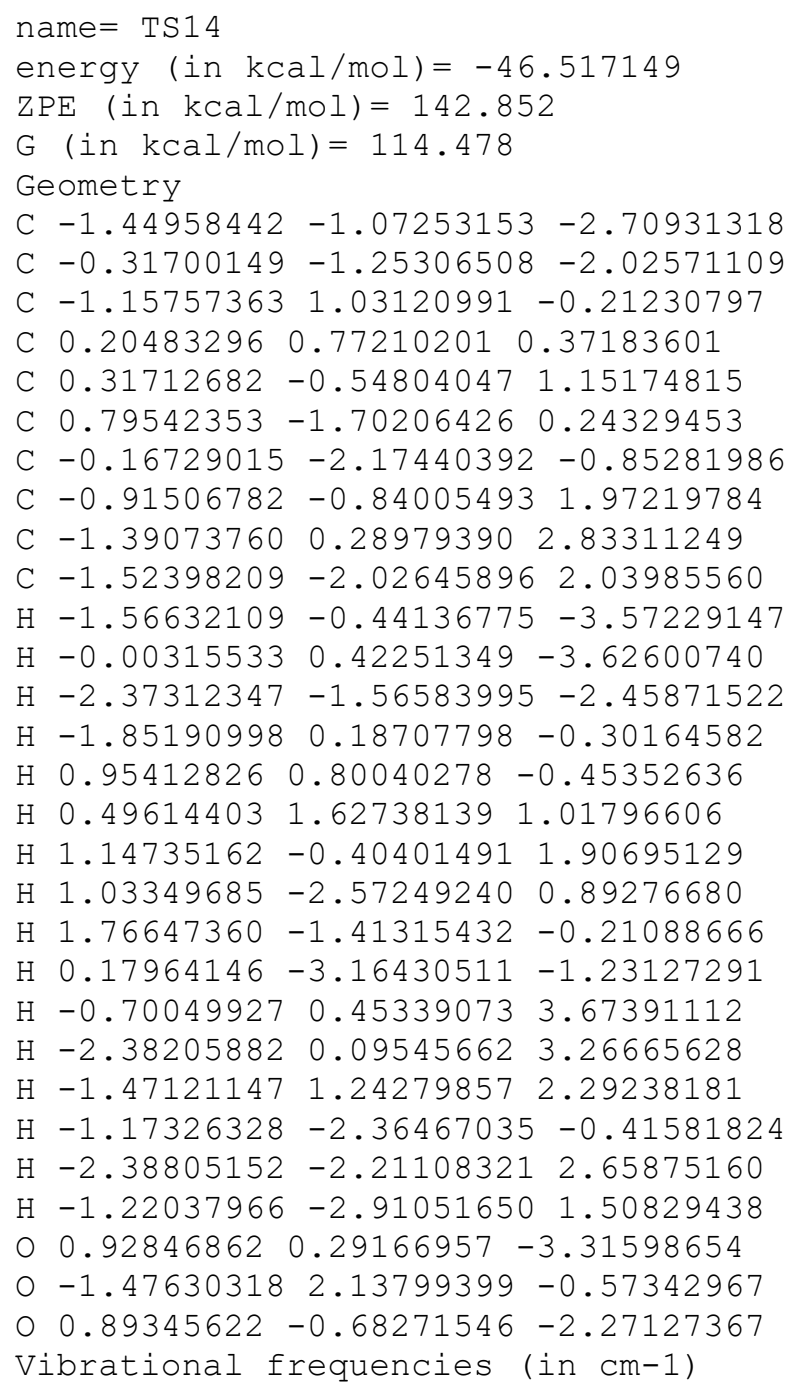

$\begin{array}{lllllllllllllll}-366.9 & 14.6 & 31.0 & 38.2 & 51.6 & 54.6 & 78.3 & 126.2 & 149.5 & 182.8 & 219.8 & 259.7 & 305.0 & 333.3\end{array}$

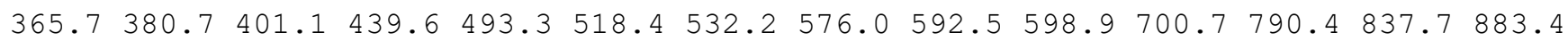
$921.7 \quad 957.6 \quad 982.9993 .1 \quad 1011.6 \quad 1034.9 \quad 1040.9 \quad 1049.1 \quad 1062.7 \quad 1076.4 \quad 1085.8 \quad 1105.6 \quad 1149.9$ $1156.31188 .91218 .3 \quad 1246.1 \quad 1264.6 \quad 1274.1 \quad 1276.4 \quad 1283.4 \quad 1286.01293 .1 \quad 1298.1 \quad 1301.5$

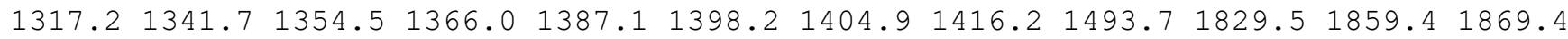
$2638.02640 .92651 .8 \quad 2652.5 \quad 2664.0 \quad 2666.92671 .42716 .62718 .82726 .12731 .72734 .1$ 2777.22790 .22792 .22800 .5

name $=\operatorname{TS} 15$

energy (in $\mathrm{kcal} / \mathrm{mol})=-41.296645$

$\mathrm{ZPE}(\mathrm{in} \mathrm{kcal} / \mathrm{mol})=138.074$

$\mathrm{G}(\mathrm{in} \mathrm{kcal} / \mathrm{mol})=109.883$

Geometry

C $-2.67322677-0.39049650-1.08052341$

C $-1.29216525-0.67650936-1.07587827$

C $0.419851391 .83539849-1.06037050$

C $1.281693071 .13016766-0.04607740$

C $0.86013123-0.23292826 \quad 0.43729219$

C $1.19864558-0.25491731-3.44048480$

C $-0.32803938-1.05437386-0.04441521$

C $-0.31897624-0.47945315 \quad 1.37074867$

C $-1.24902492 \quad 0.63692599 \quad 1.75181845$

C $-0.11762561-1.46535204 \quad 2.49915502$

$\mathrm{H}-3.39506579-1.10078115-1.42333948$

$\mathrm{H}-3.10005988 \quad 0.36236925-0.45751535$ 
$\mathrm{H}-2.13705478-0.22972835-2.59108137$

$\mathrm{H} \quad 0.932884412 .54410908-1.72693172$

$\mathrm{H} 1.38520354 \quad 1.80402008 \quad 0.83726800$

$\mathrm{H} 2.30949310 \quad 1.04508043-0.48098711$

H $1.76370301-0.837542050 .64396129$

H $0.442402150 .05866078 \quad-4.16262372$

$\mathrm{H} 1.33714053-1.32281818-3.26644869$

$\mathrm{H}-0.13631336-2.14436052-0.13845507$

$\mathrm{H}-2.23363257 \quad 0.248530632 .04787128$

$\mathrm{H}-1.43315531 \quad 1.33573671 \quad 0.92083456$

$\mathrm{H}-0.85096878 \quad 1.221878852 .58937506$

$\mathrm{H} \quad 0.27835391-0.96432547 \quad 3.39236966$

H $0.57883964 \quad-2.27166566 \quad 2.24340338$

$\mathrm{H}-1.06986515-1.93550164 \quad 2.78129748$

$\begin{array}{lllll}0 & 1.87465152 & 0.56231327 & -2.85689398\end{array}$

$\begin{array}{llll}0 & -0.77488797 & 1.68459825 & -1.14077178\end{array}$

o $-0.93385012-0.69424131-2.32422147$

Vibrational frequencies (in $\mathrm{cm}-1$ )

$\begin{array}{lllllllllllllllll}-1911.7 & 24.0 & 50.3 & 63.4 & 77.1 & 91.2 & 111.6 & 115.1 & 135.1 & 149.0 & 163.7 & 169.1 & 190.4 & 203.8\end{array}$ $\begin{array}{lllllllllllllllllll}212.9 & 230.1 & 278.7 & 303.6 & 342.9 & 381.5 & 419.7 & 464.5 & 521.7 & 546.9 & 630.2 & 713.3 & 771.8 & 784.2\end{array}$ $838.6856 .2897 .2976 .8 \quad 995.5 \quad 1001.6 \quad 1010.3 \quad 1014.7 \quad 1030.91043 .5 \quad 1050.2 \quad 1095.3 \quad 1101.3$

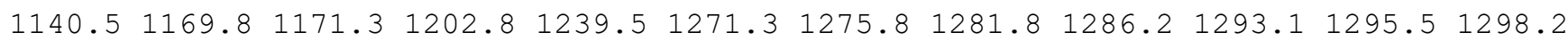
$1306.91352 .4 \quad 1361.2 \quad 1377.0 \quad 1395.9 \quad 1400.5 \quad 1418.8 \quad 1493.4 \quad 1536.7 \quad 1605.1 \quad 1817.2 \quad 1836.8$ $2134.32628 .92639 .6 \quad 2652.92660 .1 \quad 2673.7 \quad 2676.12676 .52680 .22716 .12721 .32731 .3$ $2736.52780 .02782 .3 \quad 2809.2$

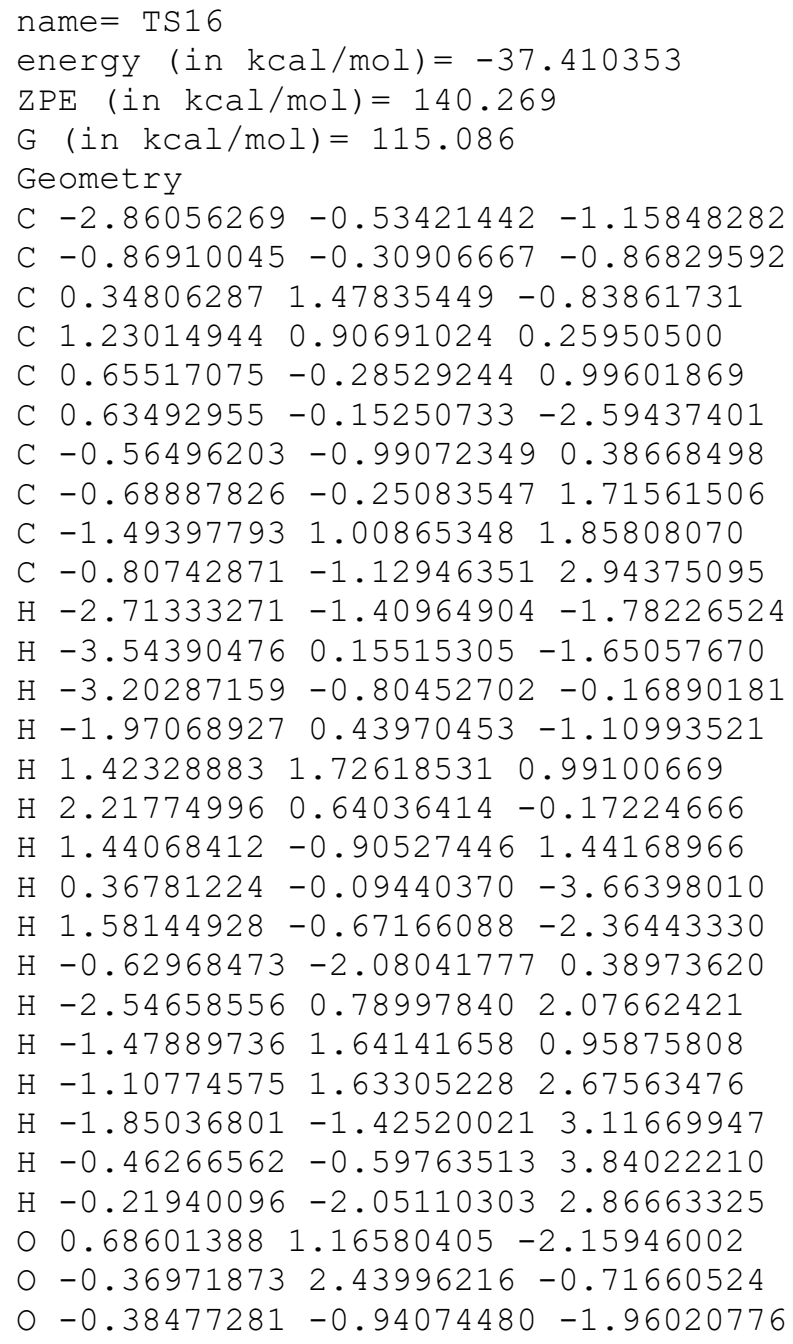


$\begin{array}{lllllllllllllll}1144.7 & 1176.1 & 1189.5 & 1211.9 & 1228.0 & 1250.4 & 1272.5 & 1276.7 & 1282.5 & 1285.5 & 1292.9 & 1294.1\end{array}$ $1295.01297 .7 \quad 1306.41320 .2 \quad 1325.7 \quad 1346.1 \quad 1373.0 \quad 1399.4 \quad 1408.11418 .01496 .91811 .9$ $1902.92635 .02655 .62662 .12663 .12674 .6 \quad 2676.32679 .22681 .72683 .02732 .92746 .0$ $2757.32763 .3 \quad 2781.5 \quad 2784.5$

name $=$ TS17

energy (in $\mathrm{kcal} / \mathrm{mol})=-36.013992$

$\mathrm{ZPE}($ in $\mathrm{kcal} / \mathrm{mol})=139.321$

$\mathrm{G}($ in $\mathrm{kcal} / \mathrm{mol})=112.737$

Geometry

C $-1.75793240-1.95683090-1.59553319$

C $-0.55950473-1.50444673-1.18047335$

C $1.886755851 .45086449-0.52943020$

C 2.034116831 .049894550 .91970805

C $1.25305656-0.09281840 \quad 1.38816228$

C $1.49152654-1.821825060 .24702741$

C $-0.02855799-1.62651066 \quad 0.20574593$

C $-0.19431559-0.33221243 \quad 1.08170695$

C $-0.93448708 \quad 0.79780694 \quad 0.37572727$

C $-0.92217677-0.652991962 .39550888$

$\mathrm{H}-0.08830264-0.78693559-2.93110008$

$\mathrm{H}-2.64940605-2.38591468-3.27418094$

$\mathrm{H}-2.49741926-2.46884775-1.00926304$

H $1.84431586 \quad 0.64989224 \quad-1.27821031$

H $1.74893334 \quad 1.91693012 \quad 1.57667309$

H $3.11877286 \quad 0.91166362 \quad 1.13582450$

H $1.93481266-1.29664731 \quad 1.38965305$

H $1.81126836-2.829917850 .49363657$

H $2.02827806-1.47254714-0.62851416$

$\mathrm{H}-0.53670648-2.48322774 \quad 0.71071640$

$\mathrm{H}-1.99393037 \quad 0.55373863 \quad 0.23060050$

$\mathrm{H}-0.526143651 .01457497 \quad-0.61987995$

$\mathrm{H}-0.88755608 \quad 1.72667608 \quad 0.95639847$

$\mathrm{H}-0.41154097-1.44516690 \quad 2.95421800$

$\mathrm{H}-1.95477839-0.96698664 \quad 2.21884693$

$\mathrm{H}-0.94746453 \quad 0.22800454 \quad 3.04825191$

o $-2.10110216-1.66097885-2.89739423$

$\begin{array}{llllll}0 & 1.83957453 & 2.61165640 & -0.85281324\end{array}$

O $0.30143825-0.86028398-2.01711253$

Vibrational frequencies (in $\mathrm{cm}-1$ )

$\begin{array}{llllllllllllllll}-1404.8 & 32.8 & 45.7 & 56.0 & 93.2 & 124.4 & 149.3 & 164.2 & 177.7 & 197.0 & 228.2 & 239.5 & 253.2 & 282.4\end{array}$ $303.2 \quad 339.3 \quad 367.4 \quad 393.6 \quad 412.4 \quad 436.7 \quad 462.9 \quad 538.9 \quad 575.5 \quad 604.6 \quad 651.4 \quad 697.2 \quad 743.1 \quad 802.7$ $836.0 \quad 858.7 \quad 898.2 \quad 986.6 \quad 995.1 \quad 1002.1 \quad 1020.4 \quad 1022.4 \quad 1063.3 \quad 1081.4 \quad 1124.4 \quad 1140.4 \quad 1156.2$ $\begin{array}{lllllllllllll}1172.4 & 1183.0 & 1200.5 & 1218.8 & 1226.6 & 1228.8 & 1235.7 & 1258.8 & 1269.4 & 1279.9 & 1289.0 & 1298.7\end{array}$ $1302.81308 .1 \quad 1315.1 \quad 1321.4 \quad 1352.4 \quad 1374.3 \quad 1387.7 \quad 1401.2 \quad 1420.5 \quad 1455.8 \quad 1831.7 \quad 1845.8$ $1986.02635 .7 \quad 2650.2 \quad 2665.8 \quad 2675.92678 .4 \quad 2679.12683 .82704 .02728 .62750 .52752 .4$ $2763.4 \quad 2780.0 \quad 2782.8 \quad 2803.4$

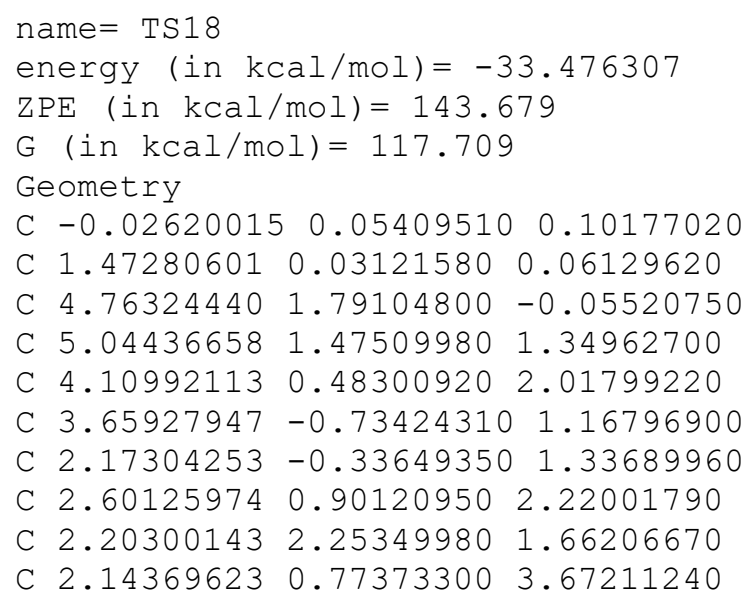


$\mathrm{H}-0.44266753 \quad 0.57329530-0.77535280$

$\mathrm{H}-0.40654920 \quad 0.55506990 \quad 1.00072870$

$\mathrm{H}-0.43330413-0.96673700 \quad 0.09205700$

$\mathrm{H} \quad 4.07785005 \quad 1.22643890-0.68168620$

H $5.073271662 .43130890 \quad 1.93927750$

H $6.10195731 \quad 1.10992500 \quad 1.42441640$

H $4.56471588 \quad 0.16404150 \quad 2.97714660$

H $3.91538613-1.70446260 \quad 1.60255420$

$\mathrm{H} \quad 4.01319920-0.73883250 \quad 0.13089660$

H $1.58997256-1.08397490 \quad 1.90007660$

H $1.12571166 \quad 2.43146160 \quad 1.76524570$

H 2.44425260 2.36330660 0.59704760

$\mathrm{H} 2.72103143 \quad 3.06628910 \quad 2.18990480$

$\mathrm{H} 1.44701294 \quad-0.05819360 \quad 3.81822730$

$\mathrm{H} 1.63115643 \quad 1.67714820 \quad 4.02523420$

$0 \quad 6.13657705 \quad 3.49835410-0.01423610$

○ $5.32284718 \quad 2.78334480 \quad-0.67142720$

$\begin{array}{lllll}0 & 2.07504783 & 0.29126460 & -0.95294160\end{array}$

H 2.98417742 $0.60913028 \quad 4.35676639$

Vibrational frequencies (in $\mathrm{cm}-1$ )

$\begin{array}{llllllllllllllll}-163.2 & 32.7 & 57.5 & 68.9 & 109.0 & 118.6 & 153.6 & 175.1 & 204.9 & 228.4 & 246.5 & 279.1 & 309.3 & 342.8\end{array}$ $380.6 \quad 408.4 \quad 434.4 \quad 450.8 \quad 558.2 \quad 578.9 \quad 601.7 \quad 690.3 \quad 731.9 \quad 764.2 \quad 822.3 \quad 832.1910 .1934 .2$ $987.0 \quad 997.4 \quad 1019.5 \quad 1024.6 \quad 1032.8 \quad 1041.5 \quad 1050.3 \quad 1054.3 \quad 1088.8 \quad 1105.01126 .01150 .6$ $\begin{array}{lllllllllllll}1166.9 & 1191.7 & 1206.9 & 1213.2 & 1237.0 & 1243.8 & 1252.1 & 1261.0 & 1270.9 & 1273.4 & 1285.6 & 1287.9\end{array}$

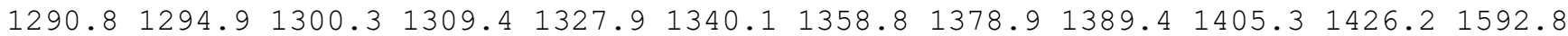
$1846.42619 .12665 .3 \quad 2667.9 \quad 2669.0 \quad 2672.1 \quad 2672.2 \quad 2673.62675 .5 \quad 2678.92710 .72718 .6$ $2728.12761 .12779 .12779 .5 \quad 2781.1$

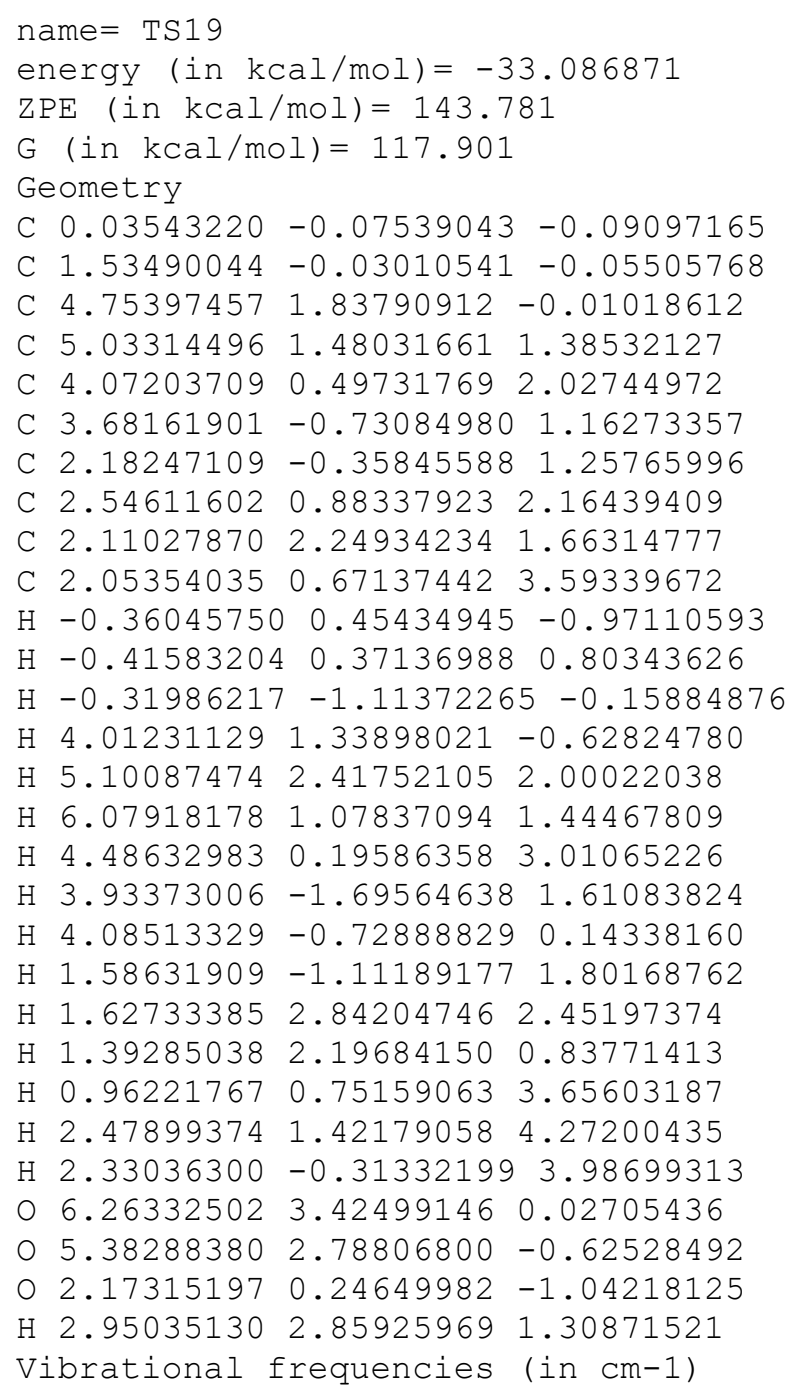


$\begin{array}{llllllllllllllllll}-165.6 & 31.9 & 58.8 & 79.6 & 119.1 & 121.9 & 151.1 & 178.5 & 205.3 & 217.9 & 230.6 & 277.3 & 314.6 & 342.9\end{array}$ $\begin{array}{llllllllllllllll}378.4 & 420.9 & 433.7 & 456.8 & 558.9 & 577.5 & 603.6 & 689.4 & 730.7 & 762.8 & 816.1 & 826.8 & 913.8 & 932.0\end{array}$ $985.91005 .91019 .5 \quad 1027.9 \quad 1035.8 \quad 1042.6 \quad 1051.5 \quad 1058.1 \quad 1089.4 \quad 1108.01129 .4 \quad 1152.6$

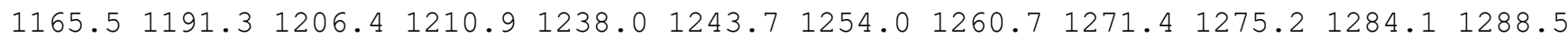

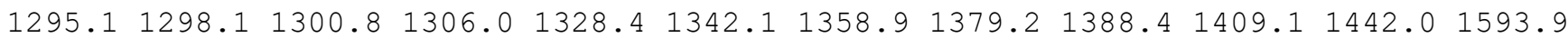
$1848.12618 .6 \quad 2662.8 \quad 2666.7 \quad 2668.3 \quad 2673.6 \quad 2674.3 \quad 2675.6 \quad 2677.8 \quad 2679.3 \quad 2711.5 \quad 2719.0$ $2726.8 \quad 2761.12779 .4 \quad 2781.1 \quad 2782.8$

name $=$ TS20

energy (in $\mathrm{kcal} / \mathrm{mol})=-32.488559$

$\operatorname{ZPE}($ in $\mathrm{kcal} / \mathrm{mol})=143.466$

$\mathrm{G}($ in $\mathrm{kcal} / \mathrm{mol})=116.693$

Geometry

C $-0.03591435 \quad 0.03794887 \quad 0.04257116$

C $1.46471370 \quad 0.00037403 \quad-0.00712028$

C 5.159082893 .794423550 .09806213

$\begin{array}{llll}\text { C } 4.96006047 & 2.34280445 & 0.12152130\end{array}$

C $3.997136531 .88970994 \quad 1.20079656$

C $3.70607845 \quad 0.36559093 \quad 1.20575579$

C $2.18303528 \quad 0.63373166 \quad 1.14958195$

C 2.459598812 .178712610 .99159975

C $2.08954667 \quad 2.72769216-0.37451619$

C $1.84379220 \quad 3.02372650 \quad 2.10173698$

$\mathrm{H}-0.47063577-0.10708578-0.95810235$

$\mathrm{H}-0.416503910 .984540850 .44289808$

$\mathrm{H}-0.42119191-0.77278508 \quad 0.67680089$

$\mathrm{H} \quad 4.64694312 \quad 4.47870914 \quad 0.75559222$

H $5.95044903 \quad 1.830119520 .24331796$

H $4.64025630 \quad 1.98894054 \quad-0.89378292$

$\mathrm{H} \quad 4.34361044 \quad 2.24424076 \quad 2.18928588$

H $4.04632310-0.165753292 .09657003$

$\mathrm{H} \quad 4.08970363-0.17233284 \quad 0.32702696$

$\mathrm{H} \quad 1.67490095 \quad 0.38810215 \quad 2.09801218$

$\mathrm{H} 1.00225648 \quad 2.78047665 \quad-0.50724641$

H $2.482004552 .10339547-1.19143622$

$\mathrm{H} \quad 2.48727832 \quad 3.73838254-0.52259903$

$\mathrm{H} \quad 1.252863393 .859182391 .70716028$

$\mathrm{H} 2.60442582 \quad 3.46046185 \quad 2.75853094$

$06.616669153 .65376595-1.53121914$

O $5.97431983 \quad 4.37945096-0.72492510$

○ $2.04998528-0.52533549-0.92046993$

H $1.170623702 .44130828 \quad 2.74054327$

Vibrational frequencies (in $\mathrm{cm}-1$ )

$\begin{array}{lllllllllllllllll}-149.9 & 29.2 & 39.4 & 53.2 & 77.0 & 113.9 & 126.6 & 163.9 & 190.6 & 237.9 & 258.8 & 277.1 & 303.2 & 341.1\end{array}$ $\begin{array}{lllllllllllllllll}396.0 & 402.9 & 433.0 & 454.1 & 485.5 & 575.7 & 594.8 & 676.4 & 728.9 & 765.9 & 812.2 & 820.4 & 917.8 & 958.3\end{array}$ $990.0 \quad 996.2 \quad 1017.0 \quad 1023.7 \quad 1035.4 \quad 1045.0 \quad 1049.8 \quad 1057.5 \quad 1093.3 \quad 1120.8 \quad 1132.4 \quad 1149.4$

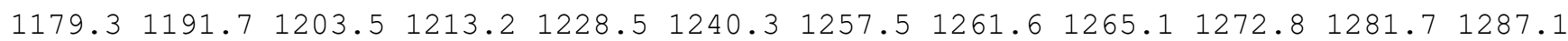

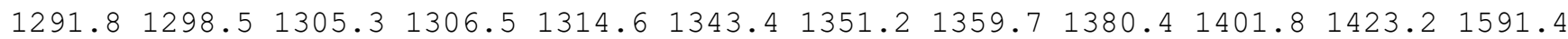
$1854.92614 .12662 .0 \quad 2668.32671 .6 \quad 2672.3 \quad 2676.2 \quad 2677.82677 .92704 .62719 .92727 .4$ $2734.4 \quad 2759.8 \quad 2778.2 \quad 2780.4 \quad 2781.6$

name $=$ TS21

energy (in $\mathrm{kcal} / \mathrm{mol})=-31.53964$

$\mathrm{ZPE}($ in $\mathrm{kcal} / \mathrm{mol})=143.597$

$\mathrm{G}($ in $\mathrm{kcal} / \mathrm{mol})=116.932$

Geometry

C $0.05871981-0.09404983-0.05359884$

C $1.56063451-0.07212534-0.01108884$

C $5.15586230 \quad 3.78753596 \quad-0.05397324$

C $4.945679962 .34338874 \quad 0.07999045$

C 3.959221651 .980587341 .17236232

C $3.70284390 \quad 0.456013451 .30101806$

C $2.17975404 \quad 0.67027744 \quad 1.13783916$ 
C $2.41619265 \quad 2.21549898 \quad 0.92260846$

C $2.007621272 .77823809-0.42945742$

C $1.78683723 \quad 3.032947192 .04606487$

$\mathrm{H}-0.30787444-0.32138049-1.06597313$

$\mathrm{H}-0.38245756 \quad 0.85859348 \quad 0.26125483$

$\mathrm{H}-0.33323250-0.87870540 \quad 0.60898070$

H $4.59841603 \quad 4.52552035 \quad 0.50090462$

H $5.93116112 \quad 1.838405550 .26533788$

$\mathrm{H} 4.646786521 .90443348-0.90809533$

H 4.279192362 .432130962 .12996148

H $4.00422035 \quad 0.01819394 \quad 2.25473682$

$\mathrm{H} 4.15034835-0.14882538 \quad 0.49910543$

$\mathrm{H} \quad 1.61751231 \quad 0.46201139 \quad 2.06593718$

$\mathrm{H} \quad 1.37821665 \quad 3.67154404-0.33240792$

$\mathrm{H} 1.437710182 .06670578-1.03800546$

$\mathrm{H} 2.10824880 \quad 4.08010498 \quad 2.00390601$

H 2.05650119 2.65112340 3.03765372

$\mathrm{H} 0.69234282 \quad 3.02131529 \quad 1.98188816$

$06.736806053 .50999296-1.54489092$

O $6.03784774 \quad 4.30051732-0.85586321$

O $2.21939389-0.64094767-0.84477314$

H 2.86741587 3.07031871 -1.04402345

Vibrational frequencies (in $\mathrm{cm}-1$ )

$\begin{array}{llllllllllllllll}-188.8 & 27.8 & 36.5 & 56.9 & 84.2 & 121.0 & 126.8 & 170.0 & 191.0 & 215.0 & 245.1 & 282.1 & 316.1 & 341.6\end{array}$ $400.6 \quad 415.0 \quad 434.4 \quad 459.9 \quad 488.8 \quad 573.5 \quad 594.7 \quad 679.5 \quad 724.4 \quad 769.1 \quad 810.1 \quad 815.8 \quad 917.6 \quad 960.5$ $990.0 \quad 1002.9 \quad 1019.5 \quad 1028.5 \quad 1037.5 \quad 1045.6 \quad 1053.1 \quad 1062.5 \quad 1094.8 \quad 1121.11134 .21149 .9$ $\begin{array}{llllllllllllll}1179.3 & 1193.5 & 1201.7 & 1213.5 & 1226.7 & 1242.5 & 1256.5 & 1261.5 & 1263.8 & 1274.3 & 1284.6 & 1288.1\end{array}$ $1295.81297 .2 \quad 1300.3 \quad 1309.0 \quad 1318.6 \quad 1343.3 \quad 1352.4 \quad 1361.91380 .6 \quad 1404.7 \quad 1444.11591 .9$ $1856.0 \quad 2612.4 \quad 2663.92667 .92668 .7 \quad 2675.3 \quad 2677.0 \quad 2677.2 \quad 2678.7 \quad 2703.6 \quad 2719.8 \quad 2725.7$ $2734.0 \quad 2759.4 \quad 2780.3 \quad 2781.7 \quad 2783.2$

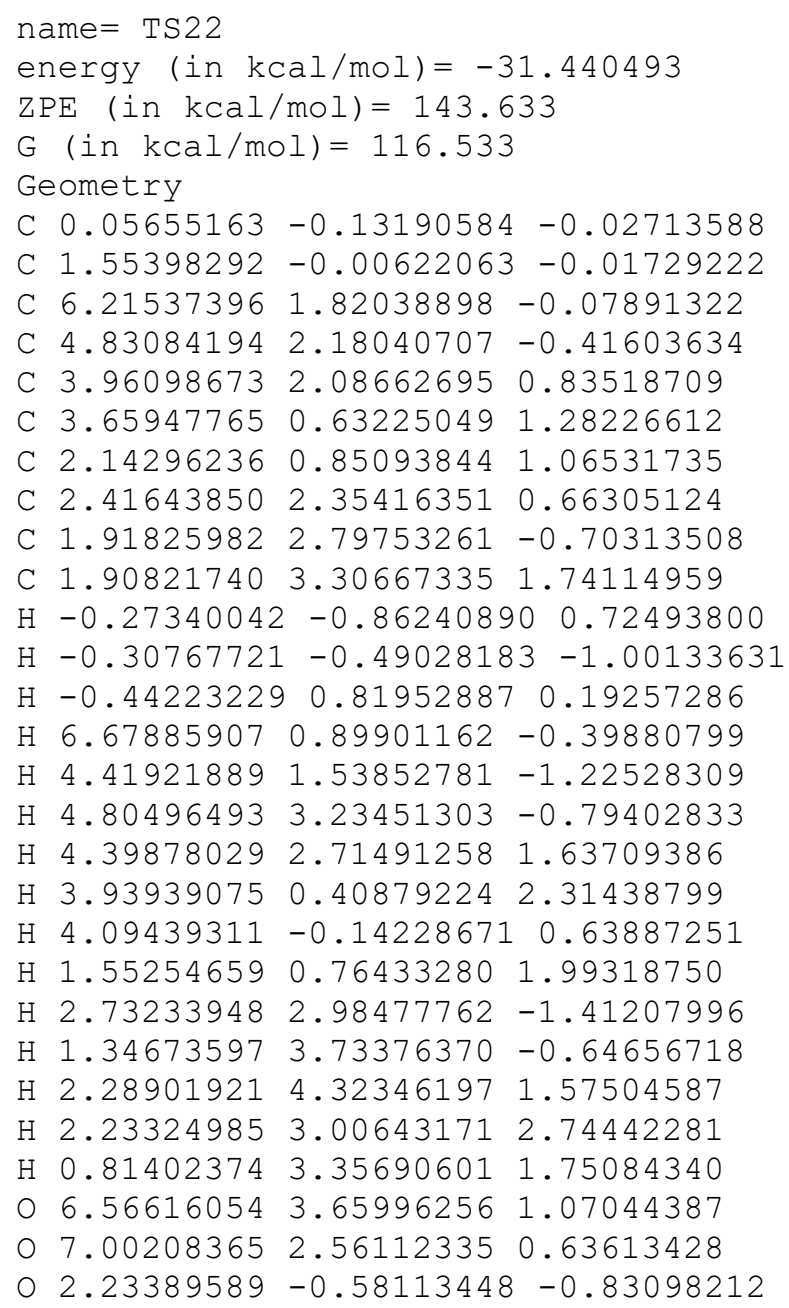


$\mathrm{H} 1.260715922 .06315746-1.18015301$

Vibrational frequencies (in $\mathrm{cm}-1$ )

$\begin{array}{lllllllllllllllll}-216.1 & 16.1 & 37.0 & 50.0 & 66.6 & 98.7 & 151.3 & 161.7 & 203.1 & 206.7 & 230.7 & 287.5 & 315.7 & 334.1\end{array}$

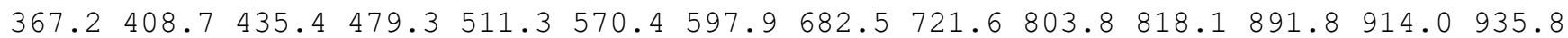
$953.8 \quad 1003.91016 .5 \quad 1025.9 \quad 1035.3 \quad 1040.9 \quad 1051.8 \quad 1071.5 \quad 1088.3 \quad 1107.5 \quad 1128.1 \quad 1140.6$

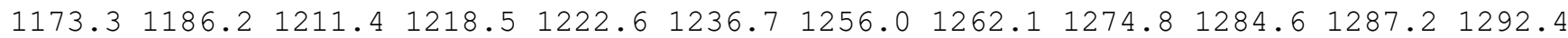

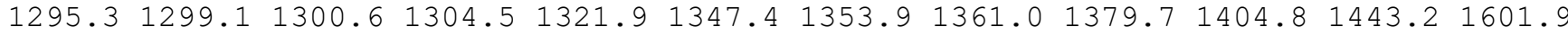
$1852.22644 .12666 .2 \quad 2668.4 \quad 2669.0 \quad 2673.0 \quad 2675.62679 .12682 .5 \quad 2701.92720 .82725 .7$ $2728.22761 .6 \quad 2779.7 \quad 2780.92783 .1$

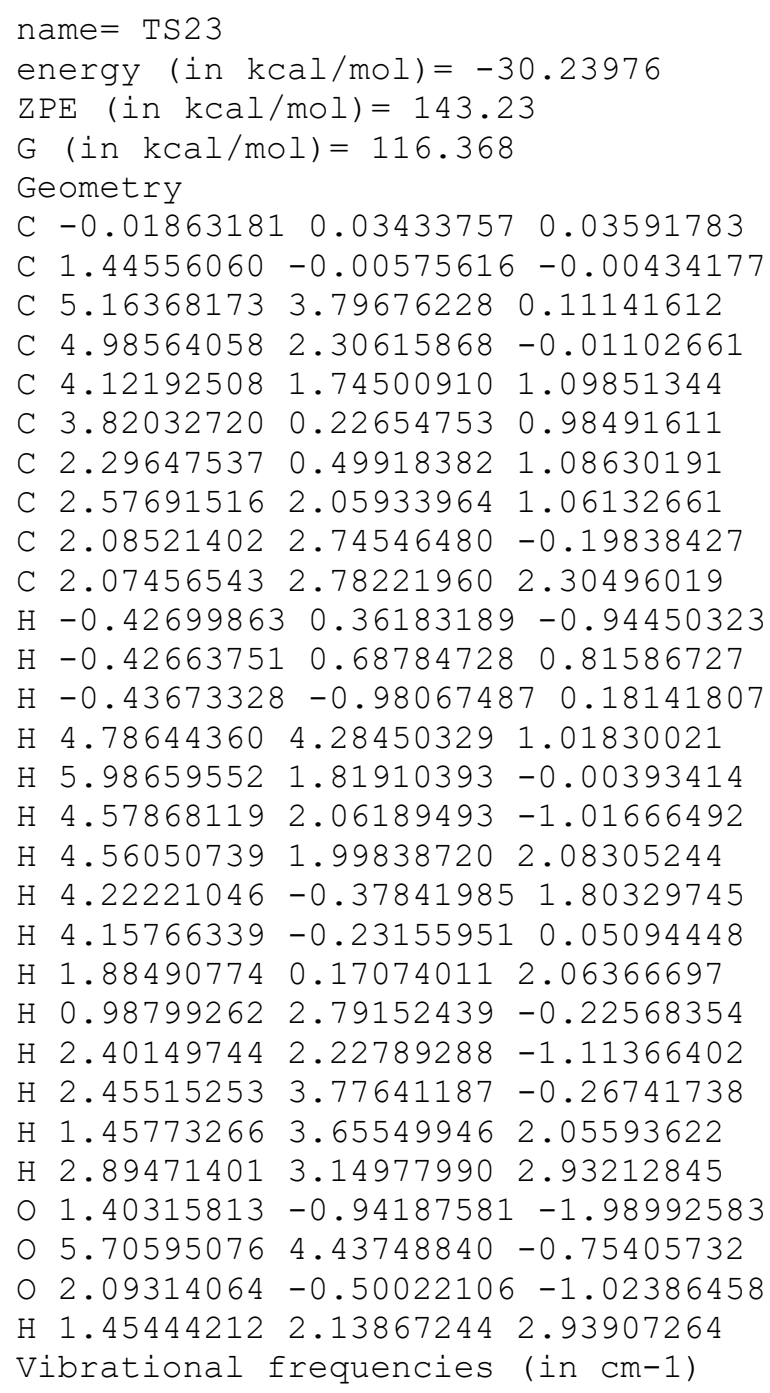


$\begin{array}{lllll}\text { C } 2.00080810 & 2.34194064 & -0.53242441\end{array}$

C $0.80020382-0.705106460 .16030438$

C $1.76892958-1.13804808 \quad 0.74975382$

C $-0.03069450 \quad 1.84030625 \quad 1.68051057$

C $2.91061133-1.630882491 .44891068$

$\mathrm{H}-2.76176735-0.62673195-1.14768408$

$\mathrm{H}-2.36937611-2.37058737-1.00198076$

$\mathrm{H}-2.30452484-1.56098927-2.61593760$

$\mathrm{H}-1.758341021 .17087252-1.17390618$

$\mathrm{H}-1.36469621 \quad 2.85005075 \quad 0.33117562$

$\mathrm{H} \quad 0.00153210 \quad 1.62331411-2.62221823$

$\mathrm{H} \quad 0.68855872 \quad 4.00673229-0.03337108$

$\mathrm{H} 2.49003852 \quad 2.35318862 \quad 0.44594517$

$\mathrm{H} 2.64981384 \quad 2.86880787-1.24683857$

H $1.94755540 \quad 1.29789478-0.87029490$

$\mathrm{H}-0.79999682 \quad 1.25685370 \quad 2.19997595$

$\mathrm{H} \quad 0.89049760 \quad 1.24662220 \quad 1.70068474$

$\mathrm{H} \quad 0.14975436 \quad 2.74962634 \quad 2.26319247$

$\mathrm{H} 3.85600943-1.25182214 \quad 1.01504606$

H $2.96560307 \quad-2.73635688 \quad 1.41914137$

H $2.90377836-1.337480832 .51539109$

$0.14251152 \quad 3.31304805-1.78648034$

$0.617295382 .38804925-2.74828053$

o $-0.74505255-1.23899445-1.40735897$

Vibrational frequencies (in $\mathrm{cm}-1$ )

$\begin{array}{llllllllllllllll}-137.5 & 15.2 & 23.8 & 34.0 & 43.1 & 80.9 & 88.3 & 140.4 & 160.9 & 174.0 & 193.4 & 202.2 & 252.7 & 265.8\end{array}$ $290.6 \quad 315.2 \quad 328.8 \quad 347.6 \quad 379.8 \quad 413.9 \quad 430.7 \quad 498.8 \quad 575.4 \quad 635.0 \quad 663.9 \quad 702.6 \quad 756.9 \quad 851.3$

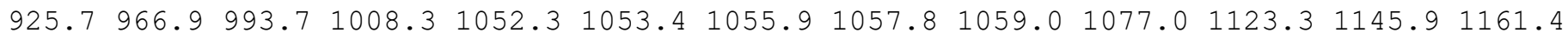

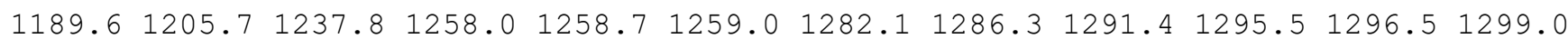

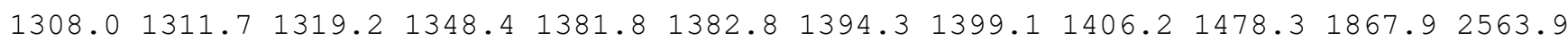
$2649.02653 .7 \quad 2657.3 \quad 2659.4 \quad 2660.5 \quad 2667.0 \quad 2676.3 \quad 2680.52684 .5 \quad 2690.72739 .6 \quad 2744.5$ $2775.8 \quad 2781.7 \quad 2782.3 \quad 2848.0$

name $=$ TS25

energy (in $\mathrm{kcal} / \mathrm{mol})=-21.906154$

$\mathrm{ZPE}($ in $\mathrm{kcal} / \mathrm{mol})=143.663$

G $($ in $\mathrm{kcal} / \mathrm{mol})=117.808$

Geometry

C $-2.36816534-0.79612775-0.95030157$

C $-0.97266838-1.28016586-0.87236002$

C $0.14735131 \quad 1.55254796 \quad-0.69378755$

C $1.06899257 \quad 1.51931381 \quad 0.50087812$

C $1.13195094 \quad 0.13633719 \quad 1.14285410$

C $1.33159676-0.99646560 \quad 0.10569155$

C $-0.14371845-1.47501268 \quad 0.32185682$

C $-0.21354333-0.54473493 \quad 1.59665763$

C $-1.39272149 \quad 0.36033176 \quad 1.87465199$

C $0.01761713-1.41154712 \quad 2.84127624$

$\mathrm{H}-2.52208908-0.08475231-1.77962049$

$\mathrm{H}-2.70730658-0.29781490-0.03301332$

$\mathrm{H}-3.06598210-1.63681129-1.11483327$

$\mathrm{H}-0.92549970 \quad 1.68439633-0.49980667$

$\mathrm{H} \quad 0.76522684 \quad 2.27892088 \quad 1.24439155$

H 2.09684127 $1.79924788 \quad 0.17337445$

$\mathrm{H} 1.88453696 \quad 0.14825456 \quad 1.95026051$

$\mathrm{H} 2.07436337-1.74943155 \quad 0.38828594$

H $1.59110615-0.67144136-0.91059139$

$\mathrm{H}-0.19920242 \quad-2.55327820 \quad 0.59887538$

$\mathrm{H}-1.628521891 .045788261 .05617458$

H $-1.19202470 \quad 0.98694573 \quad 2.75678811$

$\mathrm{H}-2.29719360-0.219938522 .10112456$

$\mathrm{H} \quad 0.18882169-0.79771672 \quad 3.73202657$

H $0.88748395 \quad-2.070269292 .73001537$

$\mathrm{H}-0.85024237-2.05021103 \quad 3.04253102$ 
$0.56484566-2.21624024-2.15711649$

○ $0.548579431 .43482235-1.82399240$

O $-0.55442065-1.65547727-2.05700185$

Vibrational frequencies (in $\mathrm{cm}-1$ )

$\begin{array}{lllllllllllllll}-110.5 & 23.4 & 54.5 & 91.7 & 101.2 & 183.4 & 197.2 & 207.8 & 212.0 & 245.7 & 249.0 & 258.2 & 279.5 & 334.7\end{array}$

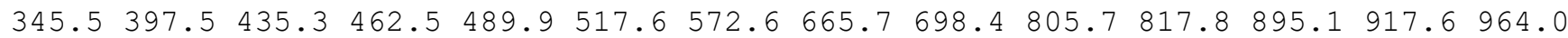
$973.7 \quad 979.4 \quad 1001.1 \quad 1011.7 \quad 1018.2 \quad 1032.0 \quad 1045.8 \quad 1079.1 \quad 1083.1 \quad 1089.11114 .71135 .8$

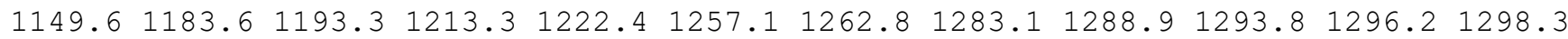

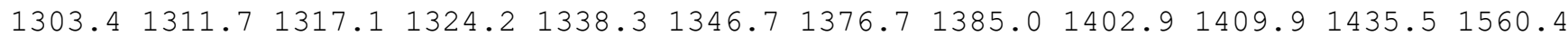
$1843.82628 .02652 .5 \quad 2659.8 \quad 2662.4 \quad 2664.5 \quad 2669.72671 .52674 .5 \quad 2678.72694 .82722 .4$ $2742.2 \quad 2755.2 \quad 2772.3 \quad 2778.7 \quad 2781.4$

name $=\operatorname{TS} 26$

energy (in $\mathrm{kcal} / \mathrm{mol})=-19.166672$

$\operatorname{ZPE}($ in $\mathrm{kcal} / \mathrm{mol})=141.519$

G $($ in $\mathrm{kcal} / \mathrm{mol})=116.134$

Geometry

C $-2.63644614 \quad-2.27445616 \quad 0.43145637$

C $-1.44251392-1.54348284-0.10793899$

C $0.528940531 .42775082-1.23953814$

C $1.23179929 \quad 1.36519693 \quad-0.00861231$

C $1.25868208 \quad 0.11258374 \quad 0.78791879$

C $1.09678698-1.21232107-0.01376475$

C $-0.20972613-1.54792093 \quad 0.74631550$

C $-0.03524291-0.247086451 .62009583$

C -1.173229240 .751027961 .54711913$

C $0.28387404-0.577834383 .07140206$

$\mathrm{H}-3.54582297-2.03414471-0.14049715$

$\mathrm{H}-2.82623888-2.02819116 \quad 1.48357995$

$\mathrm{H}-2.49281013-3.361967690 .36470749$

$\mathrm{H}-0.34731207 \quad 0.88040055-1.57288075$

$\mathrm{H} \quad 1.23923610 \quad 2.28440652 \quad 0.60116142$

$\mathrm{H} 2.402956331 .83991051-0.57619744$

H $2.16285652 \quad 0.10136664 \quad 1.42845919$

H $1.90440005-1.931216950 .14702998$

$\mathrm{H} \quad 0.97516952-1.09195085-1.09499532$

$\mathrm{H}-0.14228885-2.47356657 \quad 1.34072656$

$\begin{array}{lllll}\mathrm{H} & -2.07089451 & 0.38061701 & 2.05501210\end{array}$

$\mathrm{H}-1.45477727 \quad 0.98889943 \quad 0.51353601$

$\mathrm{H}-0.89380890 \quad 1.69898617 \quad 2.02562475$

H $1.10255745-1.30137548 \quad 3.15952264$

$\mathrm{H}-0.58471847-1.00151798 \quad 3.58788937$

$\mathrm{H} \quad 0.58513907 \quad 0.32180508 \quad 3.62286616$

O $2.25788408 \quad 2.69667852-1.68483375$

O $1.10432875 \quad 2.16003234-2.16818031$

o $-1.48589930-0.98476014-1.17853222$

Vibrational frequencies (in $\mathrm{cm}-1$ )

$\begin{array}{llllllllllllllll}-1568.2 & 53.0 & 65.3 & 87.6 & 103.0 & 112.2 & 155.8 & 174.0 & 191.1 & 220.4 & 229.5 & 270.1280 .2 & 328.9\end{array}$ $371.2 \quad 396.5 \quad 409.9 \quad 455.1 \quad 476.7 \quad 572.6 \quad 599.6 \quad 634.2 \quad 689.5 \quad 765.2 \quad 808.0 \quad 829.0 \quad 867.7 \quad 912.0$

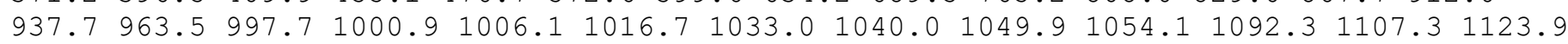

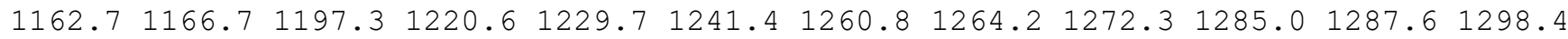
$1299.51307 .11311 .6 \quad 1318.1 \quad 1356.1 \quad 1367.8 \quad 1380.5 \quad 1403.81405 .2 \quad 1431.7 \quad 1561.5 \quad 1844.5$ $1963.72668 .72671 .2 \quad 2673.8 \quad 2675.4 \quad 2676.12677 .4 \quad 2680.62682 .62711 .4 \quad 2719.32731 .6$ $2762.6 \quad 2780.02781 .2 \quad 2783.2$

name $=\operatorname{TS} 27$

energy (in $\mathrm{kcal} / \mathrm{mol})=-16.994176$

$\operatorname{ZPE}($ in $\mathrm{kcal} / \mathrm{mol})=141.215$

$\mathrm{G}($ in $\mathrm{kcal} / \mathrm{mol})=114.809$

Geometry

C $-1.90506614-0.36115782 \quad 3.52414243$

C $-0.90034743-0.65758367 \quad 2.44675921$

C $1.94416130 \quad 2.40482079-0.75922030$ 
C $1.719203831 .10449069-0.25426810$

C $1.84824604 \quad 0.85702708 \quad 1.20309708$

C $1.54401378-0.600999651 .64916809$

C $0.47824704 \quad-0.10037118 \quad 2.65358207$

C $0.68141598 \quad 1.37249526 \quad 2.13638545$

C $-0.51858663 \quad 1.95875602 \quad 1.41875217$

$\begin{array}{llll}\text { C } 1.16867225 & 2.31561867 & 3.22552557\end{array}$

$\mathrm{H}-2.93352260-0.45029116 \quad 3.14281003$

$\mathrm{H}-1.78465409 \quad 0.644040313 .94316910$

$\mathrm{H}-1.80808264-1.08154781 \quad 4.34829057$

H 2.55140951 3.20366369 -0.36828443

H $2.01120278 \quad 0.25271694-0.89532305$

$\mathrm{H} \quad 0.50105966 \quad 0.97528876 \quad-0.89549858$

$\mathrm{H} 2.83379734 \quad 1.20187003 \quad 1.57248777$

H $2.38694900-1.12800001 \quad 2.09878937$

H $1.13119817-1.23914480 \quad 0.85564536$

$\mathrm{H} \quad 0.78881226-0.22497019 \quad 3.70529304$

$\mathrm{H}-1.336694492 .18524503 \quad 2.11207501$

$\mathrm{H}-0.919788521 .27190526 \quad 0.65736510$

$\mathrm{H}-0.26209327 \quad 2.89154285 \quad 0.90273807$

$\mathrm{H} 1.45734552 \quad 3.28765068 \quad 2.80886731$

H 2.04009390 $1.91716640 \quad 3.75763426$

$\mathrm{H} \quad 0.38791039 \quad 2.50068610 \quad 3.97274845$

○ $0.38345819 \quad 1.70281398-2.11985003$

O $1.19202664 \quad 2.73908618-1.79056902$

o $-1.19127644-1.323606661 .48509031$

Vibrational frequencies (in $\mathrm{cm}-1$ )

$\begin{array}{lllllllllllllll}-1605.6 & 25.1 & 46.1 & 51.2 & 85.4 & 109.7 & 146.2 & 167.2 & 181.7 & 223.9 & 231.6 & 266.9 & 298.2 & 317.1\end{array}$

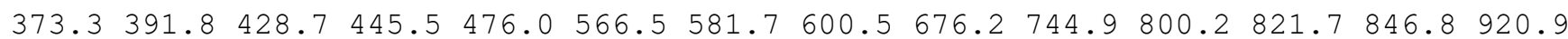
$937.5 \quad 979.7 \quad 991.8 \quad 996.0 \quad 1003.2 \quad 1014.0 \quad 1022.9 \quad 1035.7 \quad 1046.5 \quad 1050.0 \quad 1095.5 \quad 1120.7 \quad 1132.5$ $\begin{array}{lllllllllllll}1162.1 & 1171.7 & 1204.6 & 1219.6 & 1237.0 & 1252.2 & 1262.1 & 1269.1 & 1272.9 & 1288.5 & 1289.3 & 1296.2\end{array}$ $1300.01301 .91306 .8 \quad 1317.0 \quad 1348.5 \quad 1354.3 \quad 1380.6 \quad 1400.51403 .1 \quad 1429.2 \quad 1568.6 \quad 1854.7$ $1943.62660 .72668 .92676 .1 \quad 2676.6 \quad 2678.3 \quad 2680.12680 .72695 .72718 .12728 .12730 .0$ $2761.02778 .3 \quad 2780.92783 .2$

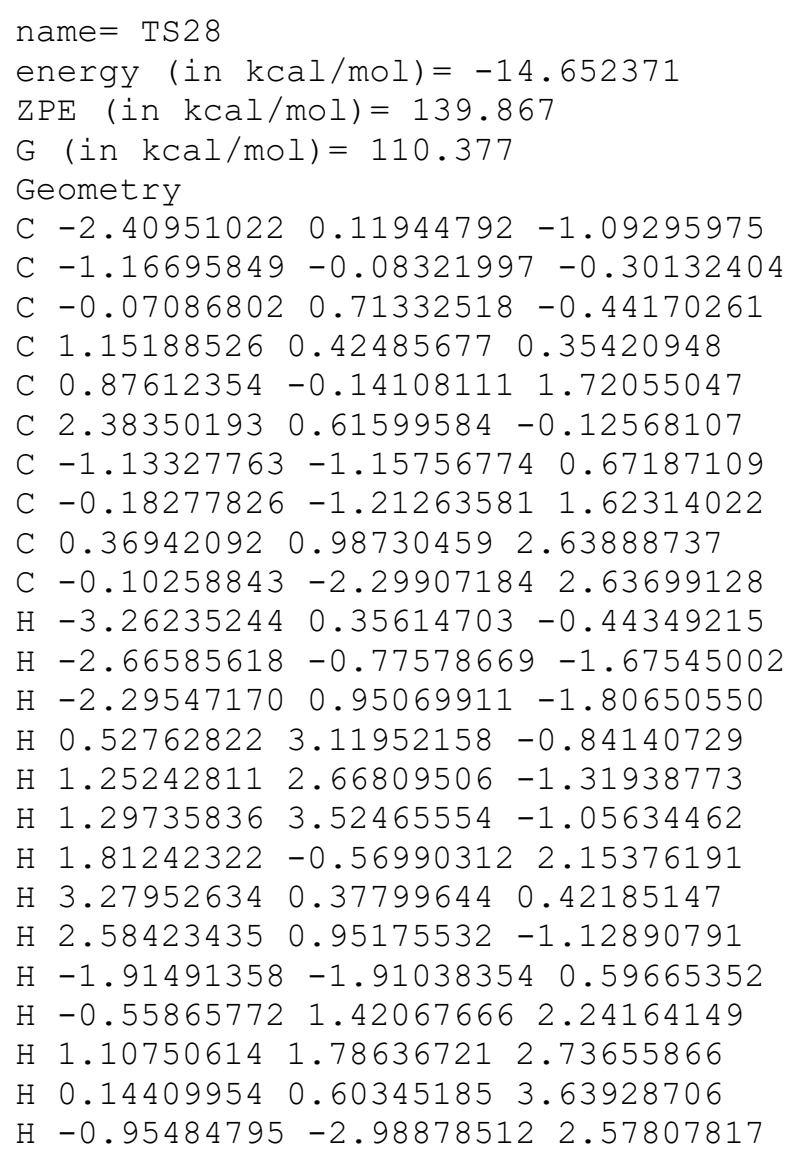


$\mathrm{H} \quad 0.80939997-2.89845235 \quad 2.49862018$

$\mathrm{H}-0.08007069-1.894296153 .65808671$

$0.290050690 .24821492-3.46615772$

$0.00718114 \quad 1.72480564-1.24969961$

$0.03347117-0.52709337-2.67605760$

Vibrational frequencies (in $\mathrm{cm}-1$ )

$\begin{array}{lllllllllllllllllll}-1553.2 & 10.5 & 37.6 & 65.6 & 69.2 & 77.1 & 100.9 & 106.1 & 110.7 & 118.8 & 147.1 & 156.9 & 175.7 & 188.2\end{array}$ $219.4 \quad 237.5 \quad 269.4 \quad 303.0 \quad 327.3 \quad 365.5 \quad 428.0 \quad 458.9 \quad 479.6 \quad 534.2 \quad 569.9 \quad 597.8 \quad 623.5 \quad 639.3$

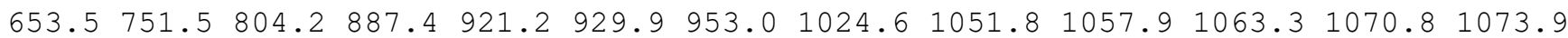
$1104.51118 .0 \quad 1134.3 \quad 1231.5 \quad 1258.7 \quad 1284.1 \quad 1290.8 \quad 1291.4 \quad 1294.1 \quad 1297.4 \quad 1300.1 \quad 1303.4$

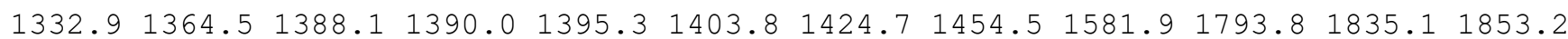
$2523.0 \quad 2665.5 \quad 2670.2 \quad 2672.4 \quad 2674.5 \quad 2677.4 \quad 2677.6 \quad 2685.12709 .72725 .2 \quad 2763.42777 .2$ $2779.12780 .02790 .3 \quad 4480.8$

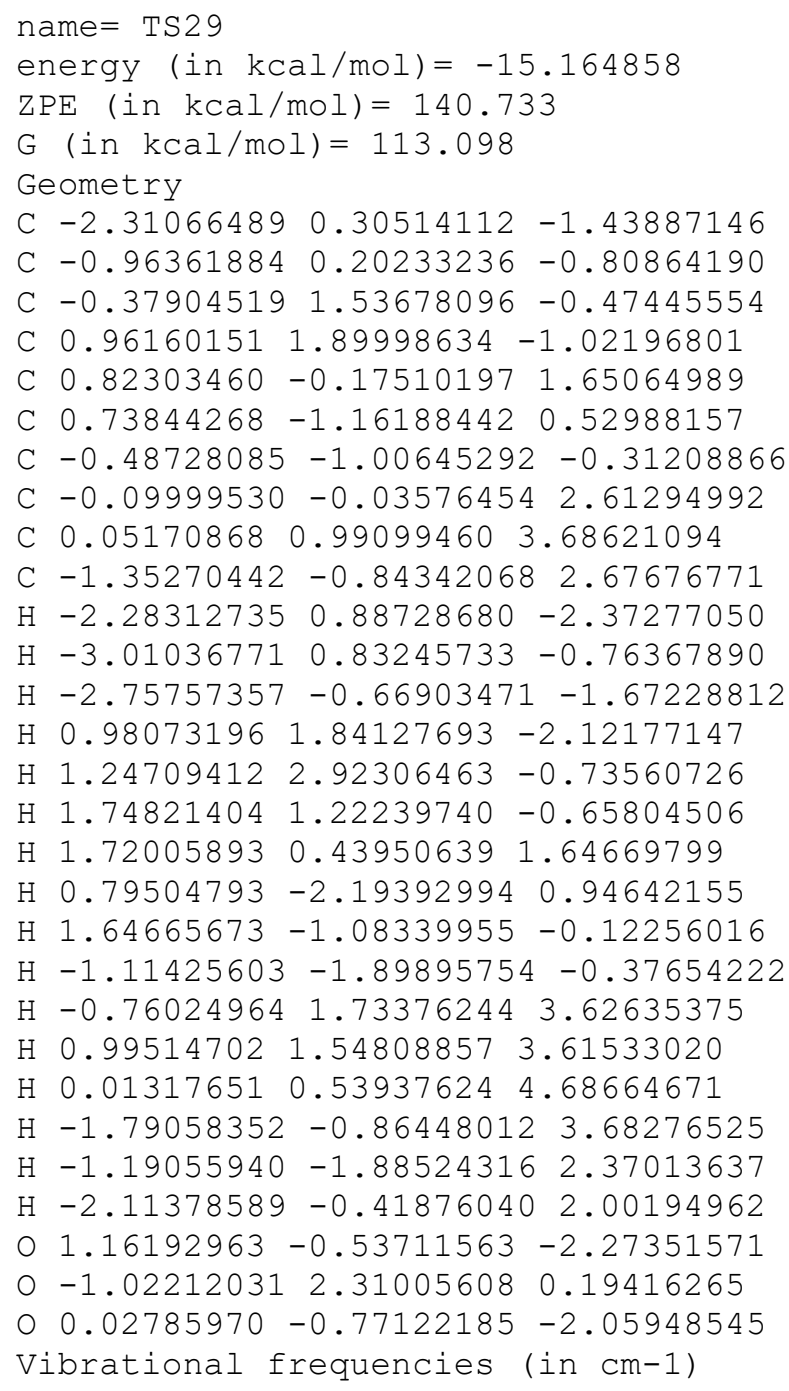


C $-2.35198303 \quad 0.76896094 \quad 1.67159338$

C $0.085769872 .08361382-0.14020555$

C $1.03269110 \quad 1.07072333 \quad 0.20599824$

C $0.56071623-0.216925690 .79690007$

C $-0.82618892-0.71684000 \quad 0.29206293$

C $-1.46069926-0.43580972 \quad 1.67749530$

C $-0.02528473-0.150113692 .26614353$

C $0.14942304 \quad 1.14796014 \quad 3.02561475$

C $0.46063037-1.318110973 .11713773$

$\mathrm{H}-4.05620823 \quad 1.71942823 \quad 2.59909372$

$\mathrm{H}-3.23163032 \quad 0.53153922 \quad 3.62911475$

$\mathrm{H}-4.26121721-0.01232495 \quad 2.29266031$

$\mathrm{H}-0.25368914 \quad 2.95154895 \quad 0.41311307$

$\mathrm{H} \quad 1.97446418 \quad 1.41393114 \quad 0.62661566$

$\mathrm{H} \quad 1.13595665 \quad 0.67609739-1.18139137$

H $1.35283779-0.98329884 \quad 0.70840676$

$\mathrm{H}-0.83686420-1.771600650 .00491083$

$\mathrm{H}-1.25222145-0.15065088-0.54387256$

$\mathrm{H}-1.95065398-1.30942817 \quad 2.13195170$

$\mathrm{H} 1.210522961 .33100802 \quad 3.24148269$

$\mathrm{H}-0.38390398 \quad 1.13095106 \quad 3.98279182$

$\mathrm{H}-0.21081872 \quad 2.01758488 \quad 2.46419764$

H $1.52401646-1.20774646 \quad 3.36305782$

$\mathrm{H} \quad 0.34469790 \quad-2.27867661 \quad 2.60174744$

$\mathrm{H}-0.08950480-1.38325115 \quad 4.06224905$

$0 \quad 0.20646755 \quad 1.00546529-2.06309818$

$\begin{array}{lllll}0 & -0.35883985 & 2.04662187 & -1.36976917\end{array}$

o $-2.13904971 \quad 1.72402988 \quad 0.95804644$

Vibrational frequencies (in $\mathrm{cm}-1$ )

$\begin{array}{llllllllllllllll}-1392.9 & 38.2 & 63.3 & 90.0 & 100.3 & 132.1 & 150.6 & 172.9 & 192.6 & 206.3 & 219.1 & 244.3 & 296.3 & 328.8\end{array}$ $\begin{array}{llllllllllllll}361.3 & 396.8 & 433.0 & 447.1 & 571.1 & 582.8 & 596.6 & 652.1 & 697.3 & 736.2 & 826.2 & 843.8 & 886.8 & 902.3\end{array}$ $930.8 \quad 950.0 \quad 995.6 \quad 1000.9 \quad 1006.2 \quad 1015.3 \quad 1034.8 \quad 1041.6 \quad 1047.3 \quad 1088.1 \quad 1097.7 \quad 1110.2 \quad 1118.8$

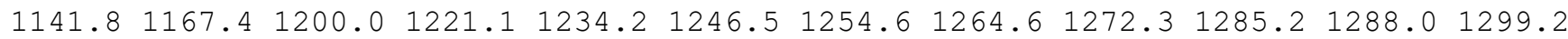

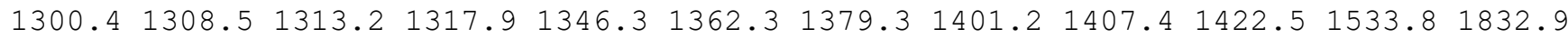
$1940.62668 .72672 .2 \quad 2673.2 \quad 2677.12677 .72678 .72681 .52693 .52721 .12735 .12759 .0$ $2762.12779 .3 \quad 2781.6 \quad 2783.8$

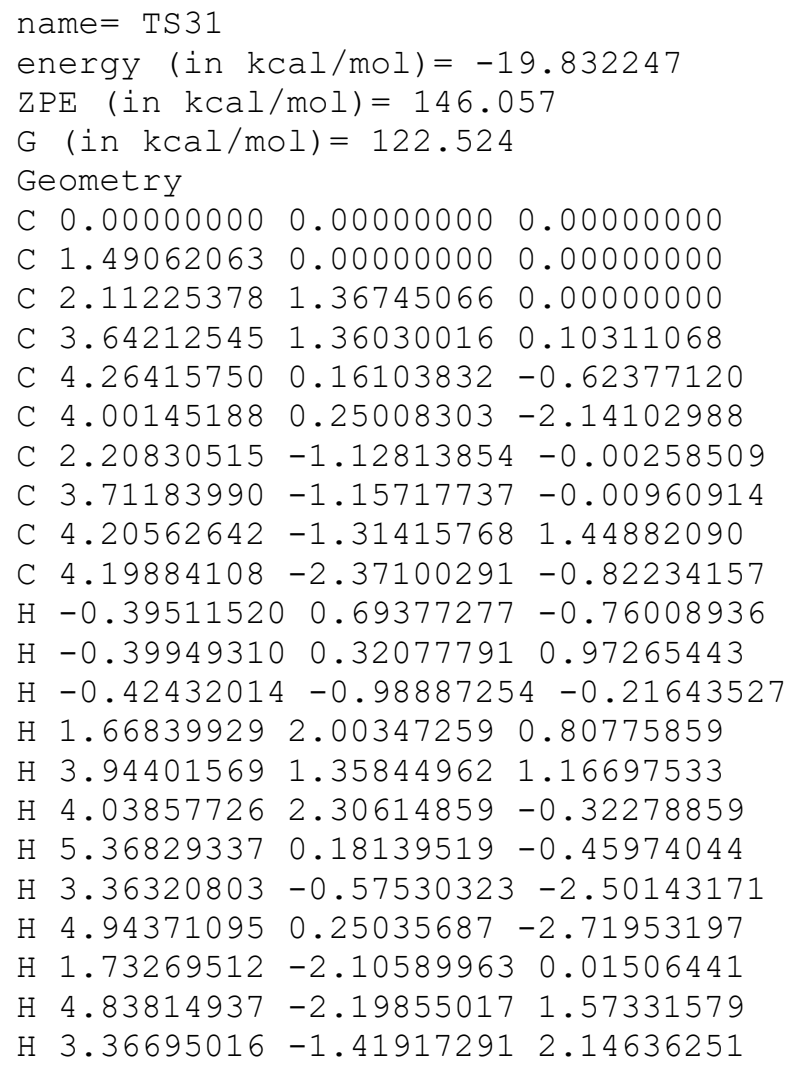


$\mathrm{H} \quad 4.79475698-0.455777341 .78423617$

$\mathrm{H} \quad 3.73981838-2.41245429-1.81580725$

$\mathrm{H} 3.94419663-3.31082300-0.31895780$

$\mathrm{H} 5.28527340-2.34934690-0.95482537$

○ $2.041224681 .41909890-2.31816191$

O $1.729231562 .11341867-1.15141781$

O $3.42443587 \quad 1.48772821-2.53003170$

Vibrational frequencies (in $\mathrm{cm}-1$ )

$\begin{array}{lllllllllllllllll}-146.4 & 79.7 & 115.8 & 129.0 & 144.7 & 207.2 & 223.1 & 262.8 & 295.3 & 311.7 & 329.8 & 367.7 & 387.7 & 412.9\end{array}$

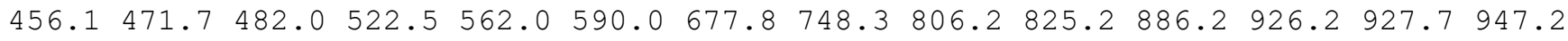
$966.8 \quad 984.6 \quad 1002.4 \quad 1011.2 \quad 1036.5 \quad 1043.5 \quad 1060.1 \quad 1071.7 \quad 1085.11108 .81137 .11159 .6$

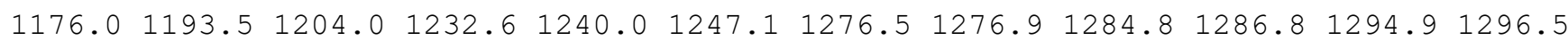
$1299.3 \quad 1303.0 \quad 1306.0 \quad 1309.2 \quad 1332.8 \quad 1345.3 \quad 1356.4 \quad 1374.8 \quad 1385.1 \quad 1401.6 \quad 1412.81430 .7$ 1875.72641 .52660 .62666 .22669 .72671 .72674 .12674 .52679 .72679 .82700 .22712 .3 $2738.6 \quad 2756.0 \quad 2777.9 \quad 2779.12780 .5$

name $=$ TS32

energy (in $\mathrm{kcal} / \mathrm{mol})=-14.897567$

$\mathrm{ZPE}($ in $\mathrm{kcal} / \mathrm{mol})=141.311$

$\mathrm{G}($ in $\mathrm{kcal} / \mathrm{mol})=115.167$

Geometry

C $-2.06157123-0.12023858-1.59210562$

C $-0.92814979-0.51714470-0.84661263$

C $2.484999181 .80606182 \quad 2.41102811$

C $2.23379448 \quad 0.547109121 .62365730$

C $0.88558526-0.063519011 .94197716$

C $0.53718464-1.327234791 .10914435$

C $-0.77437644 \quad-0.66982652 \quad 0.60710313$

C $-0.39668211 \quad 0.65995239 \quad 1.38289801$

C $-0.152543791 .85931560 \quad 0.49116822$

C $-1.40459717 \quad 0.99621499 \quad 2.46886536$

$\mathrm{H}-1.29612824 \quad 0.23784640 \quad-2.69706672$

$\mathrm{H}-2.76367774 \quad 0.58143724 \quad-1.18041251$

$\mathrm{H}-2.56595463-0.84893297-2.22432981$

$\mathrm{H} \quad 1.76438859 \quad 2.07246304 \quad 3.19367288$

$\mathrm{H} 3.04754968-0.18229343 \quad 1.83621948$

$\mathrm{H} 2.34541552 \quad 0.75876365 \quad 0.53716989$

$\mathrm{H} 0.80064955-0.250240213 .02971236$

$\mathrm{H} \quad 0.38267549-2.23282437 \quad 1.70391881$

$\mathrm{H} 1.26639096-1.57338600 \quad 0.33080393$

$\mathrm{H}-1.68054550-1.14989231 \quad 1.03223013$

$\mathrm{H}-1.08499636 \quad 2.211689690 .02823055$

$\mathrm{H} \quad 0.54562945 \quad 1.64496214 \quad-0.32966698$

$\mathrm{H} \quad 0.26428092 \quad 2.70395120 \quad 1.05440993$

$\mathrm{H}-1.053669291 .82527926 \quad 3.09622088$

$\mathrm{H}-1.60338298 \quad 0.14723048 \quad 3.13317435$

$\mathrm{H}-2.36744622 \quad 1.30282521 \quad 2.03962176$

$\begin{array}{lllll}0 & -0.07427860 & -0.38678190 & -2.86900128\end{array}$

$0 \quad 3.447229812 .499912472 .19622594$

$0.17862309-0.67382994-1.55789601$

Vibrational frequencies (in $\mathrm{cm}-1$ )

$\begin{array}{llllllllllllllll}-1540.8 & 29.9 & 43.3 & 50.7 & 103.2 & 124.6 & 134.9 & 184.7 & 216.7 & 223.5 & 246.8 & 275.5 & 302.6 & 308.7\end{array}$ $388.9 \quad 396.6 \quad 406.3 \quad 435.0 \quad 464.2 \quad 552.1 \quad 567.0 \quad 631.6 \quad 674.2 \quad 707.6 \quad 752.8 \quad 829.7 \quad 855.4 \quad 920.2$ $949.2986 .0 \quad 993.5 \quad 1005.4 \quad 1013.6 \quad 1017.6 \quad 1036.1 \quad 1051.5 \quad 1058.4 \quad 1081.2 \quad 1101.51119 .5 \quad 1128.8$

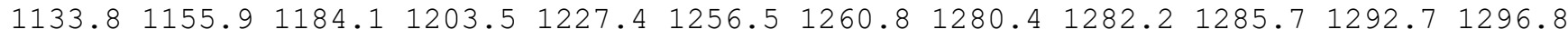
$1300.11305 .3 \quad 1310.4 \quad 1320.3 \quad 1340.8 \quad 1351.6 \quad 1360.1 \quad 1401.11404 .2 \quad 1486.11591 .7 \quad 1837.8$ $1970.52644 .92653 .1 \quad 2663.7 \quad 2670.3 \quad 2674.8 \quad 2676.02678 .92699 .3 \quad 2712.4 \quad 2719.42738 .9$ $2761.72778 .2 \quad 2781.7 \quad 2785.6$

name $=\mathrm{TS} 33$

energy (in $\mathrm{kcal} / \mathrm{mol})=-13.755833$

$\mathrm{ZPE}(\mathrm{in} \mathrm{kcal} / \mathrm{mol})=141.144$

$\mathrm{G}($ in $\mathrm{kcal} / \mathrm{mol})=113.863$ 


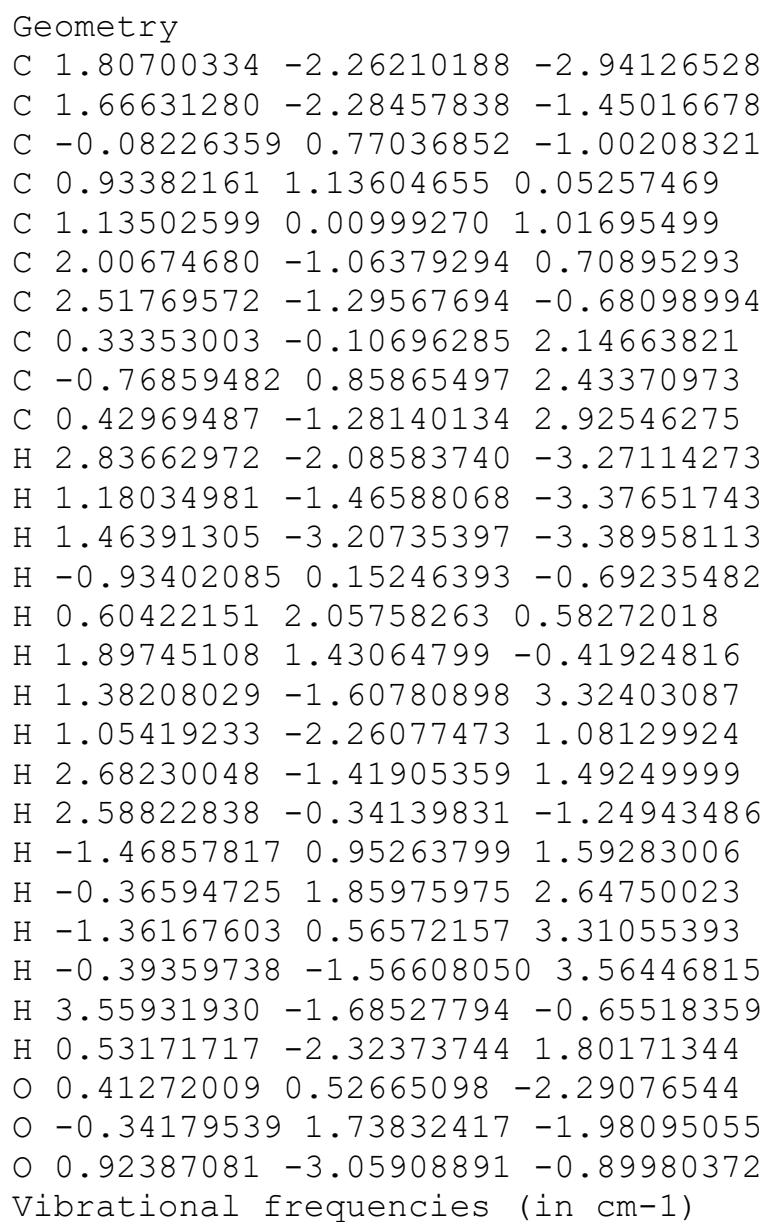

Vibrational frequencies (in $\mathrm{cm}-1$ )

$\begin{array}{llllllllllllll}-1868.2 & 27.9 & 40.0 & 53.6 & 67.5 & 77.8 & 93.5 & 102.3 & 134.4 & 162.6 & 252.9 & 289.2 & 308.5 & 345.5\end{array}$ $366.2384 .3 \quad 436.6 \quad 449.9 \quad 483.1 \quad 509.8 \quad 555.4 \quad 612.9 \quad 676.3 \quad 686.8 \quad 829.0 \quad 857.7 \quad 870.5 \quad 887.3$ $930.0 \quad 932.3 \quad 945.7 \quad 980.2 \quad 990.5 \quad 1020.4 \quad 1040.7 \quad 1050.6 \quad 1056.1 \quad 1079.9 \quad 1082.1 \quad 1112.3 \quad 1123.3$ $1147.61177 .2 \quad 1178.2 \quad 1197.6 \quad 1226.4 \quad 1246.5 \quad 1255.3 \quad 1270.2 \quad 1272.3 \quad 1276.1 \quad 1282.3 \quad 1291.5$

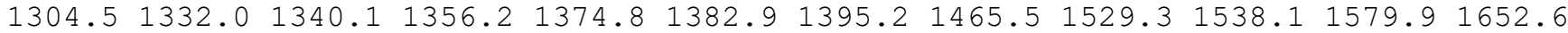
$1853.42643 .7 \quad 2649.7 \quad 2658.3 \quad 2670.4 \quad 2673.4 \quad 2676.02685 .12699 .7 \quad 2718.8 \quad 2730.4 \quad 2738.2$ $2764.12778 .32779 .2 \quad 3035.2$

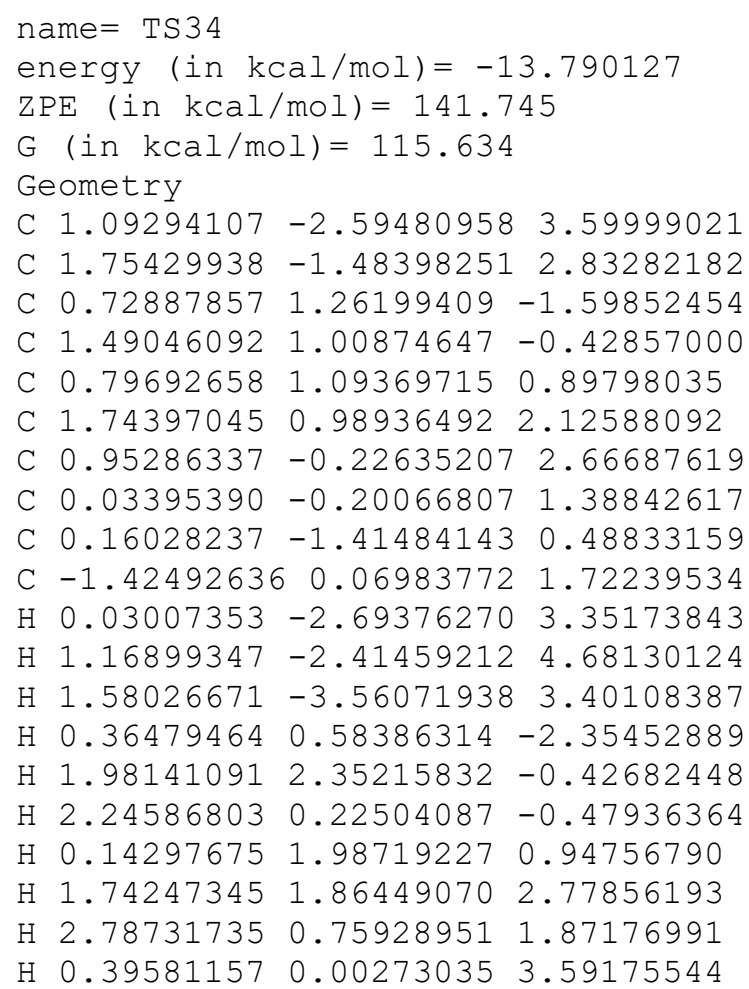


$\mathrm{H}-0.29650514-2.30321106 \quad 0.93900992$

$\mathrm{H} 1.21166493-1.65884027 \quad 0.27731251$

$\mathrm{H}-0.33153480-1.24591894-0.47593225$

$\mathrm{H}-1.54336812 \quad 0.94863501 \quad 2.36773314$

$\mathrm{H}-1.88225854 \quad-0.78060765 \quad 2.24084694$

H $-2.01134629 \quad 0.25399261 \quad 0.81443222$

$\begin{array}{llll}0 & 1.17118003 & 3.31618930 & -0.96894797\end{array}$

$0.556276882 .53203984-1.89339426$

O $2.87058803-1.607263112 .39390619$

Vibrational frequencies (in $\mathrm{cm}-1$ )

$\begin{array}{llllllllllllllllll}-1586.9 & 36.9 & 44.1 & 50.0 & 114.4 & 114.8 & 146.4 & 156.6 & 181.6 & 205.5 & 228.2 & 265.3 & 293.5 & 312.3\end{array}$ $369.3 \quad 386.9 \quad 441.4 \quad 443.6 \quad 516.4 \quad 571.9 \quad 595.0 \quad 650.4 \quad 680.6 \quad 771.0 \quad 820.6 \quad 823.8 \quad 888.2 \quad 917.8$ $947.5971 .5 \quad 995.4 \quad 1003.0 \quad 1015.5 \quad 1030.0 \quad 1034.6 \quad 1044.0 \quad 1049.3 \quad 1093.5 \quad 1108.11129 .9 \quad 1145.3$ $1163.41172 .2 \quad 1205.2 \quad 1228.2 \quad 1237.9 \quad 1260.1 \quad 1263.3 \quad 1268.1 \quad 1274.2 \quad 1286.8 \quad 1289.7 \quad 1295.2$

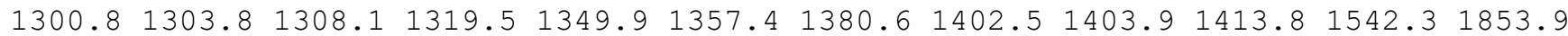
$1937.72667 .6 \quad 2669.92676 .0 \quad 2677.3 \quad 2678.2 \quad 2680.8 \quad 2682.22709 .12714 .42728 .62750 .1$ 2761.12780 .32780 .92782 .8

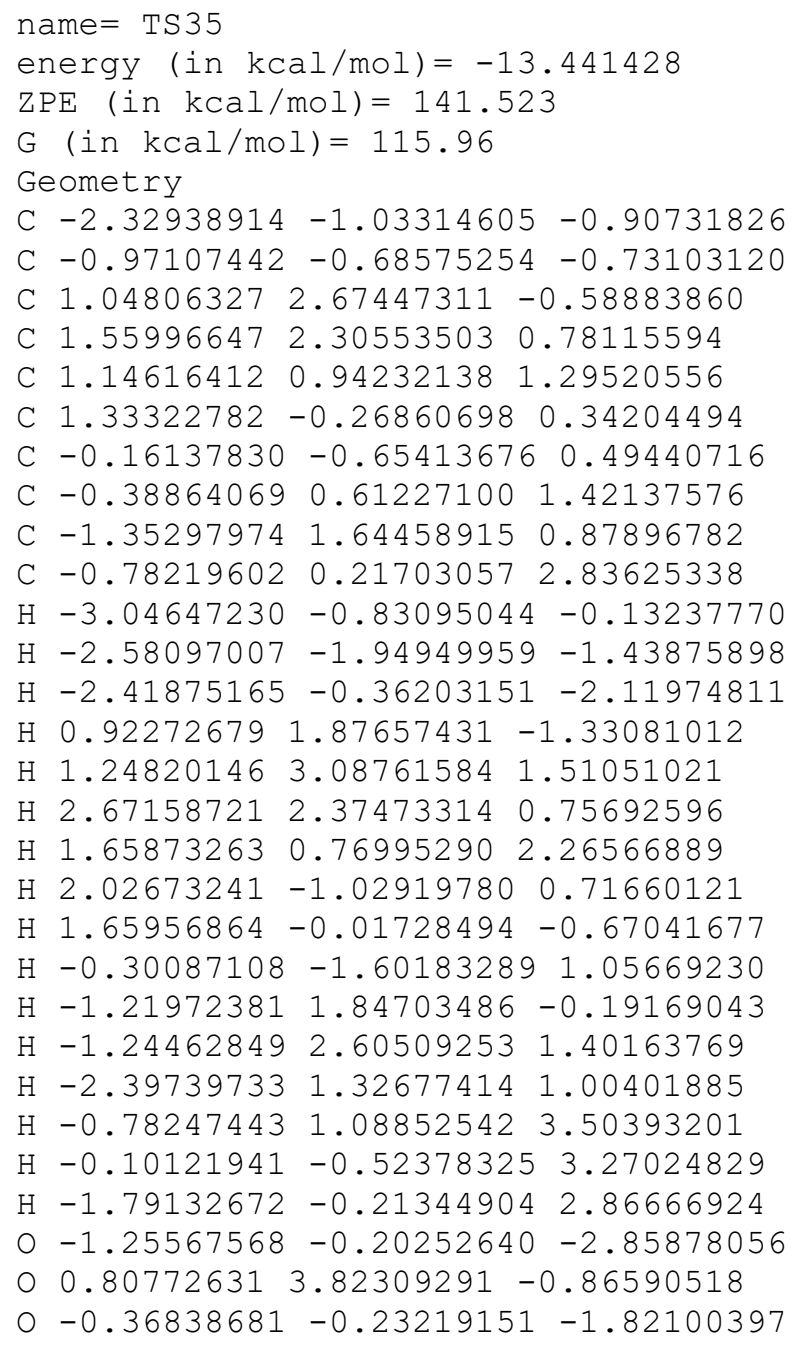


$\mathrm{ZPE}($ in $\mathrm{kcal} / \mathrm{mol})=141.545$

$\mathrm{G}($ in $\mathrm{kcal} / \mathrm{mol})=115.783$

Geometry

C $-2.44512741-1.08749910-0.92742020$

C $-1.05710774-0.84914515-0.81434806$

C $2.034175401 .54771721-0.79659442$

C $2.27147577 \quad 1.14938828 \quad 0.64138260$

C $1.64075561-0.17774398 \quad 1.02232452$

C $1.39244818-1.20248950-0.11903824$

C $-0.11631298-1.27504076 \quad 0.23157344$

C $0.10944673-0.21395729 \quad 1.38845517$

C $-0.66388002 \quad 1.07830466 \quad 1.23682706$

C $-0.14173187-0.81832004 \quad 2.76162875$

$\mathrm{H}-3.05338898-1.19410925-0.04822404$

$\mathrm{H}-2.81010707-1.72662231-1.73098403$

$\mathrm{H}-2.63674141-0.00243970-1.77016163$

$\mathrm{H} \quad 0.99665368 \quad 1.66756984-1.13304202$

H $1.90532940 \quad 1.95622640 \quad 1.31002708$

$\mathrm{H} 3.369441431 .102226370 .82402718$

$\mathrm{H} 2.24292085-0.625898521 .84031623$

$\mathrm{H} 1.92308487 \quad-2.15207096 \quad 0.01461491$

$\mathrm{H} \quad 1.62917477-0.85578931-1.12941625$

$\mathrm{H}-0.41527795-2.26601240 \quad 0.63103911$

$\mathrm{H}-0.51139114 \quad 1.56100614 \quad 0.26343088$

$\mathrm{H}-0.38124393 \quad 1.80640723 \quad 2.00813276$

$\mathrm{H}-1.74566107 \quad 0.90978809 \quad 1.33870617$

$\mathrm{H} 0.38162273-1.77143272 \quad 2.90170858$

$\mathrm{H}-1.20960057-1.00767482 \quad 2.92812467$

$\mathrm{H} \quad 0.19552318-0.144165493 .55932164$

$\begin{array}{lllll}0 & -1.56219301 & 0.42203400 & -2.53846353\end{array}$

O $2.957271321 .73856657-1.54721786$

o $-0.55995508-0.02303287-1.72384531$

Vibrational frequencies (in cm-1)

$\begin{array}{llllllllllllllll}-1544.7 & 35.6 & 39.6 & 67.7 & 99.6 & 113.7 & 169.8 & 193.8 & 216.5 & 235.3 & 253.4 & 282.1 & 301.0 & 334.3\end{array}$

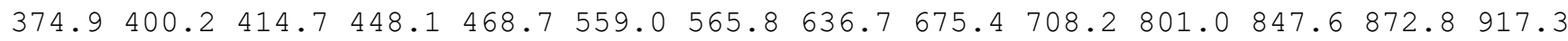
$948.4981 .9991 .9998 .1 \quad 1006.4 \quad 1017.8 \quad 1027.7 \quad 1050.51061 .8 \quad 1070.51081 .1 \quad 1103.91122 .6$ $\begin{array}{lllllllllllll}1134.0 & 1145.9 & 1193.1 & 1205.7 & 1232.5 & 1253.8 & 1274.6 & 1280.7 & 1286.8 & 1289.3 & 1290.7 & 1294.1\end{array}$

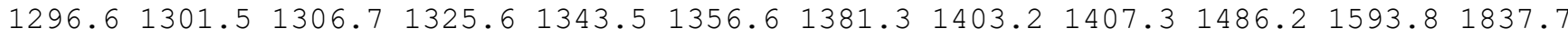
$1971.72638 .8 \quad 2658.0 \quad 2664.12670 .92672 .7 \quad 2675.12676 .22697 .42710 .12717 .22738 .4$ $2759.12778 .2 \quad 2782.02785 .5$

\section{name $=$ TS37}

energy (in $\mathrm{kcal} / \mathrm{mol})=-11.61137$

$\mathrm{ZPE}(\mathrm{in} \mathrm{kcal} / \mathrm{mol})=140.691$

$\mathrm{G}(\mathrm{in} \mathrm{kcal} / \mathrm{mol})=113.889$

Geometry

C $-1.61941749-0.71042944-2.58626591$

C $-0.79476805-0.55303467-1.35366634$

C $-0.415631960 .80060098-0.89948125$

C $-0.07710300 \quad 1.18732754 \quad 0.50373761$

C $1.36155514 \quad 0.89614647 \quad 0.78140592$

C $0.05147326-2.60126434-0.26566353$

C $-0.88141822-1.65764722-0.38368840$

C $1.80880893 \quad 0.08808530 \quad 1.75179916$

C $0.91546691-0.63796820 \quad 2.70178074$

C $3.27128241-0.145328921 .94253784$

$\mathrm{H}-0.98872092-0.59867310-3.49495886$

$\mathrm{H}-2.40503654 \quad 0.05724769-2.65607239$

$\mathrm{H}-2.10199399-1.69352538-2.65569349$

$\mathrm{H}-0.04154407 \quad 1.59431580 \quad-2.62944290$

$\mathrm{H}-0.75959481 \quad 0.68270278 \quad 1.21942799$

$\mathrm{H}-0.26771881 \quad 2.28164803 \quad 0.64278028$

H $2.05310687 \quad 1.41427527 \quad 0.11504494$

$\mathrm{H}-0.01815959-3.425104340 .42926275$ 
$\mathrm{H} \quad 0.95678092 \quad-2.63186218-0.85758011$

$\mathrm{H}-1.79342497-1.65293175 \quad 0.21745279$

$\mathrm{H} \quad 0.23953117-1.32410400 \quad 2.16866585$

H $0.29114726 \quad 0.05877970 \quad 3.27939071$

H $1.47149484-1.242236453 .43004749$

H $3.59900342 \quad 0.179873252 .94034854$

H $3.88992719 \quad 0.39219013 \quad 1.21035327$

$\mathrm{H} \quad 3.51838317-1.212832551 .84875013$

$\begin{array}{lllll}0 & 0.91734701 & 0.26501000 & -2.80400747\end{array}$

$0-0.61744251 \quad 1.82243821-1.74065197$

$0.69936190-0.18890936-1.62037102$

Vibrational frequencies (in $\mathrm{cm}-1$ )

$\begin{array}{llllllllllllllll}-272.1 & 23.9 & 63.3 & 77.5 & 87.0 & 111.6 & 130.1 & 134.4 & 141.5 & 179.7 & 205.5 & 242.2 & 259.5 & 299.5\end{array}$

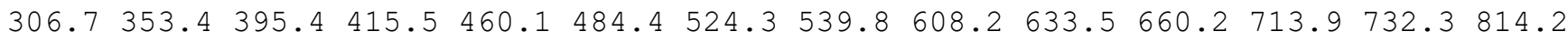

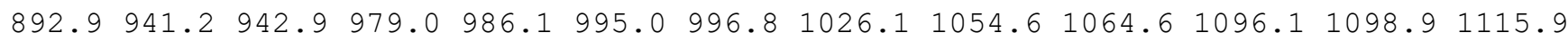
$\begin{array}{llllllllllllll}1138.7 & 1179.1 & 1225.7 & 1257.6 & 1261.7 & 1271.8 & 1277.5 & 1281.2 & 1284.7 & 1286.7 & 1287.8 & 1305.6\end{array}$

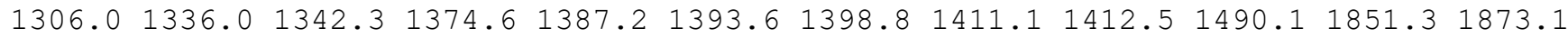
2316.02627 .22650 .12665 .32670 .62671 .52672 .22674 .42707 .02730 .22750 .22751 .9 $2767.8 \quad 2777.5 \quad 2779.8 \quad 2780.9$

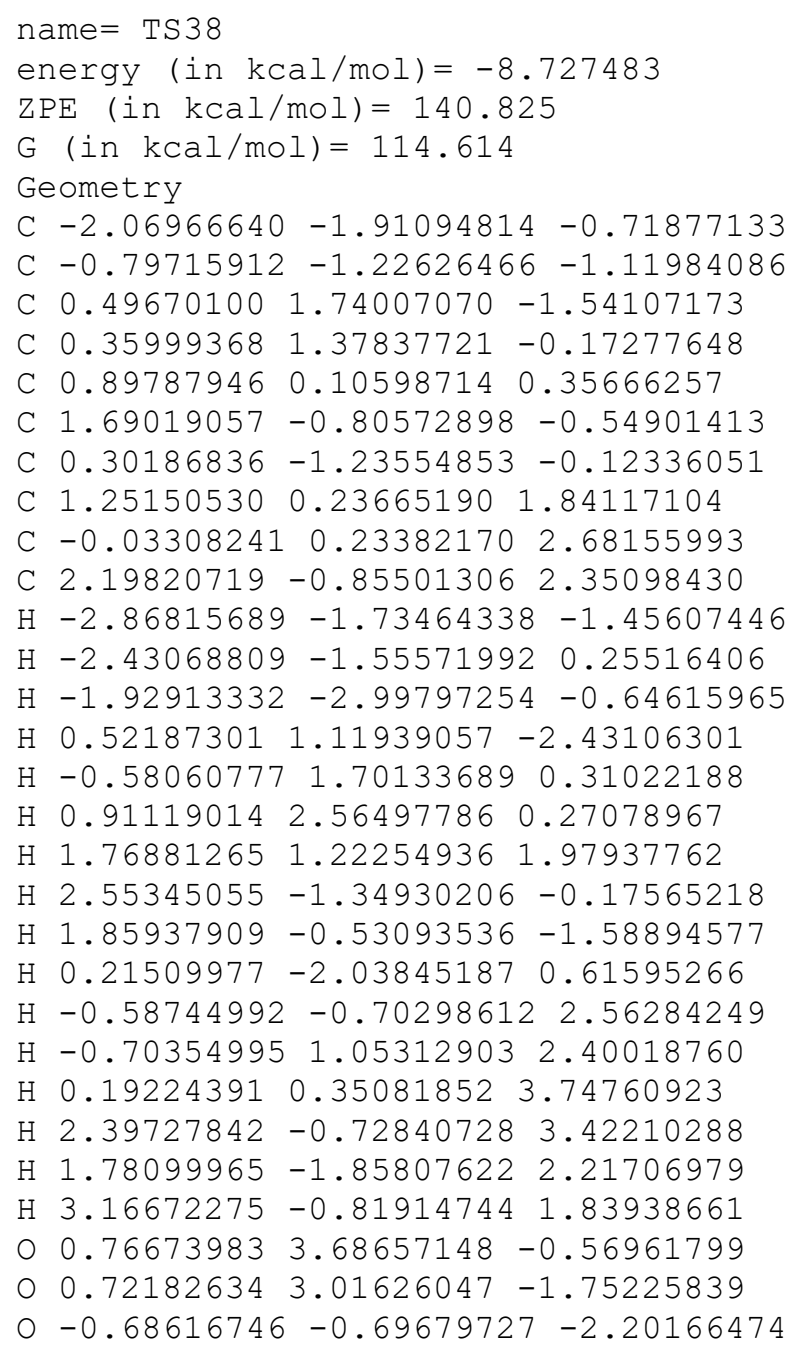




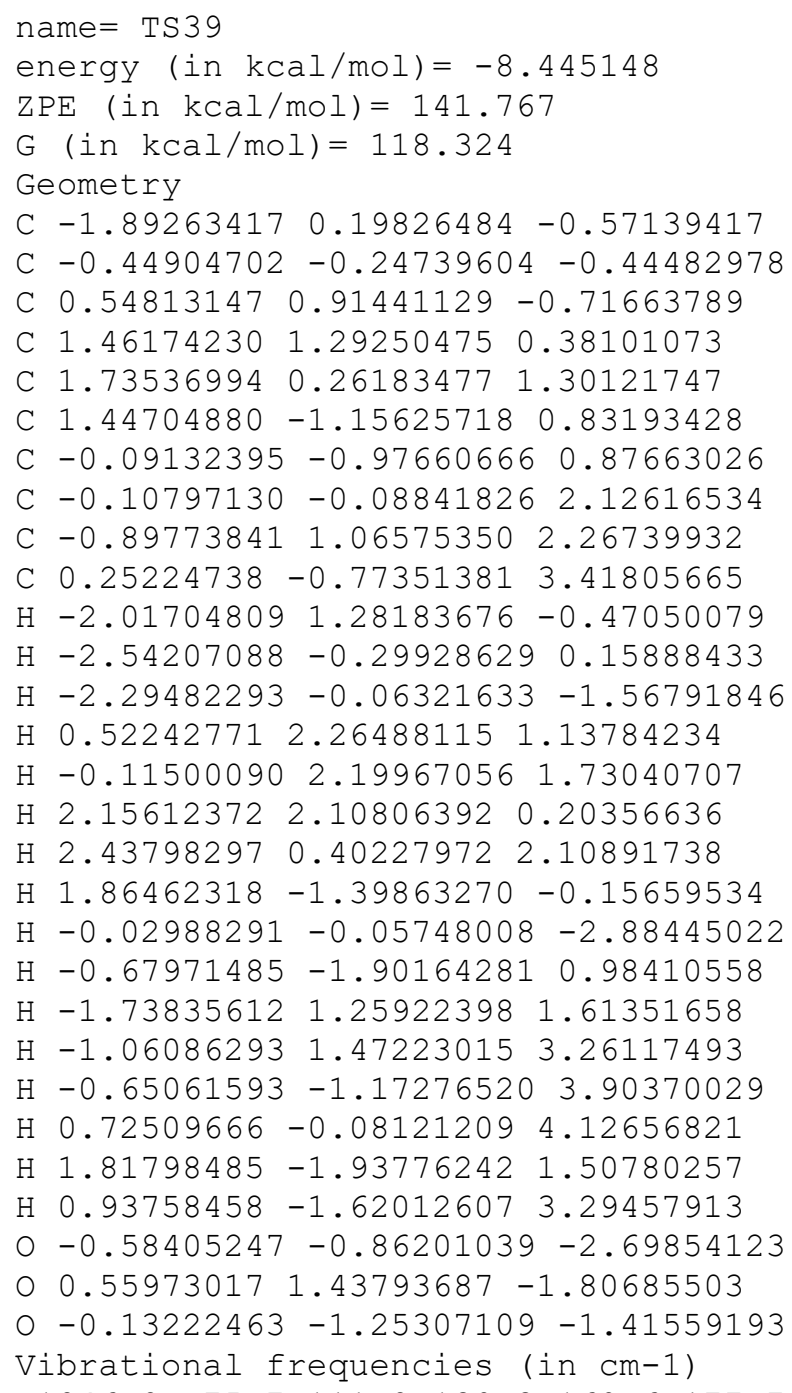


$\mathrm{H} \quad 1.69124612-2.87675027 \quad 1.70314691$

$\mathrm{H}-0.57776430-2.77605546 \quad 1.04158297$

$\mathrm{H} \quad 0.35568770-2.47958176-0.42394688$

$\mathrm{H}-1.68496108-0.408050521 .32268236$

$\mathrm{H} \quad 1.56134448 \quad 0.52243535 \quad 4.02503079$

H $1.88388740 \quad 0.83558529 \quad 2.31731384$

$\mathrm{H} 2.95304855-0.233380593 .24768960$

$\mathrm{H}-1.01257057-0.16845734 \quad 1.72026862$

$\mathrm{H}-0.13737165-1.10433820 \quad 4.46308549$

$\mathrm{H}-0.52133639-2.414581573 .31076568$

O $1.65210058-0.58661165-1.54921245$

$\begin{array}{llll}0 & 1.13737285 & 0.75018221 & 0.22762146\end{array}$

O $0.34637274-0.02225476-1.73301101$

Vibrational frequencies (in cm-1)

$\begin{array}{llllllllllllllll}-891.6 & 52.8 & 103.0 & 116.8 & 123.5 & 172.4 & 182.3 & 215.1 & 242.8 & 284.7 & 321.8 & 335.9 & 344.6 & 390.9\end{array}$

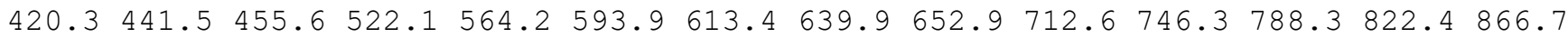
$895.0 \quad 922.3 \quad 938.9 \quad 974.6 \quad 989.0 \quad 995.8 \quad 1009.9 \quad 1016.5 \quad 1038.6 \quad 1056.7 \quad 1083.2 \quad 1092.4 \quad 1094.8$

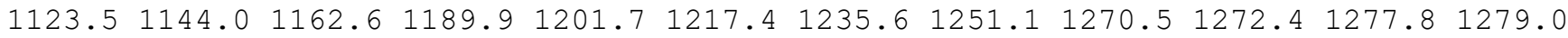

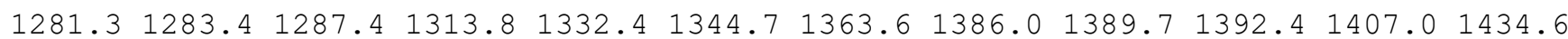
$1836.42637 .12651 .92658 .7 \quad 2666.3 \quad 2669.0 \quad 2671.2 \quad 2673.12677 .92724 .72726 .42733 .4$ $2775.52779 .02794 .7 \quad 4646.3$

name $=\operatorname{TS} 41$

energy (in $\mathrm{kcal} / \mathrm{mol})=-4.119735$

$\mathrm{ZPE}($ in $\mathrm{kcal} / \mathrm{mol})=140.614$

G $($ in $\mathrm{kcal} / \mathrm{mol})=116.692$

Geometry

C $-2.126630360 .17156389-0.29681499$

C $-0.67058255-0.10010993-0.59875606$

C $0.23070601 \quad 1.18544245-0.60172625$

C 1.142726451 .240326950 .49868660

C $1.68405635-0.009587051 .00898490$

C $1.54278521-1.13777901-0.07094175$

C $0.01247743-1.174988560 .27405520$

C $0.37610888-0.645076031 .71507147$

C $-0.53522915 \quad 0.31749888 \quad 2.44207751$

C $0.74916024-1.784356292 .65399651$

$\mathrm{H}-2.406144691 .20675535 \quad-0.53928306$

$\mathrm{H}-2.39562444-0.01895880 \quad 0.74591869$

$\mathrm{H}-2.77006612-0.47464366-0.91888257$

$\mathrm{H}-0.80728910 \quad 0.98175635 \quad-2.83200607$

H 2.29815091 2.49835872 0.22871123

H 2.54954653 2.00342733 -0.33503854

H 2.58889999 $0.01960878 \quad 1.60906917$

H $2.07506215-2.06125946 \quad 0.16458796$

H $1.80244821-0.86476660-1.09419399$

$\mathrm{H}-0.49139994-2.14099656 \quad 0.22602826$

$\mathrm{H}-0.02595574 \quad 0.74498404 \quad 3.31797070$

$\mathrm{H}-1.44480966-0.18024928 \quad 2.79917883$

$\mathrm{H}-0.84391530 \quad 1.18078079 \quad 1.83549383$

$\mathrm{H}-0.14394864 \quad-2.33977254 \quad 2.96820921$

$\mathrm{H} 1.23657985-1.40698724 \quad 3.56143228$

H $1.43378403-2.508621352 .19861738$

$0-1.28965530 \quad 0.11047371-2.83076239$

○ $0.12390513 \quad 1.99725944-1.49981848$

$0-0.51425164-0.64086200-1.91332912$

Vibrational frequencies (in $\mathrm{cm}-1$ )

$\begin{array}{lllllllllllllll}-568.6 & 78.3 & 105.7 & 145.5 & 170.4 & 188.8 & 197.8 & 220.7 & 239.5 & 262.5 & 270.7 & 311.0 & 344.4 & 351.8\end{array}$

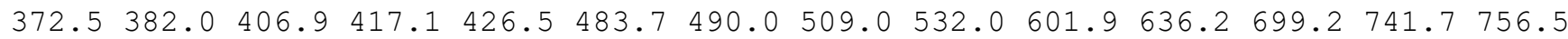
$800.4 \quad 837.9 \quad 880.9918 .3 \quad 940.6 \quad 980.7 \quad 993.4 \quad 1008.2 \quad 1010.9 \quad 1013.5 \quad 1017.6 \quad 1044.4 \quad 1060.1$

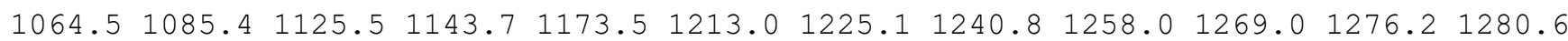

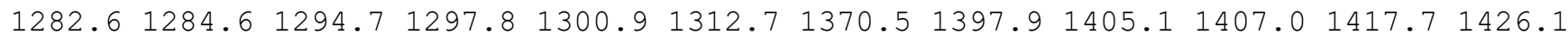
$1809.92658 .12659 .72672 .5 \quad 2674.2 \quad 2675.6 \quad 2678.42685 .82745 .2 \quad 2759.82764 .42776 .8$ $2779.32781 .92784 .9 \quad 5052.0$ 


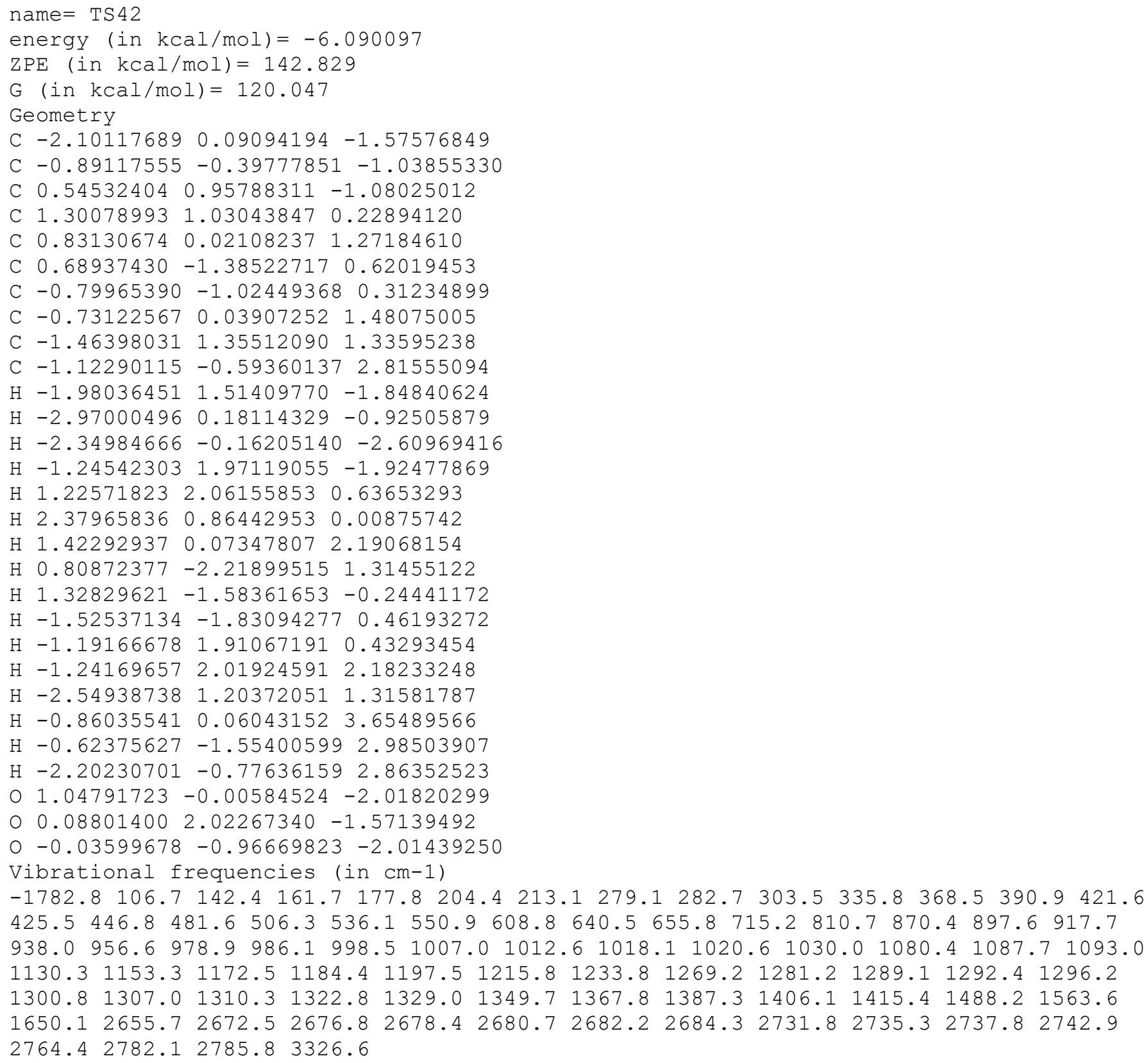


Н $2.108300891 .21059379-1.07354268$

$\mathrm{H} 2.18255515-0.48358005-0.62491680$

H $3.12075854 \quad 0.79496494 \quad 1.21177985$

$\mathrm{H} \quad 1.49110848 \quad 0.163190813 .09010880$

H $1.91878925-1.14103638 \quad 1.98012197$

$\begin{array}{lllll}\mathrm{H} & -1.65110804 & -0.41209727 & 0.30856344\end{array}$

H $1.910782292 .56894593 \quad 2.42073822$

H $0.46260365 \quad 2.35451643 \quad 1.44569717$

$\mathrm{H} \quad 1.94732564 \quad 2.99842380 \quad 0.71032522$

$\mathrm{H}-1.06167470-0.15002219 \quad 3.61621690$

$\mathrm{H}-0.41624734-1.78130553 \quad 3.37394168$

$\mathrm{H}-1.89453938-1.280136892 .54453913$

O $-2.061920991 .88880035-0.71844224$

$\begin{array}{llll}0 & -0.96261857 & 1.86718274 & -1.20377535\end{array}$

$0 \quad 0.63851266 \quad 0.11637296-2.89061892$

Vibrational frequencies (in cm-1)

$\begin{array}{llllllllllllllll}-1246.3 & 35.6 & 53.0 & 75.8 & 80.6 & 93.5 & 119.3 & 154.5 & 160.5 & 165.4 & 207.4 & 220.8 & 280.1 & 318.3\end{array}$

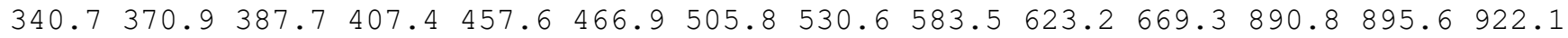

$938.4955 .3 \quad 970.8 \quad 1000.3 \quad 1038.4 \quad 1046.4 \quad 1051.7 \quad 1058.8 \quad 1081.9 \quad 1097.7 \quad 1122.1 \quad 1136.2 \quad 1168.0$

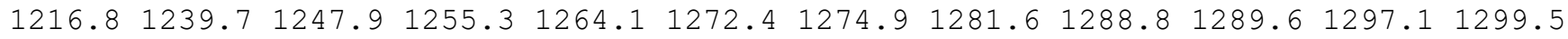
$1302.61310 .2 \quad 1347.2 \quad 1361.91374 .5 \quad 1380.7 \quad 1384.91400 .4 \quad 1414.3 \quad 1420.3 \quad 1816.2 \quad 1848.1$ $2086.62649 .62659 .02660 .2 \quad 2665.3 \quad 2667.92672 .42673 .22677 .02700 .62735 .72739 .8$ $2746.92775 .5 \quad 2777.7 \quad 2778.5$

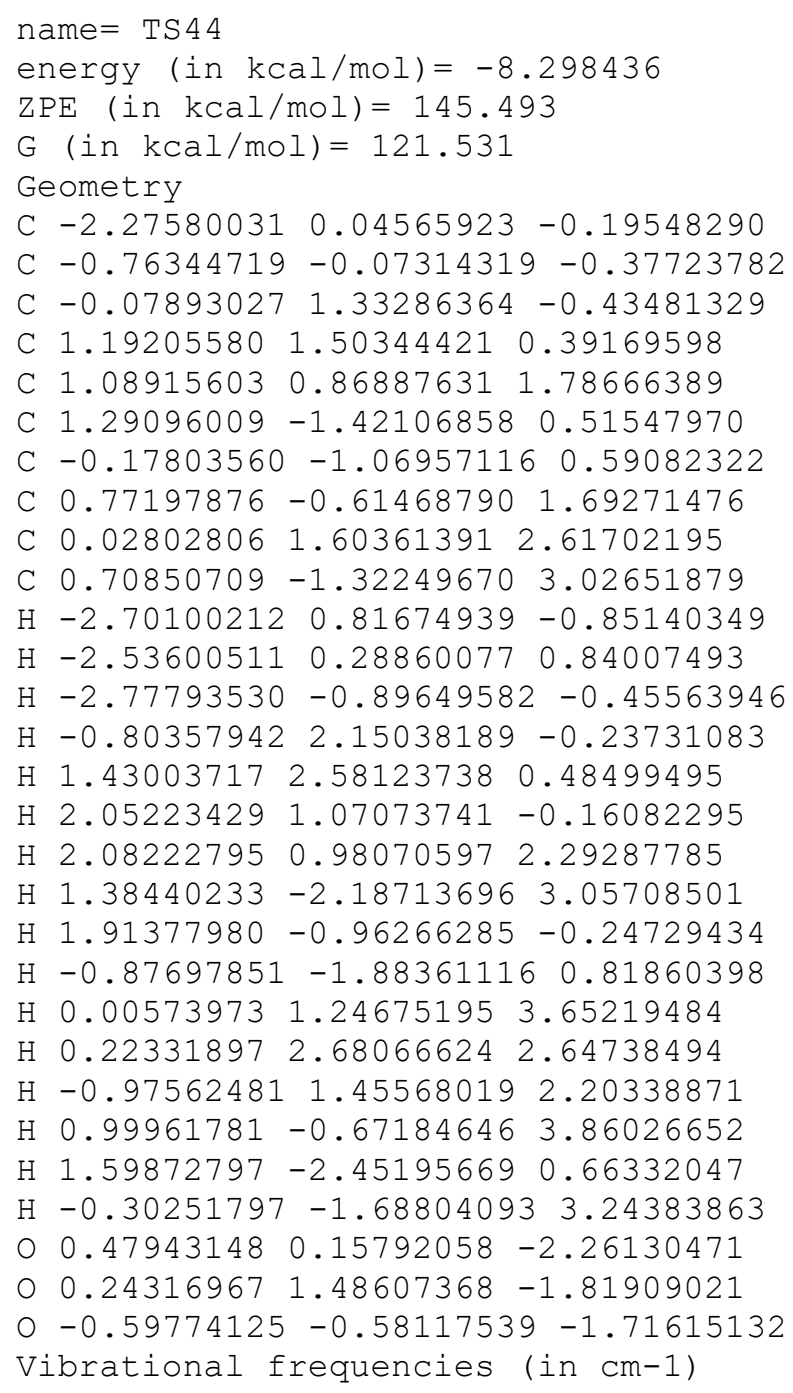


$2653.92669 .0 \quad 2670.8 \quad 2672.7 \quad 2675.3 \quad 2678.3 \quad 2679.22689 .72701 .22708 .72740 .22751 .3$ $2779.32780 .7 \quad 2782.2 \quad 2785.1$

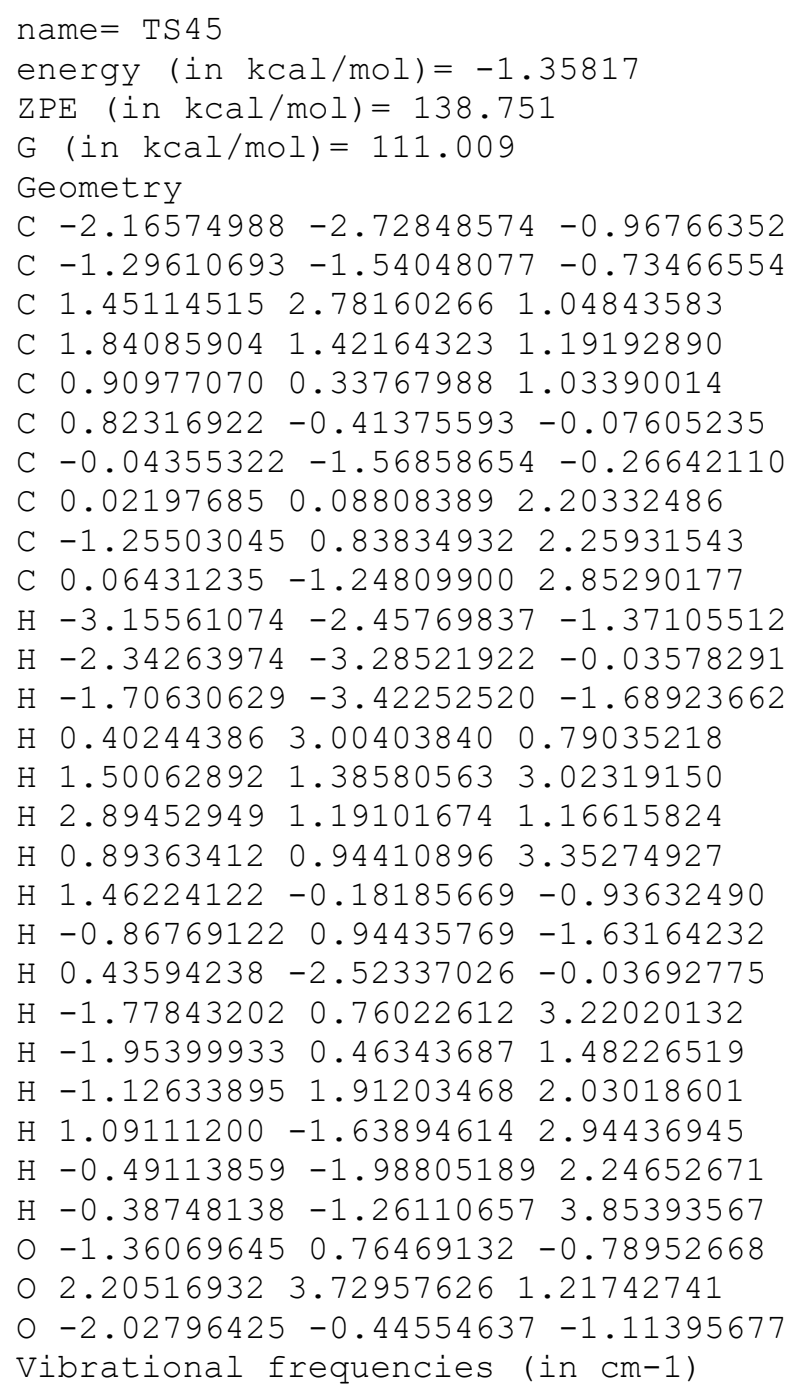


$\mathrm{H}-2.45204531-0.60534699-1.88106865$

H $0.04079575 \quad 2.82317498-1.16746757$

$\mathrm{H} 1.956837321 .86664036-0.02453948$

H $1.523819830 .39370900-1.40418888$

H $1.57307020 \quad 0.44161124 \quad 1.76971425$

H $1.93409553-1.91544098 \quad 1.07897309$

H $2.17966344-1.29196114 \quad-0.52790448$

$\mathrm{H}-0.30955375 \quad-2.62882074 \quad 0.32141352$

$\mathrm{H}-2.35653462 \quad 0.01875409 \quad 1.88930779$

H $-2.16678950 \quad 0.21302453 \quad 0.15760279$

$\mathrm{H}-1.41222992 \quad 1.36343957 \quad 1.24310721$

$\mathrm{H}-0.36941413-0.46692645 \quad 3.34999819$

H $0.58177204 \quad-1.80163404 \quad 2.70926827$

$\mathrm{H}-1.18026414 \quad-1.88885572 \quad 2.67609854$

$\begin{array}{lllll}0 & 1.45964514 & -0.48629677 & -2.22331665\end{array}$

$0-0.96106803 \quad 1.11584761-1.29223064$

$\begin{array}{lllll}0 & 0.12817572 & -0.80678469 & -2.34337815\end{array}$

Vibrational frequencies (in $\mathrm{cm}-1$ )

$\begin{array}{llllllllllllll}-786.3 & 82.4 & 108.0 & 159.7 & 180.7 & 190.1 & 194.2 & 219.6 & 241.3 & 250.0 & 269.9 & 284.1 & 329.3 & 346.0\end{array}$

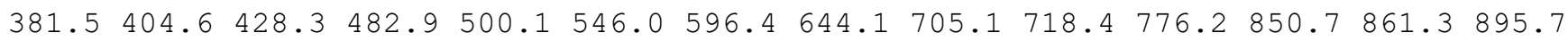
$920.5958 .4991 .8 \quad 994.4 \quad 1009.2 \quad 1022.9 \quad 1026.3 \quad 1034.3 \quad 1057.1 \quad 1071.0 \quad 1087.7 \quad 1094.5 \quad 1105.4$ $\begin{array}{llllllllllll}1157.5 & 1172.4 & 1208.5 & 1231.1 & 1246.7 & 1247.4 & 1253.1 & 1272.2 & 1284.1 & 1292.5 & 1298.9 & 1301.3\end{array}$ $\begin{array}{lllllllllllll}1303.1 & 1328.4 & 1333.2 & 1347.7 & 1351.2 & 1382.2 & 1395.1 & 1404.3 & 1411.2 & 1427.5 & 1484.3 & 1586.7\end{array}$

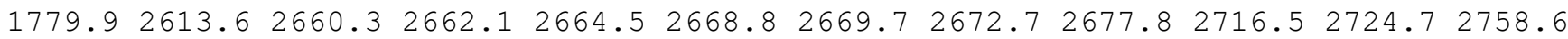
2760.62767 .02779 .62781 .6

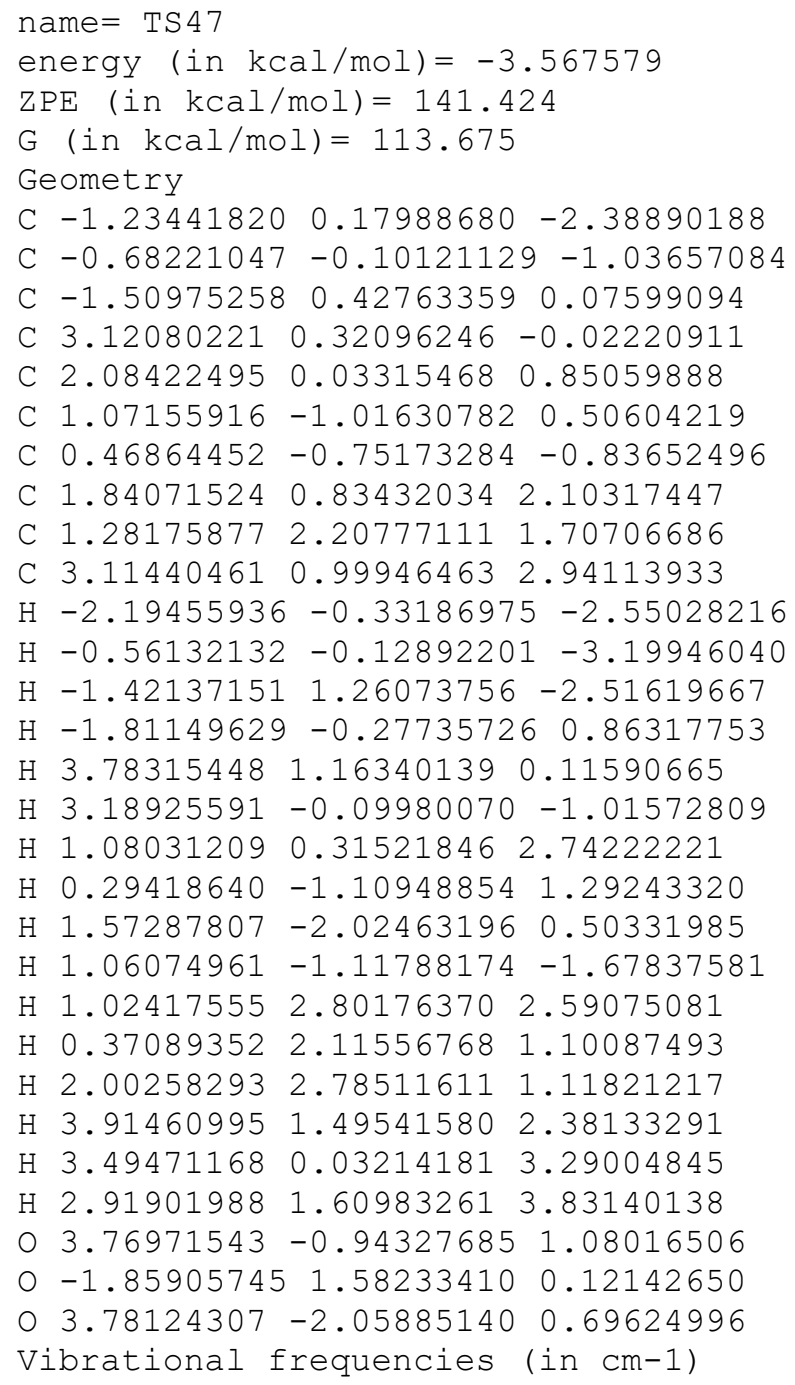


$\begin{array}{lllllllllllllll}1181.1 & 1221.4 & 1257.7 & 1260.9 & 1271.6 & 1277.3 & 1281.5 & 1290.6 & 1291.9 & 1300.3 & 1302.6 & 1306.4\end{array}$

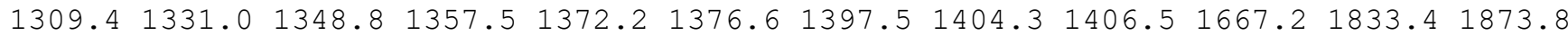
$2606.92649 .82661 .5 \quad 2668.7 \quad 2671.8 \quad 2673.4 \quad 2673.82677 .2 \quad 2702.82711 .92715 .92744 .4$ $2776.22777 .3 \quad 2779.8 \quad 2783.5$

name $=$ TS 48

energy $($ in $\mathrm{kcal} / \mathrm{mol})=-2.27341$

$\mathrm{ZPE}($ in $\mathrm{kcal} / \mathrm{mol})=140.429$

$\mathrm{G}($ in $\mathrm{kcal} / \mathrm{mol})=113.086$

Geometry

C $-1.68171610-1.86112317-1.49879269$

C $-0.45319804-1.19823565-1.62210359$

C $-0.27678077 \quad 1.12570419-0.66550755$

C $-1.301168441 .63833948 \quad 0.34937619$

C $-2.62320544 \quad 0.97786063 \quad 0.16679355$

C $1.65621812-0.29695849-0.55390109$

C $0.11964964-0.36500063-0.49039678$

C $-3.46308317 \quad 0.68167229 \quad 1.16839500$

C -3.167680120 .972083632 .60206510$

C $-4.78392283 \quad 0.03679199 \quad 0.90260745$

$\mathrm{H}-1.98956147-2.85156241-1.66465604$

$\mathrm{H}-2.42586915-1.553815010 .18812846$

$\mathrm{H}-1.93712986-2.14946035 \quad 0.32882313$

$\mathrm{H}-0.571574161 .34334856-1.71509369$

$\mathrm{H}-1.41706266 \quad 2.740367150 .22555713$

$\mathrm{H}-0.904665751 .51290262 \quad 1.37824426$

$\mathrm{H}-2.88559051 \quad 0.76194817-0.87096790$

$\mathrm{H} 2.12673246-0.56659393 \quad 0.40363416$

H 2.09522599-0.88778386-1.37990508

$\mathrm{H}-0.20858499-0.74415434 \quad 0.49777101$

$\mathrm{H}-3.10108207 \quad 2.05662700 \quad 2.77928368$

$\mathrm{H}-2.21102889 \quad 0.53374024 \quad 2.92026884$

$\mathrm{H}-3.937511190 .58651286 \quad 3.28240336$

$\mathrm{H}-4.89420422-0.90362214 \quad 1.45889627$

$\mathrm{H}-4.94243807-0.19121656-0.16074862$

$\mathrm{H}-5.610129430 .69374513 \quad 1.21267369$

O $1.990784721 .05063719-0.87432287$

O $0.91825500 \quad 1.85432434-0.36925805$

O $0.08057215-1.19366021-2.72576372$

Vibrational frequencies (in $\mathrm{cm}-1$ )

$\begin{array}{llllllllllllllll}-465.7 & 29.7 & 36.9 & 46.1 & 88.2 & 96.5 & 113.3 & 137.2 & 166.6 & 191.8 & 232.2 & 252.4 & 264.1 & 276.3\end{array}$

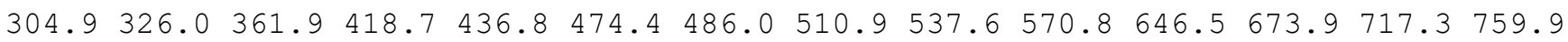

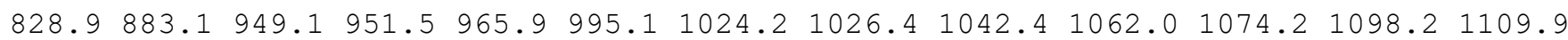

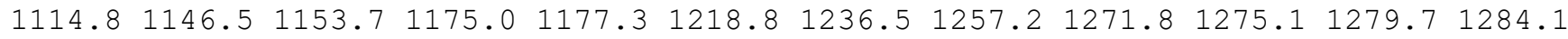
$1286.21287 .91295 .8 \quad 1309.5 \quad 1321.4 \quad 1340.7 \quad 1364.1 \quad 1378.51400 .3 \quad 1416.7 \quad 1434.31737 .5$ $1870.02643 .5 \quad 2649.6 \quad 2666.7 \quad 2669.9 \quad 2672.6 \quad 2675.02675 .42711 .5 \quad 2714.62730 .02737 .9$ $2749.6 \quad 2778.0 \quad 2780.0 \quad 5175.7$

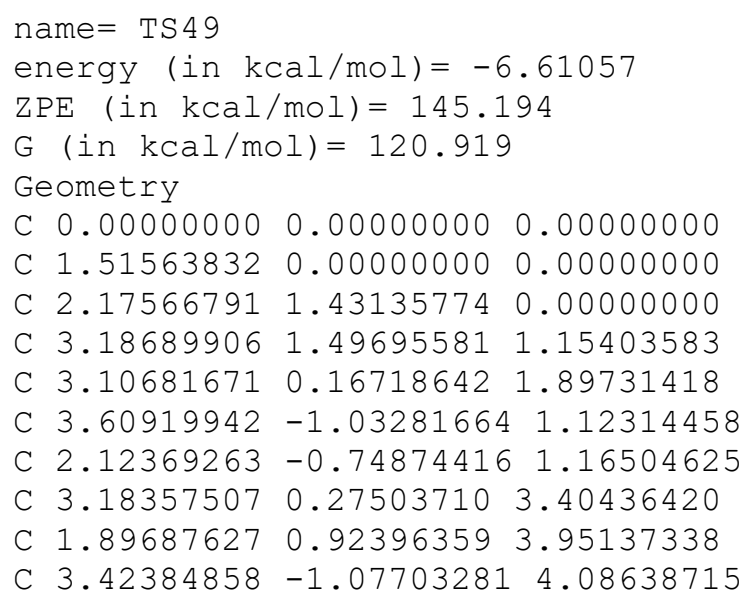


H $-0.40145035 \quad 0.59058359-0.83548815$

$\mathrm{H}-0.401029210 .411198110 .93360639$

$\mathrm{H}-0.39567139-1.01940106-0.11264416$

$\mathrm{H} \quad 1.448429292 .261171320 .00586633$

H 2.97061914 2.34778425 1.82211553

H $4.20826498 \quad 1.669942110 .76312390$

$\mathrm{H} \quad 4.05331043 \quad 0.93774848 \quad 3.65285508$

H $3.99542378-1.90382677 \quad 1.64295069$

$\mathrm{H} 4.16272152-0.88380816 \quad 0.19746400$

$\mathrm{H} 1.46427698-1.43077214 \quad 1.69253063$

H $1.381629690 .27239030 \quad 4.66459789$

$\mathrm{H} 1.178785091 .143065213 .15336978$

H $2.11226134 \quad 1.86392927 \quad 4.46766108$

H $3.44014129-0.968402495 .17684282$

H $4.38653165-1.509427543 .79066787$

H $2.63959480-1.800278273 .83811176$

$03.07844311 \quad 0.16563244-1.62419364$

$02.805262941 .51815061-1.26909311$

O $1.90419547-0.54257759-1.26593807$

Vibrational frequencies (in $\mathrm{cm}-1$ )

$\begin{array}{llllllllllllllll}-145.4 & 50.2 & 80.4 & 93.9 & 149.0 & 187.0 & 206.5 & 216.8 & 260.9 & 284.1 & 317.3 & 339.6 & 380.4 & 429.5\end{array}$ $\begin{array}{llllllllllllll}463.4 & 495.0 & 552.6 & 563.2 & 574.5 & 643.5 & 707.9 & 769.7 & 833.7 & 848.8 & 867.4 & 918.8 & 949.5 & 953.4\end{array}$ $973.9979 .6 \quad 997.9 \quad 1002.2 \quad 1004.6 \quad 1021.4 \quad 1032.9 \quad 1043.6 \quad 1079.8 \quad 1125.6 \quad 1133.5 \quad 1145.1 \quad 1164.6$

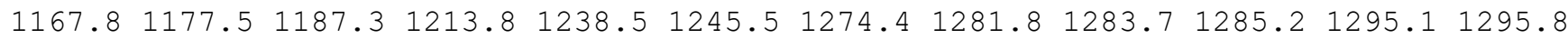
$1300.41304 .3 \quad 1306.7 \quad 1318.0 \quad 1322.0 \quad 1332.8 \quad 1338.1 \quad 1385.2 \quad 1393.6 \quad 1401.91417 .8 \quad 1487.4$ $2666.12670 .4 \quad 2670.5 \quad 2672.7 \quad 2676.5 \quad 2678.0 \quad 2678.6 \quad 2700.82703 .5 \quad 2707.6 \quad 2747.3 \quad 2770.5$ $2780.3 \quad 2780.5 \quad 2782.12783 .3$

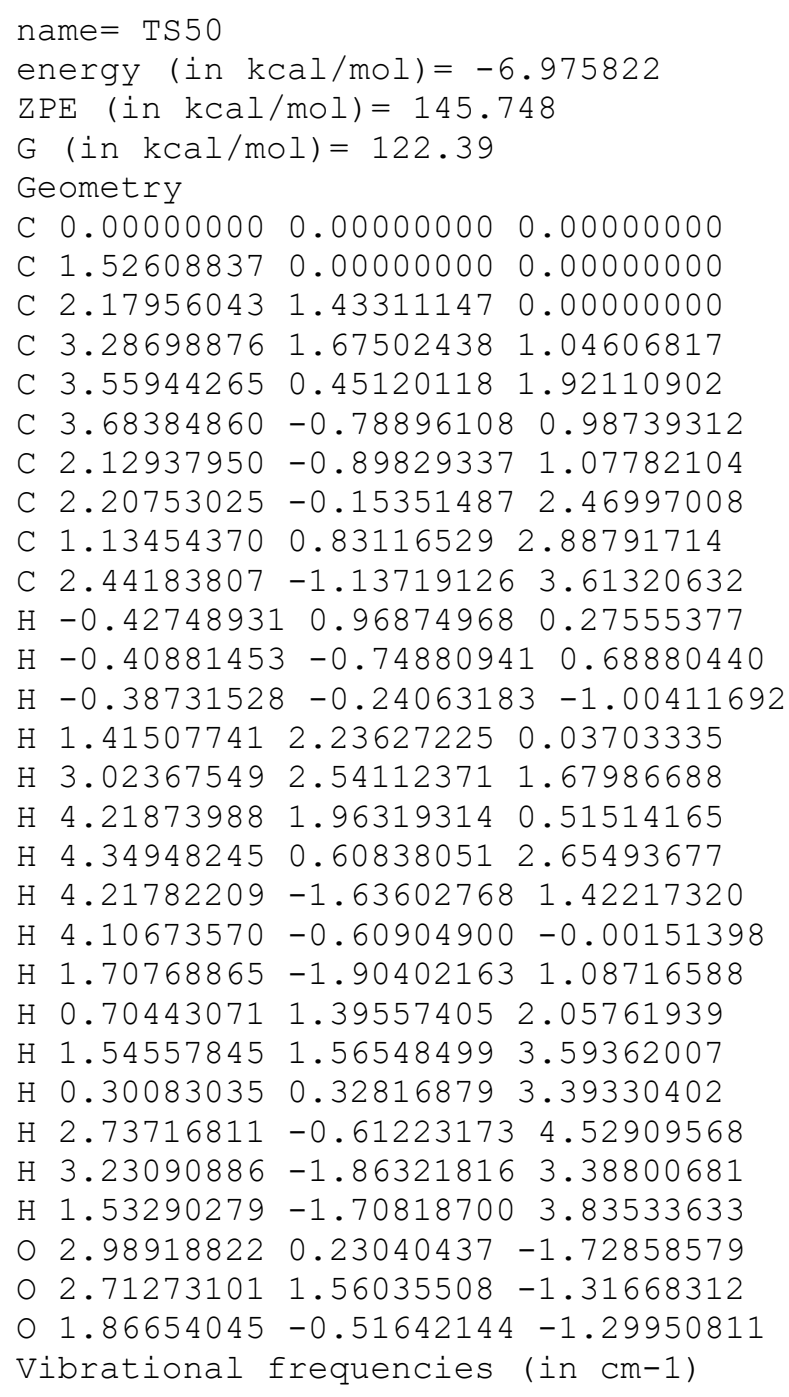


$\begin{array}{llllllllllllllllll}-111.5 & 68.0 & 125.0 & 155.0 & 174.7 & 211.9 & 222.4 & 263.7 & 290.5 & 319.8 & 356.7 & 384.6 & 417.9 & 440.3\end{array}$ $462.0 \quad 492.0 \quad 520.1 \quad 593.3 \quad 655.3 \quad 701.7 \quad 720.4 \quad 797.1 \quad 843.4 \quad 870.0 \quad 894.5 \quad 914.2 \quad 937.4 \quad 954.3$ $980.6997 .6 \quad 1003.91005 .8 \quad 1014.4 \quad 1018.3 \quad 1044.8 \quad 1059.81087 .91090 .71098 .11141 .2$

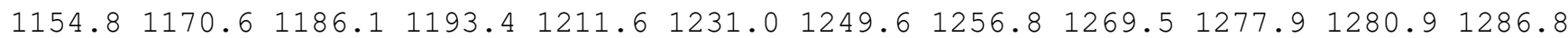
$1290.7 \quad 1292.2 \quad 1297.7 \quad 1299.8 \quad 1305.6 \quad 1308.8 \quad 1316.91322 .6 \quad 1373.4 \quad 1384.1 \quad 1405.91410 .9$ $1420.32659 .2 \quad 2660.72672 .6 \quad 2674.0 \quad 2675.7 \quad 2678.62680 .32684 .72689 .32739 .92746 .9$ $2751.22768 .4 \quad 2776.3 \quad 2781.8 \quad 2786.0$

name $=\operatorname{TS} 51$

energy (in $\mathrm{kcal} / \mathrm{mol})=-6.713037$

$\mathrm{ZPE}(\mathrm{in} \mathrm{kcal} / \mathrm{mol})=145.918$

$\mathrm{G}($ in $\mathrm{kcal} / \mathrm{mol})=122.809$

Geometry

C $0.00000000 \quad 0.00000000 \quad 0.00000000$

C $1.522620790 .00000000 \quad 0.00000000$

C $2.16058694 \quad 1.43710030 \quad 0.00000000$

C $3.31345269 \quad 1.66315380 \quad 0.99798012$

C $3.600535140 .43804252 \quad 1.86581223$

C $3.68405277-0.81283283 \quad 0.94062782$

C $2.13085326-0.90124211 \quad 1.07210178$

C $2.26692464 \quad-0.15805985 \quad 2.46470534$

C $1.196485990 .81061986 \quad 2.91777473$

C $2.54397904-1.164020293 .58709836$

$\mathrm{H}-0.39633780 \quad 0.60770027-0.82545120$

$\mathrm{H}-0.41558387 \quad 0.38165978 \quad 0.93715061$

$\mathrm{H}-0.39021800-1.01764250-0.14069332$

$\mathrm{H} \quad 1.38957858 \quad 2.230057210 .08743614$

$\mathrm{H} 3.093798832 .53592632 \quad 1.63953100$

H $4.22769248 \quad 1.93324570 \quad 0.42810266$

$\mathrm{H} \quad 4.41821764 \quad 0.59475571 \quad 2.57000377$

H $4.21534619-1.66228876 \quad 1.37472163$

H $4.08246198-0.64949527-0.06103353$

H $1.68963609-1.897754831 .09424202$

$\mathrm{H} \quad 0.27076948 \quad 0.28054353 \quad 3.17758541$

$\mathrm{H} \quad 0.94037598 \quad 1.56776467 \quad 2.17188416$

$\mathrm{H} 1.525500961 .35717775 \quad 3.81257669$

H $2.05035628-0.88553950 \quad 4.52458229$

H $3.61344992-1.248890413 .80904129$

H $2.18710824 \quad-2.16738649 \quad 3.32875991$

O $2.94266441 \quad 0.27302364-1.76091105$

o $2.631811621 .59031887-1.33729762$

O $1.85827218-0.51540151-1.30206604$

Vibrational frequencies (in cm-1)

$\begin{array}{llllllllllllllll}-115.0 & 91.3 & 123.4 & 174.6 & 188.1 & 218.0 & 230.8 & 268.7 & 283.7 & 324.5 & 350.8 & 382.5 & 417.3 \quad 443.2\end{array}$ $468.2 \quad 495.9 \quad 523.8 \quad 595.0 \quad 656.3 \quad 704.9 \quad 722.0 \quad 795.5 \quad 845.5 \quad 870.2 \quad 892.8 \quad 914.9 \quad 939.1956 .0$ $972.1995 .3 \quad 1001.8 \quad 1006.6 \quad 1022.9 \quad 1027.7 \quad 1046.9 \quad 1058.9 \quad 1087.7 \quad 1091.5 \quad 1099.8 \quad 1140.5$

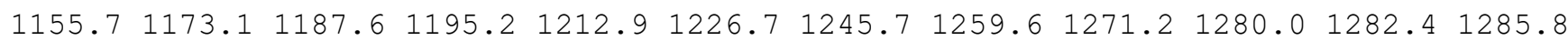

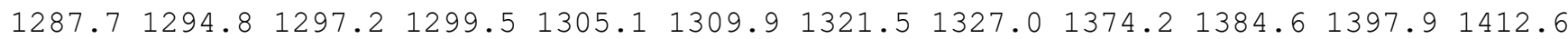
$1426.02658 .8 \quad 2670.2 \quad 2671.3 \quad 2673.3 \quad 2674.7 \quad 2676.62677 .62686 .22691 .02739 .62745 .7$ $2749.2 \quad 2768.8 \quad 2780.3 \quad 2781.4 \quad 2783.3$

name $=\operatorname{TS} 52$

energy $($ in $\mathrm{kcal} / \mathrm{mol})=-6.544514$

$\mathrm{ZPE}($ in $\mathrm{kcal} / \mathrm{mol})=145.8$

$\mathrm{G}($ in $\mathrm{kcal} / \mathrm{mol})=122.718$

Geometry

C $0.00000000 \quad 0.00000000 \quad 0.00000000$

C $1.52278110 \quad 0.00000000 \quad 0.00000000$

C 2.153060731 .440138170 .00000000

C 3.348862451 .648719420 .95034164

C $3.62264950 \quad 0.43407927 \quad 1.83681966$

C $3.68468315-0.82824330 \quad 0.92329687$

C $2.13188068-0.900576371 .07225983$ 
C $2.29069609-0.145755642 .45461136$

$\begin{array}{llll}\text { C } 1.22797816 & 0.82888065 & 2.91312312\end{array}$

C $2.58009640-1.141735093 .58425811$

$\mathrm{H}-0.39615178 \quad 0.62330664 \quad 0.81370178$

$\mathrm{H}-0.41376682 \quad 0.36483706 \quad 0.94469886$

$\mathrm{H}-0.39041340-1.01458202-0.16019389$

$\begin{array}{lllll}\mathrm{H} & 1.38516261 & 2.22885973 & 0.13314505\end{array}$

H $3.18702870 \quad 2.54677879 \quad 1.57324171$

H 4.252316231 .866732490 .34155564

H $4.44766115 \quad 0.59001931 \quad 2.53257700$

H $4.21368851-1.67718228 \quad 1.36067857$

H $4.07077181-0.67887598-0.08547614$

H $1.67944763-1.891541131 .10723272$

$\mathrm{H} 1.04228891 \quad 1.64469697 \quad 2.20963509$

H $1.519713691 .30215909 \quad 3.86115261$

$\mathrm{H} \quad 0.27230117 \quad 0.31584484 \quad 3.08280360$

$\mathrm{H} \quad 1.93882268-0.97781105 \quad 4.45706908$

H $3.61534654-1.069391513 .93521940$

H $2.41839811-2.17852397 \quad 3.26885863$

$02.909312510 .30640438-1.78572292$

$02.567934961 .61215530-1.35373601$

O $1.86054226-0.51718108-1.30079403$

Vibrational frequencies (in $\mathrm{cm}-1$ )

$\begin{array}{lllllllllllllll}-156.0 & 88.4 & 116.2 & 173.9 & 180.5 & 212.4 & 217.7 & 263.3 & 279.0 & 324.9 & 346.5 & 382.4 & 416.3 & 444.1\end{array}$

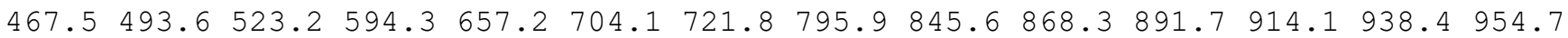

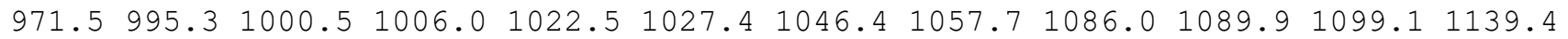

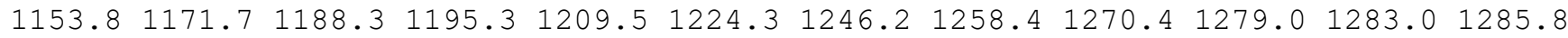

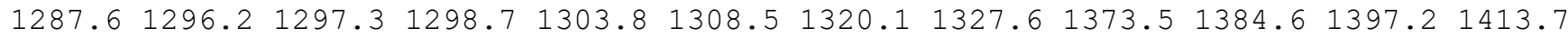
$1427.82658 .4 \quad 2670.4 \quad 2670.92673 .3 \quad 2674.1 \quad 2677.12677 .72687 .6 \quad 2691.72739 .6 \quad 2745.6$ $\begin{array}{lllll}2749.2 & 2768.8 & 2780.2 & 2781.2 & 2783.1\end{array}$

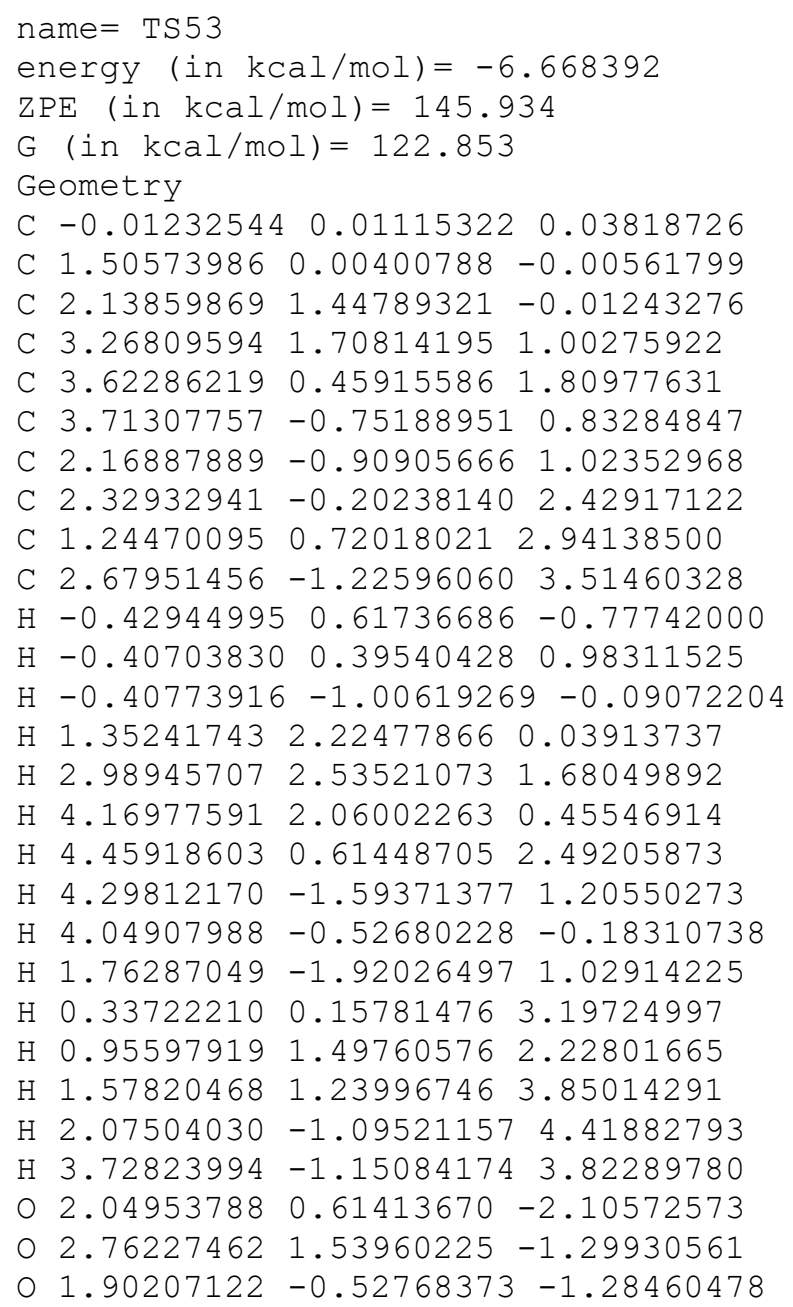


$\mathrm{H} 2.51695553-2.255242163 .17557666$

Vibrational frequencies (in $\mathrm{cm}-1$ )

$\begin{array}{lllllllllllllll}-133.1 & 85.2 & 125.7 & 173.6 & 190.0 & 212.1 & 237.6 & 271.5 & 280.1 & 325.2 & 354.9 & 373.9 & 414.8 & 441.3\end{array}$

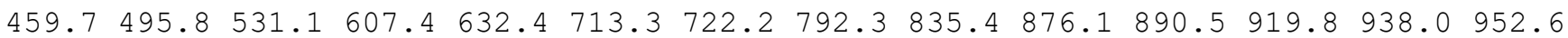
$980.4 \quad 993.5 \quad 1001.3 \quad 1012.1 \quad 1022.4 \quad 1029.3 \quad 1055.9 \quad 1072.8 \quad 1084.8 \quad 1093.5 \quad 1103.31136 .1$

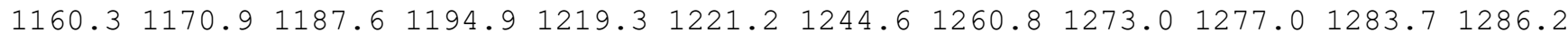
$1286.91293 .8 \quad 1297.0 \quad 1300.2 \quad 1308.6 \quad 1320.4 \quad 1325.1 \quad 1326.4 \quad 1374.8 \quad 1379.5 \quad 1398.51411 .5$ $1427.22656 .3 \quad 2670.8 \quad 2671.3 \quad 2672.9 \quad 2674.5 \quad 2676.8 \quad 2677.92684 .2 \quad 2688.12737 .42745 .7$ $2749.3 \quad 2764.8 \quad 2780.4 \quad 2781.7 \quad 2783.3$

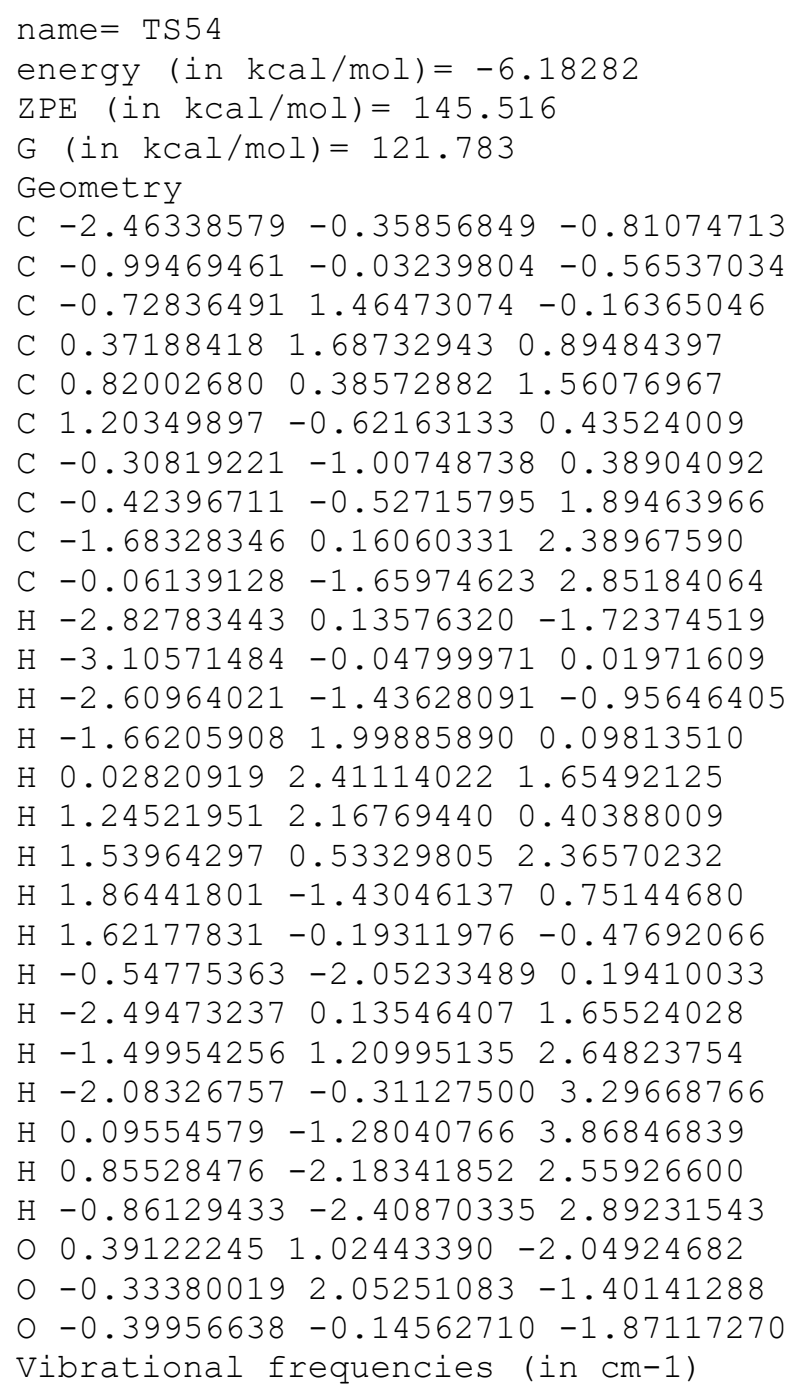


C $2.34607456-0.70054260 \quad 0.65068930$

C $-0.73985020-1.30087930 \quad 0.16777939$

C $-0.08224162-0.67552988 \quad 1.37620480$

C $-1.14089658 \quad 0.22853533 \quad 2.04836217$

C $0.35220085-1.789733962 .24068781$

$\mathrm{H}-0.46333326-1.88171451-3.00912252$

$\mathrm{H}-1.86859002-0.83118617-2.76094913$

$\mathrm{H}-1.80071095-2.41849708-1.98225823$

$\mathrm{H} \quad 0.13818439 \quad 0.77897423 \quad-2.30937350$

$\mathrm{H}-0.01191790 \quad 1.73757691-0.02622621$

$\mathrm{H} 1.69046218 \quad 1.69796777-0.49716138$

$\mathrm{H} \quad 1.43580694 \quad 0.83842598 \quad 1.82504389$

H $2.27235272-1.77688137 \quad 0.86199496$

H $3.20203234 \quad 0.33393544 \quad-1.51542274$

$\mathrm{H}-1.45673204 \quad-2.08677680 \quad 0.40043485$

$\mathrm{H}-1.55409391 \quad 0.95089218 \quad 1.33333621$

H $-0.72295382 \quad 0.799480812 .88095272$

$\mathrm{H}-1.98341386-0.361069572 .42380462$

$\mathrm{H} \quad 0.42062320-1.09784944 \quad 3.76425543$

H $1.16109927-1.268191893 .47807566$

$\mathrm{H}-0.32470352-2.542751712 .56026966$

○ $2.600976360 .32152153-2.30688938$

$01.62199549-0.59759718-1.84705803$

o $3.37628793-0.217024830 .23725606$

Vibrational frequencies (in $\mathrm{cm}-1$ )

$\begin{array}{lllllllllllllll}-980.1 & 50.2 & 95.2 & 103.8 & 128.0 & 149.5 & 165.8 & 181.4 & 199.4 & 212.4 & 252.0 & 281.1 & 315.2 & 340.3\end{array}$ $369.5 \quad 375.5 \quad 430.2 \quad 457.9 \quad 462.2 \quad 481.7 \quad 506.6 \quad 524.4 \quad 531.5 \quad 566.4 \quad 604.4 \quad 644.3 \quad 738.6 \quad 829.4$

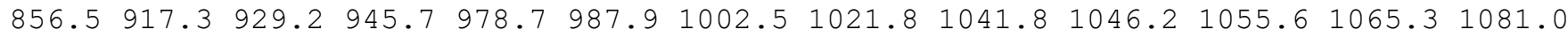

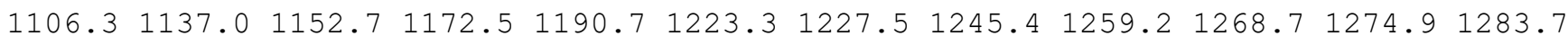
$1295.51299 .1 \quad 1307.5 \quad 1317.2 \quad 1321.3 \quad 1340.2 \quad 1364.91384 .01393 .4 \quad 1401.4 \quad 1411.21807 .6$ $1872.62633 .82661 .3 \quad 2663.3 \quad 2670.7 \quad 2673.7 \quad 2679.72685 .92700 .22721 .02741 .22751 .0$ $2776.92779 .5 \quad 2793.1 \quad 4726.4$

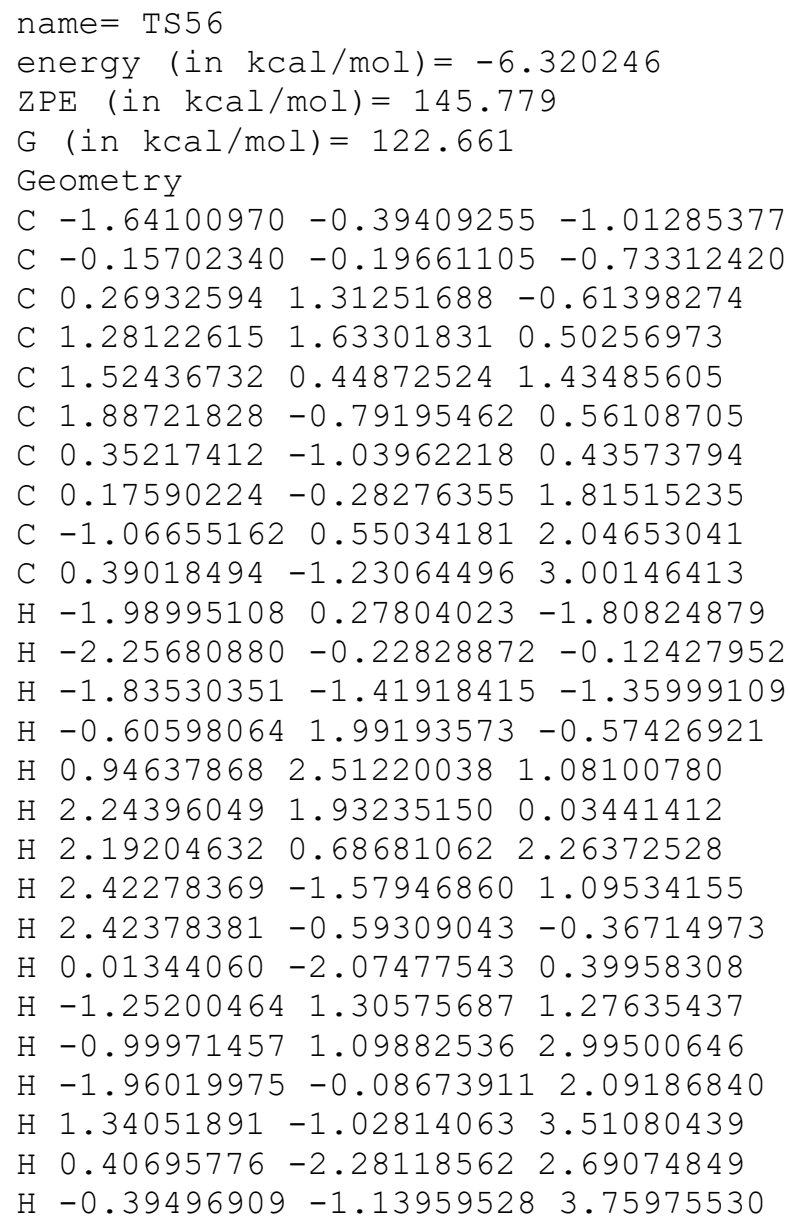


O $1.44394961 \quad 0.36117894-2.26741738$

$0.0 .839473281 .58479791-1.89108045$

O $0.47932075-0.63249883-1.94828333$

Vibrational frequencies (in $\mathrm{cm}-1$ )

$\begin{array}{lllllllllllllll}-144.8 & 91.8 & 114.2 & 169.1 & 179.1 & 211.5 & 218.3 & 262.8 & 278.4 & 326.9 & 342.7 & 382.0 & 415.2 & 443.9\end{array}$

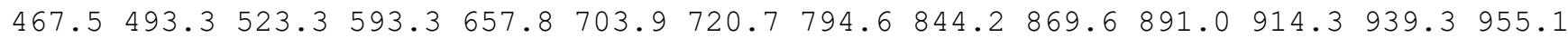
$973.9995 .4 \quad 1001.7 \quad 1007.0 \quad 1021.3 \quad 1028.3 \quad 1048.1 \quad 1057.6 \quad 1086.2 \quad 1090.2 \quad 1102.7 \quad 1139.0$

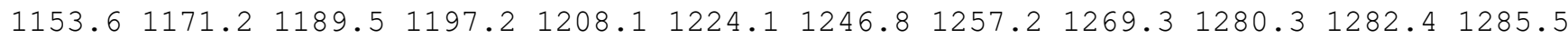

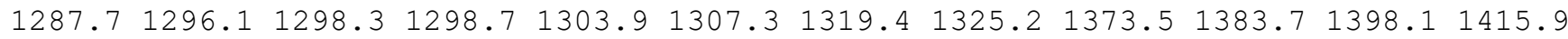
1426.62658 .32667 .82669 .92670 .62673 .22676 .32678 .22687 .62692 .12740 .22745 .3 $2749.12768 .4 \quad 2779.1 \quad 2780.7 \quad 2782.7$

name $=\operatorname{TS} 57$

energy $($ in $\mathrm{kcal} / \mathrm{mol})=-6.053573$

$\mathrm{ZPE}(\mathrm{in} \mathrm{kcal} / \mathrm{mol})=145.583$

$\mathrm{G}($ in $\mathrm{kcal} / \mathrm{mol})=121.892$

Geometry

C $-2.02690547-0.72948066-0.94496537$

C $-0.708635390 .00041691-0.70269018$

C $-0.71305674 \quad 1.51830810-1.11614244$

C $0.10934335 \quad 2.45198625 \quad-0.20467219$

C $0.55019933 \quad 1.77503631 \quad 1.09308961$

C $1.27358985 \quad 0.44250956 \quad 0.72700653$

C $-0.14391349-0.21169793 \quad 0.70132187$

C $-0.62147777 \quad 0.91353209 \quad 1.70399465$

C -2.029032391 .468380091 .63882565$

C $-0.335606190 .51557705 \quad 3.15199277$

$\mathrm{H}-2.30899147-1.37212670-0.10255061$

$\mathrm{H}-1.92834925-1.38736731-1.82881904$

$\mathrm{H}-2.85896621-0.05045481-1.14785587$

$\mathrm{H}-1.73662605 \quad 1.90856830 \quad-1.28468113$

$\mathrm{H}-0.46452027 \quad 3.36834331 \quad 0.01882271$

$\mathrm{H} 1.007892212 .78892115-0.76503775$

$\mathrm{H} 1.06519421 \quad 2.446827331 .77941609$

H $1.931512790 .05198799 \quad 1.50566223$

$\mathrm{H} \quad 1.83091805 \quad 0.43044124-0.21040204$

$\mathrm{H}-0.22481645-1.239846291 .05346121$

H $-2.09827563 \quad 2.39673448 \quad 2.22316852$

$\mathrm{H}-2.75507336 \quad 0.76070195 \quad 2.05848596$

$\mathrm{H}-2.37314873 \quad 1.706259120 .63055373$

$\mathrm{H}-0.40208060 \quad 1.38370843 \quad 3.81774274$

$\mathrm{H} \quad 0.66342811 \quad 0.08555285 \quad 3.28157993$

$\mathrm{H}-1.05582850-0.230876893 .50571006$

$\begin{array}{llllll}0 & 0.83145671 & 0.46837199 & -2.34084433\end{array}$

O $-0.146842491 .48756928-2.42292864$

O $0.18704359-0.60113664-1.65871342$

Vibrational frequencies (in $\mathrm{cm}-1$ )

$\begin{array}{lllllllllllllll}-110.1 & 60.1 & 89.2 & 147.5 & 167.7 & 185.1 & 211.6 & 257.6 & 285.5 & 329.2 & 345.1 & 386.6 & 418.0 & 439.6\end{array}$ $467.1 \quad 489.2 \quad 524.2 \quad 590.8 \quad 660.8 \quad 701.3 \quad 720.1 \quad 795.9 \quad 848.0 \quad 871.4 \quad 893.6 \quad 915.7 \quad 938.8 \quad 955.7$ $982.1998 .8 \quad 1002.8 \quad 1007.3 \quad 1015.5 \quad 1018.0 \quad 1043.3 \quad 1061.2 \quad 1085.01089 .2 \quad 1102.21139 .1$ $\begin{array}{llllllllllll}1149.5 & 1167.9 & 1185.8 & 1193.4 & 1206.5 & 1227.7 & 1246.6 & 1254.6 & 1267.8 & 1278.5 & 1279.9 & 1286.6\end{array}$

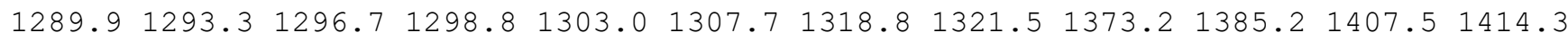
$1421.42653 .92658 .62673 .2 \quad 2675.12675 .42678 .12680 .72686 .82689 .82740 .72747 .5$ $2751.4 \quad 2767.8 \quad 2774.0 \quad 2782.0 \quad 2785.6$

name $=\operatorname{TS} 58$

energy (in $\mathrm{kcal} / \mathrm{mol})=-5.762502$

$\mathrm{ZPE}(\mathrm{in} \mathrm{kcal} / \mathrm{mol})=145.556$

$\mathrm{G}($ in $\mathrm{kcal} / \mathrm{mol})=121.775$

Geometry

C $-2.08849674-0.18204005-0.97167860$

C $-0.57747368-0.26595518-0.80464971$

C $0.145783881 .12896656-0.79136125$ 
C $1.24329340 \quad 1.30809361 \quad 0.27712653$

C $1.33361865 \quad 0.12529294 \quad 1.24189541$

C $1.41107820-1.17841387 \quad 0.39156233$

C $-0.15057409-1.146722350 .36535393$

C $-0.09495224 \quad-0.34189947 \quad 1.72862035$

C $-1.11524613 \quad 0.73592525 \quad 2.02945688$

C $0.01470970-1.31892193 \quad 2.90478545$

$\mathrm{H}-2.35948697 \quad 0.40285092-1.86122900$

$\mathrm{H}-2.57486134 \quad 0.27159387 \quad-0.10249304$

$\mathrm{H}-2.52141928-1.18331116-1.10696063$

$\mathrm{H}-0.57833477 \quad 1.96792154 \quad-0.77835730$

$\mathrm{H} \quad 1.08467752 \quad 2.24905058 \quad 0.83386873$

H $2.221219531 .43170196-0.23606731$

$\mathrm{H} 2.08051049 \quad 0.26595794 \quad 2.02328301$

H $1.83166746-2.03720437 \quad 0.91801239$

$\mathrm{H} 1.91588090-1.09838138-0.57151244$

$\mathrm{H}-0.66858227-2.10604751 \quad 0.38992310$

$\mathrm{H}-0.896247761 .684757721 .52807465$

$\mathrm{H}-1.14647657 \quad 0.965264423 .10398945$

$\mathrm{H}-2.12778307 \quad 0.43316087 \quad 1.73596491$

H $0.95290966-1.17855504 \quad 3.45348797$

$\mathrm{H}-0.00776940 \quad-2.36543969 \quad 2.57801757$

$\mathrm{H}-0.79976851-1.20272039 \quad 3.62541128$

O $1.01866996-0.13643414-2.42916179$

$00.721856661 .20844781-2.09400182$

o $-0.13374371-0.86491800-2.03714090$

Vibrational frequencies (in $\mathrm{cm}-1$ )

$\begin{array}{lllllllllllllllll}-145.3 & 38.5 & 100.3 & 116.2 & 175.5 & 206.6 & 216.3 & 260.3 & 285.5 & 325.6 & 347.1 & 385.3 & 420.8 & 447.2\end{array}$

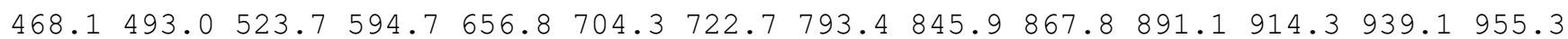
$967.5996 .0 \quad 998.5 \quad 1006.5 \quad 1019.3 \quad 1028.4 \quad 1045.6 \quad 1058.5 \quad 1085.7 \quad 1088.5 \quad 1097.01139 .5 \quad 1153.2$ $\begin{array}{llllllllllll}1171.9 & 1187.9 & 1195.5 & 1208.2 & 1226.2 & 1246.2 & 1258.4 & 1270.7 & 1277.9 & 1280.3 & 1285.3 & 1287.4\end{array}$ $1294.51297 .7 \quad 1298.7 \quad 1300.4 \quad 1309.2 \quad 1319.4 \quad 1323.1 \quad 1372.5 \quad 1386.0 \quad 1394.81418 .6 \quad 1425.8$ $2656.82664 .5 \quad 2667.12670 .4 \quad 2670.7 \quad 2674.12677 .22689 .52692 .02738 .62745 .22749 .0$ $2769.02775 .3 \quad 2780.4 \quad 2781.9$

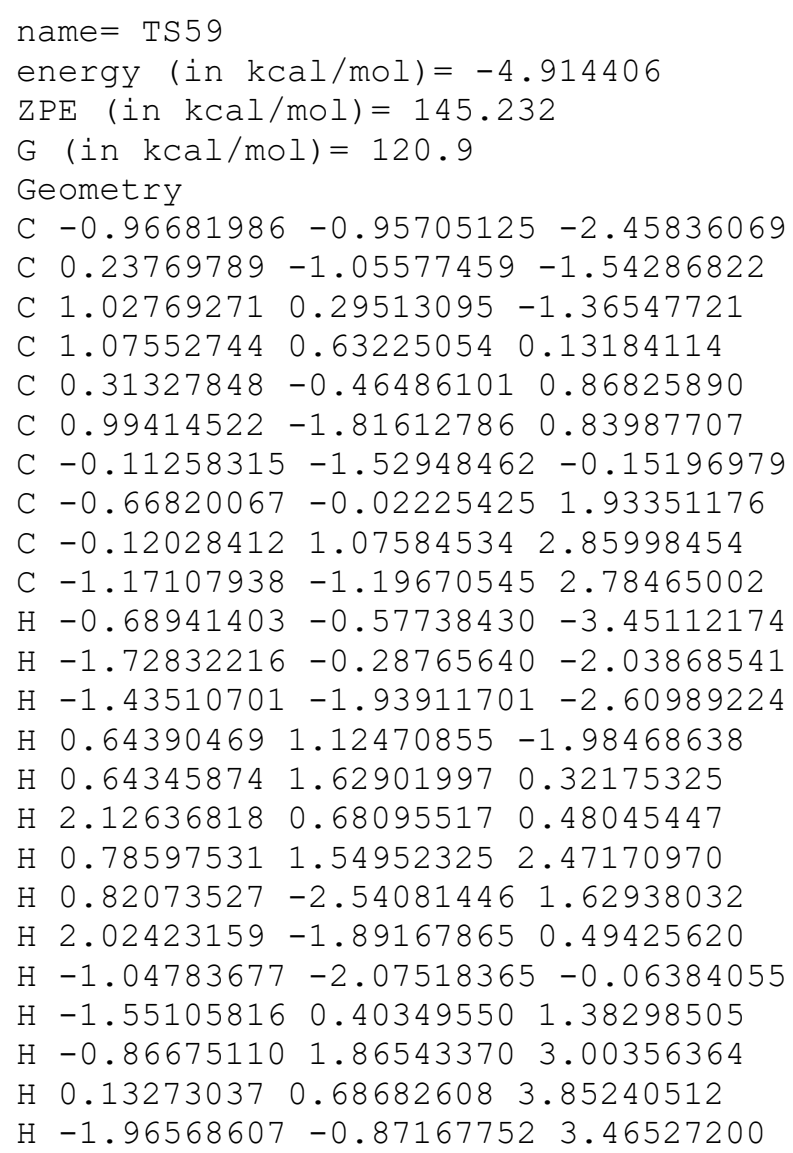


$\mathrm{H}-0.36636279-1.621359073 .39612580$

$\mathrm{H}-1.57983583-2.002831622 .16652046$

$02.44518264-1.40021528-1.81583027$

$02.312513290 .01843257-1.90141606$

O $1.17966601-1.90279123-2.20381510$

Vibrational frequencies (in $\mathrm{cm}-1$ )

$\begin{array}{lllllllllllllllll}-141.5 & 43.7 & 81.6 & 97.1 & 152.6 & 187.5 & 211.2 & 226.1 & 257.1 & 294.7 & 330.4 & 344.7 & 365.9 & 408.5\end{array}$

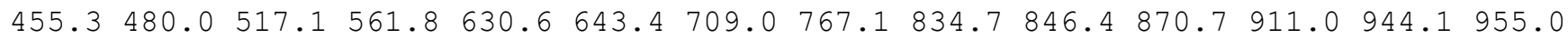
$977.6 \quad 982.2 \quad 995.1 \quad 1001.7 \quad 1006.2 \quad 1019.8 \quad 1034.7 \quad 1052.2 \quad 1074.5 \quad 1124.9 \quad 1131.8 \quad 1144.91164 .7$ $1170.51181 .7 \quad 1183.3 \quad 1218.0 \quad 1244.5 \quad 1259.9 \quad 1278.0 \quad 1283.4 \quad 1286.2 \quad 1292.2 \quad 1293.7 \quad 1300.1$

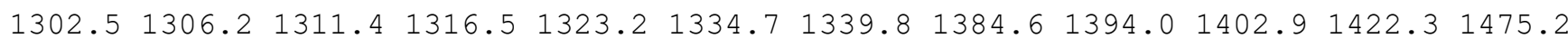
$2664.52666 .82672 .12672 .6 \quad 2675.6 \quad 2676.2 \quad 2678.42697 .52699 .4 \quad 2707.22745 .12767 .5$ $2780.42780 .6 \quad 2781.8 \quad 2783.1$

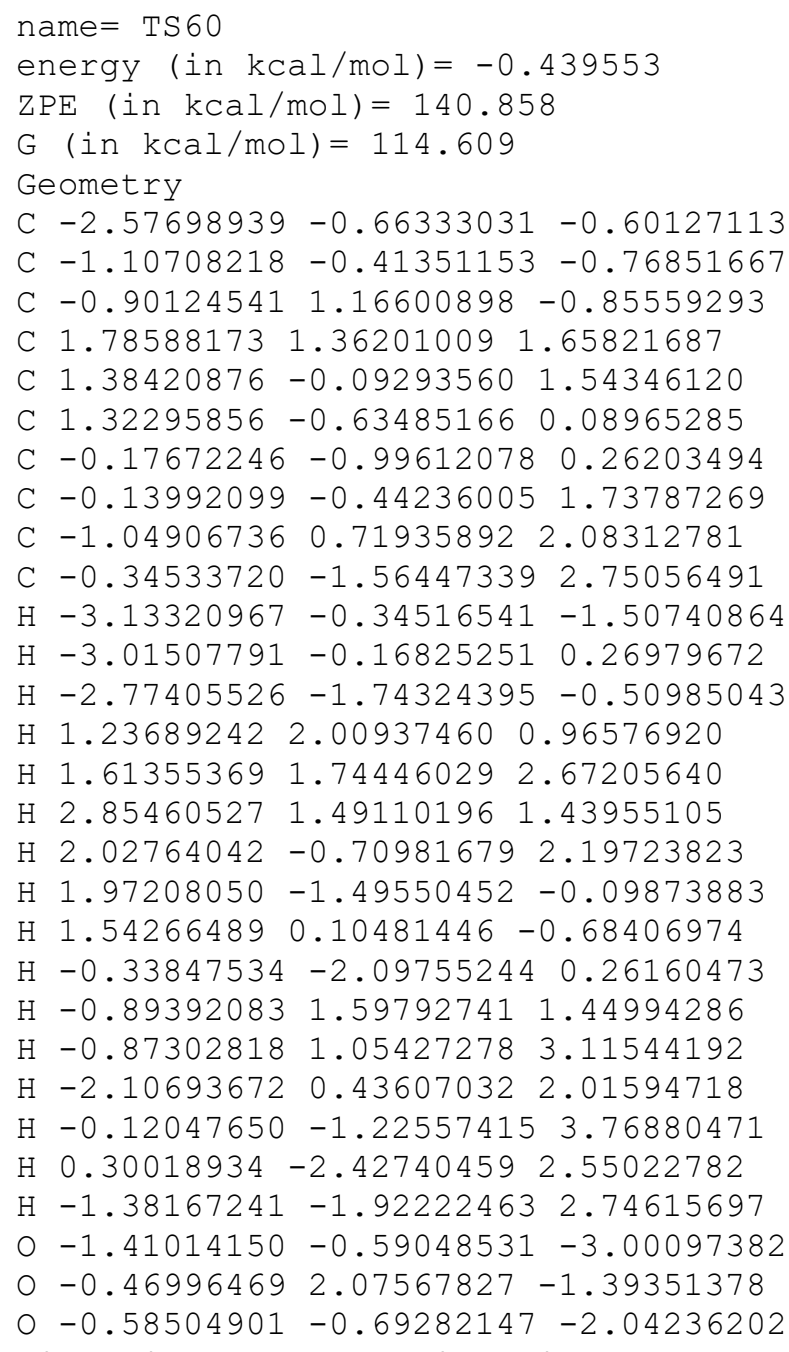


C $-0.81749893-0.30724981-0.78307003$

C $-0.23195311 \quad 1.14750333-0.68767112$

C 0.932524261 .339449720 .30385106

C $1.228112010 .08651731 \quad 1.12909033$

C $1.29932496-1.14314028 \quad 0.17495001$

C $-0.26023482-1.222111610 .30888046$

C $-0.10844225-0.51494494 \quad 1.71260708$

$\begin{array}{llll}\text { C }-1.15591806 & 0.45741168 & 2.20815841\end{array}$

C $0.17338370-1.54160662 \quad 2.81089981$

$\mathrm{H}-2.79089296 \quad 0.64477957-0.96733814$

$\mathrm{H}-2.84833151-0.99753026-0.29368861$

$\mathrm{H}-2.53810769-0.73454328-2.01309269$

$\mathrm{H}-1.01961257 \quad 1.91284211-0.53357789$

$\mathrm{H} \quad 0.71369916 \quad 2.18806091 \quad 0.97847528$

$\mathrm{H} \quad 1.83884519 \quad 1.63448611-0.26499098$

H 2.04936350 $0.21889070 \quad 1.83401721$

H $1.82733307 \quad-2.00792555 \quad 0.57932199$

H $1.69613426-0.96007519-0.82381064$

$\mathrm{H}-0.71791195-2.210089270 .32659648$

$\mathrm{H}-1.70215883 \quad 0.95687860 \quad 1.40460135$

$\mathrm{H}-0.68714410 \quad 1.23480932 \quad 2.82715215$

$\mathrm{H}-1.90610743-0.04240007 \quad 2.83476125$

$\mathrm{H} \quad 0.42085639-1.04764778 \quad 3.75718733$

H $1.01313922-2.202406212 .56615104$

$\mathrm{H}-0.69749301-2.18352897 \quad 2.98484390$

$\begin{array}{lllll}0 & 0.66772222 & 0.13933399 & -2.47827745\end{array}$

○ $0.20294961 \quad 1.39537650 \quad-2.02290346$

o - $0.31676316-0.79122627-2.05024115$

Vibrational frequencies (in $\mathrm{cm}-1$ )

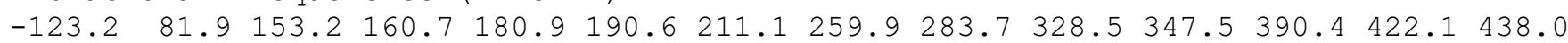
$468.7 \quad 492.3 \quad 526.8 \quad 594.2 \quad 661.1 \quad 701.3 \quad 722.6 \quad 794.3 \quad 854.5 \quad 875.4 \quad 895.5 \quad 916.6 \quad 935.7953 .9$ $976.5 \quad 992.4 \quad 1001.8 \quad 1008.9 \quad 1017.3 \quad 1022.4 \quad 1043.6 \quad 1058.8 \quad 1082.8 \quad 1088.3 \quad 1099.5 \quad 1143.3$

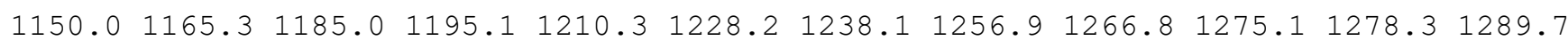
$1290.7 \quad 1292.7 \quad 1297.4 \quad 1300.4 \quad 1304.8 \quad 1306.4 \quad 1320.3 \quad 1324.01374 .6 \quad 1390.01408 .01413 .0$ $1418.52649 .5 \quad 2658.8 \quad 2671.1 \quad 2674.4 \quad 2679.3 \quad 2681.12682 .62686 .92688 .72739 .32747 .8$ $2751.7 \quad 2769.0 \quad 2778.2 \quad 2781.5 \quad 2784.3$

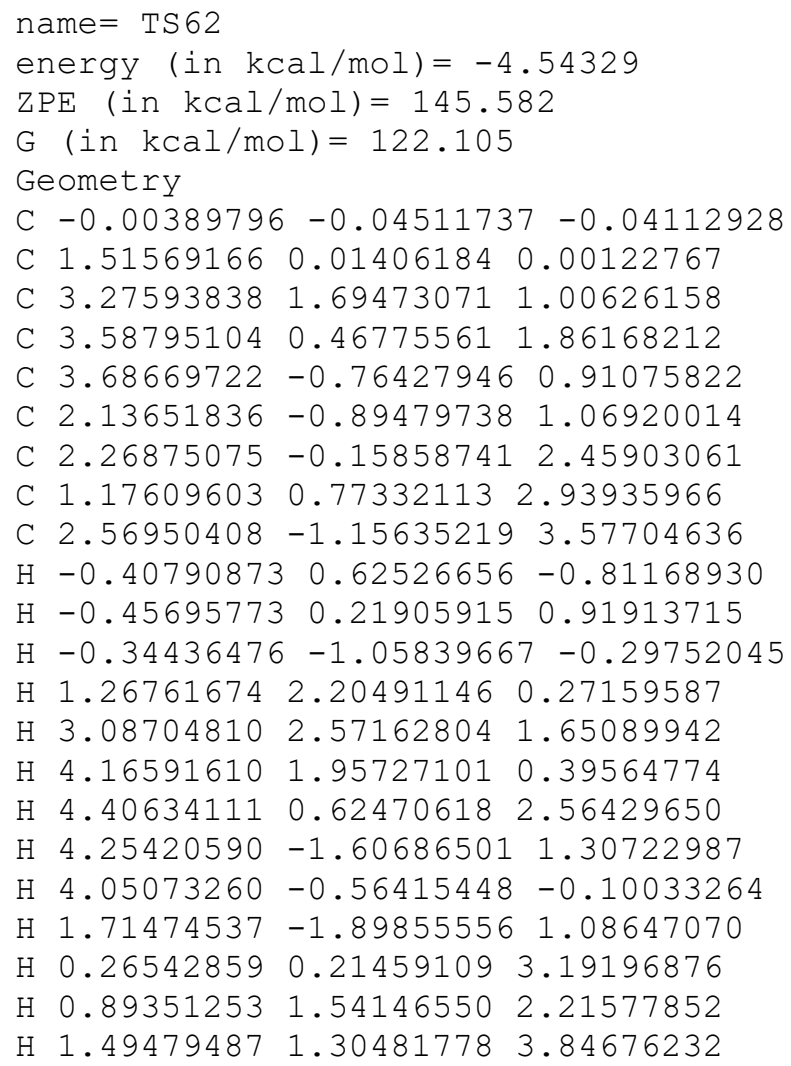


$\mathrm{H} 2.89562243-0.641121424 .48779350$

$\mathrm{H} 3.36028201-1.865044193 .30737212$

$\mathrm{H} 1.68019804-1.74570545 \quad 3.82865304$

$\begin{array}{lllll}0 & 2.47172631 & 0.57350676 & -2.03558707\end{array}$

○ $2.466533991 .77521937-1.26482772$

O $1.99125479-0.50328642-1.24500107$

C $2.07436378 \quad 1.466359290 .06126019$

Vibrational frequencies (in $\mathrm{cm}-1$ )

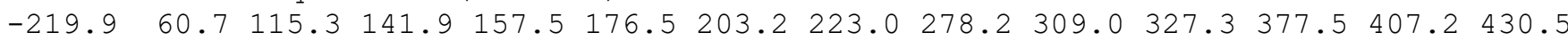
$435.8 \quad 488.7 \quad 516.8 \quad 594.2 \quad 651.0 \quad 714.4 \quad 716.8 \quad 798.6 \quad 842.6 \quad 890.8 \quad 913.4 \quad 938.8 \quad 954.5 \quad 966.6$ $988.0 \quad 996.4 \quad 1002.2 \quad 1013.2 \quad 1014.5 \quad 1025.8 \quad 1050.6 \quad 1060.5 \quad 1085.8 \quad 1088.7 \quad 1112.11151 .0$

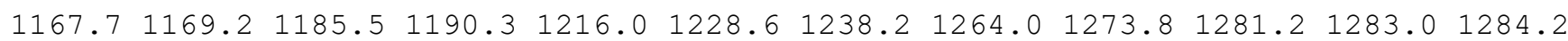

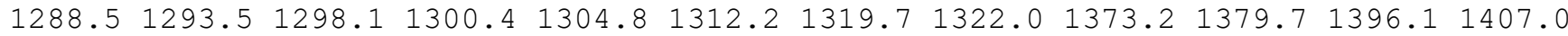
$1418.22655 .52669 .8 \quad 2672.12674 .3 \quad 2674.6 \quad 2677.22679 .02680 .12687 .02739 .62748 .4$ $2752.02764 .92780 .8 \quad 2782.0 \quad 2784.0$

name $=$ TS 63

energy $($ in $\mathrm{kcal} / \mathrm{mol})=0.602257$

$\mathrm{ZPE}($ in $\mathrm{kcal} / \mathrm{mol})=140.82$

$\mathrm{G}($ in $\mathrm{kcal} / \mathrm{mol})=113.49$

Geometry

C $0.31062530-3.105617590 .70569854$

C $0.85776951-2.27506168 \quad 1.83072508$

C $1.017851312 .12772315-1.04529329$

C $0.90566504 \quad 1.65258235 \quad 0.32491609$

C $2.04015971 \quad 0.73201475 \quad 0.74578501$

C $2.00466732-0.686196750 .11537959$

C $1.98738567-1.33680730 \quad 1.51927948$

C $1.94123616 \quad 0.090774192 .18209533$

C $0.67780952 \quad 0.40218779 \quad 2.95751044$

C $3.16912424 \quad 0.37841564 \quad 3.03266722$

$\mathrm{H}-0.45567924-2.55077581 \quad 0.14707064$

$\mathrm{H}-0.17740120-4.015009931 .09093338$

$\mathrm{H} 1.08642306-3.41649530-0.00397940$

$\mathrm{H} \quad 0.48835361 \quad 2.87219323-2.04529082$

$\mathrm{H}-0.08454720 \quad 1.14975941 \quad 0.45867870$

$\mathrm{H} 0.84994743 \quad 2.52782112 \quad 1.01995560$

H $3.01262910 \quad 1.23668376 \quad 0.59619599$

H $2.88359444-0.93616069-0.48699637$

$\mathrm{H} \quad 1.12025866-0.88514197-0.49611993$

H $2.93489173-1.86489867 \quad 1.75682974$

$\mathrm{H} \quad 0.63413589 \quad 1.45384145 \quad 3.25938474$

$\mathrm{H} \quad 0.62788917-0.20495021 \quad 3.87788197$

$\mathrm{H}-0.23383069 \quad 0.16772540 \quad 2.39782744$

H $3.20007980 \quad 1.42841035 \quad 3.34726977$

$\mathrm{H} \quad 4.10415244 \quad 0.16700113 \quad 2.50184075$

$\mathrm{H} \quad 3.16724494 \quad-0.23401400 \quad 3.94489601$

$\begin{array}{lllll}0 & 1.53411590 & 2.54247672 & -2.96384424\end{array}$

O $1.98068898 \quad 1.80173249-1.83083565$

O $0.42966153-2.397679672 .95191216$

Vibrational frequencies (in $\mathrm{cm}-1$ )

$\begin{array}{lllllllllllllll}-1758.7 & 23.3 & 26.4 & 48.1 & 81.7 & 94.3 & 110.5 & 167.4 & 182.2 & 203.9 & 232.5 & 234.5 & 261.0 & 283.2\end{array}$ $321.7 \quad 367.2 \quad 369.4 \quad 399.1 \quad 484.2 \quad 503.6 \quad 522.3 \quad 607.2 \quad 664.3 \quad 743.7 \quad 808.9 \quad 847.4 \quad 864.2 \quad 919.6$ $934.9936 .1986 .0 \quad 993.8 \quad 1007.8 \quad 1016.5 \quad 1025.91043 .2 \quad 1054.8 \quad 1078.7 \quad 1104.1 \quad 1129.01153 .2$

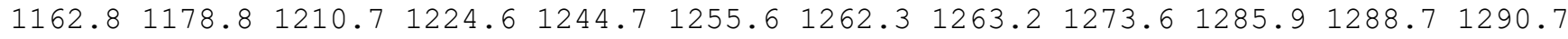
$1298.01301 .2 \quad 1305.0 \quad 1319.4 \quad 1345.5 \quad 1351.2 \quad 1356.91380 .4 \quad 1402.0 \quad 1405.6 \quad 1598.7 \quad 1844.8$ $2209.42633 .12659 .5 \quad 2666.7 \quad 2673.7 \quad 2675.2 \quad 2676.92682 .02682 .72710 .02717 .42729 .2$ $2762.8 \quad 2779.12779 .6 \quad 2781.8$

name $=\operatorname{TS} 64$

energy (in $\mathrm{kcal} / \mathrm{mol})=-1.551561$

$\mathrm{ZPE}($ in $\mathrm{kcal} / \mathrm{mol})=143.689$

$\mathrm{G}($ in $\mathrm{kcal} / \mathrm{mol})=119.679$ 


\begin{tabular}{|c|c|c|}
\hline & & \\
\hline$C$ & 0.00000000 & 0.00000000 \\
\hline & 1.50103692 & 0.00000000 \\
\hline & 2.16289391 & 0.00000000 \\
\hline & 3.64409123 & 1.143064550 .37030522 \\
\hline & 3.67272352 & -0.396526890 .60335109 \\
\hline & 3.45427558 & $-1.00780630-0.77156662$ \\
\hline & 7730173 & $-0.77066803-1.12907164$ \\
\hline & 2.24702112 & $-0.64473491 \quad 1.25$ \\
\hline & 2.03167818 & $0.08788538 \quad 2.57791772$ \\
\hline & 1.93257707 & $-2.12317691 \quad 1.44$ \\
\hline & 1022860 & $0.43865694 \quad 0.91$ \\
\hline & 0197256 & $5-1.01894221-0.07809530$ \\
\hline & 975491 & $0.57712033-0.8$ \\
\hline & 2832 & 2.105244820 .721 \\
\hline & 3.94671629 & 1.693304551 .27167578 \\
\hline & 21079 & $1.46069271-0.42$ \\
\hline & 4.5 & -0.764692321 .17251176 \\
\hline & 4.22851452 & $-1.52577706-1.29272501$ \\
\hline & 3.9 & $0.77705942-2.38116687$ \\
\hline 1 & 1.65072343 & $-1.04608038-2.01794933$ \\
\hline & 2.96140359 & 0.526770992 .95713005 \\
\hline & 1.6 & -0.580720173 .36501533 \\
\hline $\mathrm{H}$ & 1.29574198 & 0.894488362 .49170016 \\
\hline & 2.58145118 & $-2.56839197 \quad 2.21019594$ \\
\hline 1 & 2.0 & -2.706578710 .52905770 \\
\hline $\mathrm{H}$ & 0.89235838 & $-2.26736127 \quad 1.76195197$ \\
\hline & 2.18355001 & $1.42666150-2.33751828$ \\
\hline & & $2.17049220-1.18129489$ \\
\hline & 3.50298188 & $1.57671579-2.77166325$ \\
\hline & IdLOnd & Erequencies (in $\mathrm{cm}-1$ ) \\
\hline
\end{tabular}

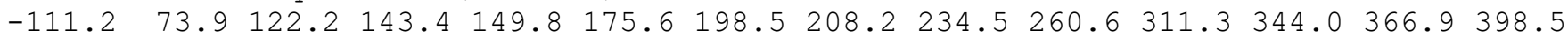

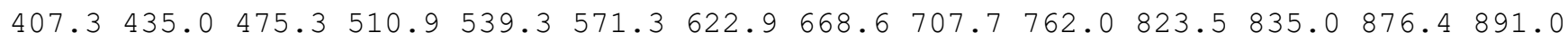
$946.7 \quad 967.9971 .5 \quad 996.3 \quad 1007.0 \quad 1012.7 \quad 1020.0 \quad 1024.9 \quad 1036.0 \quad 1066.3 \quad 1101.7 \quad 1105.8 \quad 1124.5$ $1133.31165 .5 \quad 1176.5 \quad 1200.3 \quad 1221.3 \quad 1230.6 \quad 1254.91263 .6 \quad 1280.0 \quad 1288.8 \quad 1290.1 \quad 1293.0$

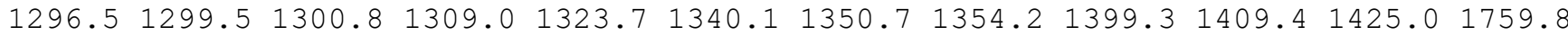
$2667.52669 .32672 .5 \quad 2673.6 \quad 2674.7 \quad 2676.5 \quad 2680.2 \quad 2687.32754 .5 \quad 2756.5 \quad 2779.72780 .1$ $2781.02781 .8 \quad 2793.8 \quad 2821.5$

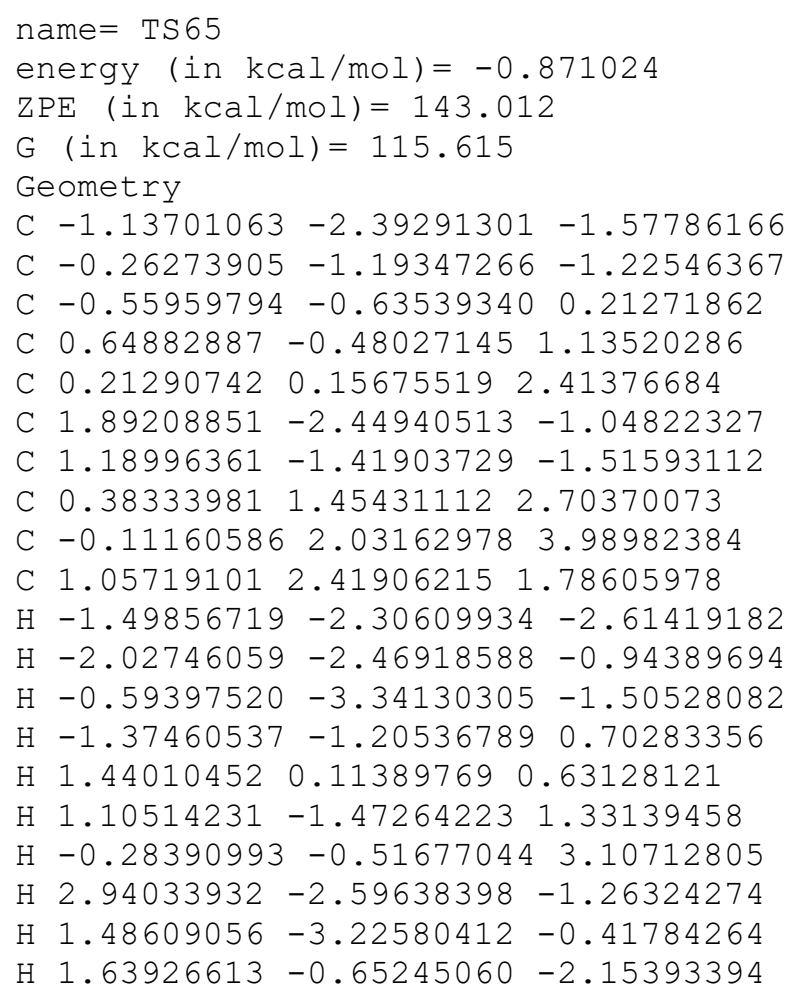


$\mathrm{H} \quad 0.714226562 .45112771 \quad 4.58125662$

$\mathrm{H}-0.62185159 \quad 1.29324707 \quad 4.62137929$

$\mathrm{H}-0.828855442 .84499920 \quad 3.80319069$

$\mathrm{H} \quad 0.54013387 \quad 2.46144146 \quad 0.81206685$

$\mathrm{H} \quad 2.09730478 \quad 2.125203291 .58616697$

$\mathrm{H} \quad 1.08035484 \quad 3.44162134 \quad 2.18132441$

$0-1.59259068 \quad 0.63169436-1.32764844$

$0-1.030320160 .69724559-0.03074571$

o $-0.63863196-0.09543237-2.08126769$

Vibrational frequencies (in $\mathrm{cm}-1$ )

$\begin{array}{llllllllllllll}-106.6 & 29.5 & 32.4 & 58.7 & 80.3 & 88.1 & 110.4 & 116.9 & 181.4 & 214.9 & 240.3 & 276.2 & 291.0 & 303.5\end{array}$ $347.0 \quad 412.0 \quad 437.9 \quad 470.0 \quad 491.4 \quad 508.5 \quad 562.0 \quad 599.0 \quad 684.3 \quad 704.8 \quad 830.9 \quad 878.5 \quad 898.6 \quad 925.5$ $960.4 \quad 973.9 \quad 980.0 \quad 993.1 \quad 994.5 \quad 1018.8 \quad 1024.5 \quad 1054.9 \quad 1062.9 \quad 1092.3 \quad 1114.8 \quad 1119.8 \quad 1141.3$ $\begin{array}{llllllllllllll}1156.9 & 1182.1 & 1187.6 & 1225.3 & 1246.3 & 1263.5 & 1268.5 & 1278.7 & 1280.0 & 1283.8 & 1286.9 & 1288.4\end{array}$ $1289.51311 .1 \quad 1327.2 \quad 1352.5 \quad 1356.8 \quad 1376.2 \quad 1385.8 \quad 1400.1 \quad 1412.3 \quad 1413.2 \quad 1854.81870 .2$ $2656.32661 .0 \quad 2665.3 \quad 2671.2 \quad 2673.5 \quad 2674.72677 .52681 .12710 .02738 .52743 .42762 .9$ 2776.92778 .22780 .22784 .9

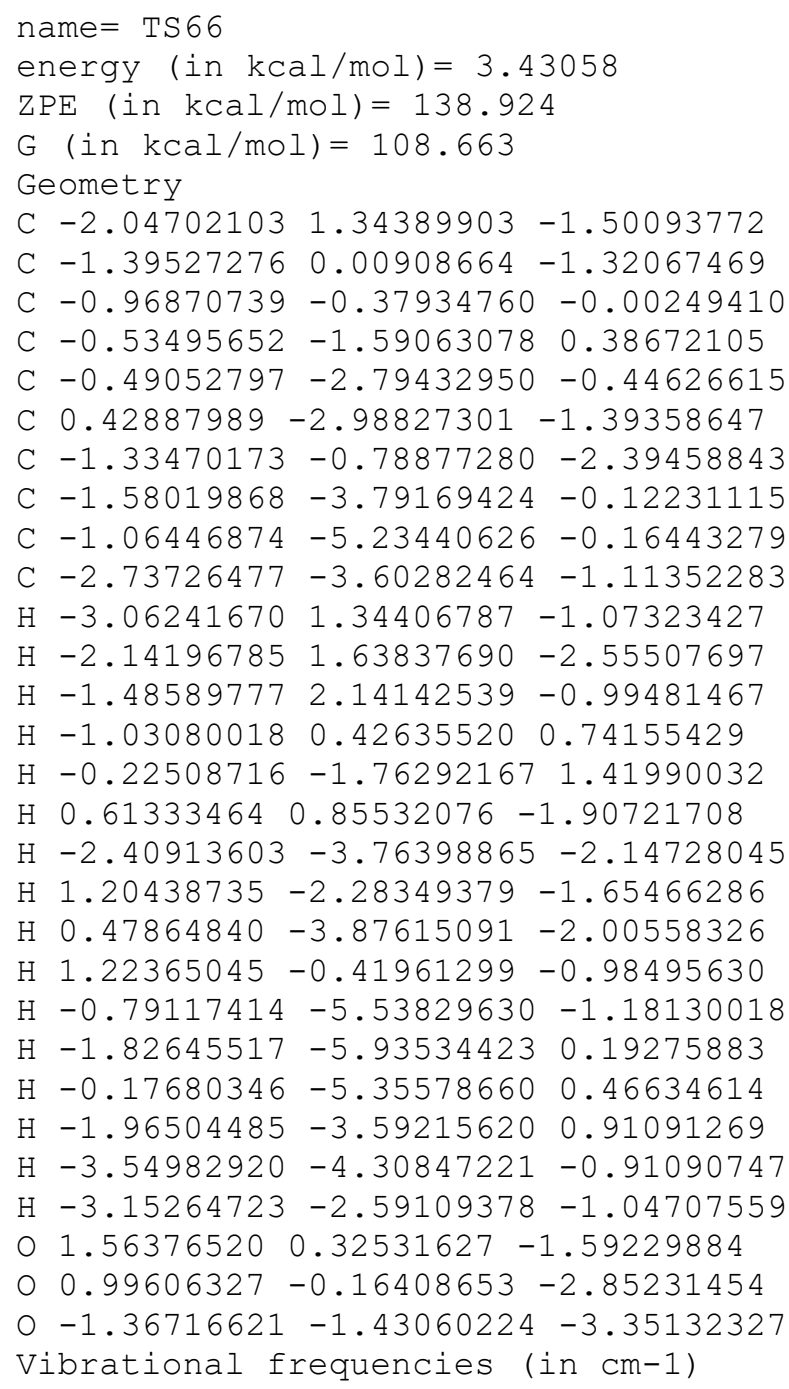


$\mathrm{ZPE}($ in $\mathrm{kcal} / \mathrm{mol})=143.216$

$\mathrm{G}($ in $\mathrm{kcal} / \mathrm{mol})=116.206$

Geometry

C $-2.17684595-0.772825490 .26492942$

C $-0.70177953-0.391875390 .15370112$

C $-0.18865055 \quad 0.42009354 \quad 1.39432284$

C $0.96905581-0.196006892 .17820426$

C $0.49125776-1.37717620 \quad 2.95463045$

C $1.30677641-1.45301097-0.87797258$

C $0.15718740-1.56386271-0.21391623$

C $-0.10150659-1.303893654 .15421175$

C $-0.34328525-0.01922526 \quad 4.87473437$

C $-0.55975122 \quad-2.53410580 \quad 4.86699621$

$\mathrm{H}-2.67763694-0.27423387 \quad 1.10273925$

$\mathrm{H}-2.31875065-1.850571150 .40319518$

$\mathrm{H}-2.72346720-0.48370197-0.64584200$

$\mathrm{H}-1.02742372 \quad 0.68632109 \quad 2.07493040$

$\mathrm{H} \quad 1.40584744 \quad 0.57714602 \quad 2.84692271$

$\mathrm{H} \quad 1.79924916-0.47427299 \quad 1.49634554$

$\mathrm{H} 0.65500253-2.334610892 .46471413$

$\mathrm{H} 1.91777588-2.30029640-1.15113240$

$\mathrm{H} \quad 1.72601004 \quad-0.50968230-1.20053852$

$\mathrm{H}-0.23287937-2.53290614 \quad 0.09510056$

$\mathrm{H}-0.85702646 \quad 0.71690293 \quad 4.24030269$

H $0.60648426 \quad 0.43759567 \quad 5.19270072$

$\mathrm{H}-0.95634033-0.14611623 \quad 5.77592205$

$\mathrm{H}-0.09255356-2.61412113 \quad 5.85916874$

$\mathrm{H}-0.31880068-3.45719147 \quad 4.32396867$

$\mathrm{H}-1.64860740-2.52244914 \quad 5.01807138$

$\begin{array}{lllll}0 & 0.41188040 & 1.44114612 & -0.53593614\end{array}$

$\begin{array}{lllll}0 & 0.21905230 & 1.67891014 & 0.85624900\end{array}$

$\begin{array}{lllll}0 & -0.65921198 & 0.59754845 & -0.89333857\end{array}$

Vibrational frequencies (in $\mathrm{cm}-1$ )

$\begin{array}{lllllllllllllll}-132.8 & 31.6 & 38.5 & 57.8 & 81.6 & 96.7 & 103.1 & 127.0 & 200.1 & 217.9 & 237.6 & 272.3 & 306.0 & 338.4\end{array}$ $377.2 \quad 391.6 \quad 425.9 \quad 459.6 \quad 491.3 \quad 549.0 \quad 568.5 \quad 628.5 \quad 673.0 \quad 721.5 \quad 814.4 \quad 869.0 \quad 907.6 \quad 919.5$

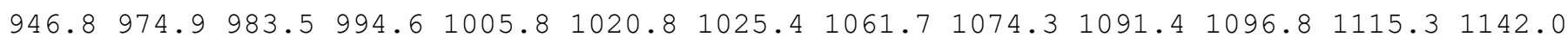

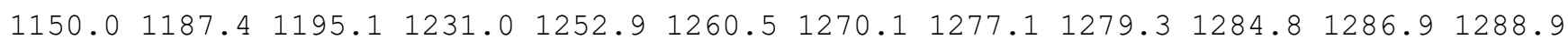

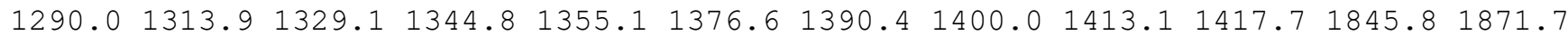
$2653.12664 .5 \quad 2666.2 \quad 2670.12672 .0 \quad 2672.92675 .62676 .52709 .72740 .82753 .12758 .5$ $2777.22778 .02780 .5 \quad 2781.7$

\section{name $=$ TS 68}

energy $($ in $\mathrm{kcal} / \mathrm{mol})=8.113889$

$\mathrm{ZPE}($ in $\mathrm{kcal} / \mathrm{mol})=139.177$

$\mathrm{G}($ in $\mathrm{kcal} / \mathrm{mol})=114.323$

Geometry

C $-2.26916697-1.60726228-1.39174969$

C $-1.12345807-0.85155173-0.80517611$

C $2.63191770 \quad 1.79414267 \quad 0.71938860$

C $2.81258538 \quad 0.52969828 \quad 1.52368143$

C $1.66398752-0.47786281 \quad 1.46814512$

C $1.28203786-0.84949036-0.00378511$

C $-0.06776215-1.51450726-0.15850320$

C $0.39701787-0.050031532 .17014983$

C $-0.05951347 \quad 1.28261582 \quad 2.11387983$

C $-0.41422707-1.107705202 .57633922$

$\mathrm{H}-3.08758611-0.95673039-1.74221816$

$\mathrm{H}-2.70701657-2.31102226-0.66616252$

$\mathrm{H}-1.94096564-2.20151407-2.26160939$

H $1.83052575 \quad 1.83671276 \quad-0.03076441$

H $3.010352920 .80658932 \quad 2.58403767$

$\mathrm{H} \quad 3.75400603 \quad 0.04334307 \quad 1.18432453$

$\mathrm{H} 2.03842722-1.418103451 .95648178$

H $2.07240647-1.51638206-0.40092769$ 
$\mathrm{H} 1.32680938 \quad 0.06146003-0.63907435$

$\mathrm{H} \quad 0.00642293-2.59540090-0.31966867$

$\mathrm{H}-0.94283044 \quad 1.55366557 \quad 2.67990395$

$\mathrm{H}-0.47289521 \quad 1.35742638 \quad 0.82431915$

$\mathrm{H} \quad 0.64231933 \quad 2.10684802 \quad 2.10144177$

$\mathrm{H}-0.57703472-1.57132376 \quad 1.14282863$

$\mathrm{H}-1.38313332-0.92580784 \quad 3.00950099$

$\mathrm{H}-0.01427102-2.05939607 \quad 2.88535808$

$\begin{array}{lllll}0 & -0.41703810 & 1.28313705 & -0.47202358\end{array}$

$03.370462612 .73249521 \quad 0.89407991$

o $-1.365639550 .43639660-0.93403839$

Vibrational frequencies (in $\mathrm{cm}-1$ )

$\begin{array}{llllllllllllllll}-1970.6 & 44.7 & 64.5 & 100.4 & 110.1 & 160.1 & 172.8 & 208.2 & 243.3 & 266.9 & 289.2 & 302.2316 .1 & 333.8\end{array}$

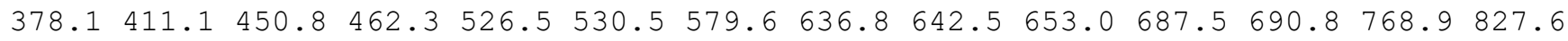
$918.4960 .6970 .6 \quad 982.1 \quad 1000.2 \quad 1024.2 \quad 1039.2 \quad 1047.4 \quad 1058.6 \quad 1073.4 \quad 1078.8 \quad 1090.7 \quad 1129.4$

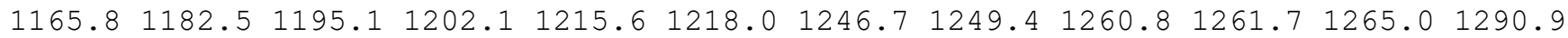

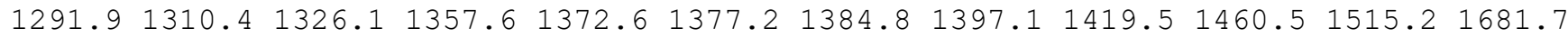
$1752.0 \quad 1828.6 \quad 2629.8 \quad 2638.7 \quad 2650.8 \quad 2654.8 \quad 2657.8 \quad 2686.4 \quad 2707.5 \quad 2723.7 \quad 2727.0 \quad 2735.8$ $2738.92770 .5 \quad 2779.4 \quad 2794.1$

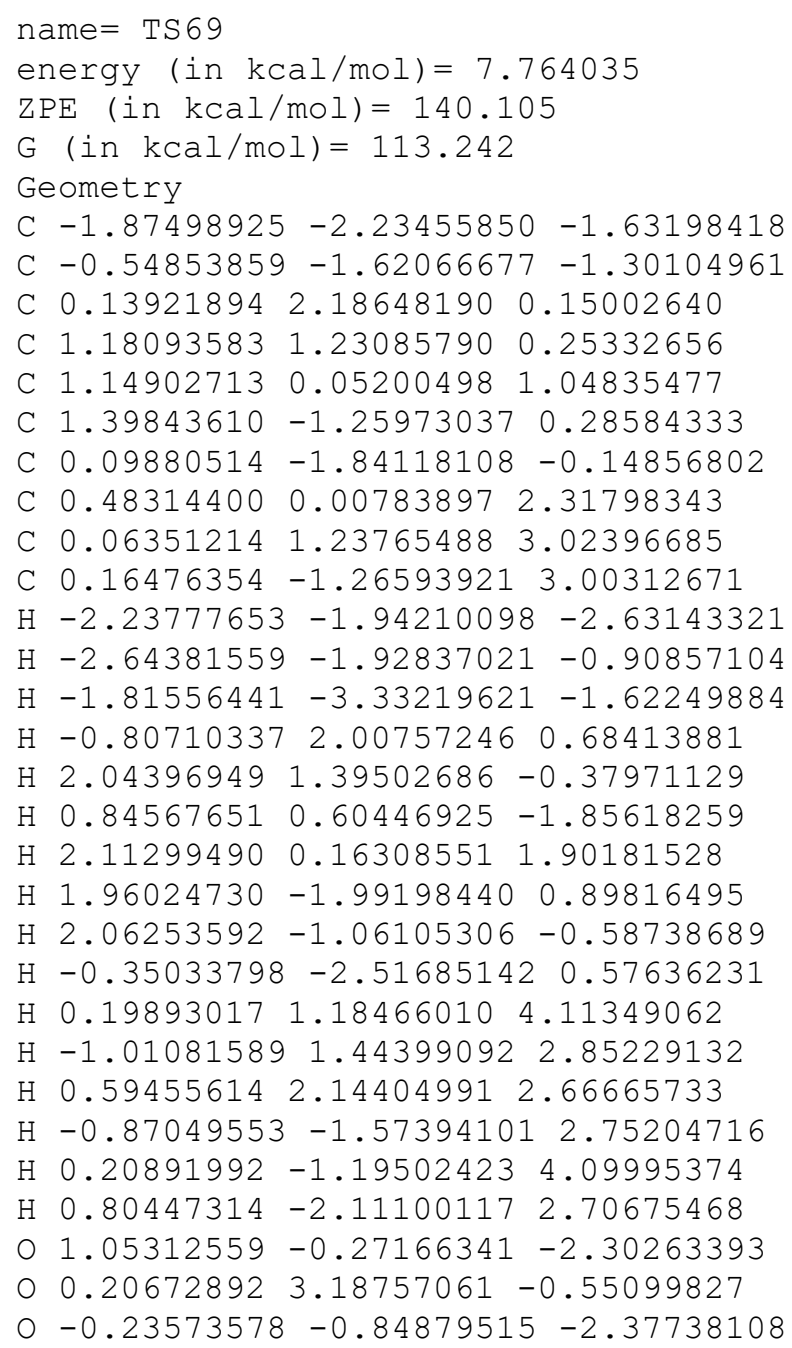




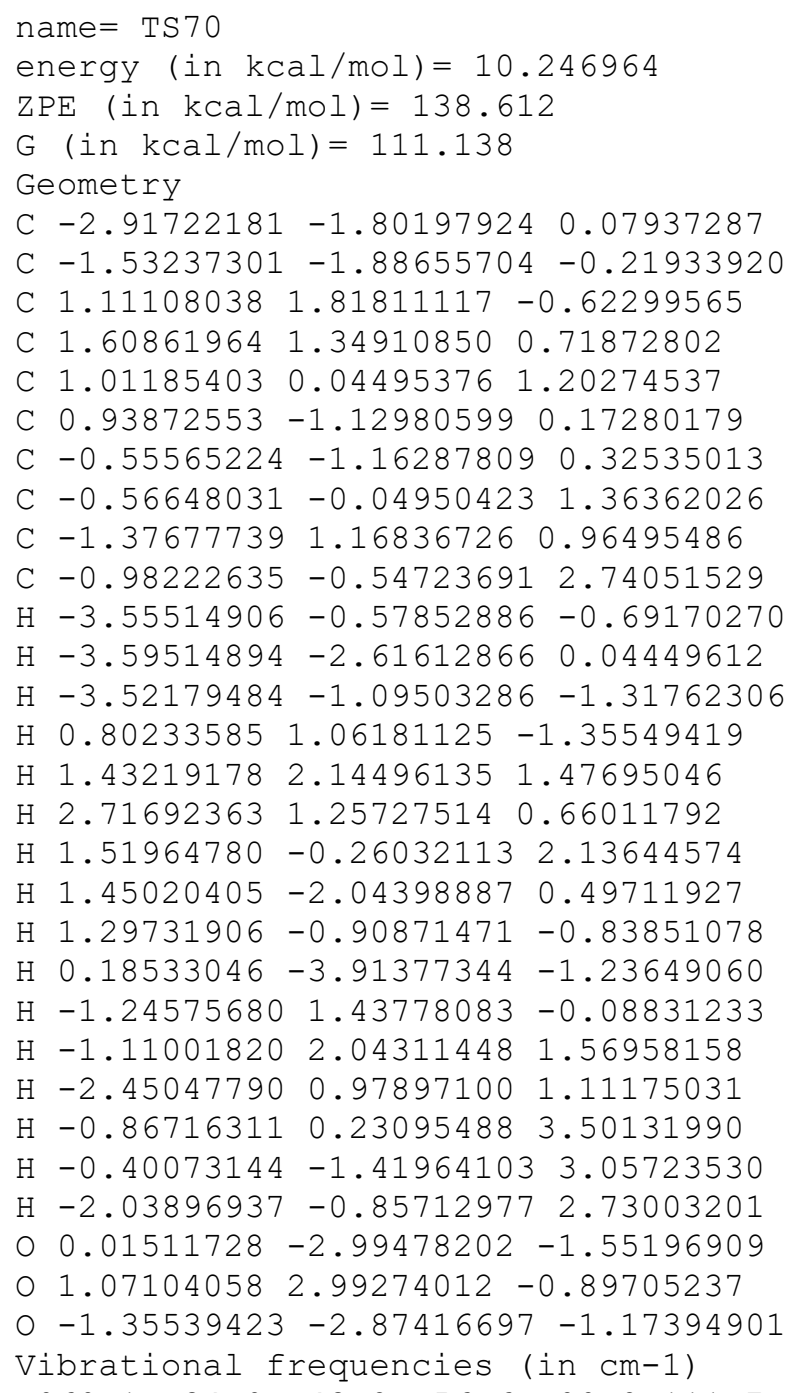


$\mathrm{H} 2.203432431 .05577523 \quad 0.54936454$

$\mathrm{H} \quad 1.02847177-1.21757474-0.51635714$

$\mathrm{H}-0.04401402-0.21823104-2.13377340$

$\mathrm{H}-0.62421271-0.351274191 .58446735$

$\mathrm{H}-0.32539846 \quad 3.56152882-0.18768084$

$\mathrm{H}-1.783563802 .75939030 \quad 0.41014666$

$\mathrm{H}-1.073313652 .26496154-1.12867109$

$\mathrm{H}-0.61240156 \quad 2.18405103 \quad 2.61729960$

$\mathrm{H} \quad 0.85926625 \quad 2.98444037 \quad 2.05234921$

$\mathrm{H} \quad 0.92154791 \quad 1.30517072 \quad 2.59269114$

$\begin{array}{lllll}0 & -0.91212428 & 0.25855631 & -2.29925866\end{array}$

$03.639647251 .72714091-2.76140320$

o $-1.82798591-0.48224111-1.45165758$

Vibrational frequencies (in $\mathrm{cm}-1$ )

$\begin{array}{llllllllllllll}-225.4 & 38.0 & 53.0 & 87.9 & 101.9 & 113.3 & 150.2 & 166.9 & 178.9 & 211.0 & 216.6 & 229.8 & 258.4 & 290.6\end{array}$

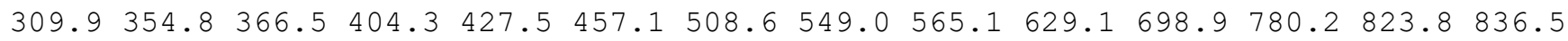
$868.8 \quad 976.9981 .3 \quad 1001.5 \quad 1008.8 \quad 1016.6 \quad 1019.2 \quad 1024.9 \quad 1051.1 \quad 1083.7 \quad 1107.01137 .9 \quad 1152.6$

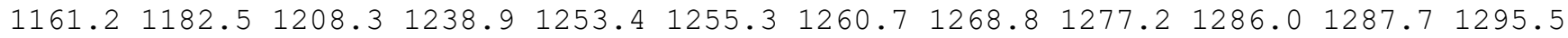
$1299.31301 .81307 .8 \quad 1315.6 \quad 1328.8 \quad 1349.2 \quad 1375.1 \quad 1380.3 \quad 1400.4 \quad 1406.01444 .31834 .1$ $2648.82651 .42655 .92657 .92659 .2 \quad 2667.3 \quad 2674.62677 .92681 .12691 .72704 .12742 .7$ $2766.4 \quad 2780.0 \quad 2782.2 \quad 2821.8$

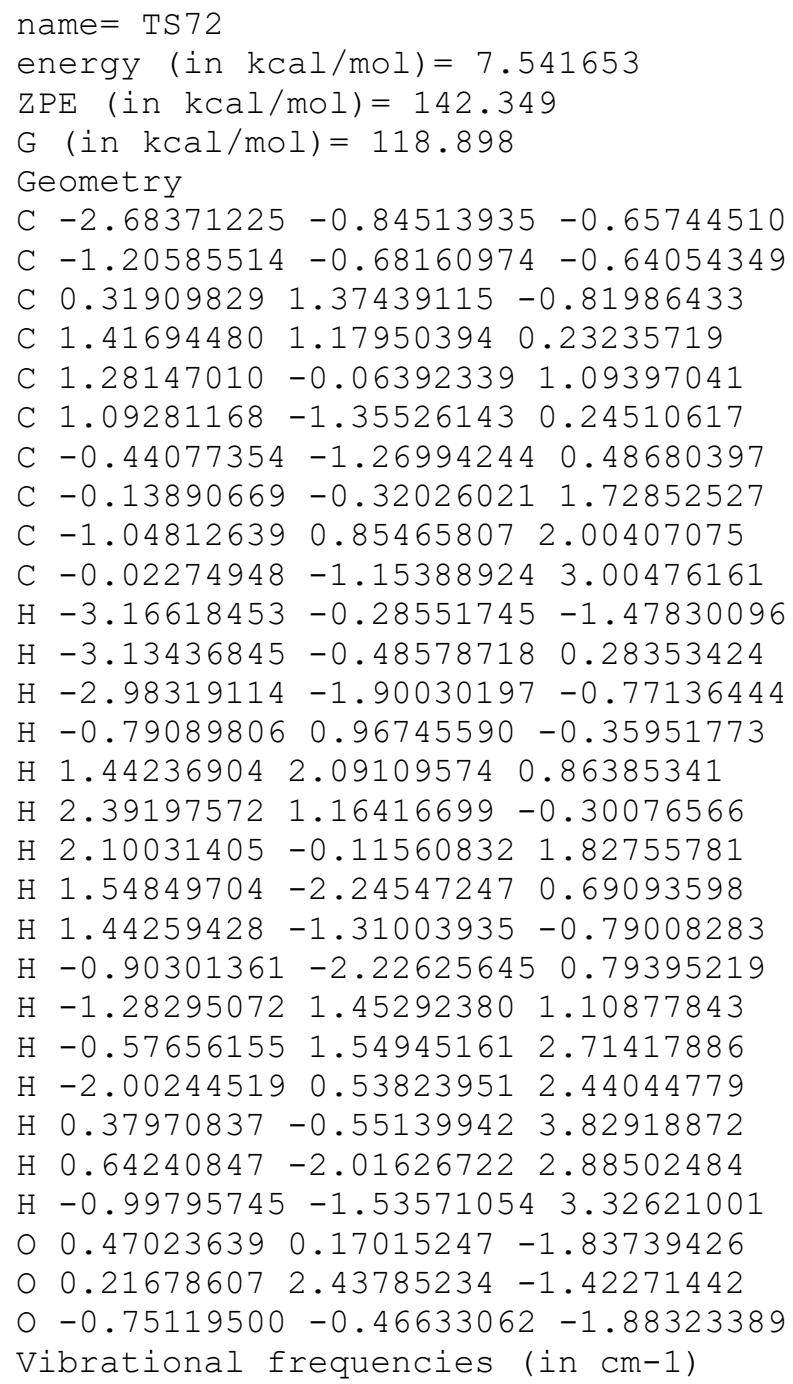




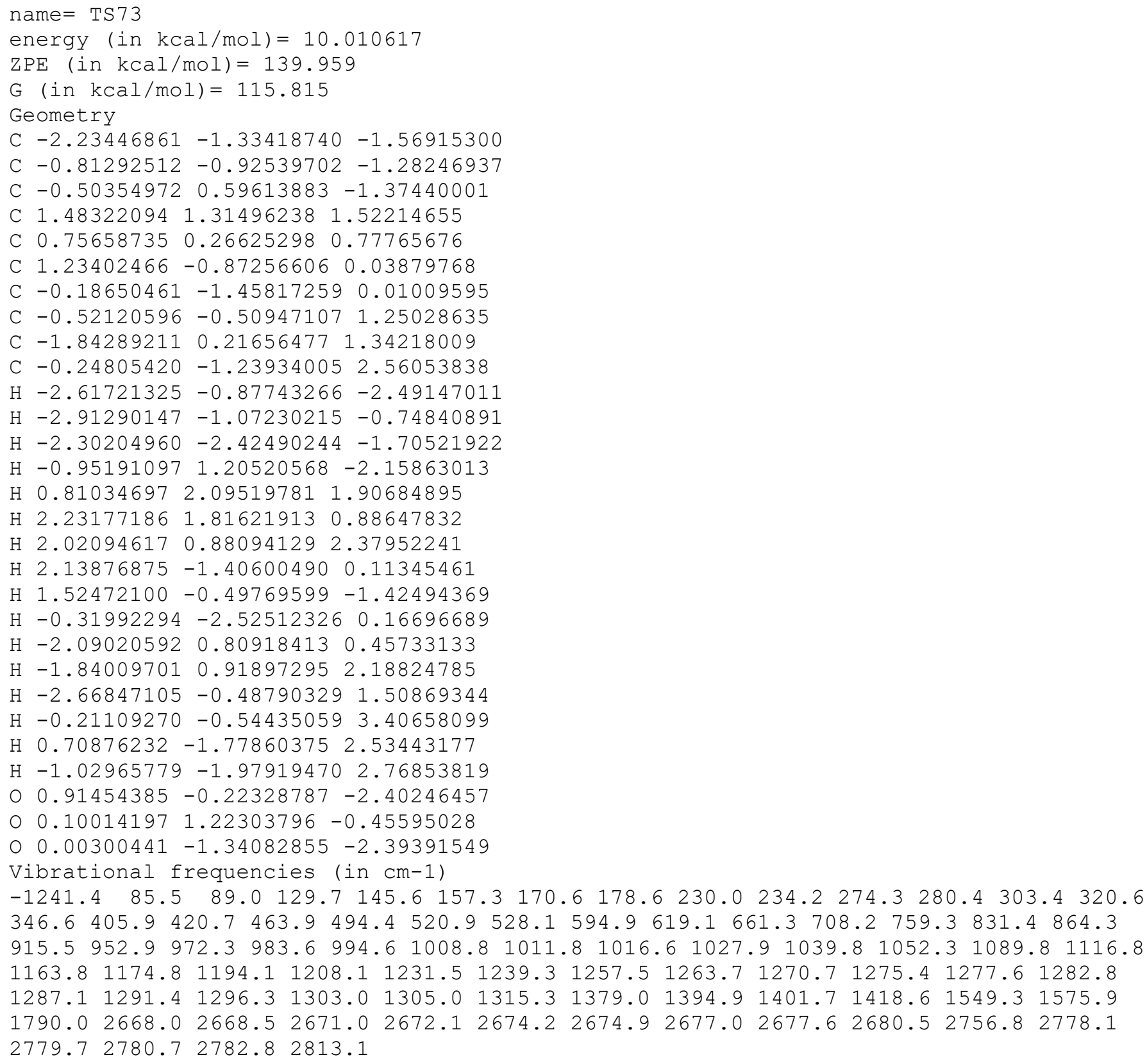


$\mathrm{H} \quad 0.78912659 \quad 1.72050495 \quad 3.37580754$

$\mathrm{H} 2.24719330 \quad 1.56276996 \quad 2.42273108$

H $1.43565025-0.655644692 .75270456$

$\mathrm{H} \quad 1.98747662-1.049735360 .50421843$

H $1.201615520 .41116093 \quad-0.07210974$

$\mathrm{H}-0.02173722-2.32420654 \quad 0.26711112$

$\mathrm{H}-2.69224402 \quad 0.24031395 \quad 2.36529217$

$\mathrm{H}-1.77025870 \quad 0.80034094 \quad 0.78042765$

$\mathrm{H}-1.49163264 \quad 1.56876560 \quad 2.34453911$

$\mathrm{H}-1.06443730-1.64349458 \quad 3.92365505$

$\mathrm{H}-0.14864995-2.49644189 \quad 2.66751107$

$\mathrm{H}-1.87579252 \quad-2.19520584 \quad 2.45308121$

O $1.28866262 \quad 4.08457626 \quad 2.60244890$

$0 \quad 0.72437066 \quad 3.68163608 \quad 1.54584954$

O $-1.501969550 .51659435-0.46867877$

Vibrational frequencies (in $\mathrm{cm}-1$ )

$\begin{array}{lllllllllllllllll}-1444.1 & 33.7 & 40.5 & 81.0 & 105.7 & 138.6 & 152.6 & 176.9 & 187.5 & 216.8 & 254.6 & 291.9 & 303.5 & 335.4\end{array}$

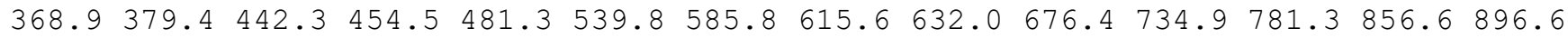
$924.6951 .2965 .7995 .8 \quad 1028.9 \quad 1040.1 \quad 1046.4 \quad 1047.1 \quad 1061.3 \quad 1069.6 \quad 1077.2 \quad 1135.2 \quad 1144.3$ $\begin{array}{lllllllllllllll}1162.2 & 1181.7 & 1203.2 & 1209.3 & 1231.8 & 1246.0 & 1253.0 & 1257.4 & 1266.3 & 1278.5 & 1278.7 & 1292.7\end{array}$

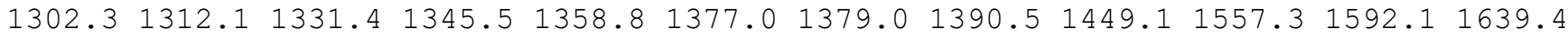
$1788.8 \quad 2618.92658 .1 \quad 2662.0 \quad 2667.3 \quad 2668.1 \quad 2671.72678 .62696 .2 \quad 2698.7 \quad 2717.92747 .2$ $2767.6 \quad 2772.1 \quad 2775.5 \quad 2781.7$

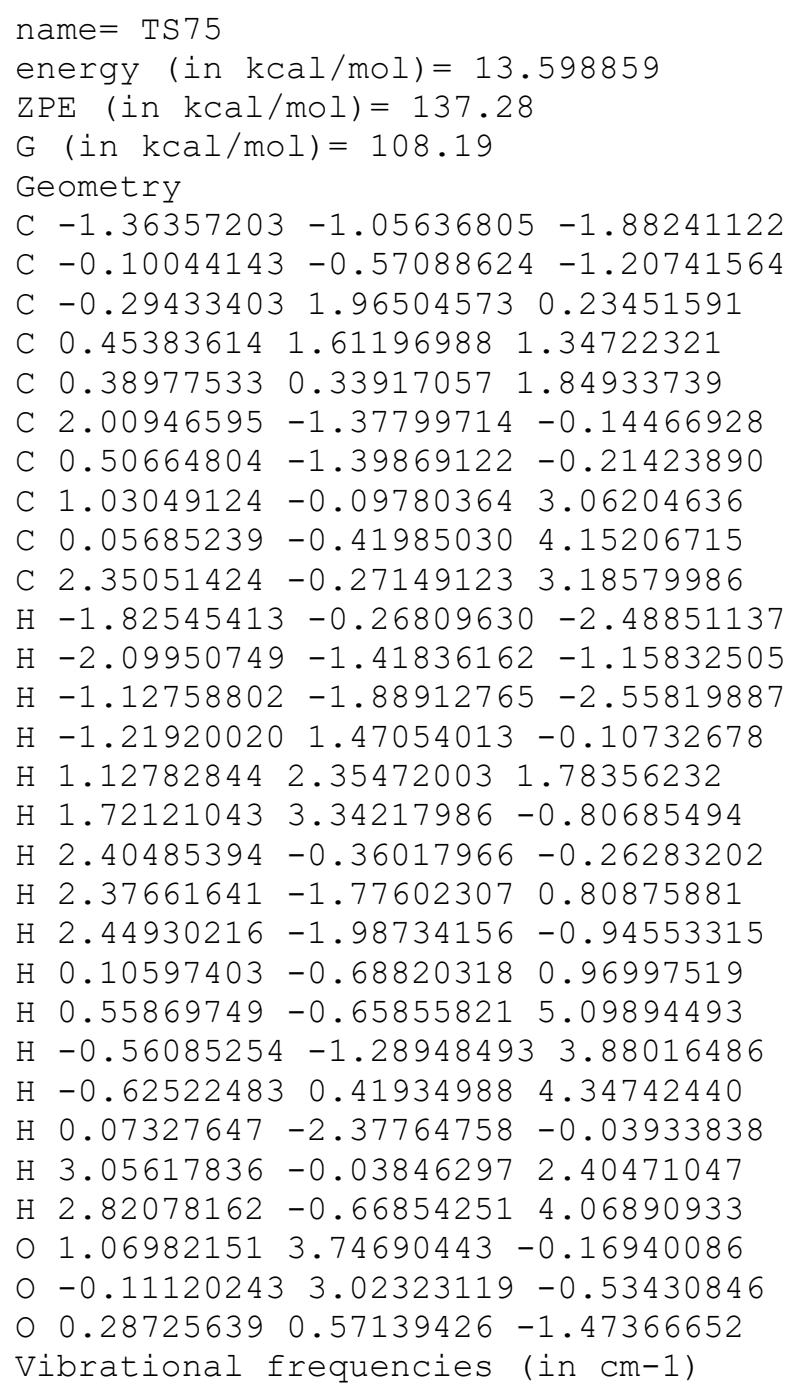


$\begin{array}{llllllllllll}1856.9 & 2655.0 & 2670.1 & 2672.7 & 2674.9 & 2676.6 & 2678.4 & 2685.6 & 2723.8 & 2741.6 & 2763.7 & 2778.0\end{array}$ 2779.32784 .62790 .62834 .7

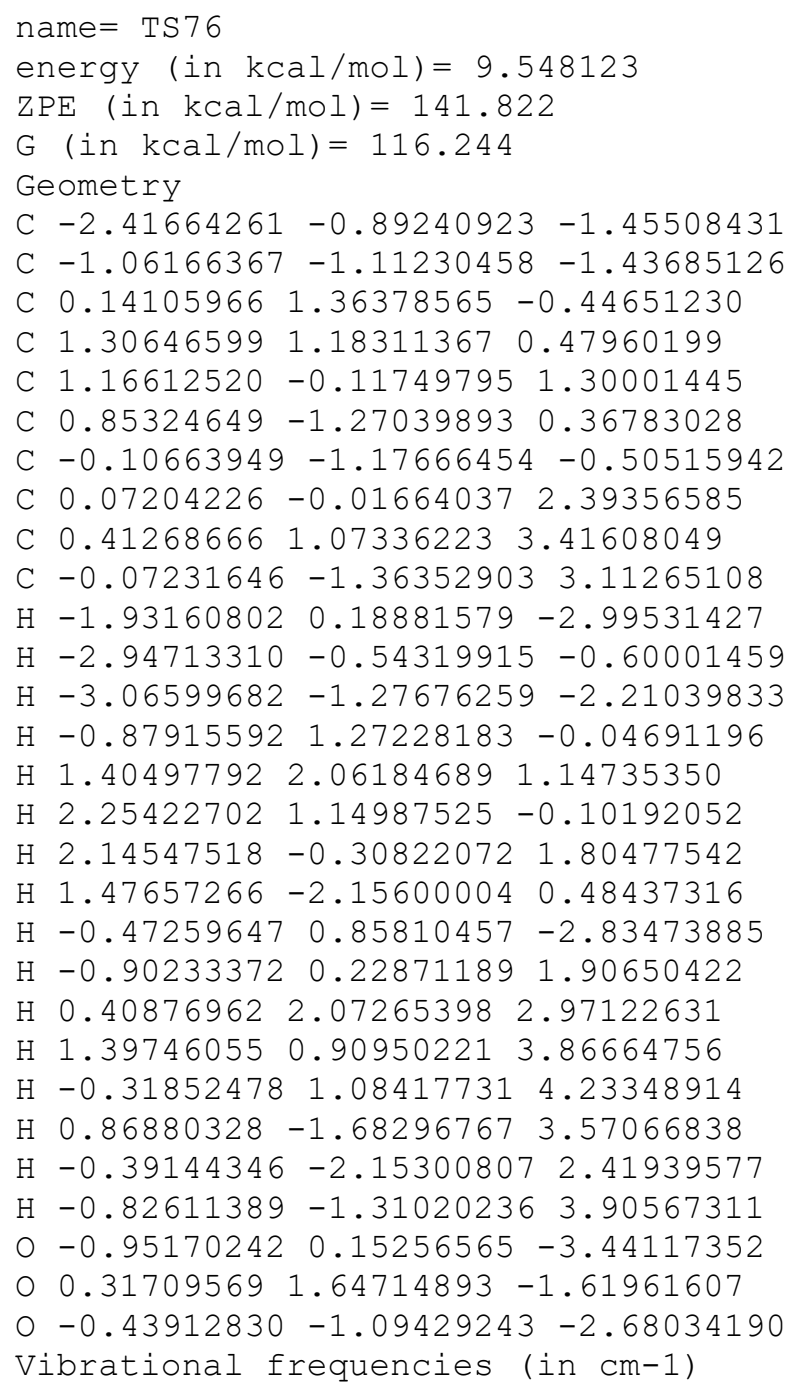


$\mathrm{H}-2.67685013-1.59458829-1.93000261$

$\mathrm{H}-1.954989160 .57556726-0.51001381$

$\mathrm{H}-0.10209995 \quad 1.22071394 \quad 0.93218089$

H $1.67382919 \quad 0.15837402-1.63434292$

$\mathrm{H} \quad 2.20165502 \quad 0.845020830 .41525209$

$\mathrm{H} 2.85264974-1.46239290-0.31056092$

$\mathrm{H} 1.17252137-1.88894649-0.62042375$

H $2.46631792-1.238588532 .10792192$

$\mathrm{H} \quad 0.58062238-3.760503713 .59941345$

$\mathrm{H}-0.30138233-2.25208768 \quad 3.89295852$

H $1.46256416-2.28170464 \quad 3.99587367$

$\mathrm{H}-0.93007410-2.112004640 .55424200$

$\mathrm{H}-0.08765485-3.66772562 \quad 0.45422200$

$\mathrm{H}-1.23692454-3.36482196 \quad 1.76144663$

$03.008718851 .64303139-1.93411452$

O $1.873400081 .33671689-1.73370802$

O $0.27863259-0.76258540-2.48631908$

Vibrational frequencies (in cm-1)

$\begin{array}{lllllllllllllllll}-1271.6 & 18.5 & 34.1 & 39.8 & 66.0 & 79.0 & 80.4 & 88.7 & 112.1 & 120.5 & 138.5 & 182.7 & 224.0 & 277.8\end{array}$ $294.3 \quad 310.5 \quad 346.1 \quad 370.6 \quad 413.0 \quad 443.6 \quad 480.9 \quad 524.8 \quad 557.8 \quad 582.3 \quad 615.4 \quad 728.6 \quad 835.2 \quad 874.4$ $\begin{array}{llllllllllllll}932.8 & 947.0 & 985.1 & 995.2 & 998.4 & 1023.4 & 1043.2 & 1055.2 & 1063.7 & 1079.0 & 1087.3 & 1113.8 & 1134.8\end{array}$

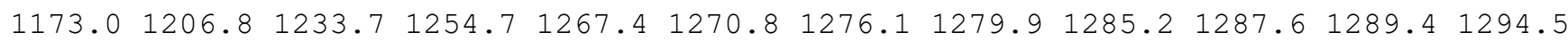
$1312.51340 .7 \quad 1361.2 \quad 1375.2 \quad 1376.8 \quad 1387.7 \quad 1400.0 \quad 1412.01428 .8 \quad 1775.1 \quad 1831.51872 .0$ $2067.72652 .8 \quad 2665.6 \quad 2669.2 \quad 2672.1 \quad 2672.7 \quad 2673.12676 .42711 .5 \quad 2723.6 \quad 2736.8 \quad 2759.5$ $2759.92777 .2 \quad 2779.6 \quad 2780.2$

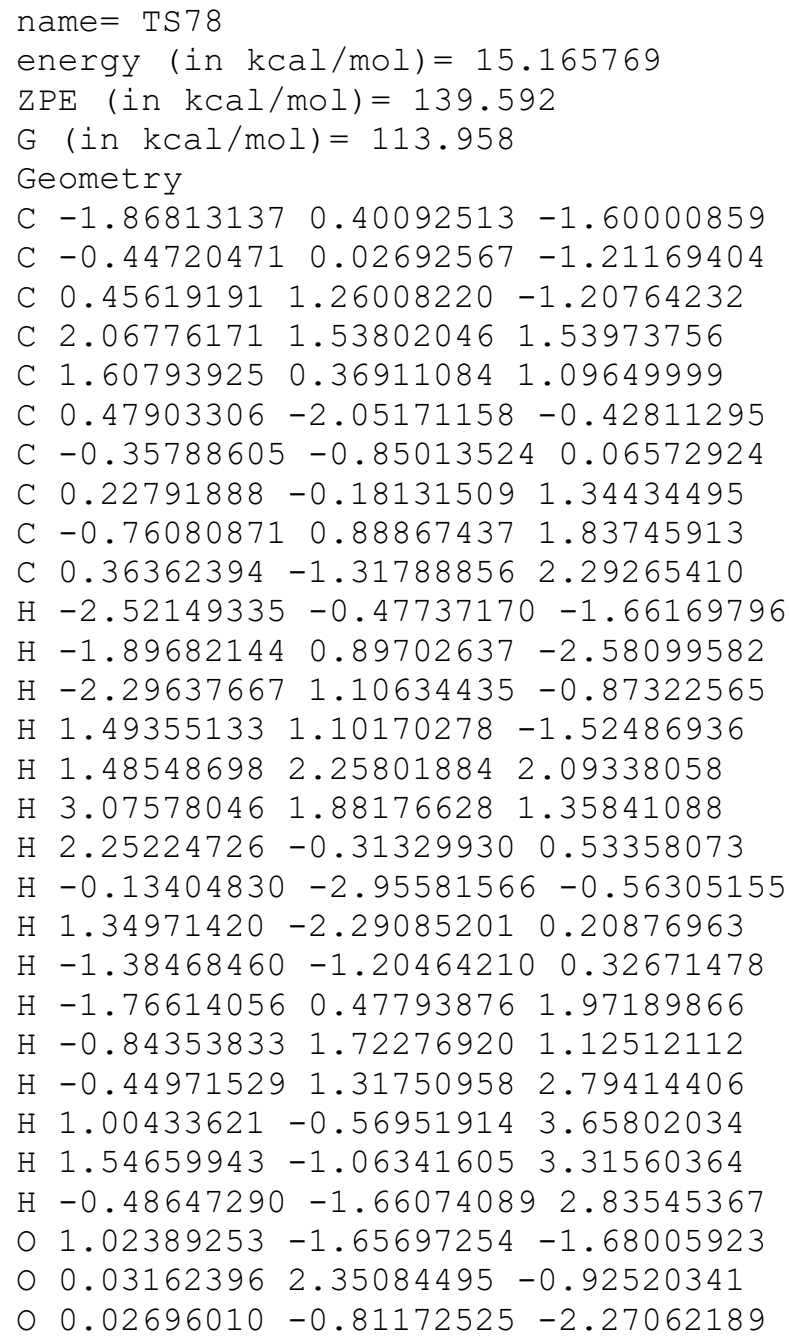


$1090.11094 .1 \quad 1116.91140 .6 \quad 1161.2 \quad 1181.4 \quad 1201.91216 .2 \quad 1222.7 \quad 1257.8 \quad 1275.11279 .2$

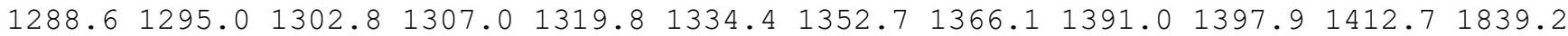
1849.82641 .22653 .92661 .32666 .92676 .12678 .92692 .02706 .52708 .02715 .62740 .9 $2778.32779 .4 \quad 2785.2 \quad 4765.9$

\section{name $=$ TS79}

energy $($ in $\mathrm{kcal} / \mathrm{mol})=18.325512$

$\mathrm{ZPE}(\mathrm{in} \mathrm{kcal} / \mathrm{mol})=137.508$

$\mathrm{G}($ in $\mathrm{kcal} / \mathrm{mol})=109.574$

Geometry

C $-2.86614192-1.61140816-0.80149164$

C $-1.41986990-1.26766529-0.79297584$

C $0.87030426 \quad 1.79693033 \quad 0.17686958$

C $1.98157543 \quad 1.06287667 \quad 0.90270698$

C $1.40928436-0.21252682 \quad 1.55995607$

C $0.67691064-0.98678387 \quad 0.51919987$

C $-0.66380149-1.102528310 .32913213$

C $0.61545952 \quad 0.02625395 \quad 2.79666299$

C $0.57660700 \quad 1.35552442 \quad 3.42960516$

C $-0.17505507-1.002820753 .30171690$

$\mathrm{H}-3.06641007-2.54514094-0.26123156$

$\mathrm{H}-3.22899438-1.75689560-1.82983392$

$\mathrm{H}-3.47937345-0.81885148-0.35243395$

$\mathrm{H} \quad 0.51949309 \quad 1.38032207 \quad-0.77933336$

H $2.47903179 \quad 1.71689013 \quad 1.63891004$

$\mathrm{H} 2.76418775 \quad 0.77466576 \quad 0.17082808$

H $2.29517767-0.84796670 \quad 1.88261727$

H $1.32777256-1.47879935-0.20056948$

$\mathrm{H}-1.63125750 \quad 0.41288923 \quad-2.84491754$

$\mathrm{H}-1.38565475-1.069653661 .35715046$

$\mathrm{H}-0.00768521 \quad 1.39214098 \quad 4.35948825$

$\mathrm{H} \quad 0.13838666 \quad 2.12432166 \quad 2.75067681$

$\mathrm{H} 1.58545216 \quad 1.735155913 .66584265$

$\mathrm{H}-1.17677846-1.02737873 \quad 2.31875817$

$\mathrm{H}-0.69791028-0.89155636 \quad 4.24527523$

$\mathrm{H} \quad 0.10885666-2.04377677 \quad 3.11797429$

$\begin{array}{lllll}0 & -0.80794650 & 0.33640318 & -2.32830340\end{array}$

$\begin{array}{llll}0 & 0.38590013 & 2.79375415 & 0.65540376\end{array}$

o - $0.83281150-1.09598358-1.99426092$

Vibrational frequencies (in $\mathrm{cm}-1$ )

$\begin{array}{llllllllllllllll}-1619.3 & 17.2 & 54.5 & 55.9 & 70.6 & 86.6 & 96.7 & 104.0 & 142.5 & 158.8 & 179.3 & 206.7 & 244.5 & 273.5\end{array}$ $299.6 \quad 314.0 \quad 360.4 \quad 401.4 \quad 434.1 \quad 463.1 \quad 490.5 \quad 503.3 \quad 558.6 \quad 581.6 \quad 606.5 \quad 717.0 \quad 741.1 \quad 817.1$ $845.3 \quad 854.0 \quad 915.4 \quad 931.8 \quad 986.2 \quad 991.8 \quad 1013.9 \quad 1029.4 \quad 1038.2 \quad 1042.2 \quad 1050.2 \quad 1057.4 \quad 1089.4$ $1110.91134 .61164 .5 \quad 1186.2 \quad 1198.5 \quad 1215.1 \quad 1243.8 \quad 1258.2 \quad 1258.91261 .3 \quad 1287.1 \quad 1290.3$

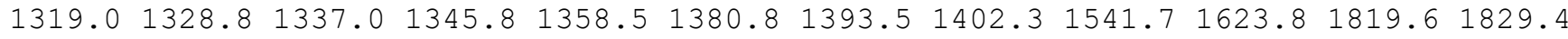
$1979.32171 .22604 .02631 .4 \quad 2648.4 \quad 2662.5 \quad 2669.12674 .02677 .7 \quad 2684.62743 .82756 .6$ $2756.8 \quad 2765.7 \quad 2779.2 \quad 2869.9$

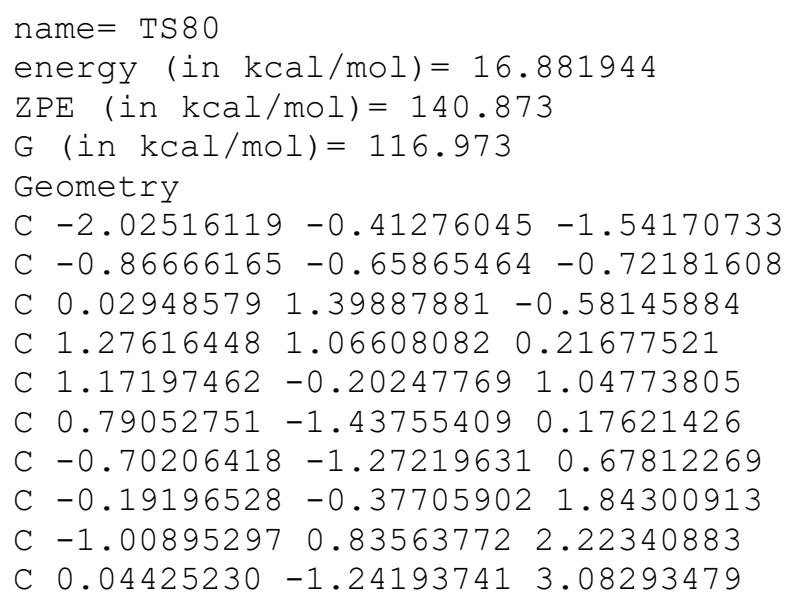


$\mathrm{H}-2.073859170 .65091105-1.91138201$

$\mathrm{H}-2.97991323-0.67083961-1.07560877$

$\mathrm{H}-1.90888316-0.90188123-2.54894473$

$\mathrm{H}-0.87914627 \quad 1.66051455-0.05191621$

H $1.51888594 \quad 1.92950206 \quad 0.86783494$

H $2.131448650 .97419416-0.48949907$

$\mathrm{H} 2.06385396-0.348943291 .67198341$

$\mathrm{H} 1.38074639-2.334324430 .15660130$

$\mathrm{H} \quad 0.52329879 \quad 0.58835293-3.50406722$

$\mathrm{H}-1.36500366 \quad-2.09044118 \quad 0.92491734$

$\mathrm{H}-1.63101766 \quad 0.65359364 \quad 3.10967344$

$\mathrm{H}-1.699830231 .15023991 \quad 1.42958012$

$\mathrm{H}-0.36307995 \quad 1.69352445 \quad 2.45671917$

$\mathrm{H} \quad 0.64788678-2.13082467 \quad 2.86992375$

$\mathrm{H}-0.90105400-1.58954625 \quad 3.51605637$

$\mathrm{H} \quad 0.56904011-0.67162257 \quad 3.85997907$

$\begin{array}{llll}0 & -0.18751476 & 0.39879623 & -2.93055722\end{array}$

$00.131598561 .73110823-1.77998860$

o $0.35355183-1.03654903-1.17056317$

Vibrational frequencies (in $\mathrm{cm}-1$ )

$\begin{array}{lllllllllllllllll}-611.1 & 74.4 & 119.0 & 129.4 & 166.3 & 188.9 & 194.7 & 208.1 & 226.6 & 252.1 & 271.4 & 286.5 & 295.2 & 333.0\end{array}$

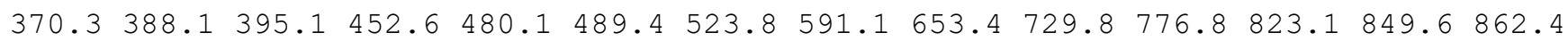
$900.7924 .6 \quad 950.3 \quad 953.1 \quad 993.3 \quad 1006.5 \quad 1013.4 \quad 1021.7 \quad 1027.5 \quad 1042.2 \quad 1063.0 \quad 1068.1 \quad 1095.6$

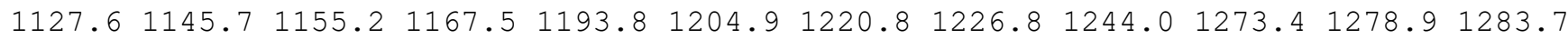
$1288.41296 .7 \quad 1299.0 \quad 1300.4 \quad 1313.1 \quad 1333.3 \quad 1366.2 \quad 1379.1 \quad 1402.8 \quad 1413.0 \quad 1562.01574 .4$ $2542.52644 .52652 .72660 .6 \quad 2670.2 \quad 2674.2 \quad 2676.12676 .62729 .62740 .32743 .12744 .7$ $2752.3 \quad 2776.2 \quad 2782.0 \quad 2834.7$

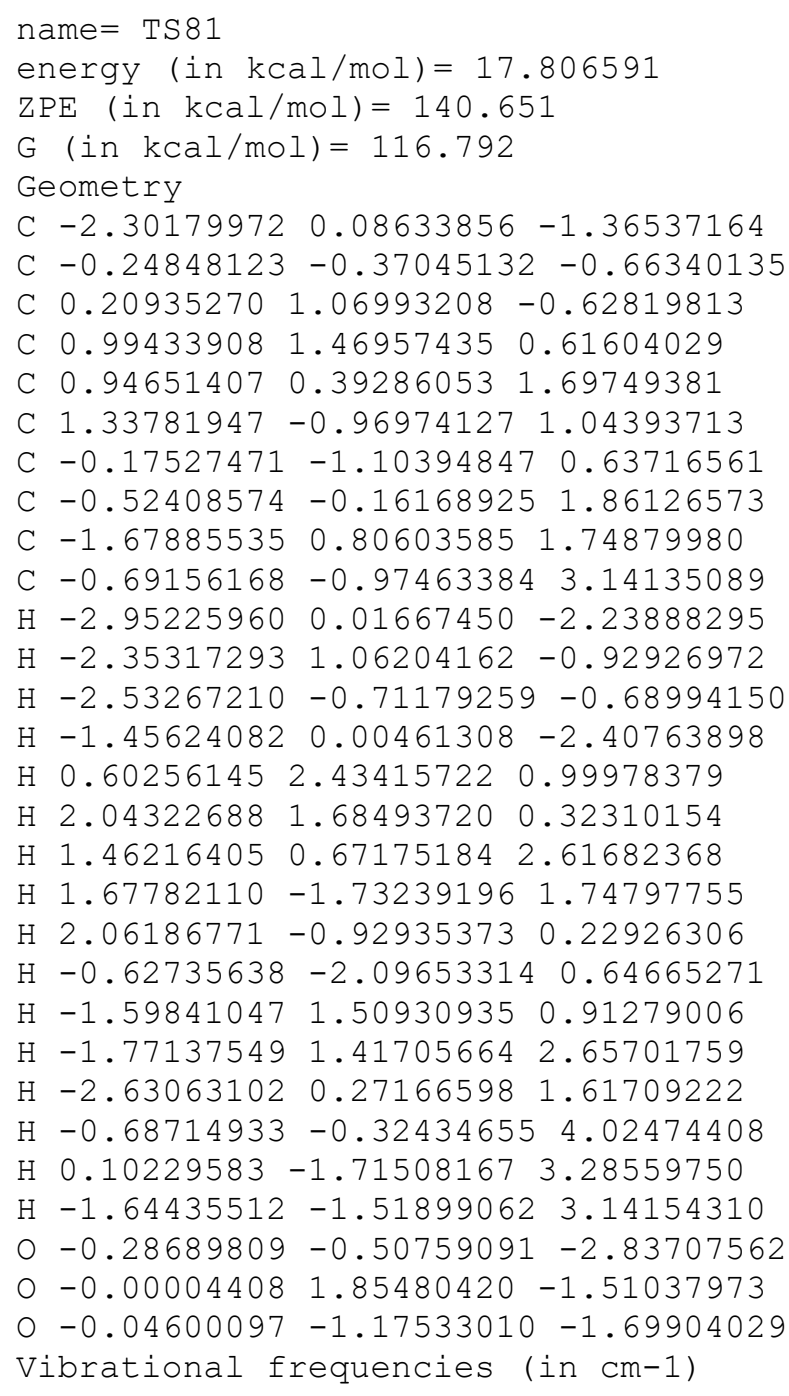


$\begin{array}{lllllllllllllllllll}-1427.5 & 59.9 & 128.8 & 135.2 & 155.4 & 171.2 & 176.8 & 208.8 & 221.6 & 256.2 & 281.7 & 309.7 & 351.1 & 383.1\end{array}$ $402.1427 .0 \quad 460.0 \quad 473.4 \quad 499.0 \quad 558.1 \quad 569.3 \quad 589.8 \quad 659.2 \quad 677.5 \quad 751.8 \quad 782.7 \quad 804.5 \quad 856.2$ $892.5923 .9943 .5990 .8 \quad 1000.3 \quad 1010.2 \quad 1013.91018 .91045 .0 \quad 1070.8 \quad 1079.91098 .7 \quad 1116.4$ $1127.61174 .7 \quad 1181.4 \quad 1186.0 \quad 1214.2 \quad 1241.3 \quad 1248.2 \quad 1270.2 \quad 1276.01280 .2 \quad 1281.2 \quad 1282.6$

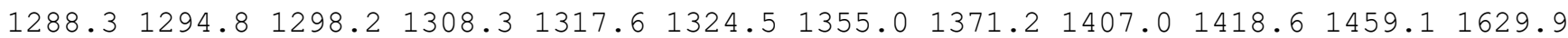
$1855.62653 .6 \quad 2663.12664 .02671 .5 \quad 2674.6 \quad 2677.12686 .12711 .72738 .32744 .72749 .8$ $2764.02778 .02780 .4 \quad 2782.9$

name $=$ TS82

energy (in $\mathrm{kcal} / \mathrm{mol})=17.832126$

$\mathrm{ZPE}($ in $\mathrm{kcal} / \mathrm{mol})=140.738$

$\mathrm{G}($ in $\mathrm{kcal} / \mathrm{mol})=115.565$

Geometry

C $-1.53861662 \quad 1.58586000 \quad 4.10764031$

C $-0.61067422 \quad 1.466290382 .96321657$

C $1.889951752 .06593133-1.05870797$

C $1.47411003 \quad 1.71261223 \quad 0.19970132$

C $0.99807430 \quad 0.37255685 \quad 0.53173650$

C $-0.55677331 \quad 0.34181617 \quad 0.73060310$

C $-0.37985065 \quad 0.19602213 \quad 2.27235571$

C $1.16944783-0.12974187 \quad 2.01261866$

C $2.18083036 \quad 0.55659053 \quad 2.90428924$

C $1.37436961-1.63681377 \quad 2.08284791$

$\mathrm{H}-2.55917551 \quad 1.25530594 \quad 3.83679715$

$\mathrm{H}-1.62647308 \quad 2.62112183 \quad 4.48652311$

$\mathrm{H}-1.22238886 \quad 0.95611074 \quad 4.95809252$

H $2.24797394 \quad 3.06451110-1.30301543$

$\mathrm{H} 0.69136860 \quad 2.48031961 \quad 1.66445479$

$\mathrm{H} \quad 1.48848034 \quad 2.54223918 \quad 0.98442046$

$\mathrm{H} \quad 1.36248797 \quad-0.36736034 \quad-0.23310777$

$\mathrm{H}-1.02792322 \quad-0.52728839 \quad 0.25912174$

$\mathrm{H}-1.076400251 .231002660 .37422218$

$\mathrm{H}-0.92835922-0.65237727 \quad 2.70804232$

$\begin{array}{llllll}\mathrm{H} & 3.18343063 & 0.47497180 & 2.44711311\end{array}$

H $2.23826730 \quad 0.08551637 \quad 3.89328330$

$\mathrm{H} 2.020781181 .62407993 \quad 3.06270110$

H $2.39075959-1.904708051 .75881832$

$\mathrm{H} \quad 0.68752758-2.17892036 \quad 1.41962479$

H $1.23717940 \quad-2.02161684 \quad 3.09844579$

O $1.53781938 \quad 0.09471813-2.10403806$

O $1.94812599 \quad 1.33360227 \quad-2.15410723$

o $-0.04273754 \quad 2.53651104 \quad 2.61438706$

Vibrational frequencies (in cm-1)

$\begin{array}{lllllllllllllll}-134.8 & 55.2 & 91.1 & 109.4 & 112.2 & 145.2 & 158.3 & 198.1 & 205.4 & 229.7 & 255.3 & 297.3 & 324.3 & 346.4\end{array}$ $365.2401 .0 \quad 430.1 \quad 474.3 \quad 510.3 \quad 545.5 \quad 582.5 \quad 681.1 \quad 701.9745 .9 \quad 775.6 \quad 794.0 \quad 864.1 \quad 893.4$ $908.4924 .8 \quad 946.2976 .1 \quad 999.1 \quad 1001.6 \quad 1013.1 \quad 1024.6 \quad 1038.7 \quad 1046.9 \quad 1076.5 \quad 1088.3 \quad 1104.8$ $1137.7 \quad 1163.3 \quad 1185.0 \quad 1212.8 \quad 1225.3 \quad 1228.4 \quad 1243.0 \quad 1247.8 \quad 1252.1 \quad 1269.4 \quad 1277.8 \quad 1284.5$ $1293.61298 .6 \quad 1304.6 \quad 1326.8 \quad 1350.0 \quad 1365.7 \quad 1402.1 \quad 1408.91414 .8 \quad 1438.5 \quad 1646.7 \quad 1704.4$ $2052.72452 .92609 .92641 .2 \quad 2648.7 \quad 2660.8 \quad 2668.2 \quad 2675.32679 .5 \quad 2686.52727 .4 \quad 2731.9$ $2763.3 \quad 2765.02779 .02781 .6$

name $=\operatorname{TS} 83$

energy $($ in $\mathrm{kcal} / \mathrm{mol})=15.127038$

$\mathrm{ZPE}(\mathrm{in} \mathrm{kcal} / \mathrm{mol})=143.756$

$\mathrm{G}($ in $\mathrm{kcal} / \mathrm{mol})=119.339$

Geometry

C $-1.91095250-0.24638854-2.06281698$

C $-0.82100413-0.43610944-1.06910546$

C $-0.07886409 \quad 1.33808722-0.88384161$

C $0.497748931 .47210732 \quad 0.52422791$

C $0.96511990 \quad 0.19133280 \quad 1.16709879$

C $0.12811370-1.078373531 .08400187$

C $-1.14159773-1.02932574 \quad 0.27073108$ 
C $0.28789315-0.362154052 .41946048$

C $-0.872783490 .34715532 \quad 3.06518523$

C $1.18148883-1.026511153 .44246192$

$\mathrm{H}-2.30547498-1.21559595-2.40619312$

$\mathrm{H}-1.55128130 \quad 0.28688608 \quad-2.96314790$

$\mathrm{H}-2.75784124 \quad 0.32634162 \quad-1.66373687$

$\mathrm{H}-1.019670831 .88047726-1.04685303$

$\mathrm{H}-0.24404230 \quad 1.98262692 \quad 1.16972285$

H $1.35727454 \quad 2.17899726 \quad 0.46292670$

H $2.053551390 .06926152 \quad 1.08946715$

$\mathrm{H}-1.58519140 \quad-2.04164310 \quad 0.16798425$

$\mathrm{H} \quad 0.65795585-2.03132393 \quad 0.97010229$

$\mathrm{H}-1.92625158-0.42500867 \quad 0.77354894$

$\mathrm{H}-1.53828555 \quad 0.83130160 \quad 2.34035716$

$\mathrm{H}-0.521102651 .12927118 \quad 3.75212038$

$\mathrm{H}-1.48518454-0.35313799 \quad 3.64883421$

H $2.03063032-1.548675192 .98507325$

$\mathrm{H} \quad 0.62602928-1.76609198 \quad 4.03283302$

$\mathrm{H} \quad 1.59568301-0.28758669 \quad 4.14083050$

○ $0.78673598-0.33294078-2.54748422$

○ $0.74692438 \quad 1.37309337-1.86288280$

o $0.39333743-0.92648137-1.50267709$

Vibrational frequencies (in $\mathrm{cm}-1$ )

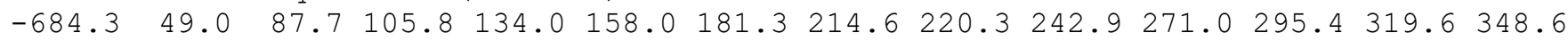

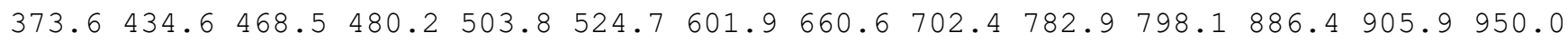
$971.2999 .2 \quad 1004.0 \quad 1007.7 \quad 1013.7 \quad 1020.4 \quad 1028.1 \quad 1066.3 \quad 1076.6 \quad 1084.11112 .01155 .3$

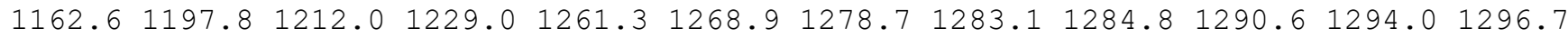

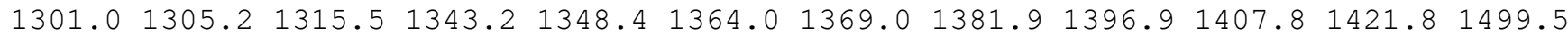
$1511.42648 .92653 .0 \quad 2653.92663 .4 \quad 2669.7 \quad 2670.92674 .3 \quad 2675.92678 .8 \quad 2734.92738 .4$ $2743.92753 .4 \quad 2773.7 \quad 2778.6 \quad 2781.9$

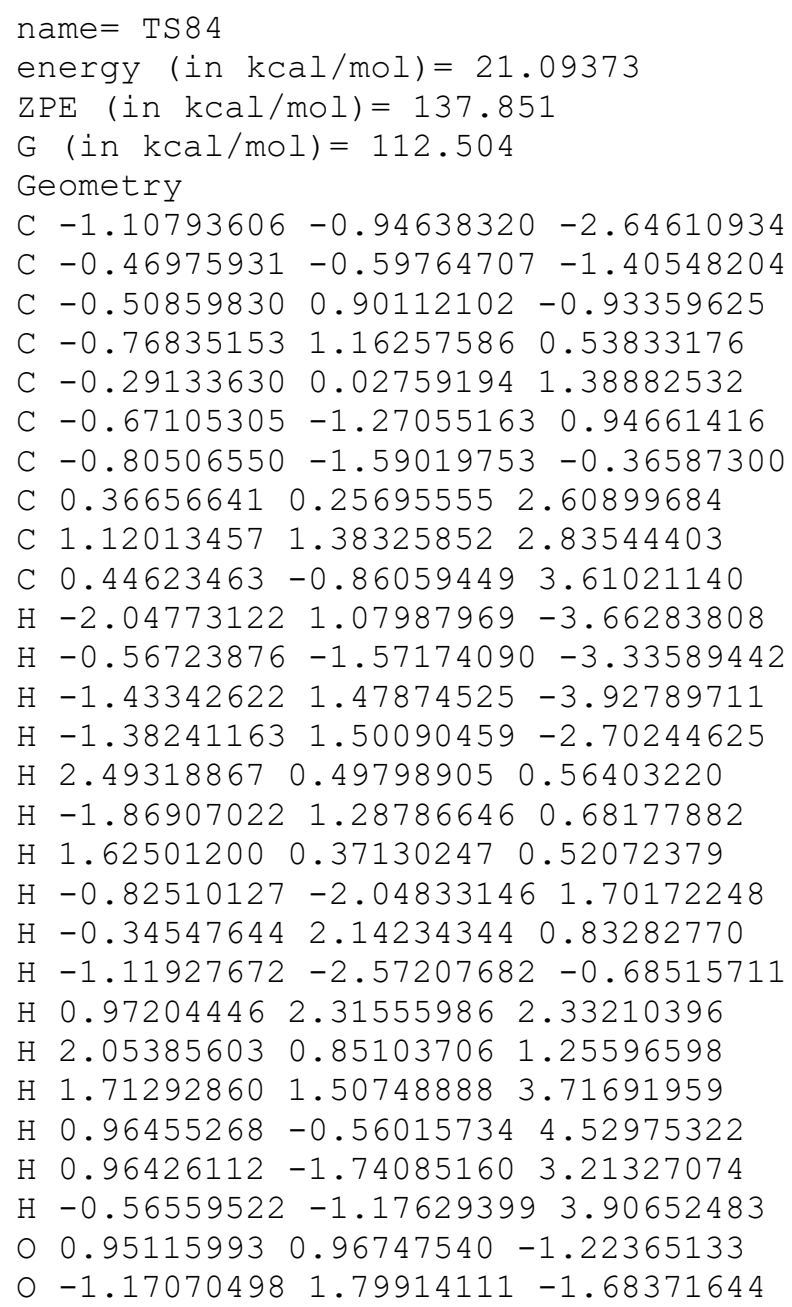


O $1.00292780-0.44634914-1.54514214$

Vibrational frequencies (in $\mathrm{cm}-1$ )

$\begin{array}{lllllllllllllllll}-416.4 & 70.4 & 84.0 & 90.3 & 114.1 & 131.0 & 146.2 & 171.0 & 222.5 & 245.2 & 261.3 & 292.0 & 295.0 & 314.1\end{array}$

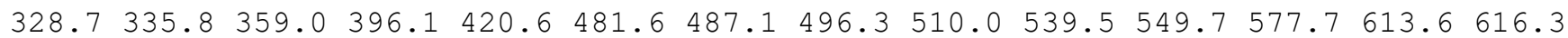

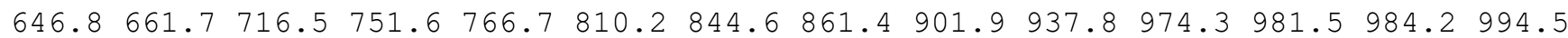

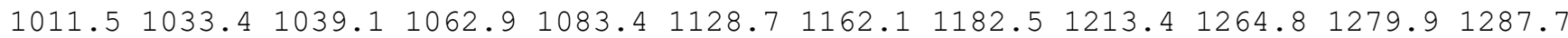

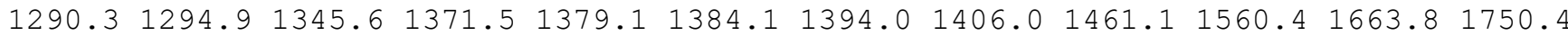
$2033.62436 .12651 .5 \quad 2671.7 \quad 2677.5 \quad 2681.92734 .82736 .72738 .7 \quad 2750.4 \quad 2778.3 \quad 2780.5$ $2808.0 \quad 4611.25303 .9$

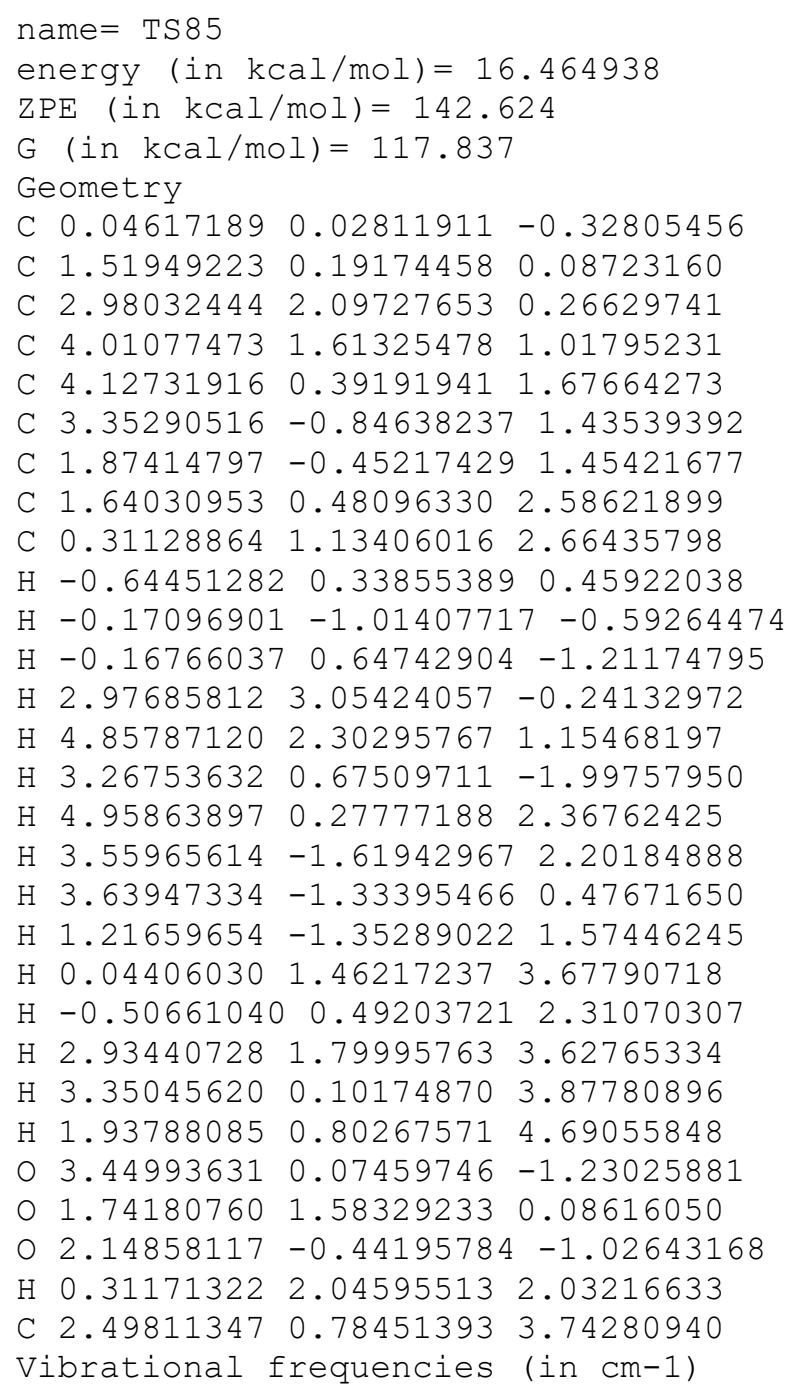


C $0.92563097-1.430856220 .40885516$

C $-0.51808569-1.10401177 \quad 0.11498664$

C $0.00113118-0.15260610 \quad 2.55725497$

C $-1.40902661 \quad 0.41390863 \quad 2.77971569$

C $0.10872515-1.51766627 \quad 3.24622877$

$\mathrm{H}-2.37690784-0.02898165-2.63309844$

$\mathrm{H}-2.98696831-0.23841662-0.98881850$

$\mathrm{H}-2.43190186-1.65050348-1.92070296$

$\mathrm{H}-0.26686947 \quad 2.23126391 \quad 0.54146392$

$\mathrm{H} 0.40393823 \quad 2.07147609 \quad 1.16607555$

H $1.70126890 \quad 1.23633180 \quad 0.15143727$

$\mathrm{H} \quad 0.74827491 \quad 0.52687925 \quad 3.04234942$

$\mathrm{H} \quad 1.16915598-2.31366326 \quad 0.99089305$

$\mathrm{H} \quad 1.67322438-1.28943640-0.37503397$

$\mathrm{H}-1.30013502-1.75271594 \quad 0.50616953$

$\mathrm{H}-2.16990122-0.23051766 \quad 2.31987480$

$\mathrm{H}-1.53733950 \quad 1.41213665 \quad 2.35587213$

$\mathrm{H}-1.64501453 \quad 0.47929198 \quad 3.84831787$

$\mathrm{H}-0.14764652-1.44418374 \quad 4.30961602$

$\mathrm{H} 1.12712315-1.918804763 .18775978$

$\mathrm{H}-0.56924728 \quad-2.25372951 \quad 2.79970791$

$\begin{array}{lllll}0 & 0.58815992 & 0.77564119 & -2.18542104\end{array}$

$\begin{array}{lllll}0 & -0.88595198 & 2.17458372 & -1.00902396\end{array}$

o $0.15796187-0.63247823-2.20288759$

Vibrational frequencies (in cm-1)

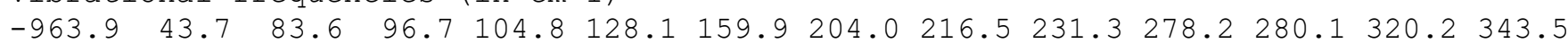

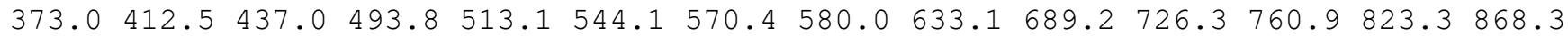
$886.3918 .9945 .2972 .8 \quad 976.2 \quad 983.2 \quad 992.9 \quad 1009.8 \quad 1011.01040 .7 \quad 1057.0 \quad 1065.01124 .7$

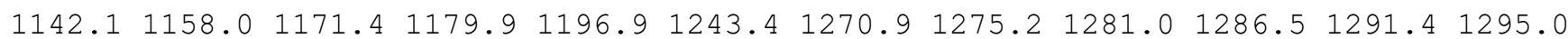

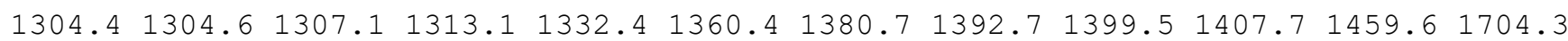
$1781.92666 .92668 .8 \quad 2673.4 \quad 2674.8 \quad 2679.5 \quad 2679.6 \quad 2700.92701 .8 \quad 2720.4 \quad 2763.5 \quad 2777.9$ $2779.9 \quad 2780.7 \quad 2783.1 \quad 3258.4$

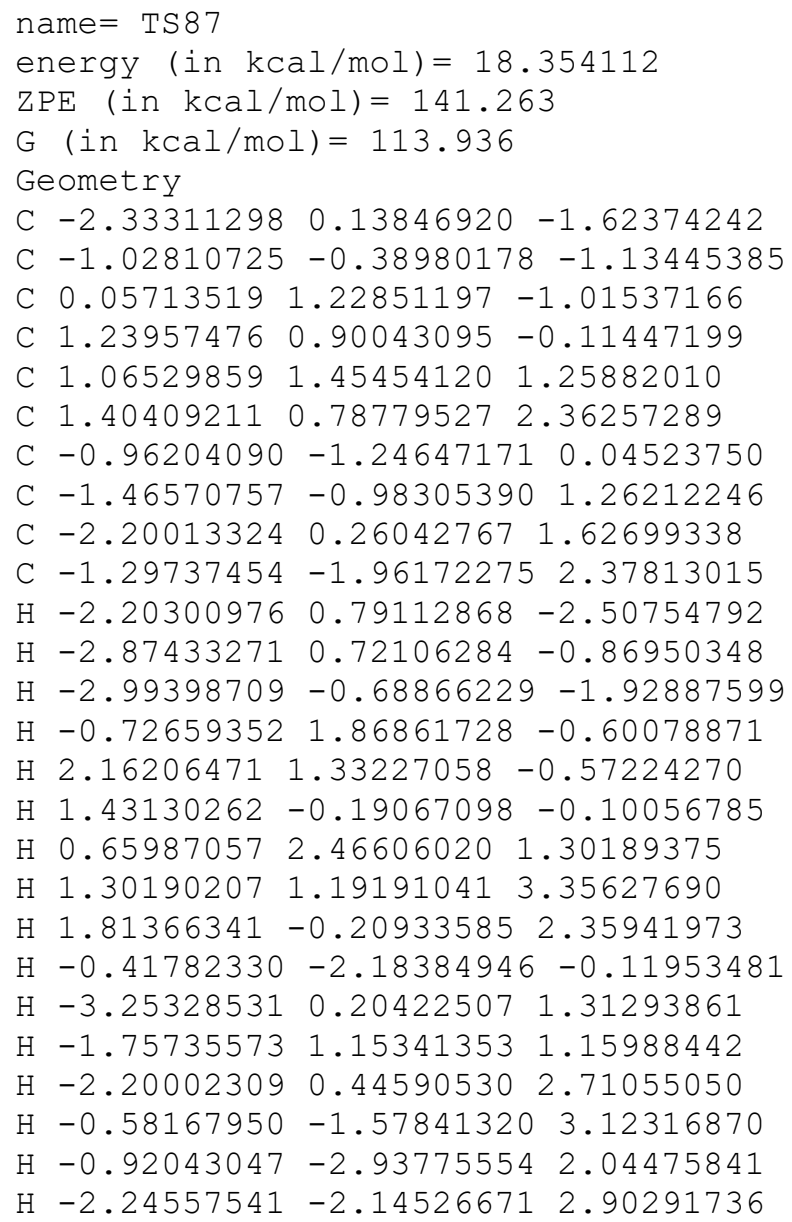


$0-0.09507847-0.11967308-3.10292575$

O $0.331308381 .41570025-2.25600383$

O $-0.15406755-0.88849743-2.08938669$

Vibrational frequencies (in $\mathrm{cm}-1$ )

$\begin{array}{llllllllllllllll}-690.8 & 21.0 & 52.0 & 67.9 & 74.0 & 87.3 & 96.5 & 116.6 & 144.7 & 168.5 & 222.9 & 247.4 & 262.4 & 284.7\end{array}$

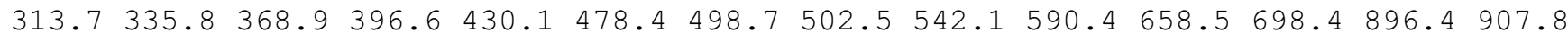
$909.1961 .7 \quad 977.8 \quad 987.8 \quad 997.7 \quad 1016.7 \quad 1021.1 \quad 1032.7 \quad 1064.9 \quad 1079.9 \quad 1097.3 \quad 1104.91117 .5$

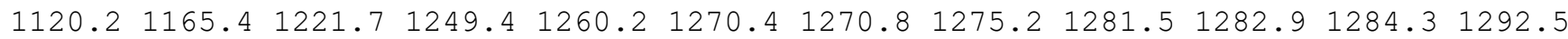

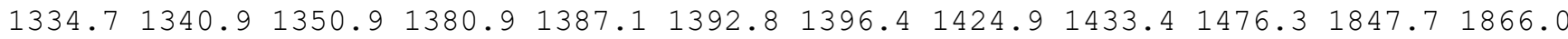
$2650.82652 .02661 .72664 .8 \quad 2665.72668 .8 \quad 2669.62673 .32718 .2 \quad 2735.82738 .32758 .0$ $2773.3 \quad 2775.12778 .4 \quad 2791.7$

name $=\operatorname{TS} 88$

energy $($ in $\mathrm{kcal} / \mathrm{mol})=15.702531$

$\mathrm{ZPE}($ in $\mathrm{kcal} / \mathrm{mol})=144.185$

$\mathrm{G}($ in $\mathrm{kcal} / \mathrm{mol})=121.059$

Geometry

C $-2.15731405-0.52362855-1.17547559$

C $-0.72559891-0.55178617-0.78298572$

C $-0.20895344 \quad 1.33155388 \quad-0.65451281$

C $0.81717401 \quad 1.45627784 \quad 0.47412086$

C $0.91612115 \quad 0.25976630 \quad 1.41636815$

C $1.27314321-1.01867805 \quad 0.59689254$

C $-0.26680542-1.265355550 .44858650$

C $-0.46849087-0.40578034 \quad 1.76405105$

C $-1.68969724 \quad 0.46866065 \quad 1.94039800$

C $-0.35714382-1.304583142 .99914058$

$\mathrm{H}-2.33671647 \quad 0.16335939-2.02230488$

$\mathrm{H}-2.82178321-0.21749530-0.35690668$

$\mathrm{H}-2.49139191-1.52263802-1.50038274$

$\mathrm{H}-1.21184370 \quad 1.71627512-0.42507438$

$\mathrm{H} \quad 0.57668650 \quad 2.36990544 \quad 1.05368488$

$\mathrm{H} 1.816270361 .645251010 .02460579$

H $1.55489478 \quad 0.47013790 \quad 2.28011034$

H $1.79196142-1.79163677 \quad 1.16819292$

$\mathrm{H} 1.84811249-0.84885741-0.31440046$

$\mathrm{H}-0.61208292-2.30121706 \quad 0.51459809$

$\mathrm{H}-1.60302190 \quad 1.070703992 .85594508$

$\mathrm{H}-2.60044001-0.136708662 .03258920$

$\mathrm{H}-1.85375404 \quad 1.17912591 \quad 1.12449241$

$\mathrm{H}-0.25586318-0.70577102 \quad 3.91186553$

H $0.50756471-1.97530184 \quad 2.95840696$

$\mathrm{H}-1.24851888-1.931867673 .11252525$

$0.028252810 .01204893-2.76337716$

$00.19518846 \quad 1.57728426-1.84609756$

$0.20465556-0.76847292-1.78097182$

Vibrational frequencies (in $\mathrm{cm}-1$ )

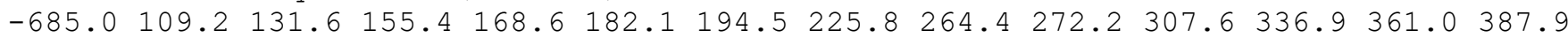

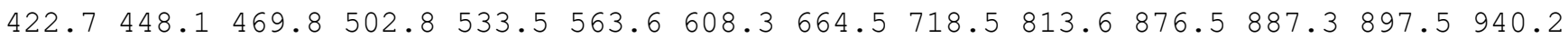
$990.2992 .1999 .4 \quad 1013.4 \quad 1016.9 \quad 1019.9 \quad 1050.1 \quad 1064.5 \quad 1073.8 \quad 1083.1 \quad 1090.01117 .7 \quad 1140.3$ $\begin{array}{llllllllllllll}1188.0 & 1199.6 & 1203.1 & 1213.2 & 1255.8 & 1263.1 & 1268.3 & 1277.2 & 1278.7 & 1285.7 & 1291.1 & 1294.3\end{array}$ $1301.01305 .41311 .3 \quad 1323.91344 .2 \quad 1370.5 \quad 1376.1 \quad 1381.81403 .2 \quad 1412.31415 .61491 .0$ $2630.22655 .52660 .02666 .42669 .4 \quad 2674.42675 .32679 .62684 .22736 .42737 .72742 .4$ $2763.32773 .0 \quad 2778.6 \quad 2782.4$

name $=\operatorname{TS} 89$

energy $($ in $\mathrm{kcal} / \mathrm{mol})=15.59352$

$\mathrm{ZPE}(\mathrm{in} \mathrm{kcal} / \mathrm{mol})=144.44$

$\mathrm{G}(\mathrm{in} \mathrm{kcal} / \mathrm{mol})=121.401$

Geometry

C $-2.22521955 \quad 0.14920922-0.59506844$

C $-0.77434786-0.27194110-0.72658960$

C $0.265788561 .40908982-0.87097548$ 
C $1.22621294 \quad 1.69544516 \quad 0.25067634$

C $1.40196947 \quad 0.47634162 \quad 1.16211392$

C $1.44394230-0.82988086 \quad 0.31443394$

C $-0.10540339-0.91308512 \quad 0.47560504$

C $0.02771308-0.009069791 .76062702$

C $-1.01285332 \quad 1.04044588 \quad 2.08609202$

C $0.23513540-0.878999653 .00227246$

$\mathrm{H}-2.69358007 \quad 0.20275480-1.59039873$

$\mathrm{H}-2.37034651 \quad 1.11814043-0.11447116$

$\mathrm{H}-2.78522227-0.59559207-0.01510231$

$\mathrm{H}-0.58886892 \quad 2.04438403-1.04261945$

$\mathrm{H} \quad 0.89069144 \quad 2.56768731 \quad 0.84664496$

$\mathrm{H} \quad 2.21904834 \quad 1.98704235-0.15818556$

H $2.20751286 \quad 0.61224682 \quad 1.88772206$

H $1.98525168-1.653139650 .78684796$

$\mathrm{H} 1.81380438-0.74938396-0.70934241$

$\mathrm{H}-0.51789049-1.91290648 \quad 0.65774889$

$\mathrm{H}-1.98581742 \quad 0.57694695 \quad 2.29542256$

$\mathrm{H}-1.163315121 .767035921 .28217662$

$\mathrm{H}-0.72459143 \quad 1.612598912 .97780280$

$\mathrm{H} \quad 0.57336697-0.27876346 \quad 3.85408277$

$\mathrm{H} \quad 0.98207987 \quad-1.66431224 \quad 2.84275501$

$\mathrm{H}-0.69828649-1.37378914 \quad 3.29468742$

$\begin{array}{llllll}0 & 0.23943398 & 0.48145447 & -2.84444979\end{array}$

$\begin{array}{lllll}0 & 0.99777591 & 0.96388469 & -1.94163602\end{array}$

O $-0.49883882-0.81243553-1.86457246$

Vibrational frequencies (in $\mathrm{cm}-1$ )

$\begin{array}{lllllllllllllllllll}-724.2 & 97.7 & 106.8 & 172.4 & 182.0 & 194.5 & 214.7 & 250.0 & 255.7 & 295.8 & 332.1 & 356.3 & 368.3 & 389.7\end{array}$

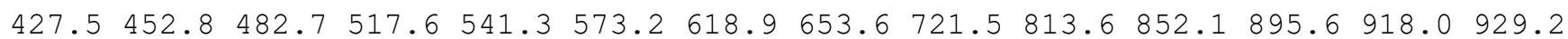

$976.0 \quad 992.4 \quad 995.9 \quad 1011.9 \quad 1017.6 \quad 1034.7 \quad 1051.5 \quad 1062.3 \quad 1083.1 \quad 1088.5 \quad 1101.8 \quad 1116.91122 .8$

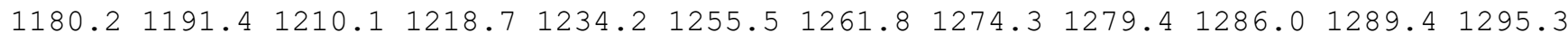
$1301.71302 .6 \quad 1315.8 \quad 1324.3 \quad 1326.4 \quad 1347.1 \quad 1372.1 \quad 1388.8 \quad 1392.2 \quad 1404.6 \quad 1413.6 \quad 1481.2$ $2654.72669 .62671 .8 \quad 2674.5 \quad 2675.6 \quad 2676.8 \quad 2680.62684 .32711 .3 \quad 2734.42735 .52744 .7$ $2765.22778 .12781 .4 \quad 2782.8$

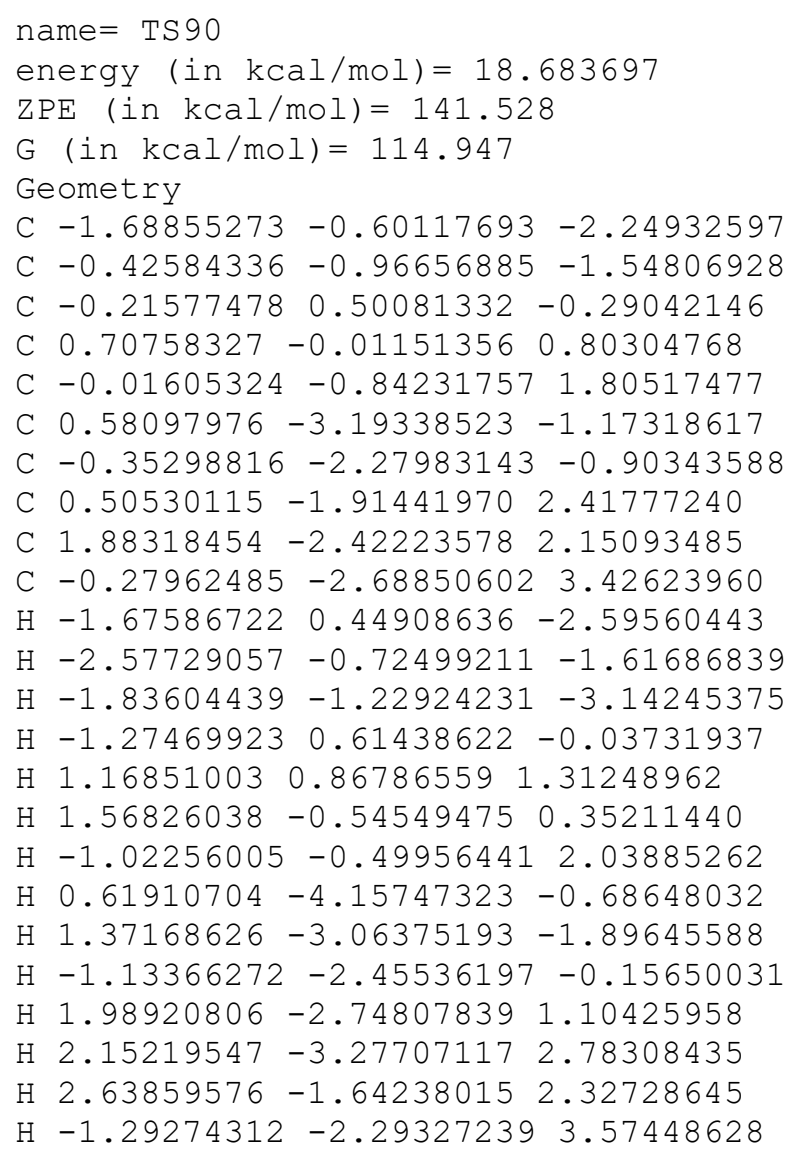


H $0.21706384 \quad-2.67482008 \quad 4.40730943$

$\mathrm{H}-0.38219969-3.74091685 \quad 3.12695098$

$0.748871920 .55519828-2.58963492$

O $0.273264201 .40272304-1.06519966$

O $0.77685302-0.63465964-2.14350309$

Vibrational frequencies (in $\mathrm{cm}-1$ )

$\begin{array}{llllllllllllllll}-691.0 & 40.1 & 55.6 & 61.4 & 83.3 & 116.1 & 134.3 & 146.1 & 170.0 & 172.8 & 207.8 & 237.9 & 245.6 & 260.3\end{array}$ $303.3 \quad 331.9 \quad 362.0 \quad 400.0 \quad 464.4 \quad 486.3 \quad 510.0 \quad 512.9 \quad 564.1 \quad 619.5 \quad 654.5 \quad 668.5 \quad 841.3 \quad 891.2$ $948.7 \quad 967.5 \quad 972.3 \quad 994.6 \quad 1004.5 \quad 1020.4 \quad 1025.7 \quad 1055.9 \quad 1072.8 \quad 1076.4 \quad 1090.5 \quad 1104.2 \quad 1108.4$

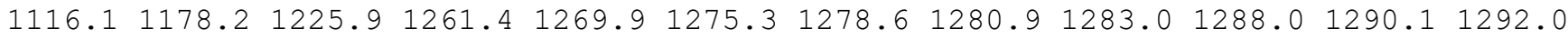

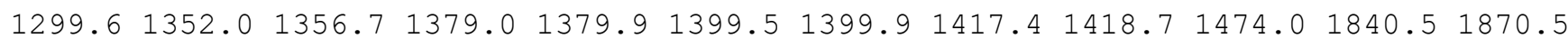
$2651.22652 .5 \quad 2663.5 \quad 2666.7 \quad 2669.5 \quad 2672.1 \quad 2673.92677 .22709 .32735 .02747 .02759 .1$ $2772.6 \quad 2777.0 \quad 2780.4 \quad 2783.0$

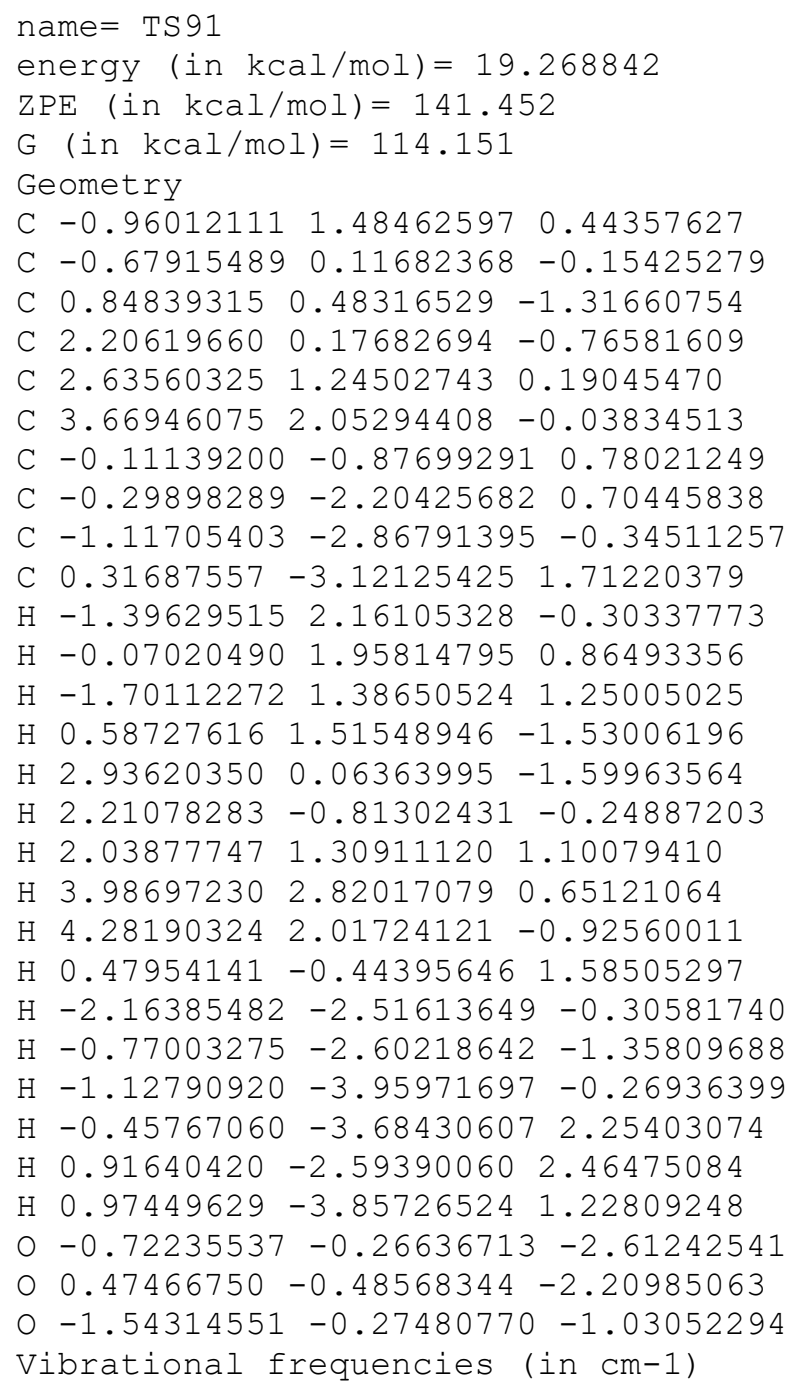


C $-0.49203194 \quad 0.05360594 \quad-0.62281899$

C $0.645997851 .53537735-1.24654884$

C $1.839240321 .61760827-0.31117591$

$\begin{array}{llll}\text { C } 1.51609947 & 2.31035033 & 0.96839655\end{array}$

C $2.16731548 \quad 2.07198150 \quad 2.10746013$

C $0.45493777-0.96206776-0.20013589$

C $0.77347692-1.364346061 .04190801$

C $0.21602942-0.801903412 .30270533$

C $1.77154569-2.46035838 \quad 1.23755617$

$\mathrm{H}-2.320787851 .17235622-0.32115180$

$\mathrm{H}-1.18737370 \quad 1.23067788 \quad 1.03490047$

$\mathrm{H}-2.14211228-0.235443620 .73908659$

$\mathrm{H}-0.108416512 .32336196-1.14091262$

$\mathrm{H} 2.648582632 .17860349-0.83900310$

H $2.28272486 \quad 0.61426265 \quad-0.14322635$

$\mathrm{H} \quad 0.72237304 \quad 3.05618876 \quad 0.91003683$

$\mathrm{H} 1.96060606 \quad 2.58747055 \quad 3.03107171$

H 2.96300916 1.350765012 .20426357

$\mathrm{H} \quad 0.95870590-1.44221018-1.05698158$

$\mathrm{H}-0.85472842-1.027200512 .40627264$

$\mathrm{H} \quad 0.33199475 \quad 0.29429568 \quad 2.33940567$

$\mathrm{H} \quad 0.71022362-1.19649770 \quad 3.20082146$

H $2.65421917-2.09703458 \quad 1.78470063$

H $2.13421275-2.88848495 \quad 0.29261661$

$\mathrm{H} 1.34364369-3.28846395 \quad 1.82133109$

O $-0.07712907-0.31151719-2.74783625$

$0 \quad 0.899804621 .16711931-2.45212954$

o - $-1.00434613-0.07996784-1.90599813$

Vibrational frequencies (in cm-1)

$\begin{array}{llllllllllllllll}-704.0 & 34.6 & 44.8 & 75.0 & 86.4 & 94.9 & 110.1 & 147.9 & 183.9 & 202.2 & 205.8 & 223.9 & 257.9 & 289.9\end{array}$

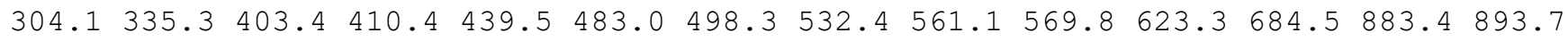
$912.2967 .6 \quad 982.5 \quad 985.8 \quad 993.5 \quad 1010.0 \quad 1021.0 \quad 1028.2 \quad 1067.8 \quad 1079.8 \quad 1101.01113 .3 \quad 1119.3$

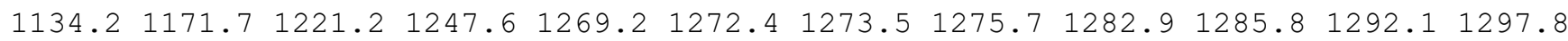

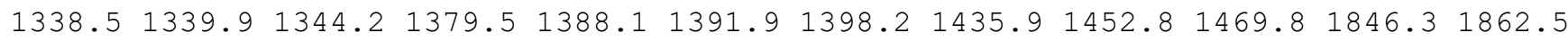
$2644.22655 .6 \quad 2662.4 \quad 2662.8 \quad 2665.3 \quad 2667.9 \quad 2669.6 \quad 2672.02714 .3 \quad 2716.92732 .2 \quad 2755.9$ $2772.6 \quad 2774.92777 .92791 .2$

name $=\operatorname{TS} 93$

energy $($ in $\mathrm{kcal} / \mathrm{mol})=16.900441$

$\operatorname{ZPE}(\mathrm{in} \mathrm{kcal} / \mathrm{mol})=144.302$

$\mathrm{G}(\mathrm{in} \mathrm{kcal} / \mathrm{mol})=121.069$

Geometry

C $-2.39680440-0.15119576-0.71002891$

C $-0.91567214-0.39662160-0.66848665$

C $0.201147161 .25516363-0.71373851$

C $0.95148438 \quad 1.44497938 \quad 0.60945972$

C $1.00822725 \quad 0.19782755 \quad 1.48415293$

C $1.19808899-1.049718170 .57493589$

C $-0.35667345-1.144000910 .48969610$

C $-0.42617725-0.331338661 .85006288$

C $-1.51427102 \quad 0.69720802 \quad 2.07336774$

C $-0.39832563-1.28142923 \quad 3.04596789$

$\mathrm{H}-2.64566722 \quad 0.89915661-0.50268145$

$\mathrm{H}-2.93729812-0.76569863 \quad 0.02365051$

$\mathrm{H}-2.82722589-0.37630297-1.70030580$

$\mathrm{H}-0.65045321 \quad 1.91643676-0.89204690$

$\mathrm{H} \quad 0.51351218 \quad 2.28903363 \quad 1.17223477$

H $1.99126440 \quad 1.75504368 \quad 0.36204540$

H $1.69868374 \quad 0.30240353 \quad 2.32499744$

H $1.66447703-1.904990721 .06853917$

$\mathrm{H} \quad 1.74137748-0.87459824-0.35794902$

$\mathrm{H}-0.76772483-2.16244432 \quad 0.54590270$

$\mathrm{H}-1.57730306 \quad 1.43861905 \quad 1.27077510$

$\mathrm{H}-1.330420501 .255973293 .00147544$ 
$\mathrm{H}-2.49926391 \quad 0.22529624 \quad 2.16776844$

$\mathrm{H}-0.20713748-0.73590927 \quad 3.97757883$

$\mathrm{H} 0.38216337-2.045227612 .95518437$

$\mathrm{H}-1.35452540-1.80410888 \quad 3.15943719$

$0.67086544-0.68702501-2.18500297$

$0.950306001 .02522316-1.73132977$

o $-0.57817185-0.70851846-1.96619563$

Vibrational frequencies (in $\mathrm{cm}-1$ )

$\begin{array}{lllllllllllllll}-710.5 & 86.0 & 96.1 & 159.1 & 175.1 & 193.8 & 203.4 & 248.2 & 262.8 & 291.0 & 303.3 & 364.4 & 384.7 & 392.9\end{array}$ $417.6 \quad 440.1 \quad 473.2 \quad 495.4 \quad 529.2 \quad 551.2 \quad 641.2 \quad 680.4 \quad 720.4 \quad 809.7 \quad 847.9 \quad 885.7 \quad 920.7946 .3$ $988.5 \quad 995.7 \quad 1005.3 \quad 1005.8 \quad 1011.9 \quad 1018.3 \quad 1037.9 \quad 1058.4 \quad 1076.8 \quad 1087.4 \quad 1093.01120 .6$

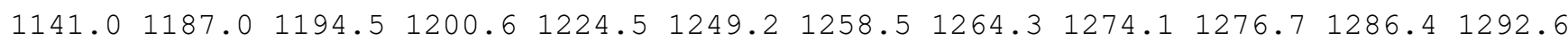

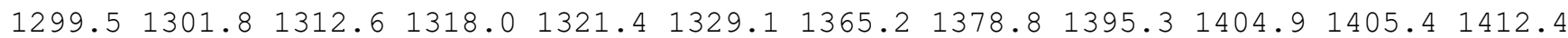
$1487.12655 .62655 .72665 .2 \quad 2671.42672 .12675 .92677 .42678 .72681 .02725 .02738 .3$ $2745.5 \quad 2761.9 \quad 2772.0 \quad 2781.2 \quad 2782.9$

name $=\operatorname{TS} 94$

energy $($ in $\mathrm{kcal} / \mathrm{mol})=20.683418$

$\mathrm{ZPE}($ in $\mathrm{kcal} / \mathrm{mol})=140.92$

$\mathrm{G}($ in $\mathrm{kcal} / \mathrm{mol})=117.051$

Geometry

C $-2.08050362-0.43315702-1.14965809$

C $-0.60736243-0.31832921-0.86316212$

C $0.032103421 .09770458-0.58024700$

C $0.94637934 \quad 1.27953335 \quad 0.62791063$

C $1.18443086-0.07227011 \quad 1.31869762$

C $1.44262662-1.03981748 \quad 0.16967600$

C $-0.05392257-1.35213898 \quad 0.10829242$

C $-0.18670720-0.81755401 \quad 1.60184677$

C $-1.379404450 .02289033 \quad 1.99883919$

C $-0.02806991-1.949861512 .61274804$

$\mathrm{H}-2.47851754 \quad 0.46650122 \quad-1.63594963$

$\mathrm{H}-2.66641914-0.59811718-0.23649644$

$\mathrm{H}-2.27703142-1.27957133-1.82433108$

$\mathrm{H}-1.20465827 \quad 2.31773790 \quad-1.51709676$

$\mathrm{H} \quad 0.50981919 \quad 2.01296541 \quad 1.33368872$

H $1.91468978 \quad 1.703421390 .28864962$

H $1.87580559-0.001275362 .15496211$

H $2.02466369-2.29519427 \quad 0.88544246$

$\mathrm{H} \quad 2.41678997-2.016454990 .18087335$

$\mathrm{H}-0.40056034-2.36374260-0.08254266$

$\mathrm{H}-1.58503709 \quad 0.847145131 .30183469$

$\mathrm{H}-1.221121350 .48528132 \quad 2.98203029$

$\mathrm{H}-2.29347424-0.579675292 .06054282$

$\mathrm{H} \quad 0.09996738-1.55259689 \quad 3.62780266$

$\mathrm{H} \quad 0.83012234 \quad-2.59563487 \quad 2.40829993$

$\mathrm{H}-0.91936982-2.59007682 \quad 2.62491329$

$\begin{array}{lllll}0 & 0.73291884 & 0.98543006 & -1.84034611\end{array}$

$\begin{array}{lllll}0 & -0.84530601 & 2.17121330 & -0.61538761\end{array}$

O $0.11995552-0.31679857-2.12743779$

Vibrational frequencies (in cm-1)

$\begin{array}{lllllllllllllll}-1506.0 & 44.0 & 106.7 & 136.7 & 168.5 & 175.4 & 216.9 & 235.7 & 244.8 & 286.3 & 294.6 & 309.7 & 333.3 \quad 358.7\end{array}$ $372.1415 .5 \quad 421.9 \quad 479.1 \quad 496.0 \quad 518.9 \quad 564.0 \quad 624.7 \quad 658.5 \quad 690.8 \quad 708.3 \quad 737.9 \quad 786.3 \quad 817.4$ $867.5 \quad 920.9954 .8 \quad 990.7 \quad 991.1 \quad 995.7 \quad 1004.5 \quad 1016.8 \quad 1038.1 \quad 1048.9 \quad 1068.9 \quad 1075.2 \quad 1096.5$ $1117.21138 .4 \quad 1150.6 \quad 1176.5 \quad 1193.7 \quad 1213.8 \quad 1241.3 \quad 1243.91264 .4 \quad 1279.0 \quad 1284.5 \quad 1286.5$

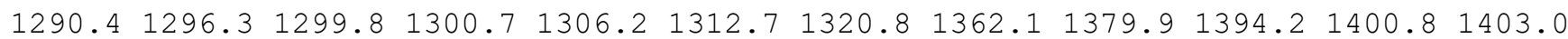
$1419.02662 .3 \quad 2663.0 \quad 2671.1 \quad 2672.6 \quad 2673.6 \quad 2674.4 \quad 2679.2 \quad 2738.2 \quad 2754.5 \quad 2758.2 \quad 2777.9$ $2780.22781 .4 \quad 2798.9 \quad 4141.0$

name $=\operatorname{TS} 95$

energy $($ in $\mathrm{kcal} / \mathrm{mol})=17.967227$

$\mathrm{ZPE}(\mathrm{in} \mathrm{kcal} / \mathrm{mol})=144.085$

$\mathrm{G}($ in $\mathrm{kcal} / \mathrm{mol})=120.702$ 


\begin{tabular}{|c|c|}
\hline & \\
\hline & $-2.22391801-0.03224892-0.42087664$ \\
\hline & $-0.79743139-0.46110853-0.68808901$ \\
\hline & $0.19200494 \quad 1.24002008-0.492$ \\
\hline & 1.521698730 .990729390 .1531 \\
\hline & $1.64960329-0.336053140 .903$ \\
\hline & $.36898890-1.50242011-0.0$ \\
\hline & $0.13381427-1.383907910 .3$ \\
\hline & $.33388163-0.731741841 .67$ \\
\hline & $\begin{array}{llll}-0.47701533 & 0.35069150 & 2.35\end{array}$ \\
\hline & $0.61588060-1.819890632 .71$ \\
\hline & $-2.89947100-0.88383942-0$ \\
\hline & $\begin{array}{llll}-2.54164899 & 0.76031831 & -1.1\end{array}$ \\
\hline & $\begin{array}{lllll}-2.38631818 & 0.30556754 & 0.6\end{array}$ \\
\hline & $-0.482006461 .94113003-0$. \\
\hline & $1.74669414 \quad 1.82428233 \quad 0.853$ \\
\hline & $6867691.02462342-0.6$ \\
\hline & $213597-0.398141221 .4 \varepsilon$ \\
\hline & $695070-2.45163782 \quad 0.17$ \\
\hline & $16698-1.30458036-1.1$ \\
\hline & $5231-2.319367610$. \\
\hline & $135780.79647458 \quad 3.180$ \\
\hline & $38448-0.06$ \\
\hline & $6907178 \quad 1.17296153 \quad 1.69883974$ \\
\hline & $1.15664484-1.41092826 \quad 3.57$ \\
\hline & $3640-2.63928468 \quad 2.31365485$ \\
\hline & $11494-2.25940222 \quad 3.08688574$ \\
\hline & $7350.53856836-2.51686480$ \\
\hline & $860321.48433146-1.83495902$ \\
\hline & $3555535-0.74941852-1.9145099$ \\
\hline
\end{tabular}

Vibrational frequencies (in $\mathrm{cm}-1$ )

$\begin{array}{llllllllllllllll}-713.7 & 87.5 & 109.7 & 136.6 & 161.0 & 182.1 & 203.4 & 207.8 & 251.3 & 283.3 & 290.6 & 341.5 & 375.2 & 390.7\end{array}$ $400.2 \quad 438.1 \quad 506.7 \quad 520.1 \quad 545.2 \quad 569.1 \quad 609.5 \quad 690.9 \quad 710.0 \quad 820.4 \quad 867.7 \quad 891.7 \quad 902.0 \quad 942.5$ $956.0 \quad 989.0 \quad 996.0 \quad 1011.3 \quad 1016.1 \quad 1030.4 \quad 1043.5 \quad 1059.8 \quad 1075.2 \quad 1082.3 \quad 1095.01103 .5 \quad 1145.3$ $1175.4 \quad 1192.6 \quad 1205.7 \quad 1218.8 \quad 1233.3 \quad 1246.1 \quad 1260.7 \quad 1277.2 \quad 1282.4 \quad 1285.1 \quad 1288.3 \quad 1293.4$

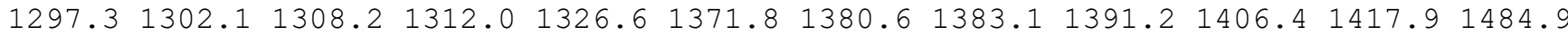
$2649.52669 .02671 .12674 .5 \quad 2675.92680 .72683 .02684 .02701 .02726 .22739 .02745 .2$ 2763.02780 .92782 .32784 .3

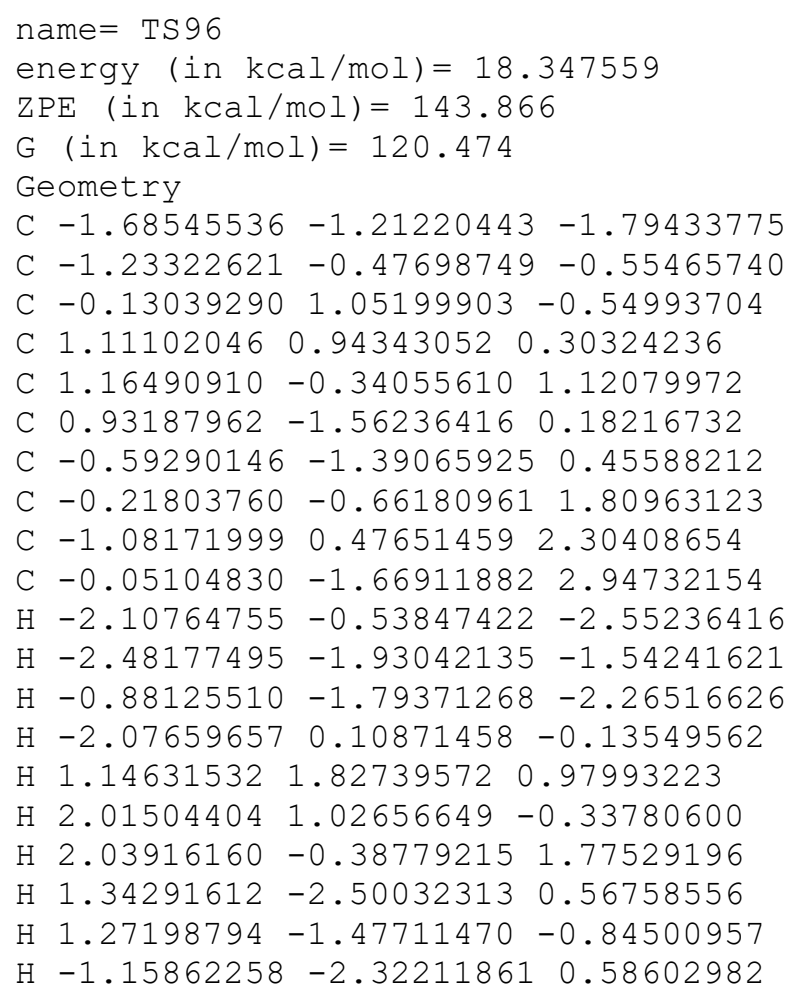


$\mathrm{H}-1.21365821 \quad 1.29322950 \quad 1.58398051$

$\mathrm{H}-0.65112482 \quad 0.93428825 \quad 3.20443866$

$\mathrm{H}-2.08806375 \quad 0.12305197 \quad 2.56272350$

$\mathrm{H} \quad 0.43467964-1.20596640 \quad 3.81430549$

$\mathrm{H} \quad 0.55625214 \quad-2.53358683 \quad 2.65999666$

$\mathrm{H}-1.02328304 \quad-2.05163018 \quad 3.27953136$

$\begin{array}{lllll}0 & 1.04329125 & 0.00733286 & -2.32938445\end{array}$

$0-0.82151708 \quad 2.05199322-0.61161056$

○ $0.02040424 \quad 0.47575782 \quad-1.98994193$

Vibrational frequencies (in $\mathrm{cm}-1$ )

$\begin{array}{lllllllllllllllllll}-526.0 & 94.8 & 122.3 & 153.6 & 178.9 & 191.5 & 198.5 & 206.4 & 257.7 & 276.7 & 284.7 & 320.7 & 354.2 & 384.4\end{array}$ $395.5 \quad 427.8 \quad 457.0 \quad 472.2 \quad 478.6 \quad 518.3 \quad 566.6 \quad 585.6 \quad 627.7 \quad 716.8 \quad 807.0 \quad 881.9 \quad 908.9 \quad 926.6$

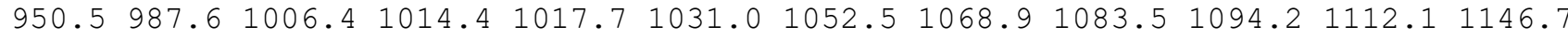

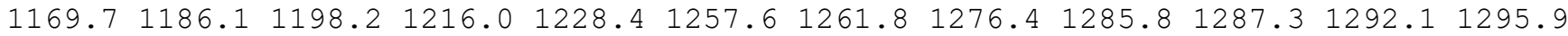

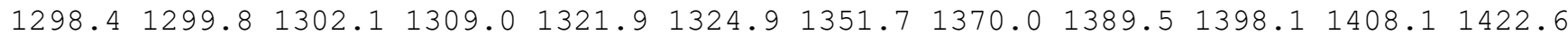
$1758.02651 .22656 .7 \quad 2664.3 \quad 2665.2 \quad 2675.8 \quad 2676.72680 .02681 .82690 .62731 .42732 .3$ $2744.32773 .7 \quad 2775.3 \quad 2781.7 \quad 2785.0$

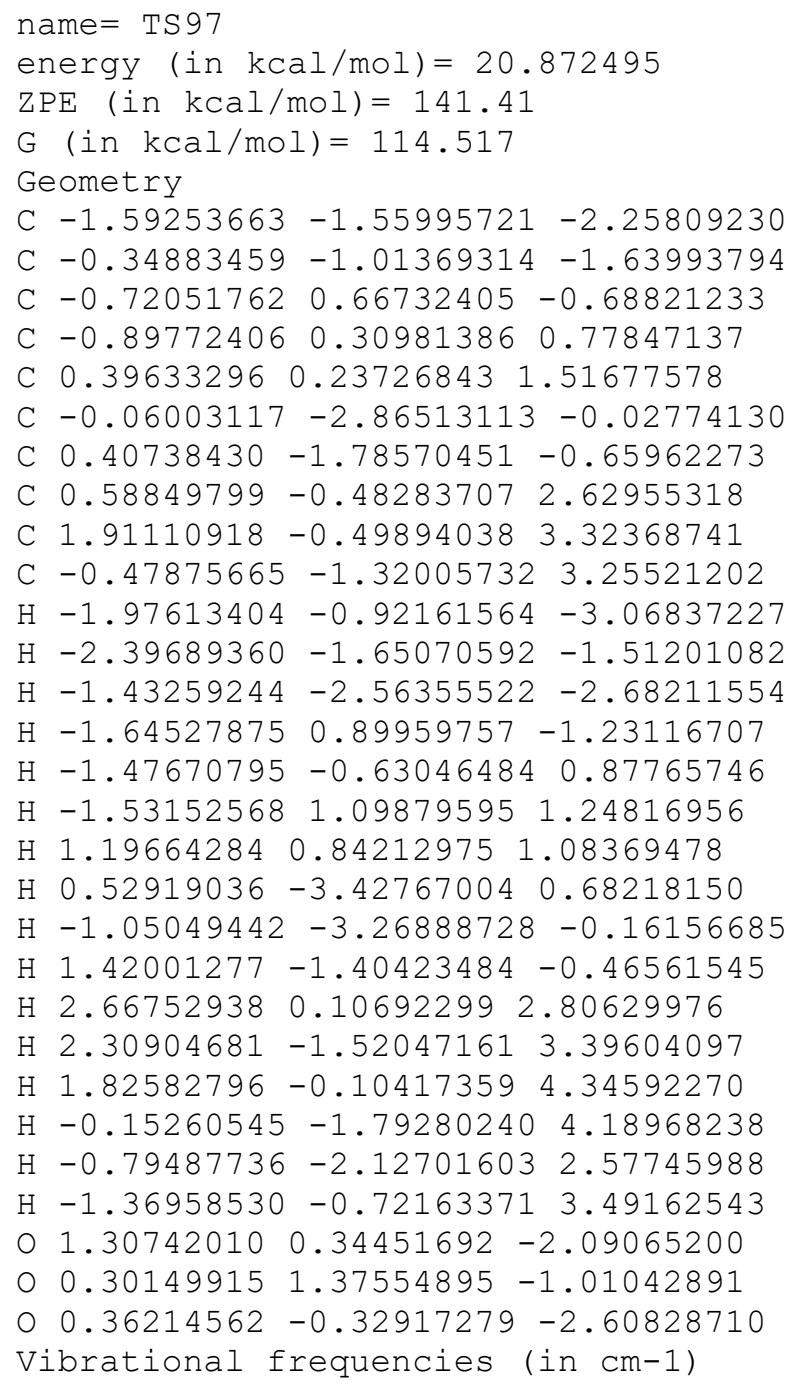
$304.4 \quad 324.2 \quad 365.0 \quad 400.9 \quad 469.9 \quad 475.6 \quad 489.9 \quad 520.2 \quad 584.2 \quad 629.6 \quad 650.3 \quad 687.0 \quad 837.4 \quad 867.0$ $951.9971 .7979 .5 \quad 996.4 \quad 1000.8 \quad 1008.4 \quad 1023.9 \quad 1048.9 \quad 1059.3 \quad 1083.4 \quad 1086.8 \quad 1094.01110 .1$

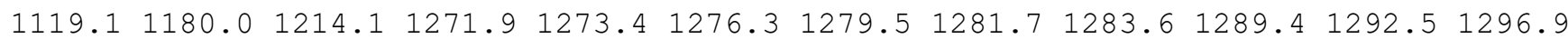

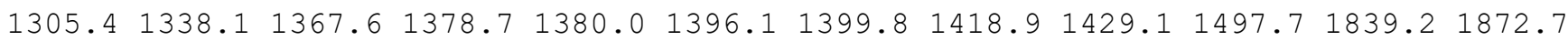
$2652.12662 .0 \quad 2664.12667 .0 \quad 2668.0 \quad 2673.2 \quad 2674.2 \quad 2677.52707 .92724 .82734 .92745 .6$ $2774.92777 .7 \quad 2779.8 \quad 2784.6$

name $=$ TS 98

energy $($ in $\mathrm{kcal} / \mathrm{mol})=23.638269$ 
$\mathrm{ZPE}($ in $\mathrm{kcal} / \mathrm{mol})=138.81$

$\mathrm{G}(\mathrm{in} \mathrm{kcal} / \mathrm{mol})=111.091$

Geometry

C $-0.46898785-0.51617570-2.09190923$

C $0.85201492-1.16265015-1.87651625$

C $-1.35779168 \quad 0.59345769 \quad 1.21949623$

C $0.03514816 \quad 0.62788974 \quad 1.14706436$

C $0.87158979-0.560354591 .36848550$

C $0.55010493-1.797711170 .50574030$

C $1.32540641-1.73885389-0.76660787$

C $0.53070793-0.770086192 .86141666$

C $1.37358111-0.043002933 .83634520$

C $-0.51594657-1.53331823 \quad 3.14299453$

$\mathrm{H}-1.28213267-1.25830898-2.07309100$

$\mathrm{H}-0.53596446 \quad 0.03096826 \quad-3.04149971$

$\mathrm{H}-0.67656406 \quad 0.20322636-1.27372341$

$\mathrm{H}-1.93458656 \quad 1.50721373 \quad 1.01716371$

$\mathrm{H} \quad 0.53660566 \quad 1.55049420 \quad 0.93134650$

H $3.38184172-0.85577165-2.84781107$

$\mathrm{H} \quad 1.96001427-0.33249118 \quad 1.26652291$

$\mathrm{H}-0.54634812-1.834653150 .31072202$

$\mathrm{H} \quad 0.79201142-2.72743302 \quad 1.05421431$

$\mathrm{H} \quad 2.31033593-2.19363391-0.73872779$

$\mathrm{H} \quad 0.97976891-0.03122924 \quad 4.85863821$

H $1.50112281 \quad 1.01426537 \quad 3.53101114$

$\mathrm{H} 2.38987965-0.47247744 \quad 3.87604256$

$\mathrm{H}-1.27591545-2.08409560 \quad 2.63166758$

$\mathrm{H}-1.32303946-1.00132978 \quad 4.40001465$

$\mathrm{H}-0.85389491-1.63674766 \quad 4.64027355$

O $2.82218408-1.66253190-2.95928405$

$0-2.01634839-0.408432921 .55321388$

o $1.53695728-1.05529937-3.06533477$

Vibrational frequencies (in $\mathrm{cm}-1$ )

$\begin{array}{lllllllllllllll}-1002.1 & 25.3 & 54.3 & 74.7 & 80.8 & 93.4 & 111.3 & 117.3 & 137.8 & 169.3 & 185.8 & 189.6 & 211.8 & 239.1\end{array}$ $265.0 \quad 295.0 \quad 333.4 \quad 359.9 \quad 398.7 \quad 414.7 \quad 468.0 \quad 472.2 \quad 490.9 \quad 497.8 \quad 529.4 \quad 616.5 \quad 675.9 \quad 753.2$

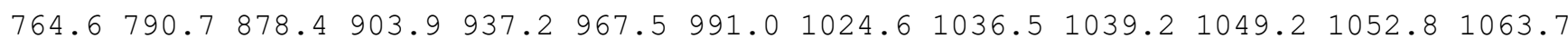

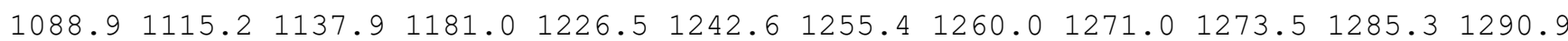

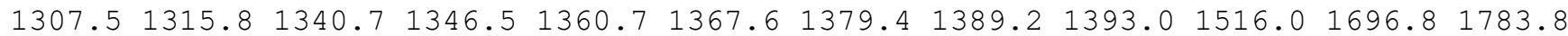
$1892.42639 .5 \quad 2648.72651 .3 \quad 2668.0 \quad 2672.3 \quad 2675.92694 .02704 .4 \quad 2735.8 \quad 2765.8 \quad 2770.2$ $2771.5 \quad 2798.12870 .7 \quad 4555.0$

\section{name $=$ TS 99}

energy (in $\mathrm{kcal} / \mathrm{mol})=24.000168$

$\mathrm{ZPE}($ in $\mathrm{kcal} / \mathrm{mol})=138.896$

$\mathrm{G}(\mathrm{in} \mathrm{kcal} / \mathrm{mol})=111.569$

Geometry

C $-2.17483098-0.86736767-2.91556368$

C $-1.26638318-1.09880755-1.75346657$

C $-0.009110861 .85282051-0.34763049$

C $1.03603600 \quad 0.896532690 .16096888$

C $0.52802062-0.24723004 \quad 0.99587448$

C $-0.68455720-1.09731566 \quad 0.67026280$

C $-1.57147380-0.85530867-0.47396310$

C $-0.71175181-0.18880008 \quad 1.91372014$

C $-1.56538343 \quad 1.05390544 \quad 1.94986368$

C $-0.69380788-0.898794353 .17248067$

$\mathrm{H}-2.565876190 .16134576 \quad-2.91879813$

$\mathrm{H}-3.03549877-1.55300410-2.88547081$

$\mathrm{H}-1.67345791-1.02267454-3.88412921$

$\mathrm{H}-0.84339437 \quad 1.44075333-0.92767097$

H $1.79958761 \quad 1.46236724 \quad 0.74220686$

$\mathrm{H} \quad 1.57905330 \quad 0.48009457 \quad-0.72263752$

$\mathrm{H} 1.38564749-0.806463991 .40629786$

$\mathrm{H}-0.58294050-2.18078932 \quad 0.86479437$ 
H $1.09205018-2.79186198-1.55579823$

$\mathrm{H}-2.56664776-0.49222061-0.20789628$

$\mathrm{H}-0.97103300 \quad 1.94224878 \quad 2.20102129$

$\mathrm{H}-2.35995296 \quad 0.95509608 \quad 2.70592266$

$\mathrm{H}-2.07408585 \quad 1.25520301 \quad 1.00051698$

H $0.30162748 \quad 0.07175523 \quad 4.10116017$

$\mathrm{H}-0.11382874-1.77161318 \quad 3.33870289$

$\mathrm{H}-0.470244090 .09260056 \quad 4.35776009$

O $0.82592186-1.89386519-1.23623814$

$00.083259153 .04176905-0.16006443$

o - $0.09260526-1.56642624-2.28106384$

Vibrational frequencies (in $\mathrm{cm}-1$ )

$\begin{array}{llllllllllllllllll}-982.3 & 47.7 & 61.5 & 74.5 & 84.7 & 87.8 & 102.4 & 122.6 & 140.4 & 144.2 & 152.7 & 189.6 & 207.6 & 231.9\end{array}$

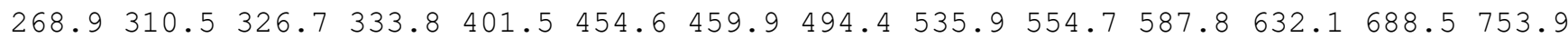

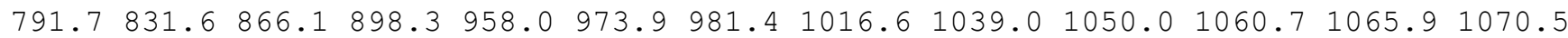
$1077.8 \quad 1102.2 \quad 1116.5 \quad 1146.4 \quad 1184.7 \quad 1240.3 \quad 1241.5 \quad 1266.3 \quad 1271.4 \quad 1271.8 \quad 1282.6 \quad 1292.8$ $1302.41305 .91316 .6 \quad 1350.8 \quad 1356.7 \quad 1378.2 \quad 1382.1 \quad 1400.4 \quad 1410.2 \quad 1427.7 \quad 1492.11832 .2$ $1896.62644 .62658 .62664 .2 \quad 2665.3 \quad 2667.12672 .02723 .22725 .52729 .52738 .52750 .6$ $2774.5 \quad 2776.8 \quad 2872.0 \quad 4696.9$

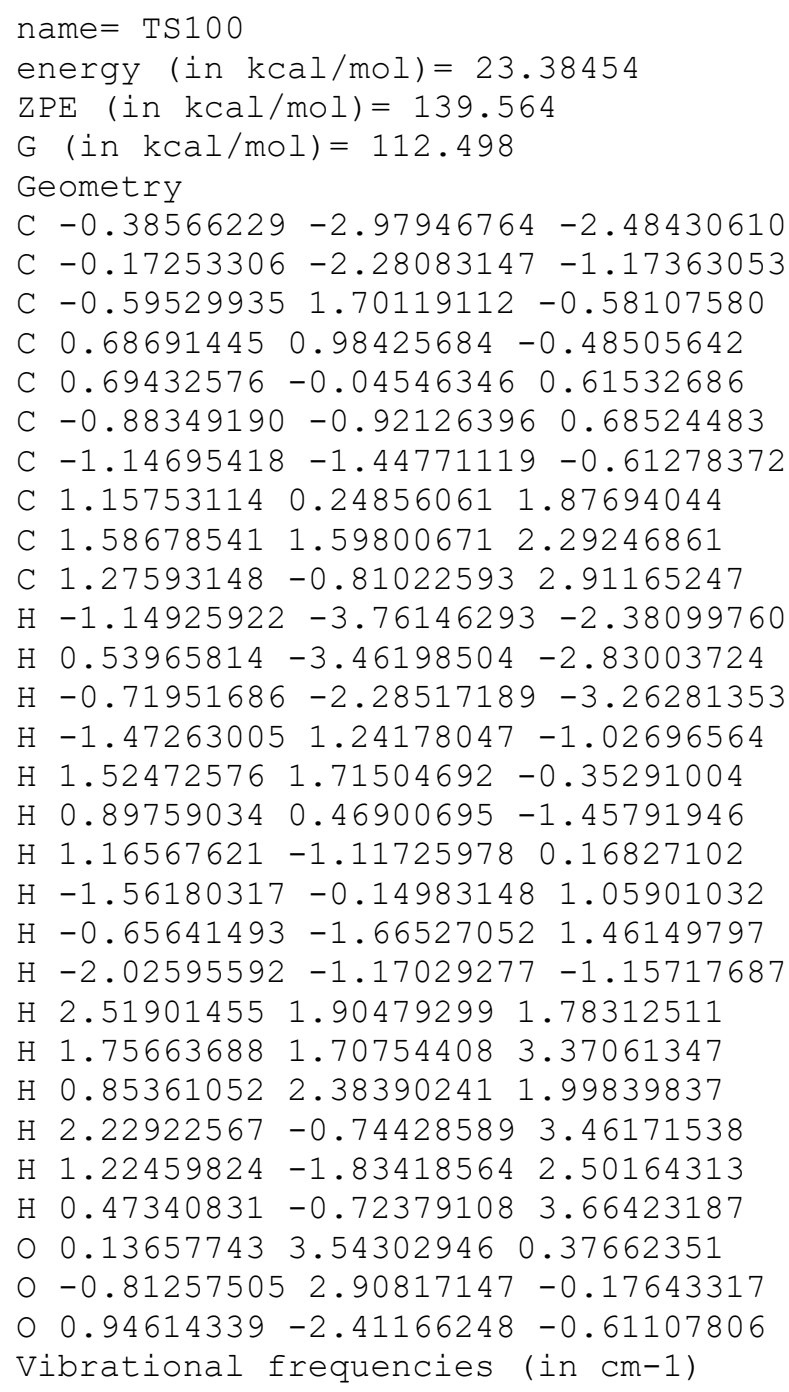


name $=$ TS101

energy (in $\mathrm{kcal} / \mathrm{mol})=24.000947$

$\mathrm{ZPE}($ in $\mathrm{kcal} / \mathrm{mol})=138.984$

$\mathrm{G}($ in $\mathrm{kcal} / \mathrm{mol})=112.07$

Geometry

C $-5.47542585 \quad 1.68285144 \quad 0.36219658$

C $-4.56128280 \quad 0.52097972 \quad 0.17340803$

C $-4.20208825-0.31919558 \quad 1.39753982$

C $-2.83666913 \quad 0.09207533 \quad 1.94743484$

C $-1.71355713-0.38703673 \quad 1.05255191$

C $-1.15699733-1.77914130 \quad 1.34059787$

C $0.08360193-1.359735321 .63775176$

C $-0.228392190 .13891682 \quad 1.44115473$

C $-0.30362184 \quad 0.98445327 \quad 2.61935881$

C $0.50368255 \quad 0.76358007 \quad 0.27036540$

$\mathrm{H}-5.018042552 .44480885 \quad 1.01284664$

$\mathrm{H}-6.41622394 \quad 1.38181298 \quad 0.85025553$

$\mathrm{H}-5.72722820 \quad 2.16358397 \quad-0.59326546$

$\mathrm{H}-4.22864398-1.409429841 .13688978$

$\mathrm{H}-2.71641626-0.30722867 \quad 2.97472024$

$\mathrm{H}-2.77844682 \quad 1.19947385 \quad 2.05823287$

$\mathrm{H}-1.93420825-0.20815395-0.01603554$

$\mathrm{H}-1.65977038 \quad-2.70855756 \quad 1.28681447$

$\mathrm{H}-4.84765008 \quad 0.79922246 \quad 3.87235140$

$\mathrm{H} \quad 0.99953520 \quad-1.80763839 \quad 1.90953937$

H $0.86824460 \quad 2.03102267 \quad 2.66964284$

H $1.30133962 \quad 1.38411403 \quad 2.89960990$

$\mathrm{H}-0.42032685 \quad 0.57474852 \quad 3.59426379$

H $1.57863303 \quad 0.84876204 \quad 0.45677117$

$\mathrm{H} \quad 0.10955049 \quad 1.766551330 .05459580$

$\mathrm{H} \quad 0.38482159 \quad 0.16664290 \quad-0.64439210$

$0-5.21221167 \quad 1.02340650 \quad 2.98151800$

$0-5.20656347-0.27162805 \quad 2.38793946$

$\begin{array}{llll}0 & -4.09773429 & 0.22833422 & -0.90092617\end{array}$

Vibrational frequencies (in cm-1)

$\begin{array}{llllllllllllllll}-951.7 & 33.3 & 38.7 & 62.2 & 91.4 & 132.8 & 133.7 & 169.0 & 182.5 & 191.5 & 197.3 & 219.6 & 232.9 & 267.8\end{array}$ $294.7 \quad 303.8 \quad 342.3 \quad 360.6 \quad 382.3 \quad 430.1 \quad 488.5 \quad 533.5 \quad 545.0 \quad 594.5 \quad 609.5 \quad 693.5 \quad 748.3 \quad 782.0$

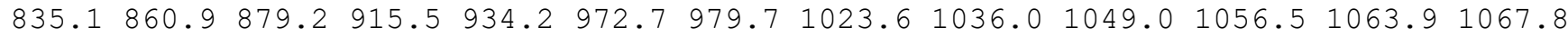

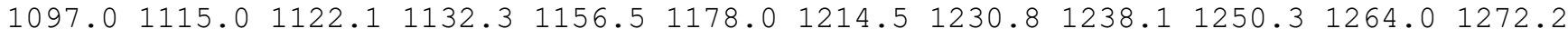

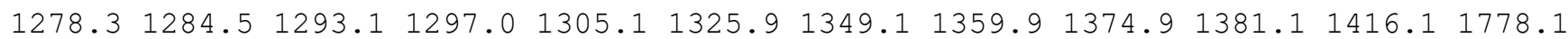
$1847.6 \quad 2647.92659 .8 \quad 2670.5 \quad 2671.6 \quad 2674.92679 .4 \quad 2702.8 \quad 2717.7 \quad 2738.3 \quad 2777.2 \quad 2779.9$ $2798.12812 .12865 .8 \quad 4727.5$

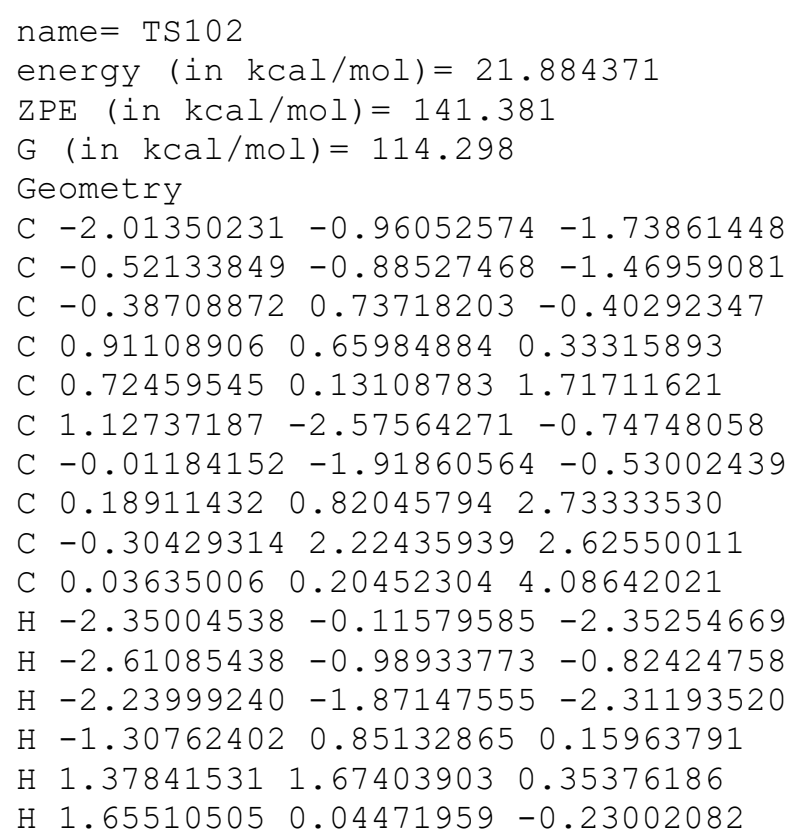


$\mathrm{H} \quad 1.06444700 \quad-0.89481287 \quad 1.85308176$

$\mathrm{H} 1.50808722-3.34627820-0.09620449$

$\mathrm{H} 1.76210114-2.38692704-1.60509046$

$\begin{array}{lllll}\mathrm{H} & -0.64699937 & -2.11287183 & 0.33187627\end{array}$

$\mathrm{H}-0.116711892 .79582176 \quad 3.54538334$

$\mathrm{H}-1.38970085 \quad 2.24608397 \quad 2.45029020$

$\mathrm{H} 0.17129300 \quad 2.78031636 \quad 1.80625442$

$\mathrm{H} \quad 0.26042507 \quad-0.87037678 \quad 4.09370602$

$\mathrm{H}-0.98645232 \quad 0.32367685 \quad 4.46966519$

$\mathrm{H} \quad 0.71338974 \quad 0.67803052 \quad 4.81255106$

O $0.549950191 .22529921-2.30022577$

$\begin{array}{lllll}0 & -0.43849887 & 1.49836630 & -1.53996049\end{array}$

O $0.21805151-0.55817892-2.47061523$

Vibrational frequencies (in cm-1)

$\begin{array}{lllllllllllllllll}-702.7 & 29.8 & 53.0 & 60.5 & 81.3 & 97.7 & 101.8 & 110.1 & 163.6 & 182.4 & 216.8 & 218.8 & 260.3 & 293.4\end{array}$ $\begin{array}{llllllllllllll}310.0 & 351.3 & 370.1 & 409.1 & 423.6 & 483.1 & 509.2 & 528.9 & 565.1 & 615.1 & 685.8 & 732.5 & 881.3 & 886.6\end{array}$ $910.6945 .2980 .1988 .5 \quad 1001.8 \quad 1017.9 \quad 1032.2 \quad 1049.0 \quad 1057.9 \quad 1079.3 \quad 1085.3 \quad 1097.9 \quad 1114.6$ $\begin{array}{lllllllllllllll}1133.2 & 1162.8 & 1201.3 & 1235.1 & 1240.0 & 1275.1 & 1277.5 & 1282.8 & 1283.4 & 1287.0 & 1288.5 & 1289.9\end{array}$ $1296.01316 .91331 .2 \quad 1368.7 \quad 1380.1 \quad 1383.7 \quad 1392.6 \quad 1400.2 \quad 1414.91509 .91838 .1 \quad 1874.3$ $2630.52669 .32670 .2 \quad 2670.42673 .12675 .5 \quad 2684.52705 .92710 .42728 .82753 .62759 .4$ $2778.4 \quad 2779.5 \quad 2780.9 \quad 2782.4$

name $=$ TS103

energy (in $\mathrm{kcal} / \mathrm{mol})=23.563591$

$\mathrm{ZPE}($ in $\mathrm{kcal} / \mathrm{mol})=139.726$

G $($ in $\mathrm{kcal} / \mathrm{mol})=112.69$

Geometry

C $-2.36040127-2.00190110-0.73664793$

C $-1.01993544-1.41311635-0.79194098$

C $1.33063280 \quad 2.50885940 \quad-0.41908725$

C 1.318848621 .684774350 .69630016

C $1.106810410 .35821392 \quad 1.15724757$

C $1.31176379-0.812685090 .14280081$

C $-0.13687266-1.308888130 .38205896$

C $-0.41728501-0.06985147 \quad 1.32226811$

C $-1.417003850 .93107660 \quad 0.78345816$

C $-0.76190548-0.47073126 \quad 2.74421872$

$\mathrm{H}-3.09053797-1.36175593-1.27654000$

$\mathrm{H}-2.73449720 \quad-2.17143130 \quad 0.27938649$

$\mathrm{H}-2.38900789-2.96628771-1.28110902$

$\mathrm{H} 0.47916682 \quad 2.39340109-1.11993587$

H 2.44445139 2.49317243 1.76338860

$\mathrm{H} 2.95900694 \quad 2.09281423 \quad 1.31183312$

H $1.65227182 \quad 0.16393097 \quad 2.10781236$

H $2.08532988-1.52975792 \quad 0.43034542$

$\mathrm{H} \quad 1.54087137-0.48276889-0.87428408$

$\mathrm{H}-0.15823193-2.26396271 \quad 0.94945019$

$\mathrm{H}-1.25915141 \quad 1.16177413-0.27724519$

$\mathrm{H}-1.35421748 \quad 1.88294187 \quad 1.33192890$

$\mathrm{H}-2.44748755 \quad 0.56606214 \quad 0.87994949$

$\mathrm{H}-0.05463094-1.19582999 \quad 3.16221766$

$\mathrm{H}-1.76033667-0.92272668 \quad 2.80185825$

$\mathrm{H}-0.76621807 \quad 0.40232718 \quad 3.41120324$

O $-1.25454731-0.99319258-2.93334071$

O $2.16053894 \quad 3.38891312-0.61428161$

o $-0.52329884-0.93848760-1.90203666$

Vibrational frequencies (in $\mathrm{cm}-1$ )

$\begin{array}{lllllllllllllll}-566.9 & 28.4 & 37.1 & 65.5 & 93.8 & 106.9 & 119.2 & 162.5 & 184.3 & 193.6 & 219.0 & 223.0 & 272.8 & 284.4\end{array}$ $289.5 \quad 321.3 \quad 359.7 \quad 378.9 \quad 422.8 \quad 428.6 \quad 431.7 \quad 480.7 \quad 534.4 \quad 583.6 \quad 665.6 \quad 684.0 \quad 724.7 \quad 800.6$

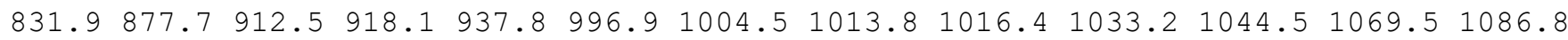

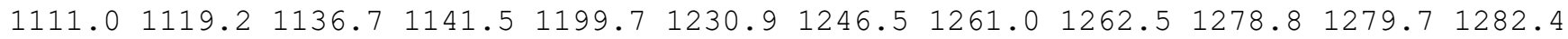

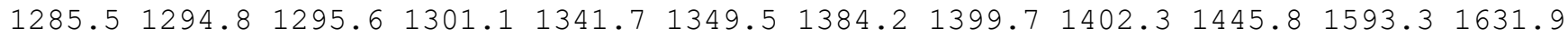
$1773.32634 .52635 .2 \quad 2667.3 \quad 2671.0 \quad 2673.6 \quad 2674.82676 .72679 .12707 .12715 .52762 .6$ $2769.32779 .2 \quad 2782.2 \quad 5022.9$ 
name $=$ TS104

energy $($ in $\mathrm{kcal} / \mathrm{mol})=27.967398$

$\mathrm{ZPE}(\mathrm{in} \mathrm{kcal} / \mathrm{mol})=136.545$

$\mathrm{G}(\mathrm{in} \mathrm{kcal} / \mathrm{mol})=108.62$

Geometry

C $-2.12396915-0.99591286-1.44945145$

C $-0.90103131-0.29199527-0.90309838$

C $0.61974544 \quad 1.33715820-1.67488449$

C $0.644088192 .61135150-1.28875276$

C $0.51642284-0.56506514 \quad 2.25786852$

C $1.43006298-0.42650019 \quad 1.07615687$

C $1.10285744-1.41113069-0.01320979$

C $-0.65361832 \quad 0.25810257 \quad 2.27147885$

C $-0.715233951 .11180993 \quad 3.37005503$

C $0.27738309-1.97875004 \quad 2.72877780$

$\mathrm{H}-2.89878374-0.27161132-1.73796808$

$\mathrm{H}-2.54926385-1.67800274-0.70209462$

$\mathrm{H}-1.87867131-1.58954074 \quad-2.34243151$

$\mathrm{H}-1.036148620 .23941195 \quad 0.06119510$

$\mathrm{H}-0.24527952 \quad 3.16942681-1.05567883$

H $1.55301190 \quad 3.18347283-1.21373648$

H 1.115357710 .025151013 .51038539

H $2.48014744 \quad-0.62359490 \quad 1.39080595$

H $1.44951058 \quad 0.61403827 \quad 0.69064720$

$\mathrm{H} 1.56522610-0.25242356-2.31813154$

$\mathrm{H}-1.610714421 .164671293 .97140225$

$\mathrm{H}-0.27765767 \quad 2.10168698 \quad 3.25714650$

$\mathrm{H} \quad 0.53667777 \quad 0.58266510 \quad 4.03236695$

$\mathrm{H}-0.32655049-2.02198972 \quad 3.64408635$

H $1.22473964 \quad-2.49792221 \quad 2.92931267$

$\mathrm{H}-0.25186496-2.56953338 \quad 1.96451723$

○ $1.754037120 .63565212-1.91339633$

$\begin{array}{llllll}0 & -0.51873547 & 0.62403218 & -1.92093806\end{array}$

O $0.13126293-1.29168395-0.80624371$

Vibrational frequencies (in cm-1)

$\begin{array}{lllllllllllllll}-2402.8 & 25.3 & 34.4 & 49.9 & 69.7 & 78.1 & 92.2 & 112.7 & 153.3 & 164.6 & 182.5 & 188.4 & 277.6 & 304.5\end{array}$

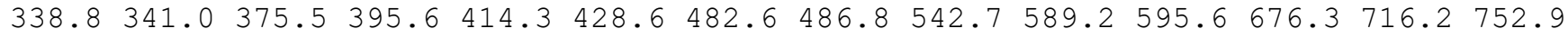

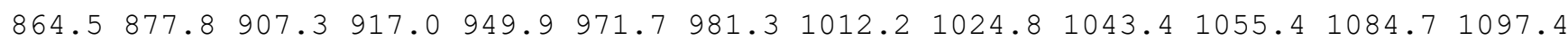

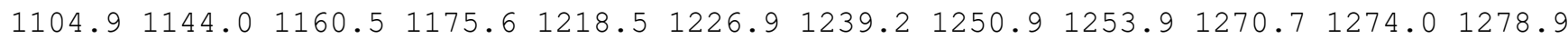
$1282.8 \quad 1288.2 \quad 1293.6 \quad 1312.5 \quad 1353.4 \quad 1363.2 \quad 1379.6 \quad 1383.3 \quad 1404.7 \quad 1455.3 \quad 1550.3 \quad 1723.8$ $1890.82582 .5 \quad 2623.8 \quad 2652.3 \quad 2661.5 \quad 2672.8 \quad 2674.02676 .32688 .5 \quad 2733.12736 .62757 .5$ 2765.12775 .92779 .02799 .9

name $=$ TS105

energy (in $\mathrm{kcal} / \mathrm{mol})=23.782541$

$\mathrm{ZPE}(\mathrm{in} \mathrm{kcal} / \mathrm{mol})=140.816$

G $($ in $\mathrm{kcal} / \mathrm{mol})=116.125$

Geometry

C $-2.006382670 .31885077-0.39288816$

C $-0.75526762-0.48964844-0.69478726$

C $0.410655890 .43682382-1.40375496$

C $0.977442331 .36363069-0.32857954$

C $1.60683294 \quad 0.82092169 \quad 0.73239986$

C $1.65283554-0.62199628 \quad 0.81436143$

C $-0.17854325-1.26702013 \quad 0.45762881$

C $-0.22857045-0.97482945 \quad 1.81597125$

C $-0.72386697 \quad 0.29616250 \quad 2.39997343$

C $0.03893132-2.044185862 .82318422$

$\mathrm{H}-2.55846862 \quad 0.48064412-1.33593989$

$\mathrm{H}-1.78966674 \quad 1.30309531 \quad 0.02572800$

$\mathrm{H}-2.69022743-0.21417712 \quad 0.27419140$

$\mathrm{H}-0.10321138 \quad 1.01046689-2.20724571$ 
$\mathrm{H} \quad 0.878530652 .42908685-0.48831910$

H $0.21953325-1.21957379-2.86028186$

$\mathrm{H} 2.06969370 \quad 1.401137951 .52121356$

H $2.12638795-1.11430606 \quad 1.65304724$

H $1.92425275-1.10117626-0.18812955$

$\mathrm{H} \quad 0.03045961-2.31406381 \quad 0.15099846$

$\mathrm{H}-1.79679779 \quad 0.21772100 \quad 2.65329635$

$\mathrm{H}-0.63786607 \quad 1.15851043 \quad 1.71262440$

$\mathrm{H}-0.197355120 .57924088 \quad 3.32406652$

$\mathrm{H} \quad 0.74154560 \quad-1.72515738 \quad 3.60881861$

H $0.42974806 \quad-2.97865450 \quad 2.39404399$

$\mathrm{H}-0.89645220-2.31419186 \quad 3.34878387$

$0-0.79901828-1.28497119-2.92949202$

O $1.32846058-0.37592696-1.84974890$

O $-1.15659029-1.53259730-1.58496554$

Vibrational frequencies (in $\mathrm{cm}-1$ )

$\begin{array}{llllllllllllllll}-175.8 & 60.9 & 106.2 & 131.0 & 155.5 & 160.8 & 182.2 & 187.3 & 228.0 & 230.4 & 243.6 & 259.5 & 311.7 & 346.7\end{array}$

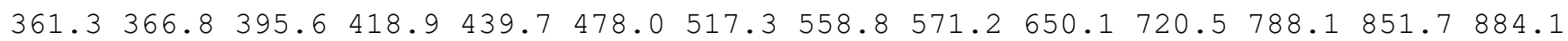

$902.9944 .4 \quad 968.3 \quad 982.0 \quad 1006.6 \quad 1013.3 \quad 1015.91018 .5 \quad 1022.5 \quad 1045.1 \quad 1069.4 \quad 1087.8 \quad 1125.2$

$\begin{array}{lllllllllllll}1162.9 & 1170.6 & 1184.8 & 1203.4 & 1243.4 & 1246.0 & 1253.2 & 1257.6 & 1263.2 & 1265.8 & 1271.6 & 1289.5\end{array}$ $1290.81299 .1 \quad 1305.91352 .91366 .2 \quad 1386.3 \quad 1389.3 \quad 1398.51409 .8 \quad 1472.0 \quad 1657.6 \quad 1757.8$ $2420.72543 .0 \quad 2633.2 \quad 2646.92652 .3 \quad 2656.5 \quad 2661.02664 .22681 .12685 .62714 .82751 .0$ $2764.62770 .5 \quad 2772.3 \quad 2778.9$

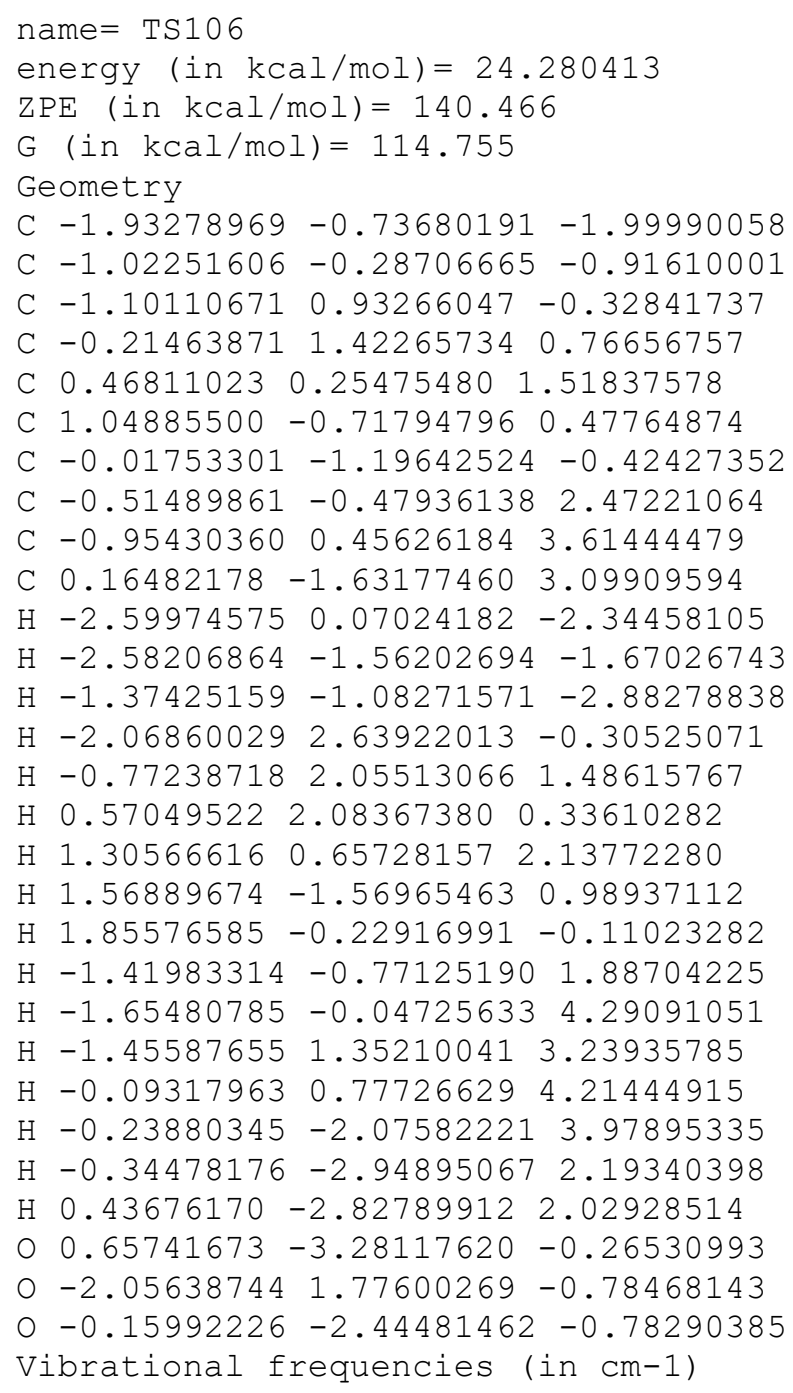
$342.5 \quad 369.8 \quad 384.8 \quad 399.8 \quad 434.7 \quad 447.7 \quad 475.4 \quad 509.3 \quad 559.6 \quad 603.8 \quad 624.3 \quad 639.8 \quad 746.5 \quad 800.0$ $827.0 \quad 889.1 \quad 920.2 \quad 934.3 \quad 968.3 \quad 975.2 \quad 1003.3 \quad 1038.5 \quad 1059.9 \quad 1066.2 \quad 1092.4 \quad 1094.1 \quad 1106.2$

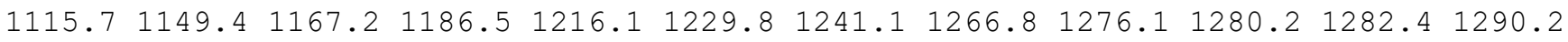

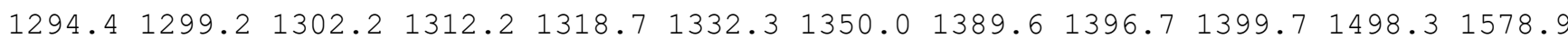


$1823.62620 .6 \quad 2649.2 \quad 2665.0 \quad 2667.8 \quad 2670.2 \quad 2674.62688 .42704 .12706 .92722 .7 \quad 2730.3$ $2774.92778 .7 \quad 2786.0 \quad 4794.6$

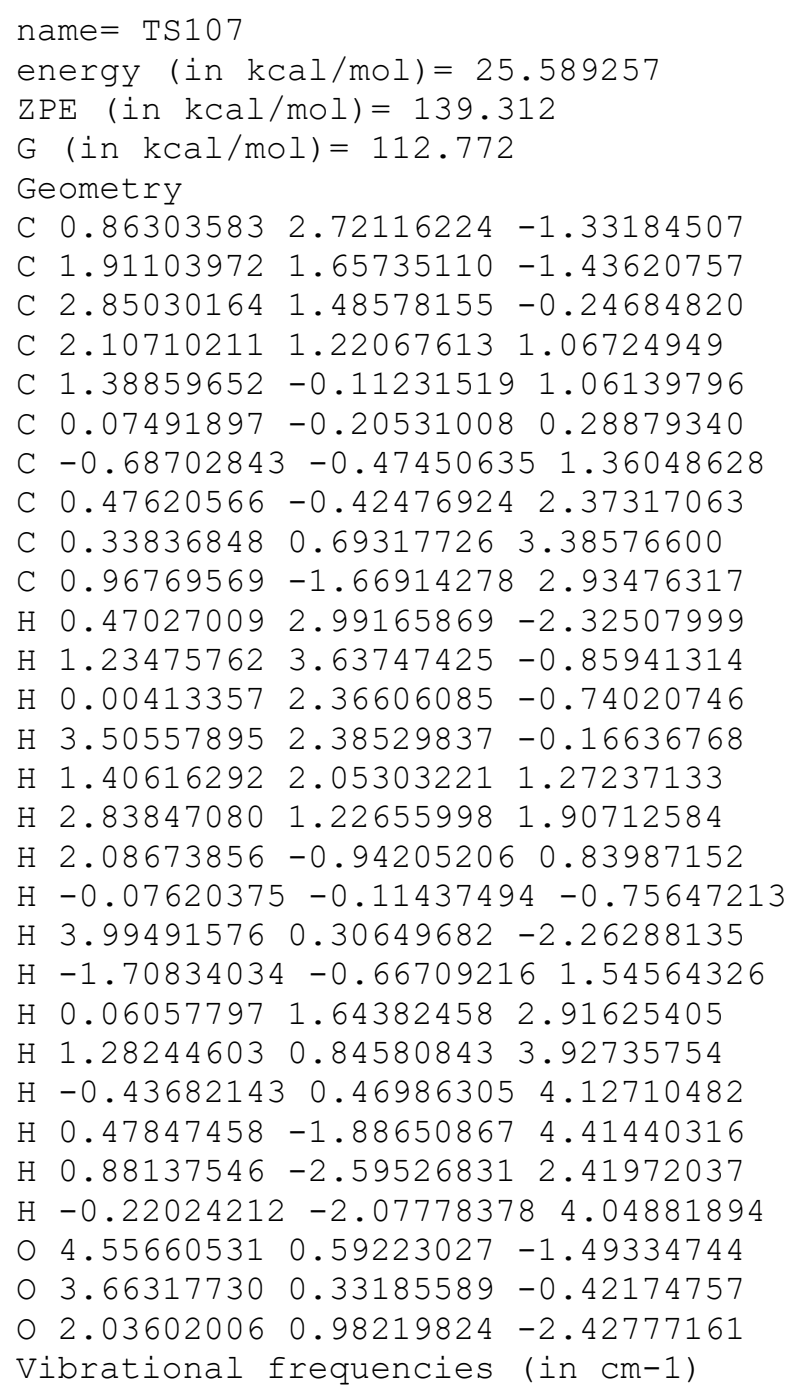


$\mathrm{H}-0.52019467 \quad 0.74166413-0.35043540$

$\mathrm{H} \quad 2.70309182 \quad 2.95986095-0.86258868$

$\mathrm{H} \quad 4.61698535 \quad 2.76638307 \quad 0.76470445$

$\mathrm{H} \quad 2.80915755 \quad 0.28242765 \quad-2.22272704$

H $4.17143245-1.126215232 .01485622$

H $4.11082550 \quad-0.64502322 \quad 0.33332902$

H $1.77620802-1.387844631 .66397811$

H $1.021644372 .30822641 \quad 3.48946696$

$\mathrm{H}-0.06243026 \quad 1.23344127 \quad 2.60865301$

$\mathrm{H} \quad 1.95012543 \quad 0.42209908 \quad 4.82723130$

H $2.78028381-0.92050410 \quad 4.03772205$

$\mathrm{H} \quad 1.01299628-0.81956214 \quad 3.99112711$

o $3.12993438-0.30713369-1.49315315$

$01.670623241 .43641905-0.26018077$

$01.88913573-0.73629101-0.96618313$

$\mathrm{H} 1.039722192 .29766065 \quad 1.72992165$

H $3.22100193 \quad 1.238983892 .87524294$

Vibrational frequencies (in cm-1)

$\begin{array}{llllllllllllll}-1391.5 & 43.0 & 73.3 & 104.4 & 117.8 & 166.0 & 181.0 & 186.0 & 193.8 & 249.8 & 255.2 & 291.1307 .4 & 312.6\end{array}$ $352.5 \quad 370.2 \quad 392.9413 .1 \quad 460.6 \quad 488.6 \quad 526.3 \quad 603.7 \quad 661.7 \quad 698.4 \quad 790.8 \quad 804.9 \quad 831.0 \quad 910.3$ $936.8959 .0 \quad 961.5 \quad 976.9 \quad 993.3 \quad 1009.0 \quad 1020.2 \quad 1035.0 \quad 1062.8 \quad 1077.8 \quad 1099.3 \quad 1116.1 \quad 1140.9$ $1146.51170 .6 \quad 1180.2 \quad 1215.91238 .2 \quad 1245.4 \quad 1270.0 \quad 1275.0 \quad 1277.4 \quad 1286.1 \quad 1293.01294 .7$ $1300.41303 .3 \quad 1312.4 \quad 1324.2 \quad 1348.2 \quad 1357.0 \quad 1362.91399 .2 \quad 1402.0 \quad 1410.3 \quad 1430.01800 .6$ $1942.82660 .12666 .5 \quad 2668.5 \quad 2669.8 \quad 2673.12675 .12676 .52699 .5 \quad 2713.3 \quad 2750.2 \quad 2762.7$ $2770.92778 .02779 .6 \quad 2863.1$

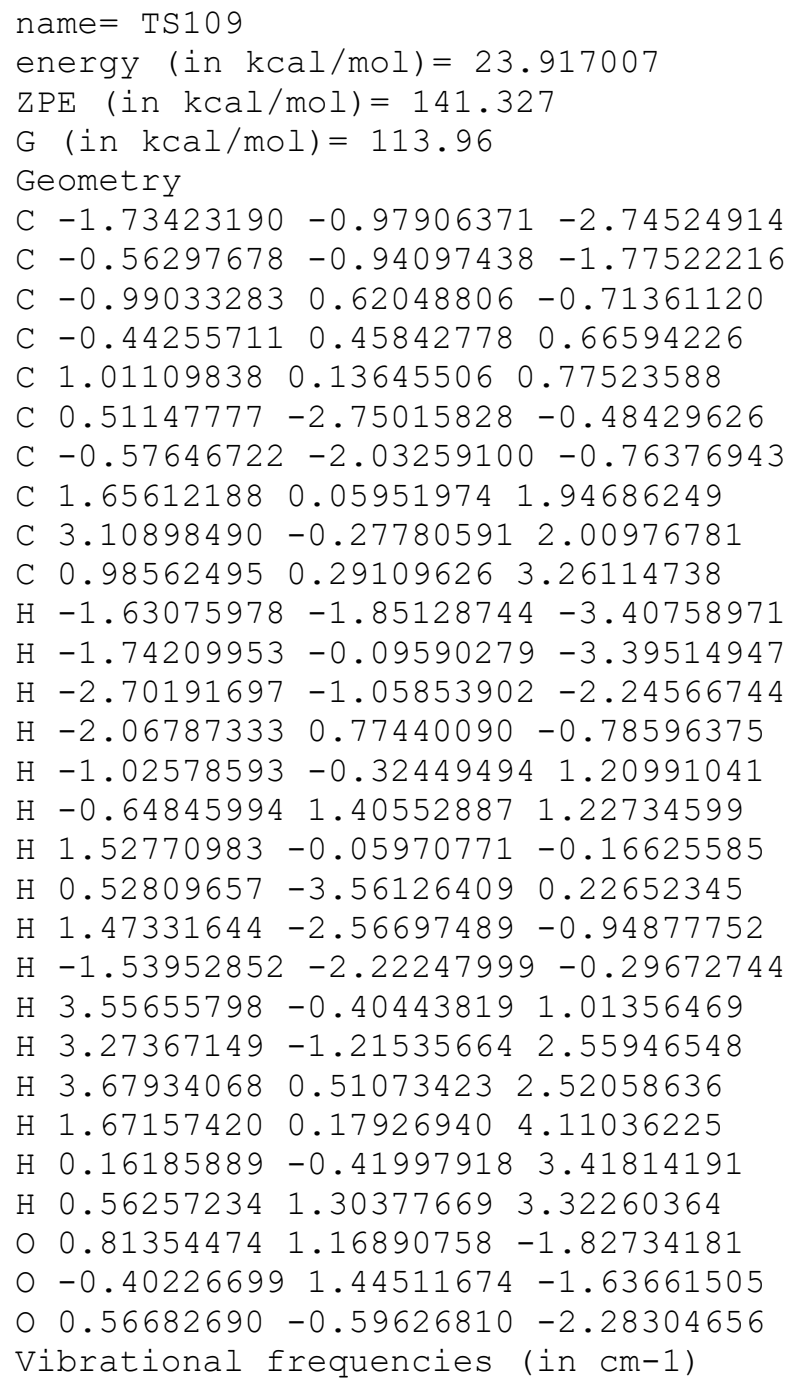


$\begin{array}{lllllllllllllll}1114.9 & 1172.9 & 1199.2 & 1230.3 & 1259.4 & 1273.8 & 1279.4 & 1282.6 & 1283.6 & 1288.4 & 1290.9 & 1291.8\end{array}$

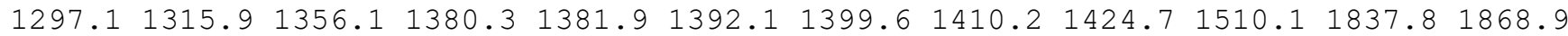
$2636.12670 .02670 .5 \quad 2671.3 \quad 2673.72676 .12686 .02704 .32710 .3 \quad 2712.62737 .72761 .8$ $2777.6 \quad 2778.7 \quad 2779.6 \quad 2782.7$

name $=$ TS110

energy (in $\mathrm{kcal} / \mathrm{mol})=24.514034$

$\mathrm{ZPE}($ in $\mathrm{kcal} / \mathrm{mol})=140.975$

$\mathrm{G}($ in $\mathrm{kcal} / \mathrm{mol})=116.987$

Geometry

C $-1.86110419-0.60704410-0.92741471$

C $-0.63122217-0.19787412-0.22200557$

C $-0.028582351 .01966890-0.35019105$

C 1.230382741 .303943120 .32255841

C 1.626790220 .233445151 .26504862

C $1.59305698-1.10839770 \quad 0.35803043$

C $0.06797913-1.15796497 \quad 0.69128959$

C $0.39733151-0.38708940 \quad 2.04162602$

C $-0.63357689 \quad 0.57414687 \quad 2.59170757$

C $0.83759961-1.33409203 \quad 3.15084538$

$\mathrm{H}-2.51238870 \quad 0.24053730-1.18026093$

$\mathrm{H}-2.46311731-1.31948677-0.34692530$

$\mathrm{H}-1.58673391-1.11457143-1.87483136$

$\mathrm{H}-0.89580413 \quad 1.73161751-1.93478859$

H $1.53084595 \quad 2.33555310 \quad 0.48185980$

H $2.31102176 \quad 1.12869866-0.77556790$

$\mathrm{H} \quad 2.52133102 \quad 0.41073027 \quad 1.85728245$

H $2.22226032-1.906112520 .76365379$

H $2.59711547 \quad 0.36811152-1.03470800$

$\mathrm{H}-0.38884537-2.14301115 \quad 0.77695556$

$\mathrm{H}-0.97375194 \quad 1.31736501 \quad 1.85834644$

$\mathrm{H}-0.23849378 \quad 1.13508509 \quad 3.44745095$

$\mathrm{H}-1.530401960 .03869556 \quad 2.92907179$

$\mathrm{H} 1.26860968-0.78230092 \quad 3.99514119$

H $1.58733397 \quad-2.06186656 \quad 2.82365523$

$\mathrm{H}-0.01632378-1.90608148 \quad 3.53631094$

○ $2.01648880-1.01939123-0.99892850$

O $-0.513980522 .06446999-1.08373126$

O $1.07863632-0.84058453-1.86208611$

Vibrational frequencies (in cm-1)

$\begin{array}{lllllllllllllll}-1342.5 & 104.5 & 119.2 & 124.1 & 135.3 & 144.5 & 164.2 & 180.2 & 203.8 & 214.1 & 249.8 & 289.6 & 324.3 & 383.3\end{array}$ $394.9 \quad 424.3 \quad 464.7 \quad 474.2 \quad 508.0 \quad 548.9 \quad 580.0 \quad 629.2 \quad 675.2 \quad 695.9 \quad 725.9 \quad 785.2 \quad 829.2 \quad 857.9$ $877.6 \quad 899.1954 .5 \quad 985.6 \quad 999.8 \quad 1008.6 \quad 1011.2 \quad 1015.9 \quad 1019.3 \quad 1043.4 \quad 1050.8 \quad 1062.9 \quad 1107.6$

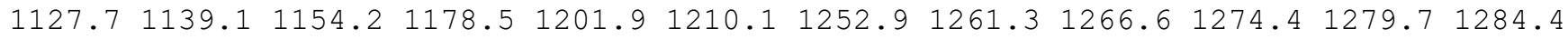
$1288.21293 .21298 .21306 .61315 .2 \quad 1335.8 \quad 1365.51388 .71393 .11407 .11417 .81456 .7$ $1755.8 \quad 2652.92662 .4 \quad 2673.9 \quad 2675.1 \quad 2675.9 \quad 2680.02707 .62731 .7 \quad 2750.22753 .7 \quad 2772.9$ $2780.5 \quad 2782.7 \quad 2789.9 \quad 3605.0$

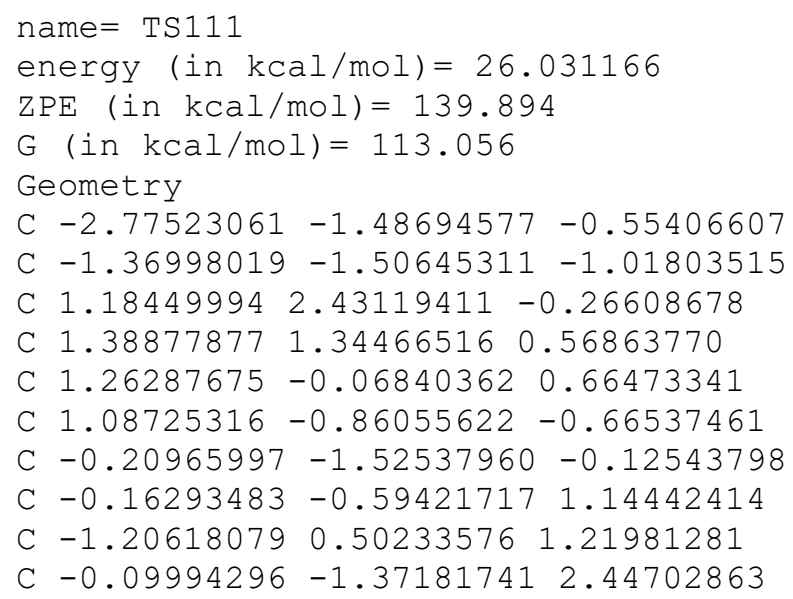


$\mathrm{H}-3.45140229-2.05869261-1.21014095$

$\mathrm{H}-3.17330498-0.45840551-0.50694302$

$\mathrm{H}-2.87238820-1.91467391 \quad 0.45705821$

$\mathrm{H} \quad 0.22073925 \quad 2.44062493-0.81391865$

H $3.11249724 \quad 1.66775520 \quad 0.96571211$

H $2.68856168 \quad 1.88789440 \quad 1.59881779$

H $2.06045196-0.515300811 .29695635$

$\mathrm{H} \quad 1.88917915-1.56048810-0.91107046$

H $0.94143433-0.22822176-1.55057379$

$\mathrm{H}-0.01727632-2.58875476 \quad 0.16036399$

$\mathrm{H}-2.16421659 \quad 0.13520494 \quad 1.60184361$

$\mathrm{H}-1.38640370 \quad 0.97099068 \quad 0.24582963$

$\mathrm{H}-0.87473427 \quad 1.30176489 \quad 1.90205762$

$\mathrm{H} \quad 0.11785903-0.70720506 \quad 3.29369870$

H $0.67422859-2.14781244 \quad 2.43555149$

$\mathrm{H}-1.05302016-1.86878706 \quad 2.66331927$

o $-0.15962398-1.71445135-2.85749875$

O $1.94145522 \quad 3.39123532-0.35594068$

o -1.29285941-1.61401641-2.31725568

Vibrational frequencies (in $\mathrm{cm}-1$ )

$\begin{array}{lllllllllllllllll}-566.0 & 24.0 & 37.4 & 63.5 & 88.2 & 130.2 & 158.0 & 185.8 & 198.9 & 208.1 & 231.0 & 257.8 & 262.3 & 280.3\end{array}$ $293.6 \quad 315.5 \quad 355.1 \quad 379.5 \quad 414.8 \quad 420.3 \quad 431.7 \quad 493.6 \quad 523.3 \quad 601.2 \quad 659.8 \quad 675.1 \quad 726.1 \quad 793.5$ $804.1876 .2911 .5 \quad 938.2 \quad 943.8 \quad 994.1 \quad 1001.2 \quad 1008.6 \quad 1014.1 \quad 1023.0 \quad 1046.4 \quad 1067.4 \quad 1085.9$

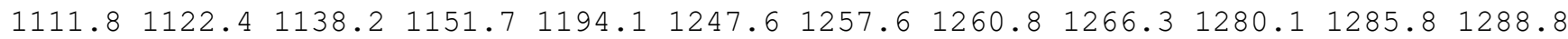
$1291.6 \quad 1297.91301 .6 \quad 1313.9 \quad 1327.4 \quad 1346.6 \quad 1378.7 \quad 1400.2 \quad 1402.7 \quad 1426.6 \quad 1588.5 \quad 1627.9$ $1777.12639 .5 \quad 2653.3 \quad 2662.0 \quad 2666.0 \quad 2671.2 \quad 2674.82676 .42680 .7 \quad 2694.22713 .72761 .9$ 2770.92779 .62782 .35021 .5

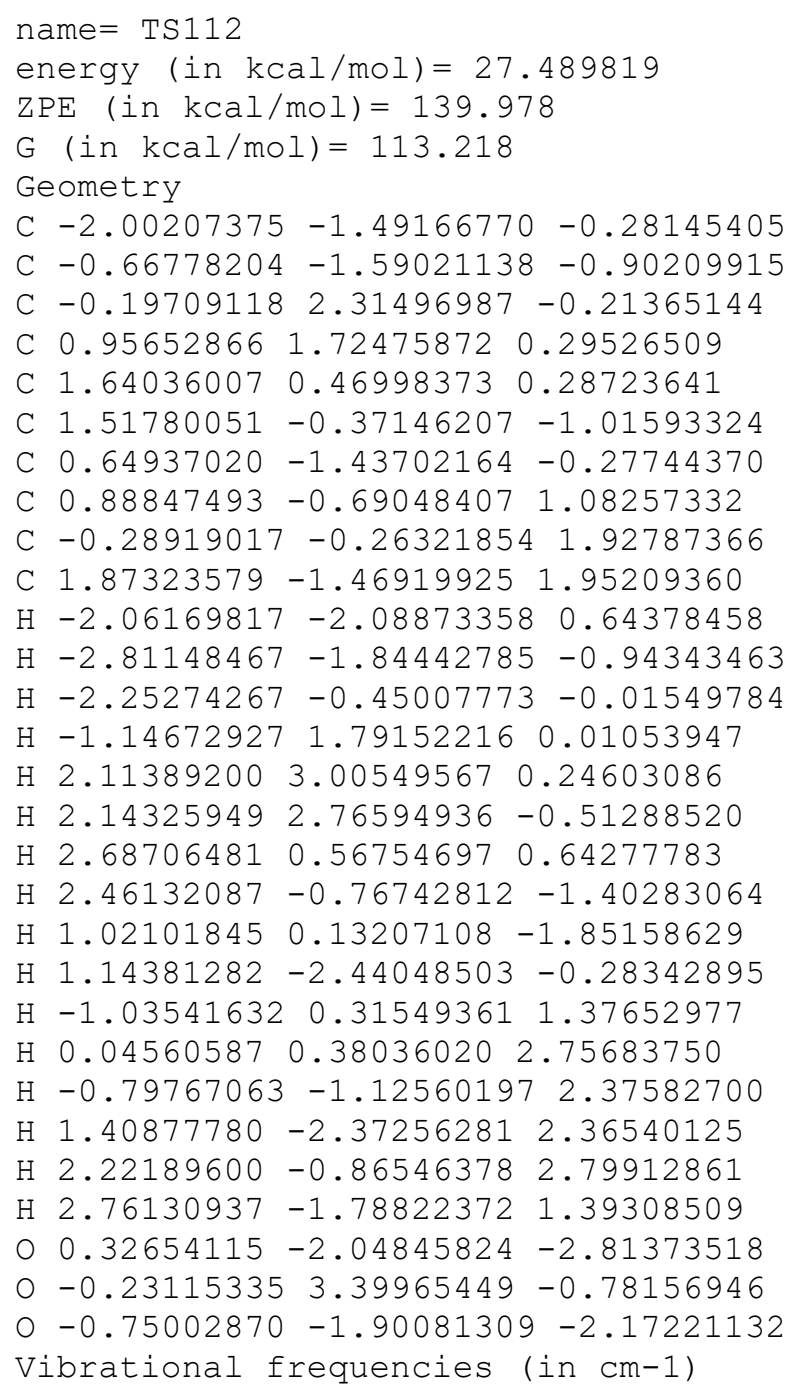


$\begin{array}{llllllllllllllllllll}-583.9 & 25.4 & 30.3 & 69.0 & 102.6 & 135.1 & 160.6 & 168.0 & 186.4 & 209.7 & 219.0 & 244.1 & 263.7 & 290.0\end{array}$

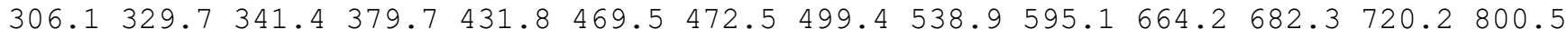
$822.1910 .6914 .2931 .3 \quad 951.3 \quad 980.7 \quad 1004.2 \quad 1013.6 \quad 1016.91018 .1 \quad 1033.0 \quad 1068.6 \quad 1083.4$

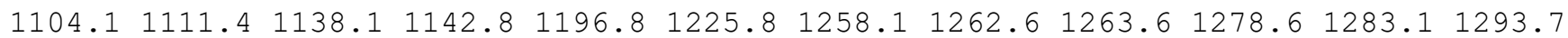
$1295.21302 .91309 .91318 .91335 .3 \quad 1353.4 \quad 1380.1 \quad 1401.01406 .01453 .6 \quad 1559.01600 .6$ $1775.2 \quad 2637.3 \quad 2649.92659 .1 \quad 2664.8 \quad 2672.92673 .6 \quad 2674.82677 .72691 .12715 .62762 .9$ $2768.22779 .02781 .5 \quad 4974.5$

name $=\mathrm{TS} 113$

energy (in $\mathrm{kcal} / \mathrm{mol})=26.790807$

$\mathrm{ZPE}(\mathrm{in} \mathrm{kcal} / \mathrm{mol})=141.198$

$\mathrm{G}($ in $\mathrm{kcal} / \mathrm{mol})=115.506$

Geometry

C $-2.33538586-0.25693645-0.78993582$

C $-0.93626616-0.41590929-0.68020300$

C $2.403177690 .49616349-2.11432635$

C $2.94163067 \quad 0.78914104 \quad-0.78171150$

C $2.08481766 \quad 0.373103490 .39936518$

C $1.36304626-0.99704403 \quad 0.28895002$

C $-0.01880317-0.317670590 .46331622$

C $0.69619408 \quad 1.089147150 .59789010$

C $0.29826176 \quad 2.11595852-0.44153747$

C $0.53948797 \quad 1.66908794 \quad 1.99569744$

$\mathrm{H}-2.764975370 .57290736-1.31592708$

$\mathrm{H}-3.02250551-0.68337003-0.09617163$

$\mathrm{H}-1.81935661-0.80566161-2.19703289$

$\mathrm{H} 1.52718036-0.12007472-2.29708859$

$\mathrm{H} \quad 3.17764387 \quad 1.88584040 \quad-0.71772157$

H $3.956746350 .31825399-0.70017966$

$\mathrm{H} 2.69788841 \quad 0.44166037 \quad 1.32081909$

H $1.61625495-1.70335616 \quad 1.08482421$

$\mathrm{H} 1.49454075-1.52033650-0.66457818$

$\mathrm{H}-0.53860067-0.614743051 .39578632$

$\mathrm{H} \quad 0.33218533 \quad 1.72219982-1.46482993$

$\mathrm{H} \quad 0.963812292 .98943390-0.41242089$

$\mathrm{H}-0.72380698 \quad 2.48074729-0.27198482$

$\mathrm{H} 1.17234406 \quad 2.55612128 \quad 2.12888846$

H $0.81432625 \quad 0.95231424 \quad 2.77801104$

$\mathrm{H}-0.49634140 \quad 1.97716264 \quad 2.18658942$

○ $3.97332002 \quad 1.68337513-3.07657489$

$\begin{array}{lllll}0 & 2.94495219 & 0.95392881 & -3.19826554\end{array}$

o $-0.53243320-0.76177042-1.85580789$

Vibrational frequencies (in cm-1)

$\begin{array}{lllllllllllllll}-1932.9 & 30.9 & 49.5 & 74.7 & 109.9 & 146.5 & 158.4 & 195.5 & 205.2 & 220.5 & 229.0 & 268.0 & 301.9 & 339.5\end{array}$ $\begin{array}{lllllllllllllllll}360.0 & 393.1 & 426.4 & 433.7 & 446.7 & 552.2 & 565.8 & 674.1 & 724.4 & 731.5 & 764.4 & 815.0 & 832.8 & 871.2\end{array}$ $925.5931 .9986 .3 \quad 998.6 \quad 1006.5 \quad 1016.7 \quad 1019.7 \quad 1039.6 \quad 1045.3 \quad 1062.5 \quad 1081.2 \quad 1119.4 \quad 1134.1$

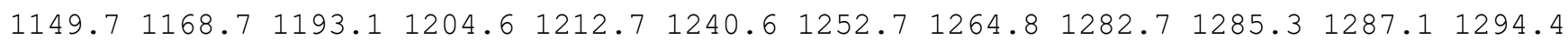
$1297.61301 .41307 .5 \quad 1328.2 \quad 1337.5 \quad 1357.6 \quad 1386.2 \quad 1405.4 \quad 1407.2 \quad 1555.2 \quad 1579.3 \quad 1592.7$ $2119.02618 .22668 .0 \quad 2670.7 \quad 2671.4 \quad 2676.0 \quad 2676.12678 .82711 .3 \quad 2717.62721 .72729 .7$ 2760.92779 .02781 .92808 .2

name $=\operatorname{TS} 114$

energy $($ in $\mathrm{kcal} / \mathrm{mol})=29.51342$

$\mathrm{ZPE}($ in $\mathrm{kcal} / \mathrm{mol})=139.251$

$\mathrm{G}($ in $\mathrm{kcal} / \mathrm{mol})=111.564$

Geometry

C $-2.17541385-0.09917497 \quad-2.09723934$

C $-1.92726385-1.42295215-1.68460784$

C $-1.51869956-1.68229730-0.28759621$

C $2.00960967 \quad 1.93069346-2.04830365$

C $1.63511214 \quad 0.79469658-2.63444222$

C $1.06951298-0.35638931-1.89113894$

C $-2.25041039-2.53511772-2.54076374$ 
C $-1.51925920-3.66155943-2.67664943$

C $-0.29336820-3.98089506-1.89322970$

C $-1.91505886-4.69930952-3.67360568$

$\mathrm{H}-0.964768130 .22680173-2.77731056$

$\mathrm{H}-2.18823953 \quad 0.70223676-1.35803673$

$\mathrm{H}-2.82673420 \quad 0.09058680 \quad-2.94994607$

$\mathrm{H}-1.74979411 \quad-2.668659640 .13647048$

$\mathrm{H} 2.42734573 \quad 2.76693782-2.58757346$

H $1.93718458 \quad 2.11417108-0.98726971$

$\mathrm{H} \quad 1.72492132 \quad 0.62623995-3.71250982$

$\mathrm{H} \quad 0.75616791-0.13040199-0.85983770$

$\mathrm{H} 1.76814294-1.20971189-1.87559040$

$\mathrm{H}-3.11674065-2.37960742-3.19083929$

$\mathrm{H}-0.53274322-4.54038583-0.97876675$

$\mathrm{H} \quad 0.27236938-3.08006047-1.61212591$

$\mathrm{H} 0.41197095-4.59624899-2.47488405$

$\mathrm{H}-2.95909863-4.60836312-4.00016167$

$\mathrm{H}-1.77826304-5.71935070-3.29135712$

$\mathrm{H}-1.29024656-4.60380777-4.57954466$

$0-0.17186613-0.79990886-2.58619225$

$0-0.96562054-0.838747220 .38210618$

$00.08419172-1.42241247-3.66304951$

Vibrational frequencies (in $\mathrm{cm}-1$ )

$\begin{array}{llllllllllllllllllll}-1391.2 & 29.6 & 48.2 & 59.1 & 74.3 & 101.6 & 104.8 & 122.4 & 128.3 & 140.0 & 152.1 & 191.3 & 223.2 & 235.9\end{array}$

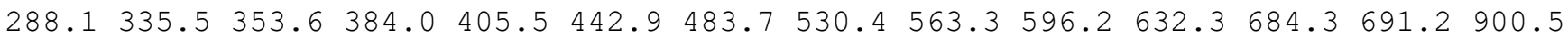

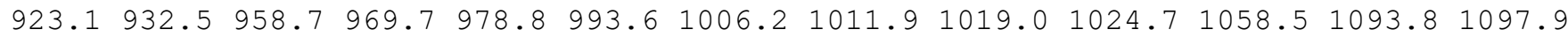

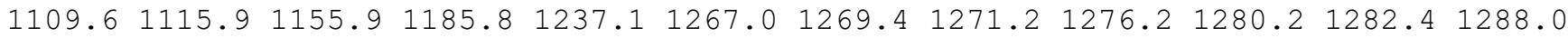

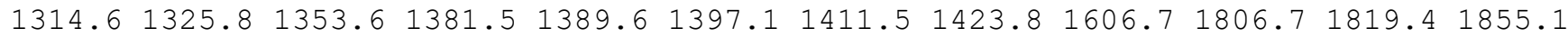
$1937.42651 .12658 .7 \quad 2660.1 \quad 2662.6 \quad 2671.5 \quad 2676.2 \quad 2685.92712 .0 \quad 2721.2 \quad 2741.0 \quad 2745.5$ $2748.6 \quad 2775.92778 .2 \quad 2787.0$

name $=\mathrm{TS} 115$

energy $($ in $\mathrm{kcal} / \mathrm{mol})=27.107498$

$\mathrm{ZPE}($ in $\mathrm{kcal} / \mathrm{mol})=141.745$

$\mathrm{G}($ in $\mathrm{kcal} / \mathrm{mol})=118.594$

Geometry

C $-2.01135044 \quad 1.17697649-1.63583654$

C $-0.75991613 \quad 0.42187101-1.21685522$

C $0.456986501 .35788019-0.87372232$

C 0.330099521 .900862760 .55411985

C $0.43328445 \quad 0.59104595 \quad 1.37572425$

C $1.21948350-0.35133010 \quad 0.45119740$

C $-0.81226735-0.62708802-0.18117427$

C $-0.88781970-0.22906991 \quad 1.26901623$

C $-2.149125450 .54648372 \quad 1.66428846$

C $-0.79111243-1.468356692 .17998056$

$\mathrm{H}-2.90414894 \quad 0.55103716-1.49314687$

$\mathrm{H}-1.98416640 \quad 1.48411570-2.69035019$

$\mathrm{H}-2.144888692 .08249904-1.03191173$

$\mathrm{H} 0.769740962 .04887450-1.65807888$

$\mathrm{H}-0.60883324 \quad 2.43963244 \quad 0.73731719$

H $1.15188885 \quad 2.59209905 \quad 0.79883822$

$\mathrm{H} \quad 0.81334398 \quad 0.72876499 \quad 2.38626580$

$\mathrm{H} \quad 1.32701884-0.57989408-1.68516660$

$\mathrm{H} \quad 0.18437244-1.72856324-1.38406307$

$\mathrm{H}-1.32929869-1.53639854-0.42894295$

$\mathrm{H}-3.03331861-0.09420042 \quad 1.57156126$

$\mathrm{H}-2.31887151 \quad 1.41484989 \quad 1.02432083$

$\mathrm{H}-2.08846160 \quad 0.89634246 \quad 2.69914124$

$\mathrm{H}-1.66363680-2.115342092 .04862306$

$\mathrm{H}-0.73698865-1.18146150 \quad 3.23380292$

$\mathrm{H} \quad 0.09850055-2.06569790 \quad 1.94976239$

O $0.57399847-1.25716738-2.27374971$

$\begin{array}{lllll}0 & 1.52826468 & 0.37382427 & -0.70187278\end{array}$ 
$0-0.43853291-0.22506984-2.48088224$

Vibrational frequencies (in $\mathrm{cm}-1$ )

$\begin{array}{llllllllllllllll}-618.7 & 60.8 & 133.0 & 158.6 & 175.1 & 196.9 & 210.4 & 241.9 & 262.2 & 290.6 & 345.6 & 356.2 & 390.4 & 435.8\end{array}$ $\begin{array}{lllllllllllllll}453.3 & 462.0 & 478.4 & 517.0 & 557.7 & 594.9 & 705.8 & 732.4 & 772.7 & 794.6 & 828.4 & 854.0 & 888.3 & 913.3\end{array}$ $929.0 \quad 952.7 \quad 963.2 \quad 975.8 \quad 992.0 \quad 1012.1 \quad 1018.9 \quad 1032.8 \quad 1044.0 \quad 1058.2 \quad 1079.3 \quad 1103.5 \quad 1117.4$

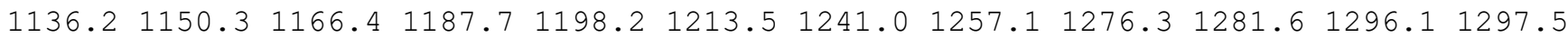

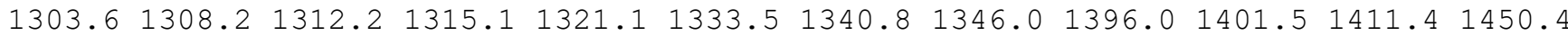
$1666.02374 .12667 .2 \quad 2669.8 \quad 2675.92677 .1 \quad 2680.42683 .32686 .72723 .0 \quad 2752.5 \quad 2759.4$ $2765.72775 .12781 .6 \quad 2783.4$

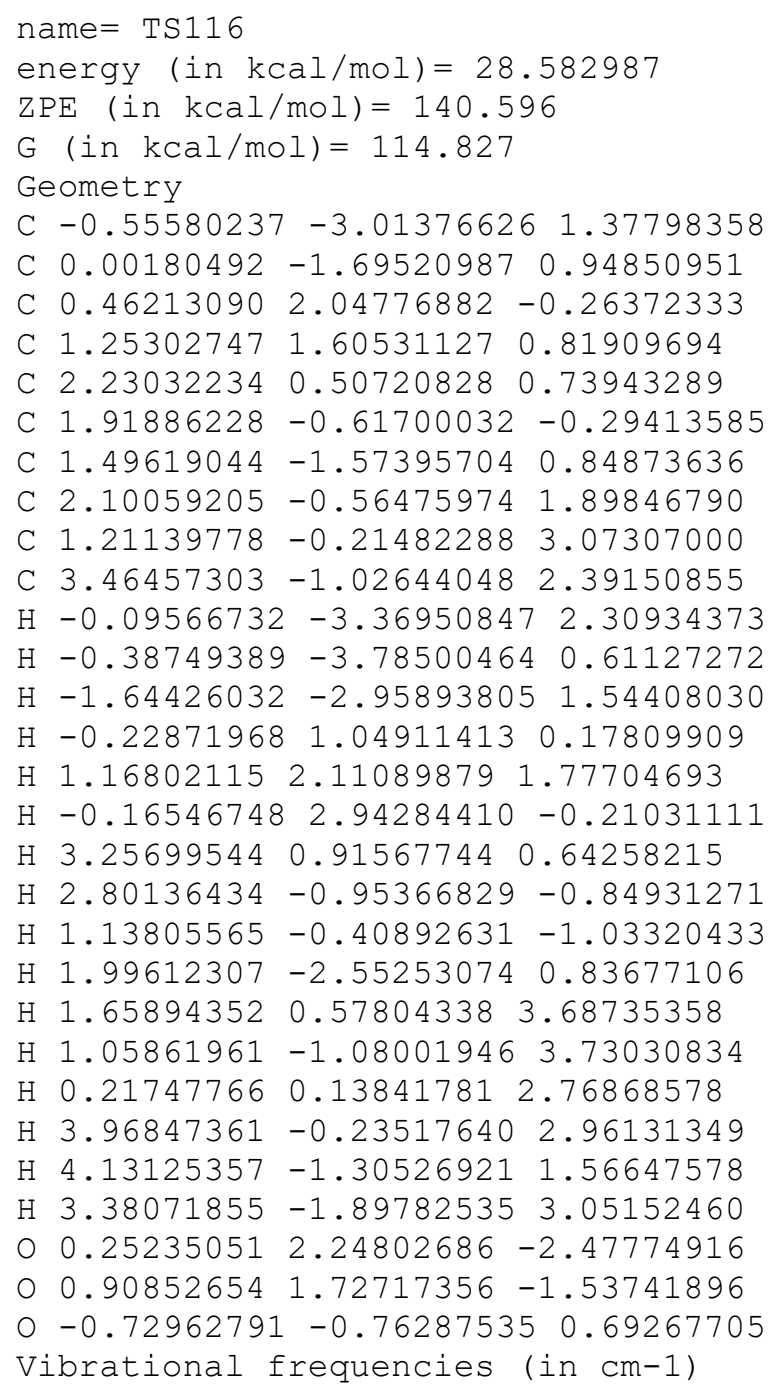


C $1.69214341-1.170071410 .77624185$

C $-0.50596468-1.10471825 \quad 0.20333593$

C $-0.44447099-0.16780674 \quad 1.29633380$

C -1.343895951 .046266951 .05240407$

C $-0.84508755-0.825238812 .62794747$

$\mathrm{H}-2.82696890-1.08524338-1.49539458$

$\mathrm{H}-2.85201276-2.48227254-0.38886519$

$\mathrm{H}-2.39194720-2.69849702-2.08874286$

H $1.47651360 \quad 0.29287775-1.57685645$

$\mathrm{H} 1.07014213 \quad 2.26417148 \quad 0.83662513$

H 2.60791354 $1.47029024 \quad 0.49418569$

H $1.43684595 \quad 0.33444092 \quad 2.33236030$

H $1.51371532-2.02365702 \quad 1.42926573$

H $2.77223524-1.109769090 .59375759$

H $1.45793546-1.49933461-0.36924569$

$\mathrm{H}-2.405056490 .78114103 \quad 1.11609727$

$\mathrm{H}-1.182973161 .499403770 .06288805$

$\mathrm{H}-1.15655146 \quad 1.82849190 \quad 1.79800532$

$\mathrm{H}-0.74220146-0.12209902 \quad 3.46210894$

$\mathrm{H}-0.22536589-1.70137532 \quad 2.85313050$

$\mathrm{H}-1.88730443-1.16234968 \quad 2.60898152$

O $1.21839891-2.00898160-1.70847041$

$0.49515867 \quad 2.04888025-1.58655221$

O $-0.06242714-2.29486415-1.82837803$

Vibrational frequencies (in $\mathrm{cm}-1$ )

$\begin{array}{llllllllllllllll}-634.2 & 36.2 & 68.0 & 73.7 & 90.7 & 112.3 & 122.7 & 176.8 & 182.2 & 199.5 & 204.8 & 243.3 & 264.7 & 277.1\end{array}$

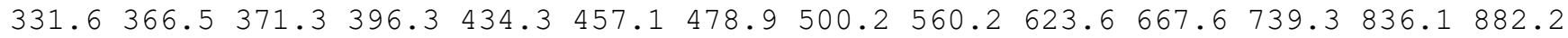
$942.6970 .6 \quad 987.4 \quad 993.6 \quad 993.8 \quad 1010.0 \quad 1014.6 \quad 1023.8 \quad 1053.2 \quad 1062.4 \quad 1121.4 \quad 1127.2 \quad 1164.5$

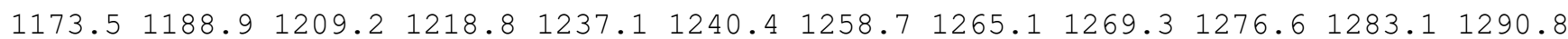

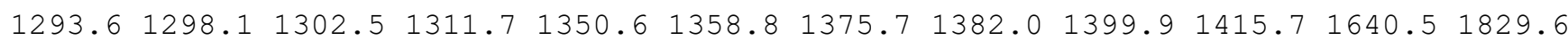
$1898.72620 .2 \quad 2652.7 \quad 2658.0 \quad 2659.5 \quad 2661.5 \quad 2669.3 \quad 2673.5 \quad 2674.5 \quad 2678.7 \quad 2696.7 \quad 2738.4$ $2748.7 \quad 2768.12778 .8 \quad 2781.9$

name $=$ TS118

energy $($ in $\mathrm{kcal} / \mathrm{mol})=32.17454$

$\mathrm{ZPE}($ in $\mathrm{kcal} / \mathrm{mol})=140.141$

G $($ in $\mathrm{kcal} / \mathrm{mol})=111.844$

Geometry

C $-2.42648837-0.06428378-1.15325437$

C $-1.25640445 \quad 0.11651894-0.23836685$

C $-0.26966790 \quad 1.18315809-0.58050160$

C $0.97393511 \quad 1.28412998 \quad 0.29659658$

$\begin{array}{lllll}\text { C } & 1.13116791 & 0.06675321 & 1.22372974\end{array}$

C $0.78683917-1.17116156 \quad 0.36589534$

C $-0.71664826-0.96805671 \quad 0.48711412$

C $-0.03881186-0.080975692 .20521571$

C $-0.791071061 .02799345 \quad 2.63300470$

C $0.00630437-1.24710100 \quad 3.15292815$

$\mathrm{H}-2.80516570 \quad 0.89785366-1.53206724$

$\mathrm{H}-3.25843082-0.59405518-0.67506337$

$\mathrm{H}-2.13490914-0.64886196-2.04026167$

$\mathrm{H}-2.01123382 \quad 0.95434749 \quad 0.83419404$

$\mathrm{H} \quad 0.94349769 \quad 2.23007300 \quad 0.86849556$

$\mathrm{H} \quad 1.863623591 .37717661-0.35966468$

$\mathrm{H} 2.11704540 \quad 0.05193202 \quad 1.71153319$

$\mathrm{H} 1.14408941-2.117604890 .79025411$

H $1.16197066-1.14025246-0.66638148$

$\mathrm{H}-1.32757031-1.812501310 .76351553$

$\mathrm{H}-1.90629649 \quad 1.17027130 \quad 1.67243503$

$\mathrm{H}-0.42889458 \quad 2.036155532 .47040726$

$\mathrm{H}-1.39371362 \quad 0.94873502 \quad 3.53039614$

$\mathrm{H} \quad 0.53538134-0.97052060 \quad 4.07671617$

H $0.52308614 \quad-2.12394828 \quad 2.74620199$

$\mathrm{H}-1.00175830-1.574411463 .43926575$ 
$0.96444617-0.47404746-3.78535385$

$0-0.44379893 \quad 1.97323799-1.47738548$

O $0.45272087-1.26754799-3.15674312$

Vibrational frequencies (in $\mathrm{cm}-1$ )

$\begin{array}{llllllllllllllllll}-1799.0 & 14.8 & 24.5 & 35.7 & 49.8 & 66.7 & 85.4 & 138.6 & 155.4 & 173.0 & 235.5 & 266.3 & 285.4 & 327.5\end{array}$

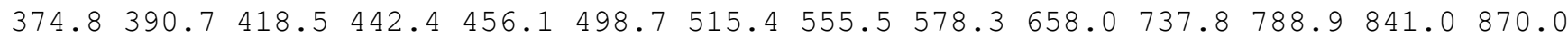
$941.6948 .0 \quad 954.1 \quad 970.4 \quad 992.5 \quad 1004.5 \quad 1007.4 \quad 1051.9 \quad 1057.0 \quad 1061.3 \quad 1073.6 \quad 1087.8 \quad 1105.7$ $\begin{array}{lllllllllllll}1126.1 & 1137.3 & 1151.7 & 1165.2 & 1189.9 & 1244.1 & 1262.8 & 1283.4 & 1284.1 & 1284.4 & 1288.5 & 1290.2\end{array}$ $1293.91304 .91331 .1 \quad 1360.4 \quad 1367.9 \quad 1379.8 \quad 1392.6 \quad 1404.4 \quad 1450.2 \quad 1538.2 \quad 1596.21597 .2$ $1838.22658 .52662 .22668 .62674 .2 \quad 2677.5 \quad 2678.42699 .42731 .5 \quad 2742.92759 .0 \quad 2760.9$ $2763.9 \quad 2777.0 \quad 2779.1 \quad 3194.0$

name $=\operatorname{TS} 119$

energy (in $\mathrm{kcal} / \mathrm{mol})=30.772434$

$\mathrm{ZPE}($ in $\mathrm{kcal} / \mathrm{mol})=141.749$

$\mathrm{G}($ in $\mathrm{kcal} / \mathrm{mol})=117.549$

Geometry

C $-2.70951626-0.63624348-0.99821583$

C $-0.37526904-0.28298421-0.97672629$

C $0.70832300 \quad 0.52701881-1.75865858$

C $1.92505021 \quad 0.96131490 \quad-0.94733051$

$\begin{array}{lllll}\text { C } 1.84908087 & 0.47101001 & 0.50359421\end{array}$

C $1.41135113-0.97853131 \quad 0.62796778$

C $-0.04744619-0.570751610 .46924765$

C $0.42338773 \quad 0.80185679 \quad 1.14594672$

C $-0.27790669 \quad 2.07945824 \quad 0.74969475$

$\begin{array}{lllll}\text { C } & 0.45541461 & 0.68136784 & 2.66402364\end{array}$

$\mathrm{H}-3.56786160-0.07103023-1.38582331$

$\mathrm{H}-2.81731072-0.815333790 .07551784$

$\mathrm{H}-2.58959925-1.57544664-1.54867936$

$\mathrm{H} 0.31400261 \quad 1.30490682 \quad-2.43100151$

H $2.017317162 .06552170 \quad-0.96094312$

H $2.84991993 \quad 0.58191540 \quad-1.42655894$

H 2.715711860 .770035061 .09758775

H $2.00198521-2.08998751-0.04181181$

$\mathrm{H} 1.86538813-1.65169551-0.73769428$

$\mathrm{H}-0.78915032-1.15812660 \quad 1.00801578$

$\mathrm{H}-1.29509958 \quad 2.109414561 .16539688$

$\mathrm{H}-0.39382260 \quad 2.21274592-0.33059502$

$\mathrm{H} \quad 0.26309488 \quad 2.95610547 \quad 1.12801370$

$\mathrm{H} \quad 0.99093851 \quad 1.52199003 \quad 3.11788222$

$\mathrm{H} \quad 0.96235893-0.24089807 \quad 2.98727542$

$\mathrm{H}-0.556005520 .65880503 \quad 3.08336950$

$0-0.07782223-1.42777130-1.83685159$

$0.97170322-0.67665451-2.53619231$

$0-1.62334362 \quad 0.24129120-1.25220365$

Vibrational frequencies (in $\mathrm{cm}-1$ )

$\begin{array}{llllllllllllllll}-1227.7 & 45.5 & 85.7 & 120.6 & 137.2 & 166.1 & 186.6 & 190.1 & 217.2 & 250.8 & 312.6 & 332.3 & 342.2 & 386.1\end{array}$ $417.2 \quad 438.5 \quad 447.3 \quad 481.4 \quad 528.7 \quad 544.9 \quad 614.8 \quad 643.6 \quad 684.5 \quad 697.4 \quad 740.4 \quad 788.6 \quad 826.8 \quad 902.7$

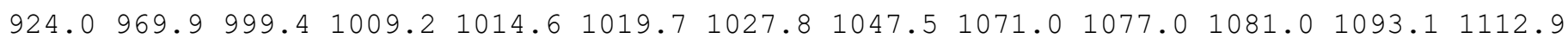

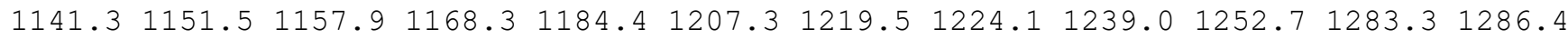

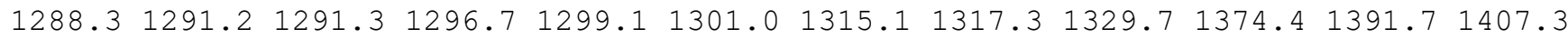
$1419.72651 .92656 .32659 .2 \quad 2666.4 \quad 2672.0 \quad 2678.22683 .52700 .72739 .32745 .52749 .3$ $2756.0 \quad 2779.7 \quad 2784.8 \quad 4416.8$

name $=\operatorname{TS} 120$

energy $($ in $\mathrm{kcal} / \mathrm{mol})=31.14166$

$\mathrm{ZPE}($ in $\mathrm{kcal} / \mathrm{mol})=141.473$

$\mathrm{G}($ in $\mathrm{kcal} / \mathrm{mol})=116.239$

Geometry

C $-1.73343445-0.53125779-0.93934005$

C $-0.44982330-0.35144340-0.22621340$

C $0.08979204 \quad 1.06963749-0.30644784$ 
C $1.56794245 \quad 1.16518046 \quad 0.12562283$

C $2.39689215-0.00420066-0.26908611$

C $1.90588438-1.07595686-0.89244297$

C $-0.25906432-1.095959821 .03690752$

C $-0.86748788-0.791019862 .19295531$

C $-1.81141147 \quad 0.34833791 \quad 2.36608244$

C $-0.62019224-1.59883045 \quad 3.42433734$

$\mathrm{H}-2.49183817 \quad 0.21839811-0.71021099$

$\mathrm{H}-2.16960226-1.52024209-0.91388394$

$\mathrm{H}-1.46943139-0.16891008-2.07299333$

$\mathrm{H}-0.53093168 \quad 1.75901385 \quad 0.30653647$

$\mathrm{H} \quad 1.63610432 \quad 1.32302164 \quad 1.22114961$

H $1.99007907 \quad 2.08641398-0.34544121$

$\mathrm{H} 3.45746788 \quad 0.06247080 \quad-0.01628045$

$\mathrm{H} 0.22405488-2.00708739-1.40044555$

H $2.42799690-1.94958301-1.23847202$

$\mathrm{H} \quad 0.42642112-1.94129256 \quad 0.99078274$

$\mathrm{H}-2.53956837 \quad 0.17130099 \quad 3.16990503$

$\mathrm{H}-2.393818150 .55380808 \quad 1.45424350$

$\mathrm{H}-1.26984729 \quad 1.272095292 .62230308$

$\mathrm{H}-1.51731400-2.17238476 \quad 3.70435090$

$\mathrm{H}-0.36608254 \quad-0.95843989 \quad 4.28167994$

$\mathrm{H} \quad 0.19866681-2.32123678 \quad 3.31055039$

$0-0.15825043 \quad 0.56939456-2.54245957$

$0-0.02276779 \quad 1.58050325-1.64091684$

$0.54915021-1.02839659-1.32583380$

Vibrational frequencies (in $\mathrm{cm}-1$ )

$\begin{array}{lllllllllllllllll}-717.3 & 48.0 & 75.0 & 90.8 & 100.4 & 118.5 & 136.0 & 144.1 & 214.6 & 257.3 & 268.2 & 298.9 & 315.3 & 338.2\end{array}$ $389.0 \quad 400.4 \quad 408.7 \quad 442.5 \quad 462.9 \quad 495.6 \quad 526.8 \quad 539.8 \quad 628.8 \quad 744.5 \quad 781.6 \quad 813.9 \quad 871.8 \quad 895.2$ $904.2936 .4966 .5 \quad 973.1 \quad 989.8 \quad 992.5 \quad 1004.0 \quad 1021.3 \quad 1042.6 \quad 1061.1 \quad 1072.0 \quad 1104.5 \quad 1117.0$

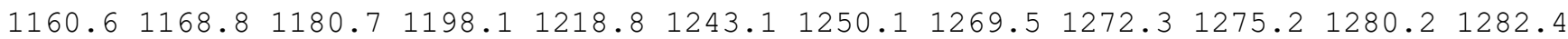

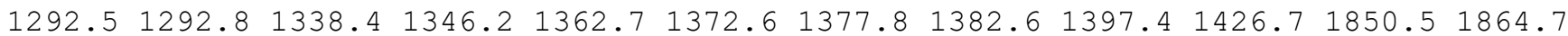
$2073.82651 .5 \quad 2660.0 \quad 2666.4 \quad 2669.5 \quad 2673.3 \quad 2682.92696 .82730 .12738 .5 \quad 2746.72751 .1$ $2770.4 \quad 2775.2 \quad 2779.9 \quad 2799.2$

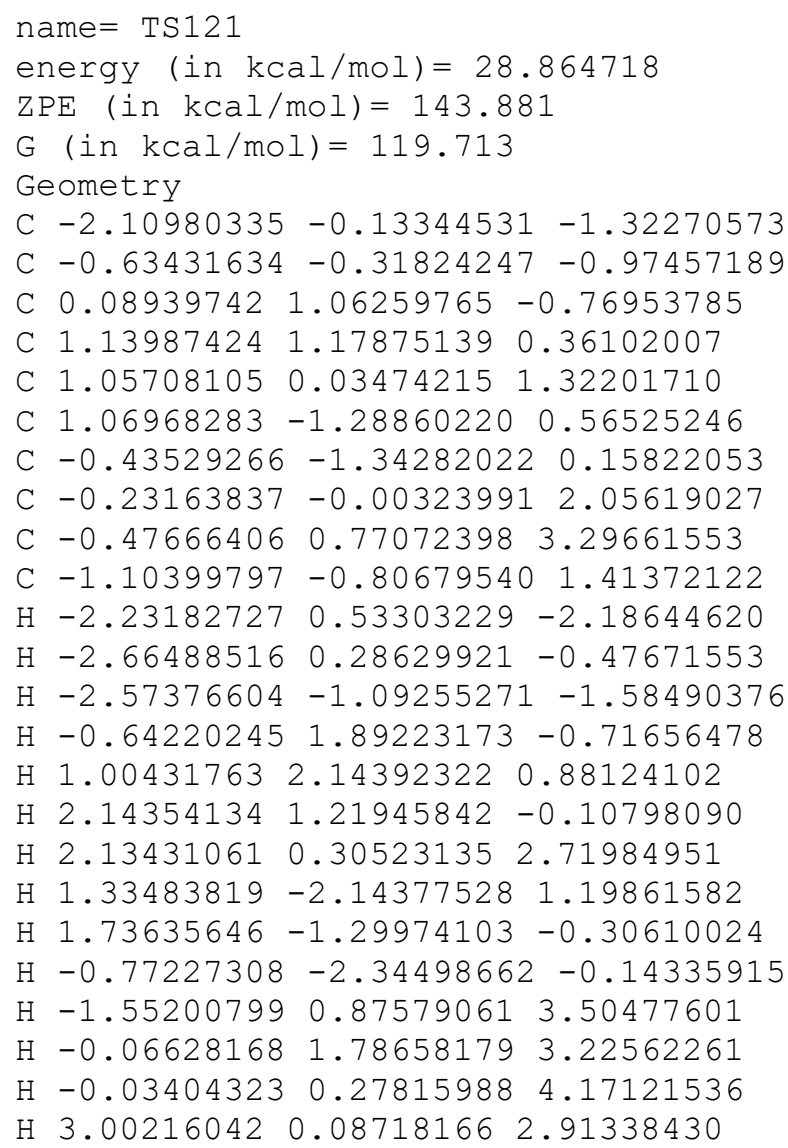


H $2.63804475-0.159206032 .10965396$

$\mathrm{H}-2.10581696-1.063803401 .66619592$

$01.11248849-0.05028405-2.42245042$

$0.736312921 .25578342-2.03076408$

O $-0.03912664-0.84652878-2.17311696$

Vibrational frequencies (in $\mathrm{cm}-1$ )

$\begin{array}{llllllllllllllll}-2571.0 & 59.2 & 98.9 & 104.8 & 116.0 & 166.2 & 174.1 & 230.7 & 236.1 & 249.8 & 264.6 & 299.0 & 336.4 & 354.2\end{array}$

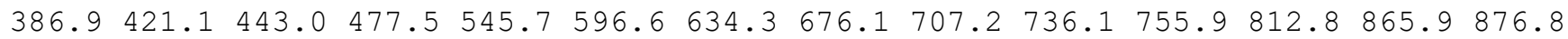
$902.1934 .2 \quad 954.7973 .2 \quad 980.0 \quad 1003.2 \quad 1018.6 \quad 1026.7 \quad 1035.9 \quad 1063.1 \quad 1068.7 \quad 1078.3 \quad 1085.9$

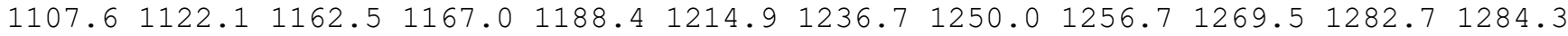

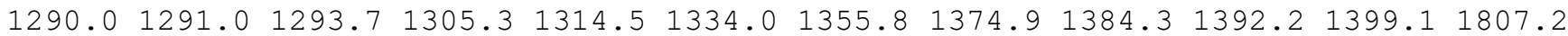
$1979.02665 .8 \quad 2673.8 \quad 2674.7 \quad 2679.4 \quad 2680.6 \quad 2688.7 \quad 2693.4 \quad 2737.5 \quad 2740.3 \quad 2757.5 \quad 2779.9$ $2780.4 \quad 2800.0 \quad 2954.7 \quad 4526.2$

name $=\operatorname{TS} 122$

energy (in $\mathrm{kcal} / \mathrm{mol})=36.709229$

$\mathrm{ZPE}(\mathrm{in} \mathrm{kcal} / \mathrm{mol})=136.741$

$\mathrm{G}($ in $\mathrm{kcal} / \mathrm{mol})=107.731$

Geometry

C $-2.31154124-1.95401212-1.18450719$

C $-0.89622324-1.63572565-0.99758523$

C $1.972907490 .87427178 \quad 3.31956004$

C $1.018793401 .78869273 \quad 3.07784461$

C $-1.24395587-0.02861350 \quad 0.83855945$

C $0.92435817-1.17609130 \quad 0.55476186$

C $-0.34300153-0.95515680 \quad 0.17081839$

C $-1.57661509-0.06187374 \quad 2.14171657$

C $-2.48489138 \quad 0.965650792 .72502700$

C $-1.08217140-1.083782773 .10346456$

$\mathrm{H}-2.94951820-1.67646491-0.33409249$

$\mathrm{H}-2.45004358-3.03806443-1.37478098$

$\mathrm{H}-2.71576847-1.45777184-2.08939051$

H $2.65681813 \quad 0.88627704 \quad 4.16924263$

$\mathrm{H} \quad 0.37525986 \quad 1.77354842 \quad 2.22372171$

$\mathrm{H} \quad 0.84720103 \quad 2.63408584 \quad 3.71186598$

$\mathrm{H}-1.66552083 \quad 0.73416870 \quad 0.17661410$

$\mathrm{H} 1.22010019-0.921555451 .87536722$

$\mathrm{H} 1.65106091-0.22202880 \quad 1.20902209$

$\mathrm{H} 1.61420817-1.864590990 .10243509$

$\mathrm{H}-1.95695971 \quad 1.54690515 \quad 3.50287502$

$\mathrm{H}-3.364323810 .51211010 \quad 3.20419819$

$\mathrm{H}-2.85557816 \quad 1.69043638 \quad 1.98751994$

$\mathrm{H}-1.83636213-1.34780438 \quad 3.85897957$

$\mathrm{H}-0.21035245-0.69165411 \quad 3.66545118$

$\mathrm{H}-0.77191901-2.02290804 \quad 2.62616384$

$0-0.38322743-2.56311379-2.91741998$

O $2.20903866-0.15848476 \quad 2.54406091$

O $0.00436193-1.96321363-1.89939008$

Vibrational frequencies (in $\mathrm{cm}-1$ )

$\begin{array}{lllllllllllllll}-2121.7 & 28.3 & 35.8 & 53.5 & 62.2 & 77.0 & 90.8 & 99.5 & 105.0 & 119.3 & 151.0 & 159.9 & 189.5 & 219.9\end{array}$ $240.1278 .6 \quad 306.0 \quad 341.2 \quad 396.0 \quad 441.6 \quad 450.4 \quad 489.1 \quad 534.3 \quad 558.0 \quad 576.7 \quad 623.0 \quad 661.5 \quad 732.5$ $859.6 \quad 883.9 \quad 899.2 \quad 935.1 \quad 965.7 \quad 978.8 \quad 997.0 \quad 1002.0 \quad 1014.4 \quad 1034.6 \quad 1038.7 \quad 1051.8 \quad 1075.0$

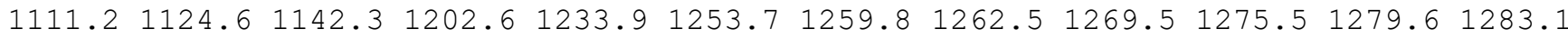

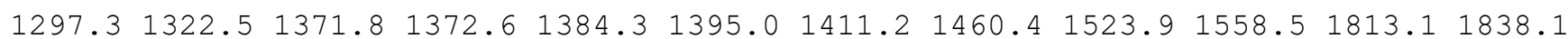
1864.32553 .62636 .22638 .72656 .92667 .42667 .62672 .42716 .42743 .52750 .22752 .3 2766.82770 .62776 .62814 .8

name $=\operatorname{TS} 123$

energy (in $\mathrm{kcal} / \mathrm{mol})=33.733021$

$\mathrm{ZPE}($ in $\mathrm{kcal} / \mathrm{mol})=139.985$

$\mathrm{G}($ in $\mathrm{kcal} / \mathrm{mol})=113.396$

Geometry

C $-0.40542327-2.99312293-1.29698080$ 
C $0.16724811-1.66334098-1.58506772$

C $1.102857542 .02386973-0.15107249$

C $1.39089477 \quad 1.291497391 .13567487$

C $0.86439516-0.15429757 \quad 1.24195171$

C $1.65062486-1.00818766 \quad 0.33426362$

C $1.49086924-1.21209355-1.10239375$

C $-0.63941942-0.29932571 \quad 1.12425261$

C $-1.42967860 \quad 0.77188764 \quad 0.46505719$

C $-1.23606567-1.38655877 \quad 1.62938911$

$\mathrm{H}-0.82925784-3.02620666-0.27437809$

$\mathrm{H} \quad 0.34792011-3.79226218-1.36867056$

$\mathrm{H}-1.22820337-3.26939075-1.97777550$

$\mathrm{H} 0.99446073 \quad 1.44787223-1.08016674$

H $1.00311936 \quad 1.88534261 \quad 1.99045981$

H $2.49867723 \quad 1.27578846 \quad 1.26884863$

H $1.12096796-0.46044150 \quad 2.30402741$

H $2.11005314-2.25219342 \quad 1.00998627$

$\mathrm{H} \quad 1.74408960-0.21977636-1.59827531$

H 2.28929857 -1.88520572 -1.49935167

$\mathrm{H}-1.15835074 \quad 0.89352481 \quad-0.60012081$

$\mathrm{H}-1.27381380 \quad 1.75117652 \quad 0.94566110$

$\mathrm{H}-2.51160570 \quad 0.58139219 \quad 0.48896721$

$\mathrm{H}-0.72618900 \quad-2.17408567 \quad 2.15152419$

H $1.31642082 \quad-2.47356463 \quad 0.90444563$

$\mathrm{H}-2.29989008-1.55171359 \quad 1.58846270$

$\begin{array}{lllll}0 & -0.19674494 & 0.24881040 & -2.61018768\end{array}$

$01.02172227 \quad 3.22852067-0.14962500$

o $-0.60848212-0.92128381-2.32241734$

Vibrational frequencies (in $\mathrm{cm}-1$ )

$\begin{array}{lllllllllllllll}-1136.4 & 32.0 & 53.1 & 66.5 & 101.0 & 111.0 & 120.5 & 133.5 & 167.7 & 188.1 & 223.9 & 253.9 & 268.5 & 285.2\end{array}$ $293.8 \quad 306.8 \quad 354.0 \quad 374.2 \quad 422.4 \quad 463.3 \quad 520.6 \quad 560.3 \quad 608.2 \quad 653.0 \quad 663.2 \quad 699.0 \quad 715.7 \quad 744.5$ $810.4 \quad 863.2915 .4 \quad 935.8 \quad 986.7 \quad 994.9 \quad 1011.1 \quad 1030.4 \quad 1039.01050 .4 \quad 1060.8 \quad 1064.1 \quad 1090.4$ $1101.81143 .0 \quad 1178.3 \quad 1197.6 \quad 1236.0 \quad 1239.2 \quad 1256.8 \quad 1258.5 \quad 1263.4 \quad 1276.2 \quad 1279.0 \quad 1281.2$

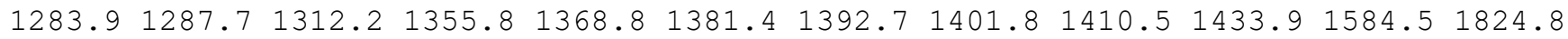
$1836.02588 .6 \quad 2626.2 \quad 2637.7 \quad 2641.5 \quad 2651.92662 .62666 .02666 .72710 .12726 .52732 .8$ $2768.22771 .12796 .7 \quad 4452.2$

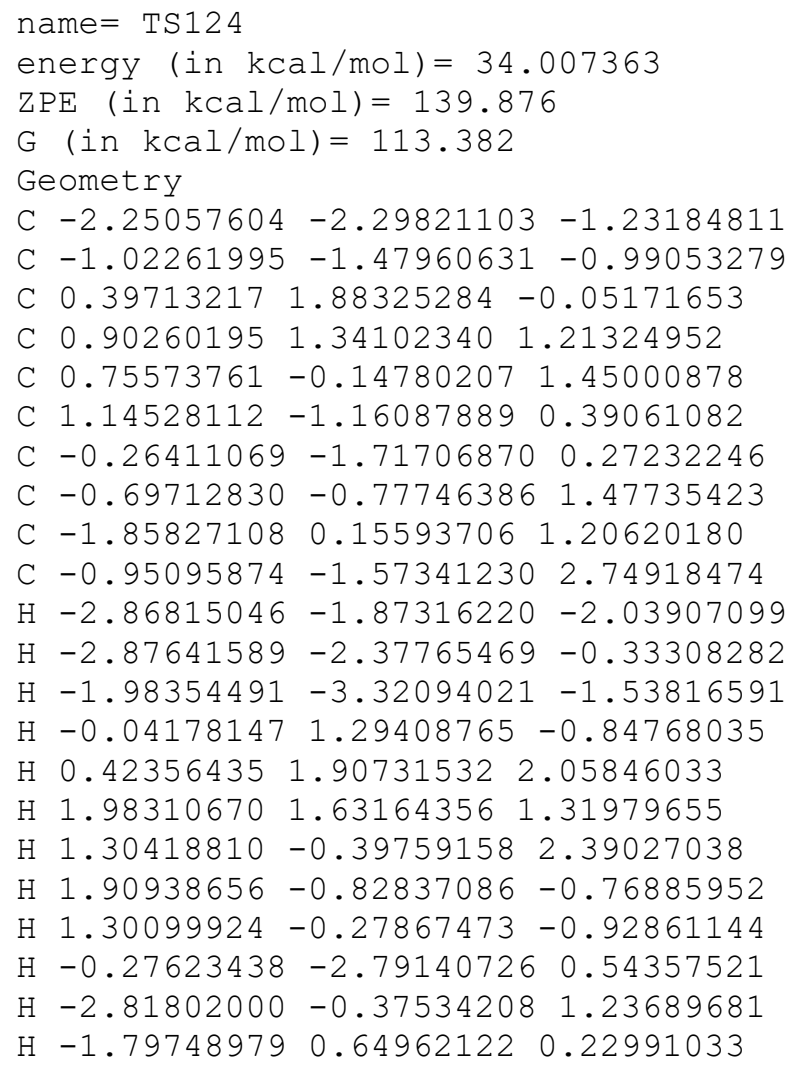


$\mathrm{H}-1.906755330 .95014135 \quad 1.96471698$

$\mathrm{H}-1.86256176 \quad-2.17659634 \quad 2.67373957$

$\mathrm{H}-1.06598292-0.90945120 \quad 3.61516051$

$\mathrm{H}-0.12451230-2.26027342 \quad 2.97419284$

$\begin{array}{lllll}0 & 0.92242553 & 3.91989074 & 0.55490900\end{array}$

$00.43143027 \quad 3.15372926-0.31600425$

o $-0.64825123-0.65566705-1.79741517$

Vibrational frequencies (in $\mathrm{cm}-1$ )

$\begin{array}{llllllllllllll}-1205.6 & 24.8 & 51.3 & 78.7 & 80.2 & 106.2 & 145.4 & 168.3 & 183.8 & 198.9 & 211.2 & 225.3 & 261.4 & 317.6\end{array}$

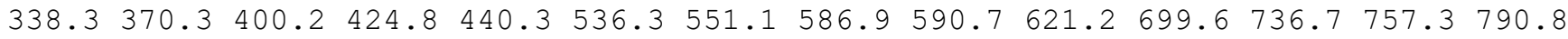
$811.2840 .2941 .5965 .2991 .6 \quad 1006.6 \quad 1013.6 \quad 1017.9 \quad 1044.9 \quad 1046.8 \quad 1060.2 \quad 1092.9 \quad 1123.5$

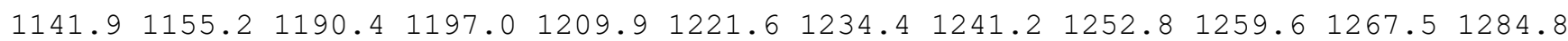

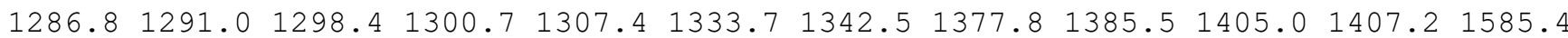
$1830.12613 .42664 .2 \quad 2668.5 \quad 2670.4 \quad 2672.4 \quad 2673.92678 .72682 .12700 .4 \quad 2716.2 \quad 2721.8$ $2777.5 \quad 2779.4 \quad 2781.9 \quad 4382.5$

name $=$ TS125

energy $($ in $\mathrm{kcal} / \mathrm{mol})=32.394555$

$\mathrm{ZPE}(\mathrm{in} \mathrm{kcal} / \mathrm{mol})=141.659$

$\mathrm{G}(\mathrm{in} \mathrm{kcal} / \mathrm{mol})=115.608$

Geometry

C $-2.51876481-0.11656562-0.04602816$

C $-1.34621743-0.75411209-0.47859519$

C $1.340284051 .90356555-1.53198008$

C $2.323563361 .40276735-0.50416954$

C $1.81312473 \quad 0.23656023 \quad 0.32352351$

C $1.22000659-0.95008899-0.47005381$

C $-0.11325891-0.85862027 \quad 0.32558995$

C $0.451728890 .39521135 \quad 1.10439157$

C $-0.24834679 \quad 1.72651763 \quad 0.91099428$

C $0.61379489 \quad 0.10645448 \quad 2.59248118$

$\mathrm{H}-3.10198812 \quad 0.50002093 \quad 0.86951094$

$\mathrm{H}-3.38587798-0.08350324-0.68758239$

$\mathrm{H}-2.24298714 \quad 0.31435225 \quad 1.14693928$

$\mathrm{H} \quad 0.80854133 \quad 1.16139529-2.14280538$

$\mathrm{H} 2.633143992 .236442330 .16192063$

$\mathrm{H} \quad 3.253717731 .09742829-1.03518930$

H 2.62253184 -0.086431691 .00902861$

$\mathrm{H} 1.74151022-1.90456095-0.35419122$

$\mathrm{H} \quad 1.12129390-0.79530827-1.55365888$

$\mathrm{H}-0.22534719-1.737886261 .00197062$

$\mathrm{H}-1.01866397 \quad 1.91981419 \quad 1.66122123$

$\mathrm{H}-0.698455091 .82556129-0.08757314$

$\mathrm{H} 0.46842005 \quad 2.56005648 \quad 1.00428395$

$\mathrm{H}-0.34843076 \quad 0.00675164 \quad 3.10156111$

H $1.16921891 \quad 0.91176671 \quad 3.09081529$

$\mathrm{H} \quad 1.17163960-0.82187734 \quad 2.77168004$

O $-0.50869194-2.03817018-2.09456245$

O $1.152418313 .08539152-1.68894995$

o - $1.49396318-1.35228496-1.64809111$

Vibrational frequencies (in cm-1)

$\begin{array}{llllllllllllll}-1191.3 & 20.9 & 34.1 & 69.6 & 109.8 & 172.8 & 187.8 & 200.4 & 220.5 & 239.9 & 248.5 & 273.5 & 294.8 & 300.2\end{array}$ $319.8 \quad 347.1 \quad 396.3 \quad 414.7 \quad 445.6 \quad 469.8 \quad 548.9 \quad 599.0 \quad 658.4 \quad 675.1 \quad 710.0 \quad 774.5 \quad 777.2 \quad 821.2$ $867.9932 .2937 .2 \quad 991.8 \quad 1003.1 \quad 1012.2 \quad 1017.8 \quad 1048.7 \quad 1053.6 \quad 1074.2 \quad 1109.01128 .01135 .4$

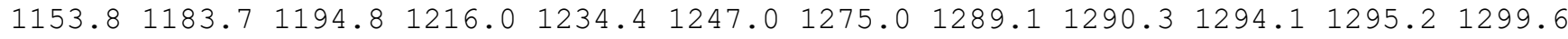
$1306.71314 .51320 .0 \quad 1328.2 \quad 1340.0 \quad 1363.0 \quad 1382.6 \quad 1406.51408 .7 \quad 1541.51570 .3 \quad 1831.4$ $1856.02638 .92651 .0 \quad 2658.1 \quad 2660.9 \quad 2670.4 \quad 2672.2 \quad 2680.72699 .82714 .62738 .42743 .8$ $2759.22772 .92782 .4 \quad 3664.6$

name $=$ TS 126

energy (in $\mathrm{kcal} / \mathrm{mol})=31.625338$

$\mathrm{ZPE}($ in $\mathrm{kcal} / \mathrm{mol})=143.391$

$\mathrm{G}($ in $\mathrm{kcal} / \mathrm{mol})=119.168$ 


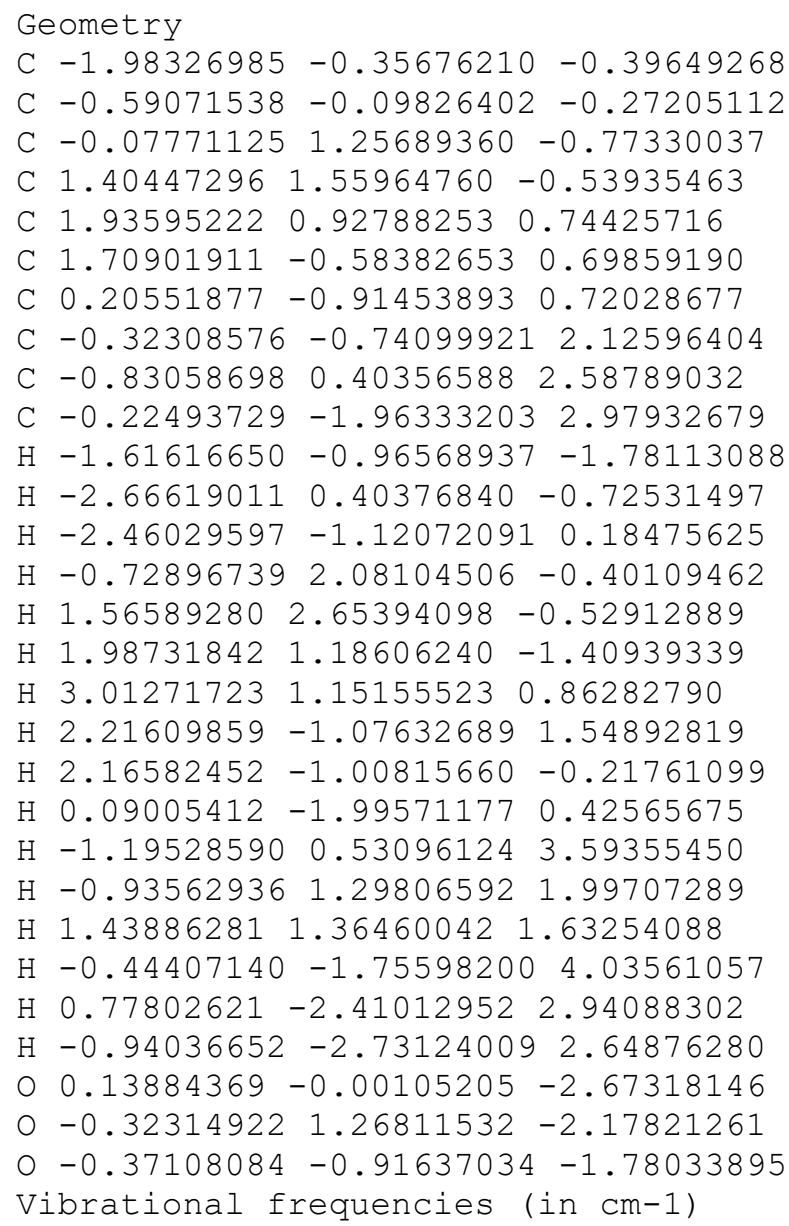

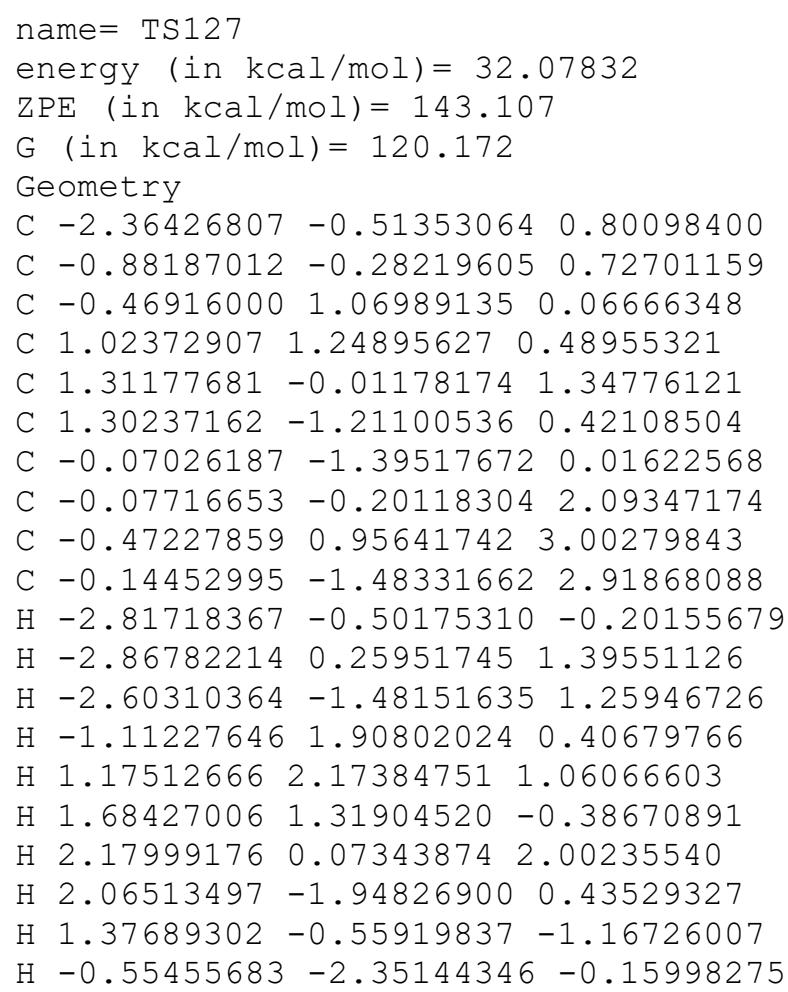


$\mathrm{H} \quad 0.21849775 \quad 1.03589632 \quad 3.85150545$

$\mathrm{H}-1.47966551 \quad 0.80735532 \quad 3.41055980$

$\mathrm{H}-0.470266251 .92891272 \quad 2.50281651$

H $0.51155156-1.41915810 \quad 3.79474003$

$\mathrm{H} \quad 0.17105754 \quad-2.36863854 \quad 2.35535129$

$\mathrm{H}-1.16284303-1.67024654 \quad 3.27720567$

$0-0.65258366-0.05112510-2.01165885$

O $-0.581212581 .18760573-1.33589085$

o $0.28113149-0.91114473-1.40352766$

Vibrational frequencies (in cm-1)

$\begin{array}{llllllllllllllll}-1155.8 & 99.8 & 117.1 & 152.1 & 161.8 & 191.7 & 202.6 & 213.8 & 261.8 & 316.3 & 341.7 & 373.2 & 408.3 & 430.0\end{array}$

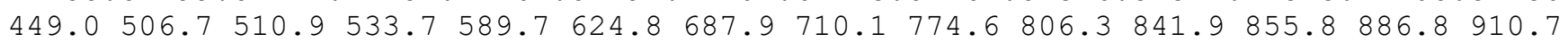
$935.3953 .4 \quad 963.9 \quad 973.2 \quad 998.7 \quad 1007.4 \quad 1016.4 \quad 1017.0 \quad 1053.0 \quad 1059.2 \quad 1079.01109 .2 \quad 1123.5$ $1130.51144 .3 \quad 1166.5 \quad 1176.7 \quad 1207.8 \quad 1223.0 \quad 1229.9 \quad 1252.51263 .1 \quad 1273.6 \quad 1288.0 \quad 1289.6$

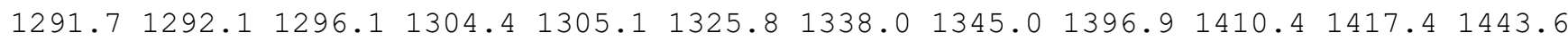
$2245.02670 .52671 .6 \quad 2673.0 \quad 2675.3 \quad 2675.8 \quad 2677.2 \quad 2681.22687 .82740 .02752 .52754 .1$ 2780.62781 .32783 .02796 .0

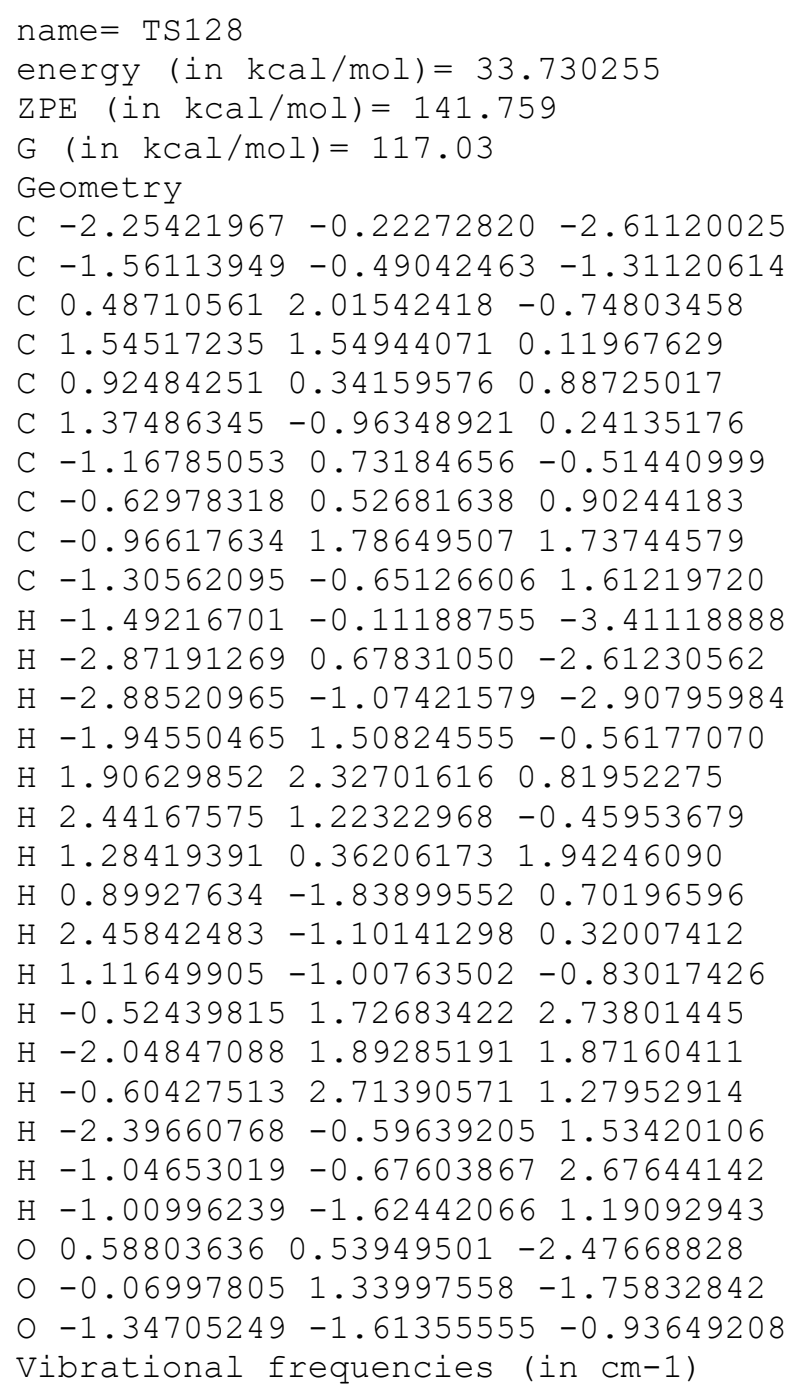


$\mathrm{ZPE}($ in $\mathrm{kcal} / \mathrm{mol})=138.979$

$\mathrm{G}($ in $\mathrm{kcal} / \mathrm{mol})=111.637$

Geometry

C $-1.27677549-2.27722556-1.96261902$

C $-0.34668478-1.29845522-1.31640157$

C $-1.34120790 \quad 1.83334684 \quad-0.00522451$

C $0.05445062 \quad 1.33499826 \quad 0.23946939$

C $0.00309012-0.018196820 .89686141$

C $1.68503939-0.85832528 \quad 0.72926086$

C $-0.32524664-1.18538695 \quad 0.07079175$

C $-0.23242757-0.05616752 \quad 2.26906129$

C -0.220343421 .178082293 .09080848$

C $-0.50773645-1.314048913 .00600552$

$\mathrm{H}-2.31796613-2.09655069-1.65392522$

$\mathrm{H}-1.02394241-3.30981395-1.67935449$

$\mathrm{H}-1.25066674-2.22819391-3.06217135$

$\mathrm{H}-2.031446231 .16014066-0.52749659$

$\mathrm{H} \quad 0.65429752 \quad 2.067343810 .81182503$

$\mathrm{H} \quad 0.59595975 \quad 1.28364307-0.76178563$

$\mathrm{H} 2.19452190-0.08940167 \quad 1.30438221$

H $1.67509689-1.833423261 .19188210$

H $2.00700525-0.84134054-0.32275906$

$\mathrm{H}-0.80967014 \quad-1.99980213 \quad 0.60942187$

$\mathrm{H}-0.87752372 \quad 1.963144692 .66796364$

$\mathrm{H} 0.78836486 \quad 1.61942008 \quad 3.14811210$

$\mathrm{H}-0.55857974 \quad 1.01690867 \quad 4.12311014$

$\mathrm{H}-0.22007402-1.258224314 .06629342$

$\mathrm{H}-0.00316616-2.19592254 \quad 2.58833847$

$\mathrm{H}-1.59192617-1.533109012 .98446332$

o $1.325007620 .09112656-1.88099640$

$0-1.694183092 .929459960 .35591847$

O $0.36526628-0.71039743-2.25762172$

Vibrational frequencies (in $\mathrm{cm}-1$ )

$\begin{array}{llllllllllllllll}-740.9 & 43.9 & 53.1 & 71.0 & 81.5 & 97.9 & 101.4 & 123.2 & 128.2 & 143.3 & 157.0 & 200.6 & 227.0 & 253.0\end{array}$ $273.3 \quad 318.6 \quad 335.0 \quad 362.0 \quad 392.7 \quad 448.3 \quad 464.6 \quad 507.4 \quad 523.3 \quad 554.7 \quad 557.1 \quad 667.6 \quad 805.0 \quad 831.8$ $899.0926 .1955 .1965 .8 \quad 992.6 \quad 1009.5 \quad 1024.6 \quad 1032.8 \quad 1050.0 \quad 1052.4 \quad 1078.1 \quad 1096.1 \quad 1118.1$

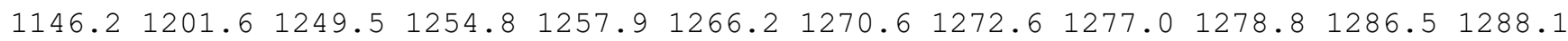

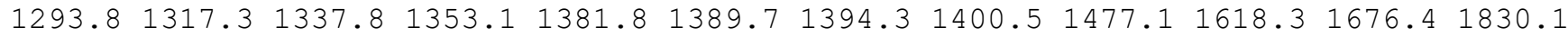

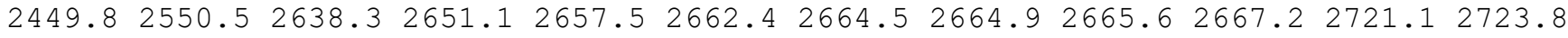
$2745.92767 .4 \quad 2769.92774 .7$

\section{name $=\operatorname{TS} 130$}

energy (in $\mathrm{kcal} / \mathrm{mol})=35.195735$

$\mathrm{ZPE}(\mathrm{in} \mathrm{kcal} / \mathrm{mol})=140.88$

$\mathrm{G}(\mathrm{in} \mathrm{kcal} / \mathrm{mol})=112.607$

Geometry

C $0.01938418-0.83359714 \quad 1.52360745$

C $1.39503503-0.67862447 \quad 0.90703867$

C $6.20572460 \quad 1.68278678 \quad 0.37285981$

$\begin{array}{llll}\text { C } 5.13699949 & 2.38058118 & -0.36024512\end{array}$

C $3.81433894 \quad 2.22281116 \quad 0.41454905$

C $3.22131662 \quad 0.76654380 \quad 0.14660234$

C $1.87960020 \quad 0.64339654 \quad 0.69292395$

C $2.742433313 .10707526-0.01850585$

C $2.44663518 \quad 3.29674517-1.44316490$

C 1.992239693 .865113570 .98858522

$\mathrm{H}-0.02236032-0.35505516 \quad 2.50642791$

$\mathrm{H}-0.22649826-1.89578520 \quad 1.65200278$

$\mathrm{H}-0.74677309-0.38371624 \quad 0.88633080$

H $6.63834528 \quad 0.74782850 \quad 0.04823860$

$\mathrm{H} \quad 5.03915610 \quad 2.01643017-1.40266745$

H $5.39264318 \quad 3.46833573-0.42630473$

H 4.018727322 .308946361 .51544060

H $3.89895677-0.01707421 \quad 0.54962521$ 
H $3.23365889 \quad 0.64051840 \quad-0.95989918$

H $3.31843852 \quad 3.64992167-2.01979943$

$\mathrm{H} 1.619502673 .99360312-1.64444388$

H $2.159945762 .32428233-1.90934459$

H 2.62580194 4.65508521 1.44188779

H $1.68768985 \quad 3.22578999 \quad 1.84830162$

$\mathrm{H} 1.08242144 \quad 4.35294374 \quad 0.61063560$

O $6.245955893 .20506160 \quad 1.94653732$

$\begin{array}{lllll}0 & 6.70138093 & 2.12695817 & 1.48572882\end{array}$

○ $2.02739814-1.68224295 \quad 0.60666607$

H 1.263559631 .467501160 .95363345

Vibrational frequencies (in $\mathrm{cm}-1$ )

$\begin{array}{llllllllllllllll}-140.1 & 28.3 & 40.6 & 58.5 & 74.8 & 82.8 & 92.1 & 111.0 & 125.0 & 158.7 & 176.6 & 204.2 & 241.3 & 256.5\end{array}$ $288.5 \quad 315.1 \quad 382.7 \quad 441.5 \quad 465.4 \quad 513.0 \quad 530.5 \quad 551.8 \quad 598.5 \quad 708.4 \quad 719.1 \quad 781.1 \quad 828.2 \quad 897.9$ $917.0 \quad 957.8 \quad 980.5 \quad 1008.4 \quad 1017.3 \quad 1029.5 \quad 1039.4 \quad 1055.8 \quad 1060.0 \quad 1085.7 \quad 1108.5 \quad 1128.6 \quad 1170.0$

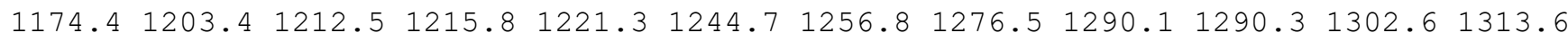

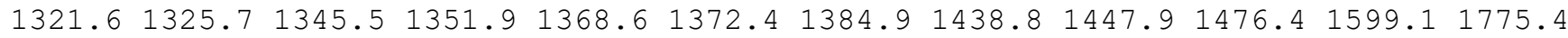
$2608.52610 .2 \quad 2624.52644 .8 \quad 2651.3 \quad 2659.5 \quad 2676.3 \quad 2683.92689 .02709 .5 \quad 2716.42728 .7$ $2753.0 \quad 2758.0 \quad 2785.2 \quad 2807.0$

name $=$ TS131

energy (in $\mathrm{kcal} / \mathrm{mol})=34.438081$

$\mathrm{ZPE}(\mathrm{in} \mathrm{kcal} / \mathrm{mol})=142.125$

$\mathrm{G}($ in $\mathrm{kcal} / \mathrm{mol})=116.392$

Geometry

C $-2.50505797-2.01126559-1.08166632$

C $-1.16469159-1.64730807-0.54504536$

C $0.68817240 \quad 1.51456728-0.77824706$

C 1.361411051 .163339890 .47647168

C $1.11037804-0.244846851 .01166592$

C $1.24521571-1.32677140 \quad-0.01589624$

C $-0.77061555-1.74283081 \quad 0.80154350$

C $-0.31100926-0.54536910 \quad 1.58702137$

C -1.309078730 .611929731 .46081445$

C $-0.18120444-0.907910543 .07762909$

$\mathrm{H}-2.69772338-3.08667787-0.94942631$

$\mathrm{H}-2.59346397-1.78967418-2.15585288$

$\mathrm{H}-3.30533189-1.47206279-0.55441301$

$\mathrm{H} \quad 0.11080516 \quad 0.81930495-1.37900992$

H $1.09084657 \quad 1.92328154 \quad 1.25864887$

H $2.46171368 \quad 1.33747353 \quad 0.34856488$

H $1.87442577-0.41911751 \quad 1.81835048$

$\mathrm{H} \quad 1.40656150 \quad-2.349583190 .30396332$

H $1.68789394 \quad-1.07681844 \quad-0.97971524$

$\mathrm{H}-1.08021364-2.59636101 \quad 1.37546047$

H $-2.29285590 \quad 0.30669226 \quad 1.83906364$

$\mathrm{H}-1.45109514 \quad 0.92076997 \quad 0.42125763$

$\mathrm{H}-0.99385260 \quad 1.48852619 \quad 2.03580851$

$\mathrm{H} \quad 0.21017176-0.06867340 \quad 3.66100379$

$\mathrm{H} \quad 0.47732588-1.76891235 \quad 3.22925867$

$\mathrm{H}-1.15796542-1.174629093 .49831227$

O $1.40484645 \quad 3.57924937-0.64028031$

$\begin{array}{lllll}0 & 0.74342668 & 2.70837059 & -1.27908602\end{array}$

O $-0.26451682-1.28771801-1.34176294$

Vibrational frequencies (in $\mathrm{cm}-1$ )

$\begin{array}{llllllllllllllllll}-844.7 & 35.1 & 47.5 & 90.4 & 105.4 & 112.5 & 154.5 & 176.6 & 220.9 & 224.2 & 246.2 & 248.6 & 304.8 & 318.6\end{array}$ $333.2412 .4 \quad 435.4 \quad 441.9 \quad 453.0 \quad 543.2 \quad 561.5 \quad 613.5 \quad 663.7 \quad 730.2 \quad 769.8 \quad 799.4 \quad 837.1 \quad 897.6$ $942.8955 .6 \quad 966.8 \quad 979.2 \quad 994.5 \quad 1022.6 \quad 1037.0 \quad 1047.0 \quad 1057.9 \quad 1088.2 \quad 1088.6 \quad 1132.8 \quad 1157.1$

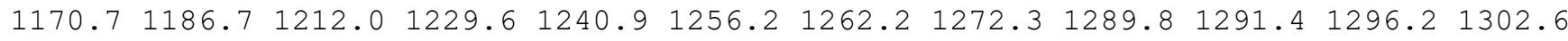
$1308.21314 .7 \quad 1317.6 \quad 1321.8 \quad 1356.4 \quad 1374.2 \quad 1381.7 \quad 1400.3 \quad 1406.1 \quad 1548.01589 .6 \quad 1612.9$ $2615.52653 .12667 .8 \quad 2673.3 \quad 2673.7 \quad 2675.12676 .82678 .72679 .72682 .72711 .62716 .5$ $2777.12780 .8 \quad 2781.8 \quad 2784.2$ 


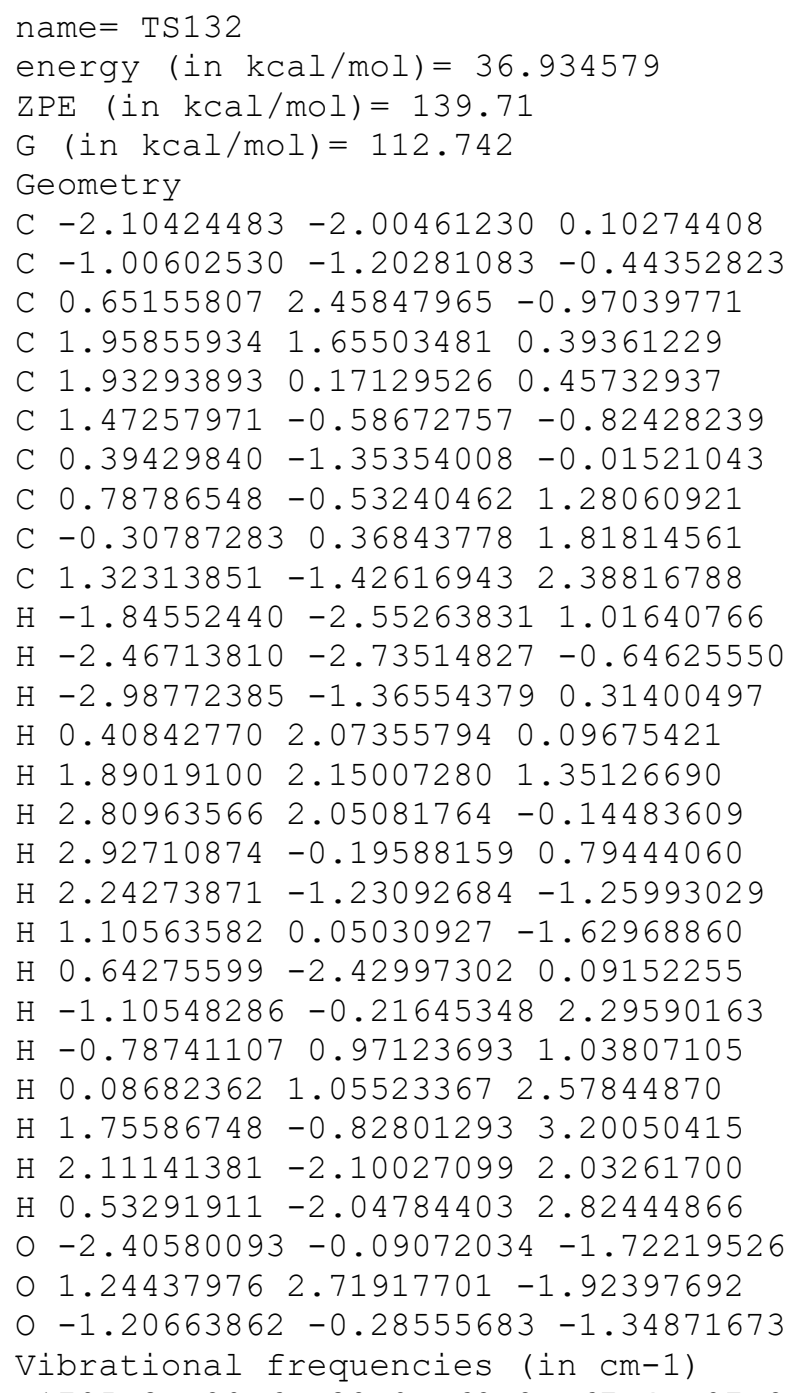


H $2.10796857 \quad 0.038877361 .58308336$

$\mathrm{H} 1.58995101-1.74079344 \quad 0.05200783$

$\mathrm{H} 0.87372236-0.62968623-1.11179526$

$\mathrm{H}-0.54212083-2.02844060 \quad 1.16521465$

$\mathrm{H}-1.42679673 \quad 1.832563390 .22454240$

$\mathrm{H}-0.95046662 \quad 2.36418781 \quad 0.58728812$

$\mathrm{H}-2.01174740 \quad 1.14660464 \quad 2.03951701$

$\mathrm{H} \quad 0.47161186 \quad 0.39444115 \quad 3.72046435$

$\mathrm{H} \quad 0.78194522-1.234682913 .09754616$

$\mathrm{H}-0.86855219-0.74107506 \quad 3.49929795$

○ $2.10530184 \quad 3.79705770-1.22916552$

O $1.25610805 \quad 2.99588296-1.71397346$

$\begin{array}{llll}0 & -1.62656647 & 0.08376755 & -1.06792527\end{array}$

Vibrational frequencies (in $\mathrm{cm}-1$ )

$\begin{array}{lllllllllllllllll}-801.1 & 36.8 & 46.1 & 66.7 & 117.1 & 131.3 & 148.5 & 165.7 & 186.4 & 200.9 & 221.2 & 238.1 & 280.2 & 309.8\end{array}$ $330.0 \quad 363.7 \quad 384.6 \quad 439.5 \quad 446.7 \quad 481.0 \quad 551.3 \quad 585.5 \quad 595.7 \quad 621.6 \quad 686.8 \quad 719.5 \quad 769.4 \quad 799.6$ $824.0 \quad 848.0 \quad 895.1 \quad 900.2 \quad 930.9 \quad 991.1 \quad 1019.7 \quad 1033.6 \quad 1040.1 \quad 1044.0 \quad 1054.2 \quad 1077.4 \quad 1086.2$ $1099.4 \quad 1117.7 \quad 1134.9 \quad 1166.2 \quad 1178.5 \quad 1187.9 \quad 1203.0 \quad 1214.4 \quad 1241.3 \quad 1249.01265 .7 \quad 1270.8$

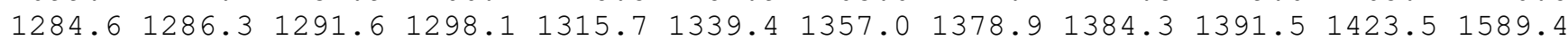
$1837.62606 .62665 .22668 .72670 .2 \quad 2676.62677 .22679 .62707 .2 \quad 2709.82714 .82731 .4$ $2761.32777 .32778 .6 \quad 4896.4$

name $=$ TS134

energy (in $\mathrm{kcal} / \mathrm{mol})=37.798258$

$\mathrm{ZPE}(\mathrm{in} \mathrm{kcal} / \mathrm{mol})=139.416$

$\mathrm{G}($ in $\mathrm{kcal} / \mathrm{mol})=113.057$

Geometry

C $-2.18827127 \quad 0.23604291-0.74686021$

C $-1.03471192-0.46369827-0.72818428$

C $2.62518623 \quad 0.32653205 \quad 0.70750564$

C $2.41353345-0.70040510 \quad 1.79485805$

C $1.10816359-1.46715790 \quad 1.71431385$

C $0.72982882-2.083695350 .34358348$

C $-0.64770078-1.371975710 .36600030$

C $-0.24414998-0.66288486 \quad 1.72725422$

C $-0.13545435 \quad 0.84488371 \quad 1.65684288$

C $-1.14688255-1.072473872 .88397467$

$\mathrm{H}-3.45599796-0.81677838-0.10754020$

$\mathrm{H}-2.81743270 \quad 0.81899806-1.33731791$

$\mathrm{H}-3.25095709-0.33907279 \quad 0.49369074$

H $2.296966120 .08547973-0.31412664$

$\mathrm{H} \quad 2.49553580 \quad-0.20741075 \quad 2.78826921$

H $3.26640459-1.414466131 .75423626$

$\mathrm{H} 1.11036298-2.236136532 .51473988$

H $0.66383948-3.174624820 .33724100$

H $1.39262286-1.80650766-0.48735518$

$\mathrm{H}-1.45732281-2.11586832 \quad 0.52753994$

$\mathrm{H}-1.122513791 .32380301 \quad 1.66202624$

H $0.36551355 \quad 1.19060032 \quad 0.74144965$

H $0.43311930 \quad 1.24625212 \quad 2.50573351$

$\mathrm{H}-1.26319145-2.15924384 \quad 2.95753455$

$\mathrm{H}-2.14640257-0.635679292 .79230518$

$\mathrm{H}-0.73149996-0.727009723 .84031502$

$0.89881570-0.52032216-1.92054437$

$03.166069481 .37588400 \quad 0.96079333$

O $-0.29866362-0.18922302-1.83949548$

Vibrational frequencies (in $\mathrm{cm}-1$ )

$\begin{array}{llllllllllllllll}-882.9 & 14.4 & 60.1 & 76.7 & 121.0 & 158.5 & 178.4 & 182.8 & 187.0 & 199.8 & 226.6 & 232.0 & 289.0 & 293.9\end{array}$ $327.4 \quad 331.3 \quad 378.2 \quad 387.6 \quad 415.8 \quad 431.3 \quad 440.8 \quad 446.6 \quad 570.0 \quad 575.0 \quad 596.6 \quad 646.3 \quad 706.5 \quad 769.6$ $811.4 \quad 826.9 \quad 859.9 \quad 910.9 \quad 934.5 \quad 993.2 \quad 1001.7 \quad 1010.2 \quad 1018.0 \quad 1047.6 \quad 1062.8 \quad 1068.3 \quad 1098.1$

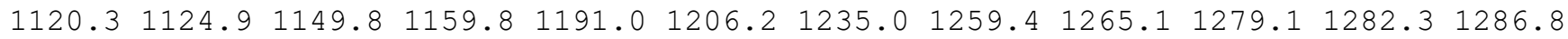
$1289.7 \quad 1298.1 \quad 1300.4 \quad 1313.7 \quad 1319.3 \quad 1330.6 \quad 1343.5 \quad 1352.6 \quad 1385.7 \quad 1405.0 \quad 1409.6 \quad 1629.2$ $\begin{array}{llllllllllll}1826.3 & 2626.3 & 2655.1 & 2658.4 & 2662.8 & 2673.6 & 2677.1 & 2680.8 & 2705.5 & 2712.6 & 2738.5 & 2755.1\end{array}$ $2772.3 \quad 2779.5 \quad 2782.5 \quad 4992.7$ 
name $=$ TS135

energy (in $\mathrm{kcal} / \mathrm{mol})=33.606348$

$\mathrm{ZPE}($ in $\mathrm{kcal} / \mathrm{mol})=143.779$

$\mathrm{G}($ in $\mathrm{kcal} / \mathrm{mol})=119.963$

Geometry

C $-2.25392336 \quad 0.02846573-0.59724008$

C $-0.96183057 \quad 0.19436950-0.02215929$

C -0.514783691 .606520720 .38541789$

C $0.67033451 \quad 1.60385587 \quad 1.34567455$

$\begin{array}{lllll}\text { C } & 1.76573931 & 0.63774884 & 0.88906712\end{array}$

C $1.25908177 \quad-0.78742988 \quad 0.60407832$

C $-0.27032683-0.96055765 \quad 0.67710785$

C $-0.80965395-1.13795310 \quad 2.07834566$

C $-2.26126045-1.488705032 .15545458$

C $-0.07367309-1.03928013 \quad 3.18769715$

$\mathrm{H}-2.94389366 \quad 0.84486948-0.67143538$

$\mathrm{H}-2.72577599-0.93013826-0.64940473$

$\mathrm{H}-1.46514676 \quad 0.26053222-1.97845225$

$\mathrm{H}-1.364610652 .20464486 \quad 0.78836176$

$\begin{array}{llllll}\mathrm{H} & 0.32253812 & 1.33707561 & 2.36378531\end{array}$

H $1.08480376 \quad 2.628862081 .42288089$

H $2.23977619 \quad 1.04406054-0.02843369$

$\mathrm{H} 1.74225832-1.50848575 \quad 1.28983031$

$\mathrm{H} 1.59094268-1.08334967-0.41322908$

$\mathrm{H}-0.51612950-1.90760808 \quad 0.11682604$

$\mathrm{H}-2.50825583-2.346342241 .51532882$

$\mathrm{H}-2.89021639-0.64719933 \quad 1.82364924$

$\mathrm{H}-2.57924645-1.74444346 \quad 3.17447319$

$\mathrm{H} \quad 2.56783398 \quad 0.60496134 \quad 1.65009421$

$\mathrm{H} \quad 0.97539108-0.79498788 \quad 3.20526326$

$\mathrm{H}-0.47005316-1.198205394 .17726316$

$0.43794341 \quad 1.36766587-1.69373885$

$0-0.19495297 \quad 2.30479965-0.81400545$

O $-0.326127410 .21373866-1.54580990$

Vibrational frequencies (in $\mathrm{cm}-1$ )

$\begin{array}{llllllllllllllll}-1397.6 & 51.8 & 69.5 & 118.1 & 149.3 & 179.3 & 190.9 & 230.6 & 285.0 & 300.3 & 334.0 & 346.9 & 377.8 & 416.0\end{array}$ $442.5 \quad 491.6 \quad 513.8 \quad 538.2 \quad 586.6 \quad 607.7 \quad 613.7 \quad 653.9 \quad 723.0 \quad 751.7 \quad 811.1 \quad 848.8 \quad 890.7 \quad 903.1$ $928.0 \quad 956.4983 .0 \quad 996.6 \quad 1004.91035 .2 \quad 1041.5 \quad 1051.1 \quad 1060.0 \quad 1070.6 \quad 1084.21097 .1 \quad 1115.8$

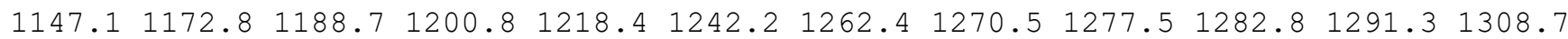
$1316.61325 .6 \quad 1328.0 \quad 1336.0 \quad 1340.2 \quad 1364.1 \quad 1379.2 \quad 1396.01403 .6 \quad 1408.6 \quad 1533.7 \quad 1862.5$ $2139.72644 .82661 .3 \quad 2663.7 \quad 2674.6 \quad 2676.7 \quad 2681.62682 .92720 .42735 .82736 .72740 .4$ $2741.7 \quad 2778.2 \quad 2791.2 \quad 2797.7$

name $=\operatorname{TS} 136$

energy (in $\mathrm{kcal} / \mathrm{mol})=37.45237$

$\mathrm{ZPE}(\mathrm{in} \mathrm{kcal} / \mathrm{mol})=139.979$

$\mathrm{G}($ in $\mathrm{kcal} / \mathrm{mol})=113.857$

Geometry

C $-0.46420299-0.58710581-1.29493814$

C $0.14066896 \quad 0.40273404 \quad-0.34381660$

C $-0.14649947 \quad 3.08884420 \quad 2.22950493$

C $0.26496315 \quad 2.17374232 \quad 3.29871407$

C $0.82054442 \quad 0.82865723 \quad 2.86799594$

C $1.74062070 \quad 0.81493909 \quad 1.61858750$

C $0.78973107-0.15828399 \quad 0.88424735$

C $-0.15870658-0.18533481 \quad 2.16239994$

C $-1.58524342 \quad 0.300350891 .92389232$

C $-0.30784135-1.45680597 \quad 2.84789954$

$\mathrm{H}-1.05111767-1.35418131-0.77572382$

$\mathrm{H} \quad 0.31584070-1.09855636-1.87643317$

$\mathrm{H}-1.12783415-0.08965395-2.02026682$

$\mathrm{H} 0.04553256 \quad 2.92121716 \quad 1.17323982$ 
$\mathrm{H}-0.60141350 \quad 2.01793836 \quad 3.99928562$

$\mathrm{H} 1.00855872 \quad 2.70329090 \quad 3.95033247$

H $1.303145490 .35969333 \quad 3.74882868$

H $2.74282657 \quad 0.418269361 .79840037$

H $1.86403736 \quad 1.78514794 \quad 1.12178077$

$\mathrm{H} 1.24274605-1.13839761 \quad 0.67518930$

$\mathrm{H}-1.61246452 \quad 1.25814228 \quad 1.39063354$

$\mathrm{H}-2.10980593 \quad 0.44331272 \quad 2.88214302$

$\mathrm{H}-2.17128746-0.41815864 \quad 1.33894686$

$\mathrm{H} \quad 0.98431699-1.87271958 \quad 3.66596355$

$\mathrm{H} 1.25982816-2.069387522 .93025858$

$\mathrm{H}-0.68216691-2.327886262 .37309818$

$0-1.04484860 \quad 4.46788944 \quad 3.67586040$

$0-0.77972513 \quad 4.193542782 .46716014$

$\begin{array}{lllll}0 & 0.10096351 & 1.58880894 & -0.56939231\end{array}$

Vibrational frequencies (in cm-1)

$\begin{array}{lllllllllllllllll}-968.5 & 33.0 & 55.9 & 71.2 & 109.9 & 117.9 & 149.0 & 168.3 & 195.2 & 200.1 & 223.8 & 226.7 & 269.5 & 306.9\end{array}$

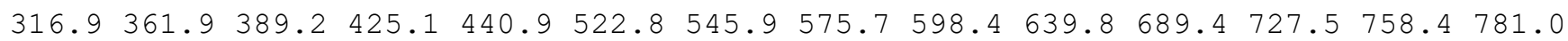
$815.2841 .2902 .0 \quad 934.6 \quad 985.4 \quad 1014.1 \quad 1028.1 \quad 1033.1 \quad 1043.2 \quad 1046.5 \quad 1052.5 \quad 1077.5 \quad 1095.4$ $1109.51120 .7 \quad 1150.7 \quad 1161.2 \quad 1179.2 \quad 1194.8 \quad 1205.5 \quad 1228.01243 .2 \quad 1257.11261 .91271 .1$

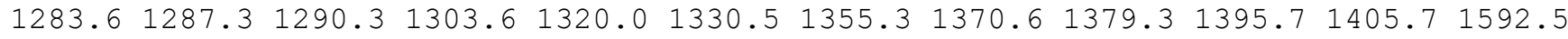
$1845.92616 .12664 .92666 .2 \quad 2670.6 \quad 2672.6 \quad 2674.72677 .92710 .2 \quad 2717.92724 .82733 .7$ $2761.3 \quad 2776.7 \quad 2779.5 \quad 4756.8$

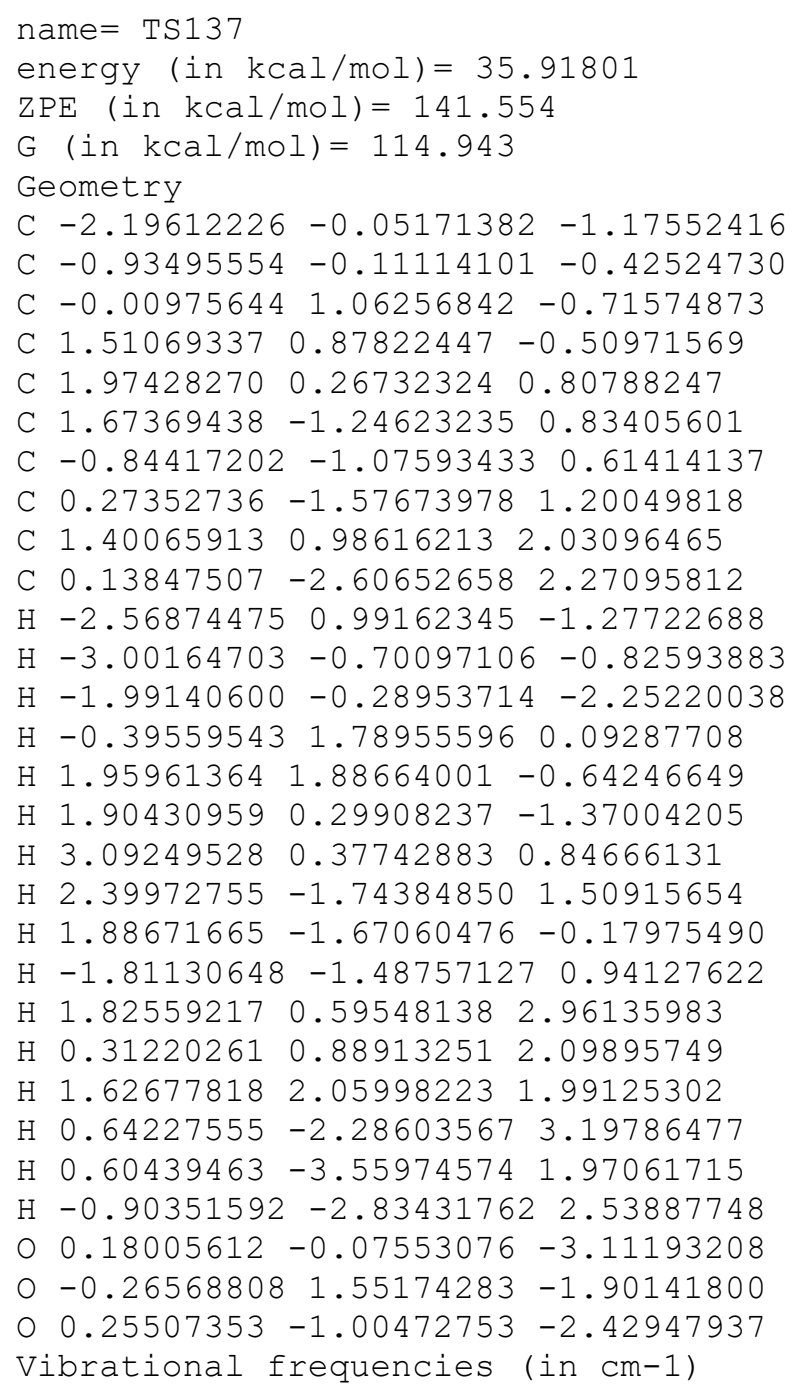
$320.2355 .3 \quad 375.4 \quad 401.6 \quad 422.2 \quad 476.3 \quad 511.6 \quad 597.0 \quad 628.6 \quad 648.0 \quad 709.2 \quad 883.1 \quad 889.9 \quad 910.4$

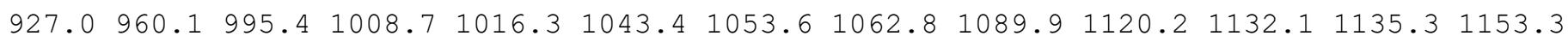
$1169.91223 .4 \quad 1236.1 \quad 1243.8 \quad 1250.4 \quad 1257.2 \quad 1271.3 \quad 1275.2 \quad 1284.2 \quad 1296.8 \quad 1300.3 \quad 1303.7$

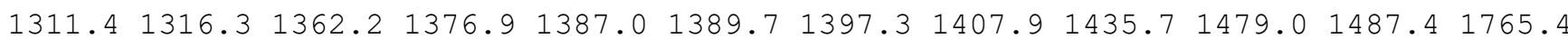


$2519.0 \quad 2586.12637 .9 \quad 2654.9 \quad 2656.7 \quad 2662.9 \quad 2668.3 \quad 2668.7 \quad 2677.7 \quad 2692.22727 .7 \quad 2728.5$ $2742.12757 .0 \quad 2773.0 \quad 2781.1$

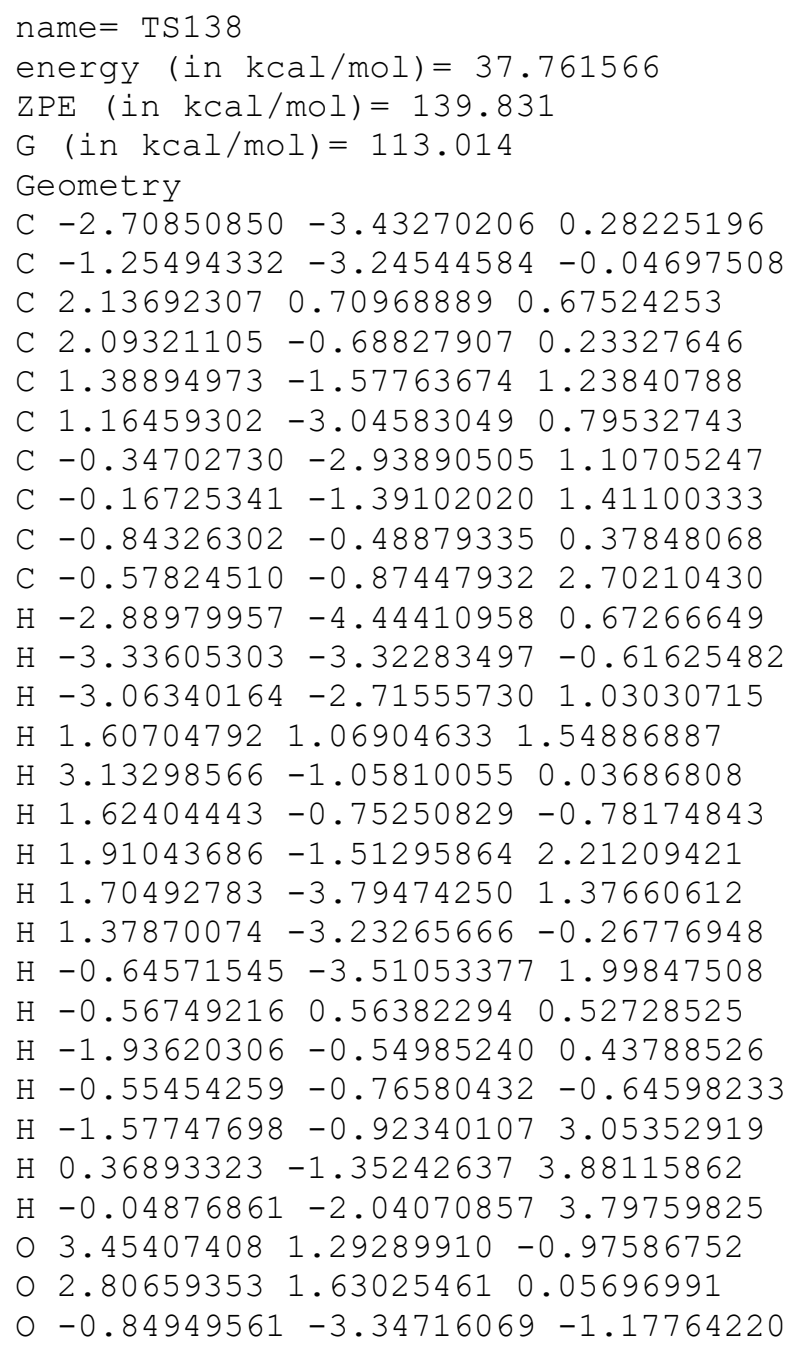


$\mathrm{H} \quad 0.81706254-0.74118101-2.01698090$

H $1.59885358 \quad 2.164101520 .39172170$

H 3.582724491 .843511351 .63597150

$\mathrm{H} \quad 4.35166191 \quad 1.781046150 .04675710$

H $5.05676241-0.253197021 .31678602$

$\mathrm{H} \quad 4.22891758-1.95304724-0.40482402$

H $3.68659819-0.50674453-1.26813330$

H $1.74941783-2.162670690 .20482877$

$\begin{array}{lllll}\mathrm{H} & 1.64052397 & 0.68371004 & 2.41322749\end{array}$

$\mathrm{H} 2.77079041 \quad 0.09853020 \quad 3.63333793$

H $1.33426142-0.85096124 \quad 3.20901295$

H $3.32917041-2.31881106 \quad 3.43016771$

H $4.55680738-2.37353548 \quad 2.17381603$

H $2.99247918-3.143736891 .91406037$

o $1.360055871 .33438226-2.13285486$

o $2.531281481 .59391564-1.37526930$

o $0.809634610 .09003800-1.40221172$

Vibrational frequencies (in $\mathrm{cm}-1$ )

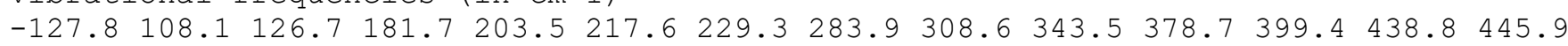

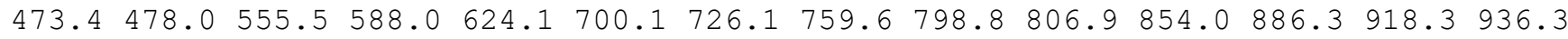
$947.7962 .6 \quad 987.8 \quad 997.0 \quad 1005.4 \quad 1020.7 \quad 1027.8 \quad 1042.6 \quad 1073.5 \quad 1083.2 \quad 1091.4 \quad 1100.01109 .4$

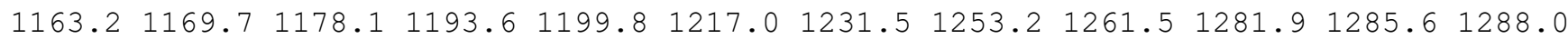

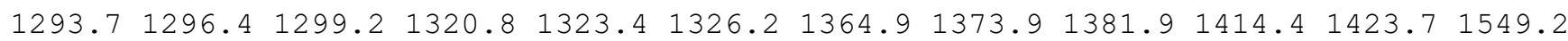
$2658.72670 .8 \quad 2674.1 \quad 2674.8 \quad 2678.8 \quad 2685.4 \quad 2687.02722 .12739 .02745 .92750 .02763 .5$ $2781.62783 .2 \quad 2786.92899 .5$

name $=$ TS 140

energy (in $\mathrm{kcal} / \mathrm{mol})=36.926433$

$\operatorname{ZPE}($ in $\mathrm{kcal} / \mathrm{mol})=141.429$

$\mathrm{G}(\mathrm{in} \mathrm{kcal} / \mathrm{mol})=115.58$

Geometry

C $-2.40409561-2.33503720-0.81036595$

C $-1.13790729-1.53593873-0.69897074$

C $0.53767378 \quad 1.76554300 \quad-0.41629197$

C $0.83104270 \quad 1.55952822 \quad 1.00565481$

C $0.60247903 \quad 0.16106003 \quad 1.54891126$

C $0.99776041-1.01949607 \quad 0.62575970$

C $-0.44669128-1.56345687 \quad 0.62554009$

C $-0.89083017-0.37934527 \quad 1.61105606$

C $-1.95273176 \quad 0.54839167 \quad 1.04237815$

C $-1.29536407 \quad-0.85443710 \quad 2.92594667$

$\mathrm{H}-3.03388501-2.22529467 \quad 0.08252659$

$\mathrm{H}-2.18097918-3.40335841-0.93105773$

$\mathrm{H}-2.99642625-2.02689213-1.68453550$

H $0.30292618 \quad 0.96481261 \quad-1.11373177$

$\mathrm{H} \quad 0.24087686 \quad 2.30157642 \quad 1.60948298$

$\mathrm{H} \quad 1.88924115 \quad 1.87655393 \quad 1.19695595$

$\mathrm{H} \quad 1.08354576 \quad 0.09597340 \quad 2.54176090$

H $1.73660844-1.700653651 .05374039$

$\mathrm{H} 1.36836232-0.72953238-0.36529939$

$\mathrm{H}-0.54982557-2.55093968 \quad 1.09701626$

$\mathrm{H}-2.93690772 \quad 0.064420391 .03039696$

$\mathrm{H}-1.73369986 \quad 0.86880897 \quad 0.01792073$

$\mathrm{H}-2.043429981 .45385722 \quad 1.65804265$

$\mathrm{H}-0.29985113-1.54585371 \quad 3.33719636$

$\mathrm{H}-2.24655873-0.58293836 \quad 3.32093119$

$\mathrm{H}-0.98943650-1.51595811 \quad 3.93827463$

$00.80264168 \quad 3.93248484-0.21833936$

$\begin{array}{lllll}0 & 0.53790141 & 2.93911924 & -0.96330643\end{array}$

o $-0.71294038-0.91118633-1.64317582$

Vibrational frequencies (in $\mathrm{cm}-1$ )

$\begin{array}{lllllllllllllllll}-1370.5 & 34.2 & 60.5 & 73.7 & 113.5 & 125.4 & 150.6 & 175.0 & 192.8 & 204.3 & 225.4 & 247.0 & 278.7 & 312.2\end{array}$ $\begin{array}{llllllllllllllll}339.7 & 369.2 & 409.0 & 432.7 & 445.9 & 547.4 & 569.1 & 597.6 & 666.0 & 674.3 & 696.1 & 736.2 & 767.6 & 812.5\end{array}$ $827.9897 .0 \quad 938.4 \quad 984.9 \quad 1022.2 \quad 1033.2 \quad 1043.1 \quad 1046.5 \quad 1053.7 \quad 1079.9 \quad 1098.81109 .3 \quad 1145.0$ 
$\begin{array}{lllllllllllllll}1152.1 & 1179.9 & 1181.2 & 1192.2 & 1206.0 & 1221.0 & 1247.1 & 1254.2 & 1264.1 & 1274.4 & 1282.8 & 1289.5\end{array}$

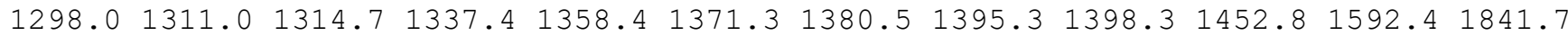
$1913.32619 .82668 .2 \quad 2671.6 \quad 2672.2 \quad 2673.7 \quad 2676.92679 .82715 .3 \quad 2723.5 \quad 2739.2 \quad 2762.8$ $2775.32780 .02782 .3 \quad 3744.3$

\section{name $=$ TS141}

energy (in $\mathrm{kcal} / \mathrm{mol})=34.471764$

$\mathrm{ZPE}($ in $\mathrm{kcal} / \mathrm{mol})=144.744$

$\mathrm{G}($ in $\mathrm{kcal} / \mathrm{mol})=121.915$

Geometry

C $-2.17583187-0.15488769-1.29814051$

C $-0.96305311-0.04808941-0.42181646$

C $-0.20997066 \quad 1.26458383-0.56843721$

C $0.912256431 .38166838 \quad 0.48157832$

C $1.35453937-0.02190991 \quad 0.93174598$

C $1.51104428-0.84840578-0.35099186$

C $-0.75329490-1.08206569 \quad 0.56058761$

C $0.20739899-0.77286950 \quad 1.68274154$

C $-0.46735698 \quad 0.07643053 \quad 2.76313489$

C $0.73917627-2.05765598 \quad 2.32939507$

$\mathrm{H}-1.97517005 \quad 0.23939022-2.31433115$

$\mathrm{H}-3.00989132 \quad 0.42001319-0.87380644$

$\mathrm{H}-2.51512789-1.19011858-1.43475825$

$\mathrm{H}-0.853373232 .16233473-0.59567179$

H $0.58524001 \quad 1.99116210 \quad 1.33972492$

$\mathrm{H} \quad 1.77018664 \quad 1.92375566 \quad 0.03523049$

H $2.27884929 \quad 0.02339011 \quad 1.53208798$

H $1.88861692-1.86747106-0.19371016$

H $2.12158770-0.34989568-1.12614478$

$\mathrm{H}-1.41494102-1.92276658 \quad 0.65808347$

$\mathrm{H}-1.20169184 \quad-0.51256992 \quad 3.32550956$

$\mathrm{H}-1.00812740 \quad 0.928931812 .33750498$

$\mathrm{H} \quad 0.26472306 \quad 0.46383091 \quad 3.47938935$

$\mathrm{H}-0.06548403-2.61112079 \quad 2.82776739$

$\mathrm{H} 1.50029680-1.83591166 \quad 3.08535820$

$\mathrm{H} \quad 1.18627395-2.73691237 \quad 1.59592299$

O $0.31604624-0.13841318-2.37107852$

$00.395705091 .18233575-1.87409968$

$0.15729792-1.01287780-0.88883025$

Vibrational frequencies (in $\mathrm{cm}-1$ )

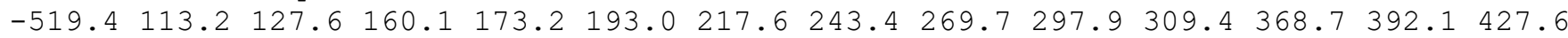
$435.4 \quad 455.5 \quad 500.1 \quad 524.3 \quad 574.7 \quad 623.8 \quad 673.4 \quad 710.8 \quad 769.9 \quad 879.5 \quad 917.7 \quad 920.4940 .9948 .7$ $959.1978 .8 \quad 991.5 \quad 1000.7 \quad 1006.3 \quad 1024.7 \quad 1036.8 \quad 1040.2 \quad 1056.4 \quad 1085.4 \quad 1101.5 \quad 1111.6 \quad 1147.6$ $\begin{array}{llllllllllll}1151.4 & 1180.3 & 1198.5 & 1219.5 & 1228.9 & 1244.9 & 1253.9 & 1266.0 & 1273.1 & 1276.7 & 1290.7 & 1296.1\end{array}$ $1300.91302 .51312 .1 \quad 1320.3 \quad 1324.8 \quad 1336.4 \quad 1340.8 \quad 1367.4 \quad 1395.6 \quad 1399.2 \quad 1410.8 \quad 1521.4$ $2637.92645 .5 \quad 2665.1 \quad 2672.2 \quad 2674.0 \quad 2676.0 \quad 2677.82681 .92701 .12713 .5 \quad 2729.5 \quad 2744.6$ $2770.6 \quad 2774.6 \quad 2781.1 \quad 2782.9$

name $=\operatorname{TS} 142$

energy (in $\mathrm{kcal} / \mathrm{mol})=38.604661$

$\mathrm{ZPE}(\mathrm{in} \mathrm{kcal} / \mathrm{mol})=140.969$

$\mathrm{G}($ in $\mathrm{kcal} / \mathrm{mol})=116.053$

Geometry

C $-2.54986374-0.78868662-0.40806485$

C $-2.44598392-1.41719340-1.63425090$

C $-0.06429745 \quad 1.12677212-0.85612976$

C $0.75892458 \quad 0.88036378 \quad-2.01877007$

C $0.49855879-0.43718786-2.75302864$

C $-0.89972545-0.56733937-3.39848390$

C $-1.24944406-1.75042250-2.44109597$

C $0.22898541-1.75521488-1.92104917$

C $0.51956935-1.74140504-0.43892566$

C $0.99819286-2.92125531-2.55055483$ 
$\mathrm{H}-2.26231701 \quad 0.92070621-1.20171376$

$\mathrm{H}-1.76279361-0.72391193 \quad 0.30464632$

$\mathrm{H}-3.51159970-0.580300190 .02145541$

$\mathrm{H}-1.71016904 \quad 1.44038693-1.45718497$

$\mathrm{H} 1.838103690 .90175855-1.70202928$

H $0.64397958 \quad 1.71543525 \quad-2.76126203$

$\mathrm{H} 1.31974093-0.58168000-3.48301721$

$\mathrm{H}-0.89176468-0.86260552-4.45515049$

$\mathrm{H}-1.56245421 \quad 0.29613595 \quad-3.32584793$

$\mathrm{H}-1.48628616-2.69256883-3.00649192$

$\mathrm{H}-0.18769498-1.144451050 .14735800$

$\mathrm{H} 1.53730451-1.40238695-0.22484563$

$\mathrm{H} 0.42668088-2.75752133-0.01922726$

$\mathrm{H} \quad 2.07143239-2.85453007-2.34855169$

$\mathrm{H} \quad 0.86384520-2.96753006-3.63835767$

$\mathrm{H} 0.64455322-3.88149933-2.15059261$

O $-3.55029740-2.07133302-3.45417935$

$\begin{array}{llll}0 & -0.15689668 & 1.29268153 & 0.28199778\end{array}$

o $-3.62344036-1.64232373-2.22830550$

Vibrational frequencies (in $\mathrm{cm}-1$ )

$\begin{array}{lllllllllllllllll}-647.7 & 38.0 & 72.2 & 88.7 & 154.8 & 182.0 & 193.8 & 200.0 & 221.5 & 243.0 & 264.0 & 270.3 & 300.8 & 334.1\end{array}$

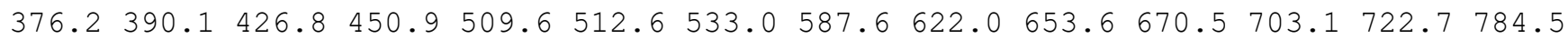
$824.4 \quad 891.7 \quad 924.9 \quad 940.3 \quad 951.5 \quad 968.5 \quad 979.6 \quad 989.4 \quad 1011.8 \quad 1018.4 \quad 1023.5 \quad 1035.0 \quad 1044.6$

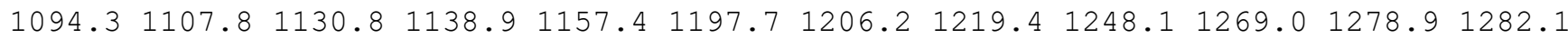

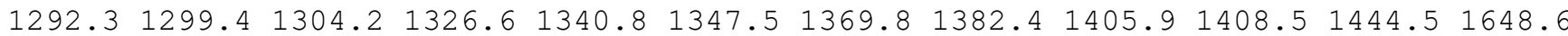
$2081.82614 .2 \quad 2638.8 \quad 2659.4 \quad 2668.8 \quad 2676.4 \quad 2679.72682 .72695 .2 \quad 2711.6 \quad 2731.72764 .7$ $2775.22780 .6 \quad 2799.0 \quad 4638.3$

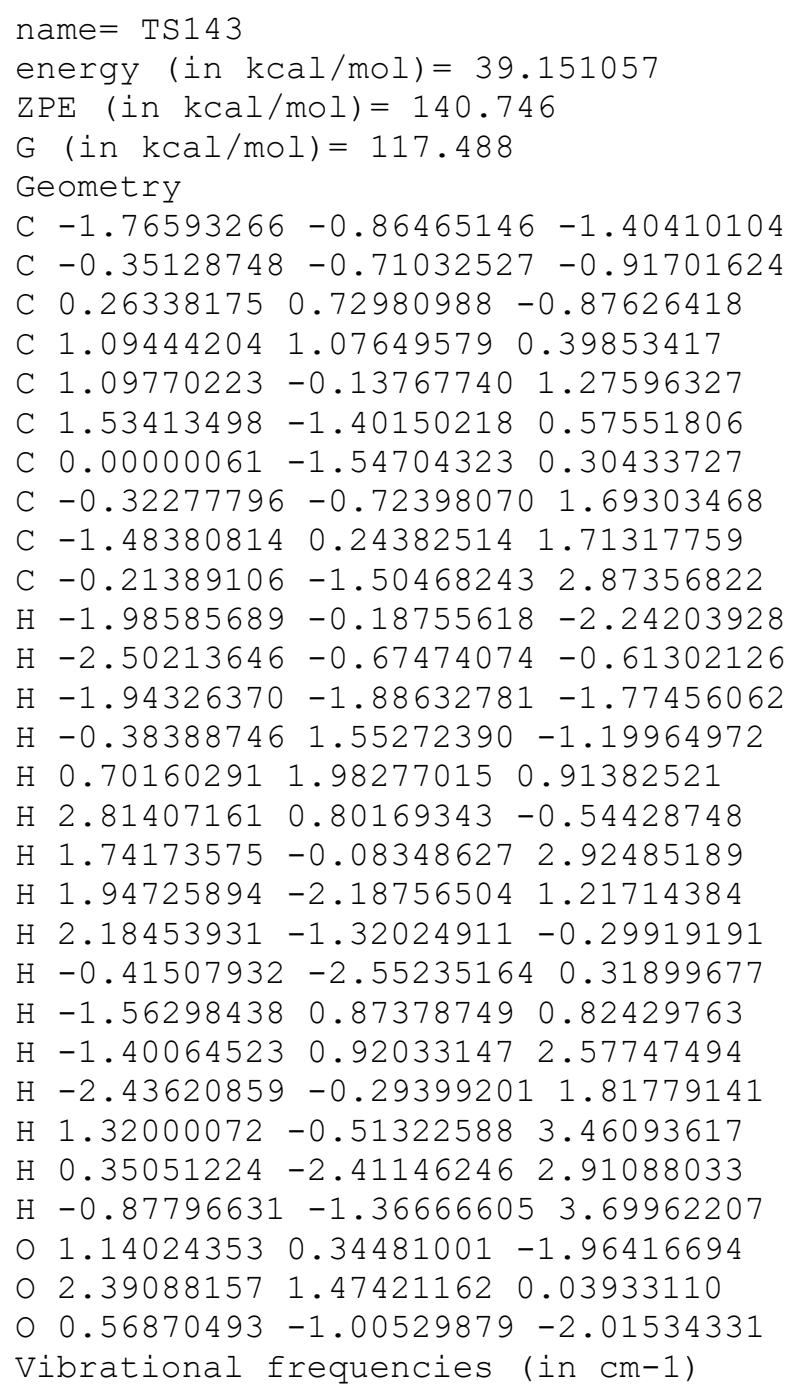


$\begin{array}{lllllllllllllllll}-802.8 & 105.6 & 118.8 & 149.1 & 180.4 & 201.5 & 219.4 & 242.7 & 261.8 & 294.4 & 313.4 & 331.6 & 341.9 & 344.5\end{array}$ $391.2 \quad 417.5 \quad 427.4 \quad 480.5 \quad 517.7 \quad 530.9 \quad 551.2 \quad 604.8 \quad 620.7 \quad 624.6 \quad 698.6 \quad 708.4 \quad 760.3 \quad 807.9$ $857.0 \quad 920.7 \quad 938.6 \quad 963.2 \quad 968.1 \quad 978.6 \quad 991.6 \quad 997.8 \quad 1013.6 \quad 1044.3 \quad 1048.8 \quad 1054.5 \quad 1061.2$

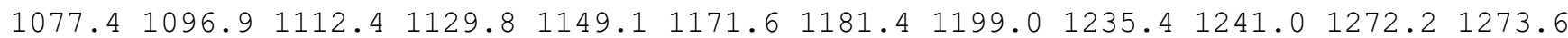

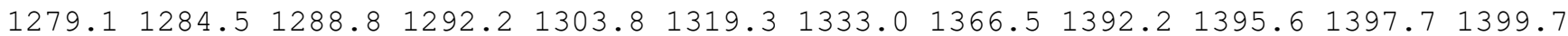
$1465.42666 .5 \quad 2671.12672 .4 \quad 2676.9 \quad 2682.6 \quad 2690.2 \quad 2705.92735 .02741 .3 \quad 2762.6 \quad 2779.2$ $2782.4 \quad 2788.6 \quad 2794.4 \quad 4561.7$

name $=\operatorname{TS} 144$

energy (in $\mathrm{kcal} / \mathrm{mol})=40.519966$

$\mathrm{ZPE}(\mathrm{in} \mathrm{kcal} / \mathrm{mol})=139.433$

$\mathrm{G}($ in $\mathrm{kcal} / \mathrm{mol})=111.94$

Geometry

C $-2.53421181-1.04129185-0.46756211$

C $-1.19520725-0.44776734-0.50654378$

C $3.05108724 \quad 2.109881321 .40287066$

C $1.59283461 \quad 1.73965951 \quad 1.52938490$

C $1.40673758 \quad 0.23540687 \quad 1.46780733$

C $1.32519134-0.35693420 \quad 0.03541253$

C $-0.08146126-0.938757610 .32367599$

C $-0.03468744-0.32448005 \quad 1.78684719$

C $-1.10660177 \quad 0.72296425 \quad 2.07147803$

C $-0.16699715-1.321032842 .83738885$

$\mathrm{H}-2.70655924-1.69134015-1.34499270$

$\mathrm{H}-3.31476165-0.25463853-0.53951156$

$\mathrm{H}-2.72345375-1.63493585 \quad 0.43860296$

H $3.28271842 \quad 3.08782297 \quad 0.96221615$

H $0.98674087 \quad 2.25141883 \quad 0.75573866$

$\mathrm{H} \quad 1.21716913 \quad 2.12971105 \quad 2.50177152$

H $2.18306857-0.25732208 \quad 2.08560908$

$\mathrm{H} 2.08694059-1.11272541-0.18234772$

H $1.37975265 \quad 0.38221943-0.76794236$

$\mathrm{H}-0.09444456-2.04784427 \quad 0.34710333$

$\mathrm{H}-2.09959800 \quad 0.25392773 \quad 2.15581105$

$\mathrm{H}-1.17213238 \quad 1.47327730 \quad 1.27442978$

$\mathrm{H}-0.91759588 \quad 1.25161948 \quad 3.01309294$

$\mathrm{H} \quad 0.63879135 \quad-2.64692194 \quad 2.51504008$

$\mathrm{H}-0.02194039-1.09159858 \quad 3.86325616$

H $1.27210240-2.201008532 .74980282$

$\begin{array}{llll}0 & -1.84048254 & 1.04775462 & -1.98301430\end{array}$

O $3.931134301 .37911809 \quad 1.78180890$

$\begin{array}{llll}0 & -0.90393551 & 0.56934354 & -1.26876022\end{array}$

Vibrational frequencies (in $\mathrm{cm}-1$ )

$\begin{array}{lllllllllllllll}-961.7 & 24.0 & 35.9 & 43.6 & 79.7 & 84.9 & 119.5 & 155.8 & 174.6 & 185.2 & 209.2 & 231.8 & 246.4 & 275.0\end{array}$ $292.8 \quad 302.2 \quad 374.7 \quad 413.7 \quad 444.3 \quad 455.1 \quad 506.3 \quad 540.5 \quad 605.6 \quad 630.4 \quad 669.2 \quad 685.5 \quad 766.7 \quad 803.0$ $851.3 \quad 867.9 \quad 921.3 \quad 937.6 \quad 973.0 \quad 1012.6 \quad 1021.1 \quad 1024.9 \quad 1043.2 \quad 1057.7 \quad 1064.8 \quad 1076.8 \quad 1096.7$ $1109.21120 .5 \quad 1130.9 \quad 1153.5 \quad 1164.2 \quad 1183.4 \quad 1203.1 \quad 1235.7 \quad 1260.8 \quad 1261.91272 .1 \quad 1287.6$ $1291.61293 .6 \quad 1298.1 \quad 1305.6 \quad 1337.6 \quad 1338.9 \quad 1354.6 \quad 1377.2 \quad 1382.8 \quad 1401.3 \quad 1439.7 \quad 1596.2$ $1841.32639 .32648 .72657 .3 \quad 2664.92667 .92673 .4 \quad 2679.32706 .5 \quad 2715.4 \quad 2725.32739 .1$ $2764.12766 .3 \quad 2776.8 \quad 4763.9$

name $=\operatorname{TS} 145$

energy $($ in $\mathrm{kcal} / \mathrm{mol})=38.496012$

$\mathrm{ZPE}($ in $\mathrm{kcal} / \mathrm{mol})=141.79$

$\mathrm{G}($ in $\mathrm{kcal} / \mathrm{mol})=117.847$

Geometry

C $-2.45659017-0.43115993-0.81334570$

C $-1.11791189-0.17244356-0.42414556$

C $-0.47621273 \quad 1.23585440-0.56865083$

C $0.71158267 \quad 1.30025795 \quad 0.41136588$

C $0.76540175-0.037185651 .14081203$

C $2.14730454-0.555130131 .39244394$

C $-0.37642964 \quad-0.92007613 \quad 0.62954647$ 
C $-0.40315333-0.393836362 .06418326$

C $-1.39978342 \quad 0.650764292 .49684533$

C $-0.17244465-1.411655333 .15818273$

$\mathrm{H}-3.10615899 \quad 0.34731729-1.15802910$

$\mathrm{H}-2.97596583-1.29679289-0.45540333$

$\mathrm{H}-1.74497620-0.77247770-2.25047932$

$\mathrm{H}-1.19941188 \quad 2.06444474 \quad-0.44136460$

$\mathrm{H} \quad 0.61313632 \quad 2.13868971 \quad 1.11983287$

H $1.647549631 .48273779-0.15171782$

H $2.68792971 \quad 0.09054685 \quad 2.09787014$

$\mathrm{H} 2.14980384-1.56922184 \quad 1.81114257$

H $2.73195506-0.59742893 \quad 0.46158210$

$\mathrm{H}-0.26126035-1.98782896 \quad 0.45839408$

$\mathrm{H}-1.65614337 \quad 1.36242651 \quad 1.70520945$

$\mathrm{H}-1.01448240 \quad 1.23001244 \quad 3.34626482$

$\mathrm{H}-2.34458982 \quad 0.18494648 \quad 2.81104119$

$\mathrm{H} \quad 0.34230651-0.95564117 \quad 4.01383667$

$\mathrm{H} \quad 0.43293832 \quad-2.26358184 \quad 2.82684406$

$\mathrm{H}-1.12427013-1.821787153 .52118872$

$00.34227777 \quad 0.08523533-2.38548390$

$0-0.060843201 .38142179-1.91535846$

o $-0.61214591-0.77102375-1.82930741$

Vibrational frequencies (in $\mathrm{cm}-1$ )

$\begin{array}{lllllllllllllll}-1352.5 & 85.5 & 96.7 & 113.8 & 131.8 & 150.0 & 164.5 & 208.0 & 226.5 & 254.2 & 278.8 & 341.9 & 359.1 & 384.9\end{array}$

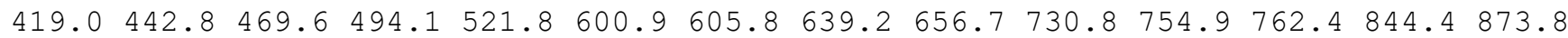

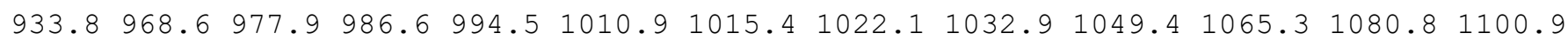
$1137.71152 .0 \quad 1158.1 \quad 1182.1 \quad 1223.2 \quad 1243.8 \quad 1273.2 \quad 1274.7 \quad 1281.6 \quad 1286.5 \quad 1289.01293 .2$ $1295.91302 .3 \quad 1306.1 \quad 1318.0 \quad 1326.1 \quad 1339.0 \quad 1367.4 \quad 1395.1 \quad 1402.8 \quad 1408.7 \quad 1504.91583 .2$ $2184.82666 .12671 .2 \quad 2671.7 \quad 2674.5 \quad 2676.2 \quad 2676.92679 .82693 .6 \quad 2736.6 \quad 2748.02766 .6$ $2780.3 \quad 2780.4 \quad 2783.8 \quad 2797.6$
name $=\operatorname{TS} 146$
energy (in $\mathrm{kcal} / \mathrm{mol})=42.109261$
$\mathrm{ZPE}($ in $\mathrm{kcal} / \mathrm{mol})=138.342$
$\mathrm{G}($ in $\mathrm{kcal} / \mathrm{mol})=110.361$
Geometry
C $-1.651339950 .12165808-0.43601149$
C $-0.27202569-0.19265519-0.97718698$
C $0.602684591 .07899135-1.30744295$
C $2.021070961 .22801140-0.88905047$
C $0.53949190 \quad-0.13444857 \quad 1.94902483$
C $0.77268304-1.28523491 \quad 1.01803573$
C $0.41973528-1.29804867-0.27110538$
C $-0.82862499-0.27444293 \quad 2.53185084$
C $-1.41190680 \quad 0.92777094 \quad 3.13864594$
C $1.54146775-0.15535308 \quad 3.11365223$
$\mathrm{H}-1.71922911 \quad 1.15804356-0.07384150$
$\mathrm{H}-1.94342482 \quad-0.55222287 \quad 0.38318206$
$\mathrm{H}-2.41606930 \quad 0.01820614-1.22041088$
$\mathrm{H}-0.30342068 \quad 1.60250807 \quad-2.83817474$
H $2.11789137 \quad 1.98847408-0.09727270$
H $2.463183130 .29093900 \quad-0.52294142$
$\mathrm{H} \quad 0.65354031 \quad 0.83260946 \quad 1.39408281$
$\mathrm{H} 1.24460486-2.156141411 .47554809$
$\mathrm{H} 2.64244944 \quad 1.58060491-1.73019384$
$\mathrm{H} \quad 0.61015941-2.17191197-0.89958420$
$\mathrm{H}-1.70523471 \quad 0.69821665 \quad 4.17852504$
$\mathrm{H}-2.36193374 \quad 1.16036819 \quad 2.62312814$
$\mathrm{H}-0.80945849 \quad 1.84151654 \quad 3.15108932$
H $1.43421216 \quad 0.72768446 \quad 3.75341625$
H $2.57255988-0.169325292 .74042791$
$\mathrm{H} 1.40917482-1.044150203 .74368483$
$\begin{array}{lllll}0 & 0.46081635 & 0.54031428 & -2.85652822\end{array}$
$\begin{array}{llll}0 & -0.09233940 & 2.14651920 & -1.59618768\end{array}$ 
$0-0.40045602-0.52805871-2.39158895$

Vibrational frequencies (in $\mathrm{cm}-1$ )

$\begin{array}{llllllllllllllll}-1565.1 & 15.6 & 41.1 & 50.6 & 73.1 & 81.8 & 110.0 & 118.3 & 137.5 & 157.4 & 185.7 & 214.4 & 257.7 & 268.8\end{array}$ $294.3 \quad 319.0 \quad 333.7 \quad 367.1 \quad 423.6 \quad 460.6 \quad 494.5 \quad 509.8 \quad 587.9611 .3 \quad 663.5 \quad 707.7 \quad 768.8 \quad 824.1$

$883.6 \quad 898.3 \quad 943.5 \quad 974.9 \quad 988.0 \quad 1012.0 \quad 1014.2 \quad 1014.9 \quad 1038.3 \quad 1051.8 \quad 1092.0 \quad 1098.3 \quad 1119.2$

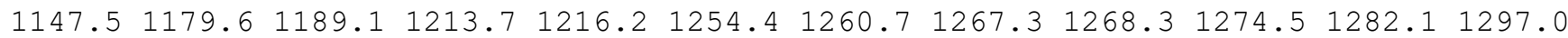

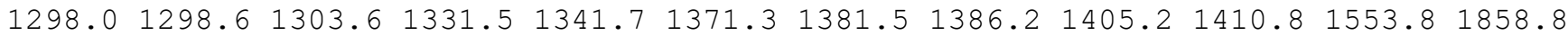
$2186.62654 .8 \quad 2655.8 \quad 2659.8 \quad 2660.4 \quad 2667.2 \quad 2668.92670 .92674 .8 \quad 2689.5 \quad 2738.92753 .6$ $2768.32770 .02775 .0 \quad 2778.6$

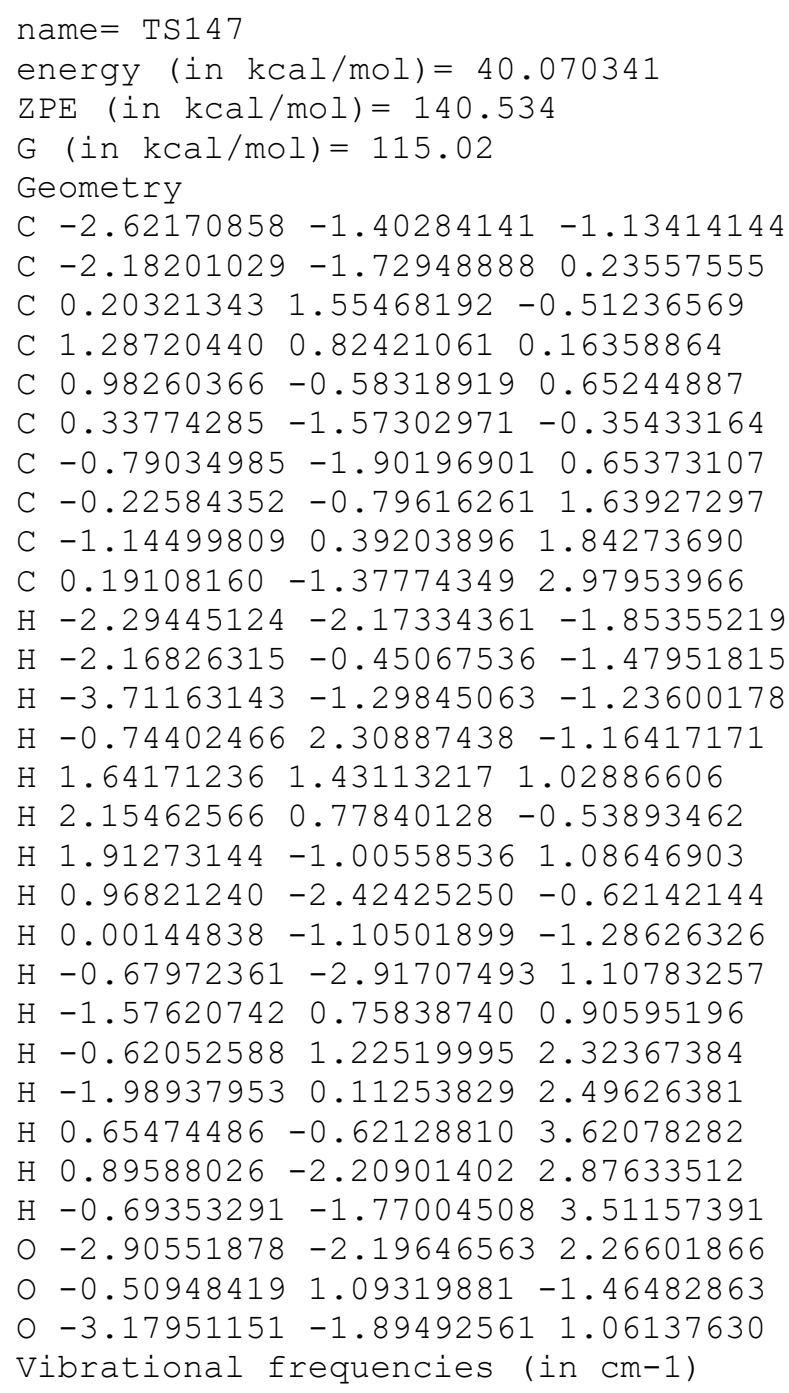


C $1.79020837-1.13344507 \quad 1.10814199$

C $-0.58404399-1.42532384 \quad 0.10663030$

C $0.27183731-1.15510647 \quad 1.27222983$

C $2.70405067-1.649769432 .18418992$

C $-0.32498894-1.679934652 .55782097$

$\mathrm{H}-3.12678618 \quad 0.02892327-0.44357953$

$\mathrm{H}-2.78721431-1.31377848-1.54495160$

$\mathrm{H}-2.55887061 \quad 0.34723979-2.10003295$

$\mathrm{H}-1.089404091 .83420532-0.38067651$

$\mathrm{H} 1.04401034 \quad 2.17860468 \quad 0.53702377$

H $1.79407773 \quad 0.99460815-0.52207877$

$\mathrm{H} \quad 1.12112990 \quad 0.66537403 \quad 2.28383947$

$\mathrm{H} 0.44806253-1.63859793-1.17867966$

$\mathrm{H} 2.18404190-1.33504060 \quad 0.10479171$

$\mathrm{H}-1.17505096-2.329294690 .15050897$

H $3.73351481-1.301407532 .02727880$

$\mathrm{H} 0.21528873-1.333921973 .44564206$

H $2.40396535-1.32405858 \quad 3.18721175$

$\mathrm{H}-1.36820461-1.35473526 \quad 2.66454762$

$\mathrm{H}-0.31422510-2.777381912 .57234662$

$\mathrm{H} \quad 2.72032893-2.74805267 \quad 2.19061941$

$0.52081203 \quad 0.15479701-2.58518939$

$0-0.053856061 .33322446-2.01991121$

o $-0.16741433-0.91264796-1.91675568$

Vibrational frequencies (in $\mathrm{cm}-1$ )

$\begin{array}{lllllllllllllll}-1318.0 & 48.7 & 96.1 & 117.4 & 131.8 & 142.5 & 172.8 & 185.9 & 226.4 & 259.8 & 281.5 & 289.3 & 321.8 & 356.7\end{array}$

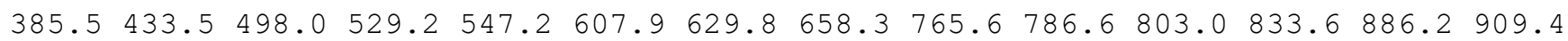
$942.8 \quad 956.9 \quad 974.7 \quad 1001.3 \quad 1012.2 \quad 1023.7 \quad 1049.2 \quad 1061.8 \quad 1074.5 \quad 1086.6 \quad 1098.8 \quad 1104.5 \quad 1130.8$

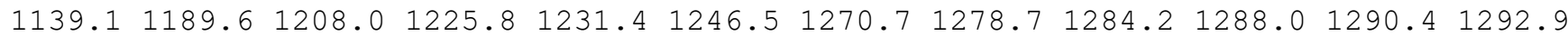
$1296.21304 .8 \quad 1308.0 \quad 1339.2 \quad 1350.0 \quad 1363.2 \quad 1383.8 \quad 1389.81398 .3 \quad 1410.1 \quad 1511.01552 .3$ $2193.12648 .72668 .5 \quad 2673.3 \quad 2674.5 \quad 2677.12677 .82679 .12693 .0 \quad 2736.4 \quad 2743.0 \quad 2760.9$ $2769.7 \quad 2778.8 \quad 2781.5 \quad 2782.2$

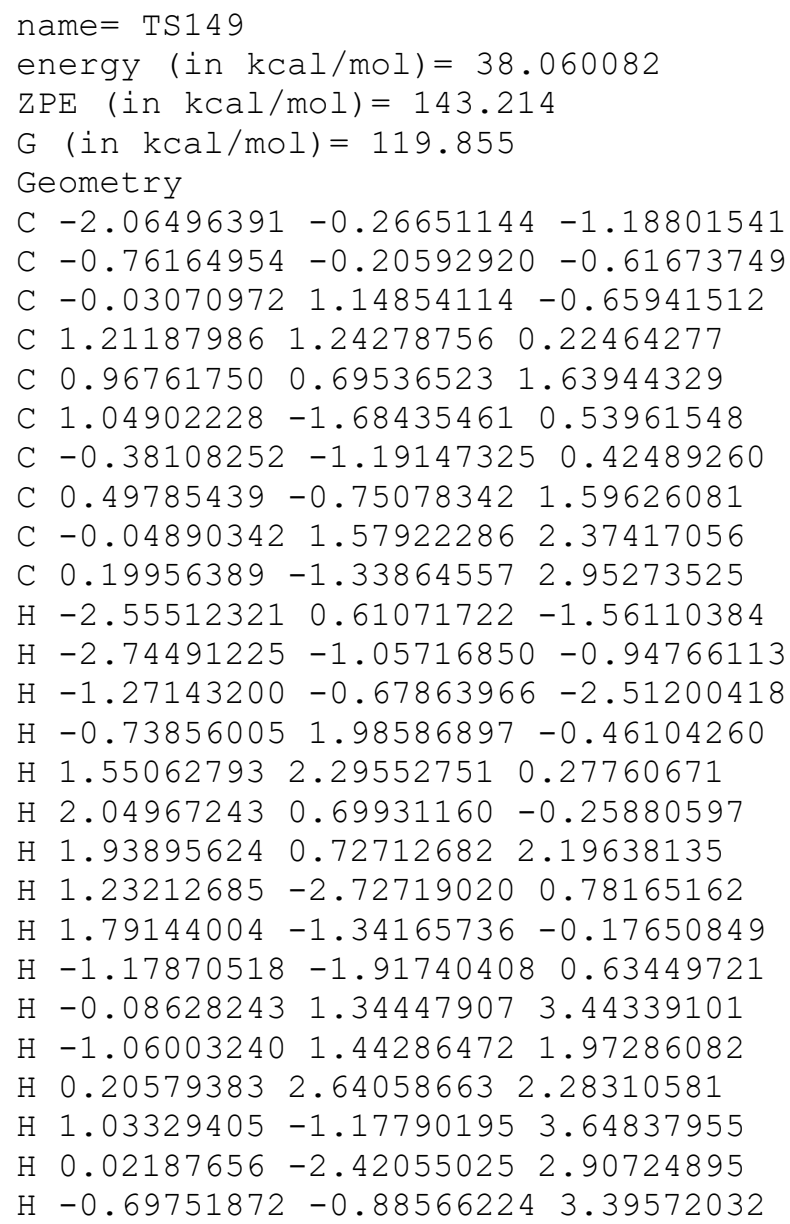


$0.80035948 \quad 0.09895196-2.52015665$

$0.344540891 .36440298-2.01601386$

- $-0.14236721-0.80012714-2.04783951$

Vibrational frequencies (in $\mathrm{cm}-1$ )

$\begin{array}{lllllllllllllll}-1437.8 & 60.2 & 113.0 & 131.1 & 162.2 & 194.0 & 225.1 & 248.8 & 277.1 & 298.2 & 341.9 & 363.7 & 372.9 & 415.7\end{array}$ $418.4 \quad 465.0 \quad 486.6 \quad 516.3 \quad 572.8 \quad 597.9 \quad 622.7 \quad 666.8 \quad 721.4 \quad 756.2 \quad 818.0 \quad 840.9872 .1889 .5$ $930.7968 .5 \quad 971.6 \quad 996.5 \quad 1001.7 \quad 1022.3 \quad 1046.3 \quad 1054.6 \quad 1069.6 \quad 1075.6 \quad 1091.4 \quad 1100.51116 .0$

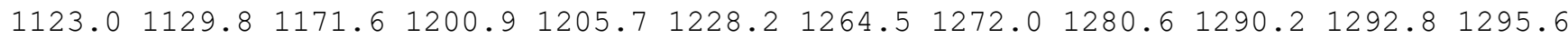
$1300.21307 .3 \quad 1318.0 \quad 1329.2 \quad 1362.8 \quad 1372.4 \quad 1380.1 \quad 1386.81398 .11409 .3 \quad 1486.81545 .0$ $2116.22654 .92672 .82673 .32676 .2 \quad 2678.02685 .52701 .62707 .72734 .32741 .92748 .6$ 2780.62781 .62784 .22797 .3

name $=\operatorname{TS} 150$

energy (in $\mathrm{kcal} / \mathrm{mol})=39.316978$

$\mathrm{ZPE}(\mathrm{in} \mathrm{kcal} / \mathrm{mol})=142.227$

$\mathrm{G}($ in $\mathrm{kcal} / \mathrm{mol})=117.191$

Geometry

C $-2.23471433-0.19269083-0.59971406$

C $-1.21744722-1.21285506-0.76421782$

C $-0.74228892-1.49503604-2.24444192$

C $0.78446814-1.33408631-2.31296680$

C $1.26335054-1.09719955-0.89409668$

C $1.03955202-2.21978770 \quad 0.08797316$

C $0.06767548-1.04893585 \quad 0.05964955$

C $0.01303466-0.15793725 \quad 1.23411884$

C $0.09178732 \quad 1.17202279 \quad 1.13575016$

C $-0.11332933-0.844302442 .55869863$

$\mathrm{H}-1.93320408 \quad 0.81484394 \quad-0.43437863$

$\mathrm{H}-2.57423482-0.34978183 \quad 1.19223564$

$\mathrm{H}-3.09151045-0.24202963-1.23330314$

$\mathrm{H}-1.28278101-0.87885199-2.98405657$

H $1.05999875-0.49126905-2.96989500$

$\mathrm{H} 1.25104517-2.23810815-2.74846402$

$\mathrm{H} 2.11298264-0.43142229-0.77809859$

$\mathrm{H} \quad 1.70620355-2.331289910 .93668418$

$\mathrm{H} \quad 0.72080609-3.19754380-0.26690123$

$\mathrm{H}-3.17003013-0.935576570 .79053700$

$\mathrm{H} \quad 0.19949163 \quad 1.68481888 \quad 0.19114717$

$\mathrm{H} \quad 0.05929797 \quad 1.84321892 \quad 1.97564303$

$\mathrm{H}-3.16563315-0.82084123 \quad 1.69154341$

$\mathrm{H}-0.61567360-0.22859038 \quad 3.31206273$

H $0.88960769-1.071700572 .95777666$

$\mathrm{H}-0.63426033-1.80761724 \quad 2.48169822$

$\begin{array}{lllll}0 & -1.29771485 & -3.44607620 & -1.23839147\end{array}$

$\begin{array}{lllll}0 & -1.15175254 & -2.82351580 & -2.51664939\end{array}$

o $-1.86700980-2.46052419-0.40878347$

Vibrational frequencies (in $\mathrm{cm}-1$ )

$\begin{array}{lllllllllllllll}-2124.2 & 29.2 & 51.7 & 93.6 & 128.3 & 135.5 & 196.8 & 215.8 & 219.8 & 257.7 & 280.8 & 298.4 & 305.3 \quad 347.3\end{array}$

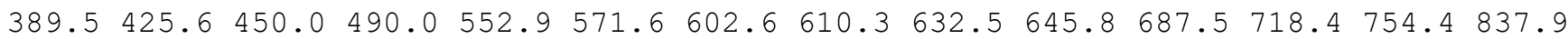

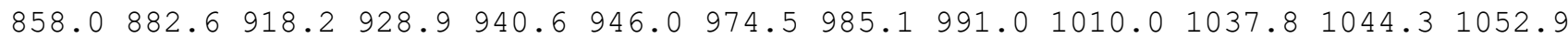

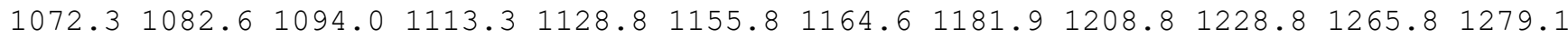

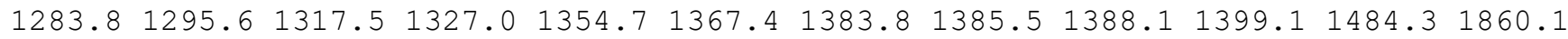
2132.02662 .62671 .22682 .22701 .62710 .52723 .72744 .12748 .92769 .62778 .12784 .6 $2790.82804 .4 \quad 2909.7 \quad 4554.0$

name $=\operatorname{TS} 151$

energy $($ in $\mathrm{kcal} / \mathrm{mol})=39.708541$

$\operatorname{ZPE}($ in $\mathrm{kcal} / \mathrm{mol})=141.927$

$\mathrm{G}($ in $\mathrm{kcal} / \mathrm{mol})=116.85$

Geometry

C $-0.76068219-1.38336413-1.24631622$

C $0.54904539-0.78498473-0.76462312$

C $0.61645367 \quad 0.79965517-0.80347396$ 
C $0.96588380 \quad 1.26558873 \quad 0.55692172$

C $1.186903520 .10631691 \quad 1.44687803$

C $2.31443823-0.86026744 \quad 1.12933439$

C $0.91321806-1.18040956 \quad 0.65384966$

C $0.76025566 \quad 0.29354990 \quad 2.85258733$

C $-0.47320944-0.01167947 \quad 3.27025083$

C $1.78821114 \quad 0.89602392 \quad 3.74883116$

$\mathrm{H}-1.01045768-1.02778349-2.25491886$

$\mathrm{H}-1.58714205-1.13439117-0.57558624$

$\mathrm{H}-0.69139680-2.47883728-1.30332737$

$\mathrm{H}-0.27871451 \quad 1.26929609-1.24811376$

$\mathrm{H}-0.29885380 \quad 2.43742821 \quad 1.19506624$

$\mathrm{H} 1.66386756 \quad 2.07680568 \quad 0.62132560$

$\mathrm{H}-1.141403332 .36939344 \quad 1.51068865$

H $2.75142774-1.46346209 \quad 1.91618888$

H $3.07257307-0.56398670 \quad 0.40665326$

$\mathrm{H} \quad 0.39253311-2.03328027 \quad 1.06992818$

$\mathrm{H}-1.20947281-0.491148712 .64917626$

$\mathrm{H}-0.68854728 \quad 1.60698617 \quad 1.29096621$

$\mathrm{H}-0.814141810 .12971896 \quad 4.28027846$

$\mathrm{H} 1.377349551 .25741884 \quad 4.69787706$

$\mathrm{H} \quad 2.30945819 \quad 1.73269334 \quad 3.25922321$

H $2.56414435 \quad 0.15100910 \quad 3.99179049$

○ $2.43791618-0.09417713-1.79885033$

o $1.614729211 .05551355-1.79858888$

o $1.53333079-1.19058141-1.71411973$

Vibrational frequencies (in cm-1)

$\begin{array}{lllllllllllllll}-2171.9 & 47.9 & 78.2 & 89.5 & 94.7 & 144.1 & 164.7 & 195.5 & 204.3 & 211.8 & 256.9 & 263.8 & 313.2 & 329.3\end{array}$

$354.2358 .5 \quad 464.2 \quad 491.1 \quad 517.4 \quad 556.0 \quad 573.1 \quad 602.9 \quad 635.6 \quad 652.2 \quad 674.4 \quad 708.2 \quad 776.9 \quad 840.5$

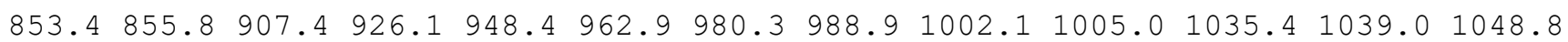

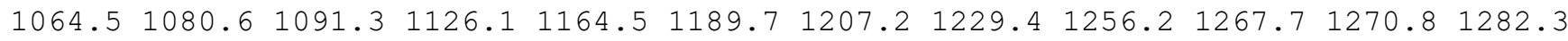

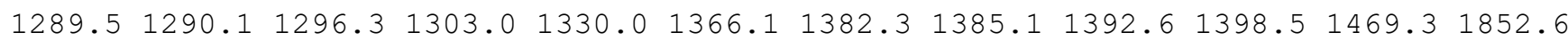

$2105.6 \quad 2663.1 \quad 2672.7 \quad 2679.92686 .0 \quad 2693.92715 .52728 .92774 .5 \quad 2778.12782 .6 \quad 2783.2$

$2786.42796 .5 \quad 2921.6 \quad 4572.4$

\author{
name $=\operatorname{TS} 152$ \\ energy $($ in $\mathrm{kcal} / \mathrm{mol})=38.8025$ \\ $\mathrm{ZPE}($ in $\mathrm{kcal} / \mathrm{mol})=142.993$ \\ $\mathrm{G}($ in $\mathrm{kcal} / \mathrm{mol})=119.738$ \\ Geometry \\ C $-1.95257754-0.36275523-1.02018472$ \\ C $-0.61217314-0.02277046-0.48080845$ \\ C $-0.092454321 .41789563-0.68642407$ \\ C $1.408848561 .43695728-0.24484087$ \\ C $1.77151197 \quad 0.20989834 \quad 0.60023462$ \\ C $1.18403630-1.09996825-0.06795480$ \\ C $-0.11353258-0.82646658 \quad 0.76091553$ \\ C $0.720944790 .01426366 \quad 1.77094451$ \\ C $0.07455339 \quad 1.23178680 \quad 2.39244544$ \\ C $1.29532751-0.87375923 \quad 2.87244475$ \\ $\mathrm{H}-2.05466819 \quad 0.00177207 \quad-2.07099831$ \\ $\mathrm{H}-2.75024501 \quad 0.13376082-0.44766733$ \\ $\mathrm{H}-2.16588302-1.43741614-1.02271564$ \\ $\mathrm{H}-0.69252667 \quad 2.19645370 \quad-0.18627821$ \\ $\mathrm{H} \quad 1.63500604 \quad 2.36433537 \quad 0.30188578$ \\ H $2.026009281 .45848659-1.17009271$ \\ H $2.82217632 \quad 0.17204720 \quad 0.88547585$ \\ $\mathrm{H} \quad 1.73665130 \quad-2.01092069-0.13166350$ \\ H $0.24429997-1.15563947-2.13618897$ \\ $\mathrm{H}-0.76220068-1.59768748 \quad 1.14467413$ \\ $\mathrm{H}-0.51373986 \quad 0.96297579 \quad 3.28000452$ \\ $\mathrm{H}-0.61294085 \quad 1.75126877 \quad 1.71707742$ \\ $\mathrm{H} \quad 0.83199170 \quad 1.95982928 \quad 2.71124444$ \\ H $1.80556703-1.758716602 .47713019$
}


$\mathrm{H} \quad 0.50929236-1.22932462 \quad 3.54903601$

$\mathrm{H} 2.02453892-0.322353623 .47880751$

$0-0.00157852 \quad 0.51100550-2.80483713$

$0-0.212895621 .68815919-2.06864228$

O $0.57516795-0.60179468-1.31233217$

Vibrational frequencies (in $\mathrm{cm}-1$ )

$\begin{array}{lllllllllllllllll}-766.9 & 73.4 & 115.3 & 156.3 & 164.3 & 174.3 & 182.3 & 220.0 & 250.3 & 285.3 & 345.7 & 367.0 & 384.2 & 422.6\end{array}$

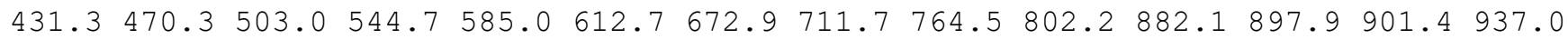

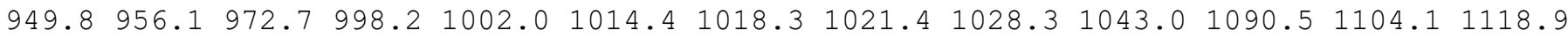
$1145.51150 .3 \quad 1155.0 \quad 1172.5 \quad 1190.6 \quad 1191.4 \quad 1210.6 \quad 1242.3 \quad 1250.3 \quad 1258.2 \quad 1270.4 \quad 1271.9$

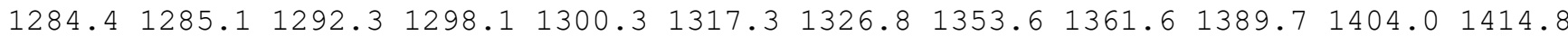
$2613.12634 .12658 .7 \quad 2667.6 \quad 2674.0 \quad 2674.5 \quad 2677.0 \quad 2678.42694 .8 \quad 2741.32744 .92757 .4$ $2762.92769 .12781 .3 \quad 2783.4$

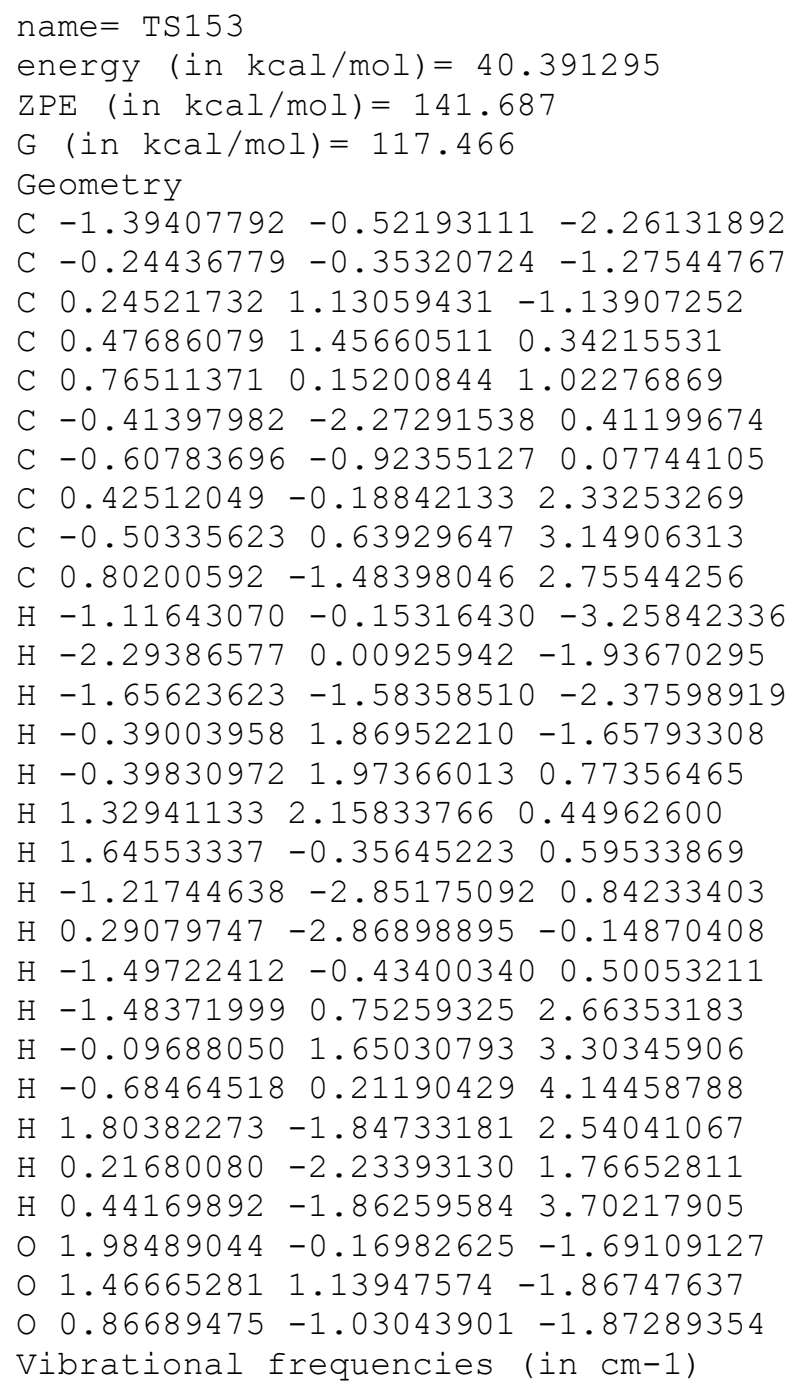


C $-1.12065110-1.04004670-0.78260169$

C $1.243508362 .03821712-0.19540275$

C $1.91303675 \quad 1.31940622 \quad 0.94995955$

C $1.40557864-0.08134023 \quad 1.22180837$

C $1.33592973-1.05564990 \quad 0.01680756$

C $-0.16253743-1.337214990 .29547523$

C $-0.13952920-0.296062321 .49180646$

C $-1.030982590 .92824910 \quad 1.30754215$

C $-0.54433493-0.863583662 .76605295$

$\mathrm{H}-2.91509221-2.06709165-1.28521650$

$\mathrm{H}-3.11957264-0.34902986-1.01058773$

$\mathrm{H}-2.88404951-1.44184195 \quad 0.38981387$

$\mathrm{H} \quad 0.97416695 \quad 1.45603141-1.08480749$

$\mathrm{H} \quad 1.82122858 \quad 1.93577458 \quad 1.87278580$

H $3.00672598 \quad 1.28882153 \quad 0.74061496$

H 2.00431148 -0.508136452 .05112880$

$\mathrm{H} 1.98107586-1.93498226 \quad 0.10983673$

H $1.56509538-0.60476574-0.95214835$

$\mathrm{H}-0.34287263-2.36974540 \quad 0.66355800$

$\mathrm{H}-2.07391165 \quad 0.70852393 \quad 1.58697712$

$\mathrm{H}-1.057456561 .27875950 \quad 0.26852376$

$\mathrm{H}-0.69589748 \quad 1.76824195 \quad 1.93005572$

$\mathrm{H}-0.01632081-2.34005128 \quad 2.98044231$

$\mathrm{H}-0.44715510-0.34633185 \quad 3.68634640$

$\mathrm{H} 0.67885009-1.95992438 \quad 3.14762757$

O $-1.37451513-0.32976445-2.84614603$

$\begin{array}{lllll}0 & 1.03495191 & 3.22413451 & -0.14100693\end{array}$

o - $0.58610398-0.59706147-1.88732066$

Vibrational frequencies (in cm-1)

$\begin{array}{lllllllllllllllll}-967.0 & 33.2 & 43.4 & 59.4 & 106.5 & 113.0 & 136.8 & 165.9 & 189.6 & 217.5 & 223.7 & 255.5 & 275.2 & 281.2\end{array}$ $294.1309 .2 \quad 379.5 \quad 399.1 \quad 446.0 \quad 453.3 \quad 510.2 \quad 540.1 \quad 584.9629 .7 \quad 671.3 \quad 685.8 \quad 743.0 \quad 784.0$

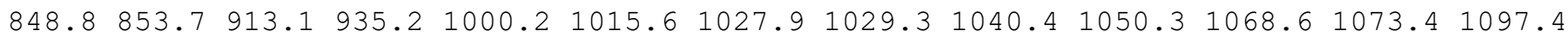

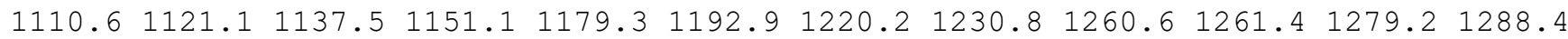
$1289.91294 .21296 .41310 .1 \quad 1319.9 \quad 1348.7 \quad 1370.3 \quad 1381.6 \quad 1389.6 \quad 1402.6 \quad 1446.2 \quad 1590.3$ $1835.22637 .92644 .3 \quad 2655.0 \quad 2659.6 \quad 2668.3 \quad 2668.82677 .32707 .92714 .42729 .12736 .4$ $2763.22766 .12774 .4 \quad 4756.2$

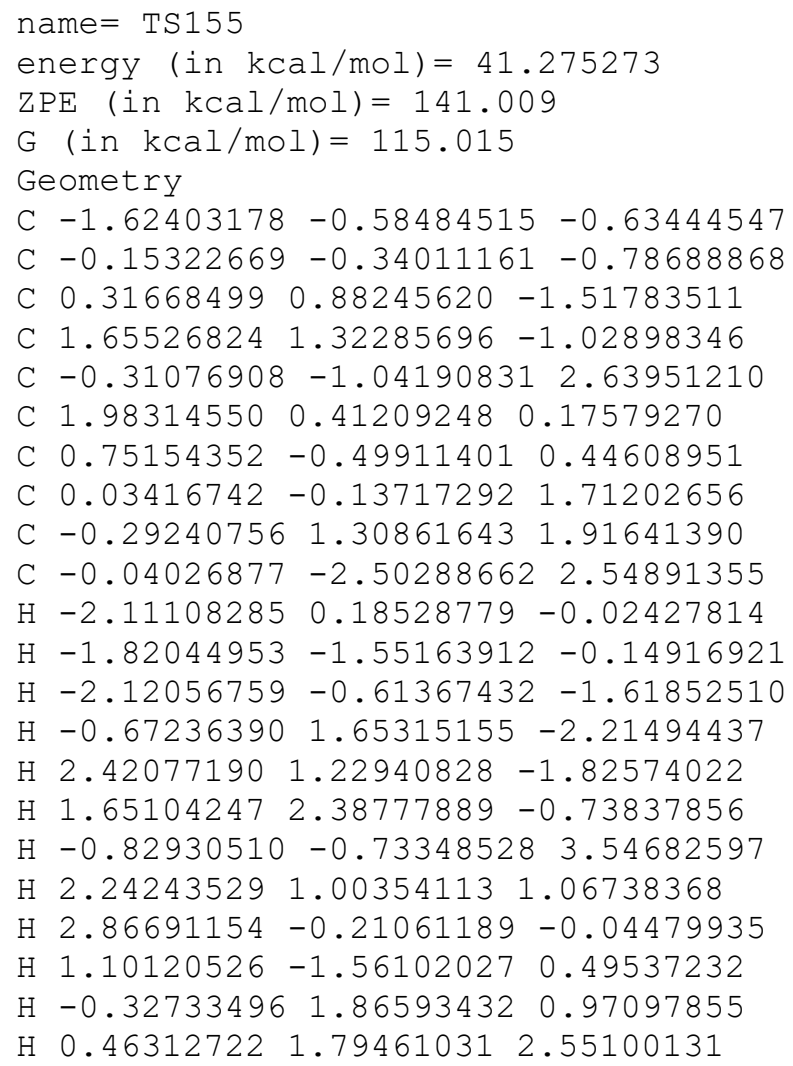


$\mathrm{H}-1.26329808 \quad 1.44687730 \quad 2.41027615$

$\mathrm{H}-0.46645934-3.05504374 \quad 3.39700415$

$\mathrm{H} 1.03959099-2.714298202 .54074815$

$\mathrm{H}-0.46807044-2.94200914 \quad 1.63523674$

$0-0.03659106-0.99824346-2.97846585$

$0.00102173 \quad 0.80861117-2.83319526$

O $0.44381625-1.25609847-1.80843291$

Vibrational frequencies (in $\mathrm{cm}-1$ )

$\begin{array}{llllllllllllllll}-1642.2 & 36.1 & 49.1 & 87.2 & 94.3 & 110.7 & 146.4 & 156.3 & 180.2 & 205.8 & 222.6 & 232.7 & 268.4 & 283.8\end{array}$ $309.7 \quad 362.6 \quad 445.6 \quad 460.7 \quad 492.7 \quad 514.9 \quad 559.1 \quad 608.0 \quad 617.7 \quad 639.8 \quad 725.2 \quad 779.7 \quad 814.8 \quad 856.9$

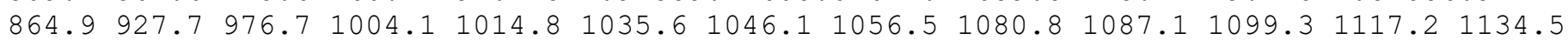

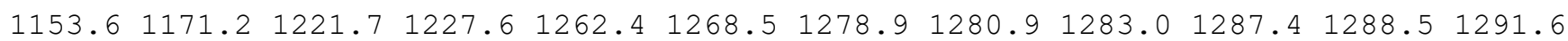

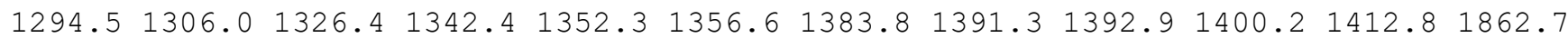
$2452.0 \quad 2654.4 \quad 2665.92667 .7 \quad 2670.4 \quad 2672.0 \quad 2675.2 \quad 2676.02678 .12695 .5 \quad 2745.92748 .9$ $2754.8 \quad 2778.12778 .8 \quad 2780.0$

name $=$ TS156

energy $($ in $\mathrm{kcal} / \mathrm{mol})=39.350581$

$\mathrm{ZPE}(\mathrm{in} \mathrm{kcal} / \mathrm{mol})=143.459$

$\mathrm{G}(\mathrm{in} \mathrm{kcal} / \mathrm{mol})=120.51$

Geometry

C $-2.11112418-0.41943896-0.88862955$

C $-0.72150483-0.21124741-0.66738623$

C $-0.148544701 .23038586-0.77175453$

C $1.122951731 .49376278 \quad 0.06323870$

C 1.575535150 .260976990 .84510608

C $1.59188260-0.94853623-0.14004583$

C $0.08096744-1.13870086 \quad 0.20785544$

C $0.36341766-0.42206856 \quad 1.59122098$

C $-0.71633972 \quad 0.45320838 \quad 2.19108569$

C $0.83391983-1.41484135 \quad 2.65029825$

$\mathrm{H}-2.787559490 .39261163-1.05878029$

$\mathrm{H}-2.59630101-1.31416278-0.55765552$

$\mathrm{H}-1.61272844-0.62074171-2.43974970$

$\mathrm{H}-0.93596321 \quad 1.98360206 \quad-0.53610474$

$\mathrm{H} \quad 0.95138018 \quad 2.33845393 \quad 0.75470795$

H $1.936056851 .82145719-0.61734122$

H 2.471089620 .430674851 .44289548

H $2.22969564-1.779042210 .16656570$

H $1.82505839-0.71835745-1.18099279$

$\mathrm{H}-0.29848066-2.15955656 \quad 0.25400319$

$\mathrm{H}-0.315384151 .06134732 \quad 3.01161316$

$\mathrm{H}-1.53399329-0.15749064 \quad 2.59780709$

$\mathrm{H}-1.17765348 \quad 1.14125588 \quad 1.47734688$

$\mathrm{H} 1.22101753-0.893206173 .53341780$

H $1.63152611-2.07173194 \quad 2.28650192$

$\mathrm{H} \quad 0.01006744 \quad-2.05887142 \quad 2.98003886$

$\begin{array}{lllll}0 & 0.42721653 & 0.25537192 & -2.79065433\end{array}$

O $0.09497515 \quad 1.49304762 \quad-2.14854982$

o $-0.43523456-0.66359329-2.18665510$

Vibrational frequencies (in cm-1)

$\begin{array}{llllllllllllllll}-1332.9 & 85.2 & 104.1 & 150.3 & 175.4 & 190.3 & 228.9 & 235.6 & 292.8 & 324.6 & 366.6 & 384.7 & 431.9 & 437.3\end{array}$ $451.7 \quad 466.9 \quad 540.1 \quad 552.8 \quad 595.7 \quad 624.7 \quad 650.0 \quad 713.0 \quad 733.2 \quad 799.0 \quad 842.6 \quad 854.0 \quad 891.2 \quad 915.7$ $939.5954 .0 \quad 979.2 \quad 1002.6 \quad 1007.3 \quad 1010.3 \quad 1017.5 \quad 1024.9 \quad 1049.5 \quad 1065.8 \quad 1078.1 \quad 1088.91092 .7$

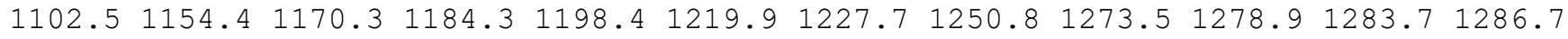

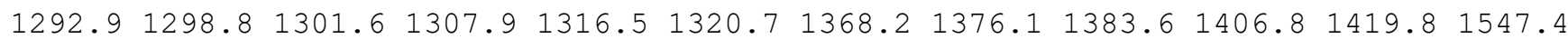
$2187.92658 .4 \quad 2672.7 \quad 2675.9 \quad 2676.5 \quad 2678.4 \quad 2681.12689 .72735 .92740 .8 \quad 2748.5 \quad 2752.2$ $2767.5 \quad 2781.8 \quad 2785.4 \quad 2797.9$

name $=$ TS 157

energy (in $\mathrm{kcal} / \mathrm{mol})=40.693119$

$\mathrm{ZPE}(\mathrm{in} \mathrm{kcal} / \mathrm{mol})=142.35$

$\mathrm{G}($ in $\mathrm{kcal} / \mathrm{mol})=117.076$ 


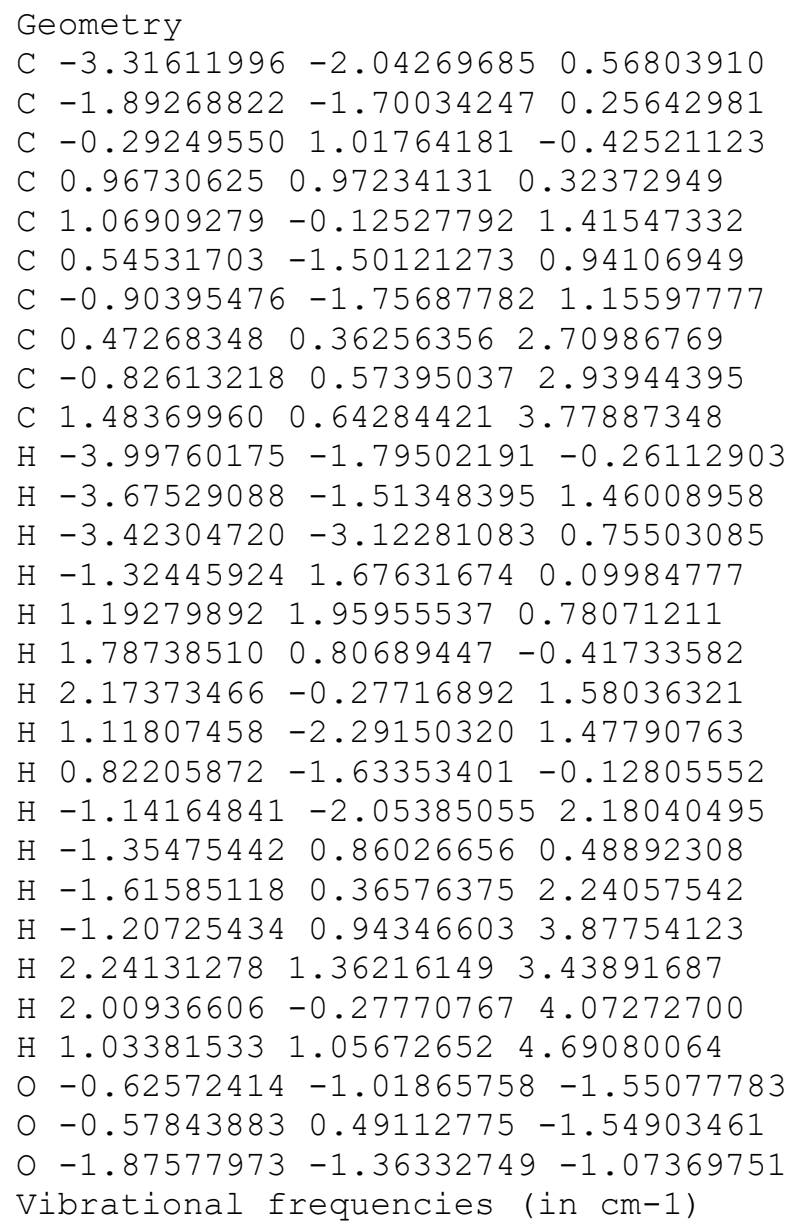

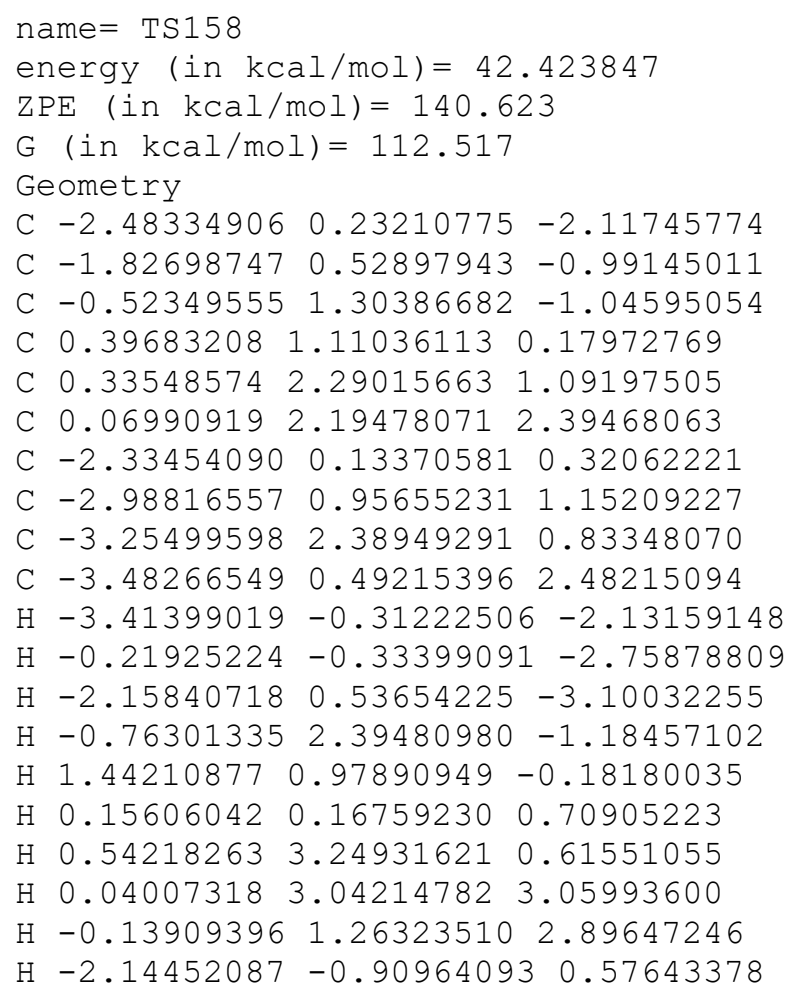


$\mathrm{H}-2.413033093 .016951161 .16939446$

$\mathrm{H}-4.161402892 .76754071 \quad 1.32199671$

$\mathrm{H}-3.37361678 \quad 2.55862402-0.24643111$

$\mathrm{H}-4.58104108 \quad 0.51847371 \quad 2.52627447$

$\mathrm{H}-3.106395631 .13599150 \quad 3.29050094$

$\mathrm{H}-3.17435799-0.53512977 \quad 2.71660607$

$\begin{array}{llll}0 & 0.70198004 & -0.67267139 & -2.17960807\end{array}$

$00.171802221 .00233636-2.20113319$

o $0.38050026-1.33672981-1.24167696$

Vibrational frequencies (in $\mathrm{cm}-1$ )

$\begin{array}{lllllllllllllllll}-1163.0 & 20.6 & 47.4 & 62.2 & 65.3 & 72.6 & 101.9 & 106.8 & 117.0 & 119.6 & 159.5 & 204.0 & 237.1 & 270.4\end{array}$ $330.0 \quad 344.7 \quad 361.5 \quad 399.2 \quad 410.4 \quad 457.0 \quad 497.2 \quad 506.1 \quad 541.5 \quad 611.1630 .6 \quad 653.3 \quad 756.1 \quad 869.9$ $915.0935 .5959 .6981 .0 \quad 994.6 \quad 1016.6 \quad 1018.6 \quad 1024.6 \quad 1076.3 \quad 1080.8 \quad 1090.6 \quad 1106.7 \quad 1118.7$ $1143.41165 .1 \quad 1202.3 \quad 1222.6 \quad 1259.0 \quad 1268.9 \quad 1271.7 \quad 1274.4 \quad 1278.8 \quad 1286.8 \quad 1287.3 \quad 1314.9$

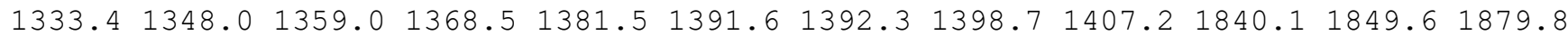
$2367.32649 .0 \quad 2655.92662 .8 \quad 2671.5 \quad 2673.8 \quad 2676.8 \quad 2717.52719 .92734 .42751 .92756 .8$ 2776.32780 .02786 .02790 .7

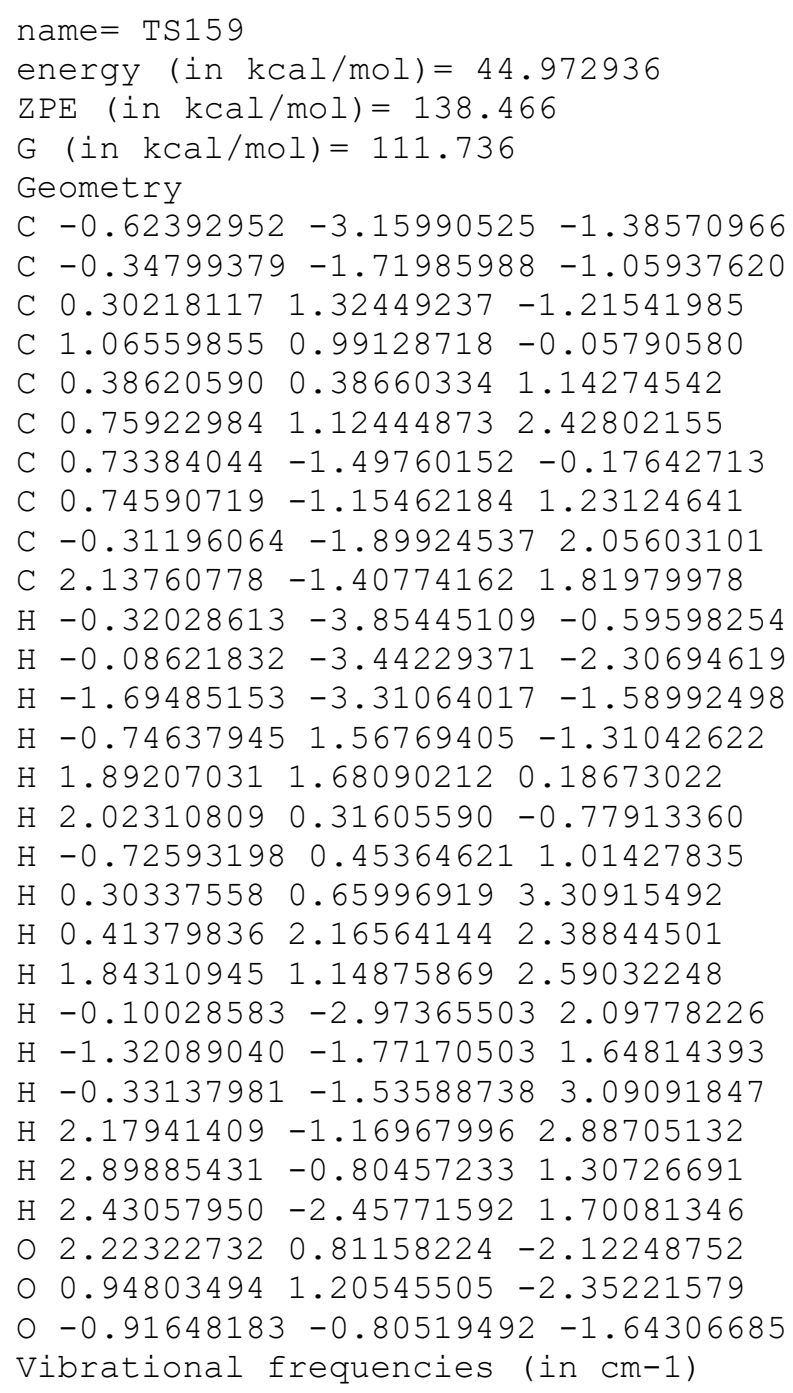


$\mathrm{ZPE}($ in $\mathrm{kcal} / \mathrm{mol})=139.956$

$\mathrm{G}($ in $\mathrm{kcal} / \mathrm{mol})=111.033$

Geometry

C $-2.67544734-0.25261278-0.76917423$

C $-1.24496996-0.28071001-0.46564001$

C $1.556001831 .87481606-0.33305196$

C $1.74562807 \quad 1.709256351 .15419616$

C $0.83641159 \quad 0.69051702 \quad 1.81373480$

C $0.69331209-0.675520821 .09163290$

C $-0.83738409-0.459655290 .92819071$

C $-0.72037286 \quad 0.90729412 \quad 1.71441156$

C $-1.19854715 \quad 2.13448561 \quad 0.96201994$

C $-1.394395090 .84131727 \quad 3.07898143$

$\mathrm{H}-2.903480810 .62527476-1.40030191$

$\mathrm{H}-3.37194440 \quad-0.25195033 \quad 0.07562433$

$\mathrm{H}-2.91640311-1.12606096-1.40445426$

$\mathrm{H} 1.057535721 .06964081-0.89273614$

$\mathrm{H} \quad 1.62928770 \quad 2.69775303 \quad 1.65044440$

H 2.808758641 .430862351 .33235235

$\mathrm{H} \quad 1.15068652 \quad 0.56777802 \quad 2.86931490$

H $0.95854982-1.53736717 \quad 1.70793342$

$\mathrm{H} \quad 1.23534696-0.766581650 .14551966$

$\mathrm{H}-1.43460936-1.21139122 \quad 1.47168753$

$\mathrm{H}-0.84998024 \quad 3.05634050 \quad 1.44669983$

$\mathrm{H}-2.29423044 \quad 2.181463320 .92670551$

$\mathrm{H}-0.84655013 \quad 2.16698072-0.07718369$

$\mathrm{H}-2.48524474 \quad 0.78619772 \quad 2.98548110$

$\mathrm{H}-1.16194650 \quad 1.73126986 \quad 3.67756244$

$\mathrm{H}-1.07358890-0.033610553 .65610626$

$0.0 .16914785-0.09989839-3.25617742$

O $1.95581323 \quad 2.86692664-0.89286907$

O $0.45511001-0.78320003-2.39546990$

Vibrational frequencies (in $\mathrm{cm}-1$ )

$\begin{array}{llllllllllllllll}-104.6 & 30.7 & 37.3 & 48.9 & 56.0 & 76.6 & 81.2 & 103.3 & 135.9 & 161.9 & 173.8 & 202.7 & 215.8 & 219.9\end{array}$ $256.0 \quad 287.1 \quad 313.2 \quad 345.1 \quad 414.6 \quad 429.2 \quad 446.4 \quad 463.4 \quad 606.5 \quad 674.8 \quad 754.1 \quad 812.9845 .1 \quad 892.2$ $908.1937 .4995 .5 \quad 998.2 \quad 1005.5 \quad 1017.7 \quad 1043.0 \quad 1057.0 \quad 1067.5 \quad 1103.2 \quad 1115.51140 .3 \quad 1157.0$ $1193.21202 .6 \quad 1214.2 \quad 1224.9 \quad 1243.7 \quad 1262.3 \quad 1266.2 \quad 1268.8 \quad 1283.5 \quad 1284.5 \quad 1291.6 \quad 1298.8$

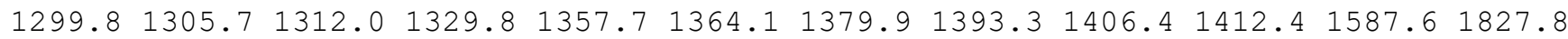
$2631.62653 .12654 .92664 .4 \quad 2671.3 \quad 2672.8 \quad 2676.42678 .12685 .0 \quad 2715.2 \quad 2723.3 \quad 2739.2$ $2764.62769 .2 \quad 2778.2 \quad 2782.1$

\section{name $=\operatorname{TS} 161$}

energy $($ in $\mathrm{kcal} / \mathrm{mol})=42.76299$

$\operatorname{ZPE}($ in $\mathrm{kcal} / \mathrm{mol})=140.956$

$\mathrm{G}($ in $\mathrm{kcal} / \mathrm{mol})=114.481$

Geometry

C $-2.82676686-2.28559411-1.90790784$

C $-1.50850698-1.73791149-1.45106716$

C $0.12701329 \quad 1.37418254 \quad-0.60878533$

C $0.94858479 \quad 0.91836000 \quad 0.48962761$

C $0.20807218-0.24653440 \quad 1.08623085$

C $0.32168615-1.52210544 \quad 0.30829449$

C $-0.99892048-1.96511692-0.23606923$

C $-0.48038219-0.120552992 .25734312$

C $-0.74714661 \quad 1.12406397 \quad 2.87434243$

C $-1.12123419-1.325846742 .88824789$

$\mathrm{H}-3.10005350-1.93063794-2.91343288$

$\mathrm{H}-3.63778579-2.00008995-1.22739376$

$\mathrm{H}-2.79483734-3.38463774-1.95539813$

$\mathrm{H}-2.166319691 .49179687 \quad 1.63512801$

$\mathrm{H} \quad 1.16367478 \quad 1.70920127 \quad 1.24062859$

$\mathrm{H} \quad 1.95694532 \quad 0.59078156 \quad 0.13321532$

$\mathrm{H}-0.03268883 \quad 1.916055592 .84793153$

$\mathrm{H} \quad 0.74234169-2.32423163 \quad 0.95684263$ 
H $1.06239905-1.42530116-0.51556489$

$\mathrm{H}-1.57852891-2.560117190 .47011296$

$\mathrm{H}-2.20998450 \quad 1.93087103 \quad 0.85298345$

$\mathrm{H}-1.431058681 .94910891 \quad 1.31603166$

$\mathrm{H}-1.319178621 .16747600 \quad 3.77226358$

$\mathrm{H}-1.29014268-1.17559784 \quad 3.96221511$

$\mathrm{H}-0.48714696-2.218039152 .78711785$

$\mathrm{H}-2.08738945-1.55443187 \quad 2.42546367$

$\begin{array}{lllll}0 & 0.14686909 & -0.37319570 & -2.28141294\end{array}$

$0-0.31006138 \quad 1.03973433-1.64876531$

O $-1.01717251-1.03463449-2.52651703$

Vibrational frequencies (in $\mathrm{cm}-1$ )

$\begin{array}{lllllllllllllllll}-1725.5 & 47.3 & 54.6 & 71.6 & 93.4 & 105.7 & 115.9 & 139.9 & 170.4 & 190.2 & 204.8 & 251.4 & 262.0 & 294.6\end{array}$

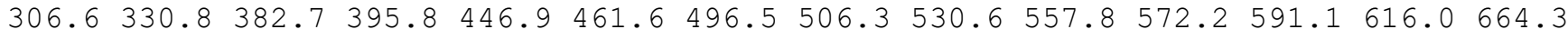
$677.2829 .9 \quad 849.6 \quad 901.8 \quad 923.3 \quad 957.2 \quad 976.1 \quad 990.4 \quad 1004.1 \quad 1021.9 \quad 1029.9 \quad 1042.0 \quad 1060.2$

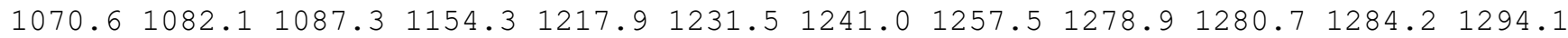
$1301.11311 .41336 .21347 .1 \quad 1376.6 \quad 1383.8 \quad 1390.1 \quad 1404.71485 .01776 .11888 .51909 .6$

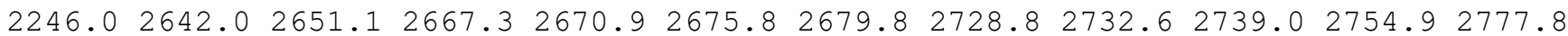
$2779.32808 .22910 .5 \quad 4621.5$

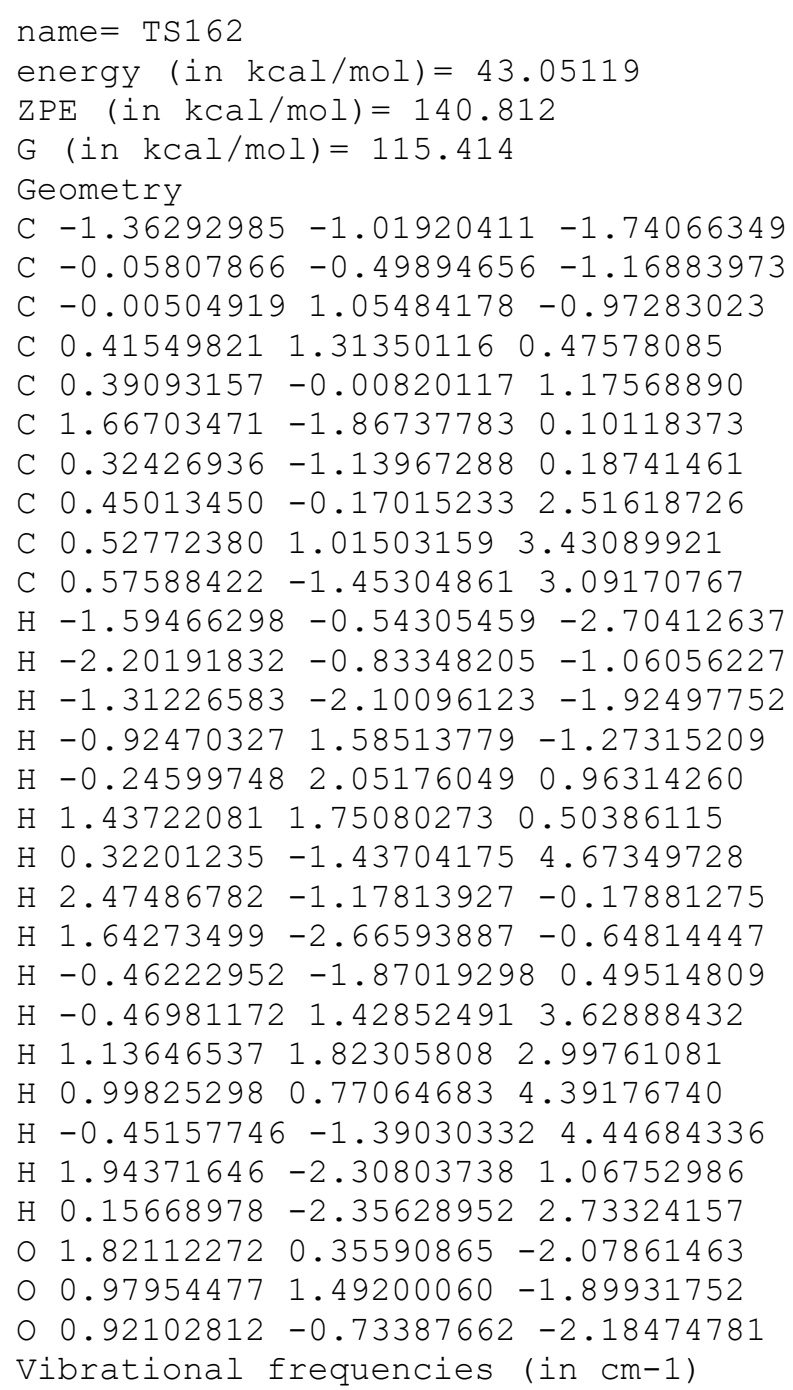


name $=\operatorname{TS} 163$

energy (in $\mathrm{kcal} / \mathrm{mol})=44.951715$

$\mathrm{ZPE}($ in $\mathrm{kcal} / \mathrm{mol})=138.953$

$\mathrm{G}($ in $\mathrm{kcal} / \mathrm{mol})=112.648$

Geometry

C $-0.55640633-0.93876014-2.36132846$

C $0.06620092-0.28242840-1.13140115$

C $-0.75072662 \quad 0.94523433-0.77089741$

C $1.71283677 \quad 1.12391907 \quad 1.45230957$

C $0.54941564 \quad 0.50823244 \quad 1.82392317$

C $1.66147449-1.72922561-0.07047794$

C $0.19405149-1.261215320 .07656210$

C $-0.09108801-0.631659671 .38989377$

C $-0.59926130 \quad 1.77866594 \quad 2.50822019$

C $-1.06599358-1.361217492 .24838480$

$\mathrm{H}-0.57942828-0.24029210 \quad-3.20885724$

$\mathrm{H}-1.57348745-1.29445113-2.17857320$

$\mathrm{H} \quad 0.05345718-1.79566649-2.68218836$

$\mathrm{H}-1.745930650 .73644493-0.35494007$

H $1.92900212 \quad 2.16350741 \quad 1.58716791$

H $2.49379508 \quad 0.59884983 \quad 0.92496040$

$\mathrm{H} \quad 0.38011418 \quad 0.70799854 \quad 3.07045757$

H $1.75105909-2.79732641-0.31963223$

H 2.26935414 $-1.50347547 \quad 0.81835906$

$\mathrm{H}-0.49171228-2.12735383-0.07837560$

$\mathrm{H}-1.55427895 \quad 1.31283112 \quad 2.72160311$

$\mathrm{H}-0.65205122 \quad 2.34647186 \quad 1.57185425$

$\mathrm{H}-0.23081610 \quad 2.41813194 \quad 3.30243795$

$\mathrm{H}-1.24534159-0.88564042 \quad 3.21736734$

$\mathrm{H}-0.71302880-2.38199707 \quad 2.46349725$

$\mathrm{H}-2.04244296-1.458705791 .75146569$

O $2.21009240-1.04488341-1.19095842$

o $-0.36949222 \quad 2.07478058-0.93511111$

o $1.394605410 .11972453-1.42195745$

Vibrational frequencies (in cm-1)

$\begin{array}{llllllllllllllllllll}-1254.9 & 40.2 & 69.1 & 76.0 & 86.3 & 112.0 & 132.6 & 152.7 & 155.4 & 169.1 & 212.2 & 222.7 & 255.7 & 266.3\end{array}$ $310.1324 .3 \quad 343.4 \quad 364.4 \quad 432.4 \quad 443.1 \quad 471.1 \quad 544.8 \quad 577.8 \quad 598.7 \quad 681.7 \quad 693.7 \quad 775.7 \quad 843.0$ $932.2945 .0 \quad 951.9970 .8 \quad 996.4 \quad 997.6 \quad 1002.6 \quad 1006.8 \quad 1021.6 \quad 1036.6 \quad 1053.8 \quad 1077.2 \quad 1091.3$ $1123.81141 .1 \quad 1161.91164 .0 \quad 1235.8 \quad 1248.5 \quad 1271.5 \quad 1280.2 \quad 1286.6 \quad 1290.3 \quad 1291.6 \quad 1295.6$

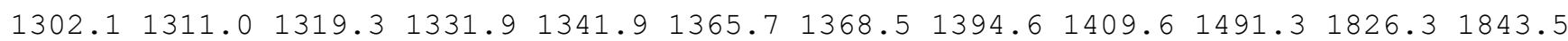
$2142.62630 .4 \quad 2650.12651 .5 \quad 2665.12668 .8 \quad 2674.2 \quad 2675.32682 .4 \quad 2704.92715 .12721 .6$ $2743.5 \quad 2774.7 \quad 2780.3 \quad 2783.6$

name $=\operatorname{TS} 164$

energy (in $\mathrm{kcal} / \mathrm{mol})=44.207053$

$\mathrm{ZPE}(\mathrm{in} \mathrm{kcal} / \mathrm{mol})=139.775$

$\mathrm{G}(\mathrm{in} \mathrm{kcal} / \mathrm{mol})=114.355$

Geometry

C $-2.30098474-2.68292534 \quad-0.69035220$

C $-1.39753564-1.63734586-0.79696686$

C $0.97825325 \quad 2.01381800 \quad-0.24063723$

C $1.20677544 \quad 1.50218201 \quad 1.16713613$

$\begin{array}{lllll}\text { C } & 0.56225824 & 0.17930387 & 1.50785157\end{array}$

C $0.80784959-1.019830590 .54192107$

C $-0.68475986-1.24072151 \quad 0.43154477$

C $-1.00050503 \quad 0.00383465 \quad 1.29013424$

C $-1.69921366 \quad 1.08308523 \quad 0.49179744$

C $-1.74627498 \quad-0.29533443 \quad 2.57649658$

$\mathrm{H}-1.36413048-3.06856924 \quad 0.83734790$

$\mathrm{H}-2.50264893-3.34166891-1.51023502$

$\mathrm{H}-3.07048132-2.72418593 \quad 0.05235920$

$\mathrm{H} \quad 0.96349687 \quad 1.29204774 \quad-1.06913569$

$\mathrm{H} 0.867129962 .27749036 \quad 1.89116431$

H 2.30745983 1.43397398 1.32122828 
$\mathrm{H} \quad 0.83606425-0.08538105 \quad 2.55026583$

$\mathrm{H} 1.41883857-1.831520250 .93858036$

$\mathrm{H} 1.26047829-0.73737948-0.43531939$

$\mathrm{H}-0.94069328-2.45133453 \quad 1.26350107$

$\mathrm{H}-2.780275920 .90752343 \quad 0.42212606$

$\mathrm{H}-1.329689331 .14598922-0.54868111$

$\mathrm{H}-1.55607427 \quad 2.07551692 \quad 0.94111574$

$\mathrm{H}-1.83051995 \quad 0.61129524 \quad 3.19469089$

$\mathrm{H}-1.25284821-1.05438787 \quad 3.19255602$

$\mathrm{H}-2.77121669-0.63742312 \quad 2.37991664$

o $-0.12399921-0.31872100-2.04496375$

$0 \quad 0.84734344 \quad 3.19795265-0.42809907$

o $-1.12355054-1.12236281-1.98096595$

Vibrational frequencies (in cm-1)

$\begin{array}{lllllllllllllll}-1373.6 & 39.6 & 61.7 & 74.5 & 120.5 & 146.6 & 165.8 & 193.1 & 210.7 & 223.7 & 234.6 & 272.4 & 285.0 & 311.4\end{array}$ $351.0 \quad 391.3 \quad 407.6 \quad 436.4 \quad 454.2 \quad 473.5 \quad 560.4 \quad 596.2 \quad 620.3 \quad 648.3 \quad 721.8 \quad 752.0 \quad 769.0 \quad 832.2$ $847.9898 .9921 .2 \quad 965.6 \quad 980.5 \quad 994.4 \quad 1001.7 \quad 1007.4 \quad 1014.7 \quad 1015.9 \quad 1041.4 \quad 1065.4 \quad 1077.3$

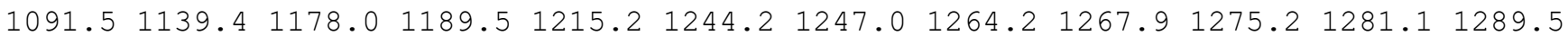

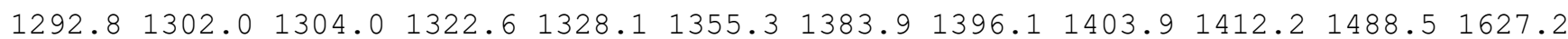
$1827.42624 .22629 .0 \quad 2635.5 \quad 2654.3 \quad 2666.42671 .82674 .72708 .82727 .72736 .12745 .9$ $2771.22779 .3 \quad 2799.2 \quad 3637.4$

name $=\operatorname{TS} 165$

energy (in $\mathrm{kcal} / \mathrm{mol})=42.629921$

$\mathrm{ZPE}($ in $\mathrm{kcal} / \mathrm{mol})=141.381$

G $($ in $\mathrm{kcal} / \mathrm{mol})=116.764$

Geometry

C $-1.00316542-2.87746623 \quad 0.16958127$

C $0.28829022-2.114630920 .37592385$

C $0.27695627-0.65164372-0.18775895$

C $0.83671222 \quad 0.23190636 \quad 0.91072148$

C 0.059526261 .350223991 .33348687

C $1.53992308-0.66589343 \quad 1.91415133$

C $0.83314173-2.03996209 \quad 1.82014472$

C $0.02191677 \quad 1.735803192 .68481224$

C $-0.01517638 \quad 2.07324309 \quad 3.84910748$

C $-0.25811097 \quad-2.15071663 \quad 2.88090734$

$\mathrm{H}-1.24833930-2.95546103-0.89984370$

$\mathrm{H}-1.84722049-2.390910920 .67278660$

$\mathrm{H}-0.92766981-3.90345038 \quad 0.55446655$

$\mathrm{H}-0.69259089-0.32417008-0.59969029$

H $2.14433634 \quad 1.76238493 \quad 0.07160868$

H $1.951588690 .86376354 \quad 0.22245606$

$\mathrm{H}-0.83163678 \quad 1.60950401 \quad 0.75358356$

H $2.61255187-0.77662496 \quad 1.67722344$

$\mathrm{H} \quad 1.49843157-0.25826204 \quad 2.93940642$

H $1.57245108-2.86133183 \quad 1.96715416$

$\mathrm{H}-0.05066908 \quad 2.35854503 \quad 4.83241020$

H $1.713455592 .57283396 \quad 0.30567142$

$\mathrm{H} 0.94496064 \quad 2.52980949 \quad 0.76024714$

$\mathrm{H}-0.78110012-3.111605612 .83511082$

$\mathrm{H} 0.16442400 \quad-2.060386693 .88951651$

$\mathrm{H}-1.00208699-1.350483922 .77468453$

○ $2.06003385-1.75193363-0.99196086$

O $1.14388186-0.71149189-1.31546965$

O $1.24839458-2.78340642-0.45962382$

Vibrational frequencies (in $\mathrm{cm}-1$ )

$\begin{array}{lllllllllllllll}-2329.8 & 40.8 & 55.6 & 89.2 & 144.0 & 191.5 & 205.2 & 214.4 & 237.3 & 262.9 & 287.8 & 314.8 & 335.5 & 363.1\end{array}$ $378.3 \quad 442.6 \quad 469.2 \quad 479.2 \quad 534.4 \quad 595.9 \quad 623.2 \quad 669.4 \quad 685.6 \quad 736.5 \quad 741.5 \quad 792.8 \quad 857.2 \quad 861.1$ $876.0 \quad 883.9 \quad 916.9938 .0 \quad 952.1 \quad 972.2 \quad 976.8 \quad 1005.5 \quad 1009.7 \quad 1018.7 \quad 1064.6 \quad 1092.0 \quad 1105.0$

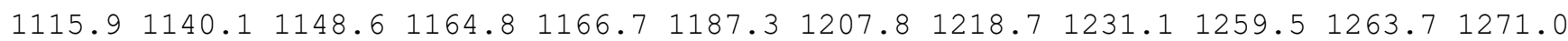
$1284.21287 .91298 .8 \quad 1300.6 \quad 1308.1 \quad 1312.5 \quad 1334.4 \quad 1364.01386 .5 \quad 1390.5 \quad 1402.01577 .8$ $2349.32437 .22664 .92670 .62671 .12675 .6 \quad 2677.52700 .82708 .12742 .72746 .12779 .4$ $2780.8 \quad 2865.8 \quad 2902.5 \quad 3481.5$ 
name $=\operatorname{TS} 166$

energy $($ in $\mathrm{kcal} / \mathrm{mol})=44.368281$

$\mathrm{ZPE}($ in $\mathrm{kcal} / \mathrm{mol})=139.734$

$\mathrm{G}($ in $\mathrm{kcal} / \mathrm{mol})=112.711$

Geometry

C $-2.70480834-1.04722800-0.23820005$

C $-1.23275882-1.27101188-0.44605300$

C $0.88071457 \quad 1.43104782-1.11255135$

C $1.655451631 .37278338 \quad 0.13333393$

C $1.41919070 \quad 0.14585882 \quad 1.00853453$

C $1.57562227-1.17299192 \quad 0.22224184$

C $-0.41326314-1.17066032 \quad 0.69938997$

C $-0.03669375-0.03945371 \quad 1.57296764$

C $-0.943808691 .18069241 \quad 1.45260001$

C $0.01397526-0.475817993 .04367919$

$\mathrm{H}-3.02066604-0.10261422-0.70208384$

$\mathrm{H}-2.99197779-1.02759182 \quad 0.81925295$

$\mathrm{H}-3.28012606-1.84832942-0.72918318$

$\mathrm{H} \quad 0.36005507 \quad 0.58475239-1.54764319$

H $1.47017906 \quad 2.310229330 .72453222$

H 2.74484867 1.45420417 -0.11793022

H $2.14305570 \quad 0.18395307 \quad 1.85434575$

H $2.42013296-1.78451400 \quad 0.55154100$

$\mathrm{H} \quad 1.60337400-1.06983298-0.86539116$

$\mathrm{H} \quad 0.72218976-2.06242965 \quad 0.55398962$

$\mathrm{H}-1.93486218 \quad 0.97825232 \quad 1.87868427$

$\mathrm{H}-1.09832384 \quad 1.49111430 \quad 0.41377952$

$\mathrm{H}-0.529224192 .03975224 \quad 1.99505219$

$\mathrm{H} \quad 0.37623910 \quad 0.32832659 \quad 3.69168539$

$\mathrm{H} \quad 0.66048693-1.34801750 \quad 3.18744323$

$\mathrm{H}-0.98372293-0.765268443 .39680028$

$\begin{array}{llll}0 & 1.33501117 & 3.55688981 & -1.37688897\end{array}$

$0.764524952 .51889801-1.80872911$

$0-0.79264857-1.60582731-1.53822133$

Vibrational frequencies (in $\mathrm{cm}-1$ )

$\begin{array}{lllllllllllllll}-1004.1 & 21.9 & 45.6 & 50.1 & 72.9 & 114.6 & 134.5 & 150.9 & 171.2 & 189.4 & 215.2 & 228.2 & 245.3 & 301.0\end{array}$

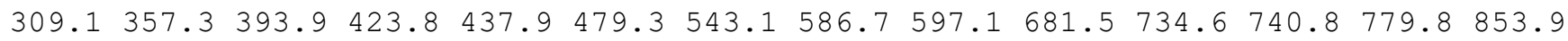

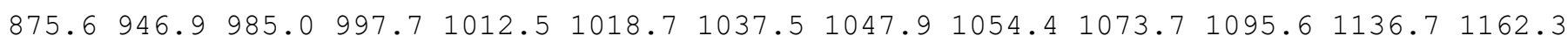

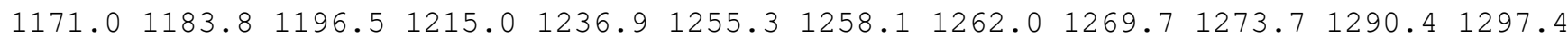
$1301.31304 .31315 .3 \quad 1320.5 \quad 1344.1 \quad 1373.8 \quad 1380.7 \quad 1401.01405 .8 \quad 1516.6 \quad 1592.01778 .1$ $2007.12616 .42655 .42666 .5 \quad 2671.92674 .12675 .92679 .22681 .8 \quad 2682.62700 .62717 .0$ $2727.0 \quad 2778.8 \quad 2780.7 \quad 2783.5$

name $=\operatorname{TS} 167$

energy (in $\mathrm{kcal} / \mathrm{mol})=43.825009$

$\mathrm{ZPE}(\mathrm{in} \mathrm{kcal} / \mathrm{mol})=140.432$

$\mathrm{G}($ in $\mathrm{kcal} / \mathrm{mol})=114.745$

Geometry

C $-2.54413333-0.50066399-0.67909907$

C $-1.17245252-0.61728873-0.41695809$

C $0.147836121 .32980678-0.57189312$

C $1.452312591 .13210526 \quad 0.11092974$

C $1.45082618 \quad 0.08566050 \quad 1.23993980$

C $0.90468746-1.28365944 \quad 0.78306036$

C $-0.56545921-1.327189420 .54039869$

C $0.80629620 \quad 0.56410345 \quad 2.56707840$

C $-0.60706934 \quad 1.13153513 \quad 2.44373047$

C $1.70903701 \quad 1.61492199 \quad 3.23123554$

$\mathrm{H}-2.52317955-0.81626589-2.53802812$

$\mathrm{H}-3.01765796 \quad 0.43560703-0.88523263$

$\mathrm{H}-2.84596606-1.43002558-2.16784671$

$\mathrm{H}-0.66209410 \quad 1.96957418 \quad 1.74094405$ 
H $1.79646230 \quad 2.11079616 \quad 0.52426697$

H $2.219339120 .85359375 \quad-0.64967891$

$\mathrm{H} 2.53959354-0.09064700 \quad 1.48288127$

H $1.18630037-2.04492561 \quad 1.54342413$

$\mathrm{H} \quad 1.44335791-1.60166357-0.13930419$

$\mathrm{H}-1.14801561-1.98318422 \quad 1.19388324$

$\mathrm{H}-1.33384992 \quad 0.37593252 \quad 2.12763888$

$\mathrm{H}-0.95801867 \quad 1.51775707 \quad 3.40968059$

$\mathrm{H} \quad 0.75909165-0.328439193 .24379457$

$\mathrm{H} \quad 1.73343101 \quad 2.54914026 \quad 2.65914937$

H $2.738089901 .26094127 \quad 3.34479405$

$\mathrm{H} \quad 1.34285180 \quad 1.86621744 \quad 4.23421142$

$\begin{array}{lllll}0 & -0.74785262 & 0.36667203 & -2.45968387\end{array}$

$0-0.55422358 \quad 2.28087806-0.65128024$

$0-0.17995204 \quad 0.05853453-1.33700154$

Vibrational frequencies (in cm-1)

$\begin{array}{lllllllllllllllllllll}-447.1 & 23.0 & 71.5 & 83.6 & 123.1 & 165.5 & 171.8 & 177.6 & 215.4 & 246.0 & 260.1 & 265.7 & 280.7 & 303.5\end{array}$ $311.2 \quad 347.0 \quad 354.5 \quad 405.2 \quad 412.8 \quad 456.4 \quad 480.0 \quad 538.4 \quad 590.6 \quad 622.5 \quad 651.1 \quad 735.7 \quad 767.9787 .2$

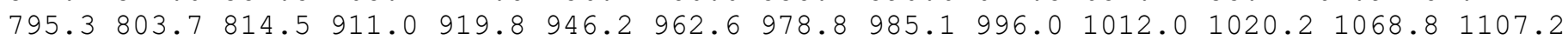

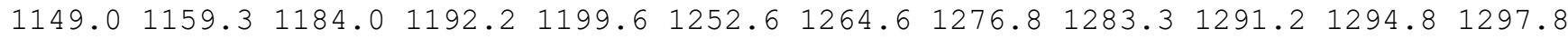
$1304.01307 .8 \quad 1313.2 \quad 1321.91334 .7 \quad 1356.3 \quad 1372.4 \quad 1382.3 \quad 1405.8 \quad 1411.5 \quad 1870.1 \quad 1922.2$ $2638.32647 .5 \quad 2665.7 \quad 2668.4 \quad 2670.3 \quad 2674.92678 .5 \quad 2700.22705 .5 \quad 2721.72732 .8 \quad 2733.9$ $2780.7 \quad 2785.4 \quad 5113.5$

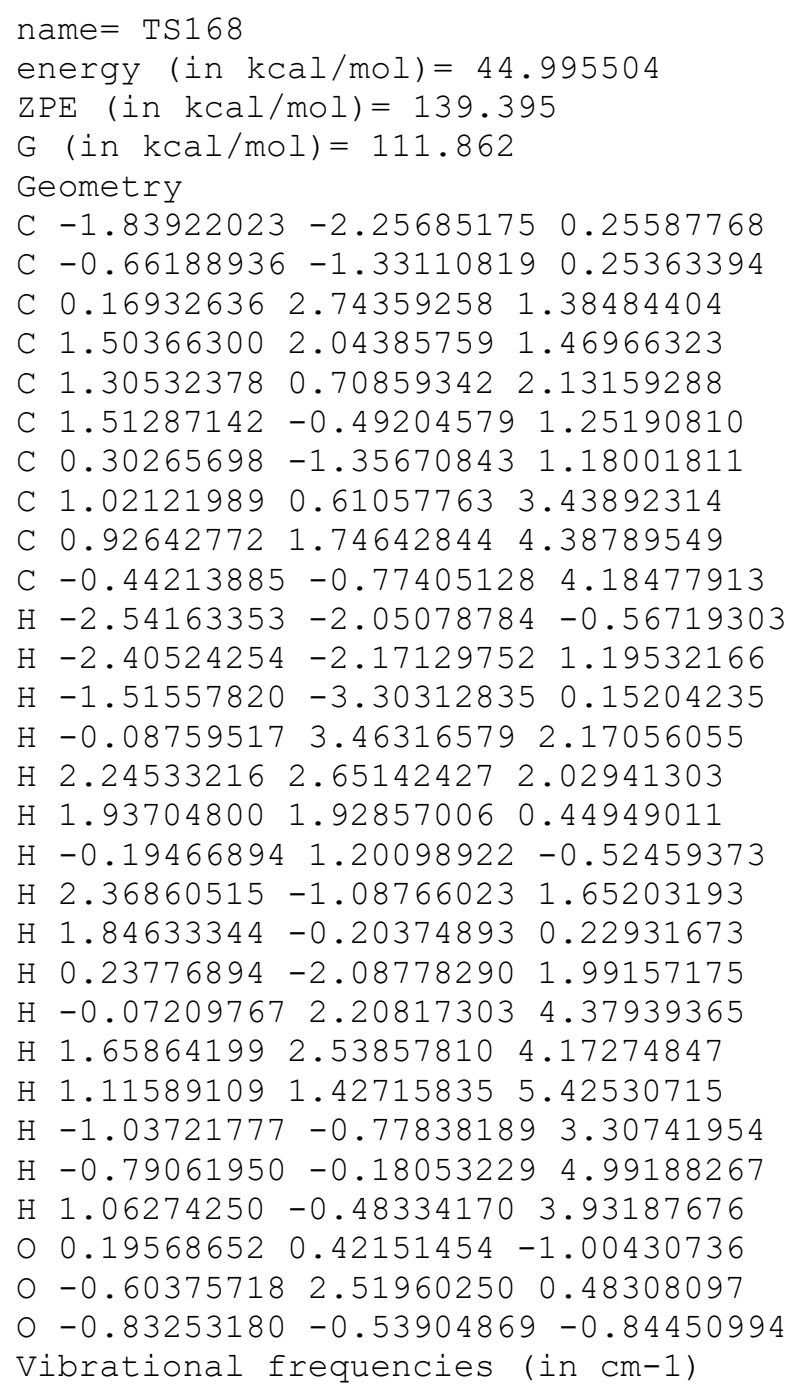


$2104.92639 .6 \quad 2643.8 \quad 2662.5 \quad 2664.1 \quad 2665.12665 .6 \quad 2667.82696 .8 \quad 2699.4 \quad 2726.8 \quad 2730.9$

$2740.32760 .12773 .8 \quad 2775.4$

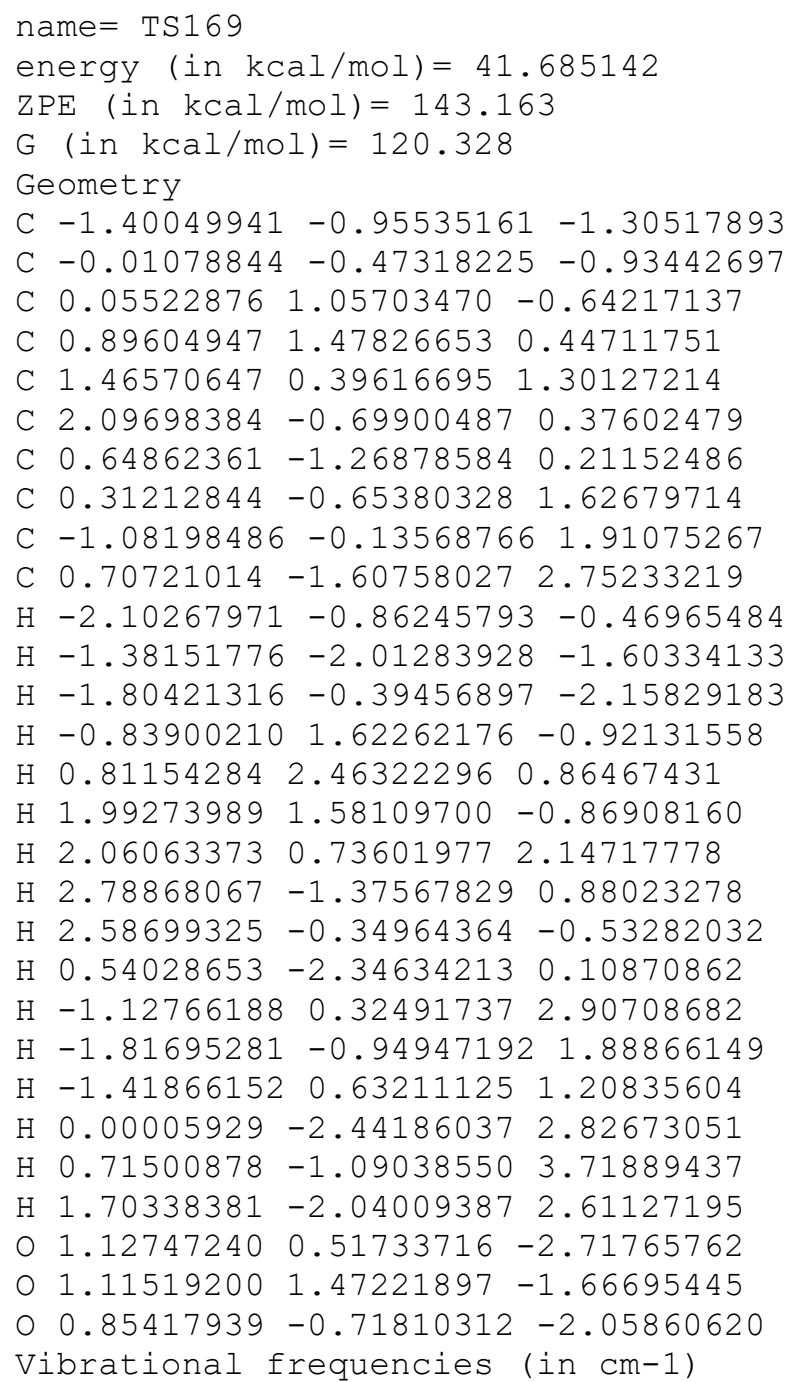

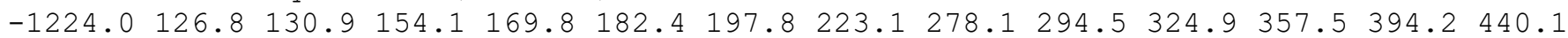
$454.1486 .4 \quad 525.8 \quad 544.8 \quad 596.1 \quad 610.5 \quad 690.4 \quad 716.3 \quad 785.8 \quad 792.2 \quad 844.7 \quad 880.1901 .8 \quad 913.8$ $938.5 \quad 946.8 \quad 959.9 \quad 974.7 \quad 985.3 \quad 1000.6 \quad 1009.6 \quad 1015.9 \quad 1017.7 \quad 1055.4 \quad 1076.1 \quad 1086.1 \quad 1103.4$

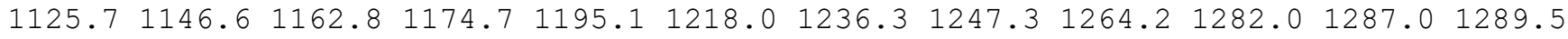
$1290.3 \quad 1296.91299 .5 \quad 1305.7 \quad 1315.7 \quad 1318.6 \quad 1328.8 \quad 1386.6 \quad 1395.2 \quad 1408.0 \quad 1420.2 \quad 1477.3$ $2220.12672 .32672 .6 \quad 2675.5 \quad 2677.4 \quad 2678.32681 .12685 .82723 .4 \quad 2750.82755 .92763 .8$ $2779.52781 .2 \quad 2782.3 \quad 2784.5$

name $=\operatorname{TS} 170$

energy (in $\mathrm{kcal} / \mathrm{mol})=43.437746$

$\mathrm{ZPE}($ in $\mathrm{kcal} / \mathrm{mol})=141.453$

$\mathrm{G}(\mathrm{in} \mathrm{kcal} / \mathrm{mol})=117.445$

Geometry

C $-2.19591161-0.42620777-1.20901907$

C $-0.72249904-0.47828917-0.91303394$

C $0.14844332 \quad 0.82875214 \quad-1.05785862$

C $1.09334674 \quad 1.05783638 \quad 0.10915312$

C $1.04202688-0.160551131 .00388140$

C $1.19536687-1.492298390 .34652267$

C $-0.28004714-1.177717350 .32509505$

C $-0.693706950 .07870202 \quad 2.09864912$

C $-1.82286129 \quad 0.89874259 \quad 1.64953570$

C $-0.95361606-0.99822912 \quad 3.07403294$

$\mathrm{H}-2.618043220 .56950517-1.01202897$

$\mathrm{H}-2.76803923-1.14928767-0.61393118$ 
$\mathrm{H}-2.39433512-0.65985500-2.26780370$

$\mathrm{H}-0.89090612 \quad 1.95970864 \quad-2.28424484$

$\mathrm{H} \quad 0.79742171 \quad 1.97137408 \quad 0.67199090$

$\mathrm{H} \quad 2.12424914 \quad 1.24148938-0.25638504$

$\mathrm{H} 1.46707703-0.045751792 .00372277$

H $1.52052960 \quad-2.33315846 \quad 0.96292777$

$\mathrm{H} 1.70993285-1.56619181-0.61941310$

$\mathrm{H}-0.96033853-1.86801620 \quad 0.79176728$

$\mathrm{H}-2.06207082 \quad 1.61555858 \quad 2.45740575$

$\mathrm{H}-2.74676937 \quad 0.35335111 \quad 1.42387906$

$\mathrm{H}-1.57014746 \quad 1.52048607 \quad 0.77548723$

$\mathrm{H}-1.00605748 \quad-0.53551324 \quad 4.07664967$

$\mathrm{H}-0.13399329-1.727344493 .12183779$

$\mathrm{H}-1.88888516-1.55309132 \quad 2.93203111$

$\begin{array}{lllll}0 & 0.78464358 & 0.24154669 & -2.21803407\end{array}$

$0-0.49141097 \quad 2.00386964-1.38513595$

o - $0.02437593-0.97479702-2.10661843$

Vibrational frequencies (in cm-1)

$\begin{array}{llllllllllllll}-693.0 & 93.8 & 96.5 & 106.8 & 170.6 & 173.5 & 184.9 & 220.2 & 233.7 & 250.7 & 267.3 & 300.0 & 309.4 & 325.2\end{array}$ $\begin{array}{llllllllllllll}330.9 & 375.9 & 397.0 & 419.9 & 438.4 & 492.1 & 557.8 & 591.3 & 625.8 & 668.8 & 786.2 & 892.8 & 911.3 & 917.8\end{array}$

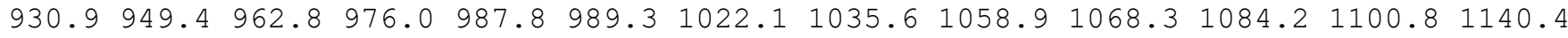

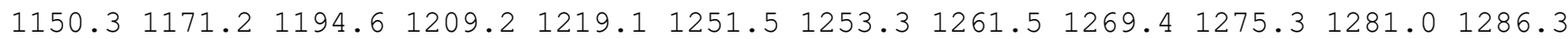

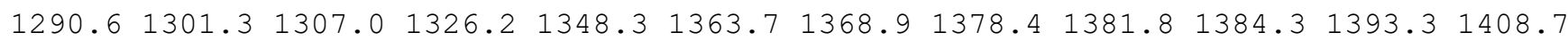
$2657.02657 .32661 .8 \quad 2663.1 \quad 2666.92670 .0 \quad 2672.42687 .62711 .82737 .02756 .12770 .6$ $2772.4 \quad 2774.2 \quad 2775.2 \quad 2794.2$

name $=\operatorname{TS} 171$

energy (in $\mathrm{kcal} / \mathrm{mol})=45.188156$

$\operatorname{ZPE}($ in $\mathrm{kcal} / \mathrm{mol})=140.594$

$\mathrm{G}($ in $\mathrm{kcal} / \mathrm{mol})=113.522$

Geometry

C $-2.01758752-1.04143366-1.68320573$

C $-0.76831447-0.86071562-1.02637469$

C $-0.52538531 \quad 0.45431634 \quad-0.25925823$

$\begin{array}{lllll}\text { C } & 0.67274802 & 0.43552528 & 0.69572765\end{array}$

C $0.34688969-0.293098051 .95575192$

C $1.05508743-2.54644430-1.18169213$

C $-0.02220961-2.05206283-0.57514463$

C $-0.44004056 \quad 0.17852774 \quad 2.93236374$

C $-1.10965612 \quad 1.51117057 \quad 2.90145840$

C $-0.71920681-0.63334636 \quad 4.15634974$

$\mathrm{H}-2.35602403-2.01566803-1.97128673$

$\mathrm{H}-2.80796530-0.32293881-1.60121596$

$\mathrm{H}-1.23923590-0.38251165-2.93319671$

$\mathrm{H}-1.45270353 \quad 0.79135398 \quad 0.25905123$

H $0.97979639 \quad 1.48282757 \quad 0.91065422$

$\mathrm{H} \quad 1.55228988-0.01407103 \quad 0.18900115$

$\mathrm{H} 0.81008843-1.274881932 .03873162$

$\mathrm{H} \quad 1.57741039-3.42716657-0.83951065$

$\mathrm{H} \quad 1.49833369-2.11234120-2.06790399$

$\mathrm{H}-0.44962712-2.51161807 \quad 0.32090445$

$\mathrm{H}-2.14769896 \quad 1.420609592 .54883388$

$\mathrm{H}-0.60099650 \quad 2.226031112 .23935853$

$\mathrm{H}-1.14767238 \quad 1.973945313 .89724850$

$\mathrm{H}-0.27677115-0.16658788 \quad 5.04811478$

$\mathrm{H}-0.31550301-1.65221756 \quad 4.09421082$

$\mathrm{H}-1.79932962-0.72427700 \quad 4.33773525$

$0.46828633 \quad 0.87800715 \quad-2.28136523$

o $-0.326742161 .46554293-1.24223796$

o $-0.11540131-0.37078105-2.46305297$

Vibrational frequencies (in $\mathrm{cm}-1$ )

$\begin{array}{llllllllllllll}-1417.2 & 24.4 & 44.0 & 48.3 & 82.5 & 88.2 & 88.8 & 106.8 & 190.9 & 205.4 & 247.9 & 273.2 & 299.4 & 302.4\end{array}$

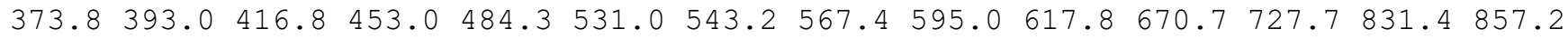
$912.5925 .3944 .4979 .8 \quad 993.3 \quad 999.6 \quad 1009.2 \quad 1016.2 \quad 1041.91064 .5 \quad 1077.3 \quad 1080.01094 .8$ 


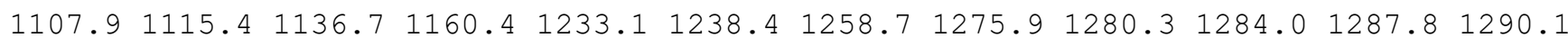

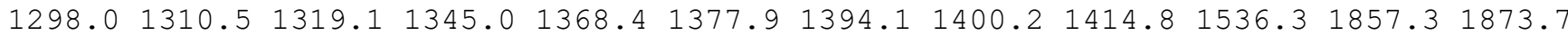
$2137.52653 .32667 .6 \quad 2670.3 \quad 2673.5 \quad 2675.92679 .12708 .52735 .52739 .82746 .8 \quad 2756.0$ $2777.5 \quad 2780.7 \quad 2781.4 \quad 2796.5$

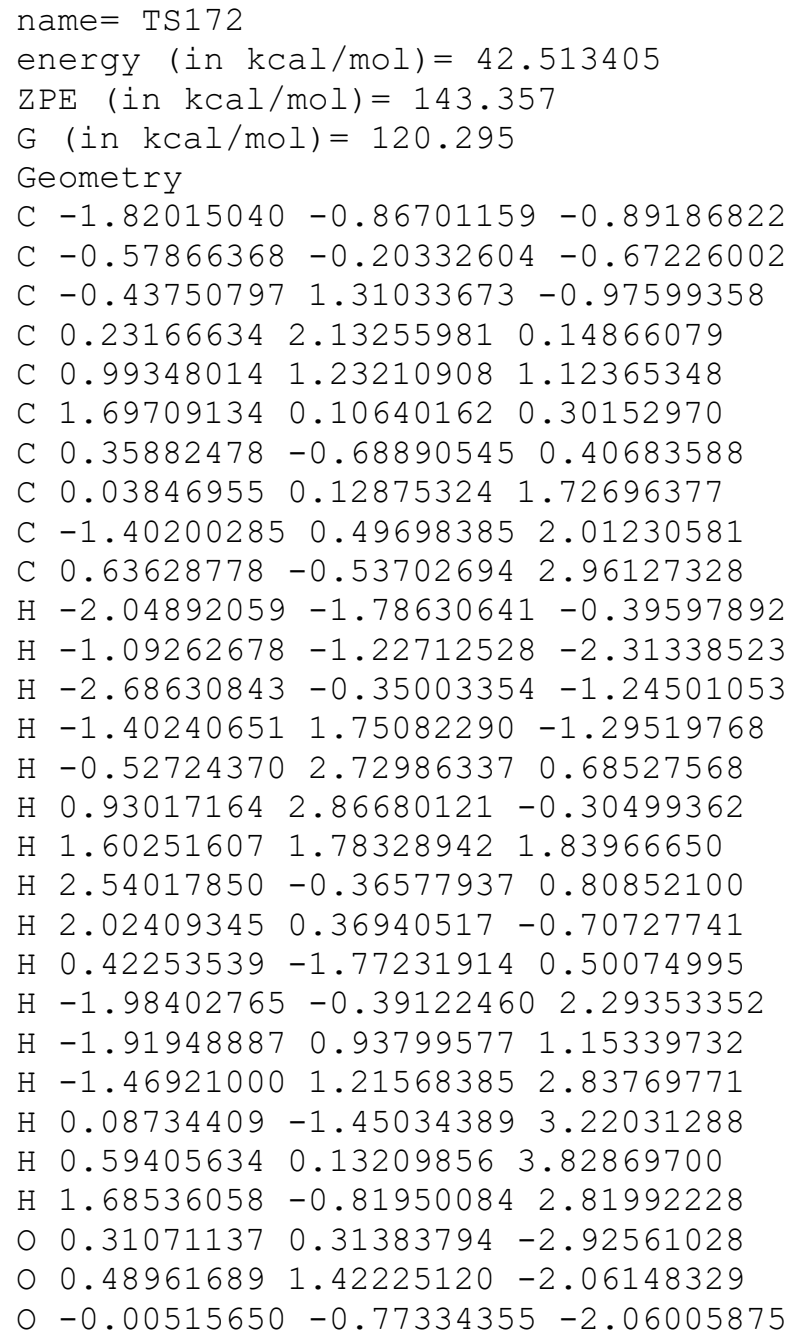


$\mathrm{H}-3.35381631-0.44870685-0.45287635$

$\mathrm{H}-2.16290867-1.21769725-1.50346825$

$\mathrm{H}-2.511911490 .50587760 \quad-1.68769982$

$\mathrm{H}-1.76294323 \quad 1.54192872 \quad 0.20672222$

$\mathrm{H} \quad 1.86044641 \quad 1.24635197 \quad 0.33431028$

H $1.68845005 \quad 0.18788197-1.07108640$

H 2.22328312 -1.14239291 1.11017851

$\mathrm{H} \quad 0.42913537 \quad-2.88617851 \quad 0.53387404$

$\mathrm{H} \quad 0.18628716-1.86647086-0.88230726$

$\mathrm{H}-1.72546512-1.71647821 \quad 1.35123096$

H $0.12889215 \quad 1.56408365 \quad 2.00796253$

$\mathrm{H} \quad 0.75317803 \quad 0.85133788 \quad 3.47875880$

$\mathrm{H}-0.982991110 .78898206 \quad 3.12948498$

H $1.32374625-1.49997136 \quad 3.65445546$

$\mathrm{H} \quad 0.46716221-2.70796971 \quad 2.69661135$

H $-0.42954088-1.68524875 \quad 3.81688024$

$\begin{array}{llll}0 & 0.84123617 & 0.15311485 & -3.60032178\end{array}$

$0-0.59310994 \quad 2.05745328-0.26659028$

O $0.86398474-0.94891231-3.33206055$

Vibrational frequencies (in cm-1)

$\begin{array}{llllllllllllllll}-2090.7 & 21.3 & 31.2 & 35.8 & 48.4 & 58.6 & 90.4 & 141.0 & 168.7 & 177.6 & 184.4 & 192.1 & 261.6 & 285.3\end{array}$ $325.7 \quad 397.6 \quad 405.9 \quad 433.1 \quad 485.7 \quad 502.1 \quad 512.5 \quad 619.3 \quad 643.4 \quad 726.4 \quad 791.7 \quad 857.2 \quad 877.7 \quad 906.3$

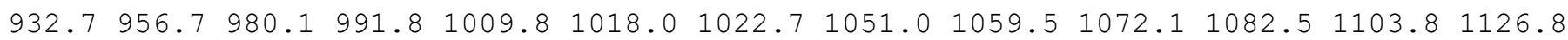

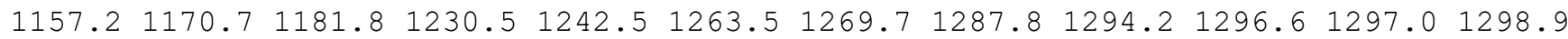
$1301.41303 .51314 .8 \quad 1321.3 \quad 1337.2 \quad 1381.3 \quad 1395.2 \quad 1408.3 \quad 1428.4 \quad 1500.01595 .3 \quad 1596.9$ $2049.12659 .6 \quad 2664.92670 .8 \quad 2675.3 \quad 2676.4 \quad 2676.62680 .72697 .7 \quad 2741.7 \quad 2749.4 \quad 2758.4$ $2771.0 \quad 2774.7 \quad 2781.8 \quad 2785.1$

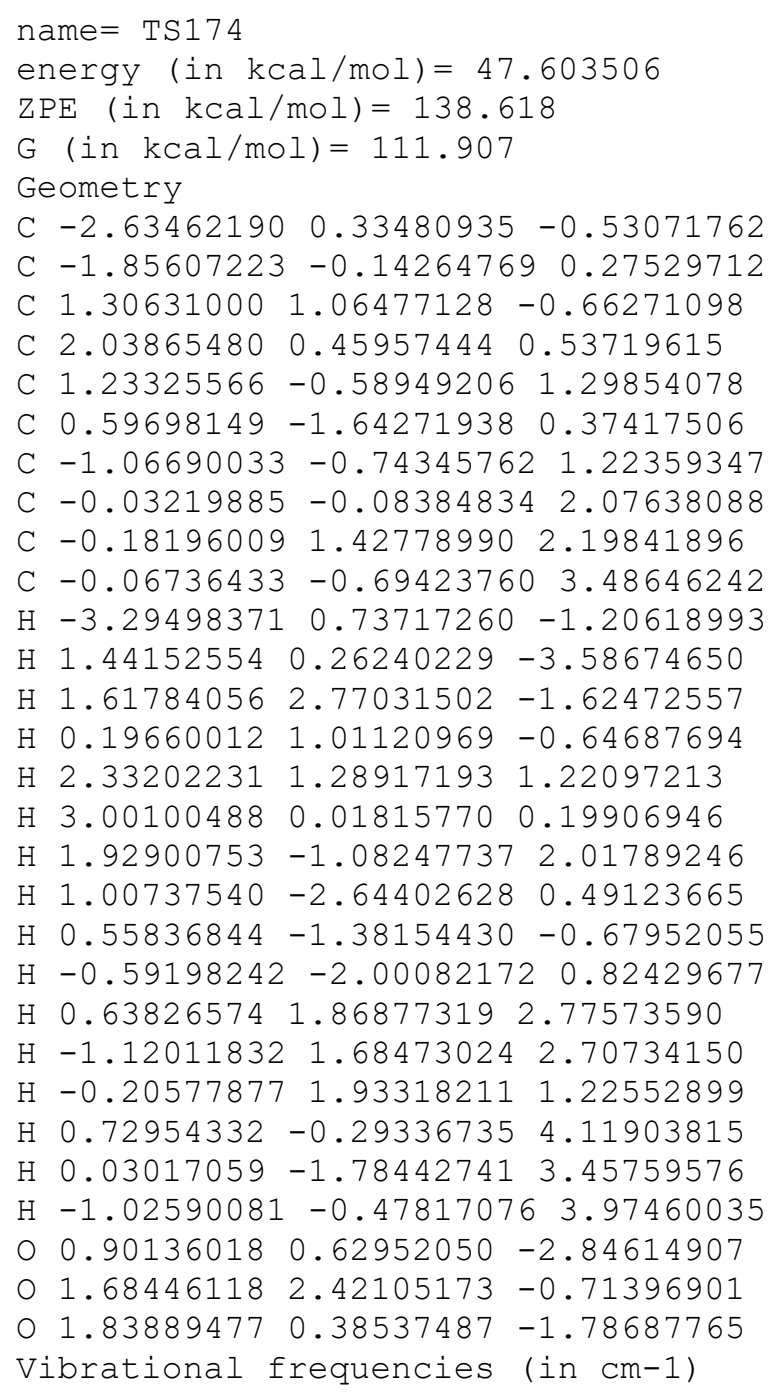


$\begin{array}{llllllllllllllllll}-1307.0 & 41.2 & 58.1 & 61.0 & 78.3 & 116.8 & 130.3 & 133.8 & 167.7 & 180.4 & 202.1 & 231.0 & 247.1 & 257.9\end{array}$ $302.3 \quad 334.4 \quad 362.5 \quad 372.2 \quad 403.2 \quad 428.0 \quad 493.7 \quad 500.7 \quad 540.9 \quad 572.0 \quad 619.0 \quad 690.0 \quad 755.7 \quad 809.1$ $827.9878 .4 \quad 897.5 \quad 915.6 \quad 984.5 \quad 999.0 \quad 1005.6 \quad 1021.1 \quad 1034.5 \quad 1052.8 \quad 1067.1 \quad 1080.8 \quad 1141.9$

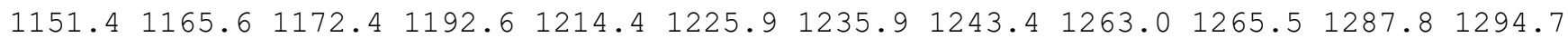

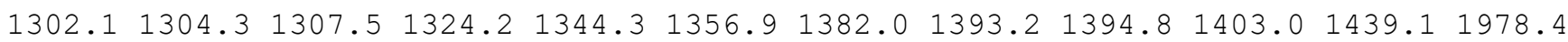
$2367.22639 .4 \quad 2648.4 \quad 2668.92671 .1 \quad 2676.1 \quad 2678.7 \quad 2684.72700 .92731 .92739 .82781 .0$ 2783.32804 .82868 .62881 .7

name $=$ TS175

energy (in $\mathrm{kcal} / \mathrm{mol})=44.933331$

$\mathrm{ZPE}($ in $\mathrm{kcal} / \mathrm{mol})=141.418$

$\mathrm{G}($ in $\mathrm{kcal} / \mathrm{mol})=116.981$

Geometry

C $-2.81739683 \quad 0.59892194 \quad 1.41461410$

C $-1.76217468-0.24234559 \quad 0.69848171$

C $-0.77459736 \quad 0.63135434 \quad-0.14057327$

C $0.70863972 \quad 0.29398703 \quad 0.07214339$

C $0.98993548 \quad 0.10857671 \quad 1.58018344$

C $2.48300236-0.089697021 .85334033$

C $-1.11508866-1.20178318 \quad 1.62675835$

C $0.15172622-1.06346592 \quad 2.06732565$

C $1.558160131 .40477410-0.54706573$

C $0.77096492-1.844533463 .04600068$

$\mathrm{H}-3.32890387 \quad 1.27744192 \quad 0.72062081$

$\mathrm{H}-2.36804114 \quad 1.19067107 \quad 2.21909617$

$\mathrm{H}-3.58652361-0.04141418 \quad 1.86597250$

$\mathrm{H}-0.97464211 \quad 1.71569561-0.02471181$

$\mathrm{H} \quad 0.92887080-0.66890816-0.45441328$

$\mathrm{H} 2.67814354-0.235607692 .92454201$

$\mathrm{H} \quad 0.64665351 \quad 1.02042866 \quad 2.13443853$

$\mathrm{H} 2.88608633-0.951148661 .31058181$

H $3.06308769 \quad 0.79012504 \quad 1.54940741$

$\mathrm{H}-1.75086235-2.02895571 \quad 1.91615014$

H $1.49461657 \quad 2.33710633 \quad 0.02332075$

H $1.229774341 .61782802-1.57416313$

$\mathrm{H} \quad 2.614479121 .11940741-0.60570281$

$\mathrm{H}-0.36794740-2.729939493 .87291850$

$\mathrm{H} 1.63399685-2.44695718 \quad 3.00723684$

$\mathrm{H}-0.27179022-3.21479386 \quad 3.24367765$

o $-1.64970522-0.97420997-1.44134734$

$\begin{array}{lllll}0 & -1.13337540 & 0.35098057 & -1.49189778\end{array}$

o $-2.49749857-0.96775434-0.31111116$

Vibrational frequencies (in cm-1)

$\begin{array}{lllllllllllllll}-786.9 & 50.5 & 78.2 & 112.3 & 160.4 & 194.7 & 203.5 & 218.6 & 239.3 & 251.4 & 284.1 & 288.4 & 315.5 & 343.0\end{array}$

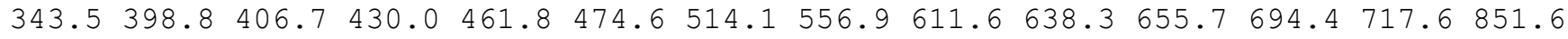

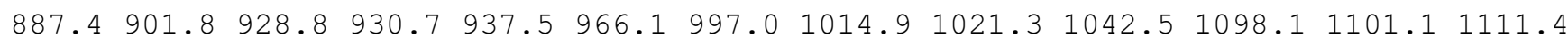

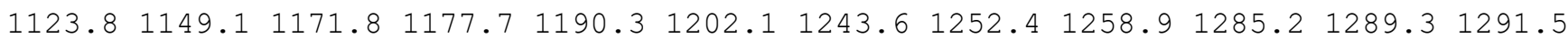
$1295.01297 .8 \quad 1299.2 \quad 1300.4 \quad 1305.8 \quad 1314.7 \quad 1326.3 \quad 1353.8 \quad 1392.4 \quad 1399.4 \quad 1405.8 \quad 1438.4$ $1829.62667 .02670 .72671 .5 \quad 2673.92674 .92681 .12684 .12693 .0 \quad 2711.62744 .12770 .2$ $2778.3 \quad 2779.5 \quad 2781.4 \quad 4913.1$

name $=\operatorname{TS} 176$

energy $($ in $\mathrm{kcal} / \mathrm{mol})=44.166493$

$\operatorname{ZPE}($ in $\mathrm{kcal} / \mathrm{mol})=142.343$

$\mathrm{G}($ in $\mathrm{kcal} / \mathrm{mol})=118.303$

Geometry

C $-2.58020289-2.53062435-0.15174621$

C $-1.75811690-1.24008166-0.20076715$

C $-2.42428134 \quad-0.12112522 \quad 0.67530277$

C $-1.52576555 \quad 0.95899102 \quad 1.29720991$

C $-0.07055116 \quad 0.63189297 \quad 1.60259439$

C $0.47355527-0.28622074 \quad 0.16969025$

C $-0.32869780-1.45617008 \quad 0.09690383$ 
C $0.26460118-0.25945623 \quad 2.65288241$

C $1.56036438-0.11297199 \quad 3.36513957$

C $-0.51247184-1.431001852 .85159815$

$\mathrm{H}-2.21564788-3.24579445-0.90099559$

$\mathrm{H}-3.63404080-2.33458381-0.38998077$

$\mathrm{H}-2.52808799-3.01310120 \quad 0.82678589$

$\mathrm{H}-3.12006768-0.550083491 .42310929$

$\mathrm{H}-2.00533784 \quad 1.32244407 \quad 2.23022794$

$\mathrm{H}-1.54484384 \quad 1.83471301 \quad 0.60540436$

$\mathrm{H} 0.545127051 .548696791 .54969112$

H $1.54931463-0.427496410 .29173574$

$\mathrm{H} \quad 0.26696237 \quad 0.49790384-0.57531124$

$\mathrm{H} \quad 0.10456023-2.42522423-0.07761369$

H $2.20108350 \quad 0.67598439 \quad 2.94899597$

H $2.14617921-1.045034453 .35243258$

H $1.38991748 \quad 0.13763369 \quad 4.42502685$

H $-0.45412870-1.95248944 \quad 1.69353784$

$\mathrm{H}-0.16326705-2.16542434 \quad 3.57304537$

$\mathrm{H}-1.59795209-1.32712160 \quad 2.91357616$

$\begin{array}{lllll}0 & -2.50706053 & 0.53890623 & -1.45764028\end{array}$

$0-3.28520645 \quad 0.50958779-0.27438425$

o $-1.91511199-0.75166108-1.55212239$

Vibrational frequencies (in $\mathrm{cm}-1$ )

$\begin{array}{lllllllllllllll}-1301.8 & 60.3 & 87.4 & 94.4 & 128.8 & 173.8 & 193.4 & 215.4 & 248.4 & 280.3 & 315.2 & 319.5 & 343.2 & 379.4\end{array}$

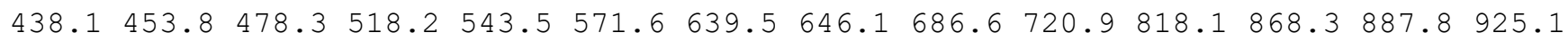
$954.1965 .2977 .9 \quad 991.4 \quad 1001.4 \quad 1010.7 \quad 1029.7 \quad 1045.6 \quad 1073.3 \quad 1085.0 \quad 1087.91117 .1 \quad 1124.7$

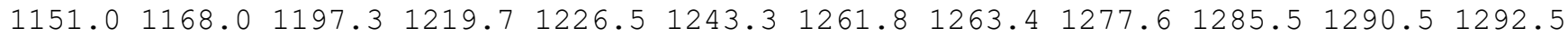

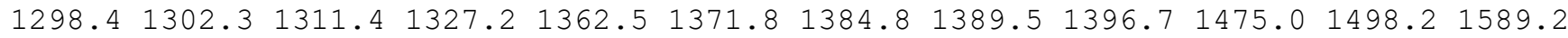
$1816.42645 .22661 .8 \quad 2670.7 \quad 2672.0 \quad 2672.6 \quad 2684.12689 .22691 .92717 .62728 .62743 .6$ $2761.6 \quad 2772.4 \quad 2776.5 \quad 2782.1$

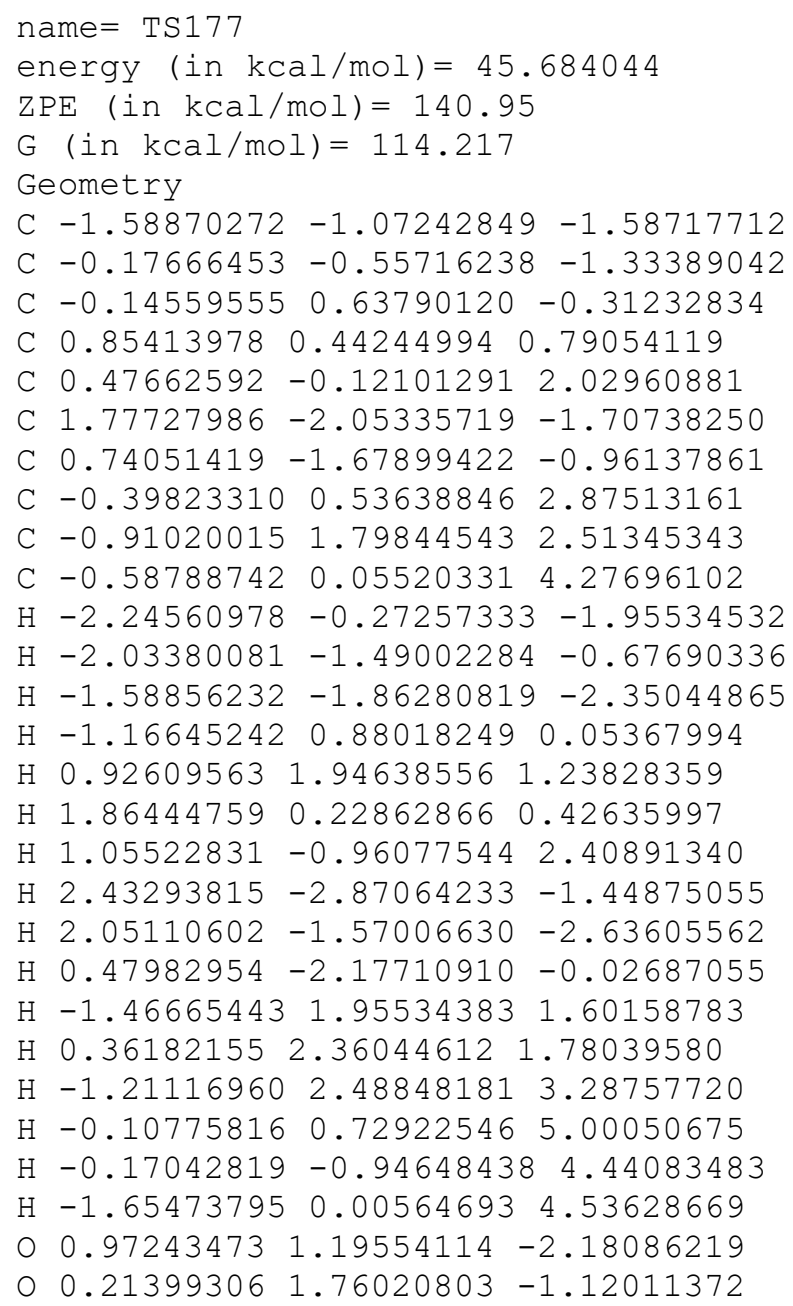


$0.22268374 \quad 0.06045043-2.56941503$

Vibrational frequencies (in $\mathrm{cm}-1$ )

$\begin{array}{llllllllllllllll}-1796.7 & 25.6 & 37.0 & 40.9 & 59.9 & 104.8 & 141.4 & 152.1 & 201.6 & 217.0 & 237.0 & 286.2 & 320.8 & 335.2\end{array}$ $392.0 \quad 434.5 \quad 443.8 \quad 505.0 \quad 550.2 \quad 558.8 \quad 594.7 \quad 644.5 \quad 682.6 \quad 703.8 \quad 734.1 \quad 817.4 \quad 852.7 \quad 867.6$ $895.6930 .3 \quad 955.3 \quad 962.2 \quad 976.7 \quad 986.3 \quad 992.2 \quad 1009.2 \quad 1046.4 \quad 1059.1 \quad 1065.5 \quad 1086.4 \quad 1095.3$

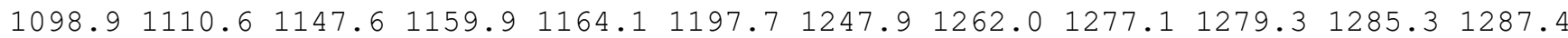
$1288.61309 .01326 .0 \quad 1335.2 \quad 1372.3 \quad 1386.0 \quad 1390.1 \quad 1394.61466 .2 \quad 1482.3 \quad 1584.91669 .6$ $1852.32668 .42671 .8 \quad 2672.4 \quad 2676.1 \quad 2676.4 \quad 2699.62710 .22729 .92752 .7 \quad 2760.7 \quad 2767.2$ $2779.02780 .6 \quad 2782.4 \quad 3092.1$

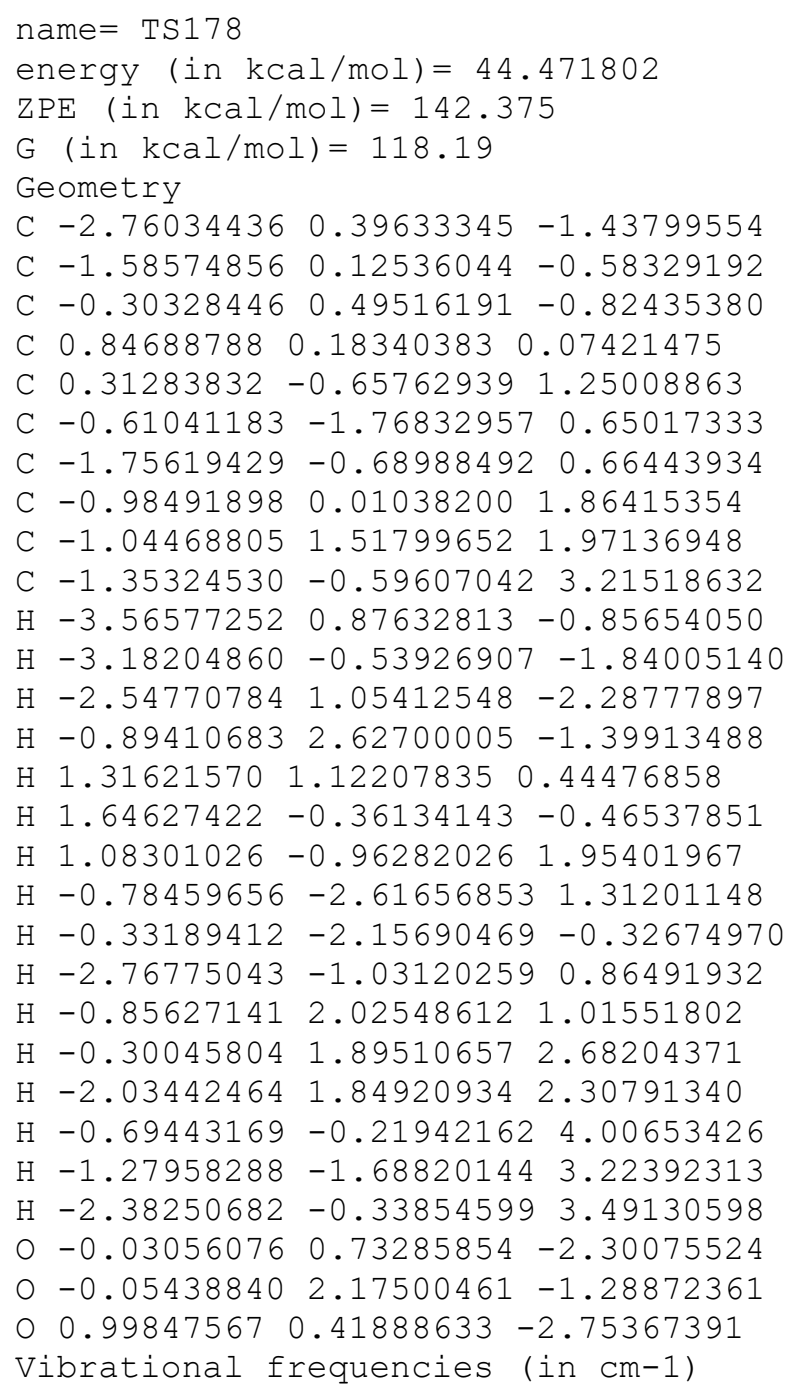


C $3.556968851 .12981741-1.54484654$

C $-1.41303128-0.27452197 \quad 1.02027325$

C $-0.37607370-0.00003180 \quad 1.75834571$

C $0.92331219 \quad 0.53066396 \quad 1.26086618$

C $-0.48250347-0.23323038 \quad 3.23564559$

$\mathrm{H}-2.00724853-0.60687617-3.35800426$

$\mathrm{H}-3.35968011-0.49085798-2.21446355$

$\mathrm{H}-2.23139563-1.85774045-2.14308707$

$\mathrm{H}-2.35937349 \quad 1.89252402-1.96548377$

$\mathrm{H} 2.28336343-0.85094027-3.26111179$

H $2.13242352-1.27841621-1.52090239$

H $1.522347291 .60969192-1.96618736$

$\mathrm{H} \quad 3.884487192 .12302240-1.27922576$

$\mathrm{H} \quad 4.34626058 \quad 0.39912938-1.47990442$

$\mathrm{H}-1.98353151-0.34440062-0.10593884$

$\mathrm{H} 1.66641617-0.27239805 \quad 1.15210862$

$\mathrm{H} \quad 1.34155084 \quad 1.28844325 \quad 1.93756807$

$\mathrm{H} \quad 0.83171736 \quad 1.01628476 \quad 0.26864452$

$\mathrm{H}-1.43683672-0.68670923 \quad 3.53590810$

$\mathrm{H}-0.38291537 \quad 0.71550179 \quad 3.78539104$

$\mathrm{H} \quad 0.32118978-0.90171495 \quad 3.58038282$

$00.47466888-0.57066768-2.53680017$

$\begin{array}{lllll}0 & -0.58377770 & 2.18673208 & -1.10108260\end{array}$

o $-0.15928881-0.56692534-1.21796258$

Vibrational frequencies (in $\mathrm{cm}-1$ )

$\begin{array}{lllllllllllllll}-1310.1 & 40.6 & 50.9 & 55.2 & 64.8 & 88.1 & 93.1 & 105.2 & 133.6 & 138.8 & 145.5 & 151.4 & 169.2 & 183.4\end{array}$ $208.1247 .6 \quad 263.2 \quad 298.7 \quad 334.8 \quad 379.2 \quad 395.5 \quad 474.6 \quad 500.3 \quad 568.2 \quad 581.4 \quad 618.2 \quad 690.1 \quad 828.6$

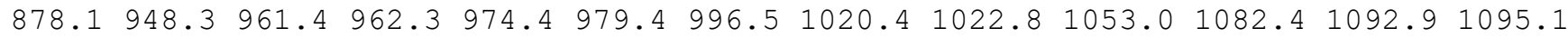

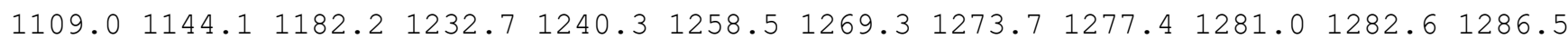

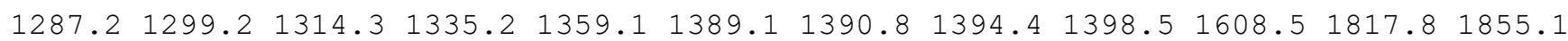
$1956.12632 .22640 .5 \quad 2652.3 \quad 2665.5 \quad 2667.92670 .12673 .22681 .62706 .72716 .12743 .6$ $2770.4 \quad 2775.12780 .2 \quad 2790.4$

name $=\mathrm{TS} 180$

energy (in $\mathrm{kcal} / \mathrm{mol})=47.588827$

$\operatorname{ZPE}($ in $\mathrm{kcal} / \mathrm{mol})=139.756$

$\mathrm{G}($ in $\mathrm{kcal} / \mathrm{mol})=114.059$

Geometry

C $-2.384135110 .42654632-1.08979353$

C $-0.91632740 \quad 0.10271643-0.95037176$

C $-0.058802521 .30854271-1.27237598$

C 2.206831881 .552974361 .67069736

C 1.873445920 .308000072 .01910016

C $0.86037761-1.08234598 \quad 0.19512930$

C $-0.51523764-0.75147981 \quad 0.17451451$

C $0.49450638-0.23661241 \quad 1.98090442$

C $-0.57936032 \quad 0.79794382 \quad 2.22437013$

C $0.30485992-1.46611178 \quad 2.84027662$

$\mathrm{H}-3.01227400-0.46470929-0.95954617$

$\mathrm{H}-2.61249055 \quad 0.82913998-2.08769374$

$\mathrm{H}-2.69949468 \quad 1.17536605-0.35064308$

H $0.49911385 \quad 1.27160014 \quad-2.21942796$

H $1.50609421 \quad 2.29514871 \quad 1.30333925$

H 3.220123861 .928047451 .71610137

H 2.63449930 $-0.39651228 \quad 2.36615161$

$\mathrm{H} 1.20101298-2.08570356 \quad 0.41281277$

$\mathrm{H} \quad 1.63020814-0.44118525-0.23181499$

$\mathrm{H}-1.27392308-1.38707622 \quad 0.62014456$

$\mathrm{H}-0.39639976 \quad 1.31885845 \quad 3.17766012$

$\mathrm{H}-1.58970030 \quad 0.37666443 \quad 2.27980349$

$\mathrm{H}-0.60311998 \quad 1.58580918 \quad 1.44945626$

$\mathrm{H} 0.34814500-1.194227613 .90813609$

H $1.07752120 \quad-2.22998867 \quad 2.68127068$

$\mathrm{H}-0.66725963-1.95117328 \quad 2.68328216$ 
$0.67078167-1.23412949-1.93518329$

$0-0.000422212 .26542295-0.53940793$

o $-0.59640733-0.95210217-1.97073866$

Vibrational frequencies (in $\mathrm{cm}-1$ )

$\begin{array}{lllllllllllllllll}-823.1 & 56.3 & 70.9 & 105.6 & 113.1 & 121.3 & 129.5 & 167.3 & 191.0 & 214.6 & 240.7 & 255.6 & 262.6 & 270.6\end{array}$ $278.6 \quad 298.4 \quad 317.4 \quad 369.7 \quad 402.3 \quad 407.7 \quad 480.3 \quad 521.0 \quad 544.2 \quad 557.3 \quad 624.4 \quad 655.8 \quad 766.6 \quad 855.9$

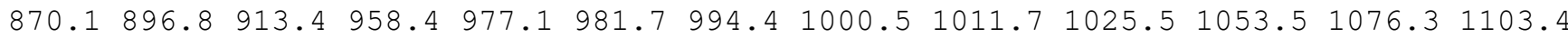
$\begin{array}{llllllllllll}1115.0 & 1141.0 & 1143.2 & 1229.4 & 1238.2 & 1269.7 & 1277.7 & 1281.2 & 1281.9 & 1282.4 & 1286.8 & 1287.4\end{array}$ $1313.81329 .5 \quad 1339.6 \quad 1360.4 \quad 1364.2 \quad 1382.8 \quad 1398.6 \quad 1401.51407 .1 \quad 1508.9 \quad 1825.5 \quad 1832.5$ $2635.42646 .02658 .92665 .62668 .6 \quad 2669.5 \quad 2674.52678 .02693 .72740 .2 \quad 2745.32749 .2$ $2769.62771 .8 \quad 2775.7 \quad 2778.9$

name $=\operatorname{TS} 181$

energy $($ in $\mathrm{kcal} / \mathrm{mol})=46.683401$

$\mathrm{ZPE}(\mathrm{in} \mathrm{kcal} / \mathrm{mol})=140.998$

$\mathrm{G}($ in $\mathrm{kcal} / \mathrm{mol})=114.594$

Geometry

C $-2.50686224 \quad 0.63087358-1.32480818$

C $-1.12742360 \quad 0.03304621-1.03656108$

C $-0.223159171 .03307849-0.28243822$

$\begin{array}{lllll}\text { C } & 0.47411877 & 0.79974990 & 0.95722387\end{array}$

C $0.46570098-0.454095961 .68845533$

C $-0.90215458-2.44535273-0.99001876$

C $-1.28430857-1.31230351-0.40483121$

C $0.28526816-0.55099690 \quad 3.01643893$

C $0.03116463 \quad 0.62737050 \quad 3.89657956$

C $0.33010827-1.87331240 \quad 3.71031132$

$\mathrm{H}-3.14010024-0.08332363-1.86854828$

$\mathrm{H}-2.434055051 .53267374 \quad-1.94653170$

$\mathrm{H}-3.024143220 .89525443-0.39537472$

$\mathrm{H}-0.532029752 .07711448-0.47900180$

$\mathrm{H} \quad 0.75570673 \quad 1.67923680 \quad 1.52851588$

$\mathrm{H} \quad 1.70083580 \quad 0.50273208-0.10662440$

$\mathrm{H} 0.64198014-1.35361507 \quad 1.09468691$

$\mathrm{H}-1.03522068-3.41909515-0.54483564$

$\mathrm{H}-0.42463854-2.48513681-1.95993439$

$\mathrm{H}-1.76320385-1.29016945 \quad 0.57600295$

$\mathrm{H}-0.34255766 \quad 0.34226078 \quad 4.88738026$

$\mathrm{H}-0.71087754 \quad 1.30795546 \quad 3.45484706$

$\mathrm{H} \quad 0.95302799 \quad 1.20711278 \quad 4.05060761$

$\mathrm{H}-0.64754018-2.12207347 \quad 4.14697611$

H $1.06311043-1.867350794 .52924065$

$\mathrm{H} \quad 0.60352124 \quad-2.69748382 \quad 3.03890841$

○ $0.88059387-0.04733628-2.16959083$

$01.08163944 \quad 0.88216122-1.07952086$

o $-0.52076289-0.05238241-2.34020521$

Vibrational frequencies (in $\mathrm{cm}-1$ )

$\begin{array}{llllllllllllllllll}-1307.9 & 31.0 & 44.1 & 86.3 & 100.1 & 106.3 & 133.8 & 139.7 & 155.0 & 180.2 & 240.7 & 242.4 & 264.3 & 297.4\end{array}$ $325.9 \quad 365.5 \quad 410.0 \quad 466.5 \quad 486.9 \quad 517.3 \quad 555.8 \quad 570.5 \quad 616.3 \quad 663.6 \quad 703.7 \quad 805.2 \quad 829.7 \quad 902.6$ $920.8 \quad 944.1 \quad 949.8 \quad 968.9 \quad 972.8 \quad 987.7 \quad 1000.4 \quad 1009.5 \quad 1024.2 \quad 1033.6 \quad 1064.0 \quad 1079.2 \quad 1094.5$

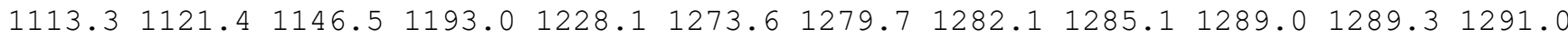

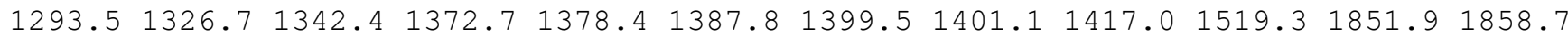
$2169.72670 .32670 .52673 .2 \quad 2674.92675 .92678 .62700 .22711 .02737 .22750 .02764 .9$ $2778.7 \quad 2779.12780 .3 \quad 2783.3$

name $=\operatorname{TS} 182$

energy $($ in $\mathrm{kcal} / \mathrm{mol})=47.74527$

$\mathrm{ZPE}(\mathrm{in} \mathrm{kcal} / \mathrm{mol})=140.82$

$\mathrm{G}($ in $\mathrm{kcal} / \mathrm{mol})=114.132$

Geometry

C $-2.11781441-0.65561133-1.17373627$

C $-0.37504082-0.09258175-1.01011690$

C $-0.457723331 .38588129-1.02790428$ 
$\begin{array}{llll}\text { C } 2.14273450 & 0.84513678 & 0.50365447\end{array}$

C $0.64871500 \quad-0.31758742 \quad 2.22137974$

C $1.36272014-0.43103416 \quad 0.84100782$

C $0.42600429-0.85669226-0.24012296$

$\begin{array}{llll}C & -0.39372788 & 0.76056314 & 2.22871412\end{array}$

C $-1.71223956 \quad 0.57041309 \quad 2.26654075$

C $0.09839743-1.68859516 \quad 2.63081435$

$\mathrm{H}-2.34829968-0.31913144-0.16201265$

$\mathrm{H}-2.21022390-1.73104407-1.24082358$

$\mathrm{H}-2.71312196-0.11895963-1.89997580$

$\mathrm{H}-0.45634863 \quad 1.90519002-0.05957814$

H $2.539116190 .79820559-0.51935675$

H $1.53697422 \quad 1.75083600 \quad 0.58216207$

H $1.43298139-0.028758752 .97309410$

H $2.12912665-1.25054970 \quad 0.95469673$

H $2.99822085 \quad 0.97265749 \quad 1.17749953$

$\mathrm{H} \quad 0.41845690-1.93917504-0.40666960$

$\mathrm{H} 0.02463837 \quad 1.77041930 \quad 2.22101166$

$\mathrm{H}-2.18524903-0.39824369 \quad 2.29444311$

$\mathrm{H}-2.42629104 \quad 1.37875915 \quad 2.29033749$

$\mathrm{H} \quad 0.89879139-2.43205606 \quad 2.71131769$

$\mathrm{H}-0.62771001-2.066464261 .90182830$

$\mathrm{H}-0.40313030-1.63809728 \quad 3.60391655$

$0-0.16220404-1.64230057-2.77339082$

$\begin{array}{llll}0 & -0.55604719 & 1.99496003 & -2.06581748\end{array}$

O $-0.65526840-0.61826762-2.43787533$

Vibrational frequencies (in cm-1)

$\begin{array}{lllllllllllllllll}-670.3 & 29.3 & 62.2 & 72.1 & 95.4 & 104.9 & 143.9 & 155.3 & 173.0 & 186.2 & 212.8 & 229.8 & 253.1 & 254.4\end{array}$ $274.6 \quad 292.6 \quad 311.1 \quad 338.6 \quad 405.2 \quad 447.6 \quad 473.2 \quad 531.1 \quad 560.4 \quad 601.1616 .7 \quad 675.5 \quad 753.7 \quad 837.7$ $889.1940 .2949 .8 \quad 958.7 \quad 984.8 \quad 996.4 \quad 1010.5 \quad 1046.3 \quad 1060.2 \quad 1074.4 \quad 1085.6 \quad 1089.0 \quad 1104.9$

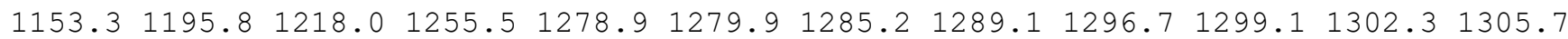

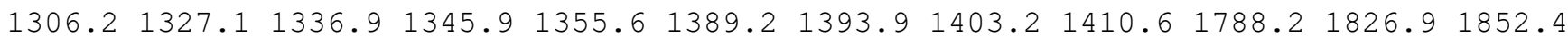
$2643.32647 .12665 .92670 .02673 .7 \quad 2678.6 \quad 2680.5 \quad 2687.02693 .4 \quad 2712.2 \quad 2731.62745 .2$ $2750.42780 .02781 .2 \quad 2787.9$

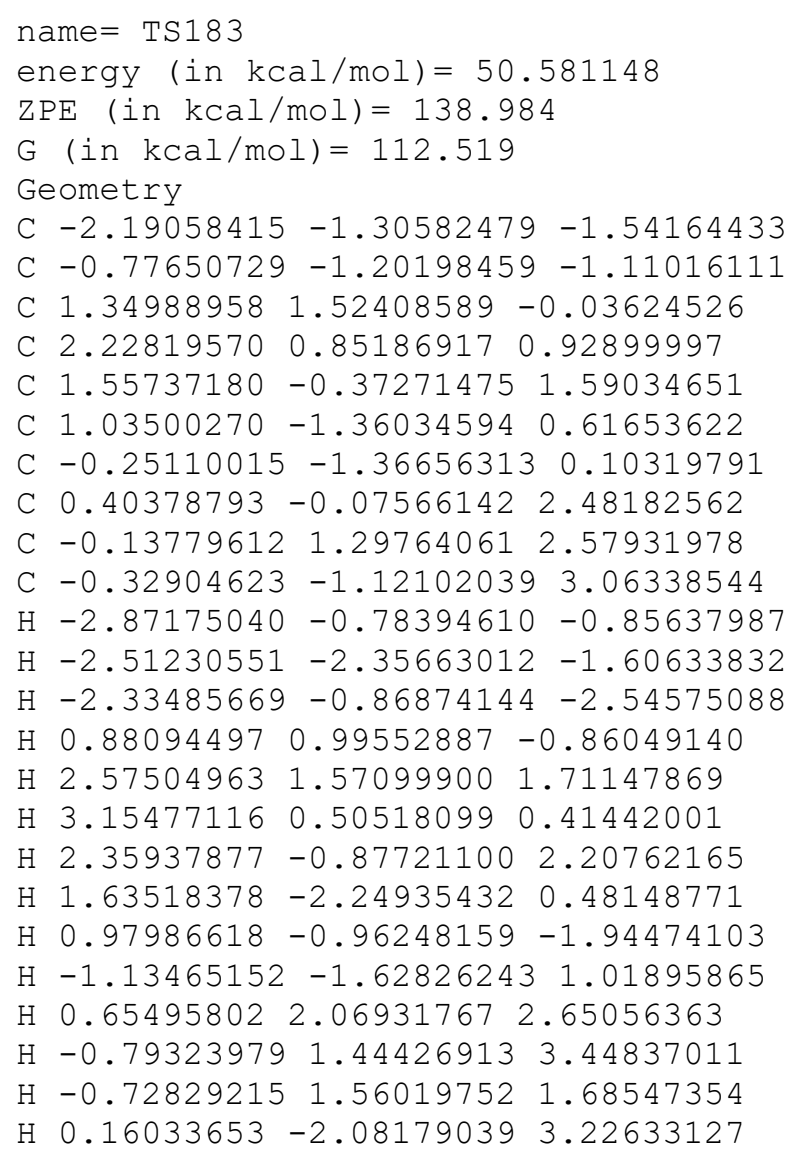


$\mathrm{H}-1.14217322-1.506670022 .00425139$

$\mathrm{H}-1.06168818-0.90384382 \quad 3.83372713$

$\begin{array}{llll}0 & 1.49202575 & 3.48881472 & 0.94421825\end{array}$

$01.036108192 .77788270-0.00252741$

O $0.01915272-0.86179107-2.17283947$

Vibrational frequencies (in $\mathrm{cm}-1$ )

$\begin{array}{lllllllllllllllll}-2020.6 & 42.3 & 50.8 & 70.6 & 78.3 & 94.8 & 112.3 & 144.1 & 179.0 & 195.0 & 220.6 & 244.3 & 301.7 & 328.6\end{array}$

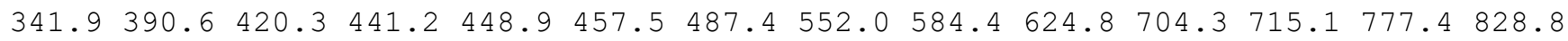

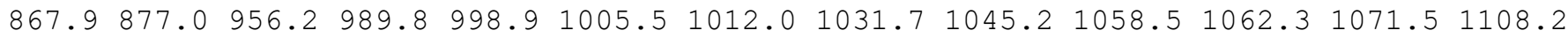

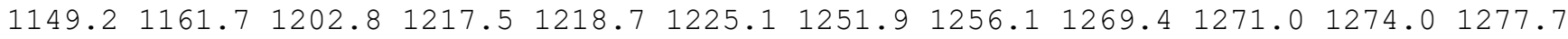

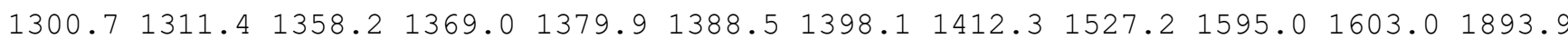
$1976.32101 .42628 .92637 .5 \quad 2664.2 \quad 2665.0 \quad 2673.12673 .12696 .32697 .5 \quad 2723.02759 .5$ $2762.7 \quad 2768.8 \quad 2776.7 \quad 2783.1$

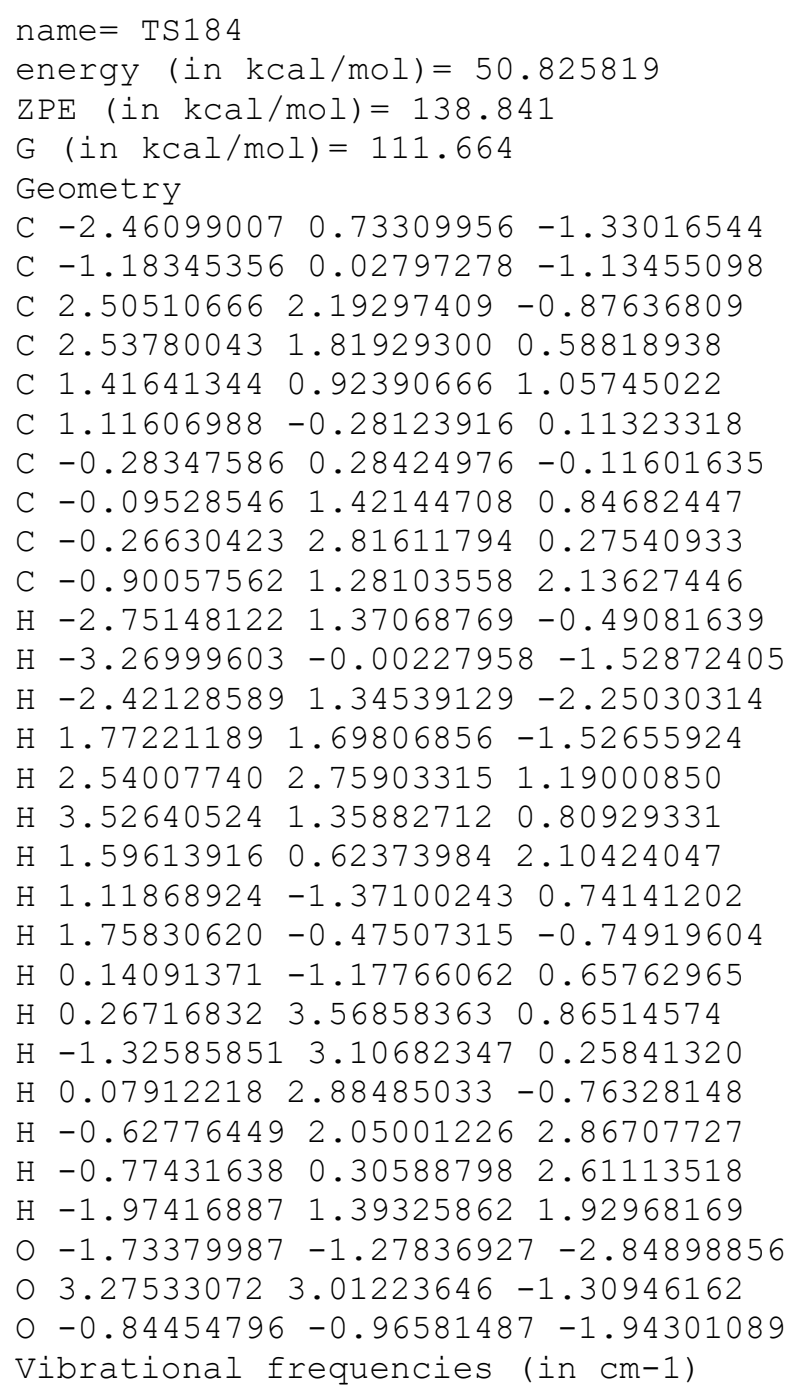


C $-0.76455631-0.93277324-0.33388526$

C $-0.02537283 \quad 1.21153790-1.34188823$

C $0.30782299 \quad 0.12190830 \quad-0.41839153$

C $0.41436955 \quad 0.62292307 \quad 1.06548354$

C $1.86134299 \quad 0.87341921 \quad 1.45686152$

C $-1.10964997-1.27999401 \quad 0.91384523$

C $-0.30386966-0.497763151 .92676580$

C $-1.24310591 \quad 0.15291077 \quad 2.95558017$

C $0.74916156-1.335904822 .52374895$

$\mathrm{H}-2.35542299-2.57140747-2.58097250$

$\mathrm{H}-3.07763123-2.07310261-1.00419525$

$\mathrm{H}-1.74454356-3.28980889-1.04360552$

$\mathrm{H}-1.04358551 \quad 1.45173782-1.61468391$

$\mathrm{H} \quad 1.28869367-0.35041237-0.71989456$

H $2.33906723 \quad 1.586447590 .76777813$

$\mathrm{H}-0.16621838 \quad 1.56567442 \quad 1.17225252$

H $2.46073137-0.050604791 .41677857$

H $1.94384434 \quad 1.27470582 \quad 2.47122583$

$\mathrm{H}-1.84189757-1.988091891 .23035120$

$\mathrm{H}-1.73772793-0.59970423 \quad 3.57513225$

$\mathrm{H}-2.030181110 .73659776 \quad 2.46626307$

$\mathrm{H}-0.69253357 \quad 0.82633741 \quad 3.62063951$

H $1.15138404 \quad-1.102437393 .48110914$

$\mathrm{H} \quad 0.37739108 \quad-2.84044613 \quad 2.48350625$

$\mathrm{H}-0.10566613-2.63781413 \quad 3.10837841$

O $2.067801921 .78149817-1.70562107$

$0 \quad 0.84622807 \quad 1.96925913-1.92873125$

o -1.20521701-1.34362999-1.53987871

Vibrational frequencies (in $\mathrm{cm}-1$ )

$\begin{array}{llllllllllllllll}-1044.4 & 33.7 & 47.3 & 77.0 & 108.6 & 138.8 & 145.6 & 166.1 & 167.7 & 177.3 & 209.9 & 234.6 & 238.3283 .1\end{array}$ $300.5 \quad 336.3 \quad 376.2 \quad 409.4 \quad 438.5 \quad 503.4 \quad 518.8 \quad 537.8 \quad 560.8 \quad 597.9 \quad 644.3 \quad 665.8 \quad 737.5 \quad 816.6$ $842.2 \quad 857.1 \quad 907.1941 .5 \quad 973.9 \quad 990.5 \quad 1013.9 \quad 1057.6 \quad 1073.8 \quad 1077.6 \quad 1087.6 \quad 1100.3 \quad 1113.9$ $1130.8 \quad 1145.4 \quad 1159.1 \quad 1175.6 \quad 1189.1 \quad 1211.3 \quad 1224.6 \quad 1241.6 \quad 1260.0 \quad 1278.2 \quad 1284.6 \quad 1286.9$ $1291.8 \quad 1299.3 \quad 1300.4 \quad 1303.6 \quad 1304.2 \quad 1325.6 \quad 1348.4 \quad 1370.2 \quad 1394.0 \quad 1396.5 \quad 1404.51601 .1$ $1857.12653 .6 \quad 2654.5 \quad 2657.3 \quad 2658.8 \quad 2675.92679 .8 \quad 2682.4 \quad 2701.5 \quad 2711.72720 .32747 .1$ $2775.4 \quad 2782.6 \quad 2800.8 \quad 4670.4$

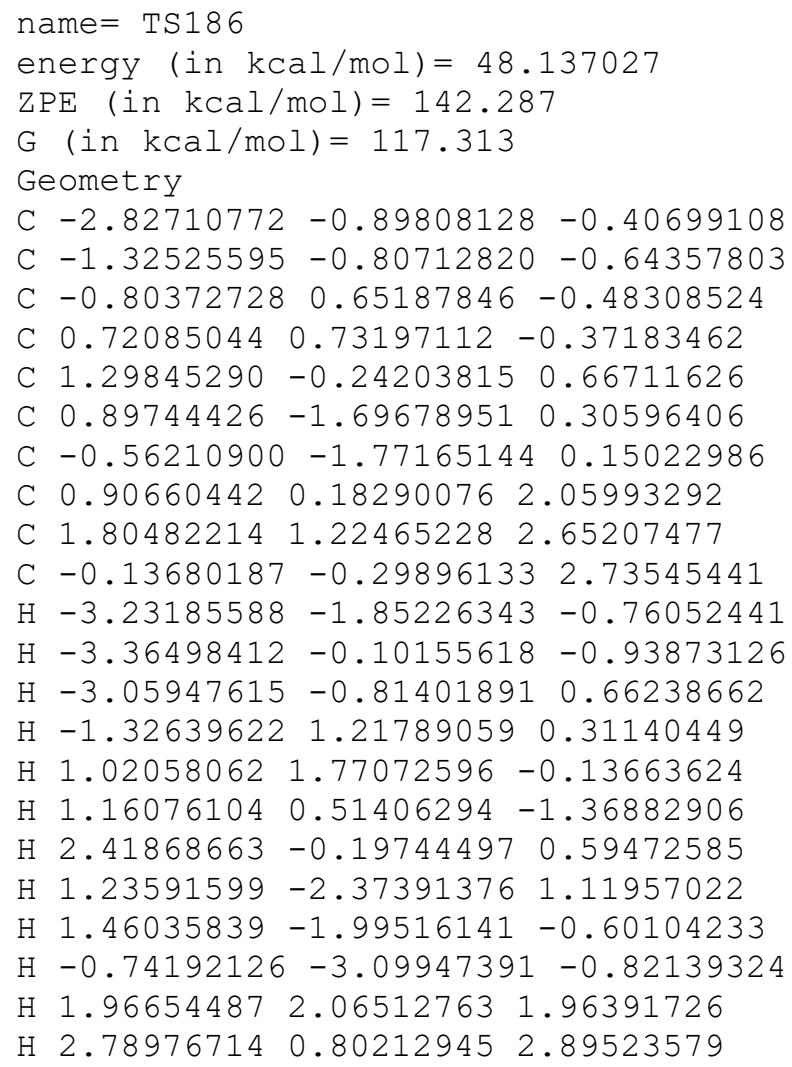


$\mathrm{H} \quad 1.39900588 \quad 1.64573101 \quad 3.58124721$

$\mathrm{H}-1.05616506-3.19764558-0.07033313$

$\mathrm{H}-0.40655634 \quad 0.02028845 \quad 3.72718807$

$\mathrm{H}-0.80422196-1.060053342 .34937928$

$0-1.42016496 \quad 0.20697087-2.66615531$

$0-1.172613861 .26813117-1.71129587$

$0-1.04892555-1.00245040-2.04568627$

Vibrational frequencies (in $\mathrm{cm}-1$ )

$\begin{array}{llllllllllllllllllll}-984.4 & 48.8 & 62.7 & 83.4 & 109.5 & 125.0 & 153.4 & 177.1 & 225.8 & 244.1 & 278.5 & 300.8 & 326.2 & 371.9\end{array}$ $397.2425 .5 \quad 463.5 \quad 526.1 \quad 551.1 \quad 592.3 \quad 597.2 \quad 615.8 \quad 646.3 \quad 677.2 \quad 721.4 \quad 766.0 \quad 839.0 \quad 870.5$ $900.9945 .3 \quad 950.8 \quad 963.2 \quad 978.4 \quad 1000.6 \quad 1004.7 \quad 1042.9 \quad 1051.5 \quad 1062.7 \quad 1075.7 \quad 1096.0 \quad 1110.0$

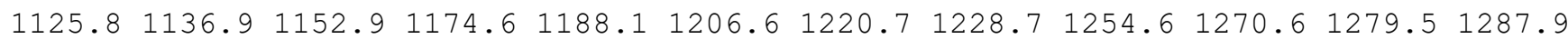

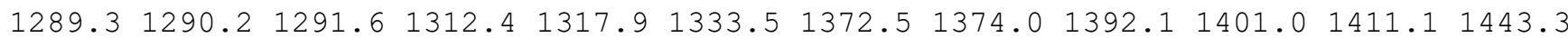
$1872.92657 .32660 .0 \quad 2672.4 \quad 2673.92674 .6 \quad 2679.2 \quad 2686.92694 .92704 .92735 .32740 .8$ $2777.92781 .6 \quad 2784.5 \quad 4600.8$

name $=$ TS187

energy (in $\mathrm{kcal} / \mathrm{mol})=52.104719$

$\mathrm{ZPE}($ in $\mathrm{kcal} / \mathrm{mol})=138.492$

$\mathrm{G}(\mathrm{in} \mathrm{kcal} / \mathrm{mol})=111.349$

Geometry

C $-1.91390430-1.56831431-2.35841447$

C $-0.74509610-1.46265881-1.46553811$

C $-0.76659942 \quad 1.40839718-0.04835493$

C $0.714974581 .08758625-0.03917116$

C $1.13457446-0.050913620 .84642613$

C $0.45746473-1.50170126 \quad 0.66078914$

C $-0.76212906-1.65908500-0.09350085$

C $0.44798168-0.50936074 \quad 2.05764581$

C $1.29039940-1.039908223 .18373306$

C $-0.89919471-0.040618332 .47955491$

$\mathrm{H}-1.84229473-0.80331929-3.16248523$

$\mathrm{H}-2.87387188-1.47055487-1.84505213$

$\mathrm{H}-1.89802703-2.53226034-2.89534827$

$\mathrm{H}-1.40860359 \quad 0.83384447 \quad-0.72910041$

$\mathrm{H} \quad 1.27746605 \quad 2.00408007 \quad 0.25834266$

$\mathrm{H} \quad 1.031885170 .87757248-1.09881936$

H $2.24489884-0.09726472 \quad 0.86581026$

H $0.86980951-2.51592071 \quad 1.14301826$

$\mathrm{H} \quad 1.36019282-2.15299634 \quad 0.18272865$

$\mathrm{H}-1.65773144-1.97380548 \quad 0.40501056$

H $0.80827262-1.87980840 \quad 3.70651267$

$\mathrm{H} 1.43069053-0.25297415 \quad 3.94862253$

H $2.29744235-1.358961552 .89223393$

$\mathrm{H}-1.28065759-0.559320913 .36863798$

$\mathrm{H}-1.66808958-0.17748797 \quad 1.69231026$

$\mathrm{H}-0.894801191 .04278007 \quad 2.70752772$

$\begin{array}{lllll}0 & 0.46464614 & -0.85524559 & -3.25103117\end{array}$

$0-1.206579332 .28504566 \quad 0.65271592$

$0.44709519-1.14310749-1.95840042$

Vibrational frequencies (in $\mathrm{cm}-1$ )

$\begin{array}{llllllllllllllll}-1055.1 & 38.6 & 46.3 & 55.8 & 68.7 & 86.7 & 101.3 & 148.7 & 173.9 & 186.0 & 215.5 & 228.9 & 249.4 & 267.4\end{array}$ $294.2306 .4 \quad 328.3 \quad 386.7 \quad 416.0 \quad 429.5 \quad 487.7 \quad 510.5 \quad 525.7 \quad 617.0 \quad 621.1 \quad 716.6 \quad 786.3 \quad 855.3$

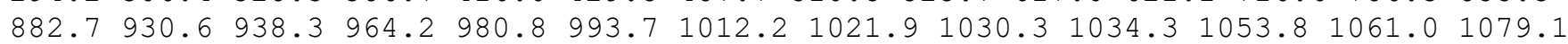
$1085.21125 .7 \quad 1182.7 \quad 1214.4 \quad 1242.2 \quad 1253.2 \quad 1259.1 \quad 1268.6 \quad 1271.6 \quad 1274.8 \quad 1278.2 \quad 1279.1$ $1284.61294 .3 \quad 1332.3 \quad 1339.7 \quad 1379.9 \quad 1387.3 \quad 1390.5 \quad 1408.7 \quad 1498.3 \quad 1503.0 \quad 1656.7 \quad 1832.0$ $2212.72606 .0 \quad 2622.1 \quad 2640.8 \quad 2644.5 \quad 2649.7 \quad 2656.42667 .82685 .42696 .92711 .72764 .5$ $2773.8 \quad 2774.7 \quad 2791.4 \quad 2805.0$

name $=\operatorname{TS} 188$

energy (in $\mathrm{kcal} / \mathrm{mol})=51.932243$

$\mathrm{ZPE}(\mathrm{in} \mathrm{kcal} / \mathrm{mol})=138.794$

$\mathrm{G}($ in $\mathrm{kcal} / \mathrm{mol})=108.58$ 


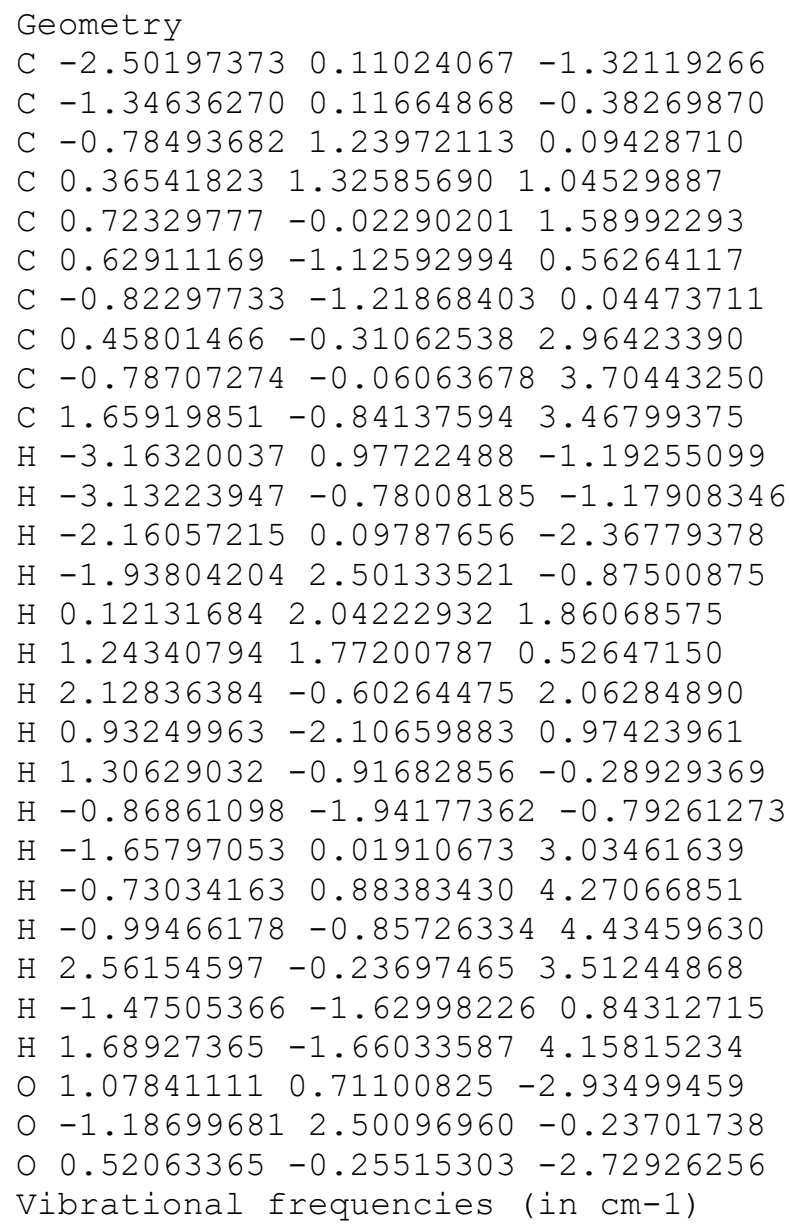

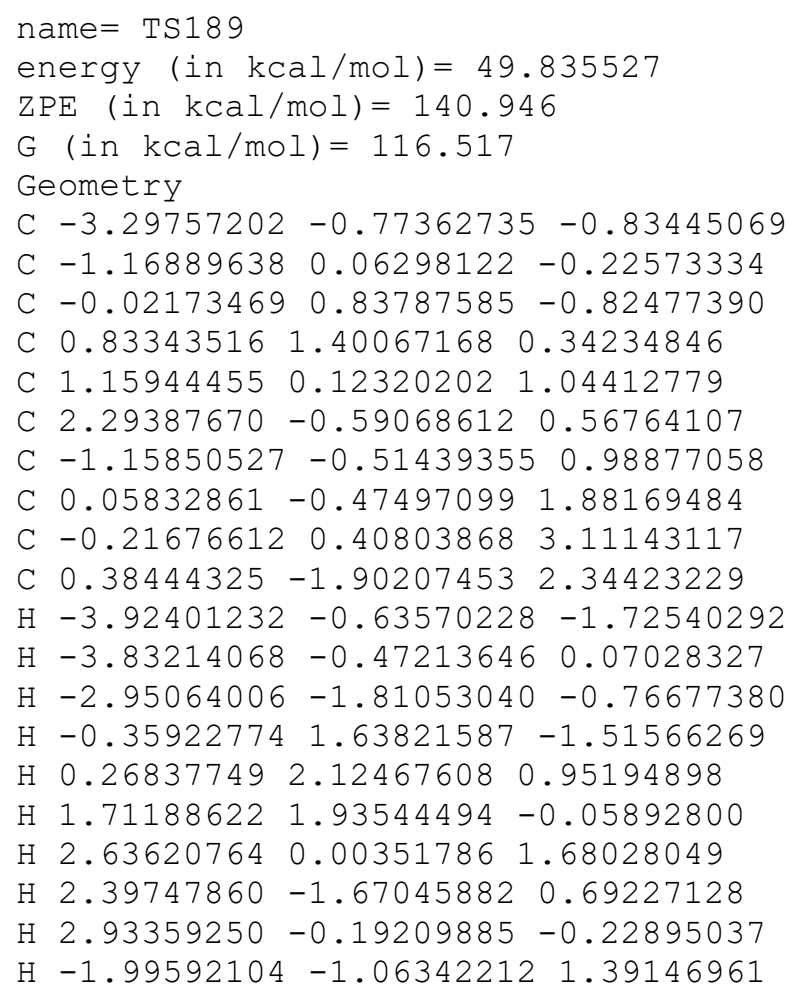


$\mathrm{H}-0.53407198 \quad 1.41641692 \quad 2.82316362$

$\mathrm{H} \quad 0.65890633 \quad 0.49229414 \quad 3.75933480$

$\mathrm{H}-1.03248452-0.01340363 \quad 3.71267869$

$\mathrm{H} \quad 0.50727495-2.56954787 \quad 1.47584380$

$\mathrm{H}-0.43699325-2.31770062 \quad 2.94021000$

$\mathrm{H} 1.28875030-1.948593262 .95404067$

O $1.20905958-1.11421783-1.11274855$

$00.79029356 \quad 0.02216835-1.68534283$

o $-2.209794820 .10663542-1.08917564$

Vibrational frequencies (in cm-1)

$\begin{array}{lllllllllllllllllll}-1461.1 & 55.6 & 84.1 & 117.0 & 146.1 & 156.9 & 179.1 & 199.1 & 229.8 & 245.0 & 248.3 & 309.8 & 318.0 & 358.7\end{array}$

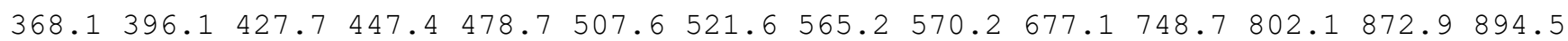
$928.9939 .4 \quad 962.6 \quad 975.2 \quad 985.3 \quad 1005.4 \quad 1025.2 \quad 1033.5 \quad 1040.3 \quad 1065.6 \quad 1071.7 \quad 1085.3 \quad 1122.5$

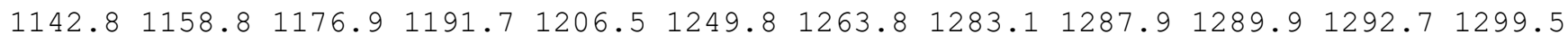

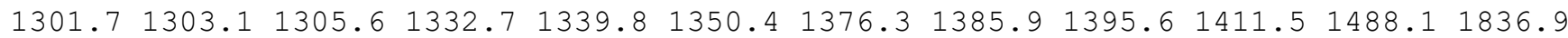
$2155.12652 .1 \quad 2658.4 \quad 2661.0 \quad 2665.1 \quad 2672.0 \quad 2674.42680 .4 \quad 2683.3 \quad 2696.4 \quad 2728.02745 .7$ $2748.92775 .7 \quad 2777.8 \quad 2782.1$

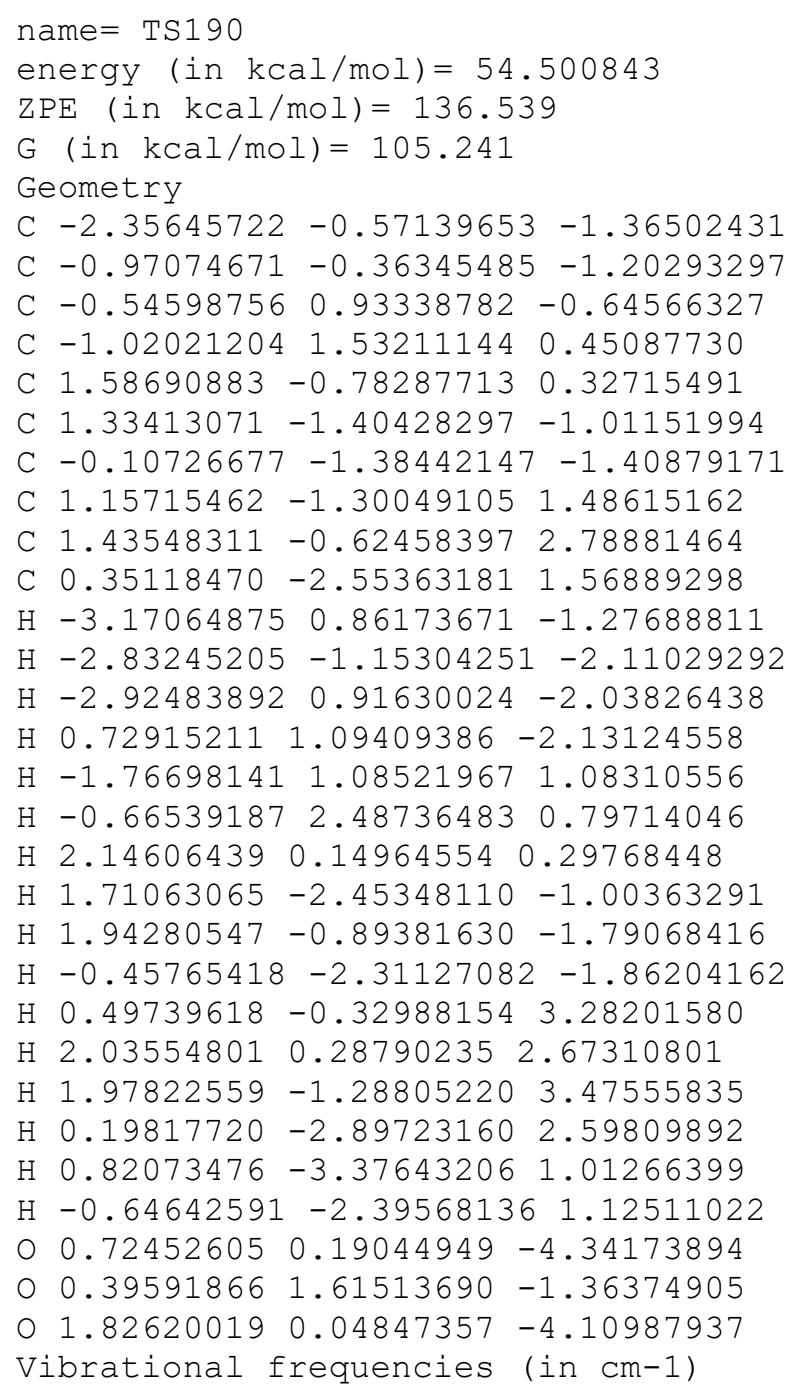

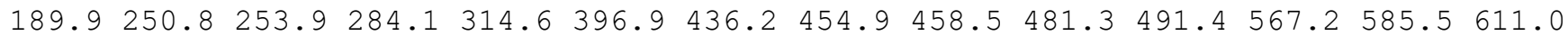

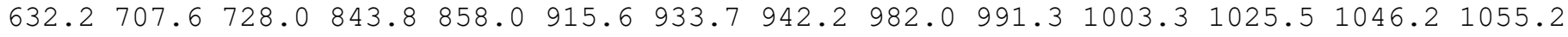
$1088.7 \quad 1115.8 \quad 1135.0 \quad 1179.3 \quad 1240.3 \quad 1269.3 \quad 1279.91284 .1 \quad 1287.7 \quad 1289.3 \quad 1309.01313 .2$ $1328.01331 .6 \quad 1364.3 \quad 1376.7 \quad 1380.9 \quad 1399.7 \quad 1414.3 \quad 1475.6 \quad 1596.2 \quad 1780.6 \quad 1866.8 \quad 1870.2$ $2651.62663 .12672 .7 \quad 2675.3 \quad 2679.8 \quad 2730.8 \quad 2733.02734 .82747 .7 \quad 2757.12777 .7 \quad 2780.0$ 2791.52804 .14880 .3

name $=$ TS191

energy $($ in $\mathrm{kcal} / \mathrm{mol})=50.839966$ 
$\mathrm{ZPE}($ in $\mathrm{kcal} / \mathrm{mol})=140.215$

$\mathrm{G}($ in $\mathrm{kcal} / \mathrm{mol})=111.48$

Geometry

C $-2.36118387-0.53298780-1.10677134$

C $-1.43514988 \quad 0.16310150-0.23543375$

C $-0.01089240 \quad 1.56546463-1.05638021$

C $1.04758324 \quad 1.64265986 \quad 0.05413747$

C $0.78863028 \quad 0.81394049 \quad 1.30579949$

$\begin{array}{lllll}\text { C } & 0.44991487 & -0.66298074 & 0.94039242\end{array}$

C $-1.07571938-0.30974003 \quad 1.09327515$

C $-0.64087870 \quad 0.94527195 \quad 1.95664173$

C $-1.417002802 .22910549 \quad 1.75698108$

C $-0.61668116 \quad 0.60026484 \quad 3.44348807$

$\mathrm{H}-3.39767117-0.48093964-0.73757210$

$\mathrm{H}-2.12739441-1.60268954-1.23513393$

$\mathrm{H}-2.34362526-0.07964383-2.11913965$

$\mathrm{H}-0.79541188 \quad 2.32958832-1.05466309$

H $1.19468199 \quad 2.70342467 \quad 0.32438909$

H $2.008448191 .29978491-0.39321013$

$\mathrm{H} 1.60959669 \quad 0.93769648 \quad 2.02587101$

$\mathrm{H} \quad 0.78735398-1.39629776 \quad 1.67783052$

$\mathrm{H} \quad 0.77760398-0.99546551-0.04897944$

$\mathrm{H}-1.69825826-1.044612421 .60430088$

$\mathrm{H}-0.91522861 \quad 3.06964388 \quad 2.25268589$

H $-2.42747245 \quad 2.15213588 \quad 2.17629341$

$\mathrm{H}-1.53288292 \quad 2.50165945 \quad 0.70159850$

$\mathrm{H}-0.06339589-0.32225848 \quad 3.64973118$

$\mathrm{H}-1.63280376 \quad 0.462204893 .83191313$

$\mathrm{H}-0.147910921 .40054627 \quad 4.02753860$

O $1.27548080-1.60689186-2.32802376$

$0.22697437 \quad 0.91571943-2.06099570$

O $0.20338930-1.54343126-2.69347818$

Vibrational frequencies (in $\mathrm{cm}-1$ )

$\begin{array}{lllllllllllllll}-120.2 & 22.5 & 49.0 & 51.1 & 59.8 & 66.0 & 74.6 & 88.9 & 141.5 & 149.6 & 183.6 & 189.4 & 199.0 & 245.3\end{array}$ $313.9334 .6 \quad 364.2 \quad 398.6 \quad 431.1 \quad 444.3 \quad 466.0 \quad 512.1 \quad 587.1 \quad 693.4 \quad 802.6 \quad 858.0 \quad 876.5 \quad 889.3$ $917.8946 .4 \quad 962.8 \quad 988.6 \quad 999.1 \quad 1009.5 \quad 1018.1 \quad 1026.4 \quad 1036.0 \quad 1074.5 \quad 1094.4 \quad 1125.7 \quad 1153.9$ $1184.61200 .7 \quad 1207.0 \quad 1214.2 \quad 1220.3 \quad 1254.0 \quad 1262.4 \quad 1270.3 \quad 1271.6 \quad 1287.0 \quad 1290.6 \quad 1294.1$ $1301.31303 .6 \quad 1314.8 \quad 1322.3 \quad 1359.4 \quad 1366.4 \quad 1378.2 \quad 1403.7 \quad 1411.5 \quad 1456.8 \quad 1596.01756 .7$ 2640.92652 .62660 .12665 .12671 .72676 .52677 .92681 .12682 .02730 .62741 .12743 .6 $2757.0 \quad 2765.8 \quad 2780.3 \quad 2783.1$

\section{name $=$ TS192}

energy (in $\mathrm{kcal} / \mathrm{mol})=49.392619$

$\mathrm{ZPE}(\mathrm{in} \mathrm{kcal} / \mathrm{mol})=141.745$

G $($ in $\mathrm{kcal} / \mathrm{mol})=117.933$

Geometry

C $-2.02067095-0.53128764-1.27442375$

C $-0.59311136-0.31716751-0.83163382$

C $-0.179702141 .11387307-0.66234404$

C $0.95992451 \quad 1.45960228 \quad 0.23157951$

C $0.80490706 \quad 0.77781563 \quad 1.60539697$

C $1.35671045-1.49835875 \quad 0.41967947$

C $-0.13091421-1.26528090 \quad 0.23161873$

C $0.58658341-0.72334027 \quad 1.46877602$

C $-0.35488185 \quad 1.43387809 \quad 2.36707816$

C $0.32222500-1.421430502 .78026742$

$\mathrm{H}-2.25834314 \quad 0.09828433 \quad-2.14920509$

$\mathrm{H}-2.73578481-0.30401566-0.47757367$

$\mathrm{H}-2.18826603-1.56978857-1.59188120$

$\mathrm{H}-1.154661892 .09838091-0.97587239$

H $1.05102447 \quad 2.55689793 \quad 0.35615041$

$\mathrm{H} 1.91958228 \quad 1.15909908-0.24638925$

$\mathrm{H} \quad 1.75083071 \quad 0.93676874 \quad 2.18261991$

Н $1.71408918-2.50011377 \quad 0.64033868$ 
H $2.07039672-0.99383363-0.22635709$

$\mathrm{H}-0.79947355-2.12307423 \quad 0.36609539$

$\mathrm{H}-1.31950106 \quad 1.18675997 \quad 1.90986996$

$\mathrm{H}-0.25795328 \quad 2.52471125 \quad 2.37983065$

$\mathrm{H}-0.390242151 .102275293 .41047284$

H $1.06675517-1.14320684 \quad 3.53734513$

$\mathrm{H} 0.35631106-2.514129852 .68378423$

$\mathrm{H}-0.66935868-1.16673157 \quad 3.17669928$

$0.06501941 \quad 0.31248307-2.93615393$

$0-0.30911050 \quad 1.76057837-1.84992991$

O $0.35651959-0.49287243-1.98174496$

Vibrational frequencies (in $\mathrm{cm}-1$ )

$\begin{array}{lllllllllllllllll}-1632.2 & 77.7 & 111.3 & 133.2 & 149.6 & 165.1 & 175.3 & 228.2 & 235.5 & 270.6 & 284.0 & 315.9 & 335.5 & 361.7\end{array}$ $370.1406 .6 \quad 435.1 \quad 477.8 \quad 480.6 \quad 518.8 \quad 571.2 \quad 628.7 \quad 644.9691 .6 \quad 738.0 \quad 780.2 \quad 823.9 \quad 876.2$ $891.1924 .9947 .6 \quad 971.3 \quad 994.1 \quad 1008.2 \quad 1024.5 \quad 1056.1 \quad 1059.1 \quad 1086.4 \quad 1094.4 \quad 1109.91120 .0$

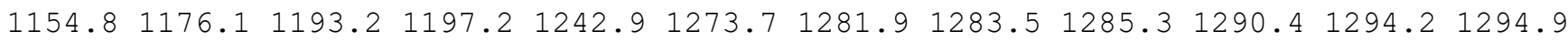
$1299.81305 .91310 .3 \quad 1328.7 \quad 1347.6 \quad 1378.2 \quad 1382.1 \quad 1393.8 \quad 1397.5 \quad 1408.01414 .4 \quad 1487.6$ $2436.52650 .72660 .2 \quad 2673.42674 .5 \quad 2676.12677 .92680 .62701 .92707 .52739 .12752 .5$ $2777.8 \quad 2780.6 \quad 2781.92784 .3$

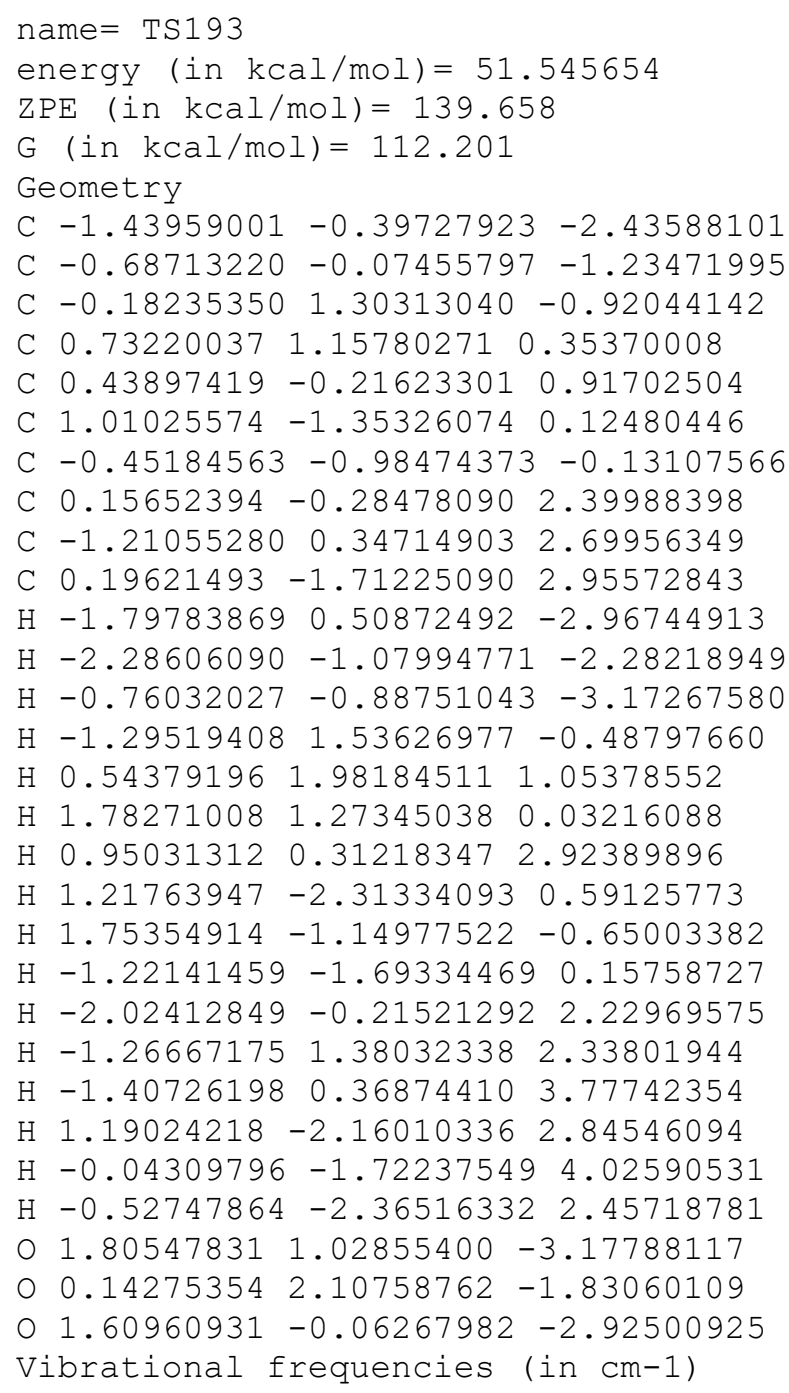




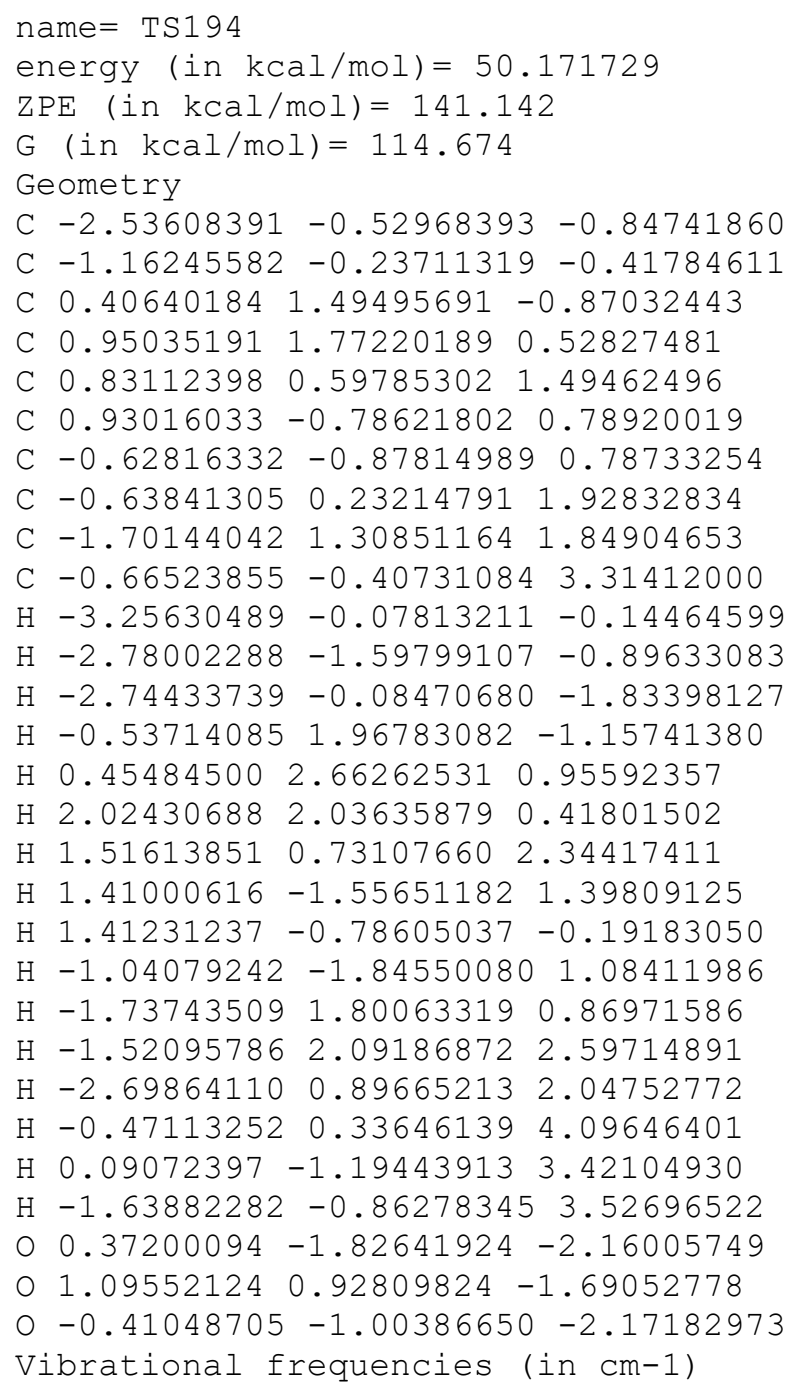


H $1.58327068-0.032650792 .82809326$

$\mathrm{H} 2.25505627-0.77323720 \quad 0.48821162$

H 2.581600591 .519126071 .04360736

$\mathrm{H} 0.43151883-1.72912442-0.60529179$

$\mathrm{H}-1.31609763-0.71672736 \quad 2.02061018$

$\mathrm{H}-0.054559341 .709009793 .48441499$

$\mathrm{H}-1.808420921 .393581893 .28082028$

$\mathrm{H} \quad 1.97736362-2.42124112 \quad 2.27496890$

$\mathrm{H} \quad 0.31822490 \quad-2.61907300 \quad 1.69879470$

H $0.62903893-2.30219474 \quad 3.40991126$

$\begin{array}{lllll}0 & -1.38425460 & 0.34850866 & -3.12856851\end{array}$

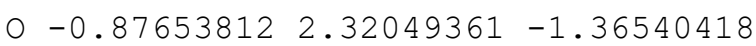

o $-1.07827304-0.42893668-2.30172222$

Vibrational frequencies (in $\mathrm{cm}-1$ )

$\begin{array}{lllllllllllllllll}-693.7 & 31.6 & 48.7 & 54.6 & 74.8 & 92.8 & 130.8 & 157.9 & 167.4 & 172.5 & 201.5 & 225.2 & 235.7 & 259.5\end{array}$ $271.7 \quad 296.6 \quad 332.0 \quad 341.8 \quad 378.2 \quad 387.5 \quad 446.1 \quad 508.7 \quad 550.4 \quad 565.3 \quad 638.5 \quad 677.6 \quad 726.5 \quad 844.8$ $889.4 \quad 924.7 \quad 940.7 \quad 961.5 \quad 991.6 \quad 995.6 \quad 1006.8 \quad 1031.2 \quad 1050.6 \quad 1071.4 \quad 1086.1 \quad 1099.01133 .7$ $\begin{array}{lllllllllllllll}1138.7 & 1204.3 & 1220.8 & 1271.5 & 1275.5 & 1284.6 & 1286.8 & 1290.1 & 1299.9 & 1301.6 & 1302.6 & 1311.7\end{array}$

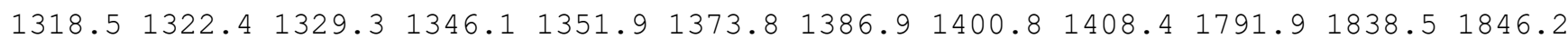
$2640.12646 .92665 .2 \quad 2672.3 \quad 2674.5 \quad 2678.5 \quad 2682.62690 .42699 .62715 .82750 .22754 .9$ $2755.52778 .12781 .8 \quad 2789.1$

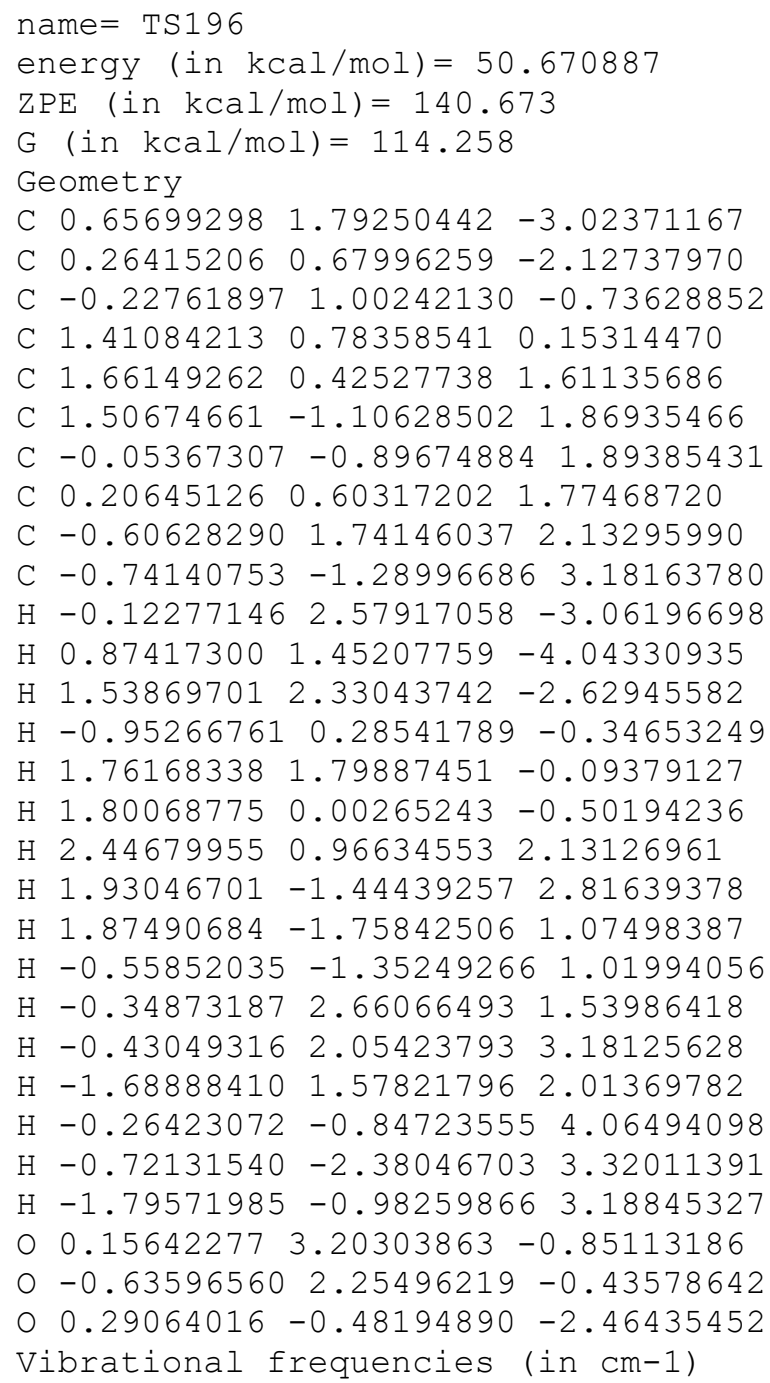

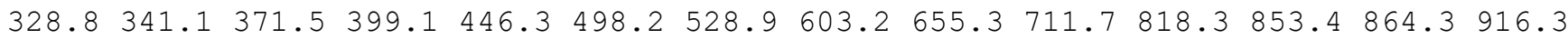
$925.6954 .8 \quad 966.9987 .3 \quad 999.3 \quad 1022.1 \quad 1039.0 \quad 1053.8 \quad 1055.3 \quad 1069.9 \quad 1101.11113 .11126 .0$ $\begin{array}{llllllllllll}1153.9 & 1172.0 & 1184.6 & 1203.8 & 1210.3 & 1228.5 & 1233.9 & 1237.7 & 1240.4 & 1248.8 & 1262.1 & 1282.9\end{array}$ $1290.0 \quad 1294.1 \quad 1295.2 \quad 1301.4 \quad 1350.8 \quad 1364.4 \quad 1370.6 \quad 1382.5 \quad 1395.4 \quad 1414.91546 .11832 .5$ $2562.62628 .62635 .72654 .72669 .2 \quad 2671.92678 .62686 .52694 .72699 .02711 .02750 .8$ $2754.8 \quad 2765.2 \quad 2771.92780 .4$ 


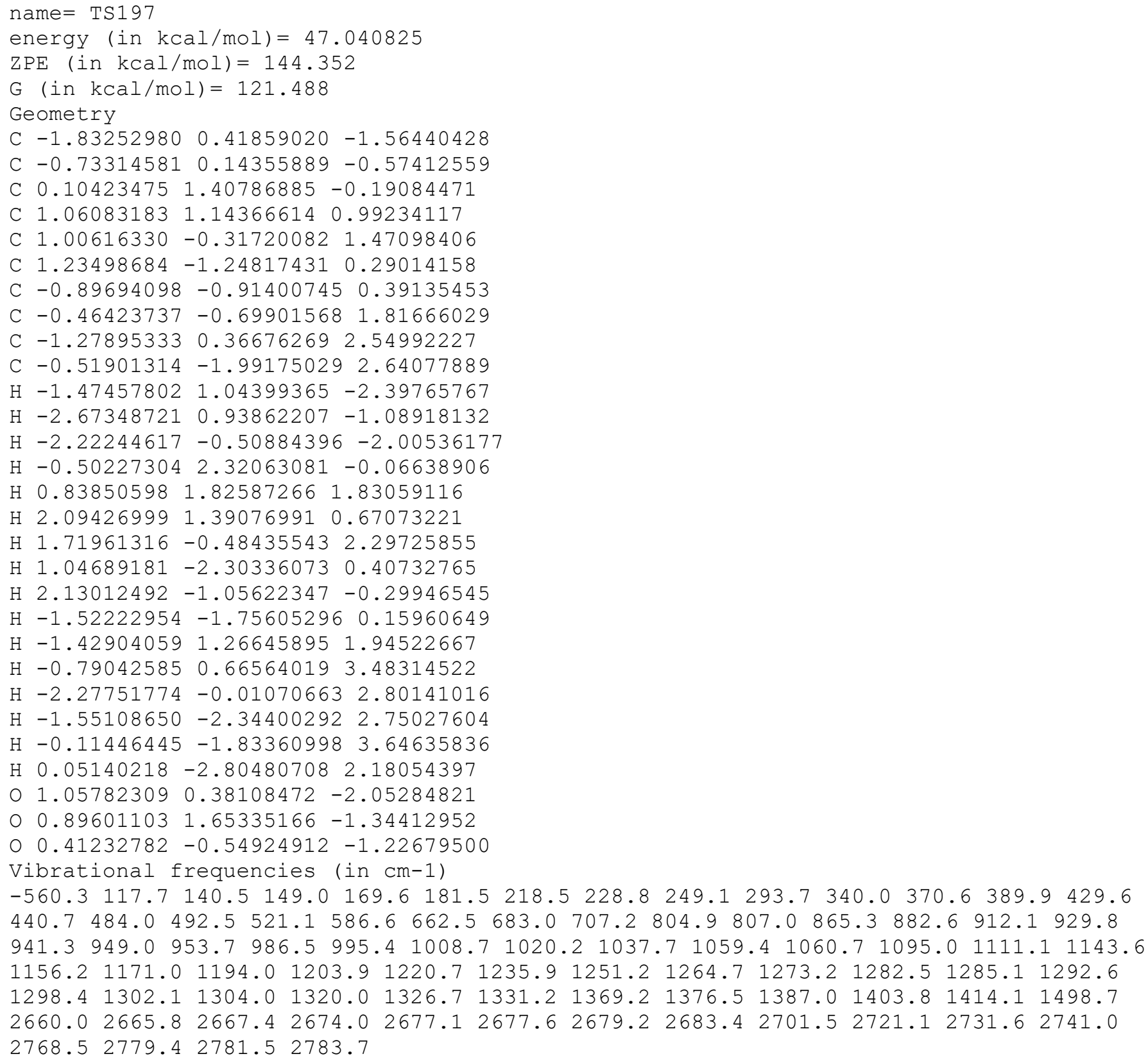


$\mathrm{H}-0.212713752 .545261351 .48794980$

$\mathrm{H} \quad 0.84032221 \quad 1.97816127 \quad 0.19885905$

H $1.46318806 \quad 0.86354300 \quad 2.28526659$

H $1.42088141-1.47433750 \quad 1.37077943$

$\mathrm{H} \quad 1.74734535-0.28821436 \quad 0.12711850$

$\mathrm{H}-1.03266083-2.00506306 \quad 1.01097281$

$\mathrm{H}-2.084620231 .444109712 .71118239$

$\mathrm{H}-0.73355590 \quad 2.03931595 \quad 3.69214848$

$\mathrm{H}-1.81958302 \quad 0.80578386 \quad 4.33447606$

$\mathrm{H}-0.73332880-2.43937026 \quad 0.37981119$

H $0.19288928-1.96418785 \quad 3.02564878$

$\mathrm{H}-1.09814064-1.46297397 \quad 4.14946615$

$\begin{array}{lllll}0 & -1.33975457 & 1.28625248 & -2.24882698\end{array}$

$0-1.15378105 \quad 2.13824104-1.10368915$

O $-0.70650476 \quad 0.08359326-1.91935890$

Vibrational frequencies (in cm-1)

$\begin{array}{lllllllllllllll}-1030.7 & 41.3 & 55.2 & 95.0 & 150.8 & 159.2 & 217.3 & 236.6 & 240.7 & 304.5 & 313.9 & 335.1 & 359.3 & 375.2\end{array}$ $424.8 \quad 450.2 \quad 471.5 \quad 498.2 \quad 558.7 \quad 601.0 \quad 617.7 \quad 634.4 \quad 676.4 \quad 704.5 \quad 744.6 \quad 770.6 \quad 808.1 \quad 854.3$

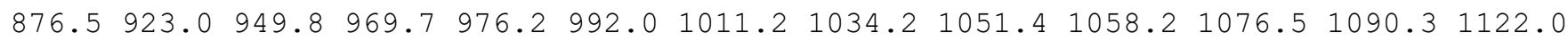
$1127.31137 .6 \quad 1143.4 \quad 1164.2 \quad 1191.5 \quad 1200.5 \quad 1218.4 \quad 1234.2 \quad 1247.2 \quad 1275.7 \quad 1279.01284 .3$

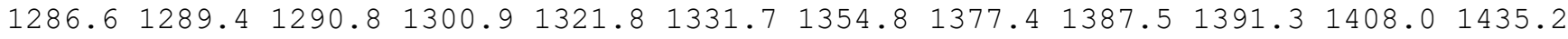
$1848.22642 .8 \quad 2652.2 \quad 2663.2 \quad 2666.5 \quad 2669.92680 .12684 .22685 .02721 .72735 .8 \quad 2738.5$ $2776.8 \quad 2778.2 \quad 2793.2 \quad 4562.8$

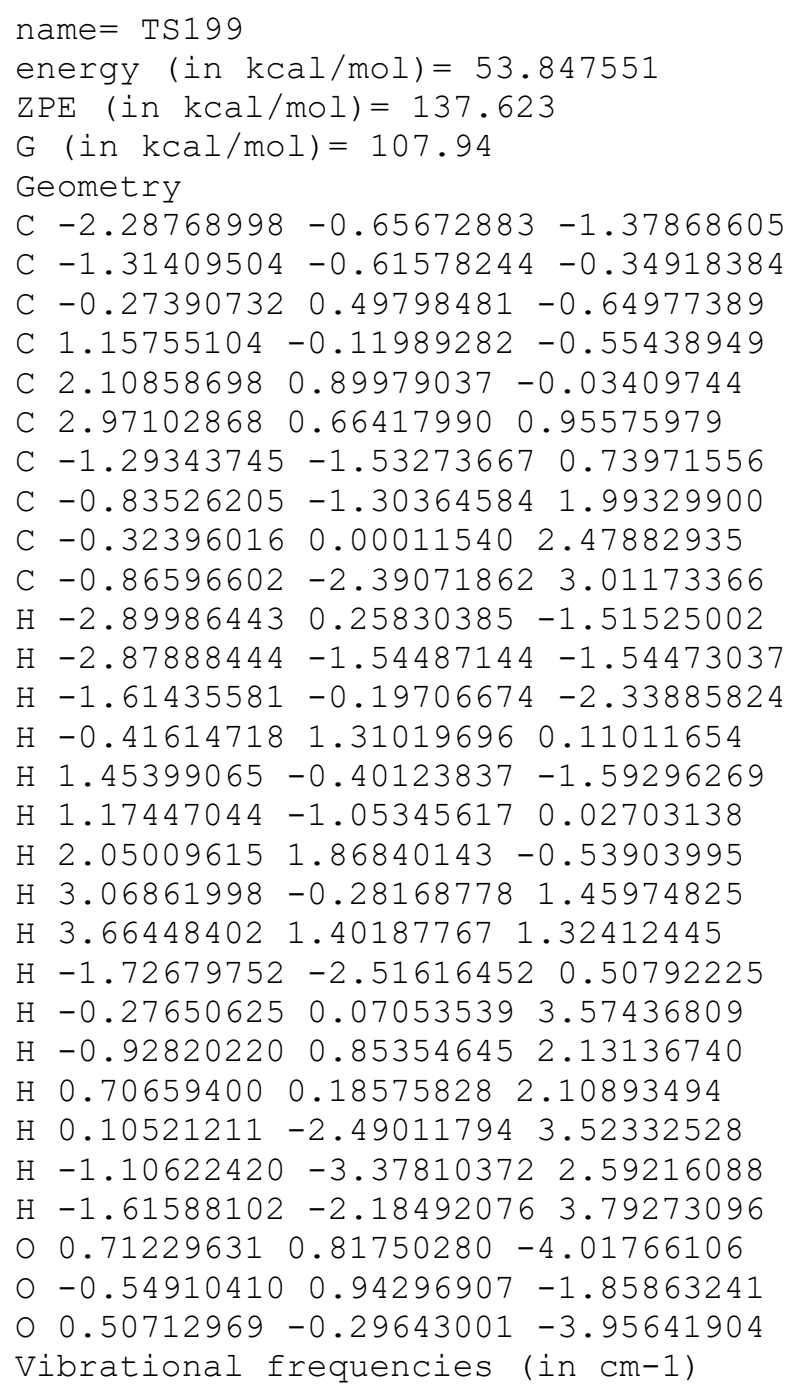


$2125.5 \quad 2613.4 \quad 2625.4 \quad 2659.2 \quad 2659.2 \quad 2661.3 \quad 2662.6 \quad 2666.8 \quad 2718.5 \quad 2733.2 \quad 2736.4 \quad 2753.7$ $2762.5 \quad 2767.2 \quad 2775.7 \quad 2794.4$

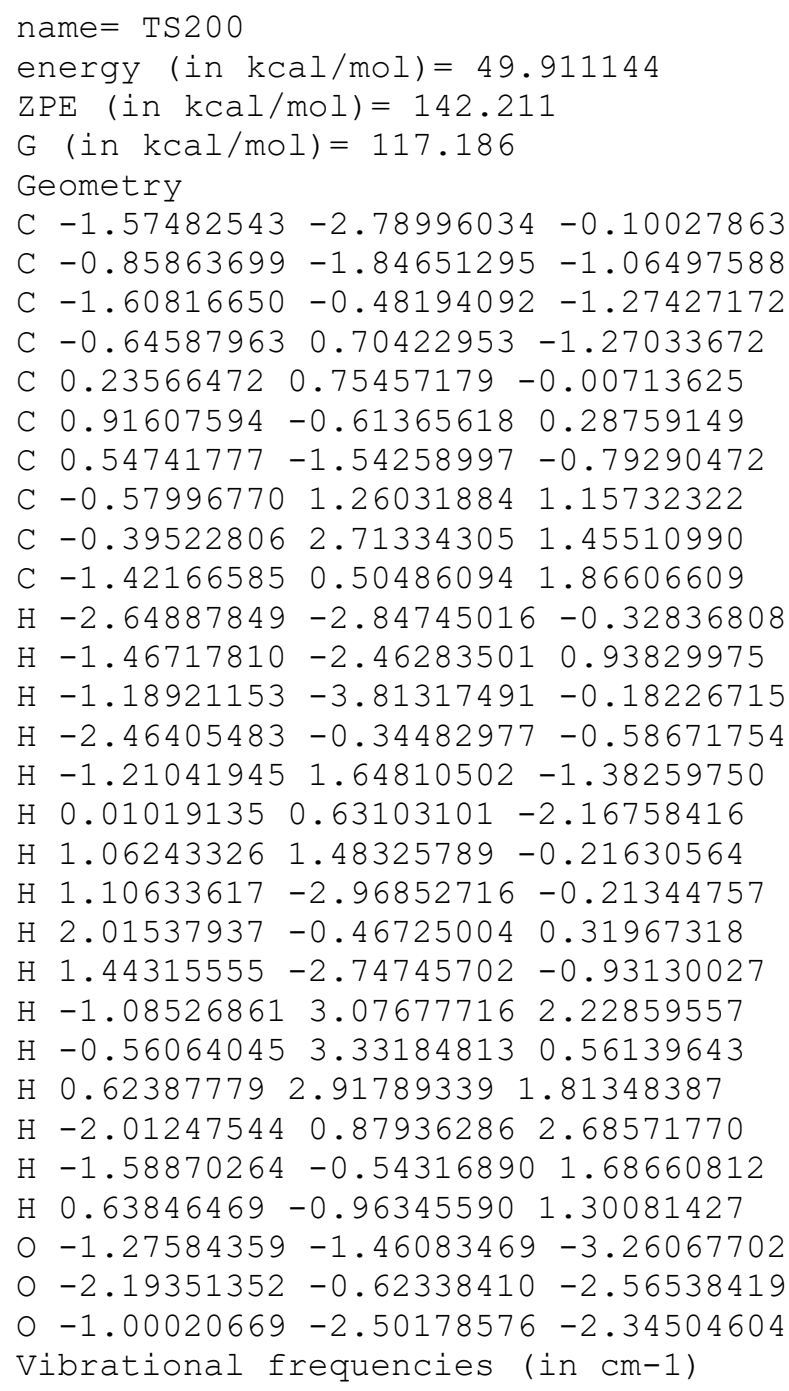


$\mathrm{H}-2.40407208 \quad 0.31364217-1.26659298$

$\mathrm{H}-0.36151856 \quad 2.07837775 \quad 0.18103625$

$\mathrm{H} \quad 0.83566188 \quad 1.73479501 \quad 2.31058050$

H $1.51118509 \quad 0.20284112 \quad 1.77904067$

$\mathrm{H}-1.57205192-1.556263973 .18035986$

$\mathrm{H} 3.08722018-1.20623578-1.98969528$

H $3.28299742 \quad 0.63834961-1.82001473$

$\mathrm{H}-1.01023250-1.98667989-0.92017382$

$\mathrm{H}-1.737956161 .98022152 \quad 2.21906004$

$\mathrm{H}-1.53830272 \quad 1.24419587 \quad 3.80975845$

H $-2.70817135 \quad 0.57079738 \quad 2.66560060$

$\mathrm{H} \quad 0.08384949-3.22712037 \quad 2.47357812$

$\mathrm{H}-0.21166946-2.14638090 \quad 0.75918832$

H $1.24149795-1.95135013 \quad 1.95845624$

$01.62041168-0.20522031-0.98530882$

O $1.202928721 .20590723-0.60385078$

O $0.94277570-1.56361601-0.80367214$

Vibrational frequencies (in cm-1)

$\begin{array}{llllllllllllll}-1820.6 & 45.6 & 69.1 & 103.1 & 120.8 & 135.2 & 160.2 & 191.7 & 244.0 & 262.9 & 278.9 & 305.7 & 307.2 & 340.5\end{array}$

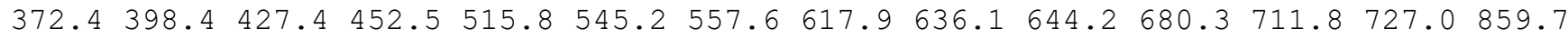

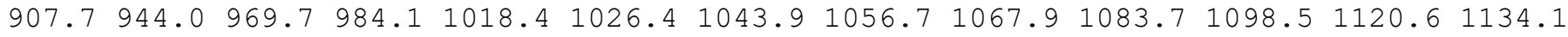

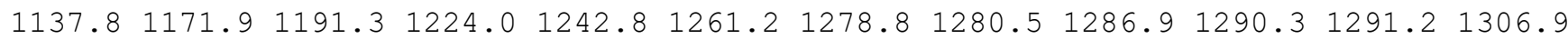
$1310.21311 .0 \quad 1339.7 \quad 1341.91352 .2 \quad 1379.4 \quad 1393.5 \quad 1401.4 \quad 1411.0 \quad 1511.5 \quad 1559.4 \quad 1694.3$ $1746.02662 .92664 .92670 .3 \quad 2672.1 \quad 2676.0 \quad 2677.4 \quad 2692.82694 .0 \quad 2704.82708 .92753 .1$ $2761.4 \quad 2771.92776 .4 \quad 2778.3$

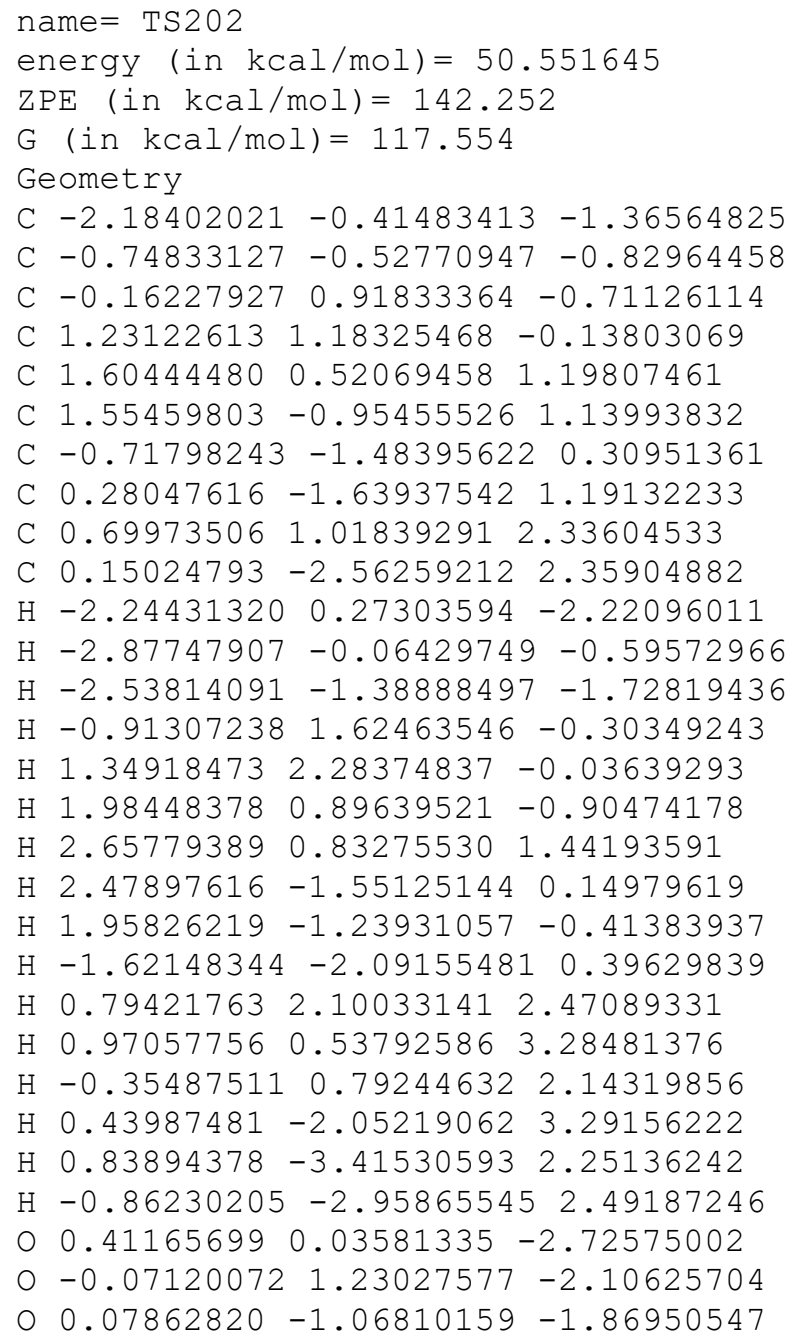


$\begin{array}{llllllllllllllll}1129.6 & 1160.9 & 1177.6 & 1188.0 & 1219.1 & 1230.1 & 1250.2 & 1267.7 & 1281.8 & 1283.8 & 1285.2 & 1290.7\end{array}$ $1296.91300 .91303 .3 \quad 1307.0 \quad 1314.3 \quad 1327.3 \quad 1370.2 \quad 1387.51400 .8 \quad 1402.7 \quad 1407.1 \quad 1472.5$ $1849.82645 .82664 .2 \quad 2665.12671 .12678 .92679 .72680 .22694 .12695 .32731 .32743 .6$ $2777.8 \quad 2779.0 \quad 2780.8 \quad 4479.3$

name $=$ TS203

energy $($ in $\mathrm{kcal} / \mathrm{mol})=49.857872$

$\mathrm{ZPE}($ in $\mathrm{kcal} / \mathrm{mol})=143.221$

$\mathrm{G}(\mathrm{in} \mathrm{kcal} / \mathrm{mol})=119.388$

Geometry

C $-2.832910330 .69346573-0.23955821$

C $-1.43464684 \quad 0.12933268-0.44328389$

C $-0.30511773 \quad 1.10699974-0.63781645$

C 1.016844070 .833267860 .00042250

C $0.95303812-0.394440670 .92347485$

C $0.30010376-1.56102540 \quad 0.11941360$

C $-1.07870568-0.97707459 \quad 0.56635398$

C $-0.34822257-0.35296737 \quad 1.81909606$

C $-0.79836824 \quad 0.98761673 \quad 2.36483842$

C $-0.27337866-1.345920932 .97643302$

$\mathrm{H}-3.023785691 .55044280-0.89391710$

$\mathrm{H}-3.01314996 \quad 0.99315470 \quad 0.79572904$

$\mathrm{H}-3.57751238-0.07131650-0.50538858$

$\mathrm{H}-0.544494192 .11228042-0.96689750$

H $1.34531468 \quad 1.72598007 \quad 0.57542817$

$\mathrm{H} \quad 1.82506875 \quad 0.67150374 \quad-0.75153481$

H $1.88976763-0.599193751 .43851741$

H $0.51534259-2.55861417 \quad 0.50332286$

$\mathrm{H} \quad 0.48036705-1.57532579-0.95925626$

$\mathrm{H}-1.88721942-1.67767345 \quad 0.75450165$

$\mathrm{H}-1.75850918 \quad 0.89315870 \quad 2.88937229$

$\mathrm{H}-0.93260832 \quad 1.75636367 \quad 1.59939154$

$\mathrm{H}-0.06840390 \quad 1.37759315 \quad 3.08563508$

$\mathrm{H} 0.41871556-0.99937640 \quad 3.75179465$

$\mathrm{H} \quad 0.06778942 \quad-2.33694254 \quad 2.65648202$

$\mathrm{H}-1.25667035-1.47979788 \quad 3.44242850$

$\begin{array}{lllll}0 & 1.15387607 & 0.27269723 & -2.76517792\end{array}$

$\begin{array}{llllll}0 & 0.17092344 & 0.82484411 & -2.55899471\end{array}$

o - $-1.20964905-0.24168260-1.74399030$

Vibrational frequencies (in $\mathrm{cm}-1$ )

$\begin{array}{lllllllllllllll}-523.0 & 59.9 & 118.5 & 142.8 & 157.3 & 178.9 & 182.3 & 196.7 & 219.1 & 286.4 & 300.8 & 328.5 & 349.4 & 366.1\end{array}$ $400.2 \quad 428.4 \quad 439.2 \quad 453.6 \quad 491.0 \quad 528.9 \quad 547.0 \quad 621.1 \quad 718.2 \quad 798.6 \quad 837.3 \quad 890.0 \quad 916.8 \quad 924.8$ $948.3 \quad 983.1 \quad 990.3 \quad 1008.0 \quad 1011.0 \quad 1017.4 \quad 1024.0 \quad 1053.7 \quad 1074.4 \quad 1087.1 \quad 1091.5 \quad 1132.4 \quad 1157.5$

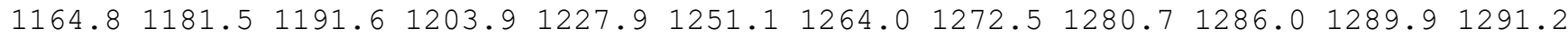
$1296.81299 .7 \quad 1302.7 \quad 1311.3 \quad 1318.0 \quad 1362.7 \quad 1376.5 \quad 1383.4 \quad 1406.3 \quad 1418.11423 .31484 .1$ $2625.52673 .12674 .6 \quad 2675.8 \quad 2677.12681 .12684 .62686 .52715 .92720 .02750 .52757 .3$ $2764.22781 .6 \quad 2783.5 \quad 2784.5$

name $=$ TS204

energy $($ in $\mathrm{kcal} / \mathrm{mol})=50.955422$

$\mathrm{ZPE}(\mathrm{in} \mathrm{kcal} / \mathrm{mol})=142.183$

$\mathrm{G}($ in $\mathrm{kcal} / \mathrm{mol})=117.234$

Geometry

C $-4.278680721 .93681385-0.57031210$

C $-2.808817551 .85532595-0.18628384$

C $-2.41090053 \quad 2.94844249 \quad 0.85563622$

C $-1.77228553 \quad 2.37954172 \quad 2.11361084$

C $-0.59760682 \quad 1.44395184 \quad 1.77838148$

C -0.924193290 .421157210 .66894259$

C $-2.39355931 \quad 0.44753755 \quad 0.24091504$

C $-0.17492277 \quad 0.73177738 \quad 3.05938963$

C $1.03393608 \quad 1.25037474 \quad 3.53558833$

C $-0.95880647-0.182605273 .65469880$ 
$\mathrm{H}-4.52834980 \quad 2.92595540 \quad-0.97727276$

$\mathrm{H}-4.93682706 \quad 1.744786350 .28316508$

$\mathrm{H}-4.524243661 .20668856-1.35248565$

$\mathrm{H}-3.25903565 \quad 3.62210517 \quad 1.09072515$

$\mathrm{H}-2.53665126 \quad 1.84235506 \quad 2.71021804$

$\mathrm{H}-1.40797916 \quad 3.200525712 .76552347$

$\mathrm{H} 0.240236762 .09002450 \quad 1.40145158$

$\mathrm{H}-0.65104990-0.60086460 \quad 0.99432445$

$\mathrm{H}-0.28801534 \quad 0.63471670-0.21522404$

$\mathrm{H}-2.55187707-0.26267197-0.59467825$

H $1.24078510 \quad 0.84905328 \quad 5.14673419$

$\mathrm{H} \quad 1.99485892 \quad 1.24722378 \quad 3.10368275$

H $1.51092188 \quad 0.17311965 \quad 4.81871795$

$\mathrm{H}-1.92090040-0.470013493 .27356490$

$\mathrm{H}-3.04263146 \quad 0.098422021 .06658757$

$\mathrm{H}-0.70412981-0.707107654 .55269117$

$0-1.71582122 \quad 3.55404767-1.19158760$

$0-1.40700088 \quad 3.71902987 \quad 0.18464418$

o $-2.00301897 \quad 2.17257450-1.33131279$

Vibrational frequencies (in $\mathrm{cm}-1$ )

$\begin{array}{lllllllllllllllll}-762.7 & 30.3 & 48.4 & 101.4 & 137.0 & 168.5 & 186.8 & 221.2 & 248.8 & 258.8 & 272.3 & 291.2 & 325.6 & 382.5\end{array}$ $405.4 \quad 425.0 \quad 449.6 \quad 507.2 \quad 530.6 \quad 566.8 \quad 582.7 \quad 598.7 \quad 616.3 \quad 679.5 \quad 687.0 \quad 705.4 \quad 814.1 \quad 833.7$

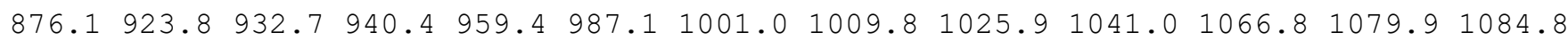

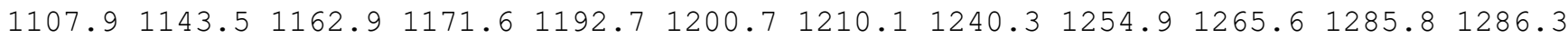

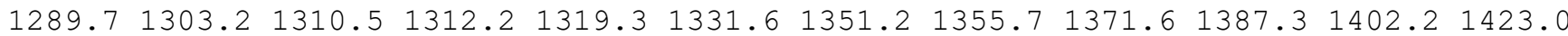

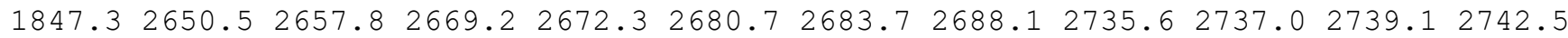
$2744.4 \quad 2782.6 \quad 2804.5 \quad 4940.2$

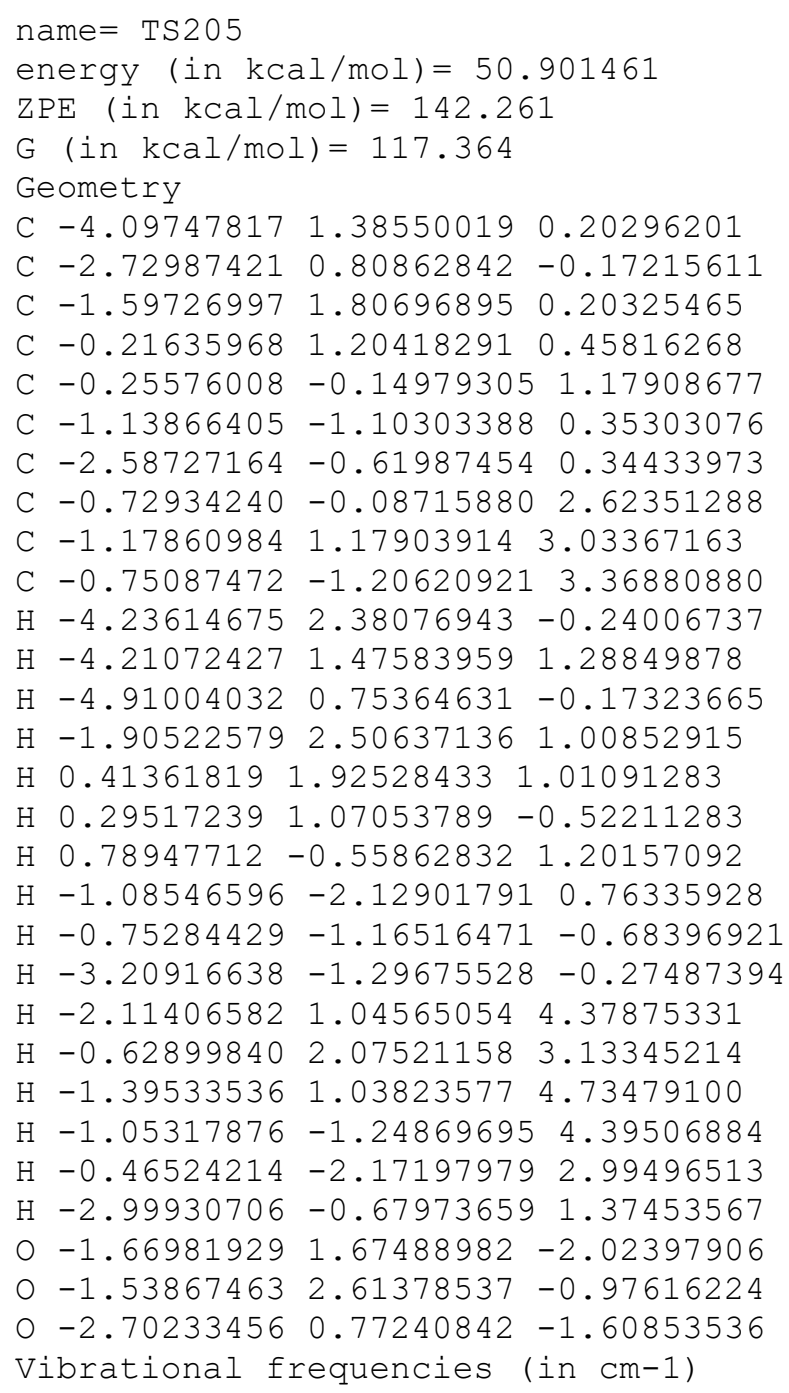


$\begin{array}{lllllllllllllllll}-826.2 & 26.1 & 49.7 & 95.5 & 136.6 & 172.4 & 183.5 & 204.5 & 262.2 & 272.8 & 313.9 & 323.3 & 346.6 & 379.5\end{array}$ $409.4 \quad 430.4 \quad 457.1 \quad 465.7 \quad 531.7 \quad 548.6 \quad 568.5 \quad 614.5 \quad 636.1 \quad 677.0 \quad 713.6 \quad 752.2 \quad 820.3 \quad 867.6$ $880.2903 .8951 .3 \quad 954.4964 .7 \quad 980.6 \quad 996.9 \quad 1004.8 \quad 1032.1 \quad 1040.8 \quad 1043.6 \quad 1077.1 \quad 1090.5$

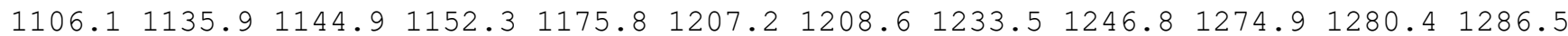
$1291.0 \quad 1294.3 \quad 1306.1 \quad 1312.2 \quad 1324.5 \quad 1334.4 \quad 1353.1 \quad 1373.4 \quad 1378.8 \quad 1384.8 \quad 1403.81431 .6$ $\begin{array}{llllllllllll}1825.6 & 2651.9 & 2656.4 & 2671.4 & 2674.6 & 2680.2 & 2687.0 & 2688.3 & 2733.1 & 2736.3 & 2738.2 & 2739.0\end{array}$ $2742.42781 .6 \quad 2803.7 \quad 4882.8$

name $=$ TS206

energy (in $\mathrm{kcal} / \mathrm{mol})=52.642077$

$\operatorname{ZPE}($ in $\mathrm{kcal} / \mathrm{mol})=140.702$

$\mathrm{G}($ in $\mathrm{kcal} / \mathrm{mol})=115.058$

Geometry

C $-2.08015402-0.51130173-0.97585164$

C $-0.85206926-0.16432432-0.27136911$

C $-0.212181681 .19632766-0.49472033$

C $1.21698140 \quad 1.28265094 \quad 0.14655394$

C $1.49572827 \quad 0.12586767 \quad 1.09927777$

C $1.22156627-1.200629160 .32482791$

C $-0.28950967-1.04499727 \quad 0.76190473$

C $0.24909885-0.20549795 \quad 2.00681326$

C $-0.60382431 \quad 0.91620879 \quad 2.55485344$

C $0.61226302-1.13669323 \quad 3.16049099$

$\mathrm{H}-2.96633200-0.51584997-0.32034679$

$\mathrm{H}-2.03328937-1.51821170-1.43556529$

$\mathrm{H}-2.280422390 .19813284 \quad-1.81592162$

$\mathrm{H}-0.93925516 \quad 1.80851689 \quad 0.18618821$

H $1.33304974 \quad 2.25827718 \quad 0.64277694$

$\mathrm{H} \quad 1.94688210 \quad 1.28781661-0.68798556$

H $2.45298360 \quad 0.20888357 \quad 1.61694993$

$\mathrm{H} 1.70100919-2.09008557 \quad 0.73608708$

$\mathrm{H} \quad 1.42356984-1.17675855-0.75133278$

$\mathrm{H}-0.83893138-1.95427751 \quad 1.00481395$

$\mathrm{H}-1.54198258 \quad 0.53582198 \quad 2.97804370$

$\mathrm{H}-0.866083521 .689965331 .82096675$

$\mathrm{H}-0.073707691 .444463913 .36154398$

$\mathrm{H}-0.28366883-1.53332697 \quad 3.65232107$

$\mathrm{H} 1.19677963-0.604833193 .92220382$

$\mathrm{H} 1.21325975-1.996443872 .84411826$

$0 \quad 0.48019347 \quad 0.15320495-2.89381413$

$\begin{array}{lllll}0 & -0.31119428 & 1.64750820 & -1.70012488\end{array}$

O $0.05582042-0.82989431-2.46790743$

Vibrational frequencies (in $\mathrm{cm}-1$ )

$\begin{array}{llllllllllllllll}-134.3 & 54.8 & 81.9 & 87.5 & 102.8 & 126.7 & 155.1 & 160.7 & 179.1 & 196.1 & 229.3 & 262.2 & 287.7 & 293.9\end{array}$

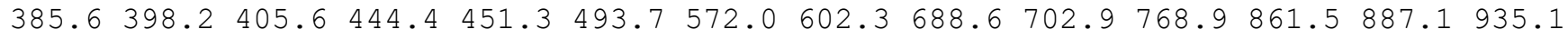
$937.9946 .9 \quad 964.9995 .0 \quad 1006.0 \quad 1013.9 \quad 1014.6 \quad 1033.9 \quad 1048.5 \quad 1066.4 \quad 1082.5 \quad 1119.8 \quad 1127.9$

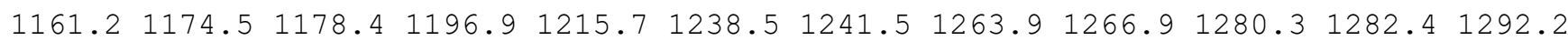
$1295.51299 .8 \quad 1310.3 \quad 1312.7 \quad 1354.2 \quad 1367.4 \quad 1372.2 \quad 1401.3 \quad 1410.5 \quad 1413.4 \quad 1433.11504 .1$ $2421.52619 .22634 .52641 .62667 .2 \quad 2671.2 \quad 2672.62673 .92677 .4 \quad 2745.12748 .12749 .5$ $2752.3 \quad 2755.8 \quad 2771.0 \quad 2781.8$

name $=\operatorname{TS} 207$

energy (in $\mathrm{kcal} / \mathrm{mol})=52.743486$

$\mathrm{ZPE}(\mathrm{in} \mathrm{kcal} / \mathrm{mol})=140.834$

$\mathrm{G}($ in $\mathrm{kcal} / \mathrm{mol})=115.055$

Geometry

C $-2.12853107-0.37030151-1.12323118$

C $-0.97588060-0.09437712-0.29223511$

C $-0.126975651 .14954183-0.59300164$

C $1.236613531 .10087584 \quad 0.18198324$

C $1.24657109 \quad 0.05258530 \quad 1.28914438$

C $0.86911282-1.31499500 \quad 0.64199667$

C $-0.64220316-0.916541290 .87532151$ 
C $-0.11945001-0.00093200 \quad 2.07566886$

C $-0.86931320 \quad 1.27537554 \quad 2.38508238$

C $-0.01136116-0.819794713 .35891093$

$\begin{array}{lllll}\mathrm{H} & -2.63389784 & 0.55638304 & -1.47225403\end{array}$

$\mathrm{H}-2.86774126-1.06797580-0.71464974$

$\mathrm{H}-1.78377328-0.78634639-2.11580802$

$\mathrm{H}-0.769569661 .97723939-0.12535079$

H $1.46651851 \quad 2.10519470 \quad 0.56972851$

$\mathrm{H} \quad 2.02778560 \quad 0.88538248 \quad-0.56512234$

H 2.148171890 .078579361 .90398229

H $1.17102601-2.19934341 \quad 1.20595470$

$\mathrm{H} \quad 1.18740562-1.45834038-0.39671914$

$\mathrm{H}-1.33170127-1.71489183 \quad 1.15016538$

$\mathrm{H}-1.938664361 .084401852 .53697609$

$\mathrm{H}-0.77381952 \quad 2.05223593 \quad 1.61673457$

$\mathrm{H}-0.49045395 \quad 1.73727180 \quad 3.30927736$

$\mathrm{H} \quad 0.58888388-0.29044357 \quad 4.11018987$

$\mathrm{H} \quad 0.46270342-1.79584597 \quad 3.20691416$

$\mathrm{H}-0.99842427-1.005791553 .79867696$

O $0.78111872-0.19490402-2.73936539$

$0-0.036424811 .37989410-1.87056362$

O $0.28696731-1.14209764-2.28941992$

Vibrational frequencies (in $\mathrm{cm}-1$ )

$\begin{array}{llllllllllllllll}-156.2 & 45.5 & 61.3 & 67.8 & 109.2 & 148.9 & 156.9 & 168.0 & 181.5 & 197.9 & 230.3 & 264.7 & 293.8 & 317.7\end{array}$

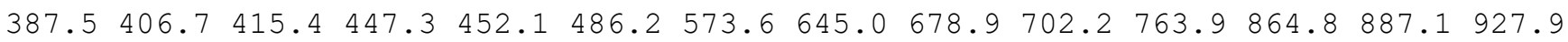
$936.6956 .4971 .2995 .0 \quad 1001.6 \quad 1012.3 \quad 1017.2 \quad 1043.3 \quad 1063.6 \quad 1071.4 \quad 1093.3 \quad 1123.01129 .3$

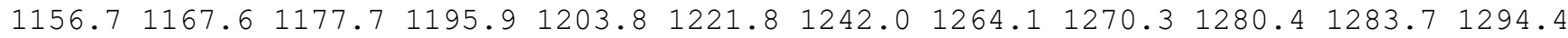
$1295.01303 .0 \quad 1310.6 \quad 1312.1 \quad 1353.9 \quad 1366.0 \quad 1373.2 \quad 1387.01405 .91415 .2 \quad 1435.91479 .3$ $2516.42545 .7 \quad 2648.2 \quad 2660.8 \quad 2662.8 \quad 2668.2 \quad 2671.12673 .12677 .12744 .12746 .92748 .2$ $2750.12753 .7 \quad 2775.2 \quad 2781.6$

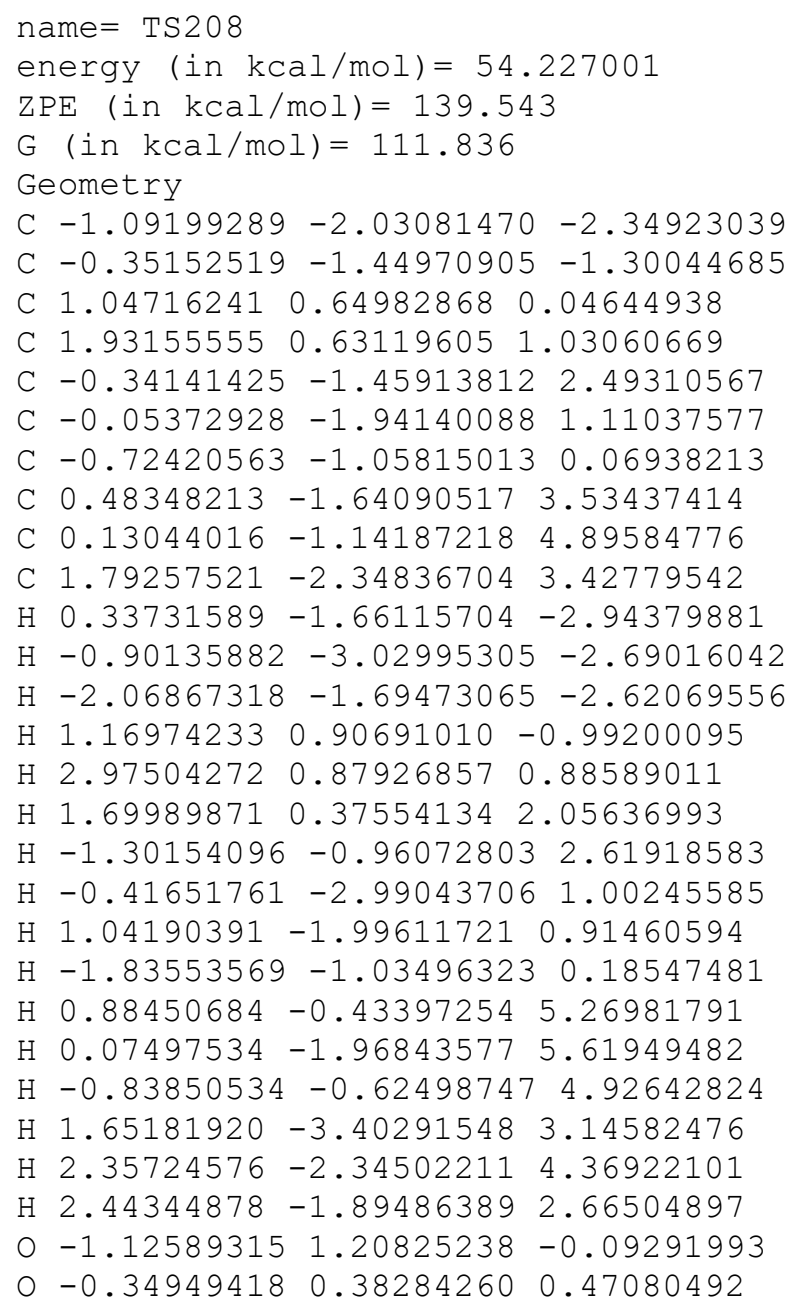


$0.83383492-1.25763379-1.76070923$

Vibrational frequencies (in $\mathrm{cm}-1$ )

$\begin{array}{lllllllllllllll}-1999.1 & 30.8 & 39.6 & 51.0 & 55.7 & 89.4 & 94.0 & 112.0 & 135.3 & 171.3 & 194.3 & 227.3 & 258.6 & 277.8\end{array}$ $316.0 \quad 334.1 \quad 390.2 \quad 426.6 \quad 444.9 \quad 474.1 \quad 489.3 \quad 510.3 \quad 554.5 \quad 637.6 \quad 663.7 \quad 716.2 \quad 807.2 \quad 852.1$

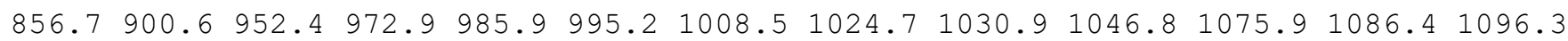

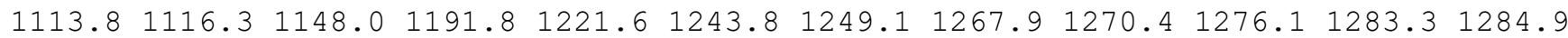

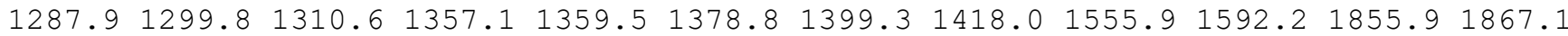
$2104.52638 .5 \quad 2663.3 \quad 2669.7 \quad 2670.6 \quad 2673.9 \quad 2675.3 \quad 2701.72721 .92725 .8 \quad 2741.42754 .2$ $2775.32777 .12780 .0 \quad 2802.2$

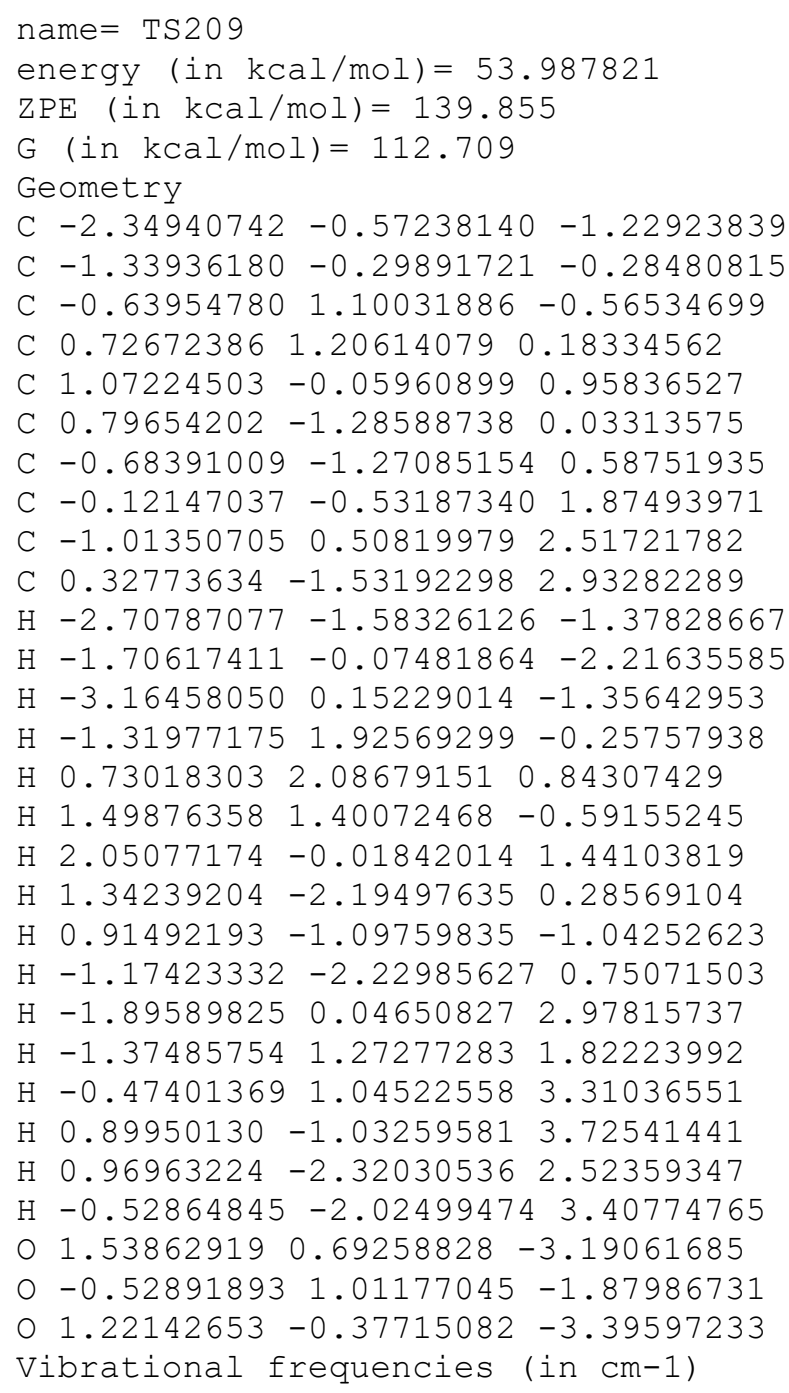


C $1.11260036-1.270820760 .17732385$

C $-0.41089306-1.207994560 .58980083$

C $0.07167681-0.44382324 \quad 1.90780318$

C $-0.84213605 \quad 0.59798018 \quad 2.51262976$

C $0.44576249-1.44608213 \quad 2.99592785$

$\mathrm{H}-2.09482360-0.22611070-2.17552997$

$\mathrm{H}-3.074774650 .02914370 \quad-0.76679160$

$\mathrm{H}-2.50344630-1.62718167-1.11824149$

$\mathrm{H}-1.11879263 \quad 1.76328802 \quad 0.15369297$

H $1.10685142 \quad 2.15354295 \quad 0.75338397$

$\mathrm{H} 1.766616991 .29911155-0.63390143$

$\mathrm{H} 2.264383190 .07773309 \quad 1.60012910$

$\mathrm{H} \quad 1.61606185-2.17219145 \quad 0.53131225$

$\mathrm{H} 1.33738639-1.15954486-0.88848975$

$\mathrm{H}-0.92369401-2.15660883 \quad 0.74992599$

$\mathrm{H}-1.034255031 .462698291 .86636081$

$\mathrm{H}-0.400459311 .014566323 .43060777$

$\mathrm{H}-1.81245014 \quad 0.16736825 \quad 2.78904021$

$\mathrm{H} 1.00879238-0.956052003 .80080325$

$\mathrm{H} \quad 1.07169058-2.266172392 .62675113$

$\mathrm{H}-0.44521098-1.898394813 .44723496$

$\begin{array}{lllll}0 & 0.48118389 & 0.22094836 & -2.87630398\end{array}$

O $-0.44898876 \quad 1.58737753-1.71010432$

O $0.02258213-0.79919539-2.58633074$

Vibrational frequencies (in cm-1)

$\begin{array}{lllllllllllllll}-157.1 & 34.7 & 56.8 & 90.9 & 129.2 & 155.4 & 173.3 & 178.7 & 189.8 & 203.8 & 230.2 & 265.0 & 292.9 & 313.9\end{array}$

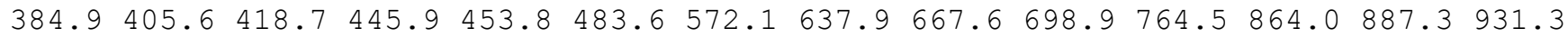
$940.6956 .9968 .5 \quad 996.9 \quad 1002.5 \quad 1014.4 \quad 1016.9 \quad 1038.7 \quad 1066.0 \quad 1072.2 \quad 1090.1 \quad 1127.3 \quad 1155.7$

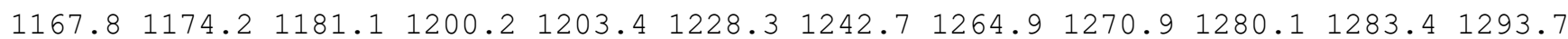

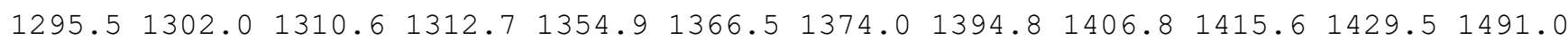
$2512.02572 .3 \quad 2647.62658 .92667 .02671 .2 \quad 2672.62673 .92677 .12744 .32747 .2 \quad 2749.5$ $2753.4 \quad 2756.6 \quad 2774.8 \quad 2781.8$

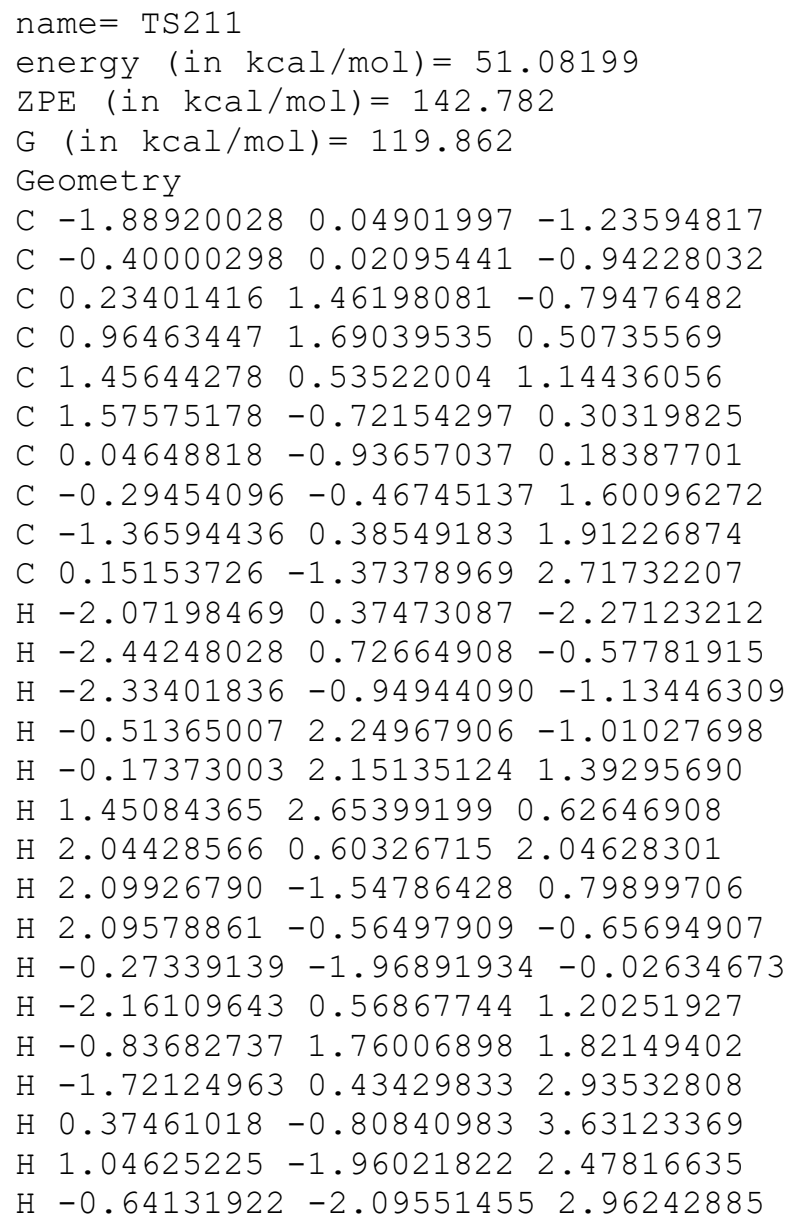


$0.69524146 \quad 0.64005985-2.82794544$

o $1.22325728 \quad 1.49648139-1.83154044$

O $0.25367717-0.50401721-2.11498060$

Vibrational frequencies (in $\mathrm{cm}-1$ )

$\begin{array}{lllllllllllllll}-1959.3 & 89.1 & 118.3 & 126.9 & 174.0 & 203.6 & 208.1 & 281.7 & 283.9 & 347.1 & 354.9 & 386.5 & 412.5 & 433.6\end{array}$ $458.8 \quad 488.9 \quad 495.6 \quad 540.9 \quad 602.2 \quad 604.4 \quad 645.5 \quad 711.6 \quad 740.0 \quad 831.6 \quad 845.1 \quad 857.5 \quad 888.8 \quad 927.4$ $940.4964 .0 \quad 974.5 \quad 985.4 \quad 987.2 \quad 1009.8 \quad 1012.4 \quad 1045.9 \quad 1053.6 \quad 1055.8 \quad 1063.2 \quad 1081.9 \quad 1112.9$ $\begin{array}{llllllllllll}1125.8 & 1129.9 & 1148.5 & 1151.7 & 1166.4 & 1179.9 & 1218.0 & 1239.5 & 1247.8 & 1272.3 & 1283.9 & 1286.3\end{array}$ $1286.71292 .5 \quad 1300.2 \quad 1303.0 \quad 1311.4 \quad 1339.0 \quad 1373.3 \quad 1380.3 \quad 1396.8 \quad 1405.7 \quad 1463.8 \quad 1535.7$ $1602.52665 .22667 .82669 .12670 .72674 .2 \quad 2684.02695 .72729 .72741 .42754 .42759 .7$ $2763.62778 .02778 .4 \quad 3059.8$

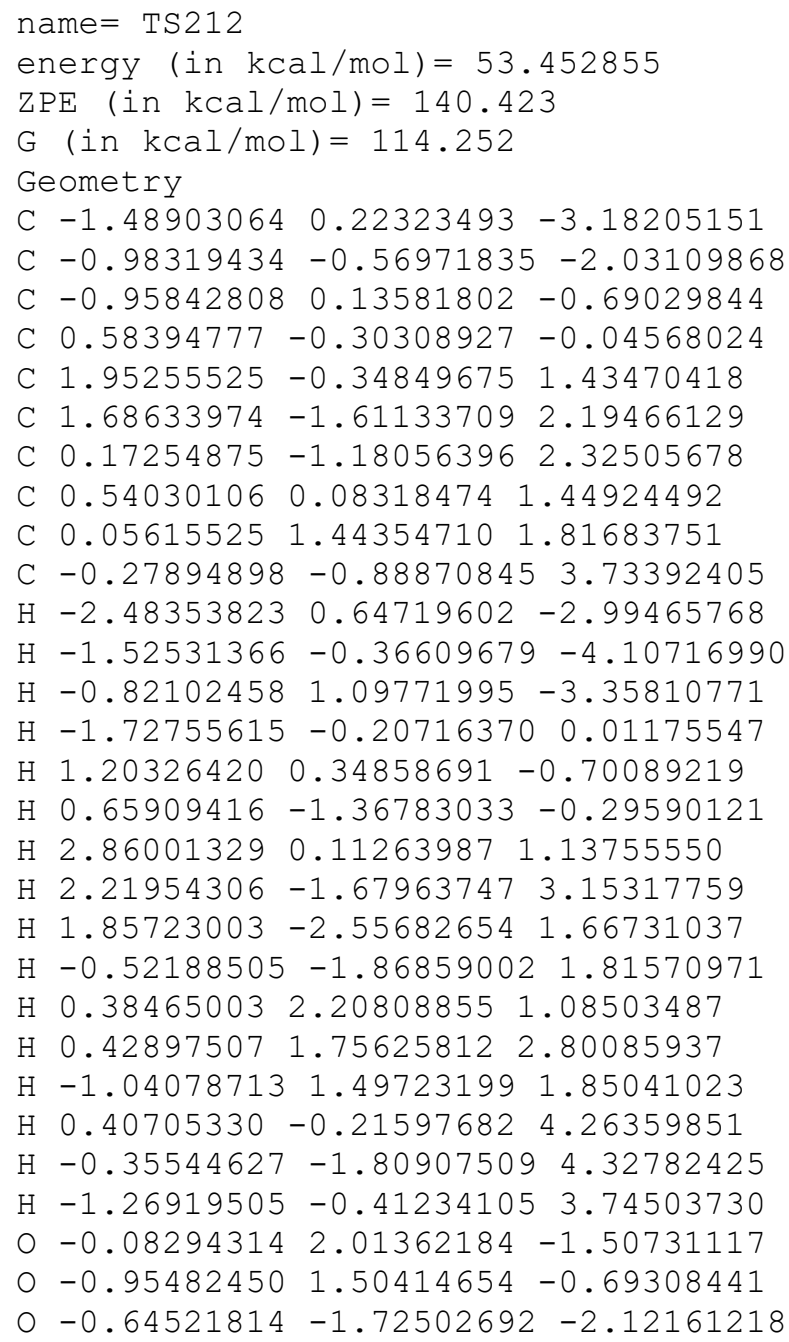


C $1.078107391 .51478154 \quad 0.39021293$

C $1.22862921 \quad 0.24914854 \quad 1.22198186$

C $1.23999024-0.96657158 \quad 0.24402110$

C $-0.32092372-0.987254090 .35353621$

C $-0.14889336-0.26717301 \quad 1.78187833$

C $-1.20434735 \quad 0.73621346 \quad 2.19140289$

C $0.02771885-1.29055275 \quad 2.89557101$

$\mathrm{H}-2.89211967 \quad 0.65607431-0.61230196$

$\mathrm{H}-2.65610793-1.04261702-1.09462501$

$\mathrm{H}-2.202397150 .31083453-2.17503759$

$\mathrm{H}-0.819802492 .23712956-0.49268506$

$\mathrm{H} \quad 0.91488773 \quad 2.39413488 \quad 1.02725940$

$\mathrm{H} \quad 2.01283250 \quad 1.71211960-0.17335440$

H $2.041256770 .30377191 \quad 1.94924140$

H $1.71833571-1.86746821 \quad 0.63201366$

$\mathrm{H} 1.65833746-0.75062267-0.74294898$

$\mathrm{H}-0.80814017-1.961327350 .36925108$

$\mathrm{H}-1.308315421 .576793301 .49609040$

$\mathrm{H}-0.951281321 .183048383 .16376254$

$\mathrm{H}-2.18894471 \quad 0.26541500 \quad 2.29551098$

$\mathrm{H} \quad 0.40742863-0.80689784 \quad 3.80562297$

H $0.73973958-2.08236636 \quad 2.63646744$

$\mathrm{H}-0.91975525-1.77698598 \quad 3.15357607$

○ $0.79752469-0.71507314-2.76342765$

O $0.337854721 .41660246-1.91214389$

o $-0.26837328-0.86999184-2.41166541$

Vibrational frequencies (in cm-1)

$\begin{array}{llllllllllllllll}-197.2 & 14.1 & 106.7 & 128.2 & 133.2 & 152.9 & 167.0 & 187.6 & 200.9 & 222.6 & 244.6 & 262.9 & 284.4 & 308.5\end{array}$ $371.2402 .2 \quad 410.3 \quad 435.6 \quad 460.0 \quad 470.6 \quad 532.7 \quad 578.9 \quad 669.4 \quad 715.7 \quad 752.3 \quad 862.4 \quad 883.6 \quad 917.7$ $945.8971 .5 \quad 997.1 \quad 1004.4 \quad 1008.2 \quad 1015.3 \quad 1021.6 \quad 1041.0 \quad 1076.1 \quad 1085.7 \quad 1116.8 \quad 1140.5 \quad 1163.3$ $1184.01193 .21201 .1 \quad 1216.4 \quad 1247.0 \quad 1250.2 \quad 1255.7 \quad 1267.2 \quad 1278.7 \quad 1283.2 \quad 1289.8 \quad 1294.0$ $1298.81303 .41311 .2 \quad 1318.6 \quad 1357.5 \quad 1364.2 \quad 1374.7 \quad 1404.51414 .51417 .7 \quad 1465.3 \quad 1570.6$ $2611.82613 .0 \quad 2662.9 \quad 2665.4 \quad 2672.6 \quad 2673.4 \quad 2675.42679 .22684 .92745 .12748 .32750 .6$ $2759.82760 .92780 .5 \quad 2782.7$

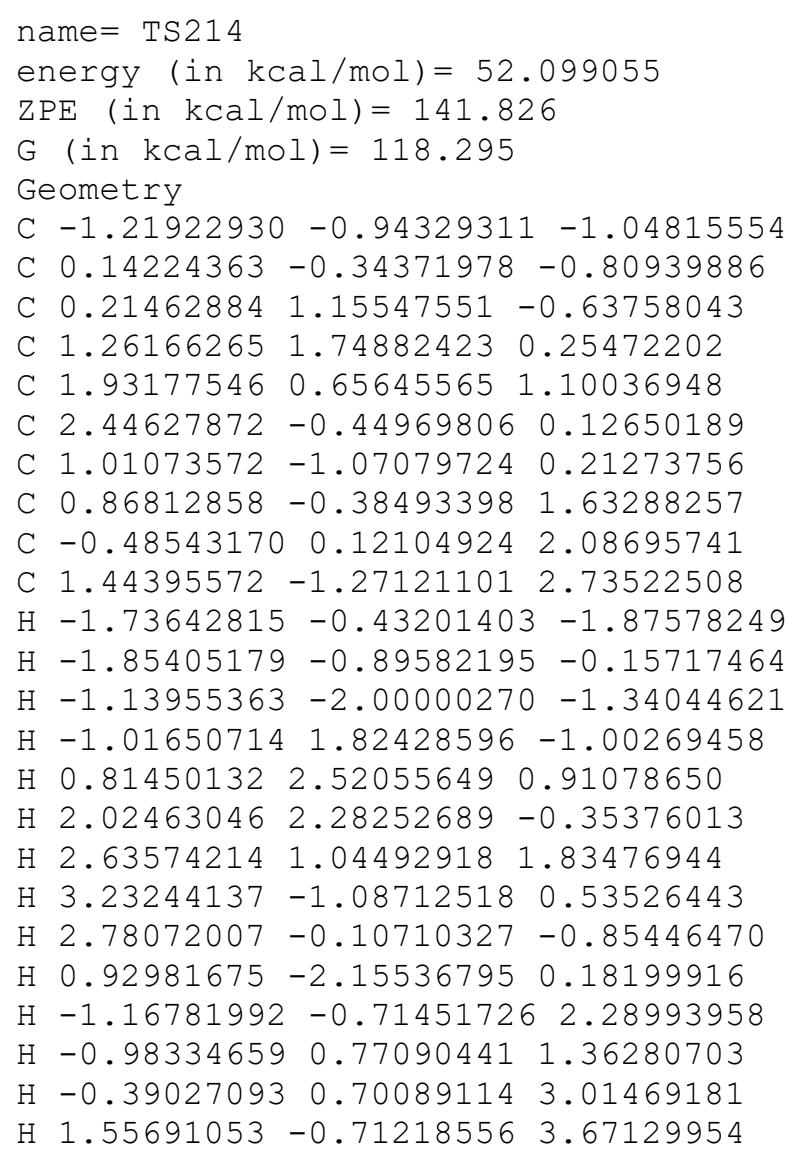


H $2.42999816-1.675138512 .48069286$

$\mathrm{H} 0.78774831-2.12597577 \quad 2.93644688$

$00.37799408 \quad 0.46341378-2.93865686$

$\begin{array}{llll}0 & -0.09342738 & 1.75038745 & -1.82394836\end{array}$

O $0.93424812-0.33168402-2.07124584$

Vibrational frequencies (in $\mathrm{cm}-1$ )

$\begin{array}{lllllllllllllllll}-1638.2 & 98.0 & 115.0 & 146.8 & 148.1 & 163.2 & 176.8 & 194.1 & 252.5 & 271.1 & 283.3 & 313.1 & 342.4 & 382.9\end{array}$

$415.3 \quad 434.6 \quad 451.9 \quad 488.6 \quad 515.3 \quad 576.7 \quad 616.0 \quad 629.6 \quad 698.0 \quad 723.5 \quad 777.0 \quad 809.6 \quad 856.4 \quad 881.5$

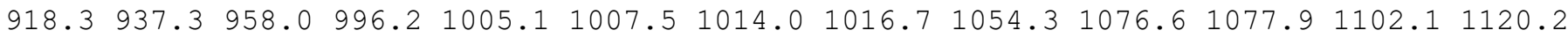
$1134.51178 .8 \quad 1180.1 \quad 1183.5 \quad 1222.0 \quad 1237.4 \quad 1264.1 \quad 1277.3 \quad 1278.7 \quad 1282.5 \quad 1287.8 \quad 1292.2$

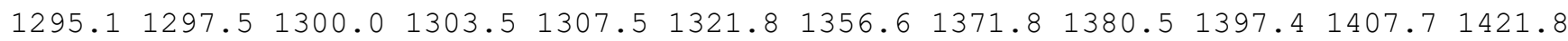
$2442.72658 .12665 .5 \quad 2673.1 \quad 2675.3 \quad 2676.8 \quad 2678.62680 .3 \quad 2684.4 \quad 2738.62748 .92752 .6$ $2763.5 \quad 2779.0 \quad 2781.8 \quad 2784.2$

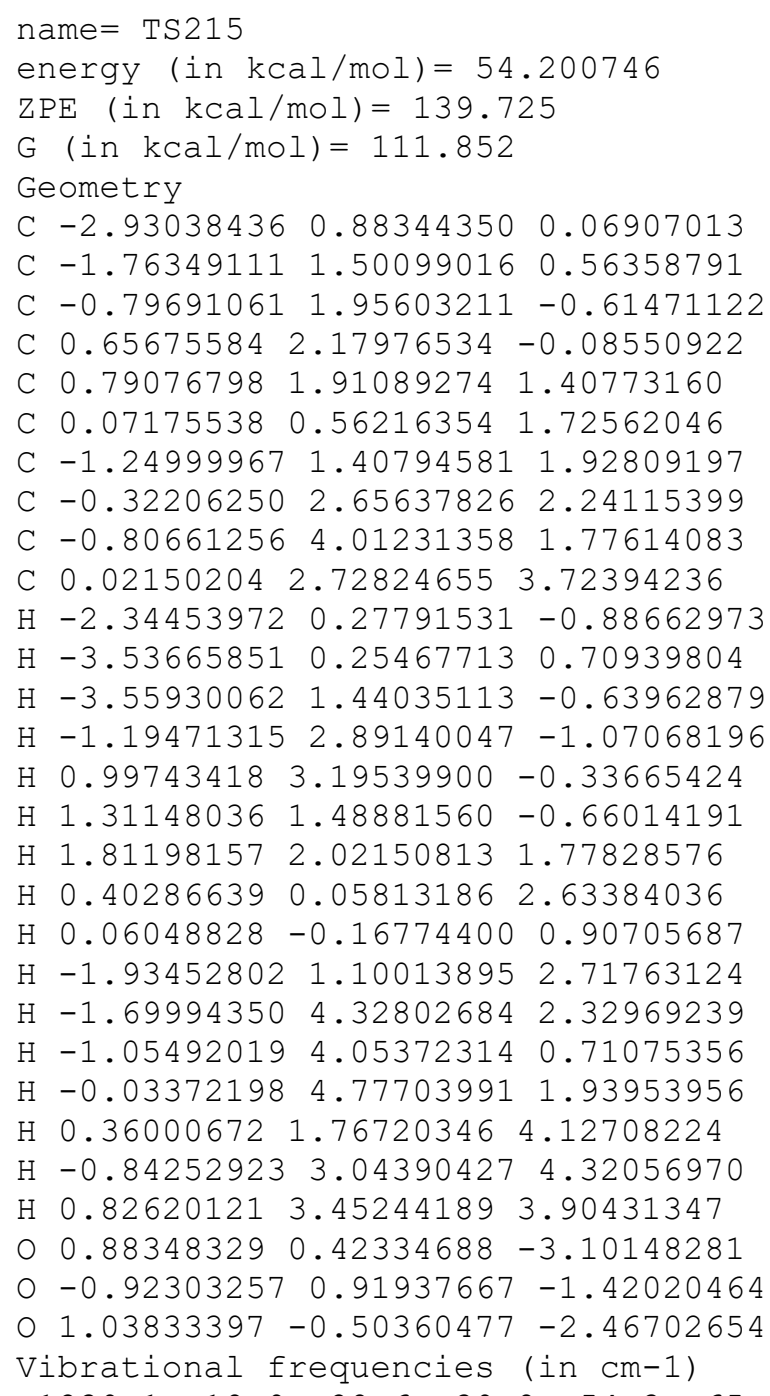


C $-0.62351602-0.70669954-1.02363084$

C $-1.03454194 \quad 0.72059399-1.13738710$

C $1.600233061 .09455828 \quad 0.33383455$

C $0.86456970-0.56174751 \quad 2.12469093$

C $1.40926795-0.38612558 \quad 0.67623169$

C $0.52742946-1.17061243-0.25729364$

C $-0.61645921-0.71850517 \quad 2.04846405$

$\begin{array}{llll}\text { C }-1.45111343 & 0.28280133 & 2.70183221\end{array}$

C $1.36459134-1.86629007 \quad 2.76573322$

$\mathrm{H}-1.36016626-2.67116201-1.58789265$

$\mathrm{H}-2.38104926-1.77590387-0.45393027$

$\mathrm{H}-2.35623560-1.32808188-2.17089232$

$\mathrm{H}-0.58683194 \quad 1.28938662-1.97127703$

H $1.14345291 \quad 1.38768844-0.61707700$

H $1.20234964 \quad 1.76223795 \quad 1.10458835$

$\mathrm{H} \quad 1.22136613 \quad 0.29369662 \quad 2.74219246$

$\mathrm{H} 2.42863878-0.86311898 \quad 0.63769380$

H 2.66616278 1.332289310 .21160088

$\mathrm{H} \quad 0.53066409-2.24280770 \quad 0.00736503$

$\mathrm{H}-1.93050728 \quad 0.88878644 \quad 1.89807069$

$\mathrm{H}-0.97165973 \quad 0.98203203 \quad 3.39312747$

$\mathrm{H}-2.29435651-0.19924414 \quad 3.22313267$

$\mathrm{H} 1.01176452-1.957657363 .80023836$

H $2.45818722-1.90725628 \quad 2.78629216$

H $1.00033022 \quad-2.74755240 \quad 2.22280732$

o $1.35716593-0.13369842-2.27675228$

$\begin{array}{lllll}0 & -1.83622375 & 1.22235552 & -0.39134332\end{array}$

O $0.70088119-1.05619016-1.79535961$

Vibrational frequencies (in $\mathrm{cm}-1$ )

$\begin{array}{lllllllllllllllll}-165.9 & 30.5 & 49.2 & 77.6 & 87.4 & 101.8 & 115.6 & 140.4 & 145.7 & 162.5 & 207.0 & 226.8 & 246.5 & 256.1\end{array}$ $293.7 \quad 302.8 \quad 348.4 \quad 355.0 \quad 399.3 \quad 451.1 \quad 521.4 \quad 551.7 \quad 603.7 \quad 627.2 \quad 698.8 \quad 721.0 \quad 848.7 \quad 877.5$

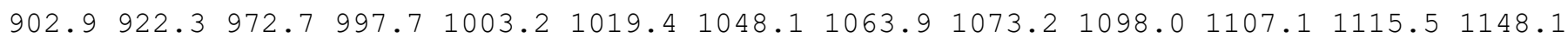
$1168.11200 .21206 .5 \quad 1232.8 \quad 1251.2 \quad 1259.9 \quad 1263.4 \quad 1273.0 \quad 1276.4 \quad 1282.8 \quad 1290.1 \quad 1297.4$ $1298.61300 .0 \quad 1307.7 \quad 1336.6 \quad 1359.7 \quad 1374.7 \quad 1386.3 \quad 1400.8 \quad 1403.9 \quad 1426.91519 .91839 .3$ $2627.32631 .52653 .22656 .62670 .2 \quad 2671.5 \quad 2672.82673 .92676 .62685 .72698 .12709 .0$ $2765.7 \quad 2774.1 \quad 2779.2 \quad 2783.1$

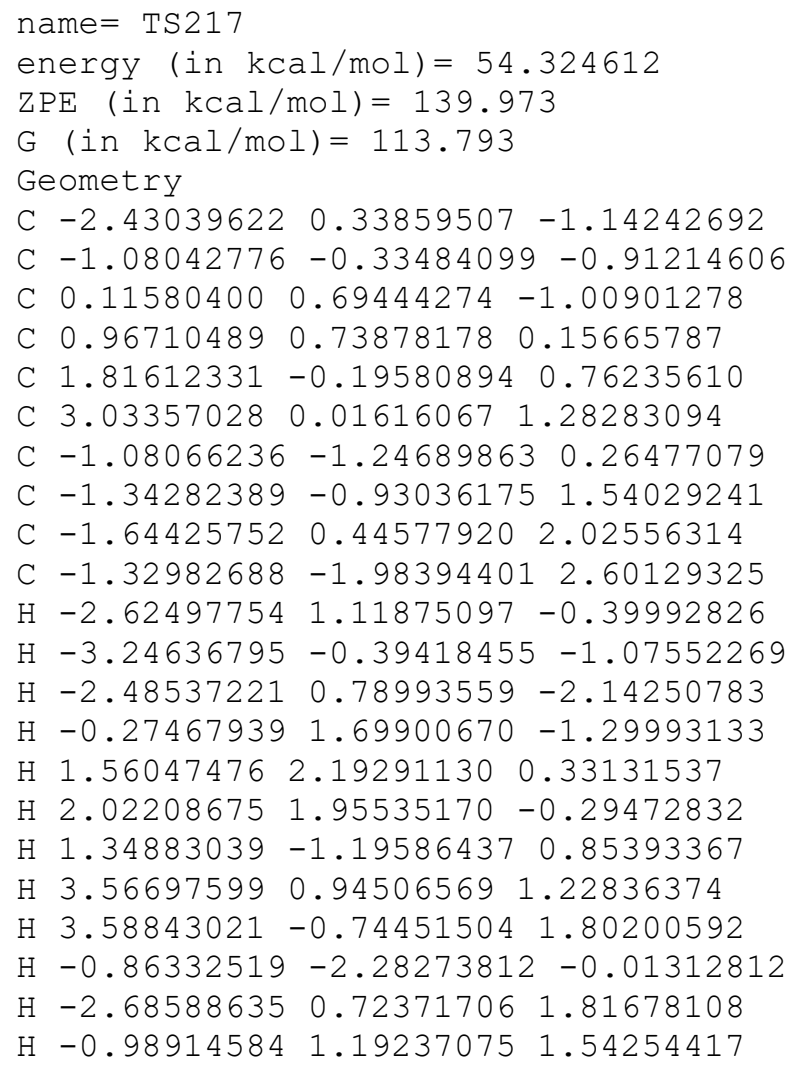


$\mathrm{H}-1.48962520 \quad 0.555202113 .10676809$

$\mathrm{H}-2.25287084-1.96246415 \quad 3.19692743$

$\mathrm{H}-0.48964728-1.827327653 .29495830$

$\mathrm{H}-1.22992844 \quad-3.00002030 \quad 2.19731345$

○ $0.50151273-1.08920231-2.34501195$

$\begin{array}{lllll}0 & 0.82324492 & 0.28200248 & -2.19537131\end{array}$

o $-0.88755798-1.13480114-2.10097827$

Vibrational frequencies (in $\mathrm{cm}-1$ )

$\begin{array}{llllllllllllllllll}-819.6 & 32.4 & 78.2 & 88.7 & 113.8 & 120.2 & 134.5 & 142.8 & 161.3 & 163.7 & 196.1 & 237.9 & 259.6 & 277.6\end{array}$

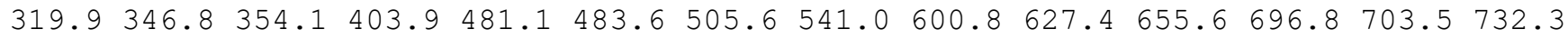
$846.9884 .8 \quad 910.1940 .4 \quad 942.4 \quad 963.2 \quad 988.5 \quad 993.2 \quad 1007.1 \quad 1021.6 \quad 1027.4 \quad 1050.5 \quad 1052.9$

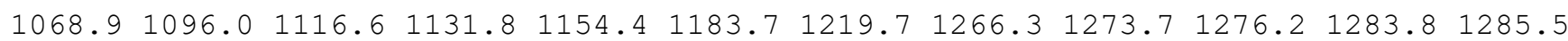

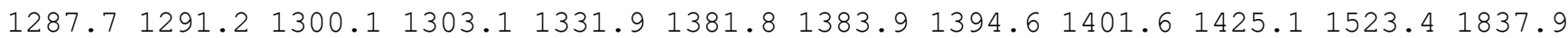
$1874.22655 .2 \quad 2669.12670 .6 \quad 2674.12675 .6 \quad 2676.6 \quad 2678.02703 .3 \quad 2738.02741 .02775 .6$ $2779.52780 .12800 .3 \quad 4713.8$

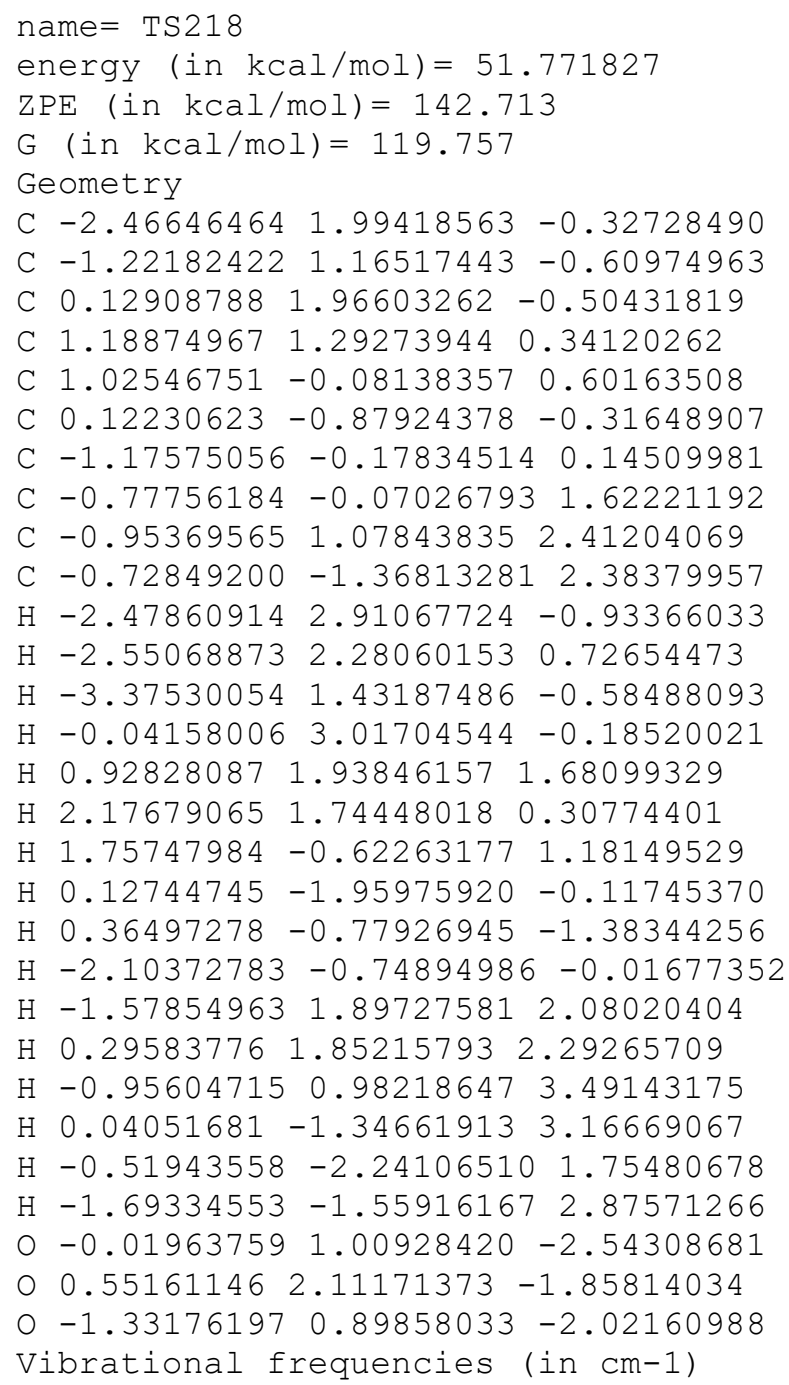


Geometry

C $-2.60544593 \quad 0.80058904 \quad 0.50672783$

C $-1.19327574 \quad 0.26806944 \quad 0.26034618$

C $-0.200650691 .45155821 \quad 0.06603986$

C $1.28537285 \quad 1.11245818 \quad 0.23390520$

$\begin{array}{llll}\text { C } & 1.41599171 & 0.17604768 & 1.36966545\end{array}$

C $0.71509867-1.12155007 \quad 1.26497930$

C $-0.80704252-0.84269896 \quad 1.25606537$

C $-1.29798981-0.53413022 \quad 2.65021949$

C $-0.81501082 \quad 0.46485980 \quad 3.39047761$

C $-2.37041838-1.44717430 \quad 3.15208535$

$\mathrm{H}-2.89144991 \quad 1.52084793-0.27226157$

$\mathrm{H}-2.69055478 \quad 1.29556531 \quad 1.48063166$

$\mathrm{H}-3.34324212 \quad-0.01005337 \quad 0.47622507$

$\mathrm{H}-0.49055020 \quad 2.35478279 \quad 0.63702496$

H $1.857198692 .04568318 \quad 0.40906781$

$\mathrm{H} \quad 1.66318154 \quad 0.70228237 \quad-0.72813293$

H $2.93869270-0.27210081 \quad 1.24277809$

H $0.95142068-1.78092785 \quad 2.12391355$

H $0.97349947-1.69371328 \quad 0.35071010$

$\mathrm{H}-1.30644795-1.78165699 \quad 0.88989557$

$\mathrm{H}-0.01783742 \quad 1.11588743 \quad 3.05014357$

H 2.73248554 $0.11451750 \quad 1.95782034$

$\mathrm{H}-1.14938178 \quad 0.69345578 \quad 4.38640991$

$\mathrm{H}-3.24351754-1.448441872 .48482239$

$\mathrm{H}-2.72766535-1.164214794 .15071942$

$\mathrm{H}-2.00698513-2.48218278 \quad 3.22278895$

$0-0.52694348 \quad 0.51894777-1.93924414$

$0-0.452171291 .77805649-1.30197022$

o $-1.23076857-0.35092281-1.03626134$

Vibrational frequencies (in $\mathrm{cm}-1$ )

$\begin{array}{lllllllllllllll}-1302.7 & 48.9 & 55.8 & 74.7 & 104.6 & 165.3 & 188.4 & 189.7 & 201.0 & 246.0 & 286.1 & 298.5 & 327.9 & 349.5\end{array}$

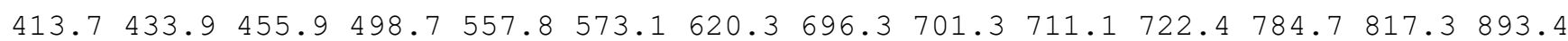
$901.6955 .5 \quad 965.4985 .0 \quad 995.9 \quad 1001.2 \quad 1017.8 \quad 1044.1 \quad 1048.9 \quad 1057.6 \quad 1084.2 \quad 1094.9 \quad 1108.9$ $\begin{array}{llllllllllll}1125.7 & 1145.3 & 1160.5 & 1169.8 & 1194.4 & 1230.7 & 1232.4 & 1268.9 & 1279.5 & 1281.6 & 1289.4 & 1292.5\end{array}$

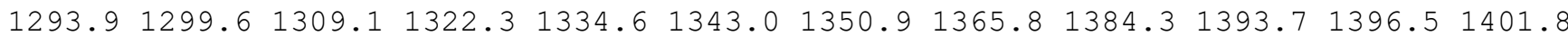
$1868.72652 .7 \quad 2660.42671 .5 \quad 2672.6 \quad 2675.2 \quad 2675.92683 .22698 .12711 .02736 .12737 .2$ $2778.12779 .5 \quad 2785.3 \quad 4337.0$

\footnotetext{
name $=$ TS220

energy $($ in $\mathrm{kcal} / \mathrm{mol})=53.410013$

$\mathrm{ZPE}($ in $\mathrm{kcal} / \mathrm{mol})=141.189$

$\mathrm{G}($ in $\mathrm{kcal} / \mathrm{mol})=117.384$

Geometry

C $-0.90405499 \quad 0.62001014 \quad 3.17062332$

C $-0.17706441 \quad 0.28887696 \quad 1.87118049$

C $-0.078493331 .52751851 \quad 0.92274826$

C $1.23358649 \quad 1.70640251 \quad 0.24094028$

C $2.37266562 \quad 1.24804774 \quad 0.76279112$

C $1.33019935-1.65411998 \quad 1.26717229$

C $1.15254265-0.46894062 \quad 2.11275707$

$\begin{array}{llll}\text { C } 2.38765105 & 0.48195041 & 2.05612850\end{array}$

C $2.35646097 \quad 1.49704525 \quad 3.21454012$

C $3.67323142-0.348683592 .17750056$

$\mathrm{H}-0.29427133 \quad 1.23982215 \quad 3.83563962$

$\mathrm{H}-1.16926890-0.29547178 \quad 3.71770256$

$\mathrm{H}-1.848168851 .14857319 \quad 2.97613554$

$\mathrm{H}-0.395647292 .46924515 \quad 1.42834612$

H $1.18723891 \quad 2.24882805-0.70153586$

$\mathrm{H} 1.02517095-1.07483882-0.28283416$

H $3.33784923 \quad 1.40756931 \quad 0.28876772$

$\mathrm{H} \quad 0.55462033-2.38116824 \quad 1.18526837$

$\mathrm{H} 1.80550253-1.26864288-0.18087614$

H $1.12676159-0.89396813 \quad 3.16176354$
} 
$\mathrm{H} \quad 2.302763490 .99202016 \quad 4.18421652$

H $1.50555548 \quad 2.18029685 \quad 3.13702799$

H $3.263912992 .11233361 \quad 3.21720747$

H $4.56886790 \quad 0.27289371 \quad 2.10542772$

$\mathrm{H} \quad 3.72221334-1.11986614 \quad 1.39417690$

$\mathrm{H} 3.70924332-0.88477328 \quad 3.13214064$

$0-1.14427760-0.11568244-0.15799664$

$\begin{array}{llll}0 & -1.11058879 & 1.29368907 & -0.03811667\end{array}$

O $-1.08994466-0.586380121 .18166284$

Vibrational frequencies (in $\mathrm{cm}-1$ )

$\begin{array}{llllllllllllllllll}-889.9 & 59.8 & 104.7 & 168.0 & 175.2 & 191.0 & 205.1 & 226.6 & 244.1 & 257.9 & 278.8 & 291.5 & 326.8 & 346.7\end{array}$ $366.5 \quad 379.6 \quad 446.2 \quad 466.8 \quad 480.9 \quad 492.9 \quad 516.4 \quad 541.4 \quad 659.4 \quad 680.7 \quad 708.0 \quad 734.8 \quad 824.0 \quad 845.6$

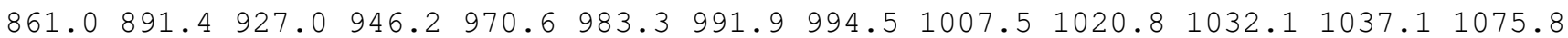

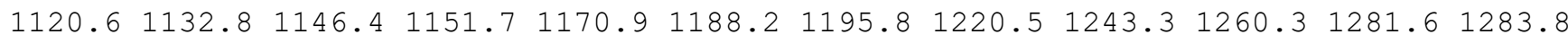

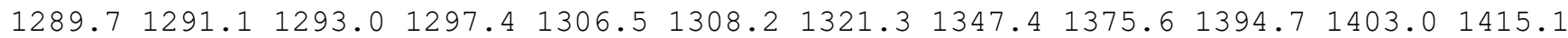
$1860.02664 .62666 .0 \quad 2669.8 \quad 2674.4 \quad 2675.8 \quad 2677.2 \quad 2679.82685 .6 \quad 2712.2 \quad 2747.92761 .3$ $2778.22780 .5 \quad 2782.2 \quad 4750.2$

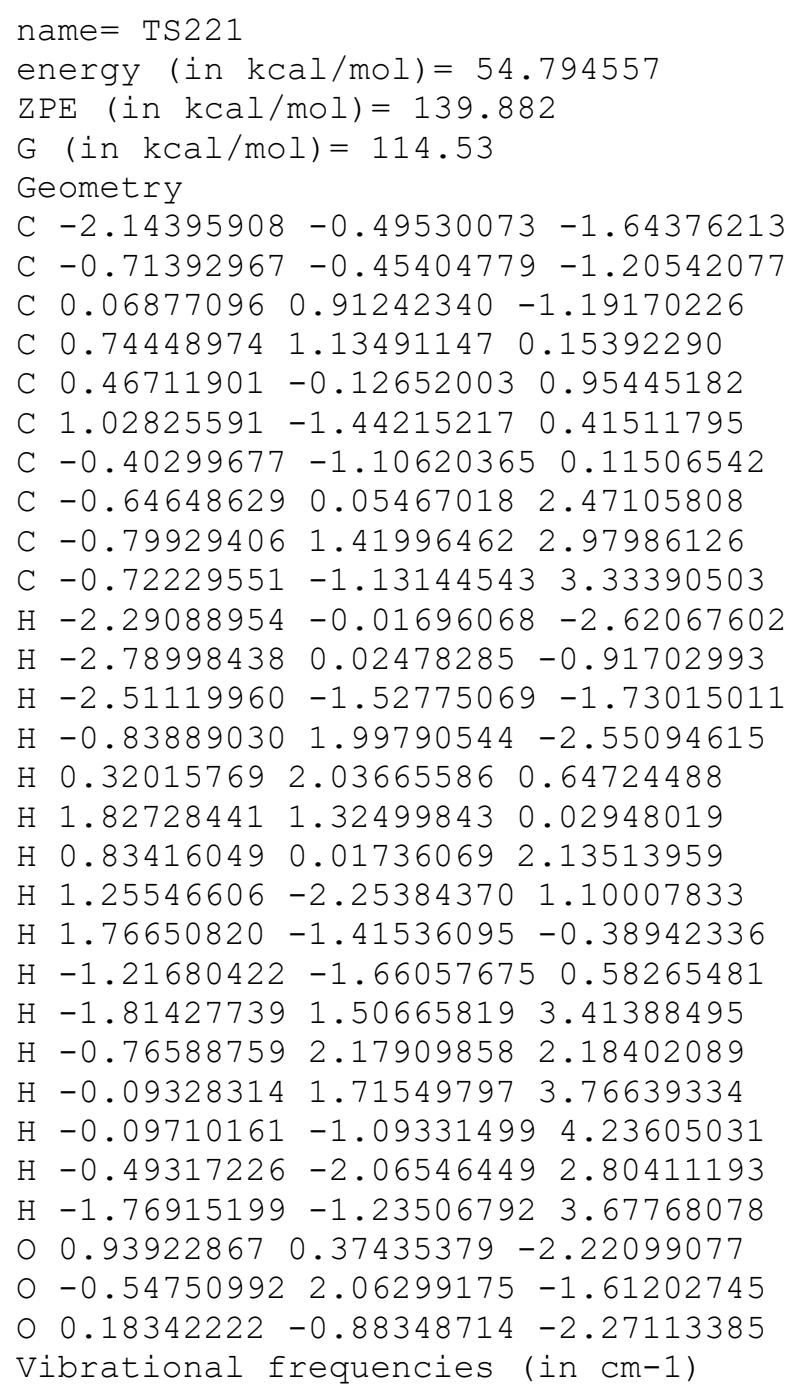


$\mathrm{ZPE}($ in $\mathrm{kcal} / \mathrm{mol})=140.852$

$\mathrm{G}(\mathrm{in} \mathrm{kcal} / \mathrm{mol})=114.673$

Geometry

C $-1.87470309-2.18312872-1.63689493$

C $-0.59009359-1.51630392-1.25325570$

C $0.334689191 .67462277-0.58185956$

C $1.294043541 .57223846 \quad 0.49310793$

C $1.12551797 \quad 0.09048057 \quad 1.37570094$

C $1.27548982-1.08327677 \quad 0.38328393$

C $0.02276168-1.72383580-0.07125036$

C $-0.05834086 \quad 0.29645921 \quad 2.12183776$

C $0.04250347 \quad 0.81143741 \quad 3.50964268$

C $-1.40929998 \quad 0.12303212 \quad 1.59303685$

$\mathrm{H}-2.24231162-1.84276290-2.61837178$

$\mathrm{H}-2.66684872-1.98106631-0.90545026$

$\mathrm{H}-1.74467796-3.27341079-1.70331232$

$\mathrm{H}-0.70284586 \quad 1.91308396-0.38053934$

H $1.22135140 \quad 2.35022891 \quad 1.26074987$

H 2.33514415 $1.49468194 \quad 0.13448164$

$\mathrm{H} 2.05058718 \quad 0.11738193 \quad 2.00037070$

$\mathrm{H} 1.91189133-1.848299520 .88490183$

$\mathrm{H} \quad 1.87993703-0.74039724 \quad-0.49319817$

$\mathrm{H}-0.38836314 \quad-2.45548004 \quad 0.61968407$

$\mathrm{H}-0.46804819 \quad 1.78389404 \quad 3.62058614$

H $1.07446301 \quad 0.95065111 \quad 3.86193335$

$\mathrm{H}-0.446440490 .12537607 \quad 4.22328312$

$\mathrm{H}-2.20949814 \quad 0.49606944 \quad 2.24326160$

$\mathrm{H}-1.63051778-0.944104951 .36965798$

$\mathrm{H}-1.51753948 \quad 0.61274262 \quad 0.59281708$

$00.96013706-0.13331781-2.18967608$

○ $0.683112691 .48548287-1.77868364$

o $-0.21939377-0.74438871-2.31077003$

Vibrational frequencies (in $\mathrm{cm}-1$ )

$\begin{array}{llllllllllllllll}-665.7 & 51.4 & 59.2 & 64.0 & 79.7 & 81.3 & 114.7 & 179.5 & 193.1 & 205.9 & 228.0 & 262.0 & 293.6 & 313.7\end{array}$

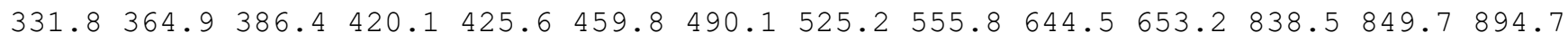
$908.9930 .0 \quad 942.2 \quad 963.1 \quad 990.6 \quad 1017.0 \quad 1028.0 \quad 1039.3 \quad 1058.6 \quad 1072.9 \quad 1081.5 \quad 1115.3 \quad 1133.8$

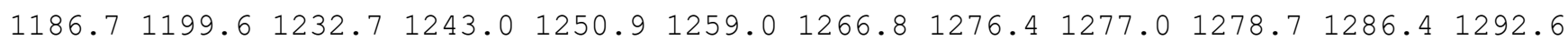

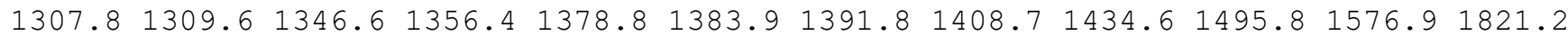
$2555.72619 .42639 .2 \quad 2649.2 \quad 2662.5 \quad 2666.12667 .52674 .52688 .62691 .72713 .02736 .6$ $2745.7 \quad 2761.4 \quad 2768.8 \quad 2777.2$

\section{name $=$ TS223}

energy (in $\mathrm{kcal} / \mathrm{mol})=56.029275$

$\mathrm{ZPE}(\mathrm{in} \mathrm{kcal} / \mathrm{mol})=139.729$

$\mathrm{G}($ in $\mathrm{kcal} / \mathrm{mol})=112.341$

Geometry

C $-1.56184186 \quad 0.04124477 \quad-2.44829673$

C $-0.70482625-0.43312131-1.27933984$

C $0.19668655 \quad 0.71671769-0.68839199$

C $-0.03776770 \quad 0.91846172 \quad 0.73050048$

C $0.318929890 .04742792 \quad 1.78411463$

C $-1.54443654-2.43916524-0.07088242$

C $-1.54871114-1.12052227-0.25177275$

C $0.29385897 \quad 0.32153624 \quad 3.10362333$

C $-0.04316783 \quad 1.66631363 \quad 3.65178838$

C $0.63233366-0.722032824 .11498458$

$\mathrm{H}-2.28437527 \quad 0.80124959-2.13042751$

$\mathrm{H}-2.12763087-0.79440135-2.88284961$

$\mathrm{H}-0.94487799 \quad 0.46260307 \quad-3.25276150$

H $0.09311902 \quad 1.64672190 \quad-1.29168833$

$\mathrm{H} \quad 0.13643853 \quad 2.40243026 \quad 1.09657461$

$\mathrm{H} \quad 0.93353217 \quad 2.23171541 \quad 1.01248212$

$\mathrm{H} \quad 0.57429235-0.97248046 \quad 1.45309097$

$\mathrm{H}-2.15837070 \quad-2.93912713 \quad 0.66212960$ 
$\mathrm{H}-0.92262252-3.11892542-0.63733996$

$\mathrm{H}-2.17445227-0.446444810 .33803238$

$\mathrm{H} 0.78000225 \quad 2.37993595 \quad 3.50969514$

$\mathrm{H}-0.25327526 \quad 1.63702112 \quad 4.72954929$

$\mathrm{H}-0.94228461 \quad 2.07844597 \quad 3.16764629$

$\mathrm{H} 1.47054740-0.40637067 \quad 4.75192113$

$\mathrm{H} 0.91561108-1.68057805 \quad 3.65932609$

$\mathrm{H}-0.22457585-0.922845294 .77452186$

O $1.44112331-1.12252079-1.11168363$

$01.53580067 \quad 0.28501786-1.00175580$

O $0.25524617-1.33106091-1.85803098$

Vibrational frequencies (in $\mathrm{cm}-1$ )

$\begin{array}{lllllllllllllllll}-987.6 & 26.9 & 36.2 & 54.3 & 72.3 & 90.2 & 116.1 & 143.7 & 151.3 & 188.8 & 204.7 & 225.6 & 240.3 & 263.3\end{array}$

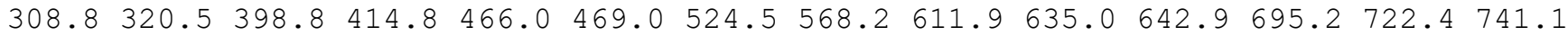

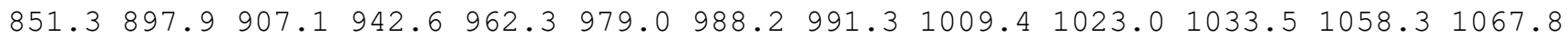

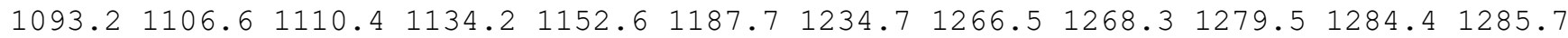
$1288.01288 .21292 .0 \quad 1311.5 \quad 1334.3 \quad 1373.7 \quad 1386.2 \quad 1394.31395 .91411 .21503 .11835 .8$ $1852.0 \quad 2667.3 \quad 2669.7 \quad 2671.2 \quad 2672.2 \quad 2674.3 \quad 2678.2 \quad 2685.52711 .42723 .12747 .42775 .1$ $2778.72779 .6 \quad 2783.6 \quad 4610.2$

name $=\operatorname{TS} 224$

energy (in $\mathrm{kcal} / \mathrm{mol})=54.45275$

$\operatorname{ZPE}($ in $\mathrm{kcal} / \mathrm{mol})=141.588$

$\mathrm{G}($ in $\mathrm{kcal} / \mathrm{mol})=116.789$

Geometry

C $-1.05095561-0.19762941-2.24459315$

C $-0.20445175-1.92079232-0.80104790$

C $-1.10728129-0.92038130-0.07120195$

C $-0.35833797 \quad 0.15403514 \quad 0.70248429$

C $0.59504367-0.48882056 \quad 1.68747147$

C $1.35358629-1.57267638 \quad 1.16611184$

C $1.17457999-2.07263872-0.22590696$

C $0.71765322-0.00736994 \quad 2.94616204$

C $-0.07180653 \quad 1.15487241 \quad 3.45606769$

C $1.66947790-0.630293863 .91577699$

$\mathrm{H}-1.66906495-0.31621785-3.14465928$

$\mathrm{H}-0.70665596-2.89662170-0.96145514$

$\mathrm{H}-0.44487634 \quad 0.71838865 \quad-2.22397113$

$\mathrm{H}-1.88055987-1.41625992 \quad 0.55834779$

$\mathrm{H}-1.093843390 .80870221 \quad 1.21405798$

H $0.20489216 \quad 0.82926011 \quad 0.03106050$

H $1.91390835-2.18525780 \quad 1.83872981$

$\mathrm{H} \quad 1.93318038-1.38669491-1.09968731$

H 2.08941184 $-0.97367789-0.21844739$

$\mathrm{H} 1.60006179-3.08561774-0.36911523$

$\mathrm{H}-1.14330957 \quad 0.91822399 \quad 3.50422745$

$\mathrm{H} \quad 0.05610924 \quad 2.04053137 \quad 2.82053758$

$\mathrm{H} \quad 0.23235157 \quad 1.44924465 \quad 4.46843178$

$\mathrm{H} \quad 1.67031602-0.13282110 \quad 4.89095681$

$\mathrm{H} 2.69809706-0.600262943 .52941910$

$\mathrm{H} 1.41645005-1.686694294 .08674441$

$\begin{array}{lllll}0 & 1.07129021 & -1.00244150 & -2.53968480\end{array}$

$0-1.87446934-0.34147762-1.13591535$

$0-0.13853296-1.41990933-2.25051913$

Vibrational frequencies (in $\mathrm{cm}-1$ )

$\begin{array}{llllllllllllllllll}-2168.1 & 48.1 & 70.2 & 84.6 & 106.3 & 118.2 & 152.1 & 174.3 & 228.9 & 277.8 & 326.3 & 352.4 & 363.6 & 423.6\end{array}$ $\begin{array}{lllllllllllllll}445.1 & 452.5 & 497.9 & 504.5 & 554.9 & 583.8 & 612.4 & 647.4 & 684.4 & 779.4 & 781.0 & 831.8 & 855.8 & 889.1\end{array}$ $903.2936 .5 \quad 990.8 \quad 993.5 \quad 997.4 \quad 1012.5 \quad 1026.8 \quad 1029.3 \quad 1045.1 \quad 1077.5 \quad 1093.8 \quad 1101.6 \quad 1131.2$

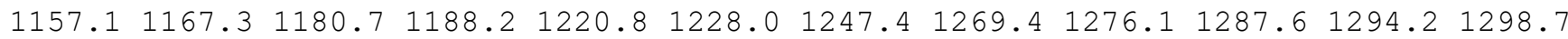
$1303.7 \quad 1304.3 \quad 1309.5 \quad 1332.6 \quad 1340.5 \quad 1347.6 \quad 1357.9 \quad 1372.4 \quad 1397.6 \quad 1405.8 \quad 1483.2 \quad 1677.3$

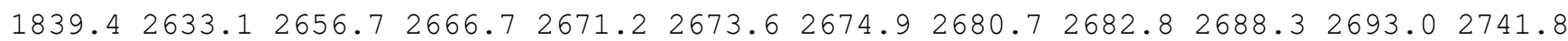
$2744.32776 .7 \quad 2777.12808 .8$ 


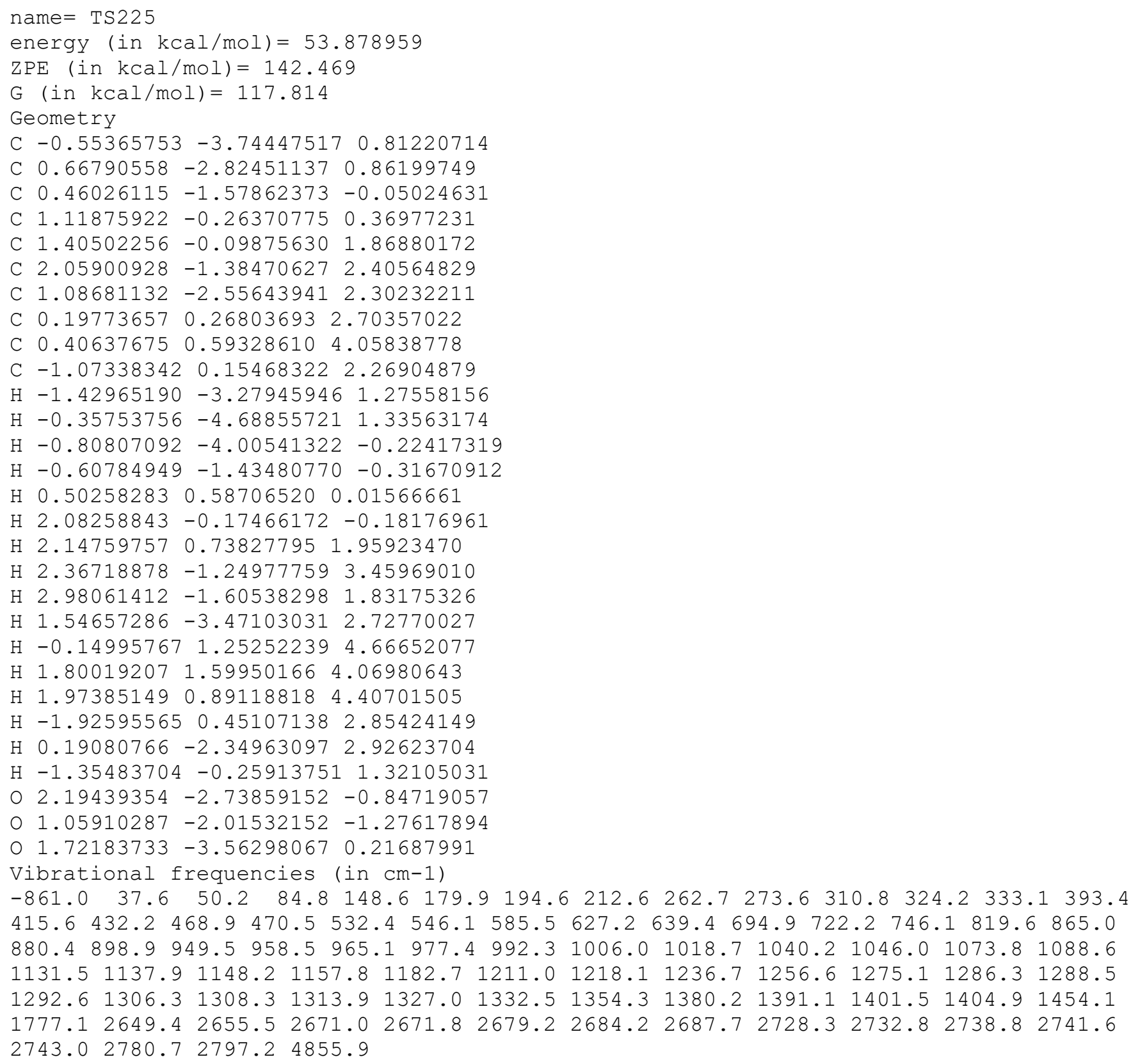


$\mathrm{H} \quad 1.90027132 \quad 0.24360292 \quad 2.14697423$

H $2.20673706-1.40473747 \quad 0.05699351$

$\mathrm{H} 1.689156610 .14038111-0.93449615$

$\mathrm{H}-0.37133807-2.14311138-0.08920962$

$\mathrm{H}-1.677605190 .757901051 .82946596$

$\mathrm{H}-1.18063769 \quad 0.10480315 \quad 3.38455331$

$\mathrm{H}-2.19338517-0.863163342 .30421836$

$\mathrm{H} 0.53216412-1.67512993 \quad 3.59393795$

$\mathrm{H} 1.20720517-2.46202924 \quad 2.16694824$

$\mathrm{H}-0.48413541-2.728130142 .59899480$

O $0.53951563-0.82030533-2.38358220$

O $1.13866714 \quad 1.14744083-1.37788128$

o $-0.59979863-0.52606448-2.05443666$

Vibrational frequencies (in cm-1)

$\begin{array}{llllllllllllll}-1614.5 & 118.6 & 142.3 & 156.8 & 168.4 & 194.4 & 202.0 & 235.0 & 275.3 & 290.2 & 324.7 & 334.8 & 365.0 & 378.4\end{array}$

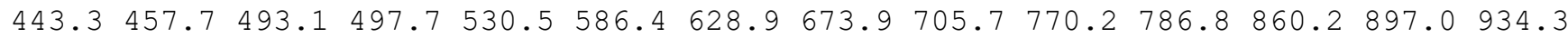
$941.3 \quad 964.6 \quad 981.0 \quad 988.3 \quad 1010.1 \quad 1016.7 \quad 1019.9 \quad 1029.8 \quad 1045.8 \quad 1064.8 \quad 1089.8 \quad 1102.2 \quad 1125.1$

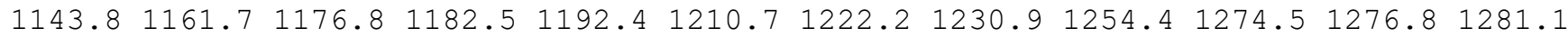

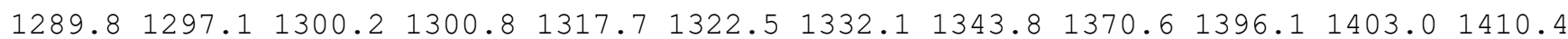
$1820.42660 .42666 .3 \quad 2667.8 \quad 2670.7 \quad 2675.3 \quad 2676.62681 .22687 .7 \quad 2738.32747 .2 \quad 2752.3$ $2771.4 \quad 2774.6 \quad 2779.9 \quad 2782.2$

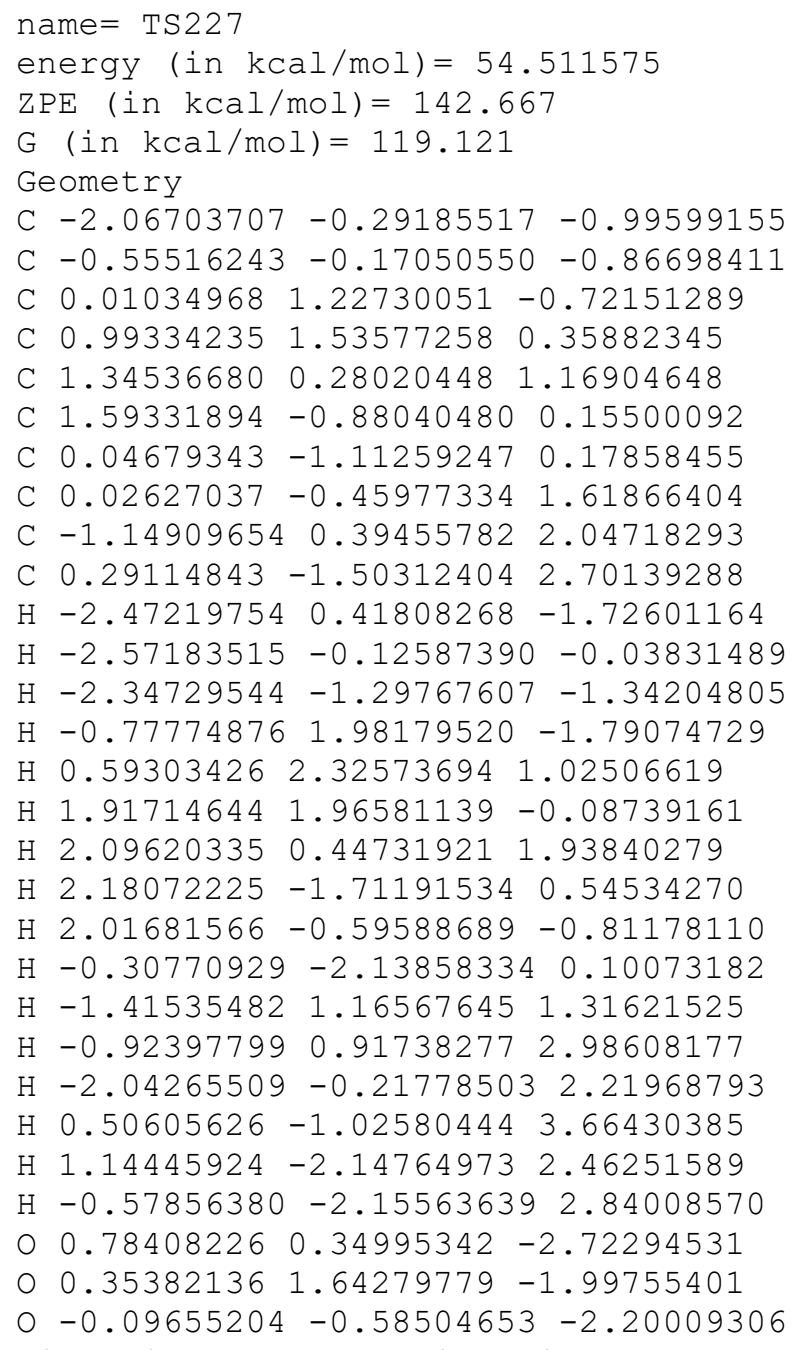


name $=\operatorname{TS} 228$

energy $($ in $\mathrm{kcal} / \mathrm{mol})=53.725661$

$\mathrm{ZPE}($ in $\mathrm{kcal} / \mathrm{mol})=143.522$

$\mathrm{G}($ in $\mathrm{kcal} / \mathrm{mol})=119.755$

Geometry

C $-2.09619779-0.80119422-1.21894073$

C $-0.82838566-0.23423546-0.68332355$

C $-0.539680451 .13108306-0.74630274$

C $0.46071617 \quad 1.71291823 \quad 0.22531655$

C $0.97077695 \quad 0.63777842 \quad 1.20040872$

C $1.42029737-0.58617117 \quad 0.34226032$

C $-0.08177601-1.027815290 .35135879$

C $-0.22351616-0.248402151 .73558185$

C $-1.53958688 \quad 0.42651636 \quad 2.06106333$

C $0.18517737-1.11811586 \quad 2.91975324$

$\mathrm{H}-2.44414000-0.26073165-2.11231758$

$\mathrm{H}-2.89611094-0.76150180-0.46569326$

$\mathrm{H}-1.97884831-1.85388858-1.51959084$

$\mathrm{H}-1.21965613 \quad 1.83496999-1.21967065$

$\mathrm{H}-0.00436151 \quad 2.545486790 .78961588$

H $1.31390542 \quad 2.16590668-0.31786939$

H $1.66600393 \quad 1.01593584 \quad 1.94663293$

H $2.08538601-1.282474160 .85928723$

H $1.87519997-0.36378167-0.61568595$

$\mathrm{H}-0.28670347-2.09597495 \quad 0.36297260$

$\mathrm{H}-1.44227441 \quad 1.06327941 \quad 2.94943320$

$\mathrm{H}-2.32102260-0.31578884 \quad 2.26711956$

$\mathrm{H}-1.91586501 \quad 1.06574745 \quad 1.25526822$

H $1.13559047-1.63716354 \quad 2.75636380$

$\mathrm{H}-0.57076864-1.88622773 \quad 3.12210011$

$\mathrm{H} \quad 0.29827040 \quad-0.51623504 \quad 3.82913150$

$\begin{array}{lllll}0 & 0.92857298 & 0.09440251 & -2.49786199\end{array}$

$\begin{array}{lllll}0 & 0.42355617 & 1.23483269 & -2.48535564\end{array}$

O $0.11746545-0.84341847-2.40687985$

Vibrational frequencies (in $\mathrm{cm}-1$ )

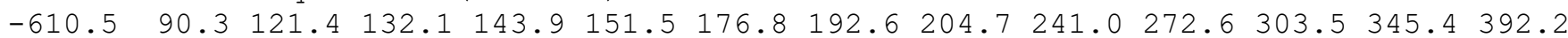
$\begin{array}{llllllllllllllll}419.1 & 440.1 & 463.8 & 496.4 & 515.8 & 602.7 & 624.7 & 658.5 & 717.9 & 782.1 & 854.9 & 896.7 & 919.3 & 931.5\end{array}$ $943.1984 .0 \quad 1005.8 \quad 1009.5 \quad 1015.7 \quad 1021.3 \quad 1052.3 \quad 1065.6 \quad 1072.1 \quad 1081.3 \quad 1083.4 \quad 1096.5$ $\begin{array}{lllllllllllllll}1124.5 & 1140.0 & 1173.9 & 1183.9 & 1190.1 & 1208.6 & 1236.9 & 1253.9 & 1267.0 & 1275.8 & 1285.7 & 1287.6\end{array}$ $1292.01294 .6 \quad 1300.4 \quad 1303.6 \quad 1312.4 \quad 1315.3 \quad 1353.1 \quad 1362.5 \quad 1388.4 \quad 1394.3 \quad 1407.81419 .7$ $1590.32660 .5 \quad 2668.4 \quad 2670.8 \quad 2671.7 \quad 2675.8 \quad 2676.5 \quad 2680.82696 .92740 .22741 .52751 .4$ $2755.8 \quad 2776.5 \quad 2779.0 \quad 2781.5 \quad 2783.4$

name $=\operatorname{TS} 229$

energy $($ in $\mathrm{kcal} / \mathrm{mol})=59.8049$

$\mathrm{ZPE}(\mathrm{in} \mathrm{kcal} / \mathrm{mol})=137.602$

$\mathrm{G}($ in $\mathrm{kcal} / \mathrm{mol})=110.672$

Geometry

C $-1.49794952-1.73120278-2.66297286$

C $-0.18387946-1.11480799-2.01812563$

C $1.103302092 .02129076-2.35461233$

C $2.135192461 .83204940-3.17413437$

C $0.86688531-2.50832510 \quad 0.47285717$

C $1.44138155-2.822982781 .63185880$

C $-1.26199582-0.91508223-1.10574626$

C $1.63573269-2.07551426-0.74488039$

C $2.69149312-3.10779828-1.16380954$

C $2.23490120-0.68632140-0.54901390$

$\mathrm{H}-1.92311745-1.17024979-3.48464764$

$\mathrm{H}-2.27186909-1.86502862-1.61550983$

$\mathrm{H}-1.38147665-2.79501256-2.87645464$

$\mathrm{H} \quad 0.86306636 \quad 2.92344276-1.79086730$ 
H $2.87822751 \quad 2.58420329-3.37485145$

H $2.314207110 .91361953-3.71037298$

$\mathrm{H}-0.21939769-2.52358047 \quad 0.33502474$

H $2.50474796-2.80559641 \quad 1.80505932$

$\mathrm{H} \quad 0.88183496-3.12125725 \quad 2.50414666$

$\mathrm{H}-1.75369059-0.02233791-0.84509198$

$\mathrm{H} 3.45602741-3.22454699-0.39056468$

H $3.18326610 \quad-2.80591904-2.09670967$

$\mathrm{H} 2.22967107-4.08706485-1.34091927$

$\mathrm{H} 1.47903550 \quad 0.02716579-0.19464829$

$\mathrm{H} 2.63506114-0.29202631-1.49302409$

$\mathrm{H} 3.04536243-0.700286220 .18617530$

$\begin{array}{llll}0 & 0.35123248 & -0.08433441 & -2.80438695\end{array}$

$0 \quad 0.12398407 \quad 1.13268351-2.04539888$

O $0.76945876-2.10508704-1.89466364$

Vibrational frequencies (in cm-1)

$\begin{array}{lllllllllllllll}-1283.1 & 23.7 & 54.4 & 61.7 & 90.9 & 108.2 & 137.5 & 145.6 & 176.9 & 203.4 & 215.9 & 231.0 & 245.2 & 270.8\end{array}$

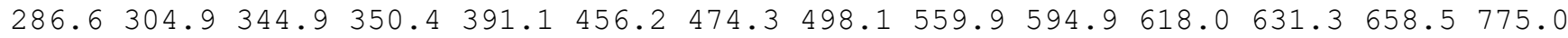

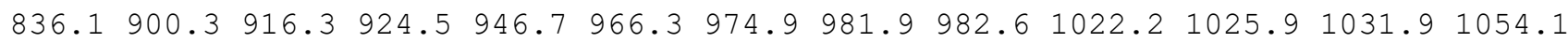

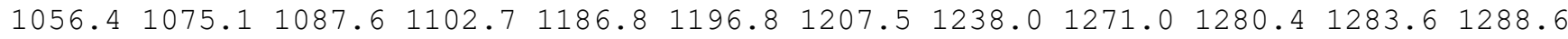
$1294.21295 .41301 .21320 .0 \quad 1355.3 \quad 1380.3 \quad 1387.4 \quad 1391.01401 .4 \quad 1507.2 \quad 1853.8 \quad 1854.1$ $2214.52667 .6 \quad 2677.8 \quad 2679.0 \quad 2683.3 \quad 2683.8 \quad 2709.22711 .22734 .82735 .42744 .82776 .0$ $2779.6 \quad 2782.4 \quad 2790.8 \quad 2795.4$

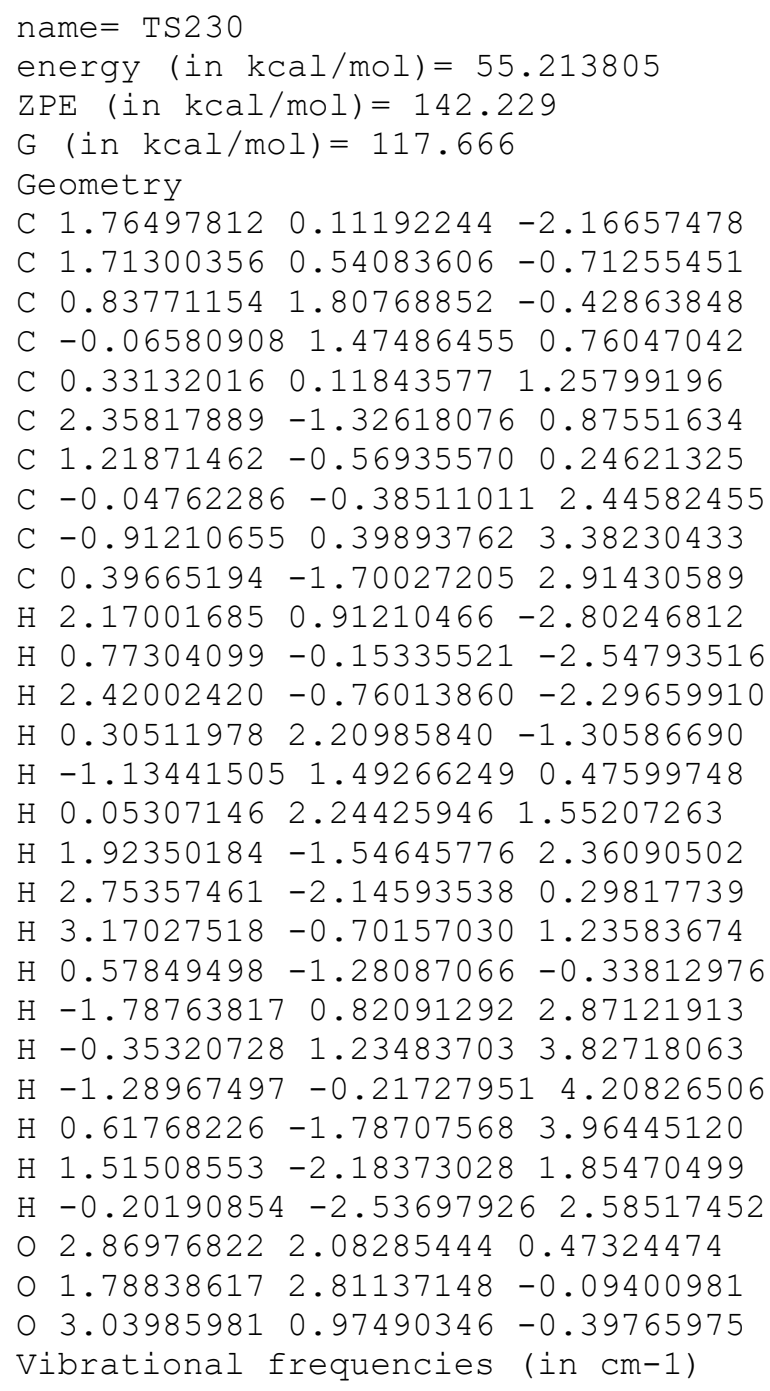


$1868.62661 .72671 .5 \quad 2671.92676 .8 \quad 2678.6 \quad 2682.42703 .52704 .62710 .02741 .62766 .2$ $2777.7 \quad 2780.3 \quad 2781.4 \quad 3615.8$

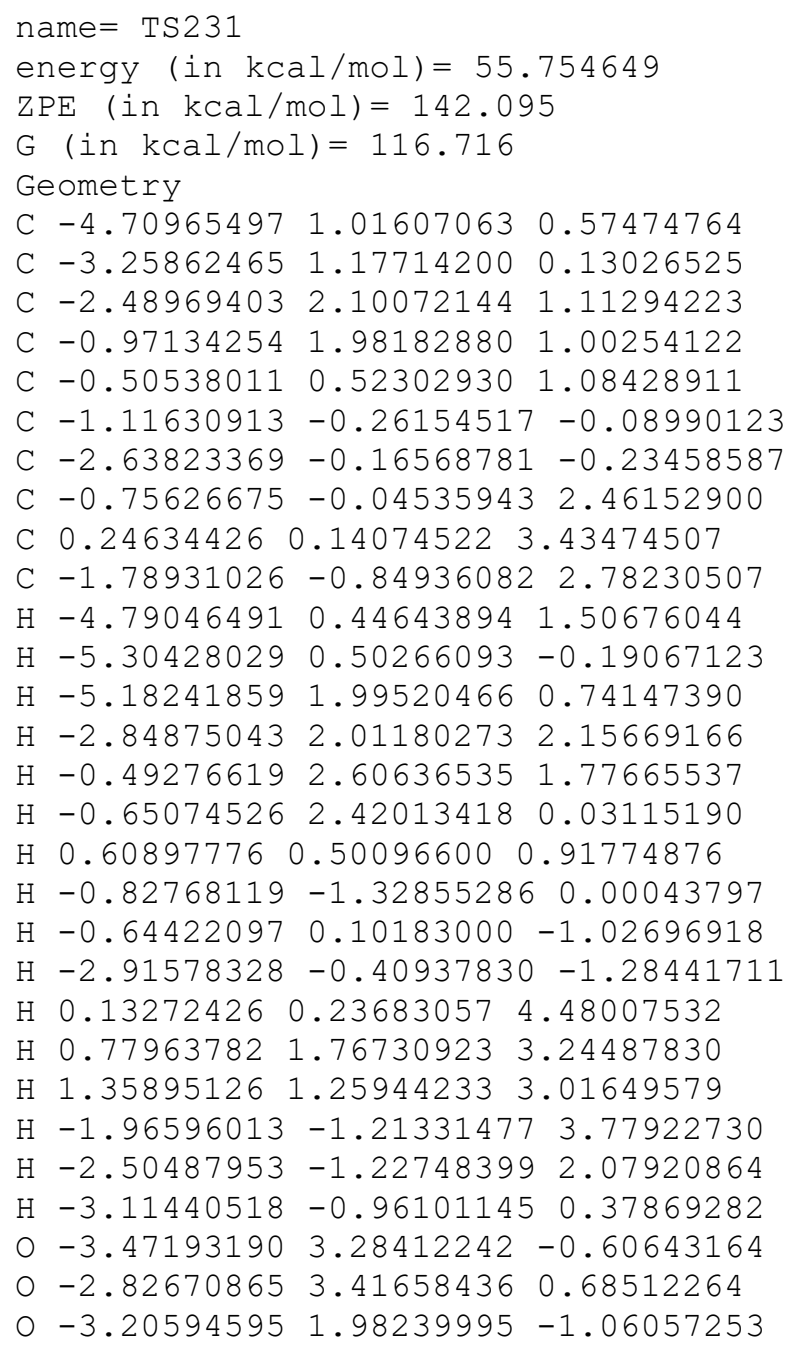


$\mathrm{H}-2.70464794-1.44324172-0.62576117$

$\mathrm{H}-1.17822071 \quad 1.92440550-0.49353552$

$\mathrm{H} 1.628081092 .49948657 \quad 0.24167856$

$\mathrm{H} \quad 1.93155225 \quad 1.90632715-0.24778297$

$\mathrm{H} \quad 1.93710513 \quad 0.54448148 \quad 2.10031084$

H $1.98645574 \quad-1.68491067 \quad 0.82241814$

$\mathrm{H} 1.70949530-0.65652305-0.58348088$

$\mathrm{H}-0.51060096-2.19452075 \quad 0.56284152$

$\mathrm{H}-2.18273612-0.190886372 .60833160$

$\mathrm{H}-1.554221091 .221867151 .73655085$

$\mathrm{H}-1.03743444 \quad 0.92336268 \quad 3.37601434$

$\mathrm{H}-0.59923461-2.056175793 .32710408$

$\mathrm{H} 1.08451863-2.06050520 \quad 2.79856817$

$\mathrm{H} \quad 0.54194783-0.87376520 \quad 3.98445869$

$\begin{array}{llll}0 & -0.23060637 & 0.36092552 & -2.56478507\end{array}$

$\begin{array}{lllll}0 & 0.37872848 & 1.31745491 & -1.70404710\end{array}$

o $-0.37782079-0.78769838-1.75873694$

Vibrational frequencies (in $\mathrm{cm}-1$ )

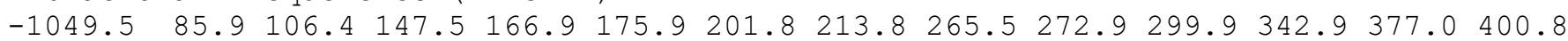

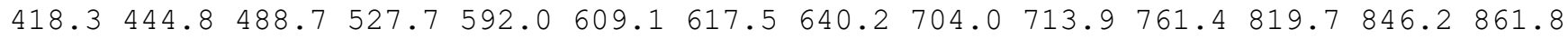
$892.9931 .6 \quad 956.4975 .5 \quad 979.3 \quad 1004.4 \quad 1007.5 \quad 1013.4 \quad 1016.5 \quad 1052.6 \quad 1061.3 \quad 1080.1 \quad 1085.3$

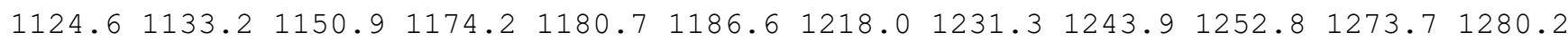
$1285.81287 .21292 .6 \quad 1295.0 \quad 1301.6 \quad 1303.5 \quad 1304.6 \quad 1315.1 \quad 1335.6 \quad 1381.2 \quad 1396.3 \quad 1407.0$ $1419.82665 .42671 .5 \quad 2675.0 \quad 2675.9 \quad 2677.2 \quad 2681.72684 .92688 .02749 .02757 .2 \quad 2764.5$ $2779.9 \quad 2781.7 \quad 2782.8 \quad 4499.7$

name $=$ TS233

energy (in $\mathrm{kcal} / \mathrm{mol})=58.816232$

$\operatorname{ZPE}($ in $\mathrm{kcal} / \mathrm{mol})=139.372$

$\mathrm{G}($ in $\mathrm{kcal} / \mathrm{mol})=113.394$

Geometry

C $-1.26310933-1.16558774-1.61825010$

C $0.02071996-0.54320900-1.06369697$

C $-0.23758416 \quad 0.96501804 \quad-0.95424681$

C 1.439361361 .531454690 .75014235

C $1.52939651 \quad 0.21582999 \quad 1.34611103$

C $1.92541974 \quad-0.86776976 \quad 0.44389579$

C $0.51122395-1.28474782 \quad 0.21732306$

C $0.17532957-0.58747679 \quad 1.62486107$

C $-1.13427961 \quad 0.12519957 \quad 1.84095426$

C $0.40657523-1.582363062 .76330622$

$\mathrm{H}-1.60848609-0.61499774-2.50718613$

$\mathrm{H}-2.06621513-1.16029095-0.87521423$

$\mathrm{H}-1.09742357-2.20332157-1.93332905$

$\mathrm{H}-0.95482330 \quad 1.28968969-0.19597996$

$\mathrm{H} \quad 0.95994198 \quad 2.34379198 \quad 1.24229463$

$\mathrm{H} \quad 2.03637547 \quad 1.77893135-0.10900502$

H $2.19539259 \quad 0.34047361 \quad 2.24614861$

H $2.83766608-1.321401050 .19520770$

$\mathrm{H} 2.79236595-0.60173604-1.74094158$

$\mathrm{H} \quad 0.26258779-2.349880650 .16148570$

$\mathrm{H}-1.047884861 .21007396 \quad 1.68749054$

$\mathrm{H}-1.50882960 \quad 0.00197327 \quad 2.86690341$

$\mathrm{H}-1.91814546-0.26108416 \quad 1.17852325$

$\mathrm{H} \quad 0.47677035-1.06412161 \quad 3.72795988$

$\mathrm{H} 1.33114722-2.15870300 \quad 2.64040069$

$\mathrm{H}-0.41468430-2.30382186 \quad 2.83509523$

○ $2.07840674 \quad 0.05600894-1.92146157$

○ $0.18659184 \quad 1.75268240-1.76313273$

O $0.96583220-0.81355373-2.09635625$

Vibrational frequencies (in $\mathrm{cm}-1$ )

$\begin{array}{llllllllllllllll}-118.2 & 22.0 & 71.6 & 114.8 & 136.9 & 154.8 & 163.7 & 189.9 & 202.5 & 221.6 & 224.1 & 255.5 & 273.0 & 299.5\end{array}$ $312.6 \quad 349.1 \quad 375.5 \quad 400.6 \quad 413.8 \quad 466.5 \quad 484.1 \quad 541.2 \quad 581.5 \quad 643.5 \quad 698.9 \quad 718.4 \quad 745.8 \quad 838.3$

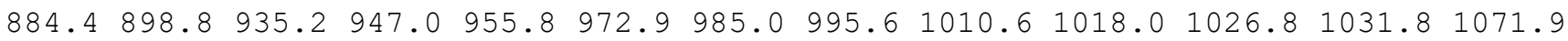




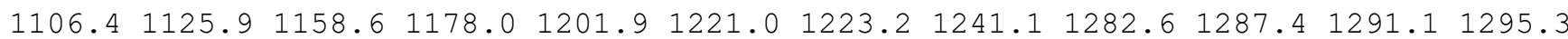

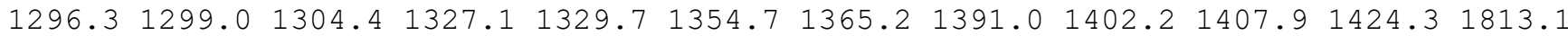
$2641.22645 .5 \quad 2663.12665 .92671 .42673 .3 \quad 2677.62678 .52718 .3 \quad 2729.92762 .32775 .9$ $2777.62780 .7 \quad 2793.6 \quad 2876.6$

\section{name $=$ TS234}

energy $($ in $\mathrm{kcal} / \mathrm{mol})=58.19544$

$\mathrm{ZPE}(\mathrm{in} \mathrm{kcal} / \mathrm{mol})=140.105$

$\mathrm{G}($ in $\mathrm{kcal} / \mathrm{mol})=113.819$

Geometry

C $-2.327527490 .71009723-0.51306033$

C $-0.932470810 .09278077-0.52209542$

C $0.200275571 .19014558-0.60244311$

C $1.03194864 \quad 1.29965958 \quad 0.57498296$

C $2.08452365 \quad 0.50666547 \quad 1.09281347$

C $2.37610785-0.72989484 \quad 0.66044112$

C $-0.74769459-0.97358271 \quad 0.49962544$

C $-0.94466361-0.882756421 .82144121$

C $-1.36434664 \quad 0.358281132 .53268093$

C $-0.74002794 \quad-2.07469496 \quad 2.70242446$

$\mathrm{H}-2.43726087 \quad 1.43845846 \quad 0.29710892$

$\mathrm{H}-3.09591567-0.06204108-0.37279658$

$\mathrm{H}-2.54622797 \quad 1.21613110-1.46329396$

$\mathrm{H}-0.267815592 .16578322-0.88425694$

H $1.28301945 \quad 2.77230022 \quad 0.90079559$

$\mathrm{H} 1.82823005 \quad 2.69958658 \quad 0.28831841$

H $2.61825246 \quad 0.92012783 \quad 1.95629771$

$\mathrm{H} 3.09455537-1.37087048 \quad 1.14058935$

H $1.89989929-1.18542133-0.19518077$

$\mathrm{H}-0.42902152-1.921848520 .05620642$

$\mathrm{H}-2.44020950 \quad 0.541384592 .41414257$

$\mathrm{H}-0.822760251 .23793135 \quad 2.14085912$

$\mathrm{H}-1.15696053 \quad 0.31832298 \quad 3.60898092$

H $0.06149714 \quad-1.88829954 \quad 3.43216437$

$\mathrm{H}-0.45912171-2.97686605 \quad 2.14286279$

$\mathrm{H}-1.65238678-2.31148557 \quad 3.26654507$

$\begin{array}{llll}0 & 0.54642354 & -0.47518710 & -2.12353452\end{array}$

$\begin{array}{lllll}0 & 0.94115718 & 0.85036963 & -1.79143344\end{array}$

o - $0.82998077-0.51088517-1.83196707$

Vibrational frequencies (in $\mathrm{cm}-1$ )

$\begin{array}{llllllllllllllll}-982.2 & 33.7 & 74.8 & 84.9 & 94.2 & 118.5 & 131.9 & 143.0 & 153.9 & 161.7 & 192.6 & 224.1 & 244.7 & 256.2\end{array}$ $322.4 \quad 349.2 \quad 369.4 \quad 411.3 \quad 481.4 \quad 483.1 \quad 496.6 \quad 542.5 \quad 628.3 \quad 660.4 \quad 682.2 \quad 701.6 \quad 712.5 \quad 751.3$

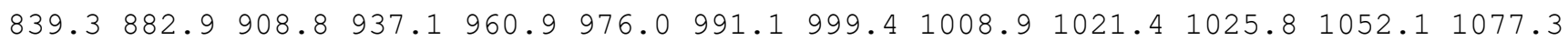
$1096.81115 .1 \quad 1125.4 \quad 1157.7 \quad 1165.2 \quad 1189.1 \quad 1221.4 \quad 1268.8 \quad 1275.3 \quad 1278.6 \quad 1287.4 \quad 1287.8$

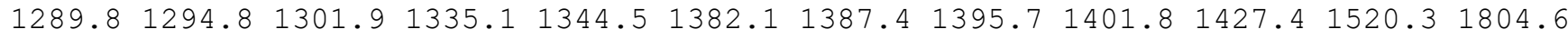
$1878.0 \quad 2655.12670 .1 \quad 2671.4 \quad 2673.6 \quad 2675.1 \quad 2676.8 \quad 2677.5 \quad 2714.8 \quad 2741.5 \quad 2750.8 \quad 2775.5$ 2779.22779 .92785 .14559 .3

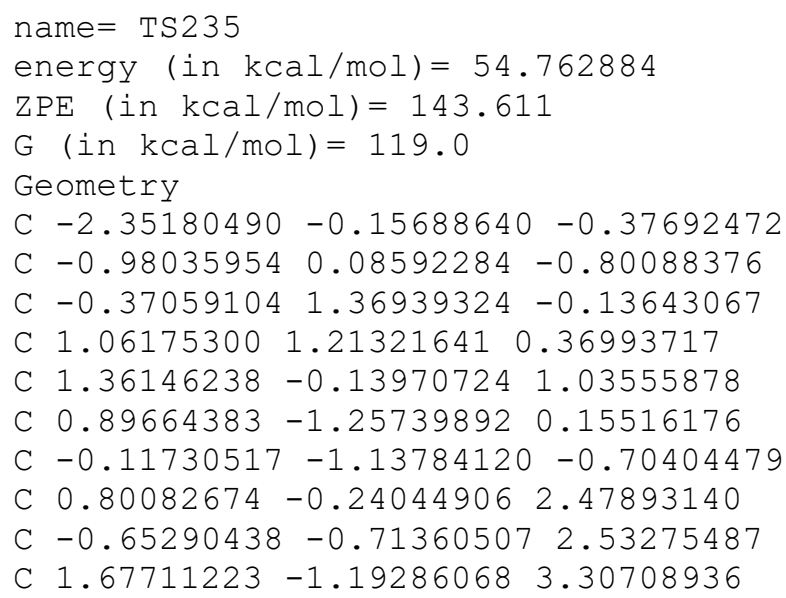


$\mathrm{H}-2.99898009 \quad 0.68825225-0.25975465$

$\mathrm{H}-3.27739353-0.96993346-0.21715057$

$\mathrm{H}-2.50605837-1.42265113-0.43359910$

$\mathrm{H}-1.04510670 \quad 1.79635713 \quad 0.63155234$

$\mathrm{H} 1.30353115 \quad 2.04245558 \quad 1.06032551$

$\mathrm{H} \quad 1.749078591 .34101383-0.49629756$

$\mathrm{H} 0.85606499 \quad 0.77799540 \quad 2.93714476$

$\mathrm{H} \quad 1.46822332-2.180050350 .24166303$

$\mathrm{H} 2.48185273-0.208693261 .11765777$

$\mathrm{H}-0.37650873-1.92725351-1.40501868$

$\mathrm{H}-0.74935383-1.753442842 .20622552$

$\mathrm{H}-1.29523511-0.10774003 \quad 1.87748855$

$\mathrm{H}-1.05474758-0.64313598 \quad 3.54789958$

H $1.29264983-1.29322648 \quad 4.32775041$

H $2.70828970-0.833005723 .38054345$

$\mathrm{H} \quad 1.70249027-2.195435512 .86789536$

o $-0.181390551 .54323420-2.36491622$

$0-0.37581192 \quad 2.33003298-1.19030464$

$0-1.069141820 .46422517-2.20611552$

Vibrational frequencies (in $\mathrm{cm}-1$ )

$\begin{array}{llllllllllllllllll}-1373.5 & 39.6 & 76.2 & 94.1 & 133.4 & 148.6 & 173.7 & 209.3 & 216.1 & 257.4 & 287.2 & 308.1 & 320.4 & 373.5\end{array}$ $409.1431 .0 \quad 468.5 \quad 472.6 \quad 555.1 \quad 565.7 \quad 632.6 \quad 675.8 \quad 710.7 \quad 730.7 \quad 790.4 \quad 821.2 \quad 828.1847 .7$ $880.7 \quad 973.6 \quad 977.5 \quad 983.6 \quad 996.8 \quad 1013.2 \quad 1018.9 \quad 1042.9 \quad 1064.2 \quad 1095.0 \quad 1100.8 \quad 1138.5 \quad 1158.4$

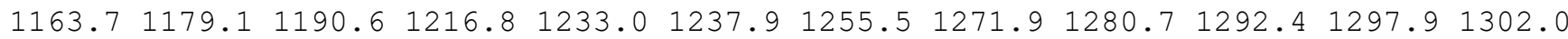
$1307.51309 .01312 .7 \quad 1331.3 \quad 1347.7 \quad 1363.2 \quad 1383.8 \quad 1396.2 \quad 1405.7 \quad 1409.01447 .3 \quad 1864.4$ $1915.92656 .2 \quad 2664.0 \quad 2675.3 \quad 2678.6 \quad 2682.0 \quad 2682.92695 .52705 .3 \quad 2738.92747 .3 \quad 2762.1$ $2764.5 \quad 2780.7 \quad 2783.2 \quad 3704.2$

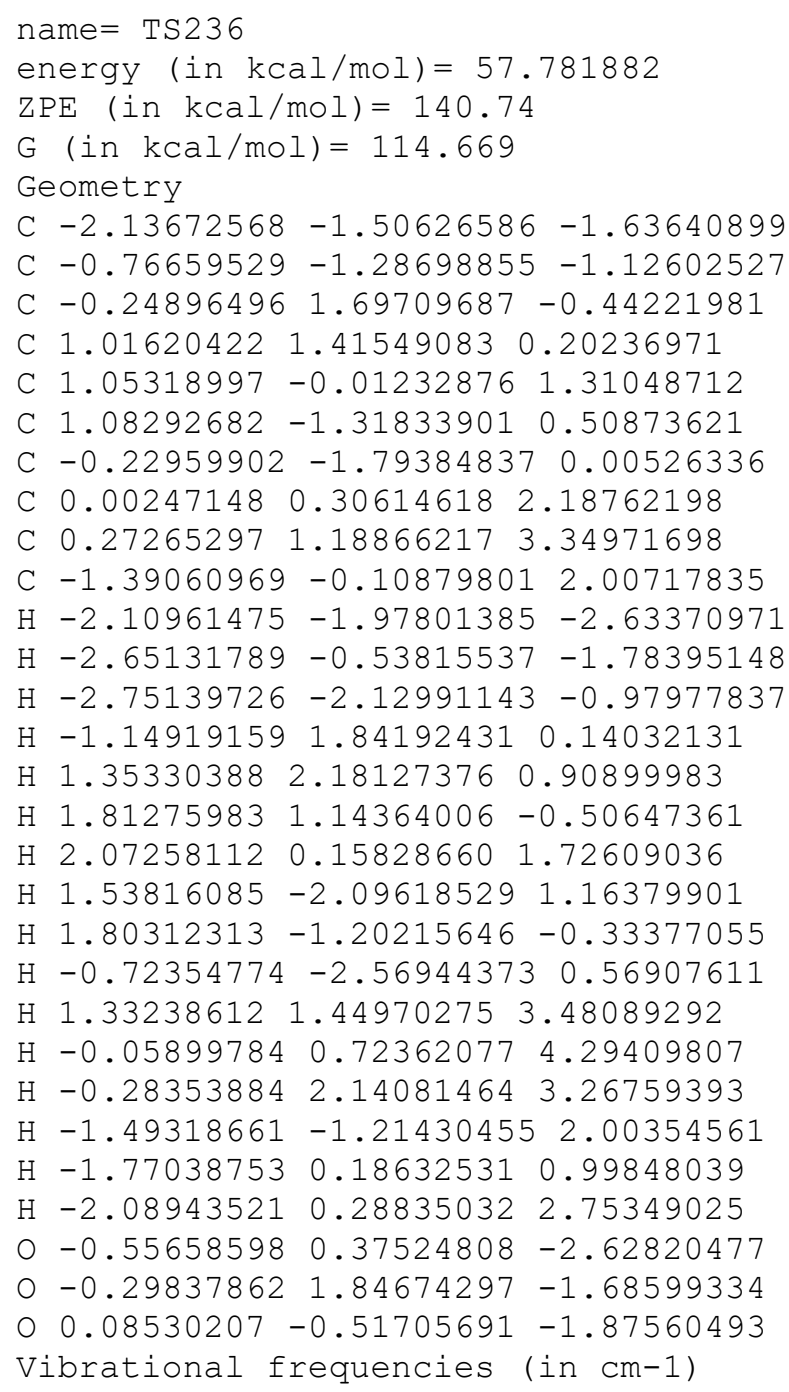


$\begin{array}{lllllllllllllllll}-684.0 & 42.8 & 69.7 & 87.7 & 91.9 & 95.8 & 113.1 & 162.3 & 191.1 & 199.4 & 219.9 & 237.9 & 274.5 & 283.0\end{array}$

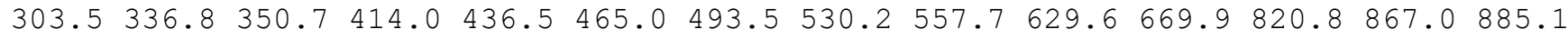

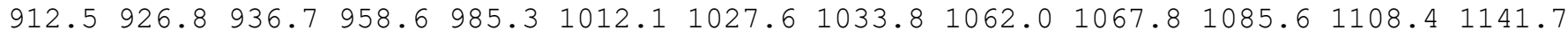

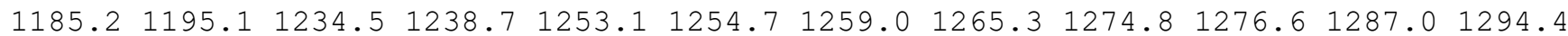
$1310.41316 .5 \quad 1345.0 \quad 1354.6 \quad 1376.6 \quad 1381.9 \quad 1388.8 \quad 1405.4 \quad 1428.1 \quad 1524.5 \quad 1602.91799 .6$ $2581.8 \quad 2645.6 \quad 2647.5 \quad 2651.4 \quad 2654.2 \quad 2661.7 \quad 2666.5 \quad 2683.02688 .82695 .6 \quad 2727.02729 .1$ $2751.32768 .2 \quad 2775.5 \quad 2781.0$

name $=$ TS237

energy (in $\mathrm{kcal} / \mathrm{mol})=58.524317$

$\mathrm{ZPE}($ in $\mathrm{kcal} / \mathrm{mol})=140.003$

$\mathrm{G}($ in $\mathrm{kcal} / \mathrm{mol})=113.142$

Geometry

C $-2.35424917-0.05768382-0.92941276$

C $-0.87179090-0.24516505-1.23887432$

C $0.01852243 \quad 0.88510122 \quad-0.61863110$

C $1.25684745 \quad 0.40101618 \quad 0.14396988$

C $0.91846883-0.19522119 \quad 1.42349523$

C $0.04271087-2.50178193-1.76540087$

C $-0.43236584-1.62866733-0.88059755$

C $1.64316970-1.07895173 \quad 2.24977909$

C $1.75124228-0.99057376 \quad 3.58705245$

C $2.24841650-2.23878276 \quad 1.50768572$

$\mathrm{H}-2.70958373 \quad 0.92851286-1.25499767$

$\mathrm{H}-2.55598628-0.16357320 \quad 0.14250035$

$\mathrm{H}-2.96187786-0.80610728-1.45620152$

$\mathrm{H}-0.57932574 \quad 1.60094005-0.01991070$

$\mathrm{H} \quad 1.99222497 \quad 1.23402725 \quad 0.20674351$

$\mathrm{H} \quad 1.79494215-0.36343502-0.47715008$

$\mathrm{H} \quad 0.72631047 \quad 1.14040955 \quad 2.38663161$

$\mathrm{H} \quad 0.34218196-3.50616388-1.50959930$

$\mathrm{H} \quad 0.16419743-2.28111749-2.81728396$

$\mathrm{H}-0.54569615-1.863978130 .18235235$

H $1.36420631-0.18772418 \quad 4.17987285$

H $0.01615688 \quad 0.75759956 \quad 2.26709917$

H 2.24285662 -1.73257191 4.18765977

H $3.21162551-1.953858231 .06212055$

$\mathrm{H} 1.59663580-2.60240543 \quad 0.70034492$

$\mathrm{H} 2.43698503-3.091695892 .17403628$

$0 \quad 0.45518041 \quad 0.69682632-2.81204717$

○ $0.44028186 \quad 1.64351535-1.75136370$

o $-0.75890839-0.01093533-2.65540683$

Vibrational frequencies (in $\mathrm{cm}-1$ )

$\begin{array}{lllllllllllllllll}-931.6 & 32.6 & 42.8 & 61.9 & 80.1 & 83.0 & 106.6 & 142.9 & 152.4 & 212.4 & 220.4 & 242.7 & 296.9 & 314.8\end{array}$

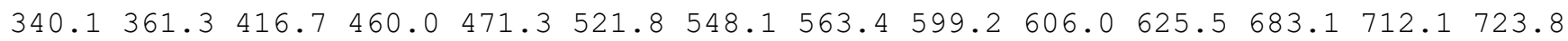

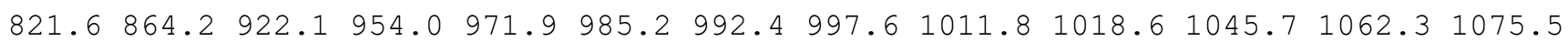
$1079.7 \quad 1083.0 \quad 1093.7 \quad 1122.3 \quad 1137.5 \quad 1183.6 \quad 1196.3 \quad 1244.91252 .2 \quad 1260.2 \quad 1276.1 \quad 1279.3$

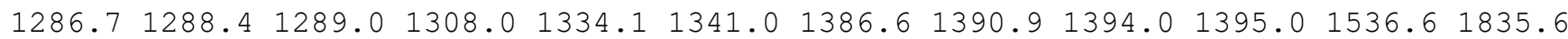
$1854.42631 .4 \quad 2667.4 \quad 2671.8 \quad 2672.5 \quad 2677.8 \quad 2688.6 \quad 2708.7 \quad 2723.3 \quad 2735.4 \quad 2738.0 \quad 2776.9$ $2779.82783 .6 \quad 2805.5 \quad 4678.8$

name $=$ TS238

energy $($ in $\mathrm{kcal} / \mathrm{mol})=56.36582$

$\operatorname{ZPE}($ in $\mathrm{kcal} / \mathrm{mol})=142.32$

$\mathrm{G}($ in $\mathrm{kcal} / \mathrm{mol})=117.857$

Geometry

C $0.48551028-3.27821140-0.68972444$

C $0.52824013-1.81925252-1.12336640$

C $-0.87955922-1.14117077-1.10269099$

C $-0.896755120 .20506522-0.38316266$

C $-0.23711815 \quad 0.07363003 \quad 0.99973901$

C $1.12369818-0.50883290 \quad 0.91641464$

C $1.60273909-1.00561920-0.39315852$ 
C $-1.03429558-0.75621343 \quad 1.94468556$

C $-2.49340886-0.63553268 \quad 2.07715848$

C $-0.25763653-1.67990640 \quad 2.69732703$

$\mathrm{H} \quad 0.24865241-3.36432349 \quad 0.37700128$

H $1.45214948-3.77000710-0.85911114$

$\mathrm{H}-0.26478066-3.84379543-1.25714771$

$\mathrm{H}-1.67025323-1.82132869-0.72966219$

$\mathrm{H}-1.92903827 \quad 0.58670416-0.30626393$

$\mathrm{H}-0.34313209 \quad 0.95286583-0.98776179$

$\mathrm{H}-0.157269261 .10632968 \quad 1.45669888$

H $1.86267433-0.201781261 .62800783$

H $1.90564042-0.15282903-1.04514357$

$\mathrm{H} 2.52359730-1.61121048-0.27617290$

$\mathrm{H}-2.85873009-0.92272376 \quad 3.07421369$

$\mathrm{H}-3.00670535-1.29245821 \quad 1.35010838$

$\mathrm{H}-2.85473990 \quad 0.38502914 \quad 1.87864682$

$\mathrm{H} 0.46906038-1.287081823 .42002416$

$\mathrm{H} \quad 0.63161021-1.97057204 \quad 1.86322505$

$\mathrm{H}-0.77569027 \quad-2.52891278 \quad 3.13766912$

O $0.07439151-0.83788437-3.11318109$

$0-1.19478169-0.95533311-2.48151389$

$0.85119089-1.86429987-2.52769838$

Vibrational frequencies (in $\mathrm{cm}-1$ )

$\begin{array}{lllllllllllllll}-1117.6 & 49.7 & 63.8 & 91.2 & 98.9 & 142.4 & 165.9 & 207.3 & 265.6 & 296.1 & 324.1 & 355.0 & 381.6 & 412.9\end{array}$

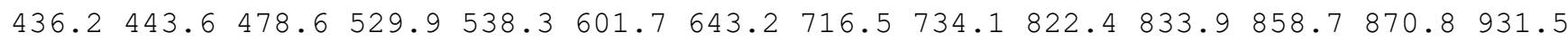
$945.6 \quad 958.0 \quad 971.7 \quad 972.6 \quad 1003.4 \quad 1019.4 \quad 1024.1 \quad 1051.3 \quad 1061.4 \quad 1093.0 \quad 1106.7 \quad 1122.7 \quad 1134.6$ $\begin{array}{llllllllllllll}1154.3 & 1185.7 & 1191.8 & 1215.6 & 1221.1 & 1230.7 & 1246.4 & 1252.6 & 1269.3 & 1270.8 & 1284.4 & 1288.6\end{array}$

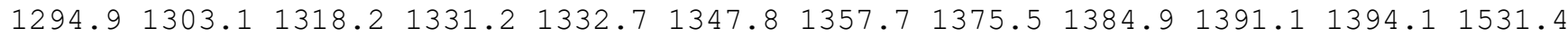
$2052.72651 .52651 .7 \quad 2657.4 \quad 2664.6 \quad 2668.3 \quad 2670.82673 .62674 .82696 .02736 .62745 .8$ $2753.32770 .2 \quad 2776.12778 .6$
name $=$ TS239
energy $($ in $\mathrm{kcal} / \mathrm{mol})=56.590335$
$\operatorname{ZPE}($ in $\mathrm{kcal} / \mathrm{mol})=142.122$
$\mathrm{G}($ in $\mathrm{kcal} / \mathrm{mol})=118.705$
Geometry
C $-1.66210281-1.05808631-0.54992257$
C $-0.32787470-0.42825716-0.91494389$
C $-0.07489864 \quad 0.99242418-0.27337989$
C $1.22875597 \quad 1.11309667 \quad 0.45204446$
C $2.18219915-0.002066470 .32991591$
C $2.16105654-0.59149826-1.12177073$
C $0.86775117-1.35950815-0.69942446$
C $1.38931661-1.302306640 .79311362$
C $0.42195795-1.15252856 \quad 1.94774487$
C $2.32727959-2.46822701 \quad 1.09528743$
$\mathrm{H}-2.49283525-0.35914231-0.72039888$
$\mathrm{H}-1.69452873-1.37581048 \quad 0.49748134$
$\mathrm{H}-1.85991816-1.94478306-1.16865596$
$\mathrm{H}-0.91912315 \quad 1.30592659 \quad 0.38169691$
$\mathrm{H} \quad 1.825915592 .47827249 \quad 0.27543953$
H $1.964950412 .23598212-0.49897507$
H $3.15548438 \quad 0.15189010 \quad 0.79432513$
H $3.00534907-1.24104877-1.36000030$
$\mathrm{H} \quad 2.06235073 \quad 0.12408130 \quad-1.93521158$
$\mathrm{H} \quad 0.72177408-2.35331833-1.12267862$
$\mathrm{H}-0.19947617-2.04688740 \quad 2.06925097$
$\mathrm{H}-0.24309147-0.28598643 \quad 1.85768780$
H $0.97083852-0.997473532 .88809203$
H $2.88904123-2.29085602 \quad 2.02011084$
H $3.06150981-2.63883698 \quad 0.30065475$
$\mathrm{H} 1.76435823-3.40055286 \quad 1.22231673$
○ $0.174888591 .10785178 \quad-2.51837997$
o $-0.16738494 \quad 1.89097729-1.37803849$ 
$0-0.47087181-0.13003238-2.31632331$

Vibrational frequencies (in $\mathrm{cm}-1$ )

$\begin{array}{lllllllllllllllll}-1043.3 & 83.6 & 98.8 & 139.6 & 162.6 & 174.8 & 204.9 & 214.4 & 252.2 & 272.6 & 313.1 & 347.5 & 381.4 & 395.7\end{array}$ $425.0 \quad 439.4 \quad 494.4 \quad 518.6 \quad 581.3 \quad 597.7 \quad 620.4 \quad 639.7 \quad 685.2 \quad 724.1 \quad 763.1 \quad 820.2 \quad 854.8 \quad 881.9$ $890.9924 .3 \quad 949.2969 .0 \quad 970.6 \quad 1003.4 \quad 1007.4 \quad 1011.7 \quad 1015.6 \quad 1032.4 \quad 1052.7 \quad 1080.3 \quad 1085.0$

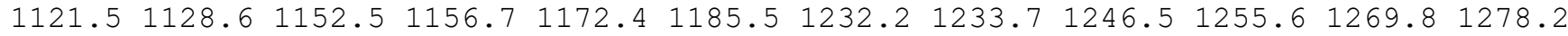

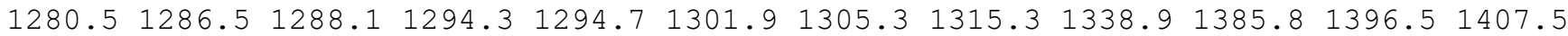
$1420.22666 .22669 .92674 .2 \quad 2675.2 \quad 2677.12680 .32681 .42689 .72748 .22756 .92768 .6$ $2779.52781 .3 \quad 2783.0 \quad 4556.0$

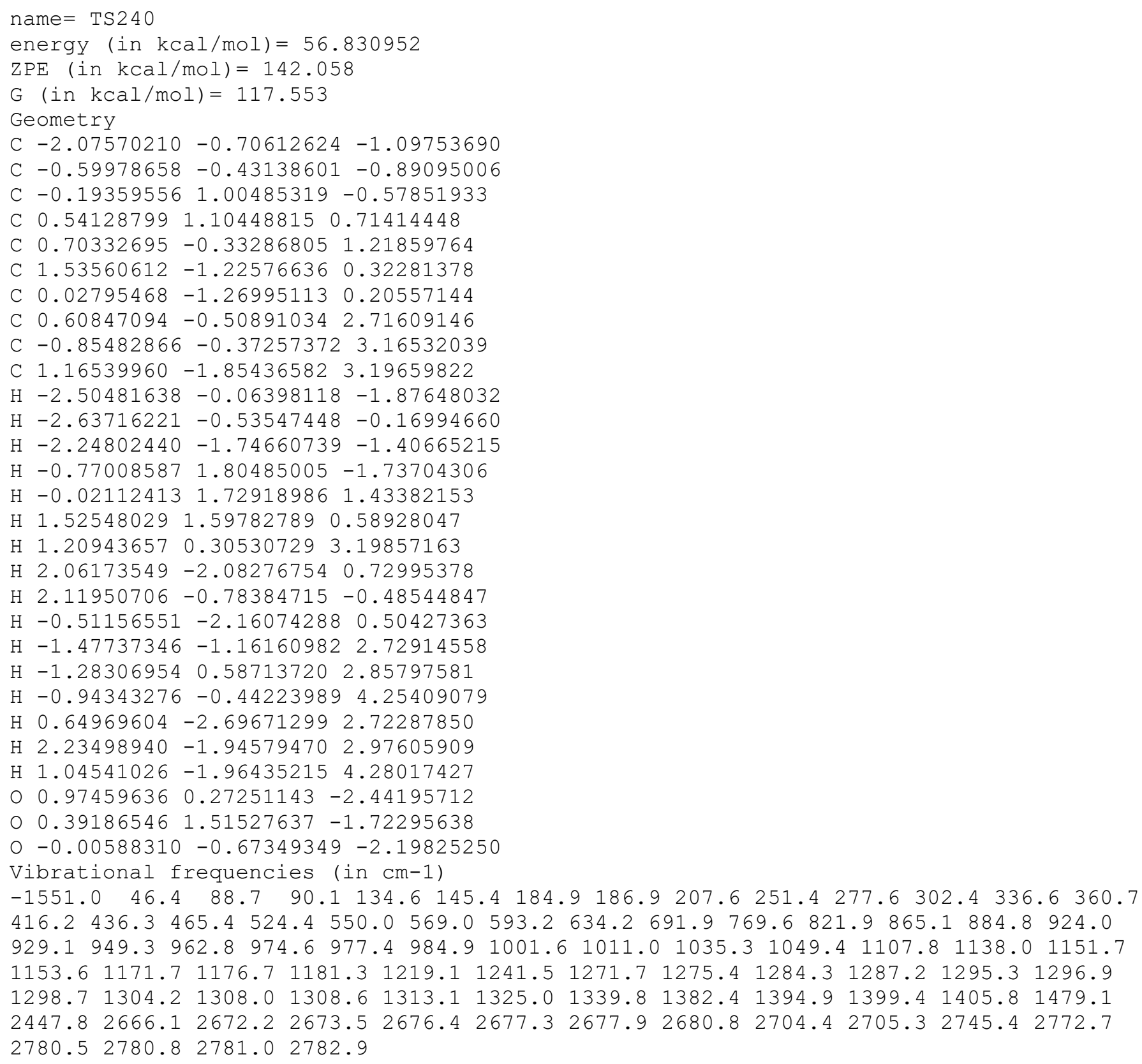


C $0.65895590-2.48816600-0.80739203$

C $-0.20394692-1.56465737-0.38983431$

C $-0.498060490 .33709723 \quad 2.53620806$

C $-1.69748494 \quad 0.78815590 \quad 2.94426353$

C $0.00153135-1.00975344 \quad 2.98173232$

$\mathrm{H}-2.34373367 \quad 0.62530286-1.97081374$

$\mathrm{H}-2.526278710 .00035831-0.31508167$

$\mathrm{H}-2.53172727-1.10795267-1.69674275$

$\mathrm{H}-0.59236877 \quad 1.78562665-0.56489372$

$\mathrm{H} \quad 1.94043034 \quad 1.55011480 \quad 0.35260535$

H $1.58300435-0.14888183 \quad 0.52854486$

$\mathrm{H} \quad 0.32466309 \quad 2.59318778 \quad 2.09692629$

$\mathrm{H} \quad 0.89903396-3.37754508-0.24472395$

$\mathrm{H} \quad 1.18658979-2.42993861-1.75082540$

$\mathrm{H}-0.73389031-1.644209750 .56067756$

$\mathrm{H}-2.11889097 \quad 1.733920522 .67330462$

$\mathrm{H}-0.29311997 \quad 2.53052373 \quad 1.56677707$

H $-2.34407286 \quad 0.23022264 \quad 3.59510464$

$\mathrm{H} \quad 0.41898174 \quad-0.93036645 \quad 3.99846342$

$\mathrm{H} 0.79981459-1.40897732 \quad 2.34356178$

$\mathrm{H}-0.80733455-1.75081882 \quad 3.01758397$

$\begin{array}{llll}0 & 1.14611877 & 0.18629205 & -2.56342307\end{array}$

$00.90763303 \quad 1.38458294-1.83398802$

o $-0.10126508-0.47467162-2.54445654$

Vibrational frequencies (in $\mathrm{cm}-1$ )

$\begin{array}{lllllllllllllll}-914.4 & 17.1 & 26.8 & 54.4 & 67.1 & 94.9 & 131.3 & 162.3 & 172.3 & 201.3 & 233.8 & 272.1 & 301.6 & 329.1\end{array}$

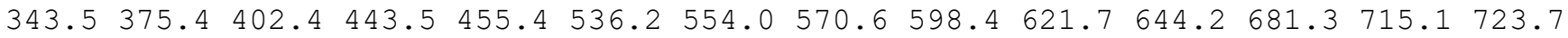

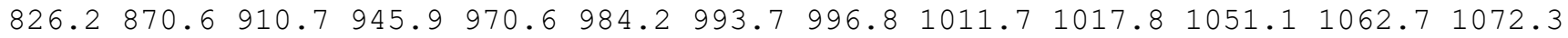

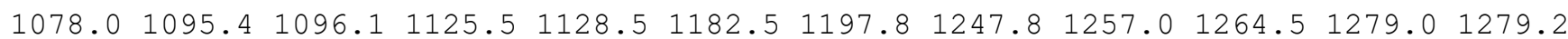

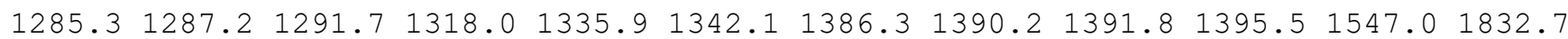
$1852.22628 .72666 .72669 .0 \quad 2672.6 \quad 2675.92677 .8 \quad 2708.72724 .4 \quad 2736.2 \quad 2748.92776 .9$ $2777.82780 .92805 .9 \quad 4663.3$

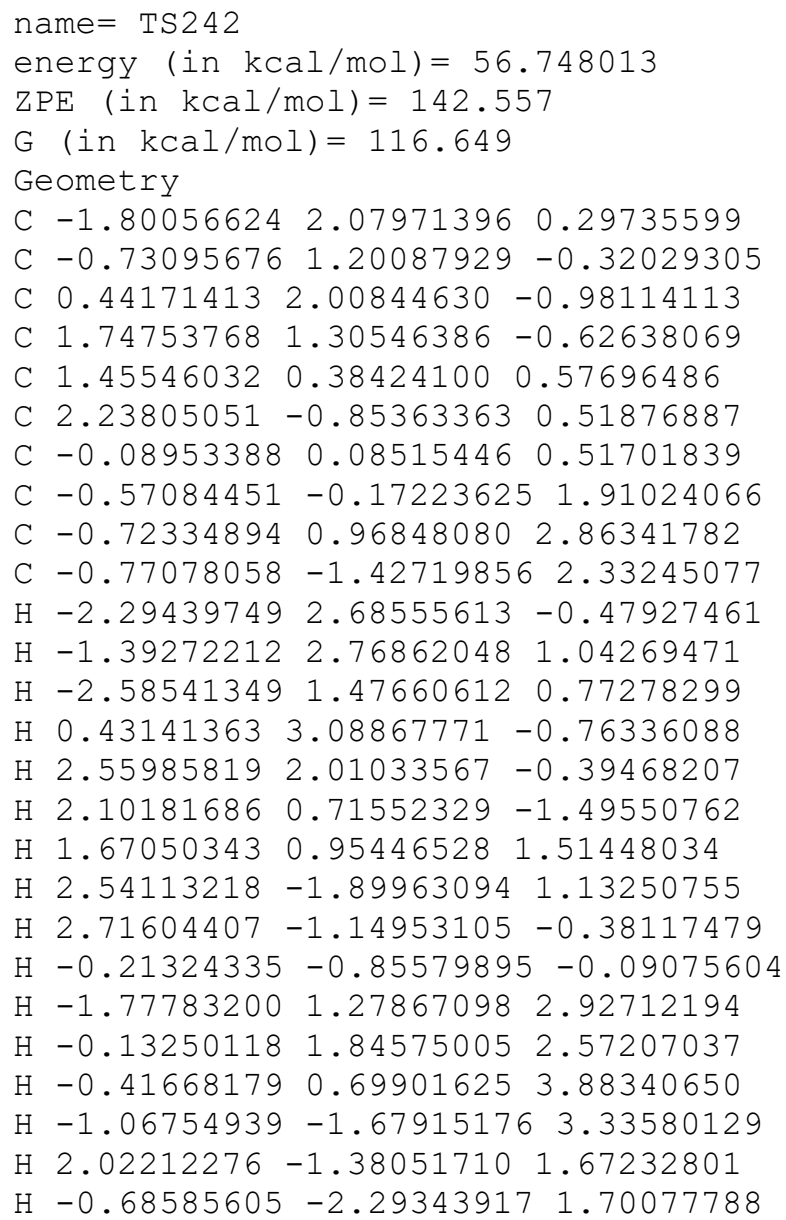


$0-0.45266127 \quad 0.64730512-2.51030115$

$0.167311721 .91793113-2.37462785$

O $-1.39645750 \quad 0.60357779-1.45181733$

Vibrational frequencies (in $\mathrm{cm}-1$ )

$\begin{array}{lllllllllllllllll}-1404.6 & 9.7 & 48.8 & 65.8 & 117.1 & 158.6 & 175.6 & 208.3 & 231.0 & 251.4 & 260.7 & 282.4 & 312.8 & 367.5\end{array}$ $409.7 \quad 444.8 \quad 472.3 \quad 499.4 \quad 516.6 \quad 574.4 \quad 585.9 \quad 613.7 \quad 651.2 \quad 686.0 \quad 713.1 \quad 721.7 \quad 825.5 \quad 860.8$

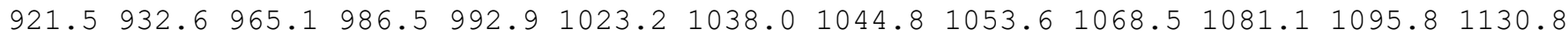

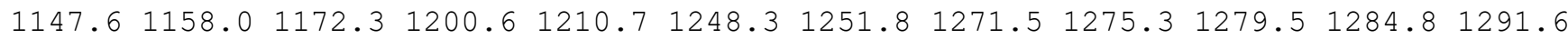
$1299.51314 .41315 .1 \quad 1323.3 \quad 1354.2 \quad 1363.2 \quad 1374.0 \quad 1393.4 \quad 1401.2 \quad 1410.01431 .01839 .2$ $1893.22663 .82666 .12667 .82673 .12674 .3 \quad 2678.92692 .92707 .5 \quad 2727.82751 .4 \quad 2777.5$ $2778.92786 .02796 .0 \quad 3774.6$

name $=\operatorname{TS} 243$

energy $($ in $\mathrm{kcal} / \mathrm{mol})=62.060769$

$\mathrm{ZPE}($ in $\mathrm{kcal} / \mathrm{mol})=137.752$

$\mathrm{G}($ in $\mathrm{kcal} / \mathrm{mol})=109.599$

Geometry

C $-2.13275730-1.81945233-1.86625841$

C $-0.77254387-1.52254986-1.38188219$

C $0.57055887 \quad 1.95707283-0.67415116$

C $-0.32749393 \quad 1.44054886 \quad 0.37851120$

C $1.16913989-0.012764321 .26622303$

C $0.97478821-1.22014644 \quad 0.41745139$

C $-0.44696249-1.36659434-0.01784304$

C $0.75103247 \quad 0.02943823 \quad 2.65541852$

C $1.34752493 \quad 1.12771223 \quad 3.47597987$

C $-0.10099721-0.856212863 .19901759$

$\mathrm{H}-2.13544970-2.78058976-2.41657294$

$\mathrm{H}-2.43884424-1.07623162-2.63031467$

$\mathrm{H}-2.89255684-1.86327745-1.08235996$

$\mathrm{H} \quad 0.22203279 \quad 1.93323816-1.71669140$

$\mathrm{H}-1.40330084 \quad 1.57325546 \quad 0.16070555$

$\mathrm{H}-0.17529330 \quad 1.87098114 \quad 1.36583341$

H $1.98729604 \quad 0.67014915 \quad 0.97588676$

H $1.28164557 \quad-2.12951877 \quad 0.99530526$

$\mathrm{H} \quad 1.66157417-1.19500409-0.46153504$

$\mathrm{H}-1.14375587-1.75411907 \quad 0.70930892$

$\mathrm{H} 2.35888150 \quad 0.85983484 \quad 3.82137369$

$\mathrm{H} \quad 0.75712080 \quad 1.35569950 \quad 4.37437669$

H $1.44576733 \quad 2.07279413 \quad 2.91812766$

$\mathrm{H}-0.679282010 .21971066 \quad 0.19127149$

$\mathrm{H}-0.41116102-0.81947830 \quad 4.23540344$

$\mathrm{H}-0.54749523-1.68126924 \quad 2.65697237$

O $-0.14721230-1.35875962-3.49730209$

O $1.67263053 \quad 2.38311474 \quad-0.40767338$

$0.20000425-1.31832681-2.24870555$

Vibrational frequencies (in $\mathrm{cm}-1$ )

$\begin{array}{lllllllllllllll}-1123.4 & 24.2 & 42.5 & 55.0 & 65.2 & 86.7 & 91.2 & 92.2 & 112.7 & 167.1 & 175.8 & 188.7 & 234.5 & 281.6\end{array}$ $289.6 \quad 314.2 \quad 323.3 \quad 441.1 \quad 473.4 \quad 502.1 \quad 512.2 \quad 543.4 \quad 576.3 \quad 588.4 \quad 622.2 \quad 675.3 \quad 722.5 \quad 830.3$ $875.5926 .0 \quad 970.0 \quad 989.3 \quad 1003.7 \quad 1005.6 \quad 1031.9 \quad 1036.6 \quad 1055.3 \quad 1059.0 \quad 1062.3 \quad 1071.2 \quad 1106.2$ $1120.21143 .21177 .9 \quad 1215.2 \quad 1232.2 \quad 1234.0 \quad 1257.1 \quad 1266.3 \quad 1269.3 \quad 1273.7 \quad 1276.8 \quad 1287.0$

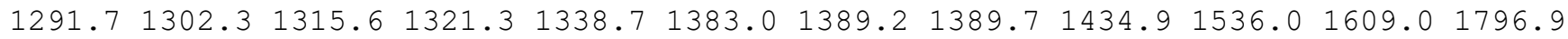
1819.52627 .12640 .02641 .22648 .02656 .62657 .72666 .52684 .42695 .02713 .02741 .1 $2761.8 \quad 2771.2 \quad 2774.8 \quad 2776.1$

name $=\operatorname{TS} 244$

energy (in $\mathrm{kcal} / \mathrm{mol})=58.387513$

$\operatorname{ZPE}($ in $\mathrm{kcal} / \mathrm{mol})=141.889$

$\mathrm{G}($ in $\mathrm{kcal} / \mathrm{mol})=118.167$

Geometry

$\begin{array}{lllll}\text { C }-2.52553071 & 0.48690164 & 0.69661767\end{array}$

C $-1.20246695 \quad 0.07727259 \quad 0.07225112$

C $-0.26592688 \quad 1.28860569-0.29781519$ 
C $0.99110399 \quad 1.29078918 \quad 0.49914230$

C $1.46418155 \quad 0.00559158 \quad 1.03658239$

C $0.96927354-1.18787819 \quad 0.15112282$

C $-0.42896075-1.00423650 \quad 0.83327546$

C $0.34187620-0.426347052 .09004119$

C $-0.25748280 \quad 0.66899973 \quad 2.94218738$

C $0.80915397-1.565072912 .99724818$

$\mathrm{H}-3.09358688 \quad 1.14426708 \quad 0.02098514$

$\mathrm{H}-2.38568098 \quad 1.02830836 \quad 1.63902645$

$\mathrm{H}-3.15808855-0.38480872 \quad 0.90601107$

$\mathrm{H}-0.799993792 .26206033-0.22906662$

H 2.02934386 2.19534268 -0.13309641

H $2.07103000 \quad 1.62039583-0.71589478$

H $2.48994939-0.02122241 \quad 1.39684545$

H $1.44084114 \quad-2.14718560 \quad 0.37425777$

$\mathrm{H} 1.02814959-1.04609118-0.92467266$

$\mathrm{H}-1.01914263-1.90532428 \quad 1.00060901$

$\mathrm{H} \quad 0.46692680 \quad 1.00720627 \quad 3.69749537$

$\mathrm{H}-1.15122727 \quad 0.32291502 \quad 3.47294946$

$\mathrm{H}-0.515420421 .569975992 .36954835$

H $1.26285614-1.18750840 \quad 3.92027010$

H $1.55829277 \quad-2.20494857 \quad 2.51761824$

$\mathrm{H}-0.03203217-2.20784008 \quad 3.28303075$

o $-0.50402735-0.15476508-2.07157138$

$0-0.03056267 \quad 1.14844764-1.70192883$

o $-1.59124910-0.41259464-1.22430513$

Vibrational frequencies (in cm-1)

$\begin{array}{lllllllllllllll}-1026.9 & 58.4 & 95.4 & 104.3 & 169.5 & 185.5 & 203.5 & 230.6 & 246.2 & 272.4 & 299.2 & 351.0 & 358.3 & 397.7\end{array}$ $420.5 \quad 446.3 \quad 499.0 \quad 519.3 \quad 563.0 \quad 590.3 \quad 604.6 \quad 627.9 \quad 682.3 \quad 719.7 \quad 754.5 \quad 803.3 \quad 849.6 \quad 881.2$ $890.6922 .4 \quad 949.1966 .5 \quad 972.8 \quad 1000.3 \quad 1006.5 \quad 1011.4 \quad 1018.5 \quad 1037.4 \quad 1053.9 \quad 1073.4 \quad 1082.2$

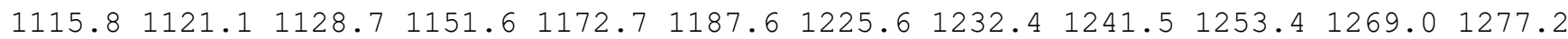
$1282.1 \quad 1286.7 \quad 1288.7 \quad 1290.8 \quad 1295.8 \quad 1301.1 \quad 1306.1 \quad 1319.6 \quad 1347.7 \quad 1391.3 \quad 1396.3 \quad 1409.4$ $1416.22661 .6 \quad 2667.92672 .7 \quad 2673.1 \quad 2677.0 \quad 2681.12684 .02688 .3 \quad 2747.2 \quad 2759.82769 .5$ $2777.5 \quad 2780.7 \quad 2781.4 \quad 4604.2$

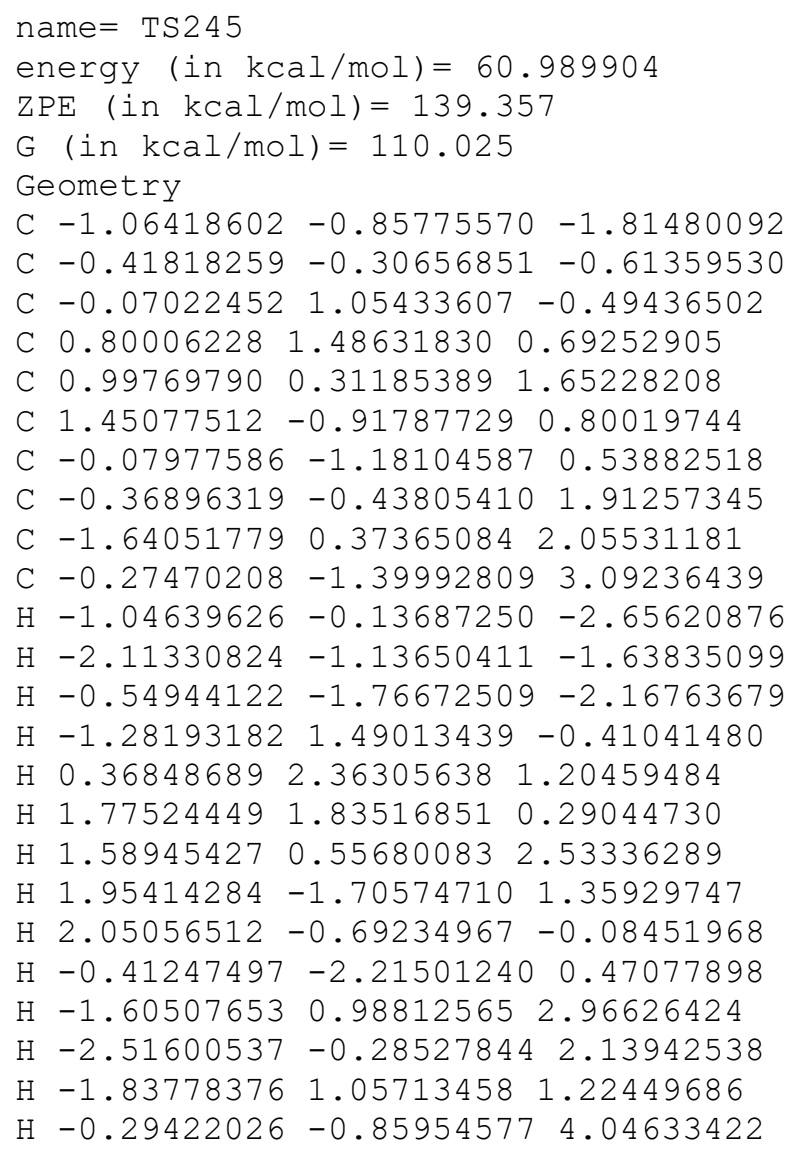


$\mathrm{H} \quad 0.64696140-1.99241088 \quad 3.08020099$

$\mathrm{H}-1.11134268-2.10858883 \quad 3.09511167$

$\begin{array}{llll}0 & 2.06028454 & 1.24966709 & -2.68491027\end{array}$

$0-0.19920834 \quad 1.89762020-1.49582479$

$\begin{array}{llll}0 & 2.24077425 & 0.13830819 & -2.54000660\end{array}$

Vibrational frequencies (in $\mathrm{cm}-1$ )

$\begin{array}{lllllllllllllll}-1426.3 & 10.2 & 21.2 & 36.0 & 42.5 & 61.6 & 86.9 & 102.5 & 118.4 & 131.4 & 171.3 & 189.2 & 267.6 & 274.8\end{array}$

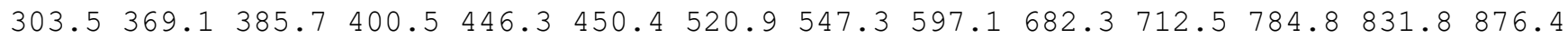

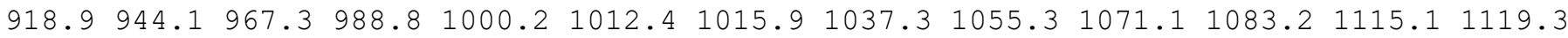
$1169.5 \quad 1176.4 \quad 1179.9 \quad 1218.0 \quad 1242.2 \quad 1261.1 \quad 1267.4 \quad 1269.6 \quad 1278.2 \quad 1286.1 \quad 1291.4 \quad 1297.4$ $1300.51306 .11310 .5 \quad 1345.3 \quad 1372.8 \quad 1378.8 \quad 1390.3 \quad 1405.21409 .5 \quad 1419.5 \quad 1592.31636 .3$ $2248.52648 .52651 .92662 .2 \quad 2662.8 \quad 2671.6 \quad 2674.02678 .62688 .6 \quad 2745.62749 .52755 .7$ $2764.02768 .32770 .5 \quad 2782.3$

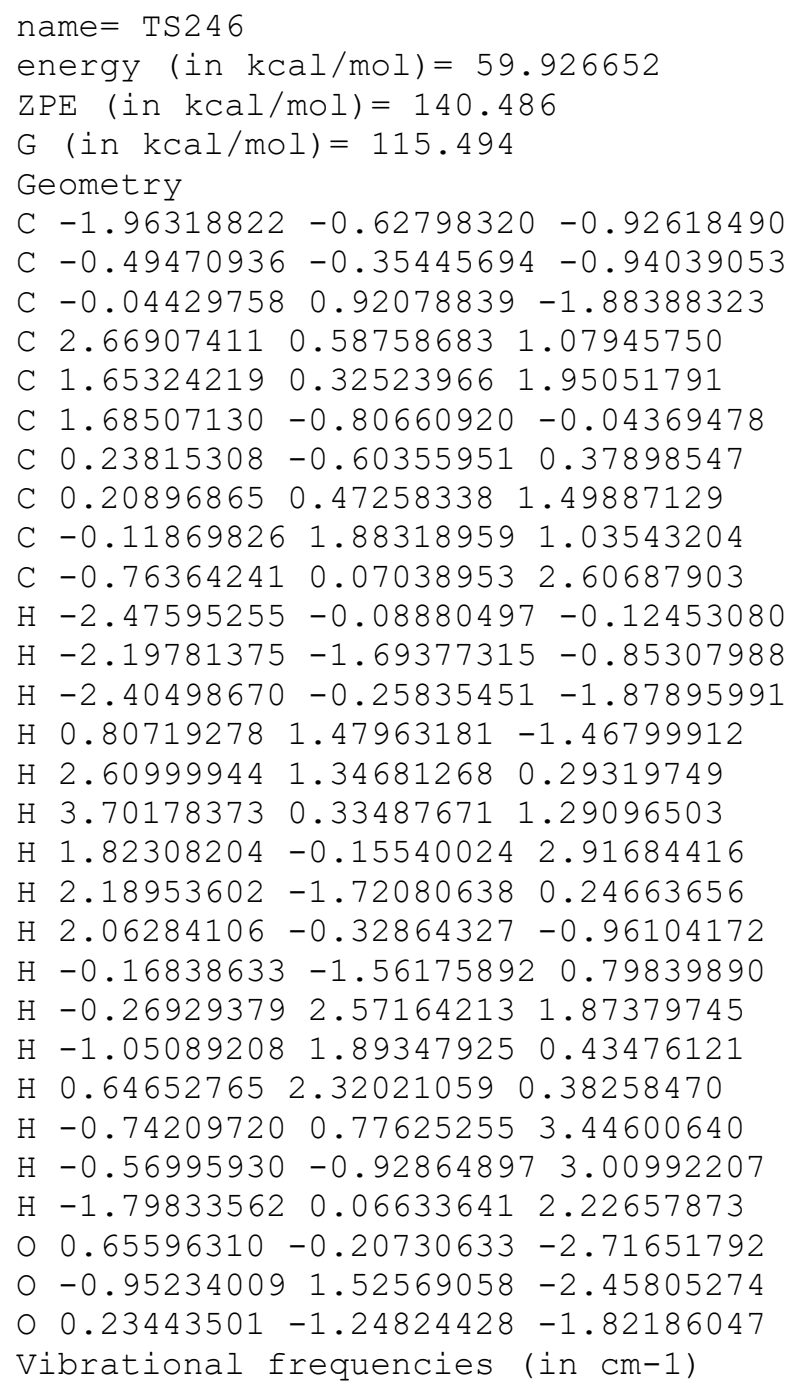


C $2.69188582-2.32219686-1.42601551$

C $2.09201369-0.91045591-1.77350965$

C $1.45939031-0.32697721-0.50165234$

C $1.71673860 \quad-1.32613198 \quad 0.62659410$

C $2.07663993-0.85632506 \quad 1.92967684$

C $2.38360186-2.58035646 \quad 0.02890852$

C $0.96439914 \quad-2.67832872 \quad 0.54536512$

C $-0.22893293-2.82226528-0.36250405$

C $0.78100924-3.44710122 \quad 1.83398982$

$\mathrm{H} 2.65897773-3.17907201-3.41669074$

$\mathrm{H} \quad 1.26769200-3.64284912-2.41364139$

H $2.85367772-4.36176485-2.11878852$

$\mathrm{H} \quad 1.42676497-0.91765492-2.65090495$

$\mathrm{H} \quad 0.38086320-0.13738505-0.62973118$

$\mathrm{H} \quad 1.91020243 \quad 0.65597423 \quad-0.26765224$

H $3.63473698-0.736932632 .05120194$

$\mathrm{H} 1.62580960-0.01427893 \quad 2.38975349$

H $3.50888540 \quad-0.05140216 \quad 1.63262219$

$\mathrm{H} \quad 3.16869621-3.11144930 \quad 0.57044587$

$\begin{array}{lllll}\mathrm{H} & -1.12670211 & -2.39710858 & 0.10888996\end{array}$

$\mathrm{H}-0.43836041-3.88147938-0.56667347$

$\mathrm{H}-0.10731758-2.32083553-1.32835333$

H $1.56661510 \quad-3.20037690 \quad 2.56884994$

$\mathrm{H} \quad 0.81057352-4.527945331 .65976858$

$\mathrm{H}-0.17405424 \quad-3.20295010 \quad 2.31405217$

O $4.24792361-1.04174822-2.42304929$

O $3.23168072-0.10452611-2.07852949$

O $4.11179084-2.08397729-1.48027512$

Vibrational frequencies (in $\mathrm{cm}-1$ )

$\begin{array}{llllllllllllllll}-987.9 & 71.6 & 107.7 & 123.9 & 132.5 & 154.8 & 173.8 & 194.2 & 196.6 & 248.7 & 255.4 & 275.0 & 310.8 & 339.2\end{array}$

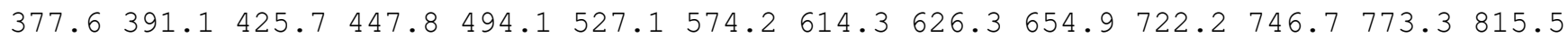
$861.5 \quad 877.7 \quad 945.3 \quad 962.2 \quad 982.1995 .5 \quad 1010.1 \quad 1012.6 \quad 1019.1 \quad 1024.3 \quad 1050.4 \quad 1059.8 \quad 1094.6$

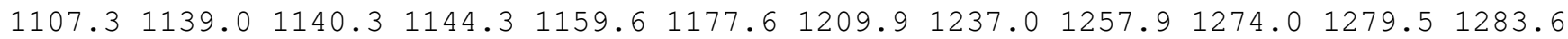
$1285.4 \quad 1289.4 \quad 1294.0 \quad 1301.0 \quad 1307.3 \quad 1325.2 \quad 1334.3 \quad 1337.7 \quad 1369.0 \quad 1395.2 \quad 1403.91405 .5$ $1499.02659 .12665 .92671 .5 \quad 2672.6 \quad 2674.8 \quad 2676.92683 .32696 .7 \quad 2733.92747 .3 \quad 2756.4$ $2778.22780 .7 \quad 2781.9 \quad 4712.2$

name $=\operatorname{TS} 248$

energy $($ in $\mathrm{kcal} / \mathrm{mol})=56.974116$

$\operatorname{ZPE}($ in $\mathrm{kcal} / \mathrm{mol})=143.934$

$\mathrm{G}($ in $\mathrm{kcal} / \mathrm{mol})=120.028$

Geometry

C $-2.266023020 .31907306 \quad 0.19648923$

C $-0.79137432 \quad 0.52565623 \quad 0.47450535$

C $-0.52027452 \quad 1.35999088 \quad 1.75053373$

C $0.66915935 \quad 0.90470734 \quad 2.62349590$

C $1.19266370-0.46654649 \quad 2.19887544$

C $1.51920788-0.42124858 \quad 0.67984167$

C $0.01800110-0.75093226 \quad 0.42173019$

C $-0.04117310-1.404758321 .86267156$

C $0.23398990 \quad 2.64401826 \quad-0.00383443$

C $0.24356386-2.83115025 \quad 1.86195638$

$\mathrm{H}-2.42680407-0.08756863-0.81443473$

$\mathrm{H}-2.84628280 \quad 1.24569411 \quad 0.28236702$

$\mathrm{H}-2.69733502-0.411353890 .89518397$

$\mathrm{H}-1.43609320 \quad 1.47613018 \quad 2.37060533$

$\mathrm{H} \quad 0.36984856 \quad 0.88969633 \quad 3.68584167$

H $1.47952927 \quad 1.65829116 \quad 2.55485126$

$\mathrm{H} 1.95166695-0.881387872 .85687000$

H $2.19402299-1.21479987 \quad 0.34665451$

H $1.89174131 \quad 0.51860864 \quad 0.27646680$

$\mathrm{H}-0.20193540-1.39149286-0.43364503$

$\mathrm{H}-0.95036133-1.15093450 \quad 2.42955460$

H $1.322816572 .53075851-0.01097082$ 
$\mathrm{H}-0.12337678 \quad 3.53346705-0.53362905$

$\mathrm{H}-0.19515683-3.24138788 \quad 2.98273836$

$\mathrm{H} \quad 0.19818527 \quad-3.905347232 .48559216$

$\mathrm{H} \quad 0.76500851-3.25568690 \quad 1.03510671$

$\begin{array}{lllll}0 & 0.37022533 & 0.94296949 & -1.53294363\end{array}$

$\begin{array}{llll}0 & -0.29409686 & 2.69058038 & 1.28838837\end{array}$

O $-0.43756596 \quad 1.46885418-0.69702346$

Vibrational frequencies (in cm-1)

$\begin{array}{lllllllllllllll}-1459.6 & 31.1 & 76.0 & 143.1 & 150.0 & 157.3 & 205.3 & 238.6 & 269.1 & 275.6 & 331.3 & 374.9 & 400.9 & 433.0\end{array}$ $461.2 \quad 491.0 \quad 540.4 \quad 585.4 \quad 599.2 \quad 637.8 \quad 647.7 \quad 666.3 \quad 781.8 \quad 810.0 \quad 843.7 \quad 871.7 \quad 896.5 \quad 924.9$ $940.9946 .6979 .6 \quad 990.2 \quad 1009.0 \quad 1013.4 \quad 1044.8 \quad 1052.4 \quad 1066.1 \quad 1088.2 \quad 1100.7 \quad 1114.6 \quad 1127.5$

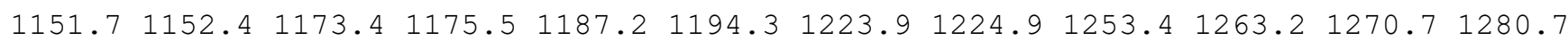

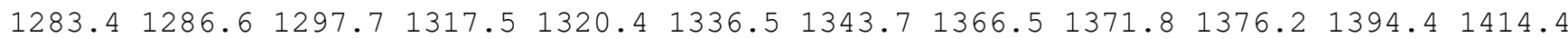
$1943.32646 .62662 .02663 .8 \quad 2673.2 \quad 2688.3 \quad 2694.02703 .72716 .82744 .62751 .02760 .4$ $2770.92776 .12780 .9 \quad 3728.9$

name $=$ TS249

energy $($ in $\mathrm{kcal} / \mathrm{mol})=58.755565$

$\mathrm{ZPE}($ in $\mathrm{kcal} / \mathrm{mol})=142.208$

$\mathrm{G}(\mathrm{in} \mathrm{kcal} / \mathrm{mol})=118.965$

Geometry

C $-1.67644633-0.15561066-1.45640310$

C $-0.24861868-0.29447010-0.95051642$

C $0.510367641 .08795327-0.75664936$

C $1.311717661 .14330290 \quad 0.47918232$

C $1.13261854 \quad 0.10615345 \quad 1.49718350$

C $1.39151193-1.218290460 .68385040$

C $-0.12140385-1.172829650 .30020077$

C $-0.37969609-0.358269291 .62994448$

C $-1.44135302 \quad 0.72183616 \quad 1.67275119$

C $-0.59698211-1.293186212 .81597445$

$\mathrm{H}-1.72730870 \quad 0.51852783 \quad-2.32223327$

$\mathrm{H}-2.35998575 \quad 0.21481594-0.68807173$

$\mathrm{H}-2.06204678-1.12889601-1.79446949$

$\mathrm{H}-0.15974437 \quad 1.95260776-0.92922202$

$\mathrm{H} \quad 0.73595760 \quad 2.46060917 \quad 1.24515868$

$\mathrm{H} 1.525634912 .526201951 .01247023$

H $1.68469623 \quad 0.23165407 \quad 2.42721740$

$\mathrm{H} \quad 1.68205232-2.06761190 \quad 1.30274708$

H $2.11069954-1.14222214 \quad-0.13706190$

$\mathrm{H}-0.64089895-2.12645832 \quad 0.20891576$

$\mathrm{H}-2.44770804 \quad 0.28187461 \quad 1.62838362$

$\mathrm{H}-1.37315224 \quad 1.44368079 \quad 0.85626088$

$\mathrm{H}-1.38213051 \quad 1.28685360 \quad 2.61244739$

$\mathrm{H}-0.54245952-0.748605593 .76545281$

$\mathrm{H} \quad 0.15012612 \quad-2.09370794 \quad 2.86200794$

$\mathrm{H}-1.58097156-1.77436237 \quad 2.76426320$

O $1.68902061-0.21768611-2.15204128$

O $1.364060321 .12797072-1.91099581$

O $0.44727098-0.91528707-2.03774094$

Vibrational frequencies (in $\mathrm{cm}-1$ )

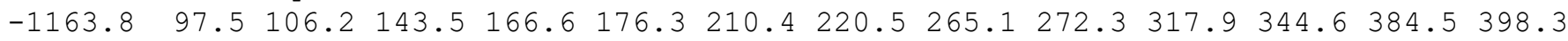
$425.7 \quad 452.9 \quad 490.2 \quad 524.9 \quad 583.9 \quad 608.6 \quad 643.2 \quad 652.7 \quad 687.6 \quad 710.0 \quad 738.8 \quad 805.9 \quad 854.5 \quad 880.5$ $897.1924 .4940 .6961 .6 \quad 978.1997 .6 \quad 1003.9 \quad 1009.2 \quad 1014.7 \quad 1024.7 \quad 1050.0 \quad 1075.4 \quad 1080.2$

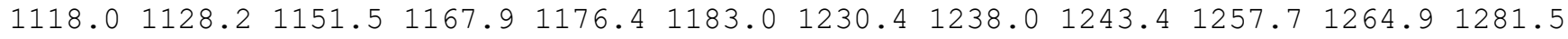
$1283.1 \quad 1287.9 \quad 1292.6 \quad 1294.7 \quad 1298.5 \quad 1304.6 \quad 1311.91314 .8 \quad 1372.2 \quad 1387.0 \quad 1396.7 \quad 1405.9$ $1414.22668 .92671 .2 \quad 2673.4 \quad 2676.92679 .2 \quad 2680.02682 .02690 .12749 .02757 .62761 .7$ $2780.12781 .92782 .9 \quad 4514.9$

name $=\operatorname{TS} 250$

energy $($ in $\mathrm{kcal} / \mathrm{mol})=58.73621$

$\mathrm{ZPE}($ in $\mathrm{kcal} / \mathrm{mol})=142.349$

$\mathrm{G}($ in $\mathrm{kcal} / \mathrm{mol})=119.26$ 


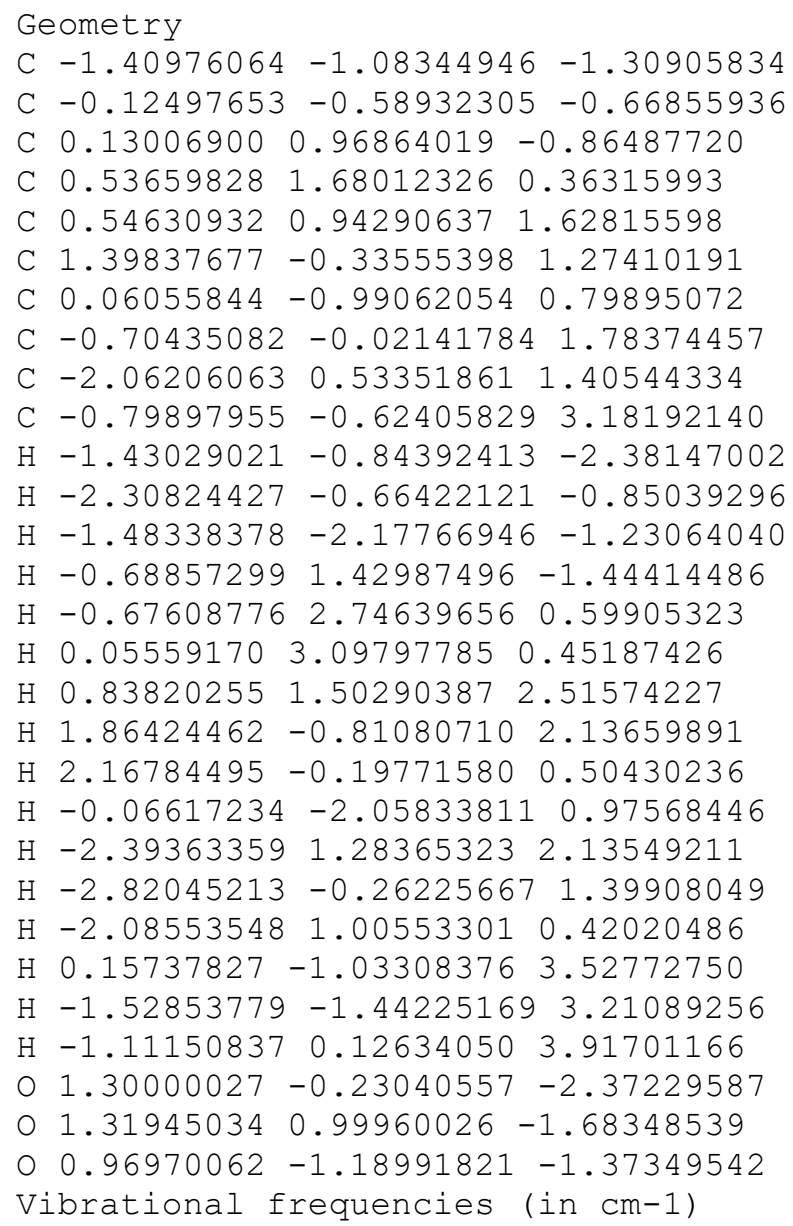

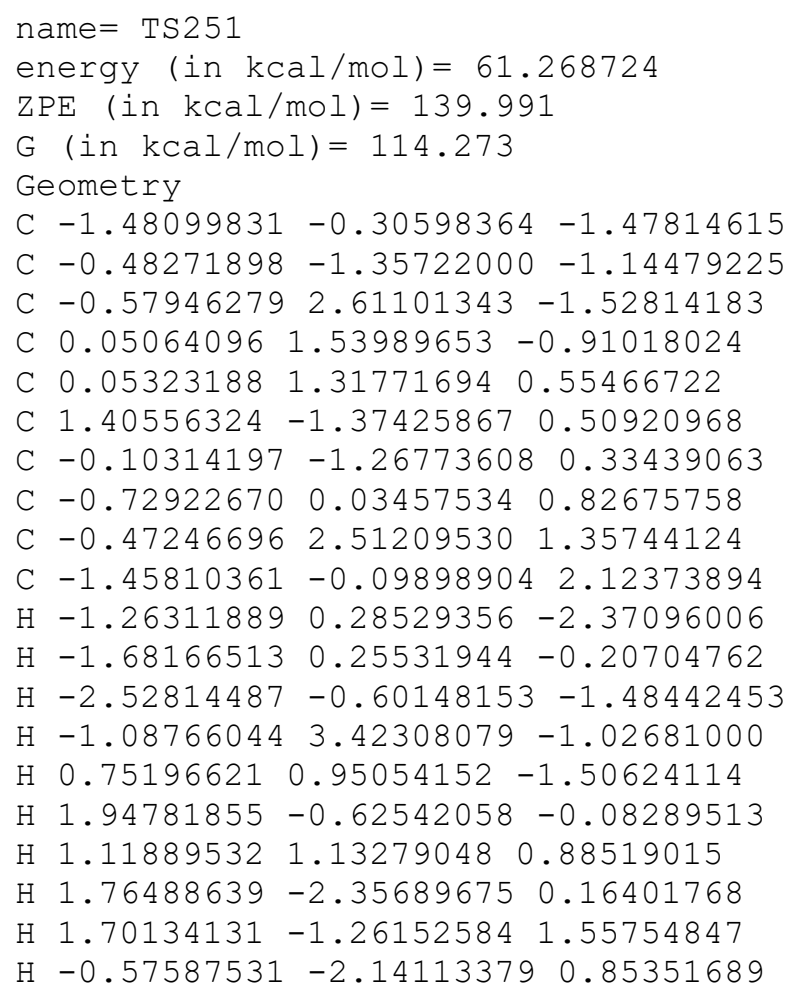


$\mathrm{H}-0.34646593 \quad 2.35854918 \quad 2.43527548$

$\mathrm{H}-1.53498907 \quad 2.69984631 \quad 1.17081319$

$\mathrm{H} 0.07829356 \quad 3.42672567 \quad 1.10250605$

$\mathrm{H}-0.75606071-0.143074772 .97175946$

$\mathrm{H}-2.06658095-1.012974842 .17298572$

$\mathrm{H}-2.13941704 \quad 0.74094347 \quad 2.31804647$

$0-0.166501041 .84025572-3.56247109$

$\begin{array}{llll}0 & -0.66845857 & 2.75557349 & -2.84155094\end{array}$

$0-0.04888571-2.17441647-1.90590663$

Vibrational frequencies (in $\mathrm{cm}-1$ )

$\begin{array}{lllllllllllllllll}-1687.6 & 40.2 & 52.0 & 77.3 & 121.2 & 150.6 & 158.2 & 179.4 & 191.5 & 224.3 & 235.6 & 250.5 & 264.6 & 270.6\end{array}$ $333.3 \quad 356.9 \quad 399.4 \quad 413.7 \quad 475.9 \quad 500.7 \quad 536.6 \quad 556.6 \quad 626.2 \quad 654.8 \quad 732.2 \quad 745.9 \quad 812.9 \quad 873.2$ $883.7923 .9951 .5971 .9 \quad 999.3 \quad 1024.2 \quad 1034.3 \quad 1064.1 \quad 1072.4 \quad 1080.01104 .2 \quad 1152.6 \quad 1160.4$ $1181.91197 .6 \quad 1217.2 \quad 1228.6 \quad 1239.3 \quad 1248.4 \quad 1255.5 \quad 1271.3 \quad 1284.5 \quad 1291.0 \quad 1297.0 \quad 1298.0$

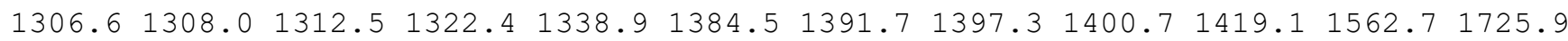
$1899.72625 .42650 .92658 .6 \quad 2661.7 \quad 2667.5 \quad 2672.4 \quad 2676.22681 .8 \quad 2696.2 \quad 2708.22716 .7$ $2741.22775 .02776 .5 \quad 2780.0$

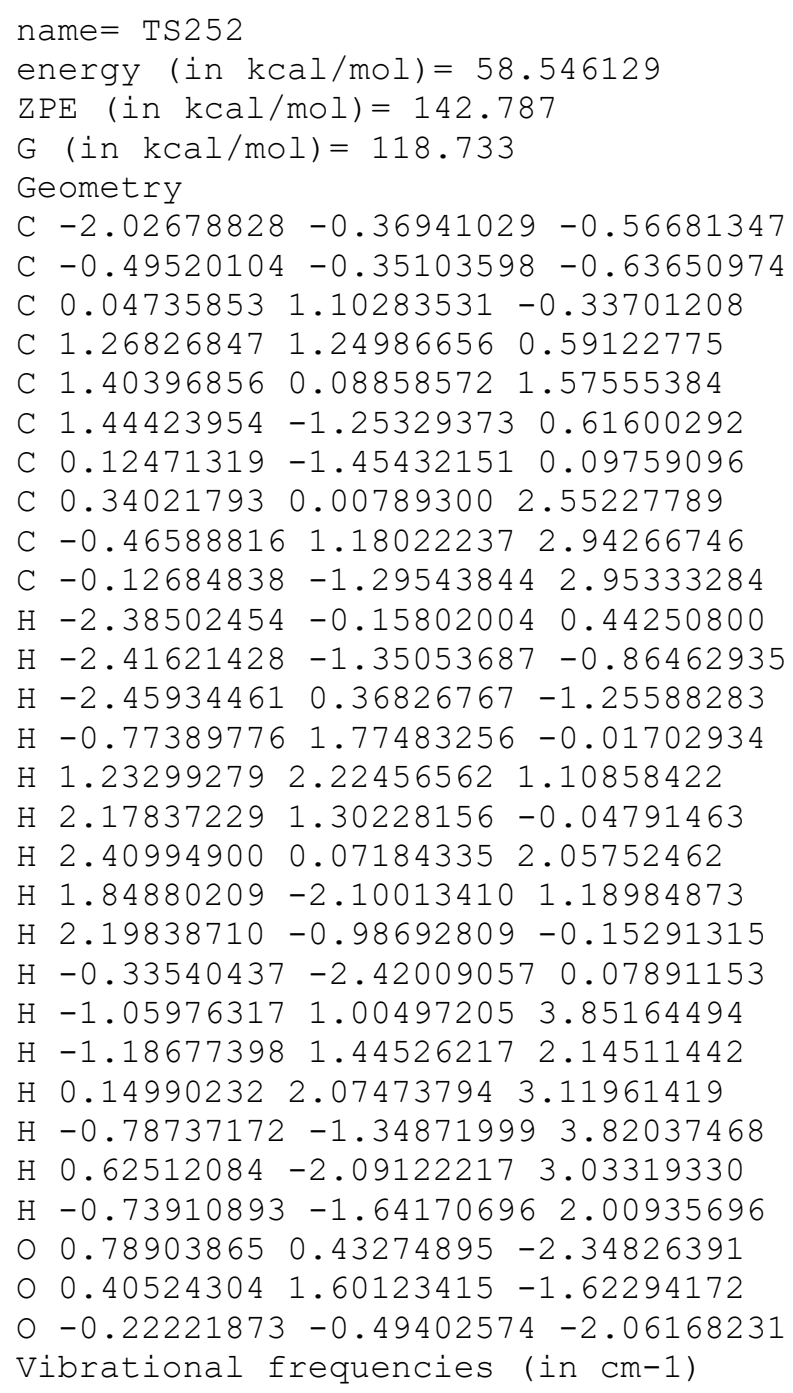
$432.1 \quad 461.9 \quad 482.8 \quad 498.3 \quad 508.8 \quad 557.2 \quad 644.0 \quad 685.9 \quad 708.6 \quad 757.4 \quad 811.2 \quad 846.4 \quad 872.1910 .4$ $933.1943 .9980 .9991 .4 \quad 1001.5 \quad 1013.1 \quad 1023.6 \quad 1037.0 \quad 1046.2 \quad 1094.6 \quad 1110.7 \quad 1118.1 \quad 1143.3$

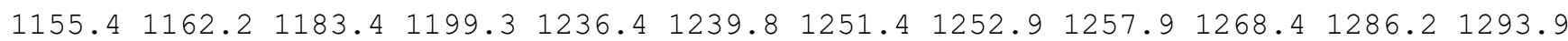

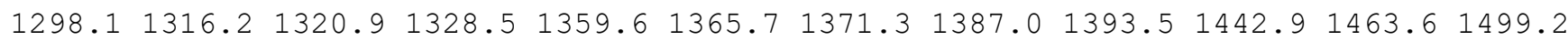
$2308.12642 .6 \quad 2649.8 \quad 2655.5 \quad 2669.12673 .8 \quad 2678.62682 .52685 .8 \quad 2687.92735 .92737 .7$ $2755.52768 .3 \quad 2782.02786 .8$ 
$\mathrm{ZPE}($ in $\mathrm{kcal} / \mathrm{mol})=138.302$

$\mathrm{G}($ in $\mathrm{kcal} / \mathrm{mol})=108.141$

Geometry

C $-2.96292342 \quad-0.69397988 \quad 0.57052631$

C $-0.91151592 \quad 0.312188190 .07455263$

C $-0.12972180 \quad 1.37695114 \quad-0.23629962$

C $1.29922594 \quad 1.03852405 \quad 0.11279012$

C $1.20652662-0.38391187 \quad 0.62990564$

C $2.42455657-1.163685150 .90582721$

C $-0.10529965-0.79998094 \quad 0.62672651$

C $-0.22936948-0.48684116 \quad 2.62463854$

C $-0.04105853 \quad 0.90046418 \quad 3.07890177$

C $0.49222697-1.53817744 \quad 3.36973116$

$\mathrm{H}-3.99861133-0.344333360 .49385168$

$\mathrm{H}-2.64539482-0.74789190 \quad 1.62081475$

$\mathrm{H}-2.83486444 \quad-1.658063690 .07176135$

$\mathrm{H}-0.415725162 .32262336-0.63528623$

H $1.71318111 \quad 1.73251618 \quad 0.86650754$

H $1.96418464 \quad 1.09967010 \quad-0.76958308$

H $2.20775138-2.207788261 .17303473$

H $3.08720848-1.191310260 .02477101$

$\mathrm{H} 3.01086472-0.72847578 \quad 1.73012482$

$\mathrm{H}-0.48155181-1.80727374 \quad 0.69661209$

H $0.962491691 .15707869 \quad 3.43457026$

$\mathrm{H}-0.74245540 \quad 1.09094640 \quad 3.90993686$

$\mathrm{H}-0.31326691 \quad 1.62383461 \quad 2.29134118$

$\mathrm{H} \quad 0.48558108-2.496723352 .83384175$

$\mathrm{H}-0.03945332-1.71272175 \quad 4.32143293$

$\mathrm{H} \quad 1.53212869-1.30373408 \quad 3.62653851$

$0.26885518 \quad 1.01647946-2.76311316$

$00.09066643 \quad 0.02817260 \quad-3.29316776$

O $-2.24181707 \quad 0.31374477-0.13719187$

Vibrational frequencies (in $\mathrm{cm}-1$ )

$\begin{array}{llllllllllllllll}-383.5 & 19.1 & 25.8 & 54.6 & 61.5 & 69.5 & 77.0 & 92.7 & 103.2 & 110.2 & 124.5 & 150.4 & 158.0 & 178.1\end{array}$ $203.7215 .1241 .0 \quad 270.6 \quad 332.1 \quad 353.4 \quad 406.8 \quad 456.7 \quad 507.0 \quad 566.7 \quad 633.4 \quad 660.6 \quad 839.2 \quad 897.5$ $926.4 \quad 944.4952 .4 \quad 966.2 \quad 985.0 \quad 999.1 \quad 1016.7 \quad 1041.3 \quad 1050.3 \quad 1075.4 \quad 1106.8 \quad 1116.2 \quad 1124.0$

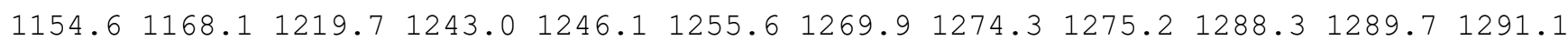

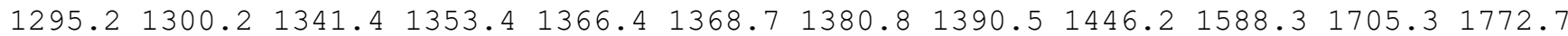
$2639.52655 .02662 .0 \quad 2662.12663 .92666 .7 \quad 2669.82671 .52673 .92744 .12744 .82769 .8$ $2771.3 \quad 2773.7 \quad 2777.3 \quad 2801.9$

\section{name $=$ TS254}

energy $($ in $\mathrm{kcal} / \mathrm{mol})=61.251718$

$\mathrm{ZPE}($ in $\mathrm{kcal} / \mathrm{mol})=140.443$

$\mathrm{G}($ in $\mathrm{kcal} / \mathrm{mol})=115.774$

Geometry

C $-3.18522751 \quad 0.52292831 \quad 2.64958267$

C $-2.30747728 \quad 0.15000173 \quad 1.47794854$

C $-1.58991291 \quad 1.35878963 \quad 0.77861901$

C $-0.11720214 \quad 0.97471260 \quad 0.54556785$

C $0.07913545-0.405803101 .16109959$

C $1.01651002-1.302732150 .41517959$

C $-1.23429834-0.88248046 \quad 1.75306222$

C $-0.08076328-0.51262772 \quad 2.69567064$

C $-0.21834223 \quad 0.70430626 \quad 3.46448232$

C $0.59821160 \quad-1.66765914 \quad 3.40353934$

$\mathrm{H}-4.02622257 \quad 1.15310398 \quad 2.33213166$

$\mathrm{H}-2.61747832 \quad 1.07909731 \quad 3.41118089$

$\mathrm{H}-3.60512444-0.36745515 \quad 3.13320543$

$\mathrm{H}-1.72485667 \quad 2.31408616 \quad 1.30940834$

H $0.56341340 \quad 1.71799722 \quad 0.98944048$

$\mathrm{H} \quad 0.101202220 .95814295-0.54062165$

H $2.03589964 \quad-0.89555491 \quad 0.40369081$

$\mathrm{H} \quad 1.07184302-2.31097603 \quad 0.84460609$ 
$\mathrm{H} \quad 0.69456694-1.42411439-0.63083551$

$\mathrm{H}-1.56335472-1.917803251 .70519154$

$\mathrm{H}-0.419182060 .68806895 \quad 4.50812366$

$\mathrm{H} \quad 0.96657321 \quad 1.66649826 \quad 3.19719546$

$\mathrm{H} 1.36412691 \quad 1.14740629 \quad 3.68529260$

$\mathrm{H} 0.18452206-1.805009864 .41085284$

$\mathrm{H} 1.67564791-1.49427903 \quad 3.50083823$

H $0.46962719-2.62300108 \quad 2.88064961$

$\begin{array}{lllll}0 & -3.43247580 & 0.73045981 & -0.37355359\end{array}$

O $-2.209048231 .45415786-0.50490238$

$0-3.11559139-0.39363607 \quad 0.41852471$

Vibrational frequencies (in $\mathrm{cm}-1$ )

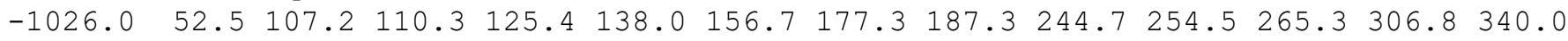

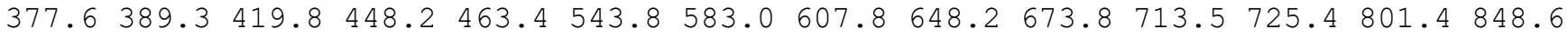
$864.3 \quad 877.8 \quad 949.5 \quad 959.3 \quad 986.2 \quad 987.1 \quad 1000.6 \quad 1004.5 \quad 1024.6 \quad 1042.6 \quad 1064.8 \quad 1080.7 \quad 1097.5$

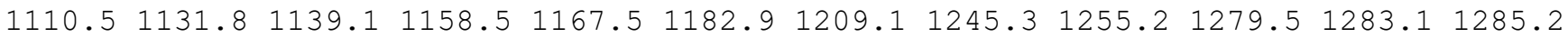

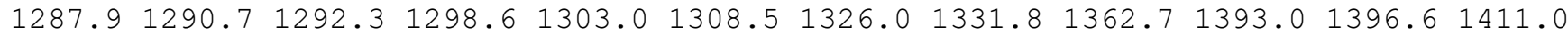
$1494.92666 .02666 .32670 .62671 .6 \quad 2676.92678 .5 \quad 2679.2 \quad 2700.4 \quad 2725.2 \quad 2746.8 \quad 2762.2$ $2779.02780 .22780 .5 \quad 4663.8$

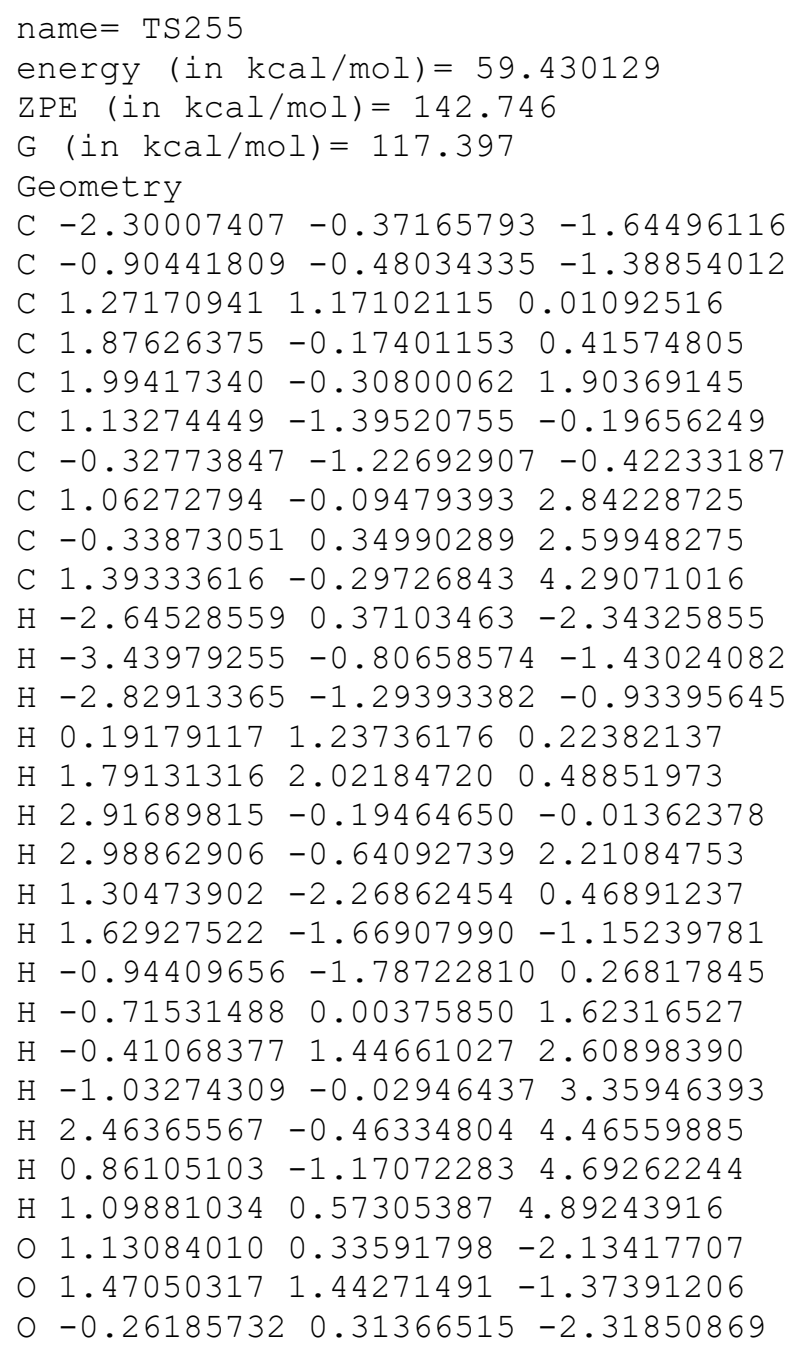




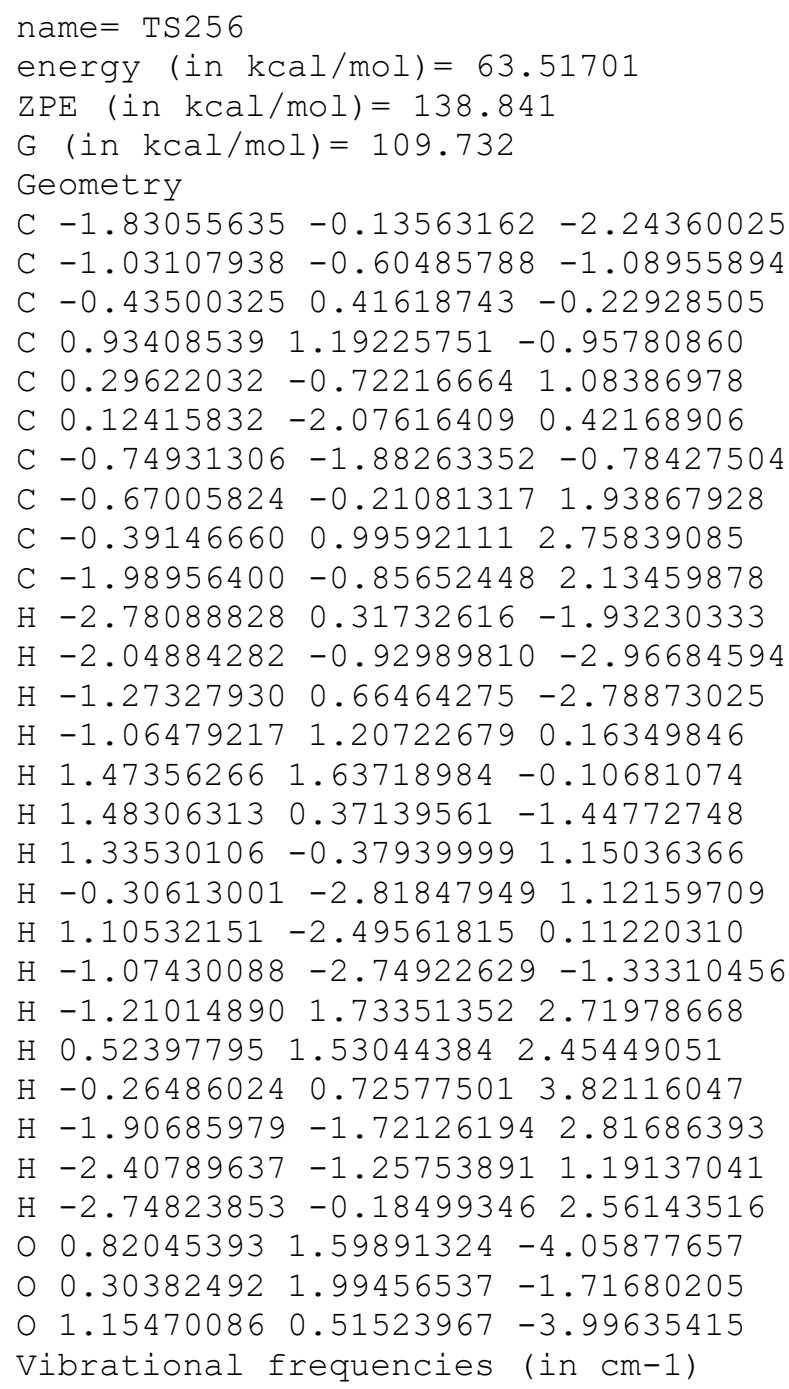


H $2.414632290 .64835509 \quad 1.73854367$

$\mathrm{H} 2.09388774-1.36350936-0.67516073$

$\mathrm{H} 1.75832010-0.69491860-1.04487607$

$\mathrm{H}-0.63248748-1.76288755 \quad 0.81589803$

$\mathrm{H}-0.98752901 \quad 1.896254361 .37566188$

$\mathrm{H}-1.708581290 .84072844 \quad 2.59497710$

$\mathrm{H}-0.32621918 \quad 1.86492524 \quad 3.00409269$

$\mathrm{H} \quad 0.89680846-0.13219692 \quad 3.91029795$

H $1.15448050-1.55737337 \quad 2.89864394$

$\mathrm{H}-0.46826772-1.18231063 \quad 3.49026272$

$\begin{array}{llll}0 & -0.16170439 & 0.11477122 & -2.73839304\end{array}$

$0 \quad 0.68454130 \quad 1.07466779 \quad-2.12318011$

o - $0.40447033-0.83325048-1.71901850$

Vibrational frequencies (in $\mathrm{cm}-1$ )

$\begin{array}{lllllllllllllll}-1182.9 & 103.2 & 125.1 & 171.3 & 173.6 & 192.4 & 207.6 & 234.2 & 271.8 & 282.6 & 323.4 & 355.0 & 370.9 & 412.9\end{array}$ $423.4 \quad 452.2 \quad 482.3 \quad 519.2 \quad 536.0 \quad 596.1 \quad 629.8 \quad 657.7 \quad 691.0 \quad 714.2 \quad 752.7 \quad 769.0 \quad 836.8 \quad 861.3$ $880.2935 .5971 .8 \quad 977.1 \quad 1001.5 \quad 1010.2 \quad 1015.6 \quad 1017.9 \quad 1026.8 \quad 1061.4 \quad 1083.3 \quad 1101.5 \quad 1109.8$

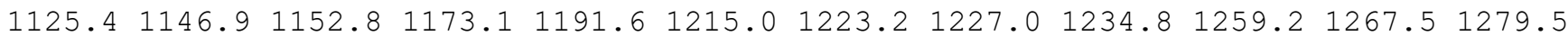

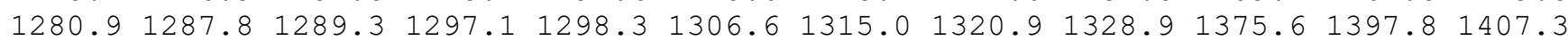
$1413.32653 .5 \quad 2665.12669 .02673 .0 \quad 2674.92676 .72683 .62684 .5 \quad 2736.82749 .4 \quad 2752.6$ $2779.12780 .7 \quad 2782.9 \quad 4407.7$

name $=$ TS258

energy $($ in $\mathrm{kcal} / \mathrm{mol})=63.164869$

$\mathrm{ZPE}(\mathrm{in} \mathrm{kcal} / \mathrm{mol})=139.255$

$\mathrm{G}($ in $\mathrm{kcal} / \mathrm{mol})=113.419$

Geometry

C $-1.64660837-1.994624390 .48100413$

C $-0.51996966-1.091711890 .01235963$

C $0.93592494 \quad 1.03491016-1.81058533$

C $1.95649561 \quad 0.52961033-0.88031772$

C $2.00990712-0.97223925-0.73869366$

C $1.27749608-1.91665153-1.66217752$

C $0.80920088-1.76047760-0.22918267$

C $-0.11544679-0.20716598 \quad 1.72476190$

C $-0.49655855 \quad 0.99872492 \quad 1.76590937$

C $0.56262358-1.153633312 .59920783$

$\mathrm{H}-2.48543121-1.403082250 .86969801$

$\mathrm{H}-1.34351754-2.70549540 \quad 1.25229546$

$\mathrm{H}-2.03109430-2.56682991-0.37576400$

H $\quad 0.62430474 \quad 0.50402479 \quad-2.70266127$

H $1.77777929 \quad 0.986942290 .13531715$

H 2.95536161 $0.92759900-1.18233525$

$\mathrm{H} \quad 2.99514892-1.29933610-0.37445272$

$\mathrm{H} \quad 1.75447060-2.84161050-1.97215866$

$\mathrm{H} \quad 0.64976041-1.53639449-2.46483930$

$\mathrm{H} \quad 1.00074687-2.58967663 \quad 0.45901804$

$\mathrm{H}-0.57821295 \quad 1.96201678 \quad 2.16861199$

$\mathrm{H}-1.29861256 \quad 1.52029338 \quad 0.56828130$

$\mathrm{H}-1.219356491 .12896818-0.15544640$

H $1.62113257-1.27474125 \quad 2.30811919$

H $0.10411190-2.15473306 \quad 2.59404836$

$\mathrm{H} \quad 0.56004912-0.81030744 \quad 3.64849040$

$\begin{array}{lllll}0 & 0.58281773 & 2.84447978 & -0.60668076\end{array}$

o $0.31235924 \quad 2.15707099-1.65672255$

o $-0.85366571-0.17859445-0.79636116$

Vibrational frequencies (in $\mathrm{cm}-1$ )

$\begin{array}{lllllllllllllllll}-1304.8 & 34.7 & 78.9 & 87.0 & 96.3 & 130.7 & 143.0 & 162.7 & 191.7 & 213.7 & 223.6 & 238.3280 .1 & 286.5\end{array}$ $331.7 \quad 348.9 \quad 395.3 \quad 422.7 \quad 433.6 \quad 441.1 \quad 446.8 \quad 501.3 \quad 533.3 \quad 587.4 \quad 613.8 \quad 707.1 \quad 781.8 \quad 804.3$ $810.2828 .3 \quad 857.8 \quad 932.2 \quad 939.5 \quad 968.5 \quad 988.9 \quad 1006.8 \quad 1025.5 \quad 1032.0 \quad 1049.5 \quad 1052.2 \quad 1061.6$

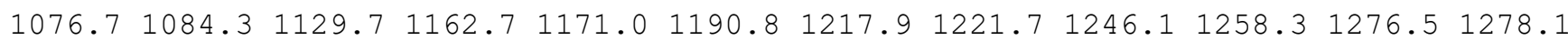
$1282.51288 .2 \quad 1298.1 \quad 1315.5 \quad 1350.0 \quad 1378.0 \quad 1381.8 \quad 1388.4 \quad 1394.8 \quad 1484.4 \quad 1596.91607 .9$ $2066.62616 .02659 .8 \quad 2662.1 \quad 2672.1 \quad 2682.4 \quad 2699.32708 .02719 .52742 .2 \quad 2756.52767 .9$ $2781.72784 .12785 .4 \quad 4156.7$ 


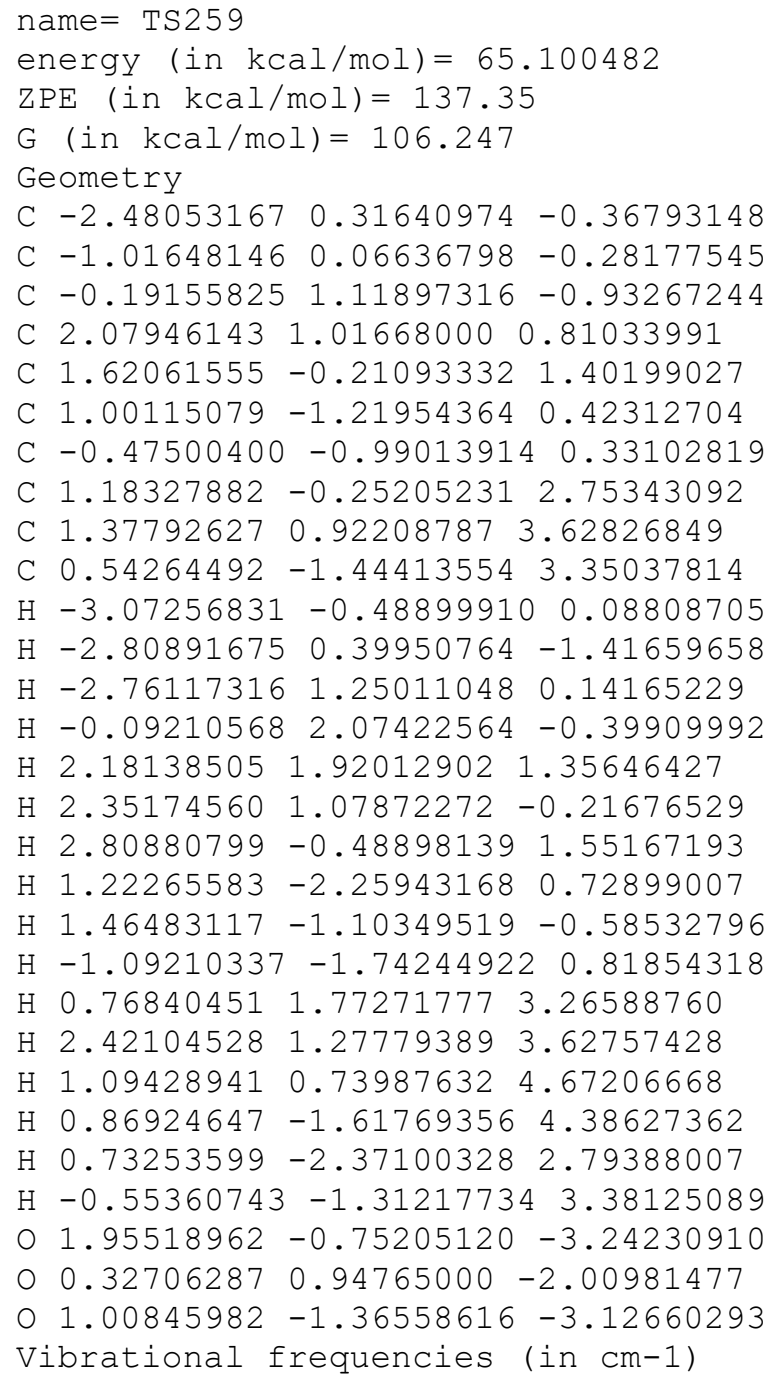

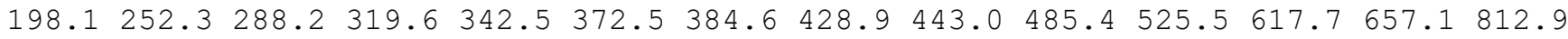
$887.9909 .4 \quad 924.5953 .2 \quad 956.2 \quad 973.9 \quad 976.7 \quad 987.7 \quad 1027.2 \quad 1029.2 \quad 1043.7 \quad 1074.0 \quad 1085.5$

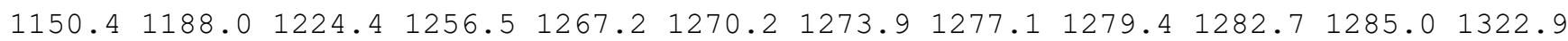
$1335.71372 .3 \quad 1373.9 \quad 1385.2 \quad 1387.7 \quad 1396.4 \quad 1401.91405 .4 \quad 1597.91613 .1 \quad 1827.6 \quad 1879.2$ $2397.12644 .12648 .5 \quad 2653.7 \quad 2657.7 \quad 2666.3 \quad 2669.72672 .32673 .0 \quad 2733.12746 .42753 .6$ $2767.42772 .0 \quad 2777.12801 .3$

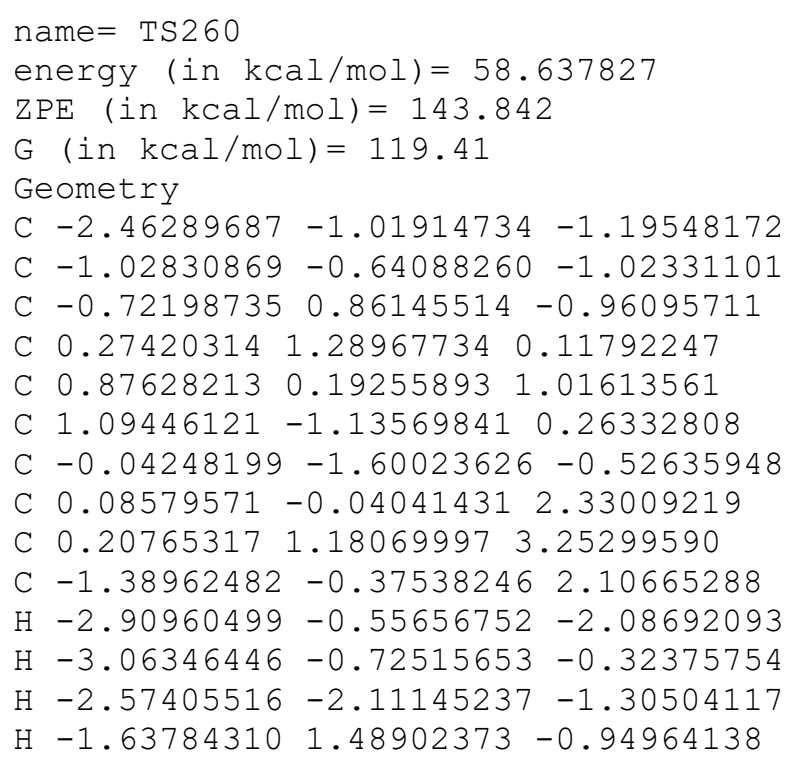


$\mathrm{H}-0.19563376 \quad 2.06018252 \quad 0.76192767$

$\mathrm{H} 1.11145041 \quad 1.81748764-0.39322154$

$\mathrm{H} \quad 0.56079726-0.91163760 \quad 2.85081302$

$\mathrm{H} \quad 1.97914586-1.04811230 \quad-0.40992946$

H $1.89117417 \quad 0.55894756 \quad 1.32831190$

H $1.38054023-1.93704867 \quad 0.98243987$

$\mathrm{H}-0.29453561 \quad 2.05955657 \quad 2.83568170$

$\mathrm{H} 1.25407254 \quad 1.44842189 \quad 3.43251864$

$\mathrm{H}-0.25041795 \quad 0.98061245 \quad 4.22828926$

$\mathrm{H}-1.91076231-0.51849563 \quad 3.05978904$

H $-1.50152747-1.30547458 \quad 1.53397481$

$\mathrm{H}-1.90891827 \quad 0.41967117 \quad 1.56356012$

$\begin{array}{lllll}0 & 0.63223814 & 0.00187099 & -2.54596111\end{array}$

$\begin{array}{llll}0 & -0.16700738 & 1.12724968 & -2.25916895\end{array}$

o - $0.17550220-1.12714353-2.16200415$

Vibrational frequencies (in $\mathrm{cm}-1$ )

$\begin{array}{lllllllllllllllllll}-540.2 & 58.5 & 63.1 & 98.0 & 120.3 & 153.6 & 178.3 & 201.4 & 232.5 & 255.8 & 275.3 & 297.7 & 326.4 & 397.0\end{array}$ $407.2 \quad 439.2 \quad 478.8 \quad 513.8 \quad 555.6 \quad 642.8 \quad 668.2 \quad 753.7 \quad 798.5 \quad 815.4 \quad 836.0 \quad 877.0 \quad 885.4 \quad 929.1$ $960.5976 .3981 .3 \quad 989.6 \quad 1011.0 \quad 1019.8 \quad 1042.3 \quad 1088.8 \quad 1099.6 \quad 1119.4 \quad 1126.6 \quad 1166.7 \quad 1175.5$ $1187.21198 .1 \quad 1233.3 \quad 1245.2 \quad 1253.6 \quad 1262.4 \quad 1269.0 \quad 1273.91281 .1 \quad 1284.91288 .4 \quad 1296.4$ $1299.61305 .1 \quad 1309.2 \quad 1312.2 \quad 1316.5 \quad 1349.8 \quad 1365.4 \quad 1388.01401 .7 \quad 1404.01408 .4 \quad 1466.5$ $2644.8 \quad 2649.4 \quad 2664.92668 .2 \quad 2671.8 \quad 2672.5 \quad 2677.12679 .02682 .92691 .62701 .12728 .5$ $2733.5 \quad 2776.92779 .3 \quad 2781.2$

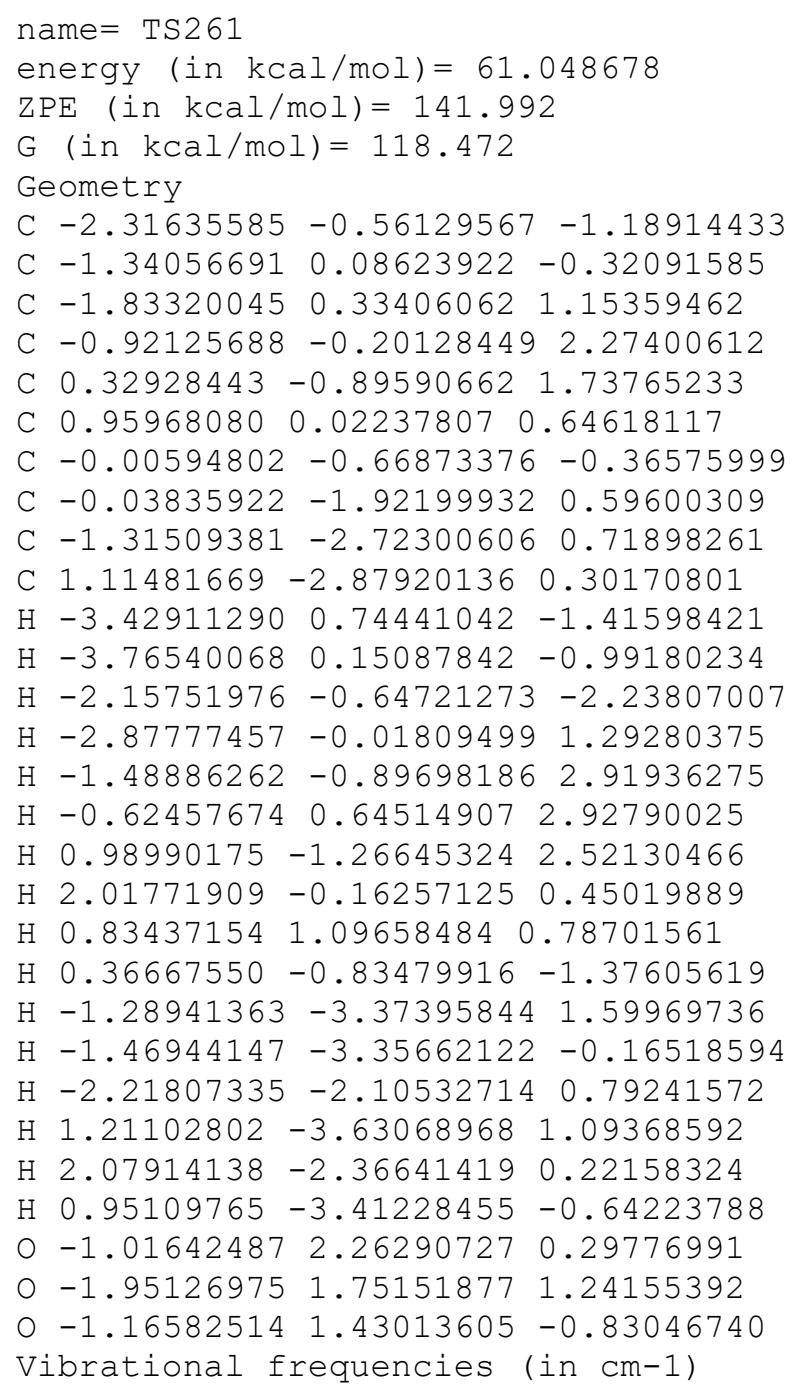
$434.9446 .8 \quad 460.0 \quad 478.8 \quad 489.9 \quad 574.7 \quad 642.2 \quad 656.6 \quad 700.0 \quad 722.3 \quad 799.6 \quad 800.7 \quad 857.9 \quad 862.6$ $893.2916 .2939 .7972 .1981 .3 \quad 992.0 \quad 1004.5 \quad 1007.2 \quad 1017.9 \quad 1020.4 \quad 1049.7 \quad 1066.2 \quad 1087.6$ $1087.91094 .0 \quad 1103.6 \quad 1155.7 \quad 1162.0 \quad 1186.4 \quad 1195.7 \quad 1201.91221 .7 \quad 1247.2 \quad 1260.4 \quad 1272.2$

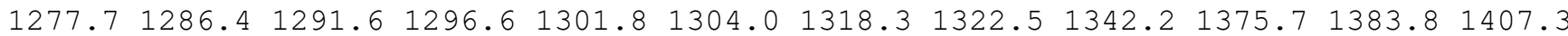


$1420.72659 .6 \quad 2667.2 \quad 2676.5 \quad 2678.6 \quad 2681.6 \quad 2684.02689 .12715 .62740 .02747 .12751 .2$ $2767.5 \quad 2781.8 \quad 2785.1 \quad 4858.4$

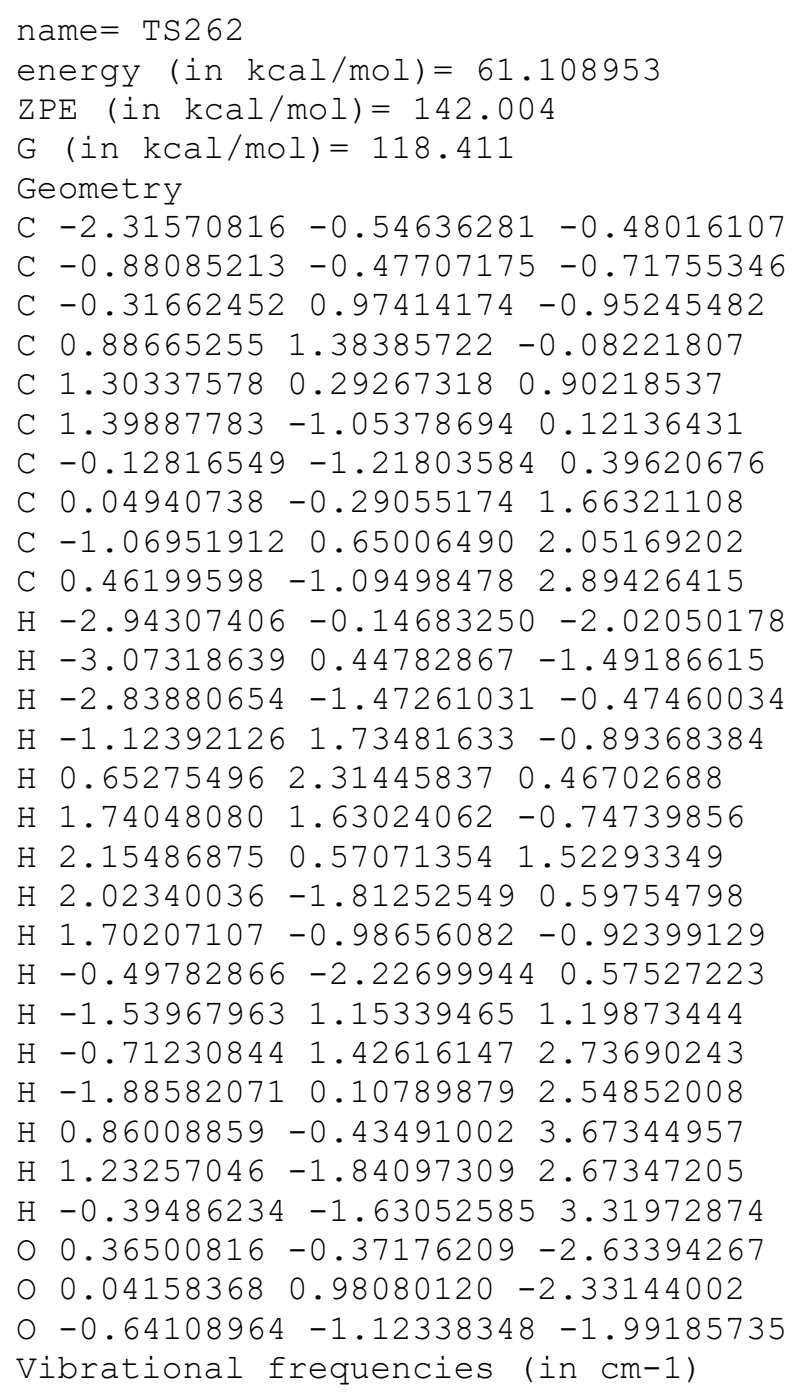
$437.6 \quad 449.1 \quad 461.5 \quad 485.8 \quad 489.1 \quad 576.4 \quad 649.1 \quad 657.5 \quad 699.7 \quad 723.1 \quad 795.9 \quad 800.2 \quad 856.5 \quad 861.9$ $893.1916 .3940 .1981 .6 \quad 982.9 \quad 992.5 \quad 1005.1 \quad 1007.4 \quad 1017.8 \quad 1020.9 \quad 1049.7 \quad 1064.5 \quad 1087.6$ $1089.61102 .6 \quad 1109.2 \quad 1155.2 \quad 1162.6 \quad 1186.8 \quad 1196.6 \quad 1202.1 \quad 1221.5 \quad 1246.91260 .7 \quad 1272.5$

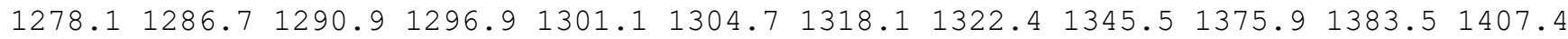
$1421.82659 .72666 .82676 .62678 .8 \quad 2682.02684 .82689 .42719 .02740 .02747 .62751 .5$ $2767.4 \quad 2781.7 \quad 2785.4 \quad 4814.0$

name $=$ TS263

energy (in $\mathrm{kcal} / \mathrm{mol})=63.777129$

$\operatorname{ZPE}(\mathrm{in} \mathrm{kcal} / \mathrm{mol})=139.35$

$\mathrm{G}(\mathrm{in} \mathrm{kcal} / \mathrm{mol})=112.589$

Geometry

C $-0.79861891 \quad 1.92672597-0.14934883$

C $-1.219249360 .45722040-0.14553478$

C $-0.58964958-0.29804383-1.37518399$

C $0.19049206-1.56328007-1.03356932$

C $0.84009195-2.12629773-2.25582577$

C $2.11820437-2.50074703-2.29151341$

C $-0.98570223-0.18177040 \quad 1.17287195$

C $-1.68984550-1.21547460 \quad 1.68773270$

C $-2.83679198-1.88388242 \quad 0.97912947$

C $-1.55034557-1.74748815 \quad 2.96633288$

$\mathrm{H}-1.281033132 .46672224 \quad 0.67610297$

$\mathrm{H}-1.106971832 .42379462 \quad-1.07793374$ 
$\mathrm{H} \quad 0.28345012 \quad 2.04284475-0.03913547$

H $0.01029345 \quad 0.39372363-2.00114562$

$\mathrm{H}-0.49052140-2.32484177-0.59133380$

$\mathrm{H} \quad 0.92861403-1.33752012-0.23679402$

$\mathrm{H} 0.18193968-2.21921618-3.12131013$

H $2.58940331-2.91874373-3.16700392$

H $2.79406062-2.42150024-1.45569939$

$\mathrm{H}-0.17438157 \quad 0.26827578 \quad 1.73401817$

$\mathrm{H}-2.63739099-1.98195306-0.10205356$

$\mathrm{H}-3.74519192-1.26260380 \quad 1.06556657$

$\mathrm{H}-3.07997853-2.87894778 \quad 1.36888202$

$\mathrm{H}-1.35303289-2.71308538 \quad 3.32730020$

$\mathrm{H}-0.62555137-0.70597433 \quad 3.94205346$

$\mathrm{H}-0.03569982-1.105699193 .58748767$

$\begin{array}{lllll}0 & -2.72101737 & 0.24909001 & -1.82119139\end{array}$

$0-1.72578272-0.71554755-2.13948064$

o $-2.63192276 \quad 0.39542150 \quad-0.41813802$

Vibrational frequencies (in $\mathrm{cm}-1$ )

$\begin{array}{lllllllllllllll}-753.0 & 37.1 & 49.2 & 55.7 & 74.3 & 83.2 & 168.2 & 173.9 & 184.9 & 197.5 & 214.6 & 245.3 & 258.3 & 303.3\end{array}$

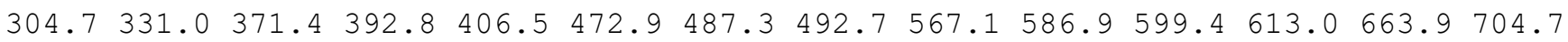
$864.8 \quad 881.8 \quad 889.6 \quad 890.9 \quad 908.8 \quad 962.2 \quad 980.6 \quad 982.0 \quad 998.6 \quad 1014.3 \quad 1040.8 \quad 1049.3 \quad 1064.2$ $1079.91090 .51106 .4 \quad 1139.2 \quad 1148.8 \quad 1179.4 \quad 1189.5 \quad 1224.6 \quad 1255.2 \quad 1266.4 \quad 1280.1 \quad 1281.6$

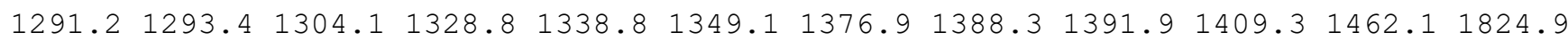
$1850.82647 .8 \quad 2655.0 \quad 2670.2 \quad 2673.5 \quad 2681.2 \quad 2682.8 \quad 2716.82736 .3 \quad 2750.92756 .42764 .0$ $2770.92781 .8 \quad 2790.8 \quad 4979.9$

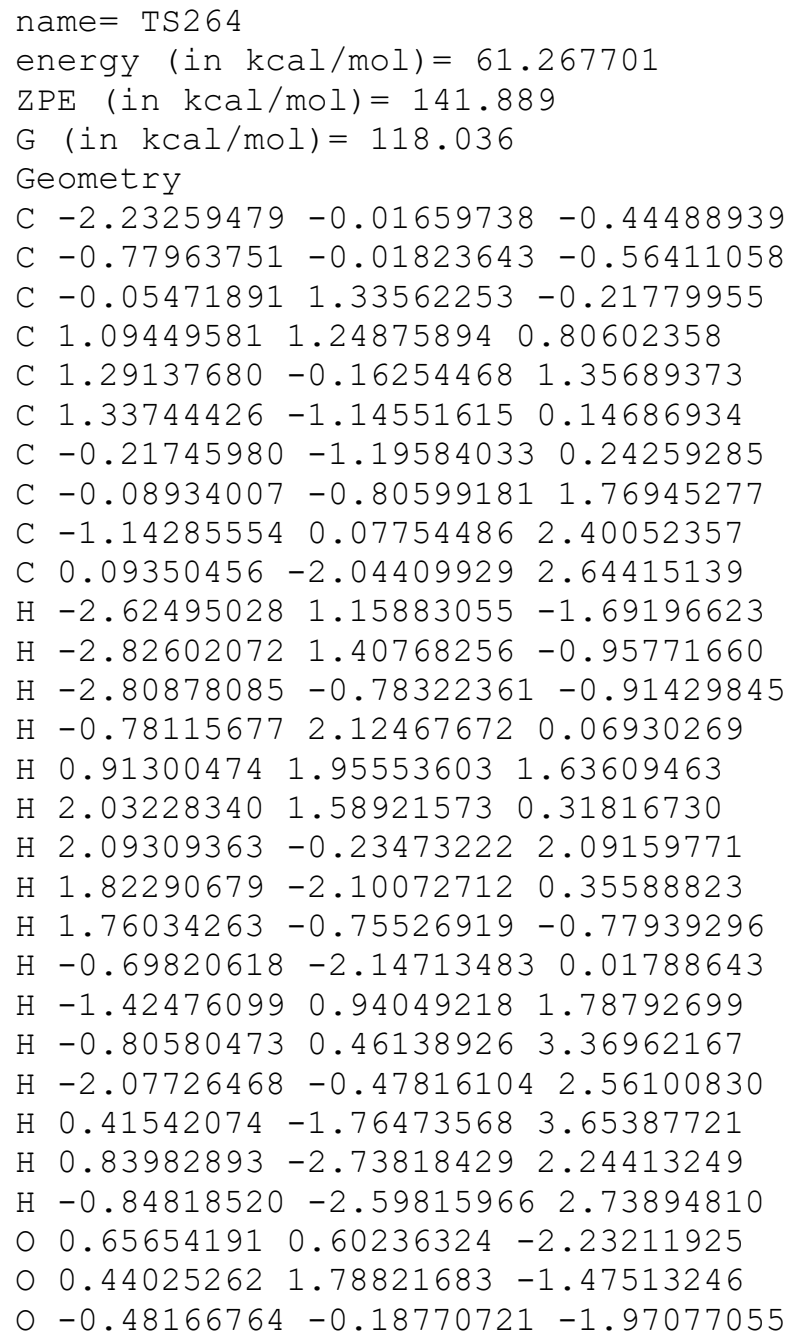




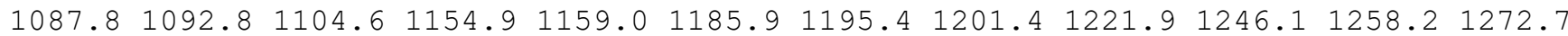

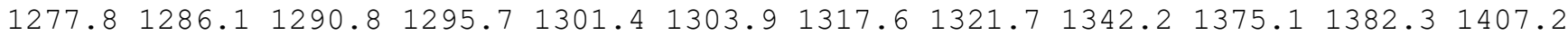
$1421.92659 .02669 .3 \quad 2677.0 \quad 2678.5 \quad 2682.2 \quad 2684.62690 .72708 .62739 .82747 .92751 .5$ $2768.4 \quad 2781.8 \quad 2786.6 \quad 4892.7$

\section{name $=$ TS265}

energy $($ in $\mathrm{kcal} / \mathrm{mol})=61.528739$

$\mathrm{ZPE}($ in $\mathrm{kcal} / \mathrm{mol})=142.029$

$\mathrm{G}($ in $\mathrm{kcal} / \mathrm{mol})=118.253$

Geometry

C $-2.38773500-0.50926172-0.83032492$

C $-0.94121063-0.37029715-0.77199611$

C $-0.39997579 \quad 1.10443390 \quad-0.67854755$

C $0.64119996 \quad 1.37816963 \quad 0.42412071$

C $0.88510116 \quad 0.16257106 \quad 1.31518297$

C $1.15759583-1.064658220 .39055290$

C $-0.38742655-1.26418330 \quad 0.34741761$

C $-0.47472722-0.518972321 .74025004$

C $-1.69268348 \quad 0.33643102 \quad 2.01417962$

C $-0.26987196-1.48148607 \quad 2.90779226$

$\mathrm{H}-2.78051066 \quad 0.35597217 \quad-2.23895346$

$\mathrm{H}-3.05109021 \quad 0.74141578-1.58289009$

$\mathrm{H}-2.84777940-1.39384969-1.20324652$

$\mathrm{H}-1.23016451 \quad 1.83913940-0.63209515$

H $0.32479872 \quad 2.24122688 \quad 1.03802229$

$\mathrm{H} 1.595214811 .68486071-0.05446009$

$\mathrm{H} \quad 1.59883884 \quad 0.34920990 \quad 2.11748966$

Н $1.69800262-1.88390206 \quad 0.86954219$

$\mathrm{H} \quad 1.64945887-0.85670825-0.56037290$

$\mathrm{H}-0.76039289-2.28734294 \quad 0.31495151$

$\mathrm{H}-2.60810579-0.18089654 \quad 1.68215996$

$\mathrm{H}-1.681779621 .296923491 .49286700$

$\mathrm{H}-1.80394774 \quad 0.560679313 .08080692$

$\mathrm{H}-0.05932037-0.93132318 \quad 3.83246572$

$\mathrm{H} \quad 0.56559421-2.17060604 \quad 2.74551304$

$\mathrm{H}-1.16598176-2.08905702 \quad 3.07874968$

$0.62976107 \quad 0.05223973-2.38624353$

O $0.175712871 .33295053-1.96186535$

o $-0.42974711-0.81677721-2.05479576$

Vibrational frequencies (in $\mathrm{cm}-1$ )

$\begin{array}{llllllllllllllll}-874.1 & 34.6 & 112.9 & 157.4 & 165.3 & 180.6 & 194.3 & 217.0 & 253.9 & 275.1 & 308.8 & 346.5 & 381.5 & 415.2\end{array}$

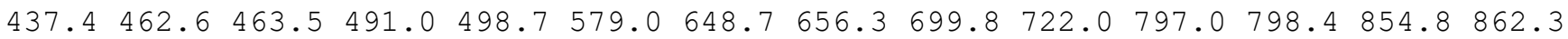
$892.8 \quad 915.3 \quad 941.3 \quad 982.7 \quad 988.2 \quad 999.8 \quad 1005.1 \quad 1011.3 \quad 1017.3 \quad 1023.0 \quad 1048.6 \quad 1061.91086 .8$ $1089.41103 .3 \quad 1117.6 \quad 1153.8 \quad 1160.2 \quad 1186.7 \quad 1196.5 \quad 1200.2 \quad 1222.4 \quad 1246.7 \quad 1259.4 \quad 1272.5$

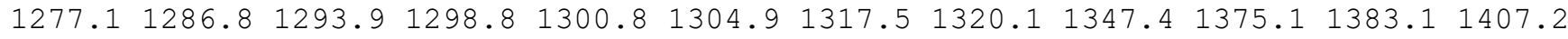
$1424.82658 .3 \quad 2664.12675 .2 \quad 2679.7 \quad 2680.4 \quad 2684.22689 .62719 .12739 .42747 .02751 .0$ $2767.4 \quad 2781.6 \quad 2783.3 \quad 4795.1$

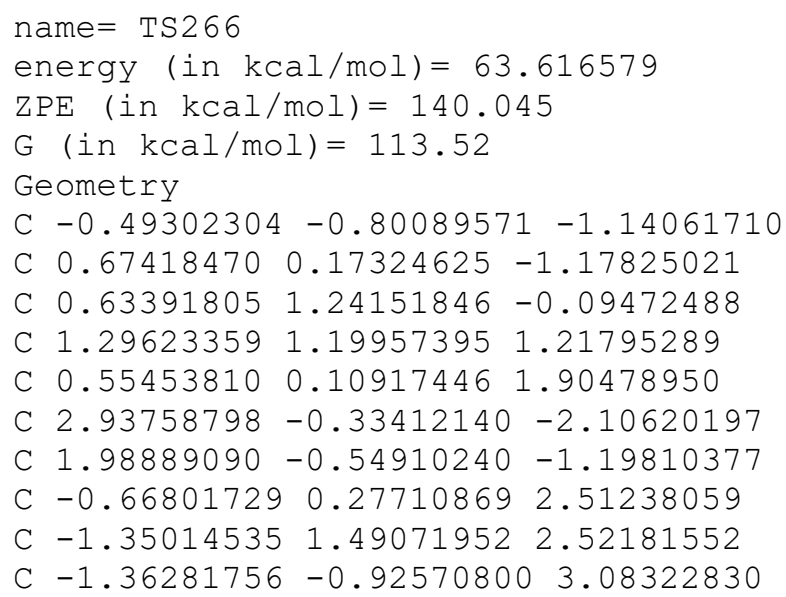


$\mathrm{H}-1.43592296-0.30914430-1.41549656$

$\mathrm{H}-0.62044235-1.23331871-0.13716432$

$\mathrm{H}-0.34747147-1.62843384-1.84735140$

$\mathrm{H}-1.048254231 .32095206 \quad 0.17466670$

$\mathrm{H} \quad 1.23888588 \quad 2.16974729 \quad 1.76053429$

H $2.38642540 \quad 0.99088201 \quad 1.13937437$

$\mathrm{H} 1.03814339-0.85704746 \quad 1.89829449$

$\mathrm{H} \quad 3.87988347-0.86265909-2.11619689$

H $2.85319296 \quad 0.38488539-2.91101720$

$\mathrm{H} 2.09861472-1.27639228-0.39244276$

$\mathrm{H}-1.563351991 .54503952 \quad 0.76432142$

$\mathrm{H}-0.845713412 .436635452 .45712436$

$\mathrm{H}-2.35479331 \quad 1.56980615 \quad 2.89002537$

$\mathrm{H}-1.48540574-0.816258824 .17025612$

$\mathrm{H}-0.81120689-1.85760648 \quad 2.91340586$

$\mathrm{H}-2.36480935-1.052378992 .65375616$

○ $0.037996852 .20165466-1.99800790$

$0 \quad 0.61668295 \quad 2.46826267-0.70490915$

O $0.59338607 \quad 0.96061697-2.38988105$

Vibrational frequencies (in cm-1)

$\begin{array}{lllllllllllllll}-1071.2 & 34.0 & 38.5 & 44.9 & 64.1 & 114.2 & 156.4 & 160.3 & 199.8 & 219.1 & 246.6 & 271.5 & 291.8 & 308.7\end{array}$ $360.1405 .2 \quad 428.4 \quad 461.5 \quad 504.2 \quad 530.4 \quad 539.4 \quad 585.3 \quad 594.6 \quad 660.2704 .3 \quad 713.2 \quad 718.6 \quad 799.8$ $847.0 \quad 865.3 \quad 872.0 \quad 918.7 \quad 959.7 \quad 968.0 \quad 974.7 \quad 981.3 \quad 985.8 \quad 1000.8 \quad 1037.4 \quad 1049.7 \quad 1061.1$ $\begin{array}{llllllllllll}1062.3 & 1097.3 & 1107.3 & 1124.6 & 1168.7 & 1184.2 & 1227.9 & 1251.7 & 1269.5 & 1279.4 & 1282.3 & 1285.3\end{array}$

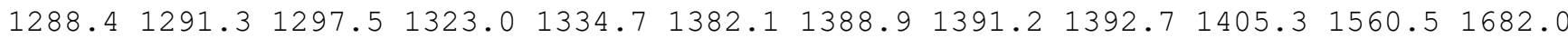
$1850.62649 .0 \quad 2662.92670 .3 \quad 2674.3 \quad 2679.2 \quad 2706.3 \quad 2727.32734 .92749 .6 \quad 2770.7 \quad 2774.8$ $2779.0 \quad 2781.3 \quad 2785.7 \quad 4212.5$

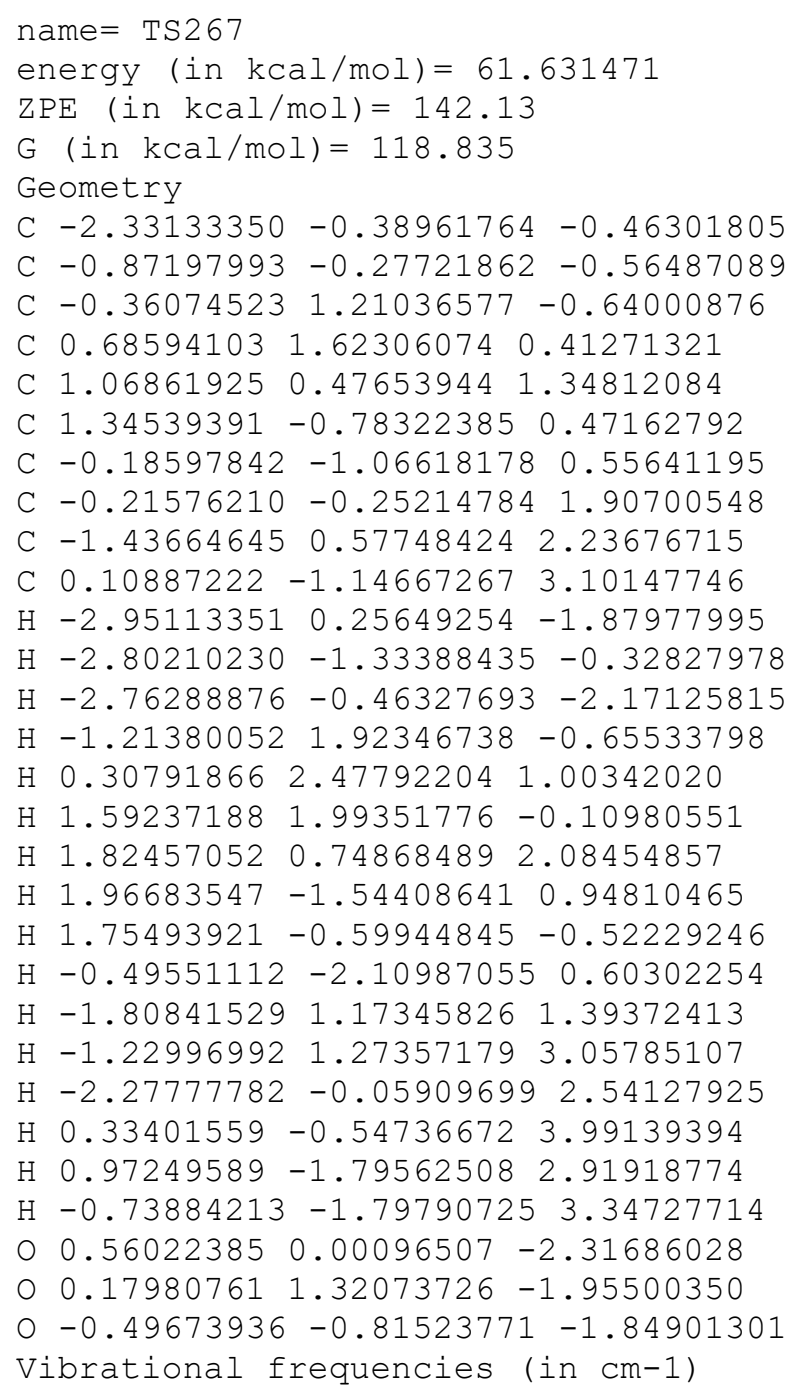


$\begin{array}{lllllllllllllllllll}-665.6 & 79.7 & 121.6 & 160.7 & 168.8 & 183.0 & 208.3 & 219.6 & 253.4 & 273.9 & 303.9 & 351.3 & 380.0 & 415.7\end{array}$

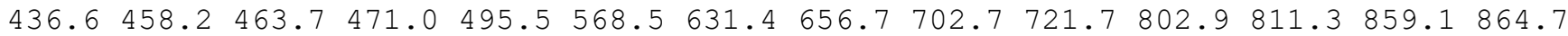
$895.3917 .6 \quad 940.7949 .9 \quad 982.6 \quad 993.0 \quad 1005.3 \quad 1008.9 \quad 1019.1 \quad 1020.8 \quad 1048.2 \quad 1052.8 \quad 1080.5$ $\begin{array}{lllllllllllllll}1089.7 & 1092.5 & 1102.7 & 1156.5 & 1162.8 & 1186.0 & 1194.3 & 1201.9 & 1223.8 & 1246.2 & 1259.9 & 1271.6\end{array}$

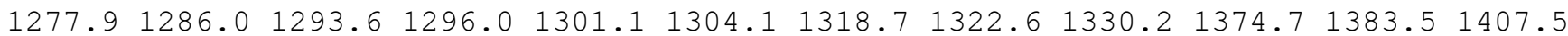
$1420.4 \quad 2659.4 \quad 2664.7 \quad 2674.8 \quad 2677.2 \quad 2680.2 \quad 2682.6 \quad 2688.92710 .82740 .12747 .32751 .1$ $2767.4 \quad 2780.7 \quad 2784.0 \quad 4929.9$

name $=$ TS268

energy (in $\mathrm{kcal} / \mathrm{mol})=63.341289$

$\operatorname{ZPE}($ in $\mathrm{kcal} / \mathrm{mol})=140.457$

$\mathrm{G}($ in $\mathrm{kcal} / \mathrm{mol})=117.373$

Geometry

C $-1.69742536-0.11880735-1.76433228$

C $-0.18219483-0.16046643-1.54480373$

C $0.376216761 .35300228-1.12885654$

C $0.68437256 \quad 1.60712003 \quad 0.35072061$

C $0.980160820 .33262971 \quad 1.14603545$

C $1.62985156-0.67097374 \quad 0.18714907$

C $0.27804092-1.11647238-0.24328971$

C $-0.29720602-0.60661228 \quad 1.12866091$

C $-1.66115482 \quad 0.03063845 \quad 1.24203089$

C $-0.18841815-1.71204608 \quad 2.18071107$

$\mathrm{H}-1.89821079-0.07180892-2.84576447$

$\mathrm{H}-2.19490335 \quad 0.73013948-1.29823557$

$\mathrm{H}-2.16534549-1.03876791-1.39995051$

$\mathrm{H}-0.315897122 .09369816-1.56199063$

H $-0.15910787 \quad 2.15174187 \quad 0.81949979$

H $1.55133425 \quad 2.29637986 \quad 0.42749367$

H $1.44190350 \quad 0.51268749 \quad 2.11582820$

H 2.43333230 $-1.33442044 \quad 0.45837333$

H 2.34852162 $-0.04214321-0.74084782$

$\mathrm{H} \quad 0.08356718-2.13639827-0.55911648$

$\mathrm{H}-1.78959382 \quad 0.90380908 \quad 0.59333200$

$\mathrm{H}-1.85191801 \quad 0.37003382 \quad 2.26887907$

$\mathrm{H}-2.45311636-0.68440647 \quad 0.98135034$

$\mathrm{H}-0.33400705-1.31280718 \quad 3.19084871$

$\mathrm{H} \quad 0.78458280 \quad-2.21443348 \quad 2.16751387$

$\mathrm{H}-0.95159495-2.48276328 \quad 2.01686533$

$\begin{array}{llll}0 & 2.29738488 & 0.47227762 & -1.89517312\end{array}$

O $1.58577205 \quad 1.56710846-1.83826331$

O $0.46363654-0.61981549-2.53270444$

Vibrational frequencies (in cm-1)

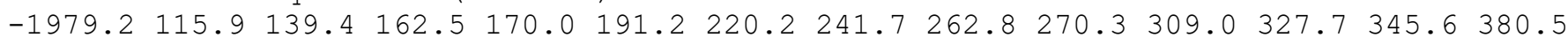

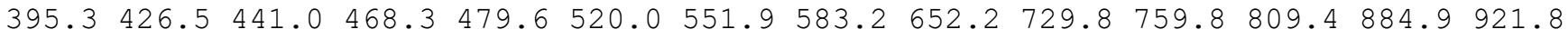
$950.8 \quad 986.9992 .5 \quad 1008.1 \quad 1017.0 \quad 1018.5 \quad 1022.2 \quad 1038.9 \quad 1046.2 \quad 1078.0 \quad 1092.7 \quad 1100.5 \quad 1110.0$

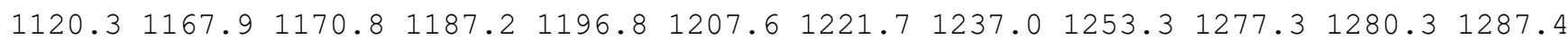
$1291.31298 .1 \quad 1299.91304 .4 \quad 1318.6 \quad 1329.2 \quad 1341.4 \quad 1373.1 \quad 1385.0 \quad 1402.7 \quad 1410.51450 .4$ $1521.52651 .82666 .02671 .2 \quad 2674.0 \quad 2676.3 \quad 2678.82683 .22691 .62731 .82733 .6 \quad 2743.5$ $2751.2 \quad 2778.5 \quad 2781.5 \quad 2782.3$

name $=\operatorname{TS} 269$

energy $($ in $\mathrm{kcal} / \mathrm{mol})=60.201049$

$\mathrm{ZPE}($ in $\mathrm{kcal} / \mathrm{mol})=143.634$

$\mathrm{G}($ in $\mathrm{kcal} / \mathrm{mol})=120.517$

Geometry

C $-2.10954178 \quad 0.20188059-0.70685672$

C $-0.81816914-0.46185094-0.70259683$

C $0.39196768 \quad 0.49475764-1.08883822$

C $1.59887240 \quad 0.49720887-0.13157785$

C $1.43390571-0.505452931 .01041730$

C $0.91703199-1.84763070 \quad 0.40831244$

C $-0.52352716-1.26282866 \quad 0.56786081$ 
C $0.03171519-0.33037616 \quad 1.71282341$

C $-0.517931521 .07151672 \quad 1.87128066$

C $-0.01553868-1.03192593 \quad 3.06926812$

H $-2.30834271 \quad 0.41575619-1.95664700$

$\mathrm{H}-2.65779532 \quad 0.29456665 \quad 0.20236940$

$\mathrm{H}-3.01048050 \quad 0.66979446-1.41709174$

$\mathrm{H} \quad 0.02132101 \quad 1.51966175-1.27527941$

H $1.75523248 \quad 1.51296513 \quad 0.27439785$

$\mathrm{H} \quad 2.51738232 \quad 0.25381166-0.70668527$

H $2.29860488-0.554888951 .67180116$

H $1.11537691-2.73005988 \quad 1.01647186$

$\mathrm{H} \quad 1.23159000-2.06568800-0.61620459$

$\mathrm{H}-1.32296871-1.951160590 .83877895$

$\mathrm{H}-0.64195103 \quad 1.59710485 \quad 0.91744424$

H $0.14323554 \quad 1.68153193 \quad 2.49767723$

$\mathrm{H}-1.507640091 .056705112 .34399373$

$\mathrm{H} \quad 0.40300507 \quad-2.04342968 \quad 3.03122594$

$\mathrm{H}-1.04725058-1.121348693 .42790752$

$\mathrm{H} \quad 0.55157937-0.47163557 \quad 3.82076623$

$0-0.21612080-0.75928696-2.86762960$

$00.87910197-0.02712695-2.32522199$

O $-0.77828708-1.44358047-1.77603297$

Vibrational frequencies (in $\mathrm{cm}-1$ )

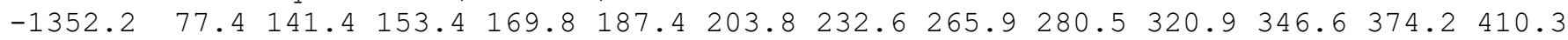
$432.0 \quad 453.5 \quad 486.6 \quad 509.9 \quad 589.4 \quad 625.0 \quad 681.9 \quad 707.0 \quad 716.6 \quad 749.1 \quad 796.6 \quad 837.7 \quad 874.8 \quad 892.6$ $923.2937 .7990 .0994 .5 \quad 1003.2 \quad 1006.0 \quad 1013.6 \quad 1018.2 \quad 1054.4 \quad 1072.6 \quad 1088.7 \quad 1091.1 \quad 1108.5$

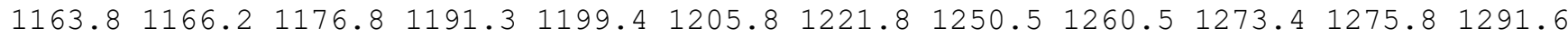

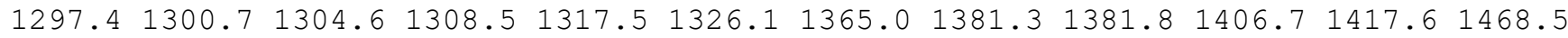
$1917.12659 .8 \quad 2671.2 \quad 2677.2 \quad 2679.4 \quad 2683.4 \quad 2687.2 \quad 2688.52738 .7 \quad 2749.4 \quad 2753.4 \quad 2765.0$ $2776.3 \quad 2781.9 \quad 2784.0 \quad 3689.5$

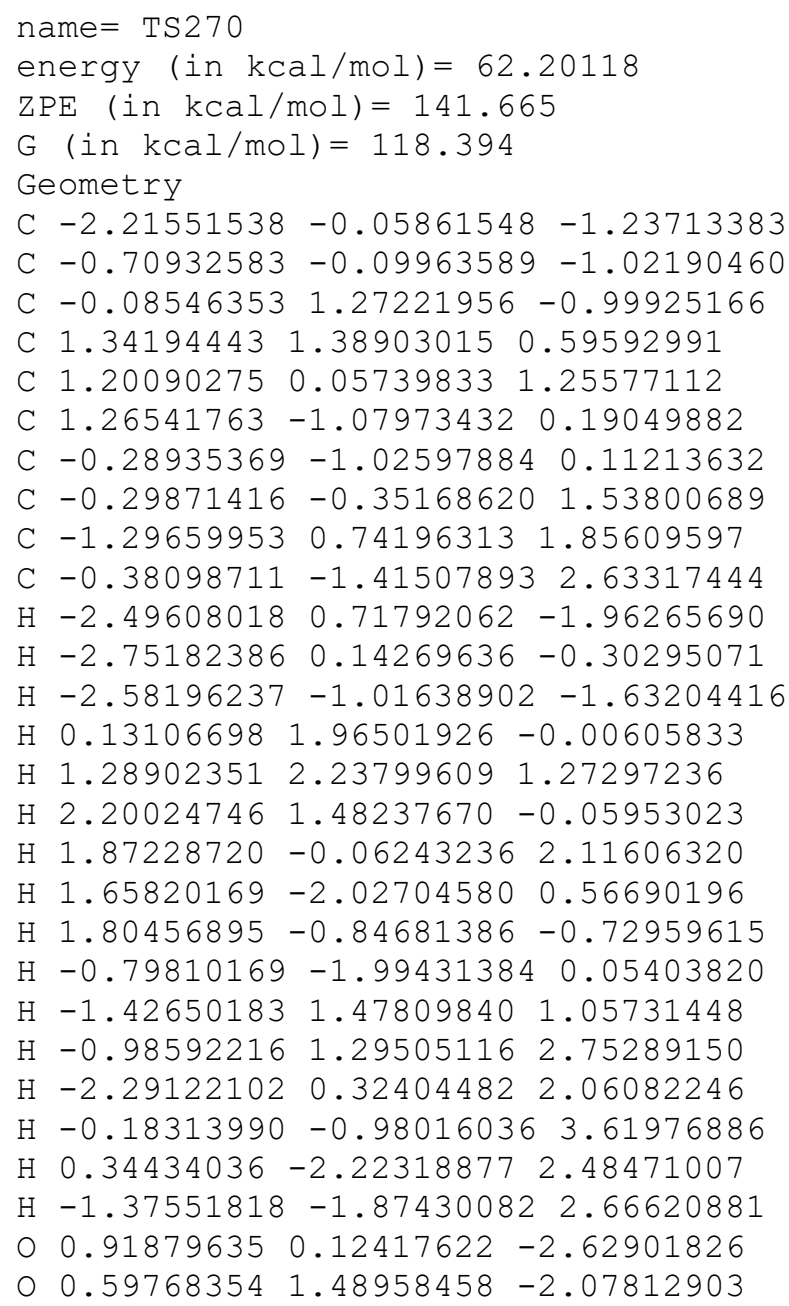


$0-0.19777308-0.63586092-2.28387191$

Vibrational frequencies (in $\mathrm{cm}-1$ )

$\begin{array}{llllllllllllllllll}-1593.1 & 98.4 & 120.9 & 157.8 & 164.6 & 185.9 & 192.4 & 210.1 & 249.4 & 271.7 & 307.4 & 325.8 & 351.3 & 380.8\end{array}$

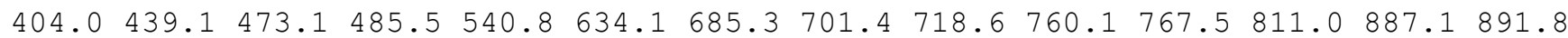

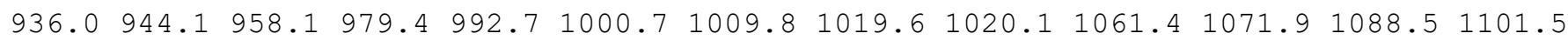

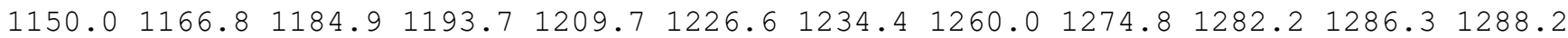
$1297.01300 .1 \quad 1306.1 \quad 1313.1 \quad 1321.2 \quad 1339.4 \quad 1357.1 \quad 1374.11387 .0 \quad 1398.91405 .81416 .8$ $1972.52667 .8 \quad 2670.3 \quad 2674.1 \quad 2674.3 \quad 2675.8 \quad 2679.72685 .82688 .8 \quad 2733.8 \quad 2740.6 \quad 2750.3$ $2766.62778 .92780 .5 \quad 2782.4$

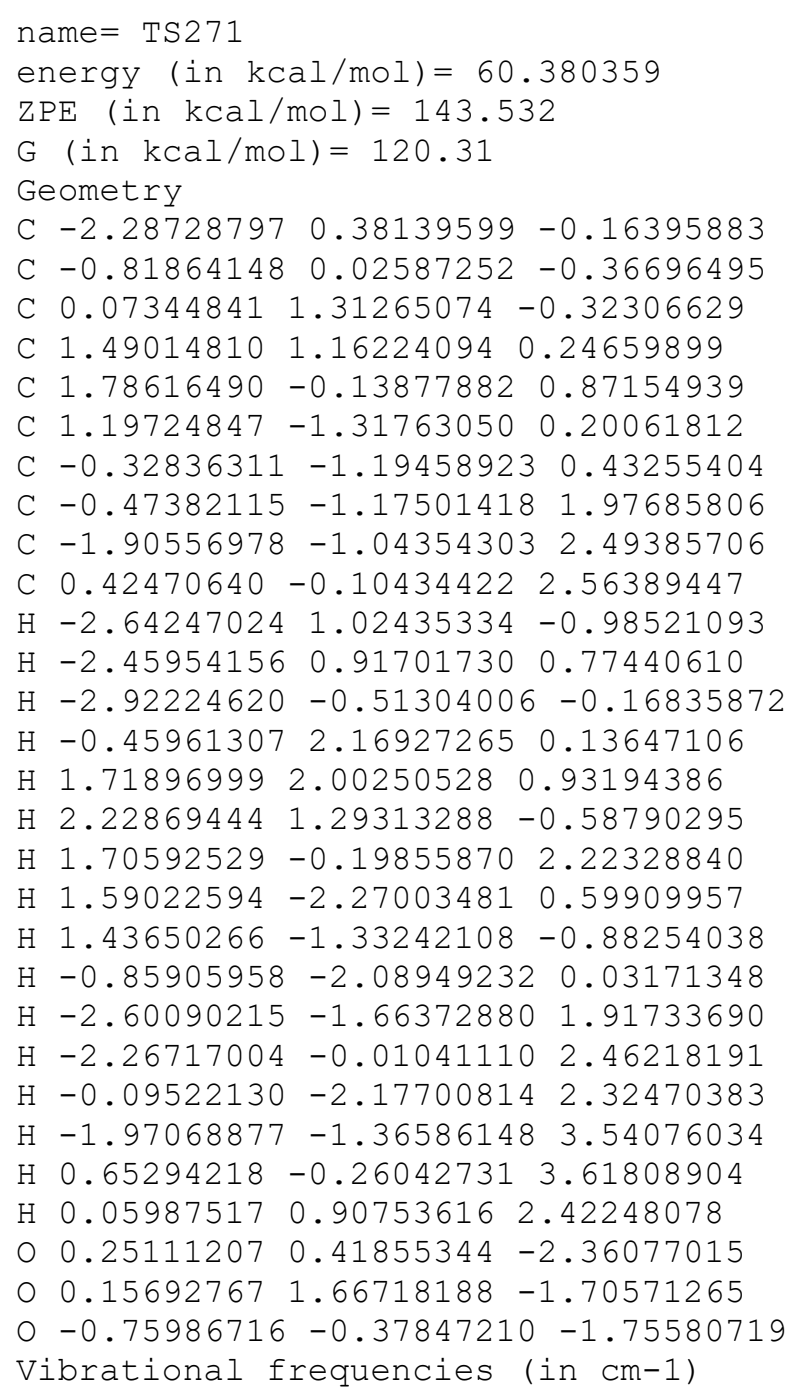


C $1.49022877 \quad 0.08189158 \quad 0.01887433$

C $0.40534223-0.97135688-0.04442160$

C $0.34761569 \quad 0.15743796 \quad 2.22589744$

C $0.54322673 \quad 1.32503013 \quad 3.08680333$

C $-1.57818842-0.635261852 .79261571$

$\mathrm{H}-1.83815068-2.60929594-0.61756504$

$\mathrm{H}-0.43058978-3.37735962-1.37193675$

$\mathrm{H}-1.63924632-2.56283124-2.37804001$

$\mathrm{H} \quad 0.62890580 \quad 2.71192546-0.80143230$

$\mathrm{H}-1.304245611 .06331071-0.63112092$

$\mathrm{H}-1.595824491 .51529948 \quad 1.04539853$

$\mathrm{H}-0.31083362-0.90627008 \quad 2.77943247$

$\mathrm{H} \quad 2.40141651-0.14125303 \quad 0.57328557$

$\mathrm{H} \quad 1.72240414 \quad 0.67348506 \quad-0.87015738$

$\mathrm{H} \quad 0.57014800 \quad-1.87933380 \quad 0.56369475$

$\mathrm{H} \quad 0.45581888 \quad 1.08489189 \quad 4.15530052$

$\mathrm{H}-0.118561892 .181839192 .87646782$

$\mathrm{H} \quad 1.57543535 \quad 1.70103506 \quad 2.94309000$

$\mathrm{H}-1.79393672-1.349263293 .57615135$

$\mathrm{H}-2.00910397-0.92775856 \quad 1.85045227$

$\mathrm{H}-1.90185957 \quad 0.34947373 \quad 3.09024064$

$\begin{array}{lllll}0 & 0.18167448 & 0.62373238 & -2.36939430\end{array}$

$0-0.27362985 \quad 3.64563472 \quad 0.73634913$

o $-0.39978089-0.49769115-2.33904799$

Vibrational frequencies (in cm-1)

$\begin{array}{lllllllllllllll}-1229.1 & 34.1 & 49.0 & 55.6 & 80.5 & 93.2 & 107.2 & 124.7 & 136.9 & 166.9 & 182.3 & 222.2 & 245.5 & 269.6\end{array}$ $270.8 \quad 291.7 \quad 323.8 \quad 381.1 \quad 412.3 \quad 419.9 \quad 470.7 \quad 508.5 \quad 596.6 \quad 615.8 \quad 669.8 \quad 686.0 \quad 769.9 \quad 814.7$ $874.7 \quad 894.3 \quad 942.3 \quad 950.9 \quad 997.5 \quad 1006.1 \quad 1013.7 \quad 1041.1 \quad 1054.11071 .3 \quad 1083.3 \quad 1093.2 \quad 1105.1$

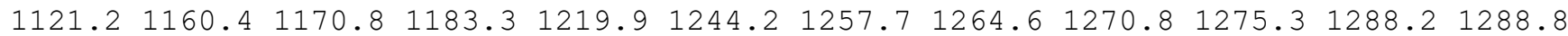

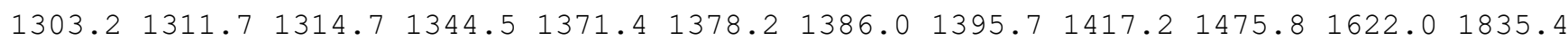
$1842.42645 .3 \quad 2646.62654 .12660 .5 \quad 2665.02668 .7 \quad 2684.52685 .92698 .2 \quad 2736.5 \quad 2743.0$ $2764.82770 .32770 .4 \quad 2778.4$

name $=\operatorname{TS} 273$

energy $($ in $\mathrm{kcal} / \mathrm{mol})=63.982832$

$\operatorname{ZPE}($ in $\mathrm{kcal} / \mathrm{mol})=140.037$

$\mathrm{G}($ in $\mathrm{kcal} / \mathrm{mol})=112.356$

Geometry

C $-2.22958089-0.32366922-1.27511884$

C $-1.31032917-0.06675080-0.18162564$

C $-0.09152928 \quad 1.69367493 \quad-0.54638550$

C $1.11861034 \quad 1.363349250 .34883980$

C $1.02539246-0.036262620 .94479199$

C $0.40757979-0.98607904-0.08726161$

C $-1.14790388-0.76781973 \quad 0.99307092$

C $-0.12371387-0.21217376 \quad 2.00147385$

C $-0.61922778 \quad 1.03831746 \quad 2.70641093$

C $0.26285767-1.26997025 \quad 3.03525869$

$\mathrm{H}-1.76776893-0.03946556-2.24387045$

$\mathrm{H}-3.110084890 .33862027-1.17831197$

$\mathrm{H}-2.59851096-1.35251883-1.35856404$

$\mathrm{H}-0.902104862 .27629692-0.10482022$

$\mathrm{H} 1.22434142 \quad 2.12527046 \quad 1.13856657$

H $2.03303807 \quad 1.44233467-0.27502399$

H $2.00731320-0.36486627 \quad 1.32737456$

$\mathrm{H} \quad 0.53897603-2.05201876 \quad 0.06320645$

$\mathrm{H} \quad 0.54365497-0.73058805-1.14933928$

$\mathrm{H}-1.77931346-1.580410571 .33175628$

$\mathrm{H}-1.02936329 \quad 1.77061364 \quad 1.99842570$

H $0.18508742 \quad 1.52819942 \quad 3.26680909$

$\mathrm{H}-1.42189661 \quad 0.80297909 \quad 3.41620172$

$\mathrm{H}-0.58715659-1.53091715 \quad 3.67683297$

$\mathrm{H} 1.06228188-0.90461482 \quad 3.69133242$

$\mathrm{H} \quad 0.61818086-2.19584422 \quad 2.57106423$ 
O $1.35844021 \quad 0.02397707-3.37210725$

$0.041495921 .59846334-1.75528767$

O $0.33457158 \quad 0.06444853-3.85785838$

Vibrational frequencies (in $\mathrm{cm}-1$ )

$\begin{array}{llllllllllllllll}-664.8 & 23.0 & 40.3 & 57.7 & 75.3 & 92.4 & 112.8 & 138.1 & 149.5 & 161.2 & 179.7 & 198.8 & 231.4 & 241.8\end{array}$ $279.6 \quad 306.5 \quad 325.3 \quad 353.6 \quad 439.7 \quad 446.9 \quad 485.3 \quad 504.0 \quad 530.5 \quad 582.2 \quad 704.1 \quad 838.0 \quad 868.0 \quad 910.1$

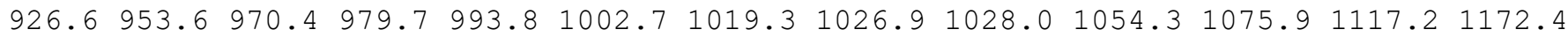

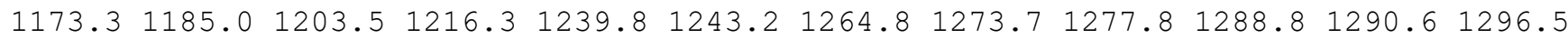

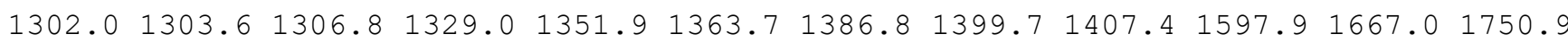
$2630.62647 .02655 .82669 .8 \quad 2670.92674 .72675 .52678 .02680 .62722 .62725 .02732 .0$ $2746.72768 .12780 .3 \quad 2782.9$

name $=$ TS274

energy $($ in $\mathrm{kcal} / \mathrm{mol})=63.103043$

$\mathrm{ZPE}($ in $\mathrm{kcal} / \mathrm{mol})=140.922$

G $($ in $\mathrm{kcal} / \mathrm{mol})=116.864$

Geometry

C 0.000000000 .000000000 .00000000

C $1.46727010 \quad 0.00000000 \quad 0.00000000$

C 2.264435511 .357520050 .00000000

C $3.444460801 .45016577 \quad 0.98803328$

C $3.53803946 \quad 0.23820342 \quad 1.91362218$

C $3.47456891-1.046922821 .03449144$

C $1.92263976-0.932064461 .13586280$

C $2.12051187-0.14199618 \quad 2.49557914$

C $1.16588641 \quad 0.98048786 \quad 2.85528803$

C $2.22624107-1.112606753 .66791303$

$\mathrm{H}-0.330399491 .28377990-1.73025751$

$\mathrm{H}-0.50083107 \quad 0.76444205 \quad 0.57203937$

$\mathrm{H}-0.47655735 \quad 0.59634969-2.03486563$

$\mathrm{H} \quad 1.58611199 \quad 2.22770914 \quad 0.09664404$

H $3.37673764 \quad 2.38176967 \quad 1.57868457$

H 4.387516551 .541126110 .40610005

H $4.35030542 \quad 0.30777966 \quad 2.63845654$

$\mathrm{H} \quad 3.88820070-1.94277467 \quad 1.50279730$

$\mathrm{H} 3.90624854-0.97276536 \quad 0.03486493$

$\mathrm{H} 1.35617201-1.86414856 \quad 1.18575069$

$\mathrm{H} \quad 0.22192896 \quad 0.92231075 \quad 2.30493310$

$\mathrm{H} \quad 1.59939569 \quad 1.97112626 \quad 2.67819569$

$\mathrm{H} \quad 0.89213864 \quad 0.96046429 \quad 3.91994390$

H $2.54926386-0.597157024 .58061260$

H 2.94645605 -1.91857415 3.48657022

$\mathrm{H} 1.25846533-1.585268453 .87507685$

$02.96035084 \quad 0.09714921-1.71925112$

○ $2.748082731 .45037276-1.33627614$

O $1.79784952-0.57810410-1.28196848$

Vibrational frequencies (in $\mathrm{cm}-1$ )

$\begin{array}{lllllllllllllll}-159.5 & 80.8 & 107.9 & 150.5 & 155.6 & 169.9 & 193.5 & 206.7 & 228.5 & 258.6 & 286.2 & 314.9 & 346.0 & 384.4\end{array}$ $\begin{array}{llllllllllllllll}413.9 & 425.4 & 438.8 & 464.8 & 495.7 & 526.8 & 558.7 & 593.9 & 616.9 & 662.7 & 704.4 & 721.4 & 790.7 & 840.8\end{array}$ $865.4 \quad 868.0 \quad 892.1921 .5 \quad 940.6 \quad 981.0 \quad 997.6 \quad 1002.0 \quad 1014.4 \quad 1018.0 \quad 1021.6 \quad 1041.8 \quad 1085.1$ $1086.1 \quad 1098.3 \quad 1106.7 \quad 1133.5 \quad 1152.3 \quad 1182.4 \quad 1188.0 \quad 1198.2 \quad 1219.7 \quad 1242.2 \quad 1255.91269 .9$

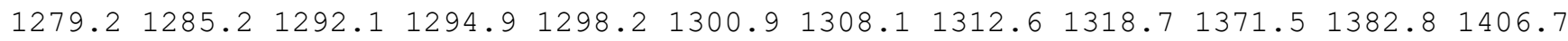
$1426.52646 .22654 .52663 .62672 .02673 .2 \quad 2677.42686 .62689 .72737 .12738 .82747 .9$ $2766.32778 .0 \quad 2781.0 \quad 5466.3$

name $=\operatorname{TS} 275$

energy $($ in $\mathrm{kcal} / \mathrm{mol})=62.194081$

$\mathrm{ZPE}(\mathrm{in} \mathrm{kcal} / \mathrm{mol})=141.935$

$\mathrm{G}($ in $\mathrm{kcal} / \mathrm{mol})=118.641$

Geometry

C $-2.03020971-0.52785819-0.92984607$

C $-0.51487815-0.48206575-0.79382456$

C $0.06739411 \quad 0.97343751-0.71982901$ 
C $1.17025138 \quad 1.204153190 .33100097$

C $1.40914237-0.009898301 .23037496$

C $1.53617336-1.302963130 .44494531$

C $0.02222050-1.38012208 \quad 0.31511297$

C $0.02077234-0.61894230 \quad 1.72214865$

C $-1.09404201 \quad 0.33498210 \quad 2.08491776$

C $0.21822718-1.61057348 \quad 2.86481352$

$\mathrm{H}-2.53658293-0.28512957 \quad 0.00990639$

$\mathrm{H}-2.36200720-1.53391329-1.22638066$

$\mathrm{H}-2.386429190 .16282885-1.70620679$

$\mathrm{H}-0.73737964 \quad 1.73464926-0.63127583$

$\mathrm{H} \quad 0.91376270 \quad 2.08265981 \quad 0.95371580$

H $2.113112321 .48082527-0.18292551$

H $2.15532921 \quad 0.17134104 \quad 2.00643731$

H 2.44674561 $-1.54261227-0.63314484$

H $2.12316358-0.84779445-0.95908525$

H $-0.41451198-2.38105416 \quad 0.30842874$

$\mathrm{H}-2.02747048-0.209739492 .28177988$

$\mathrm{H}-1.31040777 \quad 1.08680291 \quad 1.32290401$

$\mathrm{H}-0.84084614 \quad 0.88527213 \quad 3.00267909$

$\mathrm{H} 0.47005528-1.09779859 \quad 3.79868148$

$\mathrm{H} \quad 1.03660772 \quad-2.31644674 \quad 2.65274459$

$\mathrm{H}-0.68467344-2.20572408 \quad 3.03576009$

○ $0.97176377-0.07899534-2.49349572$

○ $0.57039895 \quad 1.19775097 \quad-2.03385719$

o $-0.04514690-0.97405282-2.06478202$

Vibrational frequencies (in cm-1)

$\begin{array}{lllllllllllllllll}-1223.8 & 87.2 & 111.7 & 149.3 & 167.1 & 181.1 & 208.1 & 216.0 & 256.7 & 278.1 & 321.7 & 332.8 & 375.8 & 400.8\end{array}$ $428.0 \quad 447.4 \quad 478.6 \quad 505.4 \quad 544.9 \quad 569.9 \quad 645.4 \quad 651.3 \quad 679.5 \quad 705.7 \quad 747.1 \quad 768.4 \quad 831.0 \quad 856.5$ $883.3945 .6 \quad 969.1 \quad 976.5 \quad 998.1 \quad 1005.7 \quad 1014.3 \quad 1019.2 \quad 1025.9 \quad 1052.1 \quad 1084.2 \quad 1093.2 \quad 1117.1$

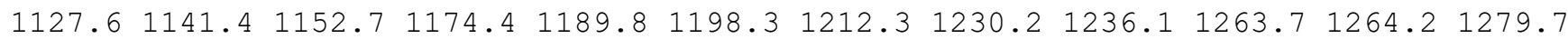

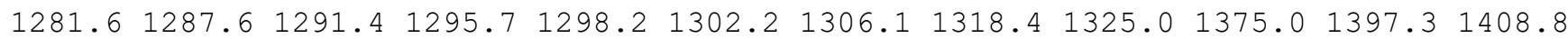
$1415.72655 .42664 .6 \quad 2667.92672 .3 \quad 2675.1 \quad 2676.92682 .52683 .5 \quad 2739.02748 .92752 .3$ $2778.82780 .02783 .3 \quad 4421.8$

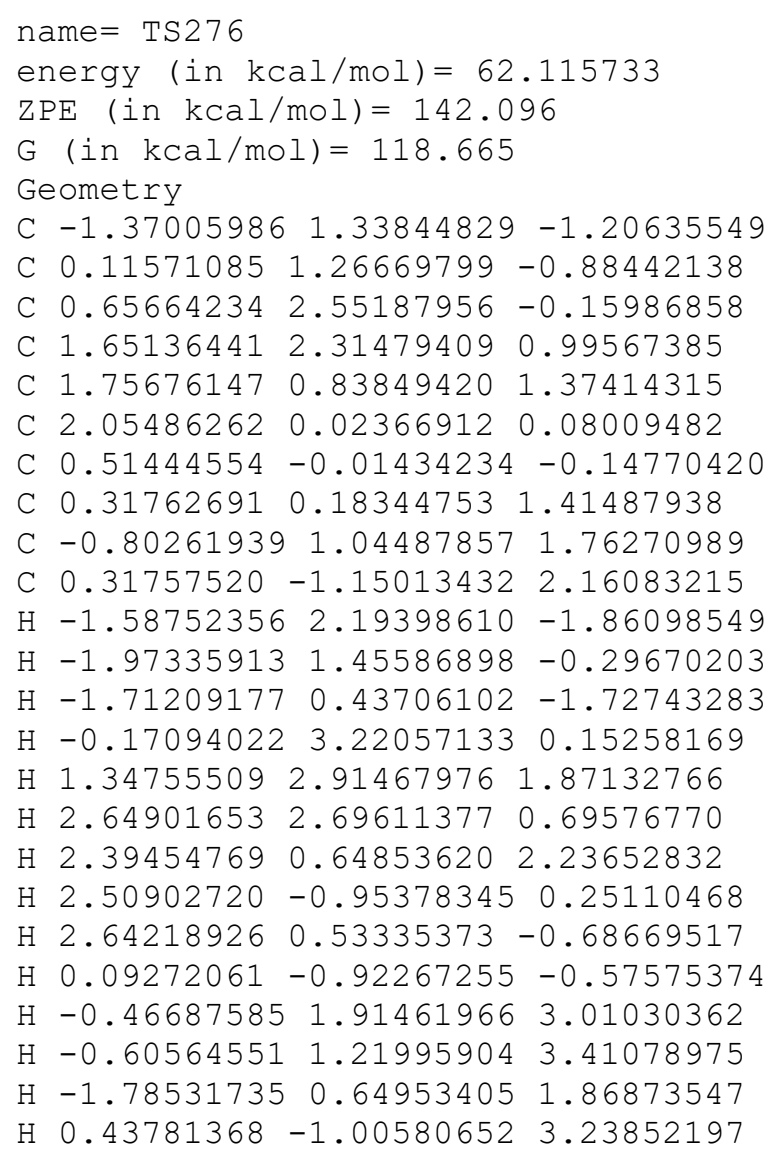


H $1.12959426-1.810294151 .83411287$

$\mathrm{H}-0.62037177-1.692180351 .99440905$

O $1.819361092 .23878969-2.05258624$

$01.304153403 .25916725-1.21706728$

O $0.76079777 \quad 1.29547420-2.16912913$

Vibrational frequencies (in $\mathrm{cm}-1$ )

$\begin{array}{llllllllllllllll}-993.1 & 69.0 & 133.3 & 134.9 & 161.1 & 177.8 & 194.5 & 204.4 & 258.4 & 269.0 & 320.2 & 339.4 & 373.2 & 395.3\end{array}$ $420.1 \quad 456.5 \quad 464.9 \quad 513.9 \quad 531.3 \quad 575.7 \quad 650.7 \quad 658.2 \quad 707.2 \quad 726.1 \quad 811.7 \quad 848.2865 .1868 .8$

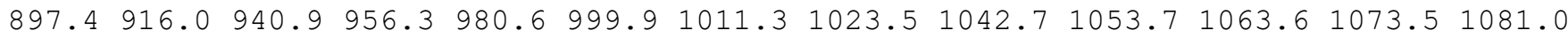

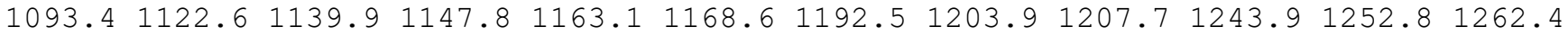

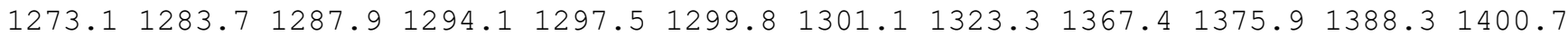
$1410.52663 .0 \quad 2669.0 \quad 2673.5 \quad 2676.4 \quad 2680.6 \quad 2689.3 \quad 2692.4 \quad 2716.72742 .4 \quad 2747.2 \quad 2750.6$ $2767.2 \quad 2779.4 \quad 2781.5 \quad 4696.7$

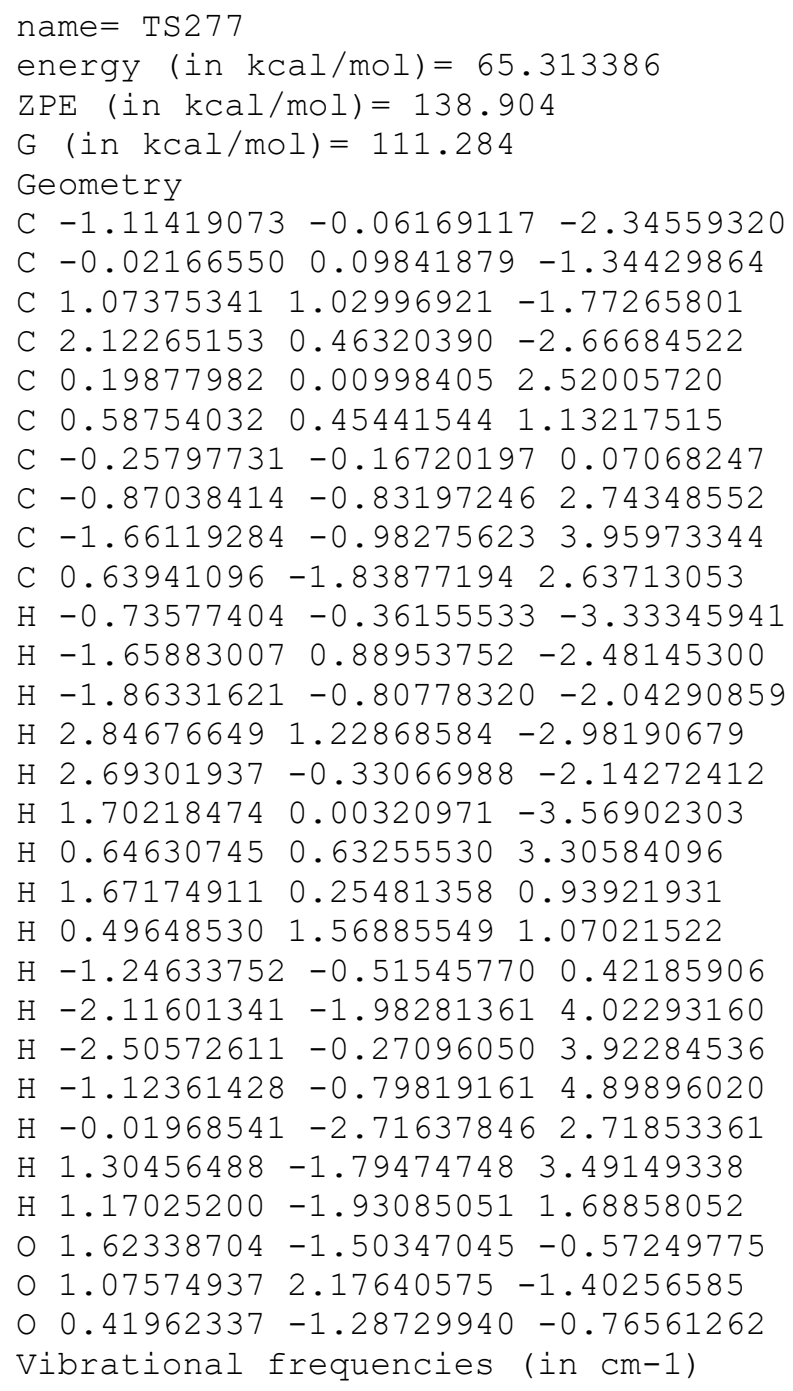
$275.4280 .1 \quad 311.9 \quad 349.7 \quad 407.6 \quad 472.0 \quad 526.8 \quad 550.0 \quad 589.7 \quad 612.6 \quad 626.8 \quad 655.9 \quad 721.2 \quad 822.4$ $872.9900 .7 \quad 957.0 \quad 980.2 \quad 997.3 \quad 1022.9 \quad 1025.7 \quad 1041.2 \quad 1052.9 \quad 1059.4 \quad 1076.9 \quad 1091.4 \quad 1127.8$ $1175.51187 .1 \quad 1198.4 \quad 1236.6 \quad 1244.8 \quad 1257.0 \quad 1264.9 \quad 1268.1 \quad 1268.4 \quad 1272.6 \quad 1277.7 \quad 1283.1$ $1288.71290 .61293 .3 \quad 1340.6 \quad 1350.6 \quad 1365.6 \quad 1378.4 \quad 1388.7 \quad 1398.6 \quad 1504.2 \quad 1670.21851 .0$ $2620.02626 .42635 .92658 .8 \quad 2666.12666 .6 \quad 2668.52672 .2 \quad 2672.62676 .02698 .02712 .3$ $2748.52773 .3 \quad 2774.2 \quad 2775.1$

name $=\operatorname{TS} 278$

energy (in $\mathrm{kcal} / \mathrm{mol})=62.201895$

$\mathrm{ZPE}($ in $\mathrm{kcal} / \mathrm{mol})=142.117$

$\mathrm{G}($ in $\mathrm{kcal} / \mathrm{mol})=118.757$

Geometry

C $-2.81546477-0.34297024-0.48568460$ 
C $-1.477667590 .22716525 \quad-0.43515307$

C $-1.596840391 .73488477 \quad 0.03349153$

C $-0.70041235 \quad 2.14318449 \quad 1.21803384$

C $0.18986952 \quad 1.00478038 \quad 1.71431691$

C $0.84842777 \quad 0.33002505 \quad 0.47378536$

C $-0.44054320-0.546347810 .37760243$

C $-0.64988233-0.313940951 .92856861$

C $-2.04869964-0.18780503 \quad 2.48970537$

C $0.11680939-1.347570512 .75181394$

$\mathrm{H}-3.42994278-0.24216305-1.35101883$

$\mathrm{H}-2.79467403-1.91915357-0.08506988$

$\mathrm{H}-2.51858507-1.99342396-0.83649816$

$\mathrm{H}-2.65636609 \quad 2.02149925 \quad 0.21765104$

$\mathrm{H}-1.32671071 \quad 2.51833204 \quad 2.04885122$

$\mathrm{H}-0.06726803 \quad 2.99966547 \quad 0.90446448$

$\mathrm{H} \quad 0.84717695 \quad 1.29419555 \quad 2.53415823$

$\mathrm{H} 1.75715706-0.23482568 \quad 0.69064806$

H $1.06297464 \quad 0.98162313-0.37407999$

$\mathrm{H}-0.30366156-1.57592511 \quad 0.05163141$

$\mathrm{H}-2.51883316-1.17016288 \quad 2.62037281$

$\mathrm{H}-2.73106086 \quad 0.38051442 \quad 1.84374668$

$\mathrm{H}-2.03469053 \quad 0.307851393 .46709519$

H $1.15529143-1.462945312 .42345595$

$\mathrm{H}-0.35593985-2.33377698 \quad 2.68738282$

H $0.14257715-1.06224534 \quad 3.80999988$

$0-0.406442351 .62403811-1.89070355$

$0-1.257180252 .47673066-1.13338024$

o $-1.01203399 \quad 0.35327600 \quad-1.80029694$

Vibrational frequencies (in $\mathrm{cm}-1$ )

$\begin{array}{llllllllllllll}-835.8 & 77.9 & 119.6 & 146.1 & 152.0 & 174.5 & 211.0 & 217.6 & 262.9 & 280.7 & 313.6 & 348.1 & 379.7 & 415.0\end{array}$

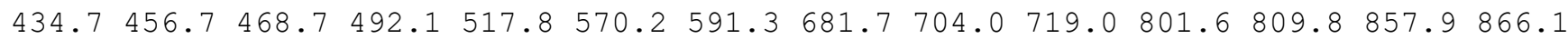
$891.5921 .9939 .1973 .0 \quad 976.4 \quad 990.3 \quad 1002.2 \quad 1006.5 \quad 1015.3 \quad 1018.8 \quad 1047.2 \quad 1068.4 \quad 1087.6$ $1090.61097 .21101 .0 \quad 1156.3 \quad 1171.8 \quad 1181.3 \quad 1197.6 \quad 1206.4 \quad 1220.8 \quad 1245.7 \quad 1257.2 \quad 1275.8$ $1280.61288 .8 \quad 1292.8 \quad 1296.3 \quad 1302.0 \quad 1304.2 \quad 1317.9 \quad 1322.3 \quad 1351.2 \quad 1374.3 \quad 1384.2 \quad 1407.2$ $1420.32657 .62663 .92675 .5 \quad 2676.3 \quad 2678.8 \quad 2683.62688 .32713 .2 \quad 2739.02747 .52751 .2$ $2767.42781 .92784 .2 \quad 4851.2$

name $=$ TS279

energy (in $\mathrm{kcal} / \mathrm{mol})=65.012383$

$\mathrm{ZPE}(\mathrm{in} \mathrm{kcal} / \mathrm{mol})=139.31$

$\mathrm{G}($ in $\mathrm{kcal} / \mathrm{mol})=110.202$

Geometry

C $-2.02188882-1.07528440-1.54924149$

C $-0.93403692-0.82035901-0.56386800$

C $-0.70736809 \quad 0.67818654 \quad-0.36883234$

C $0.92733670 \quad 0.72985733 \quad 0.41554267$

C $1.19813946-0.31863874 \quad 1.49169959$

C $0.88394301-1.723160110 .96806592$

C $-0.28411188-1.82212243 \quad 0.03788535$

C $0.22291803 \quad 0.29356702 \quad 2.39263415$

C $0.64753076 \quad 1.43676779 \quad 3.23058470$

C $-1.15820135-0.14909330 \quad 2.54478584$

$\mathrm{H}-2.18091260-2.13508772-1.76828337$

$\mathrm{H}-1.76841298-0.56471009-2.50253313$

$\mathrm{H}-2.97592146-0.64114958-1.22332023$

$\mathrm{H}-1.35400974 \quad 1.08012436 \quad 0.45771740$

H $1.16997107 \quad 1.759168320 .61406959$

H $1.41575760 \quad 0.43703153-0.52629998$

H $2.25050997-0.28322352 \quad 1.85770949$

H $0.75169321-2.41807673 \quad 1.82241174$

$\mathrm{H} \quad 1.78184119-2.097078790 .41918560$

$\mathrm{H}-0.55474571-2.85656851-0.18046104$

$\mathrm{H} 0.703508121 .14888281 \quad 4.29632712$

$\mathrm{H}-0.06049944 \quad 2.28502234 \quad 3.17663549$ 
H 1.639022901 .842642662 .96308736

$\mathrm{H}-1.624207790 .10994484 \quad 3.50474522$

$\mathrm{H}-1.30834565-1.229650462 .35638662$

$\mathrm{H}-1.80184675 \quad 0.34736593 \quad 1.74796891$

○ $0.93215491 \quad 1.14689043-3.36932832$

$0-0.65678187 \quad 1.38664792-1.39676688$

o $0.95467585 \quad 0.05013847-3.08208639$

Vibrational frequencies (in cm-1)

$\begin{array}{llllllllllllllll}-459.6 & 15.0 & 29.9 & 42.7 & 55.5 & 59.7 & 75.9 & 113.2 & 118.3 & 126.7 & 187.4 & 218.3 & 245.6 & 258.3\end{array}$

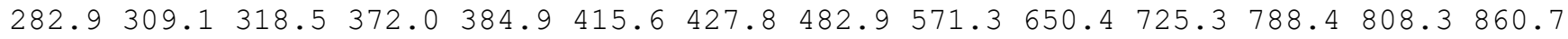
$893.5910 .1941 .2988 .8 \quad 997.6 \quad 1013.4 \quad 1033.1 \quad 1038.4 \quad 1070.2 \quad 1073.6 \quad 1117.3 \quad 1133.1 \quad 1140.0$

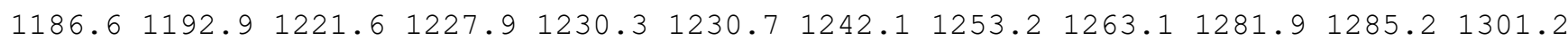
$1309.8 \quad 1326.8 \quad 1348.2 \quad 1369.5 \quad 1378.8 \quad 1386.7 \quad 1402.6 \quad 1415.1 \quad 1478.5 \quad 1597.6 \quad 1600.51875 .7$ $2382.72560 .22640 .92646 .32648 .6 \quad 2649.5 \quad 2653.72654 .02684 .42703 .12732 .12743 .1$ $2750.5 \quad 2758.0 \quad 2764.4 \quad 2775.4$

name $=\operatorname{TS} 280$

energy (in $\mathrm{kcal} / \mathrm{mol})=65.092637$

$\mathrm{ZPE}($ in $\mathrm{kcal} / \mathrm{mol})=139.292$

$\mathrm{G}($ in $\mathrm{kcal} / \mathrm{mol})=111.608$

Geometry

C $-2.38517593 \quad 0.28414178-0.87246201$

C $-1.08267326 \quad 0.00871797-0.26668077$

C $0.10001984 \quad 0.80136079-0.92708648$

C $1.117340361 .40764707 \quad 0.03800038$

C $0.72807593 \quad 2.77118532 \quad 0.50383986$

C $1.19642478 \quad 3.306796551 .63148278$

C $-1.15166037 \quad 0.12174536 \quad 1.21968281$

C $-1.74483926 \quad 1.09469678 \quad 1.92461758$

C $-2.46812902 \quad 2.25621386 \quad 1.33747988$

C $-1.70986954 \quad 1.07695386 \quad 3.42002585$

$\mathrm{H}-2.232154590 .28554749-2.47811330$

$\mathrm{H}-3.23983841-0.30465164-0.63659892$

$\mathrm{H}-2.18915109-0.51264547-2.38157783$

$\mathrm{H}-0.26839628 \quad 1.54674145-1.66274977$

$\mathrm{H} \quad 2.10536772 \quad 1.46407432-0.47735738$

$\mathrm{H} \quad 1.29087564 \quad 0.72212453 \quad 0.89482375$

$\mathrm{H} 0.040901113 .31365655-0.14552028$

$\mathrm{H} \quad 0.93816509 \quad 4.29601928 \quad 1.97136866$

$\mathrm{H} \quad 1.87175497 \quad 2.80072382 \quad 2.30222496$

$\mathrm{H}-0.65054647-0.70947483 \quad 1.72180592$

$\mathrm{H}-2.34876875 \quad 2.32876394 \quad 0.24215286$

$\mathrm{H}-2.12049843 \quad 3.20690424 \quad 1.76300194$

$\mathrm{H}-3.549085592 .18450411 \quad 1.52522651$

$\mathrm{H}-1.06424091 \quad 1.88236355 \quad 3.80132517$

$\mathrm{H}-1.32404127 \quad 0.133696813 .82849926$

$\mathrm{H}-2.70992147 \quad 1.23059149 \quad 3.84811308$

O $0.59149532-1.38689376-0.97545608$

$00.75261041-0.18706823-1.72363113$

$0-0.76880026-1.37369230-0.60995515$

Vibrational frequencies (in $\mathrm{cm}-1$ )

$\begin{array}{llllllllllllllll}-806.8 & 26.0 & 35.8 & 41.2 & 64.6 & 72.7 & 102.4 & 117.0 & 173.6 & 195.0 & 199.9 & 241.9 & 277.8 & 299.4\end{array}$

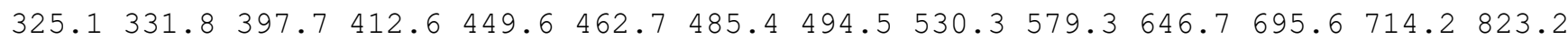
$846.2864 .0 \quad 909.4 \quad 925.0 \quad 970.9 \quad 976.5 \quad 987.5 \quad 993.1997 .6 \quad 1014.4 \quad 1025.1 \quad 1058.9 \quad 1075.7$

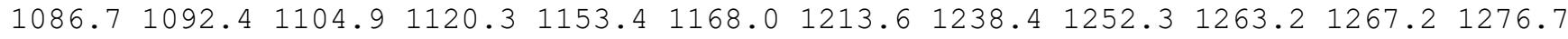

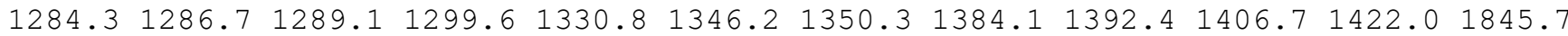
$1873.0 \quad 2648.2 \quad 2649.2 \quad 2669.5 \quad 2674.6 \quad 2675.92683 .4 \quad 2717.92718 .12731 .4 \quad 2744.22757 .3$ $2774.3 \quad 2779.92792 .5 \quad 4831.2$

name $=\operatorname{TS} 281$

energy $($ in $\mathrm{kcal} / \mathrm{mol})=64.711312$

$\mathrm{ZPE}(\mathrm{in} \mathrm{kcal} / \mathrm{mol})=139.695$

$\mathrm{G}($ in $\mathrm{kcal} / \mathrm{mol})=111.075$ 
Geometry

C $-1.65270889-0.45462935-1.58902865$

C $-0.59298258-0.49910989-0.54938154$

C $-0.04500753 \quad 0.87754688-0.19393488$

$\begin{array}{lllll}\text { C } & 1.38411606 & 0.76255531 & 0.37282864\end{array}$

C $1.38513899-0.49286003 \quad 1.69298926$

C $0.94286927-1.77141376 \quad 1.00647223$

C $-0.17524514-1.636866650 .01764831$

C $0.55854768 \quad 0.11895703 \quad 2.63593039$

C 1.058155721 .241734753 .46081083

C $-0.83847132-0.29248503 \quad 2.85968004$

$\mathrm{H}-1.30664441 \quad 0.18653372 \quad-2.42870945$

$\mathrm{H}-2.56922764 \quad 0.01696489-1.21101325$

$\mathrm{H}-1.90964440-1.43582120-1.99888913$

$\mathrm{H}-0.73705889 \quad 1.36154465 \quad 0.54282671$

$\mathrm{H} \quad 1.76811761 \quad 1.669958150 .82782769$

H $2.09091456 \quad 0.31342973-0.33070410$

H $2.47482665-0.471010391 .88463916$

$\mathrm{H} \quad 0.66684580-2.52359665 \quad 1.77788387$

$\mathrm{H} 1.81343671-2.216667390 .46808600$

$\mathrm{H}-0.61107742-2.59266764-0.26718642$

$\mathrm{H} \quad 0.78989447 \quad 1.13122848 \quad 4.52482087$

$\mathrm{H} 0.60446805 \quad 2.20034178 \quad 3.13400389$

H 2.14851953 1.38303071 3.41678506

$\mathrm{H}-0.88489692-1.24134775 \quad 3.42684406$

$\mathrm{H}-1.36692283-0.517411061 .90524160$

$\mathrm{H}-1.447678250 .44419745 \quad 3.40088232$

$\begin{array}{lllll}0 & 1.74420316 & 0.95585704 & -2.98393348\end{array}$

$0 \quad 0.23437874 \quad 1.65285045-1.21437818$

o $1.94616938-0.15743230-2.88303416$

Vibrational frequencies (in $\mathrm{cm}-1$ )

$\begin{array}{lllllllllllllllll}-557.1 & 24.4 & 38.7 & 57.6 & 69.8 & 75.1 & 88.5 & 106.4 & 116.7 & 125.3 & 140.5 & 169.8 & 213.8 & 227.8\end{array}$ $267.6 \quad 277.0 \quad 304.6 \quad 338.0 \quad 359.5 \quad 410.5 \quad 447.6 \quad 501.6 \quad 538.7 \quad 626.7 \quad 716.8 \quad 793.7 \quad 830.8 \quad 905.3$ $935.2941 .5974 .1989 .9 \quad 1001.0 \quad 1020.9 \quad 1027.0 \quad 1047.2 \quad 1051.1 \quad 1077.4 \quad 1105.7 \quad 1125.5 \quad 1189.4$ $\begin{array}{llllllllllll}1192.9 & 1211.4 & 1231.2 & 1237.1 & 1244.1 & 1250.2 & 1254.2 & 1256.9 & 1261.3 & 1272.0 & 1282.4 & 1284.7\end{array}$

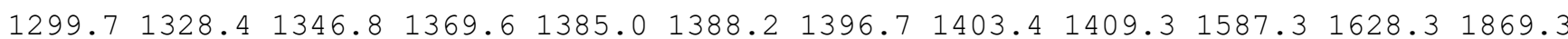
$2608.22626 .02635 .12646 .8 \quad 2649.0 \quad 2658.3 \quad 2661.02677 .82685 .02703 .22729 .12730 .0$ $2757.92759 .6 \quad 2766.8 \quad 2775.2$

\footnotetext{
name $=$ TS282

energy $($ in $\mathrm{kcal} / \mathrm{mol})=62.251237$

$\mathrm{ZPE}($ in $\mathrm{kcal} / \mathrm{mol})=142.159$

$\mathrm{G}(\mathrm{in} \mathrm{kcal} / \mathrm{mol})=118.87$

Geometry

C $-2.01765578-0.59357287-0.87855388$

C $-1.39159618-0.09336977-2.09481263$

C $-0.468778651 .16497745-1.86959308$

C $1.00930717 \quad 1.02193631-2.27737299$

C $1.27936183-0.29731071-2.99982268$

C $0.12000247-0.54196106-4.01429664$

C $-0.65170832-1.21698624-2.83659631$

C $0.74043559-1.51820991-2.15648088$

C $0.84416279-1.46063427-0.64913400$

C $1.32057683-2.84375862-2.64352309$

$\mathrm{H}-3.24849704 \quad 0.59278605-0.65734159$

$\mathrm{H}-2.70023731 \quad 0.63625651 \quad-0.07113472$

$\mathrm{H}-2.73149545-1.38164162-0.90334097$

$\mathrm{H}-0.567949391 .53848006-0.83154649$

$\mathrm{H} 1.657054251 .10666691-1.38521362$

H $1.28753955 \quad 1.87097347 \quad-2.93739127$

H $2.29877018-0.38647451-3.37485893$

$\mathrm{H} \quad 0.36530442-1.21533860-4.83654910$

$\mathrm{H}-0.34560504 \quad 0.35195611 \quad-4.43969344$

$\mathrm{H}-1.28101157-2.07386959-3.07227510$
} 
$\mathrm{H} 1.88993090-1.44619399-0.32384236$

$\mathrm{H} \quad 0.35862622-2.33062221-0.18670144$

$\mathrm{H} \quad 0.35010767-0.58448872-0.20902818$

$\mathrm{H} 1.29120476-2.94077641-3.73414192$

$\mathrm{H} \quad 0.76557054-3.69228988-2.22642849$

$\mathrm{H} \quad 2.36704393-2.94965250-2.33534922$

$\begin{array}{lllll}0 & -2.38084317 & 1.79700831 & -2.87469617\end{array}$

$0-1.013937442 .14842758-2.75650456$

O $-2.38738304 \quad 0.39089967-3.03187114$

Vibrational frequencies (in $\mathrm{cm}-1$ )

$\begin{array}{llllllllllllllllll}-805.9 & 83.7 & 116.8 & 165.1 & 170.4 & 180.2 & 205.4 & 213.4 & 260.7 & 271.6 & 319.7 & 331.7 & 372.3 & 411.0\end{array}$ $430.7 \quad 451.6 \quad 460.7 \quad 484.7 \quad 488.4 \quad 583.4 \quad 629.3 \quad 644.5 \quad 712.7 \quad 725.8 \quad 793.4 \quad 808.0 \quad 851.9 \quad 878.5$ $891.5923 .4 \quad 937.8 \quad 973.8 \quad 990.9 \quad 995.2 \quad 1007.7 \quad 1008.4 \quad 1014.4 \quad 1019.6 \quad 1062.4 \quad 1075.9 \quad 1088.0$

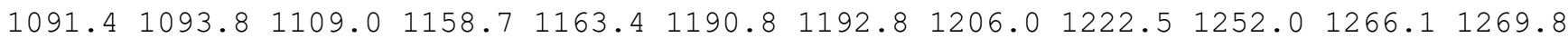

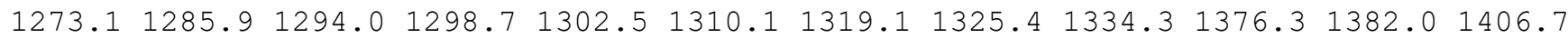
$1418.82658 .0 \quad 2664.4 \quad 2676.3 \quad 2678.7 \quad 2681.4 \quad 2684.02685 .72717 .82737 .82748 .02751 .6$ $2763.42781 .8 \quad 2784.1 \quad 4851.5$

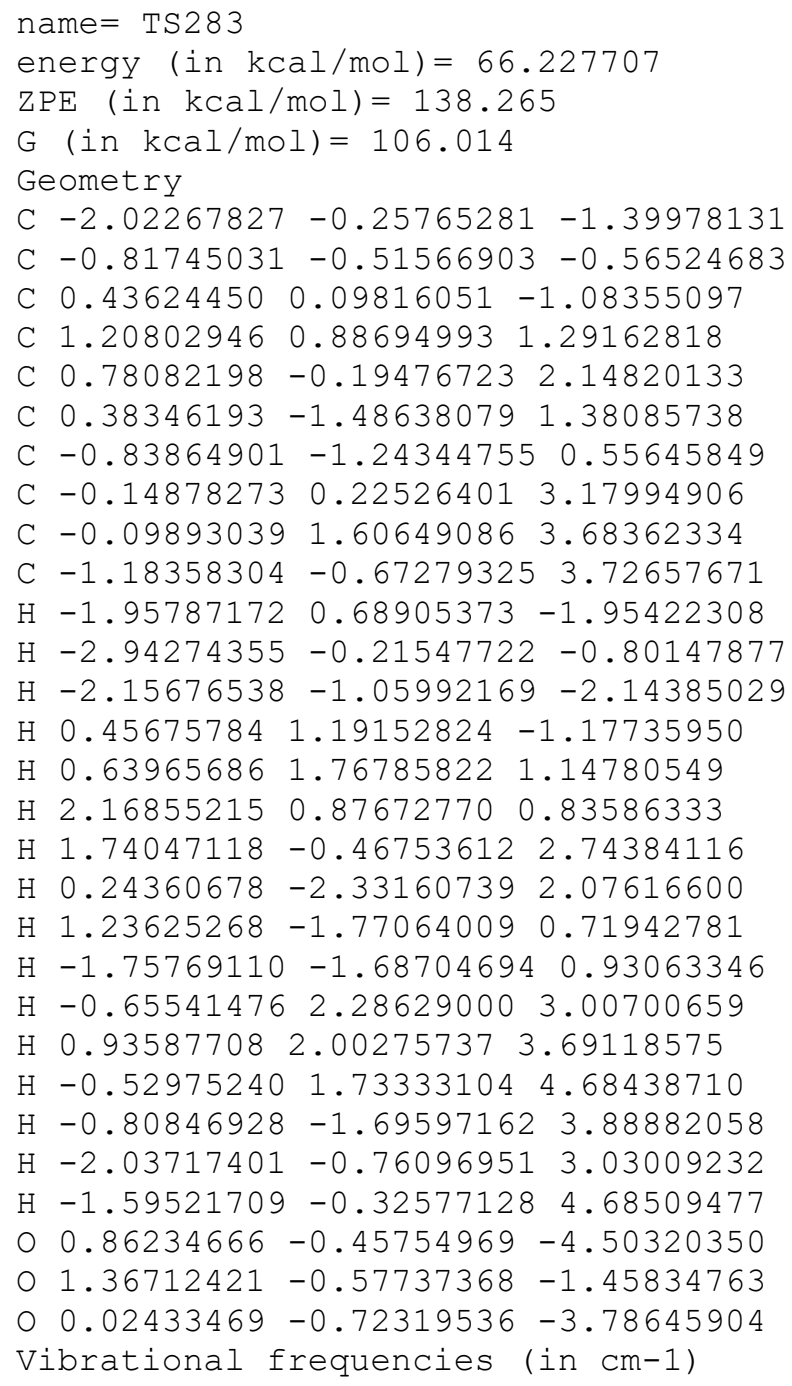
$215.4 \quad 270.1 \quad 313.3 \quad 341.3 \quad 358.1 \quad 394.4 \quad 400.9 \quad 425.4 \quad 494.3 \quad 539.2 \quad 639.9 \quad 659.0 \quad 819.6 \quad 878.4$

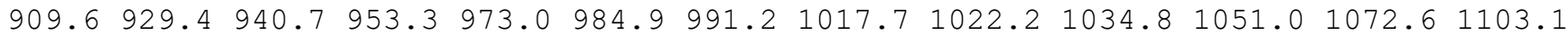

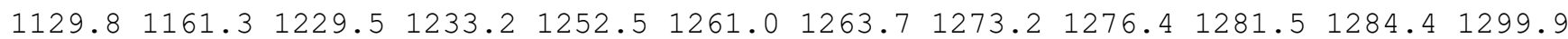
$1344.91367 .6 \quad 1374.6 \quad 1380.2 \quad 1386.3 \quad 1392.6 \quad 1398.2 \quad 1400.0 \quad 1484.5 \quad 1596.8 \quad 1812.4 \quad 1871.6$ $2574.92628 .5 \quad 2648.72651 .8 \quad 2655.6 \quad 2664.5 \quad 2665.8 \quad 2668.32673 .72735 .32751 .52755 .9$ $2760.12768 .6 \quad 2777.92801 .7$

name $=$ TS284

energy $($ in $\mathrm{kcal} / \mathrm{mol})=64.875851$ 
$\mathrm{ZPE}($ in $\mathrm{kcal} / \mathrm{mol})=139.64$

$\mathrm{G}($ in $\mathrm{kcal} / \mathrm{mol})=110.494$

Geometry

C $-0.26388329-1.08812336-2.84065434$

C $0.30603869-0.65137092-1.54054864$

C $-0.211397290 .70923921-1.08836260$

C $0.795418051 .38050966-0.13204282$

C $1.23855936 \quad 0.19335167 \quad 1.16983276$

C $1.85968659-0.97575305 \quad 0.42807703$

C $1.17926926-1.38675442-0.84252817$

C $0.01185674 \quad 0.03967195 \quad 1.81808102$

C $-0.50436428 \quad 1.09796030 \quad 2.71445802$

C $-0.83810526-1.15136018 \quad 1.64601024$

$\mathrm{H}-1.33487490-1.31496474-2.75502813$

$\mathrm{H} \quad 0.24089968-1.95586921-3.27499912$

$\mathrm{H}-0.19120389-0.24857293-3.56582719$

$\mathrm{H}-1.20874754 \quad 0.57074032 \quad-0.59644464$

$\mathrm{H} \quad 0.41723272 \quad 2.25840190 \quad 0.38237814$

$\mathrm{H} \quad 1.772631891 .55276513-0.59267358$

H $1.99640501 \quad 0.82859256 \quad 1.66724497$

H $1.92558412-1.84947177 \quad 1.11283242$

H $2.91783787 \quad-0.72650190 \quad 0.17551361$

H $1.50665050 \quad-2.35794658-1.20814361$

$\mathrm{H}-1.34559840 \quad 1.64072487 \quad 2.23505781$

$\mathrm{H} 0.24066216 \quad 1.86013913 \quad 2.98804706$

$\mathrm{H}-0.909482390 .68997769 \quad 3.65547744$

$\mathrm{H}-1.87983974-1.01604427 \quad 1.96730576$

$\mathrm{H}-0.42856617-2.01375488 \quad 2.20515272$

$\mathrm{H}-0.86385849-1.49786218 \quad 0.58767762$

O $2.063764492 .85946649-3.06214943$

o $-0.22141306 \quad 1.63906758-2.01272960$

o $1.916271991 .74209726-3.19419822$

Vibrational frequencies (in $\mathrm{cm}-1$ )

$\begin{array}{lllllllllllllll}-555.6 & 21.1 & 23.5 & 56.9 & 59.2 & 66.9 & 85.7 & 105.5 & 122.0 & 125.6 & 139.3 & 166.5 & 212.7 & 227.6\end{array}$ $267.7 \quad 277.5 \quad 305.4 \quad 338.4 \quad 359.8 \quad 410.6 \quad 447.7 \quad 501.5 \quad 539.0 \quad 627.3 \quad 717.2 \quad 793.6 \quad 830.6 \quad 905.3$ $935.9941 .4973 .6 \quad 989.6 \quad 1001.0 \quad 1020.8 \quad 1027.0 \quad 1047.2 \quad 1051.4 \quad 1077.1 \quad 1105.61125 .61189 .1$

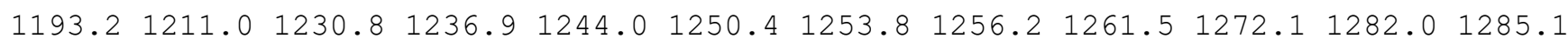

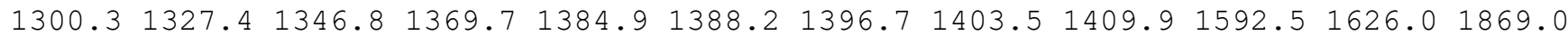
2608.22625 .12634 .52646 .52649 .62658 .42661 .12677 .72684 .92702 .72729 .32730 .2 $2758.5 \quad 2759.6 \quad 2766.6 \quad 2775.1$

\section{name $=$ TS285}

energy $($ in $\mathrm{kcal} / \mathrm{mol})=61.225676$

$\mathrm{ZPE}(\mathrm{in} \mathrm{kcal} / \mathrm{mol})=143.455$

$\mathrm{G}(\mathrm{in} \mathrm{kcal} / \mathrm{mol})=120.116$

Geometry

C $-2.50978907-0.26108609-1.03754456$

C $-0.52887186-0.00858419-0.56850188$

C $0.53386877 \quad 1.10196384 \quad-0.55903144$

C $1.524496251 .05703595 \quad 0.61341829$

C $1.32929405-0.21156780 \quad 1.44680356$

C $1.14720272-1.41356993 \quad 0.46809431$

C $-0.38502544-1.07234202 \quad 0.46238419$

C $-0.18158747-0.38844266 \quad 1.87466679$

C $-0.99877881 \quad 0.84856618 \quad 2.18356297$

C $-0.34031729-1.390052293 .01142346$

$\mathrm{H}-2.94971477 \quad 0.31096994 \quad-1.84392338$

$\mathrm{H}-2.74020920 \quad 0.16899402 \quad-0.07742391$

$\mathrm{H}-2.68621059-1.31816656-1.10822259$

$\mathrm{H} \quad 0.099601132 .10660990-0.71826502$

$\mathrm{H} \quad 1.42029147 \quad 1.956495691 .24643448$

$\mathrm{H} 2.561528501 .08900948 \quad 0.21498935$

$\mathrm{H} 2.06607462-0.325281712 .24275203$

$\mathrm{H} 1.37862642-2.391608520 .89300320$ 
$\mathrm{H} 1.66230389-1.33689650-0.49599289$

$\mathrm{H}-1.08070274-1.90920428 \quad 0.41401842$

$\mathrm{H}-0.981097151 .602599321 .39096120$

$\mathrm{H}-0.62834887 \quad 1.34116281 \quad 3.09322376$

$\mathrm{H}-2.052054620 .593780362 .36034736$

$\mathrm{H}-0.00164725-0.96301450 \quad 3.96334352$

H $0.23628765-2.30782817 \quad 2.84829518$

$\mathrm{H}-1.38854813-1.685953153 .13896969$

$\begin{array}{lllll}0 & 0.35249942 & 0.25214525 & -2.69956148\end{array}$

$\begin{array}{lllll}0 & 1.26882884 & 0.72027451 & -1.74169129\end{array}$

O $-0.93880958-0.07853330-1.80804439$

Vibrational frequencies (in $\mathrm{cm}-1$ )

$\begin{array}{lllllllllllllll}-681.8 & 110.5 & 116.7 & 145.3 & 167.9 & 179.8 & 185.9 & 194.1 & 225.6 & 253.9 & 320.2 & 347.8 & 372.9 & 401.5\end{array}$ $425.5 \quad 440.9 \quad 462.0 \quad 493.9 \quad 504.2 \quad 623.1 \quad 685.8 \quad 702.1 \quad 747.8 \quad 800.4 \quad 853.1 \quad 882.8 \quad 901.2 \quad 915.0$ $932.3950 .4 \quad 974.4976 .3 \quad 997.1 \quad 1007.4 \quad 1014.1 \quad 1017.5 \quad 1073.0 \quad 1078.5 \quad 1091.6 \quad 1098.6 \quad 1148.1$

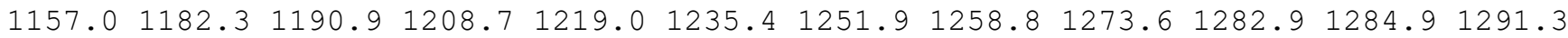
$1294.71298 .71300 .8 \quad 1302.7 \quad 1305.3 \quad 1314.3 \quad 1324.51370 .2 \quad 1376.8 \quad 1405.7 \quad 1416.51568 .4$ $2658.72672 .0 \quad 2672.8 \quad 2673.8 \quad 2675.5 \quad 2676.5 \quad 2678.42688 .32696 .72740 .12746 .22749 .6$ $2757.6 \quad 2762.5 \quad 2781.7 \quad 2783.7$

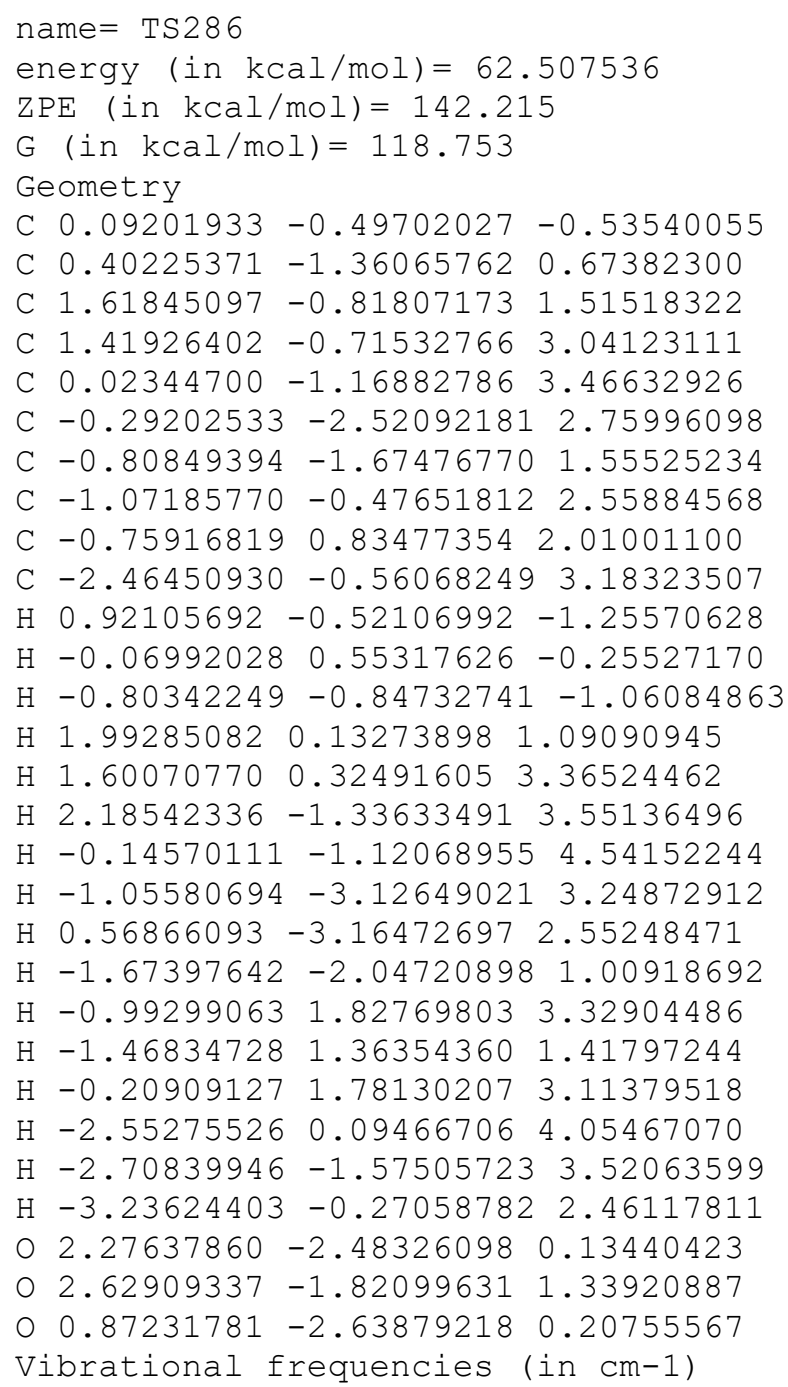




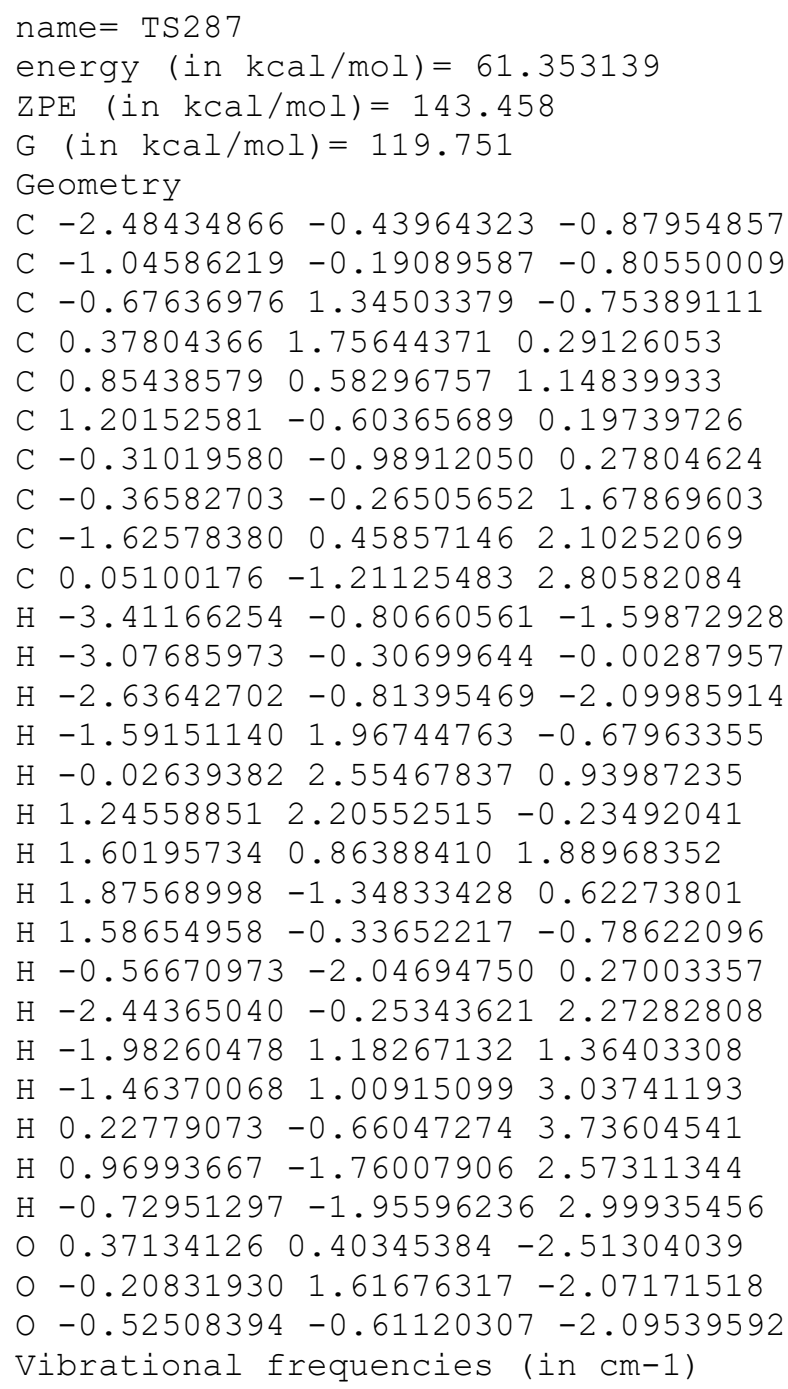


$\mathrm{H} \quad 1.965152320 .18016253 \quad 1.97796143$

H $2.03782750-1.944839150 .54618042$

$\mathrm{H} 1.79326092-0.81188334-0.77898753$

$\mathrm{H}-0.44131322-2.42957863 \quad 0.20776818$

$\mathrm{H}-1.65374480 \quad 0.77071981 \quad 1.47540180$

$\mathrm{H}-1.07255844 \quad 0.57858385 \quad 3.12615546$

$\mathrm{H}-2.15116095-0.628775062 .40958885$

$\mathrm{H} \quad 0.47758187-1.33048539 \quad 3.75640844$

$\mathrm{H} 1.11574890-2.41494098 \quad 2.51978529$

$\mathrm{H}-0.59107996-2.492806752 .95912188$

O $0.54061109-0.04783009-2.44268479$

○ $0.271233451 .24112412-1.91318956$

o $-0.56372588-0.83974968-2.03867793$

Vibrational frequencies (in $\mathrm{cm}-1$ )

$\begin{array}{lllllllllllllll}-582.6 & 83.6 & 108.0 & 140.3 & 166.0 & 181.3 & 213.7 & 228.4 & 257.6 & 281.2 & 303.4 & 355.5 & 377.9 & 415.6\end{array}$

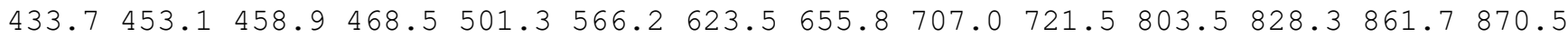
$895.6918 .5926 .5939 .5 \quad 983.1 \quad 994.3 \quad 1005.3 \quad 1008.4 \quad 1018.3 \quad 1019.2 \quad 1031.91051 .1 \quad 1088.7$

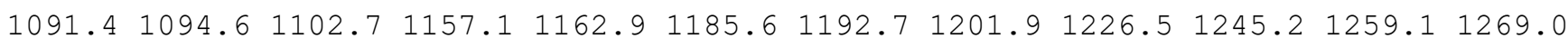

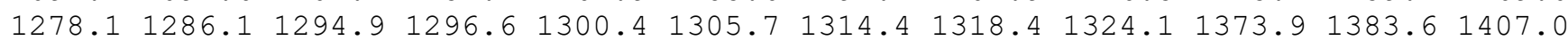
$1419.32658 .52661 .8 \quad 2675.4 \quad 2676.92677 .6 \quad 2681.32690 .72696 .92739 .4 \quad 2747.32753 .0$ $2768.92780 .5 \quad 2782.7 \quad 4973.7$

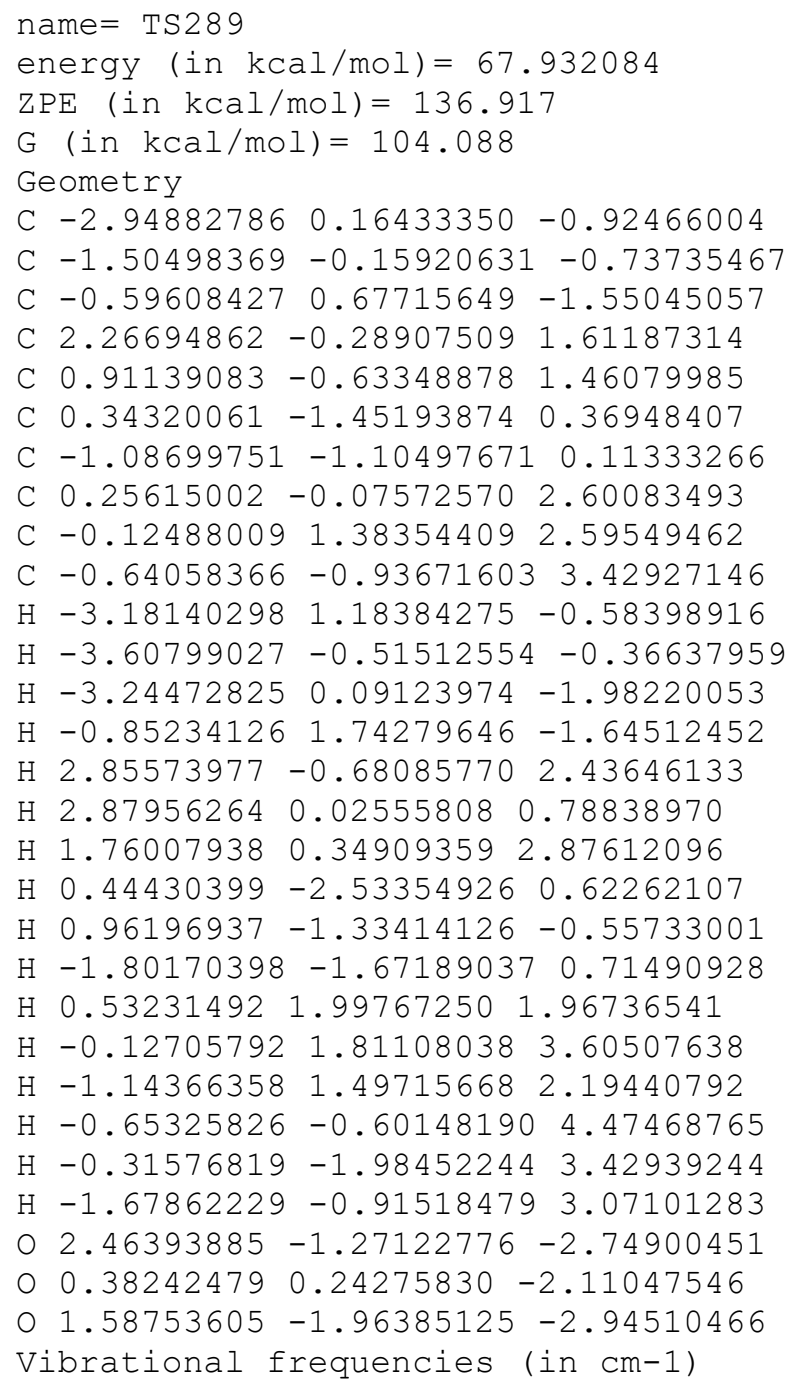




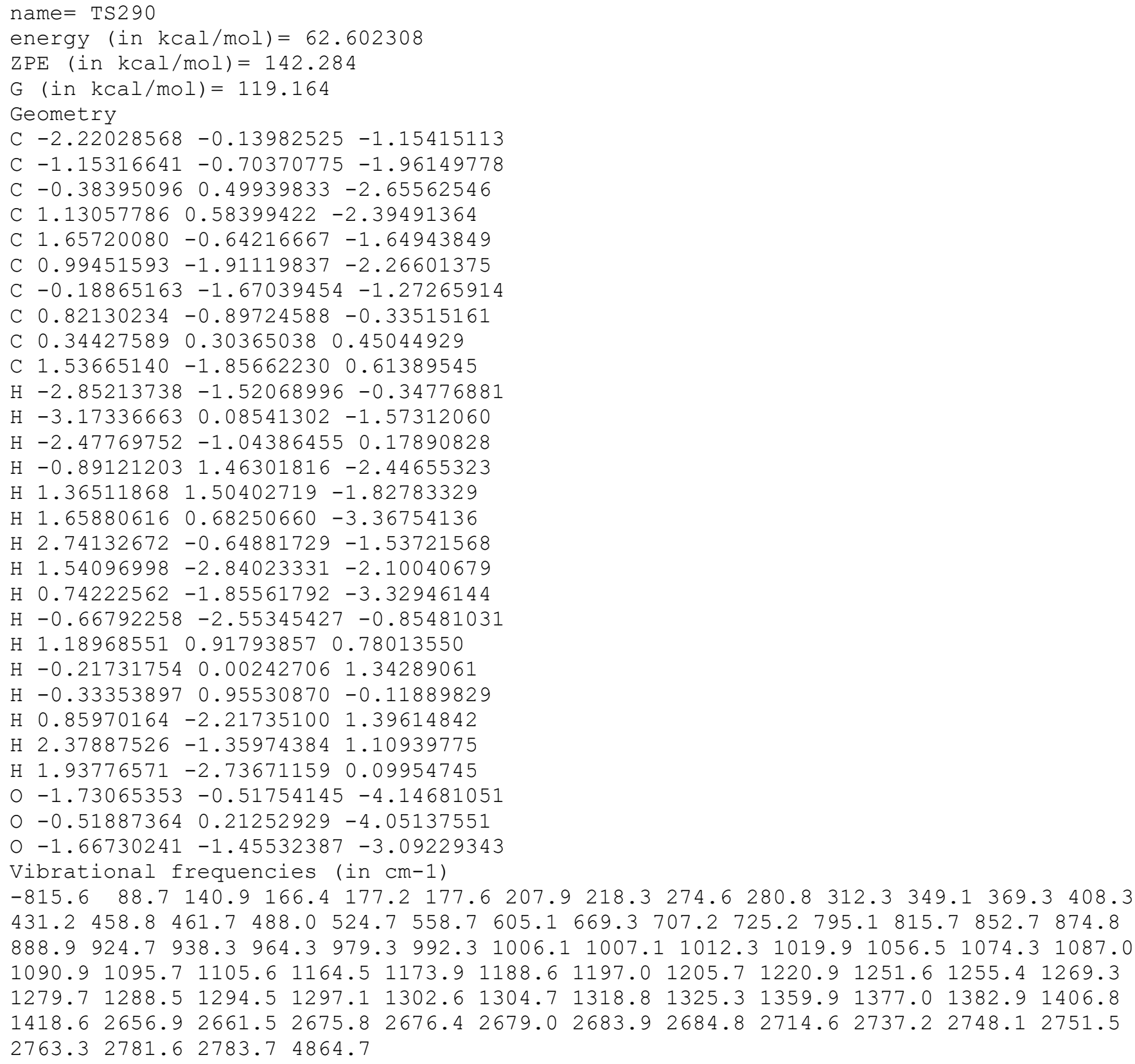
$431.2 \quad 458.8 \quad 461.7 \quad 488.0 \quad 524.7 \quad 558.7 \quad 605.1 \quad 669.3 \quad 707.2 \quad 725.2 \quad 795.1 \quad 815.7 \quad 852.7 \quad 874.8$ $888.9924 .7 \quad 938.3 \quad 964.3 \quad 979.3 \quad 992.3 \quad 1006.1 \quad 1007.1 \quad 1012.3 \quad 1019.9 \quad 1056.5 \quad 1074.3 \quad 1087.0$

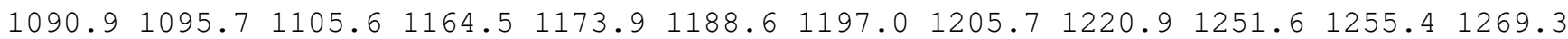
$1279.7 \quad 1288.5 \quad 1294.5 \quad 1297.1 \quad 1302.6 \quad 1304.7 \quad 1318.8 \quad 1325.3 \quad 1359.9 \quad 1377.0 \quad 1382.9 \quad 1406.8$ $1418.62656 .92661 .52675 .8 \quad 2676.4 \quad 2679.02683 .92684 .82714 .62737 .22748 .12751 .5$ $2763.3 \quad 2781.6 \quad 2783.7 \quad 4864.7$

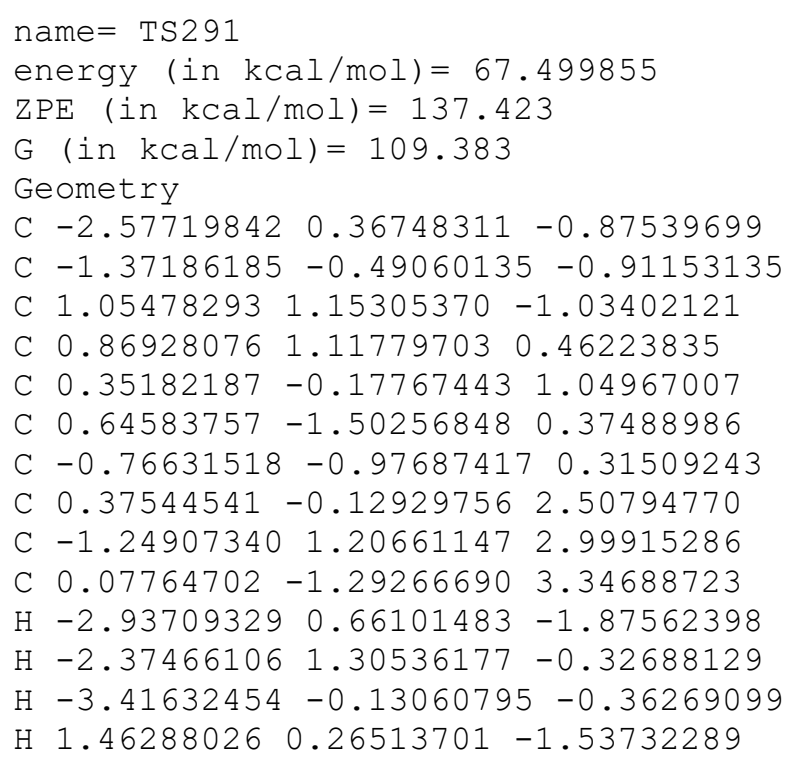


$\mathrm{H} \quad 0.20802328 \quad 1.95835728 \quad 0.76952421$

H $1.85635991 \quad 1.35179194 \quad 0.93216726$

$\mathrm{H} 0.034532401 .09150325 \quad 3.05969923$

H $0.85764675-2.38579554 \quad 0.97448668$

$\mathrm{H} 1.25909626-1.53748951-0.52986796$

$\mathrm{H}-1.50894650-1.467235350 .96858638$

$\mathrm{H}-1.82049081 \quad 0.71162914 \quad 2.23040904$

$\mathrm{H}-1.25677450 \quad 2.27677737 \quad 2.84047152$

$\mathrm{H}-1.60871891 \quad 0.940477113 .98066483$

$\mathrm{H}-0.04916658-1.020696954 .40597578$

$\mathrm{H} \quad 0.94930733-1.97633530 \quad 3.32394062$

$\mathrm{H}-0.80059311-1.88105520 \quad 3.05037763$

O $0.04047192-1.44988126-2.32109992$

O $0.77834725 \quad 2.15165610-1.65318112$

O $-0.98847430-0.75050158-2.13122061$

Vibrational frequencies (in cm-1)

$\begin{array}{llllllllllllllll}-1187.7 & 28.5 & 43.8 & 56.0 & 58.5 & 81.2 & 88.4 & 102.6 & 142.5 & 146.2 & 179.6 & 183.0 & 243.7 & 263.7\end{array}$ $270.7 \quad 298.2 \quad 337.1 \quad 378.8 \quad 390.3 \quad 434.7 \quad 450.4 \quad 533.7 \quad 552.8 \quad 608.1 \quad 660.8 \quad 679.4 \quad 743.0 \quad 805.8$ $837.4 \quad 868.6 \quad 945.5 \quad 965.2 \quad 998.5 \quad 1012.5 \quad 1019.5 \quad 1035.1 \quad 1044.7 \quad 1068.5 \quad 1087.0 \quad 1103.4 \quad 1112.3$

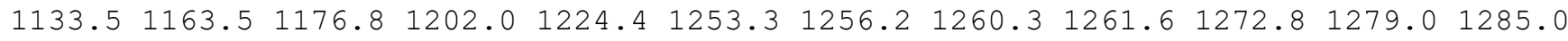

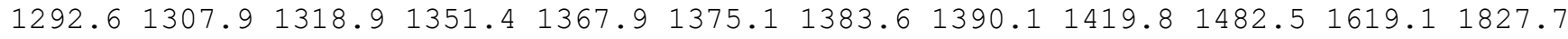

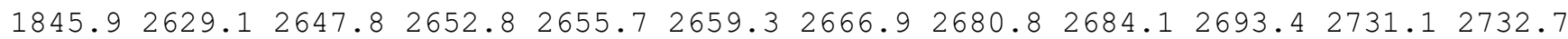
$2768.7 \quad 2769.3 \quad 2770.5 \quad 2776.0$

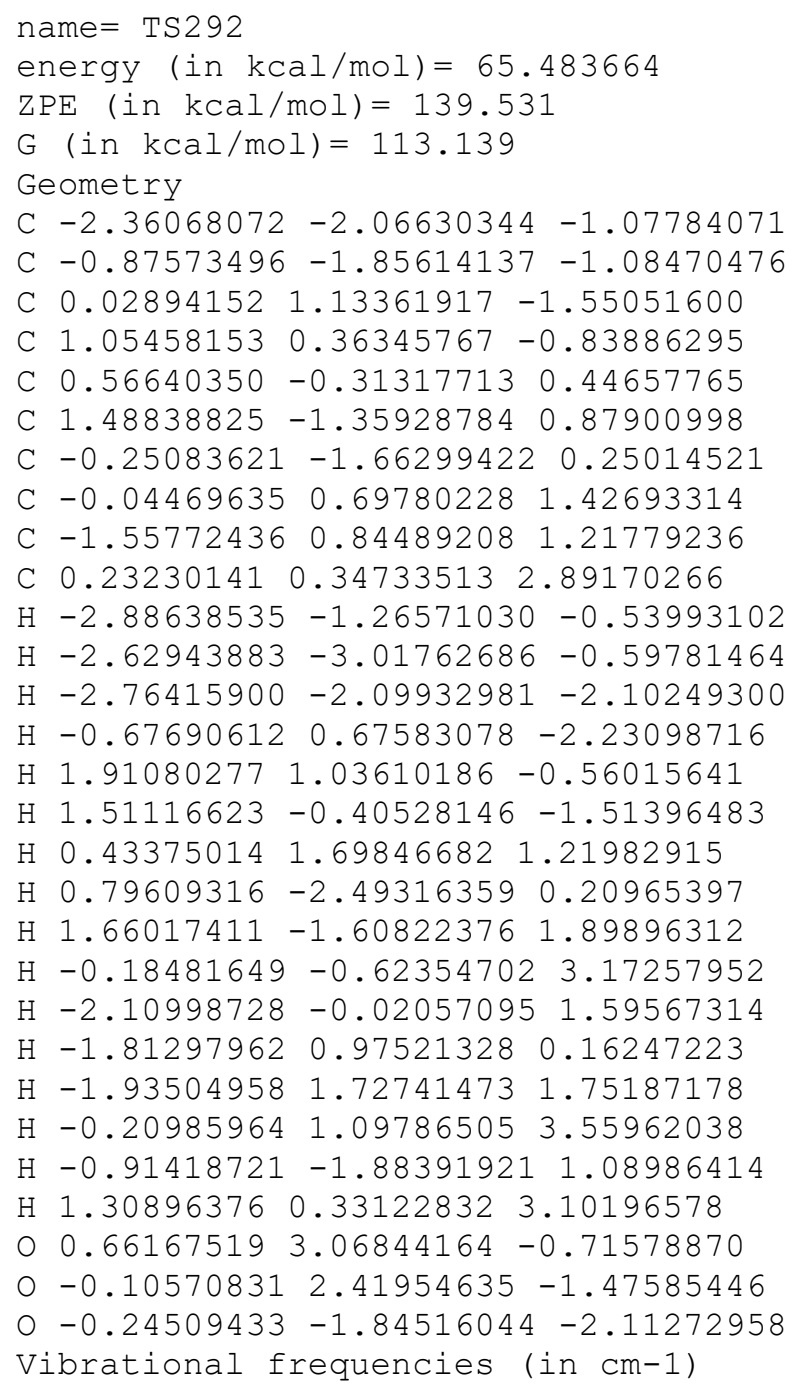


$2135.92621 .4 \quad 2652.7 \quad 2666.3 \quad 2667.8 \quad 2671.5 \quad 2674.82680 .3 \quad 2688.7 \quad 2703.62717 .12723 .6$ $2730.02778 .0 \quad 2780.0 \quad 2781.4$

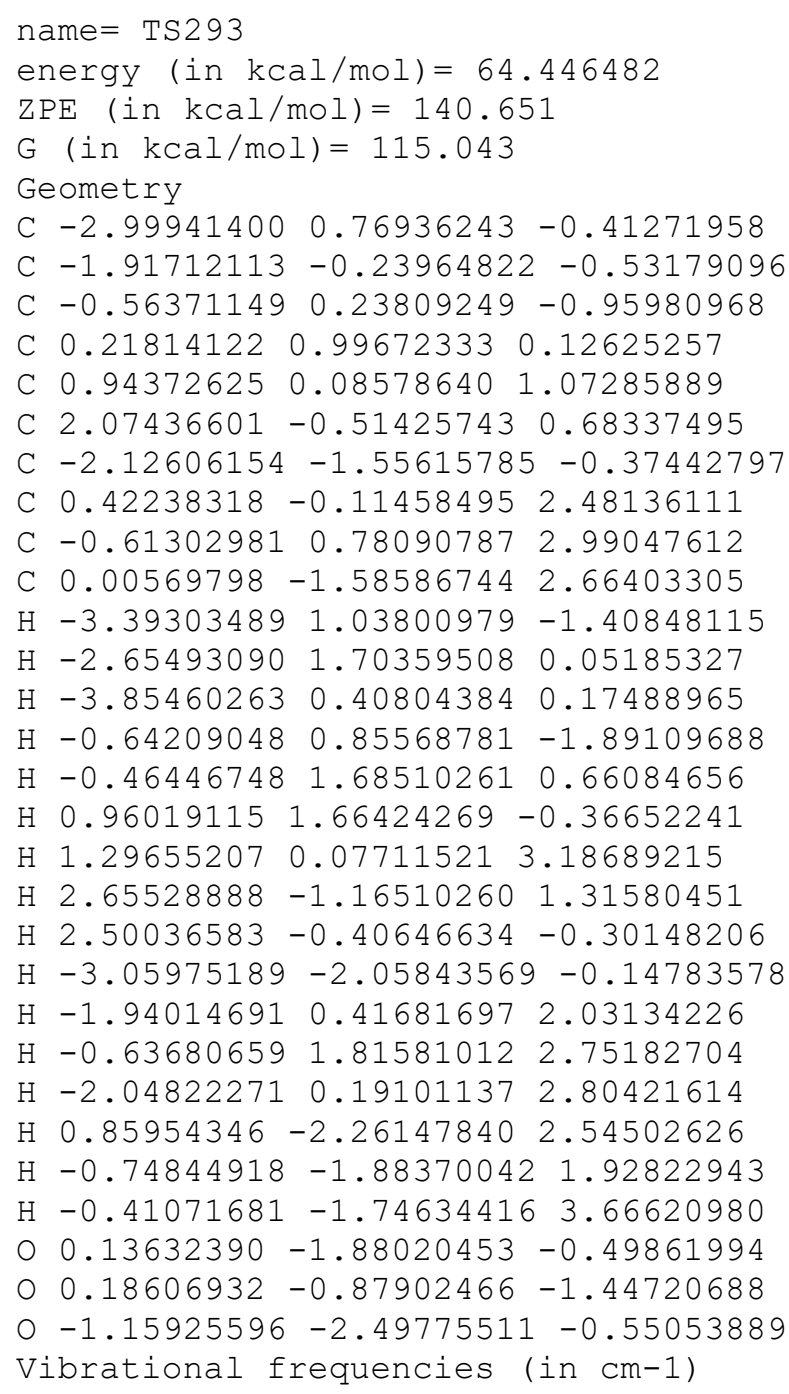


$\mathrm{H}-2.53967704-0.78399246-1.75244059$

$\mathrm{H} \quad 0.76737461 \quad 1.02442608 \quad-1.81830920$

$\mathrm{H}-0.93883715 \quad 2.20242658 \quad 2.27350062$

$\mathrm{H} \quad 3.60793579 \quad 0.65169989 \quad 1.28178338$

$\mathrm{H} \quad 1.47208322 \quad 1.79416224 \quad 2.22633879$

$\mathrm{H} 2.90095648-0.164673212 .68420485$

H 2.65746082 $-0.80784307 \quad 1.04789664$

$\mathrm{H}-1.95405909-0.892735050 .91105595$

$\mathrm{H}-1.91864534 \quad 0.82964981 \quad 0.48285739$

$\mathrm{H}-0.24144210 \quad 1.317162153 .64037780$

$\mathrm{H}-1.85829153 \quad 0.91380828 \quad 3.06786044$

$\mathrm{H}-0.72044014-1.63170926 \quad 2.82971651$

$\mathrm{H} \quad 0.83977327 \quad-0.93442261 \quad 3.26172678$

$\mathrm{H} \quad 0.63665966-1.77512368 \quad 1.71390332$

O $1.14103661-1.79395048-0.54347267$

O $1.65264778-0.64935656-1.17914135$

o $-0.26368829-1.77237992-0.80851416$

Vibrational frequencies (in cm-1)

$\begin{array}{lllllllllllllll}-394.5 & 66.3 & 91.3 & 127.7 & 158.8 & 162.8 & 213.2 & 216.1 & 229.8 & 239.7 & 271.4 & 281.9 & 294.9 & 329.7\end{array}$

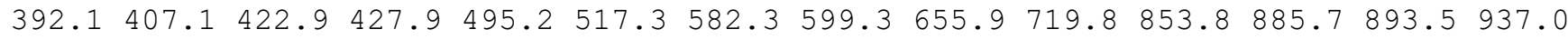
$945.2959 .0 \quad 969.9986 .5 \quad 991.6 \quad 1001.8 \quad 1015.0 \quad 1024.7 \quad 1028.8 \quad 1065.8 \quad 1096.3 \quad 1117.7 \quad 1130.3$

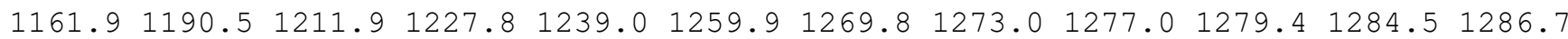
$1288.41293 .8 \quad 1295.1 \quad 1307.3 \quad 1313.2 \quad 1351.1 \quad 1379.2 \quad 1389.6 \quad 1394.6 \quad 1401.2 \quad 1416.7 \quad 1772.0$ $2654.22659 .52664 .62665 .3 \quad 2668.2 \quad 2670.3 \quad 2671.92677 .02677 .92695 .72716 .22730 .6$ $2775.22777 .2 \quad 2779.92780 .4$

name $=$ TS295

energy (in $\mathrm{kcal} / \mathrm{mol})=66.124961$

$\operatorname{ZPE}($ in $\mathrm{kcal} / \mathrm{mol})=138.998$

$\mathrm{G}($ in $\mathrm{kcal} / \mathrm{mol})=111.951$

Geometry

C $-1.05301348-0.67003481-2.84277935$

C $-0.03445985-0.57167332-1.86736658$

C $-0.53368643 \quad 0.48492330 \quad-0.62240427$

C $-0.97349597 \quad 0.10463051 \quad 0.74889002$

C $0.18889883-0.37408278 \quad 1.55996841$

C $-0.21967952-2.82321187-0.83188138$

C $0.52168368-1.78186322-1.20977767$

$\begin{array}{llll}\text { C } 1.07828300 & 0.42339487 & 2.16812624\end{array}$

C $1.04006671 \quad 1.91170278 \quad 2.10857720$

C $2.21688597-0.15035574 \quad 2.94637702$

H $-2.11434054 \quad 0.46744468 \quad-2.05535726$

$\mathrm{H}-1.01559150-0.07430829-3.73115681$

$\mathrm{H}-1.66748922-1.53819291-2.92529932$

$\mathrm{H}-1.94224692 \quad 0.89901009-1.36734337$

$\mathrm{H}-1.74775263-0.69487549 \quad 0.68010181$

$\mathrm{H}-1.480469220 .95808311 \quad 1.25330280$

H $0.27347741-1.45812255 \quad 1.61791199$

$\mathrm{H} \quad 0.19202964 \quad-3.70634400 \quad-0.36858561$

$\mathrm{H}-1.28979177-2.87747618-0.96562070$

$\mathrm{H} \quad 1.60560023-1.76017121-1.06721339$

H $0.01905615 \quad 2.309265112 .03737999$

$\mathrm{H} \quad 1.58666407 \quad 2.27525385 \quad 1.22095419$

H $1.50692422 \quad 2.37977818 \quad 2.98539844$

H $3.18003625 \quad 0.24221917 \quad 2.58738215$

H $2.27182888-1.245119842 .88150137$

$\mathrm{H} \quad 2.13702920 \quad 0.10603213 \quad 4.01282077$

$\begin{array}{llllll}0 & 0.77680704 & 1.54280382 & -2.05362136\end{array}$

○ $0.27425431 \quad 1.53644678-0.65721324$

$\begin{array}{lllll}0 & 1.10328035 & 0.22355203 & -2.28879522\end{array}$

Vibrational frequencies (in $\mathrm{cm}-1$ )

$\begin{array}{llllllllllllll}-1253.6 & 22.5 & 42.9 & 57.0 & 82.6 & 93.1 & 103.4 & 137.8 & 170.1 & 201.4 & 230.1 & 251.1 & 290.1 & 305.0\end{array}$

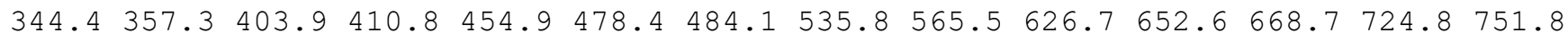

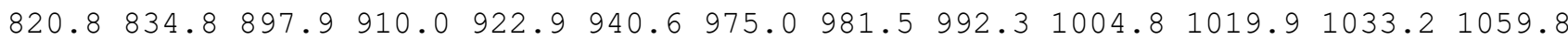


$1081.91089 .4 \quad 1115.1 \quad 1129.3 \quad 1177.5 \quad 1196.2 \quad 1210.4 \quad 1231.8 \quad 1270.3 \quad 1274.0 \quad 1280.4 \quad 1284.6$ $1286.31303 .21320 .4 \quad 1328.1 \quad 1366.2 \quad 1376.1 \quad 1389.4 \quad 1396.1 \quad 1400.1 \quad 1416.6 \quad 1512.81847 .9$ $1868.52651 .62659 .12669 .92671 .92675 .4 \quad 2712.92731 .82742 .3 \quad 2747.02754 .12775 .9$ $2779.62784 .62799 .6 \quad 4082.2$

\section{name $=$ TS296}

energy $($ in $\mathrm{kcal} / \mathrm{mol})=63.351134$

$\mathrm{ZPE}($ in $\mathrm{kcal} / \mathrm{mol})=141.794$

$\mathrm{G}(\mathrm{in} \mathrm{kcal} / \mathrm{mol})=118.09$

Geometry

C $-1.92730497-1.18573173-0.42795993$

C $-0.64166170-0.49321943-0.85919724$

C $-0.49321066 \quad 0.96632900 \quad-0.29769989$

C $0.79267244 \quad 1.20212178 \quad 0.49998922$

C 1.028672590 .169413261 .57878756

C $1.09023599-1.462107530 .62415935$

C $0.58201175-1.29257204-0.60991168$

C $0.11075380 \quad 0.04681226 \quad 2.64559556$

C $-1.12162017 \quad 0.87138456 \quad 2.76896965$

C $0.24555426-1.07709262 \quad 3.48096866$

$\mathrm{H}-2.81269537-0.60062198-0.70857753$

$\mathrm{H}-1.94568767-1.34734350 \quad 0.65479638$

$\mathrm{H}-2.02096449-2.16676900-0.91368451$

$\mathrm{H}-1.39845433 \quad 1.29462193 \quad 0.24909565$

H $0.77338098 \quad 2.22006733 \quad 0.94012433$

H $1.64932528 \quad 1.21003184-0.20999329$

H $2.09554371 \quad 0.07684369 \quad 1.84671249$

$\mathrm{H} \quad 0.31360427 \quad-2.07730788 \quad 1.55940870$

H 2.05199896 -1.92113959 0.80289968

H $1.08732556-1.65208393-1.50198066$

$\mathrm{H}-1.95114714 \quad 0.46348986 \quad 2.17198173$

$\mathrm{H}-0.95695770 \quad 1.90997612 \quad 2.44660620$

$\mathrm{H}-1.47394532 \quad 0.92148287 \quad 3.80961748$

$\mathrm{H}-0.49882845-1.27966802 \quad 4.24004500$

H $1.22813028-1.423737153 .78447403$

$\mathrm{H} \quad 0.05456811-2.092718962 .46235777$

$\begin{array}{lllll}0 & -0.08230938 & 0.92136242 & -2.51751774\end{array}$

$0-0.483872521 .79152869-1.46104453$

o $-0.80169068-0.26668649-2.27453339$

Vibrational frequencies (in $\mathrm{cm}-1$ )

$\begin{array}{lllllllllllllll}-2282.3 & 59.2 & 106.7 & 142.1 & 162.7 & 169.3 & 199.0 & 222.3 & 254.5 & 279.9 & 302.9 & 331.3 & 358.9 & 379.6\end{array}$ $399.5 \quad 439.6 \quad 456.3 \quad 482.4 \quad 572.0 \quad 573.6 \quad 638.3 \quad 684.4 \quad 707.6 \quad 718.5 \quad 820.9 \quad 829.3 \quad 859.2 \quad 899.9$ $933.9958 .6 \quad 984.1 \quad 988.2 \quad 997.7 \quad 1011.6 \quad 1026.1 \quad 1045.7 \quad 1048.8 \quad 1057.4 \quad 1077.8 \quad 1092.8 \quad 1115.0$

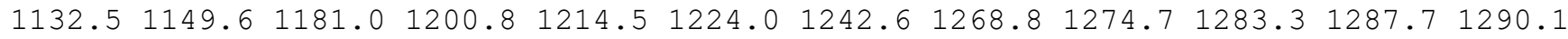

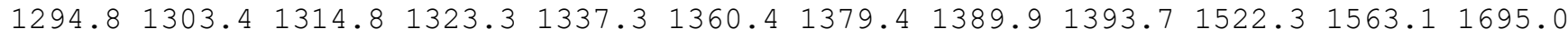

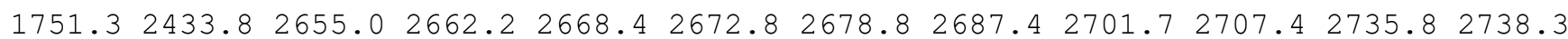
$2756.12768 .22774 .3 \quad 2779.9$

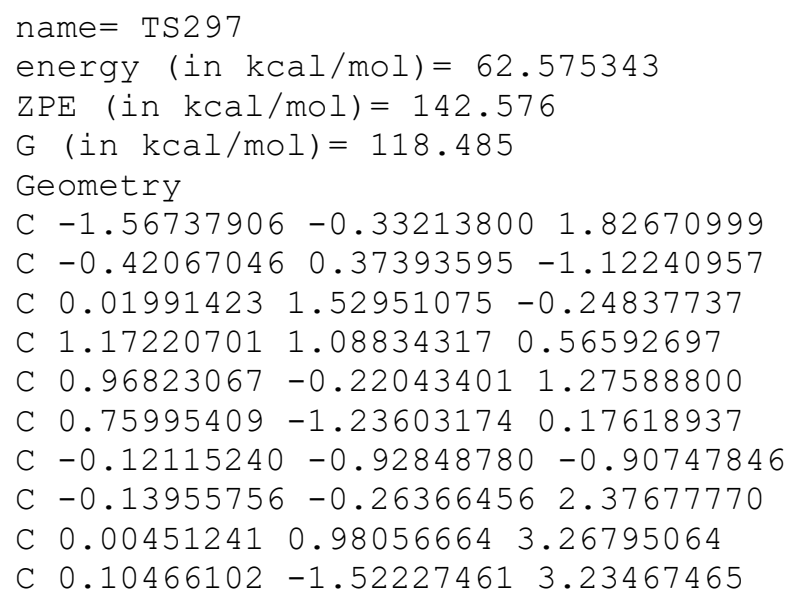


$\mathrm{H}-1.70322640-1.192824091 .16065854$

$\mathrm{H}-1.840114170 .56299028 \quad 1.26011371$

$\mathrm{H}-2.29642181-0.42972675 \quad 2.63946862$

$\mathrm{H}-0.805132261 .99780575 \quad 0.33408161$

$\mathrm{H} \quad 1.82580593 \quad 1.82877895 \quad 0.98579339$

H $2.16754437 \quad 0.32758067 \quad-0.51315097$

$\mathrm{H} \quad 1.92610829-0.466557051 .81352245$

H $0.99631506-2.266185910 .39483643$

$\mathrm{H} 2.11948554-0.52239794-0.61969003$

$\mathrm{H}-0.43366861-1.71518282-1.58623580$

$\mathrm{H}-0.69365296 \quad 0.94562986 \quad 4.11124558$

$\mathrm{H}-0.20214481 \quad 1.90042464 \quad 2.70992457$

$\mathrm{H} 1.01478540 \quad 1.06654038 \quad 3.68060572$

$\mathrm{H} 1.09502049-1.51288148 \quad 3.69963066$

H $0.02325809-2.43502256 \quad 2.63571349$

$\mathrm{H}-0.63145444 \quad-1.59560594 \quad 4.04257970$

$\begin{array}{lllll}0 & -0.62595717 & 2.31024456 & -2.22508299\end{array}$

$\begin{array}{lllll}0 & 0.40237217 & 2.47191452 & -1.27867185\end{array}$

o $-0.89837223 \quad 0.86559194-2.29833632$

Vibrational frequencies (in $\mathrm{cm}-1$ )

$\begin{array}{lllllllllllllllll}-1379.2 & 44.4 & 88.4 & 105.4 & 173.5 & 180.7 & 201.3 & 217.2 & 225.8 & 285.3 & 295.4 & 305.3 & 372.3 & 402.5\end{array}$

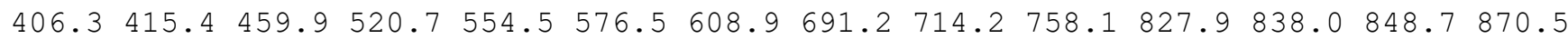

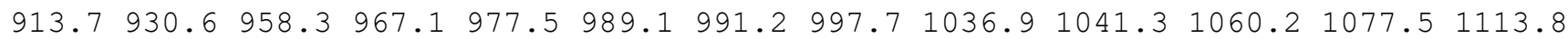
$1145.41167 .2 \quad 1196.2 \quad 1210.5 \quad 1228.4 \quad 1233.2 \quad 1246.5 \quad 1277.4 \quad 1291.6 \quad 1295.01297 .7 \quad 1301.0$

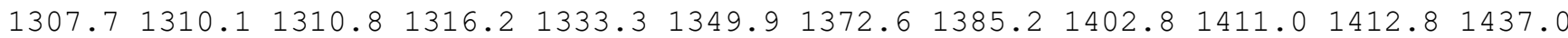
$1781.92660 .2 \quad 2672.3 \quad 2674.5 \quad 2676.3 \quad 2678.3 \quad 2680.3 \quad 2681.22685 .82750 .42764 .82771 .2$ $2780.8 \quad 2781.7 \quad 2783.7 \quad 3627.6$

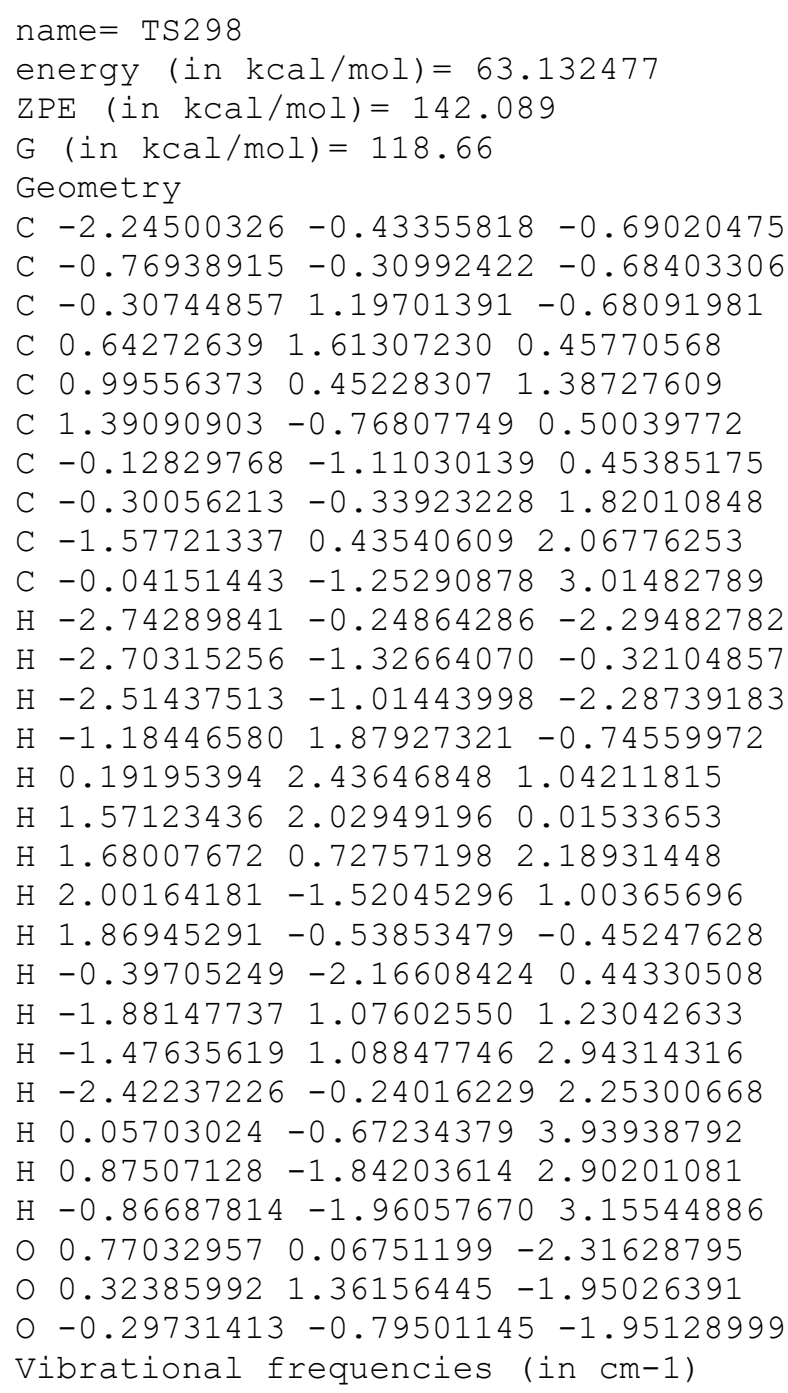


$\begin{array}{lllllllllllllllllll}-689.4 & 81.9 & 99.1 & 137.6 & 163.7 & 180.5 & 211.5 & 225.3 & 256.4 & 284.3 & 305.1 & 351.8 & 378.1 & 412.8\end{array}$ $420.4 \quad 444.9 \quad 460.2 \quad 472.6 \quad 504.1 \quad 571.7 \quad 634.5 \quad 658.0 \quad 708.6 \quad 721.8 \quad 803.2 \quad 830.7 \quad 861.7 \quad 869.9$ $895.3919 .2935 .5941 .6 \quad 985.2 \quad 995.7 \quad 1006.5 \quad 1008.5 \quad 1018.8 \quad 1022.3 \quad 1049.2 \quad 1060.2 \quad 1090.2$

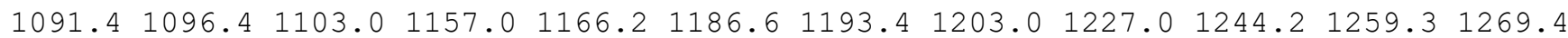
$1278.6 \quad 1286.6 \quad 1294.5 \quad 1295.91300 .3 \quad 1304.9 \quad 1314.0 \quad 1318.2 \quad 1323.2 \quad 1373.6 \quad 1383.01407 .0$ $1420.92659 .2 \quad 2663.0 \quad 2676.0 \quad 2677.2 \quad 2677.92681 .5 \quad 2689.22691 .12740 .52747 .82751 .4$ $2767.42781 .32783 .3 \quad 4929.8$

name $=$ TS299

energy (in $\mathrm{kcal} / \mathrm{mol})=64.345026$

$\operatorname{ZPE}($ in $\mathrm{kcal} / \mathrm{mol})=140.885$

$\mathrm{G}($ in $\mathrm{kcal} / \mathrm{mol})=113.867$

Geometry

C $-2.33720353-1.01925617-1.20668896$

C $-0.94079075-0.53534761-0.80191810$

C $-0.94945190 \quad 0.85265654 \quad-0.10747815$

C 0.246572331 .048729890 .83658873

C $0.74869645-0.26498714 \quad 1.35583788$

C $2.11989844-0.67400870 \quad 0.91950312$

C $-0.35362381-1.60423809-0.00231874$

C $0.08846333-0.954283392 .32487586$

C $-1.13796582-0.46212390 \quad 3.01874290$

C $0.57531150 \quad-2.27483807 \quad 2.82236162$

$\mathrm{H}-2.87087437-0.24874897-1.77399774$

$\mathrm{H}-2.92500077-1.28876826-0.32072355$

$\mathrm{H}-2.27165534-1.91095208-1.84433555$

$\mathrm{H}-1.91983714 \quad 1.09311096 \quad 0.36438312$

$\mathrm{H}-0.02488599 \quad 1.73472446 \quad 1.66336616$

$\mathrm{H} 1.050651221 .589679220 .28847046$

H 2.82444391 $-0.57394911 \quad 1.75784491$

H $2.17119238-1.72133240 \quad 0.58252372$

$\begin{array}{lllll}\mathrm{H} & 2.49946837 & -0.06954537 & 0.08495935\end{array}$

$\mathrm{H} \quad 0.47305461-2.14606984-0.42490440$

$\mathrm{H}-2.01560760-1.06247775 \quad 2.73140142$

$\mathrm{H}-1.37795356 \quad 0.58855264 \quad 2.81405265$

$\mathrm{H}-1.03546166-0.54740184 \quad 4.11309878$

$\mathrm{H} \quad 0.15015544 \quad-2.55731216 \quad 3.79281742$

H $1.66749796-2.31713855 \quad 2.92442876$

$\mathrm{H} \quad 0.27884177 \quad-3.06091273 \quad 2.10172659$

$0.092253431 .10801244-2.06610043$

$0-0.846862571 .74133646-1.21494849$

O $-0.23514188-0.28311622-2.03963581$

Vibrational frequencies (in $\mathrm{cm}-1$ )

$\begin{array}{llllllllllllllll}-145.2 & 17.9 & 53.7 & 61.9 & 85.1 & 97.0 & 124.9 & 136.3 & 172.3 & 206.5 & 213.3 & 275.3 & 291.9 & 345.5\end{array}$

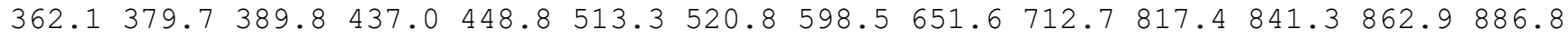
$939.8 \quad 969.8 \quad 971.8 \quad 992.0 \quad 1004.8 \quad 1008.3 \quad 1013.1 \quad 1024.0 \quad 1046.2 \quad 1062.3 \quad 1068.8 \quad 1107.4 \quad 1114.6$ $1145.01153 .7 \quad 1170.3 \quad 1227.3 \quad 1239.5 \quad 1261.3 \quad 1265.1 \quad 1274.1 \quad 1280.7 \quad 1281.8 \quad 1285.7 \quad 1287.3$ $1291.61293 .0 \quad 1305.8 \quad 1318.2 \quad 1335.2 \quad 1344.7 \quad 1358.0 \quad 1392.01394 .91401 .91408 .2 \quad 1799.7$ $2648.82654 .0 \quad 2656.12662 .0 \quad 2667.8 \quad 2673.0 \quad 2673.5 \quad 2674.52678 .0 \quad 2681.4 \quad 2700.42736 .1$ $2772.8 \quad 2774.0 \quad 2776.3 \quad 2779.6$

name $=$ TS300

energy (in $\mathrm{kcal} / \mathrm{mol})=61.094468$

$\mathrm{ZPE}($ in $\mathrm{kcal} / \mathrm{mol})=144.137$

$\mathrm{G}($ in $\mathrm{kcal} / \mathrm{mol})=119.869$

Geometry

C $-2.64553963-2.320219050 .13328193$

C $-1.68547911-1.28075938-0.45723826$

C $-2.17795871 \quad 0.18173540 \quad-0.19751970$

C $-1.06671684 \quad 1.18684988 \quad 0.10359036$

$\begin{array}{lllll}\text { C } & 0.15057814 & 0.56372150 & 0.84320507\end{array}$

C $0.72871868-0.50961939-0.10017373$

C $-0.30935291-1.54351997-0.05689596$ 
C $-0.27929807-0.07633708 \quad 2.10184050$

C $-0.87875318 \quad 0.86619010 \quad 3.09287345$

C $-0.03937244-1.42052272 \quad 2.64725579$

$\mathrm{H}-3.67732668-2.13561651-0.18686165$

$\mathrm{H}-2.60735682-2.315251001 .22611335$

$\mathrm{H}-2.37933414-3.32976718-0.20689129$

$\mathrm{H}-2.98639984 \quad 0.21302309 \quad 0.55779687$

$\mathrm{H}-1.46864547 \quad 2.03227104 \quad 0.68836992$

$\mathrm{H}-0.71129313 \quad 1.62412895 \quad-0.85382216$

$\mathrm{H} 0.87712728 \quad 1.38828357 \quad 1.05136199$

$\mathrm{H} \quad 1.71384405-0.871949090 .24386909$

$\mathrm{H} \quad 0.89863615-0.09048493-1.11257378$

$\mathrm{H}-0.00839112 \quad-2.57127368 \quad 0.04203932$

$\mathrm{H}-1.97768373 \quad 0.87599166 \quad 3.01582937$

$\mathrm{H}-0.54652810 \quad 1.90666528 \quad 2.95173589$

$\mathrm{H}-0.64091495 \quad 0.60425177 \quad 4.13476138$

$\mathrm{H} 0.48704800-1.39038821 \quad 3.61817594$

$\mathrm{H} \quad 0.53030720-2.089105721 .98474065$

$\mathrm{H}-1.00394930-1.93565937 \quad 2.83223602$

$0-2.04633124-0.13301152-2.41023643$

$0-2.81786748 \quad 0.53815666-1.41894765$

$0-1.85111360-1.43087128-1.89497449$

Vibrational frequencies (in $\mathrm{cm}-1$ )

$\begin{array}{llllllllllllllll}-177.4 & 59.5 & 72.7 & 84.2 & 157.5 & 173.9 & 192.2 & 239.4 & 256.4 & 283.5 & 311.6 & 364.8 & 371.7 & 402.1\end{array}$ $\begin{array}{llllllllllllllll}425.7 & 459.4 & 472.9 & 522.7 & 536.2 & 639.3 & 684.1 & 720.9 & 794.8 & 827.9 & 856.3 & 877.3 & 926.9 & 943.0\end{array}$ $951.8964 .6983 .1 \quad 1004.5 \quad 1019.0 \quad 1036.2 \quad 1046.0 \quad 1060.3 \quad 1066.7 \quad 1101.2 \quad 1109.3 \quad 1131.51149 .5$ $\begin{array}{lllllllllllllll}1163.0 & 1193.3 & 1210.8 & 1227.2 & 1243.6 & 1249.0 & 1253.6 & 1259.6 & 1260.6 & 1269.3 & 1286.3 & 1292.2\end{array}$

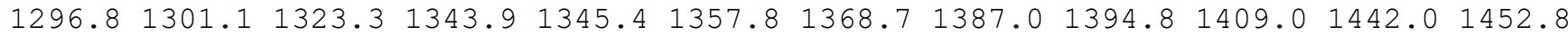
$2631.72649 .42654 .92656 .6 \quad 2661.3 \quad 2664.3 \quad 2674.52683 .22685 .42697 .82738 .12745 .5$ $2754.5 \quad 2765.22770 .3 \quad 2780.7$

name $=$ TS301

energy (in $\mathrm{kcal} / \mathrm{mol})=64.947937$

$\mathrm{ZPE}($ in $\mathrm{kcal} / \mathrm{mol})=140.378$

$\mathrm{G}($ in $\mathrm{kcal} / \mathrm{mol})=113.303$

Geometry

C $-2.44643587 \quad 0.00670481-0.49344866$

C $-1.016720050 .00680599-0.16110925$

C $-0.15093108 \quad 1.42766282-0.55787481$

C $0.94489845 \quad 1.55116763 \quad 0.58297314$

C $0.99362780 \quad 0.25088302 \quad 1.37531513$

C $1.19365004-0.908996160 .36321737$

C $-0.25883446-1.12980597 \quad 0.01684187$

C $-0.42661146-0.100146251 .84826920$

C $-1.35972907 \quad 0.97332353 \quad 2.31092345$

C $-0.60348435-1.35955290 \quad 2.64886939$

$\mathrm{H}-2.99145055 \quad 0.84128757-0.02913220$

$\mathrm{H}-2.97507513-0.92381108-0.25723294$

$\mathrm{H}-2.54402020 \quad 0.16385961-1.59185074$

$\mathrm{H}-0.90565748 \quad 2.23376068-0.47456034$

$\mathrm{H} \quad 0.75222030 \quad 2.42314278 \quad 1.21578879$

H $1.90676426 \quad 1.74371527 \quad 0.07375407$

H $1.73161388 \quad 0.27741927 \quad 2.19250125$

H $1.70324828-1.78249434 \quad 0.78239275$

$\mathrm{H} 1.76977968-0.59684267-0.54010124$

$\mathrm{H}-0.61295116-2.14036938-0.13982699$

$\mathrm{H}-2.39858173 \quad 0.625642892 .39984328$

$\mathrm{H}-1.37918486 \quad 1.86345345 \quad 1.66002497$

$\mathrm{H}-1.05205878 \quad 1.337139123 .30635358$

$\mathrm{H}-0.49762291-1.15072534 \quad 3.72674828$

$\mathrm{H} \quad 0.13270986-2.14255567 \quad 2.41738674$

$\mathrm{H}-1.60036001-1.803516202 .51608772$

$00.81438920-0.71778639-3.13845411$

$00.304036951 .20089502-1.71144438$ 
$0.34696604-1.60099647-2.59815735$

Vibrational frequencies (in $\mathrm{cm}-1$ )

$\begin{array}{lllllllllllllllll}-396.0 & 29.8 & 46.6 & 62.2 & 71.7 & 94.1 & 137.8 & 149.7 & 157.5 & 175.1 & 187.3 & 212.8 & 231.0 & 265.0\end{array}$ $301.6 \quad 323.6 \quad 370.4 \quad 405.0 \quad 425.4 \quad 439.7 \quad 468.1 \quad 517.9 \quad 548.5 \quad 635.9 \quad 714.5 \quad 858.2 \quad 861.7 \quad 921.1$ $949.5 \quad 965.3 \quad 989.7 \quad 1005.8 \quad 1008.5 \quad 1017.2 \quad 1022.4 \quad 1046.4 \quad 1055.1 \quad 1089.0 \quad 1113.1 \quad 1138.4 \quad 1149.2$

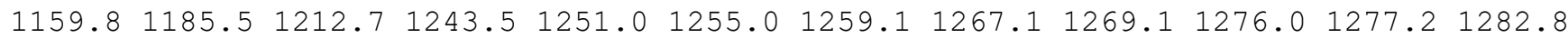

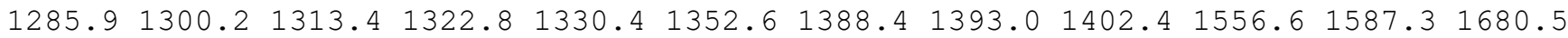
$2626.52642 .12645 .02645 .7 \quad 2655.5 \quad 2660.92665 .42676 .72679 .5 \quad 2726.92742 .42745 .6$ 2755.12769 .02771 .12775 .1

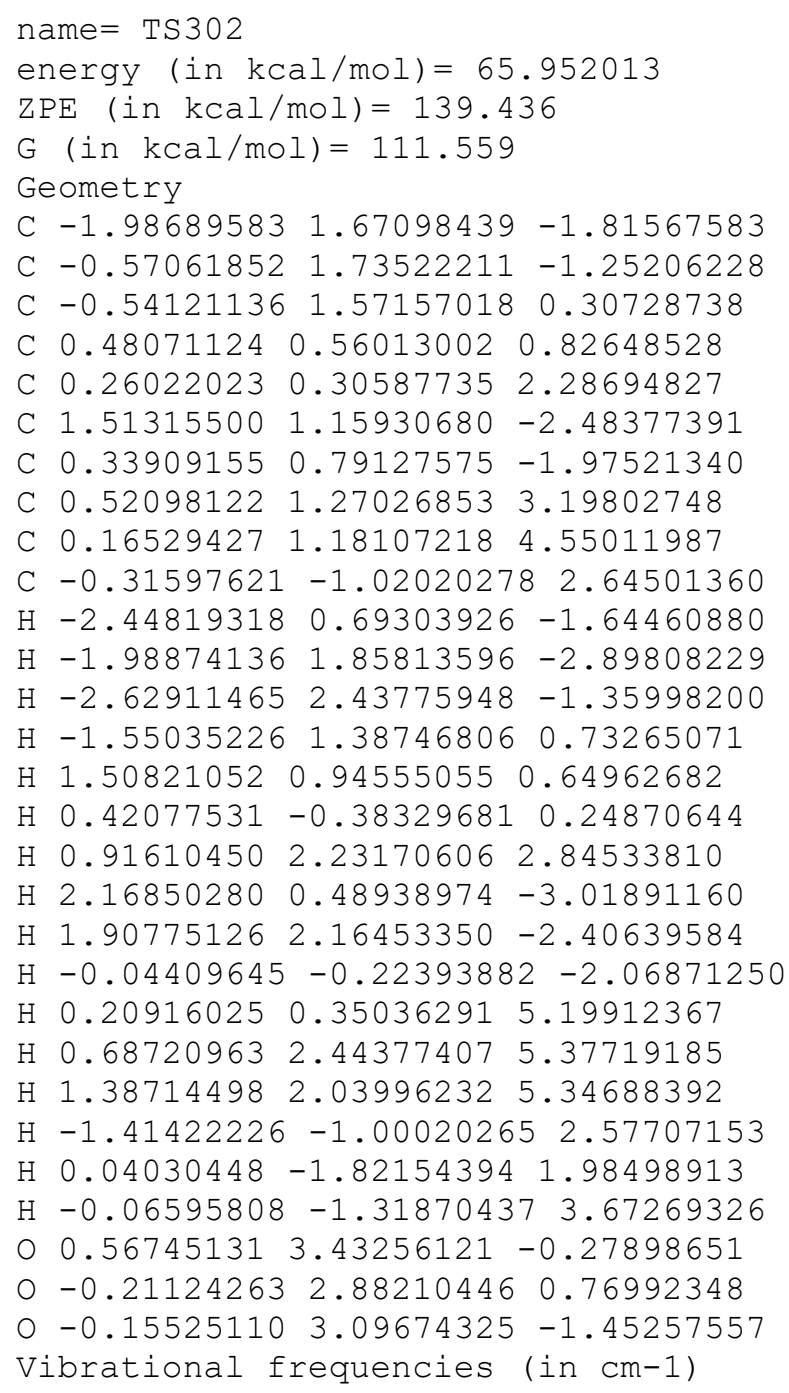


C $2.76276875-0.76600175-0.60971452$

C $-0.53958378-0.78669442 \quad 0.98149488$

C $-0.03107477-0.325544712 .14489183$

$\begin{array}{llll}\text { C }-0.26812352 & 0.98481422 & 2.59940931\end{array}$

C $0.70017441-1.265648893 .05612572$

$\mathrm{H}-3.21383630-0.18369554 \quad 0.98762215$

$\mathrm{H}-3.16878726 \quad 1.12891064-0.19516824$

$\mathrm{H}-2.380565871 .31486946 \quad 1.39030293$

$\mathrm{H}-0.85512805 \quad 2.11783601-0.59322018$

H 1.347083451 .328868110 .31987116

H $1.487712791 .76205602-1.39286639$

$\mathrm{H} \quad 0.92105006-0.94704800-1.66284542$

$\mathrm{H} 3.42713929-0.191329090 .01164261$

$\mathrm{H} \quad 3.16265859-1.73555499-0.85955966$

$\mathrm{H}-0.43309952-1.83599552 \quad 0.70530253$

$\mathrm{H}-0.13355871 \quad 1.89569098 \quad 2.08088991$

H $1.04213825 \quad 1.32982724 \quad 3.67774907$

$\mathrm{H} \quad 0.36918908 \quad 1.23632756 \quad 4.10208485$

$\mathrm{H} \quad 1.75601648-0.98726740 \quad 3.16517441$

H $0.69201628-2.29931904 \quad 2.67558356$

$\mathrm{H} \quad 0.23737414 \quad-1.29890668 \quad 4.05248632$

$0-1.89285721-0.08207446-2.16500903$

$\begin{array}{lllll}0 & -0.78468124 & 0.82456634 & -2.18551882\end{array}$

o $-1.65966707-0.90858029-1.05541678$

Vibrational frequencies (in $\mathrm{cm}-1$ )

$\begin{array}{lllllllllllllll}-840.4 & 36.9 & 51.8 & 78.9 & 92.1 & 99.7 & 115.7 & 141.5 & 146.8 & 169.0 & 195.1 & 243.5 & 266.0 & 287.1\end{array}$

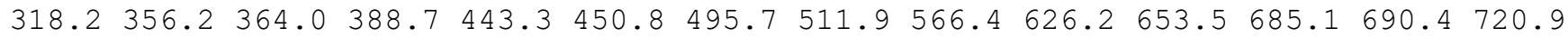
$823.5 \quad 870.4 \quad 883.0 \quad 906.7 \quad 940.6 \quad 960.6 \quad 981.6 \quad 983.0 \quad 1007.5 \quad 1012.3 \quad 1040.2 \quad 1058.0 \quad 1068.4$

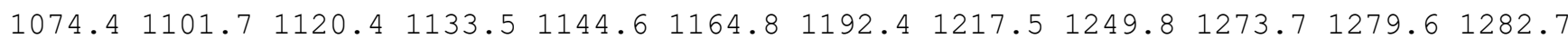

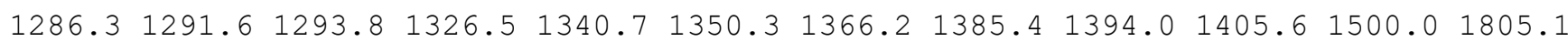
$1846.62660 .02663 .72668 .2 \quad 2674.12675 .92680 .32716 .12732 .0 \quad 2733.02748 .2 \quad 2751.0$ $2776.92779 .0 \quad 2794.14895 .4$

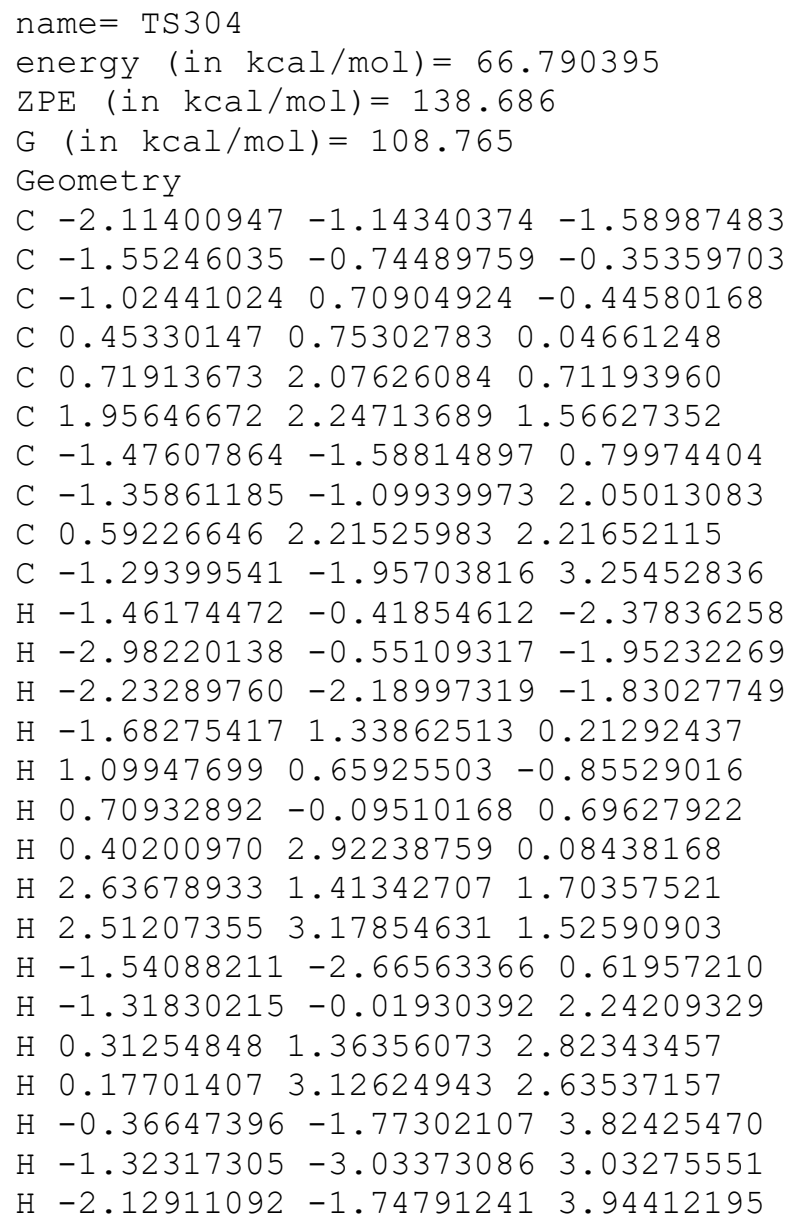


$0.471029441 .51510148-3.58341792$

$0-1.15142954 \quad 1.06679629-1.70611865$

O $0.798683880 .45057725-3.36919294$

Vibrational frequencies (in cm-1)

$\begin{array}{lllllllllllllll}-1017.8 & 15.5 & 29.3 & 46.5 & 51.6 & 55.1 & 73.5 & 77.9 & 106.0 & 122.0 & 136.2 & 149.4 & 170.9 & 190.4\end{array}$ $269.7318 .3 \quad 363.2 \quad 384.8 \quad 392.3 \quad 450.2 \quad 488.7 \quad 555.9 \quad 571.0 \quad 694.5 \quad 763.8 \quad 819.2 \quad 864.3 \quad 875.0$ $880.1901 .1939 .1950 .1 \quad 969.7 \quad 1001.3 \quad 1012.7 \quad 1016.6 \quad 1030.8 \quad 1050.1 \quad 1084.8 \quad 1087.1 \quad 1107.2$ $\begin{array}{lllllllllllll}1123.7 & 1132.1 & 1167.7 & 1188.4 & 1206.9 & 1215.0 & 1231.0 & 1253.0 & 1271.0 & 1277.3 & 1311.5 & 1316.2\end{array}$

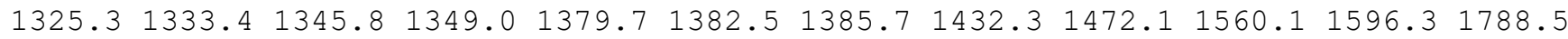
$2140.52610 .92655 .22656 .5 \quad 2664.3 \quad 2665.82710 .22710 .92725 .12740 .22747 .42754 .2$ $2760.8 \quad 2774.8 \quad 2789.5 \quad 2796.1$

name $=$ TS305

energy (in $\mathrm{kcal} / \mathrm{mol})=63.206402$

$\mathrm{ZPE}(\mathrm{in} \mathrm{kcal} / \mathrm{mol})=142.442$

$\mathrm{G}(\mathrm{in} \mathrm{kcal} / \mathrm{mol})=119.392$

Geometry

C $-3.90692039-0.63300420 \quad 3.49719674$

C $-3.20823768-1.120189032 .23839075$

C $-3.28066395-0.110790691 .03303542$

C $-1.95684499 \quad 0.15274908 \quad 0.28437111$

C $-0.76547980-0.56202807 \quad 0.91985481$

C $-1.16025626-2.050251131 .14557880$

C $-1.77881780-1.596042472 .50062382$

C $-0.77828486-0.35999407 \quad 2.49587674$

C $-1.31506709 \quad 0.84834996 \quad 3.09448111$

C $0.56079672-0.73851970 \quad 3.12476311$

$\mathrm{H}-4.88289105-0.186073113 .27598929$

$\mathrm{H}-3.29773860 \quad 0.11072386 \quad 4.03732469$

$\mathrm{H}-4.08270401-1.46389114 \quad 4.19363157$

$\mathrm{H}-3.77320070 \quad 0.83871542 \quad 1.32651433$

$\mathrm{H}-1.76539228 \quad 1.23843637 \quad 0.22507025$

$\mathrm{H}-2.07209172-0.18677454-0.76727164$

$\mathrm{H} \quad 0.18022146-0.36926905 \quad 0.41675661$

$\mathrm{H}-0.31609430-2.73541060 \quad 1.24365329$

$\mathrm{H}-1.85043176-2.482317050 .41831982$

$\mathrm{H}-1.69450345-2.27513334 \quad 3.34885424$

$\mathrm{H}-1.88774710 \quad 1.56008178 \quad 2.55226057$

$\mathrm{H} \quad 0.00068577 \quad 1.89293968 \quad 3.14922038$

$\mathrm{H}-0.23139905 \quad 1.66793568 \quad 3.89348459$

H $1.35936517-0.053380532 .82568889$

H $0.88336506-1.74559385 \quad 2.83134645$

$\mathrm{H} \quad 0.48879972 \quad-0.73800622 \quad 4.22059594$

$\begin{array}{lllll}0 & -4.08769598 & -2.10923016 & 0.38643031\end{array}$

$0-4.22384592-0.715926760 .15217289$

$0-4.01267234-2.227630021 .79633846$

Vibrational frequencies (in $\mathrm{cm}-1$ )

$\begin{array}{llllllllllllll}-971.8 & 90.2 & 134.8 & 157.7 & 175.9 & 202.5 & 208.7 & 219.8 & 264.5 & 283.3 & 328.5 & 349.8 & 382.0 & 405.8\end{array}$

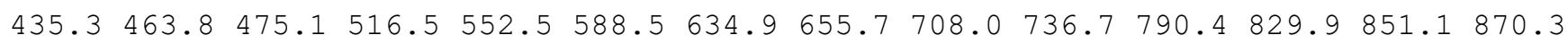
$895.6916 .6941 .5958 .1 \quad 989.6 \quad 1003.3 \quad 1009.9 \quad 1027.4 \quad 1039.0 \quad 1041.8 \quad 1056.4 \quad 1071.1 \quad 1080.5$

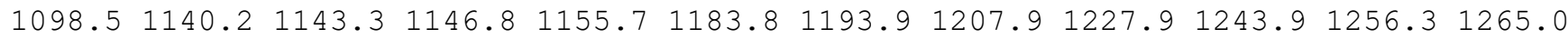

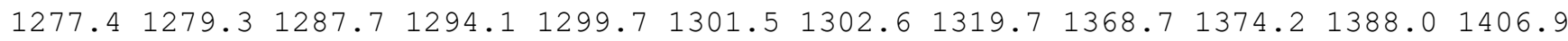
$1419.22648 .32658 .7 \quad 2668.6 \quad 2675.92679 .0 \quad 2686.5 \quad 2689.02715 .02740 .42747 .82752 .3$ $2767.6 \quad 2774.92779 .2 \quad 4731.8$

name $=\operatorname{TS} 306$

energy $($ in $\mathrm{kcal} / \mathrm{mol})=67.153643$

$\mathrm{ZPE}($ in $\mathrm{kcal} / \mathrm{mol})=138.71$

G $($ in $\mathrm{kcal} / \mathrm{mol})=108.636$

Geometry

C $-1.57625825-1.15436045-2.14958000$

C $-0.70652207-0.84494199-0.97808006$

C $-0.52278090 \quad 0.63343465-0.79134662$ 
C $1.22404516 \quad 0.75679374 \quad 0.36317403$

C $0.82172275-0.15086196 \quad 1.43988343$

C $0.74202386-1.606127370 .94245891$

C $-0.20366998-1.80453747-0.19621312$

C $-0.25865473 \quad 0.30368847 \quad 2.29139298$

C $-0.31201112 \quad 1.70806901 \quad 2.73171232$

C $-1.27275812-0.62595722 \quad 2.81432292$

$\mathrm{H}-1.18520144-0.64587185-3.05171084$

$\mathrm{H}-2.59953810-0.78359883-2.00084210$

$\mathrm{H}-1.63448798-2.22459335-2.37546267$

$\mathrm{H}-1.05554759 \quad 1.05485981 \quad 0.08226631$

H $1.20218299 \quad 1.81736464 \quad 0.48147638$

H $\quad 1.92878231 \quad 0.42429157-0.37148123$

$\mathrm{H} \quad 1.73708803-0.08432658 \quad 2.16361313$

H $0.49091152-2.29175125 \quad 1.77520586$

H $1.75644308 \quad-1.91888011 \quad 0.59769394$

$\mathrm{H}-0.42721767-2.85394567-0.39354216$

$\mathrm{H}-0.97265022 \quad 2.29699815 \quad 2.06087135$

$\mathrm{H} \quad 0.66387746 \quad 2.22014856 \quad 2.68754325$

$\mathrm{H}-0.71038740 \quad 1.83313907 \quad 3.74889747$

$\mathrm{H}-2.07462976-0.13026281 \quad 3.37983440$

$\mathrm{H}-0.83922518-1.39202760 \quad 3.47870679$

$\mathrm{H}-1.75828238-1.184744541 .98679111$

O $1.75922488 \quad 0.80983030-3.26620316$

$0-0.236890751 .34518463-1.74652933$

O $2.17526976-0.20863825-2.98765562$

Vibrational frequencies (in cm-1)

$\begin{array}{lllllllllllllllll}-221.1 & 19.6 & 20.7 & 42.0 & 52.3 & 63.0 & 68.2 & 96.7 & 102.3 & 117.1 & 126.8 & 146.9 & 196.9 & 251.2\end{array}$ $280.3 \quad 284.0 \quad 320.7 \quad 341.2 \quad 378.3 \quad 418.9 \quad 441.7 \quad 476.7 \quad 537.0 \quad 628.2 \quad 655.4 \quad 716.0 \quad 808.5 \quad 815.9$

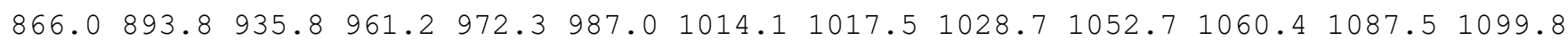
$\begin{array}{lllllllllllll}1155.7 & 1186.1 & 1230.2 & 1233.1 & 1235.9 & 1240.8 & 1253.9 & 1265.7 & 1268.5 & 1278.4 & 1286.4 & 1294.1\end{array}$ $1314.4 \quad 1360.91370 .3 \quad 1379.7 \quad 1385.3 \quad 1387.7 \quad 1398.5 \quad 1405.1 \quad 1486.1 \quad 1594.1 \quad 1723.4 \quad 1884.8$ $2544.92598 .0 \quad 2631.3 \quad 2634.1 \quad 2652.6 \quad 2657.3 \quad 2662.0 \quad 2665.02680 .0 \quad 2729.3 \quad 2732.5 \quad 2750.5$ $2762.32763 .8 \quad 2776.2 \quad 2786.9$

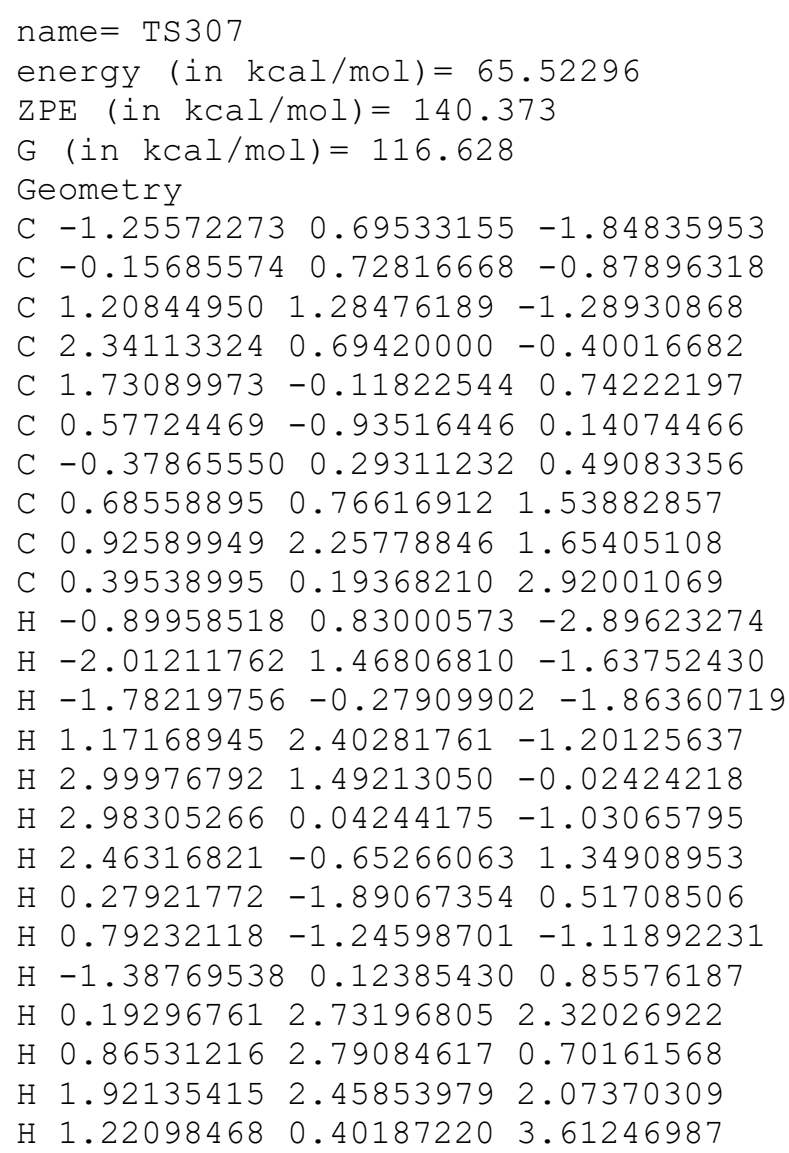


$\mathrm{H} \quad 0.25649959-0.893991792 .89883717$

$\mathrm{H}-0.51250991 \quad 0.63019470 \quad 3.35334493$

$01.60370225-0.41043496-2.90400309$

o $1.42536738 \quad 1.07630969-2.63308460$

O $0.61776734-1.08197832-2.44467927$

Vibrational frequencies (in $\mathrm{cm}-1$ )

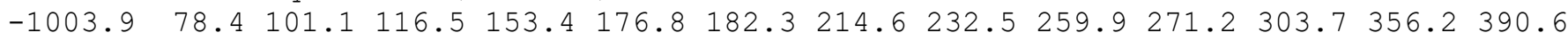
$429.1452 .8 \quad 458.6 \quad 500.2 \quad 533.4 \quad 573.9 \quad 631.6 \quad 657.7 \quad 704.4 \quad 712.6 \quad 798.1 \quad 827.0 \quad 881.6 \quad 909.9$ $950.7971 .2 \quad 991.8 \quad 1001.0 \quad 1010.0 \quad 1014.9 \quad 1018.5 \quad 1031.8 \quad 1040.4 \quad 1070.01108 .3 \quad 1133.5 \quad 1145.1$

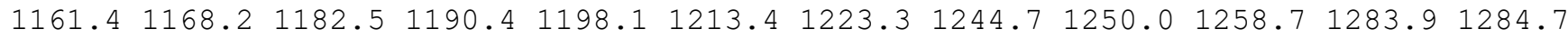

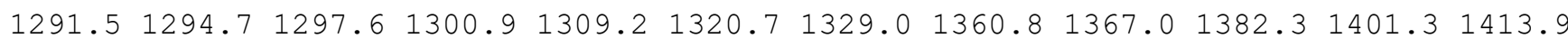
$1468.92620 .92643 .92649 .92664 .3 \quad 2670.2 \quad 2672.8 \quad 2675.82677 .2 \quad 2742.8 \quad 2749.4 \quad 2752.5$ $2754.7 \quad 2762.3 \quad 2780.5 \quad 2782.5$

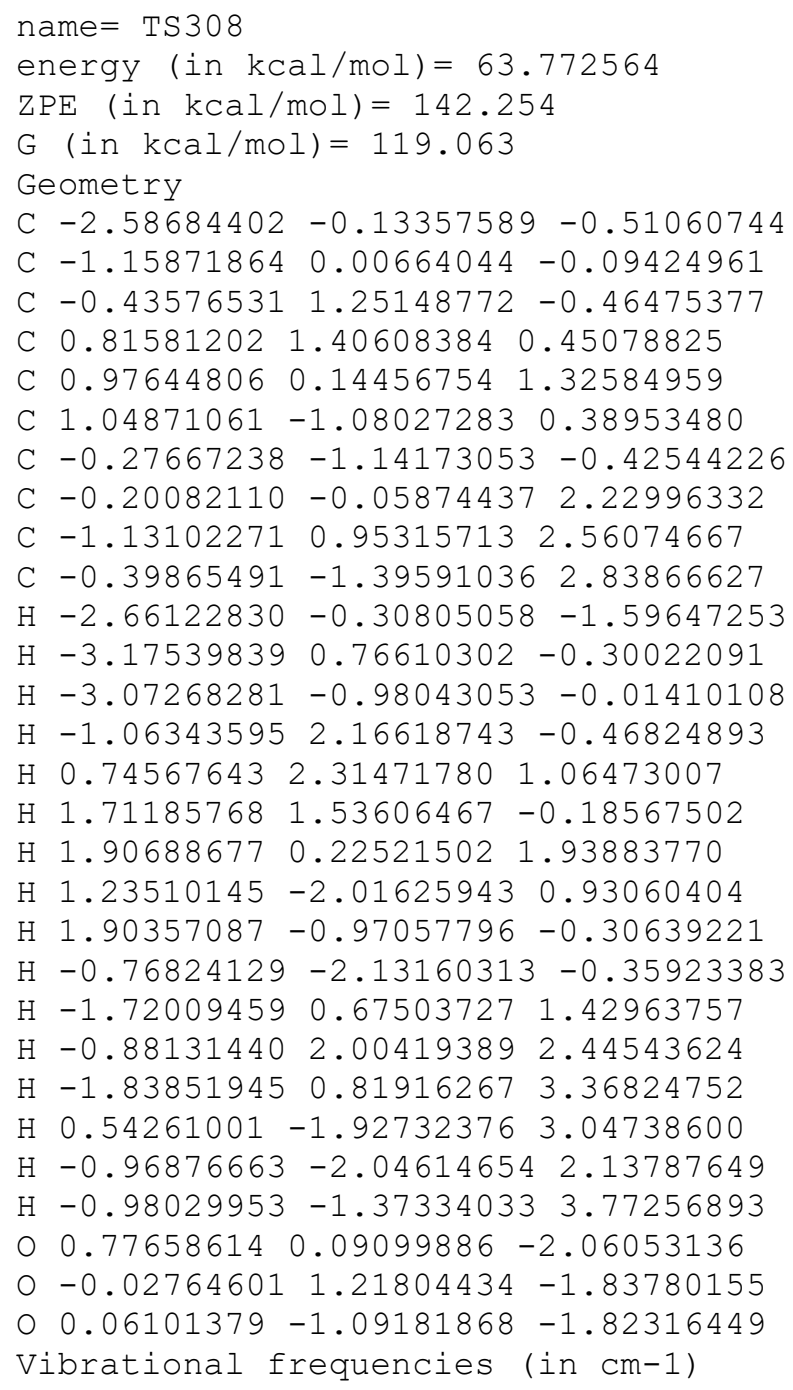


C $-0.42553493 \quad 1.36003187 \quad-0.61808991$

$\begin{array}{llll}\text { C } 0.13620499 & 2.46777289 & 0.35642169\end{array}$

C 0.980888621 .976401451 .54766068

$\begin{array}{lllll}\text { C } 1.17803692 & 0.45960677 & 1.53005081\end{array}$

$\begin{array}{lllll}\text { C } 1.53790112 & 0.02264448 & 0.08087738\end{array}$

$\begin{array}{llll}\text { C } & 0.00425275 & -0.04179447 & -0.19867685\end{array}$

C $-0.22489796-0.26696696 \quad 1.36216316$

$\begin{array}{llll}\text { C } & -1.25763780 & 0.38768488 & 2.13086263\end{array}$

C $-0.14951874-1.75375612 \quad 1.70200867$

H $-2.141509032 .50551014-1.29486899$

$\mathrm{H}-2.52601088 \quad 1.34915205-0.00647356$

$\mathrm{H}-2.23680773 \quad 0.78430105-1.65900794$

$\mathrm{H}-0.66930815 \quad 3.15308348 \quad 0.68142293$

H $0.51237130 \quad 2.28548935 \quad 2.50248333$

H $1.97136037 \quad 2.47933275 \quad 1.52249187$

H $1.802549190 .08973918 \quad 2.34376888$

H $2.05090000 \quad-0.93707787 \quad 0.00537438$

H $2.10017363 \quad 0.75049684-0.51290645$

$\mathrm{H}-0.35003527-0.83059918-0.85868606$

$\mathrm{H}-2.64641335-0.48974401 \quad 1.74643308$

$\mathrm{H}-1.71100221 \quad 1.31145728 \quad 1.88195071$

$\mathrm{H}-2.43329716-0.60433905 \quad 2.51925004$

$\mathrm{H}-0.98967772-2.30940928 \quad 1.27536832$

H $-0.15641029-1.89933616 \quad 2.79106405$

H $0.76789603-2.21983160 \quad 1.32361229$

$00.57450007 \quad 3.00401963-1.78930984$

$01.04379676 \quad 3.21078124-0.46640531$

o $0.278161641 .62133485-1.84526198$

Vibrational frequencies (in cm-1)

$\begin{array}{llllllllllllllll}-976.2 & 79.9 & 118.7 & 153.6 & 167.0 & 177.5 & 201.8 & 223.3 & 269.9 & 272.1 & 319.5 & 352.8 & 370.6 & 403.2\end{array}$

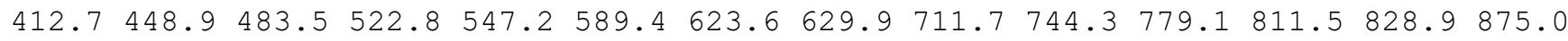

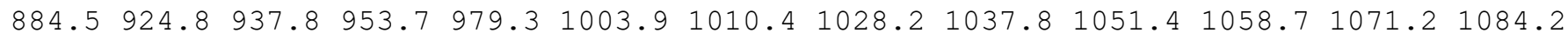
$\begin{array}{llllllllllll}1103.2 & 1128.9 & 1138.7 & 1140.5 & 1158.3 & 1172.7 & 1189.1 & 1211.7 & 1220.3 & 1239.8 & 1257.2 & 1266.5\end{array}$ $\begin{array}{lllllllllllll}1271.3 & 1283.7 & 1285.7 & 1290.6 & 1299.6 & 1300.8 & 1317.2 & 1322.0 & 1369.7 & 1374.4 & 1387.4 & 1395.5\end{array}$

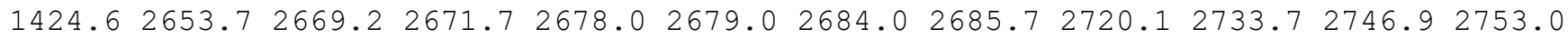
$2763.62779 .8 \quad 2780.7 \quad 4756.3$

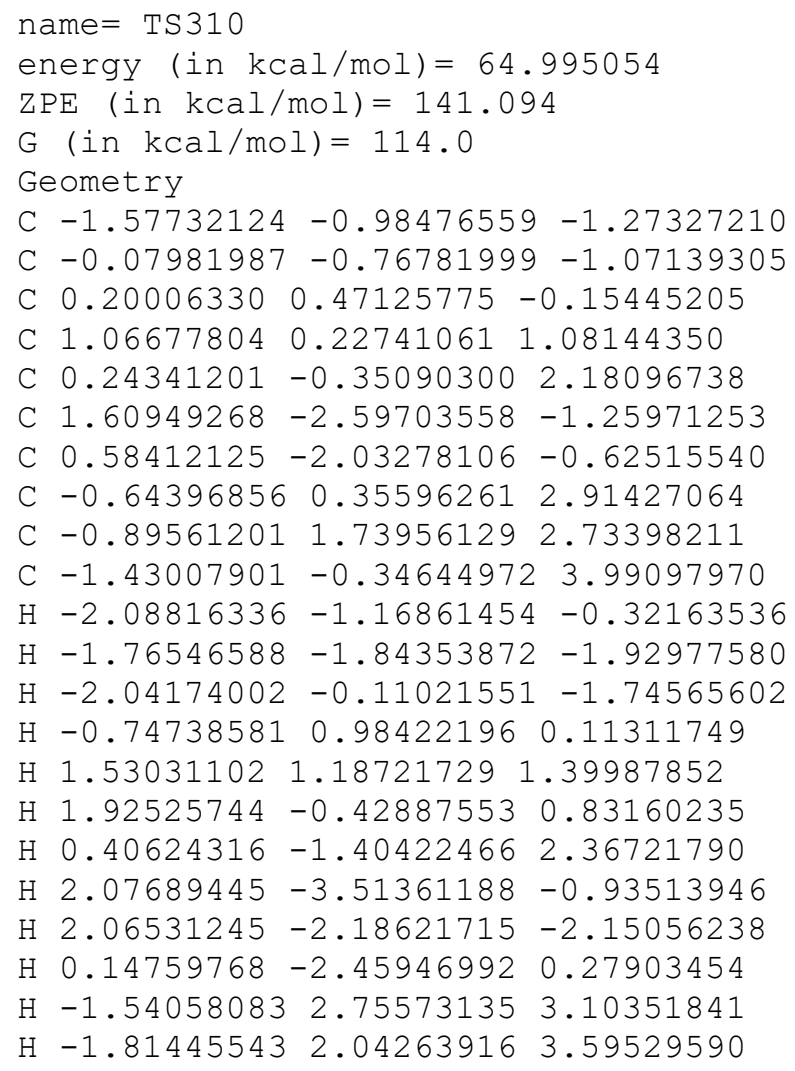


$\mathrm{H}-0.332628562 .302737492 .01616164$

$\mathrm{H}-1.23556353 \quad 0.08787963 \quad 4.97831127$

$\mathrm{H}-1.16308232-1.41074146 \quad 4.06466076$

$\mathrm{H}-2.50691786-0.31080603 \quad 3.79046361$

o $0.53390867 \quad 1.02416512-2.30495176$

$0 \quad 0.963936651 .34744513-0.99522944$

O $0.50655430-0.39415593-2.32927757$

Vibrational frequencies (in cm-1)

$\begin{array}{llllllllllllllll}-1292.0 & 30.9 & 32.8 & 45.6 & 75.9 & 91.7 & 105.2 & 160.7 & 195.0 & 205.7 & 230.1 & 232.0 & 273.1 & 310.2\end{array}$ $315.2 \quad 350.0 \quad 412.0 \quad 427.9 \quad 475.8 \quad 500.1 \quad 527.4 \quad 554.6 \quad 602.8 \quad 674.7 \quad 685.9 \quad 704.9 \quad 729.8 \quad 800.0$

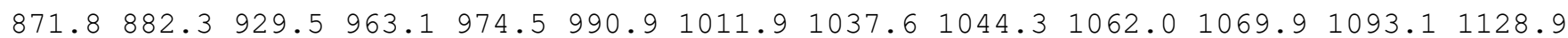

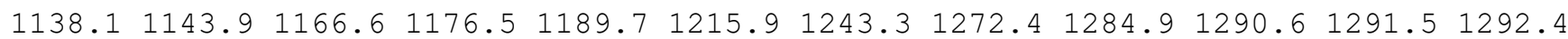
$1299.8 \quad 1326.6 \quad 1340.3 \quad 1340.7 \quad 1346.6 \quad 1386.4 \quad 1389.4 \quad 1397.6 \quad 1400.1 \quad 1486.7 \quad 1817.5 \quad 1851.8$ $1889.42655 .62669 .8 \quad 2674.7 \quad 2677.92679 .92681 .4 \quad 2710.62740 .82751 .0 \quad 2755.2 \quad 2773.3$ $2781.0 \quad 2781.4 \quad 2783.6 \quad 3792.0$

name $=\operatorname{TS} 311$

energy (in $\mathrm{kcal} / \mathrm{mol})=66.428942$

$\mathrm{ZPE}($ in $\mathrm{kcal} / \mathrm{mol})=139.691$

$\mathrm{G}(\mathrm{in} \mathrm{kcal} / \mathrm{mol})=115.572$

Geometry

C $-2.15933666-0.14219775-1.07571608$

C $-0.67254583-0.09822973-0.83838690$

C $-0.031518461 .29716599-0.67782851$

C $1.16237011 \quad 1.41069901 \quad 0.27309336$

C $1.386864590 .13846005 \quad 1.08433246$

C $1.42267202-1.05683547 \quad 0.08249227$

C $-0.14165395-1.073951030 .19439667$

C $0.02363299-0.42979496 \quad 1.63595327$

C $-0.96903577 \quad 0.49717094 \quad 2.22646592$

C $0.23731351-1.52635919 \quad 2.68831444$

$\mathrm{H}-2.49508163 \quad 0.75111187-1.63147984$

$\mathrm{H}-2.75242269-0.25543767-0.16834343$

$\mathrm{H}-2.40864225-0.98953861-1.74203133$

$\mathrm{H}-1.46342173 \quad 1.52752536 \quad 0.48091983$

$\mathrm{H} \quad 1.03130512 \quad 2.29397678 \quad 0.92292159$

H 2.06552101 $1.64214040 \quad-0.33324707$

H $2.20344778 \quad 0.21279471 \quad 1.80369335$

H $1.90998404-1.958533750 .45858895$

$\mathrm{H} \quad 1.85436683-0.84898902-0.89821071$

$\mathrm{H}-0.63347163-2.04823468 \quad 0.16903794$

$\mathrm{H}-1.542480201 .38445506 \quad 1.30889464$

$\mathrm{H}-0.56740166 \quad 1.254051212 .89764870$

$\mathrm{H}-1.89490553 \quad 0.04707361 \quad 2.58021469$

H $0.55871824-1.10644820 \quad 3.64831627$

H $1.01008730-2.243856412 .38445305$

$\mathrm{H}-0.67892204-2.10371523 \quad 2.85889773$

$0-0.58500100-0.30395415-3.14451680$

$\begin{array}{llll}0 & -0.32489180 & 2.22692323 & -1.39819112\end{array}$

o $0.04260238-0.45539269-2.09614167$

Vibrational frequencies (in $\mathrm{cm}-1$ )

$\begin{array}{lllllllllllllll}-692.8 & 74.3 & 85.3 & 129.2 & 155.8 & 164.4 & 168.6 & 200.0 & 228.9 & 250.2 & 268.4 & 294.2 & 332.7 & 380.4\end{array}$ $395.8 \quad 414.0 \quad 438.3 \quad 457.8 \quad 477.0 \quad 523.3 \quad 560.4 \quad 569.6 \quad 621.7 \quad 661.4 \quad 718.9 \quad 728.3 \quad 787.3 \quad 815.3$

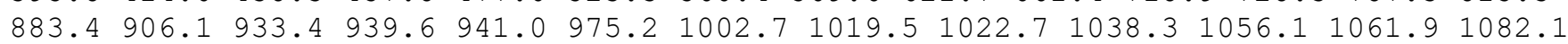

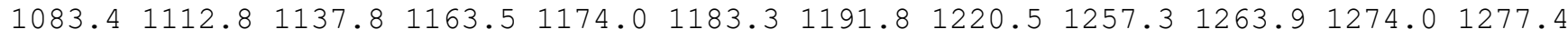
$1284.41290 .5 \quad 1292.2 \quad 1294.4 \quad 1312.9 \quad 1330.3 \quad 1345.1 \quad 1365.8 \quad 1381.8 \quad 1389.5 \quad 1404.6 \quad 1415.1$ $1776.52647 .12657 .6 \quad 2665.8 \quad 2675.1 \quad 2680.3 \quad 2681.62682 .12726 .4 \quad 2742.02744 .02747 .9$ $2763.7 \quad 2774.6 \quad 2779.2 \quad 3761.5$

name $=\operatorname{TS} 312$

energy (in $\mathrm{kcal} / \mathrm{mol})=63.994123$

$\mathrm{ZPE}($ in $\mathrm{kcal} / \mathrm{mol})=142.172$

$\mathrm{G}(\mathrm{in} \mathrm{kcal} / \mathrm{mol})=118.922$ 


\begin{tabular}{|c|c|c|}
\hline \multicolumn{3}{|c|}{ Geometry } \\
\hline & -1.79874738 & $-0.07398011-1.16869528$ \\
\hline & & $-0.38687883-0.87376659$ \\
\hline & $0.57564323 c$ & $0.89153740-0.76729216$ \\
\hline & $1.55097206 c$ & $0.93007660 \quad 0.42571007$ \\
\hline & 1.39913491 & $-0.27360603 \quad 1.35656825$ \\
\hline & 1.37945151 & $-1.56645510 \quad 0.4914$ \\
\hline & -0.14962327 & $-1.32751260 \quad 0.31]$ \\
\hline & -0.13610010 & -0.516789241 .68 \\
\hline & -0.84162460 & 0.725021811 .89970909 \\
\hline & -0.33037148 & -1.463211232 .86 \\
\hline & -2.34670159 & $-0.99038512-1.4$ \\
\hline & -1.89293289 & $0.60384176-2.02922205$ \\
\hline & -2.30401237 & $0.38333532-0.31$ \\
\hline & 1696374 & $1.82733026-0.8$ \\
\hline & 8316731 & $1.86571380 \quad 1.001$ \\
\hline & $95169 c$ & 0.971348830 .037 \\
\hline & 40715 & $-0.24764132 \quad 2.21$ \\
\hline & 1.62874680 & -2.481317121 .03286257 \\
\hline & 10812 & $-1.54849526-0.4$ \\
\hline & 8670189 & $-2.20909603 \quad 0.27729157$ \\
\hline & -1.13145691 & 1.404392671 .14203430 \\
\hline & 007966 & $0.23090618 \quad 2.17$ \\
\hline & 9987024 & $0.54594314 \quad 2.84649840$ \\
\hline & -0.17191665 & $-0.93082290 \quad 3.81650718$ \\
\hline & 758881 & -2.300125772 .85160874 \\
\hline & 511834 & -1.895633712 .87973645 \\
\hline & & $-0.49295776-2.33184709$ \\
\hline & 59015 & $0.87960081-1.99566742$ \\
\hline & & $-1.02879239-2.07644547$ \\
\hline
\end{tabular}

$\begin{array}{llllllllllllll}-984.3 & 84.9 & 115.7 & 156.2 & 167.0 & 176.9 & 208.4 & 219.8 & 264.6 & 275.5 & 318.9 & 345.2 \quad 378.2 \quad 407.2\end{array}$ $415.0 \quad 461.4 \quad 484.8 \quad 514.4 \quad 550.4 \quad 582.3 \quad 617.1 \quad 654.6 \quad 704.9 \quad 745.0 \quad 777.3 \quad 810.6 \quad 841.8 \quad 866.3$ $891.8916 .2 \quad 938.3 \quad 956.7 \quad 971.2 \quad 1002.8 \quad 1009.3 \quad 1028.6 \quad 1038.3 \quad 1042.0 \quad 1055.2 \quad 1070.8 \quad 1079.1$ $1097.4 \quad 1133.7 \quad 1139.3 \quad 1140.4 \quad 1155.6 \quad 1179.1 \quad 1189.8 \quad 1206.6 \quad 1223.6 \quad 1242.2 \quad 1255.4 \quad 1262.3$

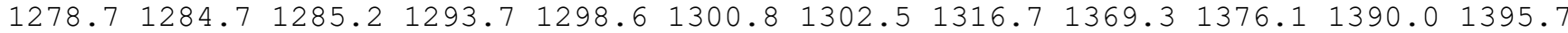
$1424.92656 .4 \quad 2668.1 \quad 2671.4 \quad 2678.8 \quad 2679.0 \quad 2685.8 \quad 2688.72721 .5 \quad 2736.5 \quad 2747.2 \quad 2752.7$ $2767.52779 .5 \quad 2780.2 \quad 4750.3$

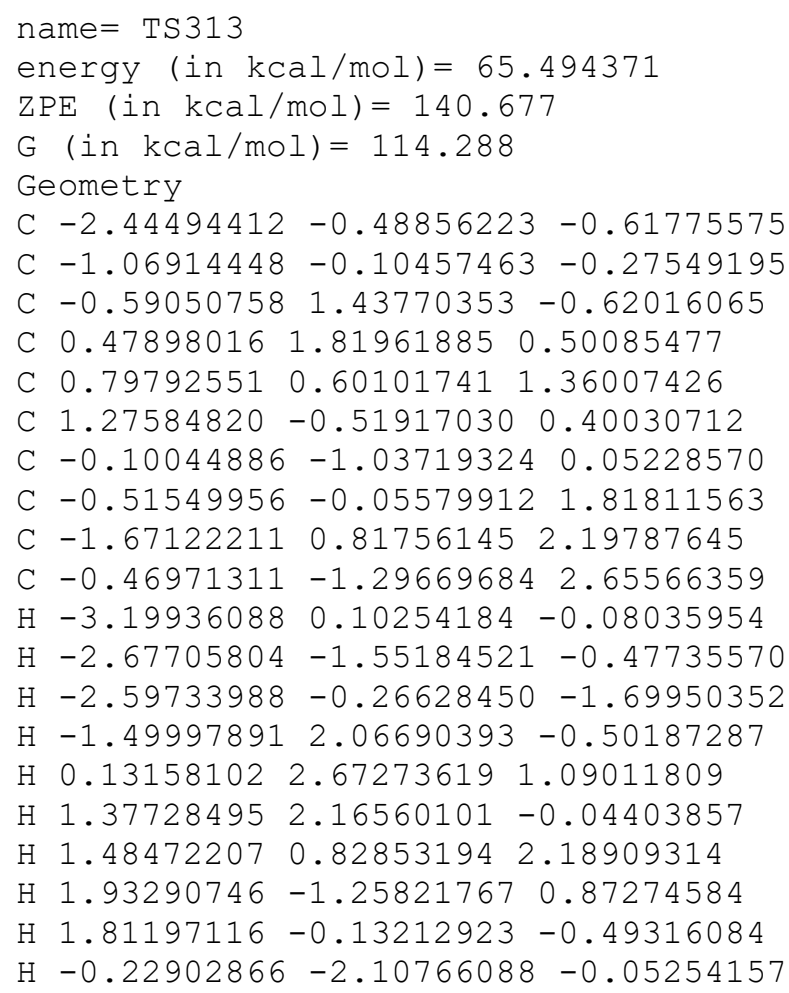


$\mathrm{H}-2.60623222 \quad 0.264197342 .35347670$

$\mathrm{H}-1.899218801 .623593961 .47869734$

$\mathrm{H}-1.437079061 .33973178 \quad 3.14345493$

$\mathrm{H}-0.47153709-1.043124303 .72776812$

$\mathrm{H} \quad 0.42094560-1.91636778 \quad 2.48128340$

$\mathrm{H}-1.34840794-1.938857952 .48705283$

O $0.12241901-0.56289367-3.38876626$

$0-0.120454761 .36154594-1.79530898$

O $-0.26552672-1.17207740-2.51267671$

Vibrational frequencies (in $\mathrm{cm}-1$ )

$\begin{array}{llllllllllllllll}-264.8 & 47.7 & 55.4 & 63.8 & 93.3 & 123.7 & 140.8 & 144.1 & 167.9 & 186.8 & 204.8 & 224.4 & 237.1 & 267.2\end{array}$ $310.4 \quad 356.2 \quad 375.5 \quad 409.5 \quad 431.2 \quad 456.7 \quad 466.0 \quad 523.9 \quad 553.7 \quad 657.8 \quad 718.1 \quad 836.7 \quad 867.8 \quad 926.8$ $949.4 \quad 962.4 \quad 989.9 \quad 1003.7 \quad 1012.5 \quad 1019.6 \quad 1020.6 \quad 1052.6 \quad 1052.8 \quad 1091.8 \quad 1118.4 \quad 1138.5 \quad 1150.9$

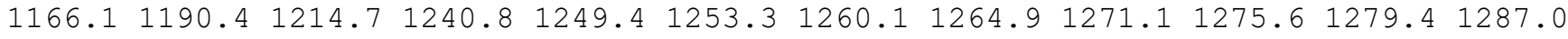

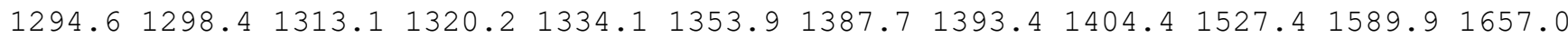
$2613.62635 .62638 .8 \quad 2656.4 \quad 2657.6 \quad 2662.6 \quad 2664.72674 .32677 .7 \quad 2729.02743 .02747 .1$ $2754.92767 .5 \quad 2769.3 \quad 2775.1$

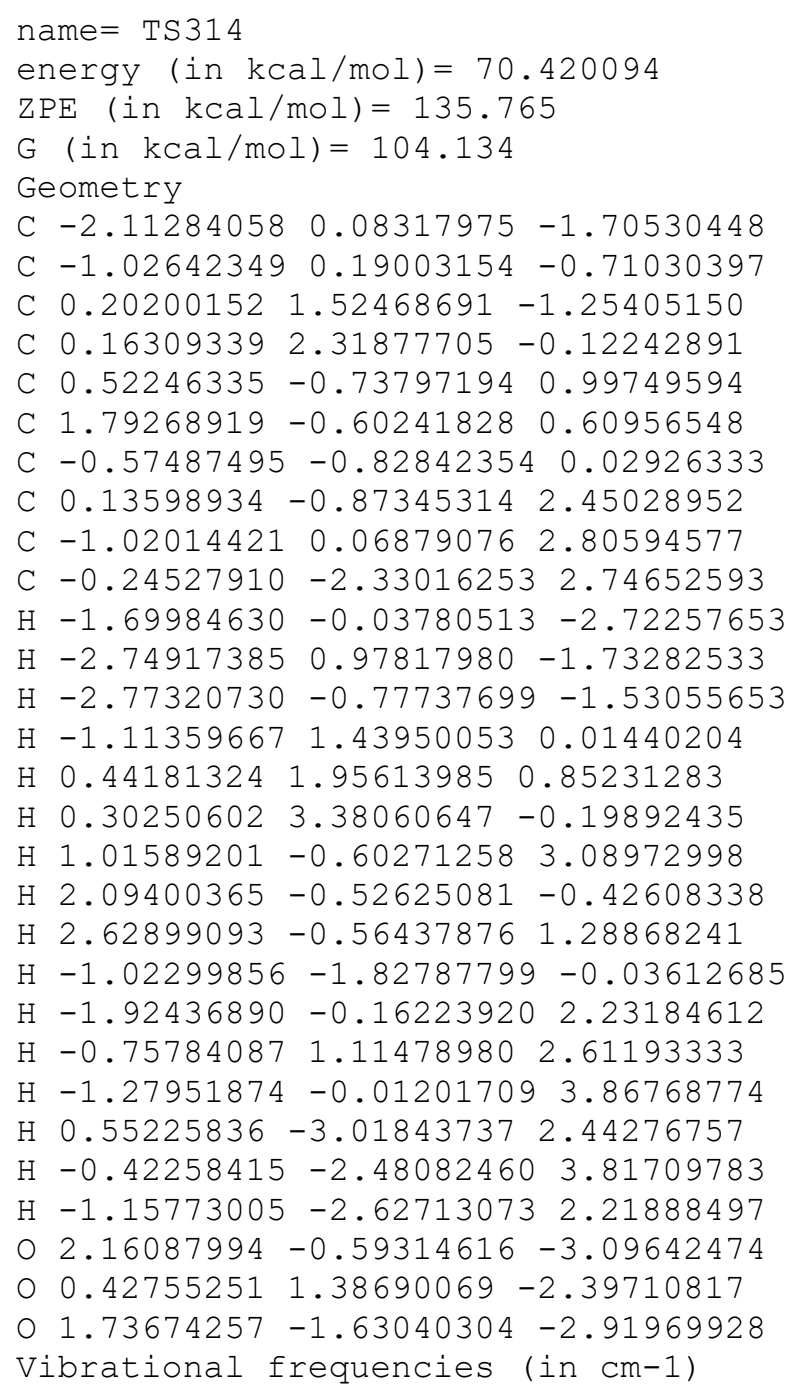


$\mathrm{ZPE}($ in $\mathrm{kcal} / \mathrm{mol})=138.648$

$\mathrm{G}($ in $\mathrm{kcal} / \mathrm{mol})=108.391$

Geometry

C $-2.35138361-0.24872309-1.10927468$

C $-1.03828199-0.22731121-0.40286175$

C $-0.605839791 .17243662-0.06795387$

C $1.45862809 \quad 0.97785369 \quad 0.21769111$

C $1.42238711-0.10956256 \quad 1.20055344$

C $0.96433359-1.428989810 .55164956$

C $-0.38161753-1.34864606-0.09078701$

C $0.83803527 \quad 0.21570244 \quad 2.48653685$

C $1.16047132 \quad 1.49431438 \quad 3.14301526$

C $-0.00764669-0.743481563 .21493692$

$\mathrm{H}-2.33036358 \quad 0.45283641-1.96545369$

$\mathrm{H}-3.16554705 \quad 0.08443124 \quad-0.45180736$

$\mathrm{H}-2.61674306-1.23647661-1.50096711$

$\mathrm{H}-0.66650760 \quad 1.42198749 \quad 1.00978393$

H $1.62632245 \quad 1.99171245 \quad 0.50583370$

$\mathrm{H} \quad 1.75136870 \quad 0.76600138-0.78982519$

$\mathrm{H} 2.55164531-0.23059991 \quad 1.46818265$

H $0.99159700 \quad-2.25710265 \quad 1.28633058$

$\mathrm{H} \quad 1.70144733-1.71046132-0.23800888$

$\mathrm{H}-0.79776756-2.32424802-0.34577367$

H $0.37247987 \quad 2.24691921 \quad 2.92905557$

H $2.09525719 \quad 1.95211164 \quad 2.77901625$

$\mathrm{H} \quad 1.22806503 \quad 1.41854681 \quad 4.23800539$

$\mathrm{H}-0.45958002-0.32762317 \quad 4.12682294$

$\mathrm{H} 0.54566239-1.64875040 \quad 3.51587718$

$\mathrm{H}-0.84152330-1.09999405 \quad 2.57397229$

O $0.89342769-0.44534991-3.30526070$

$0-0.66055758 \quad 2.05346361-0.91541276$

O $0.59796498-1.32226697-2.64769820$

Vibrational frequencies (in $\mathrm{cm}-1$ )

$\begin{array}{llllllllllllllll}-222.9 & 18.6 & 24.9 & 33.2 & 50.0 & 62.1 & 72.4 & 83.9 & 94.5 & 108.2 & 122.1 & 145.1 & 198.7 & 250.6\end{array}$ $280.2 \quad 284.0 \quad 318.4 \quad 340.2 \quad 377.9 \quad 418.9 \quad 440.0 \quad 476.9 \quad 536.8 \quad 627.2 \quad 654.3 \quad 718.7 \quad 811.8 \quad 820.1$

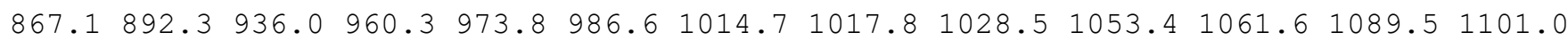
$1156.21185 .5 \quad 1230.5 \quad 1232.8 \quad 1235.2 \quad 1240.6 \quad 1253.9 \quad 1265.5 \quad 1268.6 \quad 1278.7 \quad 1286.4 \quad 1291.0$

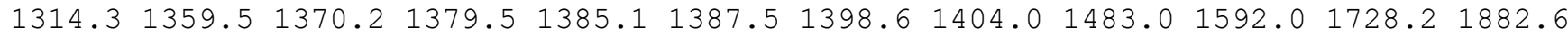
$2550.52588 .2 \quad 2631.5 \quad 2633.4 \quad 2651.92657 .2 \quad 2662.12665 .12680 .52730 .52732 .72750 .5$ $2762.52763 .5 \quad 2776.62787 .6$

\section{name $=\operatorname{TS} 316$}

energy (in $\mathrm{kcal} / \mathrm{mol})=64.422971$

$\mathrm{ZPE}(\mathrm{in} \mathrm{kcal} / \mathrm{mol})=141.839$

$\mathrm{G}(\mathrm{in} \mathrm{kcal} / \mathrm{mol})=117.852$

Geometry

C $-1.74338234-1.01118587-1.16631058$

C $-0.33137422-1.30570449-1.40715714$

C $0.48423536-0.17381984-2.13088502$

C $1.93743852-0.04509659-1.68450689$

C $2.065442370 .05106025-0.15611428$

C $1.58084836-2.52554530-0.10616826$

C $0.23509916-1.83001595-0.10873073$

C $1.44447763-1.15593800 \quad 0.52986296$

C $1.432381331 .35649004 \quad 0.34366363$

C $1.48245776-1.088201812 .03670631$

$\mathrm{H}-2.04803075-0.14451684-0.63062829$

$\mathrm{H}-2.54092804-1.07765352-2.60024500$

$\mathrm{H}-2.24929926-0.33277123-2.66485510$

$\mathrm{H}-0.04591580 \quad 0.79714885-2.08131812$

H 2.39886105 $0.84093472-2.16249509$

H 2.51954262 $-0.90971856-2.06727967$

H $3.15751055 \quad 0.06488406 \quad 0.09708538$

H $1.72451798-3.401827310 .51943360$ 
H $2.12086189-2.65340808-1.04117106$

$\mathrm{H}-0.54362294 \quad-2.23826742 \quad 0.55330365$

$\mathrm{H} 1.70757505 \quad 1.56204448 \quad 1.38430703$

$\mathrm{H} \quad 0.33918078 \quad 1.31674572 \quad 0.29536255$

$\mathrm{H} \quad 1.76321554 \quad 2.21364724-0.25342644$

$\mathrm{H} \quad 0.80588625-0.313105592 .41994648$

H 2.49303132 $-0.85943828 \quad 2.39940518$

$\mathrm{H} 1.17980010-2.034703992 .50276059$

$0-0.29214672-1.75430832-3.59686674$

$00.51608373-0.55928014-3.50096303$

O $-0.17829765-2.38482597-2.35897714$

Vibrational frequencies (in $\mathrm{cm}-1$ )

$\begin{array}{lllllllllllllllll}-813.3 & 44.5 & 112.1 & 129.9 & 157.2 & 167.6 & 189.8 & 226.4 & 254.5 & 272.0 & 291.0 & 312.3 & 347.6 & 380.3\end{array}$ $\begin{array}{llllllllllllll}386.6 & 432.4 & 444.7 & 478.7 & 486.9 & 517.5 & 617.6 & 622.5 & 682.7 & 710.5 & 755.5 & 790.1 & 806.3 & 874.7\end{array}$

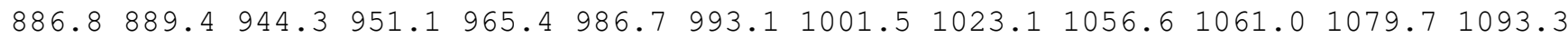
$1099.3 \quad 1114.91129 .2 \quad 1141.7 \quad 1172.5 \quad 1199.7 \quad 1204.5 \quad 1221.1 \quad 1251.0 \quad 1264.7 \quad 1280.01285 .3$ $1290.21294 .91295 .91300 .6 \quad 1308.7 \quad 1316.91327 .6 \quad 1370.6 \quad 1377.8 \quad 1389.7 \quad 1397.5 \quad 1409.6$ $\begin{array}{llllllllllll}1493.1 & 2654.1 & 2673.2 & 2673.7 & 2675.9 & 2677.2 & 2686.5 & 2699.9 & 2706.2 & 2712.3 & 2736.9 & 2741.2\end{array}$ $2780.3 \quad 2781.6 \quad 2783.7 \quad 4874.1$

\section{name $=$ TS317}

energy $($ in $\mathrm{kcal} / \mathrm{mol})=66.94627$

$\operatorname{ZPE}(\mathrm{in} \mathrm{kcal} / \mathrm{mol})=139.397$

$\mathrm{G}($ in $\mathrm{kcal} / \mathrm{mol})=112.098$

Geometry

C $-2.42942617-0.62198868-1.47403739$

C $-1.00483982-0.32458870-1.36887643$

C $-0.52944485 \quad 0.13374991-2.80214111$

C $0.45638503 \quad 1.30156375-2.84255673$

C $-0.20867848 \quad 2.60362594-3.14041632$

C $0.27358675 \quad 3.77198143 \quad-2.71591327$

C $-0.60639011 \quad 0.54326121 \quad-0.22733660$

C $-1.16836872 \quad 1.70839716 \quad 0.12405657$

C $-2.30368354 \quad 2.35893058-0.58447941$

C $-0.64979105 \quad 2.47492223 \quad 1.29949563$

$\mathrm{H}-3.14743288-0.43375432-0.01608713$

$\mathrm{H}-2.79119331-1.138799320 .12556963$

$\mathrm{H}-2.77037200-1.54819329-1.87529834$

$\mathrm{H}-1.39876950 \quad 0.29376352-3.47579526$

H $1.222820241 .09585622-3.63015156$

H $1.043027091 .34644894-1.90033572$

H $-1.111181112 .54299895-3.74968143$

$\mathrm{H} \quad 1.16143483 \quad 3.87240251 \quad-2.11245969$

$\mathrm{H}-0.18230559 \quad 4.72159580 \quad-2.94295307$

H $0.24438996 \quad 0.14480941 \quad 0.33402213$

$\mathrm{H}-3.07067810 \quad 2.726016450 .10758241$

$\mathrm{H}-2.80629196 \quad 1.68203565-1.29955817$

$\mathrm{H}-1.94987317 \quad 3.22229945-1.17041002$

$\mathrm{H}-0.37547366 \quad 3.500411821 .01197452$

$\mathrm{H} 0.24089224 \quad 2.01924000 \quad 1.75202212$

$\mathrm{H}-1.41056823 \quad 2.552714952 .08899515$

O $-0.13163570-2.08477517-2.46437415$

$0.20736750-0.98453763-3.30936150$

$0-0.20971538-1.53621688-1.17934895$

Vibrational frequencies (in $\mathrm{cm}-1$ )

$\begin{array}{llllllllllllllllll}-794.2 & 24.2 & 35.5 & 55.6 & 71.2 & 100.3 & 108.0 & 131.1 & 172.6 & 202.6 & 210.8 & 246.4 & 272.0 & 303.7\end{array}$ $328.9354 .9 \quad 365.8 \quad 386.1 \quad 437.5 \quad 466.2 \quad 486.6 \quad 488.1 \quad 549.3 \quad 581.2 \quad 590.5 \quad 701.5 \quad 728.0 \quad 828.5$

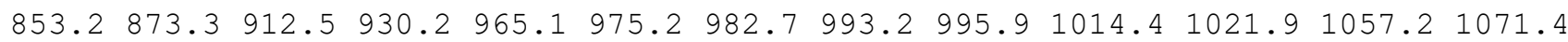
$1084.81101 .91118 .0 \quad 1122.6 \quad 1154.4 \quad 1178.4 \quad 1189.3 \quad 1203.8 \quad 1245.0 \quad 1268.8 \quad 1274.11276 .8$

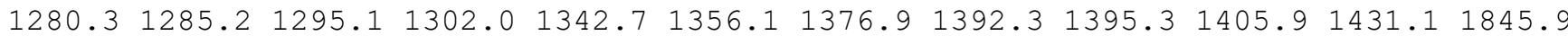

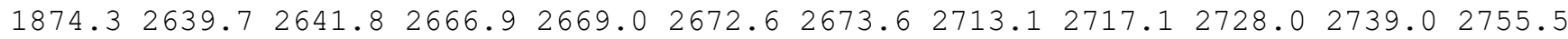
$2771.92780 .12791 .9 \quad 4875.6$ 


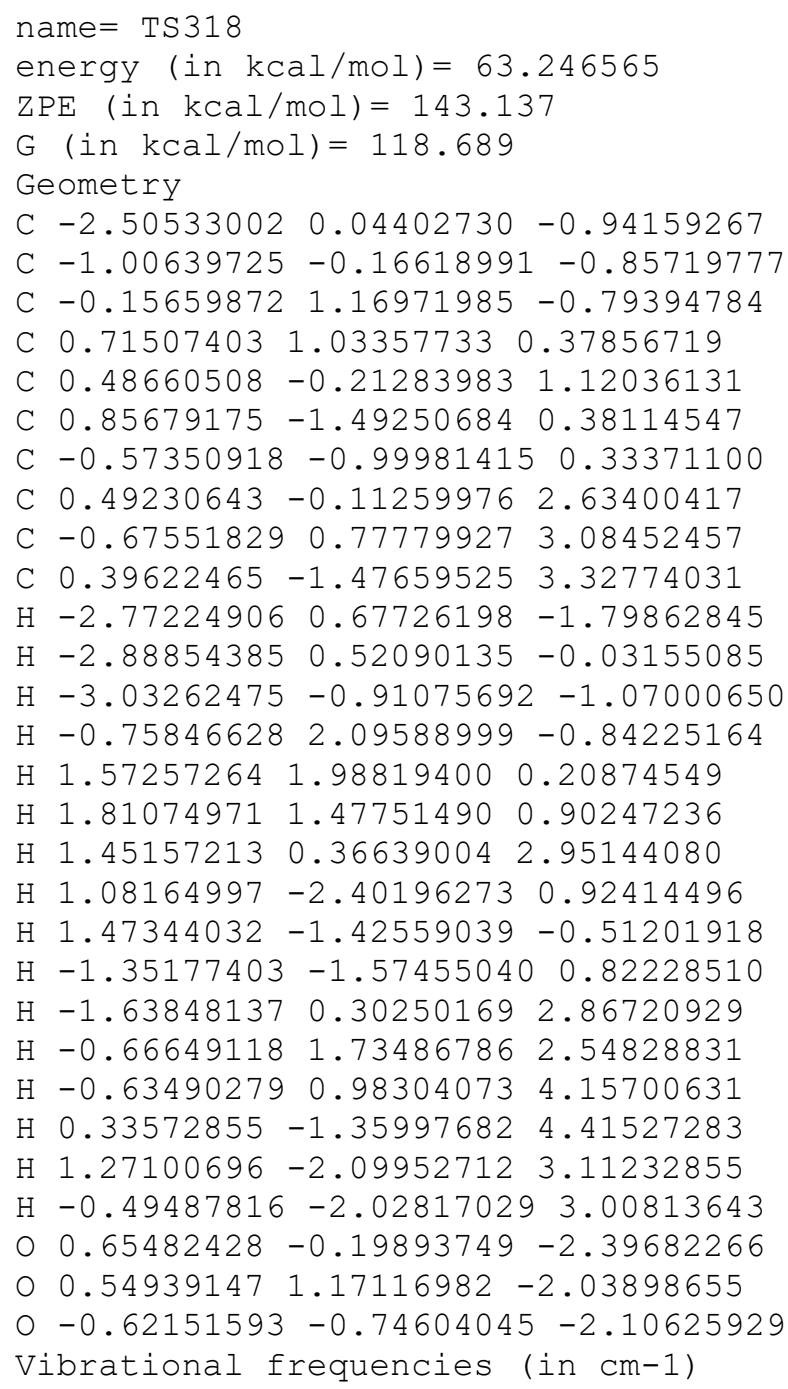


$\mathrm{H}-1.67758879-3.397593793 .12768993$

$\mathrm{H} 2.28913972-0.81223452-0.86999632$

$\mathrm{H} 1.463232190 .59784979-0.10633902$

$\mathrm{H} \quad 0.10588697-2.06832711-0.95229803$

$\mathrm{H} 1.07260070-1.03195836 \quad 4.55014265$

$\mathrm{H}-0.03686266-0.49692706 \quad 5.81927891$

$\mathrm{H}-0.29648496 \quad 0.01848882 \quad 4.14932116$

$\mathrm{H}-0.66944840 \quad-2.94725598 \quad 6.45004274$

$\mathrm{H}-2.00600873-3.57853294 \quad 5.48111968$

$\mathrm{H}-2.13307797-1.97639843 \quad 6.21606347$

$0-0.83153485 \quad 1.058704691 .63705138$

$\begin{array}{llll}0 & -1.76857733 & 0.11307789 & 2.13220194\end{array}$

$\begin{array}{lllll}0 & -0.94440433 & 0.94121718 & 0.23259091\end{array}$

Vibrational frequencies (in $\mathrm{cm}-1$ )

$\begin{array}{lllllllllllllllll}-762.2 & 30.3 & 47.9 & 70.1 & 77.8 & 100.3 & 104.4 & 124.3 & 185.3 & 190.4 & 214.1 & 237.8 & 278.6 & 287.9\end{array}$ $306.6 \quad 335.4 \quad 388.8 \quad 421.0 \quad 452.0 \quad 476.0 \quad 493.3 \quad 527.7 \quad 554.6 \quad 601.2 \quad 632.4 \quad 685.5 \quad 712.5 \quad 811.2$

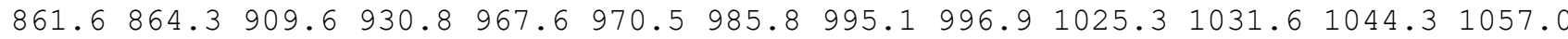

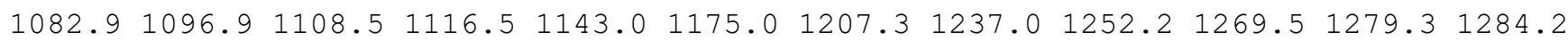

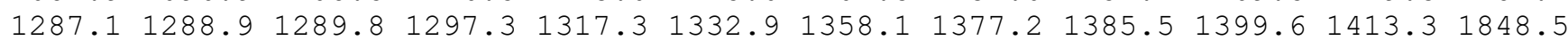
$1872.02657 .82663 .92671 .5 \quad 2674.8 \quad 2677.7 \quad 2684.72707 .92714 .32739 .22750 .12761 .8$ $2777.8 \quad 2779.4 \quad 2780.6 \quad 4867.3$

name $=$ TS320

energy (in $\mathrm{kcal} / \mathrm{mol})=64.247817$

$\mathrm{ZPE}($ in $\mathrm{kcal} / \mathrm{mol})=142.169$

$\mathrm{G}($ in $\mathrm{kcal} / \mathrm{mol})=118.691$

Geometry

C $-2.63961830 \quad 0.87284492 \quad 0.10259881$

C $-1.13090018 \quad 0.96116794 \quad-0.06780751$

$\begin{array}{lllll}C & -0.54012670 & 2.41199654 & 0.08448285\end{array}$

C $0.73115292 \quad 2.53881477 \quad 0.95133620$

C 1.137671301 .219663091 .60630093

C $1.142292960 .11116602 \quad 0.51580450$

C $-0.38190030-0.05371399 \quad 0.79672560$

$\begin{array}{llll}\text { C } & -0.13273457 & 0.52012355 & 2.25573274\end{array}$

C $-1.19797755 \quad 1.31812821 \quad 2.83467495$

C $0.26245463-0.59641603 \quad 3.22176685$

$\mathrm{H}-3.15615708 \quad 1.71428364-0.37445059$

$\mathrm{H}-2.93457080 \quad 0.83743371 \quad 1.16108688$

$\mathrm{H}-3.02831372-0.04356117-0.36625046$

$\mathrm{H}-1.31640182 \quad 3.14064254 \quad 0.39511460$

$\mathrm{H} \quad 0.58817627 \quad 3.31988534 \quad 1.71783902$

$\mathrm{H} 1.561762492 .90122003 \quad 0.30817985$

$\mathrm{H} 2.01901065 \quad 1.29598473 \quad 2.24074190$

$\mathrm{H} \quad 1.74132029-0.76779398 \quad 0.76392647$

H $1.420843920 .42409119-0.49211166$

$\mathrm{H}-0.79741688-1.06099194 \quad 0.74726656$

$\mathrm{H}-1.59588035 \quad 2.196161112 .39406386$

$\mathrm{H}-0.36990197 \quad 2.23013732 \quad 3.96563666$

$\mathrm{H}-0.82844759 \quad 1.66861556 \quad 4.33223976$

$\mathrm{H} \quad 0.73769093-0.20231738 \quad 4.12412613$

$\mathrm{H} \quad 0.95633094 \quad-1.31568880 \quad 2.77494773$

$\mathrm{H}-0.63207455-1.154542993 .53466367$

$00.067613061 .57119466-1.90812118$

$\begin{array}{llll}0 & -0.25080954 & 2.79081160 & -1.25843348\end{array}$

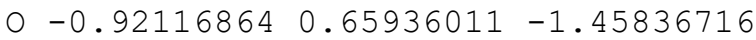

Vibrational frequencies (in $\mathrm{cm}-1$ )

$\begin{array}{llllllllllllll}-970.6 & 74.8 & 101.4 & 134.1 & 146.3 & 170.6 & 207.3 & 222.8 & 263.9 & 283.8 & 322.8 & 343.8 & 378.8 & 403.2\end{array}$

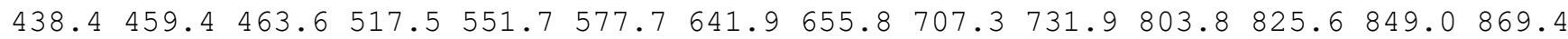

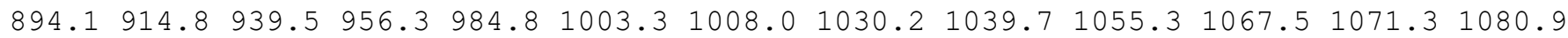
$1099.61134 .91140 .7 \quad 1146.0 \quad 1153.3 \quad 1181.0 \quad 1193.2 \quad 1203.4 \quad 1217.2 \quad 1244.2 \quad 1256.01263 .3$

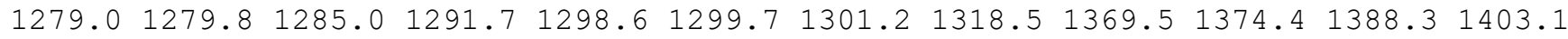
$1418.12658 .52658 .92672 .1 \quad 2674.4 \quad 2679.8 \quad 2685.82688 .62716 .92740 .32745 .52751 .3$ $2767.12775 .6 \quad 2781.2 \quad 4720.8$ 
name $=\mathrm{TS} 321$

energy $($ in $\mathrm{kcal} / \mathrm{mol})=64.488534$

$\operatorname{ZPE}($ in $\mathrm{kcal} / \mathrm{mol})=141.99$

$\mathrm{G}($ in $\mathrm{kcal} / \mathrm{mol})=118.755$

Geometry

C $-2.28106617-0.62938789-1.21634940$

C $-0.91413210-0.30079357-0.84149408$

C $-0.612690501 .09240432-0.15299429$

C $0.23390734 \quad 1.04502801 \quad 1.13392839$

C $0.60254870-0.37836876 \quad 1.55109849$

C $1.15196781-1.121641010 .29516854$

C $-0.33219261-1.47099867-0.03638292$

C $-0.65648856-1.326766371 .50296942$

C $-1.99487304-0.77328801 \quad 1.94599650$

C $-0.41204457-2.64152940 \quad 2.24055392$

$\mathrm{H}-2.64032969 \quad 0.20902958-2.58683439$

$\mathrm{H}-2.78129931 \quad 0.78649974 \quad-2.04692452$

$\mathrm{H}-3.10211982-0.58512682-0.54891106$

$\mathrm{H}-1.54316345 \quad 1.67320188 \quad 0.00351020$

$\mathrm{H}-0.29762481 \quad 1.55501727 \quad 1.95788158$

H $1.16269707 \quad 1.63212421 \quad 0.96893622$

H $1.20268474 \quad-0.42198440 \quad 2.46033816$

H $1.77618513-1.98991657 \quad 0.51520858$

$\mathrm{H} 1.68603092-0.51093526-0.43369535$

$\mathrm{H}-0.53025121-2.44276394-0.49398284$

$\mathrm{H}-2.80849277-1.46550804 \quad 1.69068275$

$\mathrm{H}-2.24149130 \quad 0.19573695 \quad 1.50710056$

$\mathrm{H}-2.01430430-0.63531758 \quad 3.03598850$

$\mathrm{H}-1.19741053-3.371848712 .01165379$

$\mathrm{H}-0.40417544-2.490157463 .32606102$

$\mathrm{H} \quad 0.54463825 \quad-3.10292351 \quad 1.97178925$

$0.73286328 \quad 0.83523425-1.93893141$

○ $0.06774145 \quad 1.82757453-1.16272750$

$0-0.22721909-0.18120177-2.11034055$

Vibrational frequencies (in $\mathrm{cm}-1$ )

$\begin{array}{lllllllllllllllll}-849.3 & 87.4 & 135.3 & 155.6 & 163.9 & 173.5 & 205.8 & 220.1 & 258.8 & 271.5 & 313.0 & 344.2 & 381.5 & 413.4\end{array}$

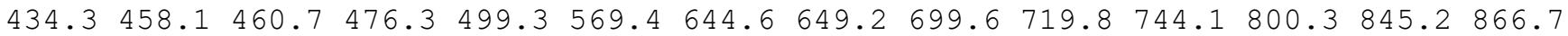
$894.2917 .9939 .2 \quad 960.3 \quad 977.6 \quad 994.5 \quad 1002.9 \quad 1007.3 \quad 1015.2 \quad 1019.6 \quad 1042.4 \quad 1051.4 \quad 1086.1$ $1087.91101 .8 \quad 1107.9 \quad 1156.5 \quad 1168.3 \quad 1185.2 \quad 1195.6 \quad 1199.3 \quad 1222.2 \quad 1243.8 \quad 1257.2 \quad 1272.6$ $1284.21287 .4 \quad 1295.2 \quad 1297.1 \quad 1300.4 \quad 1307.9 \quad 1318.8 \quad 1320.8 \quad 1342.0 \quad 1374.0 \quad 1383.8 \quad 1406.5$ $1417.42657 .92672 .92674 .0 \quad 2678.5 \quad 2679.6 \quad 2686.6 \quad 2689.0 \quad 2720.8 \quad 2738.8 \quad 2741.7 \quad 2749.4$ $2767.8 \quad 2781.4 \quad 2783.9 \quad 4866.7$

name $=$ TS322

energy $($ in $\mathrm{kcal} / \mathrm{mol})=65.371881$

$\mathrm{ZPE}(\mathrm{in} \mathrm{kcal} / \mathrm{mol})=141.294$

$\mathrm{G}($ in $\mathrm{kcal} / \mathrm{mol})=114.735$

Geometry

C $-1.81816002 \quad 0.52347900-0.48813848$

C $-0.38409710 \quad 0.08655056-0.77314166$

C $0.65451327 \quad 0.791019190 .16334355$

C $1.60203842-0.134743590 .92821129$

C $0.93345714 \quad-0.68150538 \quad 2.14199015$

C $0.12127308-2.10360133-1.84582062$

C $-0.28016691-1.40621989-0.78549791$

C $0.72527821-0.00047203 \quad 3.28969386$

C $1.173029231 .42227559 \quad 3.47993223$

C $0.04785865-0.606869094 .38321526$

$\mathrm{H}-1.931643521 .61006716-0.59629823$

$\mathrm{H}-2.12093664 \quad 0.24745846 \quad 0.52870930$

$\mathrm{H}-2.521194390 .05348880 \quad-1.18794045$

$\mathrm{H} \quad 0.16195371 \quad 1.51395661 \quad 0.84778907$ 
H $2.52258826 \quad 0.42824950 \quad 1.19457273$

H $1.96422051-0.951960130 .27095196$

$\mathrm{H} \quad 0.60559296-1.711226752 .05346507$

$\mathrm{H} 0.18567910-3.18004941-1.86126031$

$\mathrm{H} \quad 0.41776189-1.64917002-2.78155389$

$\mathrm{H}-0.57198510-1.87987974 \quad 0.15273867$

H $1.81907785 \quad 1.767922562 .65859046$

$\mathrm{H} \quad 1.76112027 \quad 1.54648333 \quad 4.39609705$

$\mathrm{H} \quad 0.31578967 \quad 2.10599721 \quad 3.51193052$

$\mathrm{H}-0.37954381-0.48538594 \quad 5.56077702$

$\mathrm{H}-0.29137825-1.61879488 \quad 4.30865233$

$\mathrm{H} \quad 0.01484442 \quad 0.29390910 \quad 5.31374251$

$\begin{array}{llll}0 & 1.28875809 & 0.98876729 & -1.99010918\end{array}$

O $1.408851891 .62113315-0.72371013$

o $-0.078899650 .63640462-2.06780756$

Vibrational frequencies (in cm-1)

$\begin{array}{llllllllllllllllll}-1294.5 & 33.6 & 38.5 & 63.4 & 88.7 & 96.5 & 120.4 & 166.8 & 201.6 & 203.7 & 217.7 & 236.5 & 288.4 & 325.6\end{array}$ $330.5 \quad 358.0 \quad 408.0 \quad 437.7 \quad 467.7 \quad 496.3 \quad 551.8 \quad 566.5 \quad 611.9675 .7 \quad 684.8 \quad 715.6 \quad 726.5 \quad 824.9$ $867.5910 .4928 .5951 .5 \quad 972.7 \quad 990.6 \quad 1004.8 \quad 1024.7 \quad 1044.91063 .9 \quad 1064.8 \quad 1080.1 \quad 1094.1$

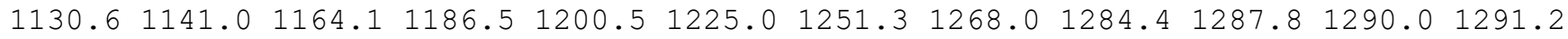

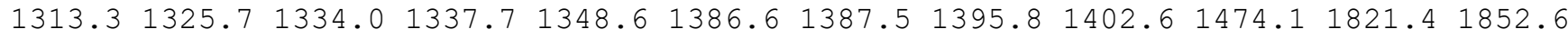
$1883.42654 .7 \quad 2661.92673 .7 \quad 2676.92680 .6 \quad 2684.3 \quad 2711.72741 .2 \quad 2750.7 \quad 2762.3 \quad 2775.4$ $2778.82779 .92784 .2 \quad 3800.6$

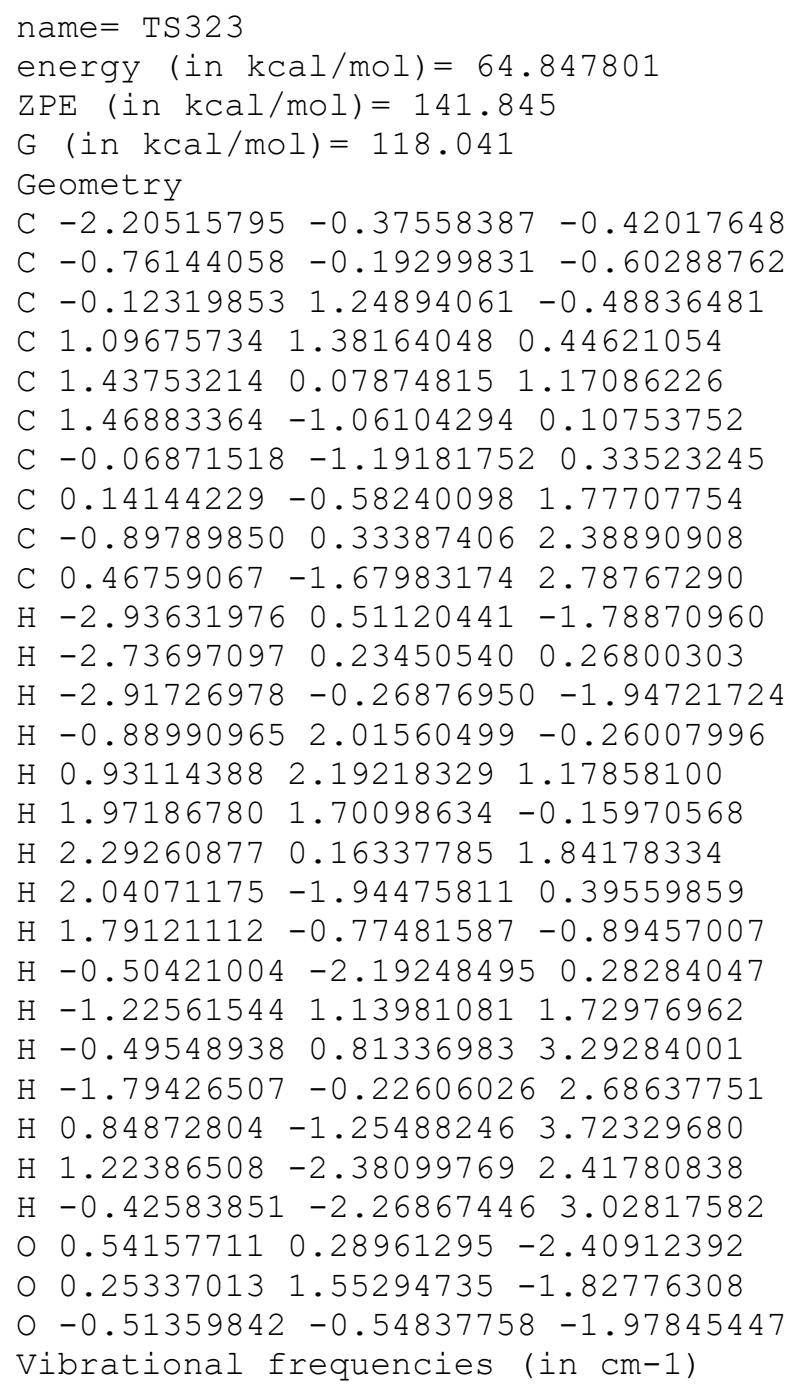

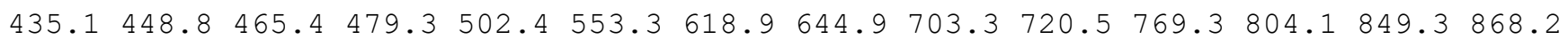
$895.6919 .3 \quad 932.9940 .9979 .2 \quad 994.4 \quad 1004.4 \quad 1006.9 \quad 1013.1 \quad 1018.7 \quad 1020.8 \quad 1041.3 \quad 1087.4$ $1088.6 \quad 1101.3 \quad 1109.3 \quad 1155.8 \quad 1159.7 \quad 1183.3 \quad 1193.3 \quad 1200.5 \quad 1221.9 \quad 1241.7 \quad 1257.8 \quad 1272.3$

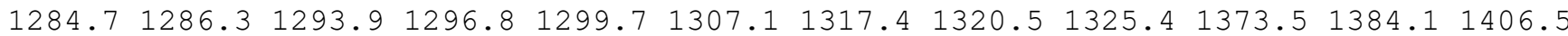


$1417.92657 .7 \quad 2673.1 \quad 2673.8 \quad 2678.5 \quad 2679.7 \quad 2686.6 \quad 2689.22710 .42739 .12741 .32749 .6$ $2768.22781 .5 \quad 2784.4 \quad 4975.6$

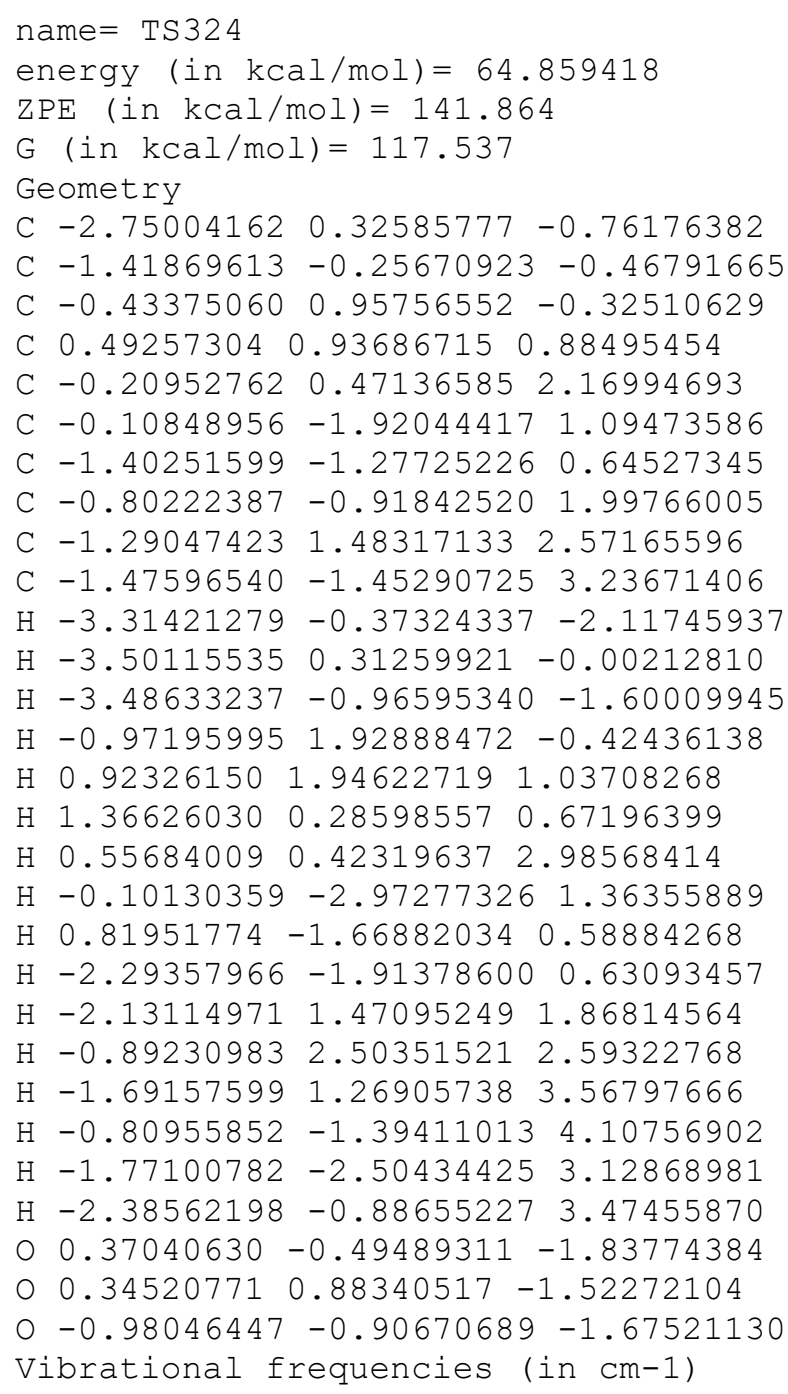


$\mathrm{H}-1.95053363-0.30136036-2.68166334$

$\mathrm{H}-0.825710612 .02335097 \quad-0.19474245$

$\mathrm{H} 0.811427962 .442183460 .67501808$

$\mathrm{H} \quad 1.53109368 \quad 1.05166669-0.19216720$

$\mathrm{H} \quad 0.98384133 \quad 0.58162795 \quad 2.43889004$

$\mathrm{H} \quad 0.24068020-1.75477377 \quad 1.75486991$

$\mathrm{H} 0.70465606-1.228927390 .13386349$

$\mathrm{H}-2.14020121-1.31796810 \quad 0.93608187$

$\mathrm{H}-1.77857950 \quad 2.38785062 \quad 0.82492291$

$\mathrm{H}-1.720957172 .51974505 \quad 2.58531990$

$\mathrm{H}-3.06143306 \quad 1.66839074 \quad 1.79556877$

$\mathrm{H}-2.60138221-0.23245585 \quad 3.38908501$

$\mathrm{H}-1.19179644 \quad 0.59986586 \quad 4.06008443$

$\mathrm{H}-1.03180521-1.03273672 \quad 3.41553638$

$\begin{array}{lllll}0 & 1.99167100 & 0.37080220 & -2.78677772\end{array}$

$\begin{array}{llll}0 & -0.17644977 & 1.48261539 & -2.06363290\end{array}$

O $1.74974180-0.62293773-2.29565777$

Vibrational frequencies (in $\mathrm{cm}-1$ )

$\begin{array}{lllllllllllllllll}-934.5 & 19.6 & 38.9 & 47.9 & 57.7 & 90.6 & 102.8 & 111.8 & 141.8 & 169.0 & 182.6 & 192.5 & 245.2 & 273.3\end{array}$ $280.8 \quad 356.6 \quad 365.7 \quad 392.0 \quad 433.0 \quad 445.2 \quad 464.8 \quad 548.3 \quad 567.7 \quad 665.2 \quad 699.7 \quad 786.9 \quad 867.6 \quad 877.6$ $895.4930 .9971 .9986 .8 \quad 990.3 \quad 1005.7 \quad 1015.1 \quad 1033.4 \quad 1043.6 \quad 1060.3 \quad 1074.3 \quad 1091.8 \quad 1112.8$

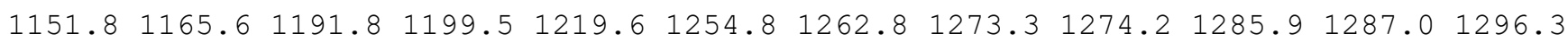
$1299.91305 .21316 .0 \quad 1341.1 \quad 1367.6 \quad 1376.2 \quad 1394.1 \quad 1405.5 \quad 1414.3 \quad 1472.2 \quad 1595.01805 .9$ $2021.02602 .3 \quad 2656.7 \quad 2664.2 \quad 2668.0 \quad 2671.8 \quad 2673.7 \quad 2678.02686 .8 \quad 2732.3 \quad 2735.8 \quad 2747.3$ $2761.7 \quad 2763.0 \quad 2768.7 \quad 2781.9$

name $=$ TS326

energy (in $\mathrm{kcal} / \mathrm{mol})=65.553411$

$\operatorname{ZPE}($ in $\mathrm{kcal} / \mathrm{mol})=141.205$

$\mathrm{G}($ in $\mathrm{kcal} / \mathrm{mol})=116.397$

Geometry

C $-2.19355083-0.68552970-1.42640296$

C $-0.71154853-0.43779536-1.28889977$

C $-0.33780932 \quad 0.80225157 \quad-0.51831377$

C $1.04592641 \quad 1.08226398 \quad 0.03301208$

C $1.39012744 \quad 0.08545182 \quad 1.13744783$

C $1.23640581-1.333813820 .47896695$

C $-0.21575656-1.202691210 .81188896$

C $0.07933927-0.26484395 \quad 1.98019409$

C $-0.89207277 \quad 0.82619935 \quad 2.38019626$

C $0.43232402-1.082266573 .22954126$

$\mathrm{H}-2.58702546-0.11708525-2.28425984$

$\mathrm{H}-2.38564019-1.74546442-1.65114222$

$\mathrm{H}-2.77533579-0.40930257-0.54473398$

$\mathrm{H}-1.15839089 \quad 1.22241513 \quad 0.07768208$

H 1.107266092 .126104220 .39555996

H $1.801518331 .01683862-0.78615155$

H $2.29375244 \quad 0.30088980 \quad 1.69820838$

H $1.77655082 \quad-2.14420580 \quad 0.98299456$

H $1.52809299-1.41840340-0.58622059$

$\mathrm{H}-1.04396770-1.84043964 \quad 0.64328290$

$\mathrm{H}-1.91062097 \quad 0.61312158 \quad 2.03418263$

$\mathrm{H}-0.59269198 \quad 1.81100734 \quad 1.99670992$

$\mathrm{H}-0.95678110 \quad 0.94771626 \quad 3.47055231$

$\mathrm{H} \quad 0.92196341-0.44681975 \quad 3.97848299$

$\mathrm{H} 1.11522792-1.913645593 .02499814$

$\mathrm{H}-0.46827670-1.50747223 \quad 3.68823087$

○ $0.49329502 \quad 2.01317053-2.39851677$

o $-0.497627021 .68126792-1.80939726$

O $0.08751030-1.06704642-1.95560412$

Vibrational frequencies (in $\mathrm{cm}-1$ )

$\begin{array}{llllllllllllllll}-517.5 & 69.5 & 100.2 & 116.3 & 123.1 & 127.9 & 149.9 & 168.6 & 181.5 & 221.4 & 244.2 & 261.6 & 276.9 & 294.1\end{array}$ $\begin{array}{lllllllllllllll}365.7 & 394.5 & 422.2 & 450.8 & 484.7 & 511.9 & 529.4 & 588.0 & 630.1 & 688.0 & 796.2 & 824.7 & 844.1 & 921.2\end{array}$ $936.0948 .7 \quad 988.4 \quad 997.3 \quad 1017.3 \quad 1022.1 \quad 1027.4 \quad 1042.8 \quad 1051.91074 .1 \quad 1089.61099 .61123 .1$ 
$\begin{array}{llllllllllllll}1152.2 & 1177.4 & 1188.2 & 1190.2 & 1200.5 & 1219.5 & 1232.2 & 1264.1 & 1268.2 & 1277.3 & 1280.5 & 1289.1\end{array}$

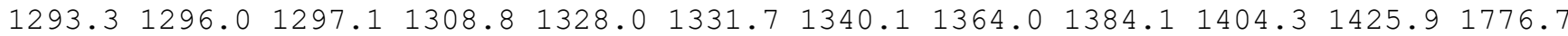
$2606.62645 .22659 .92662 .6 \quad 2670.0 \quad 2672.7 \quad 2675.12681 .72708 .72727 .52735 .2 \quad 2744.6$ 2753.92777 .12778 .92780 .1

\section{name $=$ TS327}

energy (in $\mathrm{kcal} / \mathrm{mol})=67.358951$

$\mathrm{ZPE}($ in $\mathrm{kcal} / \mathrm{mol})=139.465$

$\mathrm{G}(\mathrm{in} \mathrm{kcal} / \mathrm{mol})=112.318$

Geometry

C $-0.65284164 \quad 0.18747976-2.23060851$

C $0.640730120 .28796958-1.42007461$

C $0.582028491 .46398768-0.38953182$

C $1.32410240 \quad 1.23692687 \quad 0.93038957$

C $0.72741686 \quad 0.14803925 \quad 1.74811957$

C $2.18826572-1.61353981-0.96642131$

C $0.99518904-1.03875346-0.83153423$

C $-0.44921198 \quad 0.17088607 \quad 2.40108015$

C $-1.35315768 \quad 1.203876592 .67407727$

C $-0.92356775-1.090777173 .08980009$

$\mathrm{H}-0.86652955 \quad 1.13389387-2.74659007$

$\mathrm{H}-1.51094406-0.06657438-1.60267778$

$\mathrm{H}-0.56601640-0.58388954-3.00841317$

$\mathrm{H}-0.45130400 \quad 1.82727073-0.23515047$

H $1.37632997 \quad 2.19077433 \quad 1.49441457$

H 2.38955832 1.001491510 .70247636

$\mathrm{H} 1.34251785-0.75044081 \quad 1.78913007$

H $2.43163654-2.57796320-0.54708116$

H $3.01080911-1.16385192-1.50583368$

$\mathrm{H} \quad 0.18061953-1.50874862-0.27885044$

$\mathrm{H}-2.35612945 \quad 1.306513492 .36101378$

$\mathrm{H}-0.89517257 \quad 2.59151754 \quad 1.70612052$

$\mathrm{H}-0.638689392 .72181843 \quad 2.44869771$

$\mathrm{H}-1.68312901-0.90336170 \quad 3.86132053$

$\mathrm{H}-0.08840695-1.60178550 \quad 3.58962068$

$\mathrm{H}-1.35058200-1.78770308 \quad 2.35599011$

○ $2.26713050 \quad 1.84997843-1.79745249$

o $1.231583322 .51907740-1.10280711$

O $1.63675684 \quad 0.70802674-2.36914240$

Vibrational frequencies (in $\mathrm{cm}-1$ )

$\begin{array}{llllllllllllllll}-736.9 & 20.1 & 38.5 & 71.7 & 82.4 & 87.6 & 102.2 & 169.1 & 181.0 & 200.0 & 213.9 & 248.8 & 273.6 & 302.1\end{array}$

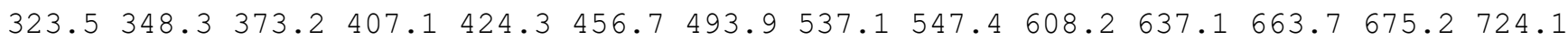
$820.3 \quad 870.0 \quad 893.4 \quad 898.3 \quad 944.4 \quad 960.1 \quad 976.6 \quad 990.8 \quad 1005.1 \quad 1037.1 \quad 1043.2 \quad 1058.9 \quad 1066.0$

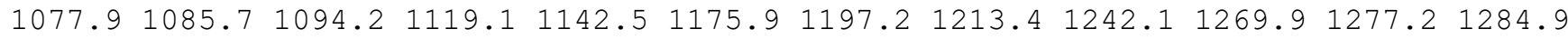

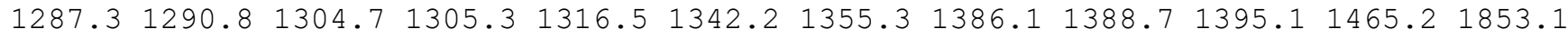
$1873.72651 .22667 .92669 .5 \quad 2671.8 \quad 2680.9 \quad 2692.92711 .82735 .6 \quad 2739.6 \quad 2752.8 \quad 2756.3$ $2776.0 \quad 2780.2 \quad 2782.7 \quad 4969.9$

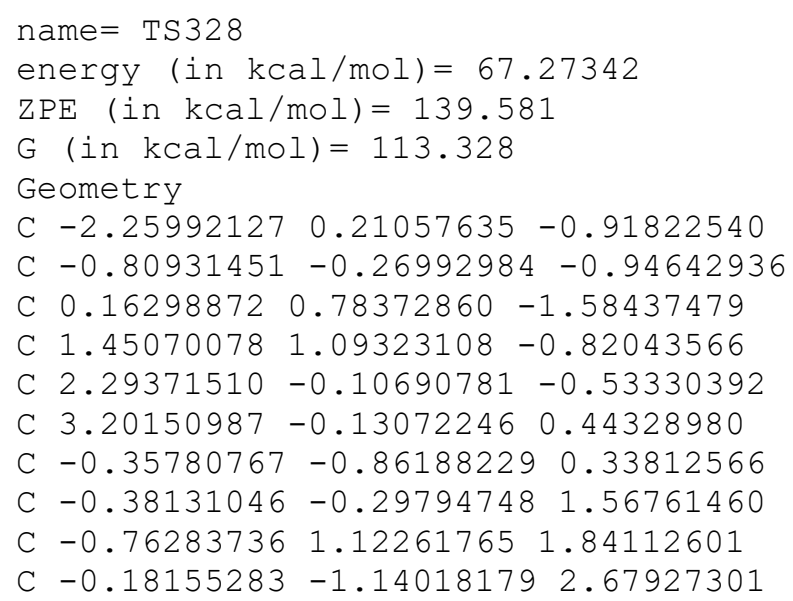


$\mathrm{H}-2.39259400 \quad 1.09597721-0.29159054$

$\mathrm{H}-2.91614561-0.57506970-0.51682674$

$\mathrm{H}-2.618247370 .44420546-1.93001180$

$\mathrm{H}-0.37545900 \quad 1.72485702-1.83317139$

$\mathrm{H} \quad 1.20685042 \quad 1.634380320 .11617364$

H $2.055631191 .80737928-1.42799955$

$\mathrm{H} 2.13158508-0.96146434-1.19282501$

H $3.83012398-0.98276924 \quad 0.64421703$

H $3.39316428 \quad 0.69208778 \quad 1.10965745$

$\mathrm{H}-0.00981147-1.892583310 .22202641$

$\mathrm{H}-0.91046518 \quad 1.70785544 \quad 0.92368163$

$\mathrm{H}-0.00005014 \quad 1.64883546 \quad 2.42820280$

$\mathrm{H}-1.70899593 \quad 1.16394271 \quad 2.40453389$

$\mathrm{H} \quad 0.73779133-0.19117704 \quad 3.79003367$

$\mathrm{H} 0.46678736-1.97064186 \quad 2.76300813$

$\mathrm{H}-0.00560966-0.29613186 \quad 4.07290089$

o $0.35567722-1.17235412-2.67897991$

$0.47810486 \quad 0.23250166-2.86119280$

o $-0.83814393-1.32130504-1.94355321$

Vibrational frequencies (in $\mathrm{cm}-1$ )

$\begin{array}{lllllllllllllllllll}-835.7 & 39.5 & 59.0 & 77.9 & 98.7 & 119.6 & 132.9 & 145.6 & 156.5 & 185.9 & 202.1 & 246.7 & 261.3 & 294.2\end{array}$ $318.4 \quad 355.3 \quad 383.8 \quad 403.2 \quad 448.0 \quad 457.8 \quad 499.0 \quad 504.4 \quad 571.0 \quad 623.9636 .9687 .8 \quad 702.0719 .4$

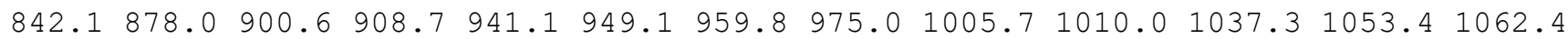

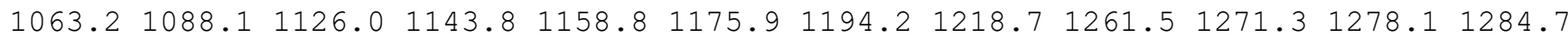

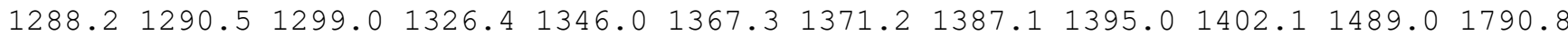
$1843.12655 .92665 .6 \quad 2669.92670 .8 \quad 2676.8 \quad 2679.92718 .7 \quad 2732.2 \quad 2734.92742 .0 \quad 2754.2$ $2775.92779 .8 \quad 2794.6 \quad 4873.2$

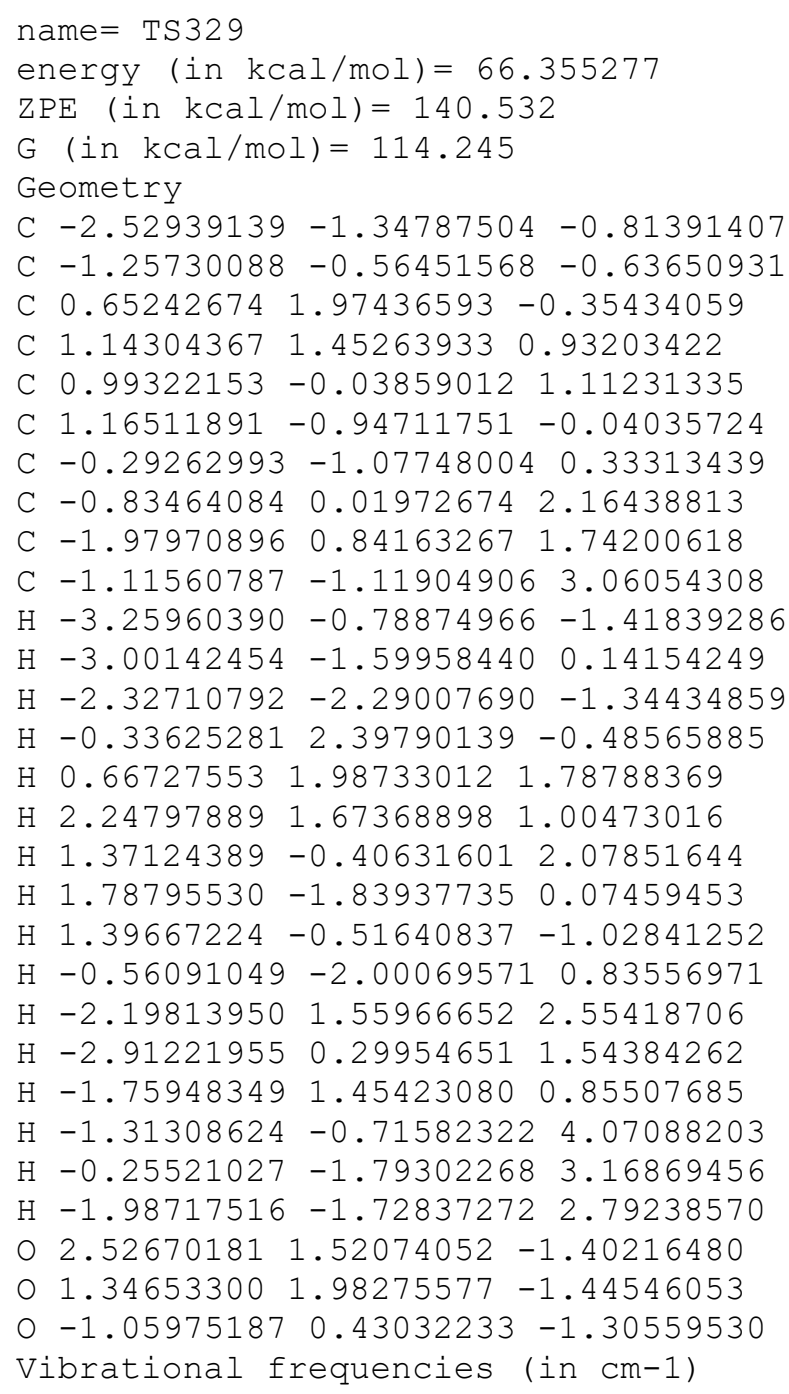


$\begin{array}{lllllllllllllllll}-598.0 & 49.4 & 69.3 & 74.5 & 90.2 & 92.3 & 138.1 & 151.3 & 163.1 & 192.0 & 200.9 & 214.8 & 254.5 & 276.6\end{array}$ $295.3 \quad 317.4 \quad 337.8 \quad 377.8 \quad 415.5 \quad 442.2 \quad 486.1 \quad 590.1606 .5 \quad 625.9 \quad 716.3 \quad 873.0 \quad 897.3 \quad 913.0$

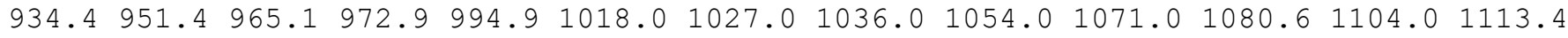

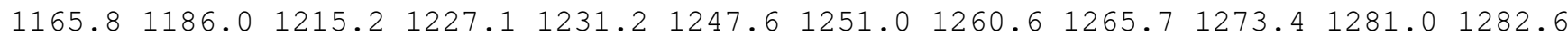

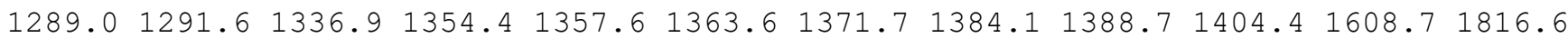
$2624.62658 .2 \quad 2662.5 \quad 2665.3 \quad 2667.3 \quad 2671.12671 .82676 .72703 .62706 .42714 .62753 .5$ 2765.02771 .62773 .32779 .6

name $=$ TS330

energy $($ in $\mathrm{kcal} / \mathrm{mol})=65.783063$

$\operatorname{ZPE}($ in $\mathrm{kcal} / \mathrm{mol})=141.274$

$\mathrm{G}($ in $\mathrm{kcal} / \mathrm{mol})=114.779$

Geometry

C $-2.44692478-1.45705142-0.20210588$

C $-1.29029793-0.66776921-0.80097363$

C $-0.99611278 \quad 0.65290636-0.01134796$

$\begin{array}{lllll}\text { C } & 0.43219871 & 0.83256109 & 0.50474541\end{array}$

C $0.62124212 \quad 0.08113427 \quad 1.77705385$

C $0.36268999-2.47866307-0.29569064$

C $-0.07616490-1.49774187-1.08199226$

$\begin{array}{llll}\text { C } & 0.19928938 & 0.48639824 & 2.99370217\end{array}$

C $-0.52013213 \quad 1.78882854 \quad 3.21037414$

C $0.40969636-0.323978654 .14390740$

$\mathrm{H}-3.37230486-0.86551720-0.19812262$

$\mathrm{H}-2.22888662-1.753659520 .83120220$

$\mathrm{H}-2.64690100 \quad-2.36918119-0.77892018$

$\mathrm{H}-1.73495488 \quad 0.80837763 \quad 0.80432313$

$\mathrm{H} \quad 0.63490935 \quad 1.916713350 .64183869$

H $1.17098036 \quad 0.51141131-0.25708785$

H $1.13998473-0.86549426 \quad 1.66582912$

$\mathrm{H}-0.10776399-2.76399108 \quad 0.63323243$

H $1.23936832-3.06785871-0.51698489$

$\mathrm{H} 0.42706167-1.23284253-2.01497261$

$\mathrm{H}-1.56086094 \quad 1.62173860 \quad 3.51352605$

$\mathrm{H}-0.55625873 \quad 2.40135042 \quad 2.29692998$

$\mathrm{H}-0.02281352 \quad 2.40599084 \quad 3.96727488$

$\mathrm{H} \quad 0.27283570-0.448388335 .38803306$

$\mathrm{H} 0.91031633-1.26475646 \quad 4.05081622$

$\mathrm{H}-0.085233820 .34685678 \quad 5.13463148$

$\begin{array}{llll}0 & -1.17072715 & 1.07825544 & -2.22245077\end{array}$

$0-1.298242031 .69257799-0.94665708$

o $-1.79797935-0.17628782-2.06078918$

Vibrational frequencies (in $\mathrm{cm}-1$ )

$\begin{array}{lllllllllllllll}-1299.2 & 33.3 & 36.2 & 64.4 & 93.9 & 107.8 & 120.8 & 177.2 & 202.7 & 205.5 & 218.7 & 238.1 & 273.7 & 317.8\end{array}$ $328.4 \quad 384.5 \quad 408.8 \quad 427.0 \quad 468.6 \quad 491.1 \quad 499.2 \quad 574.1631 .3 \quad 674.6 \quad 684.4 \quad 714.6 \quad 718.9 \quad 819.6$ $867.9915 .0 \quad 924.1946 .1 \quad 972.9 \quad 983.9 \quad 1004.3 \quad 1024.5 \quad 1044.5 \quad 1059.6 \quad 1065.1 \quad 1081.4 \quad 1092.9$

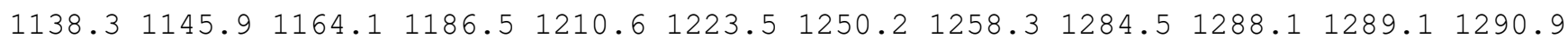

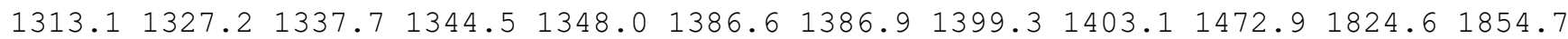
$1886.12655 .8 \quad 2662.5 \quad 2673.6 \quad 2675.0 \quad 2680.8 \quad 2682.92712 .62742 .2 \quad 2748.7 \quad 2761.4 \quad 2774.3$ $2778.92779 .2 \quad 2786.3 \quad 3796.5$

name $=\operatorname{TS} 331$

energy $($ in $\mathrm{kcal} / \mathrm{mol})=63.199714$

$\mathrm{ZPE}($ in $\mathrm{kcal} / \mathrm{mol})=143.894$

$\mathrm{G}($ in $\mathrm{kcal} / \mathrm{mol})=118.238$

Geometry

C $-1.39861157-0.39615555-1.88645975$

C $0.00697581-0.37192502-1.28818938$

C $0.49102690 \quad 1.08783677 \quad-0.98103718$

C 1.020480231 .357833590 .42634760

C $0.91220960 \quad 0.20860086 \quad 1.47041504$

C $1.31272085-1.102927120 .73017689$

C $0.18855612-1.29812146-0.17795007$ 
C $-0.415267890 .03115668 \quad 2.07649925$

C $-0.85285593 \quad 1.12280897 \quad 2.99521950$

C $-1.25729422-1.17181708 \quad 2.15147156$

$\mathrm{H}-1.46107865 \quad 0.23735075 \quad-2.78130227$

$\mathrm{H}-2.13984903-0.04847794-1.16109367$

$\mathrm{H}-1.67724460-1.41169826-2.19464501$

$\mathrm{H}-0.28060557 \quad 1.83078292-1.27118603$

$\mathrm{H} \quad 0.53897327 \quad 2.26285272 \quad 0.83764614$

H $2.10221221 \quad 1.61018562 \quad 0.33255554$

$\mathrm{H} \quad 1.64486153 \quad 0.48528930 \quad 2.27299517$

$\mathrm{H} 1.46823109-1.933860431 .43824432$

H $2.28286963-0.948094830 .21308165$

$\mathrm{H}-0.38640272-2.20028316-0.16186126$

$\mathrm{H}-0.923577892 .09806480 \quad 2.49201871$

$\mathrm{H}-0.138602641 .25142201 \quad 3.82802562$

$\mathrm{H}-1.833827490 .941036923 .45805146$

$\mathrm{H}-1.69189030 \quad-1.32202624 \quad 3.15420174$

$\mathrm{H}-0.71964183-2.10228060 \quad 1.89770796$

$\mathrm{H}-2.11073669-1.118578981 .45065852$

○ $2.02484228 \quad 0.01474106-2.25384803$

O $1.544577021 .31886976-1.91723548$

$0.86170095-0.74599190-2.41021897$

Vibrational frequencies (in $\mathrm{cm}-1$ )

$\begin{array}{lllllllllllllllll}-149.7 & 12.6 & 36.4 & 64.1 & 161.3 & 172.7 & 194.0 & 240.6 & 272.9 & 280.7 & 296.5 & 362.7 & 377.0 & 390.9\end{array}$

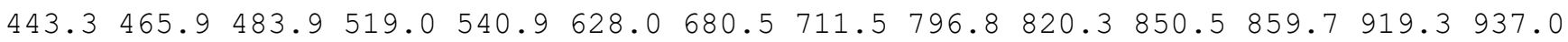
$943.3 \quad 949.7973 .1 \quad 997.1 \quad 1011.4 \quad 1017.8 \quad 1034.2 \quad 1053.4 \quad 1069.9 \quad 1094.2 \quad 1114.2 \quad 1132.01145 .8$

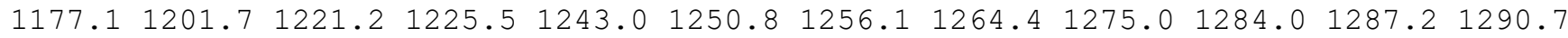

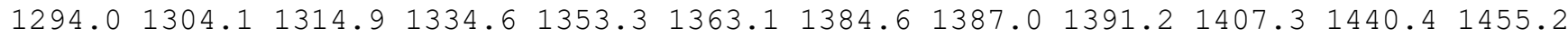
$2636.82648 .4 \quad 2649.2 \quad 2649.6 \quad 2662.4 \quad 2664.0 \quad 2673.3 \quad 2678.22683 .5 \quad 2688.12730 .02743 .9$ $2757.12768 .0 \quad 2778.4 \quad 2781.4$
name $=$ TS332
energy $($ in $\mathrm{kcal} / \mathrm{mol})=64.864486$
$\operatorname{ZPE}($ in $\mathrm{kcal} / \mathrm{mol})=142.233$
$\mathrm{G}($ in $\mathrm{kcal} / \mathrm{mol})=118.996$
Geometry
C $-0.67494674 \quad 1.08662987-0.86988691$
C $0.65379805 \quad 0.47943353-0.44033074$
C $1.660271891 .52075827 \quad 0.17298076$
C $2.37364158 \quad 1.08852458 \quad 1.46976740$
C $1.92194481-0.281461921 .97102368$
C $1.93843057-1.27403127 \quad 0.77155089$
C $0.50421578 \quad-0.76111858 \quad 0.43457231$
C $0.33477629-0.38568420 \quad 1.97271983$
C $-0.46859653 \quad 0.83946117 \quad 2.36125678$
C $-0.01188612-1.503169462 .84505567$
$\mathrm{H}-1.28691238 \quad 1.39380680 \quad-0.01756177$
$\mathrm{H}-1.26146760 \quad 0.36588531-1.45659400$
$\mathrm{H}-0.519509991 .96350567-1.51465472$
H $1.19894217 \quad 2.52389612 \quad 0.28166853$
H 2.22155324 1.84792236 2.25880915
$\mathrm{H} 3.468667051 .06766972 \quad 1.28268813$
$\mathrm{H} \quad 2.41627288-0.59381603 \quad 2.89388369$
$\mathrm{H} 1.98280610-2.32775724 \quad 1.05500657$
H 2.70736505 -1.10680521 0.01556922
$\mathrm{H}-0.20126343-1.48821283 \quad 0.03744915$
$\mathrm{H}-0.41521371 \quad 0.98587602 \quad 3.45238834$
$\mathrm{H}-1.52520794 \quad 0.73207144 \quad 2.09439836$
$\mathrm{H}-0.109068561 .76639562 \quad 1.90775355$
$\mathrm{H}-1.54301478-1.51339762 \quad 3.20443608$
$\mathrm{H} \quad 0.27683627 \quad-2.504504792 .64276376$
$\mathrm{H}-1.61634240-1.79931465 \quad 2.44852086$
O $2.63507520 \quad 0.47902167-1.56786469$
○ $2.613114691 .71327509-0.87015751$ 
O $1.271702250 .11115585-1.68796352$

Vibrational frequencies (in $\mathrm{cm}-1$ )

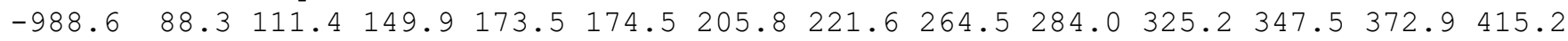

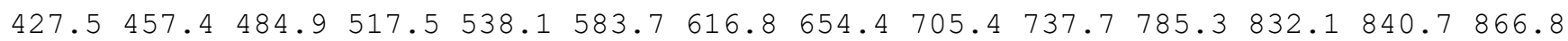

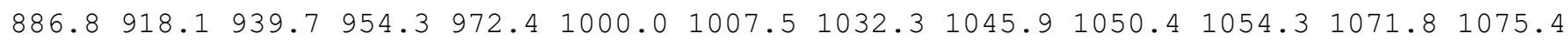

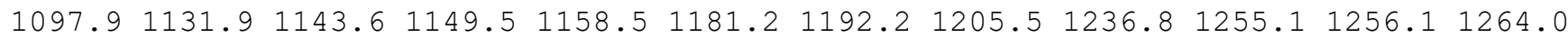
$1279.1 \quad 1282.6 \quad 1285.2 \quad 1294.5 \quad 1297.7 \quad 1300.0 \quad 1307.1 \quad 1318.8 \quad 1354.4 \quad 1373.1 \quad 1384.0 \quad 1398.1$ $1418.62657 .5 \quad 2669.3 \quad 2670.2 \quad 2677.7 \quad 2678.0 \quad 2685.92688 .12719 .2 \quad 2737.62741 .72752 .0$ $2767.22780 .02782 .6 \quad 4730.2$

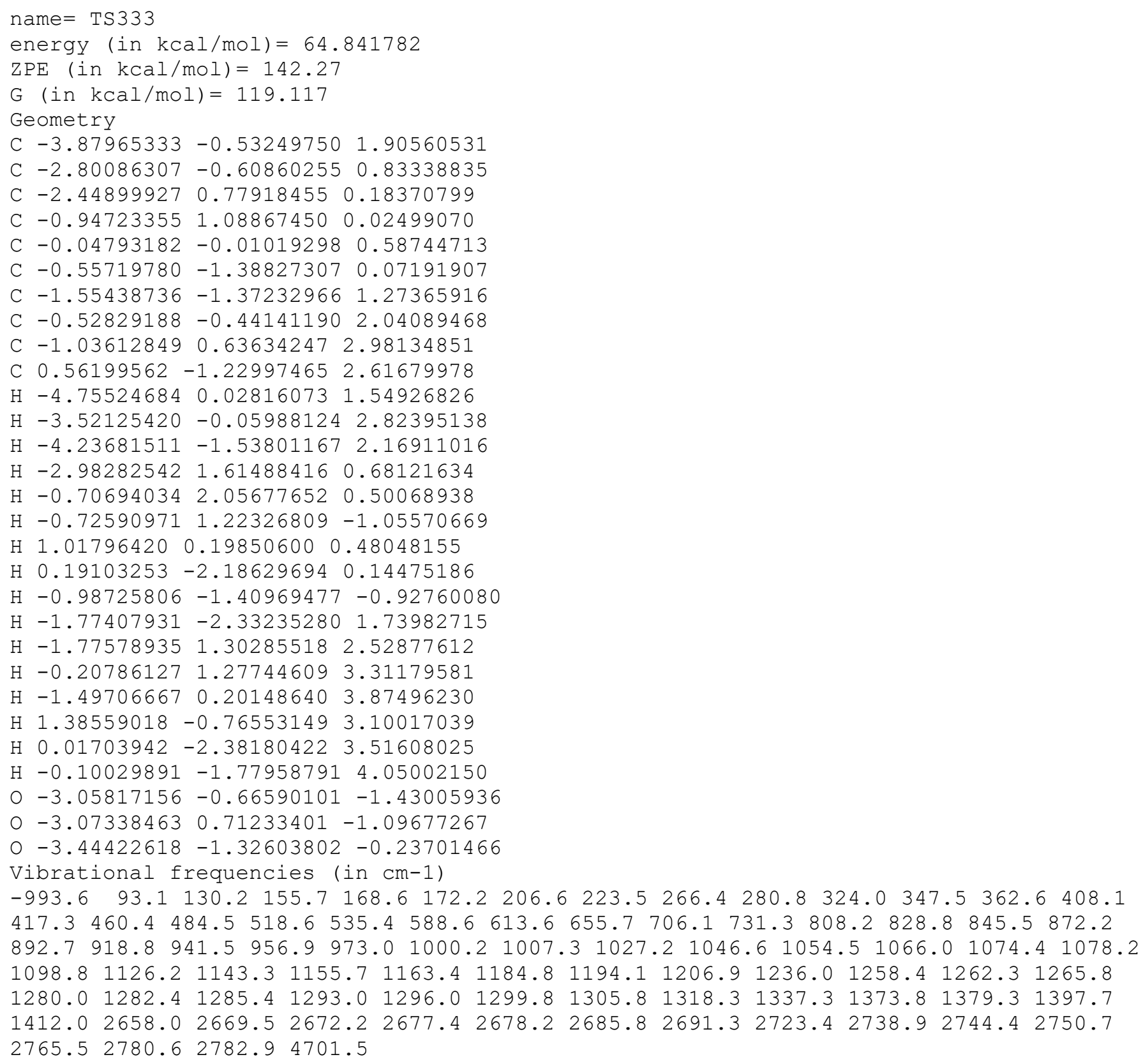


C $1.20087367-1.414979250 .31937673$

C $-0.32408336-1.74925272 \quad 0.32764232$

C $-0.49028290-0.74577150 \quad 1.53823813$

C $-1.74035825 \quad 0.12128888 \quad 1.67114132$

C $-0.41303806-1.38897607 \quad 2.84745656$

$\mathrm{H}-2.97556371-1.14177550-0.21457390$

$\mathrm{H}-2.59729004-2.53010861-1.23479374$

$\mathrm{H}-2.83325022-0.94880722-1.97863864$

$\mathrm{H}-1.39837256 \quad 0.97002881-1.33240213$

$\mathrm{H} \quad 0.03757657 \quad 1.84856376 \quad 0.26887619$

$\mathrm{H} \quad 1.39069237 \quad 1.23116434-0.68241528$

H $1.49997348 \quad 0.36486930 \quad 1.70398953$

H $1.81880900 \quad-2.06663288 \quad 0.93533824$

H $1.68011786-1.36058172-0.66098988$

$\mathrm{H}-0.61547590-2.78277993 \quad 0.51907989$

$\mathrm{H}-2.03656528 \quad 0.60102424 \quad 0.73653068$

$\mathrm{H}-1.58230160 \quad 0.92152422 \quad 2.40601742$

$\mathrm{H}-2.59436751-0.480726532 .01994613$

$\mathrm{H} \quad 0.38624226-2.74402716 \quad 2.84203911$

$\mathrm{H}-0.13487704 \quad-0.84398569 \quad 3.71652829$

$\mathrm{H} 1.02996753-2.250338722 .85604903$

$00.64876041-0.99465418-2.60240795$

$00.09076388 \quad 0.30324606-2.48123002$

o $-0.34870301-1.86273018-2.09221052$

Vibrational frequencies (in $\mathrm{cm}-1$ )

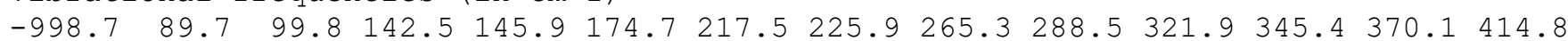
$432.5 \quad 457.5 \quad 486.3 \quad 516.3 \quad 540.9 \quad 568.2 \quad 650.4 \quad 670.9 \quad 703.9 \quad 717.8 \quad 769.2 \quad 827.3 \quad 849.6 \quad 872.7$

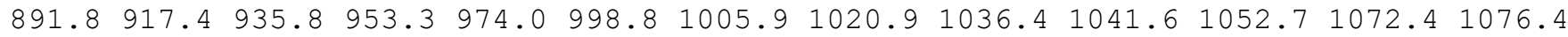
$1098.61132 .6 \quad 1139.2 \quad 1150.6 \quad 1162.0 \quad 1181.3 \quad 1191.91208 .51233 .4 \quad 1244.4 \quad 1253.8 \quad 1267.7$

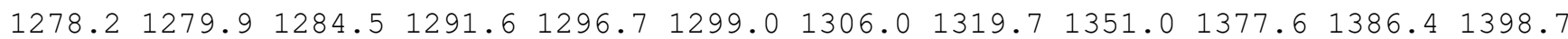
$1412.52657 .8 \quad 2669.0 \quad 2669.92674 .3 \quad 2677.2 \quad 2684.12685 .82718 .6 \quad 2739.02745 .5 \quad 2750.7$ $2767.92779 .2 \quad 2781.4 \quad 4731.6$

name $=$ TS335

energy $($ in $\mathrm{kcal} / \mathrm{mol})=65.166678$

$\mathrm{ZPE}($ in $\mathrm{kcal} / \mathrm{mol})=141.973$

$\mathrm{G}($ in $\mathrm{kcal} / \mathrm{mol})=118.61$

Geometry

C $-2.10456417-0.34404537-1.28345292$

C $-0.96274249-0.00981149-2.11726626$

C $0.47077297 \quad 0.07922307-1.43947226$

C $1.59454013-0.75257205-2.08643543$

C $1.12233985-1.48645054-3.34129514$

C $0.29312676-0.48668847-4.20453044$

C $-0.94627333-0.91749308-3.35644019$

C $-0.21066749-2.28194617-3.06199310$

C $-0.38238884-2.95087551-1.71439558$

C $-0.50350594-3.30750151-4.15453827$

$\mathrm{H}-2.12482492-1.15745263-0.60468727$

$\mathrm{H}-1.97178908 \quad 0.80977891-0.02044551$

$\mathrm{H}-2.57789138 \quad 1.01778201-0.50520590$

$\mathrm{H} \quad 0.39502480-0.11620213-0.35379738$

H $2.00807195-1.47073653-1.35532894$

$\mathrm{H} 2.43694106-0.07653593-2.35156894$

$\mathrm{H} \quad 1.91578594-2.04127059-3.84301651$

$\mathrm{H} \quad 0.22487600 \quad-0.74092753-5.26292218$

$\mathrm{H} \quad 0.58140727 \quad 0.56592688 \quad-4.13037396$

$\mathrm{H}-1.91363386-0.97528961-3.85877533$

$\mathrm{H} \quad 0.26631483-3.83371355-1.63332043$

$\mathrm{H}-1.41738417-3.29241433-1.57681759$

$\mathrm{H}-0.13742995-2.30560098-0.86736143$

$\mathrm{H}-1.52758038-3.69049611-4.06917658$

$\mathrm{H} \quad 0.17847633-4.16282755-4.08664637$

$\mathrm{H}-0.39868226-2.88917326-5.16181841$ 
$0-0.376116682 .14084835-1.77278346$

$0.85559204 \quad 1.44275229-1.61378242$

O $-1.140039871 .33123075-2.63363491$

Vibrational frequencies (in $\mathrm{cm}-1$ )

$\begin{array}{llllllllllllll}-860.7 & 80.2 & 126.6 & 145.4 & 157.6 & 170.7 & 201.3 & 222.6 & 264.3 & 267.4 & 322.8 & 339.2 & 374.6 & 411.1\end{array}$ $\begin{array}{llllllllllllll}428.5 & 452.7 & 463.5 & 488.1 & 493.2 & 574.4 & 624.8 & 651.8 & 710.9 & 722.2 & 764.1 & 794.5 & 833.3 & 875.2\end{array}$ $892.5921 .7936 .8 \quad 969.6 \quad 987.2 \quad 992.5 \quad 1001.9 \quad 1005.6 \quad 1013.0 \quad 1016.5 \quad 1043.1 \quad 1068.7 \quad 1085.6$

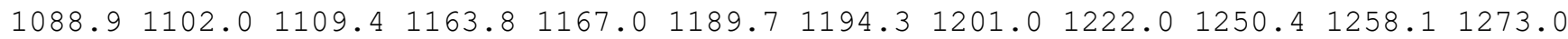

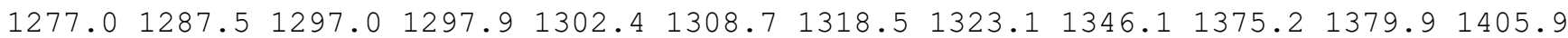
$1416.22655 .22673 .22673 .82677 .6 \quad 2679.62682 .62686 .02718 .92736 .72742 .62749 .0$ $2763.7 \quad 2781.2 \quad 2783.3 \quad 4857.7$

name $=$ TS336

energy $($ in $\mathrm{kcal} / \mathrm{mol})=67.37971$

$\mathrm{ZPE}($ in $\mathrm{kcal} / \mathrm{mol})=139.91$

$\mathrm{G}($ in $\mathrm{kcal} / \mathrm{mol})=113.693$

Geometry

C $-3.10471895-0.72655052-0.50454223$

C $-2.45485714 \quad 0.26819751 \quad 0.07326686$

C $0.31280347 \quad 1.64144258 \quad-0.50626768$

$\begin{array}{lllll}\text { C } & 1.40281781 & 0.87695766 & 0.12336228\end{array}$

C $0.94563005-0.40776510 \quad 0.80365484$

C $0.16154569-1.40851389-0.03566489$

C $-0.52790106-0.659192121 .06773828$

C $1.99262629-0.90958704 \quad 1.79603260$

C $1.46763236-1.995725592 .74263537$

C $3.20849314-1.447060371 .02836983$

$\mathrm{H}-4.01315302-0.72366612-1.04967501$

$\mathrm{H}-2.81868661-2.07342449-0.11500231$

$\mathrm{H}-2.37910662-1.91814011-0.84422490$

$\mathrm{H}-0.256249792 .39749105 \quad 0.02457552$

H $1.94992851 \quad 1.516417210 .85110793$

H $2.14976084 \quad 0.59436865 \quad-0.66922904$

H $2.31686506-0.04192674 \quad 2.42695055$

$\mathrm{H} \quad 0.36637664 \quad-2.46891946 \quad 0.05734371$

$\mathrm{H}-0.02333150-1.15336653-1.08355875$

$\mathrm{H}-0.88865887-1.13341788 \quad 1.97310047$

H $1.08808518-2.86578412 \quad 2.19743411$

$\mathrm{H} \quad 0.66646619-1.61816292 \quad 3.38704134$

H $2.26672808-2.35357126 \quad 3.40382866$

H $3.96813972-1.841242111 .71161461$

H $3.68393367-0.66799475 \quad 0.42284335$

$\mathrm{H} \quad 2.92169785-2.25743876 \quad 0.34739004$

$0.498249690 .61352594-2.44153941$

$0-0.080207531 .48785991-1.72878056$

O -1.385209710 .434859340 .74132506$

Vibrational frequencies (in $\mathrm{cm}-1$ )

$\begin{array}{lllllllllllllll}-1576.6 & 42.6 & 49.9 & 53.7 & 97.8 & 106.7 & 157.2 & 166.9 & 192.5 & 214.9 & 230.3 & 235.0 & 276.1 & 294.7\end{array}$ $302.0 \quad 346.0 \quad 388.3 \quad 421.4 \quad 450.4 \quad 487.5 \quad 507.4 \quad 556.6 \quad 620.2 \quad 650.9 \quad 712.9 \quad 749.4 \quad 794.2 \quad 813.1$

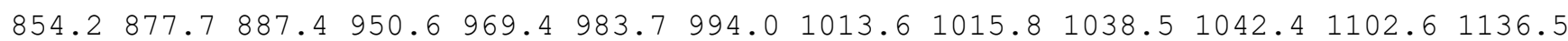

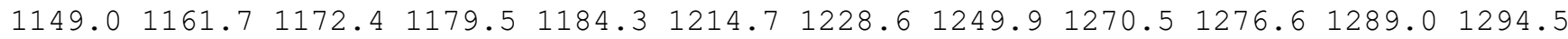
$1300.71302 .3 \quad 1306.8 \quad 1311.1 \quad 1323.1 \quad 1356.2 \quad 1372.4 \quad 1400.4 \quad 1402.4 \quad 1406.3 \quad 1495.7 \quad 1607.2$ 1754.82633 .42669 .92673 .62676 .02679 .32688 .52701 .32705 .12720 .22739 .62773 .5 $2780.3 \quad 2782.3 \quad 2784.3 \quad 4067.1$

name $=\operatorname{TS} 337$

energy $($ in $\mathrm{kcal} / \mathrm{mol})=63.707329$

$\operatorname{ZPE}($ in $\mathrm{kcal} / \mathrm{mol})=143.599$

G $($ in $\mathrm{kcal} / \mathrm{mol})=120.287$

Geometry

C $-2.80855347 \quad 1.20888816 \quad 0.82754668$

C $-1.58470478 \quad 0.68712586 \quad 0.08609916$

C $-0.44154894 \quad 1.76416698-0.08767140$ 
$\begin{array}{lllll}\text { C } & 0.88680872 & 1.25225420 & 0.26232164\end{array}$

C $1.02700361-0.135912620 .73209611$

C $0.17955529-1.04827645-0.22734731$

C $-1.05650655-0.63986334 \quad 0.63615858$

C $-0.06948308-0.41248755 \quad 1.84916269$

$\begin{array}{lllll}\text { C } & -0.33319980 & 0.68133834 & 2.86174656\end{array}$

C $0.18675644-1.71756835 \quad 2.60188481$

$\mathrm{H}-3.18032470 \quad 2.13710625 \quad 0.37327940$

$\mathrm{H}-2.595630351 .40483431 \quad 1.88267470$

$\mathrm{H}-3.62940765 \quad 0.48056126 \quad 0.78470902$

$\mathrm{H}-0.70149982 \quad 2.72619523 \quad 0.40728680$

$\mathrm{H} \quad 1.64297775 \quad 2.23786787 \quad-0.22292782$

H $2.14891854 \quad 1.57961930 \quad 0.05287627$

H $2.03313511-0.475477890 .97157471$

$\mathrm{H} \quad 0.42540266-2.10831771-0.18837368$

$\mathrm{H} \quad 0.16239671-0.74292168-1.27269925$

$\mathrm{H}-1.83444954-1.38888344 \quad 0.77651292$

$\mathrm{H} \quad 0.47818465 \quad 0.72939119 \quad 3.59917281$

$\mathrm{H}-1.267190890 .50505886 \quad 3.40634312$

$\mathrm{H}-0.38285781 \quad 1.67640626 \quad 2.40572737$

$\mathrm{H}-0.67515751-1.98409444 \quad 3.22403462$

$\mathrm{H} 1.05770368-1.627008893 .25986279$

$\mathrm{H} \quad 0.37441954 \quad-2.56147933 \quad 1.92989692$

o $-1.03823576 \quad 0.95722965-2.11579741$

$0-0.538665692 .11698763-1.47979949$

o $-2.06470140 \quad 0.49027730-1.25664600$

Vibrational frequencies (in cm-1)

$\begin{array}{lllllllllllllllll}-1221.9 & 90.7 & 107.9 & 144.2 & 171.2 & 176.6 & 203.8 & 216.9 & 239.6 & 267.1 & 307.0 & 342.8 & 359.7 & 385.8\end{array}$ $428.4 \quad 440.6 \quad 493.7 \quad 522.3 \quad 541.8 \quad 591.5 \quad 675.2 \quad 699.2 \quad 716.9 \quad 775.7 \quad 826.7 \quad 865.6 \quad 889.5 \quad 893.3$ $931.1967 .8 \quad 977.8 \quad 981.7 \quad 1000.9 \quad 1008.1 \quad 1012.2 \quad 1015.3 \quad 1031.7 \quad 1054.3 \quad 1074.01084 .4 \quad 1129.9$

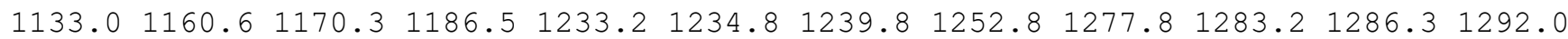

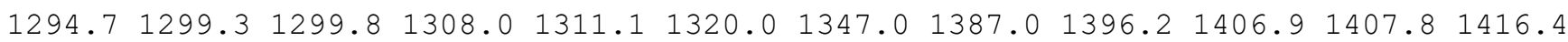
$1703.42672 .62673 .5 \quad 2676.62677 .2 \quad 2678.7 \quad 2680.42683 .62695 .62750 .42754 .02770 .5$ $2780.72782 .3 \quad 2784.0 \quad 4098.4$

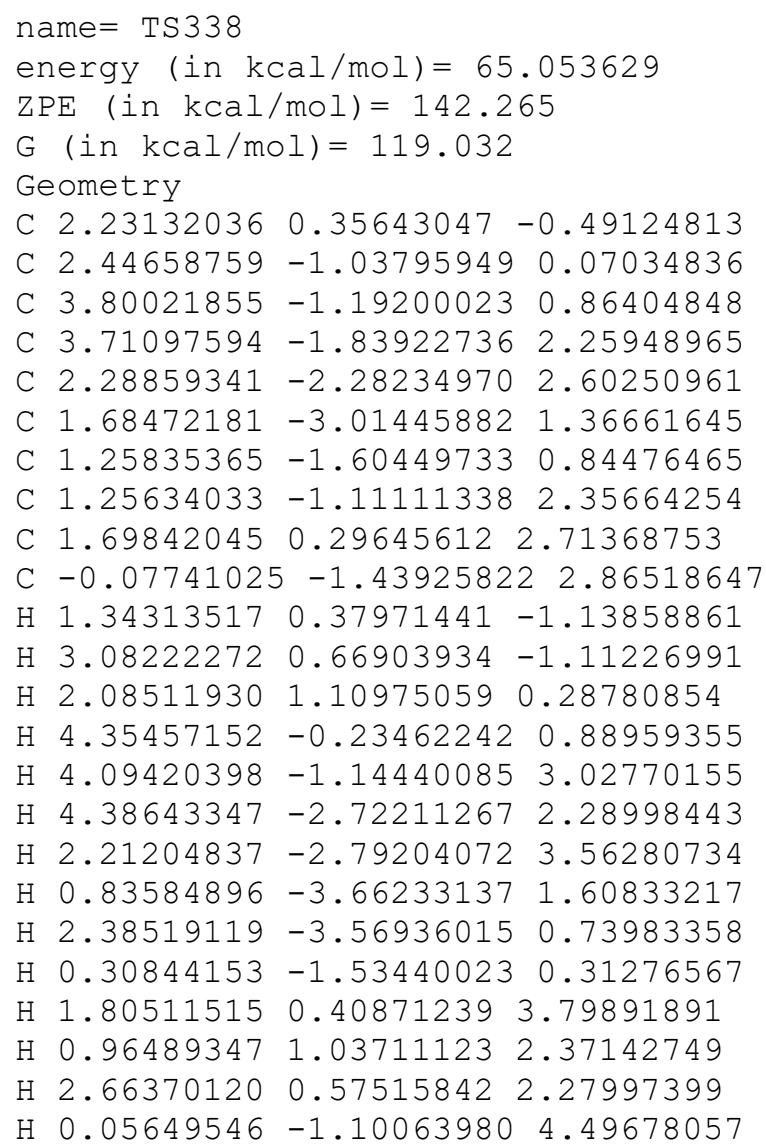


$\mathrm{H}-0.02162168-1.897575434 .35406658$

$\mathrm{H}-0.89639083-0.776184572 .73049615$

O $4.02231199-1.97825874-1.23734469$

$0 \quad 4.55592952-2.11554691 \quad 0.07056494$

O $2.62206097-1.93441761-1.04347288$

Vibrational frequencies (in $\mathrm{cm}-1$ )

$\begin{array}{lllllllllllllllll}-996.1 & 84.8 & 127.6 & 144.0 & 170.4 & 183.9 & 196.3 & 228.1 & 270.5 & 276.8 & 315.9 & 353.2 & 363.5 & 404.4\end{array}$ $422.2 \quad 455.0 \quad 463.4 \quad 528.3 \quad 535.8 \quad 595.1 \quad 626.8 \quad 642.0 \quad 712.6 \quad 723.4 \quad 801.0 \quad 831.4 \quad 839.6 \quad 876.2$ $890.7 \quad 923.3 \quad 940.1952 .3 \quad 983.1 \quad 997.6 \quad 1011.3 \quad 1025.9 \quad 1053.1 \quad 1061.7 \quad 1070.0 \quad 1073.4 \quad 1088.2$

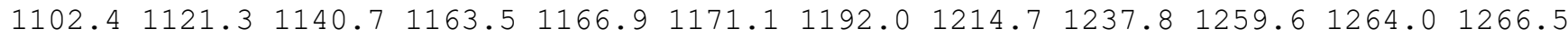

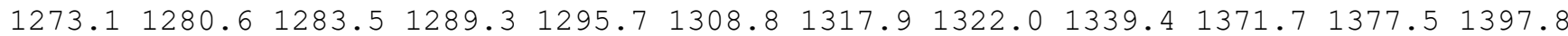
$1410.92656 .6 \quad 2670.1 \quad 2671.8 \quad 2677.3 \quad 2678.0 \quad 2683.7 \quad 2689.72720 .0 \quad 2737.92743 .8 \quad 2749.7$ $2761.32780 .62783 .0 \quad 4700.7$

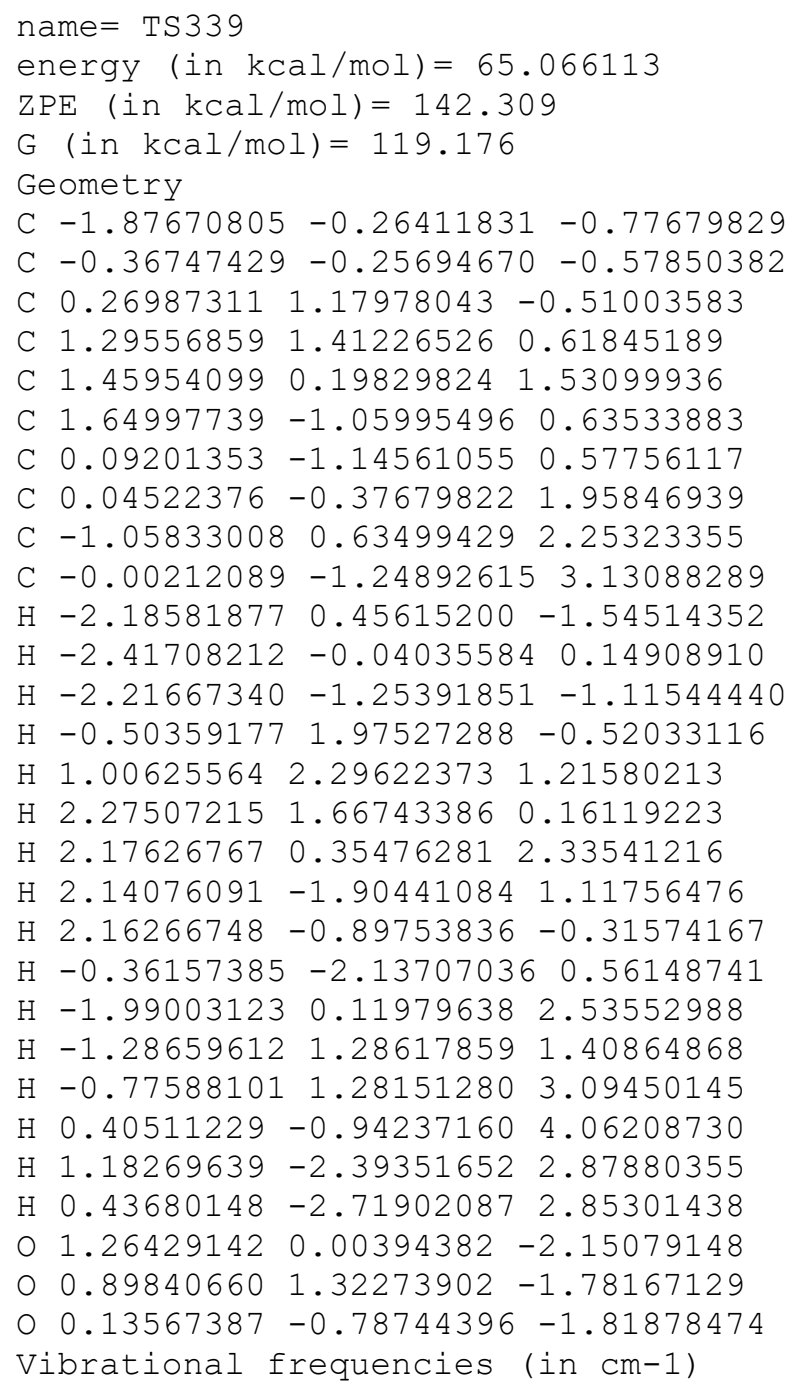


$\begin{array}{llll}C & -2.48932040 & -2.96292015 & 1.76217780\end{array}$

C $-2.22419078-2.68956457 \quad 0.23160121$

C $-0.86386049-2.06211954-0.13282305$

C $0.03341801-1.875651791 .08978260$

C $-0.02647833-3.17501068 \quad 1.94513332$

C $-1.29443056-2.57047405 \quad 2.62869611$

C $-0.74671046-1.12478198 \quad 2.25032840$

C $-1.69503879-0.01798490 \quad 1.83366773$

C $0.05381087-0.69811868 \quad 3.39593386$

$\mathrm{H}-3.85567648-1.34403866 \quad 2.30890958$

$\mathrm{H}-4.03880416-2.81432199 \quad 3.26448282$

$\mathrm{H}-4.64908639-2.771789391 .60984330$

$\mathrm{H}-3.06696289-2.13480351-0.22248702$

$\mathrm{H}-1.01602709-1.09684309-0.64827766$

$\mathrm{H}-0.35140333-2.71852659-0.87005739$

$\mathrm{H} 1.01988048-1.48472804 \quad 0.84662488$

$\mathrm{H} \quad 0.82034838-3.32197987 \quad 2.61687125$

$\mathrm{H}-0.18140615-4.10691444 \quad 1.39214564$

$\mathrm{H}-1.44063142-2.76268394 \quad 3.69387077$

$\mathrm{H}-1.15278434 \quad 0.81383267 \quad 1.37014616$

$\mathrm{H}-2.22693618 \quad 0.37779321 \quad 2.71241702$

$\mathrm{H}-2.45211686-0.336024351 .11157631$

$\mathrm{H} \quad 0.92419621-1.21867398 \quad 3.71162560$

$\mathrm{H} \quad 0.41046487 \quad 0.83121335 \quad 3.32761861$

H $\quad 0.94632475 \quad 0.57926195 \quad 2.77246012$

O $-2.96647798-4.795826750 .54048549$

$0-2.19713084-4.00005119-0.34731906$

o $-2.53678958-4.40152574 \quad 1.82890727$

Vibrational frequencies (in cm-1)

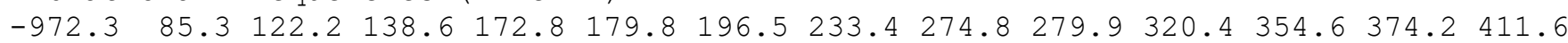

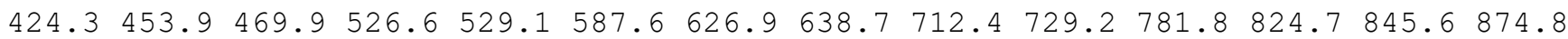
$889.5 \quad 922.4 \quad 940.7 \quad 952.0 \quad 983.0 \quad 998.4 \quad 1011.6 \quad 1031.3 \quad 1047.3 \quad 1053.6 \quad 1063.1 \quad 1072.0 \quad 1086.2$

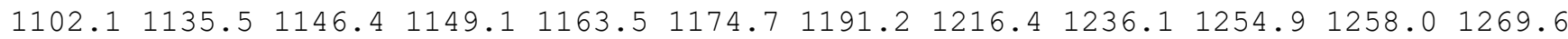
$1272.7 \quad 1280.4 \quad 1284.3 \quad 1290.7 \quad 1297.4 \quad 1309.7 \quad 1318.6 \quad 1324.2 \quad 1354.7 \quad 1375.4 \quad 1379.0 \quad 1398.3$ $1416.42655 .62670 .0 \quad 2672.3 \quad 2675.2 \quad 2677.7 \quad 2683.72685 .22717 .02737 .22739 .52750 .9$ $2763.4 \quad 2780.1 \quad 2782.8 \quad 4726.2$

name $=\operatorname{TS} 341$

energy (in $\mathrm{kcal} / \mathrm{mol})=68.677081$

$\operatorname{ZPE}(\mathrm{in} \mathrm{kcal} / \mathrm{mol})=138.853$

$\mathrm{G}(\mathrm{in} \mathrm{kcal} / \mathrm{mol})=112.44$

Geometry

C $-2.47204953-0.26934911-1.08017662$

C $-0.95332628-0.36338126-1.00143305$

C $-0.37672595 \quad 0.95212996-0.53818584$

C $1.70586735 \quad 0.63717909-0.08618824$

C $2.30074757 \quad 0.58469075 \quad 1.24911878$

C $2.47191183-0.548669681 .93807569$

C $-0.48935326-1.62598153-0.37655248$

C $-0.53409275-1.915021310 .93094727$

C $-1.00842457-0.97982500 \quad 1.98922917$

C $-0.08233018-3.24843378 \quad 1.43234763$

$\mathrm{H}-2.79059843 \quad 0.61710973-1.64741078$

$\mathrm{H}-2.93084908-0.21754487-0.08647238$

$\mathrm{H}-2.88664816-1.14870970-1.59186091$

$\mathrm{H} \quad 0.36454322 \quad 1.114689890 .42475797$

$\mathrm{H} \quad 2.06716844 \quad 1.43745336 \quad-0.72614194$

$\mathrm{H} \quad 1.69234150 \quad-0.30331160 \quad-0.63014072$

$\mathrm{H} 2.61122696 \quad 1.54687978 \quad 1.66435795$

H $2.91413504 \quad-0.586945812 .91889230$

H $2.19619196-1.521308921 .56319852$

$\mathrm{H}-0.11022909-2.35391392-1.09923441$

$\mathrm{H}-1.64461851-1.48551388 \quad 2.72852559$

$\mathrm{H}-1.58463941-0.13100523 \quad 1.59671922$ 
$\mathrm{H}-0.14970676-0.556112262 .53887013$

$\mathrm{H} \quad 0.53421945-3.15705294 \quad 2.33795585$

$\mathrm{H} \quad 0.51107929-3.80502990 \quad 0.69393067$

$\mathrm{H}-0.94633155-3.87906643 \quad 1.69162460$

$\begin{array}{llll}0 & -0.46842654 & 0.92646068 & -2.80397057\end{array}$

$0-0.298796891 .77860965-1.51483215$

o $-0.39406713-0.36963468-2.36033567$

Vibrational frequencies (in cm-1)

$\begin{array}{lllllllllllllll}-1429.0 & 40.8 & 81.2 & 84.6 & 99.7 & 116.8 & 128.2 & 130.6 & 143.5 & 149.5 & 190.9 & 201.6 & 251.2295 .0\end{array}$ $312.6 \quad 327.2 \quad 352.6 \quad 371.2 \quad 422.3 \quad 447.4 \quad 489.7 \quad 516.7 \quad 542.3 \quad 638.3 \quad 684.2 \quad 717.8 \quad 736.2 \quad 754.8$ $904.8928 .2938 .7 \quad 972.2987 .1991 .0 \quad 1000.4 \quad 1003.4 \quad 1016.0 \quad 1034.2 \quad 1041.5 \quad 1078.7 \quad 1089.8$

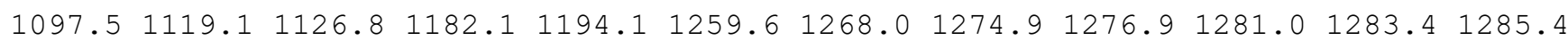

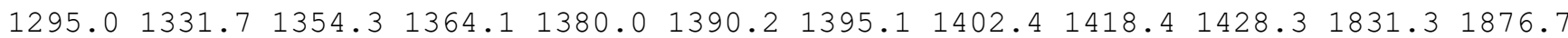
$2017.72655 .32667 .62668 .42670 .3 \quad 2672.5 \quad 2674.12685 .62717 .82742 .82747 .62759 .1$ $2774.3 \quad 2778.0 \quad 2779.4 \quad 2789.5$

\section{name $=$ TS342}

energy (in $\mathrm{kcal} / \mathrm{mol})=64.458089$

$\mathrm{ZPE}($ in $\mathrm{kcal} / \mathrm{mol})=143.113$

$\mathrm{G}(\mathrm{in} \mathrm{kcal} / \mathrm{mol})=119.419$

Geometry

C $-2.12748783 \quad 0.19146052-1.02308232$

C $-0.66717502-0.15175421-0.78779412$

C $0.246525231 .12608918-0.66296586$

C $1.321973301 .09622773 \quad 0.44526784$

C $1.17899309-0.131004441 .34538515$

C $1.11058357-1.388658690 .42760159$

C $-0.42778183-1.147428930 .34020746$

C $-0.33839600-0.380048391 .73038301$

C $-1.20789935 \quad 0.76983970 \quad 1.94987141$

C $-0.46744811-1.364199472 .89825262$

$\mathrm{H}-2.56289572 \quad 0.69791006-0.15312546$

$\mathrm{H}-2.71722776-0.71573285-1.20598841$

$\mathrm{H}-2.249888350 .84029356-1.89880737$

$\mathrm{H}-0.36860397 \quad 2.04228582-0.61110243$

$\mathrm{H} \quad 1.29711544 \quad 2.02844597 \quad 1.03041639$

H 2.32695058 $1.07964811-0.03374426$

H $1.89346165-0.157114842 .16685306$

$\mathrm{H} \quad 1.39276299-2.32334638 \quad 0.91409542$

H $1.65743630-1.32185112-0.51685210$

$\mathrm{H}-1.07581535-2.020552190 .30923682$

$\mathrm{H}-0.41127594 \quad 1.71429694 \quad 2.22234359$

$\mathrm{H}-1.30463188 \quad 1.950627532 .28706028$

$\mathrm{H}-2.26002765 \quad 0.59705503 \quad 1.95336362$

$\mathrm{H}-1.50210500-1.71475926 \quad 2.98568503$

$\mathrm{H}-0.20907879-0.875757373 .84258556$

$\mathrm{H} \quad 0.17271575-2.243047292 .78688668$

$\begin{array}{lllll}0 & 0.25865766 & 0.30401151 & -2.78006534\end{array}$

○ $0.97744168 \quad 1.16039098-1.89275041$

o $-0.15398238-0.77857874-1.98063524$

Vibrational frequencies (in $\mathrm{cm}-1$ )

$\begin{array}{llllllllllllllll}-1463.6 & 57.8 & 77.1 & 142.5 & 154.2 & 175.0 & 196.0 & 215.9 & 255.8 & 273.9 & 324.4 & 348.4 & 373.3 & 406.3\end{array}$ $421.9 \quad 443.6 \quad 480.6 \quad 525.9 \quad 598.4 \quad 633.3 \quad 666.0 \quad 690.4 \quad 710.1 \quad 735.6 \quad 804.1 \quad 837.0 \quad 874.9887 .8$

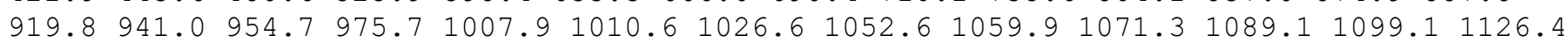

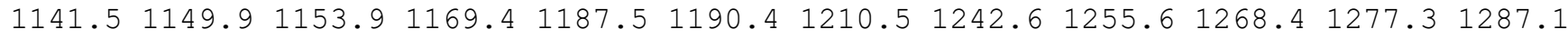

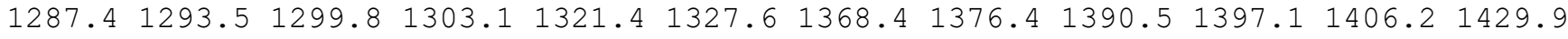
$1960.92654 .5 \quad 2675.3 \quad 2677.92681 .9 \quad 2685.0 \quad 2688.62691 .32737 .7 \quad 2749.82754 .92764 .7$ $2777.5 \quad 2781.5 \quad 2785.3 \quad 3661.8$

name $=\operatorname{TS} 343$

energy $($ in $\mathrm{kcal} / \mathrm{mol})=65.164363$

$\mathrm{ZPE}($ in $\mathrm{kcal} / \mathrm{mol})=142.925$

$\mathrm{G}(\mathrm{in} \mathrm{kcal} / \mathrm{mol})=118.34$ 


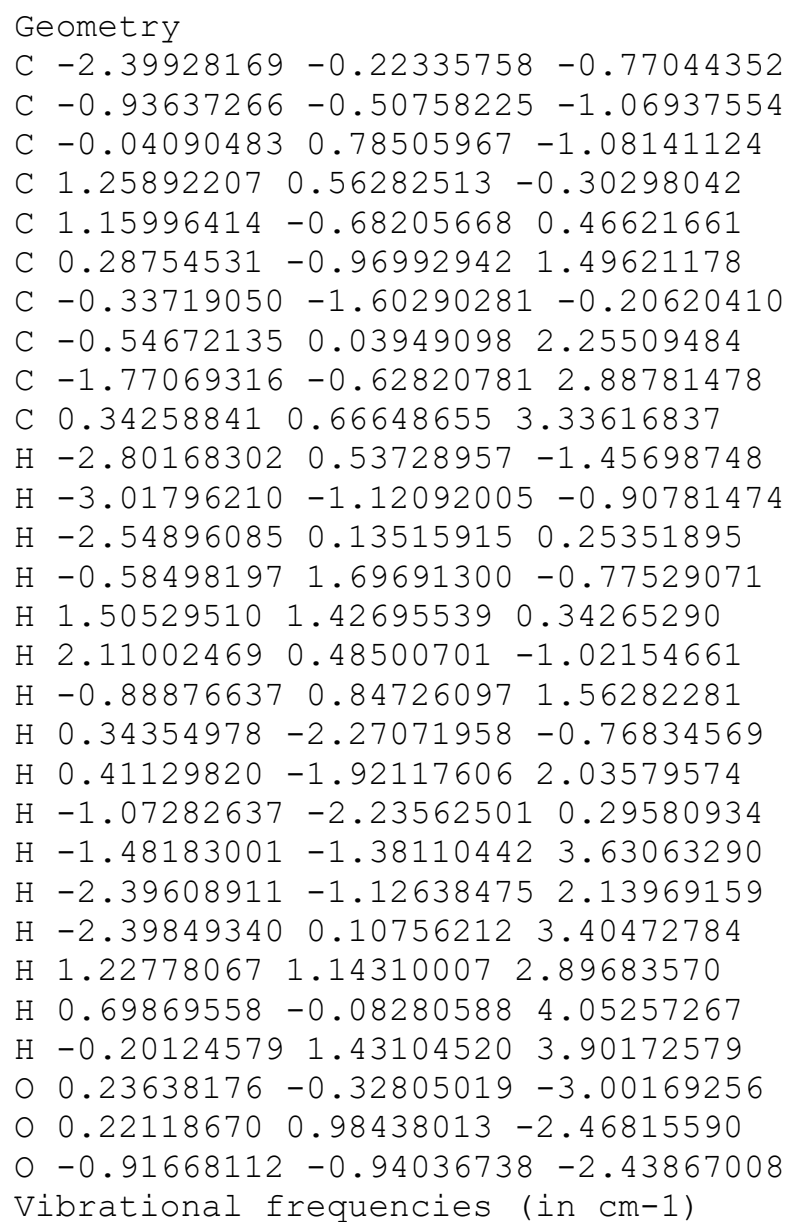

$\begin{array}{llllllllllllllllllllll}-661.9 & 59.2 & 71.2 & 88.6 & 130.3 & 164.7 & 172.2 & 180.0 & 196.0 & 247.6 & 257.8 & 320.6 & 335.1 & 348.5\end{array}$ $407.0 \quad 441.3 \quad 463.0 \quad 500.6 \quad 528.1 \quad 547.9 \quad 635.6 \quad 679.8 \quad 711.1 \quad 814.8 \quad 846.7 \quad 857.6 \quad 917.1949 .7$ $969.4 \quad 970.8 \quad 980.3 \quad 986.6 \quad 1003.3 \quad 1012.5 \quad 1017.9 \quad 1045.9 \quad 1061.5 \quad 1079.2 \quad 1124.91141 .7 \quad 1154.9$

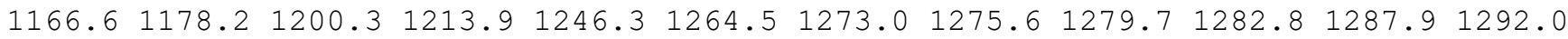

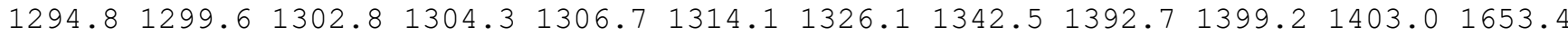
$2641.82655 .92665 .7 \quad 2670.6 \quad 2672.4 \quad 2673.7 \quad 2675.32678 .32695 .82700 .42718 .62726 .4$ $2741.22777 .92780 .3 \quad 2782.0$

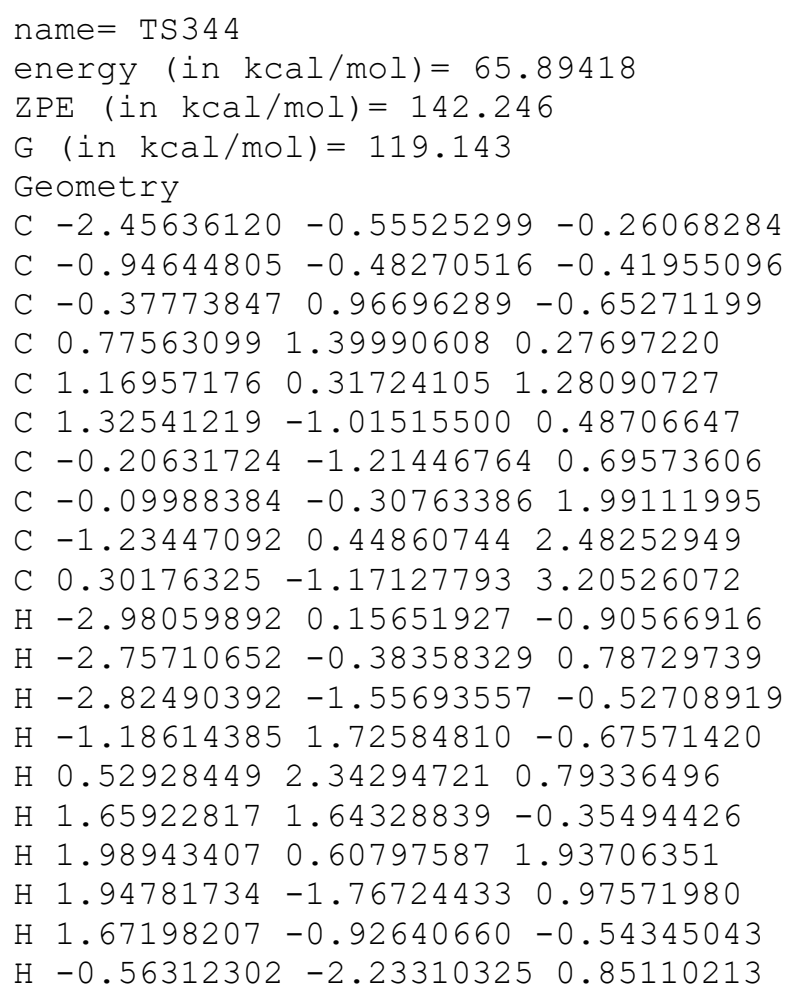


$\mathrm{H}-1.270564501 .947321191 .70066508$

$\mathrm{H}-1.19772014 \quad 0.95970764 \quad 3.41559034$

$\mathrm{H}-1.888591991 .480463961 .46683812$

$\mathrm{H} \quad 0.72590885 \quad-0.55880117 \quad 4.00731655$

H $1.04732211-1.927958412 .93956142$

$\mathrm{H}-0.56954069-1.70648413 \quad 3.60797930$

O $0.36371849-0.42059636-2.27407427$

$\begin{array}{lllll}0 & 0.07290131 & 0.94582630 & -2.00440597\end{array}$

$0-0.70126346-1.13235759-1.68076902$

Vibrational frequencies (in $\mathrm{cm}-1$ )

$\begin{array}{lllllllllllllllllllll}-962.7 & 90.5 & 123.7 & 153.5 & 166.9 & 184.0 & 213.7 & 231.8 & 275.1 & 293.2 & 324.7 & 353.2 & 379.2 & 422.1\end{array}$

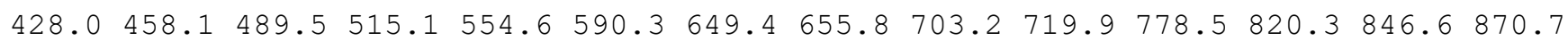
$892.4912 .0 \quad 939.0 \quad 955.5 \quad 980.6 \quad 996.1 \quad 1002.5 \quad 1007.5 \quad 1027.7 \quad 1039.7 \quad 1056.5 \quad 1068.9 \quad 1080.9$ $1098.81110 .3 \quad 1139.5 \quad 1148.3 \quad 1166.6 \quad 1178.3 \quad 1190.3 \quad 1196.91210 .7 \quad 1241.0 \quad 1255.6 \quad 1267.2$

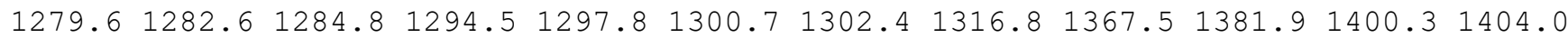
$1417.12643 .0 \quad 2652.3 \quad 2669.5 \quad 2674.8 \quad 2677.0 \quad 2682.62688 .22714 .92736 .62746 .92750 .9$ $2767.7 \quad 2773.22778 .0 \quad 4762.9$

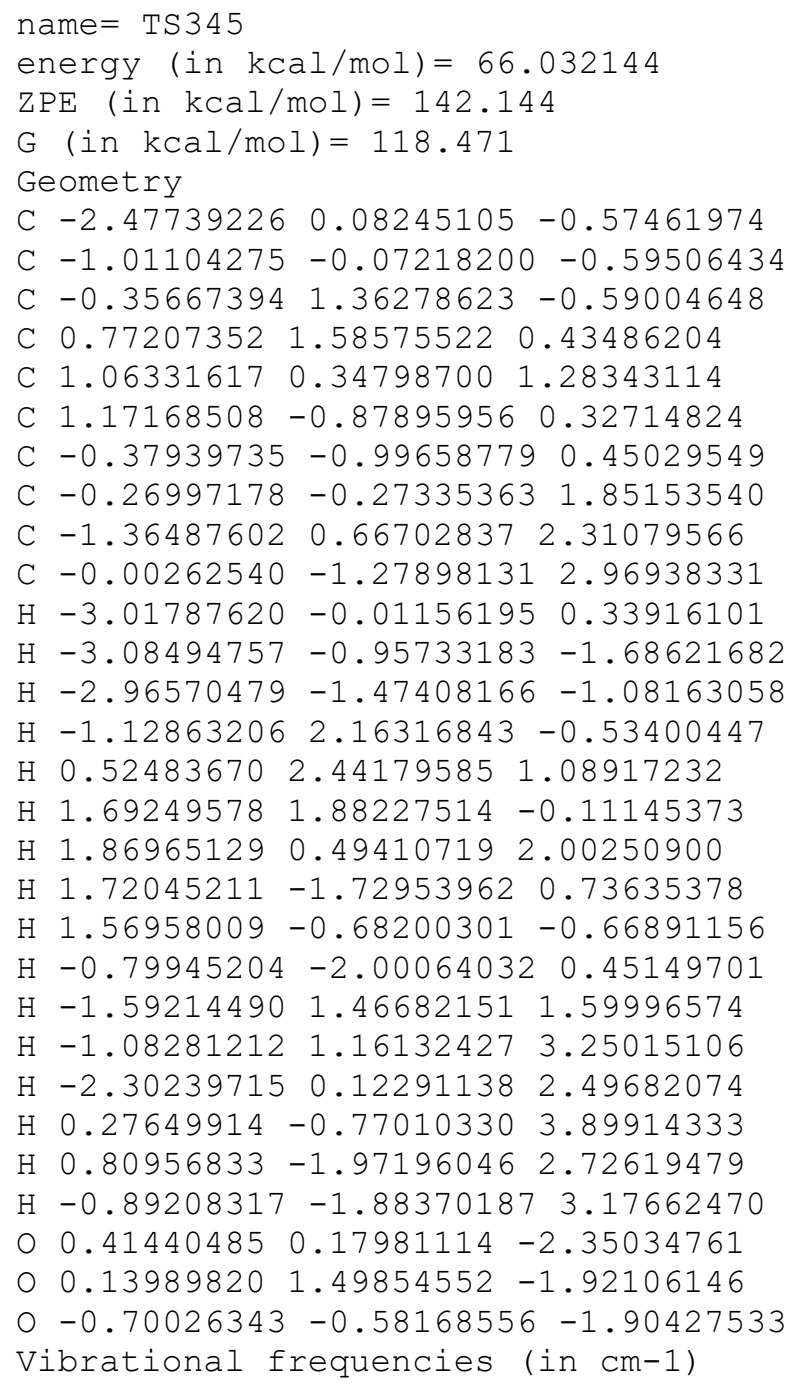
$436.8 \quad 458.2 \quad 476.1 \quad 489.6 \quad 514.5 \quad 579.1 \quad 617.3 \quad 673.3 \quad 716.0 \quad 722.9 \quad 804.2 \quad 818.2 \quad 853.8 \quad 867.1$ $895.5920 .1940 .3 \quad 980.8 \quad 988.4 \quad 999.2 \quad 1003.3 \quad 1005.8 \quad 1015.4 \quad 1019.8 \quad 1046.6 \quad 1059.5 \quad 1087.2$

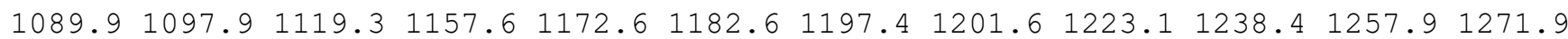
$1280.91287 .5 \quad 1294.4 \quad 1296.8 \quad 1300.9 \quad 1307.8 \quad 1315.3 \quad 1321.2 \quad 1324.1 \quad 1373.2 \quad 1384.3 \quad 1405.8$ $1416.52657 .0 \quad 2670.3 \quad 2672.0 \quad 2674.92677 .3 \quad 2681.02688 .4 \quad 2699.8 \quad 2739.4 \quad 2748.2 \quad 2752.1$ $2767.5 \quad 2780.4 \quad 2784.1 \quad 4849.2$ 
$\mathrm{ZPE}($ in $\mathrm{kcal} / \mathrm{mol})=141.741$

$\mathrm{G}($ in $\mathrm{kcal} / \mathrm{mol})=117.236$

Geometry

C $-2.21469127-0.06214592-1.38047697$

C $-0.71989989-0.19546311-1.16701674$

C $0.05585647 \quad 1.17287391-1.06058592$

C $0.89637888 \quad 1.15388445 \quad 0.22567870$

C $0.60001618-0.16299326 \quad 0.93296505$

C $1.09438279-1.41079710 \quad 0.22779116$

C $-0.34875669-0.99173214 \quad 0.06293498$

C $0.47001057-0.07538304 \quad 2.44313052$

C $-0.92794526 \quad 0.45167102 \quad 2.81045347$

C $0.68815525-1.432859952 .99046273$

$\mathrm{H}-2.44176854 \quad 0.55561923-2.26021732$

$\mathrm{H}-2.70398364 \quad 0.38840001-0.50916482$

$\mathrm{H}-2.67779648-1.04410767-1.55167296$

$\mathrm{H}-0.589751172 .06303785-1.14916156$

$\mathrm{H} \quad 0.65961757 \quad 2.02075071 \quad 0.86475136$

$\mathrm{H} \quad 1.97349118 \quad 1.23892761-0.01706382$

H $1.23095068 \quad 0.66171020 \quad 2.81206972$

$\mathrm{H} 1.30809344 \quad-2.31434599 \quad 0.79733124$

$\mathrm{H} \quad 1.78742294-1.32238214-0.60540141$

$\mathrm{H}-1.12920284-1.61177187 \quad 0.49764045$

$\mathrm{H}-1.69523829-0.301210612 .58734940$

H $-1.17781924 \quad 1.34968114 \quad 2.23465446$

$\mathrm{H}-1.00074668 \quad 0.70933755 \quad 3.86942243$

$\mathrm{H} 0.48406978-1.20553474 \quad 4.62482194$

$\mathrm{H} 1.68106488-1.798789793 .11663113$

$\mathrm{H}-0.10322813-1.66852864 \quad 4.29945167$

O $1.06449468-0.17217451-2.55351964$

$0.864534651 .20055225-2.22642632$

O $-0.20640959-0.77148434-2.37308611$

Vibrational frequencies (in cm-1)

$\begin{array}{llllllllllllllll}-1036.0 & 39.3 & 79.5 & 98.8 & 144.5 & 160.6 & 183.7 & 199.3 & 208.7 & 257.7 & 280.7 & 312.2 & 320.0 & 377.3\end{array}$ $417.4 \quad 457.2 \quad 495.0 \quad 529.5 \quad 548.0 \quad 561.9 \quad 578.2 \quad 636.4 \quad 664.2 \quad 710.4 \quad 784.8 \quad 839.3 \quad 850.3 \quad 865.8$

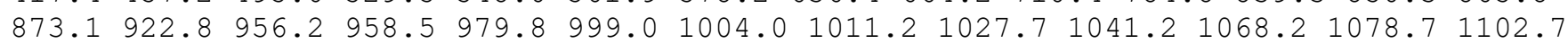

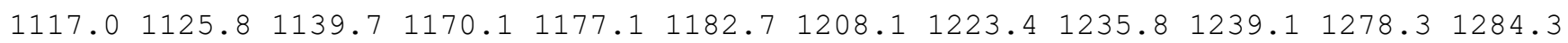

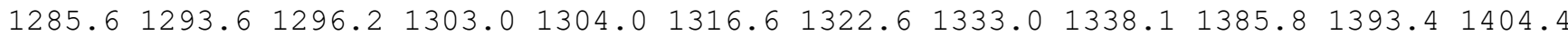
$1481.12666 .42669 .2 \quad 2671.5 \quad 2678.2 \quad 2679.12689 .22701 .02704 .52706 .32747 .32766 .8$ $2777.6 \quad 2780.5 \quad 2781.3 \quad 4668.1$
name $=\operatorname{TS} 347$
energy $($ in $\mathrm{kcal} / \mathrm{mol})=66.108341$
$\mathrm{ZPE}(\mathrm{in} \mathrm{kcal} / \mathrm{mol})=142.228$
$\mathrm{G}(\mathrm{in} \mathrm{kcal} / \mathrm{mol})=119.126$
Geometry
C $-1.48799307 \quad 3.09426554 \quad 0.00177954$
$\begin{array}{lllll}C & -0.02072507 & 2.76018194 & 0.20988647\end{array}$
C $0.56035573 \quad 3.30521306 \quad 1.57351256$
C $1.309332592 .28996175 \quad 2.45683694$
C $1.40769598 \quad 0.913916561 .79776111$
C $1.84252668 \quad 1.131368990 .31502081$
C $0.32601542 \quad 1.29247944 \quad 0.01683109$
C $0.00691423 \quad 0.41987996 \quad 1.27317805$
C $-1.18809221 \quad 0.49404488 \quad 2.08128614$
C $0.04020627-1.08327067 \quad 0.91344088$
$\mathrm{H}-1.69667129 \quad 4.15074587 \quad 0.21596070$
$\mathrm{H}-2.15525555 \quad 2.47674726 \quad 0.60829342$
$\mathrm{H}-1.76894628 \quad 2.93076073-1.05041111$
$\mathrm{H}-0.21169379 \quad 3.858933412 .14042820$
$\mathrm{H} \quad 0.84416696 \quad 2.21238959 \quad 3.45449716$
H 2.33766421 2.67266076 2.64782662
H $1.97236931 \quad 0.19130333 \quad 2.38884153$
H $2.31979086 \quad 0.27041911-0.15446192$ 
$\mathrm{H} 2.46908262 \quad 2.00682251 \quad 0.12032785$

$\mathrm{H}-0.02515120 \quad 0.89873035 \quad-0.96883657$

$\mathrm{H}-1.43675518 \quad 2.087062792 .58895785$

$\mathrm{H}-2.16147502 \quad 0.45581703 \quad 1.65650476$

$\mathrm{H}-1.202005291 .611265713 .20069552$

H $0.95604147-1.35187264 \quad 0.37855791$

$\mathrm{H}-0.80893670-1.36034117 \quad 0.27959255$

$\mathrm{H}-0.00438358-1.69869957 \quad 1.82348202$

O $1.12075850 \quad 4.67662390 \quad-0.12135034$

$0 \quad 1.55441704 \quad 4.249956801 .16077434$

O $0.73934617 \quad 3.48184537-0.77581865$

Vibrational frequencies (in $\mathrm{cm}-1$ )

$\begin{array}{lllllllllllllll}-991.8 & 93.6 & 131.3 & 148.4 & 163.7 & 197.1 & 203.3 & 237.2 & 271.5 & 280.7 & 324.3 & 357.8 & 372.5 & 415.6\end{array}$ $\begin{array}{llllllllllllllll}420.1 & 450.7 & 482.1 & 526.0 & 550.3 & 603.3 & 628.0 & 657.7 & 713.8 & 721.3 & 765.2 & 820.5 & 831.7 & 876.0\end{array}$ $884.6921 .6 \quad 937.7 \quad 952.6 \quad 980.7 \quad 1001.0 \quad 1001.6 \quad 1011.3 \quad 1027.0 \quad 1053.1 \quad 1061.4 \quad 1067.4 \quad 1085.3$ $1098.01112 .2 \quad 1136.5 \quad 1140.9 \quad 1164.4 \quad 1174.4 \quad 1182.0 \quad 1193.3 \quad 1215.91240 .5 \quad 1258.3 \quad 1270.5$

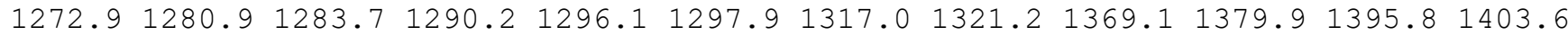
$1428.12651 .4 \quad 2665.92671 .5 \quad 2676.2 \quad 2676.92679 .92685 .42720 .92736 .5 \quad 2747.2 \quad 2752.3$ $2763.7 \quad 2777.9 \quad 2780.0 \quad 4751.9$

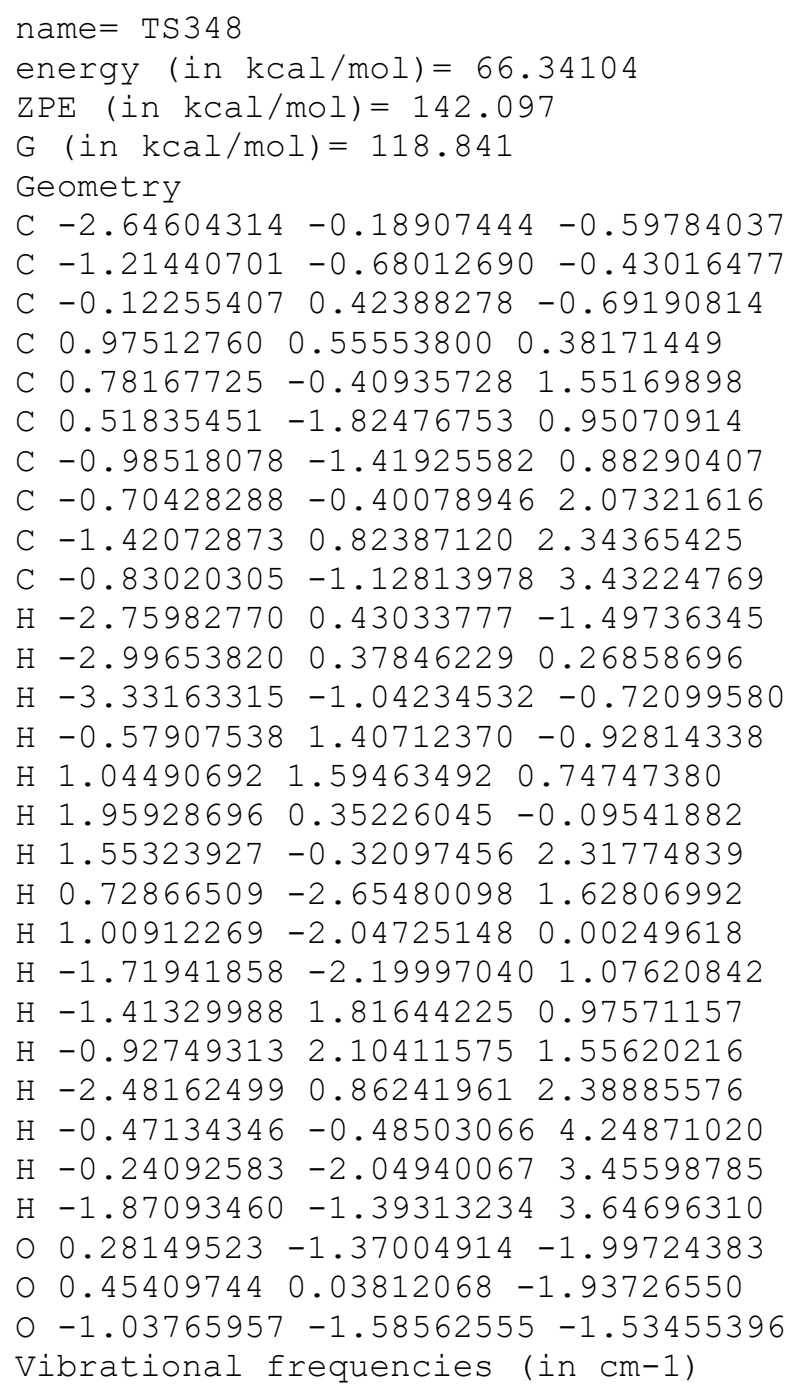




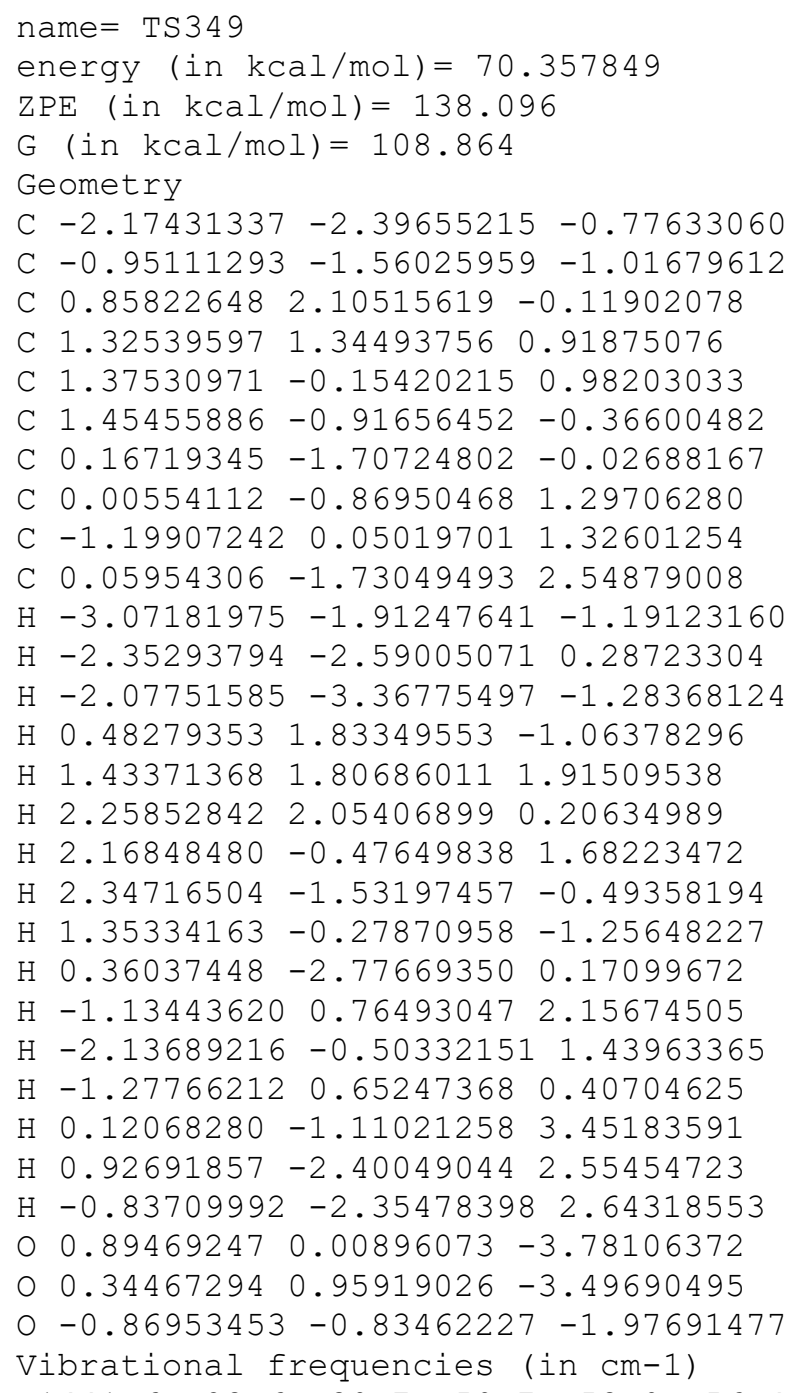


H $0.44481892 \quad 1.02497600 \quad 2.53359143$

$\mathrm{H} \quad 1.12108241-2.32757484 \quad 1.22914170$

H $1.71262179-1.58357959-0.27387091$

$\mathrm{H}-1.31517944-1.92078141 \quad 0.49504553$

$\mathrm{H}-2.21422194 \quad 0.30552539 \quad 3.63164987$

$\mathrm{H}-2.01314091 \quad 1.10118879 \quad 1.64637027$

$\mathrm{H}-1.90495440 \quad 1.06025742 \quad 3.66323085$

$\mathrm{H} \quad 0.02082179-0.66084926 \quad 4.30168919$

$\mathrm{H} 1.29752952-1.23993688 \quad 3.23023766$

$\mathrm{H}-0.34683042-1.899200343 .09689872$

O $1.19893067-0.93216076-2.49013510$

$\begin{array}{lllll}0 & 0.87129188 & 0.45539212 & -2.48024587\end{array}$

o $-0.04541240-1.59464731-2.35296264$

Vibrational frequencies (in cm-1)

$\begin{array}{lllllllllllllll}-1058.4 & 25.0 & 78.4 & 94.1 & 136.3 & 160.6 & 187.9 & 206.7 & 227.4 & 260.2 & 272.7 & 311.5 & 327.9 & 373.8\end{array}$

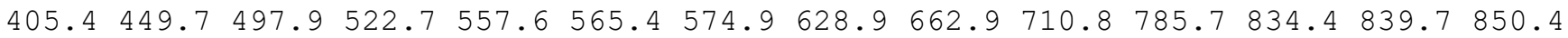
$870.6921 .7953 .9959 .2 \quad 980.4 \quad 1001.3 \quad 1003.3 \quad 1007.4 \quad 1024.4 \quad 1042.3 \quad 1068.4 \quad 1074.7 \quad 1105.9$

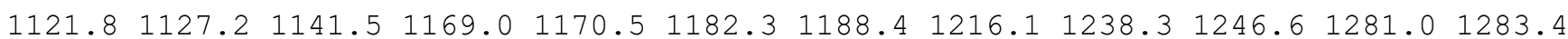

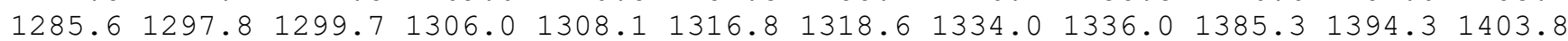
$1484.22667 .0 \quad 2669.4 \quad 2672.1 \quad 2675.9 \quad 2679.3 \quad 2689.4 \quad 2700.52707 .72711 .2 \quad 2747.92762 .5$ $2780.12780 .7 \quad 2782.8 \quad 4656.8$

name $=$ TS351

energy $($ in $\mathrm{kcal} / \mathrm{mol})=66.270867$

$\operatorname{ZPE}($ in $\mathrm{kcal} / \mathrm{mol})=142.22$

$\mathrm{G}(\mathrm{in} \mathrm{kcal} / \mathrm{mol})=119.026$

Geometry

C $-0.37555598-1.01360012-2.89101390$

C $0.66217627-0.53563631-1.88617517$

C $0.35547385 \quad 0.87857586-1.25318308$

C $0.46578377 \quad 0.97511853 \quad 0.28411219$

C $0.88740641-0.34812395 \quad 0.93573697$

C $2.02859024-0.84191473 \quad 0.05823047$

C $1.01049685-1.57314811-0.81487103$

C $-0.00914995-1.53843458 \quad 0.39954810$

C $-1.47951109-1.26983757 \quad 0.16203248$

C $0.11557264-2.79201401 \quad 1.26313123$

$\mathrm{H}-0.60858271-0.22972838-3.62426662$

$\mathrm{H}-1.30758707-1.32345380-2.41005934$

$\mathrm{H} \quad 0.00313472-1.87548979-3.45820411$

$\mathrm{H}-0.60490278 \quad 1.29254950 \quad-1.61705113$

H $-0.48678810 \quad 1.32446706 \quad 0.71695875$

$\mathrm{H} 1.224386591 .75090109 \quad 0.52748740$

$\mathrm{H} 1.00274856-0.27460360 \quad 2.01418945$

H $2.65467624-1.98900398 \quad 0.89577547$

H $3.17919744-1.420870010 .52857152$

H $1.23816757-2.55123484-1.23128118$

$\mathrm{H}-1.66729422-0.38573676-0.45478647$

$\mathrm{H}-2.00099725-1.10157717 \quad 1.11414072$

$\mathrm{H}-1.96464478-2.12163554-0.33161959$

H $1.15128130-3.05221366 \quad 1.49887587$

$\mathrm{H}-0.32837597-3.65946174 \quad 0.75853118$

$\mathrm{H}-0.41068606-2.664533382 .21733526$

○ $2.43170278 \quad 0.85319435 \quad-2.10754345$

$\begin{array}{llll}0 & 1.33544257 & 1.72233987 & -1.85614091\end{array}$

O $1.82680069-0.28407115-2.69146639$

Vibrational frequencies (in $\mathrm{cm}-1$ )

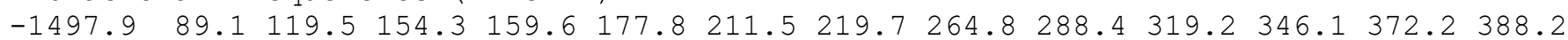

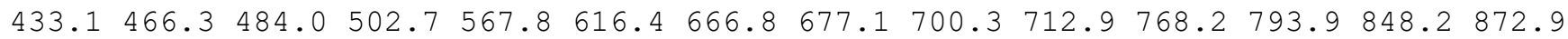
$878.5954 .9971 .0 \quad 988.9 \quad 996.2 \quad 996.9 \quad 1005.1 \quad 1016.0 \quad 1028.2 \quad 1045.7 \quad 1065.8 \quad 1090.8 \quad 1104.2$

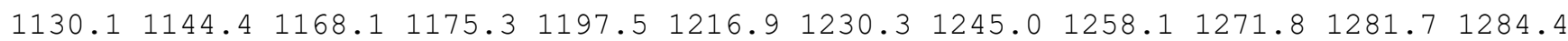

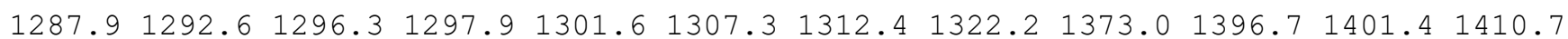
1421.72661 .22668 .72671 .72672 .72674 .82678 .02678 .62689 .62738 .52754 .82757 .7 $2777.92781 .02783 .3 \quad 4107.1$ 
name $=$ TS352

energy $($ in $\mathrm{kcal} / \mathrm{mol})=65.168193$

$\mathrm{ZPE}($ in $\mathrm{kcal} / \mathrm{mol})=143.435$

$\mathrm{G}(\mathrm{in} \mathrm{kcal} / \mathrm{mol})=120.26$

Geometry

C $-2.24826264-1.40177181-0.75778512$

C $-0.92578122-0.65148691-0.66927379$

C $-1.07700721 \quad 0.91353297 \quad-0.62887299$

C $-0.23353629 \quad 1.64790618 \quad 0.43336929$

C $0.57300365 \quad 0.69704568 \quad 1.31608006$

C $1.29943863-0.320506710 .38813485$

C $-0.00815821-1.169529350 .43077094$

C $-0.33401104-0.497819551 .84699633$

C $-1.77290705-0.166058752 .20939036$

C $0.25877977-1.23488327 \quad 2.96147107$

$\mathrm{H}-2.91106322-0.96006458-1.51274241$

$\mathrm{H}-2.77574816-1.41989207 \quad 0.20136224$

$\mathrm{H}-2.07956521-2.44695982-1.05381262$

$\mathrm{H}-2.141605591 .22226101-0.58159425$

$\mathrm{H}-0.88573336 \quad 2.28054325 \quad 1.06296763$

$\mathrm{H} \quad 0.45586976 \quad 2.34786980-0.08316586$

$\mathrm{H} \quad 1.16820370 \quad 1.20875791 \quad 2.06998316$

H $2.17465653-0.803552850 .81943697$

$\mathrm{H} \quad 1.59868481 \quad 0.05681170 \quad-0.59233814$

$\mathrm{H} \quad 0.09347162 \quad-2.25265197 \quad 0.43573179$

$\mathrm{H}-2.31165902 \quad 0.38607564 \quad 1.43834247$

$\mathrm{H}-1.79534166 \quad 0.45057754 \quad 3.11886404$

$\mathrm{H}-2.33877794 \quad-1.08258656 \quad 2.42133972$

H $1.28165222-1.78175144 \quad 3.41620643$

$\mathrm{H} 1.47463970-1.430643122 .59470911$

$\mathrm{H}-0.32868150-1.48289183 \quad 3.81134231$

$\begin{array}{lllll}0 & 0.25390428 & 0.33750526 & -2.35247338\end{array}$

$\begin{array}{llll}0 & -0.68048000 & 1.32073910 & -1.93687232\end{array}$

o $-0.32040574-0.89430484-1.95520450$

Vibrational frequencies (in $\mathrm{cm}-1$ )

$\begin{array}{lllllllllllllll}-1396.0 & 90.7 & 102.4 & 138.2 & 175.5 & 182.9 & 213.7 & 227.6 & 266.7 & 289.3 & 325.3 & 347.9 & 389.8 & 421.6\end{array}$ $442.9 \quad 461.3 \quad 490.4 \quad 519.7 \quad 581.3 \quad 652.1 \quad 657.4 \quad 688.1 \quad 706.6 \quad 733.8 \quad 796.0 \quad 840.0 \quad 865.9 \quad 882.5$ $914.8935 .3 \quad 953.7 \quad 971.0 \quad 999.8 \quad 1007.5 \quad 1032.8 \quad 1042.7 \quad 1052.0 \quad 1071.4 \quad 1076.5 \quad 1098.2 \quad 1136.0$

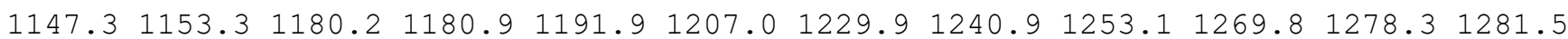
$1286.8 \quad 1295.3 \quad 1298.7 \quad 1300.7 \quad 1310.0 \quad 1322.6 \quad 1349.5 \quad 1376.81391 .3 \quad 1398.7 \quad 1406.7 \quad 1456.5$ $\begin{array}{llllllllllll}1896.1 & 2659.7 & 2671.1 & 2676.1 & 2677.5 & 2680.5 & 2685.6 & 2687.1 & 2739.9 & 2752.2 & 2755.7 & 2766.8\end{array}$ $2779.8 \quad 2782.6 \quad 2786.0 \quad 3748.1$

name $=\operatorname{TS} 353$

energy (in $\mathrm{kcal} / \mathrm{mol})=65.054246$

ZPE (in $\mathrm{kcal} / \mathrm{mol})=143.763$

G $($ in $\mathrm{kcal} / \mathrm{mol})=120.903$

Geometry

C $-2.25833845 \quad 0.13909435-1.28941785$

C $-0.88572661-0.14017200-0.72499312$

C $-0.021874131 .14289821-0.42863457$

C $0.74748544 \quad 1.10333674 \quad 0.89615169$

C $1.14001245-0.35120183 \quad 1.21335921$

C $1.65380724-1.02361524-0.03098572$

C $-0.71188978-1.24776228 \quad 0.15829347$

C $-0.15526722-1.14652842 \quad 1.53305963$

C $-1.11737194-0.450261332 .50755635$

C $0.18659250 \quad-2.52413310 \quad 2.12707618$

$\mathrm{H}-2.22174661 \quad 0.83553266-2.13736812$

$\mathrm{H}-2.913371110 .56529536-0.51960986$

$\mathrm{H}-2.73615539-0.78299040-1.64947039$

$\mathrm{H}-0.586425452 .07837832-0.58218979$ 
$\mathrm{H} \quad 0.16400424 \quad 1.54736023 \quad 1.71945541$

$\mathrm{H} 1.65616276 \quad 1.73208700 \quad 0.80600420$

$\mathrm{H} 1.88441988-0.376676152 .03500192$

$\mathrm{H} 1.53018030-2.08540766-0.15678307$

$\mathrm{H} 2.59843390-0.64247482-0.41387803$

$\mathrm{H}-1.18725208-2.17717927-0.11082290$

$\mathrm{H}-1.57676373 \quad 0.43841005 \quad 2.06390036$

$\mathrm{H}-0.60743439-0.15483560 \quad 3.42854290$

$\mathrm{H}-1.94090998-1.12081999 \quad 2.77959544$

$\mathrm{H} \quad 0.66132938-2.42235906 \quad 3.10776349$

$\mathrm{H} \quad 0.86438014-3.09528298 \quad 1.48550362$

$\mathrm{H}-0.71824200-3.128850112 .25417057$

O $1.12225537-0.30260273-1.70868015$

O $0.992569341 .13789085-1.43444459$

o $-0.12924610-0.65120696-1.99350367$

Vibrational frequencies (in cm-1)

$\begin{array}{lllllllllllllllll}-506.5 & 128.3 & 140.3 & 143.1 & 164.1 & 182.1 & 225.3 & 248.3 & 294.6 & 298.1 & 312.1 & 349.7 & 403.9 & 417.9\end{array}$

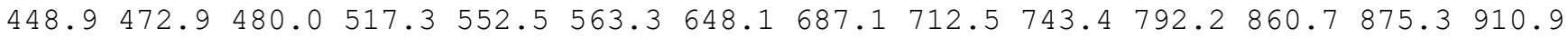

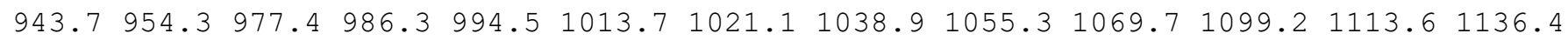
$1150.41163 .6 \quad 1192.3 \quad 1203.7 \quad 1218.7 \quad 1235.7 \quad 1244.6 \quad 1257.5 \quad 1270.3 \quad 1281.5 \quad 1288.01295 .4$ $1301.01306 .31308 .2 \quad 1330.7 \quad 1343.4 \quad 1349.5 \quad 1366.2 \quad 1380.6 \quad 1384.7 \quad 1401.01411 .7 \quad 1509.1$ $2663.12667 .12673 .0 \quad 2673.92677 .5 \quad 2677.92681 .22685 .52700 .12710 .02726 .52744 .3$ $2768.32780 .4 \quad 2781.12782 .5$

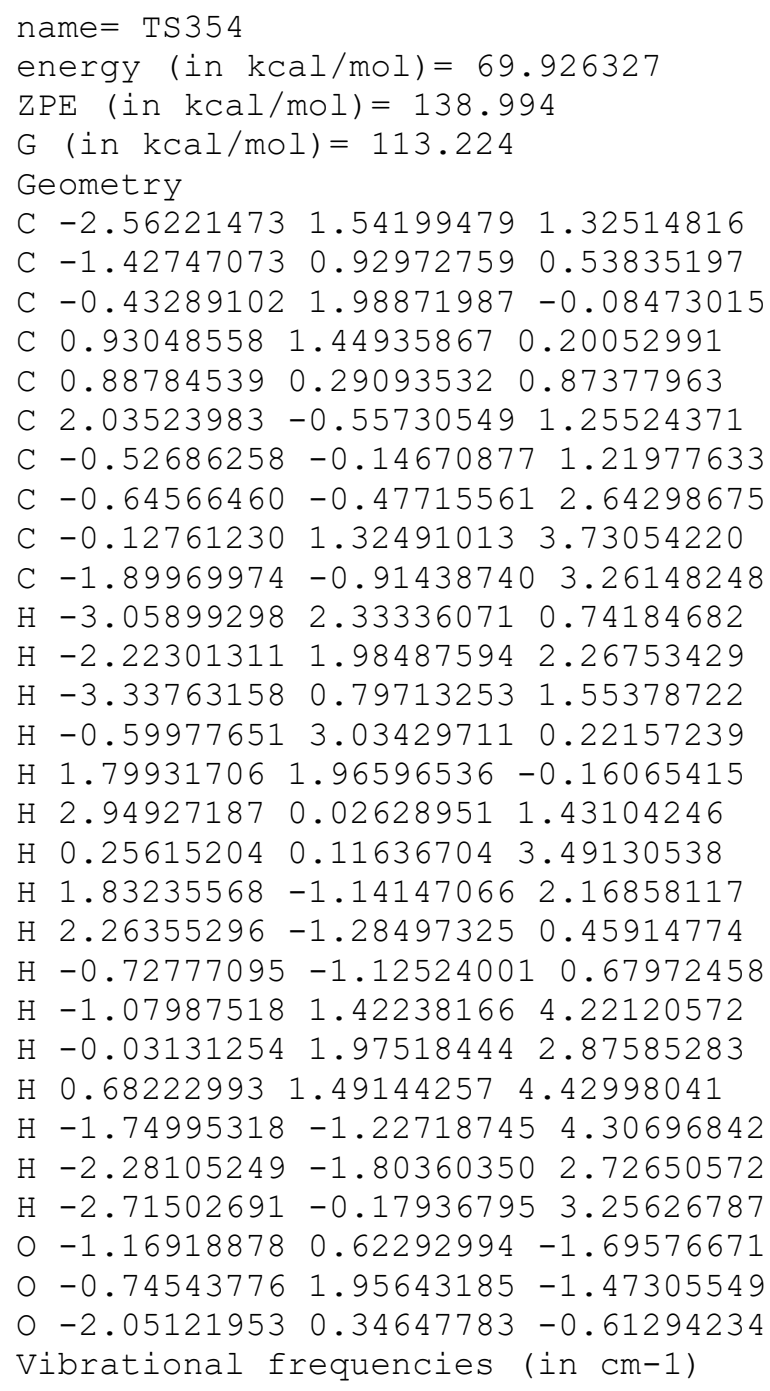


$1852.0 \quad 2654.7 \quad 2659.3 \quad 2660.92664 .8 \quad 2667.6 \quad 2672.92674 .92682 .6 \quad 2698.5 \quad 2704.5 \quad 2771.9$ $2774.92775 .8 \quad 2778.4 \quad 2789.5$

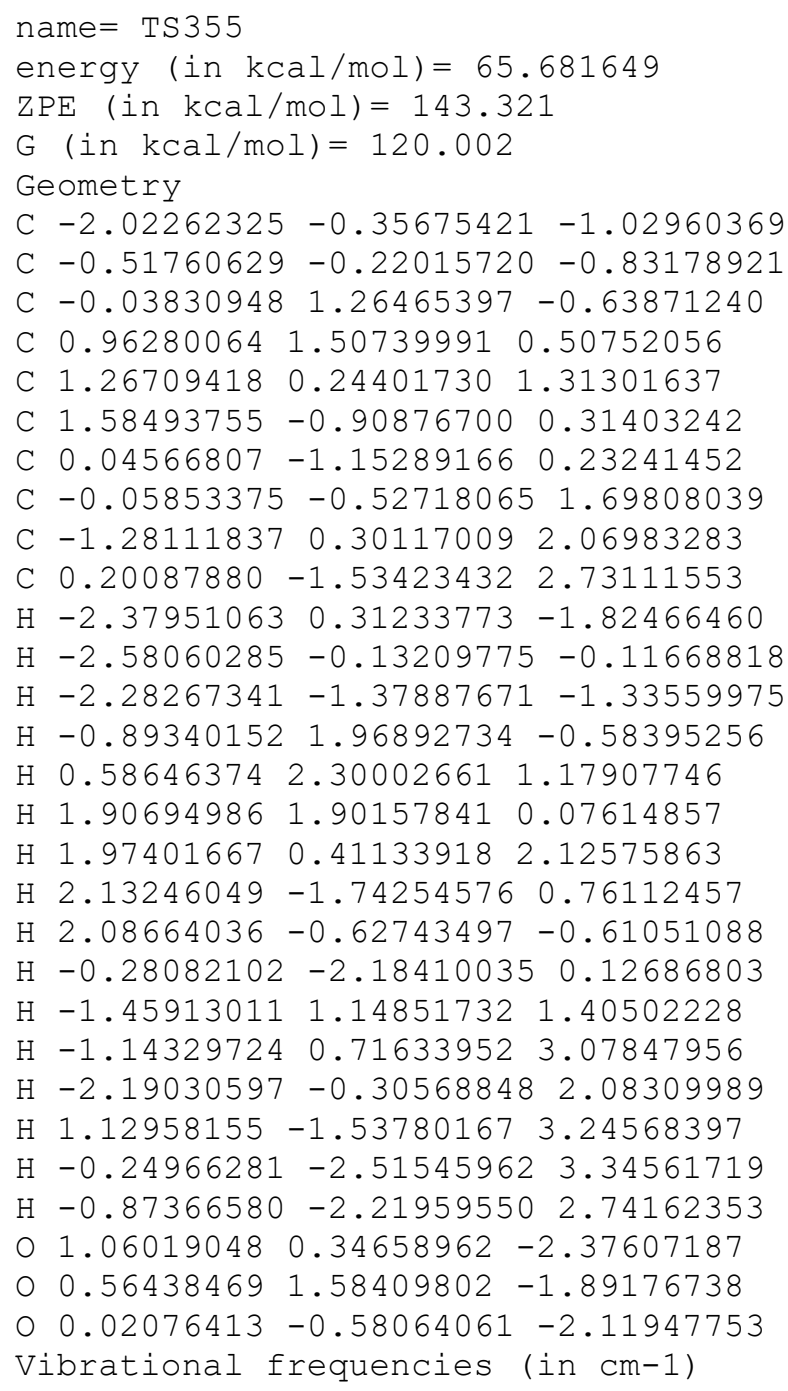


$\mathrm{H}-1.84918668-0.37410435-2.94675496$

$\mathrm{H}-0.16297374 \quad 1.45054656 \quad-2.27588903$

$\mathrm{H} 0.11214451 \quad 2.29066559 \quad 0.08107439$

$\mathrm{H} \quad 1.69980273 \quad 2.13020799-0.66543899$

H $3.29453019 \quad 1.21597957 \quad 1.28181239$

$\mathrm{H}-1.83339337-1.03683203 \quad 0.74260566$

H $2.11190425-0.37429194 \quad 0.37540441$

$\mathrm{H}-1.80052305 \quad 0.72013343 \quad 0.62806789$

$\mathrm{H} \quad 0.50050311 \quad 0.60650283 \quad 3.40038691$

$\mathrm{H}-1.19959623 \quad 0.15073844 \quad 3.19640097$

$\mathrm{H}-0.58563070 \quad 1.654477652 .48704762$

$\mathrm{H} \quad 1.36106761-1.58954273 \quad 2.38967761$

$\mathrm{H} \quad 0.60694351-2.21188002 \quad 0.90303060$

$\mathrm{H}-0.33602406-2.08045867 \quad 2.38840963$

o $1.16246272-1.26601232-1.48865072$

O $1.20906031-0.00562026-2.14178517$

o $-0.21889196-1.54013675-1.31797324$

Vibrational frequencies (in $\mathrm{cm}-1$ )

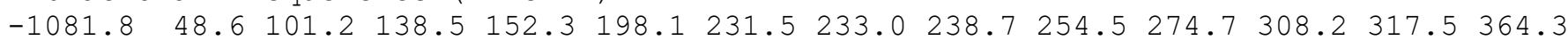
$\begin{array}{llllllllllllllll}377.5 & 419.4 & 439.1 & 478.9 & 526.3 & 569.1 & 637.9 & 675.0 & 693.3 & 719.7 & 778.9 & 798.4 & 872.8 & 900.4\end{array}$

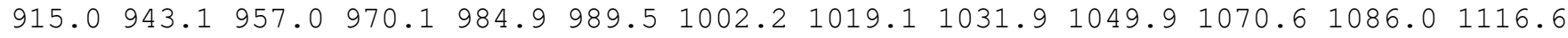

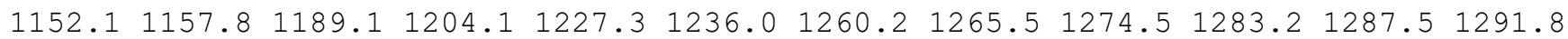
$1295.11297 .5 \quad 1306.6 \quad 1308.6 \quad 1311.1 \quad 1322.8 \quad 1352.1 \quad 1380.3 \quad 1398.2 \quad 1406.01415 .8 \quad 1617.1$ $2249.12658 .8 \quad 2663.5 \quad 2664.8 \quad 2668.4 \quad 2669.9 \quad 2676.92678 .42684 .4 \quad 2686.72739 .5 \quad 2744.7$ $2777.12779 .92780 .8 \quad 2787.7$

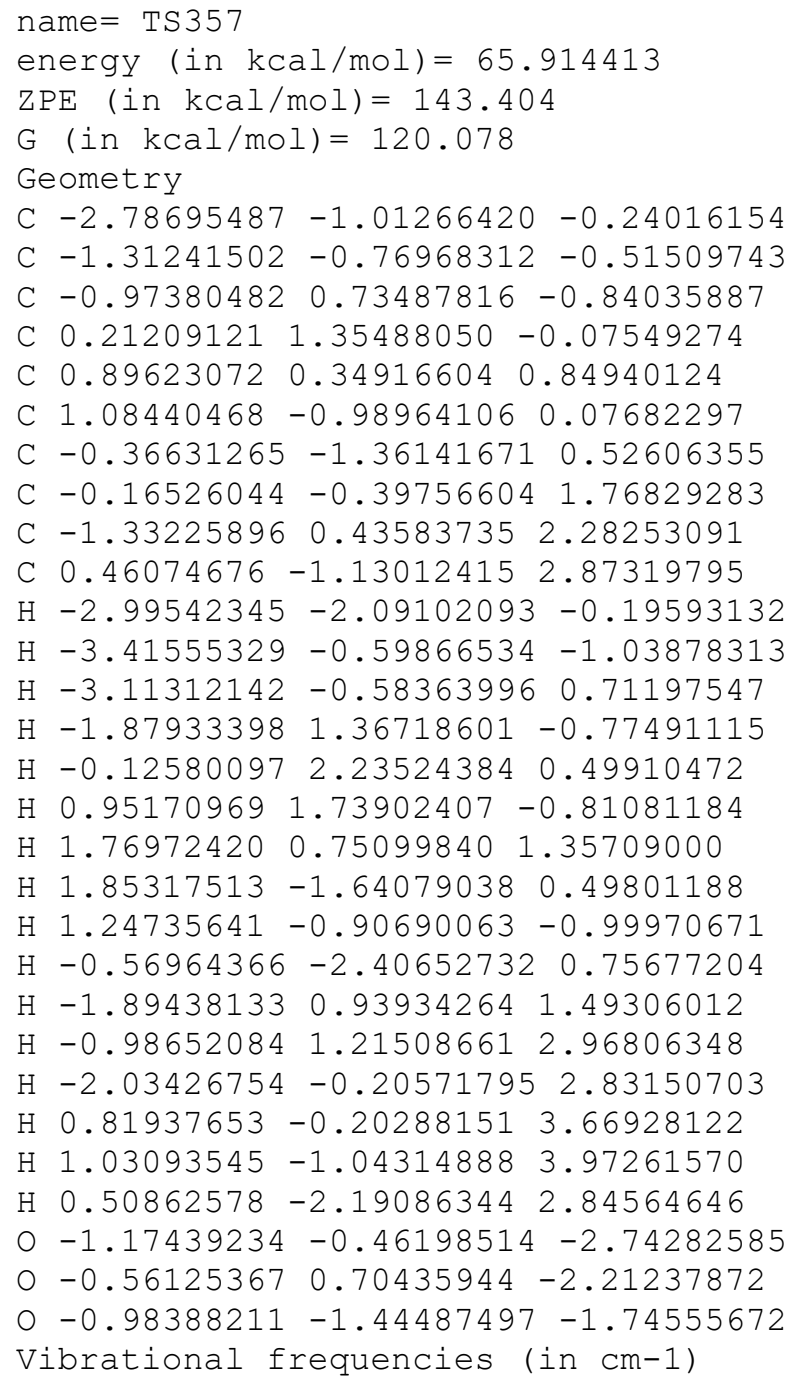




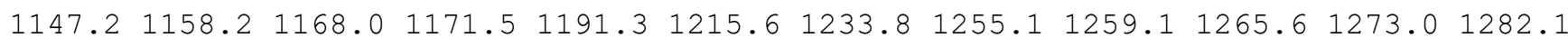

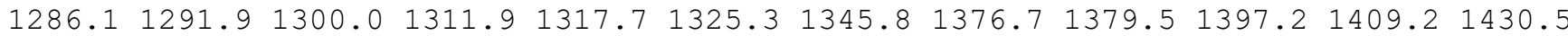
$1940.62658 .5 \quad 2672.2 \quad 2676.5 \quad 2679.5 \quad 2682.12684 .62693 .02738 .8 \quad 2750.5 \quad 2756.92765 .7$ $2781.2 \quad 2783.7 \quad 2785.5 \quad 3726.4$

\section{name $=$ TS358}

energy $($ in $\mathrm{kcal} / \mathrm{mol})=67.481631$

$\mathrm{ZPE}($ in $\mathrm{kcal} / \mathrm{mol})=141.955$

$\mathrm{G}($ in $\mathrm{kcal} / \mathrm{mol})=118.556$

Geometry

C $-2.31282667-0.00529426-0.49679558$

C $-0.93837914 \quad 0.44115127-0.31166310$

C $-0.784428391 .96028427 \quad 0.11481340$

C $0.10012125 \quad 2.23256266 \quad 1.34978326$

C $0.77500367 \quad 0.97290598 \quad 1.89070024$

C $1.35566619 \quad 0.18453814 \quad 0.67970992$

C $-0.05524442-0.47044009 \quad 0.54418061$

C $-0.27553322-0.18772644 \quad 2.08268313$

C $-1.64614911 \quad 0.19176744 \quad 2.60535629$

C $0.27223682-1.326204412 .93907813$

$\mathrm{H}-3.152581450 .43632309-0.02863184$

$\mathrm{H}-2.47577923-1.44487668 \quad 0.49471720$

$\mathrm{H}-2.44964159-1.63221140-0.28108018$

$\mathrm{H}-1.77559143 \quad 2.44800395 \quad 0.22141395$

$\mathrm{H}-0.49632101 \quad 2.71519449 \quad 2.14491242$

$\mathrm{H} 0.87800839 \quad 2.97421293 \quad 1.06754392$

$\mathrm{H} 1.441109331 .16269698 \quad 2.73316236$

H $2.15182535-0.519229510 .93100886$

H $1.70394095 \quad 0.78286721 \quad-0.16376986$

$\mathrm{H}-0.06763132-1.51245895 \quad 0.22259871$

$\mathrm{H}-2.16281293 \quad 0.93321240 \quad 1.98988505$

$\mathrm{H}-1.55930008 \quad 0.63156227 \quad 3.61024121$

$\mathrm{H}-2.30255421-0.68120526 \quad 2.69620604$

$\mathrm{H} 0.26584753-1.05966643 \quad 4.00211520$

H $1.30567952-1.58527195 \quad 2.68279629$

$\mathrm{H}-0.32718358-2.23697566 \quad 2.82207484$

$\begin{array}{lllll}0 & 0.43556129 & 1.54612311 & -1.74613787\end{array}$

O $-0.226836052 .59285236-1.02858114$

$0-0.44607442 \quad 0.45826559-1.67619837$

Vibrational frequencies (in $\mathrm{cm}-1$ )

$\begin{array}{lllllllllllllllll}-831.3 & 61.1 & 104.6 & 164.4 & 169.6 & 189.9 & 209.6 & 238.1 & 265.4 & 292.6 & 306.5 & 355.5 & 379.0 & 413.1\end{array}$

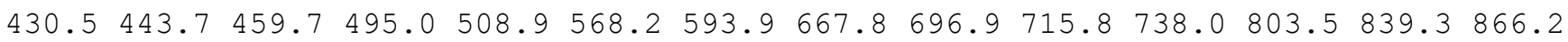

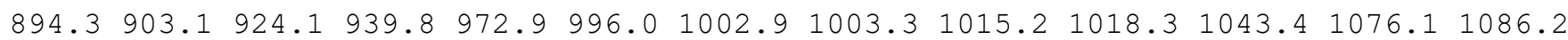
$1087.91098 .3 \quad 1104.0 \quad 1159.7 \quad 1163.1 \quad 1179.3 \quad 1199.4 \quad 1200.51222 .2 \quad 1245.8 \quad 1256.5 \quad 1276.6$

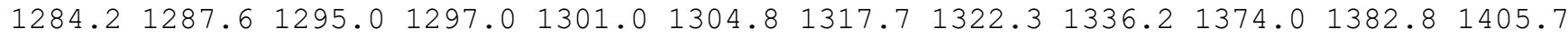
$1415.22657 .8 \quad 2669.6 \quad 2673.4 \quad 2677.6 \quad 2679.2 \quad 2681.4 \quad 2685.62717 .5 \quad 2739.12742 .72748 .7$ $2766.0 \quad 2781.4 \quad 2783.2 \quad 4926.3$

name $=$ TS359

energy $($ in $\mathrm{kcal} / \mathrm{mol})=66.685372$

$\mathrm{ZPE}(\mathrm{in} \mathrm{kcal} / \mathrm{mol})=142.778$

$\mathrm{G}($ in $\mathrm{kcal} / \mathrm{mol})=118.304$

Geometry

C $-2.11552904-0.23466153-1.24507721$

C $-0.61370649-0.17045560-1.04782166$

C $-0.03011749 \quad 1.28577243-0.89471622$

C $0.82463521 \quad 1.33104362 \quad 0.38103533$

C $0.71627752-0.03827758 \quad 1.04116938$

C $1.36778505-1.17686328 \quad 0.28487045$

C $-0.12325130-0.95238141 \quad 0.14895930$

C $0.60225637-0.03199379 \quad 2.56133287$

C $-0.81414086 \quad 0.43273951 \quad 2.96040219$

C $0.87313792-1.34835746 \quad 3.16429600$ 
$\mathrm{H}-2.43562991 \quad 0.38014596-2.09732057$

$\mathrm{H}-2.64916427 \quad 0.11129561-0.35178551$

$\mathrm{H}-2.44278093-1.26327455-1.45126794$

$\mathrm{H}-0.79244518 \quad 2.08174690 \quad-0.94118839$

$\mathrm{H} \quad 0.48337987 \quad 2.13308283 \quad 1.05682038$

H $1.87603136 \quad 1.56860410 \quad 0.13181350$

H $1.33216395 \quad 0.72893195 \quad 2.94934028$

$\mathrm{H} \quad 1.70543022 \quad-2.05728506 \quad 0.82086211$

$\mathrm{H} \quad 2.02449949-0.96623443-0.55507495$

$\mathrm{H}-0.80784381-1.685762390 .56463656$

$\mathrm{H}-1.57540414-0.241563832 .55463006$

$\mathrm{H}-1.02639364 \quad 1.43994746 \quad 2.59400576$

$\mathrm{H}-0.92163967 \quad 0.43457424 \quad 4.05017790$

$\mathrm{H} 2.14405274-1.384516353 .23979914$

$\mathrm{H} 0.06329020-1.99787253 \quad 3.38505605$

H $1.74469162-2.15044217 \quad 3.54532744$

O $1.13042895 \quad 0.14999971-2.45048360$

○ $0.74905978 \quad 1.46860511-2.06718897$

o $-0.04199793-0.62650452-2.27922910$

Vibrational frequencies (in cm-1)

$\begin{array}{lllllllllllllllll}-1440.4 & 41.9 & 79.3 & 95.7 & 140.2 & 160.6 & 179.3 & 191.3 & 207.1 & 259.1 & 282.1 & 315.8 & 330.9 & 374.5\end{array}$ $423.0 \quad 457.9 \quad 494.5 \quad 531.3 \quad 561.2 \quad 566.4 \quad 639.2 \quad 665.3 \quad 696.0 \quad 714.1 \quad 778.6 \quad 840.0 \quad 850.5 \quad 869.5$ $918.4951 .6 \quad 955.4 \quad 978.6 \quad 1000.3 \quad 1006.2 \quad 1008.6 \quad 1025.8 \quad 1042.5 \quad 1077.5 \quad 1097.3 \quad 1113.4 \quad 1125.2$

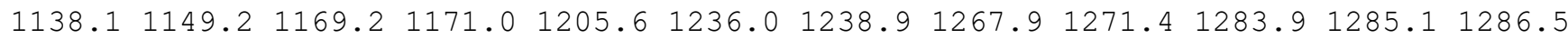
$1297.21304 .4 \quad 1306.5 \quad 1318.6 \quad 1325.2 \quad 1332.5 \quad 1341.6 \quad 1386.8 \quad 1390.6 \quad 1393.7 \quad 1421.4 \quad 1483.6$ $1933.42667 .92672 .0 \quad 2674.3 \quad 2677.8 \quad 2684.6 \quad 2693.3 \quad 2701.52716 .82748 .12772 .0 \quad 2780.5$ $2782.12787 .92789 .2 \quad 3743.0$

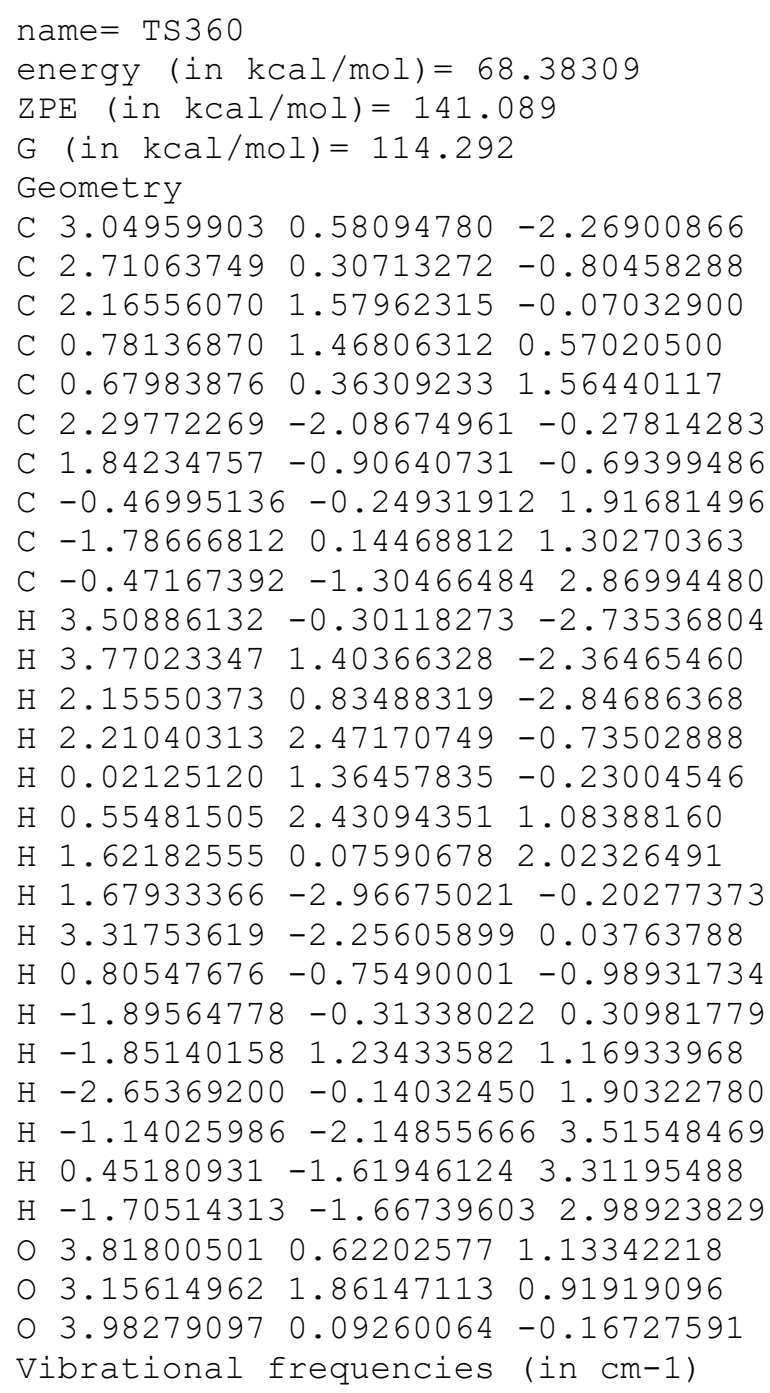


$\begin{array}{lllllllllllllll}-1328.9 & 34.3 & 43.1 & 58.7 & 69.4 & 84.9 & 127.8 & 157.9 & 180.7 & 202.3 & 215.4 & 229.7 & 248.6 & 301.6\end{array}$ $318.6 \quad 325.6 \quad 412.2 \quad 433.7 \quad 490.7 \quad 505.8 \quad 535.4 \quad 576.4 \quad 609.2 \quad 671.1696 .3 \quad 704.3 \quad 731.5 \quad 825.0$

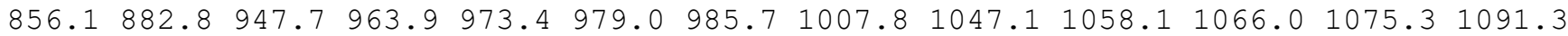
$\begin{array}{lllllllllllll}1133.3 & 1151.8 & 1161.6 & 1183.6 & 1199.7 & 1229.9 & 1266.6 & 1273.5 & 1283.9 & 1287.3 & 1289.9 & 1296.3\end{array}$

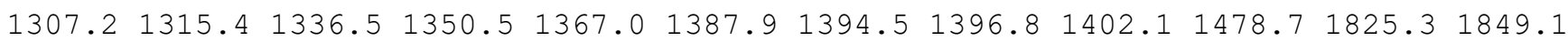
$1896.12658 .0 \quad 2670.5 \quad 2671.2 \quad 2676.5 \quad 2679.5 \quad 2680.42715 .3 \quad 2730.2 \quad 2758.7 \quad 2760.0 \quad 2773.8$ $2779.62780 .8 \quad 2786.1 \quad 3783.8$

name $=$ TS361

energy (in $\mathrm{kcal} / \mathrm{mol})=67.502755$

$\mathrm{ZPE}(\mathrm{in} \mathrm{kcal} / \mathrm{mol})=142.093$

$\mathrm{G}($ in $\mathrm{kcal} / \mathrm{mol})=116.849$

Geometry

C $-1.94580615-0.79619357-1.34765243$

C $-0.43225865-0.69555349-1.28113246$

C $-0.16814341 \quad 1.12421522-0.74947903$

C $1.20637719 \quad 1.14559141-0.17421524$

C $1.356746820 .23319270 \quad 1.05267320$

C $1.24939898-1.233191790 .59512261$

C $0.16327386-1.59421573-0.29315188$

C $0.32039678 \quad 0.54352133 \quad 2.16179247$

C $0.33493835 \quad 2.03231506 \quad 2.53028809$

C $0.61665532-0.27844446 \quad 3.42389162$

$\mathrm{H}-2.42333664-0.61860934-0.38110528$

$\mathrm{H}-2.23010341-1.80978832-1.67301440$

$\mathrm{H}-2.36289569-0.10192734-2.08588282$

$\mathrm{H}-1.005932891 .44803240 \quad-0.14019678$

H $1.48176197 \quad 2.18965824 \quad 0.09188895$

H $1.95000207 \quad 0.84104964-0.94953557$

H 2.37579307 $0.39698053 \quad 1.48284868$

H $1.24360343-1.91041214 \quad 1.48197170$

H $2.18629722-1.533265590 .04956850$

$\mathrm{H}-0.69623563 \quad 0.26946880 \quad 1.79194892$

$\mathrm{H} \quad 1.33831049 \quad 2.36458210 \quad 2.81917209$

$\mathrm{H}-0.32943643 \quad 2.23404871 \quad 3.37917951$

$\mathrm{H}-0.002277182 .66578853 \quad 1.70426445$

$\mathrm{H} 1.63370839-0.104000823 .79025982$

$\mathrm{H} \quad 0.50238727-1.35378207 \quad 3.24828429$

$\mathrm{H}-0.07173749-0.015020694 .23536092$

○ $0.51356981 \quad 1.05513282-2.81837413$

O $-0.355566561 .56022202-2.03557486$

O $0.20003122-0.69156401-2.41497243$

Vibrational frequencies (in cm-1)

$\begin{array}{lllllllllllllll}-697.2 & 44.2 & 62.7 & 63.8 & 127.1 & 136.1 & 151.8 & 175.1 & 212.9 & 263.1 & 269.1 & 294.6 & 327.7 & 354.0\end{array}$

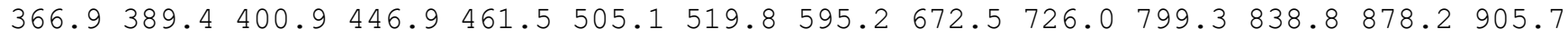
$963.5977 .0 \quad 981.0 \quad 1009.0 \quad 1013.8 \quad 1033.6 \quad 1041.7 \quad 1063.0 \quad 1082.1 \quad 1096.8 \quad 1154.7 \quad 1164.8 \quad 1166.5$

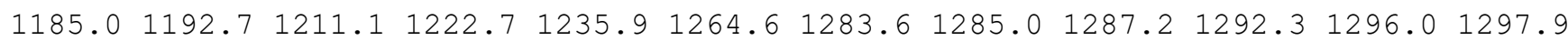
$1299.11303 .41308 .2 \quad 1315.4 \quad 1334.1 \quad 1358.9 \quad 1374.1 \quad 1392.3 \quad 1401.2 \quad 1406.6 \quad 1423.81468 .1$ $2625.12642 .12668 .92670 .5 \quad 2673.3 \quad 2675.42677 .82686 .12690 .62709 .32711 .32716 .8$ $2729.2 \quad 2779.92781 .4 \quad 2782.3$

name $=\operatorname{TS} 362$

energy $($ in $\mathrm{kcal} / \mathrm{mol})=68.277417$

$\mathrm{ZPE}($ in $\mathrm{kcal} / \mathrm{mol})=141.481$

$\mathrm{G}($ in $\mathrm{kcal} / \mathrm{mol})=117.376$

Geometry

C $-1.97427756-0.17316464-1.02869390$

C $-0.45285879-0.12706088-0.90272267$

C $0.064267631 .31619298-0.58252957$

C 1.121943721 .388103010 .51622476

C $0.80660817 \quad 0.48056037 \quad 1.71791644$

C $1.74146847-1.54102274 \quad 0.34370382$

C $-0.03271136-1.22290465-0.00924591$ 
C $0.62941449-0.97671188 \quad 1.27513433$

C $-0.44461151 \quad 0.99244502 \quad 2.44072978$

C $0.27047571-1.906085222 .41768177$

$\mathrm{H}-2.338840390 .56887075-1.74909730$

$\mathrm{H}-2.46124089-0.00261957-0.06252405$

$\mathrm{H}-2.29856606-1.16427553-1.38152289$

$\mathrm{H}-0.76681752 \quad 2.02784255-0.40365995$

H $1.229678792 .43649432 \quad 0.85845781$

H $2.11307911 \quad 1.13156394 \quad 0.09003781$

$\mathrm{H} \quad 1.67171269 \quad 0.52566188 \quad 2.42578020$

H $2.17593758-2.47920752 \quad 0.66843878$

H 2.47524056 -0.85038677 -0.04245310

$\mathrm{H} \quad 0.95987443-2.05890984-0.63662766$

$\mathrm{H}-1.35113228 \quad 0.80145877 \quad 1.85466606$

$\mathrm{H}-0.38662810 \quad 2.07101802 \quad 2.62446716$

$\mathrm{H}-0.57431854 \quad 0.505226613 .41359864$

H $0.19293228 \quad-2.94809893 \quad 2.07911834$

H $1.00853402-1.863190493 .22515786$

$\mathrm{H}-0.71159733-1.65107674 \quad 2.83733794$

O $1.10896311 \quad 0.55001494-2.40982166$

$\begin{array}{lllll}0 & 0.63341807 & 1.74789331 & -1.81754727\end{array}$

O $0.05111398-0.37787830-2.23263913$

Vibrational frequencies (in $\mathrm{cm}-1$ )

$\begin{array}{lllllllllllllll}-1412.2 & 53.4 & 100.2 & 121.6 & 131.4 & 146.7 & 170.4 & 222.1 & 242.2 & 265.6 & 294.9 & 309.3 & 334.4 & 368.8\end{array}$ $414.9 \quad 435.8 \quad 437.4 \quad 479.4 \quad 497.0 \quad 538.9 \quad 627.6 \quad 663.8 \quad 694.9 \quad 721.8 \quad 777.4 \quad 861.9 \quad 867.8 \quad 903.7$ $949.4966 .9967 .6 \quad 973.2 \quad 995.3 \quad 1004.5 \quad 1048.1 \quad 1051.4 \quad 1064.1 \quad 1086.2 \quad 1101.5 \quad 1124.0 \quad 1135.1$

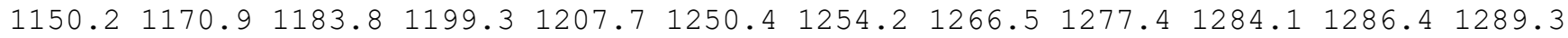

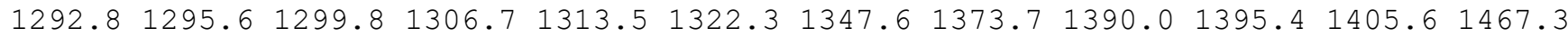
$2154.62653 .42669 .92671 .4 \quad 2672.2 \quad 2676.8 \quad 2680.02682 .62692 .6 \quad 2694.92702 .72740 .1$ $2752.92778 .92780 .8 \quad 2781.9$

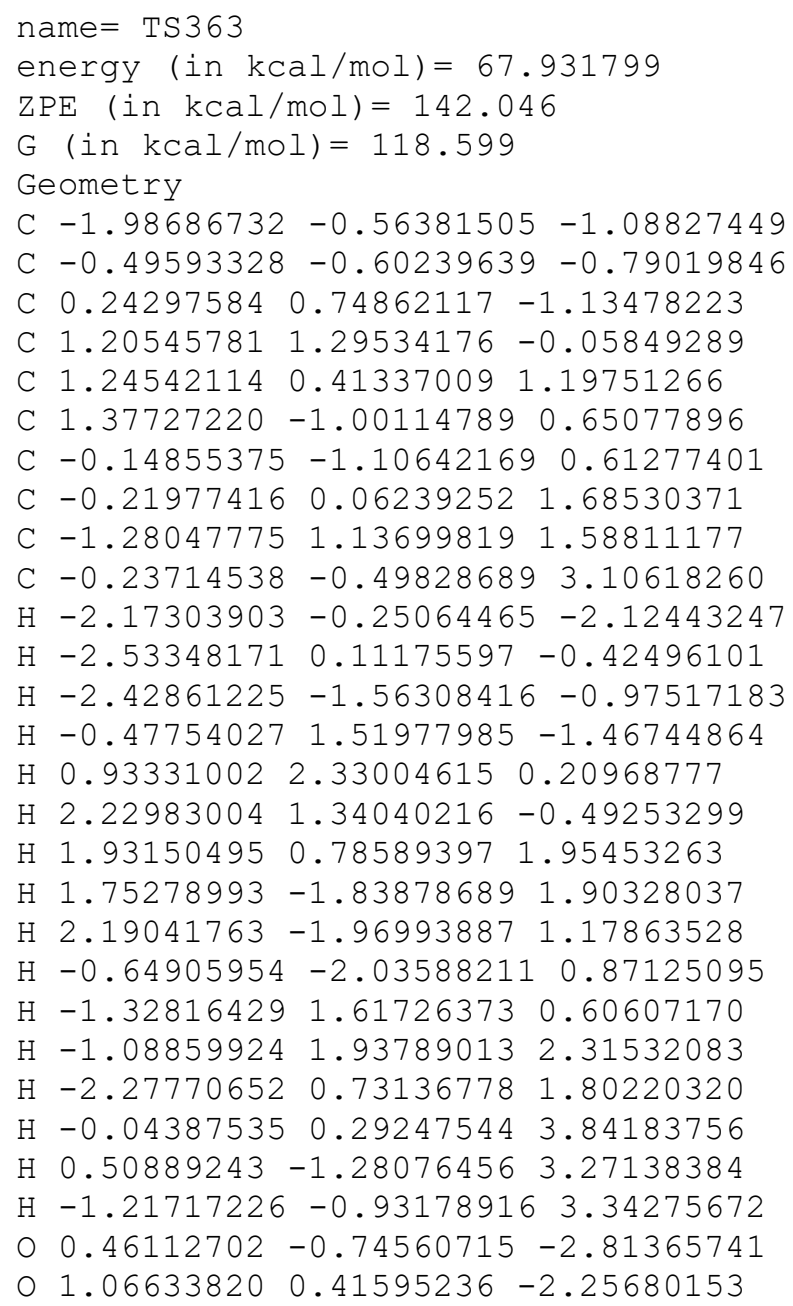


$0.11130898-1.53760748-1.69787913$

Vibrational frequencies (in $\mathrm{cm}-1$ )

$\begin{array}{lllllllllllllll}-1552.4 & 75.0 & 109.5 & 138.7 & 157.2 & 168.8 & 199.9 & 223.3 & 267.3 & 273.6 & 318.6 & 340.5 & 360.7 & 390.0\end{array}$ $421.4 \quad 440.0 \quad 479.9 \quad 512.3 \quad 578.3 \quad 600.3 \quad 662.7 \quad 673.4 \quad 708.5 \quad 716.2 \quad 763.2 \quad 788.3 \quad 858.1 \quad 870.0$ $884.0 \quad 938.2 \quad 983.4 \quad 988.0 \quad 996.2 \quad 998.3 \quad 1009.7 \quad 1015.9 \quad 1038.7 \quad 1048.4 \quad 1067.8 \quad 1092.1 \quad 1111.9$

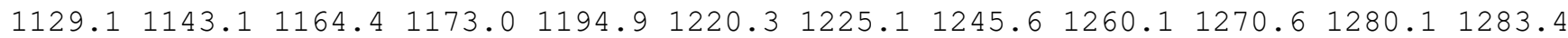
$1288.41291 .3 \quad 1297.0 \quad 1300.8 \quad 1306.6 \quad 1308.3 \quad 1316.7 \quad 1321.2 \quad 1374.6 \quad 1396.7 \quad 1401.3 \quad 1410.8$ $1421.32657 .22668 .0 \quad 2672.2 \quad 2672.7 \quad 2674.3 \quad 2677.82679 .32682 .4 \quad 2735.92754 .02756 .9$ $2777.7 \quad 2781.5 \quad 2783.7 \quad 4096.2$

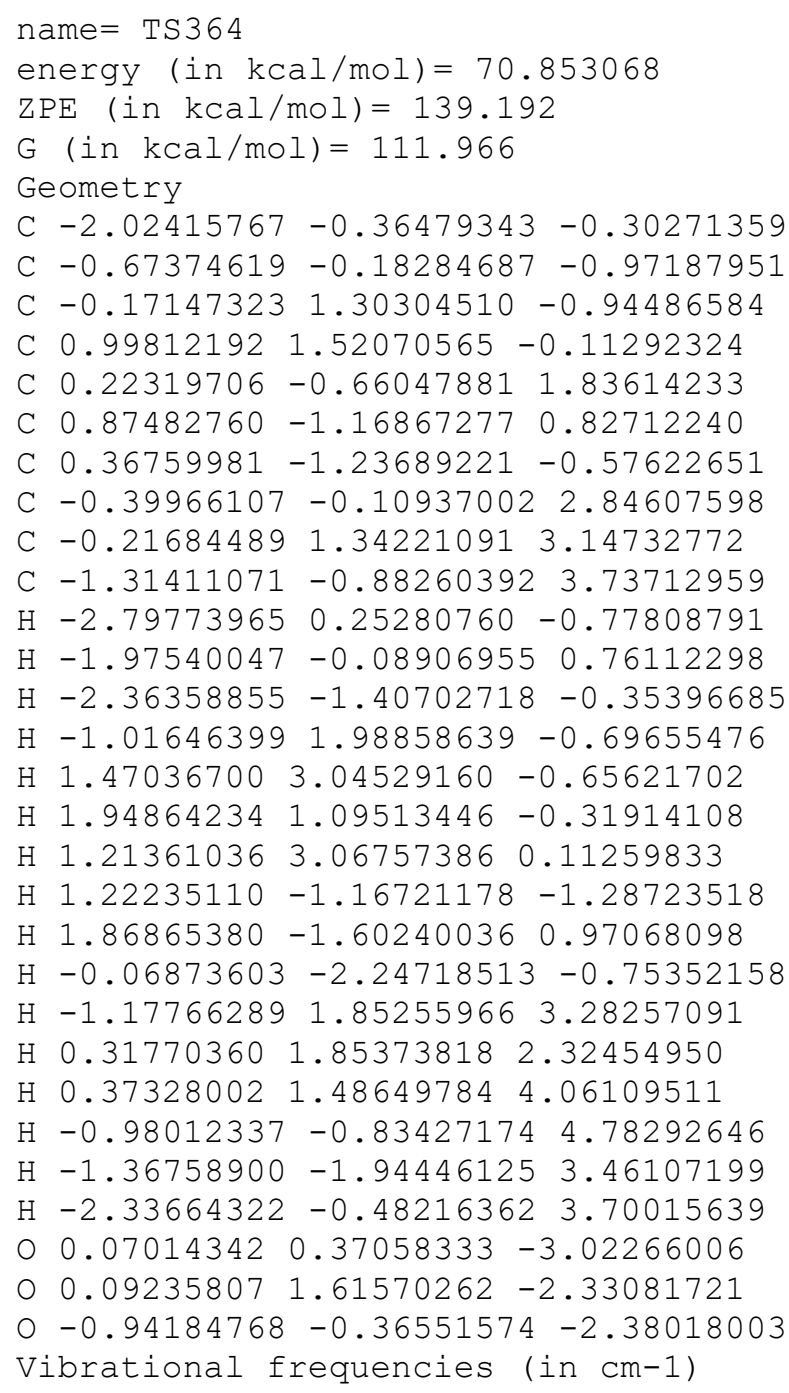
$298.3 \quad 312.5 \quad 348.6 \quad 398.1 \quad 430.5 \quad 459.4 \quad 501.5 \quad 522.0 \quad 588.8 \quad 595.8 \quad 630.9661 .6 \quad 713.4 \quad 821.4$

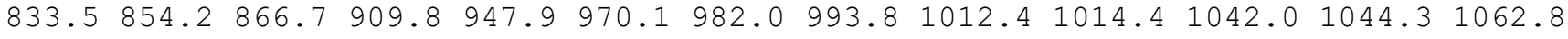

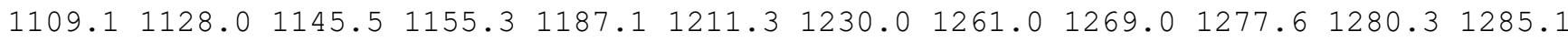

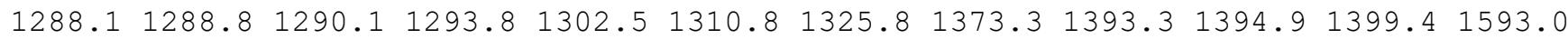
$2311.32649 .12652 .5 \quad 2663.7 \quad 2673.2 \quad 2673.4 \quad 2676.82677 .52680 .12729 .02731 .12742 .9$ $2774.12778 .12780 .4 \quad 4741.1$

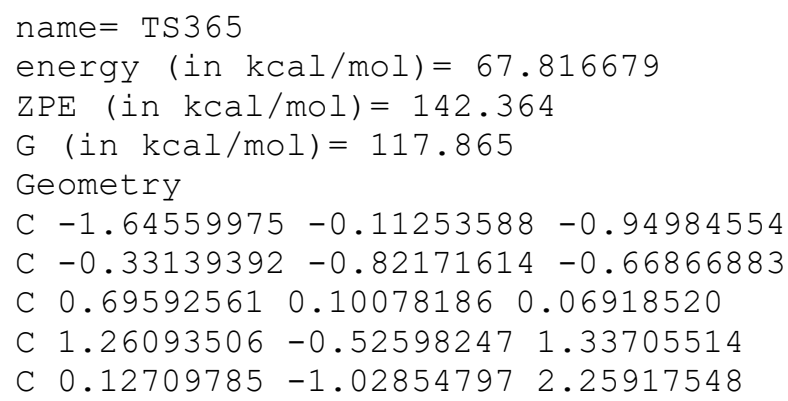


C $-0.83765372-1.98280317 \quad 1.50381311$

C $-0.45892675-2.183503920 .03153506$

C $-0.65792914-0.009197392 .91244364$

C $-0.54014355 \quad 1.30068174 \quad 3.11515038$

C $-1.47954045-3.08573956-0.66498246$

$\mathrm{H}-1.483210410 .86111478-1.43008605$

$\mathrm{H}-2.21318193 \quad 0.04946657-0.02435353$

$\mathrm{H}-2.27865181-0.69712948-1.63099357$

H $0.29083592 \quad 1.11608888 \quad 0.24275232$

H $1.90742373 \quad 0.18892655 \quad 1.87164996$

H $1.93161687-1.367667661 .06935399$

$\mathrm{H} 0.61023119-1.621574793 .09130666$

$\mathrm{H}-1.87413973-1.59377618 \quad 1.58138652$

$\mathrm{H}-0.86529715-2.959648852 .01899470$

$\mathrm{H} \quad 0.54045383-2.68808303-0.02726755$

$\mathrm{H}-1.25276311 \quad 1.89089466 \quad 3.66408352$

$\mathrm{H} 0.61417435 \quad 1.85585836 \quad 3.12520876$

H $0.12252113 \quad 2.03302236 \quad 2.29971717$

$\mathrm{H}-1.44254792-4.10454362-0.26283073$

$\mathrm{H}-1.27578693-3.14980766-1.74175445$

$\mathrm{H}-2.50502910-2.72236708-0.53932793$

O $1.65985562-0.84488668-1.73079948$

O $1.73865918 \quad 0.29804575-0.88352756$

O $0.27633391-1.01210009-1.95975102$

Vibrational frequencies (in cm-1)

$\begin{array}{llllllllllllll}-1714.6 & 30.8 & 94.2 & 105.5 & 145.2 & 172.4 & 191.6 & 206.9 & 230.1 & 258.4 & 274.3 & 291.6 & 319.7 & 366.8\end{array}$ $404.8 \quad 420.5 \quad 463.0 \quad 479.9 \quad 493.5 \quad 524.2 \quad 665.1 \quad 671.3 \quad 721.6 \quad 798.3 \quad 809.7 \quad 858.4 \quad 862.1884 .7$ $912.8 \quad 957.0 \quad 962.4 \quad 974.5 \quad 988.8 \quad 1006.9 \quad 1008.8 \quad 1065.1 \quad 1070.0 \quad 1086.6 \quad 1108.7 \quad 1128.3 \quad 1147.7$

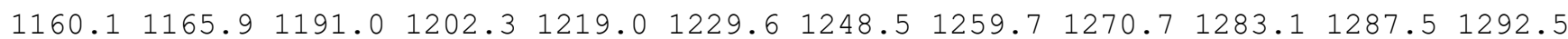

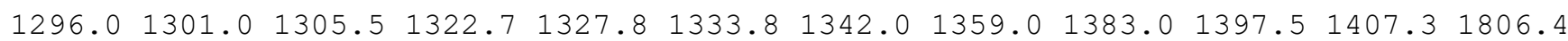
$1904.72652 .92661 .92669 .92671 .92673 .5 \quad 2676.12680 .52694 .92699 .72742 .6 \quad 2748.1$ $2756.7 \quad 2778.3 \quad 2780.5 \quad 3239.0$

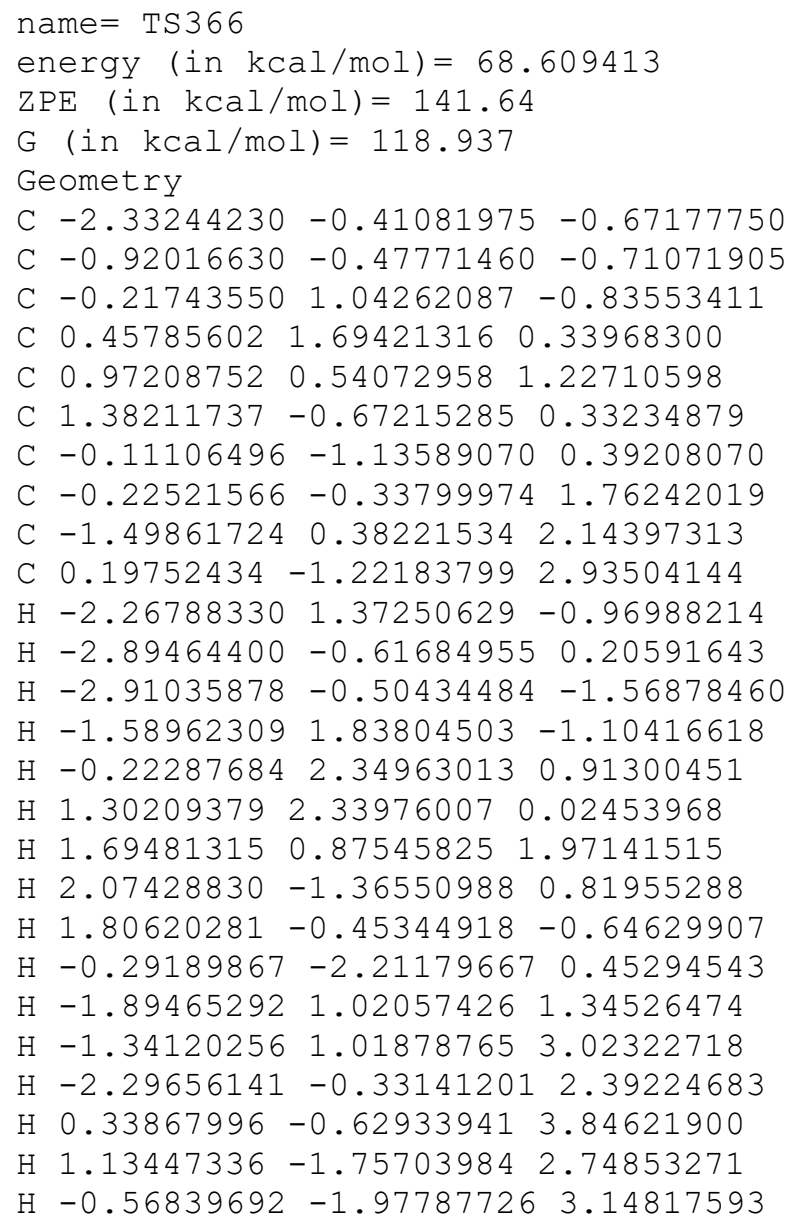


$0 \quad 0.48544333-0.27725171-2.53332445$

○ $0.538287561 .09471412-1.92647585$

O $-0.63395621-0.89105370-2.06896888$

Vibrational frequencies (in cm-1)

$\begin{array}{lllllllllllllll}-1264.6 & 127.8 & 148.7 & 164.4 & 182.3 & 191.1 & 226.0 & 248.1 & 290.5 & 332.0 & 344.9 & 387.4 & 394.8 & 402.4\end{array}$

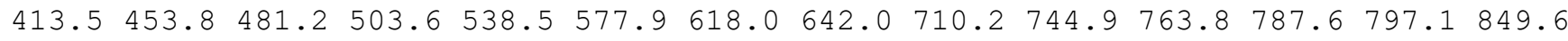

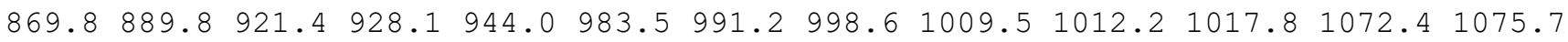

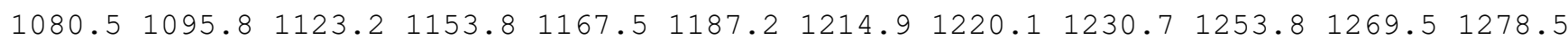

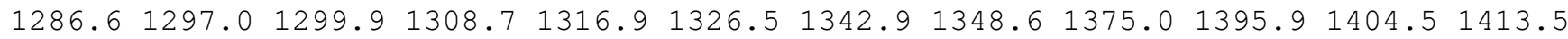
$1475.92660 .72665 .62674 .92675 .42679 .8 \quad 2682.62740 .12740 .52742 .2 \quad 2746.92767 .2$ $2779.82782 .12806 .3 \quad 4031.1$

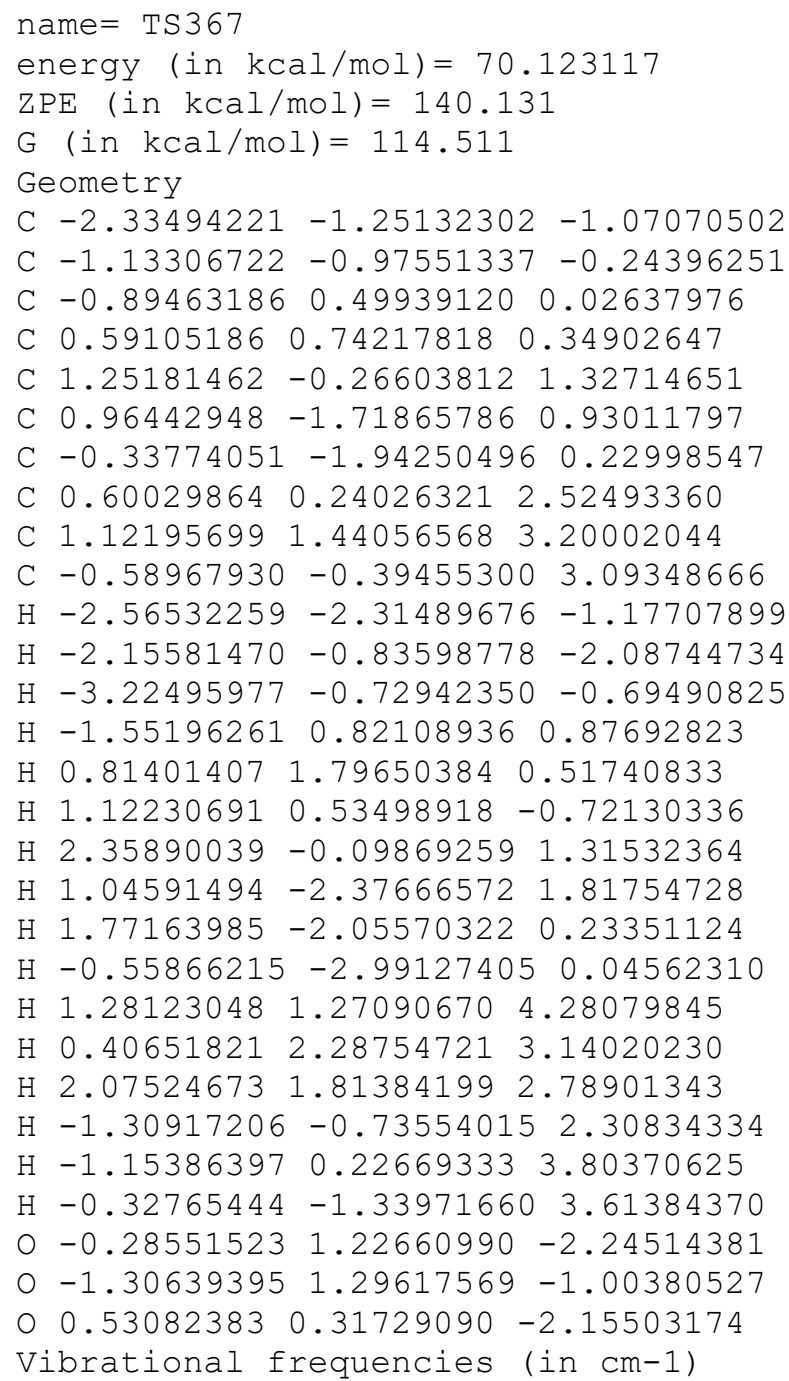


C $1.17097651 \quad 1.93814066 \quad 0.13105018$

C $1.04855106 \quad 0.74938138 \quad 1.05710306$

C $1.13908636-0.67421728 \quad 0.41687462$

C $-0.20864510-1.01668148 \quad 0.96368184$

C $-0.38666974 \quad 0.34265559 \quad 1.60939408$

C $-1.55728675 \quad 1.18558440 \quad 1.16216124$

C $-0.38910598 \quad 0.18101874 \quad 3.12615977$

$\mathrm{H}-3.49201366-0.769530250 .02805097$

$\mathrm{H}-3.54229007-1.84711855-1.38746443$

$\mathrm{H}-3.12383617-0.16027482-1.61626226$

$\mathrm{H} \quad 0.24187237 \quad 0.97980057-1.68150703$

$\mathrm{H} \quad 0.96513512 \quad 2.87257913 \quad 0.70289111$

H 2.22604112 2.04189669 -0.20990368

$\mathrm{H} 1.76940174 \quad 0.87134355 \quad 1.88880333$

$\mathrm{H} 1.94217389-1.307330230 .82786035$

$\mathrm{H} 1.28805263-0.69488814-0.66670790$

$\mathrm{H}-1.12176735-1.79930504 \quad 0.41895555$

$\mathrm{H}-1.41851621 \quad 2.239624821 .44485813$

$\mathrm{H}-2.496210790 .850204351 .62286087$

$\mathrm{H}-1.71451981 \quad 1.16952415 \quad 0.07553715$

$\mathrm{H}-0.30274221 \quad 1.15467329 \quad 3.62433283$

H $0.43620988-0.44659567 \quad 3.48223738$

$\mathrm{H}-1.32011217-0.28586313 \quad 3.47393980$

o $-1.03218345-1.16190971-2.89654459$

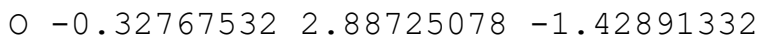

o $-0.70847359-1.43379870-1.69427318$

Vibrational frequencies (in $\mathrm{cm}-1$ )

$\begin{array}{llllllllllllllll}-1490.4 & 26.1 & 37.7 & 56.3 & 83.0 & 93.2 & 117.4 & 125.7 & 156.8 & 165.9 & 198.3 & 199.9 & 233.0 & 244.4\end{array}$ $271.4 \quad 291.1 \quad 317.0 \quad 372.2 \quad 432.6 \quad 447.8 \quad 480.1 \quad 561.1 \quad 594.6 \quad 681.2 \quad 734.5 \quad 759.7 \quad 858.5 \quad 897.5$ $917.5944 .2992 .2 \quad 1002.6 \quad 1004.6 \quad 1016.2 \quad 1018.4 \quad 1026.4 \quad 1034.5 \quad 1041.5 \quad 1078.2 \quad 1097.8 \quad 1128.0$

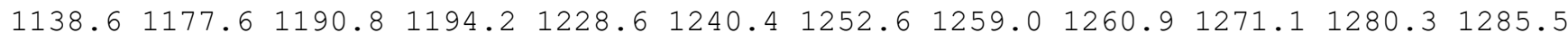

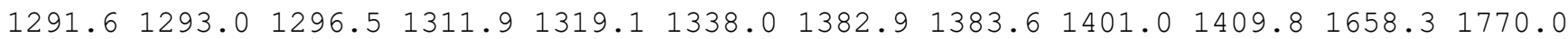
$1834.62634 .62639 .92654 .5 \quad 2661.3 \quad 2662.92668 .62671 .42674 .22676 .02714 .02735 .0$ $2754.4 \quad 2767.4 \quad 2777.5 \quad 2780.7$

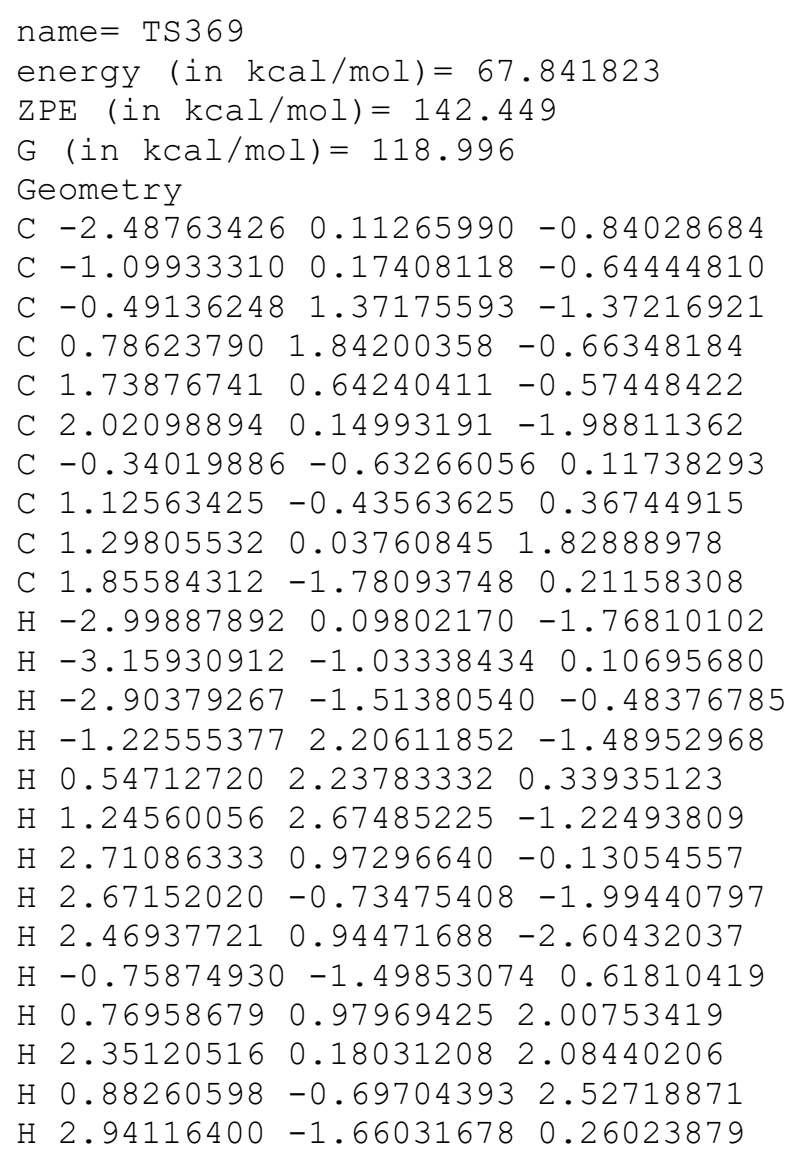


$\mathrm{H} \quad 1.59841431-2.26484260-0.73918191$

$\mathrm{H} \quad 1.56652694-2.47939528 \quad 1.00486313$

$0.79738140-0.19872500-2.77587058$

$0-0.19034571 \quad 1.01226464-2.67616521$

O $0.31712219-1.25942500-2.53102795$

Vibrational frequencies (in $\mathrm{cm}-1$ )

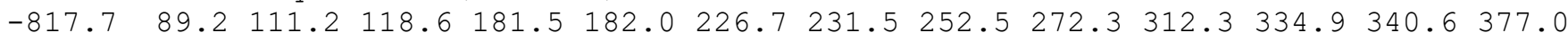

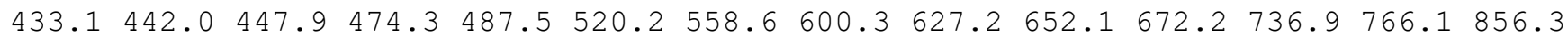

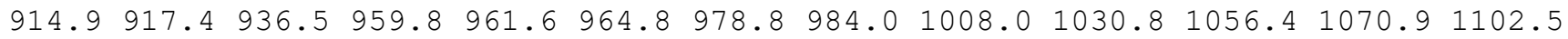

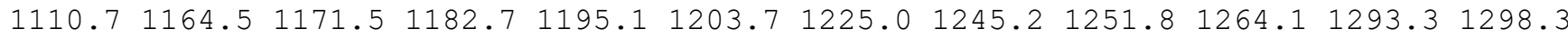
$1299.61305 .3 \quad 1305.7 \quad 1309.2 \quad 1326.3 \quad 1336.8 \quad 1350.2 \quad 1360.91365 .7 \quad 1398.4 \quad 1405.6 \quad 1416.7$ $1853.22659 .22670 .4 \quad 2673.1 \quad 2676.4 \quad 2677.8 \quad 2681.02683 .62696 .5 \quad 2724.02733 .42748 .8$ $2763.4 \quad 2780.12781 .8 \quad 4864.4$

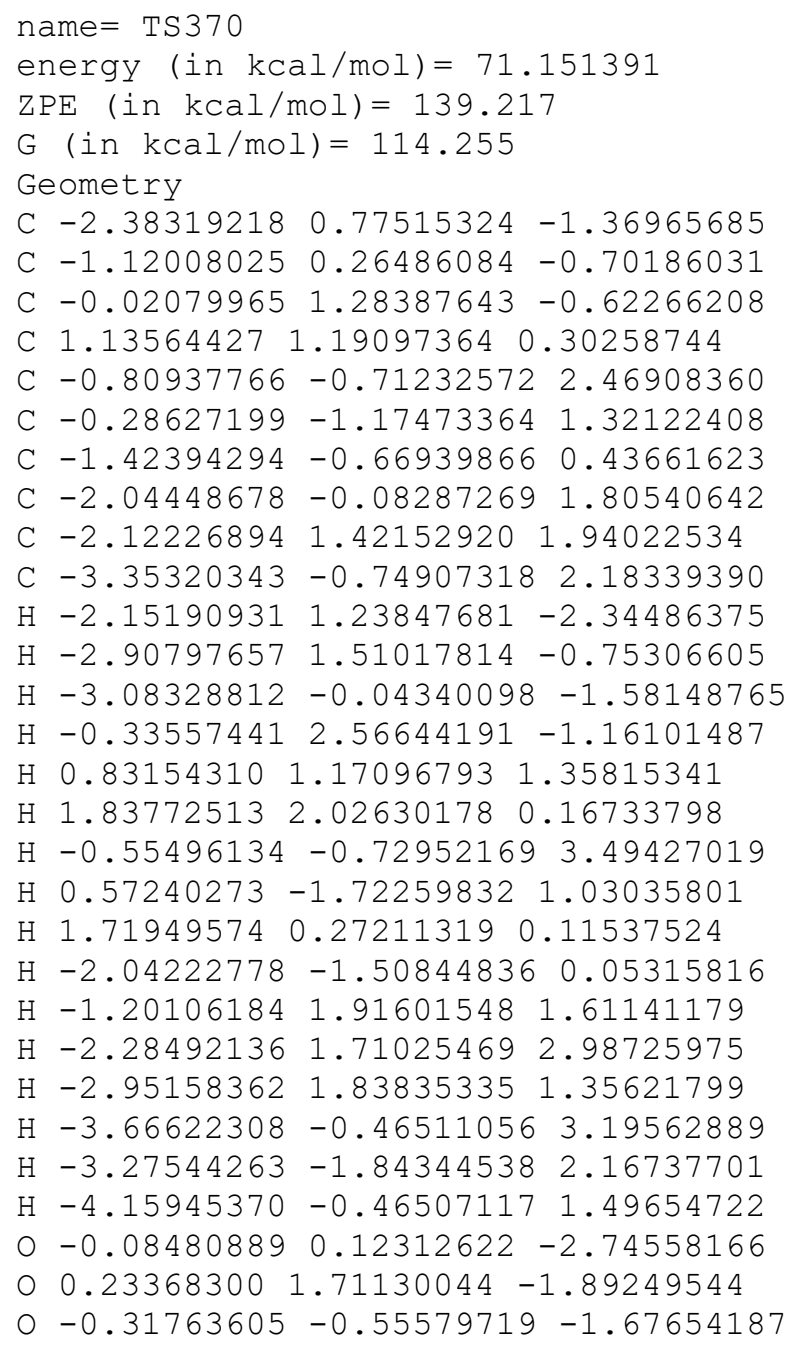


C $-0.48781714-0.15823017-0.69656511$

$\begin{array}{lllll}\text { C } & 0.51044585 & 0.92274747 & -0.57954197\end{array}$

C $0.19518864 \quad 2.35005367 \quad-0.86973188$

$\begin{array}{llll}\text { C } 0.58538109 & -0.64980240 & 2.05592700\end{array}$

C $0.88777170-1.672196620 .90179117$

C $-0.50360645-1.31672752 \quad 0.28814635$

C $-0.79254849-0.45896294 \quad 1.45055878$

$\begin{array}{llll}\text { C }-1.92665180 & 0.30290286 & 1.92064861\end{array}$

C $0.56780475-1.23299323 \quad 3.45157524$

H $-1.66002307 \quad 0.61900432-2.31848855$

$\mathrm{H}-2.39899126 \quad 0.87430318-0.72963487$

$\mathrm{H}-2.41893045-0.73147215-1.48651918$

H $1.561625920 .59361777-0.67036558$

$\mathrm{H} \quad 0.97997325 \quad 3.03339173-1.18732325$

$\mathrm{H}-0.80098285 \quad 2.66514300 \quad-1.16475490$

H $1.22380055 \quad 0.258001362 .01092378$

H $0.98517185-2.71069810 \quad 1.22046704$

H $1.74735578-1.435606550 .26263454$

$\mathrm{H}-1.12825661-2.15327072-0.03967450$

$\mathrm{H}-1.81902999 \quad 1.376906201 .63357608$

$\mathrm{H}-2.00199420 \quad 0.30738373 \quad 3.02350377$

$\mathrm{H}-2.89204961-0.03936610 \quad 1.51376007$

H $0.24056017 \quad-0.49425074 \quad 4.19432059$

H $1.57439362 \quad-1.56531164 \quad 3.74509471$

$\mathrm{H}-0.09482931-2.10387386 \quad 3.53176413$

$01.38494293-1.30098781-1.95056407$

$00.30870503 \quad 1.888067490 .47778494$

$00.21056985-1.06839961-2.02024182$

Vibrational frequencies (in cm-1)

$\begin{array}{lllllllllllllllll}-578.3 & 63.2 & 84.3 & 94.6 & 108.9 & 131.8 & 157.1 & 166.2 & 168.7 & 185.5 & 204.6 & 212.3 & 223.3 & 271.8\end{array}$ $\begin{array}{llllllllllllll}302.5 & 312.9 & 322.4 & 399.3 & 455.0 & 466.3 & 507.5 & 576.3 & 659.4 & 758.7 & 846.9 & 878.8 & 904.7 & 906.2\end{array}$

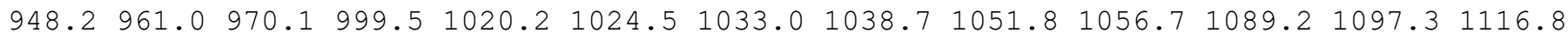
$\begin{array}{lllllllllllll}1158.6 & 1166.8 & 1187.7 & 1200.4 & 1217.8 & 1228.8 & 1245.6 & 1246.6 & 1273.1 & 1286.4 & 1289.5 & 1291.1\end{array}$

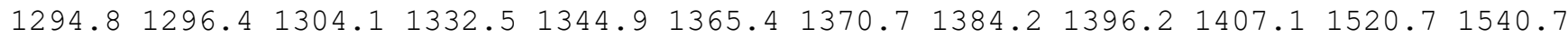

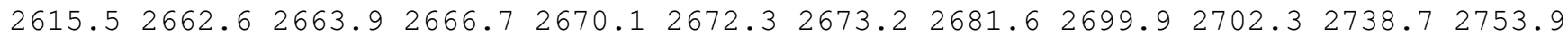
$2757.8 \quad 2759.7 \quad 2774.3 \quad 2779.8$

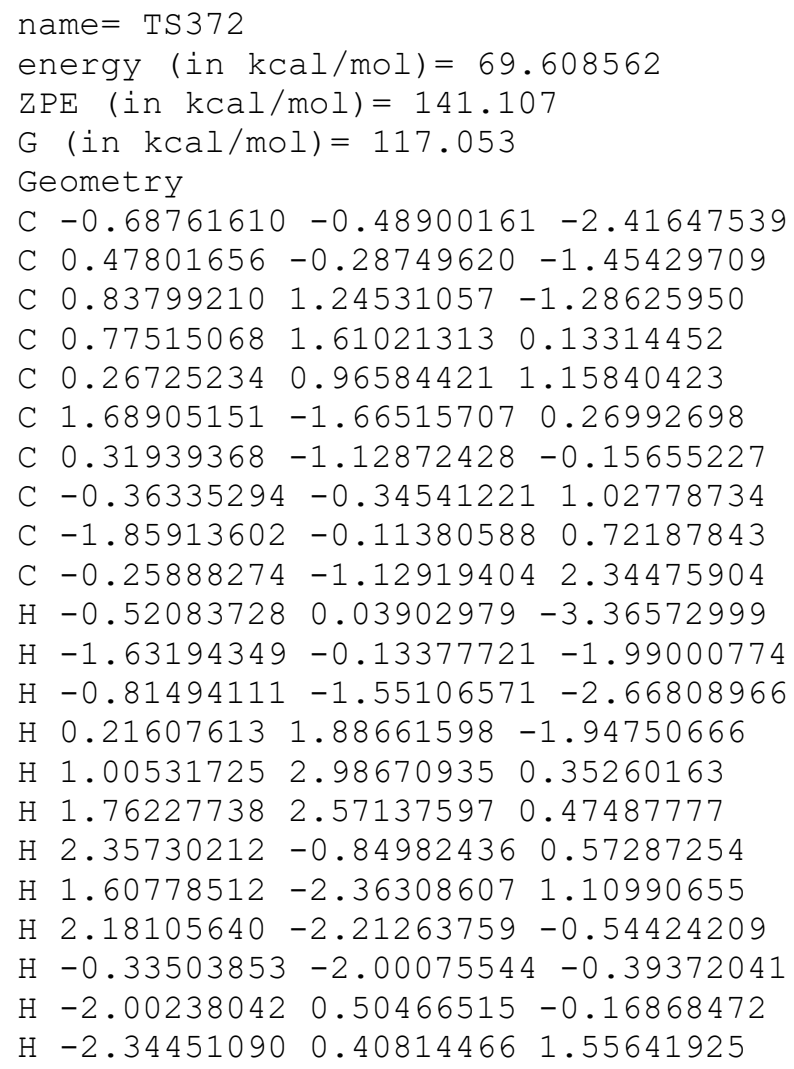


$\mathrm{H}-2.38158157-1.06329517 \quad 0.57415370$

$\mathrm{H}-0.85478815-0.64165215 \quad 3.12649424$

$\mathrm{H} \quad 0.77207834-1.14707976 \quad 2.71693063$

$\mathrm{H}-0.61107760 \quad-2.15762776 \quad 2.23568453$

$\begin{array}{lllll}0 & 2.68633014 & 0.05279832 & -1.79561674\end{array}$

$\begin{array}{llll}0 & 2.15086967 & 1.37729555 & -1.82534888\end{array}$

o $1.61310177-0.75621506-2.20933405$

Vibrational frequencies (in cm-1)

$\begin{array}{llllllllllllllll}-1257.6 & 65.1 & 86.1 & 138.1 & 167.1 & 173.6 & 196.3 & 217.0 & 221.9 & 245.4 & 258.9 & 285.0 & 331.2 & 351.4\end{array}$ $381.9 \quad 403.9 \quad 413.7 \quad 444.9 \quad 472.6 \quad 538.6 \quad 550.7 \quad 571.6 \quad 647.8 \quad 664.8 \quad 708.5 \quad 764.9 \quad 782.7 \quad 816.2$ $858.0 \quad 885.3 \quad 922.5 \quad 958.6 \quad 967.9 \quad 981.3 \quad 1000.6 \quad 1010.5 \quad 1024.0 \quad 1062.7 \quad 1087.1 \quad 1097.0 \quad 1132.2$ $1144.01168 .6 \quad 1178.8 \quad 1187.8 \quad 1199.2 \quad 1225.5 \quad 1243.8 \quad 1265.0 \quad 1279.1 \quad 1288.01294 .4 \quad 1297.6$

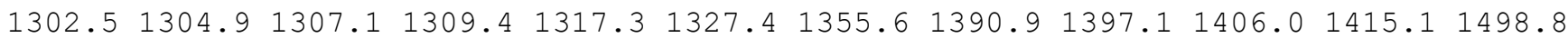
$1809.02667 .52668 .42668 .8 \quad 2672.3 \quad 2672.82675 .92680 .32681 .62686 .42699 .2 \quad 2775.6$ $2779.12779 .62781 .6 \quad 4016.7$

\section{name $=$ TS373}

energy (in $\mathrm{kcal} / \mathrm{mol})=72.686314$

$\mathrm{ZPE}($ in $\mathrm{kcal} / \mathrm{mol})=138.373$

$\mathrm{G}($ in $\mathrm{kcal} / \mathrm{mol})=111.324$

Geometry

C $-2.16083310-0.62759337-1.63586994$

C $-1.05525639-0.54670761-0.67561741$

C $-0.50006499 \quad 0.90446859-0.47901964$

C $0.96146953 \quad 0.81460980 \quad 0.12308547$

C $1.04103574-0.203386881 .27453555$

C $0.77253563-1.59331988 \quad 0.66014418$

C $-0.60832834-1.62659330 \quad 0.04895500$

C $0.02134221 \quad 0.16276810 \quad 2.30509778$

C $0.04114370 \quad 1.53042269 \quad 2.85796168$

C $-0.98191413-0.73490614 \quad 2.64120981$

$\mathrm{H}-2.93882004 \quad 0.13193187-1.44666887$

$\mathrm{H}-2.62380568-1.61008785-1.74816754$

$\mathrm{H}-1.75387674-0.30562598-2.63146802$

$\mathrm{H}-1.17488887 \quad 1.33458526 \quad 0.32190873$

H $1.27069313 \quad 1.82528191 \quad 0.41803022$

$\mathrm{H} \quad 1.63731507 \quad 0.53221526-0.70405192$

H $2.06646526-0.17866556 \quad 1.71882879$

$\mathrm{H} \quad 0.90927360-2.39776883 \quad 1.40393306$

H $1.52650434-1.79734482-0.13252990$

$\mathrm{H}-0.99857253-2.62371157-0.13424425$

$\mathrm{H} 1.06433879 \quad 1.91158454 \quad 3.01546359$

$\mathrm{H}-0.50236928 \quad 1.62902424 \quad 3.80939668$

$\mathrm{H}-0.42770810 \quad 2.25464462 \quad 2.15437512$

$\mathrm{H}-1.36126797-1.16965694 \quad 1.37141019$

$\mathrm{H}-0.77680323-1.802909682 .78183422$

$\mathrm{H}-1.81487238-0.42535509 \quad 3.26213107$

$\begin{array}{lllll}0 & 0.75905656 & 0.71594369 & -3.29176921\end{array}$

$0-0.539423341 .57371624-1.57193280$

$0.99103851-0.37500194-3.06486765$

Vibrational frequencies (in $\mathrm{cm}-1$ )

$\begin{array}{lllllllllllllllll}-1450.9 & 29.6 & 40.6 & 62.5 & 88.4 & 100.3 & 103.5 & 117.8 & 130.7 & 150.0 & 180.5 & 273.0 & 277.4 & 328.6\end{array}$ $344.4 \quad 374.5 \quad 397.0 \quad 423.6 \quad 451.5 \quad 463.0 \quad 520.2 \quad 557.6 \quad 607.7 \quad 634.8 \quad 699.8 \quad 745.8 \quad 848.8 \quad 882.1$

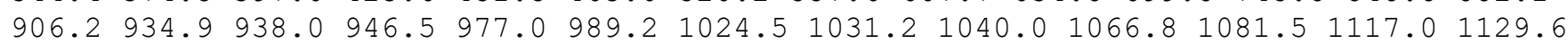

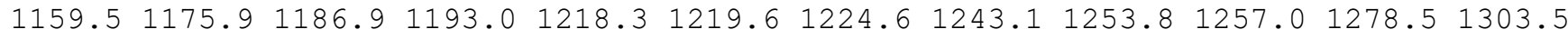

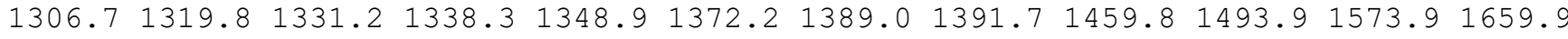
$1712.4 \quad 2557.8 \quad 2611.1 \quad 2617.6 \quad 2656.1 \quad 2662.4 \quad 2670.42672 .5 \quad 2683.72691 .32739 .02755 .0$ $2756.02757 .6 \quad 2766.92768 .7$

name $=\operatorname{TS} 374$

energy (in $\mathrm{kcal} / \mathrm{mol})=74.655952$

$\mathrm{ZPE}($ in $\mathrm{kcal} / \mathrm{mol})=136.465$

$\mathrm{G}(\mathrm{in} \mathrm{kcal} / \mathrm{mol})=107.84$ 
$\mathrm{H}-1.48178187 \quad 1.24442754 \quad 1.36787007$

$\mathrm{H}-1.58169865 \quad 0.60557144 \quad 3.00421855$

$\mathrm{H}-2.45608186-0.201764331 .70529616$

$\mathrm{H}-0.32753185-1.39372593 \quad 3.71418455$

$\mathrm{H} \quad 0.65048155-2.315374202 .57054384$

$\mathrm{H}-1.11401163-2.41825276 \quad 2.50883977$

$\begin{array}{lllll}0 & 1.00542425 & 0.19914350 & -2.50132530\end{array}$

$\begin{array}{lllll}0 & 0.56056829 & 1.48855518 & -2.12151328\end{array}$

$0-0.05384761-0.66713199-2.12414621$

Vibrational frequencies (in $\mathrm{cm}-1$ )

$\begin{array}{lllllllllllllll}-1389.0 & 59.6 & 96.6 & 131.6 & 163.2 & 191.9 & 216.5 & 224.1 & 252.6 & 265.6 & 316.5 & 350.3 & 358.6 & 386.4\end{array}$

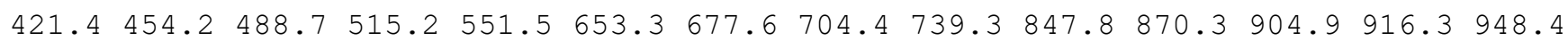

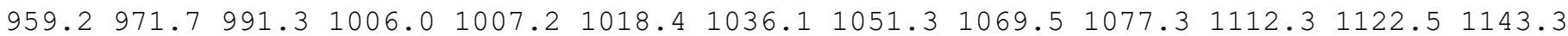
$\begin{array}{llllllllllllll}1166.1 & 1172.1 & 1185.8 & 1192.5 & 1231.0 & 1239.3 & 1262.0 & 1264.2 & 1271.8 & 1277.3 & 1281.7 & 1284.6\end{array}$ $1287.51292 .3 \quad 1294.3 \quad 1301.2 \quad 1311.5 \quad 1324.8 \quad 1334.2 \quad 1349.2 \quad 1365.2 \quad 1396.2 \quad 1401.61405 .6$ $1955.72646 .3 \quad 2664.2 \quad 2666.92670 .3 \quad 2673.3 \quad 2674.72675 .52680 .22687 .6 \quad 2717.92735 .3$ $2763.7 \quad 2776.92778 .4 \quad 2779.6$

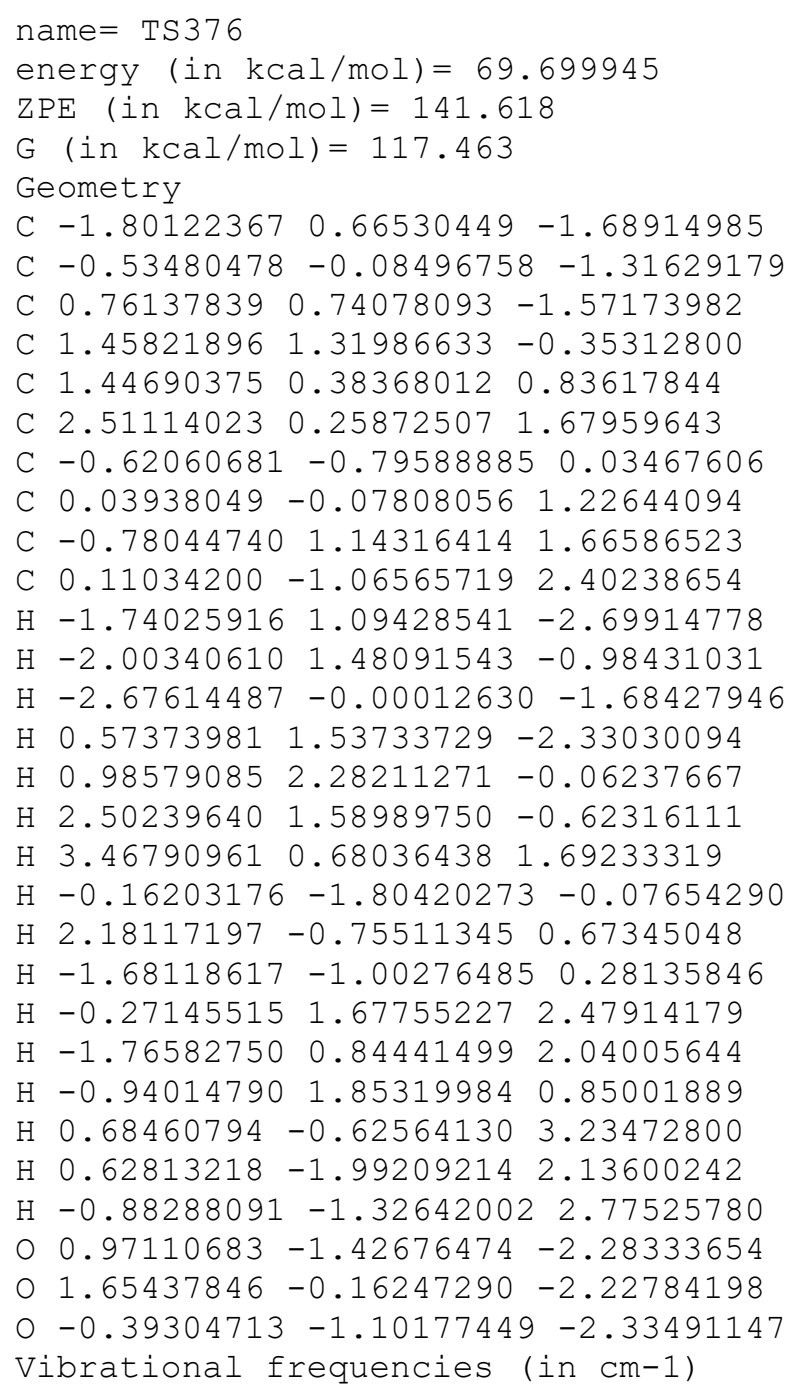


$\mathrm{ZPE}($ in $\mathrm{kcal} / \mathrm{mol})=140.851$

$\mathrm{G}($ in $\mathrm{kcal} / \mathrm{mol})=113.251$

Geometry

C $-2.41308389-0.24856391-0.16554291$

C $-0.92397917-0.22330117-0.02786594$

C $-0.263821851 .05863022-0.38585295$

C $1.16469775 \quad 1.206013170 .18695400$

C $1.53328081-0.028020320 .95766519$

C $1.92011668-1.135737130 .29850809$

C $-0.30700571-1.35123377 \quad 0.29723338$

C $1.35135246 \quad 0.05863334 \quad 2.45111965$

C $1.28540490-1.322188753 .11327700$

C $2.47950406 \quad 0.88925498 \quad 3.07421057$

$\mathrm{H}-2.705400110 .05504984-1.18391945$

$\mathrm{H}-2.88937743 \quad 0.43986969 \quad 0.54513774$

$\mathrm{H}-2.83931401-1.245862230 .00525501$

$\mathrm{H}-0.89159707 \quad 1.94365574-0.11657310$

H $1.23355841 \quad 2.11491085 \quad 0.81289114$

$\mathrm{H} \quad 1.88332239 \quad 1.38114136-0.64189208$

H $\quad 0.37357314 \quad 0.57795226 \quad 2.64554258$

H $2.19587623-2.06453980 \quad 0.77473817$

H 2.05561566 $-1.16865254 \quad-0.77519247$

$\mathrm{H} \quad 0.38787648-1.27314806-3.31161169$

$\mathrm{H} 2.25004706-1.83883975 \quad 3.07355872$

$\mathrm{H} \quad 0.54373759-1.96503343 \quad 2.61681764$

$\mathrm{H} \quad 0.99550848-1.242747034 .16566309$

$\mathrm{H} 2.35924720 \quad 0.95181142 \quad 4.16221409$

H $2.49402644 \quad 1.91340672 \quad 2.68704291$

H $3.46086791 \quad 0.44445048 \quad 2.87645399$

$0.62236173 \quad 0.32109706-2.39184859$

$0-0.247433111 .21269160-1.81402739$

O $-0.08093751-0.92363666-2.51937132$

Vibrational frequencies (in $\mathrm{cm}-1$ )

$\begin{array}{llllllllllllllll}-194.8 & 28.7 & 42.5 & 54.8 & 61.4 & 92.4 & 116.4 & 143.7 & 148.7 & 188.2 & 194.6 & 214.6 & 237.0 & 275.1\end{array}$ $291.3 \quad 329.1 \quad 367.5 \quad 401.3 \quad 436.6 \quad 465.5 \quad 492.9 \quad 516.0 \quad 553.5 \quad 668.4 \quad 684.8 \quad 733.1 \quad 848.6 \quad 863.9$ $877.8 \quad 976.9 \quad 983.3 \quad 983.5 \quad 985.0 \quad 1012.5 \quad 1021.5 \quad 1049.5 \quad 1061.3 \quad 1070.8 \quad 1080.3 \quad 1103.9 \quad 1137.7$

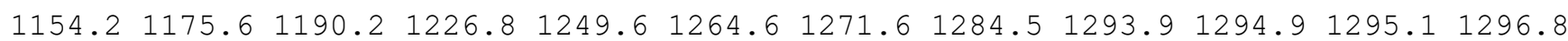
$1303.21307 .6 \quad 1325.3 \quad 1333.3 \quad 1338.8 \quad 1363.8 \quad 1390.0 \quad 1399.7 \quad 1400.4 \quad 1408.1 \quad 1812.6 \quad 1842.7$ $2654.82664 .22668 .92671 .62674 .92677 .12679 .2 \quad 2682.02699 .02711 .62741 .42775 .1$ $2778.7 \quad 2780.12782 .8 \quad 2867.3$

\section{name $=\operatorname{TS} 378$}

energy $($ in $\mathrm{kcal} / \mathrm{mol})=70.178248$

$\mathrm{ZPE}($ in $\mathrm{kcal} / \mathrm{mol})=141.335$

$\mathrm{G}($ in $\mathrm{kcal} / \mathrm{mol})=117.702$

Geometry

C $-2.28775487 \quad 0.69189538-0.77682079$

C $-0.83645450 \quad 0.24512142-0.92913163$

C $0.19498677 \quad 1.32272715 \quad-0.56946846$

C $1.21897880 \quad 1.06359172 \quad 0.26747101$

C $1.26562388-0.31061994 \quad 0.86201744$

C $1.03761078-1.29073794-0.34761345$

C $-0.49319036-1.03837655-0.14635996$

C $-0.17686631-0.71981894 \quad 1.37307281$

C $-0.965908890 .33196523 \quad 2.12407320$

C $-0.15681931-1.998351132 .20708957$

$\mathrm{H}-2.52203137 \quad 1.50493310 \quad-1.47501427$

$\mathrm{H}-2.51814855 \quad 1.031841210 .23578070$

$\mathrm{H}-2.97226289-0.13403999-1.01258125$

$\mathrm{H}-0.168577852 .83014673-1.94744266$

$\mathrm{H}-0.16984797 \quad 2.64906104-0.83625561$

$\mathrm{H} 2.018020621 .750808120 .49117683$

$\mathrm{H} 2.09823018-0.51032806 \quad 1.53076880$

H $1.35833275-2.31617179-0.16793239$ 
H $1.44806722-0.96937139-1.30857520$

$\mathrm{H}-1.17116506-1.86482607-0.34420187$

$\mathrm{H}-2.020141720 .045010992 .21831304$

$\mathrm{H}-0.92651901 \quad 1.31946013 \quad 1.65158828$

$\mathrm{H}-0.56896642 \quad 0.46293535 \quad 3.13977811$

$\mathrm{H} \quad 0.31415013-1.826443993 .18172729$

H $0.39520521-2.81051257 \quad 1.72140155$

$\mathrm{H}-1.17471337 \quad-2.36278155 \quad 2.38870847$

$\begin{array}{lllll}0 & 0.32117888 & 0.78820814 & -2.93702789\end{array}$

$00.43249456 \quad 1.84361379-2.01326489$

o - $0.70247316-0.04587009-2.32929916$

Vibrational frequencies (in $\mathrm{cm}-1$ )

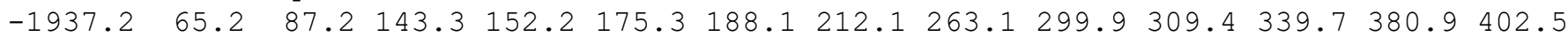

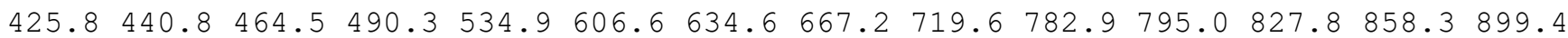
$906.4932 .9941 .1948 .3 \quad 967.1 \quad 990.6 \quad 994.2 \quad 1004.3 \quad 1009.6 \quad 1015.3 \quad 1031.8 \quad 1047.7 \quad 1077.9$

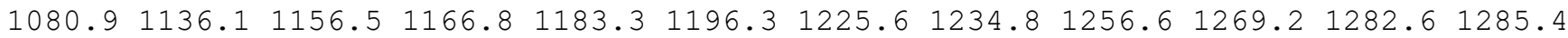
$1290.21291 .91297 .91300 .91310 .1 \quad 1315.1 \quad 1327.3 \quad 1380.3 \quad 1397.51406 .51415 .01758 .3$ $2116.02613 .2 \quad 2669.8 \quad 2674.2 \quad 2675.1 \quad 2676.8 \quad 2680.3 \quad 2681.02686 .7 \quad 2752.6 \quad 2758.3 \quad 2763.0$ $2774.3 \quad 2779.8 \quad 2782.2 \quad 2783.8$

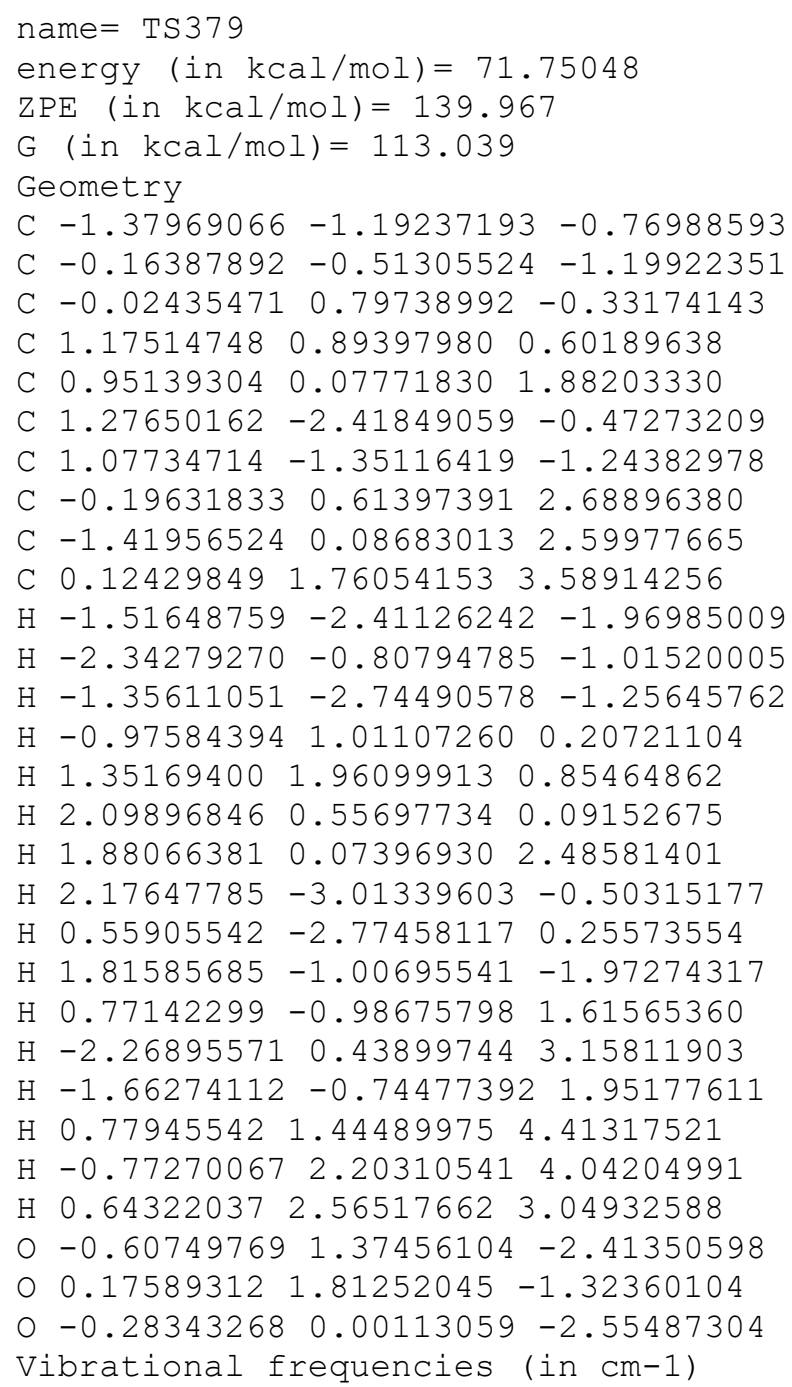

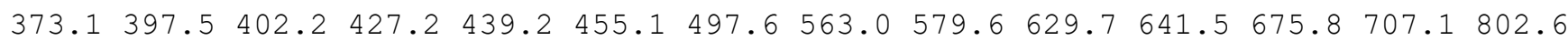
$832.8 \quad 870.7 \quad 884.7 \quad 948.3 \quad 972.2 \quad 976.9 \quad 994.8 \quad 1003.7 \quad 1013.8 \quad 1036.0 \quad 1044.6 \quad 1054.5 \quad 1080.4$ $1094.21095 .7 \quad 1098.8 \quad 1118.8 \quad 1127.6 \quad 1146.1 \quad 1166.7 \quad 1189.0 \quad 1219.8 \quad 1235.8 \quad 1277.9 \quad 1280.8$

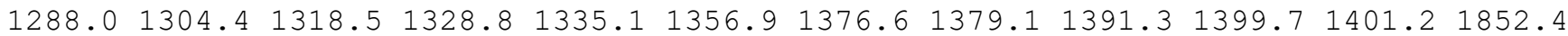
$1865.62648 .92657 .92667 .3 \quad 2672.2 \quad 2675.92706 .52713 .12714 .5 \quad 2739.22741 .42745 .5$ $2779.0 \quad 2779.4 \quad 2787.5 \quad 4854.7$ 
name $=\operatorname{TS} 380$

energy (in $\mathrm{kcal} / \mathrm{mol})=75.451966$

$\mathrm{ZPE}($ in $\mathrm{kcal} / \mathrm{mol})=136.362$

$\mathrm{G}(\mathrm{in} \mathrm{kcal} / \mathrm{mol})=106.01$

Geometry

C $-2.45314653-0.35026430-1.53459034$

C $-1.45233779 \quad 0.17736305-0.56515805$

C $-0.76283019 \quad 1.41550034-1.01468617$

C 1.699729651 .624957551 .30476271

C $1.10523613 \quad 0.378134521 .05657011$

C $1.72128619-0.56332235 \quad 0.21615393$

C $-1.20459643-0.43930878 \quad 0.59705481$

C $-0.24806204 \quad 0.00311323 \quad 1.67544072$

C -0.910628661 .164775512 .43332027$

C $-0.01571136-1.151604572 .67123743$

$\mathrm{H}-2.79382495-1.36489171-1.29814829$

$\mathrm{H}-2.03268665-0.37446187-2.55643277$

$\mathrm{H}-3.34067521 \quad 0.29883967-1.58006704$

$\mathrm{H}-0.316205412 .07024435-0.25346252$

H $1.31988834 \quad 2.34112064 \quad 1.99605706$

H 2.50916158 2.00320062 0.71466952

H 2.21257613 $0.02189035 \quad 1.79279918$

H $1.31712835-1.537471430 .04594570$

$\mathrm{H} \quad 2.54583280-0.32380692-0.42303033$

$\mathrm{H}-1.74515239-1.35727278 \quad 0.84397452$

$\mathrm{H}-0.32406907 \quad 1.46817842 \quad 3.30698905$

$\mathrm{H}-1.908310020 .88063416 \quad 2.78998709$

$\mathrm{H}-1.035133732 .048013941 .79499042$

$\mathrm{H} \quad 0.65392567-0.85756591 \quad 3.48486514$

$\mathrm{H} \quad 0.40850620-2.03364146 \quad 2.17666432$

$\mathrm{H}-0.95773018-1.467121513 .13708122$

O $1.06234047-1.10041214-3.36620929$

$0-0.69720938 \quad 1.70707633-2.18531604$

o $1.57502902-1.52633754-2.44809146$

Vibrational frequencies (in $\mathrm{cm}-1$ )

$\begin{array}{llllllllllllll}-1722.8 & 24.9 & 31.9 & 34.1 & 44.3 & 52.8 & 64.1 & 81.5 & 95.5 & 103.3 & 125.9 & 168.7 & 208.9 & 209.8\end{array}$

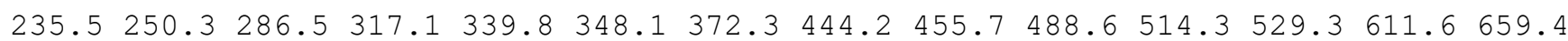

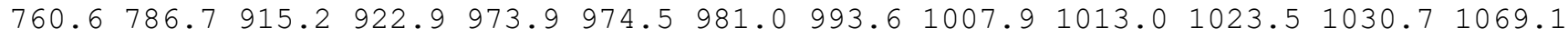

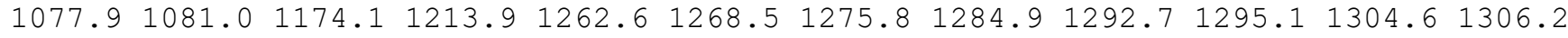
$1322.91376 .3 \quad 1379.1 \quad 1388.0 \quad 1398.4 \quad 1406.4 \quad 1423.8 \quad 1440.0 \quad 1595.5 \quad 1668.7 \quad 1825.3 \quad 1866.3$ $2088.8 \quad 2643.8 \quad 2658.0 \quad 2668.7 \quad 2671.9 \quad 2674.5 \quad 2676.92677 .62733 .82736 .5 \quad 2740.02776 .1$ $2778.32780 .3 \quad 2789.62811 .9$

name $=\operatorname{TS} 381$

energy $($ in $\mathrm{kcal} / \mathrm{mol})=71.663457$

$\mathrm{ZPE}($ in $\mathrm{kcal} / \mathrm{mol})=140.203$

$\mathrm{G}($ in $\mathrm{kcal} / \mathrm{mol})=115.404$

Geometry

C $-1.85229496-0.88602807-0.13113365$

C $-0.40799904-0.75755746 \quad 0.31509104$

C $0.42915680 \quad 0.35474417-0.44238748$

C $1.35218183 \quad 0.88540453 \quad 0.59590238$

C $0.88855038 \quad 0.60380818 \quad 1.94742312$

C $2.47984433-2.12629988-0.78467951$

C $-0.22421565-0.44827190 \quad 1.80717630$

C $-0.55454073 \quad 0.903657512 .39752977$

C $-1.39764192 \quad 1.93296774 \quad 1.69327550$

C $-0.78680503 \quad 0.93003643 \quad 3.89307800$

$\mathrm{H}-2.46168887-0.03092296 \quad 0.17138126$

$\mathrm{H}-2.30172480-1.78922303 \quad 0.30776179$

$\mathrm{H}-1.91738122-0.99754631-1.22084089$

$\mathrm{H}-0.19971967 \quad 1.09161019-0.97518490$

H $1.92453669 \quad 2.24448360 \quad 0.34422247$

H $1.157560812 .50549182 \quad 0.49329073$ 
H $1.66003623 \quad 0.53779512 \quad 2.71247441$

$\mathrm{H} 3.33511067-1.63954947-1.21991493$

H $2.64576081-2.96332462-0.12893048$

$\mathrm{H}-0.26798468-1.33649002 \quad 2.43514682$

$\mathrm{H}-1.28621007 \quad 1.92032718 \quad 0.60486359$

$\mathrm{H}-1.149744032 .94578606 \quad 2.03881880$

$\mathrm{H}-2.465339651 .77094250 \quad 1.90409489$

$\mathrm{H}-0.569455321 .92050751 \quad 4.31156407$

$\mathrm{H}-0.15696908 \quad 0.20792801 \quad 4.42753166$

$\mathrm{H}-1.83032786 \quad 0.68659698 \quad 4.13258841$

O $1.30656975-1.66156130-0.94115010$

O $1.15179109-0.27012536-1.48648835$

O $0.23015174-1.996975110 .09649651$

Vibrational frequencies (in cm-1)

$\begin{array}{llllllllllllllll}-1117.9 & 44.1 & 84.8 & 106.7 & 125.3 & 137.6 & 165.9 & 177.5 & 215.0 & 248.7 & 252.9 & 278.8 & 343.4 & 345.8\end{array}$

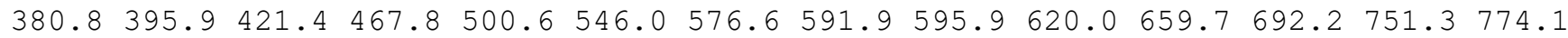

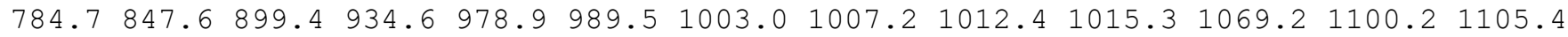

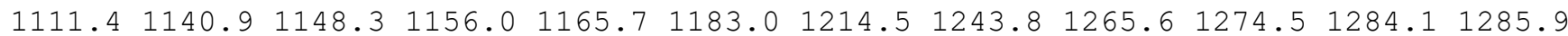

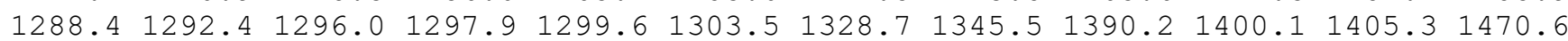
$1664.22669 .52673 .52674 .2 \quad 2675.32677 .72678 .42684 .62689 .92727 .52759 .12767 .8$ $2779.5 \quad 2781.8 \quad 2783.1 \quad 4547.5$

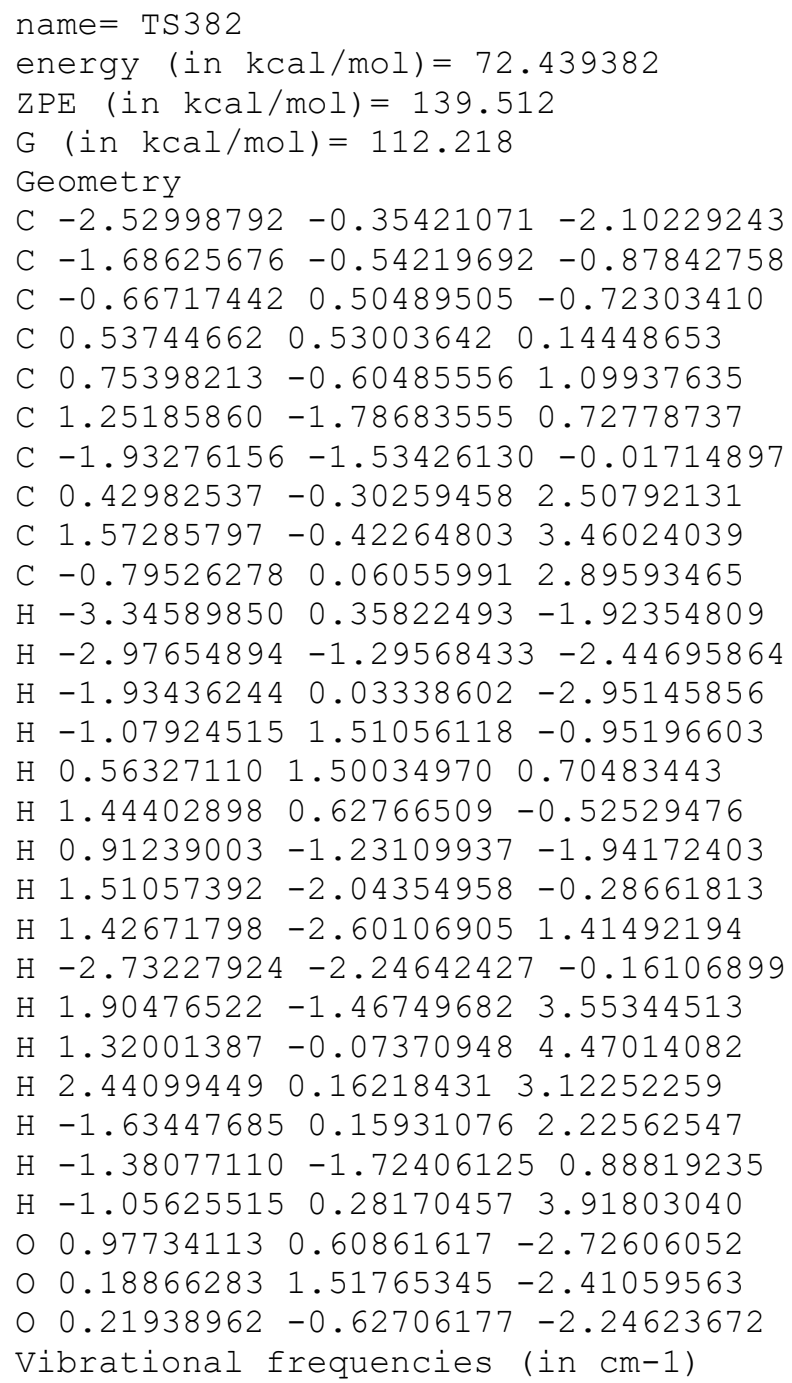


name $=\mathrm{TS} 383$

energy (in $\mathrm{kcal} / \mathrm{mol})=71.481042$

$\mathrm{ZPE}($ in $\mathrm{kcal} / \mathrm{mol})=140.482$

$\mathrm{G}($ in $\mathrm{kcal} / \mathrm{mol})=114.946$

Geometry

C $-1.44151282-3.06479516-0.02161874$

C $-0.71813371-1.90919302-0.70362388$

C $-1.44802307-0.54180394-0.48643427$

C $-0.53883748 \quad 0.57642627 \quad 0.01884839$

$\begin{array}{lllll}\text { C } & 0.41120761 & 0.05384103 & 1.12188743\end{array}$

C $1.25213238-1.03695667 \quad 0.52129362$

C $0.73343615-1.91113795-0.34373756$

C $-0.43372548 \quad-0.46683604 \quad 2.23332343$

C $-1.08055673 \quad 0.49696306 \quad 3.13603947$

C $0.97971820-1.422768863 .55438683$

$\mathrm{H}-2.47742983-3.15069140-0.37440900$

$\mathrm{H}-1.46172656-2.916889851 .06729987$

$\mathrm{H}-0.94931652-4.02344528-0.22315939$

$\mathrm{H}-2.35654697-0.65090806 \quad 0.14144342$

$\mathrm{H}-1.14010344 \quad 1.42574781 \quad 0.38787628$

$\mathrm{H} \quad 0.06310038 \quad 0.97520895 \quad-0.82414421$

H $1.06897805 \quad 0.89832305 \quad 1.44790155$

$\mathrm{H}-0.03561930-1.65572873 \quad 2.78159418$

H 2.29633178 -1.052428190 .81686825$

$\mathrm{H} \quad 1.32279259-2.68348443-0.83244072$

$\mathrm{H}-1.46880196 \quad 0.01668974 \quad 4.04606869$

$\mathrm{H}-1.96183160 \quad 0.927146632 .62508923$

$\mathrm{H}-0.45185580 \quad 1.34038162 \quad 3.44962711$

H $1.51849568-0.489840613 .53658882$

H $1.61298471-2.22723793 \quad 3.20939565$

$\mathrm{H} \quad 0.57142589-1.60917143 \quad 4.53479595$

$0-1.04358189-0.85212486-2.67424481$

$0-1.94663601-0.21175875-1.78155329$

o $-0.86317280-2.14112168-2.11753829$

Vibrational frequencies (in $\mathrm{cm}-1$ )

$\begin{array}{lllllllllllllll}-1236.3 & 35.3 & 51.4 & 81.5 & 103.3 & 134.9 & 145.1 & 172.3 & 199.7 & 208.6 & 264.1 & 273.3 & 313.2 & 333.6\end{array}$

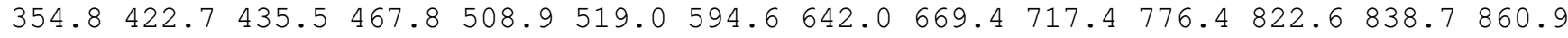
$924.8964 .7 \quad 966.8 \quad 978.0 \quad 986.4 \quad 1001.7 \quad 1019.5 \quad 1039.5 \quad 1073.5 \quad 1080.9 \quad 1101.8 \quad 1109.4 \quad 1127.6$

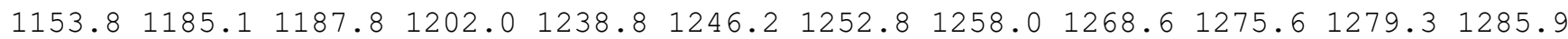

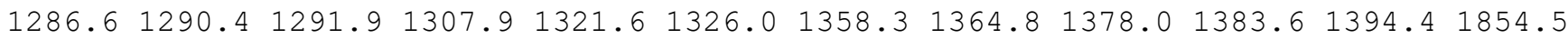
$1858.72659 .62660 .92670 .3 \quad 2671.3 \quad 2675.6 \quad 2689.22690 .52692 .12695 .92742 .62751 .5$ $2764.6 \quad 2773.2 \quad 2778.7 \quad 2780.1$

name $=\operatorname{TS} 384$

energy $($ in $\mathrm{kcal} / \mathrm{mol})=70.53864$

$\mathrm{ZPE}(\mathrm{in} \mathrm{kcal} / \mathrm{mol})=141.595$

$\mathrm{G}($ in $\mathrm{kcal} / \mathrm{mol})=117.23$

Geometry

C $-2.34510960-0.32820716-1.05748865$

C $-0.89147827-0.24463042-0.77752854$

C $-0.14547405 \quad 1.21021994-0.87352071$

C $0.35161193 \quad 1.62618638 \quad 0.59674933$

C $0.64379047 \quad 0.42121890 \quad 1.48268242$

$\begin{array}{llll}\text { C } 1.15495217 & -0.74007228 & 0.59030148\end{array}$

C $-0.16694338-1.22193749 \quad 0.04602228$

C $-0.60886847-0.23863925 \quad 1.98934150$

C $-1.91909841 \quad 0.42952724 \quad 1.98709964$

C $-0.50596800 \quad-1.42300373 \quad 2.87333924$

H $-2.84219148 \quad 0.65059374-1.01857760$

$\mathrm{H}-2.87925047-1.01497452-0.39172774$

$\mathrm{H}-2.51341903-0.70825565-2.08820036$

$\mathrm{H}-0.91239337 \quad 1.93863658-1.20819782$ 
$\mathrm{H}-0.34105791 \quad 2.33418861 \quad 1.05522508$

H $1.28887392 \quad 2.18710024 \quad 0.40320436$

$\mathrm{H} 1.34441879 \quad 0.68681242 \quad 2.30118525$

$\mathrm{H} 1.73163827-1.500780361 .12689571$

$\mathrm{H} 1.82422056-0.34328794-0.21876692$

$\mathrm{H}-0.51898844 \quad-2.21165303 \quad 0.25385742$

$\mathrm{H}-2.109496691 .02074901 \quad 1.06404662$

$\mathrm{H}-1.97576300 \quad 1.17215891 \quad 2.80805903$

$\mathrm{H}-2.77626196-0.25057932 \quad 2.10567203$

$\mathrm{H}-0.69732610-1.14693293 \quad 3.92730147$

$\mathrm{H} \quad 0.48082115-1.91758025 \quad 2.85323570$

$\mathrm{H}-1.24830679-2.20483662 \quad 2.63090882$

o $-0.52007382-1.88696333-2.39822359$

$00.846556161 .05641956-1.66944556$

o $-0.02044651-1.07781836-1.68407724$

Vibrational frequencies (in $\mathrm{cm}-1$ )

$\begin{array}{lllllllllllllll}-515.5 & 78.4 & 96.1 & 111.8 & 116.4 & 125.5 & 152.5 & 196.9 & 229.2 & 258.6 & 274.9 & 308.8 & 324.4 & 347.7\end{array}$

$366.0 \quad 396.5 \quad 426.6 \quad 455.4 \quad 495.3 \quad 520.0 \quad 529.1 \quad 631.3 \quad 677.5 \quad 753.6 \quad 780.4 \quad 817.4 \quad 858.0 \quad 919.8$

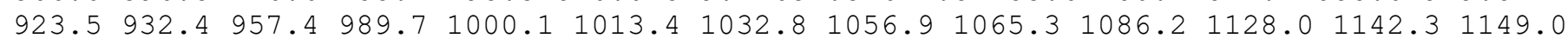

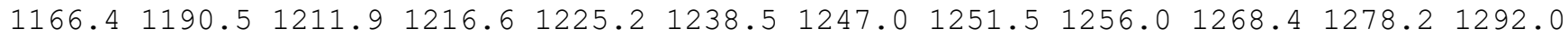

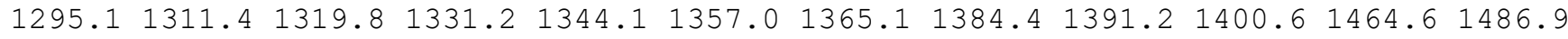
$2609.42611 .0 \quad 2633.8 \quad 2637.5 \quad 2637.8 \quad 2644.6 \quad 2650.6 \quad 2671.5 \quad 2678.2 \quad 2703.5 \quad 2737.92749 .5$ $2756.0 \quad 2763.5 \quad 2771.12772 .9$

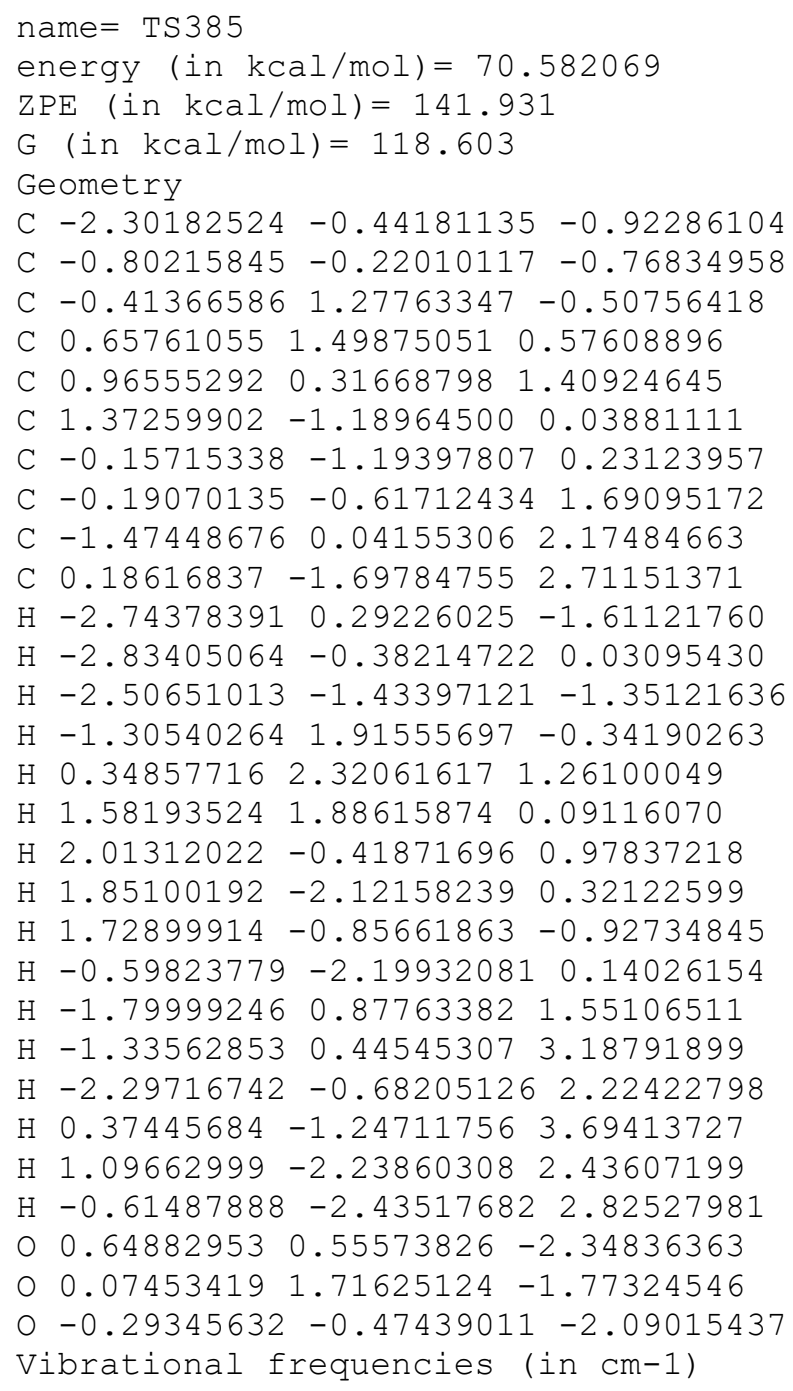


$2013.42649 .1 \quad 2667.1 \quad 2670.8 \quad 2675.3 \quad 2676.1 \quad 2677.92682 .12685 .6 \quad 2687.2 \quad 2726.8 \quad 2735.4$ $2750.8 \quad 2779.2 \quad 2781.8 \quad 2783.1$

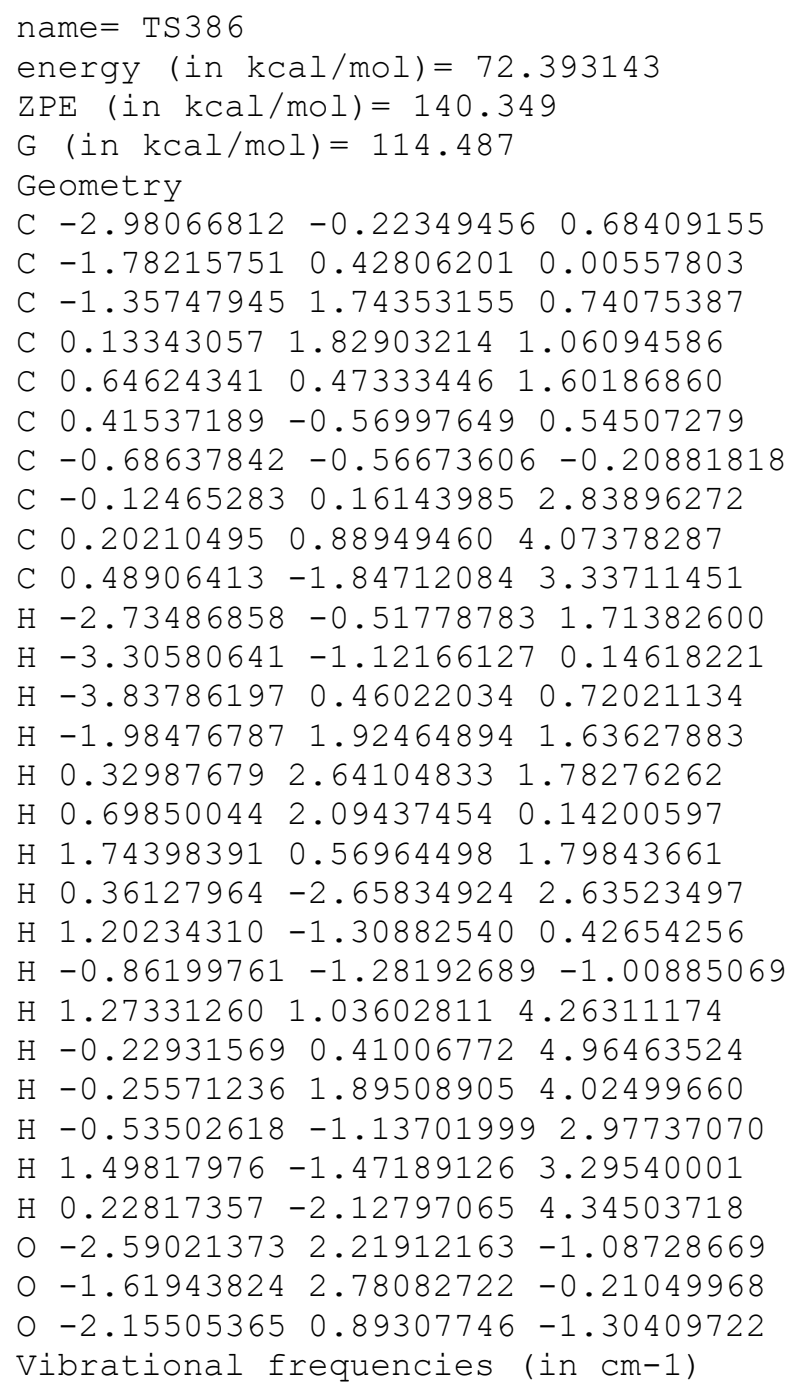


$\mathrm{H}-2.76917942-1.49993040-1.35254063$

$\mathrm{H}-0.92667588 \quad 1.63825667 \quad-1.32008632$

$\mathrm{H} \quad 0.70631930 \quad 2.25672460 \quad 0.23289275$

$\mathrm{H} \quad 1.89525441 \quad 1.40555915-0.75293348$

$\mathrm{H} \quad 1.92861525 \quad 0.53289539 \quad 1.63183159$

H $1.75378077 \quad-1.91854531 \quad 0.89410604$

$\mathrm{H} 1.72481707-1.21070576-0.71496737$

$\mathrm{H}-0.76113471-2.17481558 \quad 0.52316050$

$\mathrm{H}-1.44766868 \quad 1.494924420 .85166557$

$\mathrm{H}-0.94450387 \quad 1.57383137 \quad 2.52824388$

$\mathrm{H}-2.22788549 \quad 0.45850478 \quad 2.06624419$

$\mathrm{H} \quad 0.21588498-0.281126113 .66683467$

$\mathrm{H} \quad 0.70966928-1.731594912 .79747286$

$\mathrm{H}-0.99789392-1.455373713 .13728697$

$00.76883241-0.61247022-2.63118162$

$\begin{array}{lllll}0 & 0.39263983 & 0.74176716 & -2.51279000\end{array}$

o - $0.31790863-1.35123571-2.07946163$

Vibrational frequencies (in $\mathrm{cm}-1$ )

$\begin{array}{llllllllllllll}-1024.5 & 81.7 & 100.3 & 165.7 & 180.9 & 195.2 & 212.6 & 243.4 & 265.2 & 291.3 & 328.7 & 342.6 & 385.7 & 418.4\end{array}$

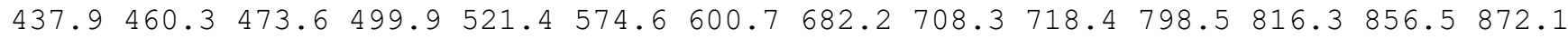
$894.4920 .9941 .3 \quad 973.1 \quad 983.4 \quad 996.2 \quad 1002.1 \quad 1006.1 \quad 1016.01023 .3 \quad 1044.8 \quad 1068.7 \quad 1085.3$

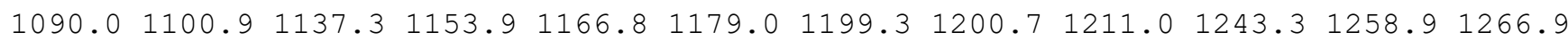

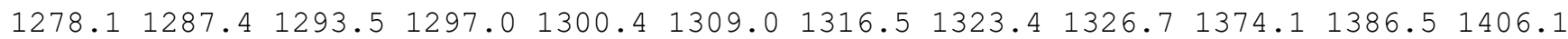
$1415.62659 .3 \quad 2667.12672 .1 \quad 2677.5 \quad 2679.9 \quad 2685.2 \quad 2686.62706 .82740 .52748 .82754 .3$ $2766.2 \quad 2779.5 \quad 2782.14772 .6$

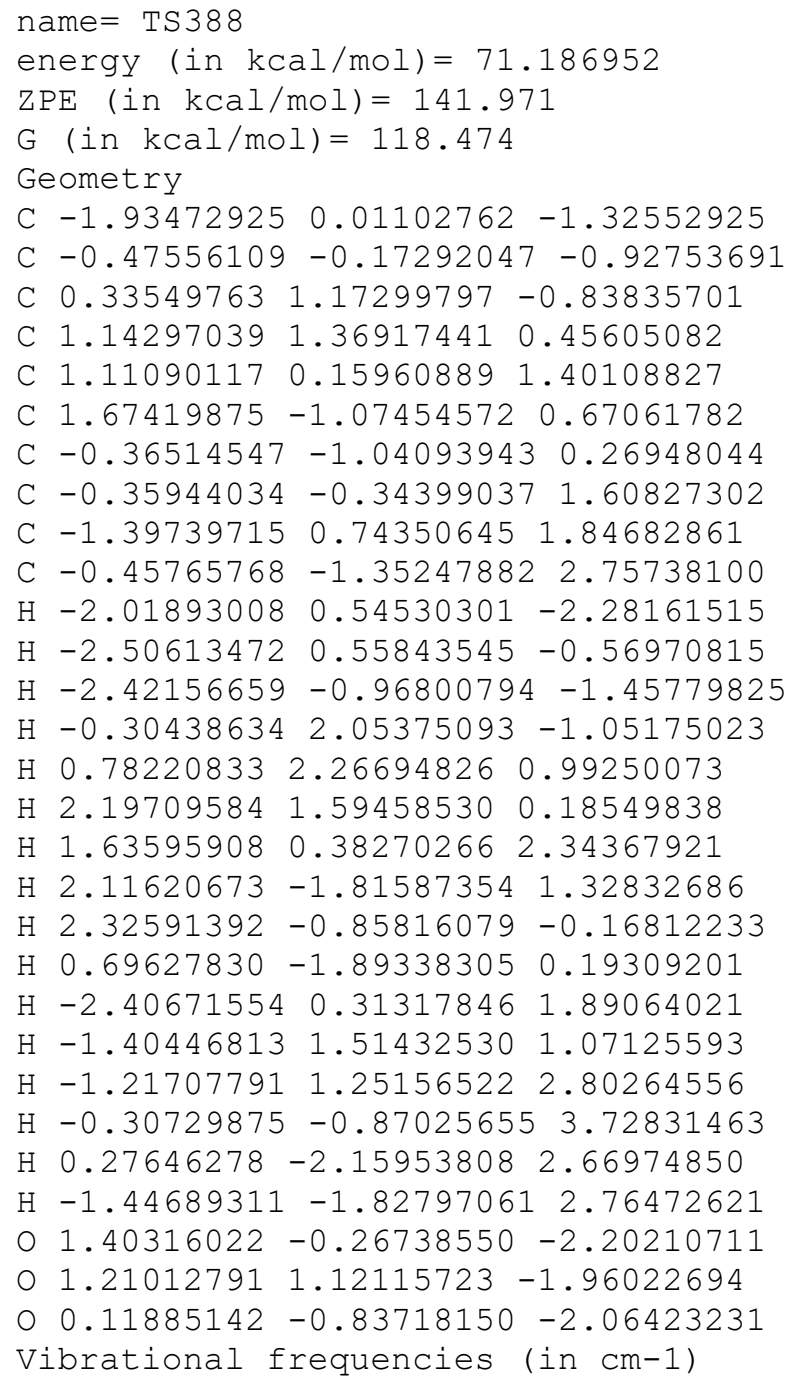




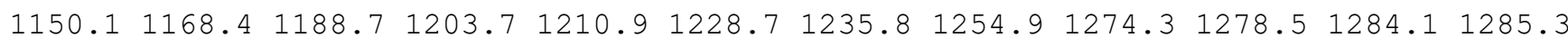

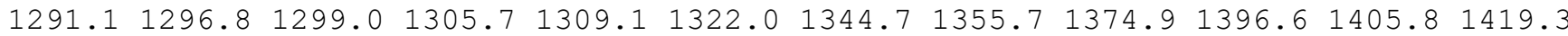
$1994.32655 .12667 .42673 .6 \quad 2675.02676 .0 \quad 2677.6 \quad 2682.42685 .2 \quad 2686.62726 .62738 .0$ $2746.62778 .12781 .3 \quad 2783.2$

\section{name $=$ TS389}

energy (in $\mathrm{kcal} / \mathrm{mol})=70.624246$

$\mathrm{ZPE}($ in $\mathrm{kcal} / \mathrm{mol})=142.551$

$\mathrm{G}($ in $\mathrm{kcal} / \mathrm{mol})=119.032$

Geometry

C $-1.61845682-0.57154099-1.70926653$

C $-0.19123903-0.39479205-1.21381418$

C $0.19665771 \quad 1.11418335-0.98374275$

C 0.854530891 .434867410 .36705392

$\begin{array}{lllll}\text { C } & 0.90807337 & 0.24986090 & 1.33906360\end{array}$

C $1.44737808-0.984976650 .57289923$

C $0.13799352-1.30468882-0.07293322$

C $-0.49866043-0.28768499 \quad 1.72742453$

C $-1.641152390 .69237821 \quad 1.69785206$

C $-0.44949015-1.06129643 \quad 3.03121739$

$\mathrm{H}-1.80110754 \quad 0.00391641-2.62638782$

$\mathrm{H}-2.35460763-0.26967079-0.95834696$

$\mathrm{H}-1.81018528-1.62903199-1.94934412$

$\mathrm{H}-0.65025883 \quad 1.79652397-1.19844101$

H $0.34611068 \quad 2.29289658 \quad 0.84441681$

$\mathrm{H} \quad 1.89289257 \quad 1.78581600 \quad 0.17385841$

H $1.50457943 \quad 0.51691758 \quad 2.22994593$

$\mathrm{H} \quad 1.81790671-1.78374913 \quad 1.23109790$

$\mathrm{H} \quad 2.26668304 \quad-0.75853986-0.12589964$

$\mathrm{H}-0.83338562-1.32293511 \quad 0.86302455$

$\mathrm{H}-2.58707322 \quad 0.22276102 \quad 2.00061950$

$\mathrm{H}-1.805613431 .12934533 \quad 0.70627381$

$\mathrm{H}-1.46447447 \quad 1.52635433 \quad 2.39303665$

$\mathrm{H}-0.22588049-0.39481272 \quad 3.87526987$

$\mathrm{H} \quad 0.32246515-1.842125593 .01920825$

$\mathrm{H}-1.40314341-1.55572073 \quad 3.25338410$

$\begin{array}{lllll}0 & 1.72227893 & 0.12638375 & -2.30640864\end{array}$

O $1.116845791 .38483555-2.03962472$

O $0.63777292-0.78022585-2.32883533$

Vibrational frequencies (in $\mathrm{cm}-1$ )

$\begin{array}{llllllllllllllllll}-1391.3 & 68.8 & 117.0 & 140.4 & 158.3 & 165.6 & 181.2 & 219.8 & 237.1 & 283.1 & 317.0 & 337.0 & 357.5 & 385.4\end{array}$

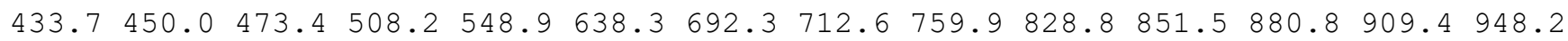

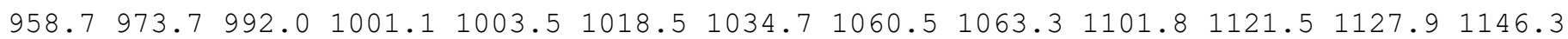
$\begin{array}{llllllllllllll}1173.8 & 1185.3 & 1195.7 & 1209.7 & 1224.0 & 1238.1 & 1252.6 & 1271.2 & 1278.6 & 1284.0 & 1286.8 & 1287.5\end{array}$

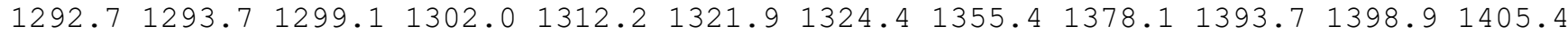
$1937.82653 .0 \quad 2668.12668 .8 \quad 2670.2 \quad 2671.8 \quad 2674.62677 .92680 .02687 .82718 .72737 .5$ $2761.2 \quad 2777.8 \quad 2778.92779 .3$

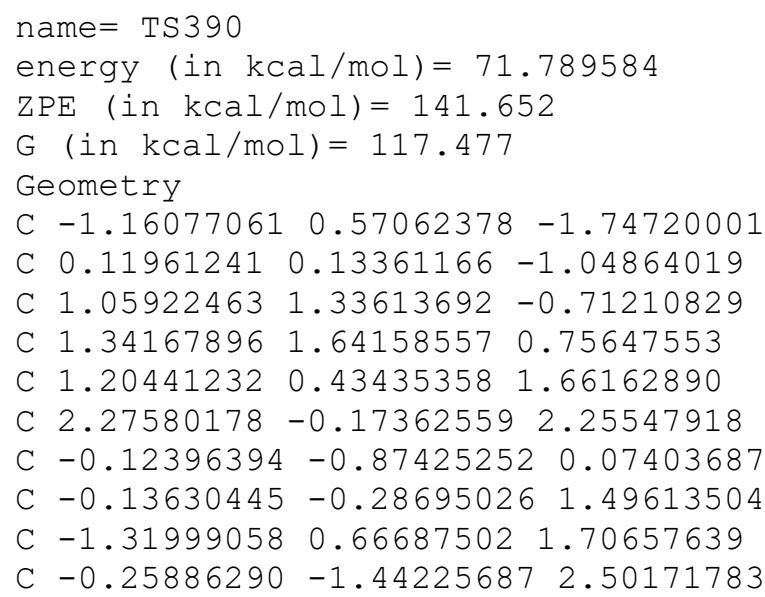


$\mathrm{H}-0.95181036 \quad 1.23083685-2.60025891$

$\mathrm{H}-1.833804241 .09710993-1.06210370$

$\mathrm{H}-1.70701117-0.29423956-2.14911661$

$\mathrm{H} \quad 0.71248543 \quad 2.26123115-1.23054356$

$\mathrm{H} \quad 0.675351412 .45560380 \quad 1.10280251$

H $2.374439892 .05221926 \quad 0.82730772$

$\mathrm{H} 1.33873866 \quad 0.66916516 \quad 2.99752178$

$\mathrm{H} \quad 0.67968974 \quad-1.64802596 \quad 0.02597618$

H $3.29896612 \quad 0.05361441 \quad 2.25667811$

$\mathrm{H}-1.06465902-1.42648488-0.10950966$

$\mathrm{H}-1.31075407 \quad 1.104998962 .70938342$

$\mathrm{H}-2.27218623 \quad 0.13334594 \quad 1.59689611$

$\mathrm{H}-1.32610245 \quad 1.48794843 \quad 0.98340697$

$\mathrm{H}-0.38136881-1.080553313 .52642743$

H $0.65591631 \quad-2.05987726 \quad 2.49636414$

$\mathrm{H}-1.10738589-2.091742812 .27227580$

O $2.21335969-0.35661087-1.69066916$

o $2.28806617 \quad 1.03360622-1.37649822$

o $0.87351059-0.54237348-2.08018889$

Vibrational frequencies (in $\mathrm{cm}-1$ )

$\begin{array}{llllllllllllllll}-1230.6 & 47.9 & 71.2 & 130.6 & 137.7 & 176.8 & 196.1 & 218.1 & 240.1 & 258.6 & 285.4 & 322.7 & 340.5 & 363.1\end{array}$

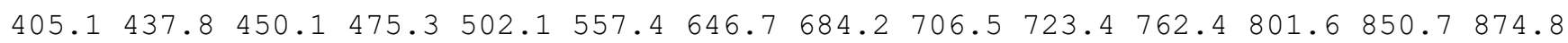

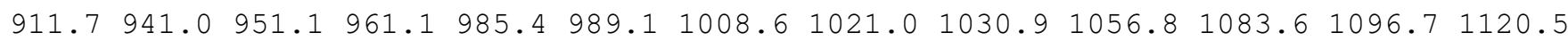

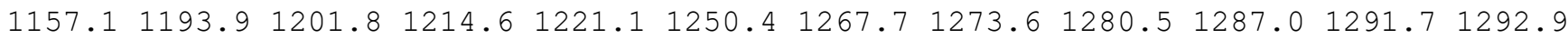
$1299.61305 .2 \quad 1306.8 \quad 1311.91318 .3 \quad 1321.2 \quad 1374.6 \quad 1388.6 \quad 1398.2 \quad 1406.2 \quad 1418.11609 .4$ $2269.52649 .22654 .6 \quad 2659.2 \quad 2667.6 \quad 2671.92674 .32675 .72678 .62684 .82733 .92734 .6$ $2778.3 \quad 2778.7 \quad 2779.4 \quad 2782.1$

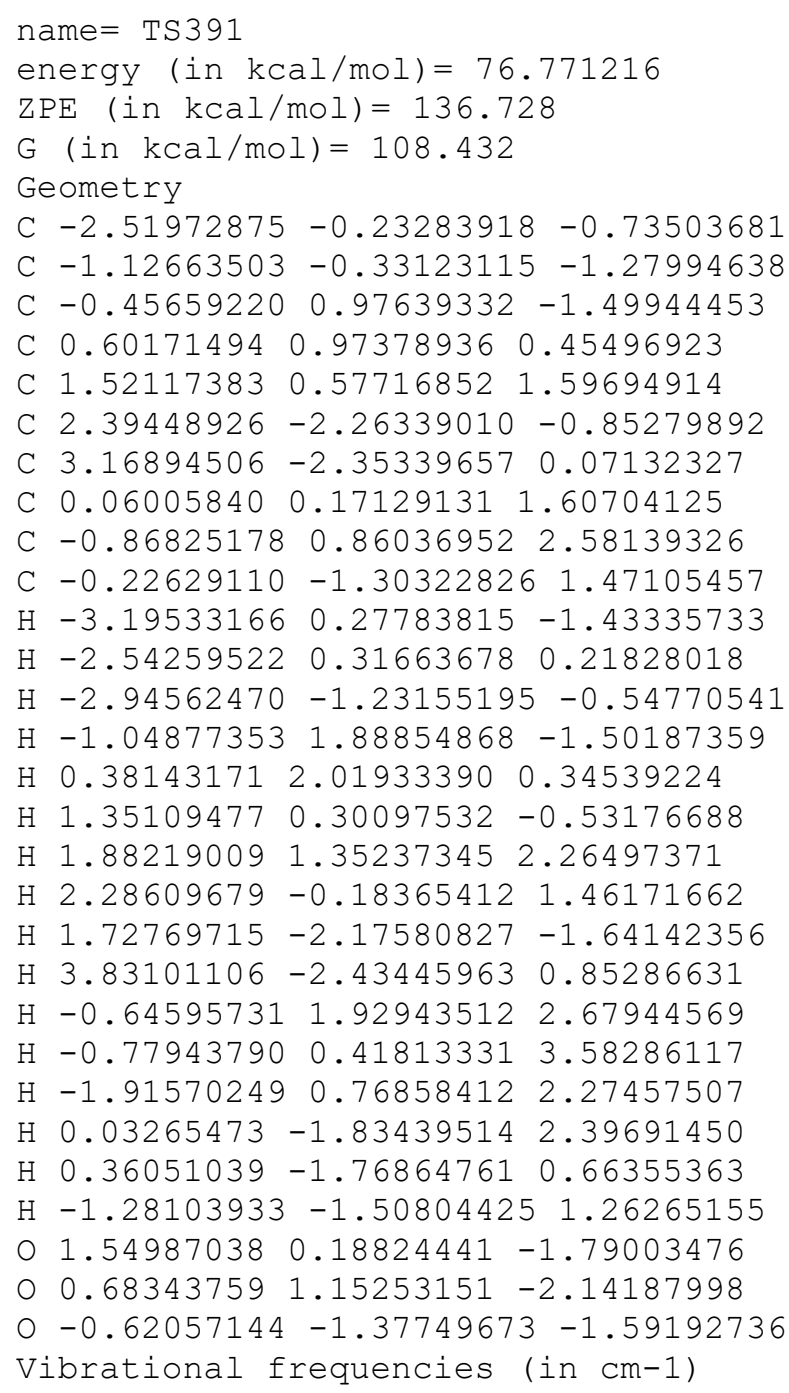


$\begin{array}{lllllllllllllllll}-1469.7 & 36.4 & 42.8 & 56.2 & 65.5 & 83.1 & 105.7 & 118.0 & 128.0 & 140.0 & 141.7 & 150.9 & 187.2 & 199.1\end{array}$ $220.1303 .2 \quad 329.8 \quad 358.9 \quad 381.2 \quad 395.8 \quad 443.5 \quad 458.9 \quad 477.6 \quad 563.7 \quad 607.2 \quad 749.9 \quad 782.3 \quad 850.1$

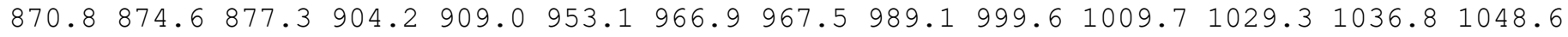
$1064.61075 .0 \quad 1127.3 \quad 1138.3 \quad 1189.5 \quad 1208.8 \quad 1250.7 \quad 1259.51260 .7 \quad 1271.0 \quad 1288.8 \quad 1294.0$ $1299.51300 .0 \quad 1312.6 \quad 1375.2 \quad 1377.8 \quad 1395.8 \quad 1402.5 \quad 1474.2 \quad 1504.6 \quad 1784.3 \quad 1847.92267 .8$ $2660.72661 .92668 .0 \quad 2675.7 \quad 2678.7 \quad 2680.8 \quad 2699.2 \quad 2707.82763 .8 \quad 2775.7 \quad 2779.6 \quad 2780.8$ 2783.32814 .92928 .7

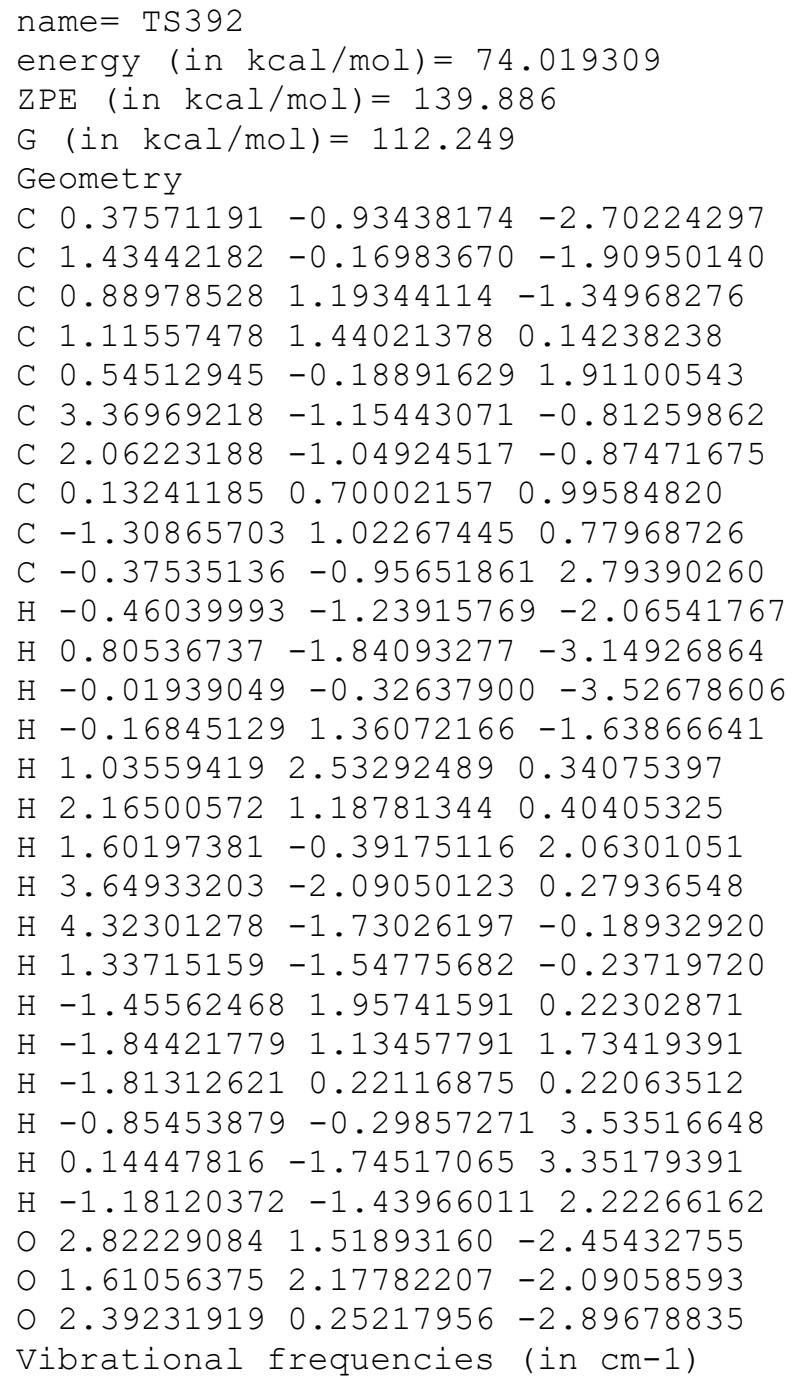


C $0.13148381-0.60131508 \quad 1.75525437$

C $-0.67151114 \quad 1.67695379 \quad 2.26452141$

C $-0.22471212-1.368328352 .95923171$

$\mathrm{H}-2.34414084 \quad 0.31848335-1.51058552$

$\mathrm{H}-2.39136650-0.00482936 \quad 0.23615545$

$\mathrm{H}-2.36302904-1.34317060-0.92536200$

$\mathrm{H}-0.56472863 \quad 1.89667403 \quad-0.65389914$

$\mathrm{H} 1.841183202 .01760926 \quad 0.50541523$

H $1.98177186 \quad-0.04692698 \quad 0.40947253$

H $1.32881661 \quad 0.93242441 \quad 2.65346611$

H $2.02917187-1.42182034-0.63794148$

H $2.10829436-1.98802950 \quad 1.05571015$

$\mathrm{H}-0.41010571-2.24625588 \quad 0.40485698$

$\mathrm{H}-1.50014583 \quad 1.57156181 \quad 1.55573850$

$\mathrm{H}-0.390980212 .73872826 \quad 2.28007931$

$\mathrm{H}-1.037791291 .410180933 .26057150$

$\mathrm{H} \quad 0.43470791-2.24881500 \quad 3.07667541$

$\mathrm{H}-1.25307729-1.765200242 .90482546$

$\mathrm{H}-0.14493378-0.788427643 .89005522$

o $1.13864330-0.10663916-2.27962764$

$00.863000221 .21956950-1.87301174$

$0-0.04189235-0.83689989-1.95931849$

Vibrational frequencies (in $\mathrm{cm}-1$ )

$\begin{array}{lllllllllllllll}-1517.9 & 69.2 & 76.9 & 99.0 & 135.0 & 150.8 & 163.9 & 224.1 & 237.3 & 246.3 & 283.3 & 312.9 & 347.3 & 401.5\end{array}$ $413.5 \quad 459.5 \quad 471.1 \quad 489.8 \quad 534.5 \quad 583.7 \quad 636.4 \quad 667.1 \quad 701.6 \quad 719.5 \quad 797.1 \quad 859.0 \quad 872.2 \quad 916.7$ $953.9974 .0 \quad 981.4 \quad 1004.0 \quad 1008.1 \quad 1015.7 \quad 1045.3 \quad 1049.7 \quad 1066.8 \quad 1076.5 \quad 1098.7 \quad 1125.3 \quad 1141.2$

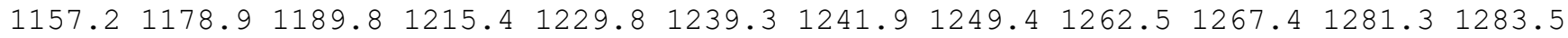

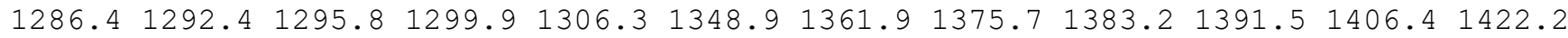
$1717.72646 .6 \quad 2665.8 \quad 2667.2 \quad 2669.1 \quad 2675.1 \quad 2676.6 \quad 2678.52692 .92694 .02724 .92765 .1$ $2771.5 \quad 2777.5 \quad 2779.9 \quad 2780.4$

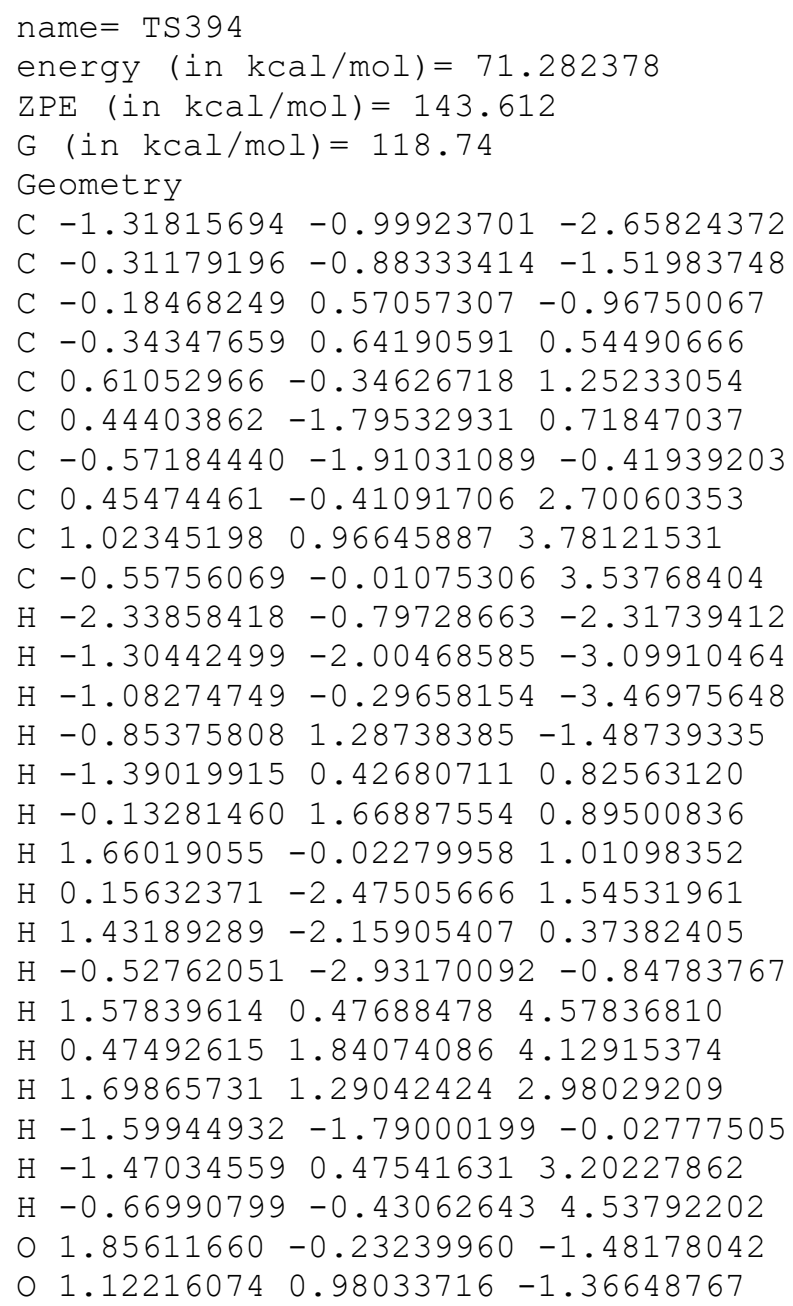


$0.96661290-1.12369474-2.12970339$

Vibrational frequencies (in $\mathrm{cm}-1$ )

$\begin{array}{llllllllllllllllll}-857.4 & 36.5 & 63.9 & 95.4 & 118.1 & 146.4 & 159.3 & 167.6 & 228.9 & 258.8 & 266.6 & 282.0 & 352.2 & 396.4\end{array}$ $419.4 \quad 427.5 \quad 486.6 \quad 537.2 \quad 586.0 \quad 603.9 \quad 677.4 \quad 715.1 \quad 808.4 \quad 821.6 \quad 843.0 \quad 874.5 \quad 934.2 \quad 938.1$ $950.1963 .2969 .8 \quad 975.8 \quad 1004.0 \quad 1010.2 \quad 1022.1 \quad 1053.6 \quad 1080.2 \quad 1095.4 \quad 1136.2 \quad 1156.2 \quad 1167.6$

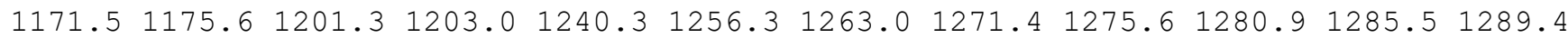
$1290.11300 .5 \quad 1312.0 \quad 1325.3 \quad 1333.0 \quad 1340.7 \quad 1352.3 \quad 1366.7 \quad 1384.5 \quad 1390.01404 .8 \quad 1691.6$ $2637.72652 .7 \quad 2669.5 \quad 2670.2 \quad 2670.5 \quad 2672.4 \quad 2678.42680 .12687 .02695 .12739 .82743 .8$ $2745.12747 .2 \quad 2752.0 \quad 2781.4$

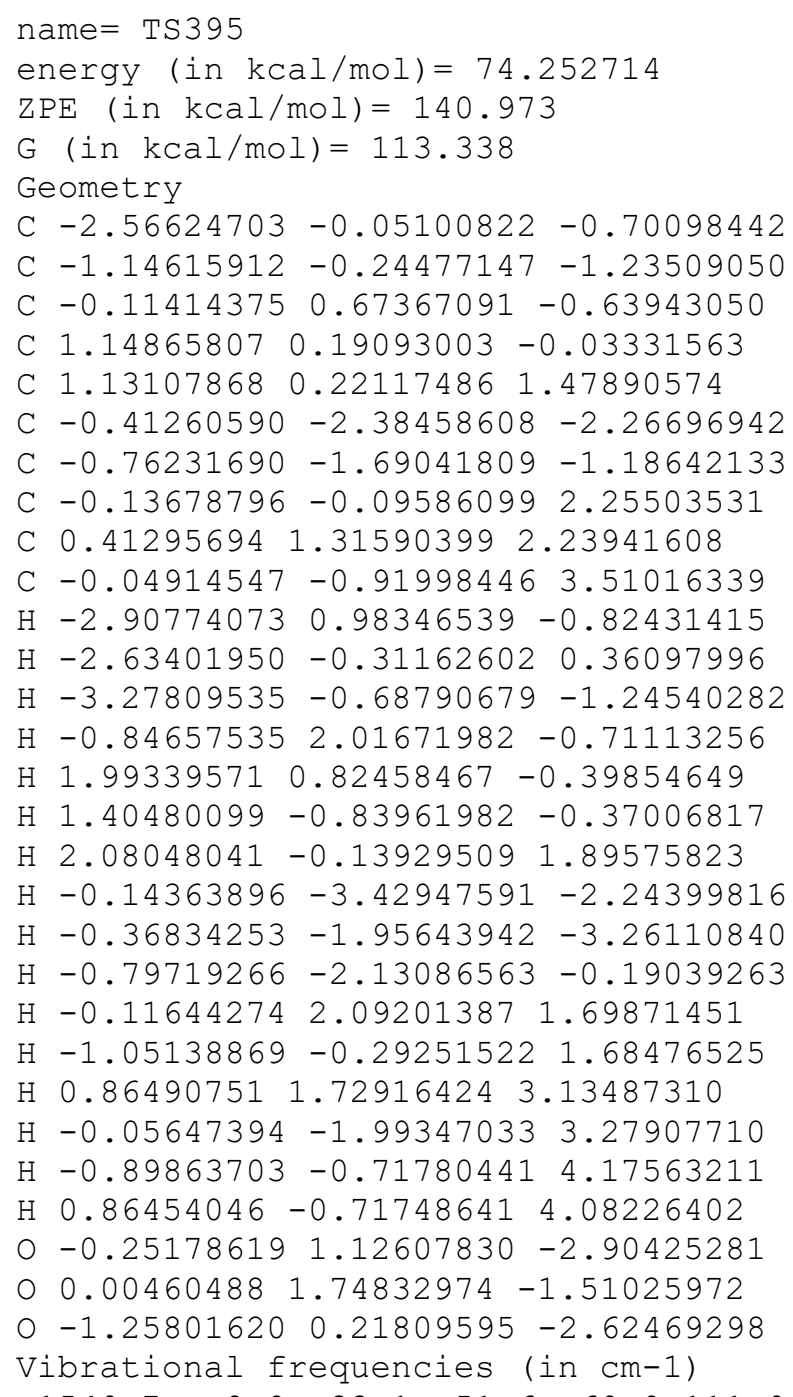


C $1.45090924-1.10846781 \quad 0.03426184$

C $-0.09325802-1.20947548 \quad 0.25185393$

C $0.11355925-0.52074663 \quad 1.67558491$

C $-0.94549377 \quad 0.43863432 \quad 2.18404335$

C $0.33215784-1.59715096 \quad 2.63553396$

$\mathrm{H}-2.238490150 .14796921 \quad-2.13950023$

$\mathrm{H}-2.25285387-1.51347279-1.45183305$

$\mathrm{H} \quad 0.46319337-0.04695277-3.97725417$

$\mathrm{H}-0.89925748 \quad 1.91542941-0.51950233$

$\mathrm{H} \quad 0.88945804 \quad 2.19746772 \quad 0.90824307$

H $1.97845826 \quad 1.66223132-0.37176111$

H $2.27847977 \quad 0.21459057 \quad 1.68540112$

H $2.00990029-1.976529200 .38602474$

$\mathrm{H} \quad 1.77558318-0.89263244-0.98570332$

$\mathrm{H}-0.53921650 \quad-2.20658042 \quad 0.26753797$

$\mathrm{H}-1.44513256 \quad 0.996878391 .38706313$

$\mathrm{H}-0.52279359 \quad 1.16968090 \quad 2.87993754$

$\mathrm{H}-1.74153405-0.11495310 \quad 2.70597178$

$\mathrm{H} \quad 0.20931316-1.090866314 .11265997$

$\mathrm{H} \quad 0.94529947 \quad-0.78055570 \quad 3.96325778$

$\mathrm{H} 1.16473489-2.253843532 .58278160$

$\begin{array}{lllll}0 & 1.31597817 & 0.86000750 & -2.56324383\end{array}$

$0.20832543 \quad 1.52339580-2.05971587$

O $0.91241560-0.34405723-3.14799813$

Vibrational frequencies (in $\mathrm{cm}-1$ )

$\begin{array}{lllllllllllllll}-1003.1 & 58.6 & 71.8 & 89.5 & 118.1 & 130.6 & 149.0 & 183.5 & 209.1 & 247.1 & 281.8 & 315.5 & 341.5 & 381.3\end{array}$ $407.4 \quad 426.0 \quad 441.0 \quad 483.2 \quad 512.8 \quad 543.5 \quad 548.1 \quad 572.7 \quad 614.6 \quad 644.9 \quad 721.7 \quad 733.0 \quad 789.3 \quad 827.2$

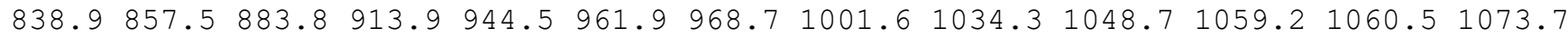

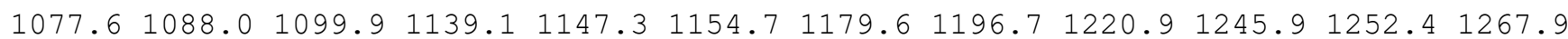

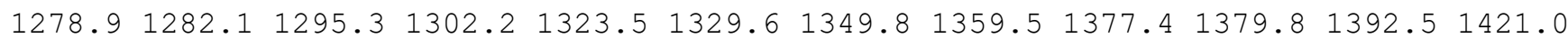
$1863.92655 .3 \quad 2669.2 \quad 2672.02681 .8 \quad 2693.3 \quad 2719.7 \quad 2723.02741 .2 \quad 2743.7 \quad 2751.2 \quad 2770.4$ $2782.22788 .6 \quad 2850.0 \quad 4712.4$

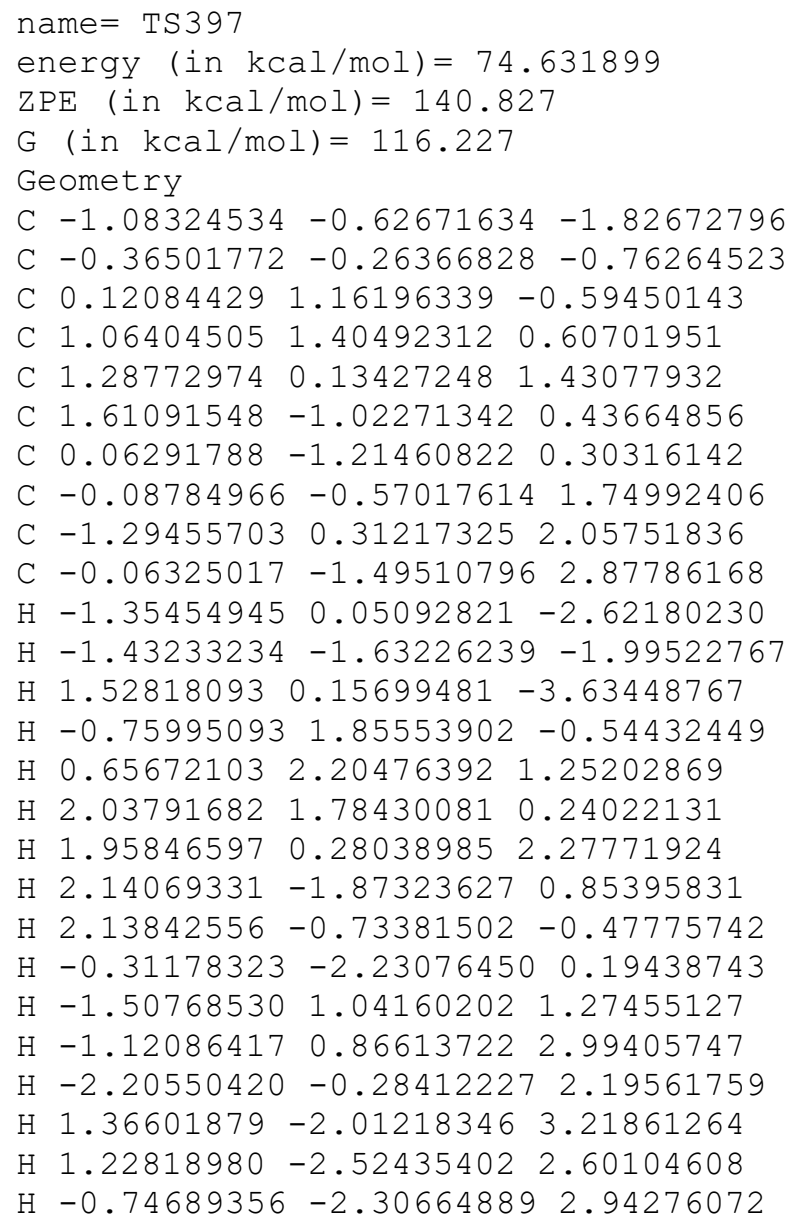


O $1.949741381 .10518417-2.06030753$

$0.697179811 .64150232-1.80745904$

O $1.81350094-0.11560301-2.72768071$

Vibrational frequencies (in $\mathrm{cm}-1$ )

$\begin{array}{lllllllllllllll}-1012.5 & 62.0 & 77.7 & 90.6 & 95.5 & 143.2 & 181.8 & 204.7 & 216.2 & 251.3 & 288.1 & 320.0 & 339.0 & 368.3\end{array}$ $411.4 \quad 437.8 \quad 439.9 \quad 482.5 \quad 512.3 \quad 549.9 \quad 560.2 \quad 570.4 \quad 628.6 \quad 673.3 \quad 711.1 \quad 732.3 \quad 780.5 \quad 827.9$ $834.6 \quad 855.3 \quad 887.7 \quad 916.5 \quad 937.5 \quad 962.1 \quad 967.5 \quad 999.4 \quad 1026.7 \quad 1046.3 \quad 1057.2 \quad 1060.0 \quad 1072.4$ $1074.21085 .5 \quad 1098.8 \quad 1133.8 \quad 1148.3 \quad 1163.0 \quad 1179.8 \quad 1198.6 \quad 1215.5 \quad 1245.4 \quad 1249.91269 .6$

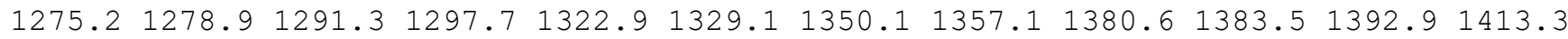
$1861.42654 .92671 .0 \quad 2672.2 \quad 2678.32690 .12719 .52722 .42741 .02747 .12754 .62773 .1$ $2781.52788 .5 \quad 2848.7 \quad 4690.1$

\section{name $=$ TS398}

energy (in $\mathrm{kcal} / \mathrm{mol})=74.504335$

$\mathrm{ZPE}($ in $\mathrm{kcal} / \mathrm{mol})=140.966$

G $($ in $\mathrm{kcal} / \mathrm{mol})=116.578$

Geometry

C $-3.01060553-0.33445066-0.59129476$

C $-1.69941787-0.44095704-0.37360621$

C $-0.69881251 \quad 0.33270376-1.21013611$

C $0.78835181 \quad 0.09613046 \quad-0.84918627$

C $0.95018881-0.88268509 \quad 0.31186116$

C $0.04499993-2.11871686 \quad 0.02329121$

C $-1.11427243-1.30925486 \quad 0.69220807$

C $-0.02311191-0.49920578 \quad 1.50520199$

C $-0.27220188 \quad 0.98235406 \quad 1.78288516$

C $0.27241926-1.038016472 .82667222$

$\mathrm{H}-3.43709482 \quad 0.28326870-1.36928678$

$\mathrm{H}-3.75798014-0.85359130-0.01235499$

$\mathrm{H}-2.11504365-2.10685273-2.19094708$

$\mathrm{H}-0.933248201 .42906300-1.15707876$

$\mathrm{H} \quad 1.27122154 \quad 1.05937891 \quad-0.60151709$

H $1.32651476-0.28321588-1.74061325$

$\mathrm{H} \quad 1.98792730-1.07240026 \quad 0.58046075$

H $0.32592558-3.03288373 \quad 0.54342437$

$\mathrm{H}-0.08825183-2.37447987-1.02880826$

$\mathrm{H}-1.83875980 \quad-1.85949013 \quad 1.29470594$

$\mathrm{H}-0.925851291 .448693021 .04080134$

$\mathrm{H} \quad 0.66907537 \quad 1.54180181 \quad 1.80850663$

$\mathrm{H}-0.77505745 \quad 1.12386462 \quad 2.75443137$

H $1.16972716-0.79173114 \quad 3.33966580$

H $0.77271371-2.62087585 \quad 2.57806238$

$\mathrm{H} \quad 0.04864253-2.58724062 \quad 2.94363403$

$\begin{array}{llll}0 & -0.66239932 & -1.18976780 & -2.96918447\end{array}$

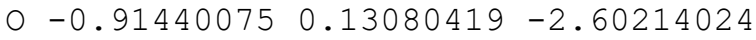

o $-1.88415226-1.84751006-3.12005526$

Vibrational frequencies (in $\mathrm{cm}-1$ )

$\begin{array}{llllllllllllllll}-985.8 & 62.1 & 76.7 & 112.7 & 125.2 & 145.5 & 157.9 & 204.7 & 216.8 & 243.5 & 299.4 & 347.1 & 366.7 & 400.8\end{array}$ $\begin{array}{llllllllllllllll}416.9 & 427.1 & 444.4 & 484.1 & 517.7 & 534.7 & 543.9 & 572.0 & 633.4 & 669.2 & 712.7 & 722.6 & 768.1 & 824.8\end{array}$

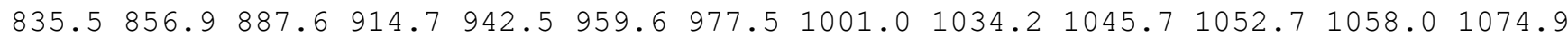

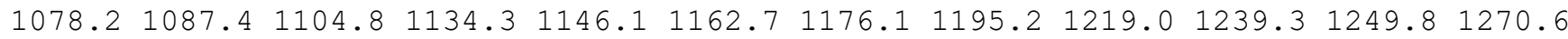
$1278.21281 .0 \quad 1290.3 \quad 1299.5 \quad 1321.2 \quad 1326.4 \quad 1357.1 \quad 1361.4 \quad 1378.8 \quad 1385.6 \quad 1389.6 \quad 1418.4$ $1863.82654 .52661 .92671 .5 \quad 2679.8 \quad 2690.52717 .02721 .12741 .12746 .92750 .62770 .1$ $2778.5 \quad 2784.7 \quad 2828.3 \quad 4724.8$

name $=\operatorname{TS} 399$

energy (in $\mathrm{kcal} / \mathrm{mol})=75.404205$

$\operatorname{ZPE}($ in $\mathrm{kcal} / \mathrm{mol})=140.439$

$\mathrm{G}($ in $\mathrm{kcal} / \mathrm{mol})=114.697$

Geometry

C $-0.26582747-0.98106660-2.56735975$

C $0.40904338-0.95890619-1.19895352$

C $1.004411310 .45373050-0.84718166$ 
C $0.25166314 \quad 1.28880138 \quad 0.07915967$

C $0.11087553 \quad 1.25167297 \quad 1.52597187$

C $-0.45746185-2.79123898 \quad 0.23960465$

C $-0.50678947-1.52238690-0.16185569$

C $0.54183832 \quad 0.30310830 \quad 2.46612294$

C $-0.06946163 \quad 0.31933532 \quad 3.82240452$

C $1.61829365-0.67422118 \quad 2.26130950$

$\mathrm{H} \quad 0.43514009-0.70250992-3.36501445$

$\mathrm{H}-1.11243983-0.28496978-2.59574080$

$\mathrm{H}-0.64643685-1.98322879-2.80425261$

$\mathrm{H} 1.178050191 .00651486-1.80966984$

$\mathrm{H}-0.32182118 \quad 2.06647071-0.40841709$

$\mathrm{H} 1.03502301 \quad 2.06333980 \quad 1.42952603$

$\mathrm{H}-0.80221471 \quad 1.80584817 \quad 1.84722145$

$\mathrm{H}-1.12470345-3.208599150 .97601852$

$\mathrm{H} \quad 0.25853182-3.50772104-0.13790311$

$\mathrm{H}-1.22939753-0.80406211 \quad 0.23167428$

$\mathrm{H}-0.76866826 \quad 1.14874863 \quad 3.98611790$

$\mathrm{H} \quad 0.68977285 \quad 0.37040333 \quad 4.61742801$

$\mathrm{H}-0.63904726-0.61168233 \quad 3.99097471$

H $2.51786639-0.221311621 .80638711$

H $1.30185924-1.463645331 .54668965$

$\mathrm{H} \quad 1.93580686-1.185265993 .17858623$

O $2.48933397-1.24484961-0.38942810$

$02.340249560 .17927658-0.40025538$

O $1.59882049-1.74359445-1.35941131$

Vibrational frequencies (in cm-1)

$\begin{array}{lllllllllllllllll}-926.7 & 38.2 & 64.2 & 96.3 & 108.9 & 123.5 & 153.0 & 163.8 & 173.1 & 196.2 & 212.5 & 255.3 & 275.4 & 284.0\end{array}$ $306.3 \quad 328.6 \quad 397.8 \quad 414.3 \quad 439.8 \quad 503.6 \quad 538.2 \quad 570.8 \quad 660.7 \quad 671.6 \quad 711.4 \quad 749.1805 .6 \quad 823.3$ $896.0 \quad 917.3 \quad 946.7 \quad 960.4 \quad 978.3 \quad 990.0 \quad 1002.0 \quad 1026.4 \quad 1034.2 \quad 1067.3 \quad 1070.7 \quad 1084.4 \quad 1089.2$

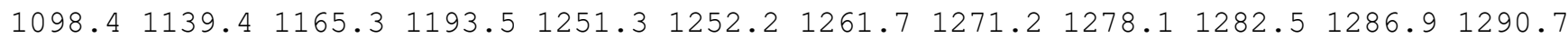

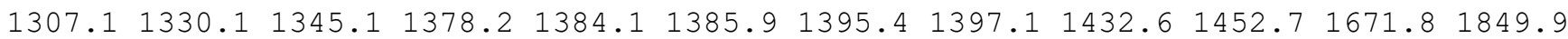
$2355.62620 .62656 .92658 .7 \quad 2662.92671 .3 \quad 2673.2 \quad 2677.62700 .92713 .92748 .12761 .6$ $2766.92774 .12779 .4 \quad 2786.3$

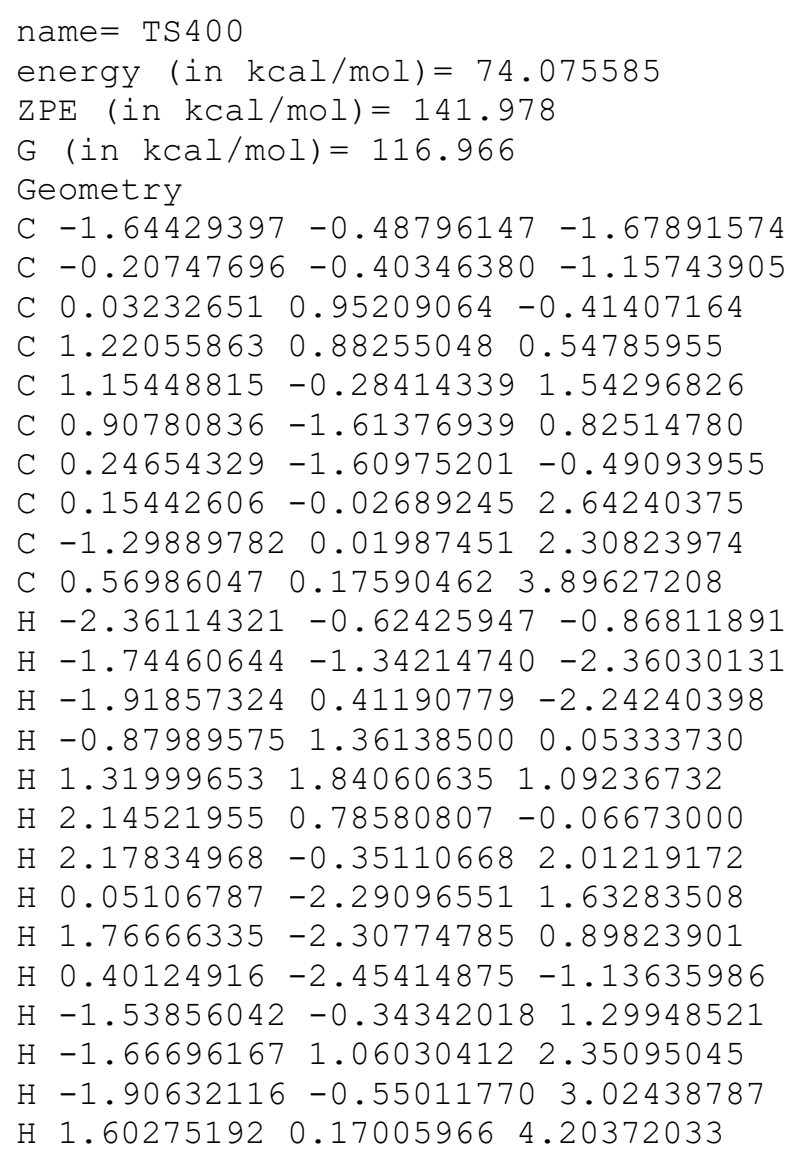


$\mathrm{H}-0.50639638-2.15478213 \quad 0.86912784$

$\mathrm{H}-0.09761588 \quad 0.37746424 \quad 4.71923774$

$0.47098124 \quad 1.11721774-2.66867972$

$0.39511564 \quad 1.87693490-1.42988779$

$0.68543179-0.20241531-2.28741882$

Vibrational frequencies (in $\mathrm{cm}-1$ )

$\begin{array}{llllllllllllllll}-3143.0 & 26.5 & 54.2 & 84.4 & 114.3 & 146.4 & 175.4 & 227.1 & 239.8 & 267.4 & 287.2 & 300.1 & 363.4 & 368.6\end{array}$ $416.4 \quad 427.5 \quad 467.2 \quad 549.2 \quad 576.9610 .4 \quad 634.6 \quad 668.0 \quad 712.6 \quad 760.0 \quad 782.6 \quad 808.8 \quad 836.1 \quad 864.3$ $891.6953 .8 \quad 956.6 \quad 974.0 \quad 989.3 \quad 1007.4 \quad 1021.3 \quad 1040.6 \quad 1060.4 \quad 1062.4 \quad 1078.2 \quad 1097.3 \quad 1126.9$

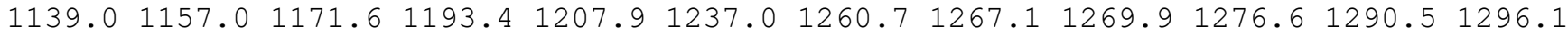

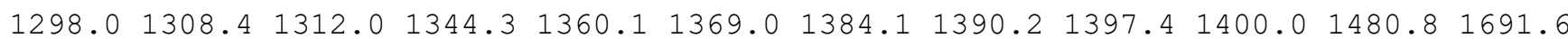
$1848.72652 .5 \quad 2655.4 \quad 2667.0 \quad 2668.2 \quad 2677.4 \quad 2681.6 \quad 2688.72696 .92719 .82738 .42772 .3$ 2783.92790 .22795 .02947 .3

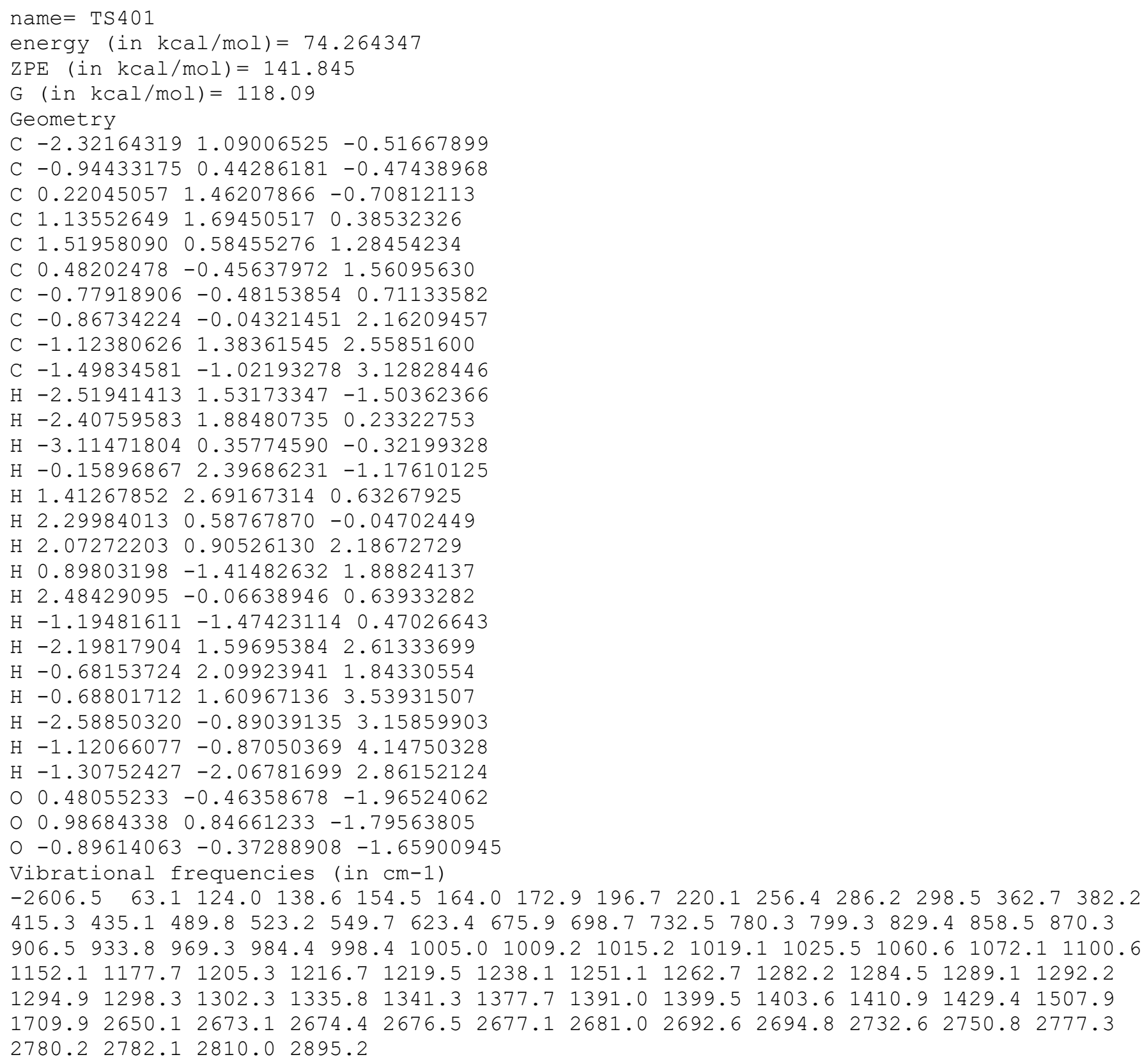


C $-0.35521429-0.19791195-0.48242037$

C $-0.45627914 \quad 0.89711730 \quad 0.63151389$

C $0.90294004 \quad 1.276887201 .22041543$

C 1.689520610 .061983391 .73560454

C $1.86232773-0.95099491 \quad 0.59912686$

C $0.88999635-0.95160366-0.50035345$

C $1.03390803-0.499711512 .97178833$

C $1.67008583-0.073471794 .25328612$

C $-0.03688653-1.296032162 .95294669$

$\mathrm{H}-2.52110608-0.49068890-0.56157751$

$\mathrm{H}-1.66269706-1.731753120 .37800284$

$\mathrm{H}-1.58542059-1.73932067-1.38861461$

$\mathrm{H}-1.20913603 \quad 0.66744370 \quad 1.40710782$

$\mathrm{H} 0.76893021 \quad 2.02048171 \quad 2.02774669$

H $1.49384716 \quad 1.78736820 \quad 0.42690191$

H $2.71707707 \quad 0.42742932 \quad 2.01158529$

$\mathrm{H} \quad 1.73645047-2.16828365 \quad 1.19111988$

H $2.91415213-1.048453710 .26906461$

$\mathrm{H} 1.22170336-1.25173138-1.47557272$

$\mathrm{H} \quad 1.12139334-0.428396025 .13701656$

$\mathrm{H} 1.71541574 \quad 1.02391367 \quad 4.33147248$

H $2.69821420 \quad-0.45087218 \quad 4.34353492$

$\mathrm{H}-0.56028299-1.599319492 .06021771$

H $0.99608279-2.186018720 .58536461$

$\mathrm{H}-0.50497355-1.682604993 .84470744$

$0-0.36923087 \quad 1.93175819-1.34438486$

$\begin{array}{lllll}0 & -0.98022928 & 2.02511997 & -0.06035290\end{array}$

o $-0.502893160 .57526702-1.70834481$

Vibrational frequencies (in $\mathrm{cm}-1$ )

$\begin{array}{llllllllllllll}-3405.4 & 47.4 & 62.8 & 93.1 & 104.0 & 114.0 & 142.4 & 191.5 & 258.7 & 270.6 & 300.1 & 305.5 & 358.6 & 391.3\end{array}$ $432.5 \quad 436.8 \quad 469.4 \quad 528.0 \quad 574.9 \quad 615.8 \quad 636.6 \quad 678.6 \quad 707.9 \quad 755.1 \quad 780.3 \quad 817.1 \quad 840.6 \quad 870.0$ $908.5944 .1959 .3 \quad 967.3 \quad 988.4 \quad 1004.8 \quad 1015.2 \quad 1041.9 \quad 1049.6 \quad 1062.5 \quad 1078.7 \quad 1094.7 \quad 1113.1$ $1140.8 \quad 1162.91165 .7 \quad 1192.3 \quad 1210.2 \quad 1240.8 \quad 1253.7 \quad 1268.6 \quad 1272.8 \quad 1278.1 \quad 1283.3 \quad 1286.5$

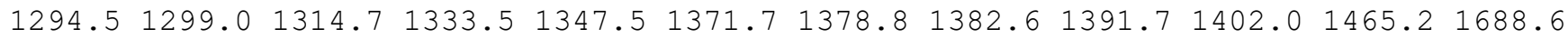

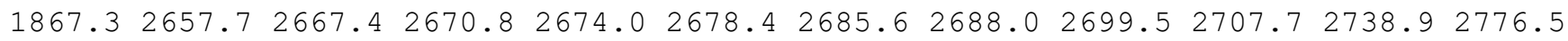
$2781.32785 .3 \quad 2797.2 \quad 2930.7$

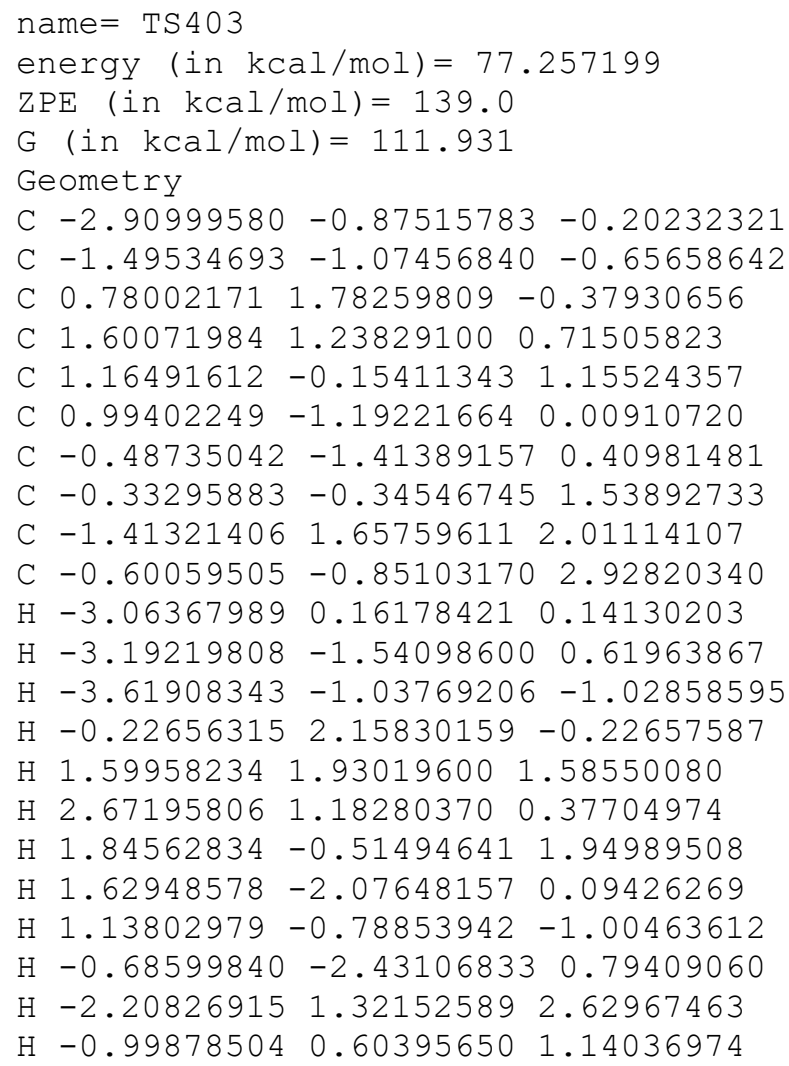


$\mathrm{H}-0.597387862 .121828332 .50680853$

$\mathrm{H}-1.66567470-1.059719543 .09478803$

$\mathrm{H}-0.27457336-0.142740943 .70127316$

$\mathrm{H}-0.06318069-1.791760313 .12697304$

O $2.323629061 .45832152-1.90771514$

$01.170767541 .86183112-1.61070017$

O $-1.18190430-0.96842210-1.81468302$

Vibrational frequencies (in $\mathrm{cm}-1$ )

$\begin{array}{llllllllllllllll}-567.0 & 33.2 & 37.5 & 52.1 & 72.7 & 93.4 & 104.1 & 142.2 & 172.4 & 202.8 & 217.8 & 235.8 & 259.8 & 283.0\end{array}$ $\begin{array}{lllllllllllllllllllll}305.1 & 353.1 & 363.6 & 387.1 & 452.7 & 534.0 & 583.9 & 600.0 & 664.0 & 703.6 & 720.2 & 794.2 & 845.1 & 855.2\end{array}$ $870.2921 .0 \quad 953.3 \quad 976.4 \quad 1024.5 \quad 1036.0 \quad 1041.5 \quad 1048.4 \quad 1079.4 \quad 1082.5 \quad 1097.4 \quad 1106.2 \quad 1113.7$

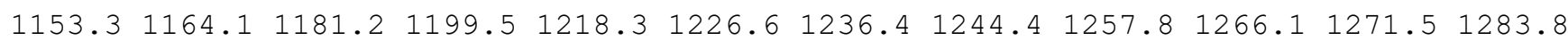

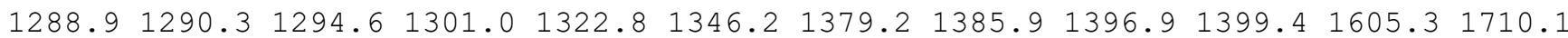
$1854.92632 .5 \quad 2654.42663 .92667 .12672 .92677 .22688 .12696 .72703 .2 \quad 2716.62721 .8$ $2725.7 \quad 2757.3 \quad 2777.7 \quad 2778.7$

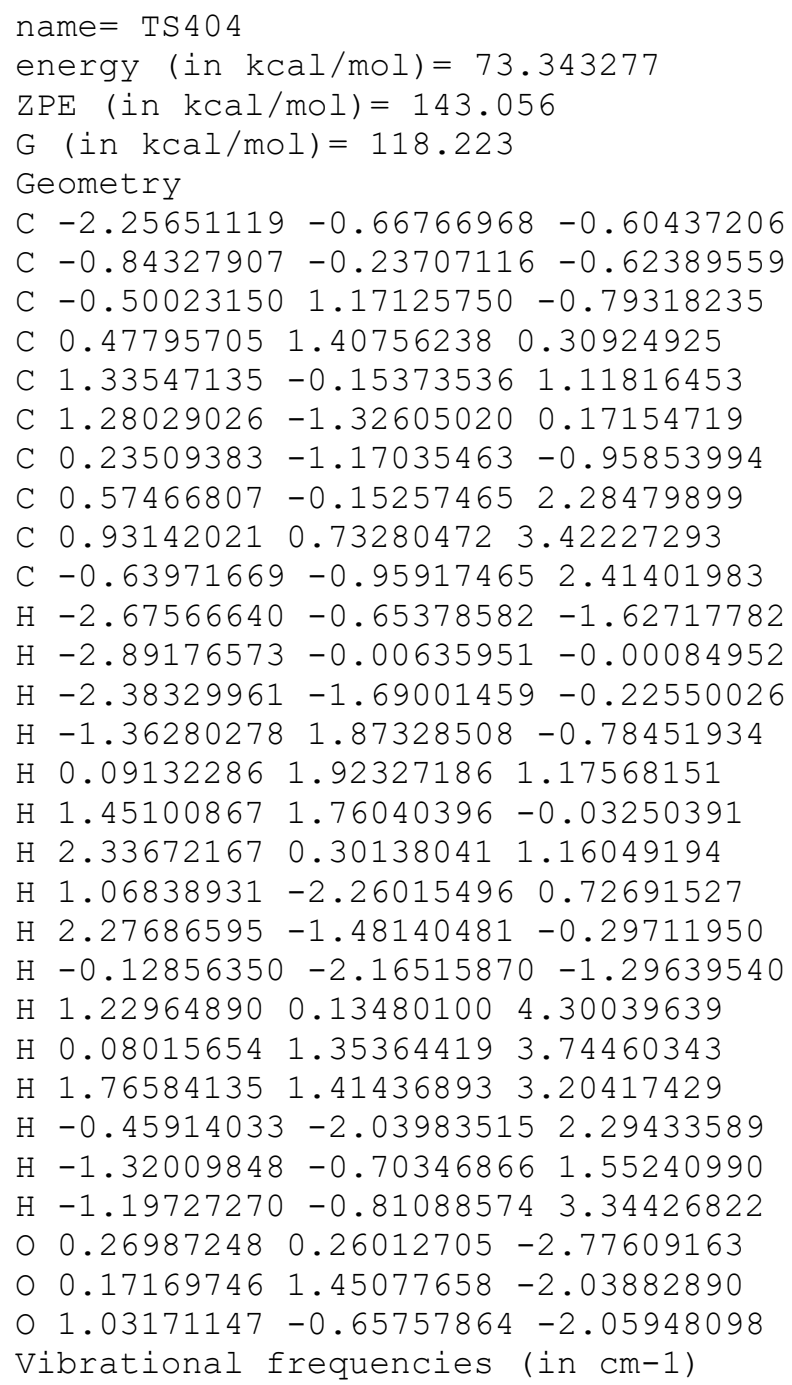




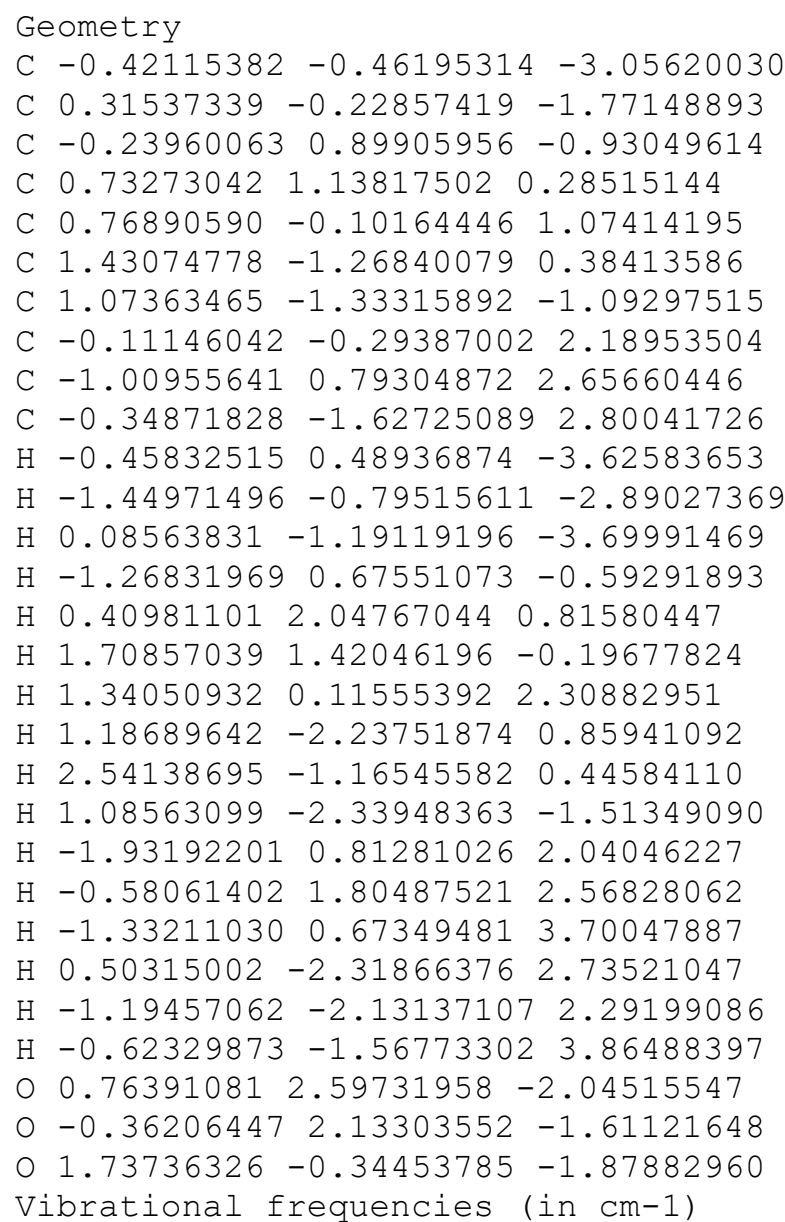

$\begin{array}{llllllllllllllll}-1374.5 & 34.4 & 61.3 & 66.6 & 79.5 & 103.1 & 119.4 & 135.9 & 167.7 & 200.7 & 259.3 & 273.4 & 296.5 & 352.9\end{array}$ $386.6 \quad 411.3 \quad 429.3 \quad 453.0 \quad 488.3 \quad 500.0 \quad 564.3 \quad 683.6 \quad 720.2 \quad 799.6 \quad 827.1 \quad 860.2890 .2 \quad 928.6$

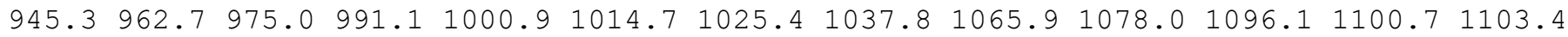
$1154.31183 .2 \quad 1206.5 \quad 1224.4 \quad 1238.2 \quad 1242.0 \quad 1253.0 \quad 1259.1 \quad 1260.7 \quad 1270.4 \quad 1275.3 \quad 1281.3$ $1284.41292 .91297 .91315 .1 \quad 1336.2 \quad 1353.9 \quad 1378.5 \quad 1387.8 \quad 1389.1 \quad 1395.3 \quad 1520.1 \quad 1555.2$ $2150.72618 .5 \quad 2634.5 \quad 2644.7 \quad 2649.0 \quad 2652.3 \quad 2656.92661 .92686 .2 \quad 2704.72727 .62731 .1$ $2733.12765 .2 \quad 2769.3 \quad 2777.8$ 
$\mathrm{H}-1.407100341 .46626258 \quad 0.84024999$

$\mathrm{H}-1.51008341 \quad 1.013731212 .52840229$

$\mathrm{H}-2.53559186 \quad 0.189765831 .34078428$

$\mathrm{H}-0.41524058-1.05699147 \quad 3.41249786$

$\mathrm{H} \quad 0.09850378-2.333941992 .31104695$

$\mathrm{H}-1.60972953-1.921070832 .43917793$

$0.87334969 \quad 0.54001848-2.99332124$

$01.375218041 .33564386-1.95101000$

O $0.36497324-0.61787498-2.32952942$

Vibrational frequencies (in $\mathrm{cm}-1$ )

$\begin{array}{llllllllllllllll}-733.2 & 91.2 & 118.8 & 149.4 & 172.5 & 181.3 & 197.9 & 215.0 & 270.9 & 281.2 & 311.7 & 334.6 & 369.8 & 378.0\end{array}$

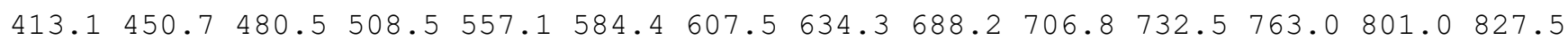
$855.8 \quad 882.2951 .0960 .7987 .6 \quad 1000.6 \quad 1007.5 \quad 1009.6 \quad 1017.61020 .3 \quad 1040.21054 .7 \quad 1079.4$

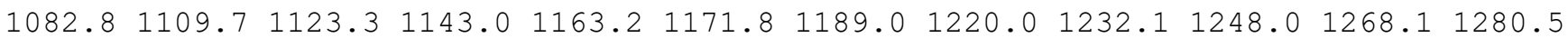

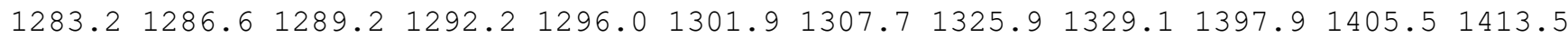
$1415.12664 .0 \quad 2669.92670 .6 \quad 2674.2 \quad 2675.8 \quad 2678.92687 .4 \quad 2735.8 \quad 2741.2 \quad 2751.92777 .9$ $2780.8 \quad 2782.8 \quad 2794.8 \quad 4576.0$

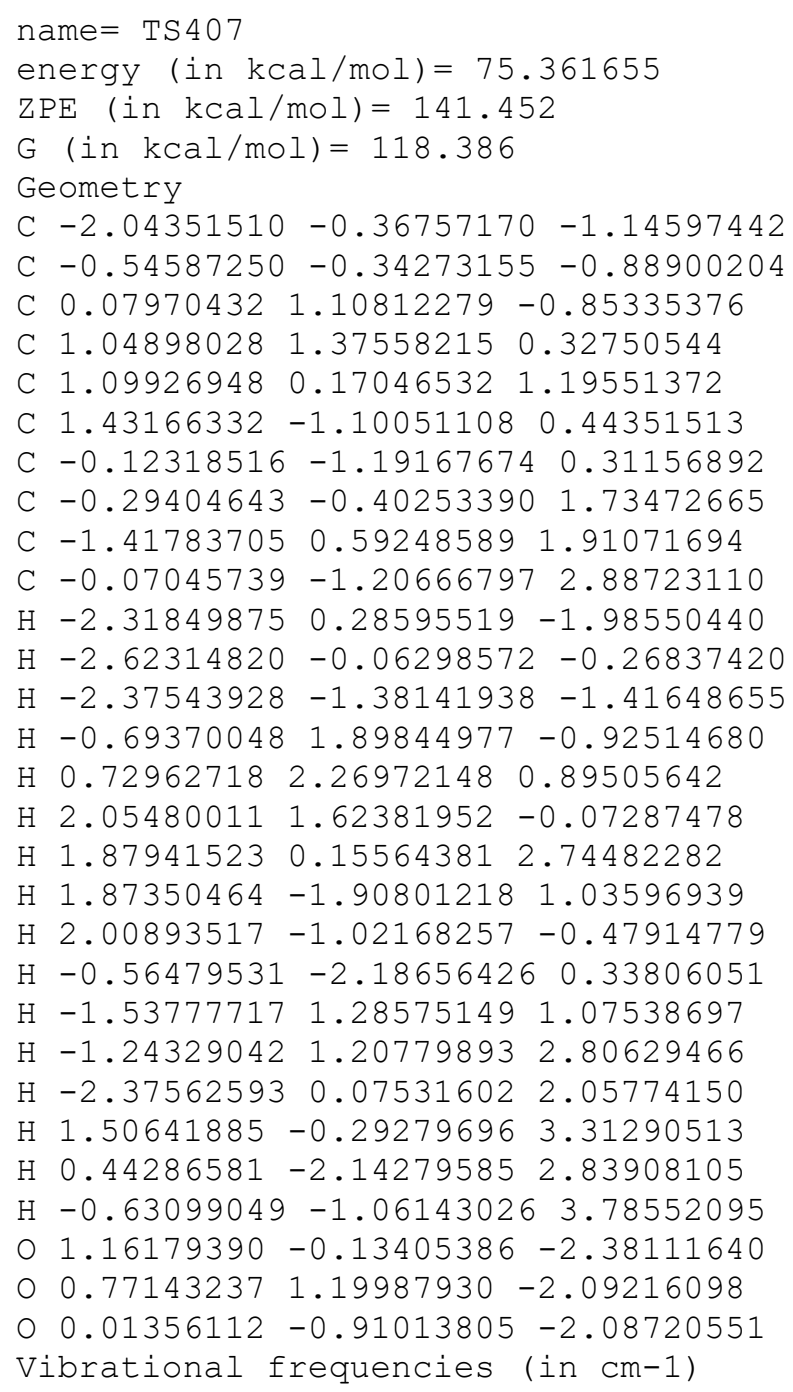


$\mathrm{ZPE}($ in $\mathrm{kcal} / \mathrm{mol})=143.981$

$\mathrm{G}($ in $\mathrm{kcal} / \mathrm{mol})=121.822$

Geometry

C $-2.28614912-0.17720123-0.73540012$

C $-0.86054035-0.59781184-0.81807588$

C $0.13094357 \quad 0.62157945-0.97706006$

$\begin{array}{lllll}\text { C } 1.12719931 & 0.84058137 & 0.17813455\end{array}$

C $1.09638687-0.29021702 \quad 1.20544137$

C $1.07727667-1.63483007 \quad 0.41583785$

C $-0.46985066-1.486128350 .36865355$

C $-0.37490623-0.600892231 .69126605$

C $-1.346600520 .52408545 \quad 1.86995509$

C $-0.37783710-1.47827434 \quad 2.94115764$

$\mathrm{H}-3.00841673-0.97671617-0.69221273$

$\mathrm{H}-2.61415030 \quad 0.56958009-1.43716671$

$\mathrm{H}-2.05181215 \quad 0.81020070 \quad 0.52668125$

$\mathrm{H}-0.41875744 \quad 1.55891139-1.20353173$

$\mathrm{H} \quad 0.93631813 \quad 1.81110199 \quad 0.66844632$

H $2.149990120 .91957606-0.24874414$

$\mathrm{H} 1.84160875-0.181279521 .99343006$

$\mathrm{H} 1.42853809-2.50107285 \quad 0.97975775$

H $1.59769749-1.63678501-0.54373270$

$\mathrm{H}-1.05802912-2.40013354 \quad 0.43341223$

$\mathrm{H}-1.97888344 \quad 0.46156648 \quad 2.74618588$

$\mathrm{H}-2.44354272 \quad 0.06212258 \quad 0.87260002$

$\mathrm{H}-0.91061657 \quad 1.51520863 \quad 1.82134763$

H $0.33133318-2.31090432 \quad 2.87143826$

$\mathrm{H}-1.36714852-1.91370494 \quad 3.11900505$

$\mathrm{H}-0.10235006-0.89503782 \quad 3.82762510$

O $0.63026649-1.03919507-2.45157701$

$\begin{array}{lllll}0 & 0.83020839 & 0.35332638 & -2.18756034\end{array}$

o $-0.69827990-1.29335500-2.06077851$

Vibrational frequencies (in $\mathrm{cm}-1$ )

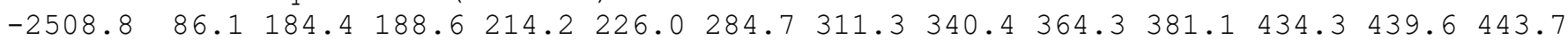
$477.9512 .7 \quad 573.1 \quad 602.6 \quad 658.1 \quad 703.0 \quad 710.9 \quad 745.3 \quad 796.7 \quad 820.8 \quad 860.7 \quad 867.1891 .1 \quad 905.4$ $939.8947 .7981 .6 \quad 993.8 \quad 1006.5 \quad 1009.6 \quad 1014.7 \quad 1027.1 \quad 1044.4 \quad 1055.8 \quad 1069.4 \quad 1086.51092 .0$

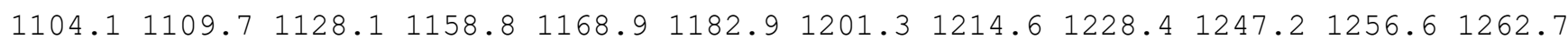

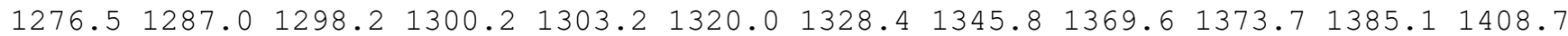
$1445.02659 .12673 .82680 .5 \quad 2682.3 \quad 2687.8 \quad 2690.2 \quad 2710.62740 .12748 .4 \quad 2752.0 \quad 2763.0$ $2767.22781 .6 \quad 2783.1 \quad 3599.1$

\section{name $=$ TS409}

energy (in $\mathrm{kcal} / \mathrm{mol})=75.634792$

$\mathrm{ZPE}($ in $\mathrm{kcal} / \mathrm{mol})=141.483$

$\mathrm{G}($ in $\mathrm{kcal} / \mathrm{mol})=118.449$

Geometry

C $-1.10675414-2.04194205-1.07029104$

C $0.29599211-1.61540954-0.68616101$

C $0.70000157-0.19369192-1.25660053$

C $1.324871790 .78391063-0.23055558$

C $1.427499330 .08907796 \quad 1.08020804$

C $2.09428559-1.266599860 .99533921$

C $0.62109433-1.76182653 \quad 0.80282564$

C $0.07223584 \quad-0.51538703 \quad 1.69683558$

C $-1.21369832 \quad 0.17476453 \quad 1.30528599$

C $0.21779383-0.68696848 \quad 3.10076230$

$\mathrm{H}-1.25562354-1.99051078-2.15803954$

$\mathrm{H}-1.88234740-1.43441924-0.59320601$

$\mathrm{H}-1.28916102-3.08663343-0.77791258$

$\mathrm{H}-0.137879390 .26928002-1.80891029$

$\mathrm{H} \quad 0.72393005 \quad 1.70942489-0.15706635$

H $2.32672786 \quad 1.10938744 \quad-0.58736866$

H $1.91371476 \quad 0.899741162 .54360996$

Н 2.57047304 -1.62943773 1.91036740 
H $2.78431310-1.44941837 \quad 0.16567720$

$\mathrm{H} \quad 0.35907067 \quad-2.72752096 \quad 1.23185675$

$\mathrm{H}-1.328356941 .11484838 \quad 1.86543609$

$\mathrm{H}-2.08107875-0.45348009 \quad 1.54882161$

$\mathrm{H}-1.27535259 \quad 0.43428234 \quad 0.24555939$

$\mathrm{H}-0.50472969-0.30228168 \quad 3.78800210$

H $1.52671084 \quad 0.65660105 \quad 3.21506040$

$\mathrm{H} \quad 0.87955318-1.413505853 .52119155$

O $1.51760144-1.83613186-2.56476758$

O $1.74516472-0.48492882-2.18766781$

O $1.22442921-2.49449849-1.34939866$

Vibrational frequencies (in $\mathrm{cm}-1$ )

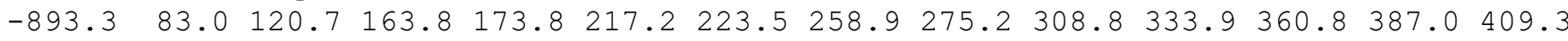

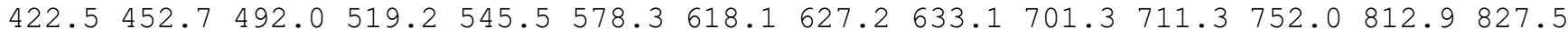
$872.7909 .5 \quad 940.3 \quad 950.1975 .7 \quad 983.3 \quad 988.8 \quad 1004.7 \quad 1009.91041 .2 \quad 1048.5 \quad 1061.6 \quad 1071.4$

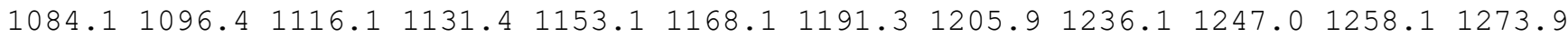
$1276.91285 .2 \quad 1288.6 \quad 1290.9 \quad 1304.8 \quad 1316.2 \quad 1326.4 \quad 1346.4 \quad 1365.1 \quad 1394.2 \quad 1396.51399 .5$ $1464.52657 .2 \quad 2668.12671 .8 \quad 2674.4 \quad 2677.5 \quad 2683.6 \quad 2685.32735 .02740 .42740 .92763 .4$ $2779.8 \quad 2782.6 \quad 2789.4 \quad 4435.6$

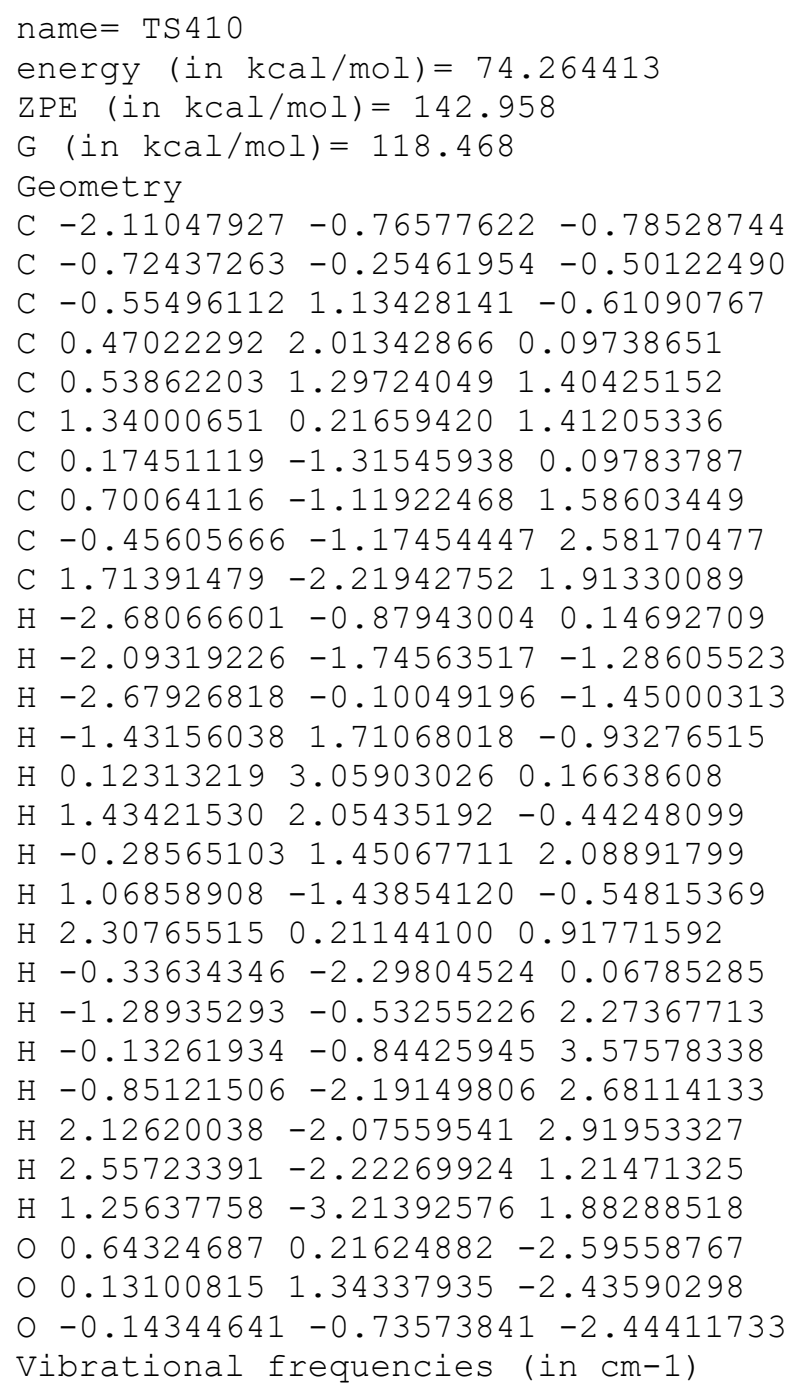


name $=$ TS411

energy $($ in $\mathrm{kcal} / \mathrm{mol})=80.713526$

$\mathrm{ZPE}($ in $\mathrm{kcal} / \mathrm{mol})=136.561$

$\mathrm{G}($ in $\mathrm{kcal} / \mathrm{mol})=107.687$

Geometry

C $-1.85769356-0.51214889-1.74831540$

C $-0.56479895 \quad 0.20839826-1.51857572$

C $-0.45402459 \quad 1.50577236-0.79359442$

C $0.76789633 \quad 1.96781761 \quad-0.04343651$

C 1.171078481 .039205861 .05597022

C $0.19073164-2.71614866 \quad 1.04114361$

C $-0.84704796-2.03360327 \quad 0.87274901$

$\begin{array}{lllll}\text { C } & 0.42297874 & 0.73439264 & 2.12553307\end{array}$

C $-0.97032449 \quad 1.21927874 \quad 2.33641822$

C $0.94556389-0.163126573 .20025913$

$\mathrm{H}-2.21776848-0.98771452-0.81501519$

$\mathrm{H}-1.76157633-1.30983797-2.50030068$

$\mathrm{H}-2.63798401 \quad 0.17509997-2.10410670$

$\mathrm{H}-1.386865461 .99755532-0.48797549$

$\mathrm{H} \quad 0.59068713 \quad 2.98510504 \quad 0.37494624$

H $1.609783112 .10640517-0.76075786$

H 2.17666585 $0.63328438 \quad 0.94402466$

$\mathrm{H} \quad 0.94824470 \quad-3.39810050 \quad 1.21215272$

H $1.90697195 \quad 0.11688076-2.33702155$

H $0.21966492 \quad-1.28647188 \quad 0.57050457$

$\mathrm{H}-1.69135155 \quad 0.52230754 \quad 1.87363416$

$\mathrm{H}-1.142526392 .21151606 \quad 1.90013936$

$\mathrm{H}-1.233658561 .28662292 \quad 3.40024287$

H $1.93760946-0.57498503 \quad 2.97555439$

$\mathrm{H} \quad 0.26937391-1.013876573 .37662836$

$\mathrm{H} \quad 1.03535300 \quad 0.37914672 \quad 4.15330866$

O $1.71653436-0.19410886-1.41275869$

$0-0.312433861 .41035012-2.23065073$

O $0.43251297-0.76520145-1.58751508$

Vibrational frequencies (in cm-1)

$\begin{array}{llllllllllllllllll}-1281.9 & 31.3 & 39.8 & 66.3 & 73.6 & 76.0 & 103.2 & 112.8 & 119.6 & 135.4 & 139.6 & 144.7 & 161.5 & 185.1\end{array}$ $204.9242 .0 \quad 262.5 \quad 294.2 \quad 326.3 \quad 330.1 \quad 373.3 \quad 408.7 \quad 454.8 \quad 482.8 \quad 491.7 \quad 571.3 \quad 629.2 \quad 793.9$

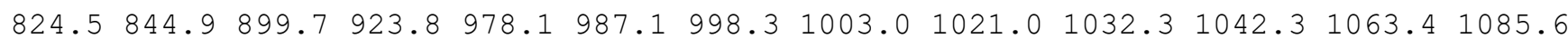
$1116.41127 .4 \quad 1161.2 \quad 1193.4 \quad 1247.5 \quad 1257.0 \quad 1266.1 \quad 1272.2 \quad 1273.7 \quad 1276.8 \quad 1282.5 \quad 1286.2$ $1290.61316 .31332 .6 \quad 1355.7 \quad 1362.0 \quad 1380.91388 .3 \quad 1400.3 \quad 1417.4 \quad 1590.6 \quad 1871.31995 .1$ $2257.7 \quad 2636.4 \quad 2649.8 \quad 2657.9 \quad 2665.8 \quad 2669.2 \quad 2672.0 \quad 2676.6 \quad 2718.92734 .6 \quad 2750.5 \quad 2769.7$ $2775.72778 .6 \quad 2840.3 \quad 2852.0$

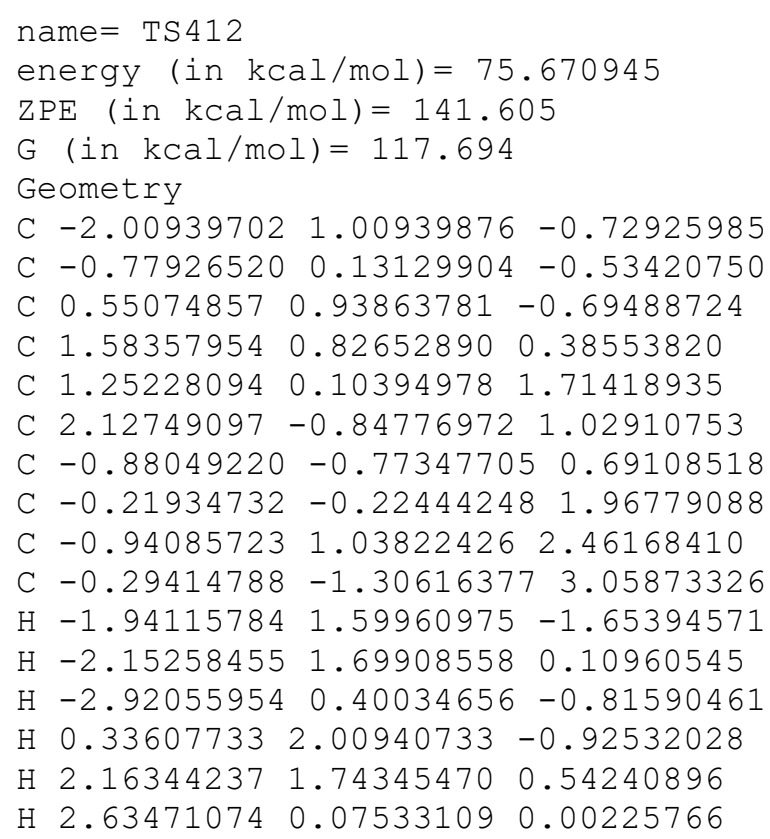


H $1.692202210 .59715447 \quad 2.60319099$

$\mathrm{H}-0.42531841-1.757943190 .44103560$

H $1.75212064-1.72542674 \quad 0.55702799$

$\mathrm{H}-1.94426342-1.00474725 \quad 0.89789487$

$\mathrm{H}-2.017329190 .86781936 \quad 2.56808271$

$\mathrm{H}-0.79571622 \quad 1.87792331 \quad 1.77438622$

$\mathrm{H}-0.56392018 \quad 1.35401281 \quad 3.44122495$

$\mathrm{H} \quad 0.15825317 \quad-0.95968234 \quad 3.99478580$

H $0.24544261-2.212995892 .76007673$

$\mathrm{H}-1.32931240-1.58675757 \quad 3.27685172$

O $0.58360389-0.87279438-2.03585154$

O $1.11168983 \quad 0.44279293-1.91560280$

o-0.77729073-0.71989422 - -1.70359735

Vibrational frequencies (in $\mathrm{cm}-1$ )

$\begin{array}{lllllllllllllll}-1351.2 & 40.9 & 114.0 & 141.4 & 144.9 & 166.6 & 201.5 & 229.6 & 247.8 & 259.5 & 266.0 & 326.4 & 348.1 & 383.1\end{array}$

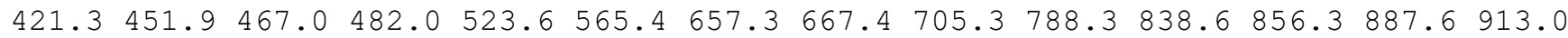

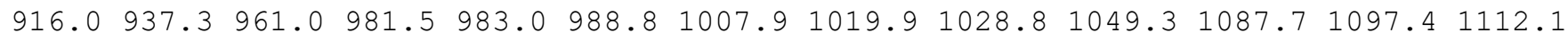
$\begin{array}{llllllllllllll}1150.6 & 1173.9 & 1202.2 & 1206.5 & 1220.4 & 1224.6 & 1250.9 & 1274.7 & 1278.9 & 1284.8 & 1285.8 & 1292.7\end{array}$

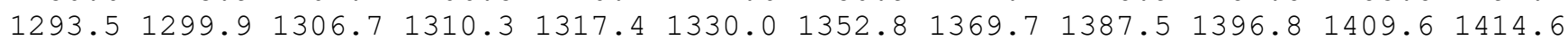
$2133.42650 .3 \quad 2666.42673 .3 \quad 2673.6 \quad 2674.12676 .82678 .82681 .72712 .42719 .92735 .7$ 2736.02778 .12780 .92782 .4

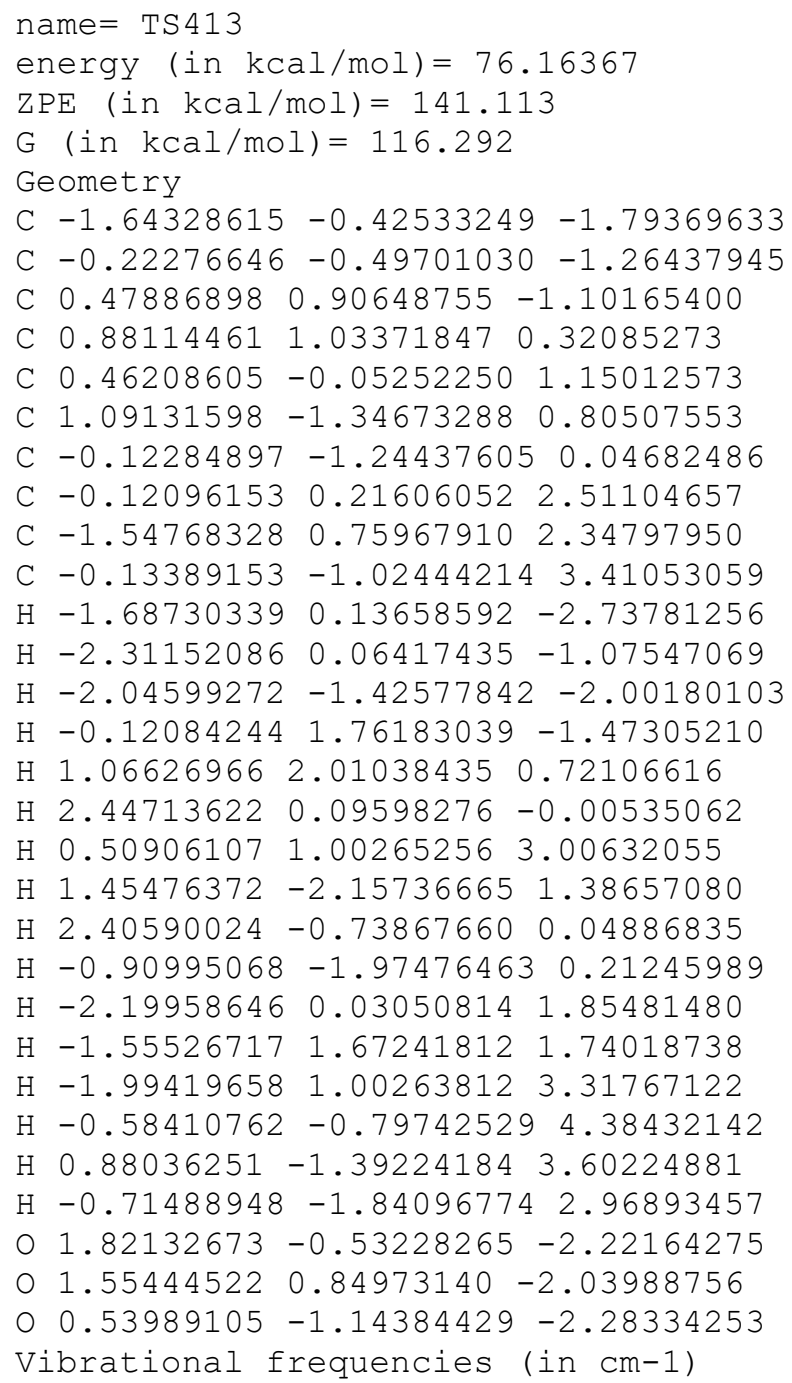




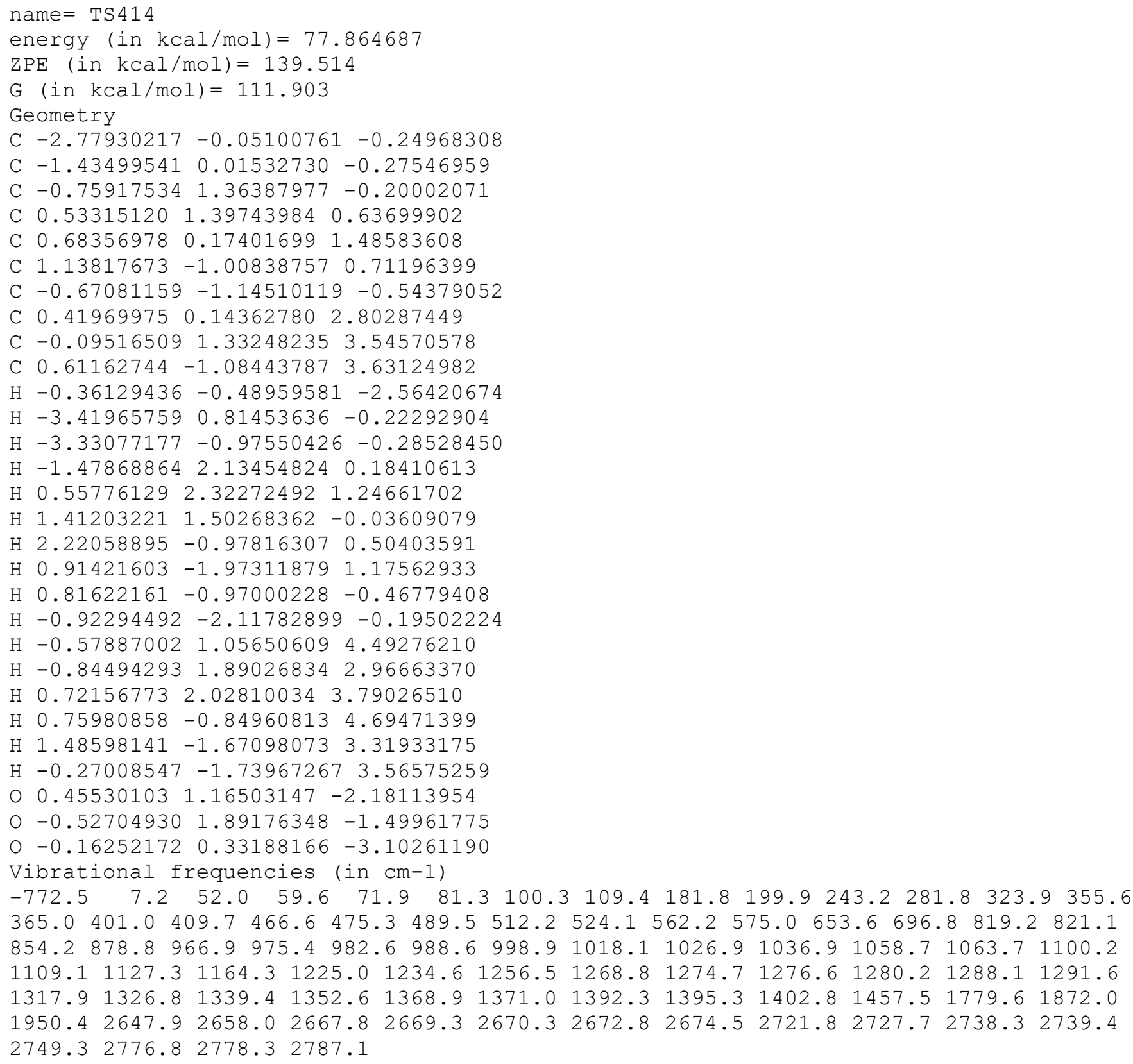

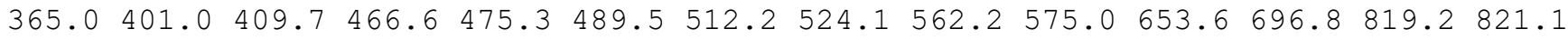
$854.2 \quad 878.8 \quad 966.9975 .4 \quad 982.6 \quad 988.6 \quad 998.9 \quad 1018.1 \quad 1026.9 \quad 1036.9 \quad 1058.7 \quad 1063.7 \quad 1100.2$ $1109.11127 .3 \quad 1164.3 \quad 1225.0 \quad 1234.6 \quad 1256.5 \quad 1268.8 \quad 1274.7 \quad 1276.6 \quad 1280.2 \quad 1288.11291 .6$ $1317.91326 .8 \quad 1339.4 \quad 1352.6 \quad 1368.9 \quad 1371.0 \quad 1392.3 \quad 1395.3 \quad 1402.8 \quad 1457.5 \quad 1779.6 \quad 1872.0$ $1950.42647 .92658 .0 \quad 2667.8 \quad 2669.3 \quad 2670.3 \quad 2672.82674 .52721 .82727 .72738 .32739 .4$ $2749.32776 .8 \quad 2778.3 \quad 2787.1$

\section{name $=$ TS4 15}

energy $($ in $\mathrm{kcal} / \mathrm{mol})=75.367943$

$\mathrm{ZPE}($ in $\mathrm{kcal} / \mathrm{mol})=142.158$

$\mathrm{G}($ in $\mathrm{kcal} / \mathrm{mol})=118.219$

Geometry

C $-1.62944790-0.54563796-1.89275561$

C $-0.60278418-1.47064554-1.24698146$

C $0.71186531-1.52345031-2.09507342$

C $1.97491477-1.96904084-1.34676652$

C $2.09580636-1.529774610 .10723198$

C $0.78441733-1.561793920 .88905774$

C $-0.46625560-1.28283090 \quad 0.19523432$

C $2.15611106-0.11073144 \quad 0.45428712$

C $1.53728000 \quad 0.94291465-0.36060447$

C $2.93007875 \quad 0.31017631 \quad 1.64971354$

$\mathrm{H}-1.35483093 \quad 0.50718850-1.78360384$

$\mathrm{H}-2.61626906-0.68356690-1.43116475$

$\mathrm{H}-1.74354936-0.76423102-2.96314725$

$\mathrm{H} \quad 0.87110347-0.58383313-2.65819294$ 
$\mathrm{H} 2.86879009-1.62505884-1.90455425$

H $2.01439331-3.08244437-1.38649091$

H $2.88647740 \quad-2.14890758 \quad 0.59484081$

H $0.07687305-2.64371378 \quad 0.68624426$

$\mathrm{H} \quad 0.83745699-1.45556403 \quad 1.96929409$

$\mathrm{H}-1.27308704-0.820482510 .73115709$

H $1.850717690 .90917270-1.41417359$

H $1.72947891 \quad 1.956623220 .01199425$

$\mathrm{H} \quad 0.42425174 \quad 0.80677950 \quad-0.36230071$

H $3.27546547 \quad-0.52906096 \quad 2.26723445$

H 2.34269435 $0.97285346 \quad 2.30417687$

$\mathrm{H} \quad 3.82787588 \quad 0.87593654 \quad 1.34949803$

o $-0.80285136-3.13960765-2.75268259$

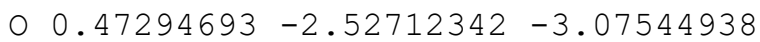

o $-1.03970344-2.84866126-1.41194677$

Vibrational frequencies (in cm-1)

$\begin{array}{llllllllllllllllll}-1536.3 & 70.4 & 100.9 & 125.9 & 138.7 & 157.7 & 175.4 & 221.8 & 236.2 & 259.4 & 283.8 & 305.3 \quad 334.7 & 360.2\end{array}$

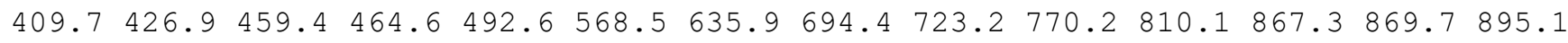
$940.7950 .2976 .6983 .9 \quad 989.1 \quad 1013.2 \quad 1027.7 \quad 1031.8 \quad 1051.2 \quad 1086.1 \quad 1092.7 \quad 1114.11129 .6$ $1157.21169 .91199 .1 \quad 1212.2 \quad 1240.3 \quad 1258.4 \quad 1264.1 \quad 1269.4 \quad 1281.8 \quad 1282.91287 .7 \quad 1291.3$ $1293.7 \quad 1298.2 \quad 1305.0 \quad 1333.4 \quad 1360.8 \quad 1368.7 \quad 1383.4 \quad 1386.91403 .3 \quad 1421.9 \quad 1441.8 \quad 1503.9$ $2213.52576 .7 \quad 2651.8 \quad 2660.5 \quad 2670.0 \quad 2672.1 \quad 2673.12682 .02688 .92708 .82736 .12746 .5$ $2759.5 \quad 2774.0 \quad 2781.12785 .7$

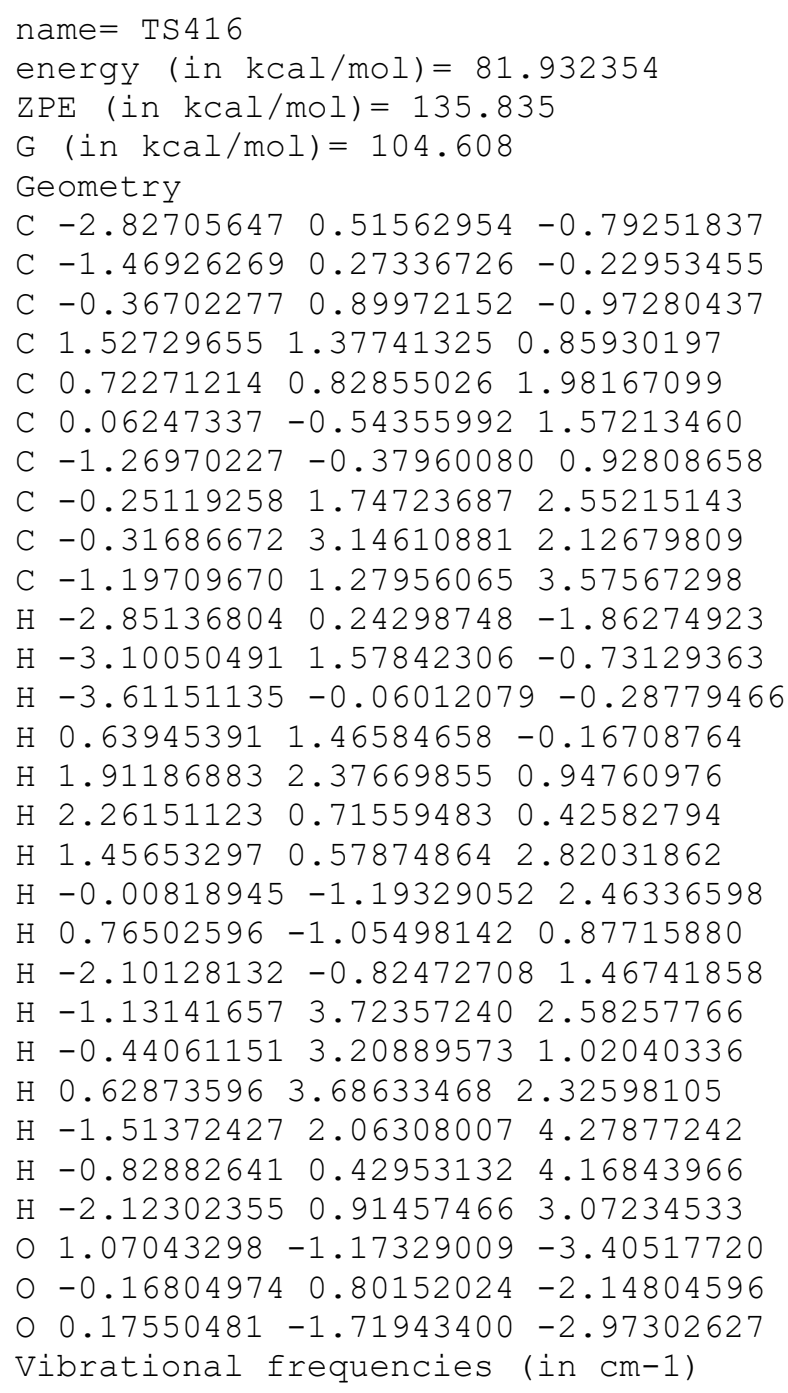
$251.6 \quad 271.4 \quad 300.1 \quad 319.7 \quad 359.6 \quad 388.0 \quad 419.9 \quad 467.5 \quad 509.7 \quad 525.1 \quad 608.3 \quad 652.6 \quad 672.3 \quad 809.4$ $849.9890 .8 \quad 914.2 \quad 936.9 \quad 959.8 \quad 994.3 \quad 1009.8 \quad 1021.7 \quad 1036.0 \quad 1060.0 \quad 1080.0 \quad 1100.0 \quad 1128.7$

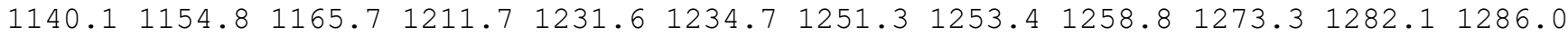
$1326.51330 .41348 .6 \quad 1372.5 \quad 1375.2 \quad 1387.9 \quad 1397.2 \quad 1399.2 \quad 1409.3 \quad 1470.2 \quad 1598.01821 .9$ 
$1866.22612 .8 \quad 2618.2 \quad 2621.0 \quad 2654.9 \quad 2660.6 \quad 2662.2 \quad 2669.72677 .8 \quad 2713.12734 .92755 .3$ $2757.4 \quad 2763.0 \quad 2776.12777 .9$

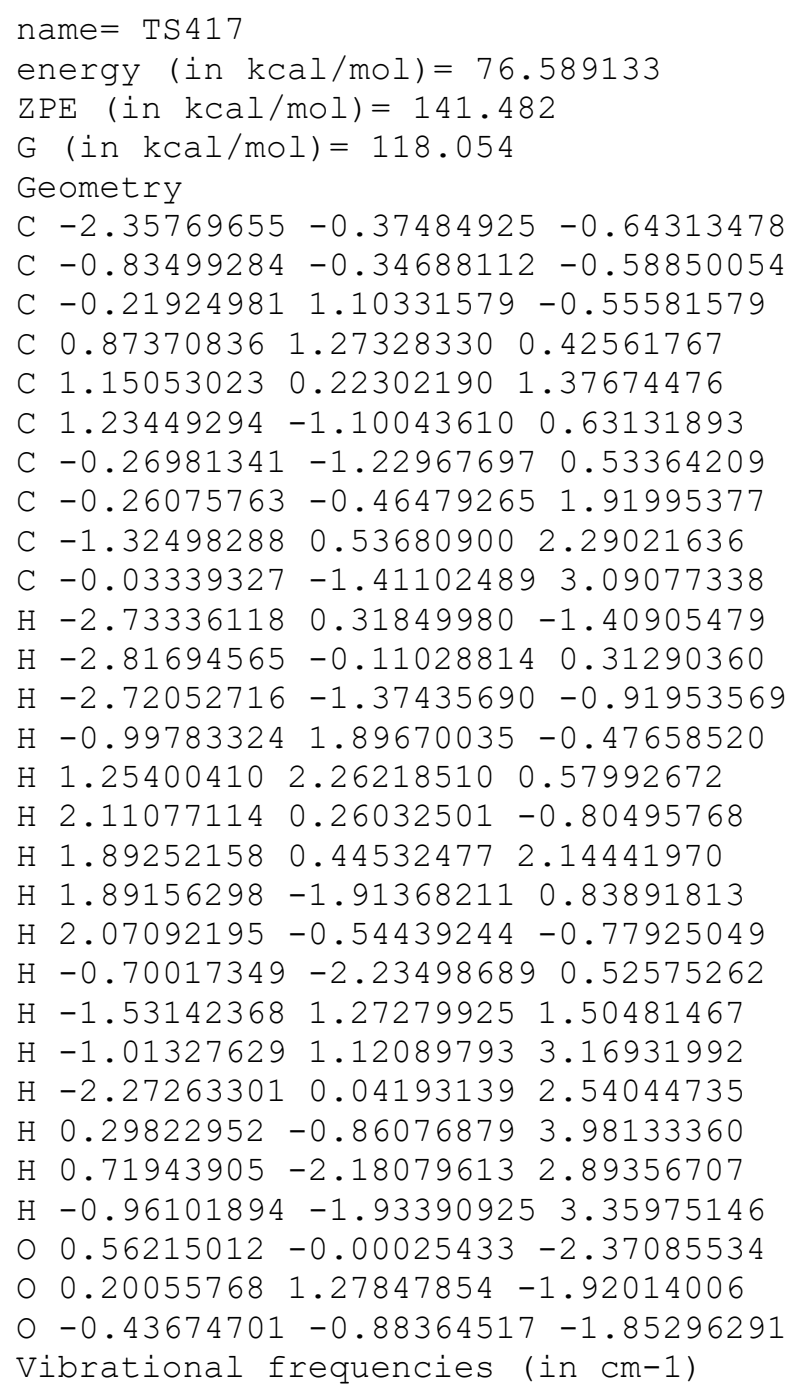


$\mathrm{H}-2.58804311-1.17898284-1.04082563$

$\mathrm{H}-1.05857010 \quad 2.10402541-0.16341542$

$\mathrm{H} \quad 1.10764941 \quad 2.39596879 \quad 1.05317024$

H $2.17752506 \quad 0.82324092 \quad-0.70456075$

$\mathrm{H} \quad 1.89853580 \quad 0.33772923 \quad 2.19804384$

H $2.08747246-1.67525761 \quad 0.41644114$

$\mathrm{H} 2.20392557 \quad 0.04531761-0.88169218$

$\mathrm{H}-0.46766809-2.12806417-0.01600416$

$\mathrm{H}-1.50901302 \quad 1.06478794 \quad 1.72717071$

$\mathrm{H}-1.12613471 \quad 0.44536209 \quad 3.31873016$

$\mathrm{H}-2.25782791-0.44387262 \quad 2.28506370$

$\mathrm{H} \quad 0.44798053-1.444641973 .66659828$

$\mathrm{H} \quad 0.91470852-2.49233905 \quad 2.32927887$

$\mathrm{H}-0.76530984-2.44241816 \quad 2.85655278$

$0.64276304 \quad 0.70409012-2.34304026$

$0 \quad 0.21394597 \quad 1.85100490-1.65402437$

o - $0.32605001-0.30175126-2.04385159$

Vibrational frequencies (in $\mathrm{cm}-1$ )

$\begin{array}{lllllllllllllll}-688.4 & 75.8 & 102.3 & 124.5 & 164.9 & 177.4 & 197.3 & 208.0 & 262.5 & 272.4 & 329.7 & 335.8 & 364.4 & 381.5\end{array}$

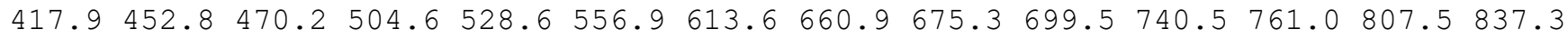

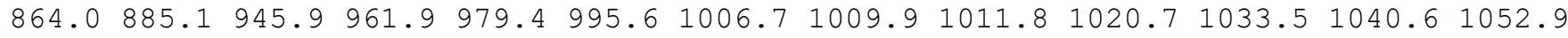
$1082.3 \quad 1117.8 \quad 1132.1 \quad 1150.5 \quad 1159.3 \quad 1177.8 \quad 1200.3 \quad 1226.2 \quad 1239.2 \quad 1250.1 \quad 1260.8 \quad 1279.6$ $1284.0 \quad 1285.0 \quad 1286.9 \quad 1290.4 \quad 1295.7 \quad 1300.9 \quad 1302.6 \quad 1308.6 \quad 1337.3 \quad 1397.9 \quad 1406.2 \quad 1414.5$ $1419.92665 .12668 .6 \quad 2670.1 \quad 2672.8 \quad 2673.6 \quad 2675.12678 .72742 .12744 .72751 .5 \quad 2778.0$ $2780.7 \quad 2781.8 \quad 2787.14765 .7$

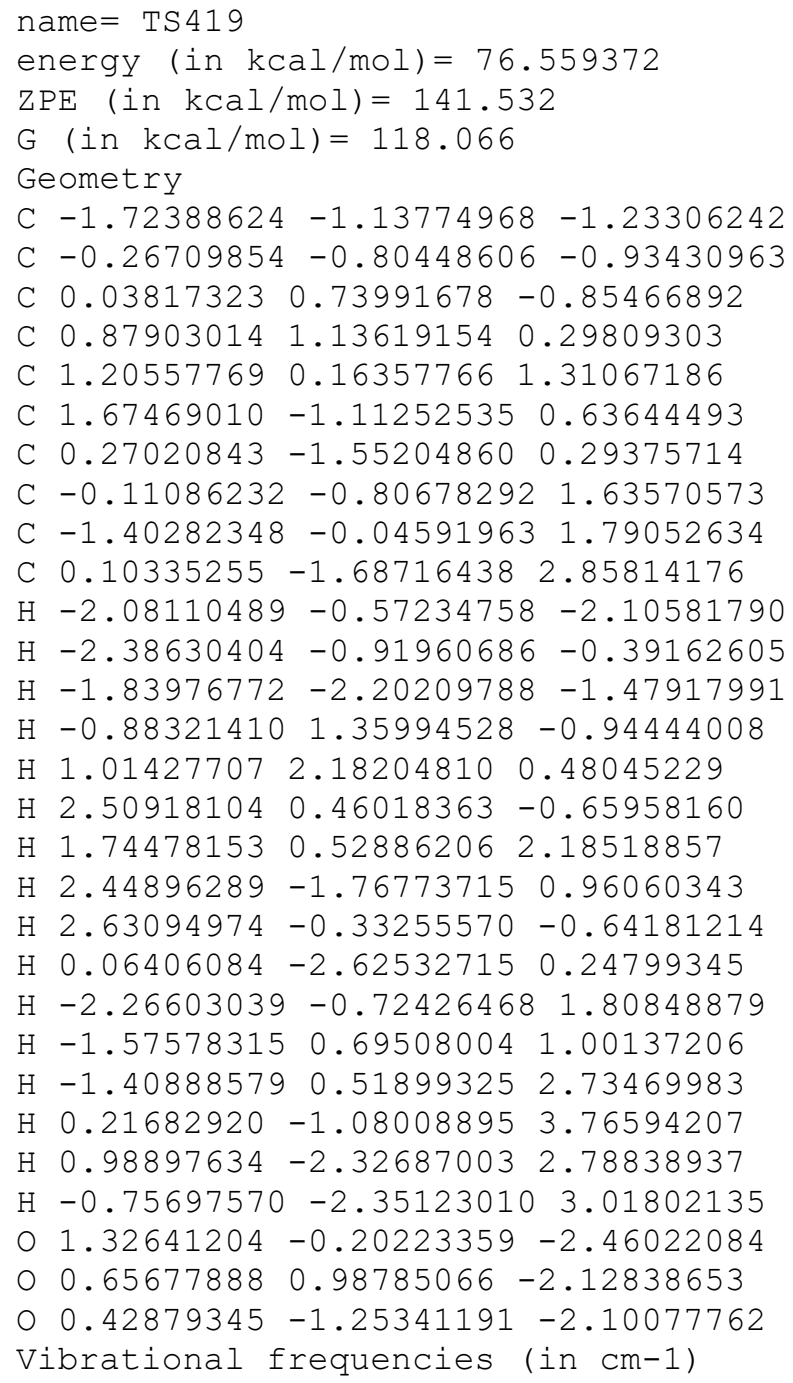


$\begin{array}{llllllllllllll}1083.3 & 1117.1 & 1130.8 & 1150.9 & 1160.1 & 1178.2 & 1198.8 & 1226.0 & 1238.5 & 1250.3 & 1258.2 & 1279.8\end{array}$

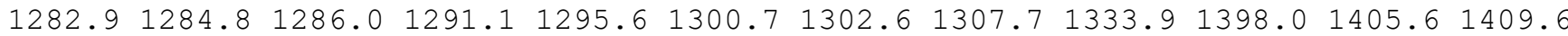
$1415.22665 .22669 .4 \quad 2670.4 \quad 2673.2 \quad 2674.3 \quad 2675.4 \quad 2678.32742 .12746 .2 \quad 2752.2 \quad 2778.4$ $2780.92782 .32786 .9 \quad 4680.9$

\section{name $=$ TS 420}

energy (in $\mathrm{kcal} / \mathrm{mol})=77.815359$

$\mathrm{ZPE}($ in $\mathrm{kcal} / \mathrm{mol})=140.282$

$\mathrm{G}($ in $\mathrm{kcal} / \mathrm{mol})=113.744$

Geometry

C $-2.08241369-0.61103700-0.56741276$

C $-0.75014754-0.74908447 \quad 0.02773477$

C $-0.089143852 .33833822-1.32993478$

C $0.79272328 \quad 2.60657149-0.36636393$

C $1.09402172-0.590303301 .45996924$

C $1.60191645-1.264247170 .24374520$

C $0.37369939-1.37923720-0.70282816$

C $-0.34206695-0.35075168 \quad 1.33496689$

C $-0.97738100 \quad 0.83335574 \quad 2.01562158$

C $-0.53929715-1.70946584 \quad 2.33284838$

$\mathrm{H}-2.69072014 \quad 0.15533420 \quad-0.06965957$

$\mathrm{H}-2.64684792-1.55600562-0.53081811$

$\mathrm{H}-2.00972341-0.32144950-1.63098510$

$\mathrm{H}-0.97217880 \quad 2.92437778-1.58615284$

H $0.72341220 \quad 3.46227850 \quad 0.27915884$

$\mathrm{H} 1.640057431 .98249469-0.14252941$

H $1.70809192 \quad-0.28195358 \quad 2.27227991$

H $2.06258536-2.246387850 .44413802$

$\mathrm{H} 2.40230326-0.65542619-0.23161387$

$\mathrm{H} \quad 0.16940835-2.42344888-1.03643981$

H $-2.06772966 \quad 0.73385688 \quad 2.07954821$

$\mathrm{H}-0.76955773 \quad 1.74624723 \quad 1.42991740$

$\mathrm{H}-0.58880690 \quad 0.99874262 \quad 3.02594391$

$\mathrm{H}-0.16296071-1.53192273 \quad 3.33664306$

$\mathrm{H}-0.13010680-2.62286157 \quad 1.90216066$

$\mathrm{H}-1.63174624-1.78878354 \quad 2.35092961$

$\begin{array}{llll}0 & 1.08780391 & 0.46494646 & -1.95902279\end{array}$

$\begin{array}{llll}0 & -0.05351276 & 1.31396382 & -2.21338361\end{array}$

O $0.64003462-0.81903249-1.99352353$

Vibrational frequencies (in $\mathrm{cm}-1$ )

$\begin{array}{llllllllllllllll}-482.7 & 34.2 & 51.9 & 57.9 & 83.5 & 100.9 & 138.0 & 149.8 & 163.8 & 187.2 & 204.8 & 226.9 & 260.5 & 277.6\end{array}$ $293.4 \quad 339.0 \quad 399.3 \quad 473.0 \quad 489.3 \quad 536.5 \quad 596.2 \quad 600.9612 .5 \quad 647.2 \quad 675.2 \quad 753.4 \quad 804.6 \quad 814.7$ $922.7 \quad 932.2 \quad 942.2 \quad 960.9 \quad 983.8 \quad 990.6 \quad 1012.0 \quad 1023.6 \quad 1030.8 \quad 1041.2 \quad 1045.1 \quad 1071.1 \quad 1072.5$

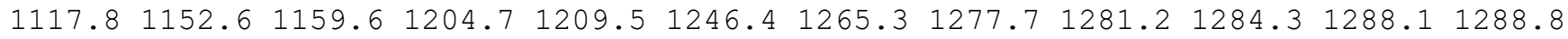

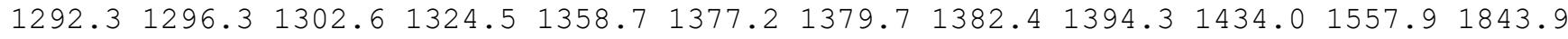
$2647.42650 .8 \quad 2659.0 \quad 2660.4 \quad 2673.8 \quad 2677.9 \quad 2679.22681 .5 \quad 2716.2 \quad 2734.52741 .22758 .2$ $2770.12776 .8 \quad 2779.0 \quad 2802.1$

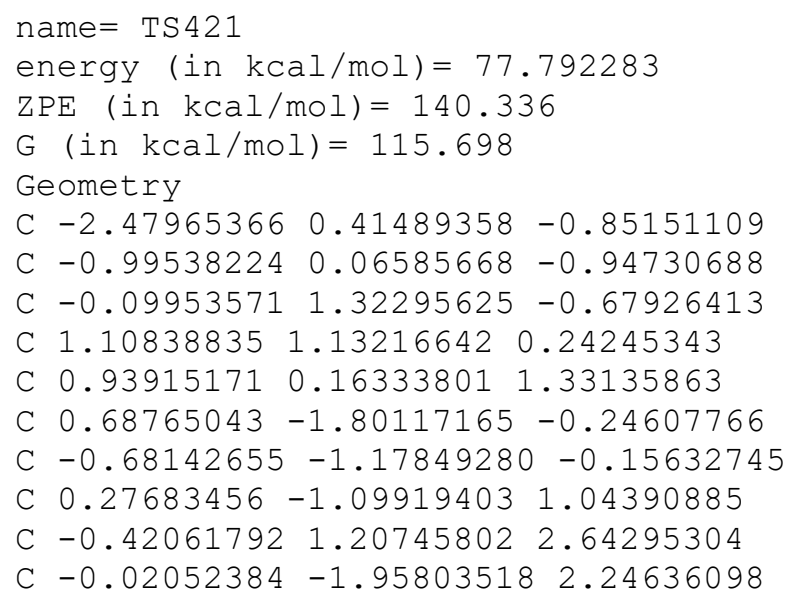


$\mathrm{H}-2.71928742 \quad 1.30892061-1.44406115$

$\mathrm{H}-2.78558414 \quad 0.59349054 \quad 0.18422864$

$\mathrm{H}-3.10348459-0.39763139-1.24989841$

$\mathrm{H}-0.70867725 \quad 2.19526411-0.36122788$

H $1.44271346 \quad 2.11983371 \quad 0.62530459$

H $\quad 1.98374375 \quad 0.78569574 \quad-0.36917409$

$\mathrm{H} \quad 0.76073745 \quad 0.67339994 \quad 2.59151183$

$\mathrm{H} 0.78801323-2.88338979-0.25032028$

$\mathrm{H} 1.43918493-1.35797204-0.89607978$

$\mathrm{H}-1.53960131-1.85560062-0.07866500$

$\mathrm{H}-0.249217312 .26742299 \quad 2.54930367$

$\mathrm{H}-0.63329579 \quad 0.91401540 \quad 3.66095863$

$\mathrm{H}-1.21155875 \quad 0.87867316 \quad 1.98734194$

$\mathrm{H}-0.79029294-1.506896262 .88456544$

$\mathrm{H} \quad 0.88367665 \quad-2.09104488 \quad 2.85856276$

$\mathrm{H}-0.37445266-2.95842284 \quad 1.96948814$

$\begin{array}{lllll}0 & 0.44112387 & 0.43930074 & -2.66592782\end{array}$

○ $0.35854471 \quad 1.68093162-1.98389338$

o $-0.77510487-0.21098062-2.34432609$

Vibrational frequencies (in $\mathrm{cm}-1$ )

$\begin{array}{lllllllllllllllll}-1227.0 & 43.1 & 81.3 & 108.5 & 120.6 & 136.9 & 163.8 & 184.3 & 226.4 & 248.9 & 280.5 & 293.5 & 322.2 & 357.5\end{array}$ $381.0 \quad 422.3 \quad 459.7 \quad 468.3 \quad 523.0 \quad 577.7 \quad 610.5 \quad 644.8 \quad 705.6 \quad 722.2 \quad 776.2 \quad 804.2 \quad 850.7 \quad 866.3$ $879.7944 .2954 .8 \quad 968.4 \quad 979.8 \quad 1004.0 \quad 1014.8 \quad 1048.1 \quad 1054.3 \quad 1070.7 \quad 1080.8 \quad 1108.3 \quad 1127.1$

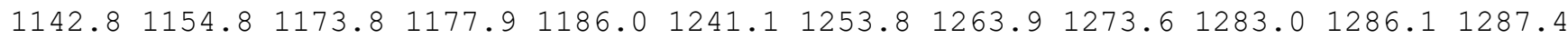

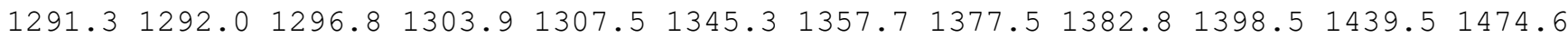

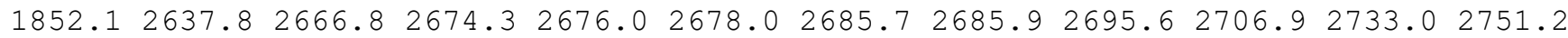
$2775.02779 .0 \quad 2779.9 \quad 2782.6$

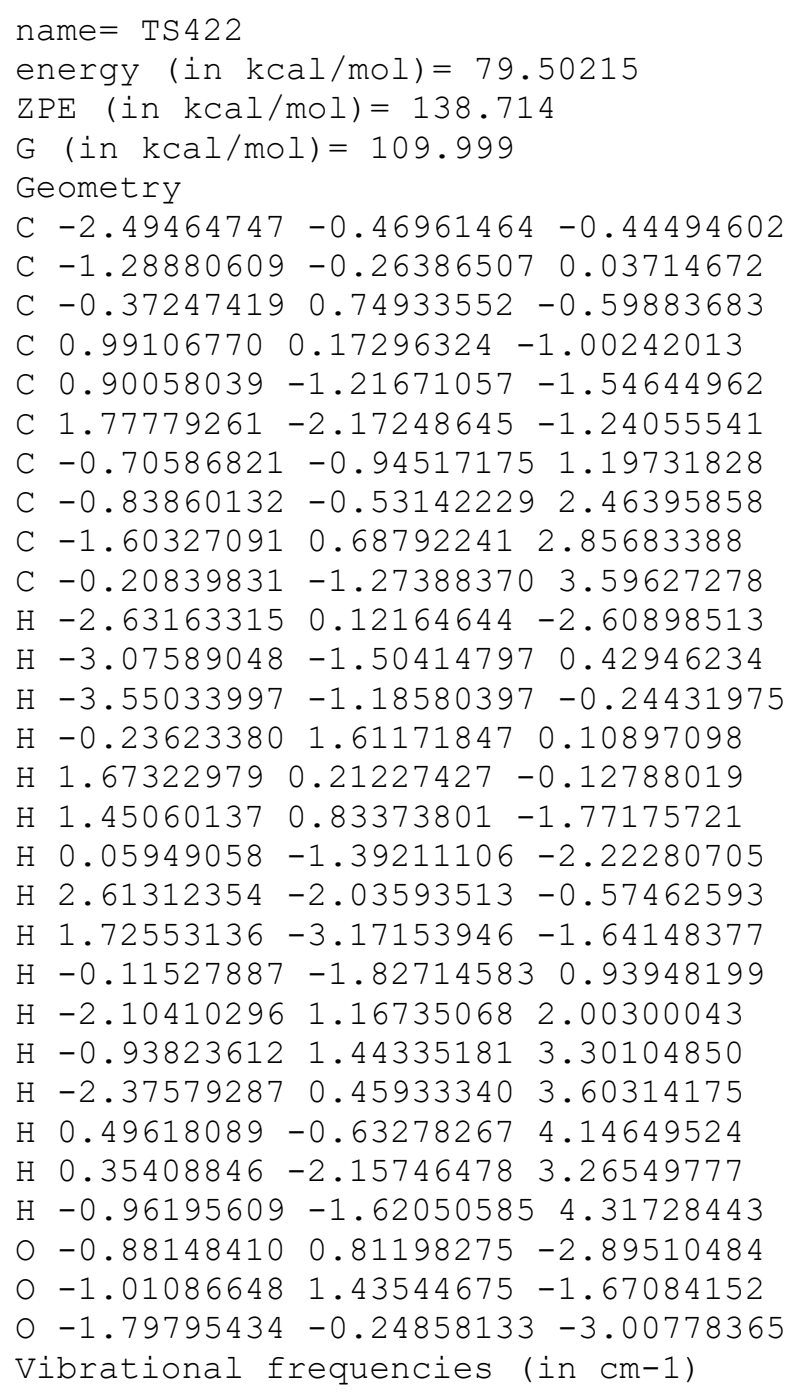


$\begin{array}{llllllllllllllll}-1570.7 & 25.3 & 30.6 & 40.7 & 53.5 & 87.2 & 94.0 & 101.9 & 107.2 & 130.8 & 159.8 & 188.6 & 229.0 & 259.6\end{array}$ $314.7330 .6 \quad 387.1 \quad 406.6 \quad 426.6 \quad 443.3 \quad 464.5 \quad 483.0 \quad 496.9 \quad 534.0 \quad 540.5 \quad 591.3 \quad 650.3 \quad 767.3$ $840.9 \quad 873.6 \quad 896.6 \quad 920.3 \quad 942.3 \quad 957.0 \quad 961.0 \quad 995.7 \quad 1006.2 \quad 1007.8 \quad 1015.7 \quad 1067.3 \quad 1080.4$ $1112.4 \quad 1117.4 \quad 1123.8 \quad 1177.6 \quad 1203.8 \quad 1258.4 \quad 1270.1 \quad 1272.1 \quad 1279.4 \quad 1283.1 \quad 1283.7 \quad 1289.2$

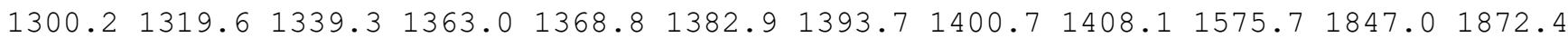
$1925.32656 .92666 .4 \quad 2667.1 \quad 2669.9 \quad 2673.2 \quad 2674.92716 .62731 .92743 .0 \quad 2750.4 \quad 2777.4$ $2780.4 \quad 2790.5 \quad 2793.0 \quad 3862.8$

name $=$ TS 423

energy (in $\mathrm{kcal} / \mathrm{mol})=76.920021$

$\operatorname{ZPE}($ in $\mathrm{kcal} / \mathrm{mol})=141.299$

$\mathrm{G}($ in $\mathrm{kcal} / \mathrm{mol})=118.103$

Geometry

C $-2.12479422-0.38298106-1.02874240$

C $-0.62373133-0.26835911-0.83800335$

C $-0.07243417 \quad 1.22068712-0.78047309$

C $0.92352931 \quad 1.49996922 \quad 0.36659766$

C $1.15872766 \quad 0.29580863 \quad 1.27729089$

C $1.42907646-0.96819108 \quad 0.40358940$

C $-0.08259026-1.00586907 \quad 0.33963073$

C $-0.25686360-0.34733405 \quad 1.78832430$

C $-1.40291895 \quad 0.60559984 \quad 2.03872988$

C $-0.13689012-1.35379118 \quad 2.78541392$

$\mathrm{H}-2.47274949 \quad 0.22680313-1.87309241$

$\mathrm{H}-2.67853828-0.08418111-0.13208175$

$\mathrm{H}-2.40622332-1.42435430-1.24739971$

$\mathrm{H}-0.89058884 \quad 1.96643889-0.80765245$

$\mathrm{H} \quad 0.57636408 \quad 2.36202575 \quad 0.96660478$

$\mathrm{H} \quad 1.891911151 .81833519-0.07704699$

H $1.84096187 \quad 0.47986080 \quad 2.10601321$

H $1.90424583-1.794735790 .94140879$

$\mathrm{H} 1.98523659-0.82174333-0.52413323$

$\mathrm{H}-0.68267261-2.59018625 \quad 0.81424877$

$\mathrm{H}-1.562952991 .333779321 .24053220$

$\mathrm{H}-1.22816683 \quad 1.17628275 \quad 2.96191338$

$\mathrm{H}-2.34524423 \quad 0.05562137 \quad 2.17370723$

$\mathrm{H}-0.85187501-1.445653313 .57504465$

$\mathrm{H} \quad 0.77519315-1.885639652 .95406607$

$\mathrm{H}-0.72109313-2.71764073 \quad 1.61233096$

o $1.01983456 \quad 0.07134754-2.37308785$

○ $0.56735490 \quad 1.37569224 \quad-2.04094962$

o $-0.07753093-0.77179171-2.06935936$

Vibrational frequencies (in $\mathrm{cm}-1$ )

$\begin{array}{llllllllllllllll}-868.0 & 86.5 & 99.5 & 149.5 & 175.3 & 212.6 & 213.7 & 249.9 & 278.6 & 299.3 & 322.1 & 352.6 & 387.5 & 409.7\end{array}$ $431.6 \quad 443.6 \quad 464.9 \quad 514.6 \quad 535.7 \quad 564.4 \quad 606.3 \quad 624.9661 .2 \quad 687.9 \quad 710.7 \quad 730.9 \quad 806.7 \quad 842.3$ $862.5903 .9931 .5937 .7 \quad 977.9980 .5 \quad 991.5 \quad 1001.4 \quad 1006.8 \quad 1029.8 \quad 1043.9 \quad 1061.2 \quad 1075.8$

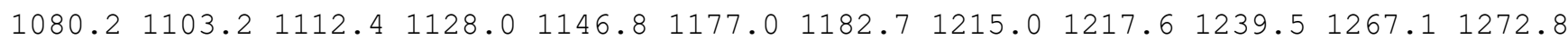

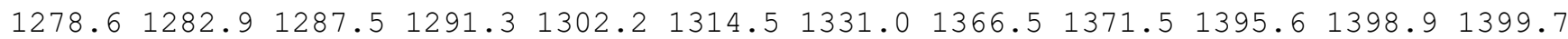
$1463.22654 .5 \quad 2668.6 \quad 2672.7 \quad 2675.5 \quad 2677.7 \quad 2686.92688 .12733 .3 \quad 2736.2 \quad 2740.12767 .7$ $2779.12782 .7 \quad 2786.3 \quad 4497.9$

name $=\operatorname{TS} 424$

energy $($ in $\mathrm{kcal} / \mathrm{mol})=76.229584$

$\mathrm{ZPE}($ in $\mathrm{kcal} / \mathrm{mol})=142.007$

$\mathrm{G}($ in $\mathrm{kcal} / \mathrm{mol})=117.631$

Geometry

C $-1.69055694-1.18132953-1.11301549$

C $-0.40410287-0.46186434-0.70492377$

C $-0.68249769 \quad 1.04148315 \quad-0.39697111$

C 0.364693391 .755216490 .45901686

C $0.98432487 \quad 0.93546101 \quad 1.58569253$

C $1.26017518-0.52303424 \quad 1.22475402$

C $0.37547414-1.201669190 .29188813$ 
$\begin{array}{lllll}\text { C } & 0.15175054 & 0.42454180 & 2.67302502\end{array}$

C $0.74078937 \quad 0.27614745 \quad 4.02772048$

C $-1.26955052 \quad 0.09342645 \quad 2.50793912$

$\mathrm{H}-2.23287195-0.61225620-1.87968372$

$\mathrm{H}-2.35479815-1.34198150-0.26011651$

$\mathrm{H}-1.46088242-2.16219102-1.55006192$

$\mathrm{H}-1.71443185 \quad 1.21374146 \quad-0.03450697$

$\mathrm{H}-0.07685237 \quad 2.67859217 \quad 0.88529756$

$\mathrm{H} 1.17937728 \quad 2.10272490 \quad-0.21704866$

H $1.90764722 \quad 1.45873545 \quad 1.93175328$

H $1.86840386-1.102819611 .91418455$

$\mathrm{H} \quad 1.81373702-0.69654950 \quad 0.05020927$

$\mathrm{H} \quad 0.20619443-2.25664848 \quad 0.39401040$

$\mathrm{H} \quad 0.55876777 \quad-0.72672942 \quad 4.44426105$

H $0.28740221 \quad 0.99727059 \quad 4.72792348$

H $1.82493841 \quad 0.44488549 \quad 4.05560069$

$\mathrm{H}-1.86221337 \quad 0.93131263 \quad 2.11394992$

$\mathrm{H}-1.75019536-0.24667111 \quad 3.43371940$

$\mathrm{H}-1.37402424-0.74159140 \quad 1.76664731$

$00.40602088 \quad 0.96342276-2.35971063$

$0-0.67465507 \quad 1.60708332-1.70480673$

$0.40940423-0.39012951-1.90634798$

Vibrational frequencies (in $\mathrm{cm}-1$ )

$\begin{array}{lllllllllllllll}-1548.6 & 51.8 & 94.3 & 105.9 & 126.6 & 142.4 & 162.3 & 189.7 & 238.7 & 252.6 & 289.0 & 295.5 & 338.2 & 357.4\end{array}$

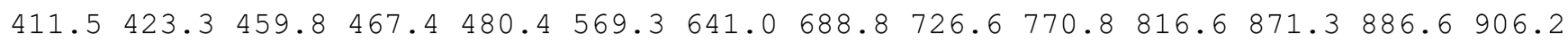
$936.0961 .2 \quad 975.4 \quad 983.3 \quad 989.3 \quad 1015.4 \quad 1023.0 \quad 1030.1 \quad 1051.2 \quad 1082.5 \quad 1085.2 \quad 1107.2 \quad 1136.3$

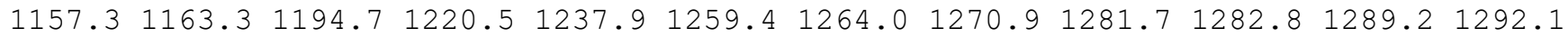

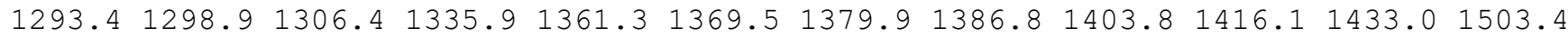
$2218.72574 .32652 .6 \quad 2661.1 \quad 2670.6 \quad 2672.8 \quad 2673.62683 .62695 .82709 .02735 .92746 .2$ 2759.22774 .02781 .12784 .5

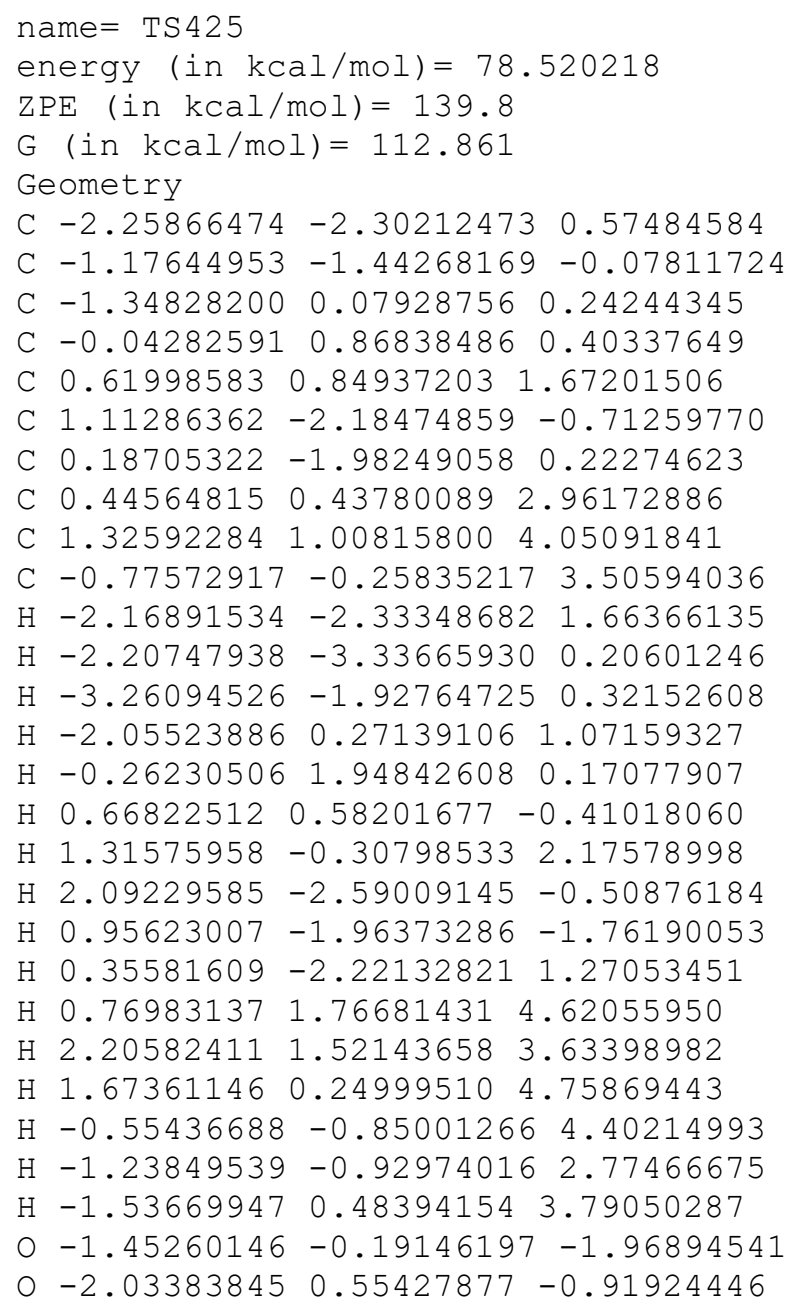


$0-1.44702187-1.53314663-1.48737175$

Vibrational frequencies (in $\mathrm{cm}-1$ )

$\begin{array}{lllllllllllllll}-1254.0 & 24.5 & 45.7 & 65.4 & 79.3 & 91.5 & 129.3 & 142.3 & 154.1 & 188.4 & 205.5 & 226.7 & 266.8 & 287.1\end{array}$ $317.3 \quad 337.4 \quad 384.2 \quad 424.1 \quad 450.0 \quad 526.2 \quad 534.0 \quad 548.9 \quad 610.7 \quad 672.2 \quad 720.8 \quad 810.6 \quad 849.8 \quad 876.9$ $906.7 \quad 946.3 \quad 969.6 \quad 977.6 \quad 984.6 \quad 994.7 \quad 1009.5 \quad 1011.1 \quad 1026.4 \quad 1056.7 \quad 1064.8 \quad 1097.8 \quad 1108.5$ $\begin{array}{lllllllllllllll}1123.3 & 1135.8 & 1181.7 & 1196.6 & 1230.5 & 1236.7 & 1252.6 & 1255.8 & 1275.0 & 1275.6 & 1282.2 & 1285.1\end{array}$

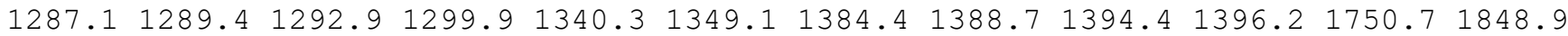
$2251.32632 .92666 .1 \quad 2667.6 \quad 2669.3 \quad 2672.3 \quad 2676.6 \quad 2679.92692 .4 \quad 2707.3 \quad 2726.3 \quad 2756.5$ 2776.02778 .22780 .12781 .5

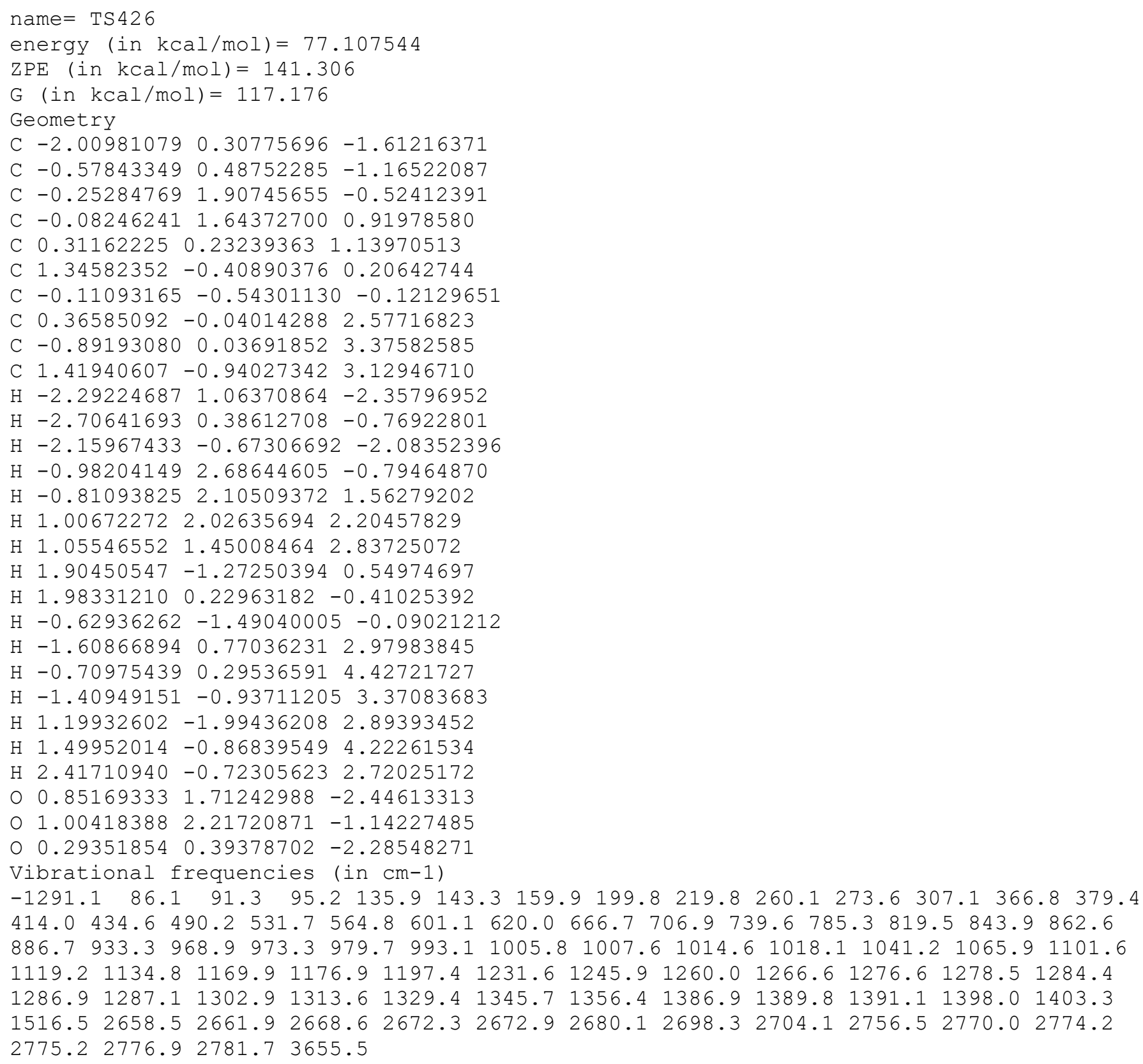


C $0.65418003-1.83557700 \quad 0.98717779$

C $-0.43374940-1.93395425-0.03560485$

C $0.08527944 \quad 0.14359332 \quad 2.54966835$

C $0.38529490 \quad 1.43317370 \quad 2.95241833$

C $-1.11202519-0.580720322 .98666783$

$\mathrm{H}-2.10895287-2.06197594 \quad-2.11816165$

$\mathrm{H}-1.72628633-0.37666246-2.57949896$

$\mathrm{H}-3.00303188-0.68904605-1.41540854$

$\mathrm{H}-1.40339190 \quad 0.80414430 \quad 0.60604517$

H $0.97229688 \quad 1.71895452 \quad 0.66116867$

$\begin{array}{lllll}\mathrm{H} & 1.47287197 & 0.43508721 & -0.53778344\end{array}$

H $2.03991695-0.398589431 .82207332$

$\mathrm{H} 0.42578443-2.48789705 \quad 1.85311416$

$\mathrm{H} \quad 1.57923429-2.26518602 \quad 0.53431745$

$\mathrm{H}-0.68747755-2.95727012-0.30041190$

H $0.537143391 .95174175 \quad 1.54297282$

$\mathrm{H} \quad 1.40169321 \quad 1.79659814 \quad 3.08573401$

$\mathrm{H}-0.29873620 \quad 2.00243636 \quad 3.56997135$

$\mathrm{H}-0.84673000-1.46172260 \quad 3.60151135$

$\mathrm{H}-1.68024645-0.99850028 \quad 2.12232121$

$\mathrm{H}-1.81560903 \quad 0.02461872 \quad 3.57829448$

O $1.14826118 \quad 0.15900337-3.01343394$

O $-0.807504821 .43701035-1.19900899$

o $0.583615641 .14255683-3.09355428$

Vibrational frequencies (in $\mathrm{cm}-1$ )

$\begin{array}{llllllllllllll}-1384.6 & 27.9 & 40.1 & 58.4 & 78.8 & 88.9 & 104.2 & 109.5 & 119.2 & 148.4 & 175.5 & 199.4 & 274.6 & 293.8\end{array}$

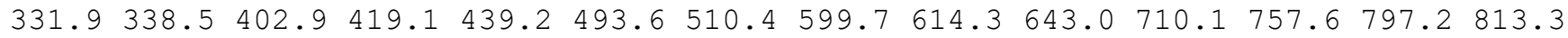

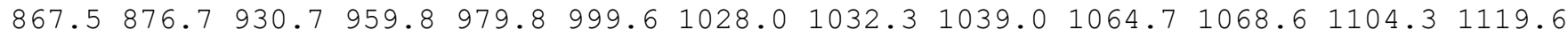

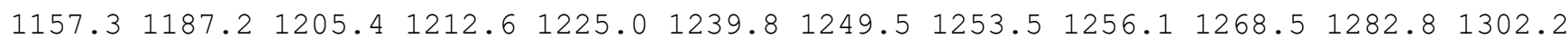

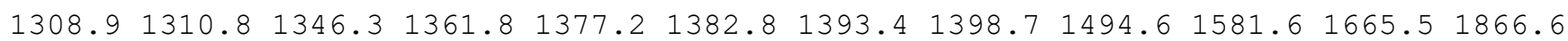
$1972.8 \quad 2263.8 \quad 2576.8 \quad 2610.3 \quad 2646.0 \quad 2650.9 \quad 2655.2 \quad 2680.52686 .92688 .02709 .2 \quad 2732.7$ $2756.4 \quad 2761.8 \quad 2771.12775 .4$

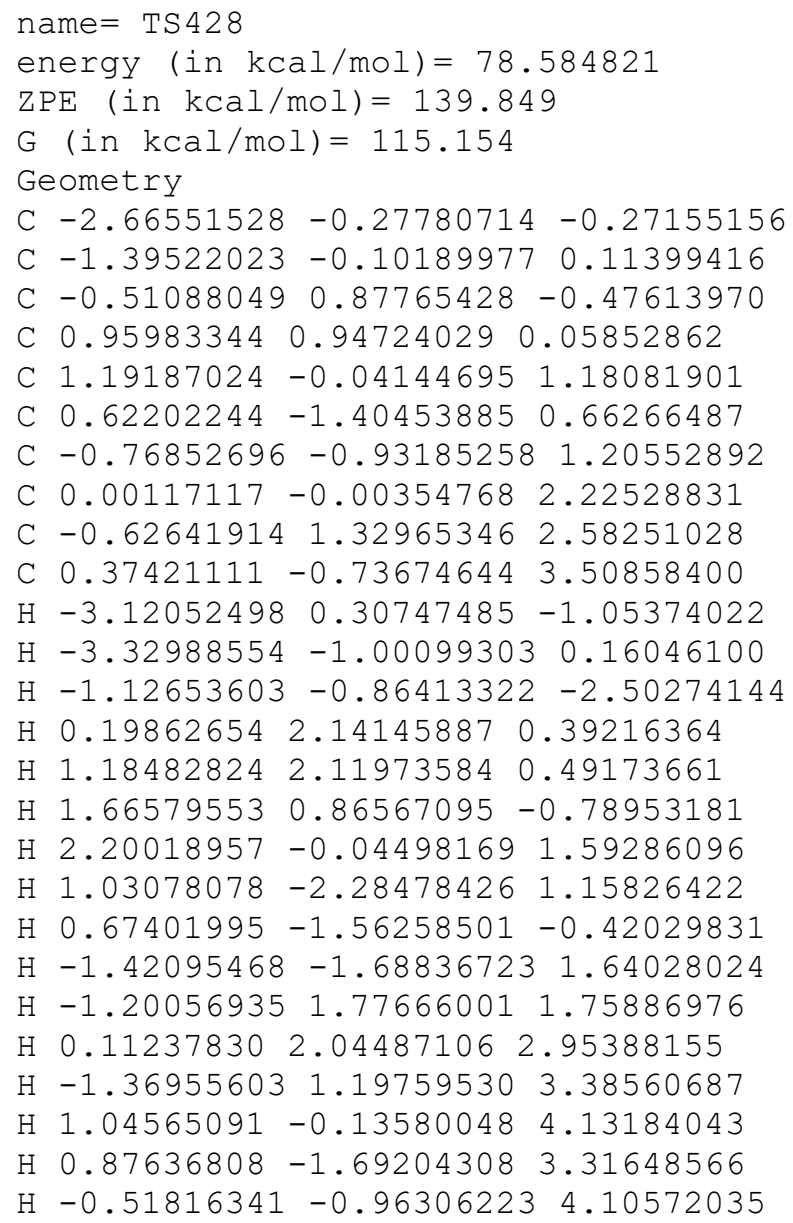


$0-0.206429850 .71047524-2.83647670$

$0-0.720660621 .47252349-1.60559300$

o $-0.16347230-0.61790488-2.50115291$

Vibrational frequencies (in $\mathrm{cm}-1$ )

$\begin{array}{lllllllllllllllll}-1604.2 & 42.6 & 75.3 & 97.6 & 126.7 & 154.6 & 167.4 & 177.0 & 198.0 & 281.5 & 288.8 & 330.7 & 372.4 & 384.0\end{array}$ $392.6 \quad 420.9 \quad 432.4 \quad 453.7 \quad 507.1 \quad 516.7 \quad 577.1 \quad 601.9619 .6 \quad 676.5 \quad 713.6 \quad 732.7 \quad 765.3 \quad 778.5$ $828.3 \quad 891.7 \quad 916.1 \quad 920.3 \quad 969.1 \quad 992.5 \quad 1002.9 \quad 1006.4 \quad 1014.6 \quad 1017.5 \quad 1027.3 \quad 1057.6 \quad 1061.6$

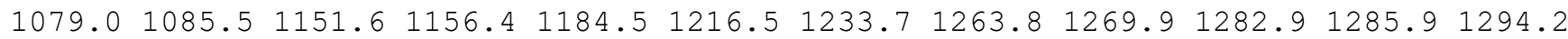
$1297.41300 .6 \quad 1310.3 \quad 1314.3 \quad 1333.5 \quad 1373.4 \quad 1373.9 \quad 1387.2 \quad 1404.8 \quad 1412.5 \quad 1488.0 \quad 1802.0$ $1845.82647 .02670 .0 \quad 2673.2 \quad 2677.0 \quad 2680.4 \quad 2683.42733 .12745 .8 \quad 2753.0 \quad 2760.4 \quad 2774.2$ 2782.12795 .92839 .62872 .0

name $=$ TS429

energy (in $\mathrm{kcal} / \mathrm{mol})=78.657707$

$\mathrm{ZPE}($ in $\mathrm{kcal} / \mathrm{mol})=139.797$

$\mathrm{G}($ in $\mathrm{kcal} / \mathrm{mol})=114.368$

Geometry

C $-1.92877096-0.07912266-1.22011667$

C $-0.45652071-0.30697521-0.94233156$

C $0.421976761 .00127600-0.92778382$

C $1.22506077 \quad 1.03257668 \quad 0.37854186$

C $0.80613077-0.17990865 \quad 1.20271958$

C $1.20333292-1.53006457 \quad 0.61712471$

C $-0.18790866-1.01966766 \quad 0.36407422$

C $0.74493616 \quad 0.01489153 \quad 2.63306423$

C $-1.00108551 \quad 1.26638147 \quad 2.89469011$

C $0.47517507-1.09423220 \quad 3.55521798$

$\mathrm{H}-2.07817454 \quad 0.46932327 \quad-2.16156061$

$\mathrm{H}-2.40726178 \quad 0.49047836-0.41523195$

$\mathrm{H}-2.46646221-1.03271760-1.32136965$

$\mathrm{H}-0.14934984 \quad 1.92671390 \quad-1.11578758$

H $1.05636894 \quad 1.97166715 \quad 0.93261971$

H 2.31277621 1.005452920 .16921282

H $0.28847767 \quad 1.24348485 \quad 3.04125778$

H $1.34728547 \quad-2.38208065 \quad 1.27606287$

$\mathrm{H} 1.92941066-1.56147238-0.19536575$

$\mathrm{H}-1.03519776-1.524536310 .81726285$

$\mathrm{H}-1.07681895 \quad 2.24922221 \quad 2.45469601$

$\mathrm{H}-1.360601521 .23712215 \quad 3.91042125$

$\mathrm{H}-1.51546567 \quad 0.54218421 \quad 2.28347883$

H $0.23034125-0.743407194 .56853660$

$\mathrm{H} 1.39947031-1.69381428 \quad 3.66347405$

$\mathrm{H}-0.32122263-1.781391123 .24018380$

$\begin{array}{llll}0 & 1.36336720 & -0.54342359 & -2.26227409\end{array}$

$01.25772588 \quad 0.86284010 \quad-2.06761535$

O $0.04541345-1.03085855-2.06971334$

Vibrational frequencies (in $\mathrm{cm}-1$ )

$\begin{array}{llllllllllllllll}-1224.6 & 40.0 & 56.3 & 79.1 & 81.1 & 149.9 & 151.8 & 166.9 & 175.3 & 243.7 & 262.5 & 271.1 & 309.7 & 350.8\end{array}$

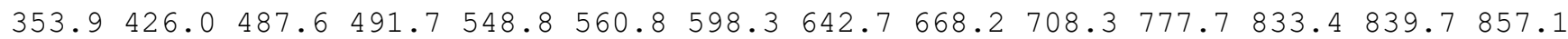
$906.0 \quad 947.3 \quad 954.9969 .5 \quad 978.6 \quad 995.3 \quad 1003.6 \quad 1028.2 \quad 1041.8 \quad 1069.5 \quad 1078.6 \quad 1102.8 \quad 1122.8$

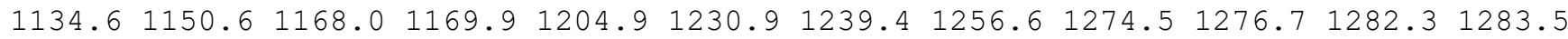
$1285.11291 .31293 .3 \quad 1305.7 \quad 1311.5 \quad 1328.0 \quad 1330.8 \quad 1373.91384 .4 \quad 1387.91400 .4 \quad 1489.5$ $1851.62659 .42664 .52668 .72669 .92677 .02688 .42696 .82700 .3 \quad 2704.62748 .52766 .6$ 2771.92777 .12779 .12780 .3

name $=\operatorname{TS} 430$

energy $($ in $\mathrm{kcal} / \mathrm{mol})=77.181953$

$\operatorname{ZPE}(\mathrm{in} \mathrm{kcal} / \mathrm{mol})=141.283$

$\mathrm{G}($ in $\mathrm{kcal} / \mathrm{mol})=118.025$

Geometry

C $-2.26989345-0.01103445-0.86721705$

C $-0.76213652-0.14153863-0.90424901$

C $0.00710410 \quad 1.25272763-0.90683854$ 
C $1.14347998 \quad 1.389836390 .12726290$

C $1.36947103 \quad 0.10981558 \quad 0.93135696$

C $1.35005902-1.11723951-0.03031159$

C $-0.14992546-0.99811793 \quad 0.15545420$

C $-0.01928261-0.39903206 \quad 1.63344387$

C $-0.99799890 \quad 0.65921965 \quad 2.08707612$

C $0.13526866-1.46079460 \quad 2.56518918$

$\mathrm{H}-2.64146285 \quad 0.58871324-1.70875751$

$\mathrm{H}-2.63192932 \quad 0.44084550 \quad 0.06198506$

$\mathrm{H}-2.74138571-1.00216233-0.94707564$

$\mathrm{H}-0.70521223 \quad 2.09598313-0.86368428$

$\mathrm{H} \quad 0.93914913 \quad 2.236648290 .80886280$

$\mathrm{H} 2.083664431 .65764265-0.40506000$

H $2.18749995 \quad 0.16407580 \quad 1.64869818$

H $1.81431158-2.02004518 \quad 0.37575342$

$\mathrm{H} 1.75054459-0.96103824-1.03711233$

$\mathrm{H}-0.84837117-2.53559487 \quad 0.66636311$

$\mathrm{H}-1.17478853 \quad 1.44748403 \quad 1.35112891$

$\mathrm{H}-0.63449608 \quad 1.149524223 .00111105$

$\mathrm{H}-1.97269814 \quad 0.21061322 \quad 2.32693973$

$\mathrm{H}-0.45674963-1.51498954 \quad 3.45396344$

$\mathrm{H} \quad 0.99363250 \quad-2.09865787 \quad 2.56734613$

$\mathrm{H}-0.77871017-2.69793736 \quad 1.45474996$

$\begin{array}{lllll}0 & -0.13972313 & 0.41647366 & -2.99349953\end{array}$

$00.65237653 \quad 1.27071987-2.18361304$

o $-0.38999165-0.70938245-2.17189727$

Vibrational frequencies (in cm-1)

$\begin{array}{llllllllllllllll}-847.9 & 76.0 & 103.7 & 155.2 & 173.1 & 205.9 & 216.0 & 254.1 & 278.2 & 289.7 & 328.4 & 356.0 & 378.9 & 402.0\end{array}$

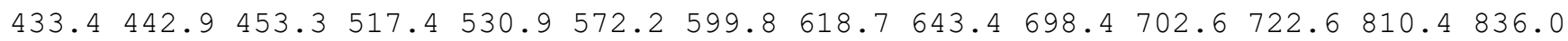

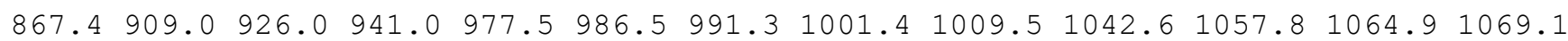

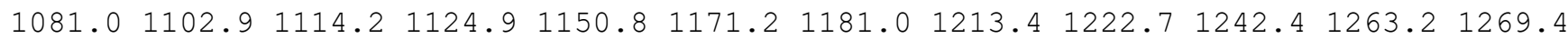
$1279.1 \quad 1284.4 \quad 1288.9 \quad 1291.5 \quad 1304.4 \quad 1316.7 \quad 1333.4 \quad 1363.1 \quad 1370.1 \quad 1394.6 \quad 1398.01400 .5$ $1464.52651 .5 \quad 2670.0 \quad 2673.1 \quad 2675.5 \quad 2677.4 \quad 2684.52686 .12733 .2 \quad 2734.2 \quad 2739.42764 .2$ $2779.92782 .5 \quad 2785.9 \quad 4522.0$

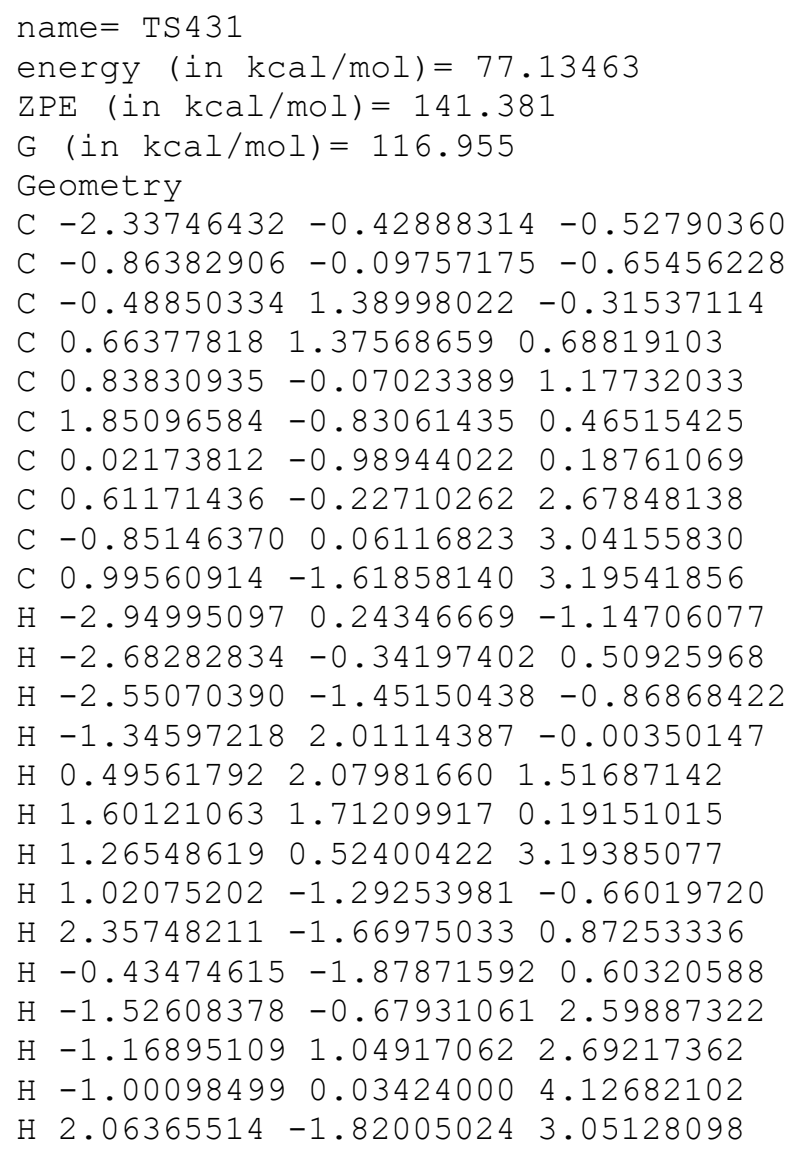


$\mathrm{H} \quad 0.79480648-1.70563046 \quad 4.26997617$

$\mathrm{H} \quad 0.43177132 \quad-2.41143266 \quad 2.69485616$

$0.401132650 .81299922-2.29065852$

$\begin{array}{llll}0 & -0.09814578 & 1.93033911 & -1.57241769\end{array}$

O $-0.55407119-0.21314604-2.04757838$

Vibrational frequencies (in $\mathrm{cm}-1$ )

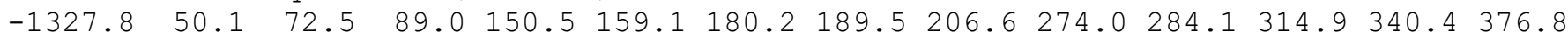
$418.2 \quad 449.6 \quad 454.4 \quad 518.1 \quad 545.7 \quad 555.3 \quad 588.2 \quad 649.0 \quad 710.1 \quad 796.5 \quad 828.8 \quad 844.2 \quad 868.0 \quad 906.4$ $947.1965 .1 \quad 972.2 \quad 979.1 \quad 984.4 \quad 1003.7 \quad 1006.8 \quad 1011.4 \quad 1060.1 \quad 1066.0 \quad 1101.2 \quad 1134.3 \quad 1150.5$

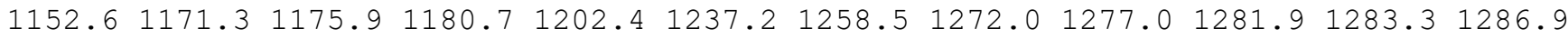

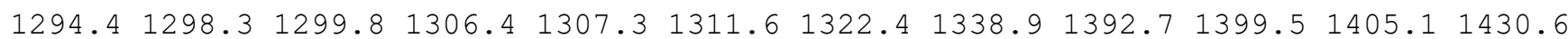
$2140.4 \quad 2658.5 \quad 2668.1 \quad 2671.4 \quad 2673.5 \quad 2674.6 \quad 2677.3 \quad 2678.3 \quad 2700.8 \quad 2703.4 \quad 2732.7 \quad 2743.5$ $2744.62779 .0 \quad 2780.4 \quad 2782.2$

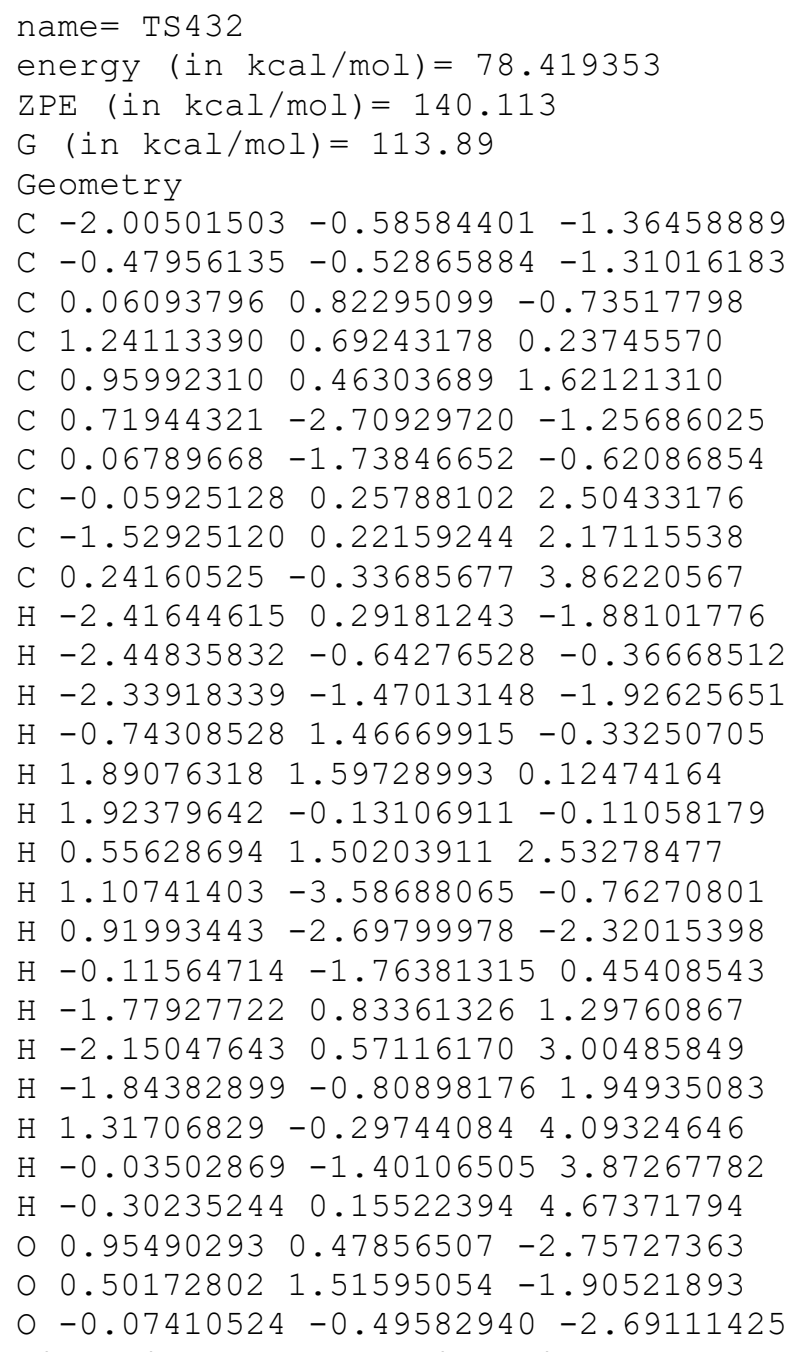


C $-1.32247753 \quad 0.21873756-0.11700302$

C $-0.31595721 \quad 1.11687800 \quad-0.62609379$

C $0.98058550 \quad 1.30428124 \quad 0.19046395$

C $0.97083906 \quad 0.47821209 \quad 1.46000124$

C $0.52442815-0.956340221 .01734211$

C $-0.94999232-0.454247931 .18202019$

C $-0.41735617 \quad 0.61543890 \quad 2.21390195$

C $-1.10864295 \quad 1.96402678 \quad 2.25199319$

C $-0.32735106 \quad 0.06283432 \quad 3.63152905$

$\mathrm{H}-0.90800729-0.73685699-2.70750638$

$\mathrm{H}-2.76190621 \quad 0.44585923-1.66087725$

$\mathrm{H}-3.23579436-0.69672169-0.35626205$

$\mathrm{H} \quad 0.14244919 \quad 2.52817507 \quad 0.20232199$

$\mathrm{H} 1.05548421 \quad 2.55154713 \quad 0.53081470$

$\mathrm{H} 1.884931821 .19608813-0.43150239$

$\mathrm{H} \quad 1.86669074 \quad 0.55815766 \quad 2.07386915$

H $0.81423526-1.75124427 \quad 1.70515400$

$\mathrm{H} \quad 0.80877534-1.257340410 .00561960$

$\mathrm{H}-1.68365094-1.16575027 \quad 1.55899734$

$\mathrm{H}-1.49061662 \quad 2.28586134 \quad 1.27223118$

$\mathrm{H}-0.470663092 .742047352 .67945650$

$\mathrm{H}-2.00973729 \quad 1.91177512 \quad 2.88468530$

$\mathrm{H} \quad 0.19319766 \quad 0.75793313 \quad 4.29971983$

$\mathrm{H} \quad 0.20527746 \quad-0.89398114 \quad 3.67817579$

$\mathrm{H}-1.32725853-0.111625864 .04914186$

$0.41850815 \quad 0.57089724-2.74601780$

$\begin{array}{lllll}0 & -0.27565154 & 1.52948290 & -1.87708338\end{array}$

o $-0.55733251-0.14954220-3.42538241$

Vibrational frequencies (in $\mathrm{cm}-1$ )

$\begin{array}{llllllllllllll}-1825.7 & 46.5 & 75.7 & 88.5 & 140.0 & 169.3 & 187.6 & 195.5 & 203.0 & 265.8 & 292.2 & 337.4 & 366.9 & 383.4\end{array}$ $403.6 \quad 419.8 \quad 442.4 \quad 482.0 \quad 508.9 \quad 550.3 \quad 582.8 \quad 620.0 \quad 626.7 \quad 701.6 \quad 715.4 \quad 740.2 \quad 770.9 \quad 786.9$

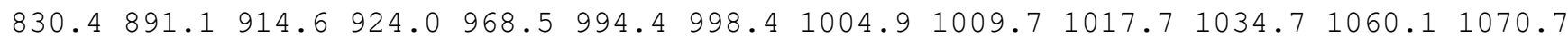

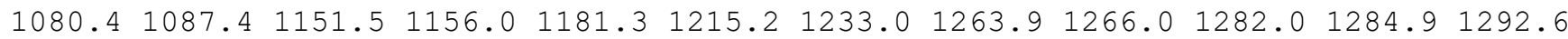
$1297.0 \quad 1299.3 \quad 1302.2 \quad 1314.4 \quad 1327.4 \quad 1371.3 \quad 1373.8 \quad 1385.0 \quad 1404.6 \quad 1412.0 \quad 1462.2 \quad 1741.7$ $1849.02644 .5 \quad 2672.8 \quad 2676.6 \quad 2679.92681 .5 \quad 2688.4 \quad 2730.8 \quad 2745.92752 .92762 .92773 .4$ 2782.22795 .22841 .02942 .0

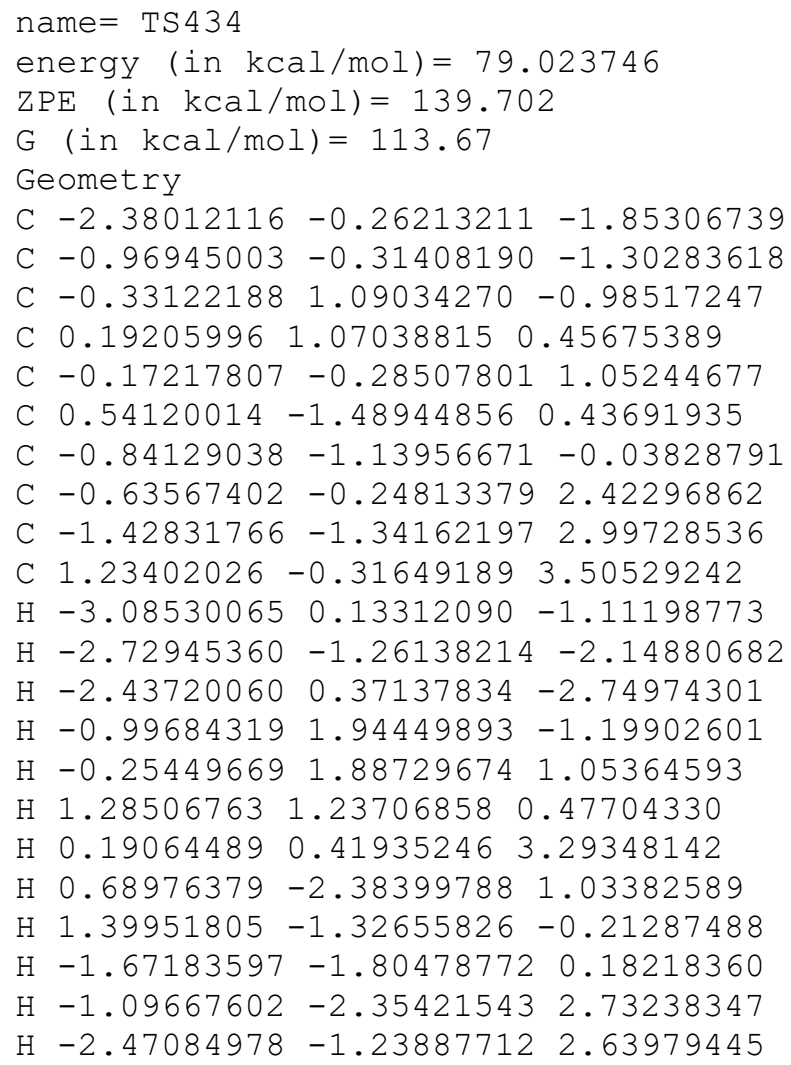


$\mathrm{H}-1.48317915-1.28425344 \quad 4.09423207$

$\mathrm{H} 1.33011378-0.08738528 \quad 4.55635377$

H $2.00862688 \quad 0.13865205 \quad 2.90824876$

$\mathrm{H} \quad 1.21208439-1.38353484 \quad 3.35493881$

$01.08186272-0.13279007-2.23458720$

$0.71742167 \quad 1.21014532-1.93578446$

o $-0.15369862-0.81854157-2.36360195$

Vibrational frequencies (in $\mathrm{cm}-1$ )

$\begin{array}{lllllllllllllll}-1219.5 & 17.5 & 48.7 & 74.6 & 85.5 & 151.2 & 152.4 & 161.9 & 184.7 & 219.5 & 254.0 & 273.5 & 305.6 & 337.4\end{array}$

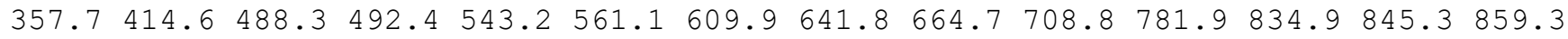

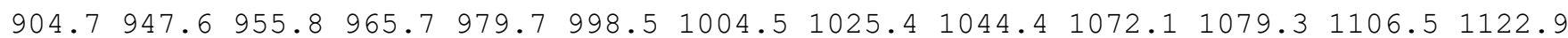

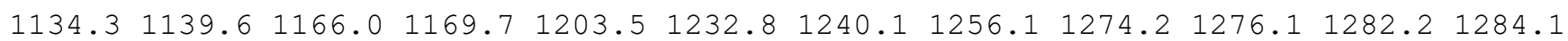
$\begin{array}{llllllllllllll}1286.7 & 1289.3 & 1291.7 & 1304.0 & 1312.4 & 1329.0 & 1330.8 & 1370.6 & 1384.3 & 1387.3 & 1398.7 & 1493.2\end{array}$ $1851.12660 .12666 .3 \quad 2669.92670 .3 \quad 2676.6 \quad 2689.02695 .22700 .62707 .72746 .42764 .6$ 2772.22776 .22780 .02782 .3

name $=\operatorname{TS} 435$

energy $($ in $\mathrm{kcal} / \mathrm{mol})=76.681364$

$\mathrm{ZPE}($ in $\mathrm{kcal} / \mathrm{mol})=142.139$

$\mathrm{G}($ in $\mathrm{kcal} / \mathrm{mol})=117.013$

Geometry

C $-2.13821760 \quad 0.41292672 \quad 0.01384365$

C $-0.75455723 \quad 0.13998240 \quad-0.45684958$

C $0.25884654 \quad 1.21140021-0.49675610$

C $1.758585120 .79958279-0.12219589$

C $1.85560606-0.27363183 \quad 0.82205785$

C $1.20664761-1.53728160 \quad 0.54577296$

C $-0.28378881-1.49409520 \quad 0.92187969$

C $-0.74073784-1.110828012 .14027478$

C $-2.06292375-1.605000712 .63351265$

C $-0.02838106-0.20050806 \quad 3.07465798$

$\mathrm{H}-2.75494338-0.49760893 \quad 0.02369782$

$\mathrm{H}-2.641950991 .12932301-0.65626420$

$\mathrm{H}-2.15306432 \quad 0.83176916 \quad 1.02850281$

$\mathrm{H} \quad 0.01001210 \quad 2.16113165 \quad 0.00166293$

H 2.29508001 1.714873790 .18692707

H $2.185018170 .49883335-1.11813643$

$\mathrm{H} 2.39792076-0.13580488 \quad 1.74338437$

$\mathrm{H} \quad 1.65117693-2.38660694 \quad 1.11070592$

$\mathrm{H} \quad 1.26451763-1.81355747-0.53580394$

H $-0.88650125-2.17141640 \quad 0.31020925$

$\mathrm{H}-2.72592569-0.77443201 \quad 2.91327734$

$\mathrm{H}-1.93257666-2.231535113 .52875856$

$\mathrm{H}-2.59883066-2.21331087 \quad 1.89335515$

$\mathrm{H} \quad 0.00175198 \quad 0.82787699 \quad 2.68538406$

$\mathrm{H} \quad 1.01600347-0.51766645 \quad 3.22743037$

$\mathrm{H}-0.49579446-0.15637402 \quad 4.06681434$

$\begin{array}{llllll}0 & 0.18911644 & 0.24989786 & -2.52600332\end{array}$

○ $0.28886234 \quad 1.49772883-1.90976069$

o - $0.63619171-0.59674531-1.57497964$

Vibrational frequencies (in $\mathrm{cm}-1$ )

$\begin{array}{llllllllllllllll}-418.1 & 49.7 & 84.7 & 91.0 & 129.5 & 134.5 & 146.8 & 181.3 & 196.4 & 202.0 & 233.4 & 258.8 & 279.7 & 323.2\end{array}$ $333.7 \quad 391.4 \quad 419.7 \quad 444.8 \quad 496.3 \quad 525.1 \quad 577.6 \quad 602.0 \quad 677.2 \quad 718.1 \quad 745.6 \quad 847.9 \quad 874.6 \quad 936.2$ $950.5954 .2974 .5 \quad 995.5 \quad 1012.5 \quad 1019.3 \quad 1021.1 \quad 1027.6 \quad 1041.2 \quad 1072.9 \quad 1100.6 \quad 1110.3 \quad 1124.5$ $1163.01176 .5 \quad 1202.4 \quad 1228.7 \quad 1269.1 \quad 1273.9 \quad 1276.2 \quad 1278.7 \quad 1282.3 \quad 1283.6 \quad 1288.1 \quad 1289.0$ $1295.41301 .1 \quad 1319.7 \quad 1343.0 \quad 1358.4 \quad 1376.8 \quad 1385.5 \quad 1399.5 \quad 1410.3 \quad 1425.8 \quad 1492.1 \quad 1760.2$ $2615.42645 .32658 .5 \quad 2662.1 \quad 2668.4 \quad 2671.3 \quad 2674.32676 .62707 .52720 .62726 .82733 .0$ $2757.22773 .6 \quad 2775.3 \quad 2777.2$

name $=\operatorname{TS} 436$

energy $($ in $\mathrm{kcal} / \mathrm{mol})=76.530353$

$\mathrm{ZPE}($ in $\mathrm{kcal} / \mathrm{mol})=142.398$

$\mathrm{G}(\mathrm{in} \mathrm{kcal} / \mathrm{mol})=119.193$ 

Geometry
C $-1.504909601 .47844931-0.06754046$
C $-0.07423588 \quad 0.96219219 \quad-0.06286594$
C $0.990898892 .01637455 \quad 0.38902903$
C $1.96765031 \quad 1.54762992 \quad 1.47468764$
C $2.05338064 \quad 0.06077320 \quad 1.64720407$
C $1.55046954-0.79969431 \quad 0.63162774$
C $0.08136021-0.376912820 .65500005$
C $0.03361710-0.32251945 \quad 2.19733233$
C -0.399955790 .849008323 .01049948$
C $-0.06800667-1.59099620 \quad 2.80641632$
$\mathrm{H}-1.91282556 \quad 1.56709163 \quad 0.94497087$
$\mathrm{H}-2.16404645 \quad 0.79724846-0.62486628$
$\mathrm{H}-1.577177492 .45823162-0.55832626$
$\mathrm{H} \quad 0.52996506 \quad 2.99128103 \quad 0.65037588$
$\mathrm{H} \quad 1.75546898 \quad 2.05318965 \quad 2.43653331$
H $2.98779103 \quad 1.90824500 \quad 1.18968763$
H $2.86321391-0.26639421 \quad 2.28649922$
$\mathrm{H} \quad 1.53042247-2.14872893 \quad 1.38585669$
H $2.06028624-1.25702275-0.18734468$
$\mathrm{H}-0.61440072-1.11960240 \quad 0.22411731$
$\mathrm{H}-1.42295146 \quad 0.68844447 \quad 3.38920844$
$\mathrm{H}-0.41466060 \quad 1.80150337 \quad 2.47299792$
H $0.24406807 \quad 0.97759664 \quad 3.89417695$
$\mathrm{H} 0.05932514-1.68553125 \quad 3.87105372$
$\mathrm{H} \quad 1.12987310-2.32835617 \quad 2.14113220$
$\mathrm{H}-0.69492819-2.36412314 \quad 2.39124059$
O $1.59343394 \quad 1.11837023-1.58525891$
O $1.709962492 .30365293-0.81219893$
o $0.23021906 \quad 0.76186220-1.45684446$
Vibrational frequencies (in $\mathrm{cm}-1$ )

$\begin{array}{llllllllllllllll}-2068.3 & 76.0 & 112.4 & 141.9 & 158.0 & 184.3 & 220.0 & 224.2 & 261.6 & 311.6 & 332.0 & 352.2 & 381.7 & 433.4\end{array}$

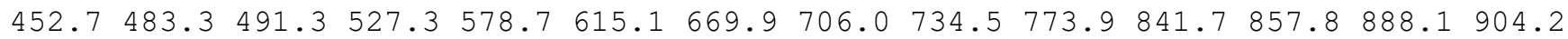
$922.9969 .3 \quad 973.4 \quad 978.9 \quad 986.6 \quad 1003.1 \quad 1007.7 \quad 1021.6 \quad 1042.8 \quad 1058.6 \quad 1071.6 \quad 1079.1 \quad 1093.0$

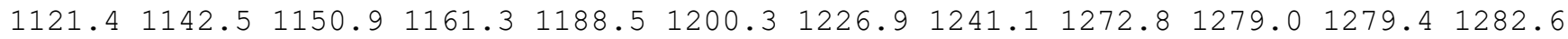

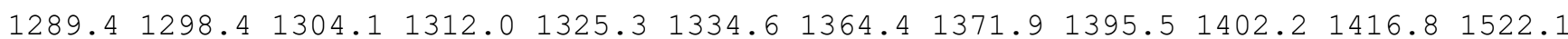

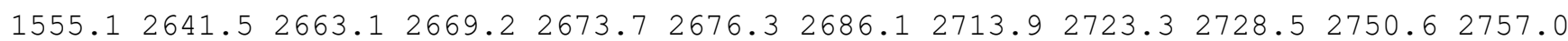
$2775.6 \quad 2779.3 \quad 2780.5 \quad 3229.0$

\footnotetext{
name $=$ TS437

energy $($ in $\mathrm{kcal} / \mathrm{mol})=77.638404$

$\mathrm{ZPE}($ in $\mathrm{kcal} / \mathrm{mol})=141.789$

$\mathrm{G}(\mathrm{in} \mathrm{kcal} / \mathrm{mol})=117.582$

Geometry

C $-1.21328274-1.73312755-1.81407989$

C $0.12322976-1.07579588-1.45958972$

C $0.013026720 .48831525-1.47352354$

C $0.72908391 \quad 1.28839240 \quad-0.37810040$

C $1.10156662 \quad 0.53364188 \quad 0.88643342$

C $1.42175699-0.90064230 \quad 0.69191166$

C $0.72743892-1.68822752-0.25155957$

C $-0.02076981-0.03677443 \quad 1.76622566$

C $0.28292209-0.15596198 \quad 3.22322809$

C $-1.42749103 \quad 0.35870809 \quad 1.45142290$

$\mathrm{H}-1.62202525-1.32392785-2.74740471$

$\mathrm{H}-1.95434712-1.59826983-1.02145037$

$\mathrm{H}-1.08579082-2.81211916-1.97215053$

$\mathrm{H}-1.04313277 \quad 0.81596296-1.57928835$

$\mathrm{H} \quad 0.11039231 \quad 2.16797089-0.10365785$

$\mathrm{H} \quad 1.65929045 \quad 1.71304250-0.82290391$

$\mathrm{H} 1.84260547 \quad 1.12219681 \quad 1.46460453$

H $2.14607062-1.36279508 \quad 1.37086515$

$\mathrm{H}-0.15517306-1.47039434 \quad 0.93459954$

$\mathrm{H} \quad 0.94873527-2.74437139-0.35233533$
} 
$\mathrm{H}-0.36992807-0.89407874 \quad 3.70780836$

$\mathrm{H} \quad 0.12103586 \quad 0.79907637 \quad 3.74556413$

$\mathrm{H} 1.31605310-0.455300023 .42770975$

$\mathrm{H}-1.71956083 \quad 0.15011870 \quad 0.41649695$

$\mathrm{H}-1.577873501 .436074411 .61877318$

$\mathrm{H}-2.14280016-0.164084002 .10121756$

$01.60879410-0.14148399-2.91346684$

$0.59596835 \quad 0.83624292-2.73194790$

$0.96624919-1.36127780-2.60124937$

Vibrational frequencies (in $\mathrm{cm}-1$ )

$\begin{array}{lllllllllllllllllll}-3216.1 & 53.3 & 84.5 & 123.0 & 140.0 & 164.7 & 168.8 & 203.9 & 213.8 & 262.6 & 292.7 & 324.6 & 333.1 & 352.0\end{array}$ $400.6 \quad 441.7 \quad 469.5 \quad 476.1 \quad 494.3 \quad 545.0 \quad 643.3 \quad 698.3 \quad 747.0 \quad 782.6 \quad 823.7 \quad 837.0 \quad 868.7 \quad 937.9$ $960.8969 .3 \quad 986.2999 .2 \quad 1004.4 \quad 1009.9 \quad 1019.5 \quad 1025.5 \quad 1029.5 \quad 1071.5 \quad 1090.01104 .6 \quad 1146.1$ $1157.41177 .6 \quad 1201.6 \quad 1221.5 \quad 1245.2 \quad 1256.7 \quad 1281.4 \quad 1287.8 \quad 1290.1 \quad 1293.2 \quad 1297.6 \quad 1300.8$

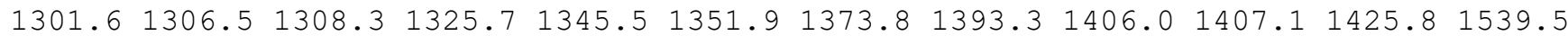
$1736.72648 .4 \quad 2666.6 \quad 2669.7 \quad 2671.8 \quad 2674.7 \quad 2679.12679 .72684 .3 \quad 2715.42724 .42733 .8$ $2770.22776 .5 \quad 2778.7 \quad 2780.9$

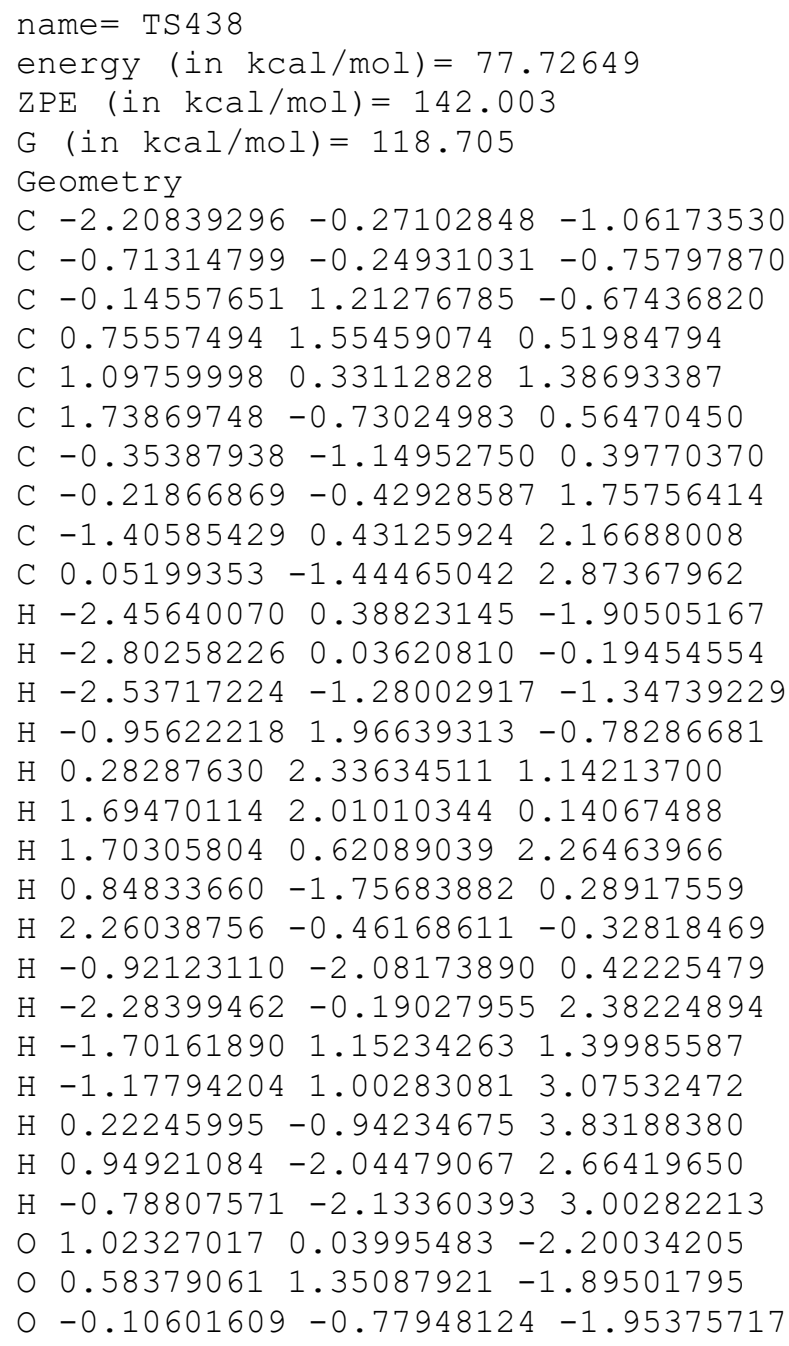


$\mathrm{ZPE}($ in $\mathrm{kcal} / \mathrm{mol})=142.325$

$\mathrm{G}(\mathrm{in} \mathrm{kcal} / \mathrm{mol})=118.968$

Geometry

C $-2.45153743 \quad 0.72309690 \quad-0.09849597$

C $-1.00195520 \quad 0.33727441-0.36928062$

C $0.01812171 \quad 1.52457855 \quad-0.29455772$

C $1.28763691 \quad 1.264827150 .52873805$

C $1.27344475-0.06533950 \quad 1.27597270$

C $0.82893410-1.20180600 \quad 0.35389955$

C $-0.56793956-0.926337850 .28831536$

C $0.08221318-0.23746917 \quad 2.23968748$

C $-0.82020729 \quad 0.86750373 \quad 2.66043163$

C $0.18132628-1.346778293 .09894281$

$\mathrm{H}-2.70393953 \quad 0.66797967 \quad 0.96422750$

$\mathrm{H}-3.13256194 \quad 0.03406219-0.62234025$

$\mathrm{H}-2.682653121 .73027121-0.46459855$

$\mathrm{H}-0.47467894 \quad 2.47370225 \quad 0.00162473$

H $1.45269803 \quad 2.09259747 \quad 1.24401527$

$\mathrm{H} 2.161959031 .28733848-0.15904253$

$\mathrm{H} 2.24889434-0.23576725 \quad 1.76841002$

$\mathrm{H} 0.84285819-2.424481951 .26055039$

$\mathrm{H} \quad 1.44516497-1.71129967-0.35776883$

$\mathrm{H}-1.28077924-1.73531974 \quad 0.20738138$

$\mathrm{H}-1.83195698 \quad 0.496022312 .88542170$

$\mathrm{H}-0.91945071 \quad 1.68637261 \quad 1.94280429$

$\mathrm{H}-0.438620261 .318922993 .59277801$

H $1.11891969-1.632041703 .54841618$

$\mathrm{H} 0.48996371-2.47595580 \quad 2.06029020$

$\mathrm{H}-0.66746926-1.65294883 \quad 3.68509542$

$\begin{array}{lllll}0 & 0.21433673 & 0.49363774 & -2.28553703\end{array}$

O $0.390008531 .75898922-1.65215546$

o $-1.025066340 .04976106-1.80132610$

Vibrational frequencies (in $\mathrm{cm}-1$ )

$\begin{array}{lllllllllllllll}-2026.7 & 75.7 & 92.7 & 139.5 & 144.9 & 157.7 & 222.4 & 236.4 & 281.6 & 310.1 & 319.2 & 378.0 & 424.2 & 432.5\end{array}$

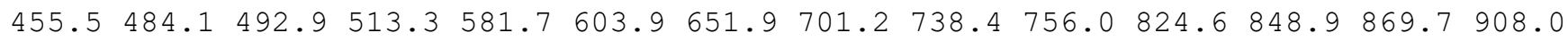
$932.9942 .2948 .0 \quad 975.9980 .0 \quad 993.7 \quad 1013.5 \quad 1034.9 \quad 1048.4 \quad 1062.7 \quad 1070.8 \quad 1077.6 \quad 1106.3$ $1117.6 \quad 1127.3 \quad 1158.4 \quad 1181.7 \quad 1189.4 \quad 1206.4 \quad 1217.2 \quad 1236.3 \quad 1272.1 \quad 1275.1 \quad 1278.7 \quad 1282.3$

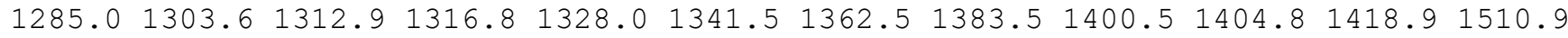
$1564.22655 .6 \quad 2660.12666 .4 \quad 2674.5 \quad 2678.4 \quad 2686.0 \quad 2713.92720 .4 \quad 2737.92747 .8 \quad 2758.0$ $2776.7 \quad 2780.7 \quad 2781.8 \quad 3212.4$

\section{name $=$ TS440}

energy $($ in $\mathrm{kcal} / \mathrm{mol})=77.270967$

$\mathrm{ZPE}(\mathrm{in} \mathrm{kcal} / \mathrm{mol})=142.523$

G $($ in $\mathrm{kcal} / \mathrm{mol})=119.426$

Geometry

C $-2.52810219-0.05994168-0.47671900$

C $-1.04035410-0.36475764-0.53671200$

C $-0.15992820 \quad 0.89626238 \quad-0.82785975$

C $1.02918562 \quad 1.112703120 .11558639$

C $1.42815598-0.10029256 \quad 0.90275575$

C $0.97696748-1.390153790 .50528476$

C $-0.53282042-1.18444703 \quad 0.64755938$

C $-0.38742202-0.41414680 \quad 1.97820326$

C $-0.93272711 \quad 0.94324317 \quad 2.26173608$

C $-0.15058944-1.249567053 .08968112$

$\mathrm{H}-3.11415816-0.98948490-0.45592502$

$\mathrm{H}-2.85710572 \quad 0.49976854 \quad-1.36219838$

$\mathrm{H}-2.801331130 .51474522 \quad 0.41403352$

$\mathrm{H}-0.780757351 .80861797-0.92506359$

$\mathrm{H} \quad 0.84950722 \quad 1.98357474 \quad 0.77528299$

$\mathrm{H} \quad 1.91233191 \quad 1.40979034-0.50538003$

$\mathrm{H} 2.36615865 \quad 0.00637251 \quad 1.43276806$

H $1.32927722-2.215316551 .75983798$ 
H $1.45396129-2.11311542-0.12039260$

$\mathrm{H}-1.11602869-2.11773288 \quad 0.74590104$

$\mathrm{H}-1.200197961 .526568561 .37593810$

$\mathrm{H}-0.21872340 \quad 1.54186078 \quad 2.84840890$

$\mathrm{H}-1.84889369 \quad 0.86282979 \quad 2.87016594$

H $0.13007993-0.81682291 \quad 4.03452374$

H $1.07420152-2.064286572 .58220561$

$\mathrm{H}-0.65630025-2.19590153 \quad 3.19897437$

$0-0.48101525-0.29182253-2.71734733$

$\begin{array}{lllll}0 & 0.41678689 & 0.62643027 & -2.11332380\end{array}$

o $-0.80057323-1.20302865-1.68362982$

Vibrational frequencies (in $\mathrm{cm}-1$ )

$\begin{array}{llllllllllllllllll}-2044.5 & 77.9 & 135.7 & 147.2 & 162.4 & 181.0 & 222.4 & 232.0 & 269.0 & 300.3 & 335.6 & 352.6 & 374.7 & 423.2\end{array}$

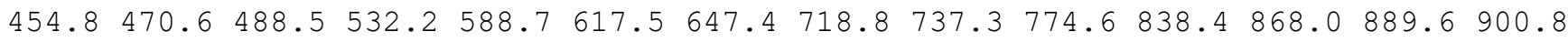
$918.3 \quad 966.4 \quad 979.5 \quad 983.4 \quad 988.7 \quad 1003.5 \quad 1016.1 \quad 1029.8 \quad 1045.3 \quad 1065.6 \quad 1071.5 \quad 1089.4 \quad 1103.2$

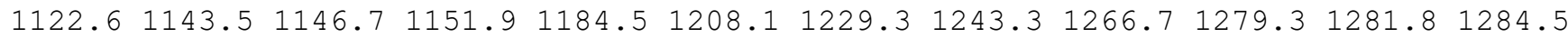

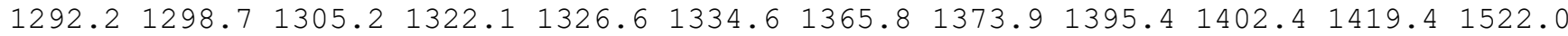
$1556.22639 .2 \quad 2662.92670 .6 \quad 2673.3 \quad 2677.12681 .92713 .72723 .4 \quad 2727.02750 .4 \quad 2754.8$ $2775.92779 .5 \quad 2781.6 \quad 3226.0$

name $=\operatorname{TS} 441$

energy (in $\mathrm{kcal} / \mathrm{mol})=81.172019$

$\operatorname{ZPE}($ in $\mathrm{kcal} / \mathrm{mol})=138.722$

$\mathrm{G}($ in $\mathrm{kcal} / \mathrm{mol})=110.833$

Geometry

C $-2.70488447 \quad 2.62581190 \quad 1.13700027$

C $-1.75707800 \quad 1.493191151 .11656865$

C $1.41984906 \quad 3.24542122 \quad 2.23615155$

C $2.27064966 \quad 2.47895791 \quad 1.56096924$

C $0.10032910-0.75022822 \quad 2.46140616$

C $-0.95910787-0.85045409 \quad 1.41824852$

C $-1.99633440 \quad 0.22348199 \quad 1.47428615$

C $0.01825806 \quad 0.18396213 \quad 3.54824070$

C $0.95052254 \quad 0.21992162 \quad 4.59723392$

C $1.26852652-1.642331722 .33168503$

$\mathrm{H}-3.72916896 \quad 2.31115075 \quad 1.38618814$

$\mathrm{H}-2.75061773 \quad 3.13437741 \quad 0.16005516$

$\mathrm{H}-2.41120846 \quad 3.38770524 \quad 1.87930656$

$\mathrm{H} \quad 1.57607054 \quad 3.73187593 \quad 3.19888005$

H $3.262754412 .24420893 \quad 1.91403509$

H 2.05363551 2.03103640 0.60356495

H $0.41979679 \quad 1.01370790 \quad 2.72357848$

$\mathrm{H}-1.44540572-1.85236670 \quad 1.51913000$

$\mathrm{H}-0.49531565-0.86885111 \quad 0.40284044$

$\mathrm{H}-2.98262991-0.08673732 \quad 1.80771193$

$\mathrm{H}-1.00306894 \quad 0.53138717 \quad 3.81264253$

$\mathrm{H} \quad 0.78267474 \quad 0.79077748 \quad 5.47887660$

H $1.86401531-0.322429824 .58957941$

$\mathrm{H} 1.34335102-2.30202958 \quad 3.21825687$

H 2.21528338 $-1.07785607 \quad 2.30136272$

$\mathrm{H} \quad 1.23867420-2.281044761 .44120855$

$\begin{array}{lllll}0 & -0.18547659 & 3.09579543 & 0.57688771\end{array}$

$\begin{array}{llll}0 & 0.14231348 & 3.56646632 & 1.86163348\end{array}$

O $-0.46717664 \quad 1.73193779 \quad 0.66217439$

Vibrational frequencies (in $\mathrm{cm}-1$ )

$\begin{array}{llllllllllllllll}-641.2 & 27.8 & 38.5 & 51.0 & 71.6 & 87.7 & 100.0 & 116.7 & 126.6 & 159.8 & 180.2 & 198.5 & 229.6 & 239.1\end{array}$ $312.5 \quad 341.6 \quad 353.1 \quad 365.4 \quad 421.3 \quad 434.9 \quad 495.5 \quad 525.3 \quad 595.2 \quad 615.1 \quad 632.7 \quad 660.0 \quad 745.0 \quad 816.5$ $846.2881 .6914 .5 \quad 930.3 \quad 940.0 \quad 947.5 \quad 990.4 \quad 1011.4 \quad 1014.5 \quad 1028.1 \quad 1046.0 \quad 1053.8 \quad 1065.5$

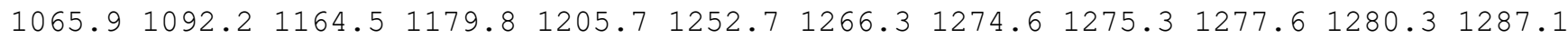
$1290.3 \quad 1329.4 \quad 1365.4 \quad 1372.4 \quad 1379.3 \quad 1390.1 \quad 1393.2 \quad 1402.7 \quad 1427.5 \quad 1615.6 \quad 1854.5 \quad 1863.3$ $2299.32640 .72645 .5 \quad 2659.8 \quad 2670.2 \quad 2673.7 \quad 2705.12708 .32724 .22731 .12757 .82759 .1$ $2767.82777 .2 \quad 2789.6 \quad 2811.2$ 


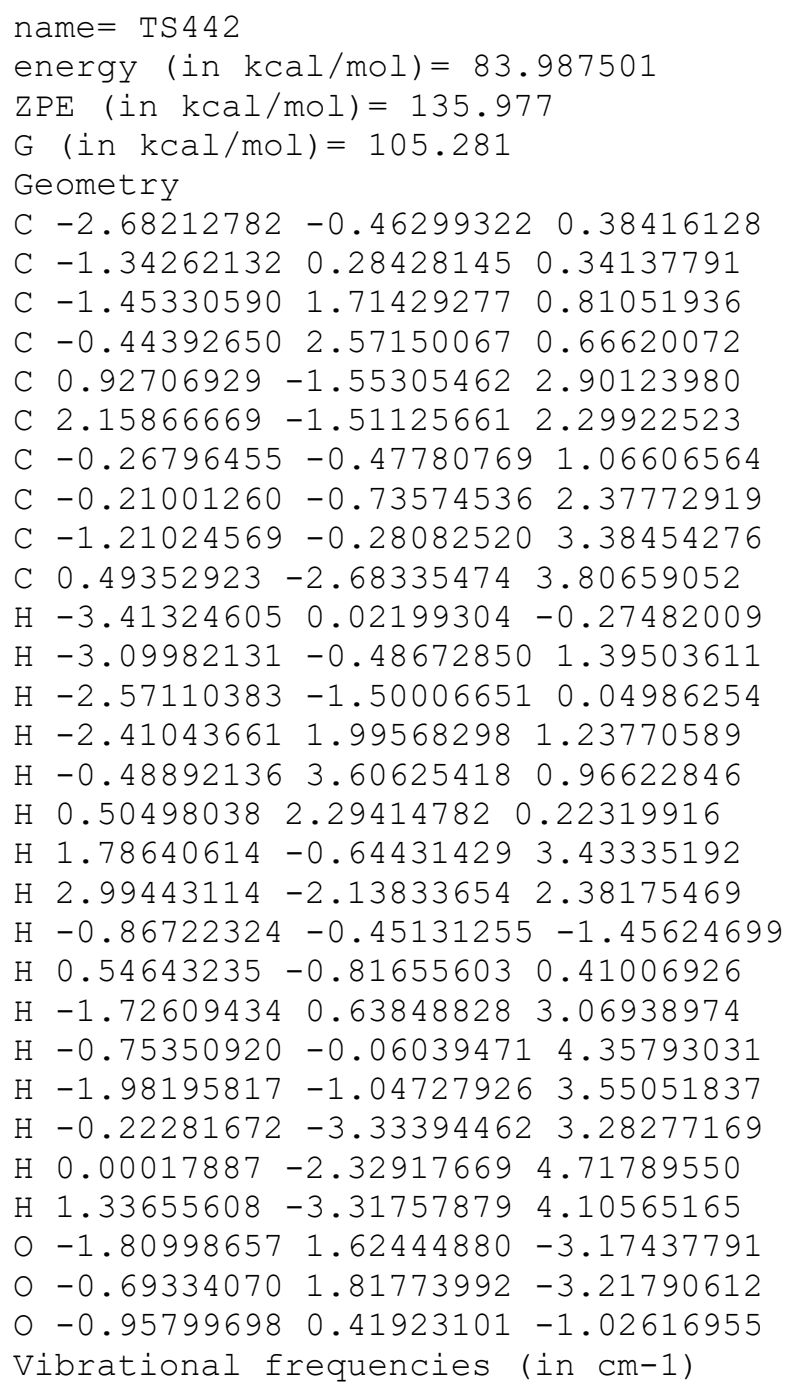


H $2.102062490 .10495182 \quad 2.07677860$

$\mathrm{H} 1.43156280-2.110949971 .13437882$

$\mathrm{H} 1.97375501-1.07860296-0.18591713$

$\mathrm{H}-0.63224943-2.27512662-0.07589368$

$\mathrm{H}-1.322032981 .363689161 .42989718$

$\mathrm{H}-0.336967112 .06667477 \quad 2.78110159$

$\mathrm{H}-1.605940490 .994838493 .44704786$

$\mathrm{H}-1.03460704-1.318402023 .87970269$

$\mathrm{H} \quad 0.53352704-1.94623843 \quad 3.31930701$

$\mathrm{H}-0.85005023-1.981147772 .23490723$

O $-0.47827716 \quad 1.14144533-2.52591829$

$0-0.242614422 .06361903-1.28086519$

o $-0.12474958-0.06024086-2.10729539$

Vibrational frequencies (in cm-1)

$\begin{array}{lllllllllllllll}-1545.8 & 83.2 & 90.3 & 140.0 & 165.2 & 179.8 & 245.2 & 259.9 & 273.7 & 306.0 & 314.7 & 356.7 & 367.1 & 391.5\end{array}$

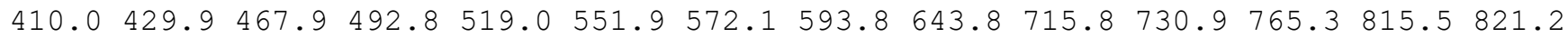
$877.1908 .2942 .9962 .5 \quad 997.3 \quad 1010.2 \quad 1024.0 \quad 1030.4 \quad 1038.3 \quad 1051.1 \quad 1070.3 \quad 1082.4 \quad 1094.3$ $1117.0 \quad 1145.0 \quad 1156.7 \quad 1162.6 \quad 1180.1 \quad 1210.4 \quad 1216.3 \quad 1244.5 \quad 1249.4 \quad 1253.8 \quad 1289.0 \quad 1291.7$

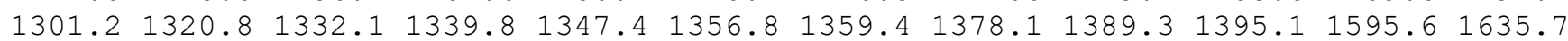

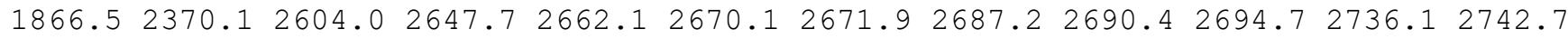
$2761.32774 .5 \quad 2782.12810 .9$

\section{name $=$ TS 444}

energy (in $\mathrm{kcal} / \mathrm{mol})=83.056607$

$\mathrm{ZPE}(\mathrm{in} \mathrm{kcal} / \mathrm{mol})=137.577$

$\mathrm{G}($ in $\mathrm{kcal} / \mathrm{mol})=108.798$

Geometry

C $-2.31924644 \quad 1.14369012-1.21741321$

C $-1.05353667 \quad 0.55990871-0.71394875$

C $0.205853731 .44180822-0.81361409$

C 0.887837361 .300409370 .72804510

C $1.08149714-0.13810364 \quad 1.17504925$

C $0.43242270-1.144175810 .17039826$

C $-0.92535549-0.68834572-0.24791955$

C $0.44752037-0.39983755 \quad 2.50788148$

C $-0.76640733 \quad 0.18680439 \quad 2.77175844$

C $1.19999968-1.190954123 .49704770$

$\mathrm{H}-3.18842320 \quad 0.49513224-1.05554723$

$\mathrm{H}-2.23735457 \quad 1.30524412-2.30869806$

$\mathrm{H}-2.534483862 .12130725 \quad-0.76920587$

$\mathrm{H}-0.05395893 \quad 2.52115599-0.89091945$

H $0.156292991 .94046281 \quad 1.48515431$

H $1.80550395 \quad 1.887806170 .69844143$

H $2.17976750-0.357371991 .19342648$

H $0.43899706 \quad-2.166249220 .57473304$

$\mathrm{H} 1.08762658-1.14846019-0.74768440$

$\mathrm{H}-1.72801489-1.40806681-0.20054527$

$\mathrm{H}-0.42552624 \quad 1.49447906 \quad 2.13438135$

H $-1.53436544 \quad 0.28848461 \quad 1.98248348$

$\mathrm{H}-1.21999987 \quad 0.18040776 \quad 3.74986284$

H $2.19377683-0.76043553 \quad 3.71347505$

$\mathrm{H} 1.39301587 \quad-2.21392150 \quad 3.11715382$

$\mathrm{H} \quad 0.68027780-1.30905701 \quad 4.46035428$

$01.17572825-0.66113288-3.40507678$

o $1.07553948 \quad 1.03325172-1.64152461$

O $0.37129435-1.45155551-3.27204503$

Vibrational frequencies (in cm-1)

$\begin{array}{lllllllllllllll}-1368.7 & 25.2 & 27.5 & 37.1 & 53.6 & 70.5 & 97.0 & 110.1 & 112.5 & 118.6 & 140.3 & 210.3 & 225.3 & 266.3\end{array}$ $296.4 \quad 326.0 \quad 349.2 \quad 411.9 \quad 469.3 \quad 502.9 \quad 527.3 \quad 557.2 \quad 609.9614 .3 \quad 639.5 \quad 697.4 \quad 729.4 \quad 798.8$ $848.2900 .9911 .4928 .5 \quad 950.1 \quad 990.2 \quad 1018.7 \quad 1023.1 \quad 1029.7 \quad 1040.3 \quad 1060.7 \quad 1079.8 \quad 1111.5$

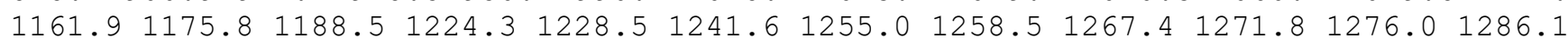

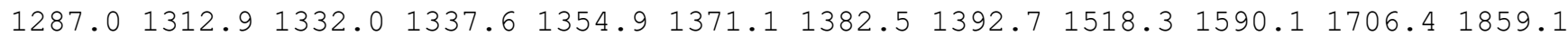
$1992.52297 .62621 .4 \quad 2624.8 \quad 2632.5 \quad 2640.6 \quad 2659.92662 .72681 .92692 .2 \quad 2714.02731 .9$ $2761.6 \quad 2772.5 \quad 2773.7 \quad 2779.0$ 


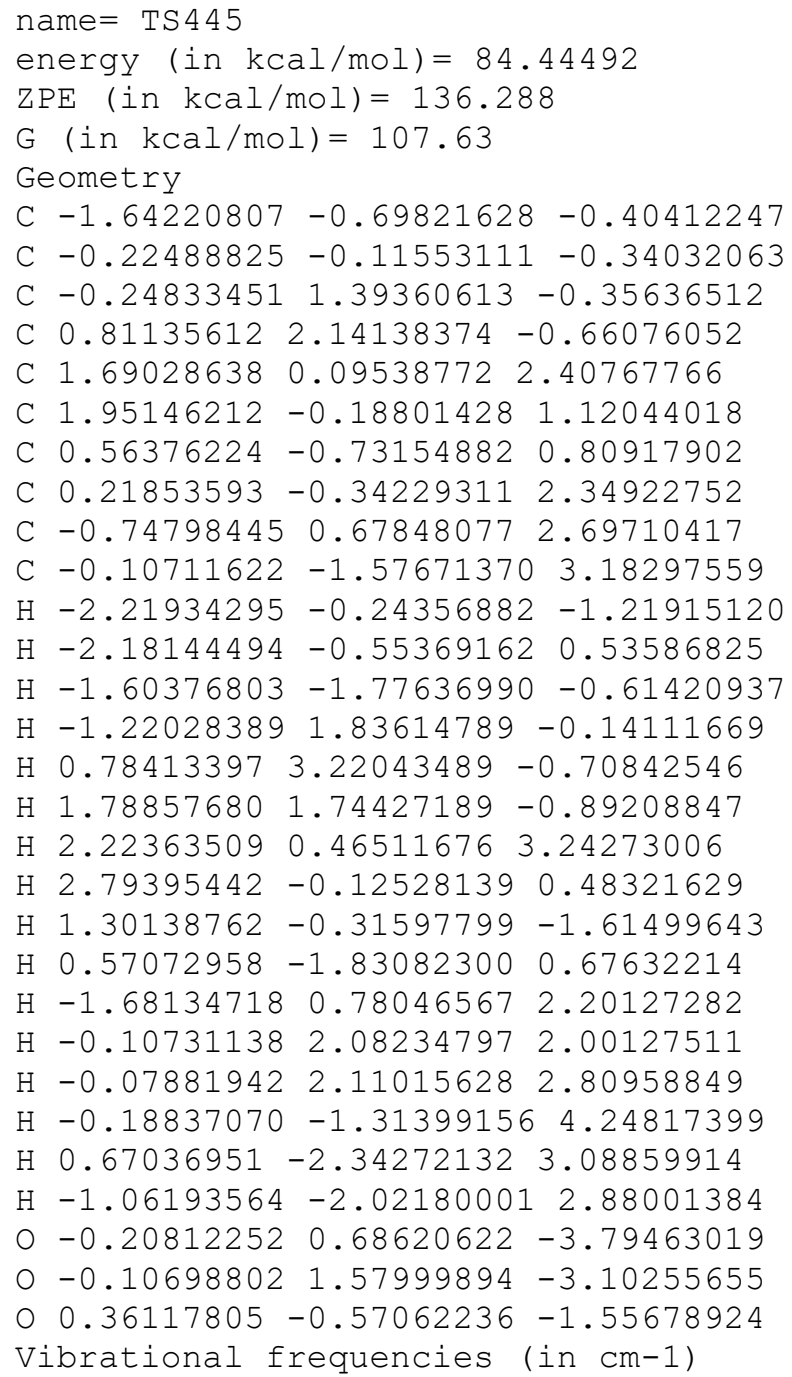

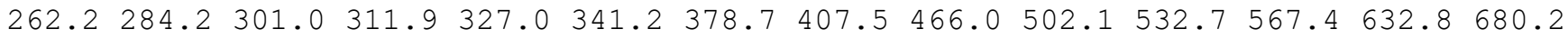

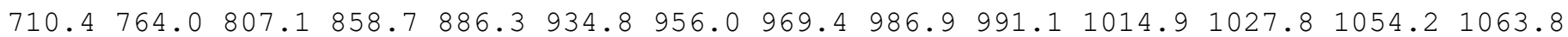

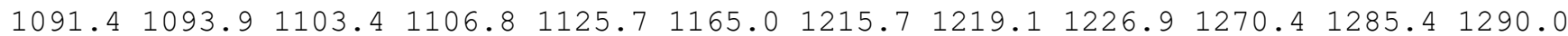
$1291.81295 .51311 .7 \quad 1344.1 \quad 1366.3 \quad 1385.0 \quad 1387.91404 .7 \quad 1413.5 \quad 1592.4 \quad 1775.6 \quad 1844.1$ $2671.52673 .4 \quad 2679.12680 .8 \quad 2708.8 \quad 2714.3 \quad 2717.2 \quad 2750.52779 .3 \quad 2780.2 \quad 2782.6 \quad 2794.7$ $2807.92816 .5 \quad 4763.0$

\section{name $=$ TS446}

energy (in $\mathrm{kcal} / \mathrm{mol})=82.257775$

$\mathrm{ZPE}(\mathrm{in} \mathrm{kcal} / \mathrm{mol})=138.604$

$\mathrm{G}($ in $\mathrm{kcal} / \mathrm{mol})=110.468$

Geometry

C $-1.72460720-1.71181112-2.79377795$

C $-1.33010259-1.77207621-1.44998186$

C $1.87916439-0.17174314-0.53190613$

C $2.38019531-1.152640840 .21444710$

C $-1.76848402 \quad 0.29248588 \quad 1.51288419$

C $-1.45388164-1.11509396 \quad 0.95618947$

C $-2.05008354-1.35479892-0.38528701$

C $-1.23819815 \quad 1.33596887 \quad 0.57246335$

C $-0.30146458 \quad 2.21892034 \quad 0.91629719$

C $-3.27222022 \quad 0.50562394 \quad 1.72347767$

$\mathrm{H}-1.33926398-3.31823084-3.36088484$

$\mathrm{H}-0.81678824-2.79783509-3.67063064$

$\mathrm{H}-2.70807886-1.72761224-3.17889786$

H $1.97679350 \quad 0.90127117-0.36964445$ 
H $2.97457401-0.98328128 \quad 1.09504041$

H $2.23243664 \quad-2.19929249 \quad 0.00276737$

$\mathrm{H}-1.26112919 \quad 0.37792596 \quad 2.50821261$

$\mathrm{H}-1.82062725-1.88439864 \quad 1.66857765$

$\mathrm{H}-0.34679374-1.24306210 \quad 0.93096701$

$\mathrm{H}-3.11762257-1.16903277-0.47266021$

$\mathrm{H} \quad 0.15314591 \quad 2.26643248 \quad 1.89226627$

H $0.07839419 \quad 2.97079930 \quad 0.24335762$

$\mathrm{H}-1.67374330 \quad 1.31278198 \quad-0.42710799$

$\mathrm{H}-3.82273975 \quad 0.49913632 \quad 0.77677918$

$\mathrm{H}-3.46560419 \quad 1.47186166 \quad 2.20432931$

$\mathrm{H}-3.69835023-0.27579022 \quad 2.36147345$

O $0.90205056-1.64293270-2.02636590$

O $1.11281696-0.30529568-1.65376562$

o $-0.03779499-2.21429531-1.16452047$

Vibrational frequencies (in cm-1)

$\begin{array}{lllllllllllllllll}-784.0 & 30.1 & 39.3 & 48.7 & 52.2 & 79.5 & 87.4 & 113.3 & 150.9 & 171.1 & 187.9 & 200.1 & 221.6 & 258.1\end{array}$

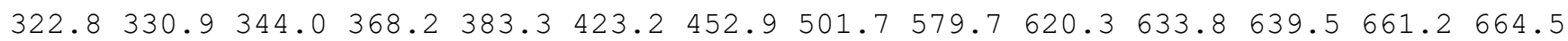

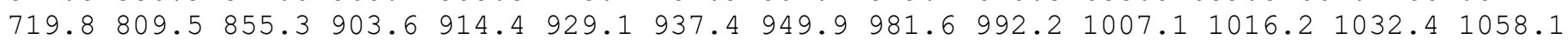

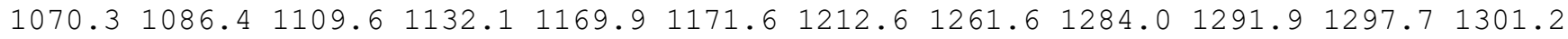

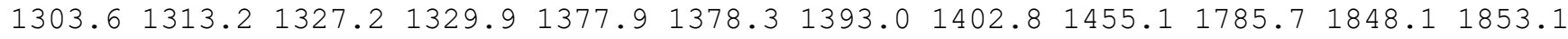
$2645.7 \quad 2672.92679 .8 \quad 2698.9 \quad 2713.9 \quad 2718.2 \quad 2725.7 \quad 2737.3 \quad 2742.0 \quad 2754.3 \quad 2755.5 \quad 2781.6$ 2790.92796 .94911 .8

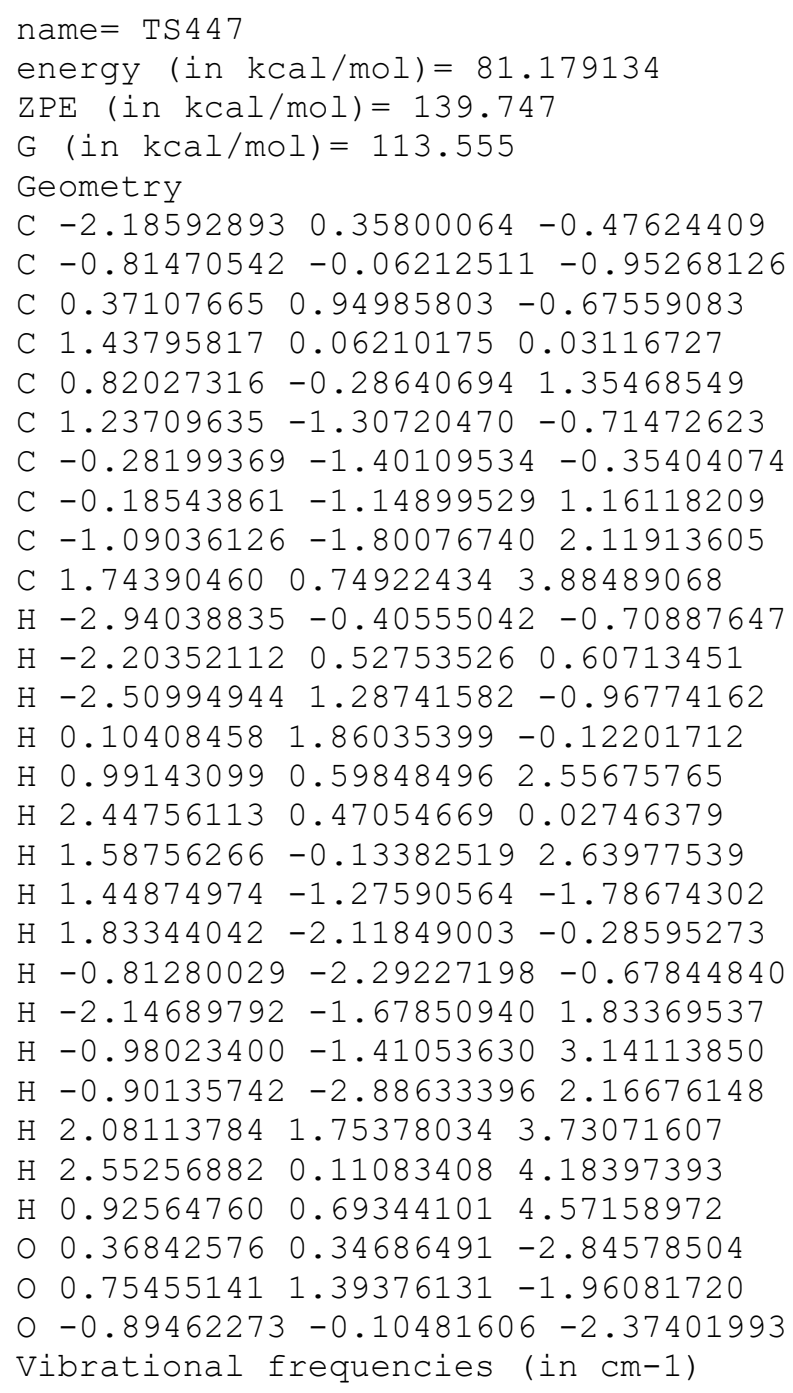


$1847.72664 .8 \quad 2670.7 \quad 2673.8 \quad 2675.8 \quad 2685.0 \quad 2706.62707 .52716 .42756 .12759 .02761 .6$ $2778.4 \quad 2779.9 \quad 2801.8 \quad 3294.6$

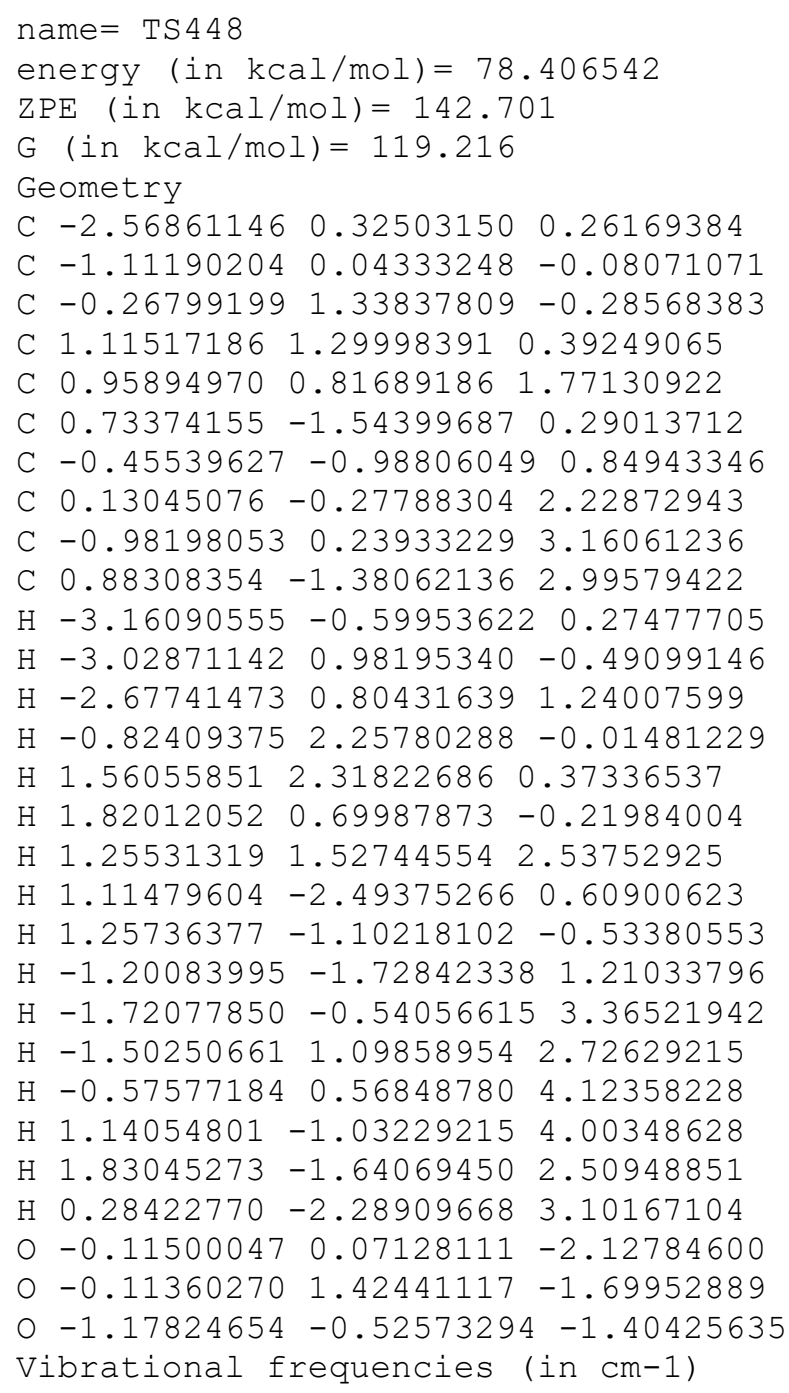


$\mathrm{H}-3.19589649-0.507447420 .55831755$

H $0.43470549 \quad 2.45596409 \quad 0.53583562$

H $1.35201898 \quad 2.41333993 \quad 0.70889360$

$\mathrm{H} \quad 1.93542092 \quad 1.21427850 \quad-0.61752744$

H $2.53147156 \quad 0.25213036 \quad 1.71055983$

H $1.49267887 \quad-2.04299033 \quad 1.23236254$

$\mathrm{H} 1.05676326-1.31424965-0.32107983$

$\mathrm{H}-0.98495239-1.611190251 .75802815$

$\mathrm{H}-0.83419805 \quad 1.95511108 \quad 1.97803837$

$\mathrm{H} \quad 0.417128992 .12887671 \quad 3.24093970$

$\mathrm{H}-1.078623201 .25289596 \quad 3.55669184$

H $1.44467695-0.011650574 .24757186$

$\mathrm{H} 1.30230831-1.548894123 .39299493$

$\mathrm{H}-0.09376609-0.88582610 \quad 4.23610298$

$\begin{array}{llll}0 & -0.57014270 & -0.24486290 & -1.99703518\end{array}$

$0-0.45724703 \quad 1.18140589-1.58928789$

o - $-1.24924821-0.86198526-0.91514266$

Vibrational frequencies (in $\mathrm{cm}-1$ )

$\begin{array}{llllllllllllll}-1910.1 & 99.2 & 124.7 & 163.7 & 169.9 & 180.1 & 194.6 & 239.0 & 271.9 & 292.8 & 312.4 & 327.3 & 382.2 & 406.5\end{array}$ $428.8 \quad 458.3 \quad 484.0 \quad 516.3 \quad 562.8 \quad 588.4 \quad 644.2 \quad 680.4 \quad 712.2 \quad 730.4 \quad 759.0 \quad 780.3 \quad 828.5 \quad 886.1$

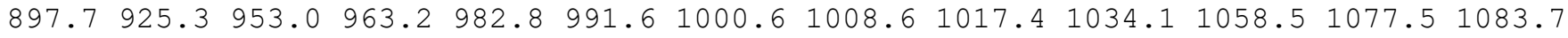

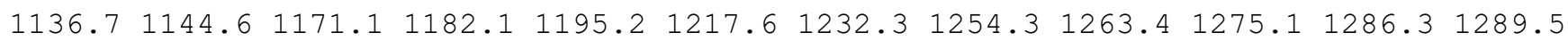
$1291.6 \quad 1294.91298 .4 \quad 1304.8 \quad 1307.7 \quad 1313.4 \quad 1319.8 \quad 1340.0 \quad 1376.4 \quad 1399.01405 .4 \quad 1412.8$ $1667.72649 .6 \quad 2672.8 \quad 2675.3 \quad 2675.7 \quad 2678.4 \quad 2680.12685 .92686 .72747 .12753 .42758 .4$ $2774.3 \quad 2782.2 \quad 2782.9 \quad 3135.5$

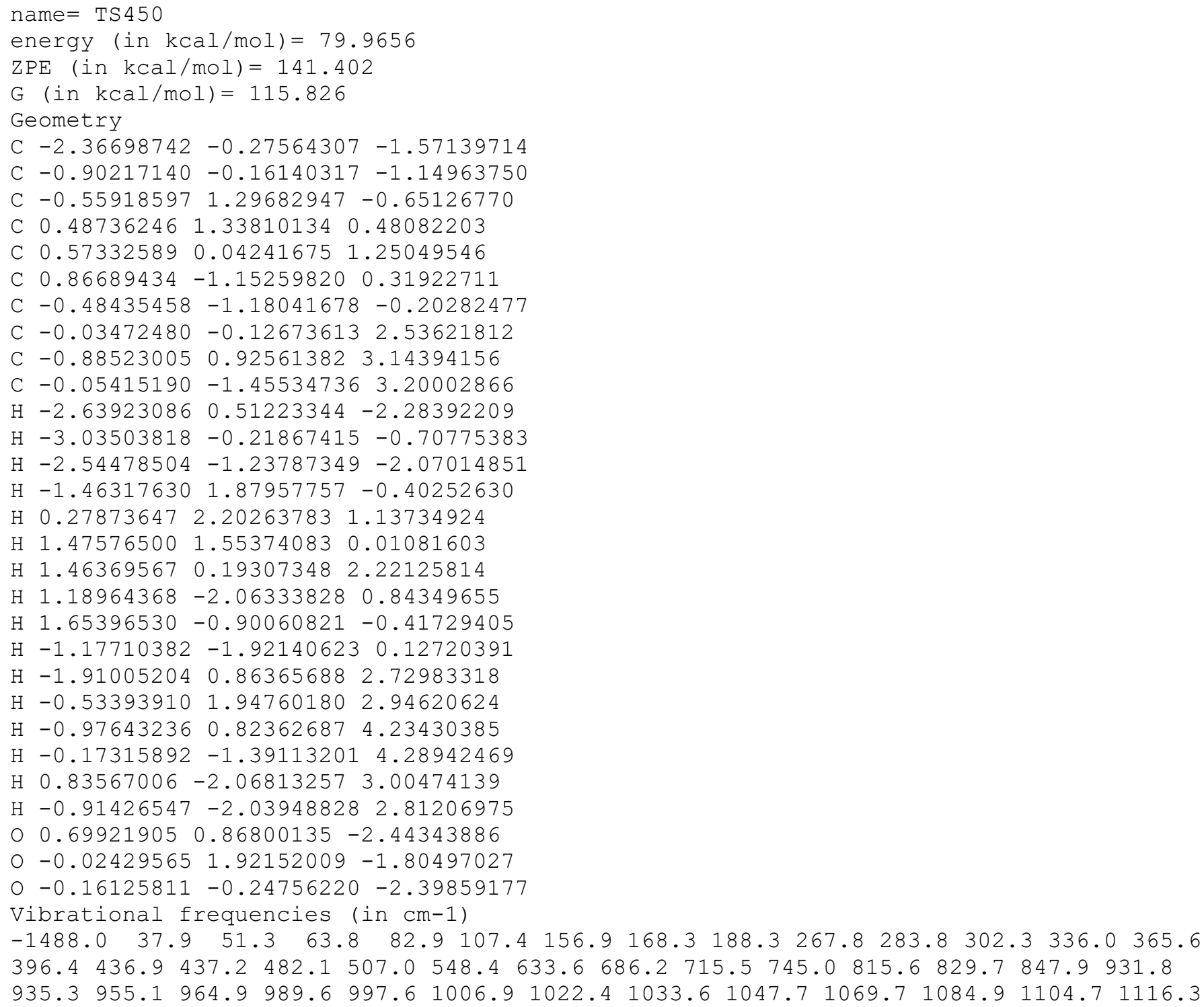


$\begin{array}{lllllllllllllll}1134.1 & 1172.1 & 1187.1 & 1223.0 & 1238.2 & 1246.9 & 1255.9 & 1266.6 & 1270.8 & 1280.7 & 1281.9 & 1292.1\end{array}$

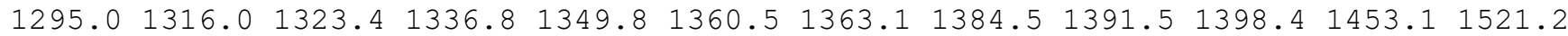
$2189.62644 .52647 .2 \quad 2651.4 \quad 2667.0 \quad 2673.12675 .92676 .52685 .82700 .82728 .52753 .1$ $2770.12770 .8 \quad 2782.2 \quad 2799.3$

\section{name $=$ TS 451}

energy $($ in $\mathrm{kcal} / \mathrm{mol})=81.00063$

$\mathrm{ZPE}($ in $\mathrm{kcal} / \mathrm{mol})=140.374$

$\mathrm{G}($ in $\mathrm{kcal} / \mathrm{mol})=114.441$

Geometry

C $-1.54294625-2.65262402-0.58451363$

C $-0.31558616-1.77582395-0.51842176$

C $0.23735357 \quad 1.67041684-0.49595026$

C $1.52235675 \quad 1.04818204 \quad 0.13191011$

C $1.382409590 .14737587 \quad 1.14636540$

C $1.84508836-1.24242864 \quad 0.82513837$

C $0.60001617-1.98546576 \quad 0.44466223$

C $0.714620290 .39804995 \quad 2.46246738$

C $0.97554050 \quad 1.83109130 \quad 2.94592226$

C $-0.79469506 \quad 0.134153792 .38047746$

$\mathrm{H}-2.09071066-2.64296479 \quad 0.36600245$

$\mathrm{H}-1.26661963-3.69118923-0.81450941$

$\mathrm{H}-2.24657591-2.32667613-1.36791567$

$\mathrm{H}-0.63097308 \quad 1.81312448 \quad 0.10040526$

H $1.38528015 \quad 2.36330273 \quad 0.08009785$

H $2.25703943 \quad 0.80382331-0.65877456$

$\mathrm{H} 1.14596571-0.30915550 \quad 3.22143987$

H 2.35346950 -1.73433054 1.67747267

$\mathrm{H} 2.58036536-1.26783557-0.00915796$

$\mathrm{H} \quad 0.42018417 \quad-2.83041250 \quad 1.11574352$

$\mathrm{H} \quad 0.46807576 \quad 2.57154612 \quad 2.31833474$

H 2.04564064 2.06518084 2.95114262

$\mathrm{H} \quad 0.60574726 \quad 1.97080199 \quad 3.96892424$

$\mathrm{H}-1.28140614 \quad 0.33072260 \quad 3.34151549$

$\mathrm{H}-1.00061529-0.908806762 .10723853$

$\mathrm{H}-1.28026631 \quad 0.76503430 \quad 1.62624775$

o $0.56788365-0.14202324-1.94055377$

○ $0.205373651 .34662020-1.74398328$

$0-0.49766581-0.92271786-1.56453691$

Vibrational frequencies (in $\mathrm{cm}-1$ )

$\begin{array}{llllllllllllllll}-1652.9 & 36.5 & 55.9 & 73.8 & 87.7 & 127.5 & 142.1 & 151.6 & 179.8 & 214.6 & 249.3 & 260.0 & 273.3 & 325.5\end{array}$ $339.7 \quad 368.9 \quad 381.5 \quad 449.0 \quad 468.3 \quad 529.5 \quad 536.6 \quad 562.6 \quad 614.8 \quad 630.0 \quad 674.7 \quad 712.7 \quad 811.8 \quad 846.9$

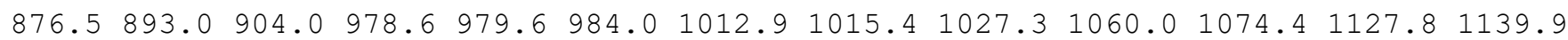

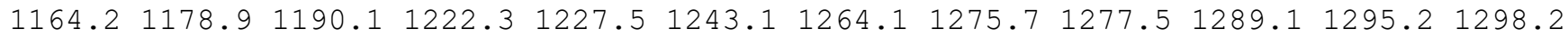
$1301.21304 .81320 .3 \quad 1333.6 \quad 1386.8 \quad 1391.4 \quad 1398.2 \quad 1409.51423 .2 \quad 1426.91702 .21853 .8$ $2196.72658 .52660 .8 \quad 2662.92671 .5 \quad 2672.8 \quad 2674.92678 .62698 .82703 .42736 .72740 .8$ $2747.4 \quad 2774.92777 .3 \quad 2781.1$

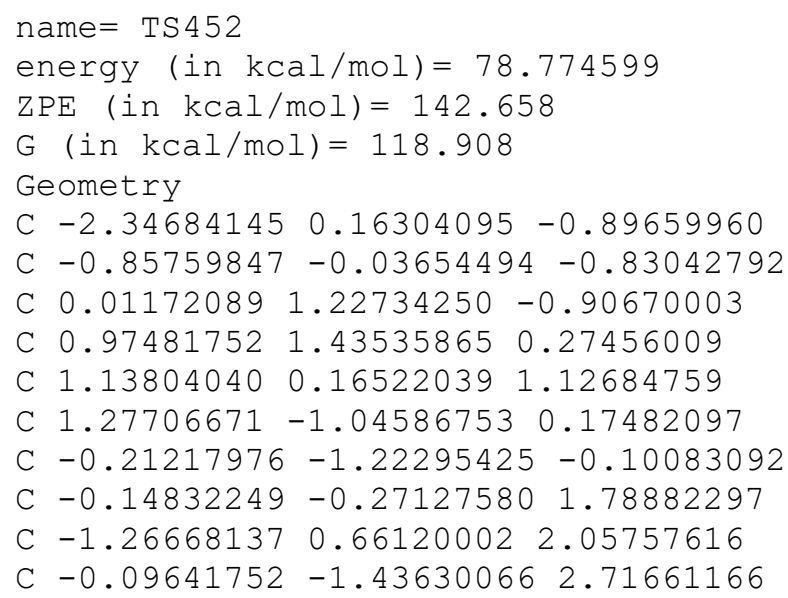


$\mathrm{H}-2.63855675 \quad 0.35499783-1.94594552$

$\mathrm{H}-2.69859261 \quad 1.00773515-0.29869785$

$\mathrm{H}-2.89453402-0.73350931-0.58135972$

$\mathrm{H}-0.60671818 \quad 2.11978834-1.10711465$

$\mathrm{H} \quad 0.64471663 \quad 2.28286104 \quad 0.90023872$

H $1.969112231 .73445399-0.12351689$

H $1.97423615 \quad 0.28301998 \quad 1.84109864$

H $1.75684132-1.92176377 \quad 0.62503745$

$\mathrm{H} 1.84724203-0.81738149-0.74912420$

$\mathrm{H}-0.73483055-2.12450056 \quad 0.16846353$

$\mathrm{H}-1.35033108 \quad 1.48311651 \quad 1.33194065$

$\mathrm{H}-1.14876526 \quad 1.13576466 \quad 3.04762827$

$\mathrm{H}-2.24109859 \quad 0.14744621 \quad 2.07031004$

$\mathrm{H} 0.09361977-1.10279030 \quad 3.75104828$

H $0.69384695-2.161168112 .47516281$

$\mathrm{H}-1.04519654-1.99341424 \quad 2.73866803$

$\begin{array}{lllll}0 & 0.27650161 & 0.37749955 & -2.98754618\end{array}$

$00.891437191 .00758093-2.04666953$

o $-0.37519592-1.03666921-1.65443764$

Vibrational frequencies (in $\mathrm{cm}-1$ )

$\begin{array}{lllllllllllllllll}-759.3 & 89.6 & 110.0 & 121.7 & 144.9 & 153.7 & 201.3 & 205.5 & 224.3 & 242.6 & 269.2 & 322.1 & 352.7 & 375.5\end{array}$

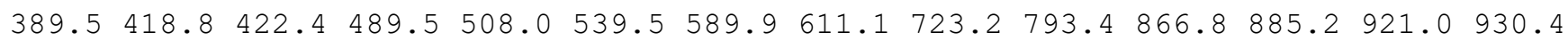
$937.6 \quad 970.8 \quad 991.9 \quad 1008.3 \quad 1018.7 \quad 1027.0 \quad 1042.4 \quad 1062.6 \quad 1068.0 \quad 1094.7 \quad 1100.7 \quad 1113.5 \quad 1133.8$ $1158.21168 .7 \quad 1183.4 \quad 1211.1 \quad 1226.7 \quad 1236.4 \quad 1251.0 \quad 1262.5 \quad 1265.4 \quad 1266.3 \quad 1273.7 \quad 1276.6$

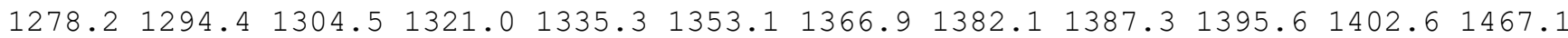
$2650.02653 .2 \quad 2655.6 \quad 2657.0 \quad 2660.3 \quad 2660.8 \quad 2665.8 \quad 2682.42694 .2 \quad 2716.82737 .82747 .0$ $2749.52769 .2 \quad 2771.8 \quad 2779.2$

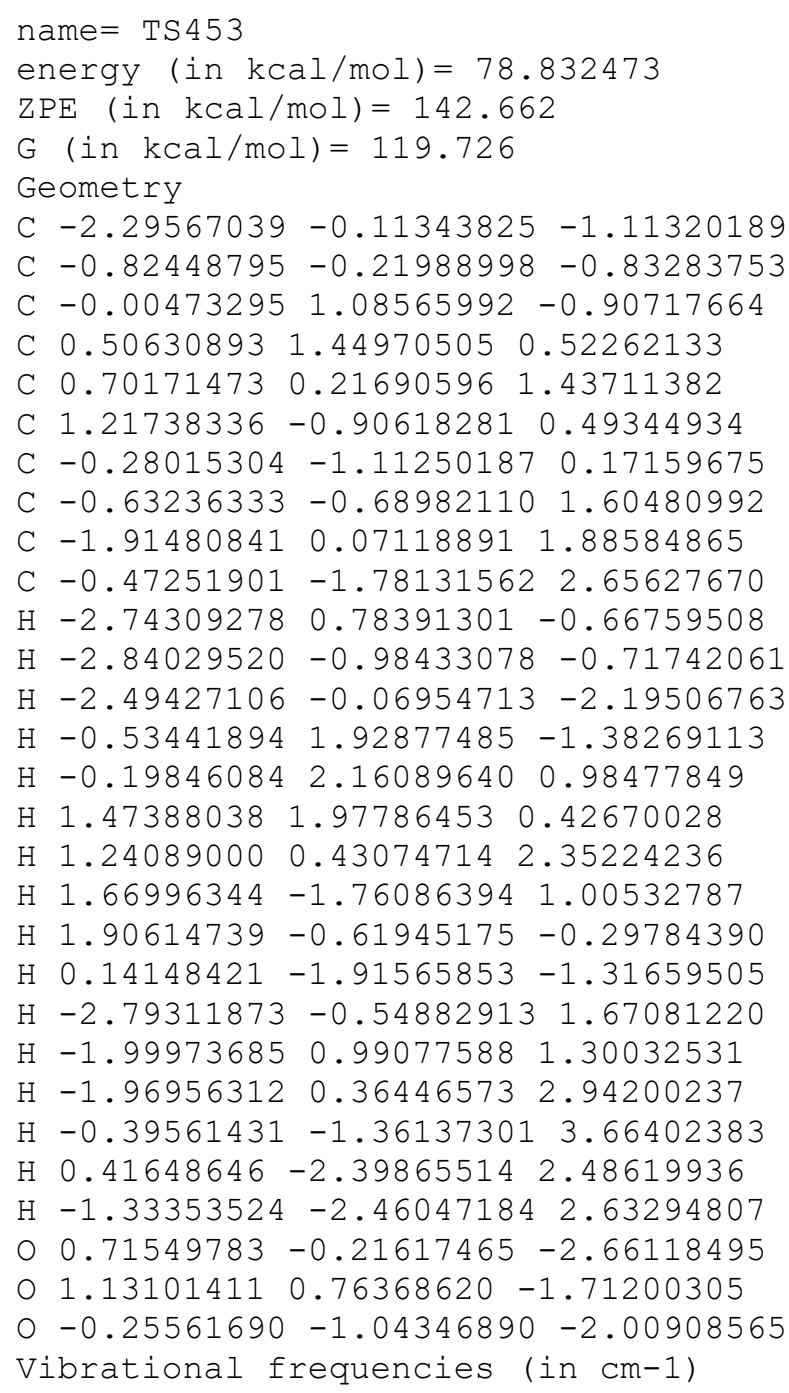


$\begin{array}{lllllllllllllllll}-1159.9 & 115.3 & 129.5 & 144.6 & 173.4 & 189.9 & 213.0 & 226.0 & 264.4 & 291.4 & 300.8 & 368.9 & 387.9 & 403.8\end{array}$

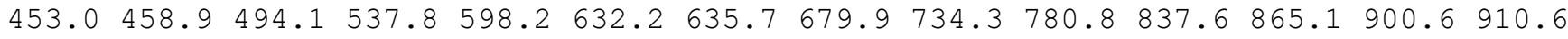
$929.1951 .6 \quad 958.2978 .1 \quad 994.4 \quad 1010.7 \quad 1013.1 \quad 1022.3 \quad 1060.4 \quad 1072.8 \quad 1084.01103 .7 \quad 1109.5$

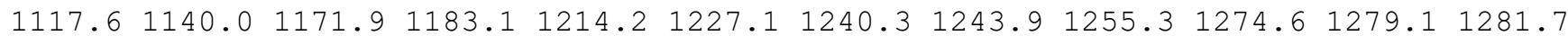
$1292.5 \quad 1298.7 \quad 1302.7 \quad 1314.0 \quad 1324.1 \quad 1324.8 \quad 1357.2 \quad 1382.5 \quad 1398.7 \quad 1406.01419 .01464 .9$ $2174.72665 .2 \quad 2666.2 \quad 2669.6 \quad 2673.2 \quad 2675.7 \quad 2679.92683 .92685 .2 \quad 2692.2 \quad 2739.5 \quad 2752.3$ $2767.02776 .2 \quad 2780.6 \quad 2782.1$

name $=$ TS 454

energy (in $\mathrm{kcal} / \mathrm{mol})=79.674356$

$\mathrm{ZPE}($ in $\mathrm{kcal} / \mathrm{mol})=141.863$

$\mathrm{G}($ in $\mathrm{kcal} / \mathrm{mol})=118.684$

Geometry

C $-0.952387551 .13190281-0.58242092$

C $0.28920226 \quad 0.33312887 \quad-0.23189452$

C 1.526497041 .223359860 .17217043

C $2.29525571 \quad 0.80339859 \quad 1.44197757$

C $1.72316915-0.456476702 .08515293$

C $1.42475009-1.505298470 .98456882$

C $0.05029547-0.794247890 .76964615$

C $0.10961783-0.27308020 \quad 2.25890784$

C $-0.29302114 \quad 1.06336208 \quad 2.62460735$

C $-0.33481921-1.27212564 \quad 3.22405544$

$\mathrm{H}-1.691716160 .49271232-1.08653702$

$\mathrm{H}-0.72260601 \quad 1.95442381-1.27170202$

$\mathrm{H}-1.444437891 .55254995 \quad 0.30286270$

$\mathrm{H} \quad 1.25139426 \quad 2.29481886 \quad 0.19904670$

$\mathrm{H} 2.30128993 \quad 1.636735332 .17007413$

$\mathrm{H} \quad 3.36162243 \quad 0.62747233 \quad 1.17559457$

H $2.24902177 \quad-0.78162611 \quad 2.98256141$

H $1.34479645-2.53400607 \quad 1.34384965$

$\mathrm{H} 2.10242142-1.50214503 \quad 0.12349479$

$\mathrm{H}-0.80653621-1.42440360 \quad 0.52523669$

H $-1.99543175 \quad 0.37264414 \quad 3.15197971$

$\mathrm{H}-0.47439171 \quad 1.82977547 \quad 1.90920363$

$\mathrm{H}-0.13686295 \quad 1.40952433 \quad 3.62461584$

$\mathrm{H}-0.56490707 \quad-2.27051364 \quad 2.92615962$

$\mathrm{H}-2.00724708-0.40036485 \quad 3.35478945$

$\mathrm{H}-0.16042441-1.12496035 \quad 4.26834152$

O $1.65514970 \quad 0.60465256-1.99766833$

O $2.45399537 \quad 1.03133672 \quad-0.90242925$

o $0.74662128-0.31746752-1.43341163$

Vibrational frequencies (in cm-1)

$\begin{array}{llllllllllllll}-732.9 & 72.9 & 106.9 & 140.4 & 182.9 & 189.6 & 232.2 & 264.5 & 275.6 & 326.1 & 346.5 & 354.3 & 376.6 & 413.9\end{array}$ $424.1 \quad 486.4 \quad 499.2 \quad 527.7 \quad 557.3 \quad 601.4 \quad 629.6 \quad 631.1656 .6 \quad 710.6 \quad 736.2 \quad 774.9 \quad 829.7 \quad 870.3$

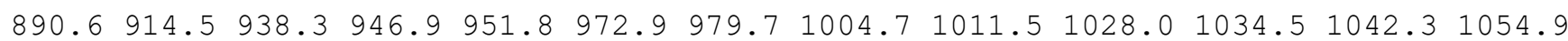
$1074.7 \quad 1086.5 \quad 1102.0 \quad 1123.0 \quad 1136.2 \quad 1154.4 \quad 1175.6 \quad 1183.6 \quad 1217.1 \quad 1233.7 \quad 1255.2 \quad 1264.1$ $1273.21278 .8 \quad 1283.0 \quad 1288.91307 .9 \quad 1313.0 \quad 1321.4 \quad 1367.5 \quad 1372.6 \quad 1376.6 \quad 1389.4 \quad 1400.6$ $1423.62651 .62665 .2 \quad 2672.5 \quad 2681.8 \quad 2684.5 \quad 2729.3 \quad 2732.62733 .0 \quad 2738.4 \quad 2746.52760 .1$ $2763.5 \quad 2777.92791 .3 \quad 4715.7$

name $=\operatorname{TS} 455$

energy $($ in $\mathrm{kcal} / \mathrm{mol})=79.56221$

$\operatorname{ZPE}($ in $\mathrm{kcal} / \mathrm{mol})=142.009$

$\mathrm{G}($ in $\mathrm{kcal} / \mathrm{mol})=118.759$

Geometry

C $-2.62057173 \quad 0.06300927 \quad 0.51089825$

C $-1.20163589-0.23487487 \quad 0.04547063$

C $-0.323394121 .04483802-0.16214328$

C $1.12252423 \quad 0.99940900 \quad 0.36927555$

C $1.44379389-0.142374751 .30646983$

C $0.73028222-1.791406190 .16882423$

C $-0.48598031-1.309283650 .88081687$ 
C $0.23356515-0.662714332 .11496935$

C $-0.49779710 \quad 0.40768431 \quad 2.91075290$

C $0.66332617-1.76778210 \quad 3.08877257$

$\mathrm{H}-3.09026290 \quad 0.83645559-0.11307603$

$\mathrm{H}-2.65978003 \quad 0.39285701 \quad 1.55267361$

$\mathrm{H}-3.25194034-0.83349788 \quad 0.42735818$

$\mathrm{H}-0.84693741 \quad 1.95967932 \quad 0.18849861$

H $1.34194705 \quad 1.96256918 \quad 0.87747356$

$\mathrm{H} \quad 1.81418203 \quad 0.97762407 \quad-0.50231190$

H 2.35114018 $0.01949621 \quad 1.89282806$

$\mathrm{H} 1.88470106-1.272843430 .72793123$

$\mathrm{H} \quad 0.80940182-1.69144458-0.89089096$

$\mathrm{H}-1.17554965-2.13077573 \quad 1.14771226$

$\mathrm{H}-1.421163690 .01194588 \quad 3.35116644$

$\mathrm{H}-0.75864388 \quad 1.28770106 \quad 2.31669011$

$\mathrm{H} \quad 0.12743072 \quad 0.76703932 \quad 3.73926344$

$\mathrm{H} 1.36862447-1.394122123 .83658929$

H $1.14444972-2.605879672 .56339512$

$\mathrm{H}-0.20032991-2.18170920 \quad 3.62030615$

$0-0.38806232-0.11936899-2.08118869$

$0-0.300197791 .20463190-1.57954705$

o $-1.39608618-0.72969329-1.29427523$

Vibrational frequencies (in $\mathrm{cm}-1$ )

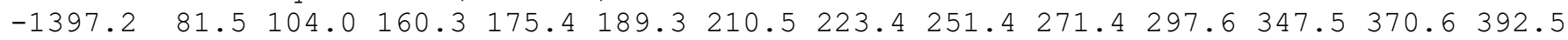

$428.4 \quad 463.2 \quad 476.2 \quad 522.5 \quad 573.1 \quad 593.7 \quad 658.7 \quad 691.6 \quad 714.0 \quad 817.7 \quad 844.3 \quad 868.8 \quad 893.9 \quad 907.2$

$947.2954 .2972 .3 \quad 993.1 \quad 1003.7 \quad 1011.7 \quad 1019.3 \quad 1029.5 \quad 1047.8 \quad 1075.4 \quad 1099.91110 .8 \quad 1144.8$

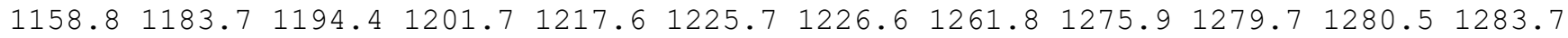

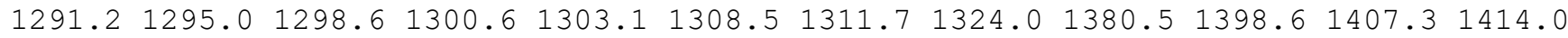
$1971.72646 .8 \quad 2665.4 \quad 2668.1 \quad 2672.6 \quad 2676.3 \quad 2678.0 \quad 2682.5 \quad 2683.92707 .7 \quad 2718.3 \quad 2729.5$ $2731.92779 .0 \quad 2780.4 \quad 2783.2$

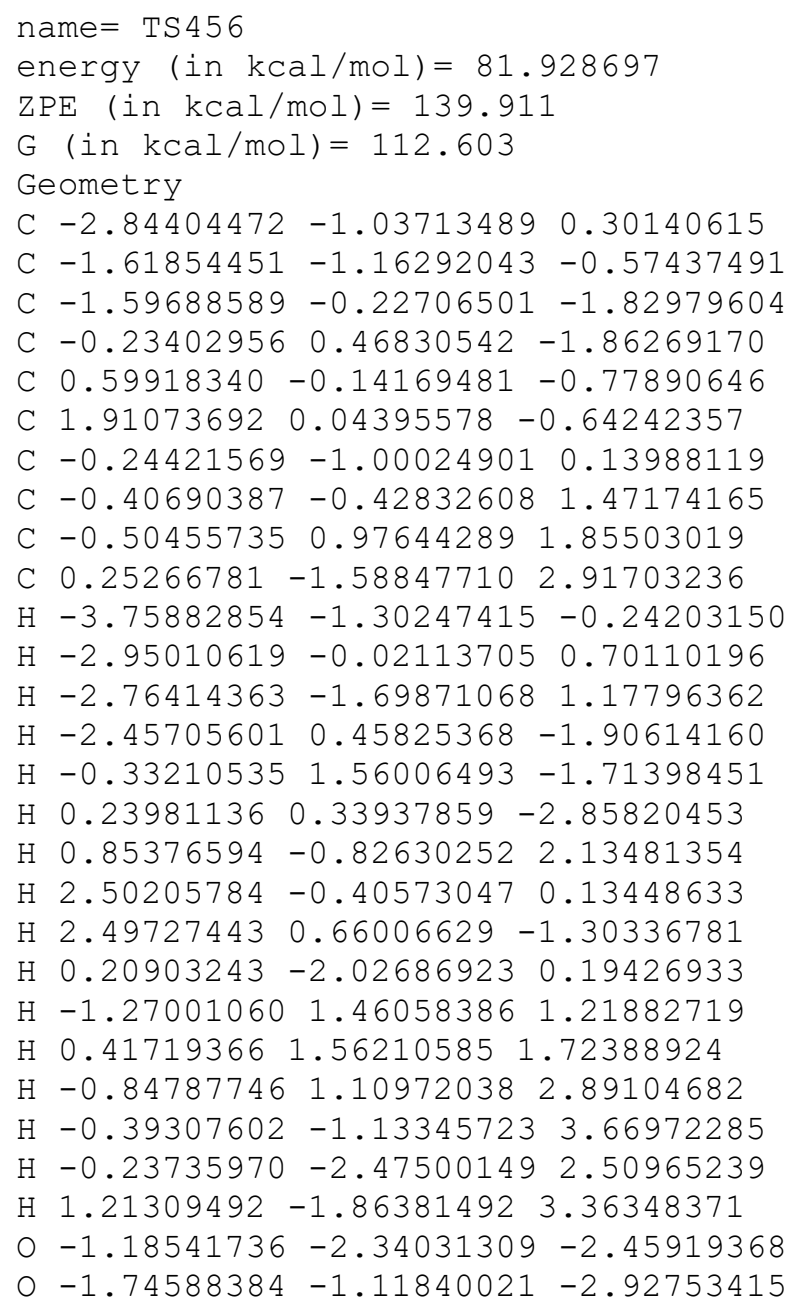


$0-1.69893850-2.47837926-1.14573578$

Vibrational frequencies (in $\mathrm{cm}-1$ )

$\begin{array}{llllllllllllllll}-1150.2 & 10.5 & 26.0 & 64.4 & 71.6 & 106.4 & 121.9 & 137.8 & 179.2 & 202.4 & 235.4 & 250.5 & 306.4 & 336.5\end{array}$

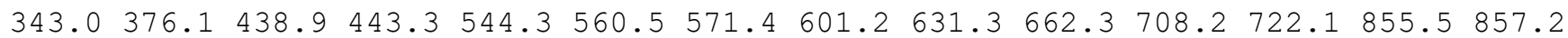
$928.6 \quad 945.0 \quad 968.1 \quad 973.5 \quad 977.9 \quad 1004.1 \quad 1010.4 \quad 1026.5 \quad 1055.0 \quad 1056.8 \quad 1083.6 \quad 1122.2 \quad 1131.5$

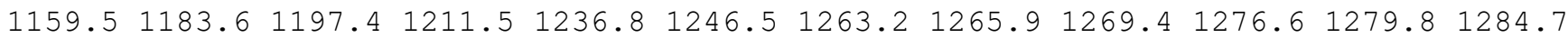

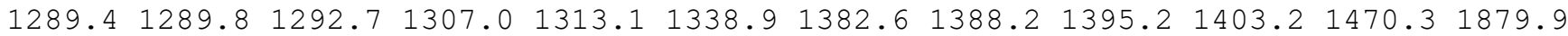
$1972.52643 .7 \quad 2657.5 \quad 2662.0 \quad 2663.2 \quad 2669.2 \quad 2671.22682 .22690 .02704 .62729 .02744 .1$ $2757.12770 .6 \quad 2782.2 \quad 2797.6$

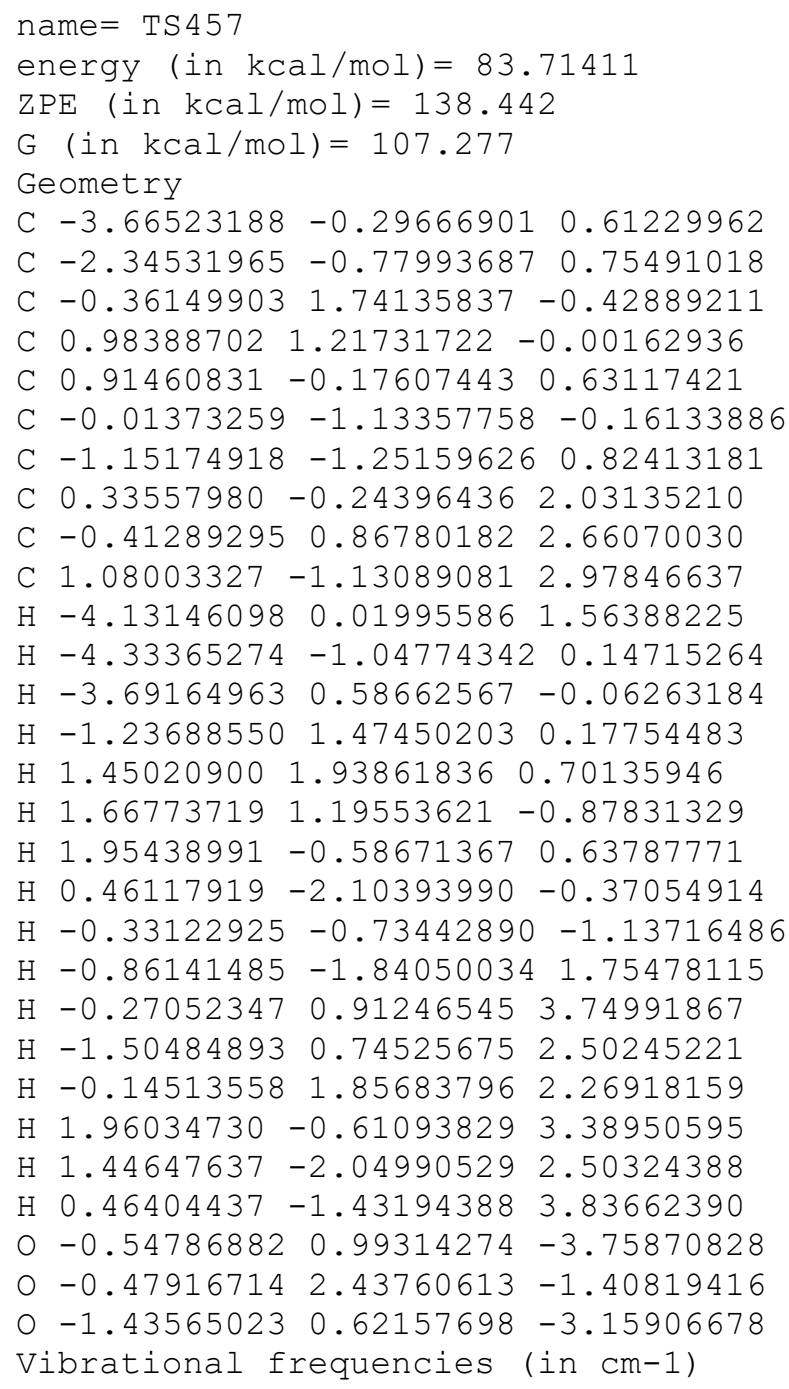


C $1.62333411-1.09779757 \quad 0.67641080$

C $-0.24506082-1.67758064-0.35414138$

C $0.94463813-0.47528873 \quad 3.03547565$

C $1.47658213 \quad 0.33082969 \quad 4.17606886$

C $0.14996277-1.52842580 \quad 3.23538268$

$\mathrm{H}-3.39600051-0.52849511-0.08384770$

$\mathrm{H}-2.69106113-1.95931134 \quad 0.70982501$

$\mathrm{H}-2.78219503-1.87118731-1.05695403$

$\mathrm{H}-1.52248341 \quad 0.09975289 \quad 1.90839516$

$\mathrm{H} \quad 0.26642024 \quad 1.89395692 \quad 1.78554717$

$\mathrm{H} \quad 0.67797497 \quad 1.40911647 \quad 0.14273588$

$\mathrm{H} \quad 2.337206390 .58493340 \quad 1.78987130$

$\mathrm{H} \quad 2.40842168-1.79032023 \quad 1.00289745$

$\mathrm{H} \quad 1.90585615 \quad-0.71953244 \quad-0.30912734$

$\mathrm{H} \quad 0.19266857-1.87061927-1.30832005$

H $1.26825193 \quad 1.40262718 \quad 4.04665489$

H $2.56611383 \quad 0.21651580 \quad 4.26924597$

$\mathrm{H} 1.04139313 \quad 0.035510465 .14029565$

$\mathrm{H}-0.26525077-2.13970304 \quad 2.44874485$

$\mathrm{H} \quad 0.71042207-1.914632320 .73409625$

$\mathrm{H}-0.15283443-1.86807420 \quad 4.21209297$

$\begin{array}{lllll}0 & -1.92428335 & 1.40502285 & -0.95772649\end{array}$

$0-1.897382291 .49346095 \quad 0.48917567$

$\begin{array}{lllll}0 & -1.16004700 & 0.27686681 & -1.32350948\end{array}$

Vibrational frequencies (in cm-1)

$\begin{array}{llllllllllllll}-823.1 & 42.1 & 55.1 & 76.3 & 95.8 & 105.8 & 133.5 & 153.3 & 227.8 & 255.1 & 260.6 & 305.8 & 324.3 & 341.6\end{array}$

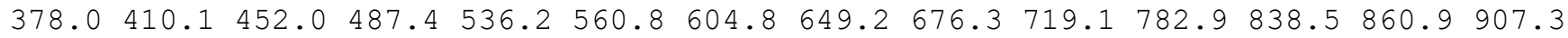
$959.9964 .2 \quad 969.4989 .2 \quad 995.4 \quad 1001.5 \quad 1025.7 \quad 1041.2 \quad 1050.8 \quad 1060.8 \quad 1090.2 \quad 1098.9 \quad 1107.6$

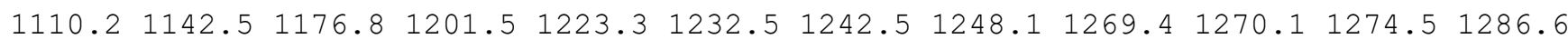

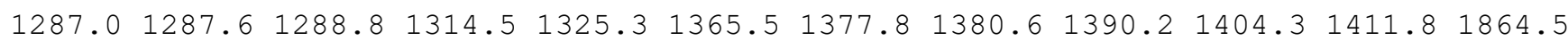
$1872.4 \quad 2652.8 \quad 2667.2 \quad 2668.92670 .5 \quad 2672.8 \quad 2676.92683 .8 \quad 2687.2 \quad 2712.7 \quad 2717.2 \quad 2737.7$ $2742.7 \quad 2778.0 \quad 2778.2 \quad 2787.2$

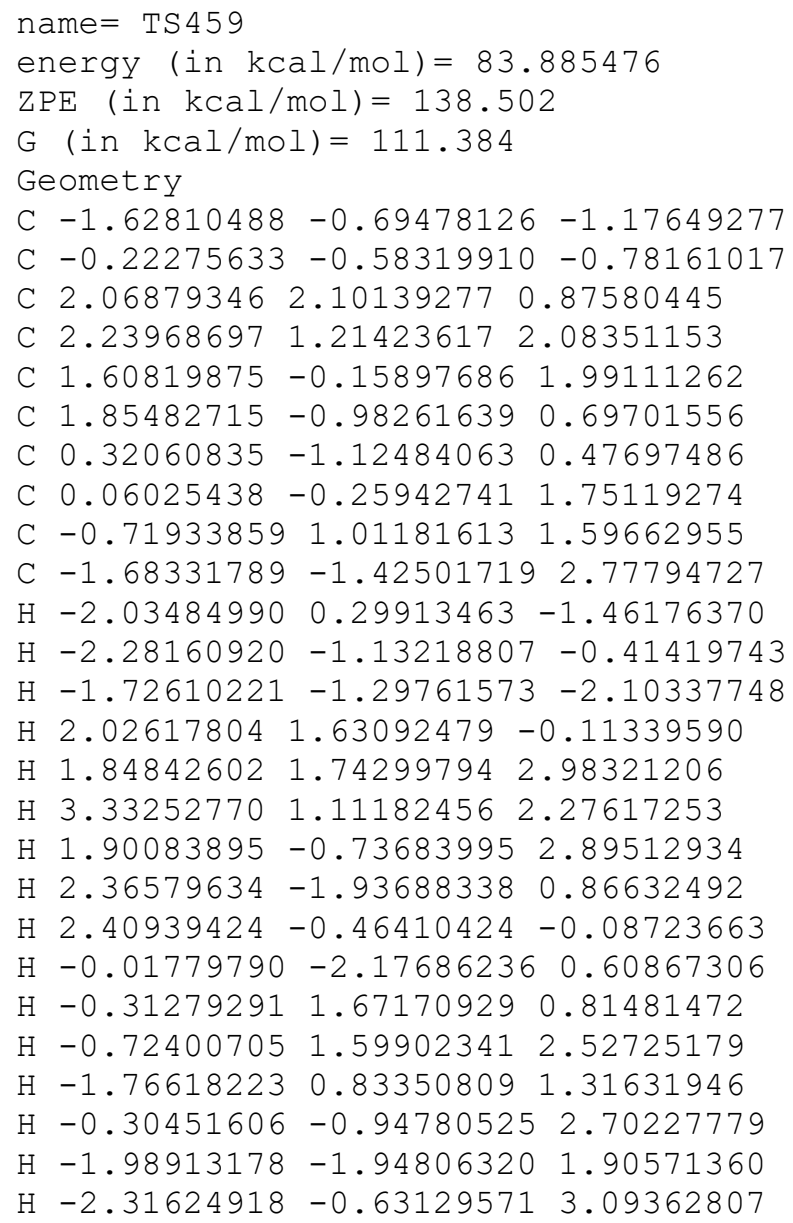


$0.24448815 \quad 0.48557832-2.64144536$

$02.003870193 .29889802 \quad 0.99665017$

O $0.65630047 \quad 0.00360207-1.54899743$

Vibrational frequencies (in $\mathrm{cm}-1$ )

$\begin{array}{lllllllllllllll}-587.6 & 35.5 & 42.1 & 54.8 & 86.3 & 99.2 & 115.7 & 128.5 & 152.8 & 180.7 & 200.6 & 220.3 & 256.4 & 269.3\end{array}$

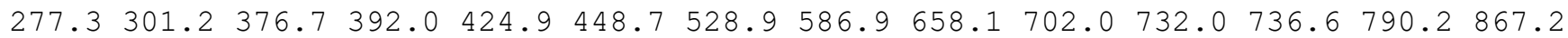
$869.5915 .4937 .2 \quad 994.0 \quad 1029.4 \quad 1039.8 \quad 1046.7 \quad 1052.7 \quad 1060.7 \quad 1082.2 \quad 1092.8 \quad 1112.7 \quad 1116.4$ $\begin{array}{llllllllllll}1135.1 & 1164.1 & 1178.9 & 1181.8 & 1208.0 & 1225.7 & 1234.5 & 1260.8 & 1261.0 & 1279.7 & 1283.9 & 1286.4\end{array}$

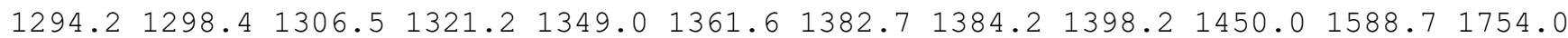
$1835.12629 .92644 .92653 .8 \quad 2658.5 \quad 2669.4 \quad 2672.5 \quad 2675.42692 .2 \quad 2694.92701 .32709 .7$ $2735.4 \quad 2761.4 \quad 2767.7 \quad 2777.6$

\section{name $=$ TS460}

energy $($ in $\mathrm{kcal} / \mathrm{mol})=84.931797$

$\mathrm{ZPE}($ in $\mathrm{kcal} / \mathrm{mol})=137.675$

$\mathrm{G}(\mathrm{in} \mathrm{kcal} / \mathrm{mol})=109.766$

Geometry

C $-2.82306464-1.47451051 \quad 0.39705040$

C $-1.62570598-0.72722340-0.06251823$

C $-0.091864672 .42780166-1.30572041$

C $1.16569395 \quad 1.99033208-1.33745908$

C $1.27252380-0.469522592 .31079008$

C $2.29238722-0.93807186 \quad 1.59252185$

C $-0.49230832-0.42994175 \quad 0.57865807$

C $-0.17876377-0.791616412 .00896209$

C $-1.08463779 \quad 0.04552403 \quad 2.93787764$

C $-0.46544765-2.236216492 .15830131$

$\mathrm{H}-3.60197363-0.791496890 .76522745$

$\mathrm{H}-2.56164463-2.16073103 \quad 1.23058708$

$\mathrm{H}-3.26698232-2.08811787-0.39997341$

$\mathrm{H}-0.43704520 \quad 3.41380489-0.99391736$

$\mathrm{H} \quad 2.005275292 .58751607 \quad-1.02361891$

$\mathrm{H} 1.462039021 .01047506-1.67111002$

H $1.43171520 \quad 0.18131144 \quad 3.16803066$

H $2.17219828-1.57242017 \quad 0.72682019$

$\mathrm{H} 3.32539725-0.71573286 \quad 1.80797201$

$\mathrm{H} \quad 0.29164401 \quad 0.14462146 \quad 0.08496887$

$\mathrm{H}-0.84278138-0.10651940 \quad 3.99164408$

$\mathrm{H}-2.13675187-0.22950634 \quad 2.79370530$

$\mathrm{H}-0.98996437 \quad 1.11406699 \quad 2.71641374$

$\mathrm{H}-0.58095295-2.63568338 \quad 3.64560021$

H $0.23318204 \quad-2.58993436 \quad 3.61949053$

$\mathrm{H} \quad 0.12404600 \quad-2.95443626 \quad 1.63871669$

$\begin{array}{lllll}0 & -0.88340416 & 0.37677885 & -1.96534448\end{array}$

$0-1.203953511 .75371314-1.70179092$

O $-1.89325115-0.35537281-1.37196972$

Vibrational frequencies (in $\mathrm{cm}-1$ )

$\begin{array}{lllllllllllllll}-1013.3 & 22.6 & 51.3 & 55.7 & 71.2 & 86.7 & 114.2 & 124.1 & 141.5 & 169.6 & 184.3 & 190.4 & 215.8 & 241.1\end{array}$

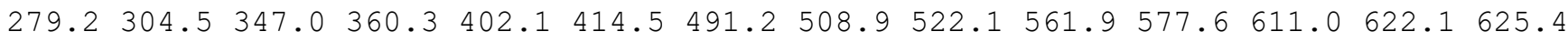

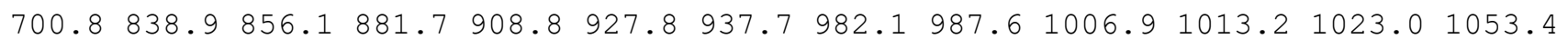
$1056.51061 .5 \quad 1078.8 \quad 1092.3 \quad 1099.6 \quad 1140.2 \quad 1181.1 \quad 1195.91235 .4 \quad 1265.8 \quad 1270.6 \quad 1278.7$

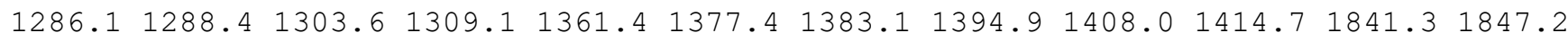
$1891.72625 .42672 .2 \quad 2677.6 \quad 2683.2 \quad 2712.3 \quad 2714.02714 .62735 .4 \quad 2739.82753 .62767 .7$ $2781.5 \quad 2786.02797 .0 \quad 4667.2$

name $=\operatorname{TS} 461$

energy (in $\mathrm{kcal} / \mathrm{mol})=83.646462$

$\mathrm{ZPE}(\mathrm{in} \mathrm{kcal} / \mathrm{mol})=139.124$

$\mathrm{G}($ in $\mathrm{kcal} / \mathrm{mol})=112.967$

Geometry

C $-1.83229939-1.08795006-1.72441321$

C $-0.48810459-1.15003505-1.18175929$

C $-0.017222291 .16578182-0.57855656$ 
C $0.71671488 \quad 1.65801950 \quad 0.59284058$

C $0.67067074 \quad 0.67167499 \quad 1.76962764$

C $1.00666198-0.76846208 \quad 1.25008331$

C $-0.49723837-0.977000551 .00164723$

C $-0.78923165 \quad 0.14609319 \quad 2.08518882$

C $-1.95936368 \quad 1.07734567 \quad 1.84377797$

C $-0.89805839-0.45329468 \quad 3.48209684$

$\mathrm{H}-1.90957288-1.67221609-2.66613539$

$\mathrm{H}-2.13998540-0.04575174-1.97920120$

$\mathrm{H}-2.60646151-1.47297032-1.03213214$

$\mathrm{H}-0.76932236-0.07649078 \quad 0.04240459$

$\mathrm{H} \quad 0.32041793 \quad 2.65527350 \quad 0.89327423$

H $1.77385436 \quad 1.87228494 \quad 0.30969240$

$\mathrm{H} 1.23566108 \quad 1.02139305 \quad 2.63516736$

H $1.43669244-1.429980012 .00713142$

$\mathrm{H} 1.67248090 \quad-0.79896896 \quad 0.38364910$

$\mathrm{H}-0.96688521-1.928075291 .10820111$

$\mathrm{H}-2.91548944 \quad 0.54831952 \quad 1.91509472$

$\mathrm{H}-1.92687744 \quad 1.57594607 \quad 0.86656337$

$\mathrm{H}-1.973299691 .88315122 \quad 2.59162858$

$\mathrm{H}-0.08131475-1.149483773 .70303721$

$\mathrm{H}-1.83650797-1.00357268 \quad 3.60866379$

$\mathrm{H}-0.86885474 \quad 0.33230953 \quad 4.24792777$

$0.29477362 \quad 2.88444726-1.93874241$

$\begin{array}{llll}0 & -0.20224478 & 1.70718412 & -1.69250770\end{array}$

O $0.59998821-1.49737786-1.38102071$

Vibrational frequencies (in cm-1)

$\begin{array}{lllllllllllllll}-1640.2 & 49.3 & 63.7 & 78.0 & 99.2 & 103.8 & 128.3 & 135.5 & 161.2 & 180.2 & 217.7 & 229.5 & 251.5 & 281.3\end{array}$

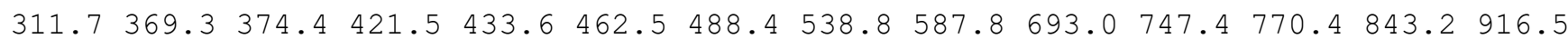
$928.7984 .91000 .2 \quad 1005.3 \quad 1010.4 \quad 1010.5 \quad 1023.1 \quad 1038.9 \quad 1056.4 \quad 1073.3 \quad 1078.8 \quad 1094.8$ $1098.51117 .1 \quad 1128.4 \quad 1176.7 \quad 1188.3 \quad 1192.8 \quad 1215.1 \quad 1219.3 \quad 1225.5 \quad 1249.01270 .81274 .6$

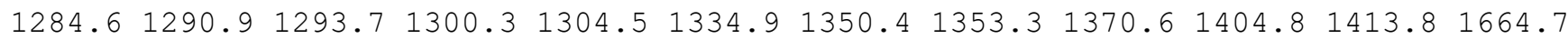
1715.9 2065.0 2607.5 2632.9 2644.3 2663.2 2675.1 2677.4 2678.5 2681.4 2731.82744 .9 $2746.9 \quad 2759.7 \quad 2765.5 \quad 2780.12782 .8$

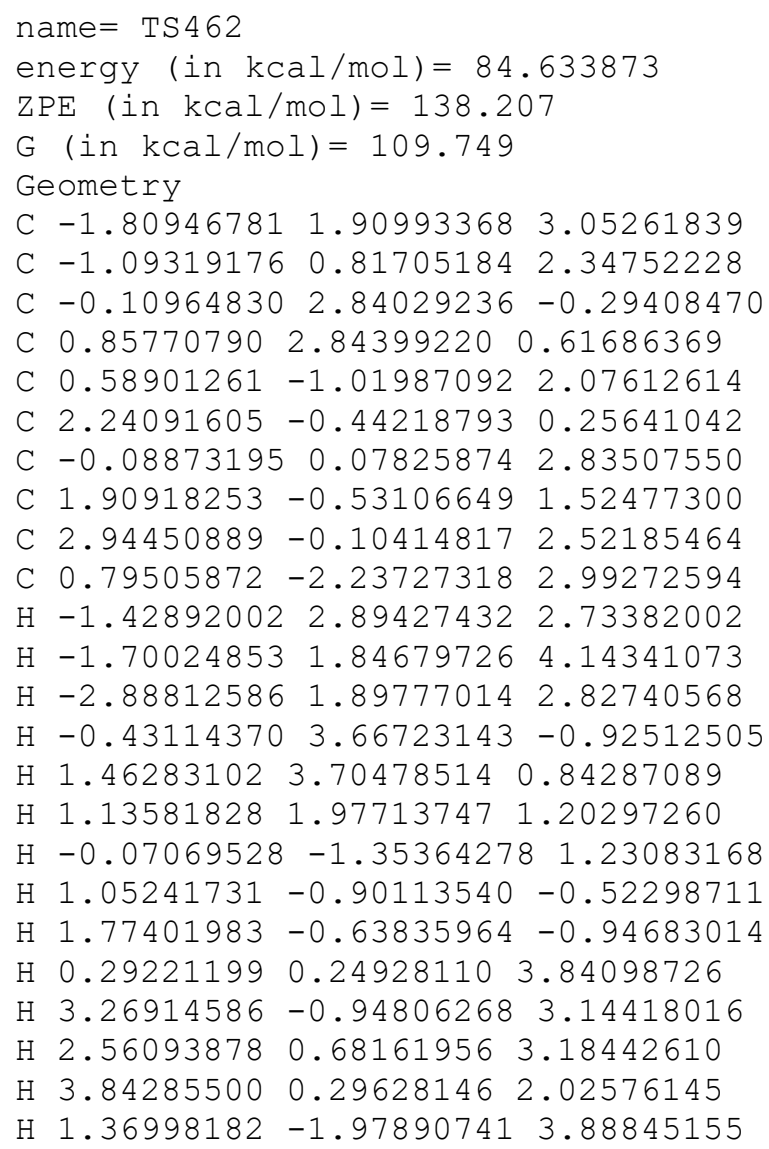


$\mathrm{H} \quad 1.34122957-3.030783512 .47085839$

$\mathrm{H}-0.16633044-2.646864313 .32227232$

$0-0.654734660 .631406710 .11606922$

$0-0.90231890 \quad 1.78347333-0.64979150$

O $-1.65820787 \quad 0.574520421 .10610254$

Vibrational frequencies (in $\mathrm{cm}-1$ )

$\begin{array}{lllllllllllllllll}-1599.5 & 27.5 & 41.8 & 55.1 & 63.3 & 65.8 & 80.9 & 96.0 & 117.1 & 144.0 & 159.5 & 169.0 & 234.0 & 237.1\end{array}$ $281.3 \quad 306.5 \quad 342.8 \quad 373.6 \quad 424.5 \quad 434.4 \quad 462.7 \quad 523.7 \quad 542.8 \quad 556.6 \quad 603.8 \quad 617.8 \quad 633.1 \quad 645.4$ $801.4 \quad 838.0 \quad 875.3 \quad 921.4 \quad 929.9 \quad 949.7 \quad 968.3 \quad 1006.2 \quad 1010.2 \quad 1053.5 \quad 1057.9 \quad 1062.0 \quad 1066.7$ $1076.1 \quad 1083.91121 .3 \quad 1166.0 \quad 1233.4 \quad 1258.7 \quad 1269.9 \quad 1275.0 \quad 1276.4 \quad 1278.2 \quad 1290.3 \quad 1294.5$

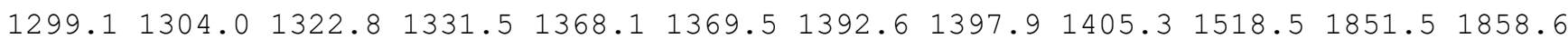
$1910.92663 .12668 .2 \quad 2671.7 \quad 2674.4 \quad 2676.2 \quad 2679.82687 .72702 .72732 .3 \quad 2754.22776 .6$ $2777.3 \quad 2781.0 \quad 2787.9 \quad 3909.3$

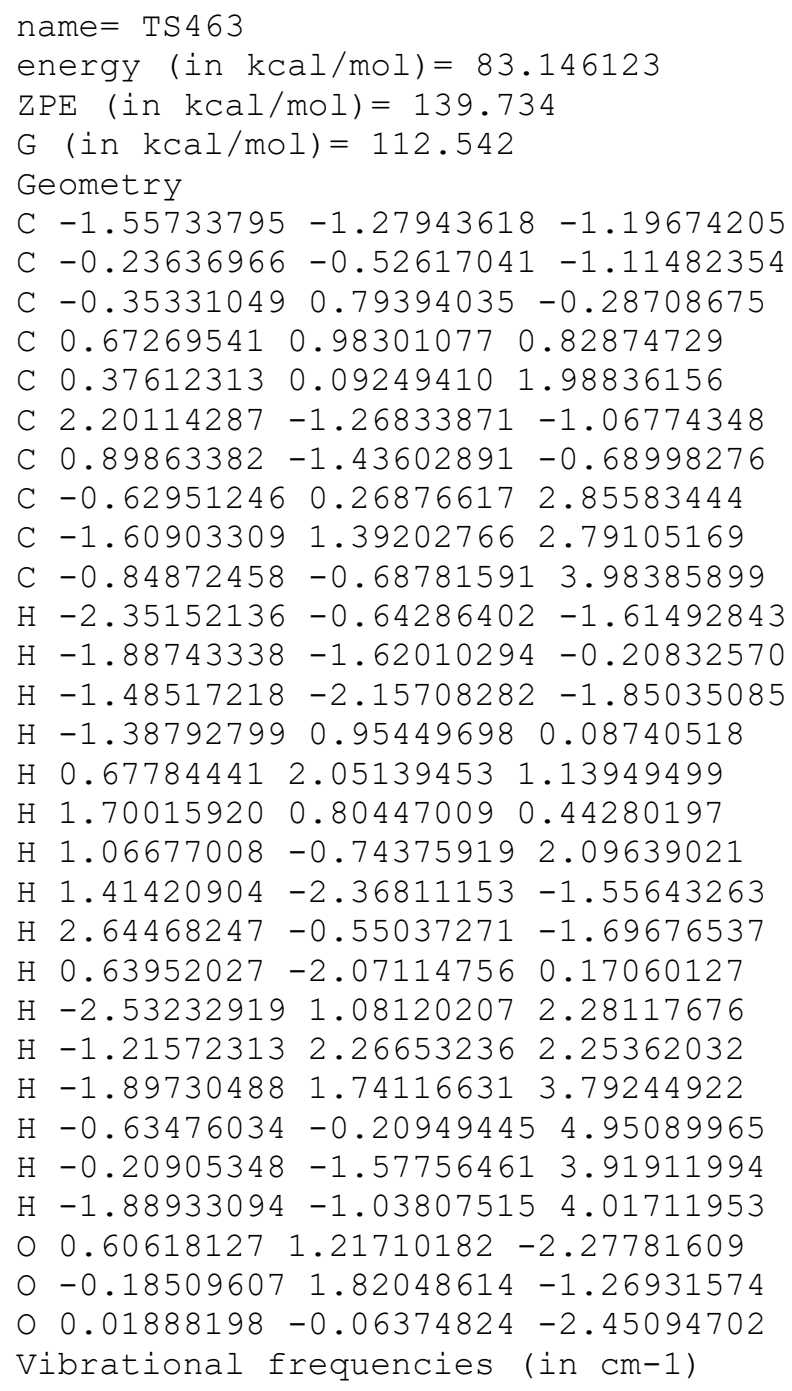


C $-0.11003715-0.39506184-1.06989381$

C $0.47911421 \quad 1.05132041-0.88832608$

$\begin{array}{lllll}\text { C } & 0.98224258 & 1.50419354 & 0.38672956\end{array}$

C $1.66322262-1.76249613 \quad 1.43605019$

$\begin{array}{llll}\text { C } 1.92053801 & -1.53995021 & 0.13540777\end{array}$

$\begin{array}{lllll}\text { C } & 0.45925480 & -1.57379361 & -0.30104067\end{array}$

C $0.13631346-1.83952091 \quad 1.25294434$

C $-0.65925985-0.802105622 .00673866$

C $-0.41158805-3.23665201 \quad 1.49386007$

H $-2.03737984 \quad 0.12470121-1.93534203$

$\mathrm{H}-2.05157178-1.39443380-1.03297833$

$\mathrm{H}-2.008420110 .14765912-0.16189301$

$\mathrm{H}-0.27042113 \quad 1.78180090-1.29126712$

H $0.66306013 \quad 2.43927965 \quad 0.78430117$

H $1.850191491 .31699163 \quad 1.25797267$

H $2.22170955-1.89870068 \quad 2.32249764$

H $2.77628202-1.41314114-0.47930855$

$\begin{array}{lllll}\mathrm{H} & 1.90832504 & 0.63208669 & 0.65210979\end{array}$

H $0.24026127-2.48927600-0.89607224$

$\mathrm{H}-1.73869308-0.94672456 \quad 1.87510981$

$\mathrm{H}-0.43696478 \quad 0.22244092 \quad 1.66964493$

$\mathrm{H}-0.45394973-0.84892467 \quad 3.08227862$

$\mathrm{H}-0.46582001 \quad-3.46133800 \quad 2.56594172$

H $0.21575070 \quad-4.00967046 \quad 1.03324864$

H $-1.42229210 \quad-3.34353976 \quad 1.08246264$

$\begin{array}{llll}0 & 1.54529116 & -0.13244858 & -2.56929143\end{array}$

$0 \quad 1.568599281 .09583351-1.84369163$

$00.22909928-0.62496312-2.45507383$

Vibrational frequencies (in cm-1)

$\begin{array}{lllllllllllllll}-1274.9 & 38.1 & 84.9 & 120.1 & 141.9 & 150.8 & 164.0 & 193.1 & 202.5 & 226.5 & 260.0 & 269.6 & 295.7 & 315.5\end{array}$

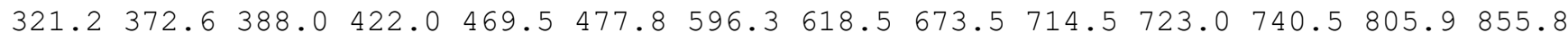

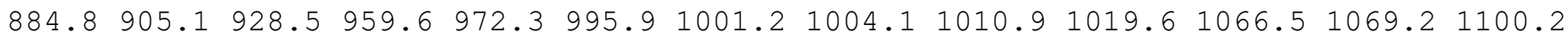

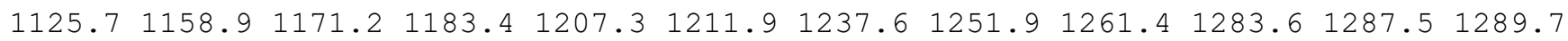

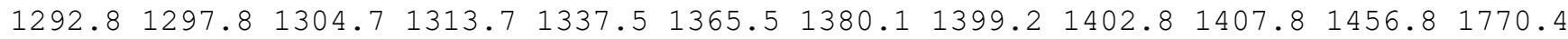

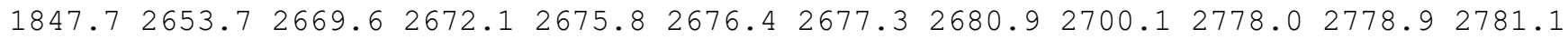
2782.22786 .92806 .13716 .5

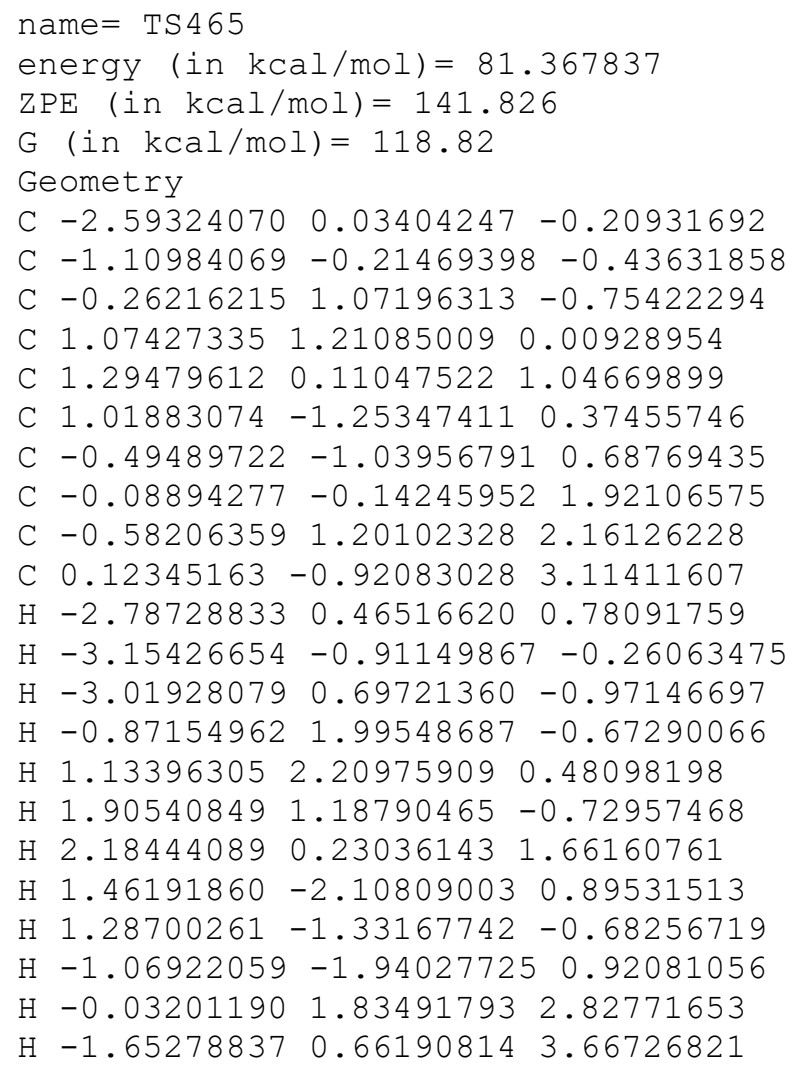


$\mathrm{H}-1.26853672 \quad 1.69364253 \quad 1.51521962$

$\mathrm{H}-1.42886174-0.03885123 \quad 3.95452872$

$\mathrm{H} 0.71204597-0.561574603 .92574409$

$\mathrm{H}-0.11787843-1.960549353 .14620830$

$0.03831093-0.43685661-2.39035561$

$0-0.009443620 .96113090-2.15158848$

O $-1.06412390-0.95742657-1.66740670$

Vibrational frequencies (in $\mathrm{cm}-1$ )

$\begin{array}{lllllllllllllll}-618.0 & 88.8 & 135.8 & 144.7 & 171.0 & 201.0 & 224.8 & 265.4 & 280.7 & 324.1 & 348.9 & 362.9 & 371.6 & 413.9\end{array}$

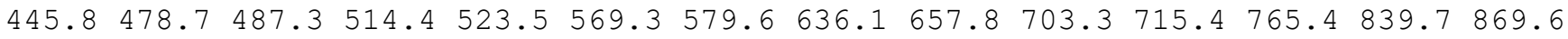

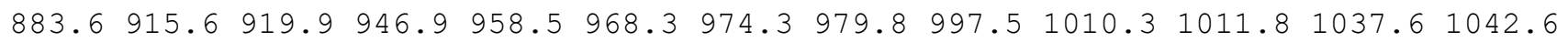

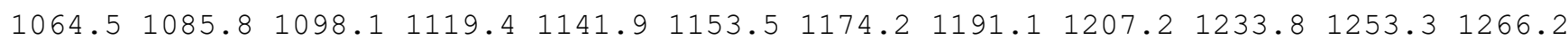
$\begin{array}{lllllllllllllll}1278.9 & 1280.7 & 1283.7 & 1295.6 & 1300.1 & 1313.4 & 1319.2 & 1366.3 & 1371.9 & 1373.4 & 1390.8 & 1398.3\end{array}$ $1422.72651 .32665 .0 \quad 2674.62684 .02685 .5 \quad 2728.62734 .62736 .2 \quad 2739.62744 .32762 .5$ $2765.32778 .2 \quad 2793.8 \quad 4914.9$

name $=$ TS 466

energy $($ in $\mathrm{kcal} / \mathrm{mol})=83.738649$

$\mathrm{ZPE}($ in $\mathrm{kcal} / \mathrm{mol})=139.518$

$\mathrm{G}($ in $\mathrm{kcal} / \mathrm{mol})=113.346$

Geometry

C $-2.52979665-1.03516783-0.84750482$

C $-1.05658133-0.73810067-0.58885216$

C $-0.79833186 \quad 0.81231411-0.55252834$

C $0.091016381 .28941660 \quad 0.50329847$

C $0.58739914 \quad 0.597226891 .51352231$

C $0.96409308-1.793502140 .69469472$

C $-0.52206316-1.53442900 \quad 0.57580610$

C $0.29568592-0.84627204 \quad 1.67335249$

C $0.94359759 \quad 4.07348548 \quad 0.17630163$

C $0.08681141-1.27911318 \quad 3.09992017$

$\mathrm{H}-3.15597571-0.746000050 .00308845$

$\mathrm{H}-2.68926799-2.10532616-1.03532521$

$\mathrm{H}-2.89228620-0.50343172-1.73738565$

$\mathrm{H}-1.761747361 .36928708-0.57275243$

$\mathrm{H} \quad 0.18082038 \quad 2.86439901 \quad 0.59575280$

$\mathrm{H} \quad 0.87546881 \quad 2.57985736 \quad 0.02796846$

$\mathrm{H} \quad 1.239554531 .02767645 \quad 2.27115468$

$\mathrm{H} \quad 1.31512681-2.764022121 .02933800$

$\mathrm{H} \quad 1.63411186-1.39008962-0.06457453$

$\mathrm{H}-1.19937991-2.33908522 \quad 0.88467574$

$\mathrm{H} \quad 0.08575696 \quad 4.66091321-0.09697521$

$\mathrm{H} \quad 1.67024897 \quad 4.07543613-0.62051922$

$\mathrm{H} 1.380118934 .39695741 \quad 1.10205200$

$\mathrm{H} 0.98890432-1.10976305 \quad 3.70295560$

$\mathrm{H}-0.16033047-2.34504340 \quad 3.18118758$

$\mathrm{H}-0.73513285-0.71866537 \quad 3.56571090$

$0-0.36787676-0.05583356-2.60961007$

$0-0.12309612 \quad 1.08724550-1.79426840$

o - $0.28331525-1.15180108-1.72861960$

Vibrational frequencies (in cm-1)

$\begin{array}{lllllllllllllll}-2793.0 & 27.0 & 38.7 & 73.6 & 80.0 & 121.2 & 132.5 & 180.4 & 199.3 & 232.6 & 269.9 & 293.2 & 313.1 & 336.9\end{array}$ $372.6 \quad 396.5 \quad 419.0 \quad 435.1 \quad 484.5 \quad 509.8 \quad 546.1 \quad 559.9600 .1 \quad 670.9 \quad 706.4 \quad 741.8 \quad 816.2 \quad 824.1$ $870.5 \quad 880.5909 .1944 .0 \quad 968.6 \quad 978.4 \quad 1000.6 \quad 1010.0 \quad 1023.6 \quad 1044.3 \quad 1055.6 \quad 1085.2 \quad 1096.0$ $1106.51124 .21148 .1 \quad 1159.7 \quad 1168.7 \quad 1181.91221 .6 \quad 1244.11278 .6 \quad 1279.6 \quad 1289.1 \quad 1290.4$

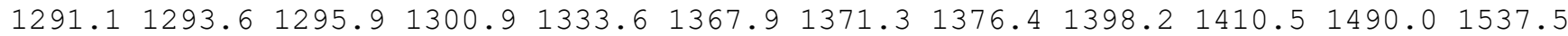
$1919.32670 .3 \quad 2675.5 \quad 2676.12677 .9 \quad 2680.5 \quad 2692.22705 .92709 .32749 .12755 .02780 .3$ $2780.6 \quad 2783.1 \quad 2793.1 \quad 3339.4$

name $=\operatorname{TS} 467$

energy (in $\mathrm{kcal} / \mathrm{mol})=82.216086$

$\mathrm{ZPE}(\mathrm{in} \mathrm{kcal} / \mathrm{mol})=141.117$

$\mathrm{G}($ in $\mathrm{kcal} / \mathrm{mol})=116.663$ 


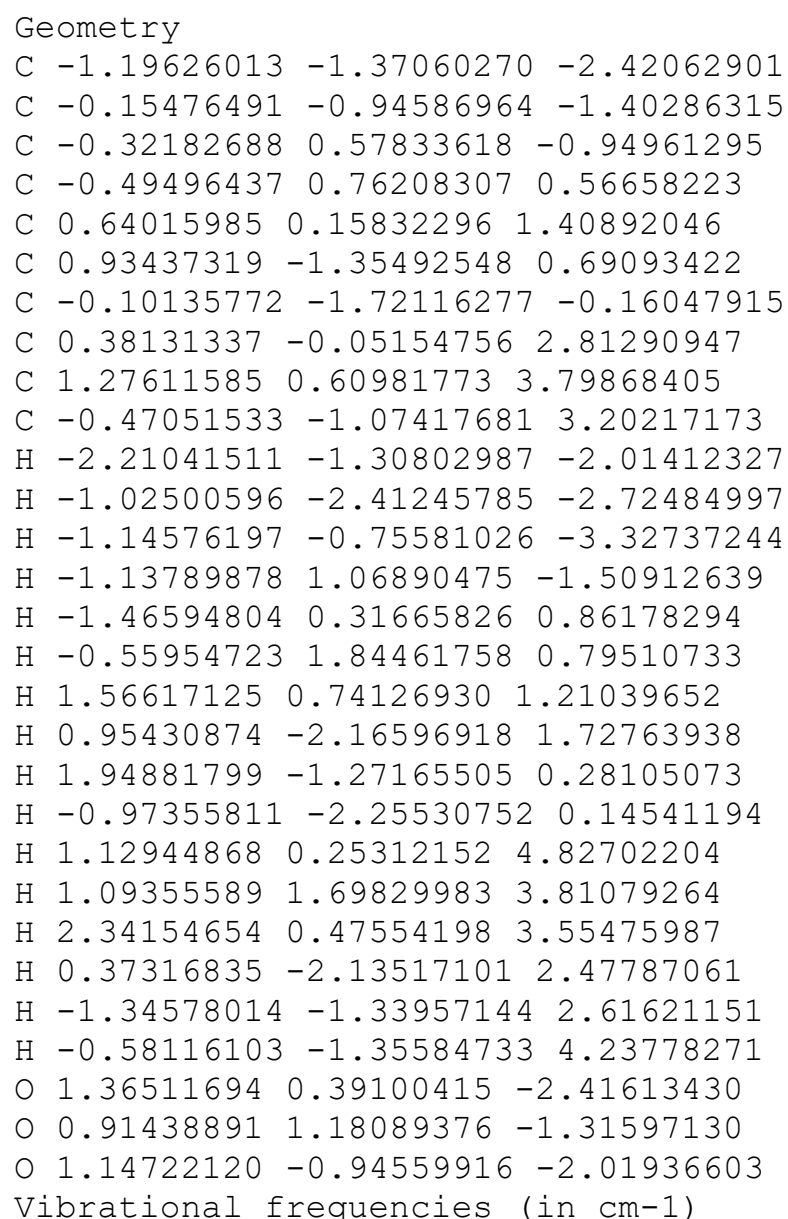

$\begin{array}{lllllllllllllllll}-2054.7 & 53.2 & 72.3 & 95.5 & 107.1 & 152.0 & 173.7 & 206.3 & 245.4 & 252.0 & 287.3 & 335.6 & 387.8 & 403.1\end{array}$ $443.1450 .4 \quad 470.8 \quad 530.9 \quad 552.3 \quad 599.8 \quad 644.2 \quad 647.8 \quad 669.5 \quad 694.3 \quad 799.5 \quad 819.9 \quad 850.0 \quad 859.3$

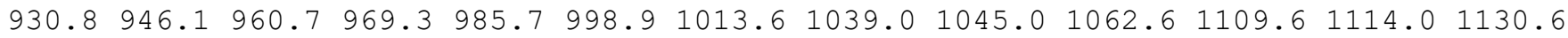
$\begin{array}{lllllllllllll}1136.6 & 1155.9 & 1176.0 & 1184.1 & 1193.7 & 1219.6 & 1243.3 & 1254.5 & 1258.8 & 1267.1 & 1276.0 & 1289.5\end{array}$

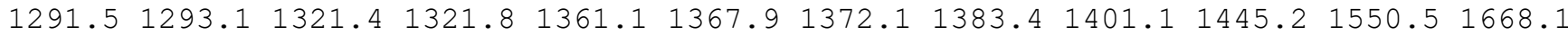
$1765.02397 .2 \quad 2657.2 \quad 2661.6 \quad 2669.8 \quad 2676.8 \quad 2684.62686 .72693 .4 \quad 2701.82708 .52736 .3$ $2771.4 \quad 2775.7 \quad 2783.3 \quad 2802.2$

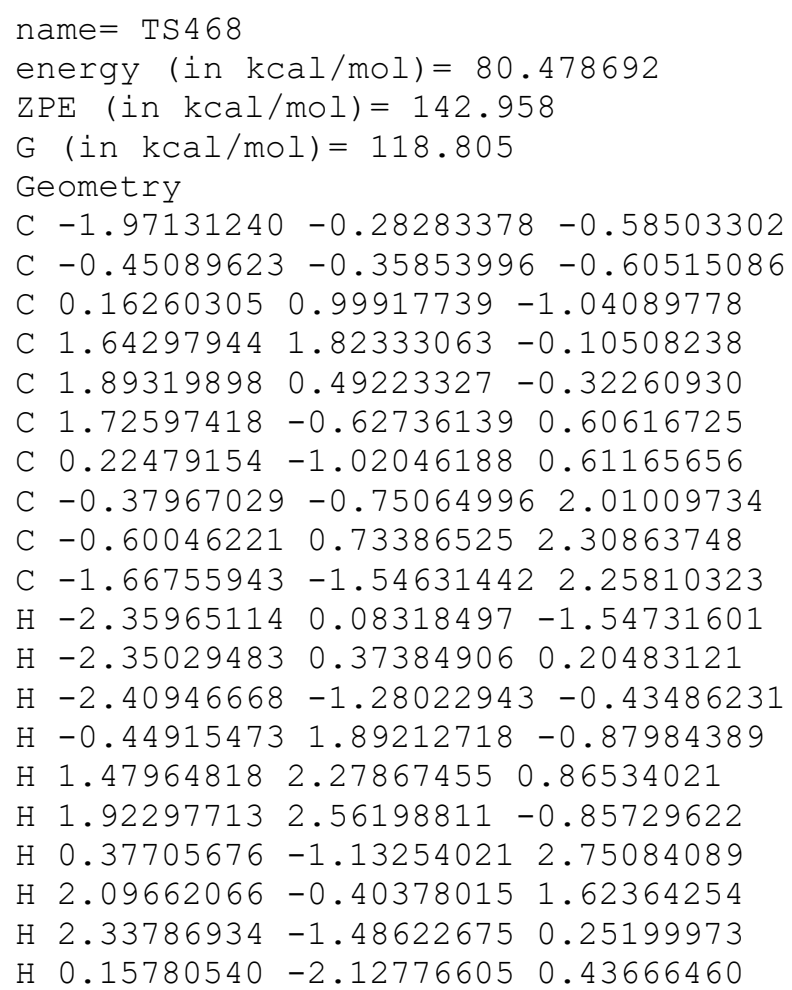


$\mathrm{H}-1.293980751 .203787391 .60623026$

$\mathrm{H} 0.34366098 \quad 1.28951500 \quad 2.27008037$

$\mathrm{H}-1.01664778 \quad 0.87181586 \quad 3.31437668$

$\mathrm{H}-1.87230945-1.624407043 .33310032$

$\mathrm{H}-1.59552357-2.567091841 .86728078$

$\mathrm{H}-2.54555410-1.077604211 .80538530$

$0.77770491-0.50591026-2.53824939$

$0.417502610 .87507615-2.40005957$

o $-0.18554640-1.19409867-1.76081589$

Vibrational frequencies (in $\mathrm{cm}-1$ )

$\begin{array}{lllllllllllllllll}-809.2 & 68.1 & 79.6 & 92.7 & 159.0 & 170.6 & 205.7 & 215.0 & 218.7 & 236.0 & 271.8 & 308.4 & 319.9 & 352.0\end{array}$ $358.9406 .5 \quad 444.0 \quad 468.2 \quad 549.8 \quad 565.0 \quad 641.1 \quad 693.1 \quad 718.7 \quad 767.9 \quad 823.6 \quad 850.7 \quad 900.9950 .6$

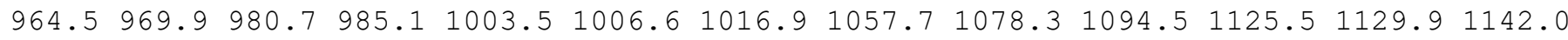

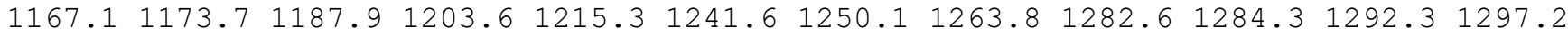
$1300.51303 .6 \quad 1309.91319 .91330 .2 \quad 1338.5 \quad 1372.1 \quad 1380.01388 .1 \quad 1406.2 \quad 1409.31671 .7$ 2655.52661 .52664 .72667 .12670 .82673 .12675 .82677 .42678 .62684 .42692 .52738 .6 $2743.4 \quad 2773.7 \quad 2779.0 \quad 2782.8$

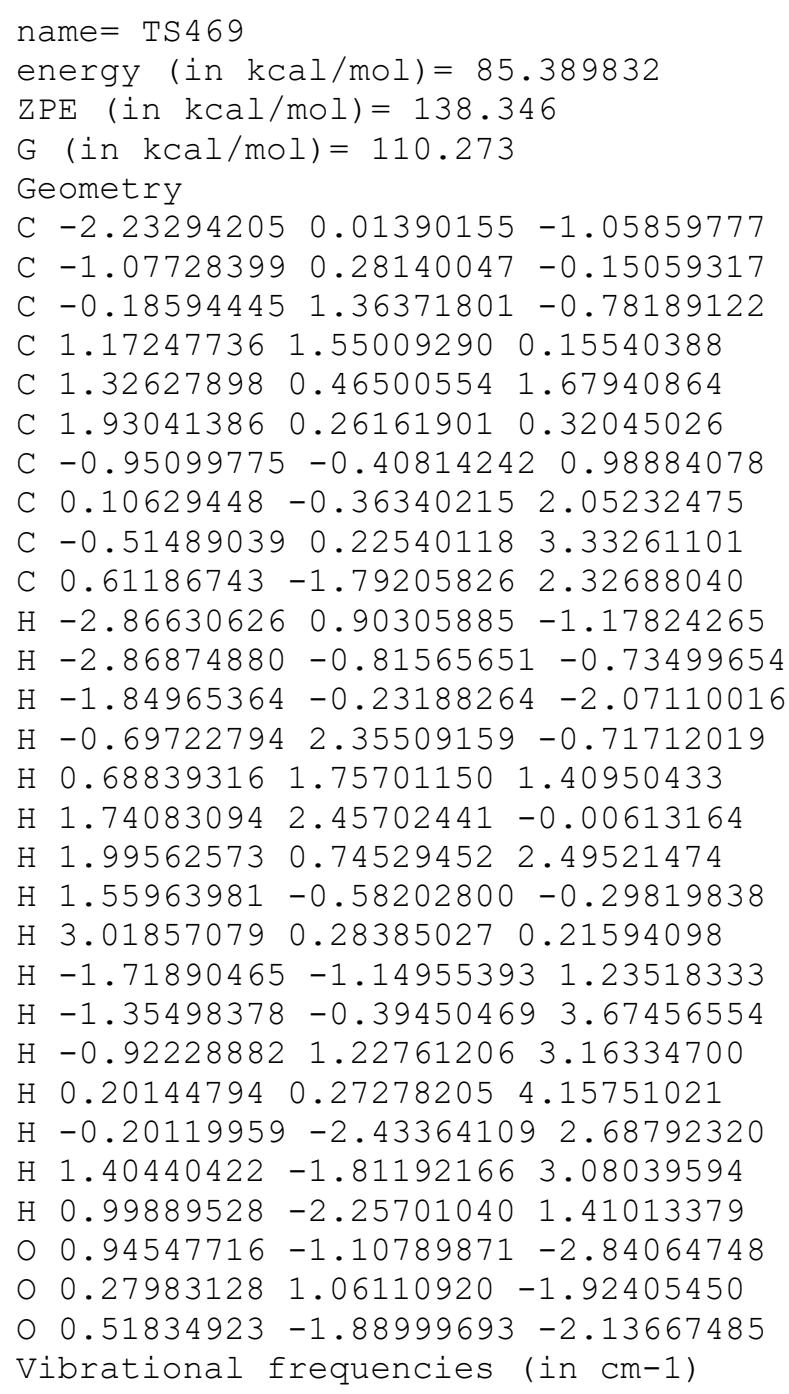


$\mathrm{ZPE}($ in $\mathrm{kcal} / \mathrm{mol})=139.14$

$\mathrm{G}($ in $\mathrm{kcal} / \mathrm{mol})=112.358$

Geometry

C $-2.18836314 \quad-2.02795748-1.09173727$

C $-1.24290305-0.96243138-0.62113732$

C $0.68522040 \quad 1.55324528-0.44949969$

C $1.46779109 \quad 0.87078103 \quad 0.59129639$

C $0.95793396-0.47629568 \quad 1.05465826$

C $1.45896397-1.49519069-0.75041697$

C $-0.29479898-1.367110110 .44377725$

C $-0.34792942-0.60396671 \quad 1.78570909$

C $-1.259807830 .58034002 \quad 1.94261598$

C $-0.35917197-1.484496353 .01857811$

$\mathrm{H}-2.88889928-1.63280653-1.84613421$

$\mathrm{H}-2.79245567-2.42283994-0.26255627$

$\mathrm{H}-1.65826525-2.87252940-1.54998825$

$\mathrm{H} \quad 0.65293838 \quad 1.20705700-1.47724400$

$\mathrm{H} \quad 1.53823087 \quad 1.538059361 .49646444$

H $2.51874922 \quad 0.74002183 \quad 0.22057431$

$\mathrm{H} 1.78773086-1.08339778 \quad 1.47172037$

$\mathrm{H} 1.52927242-2.54628439-0.66055235$

H $1.01625922-1.09028903-1.62059889$

$\mathrm{H}-0.24075646-2.45741494 \quad 0.57911189$

$\mathrm{H}-2.20362050 \quad 0.31833182 \quad 2.43400811$

$\mathrm{H}-1.51996887 \quad 1.06939177 \quad 0.99203939$

$\mathrm{H}-0.77854806 \quad 1.37491700 \quad 2.54035026$

$\mathrm{H}-0.11185428-0.89621931 \quad 3.91455232$

$\mathrm{H} \quad 0.35915010-2.31016394 \quad 2.96684984$

$\mathrm{H}-1.35192825-1.923888063 .18380736$

$0-0.02205921 \quad 3.135685350 .90377565$

$0.000180092 .63329322-0.26228390$

$0-1.28175801 \quad 0.15003015-1.09240727$

Vibrational frequencies (in $\mathrm{cm}-1$ )

$\begin{array}{llllllllllllllll}-468.7 & 26.9 & 45.6 & 73.9 & 84.1 & 131.0 & 144.9 & 151.0 & 167.9 & 199.6 & 210.4 & 222.7 & 237.2 & 247.5\end{array}$ $268.0 \quad 298.3 \quad 340.6 \quad 383.8 \quad 403.4 \quad 447.8 \quad 490.8 \quad 554.3 \quad 614.5 \quad 665.8 \quad 668.2 \quad 709.2 \quad 787.6 \quad 815.8$ $848.9896 .3941 .0 \quad 978.9 \quad 1000.6 \quad 1015.8 \quad 1017.1 \quad 1031.4 \quad 1035.1 \quad 1046.3 \quad 1051.3 \quad 1082.7 \quad 1094.7$

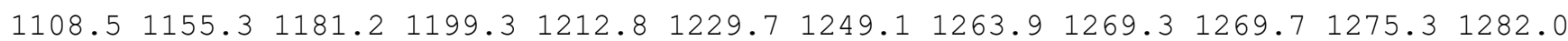
$1287.61289 .21294 .1 \quad 1332.3 \quad 1351.5 \quad 1380.8 \quad 1385.3 \quad 1388.51411 .51448 .91607 .01843 .1$ $2621.52648 .32664 .52670 .02670 .42672 .62676 .2 \quad 2689.12694 .72700 .62702 .32709 .8$ $2724.92777 .7 \quad 2778.7 \quad 2781.0$

\section{name $=$ TS471}

energy (in $\mathrm{kcal} / \mathrm{mol})=84.404165$

$\mathrm{ZPE}($ in $\mathrm{kcal} / \mathrm{mol})=139.603$

$\mathrm{G}(\mathrm{in} \mathrm{kcal} / \mathrm{mol})=113.819$

Geometry

C $-1.87228762-1.160703090 .42605068$

C $-0.51895019-0.74334054-0.14521128$

C $-0.48532199 \quad 0.79294983-0.47361994$

C $0.54372341 \quad 1.55365955 \quad 0.20333930$

C $2.26570131-0.669004231 .74574441$

C $2.04061659-0.849829660 .43347863$

C $0.62512344-1.39043815 \quad 0.60913208$

C $0.86993948-1.143079692 .18388088$

C $0.05091750 \quad-0.08045352 \quad 2.87605734$

C $0.88415165-2.441228902 .97281375$

$\mathrm{H}-2.68631642-0.93943901-0.27801080$

$\mathrm{H}-2.08414212-0.64392701 \quad 1.36890494$

$\mathrm{H}-1.90643357-2.23956464 \quad 0.62482980$

$\mathrm{H}-1.50477300 \quad 1.22750891-0.32997934$

$\mathrm{H} \quad 0.33731235 \quad 3.06435919-0.49921933$

$\mathrm{H} \quad 0.13783650 \quad 3.07450494 \quad 0.28738836$

$\mathrm{H} 3.06103115-0.329997972 .35231643$

H $2.58184749-0.72391297-0.46762822$ 
H $1.57198997 \quad 1.52362325-0.05544185$

H $0.58442449-2.48231173 \quad 0.39917384$

$\mathrm{H} \quad 0.00346968 \quad 0.84720114 \quad 2.27785252$

$\mathrm{H} \quad 0.49940463 \quad 0.19406555 \quad 3.83837018$

$\mathrm{H}-0.97390039-0.40896140 \quad 3.07159723$

H $1.23275776-2.274980293 .99900890$

$\mathrm{H} 1.55010527 \quad-3.18709828 \quad 2.52271422$

$\mathrm{H}-0.11773347-2.88134854 \quad 3.03098726$

O $0.20778992-0.41850571-2.26642164$

$\begin{array}{llll}0 & -0.33460582 & 0.84148969 & -1.91173822\end{array}$

o $-0.51228029-1.33020145-1.47036071$

Vibrational frequencies (in $\mathrm{cm}-1$ )

$\begin{array}{lllllllllllllllll}-953.5 & 10.0 & 88.7 & 105.7 & 134.6 & 156.8 & 169.9 & 181.4 & 191.2 & 221.1 & 242.3 & 271.6 & 291.9 & 304.7\end{array}$ $321.6 \quad 364.6 \quad 384.3 \quad 425.7 \quad 445.5 \quad 481.5 \quad 529.4 \quad 586.8 \quad 598.6 \quad 656.8 \quad 704.2 \quad 715.8 \quad 794.5 \quad 829.1$ $862.6 \quad 875.6 \quad 902.2934 .0 \quad 955.3 \quad 965.2 \quad 994.7 \quad 1000.2 \quad 1007.8 \quad 1012.5 \quad 1018.7 \quad 1039.3 \quad 1061.2$

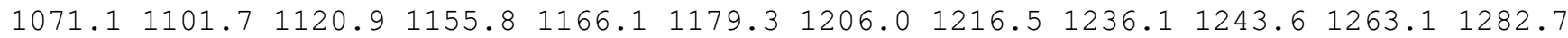
$1287.21290 .6 \quad 1291.3 \quad 1297.91299 .3 \quad 1302.4 \quad 1316.4 \quad 1333.3 \quad 1366.4 \quad 1395.51401 .51406 .2$ $\begin{array}{llllllllllllll}1775.8 & 2643.3 & 2669.8 & 2671.1 & 2674.2 & 2677.7 & 2679.4 & 2682.9 & 2703.0 & 2730.3 & 2775.3 & 2778.9\end{array}$ $2782.6 \quad 2796.32811 .4 \quad 4731.9$

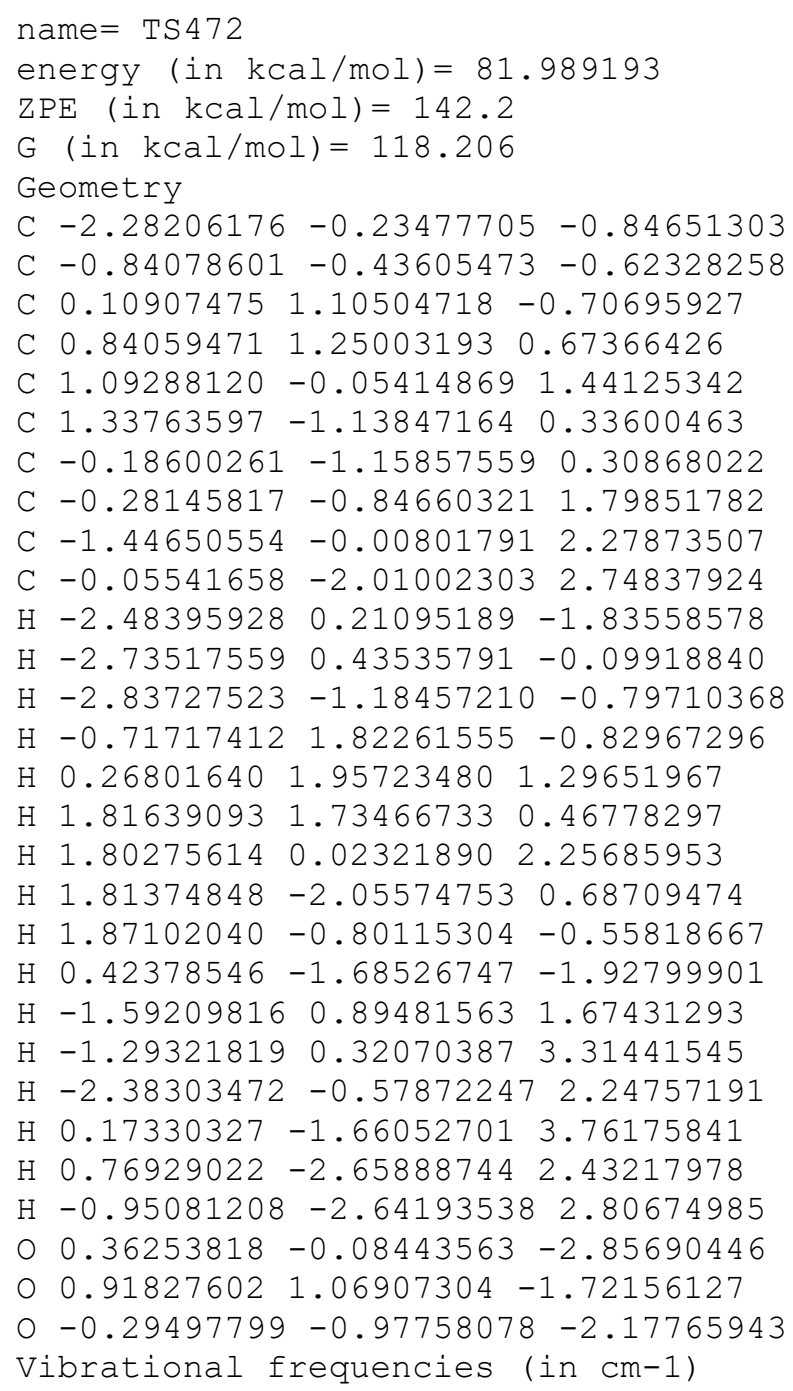




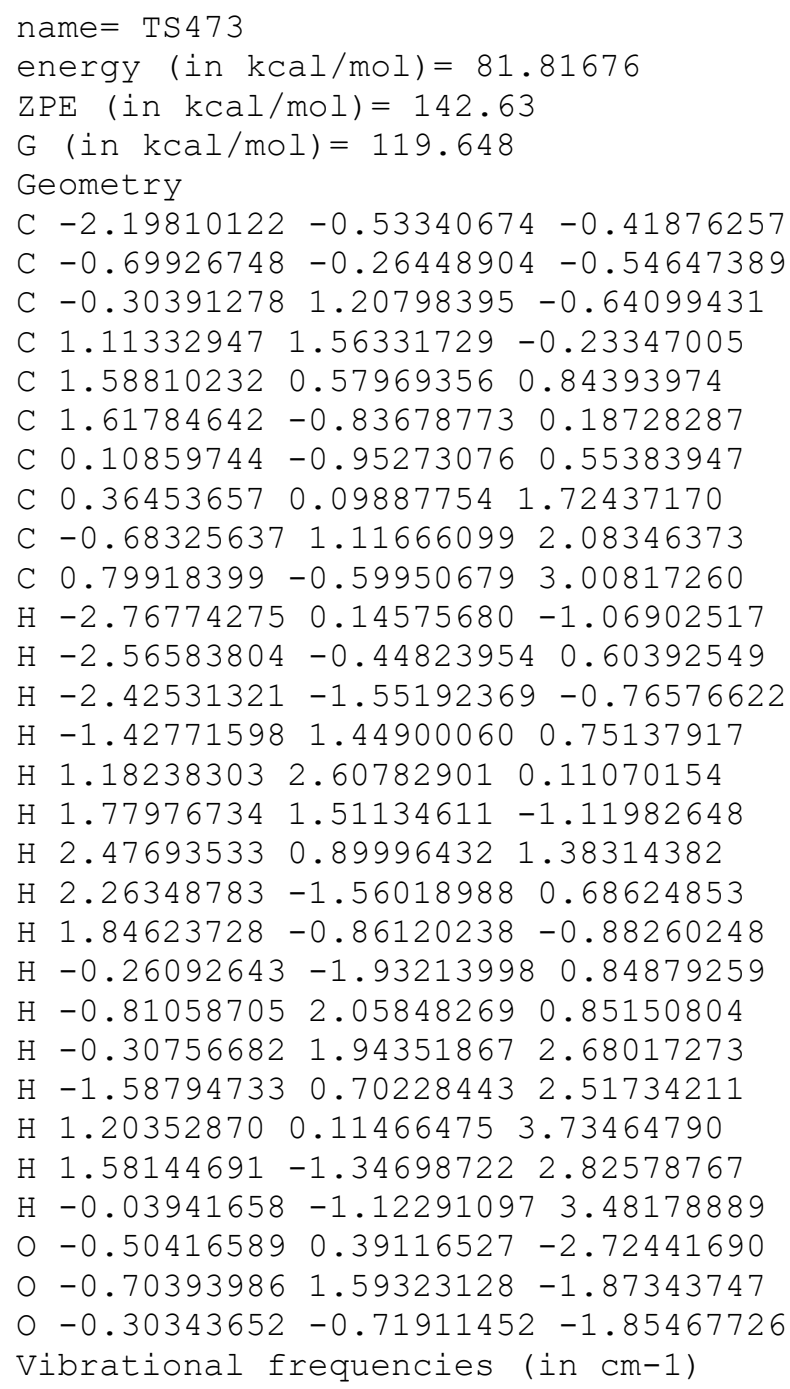


H $1.92510891 \quad 0.63093332 \quad 2.37567892$

$\mathrm{H} \quad 2.24478204-1.67138960 \quad 1.33924123$

$\mathrm{H} \quad 0.80690865-1.38572465-0.02156741$

$\mathrm{H}-0.92117132-1.92607830 \quad 0.65079730$

$\mathrm{H}-2.080009150 .203229162 .56655456$

$\mathrm{H}-1.25083102 \quad 1.52380644 \quad 1.73156743$

$\mathrm{H}-0.80976508 \quad 1.12428470 \quad 3.38296254$

$\mathrm{H} \quad 0.52532904 \quad-0.89704400 \quad 3.96088255$

$\mathrm{H} \quad 0.77764971 \quad-2.17725882 \quad 2.77527343$

$\mathrm{H}-0.85249766-1.80221207 \quad 3.32475057$

$0.72357438 \quad 0.00356966-2.15700290$

○ $0.54291926 \quad 1.36217994-1.80164663$

o $-0.49674202-0.60912414-1.77764016$

Vibrational frequencies (in cm-1)

$\begin{array}{llllllllllllll}-1342.7 & 72.8 & 118.7 & 147.5 & 166.3 & 192.3 & 210.3 & 224.8 & 266.1 & 284.3 & 300.2 & 346.6 & 385.4 & 397.5\end{array}$ $442.0 \quad 470.9 \quad 477.7 \quad 498.6 \quad 549.8 \quad 608.0 \quad 670.6 \quad 693.1 \quad 711.1 \quad 823.2 \quad 853.5 \quad 869.7 \quad 886.1 \quad 927.9$ $957.0966 .6 \quad 975.1994 .9 \quad 996.2 \quad 1006.6 \quad 1014.4 \quad 1024.8 \quad 1047.2 \quad 1071.3 \quad 1075.2 \quad 1115.5 \quad 1139.2$

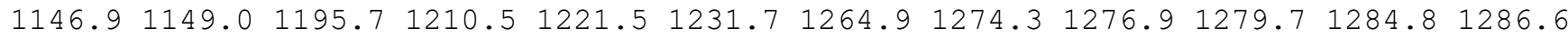

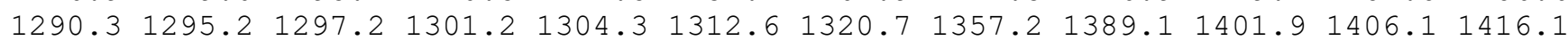
$1900.92653 .92669 .2 \quad 2670.7 \quad 2672.8 \quad 2675.3 \quad 2676.72678 .42683 .5 \quad 2691.82729 .32731 .5$ $2736.5 \quad 2779.8 \quad 2781.12782 .9$

name $=$ TS475

energy $($ in $\mathrm{kcal} / \mathrm{mol})=84.769736$

$\mathrm{ZPE}(\mathrm{in} \mathrm{kcal} / \mathrm{mol})=140.81$

$\mathrm{G}($ in $\mathrm{kcal} / \mathrm{mol})=114.529$

Geometry

C $-1.29375660 \quad 0.10281685-1.57831275$

C $0.18298437 \quad 0.08318708-1.20064776$

C $0.58980907 \quad 1.30007407-0.29664916$

C $1.24284058 \quad 0.94512924 \quad 1.04264940$

C $0.16152100 \quad 0.77014864 \quad 2.07845436$

C $1.46525553-2.05081630-1.21735616$

C $0.56837642-1.25033459-0.64714354$

C $-0.52395892-0.418492712 .22352151$

C $-1.84564394-0.63098503 \quad 2.80140883$

C $0.92800545-0.407042113 .34779087$

$\mathrm{H}-1.57353368 \quad 1.04364642-2.06887617$

$\mathrm{H}-1.92788517-0.03088529-0.69311757$

$\mathrm{H}-1.53032000-0.71062323-2.27800935$

$\mathrm{H}-0.25648246 \quad 2.00763041-0.16209895$

H $1.94807118 \quad 1.75276022 \quad 1.32839545$

$\mathrm{H} \quad 1.87487565 \quad 0.03911476 \quad 0.93075321$

$\mathrm{H}-0.22829341 \quad 1.72419660 \quad 2.45773442$

$\mathrm{H} 1.72823614-3.01957296-0.82249378$

H 2.00315268 -1.80196600 -2.12096890

$\mathrm{H} \quad 0.03696441-1.52135082 \quad 0.27876402$

$\mathrm{H}-1.91869125-1.618492293 .28314120$

$\mathrm{H}-2.58797252-0.64778922 \quad 1.98212172$

$\mathrm{H}-2.17902304 \quad 0.12449569 \quad 3.52419642$

$\mathrm{H} \quad 1.50384268 \quad 0.41598545 \quad 3.77017059$

$\mathrm{H} 1.57273296-1.134780392 .85757452$

$\mathrm{H} \quad 0.34748619-0.86267165 \quad 4.15288194$

O $2.028904431 .07747972-2.02082626$

$01.523930942 .03146514-1.08842097$

O $0.88408318 \quad 0.36424210-2.42836517$

Vibrational frequencies (in $\mathrm{cm}-1$ )

$\begin{array}{lllllllllllllllll}-781.1 & 38.0 & 41.7 & 80.4 & 97.6 & 109.7 & 132.0 & 158.6 & 163.1 & 197.3 & 234.7 & 246.6 & 273.7 & 302.3\end{array}$ $325.0 \quad 330.6 \quad 355.7 \quad 420.4 \quad 477.2 \quad 505.0 \quad 555.3 \quad 571.2 \quad 602.5 \quad 687.2 \quad 723.2 \quad 826.0 \quad 872.2 \quad 909.9$ $929.6 \quad 954.3 \quad 958.3 \quad 975.0 \quad 984.9 \quad 999.9 \quad 1010.9 \quad 1018.0 \quad 1049.4 \quad 1067.3 \quad 1083.9 \quad 1093.5 \quad 1105.0$

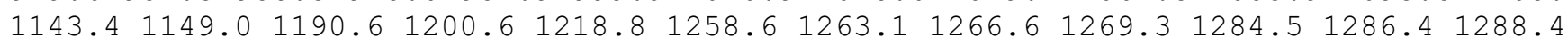

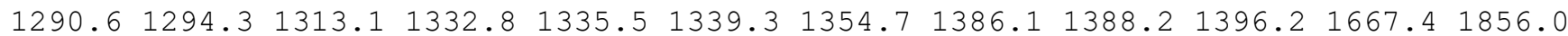
$2646.52648 .62665 .02670 .6 \quad 2671.12671 .8 \quad 2674.52679 .42680 .5 \quad 2699.2 \quad 2722.62737 .0$ $2752.8 \quad 2773.7 \quad 2779.0 \quad 2785.1$ 


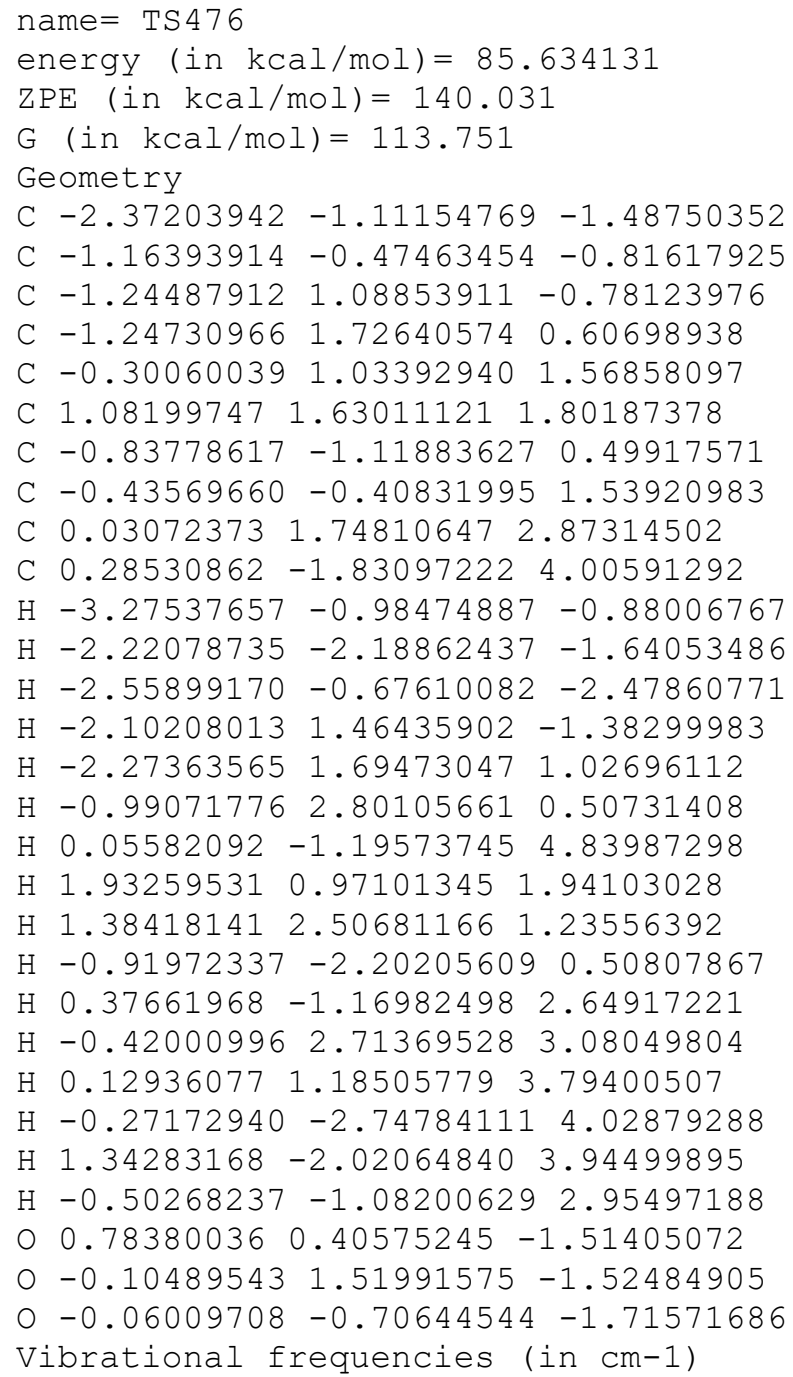


$\mathrm{H} \quad 0.76374605 \quad 1.39187469 \quad 1.21083964$

H $2.018964431 .66570651-0.37516825$

H 2.41215971 $0.19081672 \quad 2.69544951$

$\mathrm{H} \quad 1.98316979-1.42599244 \quad 2.12773650$

$\mathrm{H} \quad 2.81750280-0.31201758 \quad 1.04345141$

$\mathrm{H}-0.02439281-1.730791820 .39656357$

$\mathrm{H}-1.49083848 \quad 1.73397450 \quad 1.43388852$

$\mathrm{H}-1.48218062 \quad 1.344118693 .15298780$

$\mathrm{H}-2.64504057 \quad 0.55194458 \quad 2.08049565$

$\mathrm{H}-0.46391851-0.87424458 \quad 3.97235334$

$\mathrm{H}-0.04520266-2.12158900 \quad 2.79455342$

$\mathrm{H}-1.73693049-1.68343566 \quad 3.04179482$

$00.81250978-0.65276436-2.27775593$

$\begin{array}{lllll}0 & 0.74313587 & 0.75706282 & -2.39504059\end{array}$

o $-0.47765208-1.01479706-1.82443251$

Vibrational frequencies (in $\mathrm{cm}-1$ )

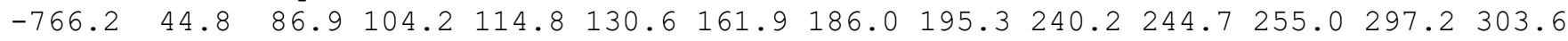
$377.5 \quad 422.9435 .6 \quad 452.5 \quad 465.2 \quad 497.6 \quad 563.7 \quad 661.8 \quad 700.3 \quad 718.9 \quad 775.9 \quad 789.7 \quad 844.0 \quad 869.6$ $933.9946 .7968 .9999 .9 \quad 1004.0 \quad 1009.91014 .2 \quad 1019.1 \quad 1032.5 \quad 1059.5 \quad 1075.01090 .4 \quad 1140.8$

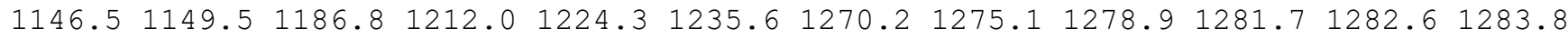

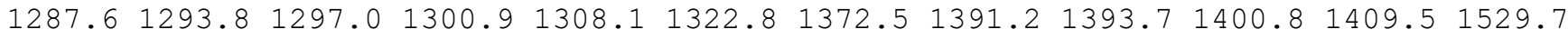
$1904.32665 .42666 .8 \quad 2668.1 \quad 2669.2 \quad 2671.6 \quad 2673.02673 .72674 .4 \quad 2677.92716 .52730 .5$ 2777.22778 .12778 .62781 .0

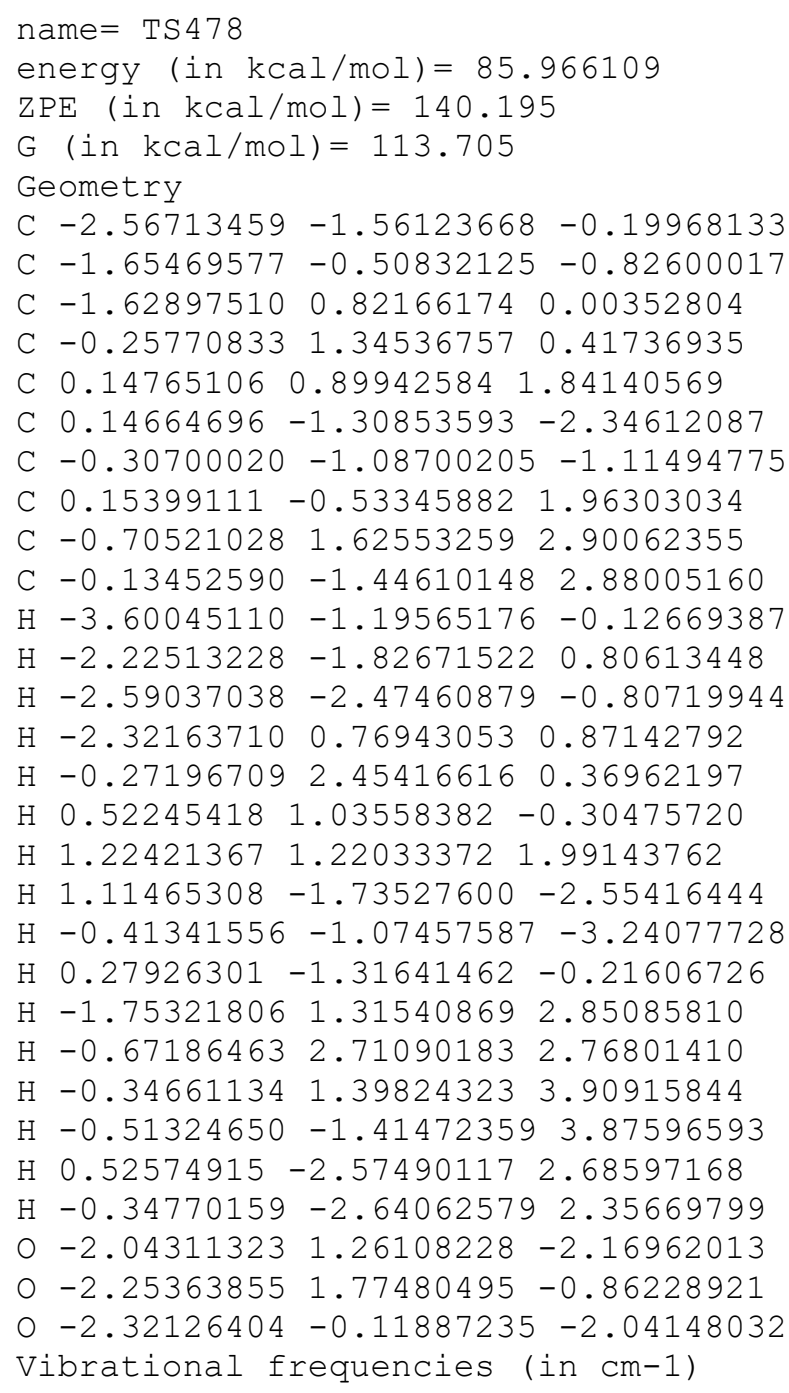


$1855.22654 .92665 .92672 .5 \quad 2677.4 \quad 2680.0 \quad 2682.6 \quad 2686.2 \quad 2697.7 \quad 2726.3 \quad 2738.5 \quad 2745.4$ $2779.2 \quad 2782.4 \quad 2785.2 \quad 3479.4$

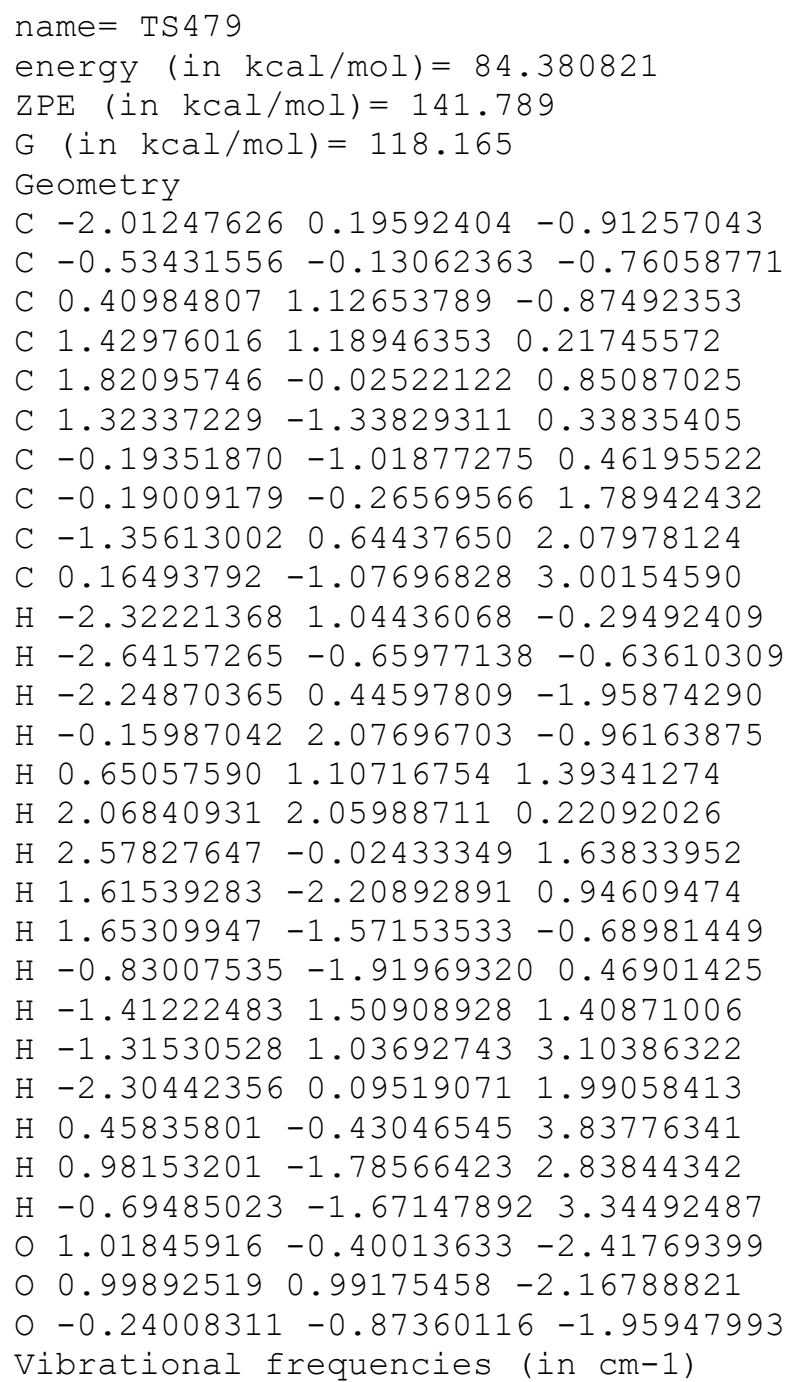

$\begin{array}{lllllllllllllllll}-3549.3 & 68.9 & 102.6 & 117.5 & 142.8 & 180.0 & 201.1 & 212.3 & 261.4 & 283.8 & 307.1 & 335.6 & 354.7 & 379.9\end{array}$ $407.9 \quad 447.7 \quad 486.2 \quad 505.2 \quad 530.2 \quad 566.7 \quad 671.2 \quad 701.3 \quad 724.9 \quad 826.8 \quad 857.8 \quad 887.6 \quad 910.3 \quad 931.4$ $942.3968 .6994 .1 \quad 1000.5 \quad 1006.2 \quad 1012.5 \quad 1017.4 \quad 1035.0 \quad 1049.9 \quad 1056.6 \quad 1086.2 \quad 1106.1 \quad 1120.5$ $1142.31160 .41171 .3 \quad 1190.8 \quad 1219.8 \quad 1241.8 \quad 1248.7 \quad 1268.4 \quad 1280.7 \quad 1283.1 \quad 1286.4 \quad 1290.5$ $1294.61300 .4 \quad 1304.5 \quad 1310.6 \quad 1315.4 \quad 1322.4 \quad 1348.2 \quad 1352.2 \quad 1394.4 \quad 1402.7 \quad 1415.7 \quad 1444.4$ $1749.72656 .62664 .0 \quad 2667.3 \quad 2668.5 \quad 2670.6 \quad 2673.32678 .22687 .72710 .22724 .32747 .0$ $2769.5 \quad 2772.4 \quad 2776.8 \quad 2778.3$

name $=\operatorname{TS} 480$

energy (in $\mathrm{kcal} / \mathrm{mol})=86.330516$

$\mathrm{ZPE}(\mathrm{in} \mathrm{kcal} / \mathrm{mol})=140.575$

$\mathrm{G}($ in $\mathrm{kcal} / \mathrm{mol})=114.038$

Geometry

C $-0.64277833-1.25010168-2.20651424$

C $0.62914138-0.77923926-1.50325529$

C $0.45633430 \quad 0.62751289 \quad-0.83677413$

C $1.24569428 \quad 0.85148740 \quad 0.44859879$

C $0.47939025 \quad 0.73124656 \quad 1.74421908$

C $2.36022796-2.36762667-0.67709174$

C $1.14409324-1.83545940-0.57796564$

C $-0.79634269 \quad 0.28020324 \quad 1.97719803$

C $-1.88220704-0.255185121 .18148016$

C $0.49185640 \quad-0.86958704 \quad 2.71201981$

$\mathrm{H}-1.00774933-0.48767140-2.90954870$

$\mathrm{H}-1.44787644-1.48114740-1.50216450$ 
$\mathrm{H}-0.44702321-2.15669558-2.79709882$

$\mathrm{H}-0.60436588 \quad 0.93571503 \quad-0.73939768$

$\mathrm{H} \quad 1.68188180 \quad 1.87956926 \quad 0.39186350$

H $2.13762826 \quad 0.18998898 \quad 0.47842522$

$\mathrm{H} \quad 0.93721121 \quad 1.37981206 \quad 2.51854833$

$\mathrm{H} 2.72766249-3.14510097-0.02456902$

$\mathrm{H} 3.08195754-2.07083665-1.42875096$

$\mathrm{H} \quad 0.42718850 \quad-2.15106308 \quad 0.17934391$

$\mathrm{H}-2.488807290 .58726896 \quad 0.79752294$

$\mathrm{H}-1.59611740 \quad-0.87907018 \quad 0.32512329$

$\mathrm{H}-2.57259772-0.847287391 .80737671$

H $1.40952769-1.23131275 \quad 2.25566265$

$\mathrm{H}-0.26353464-1.65916212 \quad 2.64419453$

$\mathrm{H} \quad 0.62158469-0.57102312 \quad 3.74789371$

$\begin{array}{lllll}0 & 2.07597167 & 0.76844204 & -2.36311256\end{array}$

○ $0.97837248 \quad 1.49438622-1.84768205$

O $1.56470930-0.54534223-2.57058660$

Vibrational frequencies (in $\mathrm{cm}-1$ )

$\begin{array}{lllllllllllllllll}-713.2 & 31.4 & 41.2 & 67.8 & 88.3 & 120.0 & 136.7 & 139.0 & 177.9 & 198.0 & 203.5 & 237.5 & 263.6 & 298.7\end{array}$ $306.2321 .1 \quad 352.9 \quad 432.5 \quad 450.3 \quad 533.6 \quad 564.1 \quad 582.0 \quad 617.6 \quad 678.1 \quad 717.6 \quad 844.8 \quad 862.8 \quad 877.8$ $919.1929 .6 \quad 960.3 \quad 972.9 \quad 985.3 \quad 987.1 \quad 1007.7 \quad 1015.7 \quad 1030.9 \quad 1051.6 \quad 1058.8 \quad 1096.4 \quad 1101.0$

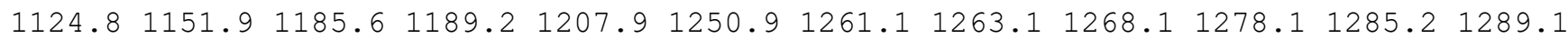

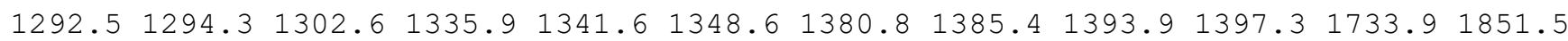
$2636.92643 .52652 .6 \quad 2662.7 \quad 2667.5 \quad 2671.92674 .92684 .62699 .12707 .02727 .92753 .0$ $2754.9 \quad 2766.7 \quad 2776.3 \quad 2779.3$

name $=$ TS 481

energy (in $\mathrm{kcal} / \mathrm{mol})=84.787166$

$\operatorname{ZPE}(\mathrm{in} \mathrm{kcal} / \mathrm{mol})=142.206$

$\mathrm{G}($ in $\mathrm{kcal} / \mathrm{mol})=117.564$

Geometry

C $-0.53028245-0.91745635-1.85265979$

C $0.410133250 .00620514-1.11186890$

C $-0.28165261 \quad 1.25360183-0.45484698$

C $0.20057523 \quad 1.352832330 .99790349$

C $1.11846880 \quad 0.16888786 \quad 1.25711259$

C $0.55349199-1.44481637 \quad 1.02744316$

C $1.25290591-0.63391361-0.02368882$

C $0.32827151-0.553344542 .93522676$

C $1.25022699-1.379294073 .73956987$

C $-1.12129422-0.767240513 .10716075$

$\mathrm{H}-1.00819104-0.40315194 \quad-2.69825324$

$\mathrm{H}-1.32216116-1.31080172-1.20596442$

$\mathrm{H} \quad 0.01228803-1.77894063-2.26769908$

$\mathrm{H}-1.37621814 \quad 1.26431308-0.57067990$

$\mathrm{H}-0.64299146 \quad 1.397228391 .70955651$

$\mathrm{H} \quad 0.75762072 \quad 2.30106254 \quad 1.15595171$

H 2.03645898 $0.37354352 \quad 1.81545248$

$\mathrm{H}-0.49572655-1.653577090 .88102605$

$\mathrm{H} 1.08650984-2.30193197 \quad 1.41334709$

$\mathrm{H} 2.23286375-0.97320691-0.37338710$

H $2.29001445-1.313422713 .39390763$

$\mathrm{H} \quad 0.98858920-2.441899613 .81339058$

$\mathrm{H} 1.24940763-0.978845894 .77002948$

$\mathrm{H}-1.72133703-0.27590824 \quad 2.33003192$

$\mathrm{H}-1.41992022-0.29921750 \quad 4.06323066$

$\mathrm{H}-1.43613707-1.81729022 \quad 3.15739666$

○ $0.72297769 \quad 1.82434604 \quad-2.39130329$

○ $0.25983196 \quad 2.37201755-1.15987736$

o $1.33435318 \quad 0.59937461 \quad-2.03747726$

Vibrational frequencies (in $\mathrm{cm}-1$ )

$\begin{array}{lllllllllllllll}-702.7 & 50.5 & 62.2 & 122.6 & 136.6 & 153.8 & 158.4 & 192.9 & 213.5 & 243.3 & 282.9 & 296.7 & 315.6 & 348.3\end{array}$

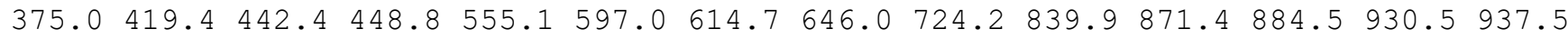
$947.7956 .4964 .9978 .3 \quad 985.7 \quad 1005.1 \quad 1008.5 \quad 1036.8 \quad 1065.8 \quad 1074.7 \quad 1104.6 \quad 1128.7 \quad 1149.4$ 


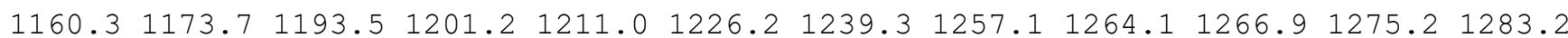

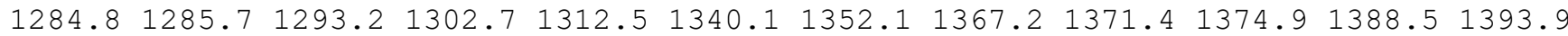

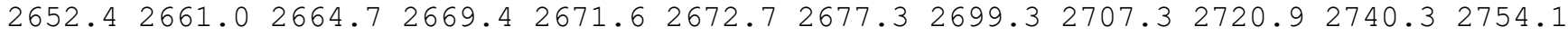
$2768.8 \quad 2771.8 \quad 2774.0 \quad 2781.3$

\section{name $=$ TS 482}

energy (in $\mathrm{kcal} / \mathrm{mol})=89.128713$

$\mathrm{ZPE}(\mathrm{in} \mathrm{kcal} / \mathrm{mol})=137.881$

$\mathrm{G}($ in $\mathrm{kcal} / \mathrm{mol})=109.801$

Geometry

C $-2.59105615-0.44466503-1.44271529$

C $-1.09012246-0.33899846-1.35706836$

C $-0.56058014 \quad 0.95268852 \quad-1.77053972$

C $1.549439731 .48599034 \quad 0.83884455$

C $0.86567895 \quad 0.359224331 .10778591$

C $0.98830474 \quad-0.82394848 \quad 0.18646883$

C $-0.35149705-1.21716033-0.37855619$

C -0.101850550 .304195092 .15995150$

C $-0.049056541 .15233091 \quad 3.35257458$

C $0.06120178-1.660276373 .04661118$

$\mathrm{H}-3.077424150 .14360247-0.65323526$

$\mathrm{H}-2.92673941-1.48646515-1.33517622$

$\mathrm{H}-2.96796763-0.09552911-2.41327232$

H $-1.13509658 \quad 1.85341434 \quad-1.58236657$

H $1.53096318 \quad 2.36172410 \quad 1.46246196$

$\mathrm{H} \quad 2.17802311 \quad 1.60554717-0.02812360$

$\mathrm{H} 1.10579763-1.40969398 \quad 3.17399666$

H $1.45772663-1.680444390 .71271431$

$\mathrm{H} \quad 1.69635168-0.60788644-0.65534766$

$\mathrm{H}-0.93488211-1.91219736 \quad 0.23397552$

$\mathrm{H}-0.33509012 \quad 2.18232667 \quad 3.06313146$

$\mathrm{H} \quad 0.94122120 \quad 1.21964027 \quad 3.82588614$

$\mathrm{H}-0.77148382 \quad 0.85010019 \quad 4.12368031$

$\mathrm{H}-0.43352651-1.71270627 \quad 4.00571699$

$\mathrm{H}-0.03492344 \quad-2.57814234 \quad 2.48220136$

$\mathrm{H}-0.72672564-0.92695349 \quad 2.36159457$

$\begin{array}{lllll}0 & 1.38812751 & 0.35902113 & -2.64602778\end{array}$

$0 \quad 0.56054363 \quad 1.23373147 \quad-2.35742720$

o $-0.38202066-1.50924022-1.77525631$

Vibrational frequencies (in $\mathrm{cm}-1$ )

$\begin{array}{lllllllllllllllll}-1106.7 & 15.1 & 41.2 & 48.6 & 79.7 & 87.0 & 114.3 & 115.6 & 137.1 & 149.4 & 175.1 & 218.3 & 225.1 & 273.4\end{array}$ $285.1 \quad 317.4 \quad 362.7 \quad 389.6 \quad 412.8 \quad 440.2 \quad 464.6 \quad 533.8 \quad 580.7 \quad 593.0 \quad 628.6 \quad 669.7 \quad 719.0 \quad 825.9$

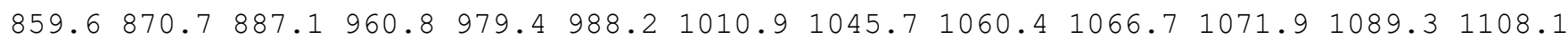
$\begin{array}{lllllllllllll}1121.3 & 1159.1 & 1169.6 & 1199.5 & 1235.5 & 1249.4 & 1257.8 & 1274.0 & 1276.1 & 1282.3 & 1285.6 & 1286.1\end{array}$

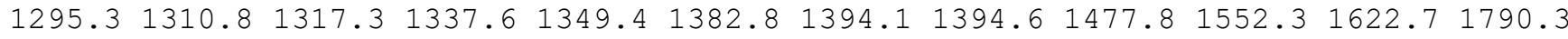
$1852.32627 .5 \quad 2657.7 \quad 2670.3 \quad 2670.5 \quad 2675.7 \quad 2679.12688 .72719 .22721 .02722 .5 \quad 2729.8$ $2769.7 \quad 2773.0 \quad 2780.1 \quad 2788.9$

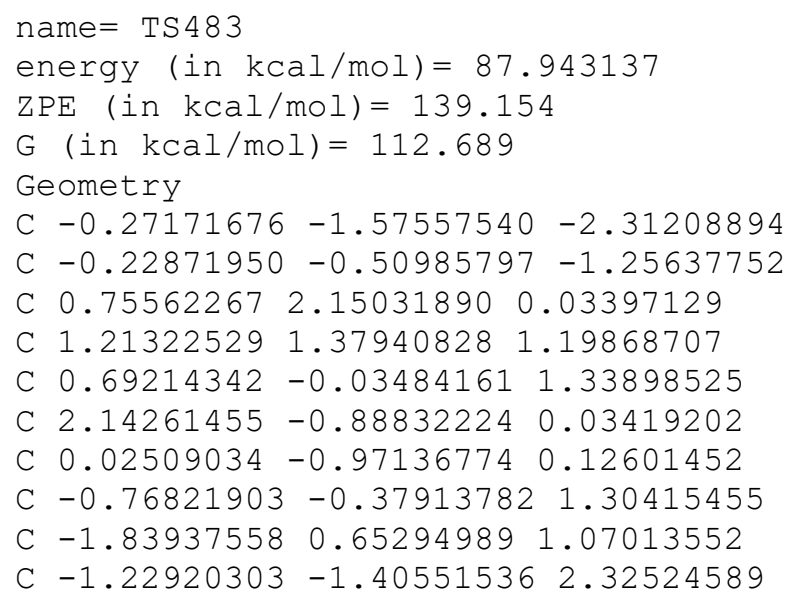


H $-0.44213541-1.13760974-3.30987441$

$\mathrm{H}-1.08404007-2.29129290-2.12708521$

$\mathrm{H} \quad 0.66952248 \quad-2.13950917-2.36221412$

$\mathrm{H}-0.26540805 \quad 2.48733465-0.09035018$

$\mathrm{H} \quad 0.97117813 \quad 1.92978476 \quad 2.13937957$

H $2.34600148 \quad 1.32750577 \quad 1.16435561$

$\mathrm{H} 1.28008013-0.591271212 .09898662$

$\mathrm{H} 2.22813553-1.93214036-0.09854362$

H 2.24212257 -0.24121539 -0.79697160

H $0.13288231-2.06366982 \quad 0.20613762$

$\mathrm{H}-1.85922944 \quad 1.38843554 \quad 1.88523768$

$\mathrm{H}-2.83647777 \quad 0.19378762 \quad 1.01712036$

$\mathrm{H}-1.71552128 \quad 1.20694585 \quad 0.12850393$

$\mathrm{H}-1.53504242-0.91532868 \quad 3.25919988$

$\mathrm{H}-0.45087650-2.13332578 \quad 2.58392680$

$\mathrm{H}-2.09291687-1.97300676 \quad 1.95501635$

○ $2.725991592 .20587989-0.94836737$

O $1.50698337 \quad 2.51288474 \quad-0.95631162$

o $-0.40440710 \quad 0.65131332-1.54829079$

Vibrational frequencies (in $\mathrm{cm}-1$ )

$\begin{array}{lllllllllllllllll}-539.0 & 37.1 & 69.3 & 72.9 & 84.3 & 106.1 & 138.8 & 149.1 & 163.2 & 188.1 & 202.6 & 220.2 & 231.6 & 262.7\end{array}$ $299.2 \quad 337.9 \quad 353.0 \quad 386.6 \quad 413.5 \quad 449.3 \quad 485.8 \quad 553.4 \quad 615.1 \quad 647.9 \quad 655.2 \quad 707.1 \quad 789.9 \quad 831.5$ $839.0 \quad 857.6 \quad 928.0 \quad 963.3 \quad 1002.1 \quad 1011.7 \quad 1017.8 \quad 1030.7 \quad 1044.7 \quad 1051.5 \quad 1054.5 \quad 1085.0 \quad 1092.6$

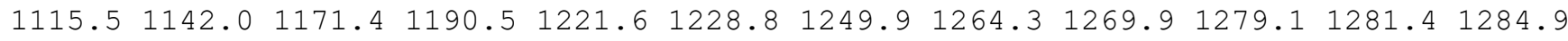
$1290.5 \quad 1292.7 \quad 1296.91332 .1 \quad 1354.5 \quad 1381.8 \quad 1386.8 \quad 1394.6 \quad 1412.9 \quad 1457.3 \quad 1606.5 \quad 1837.2$ $2604.42653 .0 \quad 2664.2 \quad 2669.6 \quad 2671.7 \quad 2672.5 \quad 2676.3 \quad 2688.52693 .92700 .7 \quad 2703.12712 .8$ $2721.8 \quad 2777.0 \quad 2777.12780 .5$

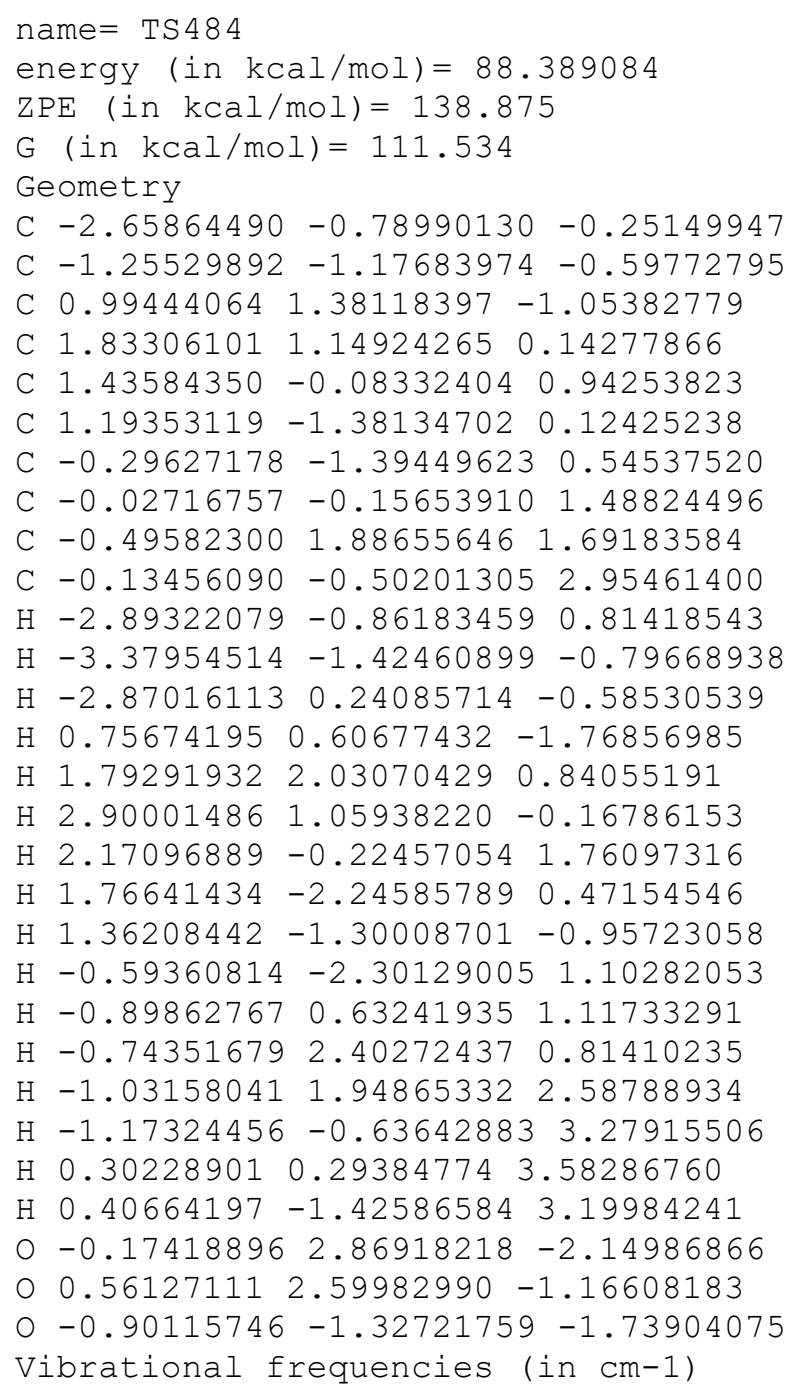


$\begin{array}{llllllllllllllllll}-939.7 & 14.1 & 48.8 & 62.5 & 79.9 & 94.6 & 102.5 & 144.9 & 160.7 & 193.0 & 196.9 & 249.6 & 265.2 & 307.7\end{array}$

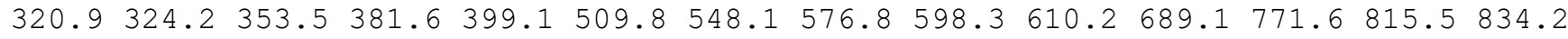
$904.9933 .3 \quad 934.2 \quad 978.7 \quad 1009.4 \quad 1027.3 \quad 1035.6 \quad 1049.9 \quad 1071.4 \quad 1088.2 \quad 1096.8 \quad 1103.5 \quad 1124.0$

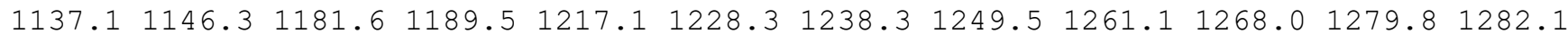
$1287.51287 .6 \quad 1293.1 \quad 1321.8 \quad 1332.2 \quad 1352.3 \quad 1379.2 \quad 1386.3 \quad 1399.4 \quad 1505.7 \quad 1567.4 \quad 1853.2$ $1907.5 \quad 2625.12648 .7 \quad 2658.5 \quad 2672.7 \quad 2673.0 \quad 2675.6 \quad 2699.4 \quad 2711.3 \quad 2717.5 \quad 2721.3 \quad 2728.2$ $2738.6 \quad 2759.4 \quad 2775.8 \quad 2777.3$

name $=$ TS 485

energy (in $\mathrm{kcal} / \mathrm{mol})=89.450114$

$\mathrm{ZPE}($ in $\mathrm{kcal} / \mathrm{mol})=137.924$

$\mathrm{G}($ in $\mathrm{kcal} / \mathrm{mol})=110.159$

Geometry

C $-2.41508140-1.079080170 .16533244$

C $-0.96086870-1.18573783-0.11327897$

C $0.335091761 .50264356-1.96745950$

C $0.981873491 .62762459-0.81358428$

C $1.49333285-0.678084812 .71071396$

C $2.42967887-0.26089706 \quad 1.89198519$

C $0.09668253-1.18493503 \quad 0.70366127$

C $0.09797225 \quad-0.98174190 \quad 2.19162797$

C $-0.78594279 \quad 0.21388456 \quad 2.59924372$

C $-0.40280848-2.275271832 .86504229$

$\mathrm{H}-3.01944676-1.48832664-0.66099048$

$\mathrm{H}-2.71581054-0.02685297 \quad 0.28812024$

$\mathrm{H}-2.69882398-1.62423715 \quad 1.07655913$

$\mathrm{H}-0.044790162 .30561755-2.59737893$

$\mathrm{H} 1.199247292 .57511098-0.35365447$

$\mathrm{H} \quad 1.34297920 \quad 0.79405408-0.22075240$

$\mathrm{H} \quad 1.58812260 \quad-0.81862630 \quad 3.78150370$

H $3.66437132 \quad 0.11700424 \quad 1.85201907$

$\mathrm{H} \quad 3.62668987-0.028293372 .72462545$

H $1.09642983-1.33605046 \quad 0.27685996$

$\mathrm{H}-1.84874159-0.001408422 .46649276$

$\mathrm{H}-0.54048140 \quad 1.09409631 \quad 1.99261615$

$\mathrm{H}-0.63399692 \quad 0.480914813 .64945487$

$\mathrm{H}-0.46720450-2.16317425 \quad 3.95087313$

$\mathrm{H} \quad 0.27472114 \quad-3.10956290 \quad 2.65029306$

$\mathrm{H}-1.39486160 \quad-2.55647313 \quad 2.49829301$

$00.39063099-0.81682845-1.93798212$

$\begin{array}{lllll}0 & 0.03125112 & 0.35320588 & -2.64009612\end{array}$

o $-0.80404645-1.38550168-1.48096599$

Vibrational frequencies (in cm-1)

$\begin{array}{lllllllllllllllll}-1687.6 & 29.5 & 44.3 & 60.0 & 71.2 & 79.3 & 116.6 & 123.9 & 138.1 & 169.5 & 175.8 & 192.6 & 221.4 & 246.1\end{array}$ $293.2 \quad 328.2 \quad 332.0 \quad 356.4 \quad 411.5 \quad 444.9 \quad 456.0 \quad 520.2 \quad 529.3 \quad 546.6 \quad 593.9604 .0 \quad 616.3 \quad 628.5$

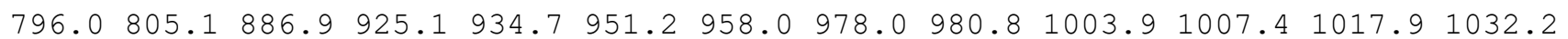
$1055.8 \quad 1069.9 \quad 1099.8 \quad 1172.2 \quad 1182.6 \quad 1217.5 \quad 1257.6 \quad 1273.8 \quad 1278.4 \quad 1281.5 \quad 1290.4 \quad 1298.1$ $1300.61303 .5 \quad 1311.5 \quad 1357.0 \quad 1367.5 \quad 1387.6 \quad 1397.9 \quad 1408.4 \quad 1440.2 \quad 1572.5 \quad 1860.7 \quad 1881.1$ $1903.92664 .8 \quad 2668.4 \quad 2677.2 \quad 2678.7 \quad 2681.5 \quad 2683.12691 .12717 .62731 .5 \quad 2750.02773 .3$ $2781.4 \quad 2783.0 \quad 2786.8 \quad 3835.3$

name $=\operatorname{TS} 486$

energy $($ in $\mathrm{kcal} / \mathrm{mol})=87.199529$

$\mathrm{ZPE}($ in $\mathrm{kcal} / \mathrm{mol})=140.272$

$\mathrm{G}($ in $\mathrm{kcal} / \mathrm{mol})=114.945$

Geometry

C $-1.30285977-1.32279444-1.17600773$

C $-0.29596889-0.52501864-0.80446933$

C $-0.34967448 \quad 0.94060015-1.22224330$

C $-0.56473124 \quad 1.95422059-0.05455300$

C $0.62416711-0.97467144 \quad 1.56217503$

C 0.246869531 .358383761 .03599215

C $0.85166080-1.08488145-0.00161562$ 
C $-0.22733557 \quad 0.26326959 \quad 1.66548843$

C -1.636056110 .076510292 .09955541$

C $1.82257505-1.037632022 .38532086$

$\mathrm{H}-2.15226947-1.00175078-1.75553280$

$\mathrm{H}-1.35327781-2.37069237-0.92649605$

H $3.26443559 \quad 0.98080196-1.87154511$

$\mathrm{H}-1.15866287 \quad 1.08935134 \quad-1.98612074$

$\mathrm{H}-1.63978450 \quad 2.035796750 .18644006$

$\mathrm{H}-0.236828392 .96465101-0.35425765$

H 2.43669032 $0.38766109 \quad 2.66059838$

$\mathrm{H} \quad 1.78773591-0.55715459-0.26251869$

$\mathrm{H} \quad 1.30774381 \quad 1.58604902 \quad 0.99032744$

$\mathrm{H} 1.02322555-2.14470325-0.25984507$

$\mathrm{H}-2.176916451 .02786616 \quad 2.18906844$

$\mathrm{H}-1.66958735-0.40648287 \quad 3.08922867$

$\mathrm{H}-2.20051692-0.561842451 .40339035$

$\mathrm{H} 2.727689890 .299735921 .90888613$

$\mathrm{H} \quad 0.01828119-1.87310039 \quad 1.88522094$

H $2.61836847-1.70269546 \quad 2.14209947$

O $1.750414911 .98751389-1.37803936$

O $0.777162891 .26541680 \quad-2.03546877$

O $2.809510111 .14204917-1.00907156$

Vibrational frequencies (in $\mathrm{cm}-1$ )

$\begin{array}{llllllllllllllll}-929.8 & 42.4 & 64.0 & 108.1 & 116.8 & 129.9 & 136.5 & 163.2 & 180.8 & 230.2 & 273.0 & 275.5 & 304.3 & 344.4\end{array}$

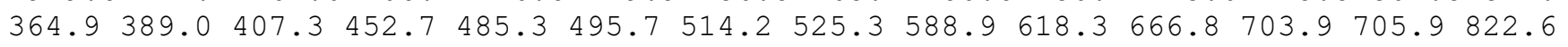
$824.2 \quad 853.3 \quad 871.3 \quad 932.4 \quad 953.8 \quad 962.9 \quad 968.3 \quad 974.7 \quad 1005.0 \quad 1019.2 \quad 1037.7 \quad 1052.7 \quad 1061.8$

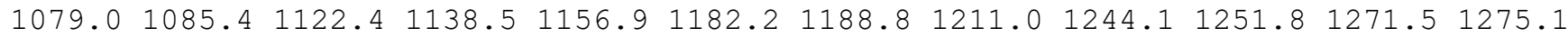

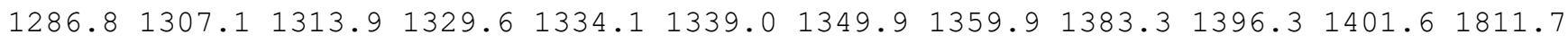
$1836.62656 .4 \quad 2659.1 \quad 2665.92672 .0 \quad 2673.5 \quad 2680.7 \quad 2712.52720 .92742 .92746 .6 \quad 2758.5$ $2775.8 \quad 2789.6 \quad 2841.2 \quad 4743.1$

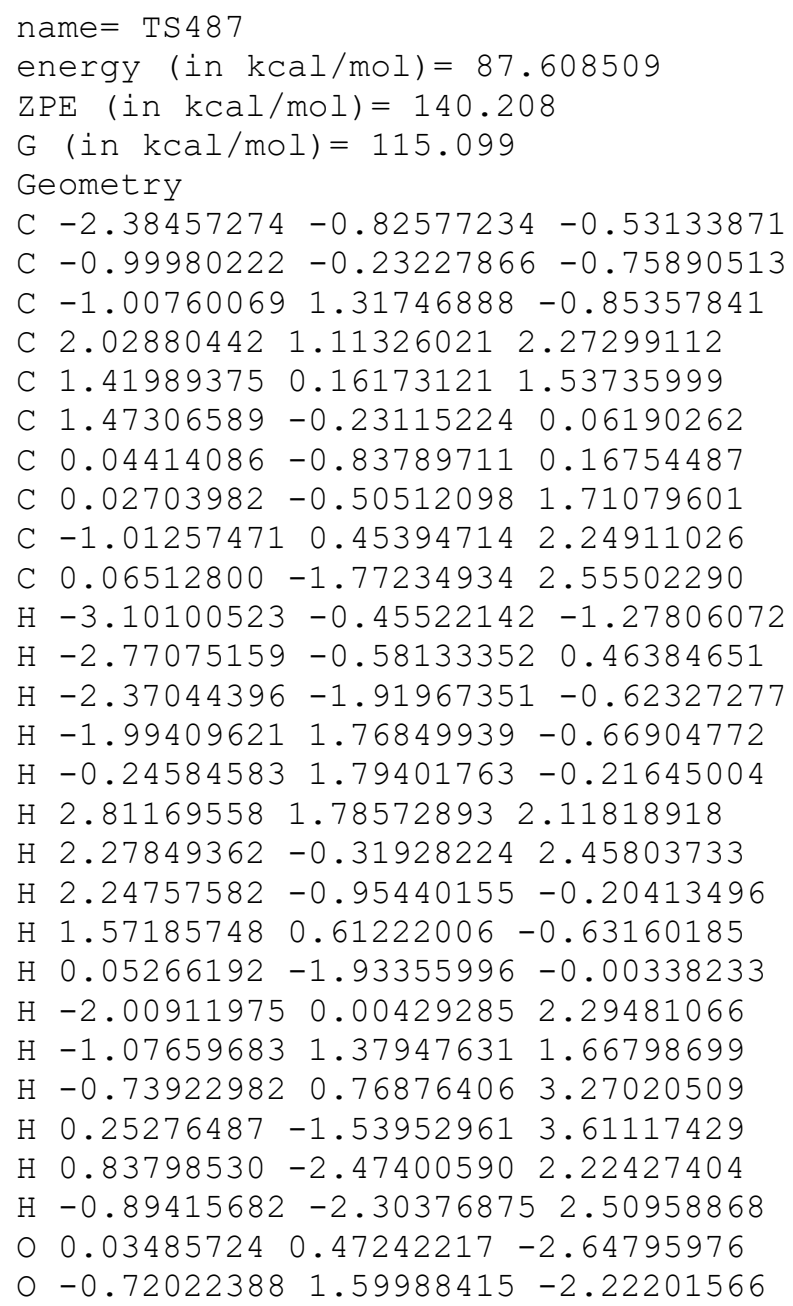


$0-0.65880487-0.63332607-2.10513878$

Vibrational frequencies (in $\mathrm{cm}-1$ )

$\begin{array}{lllllllllllllllll}-1194.7 & 42.1 & 58.8 & 86.7 & 124.8 & 145.2 & 165.6 & 190.6 & 202.6 & 249.7 & 257.3 & 270.9 & 305.7 & 331.3\end{array}$

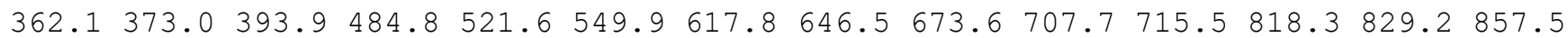
$915.1936 .4958 .5 \quad 971.9 \quad 976.9 \quad 990.3 \quad 1004.1 \quad 1007.4 \quad 1015.5 \quad 1019.3 \quad 1058.2 \quad 1070.6 \quad 1083.7$ $\begin{array}{lllllllllllllll}1103.9 & 1136.1 & 1152.7 & 1167.6 & 1205.3 & 1225.7 & 1253.9 & 1266.3 & 1278.2 & 1282.8 & 1286.9 & 1287.1\end{array}$

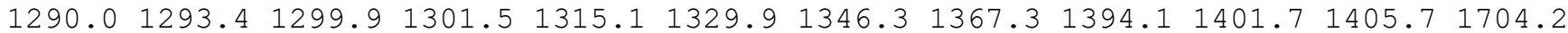
$2291.22644 .92664 .7 \quad 2670.8 \quad 2673.6 \quad 2673.8 \quad 2677.02680 .82682 .5 \quad 2714.72716 .52763 .6$ $2778.8 \quad 2780.7 \quad 2782.7 \quad 2794.6$

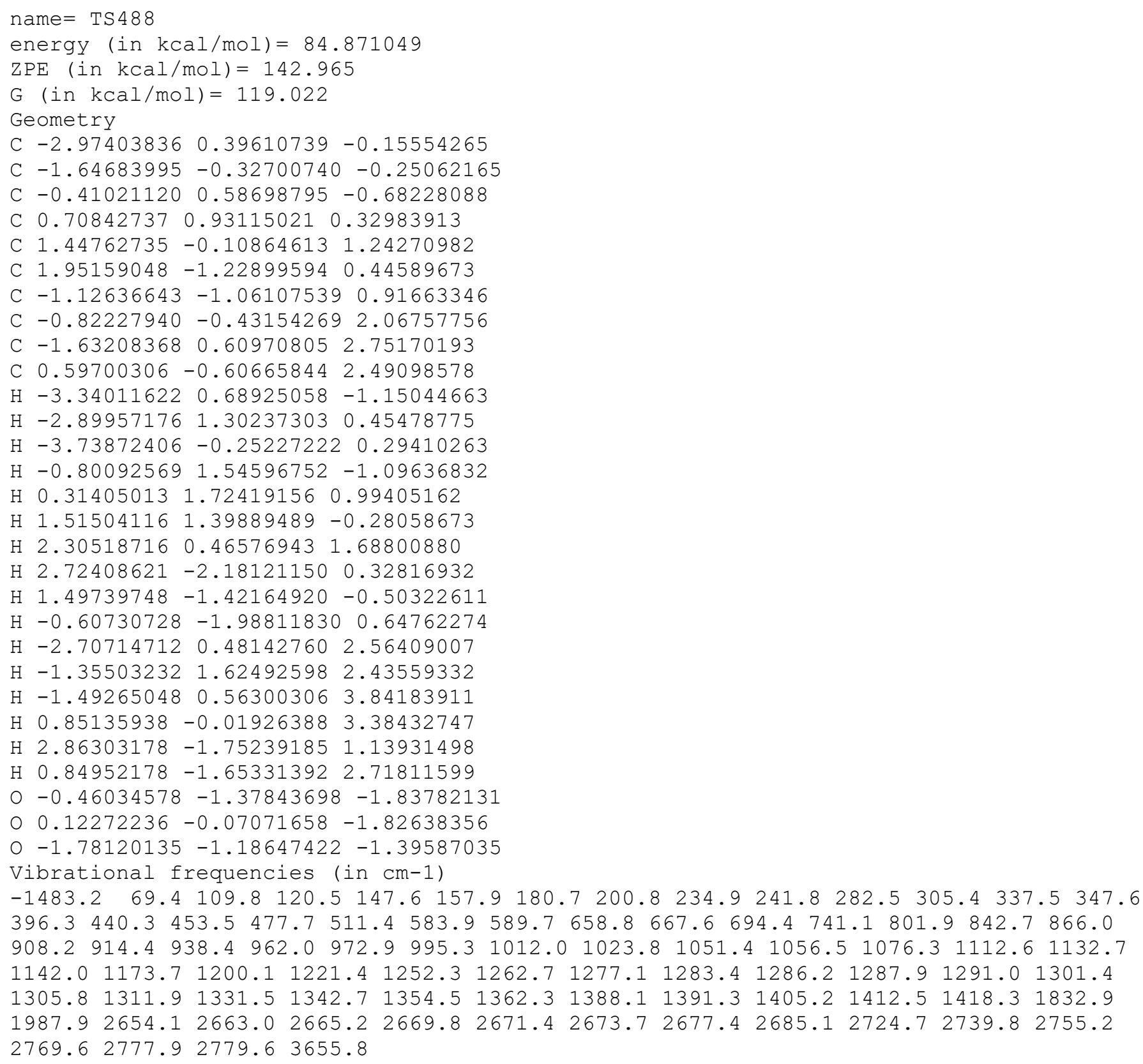


C $1.24405105-1.23432060 \quad 0.55535388$

C $-0.17969503-1.168477740 .11142803$

C $-0.14880694-0.122659952 .12662060$

C $-1.02891700 \quad 0.91548901 \quad 2.27648724$

C $-0.36394915-1.33337124 \quad 2.98744065$

$\mathrm{H}-2.33219355 \quad 0.82243724-1.58758752$

$\mathrm{H}-2.53561948 \quad 0.48121502 \quad 0.35187268$

$\mathrm{H}-2.51555819-0.91341586-1.09397975$

$\mathrm{H}-0.468356592 .08692637-0.44932075$

$\mathrm{H} 1.23637987 \quad 2.17344011 \quad 1.14189100$

H 2.31633145 $1.27522251 \quad 0.08071201$

H 2.02303204 $0.03677555 \quad 2.21546746$

$\mathrm{H} 1.51260815-2.15605353 \quad 1.08999744$

$\mathrm{H} \quad 1.95963817-1.15032768-0.28450998$

$\mathrm{H}-0.84396935-1.99112857 \quad 0.27660324$

$\mathrm{H}-2.30863783 \quad 0.68839437 \quad 1.11186034$

$\mathrm{H}-0.83315621 \quad 1.90958484 \quad 1.90901357$

$\mathrm{H}-1.83199532 \quad 0.88151760 \quad 2.99846412$

$\mathrm{H}-0.18895585-1.08548769 \quad 4.04556013$

$\mathrm{H} \quad 0.30314146 \quad-2.17031014 \quad 2.74769942$

$\mathrm{H}-1.39187156-1.71191038 \quad 2.91001986$

$\begin{array}{llllll}0 & 1.17192374 & 0.27410821 & -2.39165958\end{array}$

$0.890955551 .55448540-1.81269680$

O $0.01317174-0.47160561-2.16940222$

Vibrational frequencies (in $\mathrm{cm}-1$ )

$\begin{array}{llllllllllllll}-1267.0 & 74.1 & 103.0 & 119.5 & 142.3 & 189.2 & 212.6 & 242.0 & 298.7 & 310.3 & 344.8 & 362.4 & 397.5 & 416.1\end{array}$ $\begin{array}{lllllllllllllll}436.0 & 442.8 & 465.4 & 478.3 & 519.5 & 539.6 & 639.7 & 665.4 & 676.9 & 700.3 & 732.8 & 797.0 & 805.1 & 814.3\end{array}$

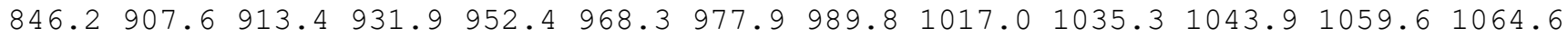

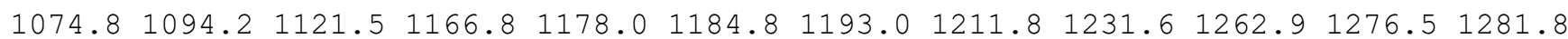

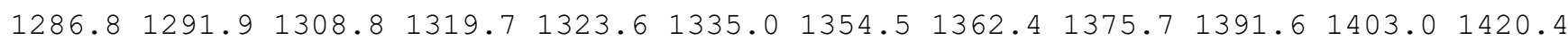
$1656.82655 .5 \quad 2665.7 \quad 2669.7 \quad 2671.8 \quad 2689.92705 .2 \quad 2712.32715 .8 \quad 2739.12747 .0 \quad 2753.5$ $2770.22773 .22778 .7 \quad 3952.0$

name $=\operatorname{TS} 490$

energy (in $\mathrm{kcal} / \mathrm{mol})=86.749329$

$\operatorname{ZPE}(\mathrm{in} \mathrm{kcal} / \mathrm{mol})=141.384$

$\mathrm{G}($ in $\mathrm{kcal} / \mathrm{mol})=116.442$

Geometry

C $-1.97422565 \quad 0.06481959-1.88408847$

C $-0.54831241-0.22424014-1.40115204$

C $0.02361284 \quad 0.97745991-0.59003078$

C $1.29494947 \quad 0.67212221 \quad 0.20661701$

C $1.11726844-0.38178595 \quad 1.31049877$

C $0.87057264-1.76454834 \quad 0.70470088$

C $-0.51488326-1.53032956-0.83172224$

C $0.06282906 \quad 0.07745077 \quad 2.29241166$

C $-1.33858776-0.402901922 .14155795$

C $0.40153981 \quad 0.91447159 \quad 3.27891017$

$\mathrm{H}-2.02877162 \quad 1.05707826-2.35354463$

$\mathrm{H}-2.69237092 \quad 0.03057924-1.05731226$

$\mathrm{H}-2.29148966-0.66773001-2.63688005$

$\mathrm{H}-0.74937031 \quad 1.47918243 \quad 0.02570664$

$\mathrm{H} 1.65828003 \quad 1.62057646 \quad 0.65750333$

H $2.09779043 \quad 0.36532766-0.49521548$

H $2.09367439-0.43911609 \quad 1.87083928$

$\mathrm{H} \quad 0.97179423-1.75339712-0.49610631$

$\mathrm{H} \quad 1.73695210-2.437148120 .82492253$

$\mathrm{H}-0.64243495-2.47401529-1.26873223$

$\mathrm{H}-1.58706554-0.67113559 \quad 1.09743098$

$\mathrm{H}-2.07160161 \quad 0.34859586 \quad 2.46211594$

$\mathrm{H}-1.51617629-1.30225146 \quad 2.74826276$

$\mathrm{H} \quad 1.39796289 \quad 1.28764160 \quad 3.44121138$

$\mathrm{H} \quad 0.01658746-2.28771006 \quad 1.17173269$

$\mathrm{H}-0.29970860 \quad 1.29411021 \quad 4.00273627$ 
$0.491677151 .18404275-2.84597186$

$0.393909231 .91389261-1.58980066$

O $0.36039124-0.17144085-2.53783860$

Vibrational frequencies (in $\mathrm{cm}-1$ )

$\begin{array}{llllllllllllll}-820.1 & 32.3 & 71.1 & 89.3 & 122.0 & 139.0 & 167.2 & 187.9 & 240.3 & 254.0 & 269.6 & 320.6 & 347.0 & 370.0\end{array}$

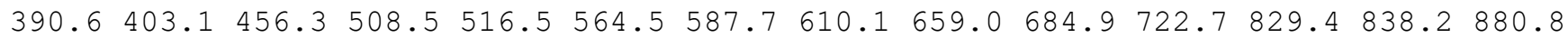

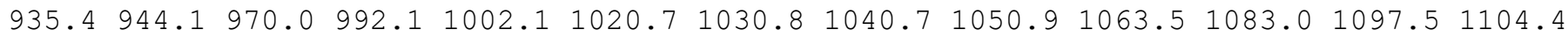

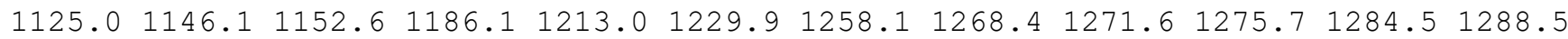

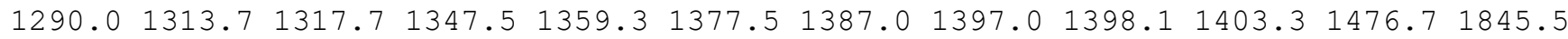
$2087.82627 .52639 .32653 .5 \quad 2669.3 \quad 2674.02678 .52679 .72690 .62712 .72726 .12738 .2$ 2764.22773 .62778 .62795 .2

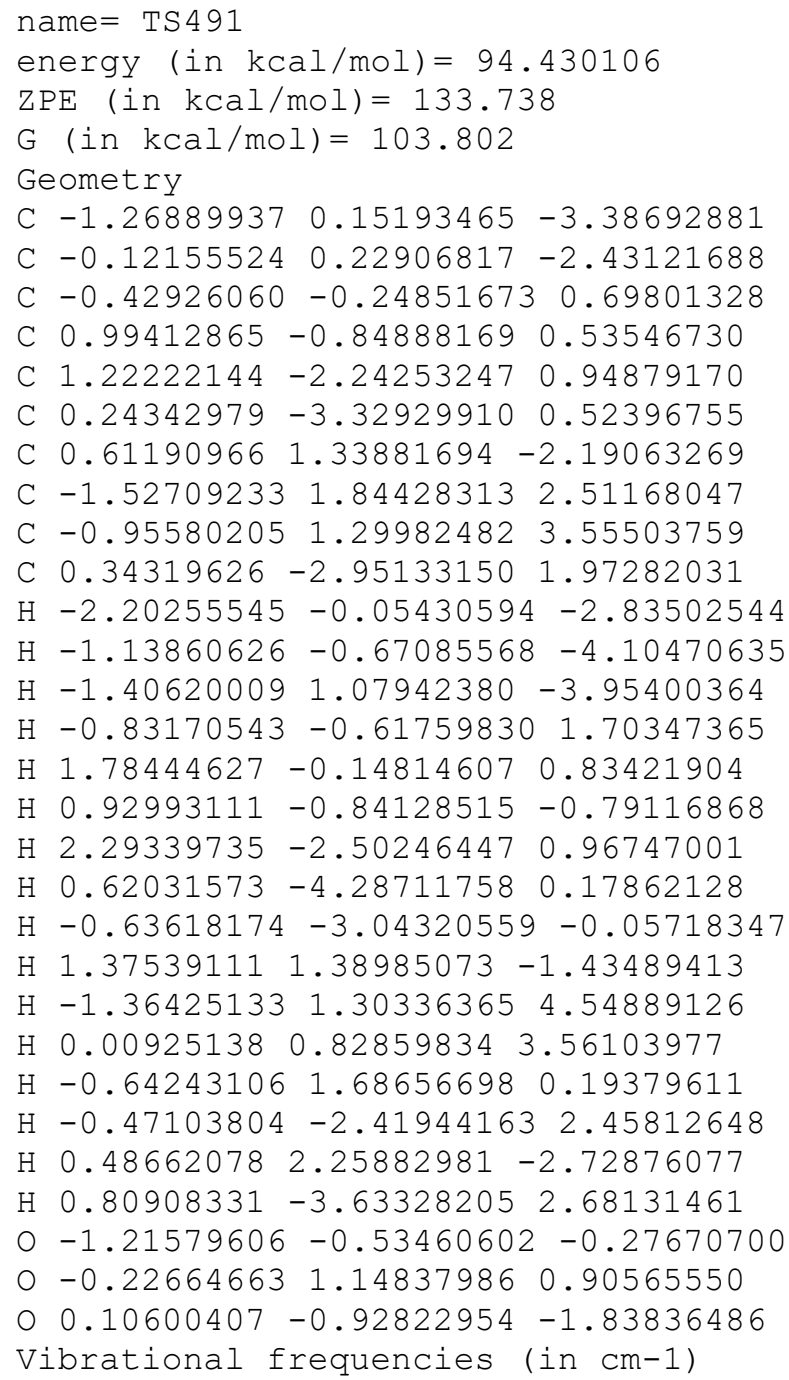


C $1.09891973 \quad 1.50110538 \quad 0.04299808$

C $1.31777290-0.24643018 \quad 1.41059766$

C $1.23564879-1.25628503 \quad 0.29506988$

C $-0.32352648-1.16741636 \quad 0.42240505$

C $-0.10305410-0.567348891 .87428701$

C $-0.96426894 \quad 0.56560787 \quad 2.38423766$

C $-0.07994512-1.68461720 \quad 2.91717582$

$\mathrm{H}-2.80067309 \quad 0.59248449-1.27720795$

$\mathrm{H}-2.75501909 \quad 0.23589834 \quad 0.46565193$

$\mathrm{H}-2.86565898-1.07517843-0.71669111$

$\mathrm{H}-0.97491605 \quad 2.02233124-0.34484155$

H $1.17919382 \quad 2.519595190 .42714410$

$\mathrm{H} \quad 1.933999201 .30728592-0.63710461$

$\mathrm{H} 1.607568961 .001001251 .18213198$

H $1.64850017-2.239734920 .54786426$

$\mathrm{H} \quad 1.65735717-0.98841446-0.67769647$

$\mathrm{H}-0.85266859-2.12612163 \quad 0.39779671$

H $-1.99707001 \quad 0.23623761 \quad 2.55742852$

$\mathrm{H}-1.00065687 \quad 1.42676070 \quad 1.71194044$

$\mathrm{H}-0.57881972 \quad 0.93779596 \quad 3.34501735$

$\mathrm{H} 0.32825433-1.32093945 \quad 3.86878905$

$\mathrm{H} \quad 0.53833282-2.53532874 \quad 2.60858843$

$\mathrm{H}-1.09018013-2.06446863 \quad 3.10838561$

$00.31053498 \quad 0.16987692 \quad-2.50778386$

$\begin{array}{lllll}0 & -0.06178673 & 1.44378770 & -2.03438547\end{array}$

$0-0.62244435-0.71979958-1.90061294$

Vibrational frequencies (in cm-1)

$\begin{array}{lllllllllllllllll}-1389.3 & 62.8 & 124.8 & 135.2 & 150.9 & 161.4 & 170.3 & 197.6 & 235.1 & 268.0 & 293.8 & 336.6 & 353.2 & 362.8\end{array}$

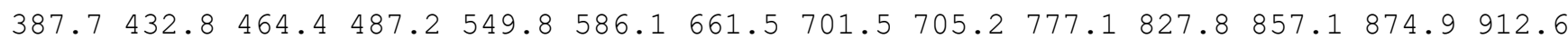

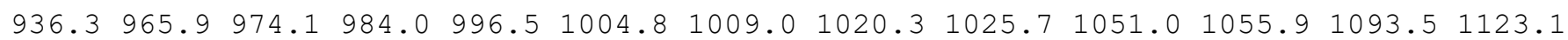

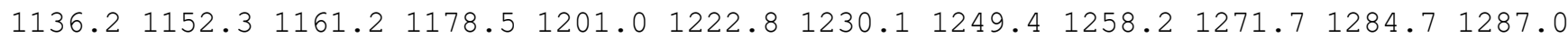
$1288.51296 .0 \quad 1298.2 \quad 1305.5 \quad 1318.7 \quad 1323.3 \quad 1329.8 \quad 1344.11349 .2 \quad 1396.81404 .2 \quad 1416.7$ $1801.92662 .8 \quad 2668.2 \quad 2670.4 \quad 2673.6 \quad 2676.6 \quad 2677.42678 .62680 .4 \quad 2686.62737 .5 \quad 2738.4$ $2764.7 \quad 2779.4 \quad 2780.6 \quad 2783.2$

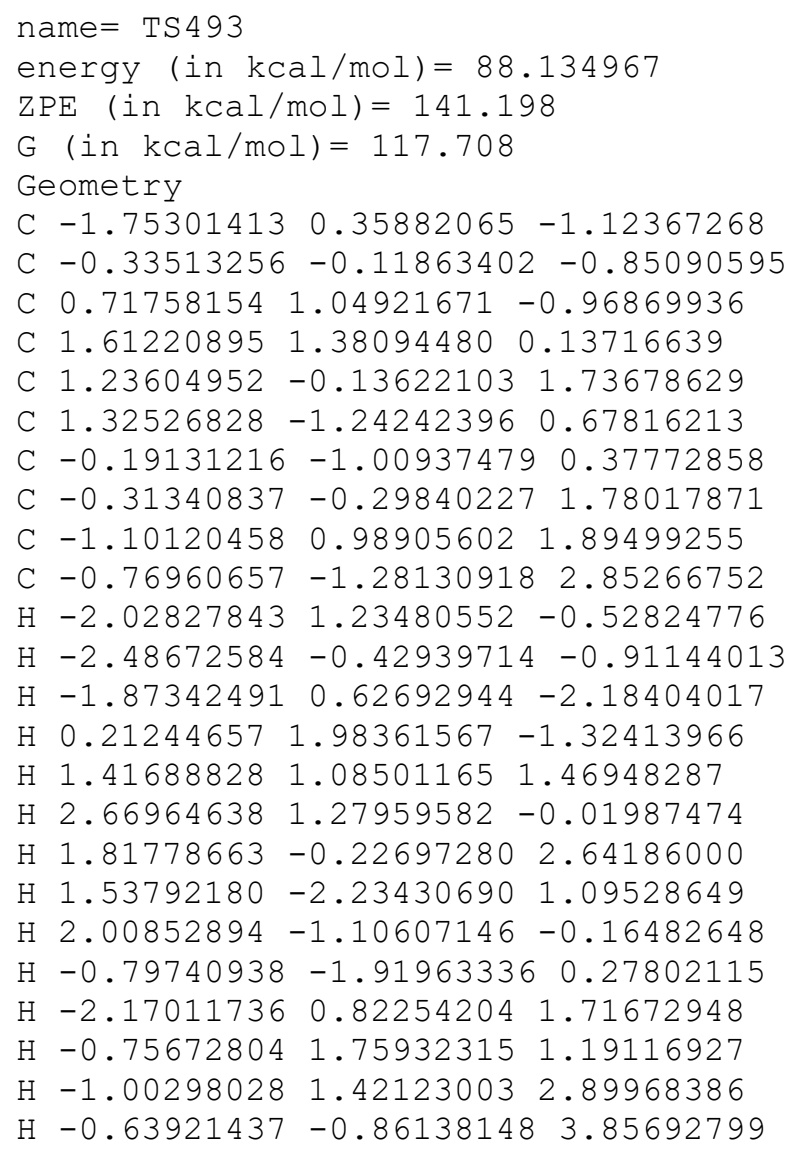


$\mathrm{H}-0.20639673-2.221373132 .81942401$

$\mathrm{H}-1.83075516-1.531111512 .73475904$

$0.72254047-0.18786026-2.85720138$

$01.56933840 \quad 0.59870623-2.04783905$

O $0.03797216-1.00675828-1.92681461$

Vibrational frequencies (in $\mathrm{cm}-1$ )

$\begin{array}{llllllllllllllllll}-1069.3 & 62.1 & 112.9 & 148.0 & 160.0 & 183.5 & 203.0 & 225.7 & 267.4 & 283.4 & 296.5 & 340.5 & 355.1 & 386.0\end{array}$ $409.7 \quad 433.6 \quad 474.4 \quad 487.5 \quad 590.1 \quad 623.2 \quad 639.0 \quad 702.8 \quad 726.6 \quad 770.1 \quad 845.3 \quad 851.5 \quad 879.1 \quad 903.5$ $935.1956 .5976 .2998 .1 \quad 1003.4 \quad 1007.6 \quad 1014.0 \quad 1018.0 \quad 1045.9 \quad 1062.5 \quad 1084.7 \quad 1100.2 \quad 1118.6$ $1121.61138 .81156 .51183 .3 \quad 1184.3 \quad 1203.7 \quad 1235.51241 .01266 .8 \quad 1280.11284 .21286 .1$

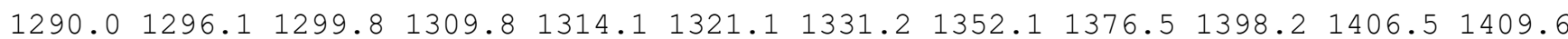
$1748.32657 .02664 .2 \quad 2669.1 \quad 2674.5 \quad 2674.7 \quad 2675.72676 .62680 .12706 .62733 .32740 .9$ $2758.92778 .32780 .4 \quad 2782.3$

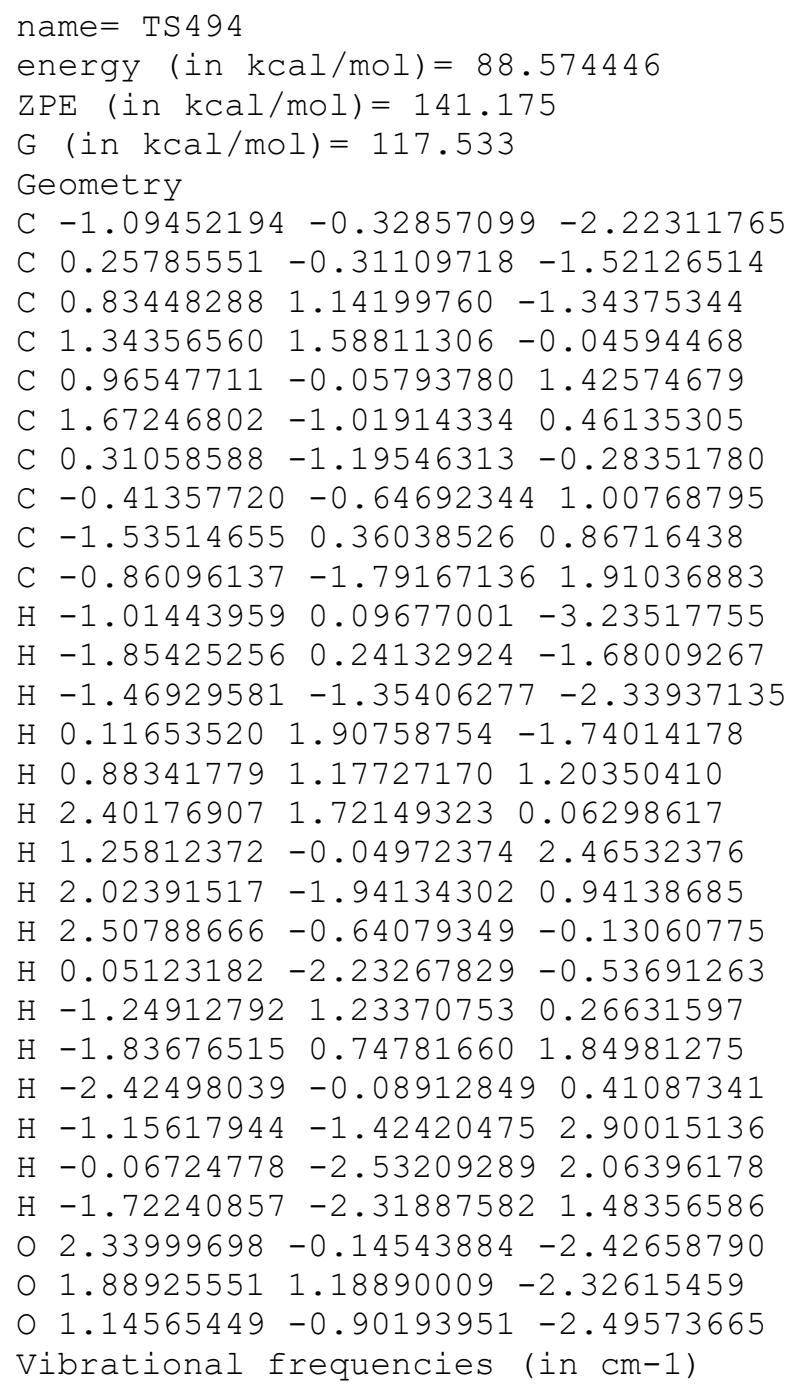


C $-1.07611513-2.660865810 .49092238$

C $-0.36990416-3.44546496-0.68172755$

C $0.68849606-2.51560664-1.28735638$

C $0.49788890-1.16124821-0.62137778$

C $1.06521452-0.922903360 .74698535$

C $-0.61392326-1.22923713 \quad 0.41181403$

C $-0.02736431-0.386226562 .32375584$

C $-0.73179443 \quad 0.90880609 \quad 2.23507500$

C $-0.50172802-1.37215493 \quad 3.29999594$

H $-2.86567929-3.886338890 .56892616$

$\mathrm{H}-3.04638516-2.40907996-0.39983866$

$\mathrm{H}-3.03110439-2.322171631 .36864113$

$\mathrm{H}-1.07629404-3.86370138-1.41561302$

H $0.58102672-2.44455025-2.38248958$

$\mathrm{H} 1.70689887-2.91294703-1.10462128$

$\mathrm{H} \quad 0.51178559-0.32481677-1.32698125$

$\mathrm{H} \quad 1.55802223 \quad 0.042356390 .87800883$

H $1.58614494 \quad-1.74283533 \quad 1.24278293$

$\mathrm{H}-1.38997880-0.479134850 .38264349$

$\mathrm{H}-1.82622362 \quad 0.85397127 \quad 2.28137507$

$\mathrm{H}-0.45782881 \quad 1.47810651 \quad 1.33751821$

$\mathrm{H}-0.40627294 \quad 1.52618448 \quad 3.09294629$

$\mathrm{H} \quad 0.17121886-2.24501557 \quad 3.36301318$

$\mathrm{H}-1.52267197-1.741384863 .13841803$

$\mathrm{H}-0.49256336-0.917955024 .30664068$

$0-0.30348791-4.629590921 .23905800$

$00.30856231-4.52743066-0.04944404$

o $-0.50803018-3.295033151 .64575370$

Vibrational frequencies (in $\mathrm{cm}-1$ )

$\begin{array}{lllllllllllllll}-719.0 & 56.6 & 65.6 & 137.0 & 148.1 & 163.1 & 177.6 & 194.5 & 251.5 & 254.5 & 270.4 & 293.4 & 323.1 & 337.1\end{array}$

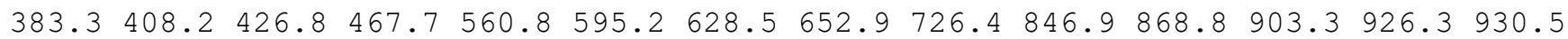
$957.7 \quad 968.5 \quad 975.9 \quad 980.7 \quad 990.3 \quad 1007.4 \quad 1010.6 \quad 1019.8 \quad 1041.0 \quad 1094.4 \quad 1095.3 \quad 1109.9 \quad 1128.1$

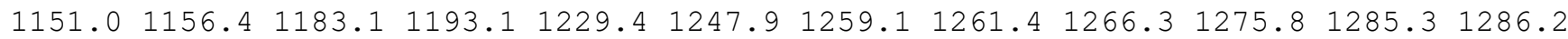

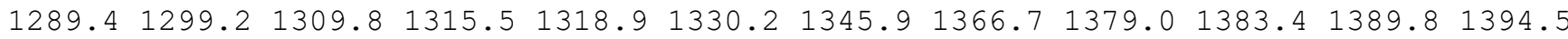
$2656.42660 .92664 .5 \quad 2665.42671 .0 \quad 2673.3 \quad 2677.92681 .52698 .4 \quad 2739.32747 .62747 .9$ 2759.12768 .12773 .32780 .5

name $=\operatorname{TS} 496$

energy (in $\mathrm{kcal} / \mathrm{mol})=89.279394$

$\mathrm{ZPE}($ in $\mathrm{kcal} / \mathrm{mol})=140.883$

$\mathrm{G}($ in $\mathrm{kcal} / \mathrm{mol})=117.341$

Geometry

C $-2.14534818-0.15940292-0.83728495$

C $-0.66450524-0.13971245-1.18262046$

C $0.11500158 \quad 1.17371349 \quad 0.87401091$

C $1.05417412 \quad 1.19275361 \quad 0.25413762$

C $1.57955651-0.15488907 \quad 0.56069081$

C $1.67168338-0.96342410-0.79296200$

C $0.15567304-1.29702136-0.60241858$

C $0.38749563-1.12924295 \quad 0.95851017$

C $-0.69899706-0.57157757 \quad 1.85324833$

C $0.88175825-2.440969831 .56693770$

$\mathrm{H}-2.73505554 \quad 0.39881849-1.58170496$

$\mathrm{H}-2.36620040 \quad 0.26203194 \quad 0.14699266$

$\mathrm{H}-2.53241564-1.18935437-0.83870777$

$\mathrm{H}-0.814505242 .11052942-0.38055714$

$\mathrm{H}-0.46342300 \quad 1.98317464 \quad 0.46757429$

H $1.64287729 \quad 2.04768714 \quad 0.48417804$

H $2.45074298-0.182117251 .21302529$

$\mathrm{H} 2.32808918-1.83272040-0.75035768$

H $1.95143526-0.39142838-1.67590949$

$\mathrm{H}-0.18620126-2.27455691-0.93992906$

$\mathrm{H}-0.91018791 \quad 0.48346763 \quad 1.65087191$

$\mathrm{H}-0.38356379-0.60851254 \quad 2.90621127$ 
$\mathrm{H}-1.62612610-1.147908851 .77081970$

H $1.32482910 \quad-2.26948440 \quad 2.55500382$

H $1.64824225-2.928456830 .95495075$

$\mathrm{H} \quad 0.05916935-3.15416156 \quad 1.68757948$

$\begin{array}{lllll}0 & 0.36569424 & 0.65062806 & -3.08073403\end{array}$

$00.601423501 .65150698-2.06470116$

o $-0.69106570-0.15709079-2.61909391$

Vibrational frequencies (in $\mathrm{cm}-1$ )

$\begin{array}{llllllllllllllll}-2213.5 & 67.6 & 115.6 & 147.5 & 149.9 & 169.6 & 189.1 & 206.4 & 217.7 & 269.9 & 318.0 & 339.5 & 386.6 & 414.0\end{array}$ $425.0 \quad 444.1 \quad 493.9 \quad 507.3 \quad 552.4 \quad 616.3 \quad 630.0 \quad 703.1 \quad 726.1 \quad 738.0 \quad 784.0 \quad 817.3 \quad 839.3 \quad 877.9$ $904.5922 .5928 .0971 .0 \quad 975.6 \quad 992.9 \quad 1003.4 \quad 1010.2 \quad 1014.2 \quad 1037.7 \quad 1051.0 \quad 1073.3 \quad 1076.0$

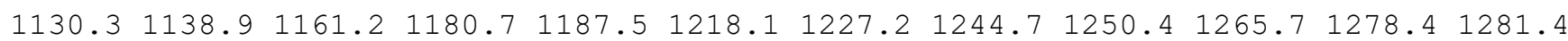
$1291.91299 .6 \quad 1300.2 \quad 1302.2 \quad 1309.2 \quad 1316.4 \quad 1320.1 \quad 1336.91396 .91398 .6 \quad 1405.11414 .0$ $1567.42660 .82662 .42668 .2 \quad 2674.7 \quad 2678.5 \quad 2682.02690 .22748 .12757 .42769 .22774 .5$ $2777.32781 .5 \quad 2801.4 \quad 3093.5$

name $=$ TS 497

energy $($ in $\mathrm{kcal} / \mathrm{mol})=89.690946$

$\mathrm{ZPE}(\mathrm{in} \mathrm{kcal} / \mathrm{mol})=140.642$

$\mathrm{G}($ in $\mathrm{kcal} / \mathrm{mol})=116.318$

Geometry

C $-2.73965267-1.12247013-0.06294176$

C $-1.25515100-0.91105192-0.37571730$

C $-1.058606020 .45820244-1.10983986$

C $0.175084401 .28995435-0.81194925$

C $1.12311799-0.299428712 .01649484$

C $1.12720094-1.20273498 \quad 0.77303070$

C $-0.43491574-1.30901170 \quad 0.84074536$

C $-0.44900390-0.372738012 .08247107$

C $-1.49462995 \quad 0.49507092 \quad 2.44762808$

C $0.45902636-0.980832113 .16972690$

$\mathrm{H}-3.38355558-0.81057375-0.89137650$

$\mathrm{H}-3.02840900-0.57089563 \quad 0.84784144$

$\mathrm{H}-2.95733593-2.181603930 .12431963$

$\mathrm{H}-1.98128483 \quad 1.07690352-1.04807497$

$\mathrm{H} \quad 0.37776949 \quad 1.39432613 \quad 0.25674801$

$\mathrm{H} \quad 0.08165990 \quad 2.29600522-1.24152515$

H $1.77228135 \quad 0.54464994 \quad 2.10907723$

H $1.51643077-0.74266967-0.13667875$

H $1.06905471 \quad 0.84173474 \quad-1.27375710$

$\mathrm{H}-0.74700390-2.34005220 \quad 1.12136042$

$\mathrm{H}-1.18928642 \quad 1.937770851 .63410077$

$\mathrm{H}-1.839818531 .55826287 \quad 1.33262883$

$\mathrm{H}-1.58366872 \quad 0.98131028 \quad 3.38383474$

$\mathrm{H} 0.47393044 \quad-0.50597811 \quad 4.14791465$

H $1.64616349-2.15781003 \quad 0.90115009$

$\mathrm{H} \quad 0.50095343-2.06190750 \quad 3.28117871$

o $-0.32643684-1.17245185-2.44950002$

$0-1.01561431 \quad 0.06181883-2.48557302$

o $-0.95407580-1.88871130-1.39732824$

Vibrational frequencies (in $\mathrm{cm}-1$ )

$\begin{array}{llllllllllllllll}-983.8 & 38.9 & 96.7 & 126.2 & 140.0 & 166.6 & 196.0 & 203.6 & 237.5 & 264.3 & 276.8 & 311.9 & 327.4 & 334.1\end{array}$

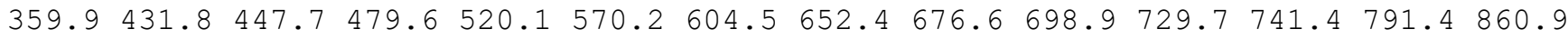

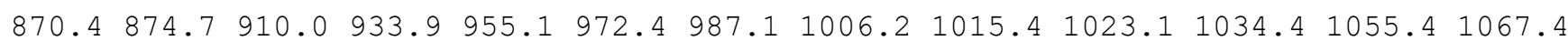
$1091.91110 .01122 .2 \quad 1139.8 \quad 1144.5 \quad 1151.5 \quad 1171.2 \quad 1180.8 \quad 1186.7 \quad 1227.4 \quad 1249.01266 .6$ $1280.21285 .0 \quad 1290.4 \quad 1291.8 \quad 1296.7 \quad 1326.5 \quad 1336.0 \quad 1361.8 \quad 1366.7 \quad 1386.4 \quad 1392.3 \quad 1404.6$ $1523.62648 .2 \quad 2658.2 \quad 2675.5 \quad 2677.3 \quad 2682.92686 .12706 .72710 .82740 .42765 .42774 .2$ $2780.12780 .8 \quad 2787.3 \quad 4732.1$

name $=\operatorname{TS} 498$

energy (in $\mathrm{kcal} / \mathrm{mol})=89.261561$

$\mathrm{ZPE}($ in $\mathrm{kcal} / \mathrm{mol})=141.197$

$\mathrm{G}($ in $\mathrm{kcal} / \mathrm{mol})=116.926$ 


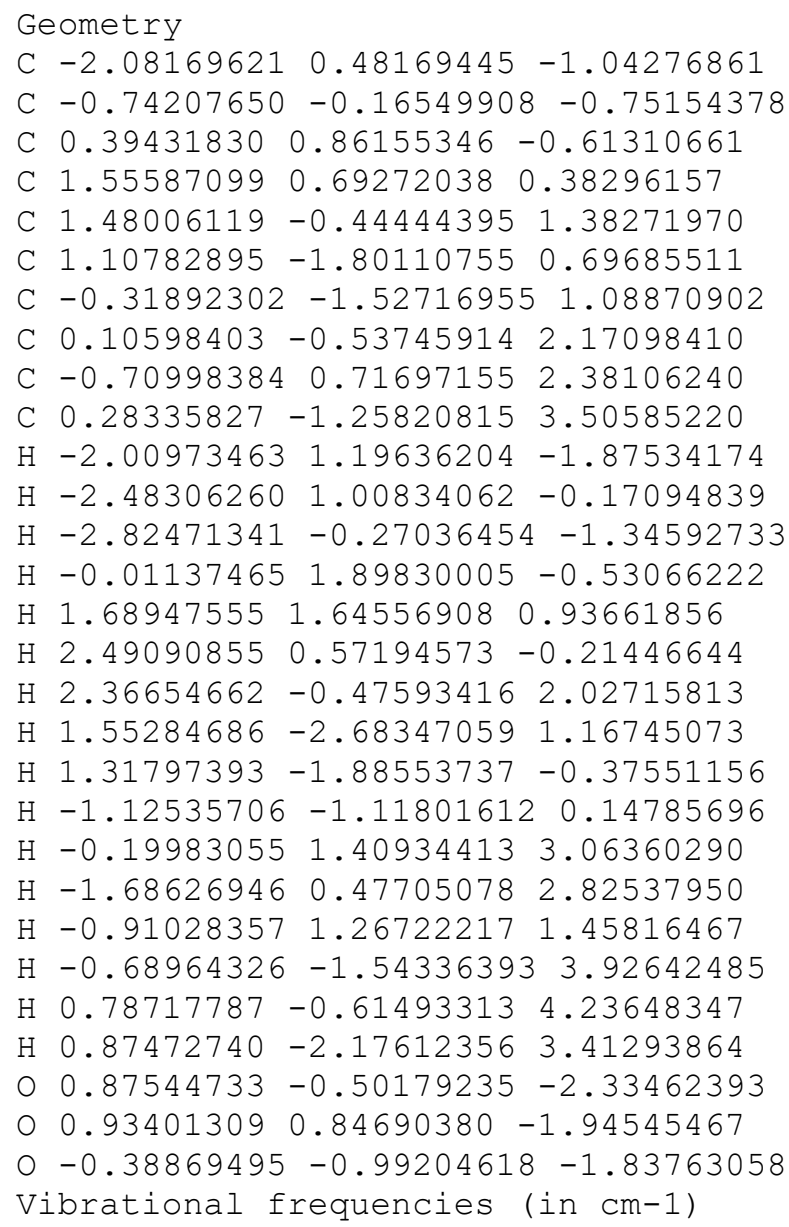

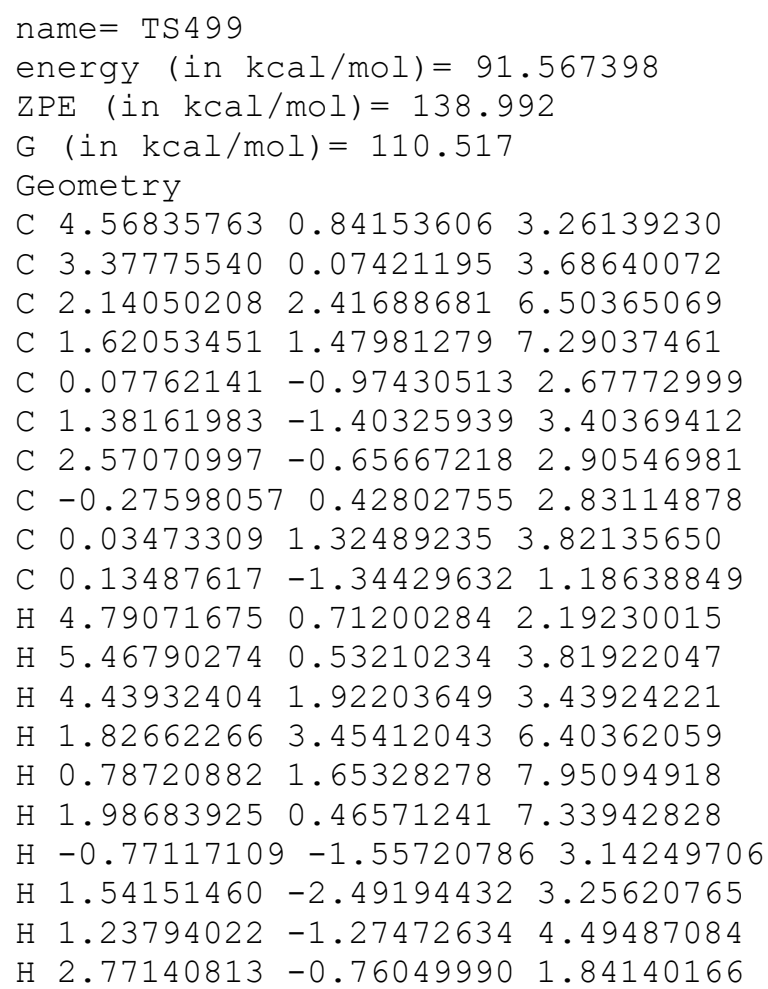


$\mathrm{H} \quad 0.60689784 \quad 1.08110509 \quad 4.71355976$

$\mathrm{H}-0.50407427 \quad 2.26389258 \quad 3.92694781$

$\mathrm{H} 0.58111240 \quad 1.49677862 \quad 2.54216955$

$\mathrm{H}-0.82082394-1.105536960 .70187205$

$\mathrm{H} \quad 0.33449141-2.409395931 .04331666$

$\mathrm{H} \quad 0.90865389-0.77869443 \quad 0.65994282$

$03.80463108 \quad 1.00028365 \quad 5.75196634$

$\begin{array}{llll}0 & 3.18839237 & 2.26220074 \quad 5.63717831\end{array}$

O 3.058946010 .067527605 .03727026

Vibrational frequencies (in cm-1)

$\begin{array}{lllllllllllllllll}-1622.1 & 18.8 & 36.1 & 49.1 & 60.5 & 83.5 & 96.4 & 114.3 & 134.1 & 146.8 & 155.2 & 167.0 & 211.0 & 228.7\end{array}$ $282.6 \quad 334.4 \quad 357.2 \quad 413.5 \quad 429.8 \quad 469.1 \quad 471.6 \quad 528.4 \quad 609.5 \quad 631.9655 .2 \quad 666.3 \quad 808.9 \quad 840.4$

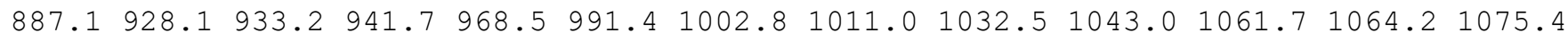
$1120.0 \quad 1126.5 \quad 1147.9 \quad 1169.4 \quad 1183.2 \quad 1202.5 \quad 1268.3 \quad 1273.2 \quad 1277.7 \quad 1280.3 \quad 1289.3 \quad 1292.0$ $1302.51306 .21324 .8 \quad 1369.6 \quad 1373.7 \quad 1379.8 \quad 1383.3 \quad 1401.6 \quad 1402.9 \quad 1663.4 \quad 1855.4 \quad 1866.8$ $2268.22660 .0 \quad 2661.3 \quad 2670.7 \quad 2671.2 \quad 2677.3 \quad 2682.8 \quad 2691.72712 .3 \quad 2735.8 \quad 2736.3 \quad 2747.5$ $2758.42777 .3 \quad 2782.3 \quad 2791.9$

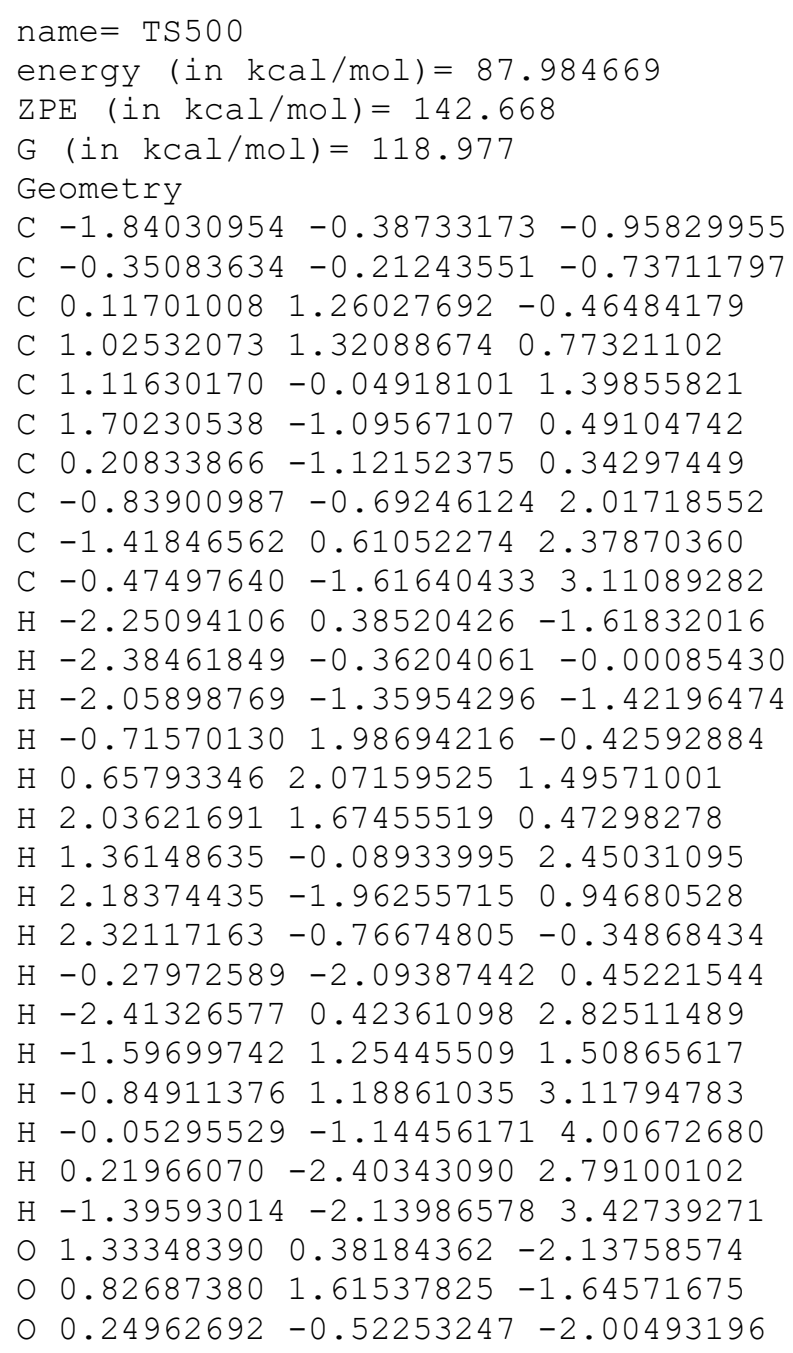


$\mathrm{ZPE}($ in $\mathrm{kcal} / \mathrm{mol})=142.165$

$\mathrm{G}($ in $\mathrm{kcal} / \mathrm{mol})=119.141$

Geometry

C $-2.45384349-0.18403595-0.62103964$

C $-0.93858973-0.27120745-0.69674740$

C $-0.230336391 .13062712-0.74610656$

C $0.88633934 \quad 1.32782804 \quad 0.29932335$

C $0.99515245 \quad 0.13273120 \quad 1.21556651$

C $1.22158391-1.114896420 .37164462$

C $-0.35910657-1.17107786 \quad 0.38639704$

C $-0.34605591-0.45652402 \quad 1.81791286$

C $-1.01074661 \quad 0.69498843 \quad 2.37008846$

C $-0.18218735-1.53979778 \quad 2.88270160$

$\mathrm{H}-2.861161290 .47851035-1.39619851$

$\mathrm{H}-2.78197738 \quad 0.186222290 .35963549$

$\mathrm{H}-2.91604986-1.16970786-0.76996124$

$\mathrm{H}-0.96002170 \quad 1.96552680 \quad-0.71784899$

$\mathrm{H} \quad 0.70882268 \quad 2.26680938 \quad 0.85489064$

H $1.854692951 .48343984-0.22386145$

$\mathrm{H} \quad 1.24827879 \quad 0.93431567 \quad 2.59593199$

$\mathrm{H} 1.70141204-1.96165940 \quad 0.86476025$

H $1.69216423-0.98842553-0.60466947$

$\mathrm{H}-0.79797715-2.17073204 \quad 0.40435526$

$\mathrm{H}-1.34432880 \quad 1.50376582 \quad 1.75421987$

H $0.49337392 \quad 1.23791355 \quad 2.93125909$

$\mathrm{H}-1.52898456 \quad 0.63172452 \quad 3.30621509$

$\mathrm{H} \quad 0.26880806-1.133415613 .79656658$

$\mathrm{H} \quad 0.45411328-2.37035734 \quad 2.56088275$

$\mathrm{H}-1.16033435-1.957446893 .15070343$

$00.50666208-0.16356001-2.43645281$

○ $0.30423477 \quad 1.19363569-2.06474010$

o $-0.66184391-0.82782976-1.99122821$

Vibrational frequencies (in $\mathrm{cm}-1$ )

$\begin{array}{lllllllllllllll}-1640.1 & 69.9 & 124.1 & 144.6 & 153.7 & 201.4 & 224.0 & 244.7 & 295.6 & 313.4 & 360.6 & 385.4 & 424.5 & 445.8\end{array}$

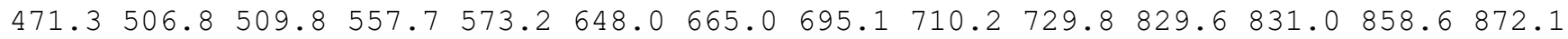
$916.1943 .0 \quad 958.3 \quad 964.7 \quad 979.5 \quad 988.3 \quad 1003.2 \quad 1036.5 \quad 1050.1 \quad 1051.6 \quad 1063.9 \quad 1079.4 \quad 1095.1$

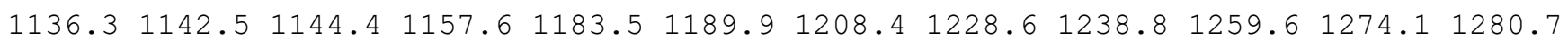

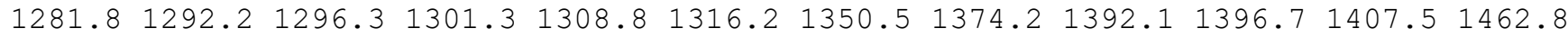
$1507.82654 .7 \quad 2668.3 \quad 2671.8 \quad 2672.5 \quad 2678.0 \quad 2686.2 \quad 2689.5 \quad 2726.8 \quad 2737.6 \quad 2739.12767 .3$ $2777.7 \quad 2779.8 \quad 2783.3 \quad 3304.3$

\section{name $=$ TS502}

energy (in $\mathrm{kcal} / \mathrm{mol})=90.800176$

$\mathrm{ZPE}(\mathrm{in} \mathrm{kcal} / \mathrm{mol})=140.282$

$\mathrm{G}($ in $\mathrm{kcal} / \mathrm{mol})=114.698$

Geometry

C $-0.92543278 \quad 1.33881695 \quad-2.53395341$

C $0.225780120 .69427653-1.78713806$

C $1.230411291 .70873497-1.11973221$

C 1.425731521 .307880050 .34572929

C $0.48360403 \quad 0.13201123 \quad 0.63081660$

C $0.82307788-1.16474277-0.09735434$

C $-0.21310887-0.25720274-0.69956810$

C $-0.23485320 \quad 0.11775388 \quad 1.84847880$

C $-1.036613321 .08379431 \quad 2.56849197$

C $0.28484377-1.429978383 .05016171$

$\mathrm{H}-0.56545240 \quad 2.05507149-3.28496188$

$\mathrm{H}-1.598812321 .86782416-1.84937611$

$\mathrm{H}-1.51918786 \quad 0.58491753-3.06973597$

H $0.95002985 \quad 2.76771751-1.25330676$

$\mathrm{H} \quad 1.20184354 \quad 2.14824660 \quad 1.02137994$

$\mathrm{H} 2.476460491 .02793586 \quad 0.53598020$

$\mathrm{H} 0.78674216-0.33390977 \quad 2.84118413$

H $0.50430227 \quad-2.11287578 \quad 0.32538230$ 
H $1.79584446-1.26816608-0.57476106$

$\mathrm{H}-1.25461966-0.56601377-0.71234962$

$\mathrm{H}-1.86013415 \quad 1.42637820 \quad 1.90857449$

$\mathrm{H}-0.48807188 \quad 1.98421678 \quad 2.88627570$

$\mathrm{H}-1.530613910 .66104944 \quad 3.45699405$

H $0.11970691-1.36759625 \quad 4.12361008$

H $1.11507788-2.07840095 \quad 2.77879675$

$\mathrm{H}-0.62950998-1.827524052 .58627603$

$\begin{array}{lllll}0 & 2.36376910 & 0.21818686 & -2.36034520\end{array}$

O $2.42142057 \quad 1.55606410-1.87891487$

o $1.02191751 \quad 0.04887135-2.78787041$

Vibrational frequencies (in $\mathrm{cm}-1$ )

$\begin{array}{lllllllllllllllllll}-981.6 & 32.9 & 71.4 & 78.6 & 105.2 & 124.2 & 136.4 & 152.4 & 185.8 & 206.3 & 243.1 & 274.7 & 309.0 & 324.3\end{array}$

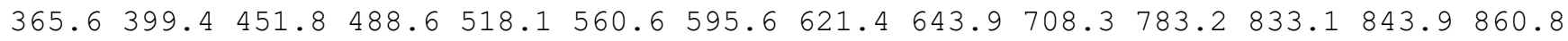
$904.8941 .9960 .4977 .4 \quad 994.1 \quad 1001.7 \quad 1004.3 \quad 1038.9 \quad 1041.2 \quad 1067.2 \quad 1078.4 \quad 1127.7 \quad 1134.3$ $1138.7 \quad 1164.0 \quad 1169.0 \quad 1201.3 \quad 1220.1 \quad 1237.5 \quad 1260.4 \quad 1264.4 \quad 1274.1 \quad 1280.01284 .2 \quad 1285.9$ $1289.51290 .4 \quad 1294.0 \quad 1312.6 \quad 1325.7 \quad 1329.1 \quad 1379.4 \quad 1388.2 \quad 1391.7 \quad 1406.2 \quad 1451.7 \quad 1617.4$ $2011.22635 .5 \quad 2653.0 \quad 2663.7 \quad 2672.1 \quad 2672.8 \quad 2675.0 \quad 2678.52702 .2 \quad 2711.32753 .82754 .6$ $2765.92769 .5 \quad 2780.8 \quad 2782.7$

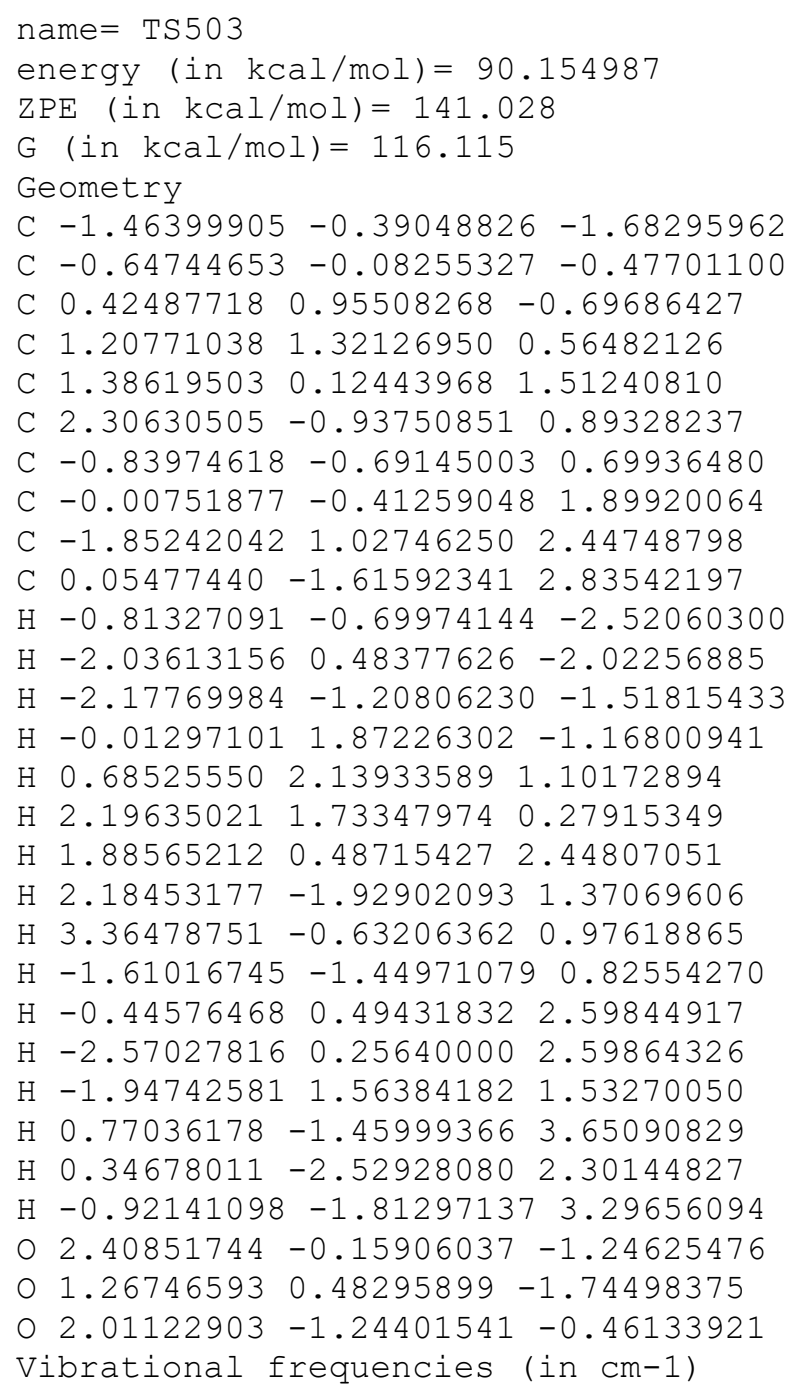




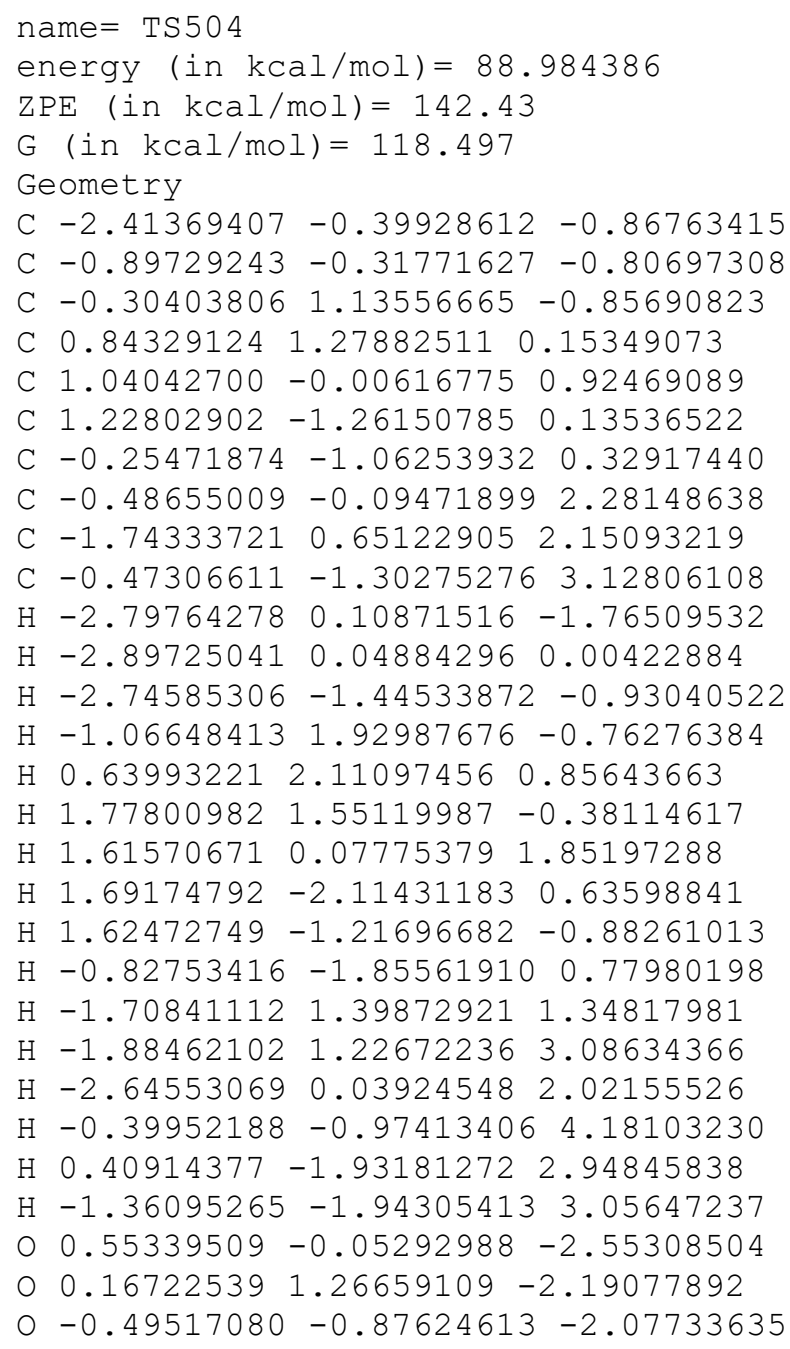


$\mathrm{H} \quad 1.46800656 \quad 0.23867330 \quad 2.28708598$

H 2.28448783 $-1.75116396 \quad 0.98753401$

$\mathrm{H} 2.43190466-0.69856524-0.42824298$

$\mathrm{H}-0.19768009-1.93048678 \quad 0.45630052$

$\mathrm{H}-2.40326291 \quad 0.66512304 \quad 2.53410421$

$\mathrm{H}-2.59189815-0.48941483 \quad 1.20942265$

$\mathrm{H}-1.77765499 \quad 1.07457342 \quad 0.94437965$

$\mathrm{H}-0.87786038-1.357557343 .88406091$

$\mathrm{H} \quad 0.38817677 \quad-2.04198488 \quad 2.87457400$

$\mathrm{H}-1.31504694 \quad-2.339312292 .47955351$

$\begin{array}{lllll}0 & 0.95155272 & 0.51431841 & -2.81376401\end{array}$

O $1.171278201 .54398927-1.84943826$

O $0.77698363-0.65555575-2.05093042$

Vibrational frequencies (in $\mathrm{cm}-1$ )

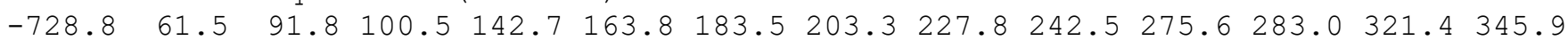
$360.4 \quad 407.6 \quad 433.0 \quad 480.4 \quad 548.2 \quad 579.6 \quad 604.1 \quad 655.6 \quad 716.1 \quad 850.4 \quad 867.8 \quad 894.4 \quad 913.9 \quad 921.2$

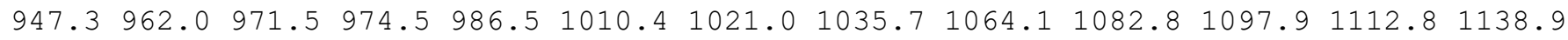

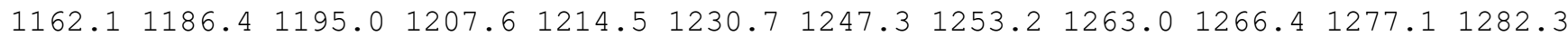

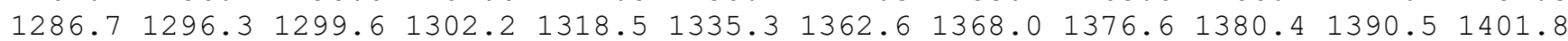
$2656.42658 .12663 .5 \quad 2668.4 \quad 2670.4 \quad 2672.3 \quad 2675.42688 .62692 .62708 .12736 .52752 .4$ $2770.62772 .4 \quad 2774.6 \quad 2781.3$

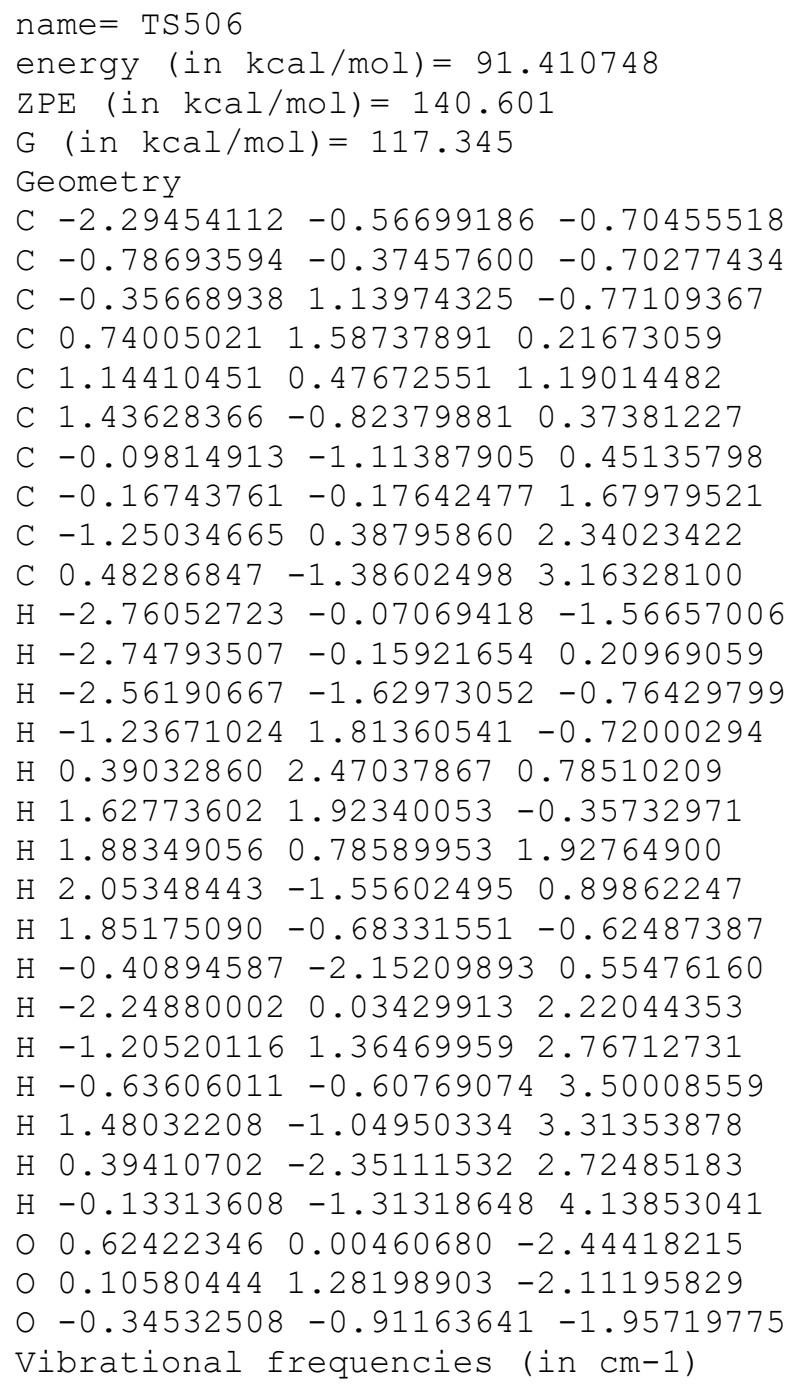




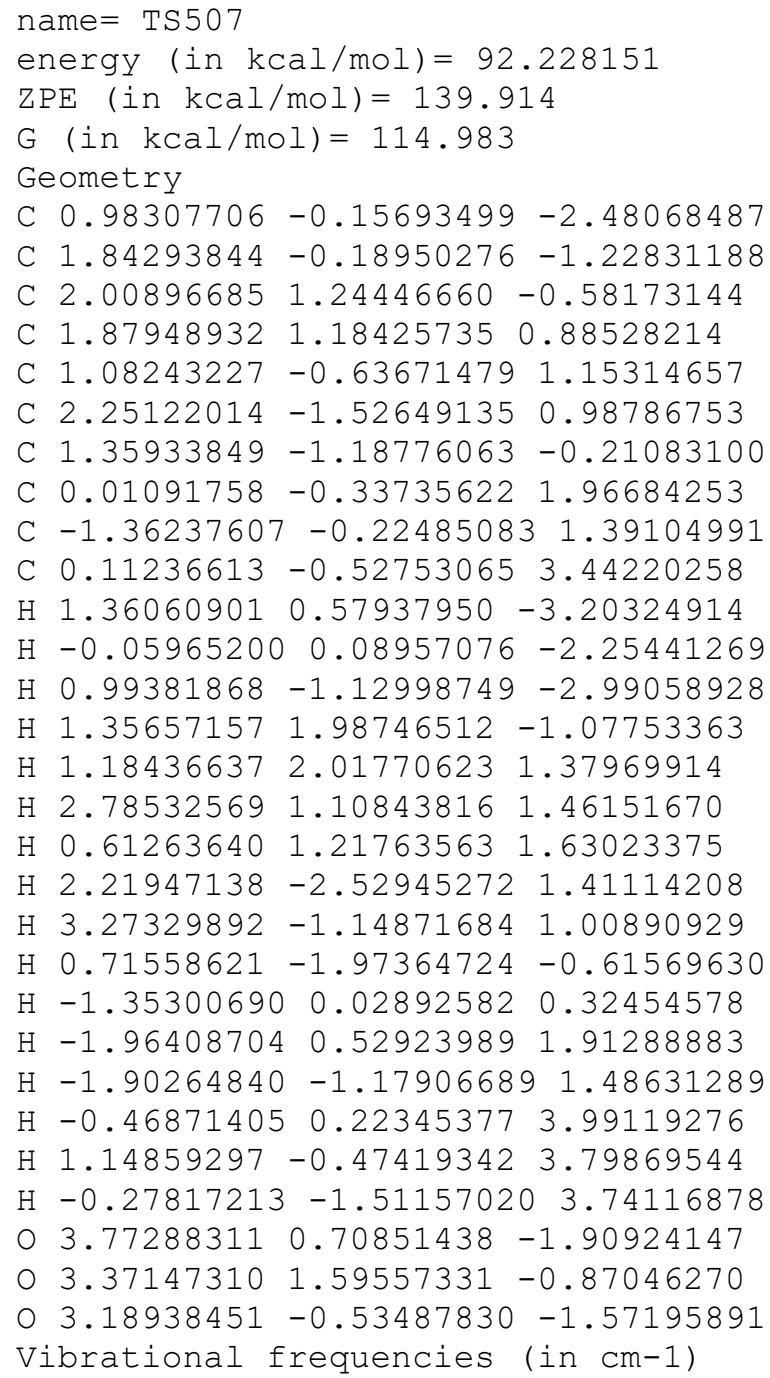


H $0.380153131 .79090537 \quad 1.08972057$

$\mathrm{H} \quad 1.55947930 \quad 1.39857229-0.14888540$

$\mathrm{H} 2.02745929-0.725442193 .11642413$

$\mathrm{H} \quad 2.05856401-1.18100714-0.57600131$

H $3.17645247-0.48265836 \quad 0.60322970$

H $0.12030502 \quad-2.48493808 \quad 0.24412236$

$\mathrm{H} \quad 0.03768775 \quad 1.63173387 \quad 3.13271419$

$\mathrm{H} \quad 1.79663963 \quad 1.85473368 \quad 3.19178952$

$\mathrm{H} \quad 0.96091584 \quad 0.97532746 \quad 4.47987571$

$\mathrm{H}-0.00016663-2.00400077 \quad 2.49675487$

H $2.37594458-2.02009811 \quad 0.95006314$

$\mathrm{H}-0.97787489-0.497134832 .32317754$

O $0.69038878-0.06581439-2.35895060$

$0-0.01377742 \quad 1.08679939-1.92356720$

o $-0.11987445-1.16184637-1.96519690$

Vibrational frequencies (in cm-1)

$\begin{array}{lllllllllllllllllll}-565.1 & 31.5 & 63.2 & 134.7 & 137.8 & 145.3 & 184.0 & 217.0 & 225.9 & 240.1 & 254.9 & 296.0 & 325.3 & 362.5\end{array}$ $398.6 \quad 441.1 \quad 444.0 \quad 480.4 \quad 507.2 \quad 575.4 \quad 601.0 \quad 662.4 \quad 705.8 \quad 768.2 \quad 826.4 \quad 856.6 \quad 872.6 \quad 919.0$ $941.8952 .1970 .2 \quad 993.2 \quad 1003.7 \quad 1010.9 \quad 1017.6 \quad 1047.4 \quad 1070.1 \quad 1085.6 \quad 1087.1 \quad 1121.51122 .5$ $1127.1 \quad 1158.6 \quad 1178.4 \quad 1187.4 \quad 1208.8 \quad 1230.2 \quad 1247.1 \quad 1282.4 \quad 1283.0 \quad 1286.1 \quad 1289.2 \quad 1290.4$

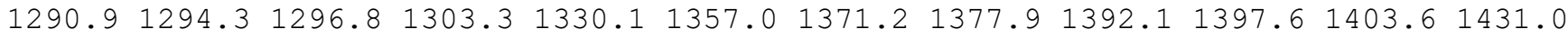
$2644.22655 .7 \quad 2669.2 \quad 2670.5 \quad 2673.0 \quad 2673.8 \quad 2681.12684 .92695 .3 \quad 2727.92731 .92738 .5$ $2753.12774 .2 \quad 2778.92780 .1$

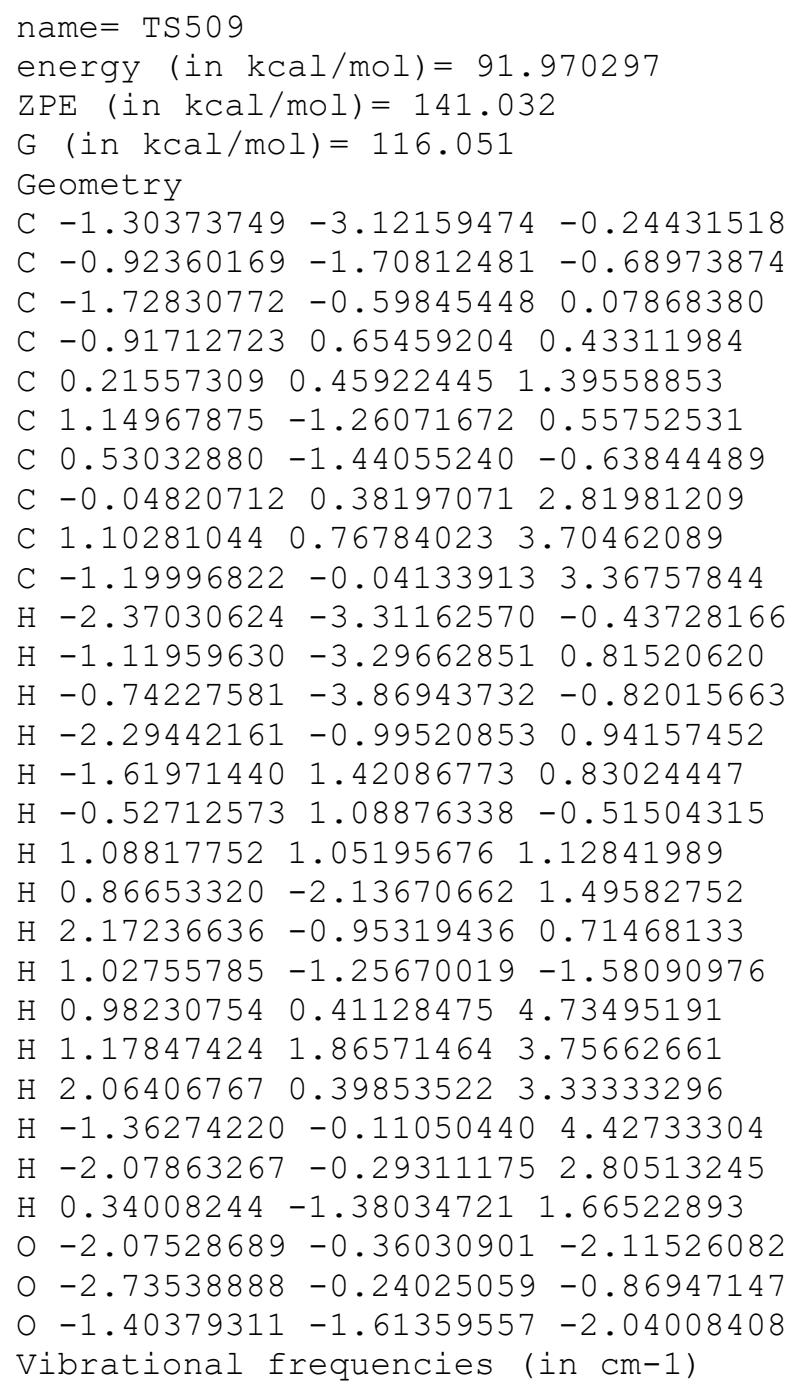


$\begin{array}{llllllllllllll}1825.3 & 2652.2 & 2668.7 & 2669.5 & 2681.1 & 2684.6 & 2687.0 & 2727.7 & 2728.7 & 2734.5 & 2748.5 & 2771.5\end{array}$ $2781.0 \quad 2782.12797 .2 \quad 3305.0$

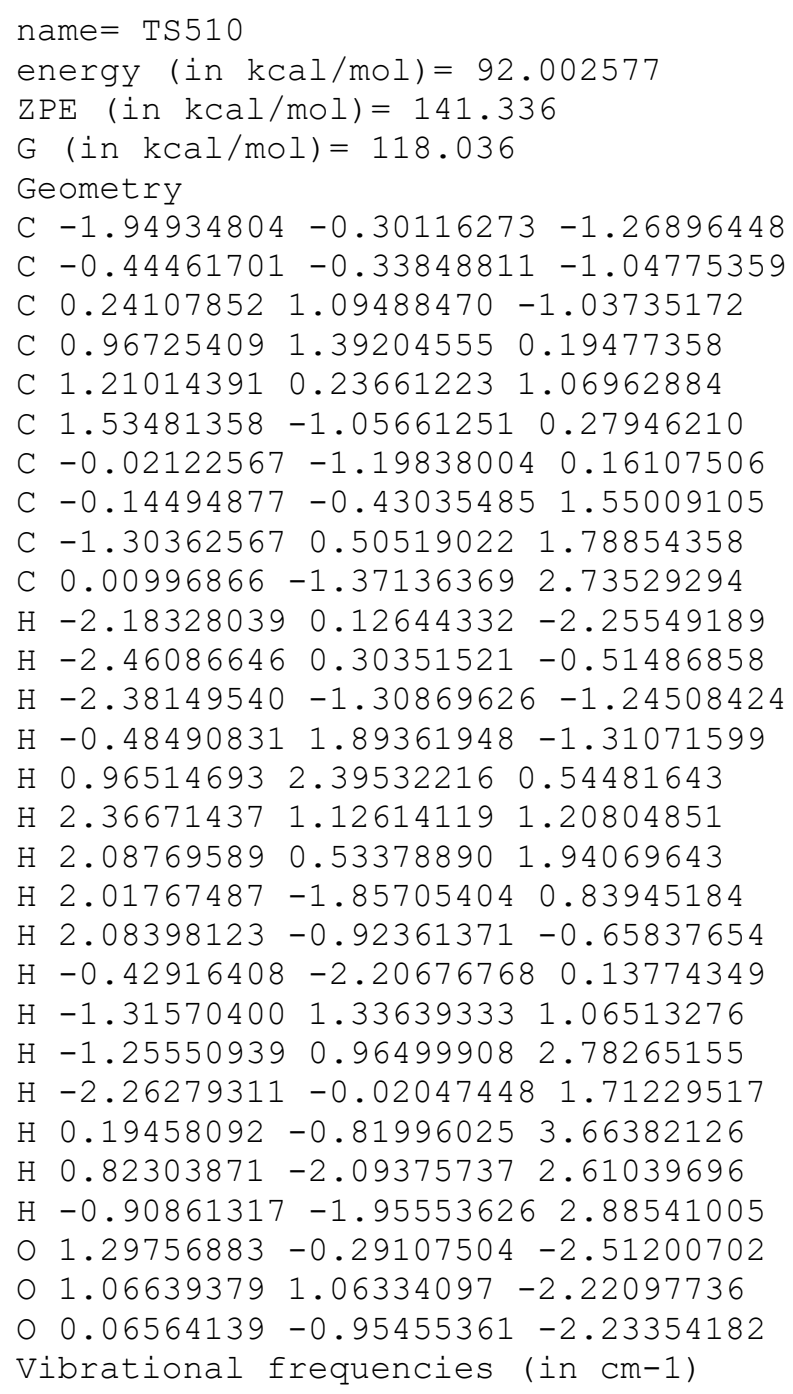


H $-1.56006095 \quad 0.29658799-3.22374285$

$\mathrm{H} \quad 0.46529901 \quad 4.38223234-0.80478848$

$\mathrm{H}-1.45051718 \quad 3.54143244 \quad 0.70564600$

$\mathrm{H}-0.87653135 \quad 1.85813042 \quad 0.48147887$

$\mathrm{H} \quad 0.67426060 \quad 0.90344660 \quad 1.54523960$

H $0.60443783-2.12264615 \quad 0.88918442$

$\mathrm{H} 1.53830754-0.91485195-0.00373971$

$\mathrm{H}-1.52352233-1.22283788 \quad 0.16303335$

$\mathrm{H}-1.158452220 .13360609 \quad 4.76303575$

$\mathrm{H}-1.90082494 \quad 0.68823618 \quad 3.26616702$

$\mathrm{H}-0.404093861 .43961372 \quad 3.82716357$

H 2.14295852 $0.20047018 \quad 3.45787345$

H $2.28457622-1.416148772 .67971596$

$\mathrm{H} \quad 0.71851450-1.29019326 \quad 3.68328178$

o $1.21710323 \quad 1.25301422-0.81777903$

O $1.236083362 .61597993-1.14881192$

o $0.50542248 \quad 0.59065689-1.84283310$

Vibrational frequencies (in $\mathrm{cm}-1$ )

$\begin{array}{llllllllllllllll}-1558.0 & 30.3 & 42.1 & 46.0 & 63.1 & 71.9 & 87.1 & 105.0 & 109.4 & 167.7 & 180.4 & 198.7 & 252.7 & 287.7\end{array}$ $\begin{array}{llllllllllllllll}306.8 & 325.0 & 335.4 & 432.6 & 437.1 & 448.8 & 551.7 & 599.9 & 622.8 & 631.2 & 638.5 & 648.8 & 806.4 & 872.5\end{array}$ $890.2934 .3940 .0945 .6 \quad 957.4 \quad 1006.9 \quad 1009.2 \quad 1012.4 \quad 1023.2 \quad 1041.4 \quad 1056.8 \quad 1070.3 \quad 1095.2$ $1113.6 \quad 1149.91163 .0 \quad 1170.3 \quad 1193.5 \quad 1205.1 \quad 1232.7 \quad 1259.1 \quad 1263.7 \quad 1271.0 \quad 1277.0 \quad 1279.3$

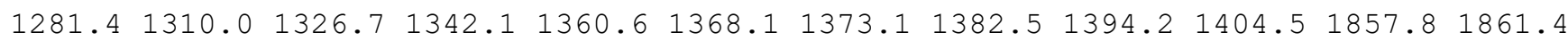
$2149.12657 .12663 .6 \quad 2664.8 \quad 2675.1 \quad 2675.4 \quad 2704.82707 .32722 .8 \quad 2731.72738 .62754 .4$ $2766.4 \quad 2776.22777 .7 \quad 2791.3$

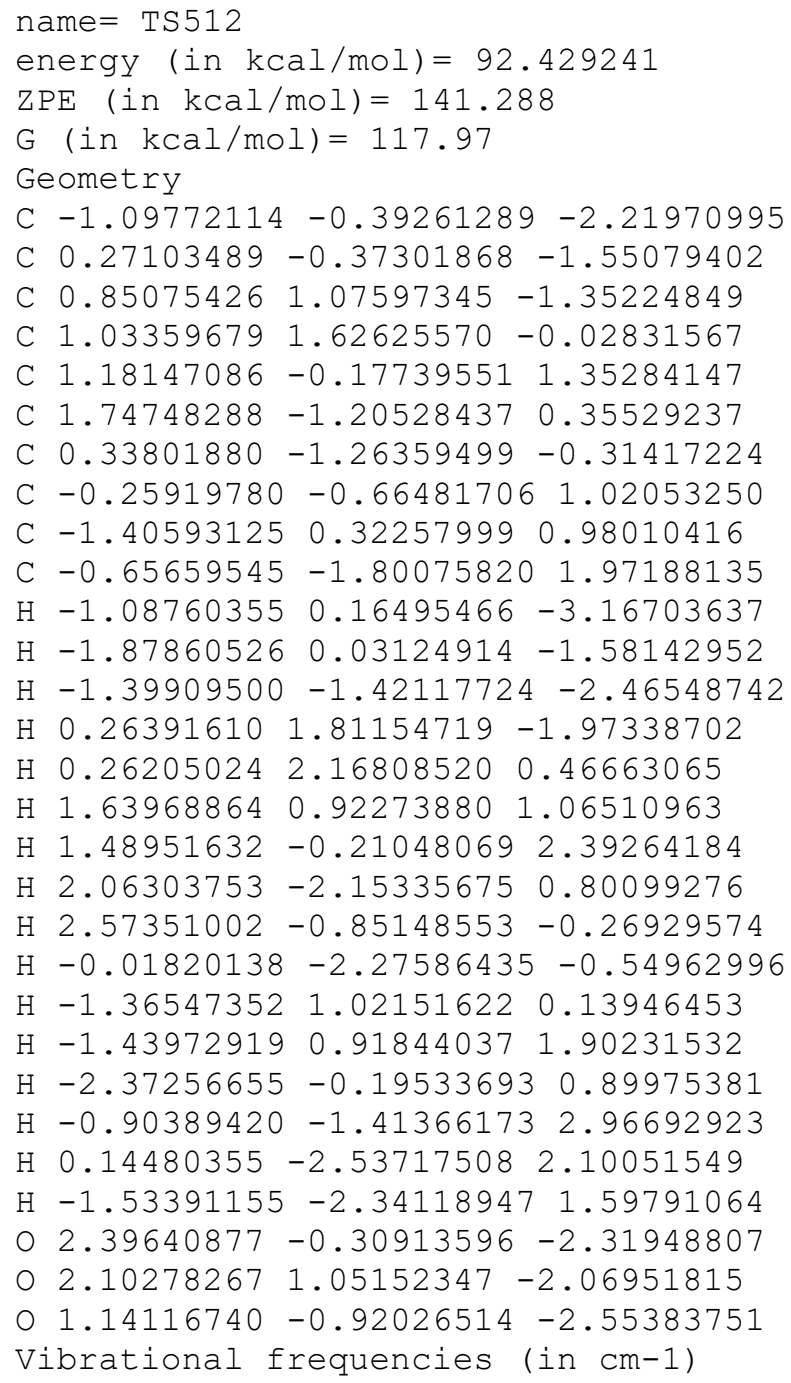




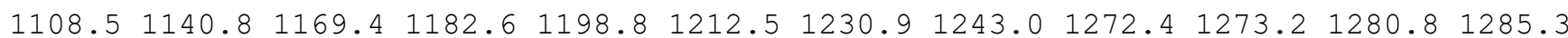

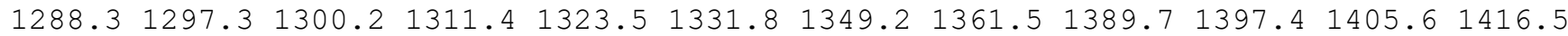
$1785.72645 .52664 .3 \quad 2667.2 \quad 2671.72673 .72674 .92677 .22678 .32712 .12729 .12733 .6$ $2760.92777 .8 \quad 2780.6 \quad 2781.7$

\section{name $=$ TS513}

energy (in $\mathrm{kcal} / \mathrm{mol})=95.045997$

$\mathrm{ZPE}($ in $\mathrm{kcal} / \mathrm{mol})=138.687$

$\mathrm{G}($ in $\mathrm{kcal} / \mathrm{mol})=112.647$

Geometry

C $-1.30058552 \quad 0.08990857-1.58889478$

C $-0.25613810 \quad 0.12382874-0.75483589$

C $0.147189721 .46139073-0.07254840$

C 1.393902951 .344312720 .71201665

C $1.440242850 .95758052 \quad 2.09713539$

C $1.92018912-0.58798739-0.10487961$

C $0.59421018-0.93619120-0.33720431$

$\begin{array}{lllll}\text { C } & 0.47471271 & 0.45614510 & 2.89409076\end{array}$

C -0.952249250 .235043412 .52967762$

C $0.80238038 \quad 0.08982420 \quad 4.31019261$

$\mathrm{H}-1.57132136-0.78803318-2.14923273$

$\mathrm{H}-1.94970098 \quad 0.92710543-1.77217174$

H $\quad 0.87230339 \quad 1.37658547 \quad-3.12043118$

$\mathrm{H}-0.721644331 .856873320 .50573156$

H $2.18311202 \quad 2.03885807 \quad 0.41628351$

H $2.42555452 \quad 0.47252498-0.64971598$

H $2.43793633 \quad 1.09049063 \quad 2.53803267$

H $2.54843668-1.09436750 \quad 0.61080266$

$\mathrm{H} 2.57632103-0.34765740-1.16934966$

$\mathrm{H} \quad 0.18179911-1.87868497-0.02635428$

$\mathrm{H}-1.05119479-0.29982386 \quad 1.57230651$

$\mathrm{H}-1.490716351 .188932642 .43798880$

$\mathrm{H}-1.49055581-0.36609946 \quad 3.27363751$

$\mathrm{H} \quad 1.83124039 \quad 0.34136748 \quad 4.59346369$

$\mathrm{H} \quad 0.67800616 \quad-0.99087122 \quad 4.46958114$

$\mathrm{H} \quad 0.13766767 \quad 0.60578414 \quad 5.01685775$

O $1.45594366 \quad 2.51817553-1.70691228$

$\begin{array}{lllll}0 & 0.22480960 & 2.44824758 & -1.11358351\end{array}$

o $1.666775861 .38480505-2.53290264$

Vibrational frequencies (in $\mathrm{cm}-1$ )

$\begin{array}{llllllllllllllll}-1136.3 & 50.0 & 52.0 & 82.5 & 93.4 & 121.6 & 130.0 & 143.1 & 166.4 & 229.1 & 235.5 & 251.0 & 283.5 & 284.6\end{array}$ $305.6 \quad 379.4 \quad 418.3 \quad 423.8 \quad 445.8 \quad 474.1 \quad 492.8 \quad 516.9 \quad 567.2 \quad 589.1 \quad 621.0 \quad 670.4 \quad 688.5 \quad 735.3$ $807.6 \quad 834.3 \quad 849.1 \quad 889.6 \quad 923.2 \quad 926.0 \quad 952.3 \quad 961.2 \quad 989.7 \quad 992.7 \quad 1023.7 \quad 1027.8 \quad 1050.6 \quad 1060.3$

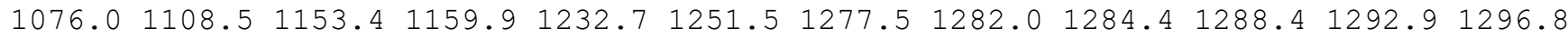

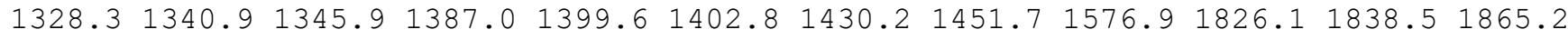
$2665.02671 .3 \quad 2672.9 \quad 2673.7 \quad 2677.1 \quad 2719.3 \quad 2731.7 \quad 2735.5 \quad 2741.0 \quad 2778.2 \quad 2779.0 \quad 2788.4$ $2795.2 \quad 2813.13134 .5$

name $=\operatorname{TS} 514$

energy $($ in $\mathrm{kcal} / \mathrm{mol})=94.667695$

$\operatorname{ZPE}($ in $\mathrm{kcal} / \mathrm{mol})=139.076$

$\mathrm{G}($ in $\mathrm{kcal} / \mathrm{mol})=110.423$

Geometry

C $-2.51880829-0.53076021-1.66642105$

C $-1.16389306-0.85301016-1.14442323$

C $-0.14421582 \quad 2.08314888-0.36421156$

C $-0.31739468 \quad 3.40341728-0.28695557$

C $0.95031098-1.049077931 .59595336$

C $0.46424623-1.90340722 \quad 0.44932329$

C $-0.90224031-1.55612968-0.03556897$

C $0.27457658 \quad 0.05253529 \quad 2.08541925$

C 0.853026451 .165284062 .83686642

C $-0.20863956-1.433474693 .04626680$ 
$\mathrm{H}-2.49344197-0.26064416-2.73405166$

$\mathrm{H}-2.948687350 .33144005-1.13086670$

$\mathrm{H}-3.21560026-1.37242036-1.55789352$

$\mathrm{H}-0.53122386 \quad 1.31984601 \quad 0.31510880$

$\mathrm{H} \quad 0.10090414 \quad 4.10519176-0.98803880$

$\mathrm{H}-0.89427024 \quad 3.87080765 \quad 0.49151501$

$\mathrm{H} \quad 2.02214507-1.203666151 .79497741$

$\mathrm{H} \quad 0.49472957-2.97978272 \quad 0.73353669$

$\mathrm{H} 1.20802325-1.81991898-0.38027030$

$\mathrm{H}-1.72035198 \quad-1.90998883 \quad 0.59028356$

$\mathrm{H} \quad 0.11195124 \quad 1.62451531 \quad 3.50831846$

H $1.14033611 \quad 1.962109592 .12487455$

$\mathrm{H} \quad 1.74633073 \quad 0.92865837 \quad 3.42813045$

$\mathrm{H}-0.48777325-0.76420315 \quad 3.86953044$

H $0.45821115-2.19560540 \quad 3.44007288$

$\mathrm{H}-1.10516545-1.846435242 .59484465$

$\begin{array}{lllll}0 & 0.83210135 & 0.25401814 & -1.30791703\end{array}$

○ $0.61772144 \quad 1.63603264-1.42275867$

o $-0.19709010-0.39308213-2.01915735$

Vibrational frequencies (in $\mathrm{cm}-1$ )

$\begin{array}{llllllllllllllll}-786.6 & 26.5 & 42.4 & 47.8 & 58.0 & 61.0 & 73.0 & 90.8 & 94.4 & 148.6 & 174.2 & 220.0 & 223.8 & 257.6\end{array}$ $286.8 \quad 305.3 \quad 341.9 \quad 346.5 \quad 392.3 \quad 414.1 \quad 539.9 \quad 558.0 \quad 571.7 \quad 587.7 \quad 613.4 \quad 648.0 \quad 814.0 \quad 860.6$

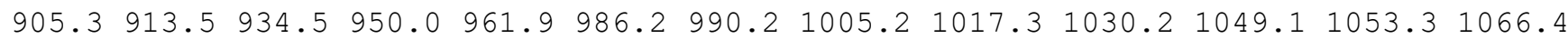

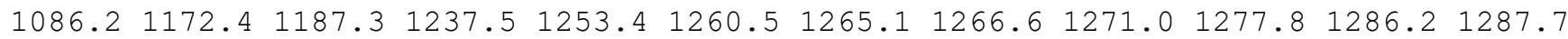

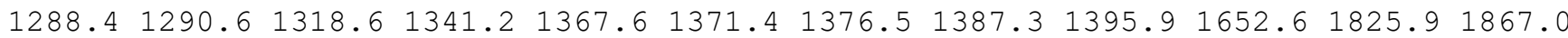
$2640.12642 .4 \quad 2655.5 \quad 2662.0 \quad 2663.6 \quad 2671.7 \quad 2673.7 \quad 2674.92689 .3 \quad 2726.3 \quad 2733.12754 .1$ $2756.0 \quad 2772.3 \quad 2776.8 \quad 2797.3$

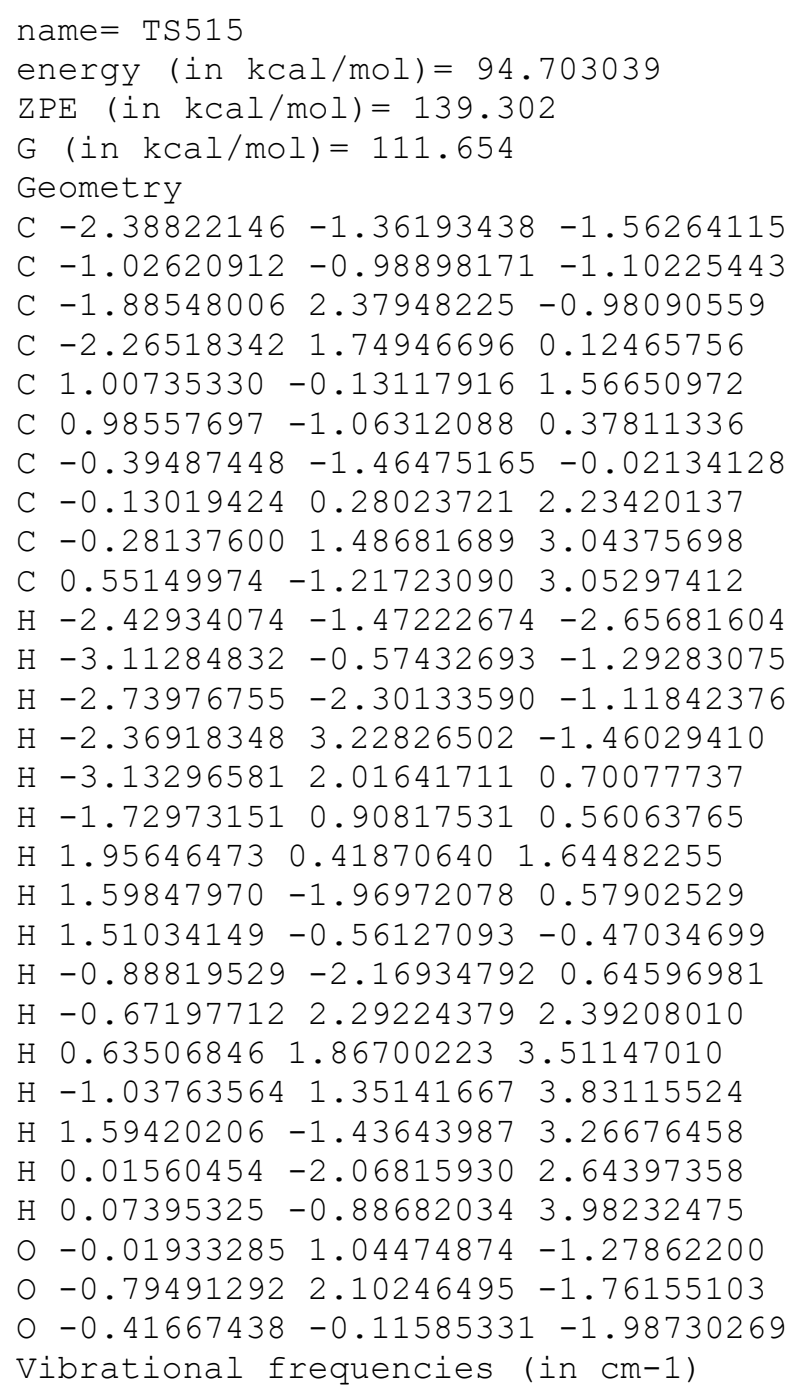


$\begin{array}{llllllllllllllllll}-794.6 & 26.7 & 48.6 & 68.4 & 80.1 & 101.3 & 102.5 & 110.2 & 112.0 & 155.7 & 175.4 & 212.4 & 219.5 & 248.1\end{array}$

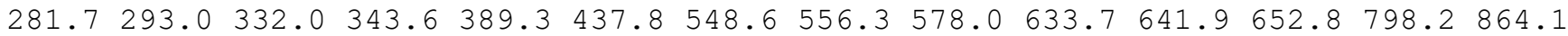
$905.8 \quad 913.6 \quad 933.6 \quad 949.1 \quad 955.6 \quad 968.0 \quad 993.0 \quad 1003.1 \quad 1013.5 \quad 1030.2 \quad 1047.7 \quad 1062.9 \quad 1087.0$

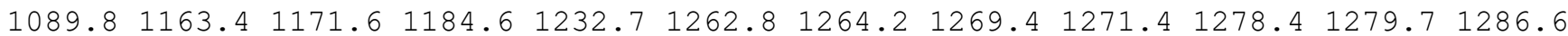

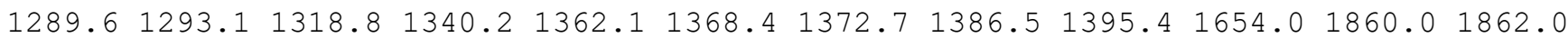
$2642.12644 .3 \quad 2660.8 \quad 2661.8 \quad 2672.5 \quad 2675.7 \quad 2677.4 \quad 2678.5 \quad 2692.5 \quad 2727.4 \quad 2731.5 \quad 2754.9$ $2757.12772 .2 \quad 2778.0 \quad 2783.7$

name $=$ TS5 16

energy (in $\mathrm{kcal} / \mathrm{mol})=92.941648$

$\mathrm{ZPE}(\mathrm{in} \mathrm{kcal} / \mathrm{mol})=141.075$

$\mathrm{G}($ in $\mathrm{kcal} / \mathrm{mol})=116.633$

Geometry

C $-2.12433766-0.65909338-1.40657578$

C $-0.64721919-0.41610894-1.04543547$

C $-0.438270421 .06022453-0.59394730$

C $0.70829355 \quad 1.40093814 \quad 0.31822053$

C $1.61094164 \quad 0.41643154 \quad 1.04385977$

C $1.46873748-1.064800810 .89794080$

C $-0.23447031-1.47727661-0.18264281$

C $0.59488255 \quad 1.24790182 \quad 1.82802987$

C $1.13284487 \quad 2.36438352 \quad 2.69005320$

C $-0.62561197 \quad 0.59442284 \quad 2.41709976$

$\mathrm{H}-2.48713898 \quad 0.12936217 \quad-2.07933335$

$\mathrm{H}-2.75622219-0.68386779-0.51261434$

$\mathrm{H}-2.24753111-1.61193912-1.93643003$

$\mathrm{H}-1.38385830 \quad 1.50437003-0.20518965$

$\mathrm{H} \quad 1.21419023 \quad 2.30965064-0.05773866$

$\mathrm{H} \quad 1.31704391-1.35864411-0.26234182$

H 2.66548474 $0.71822392 \quad 1.13487009$

H $0.82897750-1.50074991 \quad 1.69437310$

$\mathrm{H} 2.43120386-1.602234410 .97094770$

H $-0.23218668-2.51378133-0.34306856$

$\mathrm{H} \quad 1.41837711 \quad 1.99343492 \quad 3.68281120$

$\mathrm{H} \quad 0.37728470 \quad 3.14776147 \quad 2.83610738$

$\mathrm{H} 2.01725053 \quad 2.84631370 \quad 2.25660738$

$\mathrm{H}-1.01356041-0.218655451 .77616767$

$\mathrm{H}-1.440155451 .316701372 .54902142$

$\mathrm{H}-0.41021766 \quad 0.15394587 \quad 3.39783758$

$\begin{array}{lllll}0 & 0.58470874 & 0.82314809 & -2.57630067\end{array}$

$\begin{array}{lllll}0 & -0.24212674 & 1.71420795 & -1.84862816\end{array}$

O $0.08216111-0.47954492-2.30488259$

Vibrational frequencies (in $\mathrm{cm}-1$ )

$\begin{array}{lllllllllllllll}-802.2 & 46.8 & 102.8 & 121.6 & 131.2 & 148.5 & 163.7 & 187.8 & 211.7 & 259.2 & 270.8 & 276.5 & 323.6 & 361.4\end{array}$

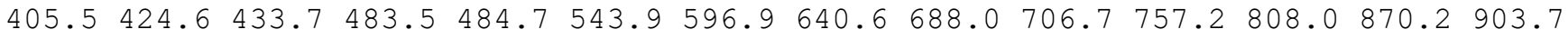
$920.4947 .5 \quad 960.0 \quad 997.0 \quad 1010.9 \quad 1014.3 \quad 1016.0 \quad 1021.2 \quad 1027.6 \quad 1052.2 \quad 1060.2 \quad 1089.3 \quad 1111.6$

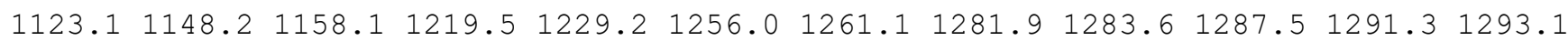
$1295.91296 .5 \quad 1299.0 \quad 1320.8 \quad 1370.9 \quad 1379.0 \quad 1384.8 \quad 1394.4 \quad 1402.4 \quad 1413.6 \quad 1462.7 \quad 1521.5$ $2117.02624 .02641 .92670 .5 \quad 2675.4 \quad 2676.7 \quad 2679.2 \quad 2681.02682 .8 \quad 2711.4 \quad 2725.2 \quad 2743.6$ $2762.7 \quad 2775.5 \quad 2778.7 \quad 2782.2$

name $=\operatorname{TS} 517$

energy $($ in $\mathrm{kcal} / \mathrm{mol})=91.581978$

$\mathrm{ZPE}($ in $\mathrm{kcal} / \mathrm{mol})=142.453$

$\mathrm{G}($ in $\mathrm{kcal} / \mathrm{mol})=118.754$

Geometry

C $-1.70735889-1.90200829-0.96530516$

C $-0.53199586-0.96315639-0.66438231$

C $-1.053072250 .49029240 \quad-0.40222481$

C $-0.16886629 \quad 1.41352272 \quad 0.44369472$

C $0.65769046 \quad 0.64761135 \quad 1.48970059$

C $1.45258911-0.442495650 .71821632$

C $0.44580045-1.50849264 \quad 0.33792047$ 
$\begin{array}{llll}C & -0.26407144 & -0.09271088 & 2.43311674\end{array}$

$\begin{array}{llll}\text { C }-1.44598918 & 0.57474381 & 3.02305793\end{array}$

C $-0.04218849-1.412467112 .42896395$

$\mathrm{H}-2.41761262-1.96982970-0.13947783$

$\mathrm{H}-1.35352039-2.90942617-1.21383500$

$\mathrm{H}-2.26001650-1.54256558-1.84780980$

$\mathrm{H}-2.10418364 \quad 0.49924070 \quad-0.05506634$

$\mathrm{H}-0.79092375 \quad 2.18521857 \quad 0.93224910$

$\mathrm{H} \quad 0.518503531 .96977596-0.22827345$

$\mathrm{H} \quad 1.33698062 \quad 1.33539084 \quad 2.03026650$

H $2.27655208-0.842751291 .32970602$

$\mathrm{H} \quad 1.92986534-0.01343421-0.18464223$

H $0.86236166-2.47824108 \quad 0.10396021$

$\mathrm{H}-1.78879656 \quad 0.06551631 \quad 3.93389151$

H $-2.29607266 \quad 0.61256933 \quad 2.32729355$

$\mathrm{H}-1.21405599 \quad 1.61126169 \quad 3.30989893$

H $0.73193071 \quad-2.09963728 \quad 2.64891211$

$\mathrm{H}-1.12090120-2.214470672 .14824156$

$\mathrm{H}-0.86860955-2.24682149 \quad 1.25717879$

$0.06676878 \quad 0.51549901-2.33219762$

$0-1.117464821 .00202808-1.73638760$

O $0.16716100-0.85137530-1.92288996$

Vibrational frequencies (in $\mathrm{cm}-1$ )

$\begin{array}{llllllllllllllll}-2154.9 & 56.1 & 90.0 & 97.1 & 161.0 & 173.1 & 194.5 & 219.9 & 283.8 & 300.9 & 316.0 & 359.0 & 381.9 & 428.2\end{array}$

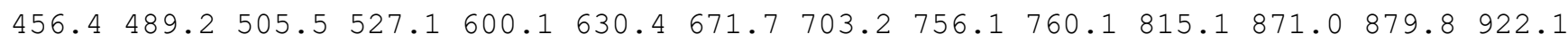
$930.1940 .9 \quad 966.3 \quad 983.5 \quad 991.5 \quad 1004.3 \quad 1036.4 \quad 1042.8 \quad 1051.8 \quad 1075.3 \quad 1083.2 \quad 1101.2 \quad 1111.7$

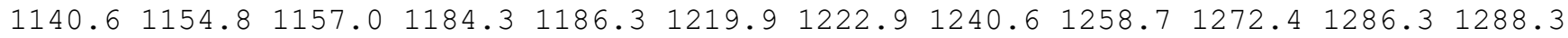

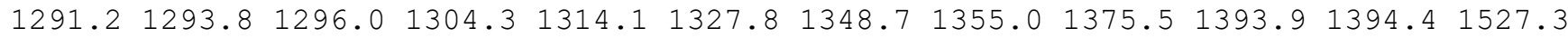
$1796.92657 .72665 .7 \quad 2667.7 \quad 2669.5 \quad 2676.7 \quad 2682.6 \quad 2697.02720 .02740 .22743 .02746 .0$ $2752.92778 .0 \quad 2779.1 \quad 3072.9$
name $=\operatorname{TS} 518$
energy $($ in $\mathrm{kcal} / \mathrm{mol})=98.860658$
$\mathrm{ZPE}(\mathrm{in} \mathrm{kcal} / \mathrm{mol})=135.419$
$\mathrm{G}($ in $\mathrm{kcal} / \mathrm{mol})=103.133$
Geometry
C $0.98228087-2.51072928-1.74344161$
C $1.39082449-1.43535431-0.76738689$
C $1.35981261-0.07876841-1.41354010$
C $1.88974828 \quad 1.12447843-0.69275816$
C $0.53687514 \quad-0.54547772 \quad 1.55574924$
C $2.43742954-1.77676072 \quad 0.27602130$
C $0.99055994 \quad-1.64860942 \quad 0.69459834$
C $-0.41553420 \quad 0.31689923 \quad 1.36295454$
C $-1.342989531 .26613416 \quad 1.86977971$
C $0.73738617-1.227893213 .37477902$
H $1.60803546-2.48381119-2.64857923$
$\mathrm{H}-0.05493003-2.35288212-2.07992765$
H $1.04786878-3.51806753-1.32110934$
$\mathrm{H} 1.058852151 .61623426-0.15515782$
H 2.67229406 $0.89658105 \quad 0.03389113$
H $2.284956361 .86343481-1.40573238$
H $1.40297577-0.193262112 .35939271$
H $2.90729127-2.75711433 \quad 0.24970210$
H $3.16492924-1.03300368 \quad 0.58309245$
H $0.43371118-2.57866930 \quad 0.90018250$
$\mathrm{H}-2.377449250 .91078590 \quad 1.66757979$
$\mathrm{H}-1.26603202 \quad 2.23635218 \quad 1.34272610$
$\mathrm{H}-1.27449194 \quad 1.456228992 .95408194$
$\mathrm{H} \quad 0.55498679-0.46503373 \quad 4.10852127$
$\mathrm{H} 1.65325011-1.77285773 \quad 3.50853998$
$\mathrm{H}-0.11005313-1.88488473 \quad 3.26612806$
○ $2.22495368-0.85468403-4.67859905$
○ $0.92139558 \quad 0.03937554 \quad-2.53381534$ 
O $1.18989740-0.64984287-5.09414616$

Vibrational frequencies (in $\mathrm{cm}-1$ )

$\begin{array}{lllllllllllllll}-1600.2 & 11.5 & 22.1 & 29.8 & 35.4 & 42.5 & 70.0 & 78.1 & 81.0 & 91.8 & 108.8 & 118.8 & 127.6 & 154.6\end{array}$

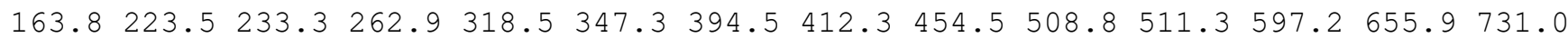
$760.9811 .2882 .7 \quad 907.4 \quad 946.2976 .2 \quad 978.6 \quad 1028.2 \quad 1030.3 \quad 1047.3 \quad 1050.6 \quad 1057.91092 .5$

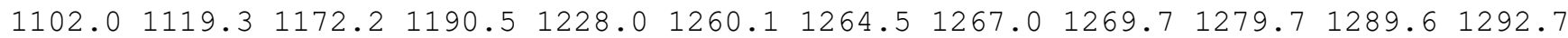
$1294.61298 .21313 .6 \quad 1364.3 \quad 1374.1 \quad 1386.1 \quad 1393.6 \quad 1406.01504 .4 \quad 1597.91831 .51952 .0$ $2218.82645 .92654 .7 \quad 2657.6 \quad 2664.4 \quad 2679.5 \quad 2679.92690 .62703 .72709 .12737 .92760 .5$ $2773.6 \quad 2777.12779 .3 \quad 2785.2$

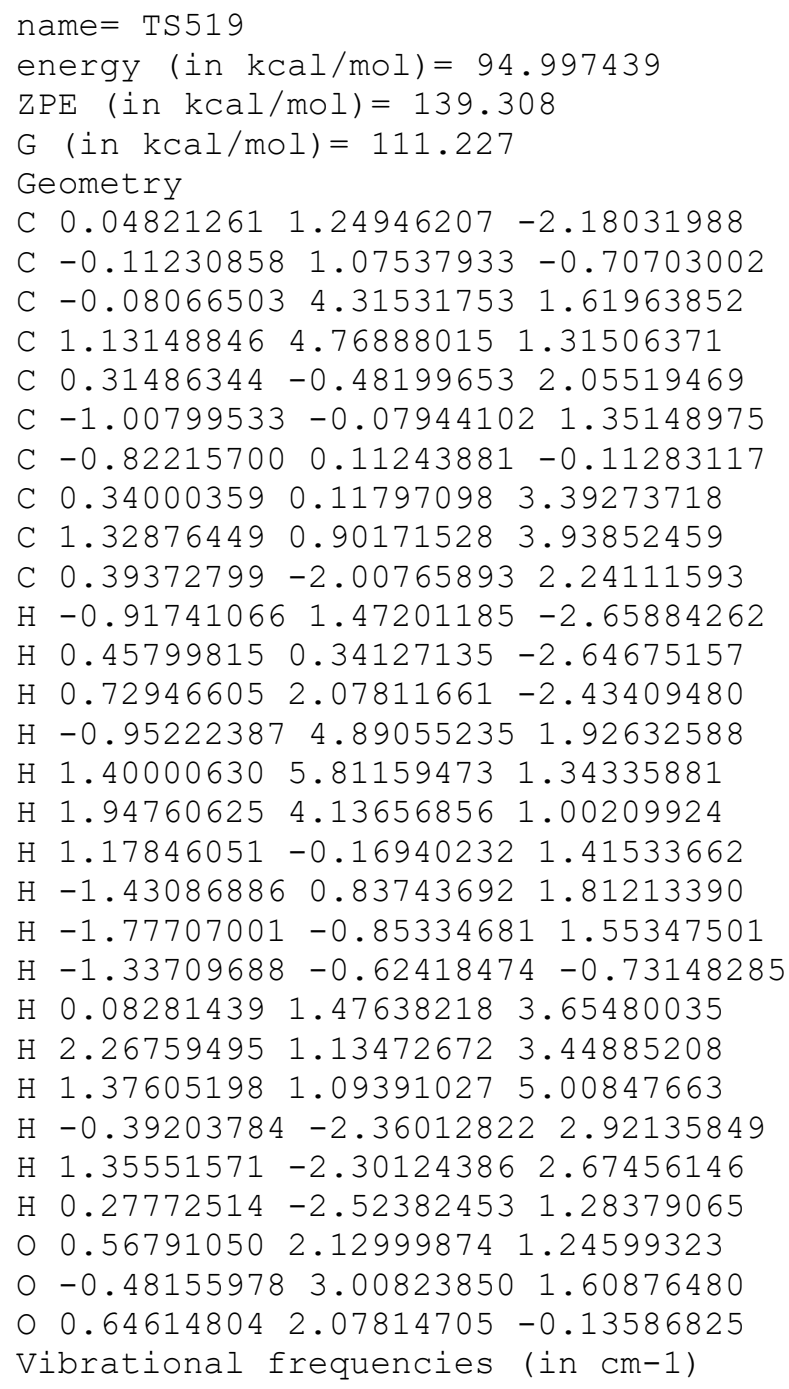


C $1.36055993-0.92973044 \quad 0.36037609$

C $-0.19099671-1.185853290 .41920576$

C $-0.19942787-0.51062243 \quad 1.85139000$

C $-1.38832513 \quad 0.29612259 \quad 2.31803814$

C $0.18136621-1.505184172 .94245683$

$\mathrm{H}-2.79336157 \quad 0.16760984-1.49838538$

$\mathrm{H}-2.86234448 \quad-0.17733742 \quad 0.24054204$

$\mathrm{H}-2.63119805-1.49202347-0.92310378$

$\mathrm{H}-1.26415901 \quad 1.93870895 \quad-0.37457643$

H $1.31250615 \quad 1.51592326 \quad 2.11373712$

$\mathrm{H} \quad 1.34699410 \quad 2.25867876 \quad 0.27493280$

H $1.819740290 .68505941 \quad 2.20101832$

$\mathrm{H} \quad 1.97101605-1.72327888 \quad 0.78293483$

H $1.76270371-0.66476127-0.62344896$

$\mathrm{H}-0.51953698-2.22152506 \quad 0.36754450$

$\mathrm{H}-1.58200776 \quad 1.17067048 \quad 1.68009020$

$\mathrm{H}-1.24306128 \quad 0.68380628 \quad 3.33321768$

$\mathrm{H}-2.30039703-0.313922672 .33164709$

$\mathrm{H} \quad 0.42437818-1.003231123 .88440686$

$\mathrm{H} \quad 1.03684774 \quad-2.13372189 \quad 2.67154239$

$\mathrm{H}-0.65488735-2.18795978 \quad 3.14713868$

$\begin{array}{lllll}0 & 0.55631412 & 0.33181571 & -2.31286256\end{array}$

○ $0.02033472 \quad 1.53759494-1.83782284$

o $-0.39418298-0.66396299-1.92097284$

Vibrational frequencies (in $\mathrm{cm}-1$ )

$\begin{array}{llllllllllllll}-1557.6 & 92.9 & 106.7 & 143.1 & 172.0 & 174.8 & 197.5 & 213.4 & 266.4 & 290.7 & 327.2 & 350.4 & 383.0 & 421.1\end{array}$

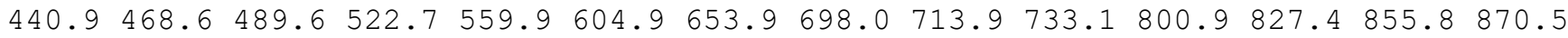
$898.8927 .5 \quad 935.0 \quad 974.7 \quad 978.8 \quad 1001.5 \quad 1004.6 \quad 1008.6 \quad 1016.5 \quad 1019.1 \quad 1044.3 \quad 1061.5 \quad 1077.4$

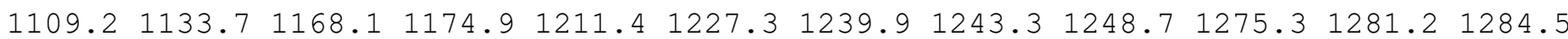
$1287.91290 .3 \quad 1293.5 \quad 1299.4 \quad 1301.7 \quad 1308.7 \quad 1330.6 \quad 1374.5 \quad 1386.7 \quad 1396.2 \quad 1406.4 \quad 1415.5$ $1737.12662 .92670 .7 \quad 2672.2 \quad 2675.2 \quad 2678.8 \quad 2680.92685 .52686 .2 \quad 2750.02762 .7 \quad 2778.8$ $2781.62782 .6 \quad 2798.92933 .2$

name $=\operatorname{TS} 521$

energy (in $\mathrm{kcal} / \mathrm{mol})=92.980128$

$\mathrm{ZPE}(\mathrm{in} \mathrm{kcal} / \mathrm{mol})=141.411$

G $($ in $\mathrm{kcal} / \mathrm{mol})=116.853$

Geometry

C $-2.19076342-0.57559971-1.07642789$

C $-0.72480843-0.19210009-0.82966759$

C $-0.468008151 .24901588-0.69679147$

C $1.791106171 .40357657 \quad 0.36459542$

C $1.74511107 \quad 0.22198252 \quad 1.00627995$

C $1.43876950-1.03277336 \quad 0.23520732$

C $-0.10948474-1.09346218 \quad 0.28019208$

C $-0.45046203-0.668450201 .65918661$

C $-1.36996587 \quad 0.42036495 \quad 1.99408932$

C $0.02310038-1.49404228 \quad 2.79481072$

$\mathrm{H}-2.67049488 \quad 0.15995418-1.73635524$

$\mathrm{H}-2.76824589-0.63480215-0.15186372$

$\mathrm{H}-2.25764620-1.54805310-1.58128570$

$\mathrm{H}-0.64436873 \quad 1.94272783 \quad 0.07608862$

H $1.88236996 \quad 2.34876902 \quad 0.87487953$

$\mathrm{H} \quad 1.873118621 .49675415-0.71051042$

H $1.91944182 \quad 0.12380163 \quad 2.06961902$

$\mathrm{H} 1.90300430-1.930306390 .67204246$

$\mathrm{H} \quad 1.80347807-0.98689501-0.81045383$

$\mathrm{H}-0.46496419-2.13346616 \quad 0.07503078$

H $-2.22359883 \quad 0.514946891 .29966091$

$\mathrm{H}-0.83870050 \quad 1.39811495 \quad 1.93477171$

$\mathrm{H}-1.78000955 \quad 0.35443342 \quad 3.01224069$

$\mathrm{H} \quad 0.37345519-0.88626114 \quad 3.64338746$

$\mathrm{H} \quad 0.83716427 \quad-2.18524260 \quad 2.53456524$

$\mathrm{H}-0.80493144 \quad-2.11756453 \quad 3.17948639$ 
$\begin{array}{lllll}0 & 0.57463126 & 0.64473424 & -2.55081284\end{array}$

$\begin{array}{llll}0 & -0.13403661 & 1.74495643 & -1.90627328\end{array}$

o $-0.07505562-0.53253736-2.08351459$

Vibrational frequencies (in $\mathrm{cm}-1$ )

$\begin{array}{llllllllllllll}-365.0 & 61.8 & 100.5 & 122.5 & 128.5 & 146.4 & 157.8 & 188.9 & 203.7 & 245.5 & 259.3 & 290.0 & 312.3 & 337.2\end{array}$ $353.4 \quad 367.2 \quad 415.5 \quad 456.2 \quad 479.2 \quad 479.6 \quad 530.0 \quad 606.1 \quad 672.6 \quad 727.1 \quad 741.3 \quad 807.9 \quad 879.5 \quad 900.9$ $941.3957 .3 \quad 960.7964 .5 \quad 975.6 \quad 1004.1 \quad 1012.7 \quad 1020.6 \quad 1038.5 \quad 1048.6 \quad 1076.5 \quad 1096.8 \quad 1110.0$

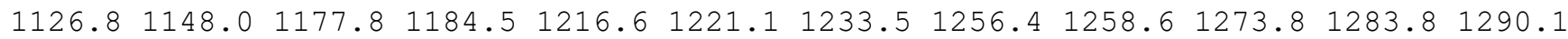

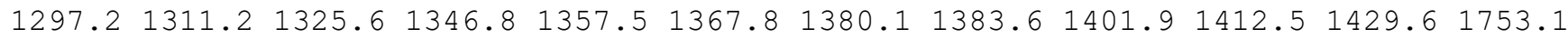
2602.22648 .72653 .12661 .92665 .62670 .52677 .32683 .32704 .52745 .82748 .92753 .0 2764.22769 .02776 .62778 .0

name $=$ TS522

energy $($ in $\mathrm{kcal} / \mathrm{mol})=93.302822$

$\mathrm{ZPE}(\mathrm{in} \mathrm{kcal} / \mathrm{mol})=141.194$

$\mathrm{G}(\mathrm{in} \mathrm{kcal} / \mathrm{mol})=117.949$

Geometry

C $-2.45821576-0.44437406-0.39474331$

C $-0.94298082-0.55082276-0.47196188$

C $-0.24244398 \quad 0.81389995-0.89959627$

C $0.86457012 \quad 1.24580479-0.02714581$

C $1.16058456 \quad 0.30931457 \quad 1.05944840$

C $1.26816300-1.14975350 \quad 0.54673618$

C $-0.29095314-1.180454530 .77005760$

C $-0.10128640-0.10276751 \quad 1.91152865$

C $-1.14535498 \quad 0.96320252 \quad 2.14647607$

C $0.25565486-0.756001653 .24147100$

$\mathrm{H}-2.87694670-0.25501602-1.39435048$

$\mathrm{H}-2.79354758 \quad 0.36262429 \quad 0.26119219$

$\mathrm{H}-2.90386596-1.37929505-0.03477216$

$\mathrm{H}-0.98985022 \quad 1.59836487-1.11636030$

H $1.59796922 \quad 1.71122948 \quad 1.46981993$

$\mathrm{H} \quad 1.59588574 \quad 1.87914264-0.47719033$

H $2.04084385 \quad 0.88231871 \quad 1.78283510$

H $1.83325807-1.853359721 .15092712$

$\mathrm{H} \quad 1.59035154-1.23816253-0.49984875$

$\mathrm{H}-0.72877373-2.13187058 \quad 1.06430407$

$\mathrm{H}-1.267723821 .62500909 \quad 1.27510397$

$\mathrm{H}-0.88528024 \quad 1.61012808 \quad 2.99206024$

$\mathrm{H}-2.123628890 .51807343 \quad 2.36769522$

$\mathrm{H} \quad 0.63302388 \quad-0.02687676 \quad 3.96552941$

H $1.00934487-1.545216353 .14238309$

$\mathrm{H}-0.63183232-1.22495758 \quad 3.68846375$

$0-0.41145678-0.58773211-2.66566355$

$00.38395643 \quad 0.45072493-2.15493725$

o - $0.63042305-1.44614724-1.54645173$

Vibrational frequencies (in $\mathrm{cm}-1$ )

$\begin{array}{lllllllllllllll}-1593.8 & 79.0 & 107.3 & 145.6 & 174.1 & 192.4 & 203.2 & 209.8 & 274.5 & 282.0 & 323.8 & 368.5 & 378.8 & 416.9\end{array}$

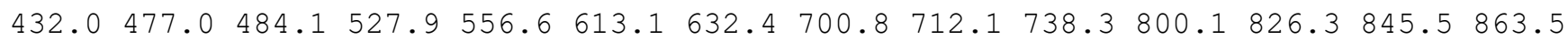
$896.5935 .7 \quad 937.7 \quad 982.0 \quad 987.8 \quad 1002.4 \quad 1007.0 \quad 1011.1 \quad 1016.3 \quad 1041.4 \quad 1045.2 \quad 1074.1 \quad 1094.7$

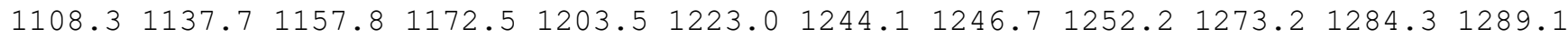
$1290.41294 .3 \quad 1298.4 \quad 1300.7 \quad 1306.3 \quad 1313.7 \quad 1329.8 \quad 1369.4 \quad 1379.7 \quad 1397.91405 .7 \quad 1413.8$ $1754.0 \quad 2660.7 \quad 2671.0 \quad 2672.4 \quad 2675.6 \quad 2679.02681 .12681 .72690 .7 \quad 2749.72759 .82777 .8$ $2781.6 \quad 2782.8 \quad 2793.7 \quad 2865.2$

name $=\operatorname{TS} 523$

energy $($ in $\mathrm{kcal} / \mathrm{mol})=95.347593$

$\operatorname{ZPE}($ in $\mathrm{kcal} / \mathrm{mol})=139.522$

$\mathrm{G}($ in $\mathrm{kcal} / \mathrm{mol})=111.671$

Geometry

C $-2.36881475-0.92964661 \quad 0.18977513$

C $-1.34342748-0.60350670-0.83218472$

C $-0.48344383 \quad 0.60262209-0.62043500$ 
C $0.69969506 \quad 0.36780892 \quad 0.33243340$

C $0.29021352 \quad 0.47722619 \quad 1.75996751$

C $1.72277162-2.108548372 .40081047$

C $-1.20769837-1.30579139-1.96660445$

C $-0.62149226 \quad 1.23204375 \quad 2.29263221$

C $-1.481861391 .56351187 \quad 3.37510181$

C $0.44459345-1.347624842 .58710789$

$\mathrm{H}-2.11264846-0.51858342 \quad 1.17892038$

$\mathrm{H}-2.50754971-2.011845690 .31531239$

$\mathrm{H}-3.34682580-0.50279024-0.08427775$

$\mathrm{H}-1.07883251 \quad 1.49956286-0.31496006$

$\mathrm{H} 1.48178160 \quad 1.13067440 \quad 0.11162026$

$\mathrm{H} \quad 1.16740509-0.611369790 .10590942$

H $1.17069119 \quad 0.13164791 \quad 2.52184037$

H $1.56800775 \quad-3.181958342 .59547527$

H 2.52039018 -1.77715049 3.07693243

$\mathrm{H}-1.78278072-2.16455916-2.28971516$

$\mathrm{H}-1.44979400 \quad 0.86559474 \quad 4.22843654$

$\mathrm{H}-2.534602201 .60986519 \quad 3.02795269$

$\mathrm{H}-1.25998110 \quad 2.57926713 \quad 3.75789904$

$\mathrm{H} \quad 0.11156732-1.22020096 \quad 3.60868921$

H $2.10553391-2.03286610 \quad 1.37306141$

$\mathrm{H}-0.36789162-1.68614040 \quad 1.94871323$

$00.66107173-0.07011186-2.47602263$

$00.02873966 \quad 1.01644565-1.89579483$

o $-0.32659809-0.98623104-2.95771682$

Vibrational frequencies (in $\mathrm{cm}-1$ )

$\begin{array}{lllllllllllllll}-1438.8 & 22.1 & 39.8 & 63.1 & 73.5 & 86.8 & 94.4 & 109.9 & 117.5 & 148.1 & 207.3 & 217.7 & 232.2 & 290.4\end{array}$ $298.1342 .5 \quad 344.4 \quad 349.3 \quad 433.8 \quad 490.1 \quad 493.2 \quad 548.6 \quad 606.8 \quad 649.1 \quad 762.7 \quad 806.1 \quad 819.7 \quad 883.3$ $894.1943 .4951 .5 \quad 963.5 \quad 1008.3 \quad 1024.0 \quad 1033.3 \quad 1040.4 \quad 1046.5 \quad 1053.7 \quad 1068.61079 .7 \quad 1136.8$

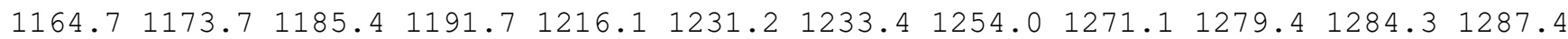
$1290.51298 .6 \quad 1306.5 \quad 1318.0 \quad 1341.4 \quad 1351.7 \quad 1380.5 \quad 1392.4 \quad 1395.1 \quad 1400.61850 .91951 .6$ $2247.22650 .52651 .0 \quad 2656.42661 .4 \quad 2662.5 \quad 2665.92671 .52674 .32696 .42733 .12739 .9$ $2745.4 \quad 2762.8 \quad 2776.7 \quad 2777.8$

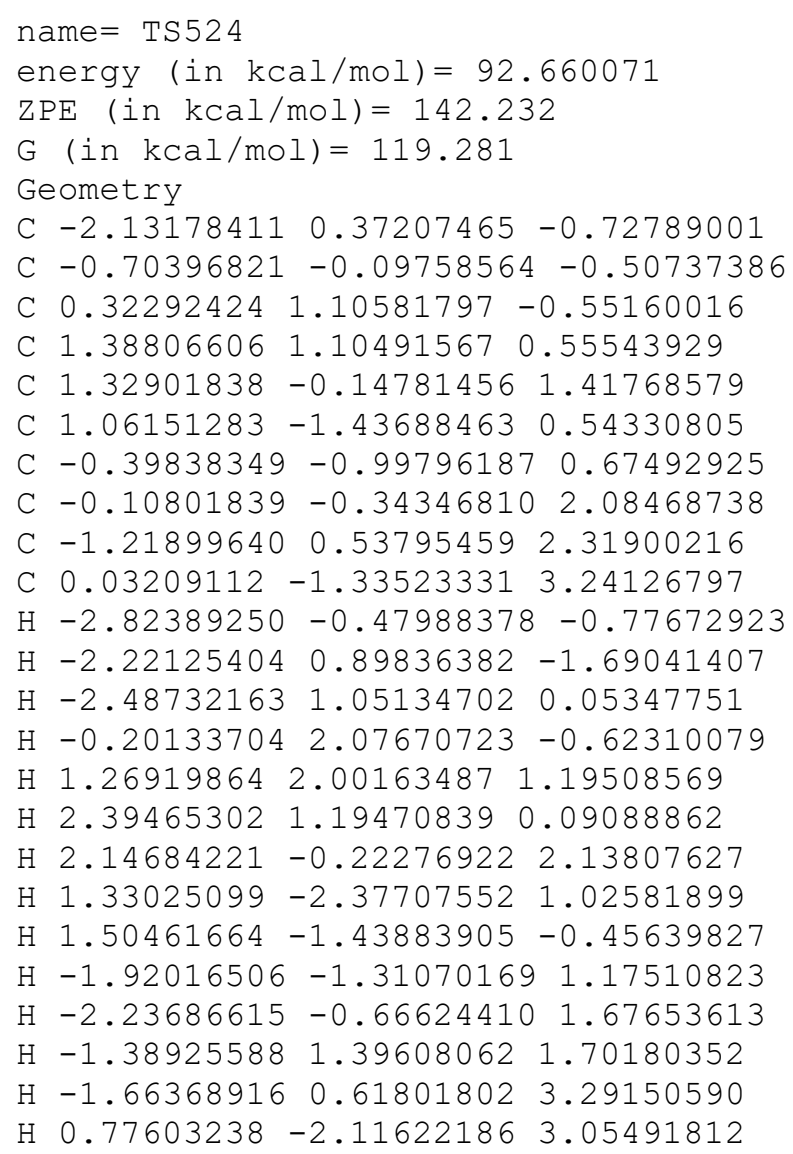


$\mathrm{H} \quad 0.32862281-0.808199334 .15603878$

$\mathrm{H}-0.91706193-1.847430683 .44241505$

O $0.17369645 \quad 0.10083070-2.56885019$

$01.04119044 \quad 0.89566189-1.77259761$

O $-0.33881817-0.86524202-1.67336690$

Vibrational frequencies (in $\mathrm{cm}-1$ )

$\begin{array}{lllllllllllllllllll}-1594.5 & 63.9 & 124.3 & 173.9 & 184.4 & 197.3 & 214.5 & 266.0 & 275.8 & 314.1 & 357.7 & 379.9 & 427.7 & 442.3\end{array}$

$454.4510 .5 \quad 533.2 \quad 546.2 \quad 607.1 \quad 628.2 \quad 637.6 \quad 686.8 \quad 705.3 \quad 742.2 \quad 815.2 \quad 845.0 \quad 867.9 \quad 881.1$

$917.2934 .2944 .2 \quad 962.6 \quad 980.4 \quad 987.9 \quad 1011.6 \quad 1037.1 \quad 1052.3 \quad 1071.2 \quad 1071.8 \quad 1084.1 \quad 1102.8$

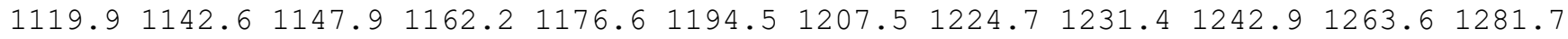

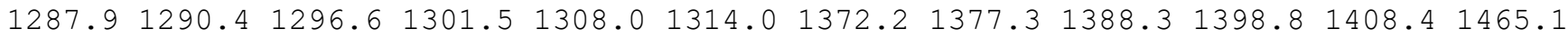
$1489.92655 .0 \quad 2665.7 \quad 2671.0 \quad 2671.6 \quad 2677.8 \quad 2683.2 \quad 2685.6 \quad 2723.5 \quad 2733.92737 .5 \quad 2763.4$ 2776.92778 .82780 .13354 .9

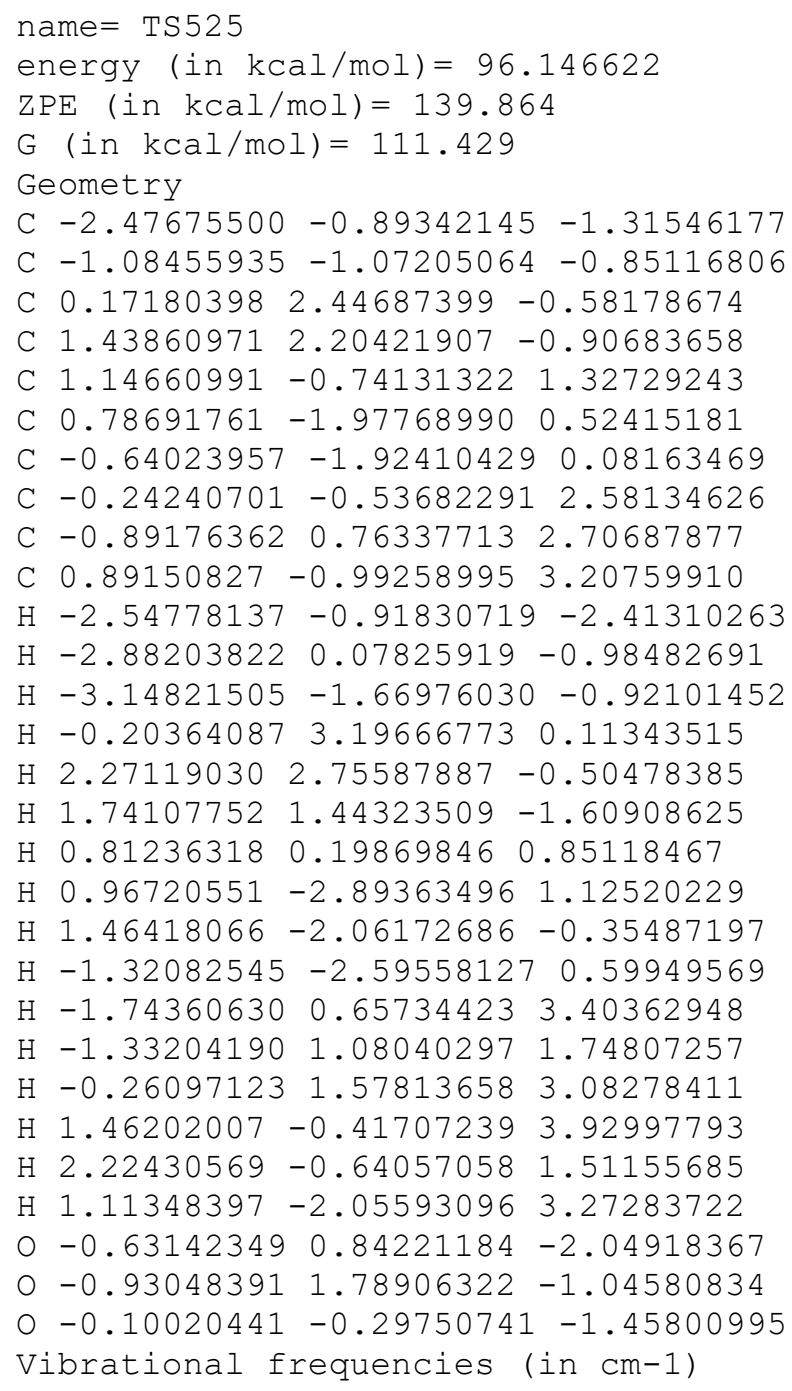
$310.7 \quad 324.0 \quad 348.5 \quad 393.9 \quad 420.8 \quad 465.4 \quad 516.2 \quad 569.3 \quad 614.5 \quad 635.4 \quad 663.1 \quad 794.9 \quad 811.2 \quad 822.1$

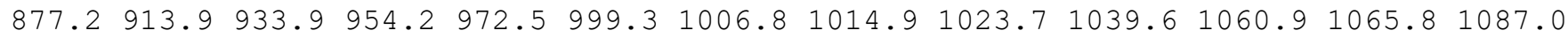
$1110.21127 .7 \quad 1173.2 \quad 1174.2 \quad 1180.5 \quad 1227.6 \quad 1262.1 \quad 1271.7 \quad 1272.8 \quad 1278.1 \quad 1283.0 \quad 1287.2$

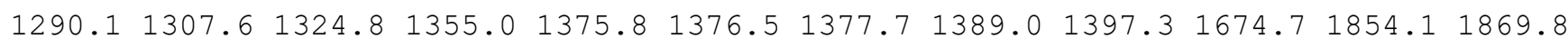
$2626.52657 .42663 .82666 .22669 .12675 .2 \quad 2680.72706 .72713 .02736 .12737 .52748 .1$ $2759.02771 .12778 .7 \quad 2794.1$

name $=\operatorname{TS} 526$

energy (in $\mathrm{kcal} / \mathrm{mol})=97.731005$

$\mathrm{ZPE}($ in $\mathrm{kcal} / \mathrm{mol})=138.607$

$\mathrm{G}(\mathrm{in} \mathrm{kcal} / \mathrm{mol})=111.573$

Geometry

C $-2.58399388-1.14288038-0.80347173$ 
C $-1.69298455-1.17181738 \quad 0.02893206$

C $0.47512681 \quad 1.94869473-0.00379160$

C $1.54057574 \quad 1.271733130 .80402491$

C $1.17440442-0.09931661 \quad 1.38160733$

C $1.18269377-1.20028960 \quad 0.31546235$

C $-0.75172839-1.315105371 .00960455$

C $-0.25506607-0.30893505 \quad 1.99044587$

C $-1.13629188 \quad 0.93261200 \quad 2.10202563$

C $-0.14229538-0.95495767 \quad 3.38025529$

$\mathrm{H}-3.35661057-1.14921345-1.47878716$

H $0.06010094 \quad 0.34725183 \quad-2.20129564$

$\mathrm{H}-0.95851682-0.78098040-2.46755070$

$\mathrm{H} \quad 0.103455692 .920451350 .36051146$

H $1.86034344 \quad 1.93576110 \quad 1.63518356$

H $2.44602524 \quad 1.14272654 \quad 0.16028239$

H $1.93721722-0.32746976 \quad 2.16639978$

$\mathrm{H} 2.03186693-1.885718170 .36682708$

$\mathrm{H} \quad 1.09512770-0.85665125 \quad-0.72684259$

$\mathrm{H} \quad 0.33861139-2.13945648 \quad 0.56688600$

$\mathrm{H}-0.61322680 \quad 1.75978474 \quad 2.58926171$

$\mathrm{H}-2.02889411 \quad 0.71663850 \quad 2.70844786$

$\mathrm{H}-1.52319197 \quad 1.26162876 \quad 1.13072002$

$\mathrm{H} \quad 0.26752980 \quad-0.26091557 \quad 4.11826995$

$\mathrm{H} \quad 0.48757673-1.85173898 \quad 3.35793879$

$\mathrm{H}-1.12882960-1.28291866 \quad 3.73314810$

$0-0.14217052-0.35799166-2.94545877$

$0 \quad 0.02738488 \quad 1.52930345-1.04637034$

o $0.82136666-1.31233087-2.61342843$

Vibrational frequencies (in $\mathrm{cm}-1$ )

$\begin{array}{llllllllllllllll}-1108.6 & 29.8 & 37.6 & 77.7 & 86.1 & 105.0 & 123.6 & 141.1 & 170.3 & 181.1 & 187.4 & 213.7 & 235.5 & 276.0\end{array}$ $296.2354 .3 \quad 369.3 \quad 375.1 \quad 403.8 \quad 424.9 \quad 468.8 \quad 514.2 \quad 547.9 \quad 594.0 \quad 629.3 \quad 679.0 \quad 749.4 \quad 791.3$

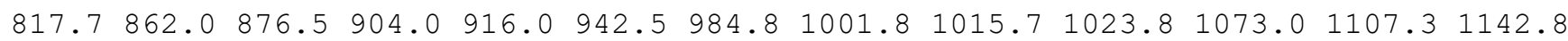

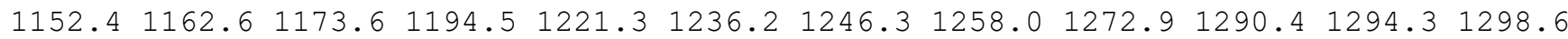
$1302.81307 .21313 .2 \quad 1325.1 \quad 1331.0 \quad 1380.8 \quad 1397.5 \quad 1402.7 \quad 1446.2 \quad 1456.6 \quad 1850.4 \quad 1986.6$ $2361.22587 .3 \quad 2596.12636 .4 \quad 2653.5 \quad 2660.6 \quad 2673.3 \quad 2682.02685 .6 \quad 2690.42712 .52728 .8$ $2777.72782 .4 \quad 2816.3 \quad 2880.6$

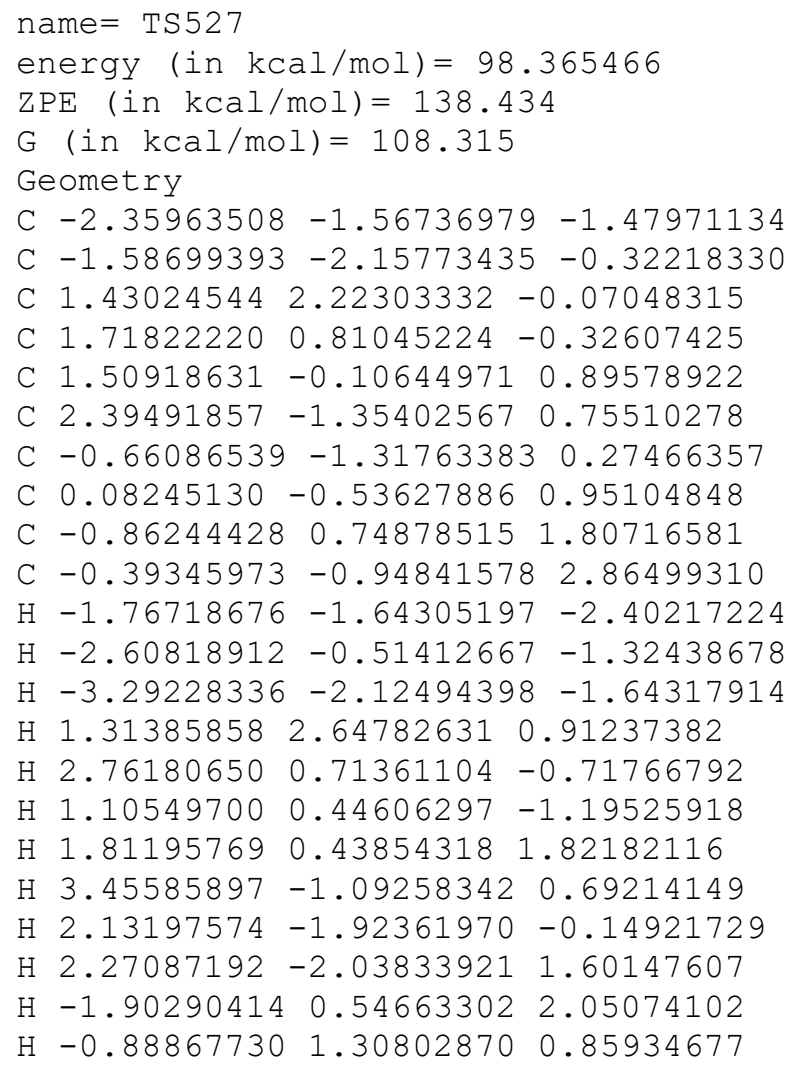


$\mathrm{H}-0.33804604 \quad 1.35834690 \quad 2.53014459$

H $0.50516258-1.55865016 \quad 2.82937897$

$\mathrm{H}-1.30060742-1.528726872 .73520960$

$\mathrm{H}-0.38162341-0.306065293 .73330631$

o $1.442573532 .70626013-2.20826205$

O $1.310306193 .09905783-1.02249968$

o $-1.77854311-3.310777160 .03238305$

Vibrational frequencies (in $\mathrm{cm}-1$ )

$\begin{array}{llllllllllllllll}-1009.3 & 14.6 & 19.5 & 26.5 & 60.1 & 66.6 & 76.4 & 83.3 & 114.7 & 152.8 & 167.5 & 175.2 & 197.9 & 215.9\end{array}$ $258.6 \quad 280.0 \quad 295.9 \quad 315.3 \quad 401.2 \quad 416.5 \quad 439.9 \quad 503.8 \quad 523.1 \quad 584.9 \quad 597.4 \quad 674.4 \quad 726.7 \quad 741.2$

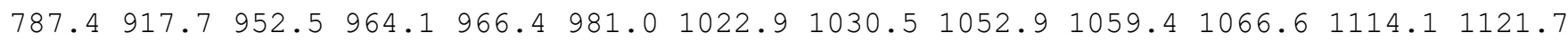
$1163.51185 .2 \quad 1211.8 \quad 1220.6 \quad 1253.8 \quad 1261.9 \quad 1269.5 \quad 1278.4 \quad 1281.2 \quad 1287.3 \quad 1291.0 \quad 1295.2$

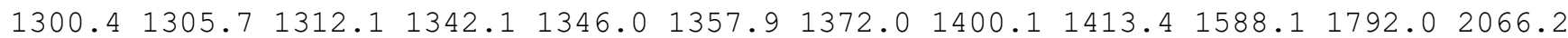
$2607.02610 .62639 .92661 .5 \quad 2664.0 \quad 2673.0 \quad 2677.5 \quad 2679.02684 .5 \quad 2699.3 \quad 2709.62725 .7$ $2735.12745 .8 \quad 2778.8 \quad 2782.2$

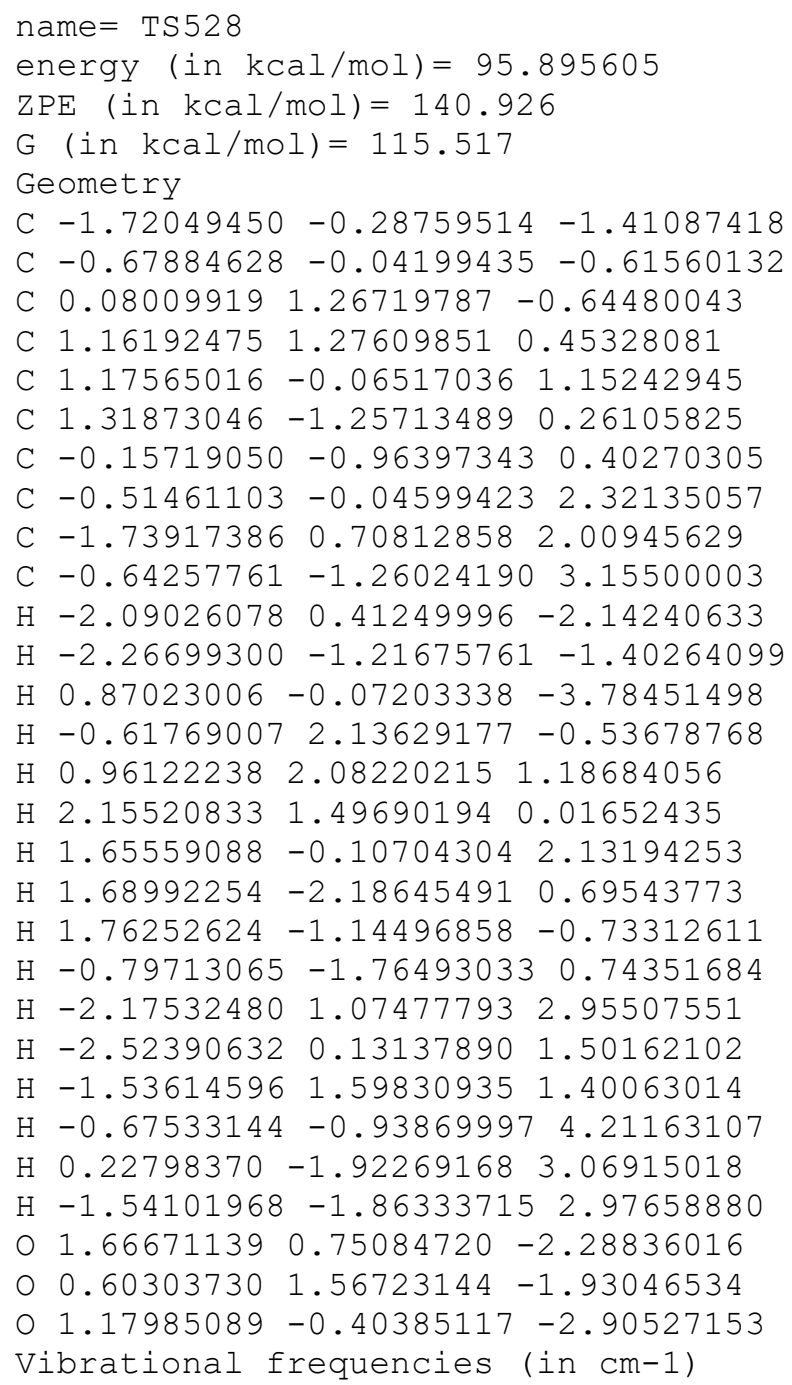




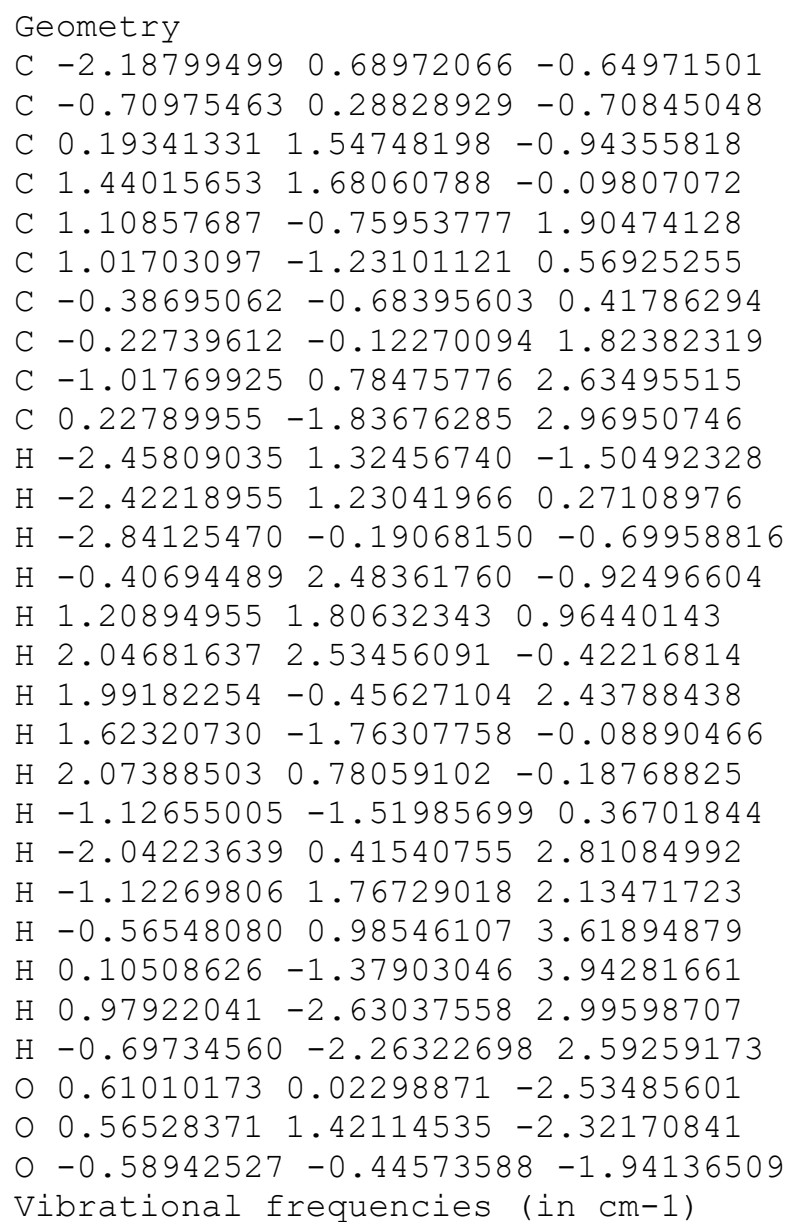

$\begin{array}{llllllllllllllllllll}-712.1 & 37.7 & 59.6 & 105.3 & 126.6 & 134.0 & 149.7 & 155.7 & 207.5 & 229.1 & 249.4 & 257.1 & 285.4 & 303.6\end{array}$ $\begin{array}{llllllllllllll}318.0 & 350.1 & 399.8 & 441.3 & 476.3 & 554.6 & 612.9 & 647.1 & 698.8 & 713.7 & 745.0 & 812.7 & 863.9 & 905.7\end{array}$ $930.6948 .1964 .5966 .0 \quad 983.6 \quad 991.2 \quad 1007.0 \quad 1008.0 \quad 1059.5 \quad 1070.1 \quad 1090.5 \quad 1097.6 \quad 1114.4$ $1127.51148 .8 \quad 1169.3 \quad 1187.0 \quad 1193.2 \quad 1234.6 \quad 1257.7 \quad 1266.0 \quad 1269.2 \quad 1270.6 \quad 1286.8 \quad 1287.6$ $1289.41291 .1 \quad 1297.4 \quad 1299.3 \quad 1308.2 \quad 1321.0 \quad 1337.1 \quad 1379.2 \quad 1390.6 \quad 1397.3 \quad 1412.11580 .5$ $2649.62650 .92653 .2 \quad 2667.7 \quad 2668.92680 .6 \quad 2681.32684 .42686 .12695 .32758 .72769 .1$ 2772.92778 .32780 .72836 .7

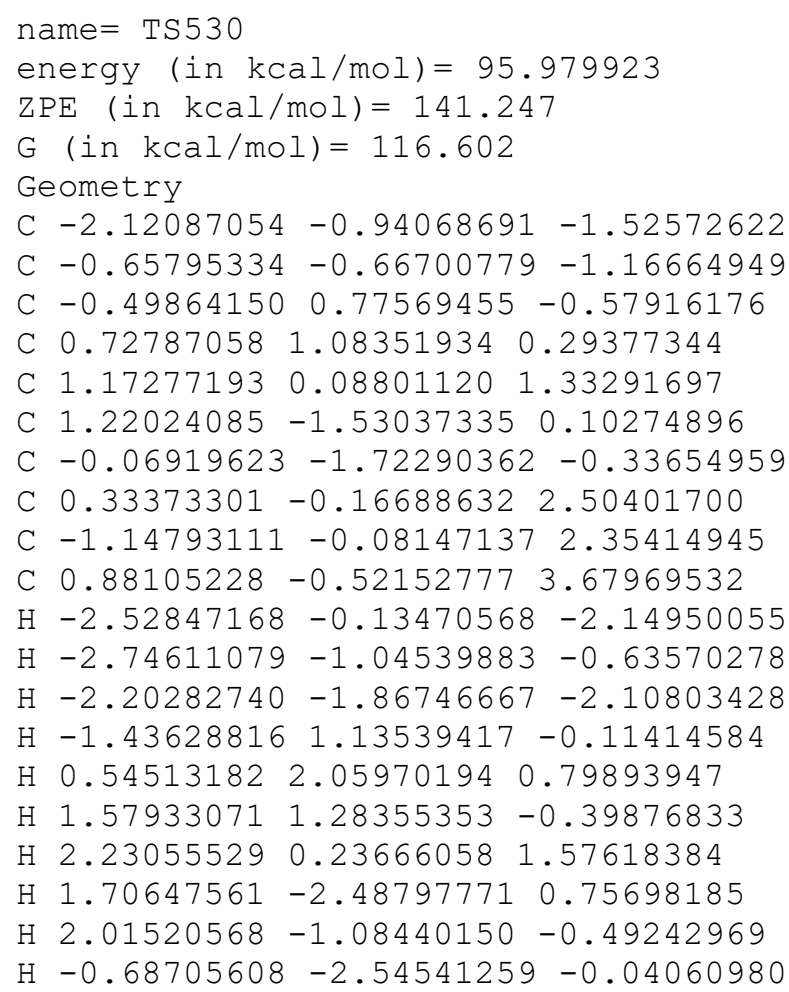


$\mathrm{H}-1.49370371-0.692029351 .50274033$

$\mathrm{H}-1.46476283 \quad 0.95493314 \quad 2.16807135$

$\mathrm{H}-1.68652200-0.426447373 .24563635$

$\mathrm{H} \quad 1.93644983-0.57737498 \quad 3.86447174$

$\mathrm{H} 1.46570057-1.773541761 .40047255$

$\mathrm{H} \quad 0.30395427 \quad-0.73843934 \quad 4.56020997$

○ $0.45673975 \quad 0.76183846-2.60595203$

$0-0.384453791 .54466379-1.77830387$

O $0.05071158-0.59601915-2.42398507$

Vibrational frequencies (in $\mathrm{cm}-1$ )

$\begin{array}{lllllllllllllllllll}-1041.8 & 44.3 & 59.6 & 92.6 & 132.1 & 173.6 & 179.4 & 201.1 & 234.6 & 263.3 & 286.0 & 292.0 & 346.3 & 386.7\end{array}$ $409.9427 .5 \quad 457.0 \quad 509.6 \quad 551.6 \quad 557.5 \quad 592.2 \quad 610.7 \quad 642.3 \quad 681.7 \quad 712.5 \quad 733.3 \quad 798.9 \quad 843.9$ $876.9909 .0927 .8 \quad 966.9976 .3 \quad 993.6 \quad 1005.7 \quad 1009.0 \quad 1028.9 \quad 1039.2 \quad 1050.6 \quad 1056.6 \quad 1083.8$ $1092.81101 .3 \quad 1146.0 \quad 1160.3 \quad 1218.3 \quad 1239.5 \quad 1246.2 \quad 1281.2 \quad 1281.6 \quad 1289.2 \quad 1291.1 \quad 1295.1$

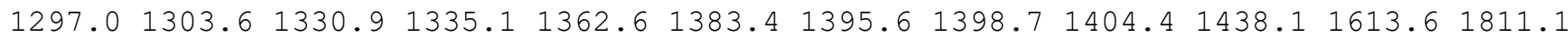
$1843.82643 .12657 .3 \quad 2674.42675 .12684 .7 \quad 2692.02695 .52719 .6 \quad 2726.22732 .42774 .3$ $2782.12796 .02801 .4 \quad 3044.3$

name $=$ TS531

energy (in $\mathrm{kcal} / \mathrm{mol})=99.304035$

$\mathrm{ZPE}(\mathrm{in} \mathrm{kcal} / \mathrm{mol})=138.13$

$\mathrm{G}($ in $\mathrm{kcal} / \mathrm{mol})=109.895$

Geometry

C $-1.19084400-0.13038480-1.42597682$

C $-0.21563312-0.49791795-0.37235968$

C $0.67441152 \quad 0.706616840 .05351941$

C $1.94920194 \quad 0.10335395 \quad 0.81771552$

C $1.53633314 \quad-0.94116717 \quad 1.85070948$

C $0.92294003-2.14676574 \quad 1.12152232$

C $-0.13463060-1.73317823 \quad 0.14694320$

C $0.54476145 \quad-0.25455524 \quad 2.78724055$

C $1.10239220 \quad 0.72683769 \quad 3.73903656$

C $-0.73648322 \quad-0.53433074 \quad 2.62897727$

$\mathrm{H}-1.80931118-0.95637652-1.78065944$

$\mathrm{H}-0.61179029 \quad 0.27443256-2.29016079$

$\mathrm{H}-1.83438629 \quad 0.70206677-1.11483308$

$\mathrm{H} \quad 0.07986894 \quad 1.28458069 \quad 0.81781312$

H $2.52443306 \quad 0.935068221 .23349353$

H $2.58030385-0.34139056 \quad 0.02750134$

H 2.41742596 $-1.27176453 \quad 2.45152830$

H $0.54317548-2.896179921 .83856713$

H $1.73204278-2.66589796 \quad 0.55427121$

$\mathrm{H}-0.76671801-2.54131158-0.20039236$

H $2.02814780 \quad 0.36631735 \quad 4.22359695$

$\mathrm{H} 0.42410604 \quad 1.04209295 \quad 4.53874440$

$\mathrm{H} \quad 1.41321604 \quad 1.64629329 \quad 3.18663530$

$\mathrm{H}-1.65465502 \quad 0.02600882 \quad 3.81957543$

$\mathrm{H}-1.43032502-1.091676562 .03828183$

$\mathrm{H}-1.68643714 \quad 0.62533396 \quad 3.26827117$

$\begin{array}{llll}0 & 2.10511464 & 0.31769303 & -2.64313706\end{array}$

$01.030898151 .44086602-0.93872653$

o $1.95204860-0.81041434-2.64945003$

Vibrational frequencies (in $\mathrm{cm}-1$ )

$\begin{array}{lllllllllllllll}-798.5 & 24.0 & 37.7 & 49.2 & 62.4 & 93.5 & 97.9 & 111.3 & 119.0 & 124.8 & 177.3 & 225.8 & 242.6 & 281.6\end{array}$

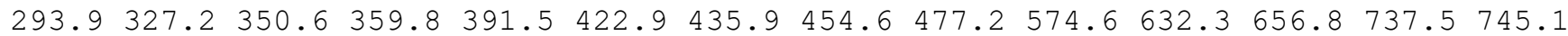

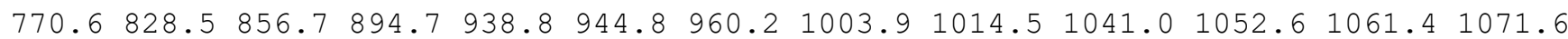

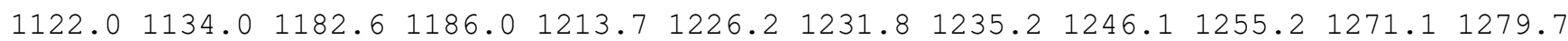
$1288.31299 .6 \quad 1312.6 \quad 1316.6 \quad 1334.9 \quad 1349.6 \quad 1375.3 \quad 1383.0 \quad 1394.4 \quad 1451.6 \quad 1571.3 \quad 1805.6$ $1831.12562 .8 \quad 2616.12638 .0 \quad 2656.0 \quad 2661.92672 .2 \quad 2676.62691 .7 \quad 2692.62737 .8 \quad 2757.2$ $2765.62768 .12775 .8 \quad 4708.3$

name $=$ TS532

energy $($ in $\mathrm{kcal} / \mathrm{mol})=97.268849$ 
$\mathrm{ZPE}($ in $\mathrm{kcal} / \mathrm{mol})=140.455$

$\mathrm{G}($ in $\mathrm{kcal} / \mathrm{mol})=117.32$

Geometry

C $-1.60885622 \quad 1.15357345 \quad-0.16312374$

C $-0.16633734 \quad 0.66971073-0.10599479$

C $0.86409417 \quad 1.734801690 .41611446$

C $1.972580101 .20188022 \quad 1.34641839$

C $1.74793700-0.24279968 \quad 1.78272086$

C $1.49254404-1.10088977 \quad 0.49008042$

C $-0.01686914-0.66939983 \quad 0.60775452$

$\begin{array}{lllll}\text { C } & 0.27621260 & -0.55441144 & 2.12381947\end{array}$

C $-0.53529047 \quad 1.09772356 \quad 3.03805520$

C $-0.17299402-1.478086093 .06356613$

$\mathrm{H}-2.11036181 \quad 1.08682273 \quad 0.80870350$

$\begin{array}{lllll}\mathrm{H} & -2.19202325 & 0.54472070 & -0.87059397\end{array}$

$\mathrm{H}-1.67236428 \quad 2.19085166-0.51725705$

$\mathrm{H} \quad 0.36187182 \quad 2.62373457 \quad 0.84781897$

H $2.07106281 \quad 1.85831644 \quad 2.23138645$

H 2.94718871 $1.27790513 \quad 0.81664986$

$\mathrm{H} 2.49823353-0.60710902 \quad 2.48853878$

$\mathrm{H} 1.65884569-2.17282658 \quad 0.62016505$

H 2.01438750 $-0.77662538-0.40995202$

$\mathrm{H}-0.76986495-1.40536736 \quad 0.31432033$

$\mathrm{H}-1.165490150 .86976177 \quad 3.97232280$

$\mathrm{H}-1.129292251 .52820420 \quad 2.27396177$

H $0.34910844 \quad 1.62642190 \quad 3.29616983$

$\mathrm{H}-0.83660795-0.117177713 .64826836$

$\mathrm{H} 0.43877556-1.81114582 \quad 3.87194919$

$\mathrm{H}-0.99669547 \quad-2.12875443 \quad 2.87376902$

O $1.46971370 \quad 1.09879442-1.64448609$

O $1.44564046 \quad 2.22701363-0.78981512$

o $0.18571693 \quad 0.50796669-1.49282541$

Vibrational frequencies (in cm-1)

$\begin{array}{llllllllllllll}-2219.0 & 84.9 & 104.1 & 135.5 & 181.1 & 203.0 & 227.7 & 260.8 & 269.7 & 301.7 & 332.1 & 340.4 & 399.6 & 408.8\end{array}$

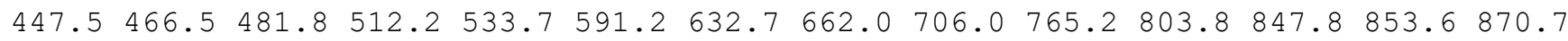
$889.5900 .0909 .1938 .1960 .4 \quad 969.1 \quad 989.1 \quad 1006.0 \quad 1035.2 \quad 1047.5 \quad 1055.6 \quad 1080.7 \quad 1093.9$

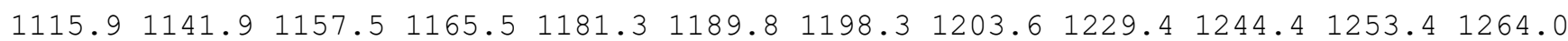

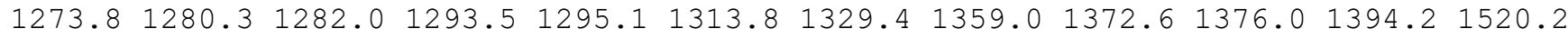
$1662.02638 .92652 .92664 .5 \quad 2670.5 \quad 2687.3 \quad 2688.8 \quad 2729.52735 .5 \quad 2742.6 \quad 2747.5 \quad 2753.3$ $2763.2 \quad 2774.4 \quad 2786.8 \quad 2819.1$

\section{name $=$ TS533}

energy $($ in $\mathrm{kcal} / \mathrm{mol})=96.714799$

$\mathrm{ZPE}(\mathrm{in} \mathrm{kcal} / \mathrm{mol})=141.088$

$\mathrm{G}(\mathrm{in} \mathrm{kcal} / \mathrm{mol})=116.014$

Geometry

C $-1.80973767-0.55260557-1.32728080$

C $-0.37893774-0.10110483-1.12304924$

C $-0.212915391 .42880765-0.78894115$

C $0.63087542 \quad 1.55847595 \quad 0.48999444$

C $0.92115305 \quad 0.15179986 \quad 0.97509800$

C $1.82829151-0.71264050 \quad 0.09149382$

C $0.34092941-0.86266652-0.02978408$

C $0.02271371-0.35882684 \quad 2.55108211$

C $-0.403560490 .72971994 \quad 3.43808267$

C $0.38217207-1.688027813 .06510747$

$\mathrm{H}-2.32590771 \quad 0.06054261 \quad-2.07726292$

$\mathrm{H}-2.37699434-0.49669362-0.38674860$

$\mathrm{H}-1.85331838-1.59331235-1.67670854$

$\mathrm{H}-1.16588249 \quad 1.98371465-0.74168080$

$\mathrm{H} \quad 0.08764737 \quad 2.14435622 \quad 1.25556953$

H $1.56405652 \quad 2.11698991 \quad 0.27828354$

H $1.39221127 \quad 0.13861896 \quad 2.12807488$

H $2.40970995-1.515523840 .53497548$ 
$\mathrm{H} 2.39656636-0.24102453-0.70947012$

$\mathrm{H}-0.14915725-1.775824790 .30878069$

$\mathrm{H}-0.65433854 \quad 1.65275410 \quad 2.89701532$

$\mathrm{H} \quad 0.29849003 \quad 0.98573677 \quad 4.24224060$

$\mathrm{H}-1.34536753 \quad 0.41483089 \quad 3.92739103$

H $1.07331233-1.684578953 .91871359$

H $0.80149638 \quad-2.35028155 \quad 2.29651087$

$\mathrm{H}-0.54602639-2.182697493 .40875516$

O $1.17744442 \quad 0.84917623-2.45748436$

$0.45836697 \quad 1.95743335-1.92181455$

O $0.26408278-0.23256990-2.39524206$

Vibrational frequencies (in $\mathrm{cm}-1$ )

$\begin{array}{lllllllllllllllll}-1127.1 & 42.6 & 66.9 & 78.9 & 117.3 & 134.7 & 158.5 & 167.0 & 199.6 & 249.7 & 281.1 & 291.8 & 310.1 & 339.6\end{array}$ $350.7 \quad 379.2 \quad 453.4 \quad 498.5 \quad 556.1 \quad 602.2 \quad 645.7 \quad 711.0 \quad 782.8 \quad 806.1 \quad 835.0 \quad 849.7 \quad 895.4 \quad 918.5$ $944.1963 .2 \quad 978.3981 .5 \quad 999.0 \quad 1001.9 \quad 1005.0 \quad 1012.5 \quad 1043.4 \quad 1066.7 \quad 1105.5 \quad 1120.6 \quad 1132.5$

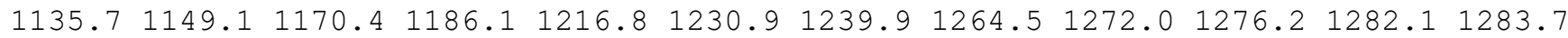

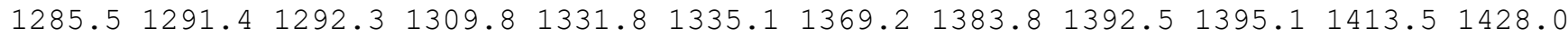
$2042.62649 .52658 .52661 .8 \quad 2664.42669 .5 \quad 2670.52674 .42699 .5 \quad 2704.62733 .92754 .0$ $2768.22771 .7 \quad 2778.7 \quad 2779.3$

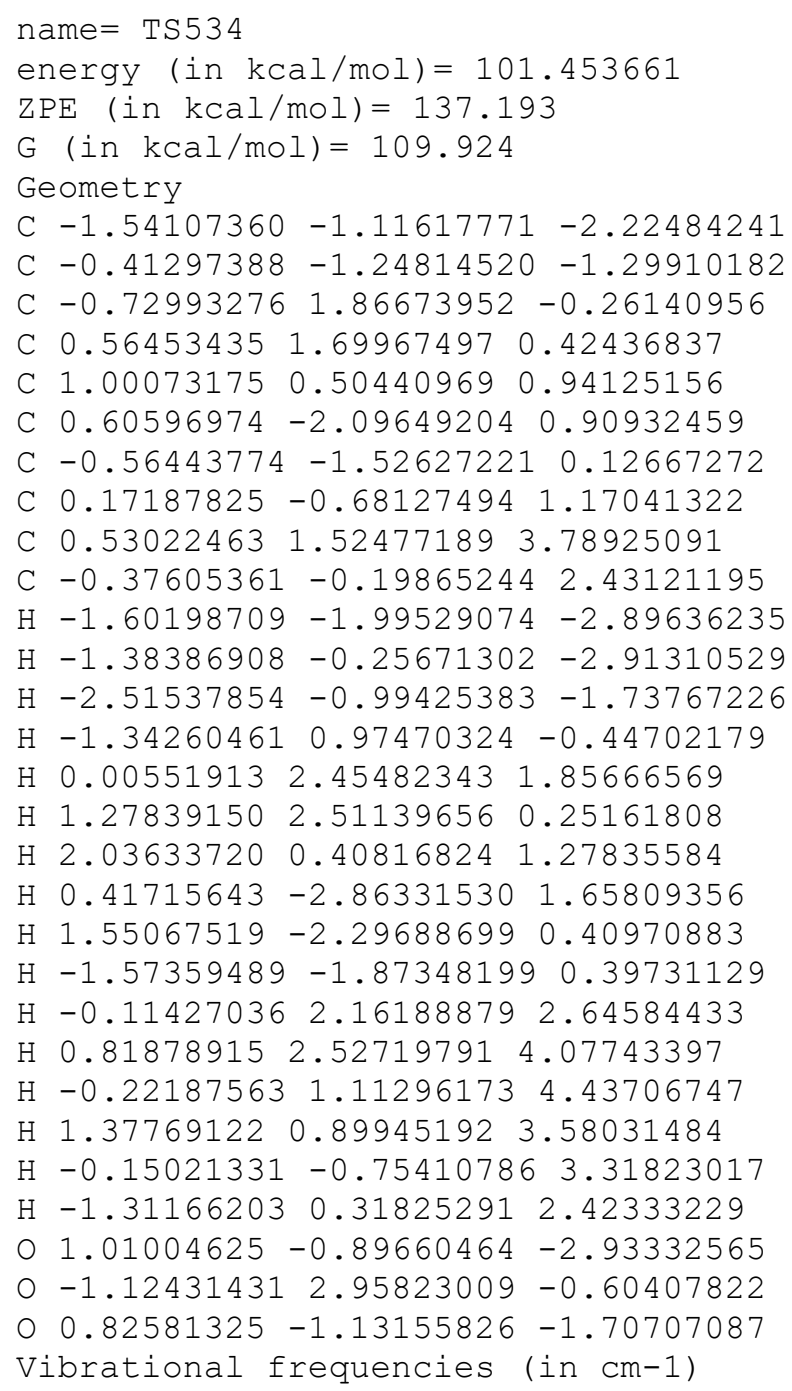




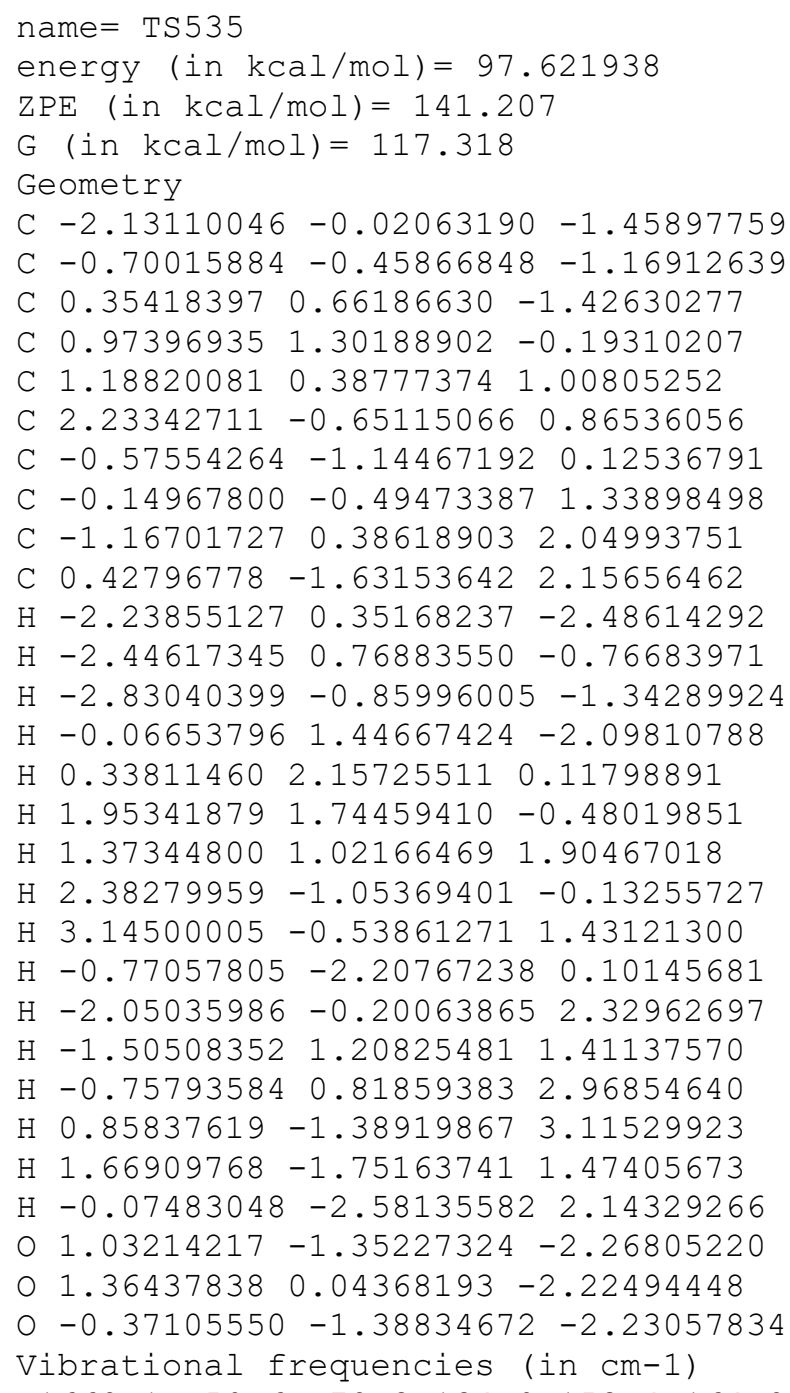


$\mathrm{H} 2.33322857-0.69821800 \quad 2.74116563$

$\mathrm{H} 2.60721707-1.25355024 \quad 0.00706218$

$\mathrm{H} 1.89541137 \quad 0.62416773-0.11742841$

H $\quad 0.26325937 \quad-2.10264390 \quad 0.34056191$

$\mathrm{H}-0.207927811 .58177347 \quad 1.85710172$

$\mathrm{H}-0.73614127 \quad 0.80077321 \quad 3.33479462$

$\mathrm{H}-1.661526820 .549642081 .84993103$

$\mathrm{H}-0.04262032-1.46656846 \quad 3.88656599$

H $0.49852970 \quad-2.595999212 .64532684$

$\mathrm{H}-1.18054723 \quad-2.03927013 \quad 2.66141194$

$\begin{array}{lllll}0 & 0.76582873 & 0.23826074 & -2.42089918\end{array}$

$\begin{array}{llll}0 & -0.01058590 & 1.39240619 & -2.13109323\end{array}$

o $-0.01452053-0.83662242-1.93157446$

Vibrational frequencies (in $\mathrm{cm}-1$ )

$\begin{array}{llllllllllllll}-1408.2 & 58.0 & 75.2 & 114.2 & 125.0 & 159.0 & 171.5 & 191.2 & 230.2 & 243.8 & 256.4 & 283.1 & 333.8 & 354.4\end{array}$

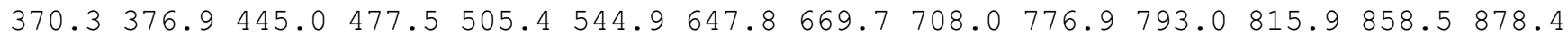

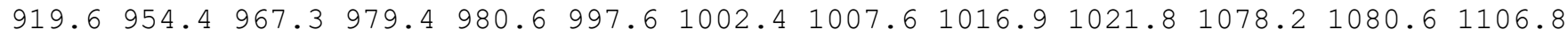
$1136.11151 .31164 .6 \quad 1184.91188 .2 \quad 1205.3 \quad 1215.2 \quad 1249.01265 .8 \quad 1280.6 \quad 1285.6 \quad 1289.1$

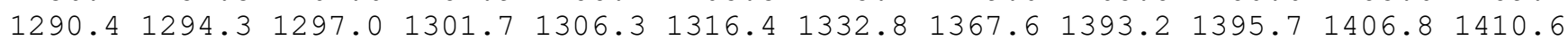
$2164.22608 .72655 .22669 .3 \quad 2673.3 \quad 2675.12678 .32680 .62701 .92709 .52756 .02758 .8$ $2765.72775 .7 \quad 2779.1 \quad 2782.2$

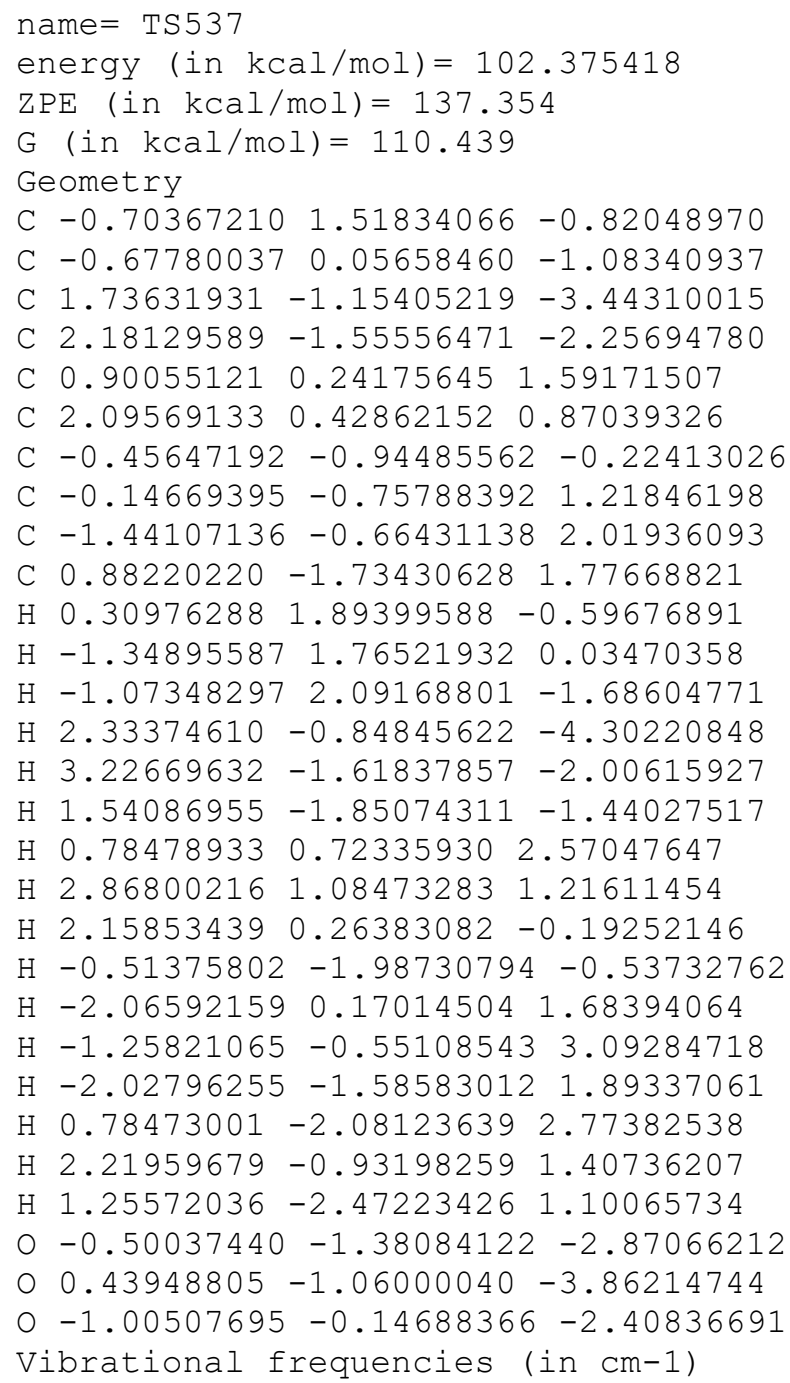


name $=$ TS538

energy (in $\mathrm{kcal} / \mathrm{mol})=100.268759$

$\mathrm{ZPE}($ in $\mathrm{kcal} / \mathrm{mol})=139.553$

$\mathrm{G}(\mathrm{in} \mathrm{kcal} / \mathrm{mol})=114.82$

Geometry

C $-2.37468882 \quad 1.20334564 \quad 0.32325628$

C $-1.05053974 \quad 0.50809866 \quad 0.09836868$

C $0.09681871 \quad 1.37419214-0.54875046$

C $1.37752563 \quad 1.170706650 .28303331$

C $1.03090746 \quad 0.20594316 \quad 1.39005464$

C $1.93647441-1.76027271 \quad 1.03551558$

C $-0.44239508-0.16273281 \quad 1.31369378$

$\begin{array}{lllll}\text { C } & 0.07147668 & 0.63961804 & 2.49757217\end{array}$

C $-0.25182645 \quad 2.09424246 \quad 2.70507355$

C $0.16621688-0.110456513 .80680150$

$\mathrm{H}-2.29754368 \quad 2.015905931 .05358647$

$\mathrm{H}-2.76939302 \quad 1.62210792 \quad-0.61436039$

$\mathrm{H}-3.12946948 \quad 0.49740816 \quad 0.70068824$

$\mathrm{H}-0.167939522 .43045447-0.71781283$

H $2.18618656 \quad 0.76997890 \quad-0.36249105$

H $1.76241612 \quad 2.12633558 \quad 0.68089228$

H $1.95923885-0.43971418 \quad 1.79094897$

H $1.12591604 \quad-2.379250391 .32918762$

$\mathrm{H} 2.07868951-1.63614213-0.00926152$

$\mathrm{H}-0.81712342-1.17223654 \quad 1.48809927$

$\mathrm{H}-1.213047352 .21506622 \quad 3.22400040$

$\mathrm{H}-0.30866068 \quad 2.66229144 \quad 1.76953500$

$\mathrm{H} 0.51599705 \quad 2.57953529 \quad 3.32494117$

$\mathrm{H} 0.904063120 .35047852 \quad 4.47677915$

$\mathrm{H} \quad 0.46939587-1.15808179 \quad 3.67446733$

$\mathrm{H}-0.79910491-0.11535402 \quad 4.32955321$

O $-0.14062970-0.53823601-1.69182401$

$\begin{array}{lllll}0 & 0.23995841 & 0.82345391 & -1.84995191\end{array}$

O $-1.32027944-0.48022699-0.90771134$

Vibrational frequencies (in $\mathrm{cm}-1$ )

$\begin{array}{llllllllllllllllllll}-626.7 & 67.8 & 92.4 & 113.0 & 114.1 & 126.8 & 156.8 & 182.8 & 189.1 & 213.7 & 233.9 & 260.4 & 292.1 & 321.0\end{array}$ $363.7 \quad 389.0 \quad 424.9 \quad 465.9 \quad 482.6 \quad 584.2 \quad 643.7 \quad 675.0 \quad 704.3 \quad 717.5 \quad 748.1 \quad 800.3 \quad 823.2 \quad 853.0$ $891.7 \quad 964.7973 .5 \quad 982.2 \quad 1000.1 \quad 1008.7 \quad 1014.0 \quad 1017.4 \quad 1030.91044 .6 \quad 1077.7 \quad 1088.3 \quad 1106.5$

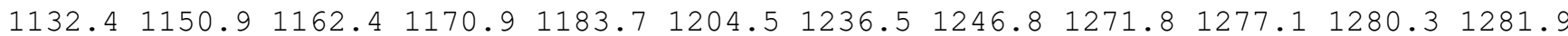
$1288.81289 .6 \quad 1292.2 \quad 1294.3 \quad 1300.91318 .4 \quad 1328.2 \quad 1337.2 \quad 1391.51400 .51403 .91491 .7$ $2015.32660 .42668 .32668 .6 \quad 2671.2 \quad 2673.7 \quad 2675.12677 .22695 .62700 .12701 .52743 .8$ $2751.8 \quad 2778.7 \quad 2780.5 \quad 2781.4$

name $=\operatorname{TS} 539$

energy $($ in $\mathrm{kcal} / \mathrm{mol})=99.78698$

$\mathrm{ZPE}(\mathrm{in} \mathrm{kcal} / \mathrm{mol})=140.123$

G $($ in $\mathrm{kcal} / \mathrm{mol})=116.676$

Geometry

C $-2.18421290 \quad 2.95916079 \quad 1.05012886$

C $-1.567709051 .83339106 \quad 0.23389648$

C $-0.09188078 \quad 2.11095694-0.24669528$

C $0.88193135 \quad 1.10311896 \quad 0.21131125$

C $0.39133095-0.18172137 \quad 0.68946323$

C $-0.97585436-0.58645139-0.02174127$

C $-1.69514072 \quad 0.45555181 \quad 0.91299934$

C $-0.56598033 \quad 0.12189425 \quad 1.88464168$

C $0.33346909 \quad 1.77931841 \quad 2.94869697$

C $-0.79944944 \quad-0.75932128 \quad 3.04133743$

$\mathrm{H}-2.24224042 \quad 3.881443490 .45061483$

$\mathrm{H}-1.605410113 .187411331 .94912859$

$\mathrm{H}-3.20994485 \quad 2.71907161 \quad 1.35595000$

$\mathrm{H} \quad 0.23321491 \quad 3.14869963-0.00185018$ 
H $0.82745993 \quad 1.65458234 \quad 1.81058838$

$\mathrm{H} 1.887527761 .19071801-0.14236264$

$\mathrm{H} 1.13709550-0.965485210 .82554302$

$\mathrm{H}-1.28729344-1.619243090 .11741170$

$\mathrm{H}-0.98285604-0.36435770-1.09131196$

$\mathrm{H}-2.72044610 \quad 0.23764555 \quad 1.21985396$

$\mathrm{H}-0.71075053 \quad 1.92770384 \quad 3.18097699$

$\mathrm{H} \quad 0.79365723 \quad 2.77262889 \quad 2.91639885$

$\mathrm{H} 0.84311796 \quad 1.16186461 \quad 3.66941934$

$\mathrm{H}-1.48828860-0.321176413 .78027341$

$\mathrm{H} \quad 0.13176617 \quad-1.01369274 \quad 3.57329197$

$\mathrm{H}-1.25206026-1.718298312 .72928976$

o $-1.398702961 .50032009-2.00922200$

$\begin{array}{llll}0 & -0.22753140 & 2.20377843 & -1.67918741\end{array}$

o $-2.330283451 .81532231-0.97406663$

Vibrational frequencies (in cm-1)

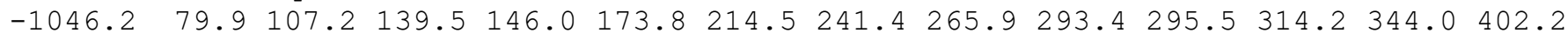
$\begin{array}{llllllllllllll}432.4 & 469.0 & 473.8 & 525.9 & 531.3 & 556.8 & 622.3 & 659.3 & 673.5 & 706.1 & 734.3 & 802.3 & 850.3 & 862.9\end{array}$

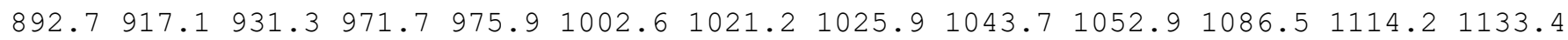

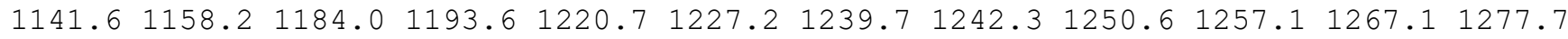
$1280.91283 .1 \quad 1287.9 \quad 1290.5 \quad 1300.7 \quad 1316.8 \quad 1357.4 \quad 1371.5 \quad 1377.3 \quad 1388.4 \quad 1392.8 \quad 1400.9$ $1497.52645 .12651 .2 \quad 2665.1 \quad 2667.4 \quad 2676.2 \quad 2677.02678 .92692 .12743 .12752 .82758 .9$ $2766.5 \quad 2772.8 \quad 2780.7 \quad 2797.5$

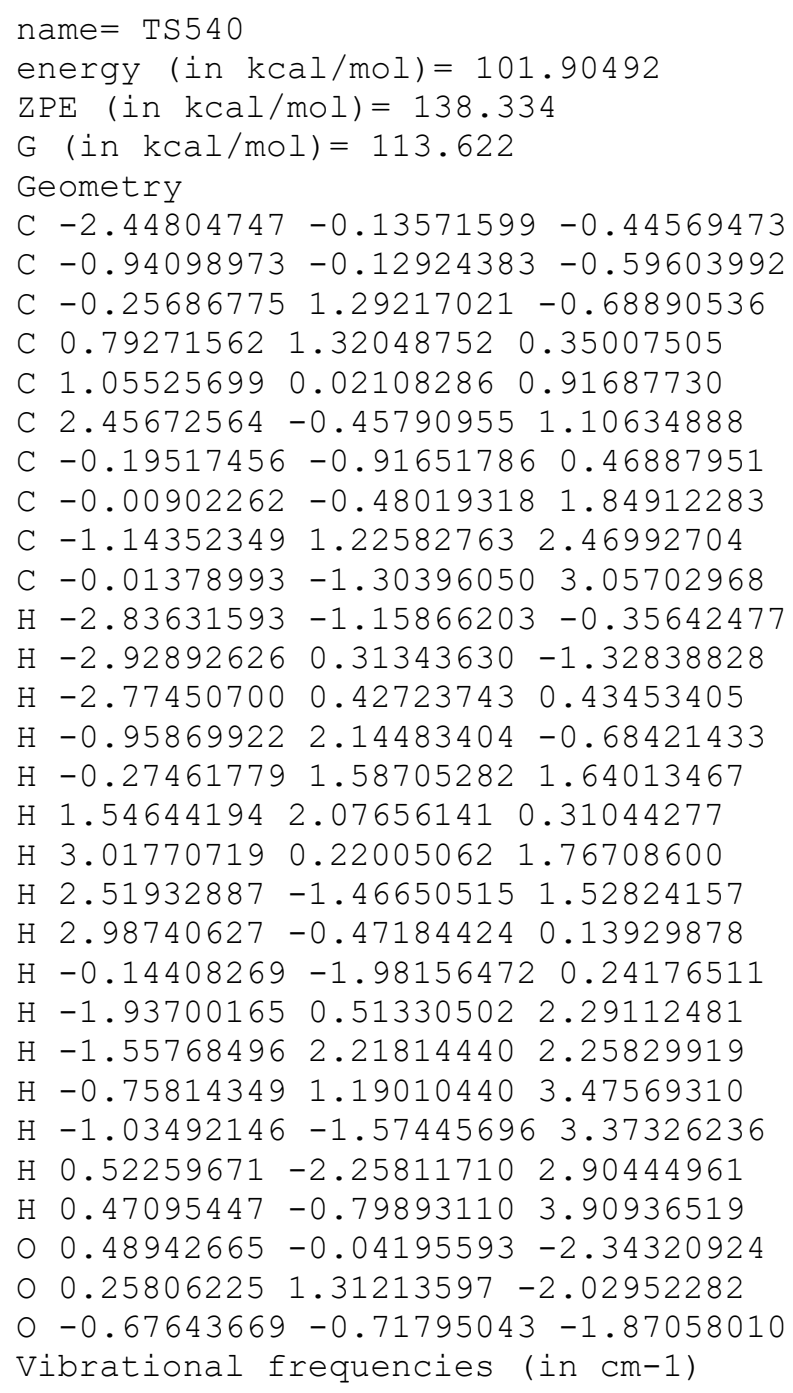


$1573.22639 .92652 .92665 .2 \quad 2666.2 \quad 2669.8 \quad 2676.5 \quad 2678.22690 .8 \quad 2700.8 \quad 2743.3 \quad 2763.9$ $2771.5 \quad 2778.8 \quad 2780.0 \quad 2799.7$

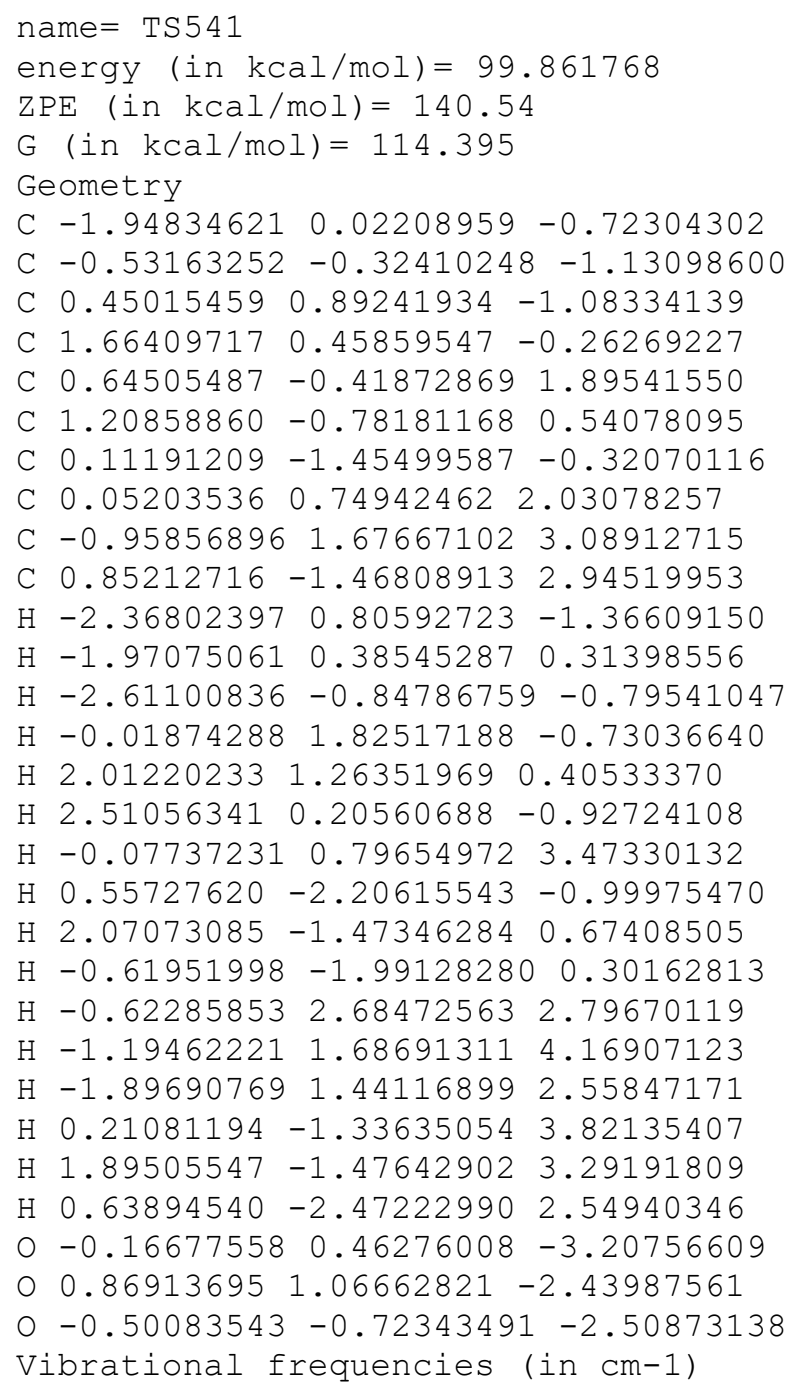


$\mathrm{H}-2.64889351-0.72863263-0.35464695$

$\mathrm{H}-0.36729990 \quad 2.21353651-0.06956777$

$\mathrm{H} 0.82440542 \quad 1.81652633 \quad 1.91795554$

H 2.19660633 $1.58083630 \quad 0.84591550$

H $2.05437659-0.380493832 .41387439$

$\mathrm{H} \quad 2.41772527-0.77082238-0.34968185$

$\mathrm{H} \quad 1.59722824-0.77903750-0.87586490$

$\mathrm{H} \quad 1.77584483-2.24385065 \quad 0.57199127$

$\mathrm{H}-1.19682023 \quad 0.99489754 \quad 2.09836733$

$\mathrm{H}-0.86396164 \quad 0.15221483 \quad 3.61007216$

$\mathrm{H}-2.12141342-0.44900806 \quad 2.51847612$

H $0.26802755-2.03350750 \quad 3.69819766$

$\mathrm{H} \quad 0.66657610 \quad-2.92142393 \quad 2.21683004$

$\mathrm{H}-1.01101182-2.699933832 .66006404$

$\begin{array}{lllll}0 & 0.95070641 & 0.27330485 & -2.05587936\end{array}$

o $1.067251411 .50071529-1.27836851$

o - $0.34840898-0.17592362-1.84920650$

Vibrational frequencies (in cm-1)

$\begin{array}{lllllllllllllll}-814.3 & 75.7 & 101.6 & 133.6 & 175.7 & 195.5 & 201.0 & 238.3 & 266.8 & 294.3 & 317.7 & 363.1 & 377.5 & 416.8\end{array}$

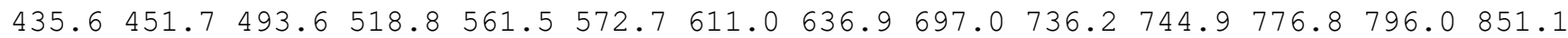
$878.8913 .5934 .6949 .3 \quad 994.1 \quad 1000.1 \quad 1011.7 \quad 1014.2 \quad 1022.0 \quad 1050.4 \quad 1069.01080 .01088 .6$

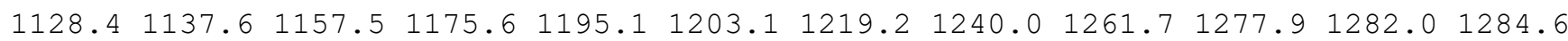

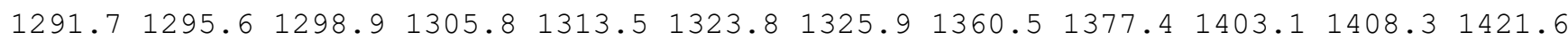
$1686.02656 .92667 .8 \quad 2673.2 \quad 2674.5 \quad 2677.8 \quad 2678.92687 .02692 .3 \quad 2713.6 \quad 2737.2 \quad 2740.4$ $2776.8 \quad 2781.0 \quad 2783.4 \quad 3149.1$

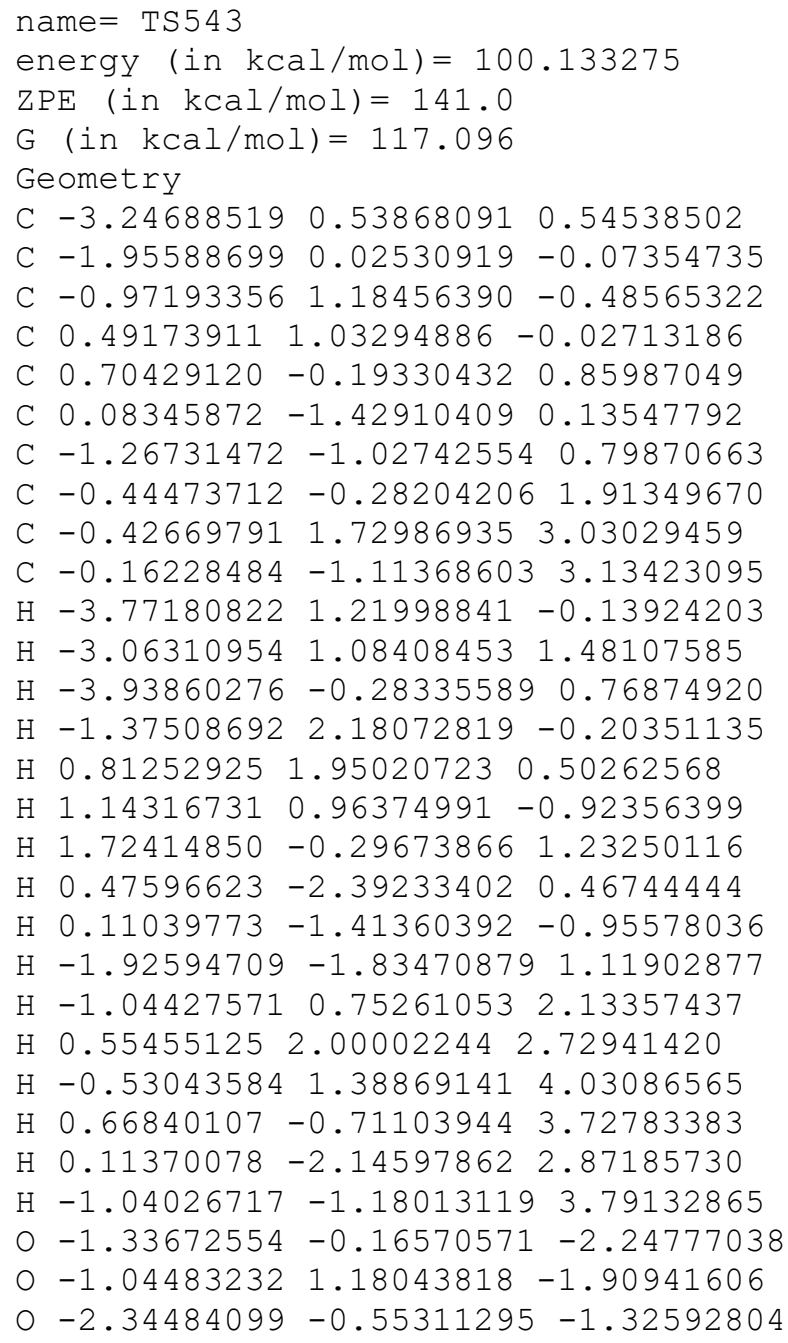




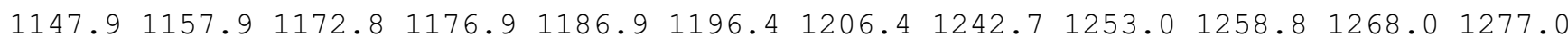

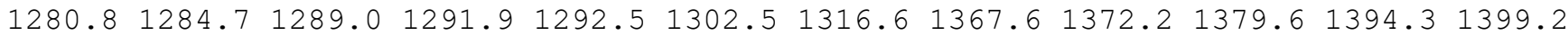
$1812.12654 .62664 .92667 .0 \quad 2672.0 \quad 2673.6 \quad 2681.92689 .32694 .12696 .52736 .82746 .9$ $2750.42766 .6 \quad 2777.3 \quad 2778.9$

\section{name $=$ TS544}

energy (in $\mathrm{kcal} / \mathrm{mol})=101.828331$

$\mathrm{ZPE}($ in $\mathrm{kcal} / \mathrm{mol})=139.534$

$\mathrm{G}($ in $\mathrm{kcal} / \mathrm{mol})=114.779$

Geometry

C $-2.40431217 \quad 0.25114037-0.29841427$

C $-0.92154429-0.03635231-0.28167995$

C $0.000729071 .13460559-0.78612048$

C $1.15141891 \quad 1.310001490 .22379310$

$\begin{array}{lllll}\text { C } & 0.93399334 & 0.27961260 & 1.30568292\end{array}$

C $2.42193712-1.324300791 .15517264$

C $-0.34121339-0.50708486 \quad 1.03596852$

C $-0.26381663 \quad 0.41029118 \quad 2.24528179$

C $-1.02659603 \quad 1.70320256 \quad 2.34294256$

C $-0.15095025-0.28221774 \quad 3.58445325$

$\mathrm{H}-2.68166462 \quad 1.07350157 \quad 0.36939425$

$\mathrm{H}-2.97637827-0.63295718 \quad 0.01985347$

$\mathrm{H}-2.74829381 \quad 0.50481937-1.31164381$

$\mathrm{H}-0.54656968 \quad 2.06192265-1.01486564$

H $1.18125525 \quad 2.334719120 .63438789$

H $2.12867407 \quad 1.16207662-0.28251733$

H $1.93890971-0.075243661 .86430081$

$\mathrm{H} 1.78933403-2.16115044 \quad 1.31665996$

$\mathrm{H} 2.70388775-1.138172020 .14829445$

$\mathrm{H}-0.42701333-1.582609431 .19870465$

$\mathrm{H}-2.05044555 \quad 1.530907762 .70364322$

$\mathrm{H}-1.100038652 .23580668 \quad 1.38781678$

$\mathrm{H}-0.54217542 \quad 2.38944789 \quad 3.05262330$

$\mathrm{H}-1.13953949-0.57198598 \quad 3.96371430$

$\mathrm{H} \quad 0.31262470 \quad 0.37375681 \quad 4.33320419$

$\mathrm{H} 0.46046717-1.19404693 \quad 3.54038978$

O $-0.30327411-0.40091955-2.40978353$

$\begin{array}{lllll}0 & 0.57769241 & 0.63943905 & -1.99425653\end{array}$

o $-0.63677832-1.08843059-1.22222616$

Vibrational frequencies (in $\mathrm{cm}-1$ )

$\begin{array}{llllllllllllllllll}-648.8 & 65.3 & 96.4 & 114.8 & 117.5 & 127.6 & 156.2 & 175.4 & 184.6 & 220.5 & 243.5 & 254.7 & 286.5 & 312.8\end{array}$ $365.1 \quad 375.5 \quad 416.4 \quad 446.3 \quad 482.0 \quad 600.4 \quad 639.4 \quad 648.5 \quad 711.1 \quad 728.7 \quad 748.8 \quad 794.5 \quad 815.9 \quad 876.0$ $891.0965 .1979 .6 \quad 984.2 \quad 998.8 \quad 1011.1 \quad 1014.1 \quad 1017.5 \quad 1029.1 \quad 1058.1 \quad 1081.7 \quad 1089.5 \quad 1115.4$

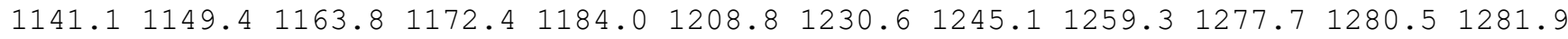
$1287.51290 .21293 .7 \quad 1299.4 \quad 1304.0 \quad 1319.5 \quad 1331.8 \quad 1339.3 \quad 1391.3 \quad 1400.4 \quad 1403.91491 .1$ $1999.42657 .7 \quad 2667.8 \quad 2669.6 \quad 2671.1 \quad 2673.6 \quad 2675.92677 .12695 .2 \quad 2696.22701 .02741 .6$ $2750.6 \quad 2778.8 \quad 2780.8 \quad 2781.6$

\section{name $=\operatorname{TS} 545$}

energy (in $\mathrm{kcal} / \mathrm{mol})=104.008623$

$\mathrm{ZPE}($ in $\mathrm{kcal} / \mathrm{mol})=137.946$

$\mathrm{G}($ in $\mathrm{kcal} / \mathrm{mol})=108.833$

Geometry

C $-3.01930265-1.57556123-0.04057713$

C $-1.62480180-1.08678411 \quad 0.23421661$

C $-1.45834463 \quad 0.29774727 \quad 0.15720037$

C $0.005550491 .99994394 \quad-0.13523819$

C $1.22840704-0.70125201 \quad 1.61423107$

C $0.69789244-1.81354436 \quad 0.82146123$

C $-0.63280624 \quad-2.02950854 \quad 0.43465582$

C $0.50554520 \quad 0.28472173 \quad 2.29365838$

C $1.16112808 \quad 1.56787822 \quad 2.65755308$

C $-0.79272343 \quad 0.034290412 .97090083$ 
$\mathrm{H}-3.21030636-2.55654250 \quad 0.41262098$

$\mathrm{H}-3.18591424-1.67629953-1.12348523$

$\mathrm{H}-3.78292581-0.88900487 \quad 0.34675514$

$\mathrm{H}-2.262806890 .98514645-0.02321045$

$\mathrm{H} 1.02102962 \quad 2.29996883-0.02969810$

$\mathrm{H}-0.73977527 \quad 2.67075006-0.47382135$

$\mathrm{H} 2.27653064-0.45146046 \quad 1.37904917$

H $1.12214586-1.84608564 \quad 2.29749351$

H $1.44209089-2.498255190 .40636548$

$\mathrm{H}-0.89701246-3.07357890 \quad 0.23011552$

$\mathrm{H} \quad 0.62495848 \quad 2.41873357 \quad 2.19148509$

H 2.20614614 $1.63342167 \quad 2.33181197$

$\mathrm{H} 1.143538131 .748367393 .74162650$

$\mathrm{H}-1.51630741 \quad 0.84041546 \quad 2.77262790$

$\mathrm{H}-0.66487186-0.01767761 \quad 4.06237317$

$\mathrm{H}-1.27526391-0.90275844 \quad 2.65905685$

O $0.67125862-1.36295274-2.56451587$

$\begin{array}{llllll}0 & -0.19215764 & 0.75453244 & 0.20556556\end{array}$

o $0.28827242-2.23391063-1.94427069$

Vibrational frequencies (in cm-1)

$\begin{array}{llllllllllllllll}-1746.1 & 22.4 & 33.6 & 52.6 & 63.4 & 66.6 & 76.2 & 103.1 & 123.3 & 134.2 & 156.9 & 164.3 & 184.9 & 216.3\end{array}$ $236.3 \quad 270.6 \quad 280.8 \quad 322.3 \quad 343.0 \quad 397.8 \quad 420.9 \quad 440.5 \quad 505.5 \quad 529.0 \quad 546.3 \quad 602.3 \quad 724.3 \quad 752.2$ $856.0 \quad 897.1911 .2 \quad 920.0 \quad 981.7 \quad 990.1 \quad 1008.5 \quad 1015.1 \quad 1032.1 \quad 1049.8 \quad 1067.1 \quad 1068.91096 .3$

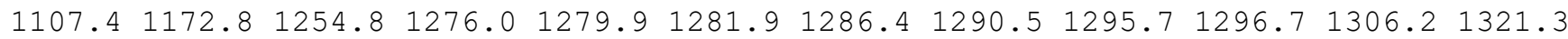

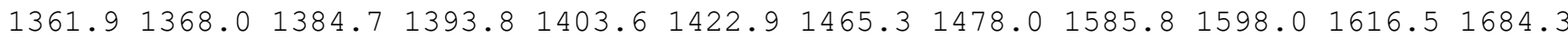
$2151.42631 .7 \quad 2663.5 \quad 2664.8 \quad 2670.8 \quad 2675.3 \quad 2676.0 \quad 2722.12729 .92737 .12745 .3 \quad 2747.9$ $2762.6 \quad 2767.7 \quad 2773.6 \quad 2779.2$

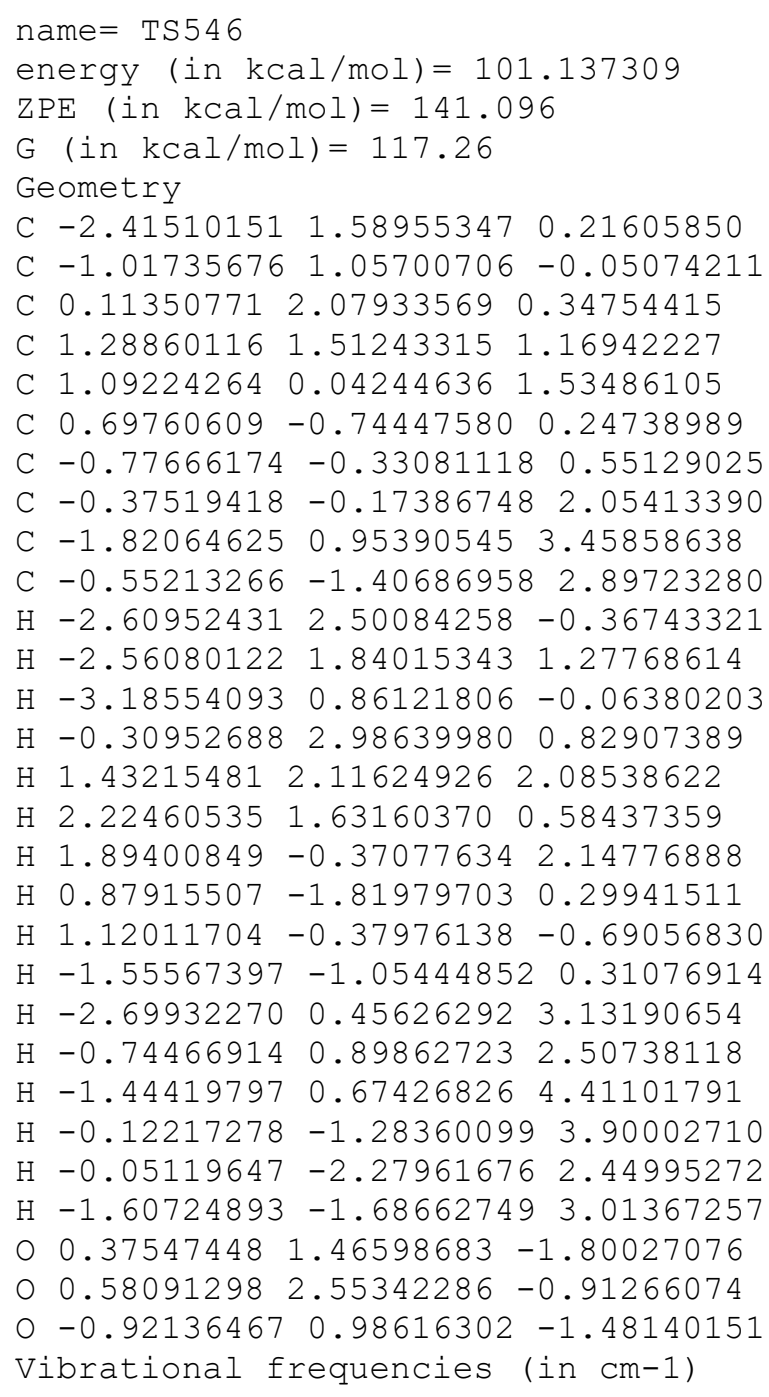


$\begin{array}{llllllllllllllllllll}-575.1 & 59.0 & 65.8 & 133.0 & 181.0 & 186.9 & 206.3 & 224.7 & 253.4 & 255.3 & 311.4 & 321.7 & 332.0 & 391.0\end{array}$

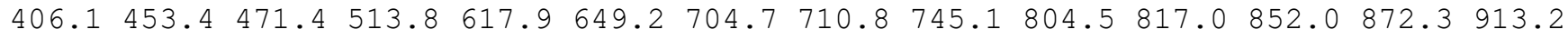

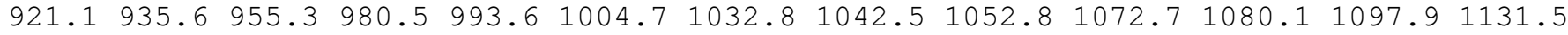

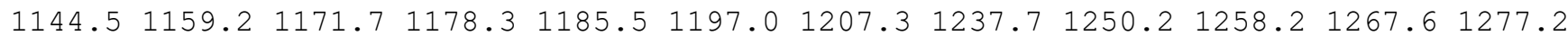

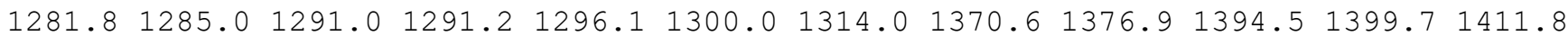
$1750.0 \quad 2654.92656 .92663 .5 \quad 2673.1 \quad 2673.5 \quad 2682.92688 .72697 .2 \quad 2698.92738 .7 \quad 2746.8$ $2750.62766 .62776 .4 \quad 2778.0$

name $=\operatorname{TS} 547$

energy $($ in $\mathrm{kcal} / \mathrm{mol})=101.559957$

$\operatorname{ZPE}($ in $\mathrm{kcal} / \mathrm{mol})=140.681$

$\mathrm{G}($ in $\mathrm{kcal} / \mathrm{mol})=116.964$

Geometry

C $-1.52255467-0.54721923-2.22736820$

C $-0.47661327-0.39230009-1.15625904$

C $0.358052190 .90235882-1.28484001$

C $0.97440074 \quad 1.42270946 \quad 0.01533509$

C $1.24101037 \quad 0.27844587 \quad 1.00588494$

C $2.20517332-0.66850568 \quad 0.41142778$

C $-0.67705553-1.03255306 \quad 0.11121694$

C $-0.10106051-0.43973386 \quad 1.35853792$

C $-1.11029545 \quad 0.55291981 \quad 1.96794372$

C $0.18363052-1.536067672 .39853809$

$\mathrm{H}-1.14854897-0.25435166-3.21739753$

$\mathrm{H}-2.407106760 .06044209-1.99962455$

$\mathrm{H}-1.85990132-1.59073468-2.31231715$

$\mathrm{H}-0.19189657 \quad 1.70137230 \quad-1.82781113$

$\mathrm{H} \quad 0.31555814 \quad 2.17974017 \quad 0.48155652$

$\mathrm{H} \quad 1.91882856 \quad 1.95704606 \quad-0.21981594$

$\mathrm{H} 1.66483316 \quad 0.70060596 \quad 1.95225280$

$\mathrm{H} \quad 0.61112140-1.89952417-0.36554608$

$\mathrm{H} 3.19861216-0.29051795 \quad 0.24425820$

$\mathrm{H}-1.49814535-1.71624391 \quad 0.24003895$

$\mathrm{H}-1.418377621 .310512791 .24060409$

$\mathrm{H}-0.69541480 \quad 1.06166255 \quad 2.84494290$

$\mathrm{H}-2.02176110 \quad 0.02983710 \quad 2.28024407$

H $0.90529331-2.26969407 \quad 2.01370805$

H $0.59647919-1.116894823 .31901702$

$\mathrm{H}-0.72941283-2.07588572 \quad 2.66423816$

O $1.87597316-0.72418490-1.77674905$

O $1.41982295 \quad 0.56223788-2.17758068$

O $0.65899489-1.43552955-1.52627068$

Vibrational frequencies (in cm-1)

$\begin{array}{llllllllllllll}-1357.8 & 46.0 & 124.6 & 148.8 & 164.6 & 181.4 & 209.1 & 234.9 & 248.7 & 254.9284 .1 & 301.0 & 337.6 & 355.0\end{array}$

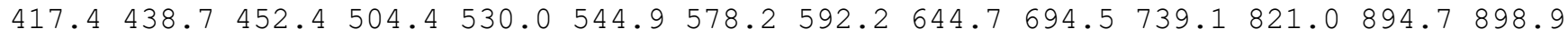

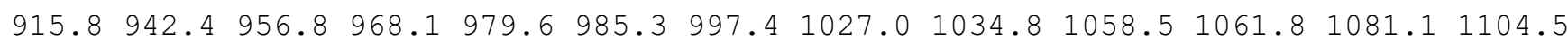

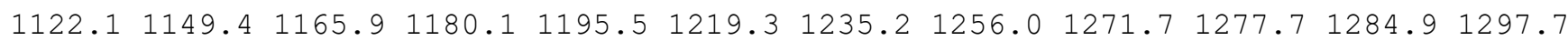
$1298.51302 .8 \quad 1310.0 \quad 1312.2 \quad 1328.3 \quad 1343.8 \quad 1350.1 \quad 1368.5 \quad 1389.0 \quad 1399.5 \quad 1410.3 \quad 1533.5$ $2029.82656 .02670 .5 \quad 2670.92672 .6 \quad 2675.8 \quad 2677.7 \quad 2678.82685 .4 \quad 2687.12691 .2 \quad 2736.0$ $2776.9 \quad 2777.7 \quad 2781.8 \quad 2783.0$

name $=\operatorname{TS} 548$

energy $($ in $\mathrm{kcal} / \mathrm{mol})=101.250219$

$\mathrm{ZPE}(\mathrm{in} \mathrm{kcal} / \mathrm{mol})=141.092$

$\mathrm{G}($ in $\mathrm{kcal} / \mathrm{mol})=117.872$

Geometry

C $-1.92478570-0.41411877-1.27241300$

C $-0.42646688-0.34523483-1.03197501$

C $0.16476809 \quad 1.11868040 \quad-0.93874953$

C $1.09636448 \quad 1.434899590 .24809867$

C $1.34312122 \quad 0.21684481 \quad 1.17041214$

C $1.57461795-0.96866043 \quad 0.30034087$

C $0.07356967-1.14624693 \quad 0.16164517$ 
$\begin{array}{llll}C & -0.06051977 & -0.42313752 & 1.57092728\end{array}$

C $-1.25096038 \quad 0.47347825 \quad 1.81725951$

C $0.08321641-1.414159622 .71770132$

$\mathrm{H}-2.21769338 \quad 0.23606714 \quad-2.11121803$

$\mathrm{H}-2.50166684-0.10631578-0.39440573$

$\mathrm{H}-2.24224071-1.42815680-1.54593079$

$\mathrm{H}-0.63301808 \quad 1.88246916-1.03907828$

$\mathrm{H} \quad 0.70618958 \quad 2.28614154 \quad 0.82639208$

$\mathrm{H} \quad 2.08007938 \quad 1.75206516-0.15676251$

H 2.03105293 $0.42713536 \quad 1.98745773$

H $\quad 0.54754695 \quad-2.43629078 \quad 0.22267779$

H 2.35169288 -1.15725116 -0.36120435

$\mathrm{H}-0.42092826-2.319602610 .14951176$

$\mathrm{H}-1.42327841 \quad 1.20256217 \quad 1.01943259$

$\mathrm{H}-1.09758968 \quad 1.05859738 \quad 2.73628275$

$\mathrm{H}-2.17471240-0.101639691 .94960148$

$\mathrm{H} 0.18488761-0.88366813 \quad 3.67385916$

H $0.99839376-2.01781556 \quad 2.61226744$

$\mathrm{H}-0.77685433-2.08176876 \quad 2.80435506$

O $1.31243606-0.07839666-2.45804932$

$0 \quad 0.878136291 .23259110 \quad-2.17497107$

O $0.15530042-0.88049200-2.22368688$

Vibrational frequencies (in $\mathrm{cm}-1$ )

$\begin{array}{lllllllllllllll}-1745.4 & 88.3 & 107.7 & 153.9 & 160.6 & 198.7 & 212.6 & 219.3 & 260.7 & 283.2 & 317.1 & 347.6 & 367.0 & 410.6\end{array}$ $421.3 \quad 463.0 \quad 483.3 \quad 505.8 \quad 544.4 \quad 599.1 \quad 648.7 \quad 680.7 \quad 701.3 \quad 724.0757 .7 \quad 803.4 \quad 844.1859 .7$

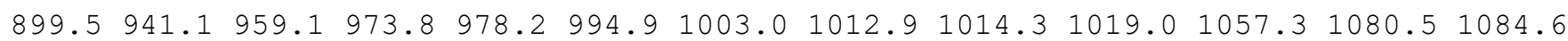

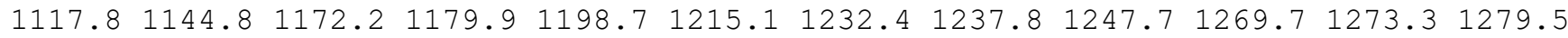

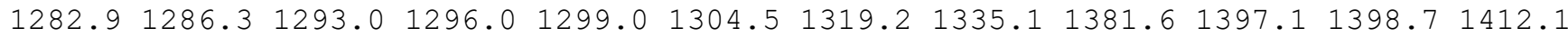
$1844.62662 .2 \quad 2664.3 \quad 2667.0 \quad 2671.3 \quad 2676.12677 .92682 .52685 .4 \quad 2741.12755 .32778 .0$ $2780.12783 .0 \quad 2844.7 \quad 3025.5$

\section{name $=\operatorname{TS} 549$}

energy $($ in $\mathrm{kcal} / \mathrm{mol})=106.342899$

$\mathrm{ZPE}($ in $\mathrm{kcal} / \mathrm{mol})=136.104$

$\mathrm{G}($ in $\mathrm{kcal} / \mathrm{mol})=104.69$

Geometry

C $-1.86069448-0.57192434-1.72220157$

C $-0.59263927-0.07245179-1.13046727$

C $0.22948783 \quad 0.74107460 \quad-2.06581241$

C $0.52628716 \quad 2.38533181 \quad 0.81348233$

C $1.28987477-0.00837495 \quad 2.16342420$

C $1.08765434 \quad 0.14468902 \quad 0.68347302$

C $-0.20469269-0.32296370 \quad 0.12522875$

C $0.13018140 \quad 0.01205205 \quad 3.14893202$

C $0.300461720 .71072402 \quad 4.47017135$

C $0.87622952-1.27695070 \quad 2.88206760$

$\mathrm{H}-1.65945135-1.19935186-2.60779206$

$\mathrm{H}-2.51171259 \quad 0.25226894 \quad-2.04957303$

$\mathrm{H}-2.44407545-1.18607143-1.02231797$

$\mathrm{H} \quad 0.19555424 \quad 1.83419527 \quad-1.93936918$

$\mathrm{H} \quad 0.82142146 \quad 2.59966091 \quad 1.81336677$

$\mathrm{H}-0.51856545 \quad 2.24197629 \quad 0.67477525$

H 2.22998988 $0.46016542 \quad 2.49116844$

$\mathrm{H} \quad 1.92769753-0.373884990 .14907116$

$\mathrm{H} 1.39339010 \quad 1.24781855 \quad 0.27705073$

$\mathrm{H}-0.82590332-0.92229874 \quad 0.79205818$

$\mathrm{H}-0.38927033 \quad 0.30320959 \quad 5.22267064$

$\mathrm{H} \quad 0.09472338 \quad 1.78553093 \quad 4.38172554$

H $1.31522030 \quad 0.60617514 \quad 4.87446723$

$\mathrm{H} 1.52511358-1.69423708 \quad 3.64666770$

$\mathrm{H} \quad 0.39430171-2.07953217 \quad 2.33195732$

$\mathrm{H}-0.88637739 \quad 0.11091674 \quad 2.75127997$

O $3.14691788-1.12176189-2.39083515$

$\begin{array}{lllll}0 & 0.90226479 & 0.23254530 & -2.92861252\end{array}$ 
$02.39448838-1.82081986-1.90974998$

Vibrational frequencies (in $\mathrm{cm}-1$ )

$\begin{array}{llllllllllllllll}-667.9 & 23.8 & 26.0 & 34.2 & 45.7 & 56.2 & 61.1 & 66.3 & 92.9 & 98.7 & 111.0 & 124.4 & 132.1 & 143.8\end{array}$

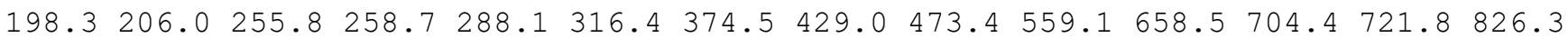
$854.2 \quad 898.7 \quad 922.9 \quad 944.7 \quad 953.9 \quad 972.6 \quad 1010.7 \quad 1024.8 \quad 1036.8 \quad 1048.5 \quad 1074.4 \quad 1083.2 \quad 1095.6$

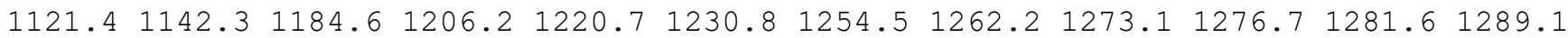

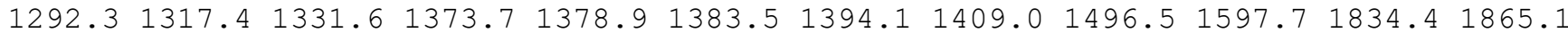
$1920.72641 .92658 .7 \quad 2662.3 \quad 2671.6 \quad 2673.4 \quad 2675.72686 .92693 .82711 .62738 .5 \quad 2743.8$ $2756.7 \quad 2776.02781 .5 \quad 2788.1$

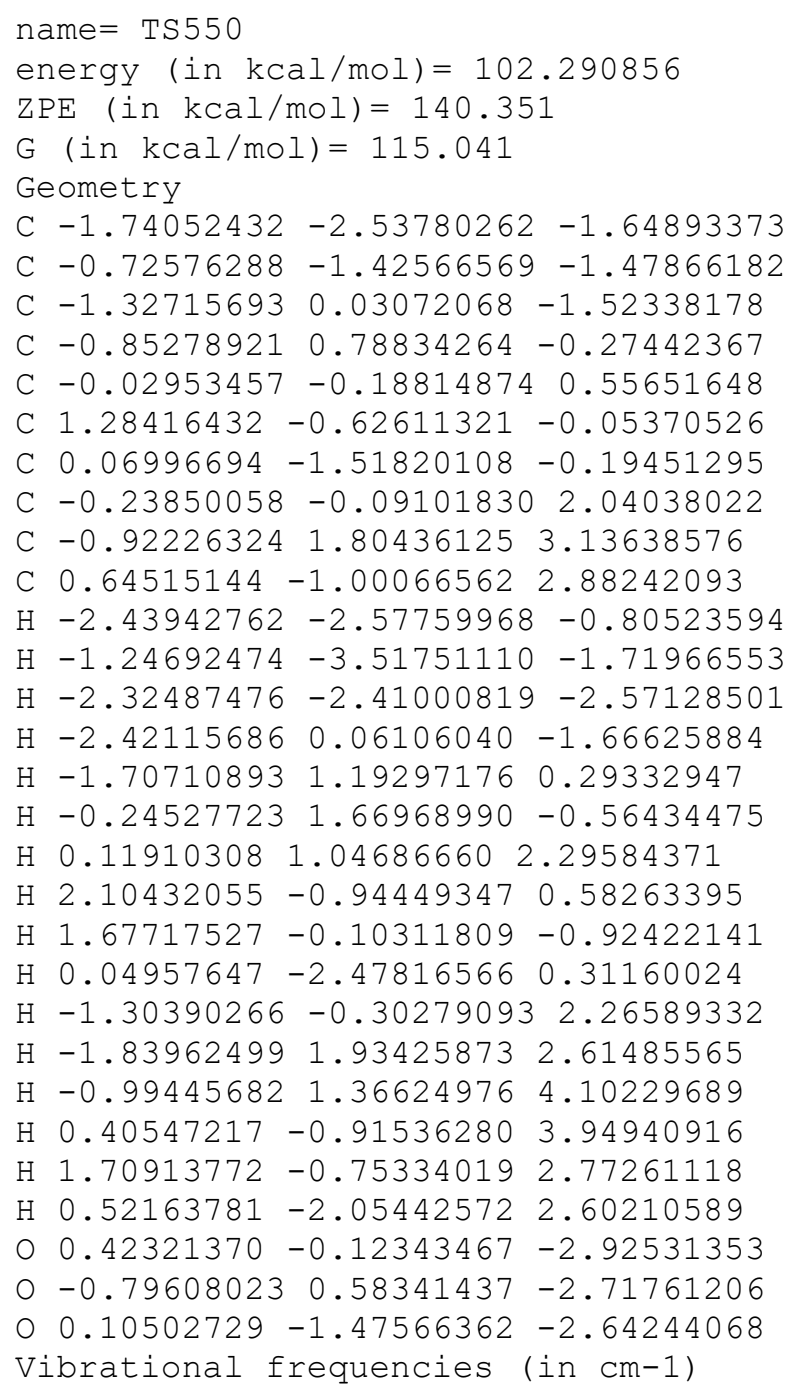


C $1.31610821-1.23397108 \quad 0.69680712$

C $-0.19011291-1.19695878 \quad 0.42614699$

C $-0.31263254-0.38095821 \quad 1.81034089$

C $-1.18761740 \quad 0.83912869 \quad 1.96532194$

C $-0.59673324-1.363459852 .94741393$

$\mathrm{H}-2.25507369 \quad 0.31239415-1.95155301$

$\mathrm{H}-2.62025153 \quad 0.03383772 \quad-0.23366971$

$\mathrm{H}-2.38980839-1.33289216-1.33452469$

$\mathrm{H}-0.69007114 \quad 1.89263529-0.54408684$

$\mathrm{H} 1.552744662 .12342206 \quad 0.55846258$

H $2.26880138 \quad 0.86683119-0.54119461$

$\mathrm{H} \quad 1.88243213 \quad 0.30162982 \quad 2.29228184$

H $1.99170295-2.008618150 .48534720$

$\mathrm{H} 1.97960942-0.07120953-0.23706278$

$\mathrm{H}-0.70663964 \quad-2.16043713 \quad 0.41311775$

$\mathrm{H}-0.74315186 \quad 1.73850164 \quad 1.52304850$

$\mathrm{H}-1.35854828 \quad 1.08006503 \quad 3.02359207$

$\mathrm{H}-2.17428728 \quad 0.68836905 \quad 1.51020105$

$\mathrm{H}-0.38721943-0.918874993 .92600857$

$\mathrm{H} \quad 0.00885791-2.275516312 .86459371$

$\mathrm{H}-1.64899125-1.67143778 \quad 2.93738298$

$0.90127031 \quad 0.06002227-2.54623213$

$0.362820491 .29699580-2.15611956$

O $0.07538650-0.94531262-1.93271220$

Vibrational frequencies (in $\mathrm{cm}-1$ )

$\begin{array}{llllllllllllll}-2120.6 & 53.4 & 113.1 & 130.2 & 140.4 & 165.8 & 183.1 & 189.1 & 254.7 & 268.9 & 286.1 & 312.6 & 340.1373 .0\end{array}$ $410.7 \quad 437.6 \quad 485.4 \quad 501.7 \quad 540.4 \quad 588.7 \quad 604.0 \quad 674.1 \quad 713.9 \quad 716.3 \quad 779.5 \quad 820.2 \quad 858.9 \quad 885.9$ $889.2915 .2 \quad 964.5 \quad 974.2 \quad 982.3 \quad 990.6 \quad 1000.0 \quad 1011.8 \quad 1012.7 \quad 1022.7 \quad 1060.8 \quad 1070.0 \quad 1088.8$ $1100.11109 .3 \quad 1147.1 \quad 1156.8 \quad 1193.2 \quad 1198.0 \quad 1201.8 \quad 1225.2 \quad 1243.4 \quad 1247.3 \quad 1269.51280 .3$

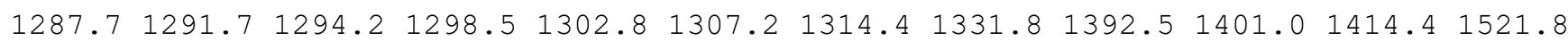
$1728.92610 .12666 .0 \quad 2667.5 \quad 2671.3 \quad 2673.12676 .3 \quad 2679.42702 .0 \quad 2742.3 \quad 2761.0 \quad 2775.4$ 2777.52780 .32780 .92816 .9

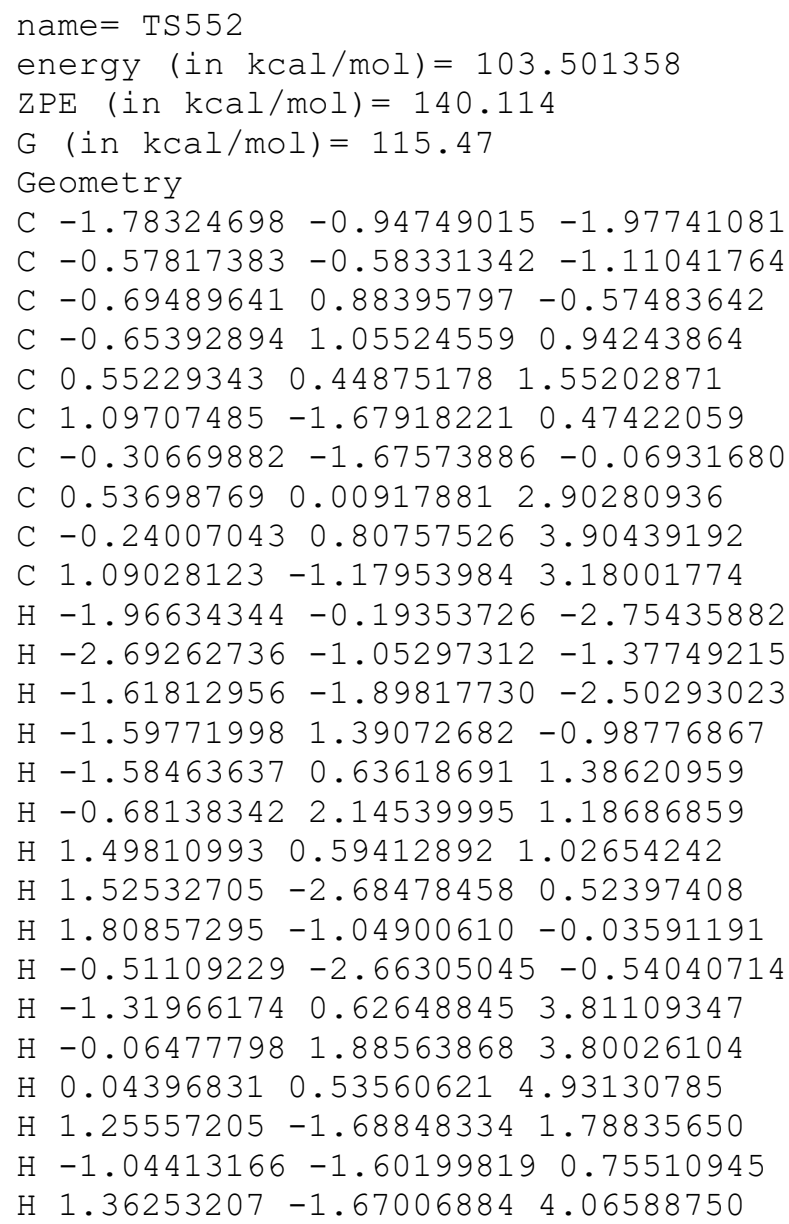


$01.31489614 \quad 0.56515414-1.56072589$

$0.386280751 .58257464-1.18084521$

O $0.51021142-0.48315630-2.04671623$

Vibrational frequencies (in $\mathrm{cm}-1$ )

$\begin{array}{lllllllllllllll}-1655.4 & 44.7 & 86.8 & 98.2 & 114.4 & 159.9 & 166.7 & 191.9 & 224.4 & 250.8 & 271.8 & 287.6 & 310.2 \quad 363.5\end{array}$

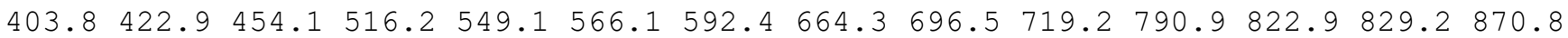

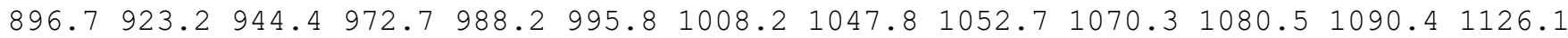

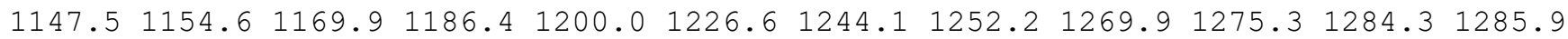
$1290.6 \quad 1292.91295 .2 \quad 1301.2 \quad 1322.1 \quad 1354.3 \quad 1369.0 \quad 1378.2 \quad 1390.8 \quad 1402.7 \quad 1438.8 \quad 1535.3$ $1683.32642 .12650 .52667 .72671 .3 \quad 2673.5 \quad 2673.82675 .62679 .6 \quad 2693.5 \quad 2729.32732 .3$ 2760.12776 .92780 .72786 .3

name $=\operatorname{TS} 553$

energy (in $\mathrm{kcal} / \mathrm{mol})=106.169727$

$\operatorname{ZPE}($ in $\mathrm{kcal} / \mathrm{mol})=138.323$

$\mathrm{G}(\mathrm{in} \mathrm{kcal} / \mathrm{mol})=110.509$

Geometry

C $-1.96276774 \quad 0.07432286 \quad-0.92299838$

C $-0.45547040-0.15544352-0.99156098$

C $0.37052287 \quad 1.05492449-0.44152004$

C $1.53322296 \quad 0.67188934 \quad 0.49027766$

C $1.16215277 \quad 0.42521461 \quad 1.82693923$

C $0.38928693-2.49733555-1.04862641$

C $-0.09468973-1.46446138-0.36290857$

C $0.51232850 \quad 0.38083841 \quad 2.94316339$

C $-1.42460324 \quad 0.59072160 \quad 2.80275505$

C $0.74069397-0.46045276 \quad 4.14869123$

$\mathrm{H}-2.24514514 \quad 1.01355785-1.41707418$

$\mathrm{H}-2.32143331 \quad 0.10489730 \quad 0.11111032$

$\mathrm{H}-2.50406410-0.73223211-1.43746812$

$\mathrm{H}-0.27093531 \quad 1.83868417 \quad 0.00483600$

H 2.31013567 1.48199939 0.45226538

$\mathrm{H} 2.08267463-0.21138473 \quad 0.06580687$

$\mathrm{H}-0.094423651 .418370413 .20049248$

$\mathrm{H} \quad 0.63818678-3.44608137-0.59821056$

$\mathrm{H} \quad 0.57573502-2.47255803-2.11443731$

$\mathrm{H}-0.27240583-1.506900170 .71282496$

$\mathrm{H}-1.663979531 .19567415 \quad 1.94938452$

$\mathrm{H}-1.89478050 \quad 0.90230384 \quad 3.71599369$

$\mathrm{H}-1.56983894-0.45967867 \quad 2.61675885$

$\mathrm{H} \quad 0.19292093-0.12025206 \quad 5.03396954$

H $1.81255501-0.46947771 \quad 4.40600118$

$\mathrm{H} \quad 0.45201625-1.50593984 \quad 3.96408053$

O $1.06118855 \quad 0.56784844-2.51549665$

O $0.89963540 \quad 1.65847364 \quad-1.62120564$

o $-0.15186506-0.15465392-2.39872655$

Vibrational frequencies (in $\mathrm{cm}-1$ )

$\begin{array}{llllllllllllll}-1553.6 & 31.4 & 42.8 & 53.6 & 62.6 & 73.4 & 75.1 & 99.7 & 153.8 & 177.9 & 206.2 & 225.1 & 246.5 & 275.2\end{array}$ $298.6 \quad 319.0 \quad 327.5 \quad 409.6 \quad 430.9 \quad 462.9 \quad 481.1 \quad 541.5 \quad 559.0 \quad 603.6 \quad 681.1 \quad 722.9 \quad 764.1 \quad 825.6$

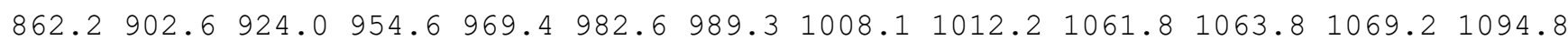

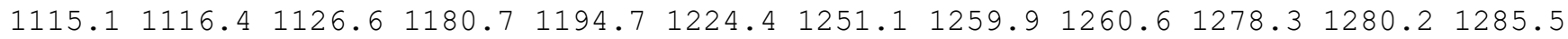
$\begin{array}{llllllllllllll}1285.9 & 1287.7 & 1299.9 & 1301.6 & 1303.4 & 1335.7 & 1373.8 & 1386.1 & 1394.2 & 1395.6 & 1853.2 & 1994.2\end{array}$ $2235.92622 .2 \quad 2668.2 \quad 2670.1 \quad 2675.5 \quad 2678.4 \quad 2692.12694 .02703 .4 \quad 2709.52718 .12748 .9$ $2771.0 \quad 2778.4 \quad 2780.5 \quad 2781.9$

name $=\operatorname{TS} 554$

energy $($ in $\mathrm{kcal} / \mathrm{mol})=104.809353$

$\mathrm{ZPE}($ in $\mathrm{kcal} / \mathrm{mol})=139.876$

$\mathrm{G}($ in $\mathrm{kcal} / \mathrm{mol})=115.598$

Geometry

C $-2.19990962-0.23239808-0.90640210$

C $-0.68221082-0.32590058-0.75533094$

C $-0.05258586 \quad 1.09855917-0.62190984$ 
C $1.12429587 \quad 1.251729210 .34351989$

C $1.03371849 \quad 0.44077299 \quad 1.57367444$

C $1.09590925-1.827737490 .44735944$

C $-0.31208103-1.339269420 .29629316$

C $0.49992316-0.91458879 \quad 1.53534477$

$\begin{array}{llll}\text { C }-0.63542290 & 1.32252591 & 2.88592208\end{array}$

C $0.10702210-1.39081908 \quad 2.84081396$

$\mathrm{H}-2.67946031 \quad 0.05212772 \quad 0.03736490$

$\mathrm{H}-2.62676130-1.19464519-1.22147347$

$\mathrm{H}-2.48194948 \quad 0.49952961-1.67520985$

$\mathrm{H}-0.82493230 \quad 1.87623687 \quad-0.44167448$

$\mathrm{H} \quad 1.25459199 \quad 2.32964644 \quad 0.59125583$

H $2.06787981 \quad 0.98588294 \quad-0.20177823$

$\mathrm{H} \quad 1.71427535 \quad 0.69473270 \quad 2.38372676$

H $1.28151429-2.86858895 \quad 0.71037888$

$\mathrm{H} 1.87700413-1.45009601-0.20814858$

$\mathrm{H}-1.11666416-2.06832816 \quad 0.47827560$

$\mathrm{H}-1.43184075 \quad 1.294662913 .62821966$

$\mathrm{H}-1.05535376 \quad 1.53106392 \quad 1.91870901$

$\mathrm{H} \quad 0.12381830 \quad 2.01892820 \quad 3.18775484$

$\mathrm{H} \quad 0.82243568-1.45570480 \quad 3.62958879$

$\mathrm{H}-0.71776972-2.05583822 \quad 2.94972400$

$\mathrm{H}-0.43866593 \quad 0.10715064 \quad 3.16235603$

$00.84209236 \quad 0.08880032-2.40108825$

O $0.40759273 \quad 1.35987082-1.94826210$

o $-0.20830557-0.79357865-2.03308890$

Vibrational frequencies (in cm-1)

$\begin{array}{lllllllllllllllll}-1249.4 & 35.7 & 82.5 & 106.0 & 148.5 & 177.7 & 193.1 & 242.9 & 251.5 & 264.6 & 305.8 & 328.2 & 338.6 & 371.9\end{array}$

$398.2434 .6 \quad 460.0 \quad 461.1 \quad 539.3 \quad 586.9614 .1 \quad 633.1 \quad 667.4 \quad 715.2 \quad 769.9 \quad 811.7 \quad 816.2 \quad 822.5$

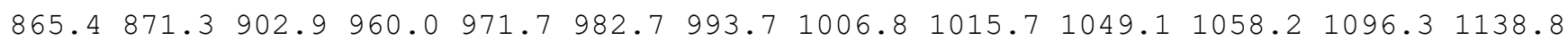
$\begin{array}{lllllllllllll}1146.2 & 1159.4 & 1169.3 & 1178.8 & 1186.8 & 1226.6 & 1250.7 & 1254.2 & 1272.3 & 1274.7 & 1285.2 & 1288.1\end{array}$

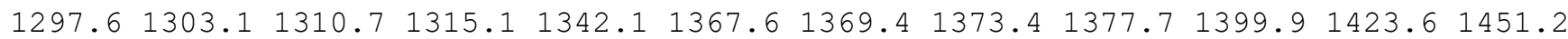

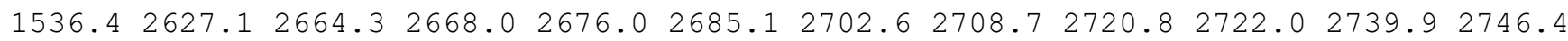
$2773.12778 .12779 .3 \quad 2803.2$

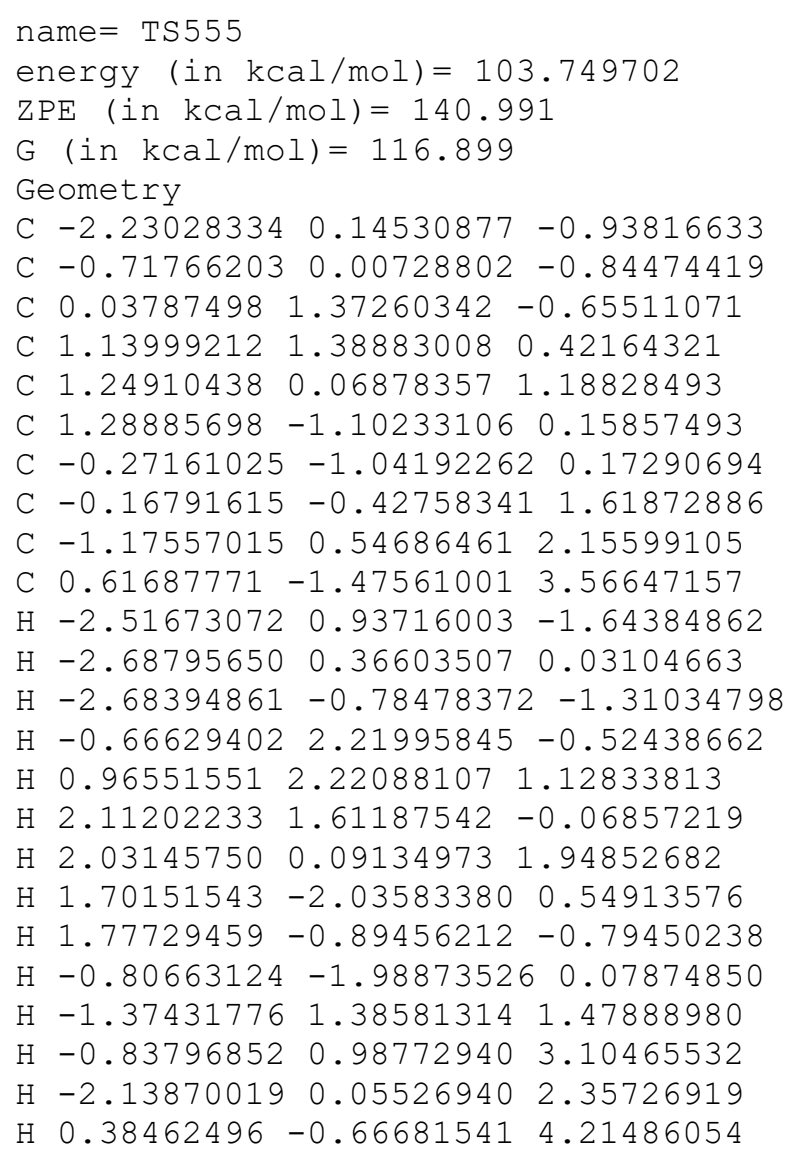


$\mathrm{H} \quad 1.64378747-1.58854864 \quad 3.31519918$

$\mathrm{H}-0.20453142-1.421863542 .32080676$

$0.84547873 \quad 0.32113473-2.47103066$

$0.60562661 \quad 1.61371221-1.94076535$

O $-0.32884914-0.41314235-2.16396745$

Vibrational frequencies (in $\mathrm{cm}-1$ )

$\begin{array}{lllllllllllllllll}-495.9 & 58.9 & 88.7 & 109.9 & 139.4 & 148.8 & 174.7 & 215.2 & 240.0 & 260.2 & 279.4 & 310.4 & 337.7 & 388.3\end{array}$ $432.3 \quad 465.4 \quad 490.7 \quad 512.2 \quad 580.0 \quad 650.6 \quad 697.6 \quad 707.2 \quad 755.6 \quad 772.7 \quad 833.5 \quad 850.7 \quad 871.1 \quad 902.4$ $917.2937 .5 \quad 955.5 \quad 975.6 \quad 997.2 \quad 1006.4 \quad 1042.6 \quad 1047.9 \quad 1064.4 \quad 1077.3 \quad 1092.7 \quad 1100.7 \quad 1128.6$

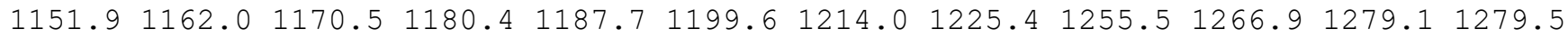

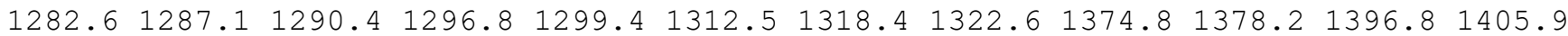
$1823.4 \quad 2656.7 \quad 2668.6 \quad 2669.12671 .12676 .5 \quad 2685.92687 .5 \quad 2687.92692 .8 \quad 2737.4 \quad 2741.3$ $2745.12765 .7 \quad 2779.0 \quad 2781.2$

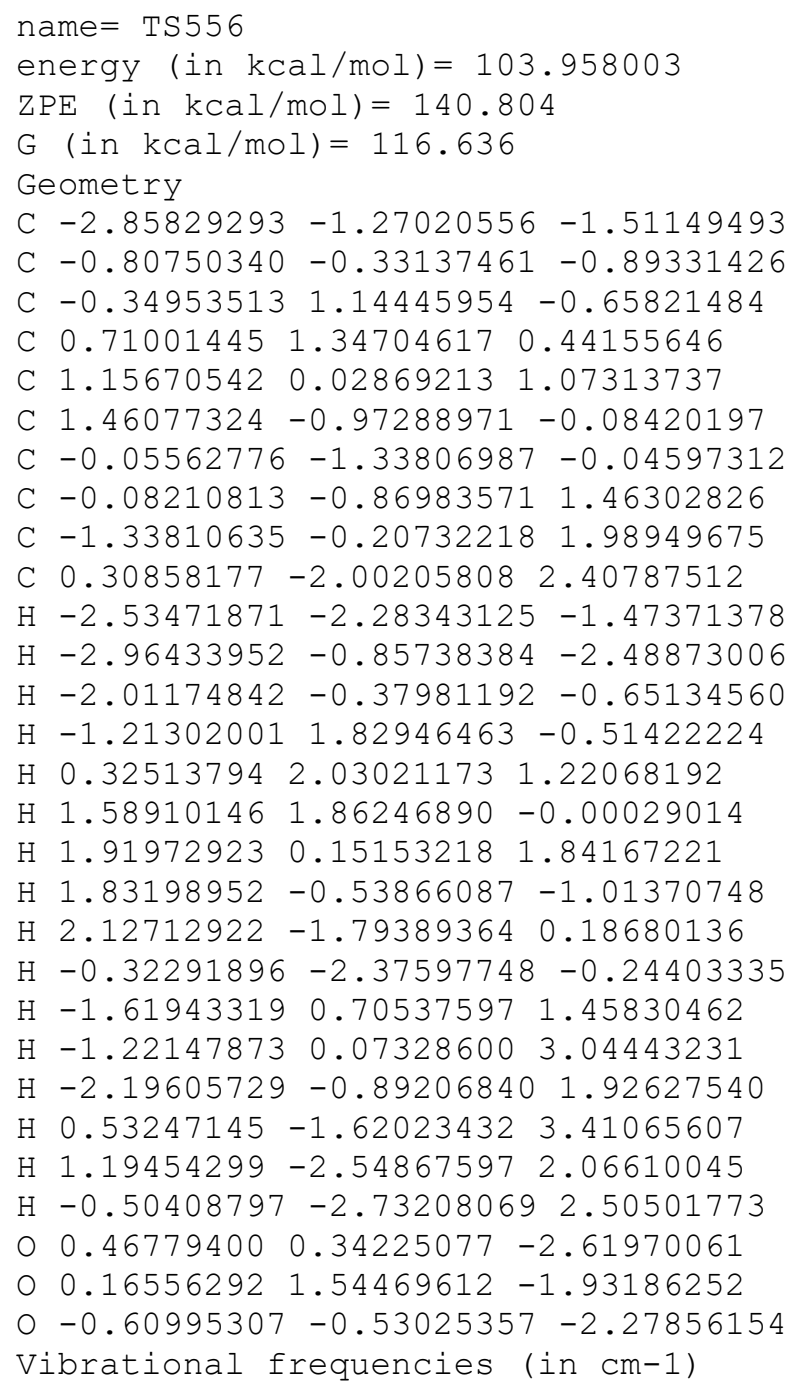
$427.4 \quad 456.2 \quad 466.1 \quad 525.0 \quad 582.7 \quad 650.0 \quad 679.4 \quad 703.1 \quad 717.7 \quad 761.3 \quad 813.8 \quad 861.1886 .2 \quad 895.7$ $921.1939 .1980 .5 \quad 986.1 \quad 992.9 \quad 1005.2 \quad 1015.0 \quad 1017.8 \quad 1043.3 \quad 1077.0 \quad 1086.3 \quad 1095.6 \quad 1103.6$

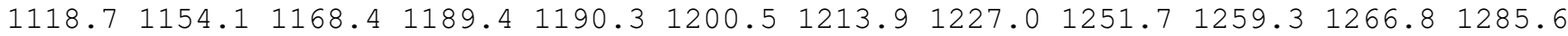

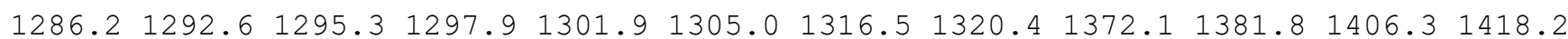
$1800.72657 .62672 .42673 .7 \quad 2676.8 \quad 2679.3 \quad 2680.0 \quad 2684.62689 .3 \quad 2692.2 \quad 2739.12747 .7$ $2751.22767 .22781 .7 \quad 2784.3$

name $=$ TS557

energy (in $\mathrm{kcal} / \mathrm{mol})=103.594963$

$\operatorname{ZPE}($ in $\mathrm{kcal} / \mathrm{mol})=141.336$

$\mathrm{G}(\mathrm{in} \mathrm{kcal} / \mathrm{mol})=117.869$

Geometry

C $-2.24989668-0.26810311-0.75600474$ 
C $-0.74829208-0.20564049-0.48882472$

C $-0.26269710 \quad 1.25487005-0.21791569$

C $0.769495291 .41004667 \quad 0.90448444$

C $0.85182933 \quad 0.27417188 \quad 1.83803376$

C $1.18729427-1.57025496 \quad 0.49556008$

C $-0.31041598-1.22597658 \quad 0.55573043$

C $-0.29131139-0.67143054 \quad 2.02622142$

C $-1.55431259-0.04754924 \quad 2.58595484$

C $0.23378982-1.810926892 .83151470$

$\mathrm{H}-2.55746862 \quad 0.48490449-1.49372757$

$\mathrm{H}-2.83218719-0.117090340 .15863079$

$\mathrm{H}-2.53297697-1.24553271-1.17158179$

$\mathrm{H}-1.10876898 \quad 1.96184348-0.09988251$

$\mathrm{H} \quad 0.56909819 \quad 2.34838537 \quad 1.47360749$

H $1.77126696 \quad 1.589203210 .43690255$

H $1.67622771 \quad 0.26068379 \quad 2.53788472$

H $1.41748702-2.58186101 \quad 0.17196515$

$\mathrm{H} \quad 1.83186346-0.87895551-0.02124116$

$\mathrm{H}-0.93921148-2.13522542 \quad 0.48242285$

$\mathrm{H}-2.37800825-0.77408516 \quad 2.57314336$

$\mathrm{H}-1.86977881 \quad 0.83608286 \quad 2.02234268$

$\mathrm{H}-1.411572450 .25310915 \quad 3.63262896$

$\mathrm{H} \quad 0.74570015-1.62166700 \quad 3.74600205$

$\mathrm{H} 1.40276021-1.94390347 \quad 1.76370428$

$\mathrm{H}-0.28117561-2.74382188 \quad 2.80658476$

$\begin{array}{lllll}0 & 0.88230071 & 0.41844272 & -1.94852935\end{array}$

$0 \quad 0.33458711 \quad 1.63361616-1.45684981$

o $-0.15158201-0.53173382-1.75847098$

Vibrational frequencies (in $\mathrm{cm}-1$ )

$\begin{array}{llllllllllllll}-1724.1 & 58.3 & 123.5 & 144.7 & 154.2 & 173.4 & 221.7 & 235.6 & 241.2 & 284.7 & 299.7 & 330.9 & 374.3 & 415.1\end{array}$ $418.0 \quad 464.2 \quad 474.9 \quad 519.7 \quad 560.2 \quad 589.4 \quad 634.9 \quad 665.1 \quad 673.6 \quad 709.9 \quad 789.0 \quad 828.5 \quad 865.7 \quad 895.1$ $913.8 \quad 959.8 \quad 971.1 \quad 977.7 \quad 1000.0 \quad 1006.4 \quad 1008.0 \quad 1024.2 \quad 1054.1 \quad 1065.5 \quad 1095.6 \quad 1105.1 \quad 1127.1$ $1158.8 \quad 1163.3 \quad 1174.4 \quad 1190.3 \quad 1208.6 \quad 1220.4 \quad 1243.7 \quad 1251.91257 .8 \quad 1265.2 \quad 1273.6 \quad 1283.5$ $1285.3 \quad 1292.8 \quad 1295.9 \quad 1300.1 \quad 1338.9 \quad 1344.0 \quad 1357.2 \quad 1371.4 \quad 1378.2 \quad 1390.4 \quad 1401.8 \quad 1412.5$ $1852.22628 .5 \quad 2668.92674 .12676 .4 \quad 2677.2 \quad 2678.42689 .42715 .52718 .32724 .52740 .9$ $2755.22779 .3 \quad 2780.2 \quad 2793.1$

name $=\operatorname{TS} 558$

energy (in $\mathrm{kcal} / \mathrm{mol})=105.069216$

$\mathrm{ZPE}(\mathrm{in} \mathrm{kcal} / \mathrm{mol})=140.705$

$\mathrm{G}(\mathrm{in} \mathrm{kcal} / \mathrm{mol})=116.356$

Geometry

C $-1.44022079-1.02675412-2.61971904$

C $-1.18912595-0.17461282-0.46443818$

C $-2.149186890 .09463556 \quad 0.74706467$

C $-1.66834770-0.39802384 \quad 2.12193061$

C $-0.22375694-0.896767992 .06714438$

C $0.60489608 \quad 0.12353272 \quad 1.22549212$

C $0.17002373-0.69156395-0.03265255$

C $-0.02737806-1.94769914 \quad 0.90398526$

C $-1.15296482-2.90858867 \quad 0.58384944$

C $1.26815507-2.73649179 \quad 1.06158771$

$\mathrm{H}-1.73204615-0.13018480-3.11446439$

$\mathrm{H}-1.79537234-0.96462427-1.18548559$

$\mathrm{H}-0.42928305-1.32351108-2.76344246$

$\mathrm{H}-3.18037092-0.23922053 \quad 0.52009935$

$\mathrm{H}-2.33623615-1.19287998 \quad 2.50176570$

$\mathrm{H}-1.74573790 \quad 0.43413172 \quad 2.85477790$

H $0.17459917-1.18171323 \quad 3.04094016$

$\mathrm{H} \quad 1.677936090 .11302973 \quad 1.42210755$

$\mathrm{H} \quad 0.26898033 \quad 1.16385531 \quad 1.25578229$

$\mathrm{H} \quad 0.89586316-0.78591900-0.83962562$

$\mathrm{H}-1.18928346-3.72989386 \quad 1.31090839$

$\mathrm{H}-1.01235265-3.35718124-0.41060710$ 
$\mathrm{H}-2.14556813-2.449997210 .58380992$

H $1.49444306-3.308605440 .15363479$

H $1.19847894-3.448492121 .89219538$

$\mathrm{H} 2.13133332-2.091449791 .26101687$

$0-2.033663691 .93100050-0.52170586$

$\begin{array}{lllll}0 & -2.13801647 & 1.52846310 & 0.81988830\end{array}$

o $-1.016343361 .08529050-1.08628189$

Vibrational frequencies (in cm-1)

$\begin{array}{lllllllllllllllll}-614.4 & 39.1 & 80.2 & 112.5 & 146.8 & 153.7 & 177.4 & 200.8 & 225.1 & 246.8 & 288.8 & 302.8 & 348.8 & 395.4\end{array}$

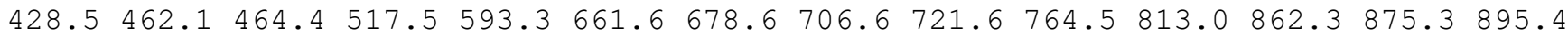
$918.0 \quad 936.3 \quad 982.7 \quad 989.0 \quad 1004.9 \quad 1008.5 \quad 1012.6 \quad 1016.6 \quad 1059.0 \quad 1077.4 \quad 1085.1 \quad 1093.91101 .2$

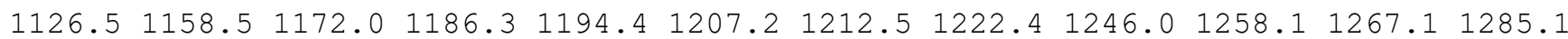
$1287.0 \quad 1290.4 \quad 1296.2 \quad 1297.6 \quad 1302.9 \quad 1309.9 \quad 1315.8 \quad 1323.4 \quad 1370.8 \quad 1381.5 \quad 1405.8 \quad 1417.0$ $1769.52656 .12670 .7 \quad 2674.02676 .7 \quad 2679.4 \quad 2681.92686 .22688 .4 \quad 2693.72737 .32747 .6$ $2751.0 \quad 2764.0 \quad 2781.5 \quad 2783.7$

name $=$ TS559

energy $($ in $\mathrm{kcal} / \mathrm{mol})=105.071844$

$\mathrm{ZPE}($ in $\mathrm{kcal} / \mathrm{mol})=140.898$

$\mathrm{G}($ in $\mathrm{kcal} / \mathrm{mol})=116.887$

Geometry

C $-2.15316414-0.59793236-0.90102415$

C $-0.67018679-0.30652995-0.74733261$

C $-0.27563159 \quad 1.21155163 \quad-0.69744520$

C $0.788854991 .40587513 \quad 0.39201432$

C $0.91117819 \quad 0.13514732 \quad 1.20907364$

C $2.09233815-1.155937320 .09112993$

C $-0.02473974-0.99043760 \quad 0.43978388$

C $-0.34024060-0.44897708 \quad 1.83161985$

C $-1.541882460 .40744422 \quad 2.13492376$

C $-0.09680754-1.44605608 \quad 2.95036393$

$\mathrm{H}-2.57577841-0.05442396-1.75862066$

$\mathrm{H}-2.72850503-0.33081513-0.00958289$

$\mathrm{H}-2.32274868-1.66822390-1.08942168$

$\mathrm{H}-1.133227621 .90181396-0.61235776$

$\mathrm{H} \quad 0.55850097 \quad 2.27071959 \quad 1.03793495$

$\mathrm{H} \quad 1.77026098 \quad 1.63041702-0.08680895$

$\mathrm{H} \quad 1.82847782 \quad 0.05850560 \quad 1.81005113$

H $2.14984349-2.12678137 \quad 0.49743231$

H 2.02166294 $-1.01123558-0.94630397$

H $0.05162285-2.073136820 .34852228$

$\mathrm{H}-1.743763351 .167869421 .37403472$

$\mathrm{H}-1.40006824 \quad 0.94519882 \quad 3.08417704$

$\mathrm{H}-2.44893912-0.20350760 \quad 2.24304086$

$\mathrm{H} 0.02599509-0.93697313 \quad 3.91482237$

$\mathrm{H} \quad 0.80746934-2.04752013 \quad 2.78954248$

$\mathrm{H}-0.93903784-2.14266053 \quad 3.04838059$

$0.83790970 \quad 0.23361509-2.37274450$

$0.241357561 .46223196-1.99636027$

$0-0.10336716-0.76231000-1.98372085$

Vibrational frequencies (in $\mathrm{cm}-1$ )

$\begin{array}{llllllllllllllllll}-625.6 & 82.3 & 104.9 & 134.6 & 142.6 & 160.9 & 191.0 & 193.9 & 211.8 & 239.1 & 270.9 & 275.1 & 285.0 & 331.2\end{array}$

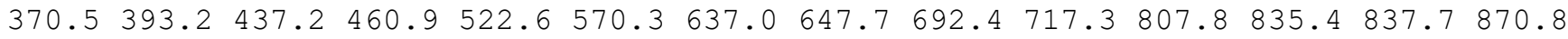
$885.6934 .7974 .8977 .6 \quad 997.1 \quad 1008.7 \quad 1011.7 \quad 1017.9 \quad 1036.2 \quad 1052.5 \quad 1063.2 \quad 1085.3 \quad 1110.5$

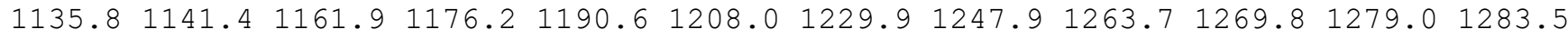
$1284.3 \quad 1292.1 \quad 1293.7 \quad 1296.3 \quad 1297.8 \quad 1304.7 \quad 1309.3 \quad 1346.0 \quad 1389.2 \quad 1398.2 \quad 1404.8 \quad 1444.8$ $2655.52668 .22669 .2 \quad 2670.7 \quad 2674.6 \quad 2676.12677 .22697 .72698 .32701 .62727 .32732 .3$ $\begin{array}{lllll}2740.1 & 2779.1 & 2780.7 & 2782.3\end{array}$

name $=\operatorname{TS} 560$

energy $($ in $\mathrm{kcal} / \mathrm{mol})=104.919231$

$\mathrm{ZPE}(\mathrm{in} \mathrm{kcal} / \mathrm{mol})=141.534$

$\mathrm{G}($ in $\mathrm{kcal} / \mathrm{mol})=117.935$ 


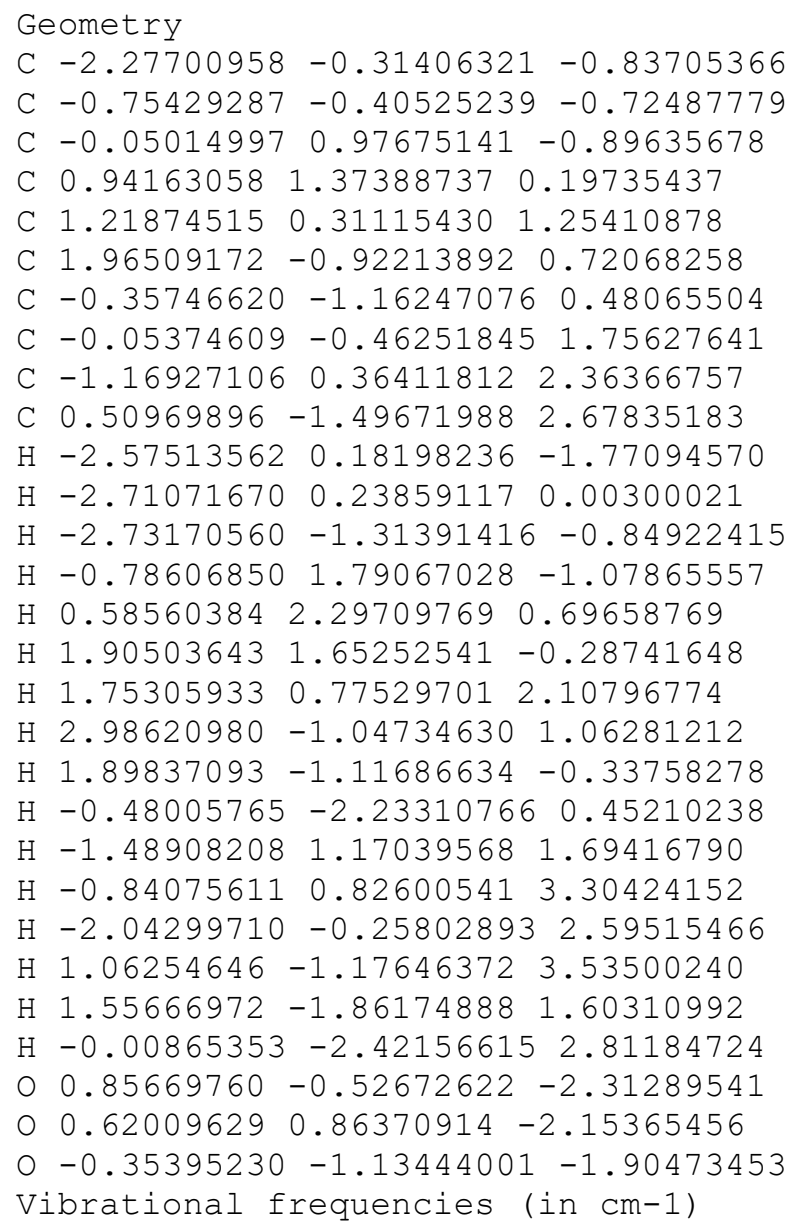

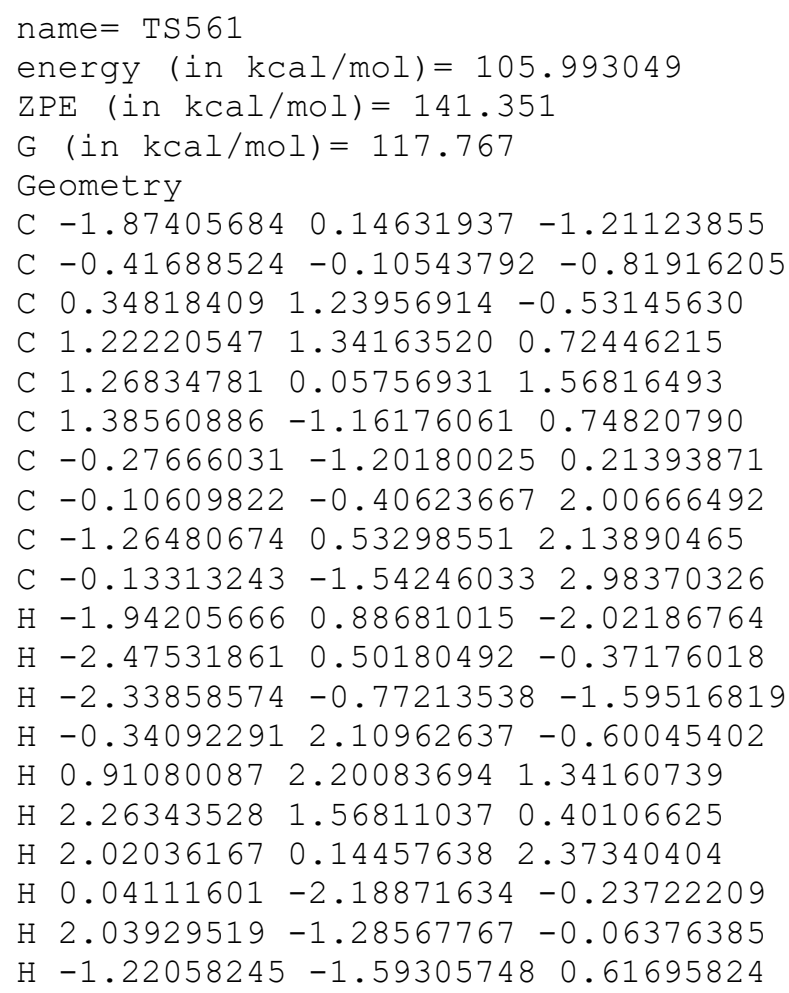


$\mathrm{H}-2.232034830 .017326252 .20600293$

$\mathrm{H}-1.34417827 \quad 1.266876321 .32670298$

$\mathrm{H}-1.14883837 \quad 1.11952221 \quad 3.06777326$

$\mathrm{H} \quad 0.20279317-1.21007238 \quad 3.97638608$

$\mathrm{H} \quad 0.58792212-2.33415616 \quad 2.67202792$

$\mathrm{H}-1.11432324-2.010959193 .09564177$

O $1.445803820 .07310779-2.10926525$

O $1.170059751 .39736018-1.69186851$

O $0.18660868-0.57126791-2.04401156$

Vibrational frequencies (in $\mathrm{cm}-1$ )

$\begin{array}{llllllllllllllllll}-732.9 & 72.4 & 107.9 & 155.1 & 172.0 & 184.9 & 198.6 & 208.5 & 239.0 & 281.1 & 292.9 & 313.0 & 334.5 & 367.3\end{array}$

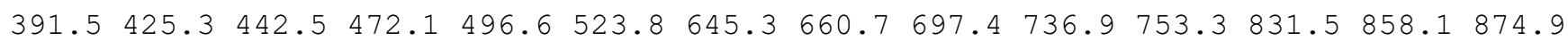
$955.4957 .7 \quad 975.6 \quad 981.2 \quad 994.1 \quad 1003.2 \quad 1012.8 \quad 1014.5 \quad 1033.5 \quad 1069.0 \quad 1075.8 \quad 1122.2 \quad 1132.5$

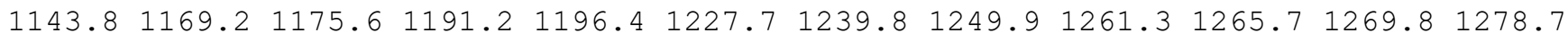

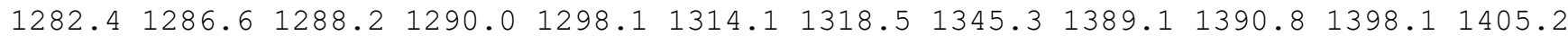
$2554.12624 .2 \quad 2656.3 \quad 2656.8 \quad 2666.0 \quad 2667.8 \quad 2677.3 \quad 2679.4 \quad 2680.8 \quad 2702.92732 .0 \quad 2739.4$ $2769.8 \quad 2772.4 \quad 2777.5 \quad 2780.0$

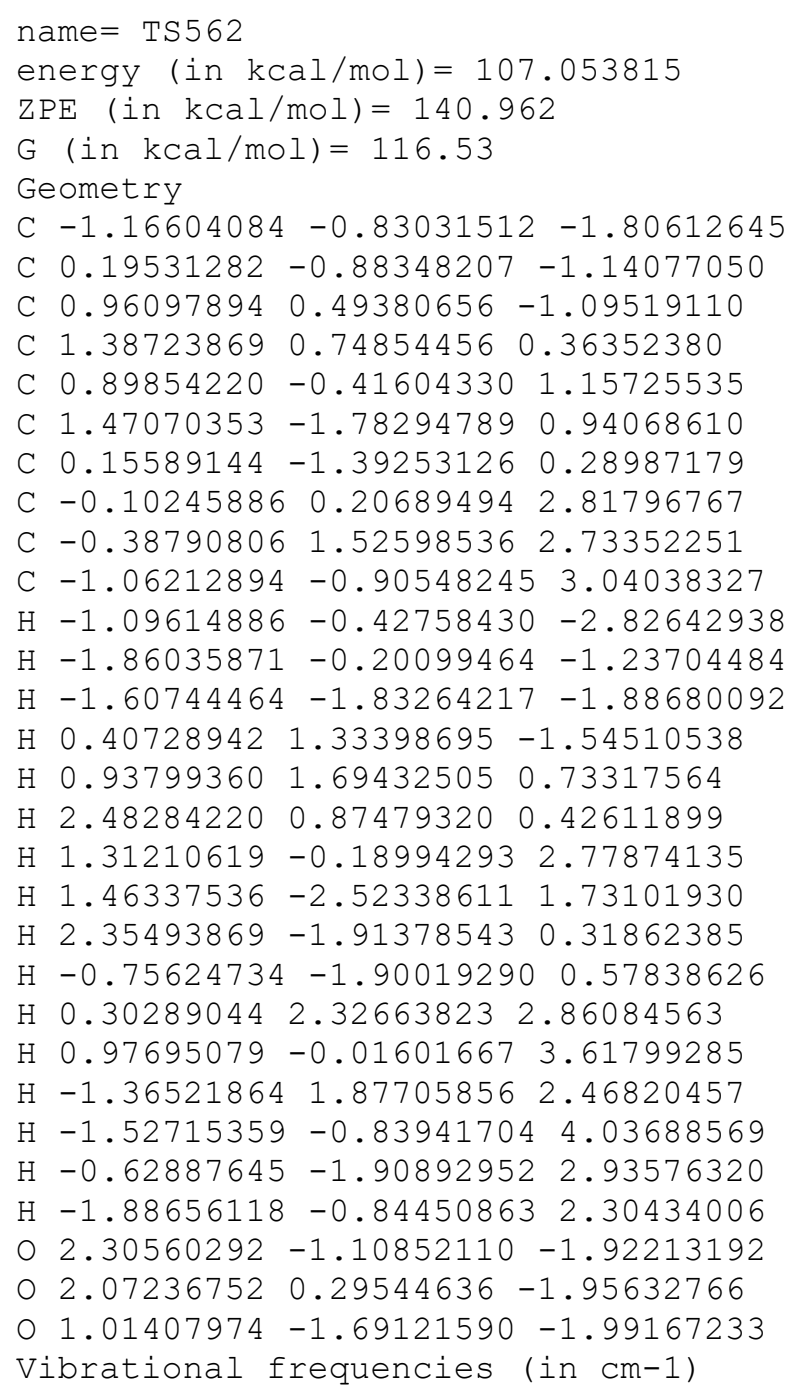


$\mathrm{ZPE}($ in $\mathrm{kcal} / \mathrm{mol})=143.064$

$\mathrm{G}($ in $\mathrm{kcal} / \mathrm{mol})=119.342$

Geometry

C $-2.62391740 \quad 1.60861107 \quad 0.31145068$

C $-1.23560674 \quad 1.03782661 \quad 0.05337372$

C $-0.08093970 \quad 2.00790182 \quad 0.45247369$

C $1.11747046 \quad 1.37462463 \quad 1.17610242$

C $1.19709068-0.13662480 \quad 1.16022753$

C $0.36189283-0.84532297 \quad 0.12217934$

C $-1.01396931-0.37859336 \quad 0.62081086$

C $-0.80520833-0.459604432 .08801068$

C $-1.39423706 \quad 0.50503537 \quad 3.02689689$

C $0.81333334-1.02807484 \quad 2.80735292$

$\mathrm{H}-2.81525401 \quad 2.47381774-0.34210267$

$\mathrm{H}-2.75708322 \quad 1.93879334 \quad 1.34523178$

$\mathrm{H}-3.40401427 \quad 0.86741942 \quad 0.09402606$

$\mathrm{H}-0.45239342 \quad 2.89792134 \quad 1.00321681$

$\mathrm{H} 1.15023114 \quad 1.73636831 \quad 2.22450670$

H 2.04158461 1.787413320 .70477839

$\mathrm{H} 2.24269461-0.47972721 \quad 1.19714225$

$\mathrm{H} \quad 0.46262037-1.94325071 \quad 0.16601399$

H $0.59725215-0.56287806-0.91628187$

$\mathrm{H}-1.85011247-1.04384484 \quad 0.31448085$

$\mathrm{H}-0.97725102 \quad 1.51886388 \quad 2.97112795$

$\mathrm{H}-1.29251260 \quad 0.17458668 \quad 4.07300445$

$\mathrm{H}-2.48002138 \quad 0.57966402 \quad 2.84225378$

$\mathrm{H} 1.61639140-0.449815573 .26711552$

H $1.10336129-2.012796992 .44931118$

H $0.17894158-1.246578313 .68592121$

O $0.113775331 .50799549-1.72966858$

O $0.34249045 \quad 2.55053769-0.79721286$

$\begin{array}{llll}0 & -1.16209361 & 0.99062493 & -1.38564317\end{array}$

Vibrational frequencies (in $\mathrm{cm}-1$ )

$\begin{array}{lllllllllllllll}-932.8 & 71.4 & 94.9 & 121.3 & 140.6 & 156.0 & 187.4 & 216.5 & 257.7 & 271.5 & 303.6 & 322.9 & 362.0 & 379.7\end{array}$ $414.6 \quad 436.0 \quad 480.1 \quad 519.6 \quad 532.0 \quad 555.1 \quad 653.9 \quad 707.6 \quad 745.0 \quad 843.6 \quad 875.8 \quad 889.9 \quad 911.5 \quad 937.1$ $952.5 \quad 969.6 \quad 973.6 \quad 987.9 \quad 1006.4 \quad 1011.7 \quad 1039.7 \quad 1070.0 \quad 1081.91092 .5 \quad 1110.91147 .2 \quad 1148.1$

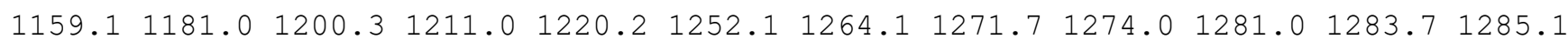

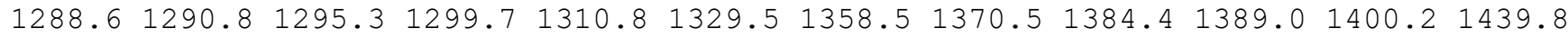
$2560.02641 .6 \quad 2656.42661 .12662 .1 \quad 2667.7 \quad 2670.4 \quad 2674.72684 .92695 .42717 .12726 .1$ $2738.6 \quad 2754.0 \quad 2765.7 \quad 2779.3$

\section{name $=\operatorname{TS} 564$}

energy (in $\mathrm{kcal} / \mathrm{mol})=109.720164$

$\mathrm{ZPE}($ in $\mathrm{kcal} / \mathrm{mol})=138.592$

$\mathrm{G}(\mathrm{in} \mathrm{kcal} / \mathrm{mol})=111.3$

Geometry

C $-2.10262841 \quad 1.01521745 \quad-0.25721264$

C $-1.21877177-0.15518112-0.65557171$

C $0.04084113 \quad 0.33299116-1.45745499$

C $1.36128434-0.38516445-1.17341884$

C $2.13317457 \quad 0.25940919-0.07166970$

C $2.80054838-0.437696590 .84769753$

C $-0.92315293-1.127868850 .43985925$

C $-0.93795519-0.85341050 \quad 1.74556387$

C $-0.20189450 \quad 1.07355504 \quad 2.46289016$

C $-0.63306439-1.850959472 .79801655$

$\mathrm{H}-2.39160480 \quad 1.61135929-1.13355270$

$\mathrm{H}-1.61050191 \quad 1.69085036 \quad 0.45842039$

$\mathrm{H}-3.03442749 \quad 0.66791964 \quad 0.20905432$

H $0.146652991 .43543003-1.39556691$

$\mathrm{H} \quad 1.98159748-0.37088765-2.10439224$

H $1.18694984-1.46441235-0.98687897$

$\mathrm{H} \quad 2.14074974 \quad 1.35020762 \quad-0.08429841$

H $2.82459444-1.515319120 .88827325$ 
H $3.38373782 \quad 0.01921845 \quad 1.63188923$

$\mathrm{H}-0.70609857-2.13980337 \quad 0.07705602$

$\begin{array}{lllll}\mathrm{H} & 0.23628045 & 0.72563961 & 3.36492087\end{array}$

$\mathrm{H}-1.41612690 \quad 0.17412588 \quad 2.12783795$

$\mathrm{H} \quad 0.45828936 \quad 1.22060228 \quad 1.64487059$

$\mathrm{H} \quad 0.43641782-1.83894326 \quad 3.06361697$

$\mathrm{H}-0.86956211-2.88017172 \quad 2.48660250$

$\mathrm{H}-1.19607913-1.662490293 .72456676$

$\begin{array}{lllll}0 & -1.65872770 & -0.25522880 & -2.85003292\end{array}$

O $-0.257075210 .00116423-2.82033879$

o $-1.91779026-0.93904070-1.64793257$

Vibrational frequencies (in $\mathrm{cm}-1$ )

$\begin{array}{lllllllllllllll}-710.2 & 22.9 & 41.5 & 61.1 & 74.2 & 89.2 & 118.3 & 131.9 & 176.7 & 201.3 & 210.7 & 231.2 & 253.4 & 278.3\end{array}$ $\begin{array}{lllllllllllllll}292.1 & 323.6 & 355.8 & 367.9 & 412.7 & 431.0 & 472.9 & 544.3 & 594.0 & 694.8 & 710.1 & 749.7 & 768.4 & 829.0\end{array}$

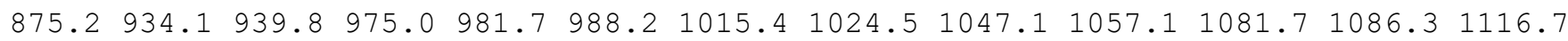

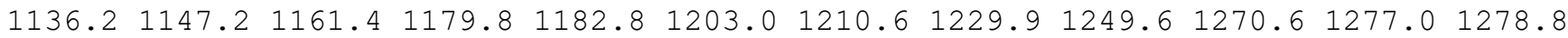
$1285.41289 .01302 .4 \quad 1326.3 \quad 1340.6 \quad 1352.3 \quad 1357.7 \quad 1388.51391 .2 \quad 1402.6 \quad 1848.31859 .6$ $2073.92643 .32655 .4 \quad 2662.8 \quad 2668.2 \quad 2670.7 \quad 2677.02696 .32698 .02714 .62728 .92731 .5$ $2755.32775 .3 \quad 2775.92788 .9$

name $=\operatorname{TS} 565$

energy $($ in $\mathrm{kcal} / \mathrm{mol})=109.12512$

$\operatorname{ZPE}(\mathrm{in} \mathrm{kcal} / \mathrm{mol})=139.245$

$\mathrm{G}($ in $\mathrm{kcal} / \mathrm{mol})=114.484$

Geometry

C $-2.609943670 .37760096-1.23393212$

C $-1.15014663-0.03352724-1.11668779$

C $-0.15972214 \quad 1.18438966-1.07063585$

C $0.33988216 \quad 1.34514460 \quad 0.29124432$

C $0.84172827 \quad 0.21663418 \quad 1.09270816$

C $2.08177026-0.468806890 .50542135$

C $-1.04334934-1.07575043-0.06369618$

C $-0.37280454-0.76792106 \quad 1.21344960$

C $-1.47957261-0.11227910 \quad 2.07405354$

C $0.05609869-2.088458121 .86624162$

$\mathrm{H}-2.79092934 \quad 0.91314513 \quad-2.17748519$

$\mathrm{H}-2.91028214 \quad 1.03545094 \quad-0.40932874$

$\mathrm{H}-3.27404498-0.50045812-1.23180532$

$\mathrm{H}-0.62411201 \quad 2.11081283-1.47794731$

H $1.24279123 \quad 2.60855660 \quad 0.42296445$

H $1.75665960 \quad 2.18754964 \quad-0.04535917$

H $1.09280094 \quad 0.56466953 \quad 2.12720914$

H $2.47077814 \quad-1.24563702 \quad 1.17247679$

H $2.89378656 \quad 0.245871550 .33734616$

$\mathrm{H} 1.86146946-0.94195223-0.45988428$

$\mathrm{H}-2.40954293-0.69123478 \quad 2.05323974$

$\mathrm{H}-1.69240438 \quad 0.91241489 \quad 1.73887124$

$\mathrm{H}-1.15706890-0.04573048 \quad 3.11930160$

$\mathrm{H} \quad 0.59900641-1.91988752 \quad 2.80062670$

$\mathrm{H} \quad 0.69647692 \quad-2.67742158 \quad 1.19814590$

$\mathrm{H}-0.81870690 \quad-2.71346258 \quad 2.08888279$

$0.60103456-0.49117818-2.43353628$

$0.84410504 \quad 0.86820277-2.04394493$

O $-0.79420179-0.63752546-2.37686565$

Vibrational frequencies (in $\mathrm{cm}-1$ )

$\begin{array}{llllllllllllllll}-876.8 & 56.5 & 75.1 & 123.4 & 131.7 & 174.0 & 178.7 & 188.0 & 199.4 & 211.8 & 236.3 & 251.4 & 295.1 & 324.2\end{array}$

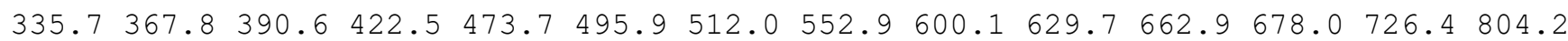
$838.2 \quad 862.4 \quad 915.5 \quad 951.6 \quad 960.7 \quad 973.4 \quad 996.1 \quad 1000.4 \quad 1019.3 \quad 1048.7 \quad 1061.91069 .2 \quad 1075.0$ $1107.51126 .61141 .1 \quad 1152.8 \quad 1180.0 \quad 1198.8 \quad 1220.2 \quad 1230.6 \quad 1247.2 \quad 1276.5 \quad 1283.3 \quad 1288.3$

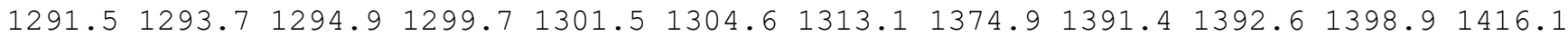
$1425.32663 .32664 .3 \quad 2669.2 \quad 2671.5 \quad 2671.8 \quad 2674.62675 .22682 .2 \quad 2684.92702 .92773 .8$ $2774.92780 .3 \quad 2780.6 \quad 4712.2$ 


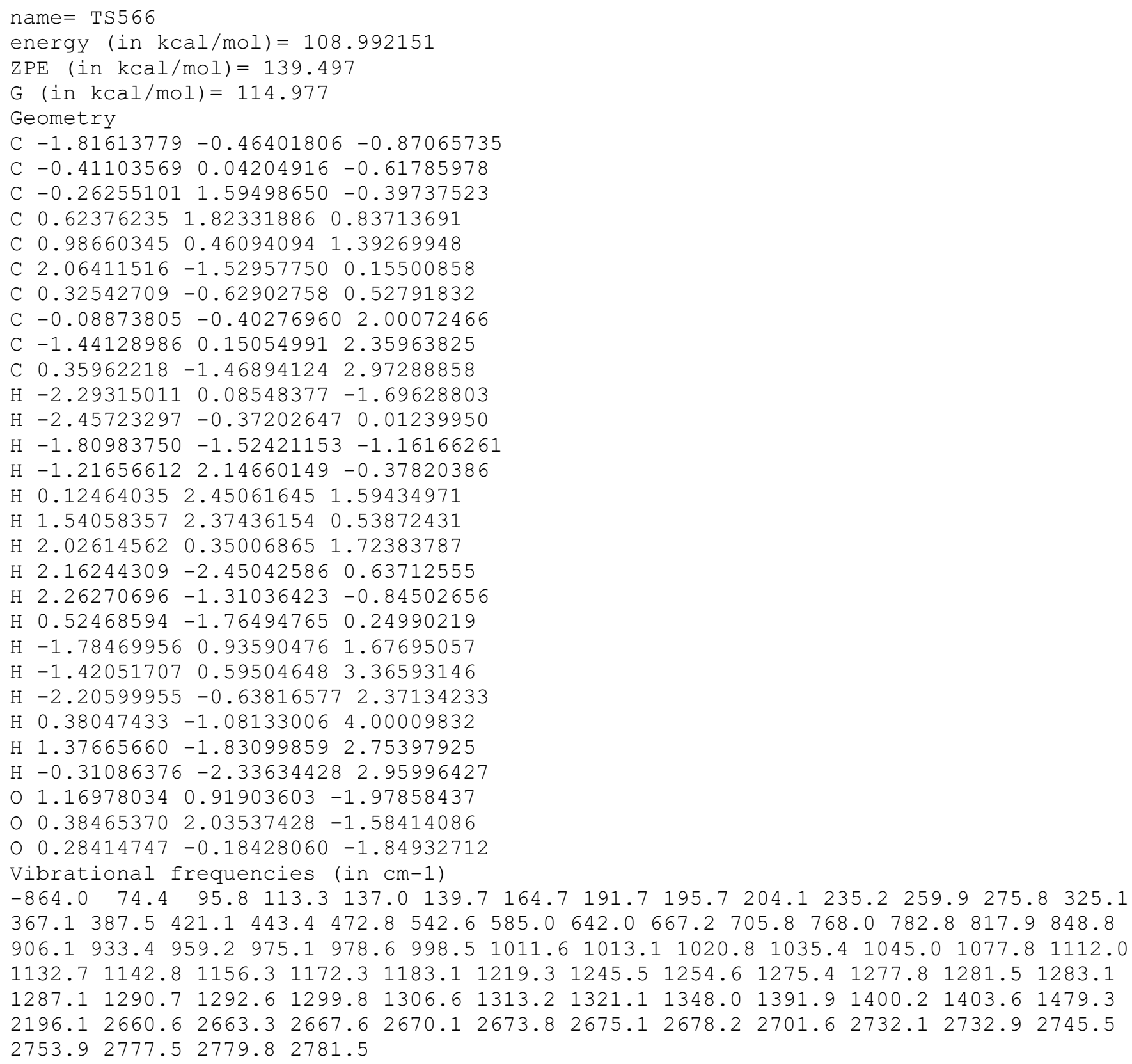


H $1.24970781 \quad 1.80506016 \quad 1.13929948$

$\mathrm{H} 2.13245830-1.15678228 \quad 1.71630512$

H $3.21008323 \quad 0.20449264 \quad 1.35018864$

$\mathrm{H}-2.30450077 \quad 0.25529064 \quad 0.61493806$

$\mathrm{H}-0.267254631 .99401552 \quad 3.12328274$

$\mathrm{H}-1.90860407 \quad 1.43923538 \quad 2.77337449$

$\mathrm{H}-1.057129352 .45862503 \quad 1.60420218$

$\mathrm{H} \quad 0.35612048 \quad-0.55714214 \quad 3.46550357$

$\mathrm{H} 0.00724318-1.707030512 .16830820$

$\mathrm{H}-1.31868576-0.95162828 \quad 3.06653824$

$\begin{array}{llll}0 & -1.20723984 & 1.34375264 & -1.94811391\end{array}$

$0-1.30671850 \quad 1.74155886-0.78546568$

o $-0.29896403 \quad 1.62855366-2.66785594$

Vibrational frequencies (in cm-1)

$\begin{array}{lllllllllllllll}-1476.6 & 44.7 & 71.5 & 95.7 & 125.3 & 176.4 & 202.6 & 230.1 & 241.8 & 266.5 & 288.7 & 307.3 & 333.3 & 344.5\end{array}$

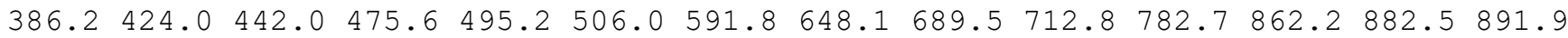
$916.7925 .5 \quad 945.0 \quad 961.3 \quad 984.5 \quad 991.5 \quad 995.3 \quad 1020.1 \quad 1029.2 \quad 1046.2 \quad 1057.8 \quad 1070.6 \quad 1083.1$

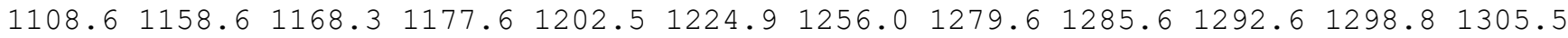

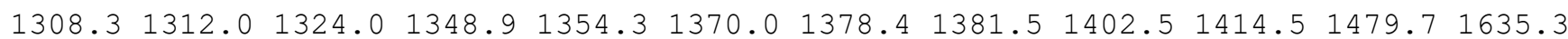
$2230.62657 .72663 .2 \quad 2674.72678 .5 \quad 2681.6 \quad 2712.72722 .12726 .82736 .72740 .12753 .0$ $2775.42777 .92781 .0 \quad 2789.4$

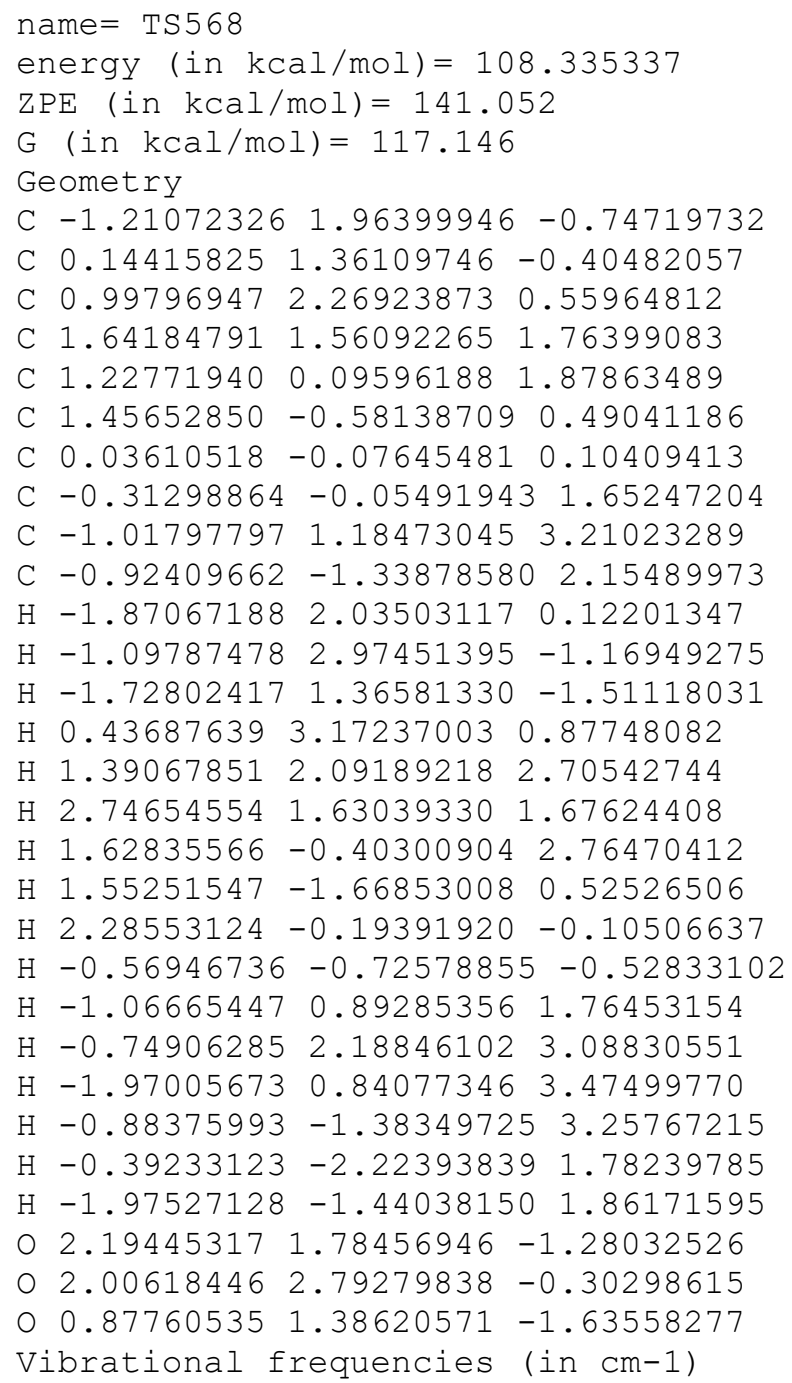
$407.1 \quad 438.6 \quad 469.8 \quad 514.2 \quad 547.4 \quad 624.3 \quad 650.9 \quad 706.9 \quad 738.7 \quad 793.2 \quad 811.8 \quad 840.5 \quad 865.1 \quad 901.4$ $914.0 \quad 936.5 \quad 957.5 \quad 979.0 \quad 999.8 \quad 1006.4 \quad 1031.4 \quad 1048.7 \quad 1052.8 \quad 1071.5 \quad 1091.4 \quad 1098.3 \quad 1108.3$ $1129.61148 .7 \quad 1158.2 \quad 1174.7 \quad 1180.4 \quad 1194.1 \quad 1209.1 \quad 1242.5 \quad 1249.4 \quad 1259.3 \quad 1265.7 \quad 1278.5$ $1281.0 \quad 1282.1 \quad 1284.2 \quad 1289.9 \quad 1294.2 \quad 1300.5 \quad 1317.1 \quad 1368.0 \quad 1374.7 \quad 1393.4 \quad 1398.8 \quad 1469.2$ $1987.72649 .82660 .4 \quad 2664.2 \quad 2674.8 \quad 2675.6 \quad 2684.72688 .52725 .2 \quad 2733.82737 .62744 .1$ $2748.0 \quad 2767.0 \quad 2777.5 \quad 2778.5$ 


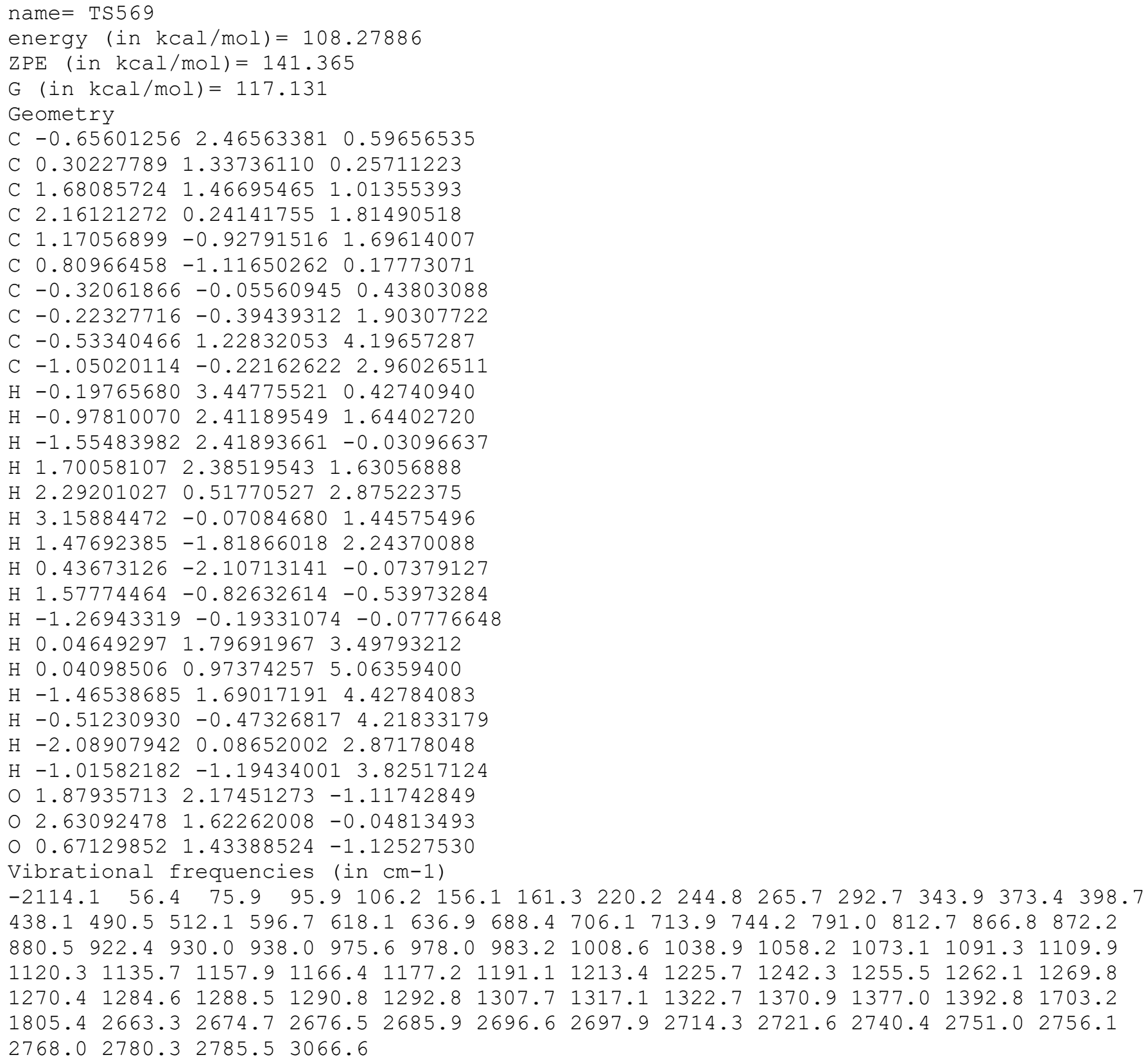


$\mathrm{H} \quad 0.90440720 \quad 2.89988089-0.69574851$

$\mathrm{H} \quad 1.21883380 \quad 2.27059463 \quad 1.60795535$

$\mathrm{H} 1.88554462 \quad 0.08163488 \quad 2.33095204$

$\mathrm{H} 1.87710434-1.85829406 \quad 0.72067884$

$\mathrm{H} \quad 1.99890644-0.60896176-0.48440522$

$\mathrm{H} \quad 0.11411497-1.86059957-1.18830943$

$\mathrm{H}-1.438456001 .15243172 \quad 1.46982693$

$\mathrm{H}-1.22331878 \quad 0.68751468 \quad 3.15647978$

$\mathrm{H}-2.31755057-0.23934875 \quad 2.11923298$

$\mathrm{H} \quad 0.12081727-1.32098172 \quad 3.80566431$

$\mathrm{H} \quad 0.74925885-2.41704857 \quad 2.56386529$

$\mathrm{H}-0.98295499-2.335406052 .85534052$

$\begin{array}{llllll}0 & 0.87980824 & 0.03783684 & -2.00071388\end{array}$

$0 \quad 0.60042148 \quad 1.30569864-1.64624441$

o $-0.29207307-0.88865689-1.85128856$

Vibrational frequencies (in cm-1)

$\begin{array}{lllllllllllllllll}-1438.2 & 81.0 & 102.6 & 153.2 & 171.0 & 183.8 & 198.0 & 210.9 & 228.0 & 251.1 & 283.4 & 318.4 & 334.1 & 377.0\end{array}$ $\begin{array}{lllllllllllllllll}383.6 & 438.1 & 454.8 & 486.0 & 523.6 & 549.0 & 589.5 & 678.0 & 693.7 & 737.6 & 775.0 & 826.4 & 851.2 & 865.9\end{array}$

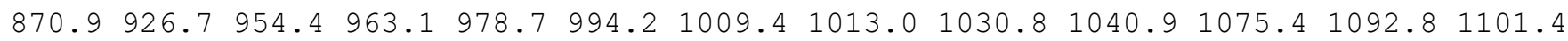
$1117.61160 .0 \quad 1164.4 \quad 1170.8 \quad 1195.2 \quad 1228.5 \quad 1261.7 \quad 1274.1 \quad 1282.8 \quad 1287.8 \quad 1292.4 \quad 1293.6$ $1299.91304 .91310 .0 \quad 1331.8 \quad 1365.6 \quad 1375.8 \quad 1383.9 \quad 1394.6 \quad 1398.4 \quad 1410.1 \quad 1428.8 \quad 1852.6$ $2045.3 \quad 2667.92677 .2 \quad 2677.9 \quad 2679.2 \quad 2681.1 \quad 2681.6 \quad 2685.92691 .4 \quad 2693.42727 .7 \quad 2742.0$ $2768.0 \quad 2779.3 \quad 2781.4 \quad 2783.3$

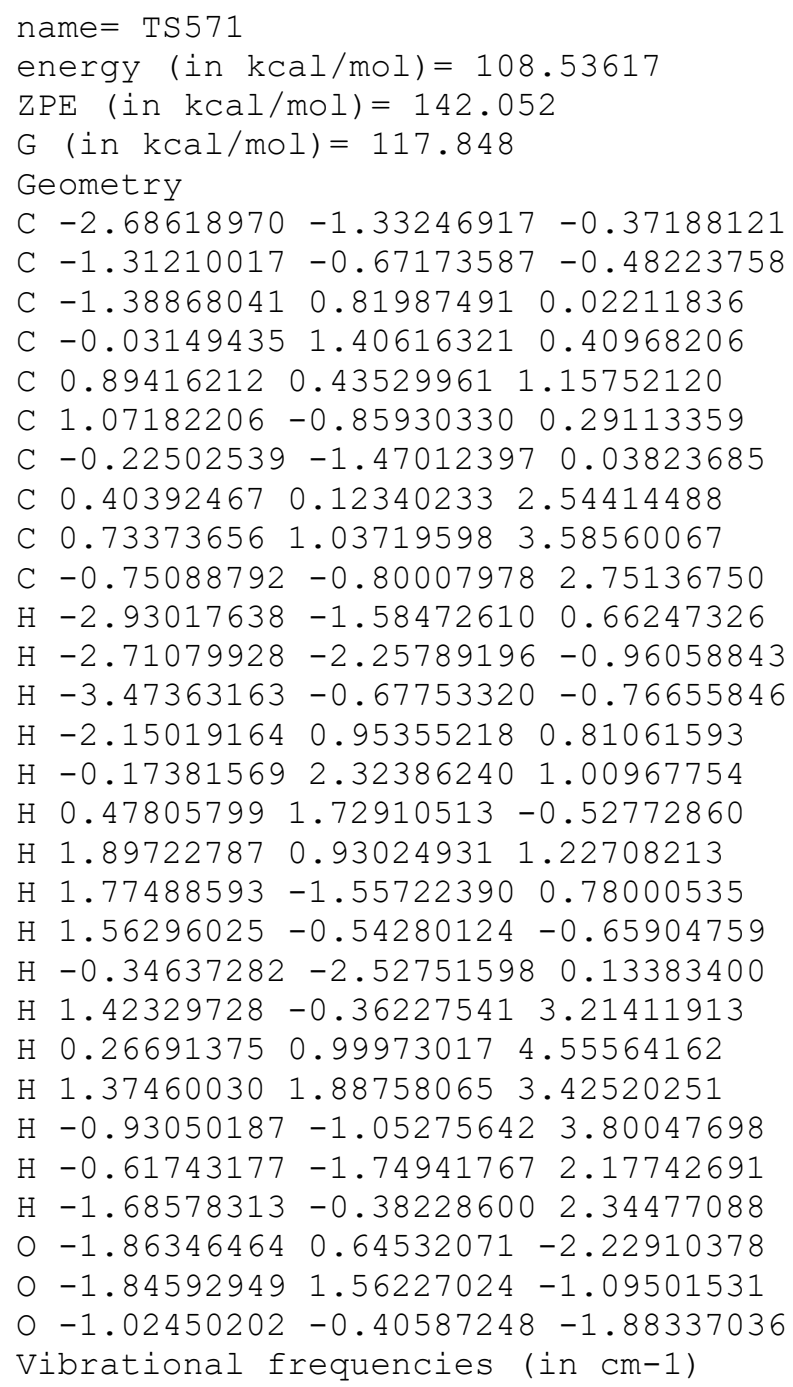


$2208.82577 .5 \quad 2647.7 \quad 2655.2 \quad 2666.12675 .3 \quad 2685.6 \quad 2686.3 \quad 2693.7 \quad 2706.72732 .5 \quad 2734.5$ $2751.32759 .5 \quad 2782.4 \quad 2791.6$

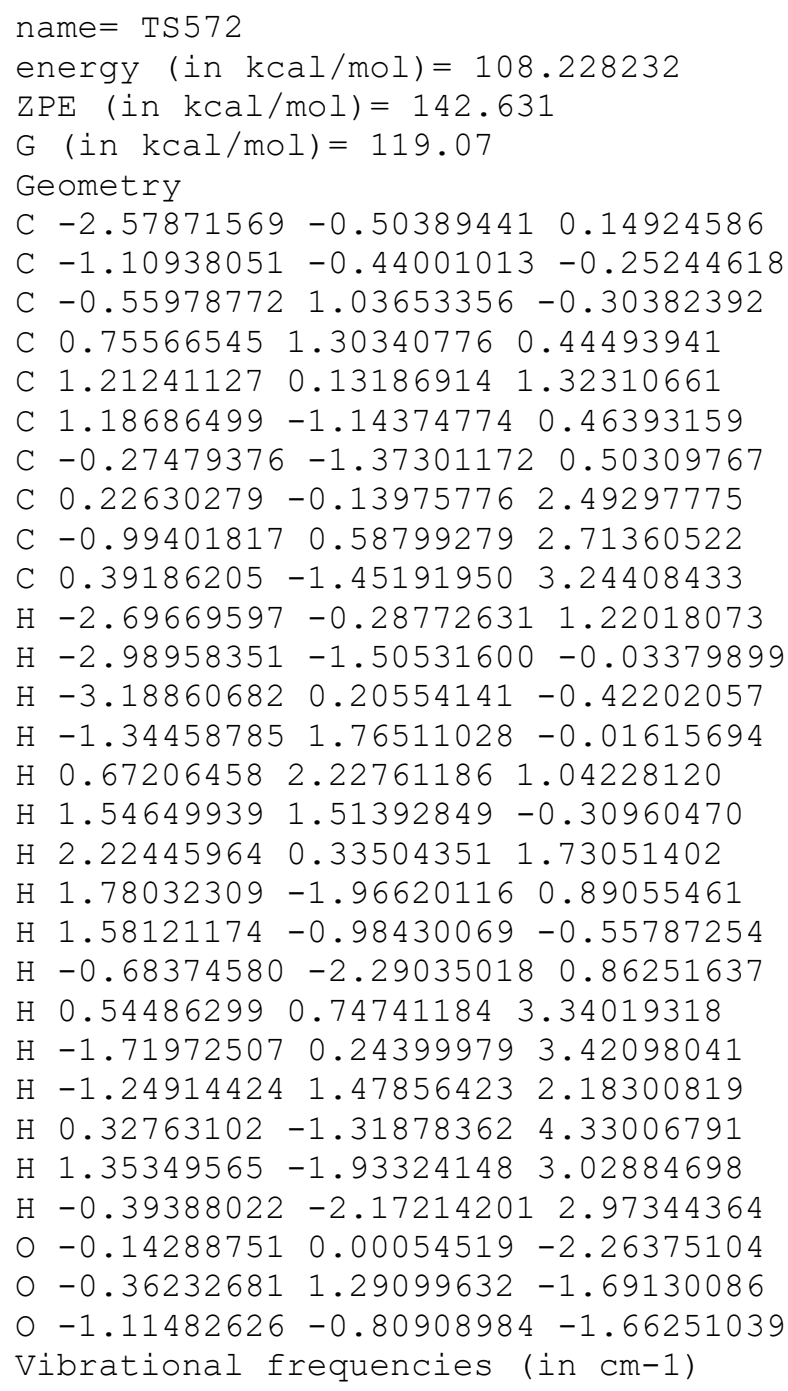


$\mathrm{H}-2.18769718-1.50773931-1.19424783$

$\mathrm{H}-1.004029331 .88927297-0.39845806$

$\mathrm{H} 0.45499431 \quad 2.29526318 \quad 1.35688573$

$\mathrm{H} \quad 1.81242045 \quad 1.96409970 \quad 0.27717907$

H $\quad 1.82947517 \quad 0.43862637 \quad 2.35523254$

H $2.29241891-1.62890506 \quad 0.87604000$

$\mathrm{H} 2.18749889-0.46312554-0.45250495$

$\mathrm{H}-0.08702402-2.24337118 \quad 0.13880552$

$\mathrm{H}-0.78585333 \quad 1.07158743 \quad 4.63654761$

$\mathrm{H}-0.72904426 \quad 1.75390034 \quad 2.96489216$

$\mathrm{H} \quad 0.81233359 \quad 1.39276225 \quad 3.86214411$

$\mathrm{H}-0.47639181-0.69356696 \quad 3.70227135$

$\mathrm{H}-1.18438843-2.052830332 .63959062$

$\mathrm{H}-1.89024261-0.435073692 .72683420$

o $1.05682149 \quad 0.47842395-2.25015266$

$00.486345361 .66259995-1.71792209$

O $0.06412298-0.51642258-2.04481056$

Vibrational frequencies (in $\mathrm{cm}-1$ )

$\begin{array}{lllllllllllllll}-337.4 & 61.0 & 96.9 & 126.6 & 153.3 & 159.2 & 185.5 & 222.7 & 252.8 & 259.6 & 281.4 & 319.7 & 368.7 & 385.5\end{array}$ $428.0 \quad 434.6 \quad 474.8 \quad 496.9 \quad 513.2 \quad 541.0 \quad 624.2 \quad 648.1 \quad 701.2 \quad 719.0 \quad 798.1 \quad 822.0 \quad 854.6 \quad 874.2$ $912.1930 .8 \quad 938.5 \quad 965.9 \quad 978.2 \quad 994.4 \quad 1006.7 \quad 1015.5 \quad 1042.4 \quad 1043.0 \quad 1087.2 \quad 1095.5 \quad 1100.7$

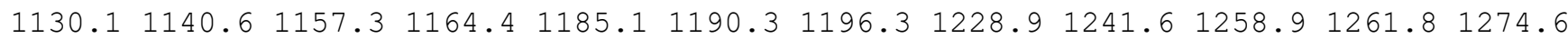

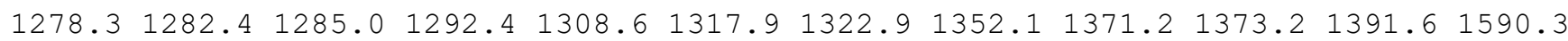
$2404.72637 .12650 .2 \quad 2668.92676 .0 \quad 2684.6 \quad 2690.82724 .52728 .2 \quad 2732.62739 .2 \quad 2748.0$ $2749.3 \quad 2766.12779 .4 \quad 2789.7$

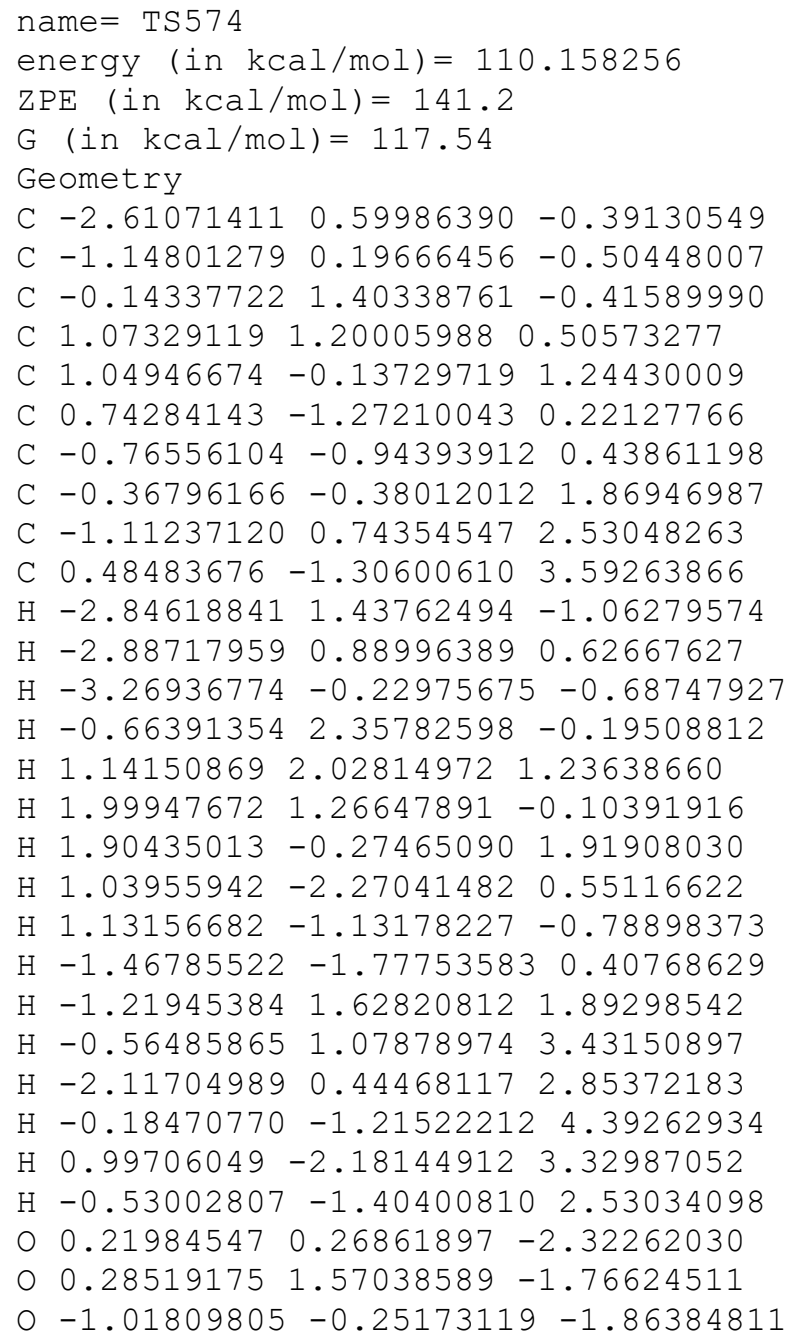


$1137.81148 .0 \quad 1157.3 \quad 1166.7 \quad 1188.4 \quad 1194.4 \quad 1211.5 \quad 1238.4 \quad 1256.7 \quad 1267.6 \quad 1279.01279 .6$

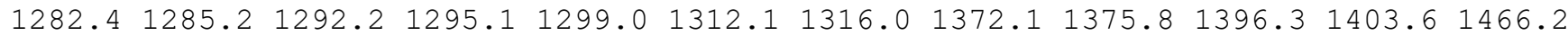
$1957.62656 .52657 .2 \quad 2667.3 \quad 2674.82676 .42686 .22687 .72720 .42722 .92736 .12739 .9$ $2743.8 \quad 2767.32777 .3 \quad 2780.9$

\section{name $=$ TS575}

energy (in $\mathrm{kcal} / \mathrm{mol})=109.26983$

$\mathrm{ZPE}($ in $\mathrm{kcal} / \mathrm{mol})=142.31$

$\mathrm{G}($ in $\mathrm{kcal} / \mathrm{mol})=118.88$

Geometry

C $-1.93070657-0.30618205-0.91830728$

C $-0.41711842-0.32426250-0.81792107$

C $0.28709787 \quad 1.07808226-0.84188841$

C $1.53186181 \quad 1.05949740 \quad 0.03259737$

C $0.99814695 \quad 0.42302081 \quad 1.78658448$

C $1.68441110-0.78055032 \quad 0.28131869$

C $0.19634656-1.135099190 .33308962$

C $-0.09122446-0.57510831 \quad 1.76604501$

C $-1.44373093 \quad 0.08331988 \quad 2.02654144$

C $0.09498868-1.71087110 \quad 2.79030144$

$\mathrm{H}-2.24659908-0.21234058-1.97072030$

$\mathrm{H}-2.38133844 \quad 0.53204635-0.37645644$

$\mathrm{H}-2.37308273-1.23288785-0.53249950$

$\mathrm{H}-0.39090273 \quad 1.92424601-0.63119894$

H $1.64265162 \quad 1.97634182 \quad 0.64209958$

H $2.474539651 .07070506-0.54127716$

$\mathrm{H} \quad 1.80352110 \quad 0.45860059 \quad 2.46115687$

$\mathrm{H} 2.33869693-1.22604318 \quad 1.02685312$

H $2.15952002-0.95277061-0.68970885$

$\mathrm{H}-0.03183832-2.20790696 \quad 0.22651916$

$\mathrm{H}-1.55669181 \quad 1.005939651 .44552920$

$\mathrm{H}-1.52681510 \quad 0.37920350 \quad 3.08064297$

$\mathrm{H}-2.27594442-0.58906736 \quad 1.80305937$

H $0.09156538-1.314897513 .81182236$

H $1.04114422 \quad-2.24069724 \quad 2.64964688$

$\mathrm{H}-0.71391239-2.44476088 \quad 2.71757105$

$01.01289779-0.08971349-2.60026861$

o $0.649501181 .22313622-2.21210184$

o $-0.00389252-0.92858150-2.06055179$

Vibrational frequencies (in $\mathrm{cm}-1$ )

$\begin{array}{llllllllllllllll}-1020.6 & 69.8 & 107.0 & 124.4 & 173.2 & 204.1 & 217.5 & 226.0 & 245.9 & 268.7 & 304.4 & 341.0 & 367.8 & 395.5\end{array}$ $416.7 \quad 449.0 \quad 493.0 \quad 523.6 \quad 547.0 \quad 587.4 \quad 662.1 \quad 679.9 \quad 705.4 \quad 753.7 \quad 823.3 \quad 869.6 \quad 877.5 \quad 917.6$ $933.8955 .5 \quad 973.5 \quad 980.9 \quad 984.5 \quad 1002.3 \quad 1013.3 \quad 1017.9 \quad 1031.5 \quad 1062.4 \quad 1065.7 \quad 1090.1 \quad 1129.3$

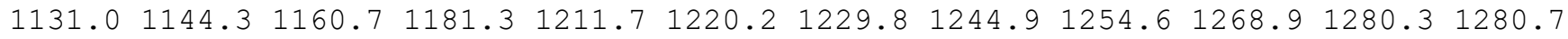
$1285.21296 .01296 .91305 .2 \quad 1307.2 \quad 1324.8 \quad 1329.3 \quad 1360.3 \quad 1387.5 \quad 1396.2 \quad 1401.31415 .2$ $2589.72651 .3 \quad 2662.7 \quad 2672.0 \quad 2673.2 \quad 2679.3 \quad 2680.4 \quad 2686.02698 .12702 .3 \quad 2726.62730 .5$ $2749.82777 .02780 .7 \quad 2782.3$

\section{name $=$ TS576}

energy $($ in $\mathrm{kcal} / \mathrm{mol})=112.082108$

$\mathrm{ZPE}(\mathrm{in} \mathrm{kcal} / \mathrm{mol})=139.909$

$\mathrm{G}($ in $\mathrm{kcal} / \mathrm{mol})=115.788$

Geometry

C $-2.12539409-0.43378382-0.82350919$

C $-0.62696512-0.68211200-0.73580376$

C $0.23889054 \quad 0.59158135-1.05402487$

C $1.30246033 \quad 0.95035276-0.00576331$

C $1.34557466-0.03278444 \quad 1.17343092$

C $1.29943178-1.46962730 \quad 0.65056191$

C $-0.22498577-1.35442572 \quad 0.58564495$

C $-0.07711004-0.28010913 \quad 1.64735537$

C $-1.02827216 \quad 0.52800833 \quad 2.38992698$

C $0.62281611-1.82718158 \quad 3.25311828$ 
$\mathrm{H}-2.47569098 \quad 0.22530050 \quad-0.02291333$

$\mathrm{H}-2.68700003-1.37598596-0.74879036$

$\mathrm{H}-2.400293430 .01743957-1.78753533$

$\mathrm{H}-0.39365451 \quad 1.46741148-1.30520220$

H $1.14432292 \quad 1.977630210 .36881862$

H 2.30062422 $0.95133144 \quad-0.49769928$

H 2.11082358 $0.22911075 \quad 1.90882341$

H $1.21339212-2.031522912 .12586565$

$\mathrm{H} 1.94654013-1.94499825-0.02094146$

$\mathrm{H}-0.82073103-2.246222290 .79776986$

$\mathrm{H}-1.97054083-0.00529928 \quad 2.59620210$

$\mathrm{H}-1.288212001 .45341140 \quad 1.84356589$

$\mathrm{H}-0.62338526 \quad 0.85407586 \quad 3.36502229$

$\mathrm{H}-0.43379895-2.00541123 \quad 3.29022601$

$\mathrm{H} 0.93197116-0.949066313 .78380006$

$\mathrm{H} 1.16286883-2.69961010 \quad 3.60883749$

O $0.94400459-1.15673513-2.28284941$

$\begin{array}{lllll}0 & 0.86606261 & 0.26336916 & -2.29249060\end{array}$

o $-0.33301869-1.56285002-1.82717235$

Vibrational frequencies (in $\mathrm{cm}-1$ )

$\begin{array}{lllllllllllllllll}-1322.5 & 64.0 & 99.6 & 116.2 & 140.3 & 150.3 & 158.1 & 205.0 & 218.9 & 236.9 & 261.5 & 297.2 & 335.0 & 374.3\end{array}$

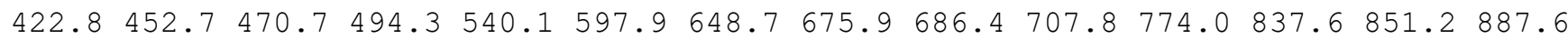
$926.2933 .9 \quad 962.9973 .0 \quad 983.2 \quad 1003.3 \quad 1009.1 \quad 1027.4 \quad 1043.4 \quad 1070.7 \quad 1086.5 \quad 1095.1 \quad 1119.0$

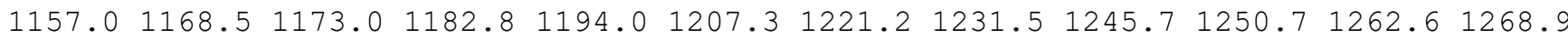

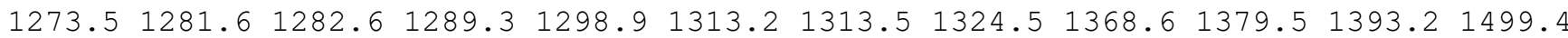
$1590.42649 .7 \quad 2655.2 \quad 2665.4 \quad 2667.5 \quad 2673.3 \quad 2678.02686 .82713 .8 \quad 2734.6 \quad 2746.12749 .3$ $2768.22779 .8 \quad 2782.0 \quad 2807.9$

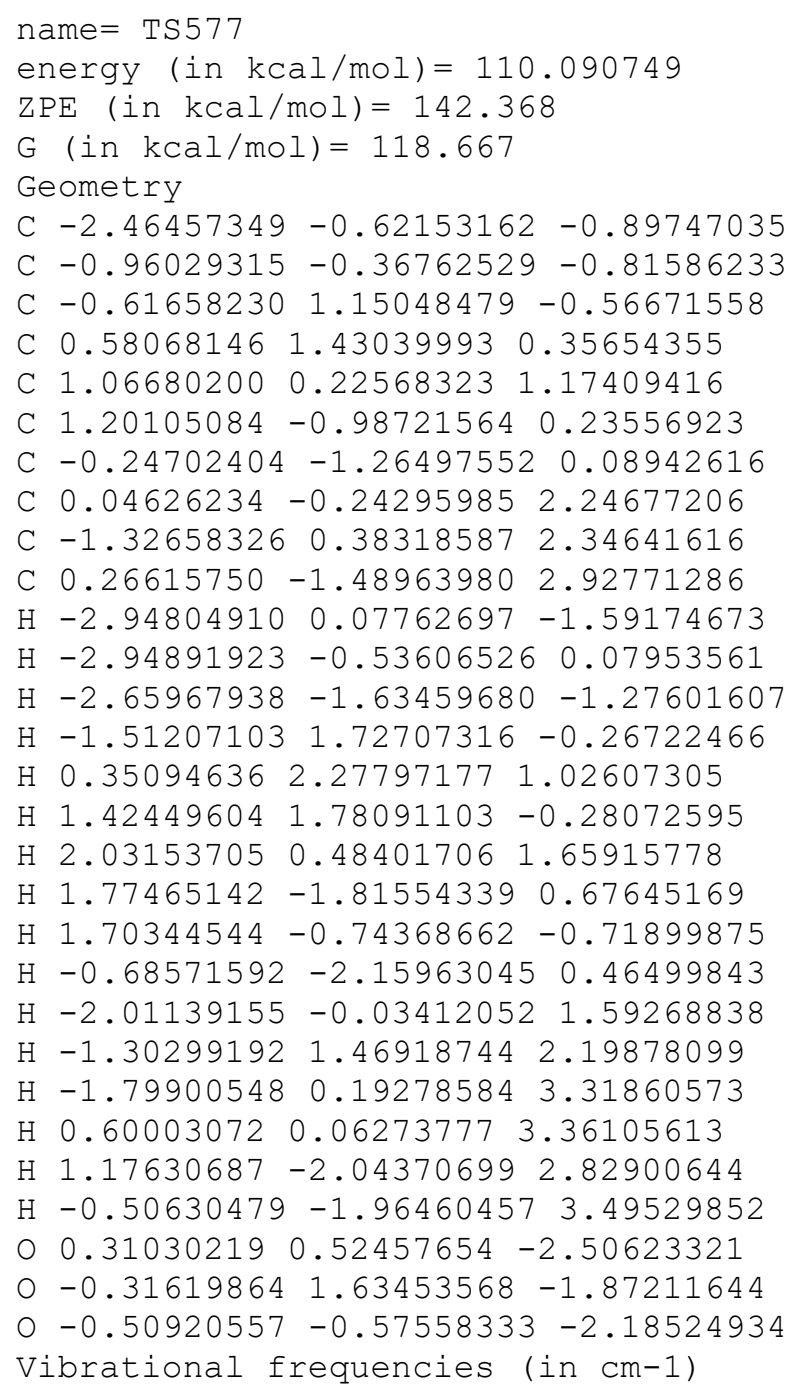


$\begin{array}{llllllllllllllllll}-1253.3 & 68.3 & 90.1 & 120.1 & 142.9 & 168.8 & 196.2 & 205.2 & 284.9 & 287.0 & 315.8 & 343.9 & 359.7 & 405.4\end{array}$

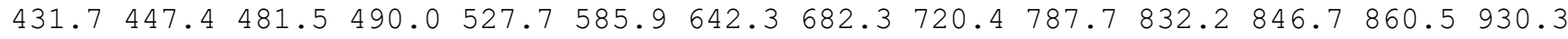
$946.2950 .6 \quad 961.9982 .4 \quad 989.7 \quad 1005.9 \quad 1025.5 \quad 1035.1 \quad 1046.3 \quad 1074.6 \quad 1081.2 \quad 1092.7 \quad 1118.2$

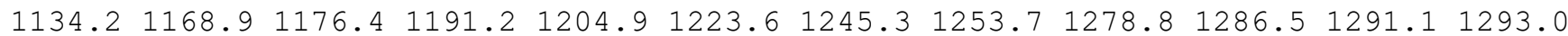
$1294.31304 .0 \quad 1313.1 \quad 1324.2 \quad 1333.1 \quad 1360.3 \quad 1363.0 \quad 1374.2 \quad 1380.3 \quad 1397.6 \quad 1412.01446 .1$ $2258.3 \quad 2645.6 \quad 2653.3 \quad 2671.8 \quad 2672.6 \quad 2674.12676 .5 \quad 2694.72700 .12728 .8 \quad 2735.7 \quad 2753.4$ $2766.62771 .6 \quad 2780.5 \quad 2789.5$

name $=$ TS578

energy $($ in $\mathrm{kcal} / \mathrm{mol})=112.108971$

$\mathrm{ZPE}($ in $\mathrm{kcal} / \mathrm{mol})=140.825$

$\mathrm{G}($ in $\mathrm{kcal} / \mathrm{mol})=117.156$

Geometry

C $-2.15467948-0.12057721-0.90625590$

C $-0.64408765-0.27601562-0.86917169$

C $0.152733941 .09176059-0.89792327$

C 1.221738131 .173456450 .07087601

C $1.20403083 \quad 0.23544878 \quad 1.40787870$

C $1.44928706-1.013785490 .16639050$

C $-0.07794916-1.18098370 \quad 0.23982741$

C $-0.13065271-0.53998501 \quad 1.66465956$

C $-1.309263290 .34982404 \quad 2.01425975$

C $0.03092842-1.58245800 \quad 2.76983346$

$\mathrm{H}-2.471950390 .15692134-1.92677801$

$\mathrm{H}-2.51452008 \quad 0.66913629-0.24065664$

$\mathrm{H}-2.67014048-1.05068860-0.64653273$

$\mathrm{H}-0.51466101 \quad 1.98256206-0.91372250$

$\mathrm{H} 1.054290391 .337398351 .79521766$

H $2.05874431 \quad 1.79368863-0.03947297$

H 2.06090720 $0.02215717 \quad 2.05927302$

H $2.06791675-1.74298519 \quad 0.68883277$

$\mathrm{H} \quad 1.91615227-0.73692462-0.78898622$

$\mathrm{H}-0.45266495-2.20889187 \quad 0.17153353$

$\mathrm{H}-1.37235676 \quad 1.23496301 \quad 1.36691531$

$\mathrm{H}-1.23961146 \quad 0.71994920 \quad 3.04493803$

$\mathrm{H}-2.25856379-0.19636151 \quad 1.93444698$

$\mathrm{H} \quad 0.23415959-1.102385963 .73549585$

$\mathrm{H} \quad 0.84692784 \quad-2.28504690 \quad 2.57845247$

$\mathrm{H}-0.88687480-2.17203825 \quad 2.89353039$

$0.82854036-0.23244874-2.61503011$

$0.634641891 .10730651-2.26460022$

o $-0.33092556-0.90991286-2.11512868$

Vibrational frequencies (in cm-1)

$\begin{array}{lllllllllllllll}-1521.5 & 71.7 & 84.7 & 115.7 & 162.9 & 181.3 & 218.7 & 228.3 & 250.8 & 273.2 & 305.0 & 327.8 & 355.2 & 398.4\end{array}$

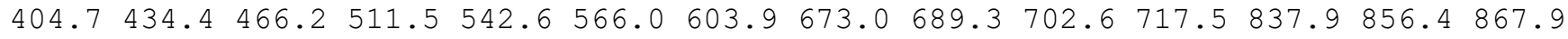

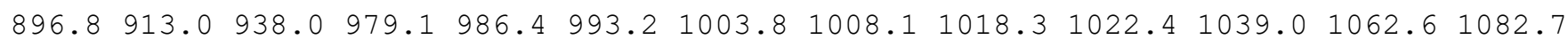

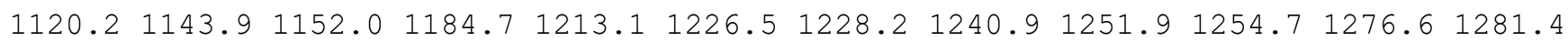
$1285.3 \quad 1289.0 \quad 1293.8 \quad 1298.2 \quad 1302.4 \quad 1305.8 \quad 1317.6 \quad 1362.91395 .4 \quad 1400.6 \quad 1403.3 \quad 1411.7$ $2419.92627 .4 \quad 2663.3 \quad 2665.3 \quad 2670.92673 .92675 .2 \quad 2678.02680 .8 \quad 2700.32715 .92738 .9$ $2777.0 \quad 2779.7 \quad 2782.5 \quad 2828.5$

name $=$ TS579

energy (in $\mathrm{kcal} / \mathrm{mol})=115.138346$

$\mathrm{ZPE}($ in $\mathrm{kcal} / \mathrm{mol})=137.82$

$\mathrm{G}($ in $\mathrm{kcal} / \mathrm{mol})=111.478$

Geometry

C $-1.26904300-0.42196388-0.81480632$

C $0.11844646-0.42900634-1.34506447$

C $0.665651461 .70014403-1.60542281$

C $1.577499442 .12123713-0.63241343$

C $0.77793314-0.34867835 \quad 1.79735293$

C $1.68617310-0.363400230 .58107886$

C $1.29766020-0.76428345-0.70885266$ 
C $-0.28924953 \quad 0.65627548 \quad 1.68138188$

C $-1.494862360 .55775422 \quad 2.50501537$

C $0.36545084-1.79154813 \quad 2.12201642$

$\mathrm{H}-2.01135728-0.55404888-1.61960853$

$\mathrm{H}-1.51622466 \quad 0.52996938-0.31504370$

$\mathrm{H}-1.42882578-1.23250468-0.08944864$

$\mathrm{H}-0.397071691 .92375754-1.55703301$

H 1.232271112 .792819230 .14943816

$\mathrm{H} 2.618413262 .26059380-0.90549308$

$\mathrm{H} \quad 1.39221614 \quad 0.036635792 .67300473$

$\mathrm{H} \quad 2.72093689-0.60089974 \quad 0.85969277$

$\mathrm{H} \quad 1.79272618 \quad 1.03642844 \quad 0.24912468$

H $2.11051364-1.12486745-1.35955051$

$\mathrm{H}-1.490589191 .39898374 \quad 3.22377597$

$\mathrm{H}-1.65737106-0.363917813 .07310252$

$\mathrm{H}-2.38961634 \quad 0.72857780 \quad 1.88168314$

$\mathrm{H}-0.16025128-1.863761153 .07830017$

H $1.24253975-2.448212122 .17846200$

$\mathrm{H}-0.28571721-2.20113513 \quad 1.34100376$

O $1.21392623 \quad 0.09465841-3.20498535$

O $1.09841172 \quad 1.52545149-2.88823011$

o $0.07636275-0.52029231-2.73559324$

Vibrational frequencies (in $\mathrm{cm}-1$ )

$\begin{array}{lllllllllllllll}-1878.6 & 34.6 & 48.3 & 68.4 & 86.4 & 114.4 & 137.9 & 153.0 & 174.5 & 200.7 & 217.0 & 246.5 & 272.6 & 301.9\end{array}$ $328.5 \quad 347.8 \quad 427.7 \quad 448.4 \quad 453.6 \quad 464.2 \quad 491.4 \quad 520.9 \quad 549.7 \quad 632.4 \quad 666.0 \quad 716.0 \quad 763.8 \quad 816.6$

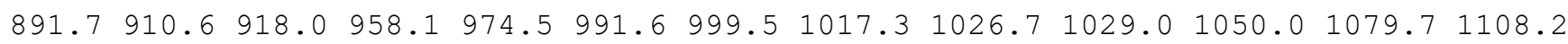
$1109.31129 .8 \quad 1189.1 \quad 1204.0 \quad 1209.5 \quad 1218.1 \quad 1234.1 \quad 1266.51267 .7 \quad 1271.7 \quad 1283.11294 .0$ $1300.91303 .6 \quad 1309.7 \quad 1333.0 \quad 1366.2 \quad 1385.2 \quad 1392.5 \quad 1405.91412 .2 \quad 1428.4 \quad 1517.81601 .8$ $1756.02647 .0 \quad 2653.1 \quad 2656.0 \quad 2664.1 \quad 2667.1 \quad 2673.7 \quad 2679.4 \quad 2694.0 \quad 2714.92720 .72733 .4$ $2760.12766 .2 \quad 2769.8 \quad 2778.5$

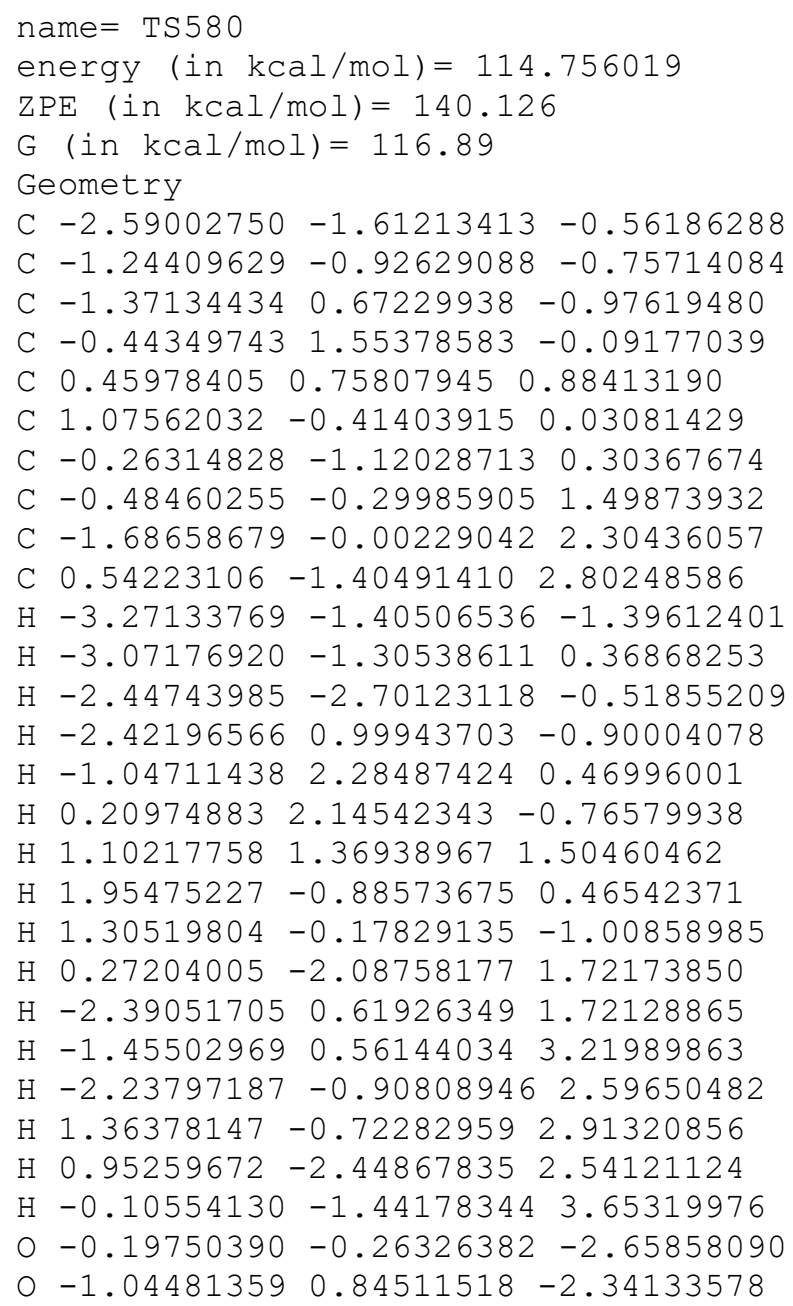


$0-0.80871693-1.37487807-2.05102531$

Vibrational frequencies (in $\mathrm{cm}-1$ )

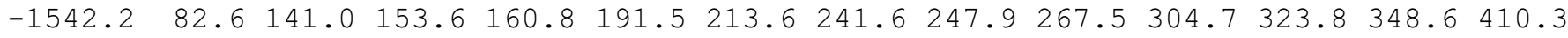

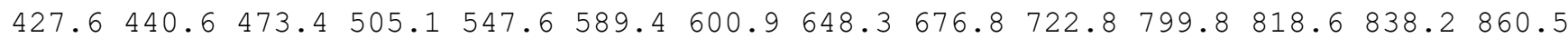
$909.8923 .4 \quad 934.9 \quad 968.3 \quad 976.1 \quad 985.1 \quad 985.7 \quad 999.6 \quad 1011.4 \quad 1058.3 \quad 1070.2 \quad 1079.11090 .9$ $1103.3 \quad 1132.8 \quad 1156.0 \quad 1178.3 \quad 1192.1 \quad 1211.4 \quad 1219.4 \quad 1222.7 \quad 1236.1 \quad 1247.2 \quad 1260.6 \quad 1267.8$

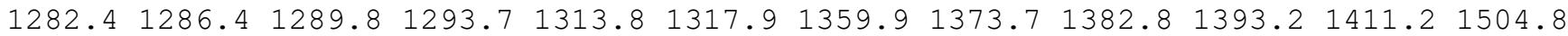
$1636.82656 .62660 .5 \quad 2667.4 \quad 2672.8 \quad 2676.6 \quad 2686.5 \quad 2692.82694 .0 \quad 2708.72738 .3 \quad 2755.3$ $2771.02773 .2 \quad 2777.9 \quad 2784.1$

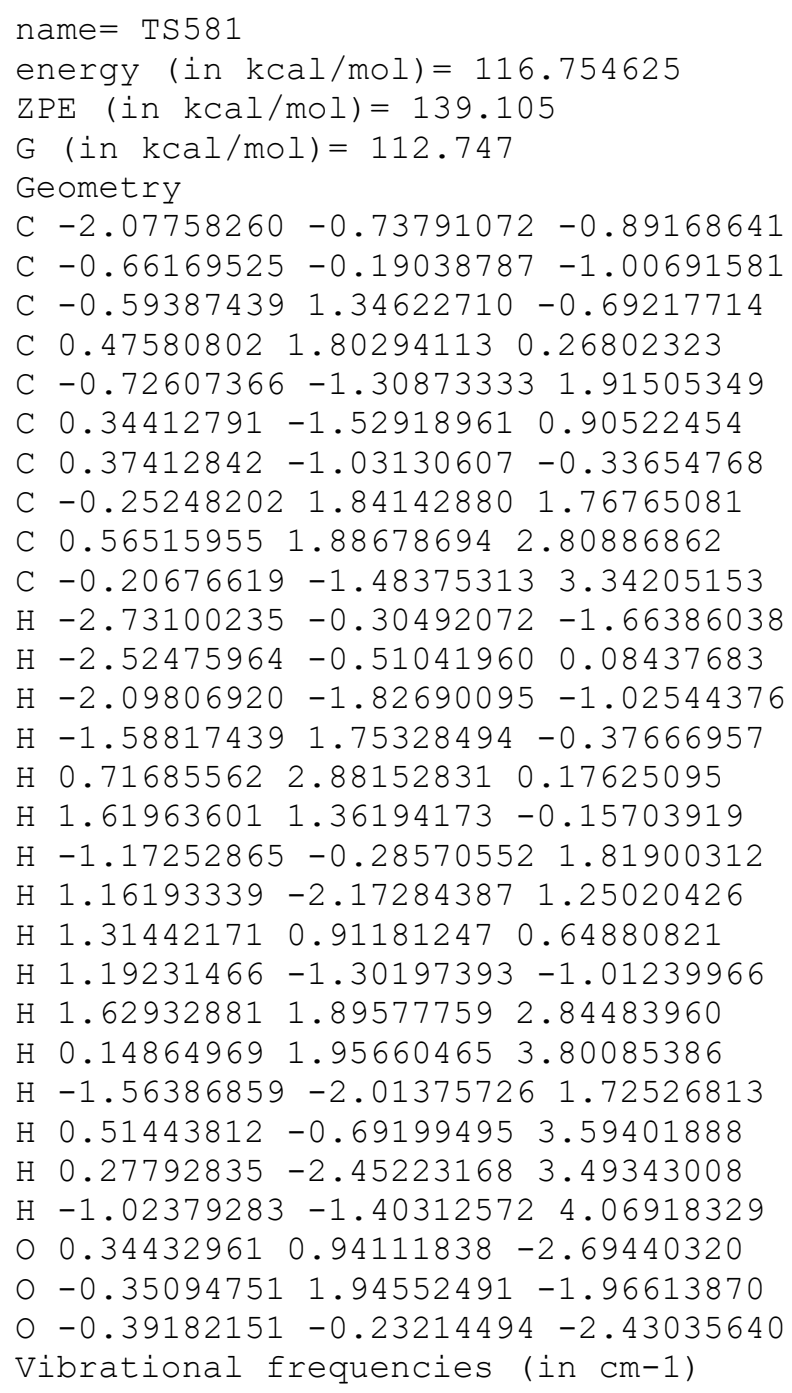


C $0.76949104 \quad-1.32835210 \quad 0.66037176$

C $-0.74333009-0.98642078 \quad 0.86667734$

C $-0.39783286-0.20825464 \quad 2.26168638$

C $-1.18356850 \quad 1.04844010 \quad 2.56173839$

C $-0.15219517-1.042502653 .38081183$

$\mathrm{H}-2.75979368 \quad 0.83544187 \quad-1.41157909$

$\mathrm{H}-3.10503551-0.79506355-0.74524927$

$\mathrm{H}-0.90251225-1.52499928-1.84637404$

$\mathrm{H}-0.78782300 \quad 2.17030782-0.01724435$

$\mathrm{H} 1.268344112 .082901051 .08014189$

$\mathrm{H} \quad 1.94918306 \quad 1.05280307-0.17887668$

H 2.03422195 -0.277408362 .76426521$

$\mathrm{H} 1.09407296-2.23722560 \quad 1.17558041$

$\mathrm{H} 1.13143961-1.37725645-0.36971032$

$\mathrm{H}-1.43147967-1.81544942 \quad 1.02618670$

$\mathrm{H}-1.943751661 .264637651 .80324957$

$\mathrm{H}-0.52592147 \quad 1.922235312 .65012870$

$\mathrm{H}-1.724367330 .96575293 \quad 3.51536313$

$\mathrm{H} 1.68080211-0.600089243 .42031477$

$\mathrm{H} 0.08346293-2.08007595 \quad 3.28129730$

$\mathrm{H}-0.43696147-0.75297216 \quad 4.36950407$

$0.15368637 \quad 0.24330474-2.35105592$

○ $0.04733194 \quad 1.48721999-1.68304594$

o-1.00310255-0.51286358-1.65853233

Vibrational frequencies (in $\mathrm{cm}-1$ )

$\begin{array}{llllllllllllll}-910.1 & 58.7 & 95.0 & 130.0 & 197.8 & 210.6 & 239.3 & 279.0 & 284.7 & 339.7 & 360.4 & 385.4 & 402.3 & 416.6\end{array}$ $\begin{array}{lllllllllllllll}438.0 & 458.3 & 487.1 & 527.9 & 554.3 & 567.4 & 608.6 & 621.0 & 631.5 & 695.9 & 722.1 & 743.8 & 749.5 & 812.0\end{array}$

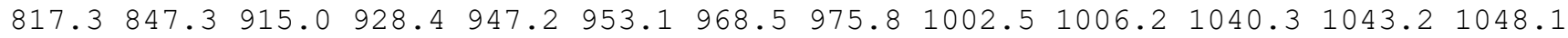

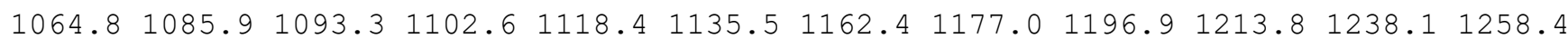

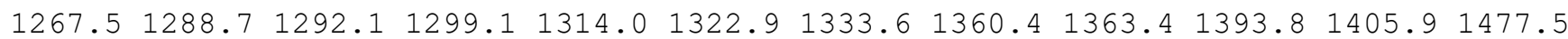
$1546.22657 .7 \quad 2667.12675 .3 \quad 2680.12686 .6 \quad 2716.8 \quad 2735.52741 .5 \quad 2743.8 \quad 2762.4 \quad 2779.5$ $2782.32790 .02902 .5 \quad 4439.2$

name $=\operatorname{TS} 583$

energy $($ in $\mathrm{kcal} / \mathrm{mol})=115.746981$

$\mathrm{ZPE}($ in $\mathrm{kcal} / \mathrm{mol})=140.62$

G $($ in $\mathrm{kcal} / \mathrm{mol})=117.034$

Geometry

C $-2.12170913-0.71141850-1.03318340$

C $-0.61227991-0.61709152-0.86371237$

C $-0.039192550 .84058469-1.01842839$

C $0.90487014 \quad 1.313483490 .10854075$

C $1.18295620 \quad 0.17996983 \quad 1.09453985$

C $1.40560081-1.149949250 .49080161$

C $-0.08630358-1.32437890 \quad 0.38037542$

C $-0.15520332-0.343812901 .70559108$

C $-1.31725745 \quad 0.60494399 \quad 1.89077469$

C $0.03606224-1.15649656 \quad 2.97699047$

$\mathrm{H}-2.45927274-0.17911580-1.93205147$

$\mathrm{H}-2.65805177-0.30752431-0.16939665$

$\mathrm{H}-2.43220971-1.75965580-1.15043023$

$\mathrm{H}-0.83838520 \quad 1.58250980 \quad-1.21336696$

$\mathrm{H} \quad 0.47008572 \quad 2.17811474 \quad 0.64154133$

$\mathrm{H} \quad 1.83271826 \quad 1.69322254 \quad-0.36227221$

$\begin{array}{lllll}\mathrm{H} & 2.39598214 & 0.38346566 & 1.48565265\end{array}$

H 2.05771892 -1.93725882 0.74447273

$\mathrm{H} \quad 2.73284043 \quad-0.15976210 \quad 0.63533976$

$\mathrm{H}-0.49302665-2.325806390 .51434360$

$\mathrm{H}-1.56173038 \quad 1.18157925 \quad 0.99401515$

$\mathrm{H}-1.10578733 \quad 1.32853373 \quad 2.68895620$

$\mathrm{H}-2.22598236 \quad 0.05991180 \quad 2.17917807$

$\mathrm{H} \quad 0.22771506-0.508109713 .83868902$

$\mathrm{H} \quad 0.87829185-1.85548778 \quad 2.89748022$

$\mathrm{H}-0.85421548-1.75578654 \quad 3.20072493$ 
$01.07195526-0.57584511-2.37008204$

$0.68622889 \quad 0.79174245-2.24448434$

o $-0.09610758-1.28647503-2.03187736$

Vibrational frequencies (in $\mathrm{cm}-1$ )

$\begin{array}{lllllllllllllll}-2307.5 & 76.3 & 106.2 & 140.9 & 144.4 & 170.1 & 200.2 & 210.7 & 233.0 & 260.2 & 297.6 & 322.5 & 353.1 & 378.6\end{array}$ $429.6 \quad 467.2 \quad 481.7 \quad 485.7 \quad 524.4 \quad 582.1 \quad 638.7 \quad 664.5 \quad 704.0 \quad 744.1757 .5 \quad 841.6 \quad 854.9884 .1$

$944.5963 .5 \quad 970.9978 .0 \quad 1001.2 \quad 1008.2 \quad 1012.2 \quad 1016.2 \quad 1054.91072 .1 \quad 1076.4 \quad 1113.01123 .8$ $\begin{array}{lllllllllllll}1140.1 & 1164.9 & 1179.3 & 1193.7 & 1207.4 & 1232.4 & 1246.3 & 1253.8 & 1273.9 & 1282.0 & 1285.2 & 1287.1\end{array}$ $1292.01297 .8 \quad 1299.4 \quad 1306.2 \quad 1308.6 \quad 1316.2 \quad 1327.3 \quad 1342.01393 .3 \quad 1403.8 \quad 1405.2 \quad 1416.9$ $1664.92513 .32659 .82670 .92672 .8 \quad 2675.42676 .02679 .02681 .82688 .62740 .32749 .5$ $2780.32781 .8 \quad 2783.12804 .0$

\section{name $=$ TS584}

energy (in $\mathrm{kcal} / \mathrm{mol})=117.158283$

$\mathrm{ZPE}($ in $\mathrm{kcal} / \mathrm{mol})=139.692$

$\mathrm{G}($ in $\mathrm{kcal} / \mathrm{mol})=115.312$

Geometry

C $-1.68114164 \quad 0.55453124 \quad-1.84709928$

C $-0.58061873-0.25819965-1.18896593$

C $0.81975891 \quad 0.43248178-1.23382248$

$\begin{array}{llll}\text { C } 1.31454124 & 0.91911824 & 0.13111584\end{array}$

C $1.00300996-0.05062978 \quad 1.23422206$

C $2.09774594-0.981955031 .59799203$

C $-1.07107496-0.82425490 \quad 0.09666179$

C $-0.43639400-0.45540911 \quad 1.37359838$

C $-1.25614097 \quad 0.74125522 \quad 1.69328481$

C $-0.65530903-1.582995762 .39107081$

$\mathrm{H}-1.46362171 \quad 0.73688770 \quad-2.90901596$

$\mathrm{H}-1.82271571 \quad 1.52251133-1.35307448$

$\mathrm{H}-2.64337197 \quad 0.01762596-1.80554312$

$\mathrm{H} \quad 0.85371111 \quad 1.25623430-1.98056263$

$\mathrm{H} \quad 0.84841114 \quad 1.90786053 \quad 0.33742677$

H $2.40583676 \quad 1.11727274 \quad 0.06620189$

$\mathrm{H} 0.883915661 .06107006 \quad 2.52044221$

$\mathrm{H} \quad 3.07750273-0.48524771 \quad 1.64842020$

$\mathrm{H} \quad 1.93868629-1.47718956 \quad 2.56648778$

H $2.19696424-1.78117092 \quad 0.83885518$

$\mathrm{H}-1.314525521 .571102491 .03002690$

$\mathrm{H} \quad 0.10856443 \quad 1.34519718 \quad 2.64126751$

$\mathrm{H}-2.07256646 \quad 0.650075332 .37164100$

$\mathrm{H}-0.32592222-1.28507087 \quad 3.39123732$

$\mathrm{H}-0.12922056-2.49804932 \quad 2.09948425$

$\mathrm{H}-1.72244910-1.839657902 .45434009$

$0.96156770-1.77973637-1.73500056$

O $1.70528843-0.54824016-1.75944581$

$0-0.35070903-1.40097403-2.04936520$

Vibrational frequencies (in $\mathrm{cm}-1$ )

$\begin{array}{lllllllllllllll}-1173.9 & 58.6 & 71.8 & 118.2 & 129.0 & 146.2 & 196.1 & 205.1 & 225.7 & 236.3 & 286.6 & 304.0 & 345.3 & 377.1\end{array}$

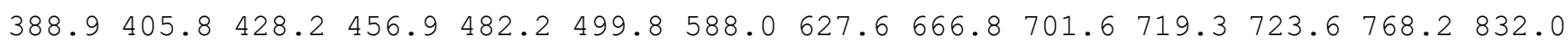
$862.8 \quad 895.5918 .9924 .9958 .7 \quad 968.8 \quad 978.9 \quad 994.6 \quad 1004.6 \quad 1021.6 \quad 1042.3 \quad 1050.4 \quad 1078.8$

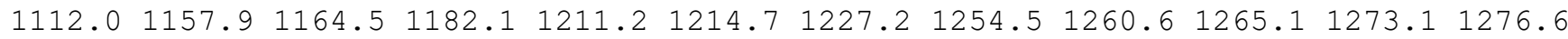

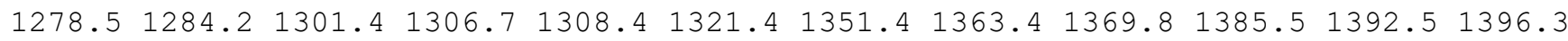
$1412.42649 .5 \quad 2651.4 \quad 2662.4 \quad 2668.7 \quad 2672.4 \quad 2673.5 \quad 2676.82683 .0 \quad 2732.5 \quad 2738.62771 .0$ $2775.3 \quad 2780.12789 .9 \quad 4029.6$

name $=\operatorname{TS} 585$

energy (in $\mathrm{kcal} / \mathrm{mol})=117.166064$

$\operatorname{ZPE}($ in $\mathrm{kcal} / \mathrm{mol})=139.732$

$\mathrm{G}($ in $\mathrm{kcal} / \mathrm{mol})=116.32$

Geometry

C $-2.31480879 \quad 0.46187189-0.69770167$

C $-0.81392924 \quad 0.20451682-0.64907540$

C $0.04824727 \quad 1.53499044-0.53902661$ 
C 1.231065691 .510133050 .48637365

C $1.14051572 \quad 0.24461090 \quad 1.23204064$

C $1.13277027-0.99357773 \quad 0.31809238$

C $-0.43801019-0.867256350 .40769783$

C $-0.20916114-0.06979091 \quad 1.71245715$

$\begin{array}{llll}\text { C }-1.20223086 & 0.73900565 & 2.44728927\end{array}$

C $0.29822759-1.36899958 \quad 3.15353191$

$\mathrm{H}-2.55951681 \quad 1.22835556-1.44682316$

$\mathrm{H}-2.71589024 \quad 0.78368695 \quad 0.26618658$

$\mathrm{H}-2.85601480-0.44778343-0.99502903$

$\mathrm{H}-0.59309198 \quad 2.42119830-0.38575367$

$\mathrm{H} 1.18468132 \quad 2.39484463 \quad 1.14243329$

H $2.19089521 \quad 1.58447411-0.06231780$

$\mathrm{H} \quad 1.31320850 \quad-0.62836570 \quad 2.77663890$

$\mathrm{H} 1.53599052-1.907990190 .74864231$

$\mathrm{H} \quad 1.57227492-0.87393723-0.67138131$

$\mathrm{H}-1.05031236-1.758898350 .38260250$

$\mathrm{H}-1.526592751 .59768467 \quad 1.83210386$

$\mathrm{H}-0.78662894 \quad 1.17728226 \quad 3.36780890$

$\mathrm{H}-2.10226603 \quad 0.17056023 \quad 2.72074002$

$\mathrm{H} 1.38563056-1.51631790 \quad 3.48492064$

$\mathrm{H}-0.04209074 \quad-2.24932141 \quad 2.64303461$

$\mathrm{H}-0.32687769-1.078123523 .97061104$

$0.69113337 \quad 0.37732876-2.35059880$

$0.536452021 .69663615-1.86742503$

O $-0.50053211-0.29423807-1.95349464$

Vibrational frequencies (in cm-1)

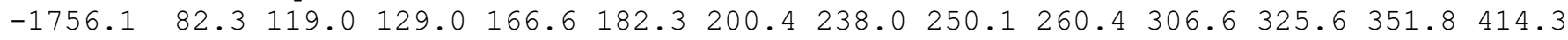

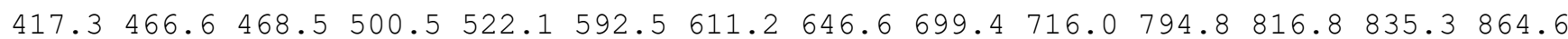
$911.3924 .1932 .4963 .4 \quad 970.7 \quad 979.2 \quad 984.8 \quad 990.1 \quad 1009.8 \quad 1034.6 \quad 1057.9 \quad 1068.2 \quad 1086.4$

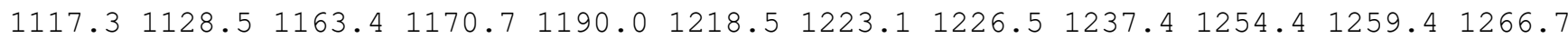

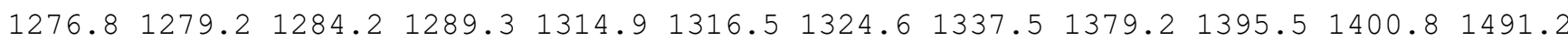
$1593.72654 .92656 .02668 .1 \quad 2672.3 \quad 2673.3 \quad 2679.02693 .22695 .42710 .02751 .22755 .8$ $2772.5 \quad 2773.22778 .02781 .0$

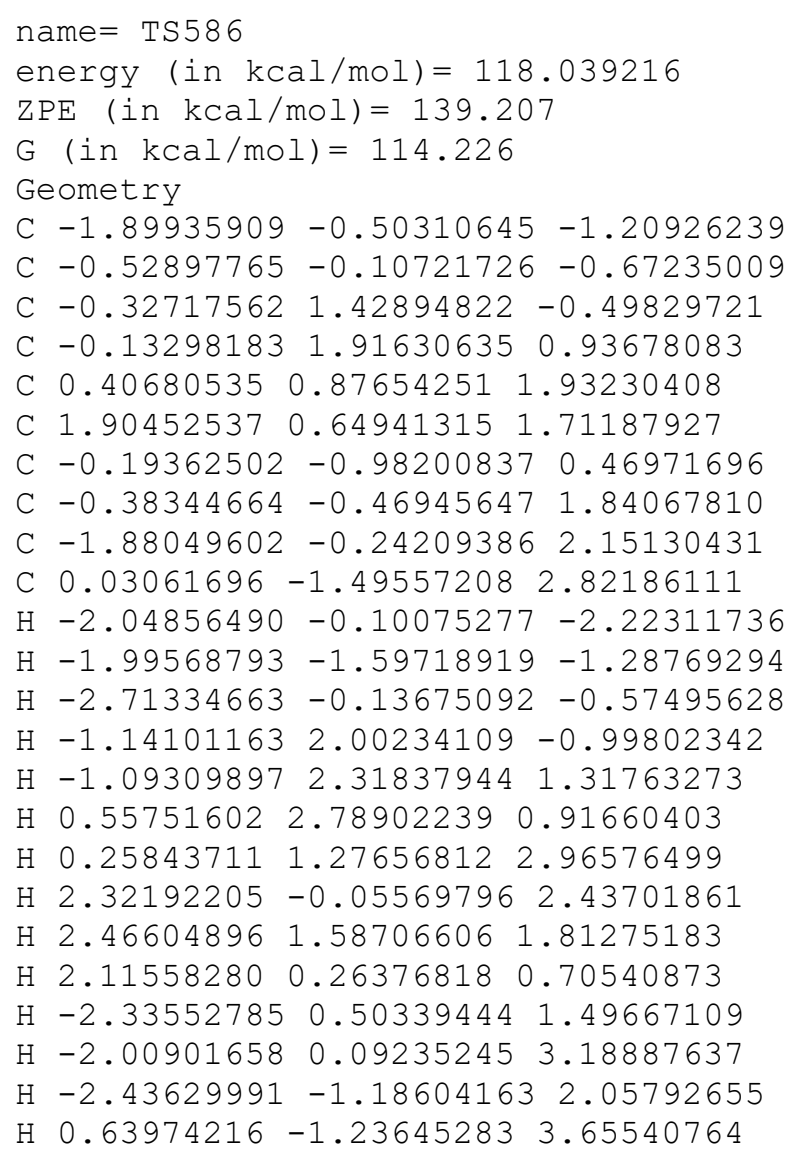


$\mathrm{H} \quad 1.30915612-2.274385352 .06407651$

$\mathrm{H} \quad 0.65031201-2.74690738 \quad 2.07159098$

$01.45765428 \quad 0.48068658-1.53422459$

$0.821553201 .73880482-1.28457410$

$0.39509199-0.41239749-1.74408458$

Vibrational frequencies (in $\mathrm{cm}-1$ )

$\begin{array}{lllllllllllllllllll}-946.1 & 38.7 & 88.9 & 107.2 & 130.9 & 152.7 & 159.9 & 186.4 & 201.4 & 210.0 & 250.7 & 262.1 & 296.8 & 309.7\end{array}$

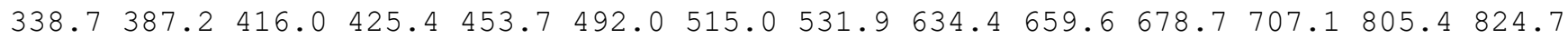
$845.0 \quad 870.3 \quad 903.7 \quad 933.0 \quad 962.6 \quad 977.3 \quad 1001.3 \quad 1013.6 \quad 1032.1 \quad 1041.3 \quad 1050.9 \quad 1064.7 \quad 1087.9$ $1097.4 \quad 1119.6 \quad 1148.7 \quad 1154.5 \quad 1176.4 \quad 1188.1 \quad 1208.7 \quad 1218.8 \quad 1256.3 \quad 1266.6 \quad 1277.6 \quad 1280.9$

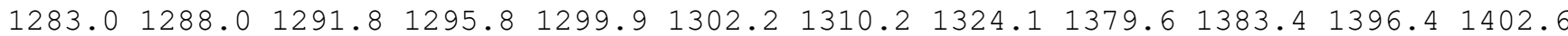
$1417.22651 .82661 .0 \quad 2663.92668 .5 \quad 2671.4 \quad 2675.32676 .3 \quad 2677.0 \quad 2698.2 \quad 2704.82733 .9$ $2774.02775 .92779 .4 \quad 4748.5$

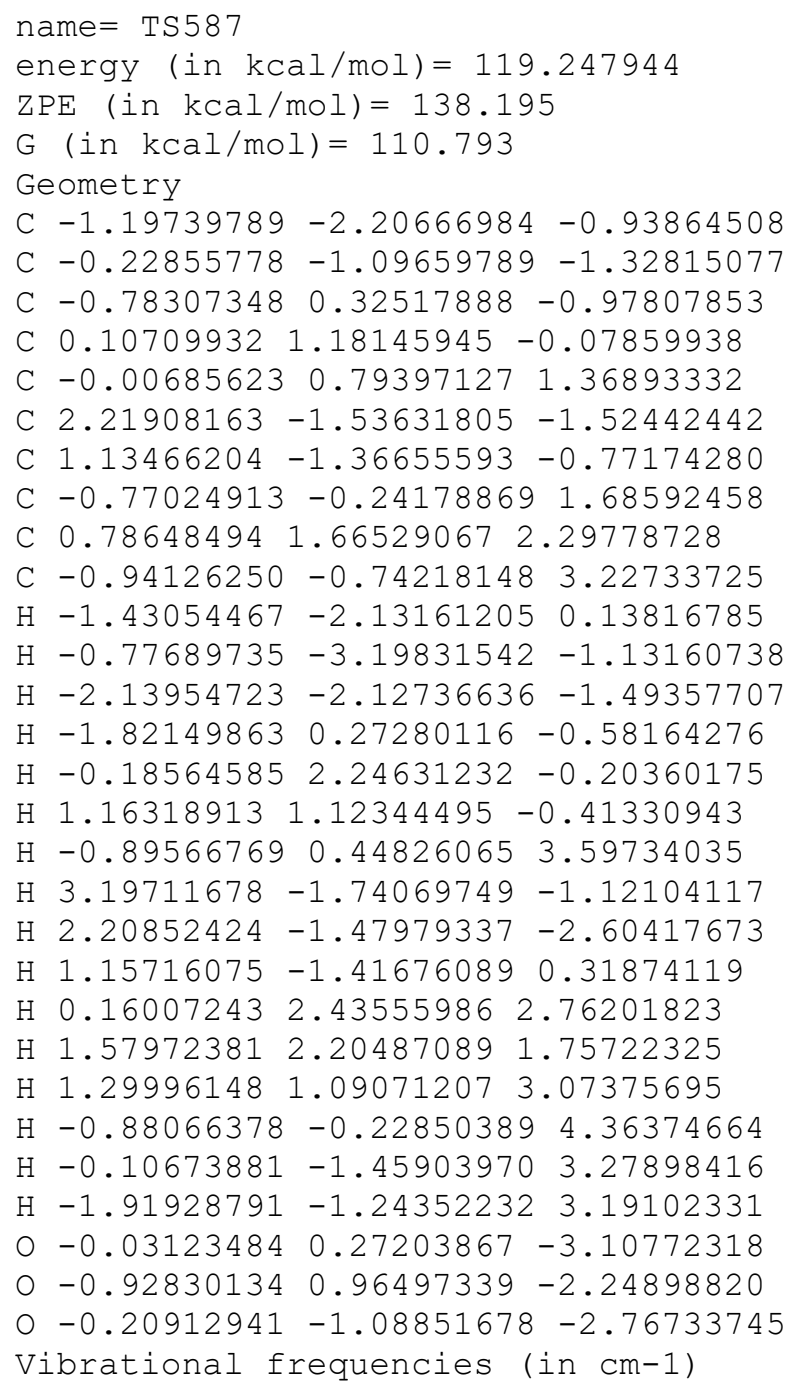


$\begin{array}{llll}\text { C }-0.88031216 & -0.86028878 & 0.74363278\end{array}$

C $-1.666230421 .04412505-0.18342100$

C $1.422726791 .46595053 \quad 0.80616298$

C $1.30143386 \quad 0.08065178 \quad 1.21496664$

C $1.44235214 \quad-0.98209497 \quad 0.09323937$

C $0.02848311-1.48452343-0.16261137$

C $-0.08800777-0.23955145 \quad 1.88344837$

C $-0.69299662 \quad 0.96100475 \quad 2.60549337$

C $0.12328302-1.35054427 \quad 2.94424551$

$\mathrm{H}-2.84085563-1.41538101 \quad 0.04832774$

$\mathrm{H}-2.78999696-0.92579649 \quad 1.74946641$

$\mathrm{H}-2.16465860-2.50291084 \quad 1.26748897$

$\mathrm{H}-2.40575627 \quad 1.00507390 \quad 0.61544632$

$\mathrm{H}-0.73522043 \quad 1.58873822-0.02313995$

$\mathrm{H} \quad 1.96966482 \quad 1.67598584-0.09936273$

H 2.10860563 $-0.05175381 \quad 1.99000736$

H $2.10430594-1.80542326 \quad 0.40876254$

$\mathrm{H} \quad 1.89506656-0.55657667-0.81789908$

$\mathrm{H}-0.07447469-2.57681250-0.32735754$

$\mathrm{H}-1.74642793 \quad 0.79425944 \quad 2.84909329$

$\mathrm{H}-0.60744080 \quad 1.89296386 \quad 2.03010911$

$\mathrm{H}-0.163451561 .16108275 \quad 3.54596768$

$\mathrm{H} \quad 0.77552227-1.01051704 \quad 3.75325332$

H $0.56905228-2.247695512 .50567940$

$\mathrm{H}-0.83262814-1.64380273 \quad 3.39127707$

$0-0.41216454-0.09819845-2.07680990$

$\begin{array}{llll}0 & -2.01595832 & 0.77109770 & -1.33698718\end{array}$

o $-0.38009806-1.19761304-1.64376744$

Vibrational frequencies (in $\mathrm{cm}-1$ )

$\begin{array}{lllllllllllllll}-597.3 & 52.0 & 78.0 & 119.3 & 156.0 & 176.5 & 187.8 & 198.9 & 204.0 & 224.7 & 239.3 & 250.8 & 269.8 & 291.3\end{array}$

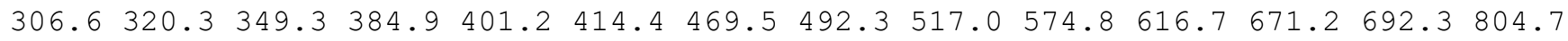
$860.5917 .3 \quad 931.3 \quad 967.6 \quad 989.1 \quad 1003.6 \quad 1021.0 \quad 1031.3 \quad 1050.6 \quad 1080.8 \quad 1092.01103 .9 \quad 1115.7$

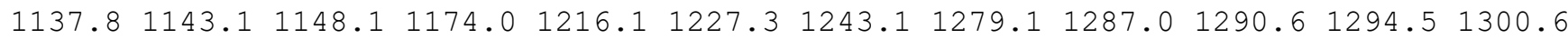
$1303.61309 .21317 .91321 .8 \quad 1339.2 \quad 1343.5 \quad 1376.0 \quad 1389.31401 .2 \quad 1417.4 \quad 1476.3 \quad 1658.9$ $2624.42657 .02657 .62660 .5 \quad 2665.0 \quad 2667.3 \quad 2669.02675 .22678 .0 \quad 2680.72682 .3 \quad 2685.4$ $2746.72771 .12777 .8 \quad 2781.6$

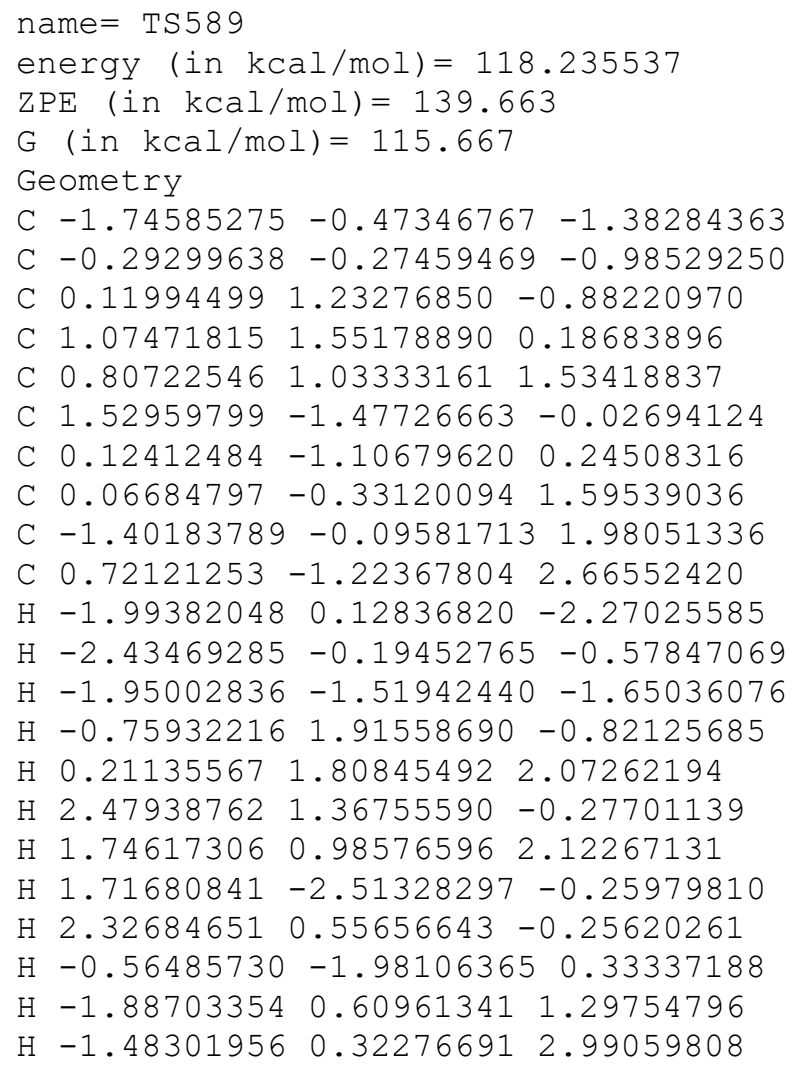


$\mathrm{H}-1.98051468-1.02540331 \quad 1.97099813$

$\mathrm{H} \quad 0.68236709-0.74889988 \quad 3.65271767$

$\mathrm{H} 1.77780887-1.416944392 .44223417$

H $0.21326149 \quad-2.18964869 \quad 2.75342514$

$\begin{array}{lllll}0 & 1.35753956 & 0.29134140 & -2.48572207\end{array}$

$0.66323281 \quad 1.47423105-2.18527877$

O $0.48072386-0.77691335-2.09078376$

Vibrational frequencies (in $\mathrm{cm}-1$ )

$\begin{array}{lllllllllllllllll}-929.5 & 49.7 & 98.0 & 159.4 & 160.1 & 187.8 & 211.8 & 216.7 & 239.8 & 253.4 & 284.6 & 302.4 & 307.8 & 341.3\end{array}$

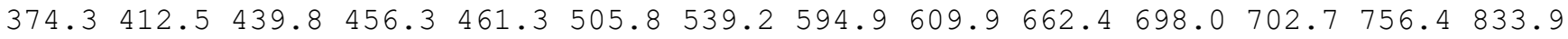
$881.3 \quad 889.9 \quad 899.7940 .1 \quad 965.8 \quad 977.3 \quad 978.8 \quad 987.1 \quad 1009.7 \quad 1025.1 \quad 1036.7 \quad 1041.9 \quad 1094.3$

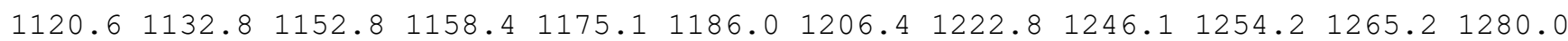
$\begin{array}{lllllllllllllll}1284.0 & 1285.9 & 1293.8 & 1302.8 & 1305.9 & 1308.6 & 1321.2 & 1330.3 & 1374.7 & 1382.7 & 1393.4 & 1402.9\end{array}$ $1407.92649 .82666 .32668 .12669 .5 \quad 2673.5 \quad 2674.32676 .22678 .02688 .02691 .92738 .8$ $2777.22778 .22780 .7 \quad 4501.9$

name $=\operatorname{TS} 590$

energy $($ in $\mathrm{kcal} / \mathrm{mol})=116.863806$

$\mathrm{ZPE}($ in $\mathrm{kcal} / \mathrm{mol})=141.372$

$\mathrm{G}($ in $\mathrm{kcal} / \mathrm{mol})=117.396$

Geometry

C $-2.23221597-0.05315948-1.00341823$

C $-0.71811746-0.13819872-0.86901862$

C $-0.01543269 \quad 1.28135200 \quad-0.81171554$

C $0.954206701 .43417847 \quad 0.27707678$

C $1.33153556-0.316532501 .52450422$

C $1.25052145-1.298540960 .34790599$

C $-0.30087176-1.002080050 .31844056$

C $-0.07277195-0.12473521 \quad 1.56958184$

C -1.029158290 .679666312 .33026806$

C $0.60276953-1.524158992 .99900899$

$\mathrm{H}-2.51272074 \quad 0.58895735-1.85145291$

$\mathrm{H}-2.70078154 \quad 0.35072105-0.10166790$

$\mathrm{H}-2.67135589-1.03931303-1.20352918$

$\mathrm{H}-0.76832894 \quad 2.09800092-0.84998878$

$\mathrm{H} \quad 0.80645492 \quad 2.22654666 \quad 0.97498039$

$\mathrm{H} 1.98702191 \quad 1.34618662-0.00003273$

H $2.17803016 \quad 0.09776893 \quad 2.02663086$

H $1.51386472 \quad-2.335500890 .56406889$

H $1.79237029-0.99071696-0.55652402$

$\mathrm{H}-0.93625160-1.88534103 \quad 0.44648736$

$\mathrm{H}-1.50657511 \quad 1.44373196 \quad 1.68691910$

$\mathrm{H}-0.56003140 \quad 1.22381756 \quad 3.16729630$

$\mathrm{H}-1.844281520 .06755785 \quad 2.75200010$

H $1.48064405 \quad-2.13358230 \quad 2.94091779$

$\mathrm{H}-0.28013633-2.102156512 .83771628$

H $\quad 0.57544184 \quad-0.91881967 \quad 3.87621082$

$\begin{array}{lllll}0 & 0.91247520 & 0.02643735 & -2.43722973\end{array}$

$\begin{array}{lllll}0 & 0.59078569 & 1.35297061 & -2.11619918\end{array}$

o $-0.25482552-0.72697037-2.07797992$

Vibrational frequencies (in $\mathrm{cm}-1$ )

$\begin{array}{lllllllllllllll}-1998.7 & 61.9 & 113.3 & 137.2 & 143.2 & 152.1 & 157.3 & 197.8 & 232.4 & 264.6 & 278.4 & 305.3 & 323.5 & 366.6\end{array}$

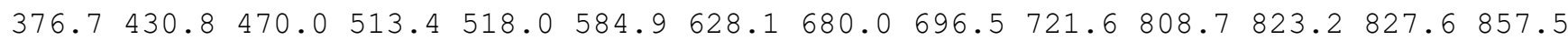
$898.8904 .6978 .2979 .7 \quad 983.7 \quad 988.6 \quad 998.9 \quad 1007.7 \quad 1019.6 \quad 1035.8 \quad 1048.7 \quad 1068.0 \quad 1087.5$

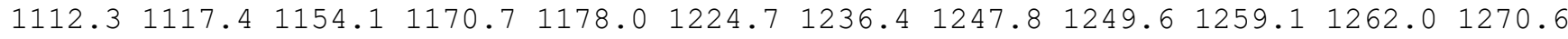

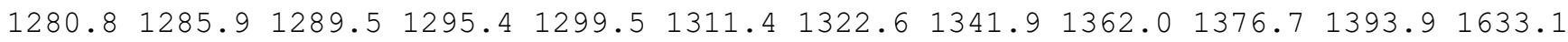
$2650.42663 .2 \quad 2668.7 \quad 2671.5 \quad 2681.4 \quad 2683.6 \quad 2702.02719 .92730 .4 \quad 2735.82751 .32756 .3$ $2771.4 \quad 2780.7 \quad 2782.9 \quad 2796.2$

name $=$ TS591

energy $($ in $\mathrm{kcal} / \mathrm{mol})=120.012255$

$\mathrm{ZPE}(\mathrm{in} \mathrm{kcal} / \mathrm{mol})=139.144$

$\mathrm{G}($ in $\mathrm{kcal} / \mathrm{mol})=114.77$ 


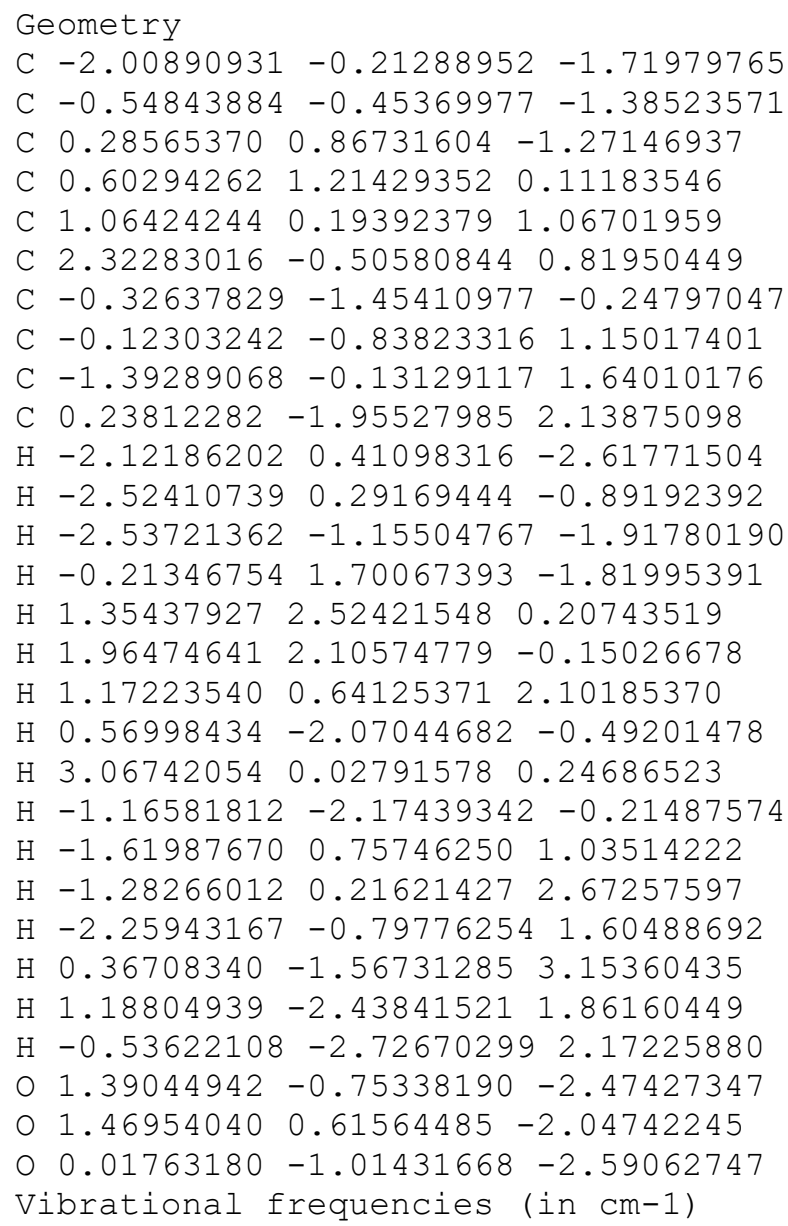

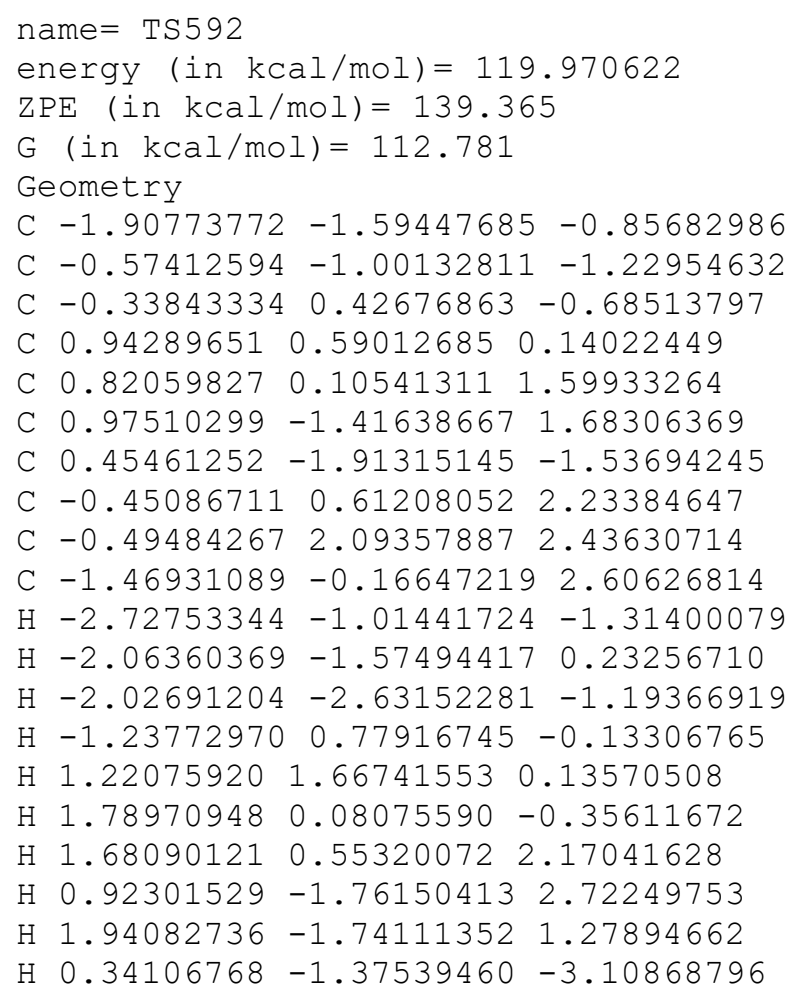


$\mathrm{H}-1.405182142 .419820232 .95748291$

$\mathrm{H}-0.46796782 \quad 2.63107815 \quad 1.47635542$

$\mathrm{H} 0.35891744 \quad 2.44507834 \quad 3.03153566$

$\mathrm{H}-2.36779658 \quad 0.21267552 \quad 3.06593063$

$\mathrm{H} \quad 0.19407830-1.940974691 .12250558$

$\mathrm{H}-1.49096986-1.23651864 \quad 2.49796217$

$\begin{array}{lllll}0 & -0.80799021 & 0.75414134 & -2.94616938\end{array}$

$0-0.151160331 .32021267-1.79321393$

o $-0.49872943-0.62771099-2.72412862$

Vibrational frequencies (in $\mathrm{cm}-1$ )

$\begin{array}{lllllllllllllllll}-1224.1 & 27.4 & 50.1 & 58.5 & 81.8 & 93.6 & 130.6 & 139.4 & 202.1 & 212.7 & 220.3 & 244.3 & 283.4 & 308.0\end{array}$

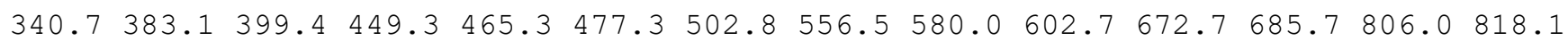
$853.6 \quad 862.2955 .9973 .3 \quad 992.7 \quad 996.6 \quad 1034.9 \quad 1038.1 \quad 1047.3 \quad 1075.8 \quad 1078.8 \quad 1088.1 \quad 1108.3$ $1125.71147 .1 \quad 1178.6 \quad 1190.9 \quad 1220.3 \quad 1229.6 \quad 1267.7 \quad 1274.4 \quad 1275.7 \quad 1280.6 \quad 1283.2 \quad 1289.6$

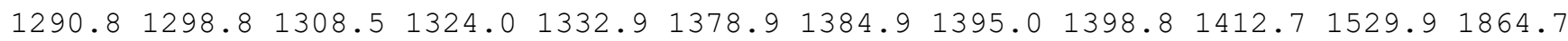
$2294.42652 .2 \quad 2657.6 \quad 2666.3 \quad 2666.8 \quad 2669.02671 .2 \quad 2674.02677 .2 \quad 2691.82722 .32738 .9$ $2772.0 \quad 2778.12781 .8 \quad 2792.9$

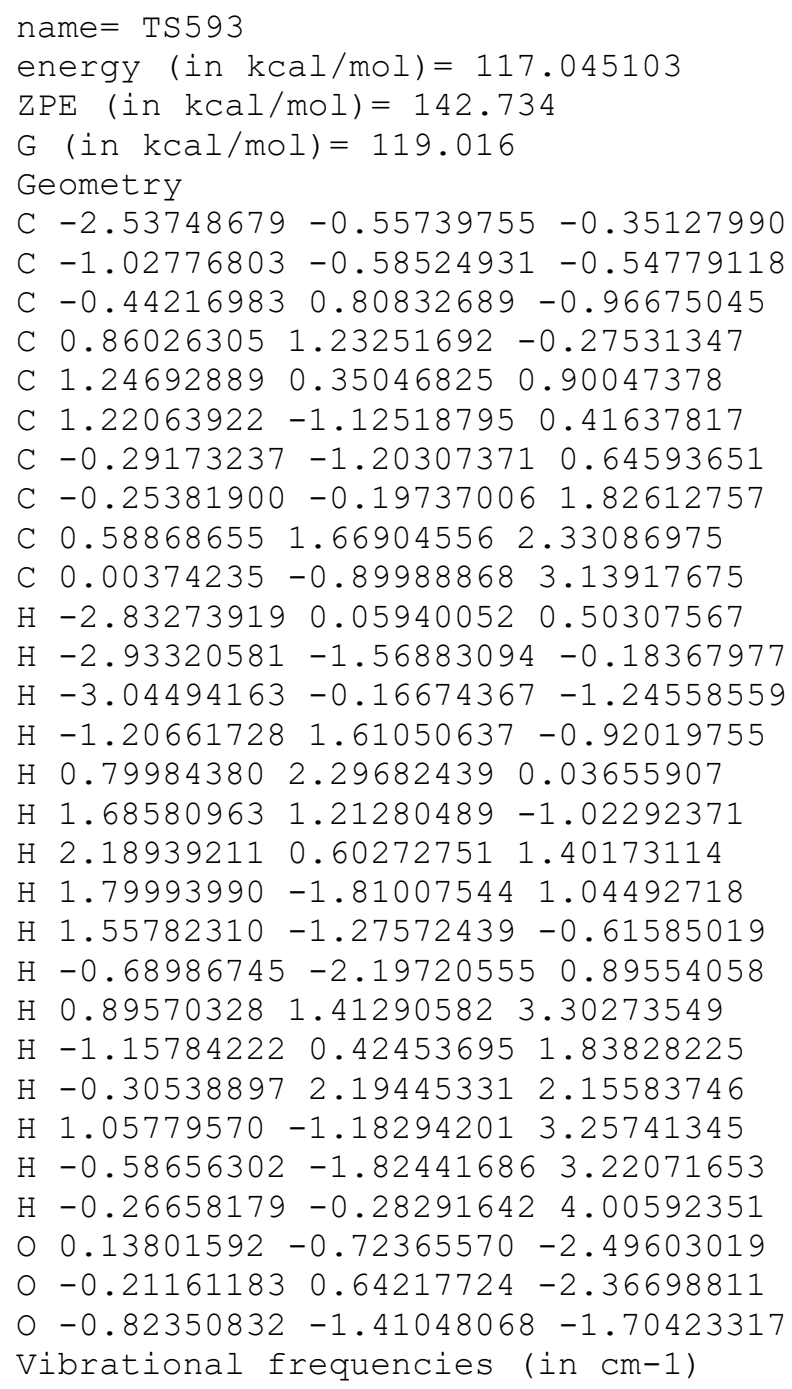
$415.0 \quad 464.3 \quad 480.1 \quad 501.5 \quad 562.6 \quad 620.0 \quad 657.9 \quad 708.1 \quad 756.2 \quad 834.4 \quad 859.6 \quad 865.7 \quad 886.2 \quad 917.4$

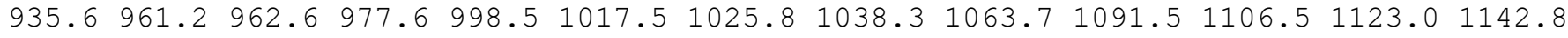

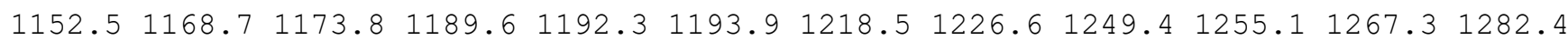
$1282.81286 .0 \quad 1290.8 \quad 1295.6 \quad 1305.2 \quad 1309.8 \quad 1314.8 \quad 1329.6 \quad 1366.1 \quad 1368.8 \quad 1394.01401 .9$ $2642.42665 .12667 .42667 .8 \quad 2676.2 \quad 2682.4 \quad 2689.42694 .52698 .52708 .72721 .2 \quad 2730.2$ $2734.02763 .02777 .3 \quad 2779.5$ 
$\mathrm{ZPE}($ in $\mathrm{kcal} / \mathrm{mol})=138.816$

$\mathrm{G}($ in $\mathrm{kcal} / \mathrm{mol})=114.763$

Geometry

C $-2.31834656-0.36925538-1.15654035$

C $-0.81779908-0.59378900-0.95060516$

C $-0.00703821 \quad 0.76919274-1.05537549$

C $1.09441944 \quad 0.92373015-0.00429098$

C $1.14357015-0.121747621 .15084129$

C $2.34755480-0.585799391 .54043818$

C $-0.45410694-1.37142298 \quad 0.21340036$

C $-0.22691368-0.79346932 \quad 1.51072868$

C $-1.23900320 \quad 0.26634474 \quad 1.99771230$

C $-0.06126407-1.83696000 \quad 2.62202083$

$\mathrm{H}-2.52938160 \quad 0.21670008-2.05608857$

$\mathrm{H}-2.77046959 \quad 0.11739195-0.28945166$

$\mathrm{H}-2.81858386-1.34230011-1.27159422$

$\mathrm{H}-0.678138391 .64543786-1.10234952$

$\mathrm{H} \quad 0.95787137 \quad 1.99952988 \quad 0.55187053$

$\begin{array}{lllll}\mathrm{H} & 2.08871867 & 0.98268121 & -0.49983347\end{array}$

$\mathrm{H} \quad 0.79176757 \quad 1.36406404 \quad 1.41477902$

H $2.48682373-1.197389912 .41404014$

H $3.25697786-0.446038810 .98632196$

$\mathrm{H}-0.25905227-2.41501984 \quad 0.05984125$

$\mathrm{H}-1.44733902 \quad 1.03222801 \quad 1.25093935$

$\mathrm{H}-0.92335816 \quad 0.73086418 \quad 2.93383727$

$\mathrm{H}-2.20098101-0.23008038 \quad 2.19528566$

$\mathrm{H} \quad 0.28671769-1.39216886 \quad 3.55691271$

H $0.65227372 \quad-2.617746192 .32887620$

$\mathrm{H}-1.01772827-2.33842597 \quad 2.81288342$

$0.0 .80261907-0.70169930-2.53730900$

$\begin{array}{lllll}0 & 0.61709250 & 0.71059526 & -2.32320298\end{array}$

o $-0.42693822-1.25921810-2.18581051$

Vibrational frequencies (in $\mathrm{cm}-1$ )

$\begin{array}{llllllllllllll}-1707.5 & 59.8 & 90.9 & 129.3 & 146.3 & 190.1 & 213.3 & 221.2 & 229.8 & 254.6 & 277.6 & 324.0 & 336.9 & 357.8\end{array}$ $397.4 \quad 419.7 \quad 426.4 \quad 467.6 \quad 476.8 \quad 503.6 \quad 518.8 \quad 591.9624 .7 \quad 657.1 \quad 687.7 \quad 690.9 \quad 722.8 \quad 784.6$

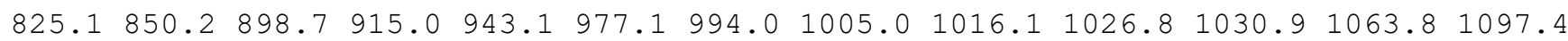

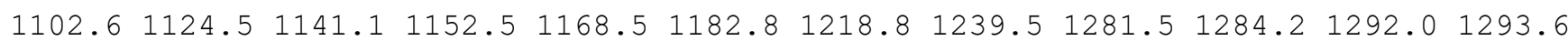
$1300.51303 .8 \quad 1308.4 \quad 1311.7 \quad 1343.4 \quad 1347.1 \quad 1383.4 \quad 1389.1 \quad 1398.4 \quad 1405.1 \quad 1510.21702 .1$ $\begin{array}{llllllllllll}1826.5 & 2562.9 & 2665.7 & 2668.4 & 2673.8 & 2675.5 & 2685.1 & 2686.9 & 2688.2 & 2690.9 & 2724.1 & 2778.8\end{array}$ $2781.7 \quad 2782.1 \quad 2785.5 \quad 2797.8$

\section{name $=\operatorname{TS} 595$}

energy (in $\mathrm{kcal} / \mathrm{mol})=123.079843$

$\operatorname{ZPE}($ in $\mathrm{kcal} / \mathrm{mol})=137.841$

G $($ in $\mathrm{kcal} / \mathrm{mol})=112.096$

Geometry

C $-2.58820649-0.60690139-1.17888139$

C $-1.13541453-0.89135066-0.95012241$

C $-0.01523297 \quad 0.25996930 \quad-0.83925724$

C $2.33743497 \quad 1.47235904 \quad 1.72769500$

C $0.47533751-0.38444287 \quad 0.47959481$

C $1.92155262 \quad-0.49603180 \quad 0.72529931$

C $-0.63920908-1.45974931 \quad 0.38714044$

C $-0.62360310-0.459544951 .53485694$

C $-1.63426877 \quad 0.64746135 \quad 1.68732558$

C $-0.24767000-1.044341392 .87987809$

$\mathrm{H}-2.73510548 \quad 0.24004842-1.86312826$

$\mathrm{H}-3.12014490-0.39207416-0.24300379$

$\mathrm{H}-3.09181627-1.47594143-1.63451527$

$\mathrm{H}-0.27997995 \quad 1.31435187-0.85465538$

$\mathrm{H} 1.33502196 \quad 1.82338597 \quad 1.77783226$

H $2.50697242 \quad 0.52956879 \quad 0.50151258$

$\mathrm{H} 2.72606813 \quad 1.06262233 \quad 2.62793274$

H $2.18137513-0.95768754 \quad 1.68280089$ 
$\mathrm{H} \quad 2.43093429-1.08357872-0.06072541$

$\mathrm{H}-0.59161492 \quad-2.52021851 \quad 0.53581772$

$\mathrm{H}-2.58938917 \quad 0.25906966 \quad 2.06789123$

$\mathrm{H}-1.845945391 .17829898 \quad 0.75250996$

$\mathrm{H}-1.28086574 \quad 1.40348342 \quad 2.40305195$

H $0.26301315-0.30305407 \quad 3.50727418$

H $0.41369585-1.915558212 .79303409$

$\mathrm{H}-1.14219171-1.378565923 .42368322$

O $0.69871027-1.38714534-2.17211418$

$0 \quad 0.83088529 \quad 0.01729314 \quad-1.92997163$

o $-0.69224853-1.65050600-2.06421072$

Vibrational frequencies (in $\mathrm{cm}-1$ )

$\begin{array}{llllllllllllll}-663.4 & 43.4 & 53.7 & 70.9 & 113.7 & 135.0 & 153.0 & 162.2 & 193.6 & 200.5 & 213.2 & 256.9 & 281.1 & 286.9\end{array}$

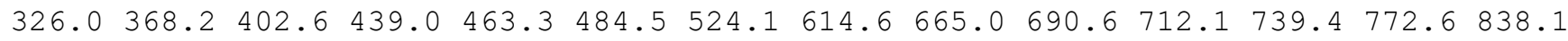

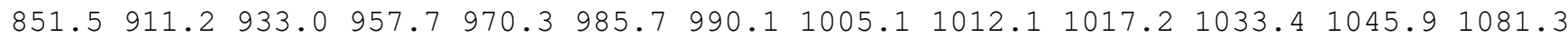

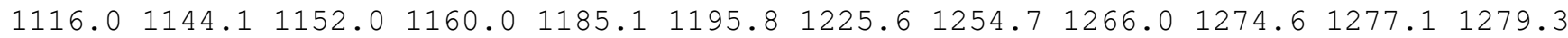

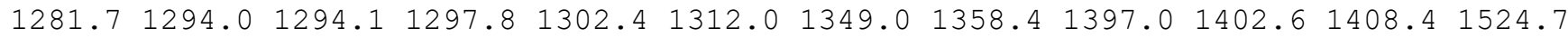
$2020.52658 .12664 .3 \quad 2669.5 \quad 2670.4 \quad 2672.92675 .3 \quad 2676.42689 .7 \quad 2690.82720 .32741 .2$ $2777.9 \quad 2778.8 \quad 2780.4 \quad 2783.4$

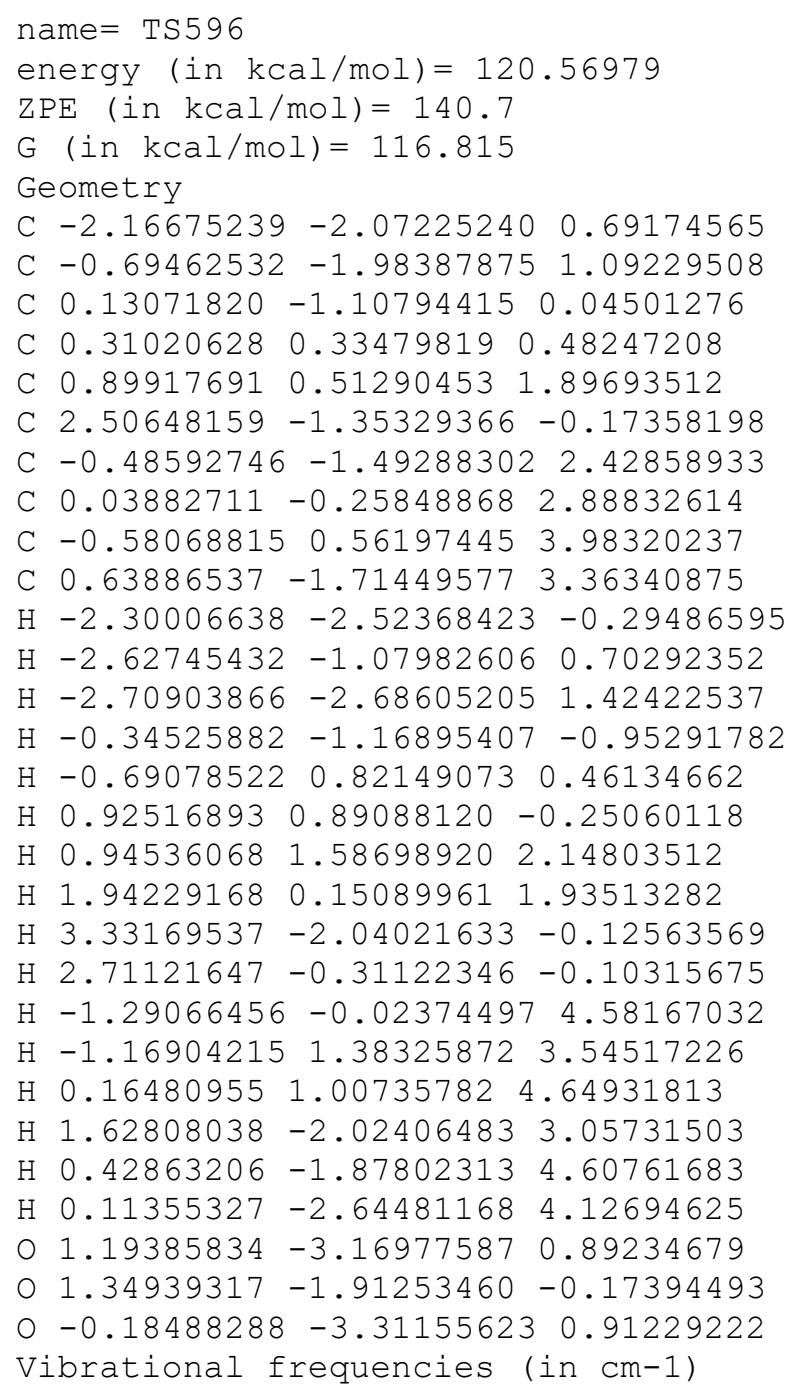




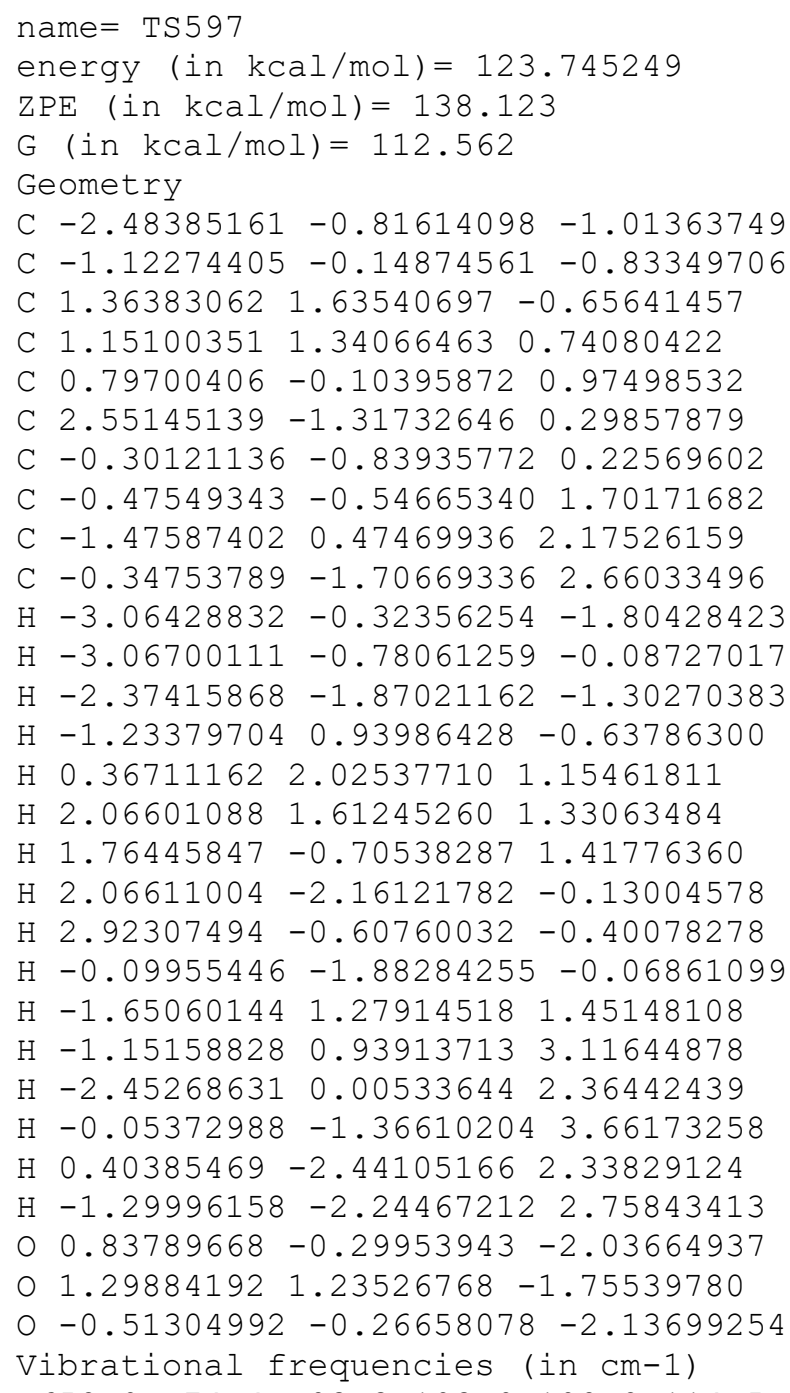


H $2.67416090 \quad 0.57986994 \quad 0.59446187$

$\mathrm{H} 1.20187217-1.394325021 .59017005$

H $1.56877620-1.52600802-0.11486173$

$\mathrm{H}-1.01702124 \quad-1.70733724 \quad 0.52477024$

$\mathrm{H}-2.02846048 \quad 0.93825137 \quad 1.91442990$

$\mathrm{H}-1.79909450 \quad 1.04078358 \quad 3.65696983$

$\mathrm{H}-2.94049500-0.136240612 .98036881$

$\mathrm{H}-0.25170942-2.646544012 .78919069$

$\mathrm{H}-2.00072873-2.39530623 \quad 2.71294694$

$\mathrm{H}-1.12344063-2.06531062 \quad 4.20948883$

o $-0.260934461 .26252552-2.41601036$

$0.095366422 .20580637-1.37504956$

O $-0.242074220 .01877306-1.80006759$

Vibrational frequencies (in $\mathrm{cm}-1$ )

$\begin{array}{lllllllllllllllll}-133.1 & 36.0 & 57.1 & 65.4 & 96.8 & 107.9 & 129.3 & 141.4 & 149.0 & 159.8 & 184.6 & 225.5 & 242.9 & 273.9\end{array}$

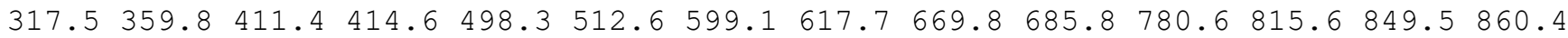

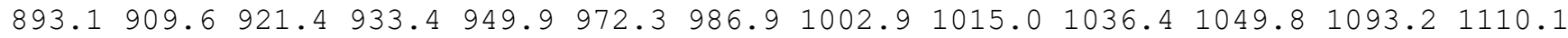

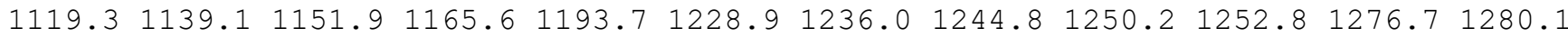

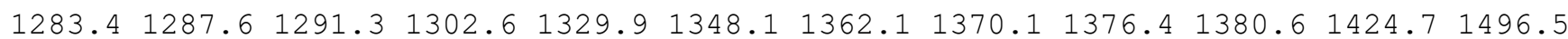
$2547.92611 .72659 .8 \quad 2667.2 \quad 2670.5 \quad 2673.2 \quad 2674.12676 .92680 .2 \quad 2685.02723 .12729 .1$ $2774.42776 .12781 .2 \quad 2796.1$

\author{
name $=$ TS599 \\ energy (in $\mathrm{kcal} / \mathrm{mol})=123.08516$ \\ $\mathrm{ZPE}(\mathrm{in} \mathrm{kcal} / \mathrm{mol})=140.748$ \\ G $($ in $\mathrm{kcal} / \mathrm{mol})=116.26$ \\ Geometry \\ C $-1.71173766-0.00544561-1.57971798$ \\ C $-0.23725961-0.08253597-1.19219111$ \\ C $0.372564961 .38020249-0.92048622$ \\ C $1.245433391 .56601241 \quad 0.21331060$ \\ C $1.12394156-0.28678671 \quad 1.55534293$ \\ C $1.51587192-1.14291487 \quad 0.33163811$ \\ C $-0.00550332-1.10385661-0.06697814$ \\ C $-0.29565161-0.43351250 \quad 1.25972717$ \\ C $-1.52840407 \quad 0.09314841 \quad 1.81160868$ \\ C $0.69418202-1.503014852 .77239582$ \\ $\mathrm{H}-1.82213267 \quad 0.56196696 \quad-2.51813412$ \\ $\mathrm{H}-2.31367955 \quad 0.49414583-0.81433239$ \\ $\mathrm{H}-2.13981007-0.99891450-1.76292282$ \\ $\mathrm{H}-0.45265602 \quad 2.12529750 \quad-0.96904577$ \\ H $0.96798731 \quad 2.260286250 .96927026$ \\ H 2.29439982 $1.47720868 \quad 0.07261877$ \\ $\mathrm{H} 1.72165385 \quad 0.44021990 \quad 2.08175472$ \\ H $1.91324077 \quad-2.13672147 \quad 0.53488748$ \\ $\mathrm{H} \quad 2.18613070-0.65478652-0.39262876$ \\ $\mathrm{H}-0.47049190-2.07135884-0.28090657$ \\ $\mathrm{H}-2.41729543-0.47673490 \quad 1.50801014$ \\ $\mathrm{H}-1.67142977 \quad 1.139229051 .44325023$ \\ $\mathrm{H}-1.53776897 \quad 0.17297271 \quad 2.90769077$ \\ $\mathrm{H} \quad 0.34045262-0.98558766 \quad 3.64978221$ \\ $\mathrm{H} 1.69606301-1.900983412 .94162019$ \\ H $0.04372690 \quad-2.32913921 \quad 2.49413969$ \\ $01.55748428 \quad 0.34038906-2.53111411$ \\ $\begin{array}{lllll}0 & 1.05448591 & 1.58795451 & -2.18341554\end{array}$ \\ O $0.45033833-0.56580377-2.33215771$ \\ Vibrational frequencies (in $\mathrm{cm}-1$ )
}

$\begin{array}{lllllllllllllllll}-945.1 & 53.9 & 60.9 & 102.2 & 138.0 & 171.5 & 179.9 & 183.6 & 238.0 & 255.1 & 270.1 & 309.1 & 322.0 & 343.9\end{array}$

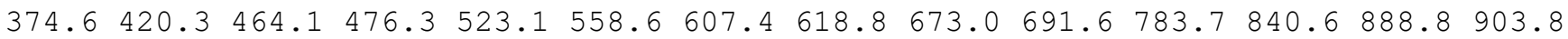

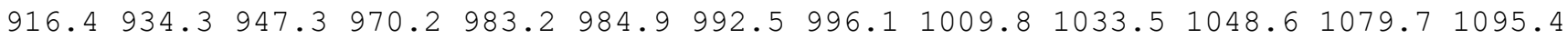

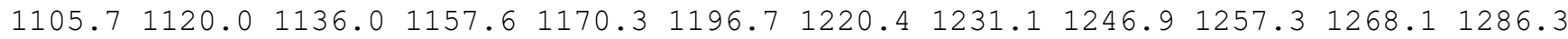

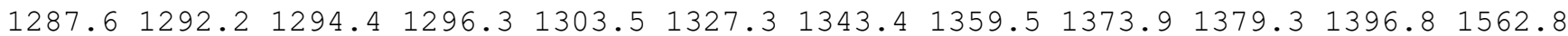
2621.22651 .62664 .72665 .72674 .62676 .42678 .42685 .52718 .92736 .72752 .62753 .0 $2763.22771 .2 \quad 2775.92806 .0$ 


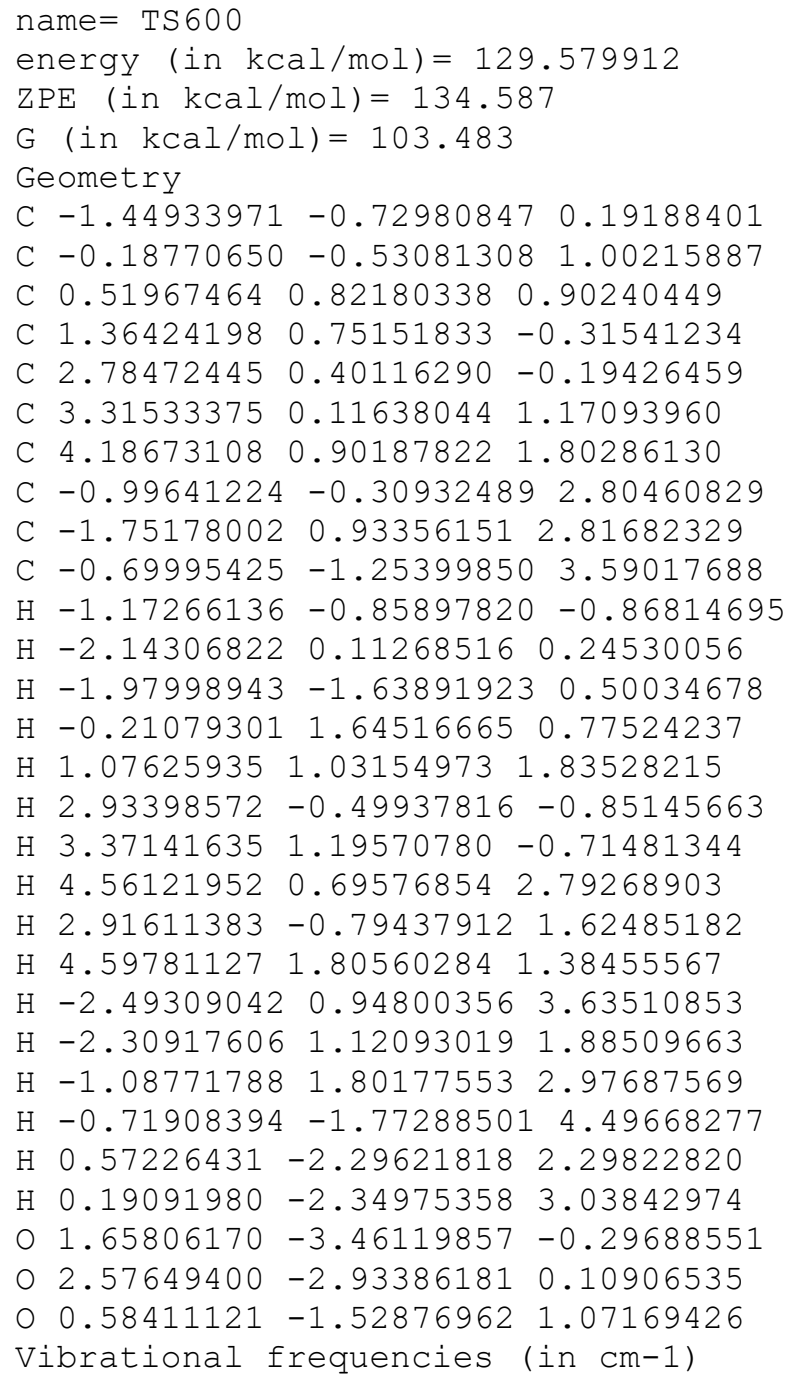


$\mathrm{H} \quad 0.80698521 \quad 2.53932303 \quad 1.20900602$

H $1.742936292 .01011946-0.19388878$

H $2.14390124 \quad 0.51423952 \quad 1.89245371$

H $1.92772201-1.605748120 .48033253$

$\mathrm{H} \quad 1.64820017-0.46628837-0.83834509$

$\mathrm{H}-0.60199258-1.902342530 .38902418$

$\mathrm{H}-2.91666269-0.08759047 \quad 1.95920683$

$\mathrm{H}-1.01386892 \quad 1.04041108 \quad 3.27310675$

$\mathrm{H}-2.74330419-0.38513080 \quad 2.64341464$

$\mathrm{H} \quad 0.65075640 \quad-0.82404708 \quad 3.82163787$

$\mathrm{H} 1.00320807 \quad-2.02610356 \quad 2.58061746$

$\mathrm{H}-0.64948181-1.82985890 \quad 3.16357264$

$0 \quad 0.31011473 \quad 0.82407317-2.33627769$

$0-0.15457637 \quad 1.99206934-1.67163569$

o $-0.57153128-0.19618093-1.90940817$

Vibrational frequencies (in $\mathrm{cm}-1$ )

$\begin{array}{llllllllllllllllllll}-802.9 & 85.0 & 123.6 & 142.0 & 147.9 & 169.6 & 179.4 & 202.1 & 215.0 & 244.8 & 254.8 & 277.2 & 309.0 & 335.7\end{array}$

$348.7 \quad 382.5 \quad 394.0 \quad 422.8 \quad 456.5 \quad 464.6 \quad 480.9 \quad 491.8 \quad 550.5 \quad 559.5 \quad 605.1 \quad 642.7 \quad 657.5 \quad 700.8$

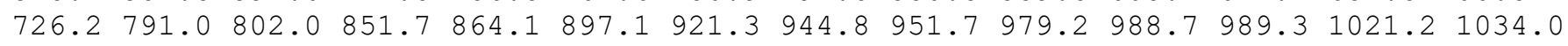
$1036.61049 .6 \quad 1070.5 \quad 1087.8 \quad 1095.3 \quad 1101.3 \quad 1132.1 \quad 1148.01168 .7 \quad 1182.3 \quad 1195.51204 .7$ $\begin{array}{llllllllllll}1234.6 & 1247.9 & 1263.0 & 1274.0 & 1287.4 & 1294.4 & 1297.1 & 1318.4 & 1343.2 & 1359.8 & 1369.4 & 1379.8\end{array}$ $1405.62657 .62663 .6 \quad 2672.8 \quad 2677.2 \quad 2683.7 \quad 2687.7 \quad 2710.82738 .4 \quad 2743.72747 .12765 .3$ $2780.4 \quad 4850.6 \quad 5461.1$

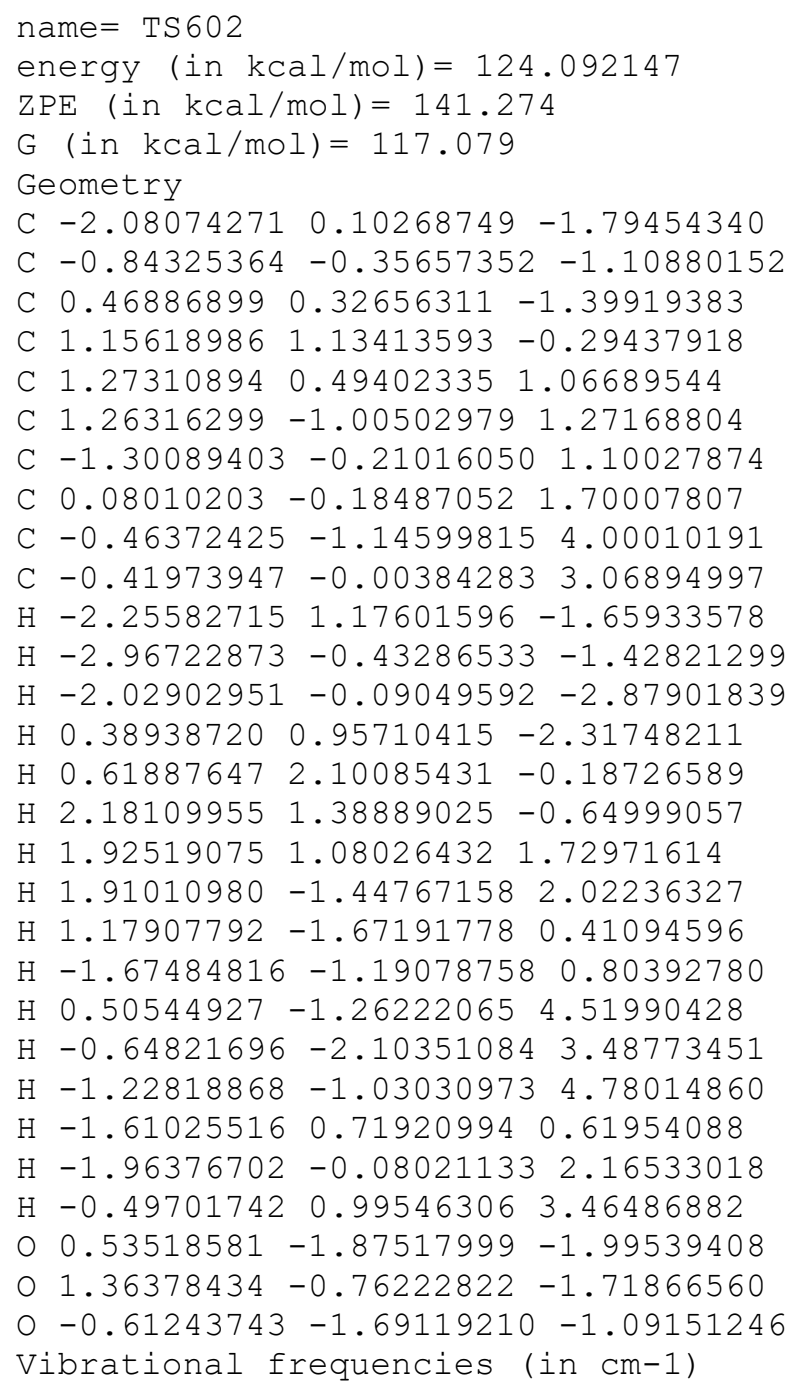


$2115.22650 .22658 .12661 .2 \quad 2666.4 \quad 2671.92673 .6 \quad 2683.02685 .0 \quad 2699.72732 .2 \quad 2747.7$ $2751.92774 .4 \quad 2777.4 \quad 2781.6$

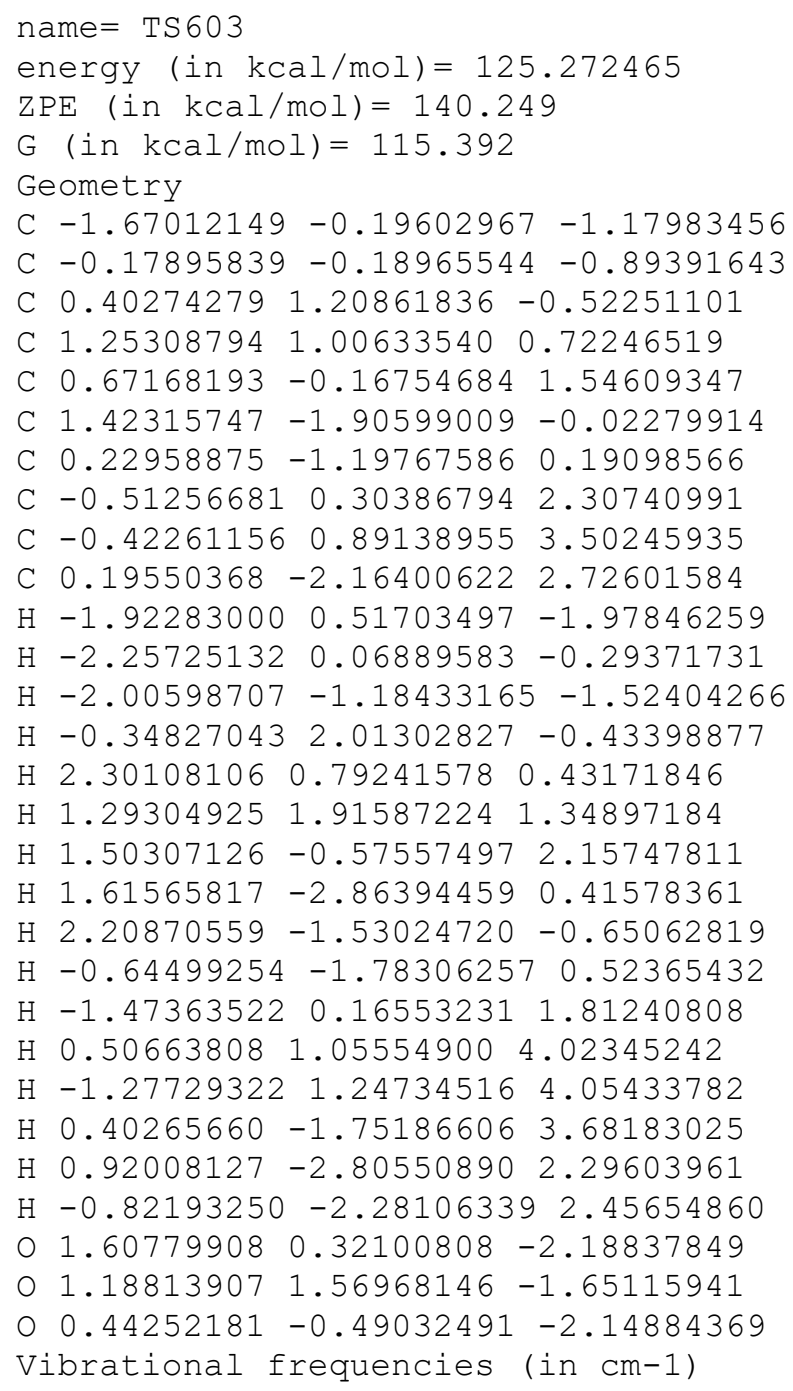


$\mathrm{H}-1.84221813-0.55826251-2.09822870$

$\mathrm{H}-0.78916752 \quad 2.36808891-0.03461039$

$\mathrm{H} \quad 1.62400841 \quad 2.89782995 \quad 0.62903242$

$\mathrm{H} \quad 2.18637612 \quad 1.42620187-0.16561088$

H $1.93539045-0.76262648 \quad 1.08089212$

$\mathrm{H} \quad 2.23727217-0.57679324-0.81171470$

$\mathrm{H} \quad 1.81770009-2.27528445-0.21607702$

$\mathrm{H}-0.61387573-1.68555080-0.15474727$

$\mathrm{H}-0.78157741 \quad 1.32904899 \quad 3.08360707$

H $0.07152035 \quad 2.79186044 \quad 2.60235707$

H $0.94684867 \quad 1.51805476 \quad 3.45259970$

$\mathrm{H} \quad 0.80952738-1.01679359 \quad 3.39246257$

$\mathrm{H} \quad 0.32107330-2.33689225 \quad 2.30792102$

$\mathrm{H}-0.84082000 \quad-1.09461718 \quad 2.76894288$

$\begin{array}{llllll}0 & 0.56687497 & 0.37039431 & -2.23465624\end{array}$

$\begin{array}{llll}0 & 1.39559746 & 1.28274087 & -2.24482485\end{array}$

O $0.97711658-0.82218349-2.43187402$

Vibrational frequencies (in cm-1)

$\begin{array}{lllllllllllllll}-1336.1 & 53.6 & 131.7 & 142.4 & 152.1 & 169.7 & 185.8 & 189.8 & 222.9 & 257.2 & 277.8 & 296.1 & 299.8 & 336.4\end{array}$ $355.2 \quad 387.2 \quad 438.2 \quad 489.8 \quad 519.3 \quad 542.8 \quad 591.8 \quad 627.1 \quad 639.3 \quad 717.8 \quad 728.1 \quad 758.8 \quad 797.8 \quad 836.5$ $905.6911 .2951 .1 \quad 972.2 \quad 1009.5 \quad 1027.8 \quad 1034.2 \quad 1048.2 \quad 1061.1 \quad 1079.4 \quad 1102.01108 .91114 .9$ $\begin{array}{llllllllllll}1123.1 & 1148.1 & 1169.3 & 1191.0 & 1214.6 & 1236.7 & 1254.1 & 1258.3 & 1264.5 & 1268.3 & 1286.4 & 1292.8\end{array}$

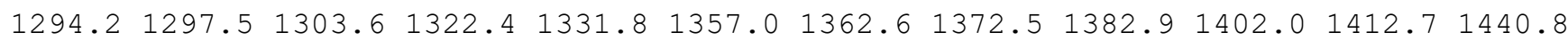
$1999.52591 .02645 .7 \quad 2663.5 \quad 2667.3 \quad 2670.0 \quad 2674.62675 .32690 .02703 .72734 .62761 .7$ $2771.12775 .7 \quad 2777.7 \quad 2780.3$

name $=$ TS605

energy $($ in $\mathrm{kcal} / \mathrm{mol})=130.887836$

$\mathrm{ZPE}(\mathrm{in} \mathrm{kcal} / \mathrm{mol})=138.586$

$\mathrm{G}($ in $\mathrm{kcal} / \mathrm{mol})=112.83$

Geometry

C $-1.83276191-1.16662932-0.60154220$

C $-0.52371914-0.42848983-0.86524645$

C $-0.53195575 \quad 1.04342382-0.34006588$

C $0.56923974 \quad 1.31338141 \quad 0.68344369$

C $0.69987253 \quad 0.14017449 \quad 1.67266992$

C $1.18488273-1.09518953 \quad 0.87702095$

C $0.57117186-1.31796174-0.42687920$

C $-0.59828602-0.188898172 .31584592$

C $-1.079905531 .57610595 \quad 3.45673657$

C $-0.64343574-1.22385794 \quad 3.36010899$

$\mathrm{H}-2.66680493-0.67616725-1.12171722$

$\mathrm{H}-2.06390806-1.18113283 \quad 0.47485590$

$\mathrm{H}-1.79355660-2.20324642-0.96275890$

$\mathrm{H}-1.53129077 \quad 1.34702601 \quad 0.03251476$

$\mathrm{H} \quad 0.36730319 \quad 2.25778197 \quad 1.22050273$

H $1.53258221 \quad 1.47883730 \quad 0.15986795$

H $1.48061227 \quad 0.40169184 \quad 2.42962099$

H $1.09338165 \quad-2.02816326 \quad 1.47881876$

H $2.28783714-1.01571005 \quad 0.70242994$

$\mathrm{H}-1.47682888 \quad 0.84855997 \quad 2.46143881$

$\mathrm{H}-1.82659916 \quad 1.37361065 \quad 4.20961765$

$\mathrm{H}-1.216453192 .544118912 .99497634$

$\mathrm{H}-0.08934516 \quad 1.49766508 \quad 3.87450782$

$\mathrm{H} \quad 0.22380788-1.24823508 \quad 4.03268790$

$\mathrm{H}-0.71174009-2.21747809 \quad 2.88091864$

$\mathrm{H}-1.54375897-1.14317274 \quad 3.98767467$

$\begin{array}{lllll}0 & 0.27599297 & 0.95157479 & -2.44359716\end{array}$

$\begin{array}{lllll}0 & -0.32899670 & 1.84197240 & -1.50105817\end{array}$

o $-0.44006361-0.25104300-2.29509773$

Vibrational frequencies (in $\mathrm{cm}-1$ )

$\begin{array}{llllllllllllll}-1243.1 & 34.3 & 59.8 & 84.0 & 92.5 & 110.8 & 134.1 & 164.5 & 183.5 & 234.6 & 254.3 & 274.2 & 303.3 & 312.4\end{array}$

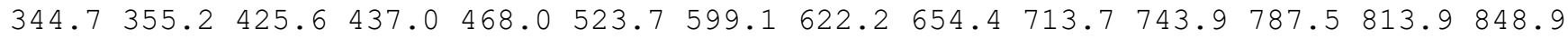
$907.8 \quad 909.5 \quad 953.8 \quad 970.1 \quad 974.8 \quad 997.7 \quad 1037.7 \quad 1048.4 \quad 1067.9 \quad 1092.1 \quad 1107.5 \quad 1124.4 \quad 1132.0$ 
$\begin{array}{llllllllllllllll}1152.7 & 1168.4 & 1182.5 & 1187.2 & 1204.5 & 1222.0 & 1234.3 & 1242.0 & 1256.9 & 1267.6 & 1275.3 & 1276.1\end{array}$

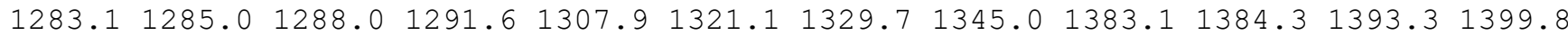
$1855.12636 .12659 .12660 .02660 .12668 .6 \quad 2669.32685 .52687 .5 \quad 2691.92692 .32726 .3$ $2744.8 \quad 2771.5 \quad 2772.12776 .7$

\section{name $=$ TS606}

energy (in $\mathrm{kcal} / \mathrm{mol})=129.940401$

$\mathrm{ZPE}(\mathrm{in} \mathrm{kcal} / \mathrm{mol})=140.79$

$\mathrm{G}(\mathrm{in} \mathrm{kcal} / \mathrm{mol})=117.487$

Geometry

C $-2.48671931-0.37029579-0.61525122$

C $-0.96131934-0.38544644-0.69722971$

C $-0.317143821 .05072364-0.82060232$

C 0.924255121 .304410860 .09084029

C $1.12771017 \quad 0.15845810 \quad 1.00010306$

C $1.25356410-1.140340030 .20111997$

C $-0.33176624-1.24686031 \quad 0.39892677$

C $-0.04870429-0.42025238 \quad 1.68883426$

$\begin{array}{llll}\text { C }-1.09713043 & 0.90846682 & 2.22401703\end{array}$

C $0.08793996-1.23687114 \quad 2.94599517$

$\mathrm{H}-2.91877280 \quad 0.35935616-1.31181306$

$\mathrm{H}-2.85166771-0.15885036 \quad 0.39151196$

$\mathrm{H}-2.89125596-1.35227894-0.90548747$

$\mathrm{H}-1.07027962 \quad 1.85186007-0.71898463$

$\mathrm{H} \quad 0.83254192 \quad 2.27580690 \quad 0.59694724$

$\mathrm{H} \quad 1.82231267 \quad 1.41225755-0.55872805$

$\mathrm{H} \quad 0.14268110 \quad 1.36323754 \quad 2.42624607$

$\mathrm{H} 1.81009479-1.94009073 \quad 0.68868782$

$\mathrm{H} \quad 1.59889820-1.05776472-0.82648095$

$\mathrm{H}-0.77343041-2.23311867 \quad 0.49221529$

$\mathrm{H}-1.67469597 \quad 1.143910201 .33410713$

$\mathrm{H}-0.66318351 \quad 1.959680112 .61836657$

$\mathrm{H}-1.709143510 .49566016 \quad 3.01724180$

$\mathrm{H} 0.50843413-0.66786555 \quad 3.78137028$

$\mathrm{H} \quad 0.80055213-2.06325838 \quad 2.77022089$

$\mathrm{H}-0.85580947-1.69331048 \quad 3.26055798$

O $0.34777006-0.21442208-2.55413463$

○ $0.05156281 \quad 1.12293596-2.19598193$

o - $0.70024707-0.98013357-1.97919383$

Vibrational frequencies (in $\mathrm{cm}-1$ )

$\begin{array}{lllllllllllllll}-775.1 & 87.6 & 109.3 & 142.1 & 162.8 & 180.9 & 215.1 & 222.5 & 255.0 & 289.1 & 314.9 & 340.4 & 373.1 & 405.4\end{array}$ $428.5 \quad 461.6 \quad 472.4 \quad 504.0 \quad 515.3 \quad 600.2 \quad 652.7 \quad 686.0 \quad 701.0 \quad 720.5 \quad 768.5 \quad 829.2 \quad 866.3 \quad 895.2$

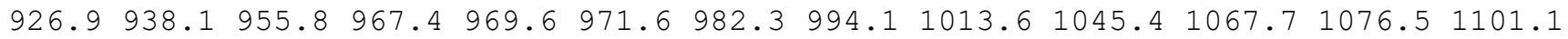

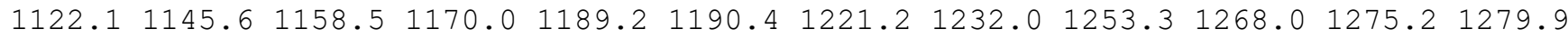

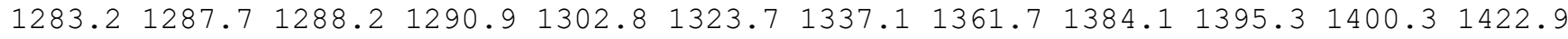
$1820.72658 .5 \quad 2660.8 \quad 2663.2 \quad 2666.7 \quad 2681.9 \quad 2685.12691 .82697 .72726 .12743 .52751 .8$ $2770.52779 .6 \quad 2780.2 \quad 2913.1$

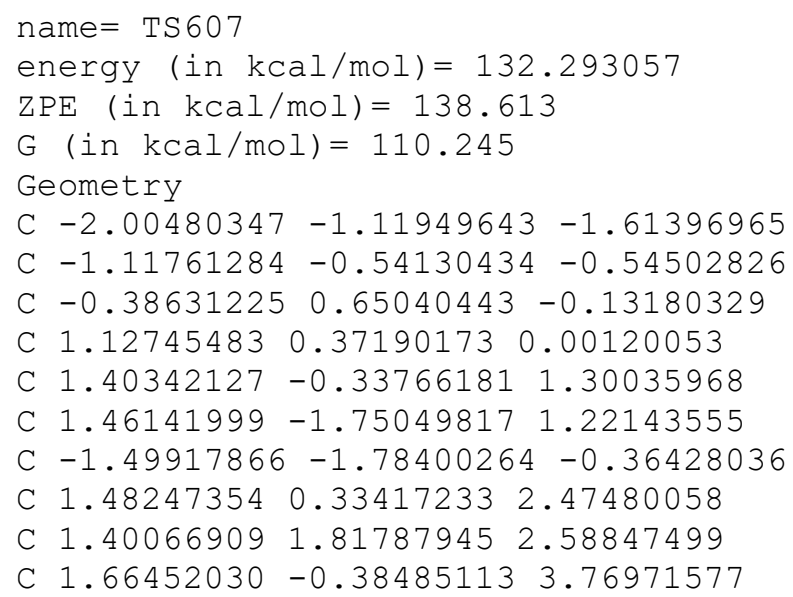


$\mathrm{H}-1.35099348 \quad 1.06058630-2.65101769$

$\mathrm{H}-3.04584404-0.82460430-1.69917855$

$\mathrm{H}-1.59857867-1.39646194-2.58421383$

$\mathrm{H}-0.78901524 \quad 1.04444162 \quad 0.84889186$

Н $3.04114553-2.105561891 .68979982$

$\mathrm{H} 1.48860254-0.22164553-0.86286627$

$\mathrm{H} 1.677485651 .33161963-0.06793244$

H $2.49608228-2.380586592 .22488488$

H $1.52339934-2.31409596 \quad 0.32785038$

$\mathrm{H}-1.51563001-2.678765320 .17077574$

H $2.07525577 \quad 2.20896011 \quad 3.36187181$

$\mathrm{H} \quad 0.37947226 \quad 2.12929174 \quad 2.85785692$

$\mathrm{H} \quad 1.65292527 \quad 2.33724997 \quad 1.65296491$

H $2.72011362-0.617141213 .96057291$

$\mathrm{H} \quad 1.10237532-1.33574618 \quad 3.77370454$

$\mathrm{H} \quad 1.30155980 \quad 0.19987754 \quad 4.62465468$

$0-0.36768134 \quad 1.42963181-2.48605797$

$0-0.62724941 \quad 1.73364420-0.96865580$

O $0.389685380 .49378300-2.72931622$

Vibrational frequencies (in $\mathrm{cm}-1$ )

$\begin{array}{llllllllllllllll}-1001.8 & 16.6 & 40.8 & 49.9 & 73.9 & 77.8 & 85.3 & 118.6 & 131.5 & 159.7 & 177.7 & 194.9 & 202.6 & 252.5\end{array}$ $295.0 \quad 305.1 \quad 367.2 \quad 383.1 \quad 437.1 \quad 452.5 \quad 496.6 \quad 505.1 \quad 527.8 \quad 583.7 \quad 662.0 \quad 685.4 \quad 724.7 \quad 766.1$

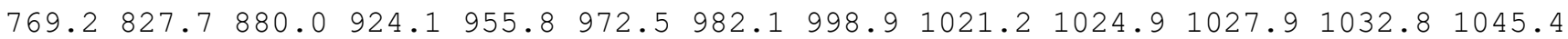

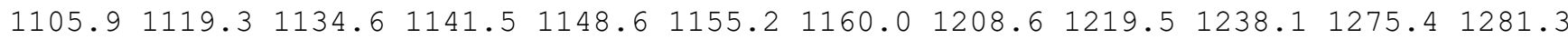

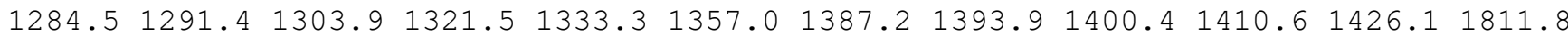
$2051.62643 .3 \quad 2657.3 \quad 2659.3 \quad 2662.7 \quad 2672.0 \quad 2673.5 \quad 2704.52737 .5 \quad 2740.62742 .32773 .1$ $2775.3 \quad 2776.4 \quad 2827.2 \quad 4719.3$

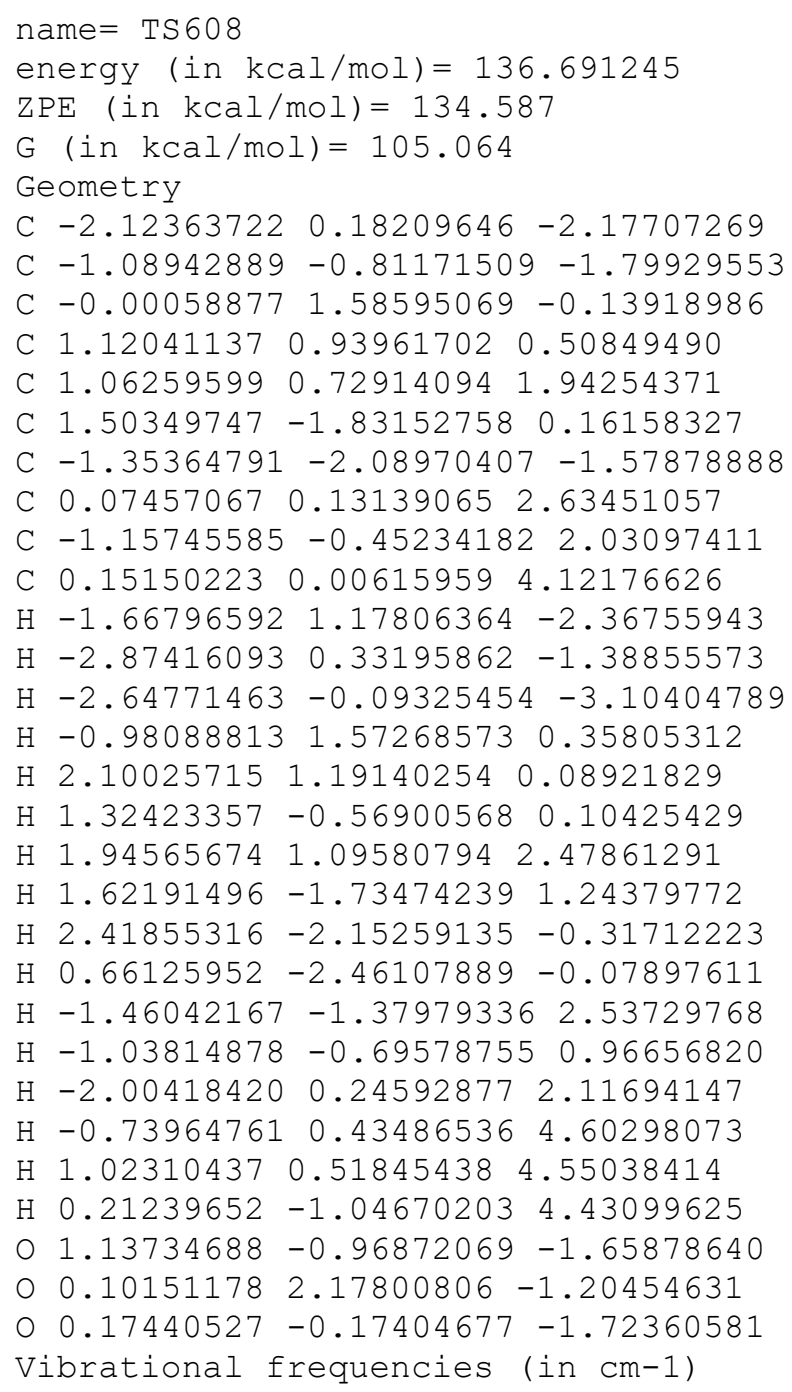


$\begin{array}{llllllllllllllllll}-1333.3 & 14.6 & 34.6 & 56.9 & 68.9 & 79.9 & 87.2 & 102.5 & 129.4 & 136.0 & 143.2 & 160.8 & 170.4 & 178.2\end{array}$ $225.5 \quad 249.8 \quad 269.2 \quad 306.7 \quad 332.4 \quad 345.5 \quad 361.8 \quad 385.5 \quad 466.5 \quad 479.0 \quad 497.3 \quad 532.6 \quad 611.2 \quad 664.3$

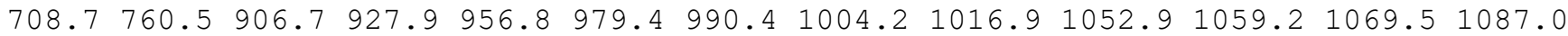
$\begin{array}{lllllllllllllll}1112.2 & 1144.7 & 1202.5 & 1249.9 & 1264.2 & 1270.0 & 1273.6 & 1276.8 & 1279.9 & 1280.1 & 1283.2 & 1286.9\end{array}$ $1295.01300 .2 \quad 1304.6 \quad 1329.9 \quad 1381.4 \quad 1390.8 \quad 1398.7 \quad 1410.5 \quad 1424.4 \quad 1440.2 \quad 1758.11831 .7$ $1843.72628 .92632 .6 \quad 2658.4 \quad 2665.3 \quad 2668.42669 .02671 .62674 .12685 .42731 .32743 .1$ 2753.32766 .02775 .12779 .3

name $=$ TS609

energy (in $\mathrm{kcal} / \mathrm{mol})=134.841143$

$\mathrm{ZPE}(\mathrm{in} \mathrm{kcal} / \mathrm{mol})=136.485$

$\mathrm{G}($ in $\mathrm{kcal} / \mathrm{mol})=106.764$

Geometry

C $-1.67275186-1.04494508-1.87593610$

C $-0.55368775-0.96559924-0.91309845$

C $0.009084162 .83686668-1.36329258$

C $1.309620692 .88986357-1.09077790$

C $0.30371334-1.04692262 \quad 2.52177825$

C $0.75874389-1.56677321 \quad 1.12763179$

C $-0.39088526-1.708874360 .19018039$

C $-0.38810345 \quad 0.25204064 \quad 2.28827756$

C $0.44371788 \quad 1.44573087 \quad 2.12187718$

C $-0.57727049-1.93943520 \quad 3.28342989$

$\mathrm{H}-2.35879666-1.87470005-1.65265021$

$\mathrm{H}-1.31411358-1.18791121-2.90825895$

$\mathrm{H}-2.27276890-0.11858948-1.86842044$

$\mathrm{H}-0.742618813 .60398284-1.18544601$

$\mathrm{H} \quad 1.790133293 .74566972-0.64702802$

H $1.998504492 .08490467-1.29699036$

$\mathrm{H} 1.22629820-0.92615366 \quad 3.15857718$

H $1.25431614-2.55151285 \quad 1.26451485$

$\mathrm{H} 1.54556180 \quad-0.89939574 \quad 0.72468999$

$\mathrm{H}-1.11342515-2.47542210 \quad 0.45465365$

$\begin{array}{lllll}\mathrm{H} & 0.02603760 & 2.27335491 & 2.71879815\end{array}$

$\mathrm{H} \quad 0.36319631 \quad 1.77562513 \quad 1.06553587$

$\mathrm{H} 1.509312021 .360681332 .35518917$

$\mathrm{H}-2.06565651-1.677708232 .58969572$

$\mathrm{H}-2.02548494-1.35281794 \quad 3.33489377$

$\mathrm{H}-0.61545650-2.98176280 \quad 3.07304187$

$\begin{array}{lllll}0 & 0.19403782 & 0.72781278 & -2.28643428\end{array}$

$\begin{array}{lllll}0 & -0.66145644 & 1.78664536 & -1.92684227\end{array}$

o $0.45296811-0.04205307-1.15443223$

Vibrational frequencies (in cm-1)

$\begin{array}{llllllllllllllll}-957.6 & 8.8 & 23.1 & 40.2 & 69.8 & 76.6 & 88.4 & 106.5 & 112.3 & 139.7 & 159.6 & 164.5 & 177.6 & 224.6\end{array}$ $259.3 \quad 275.2 \quad 332.2 \quad 357.2 \quad 401.1 \quad 434.6 \quad 476.6 \quad 499.5 \quad 525.9 \quad 564.8 \quad 611.5 \quad 634.5 \quad 654.2 \quad 702.4$ $810.4 \quad 841.4 \quad 866.6 \quad 880.5 \quad 898.4 \quad 923.6 \quad 931.9 \quad 942.8 \quad 988.6 \quad 1006.5 \quad 1010.9 \quad 1045.0 \quad 1060.0$ $1062.0 \quad 1071.2 \quad 1082.9 \quad 1129.8 \quad 1142.3 \quad 1164.3 \quad 1171.2 \quad 1198.91217 .2 \quad 1253.9 \quad 1263.6 \quad 1266.9$ $1271.7 \quad 1277.9 \quad 1280.6 \quad 1294.4 \quad 1327.8 \quad 1355.1 \quad 1371.5 \quad 1373.0 \quad 1376.8 \quad 1381.2 \quad 1404.0 \quad 1854.0$ $1868.32643 .62658 .12660 .52671 .42673 .12677 .5 \quad 2712.32712 .52735 .42736 .12764 .1$ $2767.8 \quad 2777.5 \quad 2792.3 \quad 4702.2$

name $=\operatorname{TS} 610$

energy (in $\mathrm{kcal} / \mathrm{mol})=135.137148$

$\operatorname{ZPE}($ in $\mathrm{kcal} / \mathrm{mol})=137.375$

$\mathrm{G}($ in $\mathrm{kcal} / \mathrm{mol})=113.118$

Geometry

C $-2.19266354-0.05187467-0.86251665$

C $-0.73314743-0.05159053-0.76067115$

C $-0.20594051 \quad 1.36567297 \quad-0.31398581$

C $0.69495430 \quad 1.36379082 \quad 0.93104520$

C $1.05273262-0.037991591 .44132327$

C $1.75290141-0.82970509 \quad 0.32039993$

C $-0.27556624-1.18597867 \quad 0.08851663$ 
C $-0.23466164-0.922888391 .57550467$

C $-1.44263593-0.235454332 .19309744$

C $0.04475963-2.207693562 .36048953$

$\mathrm{H}-2.61202473-0.29117181-1.82868147$

$\mathrm{H}-2.79475783-1.91197824 \quad 0.17845120$

$\mathrm{H}-2.61581146-2.22403892-0.49673815$

$\mathrm{H}-1.036634232 .09776726-0.20644590$

$\mathrm{H} \quad 0.20755505 \quad 1.93669290 \quad 1.74392915$

H $1.627646291 .92015968 \quad 0.69527048$

H $1.64601314 \quad 0.01980092 \quad 2.36806849$

H $2.42382453-1.605654110 .67652518$

$\mathrm{H} \quad 2.22418901-0.22695945-0.44805973$

$\mathrm{H} \quad 0.91126328-1.68560491-0.33464844$

$\mathrm{H}-2.27174166-0.94276032 \quad 2.32976216$

$\mathrm{H}-1.841934350 .57587966 \quad 1.57136493$

$\mathrm{H}-1.20035707 \quad 0.18301230 \quad 3.17572177$

$\mathrm{H} \quad 0.18143863-2.00065241 \quad 3.42684721$

$\mathrm{H} \quad 0.93747336-2.732376392 .00679073$

$\mathrm{H}-0.79392062-2.908713512 .25883632$

$\begin{array}{lllll}0 & 0.93520900 & 0.66201620 & -2.13529989\end{array}$

O $0.507982351 .83707063-1.44999343$

o $-0.18285411-0.19271712-2.09365216$

Vibrational frequencies (in $\mathrm{cm}-1$ )

$\begin{array}{lllllllllllllll}-1465.0 & 71.9 & 128.3 & 137.1 & 141.9 & 154.9 & 174.5 & 194.0 & 203.6 & 239.0 & 251.0 & 273.5 & 301.9 & 317.3\end{array}$

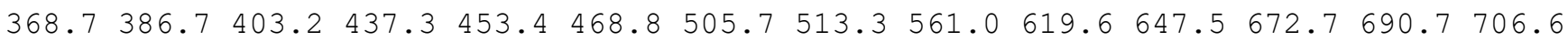

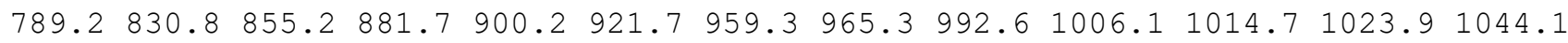

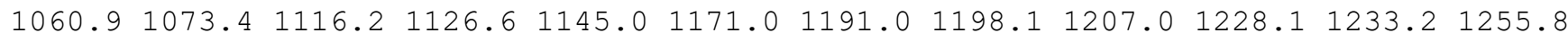

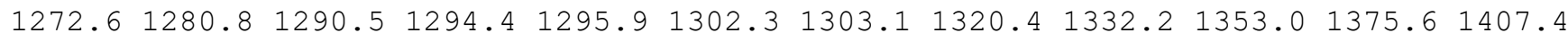

$1420.0 \quad 1994.7 \quad 2653.7 \quad 2664.5 \quad 2669.1 \quad 2675.3 \quad 2676.3 \quad 2679.4 \quad 2682.3 \quad 2683.02726 .5 \quad 2736.8$ $2743.52781 .0 \quad 2783.15482 .3$

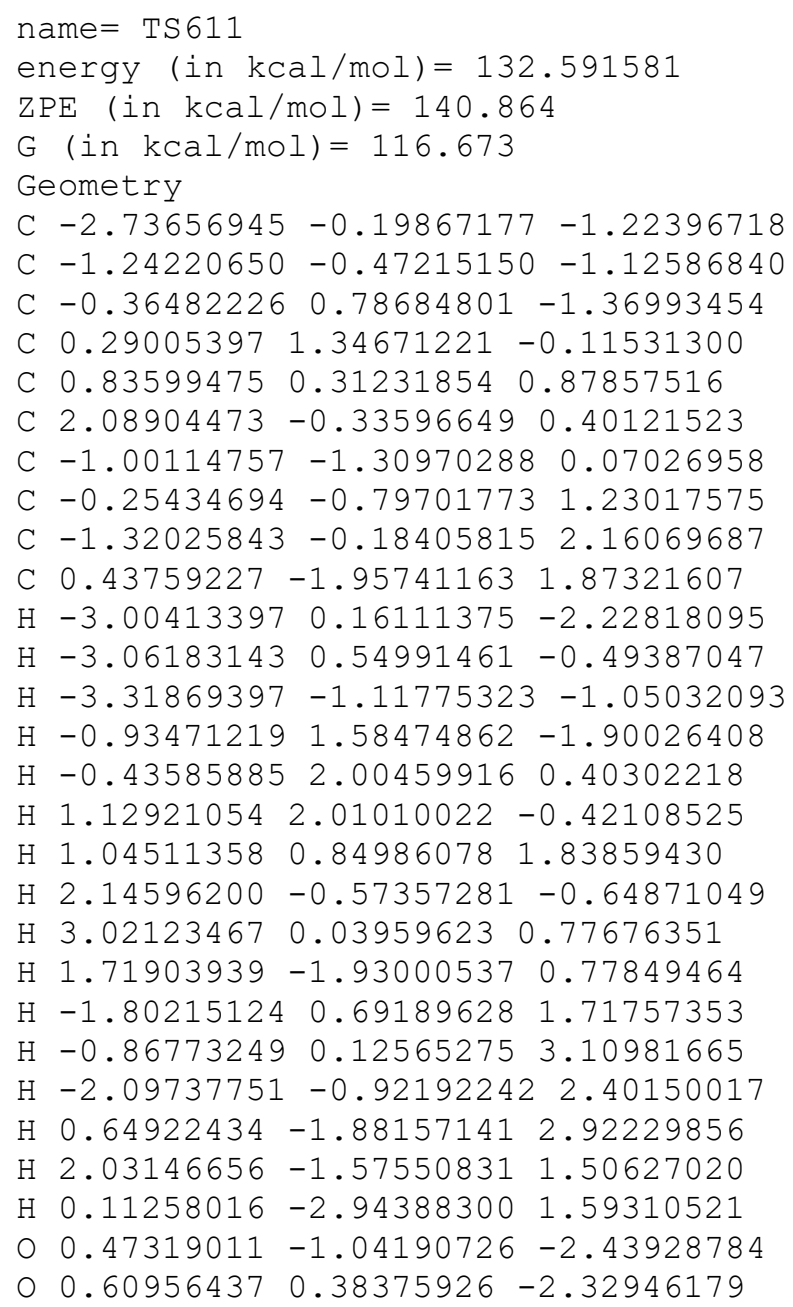


$0-0.90003968-1.28617415-2.27230065$

Vibrational frequencies (in $\mathrm{cm}-1$ )

$\begin{array}{llllllllllllllllll}-2065.9 & 46.8 & 64.3 & 127.5 & 149.3 & 178.6 & 189.8 & 213.1 & 237.6 & 272.8 & 288.4 & 313.1 & 354.8 & 382.5\end{array}$

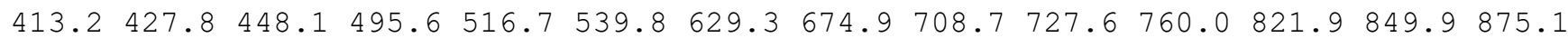
$895.8 \quad 906.1930 .7 \quad 963.4 \quad 974.9 \quad 981.0 \quad 997.3 \quad 1008.6 \quad 1022.7 \quad 1037.6 \quad 1049.8 \quad 1061.91066 .0$ $1085.3 \quad 1140.5 \quad 1162.1 \quad 1164.9 \quad 1177.3 \quad 1197.3 \quad 1213.3 \quad 1227.2 \quad 1238.0 \quad 1266.2 \quad 1274.21280 .0$ $1285.51295 .0 \quad 1298.6 \quad 1302.7 \quad 1305.2 \quad 1314.7 \quad 1344.8 \quad 1357.4 \quad 1368.0 \quad 1384.2 \quad 1390.0 \quad 1396.2$ $1421.22658 .7 \quad 2663.0 \quad 2671.3 \quad 2674.6 \quad 2674.7 \quad 2677.62687 .12715 .12717 .62736 .42770 .7$ $2775.5 \quad 2777.02781 .1 \quad 4063.0$

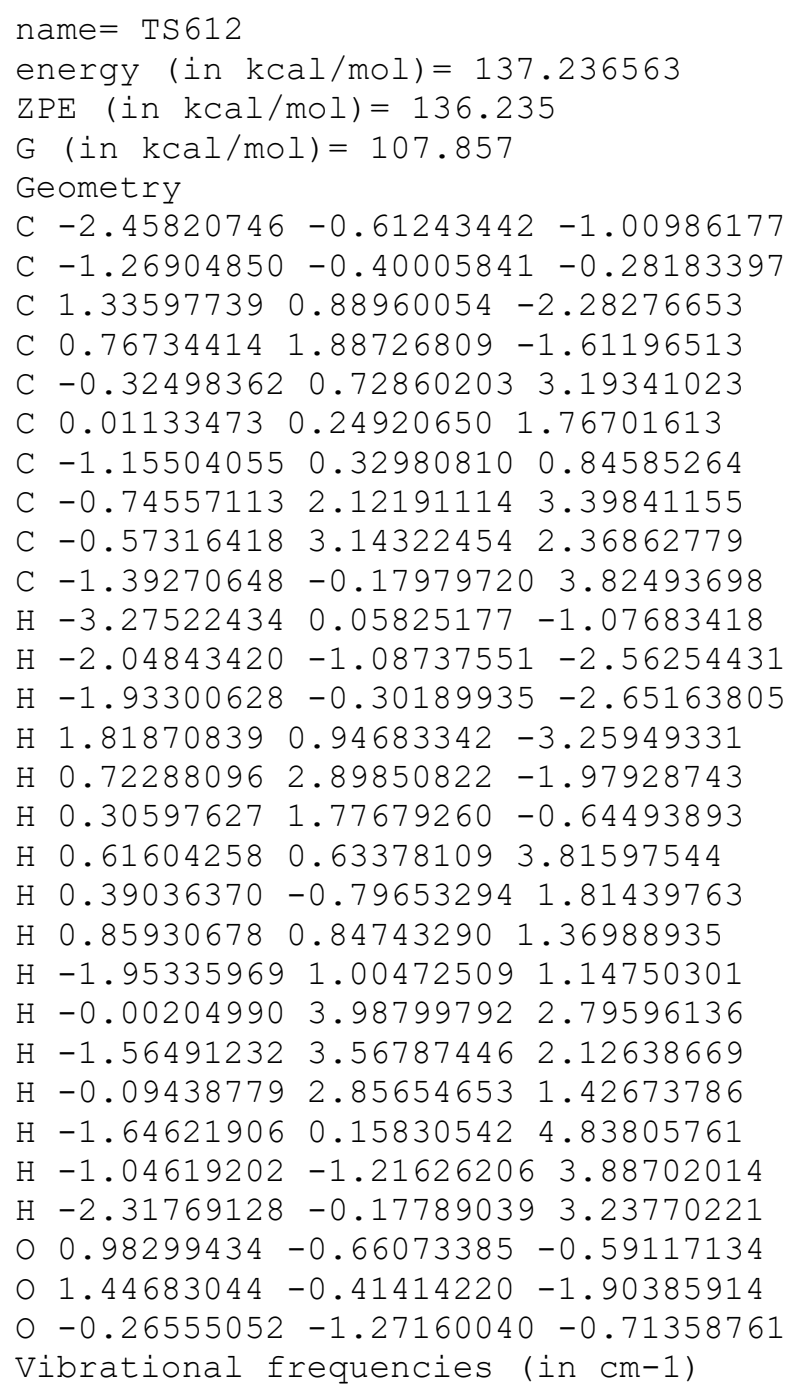


C $0.94036386-0.79608810 \quad 0.14854068$

C $-0.52439471-0.34236984 \quad 0.50855384$

C $-0.16489216-0.22814506 \quad 1.97259777$

C $-1.439251201 .02150894 \quad 2.97407976$

C $-0.27719722-1.413412542 .84907639$

$\mathrm{H}-2.39723198 \quad 2.20077762-0.91496043$

$\mathrm{H}-2.24905160 \quad 2.21855224 \quad 0.83925859$

$\mathrm{H}-2.89983303 \quad 0.78972883 \quad 0.01179759$

$\mathrm{H} 0.00708123 \quad 2.97291272 \quad 0.57145676$

$\mathrm{H} \quad 1.81028451 \quad 2.11140114 \quad 1.78025149$

H 2.52640991 $1.60705027 \quad 0.26109909$

$\mathrm{H}-0.13534199 \quad 0.93643877 \quad 2.57493747$

H $1.10613149-1.86922481 \quad 0.28991367$

$\mathrm{H} 1.26092432-0.55972510 \quad-0.86835778$

$\mathrm{H}-1.31158421-1.06303827 \quad 0.24887177$

$\mathrm{H}-1.44494048 \quad 2.09554279 \quad 2.83473115$

$\mathrm{H}-1.40381224 \quad 0.74351984 \quad 4.01874312$

$\mathrm{H}-2.28286802 \quad 0.55946150 \quad 2.47932921$

$\mathrm{H} \quad 0.10373354-1.225819893 .86375522$

H $0.36097242-2.229095552 .45345640$

$\mathrm{H}-1.29481006-1.81560262 \quad 2.92799674$

$0.637253651 .32302842-1.99806984$

$0.794290372 .47452336-1.19506549$

$\begin{array}{lllll}0 & -0.62813389 & 0.79199847 & -1.61716376\end{array}$

Vibrational frequencies (in $\mathrm{cm}-1$ )

$\begin{array}{llllllllllllll}-1331.1 & 68.3 & 80.7 & 97.7 & 130.7 & 152.5 & 191.8 & 206.6 & 218.4 & 226.2 & 260.3 & 303.9 & 329.8 & 345.2\end{array}$

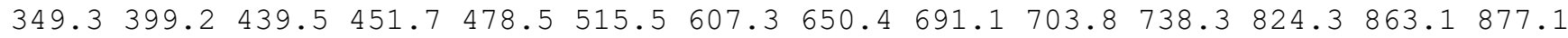

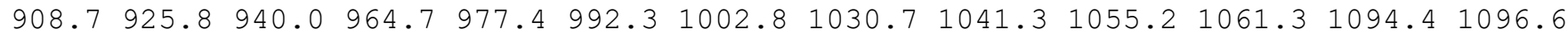

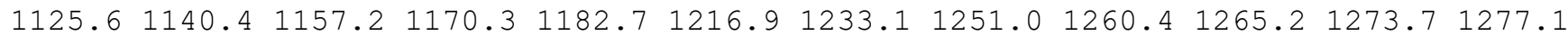

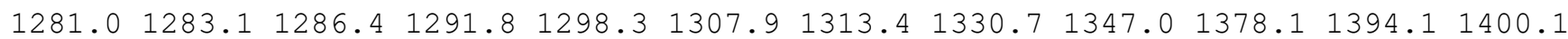
$1914.62650 .62657 .22658 .02671 .3 \quad 2674.62677 .92680 .32685 .62690 .62730 .62742 .8$ $2759.8 \quad 2765.7 \quad 2774.2 \quad 2778.6$

name $=\operatorname{TS} 614$

energy (in $\mathrm{kcal} / \mathrm{mol})=141.393948$

$\mathrm{ZPE}($ in $\mathrm{kcal} / \mathrm{mol})=132.368$

$\mathrm{G}(\mathrm{in} \mathrm{kcal} / \mathrm{mol})=101.994$

Geometry

C $-1.81534675-0.46764348-1.13271215$

C $-0.54220654-0.30282290-0.45571968$

C $0.37613206 \quad 0.94275967 \quad-0.79962509$

C $1.76466087 \quad 0.43927386-0.22552577$

C $1.46967466-0.72285171 \quad 0.66461307$

C $2.31451696-1.27482880 \quad 1.53726810$

C $0.06589556-1.148893910 .45534979$

C $-0.37297275 \quad 1.97710820 \quad 2.86604376$

C $-1.089489752 .58870891 \quad 1.83852278$

C $-0.06685399 \quad 0.70238820 \quad 3.27085202$

$\mathrm{H}-2.38570379-1.36297060-0.86695806$

$\mathrm{H}-1.60248459-0.50388088-2.23819327$

$\mathrm{H}-2.46450399 \quad 0.42019733-1.04569651$

$\mathrm{H}-0.004590791 .80414622-0.09357352$

H 2.31734327 $1.25109896 \quad 0.25638338$

H $2.37971022 \quad 0.13364118-1.09641066$

$\mathrm{H}-0.56907937-0.68078380 \quad 1.75630756$

H $2.07711440 \quad-2.12346036 \quad 2.15169070$

$\mathrm{H} 3.33144865-0.94263807 \quad 1.66270130$

$\mathrm{H}-0.19729720 \quad-2.18965584 \quad 0.59771959$

$\mathrm{H}-2.074580292 .16263851 \quad 1.58974728$

$\mathrm{H}-0.48449170 \quad 2.39136475 \quad 0.80798462$

$\mathrm{H}-1.17733404 \quad 3.68491743 \quad 1.90712250$

$\mathrm{H} 1.00000353 \quad 0.415899393 .12483360$

$\mathrm{H}-0.69256981-0.30522400 \quad 2.57158541$

$\mathrm{H}-0.37994799 \quad 0.41593421 \quad 4.28680853$ 
$0.0 .66649809 \quad 0.42228506-4.33038208$

○ $0.320318151 .25795725-2.01934654$

O $0.31205444-0.11214730-4.12302175$

Vibrational frequencies (in $\mathrm{cm}-1$ )

$\begin{array}{llllllllllllll}-1955.1 & 11.1 & 24.5 & 40.5 & 53.8 & 65.3 & 71.1 & 78.7 & 92.3 & 123.7 & 140.8 & 152.1 & 208.0 & 231.6\end{array}$ $255.1279 .2 \quad 294.6 \quad 326.6 \quad 330.9 \quad 438.5 \quad 479.0 \quad 504.3 \quad 524.7 \quad 565.8 \quad 613.1 \quad 630.7 \quad 650.9683 .5$

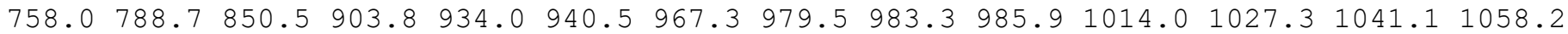
$\begin{array}{llllllllllllll}1061.1 & 1095.7 & 1113.1 & 1129.6 & 1139.6 & 1186.8 & 1213.2 & 1235.1 & 1246.3 & 1250.1 & 1258.1 & 1279.5\end{array}$ $1284.61303 .0 \quad 1335.3 \quad 1360.4 \quad 1381.5 \quad 1388.6 \quad 1477.1 \quad 1596.8 \quad 1607.8 \quad 1644.2 \quad 1669.91813 .0$ $1881.22415 .12588 .6 \quad 2599.4 \quad 2646.2 \quad 2660.6 \quad 2672.4 \quad 2679.92703 .2 \quad 2730.4 \quad 2745.3 \quad 2758.4$ $2761.0 \quad 2777.2 \quad 2800.9$

name $=$ TS615

energy (in $\mathrm{kcal} / \mathrm{mol})=133.534059$

$\mathrm{ZPE}($ in $\mathrm{kcal} / \mathrm{mol})=140.907$

$\mathrm{G}($ in $\mathrm{kcal} / \mathrm{mol})=116.842$

Geometry

C $-2.31788404-0.05679325-0.96811106$

C $-0.81291210-0.18571512-0.79425652$

C $-0.07600651 \quad 1.13743907-0.40468149$

C 1.330862051 .741132690 .79146188

C $0.99031202-0.04216092 \quad 1.19758721$

C $1.19056572-1.151990020 .18333227$

C $-0.37269022-1.271648860 .16806030$

C $-0.36393044 \quad-0.63137650 \quad 1.62833860$

C $-1.47635934 \quad 0.27395794 \quad 2.10761377$

C $-0.14561237-1.711202942 .69034645$

$\mathrm{H}-2.58705682 \quad 0.86031858-1.50864922$

$\mathrm{H}-2.85296405-0.06608374-0.01322967$

$\mathrm{H}-2.70738883-0.89831950-1.56290397$

$\mathrm{H}-0.76598757 \quad 1.93118146-0.10289420$

H $1.19548315 \quad 2.67181992 \quad 0.21993862$

H 2.37540273 $1.46996627 \quad 0.61559175$

$\mathrm{H} 1.106829592 .00504665 \quad 1.82621854$

H $1.70888399-2.02477254 \quad 0.59562373$

$\mathrm{H} 1.69491016-0.92406468-0.75425854$

$\mathrm{H}-0.82388554-2.24857107 \quad 0.04196999$

$\mathrm{H}-1.66620416 \quad 1.13624484 \quad 1.46441555$

$\mathrm{H}-1.22907090 \quad 0.68310048 \quad 3.09902205$

$\mathrm{H}-2.42084614-0.27607638 \quad 2.21167990$

H $0.18068300-1.261878013 .63698916$

H $0.61984949-2.44137863 \quad 2.40604283$

$\mathrm{H}-1.07099533-2.26666808 \quad 2.87986558$

$0.81679524 \quad 0.32881565-2.30309137$

O $0.56589318 \quad 1.53477462-1.58195969$

O $-0.35140775-0.44964666-2.13104031$

Vibrational frequencies (in $\mathrm{cm}-1$ )

$\begin{array}{lllllllllllllllll}-969.1 & 84.6 & 88.9 & 115.9 & 145.9 & 163.3 & 177.6 & 190.2 & 203.4 & 238.5 & 274.8 & 319.7 & 333.5 & 342.6\end{array}$

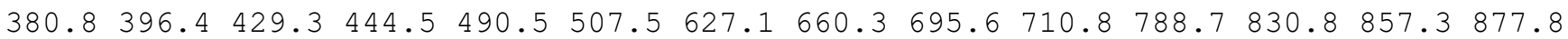
$925.4 \quad 930.3 \quad 946.2 \quad 977.6 \quad 987.7 \quad 999.1 \quad 1009.0 \quad 1014.0 \quad 1026.3 \quad 1039.2 \quad 1070.8 \quad 1094.6 \quad 1107.1$ $\begin{array}{lllllllllllll}1145.9 & 1152.2 & 1164.9 & 1177.8 & 1208.6 & 1219.4 & 1224.7 & 1255.8 & 1269.6 & 1277.7 & 1279.5 & 1284.5\end{array}$ $1287.61295 .7 \quad 1297.5 \quad 1302.3 \quad 1308.4 \quad 1322.6 \quad 1332.3 \quad 1360.8 \quad 1397.6 \quad 1406.3 \quad 1418.7 \quad 1451.3$ $2570.22660 .72664 .52667 .12672 .3 \quad 2672.82675 .12676 .32679 .12684 .12730 .52758 .0$ $2770.8 \quad 2778.0 \quad 2780.0 \quad 2780.9$

name $=\operatorname{TS} 616$

energy $($ in $\mathrm{kcal} / \mathrm{mol})=139.833762$

$\operatorname{ZPE}($ in $\mathrm{kcal} / \mathrm{mol})=137.096$

$\mathrm{G}($ in $\mathrm{kcal} / \mathrm{mol})=110.442$

Geometry

C $-3.34479650-1.07972424 \quad 0.07024379$

C $-2.01966118-0.76795673-0.61184336$

C $-1.29709260 \quad 0.48304575 \quad 0.00314385$ 
$\begin{array}{lllll}\text { C } & 0.16926800 & 0.27839018 & 0.16762327\end{array}$

C $0.80609057-0.004729691 .31143286$

C $2.29116315-0.198154601 .30561262$

C $-1.13437279-1.92118263-0.58962790$

C $0.17121793-0.19724253 \quad 2.66731192$

C $-0.90730392 \quad 0.76609229 \quad 2.94822034$

C $-0.46692841-1.60190907 \quad 2.71963377$

$\mathrm{H}-4.03269246-0.22641464-0.01344901$

$\mathrm{H}-3.20614365-1.29391515 \quad 1.13896203$

$\mathrm{H}-3.84092598-1.94432558-0.38582736$

$\mathrm{H}-1.81476002 \quad 0.83874644 \quad 0.92730630$

$\mathrm{H} \quad 0.72686051 \quad 0.40551914 \quad-0.76452881$

H $2.73227781-0.09698554 \quad 0.30444842$

$\mathrm{H} \quad 0.97437161-0.165400493 .45133412$

H $2.79634899 \quad 0.53151956 \quad 1.95207680$

H 2.55665132 -1.20204898 1.66969750

$\mathrm{H}-0.74555945-2.28002017-1.52811391$

$\mathrm{H}-0.50811763 \quad 2.20049136 \quad 2.49459494$

$\mathrm{H}-0.08290965 \quad 2.17956419 \quad 3.18977933$

$\mathrm{H}-1.36751994 \quad 0.79946304 \quad 3.90547467$

$\mathrm{H} \quad 0.29817941 \quad-2.37997853 \quad 2.62755494$

$\mathrm{H}-1.16671417-1.74854625 \quad 1.88045575$

$\mathrm{H}-1.01429778-1.76621585 \quad 3.65100760$

$0-2.477021421 .04517795-1.86169008$

$0-1.43236173 \quad 1.49779444-1.00602126$

o $-2.26405436-0.34461500-1.96741281$

Vibrational frequencies (in cm-1)

$\begin{array}{llllllllllllll}-1015.6 & 42.8 & 55.7 & 81.9 & 95.7 & 101.1 & 121.7 & 135.8 & 157.6 & 182.3 & 190.5 & 228.5 & 239.4 & 254.5\end{array}$ $279.1309 .1 \quad 341.5 \quad 391.4 \quad 402.5 \quad 446.9 \quad 495.1 \quad 523.5 \quad 558.3 \quad 568.0 \quad 598.9650 .1 \quad 669.8 \quad 700.2$

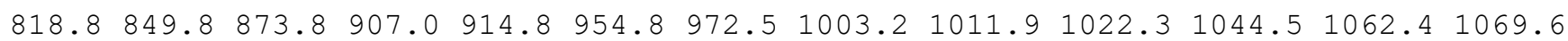

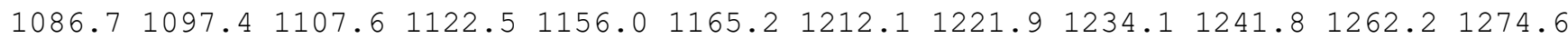

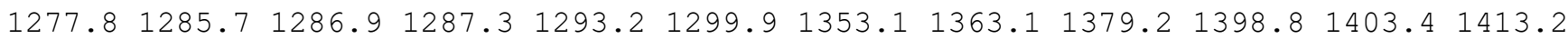
$1872.82618 .2 \quad 2652.5 \quad 2668.92669 .6 \quad 2672.4 \quad 2674.42675 .2 \quad 2683.0 \quad 2683.7 \quad 2719.3 \quad 2743.5$ $2776.82778 .3 \quad 2778.9 \quad 4680.3$

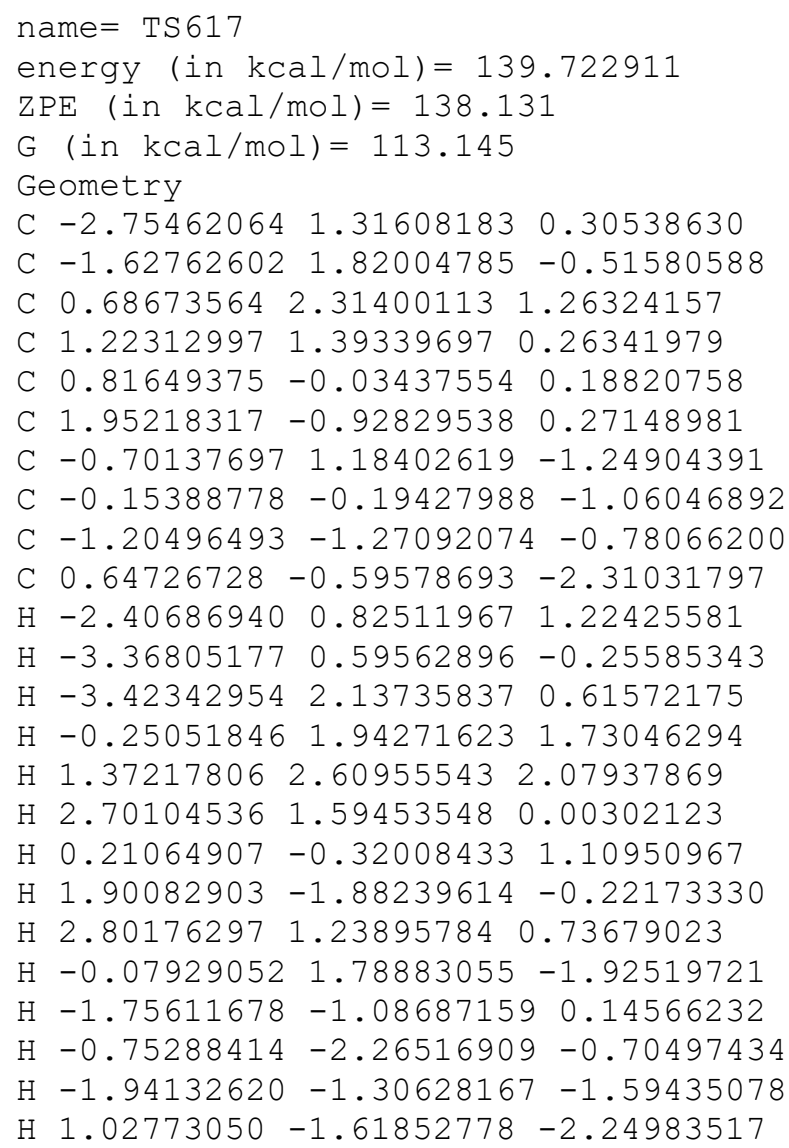


H $1.50015325 \quad 0.07672131-2.46379042$

$\mathrm{H} \quad 0.01839401-0.53361790 \quad-3.20708437$

$0-0.188703153 .57265249-0.52113204$

$\begin{array}{lllll}0 & 0.35143138 & 3.61528648 & 0.73305928\end{array}$

$0-1.573690113 .20320003-0.44770481$

Vibrational frequencies (in $\mathrm{cm}-1$ )

$\begin{array}{lllllllllllllll}-1079.4 & 43.3 & 92.2 & 99.0 & 138.0 & 162.1 & 177.3 & 205.4 & 209.6 & 231.2 & 250.5 & 258.1277 .2297 .7\end{array}$ $330.2 \quad 363.0 \quad 402.6 \quad 449.2 \quad 464.0 \quad 472.3 \quad 496.9 \quad 529.1 \quad 551.6 \quad 607.5 \quad 616.0 \quad 633.2 \quad 657.0 \quad 714.3$

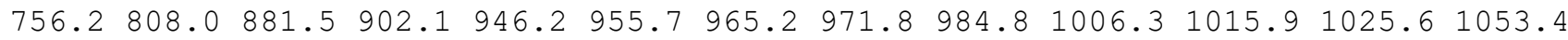
$1058.51090 .6 \quad 1097.2 \quad 1119.7 \quad 1143.3 \quad 1180.5 \quad 1183.51231 .2 \quad 1267.8 \quad 1276.2 \quad 1277.7 \quad 1287.6$

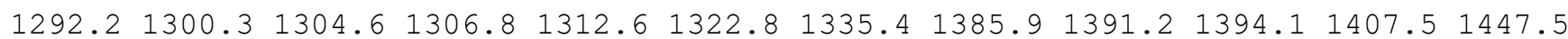
$1860.72630 .7 \quad 2637.4 \quad 2665.8 \quad 2671.3 \quad 2674.0 \quad 2676.72677 .72678 .72681 .12711 .52729 .2$ $2775.12780 .2 \quad 2782.6 \quad 4574.8$

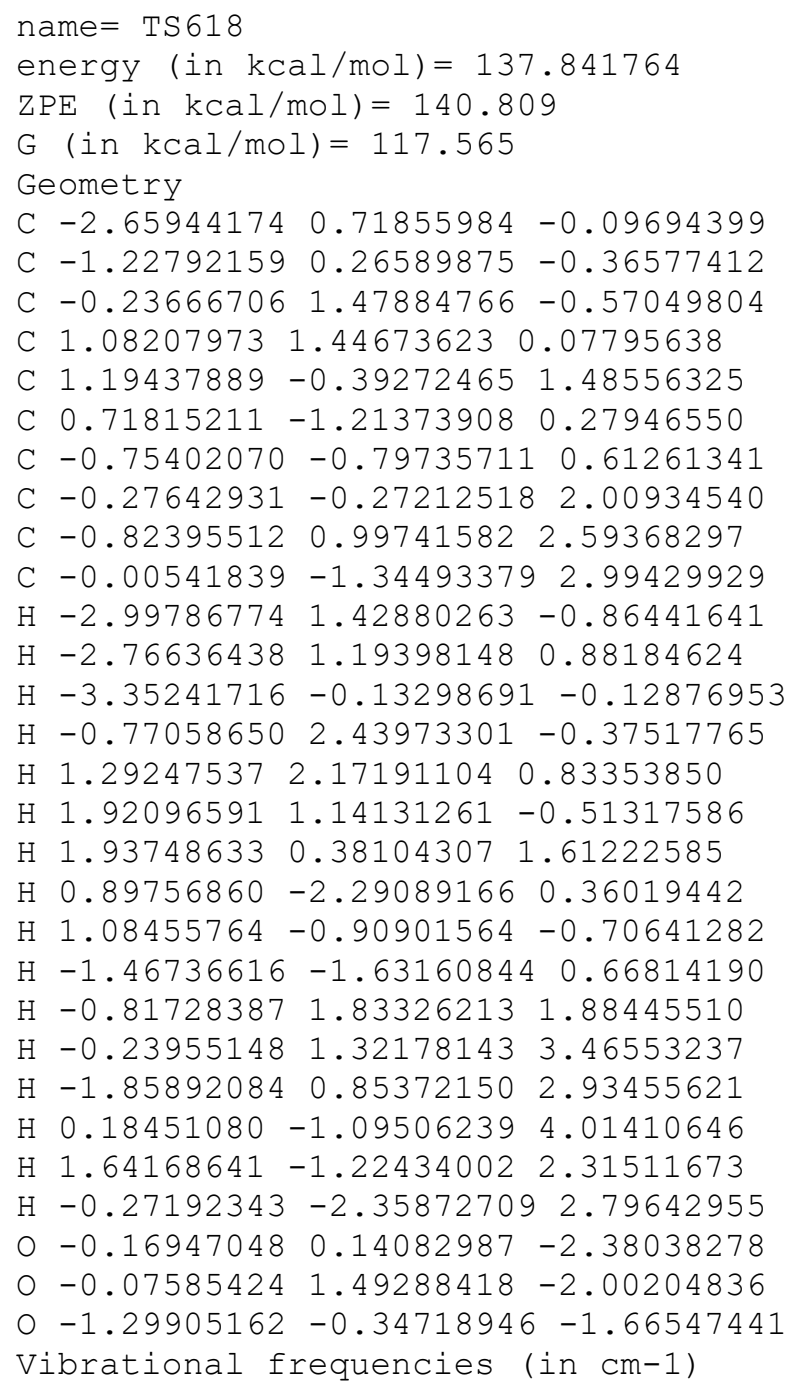


NETWORK OF THE AMK-BXDE DATABASE. ALL STRUCTURES INCLUDED

TS \# DE (kcal/mol)

$-143.932$

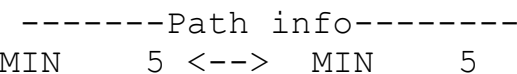

$-111.215$

$-105.117$

$-93.830$

$-87.054$

$-85.866$

$-84.752$

$-83.659$

$-81.743$

$-80.900$

$-78.036$

$-73.859$

$-69.734$

$-69.172$

$-68.791$

$-65.820$

$-62.010$

$-61.849$

$-61.409$

$-58.847$

$-58.668$

$-58.131$

$-58.076$

$-57.901$

$-57.709$

$-56.187$

$-48.837$

$-48.695$

$-48.604$

$-48.545$

$-48.327$

$-47.906$

$-45.439$

$-43.271$

$-42.750$

$-42.080$

$-32.933$

$-29.873$

$-28.960$

$-25.620$

$-25.588$

$-24.596$

$-24.209$

$-19.082$

$-17.903$

$-16.100$

$-15.721$

$\begin{array}{llll}\text { MIN } & 5<--> & \text { MIN } & 5 \\ \text { MIN } & 3<--> & \text { MIN } & 3\end{array}$

MIN $2<-->$ MIN 8

MIN $7<-->\quad \mathrm{C} 4 \mathrm{H} 6 \mathrm{O} 2+\mathrm{C} 6 \mathrm{H} 10 \mathrm{O}$

$\mathrm{CO}+\mathrm{C} 4 \mathrm{H} 6 \mathrm{O} 2+\mathrm{C} 5 \mathrm{H} 1 \mathrm{O}<-->\quad \mathrm{CO}+\mathrm{C} 9 \mathrm{H} 16 \mathrm{O} 2$

MIN $4<-->\quad$ MIN 4

MIN $16<-->\quad \mathrm{C} 2 \mathrm{H} 4 \mathrm{O} 2+\mathrm{C} 8 \mathrm{H} 12 \mathrm{O}$

MIN $10<-->\quad \mathrm{CO}+\mathrm{C} 9 \mathrm{H} 16 \mathrm{O} 2$

MIN $9<-->\quad \mathrm{H} 2+\mathrm{ClOH} 14 \mathrm{O} 3$

MIN $12<-->\quad$ MIN 14

MIN $\quad 1<-->\quad \mathrm{H} 2+\mathrm{ClOH} 4 \mathrm{O} 3$

MIN $23<-->\quad$ C4H6O2+C6H10O

MIN $11<-->$ MIN 11

MIN $35<-->\quad$ MIN 36

MIN $13<-->\quad \mathrm{CO}+\mathrm{C} 9 \mathrm{H} 16 \mathrm{O} 2$

MIN $28<-->\quad$ MIN 34

MIN $29<-->\quad \mathrm{H} 2 \mathrm{O}+\mathrm{C} 10 \mathrm{H} 14 \mathrm{O} 2$

$\mathrm{H} 2 \mathrm{O}+\mathrm{C} 10 \mathrm{H} 14 \mathrm{O} 2<-->\mathrm{H} 2 \mathrm{O}+\mathrm{C} 10 \mathrm{H} 14 \mathrm{O} 2$

$\mathrm{H} 2 \mathrm{O}+\mathrm{C} 10 \mathrm{H} 14 \mathrm{O} 2<-->\mathrm{H} 2 \mathrm{O}+\mathrm{ClOH} 14 \mathrm{O} 2$

MIN $24<-->\quad \mathrm{H} 2+\mathrm{C} 10 \mathrm{H} 14 \mathrm{O} 3$

MIN $5<-->\quad \mathrm{C} 2 \mathrm{H} 4 \mathrm{O} 2+\mathrm{C} 8 \mathrm{H} 12 \mathrm{O}$

MIN $20<-->\quad \mathrm{H} 2+\mathrm{C} 2 \mathrm{H} 2 \mathrm{O}+\mathrm{C} 8 \mathrm{H} 12 \mathrm{O} 2$

MIN $18<-->$ MIN 38

MIN $45<-->\quad$ MIN 45

MIN $19<-->$ MIN 37

$\mathrm{C} 2 \mathrm{H} 2 \mathrm{O}+\mathrm{C} 8 \mathrm{H} 14 \mathrm{O} 2<-->\quad \mathrm{C} 2 \mathrm{H} 4 \mathrm{O} 2+\mathrm{C} 8 \mathrm{H} 12 \mathrm{O}$

$\mathrm{H} 2 \mathrm{O}+\mathrm{C1OH} 14 \mathrm{O} 2<-->\mathrm{H} 2 \mathrm{O}+\mathrm{C} 10 \mathrm{H} 14 \mathrm{O} 2$

MIN $76<-->$ MIN 76

MIN $74<-->\quad$ MIN 74

MIN $22<-->\quad M I N \quad 40$

MIN $\quad 15<-->\quad$ CH2O+C9H14O2

$\mathrm{H} 2+\mathrm{H} 2 \mathrm{O}+\mathrm{C} 10 \mathrm{H} 12 \mathrm{O} 2<-->\mathrm{H} 2 \mathrm{O}+\mathrm{C} 10 \mathrm{H} 14 \mathrm{O} 2$

MIN $72<-->$ MIN 72

MIN $\quad 30<-->\quad \mathrm{H} 2+\mathrm{ClOH} 4 \mathrm{O} 3$

MIN $32<-->\quad \mathrm{H} 2 \mathrm{O}+\mathrm{C} 10 \mathrm{H} 14 \mathrm{O} 2$

$\mathrm{H} 2 \mathrm{O}+\mathrm{ClOH} 14 \mathrm{O} 2<-->\quad \mathrm{H} 2 \mathrm{O}+\mathrm{ClOH} 14 \mathrm{O} 2$

MIN $99<-->$ MIN 99

$\mathrm{CO}+\mathrm{H} 2 \mathrm{O}+\mathrm{C} 9 \mathrm{H} 14 \mathrm{O}<-->\quad \mathrm{CO}+\mathrm{H} 2 \mathrm{O}+\mathrm{C} 9 \mathrm{H} 14 \mathrm{O}$

MIN $26<-->\quad \mathrm{H} 2+\mathrm{ClOH} 4 \mathrm{O} 3$

MIN $27<-->\quad \mathrm{H} 2+\mathrm{ClOH} 1403$

MIN $39<-->\quad$ MIN 116

MIN $130<-->$ MIN 130

MIN $49<-->\quad \mathrm{H} 2 \mathrm{O}+\mathrm{C} 10 \mathrm{H} 14 \mathrm{O} 2$

MIN $155<-->\quad$ MIN 155

MIN $180<-->$ MIN 180

MIN $180<-->$ MIN 180

MIN $180<-->$ MIN 188

MIN $44<-->$ MIN 117

MIN $178<-->$ MIN 180

MIN $21<-->\quad$ MIN 97

MIN $45<-->$ MIN 119

MIN $47<-->$ MIN 118

MIN $\quad 50<-->\quad$ MIN 125

MIN $55<-->\quad$ MIN 128

MIN $54<-->\quad$ MIN 140

MIN $44<-->\quad \mathrm{H} 2 \mathrm{O}+\mathrm{C} 10 \mathrm{H} 14 \mathrm{O} 2$

MIN $65<-->\quad$ MIN 131

MIN $66<-->\quad$ MIN 135

MIN $31<-->\quad$ MIN 85 


\begin{tabular}{|c|c|c|c|c|c|c|}
\hline 60 & $-11 \cdot 327$ & MIN & 67 & $<-->$ & MIN & 145 \\
\hline 61 & -10.122 & MIN & 69 & $<-->$ & MIN & 150 \\
\hline 62 & -9.845 & MIN & 73 & $<-->$ & MIN & 162 \\
\hline 63 & -9.706 & MIN & 56 & $<-->$ & MIN & 174 \\
\hline 64 & -9.303 & MIN & 61 & $<-->$ & MIN & 158 \\
\hline 65 & -8.877 & MIN & 94 & $<-->$ & $\mathrm{C} 4 \mathrm{H} 6 \mathrm{C}$ & $\mathrm{O}+\mathrm{C} 6 \mathrm{H} 10 \mathrm{O} 2$ \\
\hline 66 & -8.531 & MIN & 59 & $<-->$ & MIN & 184 \\
\hline 67 & -8.483 & MIN & 77 & $<-->$ & MIN & 170 \\
\hline 68 & -8.136 & MIN & 59 & $<-->$ & MIN & 182 \\
\hline 69 & -7.870 & MIN & 52 & $<-->$ & MIN & 136 \\
\hline 70 & -7.616 & MIN & 63 & $<-->$ & MIN & 185 \\
\hline 71 & -7.202 & MIN & 82 & $<-->$ & MIN & 147 \\
\hline 72 & -6.764 & MIN & 85 & $<-->$ & MIN & 148 \\
\hline 73 & -6.375 & MIN & 97 & $<-->$ & MIN & 153 \\
\hline 74 & -6.228 & MIN & 91 & $<-->$ & MIN & 143 \\
\hline 75 & -5.574 & MIN & 86 & $<-->$ & MIN & 113 \\
\hline 76 & -5.260 & MIN & 104 & $<-->$ & MIN & 150 \\
\hline 77 & -5.234 & MIN & 92 & $<-->$ & MIN & 167 \\
\hline 78 & -5.214 & MIN & 102 & $<-->$ & MIN & 160 \\
\hline 79 & -4.499 & MIN & 104 & $<-->$ & MIN & 172 \\
\hline 80 & -3.937 & MIN & 104 & $<-->$ & MIN & 155 \\
\hline 81 & -3.757 & MIN & 108 & $<-->$ & MIN & 166 \\
\hline 82 & -3.225 & MIN & 109 & $<-->$ & MIN & 191 \\
\hline 83 & -1.575 & MIN & 88 & $<-->$ & MIN & 169 \\
\hline 84 & -0.469 & MIN & 83 & $<-->$ & MIN & 196 \\
\hline 85 & -0.004 & MIN & 25 & $<-->$ & MIN & 69 \\
\hline 86 & 0.318 & MIN & 107 & $<-->$ & MIN & 202 \\
\hline 87 & 1.237 & MIN & 204 & $<-->$ & MIN & 204 \\
\hline 88 & 1.387 & MIN & 75 & $<-->$ & MIN & 161 \\
\hline 89 & 1.639 & MIN & 71 & $<-->$ & MIN & 151 \\
\hline 90 & 2.001 & MIN & 48 & $<-->$ & MIN & 126 \\
\hline 91 & 2.458 & MIN & 105 & $<-->$ & $\mathrm{C} 4 \mathrm{H} 6$ & $\mathrm{O} 2+\mathrm{C} 6 \mathrm{H} 10 \mathrm{C}$ \\
\hline 92 & 3.345 & MIN & 53 & $<-->$ & MIN & 139 \\
\hline 93 & 3.887 & MIN & 45 & $<-->$ & $\mathrm{H} 2+\mathrm{C}$ & $10 \mathrm{H} 1403$ \\
\hline 94 & 3.997 & MIN & 62 & $<-->$ & MIN & 147 \\
\hline 95 & 4.563 & MIN & 60 & $<-->$ & $\mathrm{H} 2+\mathrm{O}$ & $2+\mathrm{C} 10 \mathrm{H} 14 \mathrm{C}$ \\
\hline 96 & 4.626 & MIN & 100 & $<-->$ & $\mathrm{H} 2+\mathrm{C}$ & 10H1403 \\
\hline 97 & 4.634 & MIN & 46 & $<-->$ & MIN & 156 \\
\hline 98 & 4.825 & MIN & 95 & $<-->$ & $\mathrm{H} 2+\mathrm{C}$ & $10 \mathrm{H} 1403$ \\
\hline 99 & 4.990 & MIN & 42 & $<-->$ & MIN & 163 \\
\hline 100 & 5.742 & MIN & 101 & $<-->$ & $\mathrm{H} 2+\mathrm{C}$ & $10 \mathrm{H} 1403$ \\
\hline 101 & 5.820 & MIN & 51 & $<-->$ & MIN & 177 \\
\hline 102 & 6.576 & MIN & 103 & $<-->$ & $\mathrm{H} 2+\mathrm{C}$ & $10 \mathrm{H} 1403$ \\
\hline 103 & 7.216 & MIN & 106 & $<-->$ & $\mathrm{H} 2+\mathrm{C}$ & $10 \mathrm{H} 1403$ \\
\hline 104 & 8.069 & MIN & 41 & $<-->$ & MIN & 164 \\
\hline 105 & 8.857 & MIN & 18 & $<-->$ & MIN & 149 \\
\hline 106 & 9.257 & MIN & 81 & $<-->$ & $\mathrm{H} 2 \mathrm{O} 2$ & $+\mathrm{C} 10 \mathrm{H} 14 \mathrm{O}$ \\
\hline 107 & 10.262 & MIN & 93 & $<-->$ & MIN & 138 \\
\hline 108 & 10.325 & MIN & 57 & $<-->$ & $\mathrm{H} 2+\mathrm{C}$ & $10 \mathrm{H} 1403$ \\
\hline 109 & 10.566 & MIN & 52 & $<-->$ & MIN & 86 \\
\hline 110 & 10.876 & MIN & 133 & $<-->$ & $\mathrm{C} 2 \mathrm{H} 4$ & $\mathrm{O} 2+\mathrm{C} 8 \mathrm{H} 12 \mathrm{C}$ \\
\hline 111 & 11.844 & MIN & 122 & $<-->$ & MIN & 189 \\
\hline 112 & 12.338 & MIN & 82 & $<-->$ & MIN & 114 \\
\hline 113 & 12.515 & MIN & 70 & $<-->$ & MIN & 87 \\
\hline 114 & 12.705 & MIN & 117 & $<-->$ & MIN & 190 \\
\hline 115 & 14.930 & MIN & 17 & $<-->$ & MIN & 155 \\
\hline 116 & 14.990 & MIN & 121 & $<-->$ & $\mathrm{H} 2+\mathrm{C}$ & $10 \mathrm{H} 1403$ \\
\hline 117 & 15.093 & MIN & 98 & $<-->$ & MIN & 144 \\
\hline 118 & 15.096 & MIN & 120 & $<-->$ & MIN & 183 \\
\hline 119 & 15.764 & MIN & 110 & $<-->$ & $\mathrm{H} 2+\mathrm{C}$ & $10 \mathrm{H} 1403$ \\
\hline 120 & 15.923 & MIN & 154 & $<-->$ & MIN & 192 \\
\hline 121 & 15.975 & MIN & 69 & $<-->$ & $\mathrm{H} 2+\mathrm{C}$ & $10 \mathrm{H} 1403$ \\
\hline 122 & 16.566 & MIN & 123 & $<-->$ & MIN & 187 \\
\hline 123 & 16.897 & MIN & 112 & $<-->$ & $\mathrm{H} 2+\mathrm{C}$ & $10 \mathrm{H} 1403$ \\
\hline 124 & 17.466 & MIN & 68 & $<-->$ & MIN & 194 \\
\hline
\end{tabular}




\begin{tabular}{|c|c|c|c|c|c|}
\hline 125 & 18.055 & MIN & 85 & $<-->$ & $\mathrm{H} 2+\mathrm{C} 10 \mathrm{H} 1403$ \\
\hline 126 & 18.312 & MIN & 6 & $<-->$ & MIN 175 \\
\hline 127 & 18.775 & MIN & 104 & $<-->$ & MIN \\
\hline 128 & 19.649 & MIN & 186 & $<-->$ & $\mathrm{C} 4 \mathrm{H} 6 \mathrm{O} 2+\mathrm{C} 6 \mathrm{H} 10 \mathrm{O}$ \\
\hline 29 & 20.898 & MIN & 97 & $<-->$ & $\mathrm{H} 2+\mathrm{C} 10 \mathrm{H} 1403$ \\
\hline 30 & 21.385 & MIN & 90 & $<-->$ & $\mathrm{H} 2+\mathrm{C} 10 \mathrm{H} 1403$ \\
\hline 31 & 21.477 & MIN & 58 & $<-->$ & MIN 134 \\
\hline 32 & 21.824 & MIN & 171 & $<-->$ & MIN \\
\hline 33 & 21.966 & MIN & 181 & $<-->$ & MIN \\
\hline 34 & 23.134 & MIN & 180 & $<-->$ & MIN 204 \\
\hline 35 & 23.777 & MIN & 84 & $<-->$ & $\mathrm{H} 2+\mathrm{C} 10 \mathrm{H} 1403$ \\
\hline 36 & 23.983 & MIN & 127 & $<-->$ & MIN 204 \\
\hline 37 & 24.212 & MIN & 104 & $<-->$ & $\mathrm{H} 2+\mathrm{C} 10 \mathrm{H} 1403$ \\
\hline 38 & 24.783 & MIN & 152 & $<-->$ & $\mathrm{H} 2+\mathrm{C} 10 \mathrm{H} 1403$ \\
\hline 39 & 25.205 & MIN & 165 & $<-->$ & $\mathrm{H} 2+\mathrm{O} 2+\mathrm{C} 10 \mathrm{H} 14 \mathrm{O}$ \\
\hline 40 & 25.310 & MIN & 104 & $<-->$ & $\mathrm{H} 2+\mathrm{C} 10 \mathrm{H} 1403$ \\
\hline 41 & 25.382 & MIN & 142 & $<-->$ & $\mathrm{H} 2+\mathrm{C} 10 \mathrm{H} 14 \mathrm{O} 3$ \\
\hline 42 & 26.674 & MIN & 111 & $<-->$ & MIN 137 \\
\hline 43 & 26.783 & MIN & 176 & $<-->$ & $\mathrm{H} 2+\mathrm{C} 10 \mathrm{H} 1403$ \\
\hline 44 & 28.532 & MIN & 96 & $<-->$ & MIN 96 \\
\hline 45 & 29.758 & MIN & 179 & $<-->$ & $\mathrm{C} 4 \mathrm{H} 6 \mathrm{O} 2+\mathrm{C} 6 \mathrm{H} 10 \mathrm{O}$ \\
\hline 46 & 30.118 & MIN & 146 & $<-->$ & $\mathrm{H} 2+\mathrm{C} 10 \mathrm{H} 1403$ \\
\hline 47 & 30.121 & MIN & 168 & $<-->$ & $\mathrm{H} 2+\mathrm{C} 10 \mathrm{H} 1403$ \\
\hline 48 & 30.137 & MIN & 117 & $<-->$ & MIN 193 \\
\hline 49 & 30.709 & $\mathrm{O} 2+\mathrm{C} 2 \mathrm{H} 4 \mathrm{O}+\mathrm{C} 8$ & $3 \mathrm{H} 12$ & $<-->$ & $\mathrm{O} 2+\mathrm{C} 10 \mathrm{H} 16 \mathrm{O}$ \\
\hline 50 & 32.725 & MIN & 66 & $<-->$ & $\mathrm{C} 2 \mathrm{H} 4 \mathrm{O}+\mathrm{C} 8 \mathrm{H} 12 \mathrm{O} 2$ \\
\hline 51 & 33.672 & MIN & 164 & $<-->$ & MIN 195 \\
\hline 52 & 34.269 & $\mathrm{H} 2+\mathrm{O} 2+\mathrm{C} 10 \mathrm{H}$ & 1140 & $<-->$ & $\mathrm{O} 2+\mathrm{C} 10 \mathrm{H} 160$ \\
\hline 53 & 34.283 & MIN & 79 & $<-->$ & $\mathrm{H} 2 \mathrm{O} 2+\mathrm{C} 10 \mathrm{H} 14 \mathrm{O}$ \\
\hline 54 & 34.292 & MIN & 64 & $<-->$ & MIN 217 \\
\hline 55 & 36.222 & MIN & 141 & $<-->$ & $\mathrm{H} 2+\mathrm{C} 10 \mathrm{H} 1403$ \\
\hline 156 & 36.877 & MIN & 161 & $<-->$ & $\mathrm{CO}+\mathrm{C} 9 \mathrm{H} 16 \mathrm{O} 2$ \\
\hline 157 & 36.998 & MIN & 198 & $<-->$ & MIN 199 \\
\hline 158 & 37.057 & MIN & 80 & $<-->$ & $\mathrm{C} 2 \mathrm{H} 4 \mathrm{O}+\mathrm{C} 8 \mathrm{H} 12 \mathrm{O} 2$ \\
\hline 159 & 37.156 & MIN & 78 & $<-->$ & MIN 78 \\
\hline 160 & 37.309 & MIN & 178 & $<-->$ & $\mathrm{H} 2+\mathrm{C} 10 \mathrm{H} 1403$ \\
\hline 161 & 37.854 & MIN & 155 & $<-->$ & $\mathrm{H} 2+\mathrm{C} 10 \mathrm{H} 1403$ \\
\hline 162 & 38.008 & MIN & 147 & $<-->$ & MIN 147 \\
\hline 163 & 38.927 & MIN & 117 & $<-->$ & $\mathrm{H} 2+\mathrm{C} 10 \mathrm{H} 1403$ \\
\hline 164 & 39.158 & MIN & 150 & $<-->$ & $\mathrm{H} 2+\mathrm{C} 10 \mathrm{H} 1403$ \\
\hline 165 & 39.487 & MIN & 157 & $<-->$ & $\mathrm{CO}+\mathrm{C} 9 \mathrm{H} 16 \mathrm{O} 2$ \\
\hline 166 & 40.852 & MIN & 173 & $<-->$ & $\mathrm{H} 2+\mathrm{C} 10 \mathrm{H} 1403$ \\
\hline 167 & 43.154 & MIN & 156 & $<-->$ & $\mathrm{H} 2+\mathrm{C} 10 \mathrm{H} 1403$ \\
\hline 168 & 44.074 & MIN & 155 & $<-->$ & $\mathrm{H} 2+\mathrm{C} 10 \mathrm{H} 1403$ \\
\hline 169 & 44.111 & MIN & 148 & $<-->$ & $\mathrm{H} 2+\mathrm{C} 10 \mathrm{H} 1403$ \\
\hline 170 & 44.424 & MIN & 159 & $<-->$ & $\mathrm{H} 2+\mathrm{C} 10 \mathrm{H} 1403$ \\
\hline 171 & 44.458 & MIN & 150 & $<-->$ & $\mathrm{H} 2+\mathrm{C} 10 \mathrm{H} 1403$ \\
\hline 172 & 44.745 & MIN & 204 & $<-->$ & MIN 212 \\
\hline 173 & 45.119 & $\mathrm{O} 2+\mathrm{C} 10 \mathrm{H}$ & 1160 & $<-->$ & $\mathrm{CO}+\mathrm{O} 2+\mathrm{C} 9 \mathrm{H} 16$ \\
\hline 174 & 45.432 & MIN & 220 & $<-->$ & $\mathrm{C} 4 \mathrm{H} 6 \mathrm{O}+\mathrm{C} 6 \mathrm{H} 10 \mathrm{O} 2$ \\
\hline 175 & 45.473 & MIN & 206 & $<-->$ & MIN 213 \\
\hline 176 & 46.017 & MIN & 200 & $<-->$ & $\mathrm{CO}+\mathrm{H} 2+\mathrm{C} 9 \mathrm{H} 14 \mathrm{O} 2$ \\
\hline 177 & 46.773 & MIN & 203 & $<-->$ & MIN 211 \\
\hline 178 & 46.872 & MIN & 130 & $<-->$ & MIN \\
\hline 179 & 47.800 & MIN & 203 & $<-->$ & MIN \\
\hline 180 & 47.866 & MIN & 33 & $<-->$ & MIN \\
\hline 181 & 48.194 & MIN & 129 & $<-->$ & MIN 221 \\
\hline 182 & 48.855 & MIN & 207 & $<-->$ & $\mathrm{H} 2+\mathrm{C} 10 \mathrm{H} 14 \mathrm{O} 3$ \\
\hline 183 & 49.246 & MIN & 197 & $<-->$ & MIN 209 \\
\hline 184 & 50.387 & MIN & 218 & $<-->$ & $\mathrm{O} 2+\mathrm{C} 10 \mathrm{H} 16 \mathrm{O}$ \\
\hline 185 & 51.244 & MIN & 219 & $<-->$ & $\mathrm{O} 2+\mathrm{C} 10 \mathrm{H} 16 \mathrm{O}$ \\
\hline 186 & 54.810 & MIN & 43 & $<-->$ & MIN 89 \\
\hline 187 & 54.998 & MIN & 132 & $<-->$ & $\mathrm{CH} 4+\mathrm{C} 9 \mathrm{H} 12 \mathrm{O} 3$ \\
\hline & 55.477 & MIN & 216 & $<-->$ & MIN 216 \\
\hline & 55.566 & $\mathrm{H} 2+\mathrm{O} 2+\mathrm{C} 10 \mathrm{H}$ & 1140 & $<-->$ & $\mathrm{O} 2+\mathrm{C} 10 \mathrm{H} 16 \mathrm{O}$ \\
\hline
\end{tabular}




\begin{tabular}{|c|c|c|c|c|c|}
\hline 190 & 55.858 & MIN & 41 & $<-->$ & MIN \\
\hline 191 & 56.000 & $\mathrm{O} 2+\mathrm{C} 10$ & 1160 & $<-->$ & $\mathrm{O} 2+\mathrm{C} 10 \mathrm{H} 16 \mathrm{O}$ \\
\hline 192 & 56.417 & MIN & 204 & $<-->$ & $\mathrm{H} 2+\mathrm{C} 10 \mathrm{H} 1403$ \\
\hline 193 & 56.737 & MIN & 215 & $<-->$ & $\mathrm{O} 2+\mathrm{C} 10 \mathrm{H} 160$ \\
\hline 194 & 57.415 & MIN & 128 & $<-->$ & MIN 128 \\
\hline 195 & 57.934 & MIN & 203 & $<-->$ & $\mathrm{MIN}$ \\
\hline 196 & 58.465 & MIN & 203 & $<-->$ & $\mathrm{O} 3+\mathrm{C} 10 \mathrm{H} 16$ \\
\hline 197 & 59.180 & MIN & 204 & $<-->$ & $\mathrm{O} 3+\mathrm{C} 10 \mathrm{H} 16$ \\
\hline 198 & 59.913 & MIN & 203 & $<-->$ & $\mathrm{H} 2+\mathrm{C} 10 \mathrm{H} 1403$ \\
\hline 199 & 60.648 & MIN & 204 & $<-->$ & $\mathrm{H} 2+\mathrm{C} 10 \mathrm{H} 1403$ \\
\hline 200 & 60.666 & MIN & 148 & $<-->$ & $\mathrm{CH} 4+\mathrm{C} 9 \mathrm{H}_{1} 2 \mathrm{O} 3$ \\
\hline 201 & 62.889 & MIN & 204 & $<-->$ & $\mathrm{H} 2+\mathrm{C} 10 \mathrm{H} 1403$ \\
\hline 202 & 64.978 & MIN & 204 & $<-->$ & $\mathrm{H} 2+\mathrm{C} 10 \mathrm{H} 1403$ \\
\hline 203 & 65.732 & MIN & 115 & $<-->$ & MIN 204 \\
\hline 204 & 66.238 & MIN & 204 & $<-->$ & $\mathrm{H} 2+\mathrm{C} 10 \mathrm{H} 1403$ \\
\hline 205 & 66.241 & MIN & 204 & $<-->$ & $\mathrm{H} 2+\mathrm{C} 10 \mathrm{H} 1403$ \\
\hline 206 & 66.345 & MIN & 203 & $<-->$ & $\mathrm{H} 2+\mathrm{C} 10 \mathrm{H} 1403$ \\
\hline 207 & 66.565 & MIN & 124 & $<-->$ & MIN 124 \\
\hline 208 & 66.656 & MIN & 203 & $<-->$ & $\mathrm{H} 2+\mathrm{C} 10 \mathrm{H} 1403$ \\
\hline 209 & 66.761 & $\mathrm{O} 2+\mathrm{C} 10$ & 160 & $<-->$ & $\mathrm{O} 2+\mathrm{C} 10 \mathrm{H} 16 \mathrm{O}$ \\
\hline 210 & 67.216 & MIN & 201 & $<-->$ & MIN 203 \\
\hline 211 & 68.981 & MIN & 204 & $<-->$ & $\mathrm{H} 2+\mathrm{C} 10 \mathrm{H} 1403$ \\
\hline 212 & 69.043 & MIN & 204 & $<-->$ & $\mathrm{H} 2+\mathrm{C} 10 \mathrm{H} 1403$ \\
\hline 213 & 69.275 & $\mathrm{MIN}$ & 203 & $<-->$ & $\mathrm{H} 2+\mathrm{C} 10 \mathrm{H} 1403$ \\
\hline 214 & 69.605 & $\mathrm{O} 2+\mathrm{C} 10$ & $\mathrm{H} 160$ & $<-->$ & $\mathrm{O} 2+\mathrm{C} 10 \mathrm{H} 160$ \\
\hline 215 & 69.759 & $\mathrm{O} 2+\mathrm{C} 10$ & 160 & $<-->$ & $\mathrm{O} 2+\mathrm{C} 10 \mathrm{H} 160$ \\
\hline 216 & 70.961 & $\mathrm{H} 2+\mathrm{O} 2+\mathrm{C} 10$ & $\mathrm{H} 140$ & $<-->$ & $\mathrm{O} 2+\mathrm{C} 10 \mathrm{H} 16 \mathrm{O}$ \\
\hline 217 & 75.708 & MIN & 203 & $<-->$ & MIN 224 \\
\hline 218 & 76.309 & MIN & 203 & $<-->$ & MIN \\
\hline 219 & 79.105 & MIN & 203 & $<-->$ & MIN \\
\hline 220 & 82.282 & MIN & 205 & $<-->$ & MIN 208 \\
\hline 221 & 91.676 & $\mathrm{O} 2+\mathrm{C} 10$ & 160 & $<-->$ & $\mathrm{H} 2+\mathrm{O} 2+\mathrm{C} 10 \mathrm{H} 140$ \\
\hline 222 & 98.426 & $\mathrm{O} 2+\mathrm{C} 10$ & 160 & $<-->$ & $\mathrm{O} 2+\mathrm{C} 2 \mathrm{H} 2+\mathrm{C} 8 \mathrm{H} 14 \mathrm{O}$ \\
\hline
\end{tabular}

NETWORK OF THE AMK-BXDE DATABASE. FAMILIES OF CONFORMERS FORM A NODE

TS \# DE (kcal/mol)

$3-105.117$

$4 \quad-93.830$

$7 \quad-84.752$

$8 \quad-83.659$

$9 \quad-81.743$

$11 \quad-78.036$

$-73.859$

$-68.791$

$-65.820$

$-62.010$

$-58.847$

$-58.668$

$-58.131$

$-58.076$

$-57.709$

$-48.545$

$-48.327$

$-45.439$

$-43.271$

$-29.873$

$-28.960$

$-25.620$

$-24.596$

$-15.721$

$-14.515$

$-14.008$

$-13.642$

-------Path info--------

$\begin{array}{llll}\text { MIN } & 1<--> & \text { MIN } & 8 \\ \text { MIN } & 7<--> & \text { C4 } 4602+C 6 \mathrm{H} 100\end{array}$

MIN $11<-->\quad \mathrm{C} 2 \mathrm{H} 4 \mathrm{O} 2+\mathrm{C} 8 \mathrm{H} 12 \mathrm{O}$

MIN $\quad 10<-->\quad \mathrm{CO}+\mathrm{C} 9 \mathrm{H} 16 \mathrm{O} 2$

MIN $\quad 9<-->\quad \mathrm{H} 2+\mathrm{ClOH} 14 \mathrm{O} 3$

MIN $\quad 1<-->\quad \mathrm{H} 2+\mathrm{C} 10 \mathrm{H} 1403$

MIN $13<-->\quad \mathrm{C} 4 \mathrm{H} 6 \mathrm{O} 2+\mathrm{C} 6 \mathrm{H} 10 \mathrm{O}$

MIN $\quad 13<-->\quad \mathrm{CO}+\mathrm{C} 9 \mathrm{H} 16 \mathrm{O} 2$

MIN $28<-->\quad$ MIN 34

MIN $29<-->\quad \mathrm{H} 2 \mathrm{O}+\mathrm{C} 10 \mathrm{H} 14 \mathrm{O} 2$

MIN $11<-->\quad \mathrm{H} 2+\mathrm{C} 10 \mathrm{H} 1403$

MIN $1<-->\quad \mathrm{C} 2 \mathrm{H} 4 \mathrm{O} 2+\mathrm{C} 8 \mathrm{H} 12 \mathrm{O}$

MIN $\quad 11<-->\quad \mathrm{H} 2+\mathrm{C} 2 \mathrm{H} 2 \mathrm{O}+\mathrm{C} 8 \mathrm{H} 12 \mathrm{O} 2$

MIN $13<-->$ MIN 35

MIN $13<-->\quad$ MIN 35

MIN $22<-->$ MIN 40

MIN $\quad 10<-->\quad \mathrm{CH} 2 \mathrm{O}+\mathrm{C} 9 \mathrm{H} 14 \mathrm{O} 2$

MIN $25<-->\quad \mathrm{H} 2+\mathrm{C} 10 \mathrm{H} 1403$

MIN $32<-->\quad \mathrm{H} 2 \mathrm{O}+\mathrm{ClOH} 14 \mathrm{O} 2$

MIN $25<-->\quad \mathrm{H} 2+\mathrm{ClOH} 4 \mathrm{O} 3$

MIN $25<-->\quad \mathrm{H} 2+\mathrm{ClOH} 1403$

MIN $39<-->\quad$ MIN 116

MIN $44<-->\quad \mathrm{H} 2 \mathrm{O}+\mathrm{ClOH} 14 \mathrm{O} 2$

MIN $\quad 44<-->$ MIN 117

MIN $13<-->\quad$ MIN 82

MIN $44<-->$ MIN 117

MIN $44<-->$ MIN 117

MIN $44<-->$ MIN 117 


\begin{tabular}{|c|c|c|c|c|c|c|}
\hline 54 & $-12 \cdot 314$ & MIN & 44 & $<-->$ & MIN & 117 \\
\hline 55 & -12.130 & MIN & 44 & $<-->$ & $\operatorname{MIN}$ & 117 \\
\hline 56 & -12.048 & MIN & 44 & $<-->$ & \multicolumn{2}{|c|}{$\mathrm{H} 2 \mathrm{O}+\mathrm{C} 10 \mathrm{H} 14 \mathrm{O} 2$} \\
\hline 57 & -11.636 & MIN & 65 & $<-->$ & MIN & 131 \\
\hline 58 & -11.600 & MIN & 65 & $<-->$ & MIN & 131 \\
\hline 59 & -11.476 & MIN & 25 & $<-->$ & MIN & 82 \\
\hline 60 & $-11 \cdot 327$ & MIN & 65 & $<-->$ & MIN & 131 \\
\hline 61 & -10.122 & MIN & 65 & $<-->$ & MIN & 131 \\
\hline 62 & -9.845 & MIN & 65 & $<-->$ & MIN & 131 \\
\hline 63 & -9.706 & MIN & 56 & $<-->$ & MIN & 131 \\
\hline 64 & -9.303 & MIN & 56 & $<-->$ & MIN & 131 \\
\hline 65 & -8.877 & MIN & 94 & $<-->$ & \multicolumn{2}{|c|}{$\mathrm{C} 4 \mathrm{H} 6 \mathrm{O}+\mathrm{C} 6 \mathrm{H} 10 \mathrm{O} 2$} \\
\hline 66 & -8.531 & MIN & 56 & $<-->$ & $\operatorname{MIN}$ & 131 \\
\hline 67 & -8.483 & MIN & 65 & $<-->$ & MIN & 131 \\
\hline 68 & -8.136 & MIN & 56 & $<-->$ & MIN & 131 \\
\hline 69 & -7.870 & MIN & 44 & $<-->$ & MIN & 117 \\
\hline 70 & -7.616 & MIN & 56 & $<-->$ & MIN & 131 \\
\hline 71 & -7.202 & MIN & 82 & $<-->$ & MIN & 117 \\
\hline 72 & -6.764 & MIN & 82 & $<-->$ & MIN & 117 \\
\hline 73 & -6.375 & MIN & 82 & $<-->$ & MIN & 117 \\
\hline 74 & -6.228 & MIN & 82 & $<-->$ & MIN & 117 \\
\hline 75 & -5.574 & MIN & 82 & $<-->$ & MIN & 113 \\
\hline 76 & -5.260 & MIN & 99 & $<-->$ & MIN & 131 \\
\hline 77 & -5.234 & MIN & 82 & $<-->$ & MIN & 117 \\
\hline 78 & -5.214 & MIN & 82 & $<-->$ & MIN & 117 \\
\hline 79 & -4.499 & MIN & 99 & $<-->$ & MIN & 131 \\
\hline 80 & -3.937 & MIN & 99 & $<-->$ & MIN & 131 \\
\hline 81 & -3.757 & MIN & 99 & $<-->$ & MIN & 131 \\
\hline 82 & -3.225 & MIN & 87 & $<-->$ & MIN & 191 \\
\hline 83 & -1.575 & MIN & 82 & $<-->$ & MIN & 131 \\
\hline 84 & -0.469 & MIN & 83 & $<-->$ & MIN & 131 \\
\hline 85 & -0.004 & MIN & 25 & $<-->$ & MIN & 65 \\
\hline 86 & 0.318 & MIN & 107 & $<-->$ & MIN & 202 \\
\hline 88 & 1.387 & MIN & 71 & $<-->$ & MIN & 131 \\
\hline 89 & 1.639 & MIN & 71 & $<-->$ & MIN & 131 \\
\hline 90 & 2.001 & MIN & 42 & $<-->$ & MIN & 117 \\
\hline 91 & 2.458 & MIN & 105 & $<-->$ & $\mathrm{C} 4 \mathrm{H} 6$ & $\mathrm{O} 2+\mathrm{C} 6 \mathrm{H} 10 \mathrm{O}$ \\
\hline 92 & 3.345 & MIN & 42 & $<-->$ & $\operatorname{MIN}$ & 117 \\
\hline 93 & 3.887 & MIN & 44 & $<-->$ & $\mathrm{H} 2+\mathrm{C}$ & $10 \mathrm{H} 1403$ \\
\hline 94 & 3.997 & MIN & 42 & $<-->$ & MIN & 117 \\
\hline 95 & 4.563 & MIN & 60 & $<-->$ & $\mathrm{H} 2+\mathrm{O}$ & $2+\mathrm{C} 10 \mathrm{H} 14 \mathrm{O}$ \\
\hline 96 & 4.626 & MIN & 99 & $<-->$ & $\mathrm{H} 2+\mathrm{C}$ & $10 \mathrm{H} 1403$ \\
\hline 97 & 4.634 & MIN & 42 & $<-->$ & MIN & 117 \\
\hline 98 & 4.825 & MIN & 65 & $<-->$ & $\mathrm{H} 2+\mathrm{C}$ & $10 \mathrm{H} 1403$ \\
\hline 99 & 4.990 & MIN & 42 & $<-->$ & MIN & 117 \\
\hline 100 & 5.742 & MIN & 99 & $<-->$ & $\mathrm{H} 2+\mathrm{C}$ & $10 \mathrm{H} 1403$ \\
\hline 101 & 5.820 & MIN & 42 & $<-->$ & MIN & 117 \\
\hline 102 & 6.576 & MIN & 82 & $<-->$ & $\mathrm{H} 2+\mathrm{C}$ & $10 \mathrm{H} 1403$ \\
\hline 103 & 7.216 & MIN & 99 & $<-->$ & $\mathrm{H} 2+\mathrm{C}$ & $10 \mathrm{H} 1403$ \\
\hline 104 & 8.069 & MIN & 41 & $<-->$ & MIN & 131 \\
\hline 105 & 8.857 & MIN & 13 & $<-->$ & MIN & 117 \\
\hline 106 & 9.257 & MIN & 65 & $<-->$ & $\mathrm{H} 2 \mathrm{O} 2$ & $+\mathrm{C} 10 \mathrm{H} 14 \mathrm{O}$ \\
\hline 107 & 10.262 & MIN & 93 & $<-->$ & MIN & 131 \\
\hline 108 & 10.325 & MIN & 57 & $<-->$ & $\mathrm{H} 2+\mathrm{C}$ & $10 \mathrm{H} 1403$ \\
\hline 109 & 10.566 & MIN & 44 & $<-->$ & MIN & 82 \\
\hline 110 & 10.876 & MIN & 117 & $<-->$ & $\mathrm{C} 2 \mathrm{H} 4$ & $\mathrm{O} 2+\mathrm{C} 8 \mathrm{H} 12 \mathrm{O}$ \\
\hline 111 & 11.844 & MIN & 117 & $<-->$ & MIN & 189 \\
\hline 112 & 12.338 & MIN & 82 & $<-->$ & MIN & 114 \\
\hline 113 & 12.515 & MIN & 65 & $<-->$ & MIN & 87 \\
\hline 114 & 12.705 & MIN & 117 & $<-->$ & MIN & 189 \\
\hline 115 & 14.930 & MIN & 13 & $<-->$ & MIN & 131 \\
\hline 116 & 14.990 & MIN & 117 & $<-->$ & $\mathrm{H} 2+\mathrm{C}$ & $10 \mathrm{H1} 403$ \\
\hline 117 & 15.093 & MIN & 98 & $<-->$ & MIN & 131 \\
\hline 118 & 15.096 & MIN & 120 & $<-->$ & MIN & 131 \\
\hline 119 & 15.764 & MIN & 105 & $<-->$ & $\mathrm{H} 2+\mathrm{C}$ & $10 \mathrm{H} 1403$ \\
\hline
\end{tabular}




\begin{tabular}{|c|c|c|c|c|c|}
\hline 120 & 15.923 & MIN & 117 & $<-->$ & MIN 189 \\
\hline 121 & 15.975 & MIN & 65 & $<-->$ & $\mathrm{H} 2+\mathrm{C} 10 \mathrm{H} 1403$ \\
\hline 122 & 16.566 & MIN & 120 & $<-->$ & MIN 131 \\
\hline 123 & 16.897 & MIN & 105 & $<-->$ & $\mathrm{H} 2+\mathrm{C} 10 \mathrm{H} 14 \mathrm{O} 3$ \\
\hline 124 & 17.466 & MIN & 65 & $<-->$ & MIN 194 \\
\hline 125 & 18.055 & MIN & 82 & $<-->$ & $\mathrm{H} 2+\mathrm{C} 10 \mathrm{H} 1403$ \\
\hline 126 & 18.312 & MIN & 1 & $<-->$ & MIN 131 \\
\hline 128 & 19.649 & MIN & 186 & $<-->$ & $\mathrm{C} 4 \mathrm{H} 6 \mathrm{O} 2+\mathrm{C} 6 \mathrm{H} 10 \mathrm{O}$ \\
\hline 129 & 20.898 & MIN & 82 & $<-->$ & $\mathrm{H} 2+\mathrm{C} 10 \mathrm{H} 1403$ \\
\hline 130 & 21.385 & MIN & 82 & $<-->$ & $\mathrm{H} 2+\mathrm{C} 10 \mathrm{H} 1403$ \\
\hline 131 & 21.477 & MIN & 58 & $<-->$ & MIN 117 \\
\hline 132 & 21.824 & MIN & 131 & $<-->$ & MIN \\
\hline 133 & 21.966 & MIN & 117 & $<-->$ & MIN \\
\hline 134 & 23.134 & MIN & 131 & $<-->$ & MIN 203 \\
\hline 135 & 23.777 & MIN & 65 & $<-->$ & $\mathrm{H} 2+\mathrm{C} 10 \mathrm{H} 1403$ \\
\hline 136 & 23.983 & MIN & 117 & $<-->$ & MIN 203 \\
\hline 137 & 24.212 & MIN & 99 & $<-->$ & $\mathrm{H} 2+\mathrm{C} 10 \mathrm{H} 1403$ \\
\hline 138 & 24.783 & MIN & 131 & $<-->$ & $\mathrm{H} 2+\mathrm{C} 10 \mathrm{H} 1403$ \\
\hline 139 & 25.205 & MIN & 117 & $<-->$ & $\mathrm{H} 2+\mathrm{O} 2+\mathrm{C} 10 \mathrm{H} 14 \mathrm{O}$ \\
\hline 140 & 25.310 & MIN & 99 & $<-->$ & $\mathrm{H} 2+\mathrm{C} 10 \mathrm{H} 1403$ \\
\hline 141 & 25.382 & MIN & 131 & $<-->$ & $\mathrm{H} 2+\mathrm{C} 10 \mathrm{H} 14 \mathrm{O} 3$ \\
\hline 142 & 26.674 & MIN & 111 & $<-->$ & MIN 137 \\
\hline 143 & 26.783 & MIN & 131 & $<-->$ & $\mathrm{H} 2+\mathrm{C} 10 \mathrm{H} 1403$ \\
\hline 145 & 29.758 & MIN & 131 & $<-->$ & $\mathrm{C} 4 \mathrm{H} 6 \mathrm{O} 2+\mathrm{C} 6 \mathrm{H} 10 \mathrm{O}$ \\
\hline 146 & 30.118 & MIN & 131 & $<-->$ & $\mathrm{H} 2+\mathrm{C} 10 \mathrm{H} 1403$ \\
\hline 147 & 30.121 & MIN & 131 & $<-->$ & $\mathrm{H} 2+\mathrm{C} 10 \mathrm{H} 1403$ \\
\hline 148 & 30.137 & MIN & 117 & $<-->$ & MIN 193 \\
\hline 150 & 32.725 & MIN & 65 & $<-->$ & $\mathrm{C} 2 \mathrm{H} 4 \mathrm{O}+\mathrm{C} 8 \mathrm{H} 12 \mathrm{O} 2$ \\
\hline 151 & 33.672 & MIN & 131 & $<-->$ & MIN 195 \\
\hline 153 & 34.283 & MIN & 65 & $<-->$ & $\mathrm{H} 2 \mathrm{O} 2+\mathrm{C} 10 \mathrm{H} 14 \mathrm{O}$ \\
\hline 154 & 34.292 & MIN & 64 & $<-->$ & MIN 217 \\
\hline 155 & 36.222 & MIN & 131 & $<-->$ & $\mathrm{H} 2+\mathrm{C} 10 \mathrm{H} 1403$ \\
\hline 156 & 36.877 & MIN & 131 & $<-->$ & $\mathrm{CO}+\mathrm{C} 9 \mathrm{H} 16 \mathrm{O} 2$ \\
\hline 157 & 36.998 & MIN & 198 & $<-->$ & MIN 199 \\
\hline 158 & 37.057 & MIN & 65 & $<-->$ & $\mathrm{C} 2 \mathrm{H} 4 \mathrm{O}+\mathrm{C} 8 \mathrm{H} 12 \mathrm{O} 2$ \\
\hline 160 & 37.309 & MIN & 131 & $<-->$ & $\mathrm{H} 2+\mathrm{C} 10 \mathrm{H} 1403$ \\
\hline 161 & 37.854 & MIN & 131 & $<-->$ & $\mathrm{H} 2+\mathrm{C} 10 \mathrm{H} 1403$ \\
\hline 163 & 38.927 & MIN & 117 & $<-->$ & $\mathrm{H} 2+\mathrm{C} 10 \mathrm{H} 1403$ \\
\hline 164 & 39.158 & MIN & 131 & $<-->$ & $\mathrm{H} 2+\mathrm{C} 10 \mathrm{H} 1403$ \\
\hline 165 & 39.487 & MIN & 131 & $<-->$ & $\mathrm{CO}+\mathrm{C} 9 \mathrm{H} 16 \mathrm{O} 2$ \\
\hline 166 & 40.852 & MIN & 131 & $<-->$ & $\mathrm{H} 2+\mathrm{C} 10 \mathrm{H} 1403$ \\
\hline 167 & 43.154 & MIN & 117 & $<-->$ & $\mathrm{H} 2+\mathrm{C} 10 \mathrm{H} 1403$ \\
\hline 168 & 44.074 & MIN & 131 & $<-->$ & $\mathrm{H} 2+\mathrm{C} 10 \mathrm{H} 1403$ \\
\hline 169 & 44.111 & MIN & 117 & $<-->$ & $\mathrm{H} 2+\mathrm{C} 10 \mathrm{H} 1403$ \\
\hline 170 & 44.424 & MIN & 131 & $<-->$ & $\mathrm{H} 2+\mathrm{C} 10 \mathrm{H} 1403$ \\
\hline 171 & 44.458 & MIN & 131 & $<-->$ & $\mathrm{H} 2+\mathrm{C} 10 \mathrm{H} 1403$ \\
\hline 172 & 44.745 & MIN & 203 & $<-->$ & MIN 212 \\
\hline 174 & 45.432 & MIN & 220 & $<-->$ & $\mathrm{C} 4 \mathrm{H} 6 \mathrm{O}+\mathrm{C} 6 \mathrm{H} 10 \mathrm{O} 2$ \\
\hline 175 & 45.473 & MIN & 206 & $<-->$ & MIN 213 \\
\hline 176 & 46.017 & MIN & 200 & $<-->$ & $\mathrm{CO}+\mathrm{H} 2+\mathrm{C} 9 \mathrm{H} 14 \mathrm{O} 2$ \\
\hline 177 & 46.773 & MIN & 203 & $<-->$ & MIN 211 \\
\hline 178 & 46.872 & MIN & 130 & $<-->$ & MIN \\
\hline 179 & 47.800 & MIN & 203 & $<-->$ & MIN \\
\hline 181 & 48.194 & MIN & 117 & $<-->$ & MIN 221 \\
\hline 182 & 48.855 & MIN & 207 & $<-->$ & $\mathrm{H} 2+\mathrm{C} 10 \mathrm{H} 1403$ \\
\hline 183 & 49.246 & MIN & 197 & $<-->$ & MIN 206 \\
\hline 184 & 50.387 & MIN & 218 & $<-->$ & $\mathrm{O} 2+\mathrm{C} 10 \mathrm{H} 16 \mathrm{O}$ \\
\hline 185 & 51.244 & MIN & 218 & $<-->$ & $\mathrm{O} 2+\mathrm{C} 10 \mathrm{H} 16 \mathrm{O}$ \\
\hline 186 & 54.810 & MIN & 43 & $<-->$ & MIN 65 \\
\hline 187 & 54.998 & MIN & 117 & $<-->$ & $\mathrm{CH} 4+\mathrm{C} 9 \mathrm{H} 12 \mathrm{O} 3$ \\
\hline 190 & 55.858 & MIN & 41 & $<-->$ & MIN 203 \\
\hline 192 & 56.417 & MIN & 203 & $<-->$ & $\mathrm{H} 2+\mathrm{C} 10 \mathrm{H} 1403$ \\
\hline 193 & 56.737 & MIN & 212 & $<-->$ & $\mathrm{O} 2+\mathrm{C} 10 \mathrm{H} 160$ \\
\hline 195 & 57.934 & MIN & 203 & $<-->$ & MIN 223 \\
\hline 196 & 58.465 & MIN & 203 & $<-->$ & $\mathrm{O} 3+\mathrm{C} 10 \mathrm{H} 16$ \\
\hline
\end{tabular}




\begin{tabular}{|c|c|c|c|c|c|}
\hline 197 & 59.180 & MIN & 203 & $<-->$ & $\mathrm{O} 3+\mathrm{C} 10 \mathrm{H} 16$ \\
\hline 198 & 59.913 & MIN & 203 & $<-->$ & $\mathrm{H} 2+\mathrm{C} 10 \mathrm{H} 1403$ \\
\hline 199 & 60.648 & MIN & 203 & $<-->$ & $\mathrm{H} 2+\mathrm{C} 10 \mathrm{H} 1403$ \\
\hline 200 & 60.666 & MIN & 117 & $<-->$ & $\mathrm{CH} 4+\mathrm{C} 9 \mathrm{H} 12 \mathrm{O} 3$ \\
\hline 201 & 62.889 & MIN & 203 & $<-->$ & $\mathrm{H} 2+\mathrm{C} 10 \mathrm{H} 14 \mathrm{O} 3$ \\
\hline 202 & 64.978 & MIN & 203 & $<-->$ & $\mathrm{H} 2+\mathrm{C} 10 \mathrm{H} 1403$ \\
\hline 203 & 65.732 & MIN & 111 & $<-->$ & MIN 203 \\
\hline 204 & 66.238 & MIN & 203 & $<-->$ & $\mathrm{H} 2+\mathrm{C} 10 \mathrm{H} 1403$ \\
\hline 205 & 66.241 & MIN & 203 & $<-->$ & $\mathrm{H} 2+\mathrm{C} 10 \mathrm{H} 1403$ \\
\hline 206 & 66.345 & MIN & 203 & $<-->$ & $\mathrm{H} 2+\mathrm{C} 10 \mathrm{H} 1403$ \\
\hline 208 & 66.656 & MIN & 203 & $<-->$ & $\mathrm{H} 2+\mathrm{C} 10 \mathrm{H} 1403$ \\
\hline 210 & 67.216 & MIN & 201 & $<-->$ & MIN 203 \\
\hline 211 & 68.981 & MIN & 203 & $<-->$ & $\mathrm{H} 2+\mathrm{C} 10 \mathrm{H} 1403$ \\
\hline 212 & 69.043 & MIN & 203 & $<-->$ & $\mathrm{H} 2+\mathrm{C} 10 \mathrm{H} 1403$ \\
\hline 213 & 69.275 & MIN & 203 & $<-->$ & $\mathrm{H} 2+\mathrm{C} 10 \mathrm{H} 1403$ \\
\hline 217 & 75.708 & MIN & 203 & $<-->$ & MIN 224 \\
\hline 218 & 76.309 & MIN & 203 & $<-->$ & MIN \\
\hline 220 & 82.282 & MIN & 205 & $<-->$ & MIN \\
\hline
\end{tabular}

RELATIVE ENERGIES OF THE MINS (ZPE-CORRECTED)

$\begin{aligned} \text { MIN \# } & \text { DE }(\mathrm{kcal} / \mathrm{mol}) \\ 1 & -145.650 \\ 2 & -144.990 \\ 3 & -143.728 \\ 4 & -143.520 \\ 5 & -143.475 \\ 6 & -142.390 \\ 7 & -141.558 \\ 8 & -137.856 \\ 9 & -134.149 \\ 10 & -131.104 \\ 11 & -130.949 \\ 12 & -130.242 \\ 13 & -129.811 \\ 14 & -129.466 \\ 15 & -128.636 \\ 16 & -128.400 \\ 17 & -126.884 \\ 18 & -126.346 \\ 19 & -124.571 \\ 20 & -124.024 \\ 21 & -123.998 \\ 22 & -122.304 \\ 23 & -122.302 \\ 24 & -121.897 \\ 25 & -121.059 \\ 26 & -120.535 \\ 27 & -120.092 \\ 28 & -118.294 \\ 29 & -116.290 \\ 30 & -116.176 \\ 31 & -116.043 \\ 32 & -105.766 \\ 33 & -93.426 \\ 34 & -75.250 \\ 35 & -70.549 \\ 36 & -69.922 \\ 37 & -65.371 \\ 38 & -65.207 \\ 39 & -62.450 \\ 40 & -60.776 \\ 41 & -60.503 \\ 42 & -60.395 \\ & \\ 12 & \end{aligned}$




\begin{tabular}{|c|c|}
\hline 43 & -59.642 \\
\hline 44 & -59.076 \\
\hline 45 & -58.945 \\
\hline 46 & -58.882 \\
\hline 47 & -58.172 \\
\hline 48 & -58.024 \\
\hline 49 & -57.872 \\
\hline 50 & -57.737 \\
\hline 51 & -57.555 \\
\hline 52 & -57.151 \\
\hline 53 & -57.120 \\
\hline 54 & -56.697 \\
\hline 55 & -55.499 \\
\hline 56 & -54.831 \\
\hline 57 & -54.497 \\
\hline 58 & -53.770 \\
\hline 59 & -53.334 \\
\hline 60 & -53.088 \\
\hline 61 & -52.277 \\
\hline 62 & -51.816 \\
\hline 63 & -51.509 \\
\hline 64 & -50.940 \\
\hline 65 & -50.904 \\
\hline 66 & -50.796 \\
\hline 67 & -50.785 \\
\hline 68 & -50.383 \\
\hline 69 & -50.112 \\
\hline 70 & -50.106 \\
\hline 71 & -49.952 \\
\hline 72 & -49.798 \\
\hline 73 & -49.773 \\
\hline 74 & -49.490 \\
\hline 75 & -49.179 \\
\hline 76 & -49.055 \\
\hline 77 & -48.219 \\
\hline 78 & -48.115 \\
\hline 79 & -48.010 \\
\hline 80 & -47.721 \\
\hline 81 & -47.273 \\
\hline 82 & -47.048 \\
\hline 83 & -47.029 \\
\hline 84 & -46.856 \\
\hline 85 & -46.849 \\
\hline 86 & -46.714 \\
\hline 87 & -46.620 \\
\hline 88 & -46.559 \\
\hline 89 & -46.273 \\
\hline 90 & -46.213 \\
\hline 91 & -45.754 \\
\hline 92 & -45.646 \\
\hline 93 & -45.611 \\
\hline 94 & -45.538 \\
\hline 95 & -45.350 \\
\hline 96 & -45.144 \\
\hline 97 & -45.129 \\
\hline 98 & -44.566 \\
\hline 99 & -44.532 \\
\hline 100 & -44.505 \\
\hline 101 & -44.253 \\
\hline 102 & -44.159 \\
\hline 103 & -43.982 \\
\hline 104 & -43.561 \\
\hline 105 & -43.146 \\
\hline 106 & -41.649 \\
\hline 107 & -41.612 \\
\hline
\end{tabular}




\begin{tabular}{|c|c|}
\hline 108 & -41.189 \\
\hline 109 & -40.414 \\
\hline 110 & -40.165 \\
\hline 111 & -39.369 \\
\hline 112 & -39.172 \\
\hline 113 & -38.443 \\
\hline 114 & -35.531 \\
\hline 115 & -34.368 \\
\hline 116 & -30.768 \\
\hline 117 & -29.134 \\
\hline 118 & -28.514 \\
\hline 119 & -28.440 \\
\hline 120 & $-28 \cdot 238$ \\
\hline 121 & -28.196 \\
\hline 122 & -27.815 \\
\hline 123 & -27.682 \\
\hline 124 & -27.403 \\
\hline 125 & -27.400 \\
\hline 126 & -27.297 \\
\hline 127 & -27.247 \\
\hline 128 & -27.196 \\
\hline 129 & -26.895 \\
\hline 130 & -26.837 \\
\hline 131 & -26.680 \\
\hline 132 & -26.655 \\
\hline 133 & -26.546 \\
\hline 134 & -26.429 \\
\hline 135 & -26.411 \\
\hline 136 & -26.240 \\
\hline 137 & -26.167 \\
\hline 138 & -26.153 \\
\hline 139 & -26.080 \\
\hline 140 & -25.909 \\
\hline 141 & -25.791 \\
\hline 142 & -25.770 \\
\hline 143 & -25.770 \\
\hline 144 & -25.716 \\
\hline 145 & -25.682 \\
\hline 146 & $-25 \cdot 324$ \\
\hline 147 & -25.233 \\
\hline 148 & -25.009 \\
\hline 149 & -24.999 \\
\hline 150 & -24.853 \\
\hline 151 & -24.840 \\
\hline 152 & -24.759 \\
\hline 153 & -24.651 \\
\hline 154 & -24.622 \\
\hline 155 & -24.581 \\
\hline 156 & -24.529 \\
\hline 157 & -24.480 \\
\hline 158 & -24.443 \\
\hline 159 & -24.393 \\
\hline 160 & -24.353 \\
\hline 161 & -24.328 \\
\hline 162 & -24.219 \\
\hline 163 & -24.190 \\
\hline 164 & -24.096 \\
\hline 165 & -24.013 \\
\hline 166 & -24.002 \\
\hline 167 & -23.818 \\
\hline 168 & -23.452 \\
\hline 169 & -23.415 \\
\hline 170 & -23.407 \\
\hline 171 & -23.269 \\
\hline 172 & -23.097 \\
\hline
\end{tabular}




\begin{tabular}{|c|c|}
\hline 173 & -23.038 \\
\hline 174 & -22.960 \\
\hline 175 & -22.857 \\
\hline 176 & -22.724 \\
\hline 177 & -22.641 \\
\hline 178 & -22.620 \\
\hline 179 & $-22 \cdot 302$ \\
\hline 180 & -22.130 \\
\hline 181 & -22.073 \\
\hline 182 & $-21 \cdot 932$ \\
\hline 183 & -21.901 \\
\hline 184 & -21.710 \\
\hline 185 & $-21 \cdot 427$ \\
\hline 186 & -20.268 \\
\hline 187 & -20.172 \\
\hline 188 & -19.791 \\
\hline 189 & -19.665 \\
\hline 190 & -19.296 \\
\hline 191 & -18.673 \\
\hline 192 & -18.121 \\
\hline 193 & -17.314 \\
\hline 194 & -15.078 \\
\hline 195 & -13.669 \\
\hline 196 & -13.076 \\
\hline 197 & -6.578 \\
\hline 198 & -5.388 \\
\hline 199 & -3.454 \\
\hline 200 & -2.984 \\
\hline 201 & -2.858 \\
\hline 202 & -2.068 \\
\hline 203 & -0.157 \\
\hline 204 & 0.000 \\
\hline 205 & 0.805 \\
\hline 206 & 2.729 \\
\hline 207 & 3.728 \\
\hline 208 & 4.640 \\
\hline 209 & 5.58 \\
\hline 210 & 6.011 \\
\hline 211 & 7.49 \\
\hline 212 & $7.90^{\circ}$ \\
\hline 213 & 8.292 \\
\hline 214 & 8.440 \\
\hline 215 & 8.499 \\
\hline 216 & 9.45 \\
\hline 217 & 20.77 \\
\hline 218 & 27.39 \\
\hline 219 & 28.20 \\
\hline 220 & 29.70 \\
\hline 221 & 29.75 \\
\hline 222 & 36.94 \\
\hline 223 & 39.95 \\
\hline 224 & 49.57 \\
\hline
\end{tabular}

Families of conformers

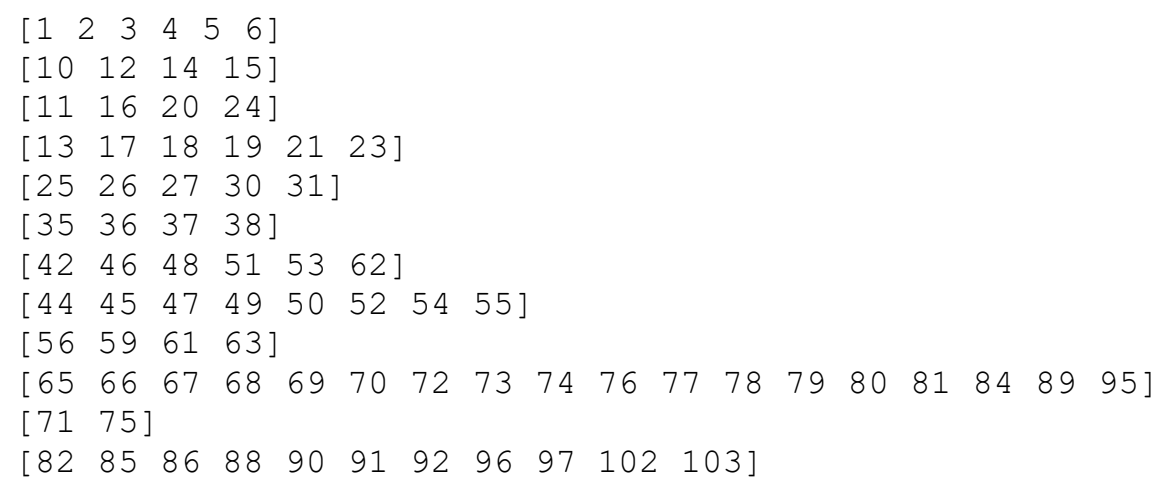


$\left[\begin{array}{ll}87 & 109\end{array}\right]$

$\left[\begin{array}{llllll}99 & 100 & 101 & 104 & 106 & 108\end{array}\right]$

$\left[\begin{array}{lll}105 & 110 & 112\end{array}\right]$

$\left[\begin{array}{ll}111 & 115\end{array}\right]$

$\left[\begin{array}{lllllllllllllllllllll}117 & 118 & 119 & 121 & 122 & 124 & 125 & 126 & 127 & 128 & 129 & 132 & 133 & 134 & 136 & 139 & 140 & 143 & 147 & 148 & 149\end{array}\right.$

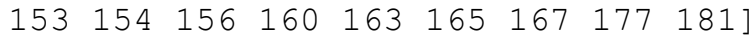

$\left[\begin{array}{ll}120 & 123\end{array}\right]$

$\left[\begin{array}{lllllllllllllllllllll}131 & 135 & 138 & 141 & 142 & 144 & 145 & 146 & 150 & 151 & 152 & 155 & 157 & 158 & 159 & 161 & 162 & 164 & 166 & 168 & 169\end{array}\right.$ $\begin{array}{lllllllllllllllll}170 & 171 & 172 & 173 & 174 & 175 & 176 & 178 & 179 & 180 & 182 & 183 & 184 & 185 & 187 & 188 & 196]\end{array}$

[ [lll 1890 192]

[ [203 204]

[ [lll 206 209 210]

$\left[\begin{array}{ll}207 & 208\end{array}\right]$

[211 216]

[ [212 214 215]

[ 218 219]

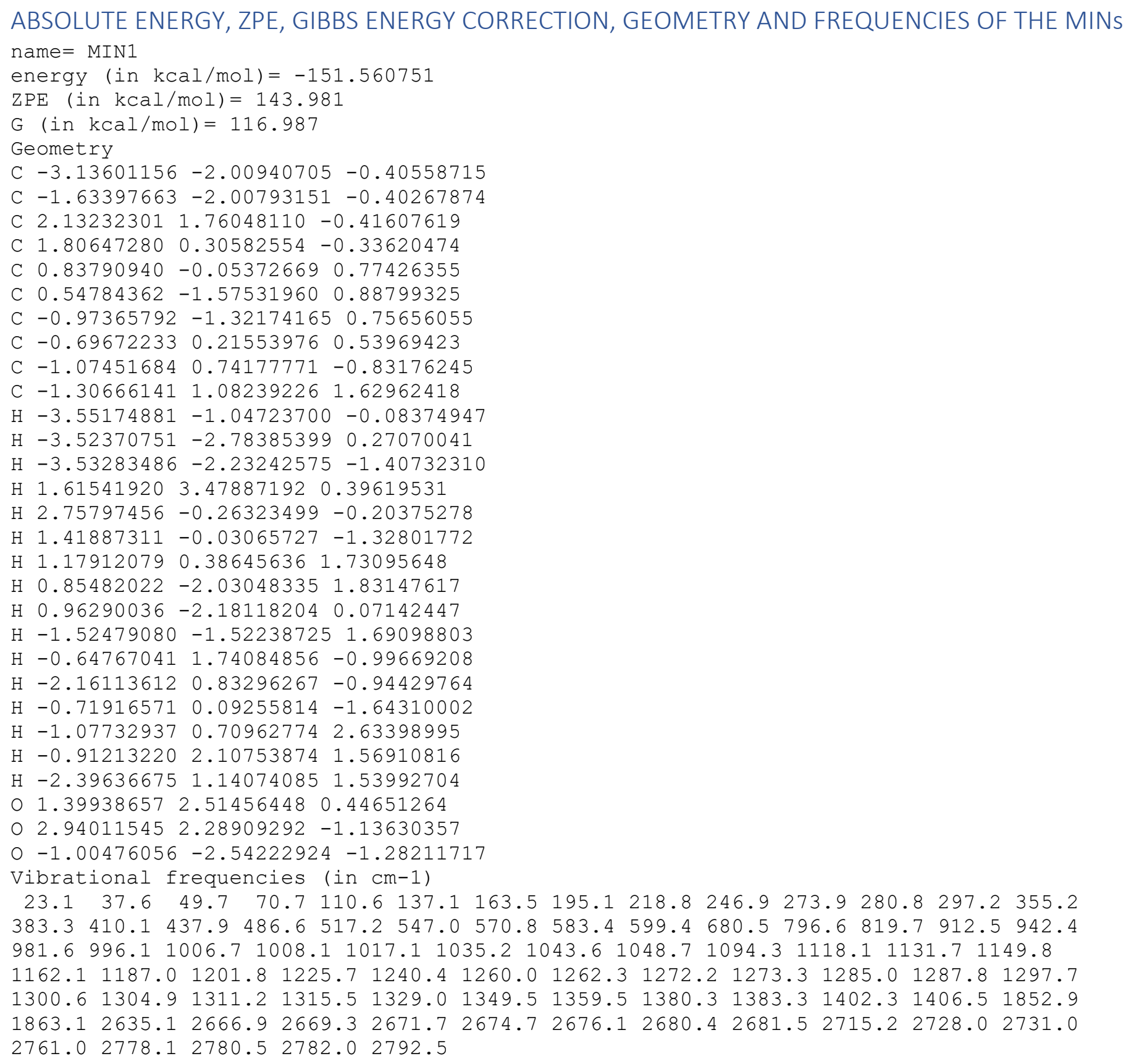

name $=$ MIN2 
energy (in $\mathrm{kcal} / \mathrm{mol})=-150.8905$

$\mathrm{ZPE}($ in $\mathrm{kcal} / \mathrm{mol})=143.971$

$\mathrm{G}($ in $\mathrm{kcal} / \mathrm{mol})=116.76$

Geometry

C $-3.77507389-1.29757622 \quad 0.43617741$

C $-2.27405153-1.27348138 \quad 0.50099617$

C $2.83311636 \quad 0.74737465 \quad 0.51861422$

$\begin{array}{lllll}\text { C } 1.78988425 & 0.02815284 & -0.27751703\end{array}$

C $0.44298445 \quad 0.71139913 \quad-0.12408384$

C $-0.372864150 .33525421 \quad 1.14194269$

C $-1.62519991 \quad 0.05911083 \quad 0.27097251$

C $-0.73884465 \quad 0.19226154-1.02750870$

C $-0.47709060-1.11596762-1.74736788$

C $-1.25411750 \quad 1.24390580 \quad-1.99583944$

$\mathrm{H}-4.21014838-0.78106394 \quad 1.30350021$

$\mathrm{H}-4.16137202-2.327687410 .44806753$

$\mathrm{H}-4.15658638-0.80851921-0.46776003$

H $3.63508193 \quad 2.55274196 \quad 0.59503478$

$\mathrm{H} \quad 1.74260144-1.036291410 .04724746$

H $2.08575415 \quad 0.00957886-1.35064073$

$\mathrm{H} \quad 0.56313068 \quad 1.80929996-0.22898357$

$\mathrm{H}-0.48684013 \quad 1.13244786 \quad 1.87788437$

$\mathrm{H} 0.00007424 \quad-0.55760550 \quad 1.66355610$

$\mathrm{H}-2.36661214 \quad 0.87527883 \quad 0.33772758$

$\mathrm{H}-0.11508135-1.89816832-1.06212342$

$\mathrm{H} \quad 0.26899361-0.99881967-2.54139193$

$\mathrm{H}-1.38901618-1.50820893-2.21354709$

$\mathrm{H}-0.514294261 .45285616-2.77954263$

$\mathrm{H}-1.474123162 .19569083-1.49899643$

$\mathrm{H}-2.17278346 \quad 0.91419343-2.49448933$

$\begin{array}{llll}0 & 2.96090137 & 2.03500897 & 0.09278827\end{array}$

o $3.52222533 \quad 0.33345177 \quad 1.41192076$

o $-1.64241568-2.270625190 .74733112$

Vibrational frequencies (in $\mathrm{cm}-1$ )

$\begin{array}{llllllllllllllll}29.6 & 36.4 & 40.1 & 63.5 & 117.0 & 131.6 & 163.7 & 180.0 & 198.6 & 222.4 & 265.5 & 268.5 & 318.5 & 352.8\end{array}$ $375.0 \quad 390.7 \quad 446.2 \quad 468.7 \quad 521.7 \quad 547.4 \quad 579.7 \quad 597.5 \quad 642.3 \quad 669.9 \quad 813.9 \quad 854.9 \quad 904.6 \quad 938.6$

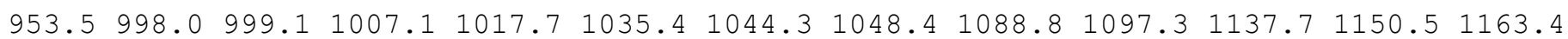

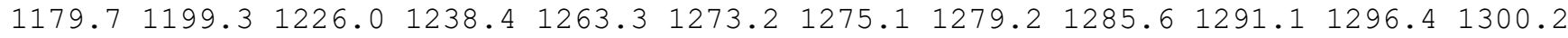
$1305.21315 .71321 .4 \quad 1332.8 \quad 1348.5 \quad 1364.6 \quad 1371.0 \quad 1381.3 \quad 1404.0 \quad 1409.3 \quad 1856.3 \quad 1877.6$ $2649.52662 .62669 .02674 .92675 .5 \quad 2677.92679 .02680 .52709 .92727 .42733 .12760 .3$ $2779.32780 .6 \quad 2783.12791 .3$

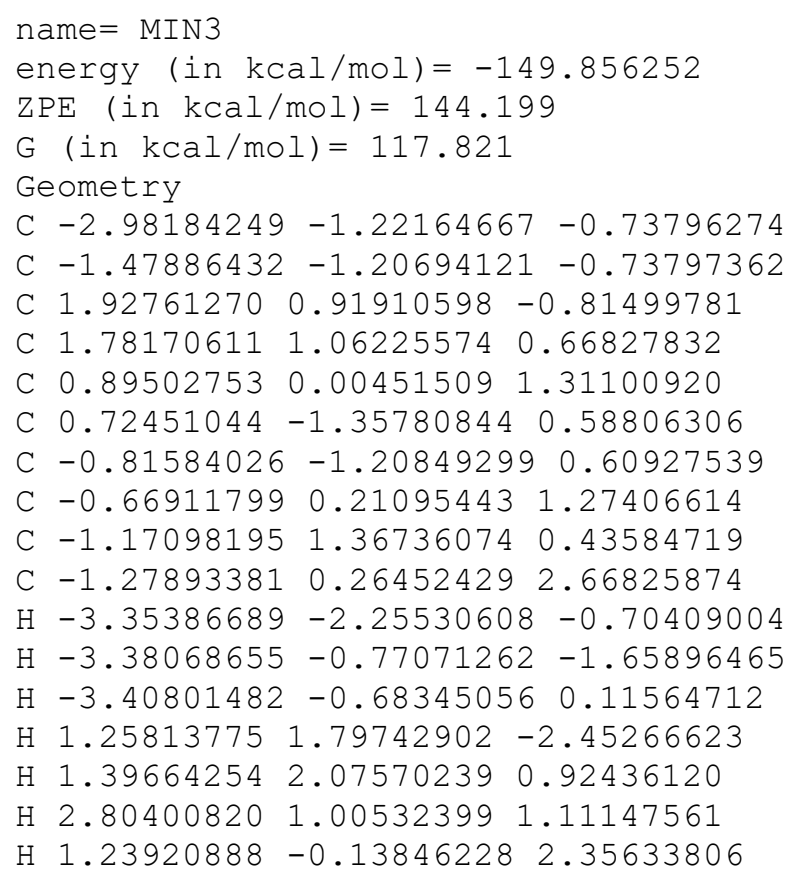


Н $1.10851726-2.21594700 \quad 1.14487253$

$\mathrm{H} 1.15203246-1.41668161-0.42409841$

$\mathrm{H}-1.30836069-1.93954394 \quad 1.27391275$

$\mathrm{H}-0.797229851 .32461613-0.59902583$

$\mathrm{H}-0.84871758 \quad 2.33287047 \quad 0.84519760$

$\mathrm{H}-2.265134291 .38837249 \quad 0.38352841$

$\mathrm{H}-0.94122221-0.56389368 \quad 3.30123365$

$\mathrm{H}-2.37333018 \quad 0.21775706 \quad 2.62732394$

$\mathrm{H}-1.01210005 \quad 1.19689540 \quad 3.18076064$

O $1.217356341 .87889518-1.46769701$

$02.60729247 \quad 0.14171191-1.43029237$

$0-0.85506129-1.21520897-1.76899718$

Vibrational frequencies (in $\mathrm{cm}-1$ )

$\begin{array}{lllllllllllllll}37.5 & 46.4 & 52.5 & 88.2 & 103.3 & 151.0 & 175.7 & 186.2 & 204.3 & 241.5 & 270.1 & 287.7 & 327.0 & 362.4\end{array}$ $\begin{array}{llllllllllllllllll}383.9 & 395.1 & 442.8 & 477.7 & 515.7 & 552.0 & 566.0 & 595.4 & 673.5 & 703.1 & 793.8 & 822.0 & 912.9 & 919.0\end{array}$ $967.5 \quad 990.5 \quad 996.3 \quad 1007.3 \quad 1016.7 \quad 1036.0 \quad 1048.0 \quad 1056.2 \quad 1093.4 \quad 1113.0 \quad 1127.9 \quad 1134.9 \quad 1163.2$ $1192.91200 .4 \quad 1225.7 \quad 1238.7 \quad 1260.0 \quad 1264.2 \quad 1273.1 \quad 1283.7 \quad 1289.9 \quad 1294.4 \quad 1296.11300 .6$ $1303.51309 .91324 .0 \quad 1336.91350 .6 \quad 1357.1 \quad 1380.6 \quad 1387.4 \quad 1403.6 \quad 1409.7 \quad 1855.8 \quad 1877.3$ $2649.62661 .52665 .92668 .92675 .7 \quad 2676.7 \quad 2677.82680 .42710 .92727 .22730 .02757 .3$ $2780.32780 .92783 .2 \quad 2788.5$

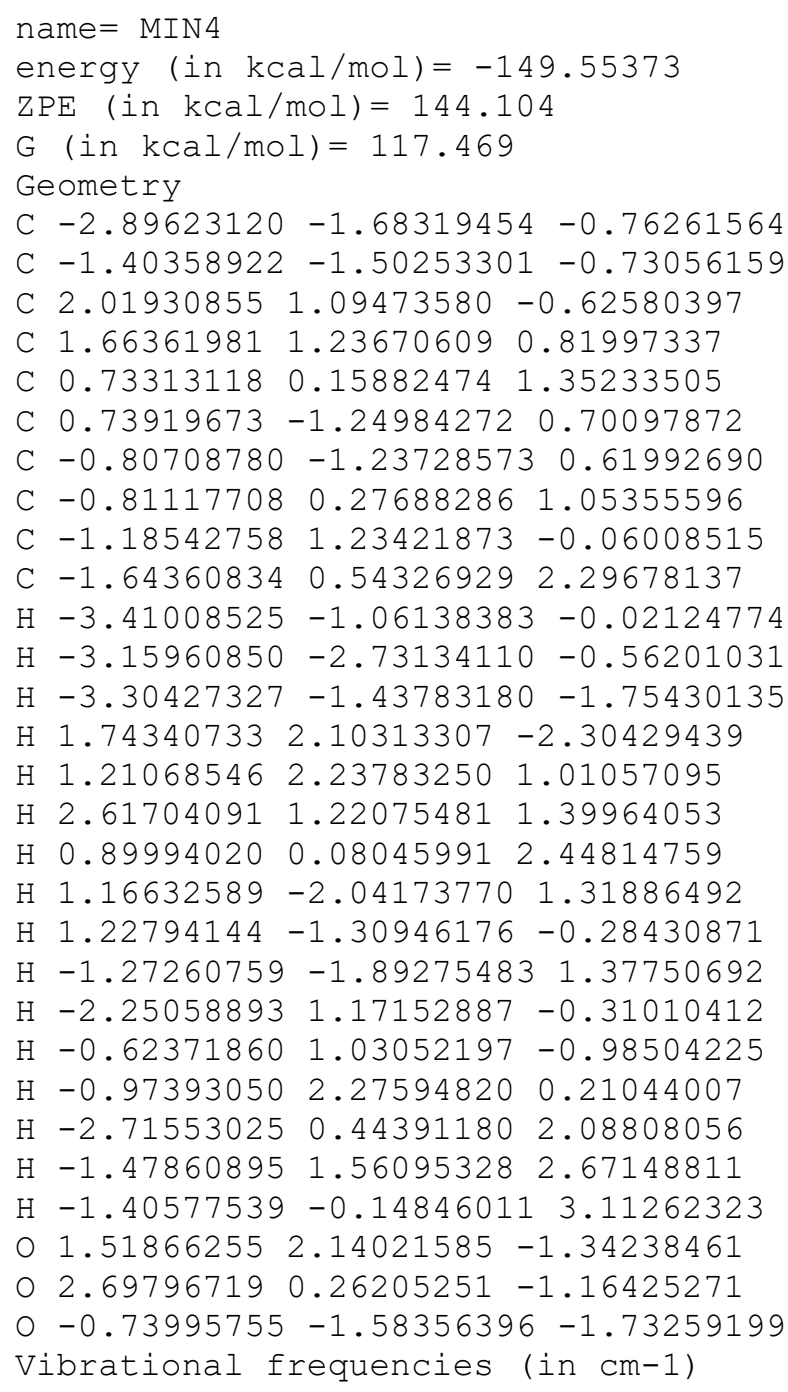


name $=$ MIN5

energy (in $\mathrm{kcal} / \mathrm{mol})=-149.652162$

$\mathrm{ZPE}(\mathrm{in} \mathrm{kcal} / \mathrm{mol})=144.247$

$\mathrm{G}($ in $\mathrm{kcal} / \mathrm{mol})=118.197$

Geometry

C $-2.99539703-0.79223272-0.86339062$

C $-1.49330493-0.76938522-0.85586187$

C $1.80731357 \quad 0.87507517 \quad-0.87014424$

C $1.842601390 .84530616 \quad 0.62903574$

C $1.01443658-0.29148599 \quad 1.21738626$

C $0.68830256-1.49093390 \quad 0.28522786$

C $-0.82967339-1.21916258 \quad 0.41535555$

C $-0.53130693-0.05298290 \quad 1.43132494$

C $-1.08061611 \quad 1.30582587 \quad 1.05620033$

C $-0.96466002-0.41625127 \quad 2.84763975$

$\mathrm{H}-3.41963169-0.42938017 \quad 0.07989234$

$\mathrm{H}-3.36558359-1.81378275-1.02656876$

$\mathrm{H}-3.39852387-0.17056415-1.67696778$

$\mathrm{H} \quad 0.66809481 \quad 1.62239297 \quad-2.29410073$

$\mathrm{H} 1.502854001 .818848961 .04585956$

H $2.90567250 \quad 0.73337714 \quad 0.94426043$

H $1.49635137 \quad-0.62127980 \quad 2.15866591$

$\mathrm{H} 1.00717516-2.45924590 \quad 0.68093181$

$\mathrm{H} 1.07459843-1.42460011-0.74089238$

$\mathrm{H}-1.38624334 \quad-2.05466197 \quad 0.87041067$

$\mathrm{H}-0.67968699 \quad 2.09488344 \quad 1.70430534$

$\mathrm{H}-2.17231082 \quad 1.34022661 \quad 1.14315371$

$\mathrm{H}-0.82989300 \quad 1.58828720 \quad 0.02122405$

$\mathrm{H}-2.05669149-0.43716347 \quad 2.93789630$

$\mathrm{H}-0.59222737 \quad 0.31611559 \quad 3.57431479$

$\mathrm{H}-0.59281315-1.40002573 \quad 3.15549623$

O $0.85735924 \quad 1.72924777-1.32583236$

$\begin{array}{lllll}0 & 2.52720794 & 0.28581334 & -1.63343693\end{array}$

o $-0.87018375-0.42296863-1.82847270$

Vibrational frequencies (in $\mathrm{cm}-1$ )

$\begin{array}{lllllllllllllll}37.1 & 47.5 & 70.3 & 102.6 & 115.4 & 154.8 & 171.1 & 193.1 & 207.0 & 233.5 & 263.3 & 295.8 & 332.4 & 363.1\end{array}$ $391.2 \quad 401.3 \quad 441.5 \quad 464.4 \quad 521.6 \quad 528.7 \quad 568.1 \quad 593.9 \quad 682.4 \quad 713.3 \quad 795.2 \quad 829.3 \quad 905.2 \quad 920.5$ $966.0 \quad 988.8996 .4 \quad 1008.1 \quad 1017.1 \quad 1035.4 \quad 1047.8 \quad 1054.0 \quad 1091.6 \quad 1113.2 \quad 1122.6 \quad 1131.5 \quad 1163.0$

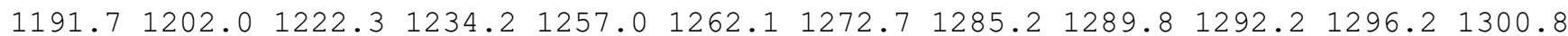

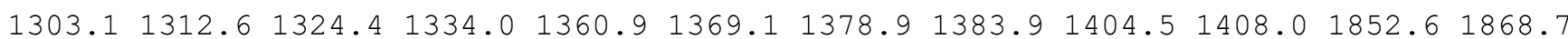
$2652.72657 .82668 .82669 .8 \quad 2675.6 \quad 2676.2 \quad 2677.62680 .4 \quad 2713.8 \quad 2730.2 \quad 2732.92757 .8$ $2778.62780 .02781 .0 \quad 2783.2$

name $=$ MIN6

energy (in $\mathrm{kcal} / \mathrm{mol})=-148.659212$

$\mathrm{ZPE}(\mathrm{in} \mathrm{kcal} / \mathrm{mol})=144.339$

$\mathrm{G}($ in $\mathrm{kcal} / \mathrm{mol})=118.116$

Geometry

C $-2.72541978-1.65768356-0.89542030$

C $-1.44382976-0.90538516-0.68037038$

C $1.18333150 \quad 1.34657794-0.99608245$

C $1.89488335 \quad 0.91465774 \quad 0.25392527$

C $1.29255483-0.283781120 .97255866$

C $0.89392662-1.49261330 \quad 0.08501985$

C $-0.59667057-1.33106951 \quad 0.48482718$

C $-0.19115856-0.196158661 .50113471$

C $-0.91427047 \quad 1.12371832 \quad 1.37938708$

C $-0.28444596-0.698726252 .94175819$

$\mathrm{H}-2.52421825-2.70646927-1.15271587$

$\mathrm{H}-3.30555396-1.22420012-1.72513254$

$\mathrm{H}-3.36258366-1.64644142-0.00348662$

$\mathrm{H} \quad 0.84262885 \quad 0.66461261 \quad-2.80711404$

H $1.92745999 \quad 1.78284171 \quad 0.95178731$ 
$\mathrm{H} \quad 2.95312208 \quad 0.68070301-0.00803300$

$\mathrm{H} \quad 1.98666604-0.57592595 \quad 1.78599331$

$\mathrm{H} 1.33714143-2.44139767 \quad 0.39756018$

$\mathrm{H} 1.09921727-1.36951864-0.98601656$

$\mathrm{H}-1.01928625-2.21397493 \quad 0.98930919$

$\mathrm{H}-0.94416807 \quad 1.50789153 \quad 0.34742370$

$\mathrm{H}-0.43264638 \quad 1.90244071 \quad 1.98413228$

$\mathrm{H}-1.95629772 \quad 1.04615846 \quad 1.71095488$

$\mathrm{H}-1.32790534-0.82648758 \quad 3.25164879$

$\mathrm{H} \quad 0.17694984 \quad 0.01286936 \quad 3.63707357$

$\mathrm{H} \quad 0.21741280-1.66271806 \quad 3.07825144$

$01.438952160 .50422625-2.03020029$

$\begin{array}{lllll}0 & 0.54962778 & 2.35595732 & -1.16327317\end{array}$

O $-1.10512919-0.03515828-1.44466035$

Vibrational frequencies (in $\mathrm{cm}-1$ )

$\begin{array}{llllllllllllll}19.0 & 46.0 & 95.9 & 111.1 & 118.5 & 158.0 & 176.9 & 193.5 & 207.9 & 215.5 & 275.1 & 307.4 & 324.9 & 352.2\end{array}$ $381.2395 .2 \quad 442.6 \quad 475.8 \quad 516.7 \quad 537.0 \quad 579.1 \quad 597.6 \quad 679.6 \quad 715.6 \quad 793.2 \quad 833.0905 .1911 .5$ $968.4 \quad 986.0 \quad 995.6 \quad 1009.7 \quad 1019.1 \quad 1036.3 \quad 1042.7 \quad 1048.2 \quad 1086.0 \quad 1103.1 \quad 1124.4 \quad 1141.3 \quad 1177.1$

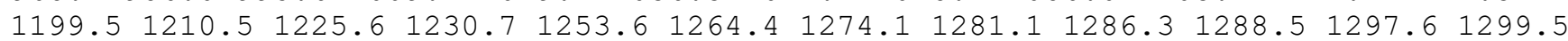
$1305.61308 .01326 .1 \quad 1343.8 \quad 1359.8 \quad 1377.0 \quad 1381.4 \quad 1386.4 \quad 1408.2 \quad 1410.21851 .01865 .7$ $2654.02655 .92669 .42674 .2 \quad 2675.5 \quad 2676.2 \quad 2678.02681 .22712 .2 \quad 2730.62732 .32758 .9$ $2780.3 \quad 2780.92783 .5 \quad 2785.2$

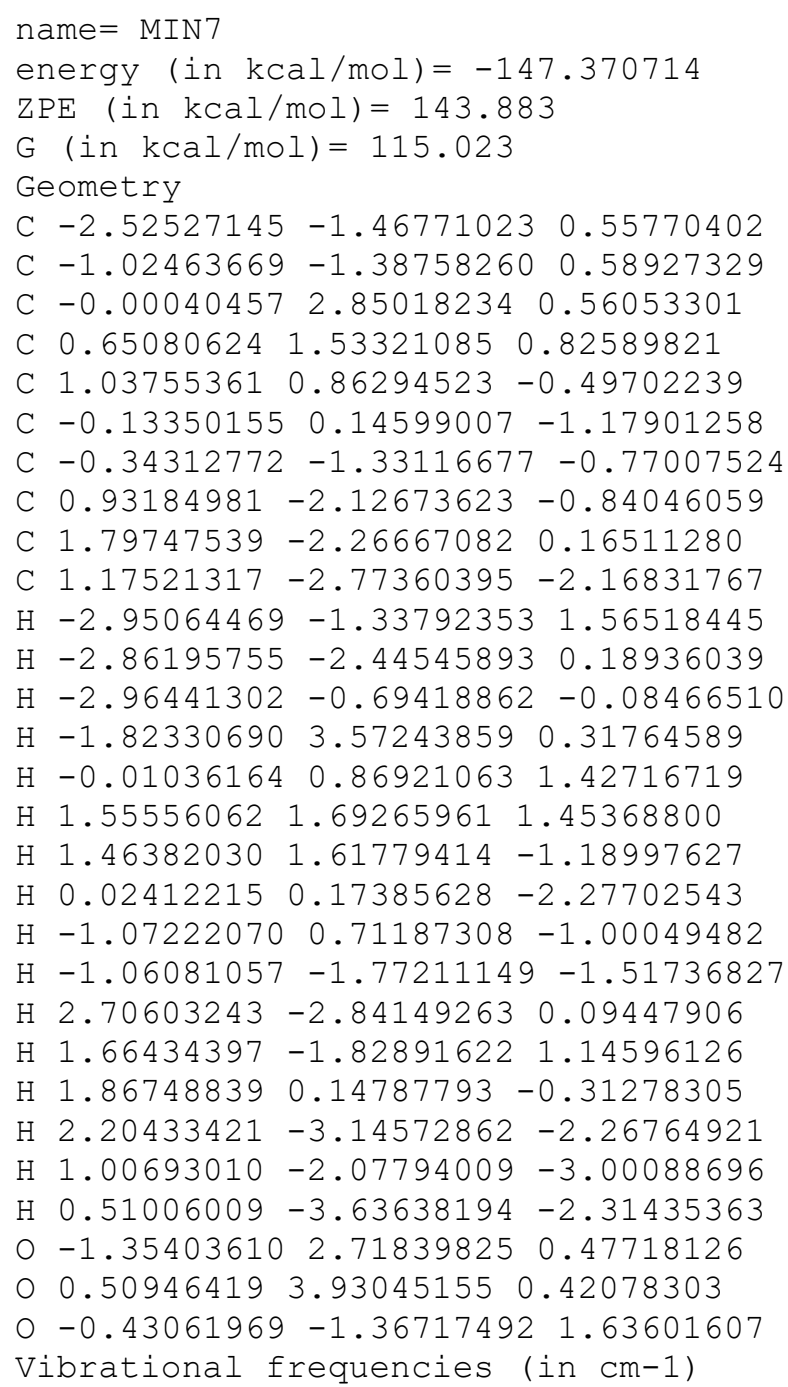


$2645.42649 .12650 .9 \quad 2668.6 \quad 2671.5 \quad 2673.4 \quad 2674.42683 .2 \quad 2709.12729 .92734 .32736 .6$ 2777.82780 .02784 .92792 .9

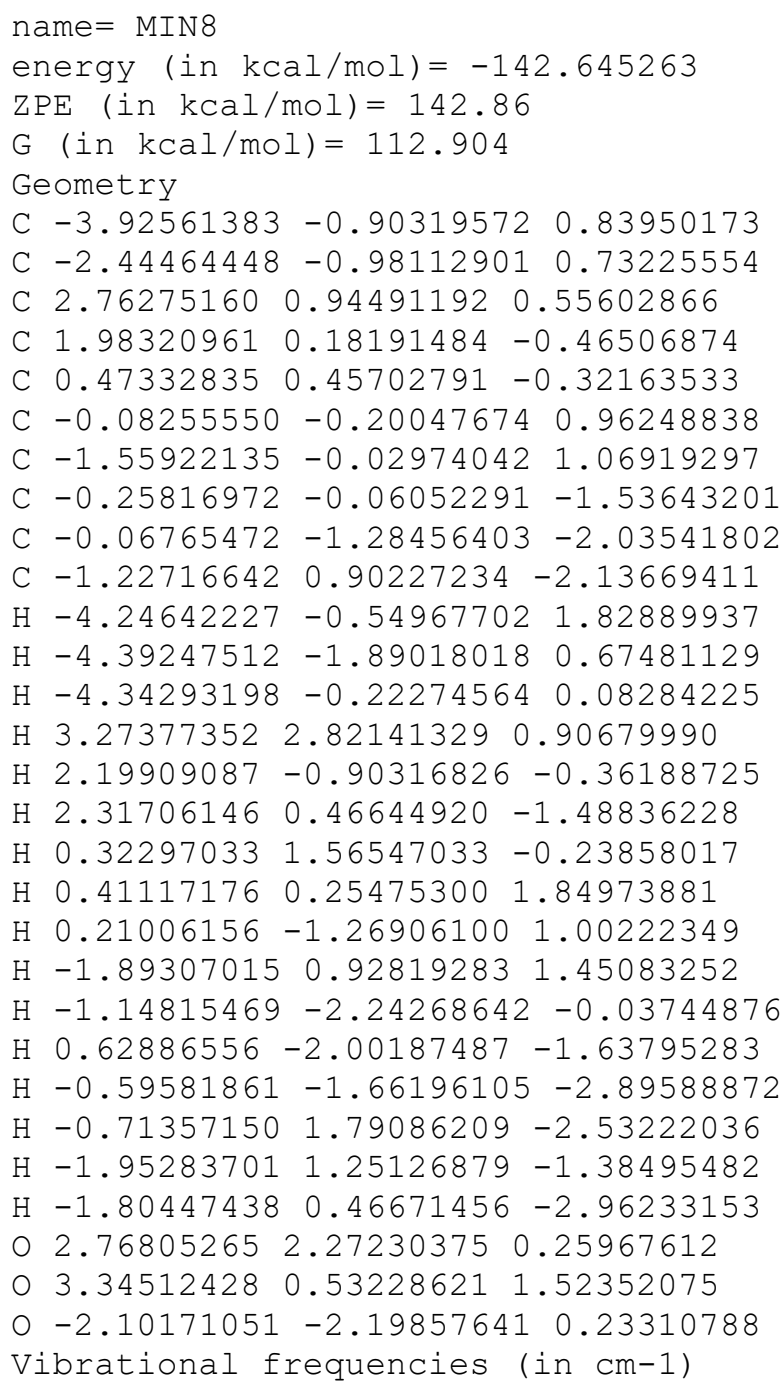

$\begin{array}{llllllllllllllll}11.4 & 18.8 & 29.1 & 38.3 & 58.8 & 89.6 & 101.1 & 116.2 & 122.0 & 180.8 & 249.0 & 259.6 & 288.3 & 336.4\end{array}$ $382.4 \quad 400.4 \quad 410.6 \quad 450.7 \quad 485.0 \quad 524.2 \quad 532.9 \quad 551.4 \quad 569.4 \quad 590.5 \quad 626.8 \quad 670.4 \quad 844.7 \quad 895.3$ $914.8967 .5 \quad 988.6 \quad 1002.5 \quad 1013.5 \quad 1034.2 \quad 1047.0 \quad 1055.3 \quad 1063.6 \quad 1079.7 \quad 1095.6 \quad 1117.0 \quad 1144.3$ $1179.4 \quad 1191.8 \quad 1196.4 \quad 1234.2 \quad 1265.4 \quad 1269.1 \quad 1272.7 \quad 1273.8 \quad 1285.91290 .1 \quad 1301.51317 .9$ $1322.01332 .91346 .4 \quad 1362.8 \quad 1376.1 \quad 1386.8 \quad 1390.2 \quad 1400.4 \quad 1440.3 \quad 1862.4 \quad 1866.01876 .5$ $2649.02652 .42665 .3 \quad 2666.8 \quad 2673.12674 .12692 .02724 .02732 .2 \quad 2734.62755 .52773 .4$ $2777.62779 .7 \quad 2790.0 \quad 2793.9$

name $=$ MIN9

energy (in $\mathrm{kcal} / \mathrm{mol})=-140.863023$

$\mathrm{ZPE}($ in $\mathrm{kcal} / \mathrm{mol})=144.784$

$\mathrm{G}($ in $\mathrm{kcal} / \mathrm{mol})=118.456$

Geometry

C $0.94129182-0.74434367-0.78471033$

C $2.43427028-0.75148244-0.75841714$

C $-1.86971010 \quad 1.94543363-0.79893199$

C $-2.12366621 \quad 1.72691163 \quad 0.66928718$

C $-1.23149680 \quad 0.70408693 \quad 1.35579523$

C $0.29822398 \quad 0.84714489 \quad 1.14971815$

C $0.38726291-0.61071924 \quad 0.62266378$

C $-1.17017484 \quad-0.76617050 \quad 0.79122798$

C $-1.96356610-1.04797084-0.46966602$

C $-1.52385803-1.79656764 \quad 1.85884807$

$\mathrm{H} 0.57482712-1.67502098-1.27032670$

$\mathrm{H} 3.90555410-0.61746423-2.07383645$ 
$\mathrm{H} \quad 0.57859078 \quad 0.08713261-1.43854566$

$\mathrm{H}-2.757174362 .00101161-1.44569676$

$\mathrm{H}-3.18387151 \quad 1.44379608 \quad 0.83297869$

$\mathrm{H}-1.99190371 \quad 2.70841824 \quad 1.18277089$

$\mathrm{H}-1.49359013 \quad 0.69443725 \quad 2.43398094$

H $0.86591581 \quad 1.03093869 \quad 2.06511140$

$\mathrm{H} 0.59464913 \quad 1.612112290 .42282437$

$\mathrm{H} \quad 0.94344542-1.27479060 \quad 1.31347818$

$\mathrm{H}-3.03646592-0.89646476-0.30338303$

$\mathrm{H}-1.83131775-2.08821053-0.79547425$

$\mathrm{H}-1.66214924-0.41390853-1.31086489$

$\mathrm{H}-1.28427039-2.81298021 \quad 1.52443522$

$\mathrm{H}-2.59229665-1.77135410 \quad 2.09866981$

$\mathrm{H}-0.97418316-1.626987112 .79249652$

○ $2.92000719-0.62809443-2.02521591$

$\begin{array}{llll}0 & -0.77314965 & 2.09561753 & -1.27695097\end{array}$

o $3.18784339-0.86157248 \quad 0.17218267$

Vibrational frequencies (in cm-1)

$\begin{array}{llllllllllllll}34.5 & 42.4 & 69.3 & 88.7 & 115.6 & 132.3 & 187.3 & 196.2 & 221.2 & 229.6 & 252.4 & 298.2 & 336.7 & 346.9\end{array}$ $397.2 \quad 439.5 \quad 469.4 \quad 487.2 \quad 515.4 \quad 564.9 \quad 574.2 \quad 624.8 \quad 670.2 \quad 789.5 \quad 866.0 \quad 877.8 \quad 905.0 \quad 929.0$ $944.0 \quad 991.8 \quad 1008.4 \quad 1009.1 \quad 1019.4 \quad 1029.1 \quad 1056.7 \quad 1072.9 \quad 1098.0 \quad 1133.9 \quad 1136.11160 .2$ $1176.81196 .21201 .6 \quad 1229.6 \quad 1234.4 \quad 1253.4 \quad 1269.1 \quad 1282.3 \quad 1285.0 \quad 1290.4 \quad 1297.3 \quad 1299.0$

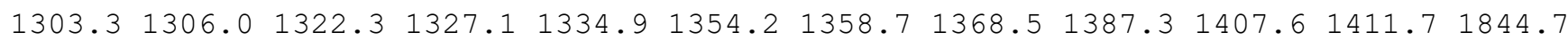
$1878.72638 .22644 .12660 .3 \quad 2670.92676 .12677 .5 \quad 2678.02681 .42709 .02714 .92731 .5$ $2736.2 \quad 2762.12780 .5 \quad 2782.8 \quad 2793.2$

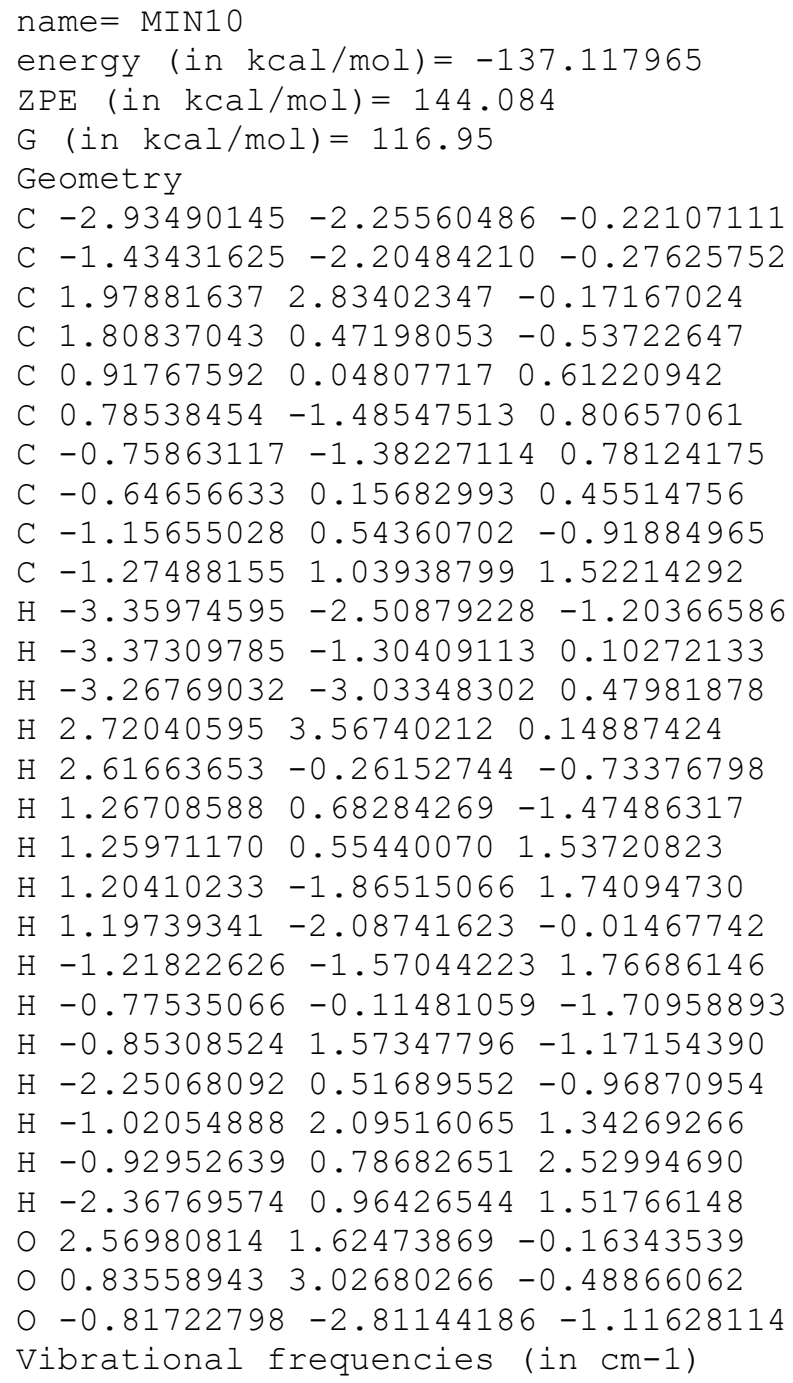




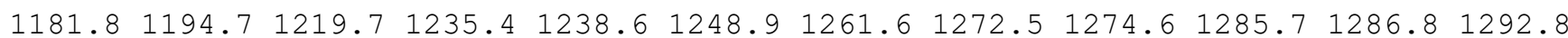
$1299.71300 .51305 .5 \quad 1318.5 \quad 1324.3 \quad 1347.8 \quad 1351.5 \quad 1366.6 \quad 1380.4 \quad 1402.8 \quad 1405.91831 .6$ $1852.32644 .52655 .62658 .8 \quad 2669.8 \quad 2670.3 \quad 2673.02676 .02679 .2 \quad 2681.3 \quad 2706.02721 .8$ $2729.42760 .2 \quad 2776.3 \quad 2780.12781 .2$

\section{name $=$ MIN11}

energy (in $\mathrm{kcal} / \mathrm{mol})=-136.902926$

$\mathrm{ZPE}($ in $\mathrm{kcal} / \mathrm{mol})=144.024$

$\mathrm{G}($ in $\mathrm{kcal} / \mathrm{mol})=116.557$

Geometry

C $-3.44241692-0.60344345-0.19042185$

C $-2.17855793-1.39288773-0.17725826$

C $0.476147122 .44784378-0.36117277$

C $1.84578230 \quad 1.83509798-0.50138907$

C $1.85862694 \quad 0.31917389-0.51999045$

C $0.90439890-0.36323911-1.53957712$

C $0.17736382-1.09463343-0.38544626$

C $1.14960255-0.44814538 \quad 0.66516438$

$\begin{array}{lllll}\text { C } & 0.48752541 & 0.38629615 & 1.73822074\end{array}$

C $2.06801637-1.48868267 \quad 1.29084663$

$\mathrm{H}-3.59951156-0.12287294-1.16936909$

$\mathrm{H}-3.43330275 \quad 0.19839908 \quad 0.56435509$

$\mathrm{H}-4.31040352-1.25188986 \quad 0.00919297$

$\mathrm{H}-0.37524684 \quad 1.91792455 \quad-0.80730917$

H $2.504087012 .20718946 \quad 0.31483827$

H 2.30434032 2.22583225 -1.43716427

$\mathrm{H} 2.90440069-0.02517155-0.64435286$

$\mathrm{H} \quad 1.39804125-1.02653065-2.25375141$

$\mathrm{H} \quad 0.26479367 \quad 0.31537830 \quad-2.11051448$

$\mathrm{H} \quad 0.16050016-2.19175623-0.41727729$

$\mathrm{H} 1.18996718 \quad 1.10566275 \quad 2.17846385$

$\mathrm{H} \quad 0.10555377-0.24162591 \quad 2.55405537$

$\mathrm{H}-0.37553045 \quad 0.95268239 \quad 1.36425009$

$\mathrm{H} 1.51146032-2.163390201 .95426052$

H 2.85169996 -1.01194210 1.89249642

H $2.56383167-2.11318156 \quad 0.53919554$

$0-1.12127863-0.54311462-0.27923277$

$0 \quad 0.32072378 \quad 3.49986247 \quad 0.20952053$

o $-2.05279231-2.58593734-0.10108383$

Vibrational frequencies (in $\mathrm{cm}-1$ )

$\begin{array}{lllllllllllllll}20.5 & 32.5 & 59.2 & 62.4 & 77.9 & 134.5 & 151.7 & 195.0 & 200.7 & 231.2 & 247.3 & 274.1 & 282.7 & 330.7\end{array}$ $349.3 \quad 408.6 \quad 433.0 \quad 468.9 \quad 490.9 \quad 549.1 \quad 575.4 \quad 617.9 \quad 684.9 \quad 768.9 \quad 845.5 \quad 871.7 \quad 915.8 \quad 933.9$ $998.01007 .0 \quad 1008.1 \quad 1020.3 \quad 1029.7 \quad 1044.1 \quad 1057.2 \quad 1063.2 \quad 1070.5 \quad 1111.8 \quad 1123.8 \quad 1157.7$

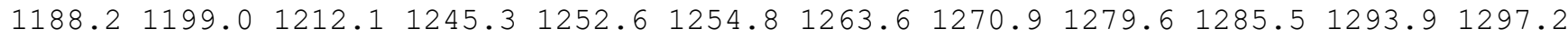
$1301.61304 .71311 .91315 .0 \quad 1328.4 \quad 1342.7 \quad 1350.0 \quad 1377.3 \quad 1382.6 \quad 1406.91409 .71831 .5$ $1873.0 \quad 2645.22657 .3 \quad 2662.3 \quad 2665.8 \quad 2667.2 \quad 2672.92676 .32678 .12683 .22695 .62716 .9$ $2739.0 \quad 2766.8 \quad 2775.3 \quad 2779.3 \quad 2781.8$

name $=$ MIN12

energy $($ in $\mathrm{kcal} / \mathrm{mol})=-136.511683$

$\mathrm{ZPE}($ in $\mathrm{kcal} / \mathrm{mol})=144.34$

$\mathrm{G}($ in $\mathrm{kcal} / \mathrm{mol})=117.843$

Geometry

C $-2.92635396-0.95406233-1.24403364$

C $-1.42725578-0.86641797-1.17185297$

C $2.02882828 \quad 1.37913504-1.20566462$

C $1.54922750 \quad 0.98411390 \quad 1.10188477$

C $0.76553995-0.29885314 \quad 1.30832891$

C $0.75101966-1.30483757 \quad 0.12934173$

C $-0.79691608-1.30918501 \quad 0.11512245$

C $-0.80620852-0.23131752 \quad 1.26488230$

C $-1.39847898 \quad 1.11043148 \quad 0.88365831$

C $-1.44900194-0.75409128 \quad 2.54221576$ 
$\mathrm{H}-3.33812232-0.14853324-1.87000446$

$\mathrm{H}-3.40319752-0.89603878-0.25963636$

$\mathrm{H}-3.23128996-1.90166168-1.71114911$

H $1.58256082 \quad 1.87874353-2.06619682$

$\mathrm{H} \quad 1.26055990 \quad 1.76365816 \quad 1.83352952$

H $2.64040397 \quad 0.80042621 \quad 1.16298791$

H $1.11873809-0.77050620 \quad 2.24722503$

H $1.20977355-2.27184017 \quad 0.34841651$

$\mathrm{H} \quad 1.20268048-0.93455486-0.80085927$

$\mathrm{H}-1.22613606-2.27509617 \quad 0.43436071$

$\mathrm{H}-1.00919392 \quad 1.47250894-0.08064224$

$\mathrm{H}-1.15698643 \quad 1.87935740 \quad 1.62701629$

$\mathrm{H}-2.48968907 \quad 1.06605792 \quad 0.79960267$

$\mathrm{H}-1.29272584-0.058619203 .37566716$

$\mathrm{H}-1.03861979-1.724418872 .84448479$

$\mathrm{H}-2.53108356-0.88077172 \quad 2.42174061$

$03.05048228 \quad 0.74809966-1.14796143$

o $1.227162111 .60054998-0.14137861$

o $-0.78044390-0.48187625-2.11463407$

Vibrational frequencies (in $\mathrm{cm}-1$ )

$\begin{array}{lllllllllllllll}24.8 & 56.7 & 61.6 & 89.2 & 103.5 & 152.7 & 178.4 & 188.5 & 197.2 & 233.7 & 269.5 & 276.4 & 295.3 & 326.0\end{array}$

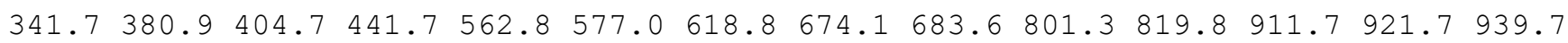
$989.2997 .5 \quad 1003.3 \quad 1016.2 \quad 1023.5 \quad 1036.4 \quad 1051.0 \quad 1061.8 \quad 1092.8 \quad 1109.4 \quad 1132.11153 .7$ $1180.11189 .0 \quad 1215.7 \quad 1238.2 \quad 1240.6 \quad 1257.7 \quad 1260.7 \quad 1271.1 \quad 1273.7 \quad 1286.3 \quad 1291.01293 .0$

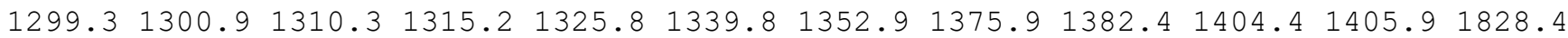
$1851.42642 .0 \quad 2655.8 \quad 2662.2 \quad 2667.6 \quad 2676.7 \quad 2677.2 \quad 2678.12678 .32681 .52708 .52715 .9$ $2727.62762 .7 \quad 2779.6 \quad 2780.7 \quad 2783.0$

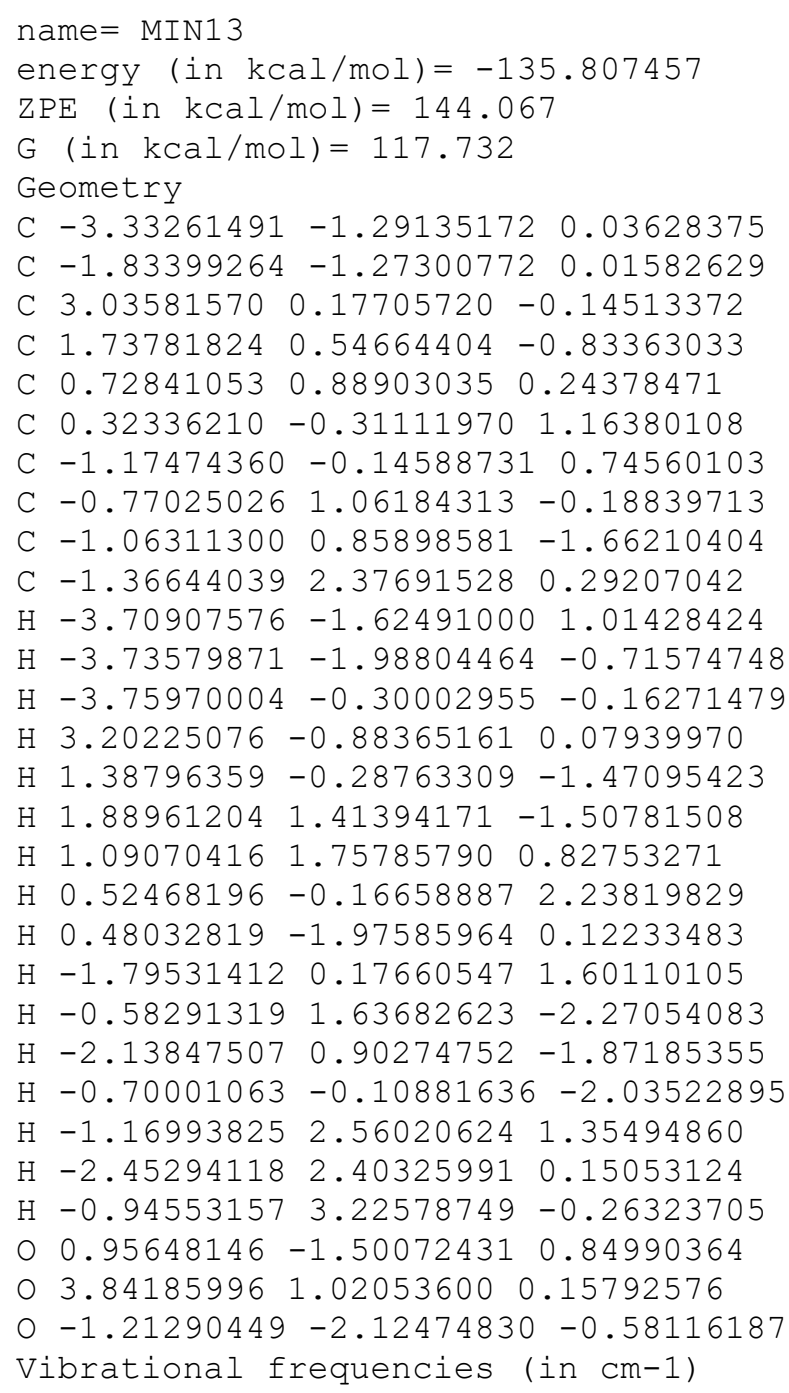


$\begin{array}{lllllllllllllllllll}35.3 & 47.6 & 59.5 & 78.5 & 114.5 & 164.9 & 184.5 & 196.7 & 210.0 & 236.5 & 249.1 & 283.1 & 320.6 & 341.1\end{array}$

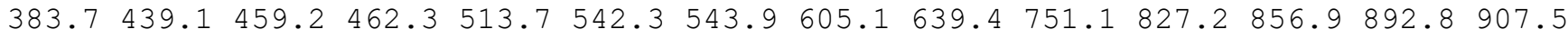
$977.3996 .91002 .3 \quad 1009.5 \quad 1018.2 \quad 1041.5 \quad 1048.01049 .51092 .4 \quad 1108.01141 .01162 .8$ $1169.7 \quad 1180.2 \quad 1205.3 \quad 1226.5 \quad 1233.4 \quad 1256.7 \quad 1260.91270 .1 \quad 1280.0 \quad 1284.7 \quad 1286.3 \quad 1296.2$

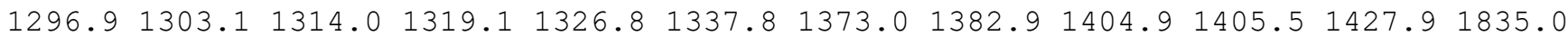
$1838.32647 .62664 .12665 .2 \quad 2670.42671 .4 \quad 2672.92675 .62678 .82689 .72709 .02714 .2$ $2723.4 \quad 2745.3 \quad 2778.4 \quad 2778.9 \quad 2782.4$

name $=$ MIN14

energy (in $\mathrm{kcal} / \mathrm{mol})=-135.765037$

ZPE (in $\mathrm{kcal} / \mathrm{mol})=144.369$

$\mathrm{G}($ in $\mathrm{kcal} / \mathrm{mol})=118.039$

Geometry

C $-2.90913471-0.84387941-1.21732259$

C $-1.40790715-0.88390279-1.16517188$

C $1.871345221 .55796368-1.16901700$

C $1.82782078 \quad 0.62550434 \quad 1.03931571$

C $0.89655774 \quad-0.55648721 \quad 1.26221721$

C $0.74011390-1.551914350 .08227005$

C $-0.79854000-1.38905446 \quad 0.10988149$

C $-0.66171872-0.31493866 \quad 1.25361508$

C $-1.12910657 \quad 1.07541419 \quad 0.87967607$

C $-1.32358568-0.76438972 \quad 2.54955452$

$\mathrm{H}-3.25993220-0.03130475-1.87160840$

$\mathrm{H}-3.36464012-0.70556391-0.23058565$

$\mathrm{H}-3.30147023-1.77974685-1.64061079$

H $2.29331817 \quad 1.32228649-2.14731437$

H $1.35493064 \quad 1.59384870 \quad 1.28757407$

$\mathrm{H} \quad 2.76429504 \quad 0.52120391 \quad 1.62254108$

H $1.20315199-1.07495727 \quad 2.19179117$

H $1.09875890 \quad-2.56222137 \quad 0.28803859$

$\mathrm{H} 1.20042723-1.22607532-0.86167816$

$\mathrm{H}-1.32183522-2.301876150 .44396988$

$\mathrm{H}-0.69695607 \quad 1.41931857-0.07379176$

$\mathrm{H}-0.84639059 \quad 1.81721427 \quad 1.63684539$

$\mathrm{H}-2.21877459 \quad 1.12435671 \quad 0.77316978$

$\mathrm{H}-1.06816402-0.091343593 .37700979$

$\mathrm{H}-1.01702379-1.77487520 \quad 2.84232404$

$\mathrm{H}-2.41613617-0.76888622 \quad 2.45802683$

$\begin{array}{lll}0 & 2.30702440 & 0.62020723-0.30313740\end{array}$

$\begin{array}{lllll}0 & 1.16918834 & 2.48282600 & -0.85815748\end{array}$

o $-0.74083422-0.55444772-2.11458003$

Vibrational frequencies (in $\mathrm{cm}-1$ )

$\begin{array}{lllllllllllllll}32.7 & 48.6 & 59.3 & 94.1 & 107.1 & 155.0 & 181.1 & 195.3 & 220.4 & 235.5 & 254.2 & 281.4 & 309.1 & 330.3\end{array}$

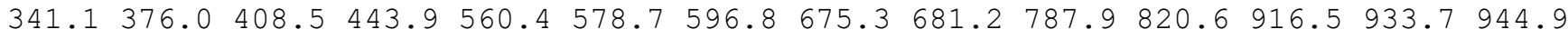
$987.4991 .3 \quad 1006.4 \quad 1015.9 \quad 1024.9 \quad 1037.3 \quad 1050.8 \quad 1058.0 \quad 1095.3 \quad 1111.8 \quad 1131.3 \quad 1152.0$ $\begin{array}{llllllllllll}1181.1 & 1196.3 & 1221.7 & 1232.1 & 1243.8 & 1258.2 & 1259.4 & 1265.8 & 1272.5 & 1285.5 & 1293.4 & 1295.8\end{array}$

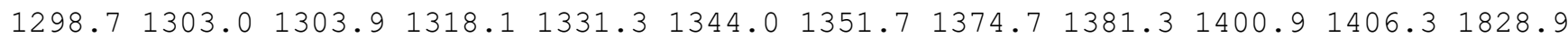
$1851.72635 .42654 .92660 .12666 .92674 .6 \quad 2675.12676 .2 \quad 2677.92679 .92706 .4 \quad 2716.3$ $2728.02760 .12780 .12780 .2 \quad 2783.1$

name $=$ MIN15

energy $($ in $\mathrm{kcal} / \mathrm{mol})=-134.723372$

$\mathrm{ZPE}(\mathrm{in} \mathrm{kcal} / \mathrm{mol})=144.158$

$\mathrm{G}($ in $\mathrm{kcal} / \mathrm{mol})=117.111$

Geometry

C $-2.89415712-1.95062684-0.59900089$

C $-1.39485843-1.85034868-0.66056447$

C $1.90915897 \quad 2.10952880-1.43687840$

C $1.64760178 \quad 1.06570414 \quad 0.67155743$

C $0.80092854-0.03970722 \quad 1.26256461$

C $0.84301938-1.42336588 \quad 0.56381420$

C $-0.70295068-1.51402272 \quad 0.62567209$ 
C $-0.76143388-0.00307814 \quad 1.07016008$

C $-1.25102644 \quad 0.93833965-0.01412126$

C $-1.522454920 .22258022 \quad 2.36550412$

$\mathrm{H}-3.34552358-1.73757910-1.57911508$

$\mathrm{H}-3.33123742-1.26275247 \quad 0.13337681$

$\mathrm{H}-3.19875539-2.97057227-0.32478164$

$\mathrm{H} 1.73538464 \quad 1.92890093-2.49777092$

H $1.34738784 \quad 2.06225674 \quad 1.04840741$

H $2.72476857 \quad 0.90894153 \quad 0.87272687$

H $1.05753103-0.123386792 .33984051$

H $1.37880791-2.205866921 .10139897$

$\mathrm{H} \quad 1.23191256-1.39024262-0.46577801$

$\mathrm{H}-1.05207784-2.19485083 \quad 1.42385337$

$\mathrm{H}-2.33742469 \quad 0.88497541-0.14305937$

$\mathrm{H}-0.79313360 \quad 0.70420918-0.98778418$

$\mathrm{H}-0.99741274 \quad 1.97986081 \quad 0.21603916$

$\mathrm{H}-1.18766411-0.44541484 \quad 3.16683183$

$\mathrm{H}-2.59817854 \quad 0.05820348 \quad 2.23200355$

$\mathrm{H}-1.39287801 \quad 1.252443792 .72221271$

O $1.488987411 .02744006-0.75161382$

$02.383576843 .08194361-0.91014969$

O $-0.79482498-2.06731548-1.68310425$

Vibrational frequencies (in $\mathrm{cm}-1$ )

$\begin{array}{llllllllllllllll}24.7 & 38.7 & 53.7 & 79.5 & 107.7 & 142.8 & 171.6 & 179.4 & 206.4 & 222.1 & 242.6 & 272.3 & 274.1 & 303.1\end{array}$ $337.7 \quad 369.4 \quad 393.4 \quad 445.3 \quad 557.0 \quad 564.8 \quad 606.5 \quad 663.4 \quad 723.6 \quad 788.1 \quad 812.7 \quad 920.9 \quad 934.1 \quad 942.4$ $985.9999 .3 \quad 1003.8 \quad 1011.8 \quad 1016.5 \quad 1034.5 \quad 1048.2 \quad 1062.2 \quad 1089.1 \quad 1114.8 \quad 1135.7 \quad 1160.1$

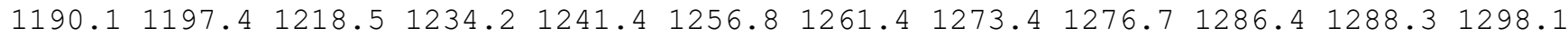
$1300.11303 .31311 .01311 .81328 .3 \quad 1344.41351 .91378 .21384 .51405 .01407 .41825 .0$ $1855.02642 .02656 .82664 .2 \quad 2668.92675 .6 \quad 2676.62677 .22678 .2 \quad 2680.62709 .52713 .2$ $2725.7 \quad 2760.4 \quad 2779.12780 .7 \quad 2782.8$

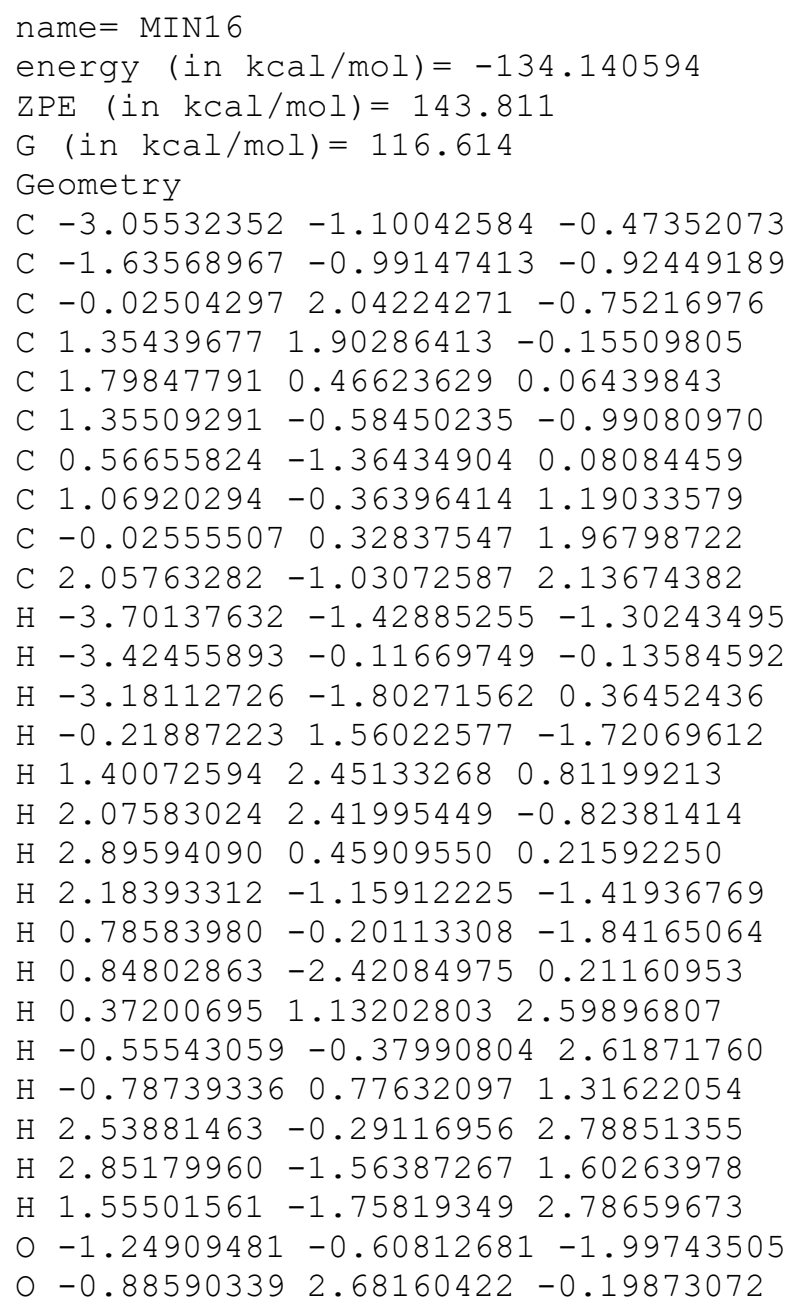


O $-0.83980538-1.401726230 .08392849$

Vibrational frequencies (in $\mathrm{cm}-1$ )

$\begin{array}{llllllllllllllll}-27.9 & 30.1 & 58.2 & 75.3 & 78.6 & 112.7 & 167.2 & 188.8 & 195.0 & 222.1 & 248.7 & 271.9 & 286.8 & 333.8\end{array}$

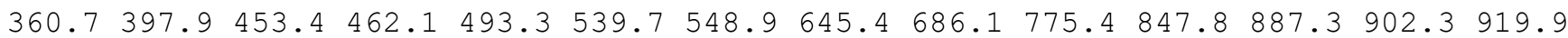
$980.7 \quad 995.7 \quad 1008.3 \quad 1017.7 \quad 1029.2 \quad 1036.9 \quad 1050.7 \quad 1063.0 \quad 1072.6 \quad 1106.6 \quad 1119.4 \quad 1152.6$ $1186.01198 .1 \quad 1219.2 \quad 1228.0 \quad 1250.2 \quad 1253.8 \quad 1261.6 \quad 1274.81279 .4 \quad 1285.0 \quad 1286.4 \quad 1295.0$

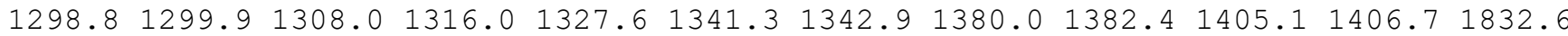
$1866.32636 .02656 .5 \quad 2660.6 \quad 2668.1 \quad 2668.7 \quad 2671.72675 .52677 .92681 .22700 .42715 .0$ $2737.9 \quad 2760.7 \quad 2773.2 \quad 2781.4 \quad 2784.7$

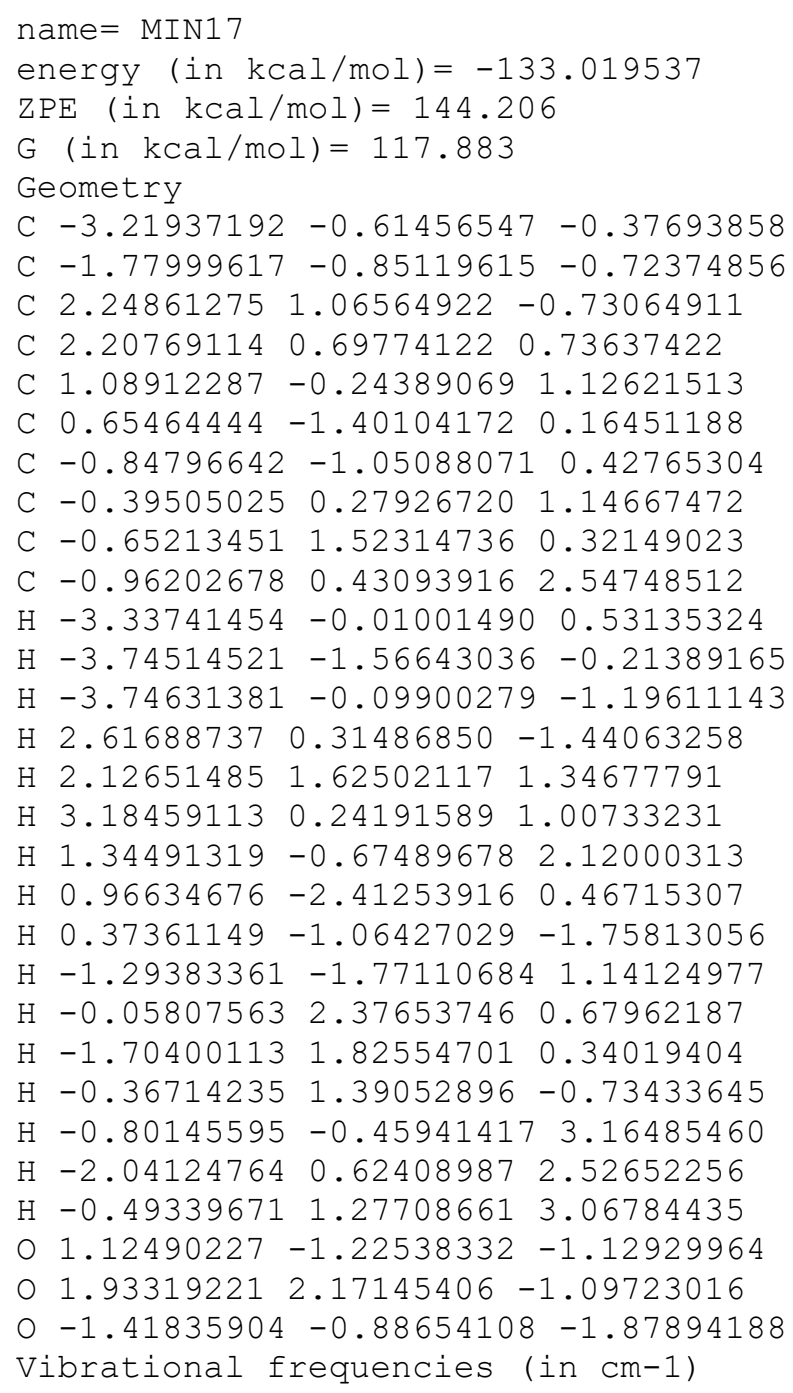


$\begin{array}{llll}C & -0.82815504 & 0.16689821 & -1.24419218\end{array}$

C $-0.33164717-1.15440237-0.56966267$

C $1.12715697-0.57781951-0.50716448$

C $1.67896356-0.34915926 \quad 0.88365023$

C $2.11587307-1.41146488-1.31648266$

$\mathrm{H}-2.90809479-2.18723207 \quad 1.51431549$

$\mathrm{H}-2.93504739-1.87239947-0.24110710$

$\mathrm{H}-2.86375636-0.52573328 \quad 0.89112396$

$\mathrm{H} \quad 1.71163527 \quad 2.69285305 \quad 1.20735535$

H $1.958820462 .38532906-1.14893234$

$\mathrm{H} \quad 0.23956920 \quad 2.79803825-1.32485657$

H $1.01166888 \quad 0.63691242 \quad-2.35293287$

$\mathrm{H}-1.32078018 \quad 0.02992563 \quad-2.22098638$

$\mathrm{H}-1.318083821 .36611540 \quad 0.25601238$

$\mathrm{H}-0.39310649-2.01269162-1.27103972$

H $1.95329541-1.309198521 .35437713$

$\begin{array}{lllll}\mathrm{H} & 0.94016841 & 0.08157818 & 1.57269987\end{array}$

H $2.57335867 \quad 0.27943988 \quad 0.87220759$

H 2.28560721 $-2.38670610-0.84054582$

H $3.08901509-0.91169298-1.38701361$

$\mathrm{H} \quad 1.76861161-1.60409119-2.33621230$

$\begin{array}{llll}0 & -1.73603379 & 0.90535995 & -0.51759153\end{array}$

$0-0.22384406 \quad 2.20665668 \quad 1.27345874$

o $-0.37811964-1.98587860 \quad 1.65022714$

Vibrational frequencies (in $\mathrm{cm}-1$ )

$\begin{array}{lllllllllllllll}34.8 & 43.8 & 101.1 & 114.4 & 173.4 & 188.5 & 198.7 & 203.7 & 223.9 & 238.3 & 289.4 & 312.7 & 335.1 & 359.6\end{array}$ $398.4 \quad 450.9 \quad 473.1 \quad 500.4 \quad 542.0 \quad 578.1 \quad 644.4 \quad 718.9 \quad 731.0 \quad 762.5 \quad 810.2 \quad 843.9 \quad 860.6 \quad 915.9$ $936.6989 .6 \quad 1005.6 \quad 1008.3 \quad 1019.8 \quad 1031.4 \quad 1045.7 \quad 1058.6 \quad 1083.7 \quad 1096.4 \quad 1156.01176 .3$ $1194.61206 .0 \quad 1221.0 \quad 1234.3 \quad 1244.4 \quad 1247.0 \quad 1262.4 \quad 1272.6 \quad 1281.2 \quad 1285.8 \quad 1293.4 \quad 1299.0$

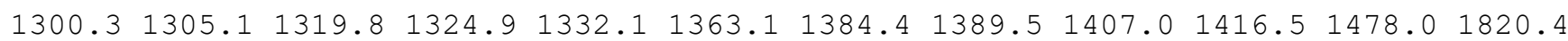
$1845.62642 .0 \quad 2649.6 \quad 2653.4 \quad 2655.92661 .2 \quad 2674.02679 .2 \quad 2681.42683 .8 \quad 2699.8 \quad 2705.8$ $2713.8 \quad 2729.3 \quad 2778.2 \quad 2778.6 \quad 2782.1$

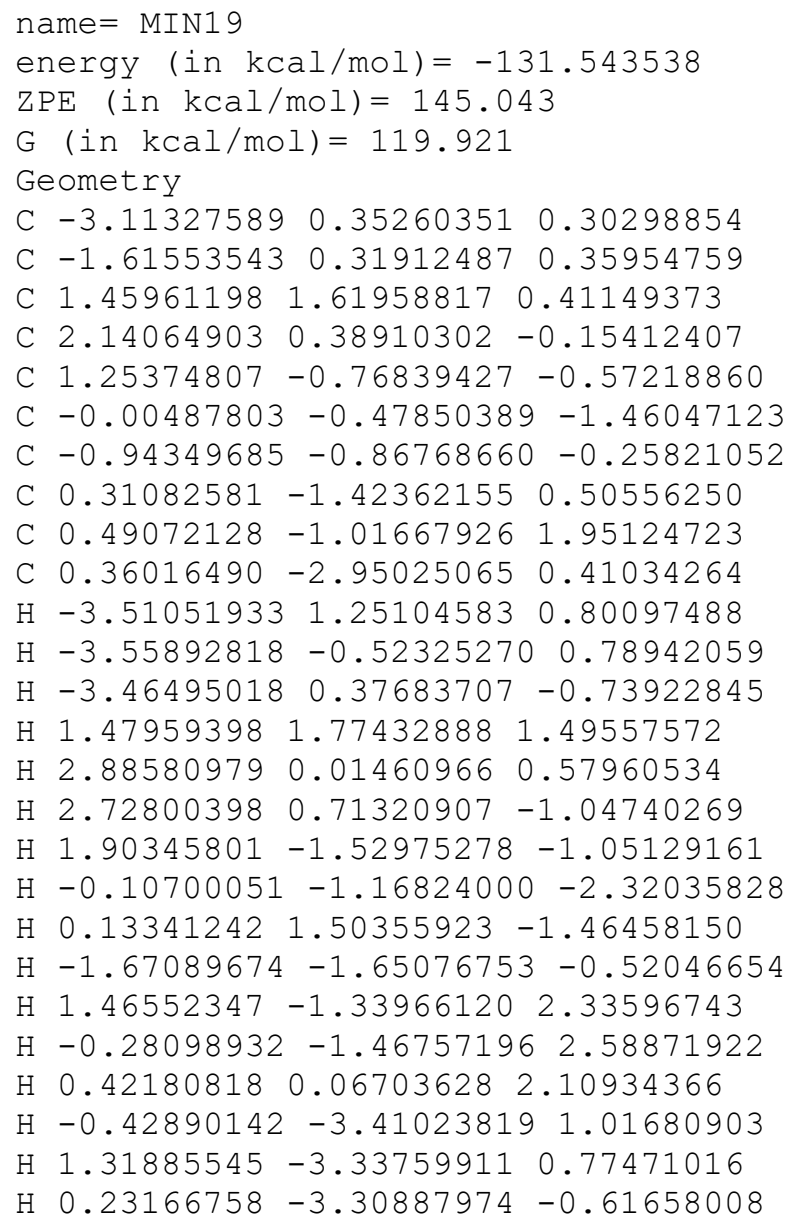


$0-0.19688778 \quad 0.75581286-2.02236509$

$0.991645052 .46192280-0.32065135$

o -0.992379461 .212608210 .88260003$

Vibrational frequencies (in $\mathrm{cm}-1$ )

$\begin{array}{lllllllllllllll}39.7 & 71.4 & 111.4 & 125.1 & 149.0 & 158.7 & 200.1 & 213.0 & 225.1 & 235.6 & 264.9 & 288.0 & 329.9 & 368.9\end{array}$ $385.6 \quad 451.4 \quad 475.2 \quad 485.0 \quad 551.5 \quad 593.4 \quad 619.1 \quad 646.2 \quad 707.6 \quad 765.8 \quad 813.9 \quad 843.5 \quad 861.7 \quad 912.8$ $973.4981 .8 \quad 993.6 \quad 1007.7 \quad 1020.5 \quad 1039.3 \quad 1044.7 \quad 1048.1 \quad 1068.91107 .4 \quad 1153.6 \quad 1171.4 \quad 1195.5$ $\begin{array}{llllllllllllll}1207.9 & 1212.7 & 1232.6 & 1245.3 & 1257.5 & 1265.9 & 1273.1 & 1277.6 & 1286.3 & 1291.8 & 1299.3 & 1300.1\end{array}$ $1304.11308 .81320 .5 \quad 1335.3 \quad 1373.7 \quad 1379.9 \quad 1391.6 \quad 1411.51418 .3 \quad 1480.2 \quad 1815.4 \quad 1845.6$ 2646.12659 .12665 .32666 .52667 .92674 .12675 .72677 .12680 .42698 .72708 .52731 .0 $2732.4 \quad 2778.6 \quad 2780.7 \quad 2783.0$

name $=$ MIN20

energy $($ in $\mathrm{kcal} / \mathrm{mol})=-130.098625$

$\mathrm{ZPE}($ in $\mathrm{kcal} / \mathrm{mol})=144.145$

G $($ in $\mathrm{kcal} / \mathrm{mol})=117.994$

Geometry

C $-2.32506420-2.07280516-0.38139973$

C $-0.83984583-2.16488978-0.28864792$

C $2.29599502 \quad 0.53664042 \quad-0.33033548$

C $1.750366321 .82743301 \quad 0.23643457$

C $0.39747716 \quad 1.70567158 \quad 0.91115026$

C $0.25880996 \quad 0.54315864 \quad 1.93320475$

C $-0.80172917-0.13771777 \quad 1.03187357$

C $-0.790352121 .10282758 \quad 0.06336666$

C $-0.51619474 \quad 0.81204565-1.39318377$

C $-2.059110891 .93188008 \quad 0.21516967$

$\mathrm{H}-2.71128737-2.80901690-1.10760959$

$\mathrm{H}-2.66486669-1.08095657-0.71025929$

$\mathrm{H}-2.80214996-2.29389830 \quad 0.58443660$

H $2.05253046-0.40351008 \quad 0.18184566$

H $1.70154368 \quad 2.59035264 \quad-0.57159183$

H 2.49670487 2.22684955 0.95815077

$\mathrm{H} 0.12539026 \quad 2.68522203 \quad 1.34805975$

$\mathrm{H}-0.11536621 \quad 0.84552240 \quad 2.91426607$

$\mathrm{H} 1.16181352-0.054548122 .09680947$

$\mathrm{H}-1.75617410 \quad-0.39708288 \quad 1.49725939$

$\mathrm{H}-1.41525086 \quad 0.47836264 \quad-1.92417311$

$\mathrm{H} \quad 0.24292020 \quad 0.02781870-1.53470331$

$\mathrm{H}-0.148611641 .70637676-1.91515781$

$\mathrm{H}-2.28364874 \quad 2.16070868 \quad 1.26323789$

$\mathrm{H}-2.93250741 \quad 1.41910982-0.20251193$

$\mathrm{H}-1.96543036 \quad 2.89087677-0.31123787$

$0-0.16200287-1.267258970 .48874743$

○ $2.99698306 \quad 0.55005288-1.31190650$

$0-0.15378586-2.99506632-0.82283436$

Vibrational frequencies (in $\mathrm{cm}-1$ )

$\begin{array}{llllllllllllllll}42.7 & 49.5 & 70.1 & 81.2 & 127.5 & 157.0 & 173.2 & 190.6 & 215.6 & 236.7 & 266.3 & 273.2 & 287.1 & 323.0\end{array}$ $382.0 \quad 407.5 \quad 430.5 \quad 470.6 \quad 492.5 \quad 541.1 \quad 563.7 \quad 604.0 \quad 690.2 \quad 771.2 \quad 838.4 \quad 859.6 \quad 900.5 \quad 932.5$ $993.11002 .8 \quad 1006.8 \quad 1019.5 \quad 1032.6 \quad 1036.4 \quad 1049.5 \quad 1058.1 \quad 1068.2 \quad 1111.01116 .11159 .2$ $\begin{array}{llllllllllll}1191.0 & 1201.5 & 1224.4 & 1238.3 & 1246.9 & 1262.0 & 1268.0 & 1270.2 & 1277.7 & 1285.7 & 1289.9 & 1296.1\end{array}$ $1298.51300 .7 \quad 1307.7 \quad 1312.7 \quad 1320.3 \quad 1335.1 \quad 1347.9 \quad 1379.6 \quad 1382.1 \quad 1405.5 \quad 1408.91830 .7$ 1858.42640 .12656 .92658 .62661 .72666 .72672 .92675 .52678 .62683 .32707 .72718 .7 $2739.02763 .92775 .8 \quad 2777.92782 .3$

name $=$ MIN21

energy $($ in $\mathrm{kcal} / \mathrm{mol})=-129.903942$

$\operatorname{ZPE}($ in $\mathrm{kcal} / \mathrm{mol})=143.976$

$\mathrm{G}($ in $\mathrm{kcal} / \mathrm{mol})=118.155$

Geometry

C $-2.13019839-1.94582447 \quad 0.42952166$

C $-0.63511945-1.94896145 \quad 0.46389927$

C $-0.53765972 \quad 2.33967298 \quad 0.57744854$ 
C $0.723545292 .18388048-0.24247079$

C $0.81962702 \quad 0.85322549-0.95987663$

C $-0.50010906 \quad 0.06380579-1.24501106$

C $0.11171890-1.21915601-0.61679251$

C $1.38181699-0.39688627-0.17980332$

C $1.53780081-0.223246631 .31666904$

C $2.66741680-0.93685740-0.78840782$

$\mathrm{H}-2.51709054-1.05165741 \quad 0.95031504$

$\mathrm{H}-2.54576205-2.82442573 \quad 0.94285571$

$\mathrm{H}-2.53188691-1.91043923-0.58926588$

$\mathrm{H}-0.64416976 \quad 1.74068626 \quad 1.48769063$

$\mathrm{H} \quad 1.60934036 \quad 2.32520017 \quad 0.41226789$

$\mathrm{H} \quad 0.77492229 \quad 3.01262200 \quad-0.98392247$

$\mathrm{H} 1.389276321 .00324386-1.90034284$

$\mathrm{H}-0.79073208-0.01463316-2.30116312$

$\mathrm{H}-2.09728630 \quad 1.20023399-0.94586252$

$\mathrm{H} \quad 0.36343590-1.96896518-1.39755617$

H 2.33512155 $0.48450361 \quad 1.56520837$

$\mathrm{H} \quad 1.79119510-1.18412558 \quad 1.79629788$

H $0.61234388 \quad 0.11758221 \quad 1.79499950$

H 2.59466909 $-1.07584617-1.87184344$

H 2.92659832 $-1.91168926-0.35216133$

$\mathrm{H} \quad 3.51098857-0.26248383-0.59868854$

$0-1.38771993 \quad 3.13277242 \quad 0.24653027$

$0-1.58205913 \quad 0.50298675-0.47556410$

O $-0.03465122-2.577376981 .30304328$

Vibrational frequencies (in $\mathrm{cm}-1$ )

$\begin{array}{llllllllllllllll}34.8 & 43.8 & 72.4 & 129.5 & 159.7 & 181.9 & 190.5 & 204.9 & 223.6 & 256.1 & 269.7 & 282.2 & 308.6 & 325.4\end{array}$

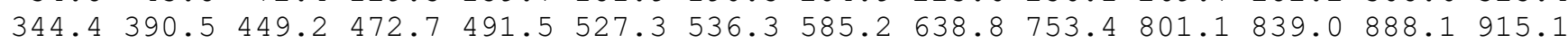
$930.1991 .4 \quad 1005.4 \quad 1014.3 \quad 1018.8 \quad 1036.9 \quad 1050.7 \quad 1068.4 \quad 1082.1 \quad 1096.01120 .8 \quad 1170.8$ $\begin{array}{lllllllllllll}1183.8 & 1199.7 & 1220.9 & 1232.0 & 1250.8 & 1259.7 & 1269.3 & 1272.6 & 1276.3 & 1276.9 & 1285.3 & 1290.3\end{array}$

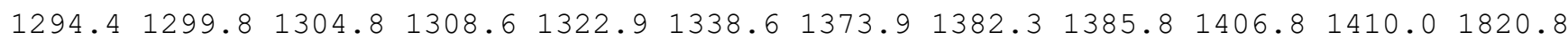
$1836.82647 .6 \quad 2657.4 \quad 2659.0 \quad 2664.5 \quad 2673.6 \quad 2678.02680 .82681 .3 \quad 2695.92706 .12711 .6$ $\begin{array}{llllll}2737.5 & 2775.8 & 2777.5 & 2779.6 & 2782.1\end{array}$

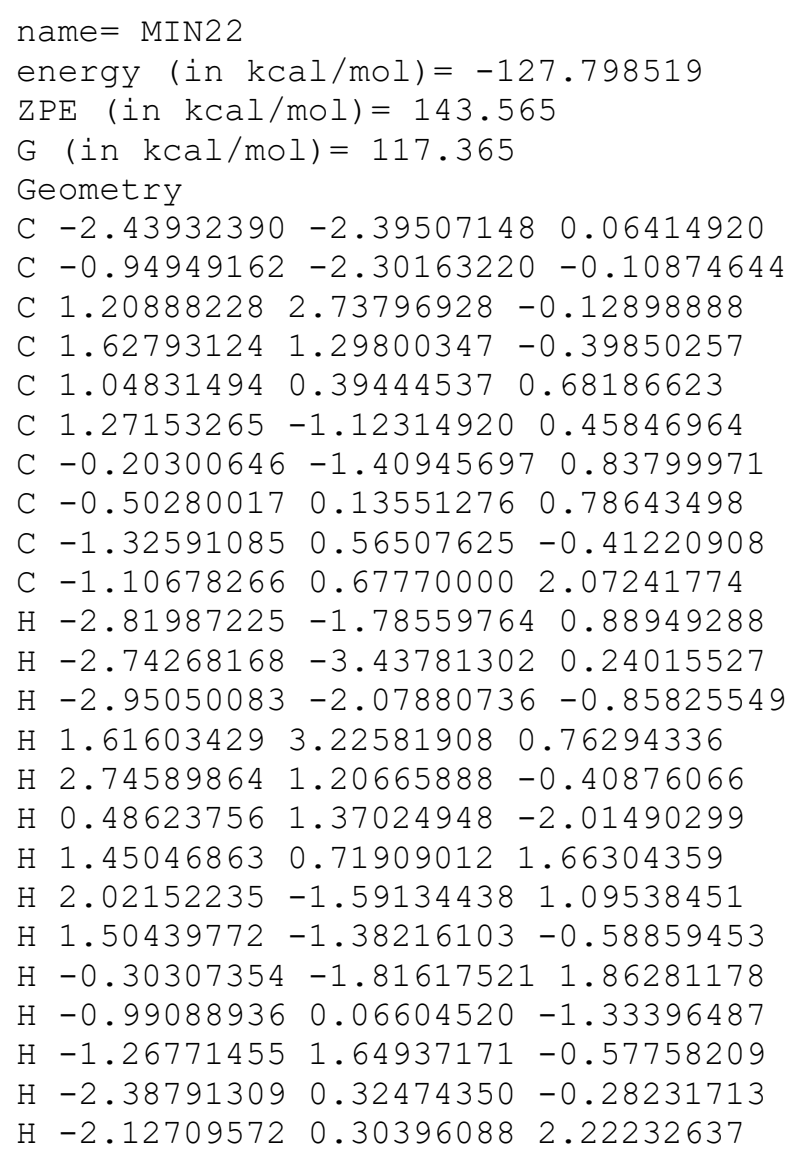


$\mathrm{H}-1.165600361 .772978802 .05181610$

$\mathrm{H}-0.52600096 \quad 0.395900352 .95760795$

O $1.288271910 .89010521-1.69482875$

$0.449312623 .31122668-0.86926335$

O $-0.38012751-2.95638127-0.94583272$

Vibrational frequencies (in $\mathrm{cm}-1$ )

$\begin{array}{llllllllllllllll}46.6 & 48.2 & 72.5 & 92.7 & 118.6 & 153.7 & 161.4 & 184.1 & 222.3 & 248.8 & 266.6 & 274.2 & 293.4 & 302.6\end{array}$ $348.1 \quad 369.0 \quad 387.3 \quad 405.0 \quad 458.3 \quad 539.7 \quad 585.9 \quad 627.2 \quad 665.2 \quad 693.9 \quad 794.8 \quad 806.8 \quad 904.7 \quad 944.2$ $984.8 \quad 993.6 \quad 1000.1 \quad 1008.3 \quad 1015.3 \quad 1032.1 \quad 1046.1 \quad 1057.5 \quad 1089.91095 .11131 .31137 .0$ $1162.11177 .2 \quad 1206.3 \quad 1216.2 \quad 1242.1 \quad 1246.9 \quad 1256.1 \quad 1266.4 \quad 1275.2 \quad 1280.21289 .11291 .8$

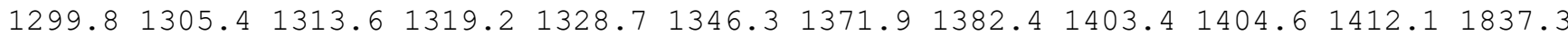
$1850.82656 .02662 .1 \quad 2664.5 \quad 2668.7 \quad 2670.6 \quad 2672.92676 .02677 .4 \quad 2680.32712 .5 \quad 2721.8$ $2758.92771 .7 \quad 2776.6 \quad 2780.4 \quad 2782.6$

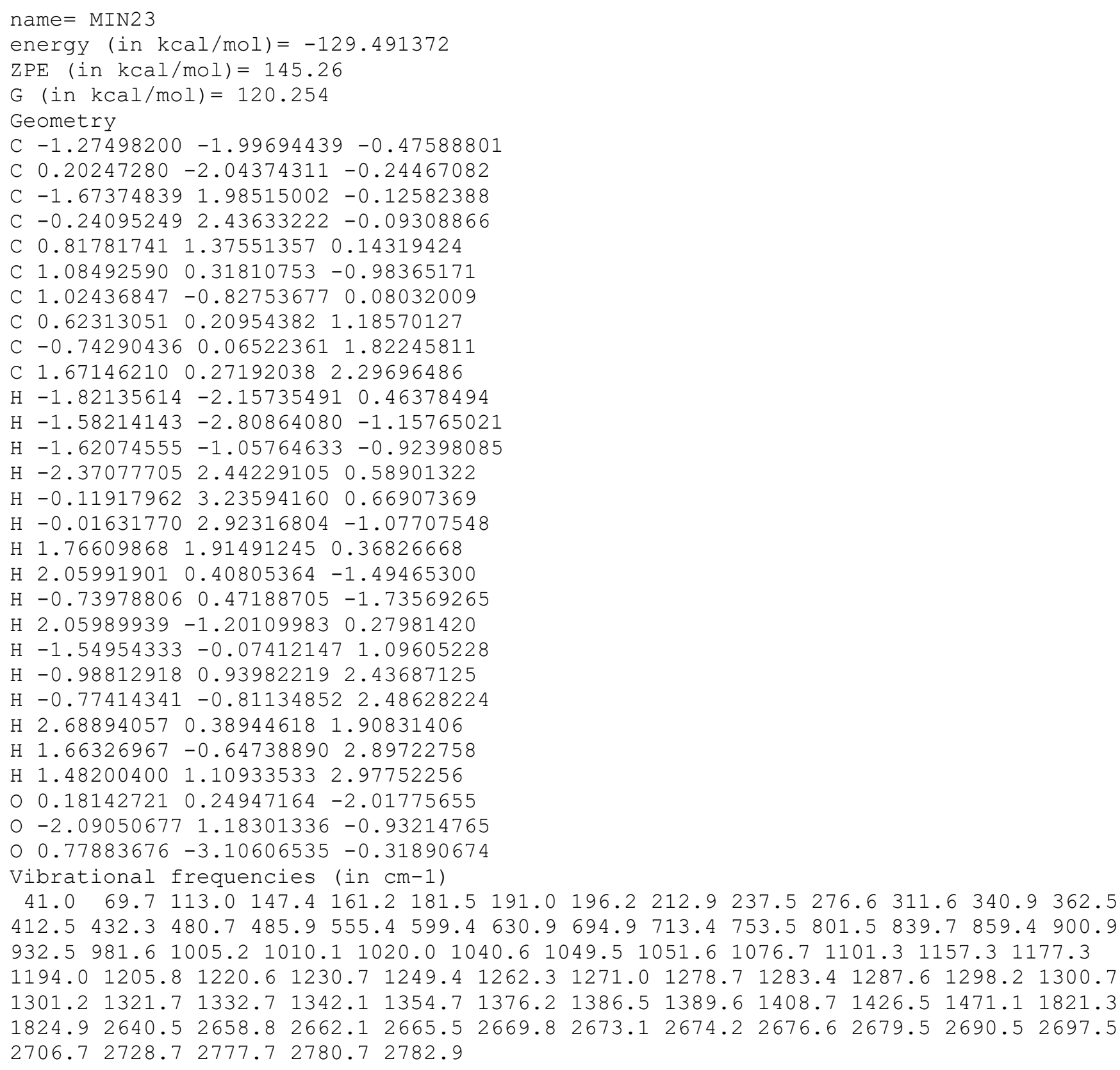


C $-0.70164058-2.32990120-0.52645251$

$\begin{array}{llll}\text { C } 2.25173522 & 1.51764718 & -0.46748077\end{array}$

$\begin{array}{llll}\text { C } 1.31674401 & 2.40965051 & 0.31626524\end{array}$

C $0.14970184 \quad 1.67706943 \quad 0.95661029$

C $0.46671472 \quad 0.35105966 \quad 1.69782892$

C $-0.63088179 \quad-0.37928948 \quad 0.88979927$

C $-0.89289325 \quad 0.92222004 \quad 0.04266455$

C $-0.539372590 .78902978-1.42218093$

C $-2.30212289 \quad 1.46708764 \quad 0.21288916$

$\mathrm{H}-2.61561681-3.03797386-1.17840380$

$\mathrm{H}-2.60854747-1.32527803-0.71879486$

$\mathrm{H}-2.56104854-2.58951428 \quad 0.53265046$

H $2.16711888 \quad 1.53469734-1.56083873$

H $0.93313226 \quad 3.22730913-0.32464766$

H $1.90549384 \quad 2.90455382 \quad 1.12329731$

$\mathrm{H}-0.37503662 \quad 2.39232262 \quad 1.62455166$

$\begin{array}{lllll}\text { H } & 0.31702246 & 0.37012326 & 2.77788053\end{array}$

H $1.47916720-0.04652016 \quad 1.51421252$

$\mathrm{H}-1.48158026-0.76130450 \quad 1.46351310$

$\mathrm{H}-1.33584354 \quad 0.29288198 \quad-1.99002251$

H $0.36697361 \quad 0.18075631-1.56995148$

$\mathrm{H}-0.37364057 \quad 1.76757133-1.88689186$

$\mathrm{H}-3.04480638 \quad 0.84814819-0.30257215$

$\mathrm{H}-2.38261360 \quad 2.47905879-0.20512260$

$\mathrm{H}-2.60107238 \quad 1.52781374 \quad 1.26567630$

$\begin{array}{lllll}0 & 0.01431908 & -1.40358380 & 0.17494054\end{array}$

$\begin{array}{llll}0 & 3.08650716 & 0.84017635 & 0.07583425\end{array}$

$0-0.04534024-3.12128163-1.15135330$

Vibrational frequencies (in cm-1)

$\begin{array}{lllllllllllllll}30.0 & 42.1 & 53.6 & 72.7 & 110.8 & 135.5 & 167.5 & 171.0 & 215.6 & 226.1 & 253.4 & 285.3 & 296.6 & 306.6\end{array}$

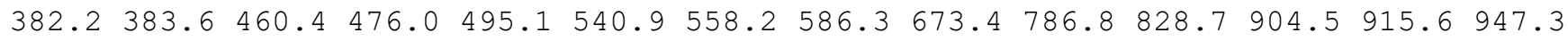

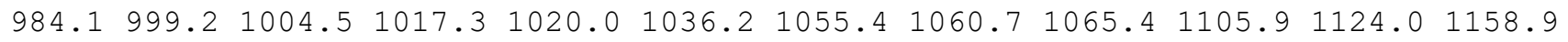
$\begin{array}{lllllllllllll}1195.4 & 1195.5 & 1222.9 & 1234.8 & 1256.9 & 1265.4 & 1269.3 & 1277.8 & 1283.1 & 1288.2 & 1294.3 & 1299.3\end{array}$ $\begin{array}{llllllllllll}1300.3 & 1305.5 & 1315.1 & 1319.9 & 1326.8 & 1339.3 & 1342.9 & 1380.3 & 1385.3 & 1405.5 & 1408.5 & 1842.2\end{array}$

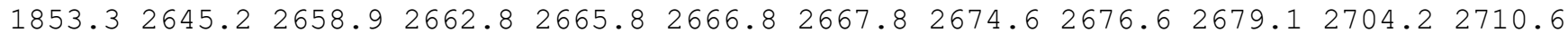
2739.42758 .12776 .22777 .32782 .0

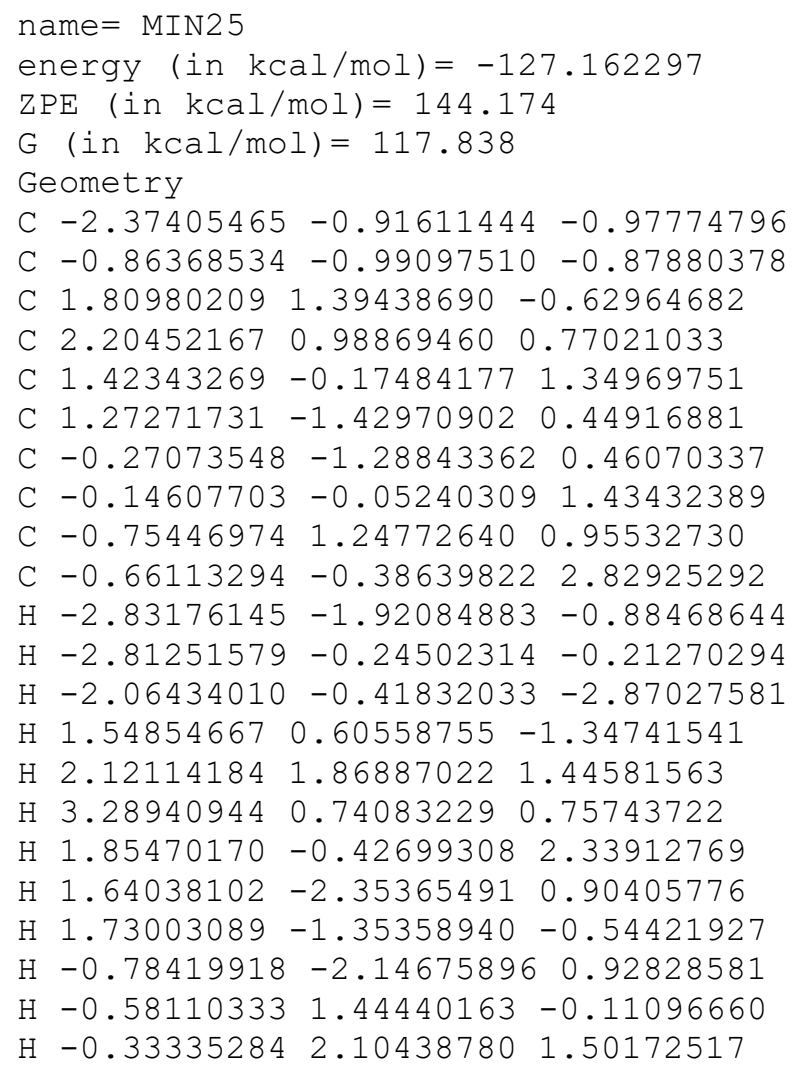


$\mathrm{H}-1.84010784 \quad 1.27718460 \quad 1.11256591$

$\mathrm{H}-1.75147520-0.498329682 .84240457$

$\mathrm{H}-0.407782450 .40846191 \quad 3.54253262$

$\mathrm{H}-0.23340486-1.317615493 .21814055$

$0-2.79819497-0.37871415-2.20793673$

$\begin{array}{llll}0 & 1.80550040 & 2.55678028-0.95247985\end{array}$

O $-0.19384483-0.83385151-1.87254302$

Vibrational frequencies (in $\mathrm{cm}-1$ )

$\begin{array}{lllllllllllllllll}34.5 & 46.6 & 66.8 & 72.9 & 122.6 & 170.2 & 185.0 & 203.5 & 223.8 & 248.7 & 271.2 & 283.4 & 297.2 & 323.0\end{array}$ $\begin{array}{llllllllllllll}353.1 & 373.0 & 413.4 & 437.7 & 446.8 & 559.2 & 593.5 & 648.4 & 687.8 & 773.8 & 835.6 & 865.1 & 916.0 & 936.0\end{array}$ $963.6994 .3 \quad 1006.5 \quad 1012.9 \quad 1019.7 \quad 1046.7 \quad 1060.5 \quad 1080.6 \quad 1113.6 \quad 1116.8 \quad 1129.5 \quad 1153.6$

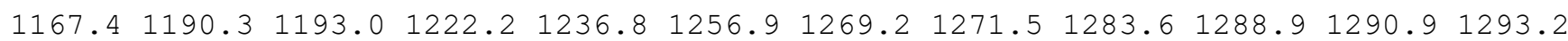

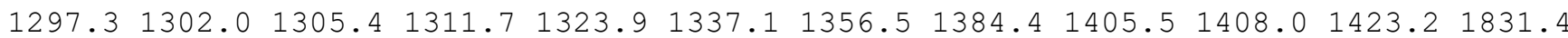
$1840.12636 .62640 .12657 .12662 .4 \quad 2669.2 \quad 2675.62677 .12677 .22710 .32714 .52725 .5$ $2738.4 \quad 2760.3 \quad 2768.9 \quad 2778.0 \quad 2782.4$

\section{name $=$ MIN26}

energy $($ in $\mathrm{kcal} / \mathrm{mol})=-126.521517$

$\mathrm{ZPE}(\mathrm{in} \mathrm{kcal} / \mathrm{mol})=144.057$

$\mathrm{G}($ in $\mathrm{kcal} / \mathrm{mol})=117.161$

Geometry

C $-2.61002866-1.25911693-0.63564784$

C $-1.10478906-1.08363746-0.56390918$

C $2.41797574 \quad 0.86128072-0.76660505$

C 2.171170991 .281193420 .66245113

C $1.172255310 .41315321 \quad 1.40227398$

C $0.99744215-1.05389620 \quad 0.92028405$

C $-0.53301761 \quad-0.85695750 \quad 0.79648937$

C $-0.36723186 \quad 0.66585352 \quad 1.17845585$

C $-0.73465512 \quad 1.64302802 \quad 0.07972473$

C $-1.09714149 \quad 1.02075488 \quad 2.46475159$

$\mathrm{H}-3.14904846-0.47133883-0.07336998$

$\mathrm{H}-2.91351900-2.25248548-0.24825228$

$\mathrm{H}-2.37360703-1.32882488-2.59892267$

H $1.55520424 \quad 0.55886161-1.37485403$

$\mathrm{H} \quad 1.84277888 \quad 2.34275884 \quad 0.67185955$

$\mathrm{H} 3.141225151 .28557840 \quad 1.20976047$

H $1.41194633 \quad 0.44986907 \quad 2.48527932$

$\mathrm{H} 1.30336244-1.808859731 .64906200$

H $1.49327333-1.30207995-0.02656220$

$\mathrm{H}-1.09403486-1.42246972 \quad 1.56162707$

$\mathrm{H}-0.26298808 \quad 1.39622293-0.88085459$

$\mathrm{H}-0.43124186 \quad 2.66582808 \quad 0.33718902$

$\mathrm{H}-1.81827924 \quad 1.66799939-0.09526852$

$\mathrm{H}-2.18489130 \quad 1.01045773 \quad 2.32787449$

$\mathrm{H}-0.82371008 \quad 2.02645162 \quad 2.80897357$

$\mathrm{H}-0.864440640 .32689357 \quad 3.28079982$

O $-3.09857095-1.15074772-1.95085534$

$\begin{array}{lllll}0 & 3.53320781 & 0.86857278 & -1.22483462\end{array}$

o $-0.43987517-1.13260690-1.57027048$

Vibrational frequencies (in $\mathrm{cm}-1$ )

$\begin{array}{llllllllllllllll}31.6 & 42.2 & 45.8 & 69.1 & 111.0 & 146.9 & 176.6 & 186.7 & 236.5 & 239.8 & 253.0 & 288.8 & 297.6 & 311.3\end{array}$

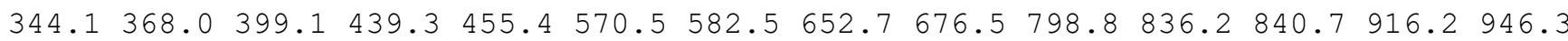
$962.7 \quad 993.7 \quad 1004.9 \quad 1013.7 \quad 1018.6 \quad 1046.2 \quad 1062.9 \quad 1083.8 \quad 1108.7 \quad 1122.1 \quad 1134.51150 .1$

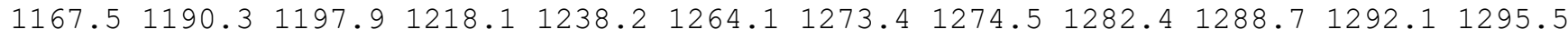
$1298.71300 .61306 .21311 .51331 .4 \quad 1344.4 \quad 1356.6 \quad 1382.81403 .0 \quad 1408.01418 .91834 .4$ $1849.12636 .8 \quad 2637.5 \quad 2654.4 \quad 2664.12671 .5 \quad 2674.2 \quad 2675.92677 .4 \quad 2711.3 \quad 2712.92726 .5$ $2738.0 \quad 2759.5 \quad 2774.7 \quad 2778.3 \quad 2782.6$

name $=$ MIN27

energy (in $\mathrm{kcal} / \mathrm{mol})=-126.051417$

$\mathrm{ZPE}($ in $\mathrm{kcal} / \mathrm{mol})=144.03$

$\mathrm{G}(\mathrm{in} \mathrm{kcal} / \mathrm{mol})=117.148$ 


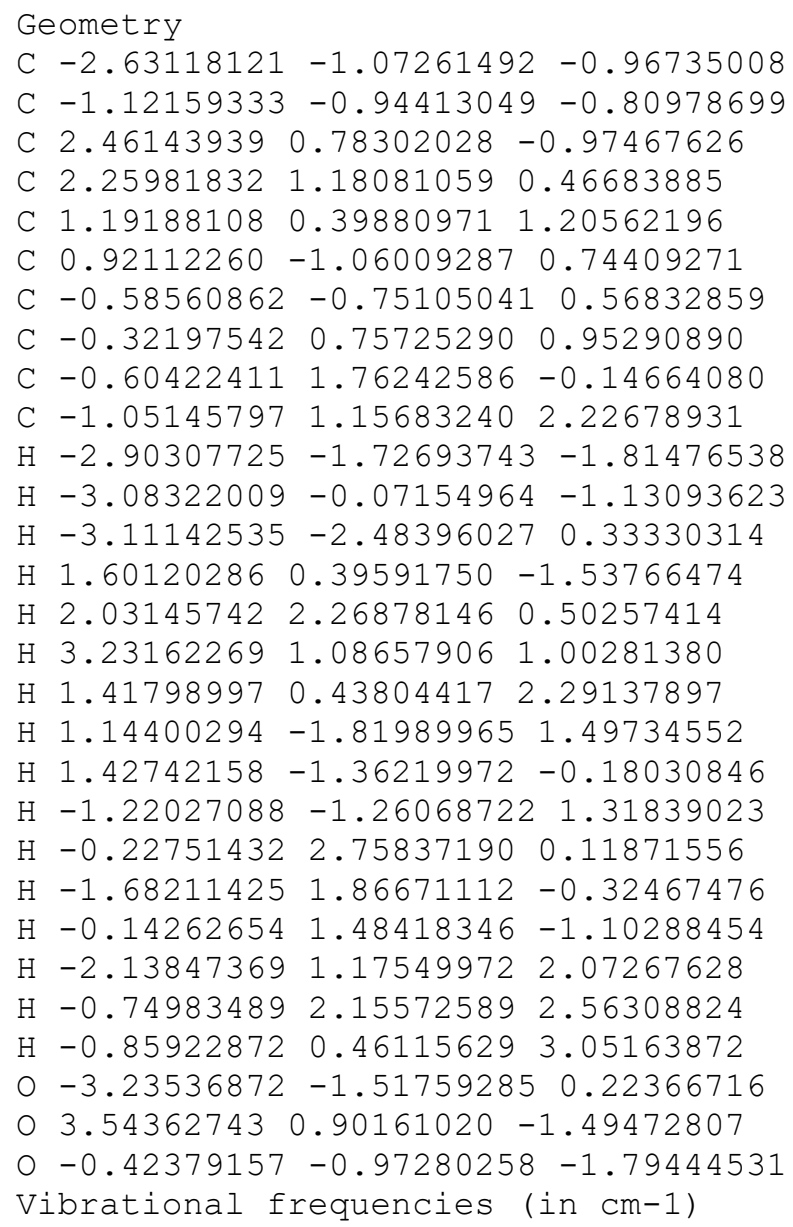

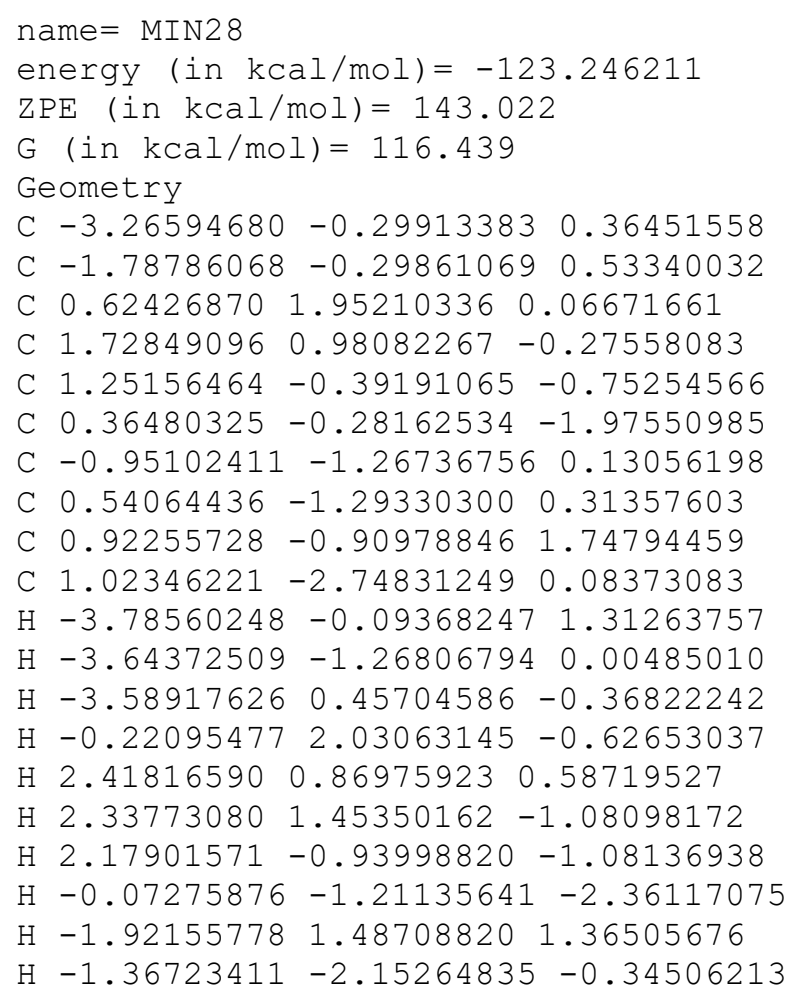


$\mathrm{H} \quad 1.99600352-1.016998831 .92723654$

H $0.39495274-1.541519122 .47149100$

$\mathrm{H} \quad 0.64920838 \quad 0.12912402 \quad 1.98699639$

$\mathrm{H} 2.09975486-2.84421771 \quad 0.25898768$

$\mathrm{H} \quad 0.81286034-3.09587139-0.93068329$

H $\quad 0.52432591-3.43470005 \quad 0.77743361$

$0.15409406 \quad 0.76747869-2.53267657$

$0.701756712 .66273001 \quad 1.04030833$

O $-1.25716328 \quad 0.794475641 .14141892$

Vibrational frequencies (in $\mathrm{cm}-1$ )

$\begin{array}{lllllllllllllllll}26.5 & 64.6 & 84.2 & 95.2 & 103.8 & 127.4 & 161.2 & 181.1 & 225.4 & 237.9 & 267.0 & 275.5 & 284.4 & 311.9\end{array}$ $323.2333 .2 \quad 360.6 \quad 376.8 \quad 420.6 \quad 464.4 \quad 501.9 \quad 525.2 \quad 565.1 \quad 595.7 \quad 699.5 \quad 777.6 \quad 878.0 \quad 886.0$

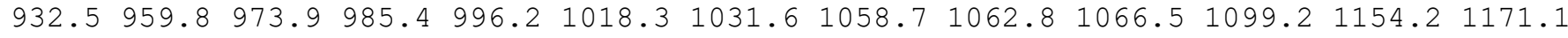

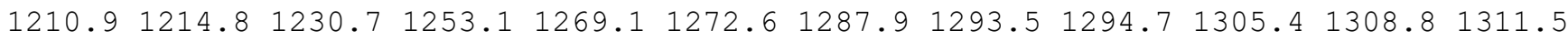

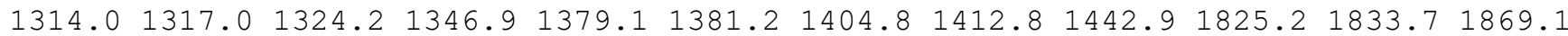
$2648.82653 .92657 .3 \quad 2660.7 \quad 2664.6 \quad 2666.92670 .42676 .12680 .92683 .32735 .12755 .9$ $2775.4 \quad 2778.7 \quad 2781.7 \quad 2787.4$

name $=$ MIN29

energy (in $\mathrm{kcal} / \mathrm{mol})=-122.045555$

$\mathrm{ZPE}(\mathrm{in} \mathrm{kcal} / \mathrm{mol})=143.826$

$\mathrm{G}($ in $\mathrm{kcal} / \mathrm{mol})=117.623$

Geometry

C $-3.11787380-0.09546247-1.37761526$

C $-1.85754598 \quad 0.36401313-0.69300289$

C $2.75903407 \quad 0.80020025-0.42021069$

C $1.78018453 \quad 0.74597598 \quad 0.48981495$

C $0.93583348-0.42706382 \quad 0.79644667$

C $0.17681543-1.13097081-0.38225816$

C $-1.19524210-0.66129880 \quad 0.18751451$

C $-0.46344587-0.13371260 \quad 1.47318155$

C $-0.76668075 \quad 1.29328090 \quad 1.87347001$

C $-0.70611886-1.065463132 .65954446$

$\mathrm{H}-2.93966886-1.00160858-1.97078183$

$\mathrm{H}-3.49738026 \quad 0.67679963-2.06267590$

$\mathrm{H}-3.90838502-0.31052064-0.64938406$

$\mathrm{H} 3.39855285 \quad 1.64529546-0.62984313$

H 1.582186821 .632375551 .09928255

H $3.72976987-0.16722335-1.82534367$

H $1.53578893-1.15528637 \quad 1.38110575$

$\mathrm{H} \quad 0.31124562-2.22580556-0.40687897$

$\mathrm{H} 1.30822000-0.44949167-1.82013496$

$\mathrm{H}-1.88187933-1.49595202 \quad 0.40281902$

$\mathrm{H}-0.19508323 \quad 1.59760425 \quad 2.75671124$

$\mathrm{H}-1.83144637 \quad 1.423279792 .10196614$

$\mathrm{H}-0.535432292 .00849178 \quad 1.06645044$

$\mathrm{H}-0.05547687-0.805530993 .50288390$

$\mathrm{H}-0.51466045-2.11452321 \quad 2.41030325$

$\mathrm{H}-1.74188105-0.99477148 \quad 3.01169253$

O $2.99217062-0.31410586-1.19335522$

$00.36027621-0.65474118-1.67464473$

o $-1.451692031 .48732026-0.84251945$

Vibrational frequencies (in cm-1)

$\begin{array}{lllllllllllllll}26.0 & 39.1 & 68.4 & 109.6 & 138.2 & 175.2 & 191.1 & 205.3 & 232.2 & 245.3 & 272.2 & 294.3 & 312.5 & 339.8\end{array}$ $362.3 \quad 409.0 \quad 440.1 \quad 459.6 \quad 511.7 \quad 523.1 \quad 585.0 \quad 592.7 \quad 616.4 \quad 667.0 \quad 751.2 \quad 816.7 \quad 850.1876 .9$ $907.6925 .9963 .2 \quad 989.4 \quad 1006.7 \quad 1019.2 \quad 1037.6 \quad 1045.2 \quad 1051.6 \quad 1099.1 \quad 1131.8 \quad 1145.4 \quad 1174.3$ $1198.31207 .7 \quad 1225.8 \quad 1240.0 \quad 1247.4 \quad 1265.0 \quad 1266.8 \quad 1278.2 \quad 1282.4 \quad 1285.8 \quad 1294.5 \quad 1299.3$ $1302.91310 .91317 .4 \quad 1336.2 \quad 1368.0 \quad 1381.5 \quad 1394.1 \quad 1400.91406 .91428 .5 \quad 1856.0 \quad 1860.8$ $2653.52673 .72677 .12677 .6 \quad 2678.6 \quad 2682.7 \quad 2687.62706 .42728 .12730 .2 \quad 2743.0 \quad 2760.7$ $2778.02781 .2 \quad 2782.6 \quad 2803.6$

name $=$ MIN30

energy $($ in $\mathrm{kcal} / \mathrm{mol})=-122.602383$ 
$\mathrm{ZPE}($ in $\mathrm{kcal} / \mathrm{mol})=144.497$

$\mathrm{G}($ in $\mathrm{kcal} / \mathrm{mol})=118.532$

Geometry

C $-1.46065323-1.84886436 \quad 0.03609255$

C $0.05755761-1.79941517-0.06136610$

C $-1.568505372 .02385721-0.08678470$

$\begin{array}{llll}C & -0.14444000 & 2.47483983 & 0.07892570\end{array}$

C $0.83263592 \quad 1.49371048-0.55878974$

C $0.26874330 \quad 0.42037257-1.53389178$

C $0.88140130-0.71651322-0.68919192$

C $1.42227733 \quad 0.34490574 \quad 0.34758316$

C $0.84610670 \quad 0.21790280 \quad 1.74262201$

C $2.94269623 \quad 0.37355765 \quad 0.40454846$

$\mathrm{H}-1.77556689-1.521048491 .04630591$

$\mathrm{H}-1.80751485-2.89461395-0.12421831$

$\mathrm{H}-2.54105324-0.31876614-0.57717466$

$\mathrm{H}-2.105827962 .36543033-0.98271306$

$\mathrm{H} \quad 0.09831302 \quad 2.59819824 \quad 1.15753410$

$\mathrm{H}-0.01223452 \quad 3.48283067-0.36364095$

$\mathrm{H} 1.651109572 .07232978-1.03134065$

$\mathrm{H} \quad 0.68594564 \quad 0.48422122 \quad-2.54563645$

$\mathrm{H}-0.81394054 \quad 0.38416819-1.65557296$

$\mathrm{H} \quad 1.71615312-1.21419588-1.23331354$

$\mathrm{H} 1.12486215-0.74450491 \quad 2.19741939$

H $-0.25117147 \quad 0.27926082 \quad 1.75128575$

$\mathrm{H} 1.21895756 \quad 1.00475105 \quad 2.40886740$

$\mathrm{H} 3.39618397 \quad 0.44979039-0.58985932$

$\mathrm{H} 3.33403193-0.54286655 \quad 0.86845931$

$\mathrm{H} \quad 3.304990391 .22099114 \quad 0.99765658$

O $-2.11037694-1.11258368-0.96868764$

$\begin{array}{lllll}0 & -2.12518848 & 1.31613574 & 0.71885460\end{array}$

o $0.64500001-2.723858910 .46057229$

Vibrational frequencies (in $\mathrm{cm}-1$ )

$\begin{array}{lllllllllllllll}-42.8 & 38.4 & 76.8 & 94.1 & 114.2 & 160.7 & 187.0 & 204.5 & 211.1 & 256.9 & 287.9 & 293.8 & 318.3 & 367.0\end{array}$

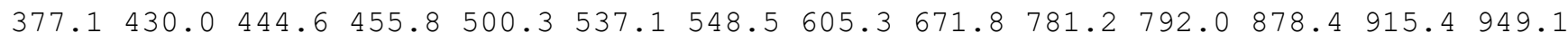
$967.0982 .8 \quad 996.3 \quad 1008.3 \quad 1009.7 \quad 1021.0 \quad 1080.3 \quad 1088.3 \quad 1117.5 \quad 1125.6 \quad 1144.71162 .01182 .6$ $1198.21203 .21221 .9 \quad 1248.5 \quad 1263.3 \quad 1279.7 \quad 1285.5 \quad 1287.8 \quad 1294.2 \quad 1297.7 \quad 1300.01302 .9$ $1311.11320 .7 \quad 1333.2 \quad 1339.3 \quad 1348.2 \quad 1356.5 \quad 1380.8 \quad 1392.1 \quad 1404.3 \quad 1407.2 \quad 1803.21833 .5$ $2631.92642 .8 \quad 2662.2 \quad 2662.92670 .6 \quad 2673.2 \quad 2676.2 \quad 2679.12703 .0 \quad 2705.72715 .8 \quad 2739.5$ $2766.2 \quad 2777.02781 .3 \quad 2793.5$

\section{name $=$ MIN31}

energy $($ in $\mathrm{kcal} / \mathrm{mol})=-122.753875$

$\mathrm{ZPE}($ in $\mathrm{kcal} / \mathrm{mol})=144.781$

$\mathrm{G}($ in $\mathrm{kcal} / \mathrm{mol})=119.681$

Geometry

C $-1.01699522-1.77406694-1.05494530$

C $0.42302201-1.84604331-0.56747587$

C $-1.40828217 \quad 1.94319054-0.46061649$

C $-0.36900087 \quad 2.33450380 \quad 0.56132786$

C $0.80239497 \quad 1.36871660 \quad 0.65146978$

C $1.24765362 \quad 0.63397138-0.64318808$

C $1.17920660-0.73056054 \quad 0.08277165$

C $0.60538935-0.02335279 \quad 1.36794363$

C $-0.80433808-0.38462950 \quad 1.78292462$

C $1.54012538-0.194024782 .56190962$

$\mathrm{H}-1.23614990-0.06367237-2.04917580$

$\mathrm{H}-1.68398390-2.23061906-0.29024426$

$\mathrm{H}-1.13810218-2.33881607-2.00008205$

$\mathrm{H}-2.33420907 \quad 1.47490601-0.10267982$

$\mathrm{H}-0.83088930 \quad 2.44735543 \quad 1.56202714$

$\mathrm{H} \quad 0.02113630 \quad 3.34237563 \quad 0.28967685$

$\mathrm{H} 1.655024881 .913530351 .10967840$

H $2.249292910 .90657415-0.99109023$ 
$\mathrm{H} \quad 0.57606890 \quad 0.74639196-1.49790443$

H $2.20716848-1.117165110 .29092700$

$\mathrm{H}-1.12422421 \quad 0.19944686 \quad 2.65483681$

$\mathrm{H}-0.87925861-1.444090002 .05819523$

$\mathrm{H}-1.53932883-0.20199790 \quad 0.98937527$

H $1.52542861-1.228325312 .92869878$

H $1.24311854 \quad 0.45372455 \quad 3.39464993$

H $2.58039788 \quad 0.04642103 \quad 2.31593353$

$0-1.51465878-0.47242483-1.19688921$

$0-1.24772340 \quad 2.16496238-1.63797478$

o $0.97655318-2.91495555-0.70843046$

Vibrational frequencies (in $\mathrm{cm}-1$ )

$\begin{array}{lllllllllllllllll}44.0 & 88.4 & 109.0 & 129.5 & 155.7 & 169.3 & 184.7 & 200.3 & 212.8 & 249.5 & 289.0 & 299.3 & 305.8 & 373.9\end{array}$ $388.8 \quad 395.6 \quad 407.7 \quad 458.2 \quad 494.8 \quad 542.6 \quad 575.0 \quad 608.6 \quad 676.6 \quad 774.2 \quad 789.5 \quad 895.7 \quad 920.2 \quad 951.4$

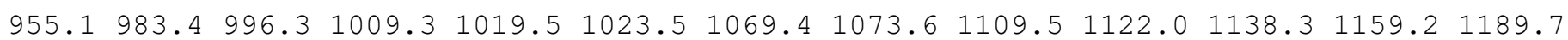

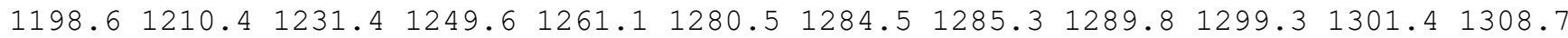

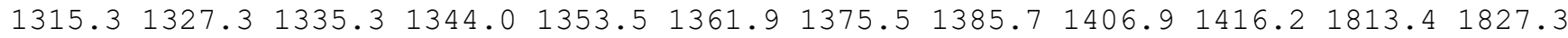
$2634.42643 .92665 .2 \quad 2669.6 \quad 2674.0 \quad 2675.4 \quad 2676.6 \quad 2679.42697 .7 \quad 2708.52711 .32739 .1$ $2764.0 \quad 2770.8 \quad 2780.8 \quad 2783.0$

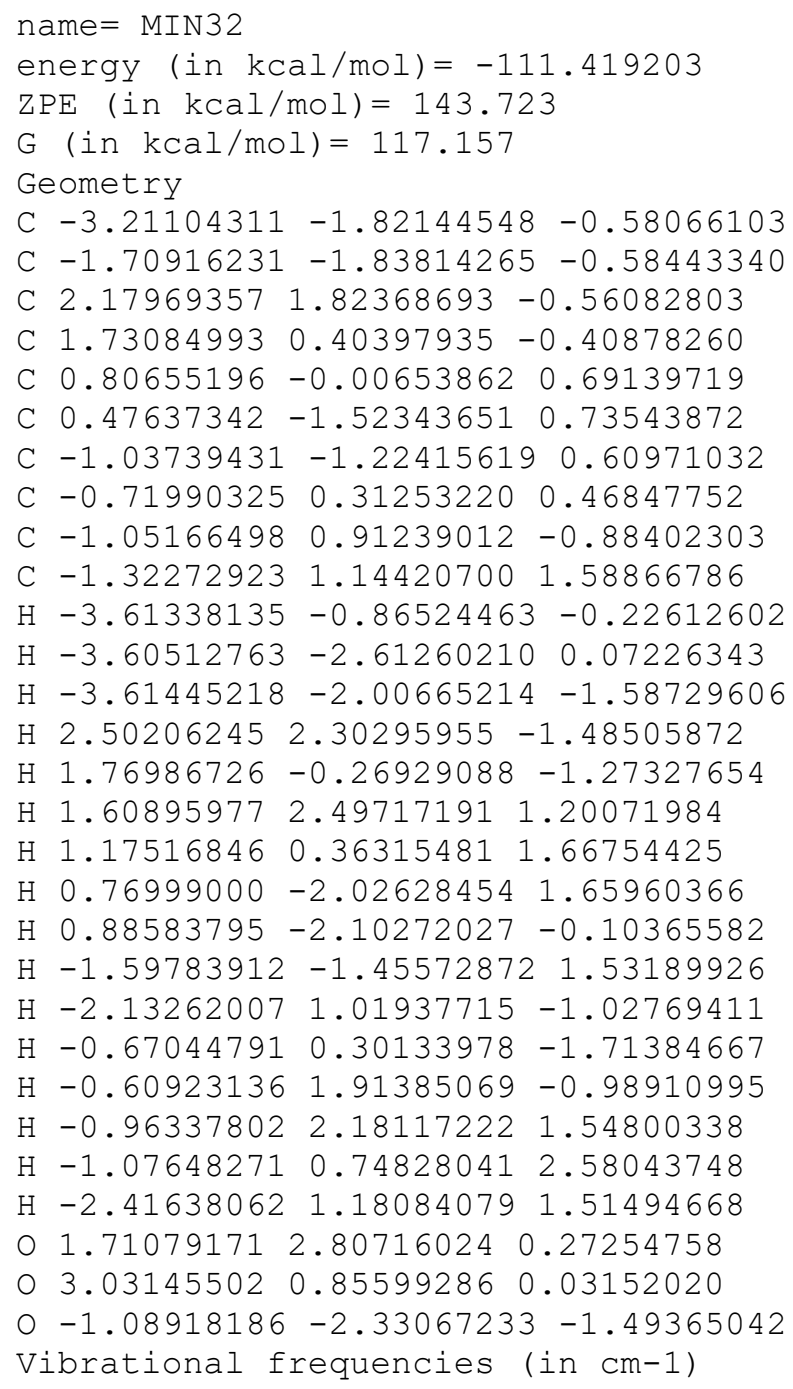


name $=$ MIN33

energy (in $\mathrm{kcal} / \mathrm{mol})=-98.157541$

$\mathrm{ZPE}($ in $\mathrm{kcal} / \mathrm{mol})=142.802$

$\mathrm{G}($ in $\mathrm{kcal} / \mathrm{mol})=117.382$

Geometry

C $-3.15022819-0.78300366-0.42284648$

C $-1.69772098-0.68563629-0.78548752$

C $2.39257766 \quad 0.96817039-0.79763422$

$\begin{array}{llll}\text { C } 2.08282527 & 0.90710133 & 0.69821408\end{array}$

C $1.16241736-0.236485021 .06248445$

C $0.78830422-1.24545404-0.07440998$

C $-0.72689275-1.098059330 .27414571$

C $-0.368202690 .05704327 \quad 1.28955258$

C $-0.84281329 \quad 1.42574264 \quad 0.84282522$

C $-0.83290483-0.238953292 .70584430$

$\mathrm{H}-3.75647008-0.08454423-1.02149234$

$\mathrm{H}-3.33411272-0.56562284 \quad 0.63692301$

$\mathrm{H}-3.53505415-1.79236479-0.62915504$

$\mathrm{H} 1.66563496 \quad 1.93220542 \quad-2.32360971$

$\mathrm{H} \quad 1.67977364 \quad 1.86947918 \quad 1.06279978$

$\mathrm{H} 3.061147720 .785291551 .20617376$

H $1.58416822-0.77652814 \quad 1.93361677$

H $1.20475155-2.25903854 \quad 0.02843980$

H $0.44726238-0.38942270-1.82172421$

$\mathrm{H}-1.09750612-2.003711510 .79168542$

$\mathrm{H}-0.479113851 .67251264-0.16957299$

$\mathrm{H}-0.47259244 \quad 2.21649936 \quad 1.50709457$

$\mathrm{H}-1.93543367 \quad 1.50004288 \quad 0.82885565$

$\mathrm{H}-1.92440117-0.17874304 \quad 2.79162482$

$\mathrm{H}-0.41133624 \quad 0.48631188 \quad 3.41454567$

$\mathrm{H}-0.53005677-1.23558569 \quad 3.04572794$

O $1.20305889-0.81084893-1.32975240$

o $1.53898395 \quad 1.81878741-1.36140176$

o $-1.36022239-0.28836027-1.87855454$

Vibrational frequencies (in cm-1)

$\begin{array}{llllllllllllllll}42.2 & 69.3 & 76.3 & 107.3 & 156.4 & 166.2 & 179.5 & 206.6 & 242.2 & 252.5 & 282.2 & 306.3 & 336.0 & 354.1\end{array}$ $389.4 \quad 430.4 \quad 446.9 \quad 460.1 \quad 482.1 \quad 530.8 \quad 588.5 \quad 603.2 \quad 628.8 \quad 683.1 \quad 767.7 \quad 790.6 \quad 845.0 \quad 895.4$ $902.0 \quad 922.2 \quad 943.5 \quad 998.0 \quad 1005.1 \quad 1016.2 \quad 1041.3 \quad 1046.7 \quad 1051.7 \quad 1101.8 \quad 1107.3 \quad 1147.6 \quad 1168.2$

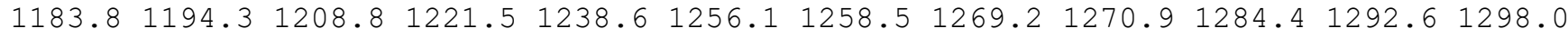
$1301.31306 .41317 .4 \quad 1324.5 \quad 1331.3 \quad 1347.2 \quad 1376.7 \quad 1385.1 \quad 1403.7 \quad 1407.7 \quad 1435.5 \quad 1836.4$ $2655.32664 .92666 .12671 .5 \quad 2674.0 \quad 2677.12677 .72679 .62703 .62713 .92720 .12743 .3$ $2777.5 \quad 2778.0 \quad 2782.2 \quad 2786.0$

\footnotetext{
name $=$ MIN34

energy (in $\mathrm{kcal} / \mathrm{mol})=-81.512821$

$\mathrm{ZPE}(\mathrm{in} \mathrm{kcal} / \mathrm{mol})=144.333$

$\mathrm{G}($ in $\mathrm{kcal} / \mathrm{mol})=119.073$

Geometry

C $-3.09977995-0.14258517 \quad 0.45042116$

C $-1.62249682-0.13896246 \quad 0.34332842$

C $0.374562391 .68200452 \quad 0.50553818$

C $1.52012868 \quad 1.02914258-0.19759237$

C $1.23832699-0.38189802-0.70794686$

C $0.35276537-0.36973901-1.93792483$

C $-0.87627613-1.23625764 \quad 0.42477468$

C $0.62102921-1.34444673 \quad 0.34784034$

C $1.19680514-1.089805031 .75010403$

C $0.99615277-2.78832875 \quad-0.06177483$

$\mathrm{H}-3.43453127 \quad 0.37727716 \quad 1.36134600$

$\mathrm{H}-3.52164836-1.15769962 \quad 0.49957123$

$\mathrm{H}-3.574265120 .35035691-0.40973373$

$\mathrm{H}-1.18963067 \quad 1.53028059-0.89327315$

H 2.40940938 $1.02196843 \quad 0.47117978$

H $1.81297638 \quad 1.68558937-1.05152742$
} 
$\mathrm{H} \quad 2.22803699-0.81374218-1.02321181$

$\mathrm{H} \quad 0.12537399-1.33795809-2.40109085$

H $-1.72065396 \quad 1.97579451 \quad 0.52579232$

$\mathrm{H}-1.36307083-2.19887134 \quad 0.60641834$

$\mathrm{H} \quad 2.29030942-1.11212127 \quad 1.75122398$

$\mathrm{H} \quad 0.84620529-1.84887766 \quad 2.45964357$

$\mathrm{H} \quad 0.87838667-0.119614692 .15321276$

$\mathrm{H} 2.08164038-2.92655540-0.09589570$

$\mathrm{H} \quad 0.58720748-3.05649483-1.03950212$

$\mathrm{H} \quad 0.60704007-3.51575820 \quad 0.66052660$

$\begin{array}{lllll}0 & -0.07949984 & 0.65009992 & -2.41865834\end{array}$

$\begin{array}{lllll}0 & 0.52379600 & 2.53586373 & 1.34017465\end{array}$

O $-1.03690868 \quad 1.23421378 \quad 0.13866055$

Vibrational frequencies (in $\mathrm{cm}-1$ )

$\begin{array}{lllllllllllllllll}62.9 & 69.7 & 83.1 & 121.0 & 134.1 & 156.9 & 200.8 & 204.4 & 219.0 & 268.7 & 277.5 & 292.6 & 323.8 & 335.7\end{array}$

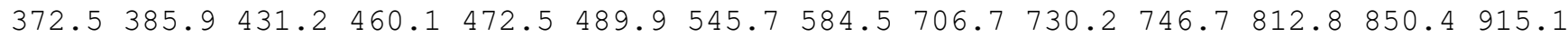

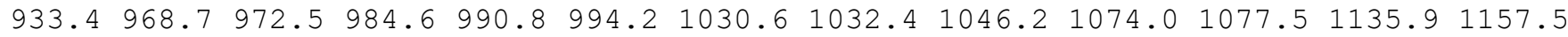

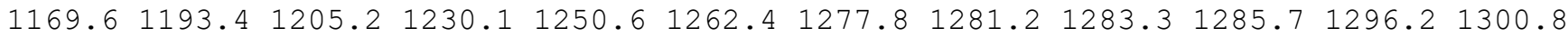

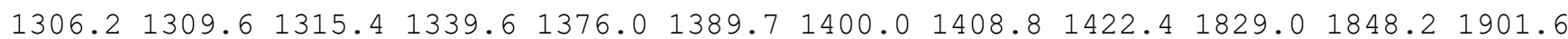
$2648.72653 .8 \quad 2666.8 \quad 2667.3 \quad 2671.0 \quad 2672.8 \quad 2678.42679 .62682 .6 \quad 2723.22731 .52738 .5$ 2776.72779 .92782 .02874 .3

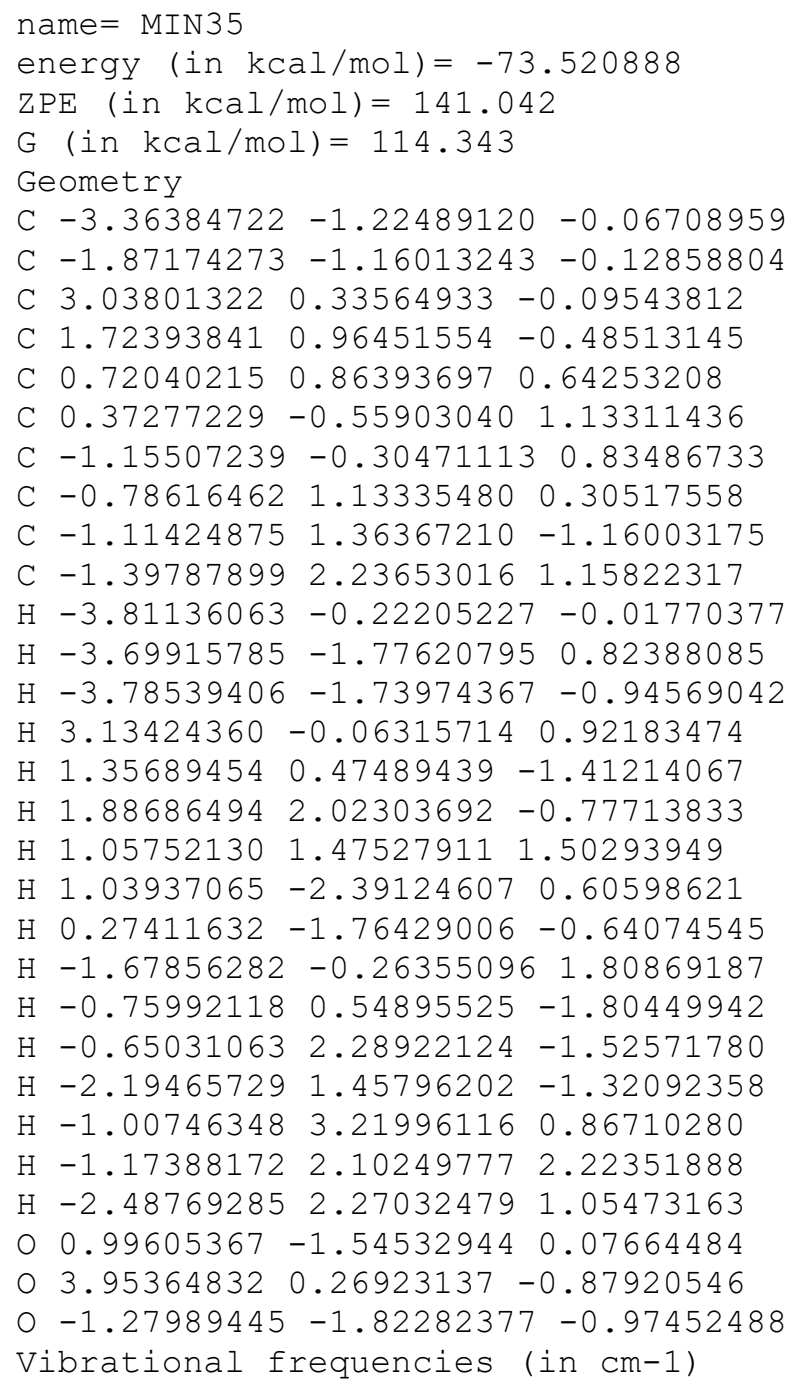


name $=$ MIN36

energy $($ in $\mathrm{kcal} / \mathrm{mol})=-73.124429$

$\mathrm{ZPE}($ in $\mathrm{kcal} / \mathrm{mol})=141.273$

$\mathrm{G}($ in $\mathrm{kcal} / \mathrm{mol})=113.773$

Geometry

C $-3.36594269-1.14075389-0.10588251$

C $-1.87102111-1.20593225-0.11648918$

C $3.07451884 \quad 0.49133010-0.09268472$

C $1.738106921 .08143377-0.47058099$

C $0.72707375 \quad 0.85935539 \quad 0.63243954$

C $0.39495457 \quad-0.58875978 \quad 1.02126219$

C $-1.12007049-0.32932147 \quad 0.81387809$

C $-0.78933438 \quad 1.12406871 \quad 0.30478585$

C $-1.11959927 \quad 1.36459877-1.15782382$

C -1.402664342 .212672821 .17204836$

$\mathrm{H}-3.73566093-0.10984437-0.03617816$

$\mathrm{H}-3.77069449-1.69531056 \quad 0.75479415$

$\mathrm{H}-3.79770573-1.59883432-1.01014866$

H $3.32054180 \quad 0.41739634 \quad 0.97341788$

H $1.39760826 \quad 0.67447370-1.44463993$

$\mathrm{H} 1.864401542 .16901238-0.66485700$

H $1.03605957 \quad 1.41418947 \quad 1.54294449$

H $0.26156918-2.21522760-0.22002399$

$\mathrm{H} 1.49189385-1.36659545-0.60453154$

$\mathrm{H}-1.61160073-0.31266067 \quad 1.80865571$

$\mathrm{H}-0.77513693 \quad 0.54985349-1.80642272$

$\mathrm{H}-0.656512512 .29155520-1.52024433$

$\mathrm{H}-2.200678801 .46530091-1.31479355$

$\mathrm{H}-1.01479611 \quad 3.201085520 .89562887$

$\mathrm{H}-1.178992862 .06299958 \quad 2.23567311$

$\mathrm{H}-2.49286017 \quad 2.24639922 \quad 1.06992665$

$0.99672350-1.636141850 .25910619$

$\begin{array}{lllll}0 & 3.83924051 & 0.10299323 & -0.94161404\end{array}$

o - $1.30301866-1.99171887-0.85788088$

Vibrational frequencies (in $\mathrm{cm}-1$ )

$\begin{array}{lllllllllllllll}25.0 & 36.5 & 51.4 & 79.7 & 84.2 & 129.2 & 156.2 & 175.7 & 186.6 & 213.4 & 223.2 & 278.4 & 297.5 & 313.6\end{array}$ $323.6 \quad 342.8 \quad 375.4 \quad 413.2 \quad 438.3 \quad 476.1 \quad 554.5 \quad 570.1 \quad 596.8 \quad 617.4 \quad 749.7 \quad 790.6 \quad 830.7 \quad 889.4$ $901.7985 .4995 .8 \quad 1003.6 \quad 1013.0 \quad 1014.3 \quad 1033.8 \quad 1044.4 \quad 1049.7 \quad 1084.2 \quad 1102.6 \quad 1105.6 \quad 1128.3$

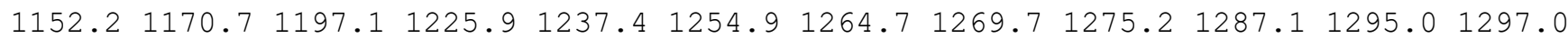

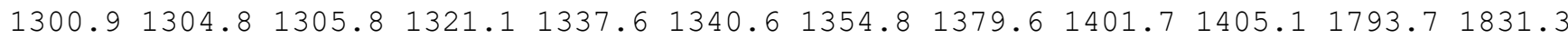
$2515.62648 .5 \quad 2657.6 \quad 2662.7 \quad 2670.4 \quad 2672.1 \quad 2673.72675 .62679 .92717 .2 \quad 2721.2 \quad 2741.8$ $2761.3 \quad 2777.4 \quad 2779.4 \quad 2782.1$

name $=$ MIN37

energy (in $\mathrm{kcal} / \mathrm{mol})=-69.115963$

$\mathrm{ZPE}(\mathrm{in} \mathrm{kcal} / \mathrm{mol})=141.815$

$\mathrm{G}($ in $\mathrm{kcal} / \mathrm{mol})=116.135$

Geometry

C $-3.11335393 \quad 0.296464840 .39117503$

C $-1.61424672 \quad 0.28039117 \quad 0.34299544$

C $1.46535801 \quad 1.625253690 .40419726$

$\begin{array}{llll}\text { C } 2.13202931 & 0.36255293 & -0.08818236\end{array}$

C $1.23926215-0.77941724-0.54325600$

C $-0.00616105-0.57095778-1.43857481$

C $-0.95415411-0.90690390-0.25302394$

C $0.29950551-1.44800010 \quad 0.53013451$

C $0.44937165-1.02139500 \quad 1.97620166$

C $0.36791646-2.97327676 \quad 0.44519688$

$\mathrm{H}-3.49029960 \quad 1.219513390 .85677676$

$\mathrm{H}-3.50530576-0.55354901 \quad 0.96361477$

$\mathrm{H}-3.53650379 \quad 0.23876056-0.62201787$

$\mathrm{H} 1.46400004 \quad 1.845882161 .47774590$ 
$\mathrm{H} 2.80619360-0.02665193 \quad 0.70677370$

$\mathrm{H} \quad 2.80036489 \quad 0.63921874 \quad-0.93675333$

$\mathrm{H} 1.88778064-1.53794194-1.03233231$

$\mathrm{H} \quad 0.26497631 \quad 0.84960534 \quad-2.79849081$

$\mathrm{H} \quad 0.09652678 \quad 1.58101051-1.39571771$

$\mathrm{H}-1.66921998-1.69475322-0.53534547$

$\mathrm{H} 1.41670239-1.33894655 \quad 2.38307429$

$\mathrm{H}-0.33372118-1.470622792 .60090739$

$\mathrm{H} 0.37243305 \quad 0.063007842 .11541024$

$\mathrm{H}-0.42291876-3.441395251 .04094992$

$\mathrm{H} \quad 1.32941569-3.34819392 \quad 0.81149271$

$\mathrm{H} \quad 0.24964029-3.32468966-0.58819936$

$\begin{array}{lllll}0 & -0.28921311 & 0.81091710 & -1.96171722\end{array}$

$\begin{array}{lllll}0 & 1.04332838 & 2.45535958 & -0.37937060\end{array}$

o $-0.97691213 \quad 1.22998397 \quad 0.75211335$

Vibrational frequencies (in cm-1)

$\begin{array}{lllllllllllllllll}40.1 & 63.6 & 113.0 & 117.3 & 137.8 & 162.3 & 180.7 & 197.6 & 221.9 & 227.6 & 252.0 & 265.8 & 297.6 & 326.3\end{array}$ $331.5 \quad 353.6 \quad 373.8 \quad 425.1 \quad 461.9 \quad 490.6 \quad 507.2 \quad 581.8 \quad 597.9 \quad 605.7 \quad 730.9 \quad 804.1819 .1 \quad 885.7$ $924.9965 .4982 .3 \quad 988.1 \quad 1003.8 \quad 1010.9 \quad 1021.9 \quad 1041.2 \quad 1042.4 \quad 1055.5 \quad 1084.01112 .61113 .9$

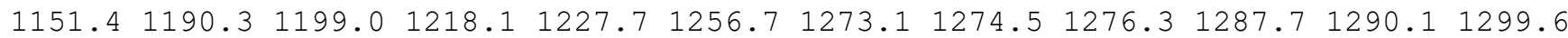
$1301.11305 .61314 .41325 .6 \quad 1341.4 \quad 1378.3 \quad 1381.5 \quad 1407.2 \quad 1409.11416 .81781 .91820 .7$ $2468.22647 .02664 .8 \quad 2668.7 \quad 2670.1 \quad 2674.0 \quad 2675.02677 .82682 .4 \quad 2709.52731 .22739 .0$ $2779.12780 .4 \quad 2782.4 \quad 2803.8$

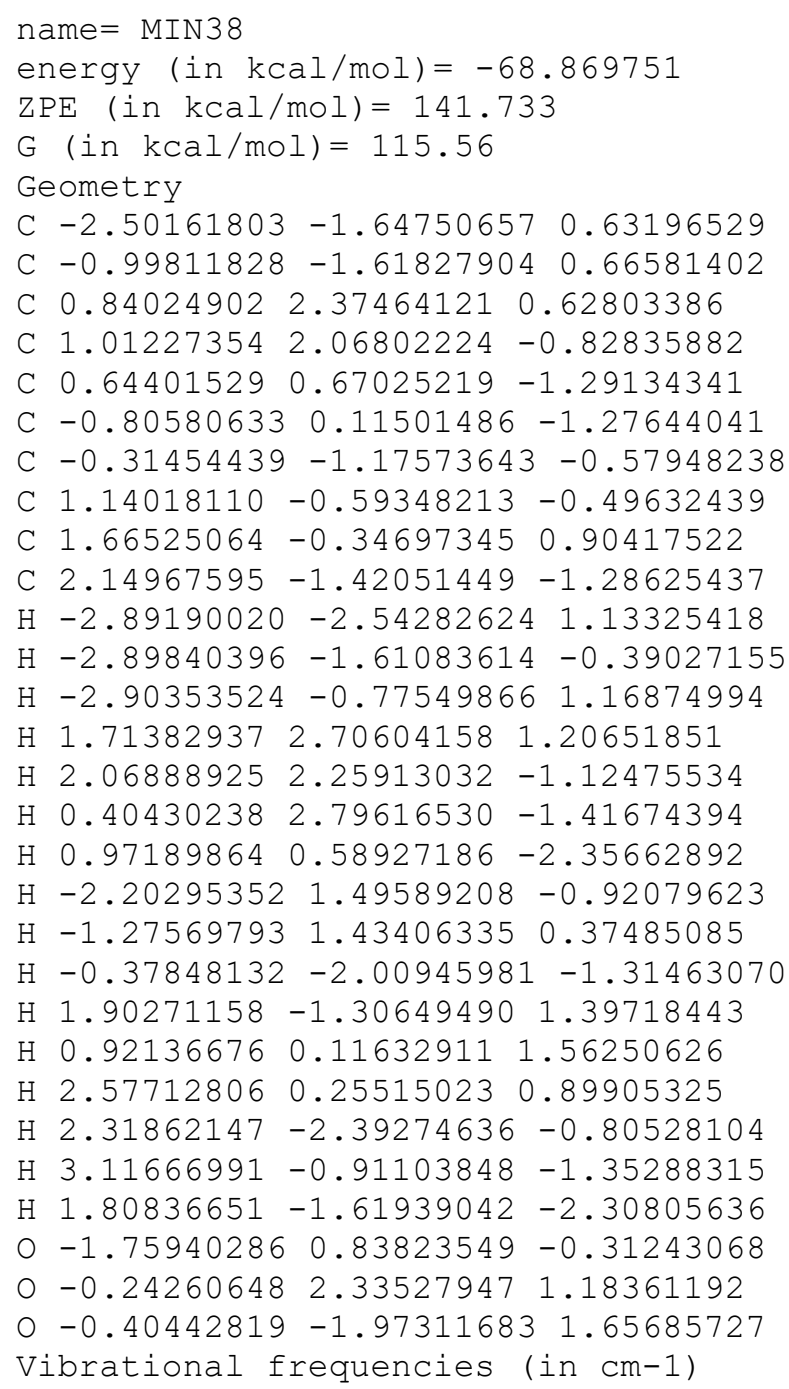


$2432.22643 .62655 .12661 .0 \quad 2669.6 \quad 2675.6 \quad 2679.72680 .42686 .6 \quad 2698.92710 .82729 .2$ $2778.12780 .02781 .4 \quad 2809.0$

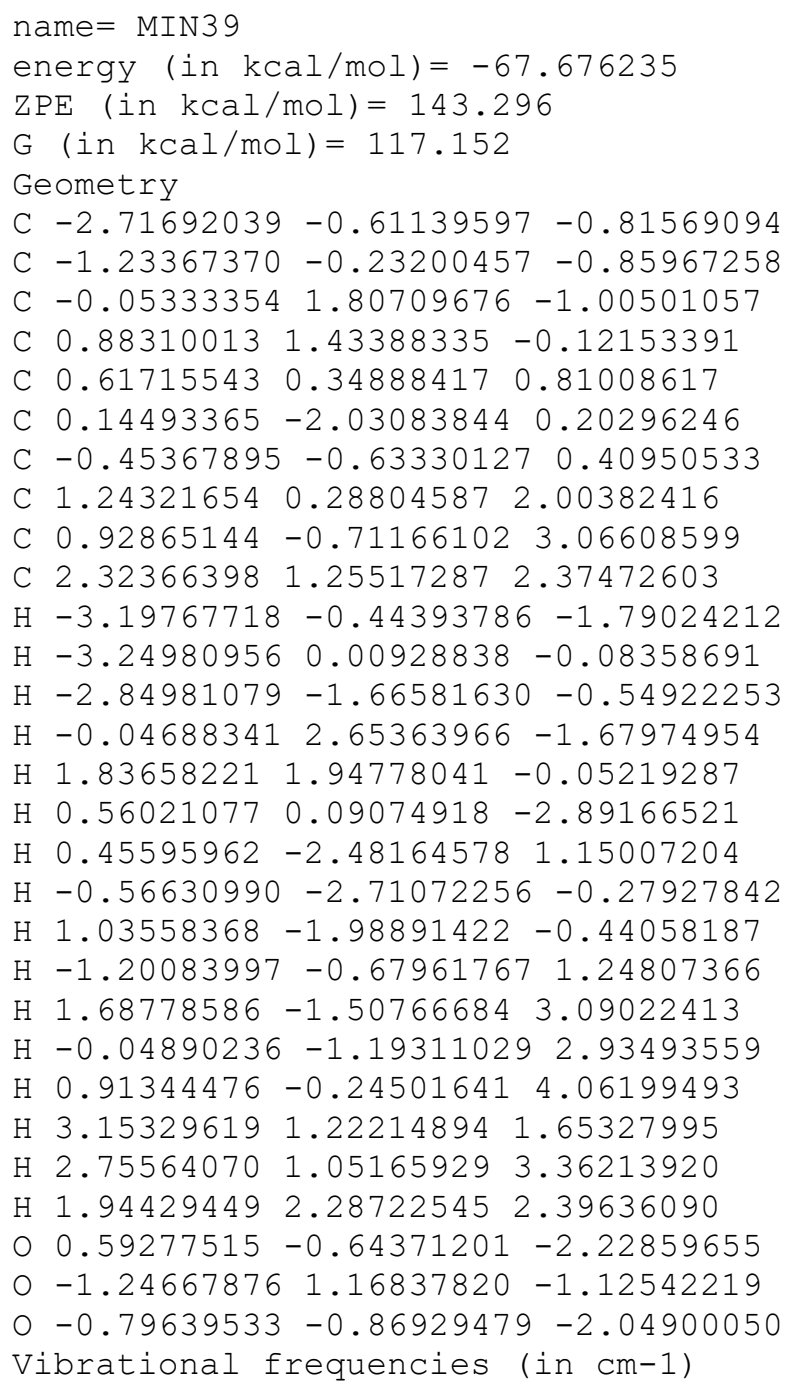

Vibrational frequencies (in $\mathrm{cm}-1$ )

$\begin{array}{llllllllllllllllll}34.1 & 83.1 & 100.7 & 111.4 & 125.0 & 125.8 & 134.9 & 173.2 & 206.3 & 219.2 & 233.9 & 271.4 & 280.0 & 310.7\end{array}$ $339.3 \quad 357.5 \quad 382.1 \quad 430.0 \quad 453.8 \quad 469.5 \quad 514.5 \quad 544.9 \quad 604.7 \quad 675.7 \quad 748.3 \quad 839.4 \quad 858.1 \quad 889.3$

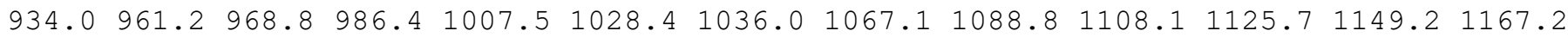

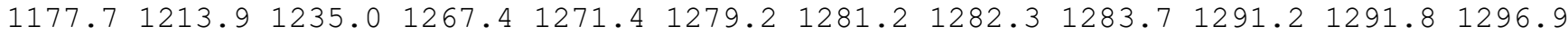

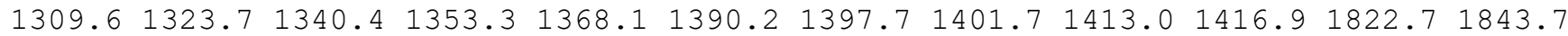
$2662.32667 .72668 .2 \quad 2669.42670 .5 \quad 2674.62676 .52678 .72685 .2 \quad 2738.72767 .52775 .8$ 2777.72778 .62780 .62867 .5

name $=$ MIN40

energy (in $\mathrm{kcal} / \mathrm{mol})=-63.180101$

$\mathrm{ZPE}(\mathrm{in} \mathrm{kcal} / \mathrm{mol})=140.474$

$\mathrm{G}($ in $\mathrm{kcal} / \mathrm{mol})=112.635$

Geometry

C $-2.63805416-2.59939620 \quad 0.17960763$

C $-1.14532488-2.39845453 \quad 0.09030482$

C $1.084564962 .70327240 \quad 0.17106837$

C $1.666784431 .36234403-0.24516356$

C $1.10545140 \quad 0.19498406 \quad 0.59472054$

C $1.75333169-1.096621490 .08579803$

C $-0.65492555-1.352664350 .91975222$

C $-0.45750618 \quad 0.057545320 .60385858$

C $-1.13000562 \quad 0.48093660 \quad-0.70558171$

C $-1.05910608 \quad 0.86719369 \quad 1.77026876$

$\mathrm{H}-3.13239998-1.94591242 \quad 0.90438365$

$\mathrm{H}-2.86124470 \quad-3.64170281 \quad 0.45790597$ 
$\mathrm{H}-3.09559342 \quad-2.43593410 \quad-0.80777947$

H $1.26354332 \quad 3.04046082 \quad 1.19740114$

$\mathrm{H} 2.784224081 .39078427-0.13106910$

$\mathrm{H} \quad 0.810769251 .70447556-2.00167270$

$\mathrm{H} 1.42668528 \quad 0.35420015 \quad 1.65619716$

H $2.84456031-1.01057538 \quad 0.02479766$

H $1.40735534-1.35364125-0.92848865$

$\mathrm{H} 1.54900961-1.962391130 .73676832$

$\mathrm{H}-0.65906634-0.00690049-1.57103045$

$\mathrm{H}-1.08303314 \quad 1.56468656-0.86593938$

$\mathrm{H}-2.19278006 \quad 0.21319989-0.71284072$

$\mathrm{H}-2.14782749 \quad 0.74456224 \quad 1.81834142$

$\mathrm{H}-0.863078601 .93778573 \quad 1.66386226$

$\mathrm{H}-0.658291120 .53591012 \quad 2.73760027$

○ $1.50137606 \quad 1.11244982-1.61589473$

$0 \quad 0.461456013 .37468811-0.61402863$

o $-0.43496351-3.16787621-0.52968609$

Vibrational frequencies (in cm-1)

$\begin{array}{llllllllllllllll}31.6 & 48.3 & 54.9 & 73.1 & 85.6 & 98.0 & 125.9 & 165.9 & 187.3 & 196.5 & 233.3 & 239.6 & 252.3 & 270.2\end{array}$ $301.5 \quad 314.0 \quad 336.7 \quad 356.5 \quad 380.2 \quad 393.3 \quad 452.1 \quad 491.5 \quad 556.1 \quad 589.5 \quad 641.5 \quad 681.7 \quad 785.7 \quad 831.9$ $902.8 \quad 966.2 \quad 983.6 \quad 991.1 \quad 1024.1 \quad 1026.6 \quad 1029.3 \quad 1041.9 \quad 1060.7 \quad 1069.8 \quad 1120.2 \quad 1126.4 \quad 1151.7$ $\begin{array}{llllllllllll}1165.1 & 1190.0 & 1216.0 & 1235.3 & 1249.3 & 1259.1 & 1267.7 & 1271.8 & 1285.8 & 1292.3 & 1293.8 & 1302.9\end{array}$ $1304.91311 .21315 .7 \quad 1341.3 \quad 1372.4 \quad 1396.7 \quad 1402.5 \quad 1403.5 \quad 1413.11476 .4 \quad 1796.2 \quad 1833.8$ $2644.82656 .42658 .62661 .3 \quad 2662.4 \quad 2664.5 \quad 2668.02670 .92676 .32678 .02688 .02765 .0$ $2769.8 \quad 2776.12776 .92780 .0$

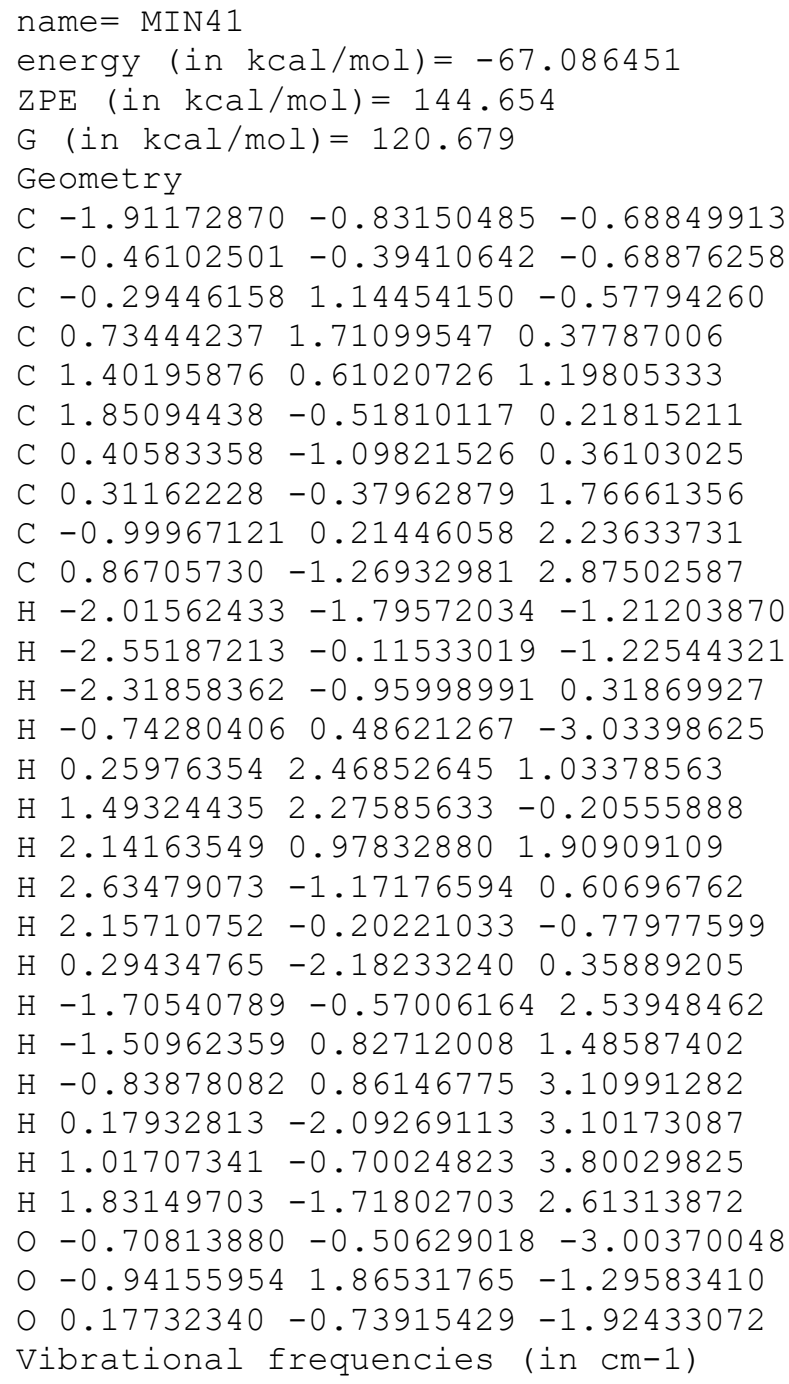


$\begin{array}{lllllllllllllll}1153.8 & 1179.3 & 1189.5 & 1193.3 & 1224.3 & 1230.0 & 1256.2 & 1268.7 & 1275.9 & 1281.0 & 1283.2 & 1286.9\end{array}$ $1289.21292 .1 \quad 1298.8 \quad 1300.3 \quad 1310.4 \quad 1317.2 \quad 1354.8 \quad 1376.41404 .4 \quad 1407.2 \quad 1414.31419 .9$ $1840.22654 .92662 .3 \quad 2668.8 \quad 2673.2 \quad 2675.02676 .12679 .02688 .02739 .42747 .12750 .8$ $2766.4 \quad 2778.12781 .0 \quad 2782.9 \quad 2833.2$

\section{name $=$ MIN42}

energy (in $\mathrm{kcal} / \mathrm{mol})=-64.475757$

$\mathrm{ZPE}($ in $\mathrm{kcal} / \mathrm{mol})=142.151$

$\mathrm{G}($ in $\mathrm{kcal} / \mathrm{mol})=115.976$

Geometry

C $-1.55781709-2.20993964 \quad 0.03868118$

C $-0.08753759-1.99315336 \quad 0.11997653$

C $-0.73837192 \quad 2.076088560 .60465830$

C $0.18859972 \quad 2.34108330-0.45595107$

C $0.719443701 .09359713-1.15911061$

C $-0.307582310 .00600052-1.56088033$

C $0.56103458-1.08744148-0.89436409$

C $1.51693760 \quad 0.02263668-0.32026978$

C $1.45898210 \quad 0.18133290 \quad 1.18607402$

C $2.95820719-0.12746809-0.78057739$

$\mathrm{H}-1.90589965-2.40543124-0.98091202$

$\mathrm{H}-2.10551260-1.29610800 \quad 0.37745152$

$\mathrm{H}-1.88908824 \quad-3.03197579 \quad 0.68647941$

$\mathrm{H}-3.49939136 \quad 0.64872949 \quad 1.05687713$

$\mathrm{H} 1.05548124 \quad 2.94225319-0.08120907$

$\mathrm{H}-0.317693642 .99087613-1.22107499$

H $1.317915421 .42636575-2.03199242$

$\mathrm{H}-0.45462846-0.11532516-2.63595166$

$\mathrm{H}-1.30808897 \quad 0.13190851-1.11127469$

$\mathrm{H} 1.07628256-1.72983964-1.63712062$

$\mathrm{H} 1.98365206-0.65574259 \quad 1.68010001$

$\mathrm{H} \quad 0.43588399 \quad 0.14337290 \quad 1.57707796$

$\mathrm{H} \quad 1.93062193 \quad 1.10919014 \quad 1.52091522$

H $3.41733870-1.02124005-0.33374077$

$\mathrm{H} \quad 3.56581800 \quad 0.73409665-0.48217195$

$\mathrm{H} 3.04191679-0.23322376-1.86737036$

$\begin{array}{lllll}0 & -2.74815785 & 0.64728336 & 0.50565190\end{array}$

o $-1.64431817 \quad 1.698505421 .19727435$

o $0.59229099-2.54786177 \quad 0.95226273$

Vibrational frequencies (in cm-1)

$\begin{array}{lllllllllllllll}33.5 & 50.9 & 89.9 & 117.0 & 133.2 & 163.6 & 189.4 & 193.4 & 200.7 & 219.5 & 243.4 & 277.7 & 296.6 & 297.5\end{array}$ $330.6 \quad 366.9 \quad 400.5 \quad 421.0 \quad 438.6 \quad 485.0 \quad 496.7 \quad 510.4 \quad 604.6 \quad 655.7 \quad 680.3 \quad 783.8 \quad 812.5 \quad 893.7$ $924.3 \quad 931.9972 .4 \quad 996.4 \quad 1005.5 \quad 1015.7 \quad 1018.7 \quad 1045.0 \quad 1048.0 \quad 1063.6 \quad 1083.91119 .8 \quad 1135.9$ $1155.21179 .91200 .6 \quad 1218.5 \quad 1236.5 \quad 1257.4 \quad 1260.8 \quad 1265.91277 .6 \quad 1285.2 \quad 1285.9 \quad 1294.7$

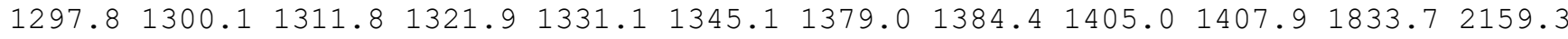
$2574.0 \quad 2633.92647 .8 \quad 2655.8 \quad 2672.2 \quad 2680.0 \quad 2683.6 \quad 2684.92709 .02715 .32720 .52752 .9$ $2771.3 \quad 2778.92782 .0 \quad 2831.7$

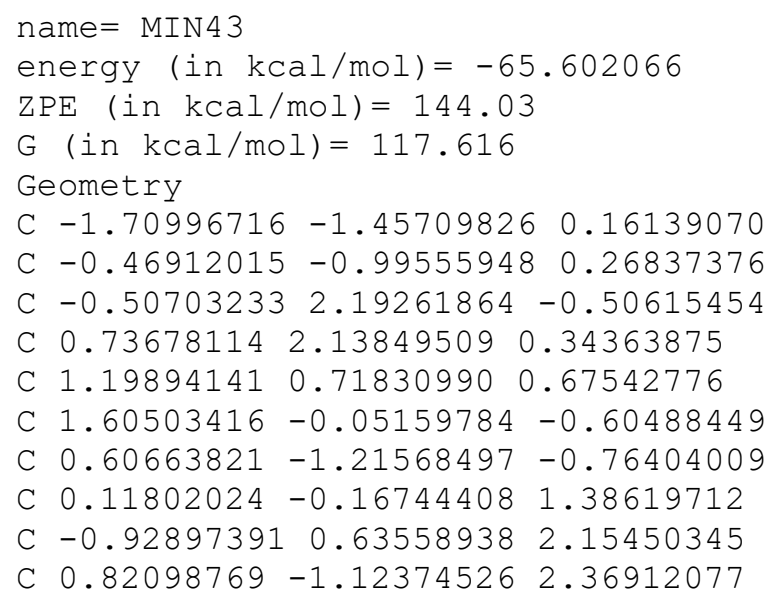


$\mathrm{H}-2.04628425-2.03809705-0.68811558$

$\mathrm{H}-2.48409611-1.288734290 .89219210$

$\mathrm{H} \quad 0.11856539-2.41544980-3.38312123$

$\mathrm{H}-0.78665698 \quad 1.29474094-1.07232143$

$\mathrm{H} \quad 0.57310075 \quad 2.71918036 \quad 1.27719235$

$\mathrm{H} \quad 1.54762828 \quad 2.69055713-0.17916569$

H 2.08345841 $0.80520757 \quad 1.34918626$

$\mathrm{H} 2.63806675-0.42550045-0.53374357$

$\mathrm{H} \quad 1.58816564 \quad 0.59960037-1.49746698$

$\mathrm{H} 1.08466836-2.21168407-0.63733061$

$\mathrm{H}-0.46354823 \quad 1.34062960 \quad 2.85213645$

$\mathrm{H}-1.57724923-0.024642422 .74480286$

$\mathrm{H}-1.587593531 .207517151 .48969229$

$\mathrm{H} \quad 0.11423287-1.85911076 \quad 2.77140897$

$\mathrm{H} \quad 1.24980671-0.58003094 \quad 3.21686803$

$\mathrm{H} 1.62835055-1.681885691 .88448713$

o $-0.28077711-2.41472347-2.48063448$

$\begin{array}{llll}0 & -1.17221419 & 3.19782144 & -0.56338558\end{array}$

o $0.09348943-1.09185522-2.08253440$

Vibrational frequencies (in cm-1)

$\begin{array}{llllllllllllllll}27.8 & 52.4 & 67.0 & 70.2 & 142.1 & 159.4 & 193.1 & 202.7 & 204.2 & 242.6 & 252.0 & 277.4 & 306.8 & 329.4\end{array}$ $337.1370 .4 \quad 417.1 \quad 440.1 \quad 480.2 \quad 546.6 \quad 588.3 \quad 619.9 \quad 670.4 \quad 711.7 \quad 772.3 \quad 818.5 \quad 890.5 \quad 903.3$ $979.2983 .7 \quad 986.3 \quad 1003.3 \quad 1026.3 \quad 1040.0 \quad 1061.5 \quad 1085.6 \quad 1091.0 \quad 1098.3 \quad 1119.81166 .1 \quad 1175.6$ $1183.51194 .91217 .8 \quad 1228.2 \quad 1228.9 \quad 1258.8 \quad 1275.3 \quad 1285.3 \quad 1292.6 \quad 1298.4 \quad 1300.4 \quad 1306.2$

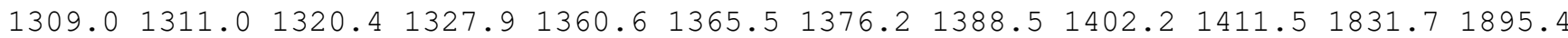
$2645.12653 .4 \quad 2660.8 \quad 2669.3 \quad 2675.6 \quad 2678.1 \quad 2680.82685 .22700 .02716 .3 \quad 2739.6 \quad 2747.3$ $2779.8 \quad 2781.4 \quad 2784.5 \quad 2872.8$

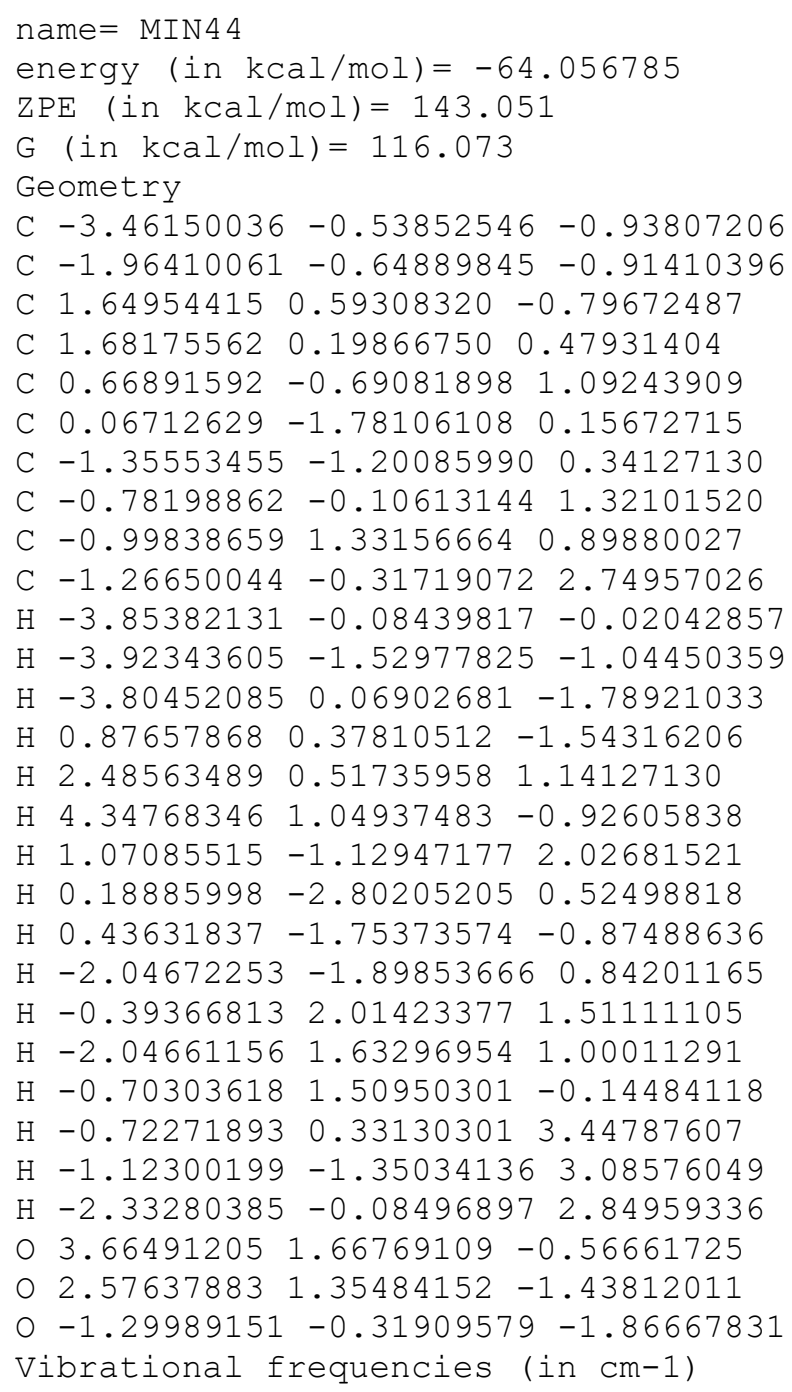


$\begin{array}{llllllllllllllllll}36.6 & 59.8 & 60.2 & 88.9 & 99.1 & 143.9 & 160.0 & 170.1 & 178.4 & 199.0 & 216.6 & 224.9 & 237.1 & 278.7\end{array}$ $329.0 \quad 343.6 \quad 374.5 \quad 397.9 \quad 458.2 \quad 538.1 \quad 575.0 \quad 597.5 \quad 677.6 \quad 701.0 \quad 814.6 \quad 827.6 \quad 908.9916 .9$ $933.2959 .2984 .2 \quad 996.7 \quad 1005.4 \quad 1015.2 \quad 1031.8 \quad 1042.1 \quad 1048.8 \quad 1091.1 \quad 1103.91124 .5 \quad 1153.9$

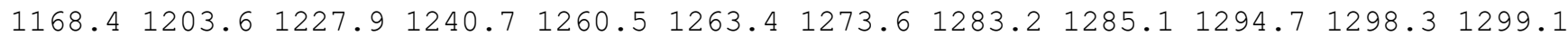
$1305.51316 .6 \quad 1324.8 \quad 1343.4 \quad 1350.2 \quad 1356.91371 .0 \quad 1381.1 \quad 1404.7 \quad 1405.7 \quad 1847.51860 .7$ $2665.12669 .5 \quad 2675.72676 .6 \quad 2677.92681 .2 \quad 2681.7 \quad 2685.32720 .6 \quad 2731.2 \quad 2758.32761 .7$ $2780.22780 .6 \quad 2782.82867 .2$

name $=$ MIN45

energy (in $\mathrm{kcal} / \mathrm{mol})=-63.958985$

$\operatorname{ZPE}($ in $\mathrm{kcal} / \mathrm{mol})=143.084$

$\mathrm{G}($ in $\mathrm{kcal} / \mathrm{mol})=115.64$

Geometry

C $-3.28634760-1.93806148-0.09098451$

C $-1.78300860-1.92875826-0.08823103$

C $2.116682421 .77632695-0.09559682$

C $1.49111826 \quad 0.59686659-0.09421595$

C $0.61940095 \quad 0.17069232 \quad 1.02316381$

C $0.40436113-1.36425998 \quad 1.15143373$

C $-1.12954812-1.18669640 \quad 1.04022574$

C $-0.93274322 \quad 0.35463700 \quad 0.78807818$

C $-1.30465855 \quad 0.82354784 \quad-0.60596967$

C $-1.60071287 \quad 1.21660694 \quad 1.84649280$

$\mathrm{H}-3.70944623-0.98344567 \quad 0.24147123$

$\mathrm{H}-3.66695862 \quad-2.724099640 .57576201$

$\mathrm{H}-3.68018939-2.15347258-1.09534501$

$\mathrm{H} 2.09752824 \quad 2.537957390 .68004559$

$\mathrm{H} 1.59648998-0.10622062-0.92046985$

H $2.502751031 .69664068-2.81299802$

$\mathrm{H} \quad 0.93919355 \quad 0.62269051 \quad 1.98109662$

$\mathrm{H} \quad 0.74937880 \quad-1.79639810 \quad 2.09228813$

$\mathrm{H} \quad 0.84128890 \quad-1.95082184 \quad 0.33163262$

$\mathrm{H}-1.65279150-1.39466208 \quad 1.98981719$

$\mathrm{H}-0.931350171 .83827841-0.79262020$

$\mathrm{H}-2.38917782 \quad 0.83749897-0.75717568$

$\mathrm{H}-0.870081630 .17682795-1.38237400$

$\mathrm{H}-1.31150422 \quad 2.26935960 \quad 1.73834228$

$\mathrm{H}-1.33001425 \quad 0.90752074 \quad 2.86250755$

$\mathrm{H}-2.69323824 \quad 1.16939026 \quad 1.76980878$

O $3.091253701 .31042286-2.11792678$

$\begin{array}{lllll}0 & 2.91518819 & 2.27629702 & -1.08284418\end{array}$

o $-1.15292466-2.50341945-0.94023451$

Vibrational frequencies (in cm-1)

$\begin{array}{llllllllllllllll}33.4 & 39.4 & 47.3 & 72.2 & 105.8 & 134.0 & 163.6 & 182.9 & 192.8 & 203.7 & 211.9 & 232.0 & 262.9 & 286.1\end{array}$ $317.0 \quad 329.8 \quad 389.4 \quad 410.8 \quad 449.5 \quad 474.6 \quad 569.2 \quad 600.3 \quad 659.2 \quad 686.8 \quad 819.9 \quad 829.5 \quad 904.0 \quad 925.4$

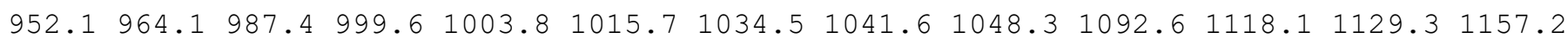
$1168.3 \quad 1204.91236 .5 \quad 1256.6 \quad 1262.4 \quad 1272.6 \quad 1274.1 \quad 1278.1 \quad 1283.4 \quad 1288.5 \quad 1298.6 \quad 1300.1$ $1305.11314 .2 \quad 1320.7 \quad 1334.5 \quad 1347.1 \quad 1352.7 \quad 1373.4 \quad 1382.1 \quad 1402.5 \quad 1405.3 \quad 1855.0 \quad 1860.8$ $2667.52669 .72676 .6 \quad 2676.8 \quad 2678.3 \quad 2679.92681 .32718 .42726 .72728 .8 \quad 2756.52761 .1$ 2779.92780 .92783 .02868 .7

name $=$ MIN4 6

energy (in $\mathrm{kcal} / \mathrm{mol})=-62.874273$

$\mathrm{ZPE}(\mathrm{in} \mathrm{kcal} / \mathrm{mol})=142.063$

$\mathrm{G}($ in $\mathrm{kcal} / \mathrm{mol})=115.219$

Geometry

C $-1.60141183-2.10316156 \quad 0.45149339$

C $-0.11664016-2.048608490 .37016727$

C $-0.962450892 .34402073-0.28879510$

C $0.36993432 \quad 2.25215462-0.80270359$

C $0.77651634 \quad 0.86156642-1.29111215$

C $-0.31550502-0.21777344-1.51830499$

C $0.52856015-1.25492033-0.73741582$ 
C $1.49516901-0.10499392-0.26914196$

C $1.32955664 \quad 0.26634837 \quad 1.18989967$

C $2.95230531-0.36125920-0.61060263$

$\mathrm{H}-2.08661026-2.22350617-0.52003320$

$\mathrm{H}-1.98504896-1.14627621 \quad 0.88773642$

$\mathrm{H}-1.94569606-2.90278500 \quad 1.12078544$

$\mathrm{H}-2.88273887 \quad 0.98473563 \quad 1.78704370$

$\mathrm{H} \quad 1.095667112 .57983776-0.00750607$

$\mathrm{H} \quad 0.50855873 \quad 2.99150383-1.63208164$

$\mathrm{H} 1.39908697 \quad 0.98661839 \quad-2.20047334$

$\mathrm{H}-0.50180969-0.46931533-2.56414852$

$\mathrm{H}-1.28234533-0.00138181-1.04402958$

$\mathrm{H} 1.04003558-1.97196861-1.41134491$

$\mathrm{H} 1.91930072 \quad 1.141885861 .47041712$

$\mathrm{H} \quad 1.65257983-0.56353224 \quad 1.84114050$

$\mathrm{H} \quad 0.27319081 \quad 0.45739751 \quad 1.44990814$

$\mathrm{H} 3.34726693-1.19428716-0.00935423$

H $3.57560984 \quad 0.51503875-0.40307382$

$\mathrm{H} 3.09488990-0.63007209-1.66263092$

o $-2.14868102 \quad 0.81214219 \quad 1.23857572$

$\begin{array}{lllll}0 & -1.94135318 & 2.16207394 & 0.27889491\end{array}$

o $0.58533149-2.65064104 \quad 1.14965307$

Vibrational frequencies (in cm-1)

$\begin{array}{llllllllllllllll}26.0 & 32.1 & 54.9 & 103.4 & 145.6 & 159.6 & 186.8 & 196.2 & 201.6 & 224.3 & 267.1 & 275.8 & 279.7 & 288.9\end{array}$ $331.2 \quad 360.7 \quad 401.6 \quad 427.0 \quad 442.9 \quad 478.7 \quad 496.0 \quad 511.8 \quad 605.1 \quad 647.3 \quad 660.9 \quad 782.1 \quad 809.7 \quad 903.9$ $927.3 \quad 929.5 \quad 976.9 \quad 1001.1 \quad 1002.9 \quad 1009.7 \quad 1017.7 \quad 1045.6 \quad 1047.6 \quad 1061.4 \quad 1088.3 \quad 1124.4 \quad 1138.8$ $\begin{array}{lllllllllllllll}1146.9 & 1184.0 & 1198.9 & 1215.7 & 1242.0 & 1259.9 & 1264.0 & 1273.0 & 1277.9 & 1285.4 & 1292.4 & 1294.6\end{array}$ $1299.01300 .61302 .6 \quad 1323.1 \quad 1335.7 \quad 1352.1 \quad 1380.2 \quad 1383.81405 .01407 .7 \quad 1831.7 \quad 2166.6$ $2572.02624 .22640 .2 \quad 2669.92673 .2 \quad 2673.92681 .42686 .12709 .02715 .32720 .32756 .9$ $2771.4 \quad 2772.2 \quad 2781.5 \quad 2832.3$

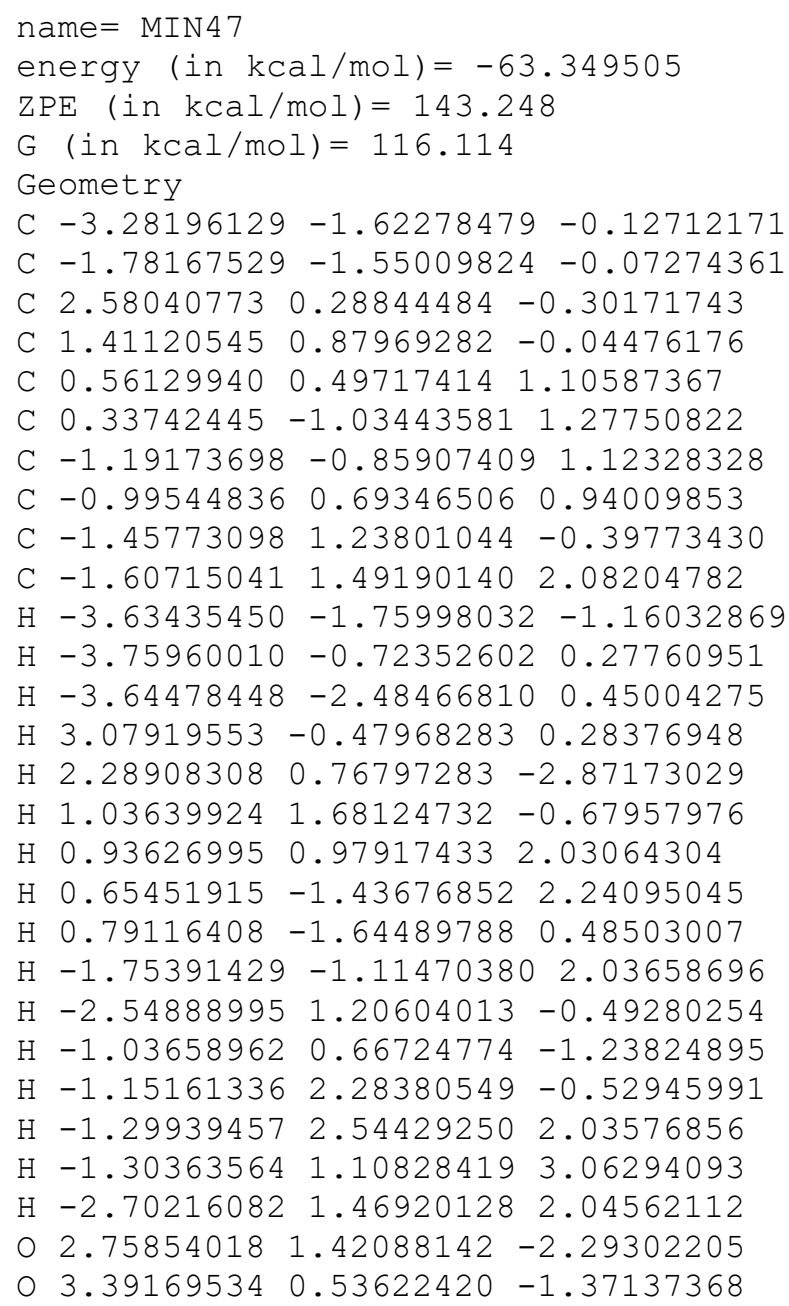


$0-1.10281741-2.03775892-0.94187976$

Vibrational frequencies (in $\mathrm{cm}-1$ )

$\begin{array}{lllllllllllllll}31.2 & 42.8 & 52.0 & 89.4 & 101.2 & 133.3 & 165.1 & 186.5 & 201.5 & 221.3 & 247.3 & 256.1 & 269.5 & 288.4\end{array}$ $319.3 \quad 334.4 \quad 377.2 \quad 402.3 \quad 470.5 \quad 501.9 \quad 570.9 \quad 598.4 \quad 674.1 \quad 677.6 \quad 825.1 \quad 871.3 \quad 894.7 \quad 920.9$ $936.7953 .4981 .5 \quad 996.2 \quad 1005.5 \quad 1015.4 \quad 1034.8 \quad 1044.2 \quad 1051.3 \quad 1097.3 \quad 1112.8 \quad 1125.01141 .1$ $\begin{array}{lllllllllllllllll}1174.1 & 1201.7 & 1229.4 & 1250.7 & 1260.0 & 1262.0 & 1273.3 & 1280.1 & 1288.7 & 1291.3 & 1298.0 & 1301.1\end{array}$

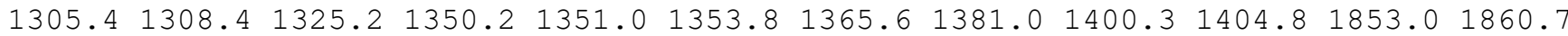
$2667.42669 .5 \quad 2674.7 \quad 2676.9 \quad 2677.2 \quad 2680.2 \quad 2680.62714 .72725 .5 \quad 2730.72754 .62759 .7$ $2778.5 \quad 2780.92782 .7 \quad 2860.8$

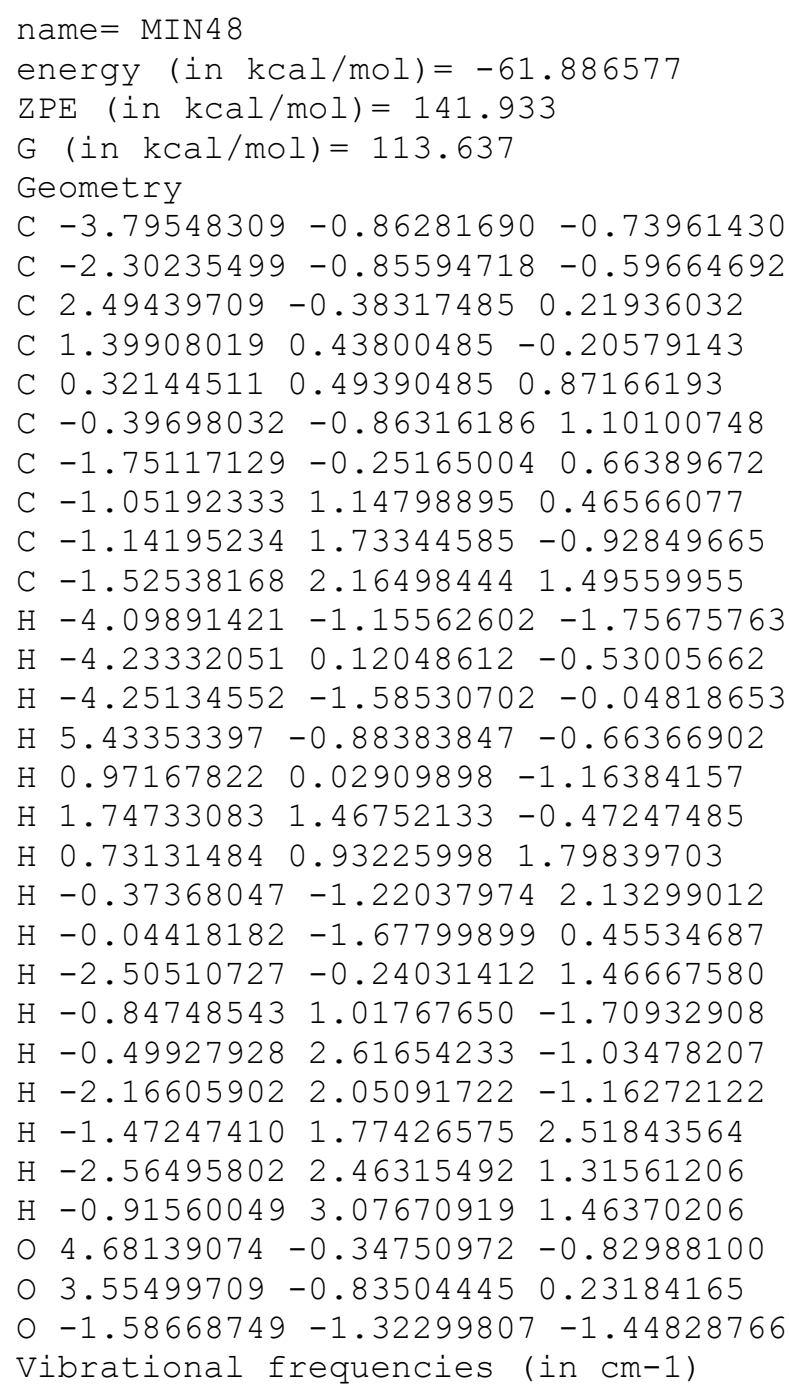


C $0.49932871-1.67528294 \quad 0.62499663$

C $-0.98978697-1.27680495 \quad 0.76936250$

C $-0.56451736 \quad 0.02689731 \quad 1.54498689$

C $-1.01872408 \quad 1.33771398 \quad 0.93718552$

C $-0.94862988-0.03818854 \quad 3.01711734$

$\mathrm{H}-3.64568045-1.90430010-0.44213929$

$\mathrm{H}-3.57421993-0.41579267-1.39137027$

$\mathrm{H}-3.53019872-0.338617120 .38309599$

$\mathrm{H} \quad 0.97616780 \quad 0.07687529-1.50369692$

H 2.40761608 1.24719799 1.02687996

H 2.66906841 2.22540280 -3.20348524

H $1.48019583-0.540589012 .26279964$

$\mathrm{H} 0.77698078-2.57638745 \quad 1.17734207$

$\mathrm{H} 0.83924792-1.80427023-0.40894976$

$\mathrm{H}-1.57264509-1.97495821 \quad 1.39194548$

$\mathrm{H}-2.09306642 \quad 1.50113140 \quad 1.07020648$

$\mathrm{H}-0.80396174 \quad 1.39269352-0.13948425$

$\mathrm{H}-0.494081632 .18433098 \quad 1.40057377$

$\mathrm{H}-0.62714538-0.97516566 \quad 3.48608301$

$\mathrm{H}-2.03293086 \quad 0.04193447 \quad 3.15262302$

$\mathrm{H}-0.48656726 \quad 0.78249108 \quad 3.58012438$

$\begin{array}{llll}0 & 2.09844127 & 1.48882826 & -2.87880381\end{array}$

○ $2.40772100 \quad 1.60650232-1.47524215$

o - $1.09265485-0.92623198-1.58063519$

Vibrational frequencies (in $\mathrm{cm}-1$ )

$\begin{array}{llllllllllllllll}-93.7 & 40.3 & 51.7 & 65.9 & 80.4 & 112.1 & 128.2 & 153.2 & 171.1 & 179.7 & 203.1 & 211.8 & 268.1 & 297.3\end{array}$

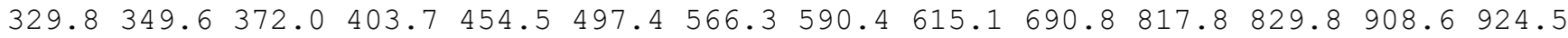
$952.5961 .8 \quad 996.4 \quad 1004.6 \quad 1014.8 \quad 1030.6 \quad 1036.8 \quad 1046.1 \quad 1056.2 \quad 1090.7 \quad 1101.8 \quad 1120.2 \quad 1159.5$

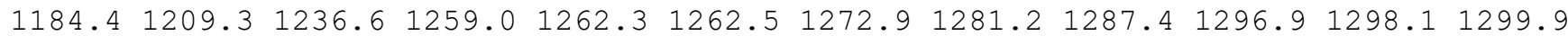
$1305.41311 .6 \quad 1314.21334 .0 \quad 1356.5 \quad 1368.6 \quad 1380.8 \quad 1403.31403 .7 \quad 1411.21830 .6 \quad 1849.6$ $2665.52669 .62676 .2 \quad 2676.92678 .2 \quad 2679.82681 .82687 .22721 .32731 .32760 .62765 .4$ $2780.0 \quad 2780.7 \quad 2782.9 \quad 2876.3$

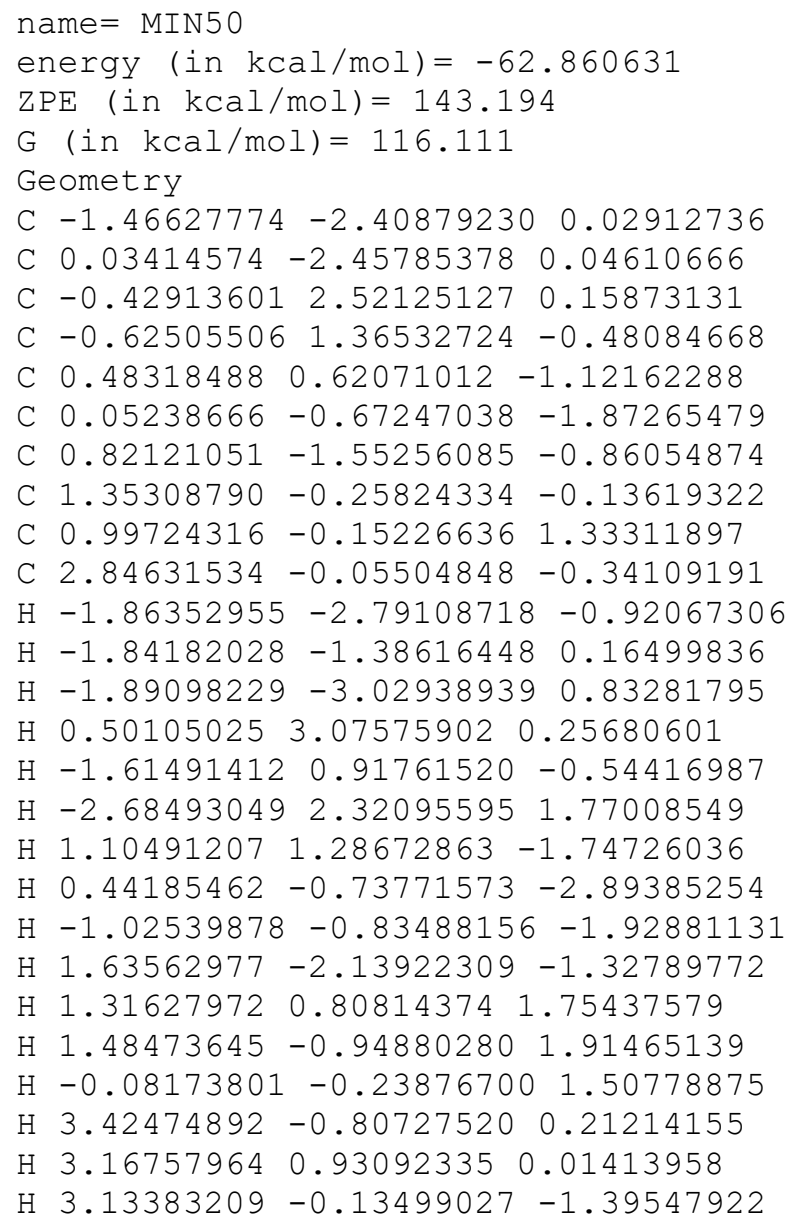


$-2.623235762 .55996297 \quad 0.81218057$

$0-1.37104475 \quad 3.24908437 \quad 0.82501425$

O $0.61351801-3.221479550 .78151253$

Vibrational frequencies (in $\mathrm{cm}-1$ )

$\begin{array}{llllllllllllllll}34.1 & 40.2 & 46.6 & 82.2 & 134.5 & 162.4 & 171.7 & 182.2 & 192.1 & 203.7 & 208.6 & 234.8 & 294.4 & 296.8\end{array}$ $320.2 \quad 328.7 \quad 406.3 \quad 435.0 \quad 461.5 \quad 475.0 \quad 520.2 \quad 599.9665 .5 \quad 693.0 \quad 821.7 \quad 826.7 \quad 896.0 \quad 911.4$ $944.3 \quad 954.3 \quad 987.4 \quad 999.1 \quad 1005.3 \quad 1018.9 \quad 1035.4 \quad 1037.4 \quad 1044.3 \quad 1075.5 \quad 1117.6 \quad 1119.4 \quad 1158.3$

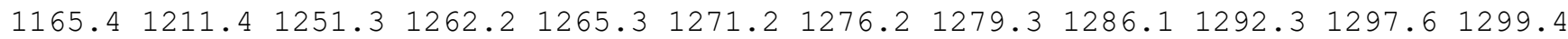

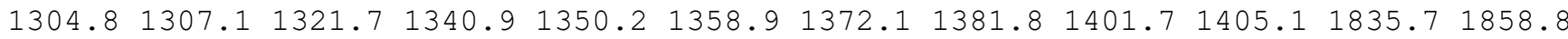
2668.32670 .82671 .02675 .12676 .62680 .12684 .72717 .92723 .12725 .92759 .92765 .9 $2778.4 \quad 2779.7 \quad 2782.0 \quad 2867.1$

name $=$ MIN51

energy $($ in $\mathrm{kcal} / \mathrm{mol})=-61.45363$

$\mathrm{ZPE}($ in $\mathrm{kcal} / \mathrm{mol})=141.969$

$\mathrm{G}($ in $\mathrm{kcal} / \mathrm{mol})=113.762$

Geometry

C $-2.76685026-1.36325947-0.68017784$

C $-1.27690824-1.30698034-0.51827523$

C $1.66088510 \quad 0.56170067-0.79132461$

C $1.78738010 \quad 0.89444400 \quad 0.60045008$

C $0.95494908-0.00919206 \quad 1.50651288$

C $0.77649709-1.469124341 .00946947$

C $-0.75492606-1.27024805 \quad 0.89109637$

C $-0.60977133 \quad 0.18452068 \quad 1.47740699$

C $-1.13985504 \quad 1.30387435 \quad 0.60705103$

C $-1.19428766 \quad 0.284518712 .88047637$

$\mathrm{H}-3.30006949-0.83498217 \quad 0.11840058$

$\mathrm{H}-3.11512296-2.40612314-0.68171571$

$\mathrm{H}-3.07834945-0.92600427-1.64232398$

$\mathrm{H} 1.395192422 .16000296-3.45118688$

H $1.505439631 .96783871 \quad 0.76970564$

$\begin{array}{lllll}\mathrm{H} & 2.86577827 & 0.83280630 & 0.89725663\end{array}$

H $1.35168376 \quad 0.04705932 \quad 2.53629606$

$\mathrm{H} 1.07622430-2.23059902 \quad 1.73328645$

$\mathrm{H} 1.27402366-1.705201550 .05909602$

$\mathrm{H}-1.33685658-1.944607201 .53943315$

$\mathrm{H}-0.84504932 \quad 2.28868634 \quad 0.99339948$

$\mathrm{H}-2.23622444 \quad 1.29588906 \quad 0.56135141$

$\mathrm{H}-0.77847521 \quad 1.25081042-0.43030643$

$\mathrm{H}-2.28864805 \quad 0.21601317 \quad 2.86365556$

$\mathrm{H}-0.935929331 .24024958 \quad 3.35272909$

$\mathrm{H}-0.83089466-0.51333550 \quad 3.53835694$

$\begin{array}{lllll}0 & 1.52523718 & 2.24229034 & -2.52975916\end{array}$

O $1.56446866 \quad 0.70733858-1.92620043$

o - $0.53895796-1.29938502-1.47258616$

Vibrational frequencies (in $\mathrm{cm}-1$ )

$\begin{array}{llllllllllllllll}-22.4 & 11.6 & 56.0 & 67.0 & 103.0 & 119.8 & 157.3 & 175.2 & 194.7 & 211.5 & 233.7 & 238.3 & 271.5 & 302.2\end{array}$ $331.9 \quad 367.7 \quad 402.6 \quad 429.1 \quad 435.0 \quad 469.1 \quad 512.5 \quad 565.2 \quad 592.9 \quad 644.6 \quad 696.0 \quad 795.2 \quad 829.3 \quad 907.5$ $915.8950 .5981 .7997 .1 \quad 1007.3 \quad 1015.8 \quad 1030.6 \quad 1043.3 \quad 1049.7 \quad 1052.9 \quad 1096.61112 .7 \quad 1124.5$ $1142.21179 .3 \quad 1201.3 \quad 1221.7 \quad 1234.8 \quad 1256.1 \quad 1259.0 \quad 1266.6 \quad 1270.01284 .01287 .6 \quad 1290.1$ $1296.91299 .5 \quad 1304.3 \quad 1319.01331 .4 \quad 1360.3 \quad 1378.7 \quad 1382.31403 .1 \quad 1406.6 \quad 1850.8 \quad 2145.3$ 2630.22660 .62665 .32672 .12674 .82675 .42676 .82678 .12714 .32722 .02731 .92759 .0 $2778.72779 .6 \quad 2783.02837 .6$

name $=$ MIN52

energy (in $\mathrm{kcal} / \mathrm{mol})=-62.005933$

$\operatorname{ZPE}($ in $\mathrm{kcal} / \mathrm{mol})=142.925$

$\mathrm{G}($ in $\mathrm{kcal} / \mathrm{mol})=114.68$

Geometry

C $-3.42415856-1.04556145-0.73030517$

C $-1.92632882-0.95715230-0.82823477$

C $2.344987911 .18060107-0.42453483$ 
C $1.284125331 .37539625 \quad 0.36974269$

C $0.54839523 \quad 0.300972331 .07206210$

C $0.36668794-1.04745769 \quad 0.31916766$

C $-1.17078279-0.95812358 \quad 0.46919443$

C $-1.02092422 \quad 0.45253378 \quad 1.15189115$

C $-1.59832458 \quad 1.60266352 \quad 0.34841790$

C $-1.54236292 \quad 0.47485470 \quad 2.58020049$

$\mathrm{H}-3.81838968-0.54843599 \quad 0.16230879$

$\mathrm{H}-3.74116079-2.09732192-0.69872839$

$\mathrm{H}-3.90316383-0.59572852-1.61265367$

H 2.91122062 $1.94769682-0.94102034$

$\mathrm{H} \quad 0.92313556 \quad 2.38579590 \quad 0.55521671$

$\mathrm{H} \quad 4.44739984-0.76574302-1.03097434$

$\mathrm{H} \quad 0.99167092 \quad 0.15430684 \quad 2.07933386$

$\mathrm{H} \quad 0.82776239-1.90776446 \quad 0.80599568$

$\mathrm{H} \quad 0.69691627-1.03130020 \quad-0.73064774$

$\mathrm{H}-1.58353559-1.71859200 \quad 1.15382190$

$\mathrm{H}-1.20830391 \quad 1.61056722 \quad-0.68078392$

$\mathrm{H}-1.34672302 \quad 2.57087958 \quad 0.79772595$

$\mathrm{H}-2.69071468 \quad 1.54790495 \quad 0.28727327$

$\mathrm{H}-1.25365083 \quad 1.402542913 .09020871$

$\mathrm{H}-1.14972353-0.357256413 .17595850$

$\mathrm{H}-2.63603754 \quad 0.41216855 \quad 2.60916888$

$03.93948831-0.02477242-1.43956524$

O $2.75770078-0.11120820-0.62236265$

o $-1.37649633-0.91482377-1.90009961$

Vibrational frequencies (in $\mathrm{cm}-1$ )

$\begin{array}{llllllllllllllll}24.3 & 38.8 & 45.0 & 58.6 & 82.6 & 86.6 & 109.3 & 153.9 & 173.0 & 194.7 & 226.4 & 236.0 & 272.8 & 292.6\end{array}$

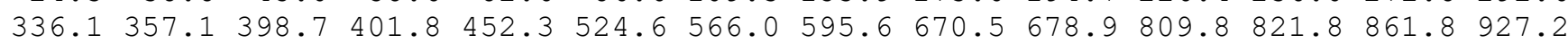
$945.5966 .9995 .9 \quad 1002.6 \quad 1014.1 \quad 1031.6 \quad 1040.7 \quad 1046.6 \quad 1054.0 \quad 1097.2 \quad 1109.81137 .1 \quad 1146.1$

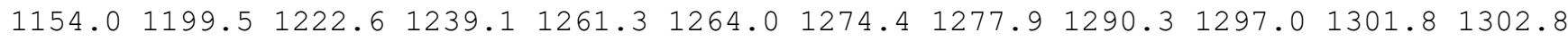
$1303.91307 .51320 .5 \quad 1347.3 \quad 1359.5 \quad 1373.5 \quad 1381.5 \quad 1399.3 \quad 1401.01404 .71840 .11853 .6$ $2667.12669 .5 \quad 2672.6 \quad 2675.6 \quad 2677.4 \quad 2678.3 \quad 2680.92711 .32726 .12729 .12756 .72757 .8$ 2778.62781 .02782 .62870 .6

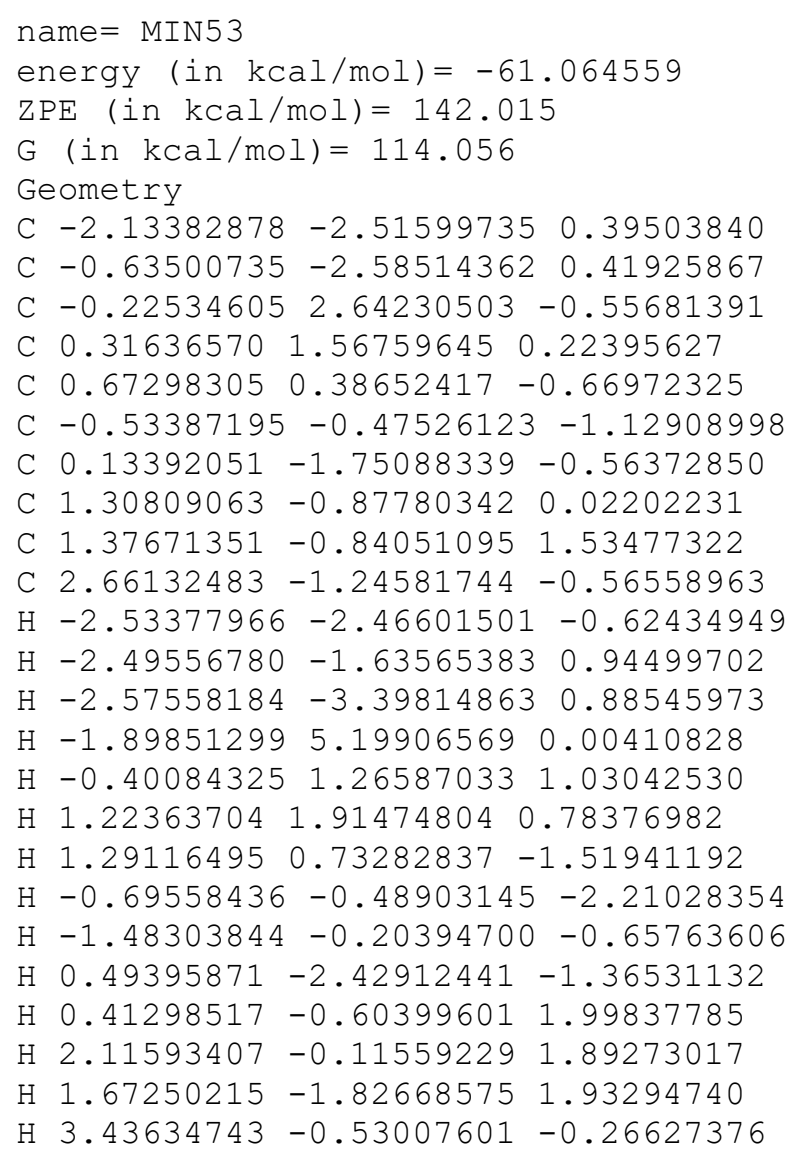


$\mathrm{H} 2.64705890-1.27533684-1.66058953$

H $2.98312916-2.23773570-0.21918183$

$\begin{array}{lllll}0 & -1.51426848 & 4.44482484 & 0.41224597\end{array}$

$\begin{array}{llll}0 & -0.80056648 & 3.61632864 & -0.78214535\end{array}$

O $-0.06378204-3.327772781 .18023455$

Vibrational frequencies (in $\mathrm{cm}-1$ )

$\begin{array}{llllllllllllllll}20.3 & 35.6 & 41.4 & 73.2 & 79.7 & 141.5 & 164.1 & 190.3 & 194.9 & 211.0 & 232.7 & 248.0 & 264.0 & 282.6\end{array}$

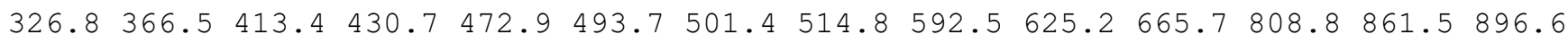
$937.7938 .2 \quad 994.0 \quad 1001.1 \quad 1008.5 \quad 1017.9 \quad 1025.7 \quad 1043.4 \quad 1055.8 \quad 1066.0 \quad 1089.7 \quad 1110.3 \quad 1131.3$

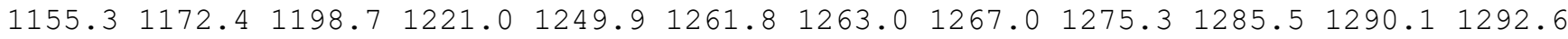
$1296.41300 .7 \quad 1304.6 \quad 1321.0 \quad 1330.5 \quad 1348.2 \quad 1359.4 \quad 1380.6 \quad 1402.2 \quad 1405.4 \quad 1841.4 \quad 2110.2$ $2626.82658 .22665 .8 \quad 2673.4 \quad 2675.3 \quad 2677.2 \quad 2681.32683 .52710 .6 \quad 2715.22725 .52764 .1$ $2778.4 \quad 2779.4 \quad 2781.9 \quad 2841.6$

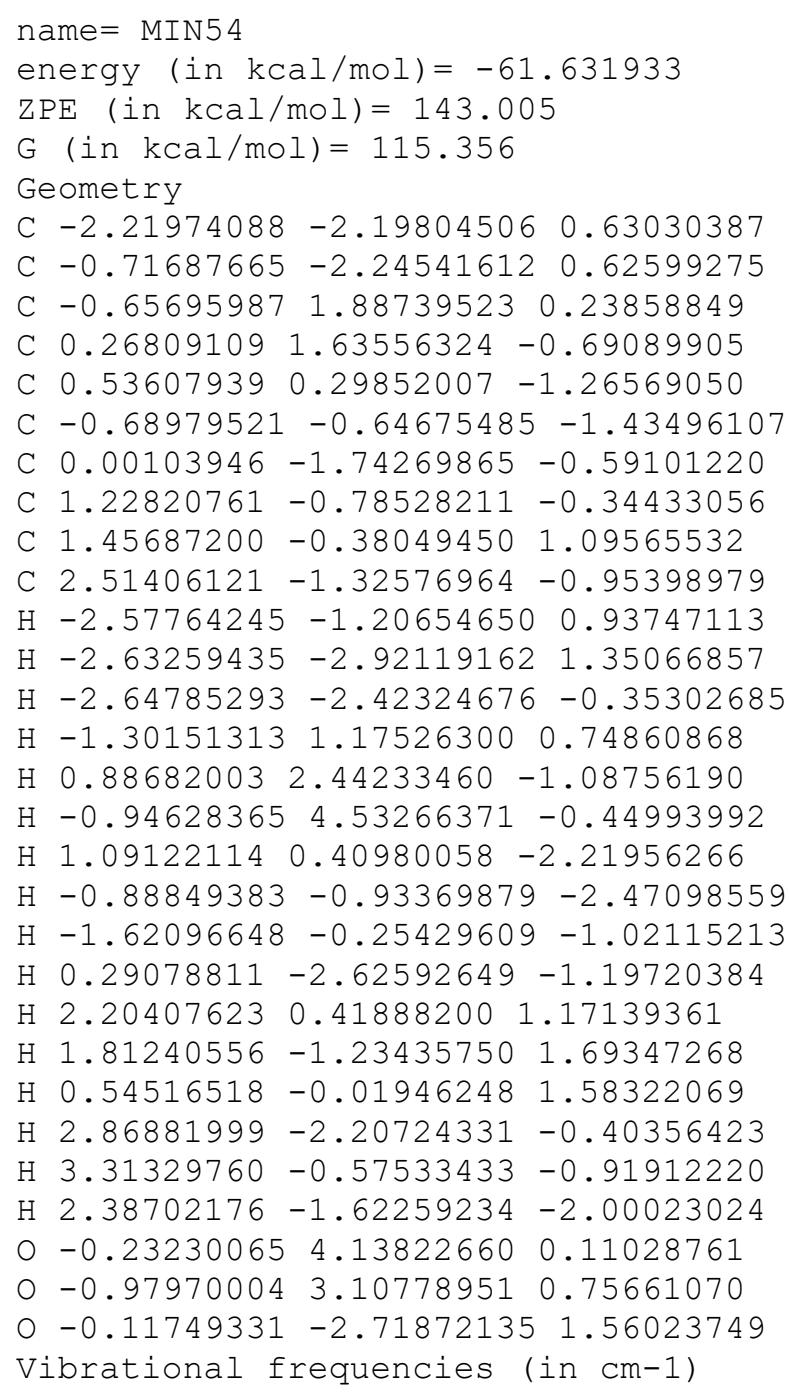


$\begin{array}{llll}\text { C } & -2.28039871 & 0.03361651 & -0.37925360\end{array}$

C $2.323431851 .49759582-0.37239562$

C $1.55013254 \quad 0.92689641 \quad 0.55489198$

C $0.91626142-0.40732867 \quad 0.46743062$

C $0.12663545-0.69823669-0.84229162$

C $-1.17877450-0.93028963-0.04681931$

C $-0.41474474-0.61682684 \quad 1.29415030$

C $-0.93843248 \quad 0.59295138 \quad 2.04500859$

C $-0.34107136-1.82490488 \quad 2.21731671$

$\mathrm{H}-3.69171313-0.75730777 \quad 1.06388810$

$\mathrm{H}-4.06031605-1.14952639-0.62566208$

$\mathrm{H}-4.36157195 \quad 0.48271346-0.01724046$

H $2.742301872 .50410193-0.30785850$

$\mathrm{H} 1.353892351 .469604351 .48218708$

H $3.41550252-0.59938813-2.09960084$

$\mathrm{H} 1.66465612-1.19388667 \quad 0.69005936$

$\mathrm{H} \quad 0.47848983-1.56732204-1.40201210$

H $0.08795516 \quad 0.15600103-1.53326034$

$\mathrm{H}-1.53853011-1.97115631-0.09608989$

$\mathrm{H}-0.29933603 \quad 0.84181006 \quad 2.90052622$

$\mathrm{H}-1.94833429 \quad 0.41963517 \quad 2.43361908$

$\mathrm{H}-0.98505000 \quad 1.48280783 \quad 1.39868136$

$\mathrm{H} \quad 0.01163072 \quad-2.72133858 \quad 1.69462666$

$\mathrm{H}-1.32061649-2.05982443 \quad 2.64880245$

$\mathrm{H} 0.34942251-1.64149564 \quad 3.04976728$

O $2.55630180-0.38172060-1.66277656$

o $2.725714421 .04002291-1.58956662$

o $-2.08728077 \quad 1.07018431-0.96357531$

Vibrational frequencies (in $\mathrm{cm}-1$ )

$\begin{array}{lllllllllllllll}31.0 & 35.7 & 42.7 & 72.2 & 87.8 & 107.2 & 143.1 & 164.1 & 175.7 & 194.8 & 213.8 & 251.3 & 276.4 & 289.3\end{array}$ $334.2341 .7 \quad 385.6 \quad 405.6 \quad 463.5 \quad 522.9 \quad 573.8 \quad 595.2 \quad 675.8 \quad 730.0 \quad 806.6 \quad 826.8 \quad 873.6 \quad 922.2$ $942.3954 .2994 .6 \quad 996.3 \quad 1002.6 \quad 1015.0 \quad 1032.9 \quad 1045.0 \quad 1048.51091 .2 \quad 1113.7 \quad 1122.3 \quad 1129.7$

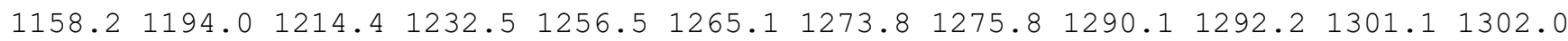
$1306.11310 .0 \quad 1323.6 \quad 1347.4 \quad 1356.1 \quad 1364.4 \quad 1380.9 \quad 1399.8 \quad 1403.4 \quad 1415.3 \quad 1854.01872 .5$ $2665.22671 .8 \quad 2676.0 \quad 2677.92678 .5 \quad 2679.02681 .2 \quad 2714.72718 .32731 .62748 .22759 .5$ $2778.02781 .5 \quad 2782.7 \quad 2873.9$

name $=$ MIN56

energy (in $\mathrm{kcal} / \mathrm{mol})=-59.016253$

$\operatorname{ZPE}(\mathrm{in} \mathrm{kcal} / \mathrm{mol})=142.256$

$\mathrm{G}(\mathrm{in} \mathrm{kcal} / \mathrm{mol})=114.185$

Geometry

C $-1.79925982-2.69626820 \quad 0.36009898$

C $-0.67085025-1.99411294-0.31229470$

C $1.09904666 \quad 3.24469222-0.65232912$

C $0.10380380 \quad 2.40161485 \quad 0.09753833$

C $0.70068569 \quad 1.09178717 \quad 0.56564714$

C $0.93571673 \quad 0.03831344-0.56710037$

C $-0.10150598-0.85443356 \quad 0.05781946$

C $-0.310400620 .07143325 \quad 1.24462313$

C $-1.73349870 \quad 0.56002809 \quad 1.44030502$

C $0.25320123-0.501920332 .53973918$

$\mathrm{H}-2.71948506-2.09251492 \quad 0.30523691$

$\mathrm{H}-1.58057506-2.87751087 \quad 1.42239803$

$\mathrm{H}-2.02517765-3.67048241-0.10102142$

H $2.053836562 .78520870-0.93459413$

$\mathrm{H}-0.77990345 \quad 2.22678107-0.55639939$

$\mathrm{H}-0.30214840 \quad 2.976220790 .95804696$

H $1.592085491 .26350128 \quad 1.18875786$

$\mathrm{H} \quad 1.94790195-0.37785989-0.59232722$

$\mathrm{H} \quad 0.68767191 \quad 0.37614006-1.58009436$

H $1.47050216-2.89252410-1.79393796$

$\mathrm{H}-2.19879645 \quad 0.85186902 \quad 0.49122011$

$\mathrm{H}-1.769705161 .423921592 .11448844$ 
$\mathrm{H}-2.36356297-0.22311276 \quad 1.87891346$

$\mathrm{H} \quad 0.29644886 \quad 0.25970373 \quad 3.32622077$

H $1.26757058-0.894377972 .39881908$

$\mathrm{H}-0.36193215-1.329160762 .91015137$

o $0.81114726-2.20367935-2.05715260$

$00.85434003 \quad 4.38939785-0.94445497$

o $-0.35078467-2.73564883-1.42635754$

Vibrational frequencies (in $\mathrm{cm}-1$ )

$\begin{array}{lllllllllllllllll}31.9 & 48.4 & 63.1 & 66.4 & 80.3 & 114.5 & 124.6 & 156.3 & 158.1 & 169.5 & 173.6 & 195.1 & 219.3 & 270.6\end{array}$ $301.2 \quad 320.9 \quad 366.5 \quad 396.3 \quad 437.9 \quad 465.3 \quad 543.7 \quad 584.2 \quad 613.6 \quad 621.9 \quad 764.5 \quad 782.5 \quad 864.6 \quad 920.7$ $951.3989 .0 \quad 994.6 \quad 1007.4 \quad 1013.7 \quad 1038.2 \quad 1041.5 \quad 1054.3 \quad 1083.5 \quad 1093.11111 .3 \quad 1137.3 \quad 1174.8$ $1200.71211 .3 \quad 1233.1 \quad 1248.0 \quad 1261.1 \quad 1268.3 \quad 1272.5 \quad 1273.4 \quad 1291.7 \quad 1297.7 \quad 1300.3 \quad 1302.6$

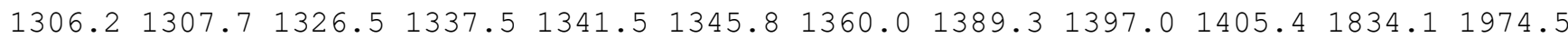
$2650.22654 .42664 .12668 .7 \quad 2675.4 \quad 2678.0 \quad 2679.02682 .02687 .2 \quad 2726.12742 .52764 .0$ $2773.92781 .2 \quad 2783.02868 .0$

\section{name $=$ MIN57}

energy (in $\mathrm{kcal} / \mathrm{mol})=-57.828012$

$\mathrm{ZPE}($ in $\mathrm{kcal} / \mathrm{mol})=141.401$

$\mathrm{G}($ in $\mathrm{kcal} / \mathrm{mol})=111.218$

Geometry

C $-1.91139563-1.00995161-0.44570712$

C $-0.38216280-1.01027715-0.47710768$

C $0.401748503 .80948256-0.49362207$

$\begin{array}{lllll}\text { C } 0.35245890 & 2.68018732 & 0.50501324\end{array}$

C $-0.29265662 \quad 1.45833445-0.05249824$

C $0.24909691 \quad 0.24399963 \quad 0.06547053$

C $0.15667663-2.25781477 \quad 0.16808238$

C $0.23539674 \quad-2.49544851 \quad 1.48261357$

C $-0.17308796-1.539303812 .55175787$

C $0.77108876-3.79619621 \quad 1.99158400$

$\mathrm{H}-2.32334289-0.24616868-1.11741386$

$\mathrm{H}-2.29765540 \quad-0.83070463 \quad 0.56168369$

$\mathrm{H}-2.30371051-1.97650163-0.78835951$

$\mathrm{H}-0.04847126 \quad 3.64366668-1.47934416$

$\mathrm{H}-0.19774230 \quad 3.01993693 \quad 1.41449822$

$\mathrm{H} \quad 1.38221918 \quad 2.481125390 .87890205$

$\mathrm{H}-1.25145651 \quad 1.62015426-0.54681856$

$\mathrm{H} \quad 1.21287580 \quad 0.08279372 \quad 0.54844986$

$\mathrm{H} \quad 0.36451035 \quad 0.44136031 \quad-2.70219986$

$\mathrm{H} \quad 0.48032931-3.01045806-0.55537495$

$\mathrm{H}-0.78104761-0.705300392 .17499402$

$\mathrm{H} 0.70967536-1.098914393 .03947038$

$\mathrm{H}-0.76284335-2.034666613 .33586446$

$\mathrm{H} \quad 1.15650174-4.442318221 .19153942$

$\mathrm{H}-0.00967356-4.365651012 .51641085$

$\mathrm{H} \quad 1.59425769-3.63840410 \quad 2.70273018$

$0-0.44831952-0.11416142-2.61569193$

$0 \quad 0.92229474 \quad 4.86098273-0.21768448$

o $0.06778352-1.17999413-1.83646269$

Vibrational frequencies (in $\mathrm{cm}-1$ )

$\begin{array}{lllllllllllllll}13.3 & 30.7 & 49.9 & 55.6 & 66.3 & 78.3 & 87.5 & 108.1 & 130.2 & 130.7 & 164.9 & 187.7 & 203.8 & 260.7\end{array}$ $281.8 \quad 301.3 \quad 335.5 \quad 347.1 \quad 400.6 \quad 446.7 \quad 465.6 \quad 484.7 \quad 506.1 \quad 569.9 \quad 679.9 \quad 755.7 \quad 896.2 \quad 905.2$ $929.9951 .1982 .8 \quad 989.5 \quad 997.5 \quad 1013.7 \quad 1018.0 \quad 1032.6 \quad 1046.3 \quad 1095.6 \quad 1107.7 \quad 1117.4 \quad 1156.3$

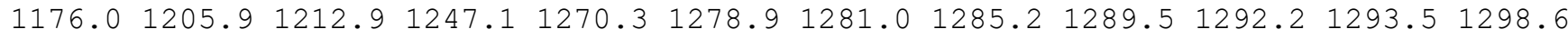

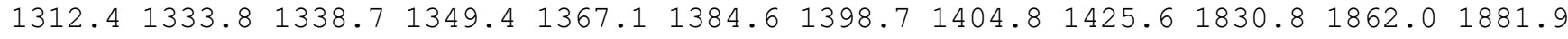
$2645.02653 .12666 .7 \quad 2669.0 \quad 2671.4 \quad 2673.2 \quad 2673.92681 .32730 .12737 .6 \quad 2740.42759 .4$ 2777.32780 .32781 .02868 .7

name $=$ MIN58

energy (in $\mathrm{kcal} / \mathrm{mol})=-58.684522$

$\mathrm{ZPE}($ in $\mathrm{kcal} / \mathrm{mol})=142.985$

$\mathrm{G}(\mathrm{in} \mathrm{kcal} / \mathrm{mol})=115.791$ 


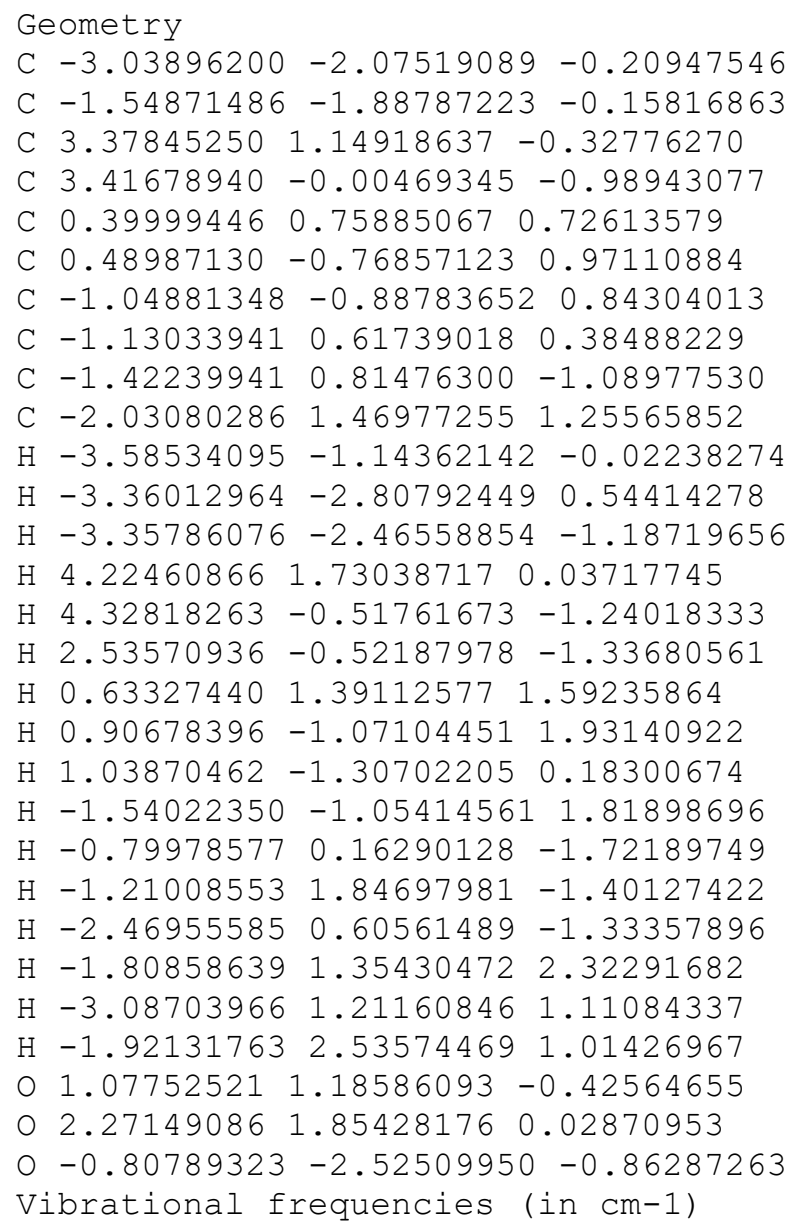

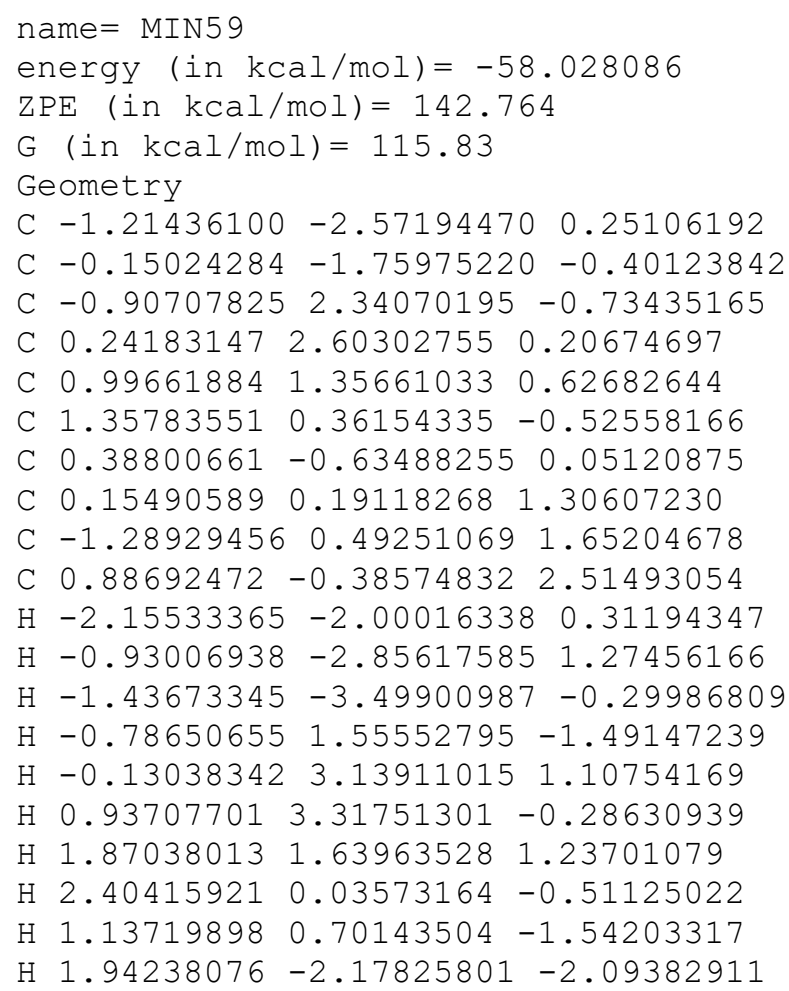


$\mathrm{H}-1.90298683 \quad 0.67985162 \quad 0.76247093$

$\mathrm{H}-1.368130341 .37680366 \quad 2.29704490$

$\mathrm{H}-1.75543118-0.343718252 .18700828$

$\mathrm{H} \quad 0.88169744 \quad 0.31730029 \quad 3.35512374$

$\mathrm{H} \quad 1.93205625-0.62022378 \quad 2.28121915$

$\mathrm{H} \quad 0.41870220-1.316230112 .85461407$

O $1.13491822-1.64965339-2.30785526$

$0-1.925764862 .98474330-0.67206309$

O $0.15497869-2.38779322-1.58604396$

Vibrational frequencies (in $\mathrm{cm}-1$ )

$\begin{array}{llllllllllllllll}40.6 & 52.7 & 71.0 & 97.0 & 115.6 & 127.0 & 144.8 & 163.9 & 183.0 & 193.8 & 210.0 & 222.9 & 252.9 & 270.9\end{array}$ $309.9 \quad 326.0 \quad 393.9 \quad 418.9 \quad 445.4 \quad 456.7 \quad 558.9 \quad 577.2 \quad 609.3 \quad 650.2 \quad 754.0 \quad 789.0 \quad 849.8 \quad 913.9$

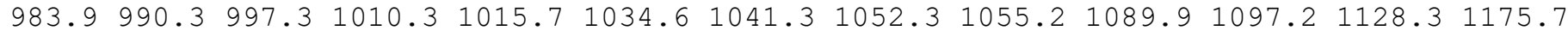
$1191.31211 .5 \quad 1238.8 \quad 1247.6 \quad 1269.6 \quad 1270.6 \quad 1272.5 \quad 1277.9 \quad 1289.6 \quad 1297.9 \quad 1300.3 \quad 1304.2$

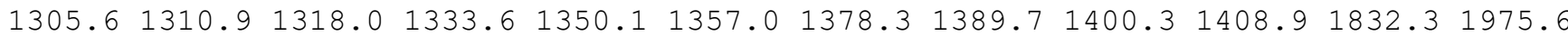
$2645.22659 .12662 .0 \quad 2668.92672 .5 \quad 2676.3 \quad 2678.72681 .82684 .12724 .32739 .62763 .1$ $2773.5 \quad 2781.4 \quad 2783.2 \quad 2868.5$

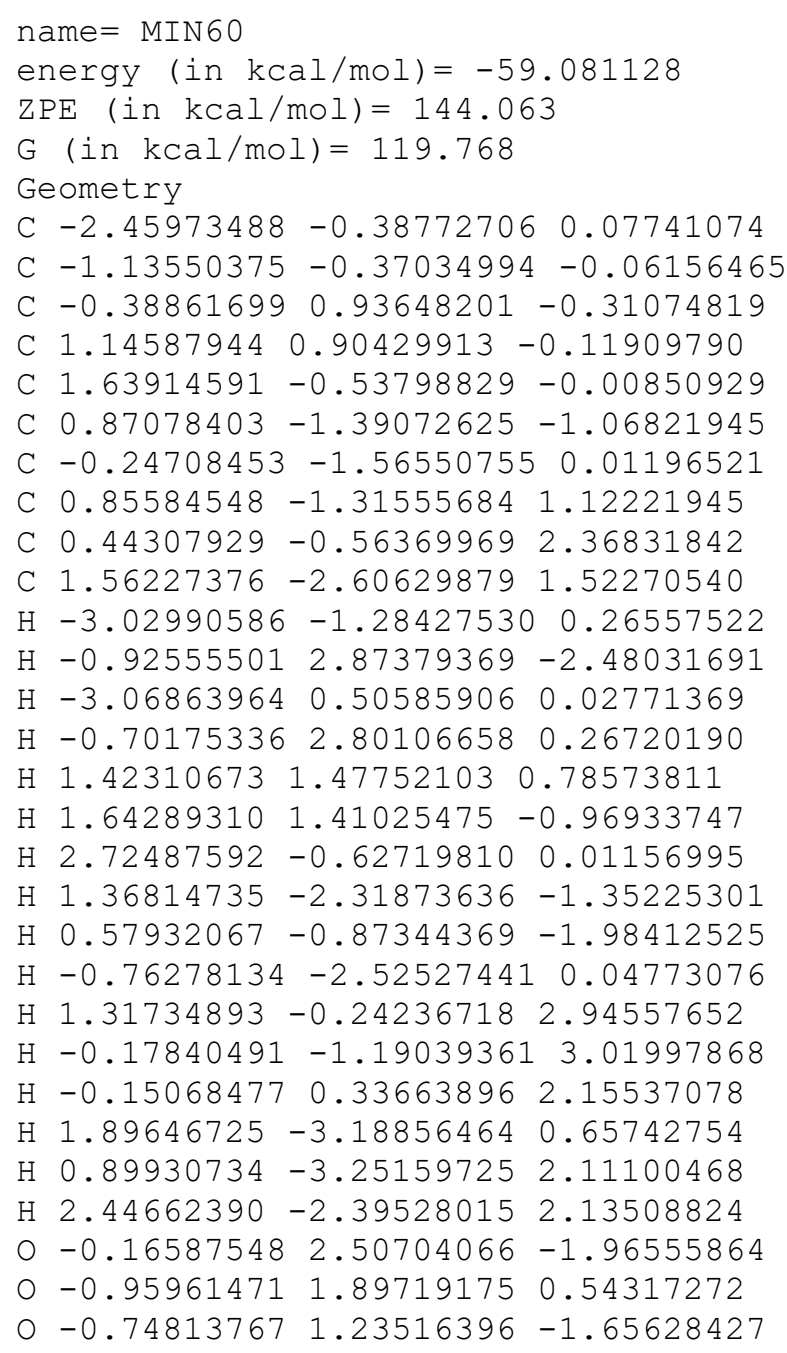


$\mathrm{ZPE}($ in $\mathrm{kcal} / \mathrm{mol})=142.337$

$\mathrm{G}($ in $\mathrm{kcal} / \mathrm{mol})=113.914$

Geometry

C $-1.62436606-2.44669835-1.13087449$

C $-0.50041523-1.85076324-0.34879690$

C $-1.40678666 \quad 2.45103684 \quad 0.29015480$

C $-0.058529592 .66095199-0.34523382$

C $0.601618251 .41293698-0.89246906$

C $-0.33303773 \quad 0.38223340-1.60094025$

C $0.01235830-0.64243123-0.54802581$

C $1.01982729 \quad 0.26764340 \quad 0.13319376$

C $0.74214607 \quad 0.53424978 \quad 1.60033822$

C $2.47437667-0.13749105-0.07591611$

$\mathrm{H}-2.58313863-1.98167495-0.85216159$

$\mathrm{H}-1.72807146-3.52891750-0.96024003$

$\mathrm{H}-1.48199130-2.29872663-2.21091177$

$\mathrm{H}-1.81929947 \quad 1.43475365 \quad 0.32072618$

$\mathrm{H} \quad 0.61390216 \quad 3.14885727 \quad 0.39691030$

$\mathrm{H}-0.16604307 \quad 3.41759864-1.15511825$

$\mathrm{H} 1.45657378 \quad 1.70304588-1.52960312$

$\mathrm{H}-0.00311981 \quad 0.11265798 \quad-2.61159983$

$\mathrm{H}-1.38797663 \quad 0.65977052-1.66601967$

H $1.60341658-3.23624790 \quad 0.86351878$

$\mathrm{H}-0.311716850 .75640322 \quad 1.79641468$

H $1.34226978 \quad 1.36950614 \quad 1.97789015$

$\mathrm{H} \quad 0.98490758-0.34910746 \quad 2.20964526$

H $2.75617644 \quad-0.96626427 \quad 0.58419811$

H $3.15045687 \quad 0.69735231 \quad 0.14227525$

H $2.66096389-0.46429679-1.10440533$

O $1.09378776-2.45474722 \quad 1.19267248$

$0-2.02522077 \quad 3.37835172 \quad 0.75384133$

o $-0.17886103-2.760925830 .62772136$

Vibrational frequencies (in $\mathrm{cm}-1$ )

$\begin{array}{lllllllllllllll}21.6 & 38.6 & 40.0 & 52.4 & 89.5 & 124.1 & 133.0 & 138.7 & 154.0 & 199.7 & 203.0 & 226.1 & 233.2 & 276.2\end{array}$ $286.0 \quad 318.4 \quad 379.9 \quad 408.5 \quad 423.6 \quad 490.0 \quad 558.4 \quad 587.0 \quad 615.4 \quad 653.5 \quad 733.7 \quad 775.5 \quad 821.1 \quad 920.0$ $977.8 \quad 989.3 \quad 993.5 \quad 1008.0 \quad 1020.2 \quad 1037.9 \quad 1046.2 \quad 1053.4 \quad 1059.3 \quad 1090.1 \quad 1096.11136 .01179 .3$

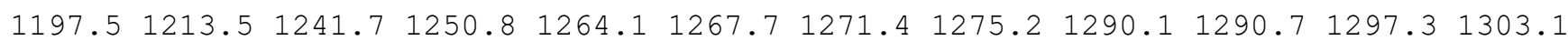

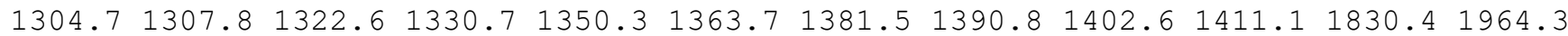
$2646.52650 .42665 .52668 .2 \quad 2670.02677 .2 \quad 2680.32682 .92684 .52720 .82737 .52762 .9$ $2775.22781 .2 \quad 2782.7 \quad 2868.4$

\section{name $=$ MIN62}

energy (in $\mathrm{kcal} / \mathrm{mol})=-56.329641$

$\mathrm{ZPE}(\mathrm{in} \mathrm{kcal} / \mathrm{mol})=142.584$

$\mathrm{G}(\mathrm{in} \mathrm{kcal} / \mathrm{mol})=116.037$

Geometry

C $-2.93163143-1.28443153-0.61854700$

C $-1.43992665-1.24632971-0.76655781$

C $1.857427601 .53549639-0.18941705$

C $2.09683349 \quad 0.75466747 \quad 0.98584320$

C $1.11776879-0.35842547 \quad 1.33125740$

C $0.90011068-1.48309358 \quad 0.28915987$

C $-0.63353006-1.39100657 \quad 0.49196751$

C $-0.43624894-0.09337038 \quad 1.36192399$

C $-0.90989286 \quad 1.18433378 \quad 0.70054759$

C $-1.02025096-0.215338262 .76115877$

$\mathrm{H}-3.42277845-0.77586163-1.46457135$

$\mathrm{H}-3.27790327-0.81277490 \quad 0.30709879$

$\mathrm{H}-3.29042820-2.32395263-0.62502298$

H $0.49355988 \quad 0.42206203 \quad-2.25103787$

$\mathrm{H} 2.171366021 .44681671 \quad 1.86734946$

$\mathrm{H} \quad 3.12295347 \quad 0.30037084 \quad 0.89794489$

H $1.44093790-0.787491432 .30592049$

H $1.34219981-2.44794650 \quad 0.54716623$ 
$\mathrm{H} \quad 1.22379732-1.23306628-0.73083806$

$\mathrm{H}-1.03113352-2.23206916 \quad 1.09078220$

$\mathrm{H}-1.99787692 \quad 1.30464234 \quad 0.78715574$

$\mathrm{H}-0.686355361 .21351351-0.37838475$

$\mathrm{H}-0.455006812 .06985347 \quad 1.16001228$

$\mathrm{H}-0.69520504-1.128730293 .27219393$

$\mathrm{H}-2.11683439-0.231352642 .73829398$

$\mathrm{H}-0.72163869 \quad 0.63299227 \quad 3.38957797$

$\begin{array}{lllll}0 & 0.82090028 & 1.27847672 & -2.47079431\end{array}$

O $1.579994691 .93060722-1.23702832$

O $-0.91879488-1.15964359-1.84962893$

Vibrational frequencies (in $\mathrm{cm}-1$ )

$\begin{array}{llllllllllllllll}-68.9 & 44.6 & 58.9 & 82.5 & 99.0 & 119.5 & 151.7 & 178.7 & 190.8 & 211.8 & 246.3 & 276.0 & 297.4 & 331.8\end{array}$ $365.1 \quad 378.2 \quad 404.2 \quad 442.3 \quad 475.6 \quad 480.4 \quad 563.0 \quad 571.1 \quad 592.2 \quad 655.8 \quad 671.8 \quad 780.4 \quad 812.2 \quad 891.4$ $933.8 \quad 976.3 \quad 994.9 \quad 1002.1 \quad 1010.5 \quad 1016.7 \quad 1038.1 \quad 1049.0 \quad 1057.3 \quad 1072.6 \quad 1092.4 \quad 1118.6 \quad 1136.2$

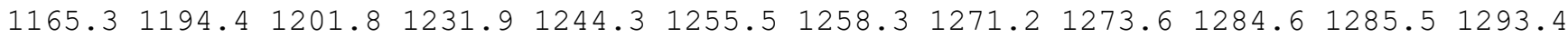
$1298.21300 .3 \quad 1315.7 \quad 1332.8 \quad 1340.6 \quad 1348.6 \quad 1380.2 \quad 1391.91404 .2 \quad 1415.5 \quad 1854.8 \quad 2147.5$ $2631.12648 .92664 .3 \quad 2673.2 \quad 2674.0 \quad 2675.5 \quad 2677.02678 .92704 .52717 .72723 .42758 .7$ $2775.92779 .4 \quad 2782.6 \quad 2813.2$

name $=$ MIN63

energy (in $\mathrm{kcal} / \mathrm{mol})=-56.058783$

$\mathrm{ZPE}($ in $\mathrm{kcal} / \mathrm{mol})=142.62$

$\mathrm{G}($ in $\mathrm{kcal} / \mathrm{mol})=114.715$

Geometry

C $-1.16210095-2.539244850 .87899663$

C $-0.24388454-1.79741325-0.02997228$

C $-0.991207692 .56309464-0.78001373$

C $0.299345592 .75209415-0.02076530$

C $0.956871991 .44994903 \quad 0.40364426$

C $1.07787155 \quad 0.34464214 \quad-0.69389416$

C $0.25874215-0.584142390 .15869562$

C $0.14509937 \quad 0.41661206 \quad 1.29718682$

C $-1.27540914 \quad 0.79144185 \quad 1.67829419$

C $0.94310181 \quad 0.00416456 \quad 2.53014269$

$\mathrm{H}-2.17875648-2.11736823 \quad 0.82576923$

$\mathrm{H}-0.82843460-2.47575385 \quad 1.92448032$

$\mathrm{H}-1.23950734-3.60640547 \quad 0.62058467$

$\mathrm{H}-1.92679999 \quad 2.72403694 \quad-0.22975017$

$\mathrm{H} \quad 0.13581205 \quad 3.39208191 \quad 0.86819521$

$\mathrm{H} \quad 1.01262456 \quad 3.30755125 \quad-0.67242124$

H $1.93469433 \quad 1.682728720 .86504867$

$\begin{array}{lllll}\mathrm{H} & 2.10263294 & 0.01988001 & -0.89450681\end{array}$

$\mathrm{H} \quad 0.61562122 \quad 0.59638296-1.66095985$

H $1.68159702-2.43510770-1.84938754$

$\mathrm{H}-1.69677719 \quad 0.07293053 \quad 2.39248754$

$\mathrm{H}-1.94196314 \quad 0.78972370 \quad 0.80732812$

$\mathrm{H}-1.31593546 \quad 1.77994736 \quad 2.14825038$

$\mathrm{H} \quad 1.01737320 \quad 0.82747507 \quad 3.24880298$

$\mathrm{H} 1.96253623-0.30268487 \quad 2.26648565$

$\mathrm{H} \quad 0.47706839-0.844895063 .04178882$

○ $0.83493172-1.96682364-2.05552314$

$0-1.012933112 .28177755-1.95166603$

o $-0.03254076-2.60360033-1.12408380$

Vibrational frequencies (in $\mathrm{cm}-1$ )

$\begin{array}{llllllllllllllll}28.2 & 40.9 & 50.2 & 54.0 & 107.3 & 121.2 & 135.9 & 165.7 & 171.8 & 176.2 & 195.8 & 217.8 & 253.1 & 265.5\end{array}$ $298.1323 .2 \quad 392.7 \quad 415.9 \quad 448.6 \quad 482.4 \quad 528.0 \quad 580.3 \quad 611.0 \quad 636.9 \quad 766.1 \quad 794.5 \quad 893.8 \quad 936.6$

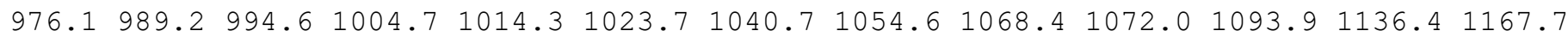
$1195.21211 .1 \quad 1239.7 \quad 1245.1 \quad 1271.4 \quad 1272.0 \quad 1274.9 \quad 1290.3 \quad 1293.1 \quad 1299.5 \quad 1301.5 \quad 1302.8$

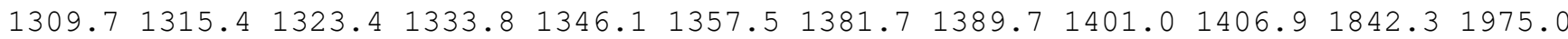
$2644.52665 .3 \quad 2667.5 \quad 2670.0 \quad 2671.6 \quad 2674.1 \quad 2677.7 \quad 2679.92682 .92716 .92739 .92758 .8$ $2774.72779 .92782 .6 \quad 2871.2$ 
name $=$ MIN64

energy (in $\mathrm{kcal} / \mathrm{mol})=-55.350225$

$\mathrm{ZPE}($ in $\mathrm{kcal} / \mathrm{mol})=142.48$

$\mathrm{G}($ in $\mathrm{kcal} / \mathrm{mol})=115.777$

Geometry

C $-2.47797826-1.10829180-0.88338626$

C $-0.99088652-1.11375023-0.89638892$

C $0.71937761 \quad 1.69704632-0.89696386$

C $0.93210072 \quad 2.146831910 .52925026$

C $0.841352090 .98449140 \quad 1.48297575$

C $1.78457640 \quad 0.81888309 \quad 2.41186756$

C $-0.10487158-0.67326840 \quad 0.00232564$

C $-0.34522887 \quad 0.04432340 \quad 1.29888772$

C $-1.63612781 \quad 0.88219484 \quad 1.34480259$

C $-0.42676690-1.01752330 \quad 2.41239491$

$\mathrm{H}-2.90356220-1.71674655-1.69933292$

$\mathrm{H}-2.88143719-0.09167124-0.99626174$

$\mathrm{H}-2.86445866-1.52564178 \quad 0.05935655$

$\mathrm{H}-0.299497131 .76148091-1.29846675$

$\begin{array}{lllll}\mathrm{H} & 0.19265988 & 2.92227918 & 0.81621190\end{array}$

$\mathrm{H} \quad 1.92681894 \quad 2.64097567 \quad 0.61186369$

H 2.63710683 $1.47126878 \quad 2.52077740$

$\mathrm{H} \quad 1.79816765 \quad 0.01966298 \quad 3.13441020$

$\mathrm{H} \quad 1.03278962-0.81692973-2.44470611$

$\mathrm{H} \quad 0.96146417-0.88455885-0.16089296$

$\mathrm{H}-1.76473273 \quad 1.490693650 .44640942$

$\mathrm{H}-1.62720561 \quad 1.55733104 \quad 2.20948419$

$\mathrm{H}-2.52233394 \quad 0.24801860 \quad 1.44797206$

H $0.46542178-1.65509681 \quad 2.42273823$

$\mathrm{H}-1.28719487-1.67721258 \quad 2.25747432$

$\mathrm{H}-0.52937577-0.55667973 \quad 3.39990762$

O $0.78247553-1.76058942-2.24191385$

O $1.626195561 .30112359-1.58743799$

o $-0.62740041-1.67010273-2.10188399$

Vibrational frequencies (in $\mathrm{cm}-1$ )

$\begin{array}{lllllllllllllll}41.5 & 60.8 & 66.3 & 87.2 & 105.3 & 131.8 & 156.5 & 168.1 & 210.8 & 220.4 & 232.2 & 255.3 & 267.9 & 283.0\end{array}$

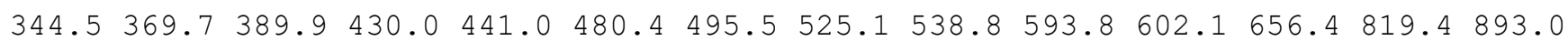
$921.0 \quad 934.3 \quad 972.7977 .3 \quad 985.5 \quad 1005.6 \quad 1007.9 \quad 1027.1 \quad 1035.1 \quad 1053.5 \quad 1076.11095 .11110 .6$

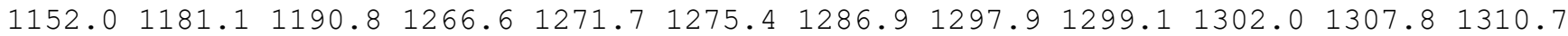

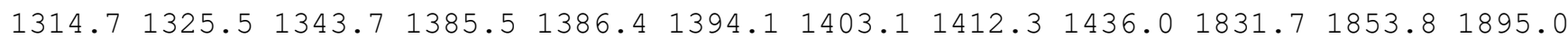
$2644.82662 .12664 .0 \quad 2667.0 \quad 2673.8 \quad 2675.3 \quad 2681.22681 .52716 .6 \quad 2719.22737 .52773 .3$ $2780.12781 .5 \quad 2788.92827 .0$

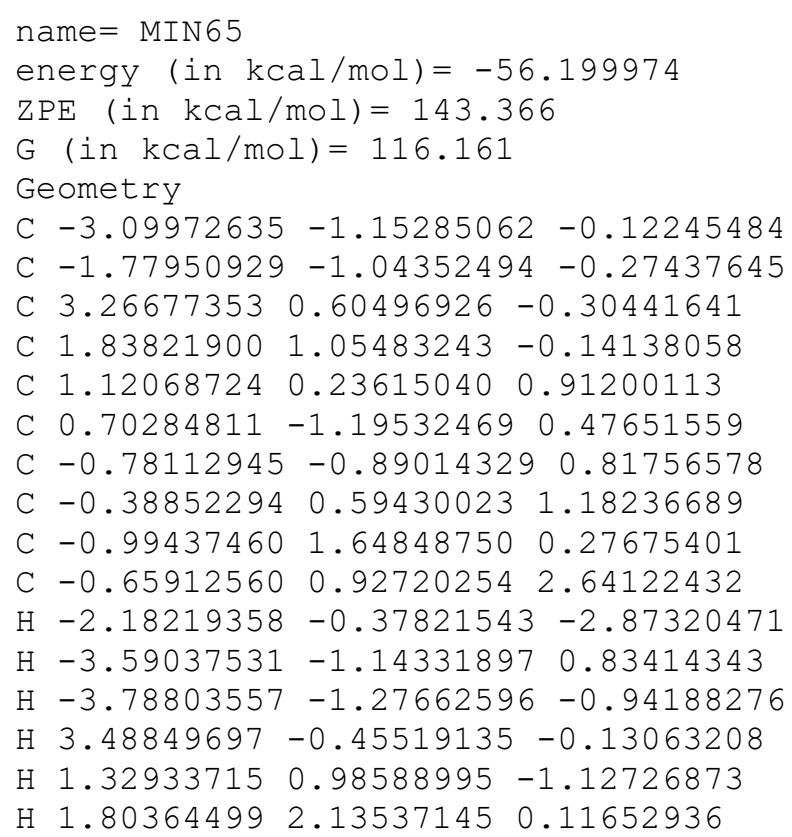


$\mathrm{H} \quad 1.70423808 \quad 0.23453528 \quad 1.85184631$

$\mathrm{H} 1.13777127-1.99981966 \quad 1.07581348$

$\mathrm{H} 0.90220903-1.43110310-0.57293116$

$\mathrm{H}-1.12487645-1.45711500 \quad 1.70651456$

$\mathrm{H}-2.08099328 \quad 1.716788320 .42466265$

$\mathrm{H}-0.83131503 \quad 1.42840211-0.78512624$

$\mathrm{H}-0.57405650 \quad 2.64095525 \quad 0.47851958$

$\mathrm{H}-0.22002922 \quad 1.89446868 \quad 2.91499592$

$\mathrm{H}-0.24395635 \quad 0.17572349 \quad 3.32248757$

$\mathrm{H}-1.73596492 \quad 0.988817832 .84184597$

o $-2.02807685-1.29765057-2.54248954$

$04.135409851 .37616096-0.62711806$

o $-1.11771647-1.03720678-1.47793327$

Vibrational frequencies (in $\mathrm{cm}-1$ )

$\begin{array}{lllllllllllllllll}27.2 & 36.5 & 60.8 & 83.0 & 93.0 & 162.1 & 170.8 & 180.4 & 191.9 & 197.9 & 231.7 & 236.5 & 285.7 & 327.4\end{array}$ $329.6 \quad 371.2 \quad 398.3 \quad 429.3 \quad 472.0 \quad 540.2 \quad 572.4 \quad 608.9644 .9 \quad 669.9 \quad 804.7 \quad 836.4 \quad 878.3 \quad 922.8$ $938.7 \quad 975.1 \quad 997.2 \quad 1007.6 \quad 1013.0 \quad 1017.5 \quad 1023.5 \quad 1048.4 \quad 1075.2 \quad 1085.8 \quad 1109.4 \quad 1129.5 \quad 1134.7$ $1168.1 \quad 1179.7 \quad 1205.4 \quad 1228.2 \quad 1260.3 \quad 1269.4 \quad 1285.1 \quad 1286.5 \quad 1292.01297 .7 \quad 1298.7 \quad 1303.3$

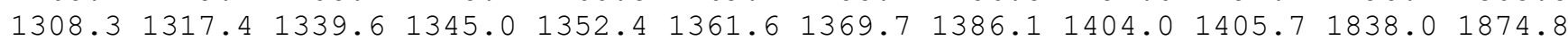
$2649.12657 .12671 .12675 .12677 .2 \quad 2679.12681 .02714 .62721 .32728 .92742 .5 \quad 2763.0$ $2779.92782 .32798 .2 \quad 2869.2$

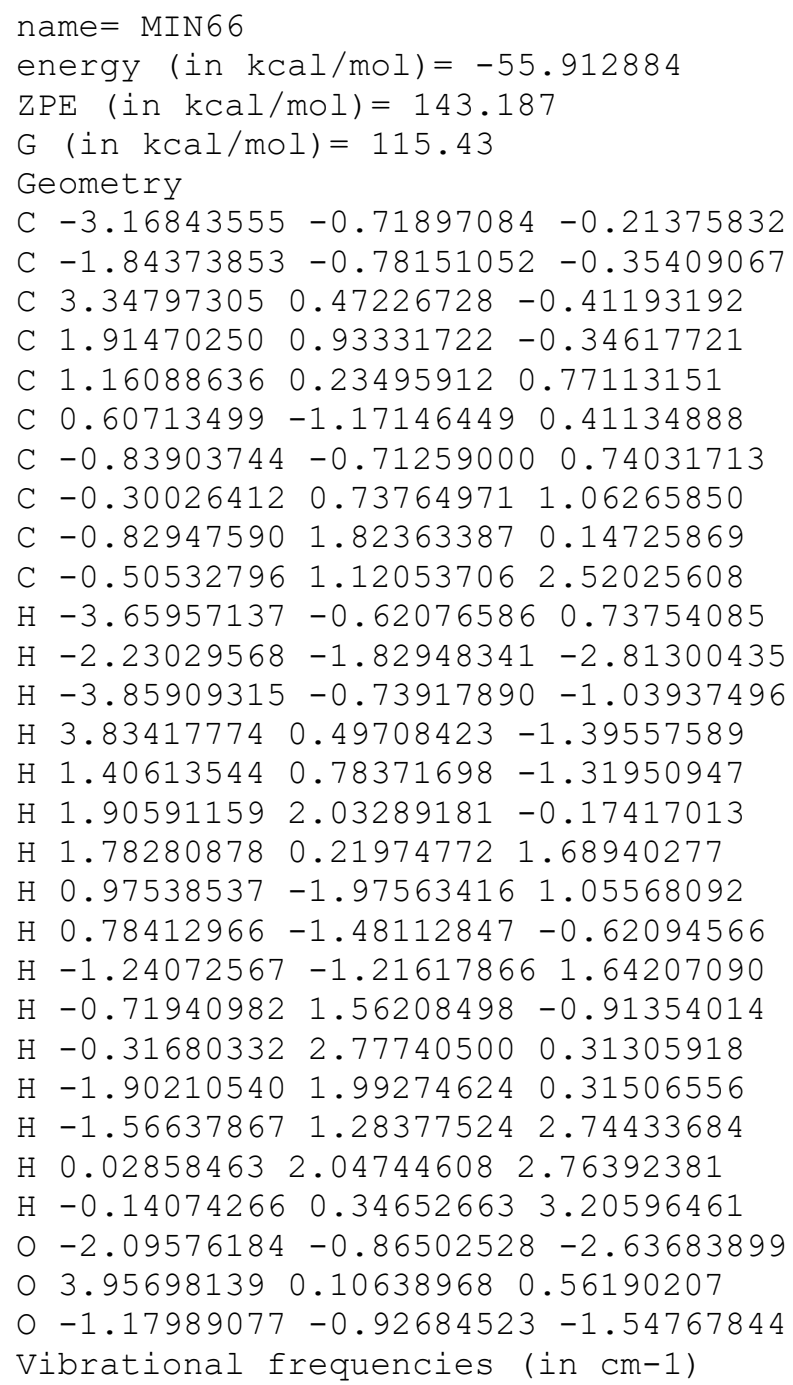




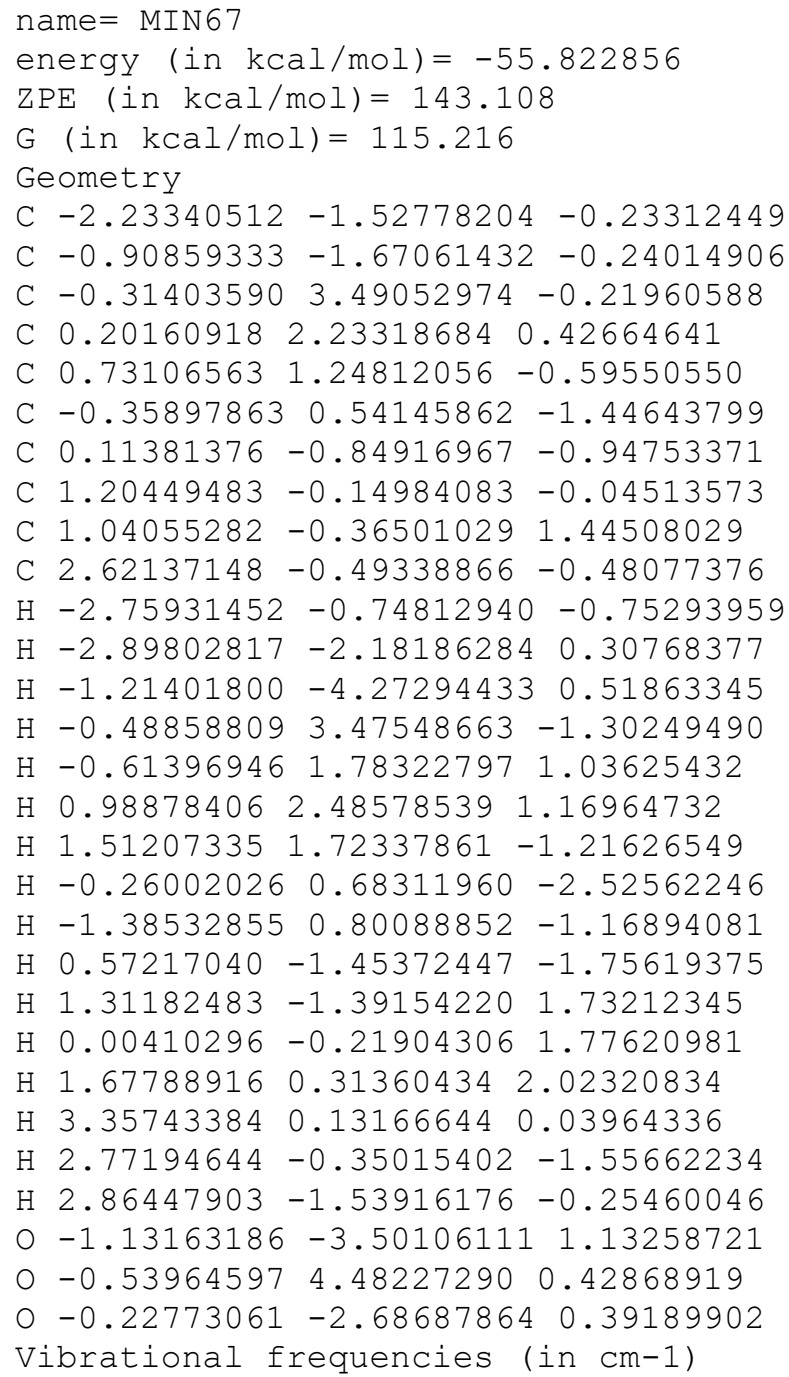
$338.3 \quad 368.6 \quad 394.1 \quad 422.9 \quad 462.1 \quad 545.2 \quad 549.0 \quad 600.5 \quad 644.4 \quad 671.0 \quad 779.5 \quad 832.7 \quad 875.6 \quad 931.1$ $940.6 \quad 985.3 \quad 995.1 \quad 1003.5 \quad 1009.3 \quad 1018.5 \quad 1022.3 \quad 1045.0 \quad 1075.9 \quad 1095.5 \quad 1118.01127 .9 \quad 1136.6$

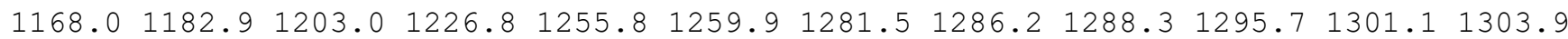
$1308.41309 .3 \quad 1331.6 \quad 1340.91343 .8 \quad 1349.0 \quad 1363.7 \quad 1385.8 \quad 1403.2 \quad 1405.0 \quad 1835.9 \quad 1880.9$ $2648.12653 .3 \quad 2665.6 \quad 2675.5 \quad 2677.1 \quad 2679.6 \quad 2682.8 \quad 2714.82722 .6 \quad 2725.4 \quad 2741.02761 .9$ $2779.22782 .3 \quad 2796.5 \quad 2867.9$

\section{name $=$ MIN68}

energy (in $\mathrm{kcal} / \mathrm{mol})=-56.262266$

$\mathrm{ZPE}(\mathrm{in} \mathrm{kcal} / \mathrm{mol})=143.95$

$\mathrm{G}($ in $\mathrm{kcal} / \mathrm{mol})=118.471$

Geometry

C $-2.66624613-0.63085017-0.70223101$

C $-1.33366744-0.63839537-0.72260172$

C $1.565531241 .31608218-0.72007470$

C $2.13033437 \quad 0.70073004 \quad 0.53456346$

C $1.51025627-0.64281514 \quad 0.89130199$

C $1.04576834-1.54707548-0.28395098$

C $-0.43498942-1.421451350 .17065191$

C $0.03782141-0.63656994 \quad 1.45712730$

C $-0.61479404 \quad 0.70749186 \quad 1.68883395$

C $-0.08459246-1.49747558 \quad 2.70957440$

$\mathrm{H}-3.26200790-0.02812376-1.37033756$

$\mathrm{H}-0.86002800 \quad 1.88299083-1.89710180$

$\mathrm{H}-3.25690490-1.21802294-0.02358817$

H $1.76235748 \quad 0.80353950-1.67196661$ 
$\mathrm{H} 2.000741201 .400067241 .39078816$

H $3.22806930 \quad 0.58534562 \quad 0.41082537$

$\mathrm{H} 2.19778824-1.175757891 .57634640$

H $1.42798707-2.57235514-0.22084677$

$\mathrm{H} \quad 1.27243695-1.19680127-1.29127740$

$\mathrm{H}-0.89759539-2.39761142 \quad 0.40705426$

$\mathrm{H}-0.15513884 \quad 1.24097168 \quad 2.52876186$

$\mathrm{H}-1.68450094 \quad 0.59560315 \quad 1.91212110$

$\mathrm{H}-0.548757951 .36819448 \quad 0.81316473$

$\mathrm{H} \quad 0.35981928-2.48991504 \quad 2.57649882$

$\mathrm{H}-1.13608731-1.645247242 .98613856$

$\mathrm{H} 0.41351610-1.02549148 \quad 3.56456305$

O $-1.34472955 \quad 1.08348224-2.23887812$

$0 \quad 0.948548562 .35455586-0.70238351$

$\begin{array}{lllll}0 & -0.55508149 & 0.07946614 & -1.59841372\end{array}$

Vibrational frequencies (in cm-1)

$\begin{array}{lllllllllllllllll}37.7 & 88.6 & 95.8 & 113.4 & 130.4 & 172.8 & 173.8 & 191.7 & 204.8 & 234.7 & 272.4 & 304.4 & 334.5 & 338.4\end{array}$

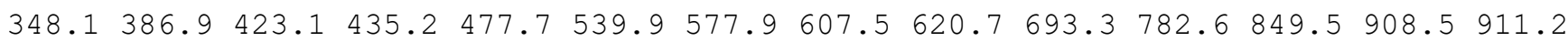
$928.7 \quad 992.5 \quad 1005.8 \quad 1007.4 \quad 1016.0 \quad 1017.0 \quad 1024.8 \quad 1037.6 \quad 1050.5 \quad 1073.4 \quad 1104.01113 .5$ $1123.11155 .0 \quad 1182.4 \quad 1208.0 \quad 1226.2 \quad 1246.2 \quad 1281.6 \quad 1283.4 \quad 1285.8 \quad 1289.6 \quad 1295.3 \quad 1299.0$ $1300.91309 .2 \quad 1318.3 \quad 1329.7 \quad 1354.4 \quad 1369.5 \quad 1375.4 \quad 1383.0 \quad 1392.7 \quad 1406.3 \quad 1407.2 \quad 1828.7$ $1875.52640 .02661 .2 \quad 2667.3 \quad 2675.4 \quad 2677.92679 .12680 .42715 .12722 .2 \quad 2728.52737 .6$ $2763.52781 .4 \quad 2783.7 \quad 2796.2 \quad 2831.6$

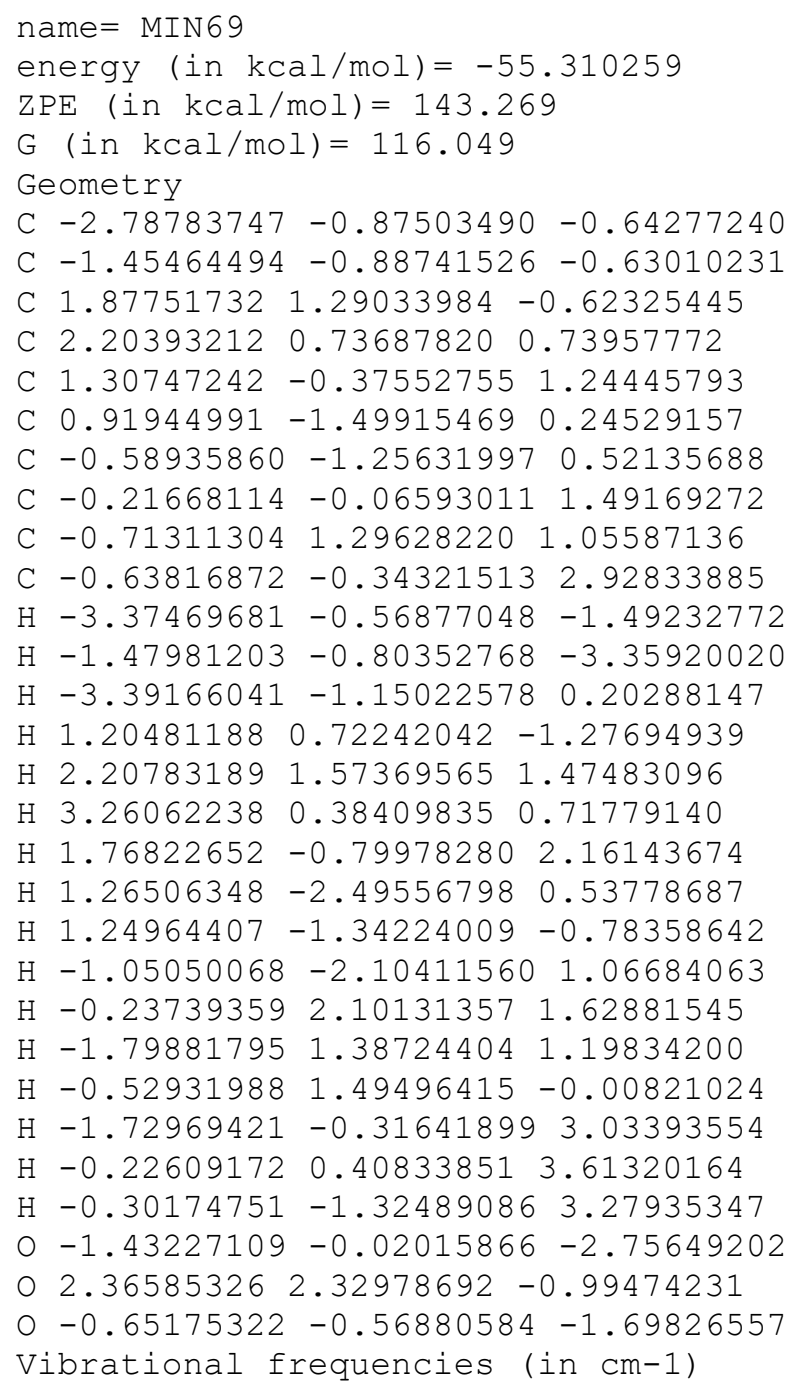


$2647.92652 .3 \quad 2665.1 \quad 2674.7 \quad 2677.5 \quad 2678.9 \quad 2679.72710 .6 \quad 2718.7 \quad 2729.82736 .6 \quad 2763.7$ $2779.72782 .3 \quad 2798.7 \quad 2869.8$

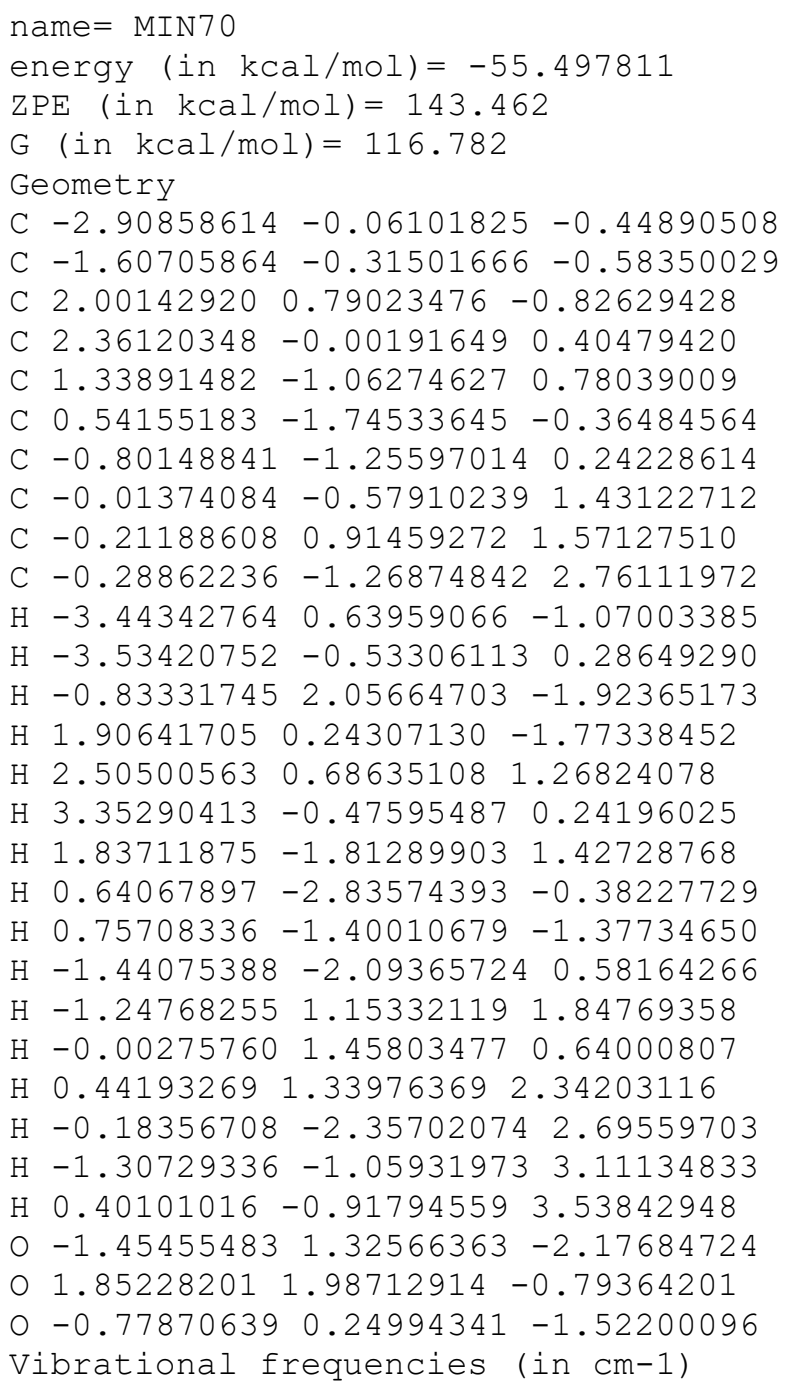

$\begin{array}{lllllllllllllll}-39.2 & 40.7 & 54.9 & 77.9 & 91.4 & 160.8 & 169.2 & 183.1 & 210.5 & 229.7 & 245.1 & 274.2 & 295.6 & 328.4\end{array}$ $\begin{array}{lllllllllllllll}336.1 & 379.0 & 415.1 & 438.7 & 471.1 & 547.4 & 578.6 & 612.9 & 617.0 & 682.9 & 776.1 & 842.8 & 899.8 & 918.2\end{array}$ $923.9993 .21005 .7 \quad 1006.2 \quad 1014.3 \quad 1017.3 \quad 1025.3 \quad 1042.3 \quad 1049.4 \quad 1075.81101 .81120 .2$ $1129.81155 .71184 .7 \quad 1208.5 \quad 1230.2 \quad 1253.0 \quad 1281.3 \quad 1283.7 \quad 1285.7 \quad 1290.2 \quad 1296.2 \quad 1300.2$ $1300.8 \quad 1309.8 \quad 1320.2 \quad 1330.9 \quad 1349.4 \quad 1362.0 \quad 1370.5 \quad 1381.8 \quad 1388.5 \quad 1405.8 \quad 1407.9 \quad 1834.6$

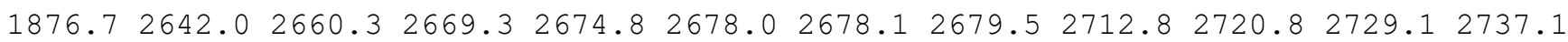
$2763.2 \quad 2781.3 \quad 2783.7 \quad 2797.0 \quad 2855.5$

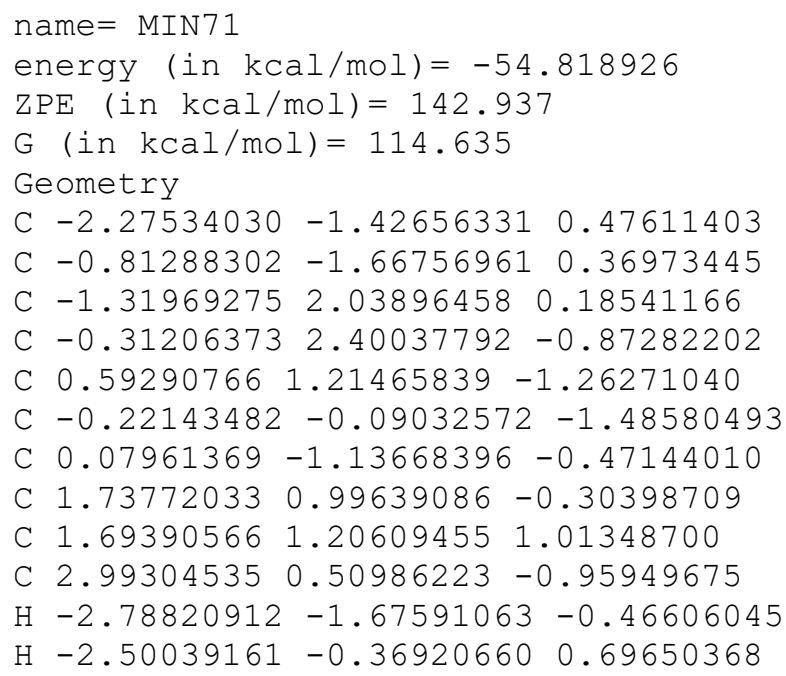


$\mathrm{H}-2.74871718-2.02696392 \quad 1.26908472$

$\mathrm{H}-0.96734494 \quad 1.94132793 \quad 1.22027580$

$\mathrm{H} \quad 0.31264964 \quad 3.24982641-0.53143981$

$\mathrm{H}-0.84397017 \quad 2.76107207-1.77985474$

$\mathrm{H} \quad 1.03829274 \quad 1.49268080 \quad-2.25856834$

$\mathrm{H} 0.01031179-0.49167263-2.49630882$

$\mathrm{H}-1.306209330 .14280151-1.52393113$

$\mathrm{H} \quad 1.11760451-1.47159834-0.45504054$

$\mathrm{H} 1.22088225-2.259587982 .00186757$

$\mathrm{H} \quad 0.83105907 \quad 1.54929021 \quad 1.55368572$

$\mathrm{H} 2.534516661 .03732785 \quad 1.66666077$

$\mathrm{H} 2.78988660-0.27178328-1.70537683$

$\mathrm{H} 3.70484937 \quad 0.08446406-0.23921567$

H $3.509885561 .33135906-1.47538196$

○ $0.88942331-2.89831858 \quad 1.32245061$

$\begin{array}{lllll}0 & -2.48417641 & 1.86964506 & -0.08287912\end{array}$

O $-0.50125498-2.59575407 \quad 1.33872738$

Vibrational frequencies (in $\mathrm{cm}-1$ )

$\begin{array}{llllllllllllllll}-17.6 & 49.8 & 63.4 & 70.2 & 72.2 & 98.9 & 117.6 & 128.8 & 148.3 & 173.7 & 213.1 & 244.5 & 250.3 & 287.6\end{array}$

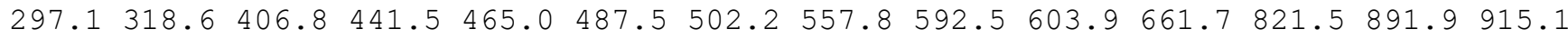

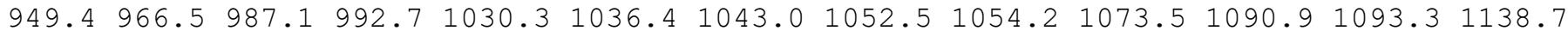

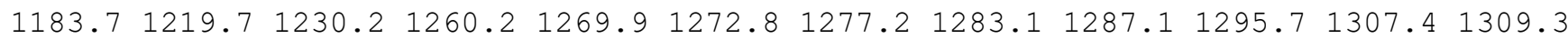
$1316.81332 .3 \quad 1347.2 \quad 1368.2 \quad 1385.2 \quad 1387.5 \quad 1397.9 \quad 1404.51409 .8 \quad 1835.5 \quad 1867.4 \quad 1899.0$ $2636.92646 .52655 .92664 .5 \quad 2665.92670 .7 \quad 2672.42681 .32720 .42730 .82739 .62751 .6$ $2773.8 \quad 2778.7 \quad 2792.8 \quad 2866.1$

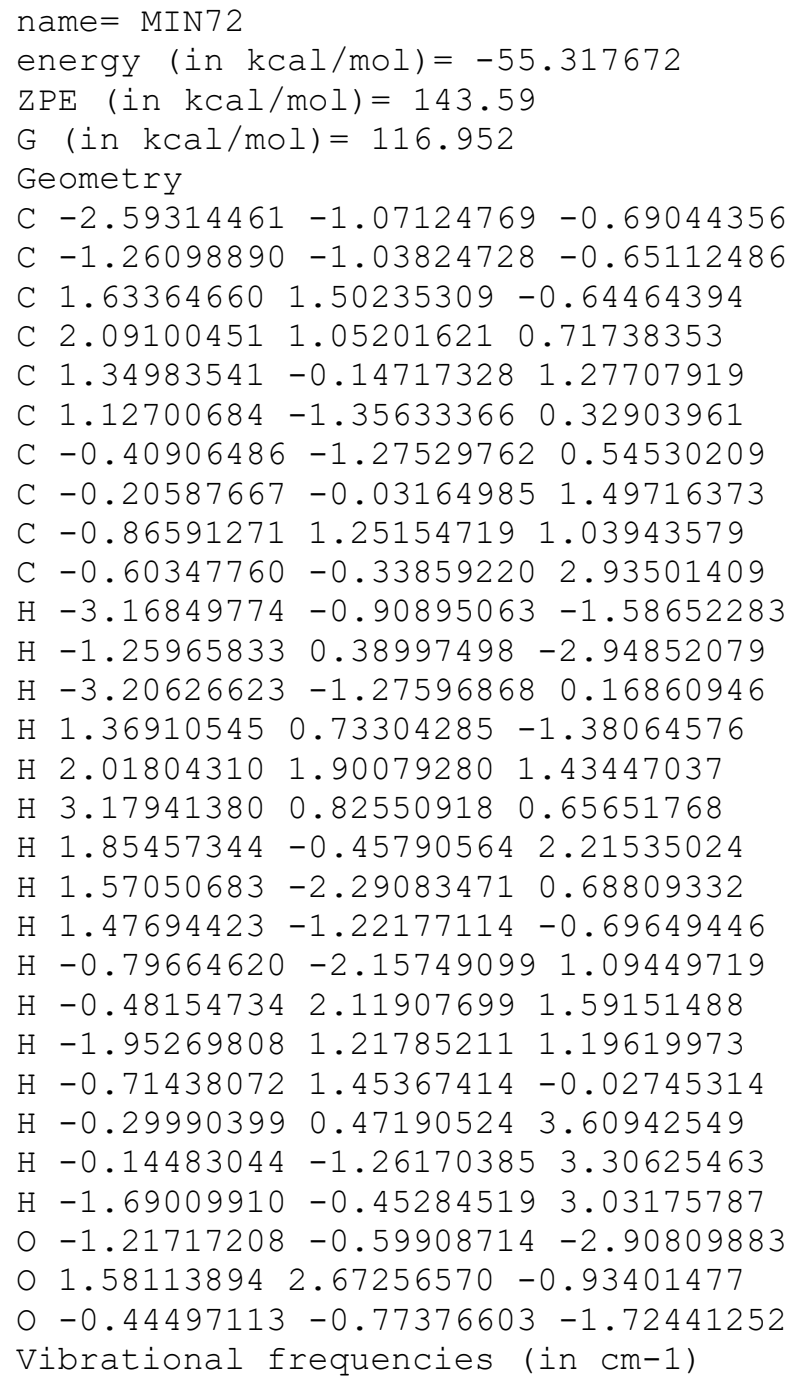


$1160.7 \quad 1189.8 \quad 1210.3 \quad 1237.2 \quad 1256.2 \quad 1271.4 \quad 1283.91286 .91290 .6 \quad 1296.6 \quad 1300.4 \quad 1301.4$ $1312.61322 .41333 .3 \quad 1348.3 \quad 1357.6 \quad 1365.5 \quad 1380.4 \quad 1387.1 \quad 1406.3 \quad 1408.91833 .7 \quad 1875.2$ $2644.92656 .72669 .8 \quad 2673.8 \quad 2677.3 \quad 2677.7 \quad 2678.2 \quad 2710.72717 .3 \quad 2729.4 \quad 2737.4 \quad 2762.9$ 2780.82782 .62798 .22864 .6

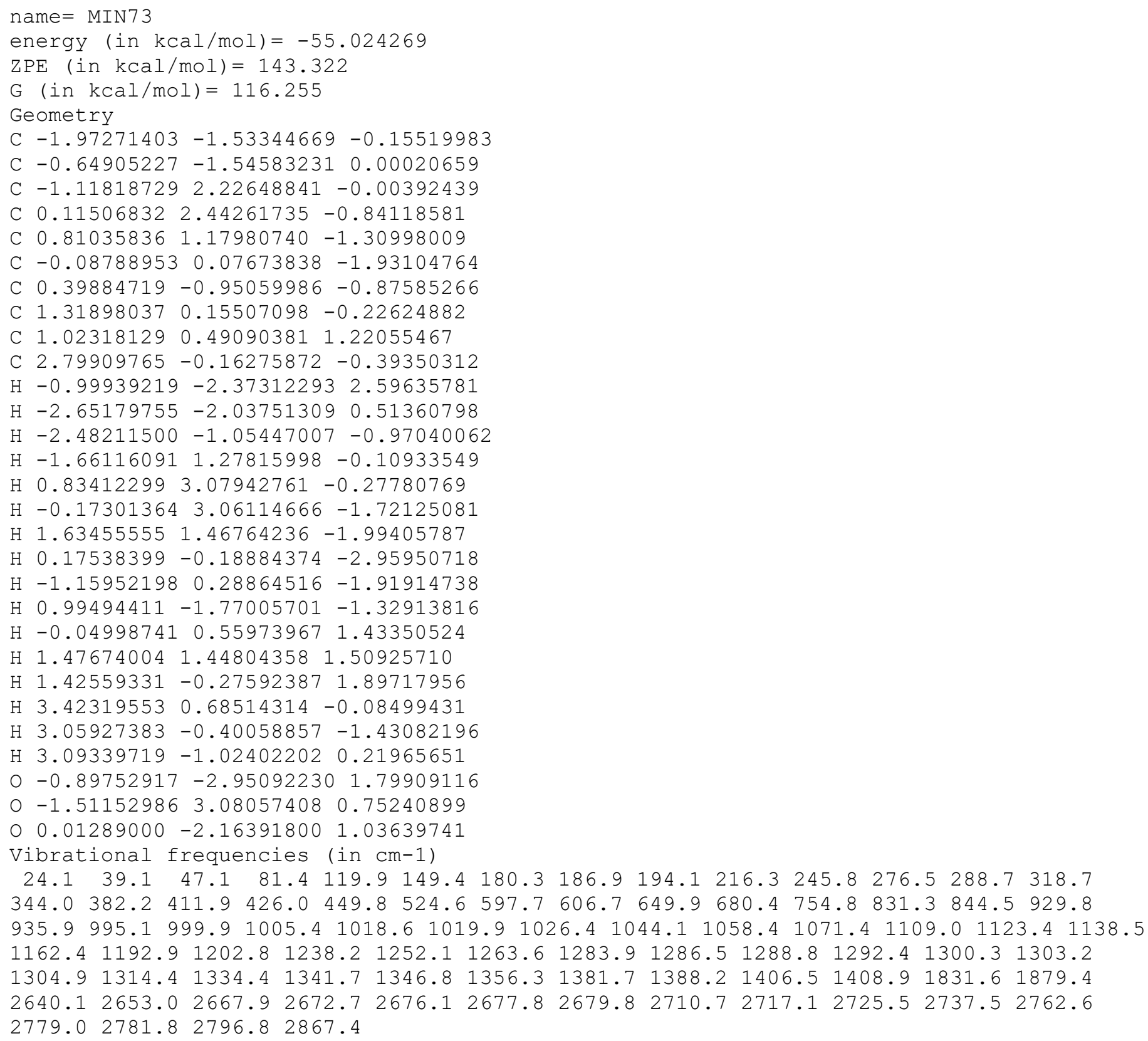




$\begin{array}{llll}\mathrm{H} & -3.41808608 & -0.05605343 & -1.34284137 \\ \mathrm{H} & -0.86343046 & 1.00390307 & -3.11194722 \\ \mathrm{H} & -3.40881147 & -1.09346144 & 0.12016623 \\ \mathrm{H} & 0.67118401 & 1.78038464 & -0.63223316 \\ \mathrm{H} & 2.10447294 & 1.47709447 & 1.48077895 \\ \mathrm{H} & 3.22603441 & 0.44997751 & 0.57454930 \\ \mathrm{H} & 1.96942175 & -1.07848652 & 1.83389985 \\ \mathrm{H} & 1.24037772 & -2.47805062 & 0.01280951 \\ \mathrm{H} & 1.15195261 & -1.10890624 & -1.07727747 \\ \mathrm{H} & -1.06059843 & -2.20129427 & 0.68715763 \\ \mathrm{H} & -1.74948579 & 1.02003314 & 1.92793986 \\ \mathrm{H} & -0.66544516 & 1.54439247 & 0.65288175 \\ \mathrm{H} & -0.15553854 & 1.66358876 & 2.34716402 \\ \mathrm{H} & 0.04384931 & -2.08674130 & 2.91281915 \\ \mathrm{H} & -1.42097379 & -1.12886146 & 3.14265777 \\ \mathrm{H} & 0.13130610 & -0.52207166 & 3.72841375 \\ \mathrm{O} & -1.53827533 & 0.84565674 & -2.40454393 \\ \mathrm{O} & 2.35346226 & 1.30825101 & -1.61339671 \\ \mathrm{O} & -0.69647217 & 0.06830570 & -1.53876515 \\ \text { Vibrational } & \text { frequencies } & \text { (in } & \mathrm{cm}-1)\end{array}$

$\begin{array}{llllllllllllllllll}-389.1 & 16.8 & 51.2 & 64.9 & 100.9 & 157.2 & 168.3 & 177.0 & 181.4 & 206.8 & 226.5 & 246.8 & 282.0 & 323.9\end{array}$ $337.5 \quad 380.1 \quad 413.7 \quad 433.2 \quad 474.9 \quad 543.1 \quad 569.6 \quad 604.6 \quad 622.9686 .7 \quad 801.8 \quad 840.7 \quad 880.9 \quad 918.6$ $955.4985 .8 \quad 991.0 \quad 993.8 \quad 1008.6 \quad 1017.2 \quad 1036.8 \quad 1047.3 \quad 1064.1 \quad 1076.4 \quad 1100.7 \quad 1122.2 \quad 1123.0$ $1145.0 \quad 1190.2 \quad 1206.3 \quad 1230.7 \quad 1248.1 \quad 1277.1 \quad 1283.7 \quad 1287.2 \quad 1288.8 \quad 1296.6 \quad 1298.8 \quad 1301.4$

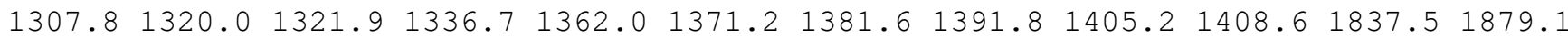
$2628.42659 .8 \quad 2664.8 \quad 2675.3 \quad 2675.6 \quad 2677.0 \quad 2679.72712 .72719 .5 \quad 2729.5 \quad 2739.62761 .1$ $2777.6 \quad 2782.12797 .92815 .4$

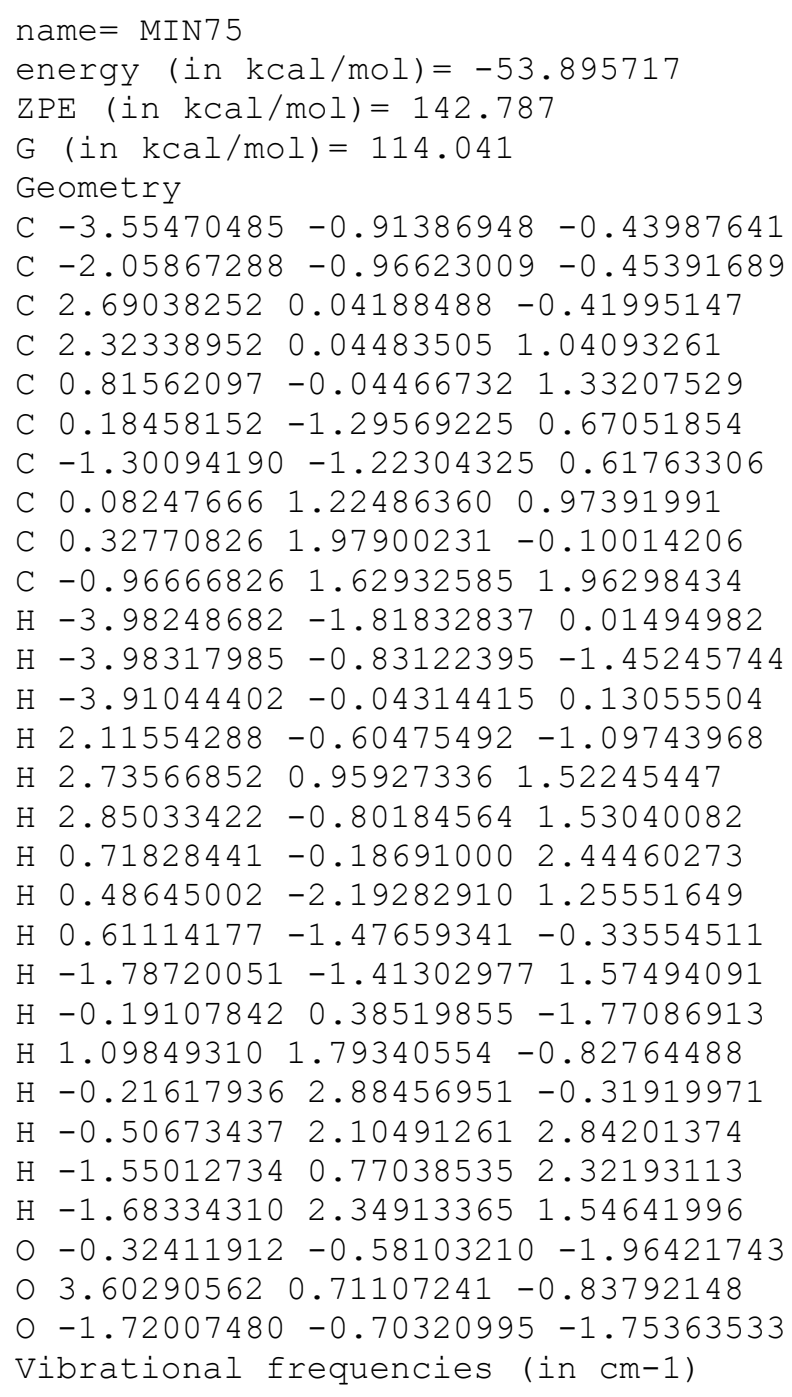


$\begin{array}{lllllllllllllllll}13.9 & 39.3 & 49.7 & 65.4 & 72.1 & 87.1 & 108.4 & 124.1 & 125.2 & 203.0 & 207.9 & 269.1 & 291.1 & 307.2\end{array}$ $318.7 \quad 334.3 \quad 343.3 \quad 420.5 \quad 446.8 \quad 485.7 \quad 524.0 \quad 572.7 \quad 613.9630 .5 \quad 654.3 \quad 792.7 \quad 837.6 \quad 899.6$ $933.2986 .7992 .1996 .1 \quad 1028.1 \quad 1039.2 \quad 1049.9 \quad 1054.6 \quad 1059.51080 .1 \quad 1093.4 \quad 1106.7 \quad 1166.8$ $\begin{array}{llllllllllll}1182.7 & 1206.0 & 1237.0 & 1254.9 & 1267.8 & 1271.5 & 1275.5 & 1275.8 & 1280.7 & 1282.9 & 1291.6 & 1298.2\end{array}$

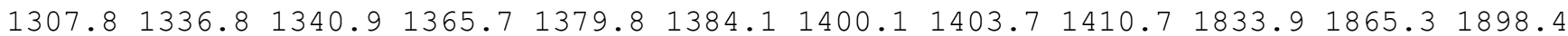
$\begin{array}{lllllllllllll}2629.9 & 2644.0 & 2656.7 & 2662.4 & 2669.1 & 2669.6 & 2672.8 & 2684.0 & 2718.2 & 2730.8 & 2737.4 & 2758.1\end{array}$ $2774.72778 .2 \quad 2791.6 \quad 2827.2$

name $=$ MIN76

energy (in $\mathrm{kcal} / \mathrm{mol})=-54.24336$

$\operatorname{ZPE}($ in $\mathrm{kcal} / \mathrm{mol})=143.259$

$\mathrm{G}(\mathrm{in} \mathrm{kcal} / \mathrm{mol})=115.9$

Geometry

C $-2.29803724-1.20860774-0.66168238$

C $-0.96656891-1.14991322-0.65666072$

C $0.923337851 .88602240 \quad-0.67771945$

C $1.461898291 .72026390 \quad 0.71925017$

C $1.26820150 \quad 0.33164737 \quad 1.30179616$

C $1.41920306-0.84334148 \quad 0.29812127$

C $-0.06280576-1.258518420 .52214625$

C $-0.19662589-0.14391508 \quad 1.63270820$

C $-1.30571641 \quad 0.87263351 \quad 1.46743445$

C $-0.27733172 \quad-0.76735611 \quad 3.02354713$

$\mathrm{H}-2.89126145-1.32164238 \quad 0.22715566$

$\mathrm{H}-2.89173873-1.15811054-1.55994849$

$\mathrm{H}-0.71021744-0.14265658-3.32332057$

$\mathrm{H} \quad 0.03698107 \quad 1.30449008-0.96175263$

$\mathrm{H} \quad 0.987464132 .48225526 \quad 1.37582615$

H 2.54442043 $1.98125526 \quad 0.72530796$

H $1.93286199 \quad 0.22060919 \quad 2.18074621$

H $2.13688789-1.60753242 \quad 0.61496073$

$\mathrm{H} \quad 1.70072574-0.55618537-0.71750132$

$\mathrm{H}-0.15592730-2.27978467 \quad 0.94496656$

$\mathrm{H}-1.393159321 .249141820 .44225280$

$\mathrm{H}-1.15618693 \quad 1.735073112 .12871078$

$\mathrm{H}-2.28304194 \quad 0.43776475 \quad 1.71916386$

H $0.52283639-1.495737123 .19840301$

$\mathrm{H}-1.22993362-1.290067413 .17054896$

$\mathrm{H}-0.19948291-0.003514693 .80639003$

o $-1.00103544-1.00980229-2.94391725$

o $1.439019192 .64223349-1.46353399$

o $-0.17862726-1.01923164-1.77529283$

Vibrational frequencies (in cm-1)

$\begin{array}{llllllllllllllll}16.6 & 34.7 & 54.7 & 95.9 & 115.7 & 129.1 & 156.7 & 182.0 & 198.1 & 207.1 & 233.5 & 240.0 & 286.5 & 328.8\end{array}$

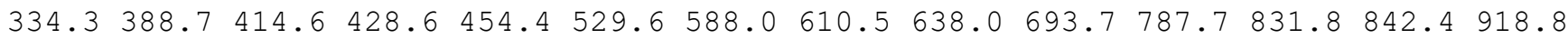
$938.6 \quad 988.1 \quad 999.1 \quad 1005.4 \quad 1014.0 \quad 1017.6 \quad 1035.0 \quad 1050.1 \quad 1067.9 \quad 1073.9 \quad 1098.7 \quad 1119.91125 .5$ $1151.7 \quad 1194.8 \quad 1208.4 \quad 1235.2 \quad 1243.7 \quad 1270.2 \quad 1286.0 \quad 1288.3 \quad 1294.5 \quad 1296.5 \quad 1299.3 \quad 1304.0$ $1306.51318 .0 \quad 1328.1 \quad 1342.6 \quad 1358.2 \quad 1361.8 \quad 1379.6 \quad 1385.7 \quad 1405.0 \quad 1407.91834 .81878 .1$ $2642.62654 .4 \quad 2668.4 \quad 2674.92676 .5 \quad 2677.5 \quad 2678.8 \quad 2711.4 \quad 2717.92728 .8 \quad 2737.92762 .2$ $2780.0 \quad 2782.2 \quad 2797.4 \quad 2866.8$

name $=$ MIN77

energy $($ in $\mathrm{kcal} / \mathrm{mol})=-53.812637$

$\operatorname{ZPE}($ in $\mathrm{kcal} / \mathrm{mol})=143.664$

$\mathrm{G}($ in $\mathrm{kcal} / \mathrm{mol})=116.745$

Geometry

C $-3.15958159-0.35707553-0.00341112$

C $-1.83274696-0.34224505 \quad 0.13910285$

C $1.54148018 \quad 1.824651730 .19916880$

C $2.11290506 \quad 0.91794579-0.86012373$

C $1.16558436-0.11173933-1.44090562$

C $-0.28158484 \quad 0.34856448-1.75650451$

C $-0.86100095-0.80442893-0.88836976$ 
$\begin{array}{lllll}\text { C } & 0.60201302 & -1.24710107 & -0.50248327\end{array}$

C $0.99386430-1.186542350 .95822012$

C $0.91538959-2.63940645-1.04492341$

$\mathrm{H}-3.843728490 .03131084 \quad 0.73238473$

$\mathrm{H}-2.202884130 .094242292 .81055235$

$\mathrm{H}-3.66068601-0.73700338-0.87479954$

$\mathrm{H} \quad 0.45356927 \quad 1.85228738 \quad 0.34166281$

$\mathrm{H} \quad 3.01313608 \quad 0.40736903-0.44872264$

H $2.512417171 .56152035-1.67773000$

$\mathrm{H} 1.64818518-0.55815362-2.33563052$

$\mathrm{H}-0.55394412 \quad 0.29475322-2.81360395$

$\mathrm{H}-0.54206598 \quad 1.35145472-1.40643455$

$\mathrm{H}-1.35075333-1.58662444-1.50698786$

H 2.03259610 -1.513759861 .10535145$

$\mathrm{H} \quad 0.35775404 \quad-1.84215471 \quad 1.56735337$

$\mathrm{H} \quad 0.93256970-0.18051346 \quad 1.39169448$

$\mathrm{H} 0.64560691-2.74610826-2.10096099$

$\mathrm{H} 0.37046020-3.41156188-0.48849678$

H $1.98476247-2.86703659-0.95798919$

$\begin{array}{lllll}0 & -2.08057548 & 0.79026001 & 2.11833661\end{array}$

$\begin{array}{llll}0 & 2.27036954 & 2.51561065 & 0.86871806\end{array}$

o $-1.17133108 \quad 0.11874841 \quad 1.24755857$

Vibrational frequencies (in cm-1)

$\begin{array}{lllllllllllllll}19.2 & 35.2 & 47.7 & 96.8 & 157.7 & 165.1 & 178.0 & 203.5 & 213.7 & 224.2 & 230.8 & 269.9 & 307.0 & 326.4\end{array}$ $336.8 \quad 381.2 \quad 386.1 \quad 422.4 \quad 490.7 \quad 578.5 \quad 610.7 \quad 624.2 \quad 628.6 \quad 675.3 \quad 744.7 \quad 816.1 \quad 836.9 \quad 936.6$ $943.0 \quad 993.6 \quad 1007.9 \quad 1009.2 \quad 1019.9 \quad 1022.8 \quad 1032.5 \quad 1048.9 \quad 1057.6 \quad 1058.4 \quad 1096.7 \quad 1124.0$ $\begin{array}{lllllllllllllll}1132.3 & 1163.9 & 1199.9 & 1208.1 & 1243.2 & 1243.8 & 1258.0 & 1282.7 & 1286.6 & 1291.6 & 1298.7 & 1301.6\end{array}$

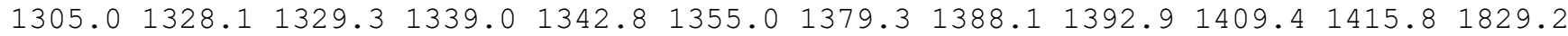
$1866.32640 .52650 .5 \quad 2662.0 \quad 2673.0 \quad 2677.3 \quad 2678.22681 .22709 .12715 .92729 .32736 .6$ $2764.02778 .0 \quad 2782.3 \quad 2799.0 \quad 2867.9$

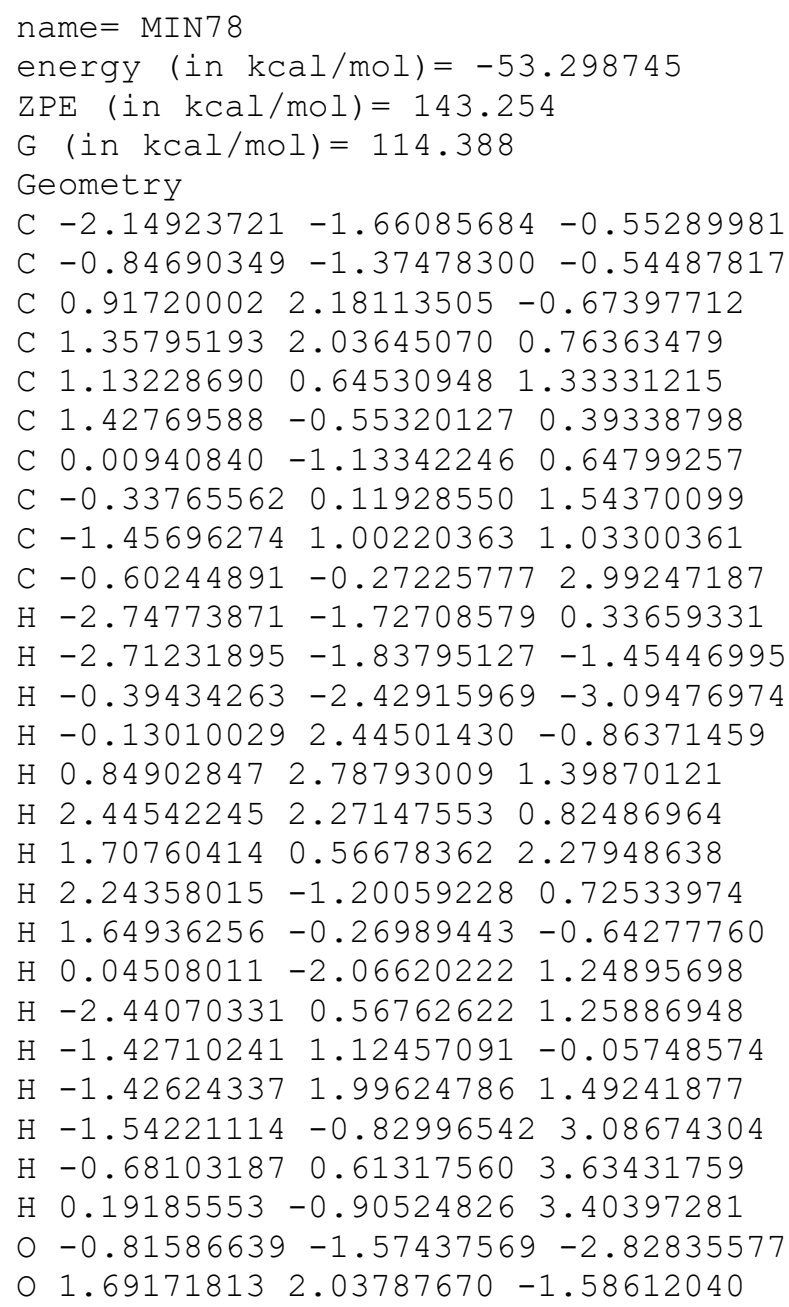


$0-0.04760034-1.29634348-1.65822323$

Vibrational frequencies (in $\mathrm{cm}-1$ )

$\begin{array}{lllllllllllllllll}-3.7 & 23.2 & 35.3 & 85.9 & 93.4 & 125.6 & 154.3 & 182.2 & 193.4 & 209.0 & 228.7 & 257.4 & 274.5 & 322.1\end{array}$ $333.4 \quad 375.6 \quad 414.6 \quad 449.0 \quad 485.7 \quad 510.3 \quad 560.2 \quad 608.4 \quad 644.0 \quad 679.1 \quad 794.7 \quad 835.2 \quad 896.1 \quad 928.7$

$964.5974 .6 \quad 991.8 \quad 1006.2 \quad 1010.8 \quad 1017.4 \quad 1026.5 \quad 1046.8 \quad 1067.9 \quad 1073.6 \quad 1088.7 \quad 1122.0 \quad 1137.4$ $1153.91193 .8 \quad 1206.91231 .1 \quad 1253.8 \quad 1282.1 \quad 1286.1 \quad 1288.3 \quad 1298.01300 .01301 .7 \quad 1306.6$ $1314.21320 .4 \quad 1333.2 \quad 1341.8 \quad 1350.6 \quad 1361.3 \quad 1380.6 \quad 1386.51405 .2 \quad 1409.41840 .11875 .6$ $2642.82663 .2 \quad 2667.0 \quad 2671.4 \quad 2676.1 \quad 2677.0 \quad 2680.5 \quad 2708.52714 .3 \quad 2730.5 \quad 2739.72758 .9$ $2778.72782 .12798 .6 \quad 2868.8$

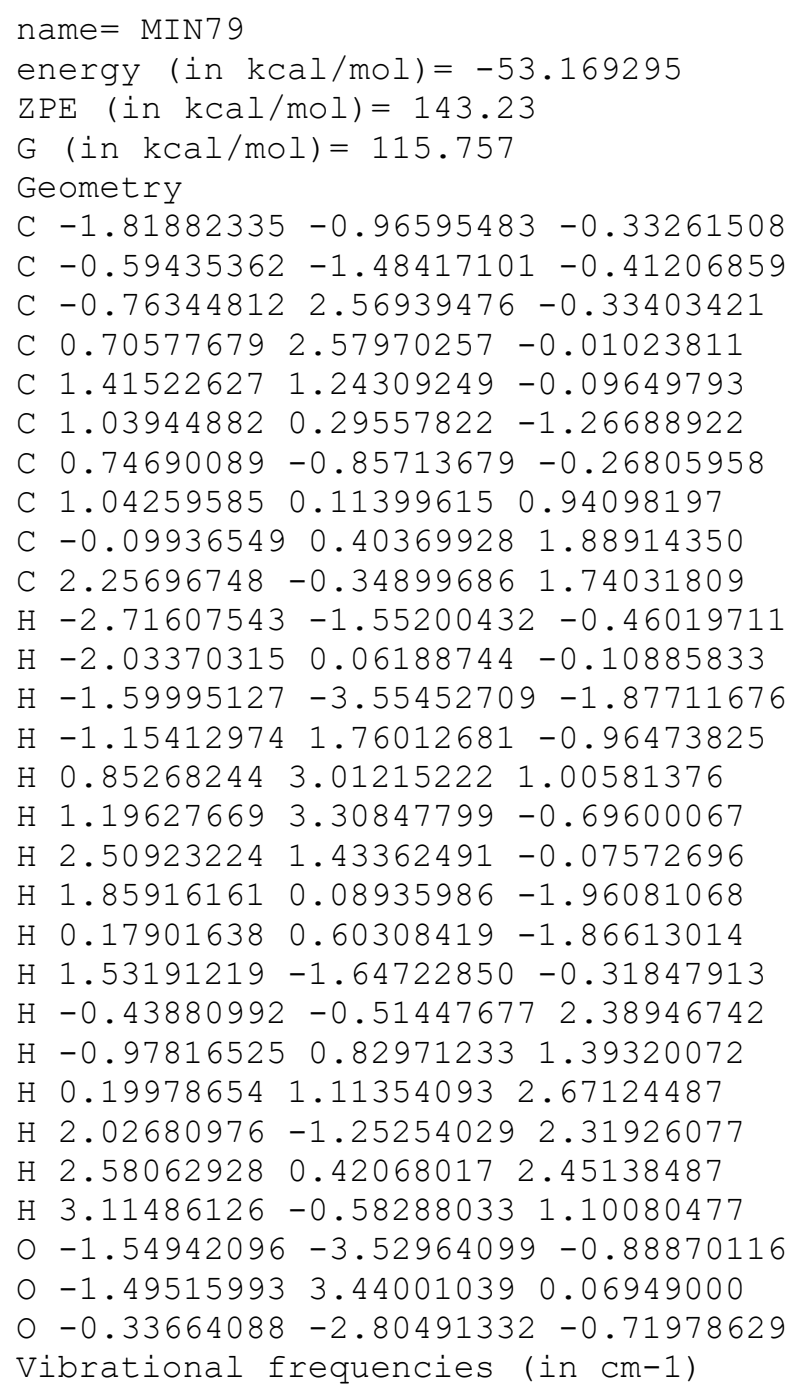


C $-0.00277390-0.15517135-1.71726372$

C $-0.70616180-1.04627403-0.65335924$

C $0.70278528-1.47559865-0.09422832$

C $0.93864075-1.30992467 \quad 1.39085919$

C $1.01877737-2.91835257-0.48900189$

$\mathrm{H}-3.52334676-0.96995195-0.39271871$

$\mathrm{H}-3.55188505 \quad 0.33227376 \quad 0.84972081$

$\mathrm{H}-1.10435498 \quad 2.38623512 \quad 0.68988287$

H $1.38839946 \quad 1.69717831 \quad 1.34828129$

H $2.98755701 \quad 0.37864943 \quad 0.08587190$

H $2.59518051 \quad 1.29372651-1.38538258$

$\mathrm{H} \quad 2.05280582-0.98857056-1.79822659$

$\mathrm{H}-0.12029819-0.51156994-2.74344350$

$\mathrm{H}-0.30196557 \quad 0.89960910-1.70538201$

$\mathrm{H}-1.25498936-1.90151620-1.09034932$

H $0.14391373-1.807964501 .96595018$

$\mathrm{H} \quad 0.95802248-0.26955650 \quad 1.72849535$

$\mathrm{H} 1.89385430-1.75417208 \quad 1.69864901$

$\mathrm{H} \quad 0.40673765-3.62678773 \quad 0.08232279$

H $2.06976027-3.16262510-0.29661745$

$\mathrm{H} \quad 0.82908602 \quad-3.10785144 \quad-1.55140277$

$\begin{array}{lllll}0 & -1.62030260 & 1.77434208 & 1.27885608\end{array}$

$00.51301167 \quad 2.46452323-0.27634450$

o $-0.882532010 .57149503 \quad 1.04637478$

Vibrational frequencies (in $\mathrm{cm}-1$ )

$\begin{array}{lllllllllllllll}48.4 & 95.9 & 130.4 & 142.6 & 154.4 & 190.0 & 195.6 & 219.6 & 240.5 & 263.0 & 287.4 & 307.4 & 317.4 & 334.0\end{array}$

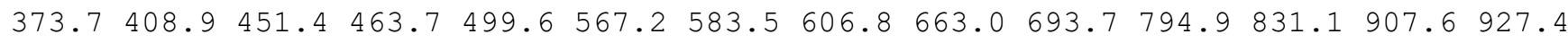
$944.6989 .2 \quad 1000.1 \quad 1009.2 \quad 1012.7 \quad 1019.8 \quad 1022.7 \quad 1050.6 \quad 1057.3 \quad 1071.7 \quad 1086.6 \quad 1106.8$

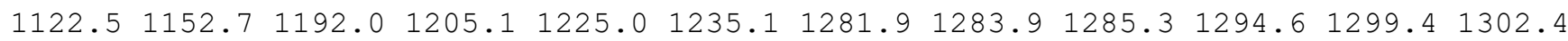

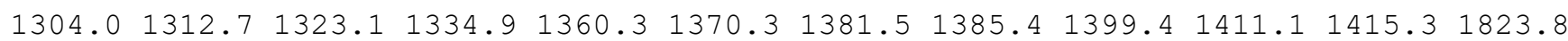
$1863.12638 .3 \quad 2663.3 \quad 2667.92673 .12675 .8 \quad 2676.82678 .72713 .12721 .52727 .52737 .6$ $2761.22780 .2 \quad 2782.2 \quad 2792.02800 .1$

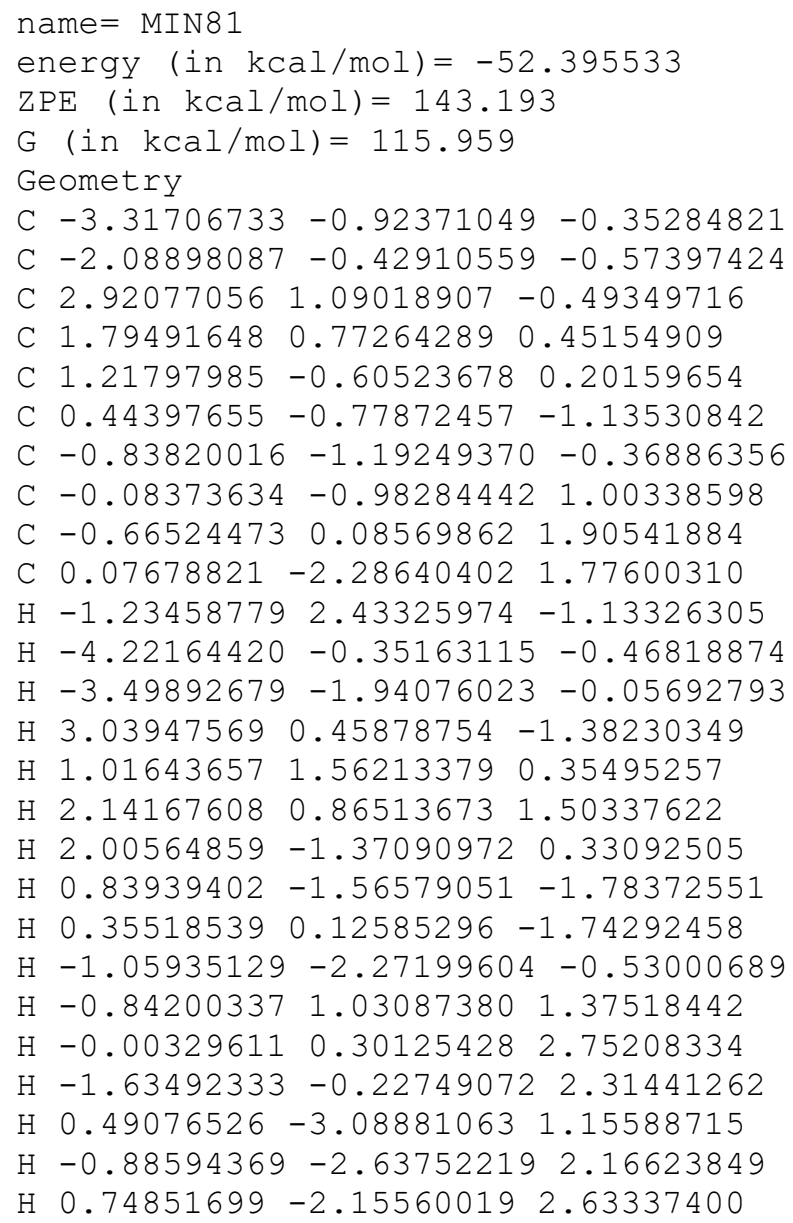


$0-0.931819621 .54766695-0.81366454$

$03.666960602 .01674881-0.29213683$

O $-2.13137427 \quad 0.82598449-1.13138713$

Vibrational frequencies (in $\mathrm{cm}-1$ )

$\begin{array}{lllllllllllllll}25.6 & 43.0 & 65.1 & 77.2 & 113.6 & 130.9 & 151.0 & 160.3 & 173.5 & 206.6 & 212.3 & 219.5 & 288.1 & 329.4\end{array}$

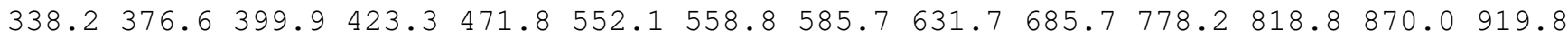
$938.6955 .2977 .8 \quad 995.8 \quad 1008.2 \quad 1016.1 \quad 1043.5 \quad 1067.8 \quad 1071.91086 .1 \quad 1116.2 \quad 1125.91137 .8$ $1169.01184 .21211 .0 \quad 1229.3 \quad 1256.1 \quad 1263.7 \quad 1279.2 \quad 1287.3 \quad 1295.2 \quad 1300.2 \quad 1302.91305 .8$ $1308.21323 .71341 .1 \quad 1353.6 \quad 1361.5 \quad 1374.8 \quad 1384.1 \quad 1405.91407 .3 \quad 1447.31814 .61834 .6$ $2648.82654 .62668 .0 \quad 2675.62678 .12679 .7 \quad 2680.32708 .02720 .2 \quad 2729.4 \quad 2742.5 \quad 2762.9$ $2780.5 \quad 2782.5 \quad 2796.2 \quad 2869.0$

name $=$ MIN82

energy $($ in $\mathrm{kcal} / \mathrm{mol})=-53.407182$

$\mathrm{ZPE}(\mathrm{in} \mathrm{kcal} / \mathrm{mol})=144.429$

$\mathrm{G}($ in $\mathrm{kcal} / \mathrm{mol})=118.292$

Geometry

C $-2.79860871-1.43926202-0.80623093$

C $-1.29992063-1.37149097-0.81841567$

C $1.36906844 \quad 1.13744234-0.91269006$

C 1.790786690 .881587890 .51410246

C $1.06927164 \quad-0.26073747 \quad 1.20753347$

C $0.93926584-1.58792098 \quad 0.41348188$

C $-0.60543299-1.52261531 \quad 0.50439185$

C $-0.49923303-0.19777086 \quad 1.35099411$

C $-1.19498676 \quad 1.01756420 \quad 0.77486321$

C $-0.94146152-0.413233512 .79397240$

$\mathrm{H}-3.22663351-0.88639087-1.65724602$

$\mathrm{H}-3.23113755-1.030499960 .11413641$

$\mathrm{H}-3.13803456-2.48010921-0.90423305$

H $1.05854975 \quad 0.27969116-1.52799920$

$\mathrm{H} \quad 1.66584556 \quad 1.81388908 \quad 1.10930857$

$\mathrm{H} \quad 2.88570154 \quad 0.67840862 \quad 0.52298265$

H $1.54590568-0.420526922 .19590616$

H $1.38122859-2.45453920 \quad 0.91192928$

$\mathrm{H} \quad 1.33414607-1.56507617-0.60915672$

$\mathrm{H}-1.04542878-2.35948750 \quad 1.07253515$

$\mathrm{H}-0.76702046 \quad 1.94666034 \quad 1.17621988$

$\mathrm{H}-2.26489961 \quad 1.02697145 \quad 1.01499435$

$\mathrm{H}-1.116012631 .07976576-0.31871216$

$\mathrm{H}-0.70796047 \quad 0.46257122 \quad 3.41208475$

$\mathrm{H}-0.44880605-1.27764494 \quad 3.25336285$

$\mathrm{H}-2.02236533-0.581640192 .86160302$

○ $0.69574275 \quad 2.34211039-1.15736632$

$\begin{array}{lllll}0 & 2.07987610 & 2.14586617 & -1.57839512\end{array}$

o $-0.68934041-1.22014980-1.84840636$

Vibrational frequencies (in $\mathrm{cm}-1$ )

$\begin{array}{lllllllllllllll}41.3 & 55.3 & 65.7 & 82.1 & 97.6 & 150.9 & 176.4 & 186.8 & 216.5 & 229.4 & 280.2 & 292.2 & 326.2 & 363.1\end{array}$ $\begin{array}{lllllllllllllllll}374.3 & 406.3 & 409.5 & 442.8 & 562.3 & 578.7 & 601.7 & 685.7 & 788.1 & 822.6 & 879.3 & 910.7 & 916.8 & 947.1\end{array}$ $985.0 \quad 999.1 \quad 1006.4 \quad 1017.1 \quad 1028.1 \quad 1039.3 \quad 1050.8 \quad 1074.4 \quad 1088.0 \quad 1098.2 \quad 1121.91127 .3$ $1169.41203 .3 \quad 1210.2 \quad 1235.1 \quad 1238.9 \quad 1258.1 \quad 1265.3 \quad 1268.01270 .8 \quad 1285.2 \quad 1288.01292 .1$

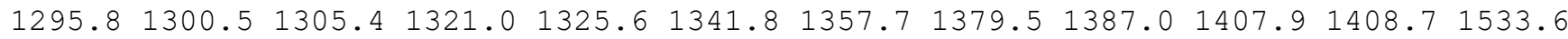
$1848.42651 .42661 .32663 .6 \quad 2666.8 \quad 2672.92675 .72677 .22677 .32679 .02713 .62728 .9$ $2736.82760 .5 \quad 2779.12780 .02782 .6$

name $=$ MIN83

energy (in $\mathrm{kcal} / \mathrm{mol})=-53.630075$

$\mathrm{ZPE}($ in $\mathrm{kcal} / \mathrm{mol})=144.671$

$\mathrm{G}($ in $\mathrm{kcal} / \mathrm{mol})=120.452$

Geometry

C $-2.33179859-0.50765090-0.67832946$

C $-0.80658494-0.35816951-0.78089521$

C $0.477990821 .75587302-0.71895706$ 
C $1.379491291 .58702586 \quad 0.25635124$

C $1.44757764 \quad 0.39515693 \quad 1.13376125$

C $1.47949921-0.88479671 \quad 0.24136053$

C $-0.06365920-0.97619658 \quad 0.38895777$

C $0.03683273-0.06724292 \quad 1.67412922$

C $-1.00544626 \quad 0.99892726 \quad 1.93189818$

C $0.18428586-0.924155812 .93173248$

$\mathrm{H}-2.65785784-1.48663638-1.05478690$

$\mathrm{H}-2.84068434 \quad 0.26251089-1.27538976$

$\mathrm{H}-2.67241065-0.40917304 \quad 0.35758590$

$\mathrm{H} \quad 0.42383114 \quad 2.62235528 \quad-1.38237155$

H $2.14921231 \quad 2.336896210 .42081139$

$\mathrm{H} \quad 0.472661890 .12004099-3.03962966$

H $2.22660051 \quad 0.46155753 \quad 1.90140316$

H $2.01333705-1.728881590 .68496486$

$\mathrm{H} 1.87086750-0.74493607-0.76759476$

$\mathrm{H}-0.44465210-1.990417320 .58139040$

$\mathrm{H}-1.94344518 \quad 0.56637526 \quad 2.29896285$

$\mathrm{H}-1.251380881 .59219356 \quad 1.04325609$

$\mathrm{H}-0.649604291 .70954648 \quad 2.68973289$

$\mathrm{H} \quad 0.47611778-0.31017661 \quad 3.79181727$

$\mathrm{H} \quad 0.94442464 \quad-1.70466248 \quad 2.81688031$

$\mathrm{H}-0.75920652-1.42075978 \quad 3.18469212$

$0.71746749-0.69205536-2.52594675$

$0-0.60705534 \quad 1.01903892-1.05867406$

O $-0.54751405-1.05156399-2.00554891$

Vibrational frequencies (in $\mathrm{cm}-1$ )

$\begin{array}{lllllllllllllll}55.2 & 115.2 & 125.0 & 160.1 & 170.2 & 190.1 & 207.9 & 227.4 & 249.5 & 288.4 & 314.2 & 328.2 & 347.2 & 364.2\end{array}$ $378.4410 .1 \quad 441.5 \quad 467.6 \quad 488.2 \quad 562.7 \quad 606.6 \quad 702.6 \quad 773.5 \quad 794.6 \quad 813.9 \quad 859.2 \quad 902.7 \quad 927.8$ $939.7 \quad 956.4 \quad 957.0 \quad 1005.1 \quad 1010.4 \quad 1016.5 \quad 1022.5 \quad 1034.1 \quad 1061.91090 .8 \quad 1096.8 \quad 1118.7 \quad 1140.4$ $\begin{array}{llllllllllll}1151.1 & 1190.1 & 1204.8 & 1226.9 & 1240.0 & 1264.2 & 1274.4 & 1278.8 & 1281.4 & 1289.2 & 1297.4 & 1301.4\end{array}$

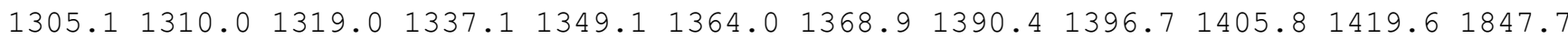
$2668.02669 .5 \quad 2672.6 \quad 2676.6 \quad 2677.3 \quad 2680.9 \quad 2687.2 \quad 2720.72731 .3 \quad 2744.2 \quad 2765.82767 .2$ $2776.02781 .5 \quad 2783.9 \quad 2861.1$

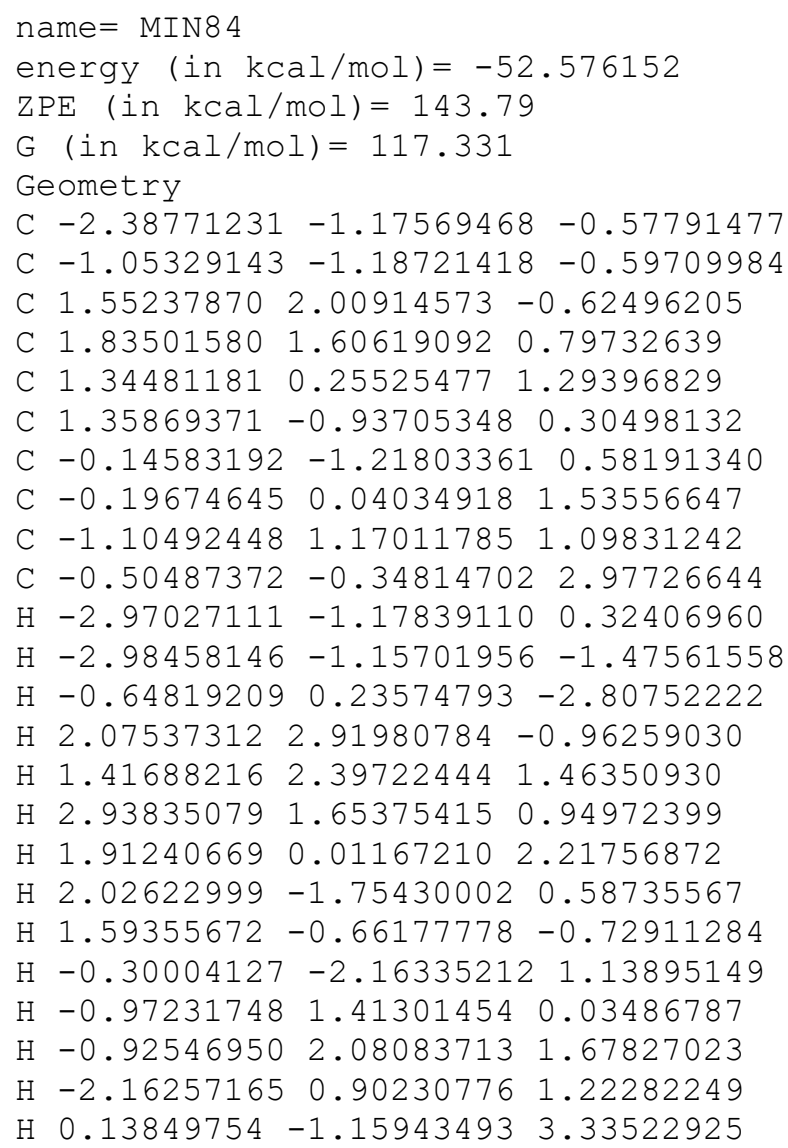


$\mathrm{H}-1.54326572-0.685988403 .08115400$

$\mathrm{H}-0.36800252 \quad 0.50269628 \quad 3.65454871$

$0-1.03327243-0.67904325-2.83022944$

$0.816807291 .43753650-1.38827438$

$0-0.29760722-1.24081700-1.74342489$

Vibrational frequencies (in $\mathrm{cm}-1$ )

$\begin{array}{llllllllllllllll}20.7 & 51.9 & 74.2 & 82.3 & 140.4 & 149.5 & 178.2 & 190.8 & 206.9 & 229.9 & 248.4 & 268.3 & 312.6 & 325.2\end{array}$ $365.7409 .1 \quad 428.1 \quad 443.3 \quad 471.0 \quad 555.6 \quad 603.2 \quad 628.9667 .5 \quad 697.0 \quad 755.3 \quad 820.4 \quad 840.7 \quad 918.5$ $937.7991 .1 \quad 1000.9 \quad 1003.0 \quad 1011.1 \quad 1016.2 \quad 1024.1 \quad 1036.3 \quad 1049.8 \quad 1075.5 \quad 1090.2 \quad 1119.5$

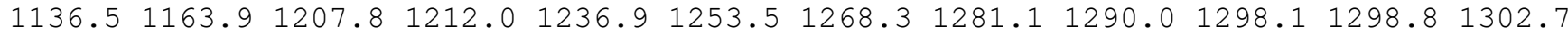

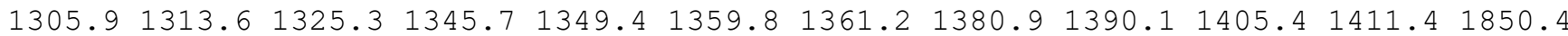
$\begin{array}{llllllllllll}1863.0 & 2639.0 & 2647.1 & 2668.1 & 2677.0 & 2678.1 & 2679.1 & 2682.1 & 2708.0 & 2717.5 & 2728.2 & 2732.0\end{array}$ $2761.72781 .12783 .0 \quad 2797.0 \quad 2853.0$

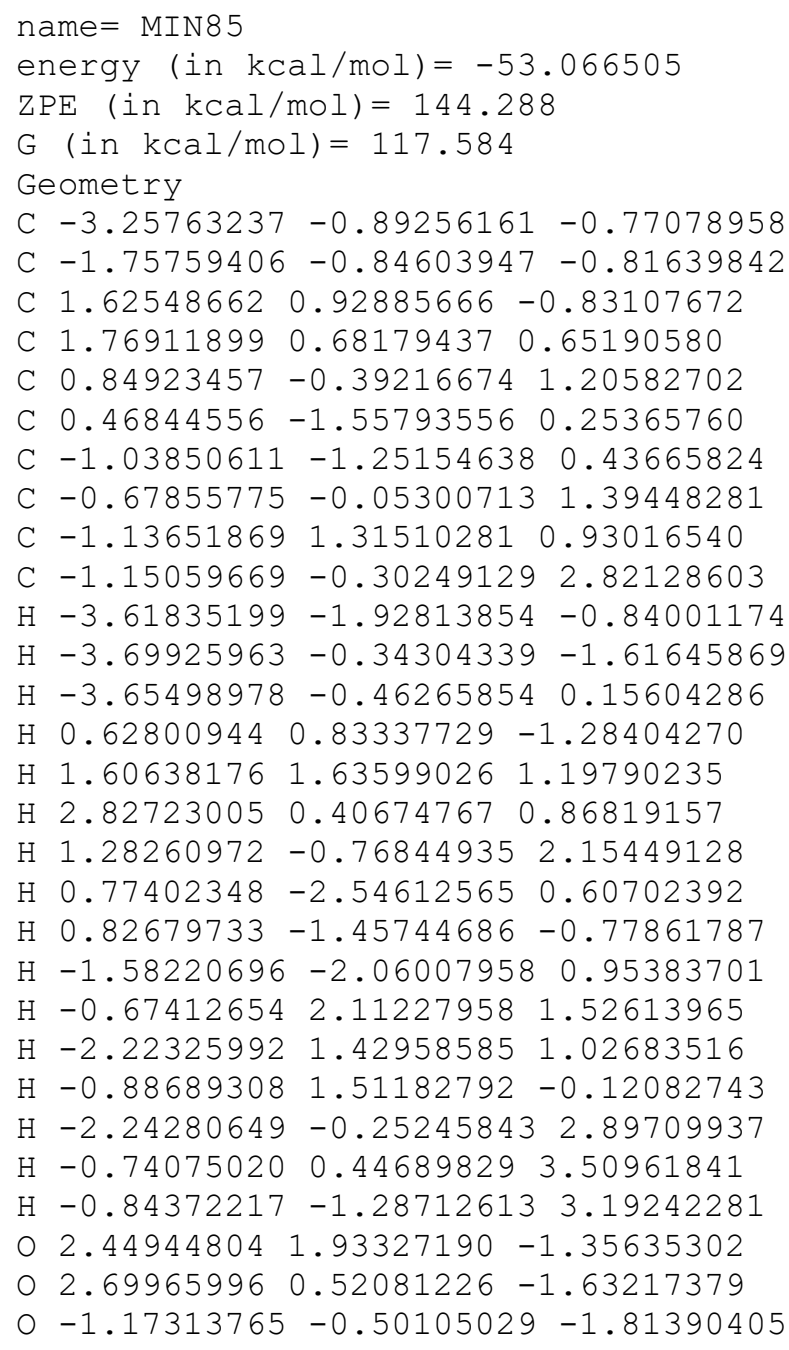


C $-2.00627623-0.44501451-0.84176101$

C $1.94959987 \quad 0.09147257-0.72227577$

C $1.92238227-0.21435709 \quad 0.75543547$

C $0.58292410-0.66668757 \quad 1.30467439$

C $-0.21111356-1.71739762 \quad 0.48359874$

C $-1.47194661-0.81841653 \quad 0.50928517$

C -0.650585360 .312401351 .24084097$

C $-0.46206287 \quad 1.590003450 .44906507$

C $-1.20946489 \quad 0.63208577 \quad 2.62084456$

$\mathrm{H}-3.71450176 \quad 0.40037804 \quad-1.86252670$

$\mathrm{H}-4.09735930-0.94736144-0.78503175$

$\mathrm{H}-3.72614615 \quad 0.64556609-0.10371521$

$\mathrm{H} \quad 1.12720212-0.28017581-1.35182823$

H $2.27166600 \quad 0.67792732 \quad 1.32291252$

H $2.68474493-0.99975284 \quad 0.96481386$

$\mathrm{H} 0.74007910-1.014425312 .34756757$

$\mathrm{H}-0.33190649-2.684932520 .97550513$

$\mathrm{H} \quad 0.17795551-1.90899543-0.52413030$

$\mathrm{H}-2.27277600 \quad-1.22860484 \quad 1.14807610$

$\mathrm{H} \quad 0.33456120 \quad 2.21133776 \quad 0.87880136$

$\mathrm{H}-1.37611765 \quad 2.19561997 \quad 0.43364431$

$\mathrm{H}-0.18959293 \quad 1.40477039-0.59950688$

$\mathrm{H}-0.52761218 \quad 1.28628149 \quad 3.17899795$

$\mathrm{H}-1.36521990-0.26814142 \quad 3.22539153$

$\mathrm{H}-2.17345873 \quad 1.14945153 \quad 2.55237052$

O $2.482582521 .33575303-1.08543924$

$03.221717620 .09683941-1.31157504$

o $-1.31968997-0.46610323-1.83371587$

Vibrational frequencies (in $\mathrm{cm}-1$ )

$\begin{array}{llllllllllllll}30.7 & 40.0 & 50.6 & 99.7 & 109.9 & 153.4 & 170.6 & 187.3 & 213.3 & 227.0 & 275.2 & 290.6 & 320.0 & 368.0\end{array}$ $371.2 \quad 391.5 \quad 398.1 \quad 445.7 \quad 572.7 \quad 590.8 \quad 603.2 \quad 673.8 \quad 785.5 \quad 818.6 \quad 884.9 \quad 900.6 \quad 920.9948 .6$ $985.61000 .5 \quad 1007.2 \quad 1017.7 \quad 1028.0 \quad 1040.8 \quad 1050.0 \quad 1074.8 \quad 1086.7 \quad 1098.1 \quad 1127.7 \quad 1136.3$ $1173.21205 .7 \quad 1220.1 \quad 1237.1 \quad 1240.2 \quad 1262.4 \quad 1266.6 \quad 1270.7 \quad 1273.7 \quad 1284.0 \quad 1287.6 \quad 1292.5$

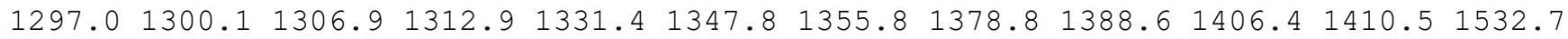
$1852.32650 .92657 .2 \quad 2662.2 \quad 2669.02674 .62674 .92677 .12677 .52679 .62711 .52729 .6$ $2735.7 \quad 2760.7 \quad 2779.8 \quad 2780.5 \quad 2783.0$

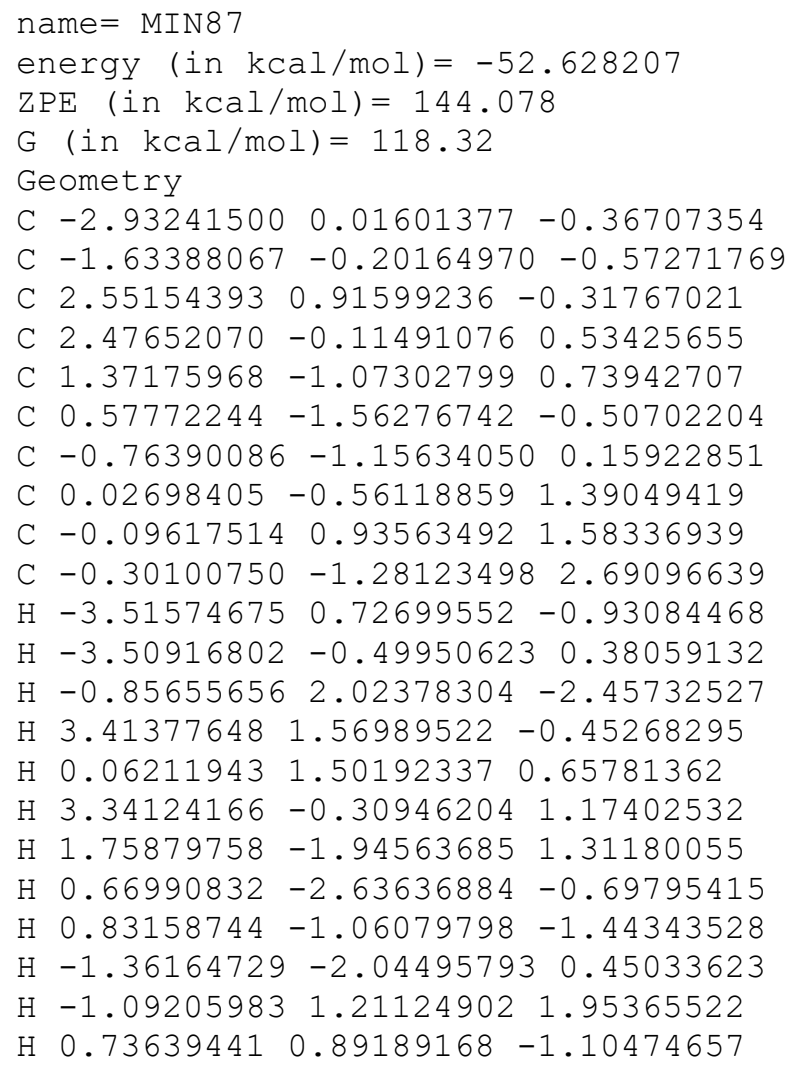


$\mathrm{H} \quad 0.64416076 \quad 1.30059432 \quad 2.30722377$

$\mathrm{H}-0.22609441-2.369778532 .59566386$

$\mathrm{H}-1.31880411-1.049612573 .02773115$

$\mathrm{H} \quad 0.38544551-0.975506593 .49086978$

$0-1.608772451 .57446791-1.99476885$

O $1.607705481 .35201041-1.17360021$

O $-0.88978894 \quad 0.40570479-1.56049068$

Vibrational frequencies (in cm-1)

$\begin{array}{llllllllllllllll}28.5 & 71.5 & 91.8 & 132.4 & 136.0 & 156.1 & 170.7 & 175.2 & 209.4 & 223.0 & 240.9 & 314.1 & 322.2 & 353.1\end{array}$ $\begin{array}{lllllllllllllllllllll}382.7 & 401.3 & 439.5 & 474.7 & 528.9 & 578.4 & 613.0 & 630.4 & 657.0 & 663.5 & 739.3 & 786.6 & 834.7 & 856.7\end{array}$ $924.2940 .8969 .8 \quad 994.2 \quad 1004.4 \quad 1007.7 \quad 1016.2 \quad 1030.3 \quad 1047.4 \quad 1052.0 \quad 1083.7 \quad 1102.7 \quad 1126.7$

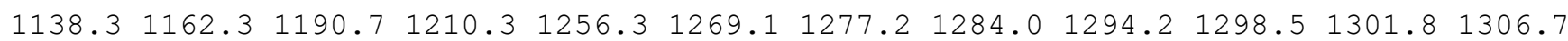

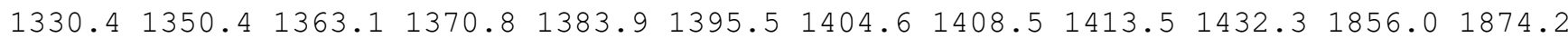
$2667.92675 .02675 .5 \quad 2678.12679 .5 \quad 2696.0 \quad 2709.52716 .82725 .92735 .2 \quad 2756.12762 .3$ $2780.8 \quad 2782.5 \quad 2794.5 \quad 2863.8$

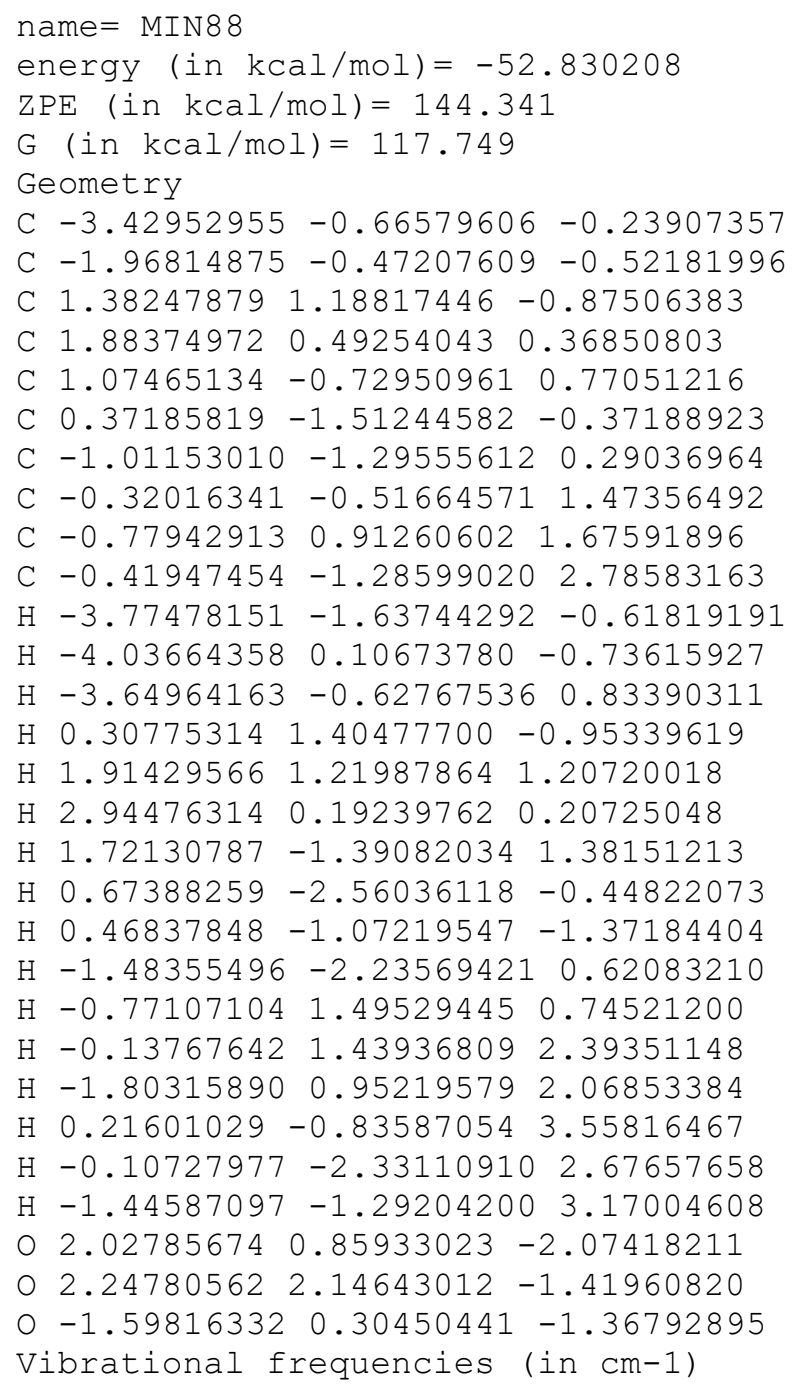




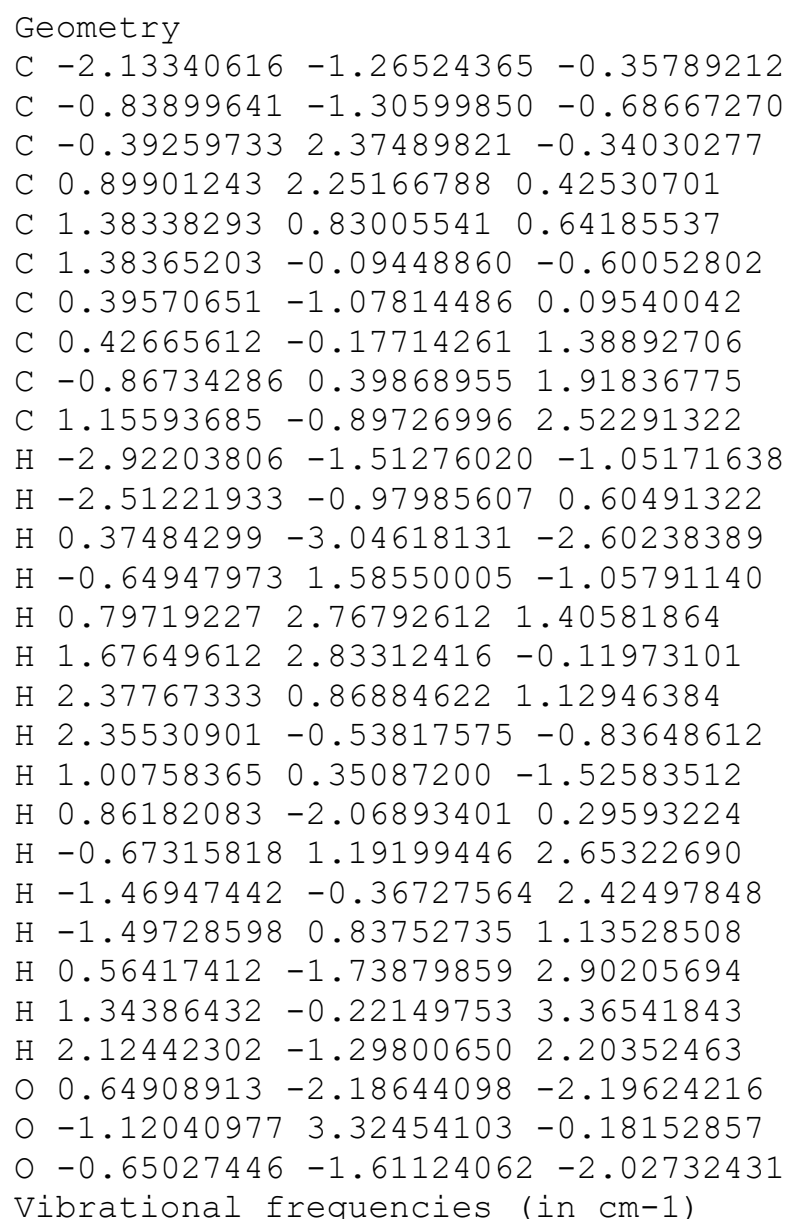

Vibrational frequencies (in $\mathrm{cm}-1$ )

$\begin{array}{llllllllllllllll}11.7 & 31.6 & 53.0 & 84.9 & 125.1 & 168.6 & 172.5 & 192.4 & 199.6 & 225.9 & 244.1 & 254.0 & 284.6 & 312.1\end{array}$

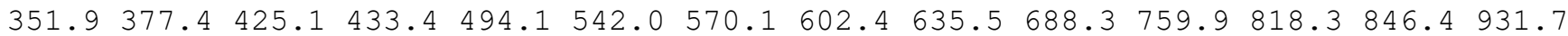
$939.9959 .0 \quad 988.0 \quad 1008.6 \quad 1017.3 \quad 1018.6 \quad 1043.3 \quad 1049.5 \quad 1055.8 \quad 1060.5 \quad 1099.5 \quad 1122.1 \quad 1128.9$ $1165.81190 .5 \quad 1212.3 \quad 1236.8 \quad 1241.9 \quad 1267.2 \quad 1286.2 \quad 1287.5 \quad 1291.7 \quad 1296.4 \quad 1300.8 \quad 1304.0$

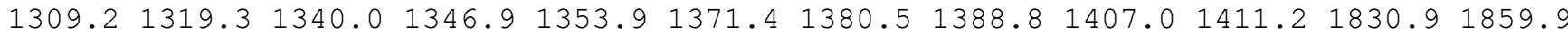
$2644.22655 .22667 .5 \quad 2671.92676 .92678 .6 \quad 2680.3 \quad 2706.12717 .2 \quad 2722.3 \quad 2738.2 \quad 2764.9$ $2778.4 \quad 2782.0 \quad 2794.4 \quad 2865.4$

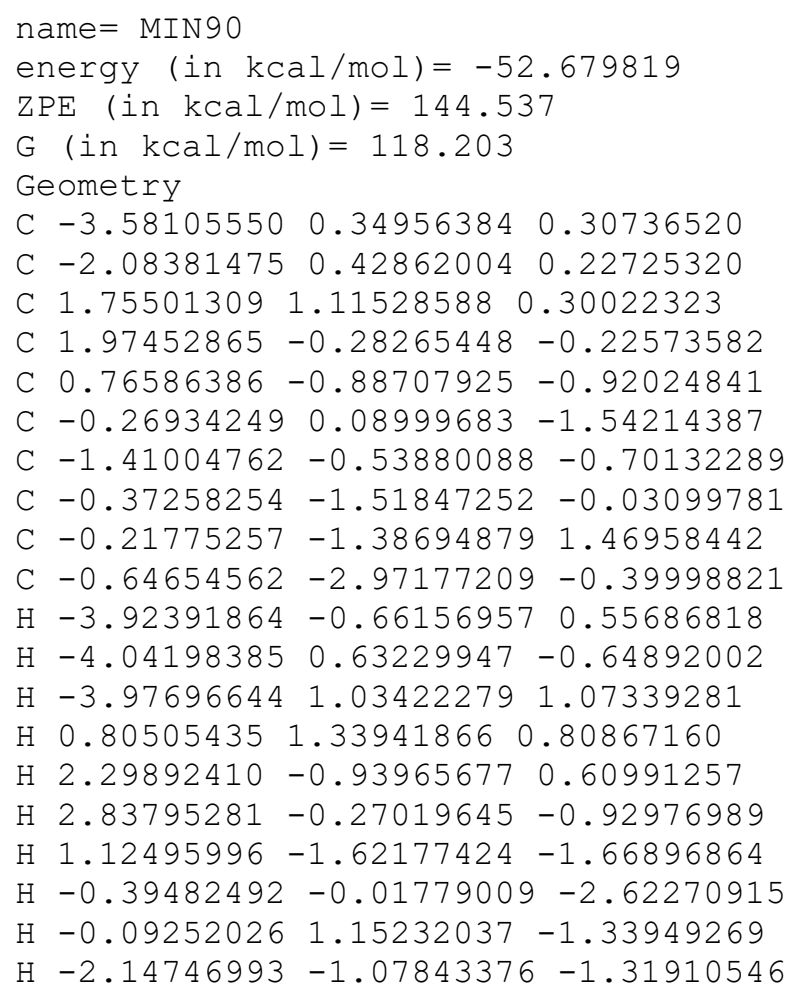


$\mathrm{H}-1.13198295-1.688485951 .99513854$

$\mathrm{H}-0.00008984-0.35542870 \quad 1.78176676$

$\mathrm{H} \quad 0.59695891-2.01807274 \quad 1.84530917$

$\mathrm{H} \quad 0.18549614-3.61895030-0.09581246$

$\mathrm{H}-0.78564770-3.10720637-1.47827064$

$\mathrm{H}-1.54905804-3.346879590 .09577580$

O 2.893934881 .755403620 .80661569

$02.300917262 .15303760-0.46754074$

o -1.470516581 .246300910 .86900276$

Vibrational frequencies (in $\mathrm{cm}-1$ )

$\begin{array}{llllllllllllllll}29.1 & 47.2 & 67.8 & 90.3 & 113.0 & 158.1 & 173.5 & 188.3 & 212.8 & 238.9 & 267.8 & 283.1 & 318.7 & 360.5\end{array}$

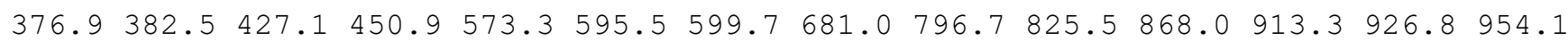
$989.0 \quad 998.3 \quad 1007.2 \quad 1018.0 \quad 1033.8 \quad 1042.3 \quad 1049.4 \quad 1072.7 \quad 1085.9 \quad 1099.2 \quad 1126.81134 .0$ $1161.71206 .7 \quad 1209.1 \quad 1236.3 \quad 1237.1 \quad 1259.5 \quad 1263.1 \quad 1268.3 \quad 1274.2 \quad 1285.5 \quad 1289.2 \quad 1294.6$

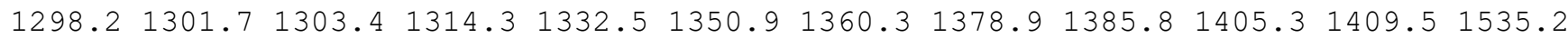
$1851.52649 .22661 .5 \quad 2663.2 \quad 2669.4 \quad 2674.8 \quad 2675.32677 .72678 .52680 .12714 .92730 .0$ $2737.12760 .92779 .4 \quad 2780.6 \quad 2783.1$

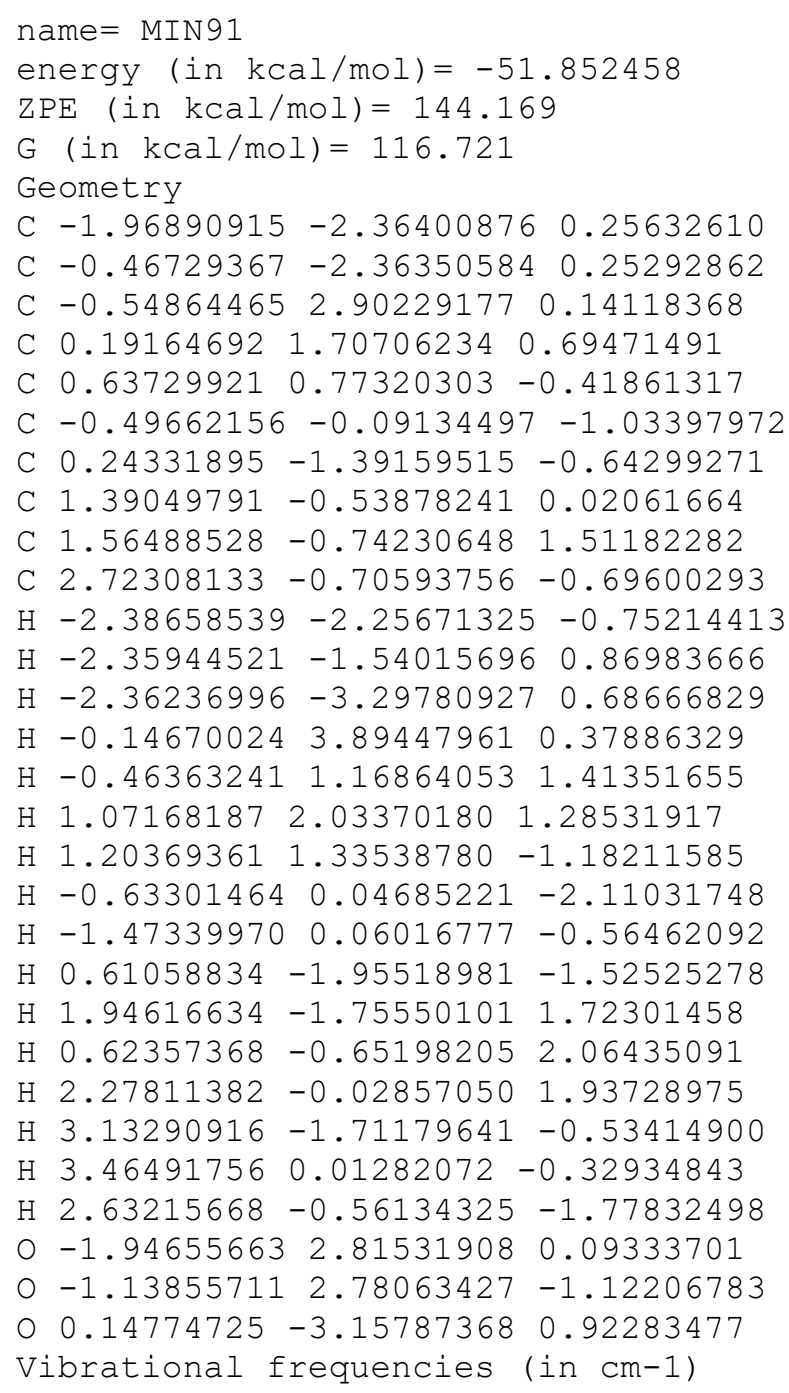


$\mathrm{ZPE}($ in $\mathrm{kcal} / \mathrm{mol})=144.564$

$\mathrm{G}($ in $\mathrm{kcal} / \mathrm{mol})=117.922$

Geometry

C $-3.48357564-0.10873298 \quad 0.57836456$

C $-1.98302179-0.05356007 \quad 0.54335807$

$\begin{array}{llll}\text { C } & 1.50832750 & 1.30036891 & 0.48342924\end{array}$

C $1.95813003 \quad 0.18338752-0.42789852$

C $0.82556512-0.51493814-1.16368589$

C $-0.45184093 \quad 0.32157909-1.45018131$

C $-1.32326615-0.68197358-0.64958758$

C $-0.04906119-1.56884951-0.38214428$

C $0.30266810-1.830090151 .06569293$

C $-0.13531602-2.89426708-1.13548969$

$\mathrm{H}-3.87969815 \quad 0.39047994 \quad 1.47632751$

$\mathrm{H}-3.85007625-1.14225468 \quad 0.58683838$

$\mathrm{H}-3.92015914 \quad 0.39474097 \quad-0.29455538$

$\mathrm{H} \quad 0.75727782 \quad 1.06983266 \quad 1.25332841$

H $2.53773622-0.558782810 .16169397$

H 2.68013266 $0.59314825-1.17064899$

$\mathrm{H} \quad 1.23689140 \quad-0.96127900 \quad-2.09125804$

$\mathrm{H}-0.71020696 \quad 0.38851388-2.51095102$

$\mathrm{H}-0.44576777 \quad 1.34104806-1.05208538$

$\mathrm{H}-2.06216309-1.20724047-1.27874609$

$\mathrm{H} 0.40660866-0.90559044 \quad 1.65086717$

H $1.24700075-2.380473261 .15536023$

$\mathrm{H}-0.47275465-2.42680592 \quad 1.56232277$

$\mathrm{H}-0.40396845-2.75656088-2.18869867$

$\mathrm{H}-0.88653573-3.55696235-0.69081538$

$\mathrm{H} \quad 0.82355887-3.42578325-1.11118003$

O $1.42876578 \quad 2.57754581-0.08849125$

$\begin{array}{lll}0 & 2.49760029 & 2.22604563 \quad 0.84214184\end{array}$

o $-1.36801968 \quad 0.49847096 \quad 1.42232235$

Vibrational frequencies (in $\mathrm{cm}-1$ )

$\begin{array}{llllllllllllll}10.9 & 61.3 & 74.1 & 102.9 & 113.5 & 161.4 & 168.5 & 189.0 & 212.5 & 236.1 & 267.0 & 280.3 & 314.4 & 355.4\end{array}$ $375.5 \quad 384.4 \quad 434.1 \quad 457.3 \quad 573.0 \quad 593.5 \quad 603.9 \quad 685.3 \quad 798.1 \quad 828.2 \quad 862.5 \quad 916.3 \quad 928.4 \quad 961.0$ $987.1995 .8 \quad 1008.8 \quad 1019.0 \quad 1035.3 \quad 1039.3 \quad 1045.5 \quad 1072.8 \quad 1085.4 \quad 1097.2 \quad 1123.7 \quad 1132.1$ $1158.51204 .0 \quad 1209.7 \quad 1233.2 \quad 1239.0 \quad 1254.1 \quad 1260.2 \quad 1265.6 \quad 1274.4 \quad 1285.3 \quad 1289.6 \quad 1295.0$

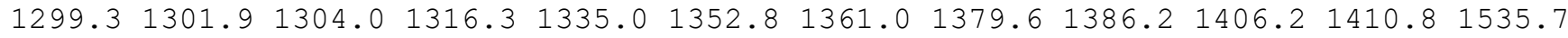

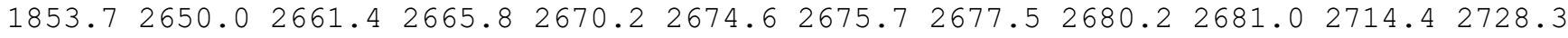
$2737.4 \quad 2762.5 \quad 2779.6 \quad 2780.5 \quad 2782.9$

\section{name $=$ MIN93}

energy (in $\mathrm{kcal} / \mathrm{mol})=-49.928182$

$\mathrm{ZPE}($ in $\mathrm{kcal} / \mathrm{mol})=142.387$

$\mathrm{G}($ in $\mathrm{kcal} / \mathrm{mol})=112.54$

Geometry

C $-2.31356254-2.04750751-1.02951929$

C $-0.90387775-1.63598015-0.84270768$

C $1.137210662 .26926630-0.55597312$

C $1.31609694 \quad 0.94416419-0.49321683$

C $1.28459242 \quad 0.09520731 \quad 0.73865224$

C $1.27319977-1.42066857 \quad 0.37251302$

C $-0.10562299-1.95748960 \quad 0.18748342$

C $0.14064915 \quad 0.36772754 \quad 1.68672912$

C $-1.02737298 \quad 0.89833290 \quad 1.31574564$

C $0.40355152-0.02811458 \quad 3.10455231$

$\mathrm{H}-2.64825441-2.76162290-0.26551772$

$\mathrm{H}-2.46659359-2.51137378-2.01740217$

$\mathrm{H}-2.98823746-1.17628378-0.98241866$

H $1.213935712 .89071612-1.44611780$

H $1.56362569 \quad 0.40573007-1.40895340$

$\mathrm{H}-1.19292661 \quad 0.69746777 \quad-2.28561878$

H 2.25132818 0.292010391 .28446916

H $1.79503489-1.98274058 \quad 1.17339432$ 
$\mathrm{H} \quad 1.88426808-1.58648614-0.53839712$

$\mathrm{H}-0.45520976-2.63064684 \quad 0.96371233$

$\mathrm{H}-1.84890100 \quad 1.07227818 \quad 1.98969069$

$\mathrm{H}-1.256208551 .18166308 \quad 0.30084149$

$\mathrm{H} \quad 0.68581927 \quad 2.61913416 \quad 1.32633684$

H $1.16613624 \quad 0.61225794 \quad 3.56800572$

$\mathrm{H} 0.76336874-1.065742993 .16748875$

$\mathrm{H}-0.496976310 .03255563 \quad 3.73071533$

$0-1.33468156-0.23134160-2.59762893$

$00.83063158 \quad 3.09464179 \quad 0.46905529$

O $-0.31697521-0.88048219-1.83366088$

Vibrational frequencies (in $\mathrm{cm}-1$ )

$\begin{array}{lllllllllllllllll}-26.9 & 5.4 & 24.1 & 66.0 & 79.3 & 88.9 & 100.4 & 129.2 & 159.0 & 177.8 & 219.7 & 228.6 & 258.0 & 296.2\end{array}$

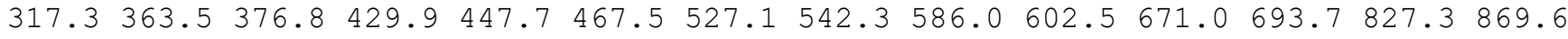
$878.1926 .6968 .6979 .5 \quad 989.0 \quad 1023.7 \quad 1040.9 \quad 1043.5 \quad 1050.0 \quad 1058.2 \quad 1066.5 \quad 1090.7 \quad 1107.6$

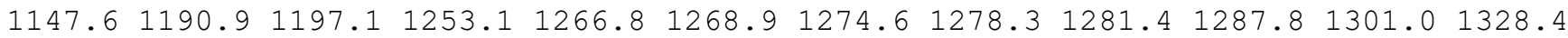

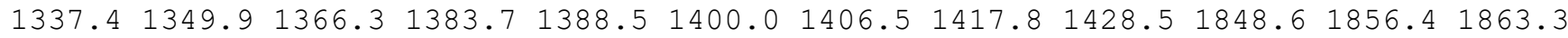
$2657.12664 .0 \quad 2670.5 \quad 2672.7 \quad 2676.92677 .3 \quad 2724.02726 .4 \quad 2738.12750 .2 \quad 2764.92768 .5$ $2778.7 \quad 2779.4 \quad 2792.2 \quad 2859.6$

name $=$ MIN94

energy (in $\mathrm{kcal} / \mathrm{mol})=-49.520509$

$\operatorname{ZPE}(\mathrm{in} \mathrm{kcal} / \mathrm{mol})=142.053$

$\mathrm{G}($ in $\mathrm{kcal} / \mathrm{mol})=112.806$

Geometry

C $-3.80769918-0.26922517-0.57441338$

C $-2.32109345-0.32480521-0.61210152$

C $1.47473888 \quad 1.07957996-0.84230795$

C $1.69881948 \quad 0.07962968 \quad 0.01221486$

C $0.77679769-1.07474881 \quad 0.27437311$

C $-0.09400037-1.45293288-0.95721668$

C $-1.57417502-1.42570024-0.78780577$

C $-0.02426595-0.84766524 \quad 1.53573694$

C $-0.16755381 \quad 0.34591535 \quad 2.11689568$

C $-0.61287634-2.08303908 \quad 2.13768551$

$\mathrm{H}-4.25226402-0.73355003-1.46549458$

$\mathrm{H}-4.16923870 \quad 0.77253789-0.53133505$

$\mathrm{H}-4.20382560-0.785585510 .31148513$

$\mathrm{H} \quad 0.60826202 \quad 1.24068808-1.48572707$

$\mathrm{H} 2.61122110 \quad 0.06123265 \quad 0.61307157$

$\mathrm{H} \quad 4.15346505 \quad 2.12285157-1.03202532$

H $1.43656856-1.96965773 \quad 0.47431750$

$\mathrm{H} \quad 0.20823100 \quad-2.47478851-1.27689543$

$\mathrm{H} \quad 0.18178473-0.81603867-1.82453018$

$\mathrm{H}-2.06014008-2.39286321-0.87704548$

$\mathrm{H}-0.873947390 .92286095-0.16712600$

$\mathrm{H} \quad 0.26514606 \quad 1.26207728 \quad 1.74834410$

$\mathrm{H}-0.72555674 \quad 0.50120412 \quad 3.02562257$

$\mathrm{H}-1.27728995-2.59483956 \quad 1.42532589$

$\mathrm{H}-1.20806752-1.869388373 .03513293$

$\mathrm{H} \quad 0.16804209-2.79830537 \quad 2.42892477$

○ $3.490495412 .01452531-0.30665061$

O $2.300687962 .13416292-1.09440084$

$\begin{array}{lllll}0 & -1.81868810 & 0.92971224 & -0.47221326\end{array}$

Vibrational frequencies (in $\mathrm{cm}-1$ )

$\begin{array}{lllllllllllllll}-37.2 & 31.8 & 38.6 & 58.2 & 60.8 & 82.1 & 84.2 & 102.9 & 123.5 & 158.3 & 198.5 & 205.5 & 225.8 & 257.4\end{array}$ $311.3 \quad 338.6 \quad 373.4 \quad 420.4 \quad 454.7 \quad 469.8 \quad 523.8 \quad 528.7 \quad 581.2 \quad 600.9659 .6 \quad 696.5 \quad 831.3 \quad 878.1$ $892.5 \quad 927.6 \quad 953.7 \quad 987.6 \quad 994.4 \quad 1011.9 \quad 1029.8 \quad 1042.6 \quad 1052.1 \quad 1062.4 \quad 1071.8 \quad 1095.7 \quad 1135.2$ $\begin{array}{llllllllllll}1192.7 & 1199.2 & 1235.4 & 1239.6 & 1269.7 & 1274.6 & 1275.4 & 1278.8 & 1283.6 & 1289.5 & 1295.4 & 1313.9\end{array}$

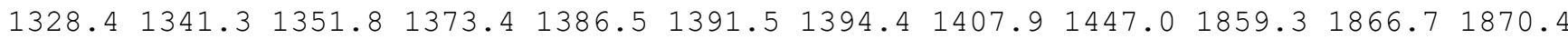
$2644.12667 .8 \quad 2668.7 \quad 2673.1 \quad 2674.2 \quad 2679.1 \quad 2700.92718 .72733 .4 \quad 2747.22754 .12768 .5$ $2778.72778 .92790 .7 \quad 2869.9$ 
name $=$ MIN95

energy (in $\mathrm{kcal} / \mathrm{mol})=-52.135038$

$\mathrm{ZPE}($ in $\mathrm{kcal} / \mathrm{mol})=144.855$

$\mathrm{G}($ in $\mathrm{kcal} / \mathrm{mol})=119.4$

Geometry

C $-2.44580512-1.24181661-0.58261100$

C $-1.11119235-1.24629340-0.61457775$

C $1.61339411 \quad 1.98238201-0.59613095$

C $1.85065700 \quad 1.52558730 \quad 0.81516248$

C $1.31706623 \quad 0.17615597 \quad 1.26830502$

C $1.30527260 \quad-0.99696790 \quad 0.25760772$

C $-0.19594021-1.27106063 \quad 0.55529025$

C $-0.23119006-0.002321791 .49636689$

C $-1.108037921 .13918570 \quad 1.02486342$

C $-0.56993797-0.36258322 \quad 2.93744024$

$\mathrm{H}-3.02424833-1.26170628 \quad 0.32324218$

$\mathrm{H}-3.04350715-1.20724082-1.48218143$

$\mathrm{H}-0.223083720 .47793503-2.31669077$

H 2.14506919 2.90542702 -0.88589077

H 2.95210973 1.539254830 .99122453

$\mathrm{H} 1.43893773 \quad 2.30758708 \quad 1.49714280$

H $1.86800950-0.10747638 \quad 2.19111191$

H $1.97297158-1.82392795 \quad 0.50725152$

H $1.51272955-0.70719848-0.77931306$

$\mathrm{H}-0.34488189-2.20926128 \quad 1.12487378$

$\mathrm{H}-0.95095482 \quad 1.36248407-0.03994926$

$\mathrm{H}-0.923717992 .05482224 \quad 1.59448368$

$\mathrm{H}-2.17322483 \quad 0.89341287 \quad 1.13171982$

$\mathrm{H} 0.04642200-1.185916413 .31497115$

$\mathrm{H}-1.61807423-0.67385302 \quad 3.02927612$

$\mathrm{H}-0.42254231 \quad 0.49302409 \quad 3.60570861$

$0-0.85832332-0.20935118-2.61900140$

○ $0.90495047 \quad 1.44806947-1.41062247$

- $-0.41593831-1.30328842-1.80917185$

Vibrational frequencies (in $\mathrm{cm}-1$ )

$\begin{array}{lllllllllllllllll}30.4 & 54.1 & 87.8 & 131.3 & 155.9 & 179.5 & 190.6 & 209.0 & 239.7 & 259.1 & 277.1 & 282.4 & 324.7 & 357.9\end{array}$ $406.2441 .9 \quad 480.8 \quad 540.7 \quad 552.7 \quad 575.3 \quad 597.3 \quad 652.8 \quad 682.9 \quad 700.2 \quad 755.6 \quad 820.9 \quad 840.5 \quad 918.3$ $940.9991 .2994 .2 \quad 1002.6 \quad 1004.7 \quad 1017.7 \quad 1026.3 \quad 1030.3 \quad 1064.0 \quad 1074.5 \quad 1089.8 \quad 1120.4 \quad 1136.4$ $1165.1 \quad 1208.91213 .91239 .4 \quad 1257.2 \quad 1265.7 \quad 1283.0 \quad 1289.7 \quad 1295.4 \quad 1298.7 \quad 1302.6 \quad 1306.5$ $1315.31326 .01348 .3 \quad 1350.8 \quad 1360.2 \quad 1376.91391 .0 \quad 1404.11412 .3 \quad 1416.5 \quad 1849.01873 .8$ $2638.72645 .22666 .3 \quad 2676.5 \quad 2678.8 \quad 2679.0 \quad 2682.7 \quad 2707.92718 .8 \quad 2726.92727 .6 \quad 2762.3$ $2781.12783 .0 \quad 2790.4 \quad 2873.7$

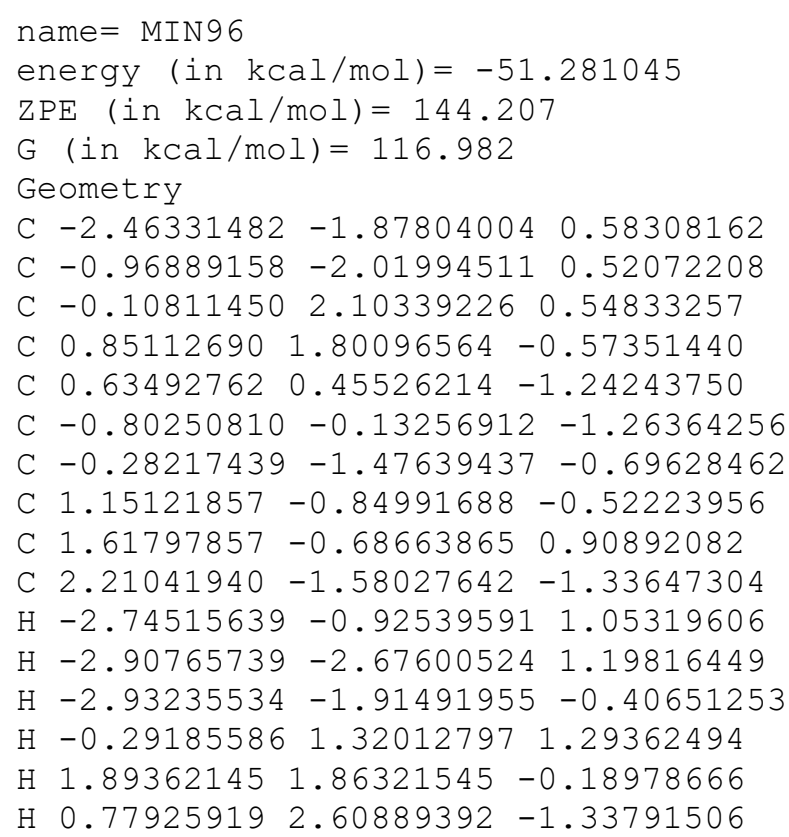


Н $1.05202631 \quad 0.51170234 \quad-2.26984814$

$\mathrm{H}-1.24627615-0.20034908-2.26064128$

$\mathrm{H}-1.52200799 \quad 0.38665447 \quad-0.62500871$

$\mathrm{H}-0.27688426-2.28258736-1.46008128$

$\mathrm{H} 1.84121694-1.668126831 .35996706$

$\mathrm{H} \quad 0.86004779-0.23121157 \quad 1.55464614$

H $2.52859702-0.08147418 \quad 0.97245763$

$\mathrm{H} 2.41700435-2.57208994-0.91312882$

$\mathrm{H} \quad 3.15637440-1.02593435-1.34374665$

$\mathrm{H} \quad 1.90908320-1.72799547 \quad-2.37901036$

$0-0.08802916 \quad 3.41675629 \quad 1.03568249$

$0-1.20472875 \quad 2.91813774 \quad 0.23724769$

o $-0.36766057-2.59529494 \quad 1.39386757$

Vibrational frequencies (in cm-1)

$\begin{array}{lllllllllllllll}24.2 & 29.0 & 41.6 & 70.1 & 95.1 & 155.2 & 177.3 & 192.3 & 202.4 & 243.4 & 261.8 & 274.5 & 323.5 & 346.0\end{array}$

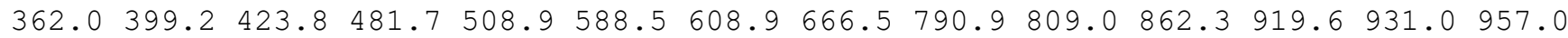
$981.9989 .9 \quad 1007.3 \quad 1013.8 \quad 1016.7 \quad 1043.2 \quad 1048.7 \quad 1076.3 \quad 1082.5 \quad 1085.3 \quad 1129.4 \quad 1137.1$ $1160.91204 .3 \quad 1210.4 \quad 1239.2 \quad 1250.5 \quad 1260.1 \quad 1262.2 \quad 1265.91273 .7 \quad 1284.8 \quad 1290.6 \quad 1293.8$

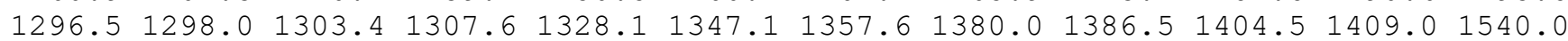
$1846.72650 .62659 .92666 .5 \quad 2674.3 \quad 2676.0 \quad 2677.0 \quad 2678.32681 .4 \quad 2683.32709 .2 \quad 2716.7$ $2736.22764 .72778 .5 \quad 2780.3 \quad 2782.0$

\author{
name $=$ MIN97 \\ energy (in $\mathrm{kcal} / \mathrm{mol})=-51.337983$ \\ $\mathrm{ZPE}(\mathrm{in} \mathrm{kcal} / \mathrm{mol})=144.279$ \\ $\mathrm{G}($ in $\mathrm{kcal} / \mathrm{mol})=117.573$ \\ Geometry \\ C $-1.90580327-1.88659096 \quad 0.57608195$ \\ C $-0.40620007-1.935286450 .54982468$ \\ C $-0.75490785 \quad 1.79452723 \quad 0.53181333$ \\ C $0.294712021 .96936582-0.53633814$ \\ C $0.71249566 \quad 0.68168749-1.22839653$ \\ C $-0.41485823-0.34559073-1.51851172$ \\ C $0.30507815-1.41865065-0.66808137$ \\ C $1.49319924-0.41630874-0.40955012$ \\ C $1.81539405-0.11548784 \quad 1.03795171$ \\ C $2.76342157-0.85378031-1.13317390$ \\ $\mathrm{H}-2.34907721-2.17780672-0.38368381$ \\ $\mathrm{H}-2.26476049-0.874130690 .81561556$ \\ $\mathrm{H}-2.30986278-2.557536851 .35000520$ \\ $\mathrm{H}-0.497437161 .17755172 \quad 1.40336263$ \\ $\mathrm{H} 1.188670462 .46044922-0.09396964$ \\ $\mathrm{H}-0.08438134 \quad 2.68392172-1.30323494$ \\ H $1.26924212 \quad 0.95139569-2.14855331$ \\ $\mathrm{H}-0.51049415-0.60888617-2.57684217$ \\ $\mathrm{H}-1.40967526-0.05402009-1.17582071$ \\ $\mathrm{H} \quad 0.62760299-2.29629797-1.26417781$ \\ H 2.28082170 -0.991109421 .52095503$ \\ H $0.93061870 \quad 0.11112982 \quad 1.64202554$ \\ H 2.51339322 $0.72401011 \quad 1.12914149$ \\ $\mathrm{H} 2.58283302-1.08280819-2.18877126$ \\ H $3.18768215-1.75528706-0.67219629$ \\ $\mathrm{H} 3.53246898-0.07365814-1.09189713$ \\ $\begin{array}{llll}0 & -2.09210929 & 1.72844368 & 0.11895262\end{array}$ \\ $\begin{array}{lllll}0 & -1.56241740 & 2.90892377 & 0.79570501\end{array}$ \\ o $0.20964180-2.41684628 \quad 1.46993640$ \\ Vibrational frequencies (in $\mathrm{cm}-1$ )
}

$\begin{array}{lllllllllllllll}22.4 & 36.2 & 46.4 & 86.9 & 143.4 & 163.1 & 183.0 & 189.5 & 203.5 & 217.8 & 259.0 & 296.7 & 325.0 & 351.6\end{array}$ $369.3 \quad 412.2 \quad 433.0 \quad 484.9 \quad 490.5 \quad 585.2 \quad 606.0 \quad 682.0 \quad 796.5 \quad 813.8 \quad 854.9 \quad 916.1 \quad 922.6 \quad 965.2$ $978.1987 .6 \quad 1008.7 \quad 1017.1 \quad 1024.0 \quad 1037.4 \quad 1041.91074 .2 \quad 1085.6 \quad 1086.11119 .91129 .9$

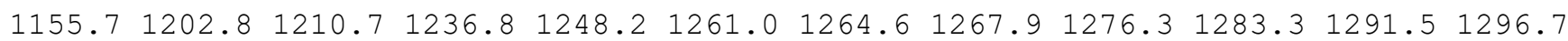

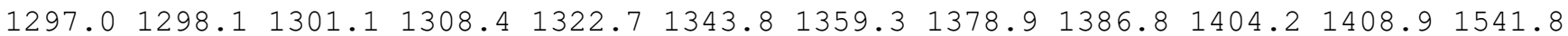
$1839.82645 .5 \quad 2656.12661 .5 \quad 2668.2 \quad 2673.9 \quad 2675.4 \quad 2677.92681 .42682 .42711 .52717 .7$ $2736.4 \quad 2766.8 \quad 2776.7 \quad 2777.8 \quad 2781.6$ 
name $=$ MIN98

energy (in $\mathrm{kcal} / \mathrm{mol})=-48.442791$

$\mathrm{ZPE}(\mathrm{in} \mathrm{kcal} / \mathrm{mol})=141.947$

$\mathrm{G}($ in $\mathrm{kcal} / \mathrm{mol})=112.694$

Geometry

C $-2.90956138-1.62812544-1.63966424$

C $-1.61362881-1.42497938-0.92469327$

C $1.75650092 \quad 2.09431285-0.94947364$

C $0.85504686 \quad 0.89228998-0.87623300$

$\begin{array}{lllll}\text { C } & 0.91020440 & 0.25423380 & 0.48553828\end{array}$

C $-0.22381891-0.620643811 .00404505$

C $-1.47652721-0.83717090 \quad 0.26762044$

C $0.04457352 \quad 0.74582791 \quad 1.63942839$

C $-0.93262558 \quad 1.87373356 \quad 1.42498823$

C $0.66981388 \quad 0.78030581 \quad 3.01262875$

$\mathrm{H}-3.30412020-2.63983473-1.45824568$

$\mathrm{H}-2.80592792-1.50868053-2.72873640$

$\mathrm{H}-3.67243049-0.91020599-1.30677303$

$\mathrm{H} 1.529561372 .83499655-1.72801289$

H $1.17508866 \quad 0.14725461-1.64423283$

$\mathrm{H}-0.183527691 .15389493-1.15975583$

$\mathrm{H} \quad 1.93523062-0.05909114 \quad 0.73685502$

H $0.07179515-1.513903631 .57486256$

$\mathrm{H} \quad 0.75204484-3.00752916-1.17054895$

$\mathrm{H}-2.36348030-0.491615290 .80471268$

$\mathrm{H}-0.417817122 .843508931 .44666903$

$\mathrm{H}-1.70105027 \quad 1.88577515 \quad 2.20803115$

$\mathrm{H}-1.454111431 .79953400 \quad 0.46311416$

$\mathrm{H}-0.09389282 \quad 0.73614108 \quad 3.79849695$

$\mathrm{H} \quad 1.24425624 \quad 1.70692316 \quad 3.15348609$

$\mathrm{H} \quad 1.36197196-0.053312443 .17938068$

$0.59515061-2.03931293-1.03989260$

- $2.698204732 .25609758-0.21421640$

$0-0.63736109-1.92209493-1.75106875$

Vibrational frequencies (in $\mathrm{cm}-1$ )

$\begin{array}{llllllllllllllll}26.7 & 32.8 & 52.6 & 56.9 & 66.2 & 96.6 & 108.6 & 110.2 & 121.7 & 130.6 & 142.0 & 176.6 & 210.3 & 260.4\end{array}$ $280.9 \quad 323.5 \quad 337.1 \quad 391.6 \quad 464.4 \quad 481.9 \quad 493.7 \quad 570.6 \quad 617.5 \quad 664.5 \quad 755.9 \quad 784.5 \quad 838.9 \quad 908.8$ $948.0 \quad 968.0 \quad 998.3 \quad 1005.4 \quad 1010.9 \quad 1022.6 \quad 1031.7 \quad 1051.8 \quad 1060.1 \quad 1063.6 \quad 1086.3 \quad 1106.7 \quad 1156.1$ $1170.21218 .6 \quad 1245.6 \quad 1262.9 \quad 1270.3 \quad 1272.3 \quad 1276.4 \quad 1277.1 \quad 1287.1 \quad 1294.3 \quad 1297.5 \quad 1298.7$ $1305.51345 .0 \quad 1349.2 \quad 1357.0 \quad 1371.0 \quad 1378.9 \quad 1400.7 \quad 1406.21410 .11514 .51842 .01901 .0$ $2644.92652 .62666 .62670 .6 \quad 2673.4 \quad 2675.3 \quad 2678.02680 .82730 .72740 .02747 .5 \quad 2753.0$ $2777.12779 .8 \quad 2781.7 \quad 2873.4$

name $=$ MIN99

energy (in $\mathrm{kcal} / \mathrm{mol})=-50.448698$

$\mathrm{ZPE}(\mathrm{in} \mathrm{kcal} / \mathrm{mol})=143.987$

$\mathrm{G}($ in $\mathrm{kcal} / \mathrm{mol})=117.567$

Geometry

C $-3.06006516-1.33805312-0.21853612$

C $-1.55637930-1.35363329-0.20979354$

C $3.18268558 \quad 0.75014025-0.21142523$

C $1.73798897 \quad 1.10870499-0.44037653$

C $0.88705428 \quad 0.75565231 \quad 0.76283436$

C $0.65517426-0.76537480 \quad 0.97207470$

C $-0.87928253-0.570682910 .87926607$

C $-0.66824691 \quad 0.96905093 \quad 0.62203778$

C $-1.148177171 .48962509-0.71805179$

C -1.239148391 .819965261 .74872225$

$\mathrm{H}-3.45874020-1.72222777-1.17061193$

$\mathrm{H}-3.46048404-0.32665551-0.07532810$

$\mathrm{H}-3.46558853-1.97524614 \quad 0.57986304$

$\mathrm{H} \quad 3.39755535-0.09101788 \quad 0.45913987$ 
$\mathrm{H} \quad 1.38313009 \quad 0.57922083-1.35345795$

H $1.642625512 .18737073-0.68627439$

$\mathrm{H} 1.280984501 .25506887 \quad 1.66680134$

$\mathrm{H} 1.00325030-1.15504940 \quad 1.93108771$

$\mathrm{H} 1.06964000-1.402689160 .18111172$

$\mathrm{H}-1.38949816-0.764705921 .84152552$

$\mathrm{H}-2.242300091 .46966923-0.78961209$

$\mathrm{H}-0.759939230 .90504022-1.56186428$

$\mathrm{H}-0.832686352 .52925614 \quad-0.87539766$

$\mathrm{H}-2.33520626 \quad 1.81080983 \quad 1.74094237$

$\mathrm{H}-0.92084495 \quad 2.86579195 \quad 1.65488223$

$\mathrm{H}-0.91700260 \quad 1.470289532 .73616575$

$0-0.96350945-2.57463394-0.56872406$

O $4.074604451 .35403319-0.75323582$

o $-0.92733292-1.41716713-1.46017651$

Vibrational frequencies (in $\mathrm{cm}-1$ )

$\begin{array}{llllllllllllllll}31.6 & 51.8 & 65.3 & 95.4 & 108.2 & 156.2 & 170.1 & 185.5 & 202.7 & 241.5 & 255.9 & 284.8 & 315.8 & 325.7\end{array}$ $345.8 \quad 388.6 \quad 414.8 \quad 434.8 \quad 530.5 \quad 534.3 \quad 564.9 \quad 670.2 \quad 777.0 \quad 805.8 \quad 865.4 \quad 882.0 \quad 918.2 \quad 933.7$

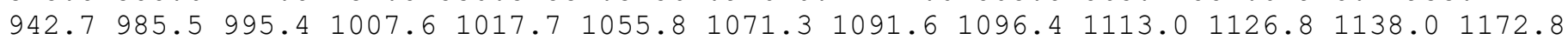
$1181.51204 .11228 .5 \quad 1252.2 \quad 1266.5 \quad 1270.0 \quad 1272.5 \quad 1275.51287 .6 \quad 1290.6 \quad 1297.11300 .3$ $1303.21311 .0 \quad 1312.8 \quad 1325.5 \quad 1339.4 \quad 1350.4 \quad 1361.91387 .6 \quad 1403.7 \quad 1406.01570 .4 \quad 1837.7$ $2647.72654 .7 \quad 2667.92669 .0 \quad 2673.3 \quad 2674.3 \quad 2676.92679 .32681 .72719 .12724 .82740 .6$ $2761.7 \quad 2779.5 \quad 2780.0 \quad 2782.9$

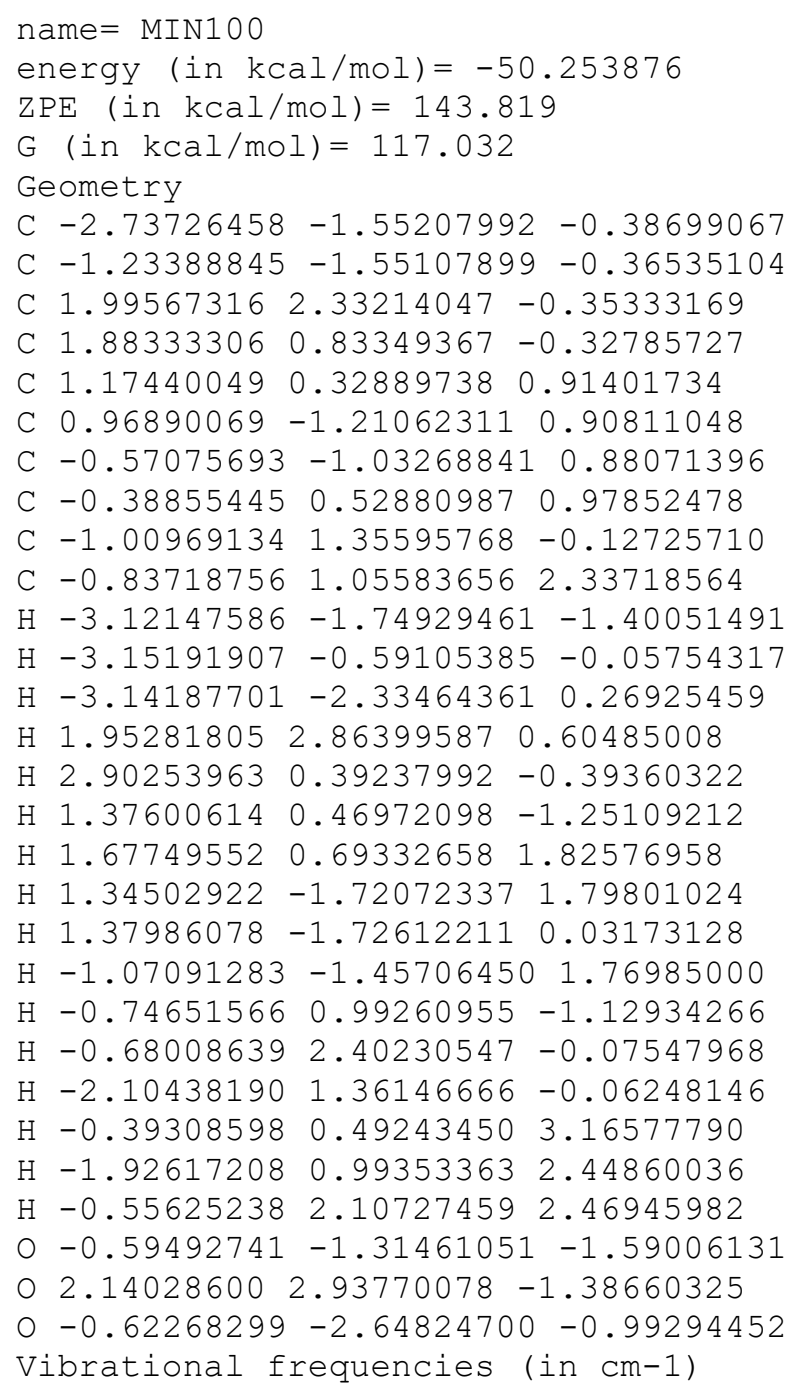


$1835.8 \quad 2642.4 \quad 2655.5 \quad 2666.7 \quad 2668.3 \quad 2672.7 \quad 2673.7 \quad 2677.5 \quad 2678.72681 .72720 .92726 .1$ $2738.5 \quad 2762.0 \quad 2779.1 \quad 2779.7 \quad 2782.7$

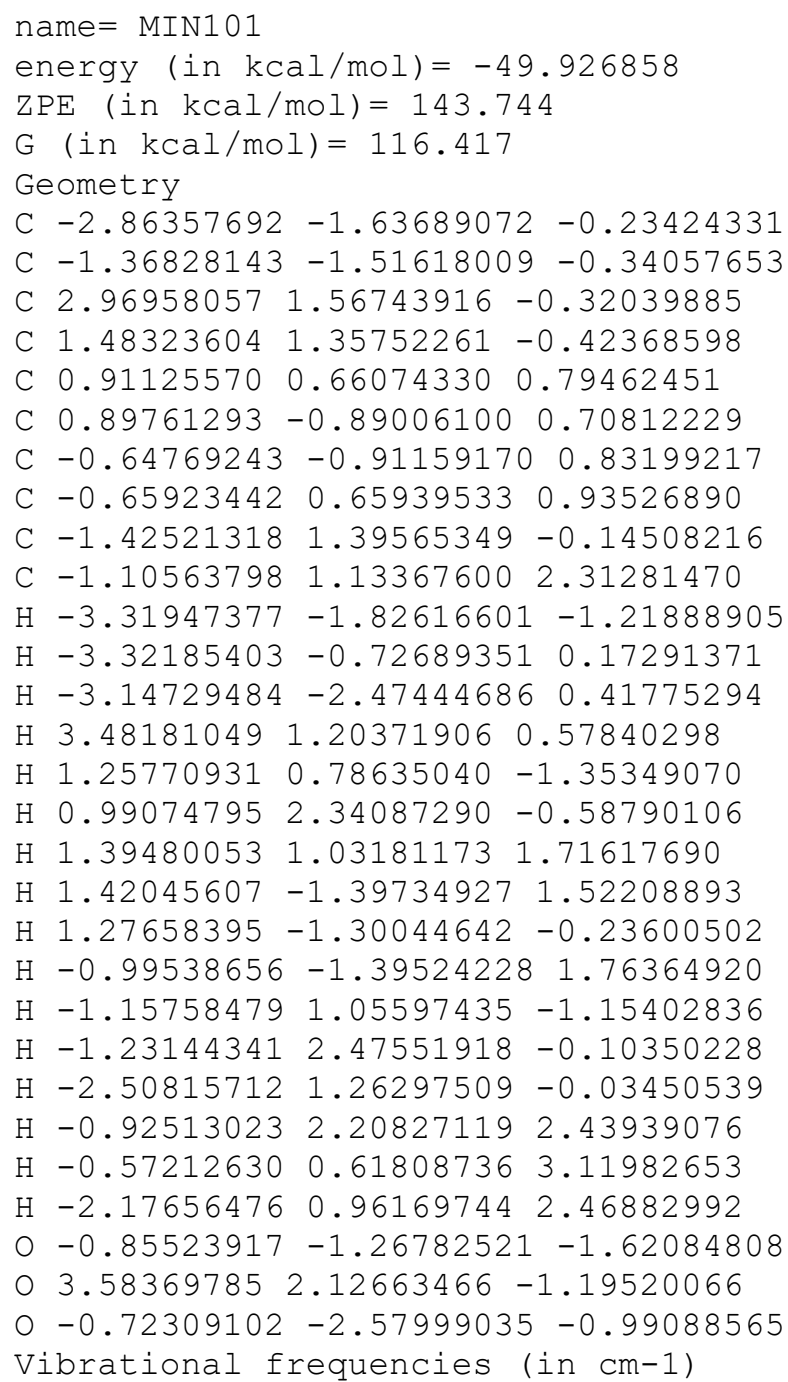

$\begin{array}{llllllllllllllll}20.4 & 35.7 & 55.0 & 69.9 & 74.4 & 145.4 & 169.0 & 188.6 & 201.4 & 235.3 & 257.1 & 286.7 & 313.9 & 327.2\end{array}$

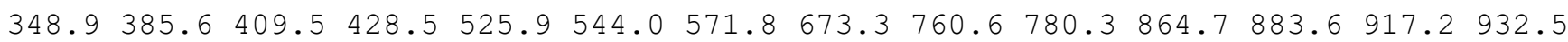
$946.2982 .4 \quad 993.5 \quad 1007.1 \quad 1015.5 \quad 1054.5 \quad 1070.3 \quad 1088.5 \quad 1101.8 \quad 1118.2 \quad 1118.7 \quad 1132.9 \quad 1168.7$

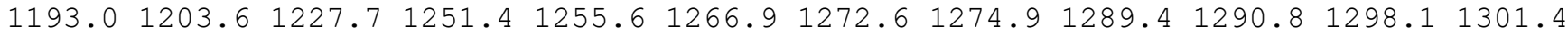
$1303.11309 .8 \quad 1314.8 \quad 1329.5 \quad 1345.2 \quad 1352.7 \quad 1365.0 \quad 1387.5 \quad 1404.7 \quad 1406.2 \quad 1568.3 \quad 1834.8$ $2643.92652 .5 \quad 2668.0 \quad 2670.6 \quad 2672.4 \quad 2672.92676 .6 \quad 2679.22680 .72716 .4 \quad 2724.52738 .6$ $2761.4 \quad 2778.8 \quad 2779.8 \quad 2782.7$

name $=$ MIN102

energy (in $\mathrm{kcal} / \mathrm{mol})=-50.653442$

$\mathrm{ZPE}(\mathrm{in} \mathrm{kcal} / \mathrm{mol})=144.565$

$\mathrm{G}($ in $\mathrm{kcal} / \mathrm{mol})=118.223$

Geometry

C $-1.71473781-2.15076069-0.08818373$

C $-0.21518117-2.14106994-0.05864753$

C $-1.35687332 \quad 2.04166256 \quad 0.02244111$

C $0.122053262 .16758238-0.23627272$

C $0.719039201 .00709323-1.02025900$

C $-0.23441305-0.04795162-1.64930035$

C $0.53378382-1.19586497-0.95486645$

C $1.43790238-0.14259372-0.20994531$

C $1.25939728-0.11733640 \quad 1.29377193$

C $2.91051975-0.27812354-0.56740986$

$\mathrm{H}-2.12304606-1.273344140 .43515366$

$\mathrm{H}-2.11254899-3.03985338 \quad 0.42660298$ 
H $-2.11090732-2.15220128-1.10991719$

$\mathrm{H}-2.026941572 .15261462 \quad-0.83948643$

$\mathrm{H} \quad 0.66564696 \quad 2.28516268 \quad 0.72900654$

$\mathrm{H} \quad 0.30642942 \quad 3.12083594 \quad-0.77754810$

$\mathrm{H} \quad 1.40609007 \quad 1.41882006-1.78709051$

$\mathrm{H}-0.20026546-0.08214263-2.74229465$

$\mathrm{H}-1.28381898 \quad 0.03599098-1.36101731$

H $1.12144202-1.80466923-1.67260386$

$\mathrm{H} \quad 1.58327617-1.069578531 .74358838$

H $0.21208964 \quad 0.03086966 \quad 1.58892577$

H $1.84979066 \quad 0.67777634 \quad 1.76235578$

$\mathrm{H} 3.07582400-0.31760663-1.64917010$

$\mathrm{H} 3.33185869-1.19909373-0.14048220$

$\mathrm{H} \quad 3.49483490 \quad 0.56171408-0.17411237$

$0-1.77513508 \quad 1.13265903 \quad 1.00146649$

$\begin{array}{llll}0 & -1.83358964 & 2.57399878 & 1.22781183\end{array}$

o $0.39065516-2.90759617 \quad 0.65160273$

Vibrational frequencies (in cm-1)

$\begin{array}{llllllllllllll}23.2 & 55.0 & 58.5 & 73.0 & 144.2 & 161.0 & 175.4 & 190.2 & 208.9 & 257.9 & 285.1 & 290.4 & 324.9 & 361.3\end{array}$ $369.2 \quad 420.3 \quad 436.4 \quad 478.6 \quad 501.6 \quad 583.5 \quad 599.5 \quad 664.2 \quad 786.0 \quad 811.1 \quad 860.4 \quad 914.3 \quad 925.8 \quad 970.7$ $988.7996 .8 \quad 1007.8 \quad 1011.8 \quad 1018.4 \quad 1041.2 \quad 1046.91069 .8 \quad 1092.1 \quad 1096.5 \quad 1130.91137 .5$ $1163.91197 .8 \quad 1211.4 \quad 1232.0 \quad 1263.3 \quad 1265.3 \quad 1271.2 \quad 1272.3 \quad 1278.7 \quad 1286.2 \quad 1292.11297 .5$ $1302.8 \quad 1302.91305 .3 \quad 1311.91324 .6 \quad 1346.1 \quad 1358.1 \quad 1379.4 \quad 1385.1 \quad 1404.2 \quad 1406.7 \quad 1546.7$ $1836.02646 .62660 .72664 .8 \quad 2673.4 \quad 2674.92675 .72680 .02682 .5 \quad 2687.8 \quad 2711.4 \quad 2718.6$ $2735.0 \quad 2764.6 \quad 2777.8 \quad 2778.8 \quad 2781.6$

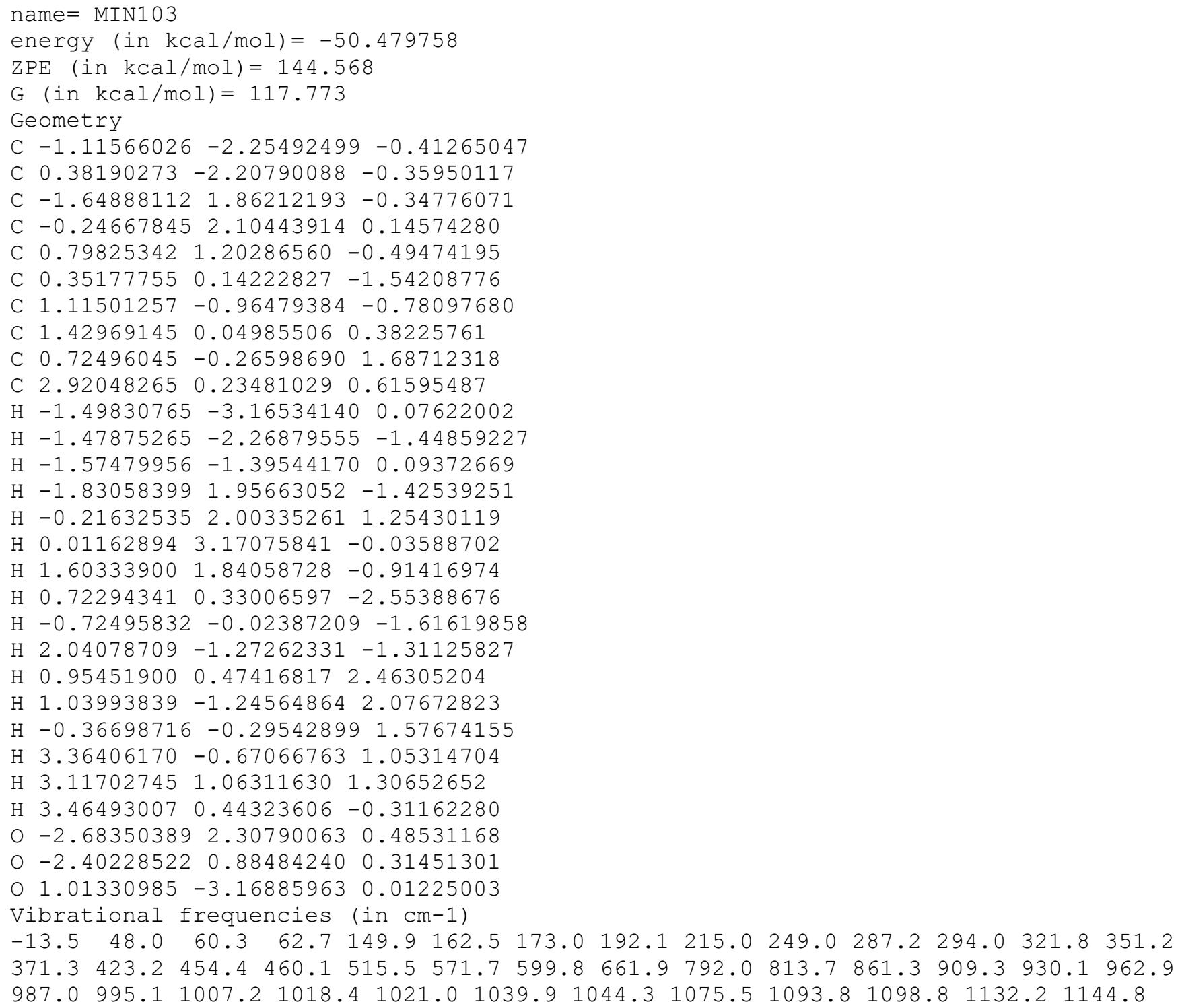


$\begin{array}{lllllllllllllll}1166.0 & 1203.6 & 1213.2 & 1230.2 & 1262.2 & 1267.8 & 1269.7 & 1271.9 & 1281.1 & 1285.3 & 1293.5 & 1299.3\end{array}$

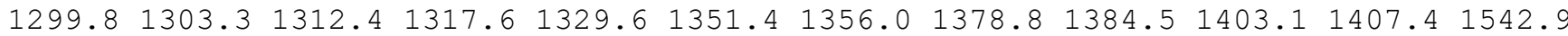
1835.12645 .42663 .82667 .82670 .82671 .72673 .72679 .12681 .82687 .62711 .12718 .7 $2736.22764 .02776 .6 \quad 2779.12781 .6$

\section{name $=$ MIN104}

energy (in $\mathrm{kcal} / \mathrm{mol})=-49.621694$

$\mathrm{ZPE}($ in $\mathrm{kcal} / \mathrm{mol})=144.131$

$\mathrm{G}($ in $\mathrm{kcal} / \mathrm{mol})=118.232$

Geometry

C $-2.67285944-1.18597073-0.79897945$

C $-1.17030849-1.18458744-0.74515166$

C $1.864749671 .33017590-0.78793600$

C $1.99990201 \quad 1.198923290 .70785566$

C $1.118166810 .15222159 \quad 1.35904114$

C $1.00031991-1.225595410 .65477972$

C $-0.54435941-1.10196914 \quad 0.61753870$

C $-0.447207320 .32502206 \quad 1.27706599$

C $-0.950940091 .48550926 \quad 0.44471985$

C $-1.09548428 \quad 0.35729467 \quad 2.65584604$

$\mathrm{H}-3.03704503-1.02102053-1.82538455$

$\mathrm{H}-3.10502102-0.40120369-0.16482578$

$\mathrm{H}-3.08092075-2.14936984-0.46422614$

H $1.54670824 \quad 0.45158926-1.36427110$

$\mathrm{H} 1.813639412 .18932560 \quad 1.18059370$

H $3.06852869 \quad 0.97826763 \quad 0.93068725$

$\mathrm{H} \quad 1.43884191 \quad 0.03741696 \quad 2.41512210$

$\mathrm{H} \quad 1.37054771-2.067882531 .24518440$

$\mathrm{H} 1.47073608-1.29286205-0.33216406$

$\mathrm{H}-1.03889666-1.83293450 \quad 1.28627128$

$\mathrm{H}-0.58742331 \quad 1.46223572-0.59020624$

$\mathrm{H}-0.631434662 .44608189 \quad 0.87130414$

$\mathrm{H}-2.046525621 .504102410 .39557238$

$\mathrm{H}-2.18661678 \quad 0.27801158 \quad 2.58623318$

$\mathrm{H}-0.86922708 \quad 1.29524965 \quad 3.17769732$

$\mathrm{H}-0.75080789-0.46208928 \quad 3.29661188$

$0-0.51408509-0.55817217-1.81338167$

O $2.12320140 \quad 2.37236952-1.33878606$

o $-0.52752881-2.01319140-1.67871601$

Vibrational frequencies (in $\mathrm{cm}-1$ )

$\begin{array}{llllllllllllll}39.7 & 50.5 & 71.2 & 100.2 & 136.8 & 168.1 & 179.5 & 195.5 & 228.8 & 240.1 & 270.1 & 289.9 & 318.3 & 320.0\end{array}$ $355.3 \quad 393.4 \quad 414.5 \quad 435.2 \quad 518.0 \quad 550.6 \quad 606.0 \quad 674.9 \quad 755.8 \quad 777.8 \quad 848.1 \quad 873.1 \quad 917.5 \quad 935.7$ $937.8 \quad 994.5 \quad 1007.8 \quad 1017.3 \quad 1018.3 \quad 1050.9 \quad 1059.8 \quad 1074.0 \quad 1090.7 \quad 1107.8 \quad 1124.4 \quad 1136.3$

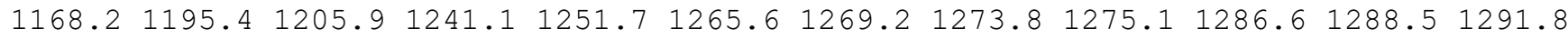
$1298.31300 .21304 .91312 .3 \quad 1334.41342 .6 \quad 1354.41384 .21389 .2 \quad 1406.91409 .91569 .5$ $1830.52639 .02654 .3 \quad 2667.5 \quad 2668.5 \quad 2672.7 \quad 2673.6 \quad 2676.92677 .92678 .92712 .5 \quad 2721.8$ $2737.7 \quad 2761.5 \quad 2779.6 \quad 2780.6 \quad 2783.1$

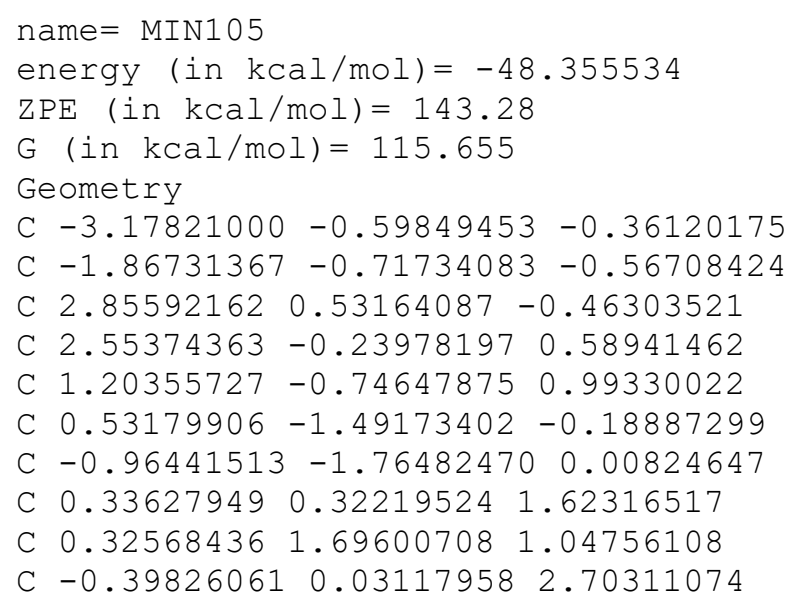


$\mathrm{H}-3.78457098 \quad 0.17557931-0.80016375$

$\mathrm{H}-3.74131028-1.255245210 .27707561$

$\mathrm{H}-2.10769061 \quad 0.97376629-2.72600033$

H $3.85157075 \quad 0.86701115 \quad-0.75107414$

$\mathrm{H} \quad 1.34229340 \quad 2.09364661 \quad 0.90978137$

$\mathrm{H} 3.35748314 \quad-0.56064477 \quad 1.25115325$

H $1.37135631-1.530696431 .78947791$

$\mathrm{H} \quad 1.06197172-2.46040627-0.31292994$

$\mathrm{H} \quad 0.72153146-0.97255382-1.14597446$

$\mathrm{H}-1.22632972-2.74570777-0.44669338$

$\mathrm{H}-0.185779831 .72185363 \quad 0.07166873$

H $1.04439172 \quad 0.83825476-1.18714335$

$\mathrm{H}-0.20644803 \quad 2.41333306 \quad 1.68881249$

$\mathrm{H}-1.18738978-1.876138291 .09439563$

$\mathrm{H}-1.03515671 \quad 0.74572384 \quad 3.19765016$

$\mathrm{H}-0.42427943-0.93358692 \quad 3.17799864$

o $-1.87418083 \quad 1.22613137-1.79738493$

O $2.001040651 .04025303-1.37440696$

o - $1.12599796 \quad 0.07992699-1.40536583$

Vibrational frequencies (in cm-1)

$\begin{array}{lllllllllllllll}14.4 & 33.2 & 46.5 & 85.8 & 112.2 & 130.3 & 162.4 & 171.2 & 190.2 & 212.2 & 228.8 & 251.8 & 296.4 & 325.7\end{array}$ $332.5 \quad 410.9 \quad 434.7 \quad 466.6 \quad 500.0 \quad 513.2 \quad 553.4 \quad 560.0 \quad 573.1 \quad 604.8 \quad 622.4 \quad 750.0793 .8 \quad 829.9$ $916.9933 .3 \quad 967.8 \quad 992.4 \quad 995.1 \quad 1010.7 \quad 1034.9 \quad 1044.1 \quad 1052.6 \quad 1058.9 \quad 1069.4 \quad 1089.1 \quad 1094.0$ $1146.41162 .5 \quad 1183.6 \quad 1218.4 \quad 1260.6 \quad 1277.2 \quad 1279.3 \quad 1292.6 \quad 1294.4 \quad 1307.4 \quad 1311.51331 .6$ $1339.01364 .7 \quad 1367.1 \quad 1382.6 \quad 1392.1 \quad 1396.3 \quad 1401.0 \quad 1406.01418 .91840 .11849 .21876 .4$ $2638.92645 .92659 .2 \quad 2667.2 \quad 2678.6 \quad 2713.4 \quad 2725.3 \quad 2727.0 \quad 2730.4 \quad 2733.3 \quad 2747.3 \quad 2760.1$ $2775.12793 .9 \quad 2799.8 \quad 2865.5$

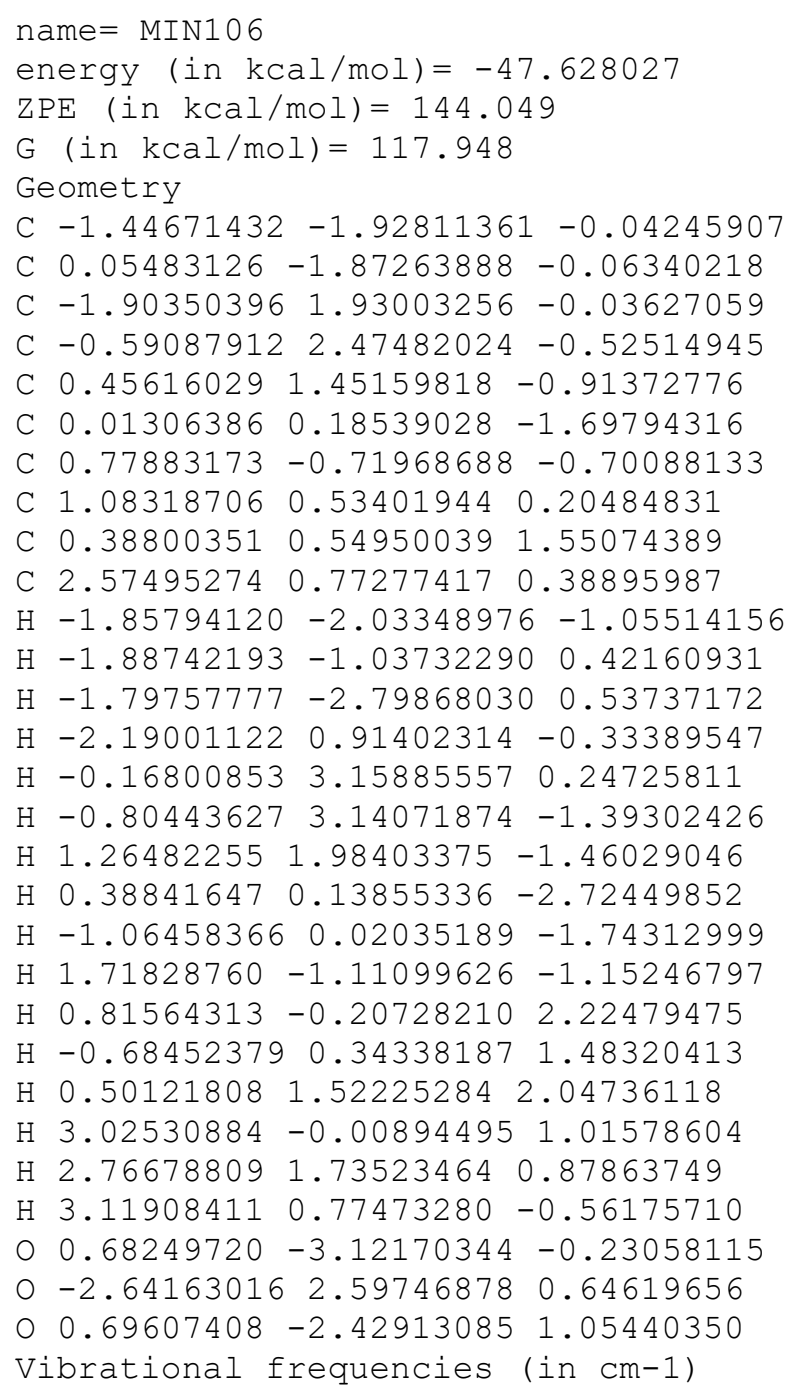


$\begin{array}{llllllllllllllllll}35.6 & 47.1 & 54.5 & 119.7 & 144.8 & 158.1 & 182.8 & 197.5 & 219.6 & 243.5 & 267.1 & 291.9 & 322.1 & 329.9\end{array}$

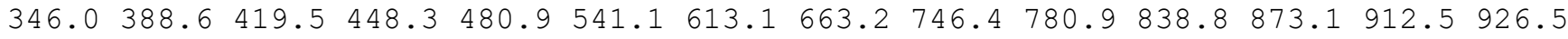

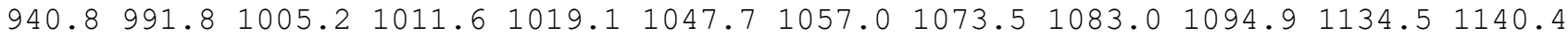

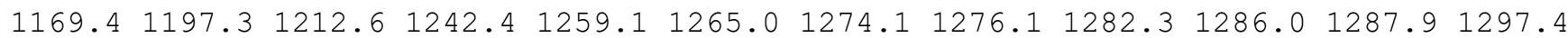
$1300.21302 .91308 .2 \quad 1327.2 \quad 1336.9 \quad 1356.8 \quad 1359.3 \quad 1385.7 \quad 1389.7 \quad 1406.2 \quad 1412.01548 .0$ $1833.4 \quad 2640.12649 .92662 .8 \quad 2667.8 \quad 2672.4 \quad 2673.6 \quad 2674.12678 .12680 .6 \quad 2703.92713 .0$ $2735.4 \quad 2765.0 \quad 2776.5 \quad 2779.7 \quad 2781.8$

\section{name $=$ MIN107}

energy (in $\mathrm{kcal} / \mathrm{mol})=-48.848722$

$\operatorname{ZPE}($ in $\mathrm{kcal} / \mathrm{mol})=145.307$

$\mathrm{G}($ in $\mathrm{kcal} / \mathrm{mol})=120.712$

Geometry

C $-2.67131284-0.15242560 \quad 0.46663888$

C $-1.37144548 \quad-0.07747042 \quad 0.13569967$

C $-0.70414543 \quad 1.26620254 \quad-0.04493382$

C $0.78421703 \quad 1.48123550 \quad 0.27537923$

C $1.70016675 \quad 0.302679490 .00012086$

C $1.23202338-0.52671060-1.22995111$

C $0.55021089-1.50852591-0.24943881$

C $1.42288904-0.94340968 \quad 0.92605455$

C $0.74558101-0.72844766 \quad 2.25917733$

C $2.68213175-1.78186570 \quad 1.11994509$

$\mathrm{H}-3.29502017 \quad 0.70095598 \quad 0.65365789$

$\mathrm{H}-3.19294992-1.090449120 .56876322$

$\mathrm{H}-2.54476749 \quad 1.18432318-1.96360074$

$\mathrm{H}-1.29233876 \quad 2.02442346 \quad 0.54137451$

$\mathrm{H} \quad 0.88637306 \quad 1.79008182 \quad 1.33601073$

H $1.132263332 .35751710-0.32019397$

H $2.75420416 \quad 0.61031903-0.02459638$

H 2.04646071 $-0.96614979-1.81143338$

$\mathrm{H} \quad 0.56618208-0.02885986-1.93780345$

$\mathrm{H} \quad 0.59713272-2.58558053-0.45484138$

$\mathrm{H} 1.39616849-0.18241240 \quad 2.95303890$

$\mathrm{H} \quad 0.49024753-1.68931785 \quad 2.72739245$

$\mathrm{H}-0.19419438-0.169313092 .18576184$

H 2.44622044 $-2.75344044 \quad 1.57098699$

H $3.39295098-1.276503601 .78468291$

H $3.20231082-1.977522840 .17556637$

o $-2.13094366 \quad 2.03737344 \quad-1.67595152$

$0-0.797881451 .61696956-1.43090782$

O $-0.82762433-1.29980578-0.11700056$

Vibrational frequencies (in $\mathrm{cm}-1$ )

$\begin{array}{llllllllllllllll}44.4 & 78.5 & 88.2 & 142.2 & 187.2 & 199.4 & 205.6 & 223.1 & 264.6 & 292.0 & 319.0 & 340.8 & 370.6 & 378.5\end{array}$

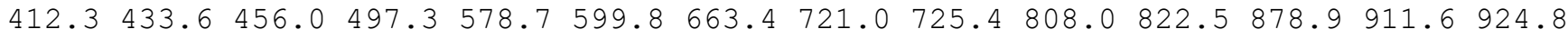
$940.8991 .2 \quad 1008.3 \quad 1014.3 \quad 1015.1 \quad 1018.4 \quad 1026.2 \quad 1047.2 \quad 1073.7 \quad 1087.3 \quad 1102.4 \quad 1117.6$ $1168.21188 .4 \quad 1201.3 \quad 1225.4 \quad 1231.1 \quad 1239.7 \quad 1262.3 \quad 1266.3 \quad 1280.2 \quad 1286.7 \quad 1290.51295 .3$ $1299.51302 .6 \quad 1315.7 \quad 1330.2 \quad 1342.0 \quad 1346.5 \quad 1370.0 \quad 1374.4 \quad 1394.5 \quad 1403.4 \quad 1412.51418 .8$ $1799.52648 .8 \quad 2657.5 \quad 2669.8 \quad 2675.92676 .8 \quad 2680.42686 .12713 .7 \quad 2729.92730 .3 \quad 2737.0$ $2767.8 \quad 2781.3 \quad 2783.8 \quad 2795.8 \quad 2854.1$

\section{name $=$ MIN108}

energy (in $\mathrm{kcal} / \mathrm{mol})=-47.552262$

$\mathrm{ZPE}($ in $\mathrm{kcal} / \mathrm{mol})=144.434$

$\mathrm{G}($ in $\mathrm{kcal} / \mathrm{mol})=118.795$

Geometry

C $-2.05774209-1.30083936 \quad 0.50323397$

C $-0.58460003-1.56742776 \quad 0.39860187$

C $-0.83786913 \quad 2.46858463 \quad 0.35321277$

C $0.131926612 .52661656-0.79857592$

C $0.603508261 .16958142-1.29912764$

C $-0.48795772 \quad 0.11278221-1.60867171$

C $0.21976472-0.92440283-0.70046814$ 
C $1.286114420 .16454805-0.29490642$

C $1.30027917 \quad 0.57685604 \quad 1.16289860$

C $2.69292947-0.21769006-0.73883226$

$\mathrm{H}-2.51015589-1.87541712 \quad 1.32609249$

$\mathrm{H}-2.58544024-1.57731574-0.41934184$

$\mathrm{H}-2.26915465-0.23722980 \quad 0.69975304$

$\mathrm{H}-0.50481255 \quad 2.88588713 \quad 1.31318012$

$\mathrm{H} 1.01501372 \quad 3.14032653-0.52833012$

$\mathrm{H}-0.36309634 \quad 3.05951546-1.64295156$

H $1.258854431 .34223722-2.17813886$

$\mathrm{H}-0.55225917-0.17917058-2.65977681$

$\mathrm{H}-1.49622730 \quad 0.40602301-1.29197479$

$\mathrm{H} \quad 0.69216785-1.73526272-1.29650799$

$\mathrm{H} \quad 0.29385762 \quad 0.67170393 \quad 1.58639059$

H $1.82644164 \quad 1.527608361 .30356049$

$\mathrm{H} 1.82116878 \quad-0.17339294 \quad 1.77883878$

H $3.06880853-1.07345920-0.16213554$

H $3.39491231 \quad 0.61094123-0.59197264$

$\mathrm{H} \quad 2.73343560-0.49999980-1.79670566$

$\begin{array}{llllll}0 & -0.20343837 & -2.88885518 & 0.70592929\end{array}$

$\begin{array}{lllll}0 & -1.95247999 & 2.02053392 & 0.24358151\end{array}$

$00.07948053-1.783530821 .61542490$

Vibrational frequencies (in $\mathrm{cm}-1$ )

$\begin{array}{lllllllllllllll}28.5 & 49.5 & 91.0 & 126.6 & 161.8 & 168.9 & 190.0 & 206.5 & 224.4 & 253.1 & 307.1 & 308.8 & 317.1 & 340.7\end{array}$

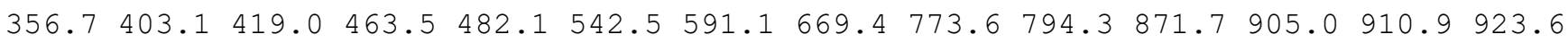
$941.3988 .8999 .5 \quad 1007.4 \quad 1018.8 \quad 1049.9 \quad 1060.0 \quad 1067.5 \quad 1082.3 \quad 1094.7 \quad 1117.5 \quad 1139.5 \quad 1164.8$ $\begin{array}{llllllllllllllll}1195.0 & 1208.9 & 1230.3 & 1258.1 & 1270.4 & 1273.5 & 1276.3 & 1289.2 & 1291.3 & 1296.1 & 1301.4 & 1302.3\end{array}$

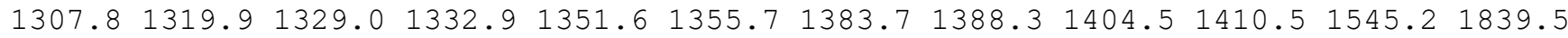
$2644.22658 .8 \quad 2662.0 \quad 2664.92672 .2 \quad 2672.5 \quad 2673.92677 .22680 .32706 .42713 .12738 .0$ $2759.8 \quad 2776.7 \quad 2777.9 \quad 2781.4$

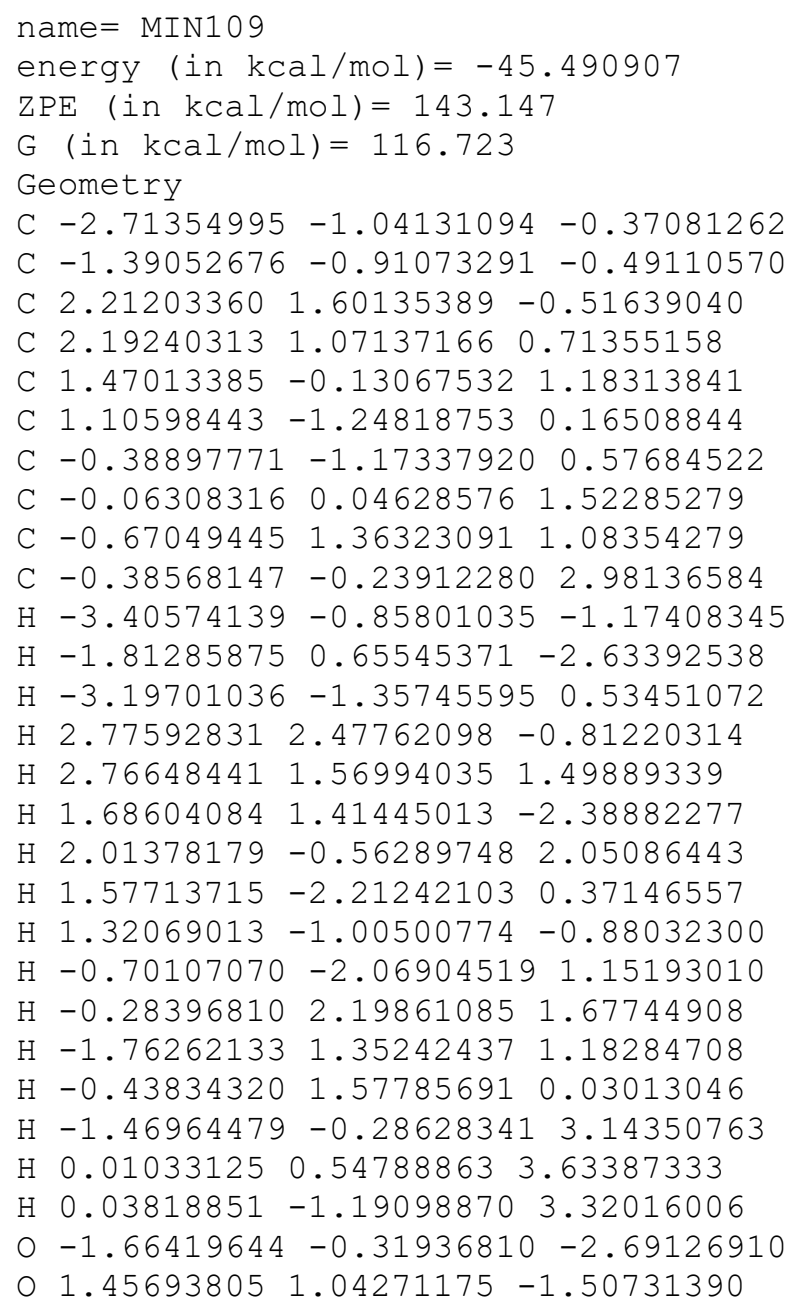


$0-0.73656682-0.49780614-1.62266011$

Vibrational frequencies (in $\mathrm{cm}-1$ )

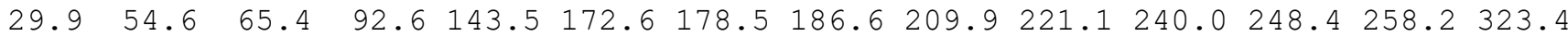
$331.4 \quad 362.1 \quad 394.6 \quad 411.4 \quad 441.4 \quad 488.2 \quad 568.8 \quad 606.8 \quad 638.2 \quad 664.6 \quad 722.7 \quad 789.3 \quad 836.9 \quad 855.7$ $923.7 \quad 939.8 \quad 965.1 \quad 999.2 \quad 1005.4 \quad 1012.0 \quad 1015.5 \quad 1018.0 \quad 1043.4 \quad 1056.7 \quad 1078.6 \quad 1117.3 \quad 1129.8$ $1138.1 \quad 1159.91207 .5 \quad 1225.2 \quad 1260.9 \quad 1274.6 \quad 1285.3 \quad 1285.91288 .2 \quad 1298.4 \quad 1300.8 \quad 1307.1$ $1309.21329 .71343 .1 \quad 1361.8 \quad 1368.3 \quad 1380.8 \quad 1390.01403 .31406 .11408 .4 \quad 1860.11868 .5$ $2669.8 \quad 2676.92677 .4 \quad 2678.8 \quad 2682.8 \quad 2711.4 \quad 2717.8 \quad 2726.82733 .5 \quad 2751.8 \quad 2762.2 \quad 2780.1$ $2782.42801 .62806 .3 \quad 2870.0$

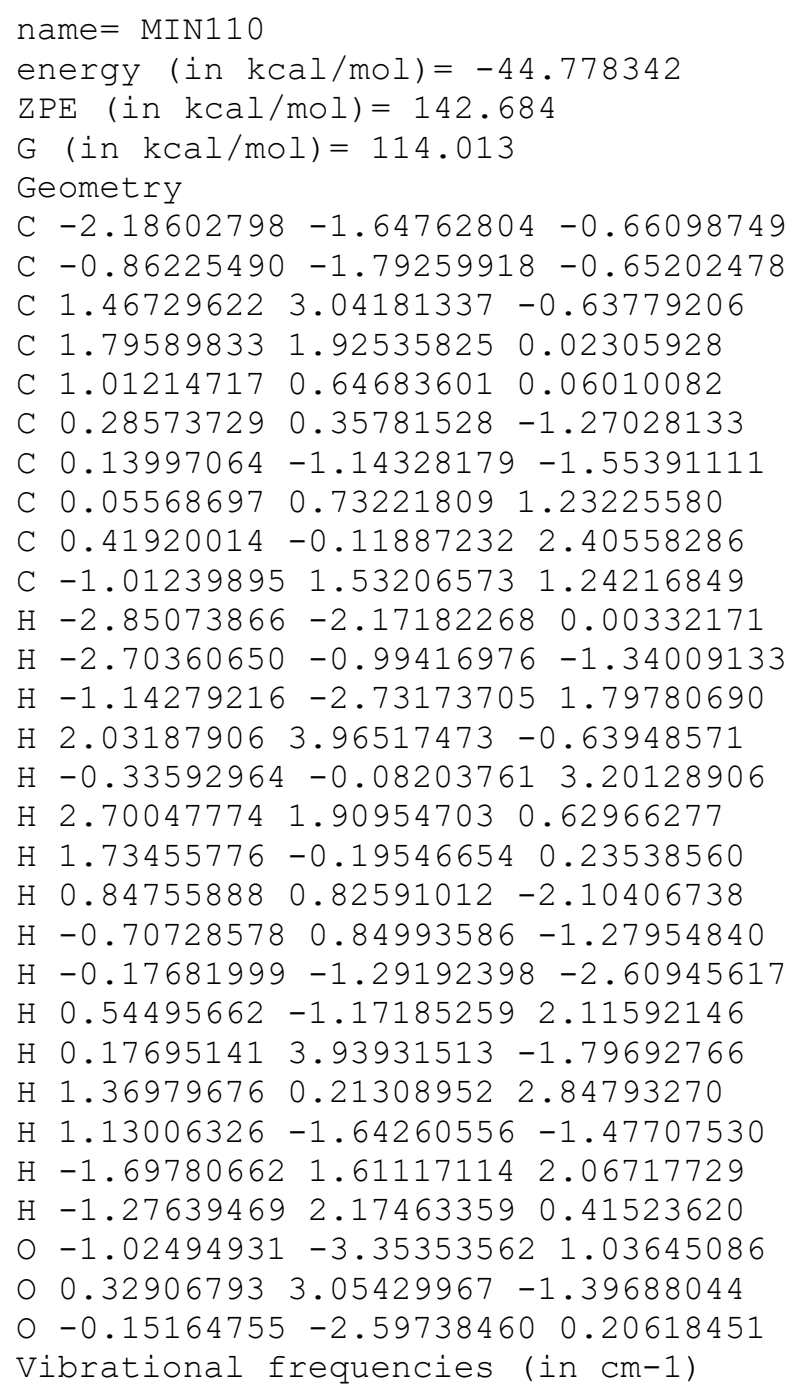


C $0.99675715-1.21167090-0.45192879$

C $2.24828163-1.39997932-0.00251926$

C $2.481461610 .07405375 \quad 0.35983805$

C $2.65994595 \quad 0.31018597 \quad 1.84488164$

C $3.55058273 \quad 0.76737215-0.45953129$

$\mathrm{H}-4.29024436 \quad 0.18893791 \quad 0.65434252$

$\mathrm{H}-4.08306240-1.45355497 \quad 0.02693621$

$\mathrm{H}-3.76082224-0.05714672-1.02160756$

$\mathrm{H}-2.02304491 \quad 1.724309690 .65137624$

$\mathrm{H}-0.03093960 \quad 0.20539286 \quad 1.68296038$

H $0.26498908 \quad 1.84583908 \quad 1.07974306$

H $1.00642817 \quad 0.89812744-1.14376169$

$\mathrm{H}-0.52682398-0.02246562-2.20407487$

$\mathrm{H} \quad 0.21111672-1.82438232-0.81252801$

H $2.90904335-2.216209170 .11389656$

$\mathrm{H} 3.66516694 \quad 0.01684973 \quad 2.17297648$

H $1.94885372-0.279869952 .43871066$

$\mathrm{H} \quad 2.52088679 \quad 1.36495120 \quad 2.10631659$

$\mathrm{H} \quad 4.554221120 .43826769-0.16218448$

$\mathrm{H} 3.508636291 .85532301-0.33087808$

H $3.44726818 \quad 0.55524060-1.53029854$

$\begin{array}{lllll}0 & -1.48347752 & 0.02409111 & -1.95238379\end{array}$

o $-1.50286038 \quad 1.21272486-1.17173146$

O $-1.73867626-1.35133024 \quad 1.05238311$

Vibrational frequencies (in cm-1)

$\begin{array}{lllllllllllllll}39.9 & 41.9 & 59.0 & 106.6 & 114.6 & 131.7 & 137.9 & 162.2 & 192.0 & 199.8 & 214.3 & 255.9 & 272.3 & 331.6\end{array}$

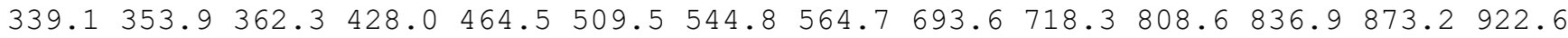
$929.5964 .9967 .5 \quad 999.1 \quad 1006.8 \quad 1016.9 \quad 1032.3 \quad 1048.0 \quad 1067.3 \quad 1080.7 \quad 1094.4 \quad 1127.7 \quad 1143.3$

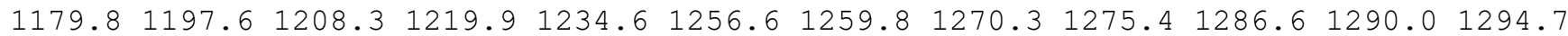

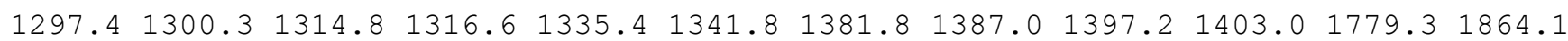
$2649.52663 .3 \quad 2667.92671 .8 \quad 2672.6 \quad 2677.1 \quad 2679.22681 .92718 .6 \quad 2734.3 \quad 2777.5 \quad 2780.6$ $2783.12792 .3 \quad 2809.5 \quad 2842.0$

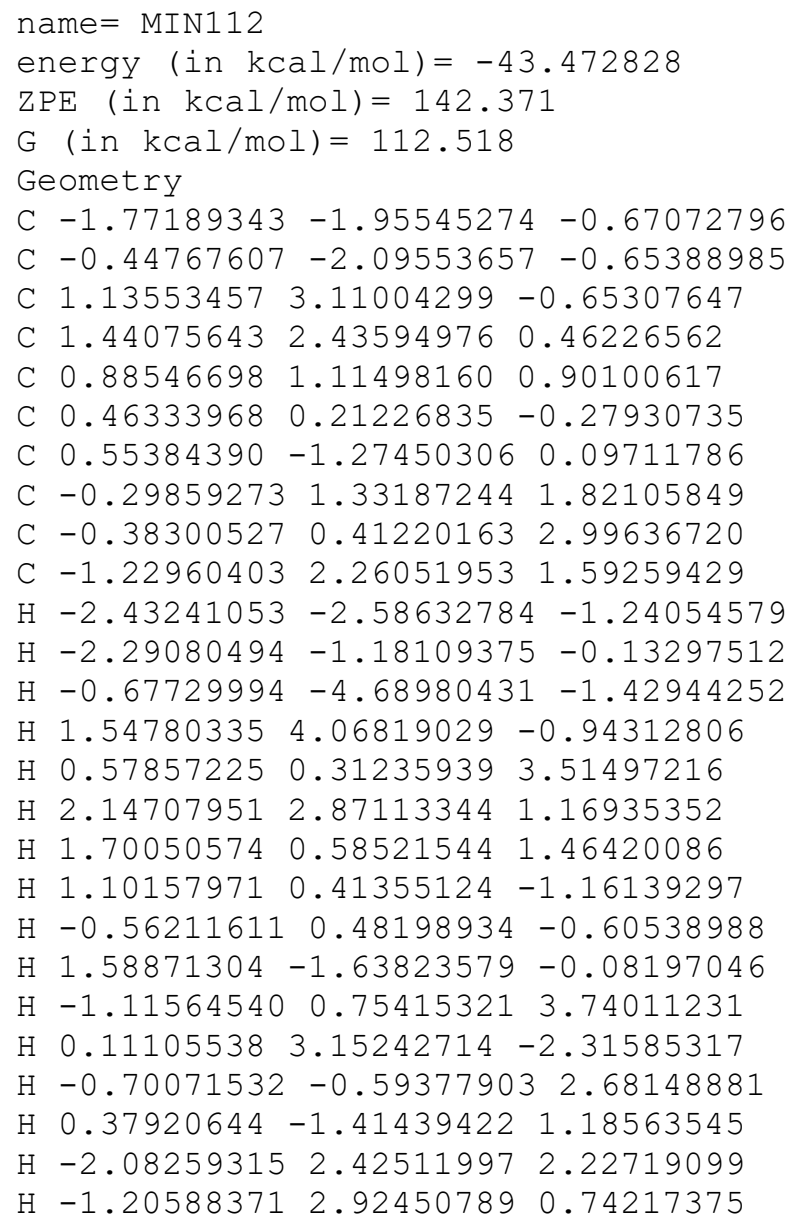


$0-0.61422973-3.91918112-2.04633974$

$0.227315432 .57834818-1.52546489$

O $0.26258996-3.07675691-1.30339758$

Vibrational frequencies (in $\mathrm{cm}-1$ )

$\begin{array}{lllllllllllllll}-26.3 & 11.8 & 26.1 & 41.6 & 67.2 & 83.4 & 111.8 & 137.0 & 179.5 & 185.5 & 205.2 & 233.1 & 266.7 & 270.7\end{array}$ $295.8 \quad 320.9 \quad 367.4 \quad 406.1 \quad 423.7 \quad 524.8 \quad 544.7 \quad 564.7 \quad 603.4 \quad 616.2 \quad 639.5 \quad 741.5 \quad 802.1825 .1$ $925.8 \quad 957.6 \quad 965.4 \quad 990.7 \quad 1003.4 \quad 1024.8 \quad 1035.9 \quad 1041.3 \quad 1049.8 \quad 1057.3 \quad 1083.0 \quad 1089.9 \quad 1105.9$ $\begin{array}{llllllllllll}1124.7 & 1169.7 & 1207.8 & 1226.1 & 1260.7 & 1274.7 & 1278.1 & 1287.5 & 1295.2 & 1300.3 & 1307.0 & 1318.7\end{array}$

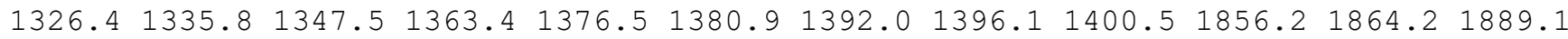
$2641.12661 .0 \quad 2665.6 \quad 2675.92689 .0 \quad 2724.2 \quad 2727.52728 .32729 .5 \quad 2743.5 \quad 2755.62777 .0$ $2791.32797 .4 \quad 2807.6 \quad 2870.4$

name $=$ MIN113

energy (in $\mathrm{kcal} / \mathrm{mol})=-43.887085$

$\mathrm{ZPE}($ in $\mathrm{kcal} / \mathrm{mol})=143.514$

$\mathrm{G}(\mathrm{in} \mathrm{kcal} / \mathrm{mol})=115.093$

Geometry

C $-4.09641517-0.98734009-0.43154793$

C $-2.62924953-0.78672528-0.56673034$

C $2.49399316 \quad 0.01306413-0.57051476$

C $1.96400989-0.40540308 \quad 0.77739042$

C $0.43002653-0.46030550 \quad 0.88664996$

C $-0.20441693-1.29972378-0.24512827$

C $-1.65891364-1.52622307-0.00627547$

C $-0.18377966 \quad 0.91736518 \quad 0.93897179$

C $0.02088592 \quad 1.86401738 \quad 0.01993708$

C $-1.08133646 \quad 1.14747738 \quad 2.11138849$

$\mathrm{H}-4.64316074-0.50510059-1.26097216$

$\mathrm{H}-4.36924183-2.05090496-0.43097622$

$\mathrm{H}-4.47320058-0.53783097 \quad 0.49935410$

$\mathrm{H} \quad 1.93414979-0.28752667-1.46355887$

H 2.35848159 $0.28293978 \quad 1.55886477$

$\mathrm{H} 2.38740215-1.401404941 .03022609$

$\mathrm{H} \quad 0.20011058-0.99208351 \quad 1.85071279$

$\mathrm{H} \quad 0.31680517-2.27953549-0.30673266$

$\mathrm{H}-0.03276883-0.82069101-1.22959810$

$\mathrm{H}-1.90205104-2.35273312 \quad 0.65062535$

$\mathrm{H} \quad 0.66390908 \quad 1.76053550 \quad-0.83616037$

$\mathrm{H}-0.43011948 \quad 2.84296513 \quad 0.06973298$

$\mathrm{H}-1.45871553 \quad 0.58731869-1.34818062$

$\mathrm{H}-0.52024214 \quad 1.11748045 \quad 3.05602863$

H $-1.86368433 \quad 0.374757542 .16706755$

$\mathrm{H}-1.593648332 .11792572 \quad 2.07054752$

$\begin{array}{lllll}0 & 3.13492215 & 1.25618189 & -0.64507222\end{array}$

$03.88931161 \quad 0.00716441-0.70178312$

$\begin{array}{llll}0 & -2.40246189 & 0.27882318 & -1.37878024\end{array}$

Vibrational frequencies (in $\mathrm{cm}-1$ )

$\begin{array}{llllllllllllllll}28.3 & 31.2 & 39.2 & 52.5 & 66.0 & 97.6 & 109.7 & 115.6 & 162.9 & 195.0 & 243.2 & 287.3 & 295.0 & 320.1\end{array}$

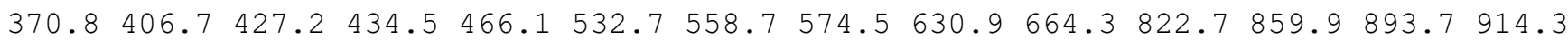
$950.7983 .0990 .1 \quad 1012.2 \quad 1016.9 \quad 1039.2 \quad 1046.3 \quad 1050.5 \quad 1064.2 \quad 1092.8 \quad 1102.1 \quad 1123.51167 .2$

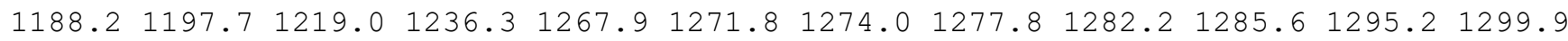
$1309.81332 .2 \quad 1348.1 \quad 1367.3 \quad 1380.3 \quad 1386.3 \quad 1394.3 \quad 1404.2 \quad 1443.5 \quad 1538.91863 .4 \quad 1867.2$ $2651.92652 .62664 .52668 .22673 .42674 .5 \quad 2682.12688 .22721 .62733 .32736 .62746 .9$ $2773.8 \quad 2777.7 \quad 2780.2 \quad 2793.6$

name $=$ MIN114

energy $($ in $\mathrm{kcal} / \mathrm{mol})=-41.73358$

$\operatorname{ZPE}($ in $\mathrm{kcal} / \mathrm{mol})=144.273$

$\mathrm{G}($ in $\mathrm{kcal} / \mathrm{mol})=118.113$

Geometry

C $-3.04693539-1.13533798-0.60228339$

C $-1.72247368-1.05814341-0.76792034$

C $1.252843801 .36161043-0.76368749$ 
C $1.91597625 \quad 0.60701011 \quad 0.36120153$

C $1.24040632-0.69503123 \quad 0.75579744$

C $0.77856382-1.63023649-0.39413726$

C $-0.68006223-1.60679250 \quad 0.13528081$

C $-0.23228278-0.65059515 \quad 1.31133259$

C $-0.91511372 \quad 0.69988138 \quad 1.34444489$

C $-0.34980438-1.32919598 \quad 2.67084399$

$\mathrm{H}-3.76807296-0.71885554-1.27960598$

$\mathrm{H}-3.50941491-1.62506707 \quad 0.23462211$

$\mathrm{H}-1.84475629-0.06152134-2.45816615$

$\mathrm{H} \quad 0.62901667 \quad 0.79786207 \quad-1.47129609$

$\mathrm{H} \quad 1.99425292 \quad 1.26745989 \quad 1.25388739$

H $2.96887417 \quad 0.395701590 .06609998$

$\mathrm{H} 1.90762876-1.22605623 \quad 1.46551408$

H $1.23789810-2.62263684-0.37646026$

$\mathrm{H} \quad 0.91172612-1.23475643-1.40552682$

$\mathrm{H}-1.00496332 \quad-2.59861530 \quad 0.50863224$

$\mathrm{H}-1.94244204 \quad 0.61823225 \quad 1.72374501$

$\mathrm{H}-0.98923330 \quad 1.15842508 \quad 0.34986637$

$\mathrm{H}-0.378428921 .405216901 .99036800$

$\mathrm{H} \quad 0.13427360-2.31185166 \quad 2.68806993$

$\mathrm{H}-1.39980615-1.47924692 \quad 2.95016793$

$\mathrm{H} \quad 0.11354514-0.72047872 \quad 3.45657642$

O $1.99795030 \quad 2.41570297-1.31169227$

$0.823194392 .66564582-0.48142090$

o-1.16600037-0.43691288-1.84794002

Vibrational frequencies (in $\mathrm{cm}-1$ )

$\begin{array}{llllllllllllllll}24.1 & 42.9 & 59.5 & 94.6 & 152.5 & 167.4 & 182.8 & 212.4 & 219.9 & 274.8 & 292.0 & 329.8 & 365.5 & 368.4\end{array}$ $390.6 \quad 403.6 \quad 414.9 \quad 442.8 \quad 531.4 \quad 574.1 \quad 588.1 \quad 620.2 \quad 682.3 \quad 787.8 \quad 831.4 \quad 882.5 \quad 904.9 \quad 923.7$ $943.2982 .6 \quad 994.9998 .1 \quad 1006.9 \quad 1017.3 \quad 1036.6 \quad 1040.4 \quad 1068.7 \quad 1084.1 \quad 1098.7 \quad 1124.51132 .2$

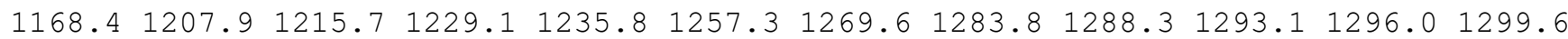
$1304.71316 .0 \quad 1323.6 \quad 1342.8 \quad 1360.5 \quad 1374.4 \quad 1386.8 \quad 1405.8 \quad 1409.11438 .4 \quad 1533.01857 .3$ $2653.62668 .12670 .0 \quad 2675.6 \quad 2677.7 \quad 2679.2 \quad 2679.92711 .92719 .12734 .12736 .8 \quad 2762.4$ $2780.8 \quad 2782.6 \quad 2788.7 \quad 2809.2$

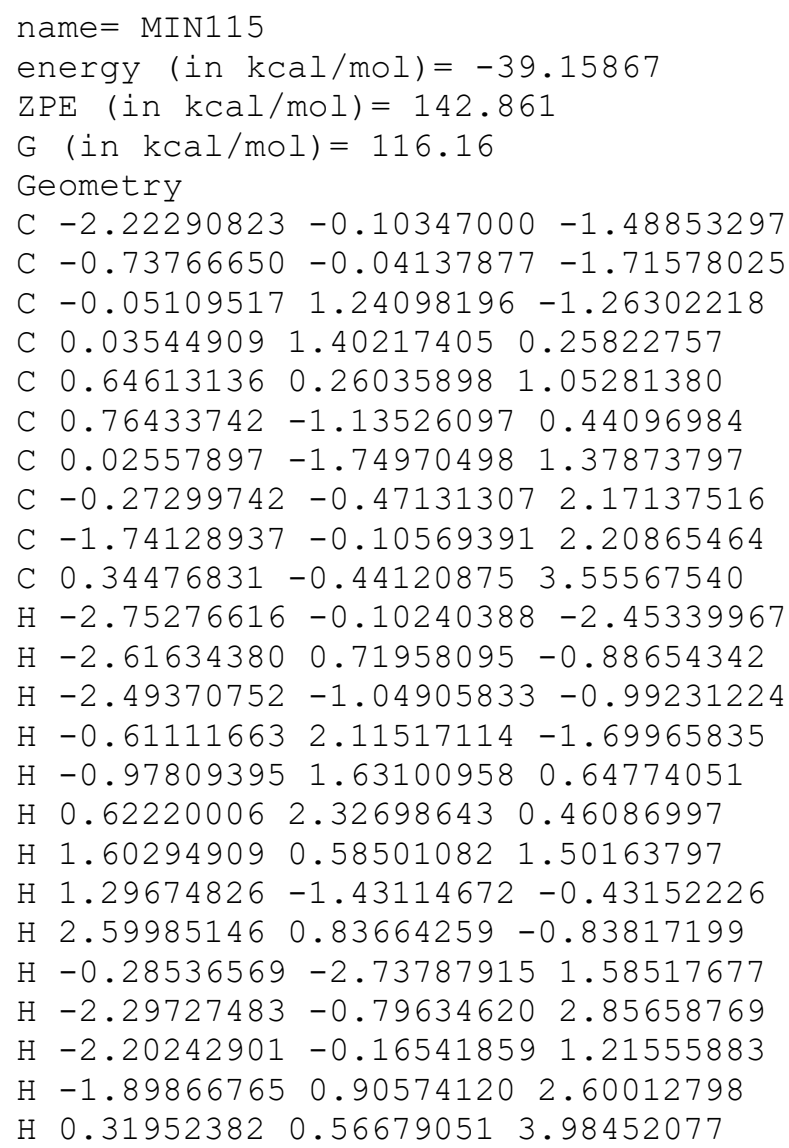


H $1.39022511-0.77293230 \quad 3.54435822$

$\mathrm{H}-0.19343691-1.10643070 \quad 4.24284276$

$02.099766890 .41384994-1.57989439$

O $1.189483651 .44816351-1.90137311$

O $-0.15412120-0.92947457-2.27430995$

Vibrational frequencies (in $\mathrm{cm}-1$ )

$\begin{array}{lllllllllllllll}23.3 & 62.6 & 86.4 & 97.4 & 114.9 & 130.9 & 158.4 & 166.8 & 180.9 & 201.2 & 240.3 & 270.4 & 273.3 & 299.9\end{array}$ $325.0 \quad 352.9 \quad 369.0 \quad 385.9 \quad 454.1 \quad 502.5 \quad 566.9 \quad 597.7 \quad 695.8 \quad 733.5 \quad 751.7 \quad 865.5 \quad 893.9 \quad 905.4$ $946.5952 .2979 .6996 .2 \quad 998.5 \quad 1011.0 \quad 1018.4 \quad 1047.7 \quad 1055.1 \quad 1082.5 \quad 1094.11114 .6 \quad 1141.4$ $\begin{array}{lllllllllllll}1184.3 & 1196.0 & 1209.7 & 1220.1 & 1240.1 & 1254.3 & 1263.6 & 1269.7 & 1278.8 & 1288.3 & 1294.8 & 1298.4\end{array}$

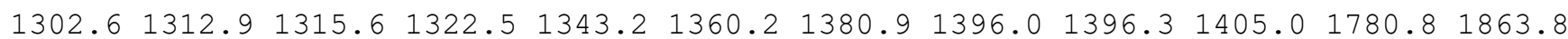
$2647.12659 .92662 .5 \quad 2673.3 \quad 2677.0 \quad 2678.4 \quad 2679.22682 .22717 .02732 .5 \quad 2771.12778 .8$ 2781.92783 .32809 .32877 .1

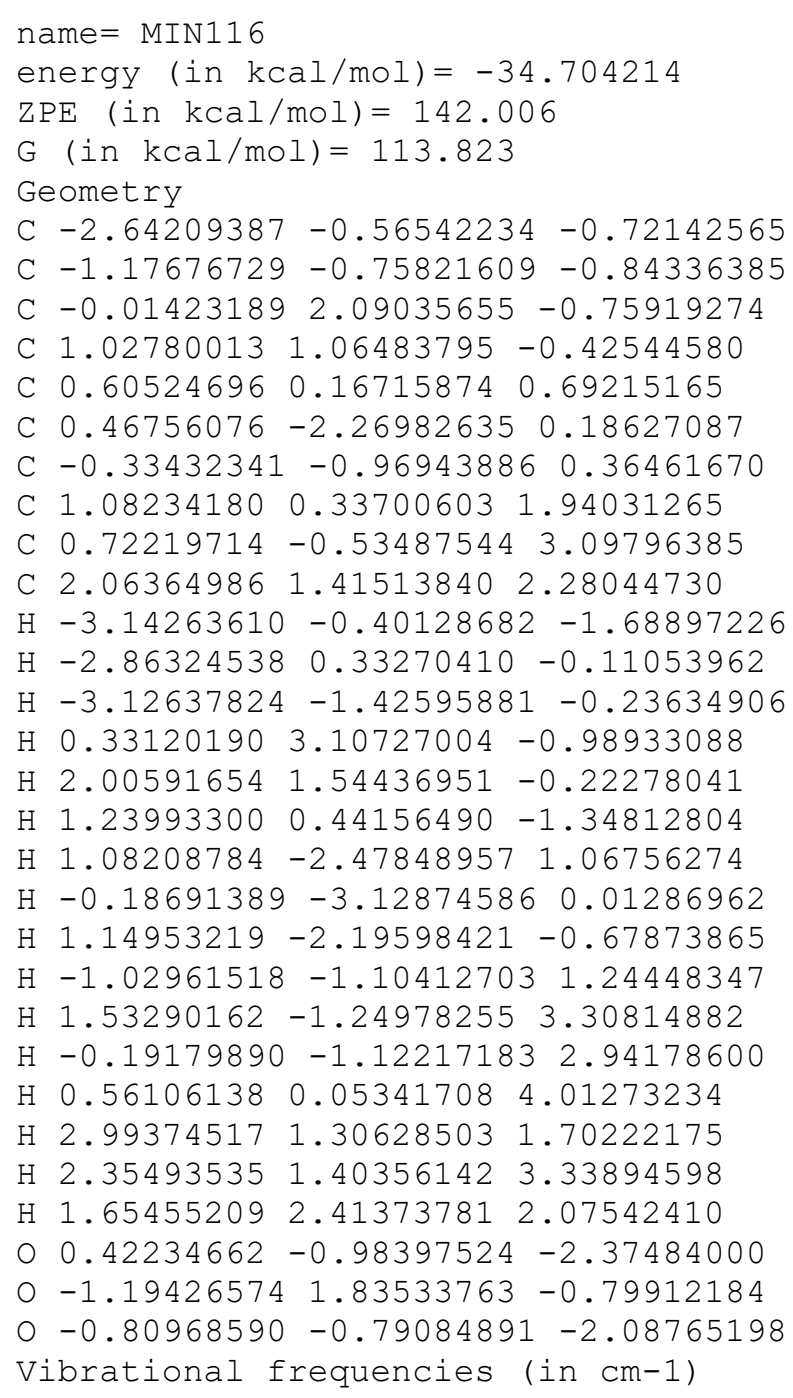


C $-2.16487853 \quad 0.00299385-0.72247584$

C $1.61520880 \quad 0.90067212 \quad-0.67859691$

C $1.88666050-0.25665423 \quad 0.18020714$

C $0.74364663-1.23028146 \quad 0.38926814$

C $-0.13680767-1.55434825-0.84757310$

C $-1.41554674-1.12776933-0.08232401$

C $-0.53544382-0.74136590 \quad 1.16946742$

C $-0.56988848 \quad 0.71697129 \quad 1.57678684$

C $-0.84312975-1.62179218 \quad 2.37441746$

$\mathrm{H}-4.05665154-0.85960006-1.27954331$

$\mathrm{H}-4.120009130 .88429240-0.98844435$

$\mathrm{H}-4.01277859-0.24006410 \quad 0.38017369$

$\mathrm{H} \quad 0.66173112 \quad 1.09381520-1.16210941$

$\mathrm{H} \quad 2.26767612 \quad 0.10849284 \quad 1.17094289$

H $2.77902109-0.79965417-0.23381519$

$\mathrm{H} 1.15068569-2.15343793 \quad 0.85051934$

$\mathrm{H}-0.12260508-2.60434423-1.15036676$

$\mathrm{H} \quad 0.07759703-0.95182681-1.73715550$

$\mathrm{H}-2.08582035-1.977520750 .13273058$

$\mathrm{H}-1.52881947 \quad 0.98398419 \quad 2.03731277$

$\mathrm{H}-0.42660985 \quad 1.39921634 \quad 0.72824542$

$\mathrm{H} \quad 0.21861198 \quad 0.94405137 \quad 2.30624802$

$\mathrm{H}-0.83577721-2.68827405 \quad 2.12381352$

$\mathrm{H}-1.82817518-1.39207376 \quad 2.79691390$

$\mathrm{H}-0.10295893-1.469367023 .17057651$

$\begin{array}{lllll}0 & 3.65831804 & 1.63660140 & -0.39819480\end{array}$

O $2.51768248 \quad 1.79613064-0.92877610$

o $-1.604673730 .90839942-1.29298317$

Vibrational frequencies (in $\mathrm{cm}-1$ )

$\begin{array}{llllllllllllll}37.9 & 47.3 & 59.5 & 111.4 & 116.3 & 149.8 & 175.3 & 188.2 & 201.9 & 221.8 & 236.3 & 270.4 & 301.7 & 337.1\end{array}$ $\begin{array}{llllllllllllll}371.8 & 388.8 & 436.6 & 448.1 & 557.4 & 590.3 & 599.9 & 679.1 & 726.7 & 771.1 & 820.8 & 828.8 & 915.9 & 929.4\end{array}$ $987.8 \quad 996.3 \quad 1007.8 \quad 1018.4 \quad 1034.0 \quad 1041.1 \quad 1049.5 \quad 1052.5 \quad 1085.4 \quad 1103.01133 .11155 .5$ $\begin{array}{lllllllllllll}1164.5 & 1195.7 & 1200.7 & 1211.8 & 1237.3 & 1240.8 & 1257.4 & 1262.8 & 1272.9 & 1279.2 & 1285.3 & 1286.1\end{array}$

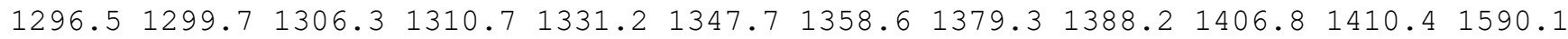
$1847.92617 .7 \quad 2664.92667 .7 \quad 2672.7 \quad 2673.6 \quad 2674.12677 .02679 .2 \quad 2681.12711 .62718 .9$ $2729.12761 .8 \quad 2779.7 \quad 2780.0 \quad 2782.7$

\section{name $=$ MIN118}

energy (in $\mathrm{kcal} / \mathrm{mol})=-34.045261$

$\operatorname{ZPE}(\mathrm{in} \mathrm{kcal} / \mathrm{mol})=143.602$

$\mathrm{G}(\mathrm{in} \mathrm{kcal} / \mathrm{mol})=116.404$

Geometry

C $-3.36101011-1.59424225-0.03633319$

C $-1.86021641-1.55308776-0.07028263$

C $2.700183920 .50404013-0.24645277$

C $1.301314320 .94297385-0.25146034$

C $0.54158786 \quad 0.50178485 \quad 0.98550228$

C $0.35813044-1.035392891 .10937433$

C $-1.18196359-0.88140013 \quad 1.08971802$

C $-1.02368364 \quad 0.67954305 \quad 0.94922692$

C $-1.59527432 \quad 1.27600721 \quad-0.32220565$

C $-1.55301718 \quad 1.42267452 \quad 2.16753735$

$\mathrm{H}-3.77689192-1.66857820-1.05283892$

$\mathrm{H}-3.79683622-0.712119190 .44522330$

$\mathrm{H}-3.70681887-2.48220616 \quad 0.51173484$

H $3.17961640 \quad 0.03786291 \quad 0.59935508$

$\mathrm{H} \quad 0.81020127 \quad 0.56176336-1.18872221$

H $1.248878602 .05236110-0.37759260$

$\mathrm{H} \quad 0.98343897 \quad 0.95543629 \quad 1.88981422$

H $0.75839286-1.472211652 .02586575$

$\mathrm{H} \quad 0.75502657-1.60791023 \quad 0.25950900$

$\mathrm{H}-1.66019676-1.17798569 \quad 2.03838394$

$\mathrm{H}-2.691269461 .30375653-0.29854520$

$\mathrm{H}-1.306344310 .70804365-1.21818649$ 
$\mathrm{H}-1.24901874 \quad 2.30732705-0.46851822$

$\mathrm{H}-1.29064404 \quad 2.48722668 \quad 2.12732782$

$\mathrm{H}-1.147337421 .023154343 .10406469$

$\mathrm{H}-2.64553687 \quad 1.35828901 \quad 2.23244958$

○ $2.992121121 .15060328-2.31956910$

$\begin{array}{lllll}0 & 3.47312409 & 0.61932014 & -1.28254257\end{array}$

o $-1.24143198-2.05463188-0.97529522$

Vibrational frequencies (in $\mathrm{cm}-1$ )

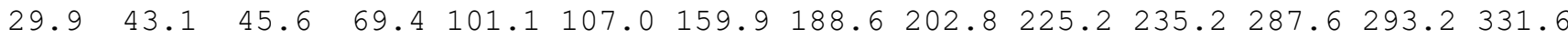
$\begin{array}{lllllllllllllllll}361.1 & 401.0 & 432.7 & 463.6 & 486.9 & 565.8 & 597.8 & 673.7 & 733.9 & 765.4 & 823.1 & 876.4 & 908.5 & 935.8\end{array}$ $959.0 \quad 994.91006 .2 \quad 1016.1 \quad 1037.2 \quad 1048.1 \quad 1053.6 \quad 1059.0 \quad 1093.6 \quad 1111.5 \quad 1128.3 \quad 1150.5$

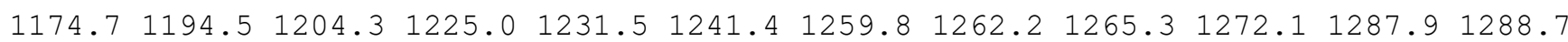
$1296.71299 .3 \quad 1304.0 \quad 1308.2 \quad 1315.2 \quad 1348.6 \quad 1350.3 \quad 1357.2 \quad 1380.7 \quad 1401.7 \quad 1405.21590 .7$ $1853.72615 .02663 .5 \quad 2667.6 \quad 2673.92676 .6 \quad 2677.32679 .52679 .8 \quad 2705.92723 .5 \quad 2729.6$ $2734.72760 .12778 .12780 .6 \quad 2782.7$

name $=$ MIN119

energy (in $\mathrm{kcal} / \mathrm{mol})=-34.078283$

$\mathrm{ZPE}(\mathrm{in} \mathrm{kcal} / \mathrm{mol})=143.709$

$\mathrm{G}(\mathrm{in} \mathrm{kcal} / \mathrm{mol})=116.489$

Geometry

C $-3.35214719-1.95636794 \quad 0.02790083$

C $-1.85149979-1.89902751-0.00501519$

C $2.737980470 .80956876-0.18190365$

C $1.30126223 \quad 0.66362610-0.43766516$

C $0.51101664 \quad 0.32396840 \quad 0.81323561$

C $0.35205283-1.191181791 .11383441$

C $-1.19082250-1.06804576 \quad 1.05732697$

C $-1.05542390 \quad 0.46017626 \quad 0.70250938$

C $-1.59126978 \quad 0.84645432 \quad-0.66259565$

C $-1.634416491 .36587487 \quad 1.77872372$

$\mathrm{H}-3.80251500-1.01581636 \quad 0.36472727$

$\mathrm{H}-3.69128216-2.75199660 \quad 0.70622145$

$\mathrm{H}-3.76138380-2.19250015-0.96624113$

H $3.26006160 \quad 0.28903388 \quad 0.60557369$

$\mathrm{H} \quad 1.13467653-0.10176704-1.23680404$

$\mathrm{H} \quad 0.91314937 \quad 1.60975360-0.90327743$

$\mathrm{H} \quad 0.90245081 \quad 0.88773674 \quad 1.67896809$

$\mathrm{H} \quad 0.74481857-1.51092036 \quad 2.08085374$

H $0.76984432-1.85566235 \quad 0.34585774$

$\mathrm{H}-1.67474633-1.23744725 \quad 2.03409930$

$\mathrm{H}-2.68649170 \quad 0.80545032 \quad-0.69373132$

$\mathrm{H}-1.218569170 .18538793-1.45777169$

$\mathrm{H}-1.30176074 \quad 1.87129237 \quad-0.93106854$

$\mathrm{H}-1.279822221 .097540382 .78045366$

$\mathrm{H}-2.72930498 \quad 1.32068797 \quad 1.79664520$

$\mathrm{H}-1.35324603 \quad 2.41275124 \quad 1.60461283$

O $2.989109312 .24467201-1.82347735$

O $3.506394851 .58608579-0.88165802$

o - $-1.22015199-2.50504541-0.83451612$

Vibrational frequencies (in $\mathrm{cm}-1$ )

$\begin{array}{llllllllllllllll}25.9 & 43.4 & 48.5 & 56.2 & 107.0 & 130.6 & 164.5 & 189.4 & 203.8 & 222.4 & 246.8 & 283.3 & 295.3 & 334.2\end{array}$ $362.7 \quad 397.6 \quad 431.2 \quad 468.1 \quad 490.3 \quad 566.0 \quad 601.9 \quad 673.0 \quad 725.9 \quad 782.5 \quad 823.8 \quad 873.0 \quad 921.4 \quad 930.5$ $958.5996 .5 \quad 1006.3 \quad 1015.7 \quad 1035.9 \quad 1047.6 \quad 1052.5 \quad 1057.1 \quad 1091.6 \quad 1110.4 \quad 1128.11145 .7$

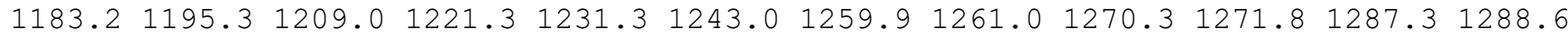
$1297.81299 .3 \quad 1306.0 \quad 1310.91318 .0 \quad 1350.8 \quad 1359.6 \quad 1362.5 \quad 1380.6 \quad 1402.7 \quad 1405.4 \quad 1591.2$ $1853.22615 .22666 .0 \quad 2667.8 \quad 2672.2 \quad 2676.0 \quad 2676.12679 .42680 .12701 .32722 .42729 .0$ $2733.4 \quad 2760.7 \quad 2778.12780 .4 \quad 2782.5$

name $=$ MIN120

energy $($ in $\mathrm{kcal} / \mathrm{mol})=-34.189015$

$\mathrm{ZPE}($ in $\mathrm{kcal} / \mathrm{mol})=144.021$

$\mathrm{G}($ in $\mathrm{kcal} / \mathrm{mol})=118.114$ 


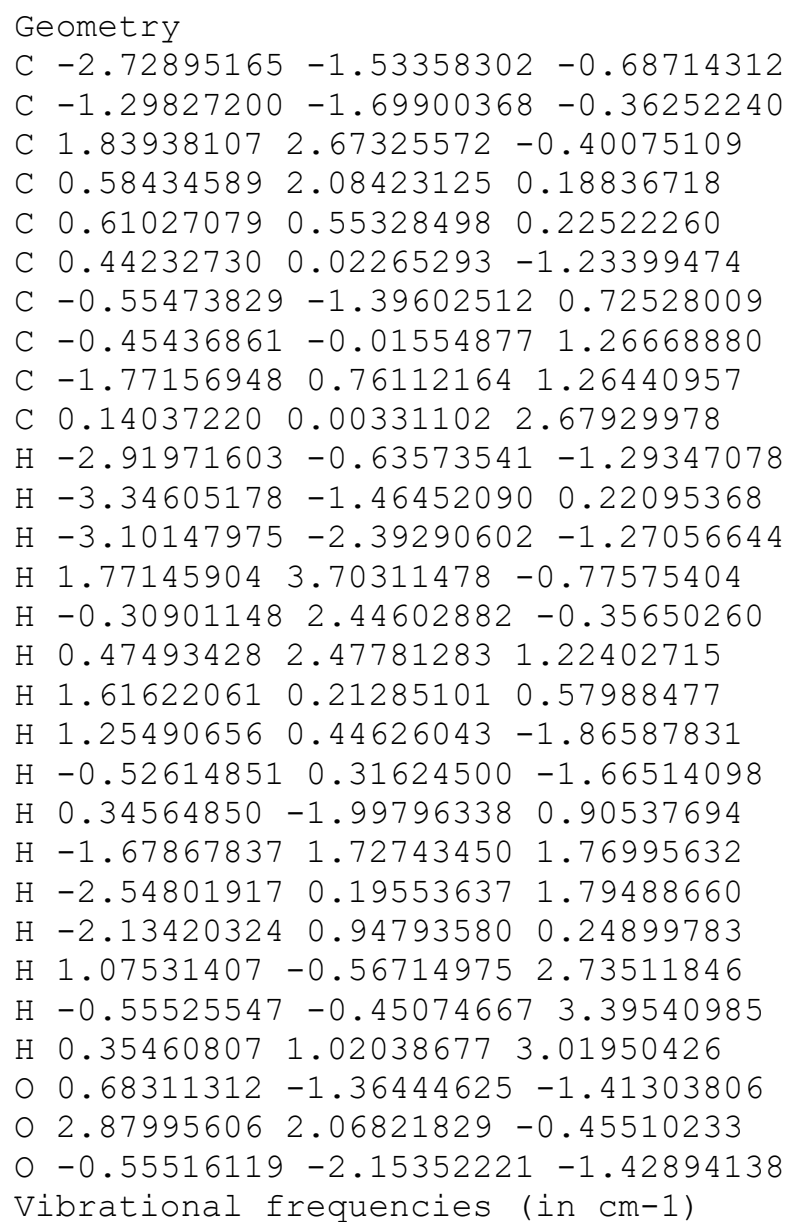

Vibrational frequencies (in $\mathrm{cm}-1$ )

$\begin{array}{llllllllllllllll}33.8 & 62.8 & 92.9 & 96.2 & 123.3 & 161.2 & 163.0 & 181.1 & 232.3 & 244.6 & 257.7 & 285.7 & 306.1 & 338.9\end{array}$ $390.6 \quad 411.3 \quad 421.3 \quad 456.0 \quad 525.0 \quad 542.2 \quad 599.1 \quad 628.3 \quad 645.9 \quad 754.4 \quad 812.1 \quad 854.3 \quad 896.6 \quad 946.5$ $966.1983 .2 \quad 990.8 \quad 1005.7 \quad 1010.0 \quad 1025.1 \quad 1049.6 \quad 1056.3 \quad 1079.3 \quad 1087.5 \quad 1128.2 \quad 1155.0 \quad 1169.0$ $1183.3 \quad 1195.6 \quad 1213.7 \quad 1265.7 \quad 1268.2 \quad 1273.5 \quad 1279.4 \quad 1285.1 \quad 1292.2 \quad 1296.7 \quad 1300.91301 .8$

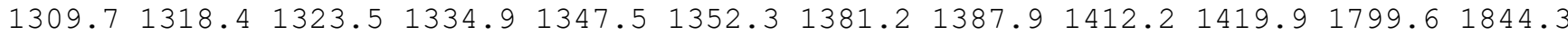
$2634.02648 .5 \quad 2654.8 \quad 2663.6 \quad 2672.3 \quad 2673.6 \quad 2676.72680 .72681 .4 \quad 2683.12708 .02733 .4$ 2737.72776 .02781 .12782 .4

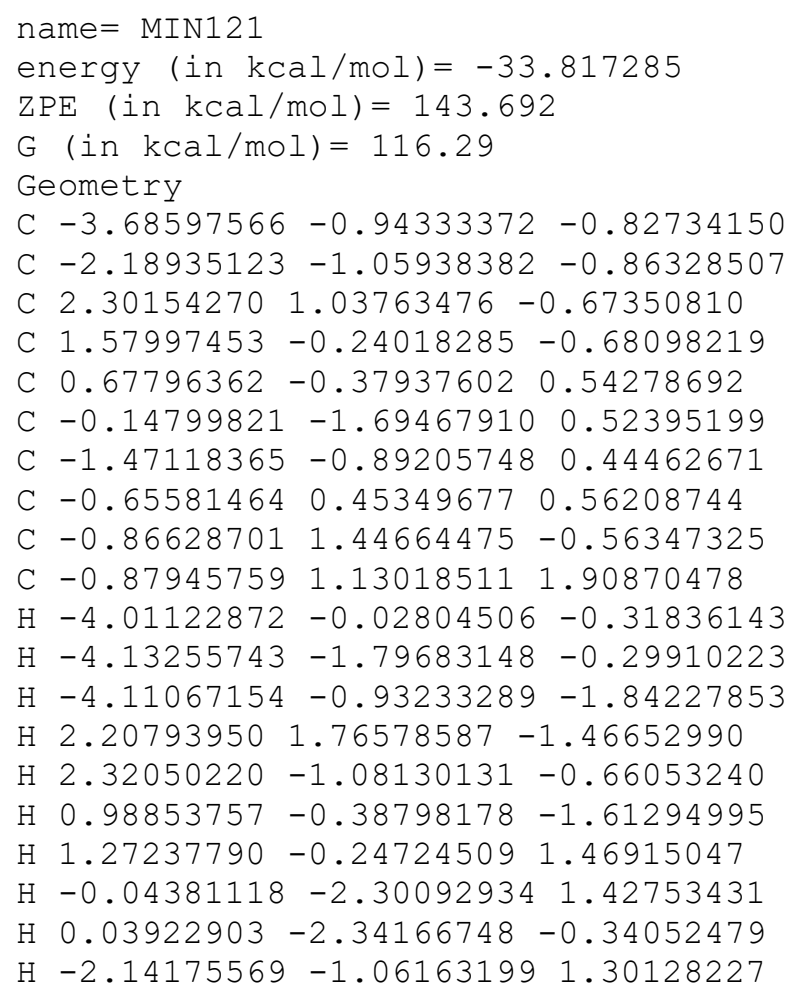


$\mathrm{H}-0.17538013 \quad 2.29357518-0.47839661$

$\mathrm{H}-1.88448550 \quad 1.85416810-0.55152168$

$\mathrm{H}-0.71094580 \quad 0.99515132-1.55298893$

$\mathrm{H}-0.74461202 \quad 0.43347126 \quad 2.74509363$

$\mathrm{H}-1.888143191 .54987464 \quad 1.98702326$

$\mathrm{H}-0.166463331 .950650712 .06065589$

○ $3.33486953 \quad 0.665294891 .23570335$

$03.12053672 \quad 1.42248076 \quad 0.25348717$

o $-1.60544383-1.28239574-1.89585846$

Vibrational frequencies (in $\mathrm{cm}-1$ )

$\begin{array}{llllllllllllllll}22.3 & 28.0 & 39.7 & 87.0 & 94.6 & 132.0 & 159.7 & 175.1 & 193.0 & 225.2 & 265.1 & 283.4 & 306.9 & 316.4\end{array}$ $370.8 \quad 391.7 \quad 433.5 \quad 451.5 \quad 501.9 \quad 577.4 \quad 594.6 \quad 688.1 \quad 721.2 \quad 801.1 \quad 828.7 \quad 846.7 \quad 900.0 \quad 947.9$ $987.51000 .0 \quad 1004.8 \quad 1016.2 \quad 1033.8 \quad 1037.7 \quad 1047.5 \quad 1053.6 \quad 1093.2 \quad 1106.51122 .5 \quad 1146.9$ $\begin{array}{lllllllllllllll}1155.6 & 1190.4 & 1204.1 & 1222.1 & 1231.1 & 1235.7 & 1260.4 & 1265.1 & 1274.6 & 1282.3 & 1285.9 & 1288.7\end{array}$

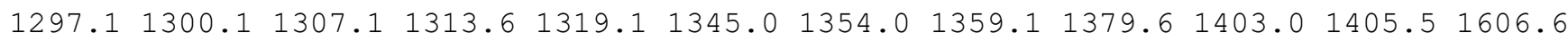

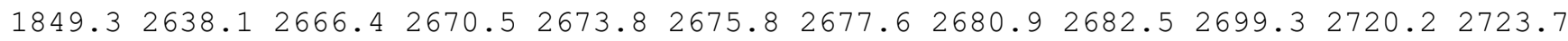
$2732.92761 .5 \quad 2779.2 \quad 2780.7 \quad 2782.4$

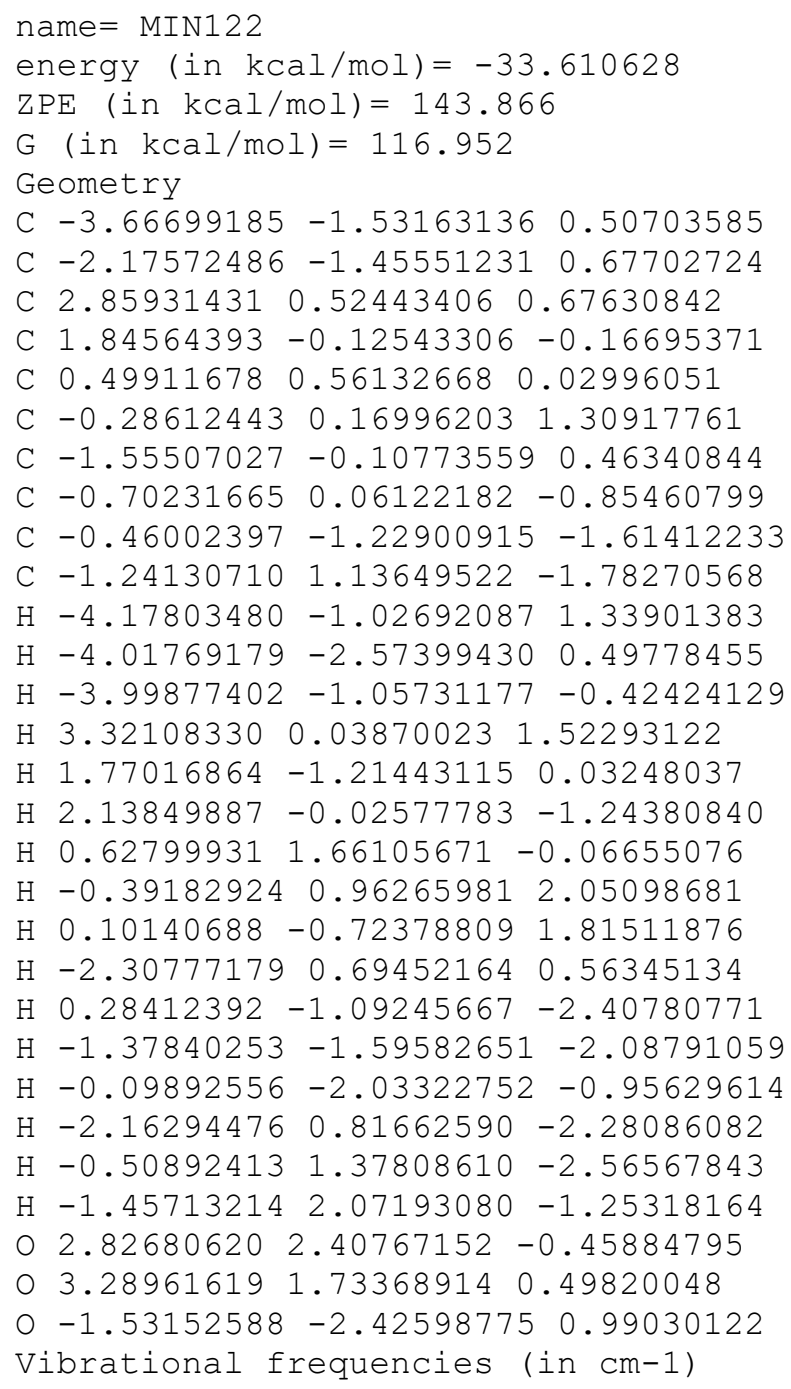


$\mathrm{ZPE}($ in $\mathrm{kcal} / \mathrm{mol})=144.477$

$\mathrm{G}(\mathrm{in} \mathrm{kcal} / \mathrm{mol})=119.582$

Geometry

C $-2.26141843-1.54001557-0.61456280$

C $-0.83602879-1.73645237-0.28589100$

C $0.00462676 \quad 2.71379079-0.31162665$

$\begin{array}{llll}\text { C } 1.18679403 & 2.01890561 & 0.31386427\end{array}$

C $1.09047521 \quad 0.48812377 \quad 0.30115142$

C $0.87891680-0.01465505-1.15970741$

C $-0.08986542-1.44973180 \quad 0.80534137$

C $0.02967081-0.07274220 \quad 1.35432487$

C $-1.27976869 \quad 0.71235594 \quad 1.38491850$

C $0.64523227-0.07318880 \quad 2.75977368$

$\mathrm{H}-2.42482985-0.64469805-1.23623693$

$\mathrm{H}-2.87975580-1.43935537 \quad 0.28904567$

$\mathrm{H}-2.65488175-2.39550090-1.18945799$

$\mathrm{H}-0.51616004 \quad 3.46607398 \quad 0.29502292$

H $1.334760292 .36429459 \quad 1.35647944$

H $2.102938992 .33119047-0.23604079$

H 2.08107170 $0.08371916 \quad 0.63194270$

$\mathrm{H} \quad 1.63732951 \quad 0.44358385-1.82813417$

$\mathrm{H}-0.113113950 .26409232-1.55630166$

H $\quad 0.79936641-2.06666381 \quad 0.98323794$

$\mathrm{H}-1.16686897 \quad 1.67624498 \quad 1.89022270$

$\mathrm{H}-2.04788884 \quad 0.15098153 \quad 1.93287435$

$\mathrm{H}-1.67036644 \quad 0.89599426 \quad 0.37743742$

$\mathrm{H} \quad 1.57766808-0.64732304 \quad 2.79836689$

$\mathrm{H}-0.04333936-0.53683558 \quad 3.47725334$

$\mathrm{H} \quad 0.86154998 \quad 0.93880688 \quad 3.11254570$

O $1.13754192-1.39685461-1.34862910$

$0-0.355751532 .49407226-1.44162600$

O $-0.09681423-2.19692753-1.35247686$

Vibrational frequencies (in $\mathrm{cm}-1$ )

$\begin{array}{llllllllllllllll}56.7 & 71.3 & 126.4 & 129.2 & 147.0 & 182.0 & 187.6 & 218.1 & 234.7 & 262.6 & 286.1 & 303.7 & 311.2 & 342.1\end{array}$ $390.4 \quad 417.5 \quad 427.5 \quad 466.1 \quad 502.1 \quad 553.5 \quad 603.4 \quad 619.1 \quad 654.9 \quad 744.6 \quad 838.0 \quad 881.0 \quad 896.3 \quad 949.1$ $967.0 \quad 978.3 \quad 989.1 \quad 992.9 \quad 1010.3 \quad 1022.8 \quad 1046.5 \quad 1055.2 \quad 1062.3 \quad 1081.0 \quad 1121.7 \quad 1160.51171 .7$ $1182.51193 .5 \quad 1221.1 \quad 1258.8 \quad 1264.7 \quad 1273.0 \quad 1282.6 \quad 1291.7 \quad 1295.7 \quad 1300.81301 .01304 .5$

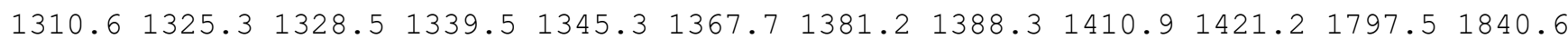
$2626.62646 .2 \quad 2659.3 \quad 2662.3 \quad 2670.6 \quad 2673.4 \quad 2675.8 \quad 2677.72682 .12684 .42707 .22736 .1$ $2737.2 \quad 2774.7 \quad 2779.4 \quad 2782.1$

\section{name $=$ MIN124}

energy (in $\mathrm{kcal} / \mathrm{mol})=-33.392353$

$\mathrm{ZPE}(\mathrm{in} \mathrm{kcal} / \mathrm{mol})=144.06$

$\mathrm{G}($ in $\mathrm{kcal} / \mathrm{mol})=116.88$

Geometry

C $-2.89641224-1.526609520 .39344325$

C $-1.51642649-1.15462601-0.06347907$

C $0.60908294 \quad 1.10467564-1.44481136$

C $1.35821667 \quad 1.42581544-0.21832090$

C $1.16629480 \quad 0.39955787 \quad 0.89619950$

C $0.99747669-1.071495890 .42657916$

C $-0.44592122-1.11264038 \quad 0.98709768$

C $-0.23762691 \quad 0.32297820 \quad 1.60627584$

C $-1.26546751 \quad 1.37198230 \quad 1.23356104$

C $-0.09528632 \quad 0.25673678 \quad 3.12302355$

$\mathrm{H}-3.14017765-1.097838391 .37245148$

$\mathrm{H}-2.99853792-2.618423210 .47147176$

$\mathrm{H}-3.65826448-1.18704902-0.32481577$

$\mathrm{H}-0.451816621 .28172784-1.56044524$

H 1.097788332 .438647210 .15544305

$\mathrm{H} 2.453284951 .46561777-0.47096284$

$\mathrm{H} \quad 1.98513909 \quad 0.52022290 \quad 1.63015966$

$\mathrm{H} 1.68470041-1.773276230 .90797007$ 
$\mathrm{H} \quad 1.08294961-1.23147659-0.65572158$

$\mathrm{H}-0.60269953-1.895881251 .74558910$

$\mathrm{H}-0.92055767 \quad 2.37664931 \quad 1.51100204$

$\mathrm{H}-2.21703917 \quad 1.20391317 \quad 1.75318219$

$\mathrm{H}-1.48496060 \quad 1.39101897 \quad 0.15970849$

H $0.64779354 \quad-0.48708320 \quad 3.43489517$

$\mathrm{H}-1.04327782-0.009046663 .60415968$

$\mathrm{H} \quad 0.22029402 \quad 1.22362264 \quad 3.53372162$

$\begin{array}{lllll}0 & 2.35857760 & 0.26889683 & -2.47914893\end{array}$

$\begin{array}{llll}0 & 1.12920814 & 0.54884229 & -2.49155576\end{array}$

$0-1.29706449-0.89182003-1.22195742$

Vibrational frequencies (in $\mathrm{cm}-1$ )

$\begin{array}{lllllllllllllllll}5.1 & 54.9 & 72.7 & 105.7 & 107.2 & 163.8 & 178.8 & 194.8 & 197.6 & 227.7 & 242.6 & 283.5 & 320.6 & 336.3\end{array}$

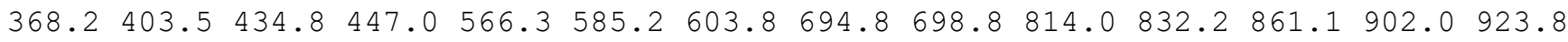
$982.7 \quad 994.4 \quad 1006.5 \quad 1016.4 \quad 1035.8 \quad 1041.3 \quad 1049.6 \quad 1060.5 \quad 1084.91105 .4 \quad 1121.01124 .2$ $1150.21198 .8 \quad 1211.7 \quad 1223.7 \quad 1230.2 \quad 1239.8 \quad 1259.7 \quad 1267.1 \quad 1271.8 \quad 1281.8 \quad 1287.11289 .2$ $1298.31298 .91305 .41311 .81327 .2 \quad 1340.6 \quad 1360.1 \quad 1379.91387 .31405 .1 \quad 1408.21605 .2$ $1845.42635 .7 \quad 2667.5 \quad 2668.1 \quad 2673.92675 .02675 .5 \quad 2676.42679 .4 \quad 2708.12716 .52726 .8$ $2731.6 \quad 2760.3 \quad 2779.7 \quad 2780.4 \quad 2782.7$

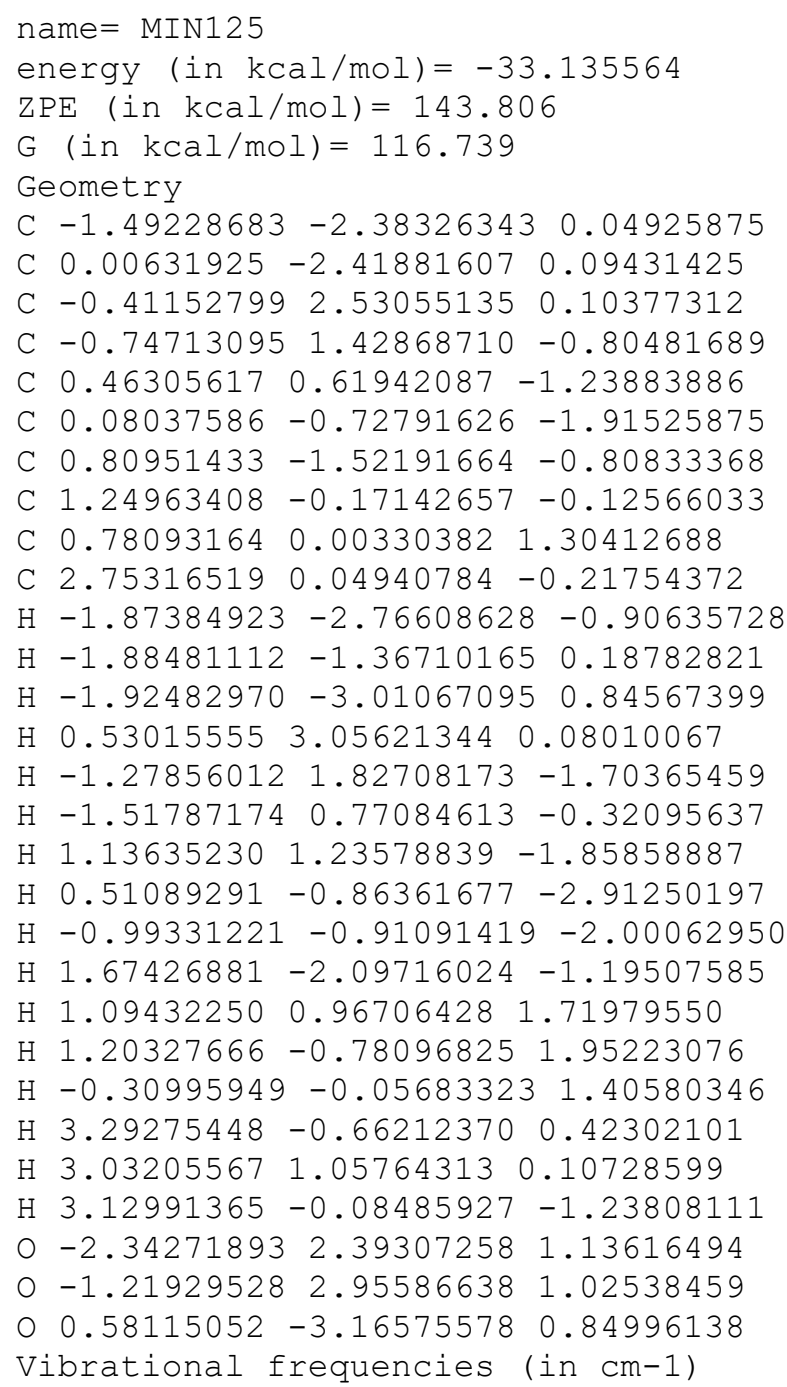
$389.8 \quad 425.1 \quad 438.8 \quad 468.7 \quad 482.8 \quad 524.3 \quad 598.0 \quad 684.2 \quad 731.4 \quad 763.6 \quad 822.3 \quad 826.0 \quad 901.6 \quad 950.1$ $982.7 \quad 999.0 \quad 1006.8 \quad 1019.1 \quad 1035.0 \quad 1037.4 \quad 1045.3 \quad 1052.8 \quad 1074.1 \quad 1119.01126 .11153 .5$

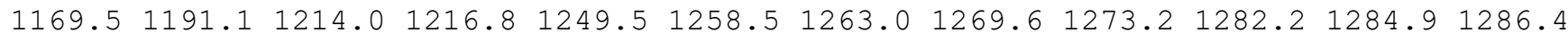

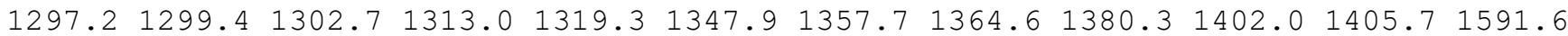
$1836.12619 .82661 .5 \quad 2665.92670 .02672 .92674 .62679 .82683 .4 \quad 2708.12717 .42724 .9$ $2733.3 \quad 2764.7 \quad 2777.1 \quad 2778.0 \quad 2781.5$ 
name $=$ MIN126

energy (in $\mathrm{kcal} / \mathrm{mol})=-32.779328$

$\mathrm{ZPE}($ in $\mathrm{kcal} / \mathrm{mol})=143.553$

$\mathrm{G}($ in $\mathrm{kcal} / \mathrm{mol})=116.149$

Geometry

C $-3.66056719-0.89134291-0.52370753$

C $-2.16101419-0.91245848-0.55831907$

C $2.68209268 \quad 0.07542488-0.76105064$

C $1.31043235 \quad 0.62114316-0.80535774$

C $0.560648820 .50744466 \quad 0.51456306$

C $0.00304254 \quad-0.91474784 \quad 0.79760625$

C $-1.45190159-0.39951871 \quad 0.66408116$

C $-0.91121414 \quad 1.07176430 \quad 0.50351392$

C $-1.352537661 .80146238-0.74970132$

C $-1.20387674 \quad 1.92026198 \quad 1.73363601$

$\mathrm{H}-4.08645044-1.09071784-1.51920440$

$\mathrm{H}-4.04921500 \quad 0.07514479-0.18056087$

$\mathrm{H}-4.04858202-1.665932220 .15234385$

$\mathrm{H} \quad 3.34293392 \quad 0.13985803-1.61178879$

$\mathrm{H} \quad 0.73286434 \quad 0.09287053-1.60951386$

$\mathrm{H} 1.34503798 \quad 1.68746401-1.12913982$

$\mathrm{H} 1.15651175 \quad 0.92218632 \quad 1.34499905$

$\mathrm{H} \quad 0.24693192-1.30780515 \quad 1.78861240$

$\mathrm{H} \quad 0.28199460-1.67409597 \quad 0.05613208$

$\mathrm{H}-2.05899987-0.54223558 \quad 1.57143559$

H $-1.19090185 \quad 1.21211394-1.66260039$

$\mathrm{H}-0.809522462 .74721259-0.87230716$

$\mathrm{H}-2.421026022 .04758299-0.71407922$

$\mathrm{H}-0.90798705 \quad 1.41875774 \quad 2.66280277$

$\mathrm{H}-2.27183224 \quad 2.15368896 \quad 1.81582175$

$\mathrm{H}-0.66496561 \quad 2.87542640 \quad 1.69382967$

$04.19776280-0.96530308 \quad 0.41861339$

$03.03140679-0.492171310 .35224272$

o $-1.56006227-1.31806436-1.52285227$

Vibrational frequencies (in $\mathrm{cm}-1$ )

$\begin{array}{lllllllllllllll}28.3 & 37.3 & 42.9 & 86.4 & 87.7 & 105.8 & 159.7 & 177.0 & 198.4 & 209.0 & 247.9 & 250.8 & 283.8 & 324.6\end{array}$

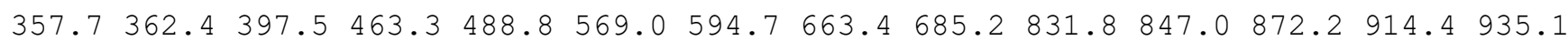
$973.0996 .1 \quad 1005.0 \quad 1015.3 \quad 1033.6 \quad 1042.1 \quad 1048.4 \quad 1063.0 \quad 1093.4 \quad 1109.2 \quad 1122.91147 .4$

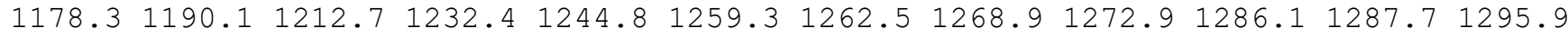

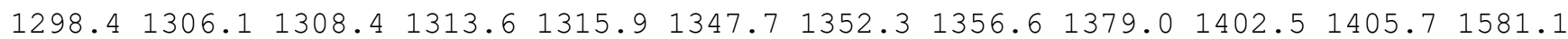
$1850.32627 .12665 .2 \quad 2668.6 \quad 2673.3 \quad 2674.7 \quad 2675.92678 .62679 .42713 .62721 .42726 .1$ $2732.92757 .92778 .9 \quad 2780.2 \quad 2782.4$

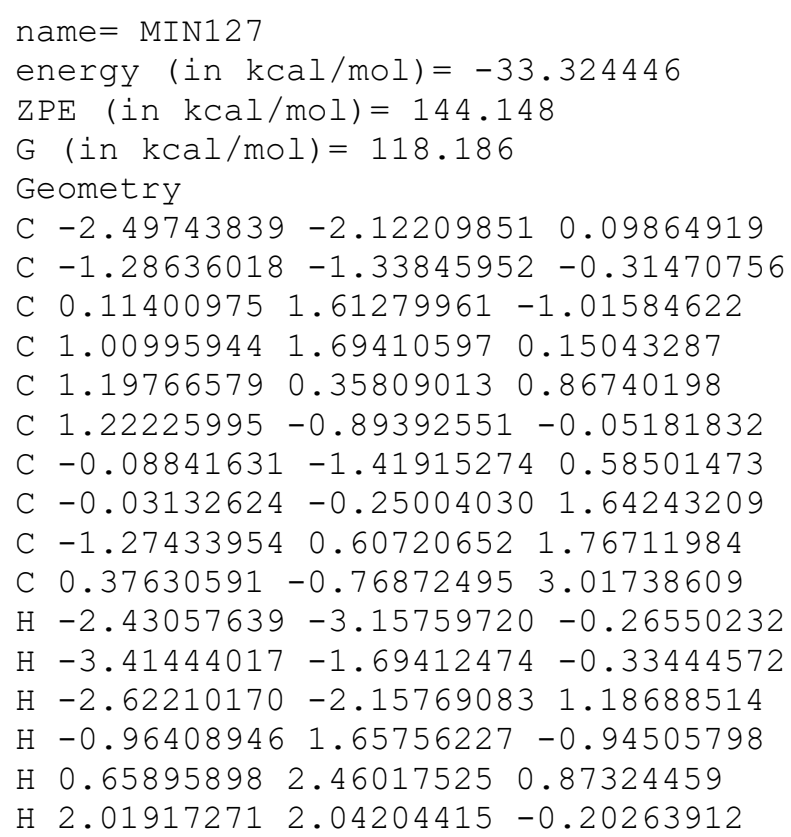


H $2.09143252 \quad 0.42915760 \quad 1.51485166$

$\mathrm{H} 2.08764063-1.543880720 .10682348$

$\mathrm{H} 1.17208088-0.68886931-1.12905809$

$\mathrm{H} \quad 0.00712319-2.420519661 .03309837$

$\mathrm{H}-2.08184543 \quad 0.069560592 .27973927$

$\mathrm{H}-1.66922838 \quad 0.92959015 \quad 0.79675725$

$\mathrm{H}-1.069577551 .51420807 \quad 2.35059656$

$\mathrm{H}-0.41668979-1.37557647 \quad 3.46875804$

$\mathrm{H} \quad 0.58764959 \quad 0.05856040 \quad 3.70570682$

$\mathrm{H} \quad 1.27722027-1.39238914 \quad 2.97169540$

o $1.757689581 .36473115 \quad-2.45185536$

O $0.51793536 \quad 1.44788867 \quad-2.23438194$

o $-1.29683270-0.66718777-1.31925996$

Vibrational frequencies (in cm-1)

$\begin{array}{llllllllllllllll}34.6 & 56.8 & 79.1 & 106.4 & 112.6 & 163.8 & 179.2 & 195.5 & 198.8 & 227.9 & 243.9 & 287.4 & 317.9 & 338.7\end{array}$ $368.7 \quad 404.8 \quad 434.9447 .0 \quad 566.3 \quad 586.4 \quad 603.8 \quad 695.5 \quad 700.6 \quad 814.2 \quad 833.5 \quad 863.6 \quad 900.1 \quad 922.2$ $983.2994 .7 \quad 1006.5 \quad 1016.6 \quad 1036.5 \quad 1039.7 \quad 1050.1 \quad 1061.5 \quad 1084.51105 .1 \quad 1120.2 \quad 1122.8$

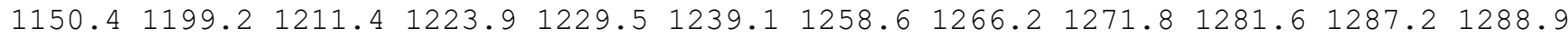

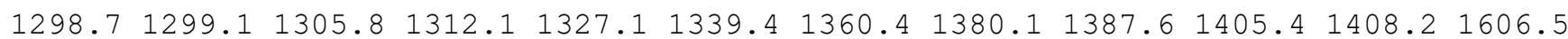
$1844.42637 .7 \quad 2666.7 \quad 2668.4 \quad 2673.92674 .4 \quad 2675.72676 .82679 .6 \quad 2710.92717 .12727 .7$ $2732.0 \quad 2760.5 \quad 2779.9 \quad 2780.4 \quad 2782.8$

\author{
name $=$ MIN128 \\ energy (in $\mathrm{kcal} / \mathrm{mol})=-33.250224$ \\ $\operatorname{ZPE}($ in $\mathrm{kcal} / \mathrm{mol})=144.124$ \\ $\mathrm{G}($ in $\mathrm{kcal} / \mathrm{mol})=117.593$ \\ Geometry \\ C $-3.38665303-0.00255844 \quad 0.42530393$ \\ C $-1.90110914 \quad 0.17361152 \quad 0.30715231$ \\ C $1.309505631 .50278017-0.05501143$ \\ C $2.00965606 \quad 0.20792625-0.03758443$ \\ C $1.09341660-0.98348271-0.31016679$ \\ C $-0.10894848-0.72110105-1.25782326$ \\ C $-1.11275754-1.03065244-0.11637117$ \\ C $0.09130549-1.42783170 \quad 0.82109610$ \\ C $0.17511193-0.71719688 \quad 2.15441297$ \\ C $0.13854261-2.938131551 .03833261$ \\ $\mathrm{H}-3.83430389-0.22695873-0.55316882$ \\ $\mathrm{H}-3.87059161 \quad 0.91135836 \quad 0.80273637$ \\ $\mathrm{H}-3.64738811-0.821248161 .10707429$ \\ $\mathrm{H} \quad 0.80153559 \quad 1.92074764 \quad 0.80623807$ \\ H 2.54281692 $0.05335141 \quad 0.92472429$ \\ $\mathrm{H} \quad 2.80467178 \quad 0.22496959-0.83207717$ \\ $\mathrm{H} 1.71800977-1.83092553-0.65210913$ \\ $\mathrm{H}-0.16692108-1.41552990-2.10145861$ \\ $\mathrm{H}-0.16825372 \quad 0.28974017-1.67562033$ \\ $\mathrm{H}-1.77262562-1.88602250-0.33474857$ \\ $\mathrm{H} 1.10209851-0.970796712 .68352126$ \\ $\mathrm{H}-0.66002123-0.997070192 .80854352$ \\ $\mathrm{H} \quad 0.14641959 \quad 0.37550733 \quad 2.05427040$ \\ $\mathrm{H}-0.68277611-3.275882591 .68016080$ \\ $\mathrm{H} \quad 1.07604567-3.239000321 .52124116$ \\ $\mathrm{H} \quad 0.06643210-3.49263597 \quad 0.09526968$ \\ $01.73475707 \quad 1.86555488-2.18169323$ \\ o $1.207524862 .25791888-1.10055672$ \\ o $-1.38835364 \quad 1.24581647 \quad 0.52409335$ \\ Vibrational frequencies (in $\mathrm{cm}-1$ )
}

$\begin{array}{llllllllllllllll}22.5 & 33.9 & 75.6 & 109.1 & 110.9 & 159.8 & 173.2 & 198.0 & 204.8 & 230.7 & 251.7 & 285.2 & 316.9 & 323.8\end{array}$

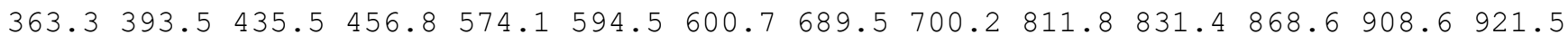
$984.3994 .0 \quad 1008.1 \quad 1018.2 \quad 1036.2 \quad 1036.7 \quad 1046.2 \quad 1057.1 \quad 1083.11103 .91122 .21129 .0$ $1153.31203 .51211 .2 \quad 1219.9 \quad 1229.2 \quad 1241.4 \quad 1260.3 \quad 1267.3 \quad 1273.7 \quad 1284.0 \quad 1286.8 \quad 1288.4$ $1299.21300 .1 \quad 1304.6 \quad 1316.6 \quad 1332.8 \quad 1347.6 \quad 1359.1 \quad 1379.3 \quad 1388.3 \quad 1406.7 \quad 1410.01603 .6$ $1847.02637 .32667 .0 \quad 2668.8 \quad 2673.5 \quad 2675.4 \quad 2676.72678 .32680 .2 \quad 2701.72715 .52725 .7$ $2730.5 \quad 2763.3 \quad 2779.8 \quad 2780.5 \quad 2782.9$ 
name $=$ MIN129

energy (in $\mathrm{kcal} / \mathrm{mol})=-32.515759$

$\mathrm{ZPE}(\mathrm{in} \mathrm{kcal} / \mathrm{mol})=143.691$

$\mathrm{G}($ in $\mathrm{kcal} / \mathrm{mol})=116.521$

Geometry

C $-3.62345570-1.18845085-0.84800643$

C $-2.12590451-1.26567242-0.82639311$

C $2.30779981 \quad 0.93592630 \quad-0.67810731$

C $1.50710676-0.30588483-0.66228218$

C $0.67192840-0.47806789 \quad 0.60039986$

C $-0.10277730-1.82561538 \quad 0.60960389$

C $-1.45615535-1.07843651 \quad 0.50623458$

C $-0.69986634 \quad 0.297764150 .65468903$

C $-0.96039551 \quad 1.31817547-0.43319772$

C $-0.94179505 \quad 0.92041414 \quad 2.02395178$

$\mathrm{H}-4.06788779-2.06795407-0.36145616$

$\mathrm{H}-4.00700019-1.16284090-1.87989744$

$\mathrm{H}-3.99621516-0.29633028-0.33018725$

$\mathrm{H} 2.492522621 .49032835-1.58462460$

H 2.18420879 $-1.18653526-0.78146838$

$\mathrm{H} \quad 0.84163252-0.35755650-1.56067679$

$\mathrm{H} \quad 1.27865478-0.32257402 \quad 1.50705927$

$\mathrm{H} \quad 0.01768076-2.405540561 .52830109$

$\mathrm{H} \quad 0.11378747-2.48722299-0.23711893$

$\mathrm{H}-2.14471318-1.28530896 \quad 1.33937500$

$\mathrm{H}-0.846943110 .90257962-1.44349350$

$\mathrm{H}-0.267239892 .16705971-0.35559042$

$\mathrm{H}-1.97750758 \quad 1.72405714 \quad-0.36633769$

$\mathrm{H}-0.28771373 \quad 1.78702263 \quad 2.18805475$

$\mathrm{H}-0.75632105 \quad 0.21309017 \quad 2.84033949$

$\mathrm{H}-1.97521917 \quad 1.27235601 \quad 2.12683765$

$\begin{array}{lllll}0 & 3.50732608 & 2.33191826 & 0.50245841\end{array}$

$\begin{array}{lllll}0 & 2.79454891 & 1.29270988 & 0.47080050\end{array}$

$0-1.49546573-1.47890334-1.83357212$

Vibrational frequencies (in $\mathrm{cm}-1$ )

$\begin{array}{lllllllllllllll}28.0 & 35.5 & 43.0 & 82.7 & 114.1 & 119.1 & 160.1 & 183.3 & 203.1 & 215.1 & 237.4 & 275.7 & 313.6 & 315.2\end{array}$ $\begin{array}{llllllllllllllll}345.2 & 388.7 & 410.1 & 431.1 & 489.3 & 575.2 & 595.8 & 655.2 & 701.7 & 787.4 & 833.4 & 888.9 & 917.8 & 938.8\end{array}$ $992.81003 .01007 .5 \quad 1017.6 \quad 1036.5 \quad 1038.3 \quad 1048.8 \quad 1061.1 \quad 1097.5 \quad 1111.91123 .6 \quad 1151.6$

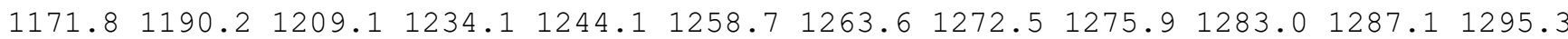
$1297.61304 .31308 .6 \quad 1310.6 \quad 1319.5 \quad 1347.5 \quad 1352.1 \quad 1360.2 \quad 1379.6 \quad 1402.81406 .11576 .1$ $1848.72626 .7 \quad 2664.0 \quad 2668.6 \quad 2671.5 \quad 2674.2 \quad 2675.3 \quad 2677.92683 .2 \quad 2713.3 \quad 2721.92728 .2$ $2734.12760 .92778 .2 \quad 2779.92782 .0$

name $=$ MIN130

energy $($ in $\mathrm{kcal} / \mathrm{mol})=-34.401593$

$\mathrm{ZPE}(\mathrm{in} \mathrm{kcal} / \mathrm{mol})=145.635$

$\mathrm{G}($ in $\mathrm{kcal} / \mathrm{mol})=121.801$

Geometry

C $-2.44471607-0.04786308-0.32802559$

C $-0.95435024-0.05418623-0.28861260$

C $-0.203011861 .28335088-0.22211698$

C $1.266165291 .17515636 \quad 0.24916979$

C $1.65436352-0.24882404 \quad 0.64071416$

C $1.20239305-1.19616540-0.51512085$

C $-0.18498440-1.25272895 \quad 0.20326693$

C $0.55129040-0.911968851 .55562686$

C $-0.16510912-0.05963288 \quad 2.58215433$

C $1.07085790-2.17128453 \quad 2.24468031$

$\mathrm{H}-3.01929192-0.90068367 \quad 0.02362284$

$\mathrm{H}-0.41878753 \quad 0.72793356-2.80750212$

$\mathrm{H}-3.00428898 \quad 0.88400941-0.33001411$

$\mathrm{H}-0.76479753 \quad 1.99569717 \quad 0.43679506$ 
$\mathrm{H} 1.445313561 .86635903 \quad 1.09129216$

H $1.92565092 \quad 1.52437194 \quad-0.57514544$

$\mathrm{H} 2.68345715-0.34004893 \quad 0.98781262$

H $1.72791375-2.15017324-0.56041162$

$\mathrm{H} \quad 1.20368857-0.76143787-1.51781956$

$\mathrm{H}-0.73492317-2.19291873 \quad 0.16301686$

$\mathrm{H}-0.63438473 \quad 0.83517514 \quad 2.16681124$

$\mathrm{H} \quad 0.53216542 \quad 0.27857503 \quad 3.35984887$

$\mathrm{H}-0.96135648-0.629671693 .07858406$

$\mathrm{H} 1.60977673-2.83372963 \quad 1.55812917$

H $\quad 0.24949162 \quad-2.75375675 \quad 2.67730951$

$\mathrm{H} 1.76163464-1.917215093 .05710426$

o $0.34304291 \quad 1.27226632-2.47990800$

O $-0.29837700 \quad 1.99323066-1.44061717$

o $-1.67084411-0.28884958-1.50737750$

Vibrational frequencies (in cm-1)

$\begin{array}{llllllllllllllllll}58.9 & 109.1 & 129.3 & 160.2 & 182.8 & 187.0 & 230.0 & 257.3 & 286.7 & 297.1 & 324.0 & 387.4 & 396.7 & 416.4\end{array}$ $441.2 \quad 444.8 \quad 470.4 \quad 521.8 \quad 579.1 \quad 672.3 \quad 705.6 \quad 783.8 \quad 826.1 \quad 851.8 \quad 892.0 \quad 913.8 \quad 945.7 \quad 949.8$ $979.1988 .4 \quad 1004.1 \quad 1008.6 \quad 1016.2 \quad 1022.1 \quad 1033.7 \quad 1053.0 \quad 1065.5 \quad 1087.9 \quad 1089.2 \quad 1117.2$ $\begin{array}{llllllllllllll}1150.7 & 1161.7 & 1176.1 & 1188.4 & 1202.8 & 1219.0 & 1222.8 & 1254.7 & 1275.8 & 1283.4 & 1287.9 & 1293.6\end{array}$

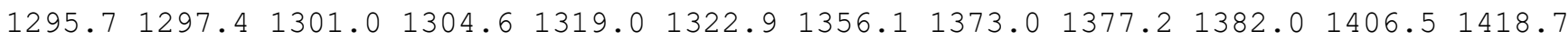
$1536.82652 .6 \quad 2669.92674 .12676 .0 \quad 2678.6 \quad 2680.82689 .22692 .32739 .12748 .62751 .6$ $2754.0 \quad 2765.8 \quad 2782.2 \quad 2785.4 \quad 2838.7$

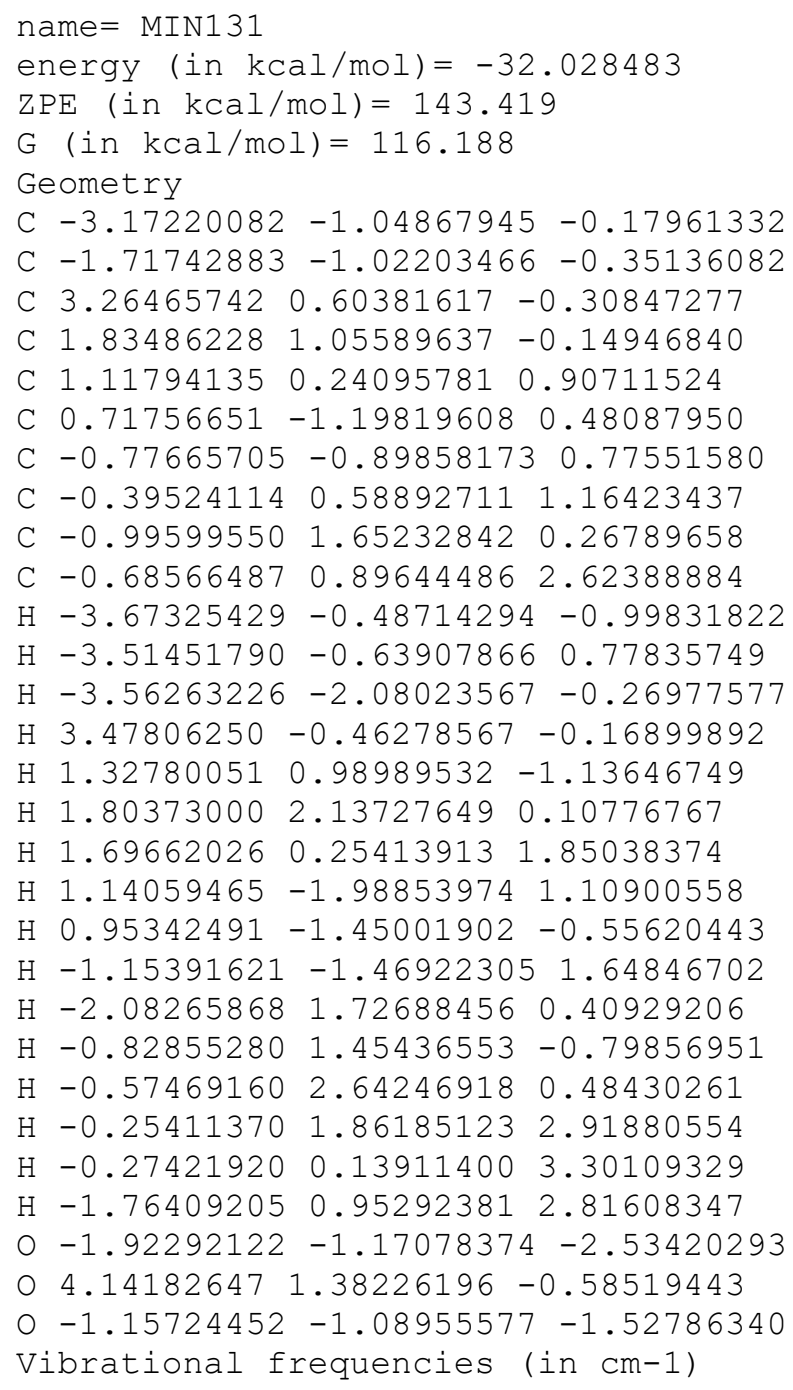


$1839.62634 .22647 .6 \quad 2656.2 \quad 2668.2 \quad 2671.6 \quad 2672.22676 .02677 .4 \quad 2680.82714 .62721 .0$ $2740.9 \quad 2762.6 \quad 2768.2 \quad 2779.8 \quad 2782.7$

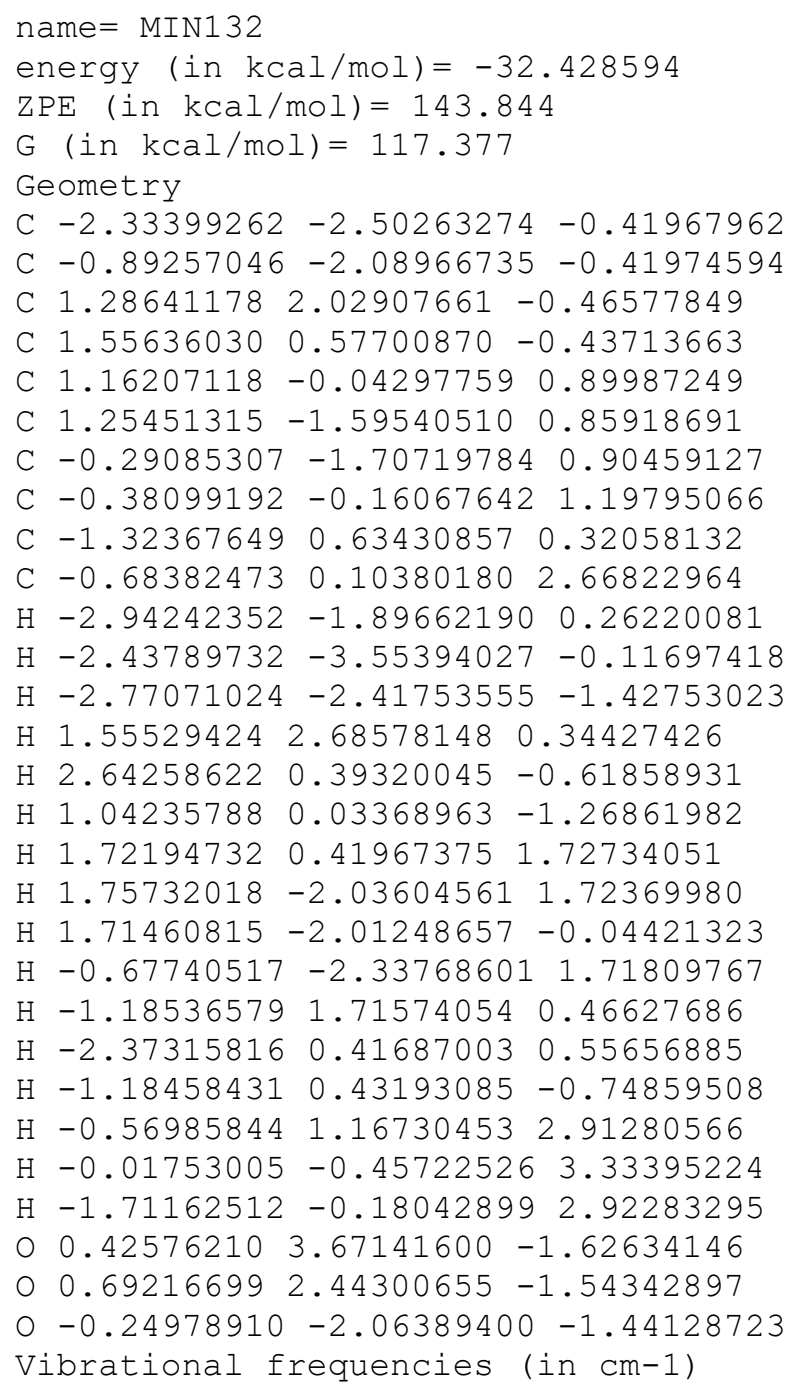


$\mathrm{H}-3.48569942-0.14000066-0.26824731$

H 1.131269641 .161889061 .68286809

$\mathrm{H} 2.80539124 \quad 0.47337633-0.07109158$

$\mathrm{H} 2.04190480 \quad 1.61009170-1.22218724$

$\mathrm{H} \quad 1.68450006-0.63610027-1.99853927$

$\mathrm{H}-0.65495058-0.27575921-2.51771172$

$\mathrm{H}-0.73654556 \quad 0.99095464 \quad-1.29952845$

$\mathrm{H}-1.48642819-1.90580636-0.89257033$

$\mathrm{H} 2.23801142-1.82632061 \quad 1.16460353$

H $0.69570052 \quad-2.53024264 \quad 1.67449502$

H $0.93922752 \quad-0.79654298 \quad 1.77722563$

$\mathrm{H} \quad 0.39959296-3.70450552-0.51079906$

$\mathrm{H} \quad 1.96720545-3.08244529-1.03487177$

$\mathrm{H} \quad 0.53560954 \quad-2.82596842 \quad-2.03661425$

$0.059699662 .93528881-0.66316668$

$\begin{array}{lllll}0 & 0.24276007 & 2.46626619 & 0.50041129\end{array}$

o $-1.00590556 \quad 0.18368030 \quad 1.55204973$

Vibrational frequencies (in $\mathrm{cm}-1$ )

$\begin{array}{lllllllllllllll}28.2 & 57.8 & 91.0 & 111.7 & 140.4 & 144.4 & 177.1 & 204.8 & 210.5 & 226.2 & 245.7 & 274.3 & 312.0 & 316.1\end{array}$

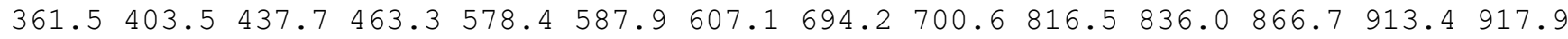
$978.3 \quad 988.9 \quad 1008.4 \quad 1019.2 \quad 1024.2 \quad 1033.3 \quad 1041.3 \quad 1055.4 \quad 1085.1 \quad 1097.0 \quad 1113.7 \quad 1124.7$ $1158.11205 .5 \quad 1207.9 \quad 1220.5 \quad 1226.3 \quad 1241.0 \quad 1255.6 \quad 1264.4 \quad 1272.8 \quad 1281.3 \quad 1285.5 \quad 1287.6$

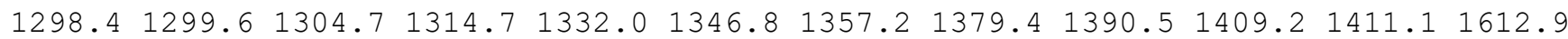
$1846.72640 .6 \quad 2662.92665 .2 \quad 2668.1 \quad 2672.8 \quad 2676.52676 .92681 .12700 .52714 .92726 .3$ $2729.5 \quad 2756.4 \quad 2779.12780 .8 \quad 2783.0$

name $=$ MIN134

energy (in $\mathrm{kcal} / \mathrm{mol})=-32.30513$

$\operatorname{ZPE}(\mathrm{in} \mathrm{kcal} / \mathrm{mol})=143.946$

$\mathrm{G}($ in $\mathrm{kcal} / \mathrm{mol})=117.367$

Geometry

C $-3.16106200-2.00223188-0.24445857$

C $-1.66096535-2.04717690-0.16750016$

C $2.78406877 \quad 1.28087418-0.17450764$

C $1.94301575 \quad 0.09064880 \quad-0.36354837$

C $0.85810563 \quad 0.00763886 \quad 0.70955249$

C $0.47082657-1.437172031 .12863206$

C $-1.02306385-1.148787350 .84983749$

C $-0.64236644 \quad 0.28407438 \quad 0.30556940$

C $-0.92333466 \quad 0.48553380-1.17031063$

C -1.259944691 .409754061 .11974427$

$\mathrm{H}-3.54804171-0.97931445-0.16176452$

$\mathrm{H}-3.60729046-2.59584284 \quad 0.56471582$

$\mathrm{H}-3.52455575-2.42691443-1.19157384$

H $3.83760766 \quad 1.21339753 \quad 0.05865052$

H $2.55082190-0.84039493-0.35573947$

$\mathrm{H} \quad 1.47948926 \quad 0.11829641-1.38079436$

H $1.14320674 \quad 0.62498371 \quad 1.58179435$

$\mathrm{H} \quad 0.70233397 \quad-1.690047412 .16470463$

$\mathrm{H} \quad 0.88855207 \quad-2.22481779 \quad 0.48767235$

$\mathrm{H}-1.63235714-1.10038678 \quad 1.76773713$

$\mathrm{H}-0.54157819-0.33713437-1.78821337$

$\mathrm{H}-0.45121711 \quad 1.41679624 \quad-1.52726083$

$\mathrm{H}-1.996671250 .57569578-1.37002182$

$\mathrm{H}-1.103234951 .285247492 .19498155$

$\mathrm{H}-2.33760886 \quad 1.49306948 \quad 0.94492770$

$\mathrm{H}-0.806282592 .37358172 \quad 0.83242348$

$\begin{array}{llll}0 & 1.18966050 & 2.76065789 & -0.53651090\end{array}$

$\begin{array}{llll}0 & 2.38809788 & 2.51056961 & -0.26032615\end{array}$

- $-1.01887471-2.77672081-0.88220074$

Vibrational frequencies (in $\mathrm{cm}-1$ )

$\begin{array}{lllllllllllllll}33.5 & 50.2 & 51.5 & 70.2 & 111.6 & 152.0 & 160.6 & 188.2 & 207.7 & 245.9 & 268.9 & 278.7 & 315.4 & 321.4\end{array}$

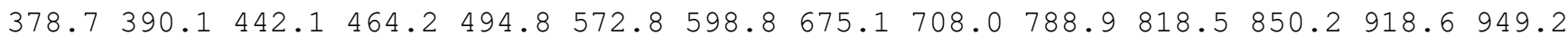
$975.2997 .2 \quad 1007.5 \quad 1017.5 \quad 1034.4 \quad 1042.0 \quad 1048.1 \quad 1065.8 \quad 1091.4 \quad 1114.3 \quad 1129.7 \quad 1134.8$ 
$\begin{array}{lllllllllllllll}1175.5 & 1183.0 & 1205.7 & 1217.3 & 1234.1 & 1238.8 & 1263.3 & 1270.4 & 1273.6 & 1279.8 & 1285.6 & 1290.5\end{array}$ $1297.61299 .61306 .1 \quad 1315.8 \quad 1323.2 \quad 1348.4 \quad 1354.01371 .51380 .8 \quad 1404.11407 .31611 .8$ $1851.72636 .72657 .6 \quad 2664.2 \quad 2671.3 \quad 2673.0 \quad 2676.22681 .32683 .82714 .02719 .22730 .3$ $2733.32761 .3 \quad 2775.12779 .8 \quad 2781.5$

\section{name $=$ MIN135}

energy (in $\mathrm{kcal} / \mathrm{mol})=-31.731979$

$\mathrm{ZPE}($ in $\mathrm{kcal} / \mathrm{mol})=143.391$

$\mathrm{G}(\mathrm{in} \mathrm{kcal} / \mathrm{mol})=116.019$

Geometry

C $-3.23311856-0.74673305-0.26705509$

C $-1.77729982-0.80975356-0.42180418$

C $3.34809707 \quad 0.47158710-0.40544163$

C $1.91355497 \quad 0.93706725-0.35084160$

C $1.15667977 \quad 0.24489340 \quad 0.76762755$

C $0.62706707-1.17338722 \quad 0.42168590$

C $-0.83267191-0.72820522 \quad 0.70606651$

C $-0.31292916 \quad 0.73072224 \quad 1.04204440$

C $-0.83861114 \quad 1.82325736 \quad 0.13305528$

C -0.539774051 .097410352 .49999009$

$\mathrm{H}-3.58436622-0.92205486 \quad 0.75650661$

$\mathrm{H}-3.72641700-1.48249345-0.93883613$

$\mathrm{H}-3.62330554 \quad 0.23417673-0.59906785$

H $3.83953437 \quad 0.48514952-1.38647335$

$\mathrm{H} 1.41418510 \quad 0.78678252-1.32773579$

$\mathrm{H} \quad 1.91008650 \quad 2.03774445 \quad-0.18298739$

$\mathrm{H} \quad 1.77183805 \quad 0.25101646 \quad 1.69174346$

$\mathrm{H} \quad 0.98989902-1.95887262 \quad 1.09378299$

$\mathrm{H} \quad 0.83481513-1.50619158-0.59720336$

$\mathrm{H}-1.25749123-1.229045761 .59935965$

$\mathrm{H}-0.71183080 \quad 1.58830514 \quad-0.93062581$

$\mathrm{H}-0.32796135 \quad 2.77689318 \quad 0.32006596$

$\mathrm{H}-1.91025832 \quad 1.99692170 \quad 0.29733063$

$\mathrm{H}-1.602889251 .255172392 .71621072$

$\mathrm{H}-0.01311083 \quad 2.02475908 \quad 2.76155164$

$\mathrm{H}-0.177684120 .32110582 \quad 3.18499215$

$\begin{array}{lllll}0 & -1.98331284 & -0.97989689 & -2.60305689\end{array}$

$\begin{array}{llll}0 & 3.94749129 & 0.11431407 & 0.57686758\end{array}$

o $-1.21549123-0.93362761-1.59311629$

Vibrational frequencies (in $\mathrm{cm}-1$ )

$\begin{array}{llllllllllllllll}27.4 & 36.4 & 53.5 & 64.5 & 102.7 & 121.1 & 159.1 & 177.7 & 190.3 & 220.6 & 229.5 & 253.8 & 276.2 & 298.2\end{array}$ $327.7 \quad 380.5 \quad 426.6 \quad 450.1 \quad 466.2 \quad 544.0 \quad 609.5 \quad 666.0 \quad 687.0 \quad 803.7 \quad 823.4 \quad 873.0 \quad 926.7 \quad 937.4$ $971.7 \quad 995.5 \quad 1008.1 \quad 1016.8 \quad 1027.9 \quad 1044.5 \quad 1058.2 \quad 1068.8 \quad 1080.3 \quad 1112.4 \quad 1123.0 \quad 1126.4$ $\begin{array}{llllllllllllll}1159.5 & 1184.0 & 1197.5 & 1219.5 & 1232.7 & 1256.6 & 1261.9 & 1274.7 & 1284.5 & 1287.4 & 1289.1 & 1293.7\end{array}$ $1297.61305 .3 \quad 1307.7 \quad 1311.41340 .5 \quad 1352.4 \quad 1360.1 \quad 1385.6 \quad 1402.7 \quad 1404.3 \quad 1440.7 \quad 1594.8$ $1843.12634 .92649 .2 \quad 2658.4 \quad 2670.7 \quad 2671.7 \quad 2673.0 \quad 2675.02677 .8 \quad 2680.12706 .32718 .2$ $2739.7 \quad 2763.9 \quad 2768.9 \quad 2779.9 \quad 2782.2$

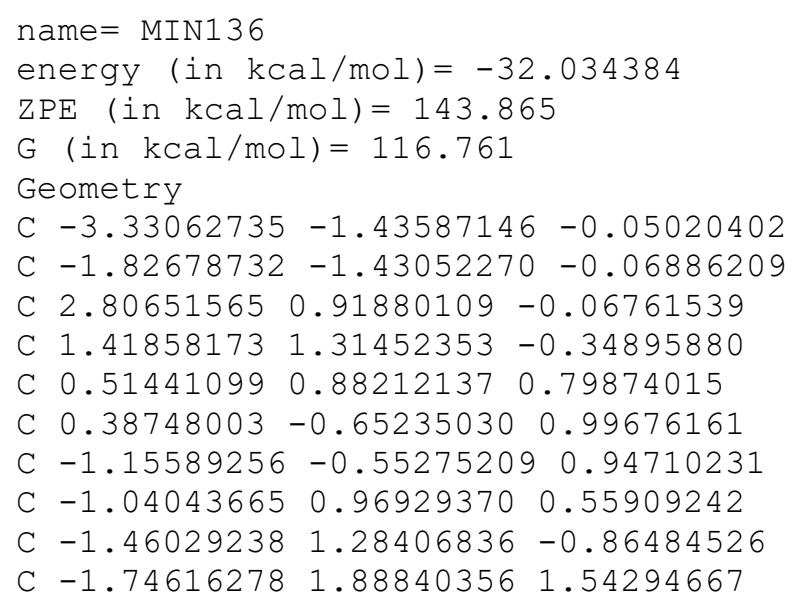


$\mathrm{H}-3.75137210-0.46121471 \quad 0.21782781$

$\mathrm{H}-3.70054195-2.173409490 .67556083$

$\mathrm{H}-3.73564302-1.72565401-1.03130540$

$\mathrm{H} \quad 3.42928831 \quad 1.42367108 \quad 0.65282127$

H $1.081792130 .83633616-1.30697960$

H $1.330363362 .40912633-0.51267725$

$\mathrm{H} 0.80739362 \quad 1.40306424 \quad 1.72759369$

H $0.79770169-1.03999288 \quad 1.92896806$

$\mathrm{H} \quad 0.81400533-1.24299786 \quad 0.16655417$

$\mathrm{H}-1.62275848-0.69834872 \quad 1.93755766$

$\mathrm{H}-2.54900787 \quad 1.23717017-0.98579829$

$\mathrm{H}-1.02655371 \quad 0.57475123-1.58655564$

$\mathrm{H}-1.14312214 \quad 2.28986091-1.16362620$

$\mathrm{H}-1.46185456 \quad 1.68013310 \quad 2.58065941$

$\mathrm{H}-2.83569092 \quad 1.78302234 \quad 1.47646909$

$\mathrm{H}-1.50708783 \quad 2.94008148 \quad 1.34401050$

O $2.78481290-0.76045767-1.48759848$

$03.40384410-0.08300379-0.63506794$

o - $1.21020634-2.11817899-0.84157853$

Vibrational frequencies (in $\mathrm{cm}-1$ )

$\begin{array}{llllllllllllllll}28.1 & 38.4 & 48.6 & 57.6 & 109.4 & 144.0 & 157.3 & 186.8 & 198.7 & 239.6 & 252.2 & 279.5 & 303.5 & 323.8\end{array}$ $364.2389 .6 \quad 434.2 \quad 464.9 \quad 517.5 \quad 565.6 \quad 601.2 \quad 665.1 \quad 722.8 \quad 803.1 \quad 816.9 \quad 881.1914 .1945 .0$ $961.5997 .4 \quad 1005.2 \quad 1015.91034 .7 \quad 1043.4 \quad 1048.4 \quad 1068.9 \quad 1089.91105 .4 \quad 1132.6 \quad 1148.1$

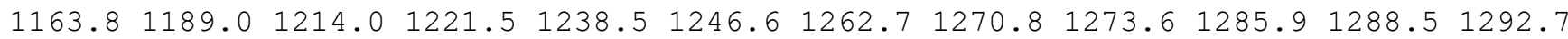

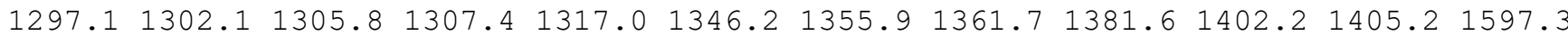
$1858.52633 .72661 .5 \quad 2664.12669 .3 \quad 2675.8 \quad 2677.3 \quad 2678.92680 .72720 .6 \quad 2723.0 \quad 2727.3$ $2734.4 \quad 2756.2 \quad 2778.02781 .4 \quad 2783.1$

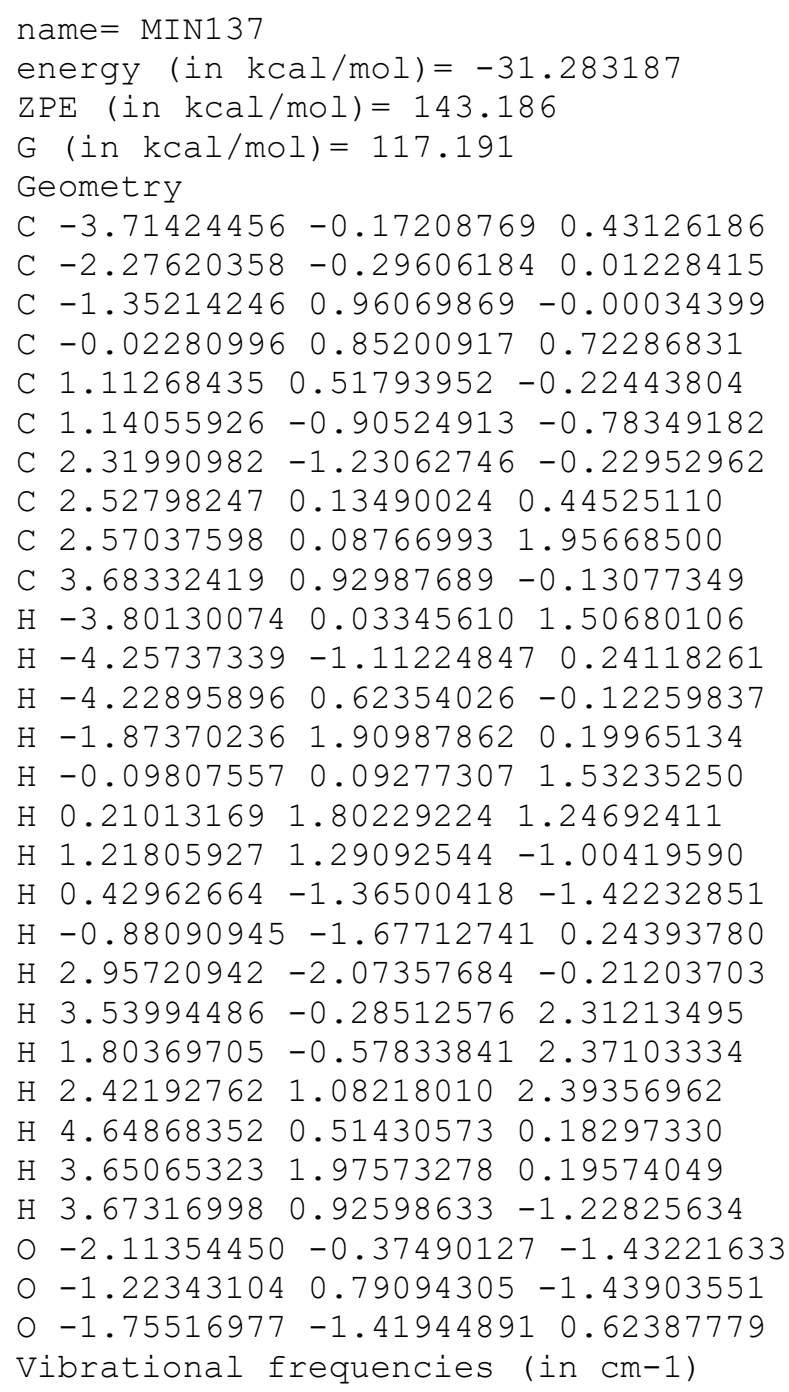


$\begin{array}{llllllllllllllllll}38.3 & 63.3 & 80.5 & 88.7 & 123.7 & 151.8 & 168.5 & 178.8 & 213.0 & 225.6 & 248.8 & 298.7 & 339.1 & 353.7\end{array}$ $359.2 \quad 387.1 \quad 417.5 \quad 453.3 \quad 480.7 \quad 551.1 \quad 587.6 \quad 697.2 \quad 709.5 \quad 811.1 \quad 827.2 \quad 879.1914 .1928 .1$ $946.1968 .4 \quad 990.8 \quad 997.5 \quad 1000.6 \quad 1008.8 \quad 1016.9 \quad 1040.9 \quad 1051.5 \quad 1072.8 \quad 1118.8 \quad 1131.7 \quad 1150.2$ $1151.51170 .21195 .3 \quad 1212.5 \quad 1218.1 \quad 1242.4 \quad 1263.1 \quad 1271.7 \quad 1274.2 \quad 1281.2 \quad 1287.01294 .3$

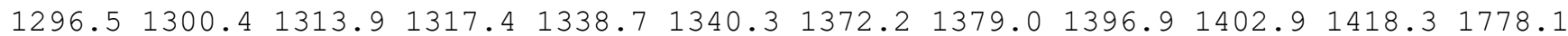
$2648.22668 .3 \quad 2673.4 \quad 2676.8 \quad 2678.0 \quad 2678.3 \quad 2681.5 \quad 2702.82723 .12737 .02767 .2 \quad 2780.5$ $2781.12782 .6 \quad 2787.4 \quad 2807.2$

name $=$ MIN138

energy (in $\mathrm{kcal} / \mathrm{mol})=-31.49255$

$\mathrm{ZPE}(\mathrm{in} \mathrm{kcal} / \mathrm{mol})=143.41$

$\mathrm{G}($ in $\mathrm{kcal} / \mathrm{mol})=116.681$

Geometry

C $-2.39348684-1.97149092-0.50266504$

C $-1.01727095-1.49887488-0.66587442$

$\begin{array}{lllll}\text { C } 1.35968667 & 2.73337538 & -0.04824933\end{array}$

C $1.448671181 .32491546-0.57458959$

$\begin{array}{llll}\text { C } 1.38194200 & 0.28470871 & 0.53012739\end{array}$

C $1.43217533-1.17380493-0.00510519$

C $-0.03878913-1.41245448 \quad 0.43370621$

C $-0.03127157-0.00211619 \quad 1.16025051$

C $-1.15997326 \quad 0.93587118 \quad 0.79144518$

C $0.05301476-0.15764130 \quad 2.67204137$

$\mathrm{H}-2.69607600-2.12594707 \quad 0.53937065$

$\mathrm{H}-2.55181539-2.91783757-1.05669630$

$\mathrm{H}-3.10607661-1.26003391-0.97295608$

$\mathrm{H} \quad 1.22064000 \quad 3.52714010 \quad-0.79529192$

H $2.40645711 \quad 1.21270804-1.13110274$

$\mathrm{H} \quad 0.65591130 \quad 1.15249446-1.33312477$

H 2.14252343 $0.50479860 \quad 1.30189318$

H $2.14949690-1.810510330 .52464446$

H $1.64148934-1.27985783-1.07088085$

$\mathrm{H}-0.15771278-2.25780074 \quad 1.13573100$

$\mathrm{H}-2.120672390 .579017291 .18457870$

$\mathrm{H}-1.28244228 \quad 1.05994976-0.29037976$

$\mathrm{H}-0.98708749 \quad 1.93557929 \quad 1.21780714$

$\mathrm{H} \quad 0.27534817 \quad 0.80856908 \quad 3.14865851$

$\mathrm{H} \quad 0.84390254 \quad-0.85273185 \quad 2.97572244$

$\mathrm{H}-0.88927415-0.52209420 \quad 3.09581386$

o $-1.38079081-1.08366119-2.79424196$

O $1.44826023 \quad 3.001394461 .12318190$

o $-0.56734223-1.08076482-1.81736200$

Vibrational frequencies (in $\mathrm{cm}-1$ )

$\begin{array}{llllllllllllllll}40.2 & 44.2 & 64.9 & 82.1 & 104.1 & 124.5 & 129.7 & 196.1 & 205.6 & 220.9 & 232.7 & 273.9 & 282.0 & 302.5\end{array}$

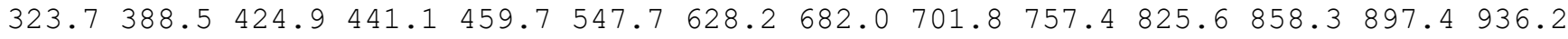
$993.31007 .91016 .7 \quad 1024.8 \quad 1031.8 \quad 1038.8 \quad 1046.7 \quad 1062.9 \quad 1081.91108 .4 \quad 1114.11121 .9$

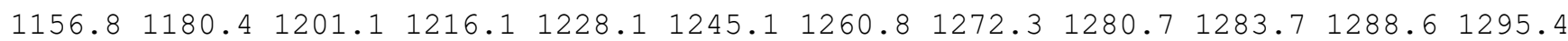
$1297.11301 .1 \quad 1305.5 \quad 1310.7 \quad 1343.7 \quad 1352.0 \quad 1361.2 \quad 1384.11401 .2 \quad 1405.6 \quad 1437.6 \quad 1592.6$ $1842.92634 .12646 .92650 .6 \quad 2666.7 \quad 2671.2 \quad 2672.5 \quad 2674.32679 .5 \quad 2682.32717 .12723 .6$ $2736.02763 .9 \quad 2769.0 \quad 2778.4 \quad 2781.2$

name $=$ MIN139

energy (in $\mathrm{kcal} / \mathrm{mol})=-31.798698$

$\mathrm{ZPE}(\mathrm{in} \mathrm{kcal} / \mathrm{mol})=143.789$

$\mathrm{G}($ in $\mathrm{kcal} / \mathrm{mol})=117.008$

Geometry

C $-2.19513741-2.46107772 \quad 0.32971600$

C $-0.69359145-2.45064418 \quad 0.31402503$

C $-0.32923103 \quad 2.81719031 \quad 0.53639223$

C $0.37868403 \quad 1.56157484 \quad 0.86370193$

C $0.57187922 \quad 0.64397840-0.33347966$

C $-0.70264644-0.10226334-0.81117987$

C $-0.01280272-1.47131714-0.59743405$ 
C $1.26739220-0.74163237-0.04322698$

C $1.59600543-1.024210561 .40818607$

C $2.49200299-0.97129103-0.91630433$

$\mathrm{H}-2.62744058-2.25884465-0.65737337$

$\mathrm{H}-2.58425944-1.70955286 \quad 1.03063490$

$\mathrm{H}-2.57706331-3.43628448 \quad 0.67115811$

$\mathrm{H}-0.48669299 \quad 3.58840441 \quad 1.27368824$

$\mathrm{H}-0.173040831 .03021600 \quad 1.67579736$

$\mathrm{H} 1.374589051 .80007873 \quad 1.30947119$

$\mathrm{H} 1.080745901 .18379942-1.15306755$

$\mathrm{H}-0.98515136 \quad 0.10015379-1.84907117$

$\mathrm{H}-1.58558307 \quad 0.07025403-0.18943154$

$\mathrm{H} \quad 0.19943158-1.99741092-1.55174524$

$\mathrm{H} \quad 0.72998159-0.91393767 \quad 2.06976898$

H $2.39537490 \quad-0.37387862 \quad 1.77870419$

$\mathrm{H} \quad 1.93648770-2.06614572 \quad 1.53397027$

$\mathrm{H} 3.32381460-0.32325260-0.61500005$

$\mathrm{H} 2.29250462-0.77861426-1.97619701$

$\mathrm{H} 2.84346625-2.00890579-0.83483366$

$\begin{array}{lllll}0 & -1.36378094 & 3.97569692 & -0.99965257\end{array}$

$\begin{array}{llll}0 & -0.75009084 & 2.92250031 & -0.68681807\end{array}$

$0-0.06612872-3.23875307 \quad 0.97780650$

Vibrational frequencies (in $\mathrm{cm}-1$ )

$\begin{array}{lllllllllllllll}31.1 & 37.6 & 57.1 & 97.0 & 116.9 & 134.6 & 173.4 & 193.0 & 212.5 & 233.6 & 249.0 & 270.1 & 286.3 & 321.6\end{array}$

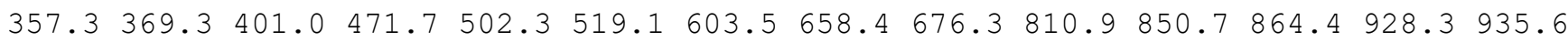

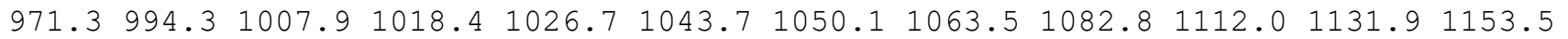

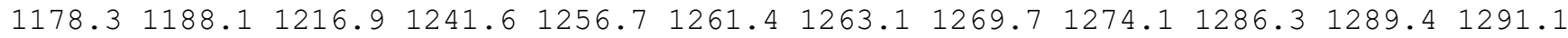
$1301.21301 .41304 .41312 .3 \quad 1320.4 \quad 1346.1 \quad 1349.3 \quad 1358.11381 .01402 .31405 .61580 .3$ $1845.22638 .4 \quad 2660.0 \quad 2666.4 \quad 2674.0 \quad 2675.3 \quad 2677.2 \quad 2681.72682 .4 \quad 2710.62717 .2 \quad 2722.9$ $2729.6 \quad 2762.2 \quad 2779.3 \quad 2779.6 \quad 2782.1$

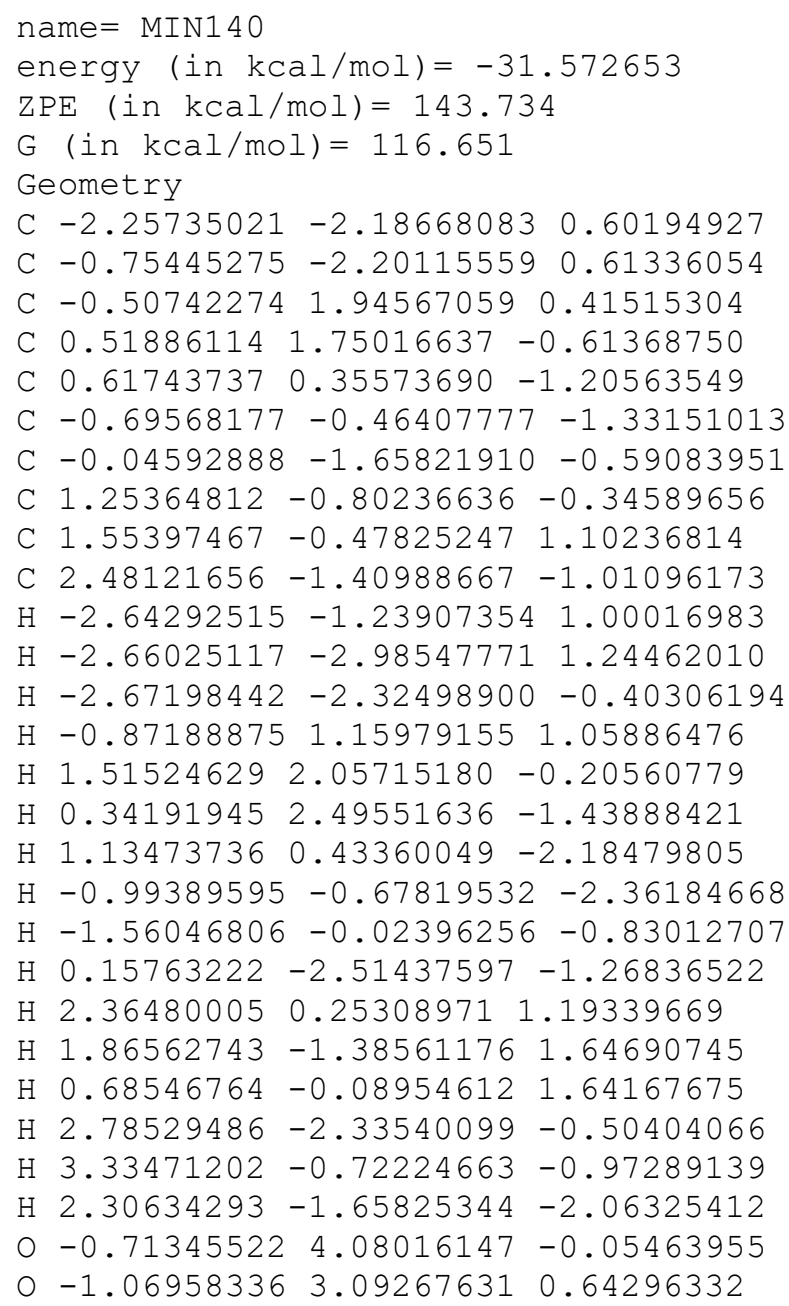


O $-0.15306710-2.681241821 .54255480$

Vibrational frequencies (in $\mathrm{cm}-1$ )

$\begin{array}{llllllllllllllll}27.1 & 32.1 & 37.5 & 86.9 & 115.8 & 158.4 & 180.0 & 192.7 & 214.6 & 220.4 & 248.0 & 270.9 & 288.0 & 330.6\end{array}$

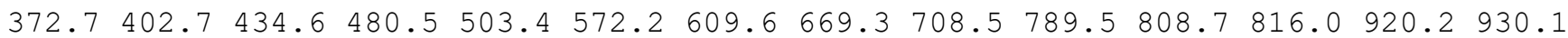
$978.7 \quad 991.3 \quad 1008.91018 .0 \quad 1020.8 \quad 1044.7 \quad 1051.0 \quad 1055.1 \quad 1082.3 \quad 1099.3 \quad 1131.91150 .6$

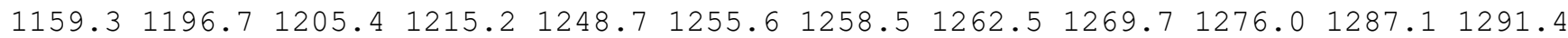

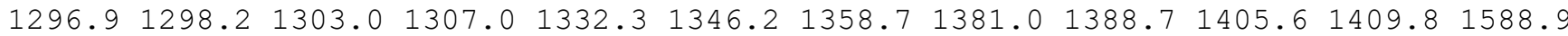
$1845.22615 .62662 .02667 .92674 .8 \quad 2677.0 \quad 2677.62682 .42683 .2 \quad 2701.62709 .12716 .6$ $2725.42765 .62780 .2 \quad 2780.92782 .3$

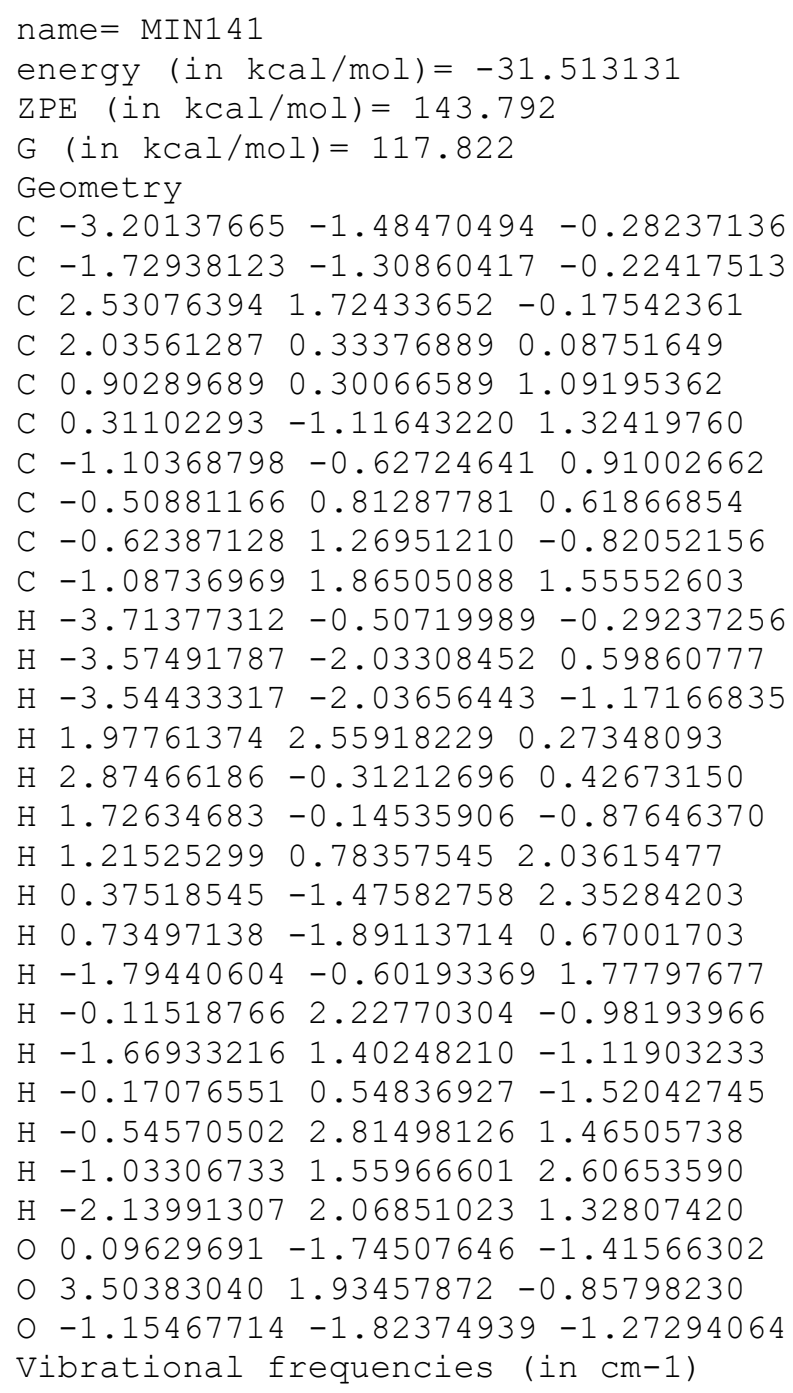


C $0.21623291-1.15156107 \quad 1.22491389$

C $-1.11965478 \quad-0.48052459 \quad 0.80520708$

C $-0.32699562 \quad 0.84879253 \quad 0.45267737$

C $-0.40818452 \quad 1.28617534-0.99497757$

C $-0.72225893 \quad 1.99796624 \quad 1.37041236$

$\mathrm{H}-3.76528424-1.56333731-1.21737391$

$\mathrm{H}-3.73387093-0.09353341-0.22817969$

$\mathrm{H}-3.73683303-1.68695788 \quad 0.54906632$

$\mathrm{H} \quad 3.376365051 .34773830-1.30744438$

$\mathrm{H} 2.77266402-0.86415824 \quad 0.19597515$

$\mathrm{H} 1.66281471-0.39996406-1.08420052$

$\mathrm{H} \quad 1.42548380 \quad 0.63017732 \quad 1.81804778$

$\mathrm{H} \quad 0.24827298-1.46356106 \quad 2.27048498$

$\mathrm{H} \quad 0.50568809-2.01063440 \quad 0.60537660$

$\mathrm{H}-1.78758949-0.32193951 \quad 1.67694133$

$\mathrm{H} \quad 0.26471519 \quad 2.13181512-1.18782650$

$\mathrm{H}-1.420560761 .60545305-1.26436905$

$\mathrm{H}-0.119360180 .48272593-1.68940495$

$\mathrm{H}-0.026443572 .84150036 \quad 1.25546242$

$\mathrm{H}-0.70072316 \quad 1.711408592 .42778747$

$\mathrm{H}-1.72827684 \quad 2.36720264 \quad 1.14558286$

O $-0.12302790-1.81029171-1.51288159$

$\begin{array}{llll}0 & 3.08298779 & 2.02205563 \quad 0.55090776\end{array}$

o $-1.37320604-1.72224555-1.34014419$

Vibrational frequencies (in $\mathrm{cm}-1$ )

$\begin{array}{lllllllllllllll}37.9 & 61.5 & 70.3 & 92.5 & 128.3 & 140.9 & 153.5 & 190.2 & 215.1 & 219.8 & 253.8 & 276.8 & 295.2 & 312.9\end{array}$ $\begin{array}{lllllllllllllll}330.8 & 382.0 & 419.0 & 436.1 & 472.6 & 584.6 & 605.7 & 656.2 & 699.7 & 764.7 & 808.9 & 862.4 & 914.3 & 943.3\end{array}$

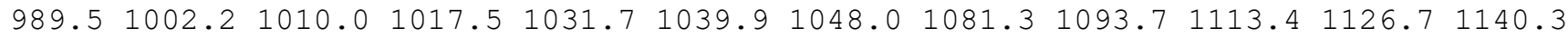

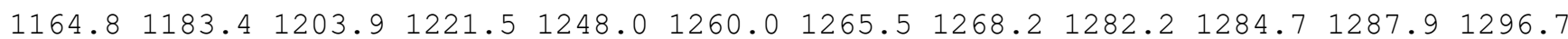

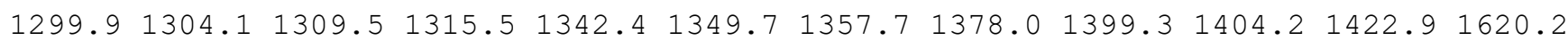
$1839.42635 .4 \quad 2652.8 \quad 2655.3 \quad 2658.12661 .7 \quad 2671.2 \quad 2676.42678 .62681 .42711 .12719 .1$ $2731.6 \quad 2758.6 \quad 2770.92777 .6 \quad 2781.3$

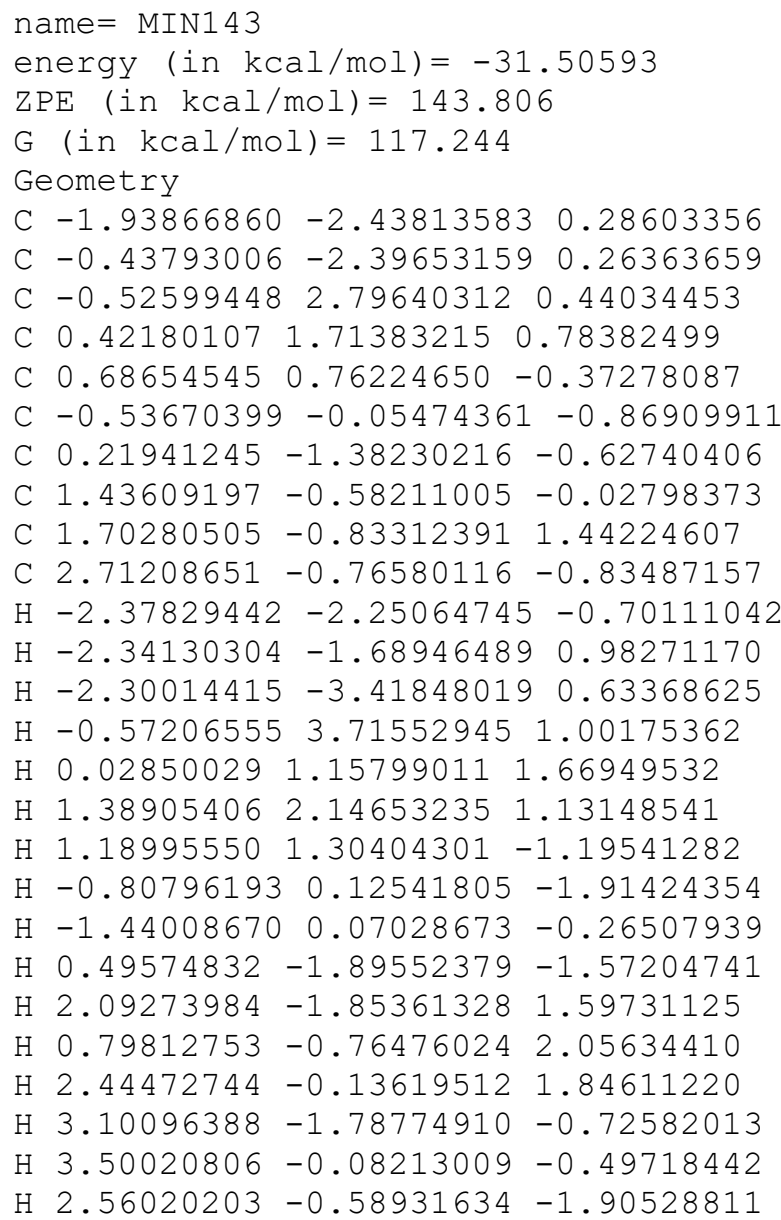


$0-2.16884053 \quad 3.41866092-0.86092947$

$0-1.312796512 .54719217-0.56158968$

O $0.21173052-3.183659760 .90692664$

Vibrational frequencies (in $\mathrm{cm}-1$ )

$\begin{array}{llllllllllllllll}38.6 & 43.3 & 58.6 & 88.2 & 129.0 & 140.1 & 173.4 & 188.6 & 210.5 & 231.7 & 252.2 & 264.5 & 286.7 & 316.6\end{array}$ $355.7 \quad 367.5 \quad 407.2 \quad 471.7 \quad 502.0 \quad 517.3 \quad 602.0 \quad 656.6 \quad 674.4 \quad 810.4 \quad 855.3 \quad 860.5 \quad 927.3 \quad 935.0$ $974.4994 .0 \quad 1007.4 \quad 1018.2 \quad 1028.9 \quad 1041.3 \quad 1048.3 \quad 1068.0 \quad 1090.4 \quad 1111.91134 .6 \quad 1154.5$

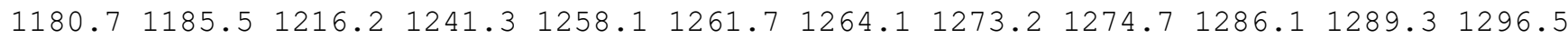
$1300.51301 .3 \quad 1304.6 \quad 1311.1 \quad 1319.9 \quad 1342.1 \quad 1349.4 \quad 1357.4 \quad 1380.4 \quad 1401.2 \quad 1405.5 \quad 1578.2$ $1844.92637 .02659 .2 \quad 2666.6 \quad 2673.4 \quad 2674.2 \quad 2677.4 \quad 2680.62681 .92711 .4 \quad 2719.2 \quad 2723.7$ $2728.4 \quad 2760.3 \quad 2778.6 \quad 2779.4 \quad 2782.0$

name $=\operatorname{MIN} 144$

energy (in $\mathrm{kcal} / \mathrm{mol})=-31.246311$

$\mathrm{ZPE}($ in $\mathrm{kcal} / \mathrm{mol})=143.601$

$\mathrm{G}($ in $\mathrm{kcal} / \mathrm{mol})=117.455$

Geometry

C $-3.07715905-1.36422839-0.26219592$

C $-1.60344503-1.27284297-0.42241237$

C $2.41242621 \quad 1.86925410 \quad-0.41996317$

C $1.99078587 \quad 0.43259878-0.48165041$

C $1.22797363-0.02437400 \quad 0.74780203$

C $0.73075321-1.49403366 \quad 0.64900383$

C $-0.74878391-1.03605758 \quad 0.74135260$

C $-0.25793223 \quad 0.46628958 \quad 0.90309721$

C $-0.78057571 \quad 1.42828405-0.14326684$

C $-0.53459192 \quad 1.000711562 .30137629$

$\mathrm{H}-3.36240567-2.24705857 \quad 0.33313320$

$\mathrm{H}-3.61985540-1.43062259-1.21796312$

$\mathrm{H}-3.46760750-0.47799346 \quad 0.26841579$

H 2.96997340 2.25526053 -1.28581953

H 2.88032907 $-0.21642078-0.63085138$

$\mathrm{H} 1.37515092 \quad 0.25544578-1.40002254$

H $1.82256195 \quad 0.17952871 \quad 1.65712693$

H $1.04572086-2.13287699 \quad 1.47700795$

$\mathrm{H} \quad 0.99748863-2.00285146-0.28612255$

$\mathrm{H}-1.23641398-1.40460453 \quad 1.66634003$

$\mathrm{H}-0.28447508 \quad 2.40651238-0.04712388$

$\mathrm{H}-1.85662681 \quad 1.59859639-0.03541938$

$\mathrm{H}-0.595465831 .07396580-1.16571143$

$\mathrm{H}-1.59944787 \quad 1.19588797 \quad 2.46042354$

$\mathrm{H} 0.00401928 \quad 1.94818567 \quad 2.45917769$

$\mathrm{H}-0.200300620 .30983312 \quad 3.08323810$

o $-0.05988839-1.30115626-2.02963077$

$\begin{array}{llll}0 & 2.17000554 & 2.59493441 & 0.51328598\end{array}$

o $-1.27045640-1.39480777-1.67433484$

Vibrational frequencies (in $\mathrm{cm}-1$ )

$\begin{array}{llllllllllllllll}40.3 & 62.8 & 75.5 & 101.3 & 108.7 & 132.6 & 155.8 & 190.8 & 218.1 & 231.6 & 247.5 & 272.5 & 286.6 & 321.5\end{array}$

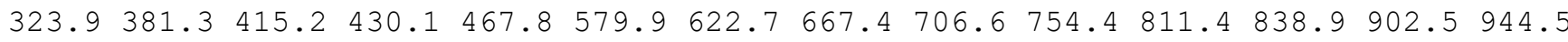
$990.0 \quad 999.3 \quad 1007.3 \quad 1014.8 \quad 1031.1 \quad 1036.3 \quad 1047.7 \quad 1077.9 \quad 1091.3 \quad 1109.4 \quad 1119.01139 .8$

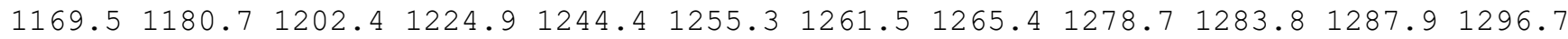
$1301.61302 .1 \quad 1309.3 \quad 1312.4 \quad 1340.8 \quad 1351.1 \quad 1360.4 \quad 1376.91398 .3 \quad 1404.3 \quad 1417.8 \quad 1619.6$ $1838.82623 .52649 .02655 .52661 .72663 .2 \quad 2669.12674 .52676 .72682 .22717 .12721 .2$ $2730.2 \quad 2758.2 \quad 2770.8 \quad 2777.02780 .3$

name $=$ MIN145

energy (in $\mathrm{kcal} / \mathrm{mol})=-31.080553$

$\mathrm{ZPE}(\mathrm{in} \mathrm{kcal} / \mathrm{mol})=143.469$

$\mathrm{G}($ in $\mathrm{kcal} / \mathrm{mol})=116.36$

Geometry

C $-2.30124541-1.64319083-0.32090508$

C $-0.84229027-1.75381404-0.24350111$

C $-0.31133222 \quad 3.48831760-0.21362844$ 
C $0.21978224 \quad 2.23687497 \quad 0.43522919$

C $0.703316621 .23355784-0.59051245$

C $-0.41962267 \quad 0.51542693-1.38696587$

C $0.11350994-0.87080143-0.94010849$

C $1.20865494-0.15585717-0.04909934$

C $1.06031878-0.37383901 \quad 1.44254814$

C $2.62533544-0.47641620-0.49644161$

$\mathrm{H}-2.66409209-1.19078705-1.25240778$

$\mathrm{H}-2.70383468-1.05613190 \quad 0.52442536$

$\mathrm{H}-2.77351307-2.64693933-0.22240760$

$\mathrm{H}-0.47994144 \quad 3.47209659-1.29725096$

$\mathrm{H}-0.57281386 \quad 1.80691708 \quad 1.08628571$

$\mathrm{H} 1.03765361 \quad 2.50014603 \quad 1.14112800$

$\mathrm{H} 1.455922231 .70114372-1.25300521$

$\mathrm{H}-0.39056084 \quad 0.67872530 \quad-2.46742316$

$\mathrm{H}-1.43180758 \quad 0.74800457-1.04203076$

H $0.57698576-1.42525998-1.78560970$

H $1.36388013-1.38763998 \quad 1.73925426$

$\mathrm{H} \quad 0.02874627 \quad-0.23438181 \quad 1.78924968$

H $1.69292986 \quad 0.32065119 \quad 2.01116955$

H $3.35531698 \quad 0.167014450 .01195619$

H 2.76588016 $-0.33423409-1.57414158$

$\mathrm{H} \quad 2.89644189-1.51482303-0.26736149$

O $-1.01150646-3.52042554 \quad 1.05566938$

$0-0.55380325 \quad 4.47475425 \quad 0.43576909$

o $-0.26303243-2.69973916 \quad 0.44763904$

Vibrational frequencies (in cm-1)

$\begin{array}{llllllllllllllll}27.0 & 32.5 & 61.7 & 100.2 & 106.7 & 120.5 & 152.9 & 179.6 & 191.9 & 245.1 & 257.5 & 280.5 & 298.0 & 301.4\end{array}$ $325.0 \quad 384.3 \quad 399.3 \quad 419.9 \quad 478.6 \quad 540.2 \quad 556.2 \quad 657.0 \quad 688.4 \quad 781.3 \quad 820.1 \quad 873.7 \quad 931.6 \quad 940.6$ $978.5995 .1 \quad 1007.3 \quad 1018.4 \quad 1025.9 \quad 1039.7 \quad 1064.4 \quad 1078.2 \quad 1100.8 \quad 1112.8 \quad 1125.6 \quad 1135.2$

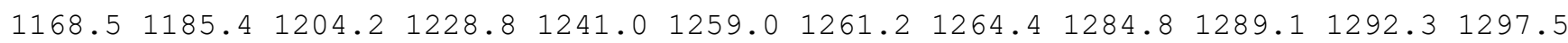
$1299.51303 .11306 .6 \quad 1314.6 \quad 1340.6 \quad 1349.1 \quad 1357.2 \quad 1386.31401 .51404 .2 \quad 1440.91576 .0$ $1837.7 \quad 2631.92649 .2 \quad 2653.2 \quad 2665.6 \quad 2669.12671 .92674 .92676 .12682 .2 \quad 2704.92720 .1$ $2741.62763 .02766 .7 \quad 2778.6 \quad 2782.1$

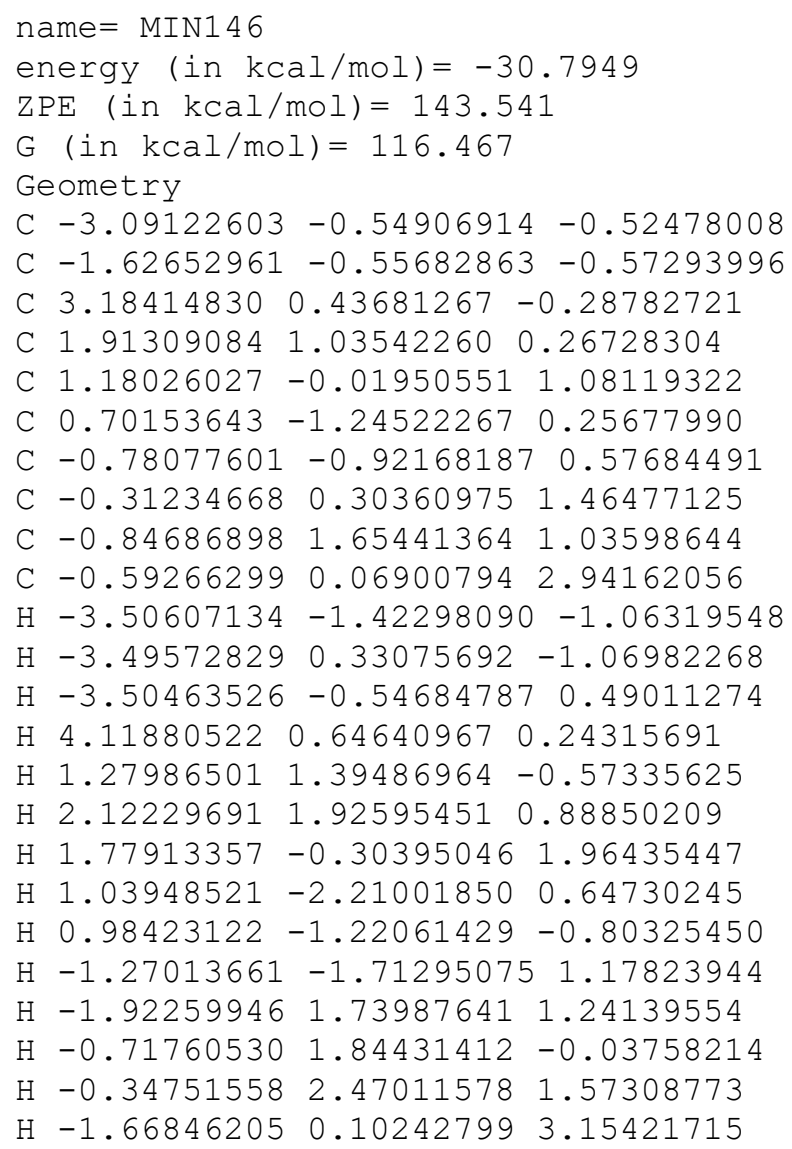


$\mathrm{H}-0.11517640 \quad 0.83562724 \quad 3.56396630$

$\mathrm{H}-0.22421236-0.904681253 .28505872$

$0-1.65316458 \quad 0.13588553-2.65491586$

$0 \quad 3.17625295-0.25188217-1.27670411$

O $-0.97025280-0.22190293-1.65114995$

Vibrational frequencies (in $\mathrm{cm}-1$ )

$\begin{array}{llllllllllllllll}31.0 & 37.2 & 64.7 & 78.5 & 87.0 & 119.2 & 169.9 & 185.8 & 194.6 & 217.5 & 229.3 & 273.6 & 282.9 & 298.8\end{array}$

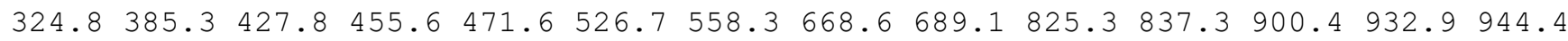
$982.7 \quad 994.8 \quad 1008.3 \quad 1017.4 \quad 1029.8 \quad 1045.5 \quad 1047.2 \quad 1068.9 \quad 1089.1 \quad 1113.6 \quad 1123.2 \quad 1131.4$

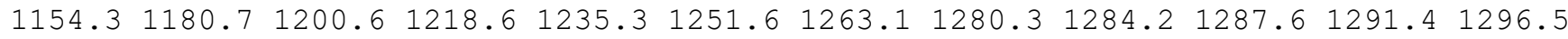

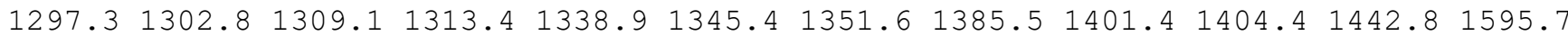
$1841.32635 .8 \quad 2649.2 \quad 2665.2 \quad 2667.7 \quad 2669.6 \quad 2673.6 \quad 2674.32676 .3 \quad 2678.62717 .72724 .1$ $2743.22757 .62769 .62779 .5 \quad 2782.5$

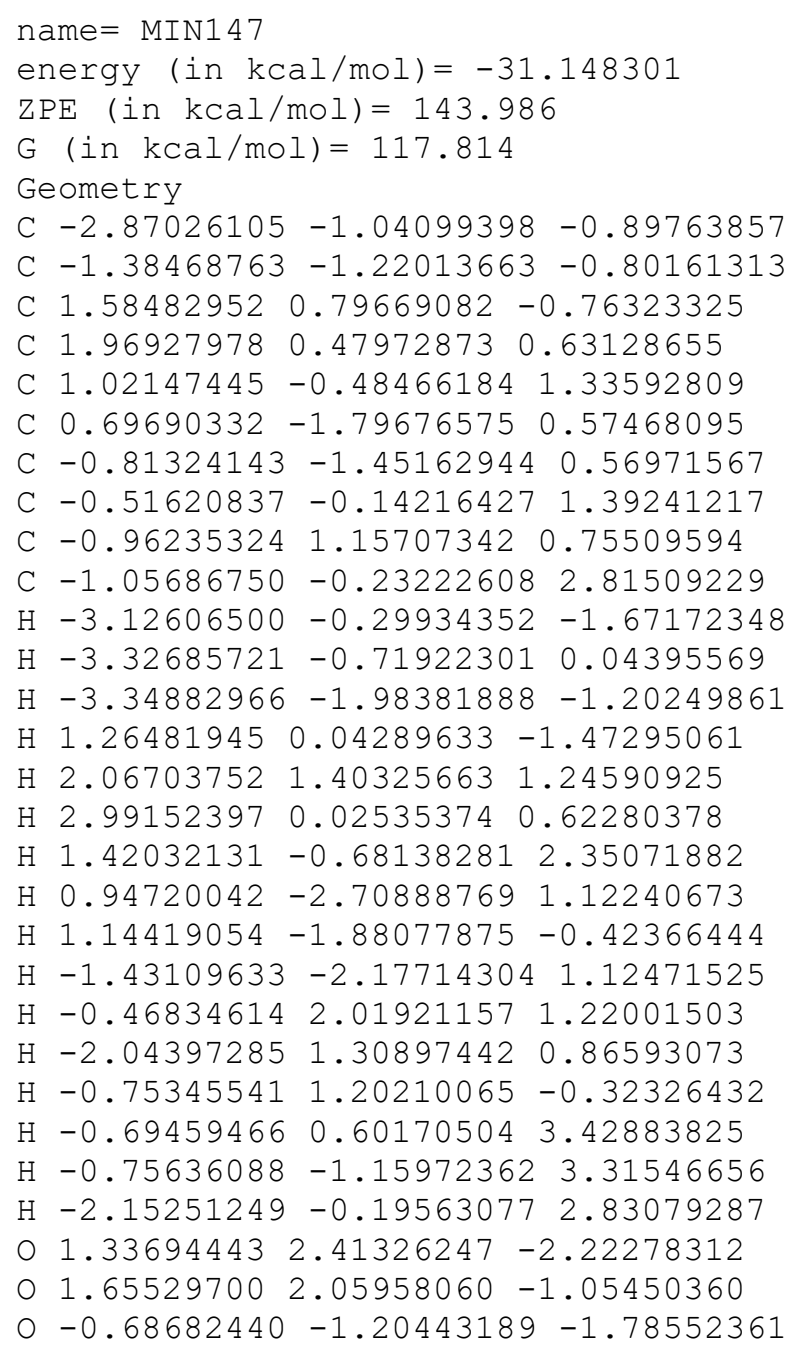


C $-1.67051640-1.05371217-0.75034625$

$\begin{array}{lllll}\text { C } & 1.65085101 & 0.75784730 & -0.72334326\end{array}$

C $1.85083704 \quad 0.48433451 \quad 0.71868590$

$\begin{array}{llll}\text { C } 0.86615329 & -0.50225634 & 1.33349794\end{array}$

C $0.47906914-1.73894286 \quad 0.48074943$

C $-1.02103437-1.36308804 \quad 0.56842639$

C $-0.65306232-0.09507746 \quad 1.42814427$

$\begin{array}{llll}\text { C }-1.03684508 & 1.23977357 & 0.82377849\end{array}$

$\begin{array}{llll}\text { C }-1.18526582 & -0.19836934 & 2.85183433\end{array}$

$\begin{array}{lllll}\mathrm{H} & -3.58065188 & -0.51183280 & 0.12096871\end{array}$

$\mathrm{H}-3.58434617-2.03155252-0.79191291$

$\mathrm{H}-3.54705255-0.49028900-1.65554944$

H $0.978086310 .18423495 \quad-1.35179874$

H $1.81703768 \quad 1.43249433 \quad 1.30538068$

$\begin{array}{lllll}\mathrm{H} & 2.88512815 & 0.08716148 & 0.87123417\end{array}$

$\begin{array}{llllll}\mathrm{H} & 1.25297801 & -0.79888017 & 2.32950851\end{array}$

H $0.73062636-2.698890240 .93873836$

H $0.88183874-1.74882587 \quad-0.54017411$

$\mathrm{H}-1.61801618-2.10008292 \quad 1.13146904$

$\mathrm{H}-0.577618492 .07248482 \quad 1.37073483$

$\mathrm{H}-2.12242948 \quad 1.39703023 \quad 0.85124331$

$\mathrm{H}-0.72865997 \quad 1.33280013-0.22749058$

$\mathrm{H}-2.27719628 \quad-0.10317966 \quad 2.87934471$

$\mathrm{H}-0.77352694 \quad 0.59479405 \quad 3.48783438$

$\mathrm{H}-0.93142049-1.15587988 \quad 3.32108971$

$\begin{array}{llll}0 & 2.27002626 & 2.01730563 & -2.40613509\end{array}$

$0 \quad 2.36841110 \quad 1.73945982-1.18027304$

$0-1.02816856-0.85926244-1.75236114$

Vibrational frequencies (in cm-1)

$\begin{array}{lllllllllllllllll}19.5 & 51.1 & 65.5 & 105.4 & 134.6 & 149.1 & 172.1 & 189.7 & 206.1 & 217.3 & 255.8 & 266.0 & 271.6 & 318.3\end{array}$

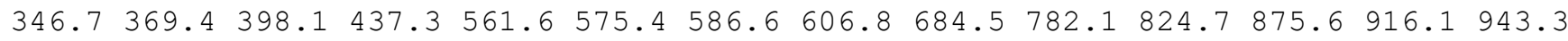
$995.81004 .1 \quad 1014.3 \quad 1016.6 \quad 1036.2 \quad 1043.0 \quad 1049.6 \quad 1071.1 \quad 1088.01108 .01128 .8 \quad 1156.2$ $\begin{array}{llllllllllllll}1174.7 & 1207.7 & 1212.8 & 1232.9 & 1239.1 & 1261.0 & 1267.2 & 1271.7 & 1273.1 & 1287.8 & 1288.6 & 1293.2\end{array}$ $\begin{array}{llllllllllll}1297.7 & 1301.9 & 1307.1 & 1311.2 & 1338.3 & 1341.8 & 1356.4 & 1379.7 & 1389.9 & 1405.6 & 1410.7 & 1556.2\end{array}$

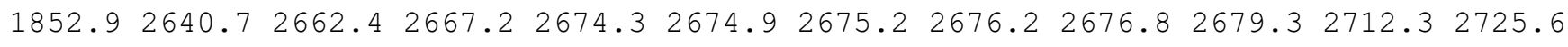
2729.92759 .02779 .02780 .12782 .9

name $=$ MIN149

energy (in $\mathrm{kcal} / \mathrm{mol})=-31.116069$

$\mathrm{ZPE}(\mathrm{in} \mathrm{kcal} / \mathrm{mol})=144.187$

$\mathrm{G}(\mathrm{in} \mathrm{kcal} / \mathrm{mol})=118.212$

Geometry

C $-2.11378997-1.74050721 \quad 0.46648467$

C $-0.61967094-1.815907690 .53215881$

C $-0.01260473 \quad 2.19742789 \quad 0.56119347$

C $0.430166262 .07878368-0.83380526$

C $0.74933800 \quad 0.66804880-1.32783562$

C $-0.47692239-0.25787352-1.54219365$

C $0.16306633-1.35883402-0.66700587$

C $1.40205817-0.42381124-0.39618687$

C $1.64479619-0.07558789 \quad 1.05699174$

C $2.68598893-0.98027207-1.00430351$

$\mathrm{H}-2.58249665-2.33961811 \quad 1.26018568$

$\mathrm{H}-2.50770127-2.08190576-0.49791330$

$\mathrm{H}-2.45832089-0.69825054 \quad 0.60360828$

$\mathrm{H} \quad 0.66335827 \quad 2.49300349 \quad 1.35580181$

H $1.315871522 .72962141-1.01088313$

$\mathrm{H}-0.38341336 \quad 2.48216454-1.49763595$

H $1.34071466 \quad 0.77348164 \quad-2.26146472$

$\mathrm{H}-0.65095402-0.54671687-2.58125407$

$\mathrm{H}-1.41969714 \quad 0.15684400-1.15838805$

$\mathrm{H} \quad 0.44754334 \quad-2.26037410 \quad-1.24605497$

$\mathrm{H} \quad 0.72318158 \quad 0.15938799 \quad 1.59959458$

H 2.34827624 $0.75578876 \quad 1.16169465$ 
$\mathrm{H} 2.06966105-0.941763451 .59356898$

$\mathrm{H} 3.50030178-0.24907624-0.94619487$

$\mathrm{H} 2.56352343-1.25673972-2.05676086$

$\mathrm{H} \quad 3.01501399-1.88048627-0.46835737$

$0-2.132073031 .71147377 \quad 0.18636918$

$0-1.218810472 .03013877 \quad 0.99704080$

o $-0.06235341-2.266235661 .50486791$

Vibrational frequencies (in $\mathrm{cm}-1$ )

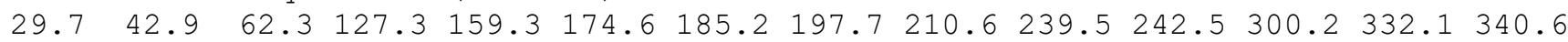
$372.5 \quad 410.4 \quad 436.0 \quad 485.1 \quad 505.5 \quad 587.2 \quad 615.1 \quad 669.4 \quad 699.8 \quad 796.2 \quad 812.3 \quad 859.1 \quad 904.5 \quad 933.7$ $965.8 \quad 989.1 \quad 1007.8 \quad 1017.7 \quad 1021.6 \quad 1040.1 \quad 1043.3 \quad 1065.9 \quad 1082.5 \quad 1109.6 \quad 1118.91132 .0$

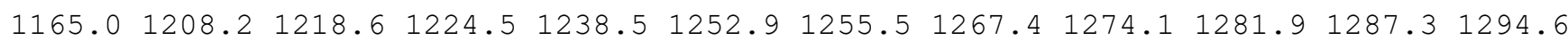
$1300.61301 .51305 .6 \quad 1323.1 \quad 1330.8 \quad 1342.5 \quad 1348.5 \quad 1380.11391 .8 \quad 1406.01413 .6 \quad 1613.7$ $1838.72637 .12642 .82657 .7 \quad 2665.92674 .6 \quad 2677.82679 .22685 .92706 .12710 .42716 .0$ $2723.5 \quad 2757.3 \quad 2775.12778 .5 \quad 2781.7$

\section{name $=$ MIN150}

energy (in $\mathrm{kcal} / \mathrm{mol})=-30.304313$

$\mathrm{ZPE}($ in $\mathrm{kcal} / \mathrm{mol})=143.522$

$\mathrm{G}(\mathrm{in} \mathrm{kcal} / \mathrm{mol})=116.657$

Geometry

C $-2.78016830-0.96041123-0.48468287$

C $-1.32537984-0.96958200-0.65554279$

C $1.689160131 .57607238-0.62461599$

C $2.22643656 \quad 0.91312032 \quad 0.61823162$

C $1.46785917-0.30891477 \quad 1.09407768$

C $1.12323108-1.394473160 .03916925$

C $-0.38081814-1.320455620 .41800016$

C $-0.05910910-0.16434423 \quad 1.45144487$

C $-0.70207093 \quad 1.17235361 \quad 1.14833874$

C $-0.35906094 \quad-0.57844787 \quad 2.88475700$

$\mathrm{H}-3.12838726-1.443236950 .43530998$

$\mathrm{H}-3.27463048-1.45271539-1.34981539$

$\mathrm{H}-3.17373654 \quad 0.07500101-0.49936142$

H $1.22121813 \quad 0.94536134-1.38940695$

H 2.27518020 1.66285459 1.44095312

H $3.28965185 \quad 0.64230147 \quad 0.42554477$

H $2.02459554 \quad-0.74706297 \quad 1.94982489$

H $1.58059189-2.36966485 \quad 0.23732781$

$\mathrm{H} \quad 1.37286319-1.13581547-0.99171941$

$\mathrm{H}-0.72574648-2.24208235 \quad 0.93197287$

$\mathrm{H}-0.65813850 \quad 1.44345430 \quad 0.08617781$

$\mathrm{H}-1.76384076 \quad 1.18054661 \quad 1.42801931$

$\mathrm{H}-0.21588227 \quad 1.98445860 \quad 1.70689755$

$\mathrm{H} \quad 0.02315870 \quad 0.16318973 \quad 3.59830064$

$\mathrm{H} \quad 0.09335006-1.54170074 \quad 3.14552823$

$\mathrm{H}-1.43820588-0.66826103 \quad 3.05877017$

$\begin{array}{llll}0 & -1.53522884 & -0.34827945 & -2.75281133\end{array}$

$\begin{array}{lllll}0 & 1.79597194 & 2.76639167 & -0.78392708\end{array}$

o-0.76790231-0.67085089-1.79746116

Vibrational frequencies (in $\mathrm{cm}-1$ )

$\begin{array}{lllllllllllllll}33.7 & 39.1 & 53.6 & 72.8 & 112.5 & 131.9 & 164.4 & 195.2 & 213.9 & 224.3 & 252.9 & 277.6 & 280.5 & 297.4\end{array}$

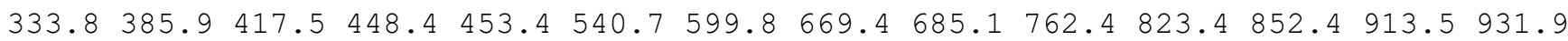
$995.11002 .2 \quad 1008.7 \quad 1016.5 \quad 1029.9 \quad 1042.3 \quad 1051.9 \quad 1068.3 \quad 1076.91114 .4 \quad 1125.7 \quad 1131.7$

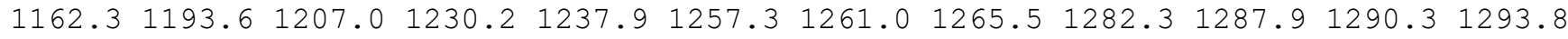
$1297.91300 .91309 .6 \quad 1312.7 \quad 1338.1 \quad 1354.2 \quad 1383.3 \quad 1385.1 \quad 1404.8 \quad 1406.3 \quad 1447.4 \quad 1590.2$ $1834.42633 .5 \quad 2645.8 \quad 2655.1 \quad 2665.92670 .92673 .5 \quad 2675.7 \quad 2677.2 \quad 2677.92708 .52715 .7$ $\begin{array}{lllll}2736.7 & 2762.9 & 2769.2 & 2779.3 & 2782.5\end{array}$

name $=$ MIN151

energy (in $\mathrm{kcal} / \mathrm{mol})=-30.827504$

$\mathrm{ZPE}($ in $\mathrm{kcal} / \mathrm{mol})=144.058$

$\mathrm{G}($ in $\mathrm{kcal} / \mathrm{mol})=118.642$ 


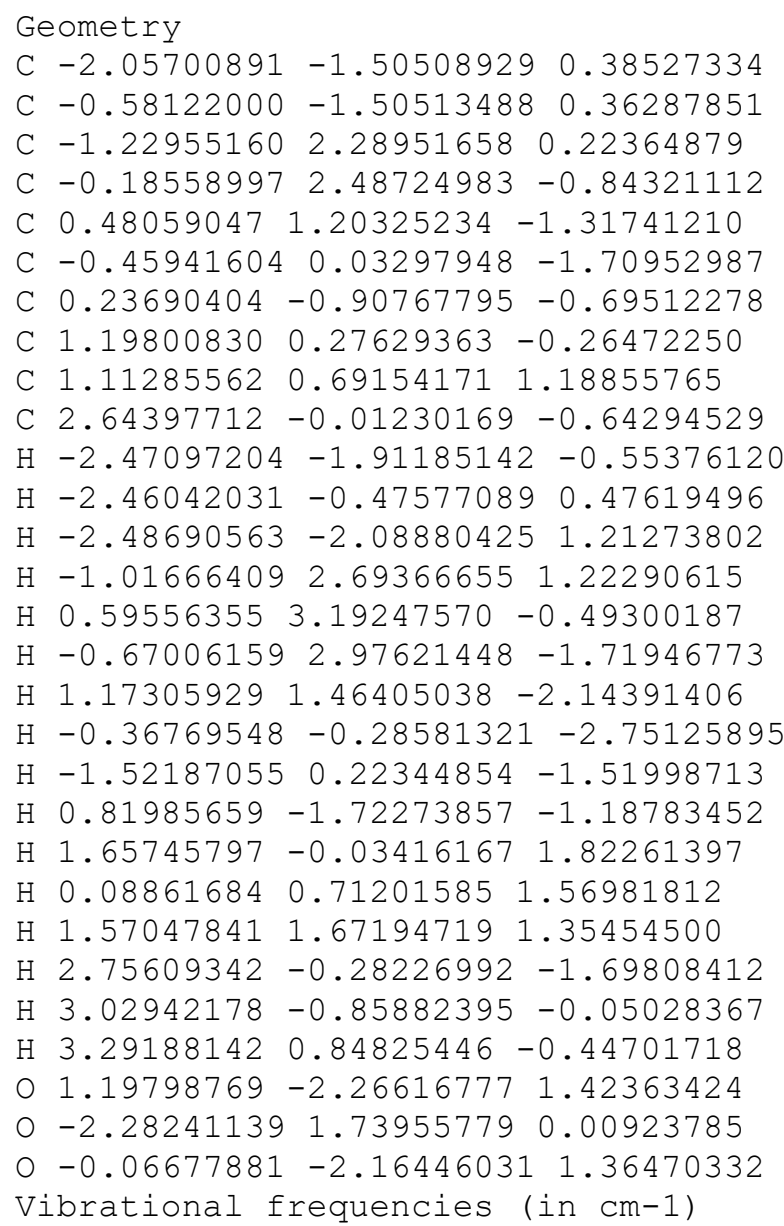

$\begin{array}{lllllllllllllll}50.9 & 55.8 & 105.1 & 123.4 & 144.8 & 156.9 & 190.2 & 214.1 & 224.8 & 245.3 & 262.7 & 279.4 & 306.9 & 309.4\end{array}$ $333.0 \quad 385.4 \quad 423.8 \quad 465.3 \quad 478.1 \quad 540.4 \quad 599.6 \quad 656.0 \quad 687.3 \quad 794.9 \quad 809.4 \quad 901.0 \quad 919.2 \quad 927.4$ $988.9996 .1 \quad 1009.5 \quad 1017.5 \quad 1019.6 \quad 1029.4 \quad 1059.4 \quad 1068.9 \quad 1082.0 \quad 1092.3 \quad 1111.3 \quad 1135.2$

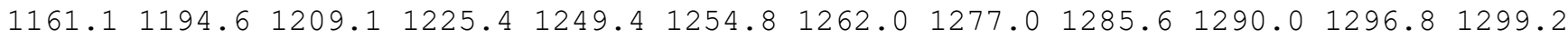
$1303.51306 .81316 .3 \quad 1322.4 \quad 1337.5 \quad 1357.3 \quad 1380.91383 .1 \quad 1405.7 \quad 1407.5 \quad 1433.8 \quad 1584.0$ $1837.42635 .6 \quad 2643.7 \quad 2648.12663 .6 \quad 2664.5 \quad 2665.12674 .12680 .62684 .62694 .42712 .7$ $2737.8 \quad 2760.7 \quad 2768.2 \quad 2775.7 \quad 2779.1$

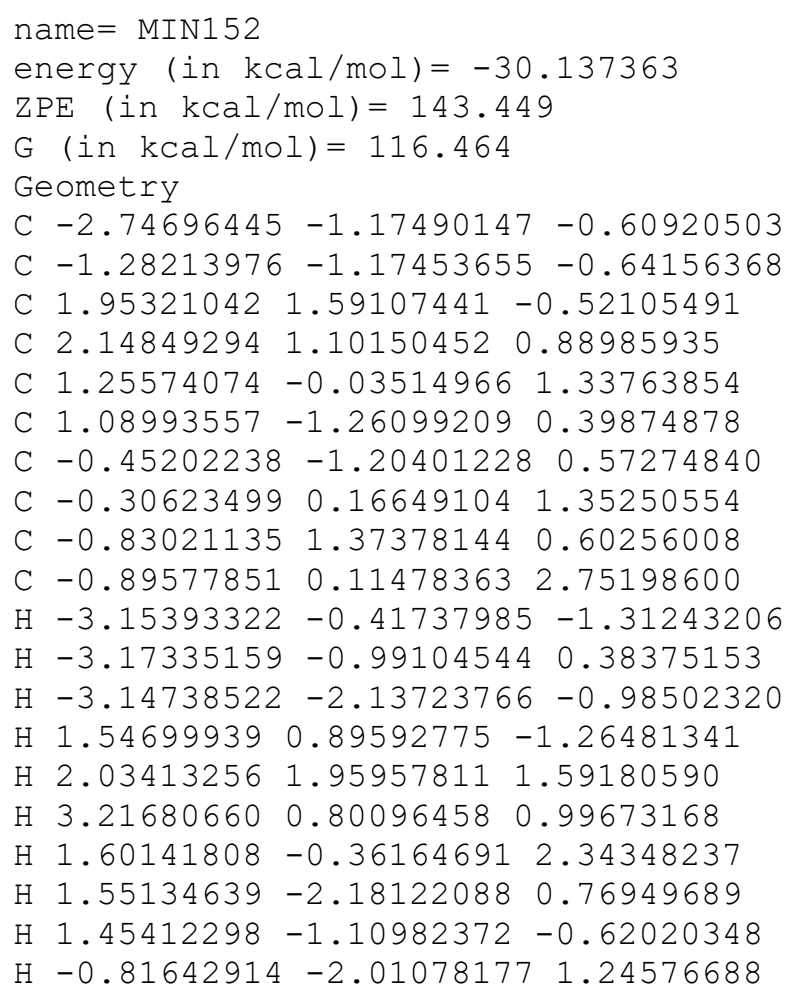


$\mathrm{H}-1.92667681 \quad 1.42637177 \quad 0.64148682$

$\mathrm{H}-0.55826921 \quad 1.36896907-0.46024407$

$\mathrm{H}-0.447899432 .309443501 .03238214$

H $-1.99078535 \quad 0.05371316 \quad 2.72461432$

$\mathrm{H}-0.63648986 \quad 1.01598619 \quad 3.32298071$

$\mathrm{H}-0.53744902-0.74635403 \quad 3.32709754$

$0-1.29699210-1.15818768-2.83709579$

$\begin{array}{llll}0 & 2.26276124 & 2.71540827 & -0.82881545\end{array}$

o $-0.61941247-1.17421968-1.76567472$

Vibrational frequencies (in $\mathrm{cm}-1$ )

$\begin{array}{lllllllllllllll}30.6 & 35.9 & 48.7 & 94.7 & 114.3 & 131.5 & 161.6 & 188.2 & 210.0 & 219.9 & 248.2 & 271.1 & 274.0 & 290.4\end{array}$ $333.5 \quad 374.7 \quad 410.6 \quad 449.6 \quad 456.7 \quad 540.3 \quad 612.6 \quad 657.3 \quad 684.0 \quad 752.5 \quad 819.9 \quad 842.9 \quad 913.6 \quad 937.2$ $995.01000 .8 \quad 1008.8 \quad 1016.3 \quad 1030.0 \quad 1043.0 \quad 1051.6 \quad 1072.1 \quad 1073.4 \quad 1112.81128 .01136 .0$

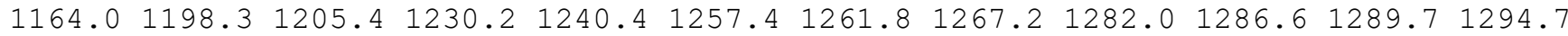

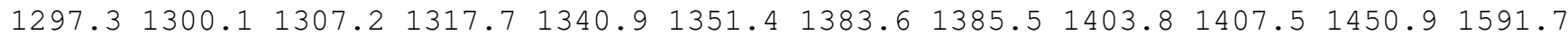
$1832.62634 .62645 .2 \quad 2652.4 \quad 2666.8 \quad 2671.12673 .4 \quad 2675.72677 .02678 .12705 .72713 .7$ $2736.12762 .5 \quad 2769.0 \quad 2779.4 \quad 2782.4$

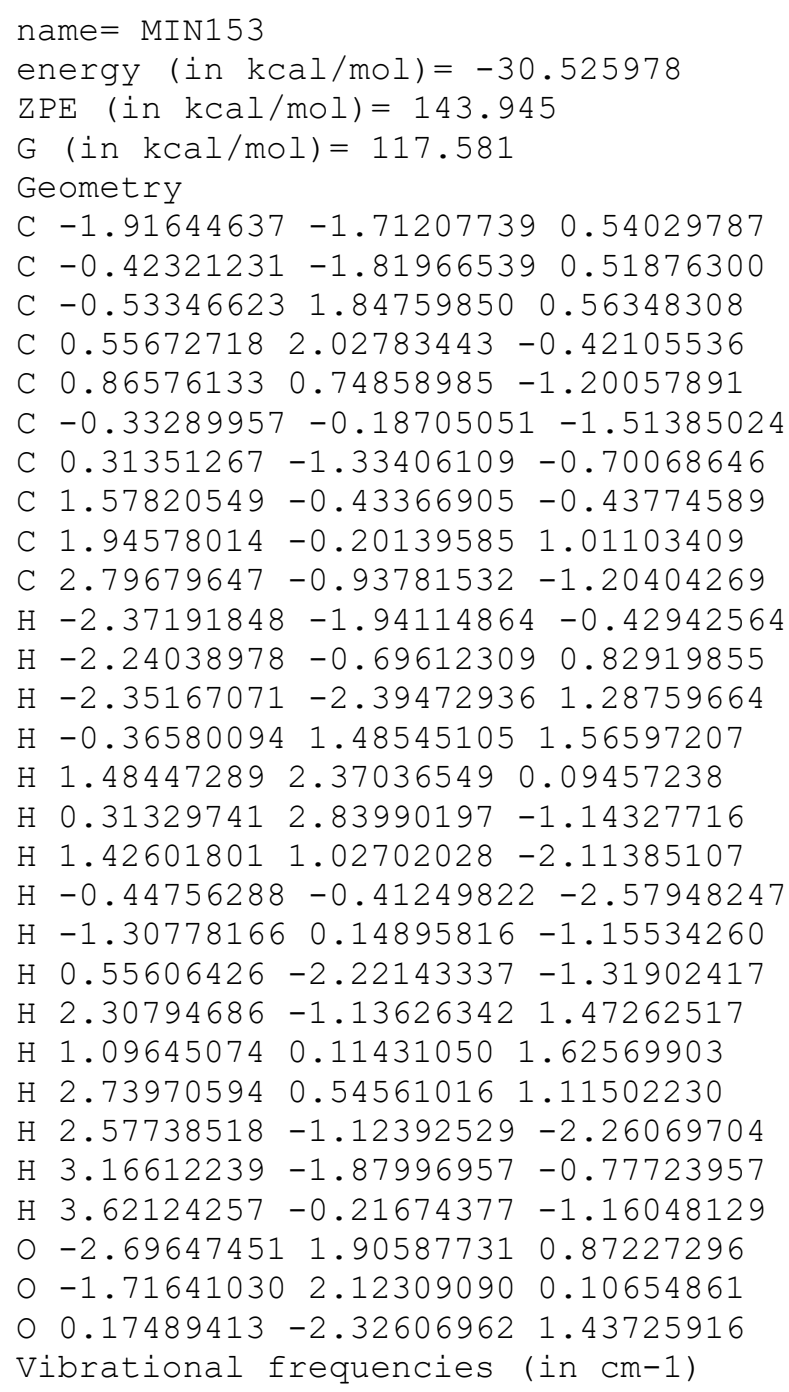

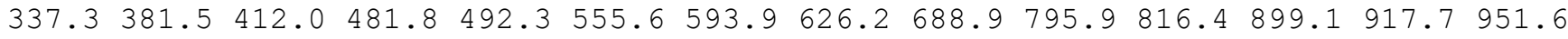
$965.3990 .3 \quad 1009.4 \quad 1019.1 \quad 1023.1 \quad 1044.4 \quad 1050.91075 .5 \quad 1081.51105 .81129 .51145 .6$

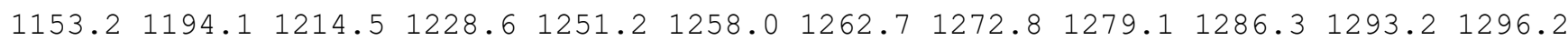

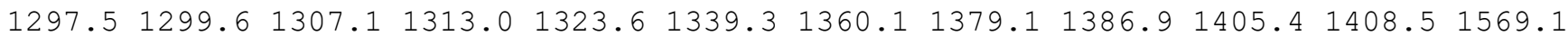
$1840.22644 .62647 .5 \quad 2659.4 \quad 2674.2 \quad 2674.92678 .02680 .02681 .72710 .52712 .52718 .3$ $2732.6 \quad 2764.2 \quad 2774.7 \quad 2779.5 \quad 2782.2$

name $=$ MIN154

energy $($ in $\mathrm{kcal} / \mathrm{mol})=-30.170767$ 
$\mathrm{ZPE}($ in $\mathrm{kcal} / \mathrm{mol})=143.619$

$\mathrm{G}(\mathrm{in} \mathrm{kcal} / \mathrm{mol})=116.064$

Geometry

C $-3.55327062-0.50041673-0.73566447$

C $-2.08648696-0.79435564-0.61029927$

C $2.04231908-0.14410519-0.50117418$

C $1.92606067-0.06921819 \quad 0.97214794$

C $0.52781219-0.30551363 \quad 1.52244081$

C $-0.24593614-1.55198980 \quad 1.01961560$

C $-1.50781686-0.68789611 \quad 0.77043013$

C $-0.66773317 \quad 0.61462515 \quad 1.06540890$

C $-0.41429771 \quad 1.49290490-0.14234659$

C $-1.240561321 .44214596 \quad 2.20633968$

$\mathrm{H}-4.15052666-1.32303159-0.31735695$

$\mathrm{H}-3.85207883-0.39001354-1.78925845$

$\mathrm{H}-3.837010910 .41785042-0.20798939$

$\mathrm{H} 1.47037653-0.83492729-1.10461388$

H $2.29929655 \quad 0.90837314 \quad 1.35677336$

$\mathrm{H} 2.61056862-0.83591176 \quad 1.41712972$

$\mathrm{H} \quad 0.59811632 \quad-0.30813728 \quad 2.63157681$

$\mathrm{H}-0.37051038-2.347665931 .75621236$

$\mathrm{H} \quad 0.16047957-2.00490696 \quad 0.10541050$

$\mathrm{H}-2.28716026-0.85306532 \quad 1.53503229$

$\mathrm{H}-1.31388879 \quad 2.04987247-0.43298578$

$\mathrm{H}-0.11042106 \quad 0.91404671-1.02880680$

$\mathrm{H} \quad 0.37364380 \quad 2.22969919 \quad 0.05294605$

$\mathrm{H}-0.54319805 \quad 2.23437712 \quad 2.50701569$

$\mathrm{H}-1.451561210 .83901134 \quad 3.09601811$

$\mathrm{H}-2.17851412 \quad 1.92998921 \quad 1.91469670$

$03.09518967 \quad 0.66089260-2.24686035$

$02.90676170 \quad 0.68649380-1.00132928$

O $-1.42264613-1.11998473-1.56254644$

Vibrational frequencies (in $\mathrm{cm}-1$ )

$\begin{array}{lllllllllllllll}22.4 & 26.6 & 39.8 & 86.6 & 91.7 & 153.3 & 159.9 & 172.9 & 200.2 & 210.0 & 254.8 & 281.3 & 288.1 & 293.5\end{array}$

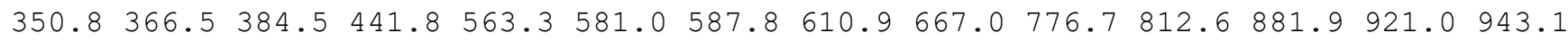
$995.4 \quad 997.7 \quad 1009.8 \quad 1014.7 \quad 1030.6 \quad 1039.9 \quad 1048.5 \quad 1075.4 \quad 1087.6 \quad 1102.9 \quad 1136.3 \quad 1162.7$

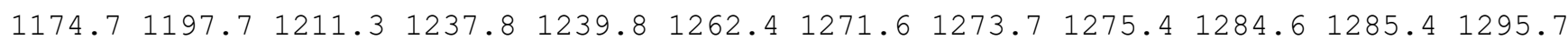

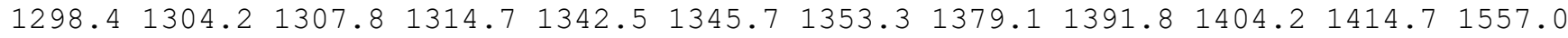
$1855.62641 .2 \quad 2655.2 \quad 2668.4 \quad 2674.6 \quad 2675.42677 .0 \quad 2677.72679 .42696 .82709 .12726 .9$ $2729.12760 .4 \quad 2778.12780 .3 \quad 2782.7$

\section{name $=$ MIN155}

energy (in $\mathrm{kcal} / \mathrm{mol})=-30.314804$

$\mathrm{ZPE}(\mathrm{in} \mathrm{kcal} / \mathrm{mol})=143.804$

G $($ in $\mathrm{kcal} / \mathrm{mol})=117.632$

Geometry

C $-3.07686411-1.01304410-0.61178487$

C $-1.59367195-0.94840103-0.64126196$

C $2.17472716 \quad 0.94750462-0.65613125$

C $2.265154120 .56034146 \quad 0.80247325$

C $1.09960456-0.246733831 .33680597$

C $0.64876592-1.50425310 \quad 0.54782191$

C $-0.83129569-1.03737561 \quad 0.60382678$

C $-0.35691487 \quad 0.34144060 \quad 1.23207911$

C $-0.535821201 .53746110 \quad 0.32233083$

C $-0.98342631 \quad 0.608496512 .59240713$

$\mathrm{H}-3.49052325-0.27091543 \quad 0.09239274$

$\mathrm{H}-3.42648778-2.00389239-0.27698162$

$\mathrm{H}-3.54455035-0.82228917-1.59047427$

H $1.93230601 \quad 0.16761444-1.38873273$

$\mathrm{H} \quad 2.38682336 \quad 1.47973628 \quad 1.41782195$

H $3.20718824-0.014781920 .94161836$

H $1.32672938-0.509751112 .39319902$

$\mathrm{H} \quad 0.83792523-2.45413523 \quad 1.05214297$ 
$\mathrm{H} 1.06492940-1.58361533-0.46516089$

$\mathrm{H}-1.40006190-1.62990142 \quad 1.35351147$

$\mathrm{H} \quad 0.07977357 \quad 2.38709704 \quad 0.65228680$

$\mathrm{H}-1.57573464 \quad 1.87939090 \quad 0.29491636$

$\mathrm{H}-0.236871591 .32538601-0.71609524$

$\mathrm{H}-0.87953324-0.24244060 \quad 3.27443872$

H $-2.05317168 \quad 0.82969054 \quad 2.50571106$

$\mathrm{H}-0.51049444 \quad 1.47342179 \quad 3.07674976$

O $0.07160549-0.71230487-2.09753055$

O $2.404135912 .08270121-0.99646792$

o $-1.16435907-0.80938694-1.86155713$

Vibrational frequencies (in $\mathrm{cm}-1$ )

$\begin{array}{llllllllllllllll}34.1 & 53.4 & 65.6 & 104.7 & 121.5 & 150.6 & 169.7 & 197.5 & 220.3 & 230.9 & 265.4 & 295.0 & 308.1 & 313.1\end{array}$ $\begin{array}{llllllllllllll}328.3 & 375.3 & 432.4 & 434.1 & 456.8 & 571.9 & 593.2 & 640.8 & 693.7 & 772.0 & 800.3 & 875.5 & 909.8 & 935.3\end{array}$ $993.21000 .4 \quad 1008.3 \quad 1015.6 \quad 1016.9 \quad 1035.5 \quad 1043.4 \quad 1069.2 \quad 1089.9 \quad 1112.01130 .8 \quad 1149.6$

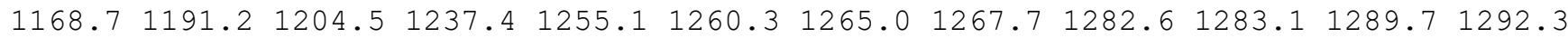

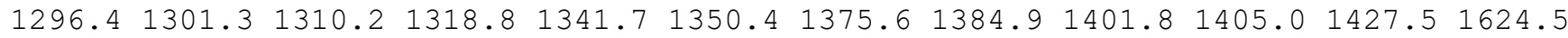
$1826.72641 .52654 .62656 .6 \quad 2660.72661 .2 \quad 2666.12673 .92676 .32679 .5 \quad 2706.42714 .3$ $2737.2 \quad 2756.92771 .12777 .12782 .0$

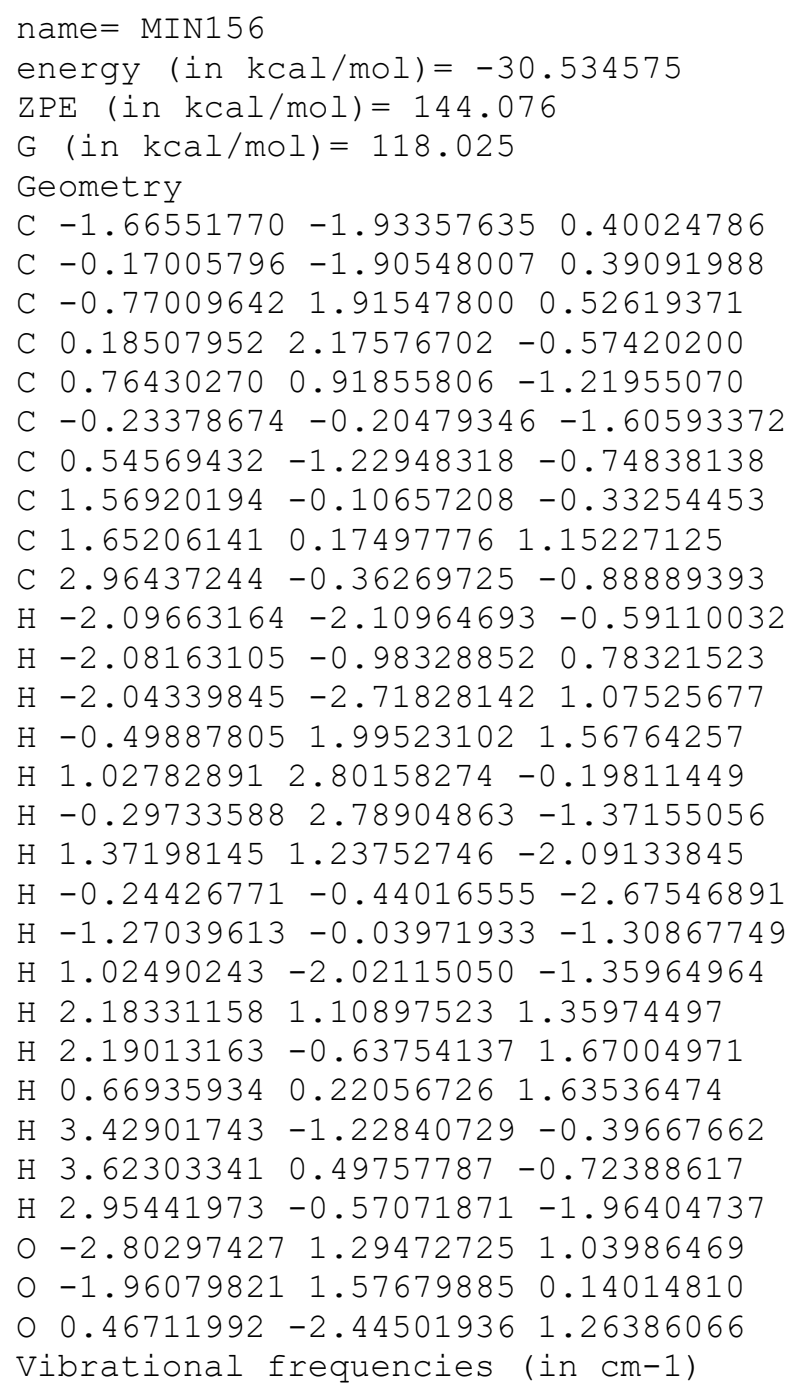


name $=$ MIN157

energy $($ in $\mathrm{kcal} / \mathrm{mol})=-30.139398$

$\mathrm{ZPE}($ in $\mathrm{kcal} / \mathrm{mol})=143.73$

$\mathrm{G}($ in $\mathrm{kcal} / \mathrm{mol})=117.331$

Geometry

C $-1.88286533-1.63026682 \quad 0.20515623$

C $-0.40671577-1.61766456 \quad 0.21325528$

C -1.653595352 .039011520 .07079032$

C $-0.34090895 \quad 2.50442220-0.50191776$

C $0.51720297 \quad 1.36154168-1.02194811$

C $-0.231390120 .16008864-1.66288017$

C $0.44141372-0.82294531-0.67606743$

C 1.205402460 .386462270 .00619613

C $0.90470651 \quad 0.59277904 \quad 1.47610765$

C $2.709102990 .28995915-0.20520271$

$\mathrm{H}-2.31980127-2.307309090 .95604221$

$\mathrm{H}-2.27301295-1.94764111-0.77905590$

$\mathrm{H}-2.30151838-0.62609600 \quad 0.39481695$

$\mathrm{H}-1.64433509 \quad 1.59652555 \quad 1.07564797$

H $0.22381364 \quad 3.08120881 \quad 0.25914493$

$\mathrm{H}-0.53901880 \quad 3.21644933-1.33495682$

H $1.27222121 \quad 1.78637753-1.71522346$

$\mathrm{H} \quad 0.02750106-0.01592200-2.71194257$

H $-1.321876190 .21186997-1.61192796$

$\mathrm{H} 1.16812340-1.51017607-1.17627557$

H $1.314129251 .54232272 \quad 1.83968195$

$\mathrm{H} \quad 1.37293142-0.21051367 \quad 2.07367162$

$\mathrm{H}-0.16315818 \quad 0.57331745 \quad 1.70623664$

H $3.11370950-0.58575930 \quad 0.32938909$

H $3.22819248 \quad 1.17642496 \quad 0.17451340$

$\mathrm{H} \quad 2.97848107 \quad 0.17104693-1.25978951$

O $1.36493728-2.52560659 \quad 1.15711610$

$\begin{array}{llllll}0 & -2.68975302 & 2.14623565 & -0.53718487\end{array}$

o $0.10146723-2.447687011 .08470475$

Vibrational frequencies (in $\mathrm{cm}-1$ )

$\begin{array}{lllllllllllllll}22.5 & 53.8 & 80.7 & 88.3 & 133.4 & 142.2 & 174.4 & 206.7 & 224.7 & 239.6 & 253.6 & 265.5 & 275.7 & 300.5\end{array}$

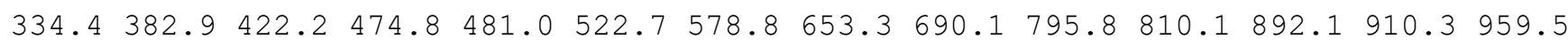
$980.1991 .2 \quad 1010.7 \quad 1012.7 \quad 1018.7 \quad 1030.9 \quad 1058.3 \quad 1077.4 \quad 1081.6 \quad 1095.1 \quad 1115.6 \quad 1137.2$ $1149.21191 .4 \quad 1209.6 \quad 1228.2 \quad 1254.7 \quad 1257.3 \quad 1263.1 \quad 1283.4 \quad 1286.5 \quad 1287.2 \quad 1290.01297 .0$ $1298.21306 .31311 .5 \quad 1326.3 \quad 1339.7 \quad 1357.4 \quad 1380.4 \quad 1381.21403 .8 \quad 1407.7 \quad 1443.6 \quad 1578.9$ $1839.8 \quad 2634.12647 .8 \quad 2654.7 \quad 2660.3 \quad 2662.7 \quad 2664.22675 .12678 .12682 .02691 .62713 .1$ $2738.8 \quad 2763.9 \quad 2769.0 \quad 2774.9 \quad 2779.2$

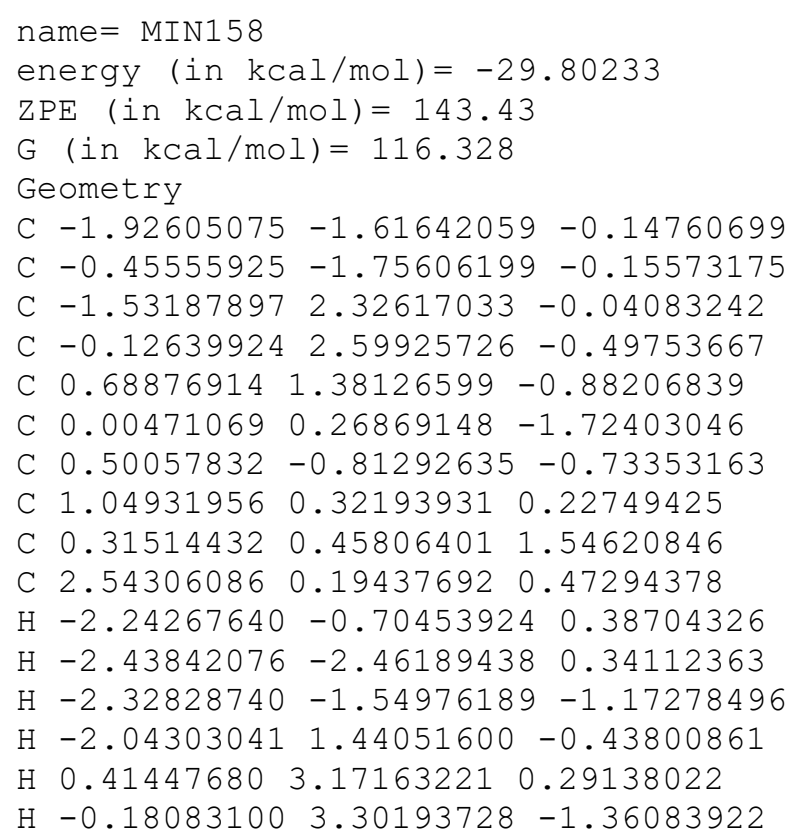


H $\quad 1.61728187 \quad 1.73455472-1.38120134$

H $0.40444491 \quad 0.16257365 \quad-2.73722767$

$\mathrm{H}-1.07951102 \quad 0.35192722-1.81229038$

H $1.35917151-1.39961131-1.15651649$

$\mathrm{H}-0.77237430 \quad 0.48597015 \quad 1.43401614$

$\mathrm{H} \quad 0.60994884 \quad 1.37635425 \quad 2.07207229$

$\mathrm{H} \quad 0.54987285-0.38332408 \quad 2.21448146$

$\mathrm{H} \quad 2.76643004 \quad-0.73591542 \quad 1.02021189$

$\mathrm{H} 2.92809266 \quad 1.02951681 \quad 1.06854065$

H $3.12062650 \quad 0.15523446-0.45705196$

O $1.18315811-3.10616736 \quad 0.43422254$

$\begin{array}{lllll}0 & -2.10257623 & 3.06870449 & 0.71994307\end{array}$

o $-0.05928398 \quad-2.86927998 \quad 0.40410781$

Vibrational frequencies (in cm-1)

$\begin{array}{llllllllllllllll}24.8 & 37.0 & 45.8 & 91.5 & 110.2 & 139.6 & 164.7 & 179.6 & 208.3 & 228.2 & 251.0 & 264.9 & 275.3 & 283.2\end{array}$

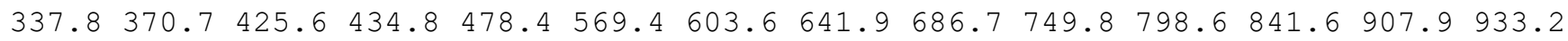
$991.9995 .8 \quad 1007.6 \quad 1014.91023 .8 \quad 1032.1 \quad 1047.8 \quad 1072.0 \quad 1082.4 \quad 1106.91130 .91145 .5$

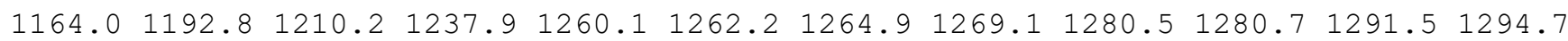

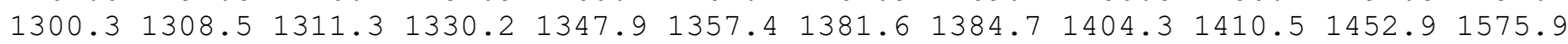
$1834.62641 .52650 .8 \quad 2653.3 \quad 2658.6 \quad 2663.3 \quad 2667.42670 .72674 .12680 .42687 .72711 .1$ $2735.4 \quad 2764.4 \quad 2768.7 \quad 2778.6 \quad 2780.6$

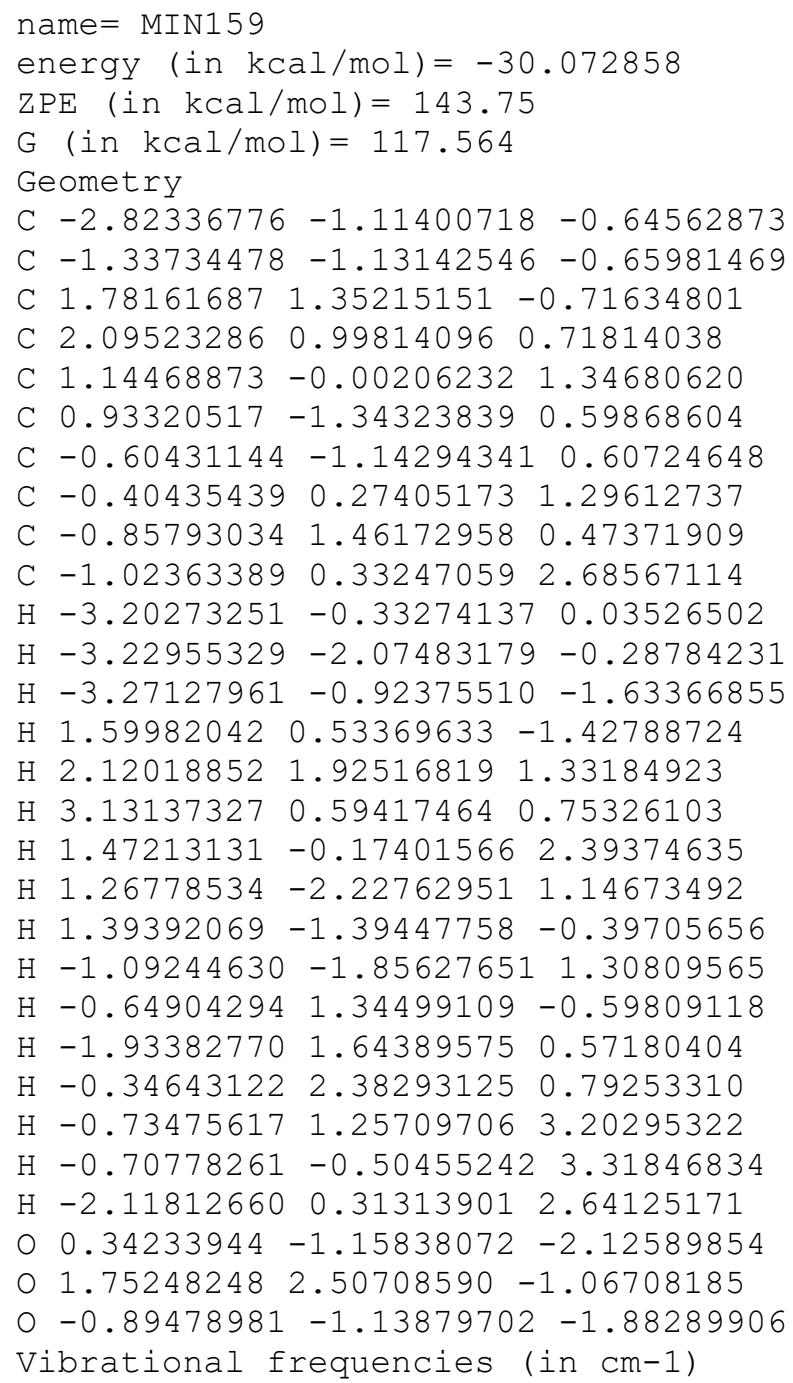


name $=$ MIN160

energy $($ in $\mathrm{kcal} / \mathrm{mol})=-30.15257$

$\mathrm{ZPE}($ in $\mathrm{kcal} / \mathrm{mol})=143.87$

$\mathrm{G}($ in $\mathrm{kcal} / \mathrm{mol})=117.36$

Geometry

C $-1.70970087-1.96302548-0.02344398$

C $-0.21553155-2.01653498 \quad 0.01327332$

C $-1.143836652 .16225454-0.08440897$

$\begin{array}{lllll}\text { C } & 0.31422991 & 2.26890159 & -0.31311690\end{array}$

C $0.877847501 .05092159-1.04593435$

C $-0.12018760 \quad 0.02336415-1.65067858$

C $0.58371950-1.13515122-0.90828973$

C $1.53022415-0.10275125-0.18918130$

C $1.34291012-0.02524907 \quad 1.31293927$

C $2.99840980-0.31615395-0.52423428$

$\mathrm{H}-2.09490659-1.04610747 \quad 0.45609882$

$\mathrm{H}-2.14948694-2.80685466 \quad 0.53302639$

$\mathrm{H}-2.10675412-1.99446923-1.04441997$

$\mathrm{H}-1.86818426 \quad 2.58721279-0.76053041$

$\mathrm{H} \quad 0.87161331 \quad 2.42002317 \quad 0.63944097$

$\mathrm{H} \quad 0.52978347 \quad 3.18854552-0.90436537$

H $1.591988281 .40744336-1.81494428$

$\mathrm{H}-0.07732824-0.04929672-2.74172958$

$\mathrm{H}-1.17046517 \quad 0.16854389-1.38348681$

$\mathrm{H} 1.14739823-1.79585654-1.59938116$

$\mathrm{H} \quad 1.68435264 \quad-0.96070004 \quad 1.79097490$

$\mathrm{H} \quad 0.29679274 \quad 0.09773876 \quad 1.60807573$

$\mathrm{H} \quad 1.92512328 \quad 0.78922724 \quad 1.75755473$

$\mathrm{H} 3.17506534-0.38814177-1.60283231$

$\mathrm{H} 3.37102698-1.24731559-0.07386120$

$\mathrm{H} \quad 3.61963508 \quad 0.50209227-0.14208135$

$\begin{array}{llll}0 & -2.70697408 & 1.28272761 & 1.16547296\end{array}$

$\begin{array}{lllll}0 & -1.47727120 & 1.48217089 & 0.96828137\end{array}$

O $0.35985442-2.779423490 .75304101$

Vibrational frequencies (in $\mathrm{cm}-1$ )

$\begin{array}{llllllllllllllll}26.1 & 45.1 & 65.1 & 73.0 & 153.2 & 158.3 & 165.1 & 184.6 & 201.7 & 244.2 & 261.8 & 282.4 & 312.1 & 320.6\end{array}$

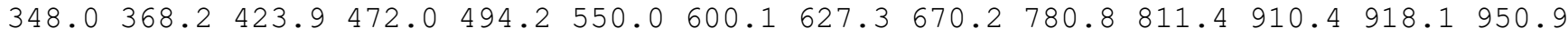
$969.7991 .0 \quad 1007.2 \quad 1014.4 \quad 1018.3 \quad 1042.3 \quad 1049.1 \quad 1075.2 \quad 1088.01111 .7 \quad 1127.4 \quad 1141.4$

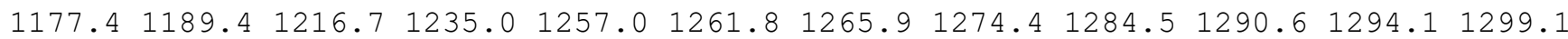
$1300.51302 .5 \quad 1305.8 \quad 1311.4 \quad 1326.7 \quad 1338.2 \quad 1357.4 \quad 1378.1 \quad 1383.7 \quad 1403.8 \quad 1407.51575 .9$

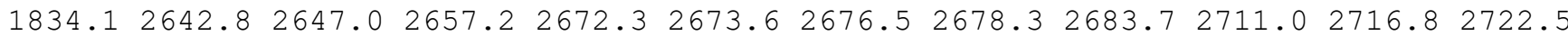
$2732.6 \quad 2761.2 \quad 2775.6 \quad 2779.7 \quad 2781.8$

name $=$ MIN161

energy $($ in $\mathrm{kcal} / \mathrm{mol})=-29.902518$

$\mathrm{ZPE}(\mathrm{in} \mathrm{kcal} / \mathrm{mol})=143.645$

G $($ in $\mathrm{kcal} / \mathrm{mol})=117.266$

Geometry

C $-3.35186554-0.55419159-0.57799736$

C $-1.86849336-0.62428143-0.56176816$

C $2.49694301 \quad 0.11174077-0.54473441$

C $2.27992912 \quad 0.16295645 \quad 0.94820094$

C $0.89447190-0.18687577 \quad 1.44593832$

C $0.16193799-1.413249950 .83918990$

C $-1.14656500 \quad-0.58398758 \quad 0.70859428$

C $-0.32567316 \quad 0.72981894 \quad 1.05569740$

C $-0.10533209 \quad 1.67041884-0.10863010$

C $-0.90594984 \quad 1.48602412 \quad 2.24157975$

$\mathrm{H}-3.79349562-1.43185801-0.07485150$

$\mathrm{H}-3.78162384-0.51779457-1.59110108$

$\mathrm{H}-3.71494305 \quad 0.33839665-0.04136761$

$\mathrm{H} 1.85725162-0.54093393-1.15205306$ 
H 2.55988329 $1.17466508 \quad 1.31932404$

H $3.02479483-0.523413251 .41276211$

$\mathrm{H} \quad 0.94876254 \quad-0.28135823 \quad 2.55344933$

$\mathrm{H} \quad 0.08622111-2.27963973 \quad 1.49811307$

$\mathrm{H} \quad 0.56941254-1.75954834-0.12038373$

$\mathrm{H}-1.84033787-0.82369988 \quad 1.54519638$

$\mathrm{H} 0.252988561 .14608211-1.00954829$

$\mathrm{H} \quad 0.64760583 \quad 2.43284955 \quad 0.13109909$

$\mathrm{H}-1.024689842 .19502741-0.38943799$

$\mathrm{H}-0.22244956 \quad 2.28049204 \quad 2.56956584$

$\mathrm{H}-1.086624320 .83788006 \quad 3.10557006$

$\mathrm{H}-1.85817444 \quad 1.96395118 \quad 1.98474122$

o $-0.15299940-0.83832967-1.95943150$

$\begin{array}{lllll}0 & 3.38538768 & 0.75925840 & -1.04419618\end{array}$

$0-1.39756004-0.74654889-1.76825591$

Vibrational frequencies (in cm-1)

$\begin{array}{lllllllllllllllll}24.1 & 60.6 & 65.4 & 111.6 & 125.6 & 155.3 & 162.9 & 187.4 & 210.5 & 226.8 & 267.3 & 274.2 & 286.5 & 307.3\end{array}$

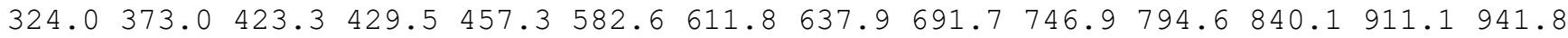
$989.81000 .91008 .91015 .6 \quad 1018.0 \quad 1037.1 \quad 1047.4 \quad 1068.3 \quad 1085.01112 .1 \quad 1136.01150 .7$ $\begin{array}{llllllllllllll}1171.1 & 1199.3 & 1205.5 & 1238.7 & 1254.5 & 1257.9 & 1265.6 & 1268.2 & 1278.0 & 1281.4 & 1284.7 & 1294.9\end{array}$ $1296.61302 .21310 .21315 .6 \quad 1345.2 \quad 1351.2 \quad 1376.6 \quad 1386.41402 .11404 .21432 .91627 .1$ $1824.72639 .92650 .12654 .0 \quad 2655.2 \quad 2661.4 \quad 2667.82674 .62678 .02680 .42705 .62713 .8$ $2736.5 \quad 2756.9 \quad 2770.8 \quad 2777.8 \quad 2782.5$

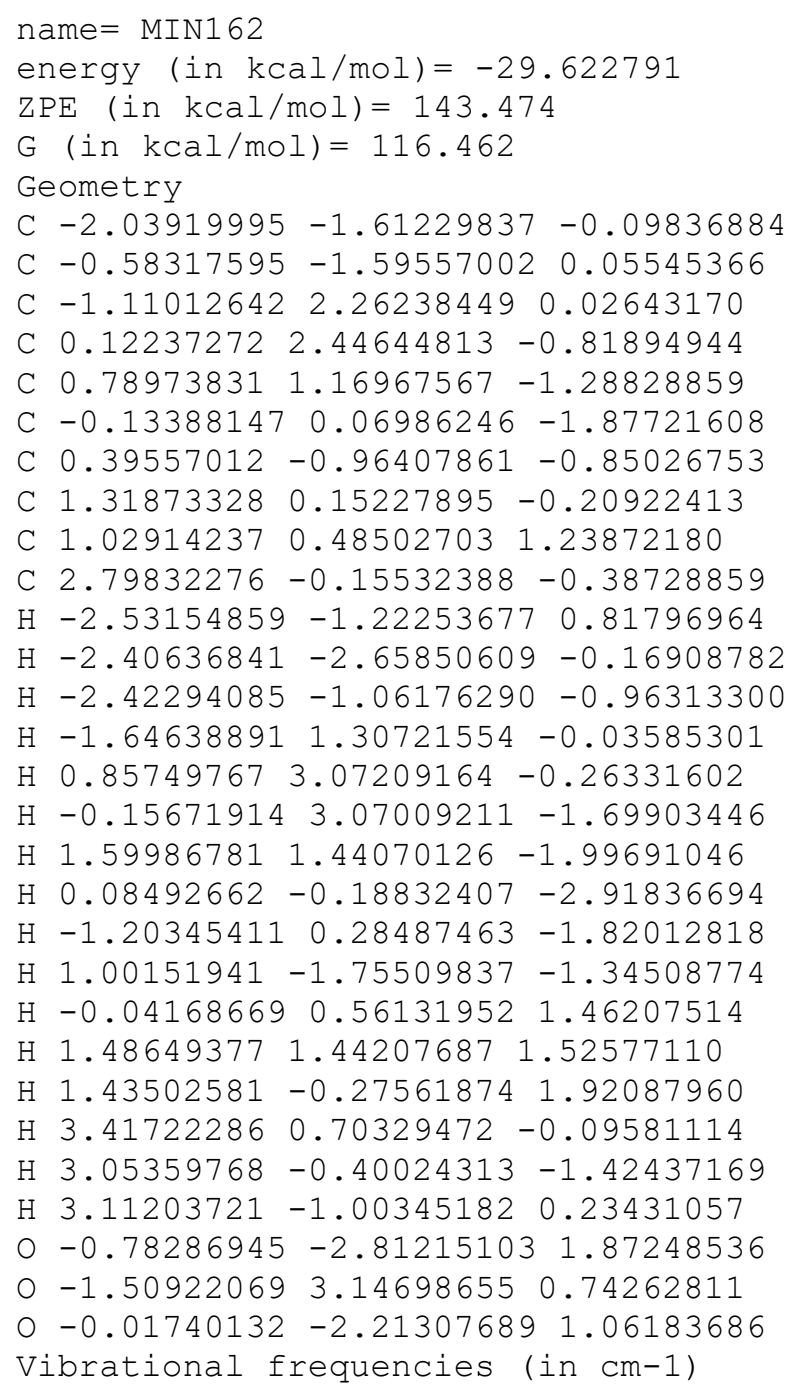


$\begin{array}{llllllllllll}1834.3 & 2629.4 & 2643.7 & 2651.4 & 2664.4 & 2669.3 & 2670.1 & 2674.7 & 2675.9 & 2676.9 & 2703.3 & 2713.9\end{array}$ $2737.12760 .92766 .02778 .6 \quad 2782.0$

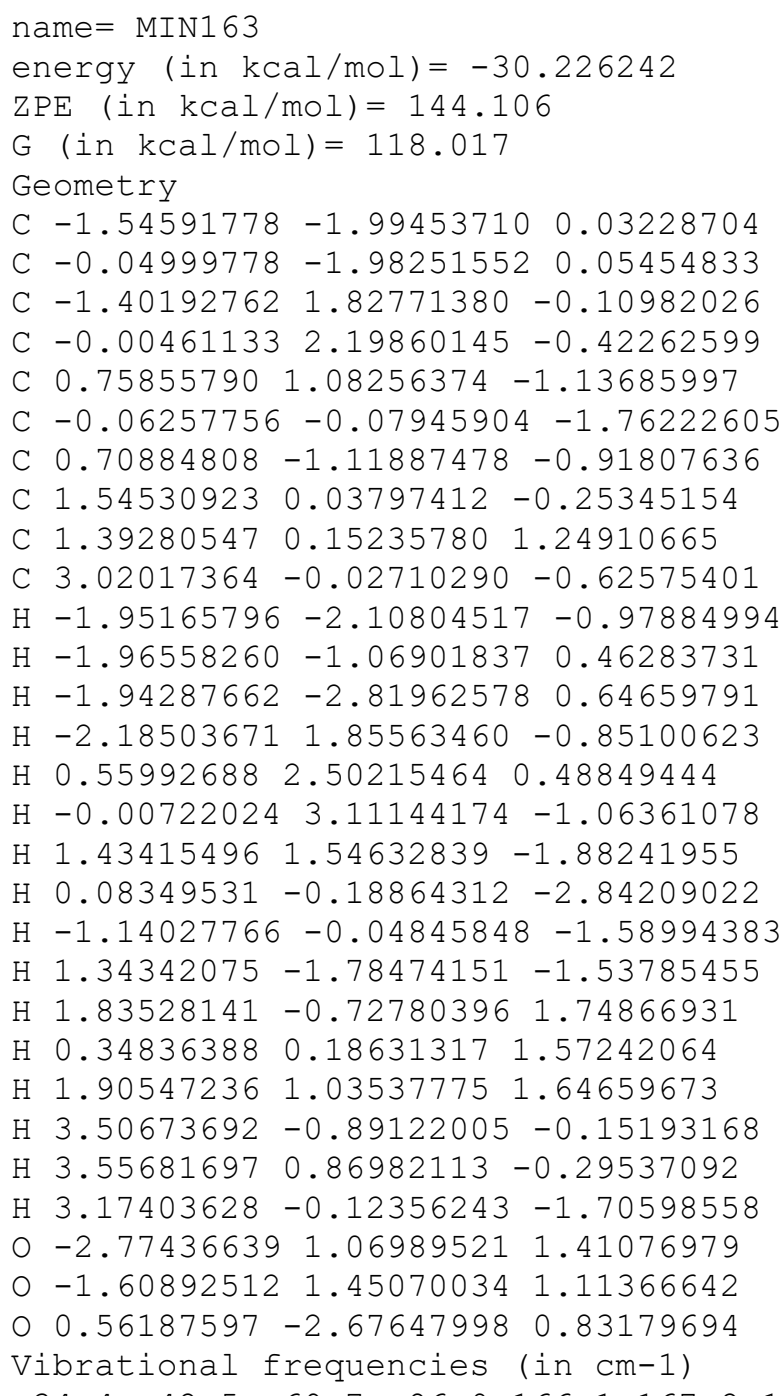

$\begin{array}{llllllllllllllll}34.4 & 42.5 & 69.7 & 86.0 & 166.1 & 167.2 & 193.5 & 203.3 & 214.0 & 241.5 & 268.3 & 282.9 & 321.3 & 325.5\end{array}$ $347.7 \quad 373.2 \quad 426.4 \quad 471.6 \quad 495.0 \quad 555.0 \quad 599.2 \quad 617.8 \quad 678.9 \quad 781.3 \quad 815.0 \quad 907.2 \quad 921.0 \quad 949.7$

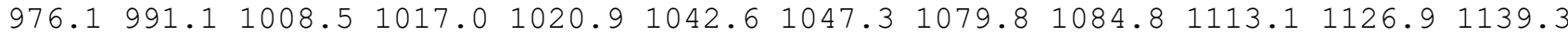
$1180.0 \quad 1190.0 \quad 1219.3 \quad 1234.7 \quad 1256.6 \quad 1259.2 \quad 1265.6 \quad 1275.8 \quad 1284.2 \quad 1291.91295 .1 \quad 1297.2$ $1299.81300 .8 \quad 1305.1 \quad 1313.7 \quad 1327.5 \quad 1339.4 \quad 1358.5 \quad 1378.5 \quad 1385.1 \quad 1403.6 \quad 1407.91575 .0$ $1834.52643 .4 \quad 2649.8 \quad 2657.8 \quad 2672.7 \quad 2674.2 \quad 2677.92678 .92683 .72711 .52716 .92720 .9$ $2732.7 \quad 2762.92776 .92780 .12782 .1$

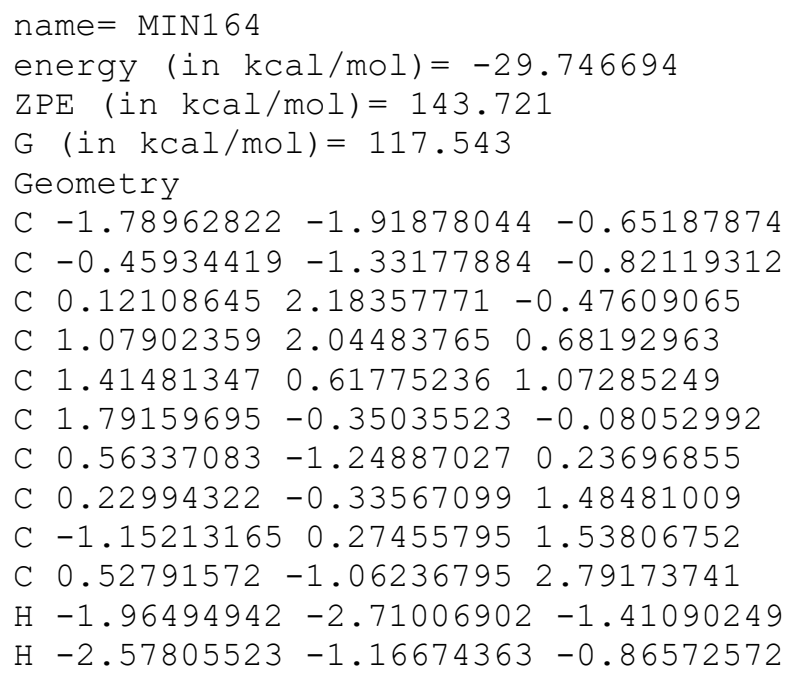


$\begin{array}{lllll}\mathrm{H} & -1.97495247 & -2.34016224 & 0.34211867\end{array}$

$\mathrm{H} 0.27175791 \quad 1.54137116-1.35210150$

$\mathrm{H} 0.66726202 \quad 2.58299452 \quad 1.56557530$

$\mathrm{H} 2.01218545 \quad 2.593273920 .42396126$

$\mathrm{H} \quad 2.19502620 \quad 0.64640548 \quad 1.85999191$

H $2.75149517 \quad-0.85780253 \quad 0.06868351$

$\mathrm{H} \quad 1.82661240 \quad 0.09751753-1.07487418$

H $\quad 0.85083921-2.27658351 \quad 0.54520484$

$\mathrm{H}-1.90608869-0.45647123 \quad 1.85714435$

$\mathrm{H}-1.48799331 \quad 0.67295303 \quad 0.57229097$

$\mathrm{H}-1.19013391 \quad 1.10737528 \quad 2.25433287$

$\mathrm{H}-0.18757571-1.87337940 \quad 2.97272447$

$\mathrm{H} \quad 0.46528820 \quad-0.37835412 \quad 3.64751664$

$\mathrm{H} 1.53052966-1.504776312 .80382956$

$0-0.89468965-0.93669952-2.93846391$

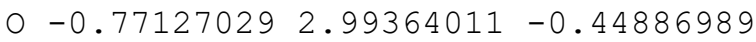

o $-0.06862896-0.87003165-1.97940661$

Vibrational frequencies (in cm-1)

$\begin{array}{lllllllllllllll}37.3 & 46.7 & 73.1 & 104.1 & 122.8 & 152.5 & 176.5 & 201.6 & 219.4 & 230.6 & 262.6 & 275.8 & 285.4 & 298.4\end{array}$ $\begin{array}{llllllllllllllllll}334.9 & 399.3 & 423.8 & 444.7 & 462.0 & 531.0 & 580.7 & 673.7 & 691.0 & 778.0 & 831.0 & 870.7 & 913.0 & 926.7\end{array}$ $992.7 \quad 1005.1 \quad 1009.5 \quad 1017.8 \quad 1028.5 \quad 1040.9 \quad 1049.9 \quad 1065.4 \quad 1078.2 \quad 1112.91119 .4 \quad 1124.0$

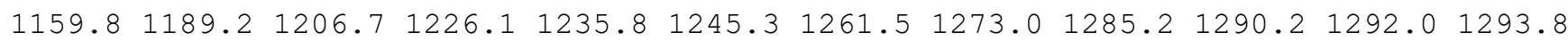
$1297.31303 .0 \quad 1308.2 \quad 1313.6 \quad 1336.8 \quad 1355.3 \quad 1382.0 \quad 1383.1 \quad 1405.91406 .6 \quad 1445.91579 .3$ $1835.62630 .8 \quad 2645.5 \quad 2658.1 \quad 2666.8 \quad 2671.7 \quad 2674.02675 .92677 .4 \quad 2678.02707 .32715 .7$ $\begin{array}{llllll}2737.6 & 2762.4 & 2768.2 & 2780.3 & 2782.7\end{array}$

name $=$ MIN165

energy (in $\mathrm{kcal} / \mathrm{mol})=-29.875807$

$\operatorname{ZPE}(\mathrm{in} \mathrm{kcal} / \mathrm{mol})=143.933$

$\mathrm{G}($ in $\mathrm{kcal} / \mathrm{mol})=117.752$

Geometry

C $-1.79568150-1.971840820 .31562134$

C $-0.30101660-1.94474253 \quad 0.30544334$

$\begin{array}{llll}\text { C }-0.75588107 & 2.32305630 & 0.31705790\end{array}$

C $0.34060665 \quad 2.27345643 \quad-0.67262521$

C $0.74740022 \quad 0.92668624 \quad-1.25159533$

C $-0.33145197-0.12985029-1.61296545$

C $0.41736998-1.20592399-0.79073010$

C $1.45834666-0.12438007-0.31517339$

C $1.44093666 \quad 0.17093960 \quad 1.16983694$

C $2.87737728-0.43813757-0.76792538$

$\mathrm{H}-2.20639048-1.06679584 \quad 0.80097783$

$\mathrm{H}-2.16975969-2.821909490 .90941392$

$\mathrm{H}-2.23141004 \quad-2.04311467 \quad-0.68578718$

$\mathrm{H}-0.93951683 \quad 3.21405909 \quad 0.90122433$

$\mathrm{H} \quad 1.25230647 \quad 2.73717838-0.21640260$

$\mathrm{H} \quad 0.068768592 .95734172-1.51674836$

$\mathrm{H} \quad 1.38982190 \quad 1.13238585-2.13674418$

$\mathrm{H}-0.38620452-0.35140414-2.68521843$

$\mathrm{H}-1.35341501 \quad 0.08056162-1.29867429$

$\mathrm{H} \quad 0.89361201-1.96906156-1.44260966$

$\mathrm{H} 2.06409191 \quad 1.03582533 \quad 1.42079391$

$\mathrm{H} 1.83191118-0.69010006 \quad 1.73971484$

$\mathrm{H} \quad 0.43570716 \quad 0.35528049 \quad 1.56257229$

H $2.93643240-0.66135880-1.83832206$

$\mathrm{H} 3.27338489-1.31334009-0.23324280$

H $3.55566387 \quad 0.39908837-0.56700349$

O $-2.44930434 \quad 1.29753066 \quad 1.24678863$

$\begin{array}{lllll}0 & -1.48888624 & 1.26209198 & 0.42688832\end{array}$

o $0.33642280 \quad-2.54173130 \quad 1.14014339$

Vibrational frequencies (in cm-1)

$\begin{array}{lllllllllllllll}31.7 & 51.7 & 68.9 & 90.6 & 135.6 & 167.6 & 185.1 & 190.2 & 204.2 & 227.7 & 260.3 & 263.4 & 292.2 & 324.2\end{array}$

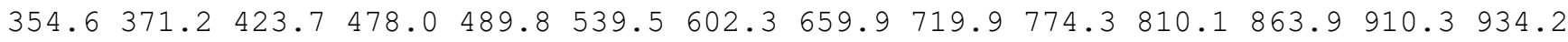
$994.5999 .5 \quad 1008.0 \quad 1018.8 \quad 1025.2 \quad 1035.4 \quad 1047.4 \quad 1054.3 \quad 1079.2 \quad 1111.91129 .81149 .1$ 
$\begin{array}{llllllllllllllll}1166.9 & 1205.7 & 1212.6 & 1255.0 & 1257.2 & 1263.3 & 1265.6 & 1266.8 & 1280.6 & 1283.9 & 1285.9 & 1292.8\end{array}$ $1295.31300 .21303 .91312 .91336 .6 \quad 1339.91373 .0 \quad 1382.3 \quad 1394.2 \quad 1407.7 \quad 1416.01596 .0$ $1835.72634 .22640 .0 \quad 2658.5 \quad 2672.4 \quad 2675.4 \quad 2676.4 \quad 2678.4 \quad 2682.3 \quad 2704.3 \quad 2712.92715 .5$ $2720.42765 .12775 .6 \quad 2780.4 \quad 2782.5$

\section{name $=$ MIN166}

energy $($ in $\mathrm{kcal} / \mathrm{mol})=-29.814472$

$\mathrm{ZPE}($ in $\mathrm{kcal} / \mathrm{mol})=143.883$

$\mathrm{G}(\mathrm{in} \mathrm{kcal} / \mathrm{mol})=117.778$

Geometry

C $-2.00977373-1.372150550 .32428437$

C $-0.56060378-1.50527761 \quad 0.17045148$

C $-0.85257723 \quad 2.494012920 .40899090$

C $0.15199773 \quad 2.62581446-0.70730508$

C $0.63322614 \quad 1.30674443-1.29041342$

C $-0.449599190 .27496700-1.70053470$

C $0.24859956-0.82965243-0.86620264$

C $1.29769888 \quad 0.23553372 \quad-0.34652272$

C $1.23653291 \quad 0.510741531 .14189915$

C $2.72202836-0.09760115-0.76329347$

$\mathrm{H}-2.38673639-2.01728307 \quad 1.14758552$

$\mathrm{H}-2.55778807-1.65435024-0.58782473$

$\mathrm{H}-2.31161226-0.33678448 \quad 0.57143398$

$\mathrm{H}-0.56291006 \quad 2.88426707 \quad 1.39400977$

$\mathrm{H} 1.02840776 \quad 3.21668260-0.37201055$

$\mathrm{H}-0.31831979 \quad 3.22035654-1.52512756$

$\mathrm{H} 1.30718886 \quad 1.53926775 \quad-2.14209374$

$\mathrm{H}-0.50021921 \quad 0.06780157 \quad-2.77199323$

$\mathrm{H}-1.46532860 \quad 0.53167463-1.37126098$

$\mathrm{H} \quad 0.73151664-1.58964495-1.51776463$

$\mathrm{H} \quad 0.20712362 \quad 0.57604127 \quad 1.51789153$

H 1.752705061 .443958031 .39684862

$\mathrm{H} \quad 1.72197795-0.29001644 \quad 1.71932268$

$\mathrm{H} 3.10345397-0.97669110-0.22824250$

$\mathrm{H} 3.40305106 \quad 0.73442340 \quad-0.54462643$

$\mathrm{H} 2.80443483-0.31058305-1.83508025$

$\begin{array}{lllll}0 & -0.42514926 & -2.91596613 & 1.86270372\end{array}$

$\begin{array}{lllll}0 & -1.94723255 & 2.01563381 & 0.24432384\end{array}$

o $0.16249536-2.27350295 \quad 0.94141182$

Vibrational frequencies (in $\mathrm{cm}-1$ )

$\begin{array}{lllllllllllllll}27.8 & 57.7 & 81.1 & 115.6 & 132.3 & 134.8 & 176.6 & 196.5 & 216.0 & 236.3 & 272.6 & 290.0 & 305.5 & 311.6\end{array}$

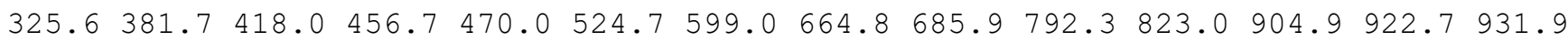
$993.11002 .0 \quad 1006.3 \quad 1018.8 \quad 1020.1 \quad 1039.8 \quad 1056.0 \quad 1067.2 \quad 1080.8 \quad 1091.01125 .7 \quad 1132.4$ $\begin{array}{llllllllllllll}1162.4 & 1195.8 & 1202.8 & 1228.7 & 1250.1 & 1256.9 & 1261.7 & 1276.7 & 1284.2 & 1290.3 & 1296.3 & 1297.2\end{array}$ $1302.91304 .61316 .21319 .8 \quad 1338.3 \quad 1353.1 \quad 1383.1 \quad 1384.71403 .8 \quad 1407.6 \quad 1435.6 \quad 1578.1$ $1840.52634 .52644 .2 \quad 2654.4 \quad 2660.92663 .5 \quad 2669.82673 .12675 .22677 .62704 .92711 .7$ $2737.6 \quad 2758.6 \quad 2762.6 \quad 2776.9 \quad 2782.1$

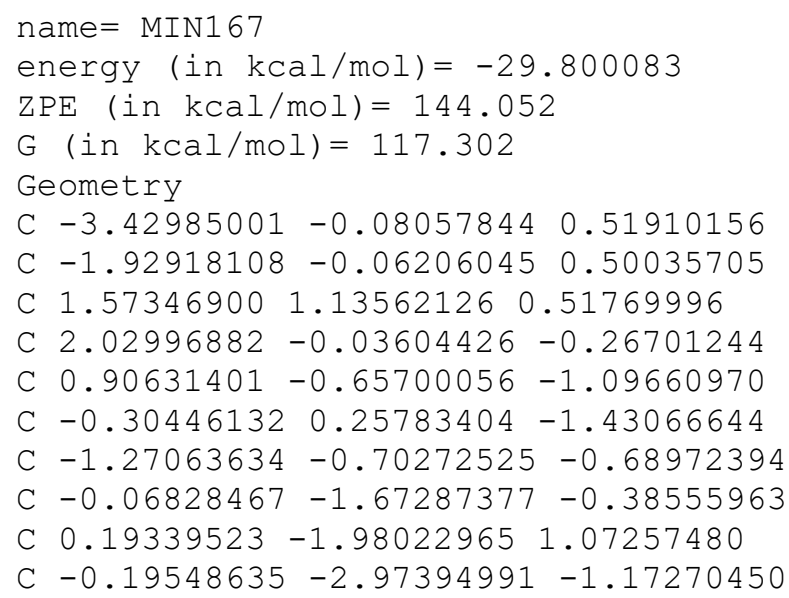


$\mathrm{H}-3.82280964 \quad 0.40303707 \quad 1.42752877$

$\mathrm{H}-3.82284098-1.10400237 \quad 0.49348071$

$\mathrm{H}-3.845011150 .45929880 \quad-0.34266106$

$\mathrm{H} \quad 0.92727722 \quad 1.04985883 \quad 1.38186380$

$\mathrm{H} 2.45507452-0.80685757 \quad 0.41734318$

H $2.87189823 \quad 0.23758242 \quad-0.94239828$

$\mathrm{H} 1.34790396-1.10608447-2.00677125$

$\mathrm{H}-0.50655308 \quad 0.35479118-2.50130802$

$\mathrm{H}-0.266910991 .27036616-1.01291496$

$\mathrm{H}-2.01705854-1.16487368-1.35698322$

$\mathrm{H} \quad 0.34612977-1.07194877 \quad 1.67427651$

H $1.08051726-2.611892121 .19883195$

$\mathrm{H}-0.65257010-2.51363505 \quad 1.52446877$

$\mathrm{H}-0.40529641-2.79932102-2.23380260$

$\mathrm{H}-1.00582926-3.59905989-0.77917628$

$\mathrm{H} 0.72694211-3.56408941-1.11592130$

$\begin{array}{lllll}0 & 1.60438536 & 3.32608654 & 0.62105346\end{array}$

O $1.97926437 \quad 2.27185881 \quad 0.04000993$

$0-1.30358260 \quad 0.46140850 \quad 1.38837743$

Vibrational frequencies (in $\mathrm{cm}-1$ )

$\begin{array}{lllllllllllllllll}16.5 & 50.1 & 56.8 & 102.1 & 115.9 & 168.6 & 176.6 & 197.1 & 211.7 & 228.5 & 257.4 & 274.9 & 312.0 & 323.1\end{array}$ $349.6 \quad 370.1 \quad 388.8 \quad 454.4 \quad 554.8 \quad 577.7 \quad 599.5 \quad 616.0 \quad 689.1 \quad 797.6 \quad 830.6 \quad 905.7 \quad 918.1958 .5$ $978.9993 .5 \quad 1007.5 \quad 1018.5 \quad 1035.9 \quad 1045.5 \quad 1049.4 \quad 1070.2 \quad 1085.3 \quad 1108.01130 .91146 .9$ $\begin{array}{lllllllllllll}1158.1 & 1197.1 & 1212.5 & 1228.7 & 1235.9 & 1259.8 & 1264.9 & 1273.6 & 1284.1 & 1287.0 & 1291.3 & 1297.1\end{array}$ $1300.21301 .61304 .11321 .2 \quad 1336.7 \quad 1348.6 \quad 1361.6 \quad 1379.3 \quad 1386.2 \quad 1405.91410 .21563 .8$ $1855.32645 .82661 .2 \quad 2668.92674 .4 \quad 2675.2 \quad 2675.82677 .32679 .7 \quad 2693.72715 .22729 .2$ $2732.12758 .12779 .2 \quad 2780.2 \quad 2783.0$

\footnotetext{
name $=\operatorname{MIN} 168$

energy (in $\mathrm{kcal} / \mathrm{mol})=-29.134467$

$\mathrm{ZPE}($ in $\mathrm{kcal} / \mathrm{mol})=143.753$

$\mathrm{G}($ in $\mathrm{kcal} / \mathrm{mol})=117.032$

Geometry

C $-2.82694334-0.87643768-0.57198884$

C $-1.35979635-0.89010802-0.57445958$

C $2.128367751 .37584586-0.57475170$

C $2.250256411 .04787928 \quad 0.89311031$

C $1.246308110 .02920953 \quad 1.40618181$

C $0.95980692-1.22464033 \quad 0.53740718$

C $-0.56829196-0.98739190 \quad 0.66179083$

C $-0.28699535 \quad 0.38645570 \quad 1.39650821$

C $-0.68266747 \quad 1.61385810 \quad 0.60023382$

C -0.890460230 .440604762 .79060008$

$\mathrm{H}-3.22776352-1.83340327-0.95827490$

$\mathrm{H}-3.21129617-0.10982193-1.27745092$

$\mathrm{H}-3.26923597-0.692810510 .41360446$

$\mathrm{H} \quad 1.860863612 .40663433-0.83973355$

H $2.176060161 .96960367 \quad 1.50465263$

H $3.27743448 \quad 0.65095140 \quad 1.06909060$

H $1.56059294 \quad-0.26692895 \quad 2.43040282$

$\mathrm{H} \quad 1.30799453-2.166138910 .97102843$

$\mathrm{H} 1.36578234-1.17848720-0.48078651$

$\mathrm{H}-1.04020402-1.72381603 \quad 1.34706958$

$\mathrm{H}-0.36166500 \quad 1.55766412-0.44781042$

$\mathrm{H}-0.25796795 \quad 2.52674457 \quad 1.03367304$

$\mathrm{H}-1.77419057 \quad 1.74078886 \quad 0.58184171$

$\mathrm{H}-0.54292176 \quad 1.32520606 \quad 3.33835163$

$\mathrm{H}-0.62909171-0.43738771 \quad 3.39237093$

H $-1.98569288 \quad 0.49209161 \quad 2.75065349$

$0-1.33941347-0.70946358-2.75652968$

$02.347603390 .56065110-1.43470437$

o $-0.67644897-0.81146654-1.68433734$

Vibrational frequencies (in $\mathrm{cm}-1$ )
} 
$\begin{array}{lllllllllllllllll}34.6 & 39.0 & 53.4 & 88.3 & 114.6 & 126.8 & 165.1 & 188.1 & 211.8 & 220.4 & 263.1 & 270.6 & 297.6 & 301.9\end{array}$ $328.8 \quad 375.0 \quad 423.4 \quad 454.7 \quad 471.6 \quad 542.4 \quad 595.0 \quad 664.1 \quad 685.5 \quad 788.0 \quad 824.4 \quad 900.5 \quad 923.6 \quad 928.2$ $993.01000 .1 \quad 1006.91017 .1 \quad 1030.6 \quad 1045.4 \quad 1049.9 \quad 1063.1 \quad 1076.5 \quad 1105.7 \quad 1126.7 \quad 1132.3$ $1160.61196 .51202 .2 \quad 1228.7 \quad 1236.2 \quad 1263.2 \quad 1266.1 \quad 1276.8 \quad 1284.8 \quad 1289.5 \quad 1295.51297 .5$ $1301.11303 .21313 .0 \quad 1317.8 \quad 1338.7 \quad 1350.6 \quad 1383.8 \quad 1386.2 \quad 1403.91407 .11450 .31597 .0$ $1842.92637 .4 \quad 2645.8 \quad 2664.0 \quad 2665.7 \quad 2667.5 \quad 2674.3 \quad 2675.42676 .5 \quad 2679.2 \quad 2707.3 \quad 2714.4$ $2738.12758 .12769 .92779 .1 \quad 2782.5$

\section{name $=$ MIN169}

energy (in $\mathrm{kcal} / \mathrm{mol})=-29.054698$

$\mathrm{ZPE}($ in $\mathrm{kcal} / \mathrm{mol})=143.71$

$\mathrm{G}($ in $\mathrm{kcal} / \mathrm{mol})=117.13$

Geometry

C $-3.18192388-0.73718386-0.38900244$

C $-1.74328907-0.53121311-0.69723940$

C $2.03852761 \quad 1.36711144-0.51023355$

C $2.24026144 \quad 0.49085765 \quad 0.70493234$

C $1.28887547-0.68048122 \quad 0.84574438$

C $0.72718801-1.32955916-0.44735458$

C $-0.72330537-1.219456370 .09426231$

C $-0.17883067-0.423439851 .35329775$

C $-0.624399531 .02252372 \quad 1.42681926$

C $-0.48192868-1.14052584 \quad 2.66102779$

$\mathrm{H}-3.49205194-1.77349588-0.60334109$

$\mathrm{H}-3.85277193-0.07791668-0.96213305$

$\mathrm{H}-3.38665202-0.55500805 \quad 0.67950563$

$\mathrm{H} 1.022279751 .50131357-0.90246206$

H 2.18394447 $1.13720602 \quad 1.60747491$

$\mathrm{H} \quad 3.28886654 \quad 0.11444747 \quad 0.69348610$

H $1.78106117-1.44361704 \quad 1.48668836$

$\mathrm{H} \quad 1.06091824-2.35561156-0.62154529$

$\mathrm{H} \quad 0.92116608-0.76935220-1.37220500$

$\mathrm{H}-1.10216734 \quad-2.22077894 \quad 0.39779691$

$\mathrm{H}-0.487087331 .54667282 \quad 0.47049099$

$\mathrm{H}-0.056900251 .576037372 .18582316$

$\mathrm{H}-1.68425344 \quad 1.10468209 \quad 1.69445879$

$\mathrm{H} \quad 0.04274895 \quad-0.66794310 \quad 3.50113254$

$\mathrm{H}-0.17304702-2.192281532 .63921039$

$\mathrm{H}-1.55250303-1.121361712 .89272526$

$\begin{array}{llll}0 & -0.46048993 & 0.62784980 & -2.09861324\end{array}$

O $2.98410761 \quad 1.92469270-1.01148757$

o $-1.60593395 \quad 0.29495132-1.69260795$

Vibrational frequencies (in $\mathrm{cm}-1$ )

$\begin{array}{llllllllllllllll}16.7 & 40.5 & 82.1 & 119.0 & 137.8 & 149.3 & 177.6 & 189.6 & 218.1 & 236.6 & 251.8 & 273.8 & 299.6 & 307.7\end{array}$ $329.0 \quad 375.6 \quad 421.6 \quad 439.2 \quad 445.7 \quad 582.3 \quad 591.6 \quad 646.6 \quad 695.9 \quad 788.2 \quad 804.6 \quad 828.8 \quad 910.8 \quad 945.3$ $990.61001 .7 \quad 1008.6 \quad 1015.8 \quad 1021.0 \quad 1036.3 \quad 1053.2 \quad 1069.4 \quad 1089.01107 .91128 .7 \quad 1153.7$ $\begin{array}{llllllllllllll}1161.9 & 1200.9 & 1205.9 & 1235.7 & 1252.6 & 1257.5 & 1265.2 & 1266.6 & 1279.0 & 1286.1 & 1288.6 & 1293.4\end{array}$ $1298.51302 .0 \quad 1304.91309 .4 \quad 1347.6 \quad 1349.7 \quad 1374.7 \quad 1384.1 \quad 1400.5 \quad 1403.4 \quad 1424.51625 .9$ $1827.62628 .72654 .5 \quad 2656.62660 .7 \quad 2661.2 \quad 2663.52674 .52675 .92679 .92707 .12712 .6$ $2737.5 \quad 2755.12771 .2 \quad 2777.7 \quad 2782.6$

\section{name $=$ MIN170}

energy (in $\mathrm{kcal} / \mathrm{mol})=-28.922114$

$\mathrm{ZPE}($ in $\mathrm{kcal} / \mathrm{mol})=143.585$

$\mathrm{G}($ in $\mathrm{kcal} / \mathrm{mol})=116.35$

Geometry

C $-3.24867189-0.35360098 \quad 0.05563613$

C $-1.79009055-0.32834363 \quad 0.20491776$

C $1.57174125 \quad 1.84748688 \quad 0.17554256$

C $2.11168038 \quad 0.91906775-0.88113078$

$\begin{array}{llll}\text { C } 1.14725287 & -0.10669960 & -1.43869700\end{array}$

C $-0.304107260 .35444797-1.73454740$

C $-0.87338092-0.79317205-0.84974901$ 
C $0.59862238-1.23695895-0.48702249$

C $1.00424268-1.166264840 .96889716$

C $0.89890163-2.63120221-1.02682870$

$\mathrm{H}-3.64132425 \quad 0.66010477-0.15359980$

$\mathrm{H}-3.73227754 \quad-0.64506271 \quad 1.01357868$

$\mathrm{H}-3.61136688-1.02537724-0.72990369$

H $0.48601851 \quad 1.91419188 \quad 0.31299823$

$\mathrm{H} 3.01363306 \quad 0.40218732-0.47941610$

$\mathrm{H} 2.507991291 .54901188-1.71137874$

$\mathrm{H} 1.61282661-0.55890654-2.34050201$

$\mathrm{H}-0.591013650 .28932995-2.78802410$

$\mathrm{H}-0.555825651 .36271238-1.39605941$

$\mathrm{H}-1.37635163-1.57557079-1.45752829$

H $2.04138716-1.50686244 \quad 1.10704690$

$\mathrm{H} \quad 0.37479553-1.814959691 .59428425$

$\mathrm{H} \quad 0.96569202-0.15922397 \quad 1.39914142$

$\mathrm{H} \quad 0.61056937-2.74470533-2.07751972$

$\mathrm{H} \quad 0.36523275-3.40216817-0.45706093$

H $1.96974808-2.86102280-0.95990233$

$0-1.99271956 \quad 0.58771196 \quad 2.18873002$

$\begin{array}{lllll}0 & 2.32073768 & 2.51535904 & 0.84453024\end{array}$

$0-1.22795788 \quad 0.145292651 .28246081$

Vibrational frequencies (in $\mathrm{cm}-1$ )

$\begin{array}{lllllllllllllll}-20.2 & 36.4 & 45.1 & 62.9 & 119.8 & 155.8 & 162.5 & 195.5 & 211.9 & 229.3 & 251.9 & 282.1 & 302.7 & 320.2\end{array}$ $\begin{array}{llllllllllllllll}326.4 & 388.7 & 397.1 & 421.5 & 491.1 & 547.8 & 614.0 & 668.1 & 693.4 & 745.0 & 819.8 & 838.1 & 929.3 & 935.5\end{array}$ $992.8 \quad 1004.2 \quad 1010.6 \quad 1018.7 \quad 1035.0 \quad 1041.2 \quad 1050.9 \quad 1059.3 \quad 1074.2 \quad 1109.7 \quad 1123.6 \quad 1133.8$

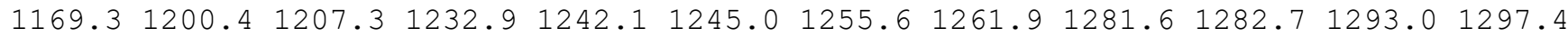

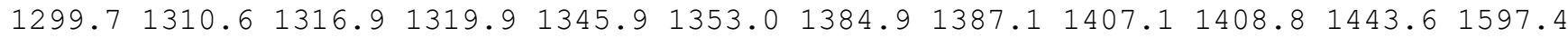
$1832.72633 .5 \quad 2644.5 \quad 2649.0 \quad 2663.1 \quad 2668.2 \quad 2672.82675 .52676 .2 \quad 2679.82708 .52715 .8$ $2735.7 \quad 2763.3 \quad 2769.5 \quad 2778.5 \quad 2782.2$

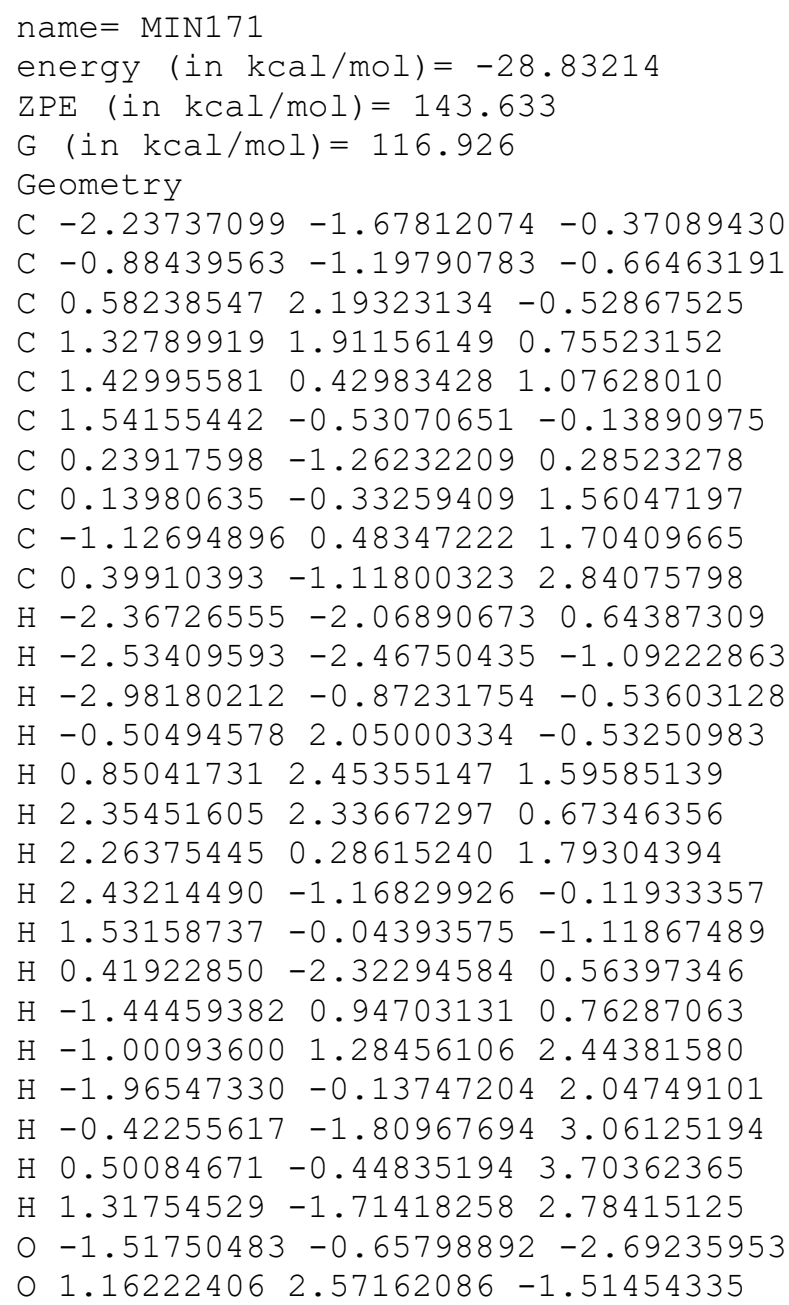


$0-0.58519363-0.70849778-1.83895790$

Vibrational frequencies (in $\mathrm{cm}-1$ )

$\begin{array}{lllllllllllllll}36.4 & 39.0 & 48.7 & 82.0 & 119.2 & 148.5 & 165.8 & 200.7 & 222.8 & 226.5 & 242.8 & 267.1 & 280.4 & 296.4\end{array}$

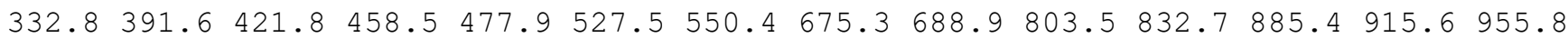
$985.7 \quad 990.3 \quad 1008.9 \quad 1017.4 \quad 1029.2 \quad 1041.3 \quad 1057.7 \quad 1068.3 \quad 1077.01105 .11122 .2 \quad 1124.9$

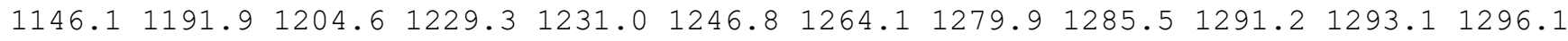

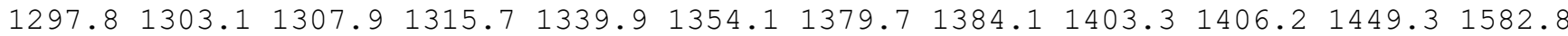
$1840.52634 .5 \quad 2637.7 \quad 2661.3 \quad 2667.7 \quad 2672.2 \quad 2672.92674 .82676 .3 \quad 2678.12706 .92714 .8$ $2739.7 \quad 2759.2 \quad 2769.8 \quad 2778.2 \quad 2782.4$

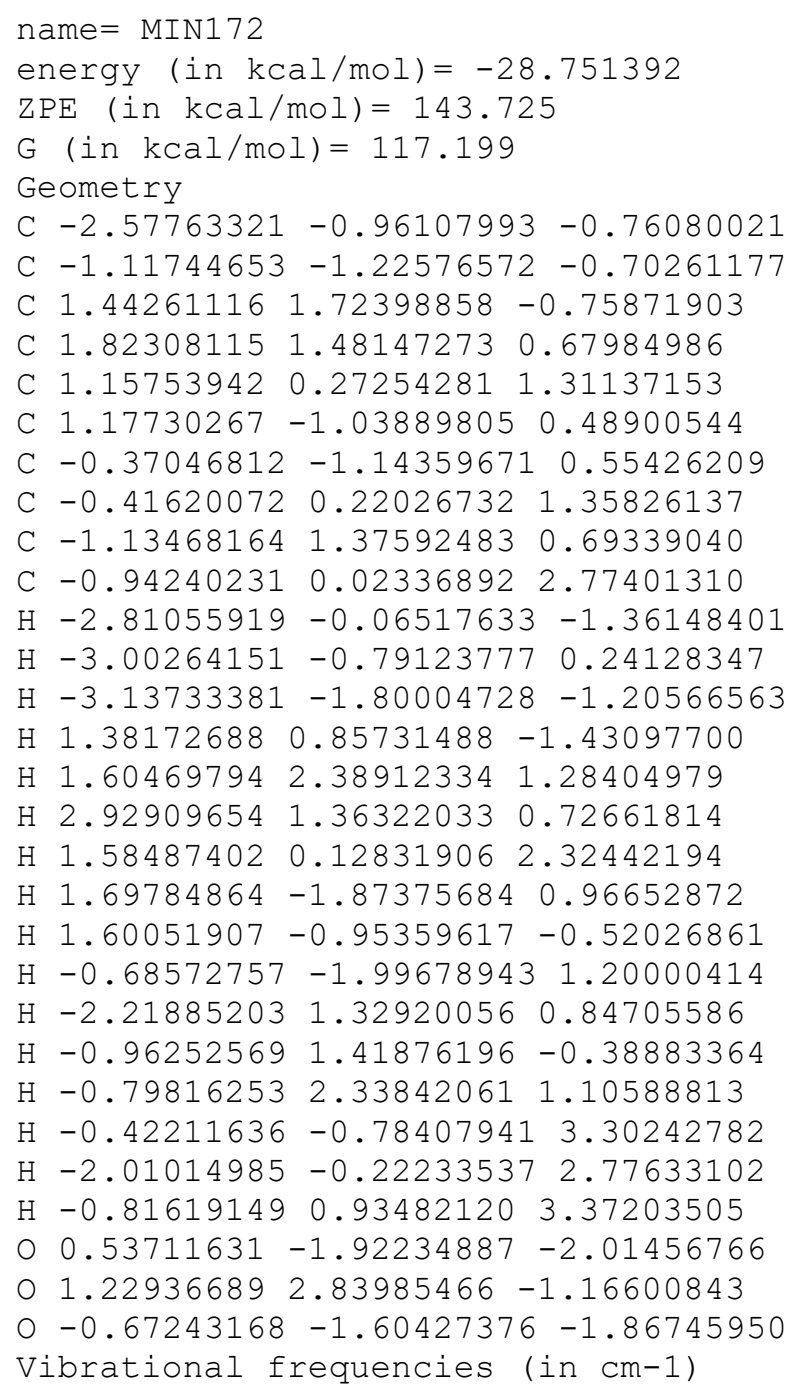


C $1.03642593-1.219114620 .59290452$

C $-0.48684113-0.963735870 .74107433$

C $-0.18196837 \quad 0.42296286 \quad 1.43892093$

C $-0.66140704 \quad 1.64175008 \quad 0.67717273$

C $-0.68598178 \quad 0.46932817 \quad 2.87459269$

$\mathrm{H}-3.19205512-1.82091196-0.71045486$

$\mathrm{H}-3.11132383-0.17019887-1.30114562$

$\mathrm{H}-3.16954920-0.47596008 \quad 0.46124925$

H $1.94380725 \quad 2.52572284 \quad-0.98007393$

H 2.38544334 $1.98525827 \quad 1.31033978$

H $3.31577182 \quad 0.62839930 \quad 0.65129115$

H $1.75865485-0.121530672 .36860767$

$\mathrm{H} 1.38888370-2.128232091 .08713625$

$\mathrm{H} \quad 1.41274681-1.24671403-0.43711239$

$\mathrm{H}-0.95967201-1.67847080 \quad 1.44795413$

$\mathrm{H}-0.15841787 \quad 2.55299384 \quad 1.01973095$

$\mathrm{H}-1.74079951 \quad 1.79591862 \quad 0.81228374$

$\mathrm{H}-0.502191951 .55201420-0.40613091$

$\mathrm{H}-1.782086890 .488727532 .91212955$

$\mathrm{H}-0.326218821 .366811393 .39227682$

$\mathrm{H}-0.35752510-0.39755187 \quad 3.45926269$

$0-0.60820469-0.94279066-1.61348139$

○ $1.65798063 \quad 0.64062984-1.57896145$

o $-1.27921885-0.92879811-2.68232004$

Vibrational frequencies (in $\mathrm{cm}-1$ )

$\begin{array}{lllllllllllllll}37.7 & 43.6 & 69.8 & 94.6 & 116.5 & 130.7 & 160.6 & 189.2 & 208.8 & 218.8 & 258.9 & 277.7 & 294.1 & 304.4\end{array}$

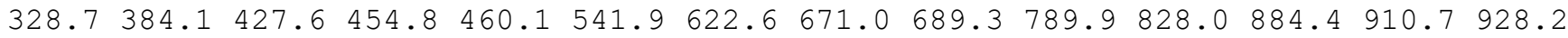
$995.01004 .1 \quad 1007.1 \quad 1017.7 \quad 1033.4 \quad 1046.9 \quad 1050.8 \quad 1062.6 \quad 1079.2 \quad 1107.6 \quad 1128.4 \quad 1131.2$

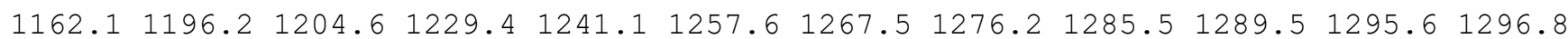
$1301.11304 .3 \quad 1316.2 \quad 1319.8 \quad 1338.0 \quad 1357.2 \quad 1384.4 \quad 1386.91405 .5 \quad 1407.2 \quad 1449.31596 .4$ $1844.62638 .7 \quad 2645.72661 .3 \quad 2662.92670 .92674 .72675 .92676 .8 \quad 2679.7 \quad 2708.32714 .6$ $2737.2 \quad 2758.3 \quad 2770.12778 .92782 .4$

name $=$ MIN174

energy (in $\mathrm{kcal} / \mathrm{mol})=-28.411661$

$\operatorname{ZPE}(\mathrm{in} \mathrm{kcal} / \mathrm{mol})=143.522$

$\mathrm{G}($ in $\mathrm{kcal} / \mathrm{mol})=116.239$

Geometry

C $-2.16027222-1.66096781-0.65712835$

C $-0.70393846-1.88350308-0.56279151$

C $0.79978899 \quad 3.36913281 \quad-0.59454524$

C $0.00707993 \quad 2.39197884 \quad 0.23536225$

C $0.75132748 \quad 1.08930591 \quad 0.43157319$

C $0.80366987 \quad 0.15077343 \quad-0.80263123$

C $0.31034313-1.041011020 .07099393$

C $0.03665608-0.04762863 \quad 1.26114102$

C $-1.406858850 .23685944 \quad 1.62370558$

C $0.81171574-0.437519762 .51285056$

$\mathrm{H}-2.39179510-0.64230360-1.00965658$

$\mathrm{H}-2.64811127-1.78743607 \quad 0.32556571$

$\mathrm{H}-2.66011722-2.36069580-1.34755899$

$\mathrm{H} 1.780705693 .05071021-0.96823809$

$\mathrm{H}-0.98361419 \quad 2.23408207-0.23993974$

$\mathrm{H}-0.232195332 .85577144 \quad 1.21773286$

$\mathrm{H} \quad 1.76178301 \quad 1.290678420 .83971101$

$\mathrm{H} \quad 1.794945290 .00546629-1.24067741$

H $0.11759451 \quad 0.42670967-1.60779585$

H $1.18014025-1.70780937 \quad 0.33930391$

$\mathrm{H}-2.00091200 \quad 0.56159158 \quad 0.76350188$

$\mathrm{H}-1.47162107 \quad 1.03559626 \quad 2.37592484$

$\mathrm{H}-1.89083835-0.64617951 \quad 2.05830341$

$\mathrm{H} \quad 0.77164364 \quad 0.35399275 \quad 3.27094783$

$\mathrm{H} 1.87090471-0.631866932 .30402742$

H $0.40198277-1.34833770 \quad 2.96650319$ 
$\begin{array}{llll}0 & 0.86361322 & -3.31129968 & -1.18098567\end{array}$

$00.36952867 \quad 4.46798563-0.84174276$

o $-0.35782116-2.98912800-1.17405123$

Vibrational frequencies (in $\mathrm{cm}-1$ )

$\begin{array}{llllllllllllllll}-26.5 & 26.0 & 52.3 & 76.4 & 133.7 & 160.4 & 162.1 & 185.7 & 189.5 & 234.2 & 242.0 & 270.9 & 274.8 & 307.8\end{array}$ $320.1359 .6 \quad 407.5 \quad 440.6 \quad 527.1 \quad 563.4 \quad 573.5 \quad 637.5 \quad 676.2 \quad 766.1 \quad 792.6 \quad 864.0 \quad 932.3 \quad 948.6$ $977.9992 .1 \quad 1005.4 \quad 1010.5 \quad 1014.3 \quad 1030.0 \quad 1059.8 \quad 1066.5 \quad 1107.9 \quad 1112.6 \quad 1132.51137 .0$ $\begin{array}{llllllllllll}1169.9 & 1185.2 & 1204.2 & 1233.0 & 1252.9 & 1259.5 & 1264.2 & 1266.8 & 1276.8 & 1287.9 & 1297.2 & 1301.7\end{array}$

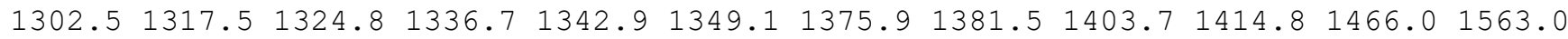

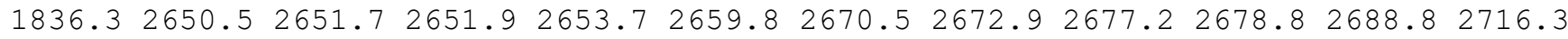
$2743.92764 .12768 .7 \quad 2779.4 \quad 2781.7$

name $=$ MIN175

energy (in $\mathrm{kcal} / \mathrm{mol})=-28.657878$

$\mathrm{ZPE}($ in $\mathrm{kcal} / \mathrm{mol})=143.871$

$\mathrm{G}($ in $\mathrm{kcal} / \mathrm{mol})=117.807$

Geometry

C $-2.51829748-0.83246566-0.76930519$

C $-1.03624072-0.91573152-0.86254256$

C $1.21492584 \quad 1.69329738 \quad-0.67413681$

C $1.69225421 \quad 1.40929162 \quad 0.73294787$

C $1.29680043 \quad 0.03528881 \quad 1.25250882$

C $1.31287106-1.11946206 \quad 0.21976068$

C $-0.23003475-1.26924458 \quad 0.30969720$

C $-0.23456903-0.229899461 .50975488$

C -1.158427620 .962216361 .40895962$

C $-0.45706529-0.945209812 .83976827$

$\mathrm{H}-3.01358269-1.60229624-1.38344371$

$\mathrm{H}-2.899501950 .14673991-1.10727481$

$\mathrm{H}-2.87381291-0.97037828 \quad 0.26382032$

H $1.61276974 \quad 1.07421561-1.49187939$

$\mathrm{H} 1.306536332 .19063875 \quad 1.42308486$

H $2.79778279 \quad 1.51160527 \quad 0.75521781$

$\mathrm{H} 1.90233993-0.18996726 \quad 2.15102650$

H $1.85078255-2.01048166 \quad 0.55981648$

$\mathrm{H} \quad 1.71938642-0.87670435-0.76878283$

$\mathrm{H}-0.52580550-2.28685404 \quad 0.64935649$

$\mathrm{H}-1.12392857 \quad 1.446976150 .42356151$

$\mathrm{H}-0.88368819 \quad 1.73400899 \quad 2.14171911$

$\mathrm{H}-2.20172416 \quad 0.69329991 \quad 1.60686208$

$\mathrm{H}-1.48399105-1.31630622 \quad 2.92947902$

$\mathrm{H}-0.27934208-0.269439213 .68539578$

$\mathrm{H} \quad 0.21316562-1.803746172 .96464786$

$0 \quad 0.56200065-0.75472187-2.40890151$

$0 \quad 0.43501257 \quad 2.58767862 \quad-0.89471681$

o $-0.65618977-0.69443906-2.08797106$

Vibrational frequencies (in $\mathrm{cm}-1$ )

$\begin{array}{llllllllllllllll}26.5 & 52.0 & 66.4 & 103.0 & 124.0 & 177.0 & 203.9 & 208.2 & 234.6 & 250.0 & 265.3 & 278.3 & 301.4 & 330.1\end{array}$ $346.2404 .5 \quad 413.4 \quad 447.3 \quad 475.1 \quad 533.6 \quad 574.6 \quad 663.2 \quad 701.3 \quad 788.3 \quad 808.7 \quad 900.4 \quad 909.5 \quad 934.3$ $988.7 \quad 998.3 \quad 1009.2 \quad 1016.91019 .7 \quad 1032.1 \quad 1043.9 \quad 1064.6 \quad 1100.8 \quad 1108.01110 .4 \quad 1142.3$

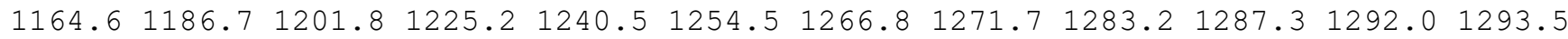

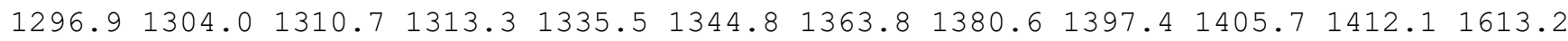
$1829.92628 .72653 .42658 .0 \quad 2659.5 \quad 2662.6 \quad 2666.5 \quad 2674.92675 .72679 .32703 .12717 .2$ $2739.4 \quad 2755.7 \quad 2770.3 \quad 2780.92783 .0$

name $=$ MIN176

energy (in $\mathrm{kcal} / \mathrm{mol})=-28.453656$

$\mathrm{ZPE}(\mathrm{in} \mathrm{kcal} / \mathrm{mol})=143.8$

$\mathrm{G}(\mathrm{in} \mathrm{kcal} / \mathrm{mol})=117.349$

Geometry

C $-1.47680910-1.58085421-0.28752853$

C $-0.01372803-1.59816613-0.30296187$

C $-1.74861318 \quad 2.49776491-0.58635436$ 
C $-0.46615778 \quad 2.61779559 \quad 0.19685107$

C $0.626057521 .72096682-0.37045876$

C $0.241546120 .68671700-1.46744814$

C $0.87145459-0.46530624-0.64478667$

$\begin{array}{lllll}\text { C } & 1.16983267 & 0.54660434 & 0.53467582\end{array}$

C $0.40930153 \quad 0.25565918 \quad 1.81144456$

$\begin{array}{lllll}\text { C } 2.65706896 & 0.67865401 & 0.82347214\end{array}$

$\mathrm{H}-1.89459333-1.93733531-1.24636402$

$\mathrm{H}-1.91402589-0.58954441-0.08544038$

$\mathrm{H}-1.87018269-2.28557971 \quad 0.47799094$

$\mathrm{H}-1.84941751 \quad 3.11433679-1.48786959$

$\mathrm{H}-0.66012356 \quad 2.37885174 \quad 1.26503761$

$\mathrm{H}-0.12579862 \quad 3.67280904 \quad 0.20625481$

$\mathrm{H} 1.46467215 \quad 2.35927318-0.71473601$

$\mathrm{H} \quad 0.72116896 \quad 0.86149839 \quad-2.43494182$

$\mathrm{H}-0.82996090 \quad 0.56377210-1.65438915$

H $1.81289222-0.83030830-1.10796806$

$\mathrm{H} \quad 0.70390892 \quad-0.71185497 \quad 2.24152189$

$\mathrm{H}-0.67771679 \quad 0.21226754 \quad 1.64930200$

$\mathrm{H} \quad 0.59759284 \quad 1.01704373 \quad 2.57815163$

H 2.85460391 $1.51929690 \quad 1.50041160$

$\mathrm{H} 3.246772920 .84858212-0.08425188$

$\mathrm{H} 3.05308604-0.22433106 \quad 1.30608649$

$0.00825115-3.710135220 .32488586$

$0-2.63652605 \quad 1.75799323-0.24235384$

O $0.66322215-2.675609590 .00086937$

Vibrational frequencies (in $\mathrm{cm}-1$ )

$\begin{array}{llllllllllllllllll}-38.6 & 41.5 & 53.0 & 92.1 & 121.7 & 148.3 & 175.2 & 194.0 & 238.3 & 247.3 & 270.0 & 278.7 & 303.1 & 310.5\end{array}$

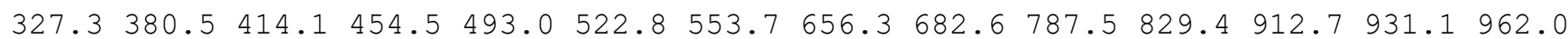
$974.4994 .8 \quad 1007.3 \quad 1018.3 \quad 1021.3 \quad 1042.5 \quad 1049.6 \quad 1074.2 \quad 1092.8 \quad 1098.4 \quad 1126.3 \quad 1137.3$

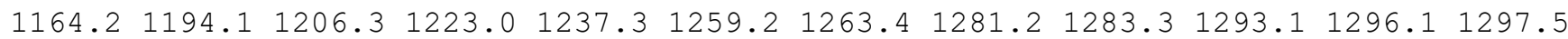
$1302.3 \quad 1305.51313 .4 \quad 1327.5 \quad 1349.1 \quad 1351.9 \quad 1375.3 \quad 1382.1 \quad 1401.91406 .2 \quad 1438.6 \quad 1579.4$ $1838.92634 .7 \quad 2643.8 \quad 2653.5 \quad 2661.6 \quad 2662.92671 .42675 .22675 .92677 .02708 .82716 .4$ $2739.8 \quad 2759.4 \quad 2761.2 \quad 2777.0 \quad 2781.9$

\footnotetext{
name $=$ MIN177

energy (in $\mathrm{kcal} / \mathrm{mol})=-28.466304$

$\mathrm{ZPE}(\mathrm{in} \mathrm{kcal} / \mathrm{mol})=143.896$

$\mathrm{G}(\mathrm{in} \mathrm{kcal} / \mathrm{mol})=116.918$

Geometry

C $-2.84412963-1.27801208-0.61410289$

C $-1.34434610-1.29666520-0.64932849$

C $1.689665891 .59098900-0.50837075$

C $1.956121241 .07159647 \quad 0.84987393$

C $1.04147336-0.01490588 \quad 1.39548883$

C $0.90678821-1.33471728 \quad 0.59477418$

C $-0.63807171-1.24837976 \quad 0.67562444$

C $-0.52440450 \quad 0.16529439 \quad 1.35862894$

C $-1.05361425 \quad 1.32289103 \quad 0.53656308$

C $-1.13722913 \quad 0.18961525 \quad 2.75258668$

$\mathrm{H}-3.24328787-0.72559400 \quad 0.24312378$

$\mathrm{H}-3.23590430-2.30438733-0.56645669$

$\mathrm{H}-3.25360449-0.82714035-1.53180757$

$\mathrm{H} \quad 1.727465172 .65089278-0.71114200$

$\mathrm{H} \quad 1.939939921 .92715347 \quad 1.56861685$

H $3.00856801 \quad 0.69151287 \quad 0.88558704$

$\mathrm{H} \quad 1.36900754 \quad-0.22599888 \quad 2.43779214$

H $1.33969045-2.21088100 \quad 1.08469572$

$\mathrm{H} \quad 1.29926024-1.31496383-0.42830128$

$\mathrm{H}-1.07188597 \quad-2.00638218 \quad 1.35284881$

$\mathrm{H}-0.64030782 \quad 2.27929404 \quad 0.88106082$

$\mathrm{H}-2.14584902 \quad 1.40147434 \quad 0.60444860$

$\mathrm{H}-0.81375514 \quad 1.23057410 \quad-0.53210803$

$\mathrm{H}-2.22947276 \quad 0.10398771 \quad 2.70969714$
} 
$\mathrm{H}-0.904150751 .127310963 .27220636$

$\mathrm{H}-0.77322883-0.63045478 \quad 3.38182552$

O $1.278101161 .19597169-2.60466449$

O $1.455151310 .72971658-1.45332410$

O $-0.73801881-1.39061371-1.68618762$

Vibrational frequencies (in $\mathrm{cm}-1$ )

$\begin{array}{lllllllllllllll}27.8 & 34.1 & 55.9 & 82.2 & 98.4 & 134.0 & 159.1 & 184.0 & 198.8 & 218.6 & 261.3 & 276.4 & 292.5 & 306.4\end{array}$

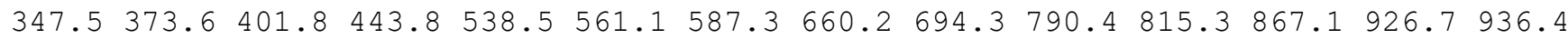
$994.2998 .3 \quad 1007.2 \quad 1016.7 \quad 1035.1 \quad 1042.5 \quad 1051.3 \quad 1070.3 \quad 1090.3 \quad 1103.4 \quad 1132.5 \quad 1156.8$ $1166.21205 .3 \quad 1208.8 \quad 1242.2 \quad 1257.3 \quad 1258.0 \quad 1271.9 \quad 1273.6 \quad 1277.7 \quad 1286.4 \quad 1287.9 \quad 1294.9$ $1299.21302 .8 \quad 1312.6 \quad 1319.3 \quad 1336.5 \quad 1342.3 \quad 1359.6 \quad 1380.91394 .8 \quad 1406.6 \quad 1417.7 \quad 1590.3$ $1855.92640 .22662 .2 \quad 2666.3 \quad 2673.9 \quad 2675.92677 .0 \quad 2677.3 \quad 2679.5 \quad 2706.12723 .32723 .7$ $2726.42762 .4 \quad 2779.0 \quad 2780.3 \quad 2782.7$

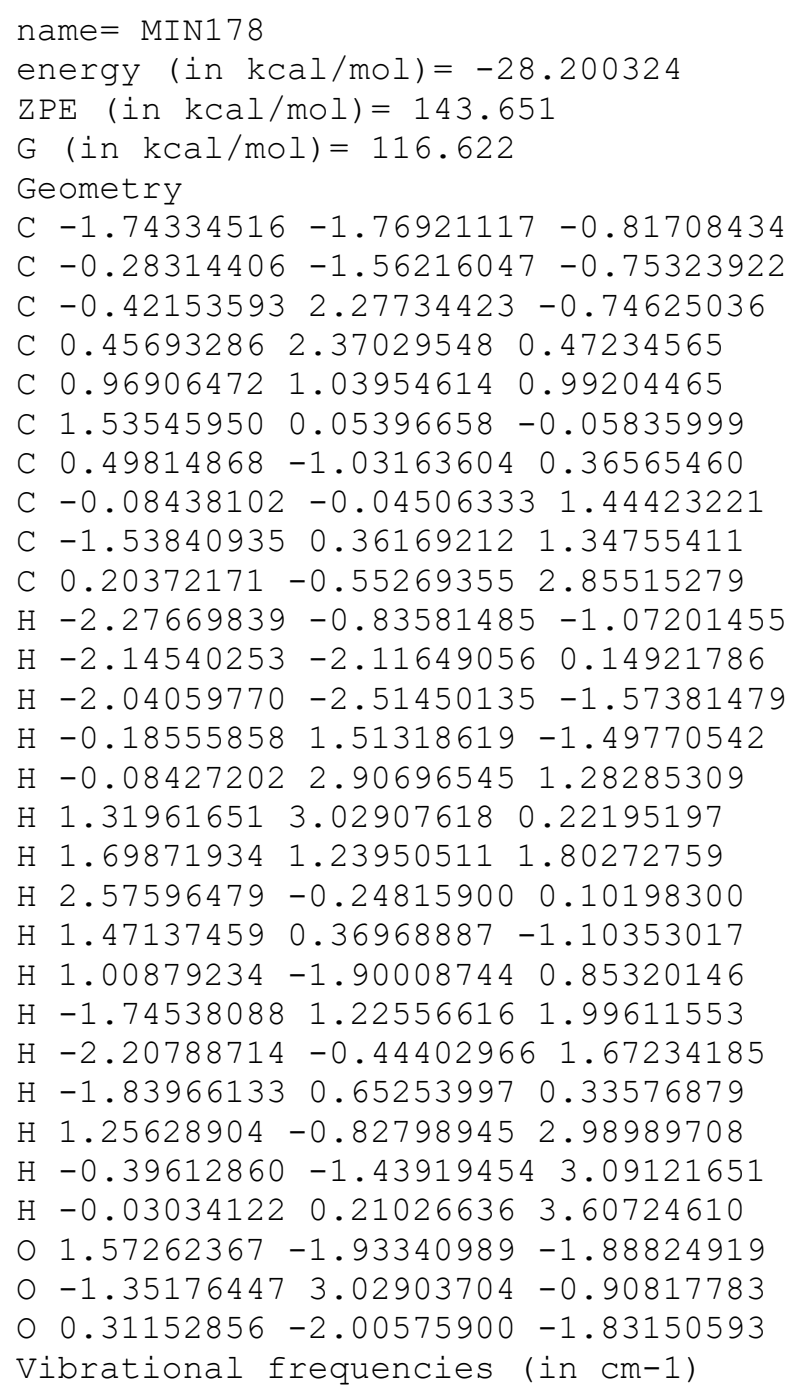


C $-0.50727748-1.26789081-0.79016314$

C $-0.14180723 \quad 2.26030031-0.77681093$

C $0.51290352 \quad 2.329300220 .58053410$

C $1.10831125 \quad 0.99286900 \quad 1.00711634$

C $1.54288374 \quad 0.04013256-0.13474888$

C $0.45350118-0.99754115 \quad 0.28416098$

C $0.11562569-0.11375501 \quad 1.54085024$

C $-1.31095384 \quad 0.31794284 \quad 1.78946775$

C $0.66396995-0.772339292 .80820268$

$\mathrm{H}-2.35564243-0.19627274-0.72938485$

$\mathrm{H}-2.35351743-1.71189745 \quad 0.20324477$

$\mathrm{H}-2.46615494-1.75202996-1.56641895$

$\mathrm{H} \quad 0.49776856 \quad 2.42302839-1.65507025$

$\mathrm{H}-0.22253709 \quad 2.66835196 \quad 1.34148132$

H $1.30712903 \quad 3.103514450 .57043375$

H $1.922032891 .18270016 \quad 1.73219519$

H 2.56402444 $-0.34651449-0.04077187$

$\mathrm{H} \quad 1.45830838 \quad 0.41438362-1.15775331$

$\mathrm{H} \quad 0.91000712-1.974123590 .57801646$

$\mathrm{H}-1.95637587 \quad-0.53387893 \quad 2.03221944$

$\mathrm{H}-1.753886320 .83942143 \quad 0.92905286$

$\mathrm{H}-1.36835632 \quad 1.01359460 \quad 2.63731170$

$\mathrm{H} \quad 0.08023280 \quad-1.66018644 \quad 3.07789401$

$\mathrm{H} \quad 0.62519928-0.08386491 \quad 3.66004725$

H $1.70687952-1.08939798 \quad 2.69237211$

o $1.16145412-1.73073697-2.15752705$

$\begin{array}{llll}0 & -1.32137264 & 2.04826661 & -0.91540392\end{array}$

o $-0.09112060-1.63724203-1.97402184$

Vibrational frequencies (in $\mathrm{cm}-1$ )

$\begin{array}{lllllllllllllll}34.5 & 65.1 & 68.7 & 115.7 & 169.0 & 177.8 & 187.5 & 204.4 & 245.4 & 247.9 & 257.8 & 270.3 & 302.3 & 319.1\end{array}$

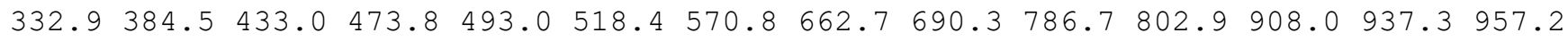
$980.8 \quad 998.7 \quad 1002.1 \quad 1012.2 \quad 1016.4 \quad 1019.1 \quad 1040.8 \quad 1053.7 \quad 1081.11107 .3 \quad 1114.81134 .7$

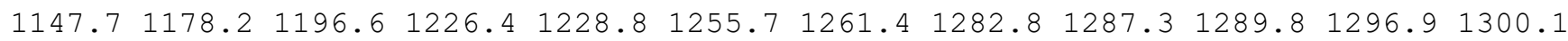
$1303.31312 .4 \quad 1324.6 \quad 1326.6 \quad 1339.1 \quad 1347.4 \quad 1377.5 \quad 1380.1 \quad 1404.6 \quad 1407.5 \quad 1446.01563 .7$ $1837.12631 .6 \quad 2643.7 \quad 2660.0 \quad 2663.0 \quad 2665.3 \quad 2673.4 \quad 2674.92675 .92679 .5 \quad 2693.02718 .1$ 2740.12764 .32765 .02778 .92782 .0

\section{name $=$ MIN180}

energy (in $\mathrm{kcal} / \mathrm{mol})=-27.69979$

$\operatorname{ZPE}(\mathrm{in} \mathrm{kcal} / \mathrm{mol})=143.64$

$\mathrm{G}($ in $\mathrm{kcal} / \mathrm{mol})=116.747$

Geometry

C $-2.06531645-1.46865983-0.60169391$

C $-0.59029708-1.48056371-0.65826873$

C $-0.23898637 \quad 2.22464188 \quad-0.78347914$

C $0.66238352 \quad 2.26862310 \quad 0.42036540$

C $1.10819600 \quad 0.91785761 \quad 0.94876551$

C $1.48571422-0.14212139-0.11481559$

C $0.35241083-1.08894866 \quad 0.39184696$

C $0.00875110-0.05448543 \quad 1.52667497$

C $-1.39579383 \quad 0.50142780 \quad 1.61872135$

C $0.41822978-0.606726812 .89152781$

$\mathrm{H}-2.43362445-1.84066478 \quad 0.36918276$

$\mathrm{H}-2.53183612-2.09412783-1.38115592$

$\mathrm{H}-2.47192296-0.45066347-0.73357909$

$\mathrm{H}-0.41539237 \quad 1.25720067-1.26999719$

$\mathrm{H} \quad 0.16099803 \quad 2.84668483 \quad 1.22855268$

H $1.55595101 \quad 2.88272012 \quad 0.16144410$

H $1.92171184 \quad 1.07815331 \quad 1.68399675$

$\mathrm{H} \quad 2.48967155-0.56660108-0.00576722$

$\mathrm{H} \quad 1.402827190 .16705938-1.16058268$

$\mathrm{H} \quad 0.78033086 \quad-2.02931363 \quad 0.82119579$

$\mathrm{H}-1.76240603 \quad 0.90903304 \quad 0.67206061$

$\mathrm{H}-1.44225642 \quad 1.31863214 \quad 2.35323733$ 
$\mathrm{H}-2.10915598-0.26209967 \quad 1.95286357$

$\mathrm{H}-0.23453531-1.43055110 \quad 3.20141791$

$\mathrm{H} \quad 0.36198603 \quad 0.16591571 \quad 3.66737815$

$\mathrm{H} \quad 1.44627197 \quad-0.98894776 \quad 2.89099697$

O $1.08808291-2.10869998-1.94464407$

$\begin{array}{lllll}0 & -0.75856518 & 3.22665131 & -1.21099398\end{array}$

O $-0.15910141-1.99047048-1.78448478$

Vibrational frequencies (in cm-1)

$\begin{array}{llllllllllllllll}22.1 & 28.4 & 37.1 & 139.7 & 149.0 & 166.7 & 185.1 & 195.8 & 224.8 & 244.3 & 246.6 & 253.2 & 290.9 & 314.0\end{array}$

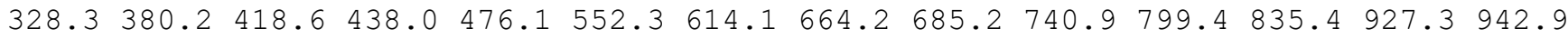
$985.91002 .6 \quad 1009.8 \quad 1011.9 \quad 1016.7 \quad 1029.1 \quad 1049.4 \quad 1062.0 \quad 1082.8 \quad 1110.21117 .11140 .0$

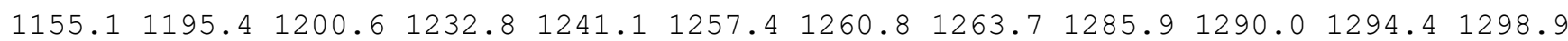
$1305.31313 .71319 .0 \quad 1327.2 \quad 1340.1 \quad 1346.5 \quad 1379.6 \quad 1381.81406 .1 \quad 1408.3 \quad 1446.2 \quad 1563.8$ 1832.32646 .02649 .32649 .92659 .12669 .82671 .82673 .62676 .62678 .92690 .52716 .1 $2737.0 \quad 2763.92768 .6 \quad 2780.12782 .2$

\section{name $=$ MIN181}

energy (in $\mathrm{kcal} / \mathrm{mol})=-28.121775$

$\mathrm{ZPE}(\mathrm{in} \mathrm{kcal} / \mathrm{mol})=144.119$

$\mathrm{G}($ in $\mathrm{kcal} / \mathrm{mol})=117.574$

Geometry

C $-2.33082325-1.88195767-0.30146490$

C $-0.88260933-1.60749550-0.58008958$

C $0.44738522 \quad 1.97923749-0.65792458$

C $1.25468717 \quad 1.76414654 \quad 0.56430359$

C $1.19264886 \quad 0.36442978 \quad 1.16930409$

C $1.42235801-0.82806366 \quad 0.20825176$

C $0.04692376-1.43443237 \quad 0.58956597$

C $-0.20282916-0.24236808 \quad 1.58275543$

C $-1.42073387 \quad 0.61998634 \quad 1.32632614$

C $-0.19955875-0.71306238 \quad 3.03434922$

$\mathrm{H}-2.66237408-2.78110667-0.84391935$

$\mathrm{H}-2.95357861-1.05493399-0.67827399$

$\mathrm{H}-2.55321888-2.02023791 \quad 0.76017397$

$\mathrm{H}-0.49992447 \quad 2.49088812-0.63957931$

$\mathrm{H} \quad 0.93965216 \quad 2.49903425 \quad 1.34223728$

H 2.32554874 2.00507218 0.36015118

$\mathrm{H} 1.89631944 \quad 0.33909404 \quad 2.02689636$

H $2.27881084-1.45754735 \quad 0.46526585$

H $1.51770299-0.57439680-0.85415017$

$\mathrm{H} 0.13161160-2.40600877 \quad 1.11275816$

$\mathrm{H}-1.32893831 \quad 1.59173780 \quad 1.82769451$

$\mathrm{H}-2.335849250 .14950090 \quad 1.70846919$

$\mathrm{H}-1.58205911 \quad 0.81101384 \quad 0.25812225$

$\mathrm{H}-1.08217404-1.32321856 \quad 3.25887258$

$\mathrm{H}-0.20459481 \quad 0.13648785 \quad 3.72739326$

$\mathrm{H} \quad 0.68189248-1.32041298 \quad 3.27083400$

$\begin{array}{lllll}0 & 0.35447193 & 1.76751229 & -2.82752827\end{array}$

○ $0.97796477 \quad 1.54749038-1.76309983$

o $-0.46544123-1.58123464-1.71111708$

Vibrational frequencies (in $\mathrm{cm}-1$ )

$\begin{array}{lllllllllllllll}24.6 & 41.9 & 56.0 & 88.2 & 127.0 & 172.1 & 182.3 & 199.8 & 208.5 & 234.3 & 275.4 & 294.6 & 306.4 & 315.0\end{array}$ $337.0 \quad 374.4 \quad 411.7 \quad 443.7 \quad 538.1 \quad 550.1 \quad 588.5 \quad 645.9 \quad 689.0 \quad 797.0 \quad 810.6 \quad 887.9 \quad 931.3 \quad 947.4$ $981.9990 .2 \quad 1008.2 \quad 1016.91032 .5 \quad 1044.1 \quad 1054.1 \quad 1082.5 \quad 1090.3 \quad 1102.5 \quad 1128.31154 .4$ $1160.21199 .3 \quad 1210.6 \quad 1240.6 \quad 1247.1 \quad 1254.3 \quad 1269.0 \quad 1274.6 \quad 1278.6 \quad 1288.8 \quad 1291.6 \quad 1298.0$

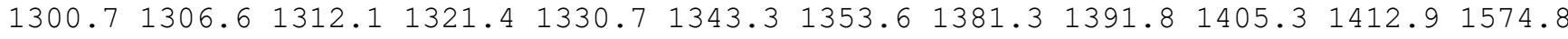

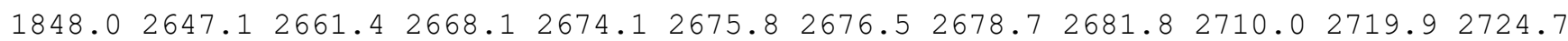
$2732.0 \quad 2761.2 \quad 2779.0 \quad 2780.2 \quad 2782.8$

name $=$ MIN182

energy (in $\mathrm{kcal} / \mathrm{mol})=-28.024385$

$\mathrm{ZPE}($ in $\mathrm{kcal} / \mathrm{mol})=144.163$

$\mathrm{G}($ in $\mathrm{kcal} / \mathrm{mol})=118.004$ 


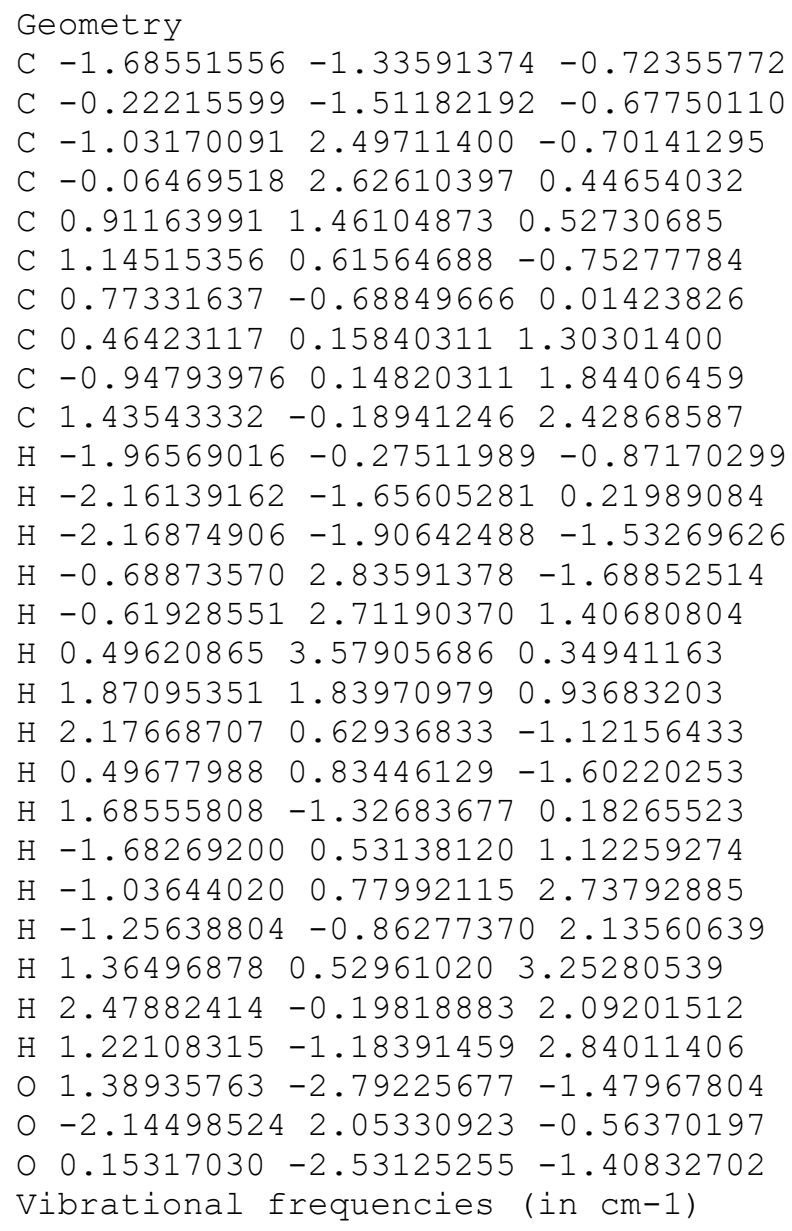

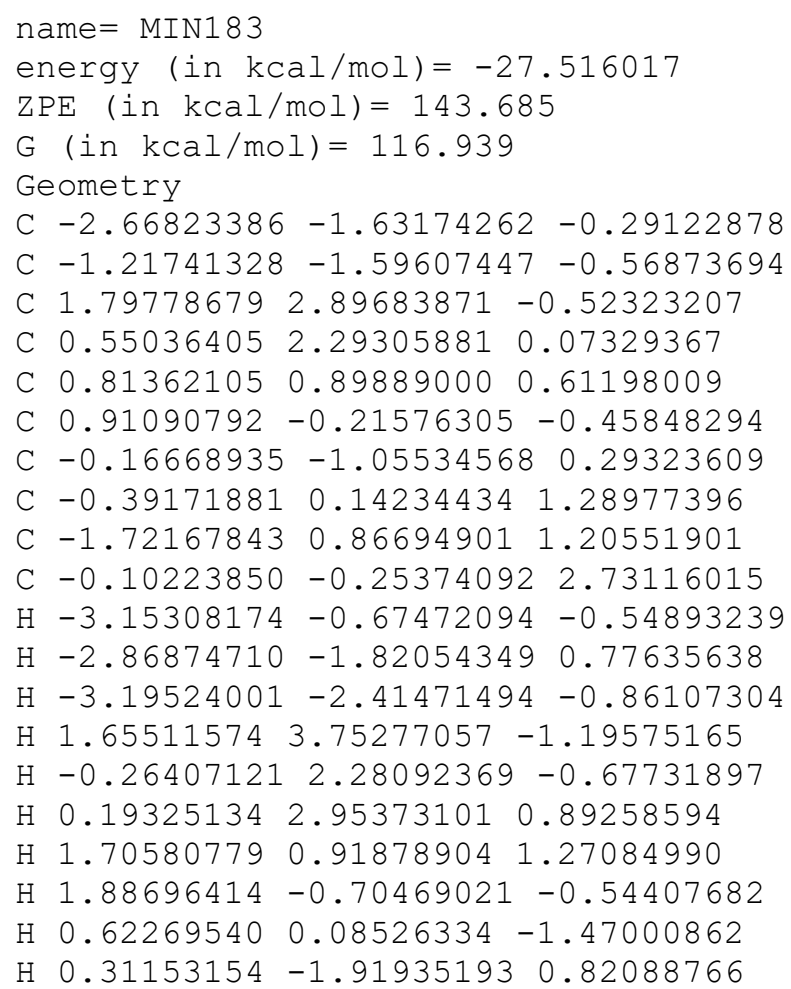


$\mathrm{H}-1.68591994 \quad 1.81630997 \quad 1.75600794$

$\mathrm{H}-2.53156753 \quad 0.27805373 \quad 1.65114594$

$\mathrm{H}-2.000446881 .09820810 \quad 0.17176490$

$\mathrm{H} \quad 0.87329836-0.74566108 \quad 2.83486901$

$\mathrm{H}-0.85931322-0.945717973 .11668009$

$\mathrm{H}-0.09066770 \quad 0.62305736 \quad 3.38968837$

$0.28617451-2.30813480-2.02007731$

○ $2.903377362 .48840150-0.27533800$

o $-0.92794855-2.21365799-1.68537079$

Vibrational frequencies (in $\mathrm{cm}-1$ )

$\begin{array}{llllllllllllllll}22.6 & 38.4 & 62.3 & 116.0 & 134.6 & 157.3 & 173.2 & 187.3 & 205.2 & 229.9 & 250.5 & 267.2 & 283.9 & 294.6\end{array}$

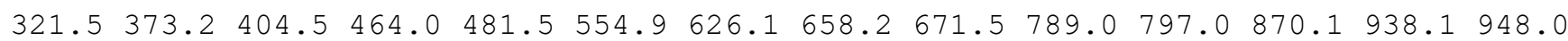

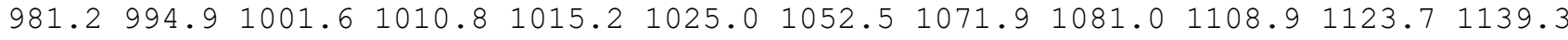
$1157.0 \quad 1182.0 \quad 1194.5 \quad 1221.2 \quad 1250.4 \quad 1263.5 \quad 1266.1 \quad 1277.2 \quad 1285.2 \quad 1292.2 \quad 1293.0 \quad 1296.6$

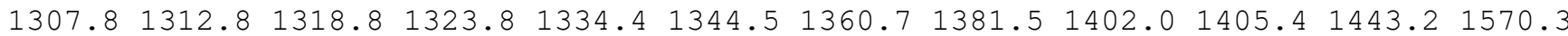
$1845.12650 .2 \quad 2654.62660 .12661 .4 \quad 2672.3 \quad 2673.2 \quad 2675.32679 .02680 .22691 .92713 .9$ $2741.92766 .6 \quad 2770.4 \quad 2780.0 \quad 2782.1$

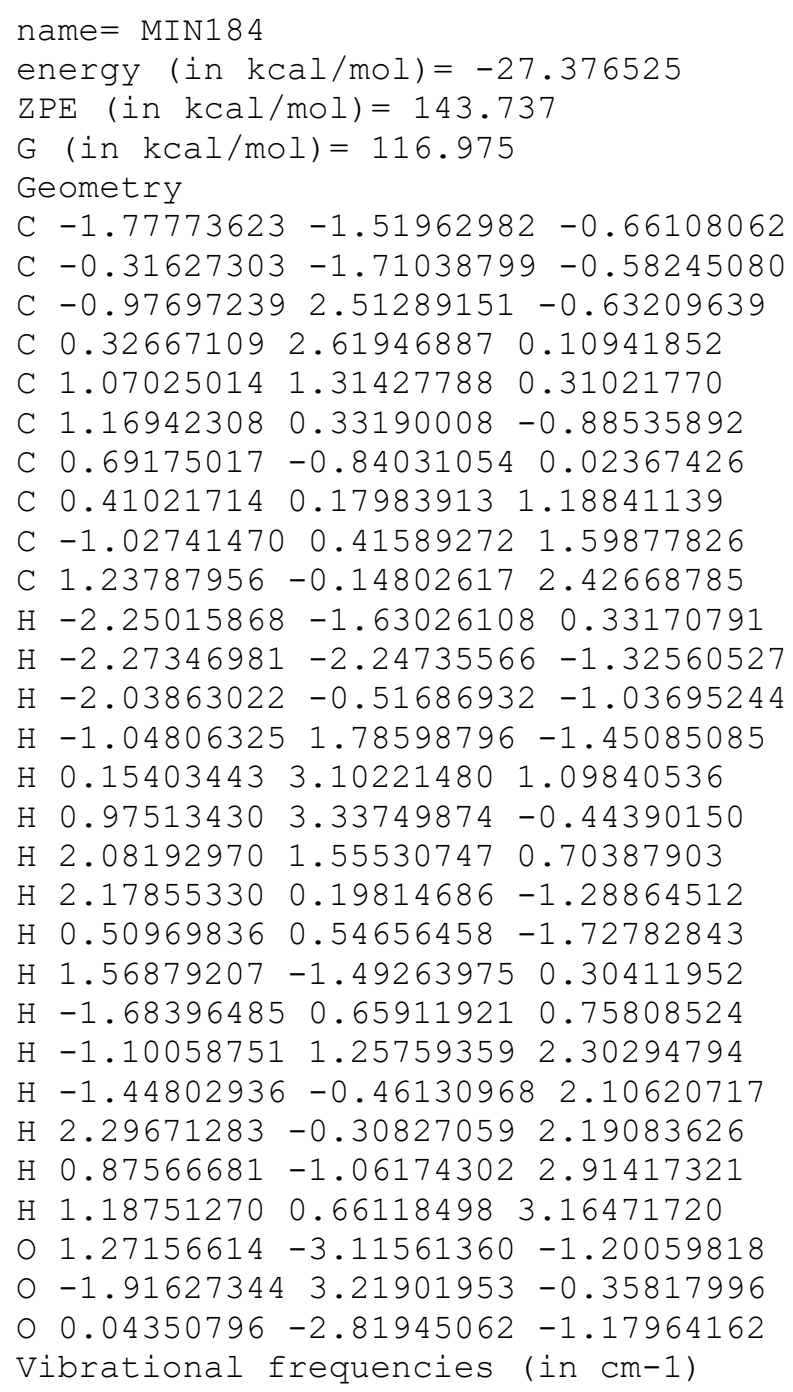


$\mathrm{ZPE}($ in $\mathrm{kcal} / \mathrm{mol})=144.01$

$\mathrm{G}($ in $\mathrm{kcal} / \mathrm{mol})=117.677$

Geometry

C $-1.73209875-1.42315457-0.52599637$

C $-0.27257263-1.61274445-0.41533322$

C $-1.364173552 .46363056-0.40183979$

C $0.04534577 \quad 2.748739790 .04114023$

C $0.93266772 \quad 1.53004280 \quad 0.23762399$

C $1.01169616 \quad 0.51141344 \quad-0.92629311$

C $0.73425206-0.66159127 \quad 0.05950172$

C $0.49248122 \quad 0.37525771 \quad 1.21930350$

C $-0.90207251 \quad 0.47685422 \quad 1.80084068$

C $1.49180650 \quad 0.17916216 \quad 2.35592609$

$\mathrm{H}-1.97400861-0.43863273-0.97278378$

$\mathrm{H}-2.22411954-1.46934418 \quad 0.45929122$

$\mathrm{H}-2.22141587-2.18080852-1.15966470$

$\mathrm{H}-2.17355933 \quad 2.88489230 \quad 0.21094900$

$\mathrm{H} \quad 0.04047741 \quad 3.345525320 .97614059$

$\mathrm{H} \quad 0.51817511 \quad 3.40022624 \quad 0.73249329$

$\mathrm{H} 1.945880161 .894652350 .51457764$

H $1.98086205 \quad 0.45499862-1.42894200$

$\mathrm{H} \quad 0.24528746 \quad 0.64123707 \quad-1.70013511$

$\mathrm{H} \quad 1.67948682 \quad-1.23999689 \quad 0.26480837$

$\mathrm{H}-1.15142063-0.419680932 .38465417$

$\mathrm{H}-1.67720752 \quad 0.58812161 \quad 1.03729341$

$\mathrm{H}-0.98254526 \quad 1.335516372 .47966000$

$\mathrm{H} 1.44650278 \quad 1.004829793 .07466262$

H $2.52537032 \quad 0.11543644 \quad 1.99477125$

$\mathrm{H} \quad 1.28794622-0.749152512 .90475558$

o $1.31273655-3.08329923-0.87640816$

$0-1.63226564 \quad 1.83773785-1.39784658$

O $0.08362683-2.78190402-0.88418061$

Vibrational frequencies (in $\mathrm{cm}-1$ )

$\begin{array}{llllllllllllll}-15.4 & 56.7 & 88.5 & 111.4 & 158.7 & 173.8 & 187.4 & 192.3 & 201.0 & 215.0 & 252.2 & 266.2 & 280.2 & 319.6\end{array}$ $343.4 \quad 377.2 \quad 426.5 \quad 464.4 \quad 506.7 \quad 578.4 \quad 601.6 \quad 647.4 \quad 680.2 \quad 783.4 \quad 795.8 \quad 894.4 \quad 912.1947 .9$ $983.6993 .0 \quad 1007.4 \quad 1016.0 \quad 1017.0 \quad 1033.8 \quad 1051.5 \quad 1065.0 \quad 1072.4 \quad 1101.4 \quad 1127.31135 .6$

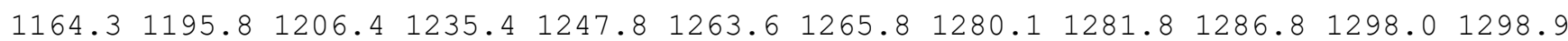

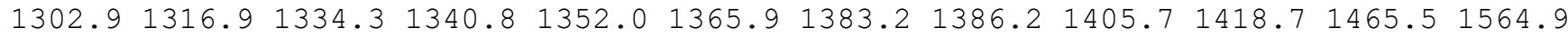
$1840.92642 .5 \quad 2643.8 \quad 2650.7 \quad 2658.2 \quad 2661.0 \quad 2671.12674 .42675 .82678 .32680 .2 \quad 2708.1$ $2735.4 \quad 2758.6 \quad 2766.8 \quad 2780.2 \quad 2782.1$

\section{name $=$ MIN186}

energy (in $\mathrm{kcal} / \mathrm{mol})=-25.772309$

$\mathrm{ZPE}(\mathrm{in} \mathrm{kcal} / \mathrm{mol})=143.575$

G $($ in $\mathrm{kcal} / \mathrm{mol})=116.416$

Geometry

C $-2.94459052-0.92088209-1.20718525$

C $-1.45966144-0.97705759-1.14436590$

C $0.640459751 .51399050-0.90800830$

C $1.73557691 \quad 1.26954224 \quad 0.10040671$

C $1.45895305 \quad 0.04387843 \quad 0.99630627$

C $0.70246238-1.07947198 \quad 0.25077592$

C $-0.81168361-0.85112009 \quad 0.16985241$

C $0.75931105 \quad 0.39152377 \quad 2.28793291$

C $-0.04429010 \quad 1.44383193 \quad 2.45190981$

C $1.03956637-0.555933123 .41162348$

$\mathrm{H}-3.31324946 \quad 0.04566471-0.81551833$

$\mathrm{H}-3.40827316-1.71293864-0.59864904$

$\mathrm{H}-3.34636460-1.01913937-2.22773131$

$\mathrm{H} \quad 0.73068304 \quad 1.01337257-1.88480130$

$\mathrm{H} \quad 1.87756279 \quad 2.170086110 .73400959$

$\mathrm{H} \quad 2.69620670 \quad 1.12237960 \quad-0.43297887$

H $2.47040474-0.37260531 \quad 1.26513309$

$\mathrm{H} \quad 0.90084716-2.044835710 .75474735$ 
H $1.15122167-1.20470668-0.76062140$

$\mathrm{H}-1.31176953-1.533991490 .89705428$

$\mathrm{H}-0.538253421 .680201923 .37914056$

$\mathrm{H}-1.05528824 \quad 0.17147772 \quad 0.57395275$

$\mathrm{H}-0.27273900 \quad 2.16138188 \quad 1.67439234$

$\mathrm{H} \quad 0.36532568-0.404070794 .26509923$

H $2.06444855-0.425501313 .78824763$

$\mathrm{H} \quad 0.93363377-1.603626593 .10076027$

O $0.29084536-1.08189794-2.52505343$

$0-0.29982802 \quad 2.23350758-0.66885381$

O $-0.96326423-1.08281494-2.33995757$

Vibrational frequencies (in $\mathrm{cm}-1$ )

$\begin{array}{llllllllllllllll}-25.4 & 57.6 & 61.9 & 82.3 & 101.0 & 119.3 & 120.1 & 131.3 & 178.0 & 213.7 & 263.9 & 280.9 & 297.8 & 320.2\end{array}$ $337.2 \quad 372.3 \quad 396.6 \quad 445.3 \quad 470.2 \quad 502.7 \quad 548.0 \quad 624.0 \quad 672.8 \quad 686.2 \quad 804.0 \quad 846.6 \quad 933.9 \quad 946.7$ $970.1995 .3 \quad 999.5 \quad 1009.6 \quad 1040.8 \quad 1046.3 \quad 1050.2 \quad 1053.9 \quad 1079.9 \quad 1102.5 \quad 1123.01148 .3 \quad 1157.0$ $\begin{array}{llllllllllllll}1183.8 & 1220.8 & 1236.0 & 1249.5 & 1259.3 & 1266.7 & 1268.6 & 1277.4 & 1278.7 & 1288.3 & 1296.8 & 1305.5\end{array}$ $1316.71321 .91328 .1 \quad 1369.5 \quad 1375.3 \quad 1380.6 \quad 1395.0 \quad 1403.61413 .0 \quad 1629.4 \quad 1830.81867 .6$ $2604.72621 .12626 .5 \quad 2650.3 \quad 2661.6 \quad 2662.4 \quad 2671.42674 .72681 .12708 .32714 .62728 .3$ $2738.6 \quad 2770.2 \quad 2778.5 \quad 2786.2$

name $=$ MIN187

energy $($ in $\mathrm{kcal} / \mathrm{mol})=-25.950438$

$\operatorname{ZPE}($ in $\mathrm{kcal} / \mathrm{mol})=143.849$

$\mathrm{G}($ in $\mathrm{kcal} / \mathrm{mol})=117.281$

Geometry

C $-2.17588676-1.57362137-0.27347674$

C $-0.72102093-1.54986745-0.52172019$

C $-0.18225125 \quad 2.72018287-0.42499757$

C $1.03913838 \quad 2.42839232 \quad 0.40698081$

C $1.25181157 \quad 0.96376233 \quad 0.75303999$

C $1.30506830-0.04025378-0.42247448$

C $0.32799814 \quad-0.99386463 \quad 0.33295040$

C $0.09563267 \quad 0.11888693 \quad 1.41985531$

C $-1.26549950 \quad 0.78450097 \quad 1.44647127$

C $0.46166192-0.358532212 .82020667$

$\mathrm{H}-2.64603886-0.61281874-0.54877992$

$\mathrm{H}-2.40003688-1.75484608 \quad 0.79019434$

$\mathrm{H}-2.69713181-2.35446587-0.85153357$

$\mathrm{H}-0.92368407 \quad 3.40553608 \quad 0.00906911$

H $1.02076529 \quad 3.02513434 \quad 1.34106876$

$\mathrm{H} \quad 1.93309687 \quad 2.78120820 \quad-0.16116475$

H $2.17226016 \quad 0.895939461 .37285259$

H 2.28438349 $-0.47397974-0.64121316$

$\mathrm{H} \quad 0.90821938 \quad 0.34245709-1.37313074$

$\mathrm{H} \quad 0.89223077-1.85633843 \quad 0.77249708$

$\mathrm{H}-1.22771028 \quad 1.73273621 \quad 1.99742123$

$\mathrm{H}-2.011395420 .15756303 \quad 1.95011808$

H $-1.64378451 \quad 0.99250506 \quad 0.43814168$

H $1.45462697-0.82272084 \quad 2.85399919$

$\mathrm{H}-0.25504765-1.100814413 .18966072$

$\begin{array}{lllll}\mathrm{H} & 0.46948954 & 0.47455940 & 3.53305542\end{array}$

$0.82171016-2.28160312-1.91645901$

$0-0.352840812 .26749195-1.52919562$

O $-0.40436000-2.17823768-1.62569447$

Vibrational frequencies (in $\mathrm{cm}-1$ )

$\begin{array}{llllllllllllllll}19.3 & 39.6 & 64.0 & 116.5 & 147.0 & 162.1 & 173.7 & 197.8 & 218.3 & 235.6 & 247.1 & 264.3 & 288.8 & 313.9\end{array}$ $339.2 \quad 386.3 \quad 409.5 \quad 462.4 \quad 492.7 \quad 541.8 \quad 611.6 \quad 660.1 \quad 663.8 \quad 782.6 \quad 792.9 \quad 900.6 \quad 915.9 \quad 954.5$ $987.3 \quad 992.0 \quad 1002.1 \quad 1010.0 \quad 1015.8 \quad 1025.0 \quad 1046.4 \quad 1068.3 \quad 1073.2 \quad 1096.11122 .11137 .7$ $1160.51192 .0 \quad 1198.2 \quad 1233.2 \quad 1249.2 \quad 1263.3 \quad 1266.2 \quad 1280.4 \quad 1282.7 \quad 1291.91299 .3 \quad 1301.7$

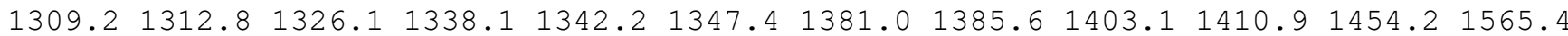
$1844.02642 .7 \quad 2652.2 \quad 2661.8 \quad 2662.4 \quad 2667.6 \quad 2669.5 \quad 2674.02675 .2 \quad 2679.62688 .42708 .9$ $2736.22759 .62769 .72777 .6 \quad 2782.2$ 
name $=\operatorname{MIN} 188$

energy (in $\mathrm{kcal} / \mathrm{mol})=-25.549102$

$\mathrm{ZPE}($ in $\mathrm{kcal} / \mathrm{mol})=143.828$

$\mathrm{G}($ in $\mathrm{kcal} / \mathrm{mol})=117.386$

Geometry

C $-2.11855201-1.33232602-0.62074755$

C $-0.64406714-1.34923972-0.69116530$

$\begin{array}{llll}\text { C }-0.34345200 & 2.24264153 & -0.65736536\end{array}$

C $0.52616696 \quad 2.32529220 \quad 0.57224643$

C $1.02903773 \quad 0.96363967 \quad 1.02965274$

C $1.41035782-0.01689945-0.10624100$

C $0.31681972-1.026461250 .36600386$

C $-0.00577044 \quad-0.08833457 \quad 1.58689062$

C $-1.42303932 \quad 0.39535064 \quad 1.80498681$

C $0.50262068-0.71855853 \quad 2.88475921$

$\mathrm{H}-2.47981859-1.732048520 .34112502$

$\mathrm{H}-2.59222675-1.93388280-1.41490302$

$\mathrm{H}-2.52018063-0.31017432-0.72366467$

$\mathrm{H}-1.375301131 .89289992-0.52658908$

$\mathrm{H}-0.01978657 \quad 2.82768497 \quad 1.39429172$

$\mathrm{H} 1.405041532 .973687090 .35060650$

$\mathrm{H} \quad 1.85846543 \quad 1.12205313 \quad 1.74688629$

H 2.42855101 $-0.41594448-0.05008662$

$\mathrm{H} \quad 1.29009953 \quad 0.35989805-1.12884481$

$\mathrm{H} \quad 0.77524106-1.98825577 \quad 0.70608297$

$\mathrm{H}-2.07495264-0.418414472 .14715998$

$\mathrm{H}-1.875875250 .82505574 \quad 0.90730866$

$\mathrm{H}-1.451420581 .171987332 .58230702$

$\mathrm{H}-0.10357463-1.585248023 .17136565$

$\mathrm{H} \quad 0.46722500-0.00366527 \quad 3.71484467$

H $1.54060143-1.06244406 \quad 2.79786881$

O $1.01738318-1.90017772-2.03244879$

$00.06071886 \quad 2.55416148-1.74891943$

o - $0.22479055-1.78125356-1.85460353$

Vibrational frequencies (in cm-1)

$\begin{array}{lllllllllllllll}26.0 & 38.7 & 61.3 & 91.6 & 157.7 & 168.6 & 188.8 & 209.6 & 229.5 & 241.8 & 242.8 & 257.6 & 288.6 & 308.1\end{array}$

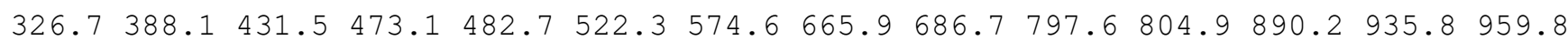
$980.1986 .01005 .1 \quad 1013.5 \quad 1018.2 \quad 1025.2 \quad 1048.2 \quad 1074.6 \quad 1079.91097 .3 \quad 1117.3 \quad 1141.6$

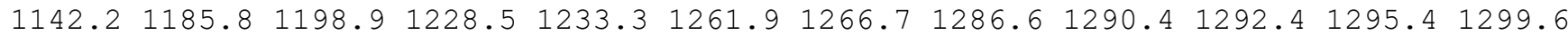
$1301.71314 .7 \quad 1321.4 \quad 1327.1 \quad 1338.8 \quad 1346.2 \quad 1380.3 \quad 1381.81403 .4 \quad 1408.81449 .51562 .6$ $1843.52632 .3 \quad 2653.92660 .2 \quad 2662.5 \quad 2667.92670 .8 \quad 2672.62674 .92679 .02689 .92715 .3$ $2740.52760 .5 \quad 2769.8 \quad 2777.7 \quad 2781.8$

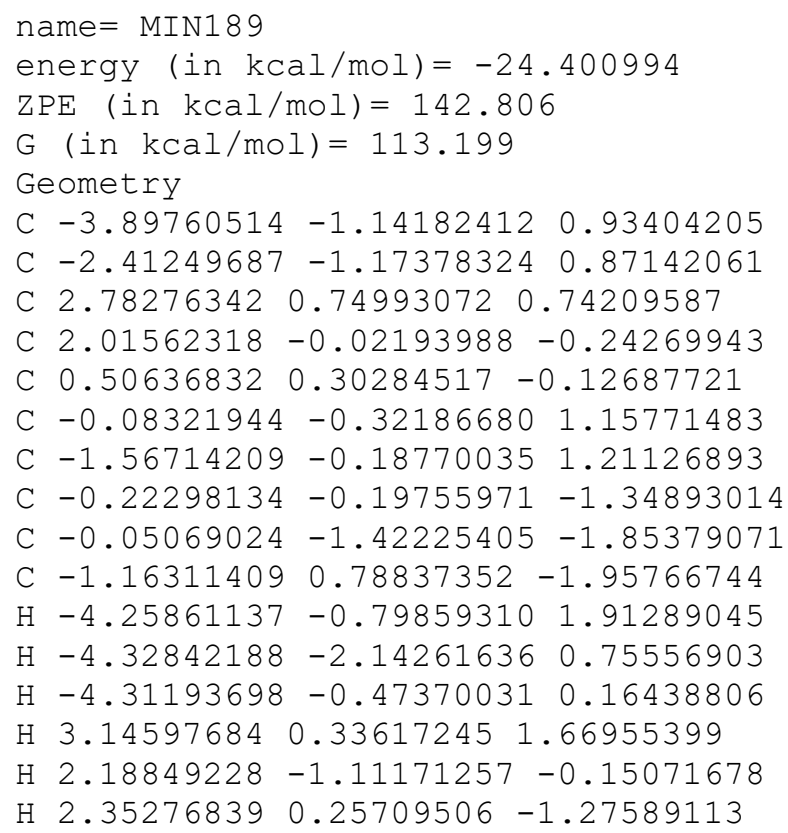


$\mathrm{H} \quad 0.39690347 \quad 1.41809589-0.06261756$

$\mathrm{H} \quad 0.36493779 \quad 0.17775424 \quad 2.04275432$

H $0.22920893-1.38208013 \quad 1.24226355$

$\mathrm{H}-1.93944824 \quad 0.77073931 \quad 1.55313120$

$\mathrm{H} \quad 0.63003817-2.15361165-1.45690740$

$\mathrm{H}-0.57712802-1.78281831-2.72273842$

$\mathrm{H}-1.06239806-2.396510690 .13441473$

$\mathrm{H}-1.74520905 \quad 0.36736719 \quad-2.78726304$

$\mathrm{H}-0.61788321 \quad 1.66109855 \quad-2.35082517$

$\mathrm{H}-1.88143646 \quad 1.16303185-1.21212252$

O $2.717506492 .58744895-0.45773463$

○ $3.093978091 .99921476 \quad 0.58650656$

o $-2.01351834-2.39208396 \quad 0.41685088$

Vibrational frequencies (in cm-1)

$\begin{array}{llllllllllllllll}15.3 & 18.9 & 30.3 & 42.5 & 56.5 & 87.4 & 108.8 & 121.6 & 145.4 & 187.6 & 227.8 & 271.9 & 285.7 & 318.1\end{array}$

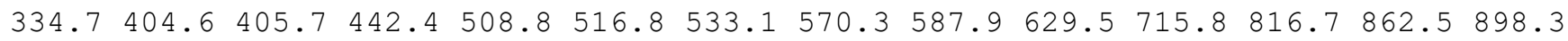
$933.1972 .0 \quad 989.3 \quad 1013.6 \quad 1035.1 \quad 1043.4 \quad 1053.1 \quad 1063.1 \quad 1067.1 \quad 1074.5 \quad 1091.7 \quad 1102.01162 .5$

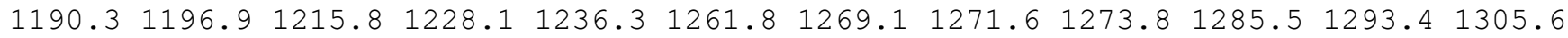

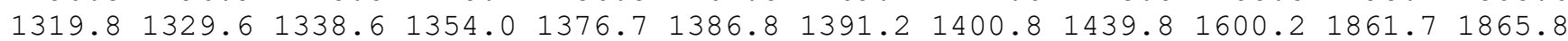
$2641.12653 .92664 .5 \quad 2665.8 \quad 2673.3 \quad 2673.92687 .5 \quad 2724.02725 .3 \quad 2731.72735 .92754 .4$ $2773.6 \quad 2776.5 \quad 2779.9 \quad 2794.5$

\author{
name $=$ MIN190 \\ energy (in $\mathrm{kcal} / \mathrm{mol})=-24.171315$ \\ $\mathrm{ZPE}($ in $\mathrm{kcal} / \mathrm{mol})=142.946$ \\ $\mathrm{G}($ in $\mathrm{kcal} / \mathrm{mol})=114.962$ \\ Geometry \\ C $-4.10667423-1.02925962-0.68563179$ \\ C $-2.69737940-0.55760247-0.72301366$ \\ C $2.17107533 \quad 0.95930304-0.50957739$ \\ C $1.99133456-0.341569350 .14054009$ \\ C $0.54420882-0.84163372 \quad 0.28364177$ \\ C $-0.22797384-0.72555472-1.05041770$ \\ C $-1.60158917-1.29756224-0.95590981$ \\ C $-0.19458414-0.14097931 \quad 1.39734855$ \\ C $-0.20650187 \quad 1.182102621 .57804056$ \\ C $-0.94405969-1.05638776 \quad 2.31041164$ \\ H $-4.30766210-1.78997765-1.45172402$ \\ $\mathrm{H}-4.81099344-0.19531497-0.85279100$ \\ $\mathrm{H}-4.35402915-1.46007466 \quad 0.29625845$ \\ H $1.369315621 .55580101-0.91518779$ \\ H $2.48254392-0.31270664 \quad 1.14954582$ \\ $\mathrm{H} \quad 2.60562494-1.10356366-0.40586173$ \\ $\mathrm{H} 0.60474486-1.936881310 .53176791$ \\ $\mathrm{H} \quad 0.34189032-1.26140940-1.84119687$ \\ $\mathrm{H}-0.26123216 \quad 0.32824362-1.39015303$ \\ $\mathrm{H}-1.67609202-2.36772628-1.10920543$ \\ $\mathrm{H}-0.74676462 \quad 1.661919912 .37935450$ \\ $\mathrm{H}-1.76250683 \quad 1.11652334 \quad-0.28725977$ \\ H $0.32795298 \quad 1.89079156 \quad 0.97351475$ \\ $\mathrm{H}-1.56753823-1.764670991 .74277026$ \\ $\mathrm{H}-1.61452038-0.51921030 \quad 2.99427301$ \\ $\mathrm{H}-0.25567751-1.648169312 .93102616$ \\ $04.340866860 .87846463-0.21958391$ \\ $03.339218921 .50664003-0.65707241$ \\ o $-2.673680140 .78256782-0.49338532$ \\ Vibrational frequencies (in $\mathrm{cm}-1$ )
}

$\begin{array}{llllllllllllllll}26.3 & 34.4 & 56.8 & 75.4 & 81.9 & 108.0 & 110.3 & 138.8 & 165.1 & 167.3 & 227.7 & 261.2 & 287.0 & 308.1\end{array}$

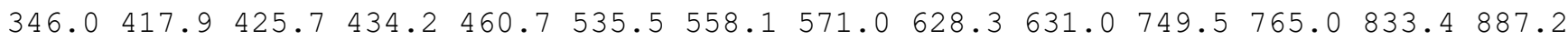
$926.9 \quad 966.4 \quad 991.1 \quad 1013.8 \quad 1027.2 \quad 1040.7 \quad 1053.0 \quad 1057.5 \quad 1064.5 \quad 1088.5 \quad 1092.91121 .5 \quad 1181.0$

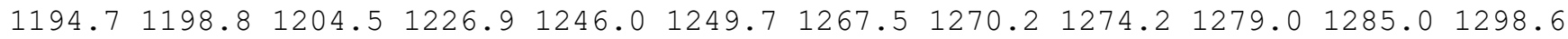

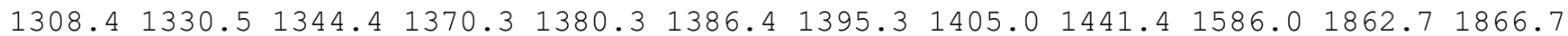
$2617.82652 .12664 .32666 .3 \quad 2672.62674 .12688 .02703 .22723 .62728 .32734 .02752 .6$ $2773.12777 .3 \quad 2779.8 \quad 2795.9$ 
name $=$ MIN191

energy (in $\mathrm{kcal} / \mathrm{mol})=-23.952016$

$\mathrm{ZPE}($ in $\mathrm{kcal} / \mathrm{mol})=143.349$

$\mathrm{G}($ in $\mathrm{kcal} / \mathrm{mol})=117.298$

Geometry

C $-2.75577992-0.90698248-0.43843742$

C $-1.29540738-0.83383544-0.56508685$

C $2.122839191 .57605105-0.56295914$

C $2.18454505 \quad 1.06467958 \quad 0.67446535$

C $1.47280209-0.12331200 \quad 1.19594965$

C $1.12789452-1.27495873 \quad 0.20846586$

C $-0.38214943-1.15529494 \quad 0.54602605$

C $-0.064188450 .05118968 \quad 1.51852118$

C -0.671244851 .377237591 .11008092$

C $-0.40174572-0.270096312 .96619916$

$\mathrm{H}-3.14713816-1.82636323-0.91224410$

$\mathrm{H}-3.23573876-0.07472502-0.99658469$

$\mathrm{H}-3.11043343-0.87828943 \quad 0.59861966$

$\mathrm{H} 2.659340742 .44914686-0.91427088$

$\mathrm{H} 2.81814479 \quad 1.56081084 \quad 1.41242024$

H $1.28974927 \quad 1.47046035 \quad-2.33953139$

H $2.01689490-0.51681600 \quad 2.08044263$

H $1.56795773-2.23838438 \quad 0.48140254$

H $1.40210971-1.08422633-0.83326326$

$\mathrm{H}-0.75859872-2.04153499 \quad 1.09641275$

$\mathrm{H}-0.26417718 \quad 2.20055310 \quad 1.70925996$

$\mathrm{H}-1.76039672 \quad 1.37929456 \quad 1.23424182$

$\mathrm{H}-0.462330551 .615731840 .05677119$

$\mathrm{H}-1.48612608-0.32707940 \quad 3.11934491$

$\mathrm{H}-0.017140890 .50519560 \quad 3.64048048$

$\mathrm{H} \quad 0.02637077-1.22505524 \quad 3.29107618$

o $-1.46448646-0.17943393-2.65312962$

$01.33316900 \quad 0.96856798-1.49258981$

o- $-0.71233138-0.46860670-1.67199246$

Vibrational frequencies (in $\mathrm{cm}-1$ )

$\begin{array}{lllllllllllllll}35.8 & 61.0 & 85.6 & 97.7 & 122.9 & 155.6 & 185.3 & 187.0 & 212.4 & 240.1 & 244.6 & 270.9 & 286.5 & 301.0\end{array}$

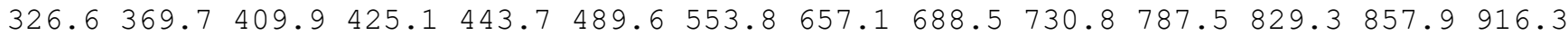
$936.9965 .3998 .6 \quad 1005.5 \quad 1017.3 \quad 1026.3 \quad 1043.9 \quad 1047.7 \quad 1066.3 \quad 1098.3 \quad 1123.6 \quad 1124.91137 .3$

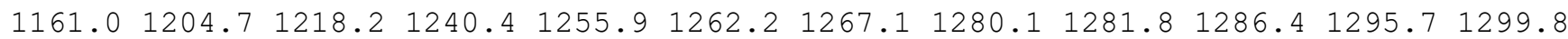
$1302.51306 .51330 .4 \quad 1363.7 \quad 1372.4 \quad 1386.0 \quad 1402.8 \quad 1403.3 \quad 1405.6 \quad 1441.3 \quad 1604.81853 .1$ $2638.62667 .22674 .3 \quad 2676.5 \quad 2676.8 \quad 2677.7 \quad 2681.82712 .92718 .72725 .12753 .52761 .6$ $2769.42780 .4 \quad 2782.6 \quad 2787.3$

name $=$ MIN192

energy (in $\mathrm{kcal} / \mathrm{mol})=-23.324971$

$\mathrm{ZPE}(\mathrm{in} \mathrm{kcal} / \mathrm{mol})=143.274$

G $($ in $\mathrm{kcal} / \mathrm{mol})=115.376$

Geometry

C $-3.98649844-1.13980468-0.24836305$

C $-2.50591865-1.03057122-0.33618763$

C $2.51907636-0.32335620-0.17028268$

C $1.96353668-0.14629224 \quad 1.18828449$

C $0.41574437-0.19228687 \quad 1.21061172$

C $-0.13652493-1.374793760 .38481760$

C $-1.61272518-1.49416888 \quad 0.55282507$

C $-0.17790481 \quad 1.11401407 \quad 0.74398103$

C $0.027382521 .63669635-0.46712835$

C $-1.05627474 \quad 1.78607906 \quad 1.74800240$

$\mathrm{H}-4.30646967-2.15181047 \quad 0.03424525$

$\mathrm{H}-4.46444547-0.89953894-1.21453644$

$\mathrm{H}-4.39220431-0.43483767 \quad 0.49222987$

$\mathrm{H} 2.55122264-1.28084455-0.66321458$ 
H $2.29715726 \quad 0.80999897 \quad 1.64922930$

H 2.35961979 $-0.94471858 \quad 1.85596175$

$\mathrm{H} \quad 0.11524696-0.36870373 \quad 2.27806356$

$\mathrm{H} \quad 0.35375921-2.31520476 \quad 0.71593517$

$\mathrm{H} \quad 0.14332502-1.26691601-0.68271492$

$\mathrm{H}-1.94083953-1.99409897 \quad 1.45622801$

$\mathrm{H}-0.409078332 .56982691-0.79001039$

$\mathrm{H}-1.21758968-0.13910141-1.52764038$

$\mathrm{H} \quad 0.63752791 \quad 1.19393200-1.23599109$

$\mathrm{H}-0.50296510 \quad 2.05065670 \quad 2.65923157$

$\mathrm{H}-1.88576447 \quad 1.12553318 \quad 2.04568993$

$\mathrm{H}-1.50973645 \quad 2.71012151 \quad 1.36427478$

$\begin{array}{lllll}0 & 3.41263271 & 0.68436848 & -1.89038061\end{array}$

$\begin{array}{lllll}0 & 2.95211417 & 0.76840225 & -0.72453478\end{array}$

o $-2.17872294-0.38397583-1.48419014$

Vibrational frequencies (in $\mathrm{cm}-1$ )

$\begin{array}{llllllllllllllll}28.6 & 38.2 & 55.7 & 67.5 & 76.3 & 101.7 & 117.0 & 131.9 & 183.3 & 201.7 & 230.7 & 257.0 & 295.2 & 311.2\end{array}$ $315.7 \quad 363.3 \quad 425.6 \quad 456.6 \quad 460.9 \quad 535.3 \quad 553.5 \quad 577.8 \quad 603.1 \quad 644.8 \quad 654.7 \quad 826.5 \quad 876.5 \quad 911.9$

$961.4974 .6 \quad 987.5 \quad 1014.9 \quad 1023.6 \quad 1044.4 \quad 1049.2 \quad 1059.2 \quad 1065.8 \quad 1094.4 \quad 1106.91116 .4 \quad 1171.8$

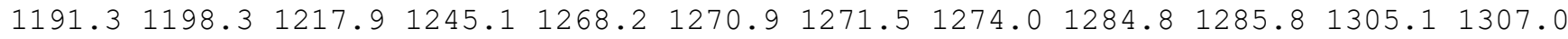

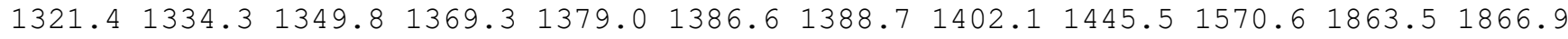
$2650.22651 .92664 .7 \quad 2668.3 \quad 2673.3 \quad 2673.9 \quad 2689.92716 .22721 .4 \quad 2733.92735 .82749 .7$ $2774.0 \quad 2777.92780 .3 \quad 2790.0$

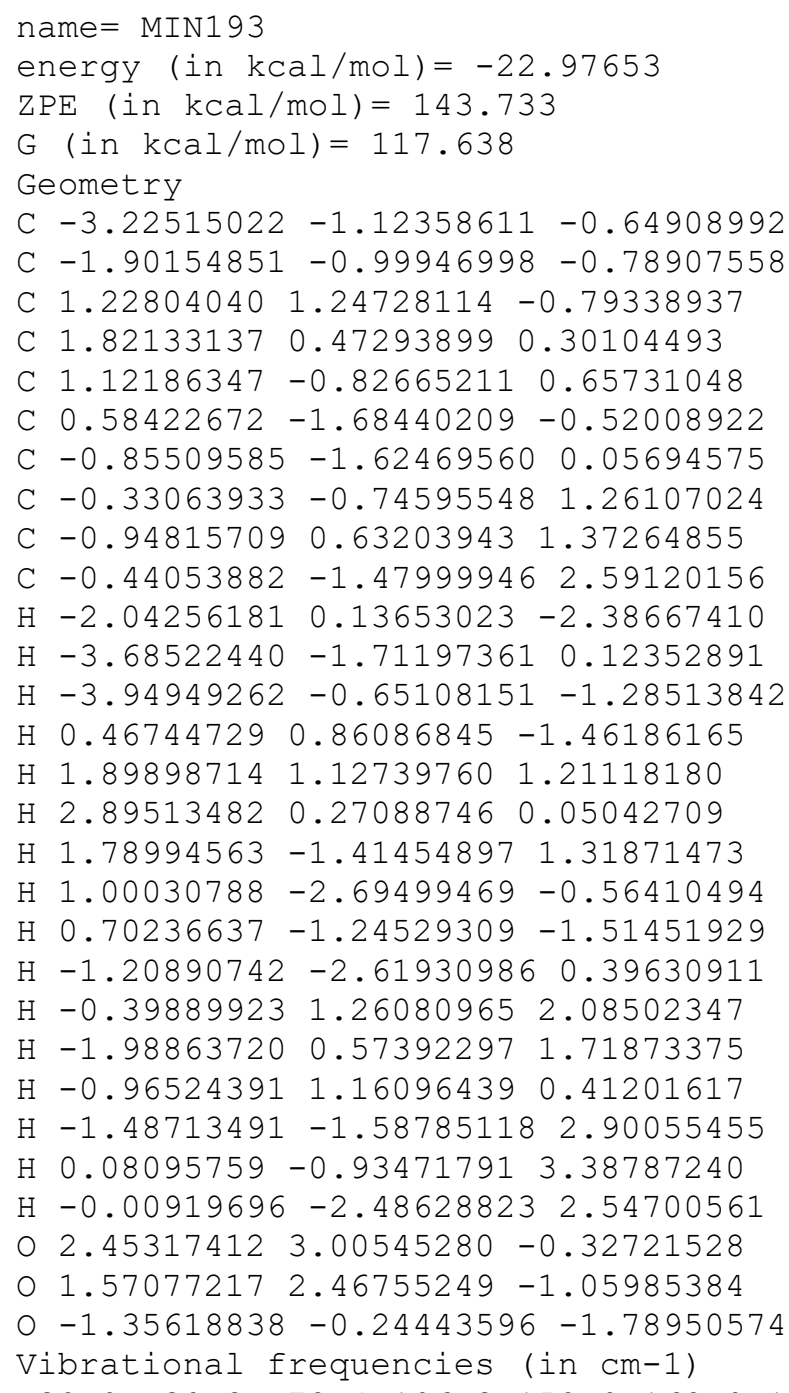


$2620.12671 .6 \quad 2674.1 \quad 2677.4 \quad 2678.4 \quad 2681.2 \quad 2691.0 \quad 2712.0 \quad 2717.8 \quad 2722.0 \quad 2733.3 \quad 2763.8$ $2780.32782 .3 \quad 2789.0 \quad 2808.8$

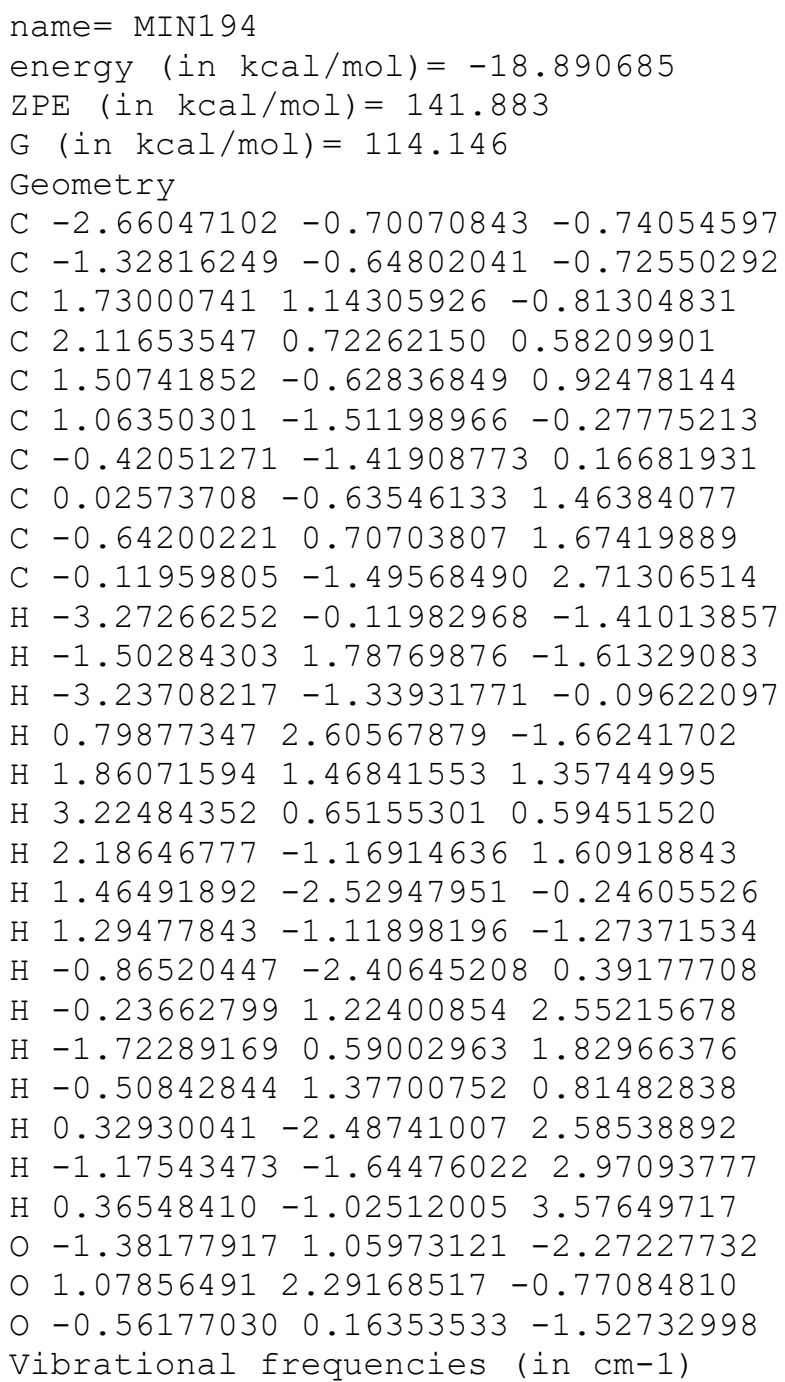

$\begin{array}{llllllllllllllll}10.1 & 31.3 & 51.7 & 65.3 & 94.6 & 153.2 & 170.0 & 181.3 & 214.9 & 217.9 & 234.5 & 276.2 & 303.2 & 333.5\end{array}$ $337.0 \quad 386.3 \quad 416.6 \quad 423.6 \quad 441.2 \quad 527.3 \quad 579.2 \quad 611.7 \quad 616.1 \quad 626.6 \quad 694.7 \quad 787.4 \quad 848.8 \quad 907.4$ $920.7927 .0 \quad 968.1 \quad 995.2 \quad 1005.0 \quad 1009.7 \quad 1013.1 \quad 1017.0 \quad 1042.2 \quad 1047.8 \quad 1080.6 \quad 1105.7 \quad 1117.2$ $1120.21171 .8 \quad 1197.9 \quad 1217.1 \quad 1227.2 \quad 1246.3 \quad 1272.51284 .91287 .0 \quad 1297.6 \quad 1299.5 \quad 1302.5$

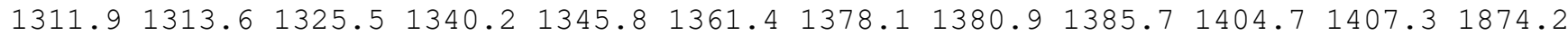
$2665.22670 .62671 .42675 .2 \quad 2677.3 \quad 2679.4 \quad 2718.12722 .32729 .72742 .02758 .02771 .7$ 2780.22782 .02798 .12862 .1

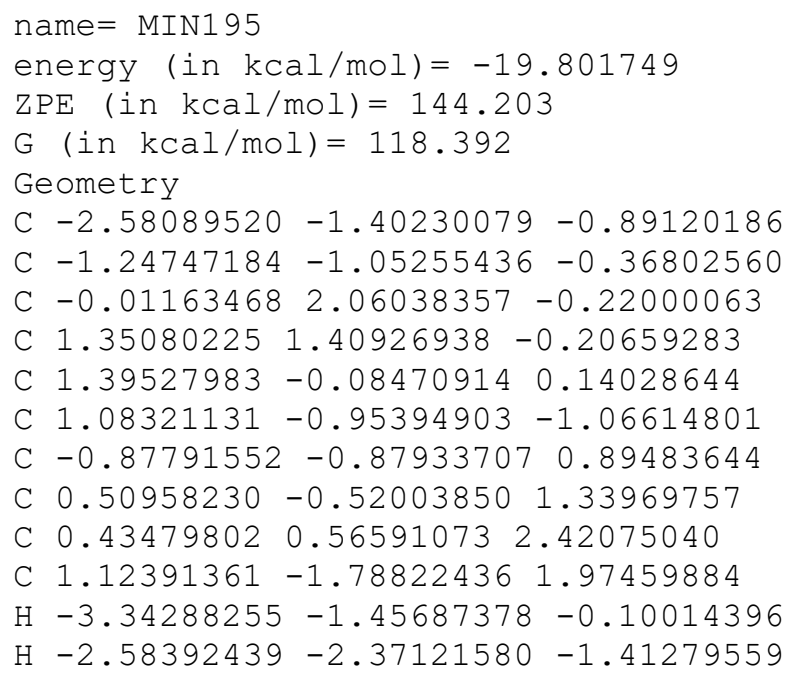


$\mathrm{H}-2.92718599-0.64337519-1.62177279$

$\mathrm{H}-0.87680537 \quad 1.48562646-0.56871073$

H $2.01097324 \quad 1.970921610 .48987536$

H $1.79551461 \quad 1.57353635-1.21555246$

H $2.46354578 \quad-0.29676788 \quad 0.42980905$

$\mathrm{H} \quad 1.25617365-2.02253717-0.87827231$

$\mathrm{H} \quad 1.65364797-0.63927240-1.95651796$

$\mathrm{H}-1.59031977-1.02548170 \quad 1.71040997$

$\mathrm{H} \quad 1.42860715 \quad 0.95610269 \quad 2.66750911$

$\mathrm{H}-0.004209920 .180345293 .34826692$

$\mathrm{H}-0.18275957 \quad 1.42005027 \quad 2.11344306$

H $1.22635269-2.601751821 .25088936$

$\mathrm{H} 0.50196113-2.16247332 \quad 2.79584192$

H $2.11585803-1.580951192 .39113548$

$0-0.59455806 \quad 0.14202995-2.18881929$

$0-0.12933835 \quad 3.20910692 \quad 0.13511195$

o $-0.34358091-0.93461170-1.53666318$

Vibrational frequencies (in $\mathrm{cm}-1$ )

$\begin{array}{lllllllllllllll}33.4 & 66.6 & 78.7 & 87.5 & 113.6 & 172.9 & 173.7 & 200.1 & 237.3 & 260.7 & 272.8 & 287.4 & 312.0 & 361.1\end{array}$ $376.2412 .4 \quad 430.9 \quad 470.2 \quad 490.3 \quad 548.0 \quad 590.8 \quad 644.3 \quad 707.5 \quad 812.1837 .1 \quad 863.9916 .4 \quad 931.3$ $956.2978 .2 \quad 980.3 \quad 986.8 \quad 1002.7 \quad 1011.0 \quad 1031.3 \quad 1062.8 \quad 1066.91076 .0 \quad 1118.4 \quad 1145.4 \quad 1173.1$ $1187.0 \quad 1202.5 \quad 1236.0 \quad 1251.2 \quad 1260.8 \quad 1265.1 \quad 1270.7 \quad 1277.5 \quad 1277.7 \quad 1291.8 \quad 1298.3 \quad 1299.7$ $1304.21306 .0 \quad 1312.1 \quad 1336.91348 .3 \quad 1365.0 \quad 1382.8 \quad 1388.1 \quad 1404.4 \quad 1415.6 \quad 1821.91913 .1$ $2642.12650 .4 \quad 2652.1 \quad 2657.7 \quad 2665.92669 .9 \quad 2672.92675 .52677 .92679 .6 \quad 2721.5 \quad 2733.4$ $2743.12772 .5 \quad 2779.92781 .9$

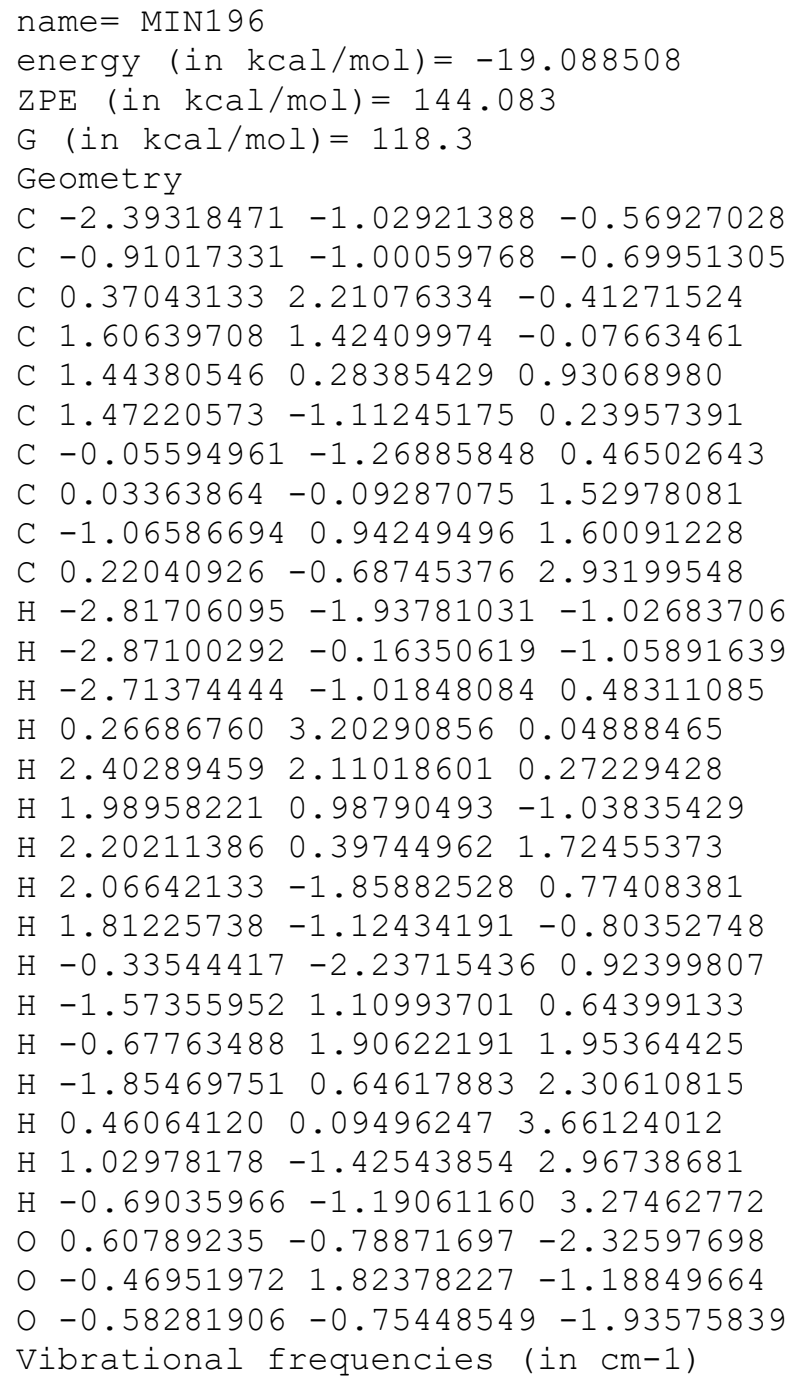




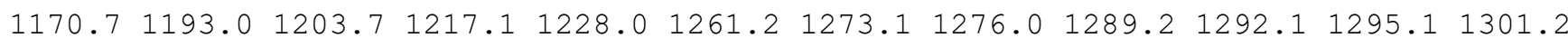
$1303.41304 .91315 .91339 .2 \quad 1345.6 \quad 1356.0 \quad 1368.4 \quad 1384.81397 .7 \quad 1410.2 \quad 1413.01616 .0$ $1837.52626 .72651 .92655 .6 \quad 2656.42661 .82665 .12673 .92675 .2 \quad 2678.72714 .72718 .2$ $2727.92752 .8 \quad 2771.4 \quad 2779.8 \quad 2781.8$

\section{name $=$ MIN197}

energy (in $\mathrm{kcal} / \mathrm{mol})=-14.686523$

$\mathrm{ZPE}($ in $\mathrm{kcal} / \mathrm{mol})=146.179$

$\mathrm{G}($ in $\mathrm{kcal} / \mathrm{mol})=121.566$

Geometry

C $-2.02913320-0.16359680-0.50932002$

C $-0.50671647-0.09383135-0.51495931$

C $-0.01275773 \quad 1.38193214-0.54118089$

C $1.44342613 \quad 1.60904524 \quad-0.12273301$

C $1.89102103 \quad 0.69341912 \quad 1.01377947$

C $1.64742901-0.76898300 \quad 0.63677087$

C $0.14235337-1.06062208 \quad 0.48553338$

C $-0.49736667-1.06824793 \quad 1.85022703$

C $-0.96030314 \quad 0.01687092 \quad 2.47495341$

C $-0.55702801-2.41716468 \quad 2.49354173$

$\mathrm{H}-2.46202783 \quad 0.20736314 \quad 0.42611907$

$\mathrm{H}-2.37650769-1.19534094-0.64550205$

$\mathrm{H}-2.45422375 \quad 0.42923049-1.33228834$

$\mathrm{H}-0.711490552 .06473223-0.01958192$

$\begin{array}{lllll}\mathrm{H} & 1.58896071 & 2.66941380 & 0.15656410\end{array}$

H $2.094562901 .44625596-1.01023069$

H $2.96423518 \quad 0.85659402 \quad 1.22948728$

$\mathrm{H} 2.08618587-1.440731121 .39758904$

$\mathrm{H} 2.16288886-1.00326502-0.31653033$

$\mathrm{H} \quad 0.03571602 \quad-2.08790728 \quad 0.03525061$

$\mathrm{H}-1.39315714-0.00538430 \quad 3.46123214$

$\mathrm{H}-0.94250083 \quad 1.00972220 \quad 2.05896041$

H $1.35844592 \quad 0.94325294 \quad 1.95199366$

$\mathrm{H}-0.63653411-2.35431683 \quad 3.58739478$

$\mathrm{H} \quad 0.33188126 \quad-3.02383934 \quad 2.27405544$

$\mathrm{H}-1.43362672-2.97936431 \quad 2.13880025$

$\begin{array}{lllll}0 & -0.34515784 & 0.52735382 & -2.66159350\end{array}$

$\begin{array}{llll}0 & -0.07988367 & 1.74132950 & -1.92226867\end{array}$

o $-0.03386753-0.53808169-1.80365102$

Vibrational frequencies (in $\mathrm{cm}-1$ )

$\begin{array}{lllllllllllllllll}58.9 & 68.5 & 98.0 & 109.4 & 148.8 & 158.9 & 216.6 & 238.2 & 269.4 & 302.1 & 313.1 & 331.0 & 344.6 & 427.2\end{array}$ $433.0 \quad 485.4 \quad 498.9 \quad 572.3 \quad 618.9 \quad 649.3 \quad 706.4 \quad 726.0 \quad 807.0 \quad 893.9 \quad 896.9 \quad 919.5 \quad 945.4 \quad 960.9$ $981.3 \quad 989.0 \quad 999.2 \quad 1019.8 \quad 1040.9 \quad 1055.8 \quad 1057.4 \quad 1084.7 \quad 1100.6 \quad 1113.6 \quad 1130.7 \quad 1156.0 \quad 1165.9$

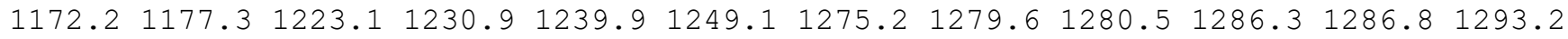

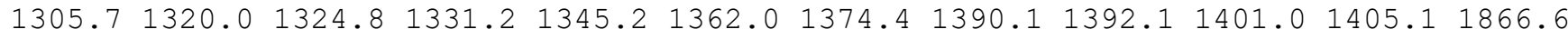
$2651.82661 .5 \quad 2668.8 \quad 2670.2 \quad 2671.8 \quad 2673.0 \quad 2674.02681 .5 \quad 2691.2 \quad 2723.5 \quad 2740.52741 .2$ $2746.0 \quad 2778.92780 .4 \quad 2793.5$

name $=$ MIN198

energy (in $\mathrm{kcal} / \mathrm{mol})=-13.620476$

$\operatorname{ZPE}($ in $\mathrm{kcal} / \mathrm{mol})=146.303$

$\mathrm{G}(\mathrm{in} \mathrm{kcal} / \mathrm{mol})=120.347$

Geometry

C $-2.34852306-0.37632074-0.98332606$

C $-0.82402221-0.38428693-0.99015333$

C $-0.24405213 \quad 1.05029258-0.78587558$

C $0.55274326 \quad 1.37915462 \quad 0.46994578$

C $1.155304590 .18729341 \quad 1.23608247$

C $1.14642101-1.104179220 .40296390$

C $-0.24899330-1.47658324-0.08919547$

C $0.49675646-0.02188563 \quad 2.57895781$

C $-0.99408246-0.00862718 \quad 2.67674451$

C $1.24113893-0.219081293 .67205273$ 
$\mathrm{H}-2.75158376-0.02359008-0.02859339$

$\mathrm{H}-2.74992233-1.38203759-1.16397965$

$\mathrm{H}-2.74798785 \quad 0.26597954-1.77930651$

$\mathrm{H}-1.04528198 \quad 1.80664291-0.94481619$

$\mathrm{H}-0.07489038 \quad 1.98529399 \quad 1.15437825$

H $1.38135824 \quad 2.06344404 \quad 0.17346555$

H 2.23743124 $0.43682859 \quad 1.42332521$

$\mathrm{H} \quad 1.57364098-1.93437537 \quad 0.99698185$

$\mathrm{H} 1.81820233-0.97185629-0.47304823$

$\mathrm{H}-0.20100786-2.42900161-0.66113696$

$\mathrm{H}-1.33860330 \quad 0.65506482 \quad 3.48234113$

$\mathrm{H}-1.37856298-1.013848592 .90791332$

$\mathrm{H}-1.48544080 \quad 0.32262995 \quad 1.75346811$

$\mathrm{H} \quad 0.82604459-0.37711134 \quad 4.65307804$

H $2.31717121-0.23893808 \quad 3.67494276$

$\mathrm{H}-0.92195925-1.68291505 \quad 0.76213917$

$0 \quad 0.05671533 \quad 0.46136883-2.91836882$

$0 \quad 0.686732391 .17299989-1.87403111$

o $-0.37749588-0.74884734-2.30930984$

Vibrational frequencies (in cm-1)

$\begin{array}{lllllllllllllllll}-6.1 & 56.2 & 64.6 & 143.0 & 163.5 & 188.0 & 203.4 & 257.9 & 275.5 & 293.4 & 314.9 & 341.7 & 404.8 & 418.9\end{array}$

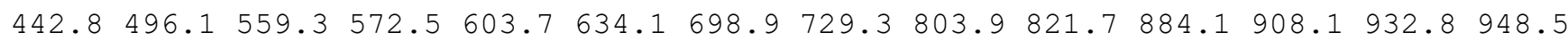
$974.6 \quad 980.7 \quad 992.6 \quad 1014.3 \quad 1039.6 \quad 1043.7 \quad 1062.3 \quad 1069.5 \quad 1092.6 \quad 1125.0 \quad 1138.01159 .6 \quad 1170.8$ $1190.91204 .0 \quad 1231.0 \quad 1240.3 \quad 1257.8 \quad 1276.8 \quad 1281.1 \quad 1283.5 \quad 1290.6 \quad 1291.6 \quad 1296.2 \quad 1315.0$

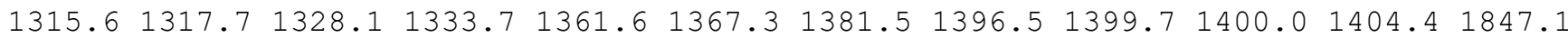
$2644.02654 .6 \quad 2667.1 \quad 2667.7 \quad 2670.4 \quad 2671.7 \quad 2673.92678 .12683 .4 \quad 2726.5 \quad 2730.3 \quad 2738.4$ $2743.3 \quad 2777.12781 .2 \quad 2795.5$

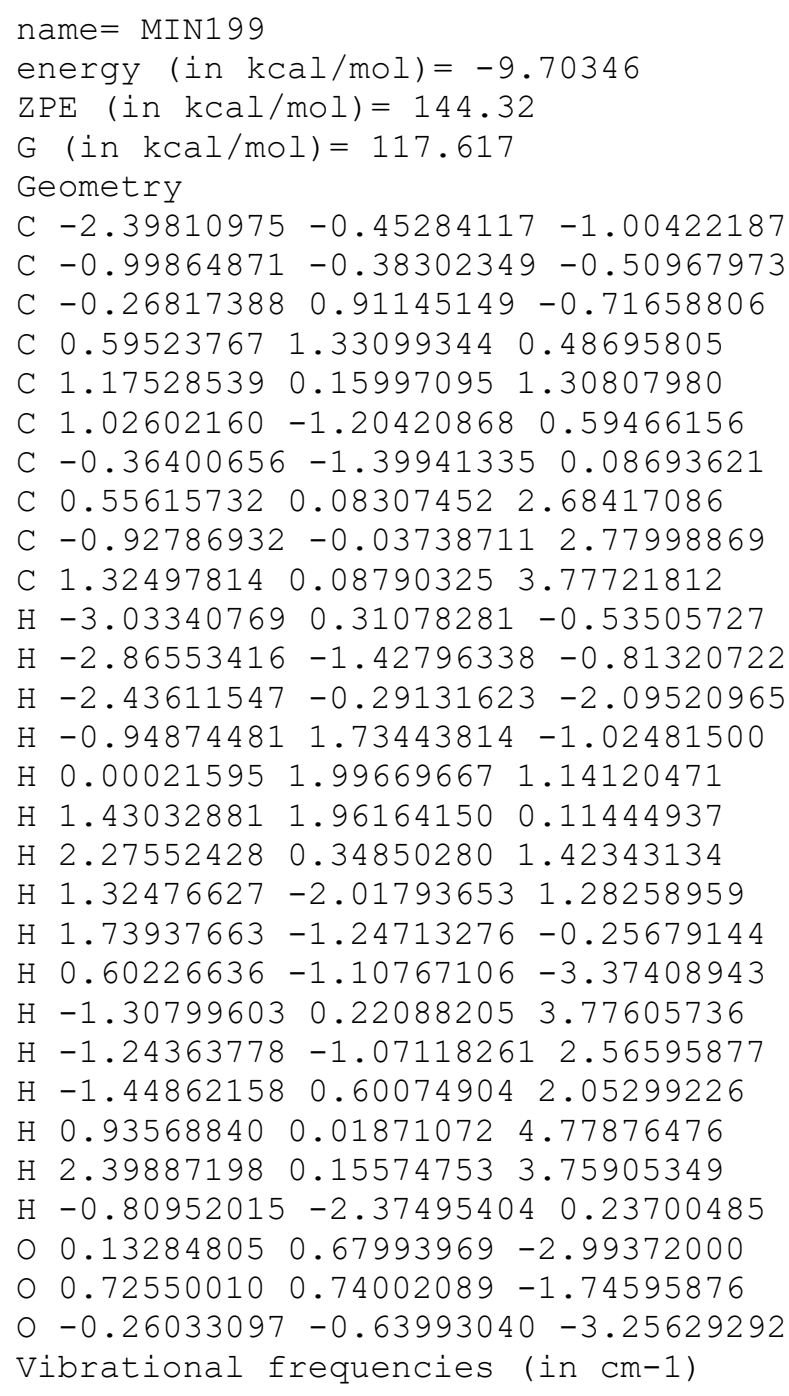


$\begin{array}{llllllllllllllll}32.1 & 39.3 & 66.6 & 76.2 & 108.4 & 121.0 & 134.5 & 175.1 & 216.1 & 239.1 & 283.2 & 292.9 & 315.1 & 320.0\end{array}$

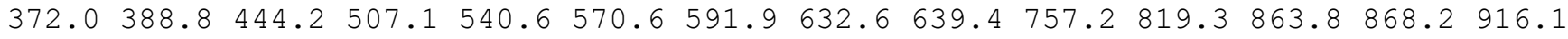
$939.5968 .2990 .7 \quad 992.7 \quad 1020.1 \quad 1039.0 \quad 1044.4 \quad 1058.4 \quad 1063.5 \quad 1073.3 \quad 1093.1 \quad 1117.2 \quad 1152.3$

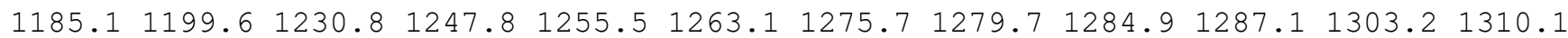

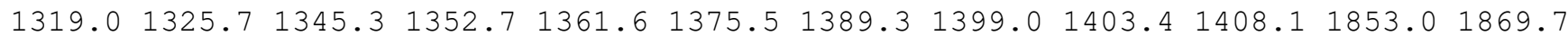
$2651.4 \quad 2663.2 \quad 2664.3 \quad 2664.7 \quad 2676.4 \quad 2677.3 \quad 2687.3 \quad 2690.62726 .72733 .82741 .62766 .5$ $2778.5 \quad 2778.8 \quad 2795.5 \quad 2848.5$

name $=$ MIN200

energy (in $\mathrm{kcal} / \mathrm{mol})=-6.804731$

$\operatorname{ZPE}($ in $\mathrm{kcal} / \mathrm{mol})=141.891$

$\mathrm{G}($ in $\mathrm{kcal} / \mathrm{mol})=113.787$

Geometry

C $-2.52159303 \quad 0.25504009 \quad 0.45694457$

C $-1.02624665 \quad 0.478796350 .44609291$

C $-0.730699651 .98192557 \quad 0.40021148$

C $1.21624113-1.31915716-2.64249432$

C $0.52206253-1.96284161-1.70364446$

C $-0.75316128-1.43984008-1.15187809$

C $-0.28900183-0.249615251 .49589995$

C $1.04483042-0.36267388 \quad 1.61604606$

C 2.060005630 .231637590 .70670563

C $1.63035882-1.13406106 \quad 2.75681781$

$\mathrm{H}-2.79330099-0.79413230 \quad 0.62397837$

$\mathrm{H}-2.96311937 \quad 0.55919038-0.51027404$

$\mathrm{H}-3.02045530 \quad 0.83677663 \quad 1.24336347$

$\mathrm{H}-1.57069423 \quad 2.637335850 .14475517$

H $2.14611760-1.68272261-3.05229875$

$\mathrm{H} \quad 0.90054746-0.37701264-3.07987484$

$\mathrm{H} \quad 0.85078022-2.91285961-1.28380424$

$\mathrm{H}-1.06092804-1.92721076-0.22043315$

$\mathrm{H}-1.58442080-1.49446212-1.87481853$

$\mathrm{H}-0.93509277-0.716102312 .24332630$

$\mathrm{H} 2.93255836-0.418822750 .56442808$

H 2.42644534 $1.18843744 \quad 1.12801461$

$\mathrm{H} \quad 1.68139920 \quad 0.48906073-0.29033572$

H $2.13733689-2.042539612 .40036645$

$\mathrm{H} 0.88276352-1.44897743 \quad 3.49600546$

H $2.38030679-0.53539894 \quad 3.29636307$

$\begin{array}{lllll}0 & -0.93874977 & 0.74428039 & -1.82512549\end{array}$

$00.36662428 \quad 2.41802892 \quad 0.62353626$

o $-0.42845386 \quad 0.02264920 \quad-0.90317604$

Vibrational frequencies (in cm-1)

$\begin{array}{llllllllllllllll}11.4 & 37.8 & 77.2 & 90.5 & 100.2 & 112.9 & 118.5 & 138.6 & 165.5 & 181.1 & 206.9 & 224.1 & 262.8 & 270.2\end{array}$ $313.8 \quad 323.6 \quad 356.5 \quad 433.8 \quad 460.0 \quad 490.1 \quad 507.9 \quad 538.8 \quad 570.9609 .7 \quad 666.7 \quad 822.6 \quad 882.6 \quad 905.3$ $921.0 \quad 945.3 \quad 963.0 \quad 972.9 \quad 991.0 \quad 993.2 \quad 1018.2 \quad 1025.8 \quad 1031.8 \quad 1051.6 \quad 1102.4 \quad 1102.6 \quad 1117.0$ $1130.4 \quad 1153.8 \quad 1195.2 \quad 1207.3 \quad 1258.9 \quad 1263.5 \quad 1267.7 \quad 1277.4 \quad 1284.0 \quad 1286.01293 .1 \quad 1301.6$ $1305.91319 .2 \quad 1334.3 \quad 1369.7 \quad 1383.8 \quad 1387.6 \quad 1404.8 \quad 1405.4 \quad 1438.0 \quad 1841.0 \quad 1843.7 \quad 1853.6$ $2651.02651 .3 \quad 2653.4 \quad 2656.1 \quad 2667.92670 .5 \quad 2671.8 \quad 2675.92699 .6 \quad 2728.12747 .5 \quad 2755.4$ $2773.0 \quad 2774.5 \quad 2777.0 \quad 2780.0$

name $=$ MIN201

energy (in $\mathrm{kcal} / \mathrm{mol})=-7.533903$

$\mathrm{ZPE}(\mathrm{in} \mathrm{kcal} / \mathrm{mol})=142.746$

$\mathrm{G}($ in $\mathrm{kcal} / \mathrm{mol})=116.412$

Geometry

C $-2.24043542 \quad 0.36701165-1.10768977$

C $-0.815024220 .48066866-1.49080989$

C $0.04688108 \quad 1.55768880 \quad-0.91179606$

C $0.21401971 \quad 1.42945893 \quad 0.61815733$

C $0.84920125 \quad 0.14282881 \quad 1.11016719$

C $0.85071324-1.08004418 \quad 0.19513440$

C $0.13712707-1.85939414 \quad 1.02391093$ 
C $-0.00002211-0.79400658 \quad 2.12038867$

C $-1.42204761-0.384031252 .43504520$

C $0.76774966-1.132601263 .38467258$

$\mathrm{H}-2.36842737-0.38147531-0.29927430$

$\mathrm{H}-2.88938804 \quad 0.04339687-1.93790652$

$\mathrm{H}-2.65144588 \quad 1.31645744-0.73055313$

$\mathrm{H}-0.421715352 .55661866-1.14493539$

$\mathrm{H}-0.76096256 \quad 1.60392949 \quad 1.10779969$

H $\quad 0.85710355 \quad 2.28384164 \quad 0.93479541$

$\mathrm{H} \quad 1.85726474 \quad 0.34925169 \quad 1.51552300$

$\mathrm{H} \quad 1.33946538-1.19610057-0.74350917$

$\mathrm{H} \quad 1.50658953 \quad 0.83866501-2.04576585$

$\mathrm{H}-0.22020592-2.85330323 \quad 1.02582215$

$\mathrm{H}-2.00169319-0.16265430 \quad 1.53280530$

$\mathrm{H}-1.45077211 \quad 0.50265264 \quad 3.08022859$

$\mathrm{H}-1.95058186-1.18835670 \quad 2.96389680$

$\mathrm{H} 0.86187538-0.260456194 .04131209$

H $1.78059749-1.491622153 .16166732$

$\mathrm{H} \quad 0.26535756-1.92487590 \quad 3.95308752$

O $0.73075388-0.48045479-2.77384473$

O $1.31475047 \quad 1.65733764-1.49182933$

o - $0.48379156-0.41997612-2.36444753$

Vibrational frequencies (in $\mathrm{cm}-1$ )

$\begin{array}{lllllllllllllll}38.9 & 44.7 & 67.8 & 79.2 & 105.9 & 159.1 & 162.1 & 186.3 & 216.6 & 262.5 & 266.6 & 299.4 & 308.8 & 337.3\end{array}$ $355.7 \quad 375.0 \quad 407.2 \quad 449.4 \quad 466.7 \quad 557.9 \quad 561.1 \quad 664.9 \quad 696.7 \quad 725.8 \quad 738.5 \quad 837.2 \quad 901.9 \quad 904.0$ $945.1958 .3978 .0 \quad 993.3 \quad 1007.8 \quad 1013.8 \quad 1018.5 \quad 1036.2 \quad 1061.7 \quad 1074.3 \quad 1098.11118 .7 \quad 1160.4$ $\begin{array}{lllllllllllllll}1176.3 & 1192.5 & 1211.0 & 1229.9 & 1246.7 & 1256.7 & 1259.8 & 1269.8 & 1282.1 & 1287.9 & 1294.3 & 1298.2\end{array}$

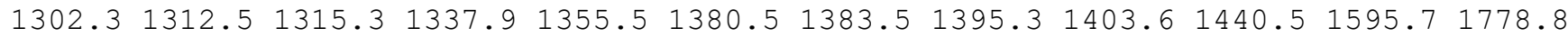
$2557.62628 .12649 .62653 .0 \quad 2660.6 \quad 2671.2 \quad 2676.02676 .42681 .42718 .62735 .62761 .5$ $2764.7 \quad 2779.6 \quad 2782.5 \quad 2808.4$

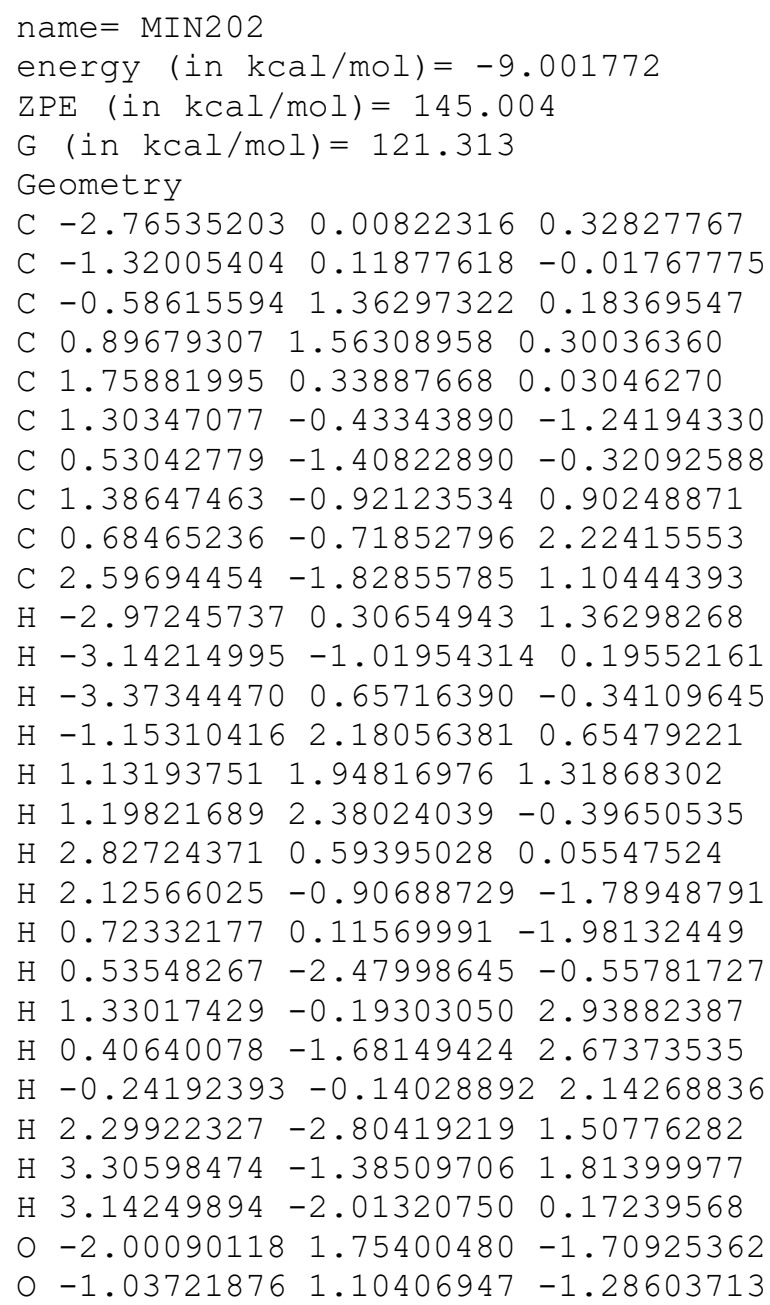


$0-0.84361161-1.13886710-0.23598086$

Vibrational frequencies (in $\mathrm{cm}-1$ )

$\begin{array}{llllllllllllllll}95.2 & 114.6 & 143.2 & 148.9 & 189.6 & 195.7 & 206.0 & 242.7 & 281.9 & 286.1 & 315.0 & 354.2 & 368.9 & 389.6\end{array}$

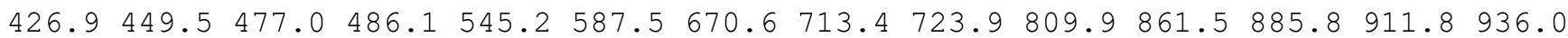
$971.7 \quad 1005.3 \quad 1008.1 \quad 1017.4 \quad 1017.8 \quad 1026.8 \quad 1053.0 \quad 1076.8 \quad 1079.3 \quad 1094.91121 .6 \quad 1147.2$ $\begin{array}{llllllllllllllll}1151.8 & 1198.0 & 1203.1 & 1212.9 & 1227.7 & 1245.1 & 1257.6 & 1265.0 & 1273.4 & 1283.4 & 1285.3 & 1288.3\end{array}$ $1291.4 \quad 1298.7 \quad 1299.2 \quad 1304.8 \quad 1322.0 \quad 1333.5 \quad 1371.7 \quad 1379.4 \quad 1391.7 \quad 1404.6 \quad 1411.91420 .1$

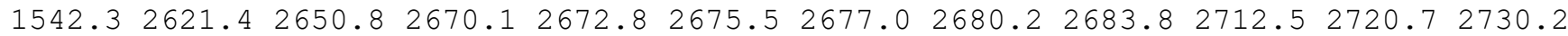
$2736.32765 .62768 .2 \quad 2781.5 \quad 2786.1$

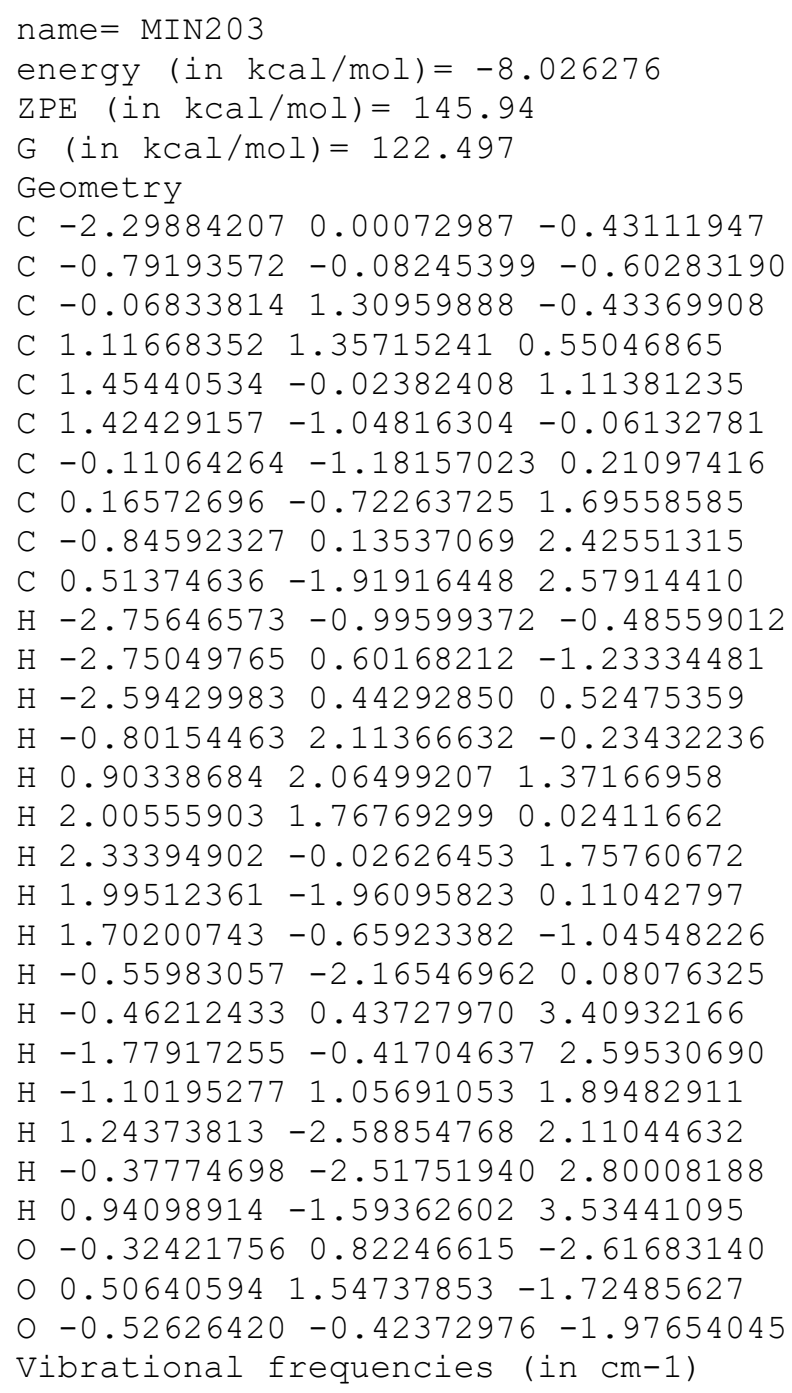


C $3.378088637-0.154390098 \quad 1.802662401$

C $2.804637074 \quad 1.2496484592 .177056833$

C $2.4233524391 .438955523 \quad 0.654476804$

C 1.1705176812 .1934415650 .260575000

C $3.5971257432 .015636405-0.135735665$

$\mathrm{H} 0.002442518 \quad 2.113309872 \quad 4.093582424$

$\mathrm{H} 0.636556267 \quad 2.964702520 \quad 2.667404022$

$\mathrm{H} \quad 1.585790823 \quad 2.886088625 \quad 4.155191922$

$\mathrm{H}-0.448470102 \quad 0.410382026 \quad 2.296213916$

$\mathrm{H} \quad 0.275391157-0.530131316 \quad 0.297430155$

H $1.112376972-1.764857135 \quad 1.241469695$

H 2.726315653 $-0.592591928-0.332903327$

H $4.433980870 \quad-0.1484913691 .526573765$

H $3.235361419-0.951285700 \quad 2.533378083$

H $3.5007373331 .975780656 \quad 2.596323767$

$\mathrm{H} \quad 0.267936498 \quad 1.852071655 \quad 0.773881794$

$\mathrm{H} \quad 0.977458054 \quad 2.082388413-0.815328482$

H $1.275792150 \quad 3.267145714 \quad 0.462737702$

$\mathrm{H} \quad 3.427784293 \quad 1.926747171-1.214904087$

$\mathrm{H} \quad 4.542618281 \quad 1.508082978 \quad 0.084774847$

H $3.740559963 \quad 3.078178669 \quad 0.092086557$

$01.649109897-0.986709818 \quad 4.116094134$

$0.417779766-0.9988813913 .412729663$

O 1.9566303350 .3874852404 .275877918

Vibrational frequencies (in $\mathrm{cm}-1$ )

$94.4 \quad 126.6 \quad 160.9 \quad 174.9 \quad 182.2 \quad 212.8 \quad 227.7 \quad 268.3 \quad 287.1 \quad 325.0 \quad 349.6 \quad 384.4 \quad 418.0 \quad 439.8$

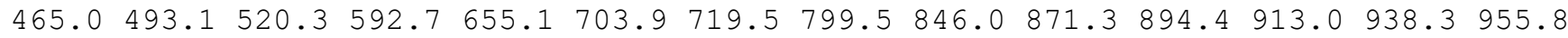
$973.3997 .31001 .8 \quad 1005.6 \quad 1014.91017 .8 \quad 1044.91058 .0 \quad 1087.11089 .01100 .2 \quad 1140.6$

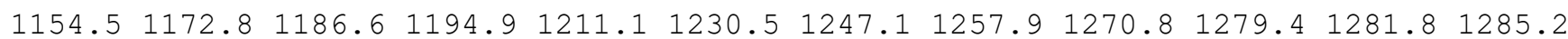

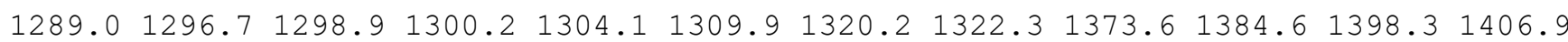
$1417.22659 .02669 .8 \quad 2672.92674 .5 \quad 2676.5 \quad 2678.12680 .32686 .0 \quad 2689.3 \quad 2740.02748 .3$ $2751.8 \quad 2768.12780 .5 \quad 2782.2 \quad 2784.0$

name $=$ MIN205

energy (in $\mathrm{kcal} / \mathrm{mol})=-6.259221$

$\mathrm{ZPE}($ in $\mathrm{kcal} / \mathrm{mol})=145.134$

$\mathrm{G}($ in $\mathrm{kcal} / \mathrm{mol})=120.046$

Geometry

C $-2.51446001-0.32594451-0.46850474$

C $-1.01718035-0.34189579-0.68442860$

C $-0.38925607 \quad 1.07586563-0.95453978$

C $0.85163085 \quad 1.23204841-0.06220587$

C $0.88410014 \quad 0.01729329 \quad 0.86227286$

C $1.22200410-1.291135360 .17668467$

C $-0.22780211-0.95307232 \quad 0.45027082$

C $1.26312953 \quad 0.29502288 \quad 2.30065303$

C $0.28320077 \quad 1.29478216 \quad 2.93463608$

C $1.29894502-0.975501703 .16044638$

$\mathrm{H}-2.79008038 \quad 0.21469185 \quad 0.44372726$

$\mathrm{H}-2.90914807-1.34844824-0.38274879$

$\mathrm{H}-3.03522908 \quad 0.14355432-1.31452901$

$\mathrm{H}-1.11427327 \quad 1.89918236-0.85677365$

$\mathrm{H} \quad 0.82250939 \quad 2.18087518 \quad 0.49734448$

$\mathrm{H} \quad 1.77073319 \quad 1.27636881-0.68137195$

H $2.28941117 \quad 0.74599042 \quad 2.30021972$

$\mathrm{H} 1.69371526-2.097982810 .72782452$

H $1.58680315-1.28180212-0.85081697$

$\mathrm{H}-0.79167693-1.53443123 \quad 1.17154399$

$\mathrm{H}-0.75119804 \quad 0.946179592 .84373902$

$\mathrm{H} \quad 0.34320501 \quad 2.28122157 \quad 2.46338788$

$\mathrm{H} \quad 0.49434540 \quad 1.433409914 .00038893$

$\mathrm{H} 0.32340572-1.473433653 .18375857$

H $1.56948142-0.74055525 \quad 4.19610956$

H $2.03555387-1.696063592 .78976850$ 
$0-0.68681752-0.05949919-2.88275176$

$0.052405701 .02042713-2.30986003$

o $-0.70072872-1.05965675-1.88536025$

Vibrational frequencies (in $\mathrm{cm}-1$ )

$\begin{array}{lllllllllllllllll}28.0 & 58.6 & 89.3 & 136.4 & 156.6 & 179.2 & 187.2 & 217.6 & 260.6 & 265.4 & 334.8 & 341.1 & 374.9 & 411.8\end{array}$ $460.3 \quad 469.7 \quad 541.7 \quad 561.8 \quad 590.9 \quad 626.0 \quad 705.9 \quad 767.8 \quad 830.7 \quad 863.2 \quad 876.5 \quad 907.0 \quad 942.1963 .3$

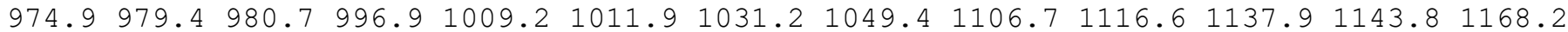

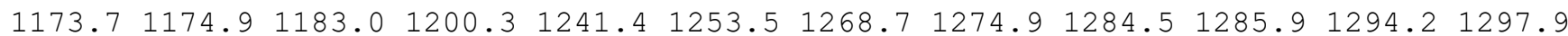
$1303.31305 .91308 .7 \quad 1314.8 \quad 1320.5 \quad 1333.4 \quad 1334.1 \quad 1377.7 \quad 1393.0 \quad 1398.9 \quad 1407.01478 .2$ $2660.12670 .82671 .5 \quad 2674.3 \quad 2676.7 \quad 2679.0 \quad 2680.0 \quad 2697.92704 .3 \quad 2706.0 \quad 2743.0 \quad 2770.4$ $2780.2 \quad 2780.7 \quad 2781.4 \quad 2782.4$

name $=$ MIN206

energy (in $\mathrm{kcal} / \mathrm{mol})=-2.052895$

$\mathrm{ZPE}($ in $\mathrm{kcal} / \mathrm{mol})=142.852$

$\mathrm{G}($ in $\mathrm{kcal} / \mathrm{mol})=114.935$

Geometry

C $-2.38114112-0.09443053-0.33522332$

C $-0.88730144-0.14767166-0.65698679$

C $-0.195557691 .24344305-0.46208369$

C $1.288356121 .19185123-0.09557651$

C $1.533851751 .39087915 \quad 1.36233022$

C $2.47398696 \quad 0.72429668 \quad 2.03245572$

C $-0.18610209-1.30906852-0.04263595$

C $-0.10719087-1.628695351 .25523250$

C $-0.69343947-0.85119862 \quad 2.38368791$

C $0.63673961-2.85410210 \quad 1.68762862$

$\mathrm{H}-2.83910971-1.08582785-0.45132664$

$\mathrm{H}-2.901223170 .58316026-1.02805400$

$\mathrm{H}-2.57987304 \quad 0.24538444 \quad 0.68471206$

$\mathrm{H}-0.77573474 \quad 1.91975373 \quad 0.19448621$

$\mathrm{H} \quad 1.820475651 .99263679-0.66438702$

$\mathrm{H} \quad 1.74334726 \quad 0.24742171-0.46354740$

$\mathrm{H} 0.90640313 \quad 2.14088771 \quad 1.84304906$

H 2.68065565 $0.87532226 \quad 3.07956849$

$\mathrm{H} 3.11111316-0.02462814 \quad 1.58745926$

$\mathrm{H} \quad 0.29077642-1.94887398 \quad-0.79307174$

$\mathrm{H}-1.19006272-1.506140473 .11320011$

$\mathrm{H}-1.43364792-0.107966272 .06323906$

$\mathrm{H} \quad 0.09665159-0.30293737 \quad 2.92242958$

$\mathrm{H} 1.19878586-3.32434148 \quad 0.86992118$

$\mathrm{H}-0.05411670-3.613826852 .08137112$

$\mathrm{H} 1.35832273-2.623619762 .48374453$

$0-0.07313324 \quad 0.72447930-2.62334056$

$0-0.323994661 .81900668-1.76405442$

$0-0.85666559-0.33872436-2.09241195$

Vibrational frequencies (in $\mathrm{cm}-1$ )

$\begin{array}{lllllllllllllll}-48.8 & 28.6 & 34.3 & 57.7 & 77.5 & 99.4 & 113.5 & 123.7 & 176.5 & 188.8 & 242.4 & 276.7 & 312.9 & 341.0\end{array}$ $343.2 \quad 378.1 \quad 405.5 \quad 466.9 \quad 485.9 \quad 494.4 \quad 557.4 \quad 601.5 \quad 699.6 \quad 715.3 \quad 850.8 \quad 880.6 \quad 907.2 \quad 930.3$ $958.3 \quad 963.9970 .6 \quad 992.4 \quad 1002.2 \quad 1011.6 \quad 1018.8 \quad 1025.2 \quad 1079.4 \quad 1088.2 \quad 1091.7 \quad 1116.91122 .8$

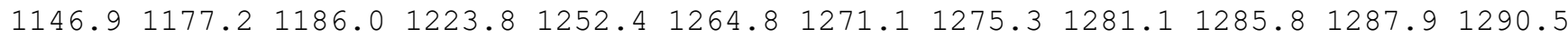
$1297.51303 .21319 .7 \quad 1339.2 \quad 1356.0 \quad 1381.6 \quad 1391.7 \quad 1392.7 \quad 1401.1 \quad 1425.6 \quad 1847.11881 .2$ $2645.02657 .12666 .3 \quad 2670.4 \quad 2671.8 \quad 2673.4 \quad 2675.52692 .22716 .02730 .02738 .92758 .1$ 2772.12779 .12780 .12789 .5

name $=$ MIN207

energy (in $\mathrm{kcal} / \mathrm{mol})=-1.335148$

$\operatorname{ZPE}($ in $\mathrm{kcal} / \mathrm{mol})=143.133$

$\mathrm{G}($ in $\mathrm{kcal} / \mathrm{mol})=115.104$

Geometry

C $-2.98023071-0.53413614-0.51589588$

C $-1.45443734-0.48230994-0.51917565$

C $-0.93495157 \quad 0.99881305-0.55005099$ 
C $0.013821361 .42525936 \quad 0.56800045$

C $1.29481927 \quad 0.65938662 \quad 0.58361961$

C $-0.03949458-2.32711457 \quad 0.38470837$

C $-0.89393248-1.32508147 \quad 0.58296451$

C $2.07576336 \quad 0.53577065 \quad 1.66506790$

C $1.73475999 \quad 1.13440766 \quad 2.99057497$

C $3.36015324-0.22450146 \quad 1.61237357$

$\mathrm{H}-3.40627275-0.059286110 .37316565$

$\mathrm{H}-3.33481493-1.57379851-0.54028610$

$\mathrm{H}-3.39616368-0.03930168-1.40334977$

$\mathrm{H}-1.79031498 \quad 1.70379683-0.62915129$

$\mathrm{H}-0.51585056 \quad 1.34260151 \quad 1.54050499$

$\mathrm{H} \quad 0.25002575 \quad 2.50810661 \quad 0.44768117$

$\mathrm{H} \quad 1.56794238 \quad 0.20610938-0.37043247$

$\mathrm{H} \quad 0.35552786-2.93639646 \quad 1.18372658$

$\mathrm{H} \quad 0.34353489-2.60914397-0.58745606$

$\mathrm{H}-1.25305490-1.05457490 \quad 1.57504049$

$\mathrm{H} \quad 1.63674201 \quad 2.22705145 \quad 2.92118537$

H 2.49431414 $0.92836991 \quad 3.75525978$

$\mathrm{H} \quad 0.78178635 \quad 0.74123728 \quad 3.37279345$

$\mathrm{H} 3.57401752-0.62643347 \quad 0.61254381$

H $3.34456003-1.076634952 .30634075$

$\mathrm{H} \quad 4.21061563 \quad 0.41362617 \quad 1.89167678$

$\begin{array}{lllll}0 & -0.81052170 & 0.10421930 & -2.60148874\end{array}$

$0-0.19137083 \quad 1.07312004-1.77140057$

o $-0.97599324-1.02487835-1.76048025$

Vibrational frequencies (in cm-1)

$\begin{array}{llllllllllllllll}25.0 & 28.4 & 39.7 & 64.9 & 70.1 & 112.2 & 127.5 & 159.0 & 196.7 & 206.3 & 227.1 & 255.9 & 276.2 & 288.0\end{array}$

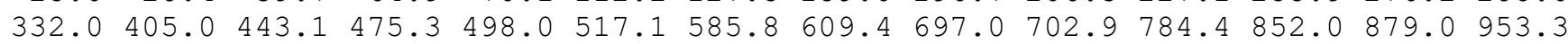

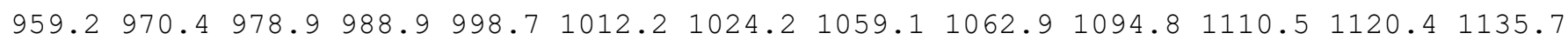
$1153.7 \quad 1162.6 \quad 1214.2 \quad 1233.1 \quad 1252.4 \quad 1272.5 \quad 1277.6 \quad 1282.5 \quad 1288.5 \quad 1289.0 \quad 1290.01290 .9$ $1293.31303 .0 \quad 1329.4 \quad 1343.0 \quad 1377.6 \quad 1379.1 \quad 1390.0 \quad 1400.01400 .7 \quad 1417.91849 .3 \quad 1870.5$ $2653.62670 .12670 .42672 .8 \quad 2673.4 \quad 2674.7 \quad 2676.62680 .32709 .22726 .72750 .22756 .9$ 2778.12779 .62780 .12782 .1

\author{
name $=$ MIN208 \\ energy (in $\mathrm{kcal} / \mathrm{mol})=-0.726059$ \\ $\mathrm{ZPE}(\mathrm{in} \mathrm{kcal} / \mathrm{mol})=143.436$ \\ $\mathrm{G}($ in $\mathrm{kcal} / \mathrm{mol})=116.482$ \\ Geometry \\ C $-2.33663681-0.61153811-0.55104690$ \\ C $-0.82201361-0.60100300-0.74431411$ \\ C $-0.24921291 \quad 0.84149648 \quad-0.56131474$ \\ C $1.21672563 \quad 0.93743167-0.12021610$ \\ C 1.499410420 .247417591 .16948450 \\ C $0.57023092-2.64895991-0.44086039$ \\ C $-0.18177353-1.67571631 \quad 0.06856015$ \\ $\begin{array}{llll}\text { C } 1.01537100 & 0.59038871 & 2.37047774\end{array}$ \\ C $0.09894522 \quad 1.74082223 \quad 2.61492114$ \\ C $1.36585637-0.20597019 \quad 3.58711150$ \\ $\mathrm{H}-2.62267449-0.38834904 \quad 0.48111849$ \\ $\mathrm{H}-2.75455726-1.59385828-0.80953585$ \\ $\mathrm{H}-2.82394223 \quad 0.12420055-1.20625074$ \\ $\mathrm{H}-0.91291704 \quad 1.46846237 \quad 0.06559923$ \\ $\mathrm{H} 1.504042962 .01015002-0.05860913$ \\ $\mathrm{H} \quad 1.85511628 \quad 0.52080566 \quad-0.93211524$ \\ $\mathrm{H} \quad 2.17366069-0.604949891 .07058015$ \\ H $1.01085087-3.43037200 \quad 0.16026341$ \\ $\mathrm{H} \quad 0.80826787-2.74375341-1.49109722$ \\ $\mathrm{H}-0.39757305-1.60408731 \quad 1.13677822$ \\ $\mathrm{H}-0.95139511 \quad 1.41581534 \quad 2.60555911$ \\ $\mathrm{H} \quad 0.20807196 \quad 2.53161841 \quad 1.85975083$ \\ $\mathrm{H} \quad 0.28129630 \quad 2.20868416 \quad 3.59240578$ \\ $\mathrm{H} \quad 0.46423074 \quad-0.55203422 \quad 4.11062225$
}


$\mathrm{H} \quad 1.94850665 \quad 0.39531694 \quad 4.29925176$

$\mathrm{H} 1.96600870-1.09597098 \quad 3.35507641$

$0-0.81031768 \quad 0.38339575-2.74503433$

$0-0.27686607 \quad 1.40156057-1.87688758$

O $-0.51371558-0.84125837-2.12791869$

Vibrational frequencies (in $\mathrm{cm}-1$ )

$\begin{array}{llllllllllllllll}27.7 & 39.3 & 76.0 & 92.6 & 99.0 & 124.8 & 131.4 & 171.6 & 193.4 & 226.8 & 247.4 & 255.4 & 269.4 & 297.3\end{array}$

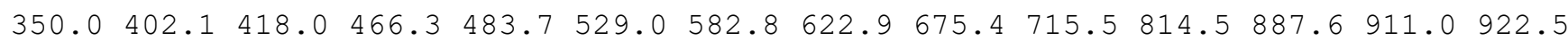

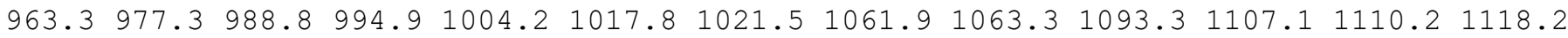
$1150.41164 .6 \quad 1195.6 \quad 1226.2 \quad 1252.3 \quad 1273.6 \quad 1278.1 \quad 1282.1 \quad 1285.4 \quad 1286.91288 .8 \quad 1291.5$

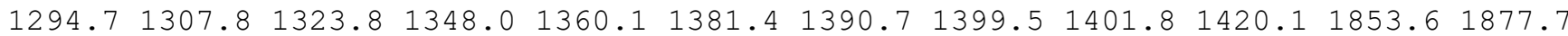
$2651.62669 .0 \quad 2670.2 \quad 2670.92674 .4 \quad 2675.2 \quad 2679.3 \quad 2685.62709 .92737 .12748 .42750 .9$ $2778.22780 .2 \quad 2780.8 \quad 2783.2$

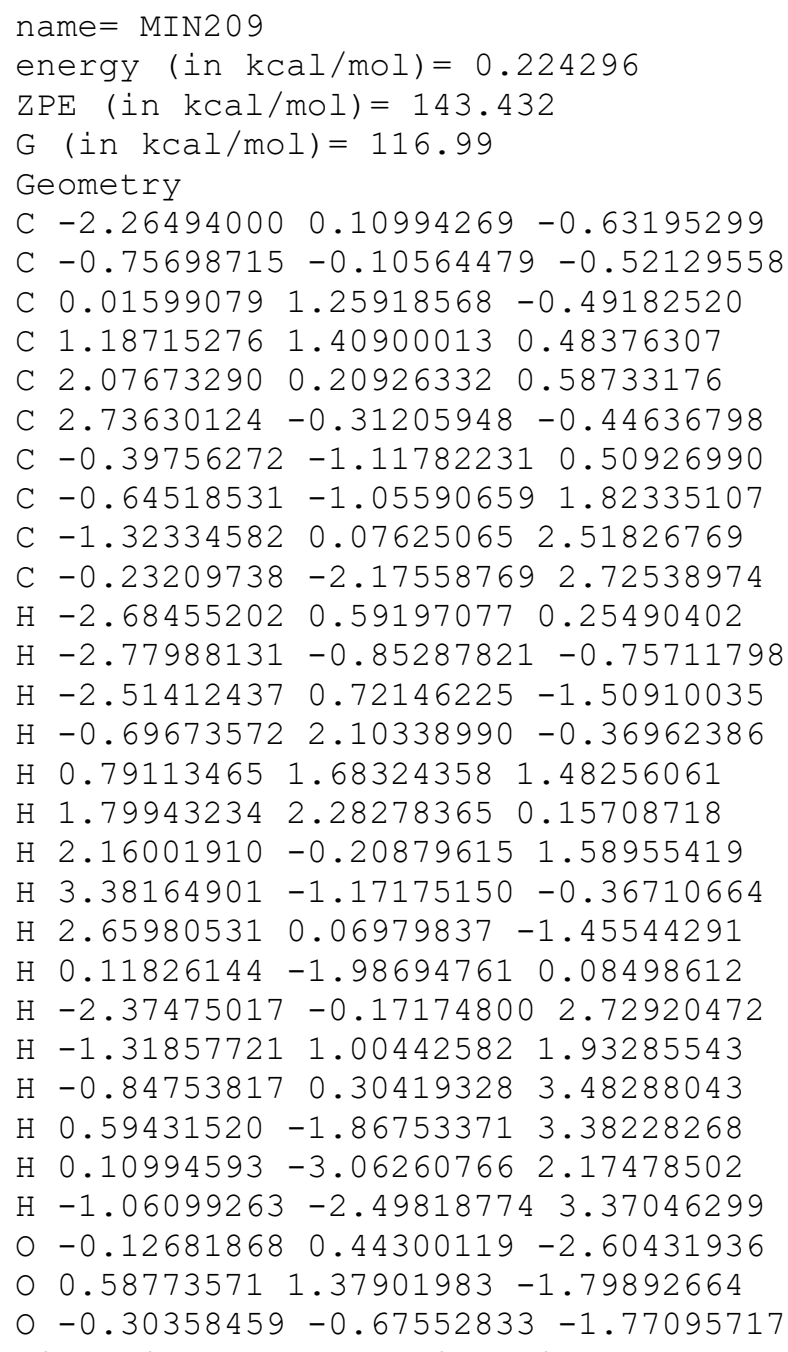


C $-0.76963262-0.22248516-0.54861079$

C $-0.17786631 \quad 1.22974167-0.63166130$

C 0.829321721 .683604330 .42528298

C $1.90958414 \quad 0.70385975 \quad 0.76243242$

C $2.67114164 \quad 0.08769894-0.14043020$

C $-0.25255357-1.10909574 \quad 0.53059532$

C $-0.48726113-1.027165931 .84548608$

C $-1.32514756 \quad 0.00896235 \quad 2.51471439$

C $0.11684944 \quad-2.02677058 \quad 2.78175073$

$\mathrm{H}-2.69039655-1.23282985-0.61412246$

$\mathrm{H}-2.63592797 \quad 0.25091926-1.56704259$

$\mathrm{H}-2.75315237 \quad 0.33122601 \quad 0.20377130$

$\mathrm{H}-1.006500451 .96880979-0.70813402$

$\mathrm{H} \quad 0.27234060 \quad 1.96937878 \quad 1.34209729$

H $1.30872116 \quad 2.62503183 \quad 0.06425729$

H $2.04702283 \quad 0.53905466 \quad 1.83097740$

H $2.55347517 \quad 0.21417773-1.20825242$

H $3.45429201-0.60607748 \quad 0.11849282$

$\mathrm{H} \quad 0.39176872-1.906497020 .13880723$

$\mathrm{H}-0.79534717 \quad 0.46500789 \quad 3.36368197$

$\mathrm{H}-2.24983112-0.434890342 .91317553$

$\mathrm{H}-1.61959997 \quad 0.82555190 \quad 1.84392946$

$\mathrm{H} \quad 0.71896882 \quad-2.78588083 \quad 2.26344698$

$\mathrm{H}-0.65931405-2.56228597 \quad 3.34574004$

H $0.77780293-1.539255653 .51193216$

$\begin{array}{llllll}0 & -0.11361105 & 0.25190037 & -2.65797554\end{array}$

$0 \quad 0.52654878 \quad 1.25246005-1.87870144$

o $-0.27740948-0.82903533-1.76734738$

Vibrational frequencies (in $\mathrm{cm}-1$ )

$\begin{array}{llllllllllllll}35.8 & 61.4 & 72.8 & 98.6 & 103.9 & 115.5 & 144.7 & 153.7 & 166.9 & 196.4 & 249.6 & 269.9 & 281.3 & 317.1\end{array}$ $351.2 \quad 387.8 \quad 416.1 \quad 465.6 \quad 484.7 \quad 507.8 \quad 573.8 \quad 662.7 \quad 690.1 \quad 728.4 \quad 811.0 \quad 874.1 \quad 900.9 \quad 907.7$ $954.4967 .6 \quad 977.3 \quad 994.5 \quad 997.2 \quad 1008.8 \quad 1011.4 \quad 1027.5 \quad 1087.4 \quad 1093.0 \quad 1101.9 \quad 1117.4 \quad 1120.3$

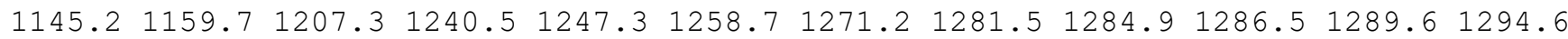

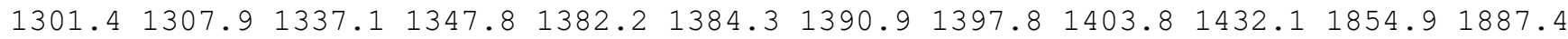
$2653.42668 .2 \quad 2668.92669 .92672 .0 \quad 2673.4 \quad 2674.32677 .32712 .0 \quad 2731.5 \quad 2733.02756 .2$ $2777.3 \quad 2780.4 \quad 2780.5 \quad 2784.4$

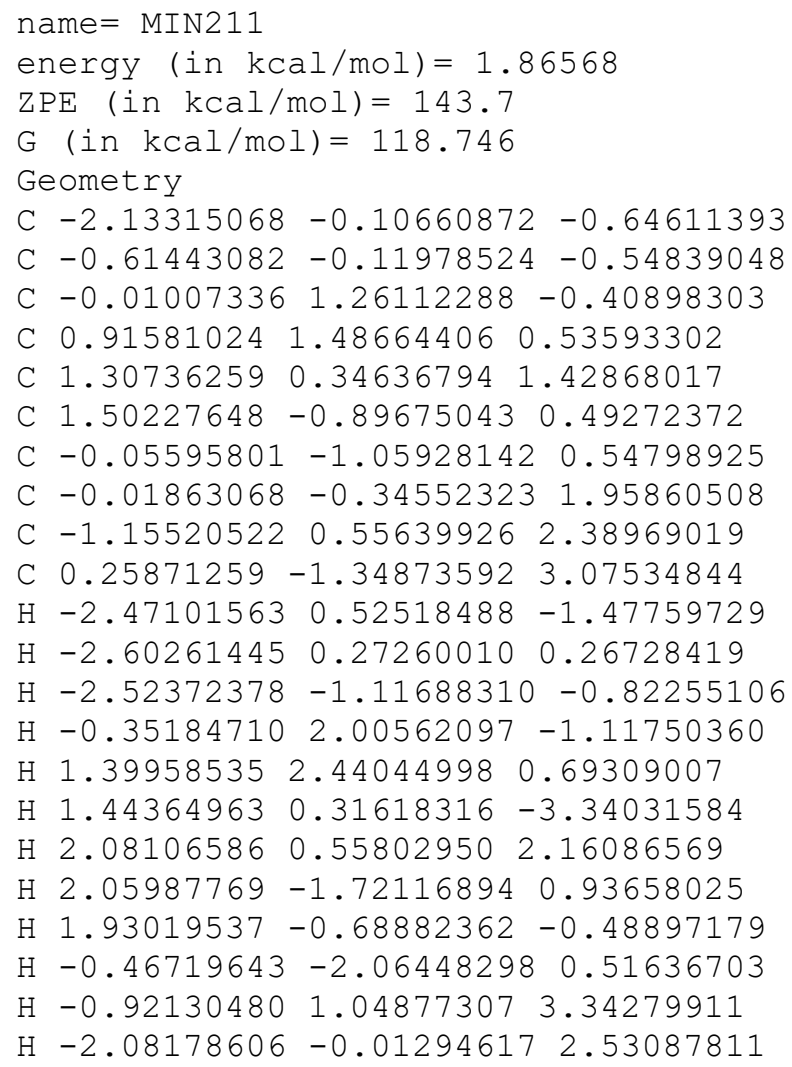


$\mathrm{H}-1.364608841 .35842604 \quad 1.67146060$

$\mathrm{H} 0.55332855-0.83749124 \quad 3.99908710$

$\mathrm{H} 1.06258404-2.049324122 .82438296$

$\mathrm{H}-0.63327832-1.94701584 \quad 3.29516059$

$0-0.35529116-0.00222663-2.86537083$

$0.701244790 .90340331-3.05802863$

O $-0.09913397-0.75602764-1.73879768$

Vibrational frequencies (in $\mathrm{cm}-1$ )

$\begin{array}{llllllllllllllll}34.1 & 90.2 & 99.8 & 140.6 & 152.8 & 166.5 & 181.5 & 206.7 & 262.5 & 277.0 & 303.6 & 315.9 & 361.5 & 385.3\end{array}$ $\begin{array}{llllllllllllll}401.8 & 424.1 & 454.8 & 468.9 & 538.6 & 559.4 & 620.9 & 645.1 & 736.8 & 791.7 & 830.6 & 863.6 & 873.8 & 909.5\end{array}$ $938.9945 .7 \quad 974.5 \quad 985.2 \quad 992.5 \quad 1003.7 \quad 1011.3 \quad 1014.3 \quad 1020.9 \quad 1052.2 \quad 1078.5 \quad 1082.8 \quad 1126.3$

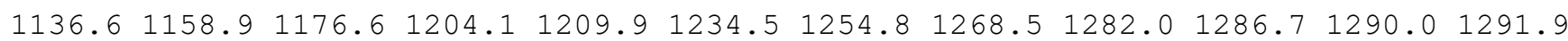

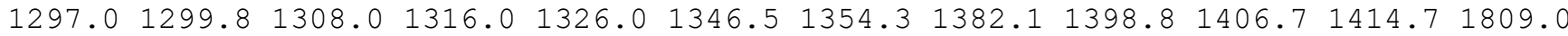
$2666.82674 .6 \quad 2675.2 \quad 2676.4 \quad 2677.0 \quad 2681.0 \quad 2691.72752 .52756 .4 \quad 2758.92766 .2 \quad 2772.3$ $2779.2 \quad 2782.2 \quad 2782.92848 .9$

\section{name $=$ MIN212}

energy $($ in $\mathrm{kcal} / \mathrm{mol})=1.528868$

$\mathrm{ZPE}($ in $\mathrm{kcal} / \mathrm{mol})=144.448$

$\mathrm{G}($ in $\mathrm{kcal} / \mathrm{mol})=119.549$

Geometry

C $-2.41595928-0.08374592-0.25213416$

C $-1.10965842-0.034277060 .01071939$

C $-0.318284021 .24008785-0.21000438$

C $1.17436897 \quad 1.17233152 \quad 0.18866305$

C $1.56377392-0.20758618 \quad 0.72034960$

C $0.99864900-1.27256763-0.27059264$

C $-0.33257117-1.19115056 \quad 0.54732209$

C $0.52241161-0.71792423 \quad 1.79194552$

C $-0.09919186 \quad 0.27541599 \quad 2.75169479$

C $1.06144006-1.901248752 .59087939$

$\mathrm{H}-3.01918378-0.96577688-0.11150775$

$\mathrm{H}-1.39954787 \quad 0.36485330-3.41313416$

$\mathrm{H}-2.97705971 \quad 0.75001613-0.64287068$

$\mathrm{H}-0.81897108 \quad 2.09194081 \quad 0.32255894$

H $1.39888078 \quad 1.94619423 \quad 0.94471238$

$\mathrm{H} 1.800279581 .42130779-0.69089105$

H 2.61451526 $-0.28818588 \quad 0.99659878$

H $1.49190921-2.24399659-0.22965339$

$\mathrm{H} \quad 0.93359988-0.97541277-1.31844607$

$\mathrm{H}-0.89922192-2.11420711 \quad 0.66931419$

$\mathrm{H}-0.56166002 \quad 1.13365647 \quad 2.25714455$

$\mathrm{H} \quad 0.65034964 \quad 0.66890311 \quad 3.44887355$

$\mathrm{H}-0.89053318-0.201434013 .34561780$

$\mathrm{H} \quad 1.54316791-2.65195211 \quad 1.95475119$

$\mathrm{H} \quad 0.25513290 \quad-2.40842688 \quad 3.13372937$

$\mathrm{H} \quad 1.80411097-1.57405390 \quad 3.32708559$

O $0.14767322 \quad 0.91053972 \quad-2.48614669$

$\begin{array}{lllll}0 & -0.47547234 & 1.71387184 & -1.54453117\end{array}$

o $-0.72344523-0.11340793-2.87260536$

Vibrational frequencies (in cm-1)

$\begin{array}{llllllllllllllll}59.7 & 73.8 & 90.4 & 92.8 & 138.6 & 160.3 & 190.3 & 209.0 & 245.1 & 284.1 & 317.2 & 344.6 & 386.4 & 413.9\end{array}$ $433.6 \quad 446.0 \quad 484.4 \quad 515.4 \quad 553.0 \quad 591.4 \quad 626.4 \quad 711.7 \quad 727.2 \quad 804.1 \quad 829.8 \quad 854.7 \quad 888.5 \quad 909.8$ $940.8 \quad 962.0 \quad 967.2 \quad 997.3 \quad 1005.1 \quad 1012.3 \quad 1015.5 \quad 1049.9 \quad 1068.0 \quad 1075.7 \quad 1087.1 \quad 1089.4 \quad 1101.7$

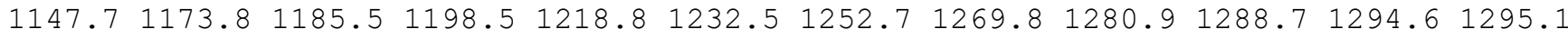
$1302.31304 .11316 .41323 .6 \quad 1330.5 \quad 1351.4 \quad 1376.3 \quad 1381.31392 .2 \quad 1406.6 \quad 1418.21864 .8$ $2656.12672 .5 \quad 2674.0 \quad 2677.0 \quad 2678.8 \quad 2682.3 \quad 2693.82724 .12741 .92749 .62753 .92771 .1$ $2781.92785 .3 \quad 2790.2 \quad 2850.0$

name $=$ MIN213

energy $($ in $\mathrm{kcal} / \mathrm{mol})=4.781458$

$\mathrm{ZPE}($ in $\mathrm{kcal} / \mathrm{mol})=141.581$

$\mathrm{G}(\mathrm{in} \mathrm{kcal} / \mathrm{mol})=111.346$ 


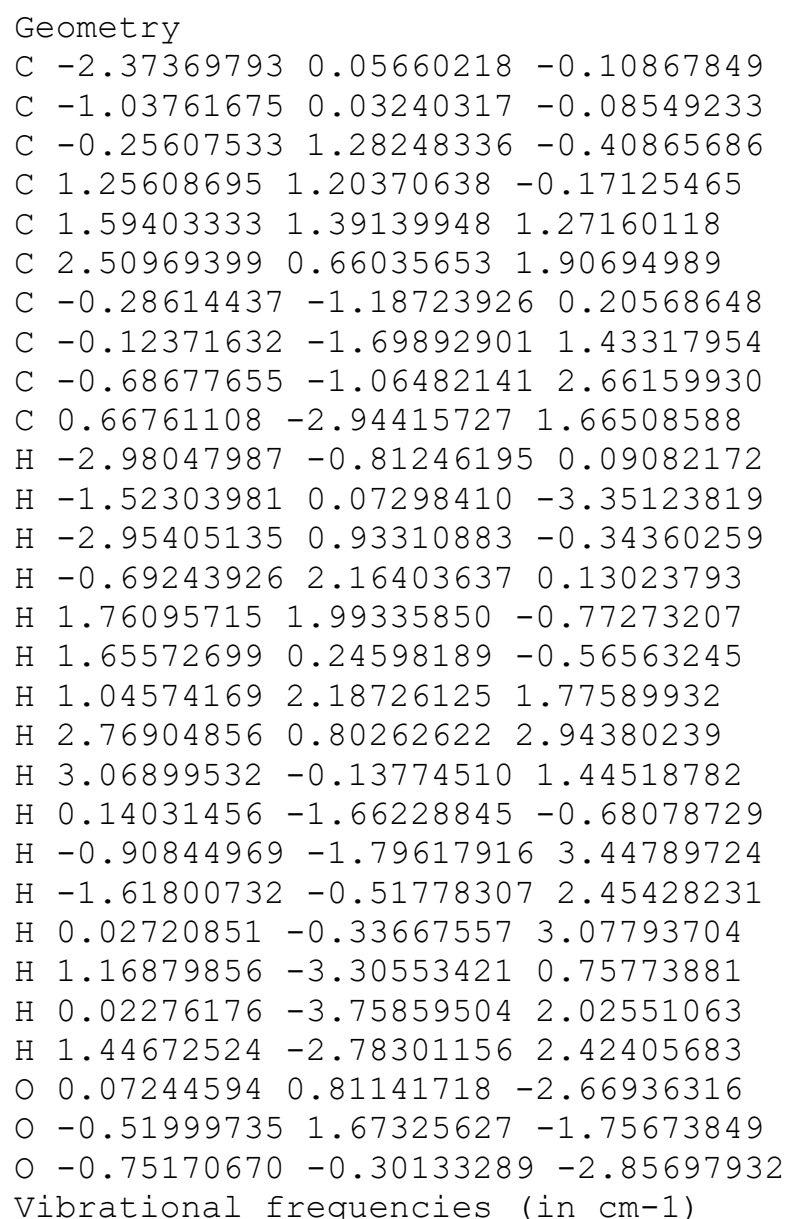

Vibrational frequencies (in $\mathrm{cm}-1$ )

$\begin{array}{llllllllllllllll}-12.2 & 18.6 & 43.4 & 47.8 & 59.9 & 72.6 & 82.8 & 90.1 & 109.2 & 154.2 & 174.1 & 240.6 & 270.7 & 281.3\end{array}$ $327.6 \quad 359.8 \quad 399.9 \quad 433.5 \quad 442.6 \quad 463.3 \quad 501.8 \quad 524.9 \quad 574.7 \quad 601.8 \quad 644.0 \quad 775.0 \quad 838.9 \quad 867.9$ $919.0 \quad 933.2 \quad 956.4 \quad 967.3 \quad 977.1 \quad 995.6 \quad 1015.4 \quad 1018.3 \quad 1028.6 \quad 1073.4 \quad 1078.5 \quad 1087.3 \quad 1098.4$ $1116.71150 .6 \quad 1157.9 \quad 1219.2 \quad 1258.1 \quad 1260.4 \quad 1274.5 \quad 1276.0 \quad 1279.2 \quad 1287.0 \quad 1288.1 \quad 1309.6$ $1339.11345 .51354 .01359 .31373 .0 \quad 1391.2 \quad 1393.6 \quad 1399.81406 .7 \quad 1842.81849 .01879 .8$ $2651.42664 .8 \quad 2671.7 \quad 2671.92674 .6 \quad 2677.5 \quad 2716.72725 .12733 .6 \quad 2747.02757 .72777 .4$ $2780.12789 .22790 .4 \quad 2847.5$

\footnotetext{
name $=$ MIN214

energy $($ in $\mathrm{kcal} / \mathrm{mol})=1.906493$

$\mathrm{ZPE}($ in $\mathrm{kcal} / \mathrm{mol})=144.604$

$\mathrm{G}($ in $\mathrm{kcal} / \mathrm{mol})=119.865$

Geometry

C $-2.24828405-0.04490241-0.51361410$

C $-0.95979244-0.00801366-0.17071997$

C $-0.124862331 .24461968-0.34575045$

C $1.12052784 \quad 1.31210944 \quad 0.57754386$

C $1.48742148-0.073615191 .11128831$

C $1.25111725-1.10927129-0.03417985$

C $-0.23652454-1.162006310 .44329410$

C $0.26506155-0.713758521 .87908753$

C $-0.64288798 \quad 0.188179812 .68865006$

C $0.68228507-1.905860572 .73204133$

$\mathrm{H}-2.87330203-0.91563578-0.39017798$

$\mathrm{H}-0.92207775-0.74228045-2.50272372$

$\mathrm{H}-2.78563616 \quad 0.79282785-0.93008792$

$\mathrm{H}-0.74913780 \quad 2.15835617-0.24136291$

H $0.93916885 \quad 2.01582223 \quad 1.40839120$

H $1.974194921 .73206945 \quad 0.00438417$

H $2.44804477-0.10506162 \quad 1.62413526$

H $1.79306218-2.04839683 \quad 0.08312760$

$\mathrm{H} 1.41989598-0.75368628-1.05435172$

$\mathrm{H}-0.74788339-2.121930410 .38532657$
} 
$\mathrm{H}-0.11630231 \quad 0.60028298 \quad 3.55795463$

$\mathrm{H}-1.51412392-0.36708614 \quad 3.06057727$

$\mathrm{H}-1.036911511 .034038342 .11541238$

$\mathrm{H} 1.34951114-2.590936642 .19682283$

$\mathrm{H}-0.19055446-2.48825716 \quad 3.04971978$

H $1.21003016-1.57892528 \quad 3.63553128$

$0-0.45270597 \quad 1.07860621-2.64623287$

○ $0.48607177 \quad 1.28884856-1.64569844$

o $-0.31440047-0.23612705-3.10146324$

Vibrational frequencies (in $\mathrm{cm}-1$ )

$\begin{array}{llllllllllllllll}35.3 & 80.7 & 118.1 & 138.8 & 156.0 & 171.1 & 181.4 & 201.4 & 238.8 & 332.9 & 344.4 & 365.0 & 393.1 & 409.5\end{array}$

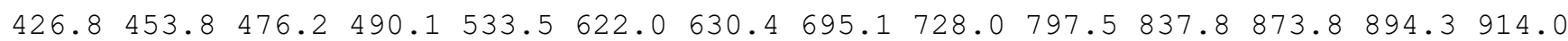
$929.4955 .2984 .0993 .5 \quad 1005.3 \quad 1009.6 \quad 1015.5 \quad 1038.2 \quad 1064.3 \quad 1076.4 \quad 1081.5 \quad 1087.91111 .2$ $1159.01169 .91185 .7 \quad 1206.2 \quad 1221.2 \quad 1236.4 \quad 1251.5 \quad 1258.2 \quad 1285.5 \quad 1287.7 \quad 1293.6 \quad 1296.3$

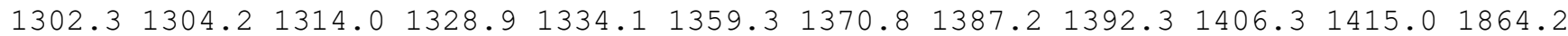
$2657.62672 .6 \quad 2676.12677 .6 \quad 2681.1 \quad 2681.5 \quad 2682.52718 .92737 .5 \quad 2749.12753 .92762 .5$ 2781.62783 .32787 .62826 .9

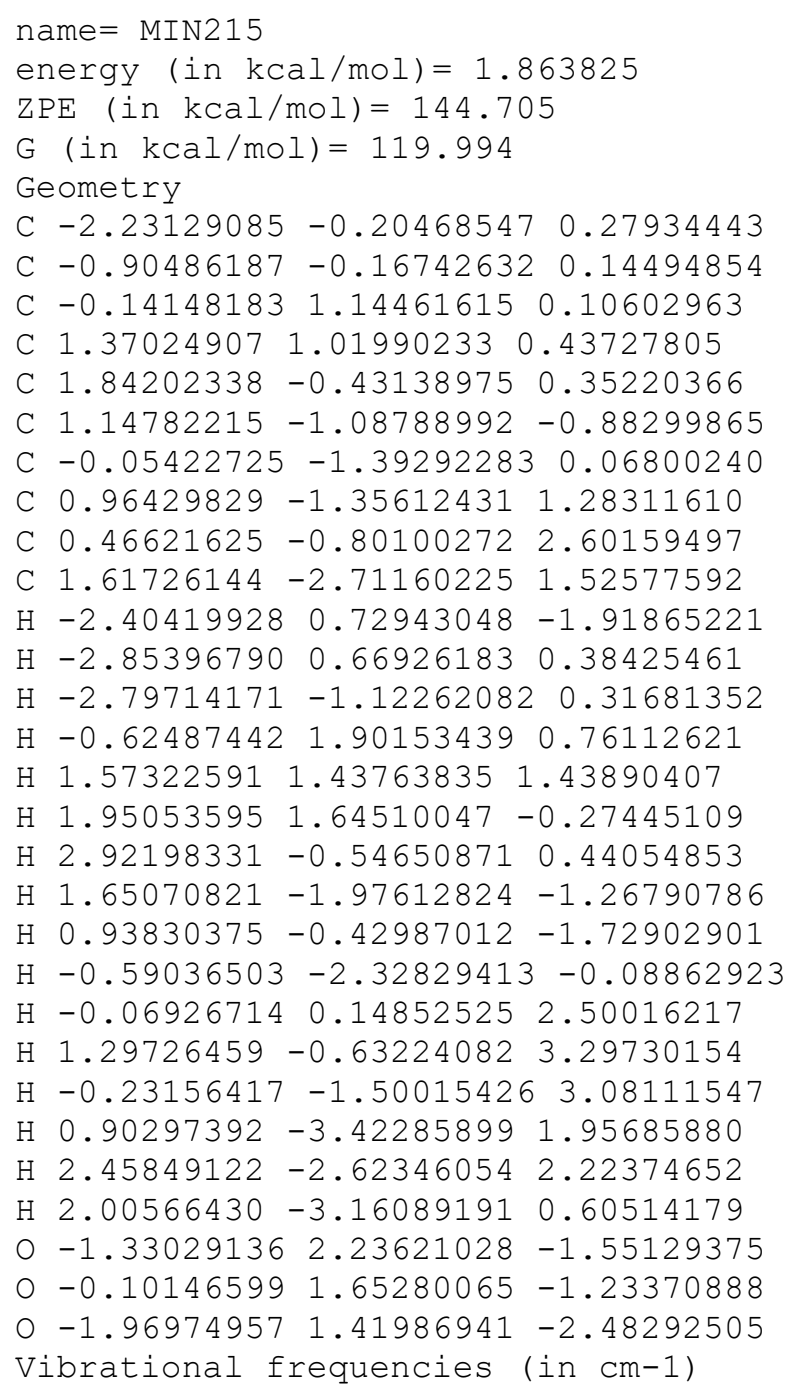


$\mathrm{ZPE}($ in $\mathrm{kcal} / \mathrm{mol})=143.612$

$\mathrm{G}($ in $\mathrm{kcal} / \mathrm{mol})=117.992$

Geometry

C $-2.303690090 .04894296-0.41848296$

C $-0.77962761-0.07260526-0.52830434$

C $-0.09307111 \quad 1.27352471 \quad-0.58828037$

C $0.89984708 \quad 1.55293244 \quad 0.27177717$

C $1.30821690 \quad 0.49752037 \quad 1.25762772$

C $1.40444611-0.83758721 \quad 0.44439756$

C $-0.15154556-0.927882120 .59177315$

C $-0.00340875-0.09044391 \quad 1.93045667$

C $-1.07417304 \quad 0.89653588 \quad 2.34448113$

C $0.30499775-0.994318373 .12088083$

$\mathrm{H}-2.75041066 \quad 0.23393225-1.40744944$

$\mathrm{H}-2.60300335 \quad 0.87586098 \quad 0.23173007$

$\mathrm{H}-2.75112919-0.87154008-0.02705530$

$\mathrm{H}-0.46953598 \quad 1.96345074-1.33244580$

$\mathrm{H} \quad 1.42777810 \quad 2.49698280 \quad 0.30146188$

$\mathrm{H} \quad 0.99545918 \quad 1.03995103-2.75673656$

H 2.13038374 $0.75433813 \quad 1.91939232$

H $1.94760784-1.63843723 \quad 0.95049359$

$\mathrm{H} 1.81065130-0.75305660-0.56111733$

$\mathrm{H}-0.58984091-1.91946778 \quad 0.68035968$

$\mathrm{H}-1.24069067 \quad 1.68837588 \quad 1.60494281$

$\mathrm{H}-0.800611351 .402533313 .27961136$

$\mathrm{H}-2.03335503 \quad 0.39115776 \quad 2.51182366$

H $1.04944541-1.76362401 \quad 2.88989464$

$\mathrm{H}-0.59938336-1.51354504 \quad 3.46091940$

$\mathrm{H} \quad 0.69227363-0.41437163 \quad 3.96670347$

$0.58711870-0.74554633-2.31966779$

$0.642727150 .27807665-3.27793813$

$0-0.68040666-0.77862373-1.77951384$

Vibrational frequencies (in $\mathrm{cm}-1$ )

$\begin{array}{llllllllllllllll}16.1 & 50.8 & 91.2 & 140.5 & 150.8 & 173.8 & 191.8 & 208.9 & 250.6 & 275.6 & 317.7 & 360.0 & 373.7 & 389.8\end{array}$

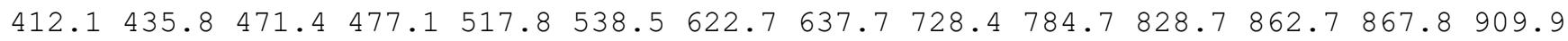
$940.2949 .7 \quad 963.4 \quad 982.9 \quad 997.5 \quad 1004.5 \quad 1010.9 \quad 1014.7 \quad 1021.6 \quad 1051.4 \quad 1079.2 \quad 1082.6 \quad 1120.9$ $1136.7 \quad 1159.3 \quad 1176.8 \quad 1198.3 \quad 1209.3 \quad 1240.6 \quad 1257.4 \quad 1266.6 \quad 1283.0 \quad 1284.4 \quad 1289.91291 .8$ $1297.41298 .91311 .3 \quad 1315.3 \quad 1319.1 \quad 1339.0 \quad 1355.1 \quad 1379.81401 .91406 .6 \quad 1415.6 \quad 1804.0$ $2666.72667 .62674 .72675 .72677 .02680 .12689 .2 \quad 2750.12754 .82758 .62767 .72769 .6$ 2778.32780 .12782 .12841 .7

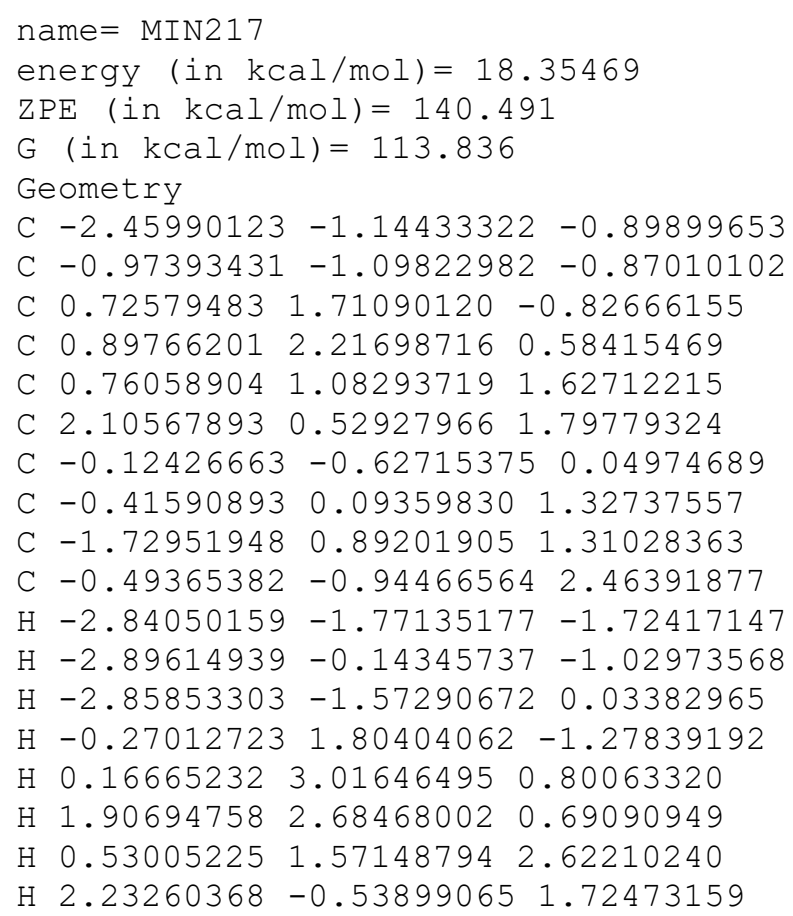


$\mathrm{H} \quad 1.08692789-0.75599534-2.37046045$

$\mathrm{H} \quad 0.94736129-0.82053706-0.09309232$

$\mathrm{H}-1.820299331 .50787010 \quad 0.41152498$

$\mathrm{H}-1.80130810 \quad 1.557814572 .17756406$

$\mathrm{H}-2.60170388 \quad 0.23066162 \quad 1.35136610$

H $0.42939133-1.529985972 .54152682$

$\mathrm{H}-1.30336654-1.66072443 \quad 2.27971119$

$\mathrm{H}-0.68151854-0.47392384 \quad 3.43282312$

$00.85347268-1.70306202-2.16336887$

○ $1.642317201 .24594181-1.46064110$

o - $0.56111928-1.64578234-2.06257298$

Vibrational frequencies (in $\mathrm{cm}-1$ )

$\begin{array}{llllllllllllllll}36.8 & 66.2 & 87.3 & 105.5 & 122.8 & 139.0 & 159.0 & 162.1 & 177.3 & 206.5 & 222.1 & 246.8 & 252.3 & 269.6\end{array}$ $301.9 \quad 351.0 \quad 373.1 \quad 375.8 \quad 443.5 \quad 460.5 \quad 481.3 \quad 502.1 \quad 522.0 \quad 537.2 \quad 605.1 \quad 628.9 \quad 819.6 \quad 896.7$ $919.1927 .9 \quad 939.8 \quad 967.8 \quad 978.0 \quad 988.8 \quad 1008.2 \quad 1026.7 \quad 1035.7 \quad 1052.9 \quad 1067.9 \quad 1078.1 \quad 1107.8$

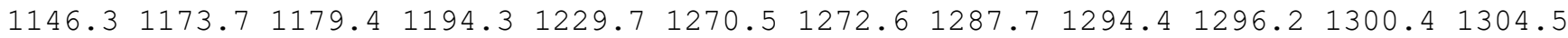

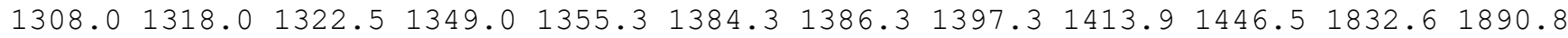
$2641.32653 .7 \quad 2660.0 \quad 2661.3 \quad 2666.4 \quad 2669.1 \quad 2671.5 \quad 2674.82680 .4 \quad 2681.92717 .2 \quad 2734.2$ $2772.3 \quad 2780.3 \quad 2782.0 \quad 2823.9$

name $=$ MIN218

energy (in $\mathrm{kcal} / \mathrm{mol})=19.894567$

$\operatorname{ZPE}($ in $\mathrm{kcal} / \mathrm{mol})=145.572$

$\mathrm{G}(\mathrm{in} \mathrm{kcal} / \mathrm{mol})=122.049$

Geometry

C $-2.29478602 \quad 0.04267214 \quad-0.04986050$

C $-0.83355557 \quad 0.05569252-0.34273063$

C $-0.10523501 \quad 1.42808202-0.28945510$

C $1.269188791 .43257530 \quad 0.40786159$

C $1.71387840 \quad 0.02496225 \quad 0.80918477$

C $1.44307060-0.93304132-0.39361003$

C $-0.00850586-1.09288640 \quad 0.17314134$

C $0.57554671-0.72539468 \quad 1.60233734$

C $-0.27833042 \quad 0.07266076 \quad 2.56371798$

C $1.09545501-1.967064432 .32052052$

$\mathrm{H}-2.50900836 \quad 0.27213543 \quad 1.00267177$

$\mathrm{H}-2.75125032-0.93459666-0.26742117$

$\mathrm{H}-2.83930131 \quad 0.78382445 \quad-0.66165026$

$\mathrm{H}-0.74124570 \quad 2.26503013 \quad 0.04935691$

H $1.25059312 \quad 2.09416241 \quad 1.29126012$

$\mathrm{H} \quad 2.00940542 \quad 1.87784798 \quad-0.29019988$

H $2.70879018-0.00579179 \quad 1.25370945$

H 2.02786511 $-1.85564619-0.37700443$

$\mathrm{H} 1.56521813-0.48959936-1.38158105$

$\mathrm{H}-0.47668673-2.07359199 \quad 0.08628841$

$\mathrm{H}-0.67344531 \quad 0.99834127 \quad 2.13325858$

H $0.30118725 \quad 0.36130991 \quad 3.45070057$

$\mathrm{H}-1.13765213-0.51595908 \quad 2.90996675$

H $1.72384386 \quad-2.59518272 \quad 1.67973970$

H $0.26844458-2.59357879 \quad 2.67538479$

$\mathrm{H} \quad 1.69989557-1.69289527 \quad 3.19326580$

$0-1.54482209-0.02753747-2.58284258$

$0.041769871 .51006940-1.69307298$

O $-0.585773110 .04214436-1.89999240$

Vibrational frequencies (in cm-1)

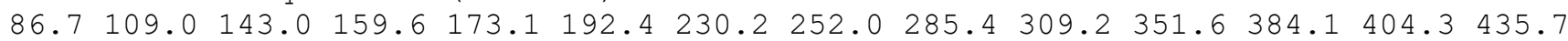
$444.6 \quad 471.6 \quad 515.3 \quad 565.1 \quad 628.6 \quad 637.1 \quad 693.2 \quad 730.9 \quad 776.2 \quad 800.3 \quad 856.6 \quad 890.5 \quad 916.1937 .3$ $962.5992 .2 \quad 1004.91012 .0 \quad 1017.2 \quad 1047.5 \quad 1059.3 \quad 1076.0 \quad 1083.6 \quad 1096.4 \quad 1113.6 \quad 1154.0$

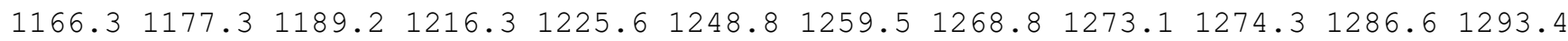
$1296.21300 .1 \quad 1307.0 \quad 1312.1 \quad 1317.3 \quad 1322.0 \quad 1329.5 \quad 1354.8 \quad 1376.8 \quad 1387.4 \quad 1403.5 \quad 1407.6$ $1416.32658 .42663 .5 \quad 2670.6 \quad 2672.6 \quad 2675.0 \quad 2676.3 \quad 2679.82684 .92695 .32742 .6 \quad 2745.8$ 2750.32763 .52776 .42781 .62783 .6 
name $=$ MIN219

energy (in $\mathrm{kcal} / \mathrm{mol})=20.638502$

$\mathrm{ZPE}($ in $\mathrm{kcal} / \mathrm{mol})=145.641$

$\mathrm{G}($ in $\mathrm{kcal} / \mathrm{mol})=122.359$

Geometry

C $-2.37922883 \quad 0.07489973-0.10224821$

C $-0.90763020 \quad 0.10009357-0.38254387$

C $-0.168135651 .46941344 \quad-0.33792587$

C 1.170260051 .500676850 .42256780

C $1.60672993 \quad 0.10194740 \quad 0.86439303$

C $1.38372070-0.86497603-0.33706076$

C $-0.08845089-1.02773148 \quad 0.17478916$

C $0.44112962-0.64412296 \quad 1.62062850$

C $-0.43997060 \quad 0.16693698 \quad 2.54791875$

C $0.93817152-1.87703626 \quad 2.37025110$

$\mathrm{H}-2.91590900 \quad 0.91656695 \quad-0.56122336$

$\mathrm{H}-2.57709434 \quad 0.13150409 \quad 0.98035902$

$\mathrm{H}-2.84865676-0.85749220-0.44873893$

$\mathrm{H}-0.81528782 \quad 2.31868430-0.05393762$

H $1.10706362 \quad 2.176774391 .29245441$

H $1.93892944 \quad 1.93682425-0.25092612$

$\mathrm{H} 2.58267663 \quad 0.08434787 \quad 1.34928845$

H $1.96833754-1.78572727-0.30160025$

H $1.52646618-0.42756063-1.32860592$

$\mathrm{H}-0.54046483-2.014527290 .07059110$

$\mathrm{H}-1.29957198 \quad-0.42063118 \quad 2.89269374$

$\mathrm{H}-0.82839168 \quad 1.08167916 \quad 2.09017991$

$\mathrm{H} \quad 0.12184453 \quad 0.47787269 \quad 3.43938631$

$\mathrm{H} \quad 0.10130368-2.502251892 .70145008$

$\mathrm{H} \quad 1.51264103-1.592008513 .25958791$

H $1.59003389-2.50781015 \quad 1.75516383$

$0-0.38950697-0.72148859-2.53380745$

$00.04003708 \quad 1.50006246-1.73663496$

o $-0.863217990 .18407376-1.94837371$

Vibrational frequencies (in $\mathrm{cm}-1$ )

$107.4123 .7 \quad 156.6 \quad 166.1 \quad 181.2 \quad 193.9 \quad 240.6 \quad 242.6 \quad 277.5 \quad 299.2 \quad 365.2 \quad 392.9421 .2432 .5$ $443.4471 .0 \quad 499.0 \quad 573.9 \quad 603.2 \quad 648.3 \quad 698.5 \quad 714.0 \quad 778.7 \quad 799.6 \quad 857.7 \quad 890.5 \quad 920.5 \quad 939.5$ $962.7996 .71003 .21011 .2 \quad 1017.8 \quad 1049.6 \quad 1061.2 \quad 1073.8 \quad 1082.1 \quad 1094.41110 .91149 .0$

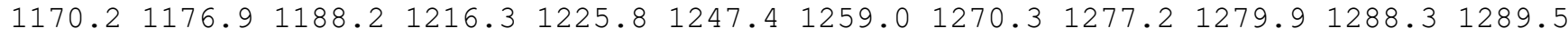

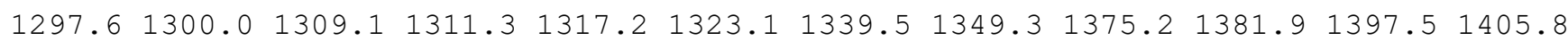
$1414.92658 .3 \quad 2663.3 \quad 2669.8 \quad 2672.6 \quad 2675.02677 .5 \quad 2680.62681 .12692 .4 \quad 2741.72747 .5$ $2751.0 \quad 2763.2 \quad 2775.8 \quad 2781.4 \quad 2783.2$

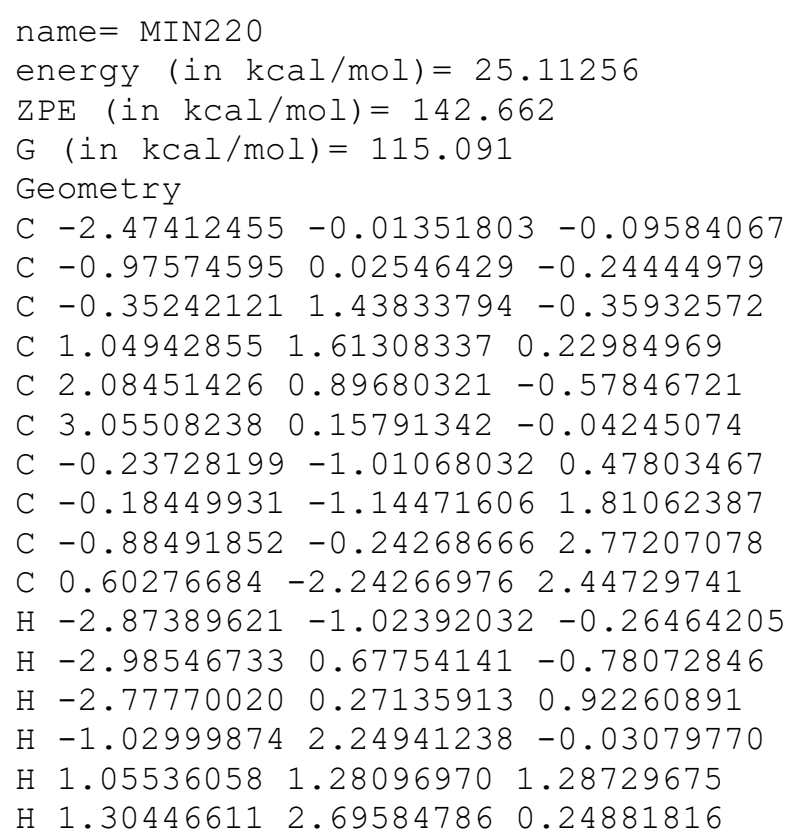


$\mathrm{H} \quad 1.98900634 \quad 1.03575910 \quad-1.65975515$

$\mathrm{H} 3.80347674-0.34619446-0.63339187$

$\mathrm{H} 3.17615722-0.009032551 .01370581$

$\mathrm{H} \quad 0.32627068-1.69566075-0.17129570$

$\mathrm{H}-1.050953170 .76347517 \quad 2.36400416$

$\mathrm{H}-0.31949172-0.11719034 \quad 3.70607919$

$\mathrm{H}-1.86774445-0.654324393 .04663187$

$\mathrm{H}-0.00533238-2.80765466 \quad 3.16773403$

$\mathrm{H} \quad 1.46815204 \quad-1.83941233 \quad 2.99456741$

$\mathrm{H} \quad 0.99539660-2.96522892 \quad 1.71865137$

$0-0.09307653-0.91947636-2.22859305$

$0-0.311510171 .41320303-1.77070656$

o - $0.83756976-0.10708076-1.81563688$

Vibrational frequencies (in $\mathrm{cm}-1$ )

$\begin{array}{lllllllllllllll}33.5 & 44.9 & 54.2 & 62.2 & 85.3 & 99.0 & 118.1 & 138.5 & 148.4 & 220.8 & 242.4 & 259.1 & 274.8 & 310.0\end{array}$

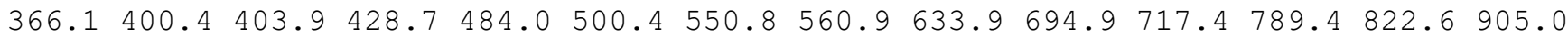
$927.2955 .3 \quad 970.7 \quad 992.8 \quad 1014.7 \quad 1016.1 \quad 1025.8 \quad 1065.2 \quad 1073.6 \quad 1086.2 \quad 1100.11115 .1 \quad 1140.7$

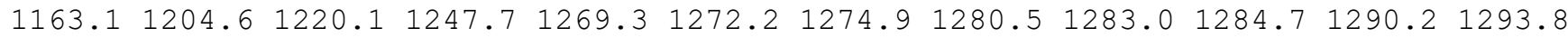

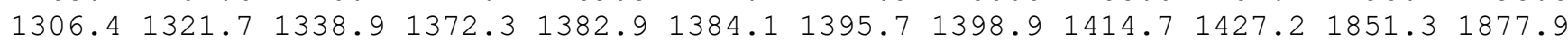
$2660.42661 .92667 .8 \quad 2669.1 \quad 2670.1 \quad 2672.0 \quad 2674.12691 .02713 .0 \quad 2716.5 \quad 2733.92748 .9$ $2775.6 \quad 2777.4 \quad 2779.8 \quad 2792.9$

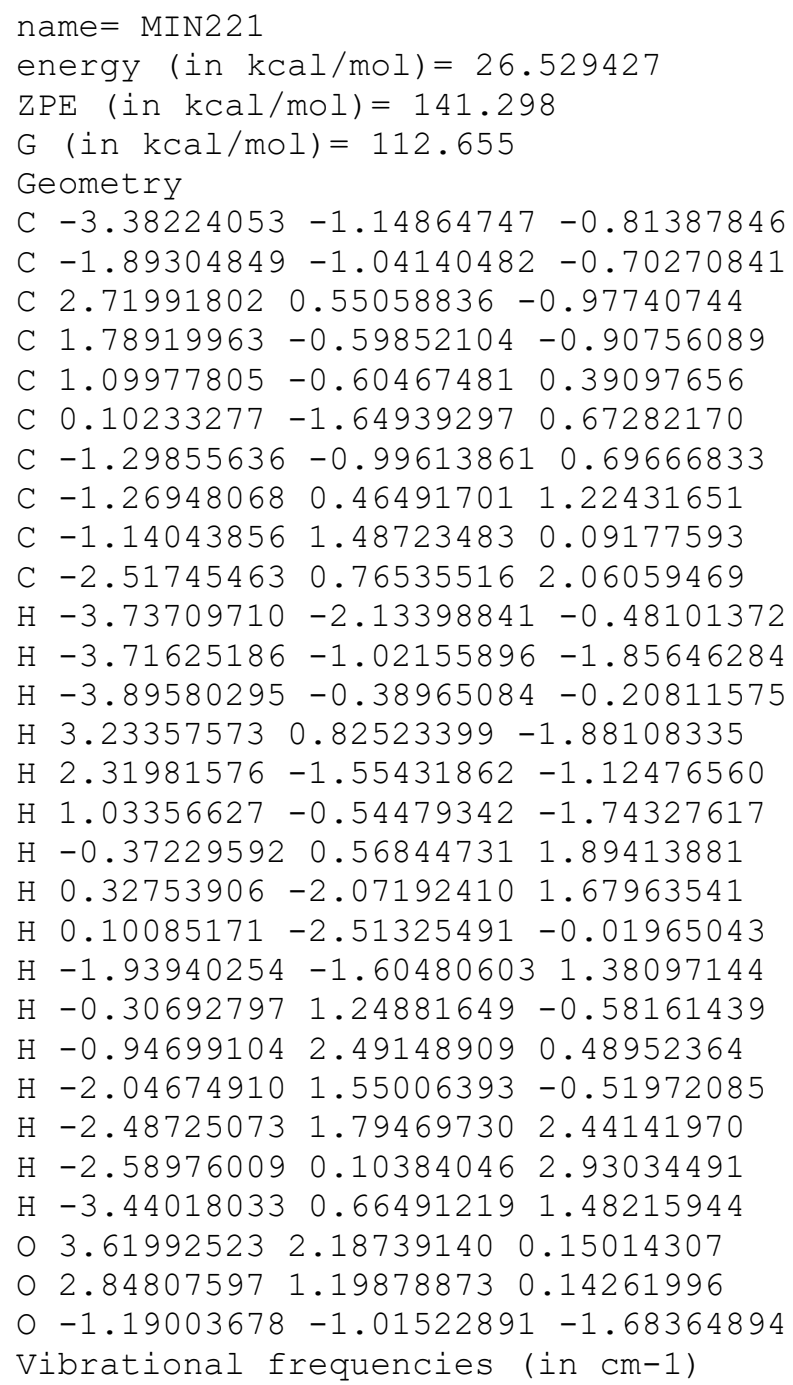


name $=$ MIN222

energy (in $\mathrm{kcal} / \mathrm{mol})=32.22062$

$\mathrm{ZPE}(\mathrm{in} \mathrm{kcal} / \mathrm{mol})=142.799$

$\mathrm{G}($ in $\mathrm{kcal} / \mathrm{mol})=118.173$

Geometry

C $-2.45553374-0.08474564-0.18479315$

C $-1.00832511-0.03648666-0.19754281$

C $-0.21078641 \quad 1.28579711-0.21934072$

C $1.26151600 \quad 1.17279493 \quad 0.23797430$

C $1.64592480 \quad-0.25482438 \quad 0.61777881$

C $1.14866463-1.19848177-0.52413314$

C $-0.21604351-1.239809610 .23103942$

C $0.56281534-0.90201104 \quad 1.56536632$

C $-0.13278688-0.04433450 \quad 2.60037999$

C $1.08257380-2.16591658 \quad 2.24426209$

$\mathrm{H}-1.73301538-1.19772167-1.78819000$

$\mathrm{H}-0.36231296 \quad 0.91938156-2.99054527$

$\mathrm{H}-3.09575520 \quad 0.75606639-0.15407929$

$\mathrm{H}-0.75597722 \quad 2.030489350 .42191099$

H $1.449592461 .85691368 \quad 1.08499640$

$\mathrm{H} \quad 1.91891735 \quad 1.52807608-0.58489470$

$\mathrm{H} \quad 2.68415787-0.35855586 \quad 0.93332609$

$\mathrm{H} \quad 1.66554199-2.15740346-0.57863111$

$\mathrm{H} 1.13645800-0.76328923-1.52534483$

$\begin{array}{lllll}\mathrm{H} & -0.78165561 & -2.17433768 & 0.23309397\end{array}$

$\mathrm{H}-0.53868566 \quad 0.89040351 \quad 2.20609925$

$\mathrm{H} \quad 0.55145740 \quad 0.22273957 \quad 3.41541713$

$\mathrm{H}-0.98279925-0.58545078 \quad 3.04168633$

H $1.60047598-2.83554616 \quad 1.54941395$

H $0.26047995 \quad-2.73762701 \quad 2.69244721$

$\mathrm{H} \quad 1.78976504-1.91968525 \quad 3.04478354$

○ $0.400984401 .25102818-2.45585925$

o $-0.314096621 .96334497-1.45312083$

o $-1.66692230-0.21223776-1.57679995$

Vibrational frequencies (in $\mathrm{cm}-1$ )

$\begin{array}{llllllllllllllll}60.6 & 92.5 & 100.3 & 128.6 & 165.7 & 181.8 & 188.4 & 213.1 & 248.4 & 260.0 & 294.2 & 300.4 & 320.9 & 386.4\end{array}$ $402.2 \quad 440.0 \quad 446.5 \quad 474.1 \quad 500.7 \quad 577.0 \quad 647.8 \quad 700.1 \quad 735.1 \quad 777.6 \quad 806.9 \quad 829.3 \quad 869.8 \quad 890.3$ $922.6947 .1965 .8 \quad 985.0 \quad 992.9 \quad 1006.9 \quad 1009.1 \quad 1017.0 \quad 1032.3 \quad 1050.7 \quad 1085.7 \quad 1089.2 \quad 1114.2$

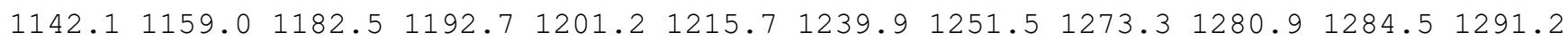
$1293.4 \quad 1299.4 \quad 1302.3 \quad 1316.8 \quad 1323.1 \quad 1340.0 \quad 1345.91376 .3 \quad 1379.6 \quad 1406.3 \quad 1418.91434 .8$ $2648.62665 .72671 .3 \quad 2675.8 \quad 2677.3 \quad 2680.6 \quad 2690.02738 .32742 .2 \quad 2743.4 \quad 2749.92766 .0$ $2781.8 \quad 2785.0 \quad 2806.6 \quad 2858.5$

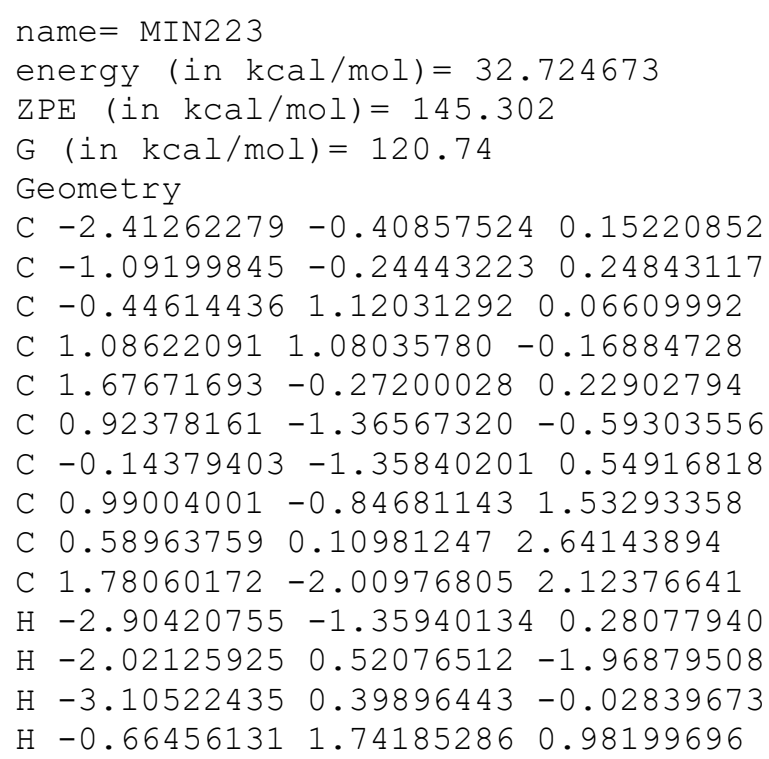


$\mathrm{H} \quad 1.57177064 \quad 1.90237882 \quad 0.38374392$

$\mathrm{H} \quad 1.299129761 .28286718-1.23895529$

$\mathrm{H} \quad 2.76594821-0.29511537 \quad 0.21199177$

$\mathrm{H} \quad 1.46181944-2.30849095-0.69869150$

$\mathrm{H} \quad 0.57648171-1.06855570-1.58418944$

$\mathrm{H}-0.60924993-2.310090630 .80509566$

$\mathrm{H} \quad 0.95778705-0.21422850 \quad 3.62330888$

$\mathrm{H}-0.50031357 \quad 0.18905135 \quad 2.73635787$

$\mathrm{H} \quad 0.99232285 \quad 1.11618983 \quad 2.48054811$

H $2.67969290-1.65104884 \quad 2.63885149$

H $2.10945515-2.72624106 \quad 1.36314807$

H $1.17606235-2.565332912 .85062253$

$\begin{array}{llll}0 & -1.29890030 & 1.23063700 & -2.29839424\end{array}$

$\begin{array}{llll}0 & -1.09370167 & 1.88624620 & -0.89155688\end{array}$

$\begin{array}{lllll}0 & -0.36331151 & 0.57978695 & -2.75221439\end{array}$

Vibrational frequencies (in cm-1)

$\begin{array}{lllllllllllllllll}42.6 & 90.2 & 104.6 & 119.3 & 164.0 & 183.9 & 188.0 & 211.2 & 256.9 & 302.0 & 346.2 & 355.3 & 399.8 & 419.2\end{array}$ $444.1457 .1 \quad 497.3 \quad 550.1 \quad 590.0 \quad 636.5 \quad 673.0 \quad 706.0 \quad 717.4 \quad 795.1 \quad 842.5 \quad 888.8 \quad 909.2 \quad 942.6$ $954.4992 .0 \quad 1002.3 \quad 1017.6 \quad 1019.1 \quad 1022.5 \quad 1046.91065 .0 \quad 1076.1 \quad 1085.7 \quad 1090.01130 .4$ $\begin{array}{lllllllllllll}1154.4 & 1170.1 & 1172.7 & 1181.7 & 1199.8 & 1216.3 & 1227.6 & 1248.5 & 1275.7 & 1281.0 & 1291.8 & 1298.3\end{array}$ $1299.11301 .91305 .91306 .8 \quad 1317.2 \quad 1329.6 \quad 1368.91376 .5 \quad 1387.5 \quad 1406.2 \quad 1417.91423 .5$ $1860.42641 .22665 .42667 .3 \quad 2673.9 \quad 2675.6 \quad 2679.12689 .32711 .92721 .62743 .42749 .0$ $2753.0 \quad 2767.5 \quad 2778.9 \quad 2781.3 \quad 2787.4$

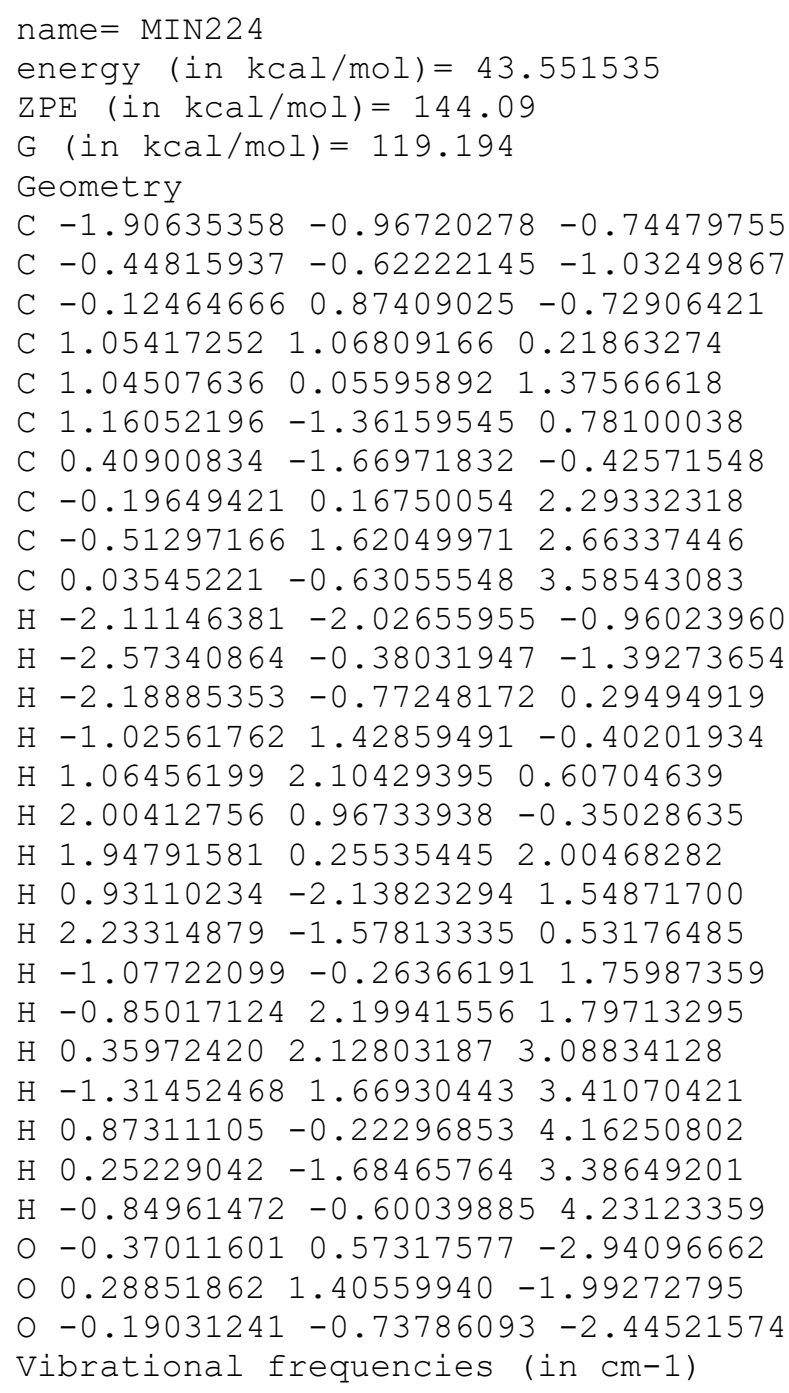


$2627.52653 .7 \quad 2662.0 \quad 2664.8 \quad 2672.2 \quad 2672.4 \quad 2676.02676 .62686 .5 \quad 2689.5 \quad 2706.42720 .1$ $2740.12773 .6 \quad 2779.92781 .8$

\section{RELATIVE ENERGIES OF THE TSS (ZPE-CORRECTED)}

\begin{tabular}{|c|c|}
\hline TS & $\mathrm{DE}(\mathrm{kcal} / \mathrm{mol}$ \\
\hline 1 & -143.932 \\
\hline 2 & -111.215 \\
\hline 3 & -105.117 \\
\hline 4 & -93.830 \\
\hline 5 & -87.054 \\
\hline 6 & -85.866 \\
\hline 7 & -84.752 \\
\hline 8 & -83.659 \\
\hline 9 & -81.743 \\
\hline 10 & -80.900 \\
\hline 11 & -78.036 \\
\hline 12 & -73.859 \\
\hline 13 & -69.734 \\
\hline 14 & -69.172 \\
\hline 15 & -68.791 \\
\hline 16 & -65.820 \\
\hline 17 & -62.010 \\
\hline 18 & -61.849 \\
\hline 19 & -61.409 \\
\hline 20 & -58.847 \\
\hline 21 & -58.668 \\
\hline 22 & -58.131 \\
\hline 23 & -58.076 \\
\hline 24 & -57.901 \\
\hline 25 & -57.709 \\
\hline 26 & -56.187 \\
\hline 27 & -48.837 \\
\hline 28 & -48.695 \\
\hline 29 & -48.604 \\
\hline 30 & -48.545 \\
\hline 31 & -48.327 \\
\hline 32 & -47.906 \\
\hline 33 & -46.658 \\
\hline 34 & -45.439 \\
\hline 35 & -43.271 \\
\hline 36 & -42.750 \\
\hline 37 & -42.080 \\
\hline 38 & -32.933 \\
\hline 39 & -29.873 \\
\hline 40 & -28.960 \\
\hline 41 & -25.620 \\
\hline 42 & -25.588 \\
\hline 43 & -24.596 \\
\hline 44 & -24.209 \\
\hline 45 & -19.082 \\
\hline 46 & -17.903 \\
\hline 47 & -16.100 \\
\hline 48 & -15.721 \\
\hline 49 & -15.566 \\
\hline 50 & -14.515 \\
\hline 51 & -14.008 \\
\hline 52 & -13.642 \\
\hline 53 & -13.240 \\
\hline 54 & -12.314 \\
\hline 55 & -12.130 \\
\hline 56 & -12.048 \\
\hline 57 & -11.636 \\
\hline 58 & -11.600 \\
\hline 59 & -11.476 \\
\hline
\end{tabular}




\begin{tabular}{|c|c|}
\hline 60 & -11.327 \\
\hline 61 & -10.122 \\
\hline 62 & -9.845 \\
\hline 63 & -9.706 \\
\hline 64 & -9.303 \\
\hline 65 & -8.877 \\
\hline 66 & -8.531 \\
\hline 67 & -8.483 \\
\hline 68 & -8.136 \\
\hline 69 & -7.870 \\
\hline 70 & -7.616 \\
\hline 71 & -7.202 \\
\hline 72 & -6.764 \\
\hline 73 & -6.375 \\
\hline 74 & -6.228 \\
\hline 75 & -5.574 \\
\hline 76 & -5.260 \\
\hline 77 & -5.234 \\
\hline 78 & -5.214 \\
\hline 79 & -4.499 \\
\hline 80 & -3.937 \\
\hline 81 & -3.757 \\
\hline 82 & -3.225 \\
\hline 83 & -1.575 \\
\hline 84 & -0.469 \\
\hline 85 & -0.004 \\
\hline 86 & 0.318 \\
\hline 87 & 1.237 \\
\hline 88 & 1.387 \\
\hline 89 & 1.639 \\
\hline 90 & 2.001 \\
\hline 91 & 2.458 \\
\hline 92 & 3.345 \\
\hline 93 & 3.887 \\
\hline 94 & 3.997 \\
\hline 95 & 4.563 \\
\hline 96 & 4.626 \\
\hline 97 & 4.634 \\
\hline 98 & 4.825 \\
\hline 99 & 4.990 \\
\hline 100 & 5.742 \\
\hline 101 & 5.820 \\
\hline 102 & 6.576 \\
\hline 103 & 7.216 \\
\hline 104 & 8.069 \\
\hline 105 & 8.857 \\
\hline 106 & 9.257 \\
\hline 107 & 10.262 \\
\hline 108 & 10.325 \\
\hline 109 & 10.566 \\
\hline 110 & 10.876 \\
\hline 111 & 11.844 \\
\hline 112 & 12.338 \\
\hline 113 & 12.515 \\
\hline 114 & 12.705 \\
\hline 115 & 14.930 \\
\hline 116 & 14.990 \\
\hline 117 & 15.093 \\
\hline 118 & 15.096 \\
\hline 119 & 15.764 \\
\hline 120 & 15.923 \\
\hline 121 & 15.975 \\
\hline 122 & 16.566 \\
\hline 123 & 16.897 \\
\hline 124 & 17.466 \\
\hline
\end{tabular}




\begin{tabular}{|c|c|}
\hline 125 & 18.055 \\
\hline 126 & 18.312 \\
\hline 127 & 18.775 \\
\hline 128 & 19.649 \\
\hline 129 & 20.898 \\
\hline 130 & 21.385 \\
\hline 131 & 21.477 \\
\hline 132 & 21.824 \\
\hline 133 & 21.966 \\
\hline 134 & 23.134 \\
\hline 135 & 23.777 \\
\hline 136 & 23.983 \\
\hline 137 & 24.212 \\
\hline 138 & 24.783 \\
\hline 139 & 25.205 \\
\hline 140 & 25.310 \\
\hline 141 & 25.382 \\
\hline 142 & 26.674 \\
\hline 143 & 26.783 \\
\hline 144 & 28.532 \\
\hline 145 & 29.758 \\
\hline 146 & 30.118 \\
\hline 147 & 30.121 \\
\hline 148 & 30.137 \\
\hline 149 & 30.709 \\
\hline 150 & 32.725 \\
\hline 151 & 33.672 \\
\hline 152 & 34.269 \\
\hline 153 & 34.283 \\
\hline 154 & 34.292 \\
\hline 155 & 36.222 \\
\hline 156 & 36.877 \\
\hline 157 & 36.998 \\
\hline 158 & 37.057 \\
\hline 159 & 37.156 \\
\hline 160 & 37.309 \\
\hline 161 & 37.854 \\
\hline 162 & 38.008 \\
\hline 163 & 38.927 \\
\hline 164 & 39.158 \\
\hline 165 & 39.487 \\
\hline 166 & 40.852 \\
\hline 167 & 43.154 \\
\hline 168 & 44.074 \\
\hline 169 & 44.111 \\
\hline 170 & 44.424 \\
\hline 171 & 44.458 \\
\hline 172 & 44.745 \\
\hline 173 & 45.119 \\
\hline 174 & 45.432 \\
\hline 175 & 45.473 \\
\hline 176 & 46.017 \\
\hline 177 & 46.773 \\
\hline 178 & 46.872 \\
\hline 179 & 47.800 \\
\hline 180 & 47.866 \\
\hline 181 & 48.194 \\
\hline 182 & 48.855 \\
\hline 183 & 49.246 \\
\hline 184 & 50.387 \\
\hline 185 & 51.244 \\
\hline 186 & 54.810 \\
\hline 187 & 54.998 \\
\hline 188 & 55.477 \\
\hline 189 & 55.566 \\
\hline
\end{tabular}




$\begin{array}{ll}190 & 55.858 \\ 191 & 56.000 \\ 192 & 56.417 \\ 193 & 56.737 \\ 194 & 57.415 \\ 195 & 57.934 \\ 196 & 58.465 \\ 197 & 59.180 \\ 198 & 59.913 \\ 199 & 60.648 \\ 200 & 60.666 \\ 201 & 62.889 \\ 202 & 64.978 \\ 203 & 65.732 \\ 204 & 66.238 \\ 205 & 66.241 \\ 206 & 66.345 \\ 207 & 66.565 \\ 208 & 66.656 \\ 209 & 66.761 \\ 210 & 67.216 \\ 211 & 68.981 \\ 212 & 69.043 \\ 213 & 69.275 \\ 214 & 69.605 \\ 215 & 69.759 \\ 216 & 70.961 \\ 217 & 75.708 \\ 218 & 76.309 \\ 219 & 79.105 \\ 220 & 82.282 \\ 221 & 91.676 \\ 222 & 98.426 \\ 210 & 56\end{array}$

Families of conformers

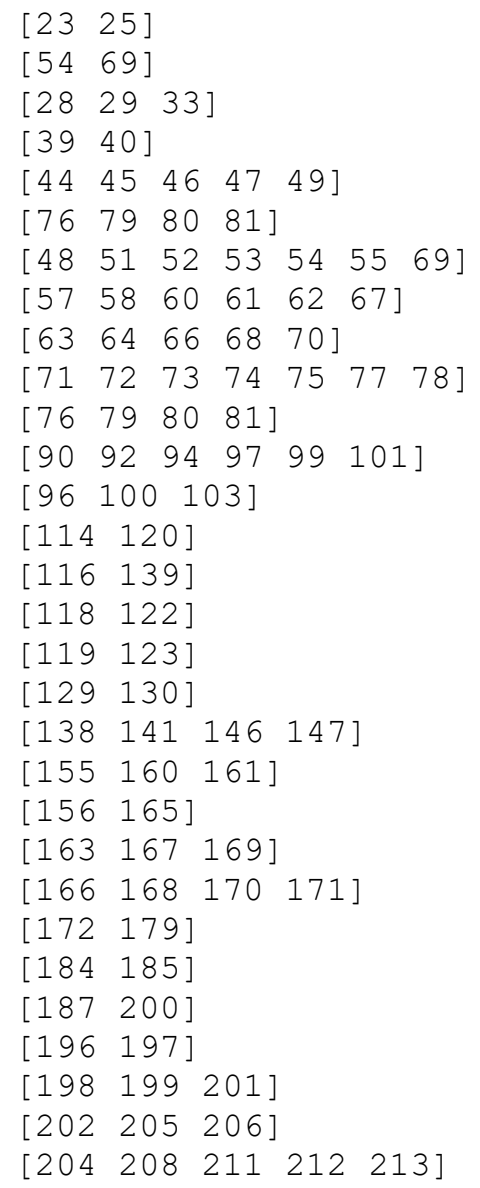


ABSOLUTE ENERGY, ZPE, GIBBS ENERGY CORRECTION, GEOMETRY AND FREQUENCIES OF THE TSS name $=$ TS 1

energy (in $\mathrm{kcal} / \mathrm{mol})=-149.562352$

$\mathrm{ZPE}($ in $\mathrm{kcal} / \mathrm{mol})=143.701$

$\mathrm{G}($ in $\mathrm{kcal} / \mathrm{mol})=118.619$

Geometry

C $1.52189919 \quad 3.49115278 \quad 2.24738404$

C $1.339080572 .26381342 \quad 3.09417712$

C $-0.19206365-0.99053803 \quad 3.90417274$

C $0.90097269-1.71377388 \quad 3.17356350$

C $2.19888277-0.915303633 .11966048$

C $2.39014404 \quad 0.19468006 \quad 4.19022550$

C $2.47605401 \quad 1.28169664 \quad 3.09226690$

C $2.36680966 \quad 0.16779072 \quad 1.98338565$

C 1.230321330 .323299090 .99713047

C $3.68229469-0.00546976 \quad 1.23085951$

$\mathrm{H} 2.00819783 \quad 3.26802891 \quad 1.29093685$

H $2.13999271 \quad 4.23304755 \quad 2.77239384$

$\mathrm{H} \quad 0.55666247 \quad 3.97388675 \quad 2.03231341$

$\mathrm{H}-1.48385903 \quad 0.45319816 \quad 3.53720893$

H $0.57371202-1.979242692 .14493205$

$\mathrm{H} \quad 1.08177850 \quad-2.68081474 \quad 3.69759255$

$\mathrm{H} 3.04620267-1.628657553 .11064254$

$\mathrm{H} \quad 3.30052148 \quad 0.08502105 \quad 4.78618925$

$\mathrm{H} \quad 1.56166743 \quad 0.33031094 \quad 4.89850658$

$\mathrm{H} \quad 3.44362744 \quad 1.810397913 .07603063$

$\mathrm{H} \quad 1.12116813-0.56657386 \quad 0.36560285$

$\mathrm{H} \quad 1.38625192 \quad 1.17757894 \quad 0.32903248$

H $0.26027906 \quad 0.48024649 \quad 1.49433542$

$\mathrm{H} \quad 3.87984869 \quad 0.84857171 \quad 0.57269676$

$\mathrm{H} 3.66088924 \quad-0.90296058 \quad 0.60095798$

$\mathrm{H} \quad 4.53858189-0.100728761 .90766276$

$0-0.93008383-0.22277868 \quad 3.06548909$

$0-0.46370208-1.06591845 \quad 5.07407465$

o $0.338937812 .10256973 \quad 3.74878556$

Vibrational frequencies (in cm-1)

$\begin{array}{lllllllllllllll}-579.1 & 53.2 & 83.3 & 99.2 & 105.7 & 116.0 & 159.9 & 189.1 & 194.7 & 205.2 & 228.3 & 260.2 & 296.8 & 335.7\end{array}$

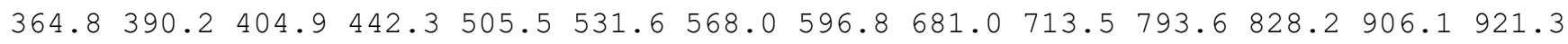
$964.6987 .6 \quad 995.5 \quad 1008.3 \quad 1017.0 \quad 1035.5 \quad 1047.8 \quad 1054.7 \quad 1091.4 \quad 1113.4 \quad 1123.5 \quad 1132.8 \quad 1166.9$

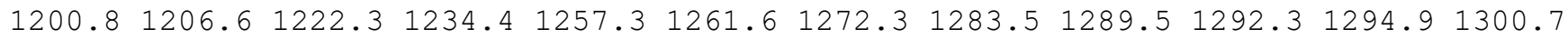
$1303.11311 .81324 .51334 .51361 .8 \quad 1375.7 \quad 1379.3 \quad 1384.51404 .8 \quad 1407.91851 .6 \quad 1867.8$ $2653.82658 .12668 .82669 .2 \quad 2676.12676 .12678 .02680 .72714 .02729 .02733 .12742 .8$ $2757.7 \quad 2780.6 \quad 2780.9 \quad 2783.5$

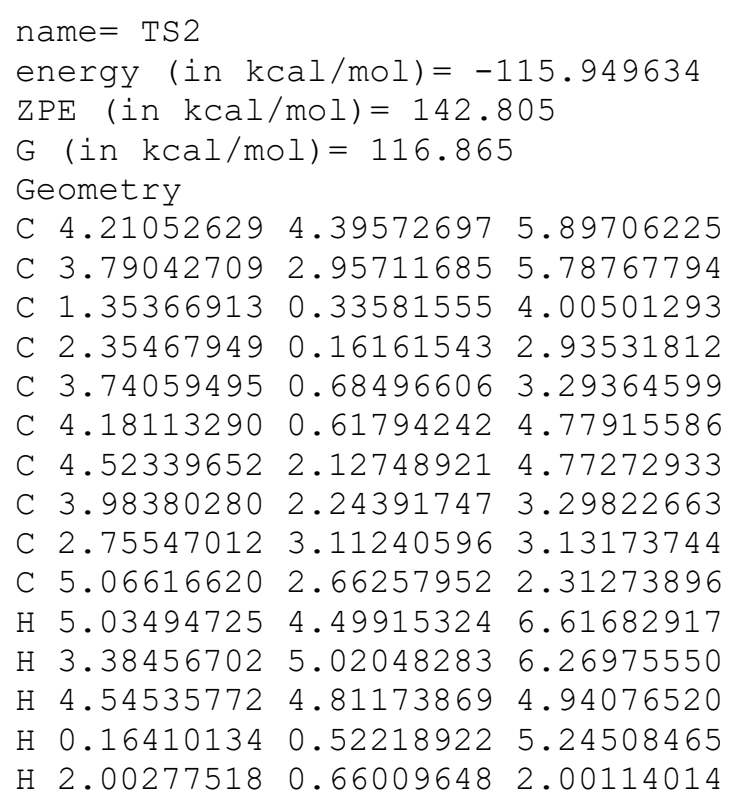


H $2.42971649 \quad-0.927486592 .69463327$

$\begin{array}{lllll}\mathrm{H} & 4.47587028 & 0.18168780 & 2.63247750\end{array}$

H $5.02792049-0.04377513 \quad 4.97421811$

$\mathrm{H} 3.38873195 \quad 0.344569975 .49376008$

$\mathrm{H} \quad 5.60874294 \quad 2.31987565 \quad 4.84194462$

$\mathrm{H} 1.979586092 .89458129 \quad 3.88366171$

H 2.28626806 2.96903094 2.15090822

H 2.99447815 4.17817742 3.22243455

H $5.973082462 .05501368 \quad 2.40803764$

H $5.35866358 \quad 3.70862498 \quad 2.46253053$

H $4.71610431 \quad 2.56834926 \quad 1.27754577$

O $0.42081460 \quad 1.21539796 \quad 4.07827114$

O $1.18149167-0.39449620 \quad 5.04488880$

O $2.93282219 \quad 2.50002684 \quad 6.49998795$

Vibrational frequencies (in $\mathrm{cm}-1$ )

$\begin{array}{lllllllllllllll}-1690.8 & 40.3 & 51.1 & 61.9 & 88.9 & 103.7 & 146.2 & 168.1 & 185.6 & 203.4 & 234.9 & 273.6 & 285.6 & 326.3\end{array}$

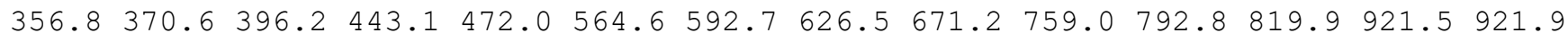
$975.7990 .4 \quad 1001.3 \quad 1008.2 \quad 1016.5 \quad 1028.3 \quad 1044.3 \quad 1050.6 \quad 1061.4 \quad 1093.5 \quad 1114.3 \quad 1134.0$ $\begin{array}{llllllllllllll}1146.3 & 1183.8 & 1198.1 & 1223.6 & 1238.7 & 1259.5 & 1264.0 & 1272.6 & 1281.4 & 1286.9 & 1292.8 & 1295.5\end{array}$

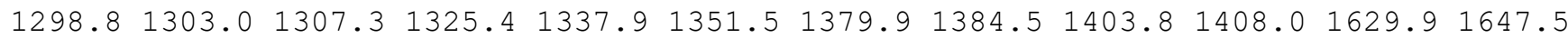
$1856.42306 .32648 .62655 .82663 .5 \quad 2668.02675 .52676 .72678 .02680 .42710 .92726 .2$ $2728.7 \quad 2756.4 \quad 2780.0 \quad 2780.7 \quad 2783.3$

name $=$ TS3

energy $($ in $\mathrm{kcal} / \mathrm{mol})=-107.986125$

$\mathrm{ZPE}(\mathrm{in} \mathrm{kcal} / \mathrm{mol})=140.939$

$\mathrm{G}($ in $\mathrm{kcal} / \mathrm{mol})=114.729$

Geometry

C $4.65802470 \quad 3.97854034 \quad 3.85573572$

C $3.45867491 \quad 3.23087598 \quad 3.35691031$

C $0.44970573-0.651897610 .67336860$

C $0.72078461 \quad 0.819831210 .64795169$

C $2.17390878 \quad 1.100345301 .03010999$

C $2.46385117 \quad 1.03019778 \quad 2.54192542$

C $3.57941175 \quad 2.028692902 .67709351$

C $2.620061472 .53852971 \quad 0.78920550$

C $1.72310923 \quad 3.59916480 \quad 0.99861459$

C $3.84641143 \quad 2.75395749 \quad-0.02935433$

$\mathrm{H} \quad 5.10135147 \quad 3.46178218 \quad 4.71907612$

$\mathrm{H} \quad 4.38547687 \quad 4.99157329 \quad 4.18870406$

$\mathrm{H} \quad 5.43462541 \quad 4.07173533 \quad 3.08848210$

$\mathrm{H} \quad 1.06413237-2.27068501-0.27966209$

$\mathrm{H} \quad 0.01721583 \quad 1.332630201 .34227473$

$\mathrm{H} \quad 0.50659804 \quad 1.22198082 \quad-0.36717513$

H $2.83579833 \quad 0.38970408 \quad 0.47598643$

$\mathrm{H} 2.75809510 \quad 0.02281265 \quad 2.87778429$

$\mathrm{H} \quad 1.58387716 \quad 1.31198625 \quad 3.15109646$

H $4.56678184 \quad 1.63283639 \quad 2.48196664$

H $1.75515085 \quad 3.86574293 \quad 2.33710395$

$\mathrm{H} 0.65332317 \quad 3.42085197 \quad 1.03424263$

$\mathrm{H} \quad 1.95669560 \quad 4.58035977 \quad 0.60213387$

H $3.58667153 \quad 2.79950794-1.10054977$

H 4.592307461 .954614810 .08655573

H $4.35048712 \quad 3.70032596 \quad 0.21691917$

O $1.20645414-1.29311178-0.25889061$

$0-0.31751218-1.26528596 \quad 1.36470174$

o $2.33147824 \quad 3.81576033 \quad 3.50381378$

Vibrational frequencies (in cm-1)

$\begin{array}{llllllllllllllll}-1455.3 & 35.2 & 40.1 & 69.4 & 88.3 & 101.8 & 132.1 & 154.7 & 176.7 & 231.0 & 260.8 & 292.5 & 328.1 & 353.1\end{array}$

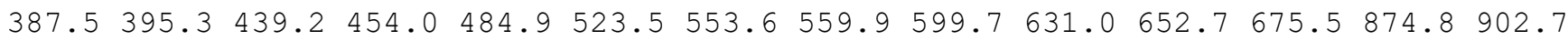
$921.1927 .2961 .8 \quad 993.5 \quad 1000.4 \quad 1029.2 \quad 1039.5 \quad 1044.5 \quad 1056.3 \quad 1063.9 \quad 1073.8 \quad 1123.5 \quad 1140.9$ $1158.01172 .6 \quad 1184.3 \quad 1204.9 \quad 1232.2 \quad 1259.4 \quad 1265.2 \quad 1276.3 \quad 1277.9 \quad 1289.3 \quad 1296.6 \quad 1309.2$ $1316.91325 .7 \quad 1335.91343 .3 \quad 1353.2 \quad 1368.9 \quad 1379.0 \quad 1387.3 \quad 1448.4 \quad 1560.6 \quad 1631.3 \quad 1792.8$ 
$1879.82649 .8 \quad 2660.2 \quad 2666.2 \quad 2668.2 \quad 2671.4 \quad 2678.6 \quad 2698.02704 .2 \quad 2730.72748 .92770 .3$ $2772.8 \quad 2776.3 \quad 2781.8 \quad 2788.8$

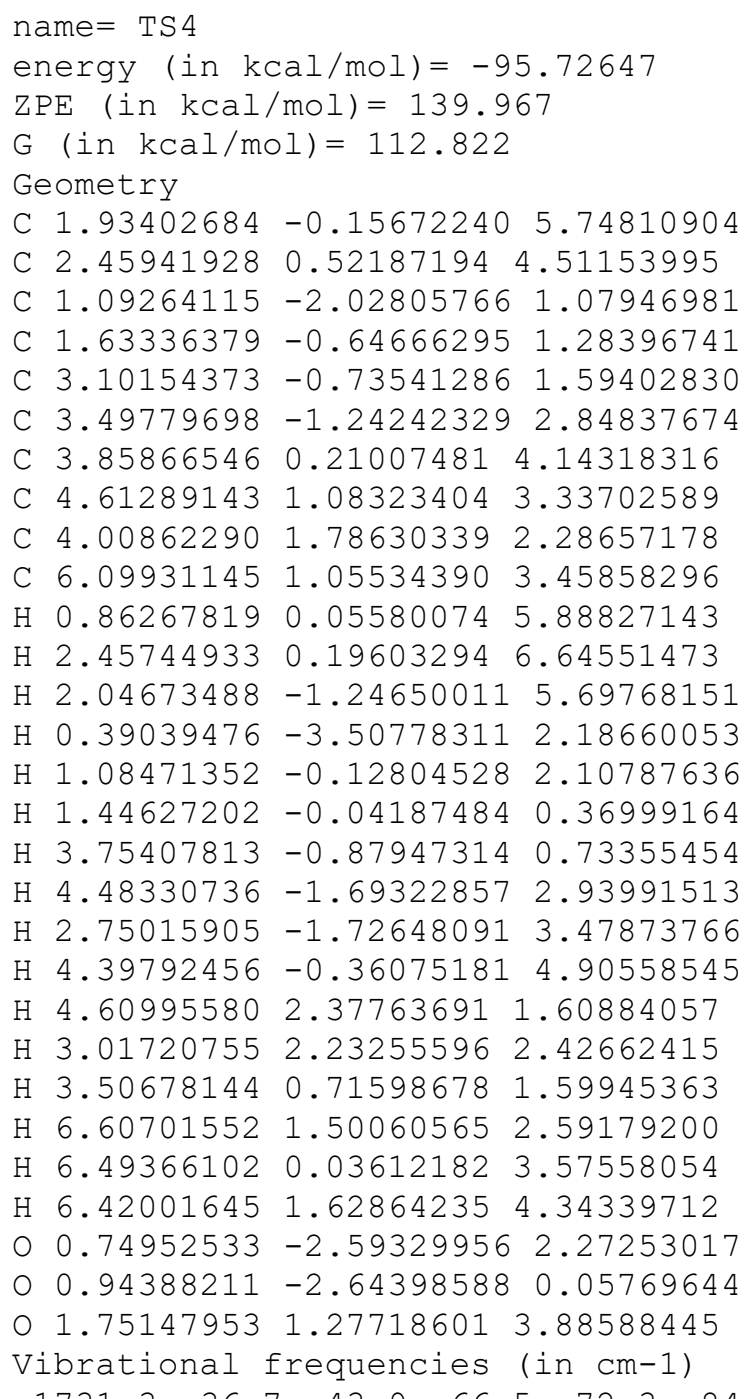

$\begin{array}{lllllllllllllllll}-1731.3 & 36.7 & 43.9 & 66.5 & 72.3 & 84.9 & 98.2 & 102.7 & 139.9 & 175.7 & 187.9 & 214.0 & 305.8 & 357.3\end{array}$

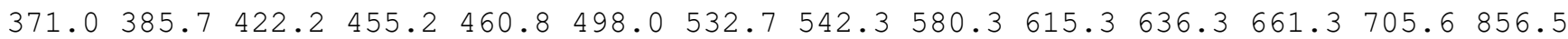
$926.7941 .2 \quad 962.4 \quad 981.2 \quad 995.5 \quad 1011.9 \quad 1032.8 \quad 1037.4 \quad 1048.3 \quad 1053.0 \quad 1070.3 \quad 1092.7 \quad 1099.6$

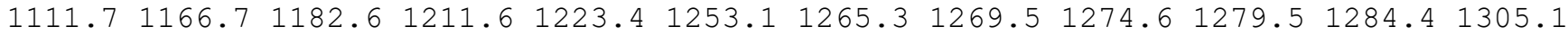

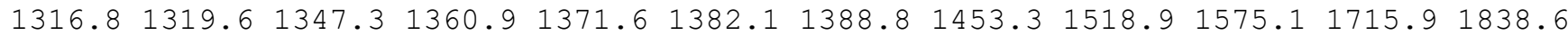
$1875.62645 .4 \quad 2664.3 \quad 2670.0 \quad 2670.7 \quad 2673.12674 .72681 .2 \quad 2729.92737 .3 \quad 2744.8 \quad 2747.2$ $2762.12777 .5 \quad 2780.7 \quad 2794.7$

name $=$ TS 5

energy (in $\mathrm{kcal} / \mathrm{mol})=-86.571799$

$\mathrm{ZPE}($ in $\mathrm{kcal} / \mathrm{mol})=137.588$

$\mathrm{G}(\mathrm{in} \mathrm{kcal} / \mathrm{mol})=109.63$

Geometry

C 2.969780463 .046545063 .25248051

C $2.102765402 .03837612 \quad 2.54080385$

C $3.49546526-0.73456978 \quad 4.98348700$

C $0.21915483-2.056507851 .21573358$

C $1.31908408-1.212970221 .84255407$

C $0.60423964 \quad 0.054637692 .62382781$

C $1.58978086 \quad 0.95126787 \quad 3.22198121$

C $2.24604656-0.623020380 .88546705$

C $1.80591971 \quad 0.06955337-0.27721584$

C $3.67778477-0.62330396 \quad 1.21888174$

H $3.53162519 \quad 3.65575836 \quad 2.53133889$

$\mathrm{H} \quad 3.67623496 \quad 2.57411145 \quad 3.93910431$ 
H 2.33981398 3.73308033 3.83440102

$\mathrm{H} \quad 0.62234221-2.83451104 \quad 0.55922859$

$\mathrm{H}-0.38121673-2.55515930 \quad 1.98836735$

$\mathrm{H}-0.48421521-1.44158124 \quad 0.63102184$

$\mathrm{H} \quad 1.83510777-1.78751074 \quad 2.64555910$

$\mathrm{H}-0.09197170-0.42477108 \quad 3.34834096$

H $0.152767151 .50509581 \quad 1.35385522$

$\mathrm{H} \quad 1.77976774 \quad 0.83247060 \quad 4.27209255$

H $1.65498205 \quad 1.19981268 \quad 0.17797561$

$\mathrm{H} \quad 0.80783606-0.16063838-0.66789452$

H $2.512589020 .23116420-1.09390627$

H $4.34672371-0.34153430 \quad 0.39386353$

$\mathrm{H} 4.03670697-1.57642269 \quad 1.64578563$

H $3.84869061 \quad 0.12609998 \quad 2.03579341$

$\begin{array}{llll}0 & -0.23566174 & 0.65008721 & 1.68040044\end{array}$

o $3.85514349-1.380312624 .11176669$

O $1.89285342 \quad 2.265913191 .31761230$

Vibrational frequencies (in $\mathrm{cm}-1$ )

$\begin{array}{lllllllllllllllll}-655.9 & 28.1 & 53.8 & 74.5 & 80.6 & 87.8 & 96.0 & 103.2 & 118.7 & 136.3 & 169.0 & 174.4 & 194.0 & 256.0\end{array}$ $270.1 \quad 297.2 \quad 329.9 \quad 363.4 \quad 371.6 \quad 388.3 \quad 426.5 \quad 438.0 \quad 531.7 \quad 556.6 \quad 572.0 \quad 612.0 \quad 633.8 \quad 707.3$

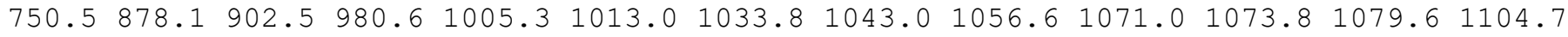

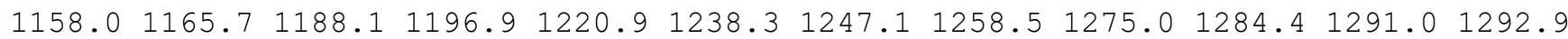

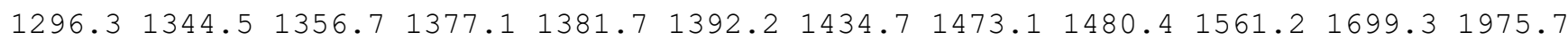
$2025.12596 .12657 .12659 .3 \quad 2668.5 \quad 2676.12676 .52679 .82688 .02689 .2 \quad 2706.82749 .6$ $2761.0 \quad 2776.7 \quad 2783.8 \quad 2797.9$

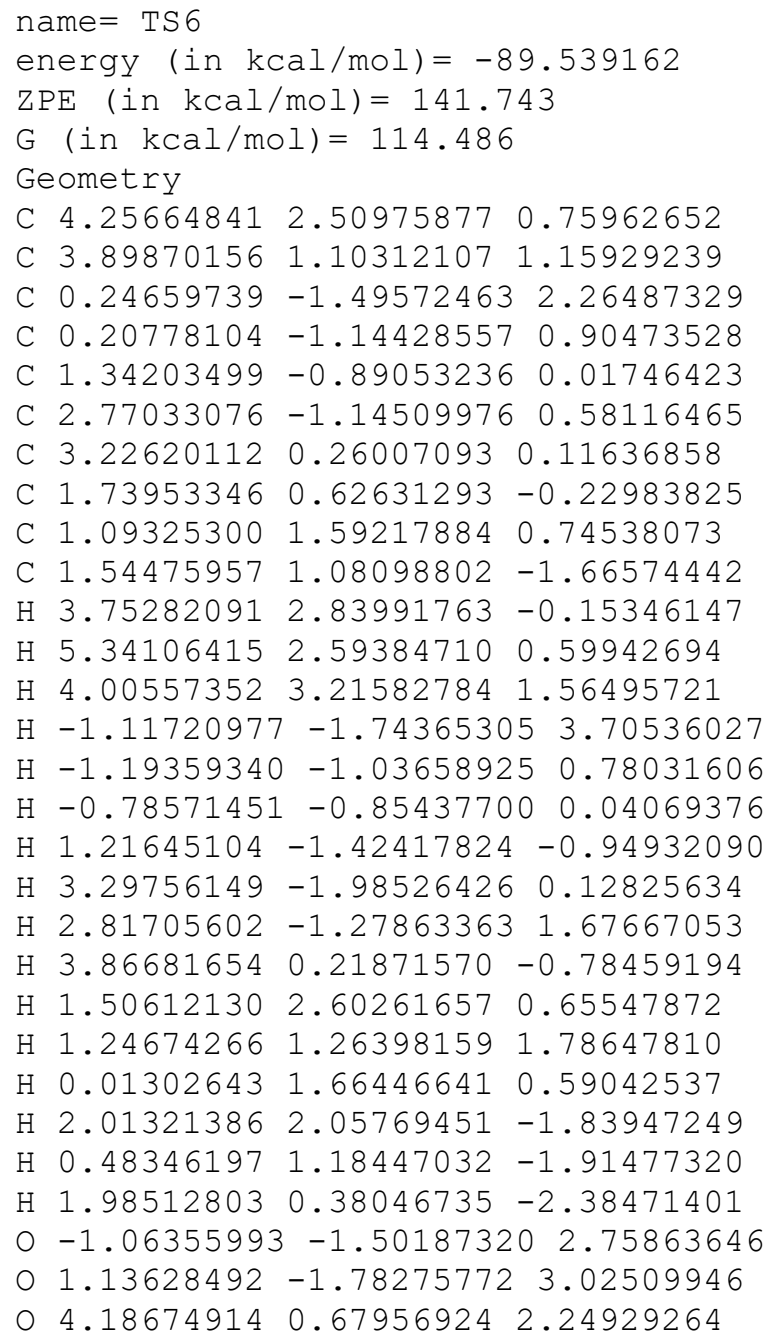


H $3.16518597 \quad 2.13520198 \quad 7.94209626$

H $3.33776361 \quad 2.77845953 \quad 6.29452347$

H $4.77260343 \quad 2.33188469 \quad 7.23574454$

$\mathrm{H} \quad 0.78935343-2.25044075 \quad 1.34616556$

H $2.28298651-2.83498556 \quad 3.62734916$

H $1.23504823-1.64279223 \quad 4.46063526$

H $3.39943753-0.76837038 \quad 2.45535322$

H $5.12661246-1.23280197 \quad 4.11323912$

H $3.92425151-1.72508800 \quad 5.31612103$

$\mathrm{H} 4.861182551 .11196870 \quad 4.73025780$

$\mathrm{H} \quad 1.278100320 .197583125 .56166639$

$\mathrm{H} \quad 0.61161724 \quad 0.78762067 \quad 4.03084375$

$\mathrm{H} 1.38081837 \quad 1.91445982 \quad 5.15039133$

H 2.20686536 1.73105711 2.23963683

H $3.95726013 \quad 1.58832027 \quad 2.44916313$

H $3.03908383 \quad 2.73911094 \quad 3.42838669$

$\begin{array}{lllll}0 & 1.07925947 & -1.73631466 & 2.43214220\end{array}$

$\begin{array}{lllll}0 & 0.06164621 & 0.25792739 & 1.68417369\end{array}$

o $3.41753089-0.290786997 .18340806$

Vibrational frequencies (in cm-1)

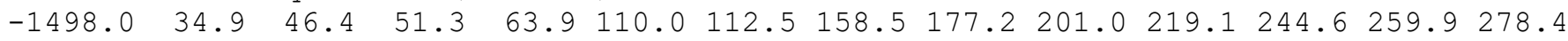

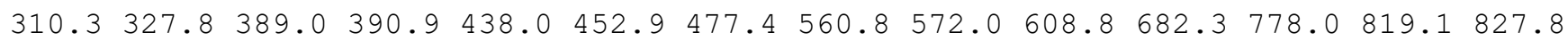
$917.7948 .6 \quad 995.5 \quad 1003.6 \quad 1016.0 \quad 1019.1 \quad 1035.8 \quad 1048.9 \quad 1049.7 \quad 1093.2 \quad 1103.3 \quad 1119.3 \quad 1133.9$ $\begin{array}{lllllllllllll}1161.9 & 1179.3 & 1200.1 & 1223.6 & 1239.2 & 1263.1 & 1265.3 & 1272.7 & 1281.0 & 1286.3 & 1293.9 & 1298.3\end{array}$ $1302.21305 .21311 .2 \quad 1316.3 \quad 1340.1 \quad 1351.7 \quad 1363.1 \quad 1380.91402 .3 \quad 1406.2 \quad 1852.81909 .8$ $2399.8 \quad 2657.7 \quad 2664.4 \quad 2669.6 \quad 2670.7 \quad 2672.7 \quad 2675.92680 .92681 .6 \quad 2718.6 \quad 2728.4 \quad 2734.4$ 2761.52777 .32780 .12781 .3

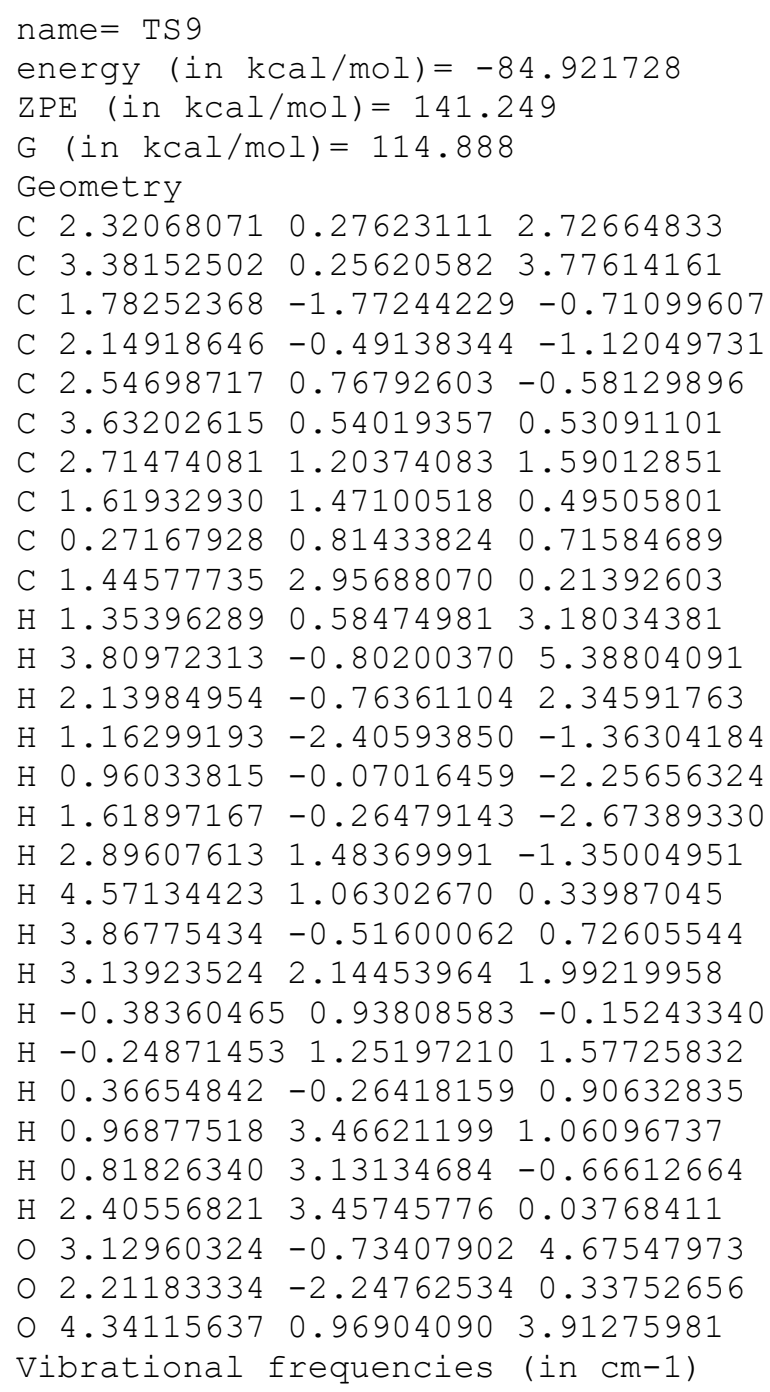


$\begin{array}{lllllllllllllllll}-774.6 & 32.2 & 39.2 & 66.4 & 77.1 & 130.3 & 161.7 & 183.9 & 196.2 & 218.4 & 227.6 & 258.3 & 272.4 & 322.1\end{array}$ $340.6 \quad 360.9 \quad 395.8 \quad 442.9 \quad 482.5 \quad 490.9 \quad 522.9 \quad 541.8 \quad 567.8 \quad 582.6 \quad 607.5 \quad 666.9 \quad 753.9 \quad 806.7$ $875.1908 .5 \quad 928.6 \quad 937.5 \quad 963.0 \quad 973.8 \quad 996.3 \quad 1003.9 \quad 1011.2 \quad 1018.6 \quad 1044.7 \quad 1086.4 \quad 1111.8$ $\begin{array}{llllllllllllll}1126.7 & 1139.4 & 1154.2 & 1185.8 & 1202.2 & 1225.5 & 1248.6 & 1263.7 & 1283.0 & 1289.5 & 1290.7 & 1297.1\end{array}$ $1303.81304 .2 \quad 1312.2 \quad 1318.6 \quad 1327.5 \quad 1348.9 \quad 1362.91370 .91403 .8 \quad 1406.0 \quad 1633.6 \quad 1735.8$ $1875.92623 .6 \quad 2661.0 \quad 2666.92670 .1 \quad 2674.8 \quad 2677.92681 .32710 .72721 .92726 .32754 .5$ $2779.5 \quad 2782.8 \quad 2793.6 \quad 4860.2$

name $=$ TS10

energy (in $\mathrm{kcal} / \mathrm{mol})=-85.372771$

$\operatorname{ZPE}($ in $\mathrm{kcal} / \mathrm{mol})=142.543$

$\mathrm{G}($ in $\mathrm{kcal} / \mathrm{mol})=116.562$

Geometry

C $1.41678355 \quad 4.222412914 .17380672$

C $1.43134832 \quad 2.72148015 \quad 4.10309164$

C $0.24629375-0.63529110 \quad 1.90982076$

C $2.59400029-0.282705551 .27528706$

C $3.27387782 \quad 0.52018366 \quad 2.31277578$

C $2.73563344 \quad 0.53190515 \quad 3.76980304$

C $2.754074362 .07833531 \quad 3.79666714$

C $3.186698862 .09821165 \quad 2.28452592$

C $2.16847944 \quad 2.69014927 \quad 1.33600789$

C 4.549873972 .744204512 .07513882

$\mathrm{H} \quad 0.47056902 \quad 4.62285063 \quad 3.77880324$

H 2.23916035 4.68565920 3.61919221

H $1.48426228 \quad 4.55370028 \quad 5.22033434$

$\mathrm{H}-0.78352417 \quad-0.79171535 \quad 2.19814090$

H 2.40522769 $0.18847644 \quad 0.30647463$

H $2.83295106-1.34811871 \quad 1.21582855$

H $4.34699356 \quad 0.19909983 \quad 2.30873865$

H $3.38690187 \quad 0.04422579 \quad 4.49827109$

$\mathrm{H} 1.72936636 \quad 0.10021299 \quad 3.89378420$

H $3.53126875 \quad 2.48884804 \quad 4.46588968$

$\mathrm{H} \quad 1.15254597 \quad 2.29650279 \quad 1.52122607$

H 2.39711468 2.45696387 0.28968921

H 2.11206709 3.78040612 1.42274420

H $4.90492692 \quad 2.59826832 \quad 1.04793714$

H $5.312598312 .33868018 \quad 2.74906441$

H $4.50612958 \quad 3.82618612 \quad 2.25011551$

$\begin{array}{lllll}0 & 1.20017507 & -1.33530207 & 2.34152970\end{array}$

○ $0.61428665 \quad 0.26246310 \quad 1.11091600$

o $0.43731420 \quad 2.07699079 \quad 4.32428074$

Vibrational frequencies (in $\mathrm{cm}-1$ )

$\begin{array}{llllllllllllllll}-646.9 & 36.2 & 47.9 & 58.2 & 95.6 & 110.6 & 157.2 & 182.0 & 185.0 & 199.0 & 247.3 & 277.7 & 284.7 & 314.1\end{array}$

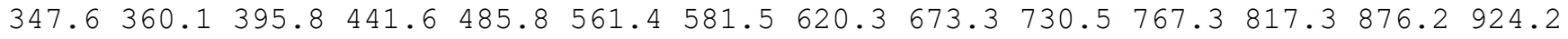

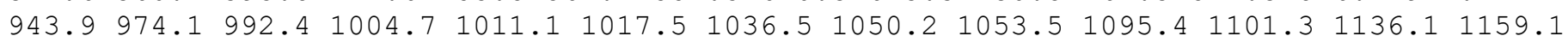
$1166.3 \quad 1185.0 \quad 1198.8 \quad 1230.5 \quad 1243.8 \quad 1255.8 \quad 1259.9 \quad 1273.3 \quad 1283.7 \quad 1295.3 \quad 1296.5 \quad 1297.2$ $1304.41306 .5 \quad 1328.0 \quad 1333.4 \quad 1338.2 \quad 1370.3 \quad 1382.1 \quad 1402.1 \quad 1406.3 \quad 1540.3 \quad 1611.01854 .2$ $2631.72645 .82661 .82666 .62675 .02677 .3 \quad 2678.62680 .82682 .0 \quad 2684.12686 .62725 .5$ $2755.92778 .3 \quad 2780.5 \quad 2782.9$

name $=$ TS11

energy $($ in $\mathrm{kcal} / \mathrm{mol})=-80.050199$

$\mathrm{ZPE}(\mathrm{in} \mathrm{kcal} / \mathrm{mol})=140.084$

$\mathrm{G}($ in $\mathrm{kcal} / \mathrm{mol})=112.801$

Geometry

C $-2.20360070 \quad 1.38421936 \quad 1.26912834$

C $-1.570062392 .68712668 \quad 1.66169865$

$\begin{array}{llll}\text { C } 3.41896058 & 4.33076701 & 2.58835384\end{array}$

C $1.97890826 \quad 4.68928886 \quad 2.44886157$

C $1.34878320 \quad 4.27548874 \quad 1.13305053$

C $-0.13354167 \quad 4.71396870 \quad 0.98386930$

C $-0.56384285 \quad 3.25395249 \quad 0.70278465$ 
$\begin{array}{lllll}\text { C } & 0.92042960 & 2.76822171 & 0.95191078\end{array}$

C $1.111625831 .88188555 \quad 2.17224124$

C $1.40300060 \quad 2.20290793-0.30994693$

$\mathrm{H}-1.48314871 \quad 0.68991926 \quad 0.82088507$

$\mathrm{H}-3.005057651 .550083050 .53494757$

$\mathrm{H}-2.66285602 \quad 0.88560150 \quad 2.13618532$

$\mathrm{H} \quad 4.83925767 \quad 3.32509104 \quad 1.66307241$

H $1.87809877 \quad 5.79479366 \quad 2.57814759$

$\mathrm{H} 1.414684124 .25955776 \quad 3.31222266$

$\mathrm{H} \quad 1.97214827 \quad 4.61098821 \quad 0.28044162$

$\mathrm{H}-0.31998370 \quad 5.40582883 \quad 0.15888147$

$\mathrm{H}-0.58098753 \quad 5.13774800 \quad 1.89192121$

$\mathrm{H}-0.88296640 \quad 3.09963852-0.34643648$

$\mathrm{H} 2.17377313 \quad 1.79337058 \quad 2.43179151$

H $0.72934867 \quad 0.86995109 \quad 1.99785845$

H $0.59138493 \quad 2.270940413 .05813695$

H $2.966766812 .54389247-0.48108856$

H $3.04527552 \quad 1.927106890 .03243163$

$\mathrm{H} \quad 1.24974143 \quad 1.17363277-0.53633570$

O $3.87593478 \quad 3.53444006 \quad 1.57686039$

$04.17533662 \quad 4.659787793 .46420076$

O $-1.86963308 \quad 3.248396932 .68645369$

Vibrational frequencies (in $\mathrm{cm}-1$ )

$\begin{array}{lllllllllllllll}-855.9 & 13.5 & 41.0 & 47.3 & 74.4 & 84.3 & 157.3 & 157.9 & 181.5 & 211.4 & 234.3 & 272.2 & 276.3 & 291.7\end{array}$

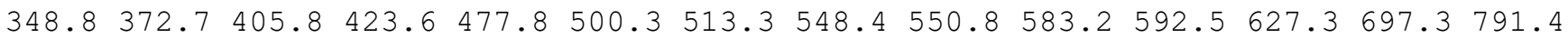

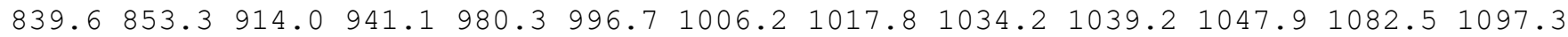

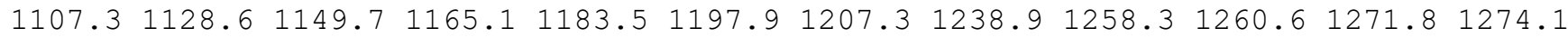

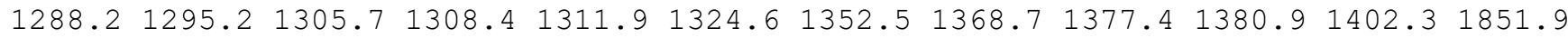
$1868.12632 .5 \quad 2665.12667 .6 \quad 2674.92676 .6 \quad 2680.72708 .02711 .52718 .52727 .12760 .2$ $2777.6 \quad 2780.12789 .0 \quad 4815.6$

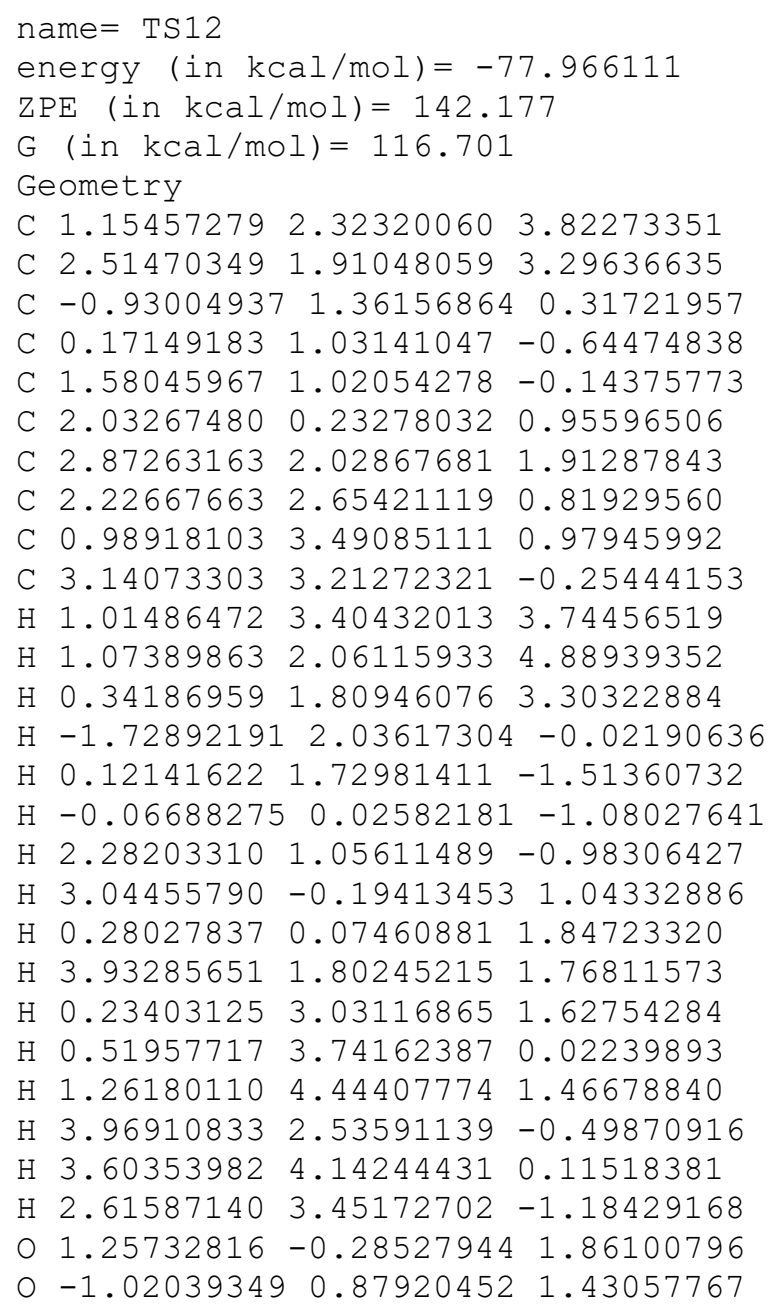


$03.306599811 .45577961 \quad 4.10637843$

Vibrational frequencies (in $\mathrm{cm}-1$ )

$\begin{array}{lllllllllllllll}-1162.5 & 40.4 & 59.7 & 94.1 & 116.3 & 156.5 & 165.2 & 184.8 & 207.4 & 211.4 & 222.4 & 233.1 & 275.8 & 315.5\end{array}$ $320.5 \quad 338.9 \quad 368.6 \quad 396.7 \quad 439.2 \quad 490.9 \quad 533.5 \quad 563.8 \quad 581.6 \quad 595.3 \quad 687.1 \quad 814.7 \quad 869.2 \quad 893.4$ $900.6925 .8 \quad 959.3 \quad 982.4 \quad 988.1 \quad 1009.1 \quad 1018.8 \quad 1029.6 \quad 1040.8 \quad 1061.3 \quad 1077.8 \quad 1100.8 \quad 1143.2$ $\begin{array}{lllllllllllllll}1184.9 & 1195.6 & 1236.8 & 1267.4 & 1273.9 & 1279.5 & 1286.3 & 1290.3 & 1291.9 & 1293.9 & 1297.4 & 1327.8\end{array}$

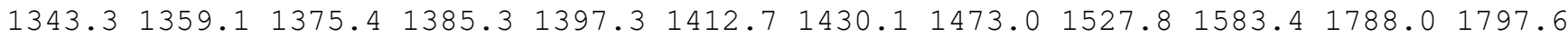
$2390.92633 .7 \quad 2654.92657 .5 \quad 2664.4 \quad 2667.5 \quad 2672.82675 .62680 .3 \quad 2687.62721 .82740 .3$ $2756.32775 .8 \quad 2779.7 \quad 2782.6$

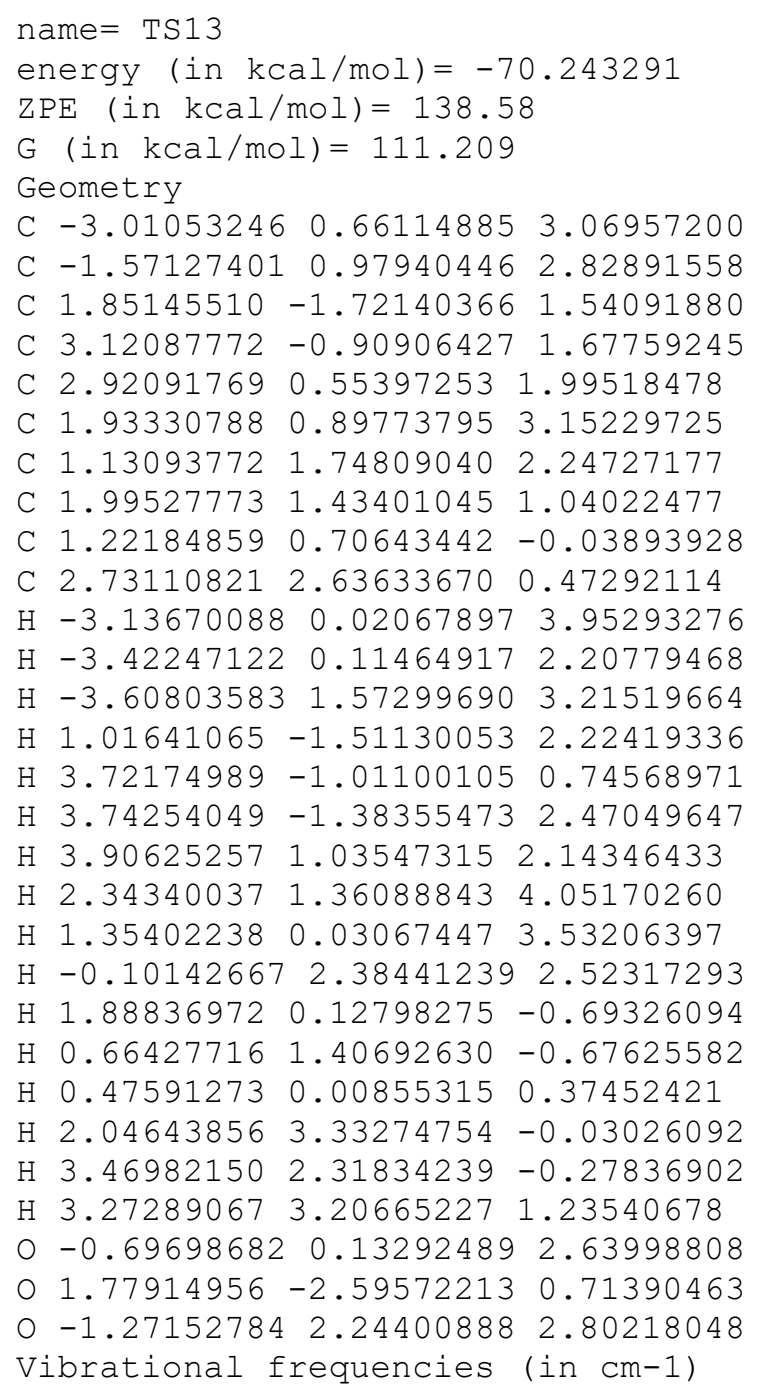


C $1.44141190-1.62302443 \quad 3.10851860$

C $2.35642704-0.526917192 .48933917$

C $1.25154923-0.23469704 \quad 1.40395458$

C $0.65343495 \quad 1.15953990 \quad 1.47350615$

C $1.72490924-0.53465810-0.01082276$

H $3.802123351 .74665001 \quad 1.83995897$

H $4.83283429 \quad 0.99274898 \quad 3.06713185$

$\mathrm{H} 3.89594856 \quad 2.43713059 \quad 3.47701221$

$\mathrm{H}-1.00128571-3.092379193 .42516148$

$\mathrm{H}-1.08554864-0.08955421 \quad 2.99446093$

$\mathrm{H}-1.69623378-0.93392923 \quad 1.59226081$

H $0.36192500-2.27277221 \quad 1.34197359$

$\mathrm{H} 1.71072035-1.119041625 .04416598$

$\mathrm{H} \quad 0.42126637-0.40812221 \quad 4.54343020$

H $3.25042683-1.00892766 \quad 2.05075617$

H $0.38293533 \quad 1.45277242 \quad 2.49520445$

$\mathrm{H}-0.25547831 \quad 1.22907904 \quad 0.86155619$

$\mathrm{H} \quad 1.35284061 \quad 1.91571461 \quad 1.09617266$

$\mathrm{H} 0.89141421-0.48847986-0.72291280$

H 2.16416909-1.53665473 -0.09147880

H $2.48365021 \quad 0.18176874-0.34406789$

○ $0.90019819-1.30657666 \quad 4.44999521$

$\begin{array}{llll}0 & -2.59682585 & -1.99929430 & 3.96902578\end{array}$

o 2.157926330 .850494524 .39434055

Vibrational frequencies (in $\mathrm{cm}-1$ )

$\begin{array}{llllllllllllllll}-149.8 & 19.4 & 39.7 & 66.4 & 80.2 & 90.8 & 128.8 & 157.4 & 175.2 & 199.5 & 219.3 & 232.3 & 279.2 & 295.4\end{array}$

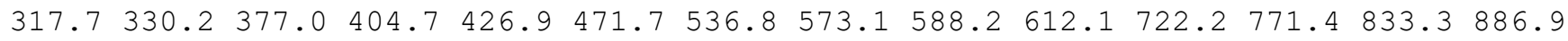
$890.9936 .8 \quad 989.8 \quad 997.6 \quad 1003.4 \quad 1013.5 \quad 1033.3 \quad 1043.6 \quad 1051.91069 .1 \quad 1078.5 \quad 1103.6 \quad 1113.5$

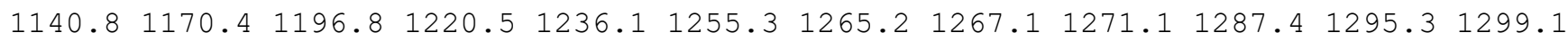

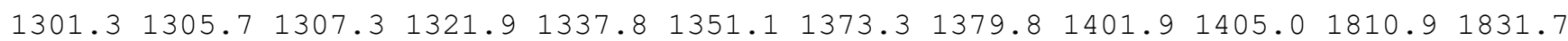
$2649.52653 .3 \quad 2665.02669 .12671 .5 \quad 2673.8 \quad 2674.3 \quad 2675.62679 .92719 .8 \quad 2727.62742 .5$ 2777.82779 .12782 .12802 .0

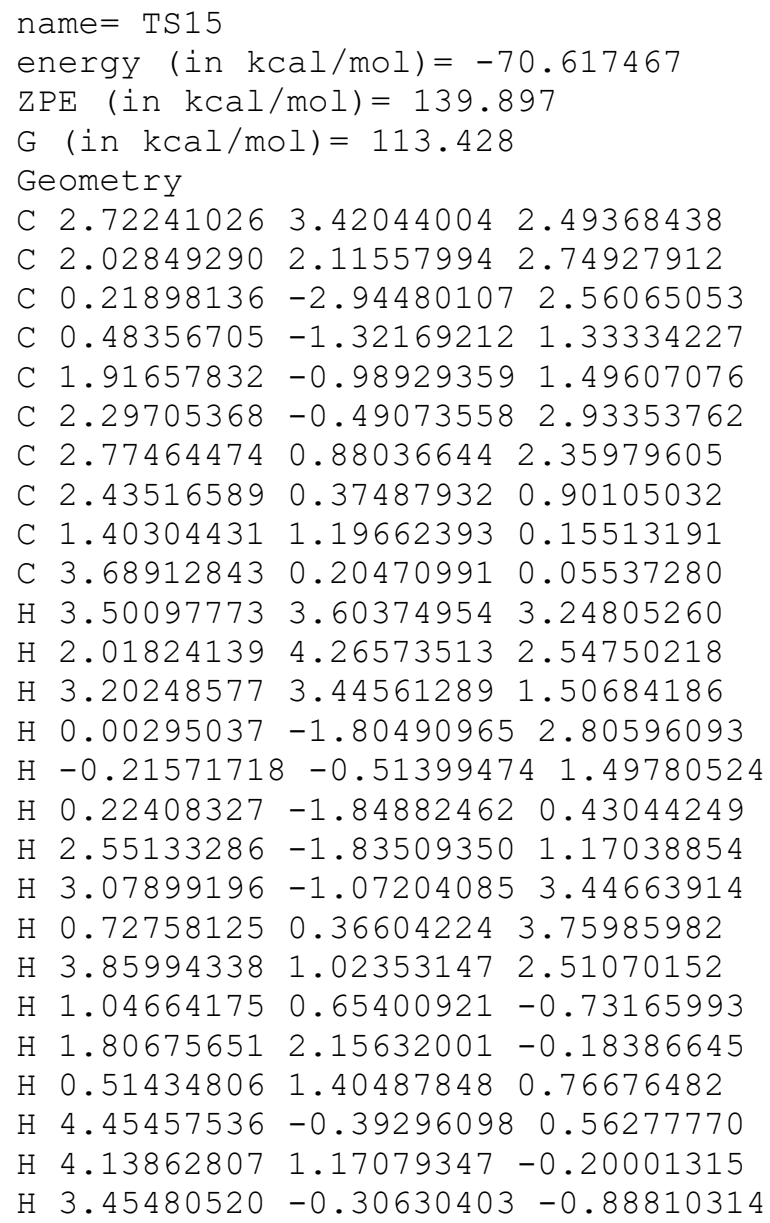


$01.22743575-0.489530523 .82183829$

○ $0.58727400-3.879258521 .99316400$

O $0.91995651 \quad 2.09588398 \quad 3.23972276$

Vibrational frequencies (in cm-1)

$\begin{array}{lllllllllllllll}-1647.4 & 33.5 & 52.9 & 54.4 & 59.5 & 115.3 & 163.0 & 183.5 & 190.1 & 208.2 & 223.1 & 242.2 & 273.1 & 311.6\end{array}$ $320.2 \quad 368.4 \quad 381.7 \quad 396.9 \quad 444.4 \quad 474.6 \quad 535.7 \quad 579.0 \quad 603.8 \quad 610.4 \quad 684.1 \quad 748.4 \quad 780.0 \quad 817.2$ $847.3 \quad 869.3 \quad 896.5 \quad 917.5 \quad 997.0 \quad 1004.6 \quad 1016.1 \quad 1041.3 \quad 1047.91048 .3 \quad 1073.7 \quad 1107.11132 .0$

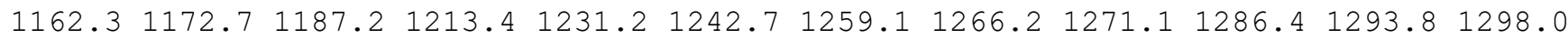
$1299.71306 .6 \quad 1315.8 \quad 1328.1 \quad 1347.2 \quad 1372.91383 .3 \quad 1404.51406 .1 \quad 1426.21831 .1 \quad 1880.7$ $2135.62665 .72666 .2 \quad 2671.42674 .8 \quad 2677.6 \quad 2678.82681 .12698 .92707 .2 \quad 2722.0 \quad 2726.5$ $2773.22778 .3 \quad 2779.5 \quad 2782.3$

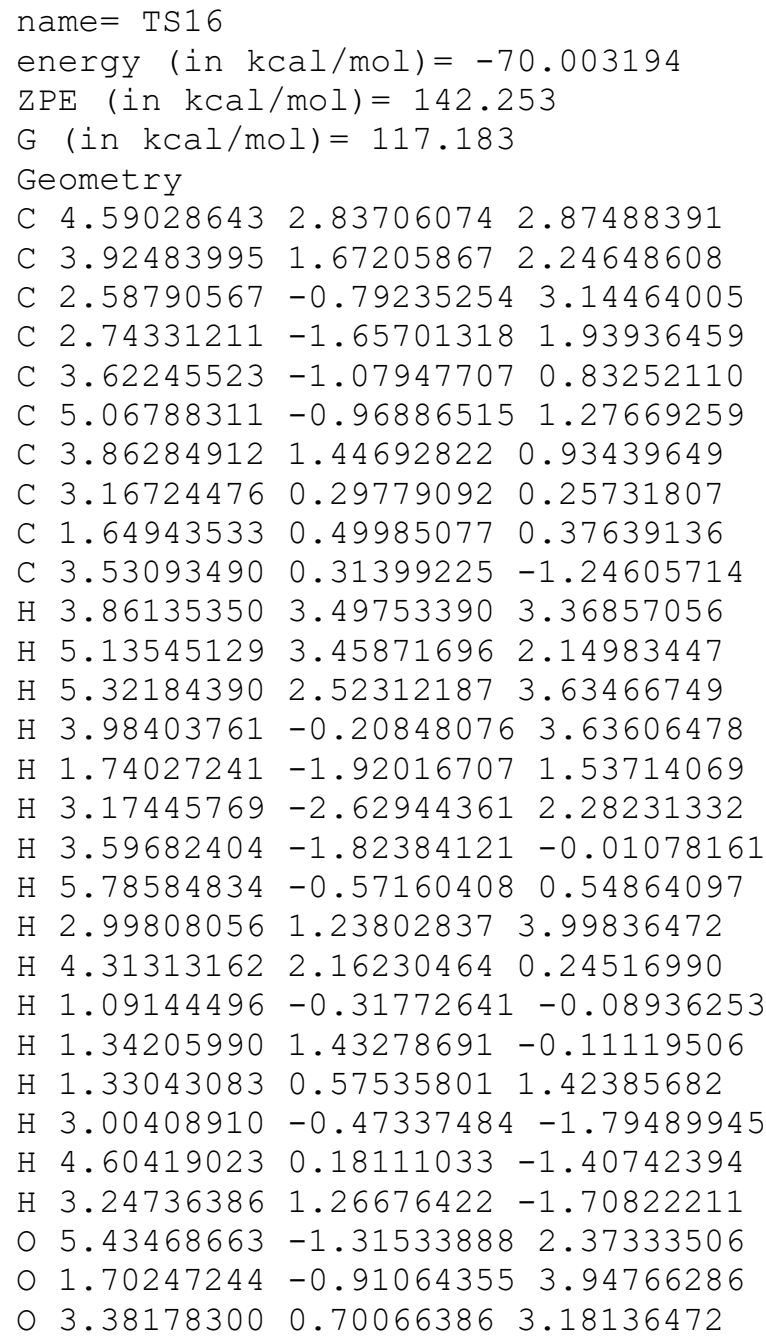


C $2.269330891 .47985424 \quad 2.21065042$

C $1.36050028 \quad 2.48950565 \quad 2.79016889$

C $-0.07120230 \quad 1.91109627 \quad 2.51483311$

C $-0.33860760 \quad 2.99205337 \quad 1.44237275$

C $1.02843928 \quad 3.69725263 \quad 1.82653153$

C $1.99476604 \quad 3.91364585 \quad 0.68276357$

C $0.79342322 \quad 4.99294828 \quad 2.58948918$

$\mathrm{H}-2.24387070 \quad 3.39650439-0.72219096$

$\mathrm{H}-1.001830993 .18461005-1.96278900$

$\mathrm{H}-0.85927653 \quad 4.50407846-0.78555259$

H $1.85422966-0.419882551 .12382142$

H $3.27292427 \quad 1.73797918 \quad 1.95683394$

H $1.39605965-1.561990792 .98420903$

H $1.58529392 \quad 2.77982983 \quad 3.82123189$

$\mathrm{H}-0.78859485 \quad 1.86181280 \quad 3.33685683$

$\mathrm{H} \quad 0.01541576-0.17026357 \quad 2.87977878$

$\mathrm{H}-1.21690984 \quad 3.61228975 \quad 1.69378669$

H 2.97326878 4.24411212 1.05415565

$\mathrm{H} \quad 1.63268831 \quad 4.66318958-0.02704945$

$\mathrm{H} 2.17636304 \quad 2.97952408 \quad 0.12635850$

$\mathrm{H} 1.73580695 \quad 5.37437230 \quad 3.00443646$

$\mathrm{H} 0.101662894 .86215982 \quad 3.42837465$

$\mathrm{H} \quad 0.38431621 \quad 5.77414320 \quad 1.93934365$

O $1.31475447-0.62482107 \quad 3.16420524$

○ $0.07069593 \quad 0.59396939 \quad 1.96905089$

O $0.107483821 .53494338-0.40674908$

Vibrational frequencies (in $\mathrm{cm}-1$ )

$\begin{array}{lllllllllllllll}-1048.1 & 37.8 & 57.0 & 95.4 & 115.9 & 157.7 & 180.2 & 186.6 & 209.3 & 229.5 & 249.7 & 293.5 & 320.9 & 372.0\end{array}$ $392.0 \quad 417.4 \quad 420.0 \quad 477.0 \quad 565.5 \quad 576.7 \quad 600.0 \quad 601.7 \quad 665.3 \quad 690.7 \quad 741.4 \quad 790.0 \quad 809.7 \quad 847.3$ $906.2913 .1 \quad 958.1 \quad 992.0 \quad 999.4 \quad 1006.2 \quad 1014.7 \quad 1018.9 \quad 1038.3 \quad 1047.6 \quad 1052.3 \quad 1092.9 \quad 1121.4$ $1156.51157 .5 \quad 1173.5 \quad 1177.3 \quad 1199.3 \quad 1226.0 \quad 1240.3 \quad 1246.8 \quad 1259.7 \quad 1267.1 \quad 1275.91283 .9$ $1290.7 \quad 1298.1 \quad 1299.5 \quad 1304.5 \quad 1308.4 \quad 1316.1 \quad 1358.8 \quad 1381.4 \quad 1401.91403 .6 \quad 1681.01860 .6$ $2195.02661 .4 \quad 2672.2 \quad 2676.0 \quad 2676.5 \quad 2680.3 \quad 2683.92712 .32718 .02724 .42737 .32779 .1$ $2781.32783 .12809 .7 \quad 2819.3$

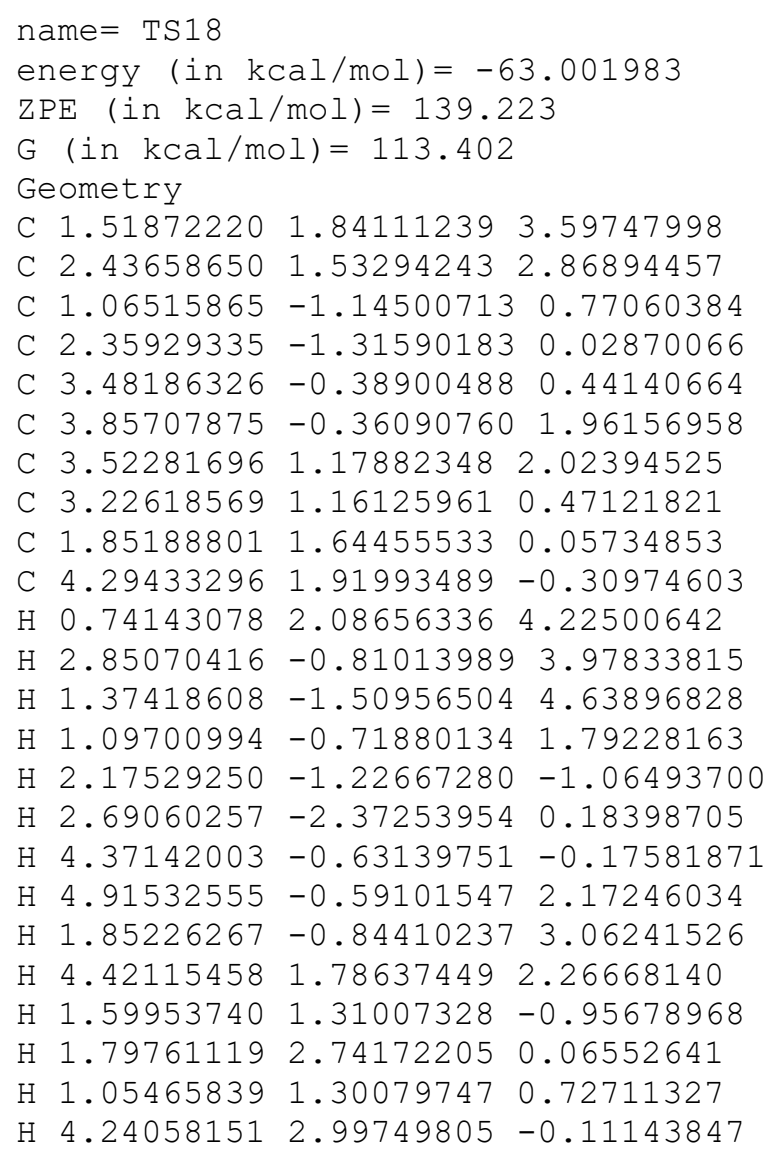


H $4.166006021 .78066575-1.39026420$

H $5.30847078 \quad 1.59354610 \quad-0.05570084$

O $1.66203751-0.66715122 \quad 4.27431185$

$00.01046820-1.481046830 .29124216$

O $3.11548099-1.205344142 .73464632$

Vibrational frequencies (in cm-1)

$\begin{array}{llllllllllllllll}-1689.1 & 35.2 & 48.3 & 78.7 & 132.1 & 146.5 & 156.5 & 175.1 & 179.1 & 204.8 & 210.9 & 245.7 & 273.6 & 297.4\end{array}$ $346.1 \quad 379.2 \quad 407.4 \quad 416.6 \quad 431.5 \quad 480.8 \quad 528.8 \quad 548.2 \quad 575.2 \quad 625.4 \quad 699.5 \quad 745.7 \quad 802.6 \quad 840.2$ $868.7 \quad 873.0 \quad 881.9 \quad 903.2 \quad 906.7 \quad 996.8 \quad 1008.3 \quad 1020.0 \quad 1035.0 \quad 1081.2 \quad 1090.7 \quad 1091.8 \quad 1131.1$ $1163.4 \quad 1179.6 \quad 1185.5 \quad 1203.5 \quad 1221.6 \quad 1249.3 \quad 1259.8 \quad 1264.8 \quad 1270.4 \quad 1285.3 \quad 1291.9 \quad 1298.0$

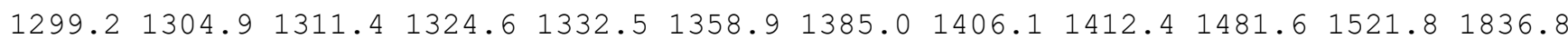
$2165.82430 .92568 .92650 .5 \quad 2666.8 \quad 2674.3 \quad 2677.3 \quad 2678.72693 .6 \quad 2710.32715 .42733 .5$ $2780.2 \quad 2782.4 \quad 2819.4 \quad 2892.3$

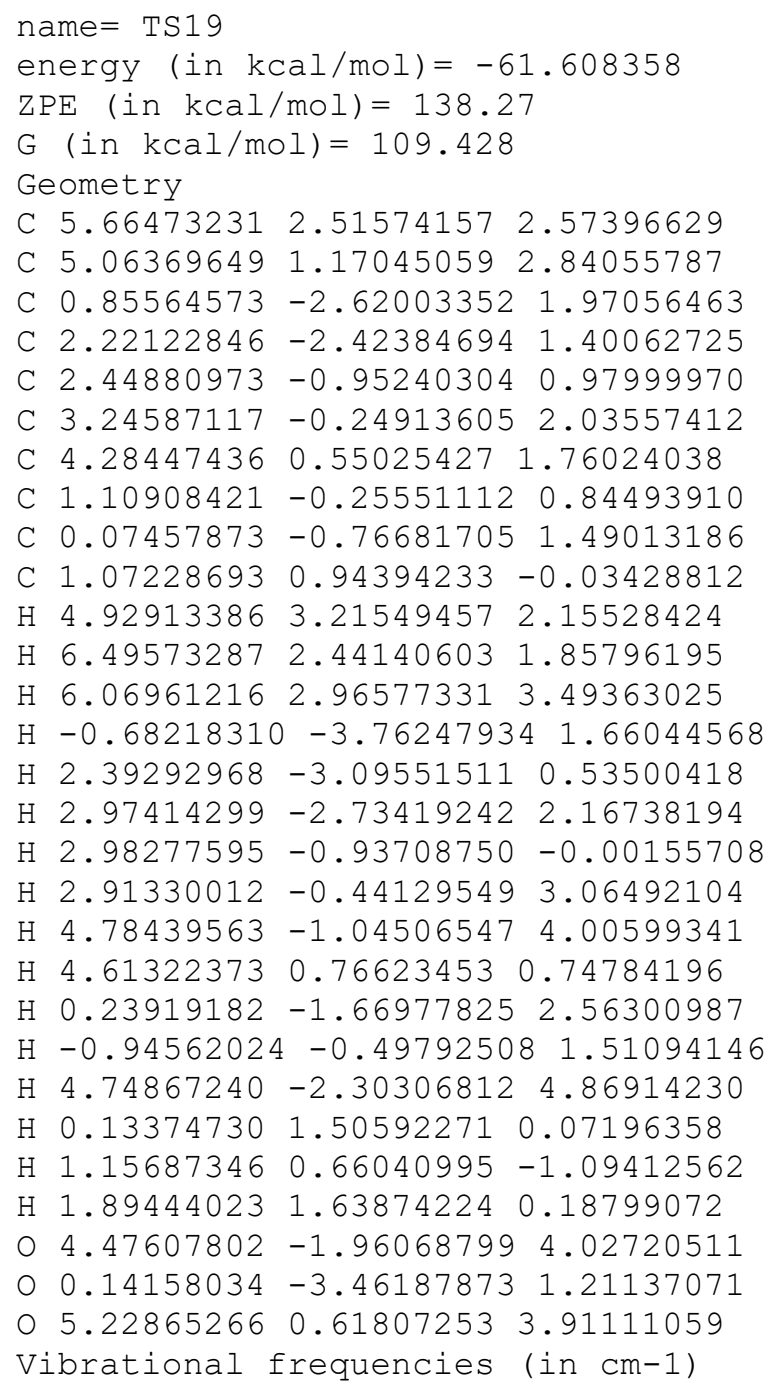


C $-0.98666545-0.19301976 \quad 0.70389725$

C $2.27894183-2.13916375-2.36981712$

C $3.18798209-0.93820208-2.34631819$

C $2.81827724 \quad 0.14046310-1.34770868$

$\begin{array}{llll}\text { C } 2.66346205 & -0.13866614 & 0.14663145\end{array}$

C $1.26615737 \quad 0.50146040 \quad 0.16196837$

C $1.381568390 .80167049-1.38442051$

C $0.34153094 \quad 0.10481455-2.23338561$

C $1.43468060 \quad 2.28728651-1.69834568$

$\mathrm{H}-2.501964391 .315805890 .84973051$

$\mathrm{H}-1.10308433 \quad 1.79888404-0.13059044$

$\mathrm{H}-0.97578926 \quad 1.744429821 .64165803$

$\mathrm{H} 1.66685476-2.30128843-3.26618288$

$\mathrm{H} 3.24918909-0.48744265-3.35900697$

$\mathrm{H} 4.22203518-1.28786956-2.11032774$

H $3.59859716 \quad 0.93986088-1.43246924$

H 2.78505646 -1.54402031 0.64225142

H $2.09723404 \quad-1.71649868 \quad 0.23288714$

$\mathrm{H} 1.17448513 \quad 1.371702320 .82066878$

$\mathrm{H}-0.62277036 \quad 0.62879705-2.20301647$

$\mathrm{H} \quad 0.14158440-0.92008364-1.88612777$

$\mathrm{H} \quad 0.64729328 \quad 0.05978400 \quad-3.28506847$

$\mathrm{H} \quad 0.45902827 \quad 2.76821331-1.56605031$

H $1.74201768 \quad 2.45941923 \quad-2.73765804$

H 2.14930435 2.81896624 -1.05766404

O $0.32618842-0.49558901 \quad 0.46510633$

o $2.24452731-2.92798628-1.45691825$

o $-1.70737384-1.142112290 .86426294$

Vibrational frequencies (in $\mathrm{cm}-1$ )

$\begin{array}{llllllllllllllll}-787.8 & 34.4 & 49.4 & 60.5 & 74.9 & 129.0 & 138.6 & 162.8 & 170.3 & 216.7 & 219.9 & 252.4 & 278.0 & 293.5\end{array}$ $301.8 \quad 364.6 \quad 380.6 \quad 434.6 \quad 466.3 \quad 476.0 \quad 513.9 \quad 540.4 \quad 549.6 \quad 569.6 \quad 620.6 \quad 689.9 \quad 753.8 \quad 798.3$ $855.7894 .2937 .3954 .4 \quad 992.1 \quad 1000.7 \quad 1009.8 \quad 1016.9 \quad 1021.6 \quad 1036.3 \quad 1056.2 \quad 1064.3 \quad 1083.5$

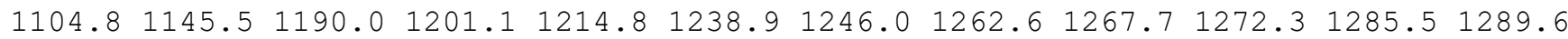
$1297.11298 .3 \quad 1300.3 \quad 1309.7 \quad 1316.2 \quad 1325.0 \quad 1338.5 \quad 1377.8 \quad 1380.5 \quad 1403.3 \quad 1406.4 \quad 1836.3$ $1857.32644 .52659 .5 \quad 2661.92662 .4 \quad 2665.12674 .42674 .92678 .62692 .22709 .12734 .2$ $2775.0 \quad 2776.6 \quad 2781.4 \quad 4612.4$

name $=\mathrm{TS} 21$

energy (in $\mathrm{kcal} / \mathrm{mol})=-58.671783$

$\mathrm{ZPE}($ in $\mathrm{kcal} / \mathrm{mol})=138.074$

$\mathrm{G}($ in $\mathrm{kcal} / \mathrm{mol})=111.522$

Geometry

C $2.84303108 \quad 2.737325115 .19062369$

C $2.47182883 \quad 1.67130245 \quad 4.20398312$

C $-0.03794343 \quad 0.16448394 \quad 1.51099968$

C $0.241931120 .99507788 \quad 0.35606589$

C $2.48076427 \quad 1.33263774 \quad 0.93110996$

C $2.96497893 \quad 0.421377092 .00293665$

$\begin{array}{llll}\text { C } 3.26538831 & 1.63534887 & 2.92952112\end{array}$

C $2.71728240 \quad 2.57716141 \quad 1.77244819$

C $1.50559624 \quad 3.40902524 \quad 2.12308460$

C $3.81364513 \quad 3.42788743 \quad 1.14718040$

H $3.65656221 \quad 2.38968847 \quad 5.84505979$

$\mathrm{H} 1.99423715 \quad 2.98240890 \quad 5.84910858$

$\mathrm{H} 3.17508620 \quad 3.664019914 .70942117$

$\mathrm{H}-0.46997745 \quad 0.41285565 \quad 3.42755660$

$\mathrm{H}-0.13223953 \quad 2.01216210 \quad 0.39455936$

H $0.00621325 \quad 0.52714341 \quad-0.59981630$

H 1.575029361 .096243990 .03073086

H $3.83461238-0.18881785 \quad 1.72097817$

H $2.23632131-0.30328603 \quad 2.41935657$

$\mathrm{H} 4.33780472 \quad 1.78101277 \quad 3.13172346$

$\mathrm{H} 0.70052036 \quad 2.80650628 \quad 2.57910550$

$\mathrm{H} \quad 1.07691990 \quad 3.88957527 \quad 1.23375520$ 
$\mathrm{H} \quad 1.75151125 \quad 4.20691567 \quad 2.83381133$

H $3.44841137 \quad 3.94003217 \quad 0.24668055$

H $4.683147262 .83092466 \quad 0.84577212$

$\mathrm{H} \quad 4.16592641 \quad 4.19790969 \quad 1.84329749$

$0-0.46690554 \quad 0.91052302 \quad 2.57044883$

$0.11018050-1.028577391 .64246858$

O 1.600037120 .871959854 .43961779

Vibrational frequencies (in $\mathrm{cm}-1$ )

$\begin{array}{lllllllllllllll}-1378.7 & 39.7 & 55.5 & 75.8 & 89.7 & 97.0 & 126.2 & 144.5 & 153.6 & 159.5 & 184.3 & 229.8 & 252.8 & 280.5\end{array}$

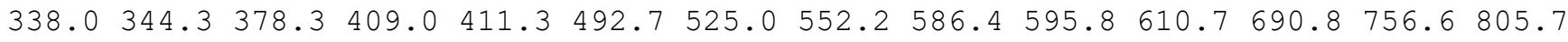
$879.7927 .5 \quad 965.6 \quad 984.4 \quad 998.0 \quad 1005.8 \quad 1009.8 \quad 1014.9 \quad 1032.3 \quad 1047.5 \quad 1057.5 \quad 1076.1 \quad 1078.3$

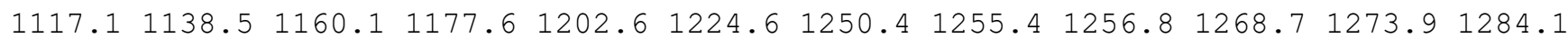

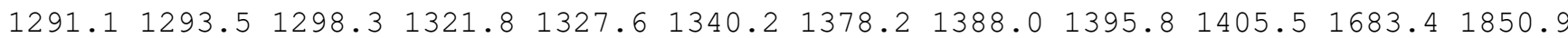
$1864.52626 .52647 .72663 .2 \quad 2672.12674 .5 \quad 2674.82678 .4 \quad 2697.2 \quad 2728.8 \quad 2740.3 \quad 2762.3$ $2770.3 \quad 2776.12778 .9 \quad 2781.4$

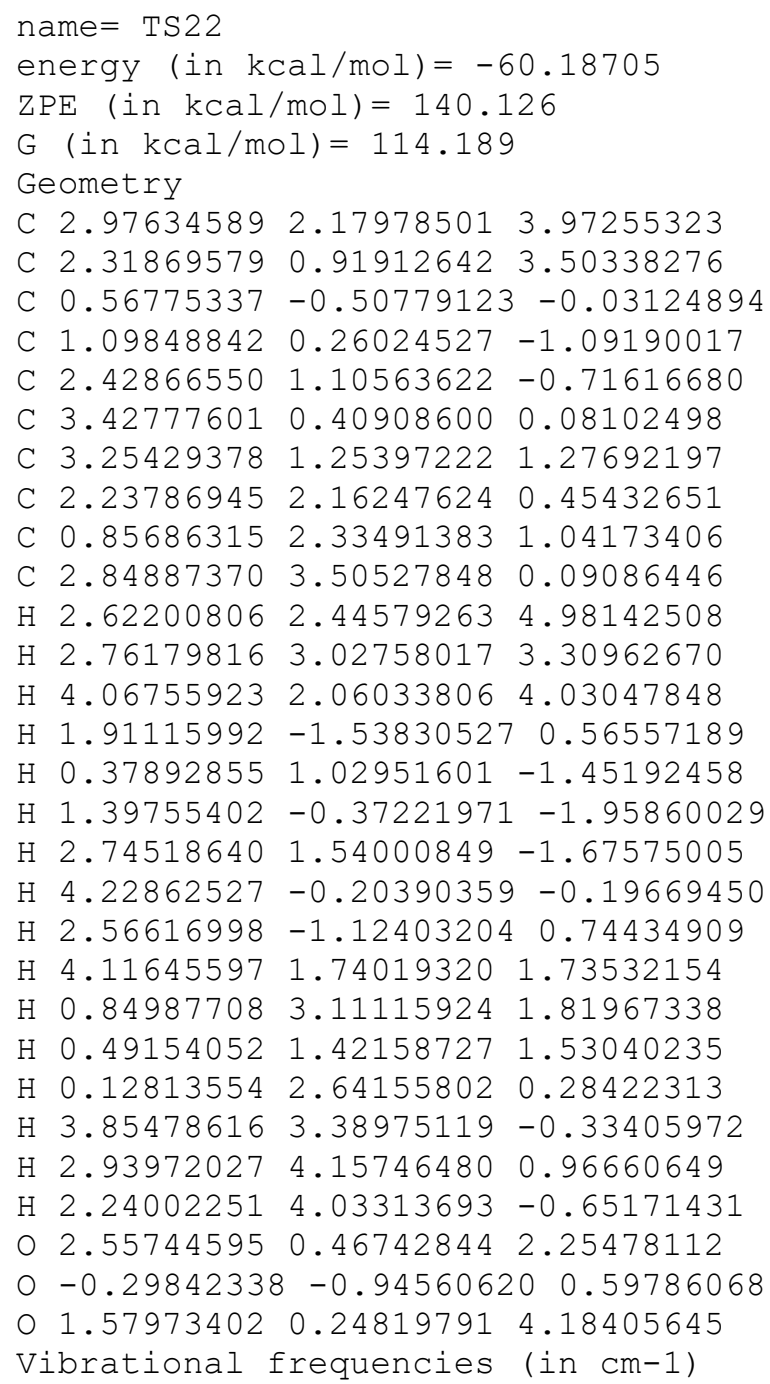




\section{Geometry}

C $3.90886193-1.028910661 .85753983$

$\begin{array}{llll}\text { C } 3.05413429 & 0.20487708 & 1.90243294\end{array}$

C $-1.00774783-0.45416930 \quad 3.46901242$

C $-1.37535312-0.38831618 \quad 2.01349589$

C $-0.25757654-0.06176184 \quad 1.03861287$

C $0.98079597 \quad-0.94265943 \quad 0.88152887$

C $1.90955259 \quad 0.26122771 \quad 0.94204784$

C $0.67812591 \quad 1.19063085 \quad 1.27334885$

C $0.66431377 \quad 1.77674565 \quad 2.66926754$

C $0.46641023 \quad 2.28753921 \quad 0.23937313$

H $4.94664106-0.807363952 .14000300$

$\mathrm{H} 3.90304047-1.50797171 \quad 0.87210832$

H $3.52620379-1.771572552 .57703032$

$\mathrm{H}-1.41723988 \quad 0.30707391 \quad 4.14635305$

$\mathrm{H}-2.18715005 \quad 0.35888039 \quad 1.86907531$

$\mathrm{H}-1.81706565-1.36928074 \quad 1.71887052$

$\mathrm{H}-0.72838968 \quad 0.02002817 \quad 0.02768489$

$\mathrm{H} \quad 0.90458883-2.49690398 \quad 0.94426740$

$\mathrm{H} \quad 0.67802371-1.97125401 \quad 2.69742095$

H $2.32346614 \quad 0.48341396-0.06641417$

H $1.42927109 \quad 2.56757523 \quad 2.76282840$

$\mathrm{H} \quad 0.93311810 \quad 1.04141602 \quad 3.43758544$

$\mathrm{H}-0.30288924 \quad 2.22309172 \quad 2.91495001$

H 1.260402063 .043362960 .30405700

$\mathrm{H}-0.489489332 .80022437 \quad 0.39272398$

$\mathrm{H} 0.472698591 .89782782-0.78438020$

O $1.27823414-1.98169974 \quad 1.88931403$

O $-0.33311337-1.35385443 \quad 3.92419244$

o $3.32083798 \quad 1.11097388 \quad 2.65504210$

Vibrational frequencies (in $\mathrm{cm}-1$ )

$\begin{array}{lllllllllllllll}-1313.0 & 38.7 & 42.8 & 89.8 & 112.0 & 165.0 & 179.2 & 192.0 & 200.9 & 218.4 & 244.5 & 282.9 & 309.8 & 331.5\end{array}$

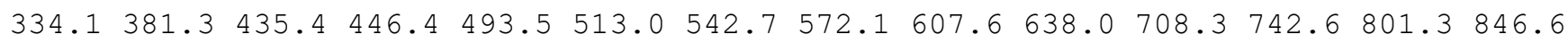
$899.7933 .6 \quad 962.1 \quad 989.3 \quad 1007.2 \quad 1018.3 \quad 1030.7 \quad 1048.4 \quad 1050.8 \quad 1068.1 \quad 1081.6 \quad 1110.1 \quad 1152.4$ $1171.4 \quad 1202.5 \quad 1213.4 \quad 1231.4 \quad 1238.3 \quad 1243.4 \quad 1261.91269 .2 \quad 1277.1 \quad 1278.6 \quad 1288.0 \quad 1294.6$

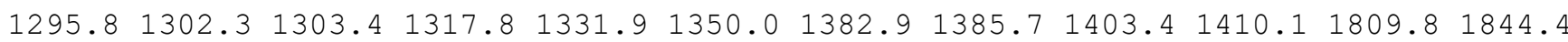
$2469.92585 .7 \quad 2645.0 \quad 2655.3 \quad 2662.92663 .6 \quad 2675.32679 .92682 .2 \quad 2685.02697 .92712 .6$ $2731.92777 .0 \quad 2779.8 \quad 2781.8$

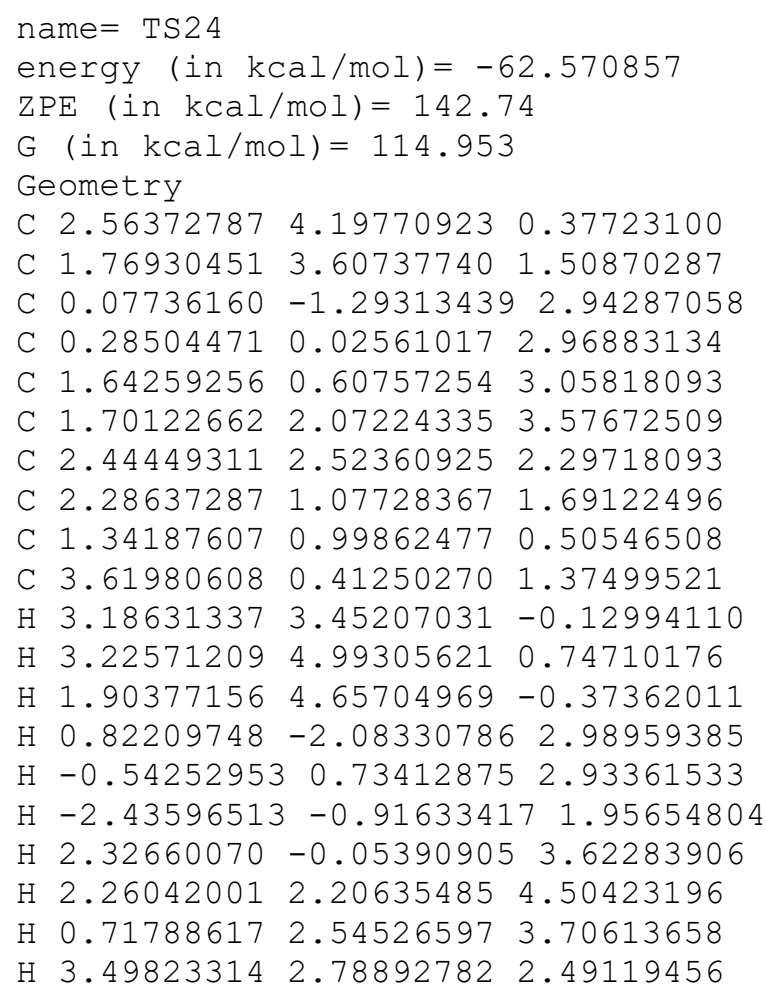


$\mathrm{H} \quad 1.08000995-0.042638790 .27902045$

$\mathrm{H} 1.78336796 \quad 1.43475390-0.39705207$

$\mathrm{H} \quad 0.39711981 \quad 1.52680209 \quad 0.70097902$

$\mathrm{H} \quad 3.54610228-0.26288726 \quad 0.51382634$

$\mathrm{H} \quad 3.99452132-0.186932592 .21231712$

H $4.394905201 .14906377 \quad 1.13530260$

o $-2.19368000 \quad-0.97793999 \quad 2.91343939$

$0-1.12854975-1.924123142 .84313348$

O 0.660118704 .003446721 .76633038

Vibrational frequencies (in $\mathrm{cm}-1$ )

$\begin{array}{llllllllllllllll}-125.9 & 24.6 & 31.8 & 44.0 & 69.6 & 88.9 & 134.4 & 165.1 & 177.7 & 199.2 & 209.8 & 235.0 & 263.1 & 286.1\end{array}$ $315.0 \quad 336.0 \quad 397.2 \quad 413.6 \quad 455.1 \quad 476.3 \quad 571.3 \quad 598.8 \quad 660.2 \quad 688.5 \quad 817.3 \quad 824.6 \quad 902.5 \quad 924.3$ $950.2963 .6 \quad 985.7 \quad 998.0 \quad 1010.7 \quad 1021.3 \quad 1033.1 \quad 1043.4 \quad 1048.6 \quad 1091.7 \quad 1121.4 \quad 1129.3 \quad 1156.3$

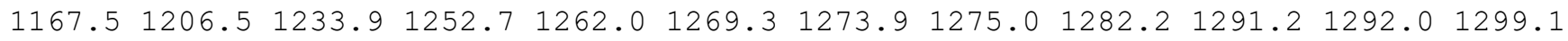

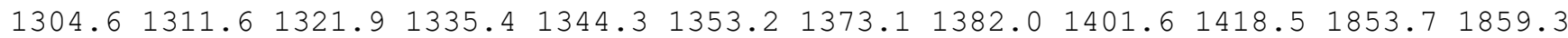
$2666.52669 .2 \quad 2672.92674 .5 \quad 2677.2 \quad 2679.6 \quad 2679.8 \quad 2716.72727 .4 \quad 2728.32756 .2 \quad 2761.1$ $2779.32780 .62781 .8 \quad 2869.2$

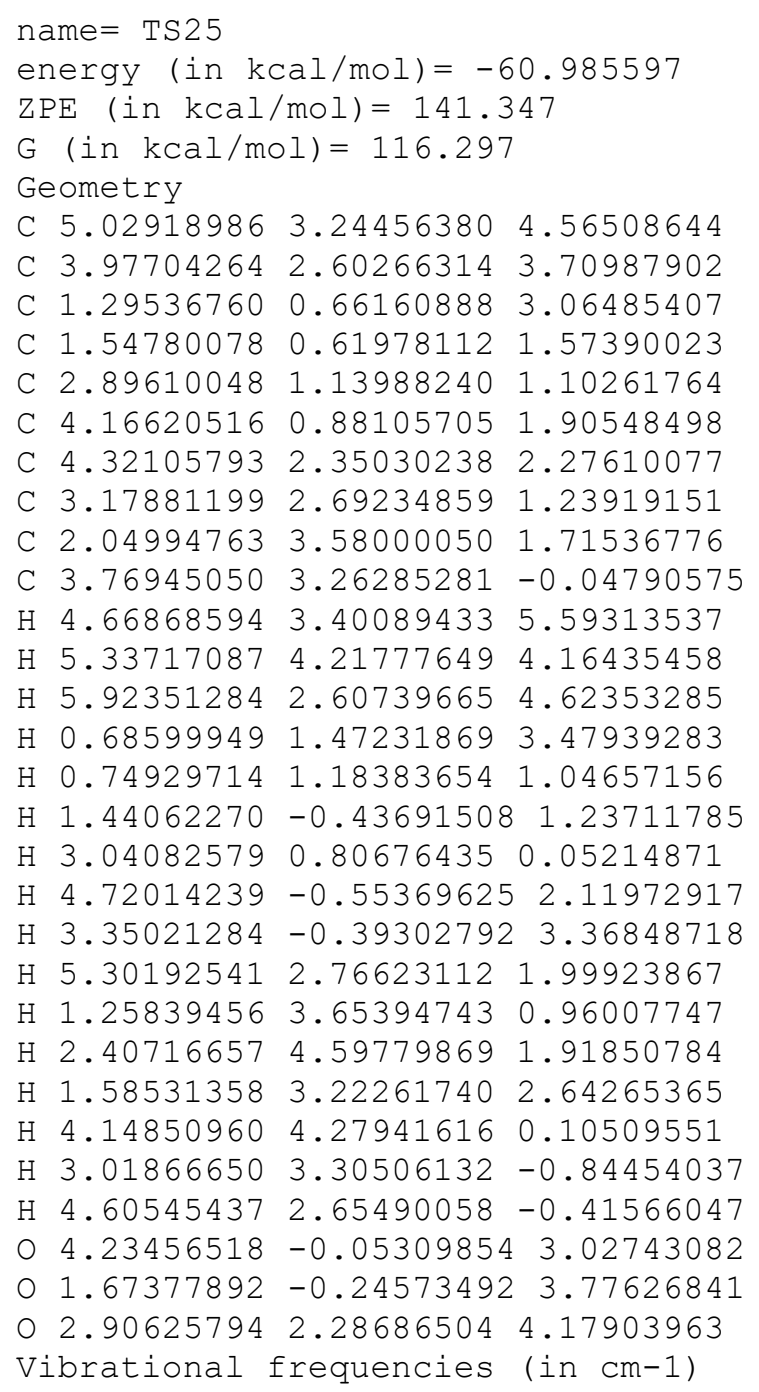


$\mathrm{ZPE}($ in $\mathrm{kcal} / \mathrm{mol})=139.524$

$\mathrm{G}($ in $\mathrm{kcal} / \mathrm{mol})=114.295$

Geometry

C $1.77891995 \quad 2.65402271 \quad 3.32157694$

C $1.676423431 .52883475 \quad 4.14084189$

C $-0.87022309 \quad 0.32977092 \quad 1.97796782$

C $-0.22764912-0.59249171 \quad 0.96605577$

C $1.28087109-0.541230640 .90938335$

C $2.13807099-0.24862207 \quad 2.15197860$

C $2.82372585 \quad 0.83972670 \quad 1.52783874$

C $2.02675363 \quad 0.70051518 \quad 0.21851267$

C $1.16312647 \quad 1.88423210-0.16126555$

C $2.880339920 .26169551-0.96249222$

$\mathrm{H} 2.31325359 \quad 3.50839790 \quad 3.70682689$

H $0.97925088 \quad 2.94155046 \quad 2.66074970$

H $2.50214794 \quad 2.00645664 \quad 2.20319560$

$\mathrm{H}-0.74893103 \quad 1.40982143 \quad 1.84552743$

$\mathrm{H}-0.63959972-0.35860187-0.04067429$

$\mathrm{H}-0.55657593-1.636414491 .17789660$

$\mathrm{H} 1.63683435-1.48707056 \quad 0.43805840$

H $2.51837419-0.990657812 .83627762$

$\mathrm{H} \quad 0.72378362-0.298254713 .91847700$

H $3.87436230 \quad 1.04331247 \quad 1.55873940$

H $0.56493350 \quad 2.25178863 \quad 0.68027218$

$\mathrm{H} \quad 0.476698591 .63876243-0.97935718$

$\mathrm{H} 1.783488202 .72646353-0.49696335$

$\mathrm{H} 2.26331812-0.06296616-1.80735428$

H $3.55263619-0.56353873-0.70304136$

H $3.512958491 .08570634-1.31584345$

$\begin{array}{llll}0 & 1.02633313 & 0.44974010 & 3.33155647\end{array}$

$\begin{array}{lllll}0 & -1.52734064 & -0.12398274 & 2.88357429\end{array}$

O 2.031612771 .135574675 .21209653

Vibrational frequencies (in $\mathrm{cm}-1$ )

$\begin{array}{llllllllllllll}-1594.5 & 51.6 & 75.3 & 83.7 & 116.3 & 139.8 & 169.2 & 181.9 & 195.0 & 206.9 & 210.9 & 244.0 & 308.6 & 344.5\end{array}$ $358.6 \quad 399.7 \quad 415.8 \quad 448.1 \quad 464.9 \quad 519.0 \quad 530.2 \quad 551.8 \quad 626.4 \quad 660.9 \quad 720.5 \quad 735.2 \quad 763.2 \quad 841.6$ $877.0895 .7912 .6950 .6987 .9 \quad 1009.5 \quad 1014.5 \quad 1016.91020 .2 \quad 1044.3 \quad 1065.11084 .4 \quad 1100.6$

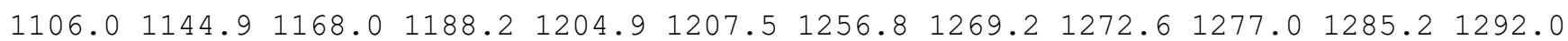

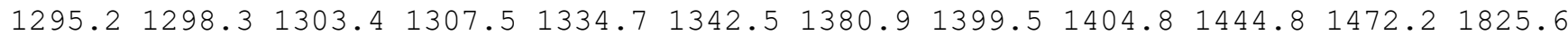
$1995.72642 .92658 .12669 .6 \quad 2676.2 \quad 2678.52681 .92694 .22719 .82723 .6 \quad 2734.42767 .5$ $2774.22779 .6 \quad 2784.02793 .5$

\section{name $=$ TS27}

energy $($ in $\mathrm{kcal} / \mathrm{mol})=-51.074477$

$\mathrm{ZPE}($ in $\mathrm{kcal} / \mathrm{mol})=140.308$

$\mathrm{G}($ in $\mathrm{kcal} / \mathrm{mol})=113.75$

Geometry

C $0.84644238 \quad 3.23103314 \quad 4.03510253$

$\begin{array}{llll}\text { C } 1.10215501 & 2.29852621 & 3.11813955\end{array}$

C $-0.16979448 \quad 0.32460763 \quad 2.82812776$

C $0.77355680-0.60689763 \quad 2.12412932$

C $2.03819623-0.584897342 .85278562$

C $2.90428770 \quad 0.53231871 \quad 3.19928401$

C $2.44930871 \quad 1.91083777 \quad 2.59098235$

C 2.562574281 .762323201 .13595296

C $1.43871621 \quad 1.89759832 \quad 0.21999498$

C $3.907951891 .56697675 \quad 0.56691350$

$\mathrm{H}-1.69429691 \quad 1.44175184 \quad 4.43662699$

$\mathrm{H}-0.16103161 \quad 3.41885705 \quad 4.40398630$

H $1.58754965 \quad 3.86427008 \quad 4.48693860$

$\mathrm{H}-2.70163734 \quad 1.49032316 \quad 5.56727387$

$\mathrm{H} \quad 0.85484965-0.31638517 \quad 1.04034462$

$\mathrm{H} 0.34051914-1.63536634 \quad 2.10833533$

H 2.27700493 -1.50475032 3.37178090

H $3.94555371 \quad 0.36554488 \quad 2.82284683$ 
$\mathrm{H} 3.02146501 \quad 0.60657119 \quad 4.30539799$

H $3.19724405 \quad 2.66955213 \quad 2.96204879$

$\mathrm{H} \quad 0.67895826 \quad 2.63213403 \quad 0.55247493$

$\mathrm{H} \quad 0.87303258 \quad 0.92107008 \quad 0.17273461$

$\begin{array}{lllll}\mathrm{H} & 1.71830363 & 2.14568541 & -0.81240704\end{array}$

$\mathrm{H} \quad 4.573557852 .42415765 \quad 0.76242327$

H $3.90773574 \quad 1.40017677-0.51959289$

H $4.41110014 \quad 0.68535652 \quad 1.02190205$

$\begin{array}{lllll}0 & -2.09189671 & 2.03285383 & 5.08058871\end{array}$

$0-1.09499176 \quad 0.05344475 \quad 3.54404945$

○ $0.02063957 \quad 1.67419752 \quad 2.54429201$

Vibrational frequencies (in cm-1)

$\begin{array}{lllllllllllllll}-254.9 & 35.1 & 59.5 & 74.1 & 81.6 & 95.8 & 108.2 & 115.1 & 177.7 & 206.6 & 258.7 & 274.3 & 304.8 & 327.0\end{array}$ $341.8 \quad 368.2 \quad 387.2 \quad 420.4 \quad 446.8 \quad 474.7 \quad 538.6 \quad 591.5 \quad 636.9666 .5 \quad 687.9 \quad 702.4 \quad 730.3 \quad 763.2$

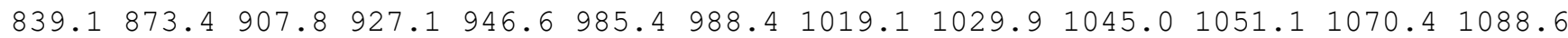

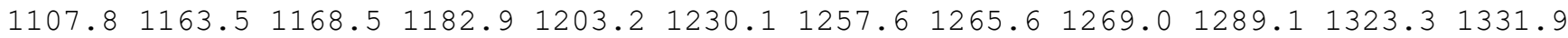

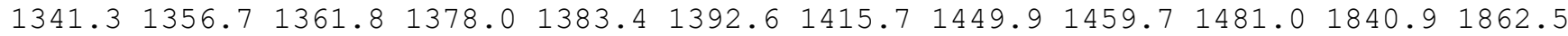
$2432.42586 .7 \quad 2593.6 \quad 2620.0 \quad 2652.4 \quad 2661.4 \quad 2670.7 \quad 2697.82698 .3 \quad 2709.02742 .5 \quad 2746.0$ $2757.7 \quad 2764.0 \quad 2781.92869 .7$

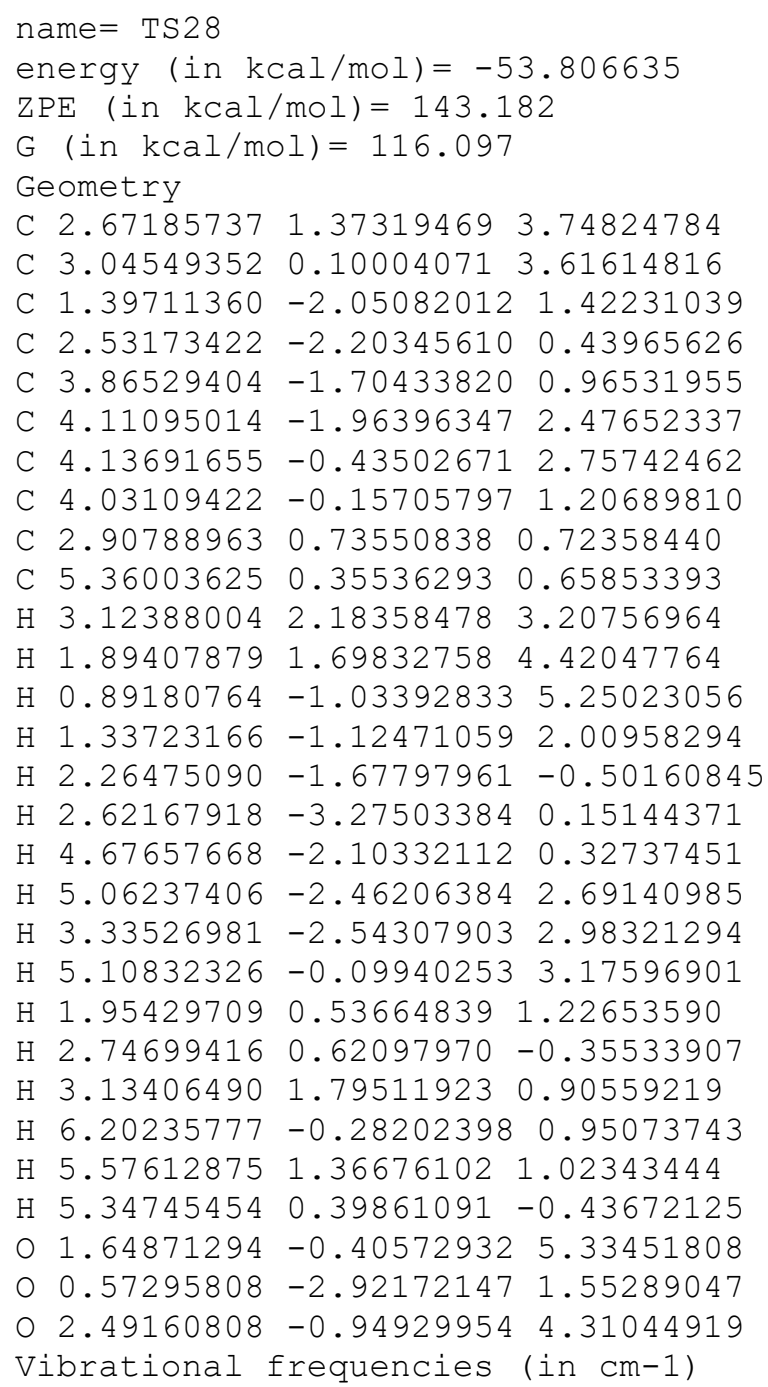




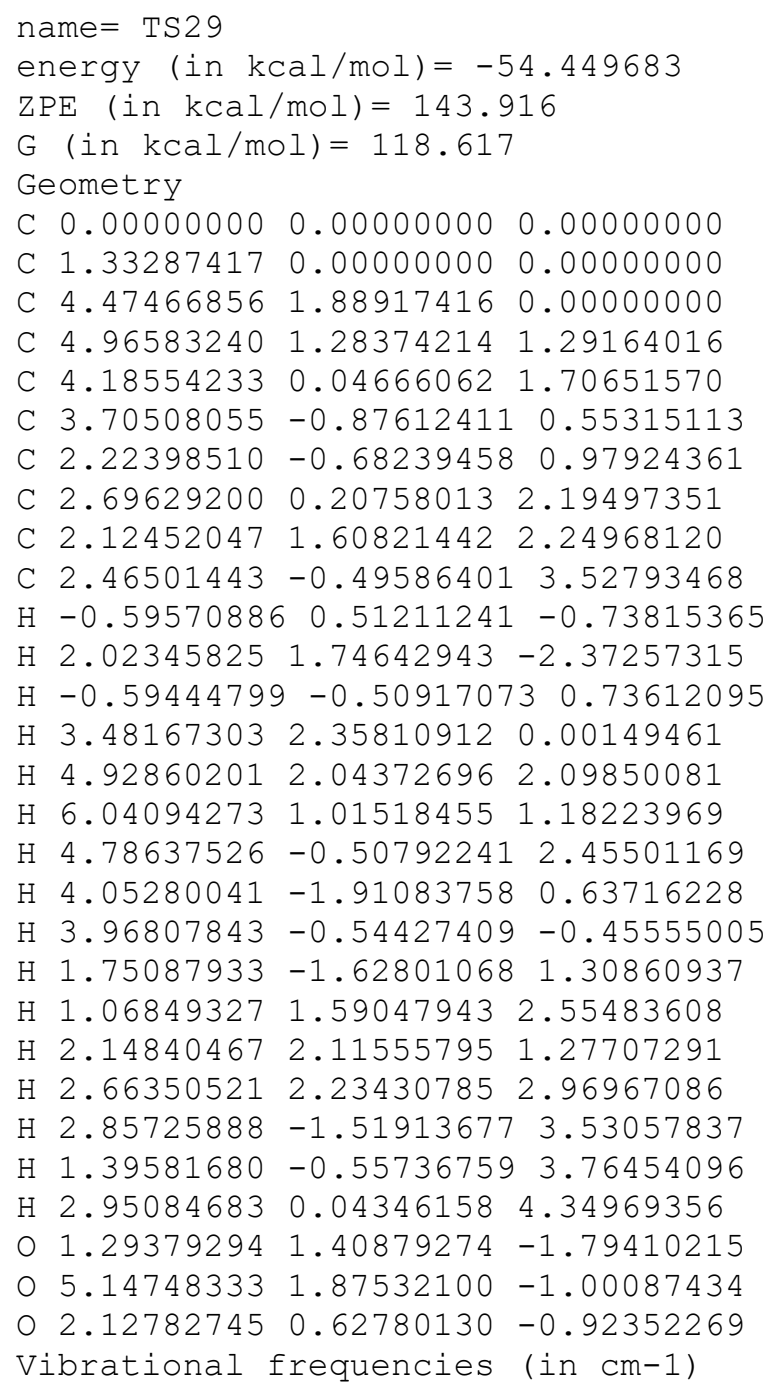


$\begin{array}{lllll}\mathrm{H} & 0.74576567 & 0.13885225 & 0.42724589\end{array}$

H $2.72887565-1.247773210 .86923641$

H $2.55936744-1.084201012 .63979567$

H $3.22930309 \quad 0.31715480 \quad 1.38435657$

$\mathrm{H} \quad 0.88256295 \quad 0.74429339 \quad 4.26947688$

$\mathrm{H}-0.57262251 \quad 1.44924158 \quad 3.52748978$

$\mathrm{H} \quad 0.76018804 \quad 2.48511814 \quad 4.02056415$

$\mathrm{H} \quad 0.92264677 \quad 3.48452913 \quad 1.72964314$

$\mathrm{H}-0.393316992 .46993033 \quad 1.12935629$

H $1.18515241 \quad 2.48167304 \quad 0.31216452$

O $0.22633897 \quad-1.40393428 \quad 3.36276957$

$0-2.15836621-0.25142272 \quad 2.91076421$

O $4.503801020 .73650613 \quad 3.50515100$

Vibrational frequencies (in $\mathrm{cm}-1$ )

$\begin{array}{llllllllllllllll}-947.8 & 30.5 & 57.3 & 72.5 & 78.4 & 84.4 & 129.2 & 138.0 & 163.2 & 202.5 & 222.1 & 251.0 & 254.9 & 280.8\end{array}$ $300.5 \quad 323.5 \quad 343.2 \quad 366.2 \quad 406.3 \quad 445.9 \quad 487.5 \quad 541.5 \quad 587.3 \quad 620.5 \quad 677.3 \quad 705.3 \quad 742.6 \quad 805.6$

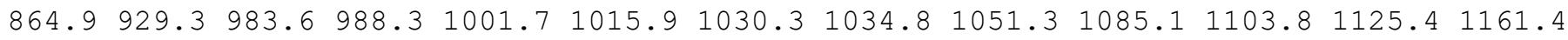

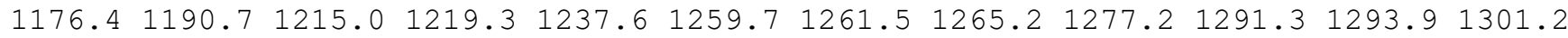
$1304.21310 .21313 .3 \quad 1335.6 \quad 1357.0 \quad 1374.1 \quad 1398.8 \quad 1402.3 \quad 1411.0 \quad 1527.91786 .2 \quad 1837.5$ $2034.42647 .22656 .62663 .92667 .0 \quad 2668.4 \quad 2673.2 \quad 2674.82680 .4 \quad 2683.92696 .2 \quad 2722.9$ $2764.12779 .12779 .3 \quad 2783.1$

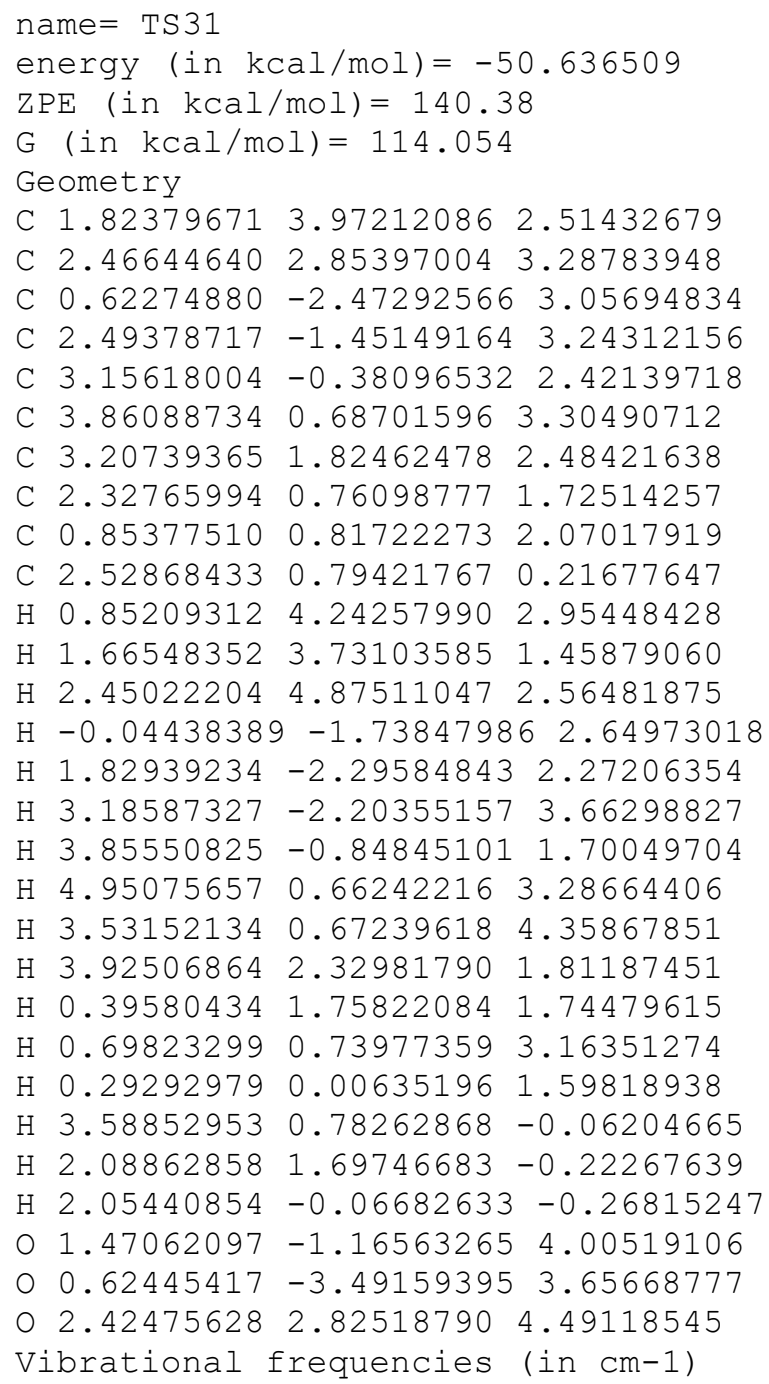




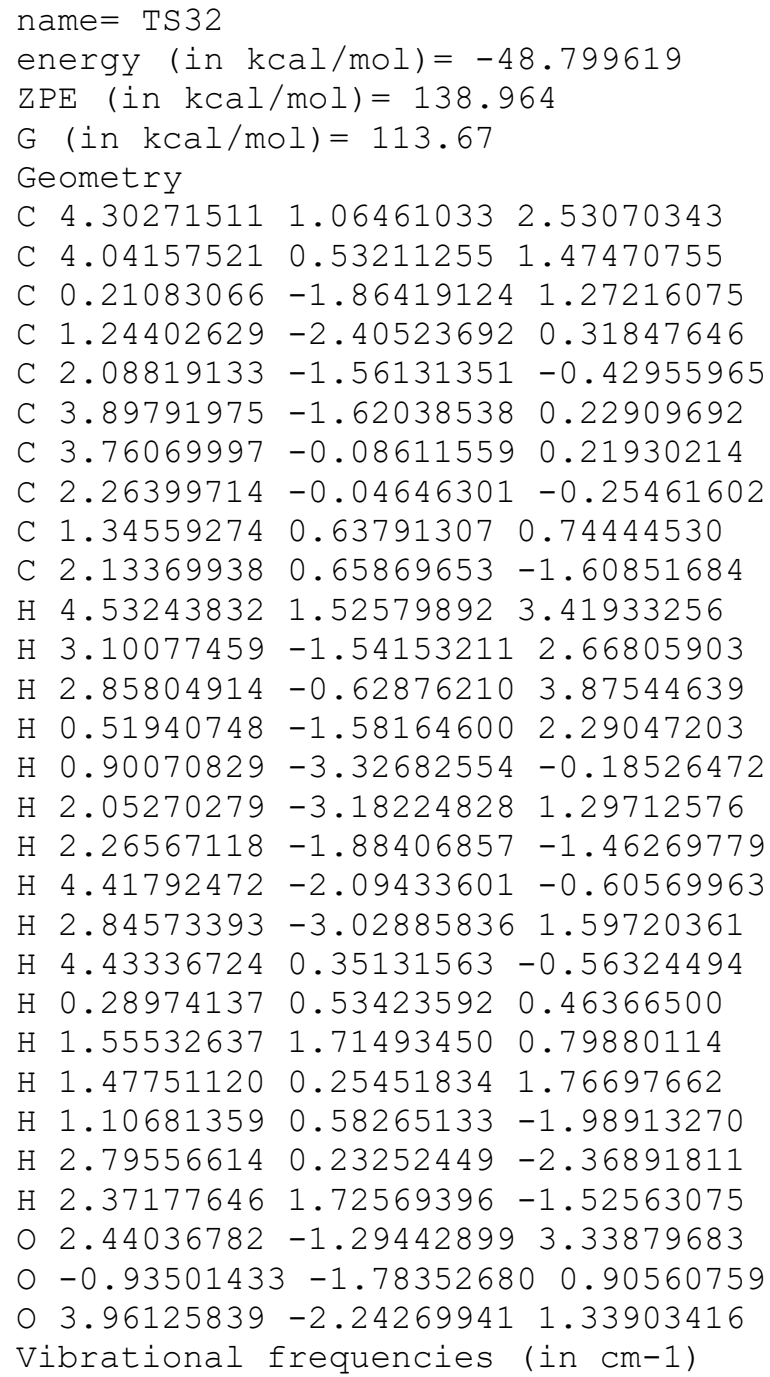


$\mathrm{H} 2.811108341 .19451608-0.55960704$

H $2.56111716-0.54732729-0.42387371$

$\mathrm{H} \quad 4.74955547 \quad 0.03746071 \quad 0.45143032$

$\mathrm{H} \quad 4.52322366-1.475016712 .30504620$

H 2.80206568 -1.171483352 .43448365$

H $5.06947407 \quad 0.34086875 \quad 3.78120286$

H 3.151076313 .110270531 .08115080

$\mathrm{H} \quad 3.52558787 \quad 3.404530592 .78576856$

$\begin{array}{lllll}\mathrm{H} & 2.20386691 & 2.33307781 & 2.35336480\end{array}$

H $5.71410755 \quad 2.53257647 \quad 0.91101098$

H $6.35720340 \quad 1.186825461 .85807059$

$\mathrm{H} \quad 5.89792325 \quad 2.690640802 .66049279$

$\begin{array}{lllll}0 & 0.94416354 & 0.11622329 & 5.06144957\end{array}$

$\begin{array}{lllll}0 & 0.48468640 & 1.29418064 & 0.40318729\end{array}$

O $1.94310290 \quad 0.09801524 \quad 4.04124281$

Vibrational frequencies (in $\mathrm{cm}-1$ )

$\begin{array}{llllllllllllllllll}-294.2 & 29.9 & 44.6 & 54.9 & 82.3 & 108.4 & 162.6 & 169.8 & 189.0 & 204.8 & 228.3 & 268.0 & 285.3 & 333.0\end{array}$

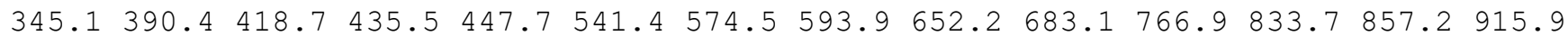
$929.2975 .3 \quad 994.0 \quad 998.4 \quad 1008.0 \quad 1016.6 \quad 1034.8 \quad 1039.1 \quad 1053.3 \quad 1074.0 \quad 1108.01123 .8 \quad 1132.5$ $1160.61191 .6 \quad 1209.3 \quad 1238.2 \quad 1255.0 \quad 1269.1 \quad 1283.0 \quad 1286.51288 .9 \quad 1294.01297 .8 \quad 1300.6$

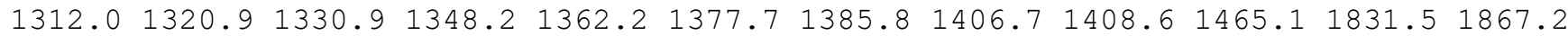
$2645.42657 .42666 .92673 .5 \quad 2677.2 \quad 2677.5 \quad 2677.92710 .32716 .12721 .12737 .82762 .6$ $2780.12782 .3 \quad 2795.8 \quad 2818.5$

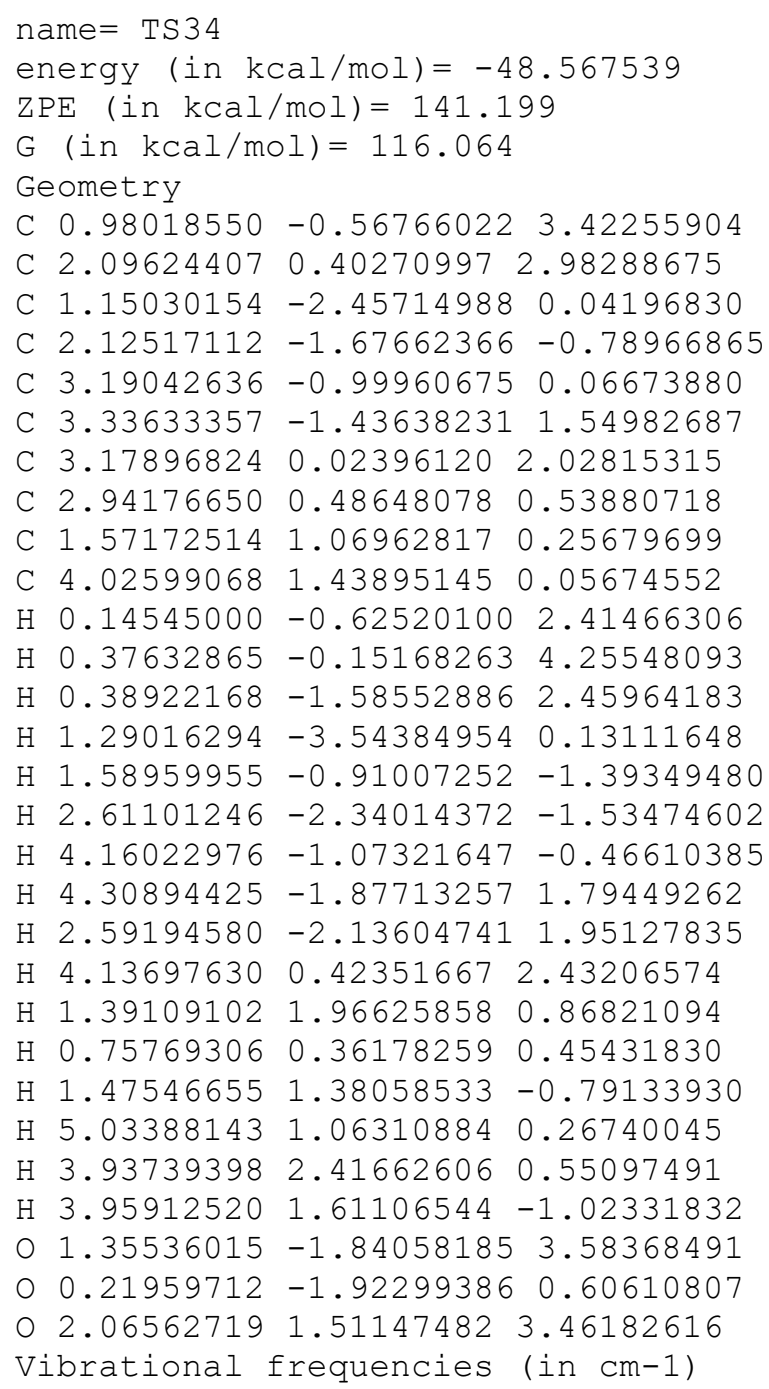




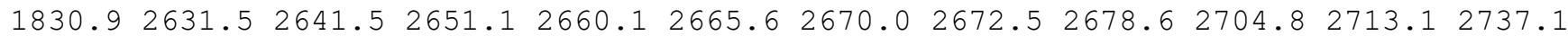
$2754.52778 .12781 .5 \quad 2802.9$

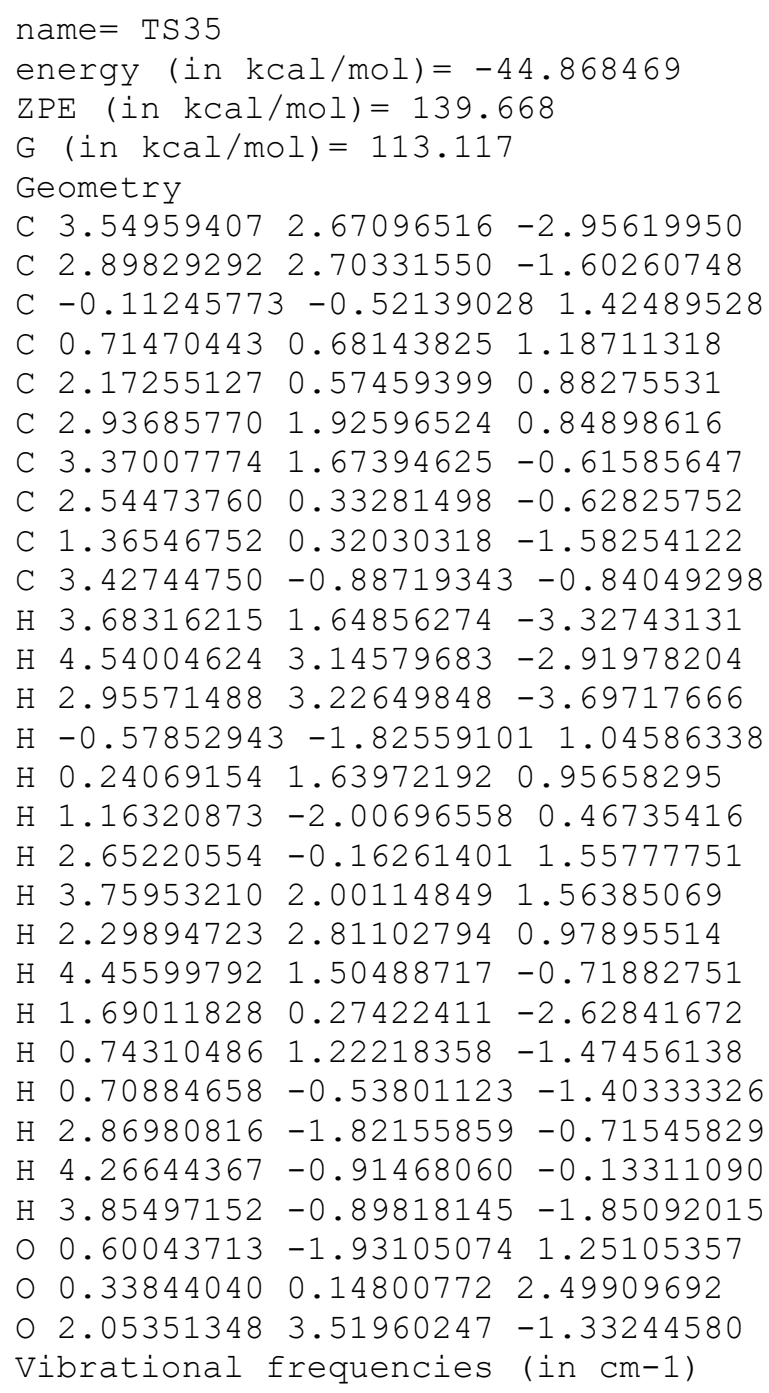


H $3.68955454 \quad 3.53093500 \quad 4.00393177$

$\mathrm{H} \quad 0.98401485-0.64476232 \quad 2.12846479$

$\mathrm{H} \quad 0.312117320 .67840706-0.60817905$

$\mathrm{H} \quad 1.17428449-0.85136143-0.36947746$

$\mathrm{H} \quad 2.695134201 .19434256-0.97663311$

H $3.86479537-1.12899602 \quad 1.88779248$

H $5.05200463-0.31985254 \quad 2.35149472$

$\mathrm{H} 4.94570206 \quad 2.00988907 \quad 0.58230730$

$\mathrm{H} 0.83287459 \quad 3.18824635 \quad 1.35311664$

$\mathrm{H} \quad 1.98549627 \quad 4.28763075 \quad 2.12709224$

$\mathrm{H} \quad 1.79070768 \quad 2.61410600 \quad 2.70067692$

$\mathrm{H} 2.03836168 \quad 3.72606800-0.92351508$

$\mathrm{H} 3.70394670 \quad 3.16693103-1.15344385$

$\mathrm{H} 3.38219771 \quad 4.61645171-0.20053568$

O $4.81880658-1.088753091 .82606476$

$\begin{array}{llll}0 & -0.20852219 & 0.95530189 & 1.88454899\end{array}$

o $5.19956388 \quad 1.19417608 \quad 3.20760668$

Vibrational frequencies (in $\mathrm{cm}-1$ )

$\begin{array}{llllllllllllllll}-326.5 & 22.7 & 40.6 & 52.4 & 73.1 & 78.7 & 119.7 & 126.7 & 152.0 & 164.8 & 180.5 & 185.9 & 208.4 & 244.9\end{array}$ $289.4 \quad 320.9 \quad 333.3 \quad 358.4 \quad 379.4 \quad 420.0 \quad 450.0 \quad 458.9 \quad 480.4 \quad 492.5 \quad 542.9 \quad 613.5 \quad 710.4 \quad 757.7$ $825.2854 .9 \quad 910.2 \quad 936.8 \quad 985.1 \quad 996.4 \quad 997.4 \quad 1008.8 \quad 1038.2 \quad 1051.1 \quad 1063.4 \quad 1072.5 \quad 1083.0$ $\begin{array}{lllllllllllll}1113.1 & 1127.7 & 1173.2 & 1243.2 & 1250.6 & 1256.2 & 1261.6 & 1267.7 & 1272.8 & 1273.6 & 1275.4 & 1277.5\end{array}$ $1280.51298 .2 \quad 1343.1 \quad 1355.2 \quad 1370.9 \quad 1383.0 \quad 1398.2 \quad 1408.81488 .7 \quad 1590.3 \quad 1830.2 \quad 1836.6$ $2636.52651 .02652 .62659 .7 \quad 2660.62661 .4 \quad 2669.62677 .52703 .4 \quad 2725.92733 .62741 .6$ $2766.8 \quad 2771.5 \quad 2779.5 \quad 2863.8$

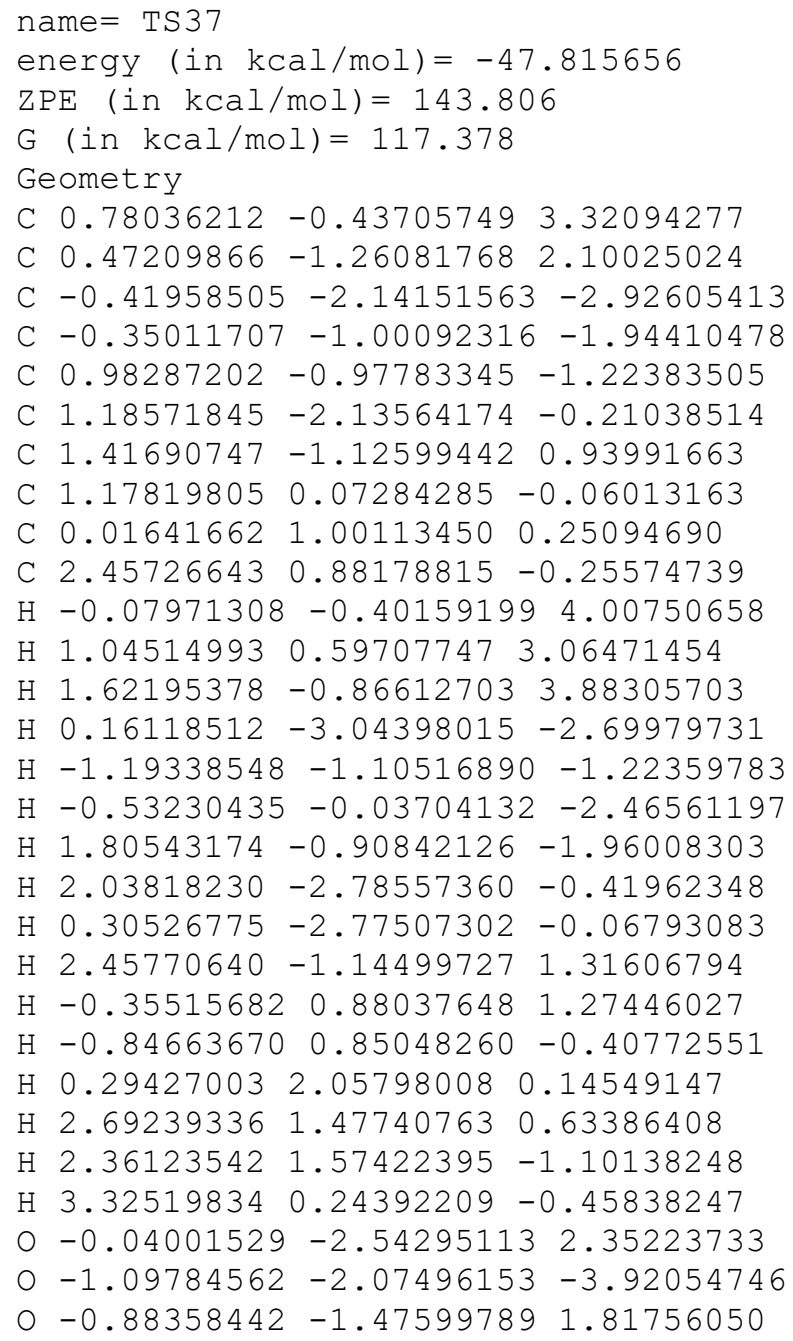


$1173.6 \quad 1178.6 \quad 1204.4 \quad 1227.9 \quad 1248.0 \quad 1264.3 \quad 1272.5 \quad 1276.7 \quad 1278.0 \quad 1287.3 \quad 1291.6 \quad 1296.2$

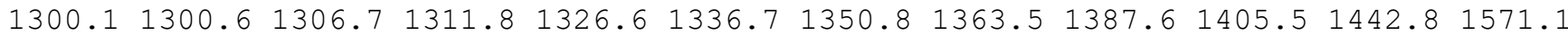
$1837.52647 .32655 .02665 .3 \quad 2668.12668 .92672 .02675 .72678 .02681 .12716 .82723 .0$ $2737.6 \quad 2761.4 \quad 2778.7 \quad 2781.2 \quad 2783.5$

name $=\operatorname{TS} 38$

energy (in $\mathrm{kcal} / \mathrm{mol})=-30.855802$

$\mathrm{ZPE}($ in $\mathrm{kcal} / \mathrm{mol})=135.993$

$\mathrm{G}(\mathrm{in} \mathrm{kcal} / \mathrm{mol})=107.849$

Geometry

C $4.04569821 \quad 1.32599577 \quad 4.51995243$

C $3.08400727 \quad 0.36414898 \quad 3.88156739$

C $-1.45531157 \quad 0.20240201 \quad 3.51826785$

C $0.75980940-0.13449380 \quad 0.29740416$

C $2.08058256-0.268132290 .64634987$

C $2.59013534-1.18288517 \quad 1.94665639$

C $3.42588441-0.07848868 \quad 2.48537272$

C $2.89165528 \quad 0.87917371 \quad 1.34824709$

C $2.030519842 .01823606 \quad 1.85316670$

C $4.009045791 .38563706 \quad 0.44861817$

$\mathrm{H} \quad 4.47561410 \quad 2.03312819 \quad 3.80145106$

$\mathrm{H} \quad 4.87631045 \quad 0.78096475 \quad 4.99214416$

H $3.55580868 \quad 1.90640561 \quad 5.31719071$

$\mathrm{H} \quad 0.83490679-0.98389904 \quad 3.84188657$

$\mathrm{H} \quad 0.25089600-0.84634975-0.33108144$

$\mathrm{H} \quad 0.14015102 \quad 0.69072888 \quad 0.62016013$

$\mathrm{H} 2.69448970-0.86702510-0.08942352$

H $2.93853790 \quad-2.17399857 \quad 1.80898893$

$\mathrm{H} \quad 0.44100508-1.477643962 .47213555$

H $4.52211832-0.24007940 \quad 2.42068634$

H $1.58451007 \quad 2.58540960 \quad 1.02586961$

$\begin{array}{llll}\mathrm{H} & 2.59949810 & 2.72692899 & 2.46420584\end{array}$

H $1.18940888 \quad 1.65065452 \quad 2.46755273$

H $4.65954249 \quad 0.58147460 \quad 0.08826135$

H $4.64516164 \quad 2.10860106 \quad 0.97482332$

$\mathrm{H} \quad 3.602100891 .89794127-0.43372079$

$0.09813789-1.399637133 .37078690$

$\begin{array}{lllll}0 & -1.10989183 & 0.83963235 & 2.62642323\end{array}$

O $2.12461050-0.035004564 .49576293$

Vibrational frequencies (in $\mathrm{cm}-1$ )

$\begin{array}{llllllllllllll}-540.7 & 36.2 & 50.5 & 61.7 & 70.9 & 83.5 & 99.8 & 112.7 & 143.5 & 158.6 & 180.3 & 187.4 & 206.9 & 244.6\end{array}$ $256.1274 .0 \quad 283.1 \quad 319.7 \quad 327.6 \quad 363.9 \quad 378.8 \quad 393.5 \quad 439.2 \quad 477.9 \quad 494.4 \quad 530.6 \quad 579.0 \quad 663.0$

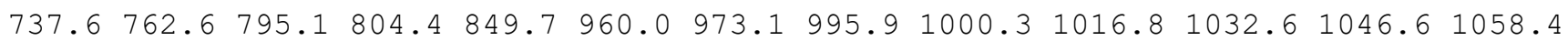
$1064.01088 .7 \quad 1145.6 \quad 1163.2 \quad 1208.0 \quad 1219.3 \quad 1245.4 \quad 1258.0 \quad 1260.7 \quad 1270.8 \quad 1282.8 \quad 1292.3$ $1295.91297 .91314 .1 \quad 1328.2 \quad 1378.4 \quad 1382.4 \quad 1397.2 \quad 1402.01490 .8 \quad 1606.4 \quad 1850.4 \quad 1987.8$ $2631.82653 .0 \quad 2665.2 \quad 2672.0 \quad 2674.6 \quad 2676.3 \quad 2678.2 \quad 2696.22703 .5 \quad 2705.02736 .3 \quad 2761.7$ $\begin{array}{lllll}2776.9 & 2779.1 & 2781.7 & 2856.1\end{array}$

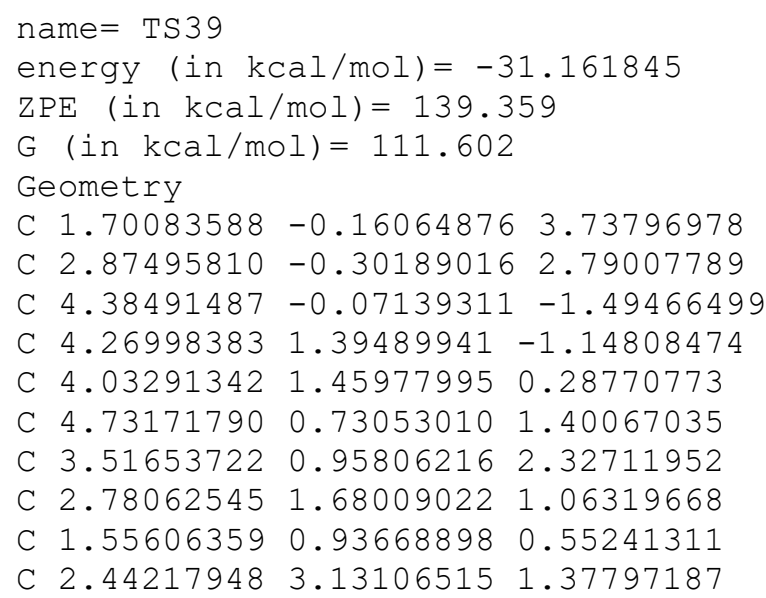


H $0.91707276 \quad 0.51067260 \quad 3.33277221$

H $2.02696708 \quad 0.21813158 \quad 4.72712354$

$\mathrm{H} 1.59037984 \quad-2.13449948 \quad 3.61399410$

$\mathrm{H} 3.46850213-0.61935374-1.73630511$

$\mathrm{H} 3.441404281 .88095834-1.70205570$

H $5.188014541 .93894490-1.45210836$

$\mathrm{H} 5.44004558 \quad 1.963093751 .13822501$

H $5.77318824 \quad 1.38958513 \quad 1.83772727$

$\mathrm{H} 5.13221175-0.27971824 \quad 1.25459071$

$\mathrm{H} 3.680959331 .660912393 .15398544$

H $1.76725948-0.129618920 .40031493$

$\mathrm{H} \quad 1.25599770 \quad 1.33728423-0.42484237$

$\mathrm{H} \quad 0.69535301 \quad 1.02607689 \quad 1.22009673$

$\mathrm{H} \quad 1.64778619 \quad 3.21922117 \quad 2.12382600$

H 2.10090563 3.63968933 0.46610392

$\mathrm{H} \quad 3.313708593 .68764372 \quad 1.73267712$

O $1.04992269-1.38699422 \quad 3.97076901$

O $5.44910024-0.64431312-1.44843425$

O $3.26738864-1.39738394 \quad 2.46717165$

Vibrational frequencies (in cm-1)

$\begin{array}{llllllllllllllll}-2478.1 & 15.1 & 38.1 & 45.0 & 60.6 & 72.5 & 133.5 & 154.9 & 174.1 & 206.7 & 210.6 & 227.6 & 239.3 & 281.7\end{array}$ $300.5 \quad 326.2 \quad 346.7 \quad 398.8 \quad 444.7 \quad 484.7 \quad 515.5 \quad 543.1 \quad 592.1 \quad 634.3 \quad 713.2 \quad 745.2 \quad 789.2 \quad 813.4$

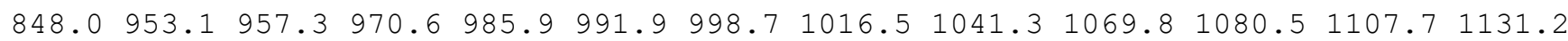

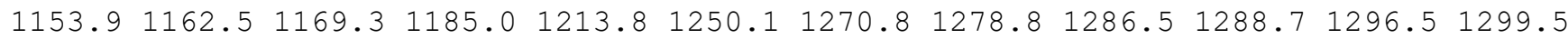

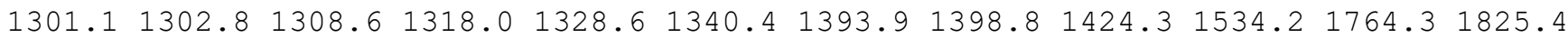
$1844.42635 .0 \quad 2655.1 \quad 2665.92673 .1 \quad 2677.6 \quad 2678.8 \quad 2681.02688 .3 \quad 2708.6 \quad 2730.7 \quad 2751.1$ $2766.62781 .22785 .7 \quad 3002.1$

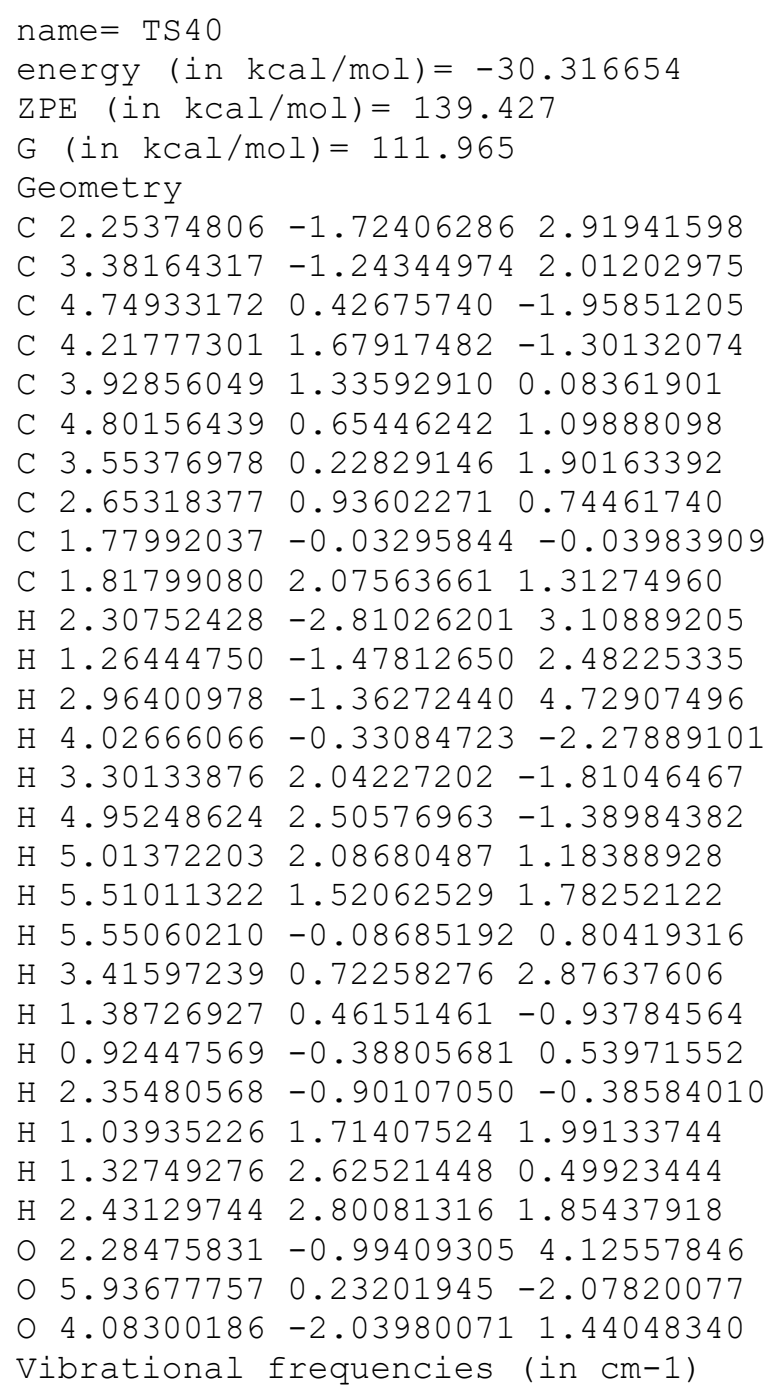


$\begin{array}{lllllllllllllllllll}-2535.6 & 27.5 & 37.4 & 42.1 & 66.7 & 70.0 & 135.8 & 159.8 & 164.3 & 198.5 & 211.6 & 228.6 & 238.9 & 276.0\end{array}$ $291.3 \quad 332.9 \quad 349.6 \quad 411.4 \quad 460.9 \quad 487.8 \quad 515.6 \quad 536.8 \quad 598.8 \quad 646.1 \quad 688.0 \quad 754.9 \quad 787.0 \quad 813.4$

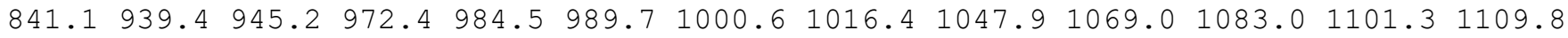
$1158.61164 .7 \quad 1173.0 \quad 1187.5 \quad 1217.0 \quad 1250.4 \quad 1279.8 \quad 1288.0 \quad 1290.2 \quad 1297.2 \quad 1299.2 \quad 1301.2$ $1302.8 \quad 1305.6 \quad 1313.2 \quad 1318.2 \quad 1328.0 \quad 1348.1 \quad 1366.1 \quad 1393.8 \quad 1399.0 \quad 1533.8 \quad 1757.5 \quad 1826.4$

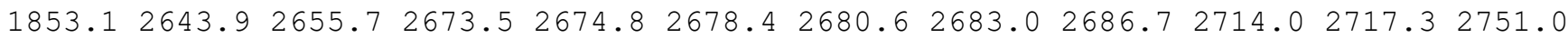
$2781.0 \quad 2785.5 \quad 2816.2 \quad 3005.3$

name $=\operatorname{TS} 41$

energy (in $\mathrm{kcal} / \mathrm{mol})=-27.641629$

$\mathrm{ZPE}(\mathrm{in} \mathrm{kcal} / \mathrm{mol})=140.092$

$\mathrm{G}(\mathrm{in} \mathrm{kcal} / \mathrm{mol})=114.4$

Geometry

C $0.09353558 \quad 1.09204758 \quad 0.80644136$

C $1.54114154 \quad 0.90480803 \quad 1.07423705$

C $2.81919864-0.69876711-0.86793943$

C $3.876815250 .02035614-0.21300643$

C $3.695399671 .47429436-0.12003568$

C $3.03485022 \quad 2.56594771 \quad 2.04048124$

C $2.532277361 .96534868 \quad 0.71633979$

$\begin{array}{llll}\text { C } 4.50864411 & 2.33915594 & -0.75689515\end{array}$

C $4.35935126 \quad 3.82480415-0.72328956$

C $5.67136458 \quad 1.86106263-1.56848291$

$\mathrm{H}-0.53386493 \quad 0.26869482 \quad 1.18712270$

$\mathrm{H}-0.090899991 .11853422-0.29029653$

$\mathrm{H}-0.284832212 .03500916 \quad 1.22851510$

$\mathrm{H} 3.03870732-1.58809066-1.47286599$

$\mathrm{H} \quad 4.89255132-0.32114754-0.39649371$

$\mathrm{H} \quad 3.71647959-0.35681198 \quad 1.12706041$

H $3.77395627 \quad 3.35526036 \quad 1.85594319$

H 2.22489341 2.99499424 2.63578590

H $3.54500053 \quad 1.80350623 \quad 2.65454661$

H $2.002295312 .76526005 \quad 0.12648892$

$\mathrm{H} \quad 5.13518484 \quad 4.27838200 \quad-0.08868144$

H $3.38790730 \quad 4.16478135 \quad-0.34719927$

$\mathrm{H} \quad 4.47033328 \quad 4.25609663-1.72940502$

H $6.411246751 .34874250 \quad-0.93577287$

$\mathrm{H} \quad 6.19663926 \quad 2.67574769-2.08065698$

H $5.35020536 \quad 1.14680984-2.34084570$

$\begin{array}{lllll}0 & 3.05826264 & -0.33711794 & 2.20960439\end{array}$

$01.63770507-0.37477722-0.73734098$

O $1.75569662-0.143909201 .81158260$

Vibrational frequencies (in $\mathrm{cm}-1$ )

$\begin{array}{llllllllllllllll}-883.5 & 56.5 & 83.2 & 99.3 & 105.3 & 111.3 & 133.9 & 151.7 & 177.3 & 210.0 & 216.3 & 230.2 & 247.6 & 261.7\end{array}$ $296.0 \quad 331.3 \quad 339.3 \quad 387.6 \quad 404.6 \quad 470.9 \quad 482.2 \quad 555.3 \quad 590.0 \quad 664.4 \quad 678.1 \quad 741.0 \quad 846.1904 .1$ $921.3956 .4987 .0 \quad 1003.2 \quad 1019.2 \quad 1027.0 \quad 1046.3 \quad 1054.8 \quad 1063.4 \quad 1096.5 \quad 1099.01104 .8 \quad 1110.7$ $\begin{array}{lllllllllllll}1188.1 & 1229.1 & 1239.6 & 1245.6 & 1247.7 & 1271.1 & 1281.8 & 1282.7 & 1286.8 & 1289.8 & 1293.0 & 1296.3\end{array}$ $1309.91322 .3 \quad 1353.2 \quad 1370.3 \quad 1382.6 \quad 1398.5 \quad 1402.2 \quad 1408.7 \quad 1431.5 \quad 1488.8 \quad 1582.1 \quad 1765.5$ $1856.92631 .52645 .62661 .62667 .92669 .3 \quad 2670.72671 .12675 .82677 .12680 .62762 .3$ $2763.92774 .5 \quad 2776.8 \quad 2778.0$

name $=$ TS 42

energy (in $\mathrm{kcal} / \mathrm{mol})=-33.010734$

$\mathrm{ZPE}(\mathrm{in} \mathrm{kcal} / \mathrm{mol})=145.493$

$\mathrm{G}($ in $\mathrm{kcal} / \mathrm{mol})=121.845$

Geometry

C $0.00000000 \quad 0.00000000 \quad 0.00000000$

C $1.491208360 .00000000 \quad 0.00000000$

C $2.237338721 .34104558 \quad 0.00000000$

C $3.71543277 \quad 1.25373275 \quad 0.44398766$

C $4.11910586-0.15351443 \quad 0.87749514$

C $3.64983991-1.14794148-0.23049784$

C $2.27601705-1.179103710 .51313606$ 
C $3.04273944 \quad-0.79781960 \quad 1.84036718$

C $2.34034128 \quad 0.08592559 \quad 2.84824397$

C $3.59176147-2.04171502 \quad 2.54497945$

$\mathrm{H}-0.56110286-0.84214651 \quad 0.39630583$

$\mathrm{H} \quad 1.97578860 \quad 0.68996686 \quad-2.56219410$

$\mathrm{H}-0.56309352 \quad 0.92953440 \quad-0.01820894$

H $1.68698927 \quad 2.07679109 \quad 0.64269546$

H $3.91002458 \quad 1.97512944 \quad 1.25702295$

$\mathrm{H} \quad 4.35667949 \quad 1.57540184 \quad-0.40570131$

H $5.15754700 \quad-0.22167430 \quad 1.20246857$

$\mathrm{H} \quad 4.17710812-2.10263291-0.23833044$

H $3.63389742-0.75662129-1.25028308$

$\mathrm{H} \quad 1.72385237 \quad-2.11930263 \quad 0.51127818$

$\mathrm{H} \quad 1.53278584 \quad-0.46135638 \quad 3.35240699$

H $1.88725050 \quad 0.98064662 \quad 2.41464676$

H $3.042810290 .42926379 \quad 3.61926156$

H $3.16934396-2.96547762 \quad 2.13409483$

$\mathrm{H} 3.36825758-2.04379390 \quad 3.61746585$

H $4.68099389-2.116511942 .45113440$

○ $2.741434541 .24595323-2.26514101$

$02.113903232 .00280809-1.24298754$

O $0.74394791-0.27838905-1.19039446$

Vibrational frequencies (in $\mathrm{cm}-1$ )

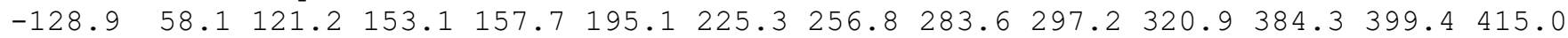
$445.2 \quad 448.4 \quad 475.6 \quad 523.3 \quad 581.0 \quad 672.6 \quad 709.6 \quad 783.9 \quad 823.8 \quad 852.6 \quad 889.9 \quad 914.7 \quad 946.5 \quad 949.4$

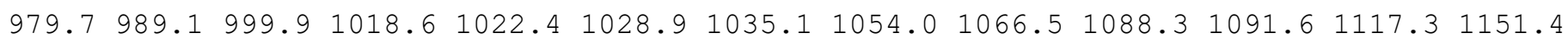

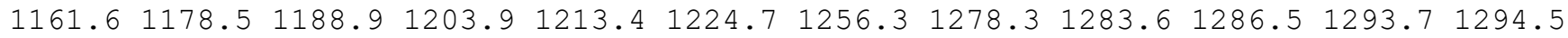

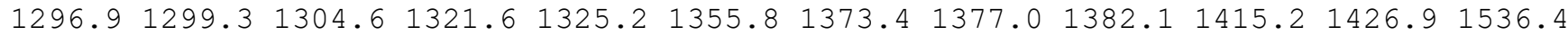
$2652.7 \quad 2669.92672 .1 \quad 2673.1 \quad 2677.3 \quad 2678.4 \quad 2691.32692 .32738 .92746 .12749 .62753 .5$ $2766.62781 .3 \quad 2784.92840 .2$

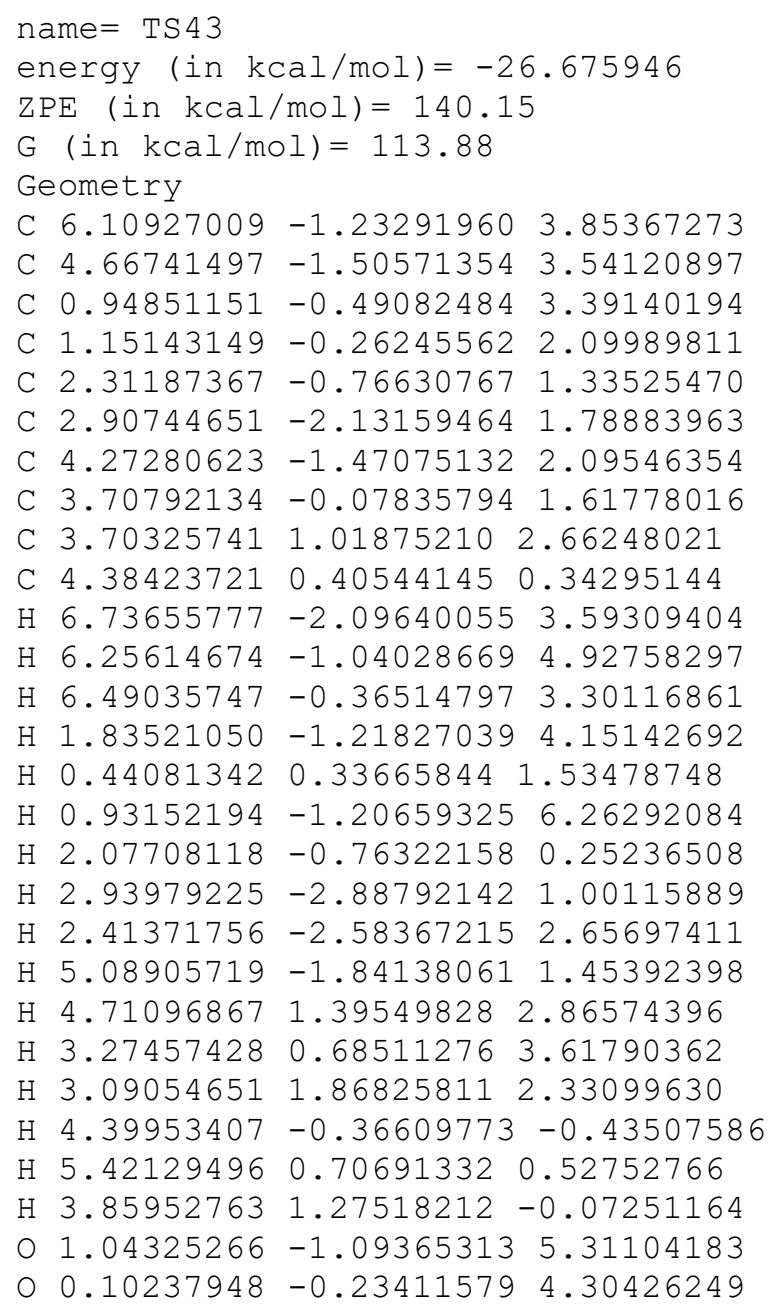


$03.87358584-1.745874164 .42001301$

Vibrational frequencies (in $\mathrm{cm}-1$ )

$\begin{array}{llllllllllllllll}-1551.7 & 40.0 & 50.7 & 60.5 & 106.2 & 121.3 & 142.5 & 161.7 & 182.2 & 188.4 & 204.7 & 225.8 & 274.5 & 309.2\end{array}$ $322.9 \quad 333.9 \quad 364.1 \quad 384.9 \quad 408.2 \quad 460.9 \quad 544.9 \quad 572.4 \quad 597.1 \quad 641.4 \quad 690.2 \quad 746.6 \quad 805.3 \quad 826.1$ $851.0 \quad 881.2 \quad 914.3 \quad 950.7 \quad 997.8 \quad 1005.2 \quad 1016.7 \quad 1031.7 \quad 1041.9 \quad 1049.9 \quad 1088.1 \quad 1103.6 \quad 1123.3$ $1143.91164 .8 \quad 1204.91232 .3 \quad 1243.4 \quad 1261.8 \quad 1264.4 \quad 1272.3 \quad 1281.4 \quad 1286.4 \quad 1294.7 \quad 1298.5$ $1299.61305 .41314 .1 \quad 1326.0 \quad 1353.0 \quad 1369.1 \quad 1381.0 \quad 1404.11405 .2 \quad 1446.2 \quad 1837.91904 .9$ $2054.92664 .12669 .3 \quad 2674.3 \quad 2676.6 \quad 2677.7 \quad 2678.32681 .82720 .5 \quad 2731.4 \quad 2759.8 \quad 2760.4$ $2779.92780 .3 \quad 2783.0 \quad 2826.8$

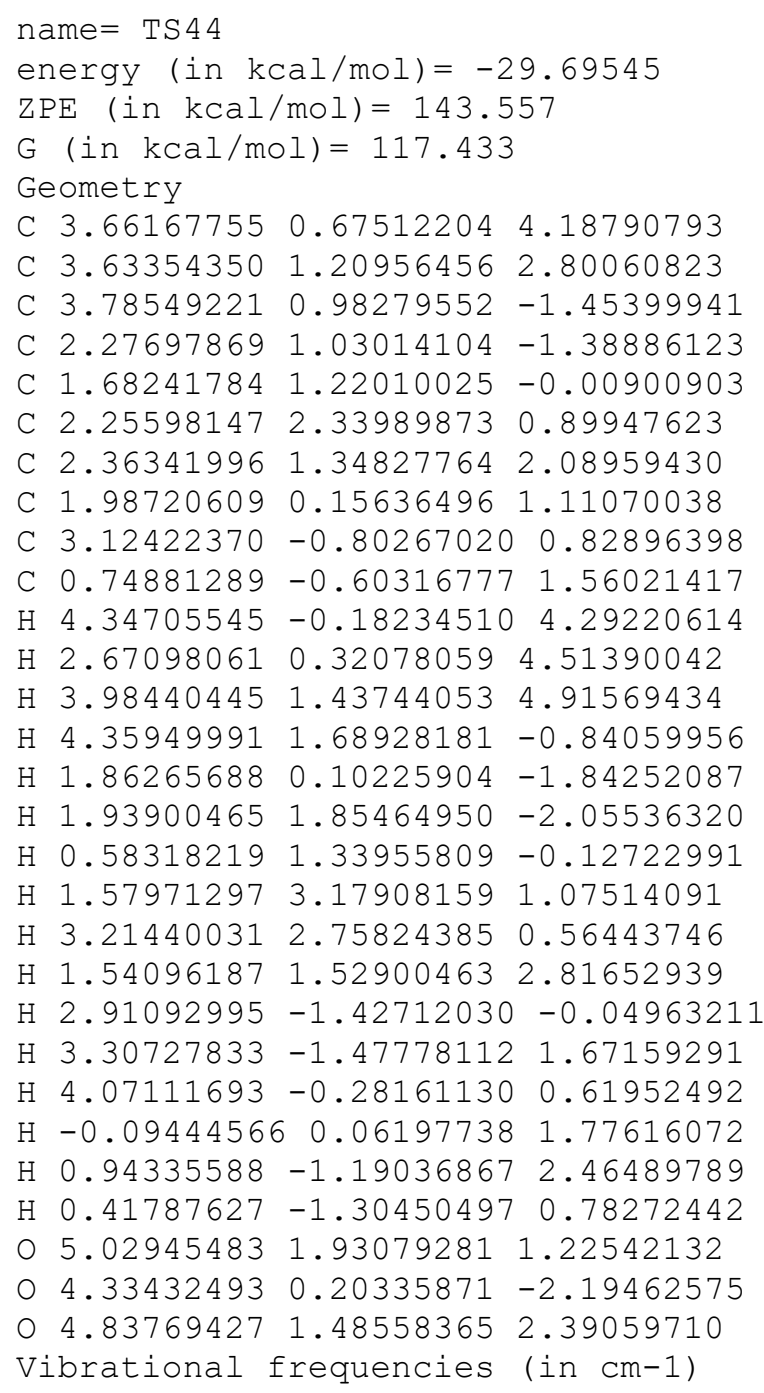


C $1.50112010 \quad 0.06705031 \quad 1.72002801$

C $2.564957541 .21179772 \quad 1.77783070$

C $1.418197322 .27006787 \quad 1.58576903$

$\begin{array}{llll}\text { C } 1.23910581 & 3.39021007 & 2.58674347\end{array}$

C $1.50295610 \quad 2.87865298 \quad 0.18397789$

$\mathrm{H} \quad 4.69731309 \quad 2.62180420 \quad 3.73175810$

H $3.94949226 \quad 1.60508323 \quad 4.97912299$

$\mathrm{H} \quad 2.995719412 .83425521 \quad 4.14823190$

$\mathrm{H} \quad 0.67241567 \quad 1.47126007 \quad 4.52941280$

$\mathrm{H}-1.18699895 \quad 2.03575910 \quad 2.83925054$

H $-1.43269065 \quad 0.30996521 \quad 2.53774990$

$\mathrm{H}-0.19790109 \quad 1.036701090 .67126825$

$\mathrm{H} \quad 1.60775463-0.584374290 .84580362$

$\mathrm{H} \quad 1.48252287 \quad-0.59744738 \quad 2.59055468$

H 3.231159501 .182603910 .87699629

$\mathrm{H} \quad 1.16476514 \quad 3.03818791 \quad 3.62063384$

$\mathrm{H} 0.31853443 \quad 3.95358572 \quad 2.37710633$

H 2.06772544 4.10872843 2.53229055

H 2.37085200 3.54116594 0.09017676

$\mathrm{H} \quad 0.61032938 \quad 3.47022530 \quad-0.04879726$

$\mathrm{H} \quad 1.594025192 .11107494 \quad-0.59445691$

$03.96916122-0.937098122 .26541054$

$0-0.24807336-0.297883704 .67115108$

O $4.10066684-0.026873063 .12956621$

Vibrational frequencies (in $\mathrm{cm}-1$ )

$\begin{array}{lllllllllllllll}-104.2 & 19.9 & 34.2 & 51.7 & 95.0 & 171.8 & 188.7 & 210.1 & 223.7 & 236.8 & 248.8 & 256.3 & 280.0 & 307.8\end{array}$

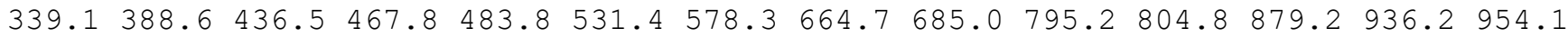

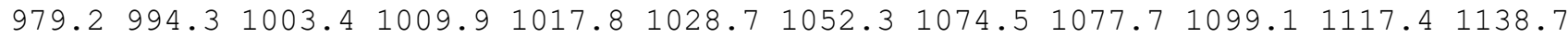

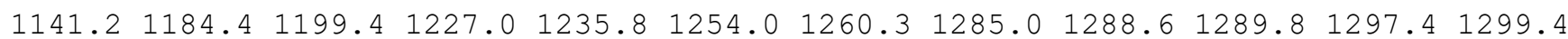

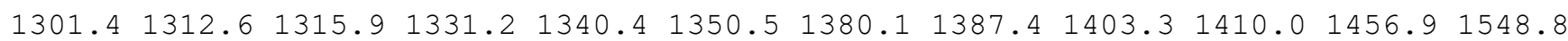
$1842.22634 .8 \quad 2652.12655 .0 \quad 2661.4 \quad 2667.6 \quad 2669.2 \quad 2671.5 \quad 2674.0 \quad 2678.82685 .5 \quad 2715.7$ $2740.62761 .12769 .8 \quad 2777.6 \quad 2781.6$

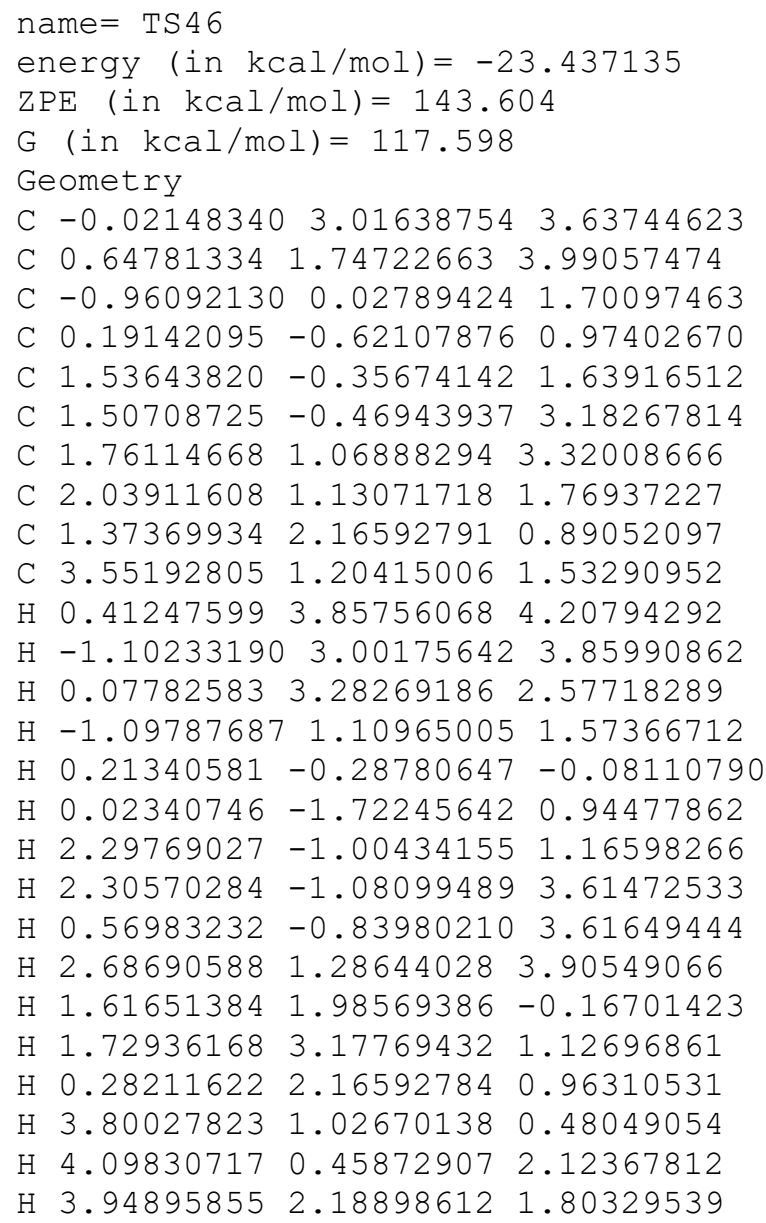


$0.72373204 \quad 0.26960716 \quad 5.63173737$

$0-1.72037588-0.603752812 .39095218$

○ $0.18203507 \quad 1.287164575 .12714545$

Vibrational frequencies (in $\mathrm{cm}-1$ )

$\begin{array}{llllllllllllllll}-120.8 & 27.6 & 48.1 & 71.6 & 95.2 & 184.6 & 192.1 & 210.0 & 211.3 & 240.3 & 249.7 & 253.5 & 278.0 & 322.8\end{array}$ $343.4 \quad 393.4 \quad 436.2 \quad 467.8 \quad 481.6 \quad 522.4 \quad 574.2 \quad 665.9 \quad 694.2 \quad 803.3 \quad 811.2 \quad 888.1924 .6 \quad 960.1$

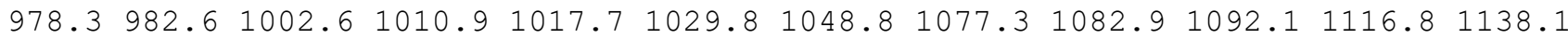

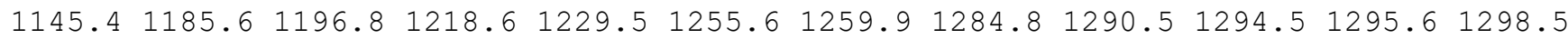

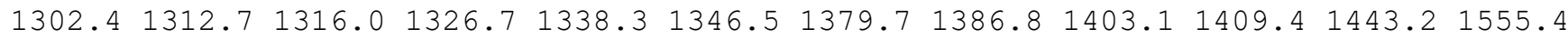
$1843.62630 .32652 .42657 .92663 .2 \quad 2666.12669 .82671 .52674 .3 \quad 2678.72691 .02718 .9$ $2741.0 \quad 2758.22771 .5 \quad 2778.6 \quad 2781.5$

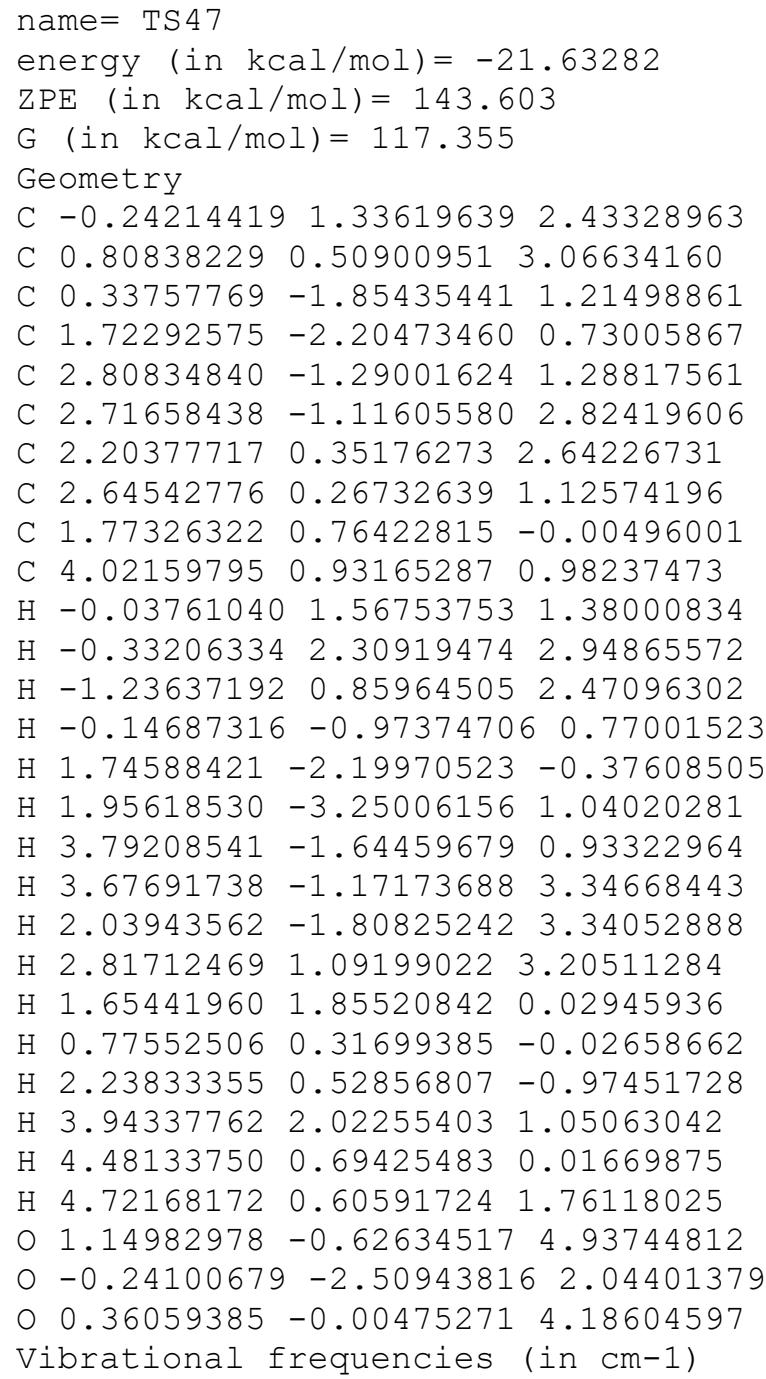

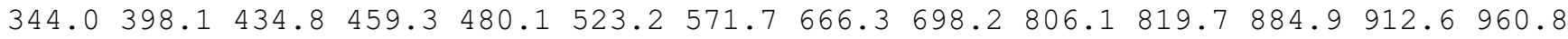
$977.2 \quad 978.3 \quad 1001.3 \quad 1012.6 \quad 1017.8 \quad 1032.4 \quad 1047.5 \quad 1080.2 \quad 1085.3 \quad 1088.3 \quad 1116.7 \quad 1138.4$ $1151.41186 .6 \quad 1195.1 \quad 1213.3 \quad 1224.7 \quad 1257.4 \quad 1262.6 \quad 1283.7 \quad 1289.0 \quad 1295.01297 .6 \quad 1298.3$ $1302.91311 .21314 .41323 .2 \quad 1335.6 \quad 1344.8 \quad 1378.91384 .91402 .7 \quad 1410.11430 .61563 .9$ $1845.12620 .7 \quad 2653.4 \quad 2659.6 \quad 2662.8 \quad 2664.3 \quad 2668.8 \quad 2671.4 \quad 2674.2 \quad 2678.5 \quad 2695.7 \quad 2721.5$ $2740.7 \quad 2755.8 \quad 2772.2 \quad 2778.2 \quad 2781.4$

name $=\operatorname{TS} 48$

energy (in $\mathrm{kcal} / \mathrm{mol})=-19.166696$

$\mathrm{ZPE}(\mathrm{in} \mathrm{kcal} / \mathrm{mol})=141.516$

$\mathrm{G}($ in $\mathrm{kcal} / \mathrm{mol})=116.131$

Geometry

C $3.17213718 \quad 0.57592202 \quad-2.63273307$

C $2.95599213 \quad 0.47803758-1.15136065$

C $1.44030356-0.834877592 .00637825$ 
C $2.64162763-1.558755452 .22059080$

C $3.90136857-1.156898891 .54558280$

C $4.02795625 \quad 0.34944363 \quad 1.17258241$

C $4.11846776-0.00002752-0.33307763$

C $4.06051790-1.53555494 \quad 0.02002857$

C $2.91299594-2.30893136-0.59773525$

C $5.38241780-2.23357886-0.26768935$

$\mathrm{H} 3.65762504-0.32158950-3.03573404$

H $3.807933191 .43764658-2.87959552$

H $2.22048830 \quad 0.71398888 \quad-3.16856784$

H $1.11045130-0.26794283 \quad 1.14132230$

H 2.54046659 $-2.64704184 \quad 2.36984197$

$\mathrm{H} 2.53968396-1.451424173 .59803160$

$\mathrm{H} \quad 4.76775404-1.51104827 \quad 2.13878781$

H $4.92235961 \quad 0.83343546 \quad 1.57342217$

H $3.16891986 \quad 0.97144113 \quad 1.44371384$

H $5.07548871 \quad 0.29657347 \quad-0.79227494$

H $2.83517726-3.31195383-0.15779731$

$\mathrm{H} 3.04416313-2.43521785-1.67838062$

H $1.94470517-1.81756375-0.43922456$

$\mathrm{H} \quad 5.39136050-3.247481180 .15199681$

H $6.23519000 \quad-1.696676190 .16363704$

H $5.56339010-2.32456124-1.34457793$

O $1.23594633-1.35956140 \quad 4.12318227$

$0.66781269-0.720095463 .06457772$

O $1.894185120 .77383252-0.65608534$

Vibrational frequencies (in cm-1)

$\begin{array}{llllllllllllllllllll}-1568.5 & 53.5 & 65.3 & 87.5 & 103.1 & 112.0 & 155.6 & 174.2 & 190.6 & 219.6 & 229.2 & 270.1 & 280.1 & 328.9\end{array}$ $371.2 \quad 396.5 \quad 409.8 \quad 455.0 \quad 476.6 \quad 572.6 \quad 599.5 \quad 634.2 \quad 689.5 \quad 765.2 \quad 807.9 \quad 829.0 \quad 867.7 \quad 912.0$

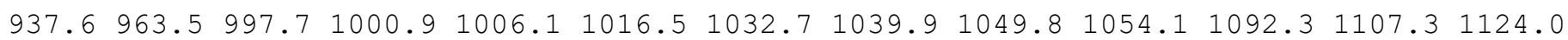
$\begin{array}{lllllllllllll}1162.7 & 1166.6 & 1197.2 & 1220.7 & 1229.7 & 1241.5 & 1260.8 & 1264.2 & 1272.4 & 1285.0 & 1287.8 & 1298.5\end{array}$ $1299.6 \quad 1307.1 \quad 1311.6 \quad 1318.1 \quad 1356.1 \quad 1367.8 \quad 1380.5 \quad 1403.81405 .2 \quad 1431.7 \quad 1561.6 \quad 1844.5$ $1963.52668 .72671 .12673 .8 \quad 2675.3 \quad 2676.12677 .42680 .62682 .62711 .42719 .32731 .6$ $2762.62780 .02781 .2 \quad 2783.2$

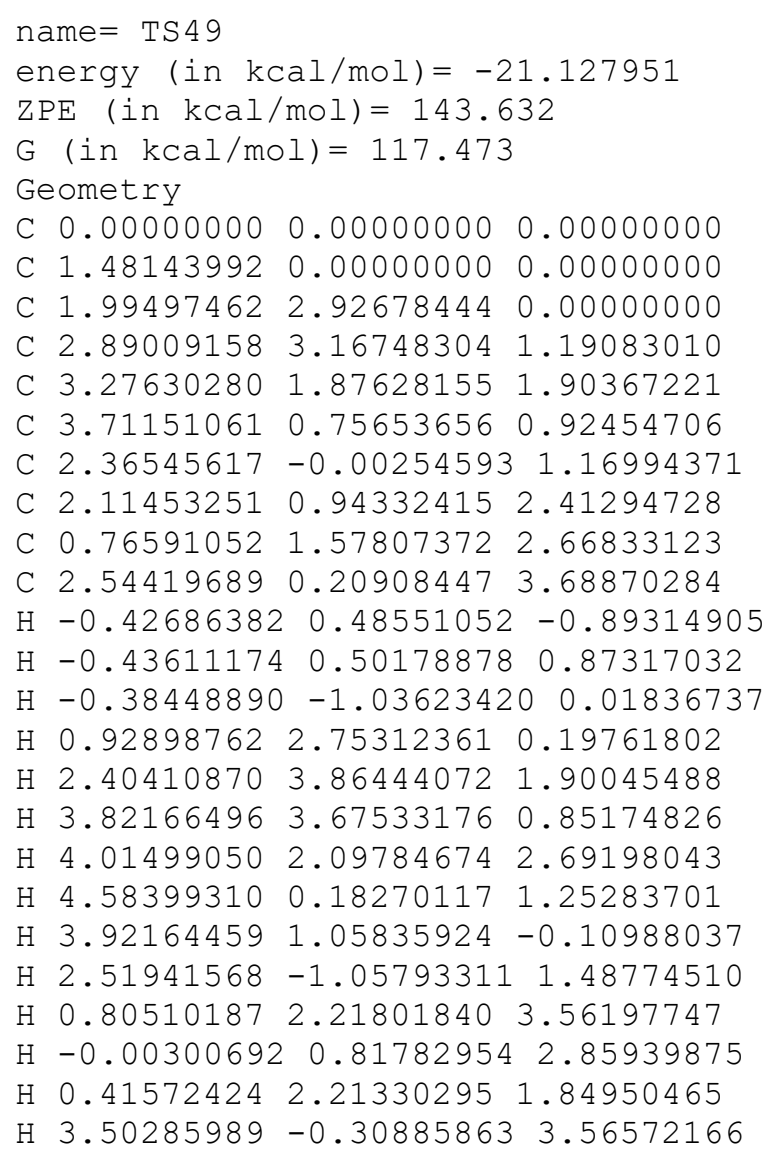


$\mathrm{H} \quad 1.80269252-0.54145003 \quad 3.98383477$

H 2.66014095 $0.90531758 \quad 4.52713255$

O $3.13153323-0.42973913-1.41851970$

o $2.40986805 \quad 2.95069841-1.13074690$

O $1.91610899-0.21205607-1.21815012$

Vibrational frequencies (in $\mathrm{cm}-1$ )

$\begin{array}{llllllllllllll}-103.3 & 15.6 & 51.4 & 88.0 & 100.9 & 186.2 & 202.1 & 205.8 & 210.3 & 241.5 & 251.5 & 262.6 & 276.4 & 337.6\end{array}$

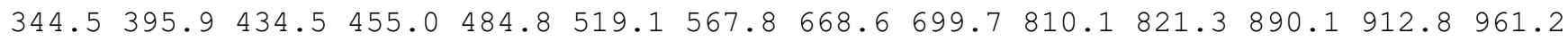

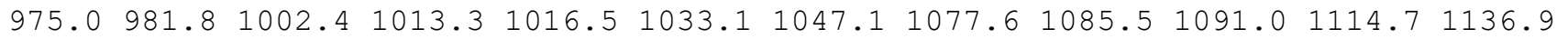
$\begin{array}{llllllllllllll}1156.8 & 1185.2 & 1195.4 & 1215.3 & 1222.5 & 1259.3 & 1262.2 & 1283.0 & 1288.7 & 1293.8 & 1295.7 & 1298.4\end{array}$

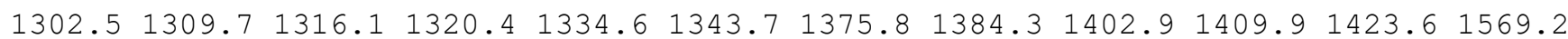

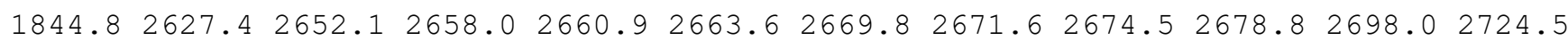
$2741.12755 .6 \quad 2772.2 \quad 2778.7 \quad 2781.5$

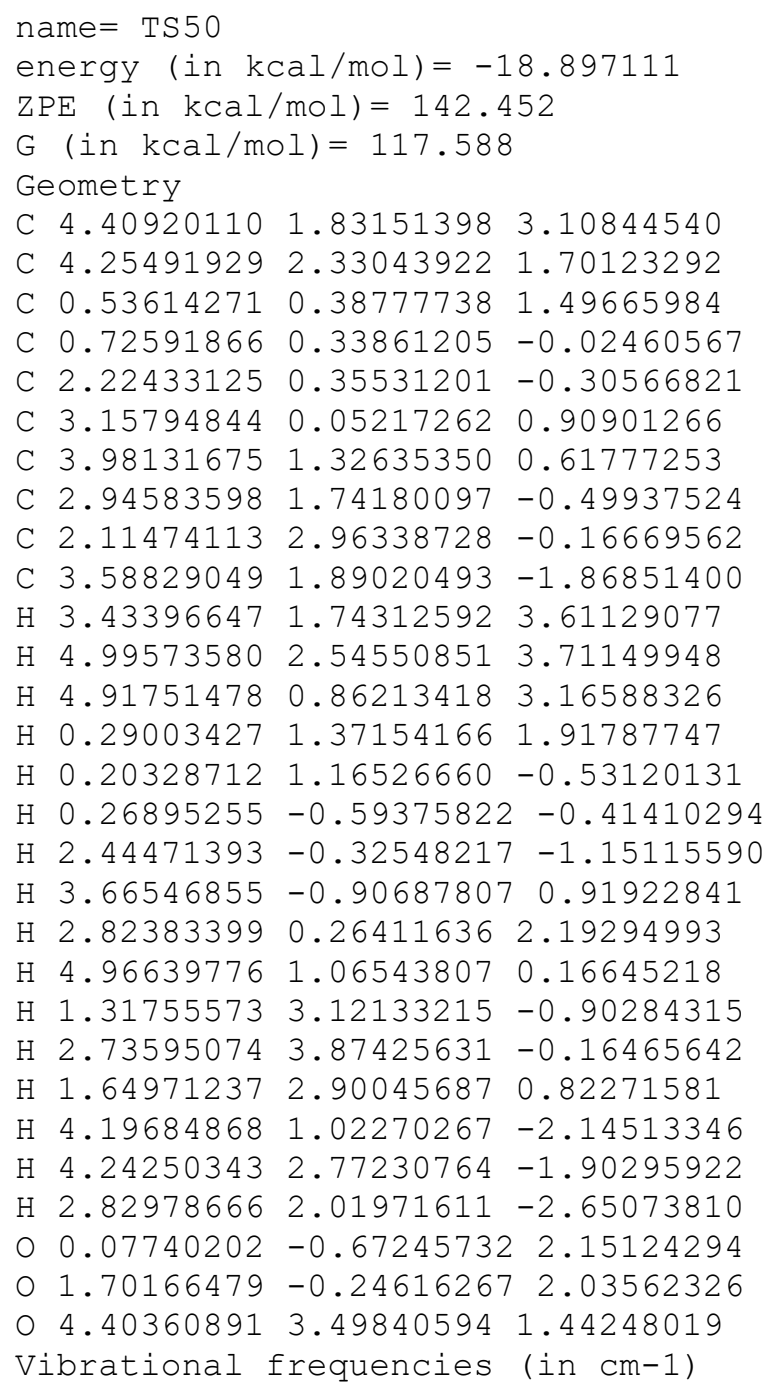


C $-0.22643308 \quad 3.75837369 \quad 0.10794111$

C $2.51016685-0.69825748 \quad 1.52809305$

C $1.859498170 .28542624 \quad 0.74934446$

C $2.33086762 \quad 1.69065064 \quad 0.79934769$

C $1.47648708 \quad 2.64875600 \quad 1.68056408$

C $1.21903241 \quad 3.57744210 \quad 0.46994915$

C $2.011994892 .58562279-0.46215886$

C $1.179643601 .92751663-1.54574626$

C $3.26037974 \quad 3.21687357-1.06004339$

$\mathrm{H} \quad 0.21682656 \quad 4.89383631-1.68748772$

$\mathrm{H}-0.56195513 \quad 5.82206974-0.39336863$

$\mathrm{H}-1.51632142 \quad 4.68759040-1.35370516$

$\mathrm{H} 3.04365481-0.606310482 .45983661$

$\mathrm{H} \quad 0.76816813 \quad 0.16706801 \quad 0.60536105$

$\mathrm{H} \quad 1.97106075 \quad-0.45342154-0.41611244$

H $3.40553675 \quad 1.74054217 \quad 1.05657457$

H $2.01483157 \quad 3.11435398 \quad 2.50674123$

$\mathrm{H} \quad 0.56542123 \quad 2.18939433 \quad 2.08635231$

$\mathrm{H} \quad 1.71802838 \quad 4.55681310 \quad 0.56607635$

$\mathrm{H} \quad 0.91269642 \quad 2.63716823-2.33788304$

H $0.24078743 \quad 1.51224834-1.15284188$

H $1.72597819 \quad 1.10307726-2.02228554$

H $3.879214523 .70896288-0.30137858$

H $3.00720158 \quad 3.97062270-1.81446394$

H $3.886819912 .46073070-1.55026670$

O $1.99431604-1.85922847-0.25401344$

o $2.58948186-1.88858563 \quad 0.96534278$

o -1.104697313 .090650210 .59435008$

Vibrational frequencies (in cm-1)

$\begin{array}{lllllllllllllll}-1600.1 & 32.1 & 36.9 & 49.4 & 103.4 & 111.3 & 146.2 & 178.1 & 185.3 & 219.5 & 244.0 & 276.9 & 285.0 & 318.6\end{array}$ $373.2374 .2 \quad 410.1 \quad 456.1 \quad 482.3 \quad 563.1 \quad 570.4 \quad 606.8 \quad 676.7 \quad 757.7 \quad 818.8 \quad 824.7 \quad 877.7 \quad 925.8$ $940.1964 .9992 .6 \quad 994.0 \quad 1005.6 \quad 1014.5 \quad 1028.2 \quad 1035.4 \quad 1047.1 \quad 1054.5 \quad 1094.2 \quad 1117.1 \quad 1130.3$

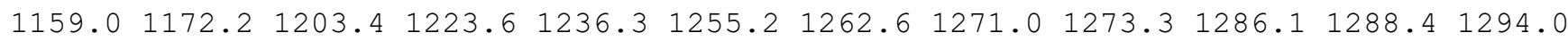

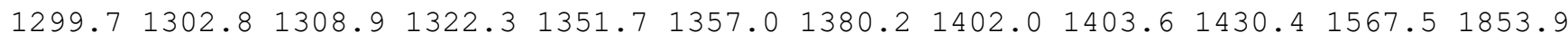
$1949.82665 .6 \quad 2669.3 \quad 2672.8 \quad 2676.3 \quad 2677.0 \quad 2679.72682 .82686 .02721 .82727 .12731 .6$ $2762.6 \quad 2777.6 \quad 2780.7 \quad 2782.6$

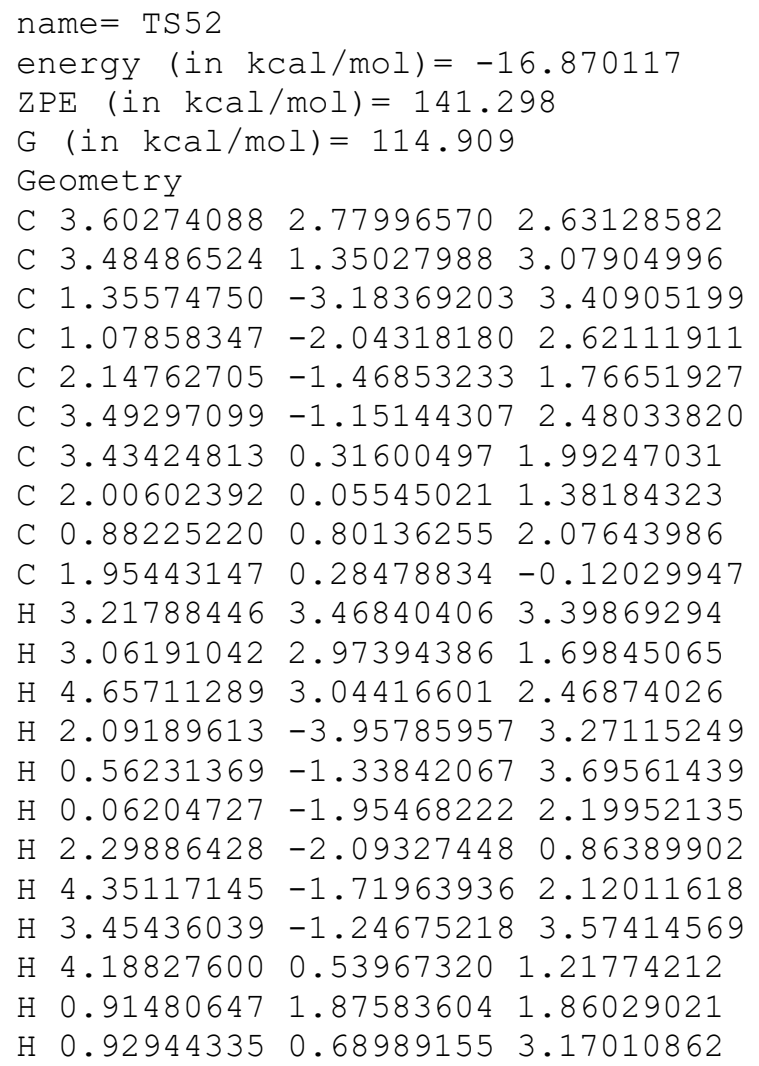


$\mathrm{H}-0.10034397 \quad 0.43647806 \quad 1.75321038$

$\mathrm{H} 1.00686097-0.07416256-0.54133885$

$\mathrm{H} 2.76119715-0.23785637-0.64654016$

$\mathrm{H} 2.039302761 .34944616-0.36698105$

$0-0.08524256-2.143480884 .67995672$

$0.71377598-3.23073408 \quad 4.56083008$

O $3.458665551 .06078604 \quad 4.24885496$

Vibrational frequencies (in $\mathrm{cm}-1$ )

$\begin{array}{llllllllllllllllll}-1606.4 & 27.5 & 37.9 & 50.6 & 108.8 & 119.4 & 129.7 & 161.5 & 184.1 & 210.0 & 243.6 & 270.8 & 304.9 & 318.2\end{array}$

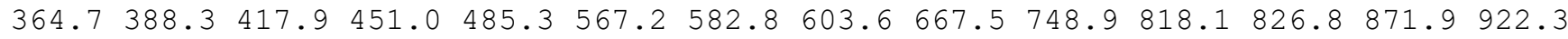
$938.2955 .7 \quad 993.2 \quad 996.9 \quad 1005.3 \quad 1014.2 \quad 1029.7 \quad 1039.4 \quad 1047.5 \quad 1059.3 \quad 1094.91114 .91133 .3$

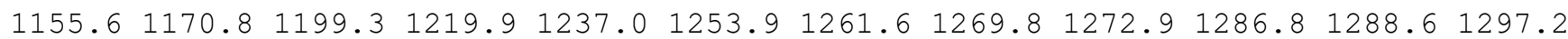
$1300.7 \quad 1303.2 \quad 1307.3 \quad 1316.7 \quad 1349.4 \quad 1358.2 \quad 1380.7 \quad 1400.8 \quad 1403.6 \quad 1427.9 \quad 1570.2 \quad 1854.7$ $1948.02662 .5 \quad 2668.6 \quad 2674.6 \quad 2676.7 \quad 2677.5 \quad 2680.02681 .32702 .12716 .92727 .12728 .9$ $2761.2 \quad 2777.4 \quad 2780.8 \quad 2782.9$

\section{name $=$ TS53}

energy $($ in $\mathrm{kcal} / \mathrm{mol})=-16.542703$

$\mathrm{ZPE}($ in $\mathrm{kcal} / \mathrm{mol})=141.373$

$\mathrm{G}($ in $\mathrm{kcal} / \mathrm{mol})=115.24$

Geometry

C $4.25430310-0.581999815 .04358503$

C $3.94735923 \quad 0.85848869 \quad 4.75796195$

C $0.84214547-0.83961434 \quad 1.12360597$

C $2.22508244-0.80988304 \quad 1.41754696$

C $2.91968698 \quad 0.495147061 .55559550$

C $4.333513860 .39385305 \quad 2.20030939$

C $3.913844461 .35108397 \quad 3.33798375$

C $2.464343801 .44940230 \quad 2.72821640$

C $1.36316667 \quad 0.93376128 \quad 3.63304209$

C $2.13469413 \quad 2.85305943 \quad 2.24233790$

H $5.28342221-0.83514411 \quad 4.75669729$

H $3.57681783-1.258384294 .50649405$

$\mathrm{H} 4.15219102-0.800690136 .11854962$

$\mathrm{H} \quad 0.22859288-0.12492537 \quad 0.59952838$

H $2.84739393-1.611345250 .98280032$

H 2.08645532 -1.65989393 2.50280305

H 2.94067708 $1.03153851 \quad 0.58738376$

H $5.14013988 \quad 0.77832624 \quad 1.56957601$

H $4.61599549-0.613754472 .51318127$

H $4.447544592 .32208884 \quad 3.29260729$

H $0.38362806 \quad 0.97470490 \quad 3.14499625$

$\mathrm{H} \quad 1.29375849 \quad 1.54078657 \quad 4.54943906$

$\mathrm{H} 1.52800562-0.103553193 .95020434$

H 2.02549222 3.54533974 3.08873414

$\mathrm{H} 1.19484862 \quad 2.87314224 \quad 1.68006311$

H $2.91642277 \quad 3.26077076 \quad 1.59167444$

○ $1.02700137-2.587230412 .42849116$

$0.17563529-1.824956301 .69339078$

O $3.73281794 \quad 1.63105247 \quad 5.66179741$

Vibrational frequencies (in $\mathrm{cm}-1$ )

$\begin{array}{lllllllllllllll}-1609.9 & 26.8 & 44.6 & 51.7 & 90.4 & 141.2 & 151.8 & 169.6 & 193.8 & 228.3 & 232.9 & 288.3 & 305.9 & 324.1\end{array}$ $371.5 \quad 405.1 \quad 441.3 \quad 464.8 \quad 490.0 \quad 506.1 \quad 588.1 \quad 601.4 \quad 678.5 \quad 744.0 \quad 804.1 \quad 818.9 \quad 843.0 \quad 912.1$ $941.1971 .8 \quad 989.9 \quad 996.3 \quad 1005.2 \quad 1017.6 \quad 1031.2 \quad 1034.8 \quad 1040.01049 .1 \quad 1080.21127 .01129 .3$ $1161.51168 .81208 .7 \quad 1220.2 \quad 1249.7 \quad 1260.1 \quad 1265.1 \quad 1273.51279 .1 \quad 1286.6 \quad 1291.91297 .6$ $1299.91302 .41311 .1 \quad 1318.6 \quad 1350.5 \quad 1358.7 \quad 1379.8 \quad 1400.81403 .9 \quad 1426.7 \quad 1567.1 \quad 1835.5$ $1959.32662 .32668 .6 \quad 2671.4 \quad 2673.4 \quad 2675.2 \quad 2680.32685 .22701 .32715 .02720 .82725 .3$ $\begin{array}{lllll}2766.1 & 2777.5 & 2778.3 \quad 2782.0\end{array}$

name $=\operatorname{TS} 54$

energy (in $\mathrm{kcal} / \mathrm{mol})=-15.907193$

$\mathrm{ZPE}(\mathrm{in} \mathrm{kcal} / \mathrm{mol})=141.663$

$\mathrm{G}($ in $\mathrm{kcal} / \mathrm{mol})=116.219$ 
Geometry

C $-0.45889182 \quad 2.94738208 \quad 0.47851595$

C $0.23400538 \quad 1.75130997 \quad 1.05833513$

C $0.60486224-1.39952561 \quad 1.99920867$

C $1.95694478-1.486759991 .54456663$

C $2.72698664 \quad-0.23952968 \quad 1.25952359$

$\begin{array}{llll}\text { C } 2.38366528 & 0.98111141 & 2.16526786\end{array}$

C $1.72956371 \quad 1.717507920 .96910970$

C $2.27300201 \quad 0.60053748-0.00325211$

C $1.26364703-0.03626580-0.93488037$

C $3.470329721 .10371534-0.80177088$

$\mathrm{H}-0.14722536 \quad 3.13219577-0.55766127$

$\mathrm{H}-0.23521216 \quad 3.85348711 \quad 1.05918007$

$\mathrm{H}-1.552459532 .82118540 \quad 0.48198349$

$\mathrm{H}-0.32893251-1.453182361 .45161290$

H $2.18271818 \quad-2.29537324 \quad 0.85404352$

H 2.40684184 -1.74522686 2.89448103

$\mathrm{H} 3.80924531-0.46435598 \quad 1.23202634$

$\mathrm{H} \quad 3.26200975 \quad 1.48893488 \quad 2.57233645$

H $1.71606775 \quad 0.76637710 \quad 3.00731642$

H $2.15370086 \quad 2.712313890 .76852237$

$\mathrm{H} \quad 1.69844047-0.91000064-1.43851115$

$\mathrm{H} \quad 0.93405481 \quad 0.66251658-1.71206578$

$\mathrm{H} \quad 0.36882136-0.39010332-0.41017491$

H $4.222583321 .57754787-0.16040809$

$\mathrm{H} \quad 3.17027976 \quad 1.83952977-1.55587978$

H $3.96539404 \quad 0.27786556-1.32745647$

O $1.65444901-1.448192853 .94101357$

$00.44403037-1.38017313 \quad 3.29713664$

$\begin{array}{lllll}0 & -0.40088721 & 0.85654190 & 1.57111273\end{array}$

Vibrational frequencies (in $\mathrm{cm}-1$ )

$\begin{array}{lllllllllllllllll}-1392.6 & 37.6 & 63.1 & 88.8 & 100.2 & 132.1 & 152.3 & 173.4 & 193.1 & 207.2 & 220.1 & 245.0 & 296.3 & 328.9\end{array}$

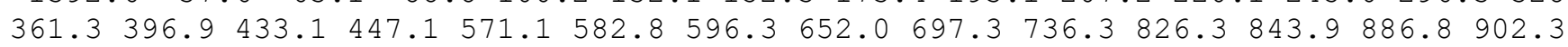
$930.8 \quad 950.0 \quad 995.7 \quad 1001.0 \quad 1006.2 \quad 1015.4 \quad 1034.9 \quad 1041.6 \quad 1047.0 \quad 1088.1 \quad 1097.7 \quad 1110.2 \quad 1118.8$ $1141.7 \quad 1167.4 \quad 1200.0 \quad 1221.2 \quad 1234.2 \quad 1246.4 \quad 1254.5 \quad 1264.4 \quad 1272.4 \quad 1285.1 \quad 1288.1 \quad 1299.2$

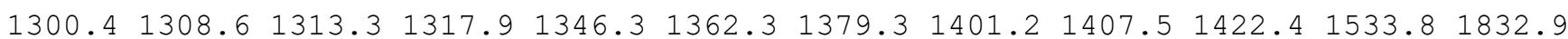
$1940.52668 .52672 .3 \quad 2673.2 \quad 2677.1 \quad 2677.7 \quad 2678.82681 .52693 .42721 .12735 .12758 .9$ $2762.12779 .3 \quad 2781.6 \quad 2783.7$

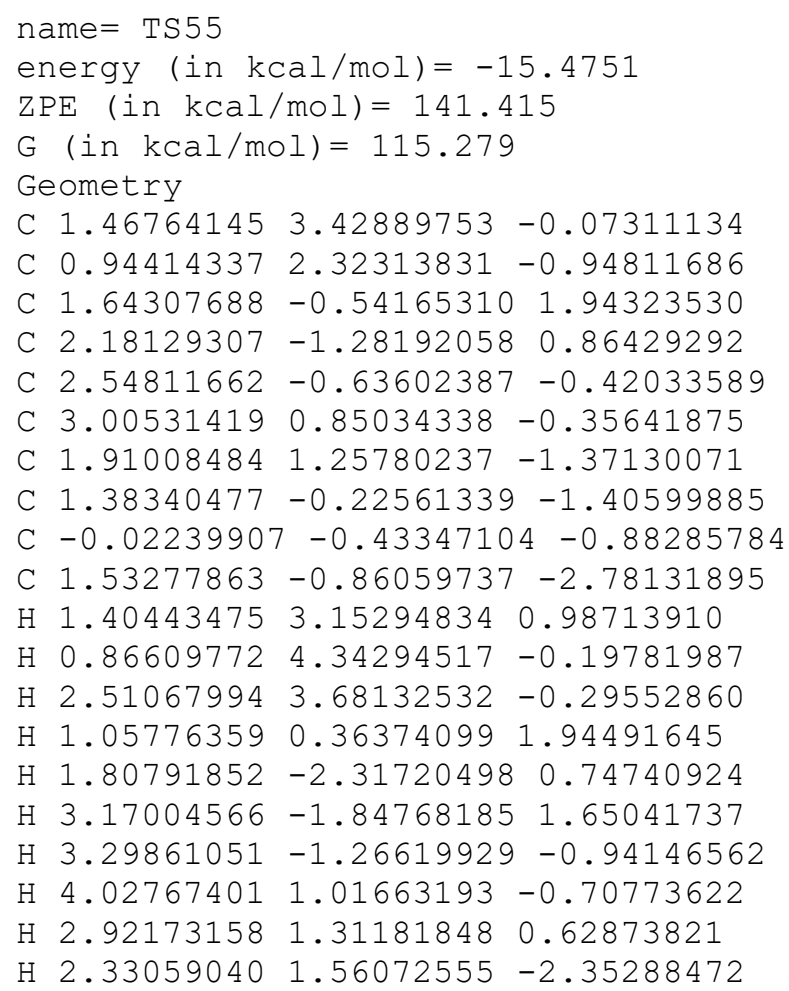


$\mathrm{H}-0.25166745-1.49896050-0.76747183$

$\mathrm{H}-0.76554271-0.00350180-1.57346595$

$\mathrm{H}-0.18969402 \quad 0.05105498 \quad 0.08329921$

$\mathrm{H} \quad 0.83057455-0.41565119-3.49841493$

H $1.32320028-1.93652365-2.74503417$

$\mathrm{H} 2.54091421-0.73618879-3.19058870$

$02.84620694-2.020716013 .01745058$

o $2.02088968-0.95066350 \quad 3.13855841$

o $-0.20155508 \quad 2.33394642-1.32558655$

Vibrational frequencies (in cm-1)

$\begin{array}{llllllllllllllll}-1602.6 & 29.2 & 34.5 & 63.3 & 102.3 & 120.6 & 161.8 & 194.7 & 199.4 & 220.5 & 241.6 & 272.5 & 283.7 & 330.1\end{array}$ $359.2390 .6 \quad 413.3 \quad 470.5 \quad 496.5 \quad 509.1 \quad 607.0 \quad 636.5 \quad 673.6 \quad 762.2 \quad 807.9 \quad 811.7 \quad 860.9 \quad 929.1$ $931.4956 .9993 .7 \quad 996.4 \quad 1008.7 \quad 1015.0 \quad 1019.0 \quad 1037.2 \quad 1044.1 \quad 1052.7 \quad 1084.7 \quad 1104.51129 .2$

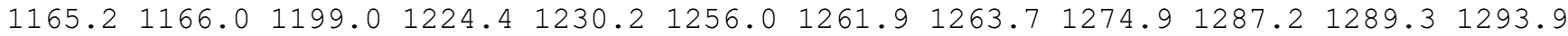
$1300.51301 .91307 .8 \quad 1321.3 \quad 1354.2 \quad 1369.7 \quad 1382.3 \quad 1403.8 \quad 1405.7 \quad 1431.7 \quad 1571.81845 .8$ $1959.92666 .52668 .92676 .02677 .4 \quad 2678.2 \quad 2684.02685 .22696 .62711 .32719 .32721 .2$ $2766.52781 .02781 .3 \quad 2783.2$

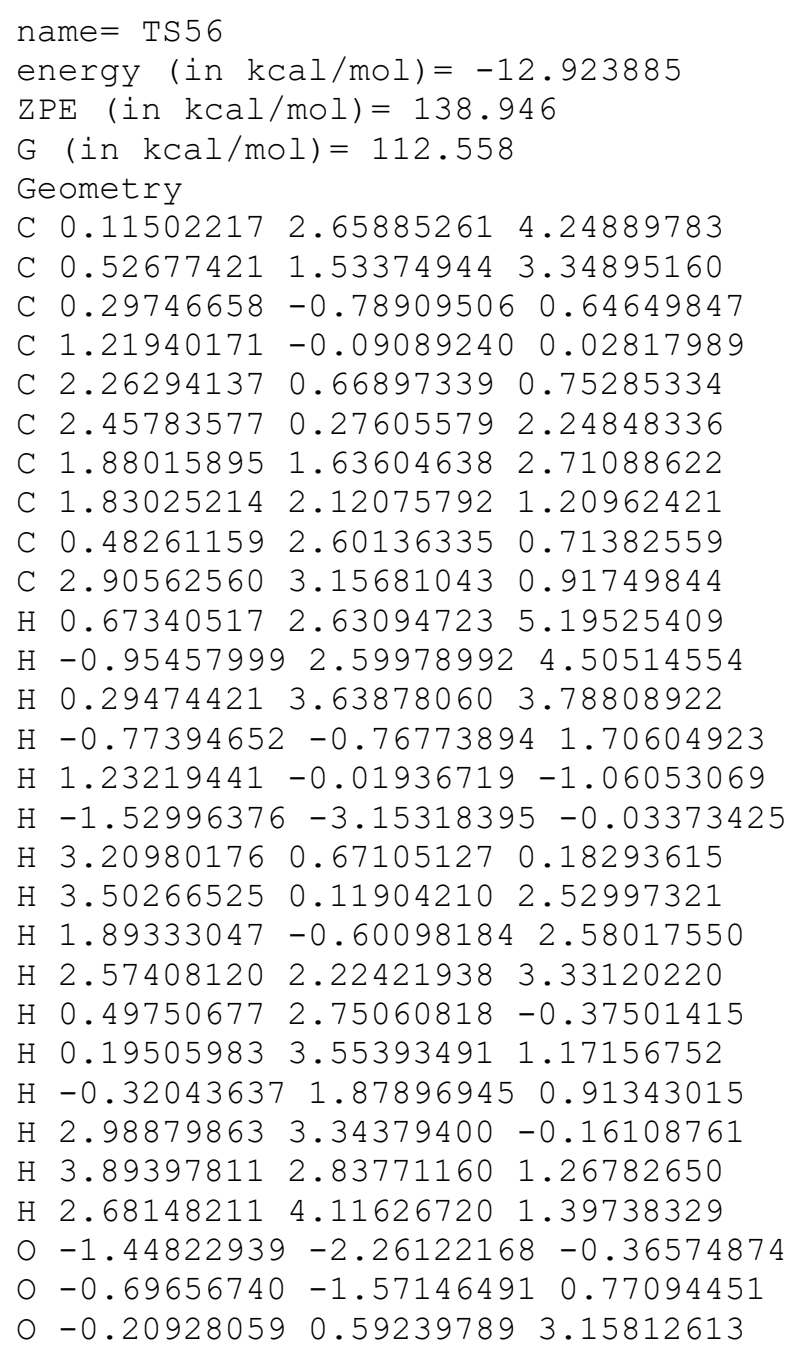


$\mathrm{ZPE}($ in $\mathrm{kcal} / \mathrm{mol})=141.35$

$\mathrm{G}($ in $\mathrm{kcal} / \mathrm{mol})=115.266$

Geometry

C $0.60848392-0.485387013 .53015348$

C $1.93289306-0.220372193 .11468788$

C $6.61441088-0.75181731 \quad 0.85124624$

C $5.22796164-1.34034166 \quad 0.92457112$

C $4.17424259-0.29163774 \quad 0.63426240$

C $3.91624160 \quad 0.72693044 \quad 1.77855960$

C $2.42006727 \quad 0.32378480 \quad 1.83916795$

C $2.67287015-0.75835360 \quad 0.70791185$

C $2.43904386-2.19126480 \quad 1.13860749$

C $1.89888130-0.44852953-0.56218040$

$\mathrm{H} 1.02131390-1.37525104 \quad 4.51625551$

$\mathrm{H}-0.13468784-0.78851617 \quad 2.81536904$

$\mathrm{H} \quad 0.16472034 \quad 0.12123159 \quad 4.31786615$

H $6.74458579 \quad 0.28267552 \quad 1.19107578$

H $5.08733324-1.79923740 \quad 1.92751983$

H $5.13642785-2.190192690 .21334347$

H $4.39167266 \quad 0.20531484-0.33065557$

H 4.081156651 .772391931 .49921263

H $4.48846003 \quad 0.54116180 \quad 2.69245555$

H 1.744886251 .133030351 .49156749

H $1.37153541-2.38991454 \quad 1.30784361$

H $2.95976659-2.447281172 .07087245$

H $2.77963724 \quad-2.89991746 \quad 0.37249405$

$\mathrm{H} 2.21427913-1.10070302-1.38734166$

$\mathrm{H} 2.03890753 \quad 0.58603024-0.89646138$

$\mathrm{H} \quad 0.82108923-0.60170293-0.42326790$

$02.29056558-1.217319835 .04251332$

$0 \quad 7.54865514-1.394327730 .44361790$

O $2.87188759-0.619394323 .96035517$

Vibrational frequencies (in $\mathrm{cm}-1$ )

$\begin{array}{llllllllllllllll}-1539.8 & 32.9 & 45.5 & 62.0 & 86.1 & 116.9 & 168.3 & 178.7 & 189.8 & 225.8 & 241.4 & 278.5 & 285.1 & 328.4\end{array}$

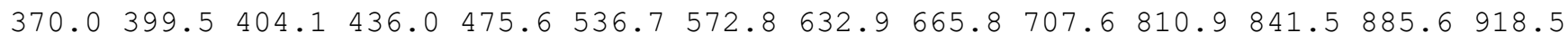
$937.3 \quad 969.8 \quad 986.8 \quad 995.7 \quad 1007.5 \quad 1017.8 \quad 1022.5 \quad 1050.0 \quad 1072.1 \quad 1078.6 \quad 1095.3 \quad 1117.2 \quad 1125.3$

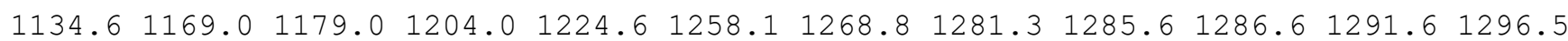

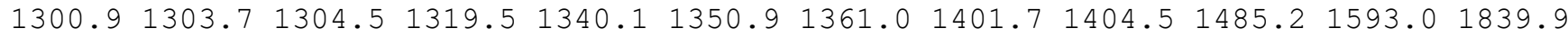
$1969.92647 .32656 .42665 .32670 .72675 .3 \quad 2675.62679 .42698 .72712 .92719 .72740 .3$ $2761.1 \quad 2778.7 \quad 2781.7 \quad 2785.1$

\section{name $=$ TS58}

energy (in $\mathrm{kcal} / \mathrm{mol})=-14.902863$

$\mathrm{ZPE}($ in $\mathrm{kcal} / \mathrm{mol})=141.373$

$\mathrm{G}($ in $\mathrm{kcal} / \mathrm{mol})=115.157$

Geometry

C $0.00520398 \quad 1.45523161 \quad 4.72605564$

C $0.890742390 .65018257 \quad 3.97327165$

C $3.91890371-1.529206830 .26604389$

C $3.00042083-0.406482330 .68249350$

C $3.49449210 \quad 0.26415916 \quad 1.95089272$

C $3.10224815-0.459960473 .26801473$

C $2.33782220 \quad 0.79414344 \quad 3.76417966$

C $2.72116575 \quad 1.54872227 \quad 2.42300890$

C 1.538129621 .973279831 .57638909

C 3.645544492 .727389162 .67758496

$\mathrm{H} \quad 0.34153785 \quad 1.93595736 \quad 5.62676469$

$\mathrm{H}-0.954030110 .45107539 \quad 4.80152249$

$\mathrm{H}-0.73113505 \quad 2.06809725 \quad 4.21073363$

H $3.46897795-2.35698862-0.29674699$

$\mathrm{H} \quad 1.96166791-0.77377514 \quad 0.80165836$

$\begin{array}{lllll}\mathrm{H} & 2.95848644 & 0.33608088 & -0.14560402\end{array}$

H $4.58708610 \quad 0.44398843 \quad 1.87708592$

$\mathrm{H} \quad 3.95670769-0.73714030 \quad 3.89519621$ 
H $2.49726751-1.36097174 \quad 3.13675914$

H $2.807605261 .26154011 \quad 4.65394090$

H $0.841905461 .15015031 \quad 1.37285721$

$\mathrm{H} \quad 1.86371160 \quad 2.37106400 \quad 0.60674812$

$\mathrm{H} \quad 0.96019392 \quad 2.76724259 \quad 2.06979915$

H $3.12310754 \quad 3.54537145 \quad 3.18925892$

H $4.04108748 \quad 3.13101877 \quad 1.73646486$

H $4.508548152 .45297233 \quad 3.29655508$

O $-0.97657182-0.49833734 \quad 3.79427771$

O $5.09750042-1.52455108 \quad 0.51630039$

o $0.34591064-0.44139953 \quad 3.45523011$

Vibrational frequencies (in $\mathrm{cm}-1$ )

$\begin{array}{llllllllllllllll}-1529.2 & 29.0 & 39.0 & 51.8 & 109.2 & 117.7 & 158.4 & 179.3 & 191.5 & 221.3 & 243.8 & 255.9 & 276.4 & 328.5\end{array}$

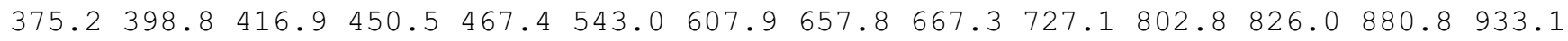
$938.3970 .1986 .1 \quad 996.2 \quad 1005.3 \quad 1016.8 \quad 1023.7 \quad 1054.6 \quad 1068.9 \quad 1074.2 \quad 1081.31106 .91119 .4$

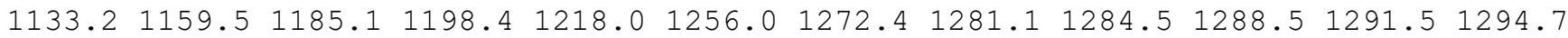

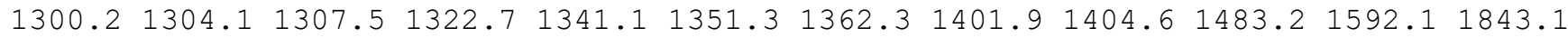
$1970.32648 .92657 .7 \quad 2667.8 \quad 2671.6 \quad 2674.6 \quad 2676.5 \quad 2678.82700 .72706 .5 \quad 2716.5 \quad 2738.7$ $2762.4 \quad 2779.0 \quad 2781.5 \quad 2785.4$

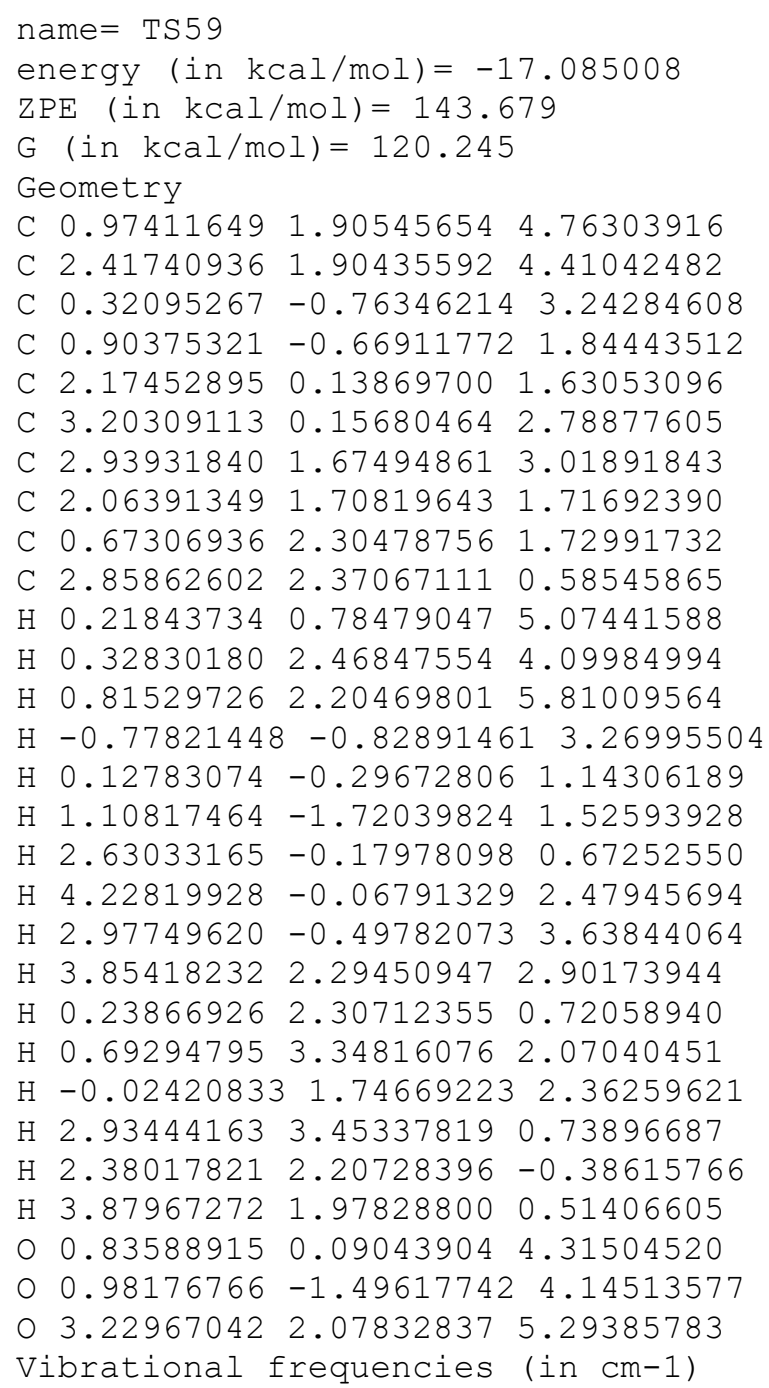




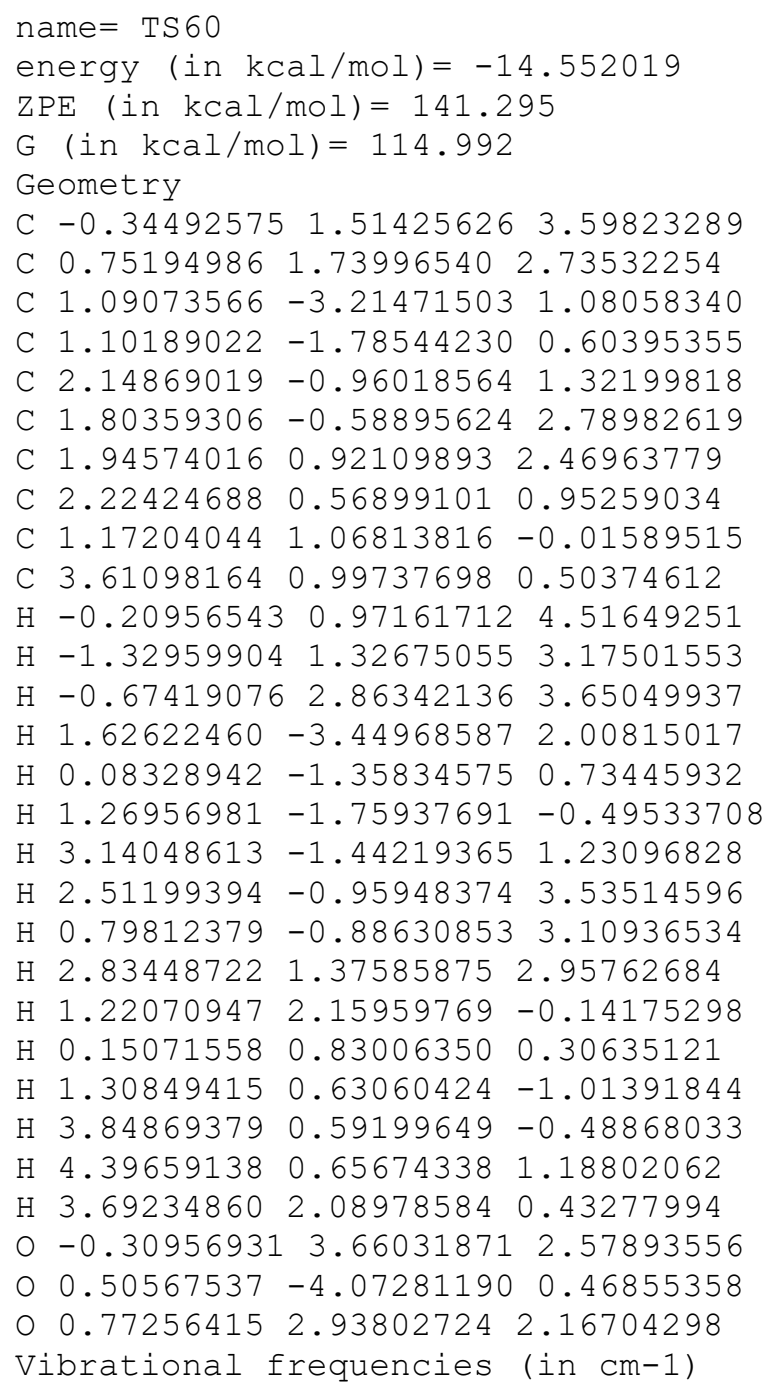


$\mathrm{H}-1.69037470 \quad 0.11702305-0.05991242$

$\mathrm{H} \quad 0.60461532-0.57481430-0.21691470$

$\mathrm{H} 0.49286110-1.554455791 .23485861$

$\mathrm{H} 1.155613681 .419405551 .04191656$

$\mathrm{H}-1.09156692 \quad 0.14743137 \quad 3.81557882$

$\mathrm{H}-1.08186311 \quad 1.90592096 \quad 3.79015945$

$\mathrm{H}-2.52281161 \quad 1.02281992 \quad 3.27217745$

$\mathrm{H}-2.350602242 .50991542 \quad 1.12533819$

$\mathrm{H}-0.916553812 .393110170 .10162753$

$\mathrm{H}-0.81764480 \quad 3.19683694 \quad 1.67046004$

o $2.28276392-0.93113572 \quad 4.34962277$

O $-2.57198818-2.312932073 .57145419$

o $1.51887562-0.84859994 \quad 3.22156668$

Vibrational frequencies (in $\mathrm{cm}-1$ )

$\begin{array}{lllllllllllllll}-1534.5 & 36.0 & 40.5 & 55.5 & 111.9 & 129.7 & 166.0 & 200.6 & 211.8 & 231.3 & 263.6 & 274.5 & 289.1 & 335.1\end{array}$ $\begin{array}{llllllllllllllll}376.7 & 396.0 & 414.8 & 445.5 & 454.1 & 533.9 & 598.9 & 656.5 & 671.7 & 728.4 & 763.7 & 828.3 & 856.0 & 924.0\end{array}$ $932.0980 .1991 .7 \quad 997.0 \quad 1007.2 \quad 1018.7 \quad 1036.0 \quad 1051.9 \quad 1055.6 \quad 1071.7 \quad 1081.01109 .5 \quad 1124.0$

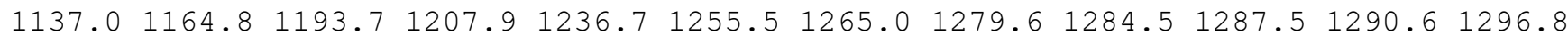

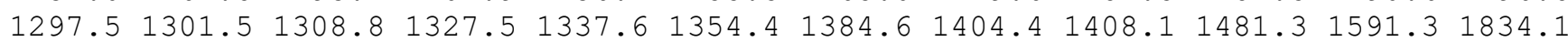
$1971.82644 .3 \quad 2654.2 \quad 2664.3 \quad 2669.7 \quad 2675.2 \quad 2675.82676 .42698 .02707 .72714 .42736 .6$ $2761.62778 .62781 .8 \quad 2785.1$

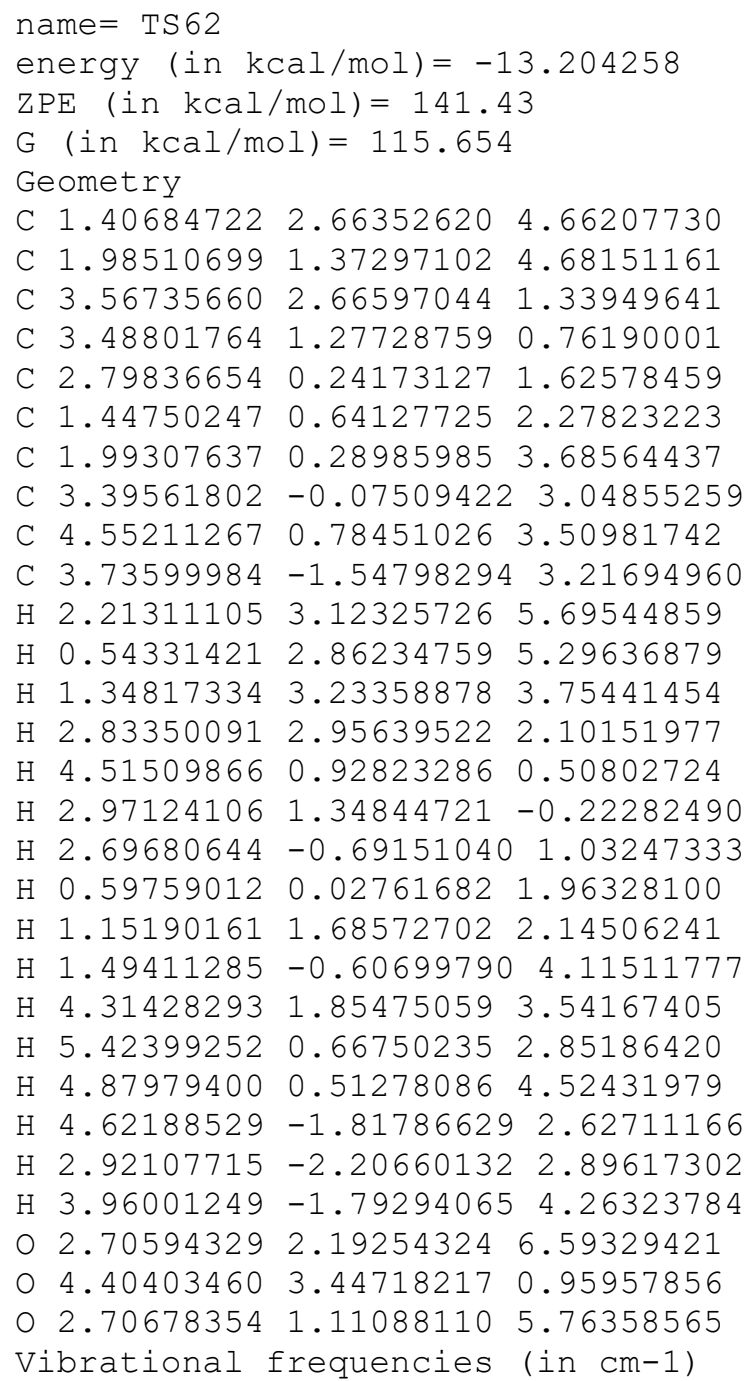




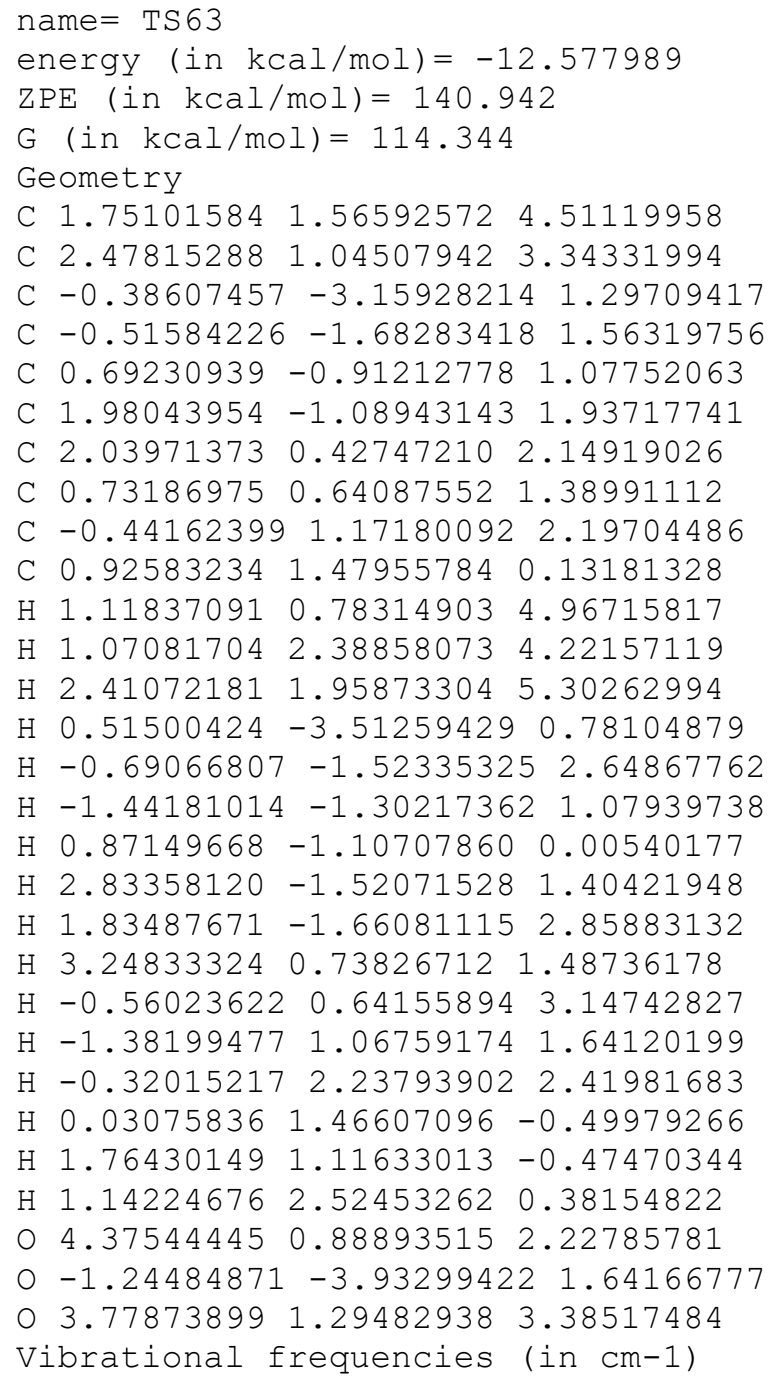


$\mathrm{H}-0.16499753 \quad 2.80450196 \quad 1.04413796$

H $1.29263327 \quad 2.72667982 \quad 0.05806417$

H $1.86762761 \quad 3.72907714 \quad 2.17957907$

$\mathrm{H} 3.87189978 \quad 2.42991793 \quad 2.45757837$

H $3.14653424 \quad 1.01518561 \quad 1.69689704$

H $2.98593294 \quad 1.93437507 \quad 4.97053147$

$\mathrm{H}-0.00598163 \quad 0.39767960 \quad 3.07430017$

$\mathrm{H}-0.96800561 \quad 1.87088917 \quad 3.22777608$

$\mathrm{H}-0.36834928 \quad 1.04821491 \quad 4.67138252$

$\mathrm{H} 0.90402578 \quad 2.99880576 \quad 5.61012423$

H $0.11439218 \quad 3.95336410 \quad 4.34161903$

$\mathrm{H} \quad 1.87039700 \quad 4.019834994 .54767757$

$\begin{array}{lllll}0 & 2.82005808 & 1.25243581 & 6.13299379\end{array}$

$\begin{array}{lllll}0 & -0.36389105 & 0.40835580 & 0.17227864\end{array}$

o $2.76564511-0.00487444 \quad 5.60120205$

Vibrational frequencies (in cm-1)

$\begin{array}{llllllllllllllllll}-1510.5 & 37.3 & 53.0 & 76.0 & 110.2 & 119.6 & 147.4 & 167.2 & 206.2 & 214.9 & 238.1 & 262.3 & 265.7 & 279.0\end{array}$

$335.4 \quad 341.2 \quad 394.6 \quad 436.8 \quad 452.7 \quad 514.4 \quad 596.0 \quad 607.9690 .7 \quad 701.1 \quad 754.7 \quad 802.1 \quad 843.0 \quad 920.1$

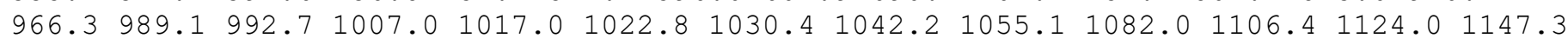

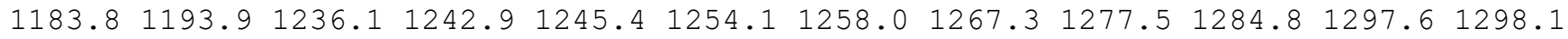

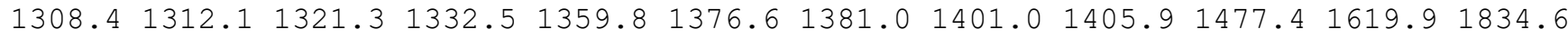
$1955.72644 .5 \quad 2648.32654 .2 \quad 2657.5 \quad 2666.2 \quad 2673.32676 .72681 .02685 .12716 .72737 .3$ $2763.4 \quad 2767.6 \quad 2779.2 \quad 2781.6$

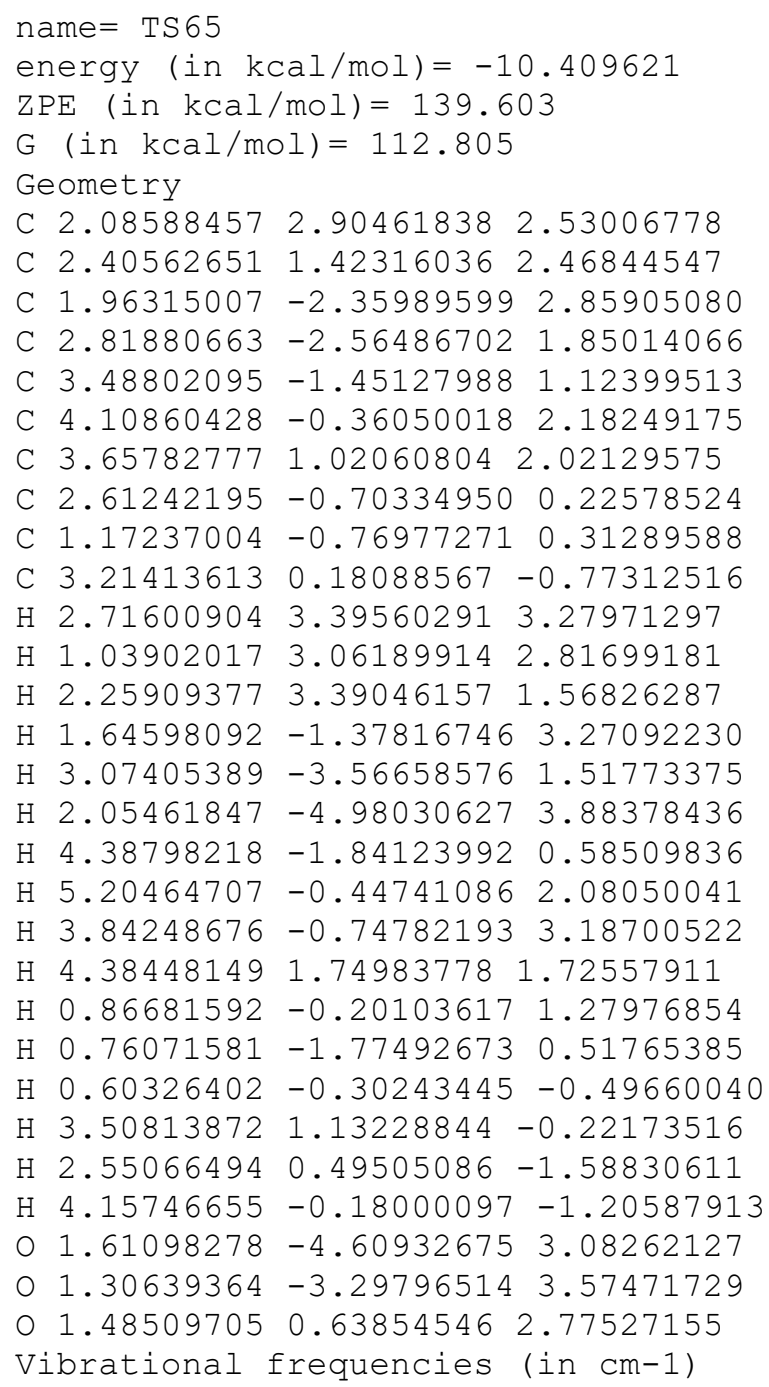


$2310.8 \quad 2518.5 \quad 2593.8 \quad 2653.0 \quad 2657.1 \quad 2670.1 \quad 2684.3 \quad 2691.3 \quad 2692.0 \quad 2728.82744 .12759 .4$ $2763.22786 .5 \quad 2805.52866 .7$

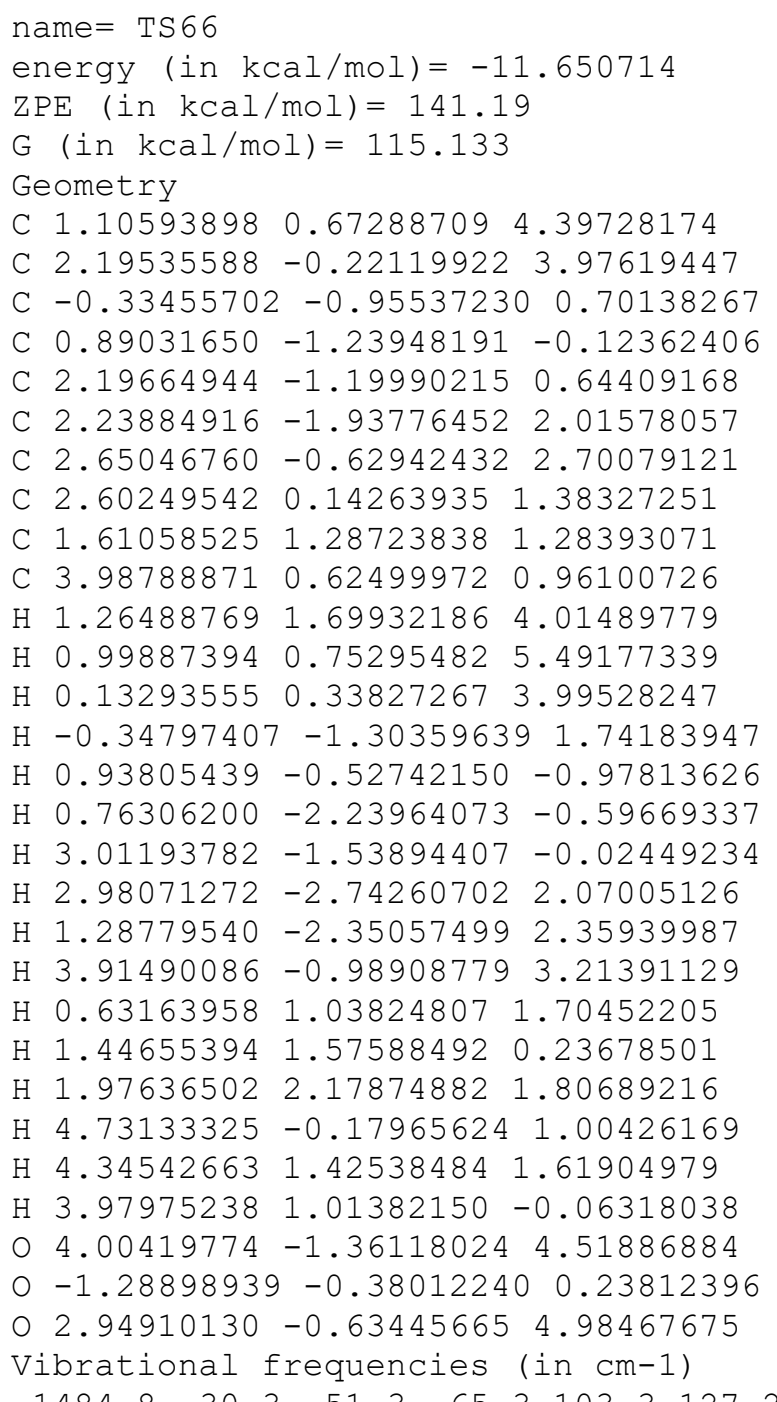

$\begin{array}{llllllllllllllllll}-1484.8 & 30.3 & 51.3 & 65.3 & 103.3 & 127.2 & 155.0 & 179.3 & 185.9 & 217.3 & 239.5 & 247.1 & 274.8 & 298.6\end{array}$

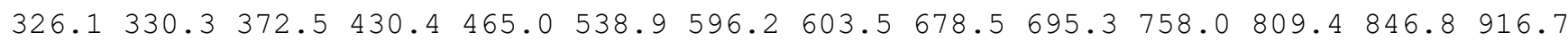
$982.1985 .8 \quad 1001.0 \quad 1005.9 \quad 1015.8 \quad 1018.4 \quad 1036.0 \quad 1040.8 \quad 1060.4 \quad 1068.5 \quad 1105.4 \quad 1123.2$ $1139.81174 .6 \quad 1185.2 \quad 1211.91240 .3 \quad 1247.5 \quad 1251.4 \quad 1256.7 \quad 1269.2 \quad 1275.91289 .4 \quad 1300.3$ $1300.81305 .8 \quad 1318.0 \quad 1321.8 \quad 1343.1 \quad 1359.5 \quad 1376.3 \quad 1381.1 \quad 1400.7 \quad 1407.8 \quad 1472.1 \quad 1621.3$ $1834.1 \quad 1957.8 \quad 2644.2 \quad 2644.7 \quad 2655.4 \quad 2657.8 \quad 2673.2 \quad 2675.2 \quad 2678.8 \quad 2680.3 \quad 2681.2 \quad 2716.7$ $2737.5 \quad 2760.0 \quad 2766.7 \quad 2781.12782 .7$

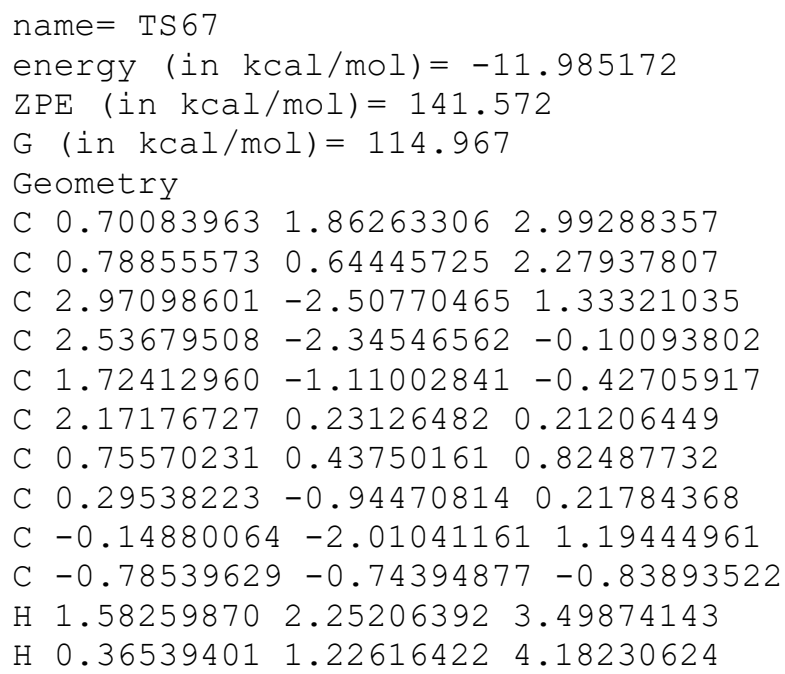


$\mathrm{H} \quad 0.083222512 .66311775 \quad 2.62924642$

H $2.89365687-1.64656425 \quad 2.00758977$

$\mathrm{H} 1.97139246-3.25477854-0.41014919$

$\mathrm{H} 3.45357342-2.36782803-0.73500844$

$\mathrm{H} \quad 1.65857564-1.02351837-1.53280509$

H $2.45810944 \quad 1.00183350-0.51072798$

H $2.98430110 \quad 0.15838330 \quad 0.93979313$

$\mathrm{H} \quad 0.21130372 \quad 1.28340792 \quad 0.34971256$

$\mathrm{H}-0.45164174-2.92813147 \quad 0.66958358$

$\mathrm{H}-1.01672480-1.68016883 \quad 1.78308377$

$\mathrm{H} \quad 0.62447708-2.31667944 \quad 1.90858749$

$\mathrm{H}-0.52346207 \quad 0.03369343-1.56492746$

$\mathrm{H}-1.74062073-0.45528608-0.38246173$

$\mathrm{H}-0.96301910-1.66697305-1.40524418$

$00.79597018-0.07818569 \quad 4.35498875$

$03.40694320-3.56181395 \quad 1.72454822$

o $0.83533926-0.437650423 .03976079$

Vibrational frequencies (in $\mathrm{cm}-1$ )

$\begin{array}{lllllllllllllll}-1542.6 & 9.0 & 36.9 & 44.7 & 117.8 & 158.5 & 165.9 & 194.7 & 212.3 & 230.1 & 252.8 & 300.0 & 312.1 & 325.1\end{array}$

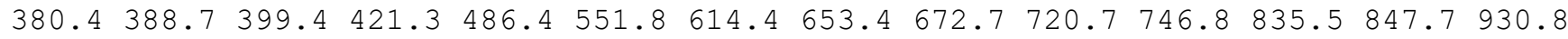
$938.0986 .5988 .9995 .1 \quad 1009.2 \quad 1019.9 \quad 1034.9 \quad 1046.6 \quad 1055.8 \quad 1060.6 \quad 1080.8 \quad 1113.9 \quad 1128.3$

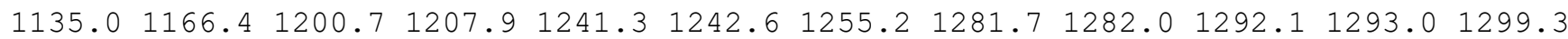
$1300.51307 .91318 .5 \quad 1330.6 \quad 1343.3 \quad 1353.4 \quad 1385.6 \quad 1407.3 \quad 1410.5 \quad 1499.7 \quad 1587.0 \quad 1832.9$ $1971.72644 .82649 .12663 .3 \quad 2668.2 \quad 2675.3 \quad 2675.62677 .62698 .62706 .82713 .72735 .6$ $2761.62778 .92782 .0 \quad 2785.5$

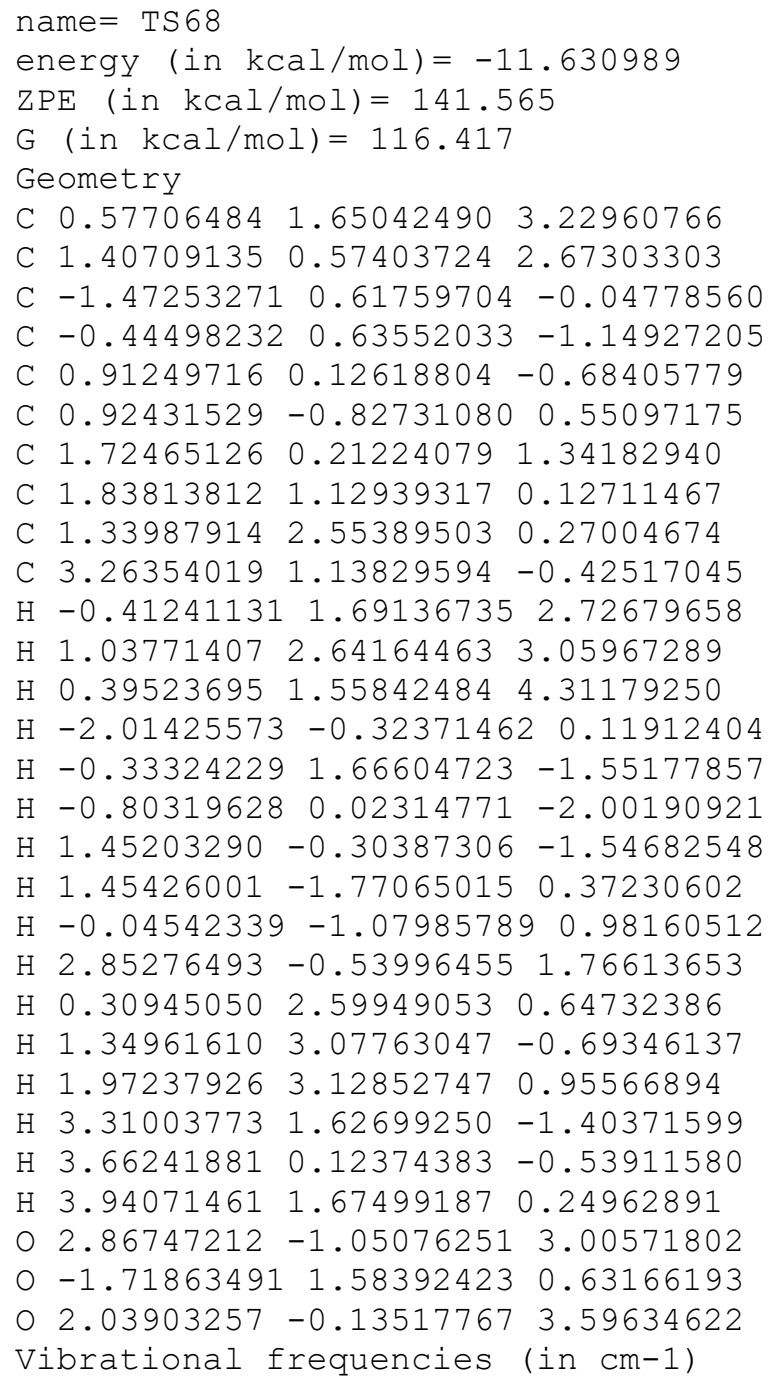


$\begin{array}{lllllllllllllll}1169.5 & 1177.3 & 1210.9 & 1231.8 & 1236.1 & 1242.4 & 1252.5 & 1274.4 & 1289.9 & 1291.8 & 1299.7 & 1302.9\end{array}$

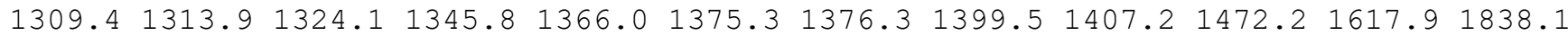
$1960.72629 .92643 .6 \quad 2657.12664 .5 \quad 2668.7 \quad 2676.72678 .92682 .2 \quad 2682.92720 .02739 .9$ $2762.12763 .2 \quad 2781.02783 .1$

\section{name $=$ TS 69}

energy (in $\mathrm{kcal} / \mathrm{mol})=-11.069014$

$\mathrm{ZPE}($ in $\mathrm{kcal} / \mathrm{mol})=141.269$

$\mathrm{G}(\mathrm{in} \mathrm{kcal} / \mathrm{mol})=114.645$

Geometry

C $4.31284777-0.95035653 \quad 3.21337132$

C $2.92641550-1.503231463 .01968729$

C $-1.70378697-1.763404630 .43091202$

C $-0.50223246-1.009096790 .44564115$

C $0.81667989-1.704999810 .34730376$

C $1.23711966-2.695093251 .47088309$

C $2.59358171-1.967399601 .63289918$

C $2.10072610-0.83898577 \quad 0.65169737$

C $1.82945434 \quad 0.49348968 \quad 1.32545975$

C $3.00449966-0.66006071-0.55730791$

$\mathrm{H} \quad 4.65624047-0.37418456 \quad 2.34735312$

H $5.02972072-1.765750423 .38036918$

H $4.35854101-0.29820548 \quad 4.09771292$

$\mathrm{H}-2.35583000-2.02633877-0.38966651$

$\mathrm{H}-0.61573898-0.830764451 .89180640$

$\mathrm{H}-0.51628883-0.07427794-0.11500239$

$\mathrm{H} 0.89858612-2.18148036-0.65197285$

$\mathrm{H} 1.30706886-3.73876576 \quad 1.16479871$

$\mathrm{H} \quad 0.61000101-2.65208917 \quad 2.37615739$

H $3.43568072-2.54685200 \quad 1.21423125$

H $2.75610318 \quad 0.98743341 \quad 1.63848189$

H $1.20176480 \quad 0.37457250 \quad 2.22133503$

$\mathrm{H} \quad 1.30198170 \quad 1.180002120 .65268504$

H $3.22297505-1.61195109-1.05432757$

H $3.96310779-0.20812360-0.27780695$

$\mathrm{H} 2.53724684-0.00462884-1.30262598$

$0-1.39267200-1.622912202 .60852868$

$0-2.18880309-2.087348541 .60429724$

o $2.15269588-1.580319513 .93959149$

Vibrational frequencies (in $\mathrm{cm}-1$ )

$\begin{array}{llllllllllllllll}-1522.7 & 20.5 & 44.1 & 46.9 & 88.9 & 131.0 & 136.1 & 153.3 & 173.1 & 192.7 & 218.0 & 267.5 & 272.6 & 318.6\end{array}$ $355.1385 .8 \quad 432.3 \quad 458.9 \quad 514.8 \quad 564.9 \quad 594.1 \quad 644.3 \quad 661.1 \quad 748.5 \quad 816.6 \quad 821.7 \quad 872.4 \quad 924.1$ $937.8 \quad 947.1 \quad 984.6 \quad 995.8 \quad 1004.1 \quad 1013.8 \quad 1033.0 \quad 1045.8 \quad 1050.8 \quad 1083.6 \quad 1106.7 \quad 1118.7 \quad 1137.9$ $1150.21163 .5 \quad 1201.8 \quad 1232.6 \quad 1235.1 \quad 1246.4 \quad 1264.0 \quad 1273.91275 .4 \quad 1287.6 \quad 1290.7 \quad 1297.9$ $1302.01304 .21308 .91322 .2 \quad 1346.1 \quad 1362.8 \quad 1381.51402 .51404 .41416 .01544 .31857 .7$ $1921.52664 .72666 .2 \quad 2670.7 \quad 2676.8 \quad 2677.5 \quad 2678.72681 .32706 .72708 .82727 .32749 .3$ $2757.4 \quad 2778.5 \quad 2781.3 \quad 2782.8$

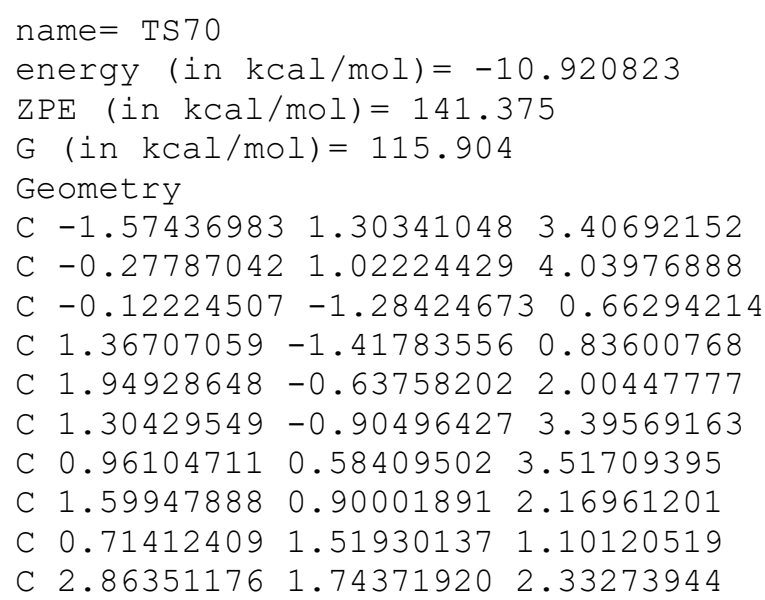


$\mathrm{H}-1.91313997 \quad 0.41987227 \quad 2.82357263$

$\mathrm{H}-1.50353504 \quad 2.14231627 \quad 2.69292359$

$\mathrm{H}-2.375204991 .54968758 \quad 4.12219204$

$\mathrm{H}-0.47859677-0.95586072-0.32406825$

$\mathrm{H} 1.89007607-1.11998562-0.09532392$

H $1.60219770-2.49767907 \quad 0.98506165$

H $3.04463302-0.795038792 .02060635$

H $1.99683430-1.28648643 \quad 4.15151818$

$\mathrm{H} \quad 0.42970349-1.56664640 \quad 3.37372631$

$\mathrm{H} \quad 1.65032280 \quad 0.96457330 \quad 4.70951090$

$\mathrm{H} 0.57380770 \quad 2.59265797 \quad 1.28216400$

$\mathrm{H}-0.28181370 \quad 1.06834068 \quad 1.06973249$

H $1.16636898 \quad 1.41649118 \quad 0.10813229$

H $3.418325331 .81994534 \quad 1.39245661$

$\mathrm{H} 3.53863361 \quad 1.31974483 \quad 3.08587857$

$\mathrm{H} \quad 2.61799677 \quad 2.75972681 \quad 2.66291603$

○ $0.98874347 \quad 1.06523259 \quad 5.85526284$

O $-0.92159499-1.54348347 \quad 1.52884977$

o $-0.26405958 \quad 1.27532222 \quad 5.33931001$

Vibrational frequencies (in cm-1)

$\begin{array}{lllllllllllllllll}-1437.8 & 47.1 & 60.9 & 70.8 & 118.8 & 157.6 & 161.4 & 180.3 & 186.7 & 203.4 & 222.7 & 250.1 & 275.5 & 310.4\end{array}$ $323.1333 .6 \quad 377.7 \quad 430.3 \quad 484.2 \quad 529.7 \quad 598.1 \quad 619.2 \quad 686.5 \quad 696.0 \quad 786.8 \quad 816.0 \quad 891.7 \quad 902.6$ $978.7 \quad 986.8 \quad 999.6 \quad 1002.4 \quad 1015.4 \quad 1017.1 \quad 1024.5 \quad 1039.3 \quad 1057.4 \quad 1065.6 \quad 1077.7 \quad 1110.91138 .4$ $1166.21191 .1 \quad 1209.8 \quad 1235.0 \quad 1239.8 \quad 1247.0 \quad 1257.1 \quad 1275.3 \quad 1290.7 \quad 1291.4 \quad 1301.51301 .9$ $1305.41321 .8 \quad 1333.2 \quad 1345.4 \quad 1362.0 \quad 1376.91382 .4 \quad 1400.6 \quad 1407.4 \quad 1469.6 \quad 1620.8 \quad 1840.8$ $1959.32633 .02643 .1 \quad 2658.6 \quad 2662.5 \quad 2673.3 \quad 2677.0 \quad 2678.92679 .82683 .5 \quad 2716.3 \quad 2736.9$ $2757.32763 .12780 .8 \quad 2782.4$

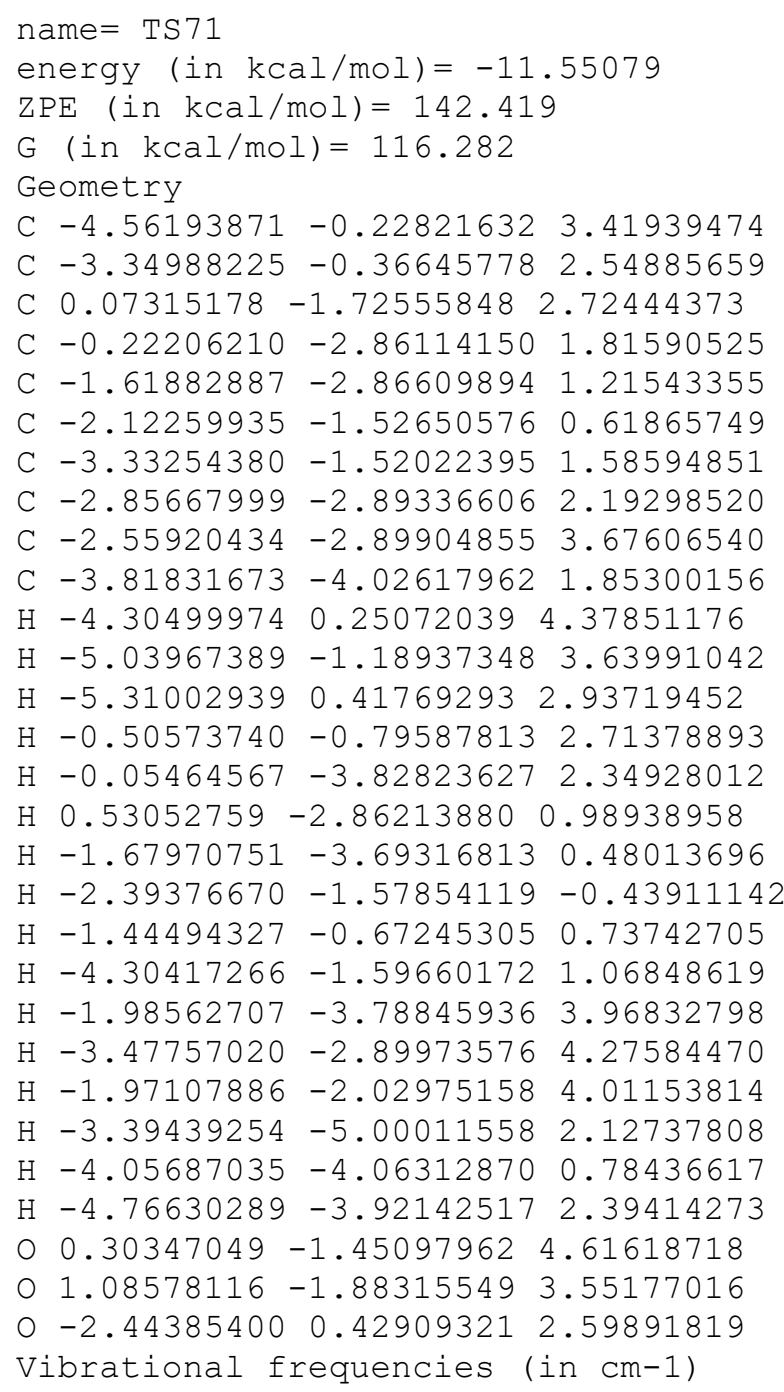


$\begin{array}{llllllllllllllllll}-581.6 & 32.2 & 53.1 & 68.7 & 79.2 & 99.0 & 155.6 & 174.0 & 187.3 & 210.8 & 222.0 & 270.6 & 293.7 & 328.1\end{array}$ $347.8 \quad 373.6 \quad 391.1 \quad 410.2 \quad 438.2 \quad 562.0 \quad 583.7 \quad 605.3 \quad 684.6 \quad 780.2 \quad 817.9 \quad 823.2 \quad 899.5 \quad 917.0$

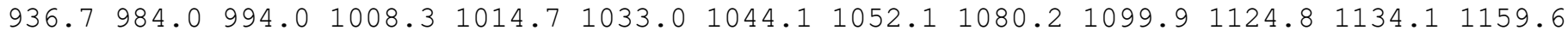
$\begin{array}{llllllllllllll}1189.5 & 1206.3 & 1232.8 & 1238.9 & 1253.0 & 1255.7 & 1266.0 & 1269.4 & 1278.7 & 1284.3 & 1288.0 & 1291.4\end{array}$ $1295.81301 .2 \quad 1305.8 \quad 1324.2 \quad 1334.8 \quad 1356.7 \quad 1378.3 \quad 1382.81404 .4 \quad 1407.4 \quad 1523.91848 .6$ $2632.6 \quad 2639.92647 .5 \quad 2663.9 \quad 2673.4 \quad 2675.2 \quad 2677.12677 .2 \quad 2678.6 \quad 2713.5 \quad 2724.4 \quad 2728.4$ 2760.52778 .02779 .12782 .6

name $=\operatorname{TS} 72$

energy (in $\mathrm{kcal} / \mathrm{mol})=-11.281429$

$\operatorname{ZPE}($ in $\mathrm{kcal} / \mathrm{mol})=142.588$

$\mathrm{G}($ in $\mathrm{kcal} / \mathrm{mol})=116.543$

Geometry

C $2.33948710 \quad 3.381509892 .81941858$

C $2.163412531 .91947232 \quad 3.10097874$

C $-0.03274286-0.917417194 .14905728$

C $-0.06823915-1.49175346 \quad 2.78211713$

C $0.83575301-0.81734301 \quad 1.76510694$

C $2.28196629-0.488697662 .22061323$

C $2.067316671 .01297760 \quad 1.90698883$

C $0.59301532 \quad 0.70648149 \quad 1.44311188$

C $-0.49860470 \quad 1.402112692 .23080293$

C $0.39911548 \quad 0.95275391 \quad-0.04696963$

H $1.68085550 \quad 3.73080550 \quad 2.01547738$

H $3.37432833 \quad 3.600073292 .52061283$

H $2.13098682 \quad 3.98862021 \quad 3.71462651$

$\mathrm{H} \quad 0.85646025-0.43432323 \quad 4.56584723$

$\mathrm{H}-1.11239556-1.514969362 .39168260$

$\mathrm{H} \quad 0.21913617 \quad-2.57294188 \quad 2.86728081$

H $\quad 0.82731374-1.42116999 \quad 0.83504089$

$\mathrm{H} 3.06231841-0.96615756 \quad 1.62184706$

$\mathrm{H} 2.50362990-0.70326595 \quad 3.27359605$

H 2.70473986 $1.37889714 \quad 1.08503427$

$\mathrm{H}-1.48283633 \quad 0.964965132 .01960946$

$\mathrm{H}-0.56102782 \quad 2.46790634 \quad 1.97567789$

$\mathrm{H}-0.34363604 \quad 1.350049823 .31733840$

H $0.42912208 \quad 2.02275399-0.28433757$

$\mathrm{H}-0.57114840 \quad 0.57156148-0.38908418$

H $1.17165380 \quad 0.46545575-0.65284659$

O $-0.40369285-1.56862038 \quad 5.92532558$

o $-1.13162346-1.05048253 \quad 4.86127833$

o $2.10632670 \quad 1.49669558 \quad 4.22943575$

Vibrational frequencies (in $\mathrm{cm}-1$ )

$\begin{array}{llllllllllllllll}-564.6 & 36.1 & 53.7 & 61.3 & 102.0 & 105.3 & 141.1 & 172.4 & 188.4 & 211.1 & 224.8 & 272.4 & 292.9 & 320.7\end{array}$

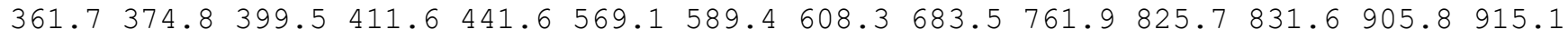
$927.5 \quad 987.4 \quad 999.4 \quad 1007.9 \quad 1016.8 \quad 1034.1 \quad 1044.6 \quad 1050.2 \quad 1079.9 \quad 1101.5 \quad 1127.3 \quad 1137.01159 .5$

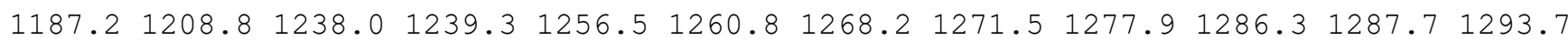
$1298.31301 .91311 .31326 .3 \quad 1337.6 \quad 1354.7 \quad 1378.7 \quad 1384.6 \quad 1406.2 \quad 1407.91526 .7 \quad 1851.8$ $2632.22638 .92661 .82667 .2 \quad 2673.12674 .72674 .82676 .42678 .4 \quad 2712.92724 .22730 .6$ $2758.8 \quad 2778.5 \quad 2780.3 \quad 2782.9$

name $=\operatorname{TS} 73$

energy $($ in $\mathrm{kcal} / \mathrm{mol})=-10.901584$

$\mathrm{ZPE}(\mathrm{in} \mathrm{kcal} / \mathrm{mol})=142.597$

$\mathrm{G}($ in $\mathrm{kcal} / \mathrm{mol})=116.58$

Geometry

$\begin{array}{llll}\text { C } 2.12756934 & 2.31510617 & 3.88126128\end{array}$

$\begin{array}{llll}\text { C } 2.25548906 & 2.64949021 & 2.42881458\end{array}$

C $-0.57384965 \quad 0.21748530 \quad 1.74437367$

C $0.08483413-0.346145190 .54030267$

C $1.57396076-0.015181440 .47505508$

C 2.315733320 .116239321 .83139896

C $2.729934361 .56870087 \quad 1.49592224$ 
C $2.00209681 \quad 1.45705054 \quad 0.10517701$

C $0.89128834 \quad 2.45268731-0.15047730$

C $2.98994518 \quad 1.47926432-1.05611019$

$\mathrm{H} 2.93782020 \quad 1.67102806 \quad 4.24142574$

H $1.17894295 \quad 1.78203371 \quad 4.07966124$

H $2.10937313 \quad 3.22358493 \quad 4.50192845$

$\mathrm{H}-0.67818843 \quad 1.29506586 \quad 1.88551700$

$\mathrm{H}-0.41380203 \quad 0.03590570-0.38118988$

$\mathrm{H}-0.03952804-1.452968330 .50559245$

$\mathrm{H} 2.06288502-0.74913600-0.19497129$

H $3.15631387-0.57403931 \quad 1.94932644$

$\mathrm{H} \quad 1.68725893-0.00009778 \quad 2.72499054$

$\mathrm{H} 3.82632003 \quad 1.68859666 \quad 1.37954217$

$\mathrm{H} \quad 1.306379873 .46856569-0.27350586$

$\mathrm{H} \quad 0.18302125 \quad 2.53355908 \quad 0.68002391$

$\mathrm{H} \quad 0.33230292 \quad 2.21483014-1.06110958$

H $3.81517179 \quad 0.77287141-0.91497851$

H $3.43344766 \quad 2.47699456-1.17441814$

H $2.49886586 \quad 1.22768415-2.00298197$

$0-0.40516404 \quad 0.09129306 \quad 3.63999009$

O $-1.04624236-0.630475312 .63373690$

O $2.02669067 \quad 3.76369294 \quad 2.02310755$

Vibrational frequencies (in $\mathrm{cm}-1$ )

$\begin{array}{llllllllllllllll}-592.4 & 25.3 & 43.4 & 77.5 & 83.9 & 147.8 & 160.5 & 179.7 & 198.8 & 205.9 & 234.9 & 264.7 & 297.4 & 328.2\end{array}$

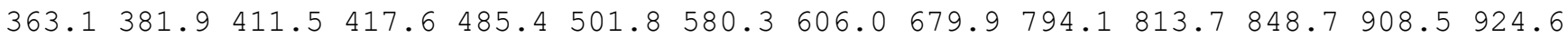
$934.3 \quad 969.1 \quad 988.4 \quad 1008.4 \quad 1016.7 \quad 1023.4 \quad 1043.7 \quad 1058.0 \quad 1080.8 \quad 1096.4 \quad 1114.91132 .4 \quad 1144.9$

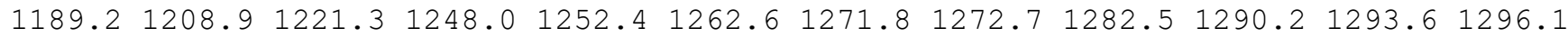
$1296.91301 .11308 .01318 .91338 .0 \quad 1354.5 \quad 1377.1 \quad 1382.11404 .11407 .51530 .91840 .3$ $2642.02644 .92655 .92658 .2 \quad 2663.2 \quad 2674.3 \quad 2676.72677 .92682 .2 \quad 2711.62715 .92729 .5$ $2754.42776 .3 \quad 2778.3 \quad 2781.9$
name $=$ TS74
energy (in $\mathrm{kcal} / \mathrm{mol})=-10.593094$
$\mathrm{ZPE}($ in $\mathrm{kcal} / \mathrm{mol})=142.435$
$\mathrm{G}($ in $\mathrm{kcal} / \mathrm{mol})=115.526$
Geometry
C $3.52234603 \quad 2.55110484 \quad 0.67024939$
C $3.40093348 \quad 1.72026664-0.57317453$
C $0.81532292-1.89389155 \quad 2.13244220$
C $0.91255043-1.36635547 \quad 0.75237404$
C $2.33232968-1.41296285 \quad 0.21051238$
C $3.32790267 \quad-0.43691840 \quad 0.89350871$
C $3.63553856 \quad 0.24115738-0.46170585$
C $2.58561578-0.69532108-1.16963312$
C $1.39387263 \quad 0.01752166-1.77551242$
C $3.22651203-1.61957102-2.19420176$
$\mathrm{H} \quad 4.35999854 \quad 2.23431961 \quad 1.30397716$
H 2.60586953 2.49167288 1.27455107
H $3.67070550 \quad 3.61373651 \quad 0.42202667$
$\mathrm{H}-0.14867137-2.16883972 \quad 2.56083722$
$\mathrm{H} \quad 0.53092675 \quad-0.31370214 \quad 0.74987399$
$\mathrm{H} \quad 0.22657675-1.91213803 \quad 0.06632186$
H $2.69955525-2.455000620 .20084391$
$\mathrm{H} \quad 4.18837353-0.92393937 \quad 1.36243943$
H $2.87375463 \quad 0.21017460 \quad 1.65147279$
H $4.66778342 \quad 0.03891715-0.81568412$
$\mathrm{H} \quad 1.70315367 \quad 0.61119557 \quad-2.65293205$
$\mathrm{H} \quad 0.92706367 \quad 0.72864867 \quad-1.08549109$
$\mathrm{H} \quad 0.62694017-0.68678442-2.11359750$
H $3.57479366-1.05407296-3.06933666$
H 2.51454888 $-2.37075132-2.55538701$
H $4.09227125 \quad-2.15493804-1.78882045$
o $1.31646511-1.33262900 \quad 3.89226156$
O $1.92084111-2.00449718 \quad 2.83722308$ 
$03.164427692 .23495795-1.63911372$

Vibrational frequencies (in $\mathrm{cm}-1$ )

$\begin{array}{llllllllllllllll}-591.0 & 21.9 & 35.1 & 38.8 & 70.0 & 133.5 & 140.4 & 175.7 & 191.6 & 209.8 & 235.6 & 261.8 & 282.8 & 320.1\end{array}$ $353.3 \quad 369.0 \quad 408.2 \quad 418.5 \quad 491.8 \quad 513.4 \quad 573.2 \quad 621.0 \quad 668.4 \quad 790.5 \quad 811.9 \quad 868.2 \quad 885.3 \quad 926.9$ $935.5955 .6 \quad 993.3 \quad 1007.9 \quad 1018.6 \quad 1026.9 \quad 1043.1 \quad 1056.1 \quad 1075.6 \quad 1106.6 \quad 1114.5 \quad 1129.6 \quad 1160.6$

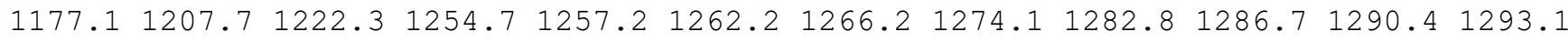

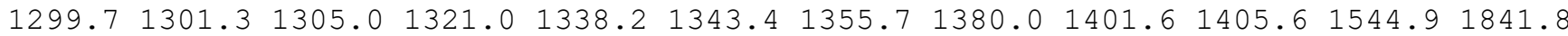
$2632.72659 .2 \quad 2666.1 \quad 2673.3 \quad 2674.0 \quad 2674.6 \quad 2677.82678 .42682 .5 \quad 2712.42720 .02728 .9$ $2761.92778 .8 \quad 2779.1 \quad 2782.1$

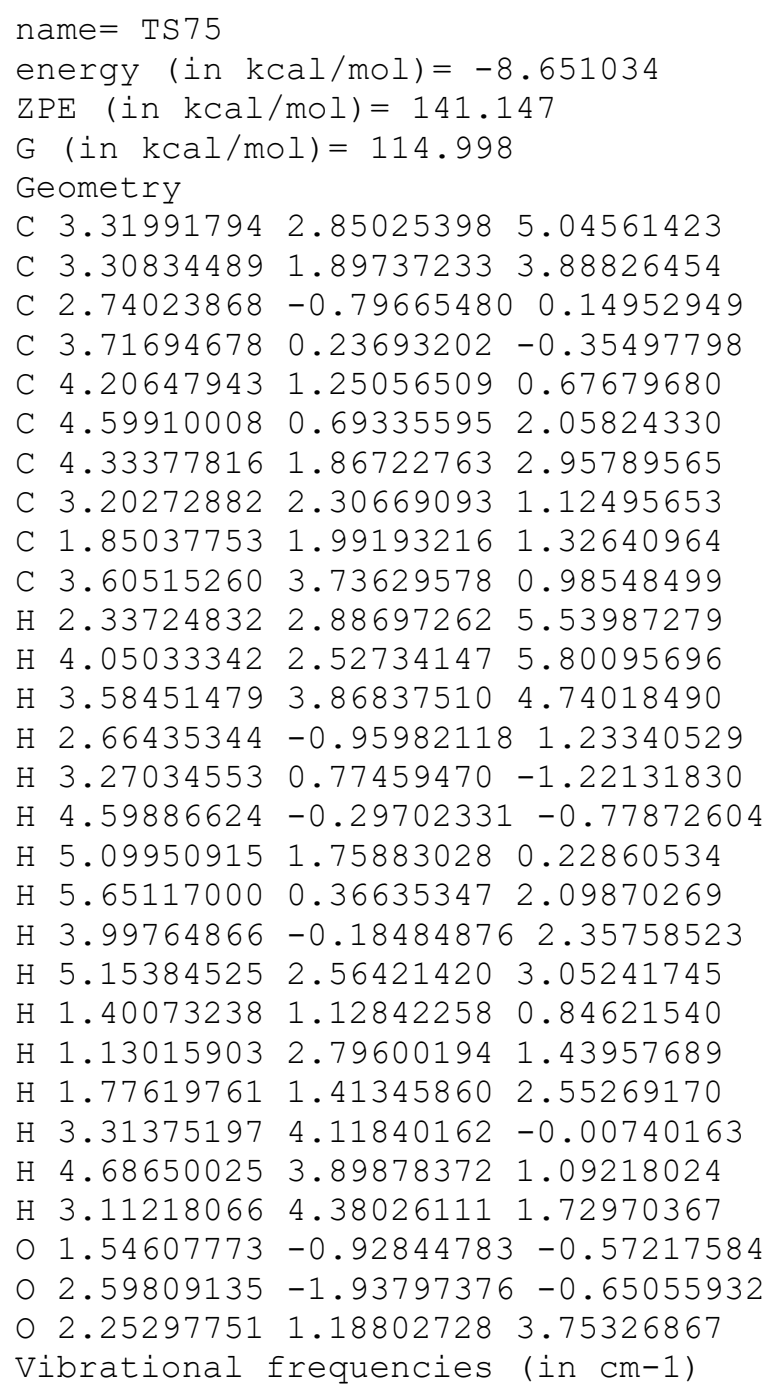


C $3.06641526 \quad 0.03336473 \quad 2.67442016$

C 2.118044251 .258603462 .72649575

C $1.10535548 \quad 0.50737335 \quad 1.76131148$

$\begin{array}{lllll}C & -0.26665875 & 0.22517338 & 2.33155174\end{array}$

C $0.98008104 \quad 1.21532574 \quad 0.41912066$

H $0.56065640 \quad 3.02537777 \quad 5.31077798$

$\mathrm{H}-0.24658200 \quad 2.69981260 \quad 3.78289748$

H $1.21707639 \quad 3.70434970 \quad 3.78939709$

H $1.31119233-1.42075859 \quad 4.26192377$

H $0.86084534-2.44136425 \quad 1.40048187$

H $2.46046621-2.808252062 .03385343$

H $2.55272031-0.790549230 .68753028$

H $4.05355335 \quad 0.25021794 \quad 2.25568012$

H $3.22746839-0.474995923 .63084471$

H $2.55249281 \quad 2.15406837 \quad 2.24031492$

$\mathrm{H}-0.93113827 \quad 1.09426234 \quad 2.25667875$

$\mathrm{H}-0.24545512-0.056619173 .39850713$

$\mathrm{H}-0.75834227-0.59901949 \quad 1.79709943$

H $1.95374295 \quad 1.42187945-0.03871652$

$\mathrm{H} \quad 0.45490769 \quad 2.17288961 \quad 0.51529230$

$\mathrm{H} \quad 0.41008635 \quad 0.60464030 \quad-0.29373455$

$0 \quad 0.61871039 \quad 0.63049517 \quad 5.44510224$

$00.37414633-3.13964336 \quad 3.81003336$

o $1.94568418 \quad 0.831462525 .06737030$

Vibrational frequencies (in $\mathrm{cm}-1$ )

$\begin{array}{llllllllllllllll}-532.1 & 21.4 & 53.7 & 68.0 & 122.0 & 132.3 & 159.7 & 170.1 & 193.0 & 222.1 & 237.3 & 270.6 & 287.3 & 325.8\end{array}$

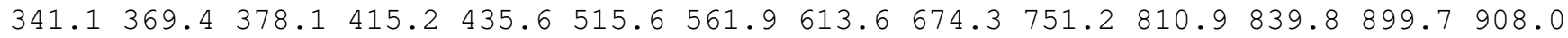
$937.1998 .3 \quad 1004.1 \quad 1011.6 \quad 1015.0 \quad 1032.3 \quad 1035.2 \quad 1053.8 \quad 1070.8 \quad 1089.51119 .21121 .2$ $1163.51199 .0 \quad 1204.2 \quad 1235.2 \quad 1243.3 \quad 1243.6 \quad 1253.6 \quad 1257.9 \quad 1280.2 \quad 1283.5 \quad 1287.5 \quad 1289.6$

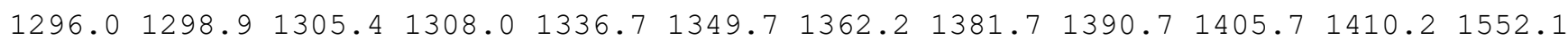
$1828.02636 .62640 .52641 .6 \quad 2652.12662 .7 \quad 2671.72675 .22677 .12677 .62710 .32718 .1$ $2737.0 \quad 2760.7 \quad 2766.8 \quad 2774.1 \quad 2782.2$

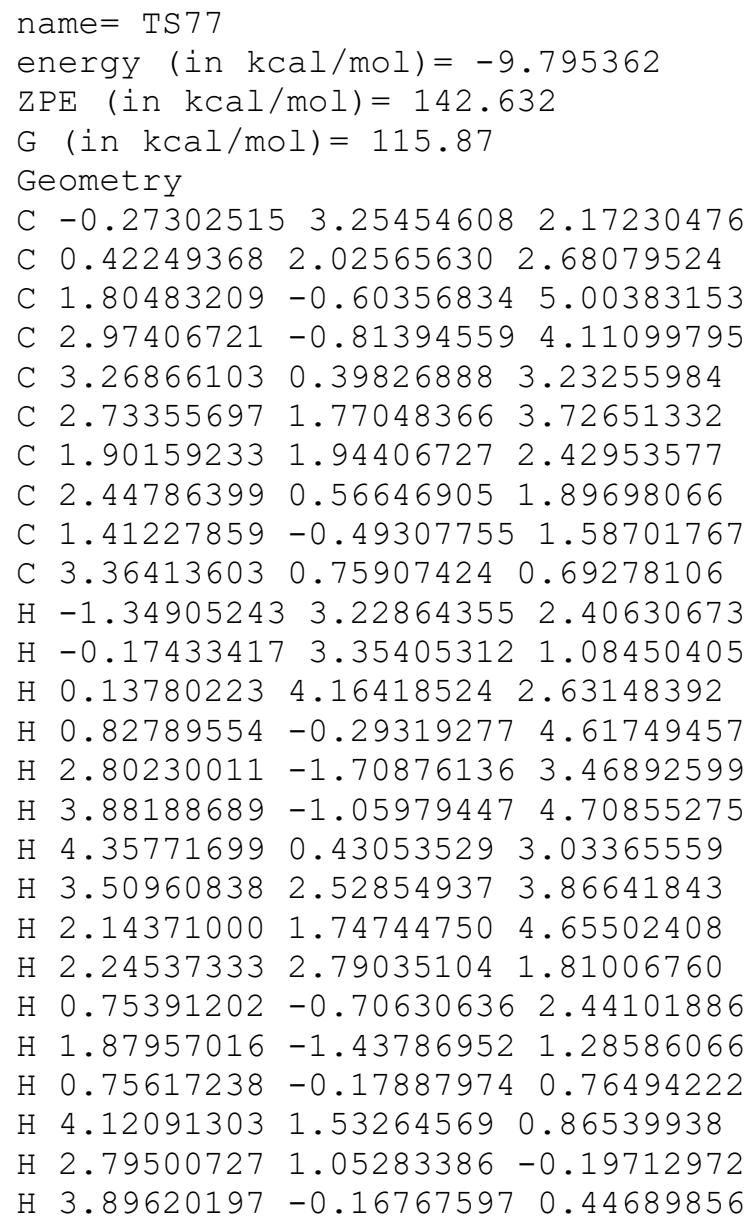


$01.40634078 \quad 0.429171676 .57556035$

O $2.00186197-0.794123956 .29144746$

O -0.187563791 .166615393 .26814252$

Vibrational frequencies (in $\mathrm{cm}-1$ )

$\begin{array}{llllllllllllllll}-588.5 & 10.8 & 46.3 & 71.4 & 88.8 & 94.9 & 162.0 & 169.3 & 181.4 & 207.0 & 239.7 & 274.9 & 287.3 & 316.3\end{array}$

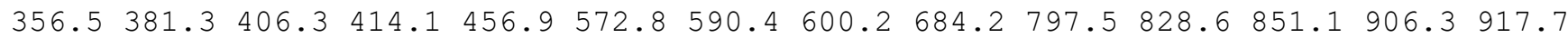
$932.8980 .1 \quad 994.3 \quad 1006.4 \quad 1017.0 \quad 1034.2 \quad 1044.0 \quad 1049.1 \quad 1079.01097 .5 \quad 1119.4 \quad 1132.7 \quad 1146.0$ $1191.61210 .0 \quad 1226.2 \quad 1233.8 \quad 1256.8 \quad 1263.5 \quad 1267.8 \quad 1273.5 \quad 1284.2 \quad 1287.9 \quad 1290.5 \quad 1297.6$ $1299.61300 .7 \quad 1314.4 \quad 1332.4 \quad 1347.3 \quad 1357.2 \quad 1376.6 \quad 1382.3 \quad 1406.0 \quad 1408.8 \quad 1524.2 \quad 1853.4$ $2631.02649 .12657 .5 \quad 2662.8 \quad 2668.6 \quad 2673.92675 .3 \quad 2676.92679 .72713 .8 \quad 2726.6 \quad 2730.0$ $2752.22779 .8 \quad 2779.8 \quad 2782.8$

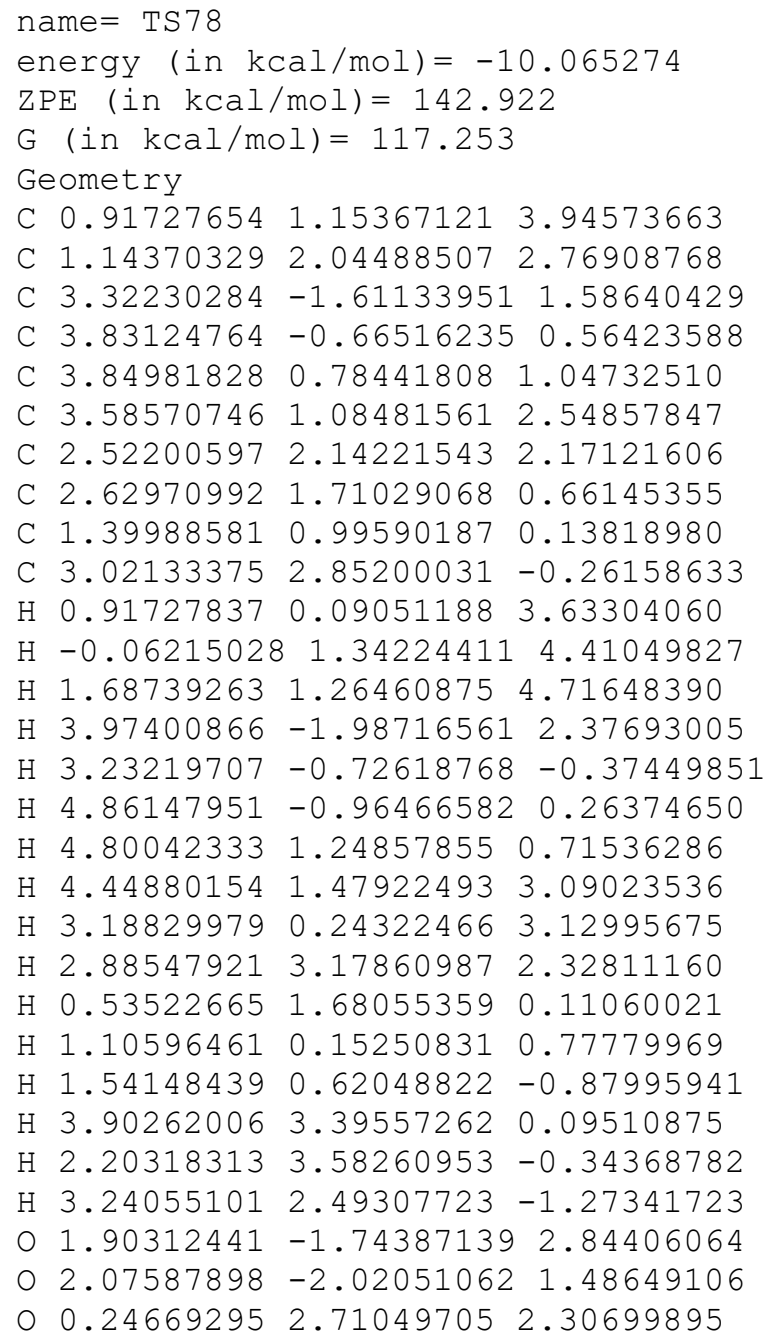


C $1.12877157 \quad 1.42332755-1.06887432$

C $2.356562281 .85956774-0.29184778$

C $3.04493577 \quad 0.78315728 \quad 0.58444104$

C $2.89423881 \quad 1.66750257 \quad 1.84872975$

C $2.15826693 \quad 2.770055690 .97801529$

C $0.72491336 \quad 3.07377378 \quad 1.36280971$

C 2.964079834 .058256460 .89717526

$\mathrm{H} \quad 0.79449179 \quad 1.87010254 \quad 4.52587910$

H 2.00560192 3.05443063 3.97654142

$\mathrm{H} 2.48606400 \quad 1.68076258 \quad 4.99281185$

$\mathrm{H} \quad 0.50448451-0.117843560 .43899436$

$\mathrm{H} 0.632882532 .31122573-1.51881107$

$\mathrm{H} 1.440638620 .80008405-1.93751255$

H $3.06980660 \quad 2.32773282-1.00066234$

$\begin{array}{lllll}\mathrm{H} & 4.08398894 & 0.56762696 & 0.31688510\end{array}$

H $2.53814884 \quad-0.18921975 \quad 0.63798063$

H $3.87950383 \quad 2.02009248 \quad 2.22135170$

H $0.66399239 \quad 3.76193299 \quad 2.21412867$

$\mathrm{H} \quad 0.14952985 \quad 2.17872948 \quad 1.62878284$

H $0.18815562 \quad 3.55372375 \quad 0.53094596$

$\mathrm{H} \quad 4.00437219 \quad 3.87978747 \quad 0.59976812$

H 2.98646056 4.58282166 1.85922423

H 2.53175176 4.74806849 0.16088496

$\begin{array}{lllll}0 & 2.79653357 & -0.68488239 & 2.79475484\end{array}$

$\begin{array}{lllll}0 & -1.05978999 & 0.80497481 & -0.41143264\end{array}$

O $1.56937898-0.074140763 .01869953$

Vibrational frequencies (in $\mathrm{cm}-1$ )

$\begin{array}{lllllllllllllllll}-533.8 & 25.3 & 36.8 & 62.0 & 98.5 & 113.4 & 162.5 & 186.5 & 194.3 & 228.2 & 247.4 & 268.0 & 280.1 & 303.6\end{array}$

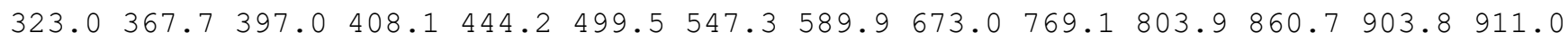

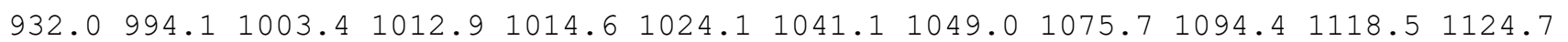
$\begin{array}{llllllllllll}1156.9 & 1188.1 & 1200.1 & 1230.2 & 1236.9 & 1247.7 & 1259.3 & 1271.7 & 1278.8 & 1286.1 & 1290.1 & 1292.8\end{array}$

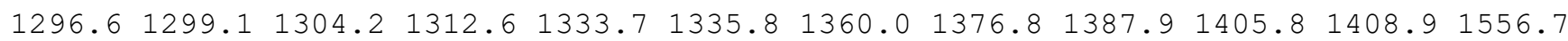
$1832.7 \quad 2636.8 \quad 2643.0 \quad 2656.4 \quad 2664.8 \quad 2665.8 \quad 2666.42671 .82674 .92677 .8 \quad 2707.2 \quad 2714.9$ $2738.22759 .12768 .72779 .3 \quad 2782.3$

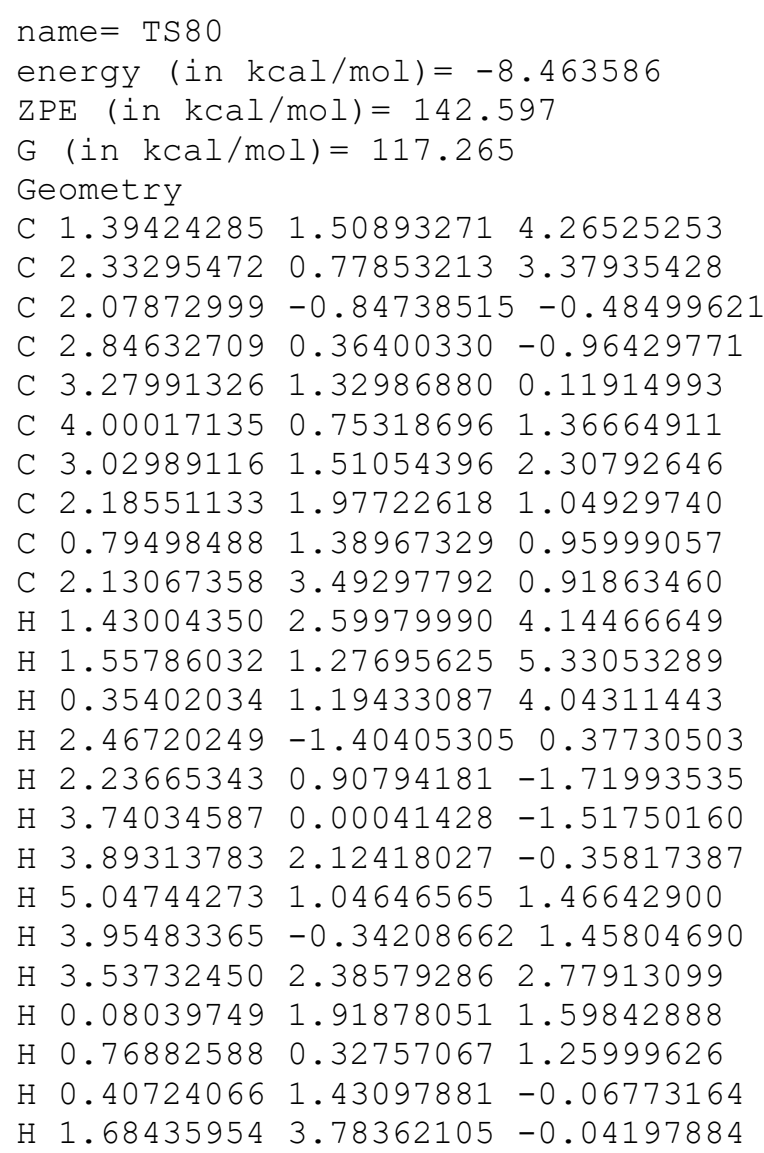


$\mathrm{H} \quad 3.12298832 \quad 3.95431952 \quad 0.96605030$

$\mathrm{H} \quad 1.51953472 \quad 3.944457351 .70803303$

O $2.18339039-0.954535162 .47994264$

$01.08712706-1.20654181-1.07155401$

O $2.55163624-0.478357623 .72938459$

Vibrational frequencies (in $\mathrm{cm}-1$ )

$\begin{array}{llllllllllllllllll}-526.7 & 51.5 & 57.5 & 82.3 & 104.2 & 132.7 & 165.6 & 184.9 & 204.7 & 237.5 & 244.4 & 284.2 & 302.2315 .4\end{array}$

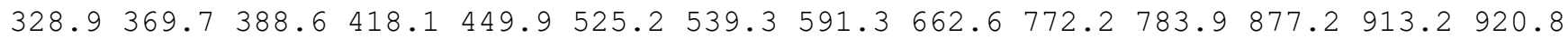

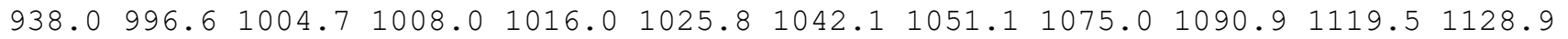
$1160.31191 .0 \quad 1199.8 \quad 1234.3 \quad 1237.5 \quad 1255.3 \quad 1258.3 \quad 1264.1 \quad 1281.8 \quad 1286.1 \quad 1288.01290 .2$

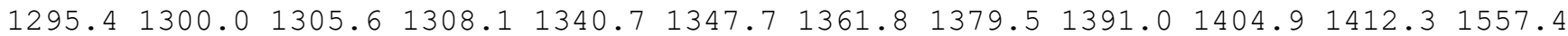

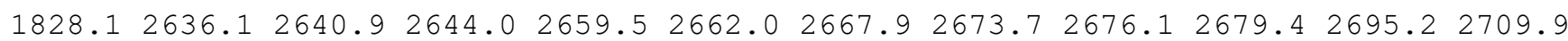
$2737.9 \quad 2754.0 \quad 2769.5 \quad 2775.5 \quad 2782.3$

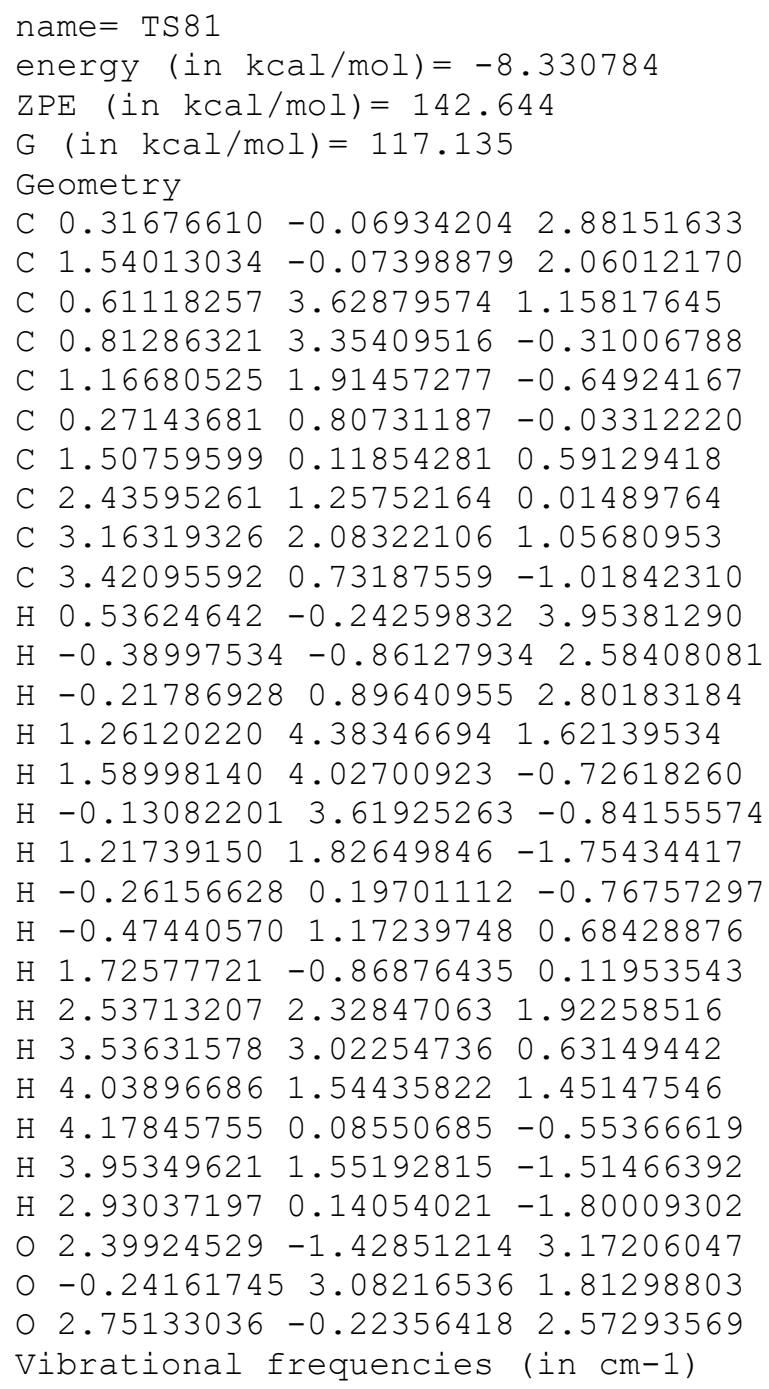


$\begin{array}{llll}\text { C } 2.31917261 & 1.63928511 & 0.89007254\end{array}$

C $2.24823723-2.54311797 \quad 1.42910911$

C $2.95174844 \quad-2.44176255 \quad 0.29267433$

C $3.62878838-1.24098842-0.24437450$

C $4.15453116-0.169601250 .75381150$

C 3.311382170 .934205130 .06556570

C $2.70789689-0.14900931-0.92003627$

C $1.21733424-0.37457472-0.77972263$

C $3.07850016 \quad 0.12317555-2.36884481$

$\mathrm{H} 1.73807096 \quad 3.72075981 \quad 1.03517069$

$\mathrm{H} \quad 0.61427016 \quad 2.50151842 \quad 1.58555893$

H $1.178846702 .90324348-0.44639896$

$\mathrm{H} 1.76114999-3.440168631 .79200156$

$\mathrm{H} 3.05043690-3.32617246-0.33971183$

$\mathrm{H} 1.50322009-1.600491892 .98087911$

H $4.43645986-1.55652920-0.93875255$

$\mathrm{H} 5.23432486-0.00418176 \quad 0.69847319$

H $3.91415096-0.362901461 .80446507$

$\mathrm{H} 3.93993157 \quad 1.67414350-0.47283764$

$\mathrm{H} \quad 0.89526501-1.25645158-1.34654960$

$\mathrm{H} \quad 0.64048729 \quad 0.48472899-1.14294731$

$\mathrm{H} \quad 0.92498877 \quad-0.54498129 \quad 0.26730877$

H $2.55983005 \quad 1.01060281-2.75279388$

H 2.79911495 -0.72136636 -3.01118085

H $4.15362925 \quad 0.28995078 \quad-2.49981443$

01.039210391 .794703402 .67249450

$\begin{array}{llll}0 & 2.12179380 & -1.44838759 & 2.22942517\end{array}$

O 2.054228591 .100386042 .06887855

Vibrational frequencies (in $\mathrm{cm}-1$ )

$\begin{array}{lllllllllllllll}-1538.8 & 29.9 & 53.7 & 92.7 & 117.7 & 149.7 & 175.4 & 182.2 & 210.6 & 234.5 & 256.3 & 259.1 & 281.8 & 325.7\end{array}$

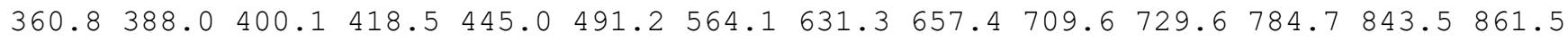
$923.4 \quad 936.3 \quad 963.9986 .7 \quad 998.2 \quad 1004.7 \quad 1007.4 \quad 1016.5 \quad 1048.6 \quad 1057.4 \quad 1075.1 \quad 1088.7 \quad 1121.7$

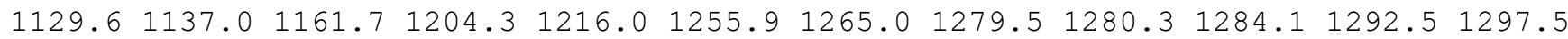
$1299.51305 .3 \quad 1315.91329 .7 \quad 1362.9 \quad 1378.1 \quad 1402.9 \quad 1404.81407 .5 \quad 1490.91586 .8 \quad 1853.5$ $1967.12665 .12675 .1 \quad 2675.3 \quad 2677.2 \quad 2680.12706 .52711 .22717 .12725 .22754 .12760 .4$ 2779.52781 .92789 .12791 .0

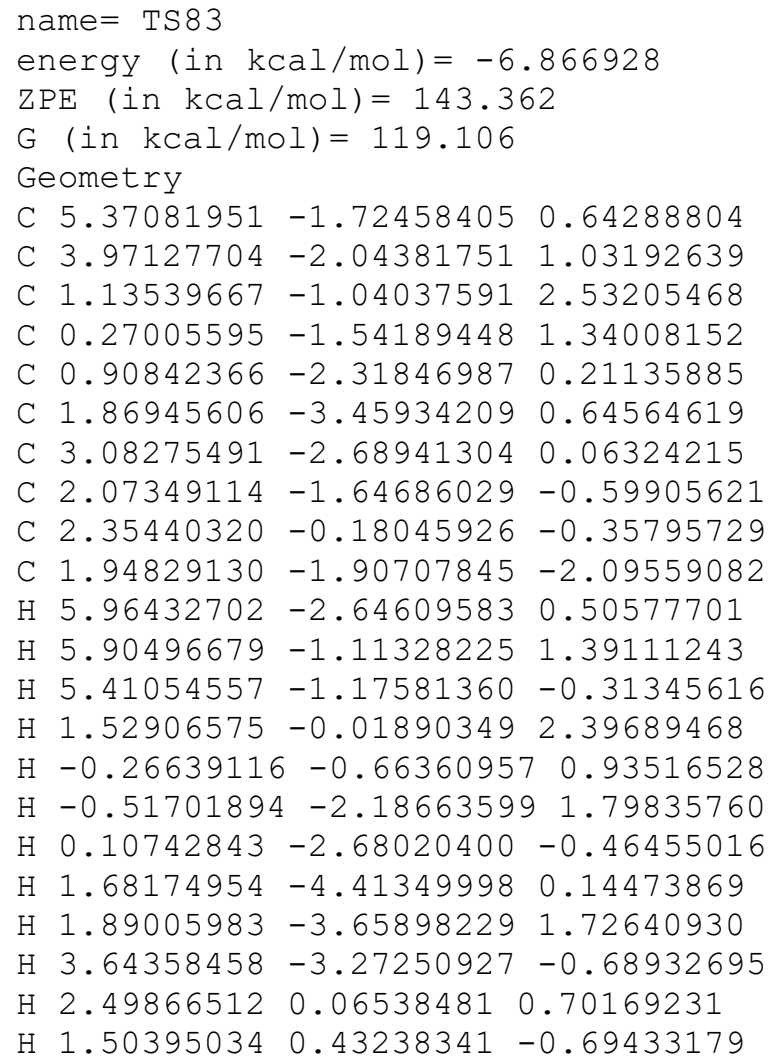


$\mathrm{H} \quad 3.23636650 \quad 0.16585739-0.90731251$

H $1.11877024-1.32865742-2.52417051$

$\mathrm{H} 1.75019015-2.96234914-2.31852561$

$\mathrm{H} 2.85628579-1.62152268-2.63712389$

o $2.40656391-1.91547420 \quad 2.73408027$

$0 \quad 0.70727267-1.38543045 \quad 3.67142574$

o $3.69375127-1.65020510 \quad 2.21378593$

Vibrational frequencies (in $\mathrm{cm}-1$ )

$\begin{array}{lllllllllllllllll}-417.7 & 70.1 & 88.1 & 105.9 & 145.6 & 167.6 & 184.8 & 200.5 & 208.4 & 245.6 & 272.9 & 303.1 & 320.0 & 351.8\end{array}$ $391.7 \quad 401.0 \quad 436.5 \quad 456.8 \quad 482.3 \quad 577.6 \quad 587.2 \quad 676.5 \quad 700.4 \quad 761.0 \quad 800.5 \quad 831.3 \quad 878.5 \quad 905.4$ $966.6987 .4995 .6 \quad 1006.6 \quad 1014.3 \quad 1034.7 \quad 1039.4 \quad 1043.0 \quad 1085.6 \quad 1101.0 \quad 1113.6 \quad 1125.9 \quad 1162.5$

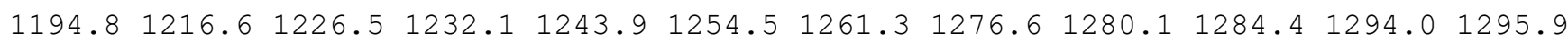

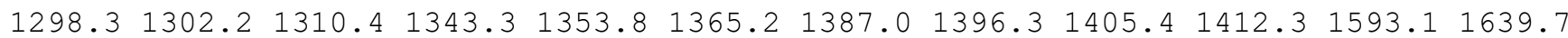
$2627.02647 .8 \quad 2652.5 \quad 2656.92660 .8 \quad 2663.7 \quad 2672.8 \quad 2675.4 \quad 2679.3 \quad 2712.5 \quad 2723.5 \quad 2736.9$ $2752.92766 .4 \quad 2778.0 \quad 2782.1$

\section{name $=$ TS 84}

energy $($ in $\mathrm{kcal} / \mathrm{mol})=-4.066861$

$\mathrm{ZPE}($ in $\mathrm{kcal} / \mathrm{mol})=141.668$

$\mathrm{G}(\mathrm{in} \mathrm{kcal} / \mathrm{mol})=118.109$

Geometry

C $0.43173334 \quad 1.63174796 \quad 2.98486607$

C $1.53428131 \quad 0.64542598 \quad 3.15537914$

C $0.81911420-2.143376652 .02939478$

C $2.23809402-2.226521582 .08141362$

C $3.19196697-1.22854430 \quad 1.51617313$

C $3.84051211-0.31651242 \quad 2.62179742$

$\begin{array}{llll}\text { C } 2.74878331 & 0.74746741 & 2.33264800\end{array}$

C $2.61396926 \quad 0.09374295 \quad 0.86948424$

C $1.29892888 \quad 0.08674686 \quad 0.13003893$

C $3.66203100 \quad 0.73445468 \quad-0.05237223$

$\mathrm{H} 0.14686842 \quad 2.13264623 \quad 3.92298687$

$\mathrm{H}-0.48638281 \quad 1.11161854 \quad 2.61897302$

$\mathrm{H} \quad 0.66814582 \quad 2.40782736 \quad 2.24201627$

$\mathrm{H} \quad 0.23227237-3.07217931 \quad 1.94258879$

H $2.65026134-3.232033492 .08414277$

H 2.24397752 -1.83870122 3.55205300

H $3.92902517-1.72454368 \quad 0.86376014$

H $4.84921409 \quad 0.02473154 \quad 2.36581955$

$\mathrm{H} \quad 3.91417357-0.72334187 \quad 3.62956584$

H 3.116110551 .788027892 .30254897

H $0.44046631 \quad 0.38384355 \quad 0.74385204$

$\mathrm{H} \quad 1.06858454-0.92453651-0.23837431$

$\mathrm{H} \quad 1.296007610 .75291625-0.74082910$

H $3.73113786 \quad 0.17340679-0.99364730$

$\mathrm{H} \quad 4.66703638 \quad 0.74980532 \quad 0.38065107$

$\mathrm{H} \quad 3.39662843 \quad 1.76745697 \quad-0.30041942$

○ $2.14660987-1.11062416 \quad 4.50578557$

○ $0.17074626-1.10446205 \quad 2.18438638$

o $1.24692983-0.132035414 .15519804$

Vibrational frequencies (in $\mathrm{cm}-1$ )

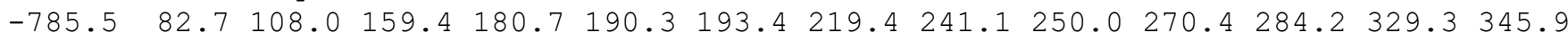
$381.6 \quad 404.6 \quad 428.3 \quad 482.9 \quad 500.0 \quad 546.1 \quad 596.5 \quad 644.2 \quad 705.0 \quad 718.5 \quad 776.2 \quad 850.6 \quad 861.3 \quad 895.7$ $920.5958 .4991 .8 \quad 994.3 \quad 1009.2 \quad 1022.7 \quad 1026.2 \quad 1034.2 \quad 1057.0 \quad 1071.0 \quad 1087.7 \quad 1094.4 \quad 1105.4$

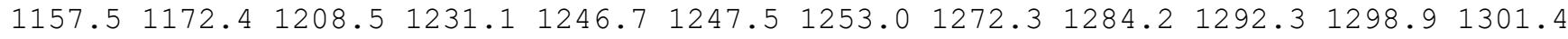
$1303.11328 .41333 .2 \quad 1347.7 \quad 1351.4 \quad 1382.2 \quad 1395.1 \quad 1404.31411 .2 \quad 1427.4 \quad 1484.4 \quad 1586.8$ $1780.02613 .5 \quad 2660.3 \quad 2662.0 \quad 2664.6 \quad 2668.9 \quad 2669.8 \quad 2672.72677 .82716 .52724 .72758 .7$ 2760.62767 .02779 .62781 .6

name $=\operatorname{TS} 85$

energy (in $\mathrm{kcal} / \mathrm{mol})=-2.833226$

$\mathrm{ZPE}(\mathrm{in} \mathrm{kcal} / \mathrm{mol})=140.899$

$\mathrm{G}($ in $\mathrm{kcal} / \mathrm{mol})=114.467$ 
Geometry

C $2.96903692 \quad 2.33715533 \quad 3.78366199$

C $2.792396981 .01592904 \quad 3.33094356$

C $-0.07763761-1.31489980 \quad 2.52821008$

C $0.32587093-1.38895276 \quad 1.07562945$

C $1.60650012-0.66648027 \quad 0.71071042$

C $2.83604995-0.908580191 .62706653$

C $2.95835674 \quad 0.61072149 \quad 1.90862138$

C $1.70379414 \quad 0.88144969 \quad 0.98730568$

C $0.520634061 .52910324 \quad 1.67449159$

C $2.075778561 .66556993-0.26362026$

$\mathrm{H} \quad 3.83083518 \quad 2.92822269 \quad 3.54462081$

H $2.15952585 \quad 2.88156913 \quad 4.24672532$

H $2.80138421 \quad 0.95243457 \quad 6.08135332$

$\mathrm{H} 0.70424808-1.16381796 \quad 3.28423284$

$\mathrm{H}-0.51000925-1.01048278 \quad 0.44547094$

$\mathrm{H} 0.41410923-2.465691750 .80490072$

$\mathrm{H} \quad 1.84178726-0.88837750-0.35060951$

$\mathrm{H} 3.70045598-1.339756531 .11422596$

H 2.64474599 -1.52588704 2.51113272

$\mathrm{H} 3.887163581 .051789291 .49981416$

$\mathrm{H} \quad 0.30304481 \quad 1.09627373 \quad 2.66074238$

$\mathrm{H}-0.393314691 .42865452 \quad 1.07373677$

$\mathrm{H} \quad 0.68536003 \quad 2.60225707 \quad 1.83642416$

H $2.33699073 \quad 2.70345898-0.02470558$

$\mathrm{H} \quad 1.237863121 .69739110-0.97195022$

H $2.93020874 \quad 1.22785420 \quad-0.79175999$

○ 2.066164051 .310404695 .57381538

O $-1.23494701-1.440669172 .84513243$

o 2.49092939 $0.17073744 \quad 4.20525584$

Vibrational frequencies (in $\mathrm{cm}-1$ )

$\begin{array}{llllllllllllllll}-1287.7 & 28.2 & 44.8 & 49.0 & 90.7 & 122.5 & 161.8 & 184.3 & 195.2 & 212.4 & 229.7 & 242.9 & 266.5 & 287.7\end{array}$ $331.2 \quad 364.9400 .9 \quad 425.0 \quad 439.8 \quad 444.3 \quad 540.8 \quad 582.4 \quad 606.7 \quad 647.6 \quad 683.4 \quad 755.8 \quad 823.1 \quad 846.9$ $923.1933 .5 \quad 945.1986 .4 \quad 996.2 \quad 1004.6 \quad 1007.7 \quad 1017.7 \quad 1032.3 \quad 1054.7 \quad 1069.7 \quad 1110.5 \quad 1122.1$

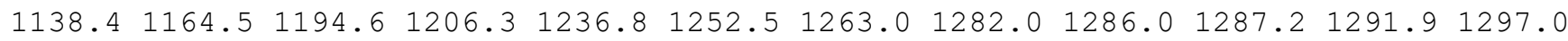
$1299.91308 .51315 .81335 .5 \quad 1344.6 \quad 1355.3 \quad 1383.91405 .21406 .6 \quad 1444.4 \quad 1538.6 \quad 1829.9$ $2638.62653 .7 \quad 2660.92672 .2 \quad 2676.4 \quad 2677.5 \quad 2678.12695 .72712 .5 \quad 2720.8 \quad 2737.5 \quad 2752.2$ $2761.02778 .3 \quad 2782.4 \quad 2881.1$

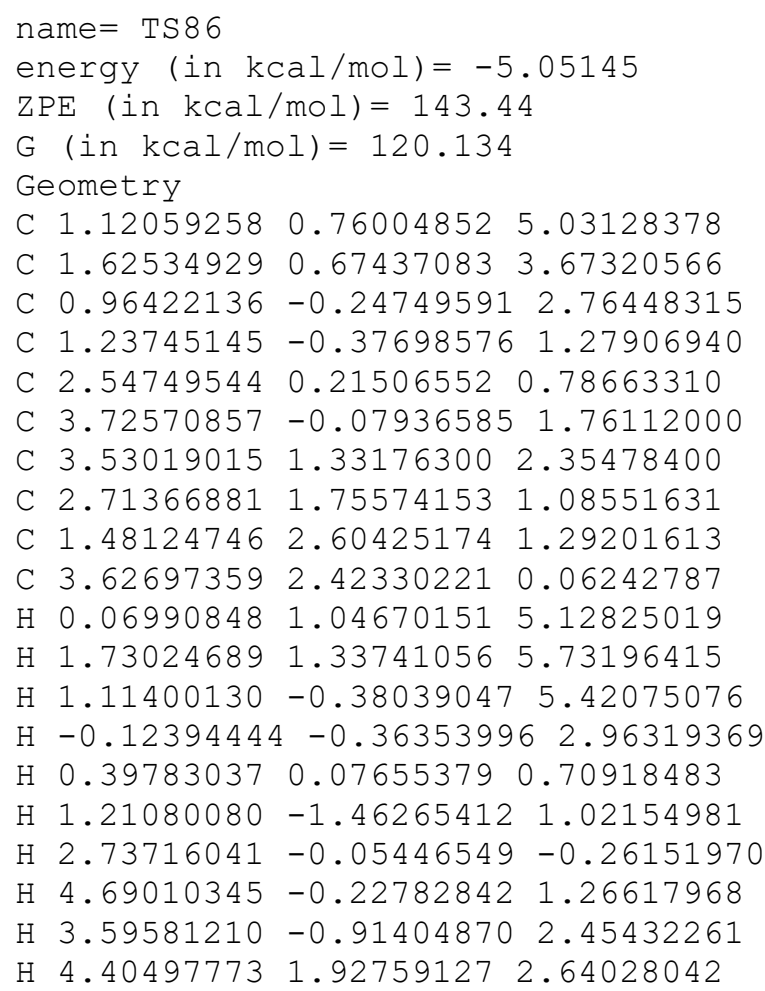


H $0.935983892 .74444663 \quad 0.34922257$

$\mathrm{H} 1.74909868 \quad 3.60110036 \quad 1.66885647$

$\mathrm{H} 0.76844565 \quad 2.18236743 \quad 2.00791364$

$\mathrm{H} \quad 3.91928064 \quad 3.43000783 \quad 0.38516955$

$\mathrm{H} \quad 3.12309214 \quad 2.52599552-0.90667491$

$\mathrm{H} \quad 4.54785174 \quad 1.85502942 \quad-0.11154475$

$\begin{array}{lllll}0 & 1.12172714 & -1.75967878 & 4.54419264\end{array}$

$01.68990668-1.384928393 .46978148$

$\begin{array}{llll}0 & 2.78538777 & 1.31604153 & 3.55659821\end{array}$

Vibrational frequencies (in $\mathrm{cm}-1$ )

$\begin{array}{lllllllllllllllll}-506.1 & 90.7 & 106.4 & 132.9 & 175.8 & 193.9 & 207.2 & 244.0 & 274.2 & 290.6 & 322.0 & 351.3 & 365.8 & 380.8\end{array}$

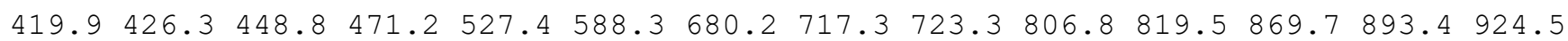
$946.2977 .21001 .2 \quad 1008.4 \quad 1016.4 \quad 1017.4 \quad 1029.6 \quad 1075.2 \quad 1095.11107 .7 \quad 1119.41132 .9$

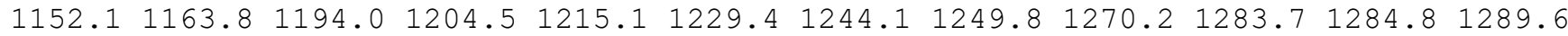

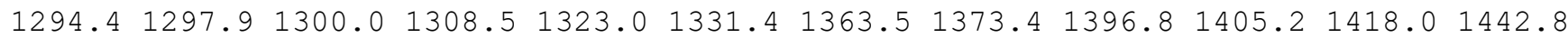
$1540.92104 .62650 .8 \quad 2671.92673 .6 \quad 2676.3 \quad 2678.12681 .92683 .6 \quad 2694.22713 .12730 .2$ $2736.22756 .8 \quad 2765.52781 .2 \quad 2784.5$

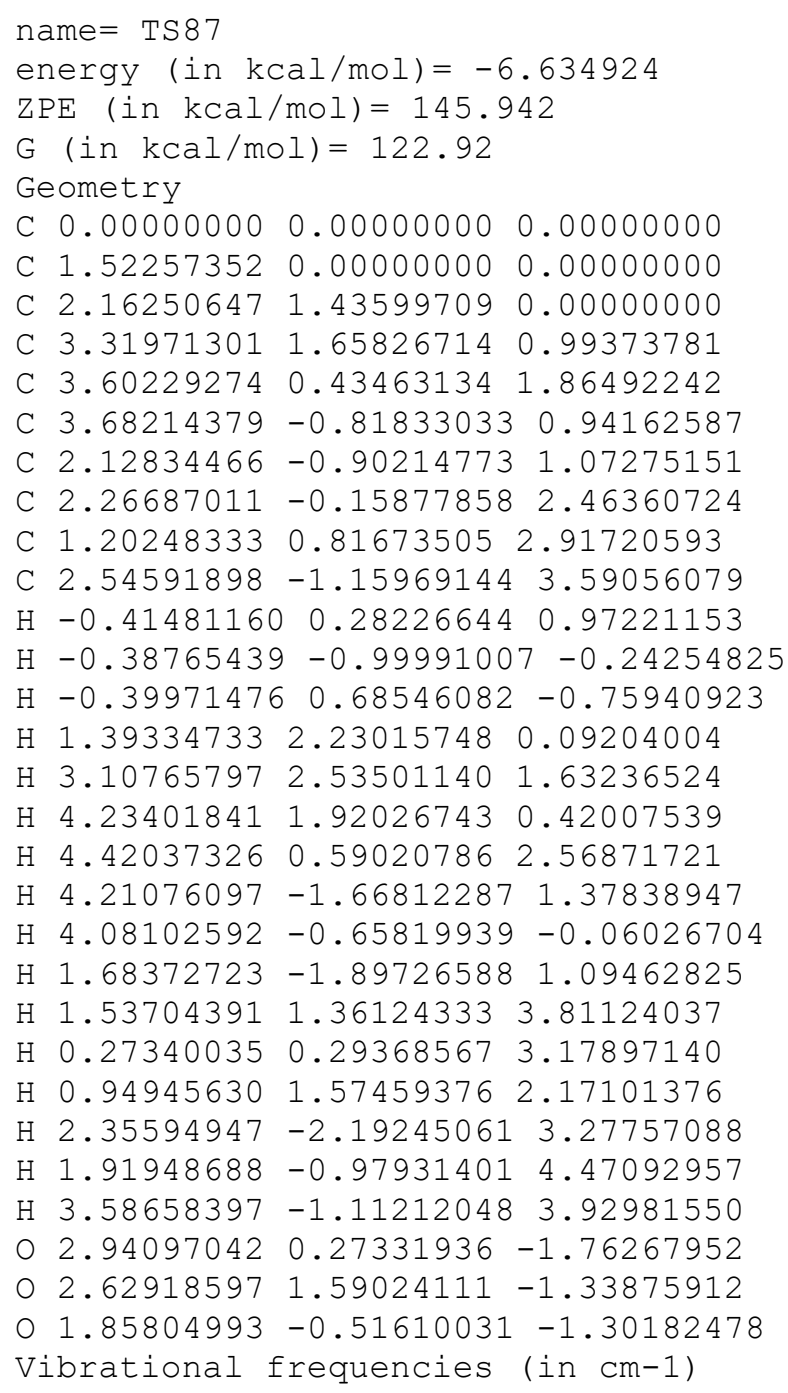


$\mathrm{ZPE}($ in $\mathrm{kcal} / \mathrm{mol})=140.343$

$\mathrm{G}($ in $\mathrm{kcal} / \mathrm{mol})=115.021$

Geometry

C $2.817903132 .63731982 \quad 3.34449450$

C $2.60056741 \quad 1.35385433 \quad 2.60994613$

C $1.57261374-2.13345559-0.21170489$

C $2.90181025-1.72022557-0.80399444$

C $3.60187590-0.55673097-0.11988219$

C $3.72273806-0.62412713 \quad 1.41761546$

C $3.56088750 \quad 0.84440918 \quad 1.74190630$

C $2.89701850 \quad 0.80649777-0.16292891$

C $1.473200030 .87026510-0.19889843$

C $3.669570591 .95585295-0.73412363$

$\mathrm{H} 3.70136140 \quad 2.57177994 \quad 3.99997069$

H $1.96366827 \quad 2.92392673 \quad 3.97900196$

$\mathrm{H} 2.99407409 \quad 3.469130992 .64399813$

H $1.42365881-2.03294228 \quad 0.87176169$

H 2.76438728 -1.49274082 -1.88416805

H $3.56573644-2.61383807-0.78337005$

$\mathrm{H} 4.62067293-0.47104860-0.57136118$

$\mathrm{H} 4.68697413-1.027673691 .75964193$

H 2.93918636 $-1.24618661 \quad 1.89037857$

H $4.493019291 .40422058 \quad 1.67039708$

H $1.03374790 \quad 0.32118668 \quad 0.92551597$

H $0.94061644 \quad 0.16593977-0.83362832$

$\mathrm{H} \quad 1.00122407 \quad 1.84037598-0.24532778$

$\mathrm{H} 3.504505692 .00264479-1.82485252$

H $4.752069891 .88973410-0.57242168$

H $3.333887892 .92065383-0.32874498$

O $1.01289579-0.19187150 \quad 2.15109543$

O $0.71460717-2.59236715-0.92503611$

o $1.39755775 \quad 0.90227342 \quad 2.89071897$

Vibrational frequencies (in $\mathrm{cm}-1$ )

$\begin{array}{lllllllllllllll}-1608.0 & 45.8 & 64.2 & 89.7 & 95.0 & 139.2 & 161.0 & 182.6 & 209.7 & 222.9 & 263.1 & 272.8 & 296.4 & 316.7\end{array}$

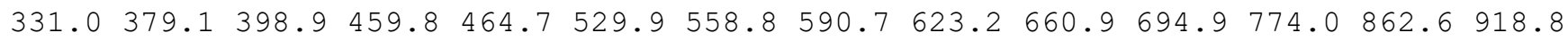
$942.1951 .5984 .5 \quad 988.3 \quad 1026.2 \quad 1028.1 \quad 1037.1 \quad 1051.4 \quad 1057.3 \quad 1088.9 \quad 1109.31122 .91140 .1$ $1161.6 \quad 1185.8 \quad 1203.1 \quad 1207.2 \quad 1235.3 \quad 1250.2 \quad 1259.4 \quad 1262.91264 .9 \quad 1272.4 \quad 1282.3 \quad 1282.6$

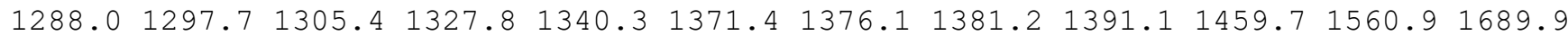

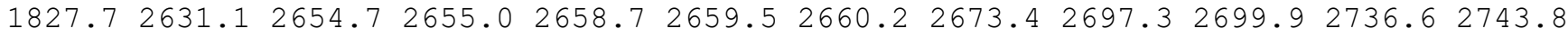
$2755.12771 .3 \quad 2775.2 \quad 2780.6$

\section{name $=$ TS 89}

energy (in $\mathrm{kcal} / \mathrm{mol})=-0.735153$

$\mathrm{ZPE}($ in $\mathrm{kcal} / \mathrm{mol})=140.444$

$\mathrm{G}($ in $\mathrm{kcal} / \mathrm{mol})=115.407$

Geometry

C $1.01058796 \quad 3.26084931 \quad 4.45561209$

C $1.723472163 .90251193 \quad 3.32462485$

C $0.48625514 \quad 0.86007468 \quad 1.60908541$

C 1.800641730 .567303290 .93897900

C $2.89365061 \quad 1.585258331 .25148588$

C $3.14685161 \quad 1.89425195 \quad 2.74472373$

C $2.853648863 .37851903 \quad 2.66350142$

C $2.62582522 \quad 3.05302467 \quad 0.82426395$

C $1.37902370 \quad 3.57780045 \quad 0.39146968$

C $3.83138591 \quad 3.67144024 \quad 0.14399218$

$\mathrm{H} 1.71403862 \quad 2.882098125 .21593053$

$\mathrm{H} \quad 0.42917631 \quad 2.37623977 \quad 4.11451479$

$\mathrm{H} \quad 0.29359783 \quad 3.92610520 \quad 4.96215018$

$\mathrm{H}-0.33903060 \quad 1.224409290 .98215957$

$\mathrm{H} \quad 1.66499847 \quad 0.51591564-0.16186800$

$\mathrm{H} 2.14928372-0.440104231 .25842413$

H $3.826693891 .21582695 \quad 0.76583958$

H 4.174211131 .668285433 .06187261 
H $2.48453693 \quad 1.33151807 \quad 3.42072498$

H $3.70861326 \quad 4.061546792 .61310082$

$\mathrm{H} 1.41859950 \quad 4.88527745 \quad 1.04806059$

$\mathrm{H} \quad 0.45235775 \quad 3.15894683 \quad 0.71181850$

H $1.30137624 \quad 4.02803560 \quad-0.58201462$

H $4.78436150 \quad 3.37345716 \quad 0.59272494$

H $3.78384357 \quad 4.77073100 \quad 0.17676769$

$\mathrm{H} \quad 3.85614108 \quad 3.38415152-0.91627320$

$\begin{array}{llll}0 & 1.69478621 & 5.67692298 & 1.91990741\end{array}$

$\begin{array}{lllll}0 & 0.31476915 & 0.68069366 & 2.79147077\end{array}$

O $1.13837480 \quad 5.04076770 \quad 3.03198631$

Vibrational frequencies (in cm-1)

$\begin{array}{lllllllllllllllll}-1539.0 & 41.1 & 70.2 & 104.0 & 116.1 & 145.3 & 173.2 & 197.3 & 202.8 & 224.0 & 235.2 & 256.3 & 305.1 & 317.5\end{array}$

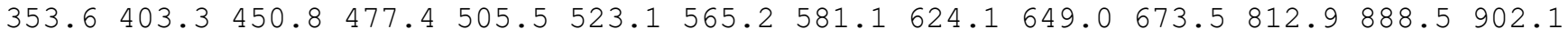
$927.5933 .6961 .8 \quad 966.3 \quad 1004.5 \quad 1024.0 \quad 1034.5 \quad 1041.91057 .1 \quad 1079.2 \quad 1083.3 \quad 1109.3 \quad 1122.5$

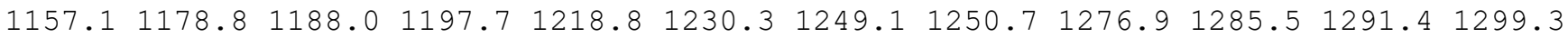

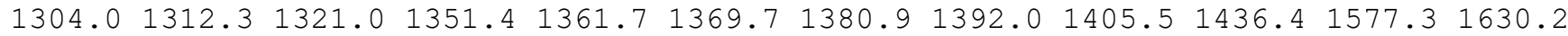
$1832.32617 .42643 .62660 .2 \quad 2666.02666 .6 \quad 2669.62676 .32700 .52724 .2 \quad 2735.92737 .6$ $2755.12763 .4 \quad 2778.0 \quad 2805.7$

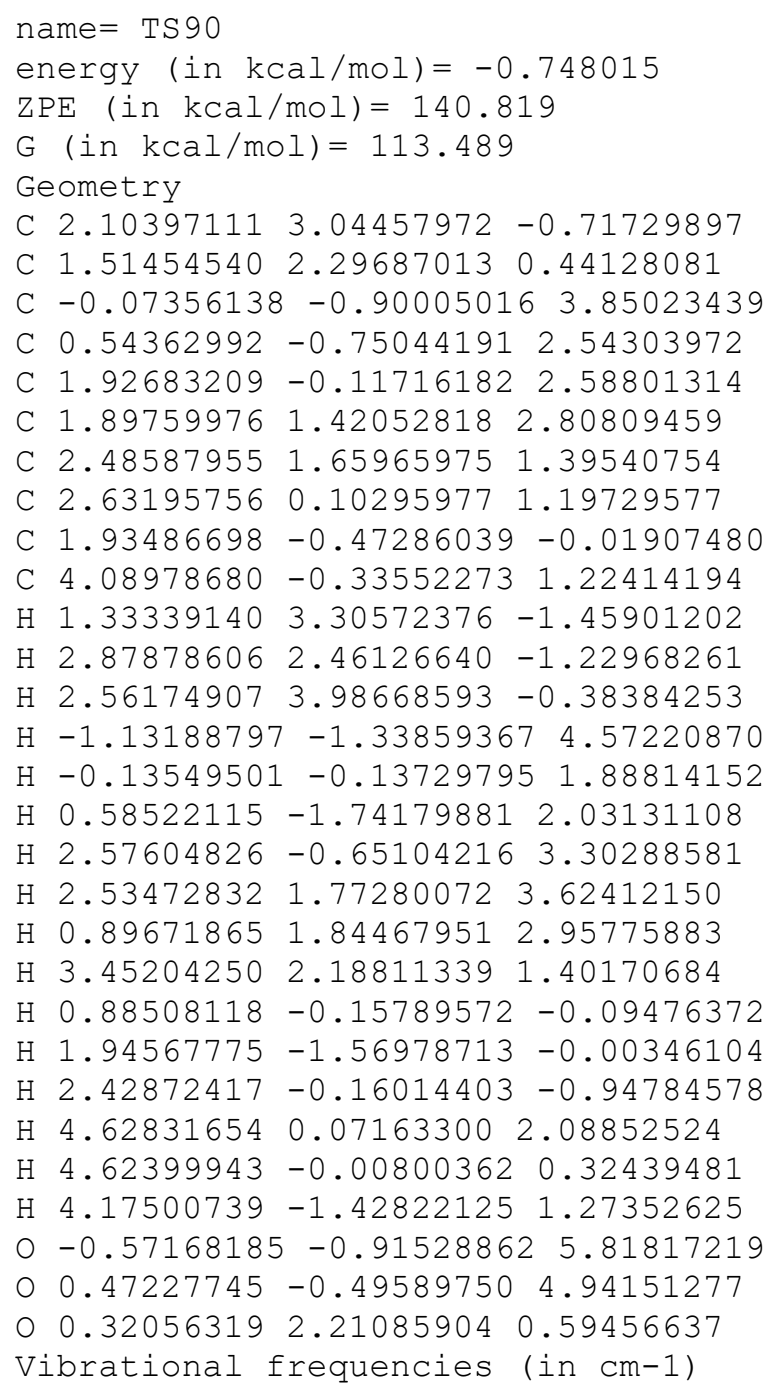




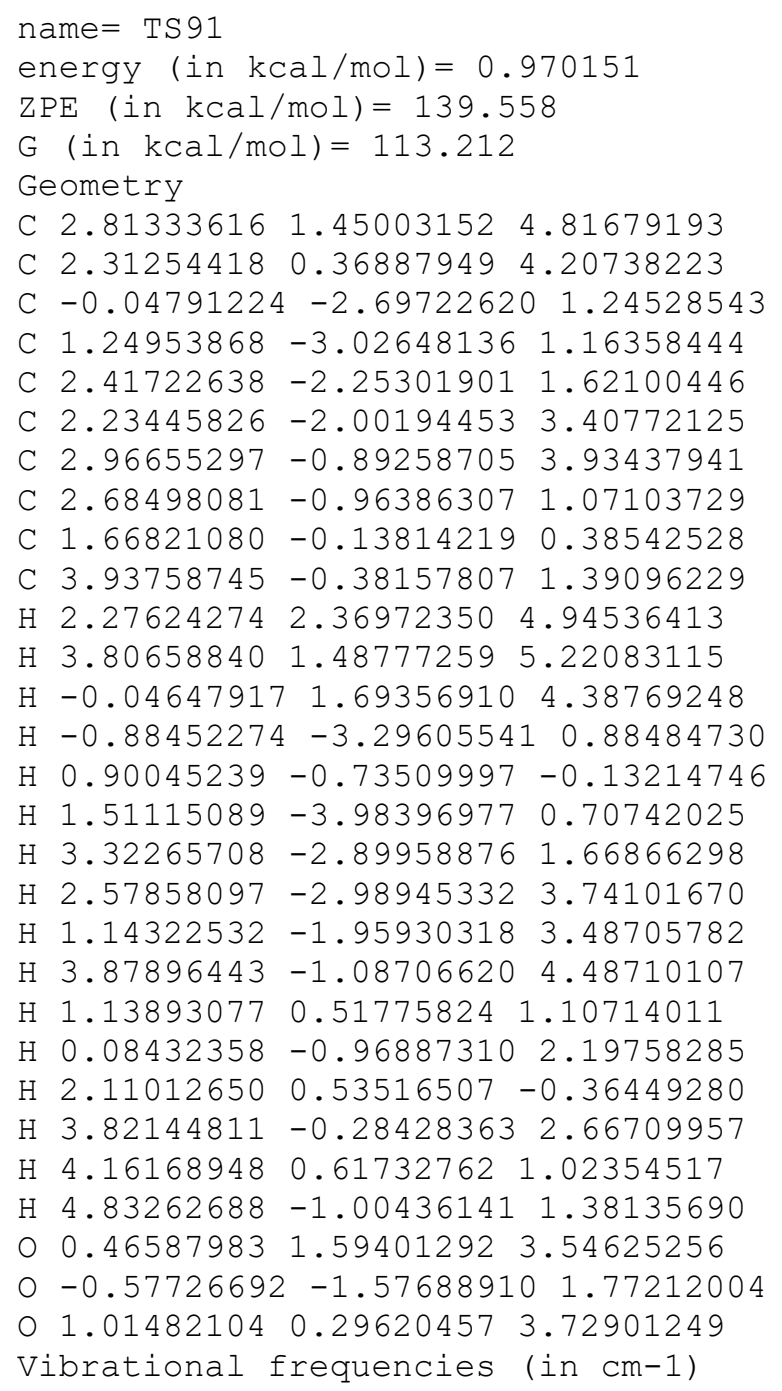


$\mathrm{H} \quad 2.65551848-0.757351851 .78751356$

$\mathrm{H} \quad 3.28109520 \quad 0.94736469 \quad 3.40408414$

H $1.889010831 .94141578 \quad 2.97297863$

$\mathrm{H} \quad 4.626997301 .89841218 \quad 1.61995938$

H $1.36272231 \quad 2.19936643-0.33812928$

$\mathrm{H} \quad 1.68311682 \quad 0.74867144-1.30891917$

$\mathrm{H} 2.765164862 .14761846-1.38830420$

$\mathrm{H} 3.71147431-0.73021083-0.76771323$

$\mathrm{H} 4.75236785-0.33985525 \quad 0.60613873$

$\mathrm{H} \quad 4.76649608 \quad 0.68567787-0.83228158$

$0-0.47326929-1.28649228 \quad 4.34487156$

$0 \quad 0.67158512-0.83823433 \quad 3.62361689$

o $3.41668434 \quad 3.92428068 \quad 0.07152992$

Vibrational frequencies (in $\mathrm{cm}-1$ )

$\begin{array}{llllllllllllll}-1758.8 & 23.1 & 25.9 & 48.3 & 81.2 & 94.0 & 110.2 & 166.9 & 181.6 & 203.8 & 232.3 & 234.2 & 260.8 & 283.2\end{array}$ $321.6 \quad 367.0 \quad 369.4 \quad 399.7 \quad 484.1 \quad 503.7 \quad 522.1 \quad 607.2 \quad 664.2 \quad 743.7 \quad 808.9 \quad 847.5 \quad 864.1 \quad 919.7$ $935.0 \quad 935.9 \quad 986.0 \quad 993.8 \quad 1007.8 \quad 1016.5 \quad 1026.0 \quad 1043.1 \quad 1054.7 \quad 1078.6 \quad 1104.2 \quad 1128.91153 .2$ $\begin{array}{llllllllllllllll}1162.8 & 1178.6 & 1210.7 & 1224.6 & 1244.6 & 1256.0 & 1262.3 & 1263.4 & 1273.6 & 1285.8 & 1288.8 & 1290.6\end{array}$

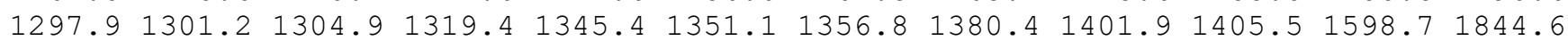
$2209.32633 .0 \quad 2659.4 \quad 2666.7 \quad 2673.6 \quad 2675.12676 .92681 .92682 .72710 .12717 .52729 .2$ $2762.8 \quad 2779.12779 .6 \quad 2781.7$

\section{name $=$ TS93}

energy (in $\mathrm{kcal} / \mathrm{mol})=2.625517$

$\mathrm{ZPE}($ in $\mathrm{kcal} / \mathrm{mol})=139.332$

$\mathrm{G}($ in $\mathrm{kcal} / \mathrm{mol})=111.598$

Geometry

C $4.88339232 \quad 1.76842850 \quad 4.46140437$

C $3.72610028 \quad 0.89235791 \quad 4.09123608$

C $-0.90034818 \quad 0.34831774 \quad 1.36572070$

C $0.17092674 \quad 0.66986848 \quad 2.09624520$

C $1.54364931 \quad 0.57147019 \quad 1.56202232$

$\begin{array}{llll}\text { C } 2.48305624 & -0.42306554 & 2.23601759\end{array}$

C $3.47432064 \quad 0.65918972 \quad 2.63832253$

C $2.54948668 \quad 1.73757638 \quad 1.93915062$

C 1.998290912 .807092192 .86158820

C $3.20289192 \quad 2.36401456 \quad 0.71870977$

H $4.971407892 .63932002 \quad 3.79995418$

H $5.82771957 \quad 1.20790221 \quad 4.39841462$

$\mathrm{H} \quad 4.79640994 \quad 2.13329057 \quad 5.49650107$

$\mathrm{H}-0.92006449-0.00368931 \quad 0.33640278$

$\mathrm{H} 0.06855930 \quad 1.00340548 \quad 3.12813924$

$\mathrm{H}-2.688985691 .64021302 \quad 3.06688268$

$\begin{array}{lllll}\mathrm{H} & 1.54235253 & 0.38737452 & 0.46579841\end{array}$

H $2.01680515-1.359436403 .18152710$

$\mathrm{H} \quad 1.71556193-0.69089350 \quad 3.59286472$

H $4.431119590 .50901044 \quad 2.09791452$

H 2.75849654 3.55489528 3.11584434

H $1.61900732 \quad 2.39361848 \quad 3.80537718$

$\mathrm{H} 1.16296830 \quad 3.33861627 \quad 2.38588859$

H 2.48098092 2.96278594 0.14941734

H $3.60512300 \quad 1.60498858 \quad 0.03566439$

$\mathrm{H} \quad 4.03149843 \quad 3.02406801 \quad 0.99843307$

$\begin{array}{llll}0 & -2.27784125 & 0.74454029 & 3.15095205\end{array}$

$0-2.201711130 .387689921 .76973506$

$\begin{array}{llll}0 & 3.03008224 & 0.37921087 & 4.93812983\end{array}$

Vibrational frequencies (in $\mathrm{cm}-1$ )

$\begin{array}{lllllllllllllll}-1176.2 & 22.4 & 32.9 & 53.0 & 73.5 & 85.2 & 132.4 & 158.5 & 159.3 & 177.3 & 195.5 & 215.8 & 230.3 & 261.8\end{array}$

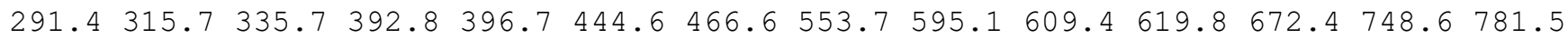
$819.2 \quad 867.9 \quad 902.9950 .2 \quad 964.2 \quad 982.4 \quad 994.6 \quad 1001.3 \quad 1014.2 \quad 1041.0 \quad 1045.7 \quad 1058.2 \quad 1094.2$

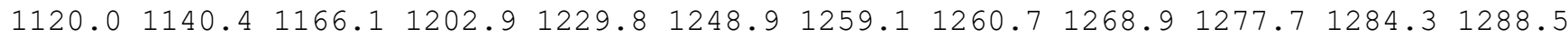
$1297.11299 .81305 .2 \quad 1307.2 \quad 1332.8 \quad 1338.91347 .3 \quad 1371.4 \quad 1380.2 \quad 1401.91405 .7 \quad 1838.2$ $1858.12666 .52667 .62673 .5 \quad 2675.2 \quad 2675.6 \quad 2680.52713 .32721 .2 \quad 2722.92755 .12778 .7$ $2779.02781 .92867 .3 \quad 4317.3$ 


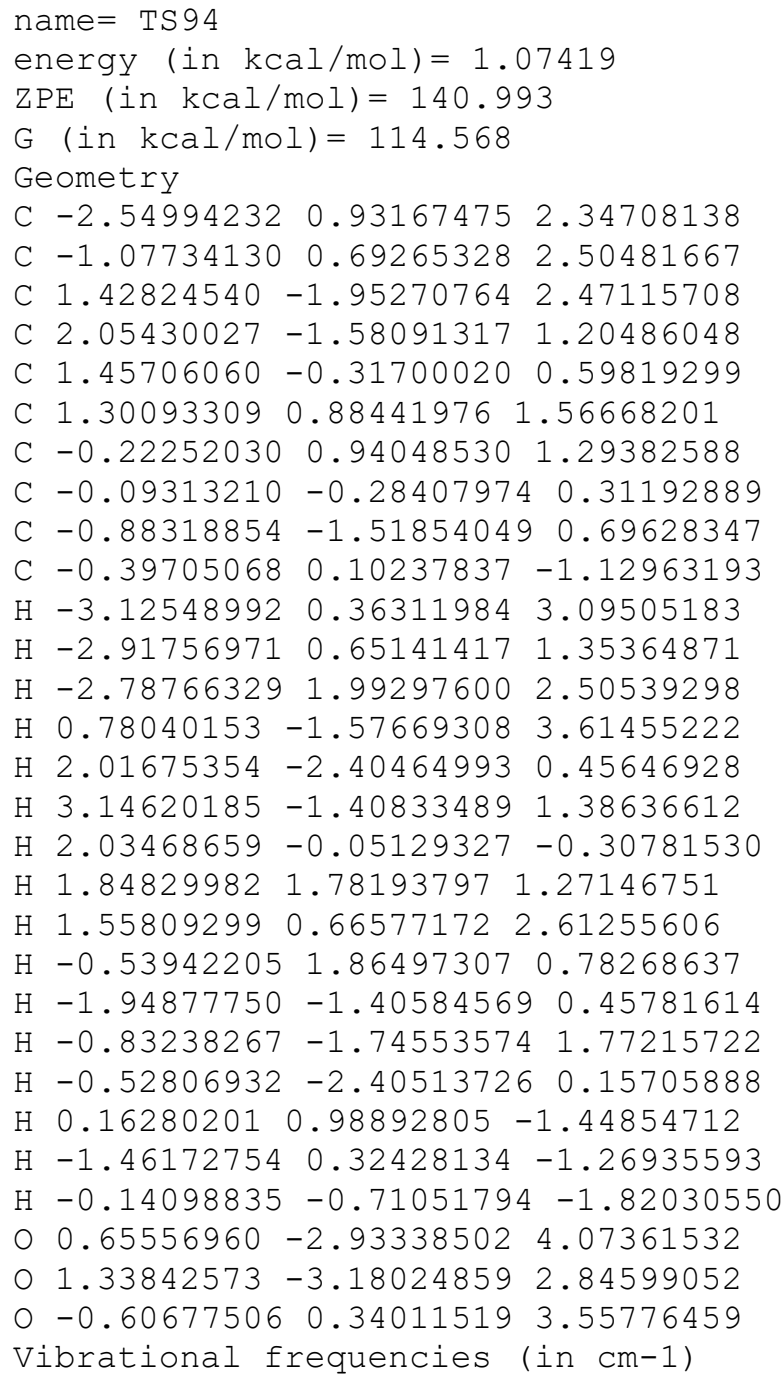


$\mathrm{H}-1.28310411-1.53524384 \quad 1.52039019$

$\mathrm{H}-0.15814287-2.18272148 \quad 0.32388491$

$\mathrm{H}-1.25133341-0.06451162-0.58668649$

$\mathrm{H} \quad 0.98692890 \quad 1.03036972-1.18804335$

$\mathrm{H} \quad 1.66388868-0.44814702-0.51327255$

$\mathrm{H} 1.695261822 .041275831 .05168652$

$\mathrm{H}-2.20488627 \quad 0.99981096 \quad 2.34644725$

$\mathrm{H}-0.97107152 \quad 2.06925950 \quad 3.03575122$

$\mathrm{H}-0.75176748 \quad 0.330429693 .08433366$

$\mathrm{H}-0.497171752 .60204813-0.61154280$

$\mathrm{H}-0.69239478 \quad 3.39502198 \quad 0.95184038$

$\mathrm{H}-2.02775692 \quad 2.46673402 \quad 0.25585982$

$\begin{array}{llll}0 & 2.28197928 & -3.23070474 & 1.46594475\end{array}$

○ $0.77487458-1.930635353 .00877135$

○ $2.16250625-2.093449250 .98973921$

Vibrational frequencies (in cm-1)

$\begin{array}{llllllllllllllllll}-1327.2 & 69.6 & 82.9 & 124.2 & 142.2 & 159.4 & 179.2 & 194.3 & 198.3 & 294.3 & 305.1 & 335.2 & 362.3 & 372.5\end{array}$

$392.9 \quad 404.1 \quad 441.4 \quad 447.9 \quad 469.9 \quad 518.9 \quad 524.6 \quad 601.6 \quad 623.6 \quad 669.5 \quad 722.6 \quad 764.0 \quad 803.9 \quad 819.8$

$882.6 \quad 921.9945 .5 \quad 962.0 \quad 1002.4 \quad 1013.5 \quad 1015.8 \quad 1020.0 \quad 1043.1 \quad 1048.2 \quad 1080.7 \quad 1083.5 \quad 1091.1$

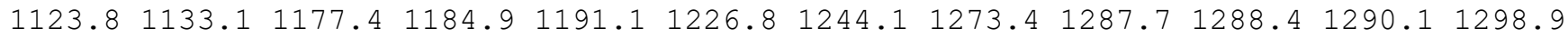
$1302.21303 .81304 .91314 .5 \quad 1328.5 \quad 1359.4 \quad 1385.4 \quad 1389.2 \quad 1407.5 \quad 1421.91625 .01656 .8$ $1862.82659 .12666 .6 \quad 2676.7 \quad 2677.1 \quad 2681.3 \quad 2690.32717 .92740 .02749 .62753 .72767 .9$ $2781.4 \quad 2781.6 \quad 2783.7 \quad 3521.7$

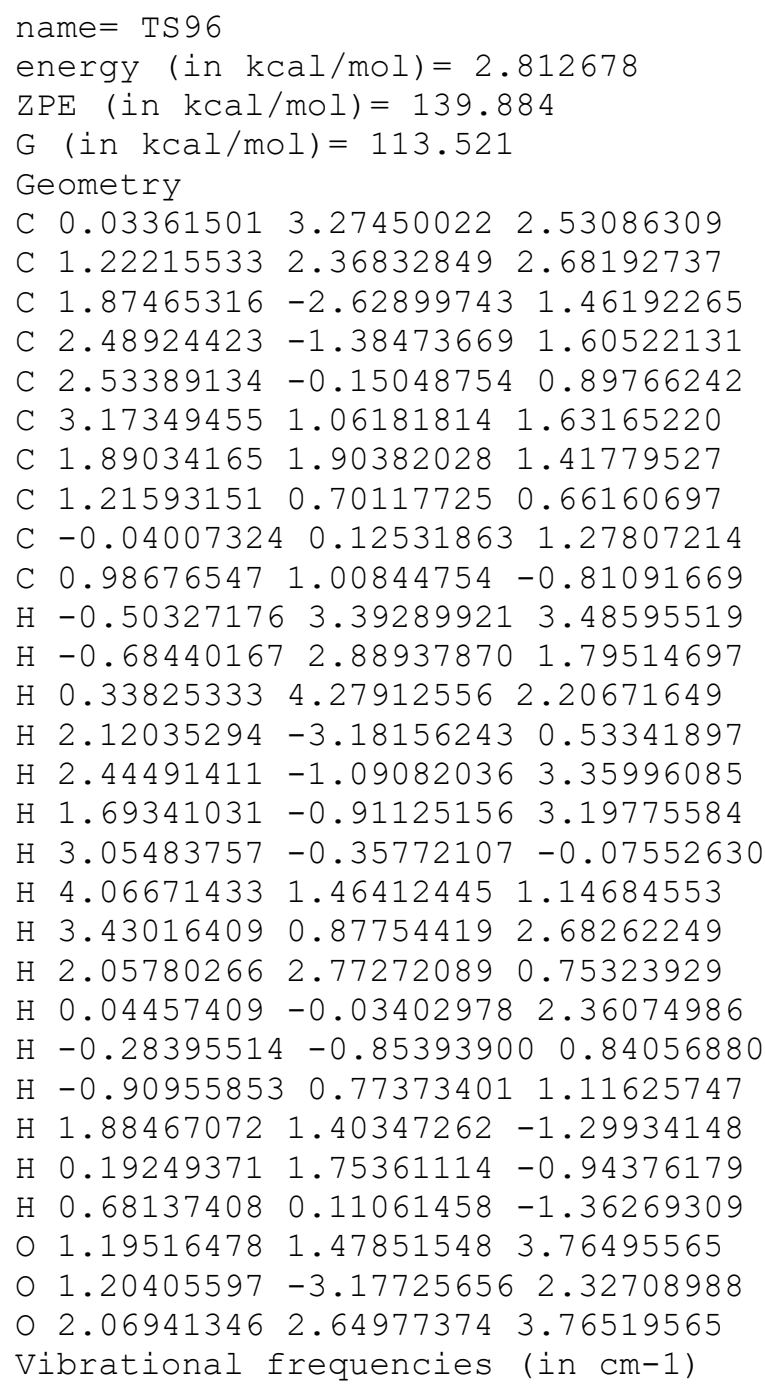
$316.9355 .2 \quad 370.0 \quad 375.6 \quad 394.0 \quad 429.6 \quad 468.3 \quad 492.8 \quad 536.0 \quad 575.7 \quad 639.1 \quad 687.4 \quad 777.9 \quad 802.5$ $825.0 \quad 874.1917 .5 \quad 920.2 \quad 928.3 \quad 947.6 \quad 991.2 \quad 1006.1 \quad 1014.91034 .0 \quad 1053.5 \quad 1085.7 \quad 1093.8$ $1098.31111 .4 \quad 1136.3 \quad 1164.5 \quad 1194.3 \quad 1215.6 \quad 1252.8 \quad 1263.8 \quad 1269.3 \quad 1273.5 \quad 1280.91283 .8$ $1285.1 \quad 1293.7 \quad 1297.1 \quad 1302.2 \quad 1311.8 \quad 1341.6 \quad 1351.6 \quad 1386.51400 .6 \quad 1404.4 \quad 1568.01596 .5$ 
$1772.42640 .92662 .2 \quad 2666.8 \quad 2670.0 \quad 2670.9 \quad 2672.4 \quad 2676.4 \quad 2676.7 \quad 2687.12722 .3 \quad 2759.8$ $2778.4 \quad 2779.12782 .1 \quad 5072.4$

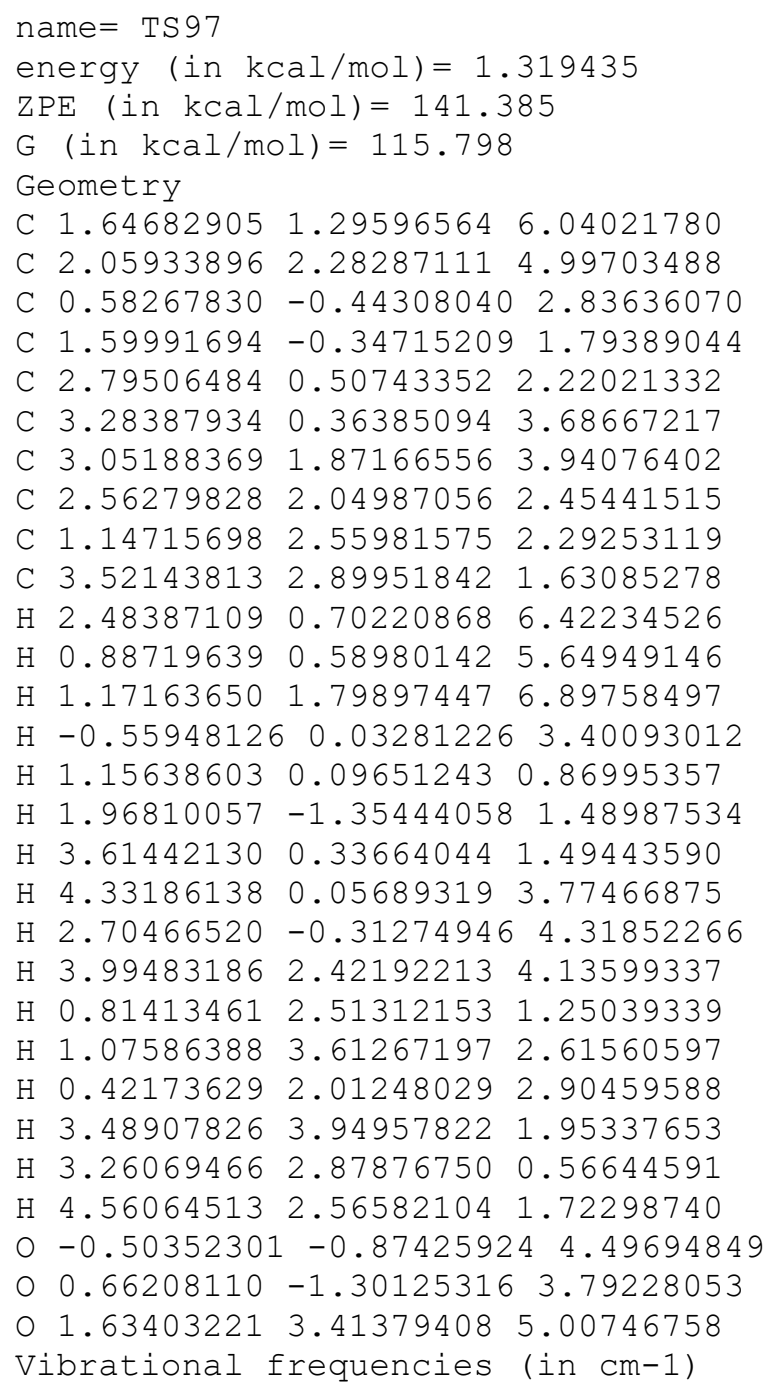


$\mathrm{H}-0.689322090 .55947893 \quad 3.21993613$

$\mathrm{H}-1.10935720-1.43856582 \quad 0.20748633$

$\mathrm{H} 1.17766833-1.92645103-0.67132576$

$\mathrm{H} \quad 1.06957770-1.18264694-0.95632899$

$\mathrm{H} 3.07049908-1.067918130 .65416846$

$\mathrm{H} 3.18987335-1.112716013 .05995095$

$\mathrm{H} 1.44780103-0.833296013 .21441022$

$\mathrm{H} 3.63236297 \quad 1.28328487 \quad 2.97438252$

$\mathrm{H} \quad 0.32590809$ 1.57324860 1.11658919

$\mathrm{H} \quad 1.07757999 \quad 1.63922228 \quad-0.48847104$

H $1.40407386 \quad 2.90306998 \quad 0.70501648$

H $4.58050079 \quad 0.85968767 \quad 0.72516983$

H $3.941347432 .49445040 \quad 0.54628683$

$\mathrm{H} \quad 3.62983280 \quad 1.28752514 \quad-0.70281465$

$0-0.54353043 \quad 1.30348420 \quad 3.86527475$

$\begin{array}{lllll}0 & -0.90757334 & -0.62116711 & 2.01452866\end{array}$

○ 0.811353931 .037478314 .23425327

Vibrational frequencies (in cm-1)

$\begin{array}{llllllllllllll}-756.6 & 33.5 & 55.4 & 99.1 & 140.3 & 163.6 & 168.3 & 184.6 & 214.9 & 226.6 & 266.1 & 272.0 & 279.9 & 312.7\end{array}$ $328.5 \quad 361.2 \quad 408.0 \quad 469.3 \quad 481.9 \quad 543.5 \quad 566.0 \quad 583.0 \quad 615.6 \quad 639.6 \quad 654.6 \quad 693.0 \quad 703.3 \quad 761.1$ $805.5 \quad 843.9924 .3 \quad 929.8 \quad 945.3 \quad 978.2 \quad 995.4 \quad 996.4 \quad 1002.2 \quad 1006.3 \quad 1015.1 \quad 1035.8 \quad 1062.3$ $1074.11107 .6 \quad 1119.5 \quad 1130.8 \quad 1157.2 \quad 1206.6 \quad 1252.0 \quad 1281.4 \quad 1286.4 \quad 1288.2 \quad 1291.4 \quad 1299.0$ $1301.21303 .91324 .8 \quad 1338.91345 .3 \quad 1358.8 \quad 1376.4 \quad 1401.91403 .6 \quad 1443.6 \quad 1654.2 \quad 1749.2$ $1850.32658 .7 \quad 2658.92666 .1 \quad 2674.8 \quad 2679.4 \quad 2682.7 \quad 2715.32720 .4 \quad 2726.8 \quad 2753.3 \quad 2770.1$ $2778.8 \quad 2782.5 \quad 2790.7 \quad 4858.1$

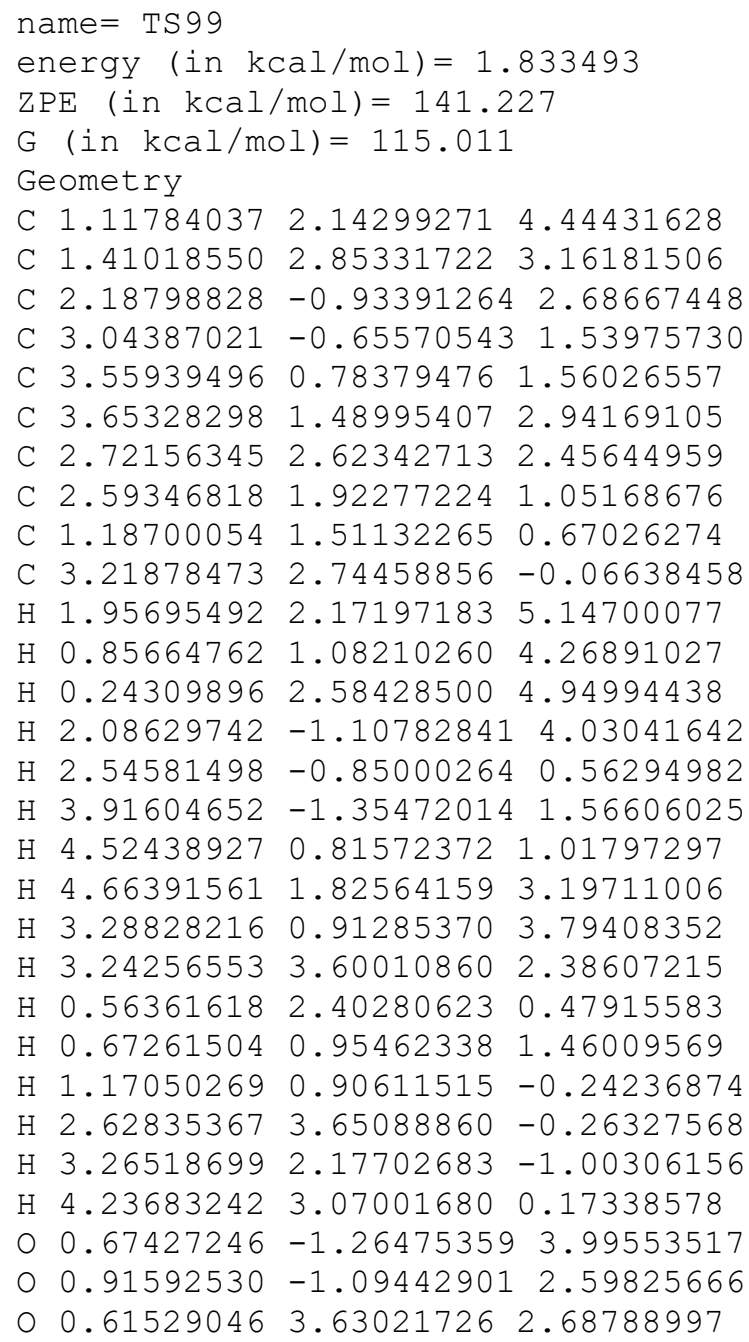




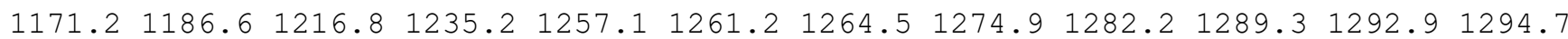

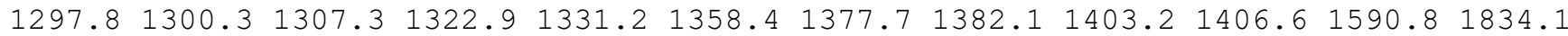
$2208.02635 .12646 .6 \quad 2657.6 \quad 2672.0 \quad 2674.5 \quad 2678.0 \quad 2678.42683 .0 \quad 2711.92717 .92728 .8$ $2762.7 \quad 2773.7 \quad 2779.9 \quad 2782.0$

\section{name $=$ TS100}

energy $($ in $\mathrm{kcal} / \mathrm{mol})=4.087747$

$\mathrm{ZPE}(\mathrm{in} \mathrm{kcal} / \mathrm{mol})=139.725$

$\mathrm{G}($ in $\mathrm{kcal} / \mathrm{mol})=112.857$

Geometry

C $0.307589493 .57599424 \quad 2.94758075$

C $1.289752492 .46283346 \quad 3.17987648$

C $2.37133445-2.513160341 .55569146$

C $2.13206689-1.163656421 .43776169$

C $2.62284795 \quad 0.12764791 \quad 1.16737142$

C $3.213269130 .97568462 \quad 2.33403500$

C $2.20957368 \quad 2.119553362 .04139535$

C $1.58941610 \quad 1.28903810 \quad 0.85829445$

C $0.13663813 \quad 0.88395976 \quad 0.98105133$

C $1.84457698 \quad 1.93994247 \quad-0.49346154$

$\mathrm{H}-0.45008180 \quad 3.61352448 \quad 3.74649111$

$\mathrm{H}-0.22475212 \quad 3.45868641 \quad 1.99461689$

$\mathrm{H} \quad 0.80906748 \quad 4.55305294 \quad 2.93234153$

H $2.86860372 \quad-2.96120435 \quad 0.66511732$

$\mathrm{H} 1.48080533-0.99297108 \quad 3.33781506$

$\mathrm{H} \quad 0.82840686-0.96389658 \quad 2.91943636$

H $3.35938126 \quad 0.02357511 \quad 0.32235245$

H $4.26365250 \quad 1.25003740 \quad 2.21043386$

$\mathrm{H} \quad 3.10631408 \quad 0.52155398 \quad 3.32801544$

$\mathrm{H} 2.70817459 \quad 3.04188901 \quad 1.68665750$

$\mathrm{H}-0.129101870 .551949201 .99291625$

H $-0.08416524 \quad 0.03681969 \quad 0.31311904$

$\mathrm{H}-0.54496172 \quad 1.69823055 \quad 0.71348367$

$\mathrm{H} \quad 1.576529861 .26305895-1.31487865$

$\mathrm{H} \quad 2.893281592 .22477240 \quad-0.63100764$

H $1.24358771 \quad 2.84913140 \quad-0.61688071$

o $0.86361118 \quad 1.41487558 \quad 4.00660211$

O $2.00001510 \quad-3.24439416 \quad 2.46548763$

O $1.844547392 .39630732 \quad 4.46699679$

Vibrational frequencies (in $\mathrm{cm}-1$ )

$\begin{array}{lllllllllllllll}-150.5 & 23.3 & 48.9 & 69.2 & 97.2 & 151.8 & 162.0 & 185.0 & 200.0 & 201.6 & 247.2 & 253.9 & 275.5 & 295.9\end{array}$

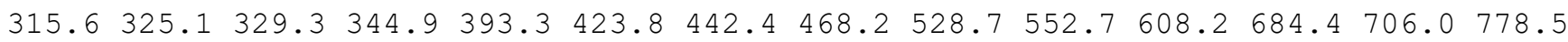

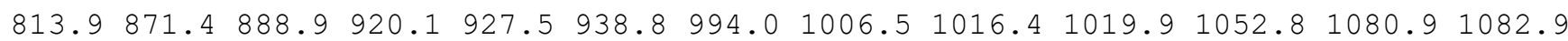

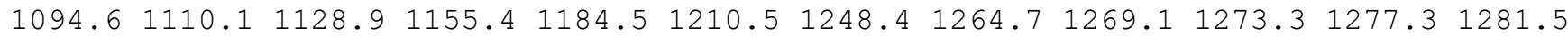

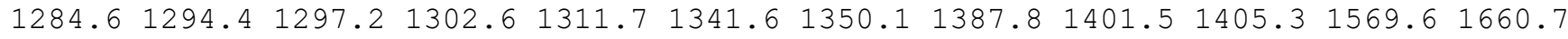
$1796.02621 .3 \quad 2662.2 \quad 2662.5 \quad 2667.7 \quad 2672.3 \quad 2673.6 \quad 2676.92678 .0 \quad 2680.7 \quad 2721.3 \quad 2756.7$ $2778.6 \quad 2779.8 \quad 2782.6 \quad 5297.1$

name $=$ TS101

energy $($ in $\mathrm{kcal} / \mathrm{mol})=2.692482$

$\operatorname{ZPE}($ in $\mathrm{kcal} / \mathrm{mol})=141.198$

$\mathrm{G}($ in $\mathrm{kcal} / \mathrm{mol})=114.881$

Geometry

C $4.56419162 \quad 3.51196188 \quad 4.53472728$

C $4.80445307 \quad 2.12371305 \quad 4.02023482$

C $2.72268338-1.30148327 \quad 3.28991302$

C $2.75395533-0.916025651 .88856896$

C $3.54470765 \quad 0.31322931 \quad 1.47119406$

C $4.97384263 \quad 0.48750355 \quad 2.04376377$

C $4.62347637 \quad 1.91165419 \quad 2.54344060$

C 3.139695431 .720304262 .05656416

C 2.089040141 .718029493 .14880875

C $2.75510644 \quad 2.70950850 \quad 0.96463148$ 
H $3.81917128 \quad 4.06012613 \quad 3.94836374$

H $5.49867996 \quad 4.092186324 .51935972$

$\mathrm{H} \quad 4.22568384 \quad 3.49002981 \quad 5.58295564$

H $2.06509095-2.070448394 .18899939$

$\mathrm{H} \quad 1.69850085-0.79631633 \quad 1.53249774$

H $3.13512598-1.79990837 \quad 1.30956453$

H $3.55483100 \quad 0.35109208 \quad 0.36120636$

H $5.77082644 \quad 0.45265832 \quad 1.29614164$

H $5.25364460 \quad-0.21007292 \quad 2.84272796$

H $5.15215688 \quad 2.70422686 \quad 1.98381844$

H $1.14816901 \quad 1.27643434 \quad 2.79835527$

H $1.85753760 \quad 2.73616715 \quad 3.48776889$

H 2.39985482 $1.15809414 \quad 4.04153074$

$\mathrm{H} \quad 2.67537100 \quad 3.72983917 \quad 1.35897864$

H $1.78420571 \quad 2.45456900 \quad 0.52279900$

H $3.48745510 \quad 2.73456027 \quad 0.14948496$

○ $2.92386011-1.691599315 .26586802$

O $3.52387672 \quad-0.93475578 \quad 4.22052476$

O $5.15553170 \quad 1.23059534 \quad 4.74887851$

Vibrational frequencies (in cm-1)

$\begin{array}{llllllllllllllll}-1757.7 & 36.5 & 48.5 & 64.1 & 92.0 & 101.4 & 120.4 & 160.9 & 186.6 & 199.4 & 207.2 & 252.9 & 263.7 & 287.6\end{array}$

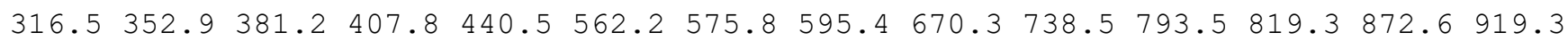
$926.4952 .1 \quad 996.2 \quad 1006.8 \quad 1016.4 \quad 1019.6 \quad 1036.5 \quad 1048.1 \quad 1055.1 \quad 1085.5 \quad 1102.7 \quad 1130.91134 .8$ $\begin{array}{lllllllllllll}1157.9 & 1194.8 & 1204.1 & 1233.8 & 1242.6 & 1252.2 & 1257.8 & 1265.6 & 1271.2 & 1286.3 & 1287.2 & 1292.5\end{array}$ $1297.3 \quad 1302.0 \quad 1307.7 \quad 1327.7 \quad 1340.8 \quad 1360.8 \quad 1380.6 \quad 1394.6 \quad 1406.4 \quad 1414.4 \quad 1636.1 \quad 1855.6$ $2218.02633 .92662 .6 \quad 2665.0 \quad 2673.9 \quad 2675.2 \quad 2675.62677 .42679 .12709 .32721 .52725 .9$ $2760.72779 .8 \quad 2780.0 \quad 2782.9$

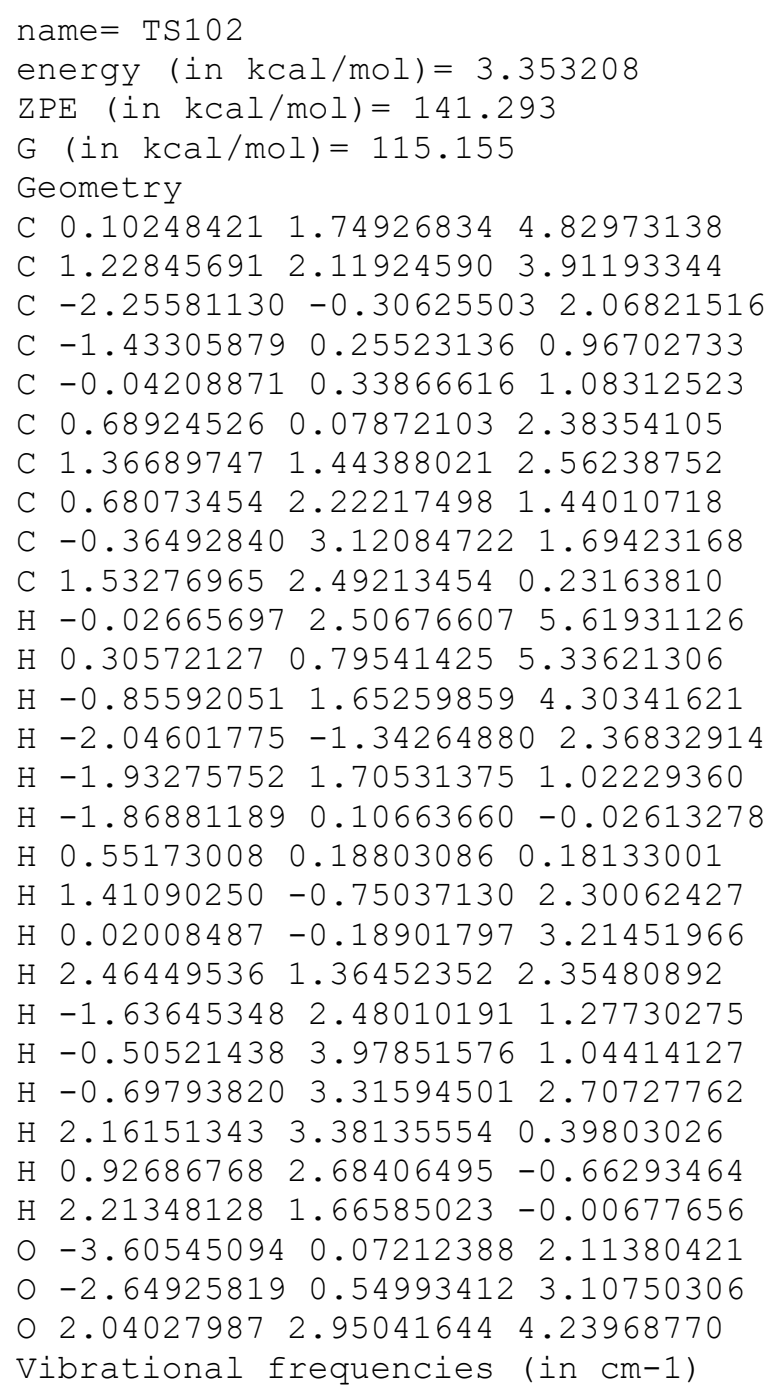


$\begin{array}{lllllllllllllll}-1791.2 & 31.5 & 38.6 & 53.0 & 78.5 & 141.5 & 144.6 & 169.9 & 183.2 & 206.9 & 280.6 & 311.4 & 339.3 & 344.4\end{array}$

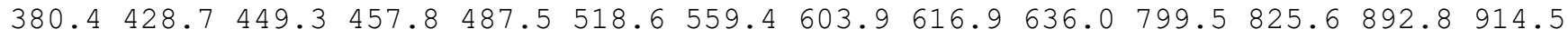
$935.6964 .8 \quad 972.6 \quad 978.8 \quad 995.8 \quad 1009.0 \quad 1018.0 \quad 1037.7 \quad 1043.4 \quad 1051.6 \quad 1061.3 \quad 1072.6 \quad 1091.8$

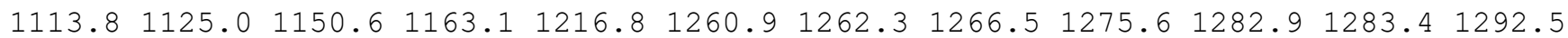

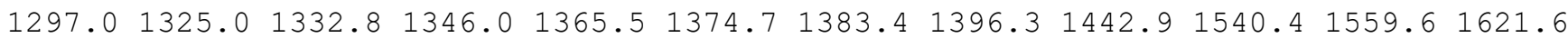
$1838.62666 .0 \quad 2667.0 \quad 2668.7 \quad 2671.4 \quad 2676.5 \quad 2683.5 \quad 2697.82699 .62730 .22741 .12754 .9$ $2759.0 \quad 2778.0 \quad 2778.8 \quad 3218.7$

name $=$ TS103

energy $($ in $\mathrm{kcal} / \mathrm{mol})=5.108035$

$\mathrm{ZPE}($ in $\mathrm{kcal} / \mathrm{mol})=140.178$

$\mathrm{G}($ in $\mathrm{kcal} / \mathrm{mol})=113.747$

Geometry

C $-0.72370770 \quad 0.82364729-1.38913092$

C $0.731228191 .17215271-1.25103212$

C $-0.46968665-3.08909110-1.21170629$

C $0.73316086-2.89780201-0.55108327$

C $1.71076951-1.92689124-0.19457412$

C $1.05309969-0.705842290 .53654057$

C $1.668617890 .27051702-0.49288157$

C $2.28895750-0.93511399-1.29042546$

C $1.76341473-1.14185456-2.69390432$

C $3.80879602-0.90260448-1.29283757$

$\mathrm{H}-1.261997650 .97403593-0.44314765$

$\mathrm{H}-0.87434691-0.21382182-1.71219338$

$\mathrm{H}-1.212649641 .47039889-2.13752231$

$\mathrm{H}-1.28771331-2.39284487-0.93775627$

$\mathrm{H} 1.73460911-4.09575588-1.47005274$

$\mathrm{H} \quad 1.55191847-4.44243866-0.78259849$

H $2.53131012-2.367208210 .41329490$

$\mathrm{H} 1.41244059-0.56863490 \quad 1.56164586$

$\mathrm{H}-0.03900683-0.72642192 \quad 0.57486030$

$\mathrm{H} 2.46559801 \quad 0.90020036-0.03846553$

H $2.05010110-0.31026617-3.35374360$

$\mathrm{H} \quad 0.67077181-1.22251030 \quad-2.73261074$

$\mathrm{H} 2.16393358-2.05985330-3.14333124$

$\mathrm{H} \quad 4.18282413-0.07096613-1.90674908$

$\mathrm{H} \quad 4.23099552-1.82533947-1.70815018$

H $4.22707726-0.77241633-0.28844903$

○ $0.997920222 .54609691-1.11141909$

o $-0.70483881-4.02188949-1.97210664$

o $1.308018351 .83217854-2.34670631$

Vibrational frequencies (in $\mathrm{cm}-1$ )

$\begin{array}{lllllllllllllll}-524.4 & 33.3 & 39.7 & 73.3 & 87.9 & 128.4 & 149.1 & 173.6 & 183.8 & 211.2 & 231.6 & 274.4 & 279.4 & 307.0\end{array}$

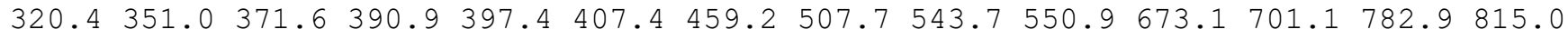

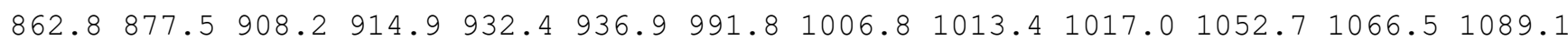

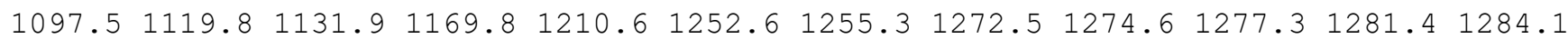

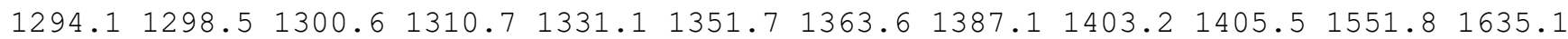
$1775.12637 .8 \quad 2662.4 \quad 2667.3 \quad 2670.5 \quad 2671.6 \quad 2672.92677 .5 \quad 2678.3 \quad 2704.82713 .4 \quad 2760.9$ $2776.4 \quad 2779.4 \quad 2781.5 \quad 5051.0$

name $=$ TS104

energy $($ in $\mathrm{kcal} / \mathrm{mol})=4.905012$

$\mathrm{ZPE}($ in $\mathrm{kcal} / \mathrm{mol})=141.234$

$\mathrm{G}($ in $\mathrm{kcal} / \mathrm{mol})=117.642$

Geometry

C $1.25211534 \quad 1.55380270 \quad 5.11998736$

C $1.916047741 .83642162 \quad 3.84624749$

C $2.08818539-0.465540513 .18362865$

C $1.83820935-0.469525851 .68880748$

C $1.25418646 \quad 0.82187236 \quad 1.12934775$

C $2.173131892 .05198044 \quad 1.38848253$

C 1.289578982 .411942392 .63000596 
C $0.13662495 \quad 1.52499991 \quad 1.99189761$

C $-0.76410791 \quad 0.69381854 \quad 2.86943675$

C $-0.74783412 \quad 2.410183291 .10763310$

$\mathrm{H} \quad 1.86256504 \quad 1.91194843 \quad 5.97319707$

H $1.13842084 \quad 0.46235760 \quad 5.32692188$

$\mathrm{H} \quad 0.24596345 \quad 1.99027724 \quad 5.19077583$

$\mathrm{H} \quad 3.26806872-0.006537493 .89397437$

$\mathrm{H} 1.15724857-1.313772951 .44428898$

H $2.79336866-0.698496361 .17486463$

$\mathrm{H} \quad 0.96524935 \quad 0.68347274 \quad 0.07635433$

H 2.14078832 2.80640739 0.59734659

H $3.22470907 \quad 1.82013670 \quad 1.56797284$

H $1.02318435 \quad 3.47150305 \quad 2.74842635$

$\mathrm{H}-1.42508448 \quad 1.318627693 .48149455$

$\mathrm{H}-0.23612418 \quad 0.01832028 \quad 3.55313834$

$\mathrm{H}-1.40363235 \quad 0.04007070 \quad 2.25793633$

$\mathrm{H}-1.38426941 \quad 3.06682445 \quad 1.71153281$

$\mathrm{H}-1.41092138 \quad 1.79741984 \quad 0.48385551$

$\mathrm{H}-0.16900964 \quad 3.04697207 \quad 0.43075732$

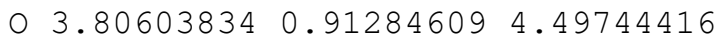

o $1.50200969-1.23922910 \quad 3.89624864$

o 3.239819111 .906115413 .74822939

Vibrational frequencies (in $\mathrm{cm}-1$ )

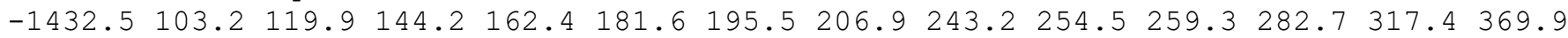

$370.2394 .7 \quad 431.5 \quad 466.3 \quad 486.0 \quad 529.2 \quad 552.5 \quad 602.8 \quad 656.0 \quad 706.9 \quad 802.2 \quad 855.9 \quad 891.6 \quad 917.0$

$935.6 \quad 980.9 \quad 992.7 \quad 1003.8 \quad 1012.9 \quad 1018.4 \quad 1028.6 \quad 1029.7 \quad 1045.2 \quad 1067.9 \quad 1077.9 \quad 1090.8 \quad 1100.4$

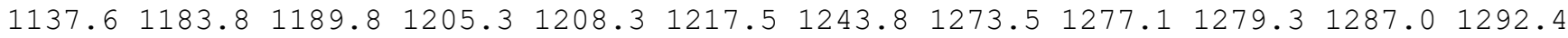

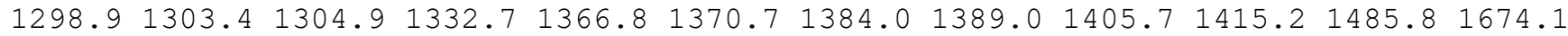

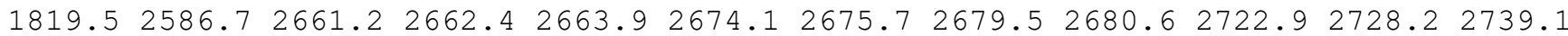
$2755.92761 .3 \quad 2781.4 \quad 2787.5$

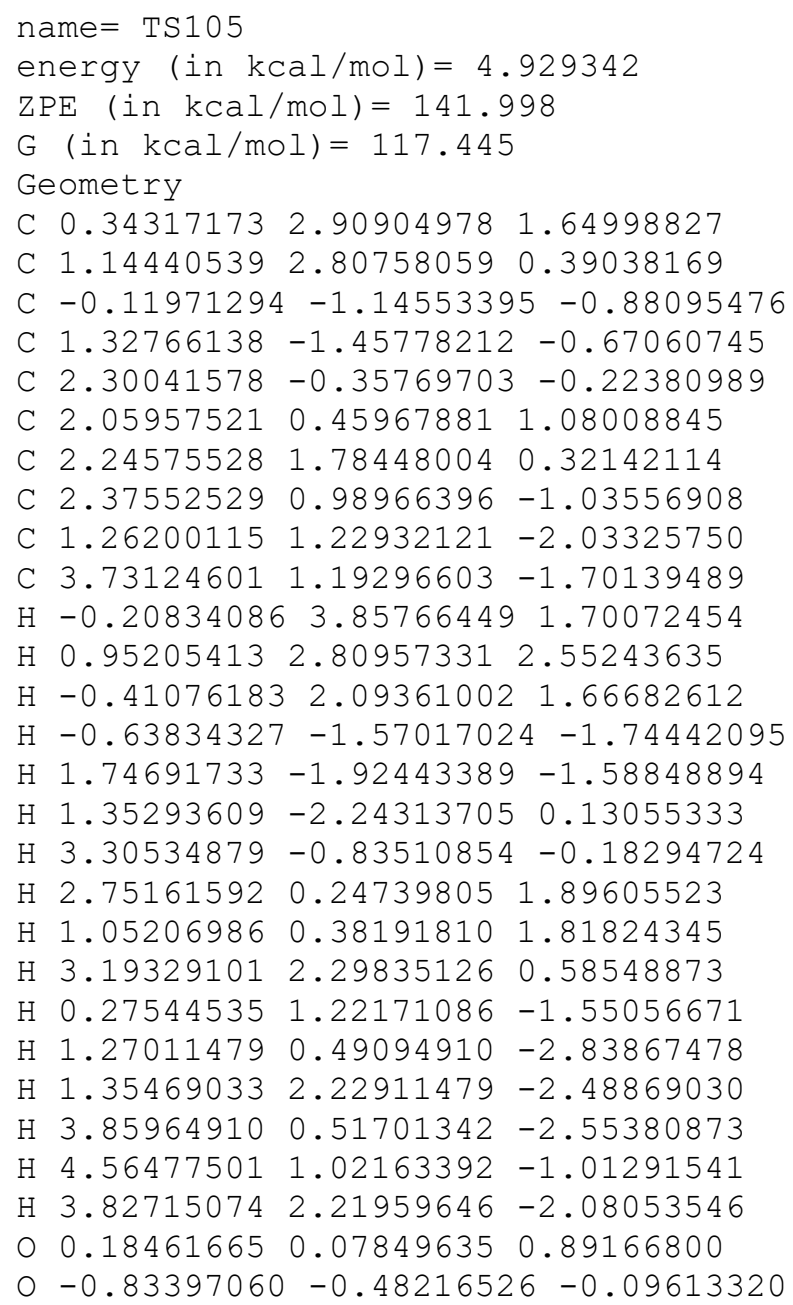


$0.954639233 .56988565-0.52809150$

Vibrational frequencies (in $\mathrm{cm}-1$ )

$\begin{array}{llllllllllllllll}-1117.5 & 36.6 & 74.2 & 111.3 & 158.8 & 181.4 & 198.2 & 198.8 & 214.9 & 236.8 & 262.7 & 309.6 & 325.3 & 350.3\end{array}$ $389.1400 .2 \quad 431.7 \quad 444.5 \quad 480.6 \quad 508.4 \quad 559.3 \quad 598.6 \quad 659.3 \quad 711.3 \quad 765.9 \quad 812.2 \quad 845.6 \quad 870.9$ $902.5948 .3989 .8 \quad 996.9 \quad 1008.6 \quad 1018.2 \quad 1025.8 \quad 1043.1 \quad 1053.1 \quad 1062.6 \quad 1100.2 \quad 1118.51164 .1$

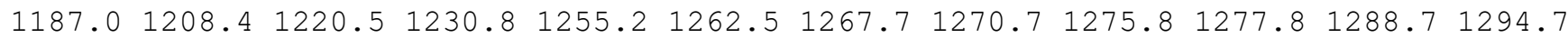

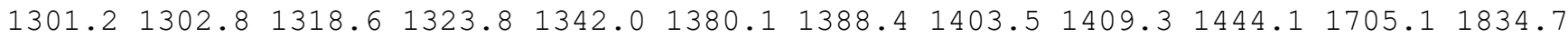
$2064.12631 .62643 .4 \quad 2659.7 \quad 2674.7 \quad 2675.12680 .82684 .02686 .3 \quad 2692.92705 .12712 .6$ $2720.62774 .6 \quad 2779.0 \quad 2782.1$

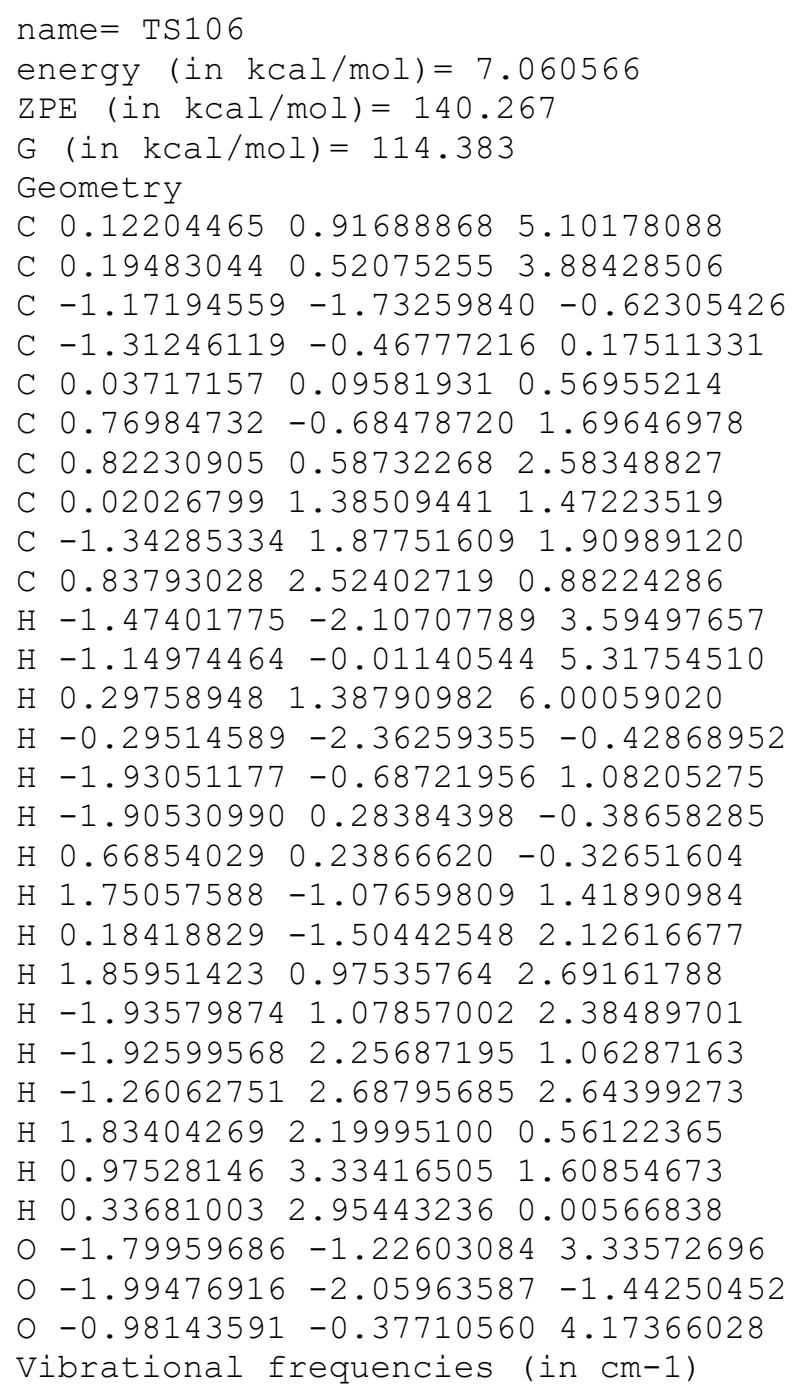


C 1.787556221 .944003030 .40710305

C $0.908019612 .74021337 \quad 1.40123537$

C -0.262061181 .520688161 .14200901$

C $-1.01500430 \quad 1.01178399 \quad 2.33296017$

C $-1.21140354 \quad 2.05174987 \quad 0.08646164$

$\mathrm{H}-0.39134400 \quad 3.47711689 \quad 3.75526448$

$\mathrm{H} 1.19730852 \quad 3.73084766 \quad 4.51714070$

$\mathrm{H} 0.38503145 \quad 2.17988433 \quad 4.70329864$

$\mathrm{H} \quad 1.22753238-2.45174865 \quad 2.08718139$

H $2.55175655-0.48350893 \quad 1.41343096$

$\mathrm{H} 1.72661133-0.03617828 \quad 3.10249763$

$\mathrm{H} \quad 0.61900881 \quad 0.21319341-0.41635456$

$\mathrm{H} \quad 1.76364200 \quad 2.35619743 \quad-0.60777544$

$\mathrm{H} 2.84008453 \quad 1.820607790 .66868795$

H $0.56791313 \quad 3.72783151 \quad 1.09896826$

$\mathrm{H}-1.68412738 \quad 1.74568143 \quad 2.78343448$

$\mathrm{H}-0.37545286 \quad 0.56276547 \quad 3.10387597$

$\mathrm{H}-1.63979068 \quad 0.14280010 \quad 2.00923906$

$\mathrm{H}-1.85106774 \quad 1.22992962-0.28043170$

$\mathrm{H}-0.694026862 .45870523-0.78976034$

$\mathrm{H}-1.87824451 \quad 2.82776724 \quad 0.47648452$

$\begin{array}{llll}0 & 2.09926481 & 0.72899010 & 3.85171579\end{array}$

$0-0.50388059-1.651710211 .53443774$

O 2.307386491 .693675192 .84625121

Vibrational frequencies (in cm-1)

$\begin{array}{llllllllllllll}-484.1 & 67.9 & 105.6 & 111.5 & 139.7 & 146.5 & 196.8 & 209.9 & 221.3 & 254.9 & 263.3 & 295.6 & 298.1 & 320.0\end{array}$

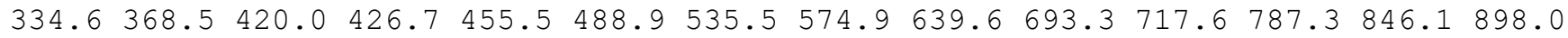
$923.8 \quad 948.5 \quad 984.4 \quad 994.5 \quad 997.6 \quad 1002.2 \quad 1012.9 \quad 1018.7 \quad 1026.2 \quad 1045.9 \quad 1068.7 \quad 1082.0 \quad 1102.3$

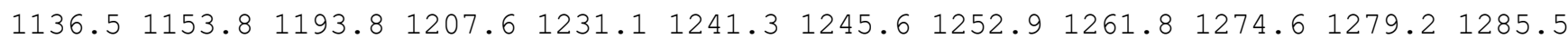

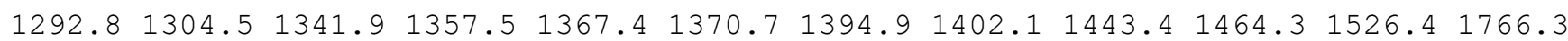
$1980.12610 .92619 .3 \quad 2650.0 \quad 2655.8 \quad 2660.6 \quad 2678.42680 .82684 .6 \quad 2707.72745 .5 \quad 2758.0$ $2762.4 \quad 2771.0 \quad 2778.4 \quad 2778.8$

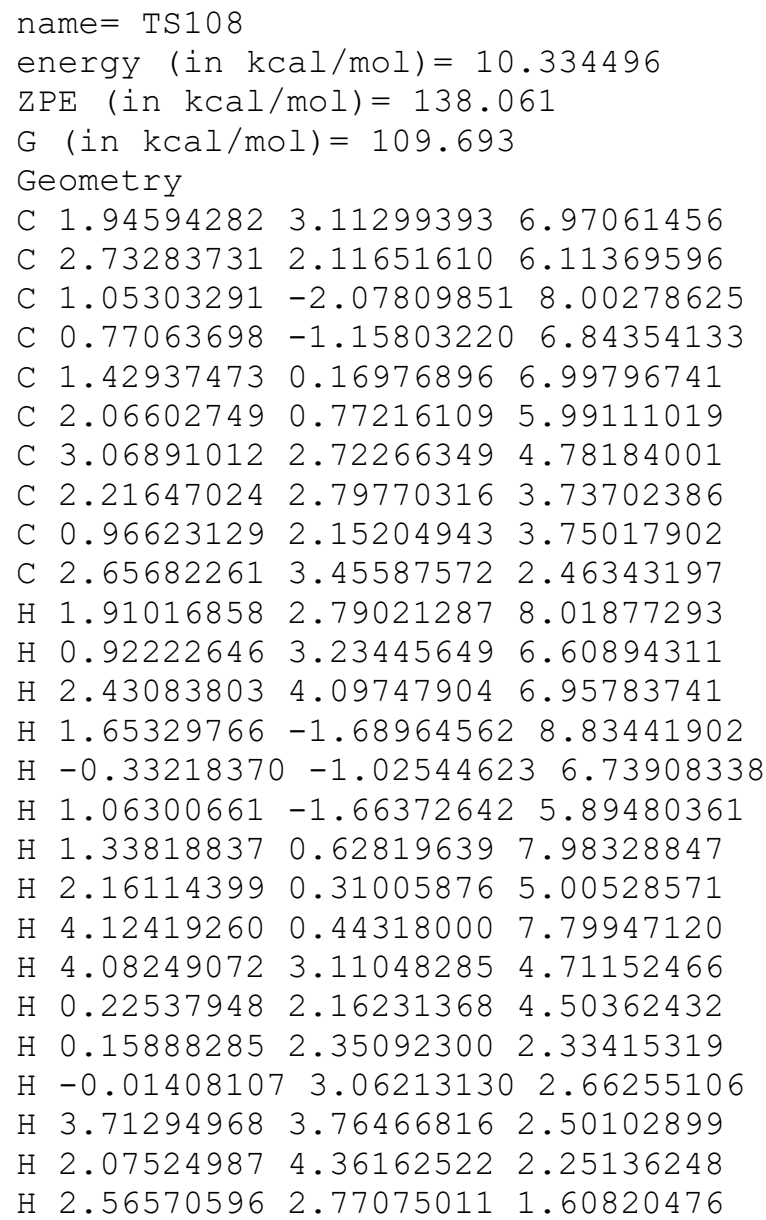


03.892802791 .385972987 .98453108

$0.63309602-3.208248058 .01794286$

O 4.041992751 .928473876 .68270637

Vibrational frequencies (in $\mathrm{cm}-1$ )

$\begin{array}{llllllllllllllll}-832.8 & 27.8 & 46.6 & 60.4 & 88.8 & 92.1 & 95.7 & 120.1 & 128.7 & 137.3 & 166.6 & 177.6 & 200.6 & 243.9\end{array}$

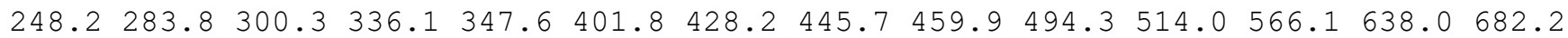
$704.9758 .1 \quad 881.6 \quad 902.0 \quad 933.2 \quad 958.6 \quad 965.4 \quad 992.5 \quad 1002.6 \quad 1021.9 \quad 1040.9 \quad 1048.91059 .9$ $1070.71110 .41130 .2 \quad 1157.6 \quad 1185.0 \quad 1206.5 \quad 1208.2 \quad 1243.91280 .7 \quad 1281.6 \quad 1287.2 \quad 1293.1$ $1293.91301 .91334 .8 \quad 1335.7 \quad 1342.1 \quad 1360.5 \quad 1373.2 \quad 1386.1 \quad 1400.8 \quad 1498.6 \quad 1801.7 \quad 1830.6$ $1861.52644 .4 \quad 2652.12665 .1 \quad 2672.4 \quad 2674.0 \quad 2685.6 \quad 2725.52731 .6 \quad 2733.4 \quad 2752.8 \quad 2755.2$ $2777.62781 .92868 .1 \quad 4876.4$

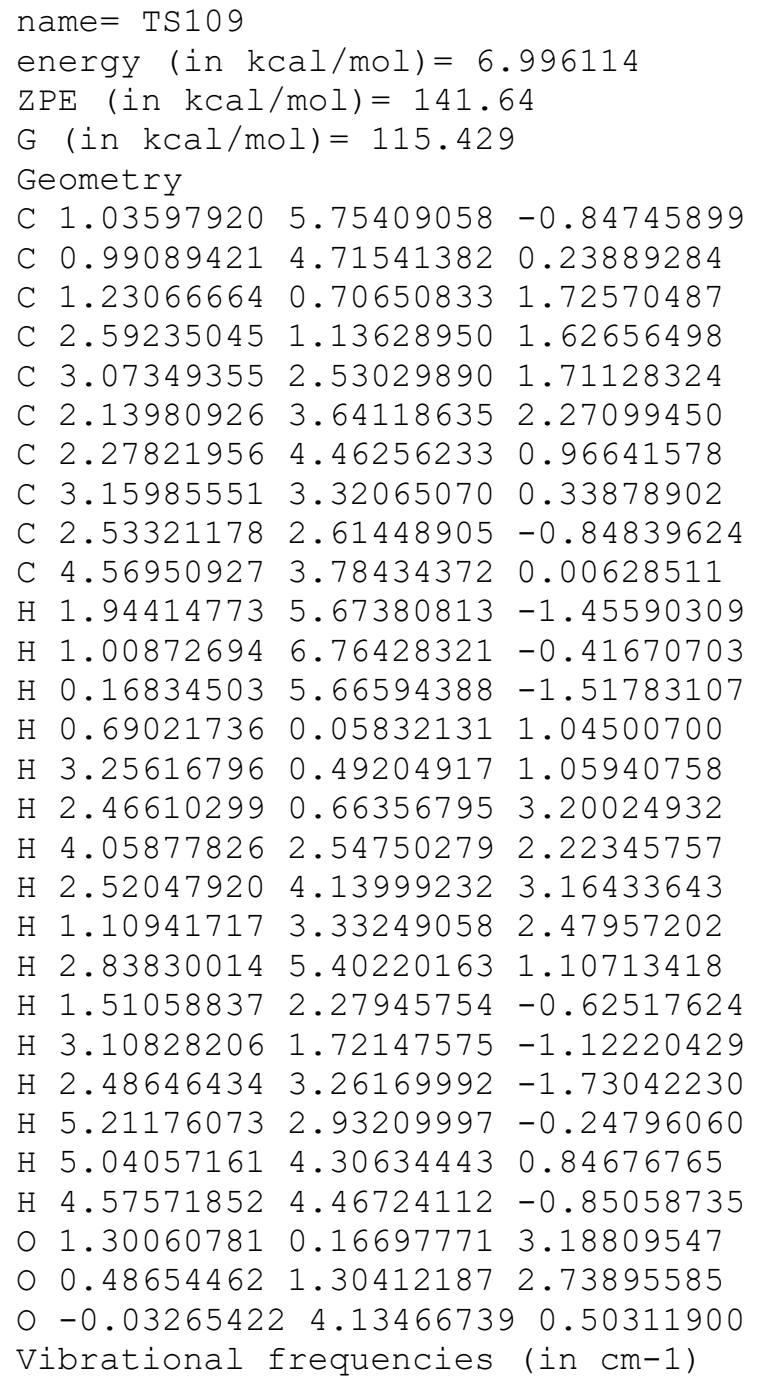


C $1.05157105-0.86016926 \quad 2.17131614$

C $2.74228084-0.718051632 .06377985$

C $3.39483280-0.38098497 \quad 3.30268387$

C $3.77747208 \quad 1.01001526 \quad 2.86655461$

C $3.24301211 \quad 0.63112713 \quad 1.41929106$

C $2.26016604 \quad 1.555572150 .73972326$

C $4.41050340 \quad 0.348516590 .47465460$

$\mathrm{H} 3.10566981 \quad 3.34862805 \quad 5.36542500$

H $4.54120721 \quad 3.39023088 \quad 4.32531721$

H $4.27641986 \quad 2.01323457 \quad 5.41002828$

$\mathrm{H} \quad 0.07372850 \quad 1.08247414 \quad 2.71473498$

$\mathrm{H} \quad 0.69822468-0.81732645 \quad 1.13343376$

H $0.94877290-1.89165962 \quad 2.55743199$

H $2.98579505-1.640015331 .52738047$

$\mathrm{H} 3.88406575-0.980465794 .01007131$

H $2.22606367-0.91043101 \quad 4.63337082$

H $4.85950577 \quad 1.21961848 \quad 2.87201632$

H $1.81206218 \quad 1.08158024 \quad 0.14073489$

H 2.75879355 2.47456878 0.40220177

$\mathrm{H} 1.44781874 \quad 1.88355738 \quad 1.39670543$

H $4.94226194 \quad 1.27018880 \quad 0.21488543$

H $4.06540430 \quad-0.11319304-0.45622377$

H $5.14056355-0.33269267 \quad 0.93091008$

O $1.21310853-1.04985271 \quad 4.86219302$

$\begin{array}{lllll}0 & 0.66770354 & 0.15887265 & 4.33569661\end{array}$

o 1.832197362 .245000663 .50826172

Vibrational frequencies (in cm-1)

$\begin{array}{lllllllllllllllll}-626.0 & 48.3 & 90.9 & 108.4 & 115.1 & 143.0 & 163.1 & 197.9 & 203.4 & 211.4 & 228.5 & 239.4 & 295.7 & 306.0\end{array}$

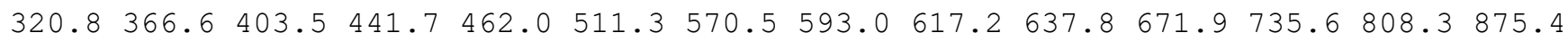
$907.6947 .5 \quad 972.8 \quad 982.4 \quad 992.3 \quad 1009.0 \quad 1019.7 \quad 1035.2 \quad 1041.6 \quad 1067.6 \quad 1081.9 \quad 1089.8 \quad 1111.4$

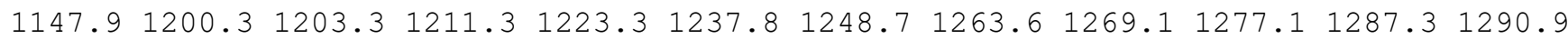
$1299.71300 .6 \quad 1305.0 \quad 1310.4 \quad 1336.1 \quad 1371.3 \quad 1378.0 \quad 1400.01402 .5 \quad 1415.1 \quad 1563.4 \quad 1816.3$ $2272.62637 .92667 .8 \quad 2673.2 \quad 2675.2 \quad 2676.2 \quad 2676.32679 .52683 .2 \quad 2715.32729 .32745 .8$ $2780.42781 .2 \quad 2783.2 \quad 2819.3$

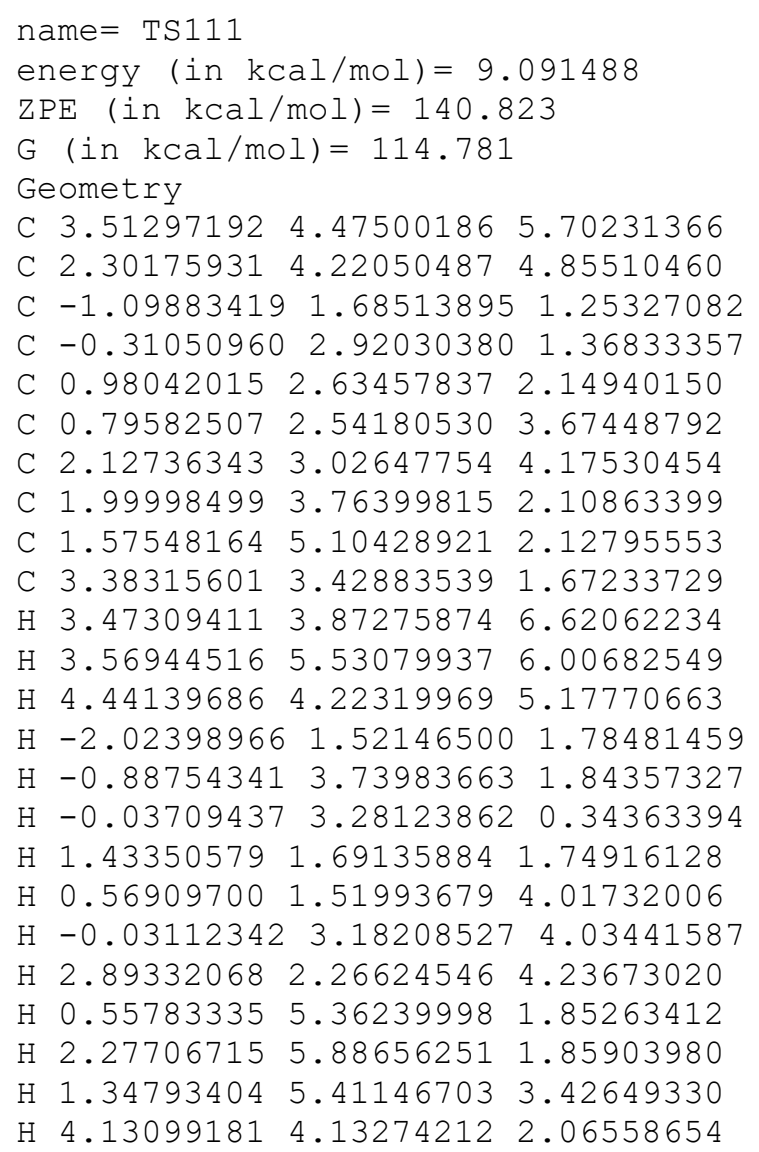


$\mathrm{H} \quad 3.44987254 \quad 3.46621846 \quad 0.57015963$

H $3.69958957 \quad 2.41861458 \quad 1.97175021$

$0.31477257 \quad 0.75888068-0.14743300$

$0-0.75379437 \quad 0.67886857 \quad 0.51259886$

O $1.496086495 .20277008 \quad 4.71653128$

Vibrational frequencies (in $\mathrm{cm}-1$ )

$\begin{array}{lllllllllllllllllll}-1410.8 & 37.4 & 49.5 & 78.2 & 80.4 & 101.8 & 141.9 & 150.4 & 174.1 & 230.7 & 258.2 & 288.8 & 301.2 & 341.1\end{array}$

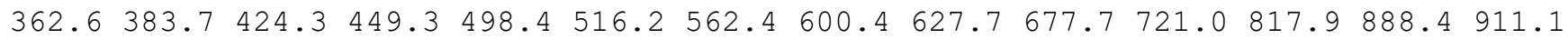
$932.4938 .2 \quad 968.1 \quad 998.2 \quad 1028.0 \quad 1036.9 \quad 1041.7 \quad 1049.3 \quad 1058.8 \quad 1065.5 \quad 1085.4 \quad 1128.3 \quad 1139.4$ $1150.81176 .0 \quad 1204.8 \quad 1213.8 \quad 1222.4 \quad 1235.3 \quad 1256.1 \quad 1266.3 \quad 1273.6 \quad 1279.4 \quad 1290.1 \quad 1297.6$

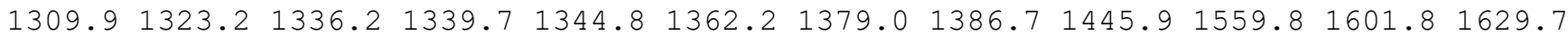
$1796.22644 .32655 .7 \quad 2666.8 \quad 2669.2 \quad 2673.2 \quad 2679.12688 .12703 .32721 .5 \quad 2729.02750 .3$ 2768.92773 .12775 .92782 .3

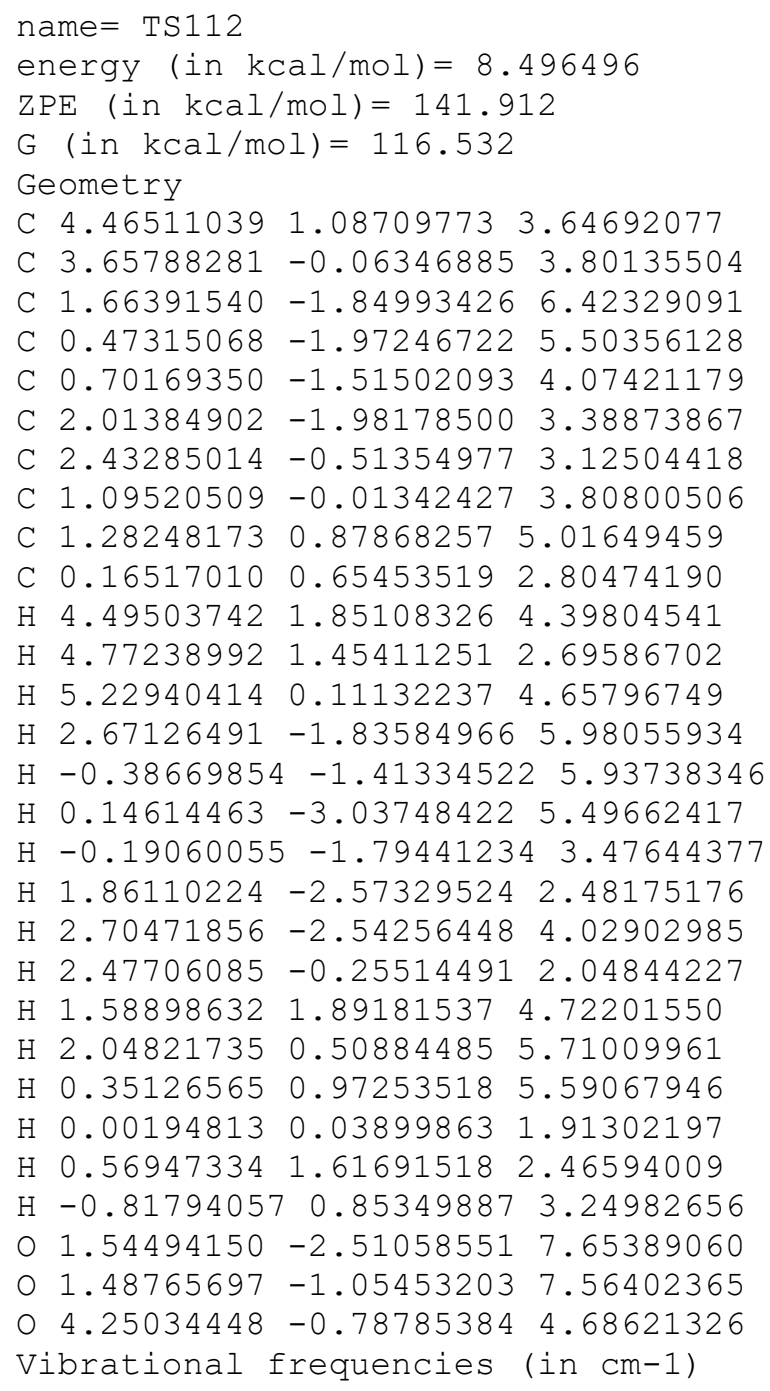


C $2.56373048 \quad 0.56601993 \quad 3.61025786$

C $0.02457647 \quad-2.39523769 \quad 1.68588745$

C $1.27182850-2.625325551 .08720364$

C $2.57673671-2.22478885 \quad 1.64197555$

C $2.66567741-1.981241293 .17833111$

C $3.27221643-0.569864852 .95627170$

C $3.06646634-0.71475654 \quad 1.40809803$

C $1.95081254 \quad 0.12888974 \quad 0.81458527$

C $4.34449686-0.58754005 \quad 0.61025518$

H 2.55421382 2.46722395 4.56154600

H $4.17779820 \quad 1.86540411 \quad 4.09114882$

$\mathrm{H}-0.37708874 \quad 0.99144328 \quad 4.12528811$

$\mathrm{H}-0.79274358-3.12182401 \quad 1.61541946$

$\mathrm{H} 1.03606790 \quad-0.89109176 \quad 0.52266690$

H $1.32603332-3.41759821 \quad 0.35485221$

H $3.36429491-2.92657458 \quad 1.30265119$

$\mathrm{H} 3.31521279-2.67698090 \quad 3.71168333$

H $1.69146745-1.98956102 \quad 3.68260424$

$\mathrm{H} \quad 4.34815837-0.537013693 .22427452$

$\mathrm{H} \quad 1.79897263 \quad 1.09418617 \quad 1.33558090$

$\mathrm{H} 0.78177113-0.284618321 .30585456$

H $2.08837567 \quad 0.35881350-0.25138995$

H $5.15093852-1.20096887 \quad 1.03467114$

$\mathrm{H} \quad 4.70690638 \quad 0.44787944 \quad 0.58831763$

H $4.21059120 \quad-0.92159133-0.42630228$

o $0.50391908 \quad 1.43945529 \quad 4.04344432$

o $-0.25784298-1.29240246 \quad 2.20919657$

O 1.225778220 .279465313 .60276113

Vibrational frequencies (in $\mathrm{cm}-1$ )

$\begin{array}{llllllllllllllll}-1371.9 & 41.3 & 56.9 & 76.3 & 129.0 & 159.6 & 174.8 & 193.6 & 210.0 & 238.5 & 244.7 & 282.7 & 313.9 & 327.1\end{array}$

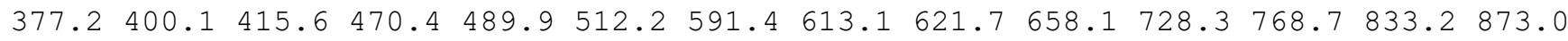
$926.7934 .2 \quad 956.3 \quad 980.3 \quad 988.9 \quad 1002.4 \quad 1003.3 \quad 1026.6 \quad 1048.5 \quad 1061.0 \quad 1086.5 \quad 1098.9 \quad 1114.0$

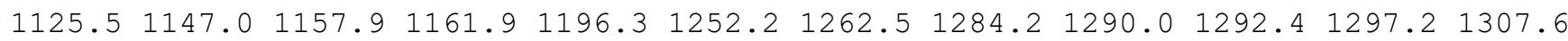

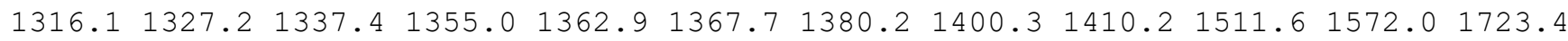

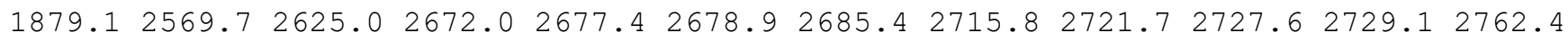
$2781.0 \quad 2784.12797 .5 \quad 2852.8$

name $=\operatorname{TS} 114$

energy $($ in $\mathrm{kcal} / \mathrm{mol})=9.942461$

$\mathrm{ZPE}(\mathrm{in} \mathrm{kcal} / \mathrm{mol})=140.833$

$\mathrm{G}(\mathrm{in} \mathrm{kcal} / \mathrm{mol})=115.015$

Geometry

$\begin{array}{lllll}\text { C } 0.67854871 & 2.89738401 & 2.28892690\end{array}$

C $1.36038637 \quad 1.59397520 \quad 2.57581963$

C $2.24042188-2.35383041 \quad 3.17220894$

C $3.55430425-2.08148843 \quad 2.57549518$

C $3.83195637-0.62159866 \quad 2.21711634$

C $3.456102990 .37352708 \quad 3.33157732$

C $2.73698043 \quad 1.48528027 \quad 2.62517005$

C $3.01752162-0.05772306 \quad 1.06123124$

C $1.78676551-0.62070420 \quad 0.69540395$

C $3.72196072 \quad 0.84732556 \quad 0.10829884$

$\mathrm{H} 0.75561503 \quad 3.57040386 \quad 3.15471272$

$\mathrm{H}-0.39333418 \quad 2.74925765 \quad 2.08779999$

$\mathrm{H} 1.117040123 .412316961 .42691532$

H $1.52469024-1.58140788 \quad 3.44309402$

H $3.69790025-2.74123994 \quad 1.68049803$

$\mathrm{H} \quad 4.34604764 \quad-2.45201874 \quad 3.27899458$

H $4.92194177-0.53575820 \quad 1.98149218$

H $4.34888747 \quad 0.74514390 \quad 3.86509780$

$\mathrm{H} \quad 2.81853741 \quad-0.07991548 \quad 4.11319926$

H $3.34008305 \quad 2.35814266 \quad 2.41583780$

$\mathrm{H} \quad 1.30299992-0.29241337-0.21836728$

$\mathrm{H} \quad 0.91179084 \quad-0.21267767 \quad 1.65657581$ 
H $1.56884475-1.66571208 \quad 0.89964597$

H 4.552649891 .403335350 .56550789

$\mathrm{H} 3.045049181 .59348984-0.33491412$

$\mathrm{H} \quad 4.14139425 \quad 0.26392987 \quad-0.72934803$

$02.60802238-4.511298933 .14271161$

$\begin{array}{lllll}0 & 1.83222350 & -3.55628747 & 3.43119124\end{array}$

o $0.59601893 \quad 0.56553943 \quad 2.66001883$

Vibrational frequencies (in $\mathrm{cm}-1$ )

$\begin{array}{lllllllllllllllll}-1444.8 & 33.2 & 40.4 & 79.5 & 105.7 & 138.8 & 152.8 & 176.6 & 187.3 & 216.5 & 254.6 & 292.0 & 303.3 & 335.4\end{array}$

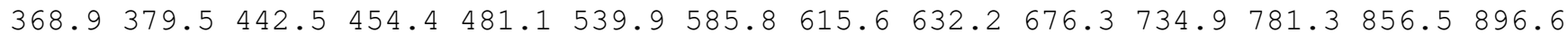
$924.6951 .1965 .6 \quad 995.7 \quad 1028.9 \quad 1040.1 \quad 1046.4 \quad 1047.3 \quad 1061.3 \quad 1069.7 \quad 1077.3 \quad 1135.2 \quad 1144.2$

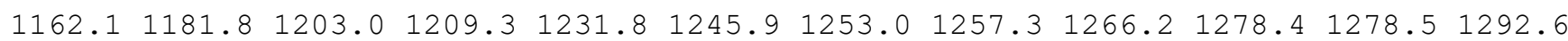

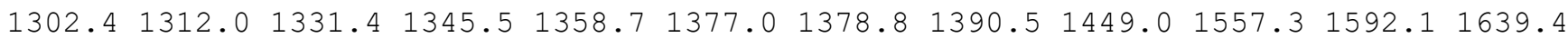
$\begin{array}{lllllllllllll}1788.7 & 2618.9 & 2658.1 & 2662.0 & 2667.3 & 2668.3 & 2671.8 & 2678.7 & 2696.3 & 2698.7 & 2717.9 & 2747.1\end{array}$ $2767.6 \quad 2772.12775 .5 \quad 2781.8$

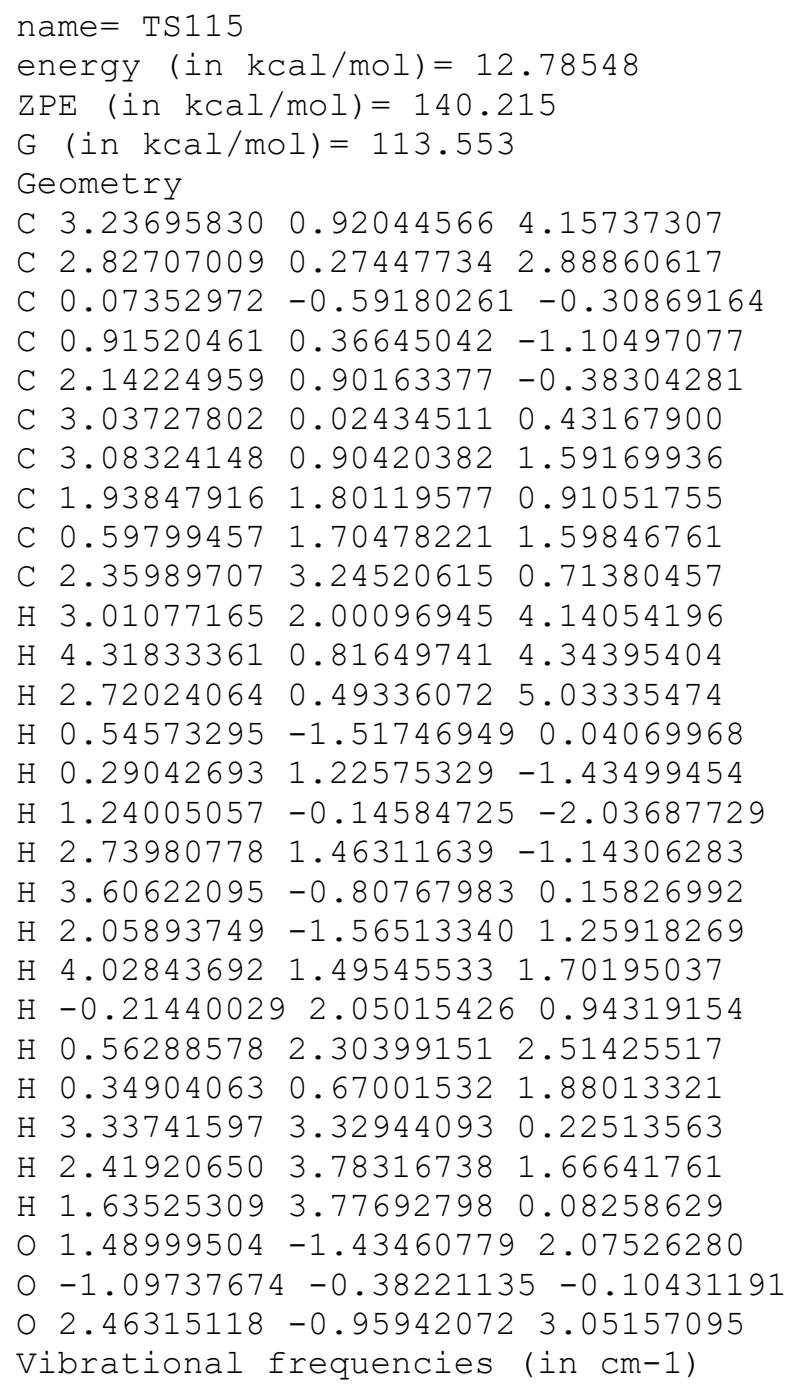




\begin{tabular}{|c|c|c|}
\hline \multicolumn{3}{|c|}{ Geor } \\
\hline & 3.578858460 & $0.91220652 \quad 3.25688755$ \\
\hline & & $0.11374748 \quad 2.7274722$ \\
\hline & & $-1.22100082-0.1894$ \\
\hline & 0.39382859 & $-1.11048794 \quad 0.4$ \\
\hline & & -1.374783460 .28 \\
\hline & 11 & $-1.99443461 \quad 1.4$ \\
\hline & & -0.751008511 .5 \\
\hline & 52 & $-0.19995100 \quad 0.2$ \\
\hline & 49191 & 1.203970220 .260 \\
\hline & & $-0.34964439-0$. \\
\hline & 378 & $1.49426668 \quad 2.46$ \\
\hline & 22866 & 0.259849613 .70 \\
\hline & 723371 & $1.61747021 \quad 4.03$ \\
\hline & -2.5 & -0.887124080 \\
\hline & 5352609 & -0.688534202 . \\
\hline & 7652337 & 0.016747861 .7 \\
\hline & & $-2.02319262-0$. \\
\hline & 2.28 & $-2.92603490 \quad 1.22$ \\
\hline & 1.1 & -2.173058832 .3 \\
\hline & 3.75800321 & -0.983510421 .4 \\
\hline & 0.83010206 & $1.39819072-0.6$ \\
\hline & 2.25714134 & 1.958930160 .26 \\
\hline & 0.84239711 & $1.38812748 \quad 1.145$ \\
\hline & 3.38979692 & $-1.35328074-1$. \\
\hline & 3.77542821 & $0.37125962-0.9636$ \\
\hline & $2.40174743-$ & $\begin{array}{lll}-0.18750250 & -1.93159728\end{array}$ \\
\hline & & $-2.28566127-1$ \\
\hline & -1.63146918 & $-1.84137427-1.38532926$ \\
\hline & $\begin{array}{l}1.33409603 \mathrm{f} \\
\text { brational fr }\end{array}$ & $\begin{array}{ll}0.17091800 & 3.24070195 \\
\text { requencies } & \text { (in cm-1) }\end{array}$ \\
\hline
\end{tabular}

$\begin{array}{lllllllllllllllllll}-723.4 & 32.8 & 50.6 & 76.9 & 104.2 & 106.9 & 114.7 & 157.0 & 177.7 & 199.7 & 217.6 & 261.3 & 289.7 & 310.4\end{array}$

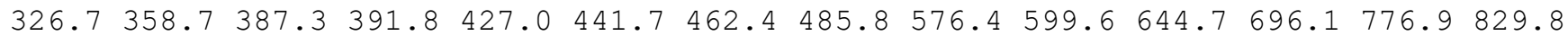
$846.9 \quad 859.4 \quad 881.9 \quad 903.8 \quad 942.1 \quad 988.4 \quad 998.2 \quad 1007.4 \quad 1017.1 \quad 1034.9 \quad 1035.6 \quad 1047.7 \quad 1067.5$ $1089.21107 .91125 .2 \quad 1152.1 \quad 1183.3 \quad 1209.8 \quad 1226.91241 .2 \quad 1259.5 \quad 1268.4 \quad 1271.7 \quad 1278.3$

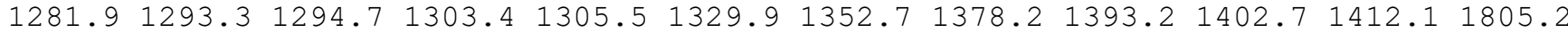
$1840.62463 .02665 .5 \quad 2668.2 \quad 2669.12671 .5 \quad 2673.82679 .62683 .8 \quad 2733.2 \quad 2744.62760 .5$ $2778.62779 .22781 .2 \quad 4898.3$

\footnotetext{
name $=\operatorname{TS} 117$

energy (in $\mathrm{kcal} / \mathrm{mol})=14.405961$

$\mathrm{ZPE}($ in $\mathrm{kcal} / \mathrm{mol})=138.757$

$\mathrm{G}($ in $\mathrm{kcal} / \mathrm{mol})=111.578$

Geometry

C 1.032522064 .142787515 .24545385

C $1.14463978 \quad 2.66023470 \quad 5.10797896$

C $0.69895051-1.22552281 \quad 1.61716845$

C $0.88649938-0.85680201 \quad 3.05432521$

C $2.14591946-0.01450538 \quad 3.27188624$

C $2.30337316 \quad 0.52871464 \quad 4.66589675$

C $2.272188292 .00409013 \quad 4.72033671$

C $2.17109344 \quad 1.322287892 .62035780$

C $0.94315686 \quad 2.00323492 \quad 2.16327496$

C 3.464171861 .844665122 .11980283

$\mathrm{H} 1.53624227 \quad 4.476569796 .16802650$

$\mathrm{H}-0.01199328 \quad 4.484771125 .31198384$

$\mathrm{H} 1.50271839 \quad 4.66742893 \quad 4.40436402$

$\mathrm{H} \quad 0.01766010-2.05285472 \quad 1.38092671$

H $0.93977202-1.75727435 \quad 3.70289631$

$\mathrm{H}-0.01509188-0.31719473 \quad 3.43801833$

$\mathrm{H} \quad 3.03215915 \quad-0.63708548 \quad 2.98653094$

H 2.98441475 $0.02620048 \quad 5.32237471$

$\mathrm{H} 0.94241640 \quad 0.34030627 \quad 5.31075289$

H 3.219994962 .529026124 .68375602
} 
$\mathrm{H} \quad 0.64245250 \quad 1.60636476 \quad 1.17032432$

$\mathrm{H} 1.05041806 \quad 3.09122580 \quad 2.05599195$

$\mathrm{H} 0.080231081 .826605152 .83635070$

$\mathrm{H} 3.518096262 .94298343 \quad 2.09910728$

$\mathrm{H} \quad 3.628021331 .49424364 \quad 1.08061316$

H $4.32826421 \quad 1.48957852 \quad 2.70819278$

$0-0.11421822 \quad 0.74451791 \quad 5.53263895$

$01.26426033-0.630908700 .72808727$

O $-0.07017527 \quad 2.13094344 \quad 5.33822254$

Vibrational frequencies (in $\mathrm{cm}-1$ )

$\begin{array}{llllllllllllllll}-947.8 & 18.1 & 51.1 & 74.4 & 82.5 & 92.2 & 124.5 & 137.9 & 150.1 & 164.3 & 195.6 & 222.0 & 237.5 & 270.5\end{array}$ $293.9 \quad 321.5 \quad 340.7 \quad 396.1 \quad 457.4 \quad 467.7 \quad 525.1 \quad 582.6 \quad 617.0 \quad 645.6 \quad 710.7 \quad 784.4 \quad 808.4 \quad 839.0$ $897.9928 .9 \quad 944.3 \quad 974.6 \quad 997.5 \quad 1008.3 \quad 1038.3 \quad 1043.4 \quad 1051.0 \quad 1062.2 \quad 1094.8 \quad 1127.4 \quad 1143.0$ $1158.21173 .6 \quad 1201.91230 .4 \quad 1238.0 \quad 1248.2 \quad 1252.8 \quad 1259.01268 .1 \quad 1269.7 \quad 1273.3 \quad 1276.6$ $1290.61296 .8 \quad 1315.0 \quad 1334.7 \quad 1365.2 \quad 1380.5 \quad 1381.1 \quad 1392.91410 .4 \quad 1439.01645 .2 \quad 1762.2$ $1826.32617 .22623 .5 \quad 2640.8 \quad 2652.12656 .8 \quad 2660.4 \quad 2662.22674 .02695 .42728 .02755 .5$ 2758.32766 .22776 .12783 .0

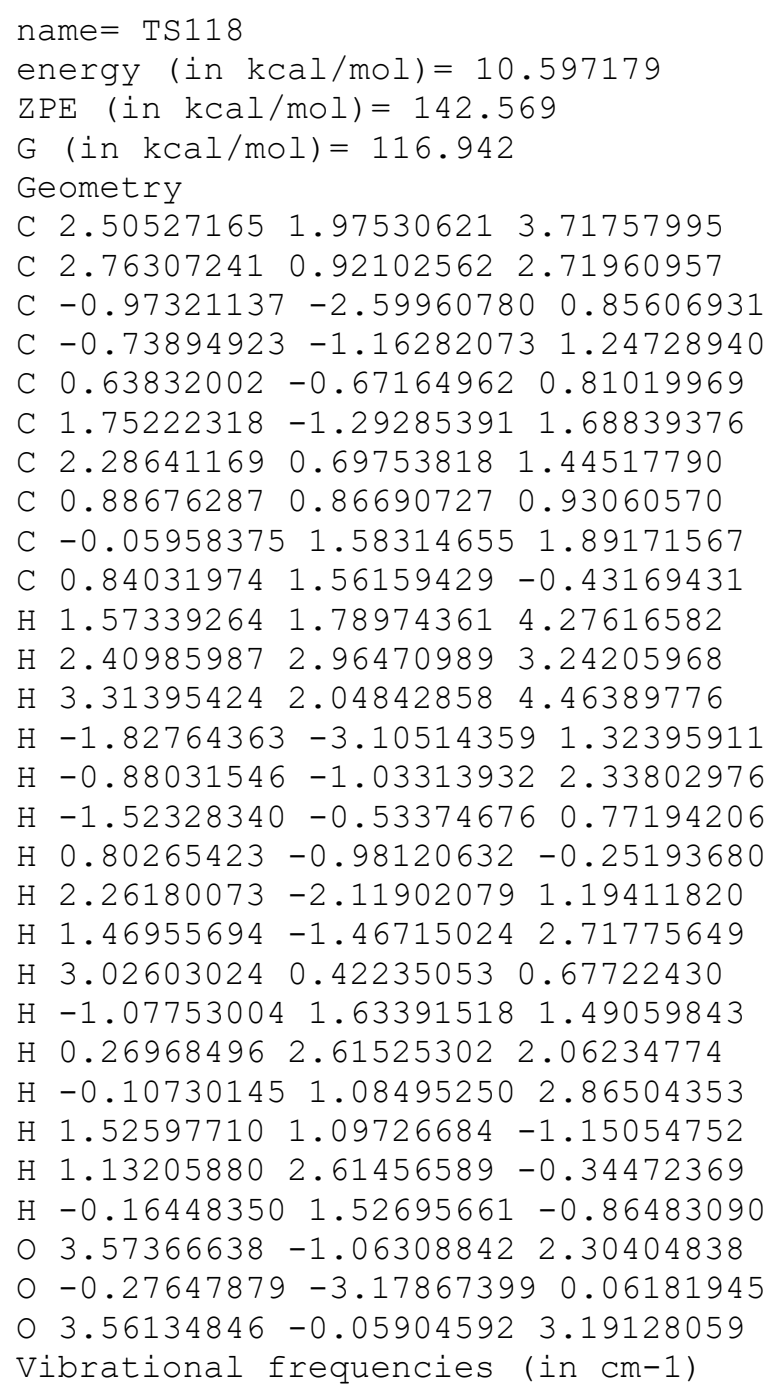


$\mathrm{ZPE}($ in $\mathrm{kcal} / \mathrm{mol})=139.14$

$\mathrm{G}($ in $\mathrm{kcal} / \mathrm{mol})=111.28$

Geometry

C $1.78779261-1.23850617 \quad 6.07098562$

C $2.46251065-1.541565094 .95900870$

C $-0.03431074 \quad-4.73477229 \quad 1.36070272$

C $0.73954791-3.66342137 \quad 1.14886210$

C $1.31967898-2.76132105 \quad 2.19563373$

C $1.70977698-3.522303913 .48705766$

C $2.62207993-2.84036400 \quad 4.38570480$

C $0.32030817-1.65604656 \quad 2.46462727$

C $0.69847495-0.31804013 \quad 1.91801446$

C $-0.82658201-1.85772170 \quad 3.11601412$

$\mathrm{H} 1.68457975-0.24124734 \quad 6.45512825$

$\mathrm{H} 1.30443260-1.98269406 \quad 6.67959493$

H $2.45453299 \quad 1.08389138 \quad 4.53088699$

$\mathrm{H}-0.46583972 \quad-5.36462813 \quad 0.59313917$

$\mathrm{H}-0.05495305 \quad 0.45109458 \quad 2.13392288$

$\mathrm{H} \quad 0.96787872 \quad-3.36367126 \quad 0.12655359$

H $2.26179757-2.31222513 \quad 1.78244004$

$\mathrm{H} 2.08048961-4.535933373 .21890236$

$\mathrm{H} \quad 0.78137184 \quad-3.74923923 \quad 4.08252523$

$\mathrm{H} 4.09159784 \quad-3.088095794 .04736069$

H $1.65120460 \quad 0.03147152 \quad 2.34184917$

$\mathrm{H}-0.92460593-5.88094036 \quad 2.66955985$

$\mathrm{H} \quad 0.81708009-0.356130220 .82587111$

H $3.94401580 \quad-2.57346697 \quad 3.42344798$

$\mathrm{H}-1.54941502-1.08515663 \quad 3.31266262$

$\mathrm{H}-1.12812148 \quad-2.82032574 \quad 3.50068327$

$\begin{array}{llll}0 & 3.28677421 & 0.62964403 & 4.81419940\end{array}$

O $-0.32118724-5.10514398 \quad 2.64520073$

O $3.13317654-0.626525814 .16542921$

Vibrational frequencies (in $\mathrm{cm}-1$ )

$\begin{array}{lllllllllllllllll}-933.1 & 25.6 & 38.0 & 55.8 & 60.5 & 76.7 & 116.9 & 139.6 & 141.1 & 179.5 & 198.3 & 237.1 & 248.8 & 251.5\end{array}$

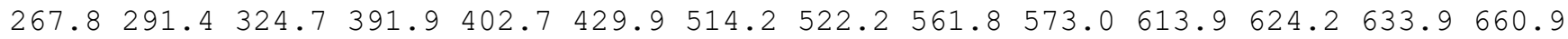

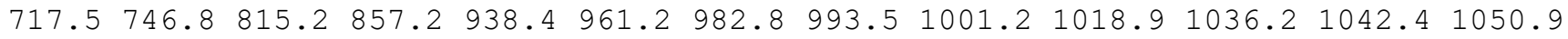
$1072.21084 .2 \quad 1098.5 \quad 1151.6 \quad 1174.3 \quad 1208.8 \quad 1219.4 \quad 1250.3 \quad 1260.4 \quad 1276.2 \quad 1282.4 \quad 1289.2$

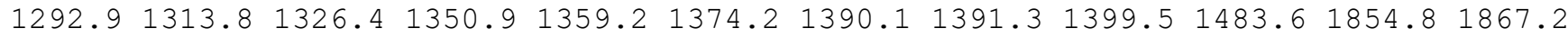
$1875.02625 .32670 .0 \quad 2674.8 \quad 2688.0 \quad 2722.1 \quad 2727.2 \quad 2728.62735 .0 \quad 2755.92778 .0 \quad 2789.7$ 2800.22806 .62868 .34569 .5

\section{name $=\operatorname{TS} 120$}

energy $($ in $\mathrm{kcal} / \mathrm{mol})=13.434604$

$\mathrm{ZPE}($ in $\mathrm{kcal} / \mathrm{mol})=140.559$

$\mathrm{G}(\mathrm{in} \mathrm{kcal} / \mathrm{mol})=114.159$

Geometry

C $1.674176193 .70909015 \quad 4.45033020$

C $1.26294964 \quad 2.44534876 \quad 3.75769451$

C $-0.40855849-1.153806591 .21847445$

$\begin{array}{lllll}\text { C } & 0.67427344 & -0.63571318 & 0.35514221\end{array}$

C $1.46051667 \quad 0.54208973 \quad 0.93965140$

C $1.94009584 \quad 0.40169864 \quad 2.39662112$

C 2.064370011 .845774552 .79702348

C $0.718812521 .86811740 \quad 1.07959876$

C $-0.60623976 \quad 1.91839655 \quad 1.53829524$

C $1.28846371 \quad 3.04770627 \quad 0.36560992$

$\mathrm{H} 2.47651553 \quad 3.50655105 \quad 5.17423525$

$\mathrm{H} 0.83395492 \quad 4.14660202 \quad 5.01135419$

H 2.04249320 4.46436228 3.74735658

$\mathrm{H}-0.28620692-1.320872712 .27768867$

$\mathrm{H} \quad 0.29091679-0.34747398-0.65003496$

$\mathrm{H} 1.39480170-1.47065028 \quad 0.16158808$

H $2.35502861 \quad 0.68555852 \quad 0.27994151$

H 2.89306301 $-0.14349142 \quad 2.48533190$ 
$\mathrm{H} \quad 1.21627879-0.13482590 \quad 3.03764583$

H $2.99927304 \quad 2.31454183 \quad 2.52476862$

$\mathrm{H}-1.17975600 \quad 2.83019990 \quad 1.40657374$

$\mathrm{H}-0.54946891 \quad 1.86128277 \quad 2.89660189$

$\mathrm{H}-1.24600514 \quad 1.04167341 \quad 1.51349535$

$\mathrm{H} \quad 0.88379237 \quad 3.10810448-0.65824940$

$\mathrm{H} 2.383447193 .02155758 \quad 0.28205138$

$\mathrm{H} \quad 1.03164471 \quad 3.99559431 \quad 0.86354736$

$0-2.47579021-1.872073091 .25423615$

O $-1.51652281-1.408520290 .58805933$

o $0.09388066 \quad 2.00912914 \quad 4.02731060$

Vibrational frequencies (in $\mathrm{cm}-1$ )

$\begin{array}{lllllllllllllll}-1479.8 & 43.1 & 46.5 & 48.1 & 70.4 & 98.9 & 139.9 & 160.8 & 187.1 & 239.1 & 249.2 & 268.1 & 284.3 & 326.7\end{array}$

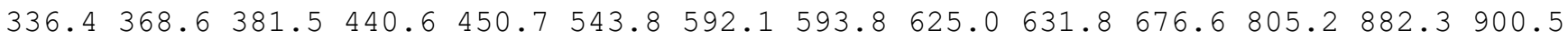
$922.7947 .8 \quad 974.5 \quad 995.5 \quad 1027.5 \quad 1038.4 \quad 1039.9 \quad 1047.6 \quad 1060.7 \quad 1069.5 \quad 1093.7 \quad 1125.2 \quad 1152.1$

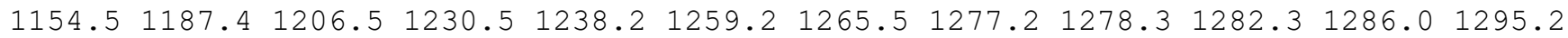
$1304.51331 .31336 .6 \quad 1339.4 \quad 1348.0 \quad 1378.2 \quad 1384.1 \quad 1398.51445 .7 \quad 1558.11561 .91633 .7$ $1789.52641 .22660 .72665 .5 \quad 2667.7 \quad 2670.8 \quad 2678.6 \quad 2695.02699 .7 \quad 2706.92729 .3 \quad 2748.5$ $2769.22773 .92776 .4 \quad 2781.8$

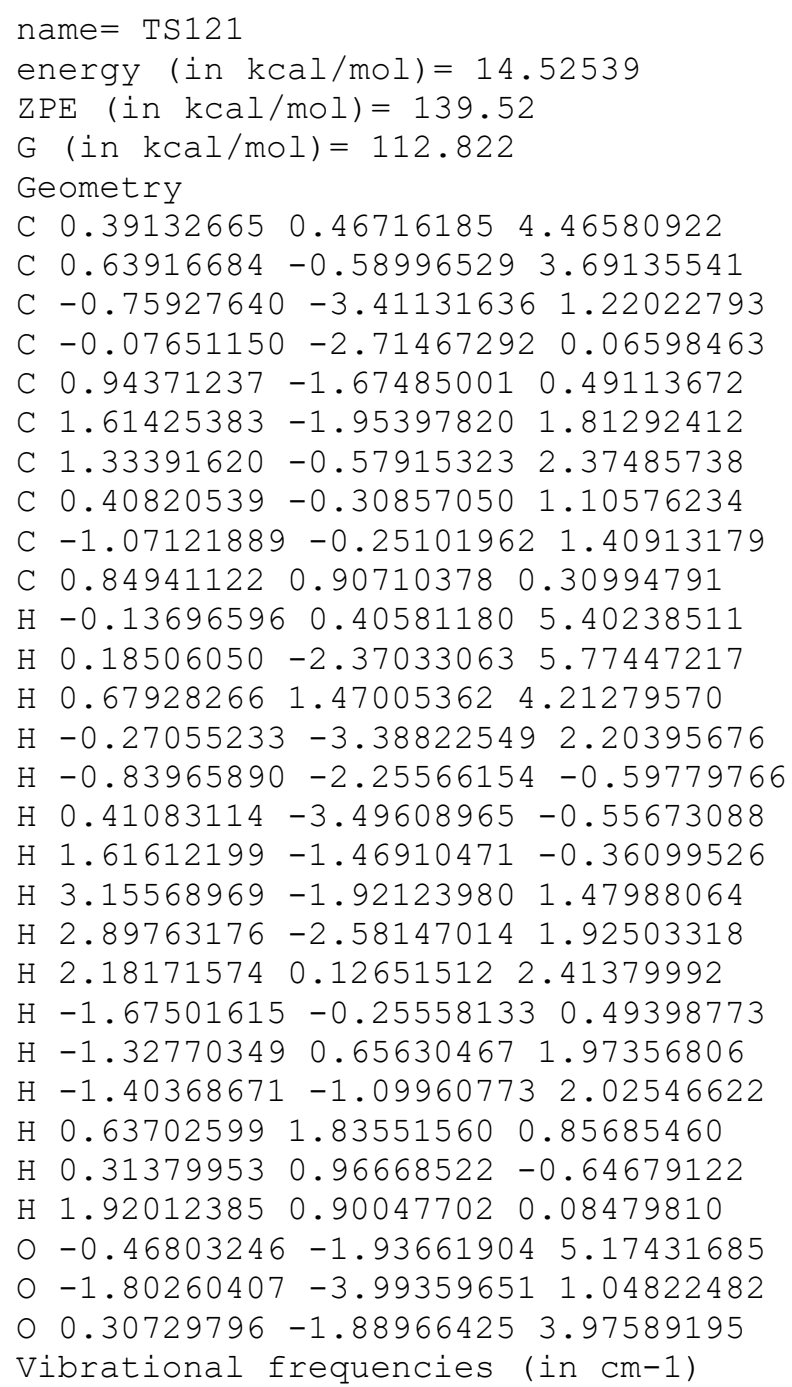




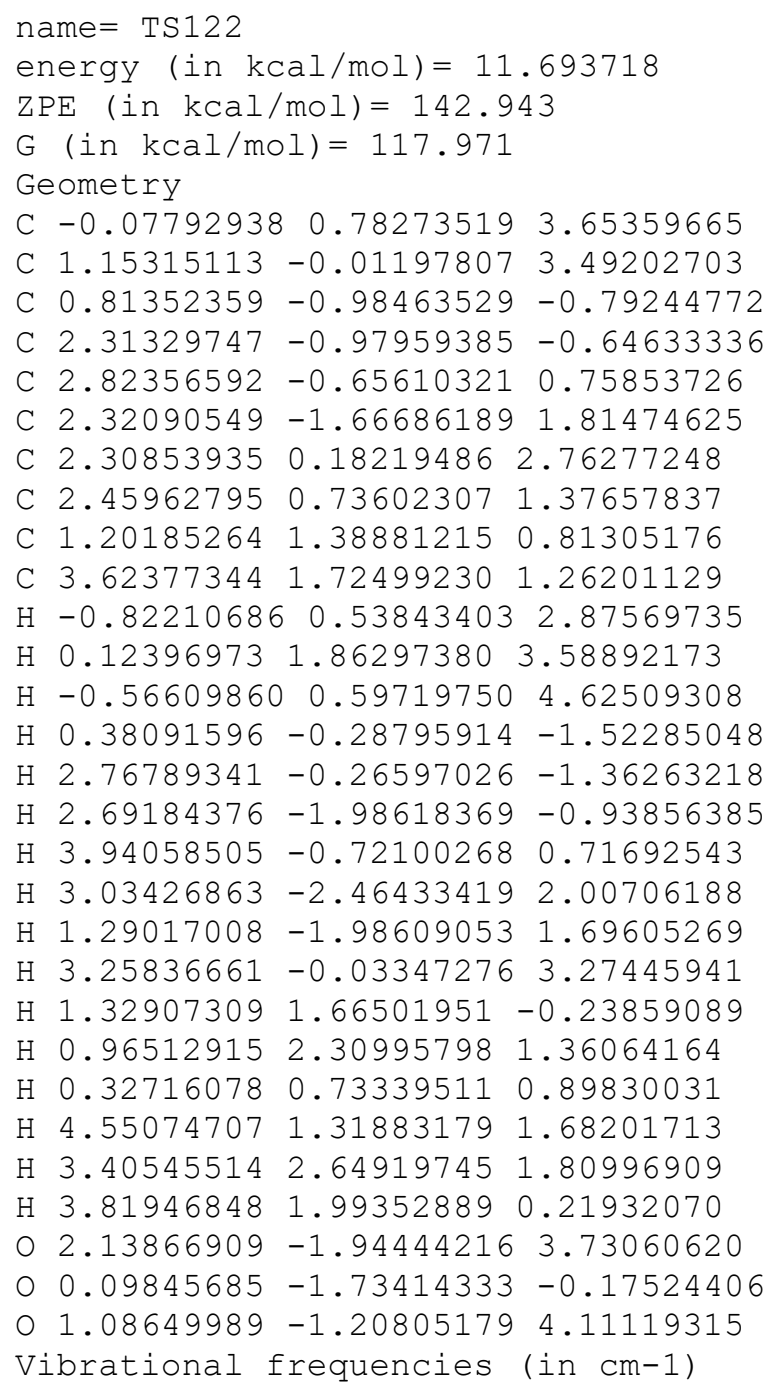


$\mathrm{H} \quad 3.01707533-4.260809891 .92951844$

$\mathrm{H} 1.24805588-3.53069153 \quad 3.82060186$

$\mathrm{H} \quad 0.93336770 \quad-2.15719288 \quad 2.79115816$

$\mathrm{H} \quad 3.57942989-3.238514394 .59650783$

$\mathrm{H} 3.49506452-1.53683502-0.46639246$

$\mathrm{H}-1.71589532-3.876700002 .85179588$

$\mathrm{H} 3.88700267-1.82439985 \quad 1.23782514$

H $4.04021772-3.126263733 .92327345$

H $1.10939347-1.82093519-0.99291336$

H $0.06083352-2.85121654 \quad 0.03395182$

$03.55974142-0.04045278 \quad 6.27889294$

$0-0.75355810-3.781784662 .67270760$

O $3.47161970-1.228186365 .50452079$

Vibrational frequencies (in $\mathrm{cm}-1$ )

$\begin{array}{lllllllllllllllll}-978.2 & 30.4 & 38.5 & 43.5 & 54.4 & 71.7 & 108.1 & 120.7 & 158.8 & 184.9 & 206.7 & 233.9 & 245.8 & 263.8\end{array}$ $269.5 \quad 295.1 \quad 309.3 \quad 347.0 \quad 405.8 \quad 413.4 \quad 522.0 \quad 532.7 \quad 566.6 \quad 579.7 \quad 609.2 \quad 624.3 \quad 638.1 \quad 650.4$ $710.4 \quad 748.7 \quad 813.8 \quad 861.8 \quad 945.3 \quad 971.0 \quad 977.8 \quad 995.8 \quad 1002.6 \quad 1027.7 \quad 1033.6 \quad 1041.91052 .8$

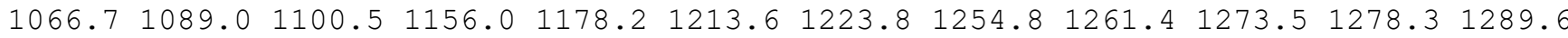

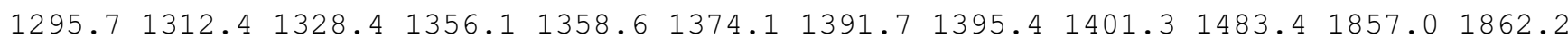
$1867.22626 .92651 .82681 .0 \quad 2691.2 \quad 2722.12727 .82728 .52734 .42755 .0 \quad 2775.8 \quad 2794.8$ $2801.02805 .4 \quad 2869.6 \quad 4550.7$

name $=$ TS124

energy (in $\mathrm{kcal} / \mathrm{mol})=14.903045$

$\mathrm{ZPE}(\mathrm{in} \mathrm{kcal} / \mathrm{mol})=140.633$

G $($ in $\mathrm{kcal} / \mathrm{mol})=115.36$

Geometry

C $2.09815387 \quad 0.83635817 \quad 0.39451456$

C $1.23532653-0.17777788 \quad 0.44723583$

C $-1.28278464-1.796723812 .20376825$

C $-2.31708259-1.64936670 \quad 1.15822627$

C $-1.71344139-1.42706214-0.22373587$

C $-0.30445266-2.04006746-0.46173177$

C $0.31146092-0.62383414-0.63078809$

$\begin{array}{llll}\text { C } & -1.13497484 & 0.00558364 & -0.53572402\end{array}$

C $-1.320487301 .07280675 \quad 0.52082672$

C $-1.608022010 .52306908-1.88890273$

H 2.73252246 $1.11627060 \quad 1.22057590$

$\mathrm{H} \quad 0.85838439 \quad 0.02996912 \quad 3.09205680$

H $2.23686544 \quad 1.45592317-0.47279646$

$\mathrm{H}-0.31938379-1.941642243 .18264969$

$\mathrm{H}-3.02755550-0.82759794 \quad 1.39372732$

$\mathrm{H}-2.92879105-2.579911131 .14902069$

$\mathrm{H}-2.44487884-1.74314925-0.98998638$

$\mathrm{H}-0.23851841-2.65266327-1.36616374$

$\mathrm{H} \quad 0.09184186-2.64633597 \quad 0.35694308$

$\mathrm{H} \quad 0.78236420-0.47302255-1.61932228$

H $-2.36581068 \quad 1.39819518 \quad 0.58490228$

$\mathrm{H}-0.71076703 \quad 1.95920209 \quad 0.30111581$

$\mathrm{H}-1.03211798 \quad 0.72831616 \quad 1.52343172$

$\mathrm{H}-1.47014275-0.21596057-2.68590044$

$\mathrm{H}-1.05947400 \quad 1.42705041-2.18100471$

$\mathrm{H}-2.67347625 \quad 0.78203150 \quad-1.86230023$

$01.64866839-0.430589632 .70059180$

$0-1.16378854-0.976750373 .17422500$

o $1.04926671-0.995861521 .53748007$

Vibrational frequencies (in $\mathrm{cm}-1$ )

$\begin{array}{lllllllllllllll}-1992.0 & 45.7 & 86.8 & 95.0 & 103.4 & 128.1 & 172.4 & 178.1 & 188.4 & 208.6 & 232.8 & 274.7 & 292.4 & 317.8\end{array}$

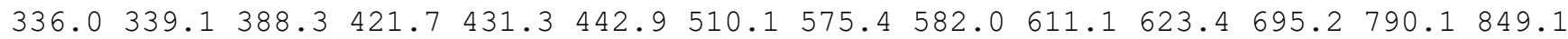
$910.8 \quad 925.0 \quad 951.2 \quad 995.7 \quad 1005.6 \quad 1008.0 \quad 1012.0 \quad 1017.2 \quad 1037.8 \quad 1050.6 \quad 1071.0 \quad 1102.4 \quad 1112.8$ $1119.8 \quad 1149.3 \quad 1175.2 \quad 1206.0 \quad 1222.1 \quad 1244.5 \quad 1274.1 \quad 1284.2 \quad 1285.3 \quad 1296.01298 .6 \quad 1300.7$

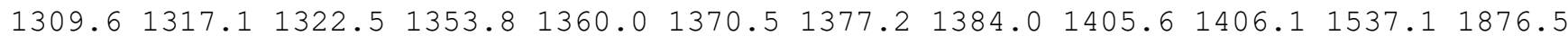
$2482.62658 .72668 .0 \quad 2674.92678 .0 \quad 2679.3 \quad 2679.4 \quad 2717.82723 .5 \quad 2729.02735 .52762 .5$ $2780.7 \quad 2782.6 \quad 2796.2 \quad 2830.1$ 


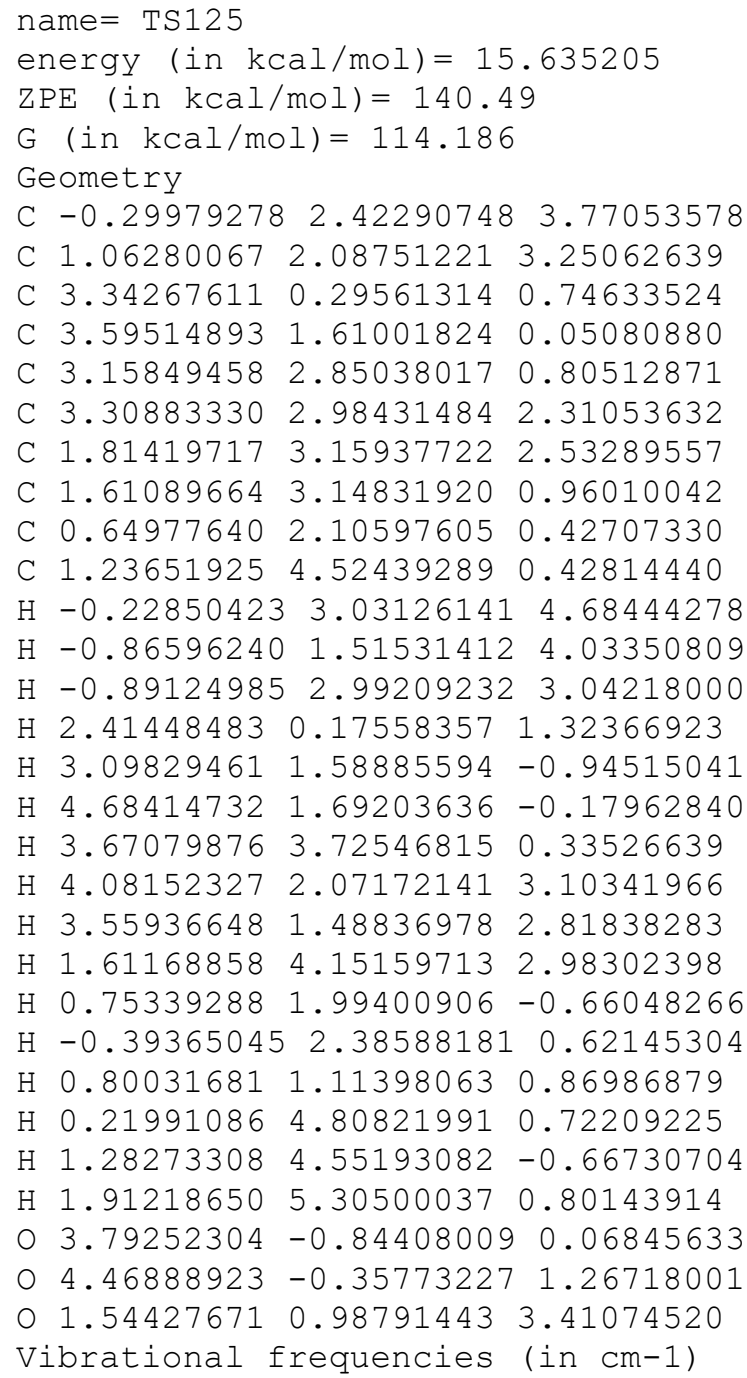


H $1.57794176-0.596809320 .30842744$

$\mathrm{H} 2.63190025-1.778213201 .10189494$

$\mathrm{H} 4.04143086-0.06707154 \quad 0.06288559$

H $5.43962932 \quad 0.37286456 \quad 2.01890115$

$\mathrm{H} \quad 4.21632886-0.41697386 \quad 2.99684918$

H $4.56881156 \quad 2.53230865 \quad 2.64810677$

$\mathrm{H} 1.03998807 \quad 1.72310647 \quad 1.54911581$

H $1.38097878 \quad 1.88855029-0.15722215$

$\mathrm{H} 1.61897013 \quad 3.273499690 .91076250$

H $4.02915930 \quad 3.61941304 \quad 0.29817689$

$\mathrm{H} \quad 3.78660838 \quad 2.39381199 \quad-0.94929948$

$\mathrm{H} \quad 5.13138328 \quad 2.24194683 \quad 0.18192693$

$\begin{array}{llll}0 & 2.98372442 & -0.13781260 & 4.57452643\end{array}$

O $0.44658451-0.17965072 \quad 2.57101896$

$\begin{array}{llll}0 & 2.15432590 & 1.01128071 & 4.25565404\end{array}$

Vibrational frequencies (in cm-1)

$\begin{array}{lllllllllllllllllll}-849.9 & 51.3 & 74.6 & 114.5 & 133.0 & 168.8 & 193.3 & 205.8 & 217.7 & 220.2 & 242.7 & 275.3 & 301.3 & 329.1\end{array}$ $\begin{array}{lllllllllllllll}357.1 & 368.6 & 394.8 & 431.0 & 441.0 & 495.7 & 538.1 & 575.6 & 590.6 & 686.2 & 768.2 & 791.3 & 815.9 & 883.1\end{array}$ $911.6936 .9948 .0 \quad 994.3 \quad 1005.7 \quad 1013.3 \quad 1027.7 \quad 1031.3 \quad 1043.6 \quad 1078.2 \quad 1084.9 \quad 1107.9 \quad 1124.5$

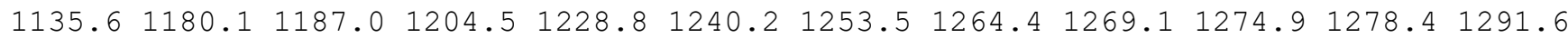

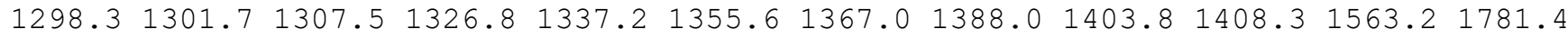
$2224.32642 .5 \quad 2650.3 \quad 2653.4 \quad 2671.2 \quad 2672.9 \quad 2673.7 \quad 2675.72679 .3 \quad 2715.42722 .6 \quad 2742.8$ $2760.8 \quad 2762.6 \quad 2778.5 \quad 2782.0$

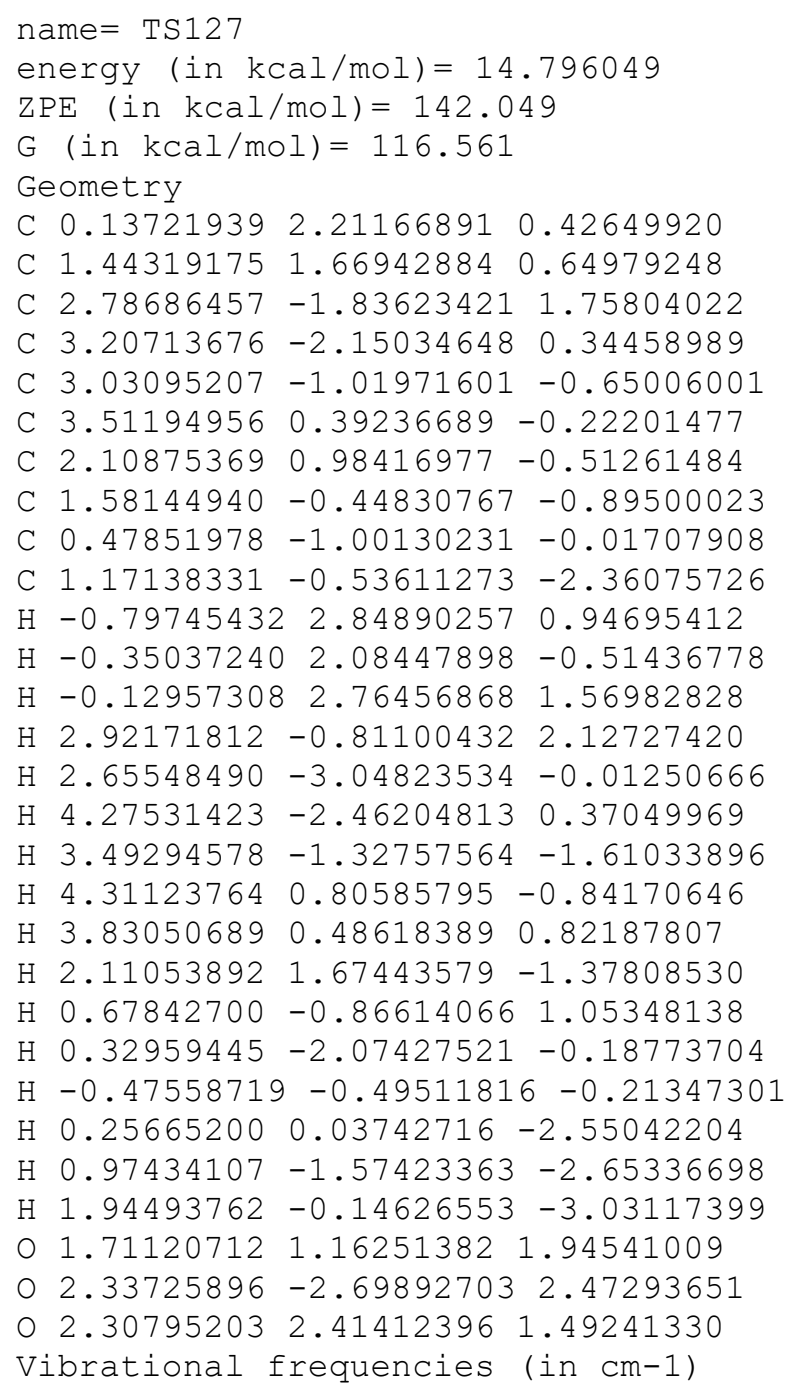


$\begin{array}{llllllllllllll}1881.0 & 2638.5 & 2657.3 & 2668.4 & 2677.2 & 2678.7 & 2679.6 & 2681.3 & 2713.5 & 2723.5 & 2738.4 & 2762.7\end{array}$ $2775.5 \quad 2781.3 \quad 2783.3 \quad 3713.7$

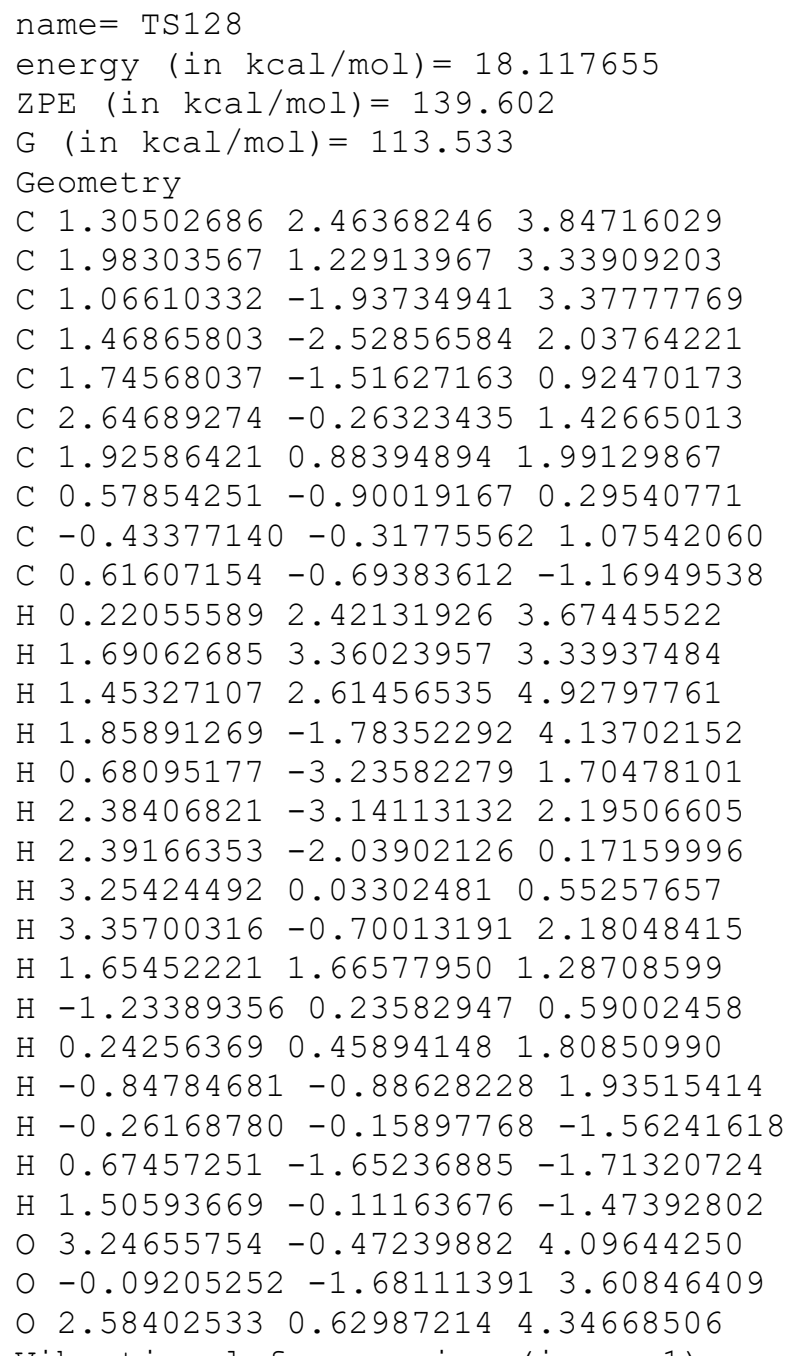

Vibrational frequencies (in $\mathrm{cm}-1$ )

$\begin{array}{lllllllllllllllll}-884.7 & 42.9 & 69.6 & 80.2 & 90.2 & 98.6 & 105.3 & 124.1 & 159.9 & 216.8 & 252.1 & 264.3 & 312.6 & 328.8\end{array}$ $332.4 \quad 353.6 \quad 401.2 \quad 423.9 \quad 467.3 \quad 488.8 \quad 508.4 \quad 560.9 \quad 604.0 \quad 649.5 \quad 673.3 \quad 727.8 \quad 832.8 \quad 878.5$ $918.4 \quad 950.8 \quad 972.0 \quad 974.6 \quad 988.9 \quad 1003.7 \quad 1025.1 \quad 1035.5 \quad 1052.8 \quad 1058.0 \quad 1066.5 \quad 1090.5 \quad 1151.0$

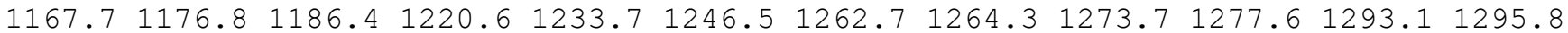
$1303.81309 .51331 .61346 .2 \quad 1367.2 \quad 1378.0 \quad 1384.2 \quad 1440.51458 .0 \quad 1557.7 \quad 1673.21817 .8$ $1858.12537 .72574 .0 \quad 2600.12645 .42658 .72661 .02665 .72669 .72686 .52710 .52734 .7$ $2746.92757 .5 \quad 2769.3 \quad 2776.2$

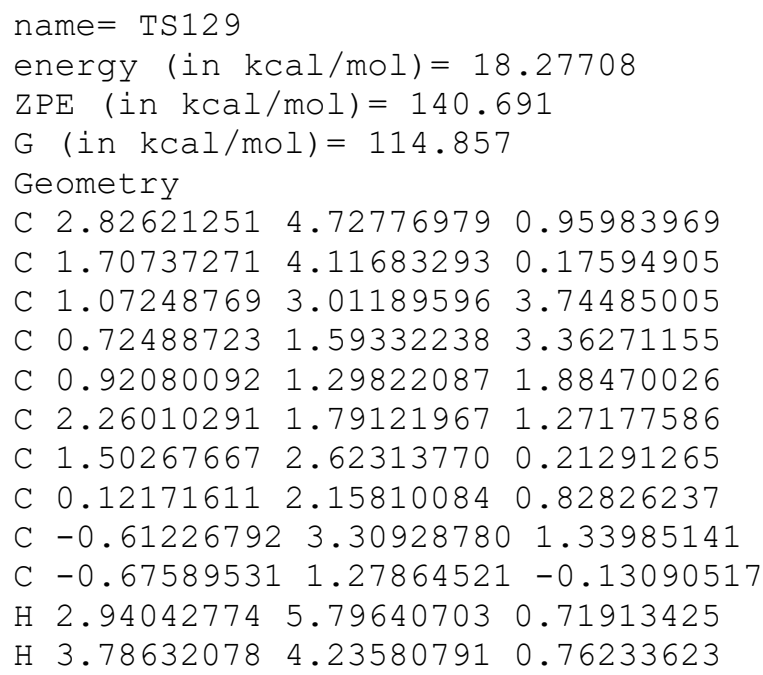


$\mathrm{H} 2.63300046 \quad 4.664888392 .04281293$

$\mathrm{H} 0.61926128 \quad 3.82366132 \quad 3.14836221$

$\mathrm{H}-0.32411917 \quad 1.38635883 \quad 3.65685112$

H $1.34358171 \quad 0.88695467 \quad 3.96076605$

$\mathrm{H} \quad 0.76363541 \quad 0.21593148 \quad 1.71400412$

H $2.88221023 \quad 0.99454977 \quad 0.85360646$

H $2.88893498 \quad 2.36296428 \quad 1.96038246$

$\mathrm{H} 1.64145690 \quad 2.23440039-0.81614728$

$\mathrm{H}-1.72032415 \quad 2.85984887 \quad 2.35823272$

$\mathrm{H}-2.12667611 \quad 2.67895934 \quad 1.67964886$

$\mathrm{H}-1.01980632 \quad 4.04376190 \quad 0.68522159$

$\mathrm{H}-1.08274350 \quad 1.87151006-0.96041962$

H $-1.51106625 \quad 0.78254897 \quad 0.37280621$

$\mathrm{H}-0.05783451 \quad 0.49045495 \quad-0.57744269$

$\begin{array}{llll}0 & 2.37543479 & 3.23508939 & 4.21219270\end{array}$

O $1.22867416 \quad 3.25643248 \quad 5.11615348$

o $0.98938400 \quad 4.79623584 \quad-0.51870953$

Vibrational frequencies (in $\mathrm{cm}-1$ )

$\begin{array}{llllllllllllllll}-951.2 & 33.7 & 53.9 & 81.2 & 103.4 & 150.8 & 152.5 & 179.1 & 183.7 & 194.8 & 211.0 & 255.7 & 296.4 & 320.6\end{array}$

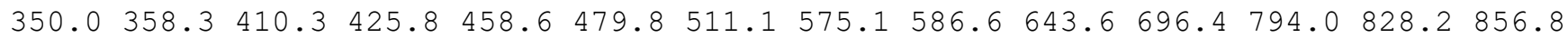

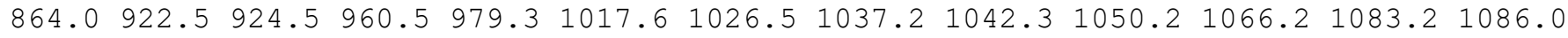

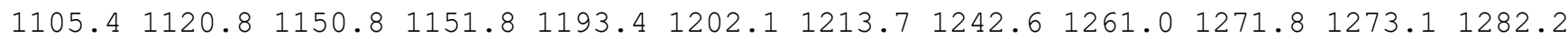

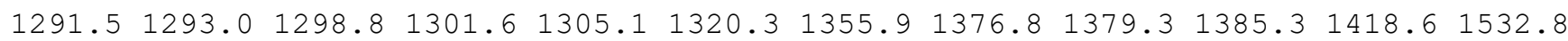

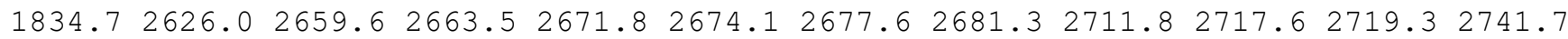
$2764.32777 .12780 .0 \quad 4735.6$

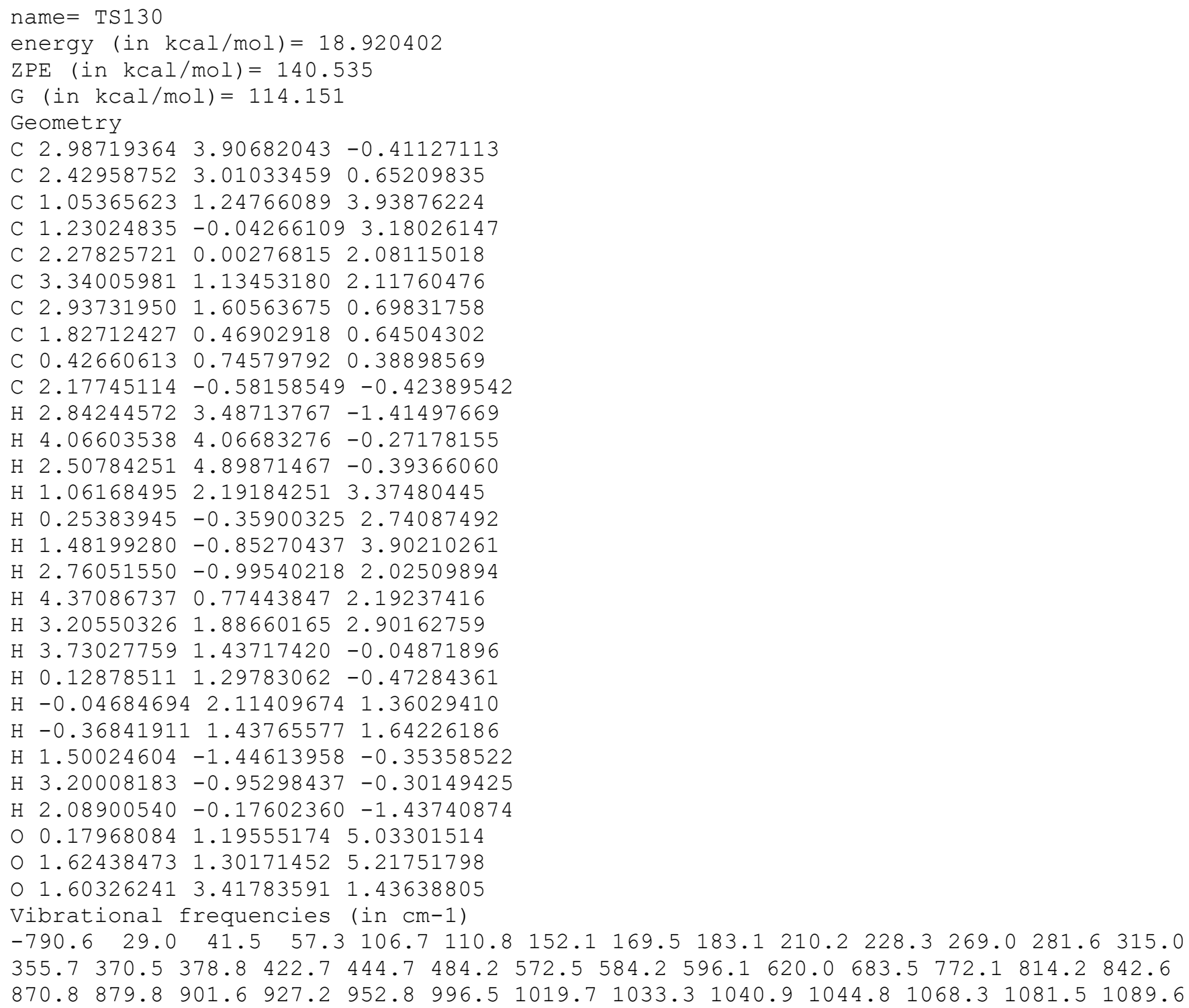




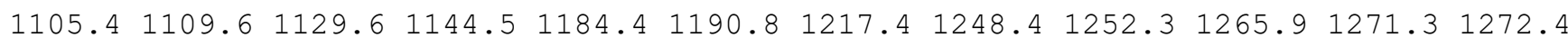
$\begin{array}{lllllllllllll}1288.1 & 1292.3 & 1295.1 & 1299.1 & 1322.6 & 1337.5 & 1357.3 & 1377.9 & 1383.1 & 1392.2 & 1422.6 & 1537.5\end{array}$ $1840.82639 .22663 .62666 .2 \quad 2669.92670 .92676 .82678 .02710 .12711 .72729 .42731 .2$ $2761.0 \quad 2777.7 \quad 2779.1 \quad 4899.3$

name $=$ TS131

energy $($ in $\mathrm{kcal} / \mathrm{mol})=17.385282$

$\mathrm{ZPE}($ in $\mathrm{kcal} / \mathrm{mol})=142.162$

$\mathrm{G}(\mathrm{in} \mathrm{kcal} / \mathrm{mol})=116.833$

Geometry

C $2.68458702 \quad 3.42824972 \quad 4.32268282$

C $2.575194961 .98872025 \quad 3.90803514$

C $0.32918742-1.622648740 .18573938$

C $0.81900982-1.39450941 \quad 1.45041161$

C $1.69042604 \quad 0.44821375 \quad 1.00079772$

C $2.78942379 \quad 0.204612392 .06361763$

C $2.753427591 .70501255 \quad 2.44584252$

C $1.45016278 \quad 1.87815413 \quad 1.56652461$

C $0.19124408 \quad 2.04377981 \quad 2.39747283$

C $1.56375988 \quad 2.960227390 .50889335$

$\mathrm{H} \quad 2.26939948 \quad 4.11258225 \quad 3.57334560$

H $3.73637761 \quad 3.70392296 \quad 4.48137848$

H 2.16191183 3.60804391 5.27410348

$\mathrm{H} \quad 0.42045845-2.49352012-0.44167295$

$\mathrm{H} 1.52456106-2.06546482 \quad 1.91168304$

$\mathrm{H} 0.23584271-0.83088616 \quad 2.18163723$

$\mathrm{H} \quad 1.98876096 \quad 0.33129664 \quad-0.03324968$

H $3.73371601-0.15536318 \quad 1.64867620$

H $2.53892570-0.442155572 .91639186$

H $3.60887106 \quad 2.273710912 .04354164$

$\mathrm{H} 0.06506416 \quad 1.22505303 \quad 3.11844232$

$\mathrm{H}-0.711051492 .04576658 \quad 1.76538812$

$\mathrm{H} \quad 0.19190778 \quad 2.98708148 \quad 2.95414823$

H $2.48970803 \quad 2.88519070 \quad-0.07037957$

H $1.52442215 \quad 3.96263702 \quad 0.94886317$

$\mathrm{H} \quad 0.72811827 \quad 2.88033817 \quad-0.20478191$

$\begin{array}{lllll}0 & -0.15012323 & 0.48111335 & 0.35967585\end{array}$

$0-0.23409064-0.58691618-0.44297664$

o 2.378183931 .115101514 .71525051

Vibrational frequencies (in $\mathrm{cm}-1$ )

$\begin{array}{llllllllllllllllll}-647.1 & 50.0 & 54.4 & 76.0 & 110.1 & 136.8 & 172.0 & 182.0 & 196.8 & 220.0 & 260.0 & 278.2 & 294.0 & 331.8\end{array}$ $354.0 \quad 404.2 \quad 421.4 \quad 447.7 \quad 482.5 \quad 543.1 \quad 573.8 \quad 606.6 \quad 671.9 \quad 713.4 \quad 774.5 \quad 813.6 \quad 834.1924 .6$ $926.5942 .2994 .3 \quad 999.5 \quad 1002.4 \quad 1011.4 \quad 1022.2 \quad 1036.7 \quad 1046.0 \quad 1049.7 \quad 1078.7 \quad 1102.6 \quad 1115.0$ $1135.71143 .41189 .9 \quad 1201.0 \quad 1223.3 \quad 1240.1 \quad 1261.5 \quad 1273.1 \quad 1278.4 \quad 1284.4 \quad 1290.01293 .1$ $1297.71301 .91307 .91319 .5 \quad 1331.1 \quad 1336.4 \quad 1380.8 \quad 1392.7 \quad 1400.2 \quad 1409.8 \quad 1620.8 \quad 1853.6$ $2659.12669 .2 \quad 2669.3 \quad 2669.7 \quad 2674.3 \quad 2675.6 \quad 2682.0 \quad 2682.7 \quad 2713.8 \quad 2728.92740 .2 \quad 2755.0$ $2766.32776 .0 \quad 2780.12781 .1$

name $=$ TS132

energy $($ in $\mathrm{kcal} / \mathrm{mol})=15.702516$

$\mathrm{ZPE}(\mathrm{in} \mathrm{kcal} / \mathrm{mol})=144.192$

$\mathrm{G}($ in $\mathrm{kcal} / \mathrm{mol})=121.079$

Geometry

C 1.789316163 .000190463 .40187397

C $2.27483308 \quad 1.83692399 \quad 2.61716992$

C $0.82794257 \quad 0.52383704 \quad 2.73004530$

C $0.78797554 \quad-0.23916390 \quad 1.40396668$

C $1.56278178 \quad 0.39300560 \quad 0.25100787$

C 3.060849360 .550664120 .65641923

C $2.69160788 \quad 1.97740962 \quad 1.18778096$

C $1.462199381 .96367105 \quad 0.18852847$

C $0.15888820 \quad 2.63946823 \quad 0.55155896$

C $1.895190452 .49303508-1.18189212$ 
$\mathrm{H} 1.00163057 \quad 3.566848852 .88830753$

$\mathrm{H} 2.61211547 \quad 3.70643299 \quad 3.60028613$

H $1.38900298 \quad 2.69311423 \quad 4.38508654$

$\mathrm{H} \quad 0.01770074 \quad 1.24985006 \quad 2.88127493$

$\mathrm{H}-0.27471258-0.36563026 \quad 1.11566702$

H $1.17248530-1.268013581 .57604226$

$\mathrm{H} 1.37371513-0.11791720-0.69849003$

H $3.75826191 \quad 0.55845842-0.18415644$

H $3.43256746-0.17734595 \quad 1.37871352$

H $3.419937792 .77510444 \quad 1.01531562$

$\mathrm{H}-0.26314544 \quad 2.31506557 \quad 1.50761442$

$\mathrm{H}-0.60808726 \quad 2.42915950-0.20719932$

$\mathrm{H} \quad 0.27833063 \quad 3.72923648 \quad 0.60191082$

H $2.03602598 \quad 3.57955433-1.15890866$

H $1.138111532 .27378530-1.94391602$

H $2.835715402 .05204531-1.52854530$

$\begin{array}{lllll}0 & 2.71329075 & 0.59268233 & 4.36871178\end{array}$

O $1.15183223-0.137751013 .77932650$

$\begin{array}{llll}0 & 3.14999721 & 0.96843505 & 3.24015165\end{array}$

Vibrational frequencies (in $\mathrm{cm}-1$ )

$\begin{array}{lllllllllllllll}-685.0 & 109.5 & 133.0 & 156.5 & 168.8 & 182.7 & 195.2 & 226.2 & 264.5 & 272.3 & 307.6 & 336.9 & 360.9 & 388.1\end{array}$

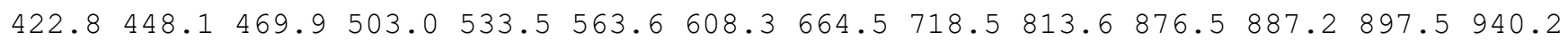
$990.1992 .2999 .5 \quad 1013.3 \quad 1016.9 \quad 1019.8 \quad 1050.1 \quad 1064.5 \quad 1073.8 \quad 1083.1 \quad 1089.91117 .6 \quad 1140.3$ $1188.01199 .6 \quad 1203.1 \quad 1213.2 \quad 1255.8 \quad 1263.1 \quad 1268.4 \quad 1277.2 \quad 1278.7 \quad 1285.7 \quad 1291.1 \quad 1294.3$

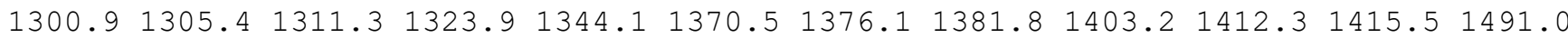
$2630.12655 .52660 .0 \quad 2666.5 \quad 2669.4 \quad 2674.4 \quad 2675.3 \quad 2679.62684 .2 \quad 2736.5 \quad 2737.82742 .4$ 2763.22773 .12778 .62782 .4

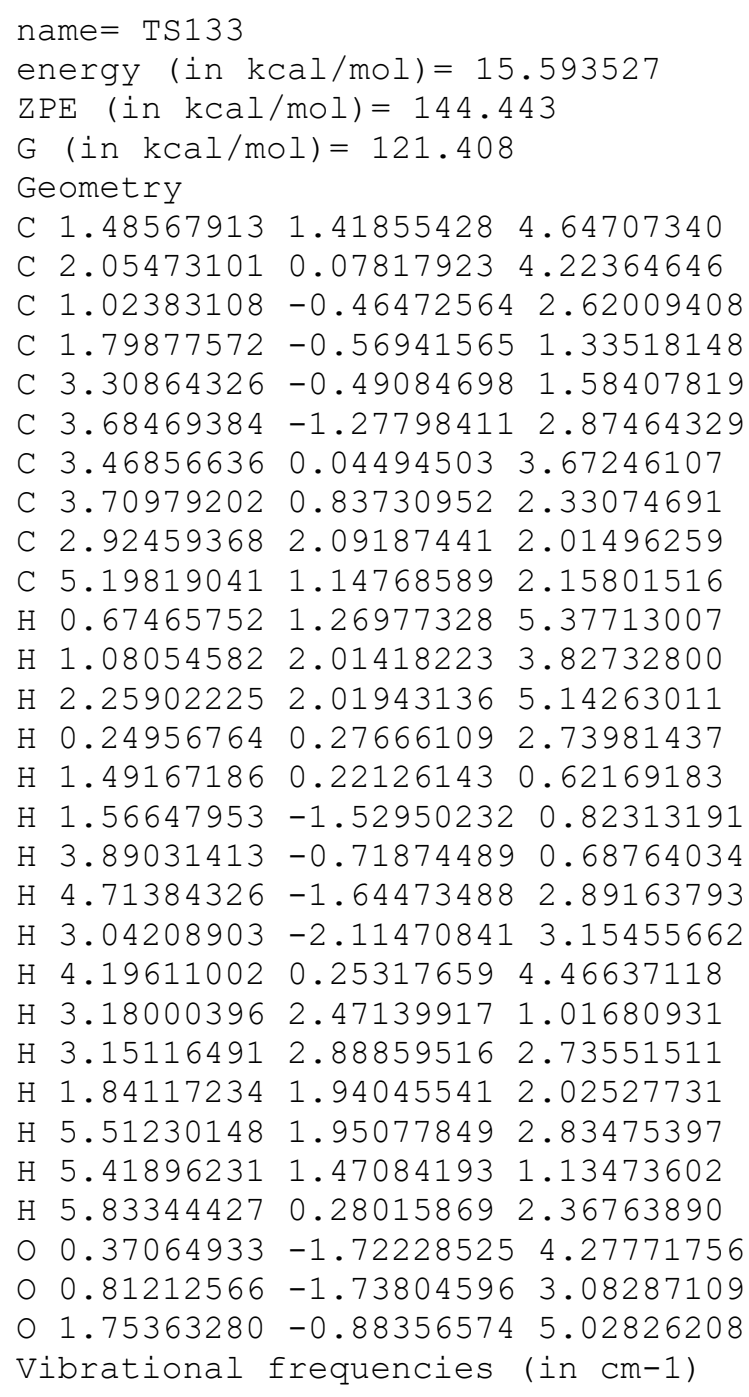


$\begin{array}{lllllllllllllllllll}-724.2 & 97.8 & 107.0 & 172.6 & 183.1 & 194.2 & 215.0 & 250.0 & 255.7 & 295.9 & 332.1 & 356.3 & 368.4 & 389.7\end{array}$ $427.5 \quad 452.9 \quad 482.7 \quad 517.5 \quad 541.3 \quad 573.2 \quad 618.9 \quad 653.6 \quad 721.5 \quad 813.5 \quad 852.1 \quad 895.6 \quad 918.0 \quad 929.3$

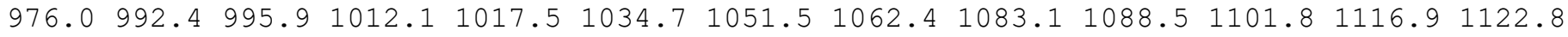

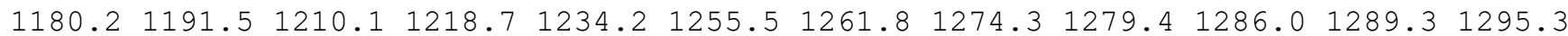
$1301.7 \quad 1302.6 \quad 1315.91324 .3 \quad 1326.3 \quad 1347.1 \quad 1372.1 \quad 1388.8 \quad 1392.2 \quad 1404.6 \quad 1413.7 \quad 1481.2$ $2654.72669 .62671 .82674 .5 \quad 2675.6 \quad 2676.8 \quad 2680.62684 .32711 .3 \quad 2734.4 \quad 2735.52744 .7$ $2765.22778 .12781 .4 \quad 2782.8$

name $=$ TS134

energy (in $\mathrm{kcal} / \mathrm{mol})=16.900373$

$\operatorname{ZPE}($ in $\mathrm{kcal} / \mathrm{mol})=144.304$

$\mathrm{G}($ in $\mathrm{kcal} / \mathrm{mol})=121.071$

Geometry

C $-0.94779018 \quad 1.68160057 \quad 4.13045782$

C $-0.22552140 \quad 0.72711076 \quad 3.22330621$

C $-1.37596957-0.13785350 \quad 1.84281083$

C $-0.98288321 \quad 0.32386043 \quad 0.43490090$

C $0.38491820 \quad 0.99154893 \quad 0.35160899$

C $1.36722654 \quad 0.25335561 \quad 1.30513652$

C $0.91988411 \quad 1.24168149 \quad 2.42609718$

C $0.48285073 \quad 2.25276786 \quad 1.28491475$

C $-0.76105962 \quad 3.10015724 \quad 1.44708263$

C $1.64737110 \quad 3.16258807 \quad 0.89786415$

$\mathrm{H}-1.13043971 \quad 1.24837391 \quad 5.12820459$

$\mathrm{H}-1.93509577 \quad 1.95803649 \quad 3.73435613$

$\mathrm{H}-0.38428958 \quad 2.61309034 \quad 4.28147811$

$\mathrm{H}-2.30317072 \quad 0.27225490 \quad 2.25072325$

$\mathrm{H}-1.76215504 \quad 0.99293619 \quad 0.02745062$

$\mathrm{H}-0.98247011-0.57385170-0.22280263$

$\mathrm{H} \quad 0.71977913 \quad 1.14213243 \quad-0.67788560$

H 2.42065266 $0.34512815 \quad 1.03216073$

H $1.16182004 \quad-0.80921980 \quad 1.46270459$

$\mathrm{H} 1.72753668 \quad 1.60932965 \quad 3.07566014$

$\mathrm{H}-0.98644811 \quad 3.63458977 \quad 0.51381042$

$\mathrm{H}-0.63263597 \quad 3.85533018 \quad 2.23134372$

$\mathrm{H}-1.65211855 \quad 2.51434174 \quad 1.69340395$

H $1.83582895 \quad 3.91586500 \quad 1.67080545$

$\mathrm{H} 1.43725488 \quad 3.69420136-0.03769100$

H 2.58032050 2.60736985 0.74911525

○ $0.19729922-1.44587358 \quad 3.24690358$

O $-1.16455003-1.38192484 \quad 2.08188242$

o $-0.14209076-0.442574013 .94446031$

Vibrational frequencies (in $\mathrm{cm}-1$ )

$\begin{array}{lllllllllllllll}-710.5 & 85.9 & 95.7 & 159.8 & 175.2 & 193.9 & 203.5 & 248.4 & 262.9 & 291.4 & 303.5 & 364.4 & 384.8 & 392.9\end{array}$

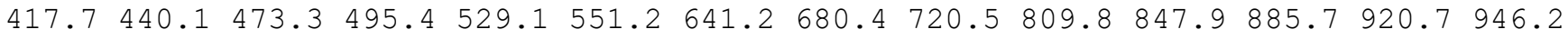
$988.4995 .6 \quad 1005.2 \quad 1005.8 \quad 1011.91018 .2 \quad 1037.9 \quad 1058.5 \quad 1076.8 \quad 1087.3 \quad 1093.01120 .6$ $1141.01187 .0 \quad 1194.6 \quad 1200.6 \quad 1224.5 \quad 1249.2 \quad 1258.4 \quad 1264.4 \quad 1274.1 \quad 1276.6 \quad 1286.4 \quad 1292.6$ $1299.51301 .91312 .7 \quad 1318.0 \quad 1321.5 \quad 1329.2 \quad 1365.3 \quad 1378.8 \quad 1395.2 \quad 1405.0 \quad 1405.5 \quad 1412.4$ $1487.22655 .72655 .8 \quad 2665.3 \quad 2671.4 \quad 2672.12675 .92677 .4 \quad 2678.72681 .02725 .02738 .4$ $2745.5 \quad 2761.9 \quad 2772.1 \quad 2781.2 \quad 2782.9$

name $=\operatorname{TS} 135$

energy (in $\mathrm{kcal} / \mathrm{mol})=21.605162$

$\mathrm{ZPE}(\mathrm{in} \mathrm{kcal} / \mathrm{mol})=140.242$

$\mathrm{G}($ in $\mathrm{kcal} / \mathrm{mol})=114.735$

Geometry

C $2.37897344 \quad 2.942405835 .73108630$

C $2.549694192 .01218735 \quad 4.78984341$

C $1.198521251 .98821181 \quad 0.98629855$

C $2.56719810 \quad 2.20357732 \quad 0.65911493$

C $3.67315160 \quad 2.01627690 \quad 1.61973060$

C $3.51437979 \quad 0.88667248 \quad 2.67459677$

C $3.687339241 .90518916 \quad 3.83590348$ 
C $3.81802467 \quad 3.07474699 \quad 2.78358104$

C $2.73257976 \quad 4.13048788 \quad 2.82982390$

C $5.19427857 \quad 3.72458099 \quad 2.81565654$

$\mathrm{H} 3.06673813 \quad 3.74914273 \quad 5.90305065$

$\mathrm{H} 1.52541080 \quad 2.95587855 \quad 6.39065708$

$\mathrm{H}-0.00711648 \quad 1.48795510 \quad 4.13738565$

$\mathrm{H} \quad 0.81967988 \quad 1.58287060 \quad-0.22902646$

$\mathrm{H} \quad 2.79641430 \quad 2.91439575-0.13358698$

$\mathrm{H} \quad 1.812519051 .25504432-0.33928315$

H $4.62484432 \quad 1.91179308 \quad 1.05771275$

H $4.29115979 \quad 0.11997661 \quad 2.63582196$

H 2.54607652 $0.37701545 \quad 2.64361311$

$\mathrm{H} \quad 4.62753597 \quad 1.74713800 \quad 4.39956155$

$\mathrm{H} 1.73036280 \quad 3.68447301 \quad 2.89629345$

H $2.75476195 \quad 4.76414531 \quad 1.93678997$

H $2.84331868 \quad 4.78225481 \quad 3.70588377$

H $6.00175791 \quad 2.98566407 \quad 2.76800960$

$\mathrm{H} 5.33570450 \quad 4.30489802 \quad 3.73591140$

$\mathrm{H} 5.32693703 \quad 4.410996291 .97145242$

○ 0.388263961 .296109535 .02829705

O $0.49548899 \quad 1.68166775 \quad 1.89661327$

o $1.703713310 .94591457 \quad 4.59733969$

Vibrational frequencies (in cm-1)

$\begin{array}{lllllllllllllllll}-2553.4 & 43.8 & 61.7 & 70.5 & 93.2 & 142.6 & 176.7 & 186.3 & 199.1 & 219.1 & 245.0 & 254.3 & 307.2 & 318.2\end{array}$ $352.1 \quad 377.4 \quad 408.2 \quad 424.4 \quad 437.2 \quad 487.6 \quad 547.7 \quad 583.9 \quad 602.3 \quad 635.5 \quad 669.1 \quad 703.9762 .6 \quad 825.0$ $844.2924 .4938 .3 \quad 960.5 \quad 996.2 \quad 1005.6 \quad 1007.5 \quad 1016.7 \quad 1018.9 \quad 1050.9 \quad 1063.0 \quad 1064.5 \quad 1102.8$

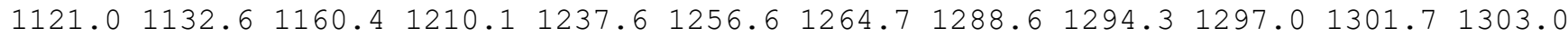
$1308.8 \quad 1315.8 \quad 1343.5 \quad 1352.6 \quad 1367.6 \quad 1372.9 \quad 1383.3 \quad 1403.7 \quad 1404.9 \quad 1416.6 \quad 1819.4 \quad 1863.9$ $1960.22520 .12668 .4 \quad 2677.0 \quad 2678.8 \quad 2681.4 \quad 2682.5 \quad 2714.52720 .5 \quad 2730.72745 .8 \quad 2762.5$ $2780.92782 .7 \quad 2796.12849 .4$

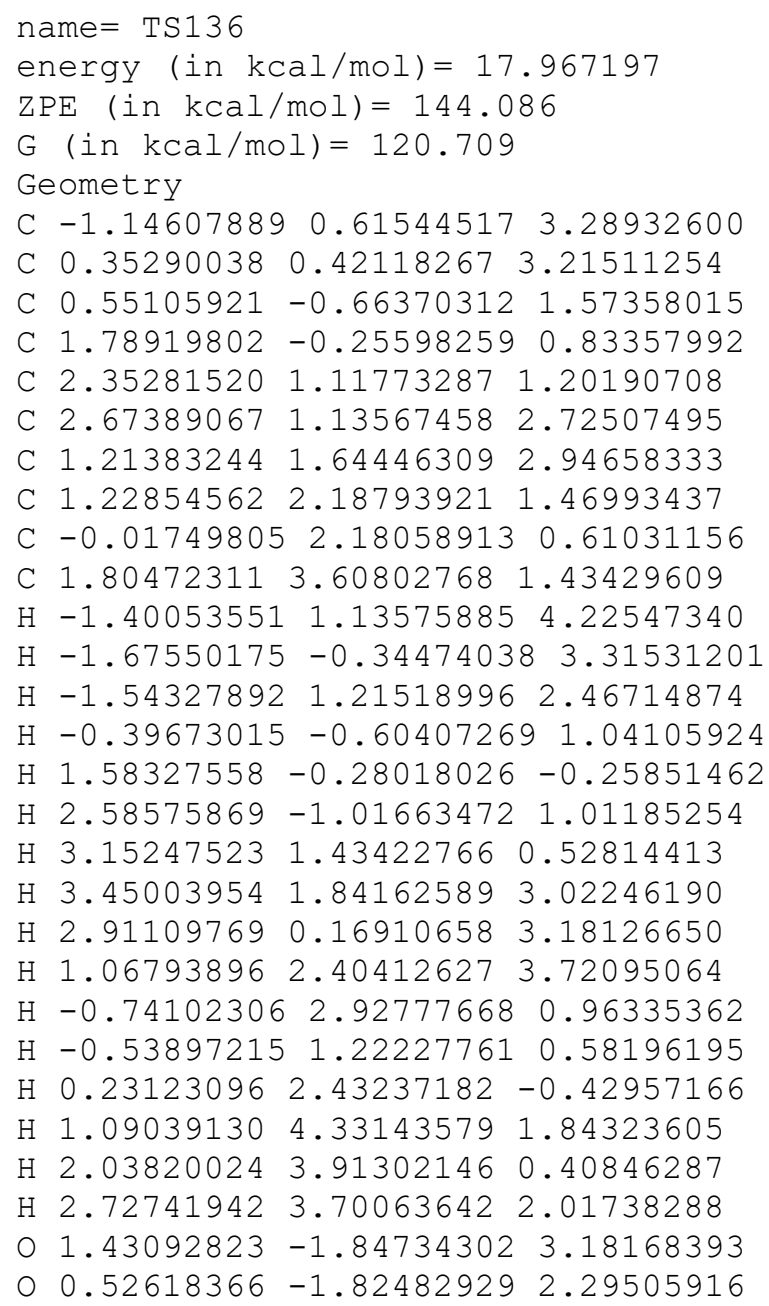


$0.84246558-0.382628304 .09263810$

Vibrational frequencies (in $\mathrm{cm}-1$ )

$\begin{array}{lllllllllllllll}-713.5 & 88.2 & 110.0 & 136.9 & 160.7 & 182.1 & 203.4 & 207.8 & 251.4 & 283.3 & 290.5 & 341.5 & 375.2 & 390.8\end{array}$ $400.1438 .2 \quad 506.7 \quad 520.2 \quad 545.2 \quad 569.2 \quad 609.6 \quad 690.9 \quad 710.0 \quad 820.4 \quad 867.7 \quad 891.8 \quad 902.0 \quad 942.5$ $956.1989 .0 \quad 996.2 \quad 1011.3 \quad 1016.2 \quad 1030.4 \quad 1043.5 \quad 1059.8 \quad 1075.2 \quad 1082.3 \quad 1095.01103 .51145 .3$ $\begin{array}{llllllllllllllll}1175.4 & 1192.6 & 1205.7 & 1218.7 & 1233.3 & 1246.1 & 1260.7 & 1277.2 & 1282.3 & 1285.2 & 1288.3 & 1293.5\end{array}$

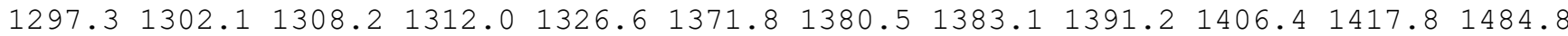
$2649.52669 .02671 .02674 .5 \quad 2675.92680 .7 \quad 2682.92684 .02700 .92726 .12739 .02745 .2$ $2762.92780 .8 \quad 2782.2 \quad 2784.2$

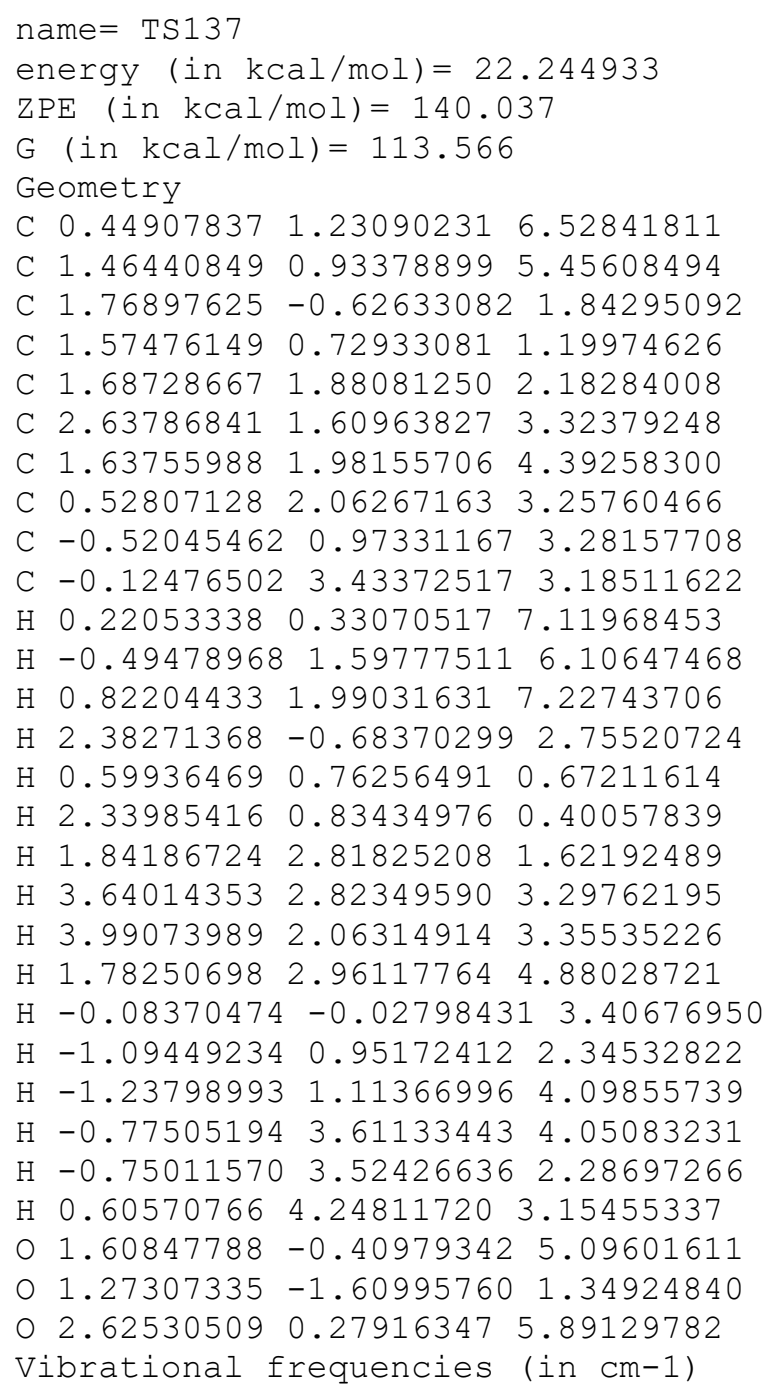


C $1.06764733 \quad 0.41774401 \quad 1.14801716$

C $-0.16516550 \quad 1.27069592 \quad 0.75127249$

C -1.120498850 .051616261 .06574218$

C $-2.05369472 \quad 0.25882014 \quad 2.23829928$

C $-1.88336272-0.42314386-0.15730659$

$\mathrm{H}-2.04911634 \quad 3.72389293 \quad 2.12767070$

$\mathrm{H}-2.19299444 \quad 3.09020001 \quad 0.45949178$

$\mathrm{H}-1.05277008 \quad 4.40485862 \quad 0.85929131$

$\mathrm{H} \quad 0.59442490 \quad-0.19449071 \quad 4.39912087$

$\mathrm{H}-0.63315796-2.911046632 .56426544$

$\mathrm{H} \quad 0.12627515-3.13701892 \quad 2.56700016$

$\mathrm{H} \quad 0.31394323-1.589241620 .57036068$

H $1.80432537 \quad 0.28706323 \quad 0.34871618$

$\mathrm{H} 1.61492350 \quad 0.74638573 \quad 2.03662164$

$\mathrm{H}-0.16553097 \quad 1.52042202-0.33154550$

$\mathrm{H}-2.86122934 \quad 0.96143011 \quad 1.99404493$

$\mathrm{H}-1.54107185 \quad 0.65871534 \quad 3.12314620$

$\mathrm{H}-2.52717874 \quad-0.683429462 .54630176$

$\mathrm{H}-2.63800654 \quad 0.31115694-0.46814422$

$\mathrm{H}-2.41578322-1.361798550 .04347772$

$\mathrm{H}-1.22959102-0.59801585-1.01937572$

O $0.19395922 \quad 3.70074023 \quad 3.26290448$

$0-0.28021222-1.964182094 .81824062$

O 0.377670472 .667691122 .55465238

Vibrational frequencies (in cm-1)

$\begin{array}{llllllllllllllll}-537.5 & 26.5 & 38.6 & 70.1 & 100.4 & 105.6 & 126.6 & 157.8 & 184.6 & 201.1 & 218.5 & 240.2267 .1270 .6\end{array}$ $289.8 \quad 317.8 \quad 365.9 \quad 371.7 \quad 405.8 \quad 421.5 \quad 439.8 \quad 491.7 \quad 550.1 \quad 561.8 \quad 671.7 \quad 683.8 \quad 710.0 \quad 809.2$ $835.1872 .1914 .6 \quad 916.2 \quad 936.0 \quad 995.1 \quad 1005.1 \quad 1011.6 \quad 1015.5 \quad 1031.5 \quad 1044.4 \quad 1061.5 \quad 1082.9$

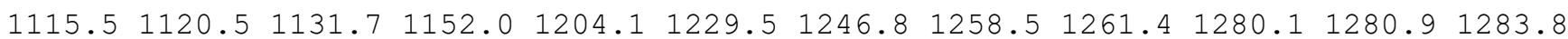

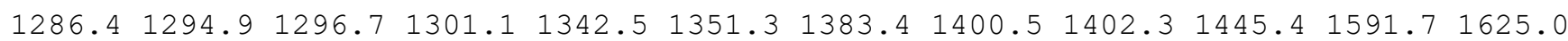
$1771.02633 .4 \quad 2636.4 \quad 2667.5 \quad 2670.92672 .92674 .82675 .92676 .0 \quad 2707.12714 .52759 .0$ $2768.62780 .12782 .5 \quad 5034.9$

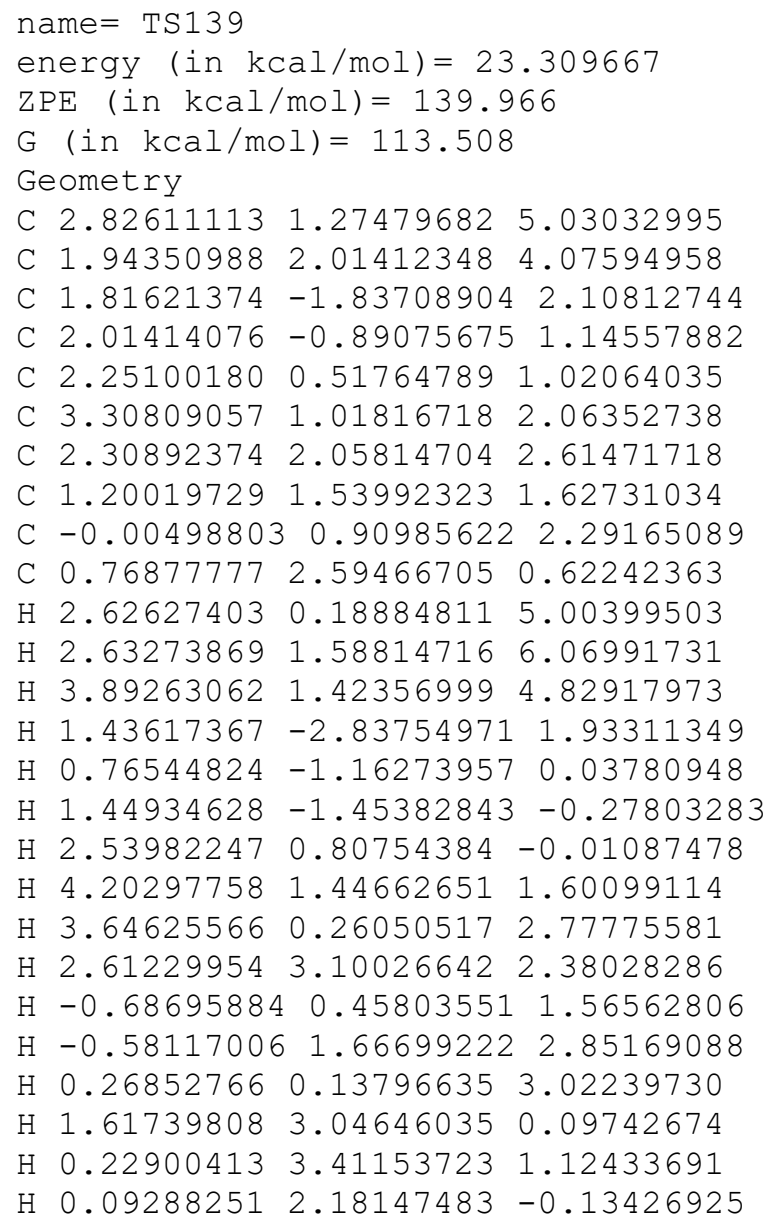


O $2.13935196-2.43332953 \quad 4.21938931$

○ $2.20338908-1.518951103 .34680937$

○ $0.96595770 \quad 2.60781156 \quad 4.46331101$

Vibrational frequencies (in $\mathrm{cm}-1$ )

$\begin{array}{llllllllllllllll}-864.7 & 28.7 & 45.0 & 65.0 & 111.0 & 121.9 & 154.5 & 162.4 & 184.5 & 193.3 & 218.4 & 229.6 & 247.7 & 288.6\end{array}$ $307.0 \quad 348.6 \quad 353.9 \quad 400.1 \quad 442.6 \quad 475.5 \quad 511.8 \quad 535.5 \quad 553.7 \quad 599.9 \quad 640.5 \quad 660.5 \quad 728.8 \quad 802.8$ $810.4921 .6 \quad 935.8 \quad 946.7 \quad 976.6 \quad 995.4 \quad 1004.9 \quad 1012.8 \quad 1016.8 \quad 1038.4 \quad 1045.0 \quad 1055.2 \quad 1078.6$

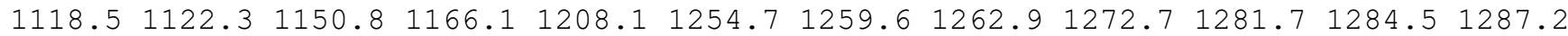

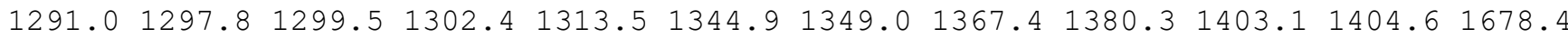
1837.32645 .92656 .02670 .52673 .62673 .92678 .12681 .02710 .62717 .72720 .92758 .3 $2775.2 \quad 2778.7 \quad 2781.8 \quad 4797.3$

name $=\operatorname{TS} 140$

energy $($ in $\mathrm{kcal} / \mathrm{mol})=23.10594$

$\mathrm{ZPE}($ in $\mathrm{kcal} / \mathrm{mol})=140.274$

$\mathrm{G}($ in $\mathrm{kcal} / \mathrm{mol})=114.325$

Geometry

C $0.11118630 \quad 5.40834644 \quad 3.16216499$

C $1.25364734 \quad 4.62139585 \quad 3.73832991$

C $1.98893286 \quad 0.80958092 \quad 4.24762610$

C $2.96935277 \quad 0.80850229 \quad 3.10061412$

C $3.187415262 .15739302 \quad 2.44602358$

C 3.480483083 .358900563 .38074948

C $2.30384061 \quad 4.15721075 \quad 2.76961693$

C $1.93647413 \quad 2.92923804 \quad 1.85050312$

C $0.57706654 \quad 2.28111887 \quad 2.09630681$

$\begin{array}{lllll}\text { C } 1.94362741 & 3.23961423 & 0.42884541\end{array}$

$\mathrm{H}-0.167783475 .04654122 \quad 2.16039693$

$\mathrm{H} 0.37242876 \quad 6.47080658 \quad 3.06494686$

$\mathrm{H}-0.78237376 \quad 5.35080765 \quad 3.80094835$

$\mathrm{H} 2.00186459 \quad 1.66178795 \quad 4.93915037$

H 2.64214476 $0.06917954 \quad 2.33609032$

H $3.93896174 \quad 0.41556128 \quad 3.48150383$

H $3.97280365 \quad 2.042901331 .67368192$

$\mathrm{H} \quad 4.46272403 \quad 3.816890253 .24226958$

$\mathrm{H} \quad 3.37341021 \quad 3.15386677 \quad 4.45251911$

$\mathrm{H} 2.63932237 \quad 5.02827743 \quad 2.17367425$

$\mathrm{H}-0.22850390 \quad 2.82936728 \quad 1.58503162$

$\mathrm{H} \quad 0.31203144 \quad 2.24865831 \quad 3.15961759$

$\mathrm{H} \quad 0.55413453 \quad 1.24761295 \quad 1.72576240$

H $3.54567912 \quad 3.61592615 \quad 0.09276987$

H $3.06602532 \quad 4.26749938 \quad 0.03267681$

H $1.90288203 \quad 2.48947012 \quad-0.31998578$

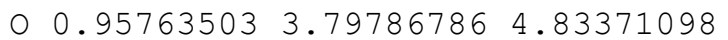

O $1.23015372-0.11388626 \quad 4.41062170$

o $1.71377931 \quad 5.03723463 \quad 4.99863705$

Vibrational frequencies (in $\mathrm{cm}-1$ )

$\begin{array}{lllllllllllllll}-1000.3 & 43.0 & 47.5 & 63.4 & 88.3 & 120.8 & 165.5 & 178.9 & 192.7 & 227.3 & 243.4 & 269.6 & 285.5 & 310.1\end{array}$ $316.6 \quad 337.9 \quad 386.5 \quad 407.1 \quad 439.0 \quad 501.9 \quad 534.0 \quad 543.7 \quad 587.3 \quad 644.1678 .4 \quad 747.9 \quad 767.0 \quad 814.9$ $857.3 \quad 879.2 \quad 913.9936 .5 \quad 938.4 \quad 1011.9 \quad 1017.2 \quad 1041.2 \quad 1047.7 \quad 1060.8 \quad 1071.0 \quad 1088.6 \quad 1103.7$

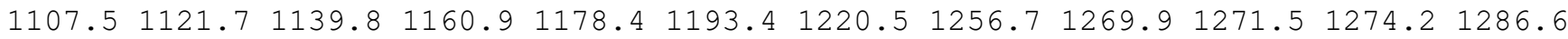

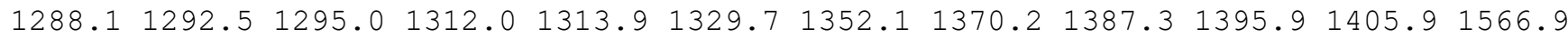
1832.22640 .62656 .52662 .52666 .12668 .92671 .82677 .82712 .32720 .12727 .52737 .2 $2762.6 \quad 2776.32778 .0 \quad 4732.6$

name $=\operatorname{TS} 141$

energy $($ in $\mathrm{kcal} / \mathrm{mol})=23.42184$

$\operatorname{ZPE}($ in $\mathrm{kcal} / \mathrm{mol})=140.03$

$\mathrm{G}($ in $\mathrm{kcal} / \mathrm{mol})=114.314$

Geometry

C $0.31068195 \quad 2.13631813 \quad 5.04944481$

C $1.01667716 \quad 0.96633049 \quad 4.47162349$

C $1.81541268-2.95983690 \quad 0.84038632$ 
C $2.34492544-1.93980611 \quad 1.64020423$

C $2.23919145-0.525434061 .69730996$

C $2.90389648 \quad 0.23089724 \quad 2.87839851$

C $1.60157317 \quad 1.03922972 \quad 3.13239843$

C $0.87154557 \quad 0.21025737 \quad 1.99606503$

C $-0.25991569-0.68038000 \quad 2.46010173$

C $0.417872601 .08899251 \quad 0.84204088$

$\mathrm{H}-0.16816663 \quad 1.92889011 \quad 6.01948929$

$\mathrm{H}-0.48179998 \quad 2.49639439 \quad 4.37047730$

$\mathrm{H} 1.00380927 \quad 2.98066126 \quad 5.20811214$

$\mathrm{H} \quad 1.53344502-3.92688001 \quad 1.28100240$

H $2.53382326-2.55690503 \quad 3.30490663$

H $1.74606715-2.52506677 \quad 3.25743921$

$\mathrm{H} \quad 2.65680977-0.182802420 .70052632$

H $3.76591891 \quad 0.84233164 \quad 2.60173736$

H $3.21211795-0.418577713 .70960223$

$\mathrm{H} 1.72337963 \quad 2.09959619 \quad 2.82250608$

$\mathrm{H}-0.59383171-1.34324236 \quad 1.64901784$

$\mathrm{H}-1.13095056-0.09769828 \quad 2.77990341$

$\mathrm{H} \quad 0.02999104 \quad-1.32411908 \quad 3.30314712$

$\mathrm{H} \quad 0.11656723 \quad 0.47452147 \quad-0.01973109$

H $1.20728323 \quad 1.76339651 \quad 0.49175770$

$\mathrm{H}-0.44495130 \quad 1.70570696 \quad 1.11730355$

O $1.56841194-1.12729193 \quad 4.96574615$

$\begin{array}{llll}0 & 1.78722681 & -2.82884574 & -0.38047857\end{array}$

O $1.02134903-0.033473645 .30346099$

Vibrational frequencies (in cm-1)

$\begin{array}{lllllllllllllllll}-530.7 & 45.5 & 72.7 & 83.1 & 123.8 & 136.1 & 150.9 & 185.4 & 198.4 & 219.7 & 223.9 & 251.8 & 278.5 & 299.7\end{array}$

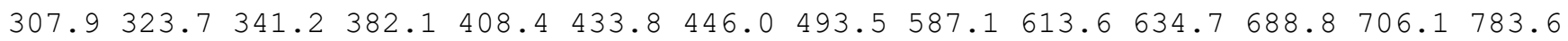
$804.2816 .9909 .4 \quad 931.4 \quad 940.5 \quad 990.0 \quad 1002.9 \quad 1010.9 \quad 1016.6 \quad 1034.2 \quad 1042.6 \quad 1066.8 \quad 1088.5$

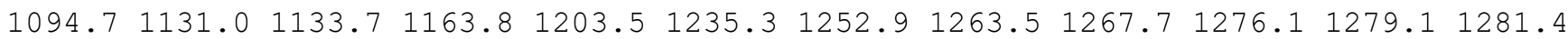

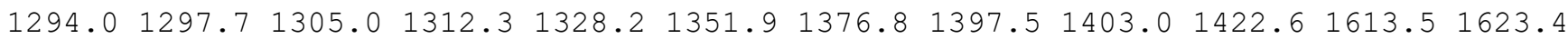
$1728.52647 .7 \quad 2653.2 \quad 2656.8 \quad 2659.6 \quad 2662.1 \quad 2668.3 \quad 2672.42675 .12679 .12714 .32755 .5$ $2769.62776 .7 \quad 2780.6 \quad 5069.4$

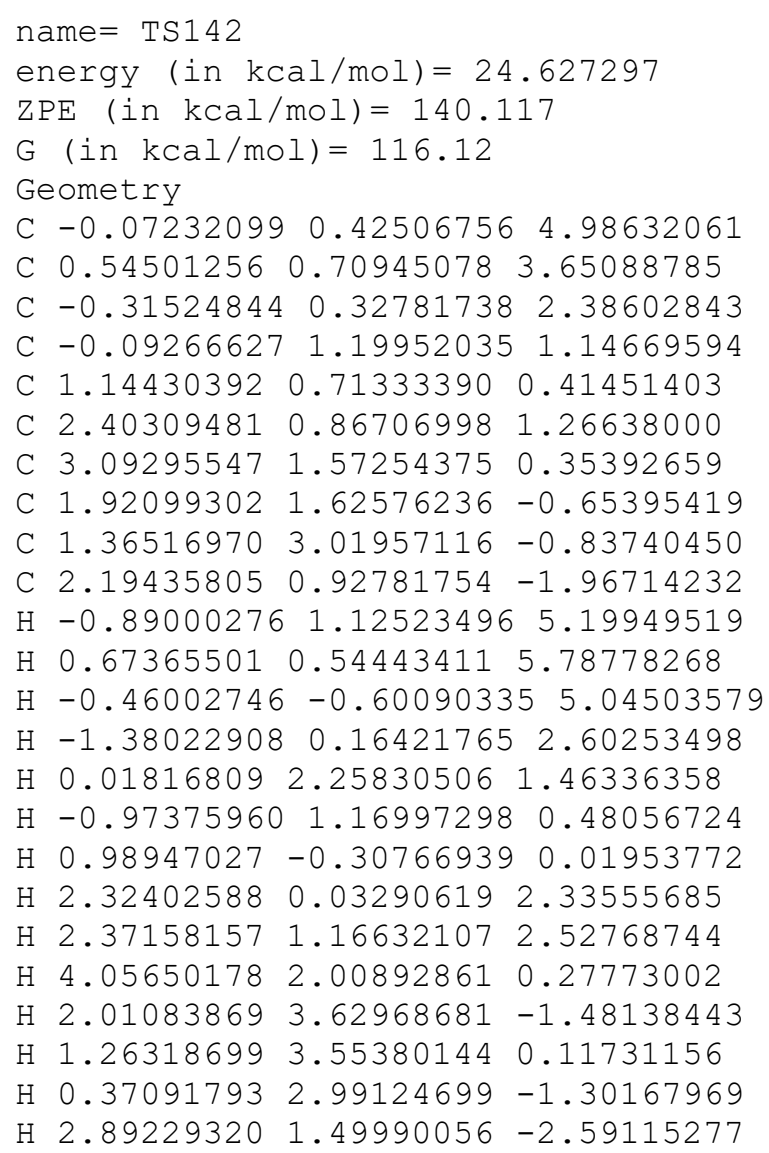


$\mathrm{H} \quad 1.27144641 \quad 0.79582325-2.54678586$

$\mathrm{H} 2.63117241-0.06871155-1.82139153$

$01.37985746-0.690833873 .19005587$

$0.32646940-0.950233412 .23553062$

O $1.426067771 .61681423 \quad 3.56354597$

Vibrational frequencies (in $\mathrm{cm}-1$ )

$\begin{array}{lllllllllllllllllll}-1865.9 & 81.4 & 114.5 & 122.9 & 156.7 & 164.1 & 174.4 & 210.7 & 219.1 & 232.2 & 259.3 & 304.4 & 323.1 & 345.7\end{array}$ $361.2400 .8 \quad 451.3 \quad 463.6 \quad 504.8 \quad 527.8 \quad 572.4 \quad 624.4 \quad 701.1 \quad 721.5 \quad 800.2 \quad 837.9 \quad 869.9895 .4$

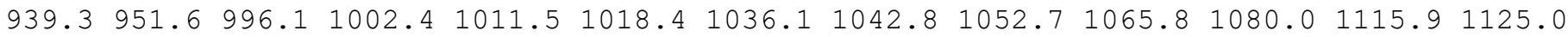

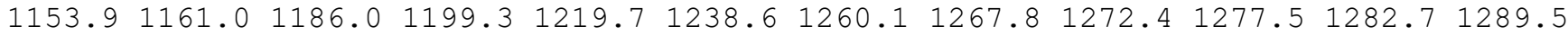

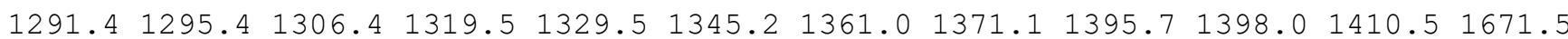
$1759.42069 .3 \quad 2660.7 \quad 2669.8 \quad 2672.1 \quad 2674.3 \quad 2674.72678 .42678 .6 \quad 2706.4 \quad 2717.3 \quad 2742.9$ $2779.82780 .5 \quad 2781.7 \quad 2785.3$

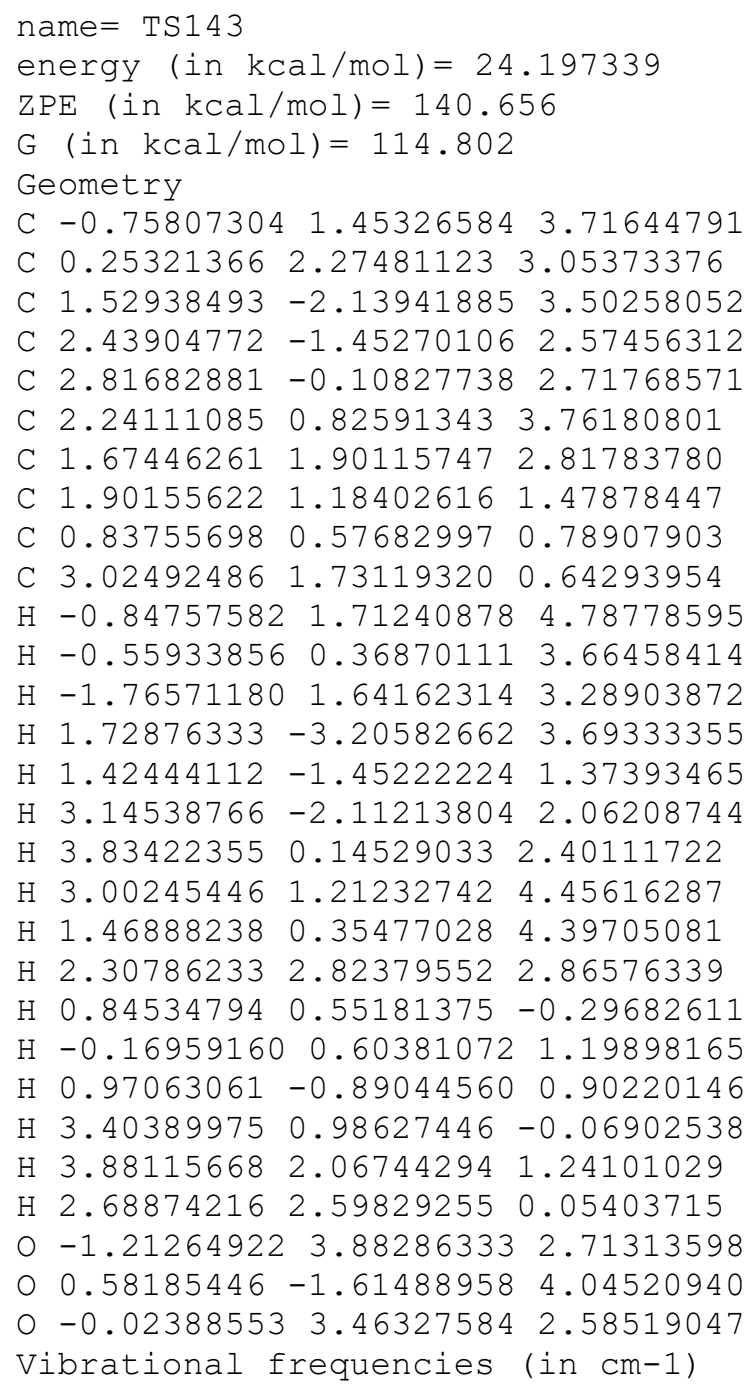


C $-0.00672491 \quad 1.51258404 \quad 3.51147153$

C $1.64473989-1.20950350 \quad 0.77438836$

C $2.74687711-0.20573303 \quad 0.53035392$

C $2.849116420 .84652185 \quad 1.61707871$

C $2.36312586 \quad 0.41530001 \quad 3.03871768$

C $1.26728857 \quad 1.41190805 \quad 2.92491192$

C $1.74002300 \quad 2.00451088 \quad 1.60099311$

C $0.61791030 \quad 1.93727532 \quad 0.55110373$

C $2.26848344 \quad 3.33568285 \quad 1.59019385$

$\mathrm{H}-0.43853648-0.41708060 \quad 4.35355986$

$\mathrm{H}-1.20582199 \quad 0.92827822 \quad 5.21240076$

$\mathrm{H} \quad 0.53071766 \quad 0.61586900 \quad 5.41915669$

$\mathrm{H} \quad 0.67118592-0.82659040 \quad 1.11088419$

$\mathrm{H} \quad 2.60117606 \quad 0.26994213-0.46242891$

H $3.71847505-0.746692520 .44907129$

$\mathrm{H} \quad 3.86457741 \quad 1.27712316 \quad 1.63724478$

H $3.08732703 \quad 0.63621655 \quad 3.83630123$

$\mathrm{H} 2.07903778-0.63544674 \quad 3.15682625$

$\mathrm{H} 1.88987648 \quad 3.408361493 .89837061$

$\mathrm{H}-0.21616693 \quad 2.60552790 \quad 0.83867944$

$\mathrm{H} \quad 0.17218055 \quad 0.93871020 \quad 0.48994115$

H $0.97336826 \quad 2.21237600-0.44536589$

$\mathrm{H} \quad 1.63983851 \quad 4.18118270 \quad 1.33620253$

H $3.33003200 \quad 3.52690013 \quad 1.64286381$

H $2.17253690 \quad 4.01109402 \quad 3.50605924$

o $1.61275451-2.31231247-0.08611356$

$\begin{array}{llll}0 & 2.02980697 & -2.44547782 & 1.30796718\end{array}$

o - $0.865916552 .29635068 \quad 3.12400686$

Vibrational frequencies (in cm-1)

$\begin{array}{lllllllllllllll}-126.9 & 9.1 & 46.5 & 65.3 & 74.5 & 91.7 & 109.0 & 129.9 & 152.5 & 188.4 & 208.3 & 250.6 & 267.0 & 283.2\end{array}$ $296.5 \quad 321.4 \quad 364.7 \quad 370.1 \quad 401.2 \quad 425.5 \quad 458.6 \quad 495.3 \quad 538.6 \quad 583.9 \quad 600.8 \quad 643.2 \quad 658.7 \quad 718.2$

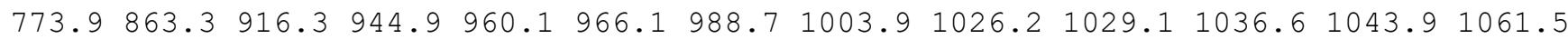
$1073.3 \quad 1098.21110 .5 \quad 1137.4 \quad 1173.1 \quad 1192.3 \quad 1212.4 \quad 1240.2 \quad 1263.3 \quad 1269.6 \quad 1273.5 \quad 1279.5$

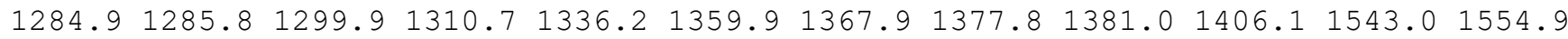
$1760.32640 .72650 .92667 .6 \quad 2673.3 \quad 2676.12677 .42684 .92686 .42711 .62718 .62737 .2$ $2756.92770 .6 \quad 2783.4 \quad 5300.5$

\section{name $=$ TS145}

energy $($ in $\mathrm{kcal} / \mathrm{mol})=25.715291$

$\mathrm{ZPE}(\mathrm{in} \mathrm{kcal} / \mathrm{mol})=142.113$

G $($ in $\mathrm{kcal} / \mathrm{mol})=117.546$

Geometry

C $0.751468462 .14154408 \quad 3.91178068$

C $1.90809174 \quad 1.26091917 \quad 3.77724447$

C $0.45330989-1.186655821 .54802433$

$\begin{array}{llll}\text { C } 1.35168958 & -0.87151297 & 0.38352213\end{array}$

C $2.61235663-0.20129068 \quad 0.82011330$

C $3.15340657-0.47345216 \quad 2.83701079$

C $2.89081144 \quad 1.07246583 \quad 2.72168259$

C $2.61448040 \quad 1.24158654 \quad 1.18887274$

C $1.35722820 \quad 2.04353190 \quad 0.86551721$

C $3.81520721 \quad 1.97898272 \quad 0.55669412$

$\mathrm{H}-0.10476566 \quad 1.72496376 \quad 3.32015843$

$\mathrm{H} \quad 0.93632531 \quad 3.15208774 \quad 3.50940200$

$\mathrm{H} \quad 0.38544487 \quad 2.24658979 \quad 4.94617358$

$\mathrm{H} \quad 0.44856522 \quad-2.22058938 \quad 1.92395220$

H $0.80883032-0.22474032-0.34179025$

H $1.59231383-1.80043632-0.17300162$

H $3.50116214-0.580452020 .35095849$

H $4.16536349-0.819871992 .65975562$

H 2.37096198 $-1.22491890 \quad 2.81423706$

$\mathrm{H} 3.83117110 \quad 1.62122572 \quad 2.98236106$

$\mathrm{H} 1.40894757 \quad 3.06183184 \quad 1.25705889$

$\mathrm{H} \quad 0.45014445 \quad 1.56252264 \quad 1.25373358$ 
$\mathrm{H} \quad 1.22414180 \quad 2.11667783-0.22169530$

$\mathrm{H} \quad 3.88758152 \quad 3.012980360 .90123288$

H $3.709984861 .99584094-0.53444029$

$\mathrm{H} \quad 4.76109891 \quad 1.47436685 \quad 0.77605105$

$03.18767886-0.391786514 .47352286$

$0-0.25360585-0.366458592 .08969061$

O $2.082997290 .42029705 \quad 4.78357558$

Vibrational frequencies (in cm-1)

$\begin{array}{lllllllllllllllll}-820.8 & 60.0 & 74.2 & 115.7 & 134.3 & 160.9 & 199.3 & 208.7 & 220.9 & 238.6 & 250.9 & 262.2 & 306.8 & 318.1\end{array}$

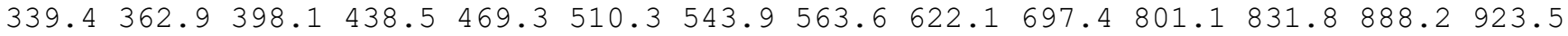
$934.2958 .5 \quad 975.9980 .4 \quad 992.0 \quad 1012.2 \quad 1014.4 \quad 1021.7 \quad 1029.3 \quad 1067.6 \quad 1087.8 \quad 1103.4 \quad 1128.4$

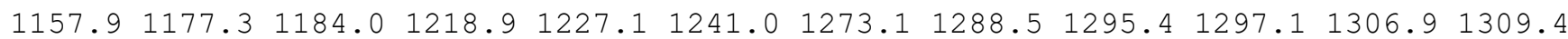

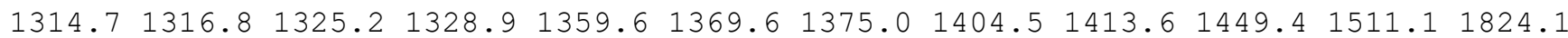
$2598.02640 .62659 .92663 .6 \quad 2665.2 \quad 2669.32678 .52682 .22685 .52691 .62729 .2 \quad 2737.8$ $2755.9 \quad 2774.3 \quad 2780.0 \quad 2782.5$

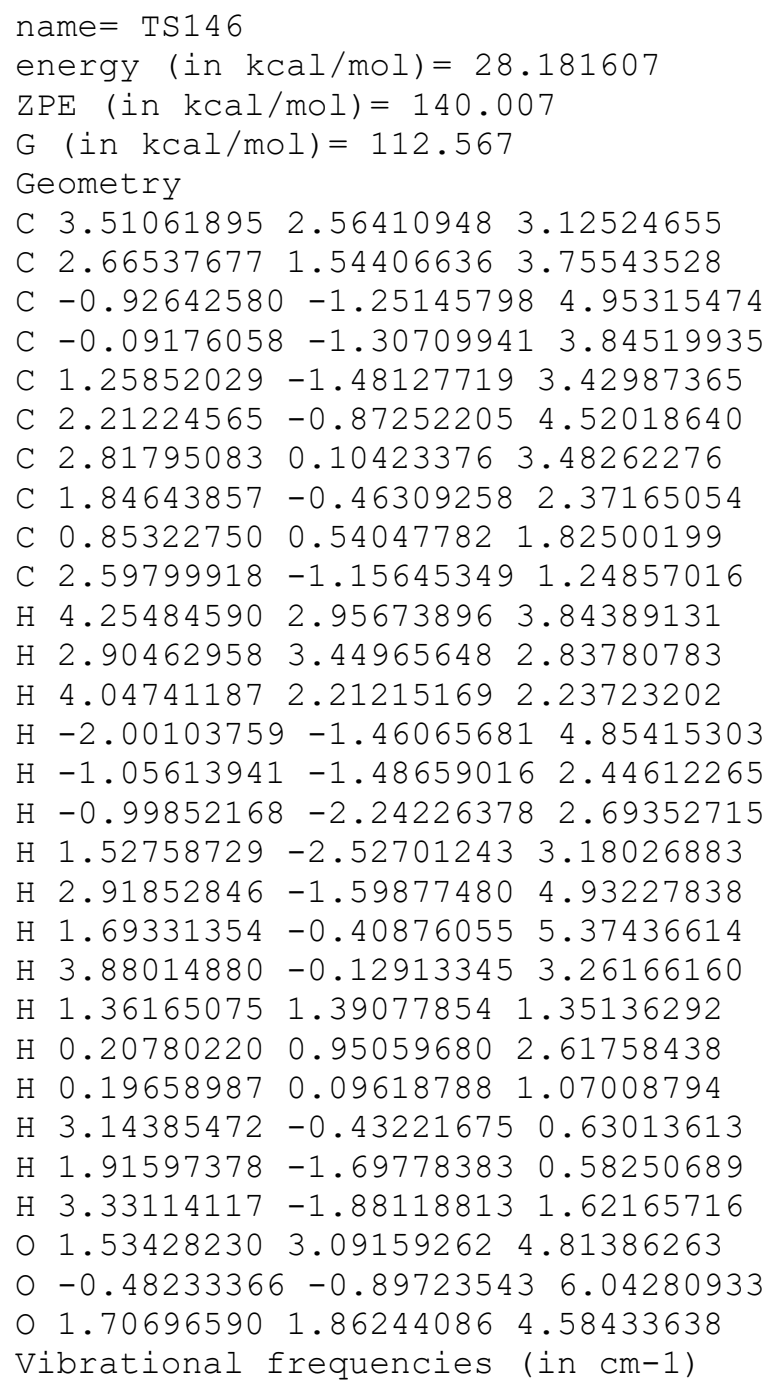




\section{Geometry}

C $6.26103100 \quad 2.15292974 \quad 3.78179542$

C $5.10498428 \quad 1.27455256 \quad 3.99674329$

C $0.80899457 \quad 0.75622597 \quad 5.08674402$

C $1.01192043 \quad 0.755256793 .71001302$

$\begin{array}{lllll}\text { C } 2.01840638 & 0.67228411 & 2.70685527\end{array}$

C $3.18514548-0.26091268 \quad 3.19112725$

C $4.22409169 \quad 0.84957207 \quad 2.89648407$

C $3.05975894 \quad 1.854300392 .53882075$

C $2.89037075 \quad 2.99298791 \quad 3.52299610$

$\begin{array}{llll}\text { C } 3.14482611 & 2.36998345 & 1.11387474\end{array}$

$\mathrm{H} 7.20699056 \quad 1.58575275 \quad 3.87372135$

H $6.31915981 \quad 2.92217396 \quad 4.58080477$

$\mathrm{H} \quad 6.25971850 \quad 2.66203930 \quad 2.81171404$

$\mathrm{H}-0.08549083 \quad 1.22827063 \quad 5.51819395$

$\mathrm{H} \quad 0.01174834 \quad 2.04383921 \quad 3.13773104$

$\mathrm{H}-0.38233071 \quad 1.36696975 \quad 2.97857385$

H $1.62290762 \quad 0.34805120 \quad 1.72180614$

$\mathrm{H} 3.29718104-1.169251392 .59398766$

$\mathrm{H} 3.10387092-0.568675194 .24609996$

H $4.84014056 \quad 0.60637476 \quad 2.00343767$

$\mathrm{H} \quad 2.78007060 \quad 2.62903671 \quad 4.55604910$

H 2.01223148 $3.60445554 \quad 3.29330391$

H $3.76219088 \quad 3.66149433 \quad 3.51494357$

H 2.24577085 2.93150499 0.83457731

$\mathrm{H} \quad 3.263040631 .56109708 \quad 0.38331240$

H $3.99958823 \quad 3.04737009 \quad 0.98713091$

○ 5.548130431 .182456146 .13583280

O $1.582555620 .15469967 \quad 5.82697013$

O 4.799307830 .827269415 .18608245

Vibrational frequencies (in $\mathrm{cm}-1$ )

$\begin{array}{lllllllllllllllll}-712.5 & 32.7 & 37.8 & 48.5 & 96.3 & 120.6 & 140.5 & 167.2 & 181.9 & 214.5 & 223.2 & 227.5 & 270.1 & 279.0\end{array}$ $293.7 \quad 315.8 \quad 331.5 \quad 374.3 \quad 419.1 \quad 450.1 \quad 486.3 \quad 513.1 \quad 554.3 \quad 592.0 \quad 661.2 \quad 688.0 \quad 745.5 \quad 801.4$ $831.8 \quad 922.8 \quad 931.4 \quad 938.5 \quad 940.6 \quad 997.5 \quad 1002.2 \quad 1016.2 \quad 1019.5 \quad 1036.5 \quad 1047.4 \quad 1063.2 \quad 1099.1$

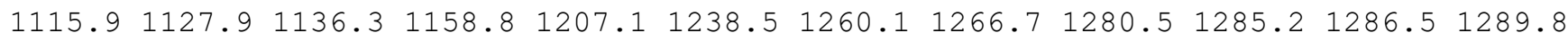

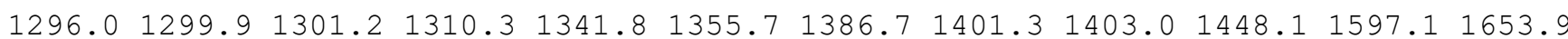
$1734.82637 .7 \quad 2654.0 \quad 2662.1 \quad 2663.3 \quad 2673.9 \quad 2676.0 \quad 2676.52679 .72709 .72715 .72748 .6$ $2770.12779 .0 \quad 2782.8 \quad 4927.7$

\footnotetext{
name $=\operatorname{TS} 148$

energy $($ in $\mathrm{kcal} / \mathrm{mol})=27.009542$

$\mathrm{ZPE}($ in $\mathrm{kcal} / \mathrm{mol})=141.198$

$\mathrm{G}($ in $\mathrm{kcal} / \mathrm{mol})=115.484$

Geometry

C -0.810394132 .518848853 .41776806$

C $0.01699097 \quad 1.42384758 \quad 3.08471853$

C $0.27103395-1.619018320 .88638486$

C $1.62743924-1.25040198 \quad 0.46694508$

C $2.28165599-0.110119431 .22471149$

C $2.06295143-0.07395592 \quad 2.76123013$

C $1.42379421 \quad 1.33544024 \quad 2.66843052$

C $1.64574987 \quad 1.32538210 \quad 1.09984540$

C $0.38649200 \quad 1.44784610 \quad 0.26858008$

C $2.66326107 \quad 2.37172171 \quad 0.66951924$

$\mathrm{H}-1.74557982 \quad 1.22355530 \quad 3.38452725$

$\mathrm{H}-0.84730202 \quad 3.41302700 \quad 2.83966962$

$\mathrm{H}-1.14230992 \quad 2.68157481 \quad 4.42444777$

$\mathrm{H}-0.20101504-1.268973891 .79980599$

H $1.61872336-1.02351629-0.63386303$

H $2.27496298-2.16451327 \quad 0.52169884$

H $3.35915752-0.086787090 .96316644$

$\mathrm{H} 2.98601434-0.10590950 \quad 3.34731767$

$\mathrm{H} 1.39808782-0.849239523 .15778017$

$\mathrm{H} 2.02310537 \quad 2.12088113 \quad 3.16972893$
} 
$\mathrm{H} \quad 0.58609599 \quad 1.23497492-0.79031956$

H $-0.033225332 .46138631 \quad 0.32308888$

$\mathrm{H}-0.405119350 .75941825 \quad 0.59023333$

$\mathrm{H} 2.26526652 \quad 3.38781971 \quad 0.78405852$

H $2.93561427 \quad 2.24656757-0.38655353$

H $3.58933078 \quad 2.31832764 \quad 1.25335959$

$0-0.01075460-2.86556777-0.89383800$

$\begin{array}{lllll}0 & -0.48231482 & -2.40961306 & 0.18973074\end{array}$

o $-0.74196329 \quad 0.38252067 \quad 3.15520493$

Vibrational frequencies (in cm-1)

$\begin{array}{lllllllllllllll}-1922.9 & 30.8 & 51.9 & 67.7 & 109.3 & 146.8 & 160.8 & 194.1 & 205.3 & 220.6 & 228.8 & 265.6 & 300.0 & 341.3\end{array}$

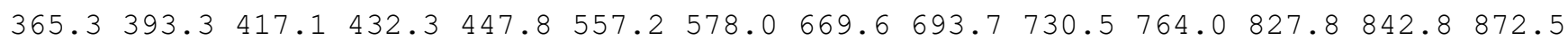

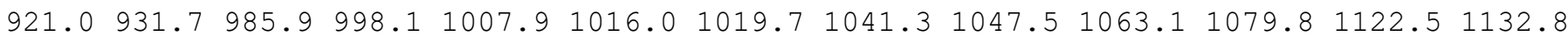
$1150.01169 .2 \quad 1192.9 \quad 1205.2 \quad 1213.0 \quad 1241.6 \quad 1252.4 \quad 1266.1 \quad 1282.5 \quad 1285.4 \quad 1288.8 \quad 1293.6$

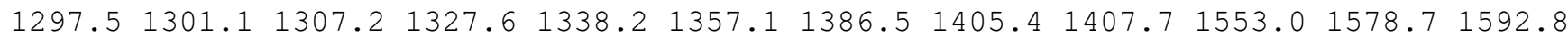
$2120.82618 .32667 .52671 .2 \quad 2674.8 \quad 2675.92676 .52678 .62711 .2 \quad 2718.02722 .2 \quad 2729.2$ $2760.5 \quad 2779.3 \quad 2782.12807 .5$

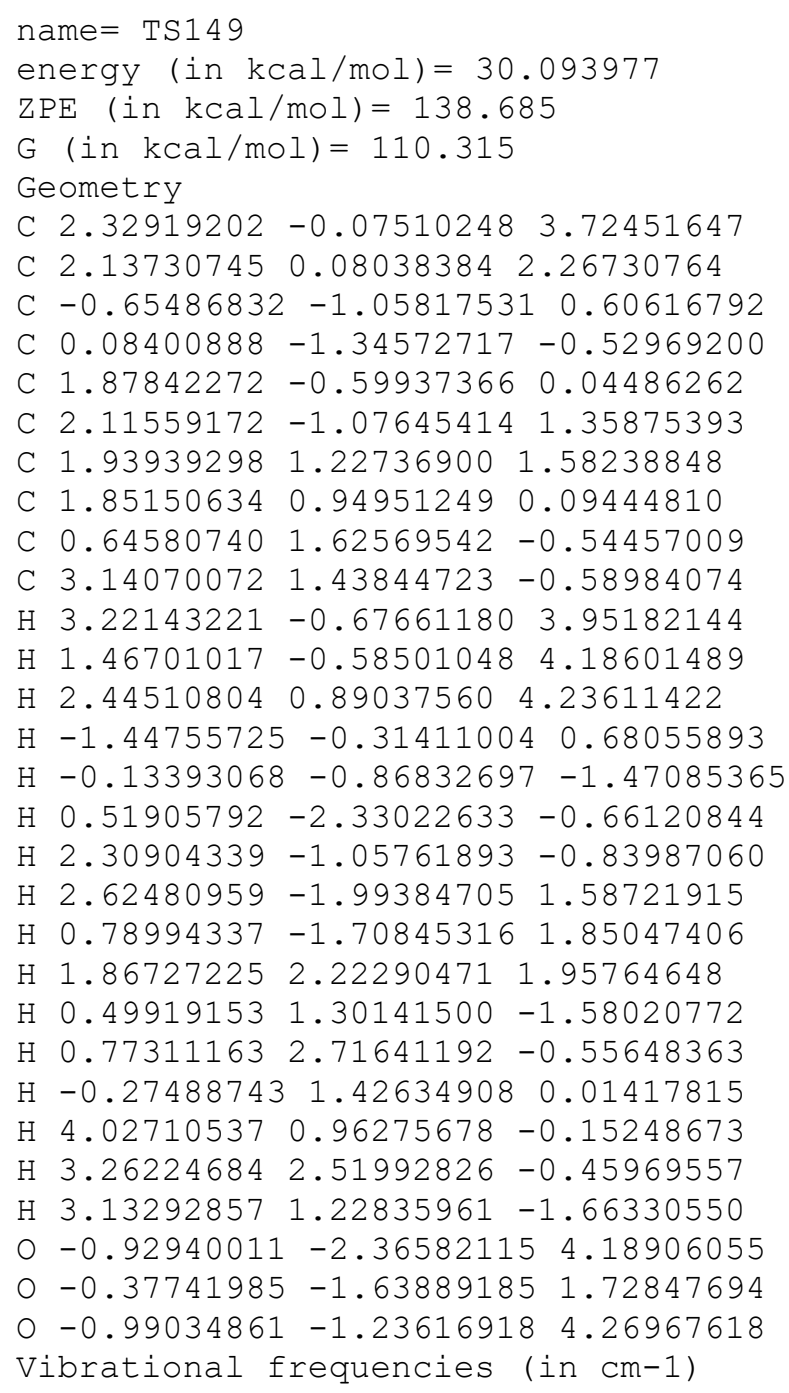


$\mathrm{ZPE}($ in $\mathrm{kcal} / \mathrm{mol})=139.347$

$\mathrm{G}($ in $\mathrm{kcal} / \mathrm{mol})=112.667$

Geometry

C $1.57313443 \quad 4.01115056 \quad 0.54995663$

C $1.78564053 \quad 2.93769564 \quad 1.31207355$

C $2.70416290-1.797555813 .18172286$

C $1.93935076-1.26030027 \quad 2.17952089$

C $3.39961445 \quad 0.05811453 \quad 1.81319638$

C $3.222481791 .27639365 \quad 2.69660927$

C $3.05927470 \quad 2.18468937 \quad 1.44124940$

C $3.17015635 \quad 0.87958094 \quad 0.54014201$

C $1.94707656 \quad 0.59635566-0.30446056$

C $4.43464624 \quad 0.87255753-0.30907403$

$\mathrm{H} 2.33394210 \quad 4.46421177 \quad-0.05997327$

$\mathrm{H} \quad 0.61878697 \quad 4.50286318 \quad 0.46210400$

$\mathrm{H}-0.44329447 \quad 3.58163631 \quad 2.68632519$

H 2.41998249 $-1.95174605 \quad 4.22176398$

H $0.96331205 \quad-0.84470967 \quad 2.36779517$

H 2.05941770 $-1.53794772 \quad 1.13502514$

H $4.35345277-1.53708615 \quad 2.18268744$

$\mathrm{H} \quad 4.10702337 \quad 1.50221127 \quad 3.30337823$

H 2.37228449 1.25929585 3.38017833

H $3.91765125 \quad 2.86641875 \quad 1.29785189$

$\mathrm{H} 1.80785027 \quad 1.37096169-1.07151482$

H $1.02647156 \quad 0.58024871 \quad 0.29520236$

H 2.02813701 $-0.36662518-0.82141975$

H $4.39748748 \quad 1.64701692-1.08373032$

$\mathrm{H} \quad 4.56239604-0.09515197-0.80954077$

$\mathrm{H} \quad 5.33352185 \quad 1.04506607 \quad 0.29449584$

$\begin{array}{llll}0 & -0.42944651 & 2.94564092 & 1.92823421\end{array}$

$03.96412590-2.13468904 \quad 2.97895636$

$\begin{array}{lllll}0 & 0.84689180 & 2.34431292 & 2.12186486\end{array}$

Vibrational frequencies (in $\mathrm{cm}-1$ )

$\begin{array}{lllllllllllllll}-500.6 & 30.8 & 42.0 & 65.7 & 87.2 & 153.7 & 161.8 & 171.1 & 178.0 & 193.0 & 216.5 & 218.2 & 229.1 & 247.4\end{array}$ $288.6 \quad 330.3 \quad 351.2 \quad 401.2 \quad 421.0 \quad 440.4 \quad 488.4 \quad 569.2 \quad 610.5 \quad 629.0 \quad 643.1 \quad 674.5 \quad 756.5 \quad 819.5$ $871.0 \quad 922.6 \quad 929.3 \quad 944.2 \quad 997.2 \quad 999.1 \quad 1006.7 \quad 1011.6 \quad 1015.1 \quad 1020.8 \quad 1042.3 \quad 1047.4 \quad 1077.6$ $1093.6 \quad 1118.5 \quad 1137.5 \quad 1158.0 \quad 1198.1 \quad 1227.4 \quad 1244.91279 .6 \quad 1288.9 \quad 1296.3 \quad 1298.8 \quad 1303.7$

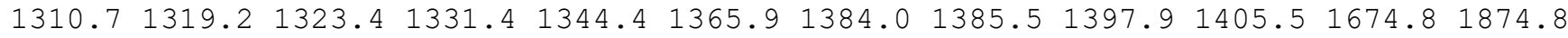
$2392.92666 .62675 .8 \quad 2678.2 \quad 2680.2 \quad 2685.72693 .02717 .12721 .52729 .52766 .82777 .6$ $2778.0 \quad 2781.4 \quad 2797.92866 .9$

\section{name $=$ TS151}

energy $($ in $\mathrm{kcal} / \mathrm{mol})=29.618965$

$\mathrm{ZPE}($ in $\mathrm{kcal} / \mathrm{mol})=142.123$

$\mathrm{G}($ in $\mathrm{kcal} / \mathrm{mol})=115.95$

Geometry

C $0.67068727 \quad 2.60498031 \quad 3.64038937$

C $1.823044471 .92964562 \quad 3.06142306$

C $1.85388948-1.70443513 \quad 3.04831588$

C $2.90170046-1.67915368 \quad 1.95860250$

C $3.38589550 \quad-0.29273703 \quad 1.53555714$

C $3.92617376 \quad 0.560444192 .65594857$

C 2.414637851 .878310561 .80191445

C $2.33793188 \quad 0.652650110 .89629553$

C $0.90265229 \quad 0.13627173 \quad 0.84737822$

C $2.769310801 .03494074-0.52671193$

$\mathrm{H}-0.20853347 \quad 2.57689374 \quad 2.97792394$

$\mathrm{H} \quad 0.87821222 \quad 3.66377323 \quad 3.87003115$

$\mathrm{H} 0.37468555 \quad 2.12822256 \quad 4.60022219$

$\mathrm{H} 2.04182600-1.140898223 .97260993$

H $2.51738015-2.221056131 .06657636$

$\mathrm{H} \quad 3.76892752-2.280223262 .31086002$

H $4.21672451-0.47767151 \quad 0.79914518$

H $4.65307768 \quad 1.31284275 \quad 2.38535237$ 
H $4.26262473 \quad 0.01823857 \quad 3.54127262$

H $2.734815342 .80297400 \quad 1.32461724$

$\mathrm{H} \quad 0.83272127 \quad-0.81278894 \quad 0.30068480$

$\mathrm{H} \quad 0.23511217 \quad 0.84988641 \quad 0.35041946$

$\mathrm{H} \quad 0.48502760 \quad-0.041342291 .84801956$

H $3.784646231 .44403558-0.55637484$

H $2.099347121 .78458253-0.96366787$

H $2.74624450 \quad 0.16089627-1.18954467$

$0 \quad 2.41049197 \quad 0.75142124 \quad 4.92526094$

○ $0.85337966-2.364833932 .91087725$

o $2.710382321 .26028684 \quad 3.83018179$

Vibrational frequencies (in $\mathrm{cm}-1$ )

$\begin{array}{llllllllllllllllll}-634.1 & 20.4 & 52.6 & 67.3 & 74.8 & 131.1 & 139.5 & 200.9 & 220.3 & 237.9 & 258.7 & 271.9 & 287.7 & 298.8\end{array}$

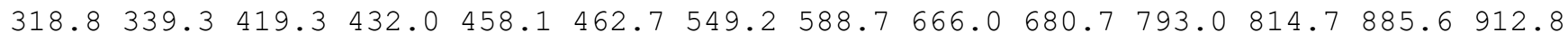

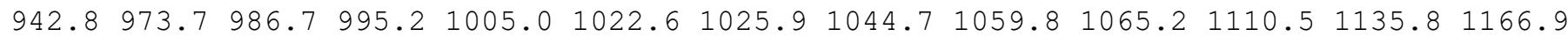

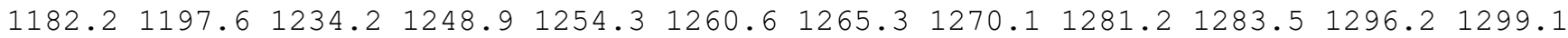
$1303.11311 .61312 .61331 .41348 .61381 .41384 .41396 .81405 .6 \quad 1408.4 \quad 1679.01828 .3$ $2626.12642 .7 \quad 2653.2 \quad 2658.0 \quad 2663.12664 .5 \quad 2671.82675 .92676 .6 \quad 2679.02721 .2 \quad 2735.9$ $2739.32766 .6 \quad 2779.02781 .7$

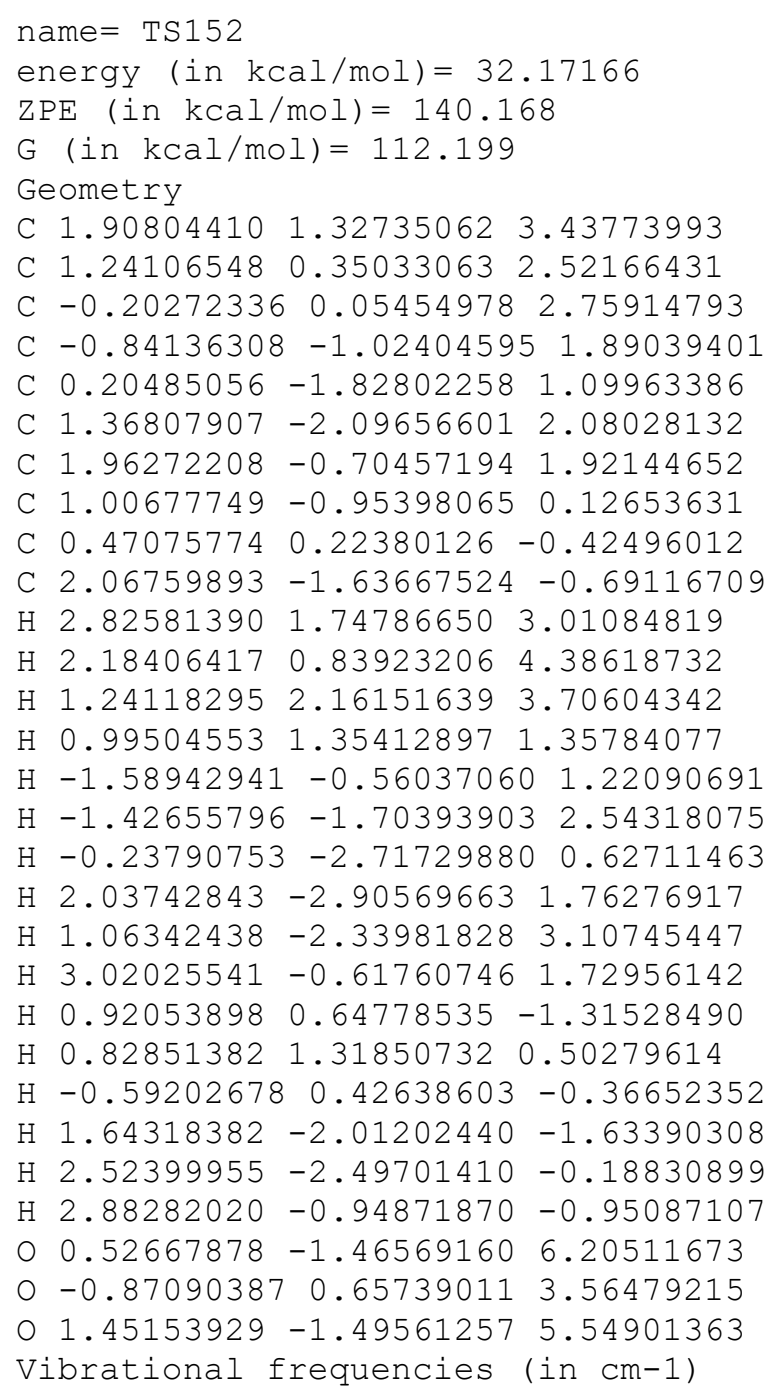




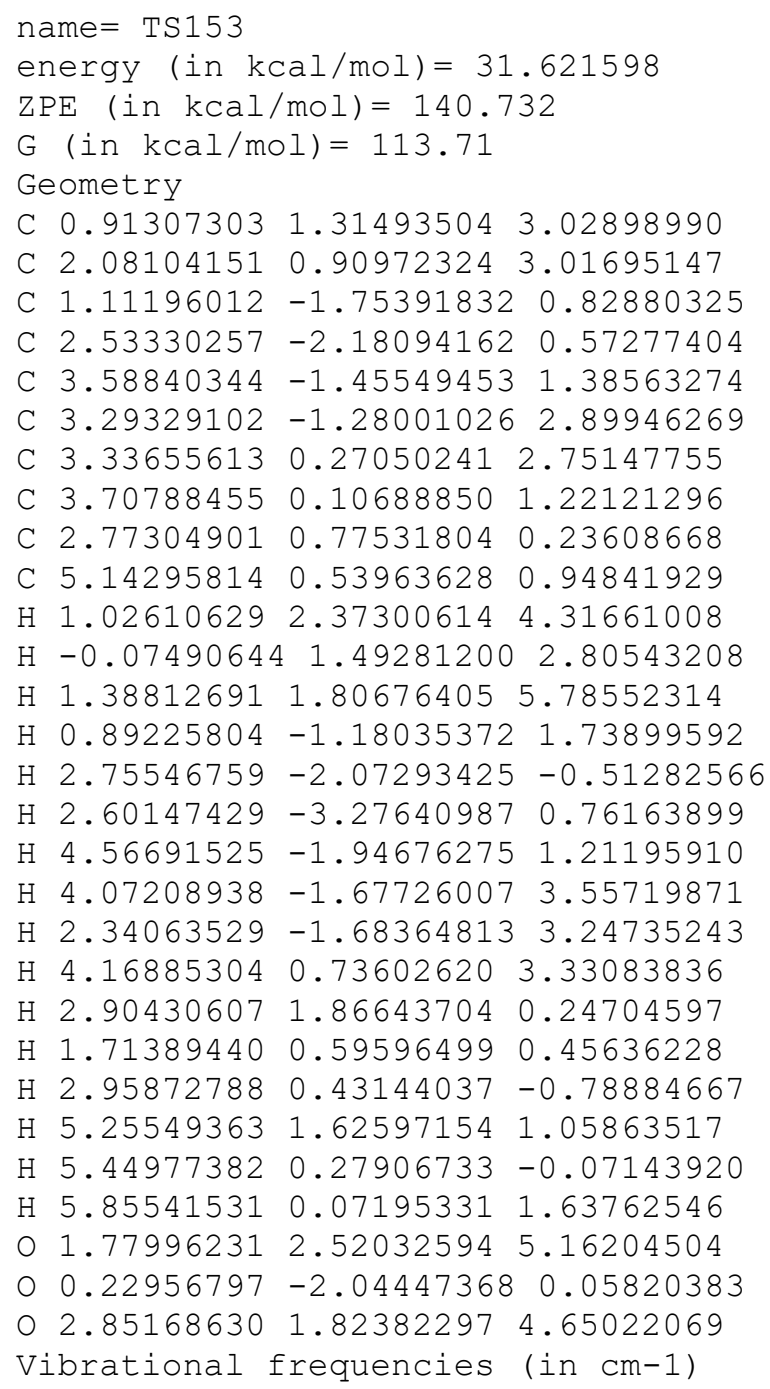


$\mathrm{H} \quad 3.32045061-1.499757170 .27873083$

H $5.26622703-2.10865757 \quad 1.02876207$

H $3.45627139-0.76870908 \quad 5.94522894$

$\mathrm{H} \quad 4.32011389-0.65837904 \quad 3.83955453$

$\mathrm{H} \quad 1.597459901 .03522837 \quad 2.06246111$

$\mathrm{H} \quad 2.27418194 \quad 0.93775188 \quad 0.43112699$

H $2.79990698 \quad 2.20895887 \quad 1.52264294$

H $5.79886864 \quad 0.05312991 \quad 1.64541663$

H 5.216086731 .712281221 .61094764

H $4.90394830 \quad 0.64790790 \quad 0.23364046$

$04.25329056-0.179267736 .05191731$

○ $2.12728316-2.06245509 \quad 4.47086676$

03.729688961 .094099565 .70692666

Vibrational frequencies (in cm-1)

$\begin{array}{lllllllllllllllll}-1208.4 & 43.3 & 66.2 & 75.7 & 90.8 & 112.3 & 144.7 & 160.7 & 168.8 & 198.1 & 218.3 & 228.5 & 260.3 & 268.1\end{array}$

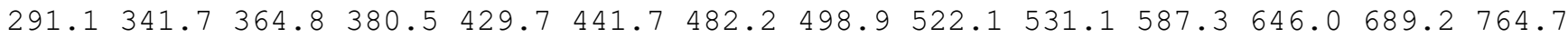
$810.3 \quad 894.4924 .5 \quad 940.7974 .1 \quad 979.6 \quad 986.2 \quad 1008.3 \quad 1027.3 \quad 1036.2 \quad 1054.2 \quad 1070.4 \quad 1094.4$

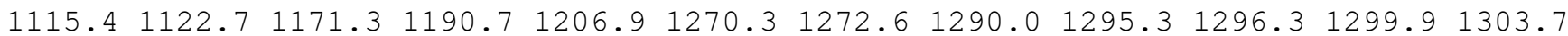

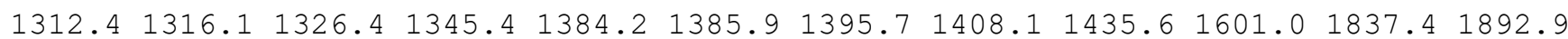
$2269.32640 .82660 .12660 .6 \quad 2666.8 \quad 2669.2 \quad 2673.92679 .42682 .32714 .22734 .52772 .9$ $2780.22781 .02782 .2 \quad 2827.8$

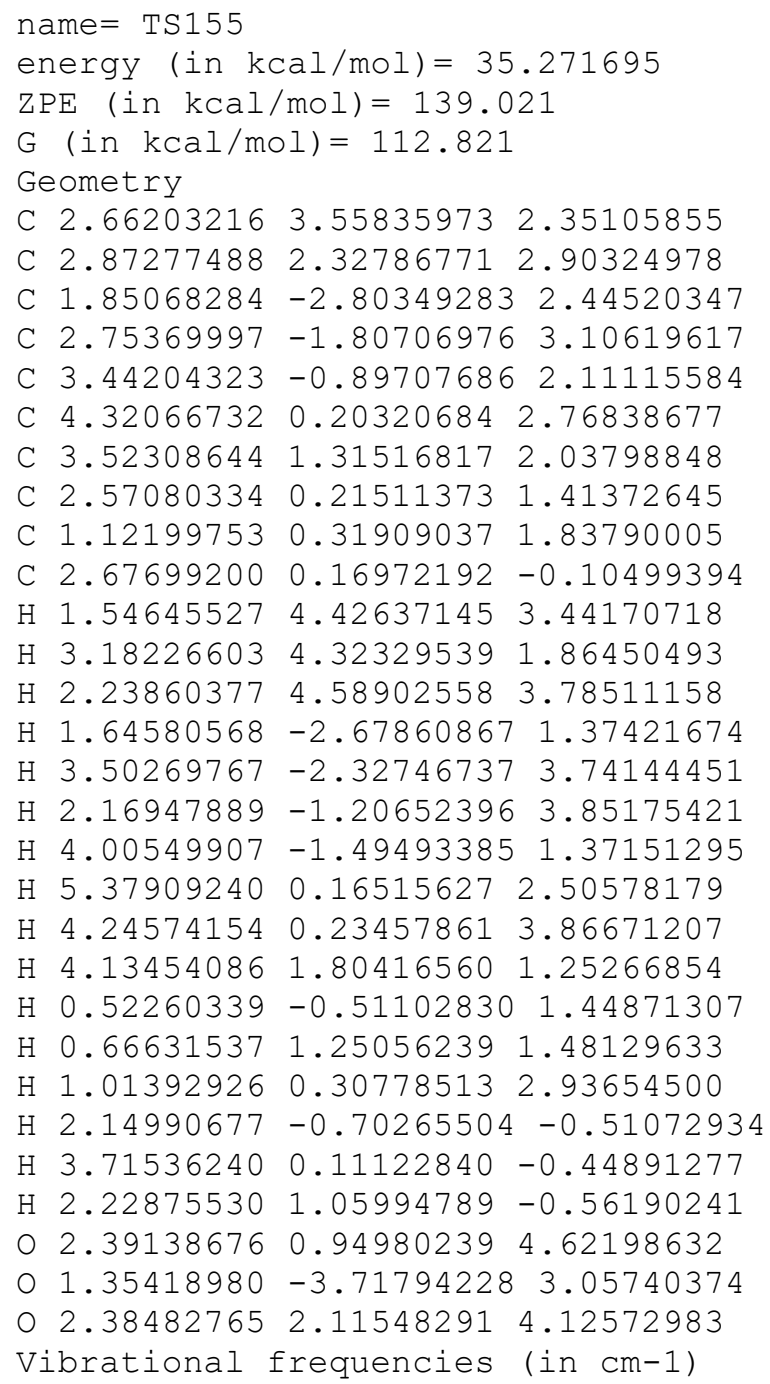




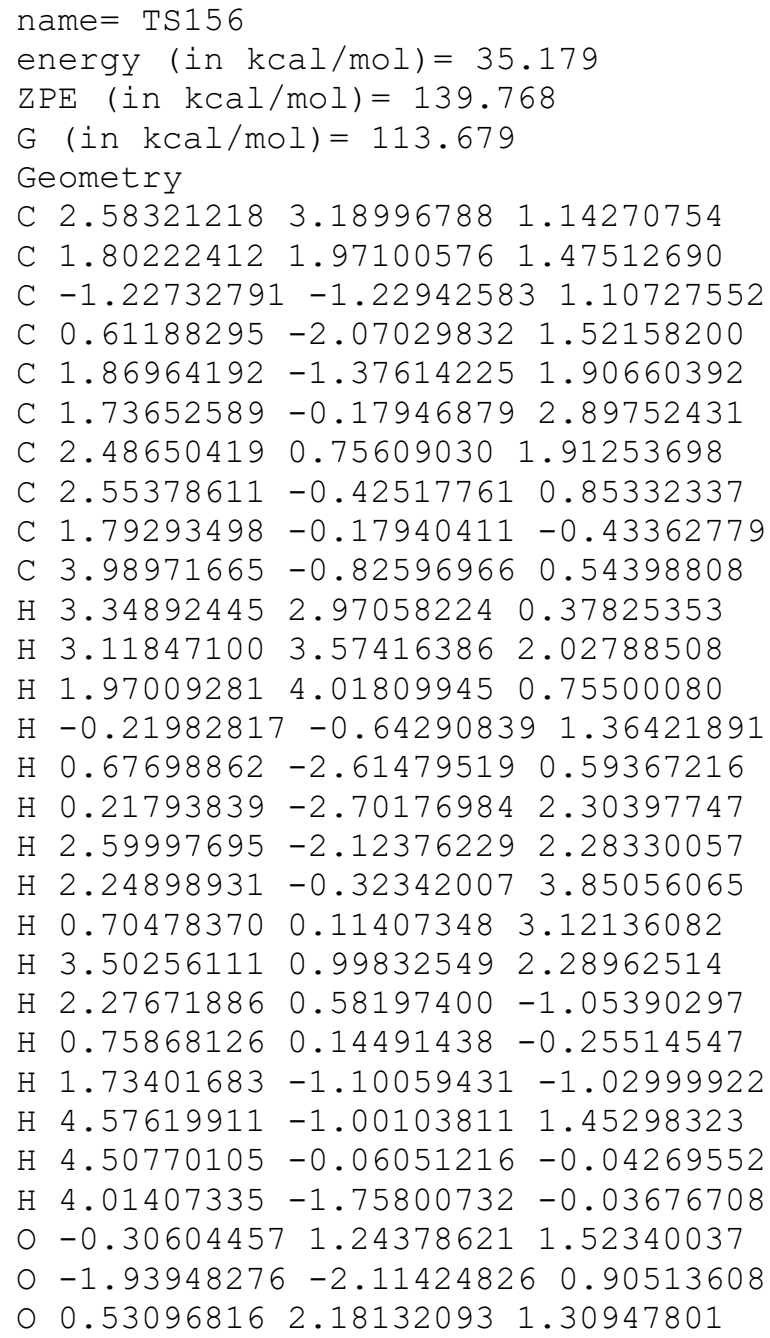


$\mathrm{H} \quad 2.90440737-0.67673292-0.49618930$

H $4.20261926 \quad 0.12499116 \quad 0.38257390$

H $3.18579927 \quad 2.05560843-0.07725654$

H $1.20566348 \quad 2.766268391 .33436631$

H 2.63495762 2.17762410 2.19474435

H $1.60291717 \quad 0.83133109 \quad 3.74201978$

$\mathrm{H}-0.39329780 \quad 0.27551446-1.65480705$

$\mathrm{H}-0.34057827 \quad 0.39776722 \quad 0.11063400$

$\mathrm{H} \quad 0.69656901-0.76466633-0.72970197$

$\mathrm{H} \quad 0.65547143 \quad 2.21287537 \quad-2.65076042$

H $2.125240642 .95049588-1.95024388$

$\mathrm{H}-0.09562264 \quad 1.08094527 \quad 2.38463702$

O $3.18973663-0.75333594 \quad 3.90415803$

$03.72926495-0.41122262 \quad 2.62691971$

o $1.84757620-0.36138024 \quad 3.80349235$

Vibrational frequencies (in cm-1)

$\begin{array}{llllllllllllllll}-1388.7 & 33.9 & 56.9 & 94.8 & 114.7 & 130.5 & 183.6 & 208.7 & 237.1 & 262.7 & 288.6 & 310.9 & 353.4 & 389.0\end{array}$ $445.7 \quad 465.6 \quad 517.0 \quad 546.2 \quad 563.3 \quad 569.6 \quad 588.2 \quad 628.2 \quad 696.6 \quad 763.2 \quad 815.0 \quad 844.8 \quad 869.5 \quad 908.2$ $932.7958 .0 \quad 986.6 \quad 995.5 \quad 1008.5 \quad 1037.6 \quad 1040.3 \quad 1047.9 \quad 1055.1 \quad 1070.2 \quad 1090.4 \quad 1094.01128 .7$

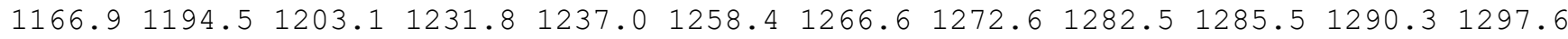
$1313.41321 .5 \quad 1324.2 \quad 1335.7 \quad 1365.5 \quad 1374.5 \quad 1386.8 \quad 1397.0 \quad 1400.7 \quad 1405.8 \quad 1558.8 \quad 1850.3$ $2143.42648 .5 \quad 2661.1 \quad 2664.7 \quad 2669.8 \quad 2670.7 \quad 2676.4 \quad 2679.92684 .4 \quad 2726.12732 .02738 .8$ $2774.32777 .3 \quad 2779.5 \quad 2795.0$

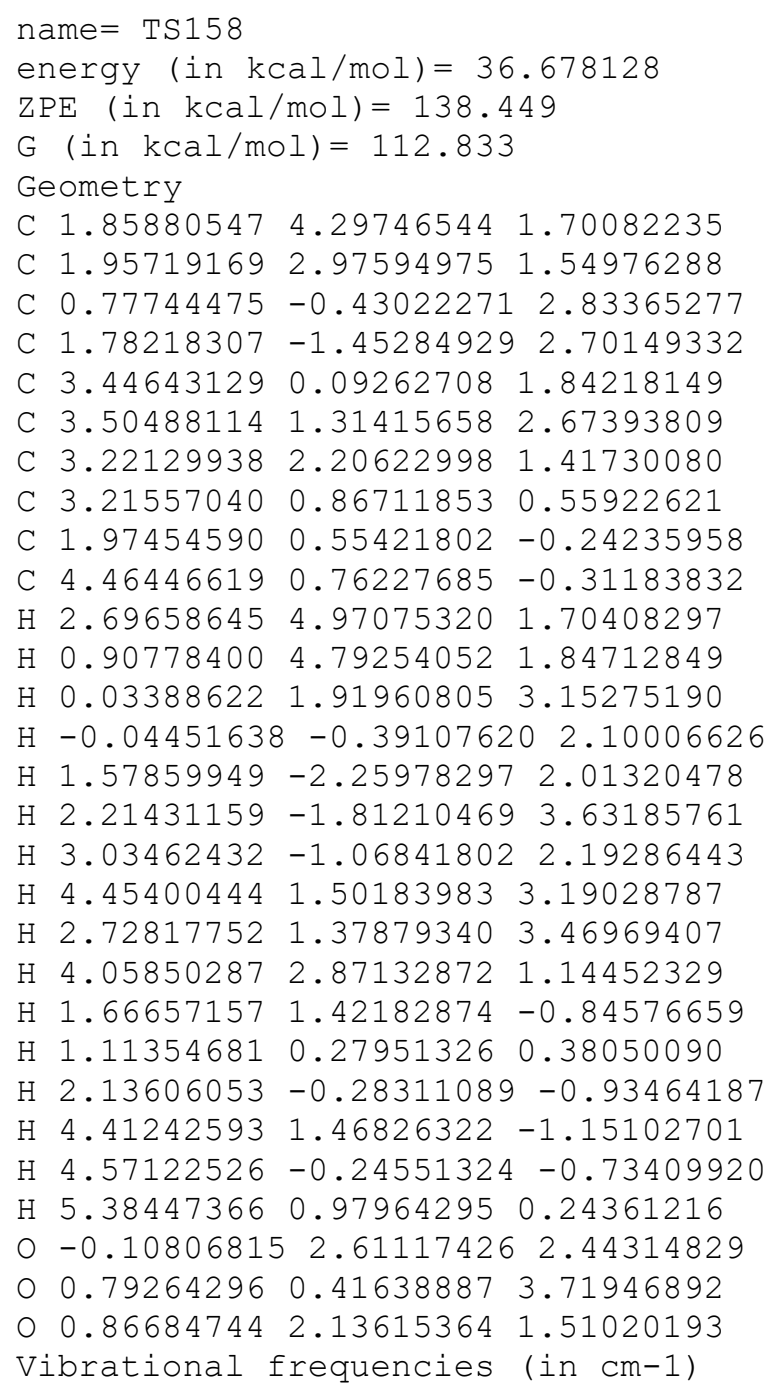


$1860.7 \quad 2608.4 \quad 2649.4 \quad 2662.5 \quad 2668.3 \quad 2673.4 \quad 2673.8 \quad 2711.12720 .72725 .12732 .7 \quad 2737.2$ $2770.12776 .7 \quad 2779.6 \quad 2790.1$

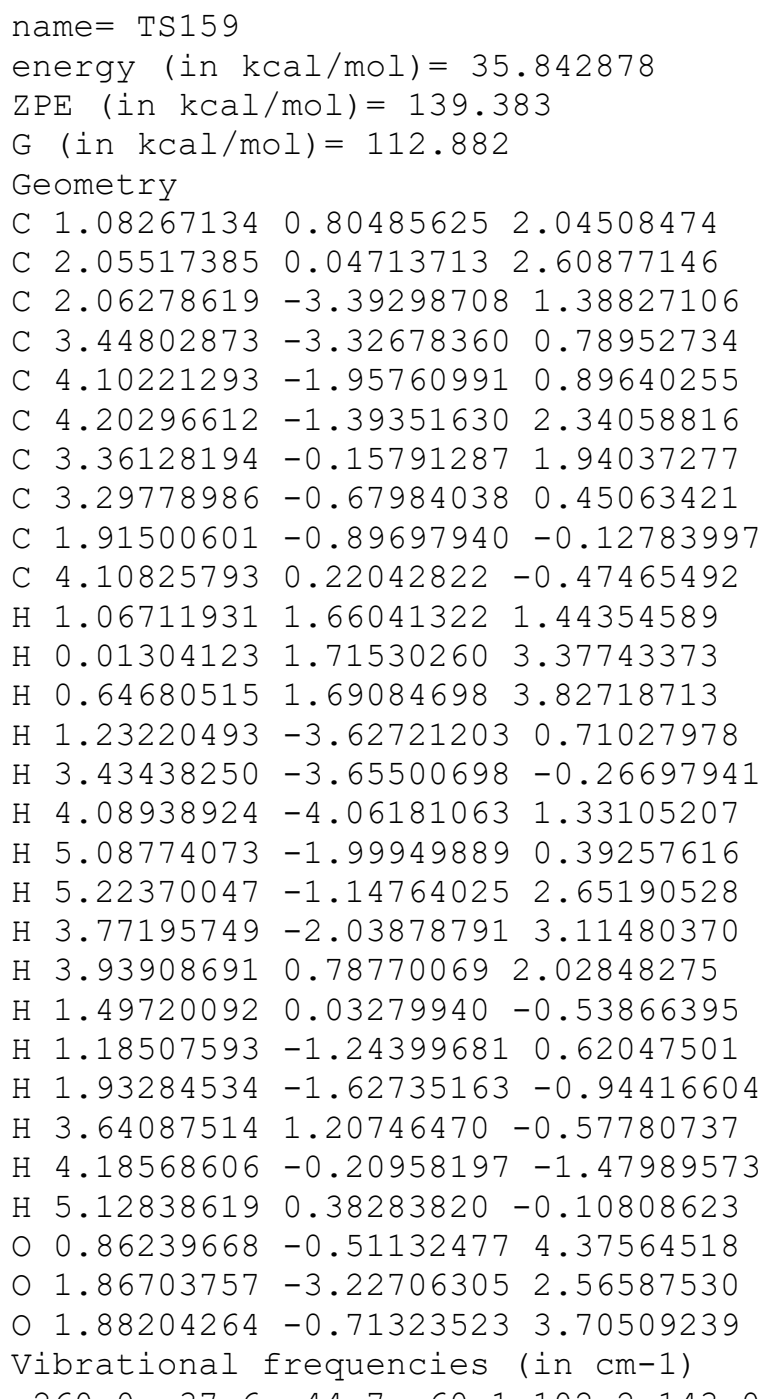

$\begin{array}{lllllllllllllllllll}-260.0 & 37.6 & 44.7 & 60.1 & 102.2 & 143.0 & 164.9 & 182.3 & 193.9 & 209.7 & 215.1 & 226.7 & 265.2 & 273.4\end{array}$ $314.2 \quad 332.7 \quad 345.8 \quad 383.6 \quad 391.1 \quad 394.9 \quad 429.9 \quad 459.4 \quad 480.6 \quad 560.6 \quad 603.0 \quad 678.1 \quad 710.4 \quad 747.2$ $804.0 \quad 859.3 \quad 892.1 \quad 906.2 \quad 915.7 \quad 947.5 \quad 996.8 \quad 1003.0 \quad 1006.8 \quad 1020.3 \quad 1056.4 \quad 1071.1 \quad 1082.0$ $1120.01124 .8 \quad 1156.91181 .3 \quad 1193.9 \quad 1203.6 \quad 1226.5 \quad 1250.61273 .1 \quad 1287.6 \quad 1290.6 \quad 1297.3$ $1299.81302 .3 \quad 1308.3 \quad 1319.3 \quad 1328.4 \quad 1335.91355 .91376 .6 \quad 1387.4 \quad 1407.01408 .6 \quad 1541.6$ $1840.22645 .92649 .6 \quad 2665.4 \quad 2672.7 \quad 2674.12676 .5 \quad 2679.12706 .02716 .02739 .52755 .9$ $2765.22775 .02782 .3 \quad 5266.7$

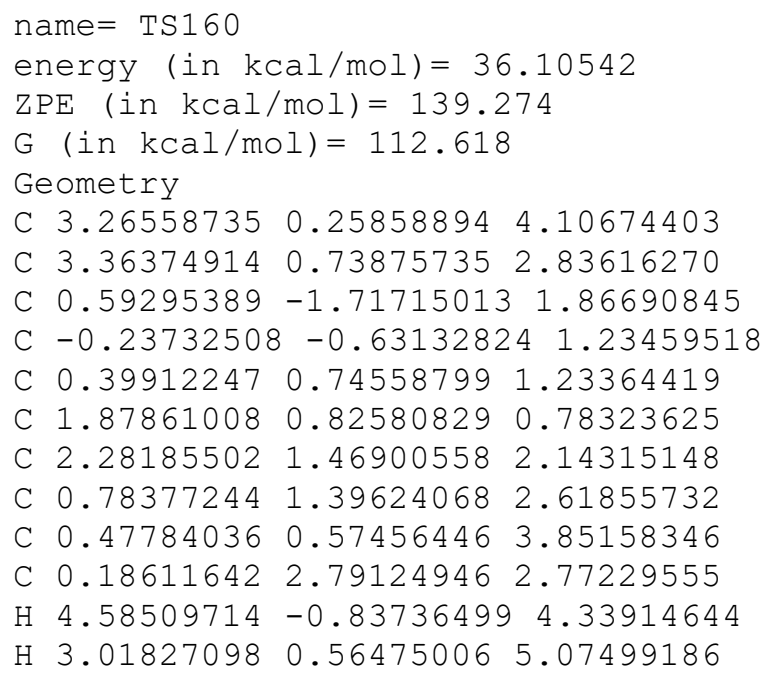


$\mathrm{H} \quad 4.93862833-0.14667063 \quad 4.51419623$

H $1.68598709-1.62782794 \quad 1.82414530$

$\mathrm{H}-1.23152523-0.58709986 \quad 1.73113463$

$\mathrm{H}-0.45900007-0.93885467 \quad 0.18686251$

$\mathrm{H}-0.24641810 \quad 1.428888210 .64537226$

H $2.06984731 \quad 1.47139895-0.08091569$

H $2.37212797 \quad-0.12556290 \quad 0.56011947$

H 2.627821462 .521656832 .00968977

$\mathrm{H}-0.59858378 \quad 0.36229841 \quad 3.92497108$

$\mathrm{H} \quad 0.75696912 \quad 1.10099470 \quad 4.77161691$

$\mathrm{H} \quad 0.98407521-0.400944733 .85907358$

H $0.36297961 \quad 3.41726218 \quad 1.88940614$

$\mathrm{H} 0.61162481 \quad 3.31740454 \quad 3.63430053$

H $-0.89929146 \quad 2.74176638 \quad 2.92252878$

$\begin{array}{lllll}0 & 4.55146837 & 0.84085880 & 0.94354686\end{array}$

$0.07310845-2.672135602 .39114444$

$\begin{array}{llll}0 & 4.49180745 & 0.47871123 & 2.16042350\end{array}$

Vibrational frequencies (in cm-1)

$\begin{array}{lllllllllllllll}-711.3 & 22.7 & 34.8 & 52.3 & 117.2 & 160.6 & 164.5 & 184.8 & 204.3 & 221.3 & 229.6 & 249.3 & 251.8 & 286.0\end{array}$ $299.1328 .3 \quad 358.7 \quad 385.9 \quad 418.6 \quad 420.2 \quad 429.3 \quad 478.3 \quad 537.0 \quad 603.9614 .2 \quad 669.1691 .1 \quad 758.9$ $829.3 \quad 846.0 \quad 847.4 \quad 927.7 \quad 945.3 \quad 991.1 \quad 1005.3 \quad 1011.3 \quad 1017.5 \quad 1036.3 \quad 1052.4 \quad 1056.2 \quad 1071.2$

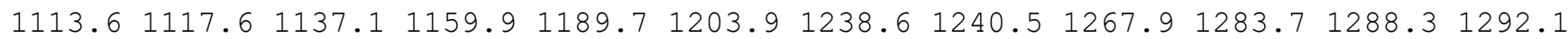
$1297.21302 .8 \quad 1304.3 \quad 1317.4 \quad 1331.3 \quad 1333.4 \quad 1342.4 \quad 1377.0 \quad 1386.8 \quad 1404.91409 .2 \quad 1577.1$ $1830.52643 .92655 .2 \quad 2658.0 \quad 2672.1 \quad 2672.5 \quad 2674.92678 .72695 .8 \quad 2715.12738 .2 \quad 2756.2$ $2763.7 \quad 2776.12782 .1 \quad 4985.1$

name $=\operatorname{TS} 161$

energy (in $\mathrm{kcal} / \mathrm{mol})=36.535582$

$\mathrm{ZPE}($ in $\mathrm{kcal} / \mathrm{mol})=139.389$

$\mathrm{G}($ in $\mathrm{kcal} / \mathrm{mol})=113.275$

Geometry

C $1.77546931 \quad 1.52752702 \quad 4.37097777$

C $1.612608890 .17033964 \quad 4.44536450$

C $-0.29362083-3.59007504 \quad 4.32027870$

C $0.12807511-3.64940886 \quad 2.86922830$

C $0.95651898-2.47927200 \quad 2.37998606$

C $2.19750455-2.05338990 \quad 3.20820318$

C $1.74054075-0.571014373 .16928110$

C $0.37488806-1.02051408 \quad 2.50229749$

C $-0.84016931-0.856459263 .38907186$

C $0.14093654 \quad-0.36611150 \quad 1.14901284$

H $1.213047392 .11502233 \quad 5.97993883$

H $1.39119860 \quad 2.35268517 \quad 3.85753440$

$\mathrm{H} 2.00277537 \quad 2.08540034 \quad 6.02717046$

$\mathrm{H} \quad 0.46349468-3.34371282 \quad 5.07613844$

$\mathrm{H}-0.77722699-3.750260972 .23008442$

$\mathrm{H} \quad 0.69854187 \quad-4.59319337 \quad 2.72288505$

H $1.24283212-2.68539755 \quad 1.32551477$

H $3.15961552 \quad-2.23848084 \quad 2.72641715$

H $2.24992115-2.48985483 \quad 4.21710585$

$\mathrm{H} 2.36277297 \quad 0.00567504 \quad 2.45022626$

$\mathrm{H}-1.18285651 \quad 0.18196016 \quad 3.42991668$

$\mathrm{H}-0.64730109-1.177687394 .42587693$

$\mathrm{H}-1.68247607-1.465377593 .02923926$

$\mathrm{H}-0.07767830 \quad 0.70260631 \quad 1.24843074$

$\mathrm{H}-0.71566868-0.82632595 \quad 0.63805708$

$\mathrm{H} \quad 1.00501010 \quad-0.46358906 \quad 0.48239194$

O $1.41848210-1.59728782 \quad 5.83750661$

$\begin{array}{llll}0 & -1.43298407 & -3.84488303 & 4.62817219\end{array}$

o $1.45208967-0.34145588 \quad 5.66305365$

Vibrational frequencies (in $\mathrm{cm}-1$ )

$\begin{array}{llllllllllllllll}-626.0 & 24.0 & 59.1 & 68.8 & 122.3 & 151.8 & 171.9 & 197.2 & 215.4 & 221.6 & 230.0 & 234.8 & 273.0 & 298.7\end{array}$ $311.8 \quad 327.0 \quad 342.2 \quad 375.5 \quad 401.8 \quad 422.4 \quad 440.3 \quad 460.8 \quad 547.1 \quad 596.6 \quad 606.1 \quad 654.8 \quad 702.9771 .8$ $808.9817 .1873 .5911 .6 \quad 935.3 \quad 994.7 \quad 1002.7 \quad 1005.7 \quad 1017.7 \quad 1024.5 \quad 1042.6 \quad 1060.4 \quad 1078.9$ 


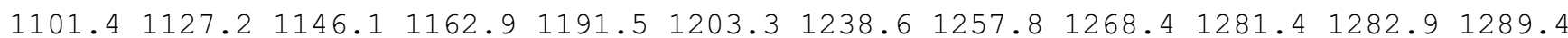

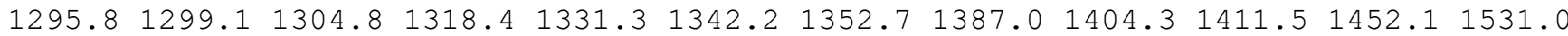

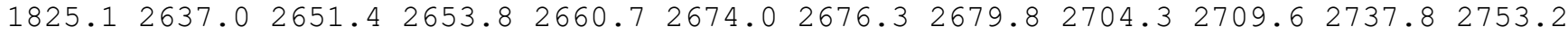
$2754.62776 .5 \quad 2782.3 \quad 5043.8$

name $=\operatorname{TS} 162$

energy (in $\mathrm{kcal} / \mathrm{mol})=36.660413$

$\mathrm{ZPE}($ in $\mathrm{kcal} / \mathrm{mol})=139.418$

$\mathrm{G}(\mathrm{in} \mathrm{kcal} / \mathrm{mol})=112.765$

Geometry

C $1.839308842 .50350177 \quad 4.04532138$

C $0.863886081 .49863828 \quad 3.90867868$

C $-2.12921123 \quad 0.45547035 \quad 2.24386587$

C $-1.49123982-0.60034067 \quad 1.42464785$

C $0.00355951-0.76691771 \quad 1.67577553$

C $0.43354821-0.912279263 .15970507$

C 1.335528890 .341548123 .04940683

C $0.94916596 \quad 0.48510695 \quad 1.52842803$

C $0.301393251 .79622661 \quad 1.13226883$

C $2.130697690 .19532626 \quad 0.61106815$

$\mathrm{H} \quad 0.42972561 \quad 3.72605490 \quad 5.04196669$

H 2.13082268 $3.23827726 \quad 3.34320392$

H $1.13917273 \quad 4.00592118 \quad 5.15594960$

$\mathrm{H}-1.93660400 \quad 0.57595805 \quad 3.30422945$

$\mathrm{H}-1.65867406-0.42913124 \quad 0.33700328$

$\mathrm{H}-1.99583069-1.57406840 \quad 1.64730606$

$\mathrm{H} 0.36606646-1.610369731 .05471233$

$\mathrm{H} \quad 0.97273484-1.83595109 \quad 3.38552128$

$\mathrm{H}-0.37847258-0.81846700 \quad 3.89321450$

H 2.40391463 $0.12590691 \quad 3.21360477$

$\mathrm{H}-0.20782475 \quad 1.70979634 \quad 0.16349947$

$\mathrm{H} 1.04052176 \quad 2.59999923 \quad 1.03142399$

$\mathrm{H}-0.44831428 \quad 2.14200739 \quad 1.85889731$

$\mathrm{H} \quad 1.81122283 \quad 0.13331153-0.43709894$

H $2.62467578-0.75199627 \quad 0.85617360$

$\mathrm{H} \quad 2.89174470 \quad 0.98196444 \quad 0.67169650$

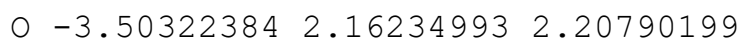

$\begin{array}{llll}0 & -2.93051951 & 1.23037000 & 1.57918034\end{array}$

$0-0.206988831 .471603254 .49072741$

Vibrational frequencies (in $\mathrm{cm}-1$ )

$\begin{array}{llllllllllllllll}-161.1 & 27.5 & 55.8 & 66.4 & 114.2 & 148.0 & 172.5 & 190.2 & 193.8 & 206.1 & 215.5 & 236.8 & 271.5 & 298.4\end{array}$ $302.9307 .2 \quad 327.9 \quad 357.5 \quad 373.5 \quad 398.4 \quad 445.2 \quad 541.4 \quad 570.2 \quad 576.8 \quad 593.3 \quad 647.7 \quad 671.9 \quad 712.1$ $790.4 \quad 837.6 \quad 890.0 \quad 901.2 \quad 926.3 \quad 942.9 \quad 997.0 \quad 1004.3 \quad 1011.6 \quad 1018.5 \quad 1035.4 \quad 1072.2 \quad 1089.0$

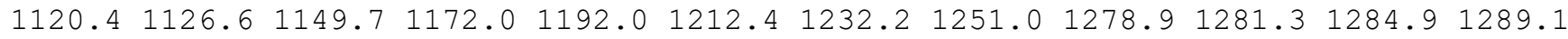

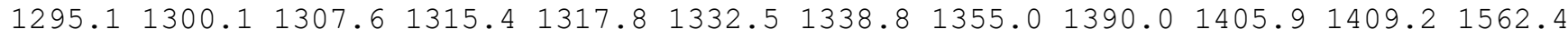
$1803.12643 .5 \quad 2657.4 \quad 2673.1 \quad 2673.8 \quad 2675.7 \quad 2677.6 \quad 2682.12712 .12720 .72724 .82728 .4$ $2758.12777 .6 \quad 2782.4 \quad 5375.0$

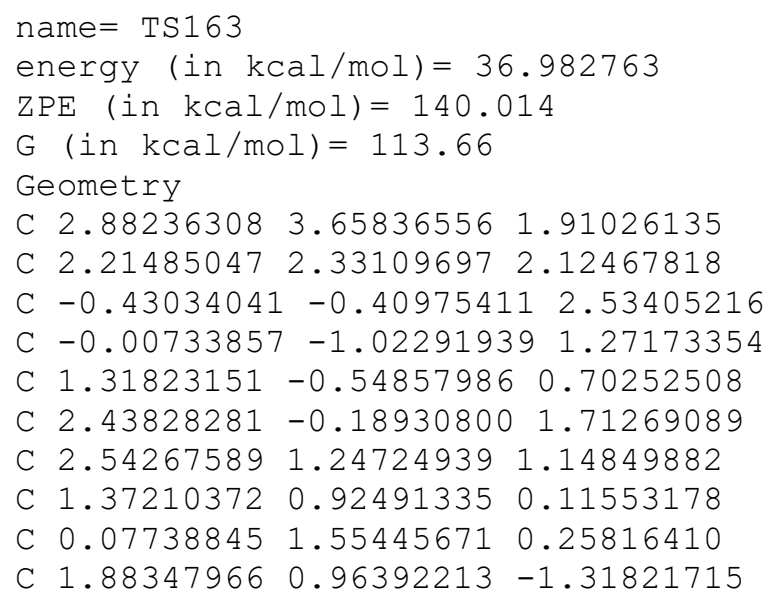


H $2.39214127 \quad 4.45295866 \quad 2.49416542$

H $2.884562793 .96199756 \quad 0.85809323$

H $3.93005082 \quad 3.62145254 \quad 2.24590766$

$\mathrm{H} \quad 0.02415053 \quad 0.47973221 \quad 2.96062646$

$\mathrm{H}-0.81925611-0.86223152 \quad 0.50739194$

$\mathrm{H} \quad 0.00123696-2.13827157 \quad 1.39764759$

$\mathrm{H} 1.66048484-1.27744324-0.05835585$

$\mathrm{H} 3.35086418-0.78201786 \quad 1.60097452$

H $2.14392423-0.23994172 \quad 2.76766621$

H $3.50228598 \quad 1.43703546 \quad 0.64211360$

$\mathrm{H}-0.19366704 \quad 2.65534224-0.85717913$

$\mathrm{H} \quad 0.30324258 \quad 3.09377239-0.39263733$

$\mathrm{H}-0.294443551 .94320383 \quad 1.17357509$

$\mathrm{H} 2.172355661 .97322239-1.62560393$

H $1.10878718 \quad 0.60663504 \quad-2.01283021$

H $2.76122534 \quad 0.32123806-1.45904606$

$\begin{array}{lllll}0 & -1.99296361 & -1.92068660 & 2.82915378\end{array}$

$0-1.40131745-0.88112166 \quad 3.24947470$

O 1.447565662 .166529513 .04345360

Vibrational frequencies (in $\mathrm{cm}-1$ )

$\begin{array}{lllllllllllllllll}-955.6 & 34.1 & 43.4 & 70.7 & 102.8 & 121.0 & 145.0 & 161.0 & 187.2 & 205.6 & 206.8 & 221.2 & 248.2304 .0\end{array}$

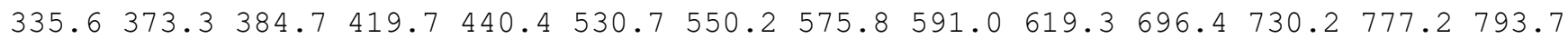
$820.9855 .6 \quad 903.1931 .9 \quad 994.4 \quad 1021.7 \quad 1027.8 \quad 1034.7 \quad 1042.5 \quad 1046.1 \quad 1052.7 \quad 1078.1 \quad 1093.0$ $1101.81123 .21158 .6 \quad 1161.0 \quad 1183.2 \quad 1197.9 \quad 1206.91220 .3 \quad 1245.4 \quad 1247.6 \quad 1260.91273 .1$

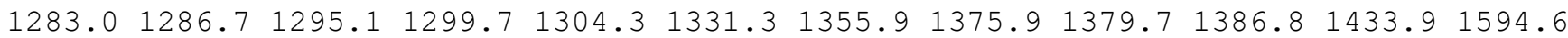
$1846.02612 .5 \quad 2663.8 \quad 2671.0 \quad 2675.8 \quad 2676.3 \quad 2677.92679 .02708 .4 \quad 2716.2 \quad 2717.5 \quad 2732.3$ $2760.52779 .22780 .3 \quad 4765.9$

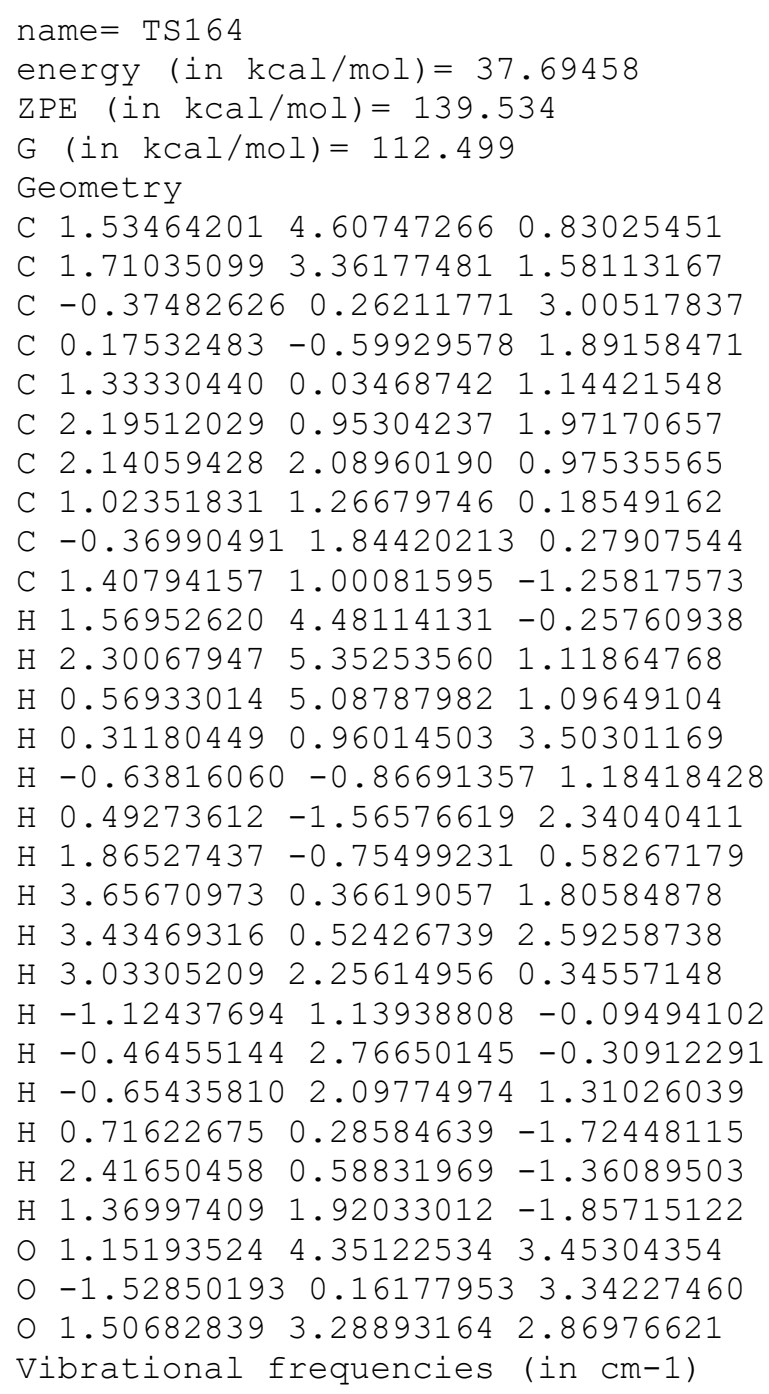


$\begin{array}{llllllllllllll}-1212.3 & 31.3 & 40.8 & 52.7 & 71.8 & 95.8 & 121.4 & 152.8 & 171.8 & 207.8 & 217.4 & 240.4 & 264.2 & 269.2\end{array}$ $285.0 \quad 325.7 \quad 382.3 \quad 406.5 \quad 436.0 \quad 443.9 \quad 524.4 \quad 566.1 \quad 578.1600 .1 \quad 639.7 \quad 688.5 \quad 730.6 \quad 771.7$ $819.7 \quad 849.7 \quad 963.0 \quad 987.7 \quad 998.0 \quad 1012.2 \quad 1024.0 \quad 1033.1 \quad 1039.7 \quad 1047.6 \quad 1079.91097 .1 \quad 1113.6$

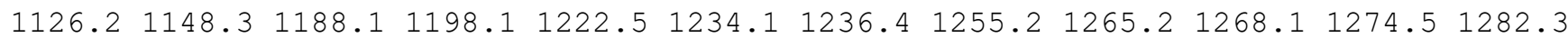

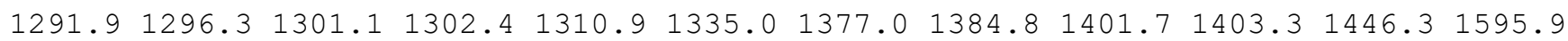
$1831.0 \quad 2638.3 \quad 2640.3 \quad 2658.7 \quad 2664.7 \quad 2670.92675 .4 \quad 2676.72678 .22716 .22719 .32741 .0$ $2770.82779 .0 \quad 2782.2 \quad 4436.0$

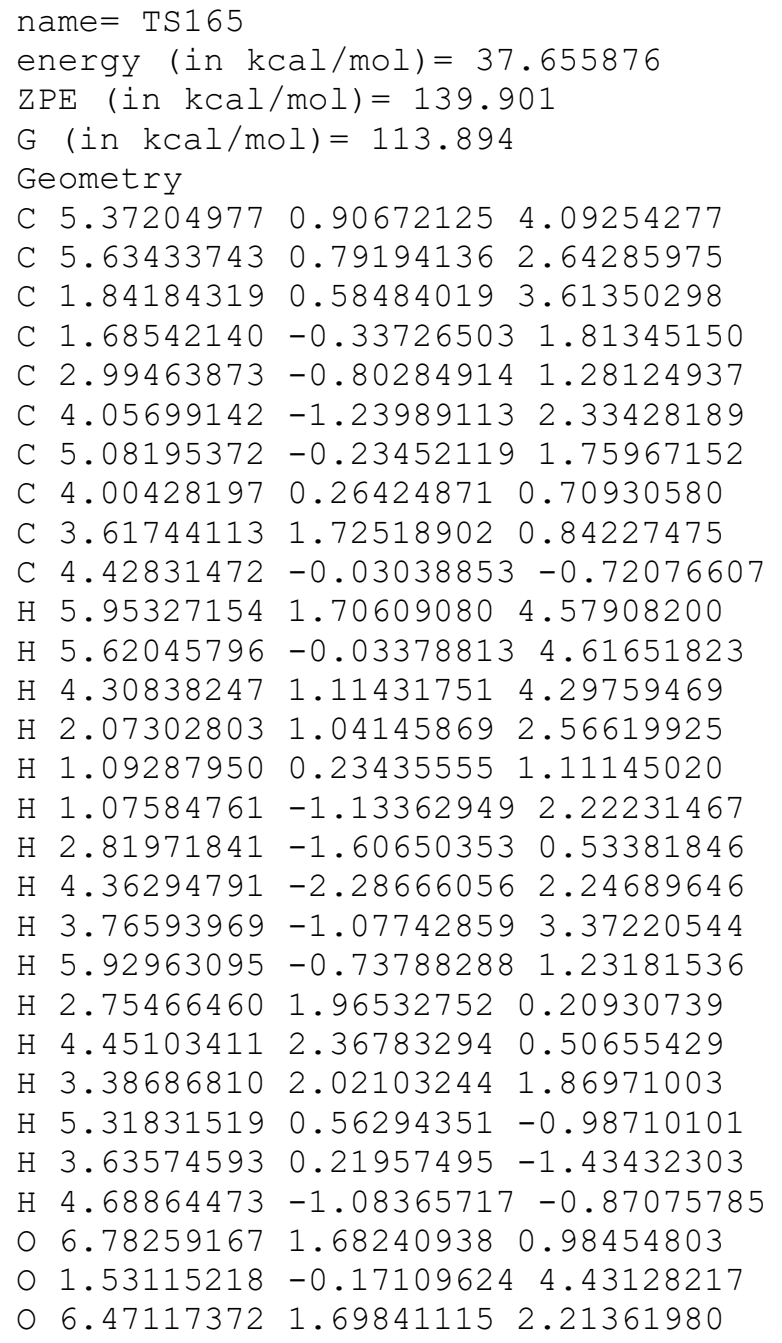


C $2.23225987 \quad 1.55315038 \quad 1.09041528$

C 0.772965631 .590544161 .00151952

C $2.93543811 \quad 1.62517304 \quad-0.25305273$

H $3.12268916 \quad 5.48210715 \quad 2.72672824$

H $1.39687425 \quad 5.45119121 \quad 2.41547748$

H $2.53890362 \quad 4.89893194 \quad 1.15279801$

$\mathrm{H}-0.81526648 \quad 0.20121213 \quad 2.10671336$

H $1.03884730-1.26237316 \quad 1.57658271$

$\mathrm{H} 1.47192207-1.074541133 .28140506$

H $3.29658157-0.40615990 \quad 1.45411486$

H $4.19469146 \quad 0.904096013 .33058042$

$\mathrm{H} 2.56327911 \quad 1.110850993 .99215445$

H 3.950414862 .761411861 .80586073

$\mathrm{H} 0.28037473 \quad 1.590119850 .05139469$

H $0.30312281 \quad 3.22596409 \quad 1.20221880$

$\mathrm{H} 0.05291470 \quad 2.92310411 \quad 1.89576769$

H 2.81694619 2.61154065 -0.71690089

H $2.54315850 \quad 0.87864817-0.95511458$

$\mathrm{H} \quad 4.01296937 \quad 1.43572415-0.16553105$

O $1.60934712 \quad 3.297423693 .91641220$

$\begin{array}{lllll}0 & 0.17918866 & 1.25657310 & 3.50310648\end{array}$

o $1.072215324 .27569837 \quad 4.47350652$

Vibrational frequencies (in $\mathrm{cm}-1$ )

$\begin{array}{lllllllllllllll}-505.1 & 58.9 & 81.7 & 95.4 & 105.4 & 127.2 & 147.2 & 169.0 & 193.6 & 222.1 & 267.9 & 290.6 & 311.5 & 325.2\end{array}$ $\begin{array}{llllllllllllllll}363.4 & 384.7 & 423.9 & 424.7 & 448.0 & 469.4 & 505.3 & 530.4 & 614.5 & 659.1 & 678.4 & 702.3 & 809.0 & 822.7\end{array}$ $837.3 \quad 905.5 \quad 925.3 \quad 945.6 \quad 954.6 \quad 994.6 \quad 1019.5 \quad 1029.8 \quad 1040.4 \quad 1049.4 \quad 1063.7 \quad 1067.2 \quad 1086.7$

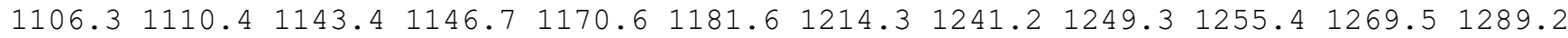

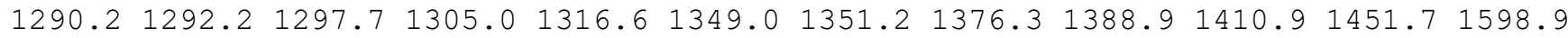
$1717.32639 .22653 .92671 .3 \quad 2672.0 \quad 2673.8 \quad 2676.62676 .92701 .02702 .02731 .42750 .1$ $2756.82770 .2 \quad 2780.9 \quad 4960.5$

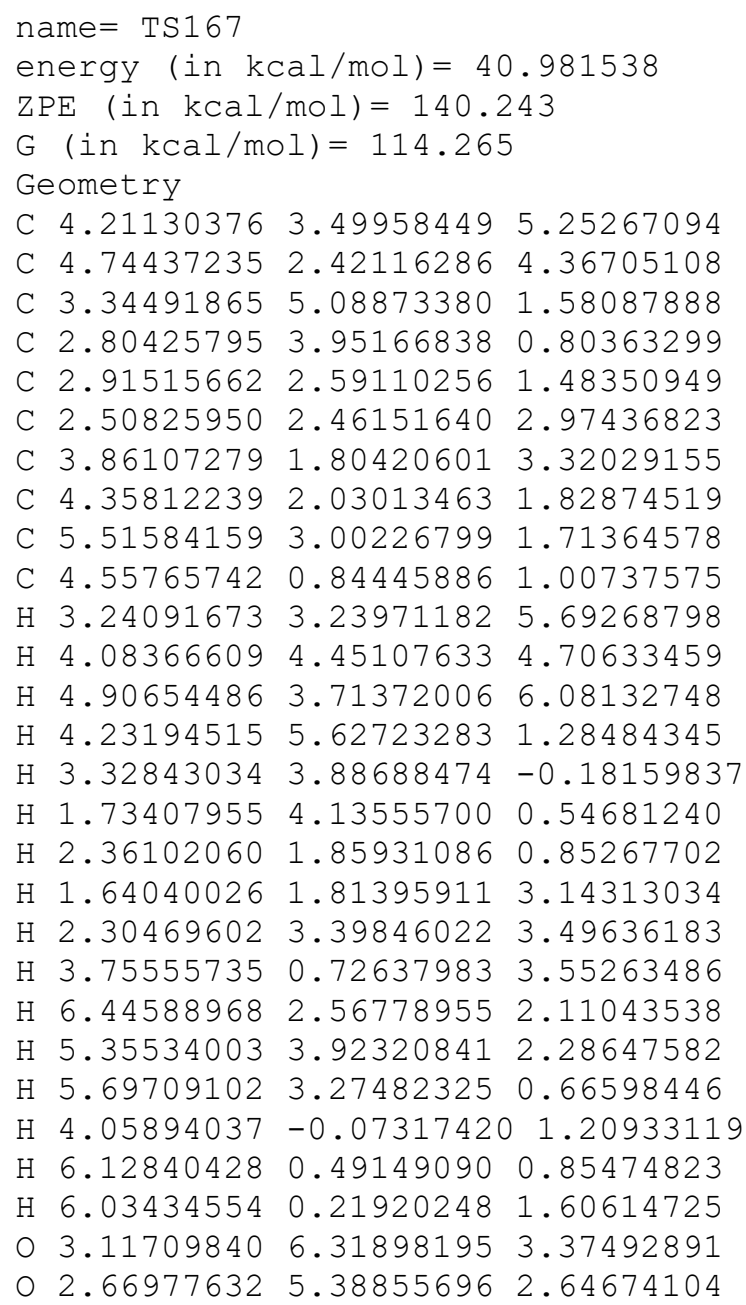


O 5.877039932 .013876324 .48888676

Vibrational frequencies (in $\mathrm{cm}-1$ )

$\begin{array}{lllllllllllllllll}-844.7 & 36.3 & 45.1 & 73.4 & 105.7 & 134.9 & 167.1 & 173.1 & 179.7 & 203.4 & 228.9 & 272.8 & 301.0 & 309.4\end{array}$ $\begin{array}{llllllllllllllll}316.7 & 353.6 & 368.6 & 421.6 & 467.0 & 474.0 & 502.5 & 545.0 & 585.7 & 609.1 & 644.3 & 693.7 & 784.9 & 805.7\end{array}$ $850.1885 .6 \quad 902.2 \quad 941.6 \quad 983.7 \quad 987.1 \quad 1017.0 \quad 1023.3 \quad 1042.4 \quad 1047.7 \quad 1068.5 \quad 1083.6 \quad 1095.6$

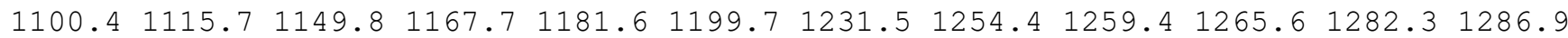

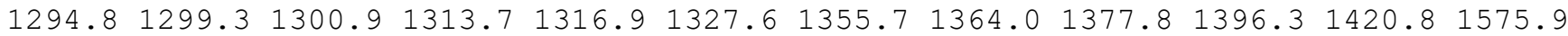
$1830.02642 .7 \quad 2645.0 \quad 2658.8 \quad 2671.6 \quad 2676.9 \quad 2677.12699 .02712 .2 \quad 2717.4 \quad 2718.92727 .4$ $2764.0 \quad 2774.4 \quad 2776.5 \quad 4838.9$

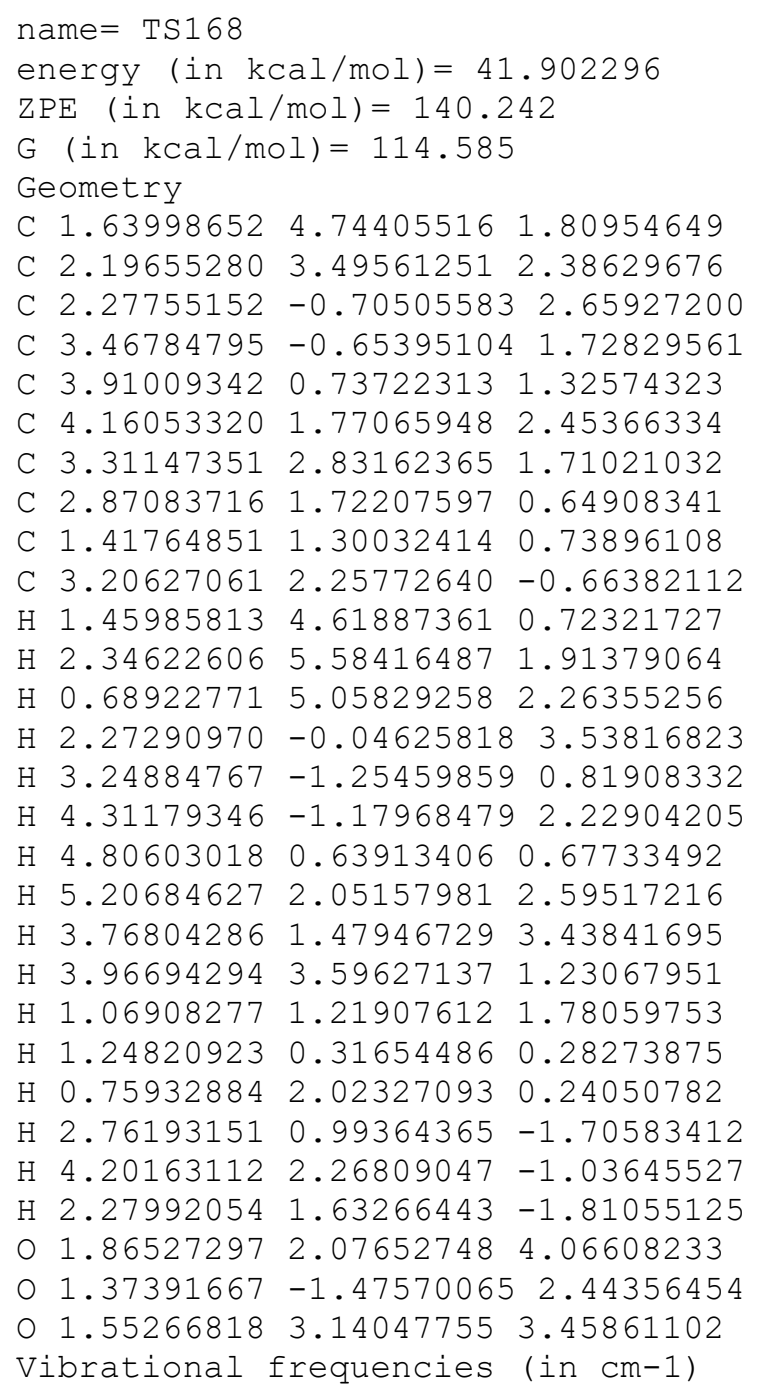


C $3.55191318 \quad 0.88684173 \quad 2.18901715$

C $3.626852292 .41597108 \quad 1.96810842$

C $3.872509792 .18114257 \quad 0.41550193$

$\begin{array}{llll}\text { C } 2.78946045 & 2.75253868 & -0.47896113\end{array}$

C $5.205972732 .46102159-0.08635848$

H $3.14978672 \quad 5.13819951 \quad 1.92741558$

H $2.90559830 \quad 4.733204293 .63614056$

H $1.51355993 \quad 5.10933162 \quad 2.61511390$

$\mathrm{H} \quad 0.98181137 \quad 0.74379034 \quad 0.86014155$

$\mathrm{H} 2.78156850 \quad 0.15725578-1.14680240$

$\mathrm{H} \quad 3.16890174-1.25669090 \quad-0.16385008$

H $4.82876462 \quad 0.16289717 \quad 0.52822572$

H $4.31505695 \quad 0.49045304 \quad 2.86485651$

H $2.57749591 \quad 0.53723255 \quad 2.55766322$

$\begin{array}{lllll}\mathrm{H} & 4.49453194 & 2.87997381 & 2.46089488\end{array}$

H $2.74721576 \quad 3.84550868-0.42505555$

$\mathrm{H} \quad 1.78825547 \quad 2.38644320-0.20829667$

H $2.96334970 \quad 2.47739740-1.52886050$

H $5.23493427 \quad 4.14654956-0.22852376$

H $5.26016598 \quad 3.79684258-0.95687311$

H $6.07213068 \quad 2.44864620 \quad 0.52653436$

$0-0.35105413-1.358235590 .90078534$

$0.82957605-1.309407740 .46581417$

O 1.280389572 .610571512 .26863894

Vibrational frequencies (in cm-1)

$\begin{array}{lllllllllllllll}-952.1 & 24.6 & 42.4 & 54.1 & 88.9 & 101.2 & 147.3 & 165.4 & 180.9 & 201.1 & 224.4 & 251.8 & 257.9 & 289.0\end{array}$ $\begin{array}{llllllllllllllll}323.8 & 354.7 & 361.6 & 389.7 & 425.6 & 518.2 & 550.4 & 570.4 & 584.0 & 601.5 & 617.7 & 694.6 & 777.5 & 813.4\end{array}$ $831.1886 .9907 .2 \quad 950.6 \quad 1000.7 \quad 1015.1 \quad 1018.6 \quad 1033.1 \quad 1047.2 \quad 1052.4 \quad 1069.1 \quad 1083.6 \quad 1097.4$

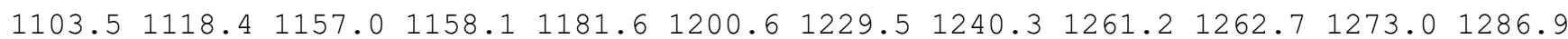

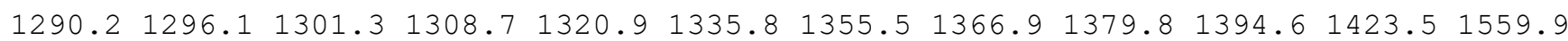
$1852.12637 .32660 .72665 .2 \quad 2671.6 \quad 2675.12679 .2 \quad 2685.62702 .02721 .62726 .02734 .0$ $2756.32777 .12781 .0 \quad 4780.3$
name $=$ TS170
energy (in $\mathrm{kcal} / \mathrm{mol})=42.145961$
$\operatorname{ZPE}(\mathrm{in} \mathrm{kcal} / \mathrm{mol})=140.348$
$\mathrm{G}($ in $\mathrm{kcal} / \mathrm{mol})=115.046$
Geometry
C $2.644664872 .73976592 \quad 1.99951808$
C 3.020646811 .963658460 .78722426
C $1.42820690-0.59912340-1.59059695$
C $1.58873271 \quad 0.30776735-2.80909409$
C $2.00713250 \quad 1.72238090-2.45195280$
C $3.399940371 .86093911-1.78847096$
C $2.776248452 .53484187-0.53811359$
C $1.32918125 \quad 2.32659233-1.14657943$
C $0.51010074 \quad 1.25534854 \quad-0.60161794$
C $0.61401010 \quad 3.65023340-1.37216362$
$\mathrm{H} 1.72965618 \quad 3.33056023 \quad 1.84480261$
H $3.43945516 \quad 3.46324005 \quad 2.25804361$
H 2.48828854 2.11349260 2.89116711
$\mathrm{H} 2.22410145-0.59558339-0.83961082$
$\mathrm{H} \quad 0.63774304 \quad 0.31765739-3.37981760$
H $2.33611741-0.15361780-3.48574647$
$\mathrm{H} \quad 1.87825253 \quad 2.39120701 \quad-3.32041921$
H $4.105790232 .49362980-2.33091710$
$\mathrm{H} \quad 3.90447194 \quad 0.90245226-1.59730764$
H $3.03130913 \quad 3.62000660-0.51713350$
H $0.52546667 \quad 1.15631843 \quad 0.97203386$
$\mathrm{H} \quad 0.04451056 \quad 1.80630153 \quad 0.91188744$
$\mathrm{H}-0.50140620 \quad 1.11998313-0.91111997$
$\mathrm{H}-0.30029393 \quad 3.50804571-1.96317099$
$\mathrm{H} 1.23461927 \quad 4.36929329-1.92015604$
$\mathrm{H} \quad 0.32716479 \quad 4.12130142-0.42715369$ 
$04.05534781 \quad 0.05780175 \quad 0.26891030$

O $0.51010910-1.38757676-1.55333557$

O 3.596258790 .845654001 .12615334

Vibrational frequencies (in $\mathrm{cm}-1$ )

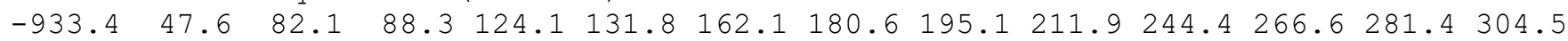

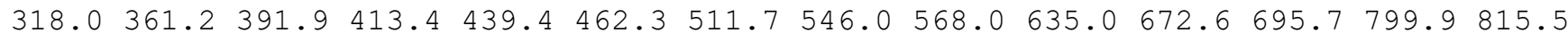
$863.8 \quad 882.8 \quad 923.6 \quad 939.5 \quad 996.8 \quad 1008.1 \quad 1016.6 \quad 1027.8 \quad 1039.1 \quad 1039.3 \quad 1062.4 \quad 1090.3 \quad 1092.4$ $\begin{array}{lllllllllllll}1110.7 & 1139.9 & 1154.9 & 1162.6 & 1175.7 & 1184.6 & 1218.5 & 1247.9 & 1261.4 & 1262.2 & 1266.9 & 1282.8\end{array}$

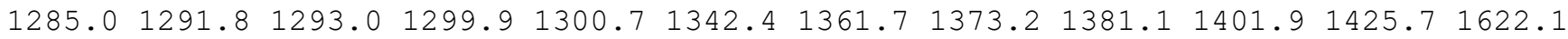
1798.02651 .22653 .22661 .52665 .12668 .42672 .62679 .72703 .72720 .12722 .32744 .5 $2756.22771 .6 \quad 2781.7 \quad 4745.0$

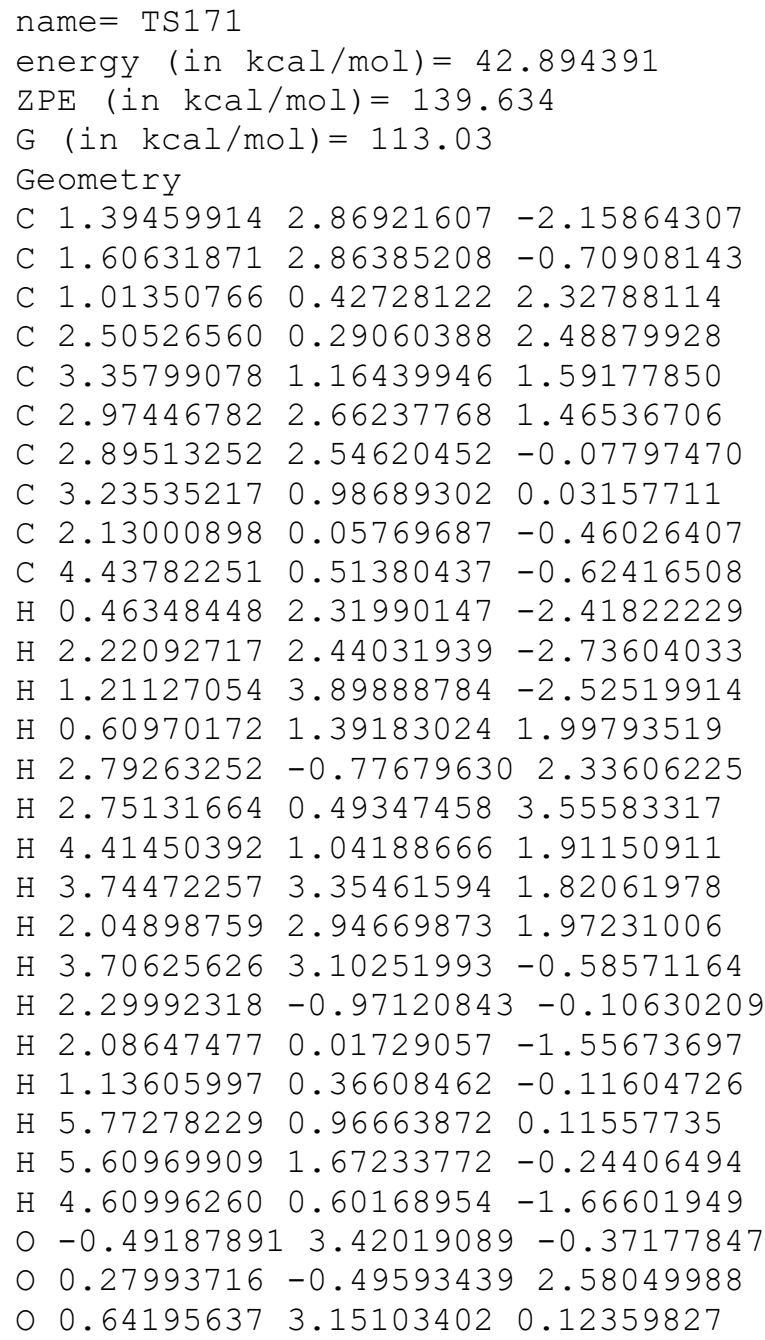
$291.8 \quad 312.1 \quad 377.3 \quad 400.3 \quad 443.3 \quad 449.7 \quad 511.3 \quad 541.9 \quad 595.0 \quad 632.8 \quad 672.2 \quad 685.6 \quad 741.5 \quad 781.3$ $801.7 \quad 855.9908 .8 \quad 933.4 \quad 1001.8 \quad 1010.2 \quad 1023.0 \quad 1028.6 \quad 1040.2 \quad 1051.9 \quad 1065.5 \quad 1074.7 \quad 1092.1$ $1109.4 \quad 1122.1 \quad 1132.8 \quad 1161.3 \quad 1176.5 \quad 1199.3 \quad 1218.9 \quad 1228.3 \quad 1256.4 \quad 1261.6 \quad 1275.7 \quad 1287.3$ $1288.51294 .0 \quad 1300.3 \quad 1304.9 \quad 1324.8 \quad 1350.7 \quad 1371.0 \quad 1383.3 \quad 1392.2 \quad 1404.7 \quad 1450.61588 .7$ $1833.52631 .92644 .2 \quad 2652.8 \quad 2664.92667 .92673 .8 \quad 2676.72708 .3 \quad 2719.02728 .02734 .8$ $2762.92768 .6 \quad 2776.2 \quad 4773.6$

name $=$ TS172

energy $($ in $\mathrm{kcal} / \mathrm{mol})=39.35058$

$\mathrm{ZPE}($ in $\mathrm{kcal} / \mathrm{mol})=143.465$

$\mathrm{G}($ in $\mathrm{kcal} / \mathrm{mol})=120.528$

Geometry

C $3.54563630 \quad 1.786990892 .86815060$

C 2.666543510 .812917522 .31883105

C $1.26564681 \quad 1.26019337 \quad 1.81396536$ 
$\begin{array}{lllll}\text { C } & 0.65503730 & 0.37769951 & 0.70418736\end{array}$

C $1.56733980-0.78405095 \quad 0.31183939$

C $2.03422847-1.487217381 .62382177$

C $3.19250193-0.440101871 .66808673$

C $3.05423958-0.298756490 .09713889$

C $3.300536091 .05415854-0.53591716$

C $3.89307888-1.34229548-0.63493108$

H $4.59442603 \quad 1.59555252 \quad 2.96234104$

H 2.79418994 1.36186519 4.26454200

H $3.30084862 \quad 2.82898092 \quad 2.88421412$

$\mathrm{H} 1.28498573 \quad 2.32948696 \quad 1.49911003$

H $0.42449944 \quad 0.99424363-0.18337916$

$\mathrm{H}-0.31958239-0.01813357 \quad 1.05793878$

$\mathrm{H} 1.15220806-1.41600942-0.47330026$

H 2.36766844 $-2.51823427 \quad 1.49594711$

H $1.33002693-1.467591252 .45727176$

H $4.15813818 \quad-0.775992292 .04619924$

$\mathrm{H} 2.80563305 \quad 1.88207836-0.02088837$

H 2.95243692 1.06800410 -1.57633374

H $4.372435091 .29486396-0.53994513$

$\mathrm{H} 3.74931274-2.35261066-0.23663371$

H $4.96252087-1.11329114-0.55685838$

H $3.63775444-1.37454798-1.70059803$

$\begin{array}{lllll}0 & 0.83710740 & 0.32295238 & 3.88672521\end{array}$

○ $0.40217540 \quad 1.31068845 \quad 2.94345385$

O $2.230735350 .39719713 \quad 3.81268595$

Vibrational frequencies (in $\mathrm{cm}-1$ )

$\begin{array}{lllllllllllllllllll}-1332.4 & 84.8 & 106.3 & 150.6 & 177.6 & 190.3 & 228.9 & 235.6 & 292.8 & 324.6 & 366.6 & 384.7 & 431.9 & 437.3\end{array}$

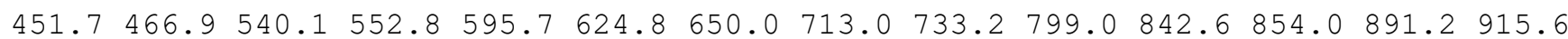

$939.5954 .0 \quad 979.3 \quad 1002.6 \quad 1007.4 \quad 1010.3 \quad 1017.5 \quad 1024.8 \quad 1049.5 \quad 1065.8 \quad 1078.01088 .9 \quad 1092.6$

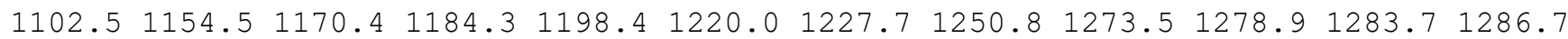
$1292.91298 .81301 .6 \quad 1307.91316 .5 \quad 1320.7 \quad 1368.2 \quad 1376.1 \quad 1383.6 \quad 1406.8 \quad 1419.7 \quad 1547.3$ $2188.22658 .42672 .7 \quad 2675.92676 .5 \quad 2678.4 \quad 2681.2 \quad 2689.72735 .92740 .8 \quad 2748.5 \quad 2752.2$ $2767.5 \quad 2781.8 \quad 2785.4 \quad 2797.9$

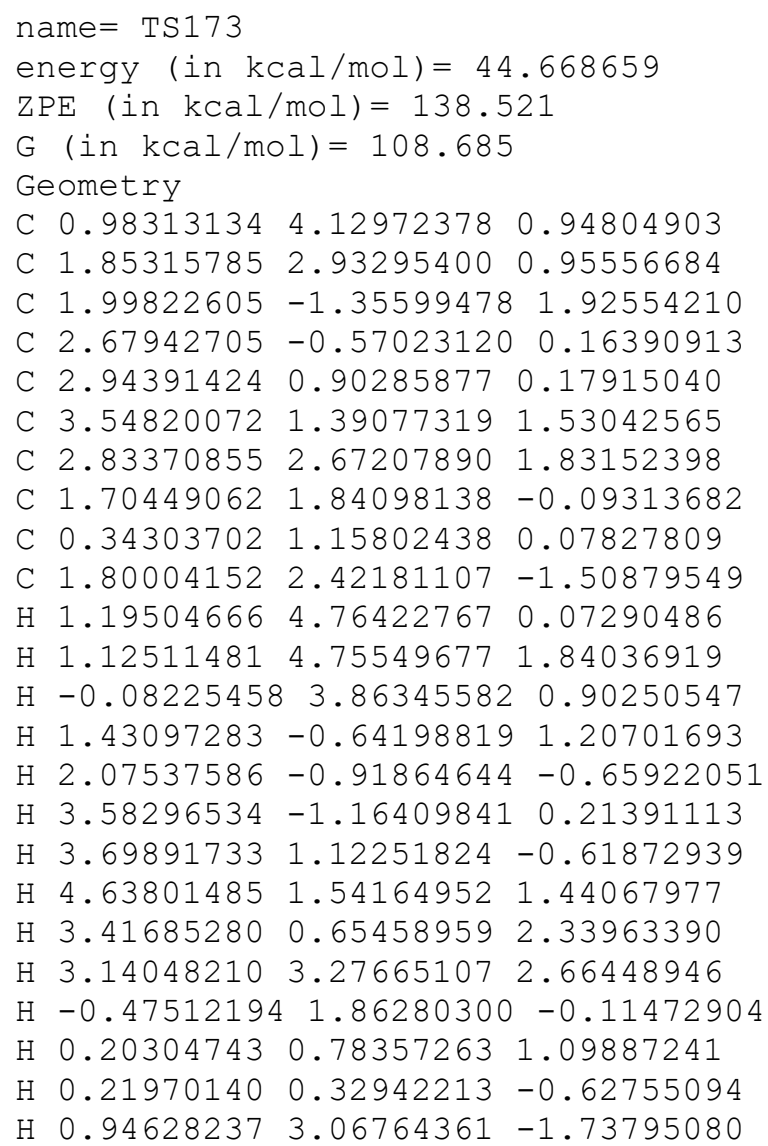


$\mathrm{H} \quad 1.82417504 \quad 1.62421105-2.25965923$

H 2.70767983 $3.02411810-1.63197766$

o $1.344485491 .57443735 \quad 3.68531649$

$\begin{array}{lllll}0 & 2.84210912 & -2.00854190 & 2.36881207\end{array}$

O $0.21384701 \quad 1.47850180 \quad 3.64580881$

Vibrational frequencies (in $\mathrm{cm}-1$ )

$\begin{array}{llllllllllllll}-1698.3 & 13.1 & 27.2 & 42.1 & 50.6 & 60.2 & 71.1 & 82.0 & 97.5 & 106.5 & 192.6 & 207.3 & 216.9 & 228.9\end{array}$ $263.3 \quad 267.9 \quad 285.8 \quad 314.9 \quad 350.5 \quad 378.0 \quad 472.3 \quad 499.2 \quad 567.0 \quad 608.0 \quad 647.7 \quad 681.1 \quad 759.8 \quad 796.5$

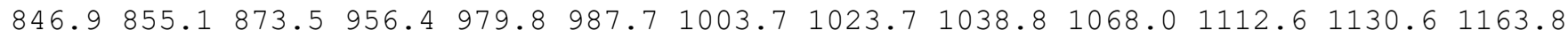
$1172.81180 .3 \quad 1206.2 \quad 1226.2 \quad 1238.0 \quad 1271.6 \quad 1276.8 \quad 1285.4 \quad 1298.5 \quad 1300.1 \quad 1301.8 \quad 1308.3$

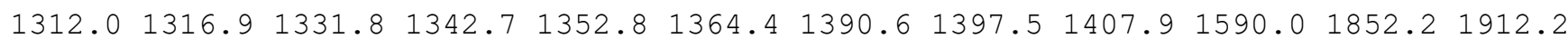
$2192.72665 .32668 .32668 .92675 .0 \quad 2675.5 \quad 2678.32680 .52689 .22699 .62751 .62766 .1$ $2774.72777 .7 \quad 2781.6 \quad 2786.2$

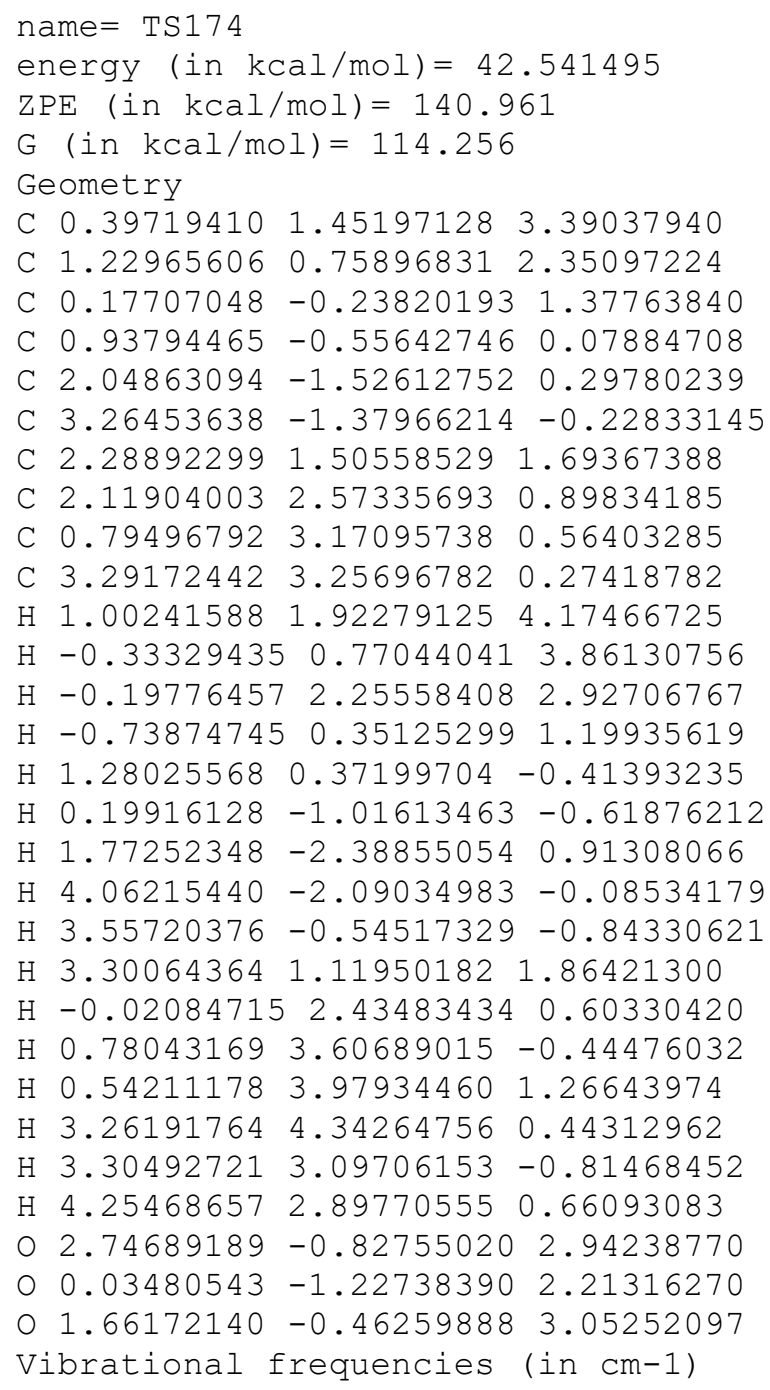


C $0.36693551 \quad 1.76291735 \quad 3.58905980$

C $0.19845942 \quad 0.26094880 \quad 3.27521067$

C $1.42748476-0.42420390 \quad 2.67070235$

C $1.41536304-0.37832780 \quad 1.17886241$

C $2.50432609-0.13263488 \quad 0.45051749$

C $1.58871579 \quad 2.43238618 \quad 3.11704439$

C $1.71041728 \quad 3.01631699 \quad 1.91635988$

C $0.62435840 \quad 3.03294755 \quad 0.89526188$

C $2.98250622 \quad 3.68138868 \quad 1.50493835$

$\mathrm{H}-0.79376280 \quad 3.58957765 \quad 3.82182846$

$\mathrm{H}-0.50030835 \quad 2.10198609 \quad 5.38433823$

$\mathrm{H}-1.78590327 \quad 2.09707221 \quad 3.78649128$

$\mathrm{H}-0.71436937 \quad 0.08579258 \quad 2.66020050$

$\mathrm{H} 1.44272721-1.48894083 \quad 3.00380579$

H 2.35779564 $0.01063813 \quad 3.09090116$

$\mathrm{H} \quad 0.45000334 \quad-0.58395573 \quad 0.71579842$

$\mathrm{H} 2.50901493-0.11963410 \quad-0.62747196$

H $3.47535792 \quad 0.07395941 \quad 0.87219226$

H $2.41191278 \quad 2.43154137 \quad 3.83344366$

$\mathrm{H} \quad 0.67883382 \quad 3.90866446 \quad 0.23671184$

$\mathrm{H}-0.37719357 \quad 3.03585627 \quad 1.35312237$

$\mathrm{H} \quad 0.68489576 \quad 2.13752230 \quad 0.25603977$

$\mathrm{H} \quad 3.79348421 \quad 3.54141439 \quad 2.23196194$

H 2.838610424 .765736951 .38748359

H $3.34094975 \quad 3.29273552 \quad 0.54072424$

$0.68349096 \quad 0.22909698 \quad 5.51833886$

$0-0.13272974-0.37647411 \quad 4.50480753$

O $0.59907666 \quad 1.58204167 \quad 5.23450730$

Vibrational frequencies (in $\mathrm{cm}-1$ )

$\begin{array}{llllllllllllll}-1445.5 & 32.1 & 47.1 & 53.2 & 76.0 & 84.2 & 100.0 & 105.0 & 151.6 & 198.1 & 246.2 & 271.5 & 286.6 & 346.3\end{array}$ $360.4 \quad 398.9 \quad 412.0 \quad 428.0 \quad 476.1 \quad 490.2 \quad 503.8 \quad 597.8 \quad 602.9 \quad 609.0 \quad 653.8 \quad 767.7 \quad 836.5 \quad 866.2$ $911.2935 .6 \quad 956.7 \quad 972.1 \quad 990.4 \quad 1002.1 \quad 1007.9 \quad 1019.4 \quad 1024.7 \quad 1064.6 \quad 1079.4 \quad 1083.4 \quad 1092.1$ $1103.11119 .7 \quad 1145.21151 .2 \quad 1218.2 \quad 1250.1 \quad 1267.2 \quad 1269.1 \quad 1273.2 \quad 1278.1 \quad 1284.01286 .0$

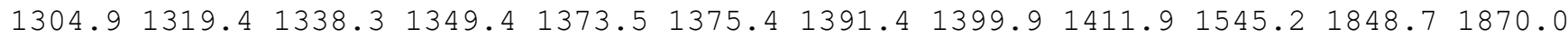
$2117.72649 .6 \quad 2659.8 \quad 2669.7 \quad 2672.5 \quad 2674.7 \quad 2678.42716 .62733 .12736 .22749 .12757 .3$ $2775.5 \quad 2779.8 \quad 2790.3 \quad 2796.1$

name $=\mathrm{TS} 176$

energy (in $\mathrm{kcal} / \mathrm{mol})=46.083952$

$\mathrm{ZPE}(\mathrm{in} \mathrm{kcal} / \mathrm{mol})=138.003$

$\mathrm{G}(\mathrm{in} \mathrm{kcal} / \mathrm{mol})=110.479$

Geometry

C $0.73431428 \quad 0.86811743 \quad 5.58111206$

C $1.14591305 \quad 0.05934693 \quad 4.49600172$

C $0.19666975 \quad 0.66771207 \quad 3.17841671$

C $0.59775344 \quad-4.09591026 \quad 4.36646826$

C $1.42463352-3.409969295 .15673610$

C $1.06682489-2.08034248 \quad 5.70948676$

C $2.53242807 \quad 0.00544433 \quad 4.03675152$

C $3.02980882-0.713767893 .01618098$

C $2.24824114-1.57745986 \quad 2.09134465$

C $4.49391867-0.67092412 \quad 2.71394219$

$\mathrm{H} 1.367920991 .645290495 .96953700$

$\mathrm{H} \quad 0.02807106 \quad 0.47231256 \quad 6.29935775$

$\mathrm{H}-0.32930350 \quad 1.78019042 \quad 4.86252729$

$\mathrm{H}-0.54017417 \quad 1.71087871 \quad 4.01299124$

$\mathrm{H} \quad 0.83407503-5.06108678 \quad 3.94579622$

$\mathrm{H}-0.38915825-3.73936284 \quad 4.08632331$

H 2.40914507 $-3.78159962 \quad 5.43672323$

H $1.90603967-1.54045284 \quad 6.16195336$

$\mathrm{H} \quad 0.24082283-2.12420555 \quad 6.43852018$

$\mathrm{H} \quad 3.20650984 \quad 0.63376923 \quad 4.62792382$

H $2.78448661-2.50017475 \quad 1.82867412$

H $2.04727690-1.03849974 \quad 1.14615624$ 
$\mathrm{H} \quad 1.26286227-1.88196398 \quad 2.46562541$

$\mathrm{H} \quad 4.97108002-1.634582032 .94745485$

$\mathrm{H} \quad 5.03030395 \quad 0.09975476 \quad 3.28276194$

H $4.67807544-0.46851224 \quad 1.64827297$

$0-0.70604082-1.265379724 .47787897$

$0.15782165 \quad 0.428988812 .04576496$

O $0.57525114-1.343147914 .47196424$

Vibrational frequencies (in $\mathrm{cm}-1$ )

$\begin{array}{llllllllllllllll}-1637.2 & 31.3 & 40.9 & 75.6 & 82.7 & 86.5 & 109.9 & 117.1 & 118.4 & 152.3 & 183.1 & 203.2 & 227.2 & 248.4\end{array}$ $294.7318 .3 \quad 354.8 \quad 384.0 \quad 402.2 \quad 454.1 \quad 466.8 \quad 487.5 \quad 497.7 \quad 540.9 \quad 593.8 \quad 623.3 \quad 657.6 \quad 769.9$

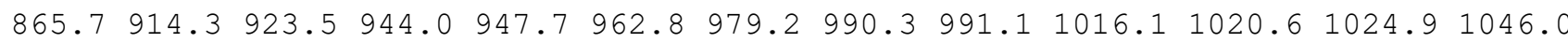

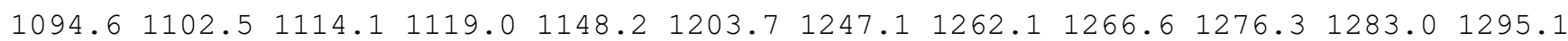

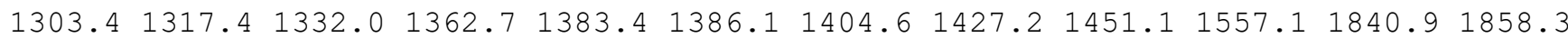
$1946.62652 .32656 .12667 .12669 .5 \quad 2673.2 \quad 2699.52712 .12729 .82744 .2 \quad 2756.42771 .0$ $2774.0 \quad 2776.3 \quad 2779.6 \quad 3341.8$

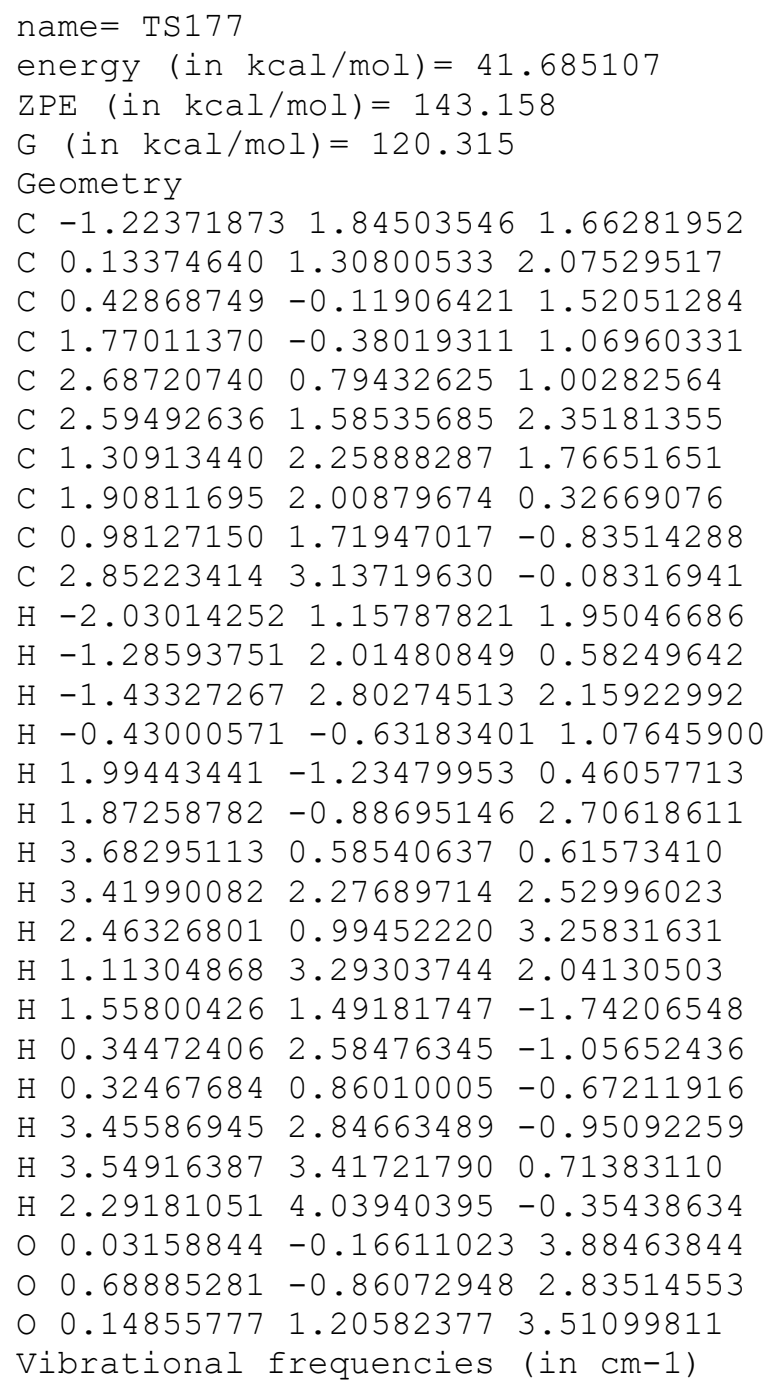




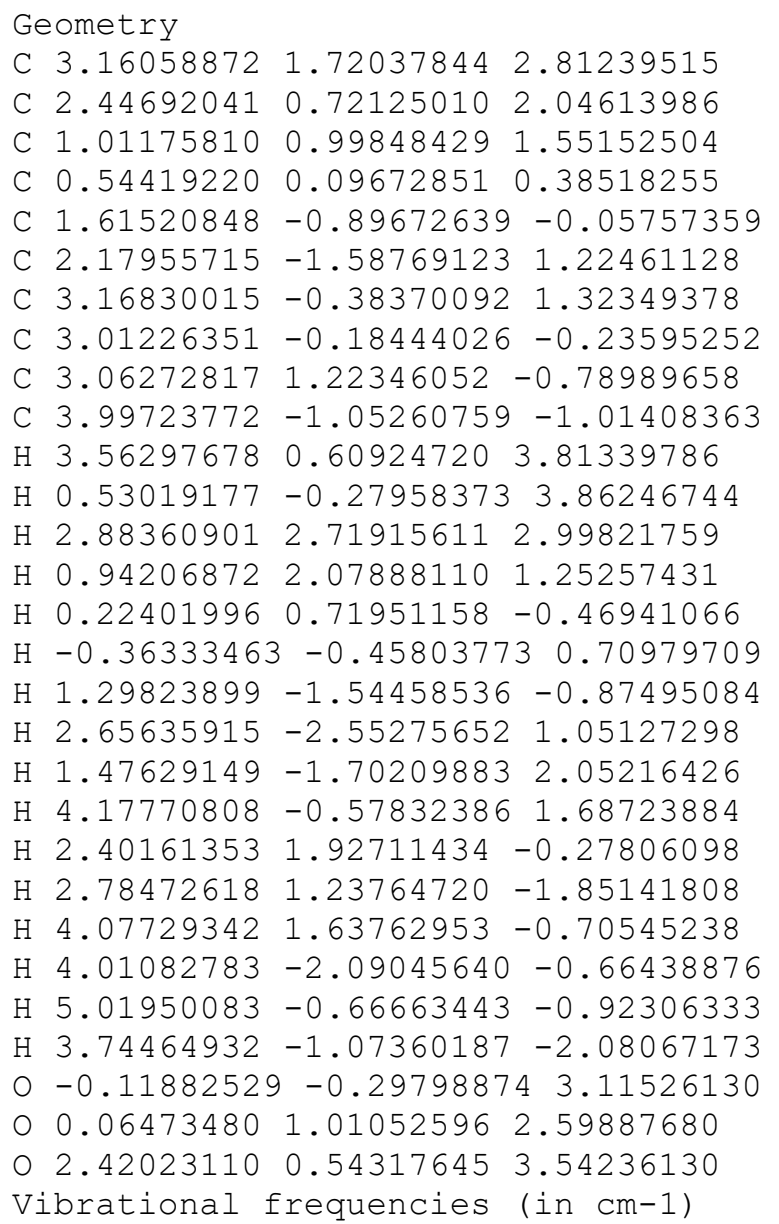

$\begin{array}{llllllllllllllllllll}-1435.4 & 54.8 & 76.0 & 129.2 & 163.0 & 168.4 & 187.9 & 223.1 & 259.8 & 272.3 & 297.5 & 322.1 & 342.7 & 380.4\end{array}$ $387.2 \quad 416.8 \quad 439.8 \quad 450.0 \quad 507.5 \quad 511.3 \quad 583.3 \quad 669.9 \quad 711.1 \quad 775.5 \quad 793.1 \quad 840.6 \quad 888.2 \quad 893.8$ $917.0 \quad 944.3 \quad 959.1 \quad 983.1 \quad 1005.3 \quad 1006.9 \quad 1014.7 \quad 1018.2 \quad 1042.5 \quad 1045.2 \quad 1054.8 \quad 1085.0 \quad 1088.5$ $1115.0 \quad 1149.1 \quad 1173.1 \quad 1186.8 \quad 1201.8 \quad 1216.1 \quad 1222.4 \quad 1250.6 \quad 1274.2 \quad 1278.2 \quad 1285.9 \quad 1291.3$ $1294.81297 .51301 .3 \quad 1306.2 \quad 1317.91322 .7 \quad 1346.8 \quad 1376.4 \quad 1380.4 \quad 1406.5 \quad 1418.7 \quad 1473.9$ $2467.72649 .22666 .2 \quad 2673.12675 .92678 .12680 .72690 .82737 .4 \quad 2747.92751 .5 \quad 2766.9$ $2767.12781 .92785 .2 \quad 2846.5$

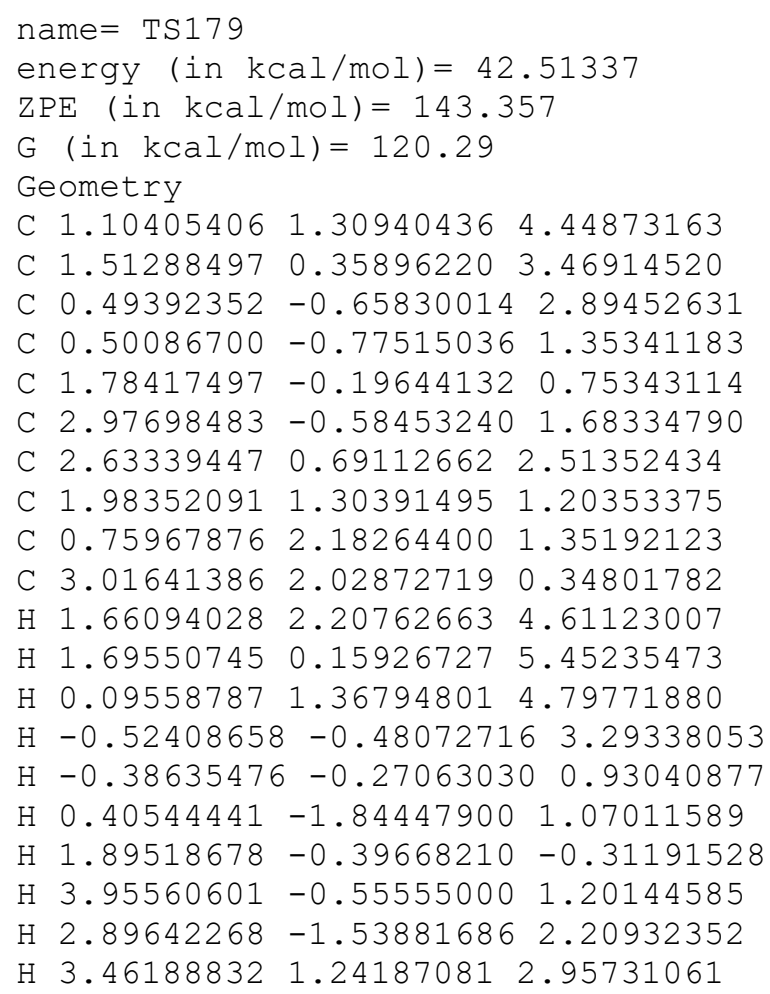


H $0.29626187 \quad 2.38089109 \quad 0.37814189$

$\mathrm{H} 1.02510973 \quad 3.15005121 \quad 1.79956870$

$\mathrm{H}-0.012594121 .752071551 .99861105$

$\mathrm{H} \quad 3.92649608 \quad 1.43771644 \quad 0.19708573$

$\mathrm{H} \quad 3.31842796 \quad 2.97476904 \quad 0.81317888$

$\mathrm{H} 2.61206676 \quad 2.26166866-0.64404988$

o $1.49415002-1.83618893 \quad 4.61720264$

$0.92626611-1.953876343 .32442241$

O $2.15232647-0.572305624 .61058619$

Vibrational frequencies (in $\mathrm{cm}-1$ )

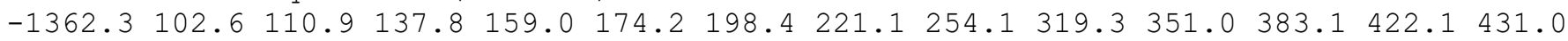

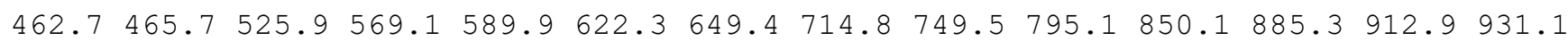
$942.4950 .6 \quad 975.8 \quad 988.6 \quad 1005.5 \quad 1009.5 \quad 1015.9 \quad 1029.8 \quad 1065.2 \quad 1074.2 \quad 1074.6 \quad 1088.8 \quad 1095.8$

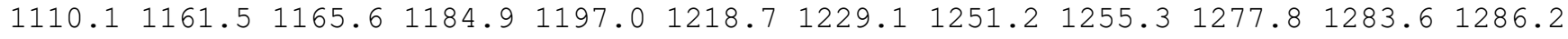

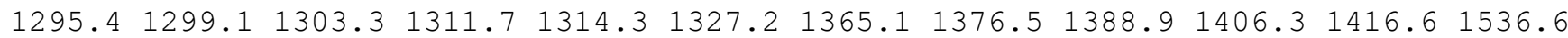
$2166.22658 .92670 .8 \quad 2676.2 \quad 2677.3 \quad 2681.6 \quad 2684.3 \quad 2685.82738 .12739 .72749 .02753 .4$ $2764.92781 .4 \quad 2783.7 \quad 2801.5$

name $=\operatorname{TS} 180$

energy (in $\mathrm{kcal} / \mathrm{mol})=47.252832$

$\operatorname{ZPE}($ in $\mathrm{kcal} / \mathrm{mol})=138.683$

$\mathrm{G}(\mathrm{in} \mathrm{kcal} / \mathrm{mol})=112.506$

Geometry

C $0.76891796 \quad 3.63823827 \quad 3.33983164$

C $1.26633561 \quad 2.24845484 \quad 3.47303108$

C $1.07156136-1.69019568 \quad 3.73541754$

C $1.25326765-1.80563360 \quad 2.25105821$

C $1.94471664-0.54448571 \quad 1.73636063$

C $2.66150045 \quad 0.353662392 .69894581$

C $1.91961872 \quad 1.55806908 \quad 2.35309887$

C $1.01922968 \quad 0.69362547 \quad 1.35518011$

C $-0.44032287 \quad 0.59862990 \quad 1.73445151$

C $1.18168147 \quad 1.14473957-0.08623886$

$\mathrm{H} 0.11830556 \quad 3.94366398 \quad 4.17516571$

$\mathrm{H} \quad 0.18119722 \quad 3.75270372 \quad 2.41089255$

$\mathrm{H} \quad 1.59188391 \quad 4.36991606 \quad 3.29187349$

$\mathrm{H}-0.39254889-1.59882474 \quad 4.99204710$

H $0.31391129-1.985494891 .69722057$

H $1.89292844 \quad-2.689614312 .05224643$

H $2.59726340 \quad-0.82807340 \quad 0.88387968$

H $3.55621990 \quad 0.19786410 \quad 3.21556151$

H $2.09518614 \quad 0.06894133 \quad 4.49372836$

$\mathrm{H} 2.51049070 \quad 2.33181516 \quad 1.80195082$

$\mathrm{H}-0.56963425 \quad 0.25293149 \quad 2.77108044$

$\mathrm{H}-0.97092088-0.11666231 \quad 1.09246322$

$\mathrm{H}-0.94835220 \quad 1.56388615 \quad 1.64158405$

$\mathrm{H} \quad 0.71914083 \quad 2.12371384 \quad-0.25419474$

$\mathrm{H} \quad 0.70893663 \quad 0.43398572 \quad-0.77527005$

H 2.23684411 $1.22242507-0.37527943$

o $1.20477377 \quad 0.40532711 \quad 4.88341853$

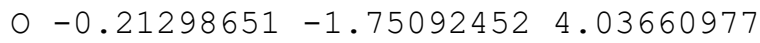

o $1.286392881 .83847196 \quad 4.70356798$

Vibrational frequencies (in $\mathrm{cm}-1$ )

$\begin{array}{llllllllllllll}-483.1 & 39.3 & 50.2 & 68.0 & 87.8 & 117.2 & 130.3 & 166.7 & 187.6 & 214.2 & 224.8 & 268.5 & 280.1 & 305.8\end{array}$ $326.4 \quad 352.5 \quad 386.5 \quad 417.6 \quad 431.8 \quad 436.0 \quad 521.6 \quad 530.9 \quad 567.6 \quad 609.1 \quad 640.3 \quad 734.2 \quad 748.9 \quad 810.2$

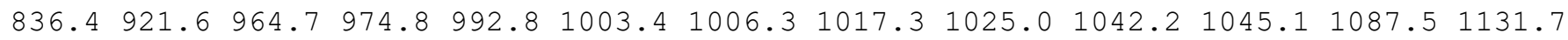
$1146.01163 .2 \quad 1198.4 \quad 1213.1 \quad 1230.0 \quad 1248.4 \quad 1256.7 \quad 1260.8 \quad 1270.0 \quad 1285.2 \quad 1294.51296 .6$ $1300.21304 .11306 .0 \quad 1317.91322 .0 \quad 1341.7 \quad 1369.8 \quad 1377.6 \quad 1391.8 \quad 1400.7 \quad 1408.8 \quad 1447.3$ $2420.32651 .42659 .5 \quad 2666.92668 .5 \quad 2675.6 \quad 2678.42679 .52682 .2 \quad 2703.82743 .12766 .9$ $2779.42779 .8 \quad 2782.0 \quad 2818.5$

name $=$ TS181

energy $($ in $\mathrm{kcal} / \mathrm{mol})=46.464826$ 
$\mathrm{ZPE}($ in $\mathrm{kcal} / \mathrm{mol})=139.799$

$\mathrm{G}($ in $\mathrm{kcal} / \mathrm{mol})=112.485$

Geometry

C $1.48912947 \quad 4.090482213 .04477820$

C $1.22610422 \quad 2.716663562 .51032347$

C $-1.24806534-0.70061326-0.04364406$

C $-0.13886357-0.30028128 \quad 0.84872069$

C $0.89619522 \quad 0.46922873 \quad 0.13252007$

C 2.074068690 .954061190 .90861114

C $1.690100142 .43398665 \quad 1.09458358$

C $0.53424720 \quad 2.62016240 \quad 0.07884200$

C $-0.82005405 \quad 2.94351698 \quad 0.65043333$

C $0.921901393 .55312616-1.05089383$

H $2.55102595 \quad 4.21443927 \quad 3.30300391$

$\mathrm{H} 0.91306636 \quad 4.27983596 \quad 3.96494650$

H $1.23023674 \quad 4.87342463 \quad 2.32077713$

$\mathrm{H}-2.21296634-0.99762938 \quad 0.32965745$

H $0.33610725-1.21405000 \quad 1.29644177$

$\mathrm{H}-0.52500604 \quad 0.23969893 \quad 1.75318066$

$\mathrm{H} \quad 0.43900983 \quad 1.45250761-0.65650235$

$\mathrm{H} \quad 3.009520210 .83442401 \quad 0.33067173$

H 2.24264545 $0.43467713 \quad 1.86733716$

H 2.54632496 3.09741281 0.84407664

$\mathrm{H}-1.13776671 \quad 2.23114891 \quad 1.42528627$

$\mathrm{H}-1.598779092 .93640888-0.12607500$

$\mathrm{H}-0.84425505 \quad 3.94218617 \quad 1.10819118$

$\mathrm{H} \quad 0.14318989 \quad 3.60500518-1.82422528$

$\mathrm{H} \quad 1.84783856 \quad 3.23639509-1.54867294$

$\mathrm{H} 1.07816993 \quad 4.57649199-0.68545856$

o $-1.86143252-0.99005671-2.11806931$

$0-0.95481359-0.66988033-1.30902559$

o $0.67596503 \quad 1.87267135 \quad 3.17379287$

Vibrational frequencies (in cm-1)

$\begin{array}{llllllllllllllll}-1320.9 & 28.6 & 37.7 & 50.1 & 82.6 & 91.0 & 103.0 & 147.5 & 167.7 & 174.9 & 207.4 & 212.6 & 242.6 & 271.5\end{array}$ $\begin{array}{llllllllllllllll}313.6 & 335.9 & 369.8 & 381.1 & 427.0 & 452.2 & 550.4 & 601.9 & 653.8 & 659.6 & 683.4 & 842.4 & 862.3 & 922.7\end{array}$ $948.3 \quad 978.7 \quad 992.1 \quad 1005.5 \quad 1017.8 \quad 1036.3 \quad 1051.5 \quad 1057.5 \quad 1102.2 \quad 1110.9 \quad 1122.3 \quad 1130.5 \quad 1134.1$

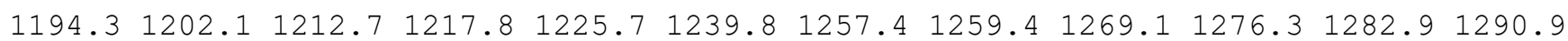

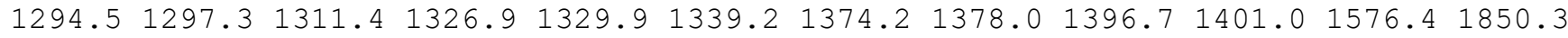
$1918.52608 .8 \quad 2659.2 \quad 2664.7 \quad 2666.42667 .3 \quad 2670.72671 .62673 .6 \quad 2707.32709 .32727 .5$ $2749.12774 .92777 .4 \quad 2778.8$

\section{name $=\mathrm{TS} 182$}

energy (in $\mathrm{kcal} / \mathrm{mol})=45.733236$

$\mathrm{ZPE}(\mathrm{in} \mathrm{kcal} / \mathrm{mol})=141.192$

$\mathrm{G}(\mathrm{in} \mathrm{kcal} / \mathrm{mol})=115.295$

Geometry

C $-1.729772170 .31582945 \quad 1.13757078$

C $-0.71994355 \quad 1.37326294 \quad 1.57713477$

C $0.75265903 \quad 0.82587269 \quad 1.47184582$

C $1.74183284 \quad 1.66436944 \quad 0.72540549$

$\begin{array}{llll}\text { C } 2.10467664 & 2.92565717 & 1.24755346\end{array}$

C $-1.04881481 \quad 3.84348100 \quad 1.51123488$

$\begin{array}{lllll}\text { C }-0.97335340 & 2.67257281 & 0.88107976\end{array}$

C $2.61619557 \quad 3.88832515 \quad 0.39892250$

C $2.75679218 \quad 3.57570699 \quad-0.97412502$

C $2.78043948 \quad 5.299569210 .85241037$

$\mathrm{H}-1.60088469 \quad 0.03123304 \quad 0.08929125$

$\mathrm{H}-2.75740937 \quad 0.68404338 \quad 1.26053144$

$\mathrm{H}-1.64556713-0.59022026 \quad 1.75248887$

H $0.74094549-0.22337043 \quad 1.10502168$

H $1.142567562 .13875232-0.64361364$

$\mathrm{H} 2.48484361 \quad 1.08854368 \quad 0.16869487$

$\mathrm{H} \quad 1.80583613 \quad 3.18305175 \quad 2.26325529$

$\mathrm{H}-1.24617103 \quad 4.777748421 .00730388$ 
$\mathrm{H}-0.91363034 \quad 3.96168830 \quad 2.57892005$

$\mathrm{H}-1.11117075 \quad 2.58318358-0.19556857$

$\mathrm{H} 3.35784208 \quad 2.72988018-1.28501101$

$\mathrm{H} \quad 2.77151712 \quad 4.37597310-1.69971516$

H $1.477171612 .76918489-1.18865324$

$\mathrm{H} 2.58532343 \quad 5.421493391 .92652007$

H $2.09944416 \quad 5.97731522 \quad 0.31959494$

$\mathrm{H} 3.80519320 \quad 5.65375962 \quad 0.67030866$

$0 \quad 0.00942397 \quad 0.68742942 \quad 3.59344740$

$01.20142683 \quad 0.80600047 \quad 2.83632270$

o $-0.88107112 \quad 1.60662923 \quad 2.98495112$

Vibrational frequencies (in $\mathrm{cm}-1$ )

$\begin{array}{lllllllllllllllll}-1947.9 & 32.1 & 41.4 & 69.1 & 98.4 & 111.2 & 143.5 & 177.0 & 209.7 & 227.4 & 261.1 & 269.2 & 308.8 & 316.9\end{array}$

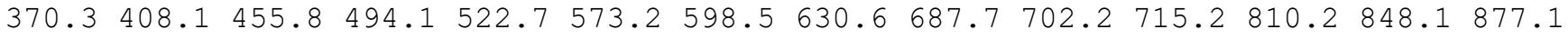
$914.9951 .9965 .3 \quad 973.2 \quad 975.5 \quad 991.0 \quad 1013.1 \quad 1027.8 \quad 1048.0 \quad 1061.1 \quad 1061.91097 .1 \quad 1101.6$

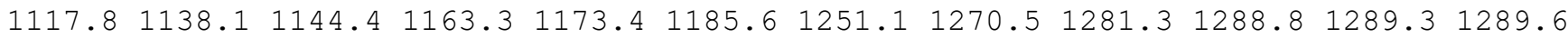

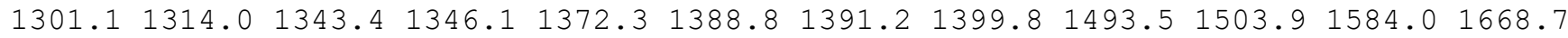

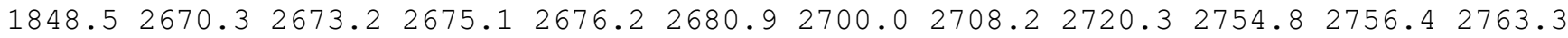
$2779.22780 .5 \quad 2782.02953 .6$

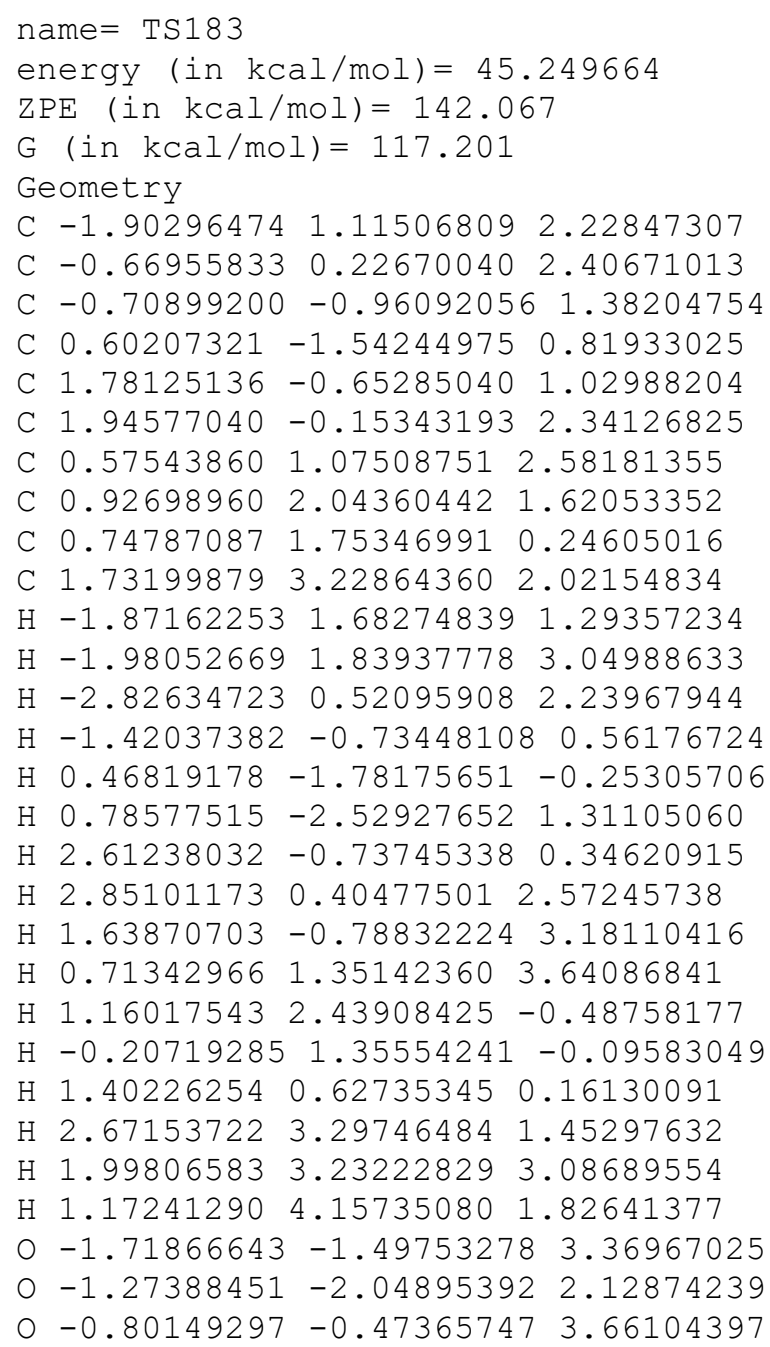


name $=\operatorname{TS} 184$

energy (in $\mathrm{kcal} / \mathrm{mol})=45.23258$

$\mathrm{ZPE}($ in $\mathrm{kcal} / \mathrm{mol})=143.225$

$\mathrm{G}($ in $\mathrm{kcal} / \mathrm{mol})=119.415$

Geometry

C $0.86533651 \quad 1.09363337 \quad 3.51040962$

C $1.03801155 \quad 0.54575609 \quad 2.15395396$

C $-0.17492590 \quad 0.24943792 \quad 1.30565779$

C $0.05403942 \quad 0.38180755 \quad-0.22702671$

C $1.53892766 \quad 0.53735859 \quad-0.55697941$

C $2.32411885-0.496275290 .31047428$

C $2.34273673 \quad 0.58533792 \quad 1.44340786$

C $2.19971254 \quad 1.687283620 .29612846$

C $1.38606010 \quad 2.926800630 .60367000$

C $3.56193838 \quad 2.10384921-0.24304970$

$\mathrm{H} \quad 0.56059182 \quad 2.15399730 \quad 3.46942381$

$\mathrm{H} \quad 1.77153418 \quad 1.03501844 \quad 4.13112471$

$\mathrm{H} \quad 0.06426687 \quad 0.56404754 \quad 4.06538040$

$\mathrm{H}-1.11000416 \quad 0.76450815 \quad 1.60513954$

$\mathrm{H}-0.528911191 .22149995-0.63514885$

$\mathrm{H}-0.34488196-0.53757791-0.70234261$

H $1.74972967 \quad 0.55407662-1.62633766$

H $3.31234372-0.75868787-0.06906996$

H $1.78285439-1.42591845 \quad 0.52379624$

H $3.23527862 \quad 0.64376911 \quad 2.06448993$

$\mathrm{H} \quad 0.36806454 \quad 2.71096468 \quad 0.94426142$

H $1.283918523 .55524752-0.29179496$

$\mathrm{H} \quad 1.86833682 \quad 3.53639845 \quad 1.37744406$

H $4.20559667 \quad 1.24659580 \quad-0.47134734$

$\mathrm{H} \quad 4.10078258 \quad 2.73163774 \quad 0.47609196$

$\mathrm{H} 3.456929012 .68079601-1.17024727$

O $1.26094603-1.55777173 \quad 3.92871558$

O $-0.15463172-1.03514603 \quad 1.75334669$

o $1.48080805-1.35319585 \quad 2.82536654$

Vibrational frequencies (in cm-1)

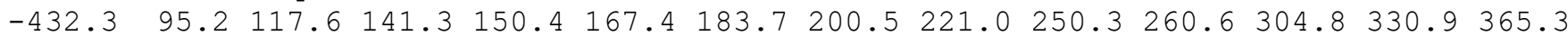

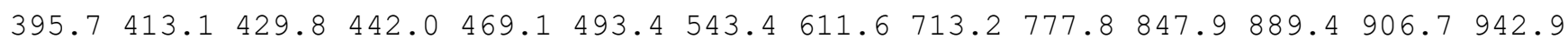
$957.8 \quad 986.8 \quad 1006.4 \quad 1009.8 \quad 1015.1 \quad 1027.6 \quad 1036.5 \quad 1062.4 \quad 1078.5 \quad 1087.8 \quad 1113.51155 .6$

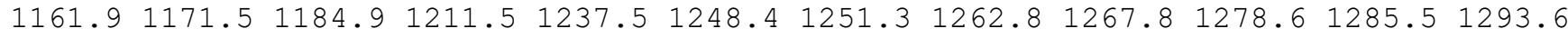

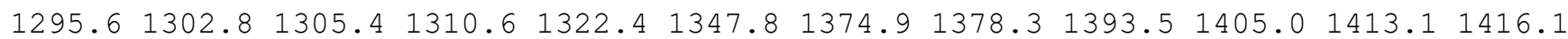
$1499.62642 .0 \quad 2663.4 \quad 2667.8 \quad 2670.9 \quad 2672.6 \quad 2675.2 \quad 2676.02680 .02683 .3 \quad 2746.02748 .3$ $2751.42755 .6 \quad 2767.3 \quad 2781.6 \quad 2783.6$

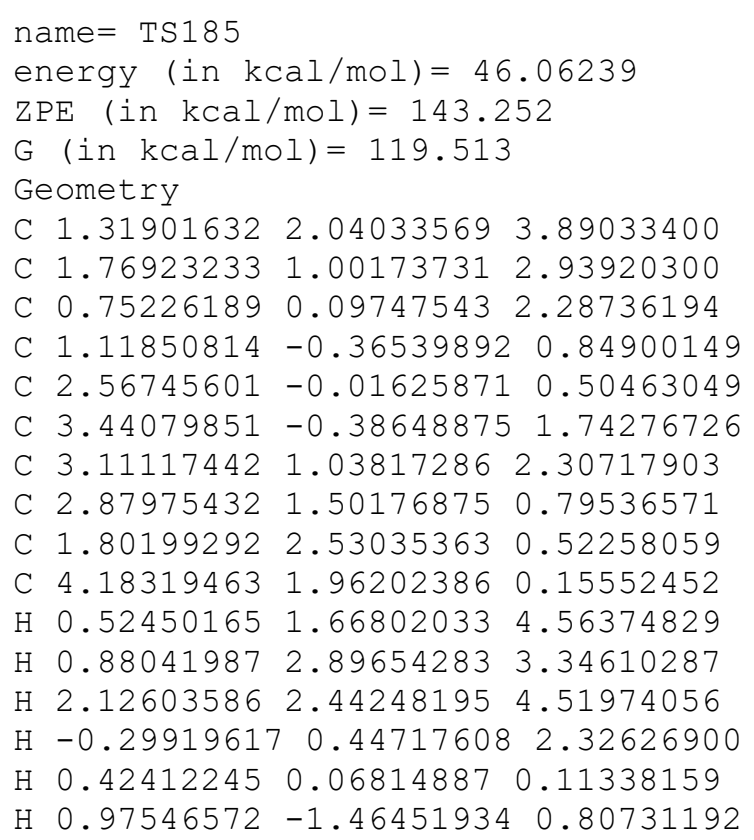


$\mathrm{H} 2.88861330-0.39265384-0.46648159$

$\mathrm{H} \quad 4.49919612-0.544998691 .53313475$

H $3.07846957-1.241014492 .32715221$

$\mathrm{H} \quad 3.89937642 \quad 1.54511064 \quad 2.86179651$

H $2.05453925 \quad 3.49896176 \quad 0.97030827$

$\mathrm{H} \quad 0.81476908 \quad 2.24137314 \quad 0.89752087$

$\mathrm{H} 1.68498610 \quad 2.69020897-0.55828969$

$\mathrm{H} \quad 4.495365292 .93815794 \quad 0.54423166$

$\mathrm{H} \quad 4.07405165 \quad 2.05877404 \quad-0.93168867$

H $5.009098261 .26336975 \quad 0.33329239$

O $3.33911149-0.59694403 \quad 4.56123270$

$00.99820856-0.838159693 .24548292$

o $2.29709316-0.12742963 \quad 4.56267699$

Vibrational frequencies (in cm-1)

$\begin{array}{llllllllllllllllll}-433.9 & 100.4 & 117.7 & 147.3 & 163.3 & 168.5 & 187.0 & 190.3 & 213.0 & 252.3 & 276.5 & 289.7 & 328.3 & 358.1\end{array}$

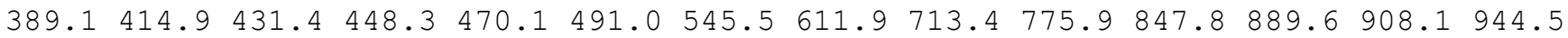

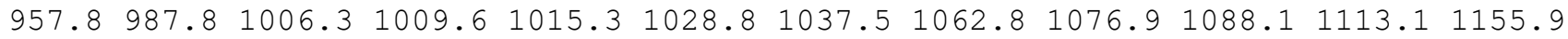

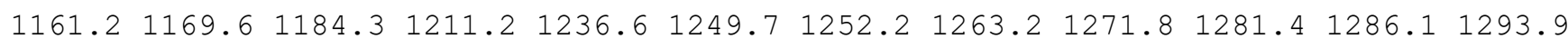

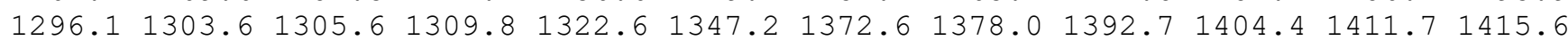
$1499.3 \quad 2646.4 \quad 2662.6 \quad 2668.1 \quad 2670.7 \quad 2673.1 \quad 2675.0 \quad 2676.42680 .3 \quad 2682.92746 .02748 .9$ $2752.3 \quad 2756.62767 .6 \quad 2781.5 \quad 2783.4$

name $=$ TS186

energy (in $\mathrm{kcal} / \mathrm{mol})=52.901582$

$\mathrm{ZPE}($ in $\mathrm{kcal} / \mathrm{mol})=139.979$

$\mathrm{G}($ in $\mathrm{kcal} / \mathrm{mol})=113.132$

Geometry

C $1.812450812 .01852757 \quad 1.79772963$

C $2.60898216 \quad 1.17658366 \quad 2.38057254$

C $1.56996176-1.53541758 \quad 0.50972593$

C $2.95688803-2.114196730 .60307779$

C $3.90352855-1.36466217 \quad 1.53774850$

C $3.34017165-1.17632222 \quad 2.96445148$

C $3.458605460 .32912736 \quad 3.06712877$

C $4.21118057 \quad 0.09525528 \quad 1.18281215$

C $3.701342120 .63333102-0.11605440$

C $5.63193392 \quad 0.51674605 \quad 1.43849164$

$\mathrm{H} 0.96878957 \quad 2.46100834 \quad 2.33324363$

$\mathrm{H} \quad 1.88210990 \quad 2.36927906 \quad 0.77650661$

H $1.96975746 \quad 1.54165664 \quad 5.83608586$

H $1.17976018-0.97837354 \quad 1.37140232$

H $3.40324929-2.17382273-0.41401145$

$\mathrm{H} \quad 2.86793133-3.173860730 .93087307$

H $4.85733041-1.946009621 .57263941$

H $3.93419441-1.693462873 .73472536$

H 2.30837925 -1.53830065 3.10384675

$\mathrm{H} \quad 4.27936609 \quad 0.74569523 \quad 3.66520184$

H $4.21003791 \quad 0.14316979-0.96313914$

H $3.897956151 .70910371-0.22174235$

$\mathrm{H} \quad 2.62839231 \quad 0.47695409-0.27817502$

H $5.71376410 \quad 1.60454746 \quad 1.56393412$

H $6.28530835 \quad 0.24761558 \quad 0.59473362$

H $6.06713093 \quad 0.05043807 \quad 2.33028884$

○ $2.472778591 .75989516 \quad 5.01716683$

$\begin{array}{lllll}0 & 0.89679960 & -1.68702065 & -0.48013884\end{array}$

$\begin{array}{lllll}0 & 2.04191877 & 0.69986985 & 4.22036944\end{array}$

Vibrational frequencies (in $\mathrm{cm}-1$ )

$\begin{array}{lllllllllllllllll}-1260.2 & 29.9 & 46.9 & 75.9 & 97.0 & 127.6 & 135.6 & 151.8 & 177.1 & 186.3 & 216.0 & 222.2 & 246.7 & 261.4\end{array}$ $270.1 \quad 279.2 \quad 313.9 \quad 337.9 \quad 347.9 \quad 418.1 \quad 423.3 \quad 495.9 \quad 515.7 \quad 527.3 \quad 606.5 \quad 642.8 \quad 770.7 \quad 856.4$ $930.5944 .8 \quad 971.3 \quad 996.3 \quad 1010.3 \quad 1015.5 \quad 1024.1 \quad 1038.5 \quad 1047.7 \quad 1064.1 \quad 1072.3 \quad 1105.5 \quad 1111.5$

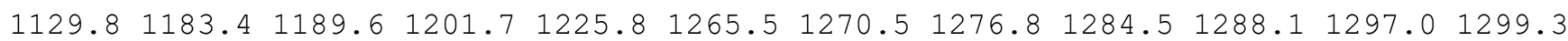

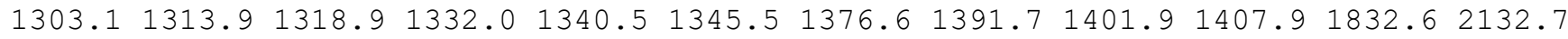
$2642.42654 .12658 .6 \quad 2664.6 \quad 2665.7 \quad 2670.8 \quad 2675.52678 .72697 .32710 .82738 .32754 .1$ $2758.7 \quad 2774.8 \quad 2776.7 \quad 2874.4$ 


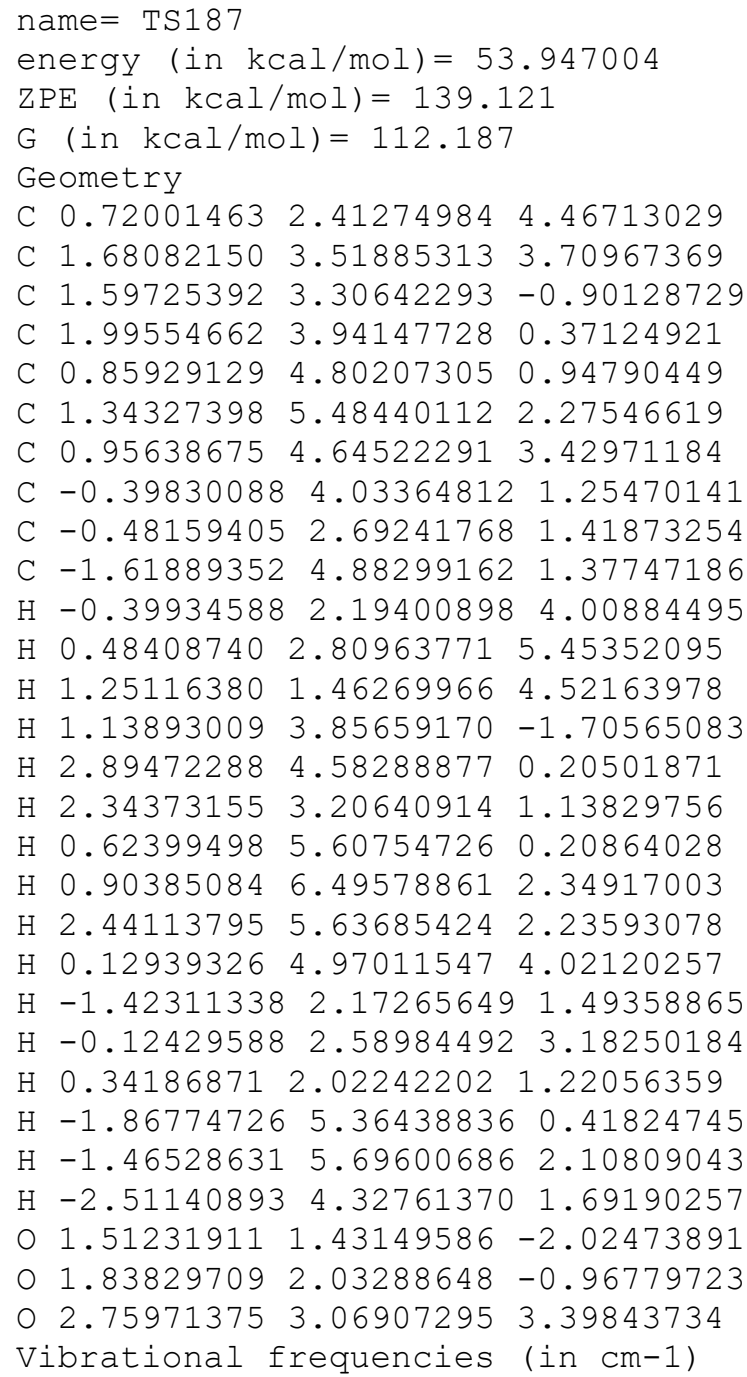

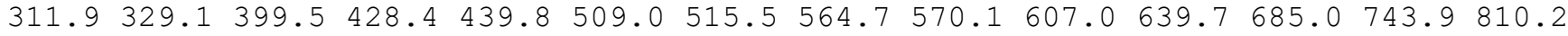

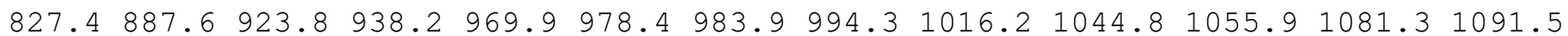

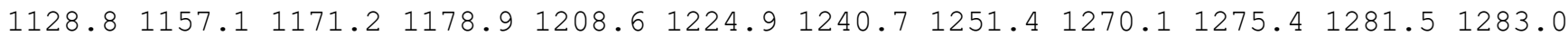
$1290.61317 .8 \quad 1327.7 \quad 1331.2 \quad 1342.1 \quad 1376.8 \quad 1382.1 \quad 1399.2 \quad 1484.91566 .7 \quad 1755.3 \quad 1848.9$ $1957.42621 .42656 .0 \quad 2663.8 \quad 2666.3 \quad 2669.3 \quad 2694.22714 .12715 .02725 .4 \quad 2733.02742 .2$ $2774.22782 .2 \quad 2812.5 \quad 2987.2$

\section{name $=\operatorname{TS} 188$}

energy (in $\mathrm{kcal} / \mathrm{mol})=51.744217$

$\mathrm{ZPE}(\mathrm{in} \mathrm{kcal} / \mathrm{mol})=141.803$

$\mathrm{G}($ in $\mathrm{kcal} / \mathrm{mol})=116.796$

Geometry

C $1.58941001 \quad 0.90425004 \quad 5.13394334$

C $1.22878828 \quad 0.29311880 \quad 3.76720485$

C $-0.21853655 \quad 0.11363858 \quad 3.56728677$

C $-0.73543680 \quad 0.07218857 \quad 2.28071434$

C $0.03787133 \quad 0.26033129 \quad 1.15842704$

C $1.526642110 .33160043 \quad 1.25314790$

C $1.85063194 \quad 1.09142970 \quad 2.56282021$

C $1.24663027 \quad 2.42426231 \quad 2.34871978$

C $0.30488518 \quad 3.01735835 \quad 3.27911805$

C $1.71381740 \quad 3.27015734 \quad 1.23217453$

$\mathrm{H} 1.73898791 \quad 0.09508349 \quad 5.86880981$

$\mathrm{H} 0.78234024 \quad 1.52697540 \quad 5.52807726$

H 2.513076461 .483244175 .10326317

$\mathrm{H}-0.83588287-0.03588455 \quad 4.43185231$ 
$\mathrm{H}-1.81044271-0.08984670 \quad 2.15722978$

$\mathrm{H}-0.24230355-2.222627493 .72110939$

$\mathrm{H}-0.37723320 \quad 0.15579444 \quad 0.17136161$

$\mathrm{H} \quad 1.96514015 \quad 0.83628962 \quad 0.37393633$

$\mathrm{H} \quad 1.97567331-0.68173086 \quad 1.25034452$

H 2.95264899 $1.14325466 \quad 2.72317306$

$\mathrm{H}-0.52946653 \quad 2.26400001 \quad 3.48586825$

$\mathrm{H}-0.15322585 \quad 3.957115592 .94888525$

$\mathrm{H} \quad 0.72075904 \quad 3.18214979 \quad 4.29208826$

H 2.49930959 2.80738519 0.61307776

H $2.12120633 \quad 4.232644591 .59234219$

$\mathrm{H} \quad 0.88299768 \quad 3.52832658 \quad 0.54860101$

O $1.46858039-1.92294764 \quad 3.02027053$

○ $0.61222614 \quad-2.72404748 \quad 3.78317277$

o $1.99170675-0.932140753 .82711602$

Vibrational frequencies (in cm-1)

$\begin{array}{lllllllllllllllll}-167.1 & 53.1 & 77.8 & 94.5 & 102.8 & 124.8 & 153.8 & 211.0 & 254.6 & 274.0 & 302.9 & 331.4 & 339.3 & 358.3\end{array}$ $\begin{array}{llllllllllllllllll}383.6 & 410.3 & 428.0 & 443.8 & 467.1 & 523.6 & 547.9 & 596.0 & 624.7 & 739.3 & 791.5 & 801.2 & 850.9 & 871.4\end{array}$

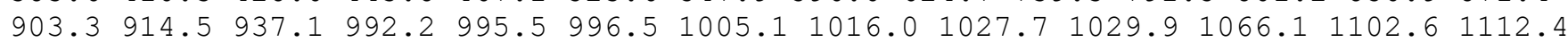
$\begin{array}{lllllllllllll}1138.8 & 1145.8 & 1160.4 & 1189.7 & 1212.8 & 1227.5 & 1243.7 & 1252.3 & 1256.1 & 1274.3 & 1288.6 & 1299.3\end{array}$

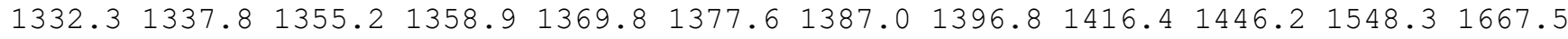
$2457.52640 .6 \quad 2647.5 \quad 2652.1 \quad 2654.6 \quad 2661.6 \quad 2685.12689 .42739 .5 \quad 2743.5 \quad 2750.5 \quad 2761.3$ $2778.5 \quad 2783.12791 .5 \quad 2816.8$

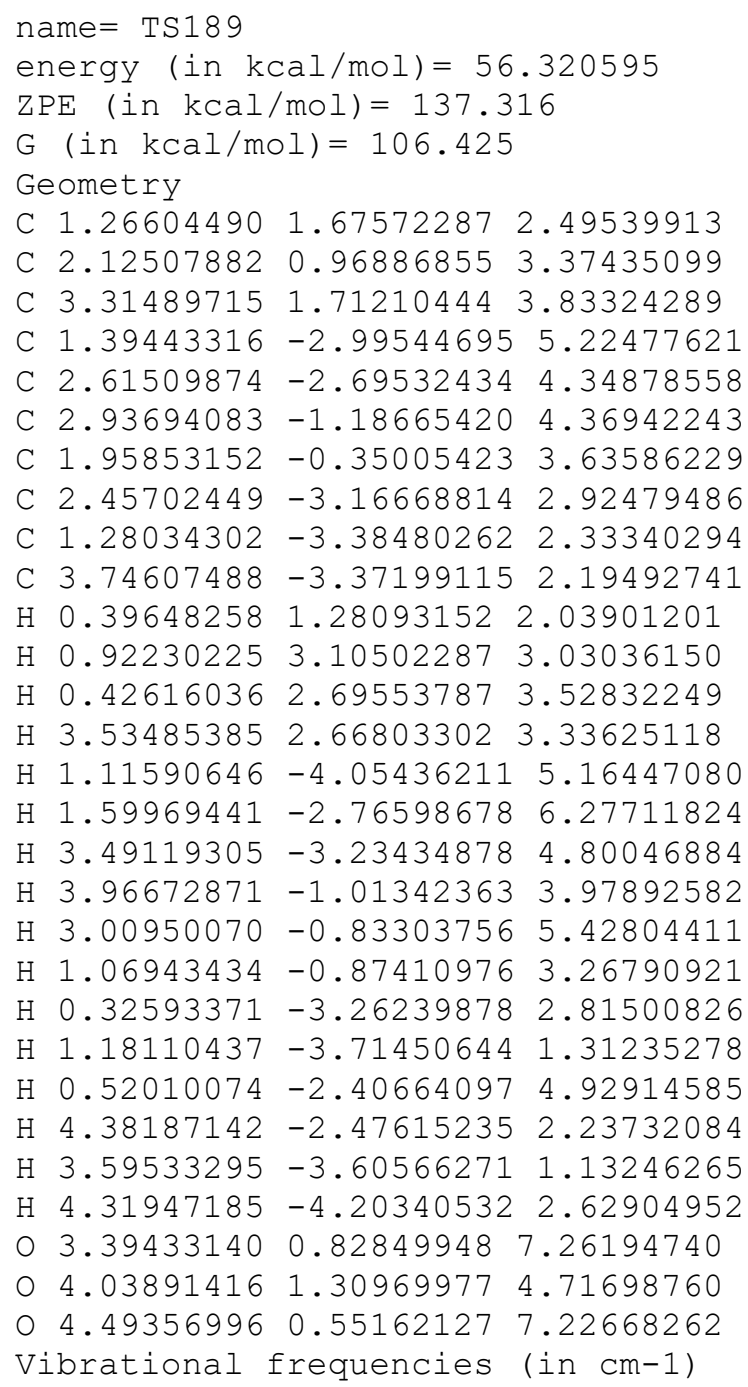
$201.5 \quad 227.6 \quad 277.4 \quad 319.7 \quad 338.2 \quad 380.5 \quad 401.2 \quad 433.0 \quad 454.5 \quad 519.8 \quad 543.5 \quad 594.2 \quad 623.0 \quad 653.3$ $690.6 \quad 771.6 \quad 814.1 \quad 867.4 \quad 978.6 \quad 990.0 \quad 992.9 \quad 997.0 \quad 1005.4 \quad 1039.3 \quad 1047.6 \quad 1048.5 \quad 1087.2$ $1093.4 \quad 1126.1 \quad 1138.1 \quad 1161.5 \quad 1213.4 \quad 1219.4 \quad 1250.1 \quad 1273.4 \quad 1276.7 \quad 1287.5 \quad 1289.2 \quad 1296.1$ $1300.0 \quad 1303.5 \quad 1334.1 \quad 1346.7 \quad 1381.7 \quad 1394.8 \quad 1398.6 \quad 1410.6 \quad 1455.2 \quad 1597.7 \quad 1752.3 \quad 1821.0$ 


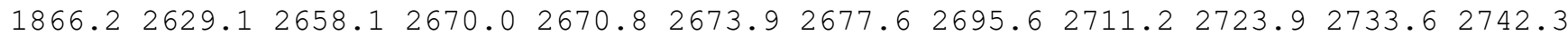
$2778.82781 .12794 .3 \quad 4697.2$

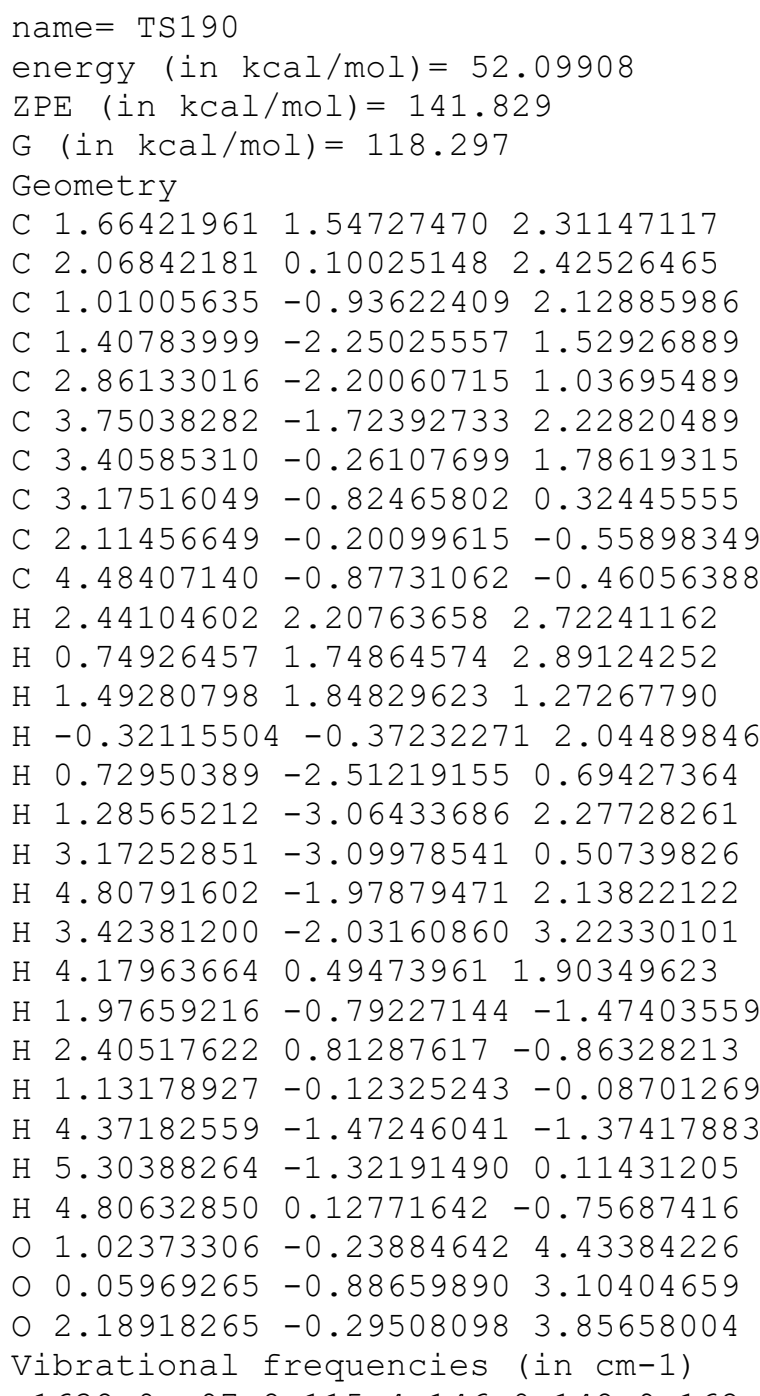

$\begin{array}{llllllllllllllllll}-1638.0 & 97.8 & 115.4 & 146.0 & 148.9 & 162.9 & 176.8 & 194.0 & 252.5 & 271.1 & 283.4 & 313.0 & 342.4 & 382.9\end{array}$ $415.2 \quad 434.6 \quad 451.9 \quad 488.7 \quad 515.4 \quad 577.0 \quad 616.3 \quad 629.8 \quad 699.0 \quad 723.7 \quad 777.2 \quad 809.6 \quad 856.5 \quad 881.5$ $918.3937 .3958 .2 \quad 996.2 \quad 1005.0 \quad 1007.5 \quad 1013.9 \quad 1016.7 \quad 1054.4 \quad 1076.6 \quad 1077.8 \quad 1102.1 \quad 1120.2$

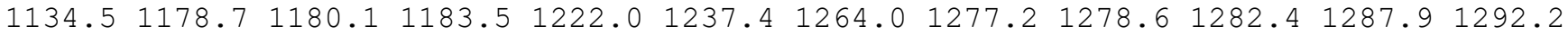
$1295.11297 .41300 .0 \quad 1303.5 \quad 1307.6 \quad 1321.8 \quad 1356.6 \quad 1371.7 \quad 1380.5 \quad 1397.5 \quad 1407.7 \quad 1422.0$ $2442.92658 .12665 .5 \quad 2673.12675 .3 \quad 2676.8 \quad 2678.7 \quad 2680.32684 .3 \quad 2738.6 \quad 2749.02752 .7$ 2763.42779 .12781 .92784 .2

name $=\operatorname{TS} 191$

energy (in $\mathrm{kcal} / \mathrm{mol})=54.303688$

$\mathrm{ZPE}($ in $\mathrm{kcal} / \mathrm{mol})=139.767$

$\mathrm{G}(\mathrm{in} \mathrm{kcal} / \mathrm{mol})=112.698$

Geometry

C $2.61333096 \quad 0.69895917 \quad 4.84230983$

C $2.60999707 \quad 0.043210313 .59485887$

C $1.26800924-0.76340773 \quad 3.36700313$

C $1.52578763-1.959301362 .38832329$

C $2.89931111-1.90329469 \quad 1.73083934$

C $3.96441930-1.746185932 .86203733$

C $3.76000228-0.18312610 \quad 2.72353024$

C $3.27073493-0.449561491 .23642969$

$\begin{array}{llll}\text { C } 2.18481767 & 0.46144891 & 0.70234175\end{array}$

C $4.44949529-0.45866268 \quad 0.27060931$

$\mathrm{H} 1.99195282-0.18888595 \quad 5.48559745$

$\mathrm{H} \quad 1.78928197 \quad 1.39968568 \quad 5.06064838$ 
H $3.53999468 \quad 1.04583897 \quad 5.28263326$

$\mathrm{H} \quad 0.52175008-0.05728434 \quad 2.92522212$

$\mathrm{H} \quad 0.72181260-2.00194114 \quad 1.63815928$

H $1.42235117-2.889577292 .98566053$

H $3.06637257-2.70352396 \quad 1.00707795$

H $4.97278092 \quad-2.071151632 .60285862$

H $3.69912772-2.19132970 \quad 3.82754900$

$\mathrm{H} \quad 4.633735390 .456309832 .84241030$

H $1.17292628 \quad 0.12065046 \quad 0.95406709$

$\begin{array}{lllll}\mathrm{H} & 2.20869968 & 0.52088782 & -0.39498191\end{array}$

H 2.29749866 1.48518755 1.07720884

H $4.81836150 \quad 0.55682896 \quad 0.08267132$

$\mathrm{H} \quad 4.15911921-0.88860332-0.69669281$

H $5.29677447-1.04786450 \quad 0.63863972$

O $1.51019543-3.22668083 \quad 5.63162069$

○ $0.92693246-1.11910329 \quad 4.58459954$

o $2.55663176-2.887184715 .91250237$

Vibrational frequencies (in cm-1)

$\begin{array}{lllllllllllllll}-1079.4 & 22.7 & 39.9 & 52.0 & 75.5 & 81.3 & 89.8 & 134.7 & 177.8 & 193.2 & 196.5 & 256.0 & 312.6 & 373.2\end{array}$ $381.9 \quad 397.1 \quad 439.4 \quad 446.9 \quad 464.1 \quad 480.0 \quad 539.4 \quad 584.2 \quad 659.2 \quad 696.8 \quad 731.4 \quad 786.0 \quad 871.4 \quad 894.7$ $921.4942 .4 \quad 948.4 \quad 964.1 \quad 994.7 \quad 1002.3 \quad 1015.3 \quad 1020.1 \quad 1024.4 \quad 1066.0 \quad 1081.4 \quad 1093.3 \quad 1118.1$

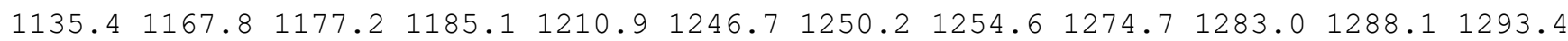
$1296.41299 .21308 .2 \quad 1314.3 \quad 1320.7 \quad 1357.4 \quad 1369.0 \quad 1405.6 \quad 1418.3 \quad 1437.0 \quad 1564.7 \quad 1589.9$ $2108.02621 .32661 .0 \quad 2666.3 \quad 2672.8 \quad 2673.4 \quad 2674.92676 .12677 .3 \quad 2744.22747 .42749 .9$ $\begin{array}{lllll}2754.5 & 2761.7 & 2779.7 & 2782.2\end{array}$

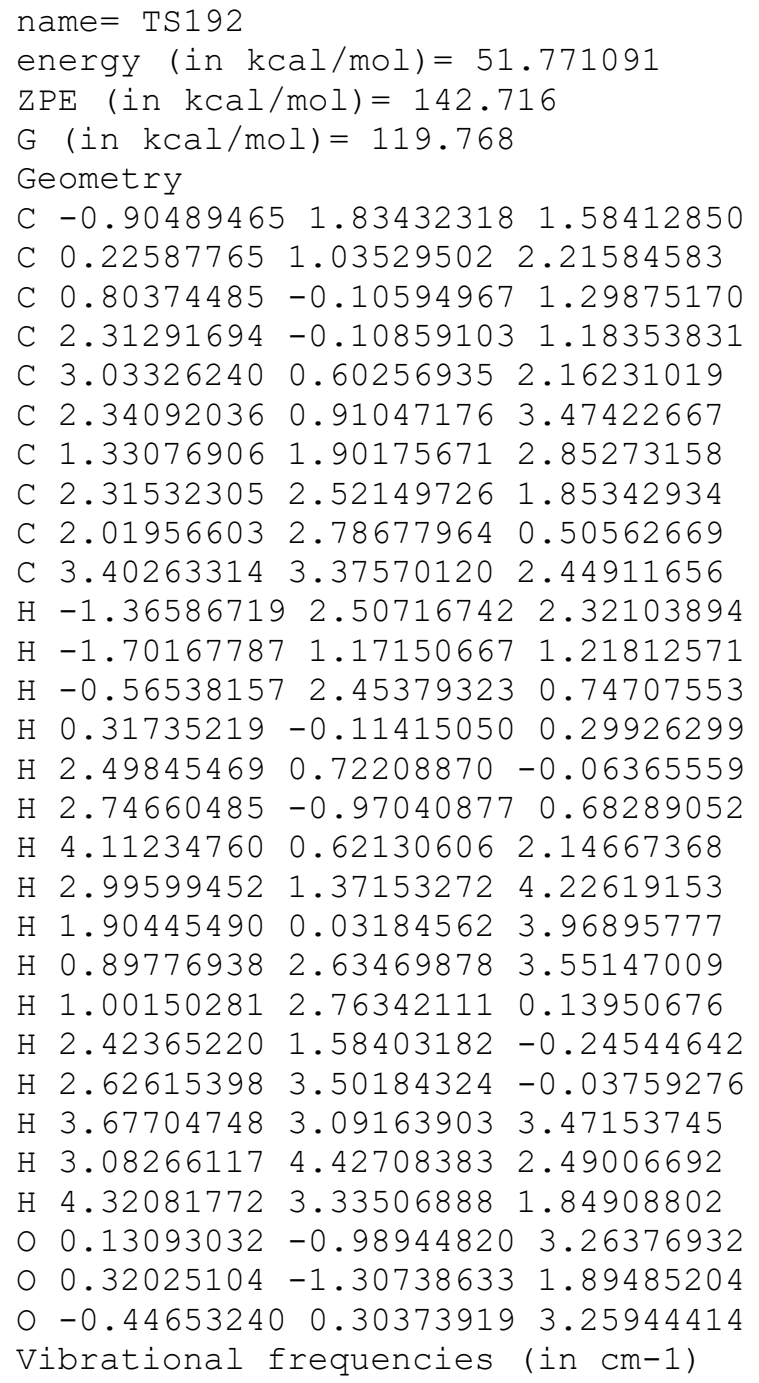




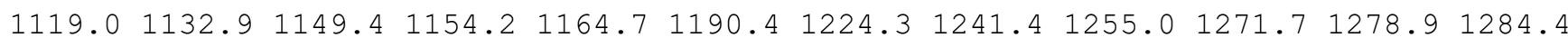

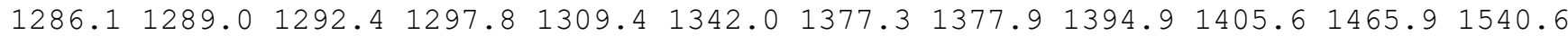
1601.62654 .02667 .72669 .62671 .72674 .12681 .92692 .62729 .92738 .72756 .02759 .2 $2763.12776 .32778 .3 \quad 3030.7$

\section{name $=$ TS193}

energy (in $\mathrm{kcal} / \mathrm{mol})=53.571091$

$\mathrm{ZPE}($ in $\mathrm{kcal} / \mathrm{mol})=141.236$

$\mathrm{G}($ in $\mathrm{kcal} / \mathrm{mol})=117.38$

Geometry

$\begin{array}{lllll}\text { C } & 0.02405839 & 0.64403720 & 2.67707740\end{array}$

C $1.10063844 \quad 1.56298370 \quad 2.72135370$

C $2.549674331 .10703521 \quad 2.53193571$

$\begin{array}{llll}\text { C } 3.51004769 & 2.27050670 & 2.15280537\end{array}$

$\begin{array}{lllll}\text { C } 2.88859469 & 3.62680601 & 2.48536499\end{array}$

C $2.14734224 \quad 3.49346973 \quad 3.85280043$

C $0.90354070 \quad 2.98877431 \quad 3.04802719$

C $1.48331690 \quad 3.79303111 \quad 1.78957582$

C $1.26660192 \quad 3.18322920 \quad 0.42064627$

C 0.998183225 .234480291 .78460993

$\mathrm{H} \quad 0.15208436-0.07918872 \quad 3.66938553$

$\mathrm{H} \quad 0.07446304-0.14821717 \quad 1.91908502$

$\mathrm{H}-0.99095767 \quad 1.03734748 \quad 2.75689453$

H 2.65457400 $0.25632959 \quad 1.82825629$

H $3.79741957 \quad 2.21418508 \quad 1.09188107$

$\mathrm{H} \quad 4.44423410 \quad 2.13172014 \quad 2.73771318$

H $3.57924463 \quad 4.46314255 \quad 2.37223906$

$\mathrm{H} \quad 1.97962830 \quad 4.43396333 \quad 4.37980613$

$\mathrm{H} 2.58022106 \quad 2.77886546 \quad 4.56769270$

$\mathrm{H}-0.07933138 \quad 3.28687450 \quad 3.41179485$

$\mathrm{H} \quad 1.61086034 \quad 2.14729562 \quad 0.33497587$

$\mathrm{H} \quad 1.80961070 \quad 3.75254805-0.34713601$

$\mathrm{H} \quad 0.20500815 \quad 3.19652425 \quad 0.14192934$

$\mathrm{H}-0.06368001 \quad 5.30010527 \quad 1.51867810$

H $1.55707313 \quad 5.83409856 \quad 1.05465051$

$\mathrm{H} \quad 1.11834588 \quad 5.72416660 \quad 2.75851054$

$\begin{array}{lllll}0 & 2.10574518 & -0.88248689 & 4.02549994\end{array}$

$\begin{array}{lllll}0 & 2.75176188 & 0.74007071 & 3.82847190\end{array}$

o $1.07813823-0.833781694 .62802301$

Vibrational frequencies (in $\mathrm{cm}-1$ )

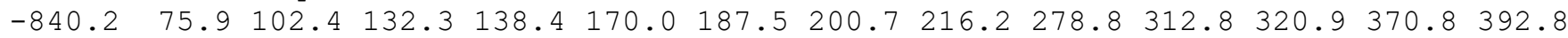

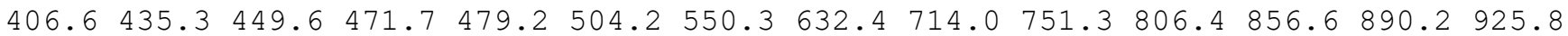
$943.5947 .9 \quad 982.2 \quad 1005.1 \quad 1010.1 \quad 1012.8 \quad 1019.3 \quad 1033.0 \quad 1046.1 \quad 1076.3 \quad 1083.8 \quad 1109.3 \quad 1141.6$

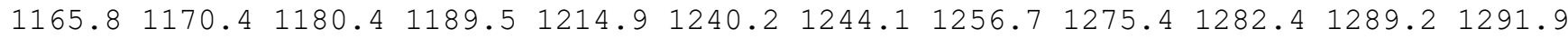

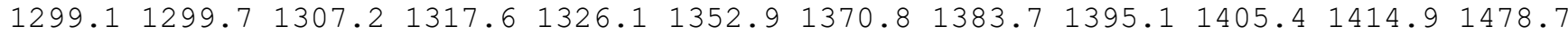
$1772.52660 .2 \quad 2665.4 \quad 2669.1 \quad 2672.9 \quad 2673.5 \quad 2677.4 \quad 2677.92681 .8 \quad 2743.7 \quad 2746.92749 .4$ $2751.8 \quad 2755.0 \quad 2780.5 \quad 2782.7$

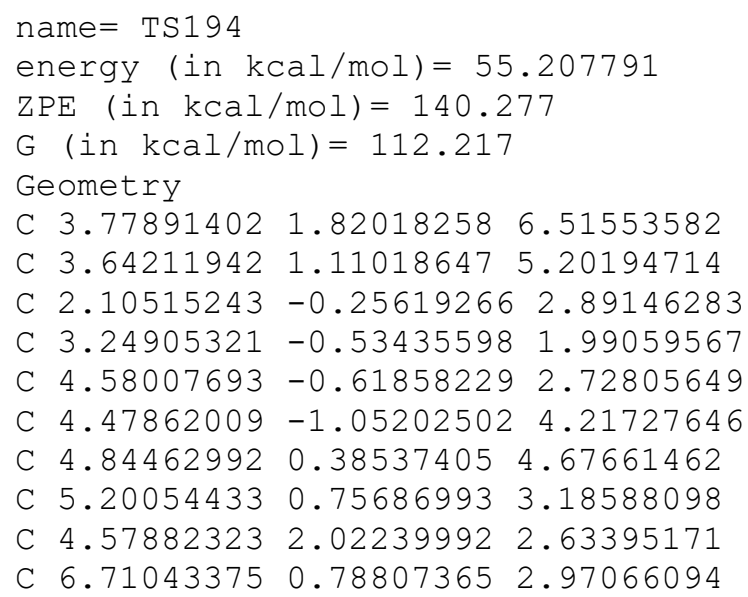


$\mathrm{H} 4.008078461 .11743371 \quad 7.32726028$

H $2.84455661 \quad 2.33850498 \quad 6.78438542$

H $4.57765965 \quad 2.57191620 \quad 6.48361527$

$\mathrm{H} \quad 1.49576917 \quad 0.59269272 \quad 2.60083888$

H $3.36063065 \quad 0.14718924 \quad 1.12511952$

H $3.01264968-1.53647964 \quad 1.55137249$

H $5.29247380-1.22530125 \quad 2.14433714$

H $5.20796797-1.81008647 \quad 4.51138840$

H $3.48185709-1.400539124 .52236371$

H $5.702881990 .44044975 \quad 5.35975608$

$\mathrm{H} 4.821645092 .15183163 \quad 1.57173428$

$\mathrm{H} \quad 4.93698511 \quad 2.91122913 \quad 3.16626730$

H $3.48206468 \quad 2.02271678 \quad 2.70787624$

H 7.164513631 .664335493 .44775025

H $6.95371327 \quad 0.83473075 \quad 1.90181822$

$\mathrm{H} \quad 7.20423169-0.101375623 .37800587$

O $1.73746094-3.302424313 .66008662$

o $1.24482798 \quad-2.42924276 \quad 4.19561414$

o $2.590214731 .13729755 \quad 4.60218798$

Vibrational frequencies (in $\mathrm{cm}-1$ )

$\begin{array}{llllllllllllllll}-127.0 & 26.6 & 43.1 & 52.2 & 71.0 & 82.7 & 108.5 & 115.7 & 158.3 & 169.1 & 184.8 & 207.8 & 243.5 & 279.5\end{array}$ $288.4 \quad 319.9 \quad 338.5 \quad 380.5 \quad 409.9 \quad 443.2 \quad 537.6 \quad 591.6 \quad 598.4 \quad 631.6 \quad 702.8 \quad 796.9 \quad 846.9 \quad 885.0$ $906.0 \quad 938.0 \quad 1000.6 \quad 1005.3 \quad 1011.6 \quad 1018.9 \quad 1036.8 \quad 1045.1 \quad 1067.4 \quad 1093.2 \quad 1099.3 \quad 1114.0$ $1120.6 \quad 1133.6 \quad 1177.7 \quad 1205.9 \quad 1225.8 \quad 1238.6 \quad 1255.9 \quad 1265.8 \quad 1273.7 \quad 1284.8 \quad 1286.4 \quad 1288.0$ $1298.61300 .4 \quad 1304.0 \quad 1313.1 \quad 1325.3 \quad 1334.0 \quad 1358.6 \quad 1377.3 \quad 1383.4 \quad 1406.2 \quad 1409.51582 .6$ $1826.92639 .8 \quad 2658.8 \quad 2662.5 \quad 2669.6 \quad 2674.2 \quad 2675.12675 .42677 .8 \quad 2680.7 \quad 2720.92726 .8$ $2738.12756 .92779 .4 \quad 2779.6 \quad 2782.3$

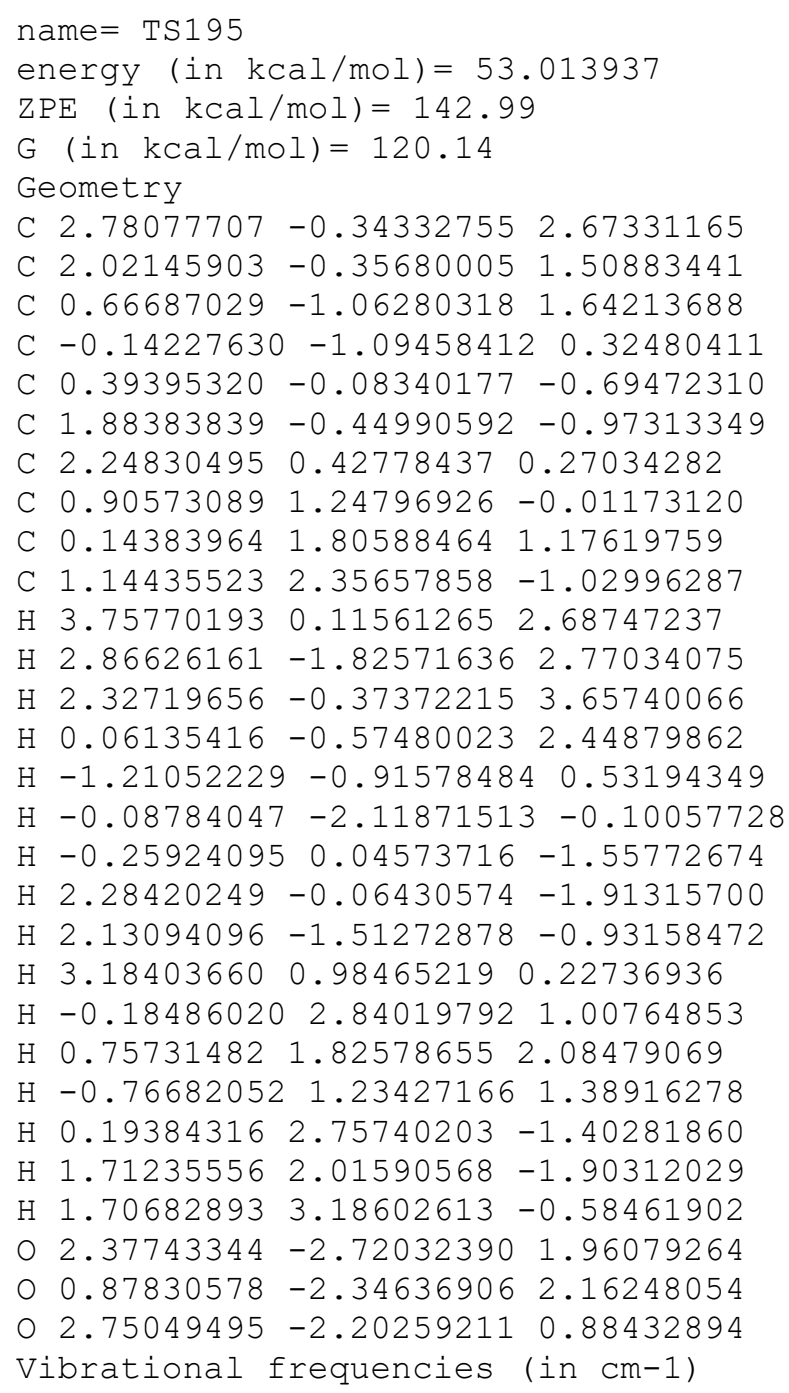


$\begin{array}{llllllllllllllllll}-1350.8 & 87.8 & 122.2 & 159.1 & 188.3 & 194.1 & 201.5 & 250.4 & 298.7 & 342.3 & 362.0 & 389.8 & 411.3 & 437.6\end{array}$ $442.4 \quad 473.7 \quad 502.6 \quad 555.1 \quad 595.2 \quad 620.1 \quad 664.4 \quad 701.3 \quad 708.9 \quad 725.8 \quad 781.9 \quad 838.2 \quad 877.8 \quad 922.9$

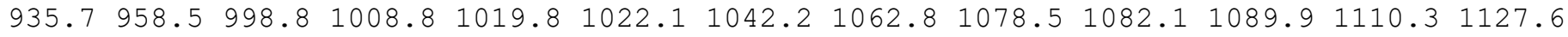
$1156.11164 .4 \quad 1175.4 \quad 1187.8 \quad 1195.7 \quad 1219.5 \quad 1231.91248 .7 \quad 1270.1 \quad 1280.6 \quad 1284.91295 .3$

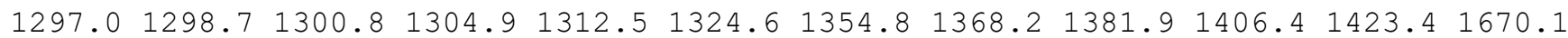
$1737.0 \quad 2650.0 \quad 2665.3 \quad 2670.0 \quad 2673.5 \quad 2673.6 \quad 2678.2 \quad 2678.7 \quad 2707.4 \quad 2743.4 \quad 2748.2 \quad 2752.5$ $2761.4 \quad 2777.6 \quad 2779.3 \quad 2781.3$

name $=$ TS196

energy (in $\mathrm{kcal} / \mathrm{mol})=52.965751$

$\operatorname{ZPE}(\mathrm{in} \mathrm{kcal} / \mathrm{mol})=143.57$

$\mathrm{G}(\mathrm{in} \mathrm{kcal} / \mathrm{mol})=119.877$

Geometry

C $-0.73070685 \quad 2.10503114 \quad 3.17691921$

C $0.12180522 \quad 0.98647049 \quad 2.68741955$

C $-0.39228770-0.05686237 \quad 1.91446284$

$\begin{array}{lllll}\text { C } & 0.54588477 & -0.85337207 & 1.03814948\end{array}$

C $1.98871596-0.33870974 \quad 1.17440240$

C $2.28378321-0.217937192 .70247069$

C $1.60733793 \quad 1.19403385 \quad 2.62781247$

C $2.03037334 \quad 1.23844117 \quad 1.09466902$

C $1.11587467 \quad 1.93947475 \quad 0.11068277$

C $3.44803149 \quad 1.77065480 \quad 0.91399270$

$\mathrm{H}-0.343546612 .53270102 \quad 4.11519425$

$\mathrm{H}-1.76827726 \quad 1.801900013 .36347282$

$\mathrm{H}-0.75377078 \quad 2.92379258 \quad 2.44020559$

$\mathrm{H}-1.44800814-0.13161890 \quad 1.67768348$

$\mathrm{H} \quad 0.21702159-0.80365297-0.01729903$

H $0.50847952-1.928316191 .31722815$

H $2.70708883-0.87509799 \quad 0.55903976$

H $3.34044627 \quad-0.19502127 \quad 2.96796552$

$\mathrm{H} \quad 1.79078163-0.951798793 .34378357$

H 2.00180054 1.97354333 3.27433024

H $1.13942670 \quad 3.026133110 .25825035$

$\mathrm{H} \quad 0.06897721 \quad 1.63010857 \quad 0.19309667$

$\mathrm{H} \quad 1.426264661 .73601653-0.92191357$

$\mathrm{H} 3.807875561 .59192782-0.10586523$

$\mathrm{H} 4.163294951 .299055251 .59701903$

$\mathrm{H} 3.48936816 \quad 2.85040787 \quad 1.09802698$

$0-0.83069970-0.70579835 \quad 4.30340071$

o $-0.67231158-1.45617873 \quad 3.31930134$

o $0.11412169 \quad 0.06689183 \quad 4.53616936$

Vibrational frequencies (in cm-1)

$\begin{array}{llllllllllllll}-595.1 & 96.7 & 123.3 & 138.7 & 151.8 & 152.8 & 180.6 & 193.2 & 204.8 & 236.9 & 262.2 & 309.2349 .6 & 387.0\end{array}$

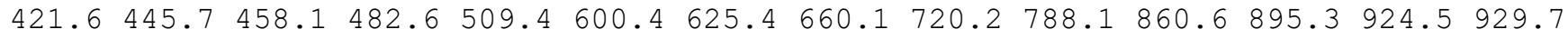
$948.5986 .5 \quad 1002.7 \quad 1008.1 \quad 1015.9 \quad 1020.2 \quad 1053.0 \quad 1062.6 \quad 1075.8 \quad 1077.11085 .2 \quad 1096.5$

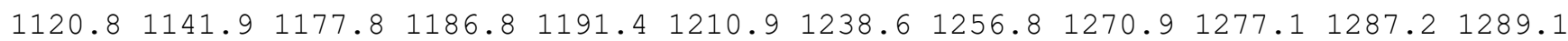
$1293.91296 .1 \quad 1301.8 \quad 1304.8 \quad 1312.3 \quad 1318.0 \quad 1355.7 \quad 1367.1 \quad 1383.7 \quad 1393.4 \quad 1406.6 \quad 1417.9$ $1590.22657 .5 \quad 2664.5 \quad 2672.6 \quad 2674.8 \quad 2676.4 \quad 2677.5 \quad 2681.72690 .0 \quad 2736.4 \quad 2746.5 \quad 2752.4$ $2758.2 \quad 2766.5 \quad 2777.12781 .5 \quad 2783.5$

name $=\operatorname{TS} 197$

energy $($ in $\mathrm{kcal} / \mathrm{mol})=53.725732$

$\mathrm{ZPE}(\mathrm{in} \mathrm{kcal} / \mathrm{mol})=143.525$

$\mathrm{G}($ in $\mathrm{kcal} / \mathrm{mol})=119.76$

Geometry

C $1.00641903 \quad 1.40940865 \quad 3.55346284$

C $1.80618655 \quad 0.18603796 \quad 3.27163928$

C $1.33150527-0.83915355 \quad 2.45005492$

C $2.32454247-1.77087107 \quad 1.79502838$

C $3.76874568-1.361457152 .13196326$

C $3.84909874-1.18905927 \quad 3.68127368$

C $3.29552775 \quad 0.25818298 \quad 3.45701751$ 
$\begin{array}{llll}\text { C } 3.95375535 & 0.20308181 & 2.00533098\end{array}$

C 3.266883810 .925309890 .86509296

$\begin{array}{lllll}\text { C } 5.42102721 & 0.61772491 & 2.03264483\end{array}$

H $1.17009196 \quad 2.17489110 \quad 2.78140728$

$\mathrm{H} 1.27231080 \quad 1.85775177 \quad 4.52329615$

$\mathrm{H}-0.072334891 .19568833 \quad 3.59619973$

$\mathrm{H} 0.34021637-0.79891417 \quad 2.00553045$

H 2.17628483 -1.76592537 0.69690023

H $2.14472015-2.81756527 \quad 2.11168986$

$\mathrm{H} \quad 4.52392748 \quad-1.97933348 \quad 1.65123496$

$\mathrm{H} \quad 4.86327500-1.212643964 .08794720$

$\mathrm{H} \quad 3.25235676-1.87120670 \quad 4.27510994$

$\mathrm{H} 3.648641431 .03624978 \quad 4.13008737$

H $3.72223918 \quad 0.65766614 \quad-0.09674130$

H $3.35040751 \quad 2.01313826 \quad 0.98226165$

H $2.19876040 \quad 0.69794458 \quad 0.78171666$

H $5.98585009 \quad 0.125262712 .83129716$

H $5.52059499 \quad 1.69879044 \quad 2.18694905$

H $5.91721137 \quad 0.37054883 \quad 1.08672622$

o $1.15741386-1.763897434 .77651283$

$\begin{array}{lllll}0 & 0.44056629 & -2.01470437 & 3.78712748\end{array}$

O $1.21632490-0.563749015 .09549643$

Vibrational frequencies (in $\mathrm{cm}-1$ )

$\begin{array}{llllllllllllllll}-610.1 & 90.1 & 121.8 & 132.0 & 143.8 & 151.6 & 177.2 & 192.7 & 204.8 & 241.1 & 272.6 & 303.4 & 345.4 & 392.2\end{array}$ $419.1440 .1 \quad 464.0 \quad 496.5 \quad 515.8 \quad 602.7 \quad 624.7 \quad 658.5 \quad 718.0 \quad 782.1 \quad 854.8 \quad 896.7 \quad 919.3 \quad 931.5$ $942.9984 .0 \quad 1005.8 \quad 1009.6 \quad 1015.8 \quad 1021.4 \quad 1052.3 \quad 1065.7 \quad 1072.11081 .4 \quad 1083.4 \quad 1096.5$ $\begin{array}{llllllllllllllll}1124.5 & 1139.9 & 1173.9 & 1183.9 & 1190.2 & 1208.6 & 1236.8 & 1254.0 & 1267.0 & 1275.7 & 1285.7 & 1287.6\end{array}$

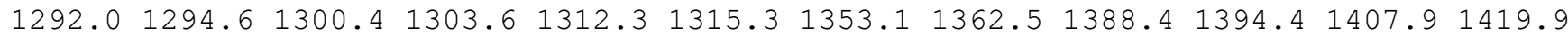
$1590.42660 .5 \quad 2668.4 \quad 2670.8 \quad 2671.7 \quad 2675.8 \quad 2676.5 \quad 2680.92696 .92740 .2 \quad 2741.5 \quad 2751.4$ $2755.8 \quad 2776.6 \quad 2779.0 \quad 2781.5 \quad 2783.4$
name $=$ TS198
energy $($ in $\mathrm{kcal} / \mathrm{mol})=55.783845$
$\operatorname{ZPE}($ in $\mathrm{kcal} / \mathrm{mol})=142.199$
$\mathrm{G}($ in $\mathrm{kcal} / \mathrm{mol})=118.85$
Geometry
$\begin{array}{llll}\text { C } 2.13634312 & 2.07667921 & 2.55605832\end{array}$
C $2.43156296 \quad 0.76431357 \quad 3.25403869$
C $1.32604474-0.34393934 \quad 3.01570531$
C $1.85885435-1.64145187 \quad 2.49306197$
C $3.32122992-1.82174410 \quad 2.52863987$
C $3.93154381-1.140056853 .80040202$
C $3.84039630 \quad 0.21169268 \quad 3.01760224$
C $3.92424561-0.619575331 .67687835$
C $3.15218567-0.18003982 \quad 0.45152254$
C $5.37515371-0.87322591 \quad 1.27712307$
$\mathrm{H} \quad 1.15500711 \quad 2.47149829 \quad 2.85361702$
H $2.14414137 \quad 1.98192638 \quad 1.46518578$
H 2.88044579 2.83918662 2.82504141
$\mathrm{H} \quad 0.49786091 \quad 0.04254410 \quad 2.38763240$
H $1.06819588 \quad-2.79592968 \quad 2.99252439$
H $1.40386617-2.62456573 \quad 3.72847404$
H $3.68460731-2.815087652 .26859093$
$\mathrm{H} \quad 4.94808472-1.450604654 .04264236$
$\mathrm{H} \quad 3.33559715-1.20224553 \quad 4.71278074$
$\mathrm{H} \quad 4.63043845 \quad 0.94257621 \quad 3.18597778$
$\mathrm{H} 2.07383213-0.07939790 \quad 0.62510276$
H $3.24659726-0.92643530-0.35016569$
H $3.52535292 \quad 0.77459791 \quad 0.06419621$
H $5.99691742-1.191311322 .12104469$
$\mathrm{H} \quad 5.83085048 \quad 0.03321023 \quad 0.86115404$
$\mathrm{H} 5.44091588-1.657961450 .51412253$
O $1.04089663 \quad 0.63024650 \quad 5.03206140$
○ $0.80653634 \quad-0.58536569 \quad 4.32891196$ 
$02.358531190 .98851300 \quad 4.67677307$

Vibrational frequencies (in $\mathrm{cm}-1$ )

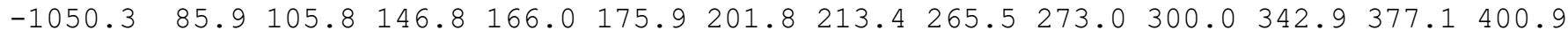
$418.4 \quad 444.8 \quad 488.6 \quad 527.7 \quad 592.0 \quad 609.4 \quad 617.7 \quad 640.5 \quad 704.1 \quad 714.0 \quad 761.7 \quad 819.9 \quad 846.3 \quad 861.9$ $893.0931 .6 \quad 956.5 \quad 975.6 \quad 979.3 \quad 1004.4 \quad 1007.7 \quad 1013.4 \quad 1016.5 \quad 1052.6 \quad 1061.5 \quad 1080.1 \quad 1085.4$

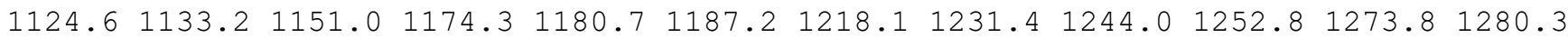
$1285.81287 .2 \quad 1292.6 \quad 1295.1 \quad 1301.6 \quad 1303.6 \quad 1304.7 \quad 1315.2 \quad 1335.6 \quad 1381.2 \quad 1396.4 \quad 1407.0$ $1419.82665 .42671 .5 \quad 2675.1 \quad 2675.8 \quad 2677.2 \quad 2681.72685 .02688 .02749 .02757 .2 \quad 2764.5$ $2779.92781 .8 \quad 2782.8 \quad 4498.3$

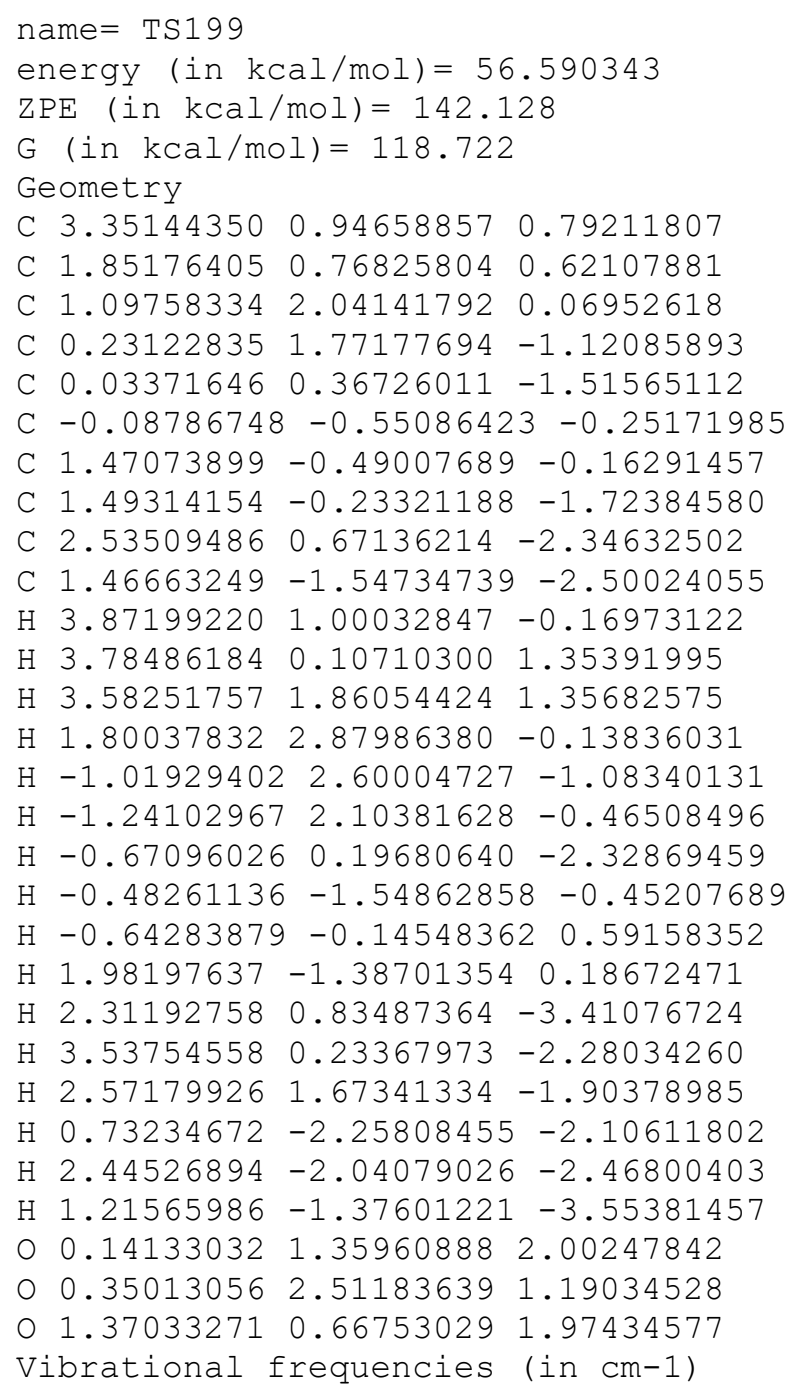


C $2.31857322-1.013609093 .68722498$

$\begin{array}{llll}\text { C } 2.48850381 & 0.37195068 & 4.17490247\end{array}$

C $2.82969870 \quad 0.32511950 \quad 1.65614592$

C 2.063327651 .368241951 .25085706

C $4.31913447 \quad 0.44864122 \quad 1.62633089$

H 2.35454525 $3.03278138 \quad 5.03292471$

$\mathrm{H} 0.72941397 \quad 3.25602207 \quad 4.27066778$

H 2.27829642 3.04221579 3.23930071

$\mathrm{H}-0.25860660 \quad 0.04375840 \quad 2.71652337$

H $0.93349364-1.06138063 \quad 0.44120094$

H $0.94256406-2.53025260 \quad 1.42263344$

$\mathrm{H} \quad 3.06300907-1.79501880 \quad 1.79323006$

H $3.12700753-1.67155107 \quad 4.05557005$

$\mathrm{H} 1.37709791-1.462323894 .06642884$

H $3.48421406 \quad 0.73243862 \quad 4.30744674$

H $2.06024227 \quad 2.21869834 \quad 2.80503899$

H $0.990369531 .29732540 \quad 1.14843719$

$\begin{array}{lllll}\mathrm{H} & 2.46761981 & 2.23170387 & 0.74882871\end{array}$

H $4.71014815 \quad 0.17266297 \quad 0.63393242$

$\mathrm{H} \quad 4.80986900-0.20930082 \quad 2.36336450$

H $4.67213771 \quad 1.46625373 \quad 1.83586963$

O $-2.29838815-1.39126228 \quad 2.73924181$

$0-1.21637523-1.747317562 .20905818$

O 0.223039880 .883691554 .75885903

Vibrational frequencies (in $\mathrm{cm}-1$ )

$\begin{array}{lllllllllllllll}-846.0 & 22.2 & 45.1 & 77.1 & 102.0 & 123.0 & 126.8 & 160.7 & 181.8 & 198.4 & 218.7 & 229.1 & 277.2 & 287.0\end{array}$ $322.4 \quad 330.1 \quad 371.4 \quad 419.9 \quad 455.6 \quad 526.3 \quad 568.1 \quad 577.2 \quad 586.4 \quad 597.4 \quad 635.4 \quad 684.6 \quad 756.6 \quad 797.6$

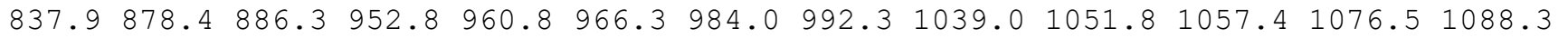

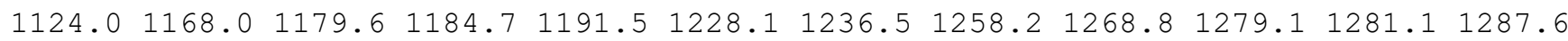

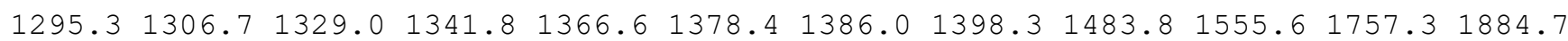
$1963.82640 .42657 .6 \quad 2658.5 \quad 2666.4 \quad 2667.8 \quad 2685.5 \quad 2697.0 \quad 2715.0 \quad 2726.2 \quad 2727.12739 .2$ $2774.6 \quad 2782.0 \quad 2812.5 \quad 2957.1$

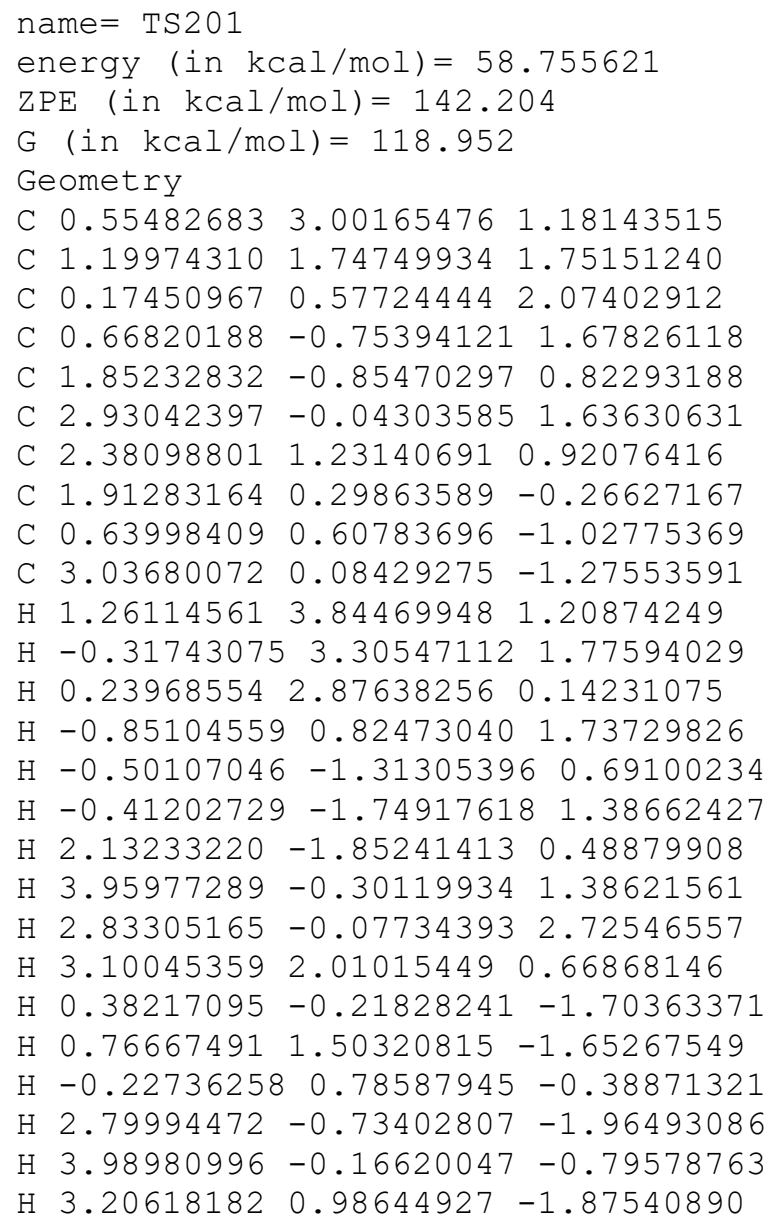


01.324844601 .090355463 .93249870

$0.05654223 \quad 0.66468978 \quad 3.50276587$

O 1.669983032 .153334813 .04217156

Vibrational frequencies (in $\mathrm{cm}-1$ )

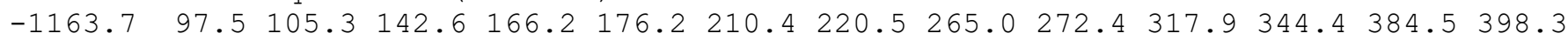

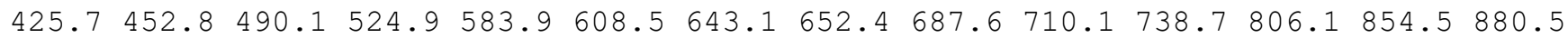
$897.1924 .4940 .7961 .6 \quad 978.1997 .7 \quad 1003.9 \quad 1009.2 \quad 1014.7 \quad 1024.6 \quad 1050.1 \quad 1075.4 \quad 1080.3$

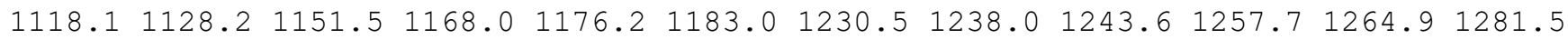

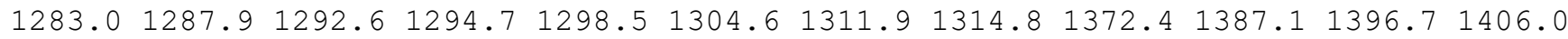
$1414.32668 .82671 .22673 .42676 .8 \quad 2679.3 \quad 2680.02682 .02690 .12749 .02757 .72761 .7$ $2780.12781 .92782 .9 \quad 4515.0$

name $=\operatorname{TS} 202$

energy (in $\mathrm{kcal} / \mathrm{mol})=61.048779$

$\mathrm{ZPE}(\mathrm{in} \mathrm{kcal} / \mathrm{mol})=141.999$

$\mathrm{G}($ in $\mathrm{kcal} / \mathrm{mol})=118.489$

Geometry

C $-0.69965756 \quad 1.199789493 .37352492$

C $0.40953973 \quad 0.41273780 \quad 2.84850420$

C $-0.01802214-0.85609984 \quad 2.02048708$

$\begin{array}{lllll}\text { C } & 0.59056704 & -0.98488491 & 0.61104973\end{array}$

C $1.52303126 \quad 0.17362282 \quad 0.26119386$

C $2.48768715 \quad 0.39644354 \quad 1.46605113$

C $1.37808961 \quad 1.31207354 \quad 2.06987128$

C $0.85313499 \quad 1.56194281 \quad 0.60058464$

C $-0.631376161 .73102395 \quad 0.36720944$

C $1.58956524 \quad 2.72372245-0.06320302$

$\mathrm{H}-0.54152683 \quad 1.99107878 \quad 4.06753308$

$\mathrm{H}-1.24966437 \quad 0.14526102 \quad 4.63041100$

$\mathrm{H}-1.79439415 \quad 0.19917718 \quad 4.04249878$

$\mathrm{H}-1.12382462-0.958010101 .98371425$

$\mathrm{H}-0.21689281-1.05637782-0.14099280$

$\mathrm{H} 1.14830931-1.942547190 .54910903$

$\mathrm{H} \quad 1.97140164 \quad 0.08635908 \quad-0.72834324$

H 3.412707120 .921004491 .21858437

$\mathrm{H} 2.75657046-0.49083048 \quad 2.04041802$

$\mathrm{H} 1.70488401 \quad 2.18770682 \quad 2.62986504$

H $-1.25011911 \quad 1.02399354 \quad 0.93263886$

$\mathrm{H}-0.88031817 \quad 1.61133122-0.69286280$

$\mathrm{H}-0.96923342 \quad 2.72751893 \quad 0.68311234$

H 2.67702027 2.65017679 0.04313078

H $1.28467364 \quad 3.68212459 \quad 0.37327182$

H $1.369849272 .76401485-1.13619794$

O $1.47869836-1.460349113 .60621800$

○ $0.38448539-1.950846412 .83926025$

o $1.08216543-0.163627393 .99371739$

Vibrational frequencies (in $\mathrm{cm}-1$ )

$\begin{array}{llllllllllllll}-787.7 & 66.8 & 100.2 & 153.6 & 169.6 & 179.6 & 201.3 & 216.0 & 252.4 & 270.2 & 302.7 & 347.5 \quad 380.3 \quad 413.2\end{array}$

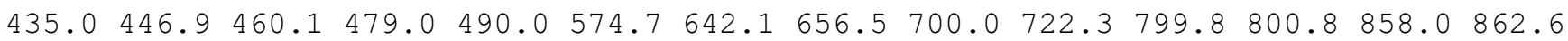
$893.2916 .3939 .7 \quad 971.8 \quad 981.3 \quad 992.0 \quad 1004.4 \quad 1007.4 \quad 1018.2 \quad 1020.5 \quad 1049.7 \quad 1066.3 \quad 1087.5$

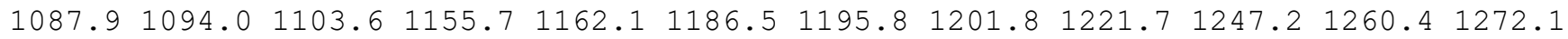

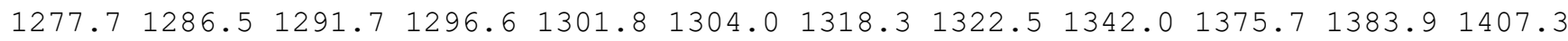
1420.72659 .62666 .92676 .52678 .62681 .62684 .02689 .12715 .32739 .92747 .12751 .2 $2767.4 \quad 2781.8 \quad 2785.0 \quad 4858.7$

name $=$ TS203

energy $($ in $\mathrm{kcal} / \mathrm{mol})=63.34127$

$\mathrm{ZPE}(\mathrm{in} \mathrm{kcal} / \mathrm{mol})=140.461$

$\mathrm{G}($ in $\mathrm{kcal} / \mathrm{mol})=117.381$

Geometry

C $-0.18164227 \quad 1.77961251 \quad 0.44972189$

C $0.84273407 \quad 1.41942951 \quad 1.52987927$

C $1.19064813-0.208805891 .46649334$ 
C $2.50882445-0.621204770 .80246446$

$\begin{array}{lllll}\text { C } 3.56673561 & 0.48562041 & 0.79379400\end{array}$

C $3.34734880 \quad 1.34523381 \quad 2.04336959$

C $2.33844652 \quad 2.14623992 \quad 1.30056547$

C $3.030523121 .71930069-0.04553541$

C $2.242474861 .44564135-1.30383187$

$\begin{array}{llll}\text { C } 4.15925624 & 2.69575217 & -0.38174408\end{array}$

$\mathrm{H}-1.17746759 \quad 1.84916097 \quad 0.91397348$

$\mathrm{H}-0.24593207 \quad 1.06034215-0.36509304$

$\mathrm{H} \quad 0.03469514 \quad 2.76563927 \quad 0.02659940$

$\mathrm{H} \quad 0.31143238-0.72059486 \quad 1.04200317$

$\mathrm{H} \quad 2.31458384-0.95938652-0.23470106$

H $2.91519182-1.51230750 \quad 1.32534867$

H $4.571811890 .13599900 \quad 0.56204089$

H $4.14178555 \quad 1.76390587 \quad 2.63739838$

H 2.87032158 $0.60997982 \quad 3.04611150$

$\mathrm{H} 2.17614518 \quad 3.19906784 \quad 1.50815639$

H $1.733999812 .35341293-1.65568828$

$\mathrm{H} \quad 1.47457871 \quad 0.67474570-1.17813673$

H $2.90147863 \quad 1.10505981-2.11360159$

$\mathrm{H} \quad 4.816738312 .28862262-1.15819473$

H $4.78696976 \quad 2.931331990 .48414229$

$\mathrm{H} \quad 3.75530150 \quad 3.64483070-0.75504584$

01.806588320 .196014843 .59026737

$\begin{array}{lllll}0 & 1.24353670 & -0.68204880 & 2.80262621\end{array}$

o $0.49071952 \quad 1.75630186 \quad 2.69895758$

Vibrational frequencies (in $\mathrm{cm}-1$ )

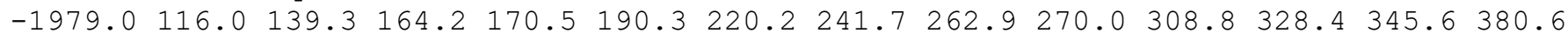

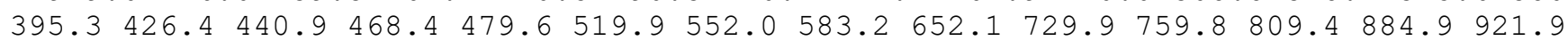

$950.8 \quad 986.8 \quad 992.7 \quad 1008.1 \quad 1017.0 \quad 1018.5 \quad 1022.2 \quad 1038.9 \quad 1046.2 \quad 1077.9 \quad 1092.7 \quad 1100.5 \quad 1110.0$

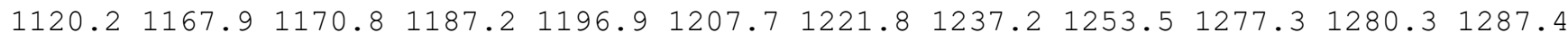

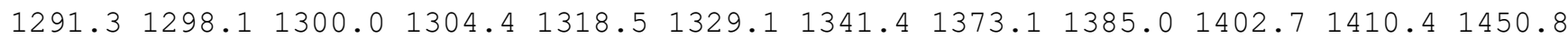
1521.4 2651.7 2666.0 2671.2 2674.1 2676.3 2678.9 2683.2 2691.7 2731.8 $2733.6 \quad 2743.5$ $2751.22778 .5 \quad 2781.62782 .4$

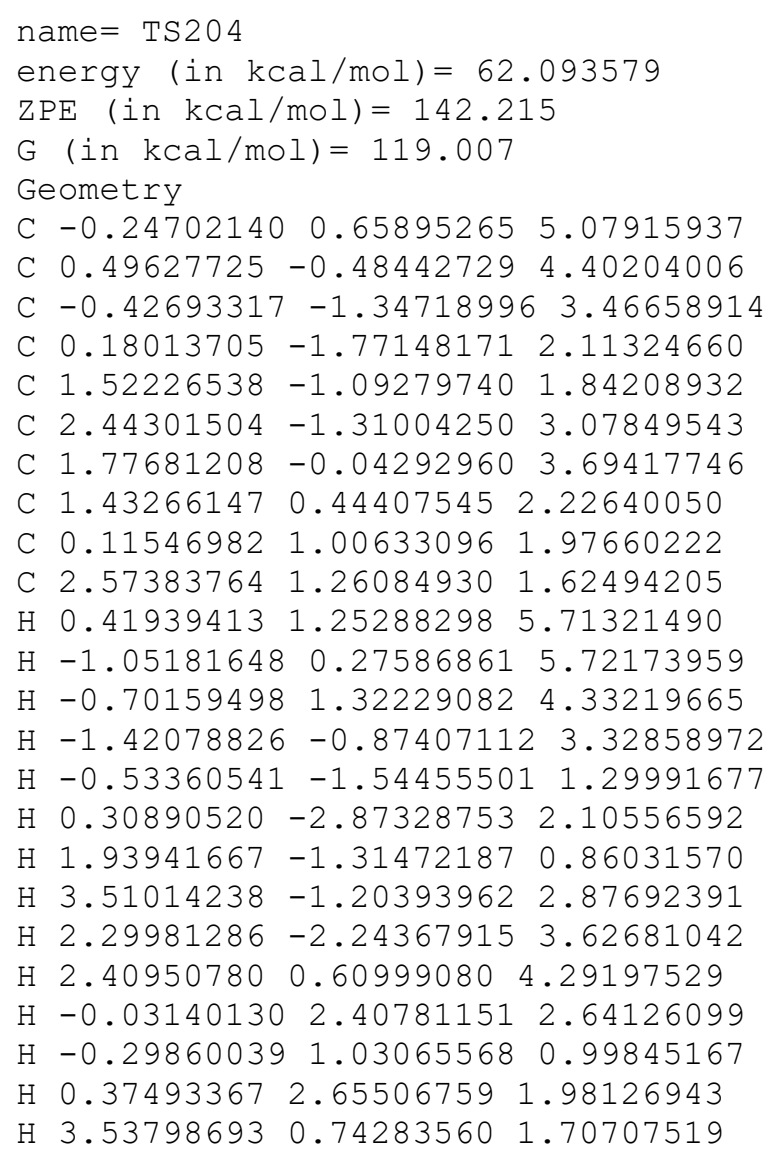


H $2.69188200 \quad 2.22217807 \quad 2.13509580$

H 2.407142331 .451909710 .55937576

$0.48856422-2.681822025 .02138670$

$0-0.69326772-2.501269624 .26162993$

O $0.82947104-1.379585585 .47814104$

Vibrational frequencies (in $\mathrm{cm}-1$ )

$\begin{array}{lllllllllllllll}-976.0 & 73.9 & 144.0 & 161.3 & 176.4 & 189.9 & 203.0 & 207.9 & 258.8 & 268.3 & 322.4 & 344.3 & 364.9 & 405.7\end{array}$ $411.9 \quad 464.7 \quad 473.5 \quad 514.7 \quad 536.0 \quad 583.9 \quad 626.3 \quad 655.6 \quad 706.5 \quad 748.9 \quad 801.3 \quad 828.4 \quad 854.1869 .2$ $897.1919 .3 \quad 942.0 \quad 958.7 \quad 971.8 \quad 1000.8 \quad 1011.2 \quad 1023.3 \quad 1042.0 \quad 1055.1 \quad 1065.0 \quad 1072.9 \quad 1081.3$ $1093.91129 .0 \quad 1139.4 \quad 1148.2 \quad 1164.3 \quad 1169.9 \quad 1190.8 \quad 1202.4 \quad 1210.1 \quad 1244.5 \quad 1253.5 \quad 1263.5$

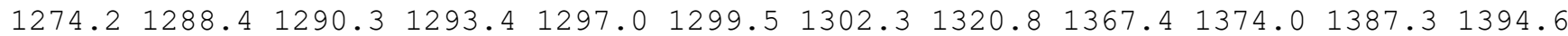
$1413.72660 .72669 .92670 .8 \quad 2678.5 \quad 2680.5 \quad 2689.32690 .92724 .12740 .32747 .3 \quad 2753.2$ $2767.52779 .2 \quad 2780.4 \quad 4699.6$

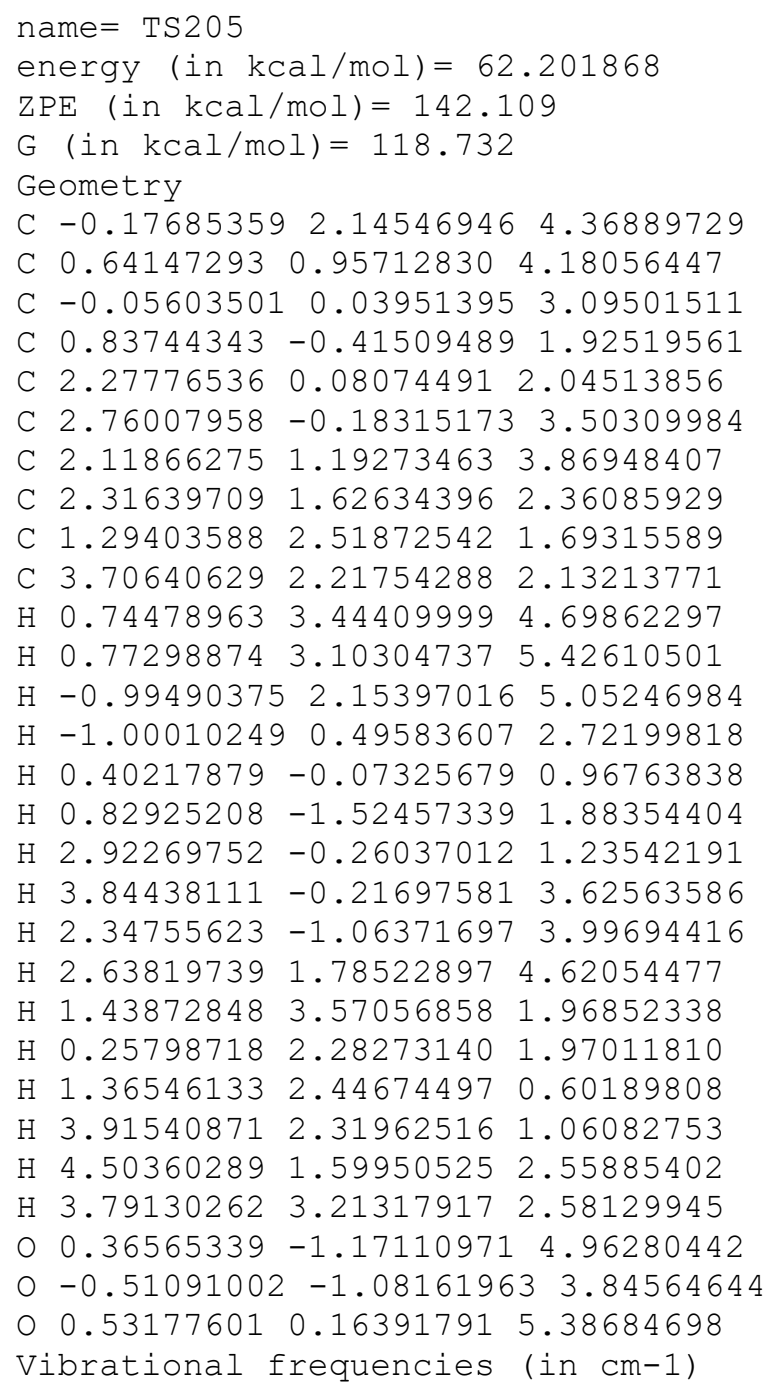


C $2.89210643 \quad 1.93037741 \quad 3.18279135$

C $2.581929920 .60452155 \quad 3.97757953$

C $2.07852907-0.595256423 .15381945$

C $2.13342868-0.32206403 \quad 1.65127176$

C $3.48915606 \quad 0.38143840 \quad 1.33466340$

C $2.76869365 \quad 1.72676120 \quad 1.66504597$

C 1.399562731 .031029811 .30073085

C $0.15983587 \quad 1.40715008 \quad 2.08032743$

C $1.10005100 \quad 1.14326177-0.19209464$

H $2.18482750 \quad 3.20218598 \quad 5.23693600$

H 2.28496805 4.05265265 3.22826234

$\mathrm{H} \quad 2.92987084 \quad 3.46496219 \quad 5.08873498$

H $1.91513115 \quad 0.81382459 \quad 4.83690585$

$\mathrm{H} \quad 1.04692337 \quad-0.85455798 \quad 3.45593193$

H $2.69857482-1.485394353 .39274677$

$\mathrm{H} 1.86407132-1.185099431 .04265754$

H $3.82146750 \quad 0.29088848 \quad 0.29973530$

$\mathrm{H} \quad 4.33134814 \quad 0.11967202 \quad 1.98199110$

H 3.046829732 .603446321 .08206025

$\mathrm{H} 0.321480121 .45273573 \quad 3.16531688$

$\mathrm{H}-0.65368526 \quad 0.69656994 \quad 1.89884465$

$\mathrm{H}-0.195770792 .40717076 \quad 1.79740455$

$\mathrm{H} \quad 0.81799666 \quad 2.16825173-0.46015739$

$\mathrm{H} \quad 0.26894099 \quad 0.48705550-0.47482669$

$\mathrm{H} \quad 1.95788349 \quad 0.86874123 \quad-0.81538918$

O $4.58104514 \quad 1.41936771 \quad 4.61365181$

$03.86056237 \quad 0.20684559 \quad 4.48550692$

o $4.306936772 .13803007 \quad 3.42598495$

Vibrational frequencies (in $\mathrm{cm}-1$ )

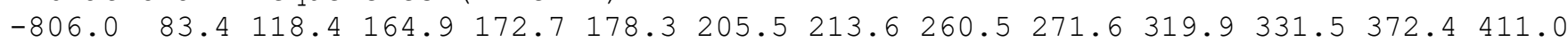
$430.7 \quad 451.8 \quad 460.8 \quad 484.8 \quad 488.0 \quad 583.3 \quad 629.2 \quad 644.3 \quad 712.7 \quad 725.8 \quad 793.4 \quad 808.0 \quad 851.8 \quad 878.5$ $891.5 \quad 923.5 \quad 937.9 \quad 974.0 \quad 990.8 \quad 995.2 \quad 1007.8 \quad 1008.4 \quad 1014.5 \quad 1019.7 \quad 1062.5 \quad 1075.9 \quad 1088.0$

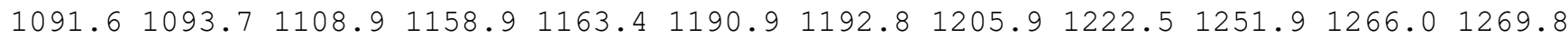

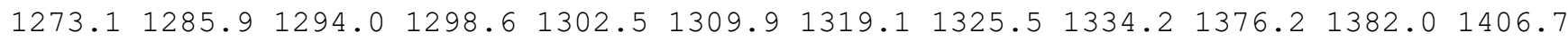
$1418.72658 .0 \quad 2664.5 \quad 2676.3 \quad 2678.6 \quad 2681.4 \quad 2684.02685 .72717 .92737 .8 \quad 2748.02751 .6$ $2763.4 \quad 2781.7 \quad 2784.1 \quad 4851.7$

name $=$ TS207

energy $($ in $\mathrm{kcal} / \mathrm{mol})=63.308146$

$\operatorname{ZPE}(\mathrm{in} \mathrm{kcal} / \mathrm{mol})=141.327$

$\mathrm{G}($ in $\mathrm{kcal} / \mathrm{mol})=115.97$

Geometry

C $-0.60963142 \quad 2.23666552 \quad 1.82057778$

C $0.56285701 \quad 1.70642670 \quad 2.60222587$

C $0.96488385 \quad 0.24969354 \quad 2.36711171$

C $2.44622602-0.100380932 .35340017$

C $3.34402176 \quad 0.81133144 \quad 1.54064343$

C $3.28999094 \quad 2.280492162 .10067452$

C $2.307860462 .56769228 \quad 1.04024416$

C 2.706407161 .392986450 .18996763

C $1.65699886 \quad 0.59085922-0.54765622$

C $3.811946351 .79692590 \quad-0.79590030$

$\mathrm{H}-0.56261822 \quad 1.99430148 \quad 0.75645442$

$\mathrm{H}-0.665078513 .33209623 \quad 1.92277826$

$\mathrm{H}-1.55754258 \quad 1.84648603 \quad 2.21822647$

H $0.41725999-0.20454037 \quad 1.52162719$

$\mathrm{H} 2.56031272-1.14978008 \quad 2.01471810$

H $2.80499994-0.09650603 \quad 3.41799298$

H $4.34821913 \quad 0.39648452 \quad 1.40842587$

H $4.245452592 .82172526 \quad 2.06231624$

$\mathrm{H} \quad 2.95303760 \quad 2.39496373 \quad 3.15480260$

H $1.63300778 \quad 3.36415781 \quad 0.87651391$

$\mathrm{H} \quad 1.82899398-0.48686796-0.41458891$

H $1.670727310 .77776172-1.62998713$ 
$\mathrm{H} \quad 0.63743807 \quad 0.81433755 \quad-0.21474486$

$\mathrm{H} \quad 4.62769622 \quad 2.35038370-0.31858595$

H $3.411724672 .43357219-1.59508342$

H $4.25351073 \quad 0.91142974-1.27041689$

$\begin{array}{lllll}0 & 0.92354170 & -0.28538221 & 4.59399556\end{array}$

$00.30618663-0.349516773 .52375552$

$\begin{array}{lllll}0 & 0.97514700 & 2.30688242 \quad 3.57158717\end{array}$

Vibrational frequencies (in cm-1)

$\begin{array}{llllllllllllllllll}-121.2 & 55.5 & 97.5 & 108.8 & 118.5 & 128.5 & 135.1 & 178.9 & 198.5 & 218.1 & 225.3 & 262.2 & 275.6 & 342.8\end{array}$

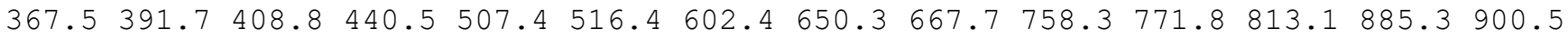

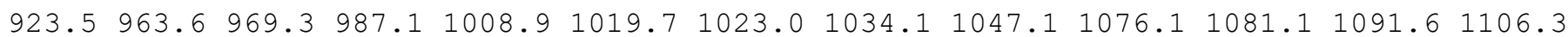
$1167.31184 .4 \quad 1190.2 \quad 1200.1 \quad 1216.3 \quad 1219.3 \quad 1237.2 \quad 1262.2 \quad 1265.2 \quad 1271.91277 .11287 .8$

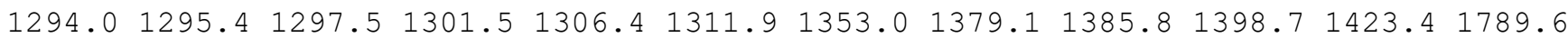
$2582.52627 .52660 .2 \quad 2664.3 \quad 2668.0 \quad 2672.2 \quad 2672.82676 .82698 .82715 .92724 .32736 .1$ $2755.7 \quad 2773.6 \quad 2778.2 \quad 2778.7$

name $=\operatorname{TS} 208$

energy (in $\mathrm{kcal} / \mathrm{mol})=62.507515$

$\mathrm{ZPE}(\mathrm{in} \mathrm{kcal} / \mathrm{mol})=142.219$

$\mathrm{G}(\mathrm{in} \mathrm{kcal} / \mathrm{mol})=118.758$

Geometry

C $-1.67183645 \quad 0.71512518 \quad 2.43610254$

C $-1.13573528-0.63426035 \quad 1.99328182$

C $-1.58164592-1.018033200 .53199048$

C $-0.47994870-1.52066766-0.42328695$

C $0.89094343-1.548319560 .25093678$

C $0.74106122-2.22692704 \quad 1.64508776$

C $0.36970835-0.81625663 \quad 2.19843612$

C $1.16219370-0.17373532 \quad 0.98541474$

C $0.535019191 .01830804 \quad 0.43387664$

C $2.64292566 \quad 0.012589931 .31576863$

$\mathrm{H}-2.76754053 \quad 0.69443064 \quad 2.51177671$

$\mathrm{H}-1.406158651 .511646891 .72714280$

$\mathrm{H}-1.28470058 \quad 0.99831252 \quad 3.42125613$

$\mathrm{H}-2.17009367-0.19937756 \quad 0.07618536$

$\mathrm{H}-0.45338291-0.87892195-1.32189228$

$\mathrm{H}-0.74239680-2.53945376-0.77830184$

$\mathrm{H} 1.69028673-1.91245928-0.39358835$

$\mathrm{H} 1.66165418 \quad-2.64554680 \quad 2.05279484$

$\mathrm{H}-0.04134394-2.988353391 .72509835$

H $0.71919641-0.55595063 \quad 3.19646547$

H $0.76247205 \quad 1.10453531-1.10158858$

$\mathrm{H} \quad 1.50773517 \quad 1.34639920-0.88007090$

$\mathrm{H} \quad 0.70463226 \quad 1.97590551 \quad 0.86679264$

H $3.07556629-0.87136452 \quad 1.79924290$

H 2.78880831 $0.85473845 \quad 2.00175842$

$\mathrm{H} \quad 3.23313519 \quad 0.20369743 \quad 0.41477878$

O $-2.90148499-2.027572502 .06503896$

$0-2.44831204-2.14490626 \quad 0.72517913$

$0-1.75311313-1.653035782 .80130086$

Vibrational frequencies (in $\mathrm{cm}-1$ )

$\begin{array}{llllllllllllllll}-986.8 & 56.9 & 135.5 & 142.2 & 168.9 & 183.6 & 200.2 & 204.0 & 258.3 & 275.1 & 313.6 & 352.2 & 372.0 & 388.1\end{array}$

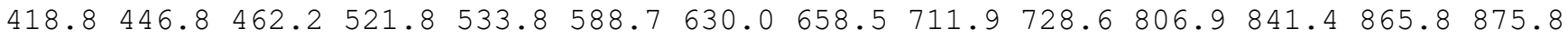
$899.5920 .4940 .2954 .8 \quad 987.3 \quad 1003.8 \quad 1011.5 \quad 1024.5 \quad 1052.2 \quad 1061.2 \quad 1071.8 \quad 1076.7 \quad 1089.6$

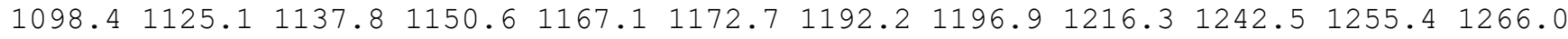
$1275.31284 .5 \quad 1286.5 \quad 1295.0 \quad 1296.2 \quad 1300.1 \quad 1320.4 \quad 1325.2 \quad 1367.5 \quad 1376.7 \quad 1386.4 \quad 1401.8$ $1410.72660 .92666 .4 \quad 2673.0 \quad 2677.0 \quad 2680.6 \quad 2686.22688 .92717 .4 \quad 2739.72747 .22750 .5$ $2763.92779 .12781 .6 \quad 4688.1$

name $=$ TS209

energy (in $\mathrm{kcal} / \mathrm{mol})=62.866777$

$\mathrm{ZPE}(\mathrm{in} \mathrm{kcal} / \mathrm{mol})=141.964$

$\mathrm{G}($ in $\mathrm{kcal} / \mathrm{mol})=115.526$ 


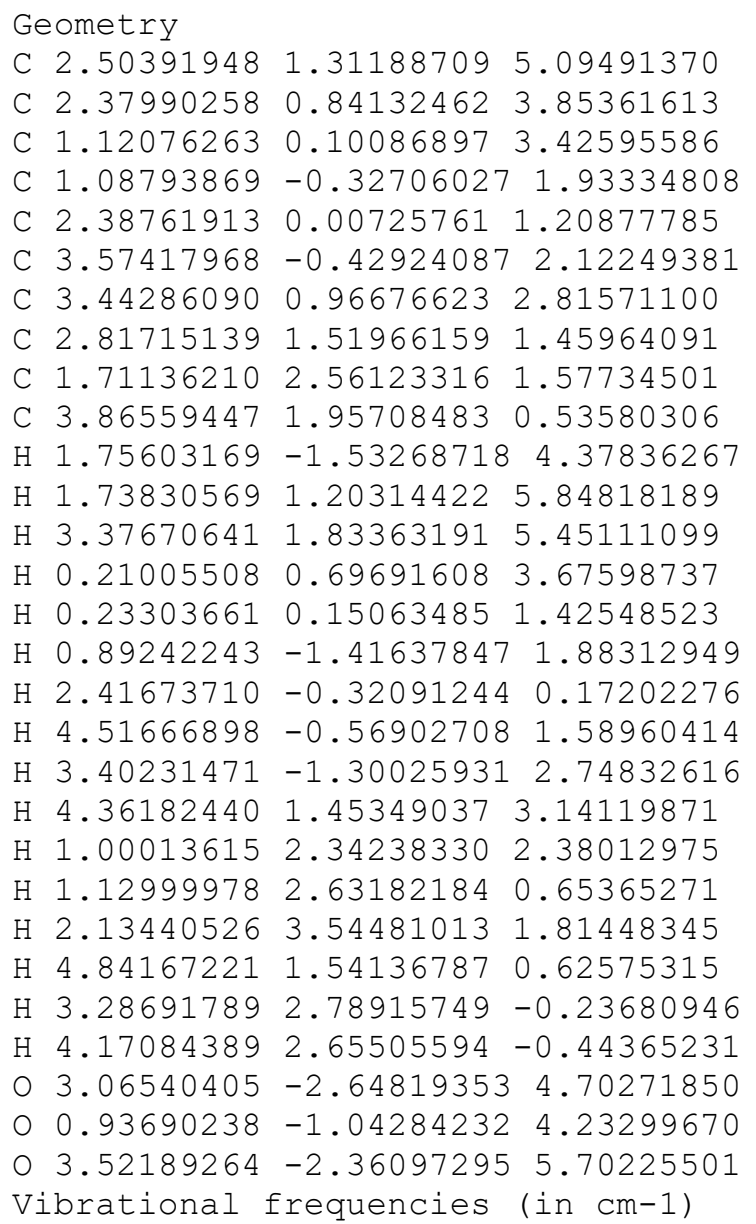

Vibrational frequencies (in $\mathrm{cm}-1$ )

$\begin{array}{lllllllllllllll}-1435.3 & 31.5 & 38.8 & 61.8 & 73.1 & 99.6 & 121.5 & 147.5 & 172.3 & 198.5 & 246.0 & 280.3 & 336.3 & 369.0\end{array}$

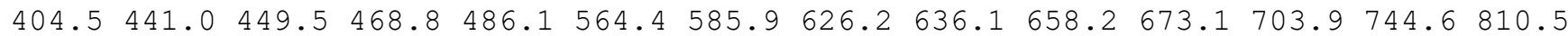

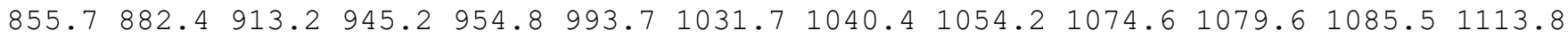

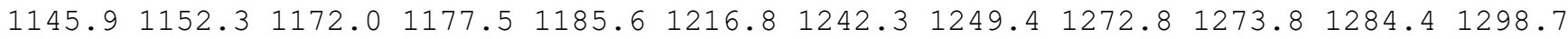
$1301.81323 .51330 .8 \quad 1350.1 \quad 1373.1 \quad 1383.7 \quad 1392.4 \quad 1408.61416 .01445 .7 \quad 1595.4 \quad 1865.1$

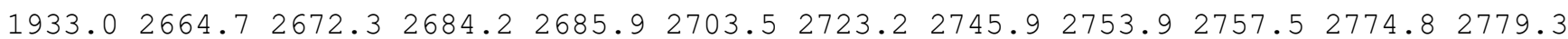
$2784.5 \quad 2788.2 \quad 2811.7 \quad 3732.2$

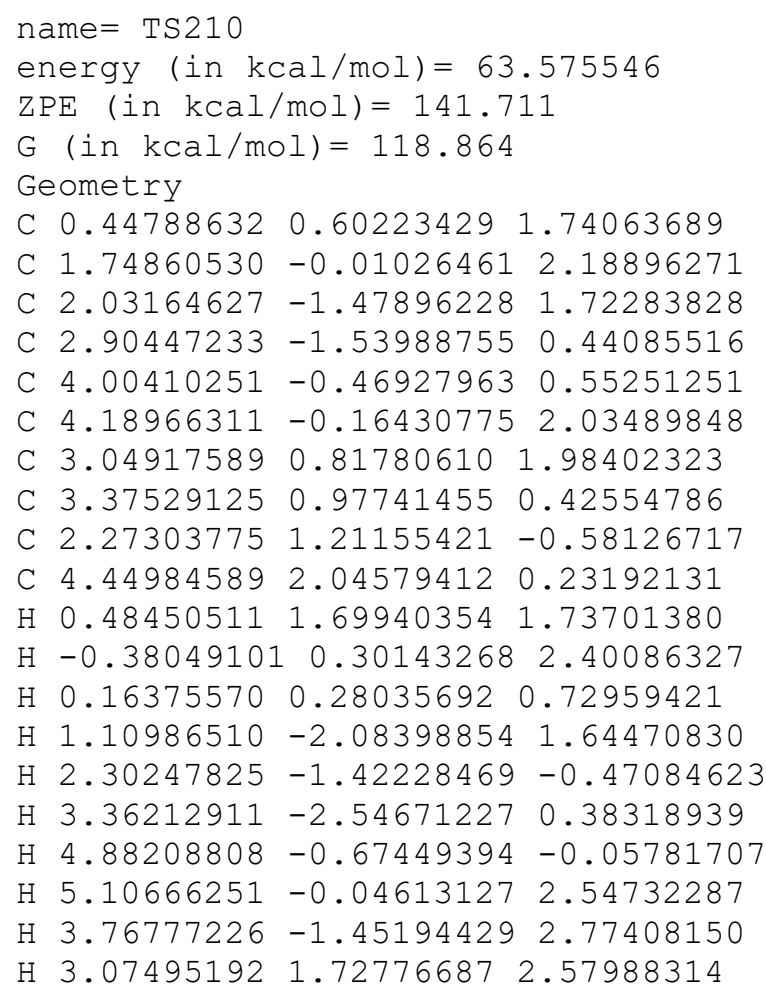


H $1.54241756 \quad 0.39697538-0.62306862$

H 2.68829787 $1.30406635-1.59482471$

H $1.728464262 .13898002-0.36649222$

$\mathrm{H} \quad 4.87563453 \quad 1.99828990-0.77703272$

H $5.28190392 \quad 1.93123317 \quad 0.93721900$

H $4.03866115 \quad 3.05137723 \quad 0.37351845$

O $1.40722294-1.13984762 \quad 4.12265707$

O $2.77835113-2.021215542 .77253055$

O $1.69597416-0.044995053 .66233576$

Vibrational frequencies (in $\mathrm{cm}-1$ )

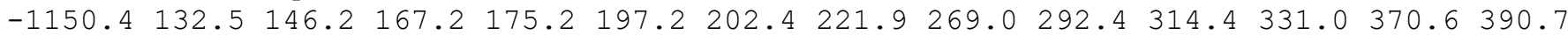
$446.8 \quad 454.7 \quad 482.5 \quad 504.7 \quad 549.8 \quad 564.3 \quad 639.8 \quad 681.8 \quad 735.5 \quad 759.1 \quad 813.6 \quad 836.7 \quad 873.8 \quad 893.7$ $949.1955 .3 \quad 968.2988 .3 \quad 1013.4 \quad 1017.3 \quad 1025.6 \quad 1034.2 \quad 1054.11069 .01086 .11092 .11116 .6$ $1136.0 \quad 1158.3 \quad 1180.0 \quad 1187.1 \quad 1202.0 \quad 1209.8 \quad 1215.7 \quad 1233.4 \quad 1252.0 \quad 1264.3 \quad 1278.4 \quad 1281.8$ $\begin{array}{lllllllllllll}1286.9 & 1290.1 & 1296.6 & 1299.8 & 1301.8 & 1317.9 & 1325.1 & 1335.4 & 1362.9 & 1393.1 & 1402.5 & 1409.9\end{array}$ $1939.52659 .12663 .8 \quad 2669.02670 .5 \quad 2674.5 \quad 2676.12680 .82696 .62745 .02746 .62752 .3$ 2774.72778 .32781 .12782 .3

name $=\operatorname{TS} 211$

energy (in $\mathrm{kcal} / \mathrm{mol})=64.948654$

$\mathrm{ZPE}($ in $\mathrm{kcal} / \mathrm{mol})=142.103$

$\mathrm{G}($ in $\mathrm{kcal} / \mathrm{mol})=118.753$

Geometry

C 3.894200692 .123639863 .99936203

C 3.095456541 .024503693 .31269723

C $1.543253091 .28068278 \quad 3.28919779$

C $0.836330721 .03367810 \quad 1.94066170$

C $1.80448393 \quad 0.63791016 \quad 0.82530166$

C $2.72982948-0.48941608 \quad 1.37458051$

C $3.63588493 \quad 0.64911136 \quad 1.93593351$

C $3.054741291 .59791185 \quad 0.79936723$

C $2.826775313 .08112461 \quad 1.08085410$

C $3.779267121 .62751390 \quad-0.46772748$

H $3.94141657 \quad 3.03864083 \quad 3.40185593$

$\mathrm{H} \quad 4.92813267 \quad 1.79712219 \quad 4.18134449$

H $3.46606595 \quad 2.37335250 \quad 4.98026543$

$\mathrm{H} \quad 1.28510260 \quad 2.27751139 \quad 3.70267078$

$\mathrm{H} 0.26487861 \quad 1.93145310 \quad 1.64143765$

$\mathrm{H} \quad 0.08140600 \quad 0.23093717 \quad 2.07690815$

$\mathrm{H} \quad 1.31319960 \quad 0.47014329-0.13413804$

H $3.19891341-1.11984579 \quad 0.62073854$

H 2.28161451 $-1.16028768 \quad 2.11133227$

H $4.71174903 \quad 0.48506410 \quad 1.92390731$

H 2.20956135 $3.26477664 \quad 1.96207757$

H $2.31021786 \quad 3.54978486 \quad 0.22683214$

H $3.77587384 \quad 3.61406964 \quad 1.21789084$

$\mathrm{H} \quad 4.80132964 \quad 1.91506491-0.51474974$

H $3.59496983 \quad 0.32670012-1.33334638$

H $4.15447910 \quad 0.02850772 \quad-0.82731365$

O $1.95666664-0.70412417 \quad 4.26842784$

$\begin{array}{lllll}0 & 1.04455020 & 0.38162787 & 4.27693761\end{array}$

o $3.23587802-0.097593624 .20412316$

Vibrational frequencies (in cm-1)

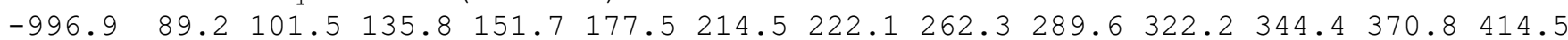
$434.6 \quad 458.0 \quad 481.6 \quad 517.4 \quad 537.4 \quad 569.2 \quad 650.0 \quad 667.9 \quad 703.6 \quad 720.6 \quad 774.4 \quad 827.2 \quad 842.2 \quad 867.1$ $891.7 \quad 917.4 \quad 935.1953 .7 \quad 973.2 \quad 999.0 \quad 1006.9 \quad 1021.8 \quad 1035.7 \quad 1044.0 \quad 1050.5 \quad 1070.11077 .5$ $1098.21124 .8 \quad 1143.6 \quad 1153.6 \quad 1161.4 \quad 1179.4 \quad 1191.4 \quad 1207.7 \quad 1231.5 \quad 1247.4 \quad 1254.91270 .2$

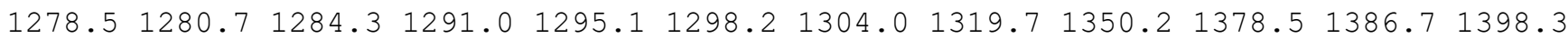
$1412.82658 .42667 .92669 .2 \quad 2676.4 \quad 2677.5 \quad 2684.12686 .12718 .62739 .22746 .12751 .3$ $2768.0 \quad 2779.2 \quad 2781.7 \quad 4734.0$

name $=$ TS212

energy $($ in $\mathrm{kcal} / \mathrm{mol})=64.841692$ 
$\mathrm{ZPE}($ in $\mathrm{kcal} / \mathrm{mol})=142.272$

$\mathrm{G}($ in $\mathrm{kcal} / \mathrm{mol})=119.117$

Geometry

C 2.727010931 .180628164 .89941533

C $2.157841790 .33182819 \quad 3.77036235$

C $0.61246723 \quad 0.51854391 \quad 3.54964390$

C $0.15543415 \quad 0.68981093 \quad 2.08744319$

C $1.316768920 .69947552 \quad 1.09558485$

C $2.26286705-0.494048361 .41902526$

C 2.944544120 .442212362 .46664852

C $2.47166500 \quad 1.67411804 \quad 1.59099873$

C $2.076665152 .97685752 \quad 2.26227179$

C 3.361241971 .872358610 .44575733

$\mathrm{H} 2.13212507 \quad 1.07154309 \quad 5.81714664$

H $2.767560462 .24243365 \quad 4.64225205$

H $3.74896816 \quad 0.86160930 \quad 5.14794505$

H $0.19652121 \quad 1.31662917 \quad 4.19843343$

$\mathrm{H}-0.44158568 \quad 1.61379720 \quad 1.98224302$

$\mathrm{H}-0.53474362-0.143021251 .83248701$

$\mathrm{H} \quad 1.004687110 .82018430 \quad 0.05676136$

$\mathrm{H} 2.92564115-0.76440856 \quad 0.58858316$

H $1.79601927-1.40179486 \quad 1.79652009$

$\mathrm{H} \quad 4.02046935 \quad 0.32945954 \quad 2.59658878$

H $1.63926312 \quad 3.66800739 \quad 1.52934624$

H 2.94378599 $3.47345315 \quad 2.71126813$

$\mathrm{H} 1.329338932 .856519813 .05169584$

H $4.84895228 \quad 1.99792669 \quad 0.89711273$

H $4.62090162 \quad 2.74991677 \quad 1.10697120$

H $3.154733792 .60442329-0.29521613$

O $1.05570215-1.65749963 \quad 3.91343190$

$00.05363112-0.67191688 \quad 4.10146162$

o $2.26389109-1.012234614 .27690997$

Vibrational frequencies (in $\mathrm{cm}-1$ )

$\begin{array}{lllllllllllll}-993.7 & 92.9 & 128.6 & 156.9 & 169.2 & 171.8 & 206.2 & 223.3 & 266.5 & 280.7 & 324.0 & 347.5 \quad 362.7 & 408.2\end{array}$

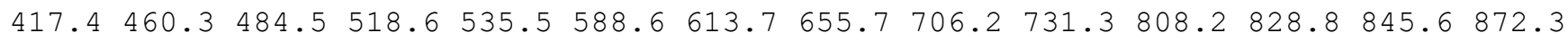
$892.7918 .8 \quad 941.4956 .9973 .0 \quad 1000.2 \quad 1007.3 \quad 1026.9 \quad 1046.6 \quad 1054.6 \quad 1066.3 \quad 1074.4 \quad 1078.3$

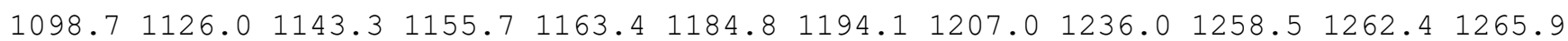

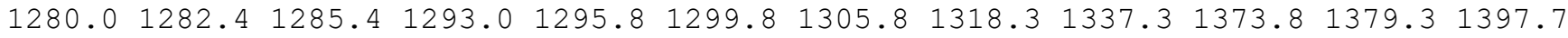
$1412.0 \quad 2658.12669 .7 \quad 2672.2 \quad 2677.4 \quad 2678.2 \quad 2685.8 \quad 2691.3 \quad 2723.5 \quad 2738.92744 .4 \quad 2750.7$ $2765.52780 .62783 .0 \quad 4701.5$

\section{name $=$ TS213}

energy $($ in $\mathrm{kcal} / \mathrm{mol})=65.053671$

$\mathrm{ZPE}(\mathrm{in} \mathrm{kcal} / \mathrm{mol})=142.292$

$\mathrm{G}($ in $\mathrm{kcal} / \mathrm{mol})=119.088$

Geometry

$\begin{array}{llll}\text { C } 3.23404014 & 3.29802580 & 2.67622035\end{array}$

C $3.148847161 .87199464 \quad 2.16122945$

C $1.91192007 \quad 1.07412884 \quad 2.72641344$

C $1.04186100 \quad 0.33044484 \quad 1.69491955$

C $1.59275081 \quad 0.45638708 \quad 0.27442328$

C $3.13113046 \quad 0.21176154 \quad 0.31456835$

C $3.279797561 .73169167 \quad 0.64602379$

C $1.887666191 .96665571-0.08606361$

C $0.91007928 \quad 3.01033627 \quad 0.42307873$

C $2.222623172 .13744903-1.50160131$

$\mathrm{H} \quad 4.17760098 \quad 3.76664733 \quad 2.36226104$

H $3.21937125 \quad 3.32428840 \quad 3.77458426$

H 2.42021039 3.93042173 2.31083976

H $1.30254960 \quad 1.71140027 \quad 3.39505575$

$\mathrm{H} \quad 0.00090057 \quad 0.69692998 \quad 1.73866007$

$\mathrm{H} \quad 0.99319885 \quad-0.74481226 \quad 1.97435335$

H $1.02342516-0.10795851-0.46395170$

H $3.55978499-0.06479116-0.65420128$ 
$\mathrm{H} \quad 3.49318915-0.48503511 \quad 1.07274244$

H $4.14063759 \quad 2.25344334 \quad 0.22532635$

$\mathrm{H} 0.70777488 \quad 2.93382549 \quad 1.49581546$

$\mathrm{H}-0.059214712 .91571251-0.08011452$

$\mathrm{H} \quad 1.28582177 \quad 4.02422000 \quad 0.23701503$

$\mathrm{H} 2.49841345 \quad 3.08625547 \quad-1.89167395$

$\mathrm{H} 0.72123348 \quad 2.16151866-2.23562525$

$\mathrm{H} \quad 1.14341458 \quad 1.49194722 \quad-2.42297140$

$\begin{array}{llll}0 & 3.77658819 & 0.56758952 & 3.88809652\end{array}$

$\begin{array}{lllll}0 & 2.50826131 & 0.04444927 & 3.52505642\end{array}$

O 4.272428461 .147060062 .69703650

Vibrational frequencies (in $\mathrm{cm}-1$ )

$\begin{array}{llllllllllllllll}-995.1 & 84.7 & 126.6 & 152.8 & 171.0 & 185.2 & 197.4 & 229.2 & 270.4 & 276.9 & 316.0 & 353.1 & 363.2 & 404.8\end{array}$

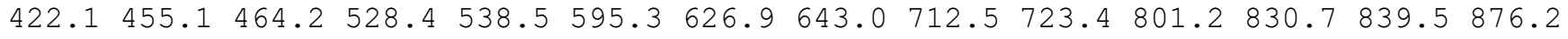

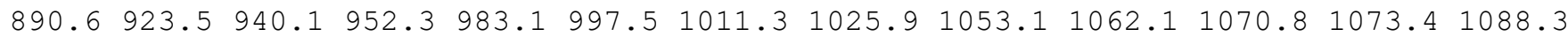

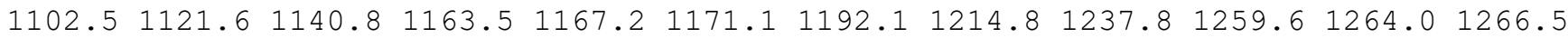
$1273.21280 .5 \quad 1283.4 \quad 1289.2 \quad 1295.8 \quad 1308.8 \quad 1317.91322 .01339 .3 \quad 1371.7 \quad 1377.6 \quad 1397.8$ $1410.82656 .6 \quad 2670.2 \quad 2671.8 \quad 2677.4 \quad 2678.1 \quad 2683.72689 .72720 .4 \quad 2737.92743 .82749 .7$ $2761.32780 .6 \quad 2783.0 \quad 4700.1$

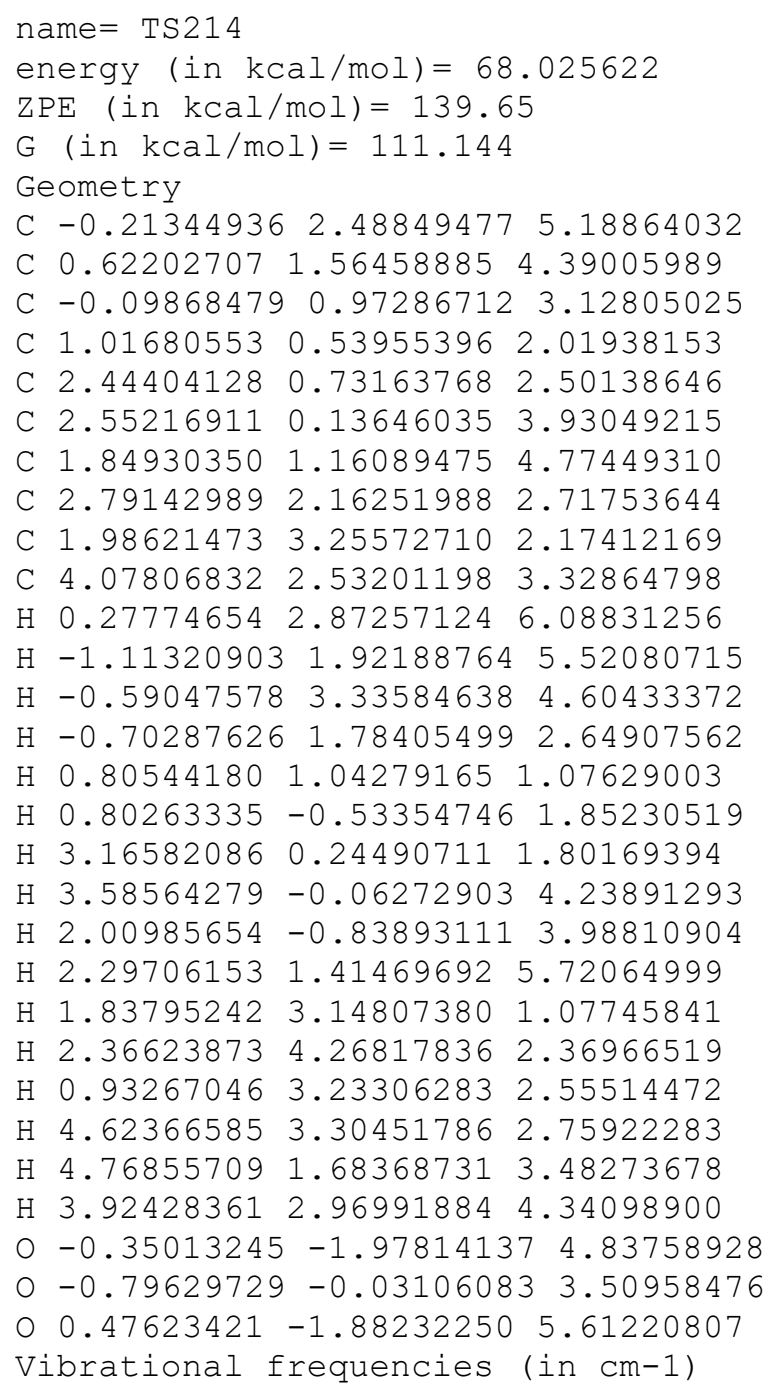




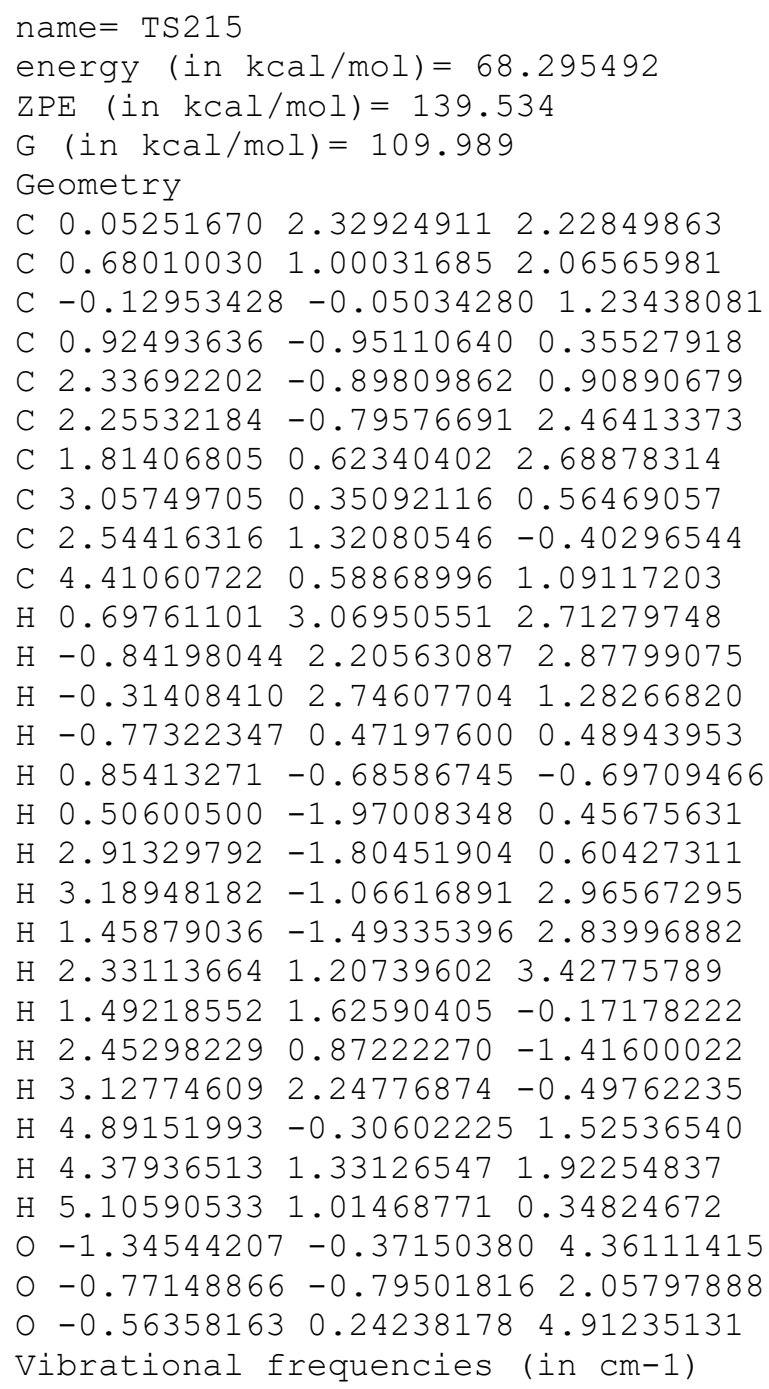


$\mathrm{H}-0.80990799 \quad 0.19810854-0.19969405$

$\mathrm{H} 1.42509176 \quad 0.62523498 \quad 0.57549192$

$\mathrm{H} \quad 0.89926058 \quad 0.65382092 \quad 2.25277808$

$\mathrm{H} \quad 1.02631720 \quad 2.99210668 \quad 0.32374211$

$\mathrm{H}-3.14240834 \quad 2.40221340 \quad 1.28201576$

$\mathrm{H}-2.23504366 \quad 3.92854175 \quad 1.31649233$

$\mathrm{H}-1.954048712 .749105952 .56595704$

$\mathrm{H}-2.26616238 \quad 2.29976697-1.18914670$

$\mathrm{H}-0.52374262 \quad 2.20809397-1.48540416$

$\mathrm{H}-1.244526793 .72496146-0.94537280$

o $1.77204631-0.33310797 \quad 4.46301120$

$0-0.689575690 .382268023 .86725818$

O $1.36438388 \quad 0.00830688 \quad 5.46510772$

Vibrational frequencies (in $\mathrm{cm}-1$ )

$\begin{array}{llllllllllllllll}-1519.1 & 28.6 & 45.5 & 56.4 & 66.1 & 103.1 & 109.0 & 117.2 & 137.8 & 145.4 & 178.3 & 201.3 & 220.7 & 232.7\end{array}$ $258.9 \quad 292.0 \quad 300.5 \quad 338.8 \quad 384.5 \quad 423.6 \quad 465.8 \quad 481.9 \quad 528.7 \quad 588.1 \quad 670.4 \quad 781.2 \quad 795.5 \quad 838.4$

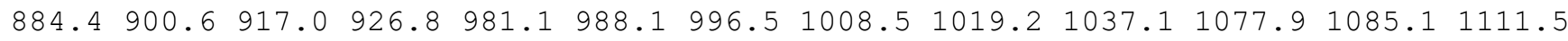
$\begin{array}{lllllllllllllll}1148.1 & 1152.3 & 1189.7 & 1196.2 & 1217.9 & 1251.1 & 1274.9 & 1277.1 & 1286.4 & 1289.5 & 1298.6 & 1301.6\end{array}$

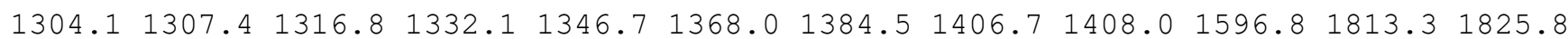
$1868.62642 .92661 .7 \quad 2663.2 \quad 2675.6 \quad 2681.0 \quad 2686.2 \quad 2689.72711 .12718 .82736 .12761 .5$ $2771.62778 .8 \quad 2781.9 \quad 3296.6$

name $=\operatorname{TS} 217$

energy $($ in $\mathrm{kcal} / \mathrm{mol})=71.263566$

$\mathrm{ZPE}($ in $\mathrm{kcal} / \mathrm{mol})=142.515$

$\mathrm{G}($ in $\mathrm{kcal} / \mathrm{mol})=118.929$

Geometry

C $0.28589277 \quad 1.037946953 .15258649$

C $1.05066411-0.23220438 \quad 3.48629623$

C $1.43499644-1.04838530 \quad 2.19257350$

C $2.89989781-1.490429632 .07250430$

C $3.79916489-0.948968063 .19124393$

C $3.09006194-1.184544914 .54866721$

C $2.192961990 .00686496 \quad 4.43175271$

C $3.88043675 \quad 0.60385163 \quad 3.21250418$

C $3.682744571 .32055476 \quad 1.90341075$

C $5.12660791 \quad 1.07765237 \quad 3.93592513$

$\mathrm{H}-0.022461121 .54731471 \quad 4.07878358$

$\mathrm{H}-0.63019071 \quad 0.81870624 \quad 2.58917291$

$\mathrm{H} \quad 0.88430479 \quad 1.74754521 \quad 2.57426975$

$\mathrm{H} 1.08316928-0.531049721 .27951904$

$\mathrm{H} 3.30597165-1.20640027 \quad 1.08412139$

H $2.93801885-2.603964292 .09179208$

$\mathrm{H} \quad 4.80161211-1.40988094 \quad 3.12653645$

H $3.76764272-1.14051068 \quad 5.41282174$

H 2.56198260 $-2.14903024 \quad 4.61794628$

H 2.91503401 $1.09073450 \quad 4.08388993$

H 2.70229850 $1.12777970 \quad 1.45258422$

$\mathrm{H} \quad 4.44204673 \quad 1.01670186 \quad 1.16771029$

$\mathrm{H} 3.771783162 .40956165 \quad 2.01879868$

$\mathrm{H} \quad 6.03200354 \quad 0.81535187 \quad 3.37194187$

H $5.22168668 \quad 0.62730499 \quad 4.93299870$

$\mathrm{H} \quad 5.13504170 \quad 2.16598333 \quad 4.07310951$

O $-0.40496177-1.914889663 .16134986$

$\begin{array}{lllll}0 & 0.68546986 & -2.26327109 & 2.32302142\end{array}$

o $0.18093981-1.135820394 .18894440$

Vibrational frequencies (in $\mathrm{cm}-1$ )

$\begin{array}{lllllllllllllll}-1391.2 & 63.8 & 113.9 & 144.3 & 157.6 & 165.0 & 180.3 & 218.8 & 241.5 & 274.0 & 307.9 & 343.0 & 351.3 & 381.7\end{array}$ $436.8 \quad 450.5 \quad 457.4 \quad 522.5 \quad 561.0 \quad 614.9 \quad 686.5 \quad 717.0 \quad 750.6 \quad 814.9 \quad 866.2 \quad 874.0 \quad 921.0 \quad 949.0$ $952.5 \quad 980.7 \quad 991.4 \quad 1002.6 \quad 1009.6 \quad 1018.7 \quad 1042.2 \quad 1057.6 \quad 1092.11101 .4 \quad 1125.11131 .3 \quad 1144.1$ $1165.21185 .91189 .0 \quad 1203.4 \quad 1226.3 \quad 1238.6 \quad 1252.3 \quad 1268.2 \quad 1274.6 \quad 1284.01287 .8 \quad 1288.7$

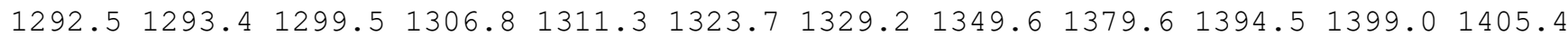
$1933.22649 .52667 .82669 .5 \quad 2669.82671 .32674 .32679 .12679 .52683 .22718 .12735 .2$ $\begin{array}{lllll}2760.1 & 2777.7 & 2778.8 & 2780.2\end{array}$ 
name $=$ TS218

energy $($ in $\mathrm{kcal} / \mathrm{mol})=71.589823$

$\mathrm{ZPE}($ in $\mathrm{kcal} / \mathrm{mol})=142.789$

$\mathrm{G}($ in $\mathrm{kcal} / \mathrm{mol})=118.868$

Geometry

C $-0.66701628 \quad 2.71492422 \quad 3.94131092$

C $0.33460886 \quad 1.59372614 \quad 3.68691565$

C $-0.35975805 \quad 0.28374848 \quad 3.16369529$

C $0.09888494 \quad-0.24170528 \quad 1.81432101$

C $1.49067015 \quad 0.24310821 \quad 1.31279615$

C $2.57429717-0.20799612 \quad 2.13338211$

C $1.49006404 \quad 1.98904843 \quad 2.87227692$

C $1.48902470 \quad 1.89071341 \quad 1.43420949$

$\begin{array}{lllll}\text { C } & 0.28332241 & 2.45298352 & 0.68220920\end{array}$

C $2.76927279 \quad 2.42779594 \quad 0.78688090$

$\mathrm{H}-0.16322183 \quad 3.58571433 \quad 4.38324687$

$\mathrm{H}-1.444124292 .40149195 \quad 4.64985564$

$\mathrm{H}-1.15105390 \quad 3.05017300 \quad 3.01906129$

$\mathrm{H}-1.46400564 \quad 0.39087913 \quad 3.20362983$

$\mathrm{H}-0.65755782-0.00469640 \quad 1.04197758$

$\mathrm{H} \quad 0.14333533-1.35346144 \quad 1.85818813$

H $1.58622138 \quad-0.08268595 \quad 0.25265735$

H $3.04262620 \quad 0.35152043 \quad 2.91553566$

H $2.99189837 \quad-1.18578260 \quad 1.98538133$

H 2.35995888 2.35605295 3.39614321

H $0.26966355 \quad 2.11836701-0.36083686$

$\mathrm{H} \quad 0.32111140 \quad 3.55021434 \quad 0.67571608$

$\mathrm{H}-0.67106427 \quad 2.16928462 \quad 1.13581214$

H $3.66879401 \quad 1.99274744 \quad 1.23961595$

H $2.83861800 \quad 3.51579567 \quad 0.89812491$

H $2.80348298 \quad 2.19986262 \quad-0.28363308$

$\begin{array}{lllll}0 & 0.14497269 & 0.04335538 & 5.33233038\end{array}$

$00.02176872-0.700336314 .13237760$

O $0.88516855 \quad 1.19289102 \quad 4.96343533$

Vibrational frequencies (in $\mathrm{cm}-1$ )

$\begin{array}{llllllllllllllllllllll}-583.6 & 51.8 & 99.7 & 129.0 & 162.3 & 177.1 & 197.5 & 216.4 & 258.4 & 261.1 & 277.3 & 323.6 & 347.7 & 355.0\end{array}$

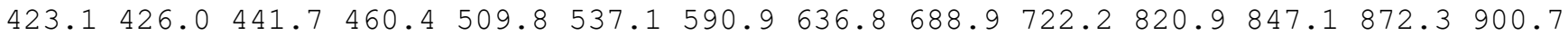
$930.1940 .5 \quad 973.6 \quad 994.3 \quad 997.4 \quad 1007.3 \quad 1017.7 \quad 1023.4 \quad 1040.4 \quad 1070.8 \quad 1102.2 \quad 1118.7 \quad 1128.2$ $\begin{array}{llllllllllll}1139.4 & 1173.8 & 1193.2 & 1212.8 & 1225.9 & 1246.4 & 1266.7 & 1282.8 & 1288.8 & 1291.2 & 1295.8 & 1302.9\end{array}$ $1303.6 \quad 1306.41318 .7 \quad 1337.8 \quad 1347.6 \quad 1353.8 \quad 1385.2 \quad 1402.4 \quad 1406.8 \quad 1421.6 \quad 1433.7 \quad 1503.1$ $2650.12671 .32671 .7 \quad 2674.2 \quad 2677.3 \quad 2678.0 \quad 2678.72680 .92688 .32711 .72730 .12742 .9$ $2765.22779 .3 \quad 2780.7 \quad 2781.9$

name $=\operatorname{TS} 219$

energy (in $\mathrm{kcal} / \mathrm{mol})=73.177244$

$\mathrm{ZPE}(\mathrm{in} \mathrm{kcal} / \mathrm{mol})=143.998$

$\mathrm{G}($ in $\mathrm{kcal} / \mathrm{mol})=121.834$

Geometry

C $-0.460887651 .33403356 \quad 3.27850289$

C $0.77548750 \quad 0.50799059 \quad 3.20712275$

C $0.44918693-1.021527212 .96240395$

C $0.92478828-1.620962491 .62903805$

C $1.85097553-0.668317290 .87317140$

C $2.83867298-0.049318651 .91049561$

C 1.761159931 .044697792 .15925121

$\begin{array}{lllll}\text { C } 1.20026444 & 0.76234574 & 0.69615525\end{array}$

C $-0.27202597 \quad 0.88205728 \quad 0.46208251$

C $1.95266477 \quad 1.58873117-0.34509391$

$\mathrm{H}-0.661121861 .72617229 \quad 1.72060847$

$\mathrm{H}-0.308195422 .37293922 \quad 3.52828143$

$\mathrm{H}-1.27756057 \quad 0.942234113 .85928405$

$\mathrm{H}-0.62361332-1.226756103 .14109149$ 
$\mathrm{H} \quad 0.05936300-1.89994974 \quad 1.00247120$

$\mathrm{H} 1.46369795-2.57172440 \quad 1.83320029$

$\mathrm{H} 2.28038774-1.10588608-0.02814860$

H $3.77375814 \quad 0.321094291 .48861653$

H $3.08698540 \quad-0.674029812 .77434736$

H $2.109213212 .05483422 \quad 2.36504364$

$\mathrm{H}-0.57265305 \quad 1.63856174 \quad-0.24995924$

$\mathrm{H}-1.00544457 \quad 0.88926183 \quad 1.83491446$

$\mathrm{H}-0.77644860 \quad-0.04562037 \quad 0.22447586$

H $3.040236261 .52028676-0.22938578$

$\mathrm{H} \quad 1.68802310 \quad 2.64954952-0.27678152$

H $1.71379511 \quad 1.24737945-1.35913495$

O $1.18827777-0.74128545 \quad 5.05554524$

O $1.19995154-1.67582627 \quad 3.98905524$

O $1.49207498 \quad 0.50605472 \quad 4.44947223$

Vibrational frequencies (in cm-1)

$\begin{array}{llllllllllllllll}-2532.4 & 90.1 & 184.8 & 192.9 & 206.8 & 219.9 & 296.3 & 304.0 & 335.6 & 357.3 & 371.5 & 432.1 & 437.2 & 440.7\end{array}$

$481.3 \quad 515.9 \quad 572.5 \quad 615.9 \quad 638.6 \quad 710.0 \quad 716.9 \quad 742.4 \quad 794.0 \quad 820.4 \quad 856.6 \quad 871.5 \quad 883.6 \quad 915.2$

$936.2943 .5983 .6 \quad 1002.0 \quad 1004.4 \quad 1009.4 \quad 1017.1 \quad 1030.1 \quad 1044.4 \quad 1062.9 \quad 1079.3 \quad 1087.6 \quad 1091.3$ $1106.51111 .0 \quad 1124.2 \quad 1159.0 \quad 1175.6 \quad 1185.7 \quad 1198.8 \quad 1216.8 \quad 1220.7 \quad 1239.5 \quad 1254.51267 .1$ $1272.41283 .8 \quad 1299.3 \quad 1300.91308 .3 \quad 1322.5 \quad 1331.2 \quad 1344.61370 .8 \quad 1373.4 \quad 1384.7 \quad 1408.6$ $1452.0 \quad 2657.8 \quad 2674.4 \quad 2680.6 \quad 2682.8 \quad 2685.4 \quad 2693.7 \quad 2708.7 \quad 2738.7 \quad 2748.4 \quad 2753.0 \quad 2763.9$ $2766.0 \quad 2780.92782 .5 \quad 3603.3$

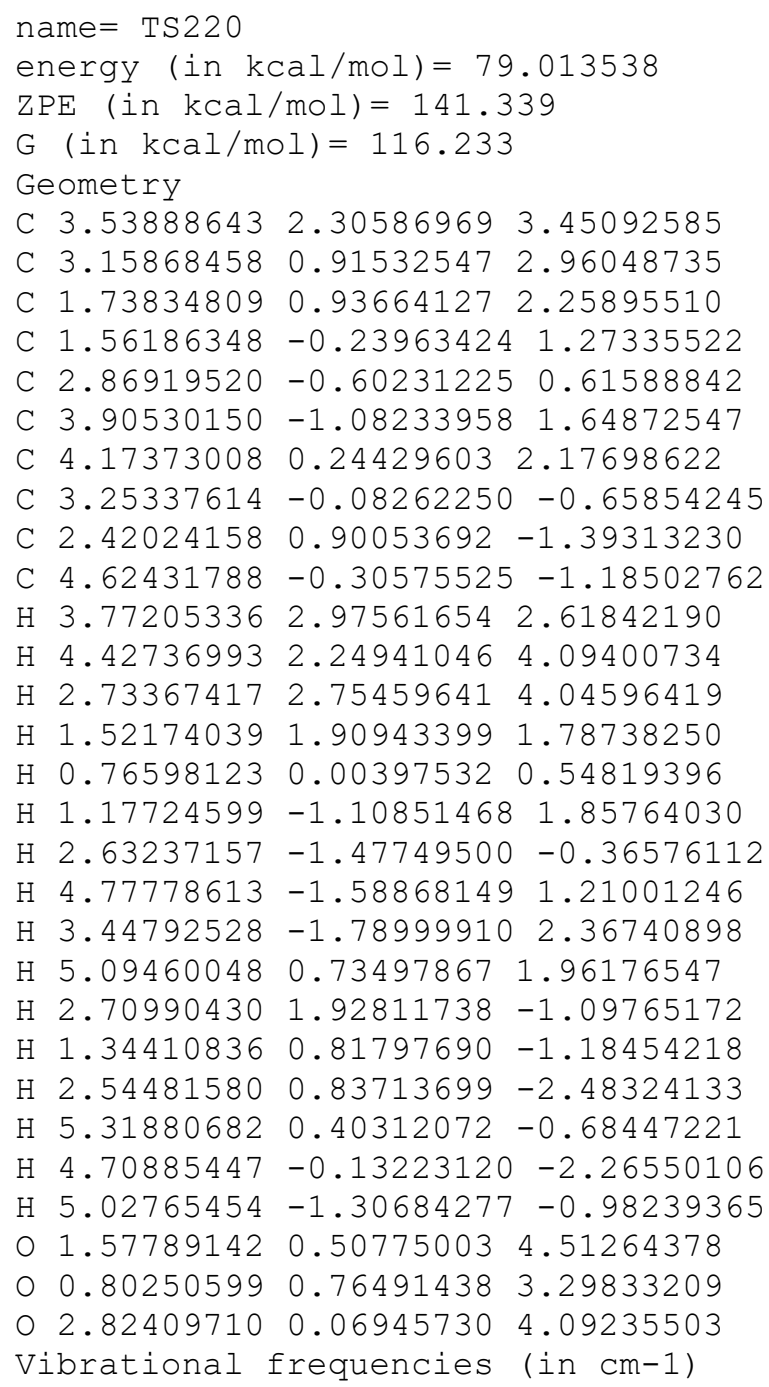


$2178.22639 .12647 .92650 .12665 .2 \quad 2672.4 \quad 2674.3 \quad 2676.0 \quad 2685.6 \quad 2698.12732 .0 \quad 2752.0$ 2769.32770 .02782 .62803 .8

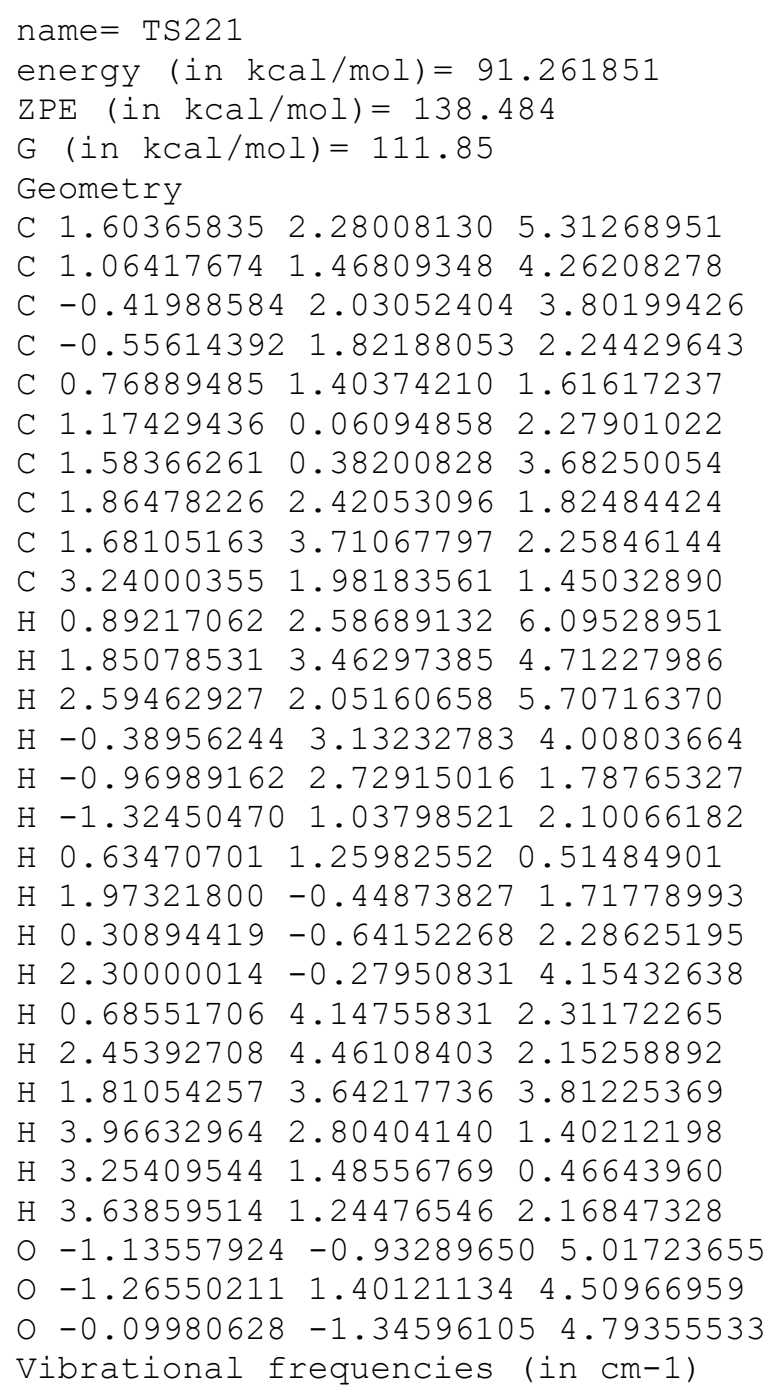


H $5.74290651 \quad 0.27711685 \quad 4.17140758$

$\mathrm{H} \quad 0.10254659-0.35088257 \quad 3.22395432$

$\mathrm{H} \quad 0.20138038 \quad 0.91021374 \quad 0.30860760$

$\mathrm{H} \quad 0.50829441-0.89795063 \quad 0.38994774$

H 2.12603578 $-0.91718258-0.58073067$

H $3.05914816-2.51956243 \quad 1.17013887$

$\mathrm{H} 1.99097991-1.72258992 \quad 2.34468611$

$\mathrm{H} 4.82196398-0.87032004 \quad 1.58874802$

H 2.04199482 $1.60608738 \quad 2.29244825$

H $2.07053538 \quad 2.33342445 \quad 0.68528516$

H $3.496638592 .43676991 \quad 1.72302117$

$\mathrm{H} \quad 4.55026886-0.13968976-0.77852775$

H 5.014242651 .369053870 .01615647

H $3.65968951 \quad 1.34644801-1.11917453$

o $1.23888751-2.26068045 \quad 4.76753185$

○ $0.97339161-1.41021311 \quad 5.46924990$

o $2.96746909-0.381360224 .09137724$

Vibrational frequencies (in cm-1)

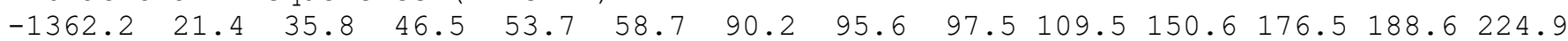
$251.9266 .3 \quad 284.0 \quad 332.8 \quad 352.6 \quad 400.2 \quad 416.4 \quad 447.5 \quad 484.0 \quad 558.0 \quad 566.4 \quad 603.6 \quad 676.4 \quad 761.7$ $810.9873 .5 \quad 895.2 \quad 926.1 \quad 978.8 \quad 989.7 \quad 991.8 \quad 1007.2 \quad 1015.6 \quad 1038.0 \quad 1047.5 \quad 1053.2 \quad 1084.9$

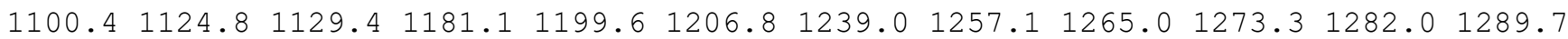
$1300.4 \quad 1302.1 \quad 1311.0 \quad 1318.8 \quad 1329.4 \quad 1346.9 \quad 1381.0 \quad 1398.2 \quad 1405.8 \quad 1597.6 \quad 1835.2 \quad 1849.8$ $2280.82658 .32663 .72666 .5 \quad 2676.8 \quad 2677.2 \quad 2678.52681 .22728 .2 \quad 2740.62746 .72751 .4$ $2778.7 \quad 2780.12782 .6 \quad 2819.2$ 
TSS FOR THE CYCLOADDITION REACTION OPTIMIZED AT THE MP2/6-31G(D) LEVEL OF THEORY

\section{TS 196. Optimized structure at the MP2/6-31G(d) level of theory}

$\begin{array}{lccl}\text { C } & -0.74168 & 2.11437 & 3.11247 \\ \mathrm{C} & 0.14013 & 0.94997 & 2.74787 \\ \mathrm{C} & -0.3734 & -0.09415 & 1.96309 \\ \mathrm{C} & 0.55112 & -0.85078 & 1.03607 \\ \mathrm{C} & 1.98339 & -0.32571 & 1.159 \\ \mathrm{C} & 2.31936 & -0.23893 & 2.66383 \\ \mathrm{C} & 1.62941 & 1.14753 & 2.63681 \\ \mathrm{C} & 2.02693 & 1.23269 & 1.11723 \\ \mathrm{C} & 1.11937 & 1.96852 & 0.14069 \\ \mathrm{C} & 3.45072 & 1.75887 & 0.94215 \\ \mathrm{H} & -0.31789 & 2.68592 & 3.94239 \\ \mathrm{H} & -1.73467 & 1.75864 & 3.40866 \\ \mathrm{H} & -0.8641 & 2.79009 & 2.25699 \\ \mathrm{H} & -1.43533 & -0.09064 & 1.71558 \\ \mathrm{H} & 0.1823 & -0.74774 & 0.00617 \\ \mathrm{H} & 0.5288 & -1.92495 & 1.26926 \\ \mathrm{H} & 2.66893 & -0.86298 & 0.49051 \\ \mathrm{H} & 3.38963 & -0.17441 & 2.86911 \\ \mathrm{H} & 1.86769 & -0.98689 & 3.31738 \\ \mathrm{H} & 1.99445 & 1.93699 & 3.30637 \\ \mathrm{H} & 1.15814 & 3.04915 & 0.32296 \\ \mathrm{H} & 0.0758 & 1.6535 & 0.20475 \\ \mathrm{H} & 1.46013 & 1.7947 & -0.88724 \\ \mathrm{H} & 3.78727 & 1.59499 & -0.08817 \\ \mathrm{H} & 4.16926 & 1.27723 & 1.60887 \\ \mathrm{H} & 3.48134 & 2.8369 & 1.13738 \\ \mathrm{O} & -0.94929 & -0.68924 & 4.3953 \\ \mathrm{O} & -0.69443 & -1.50949 & 3.38454 \\ \mathrm{O} & 0.10377 & 0.09806 & 4.58006\end{array}$
$-0.61413777347 \mathrm{D}+03$

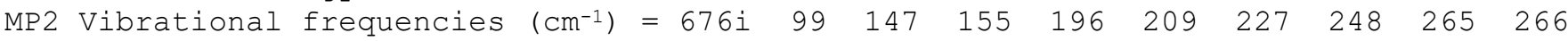

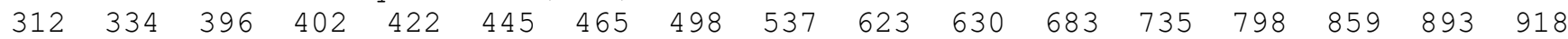

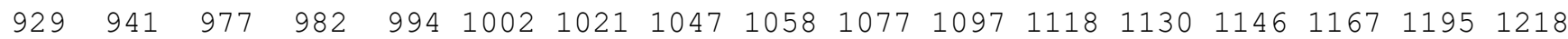
$\begin{array}{llllllllllllllllll}1246 & 1274 & 1281 & 1289 & 1316 & 1340 & 1374 & 1400 & 1410 & 1450 & 1460 & 1468 & 1517 & 1534 & 1541 & 1545 & 1550\end{array}$

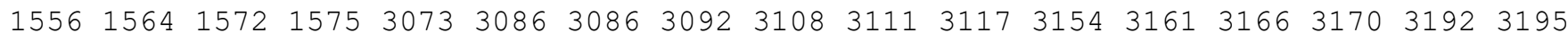
320132033220

$\operatorname{CCSD}(\mathrm{T}) / 6-311++\mathrm{G}(\mathrm{d}, \mathrm{p})$ energy $(\mathrm{Eh})=-0.61469876150 \mathrm{D}+03$

TS 197. Optimized structure at the MP2/6-31G(d) level of theory

$\begin{array}{llll}\mathrm{C} & 0.99959 & 1.40817 & 3.51995 \\ \mathrm{C} & 1.8054 & 0.15381 & 3.33257 \\ \mathrm{C} & 1.33159 & -0.85996 & 2.485 \\ \mathrm{C} & 2.33156 & -1.77221 & 1.80289 \\ \mathrm{C} & 3.76912 & -1.34491 & 2.12263 \\ \mathrm{C} & 3.88238 & -1.19481 & 3.65384 \\ \mathrm{C} & 3.30746 & 0.22835 & 3.45783 \\ \mathrm{C} & 3.94403 & 0.20119 & 2.0176 \\ \mathrm{C} & 3.26536 & 0.9401 & 0.87196 \\ \mathrm{C} & 5.41481 & 0.61568 & 2.0495 \\ \mathrm{H} & 1.20804 & 2.12207 & 2.71412 \\ \mathrm{H} & 1.24562 & 1.889 & 4.47177 \\ \mathrm{H} & -0.0685 & 1.17453 & 3.5194 \\ \mathrm{H} & 0.36728 & -0.7212 & 1.99669 \\ \mathrm{H} & 2.14813 & -1.74491 & 0.71995 \\ \mathrm{H} & 2.1821 & -2.81463 & 2.11523\end{array}$




$\begin{array}{llll}\mathrm{H} & 4.50065 & -1.97083 & 1.59477 \\ \mathrm{H} & 4.91153 & -1.1832 & 4.01723 \\ \mathrm{H} & 3.30519 & -1.88981 & 4.26728 \\ \mathrm{H} & 3.62551 & 1.02319 & 4.14411 \\ \mathrm{H} & 3.7466 & 0.67367 & -0.07686 \\ \mathrm{H} & 3.36893 & 2.02407 & 1.001 \\ \mathrm{H} & 2.2014 & 0.71186 & 0.78221 \\ \mathrm{H} & 5.98132 & 0.12952 & 2.84666 \\ \mathrm{H} & 5.49726 & 1.69916 & 2.19218 \\ \mathrm{H} & 5.89601 & 0.3656 & 1.09686 \\ \mathrm{O} & 1.13688 & -1.84596 & 4.85194 \\ \mathrm{O} & 0.30033 & -1.98663 & 3.83157 \\ \mathrm{O} & 1.24697 & -0.55171 & 5.13441\end{array}$

MP2/6-31G(d) energy (Eh) =

$-0.61413421150 D+03$

MP2 Vibrational frequencies $\left(\mathrm{cm}^{-1}\right)=\begin{array}{llllllllll}686 i & 86 & 154 & 156 & 195 & 204 & 213 & 230 & 259 & 273\end{array}$

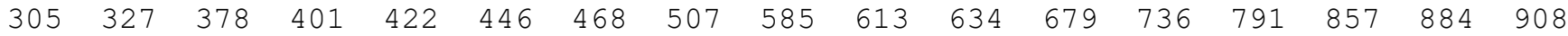

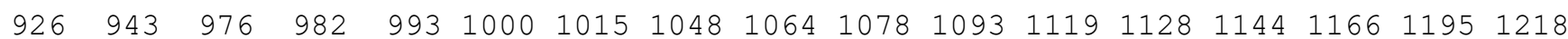

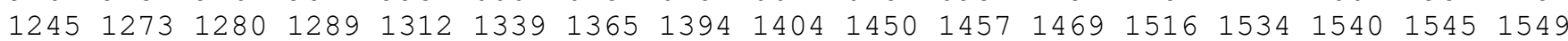
$15571562 \quad 1572 \quad 158130793086 \quad 3092 \quad 3093 \quad 3108 \quad 31163123315431623170317531973200$ 320132033214

$\operatorname{CCSD}(T) / 6-311++G(d, p)$ energy $(E h)=-0.61469629322 D+03$ 